\title{
Calculation of Hazard Category 2/3 Threshold Quantities Using Contemporary Dosimetric Data
}

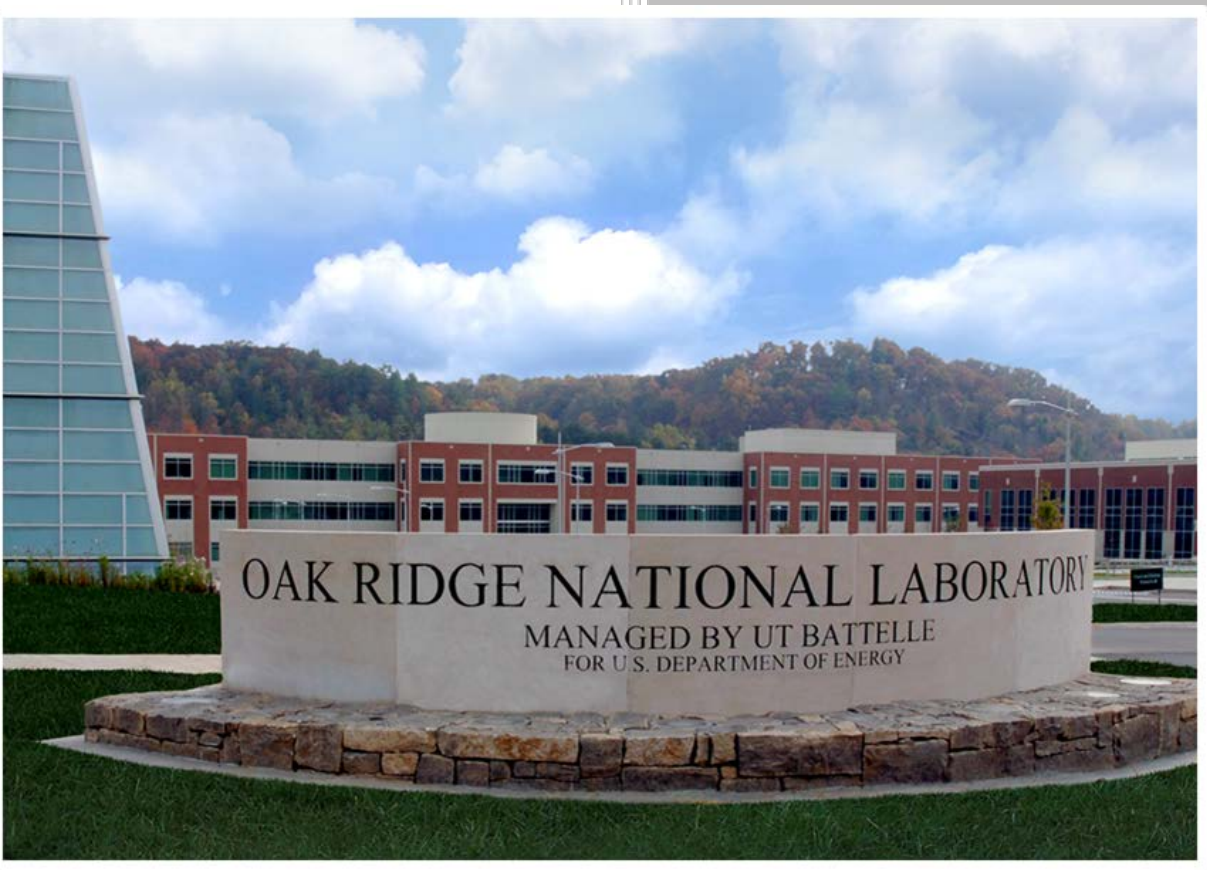

William C. Walker 


\title{
DOCUMENT AVAILABILITY
}

Reports produced after January 1, 1996, are generally available free via US Department of Energy (DOE) SciTech Connect.

\section{Website http://www.osti.gov/scitech/}

Reports produced before January 1, 1996, may be purchased by members of the public from the following source:

\author{
National Technical Information Service \\ 5285 Port Royal Road \\ Springfield, VA 22161 \\ Telephone 703-605-6000 (1-800-553-6847) \\ TDD 703-487-4639 \\ Fax 703-605-6900 \\ E-mail info@ntis.gov \\ Website http://classic.ntis.gov/
}

Reports are available to DOE employees, DOE contractors, Energy Technology Data Exchange representatives, and International Nuclear Information System representatives from the following source:

Office of Scientific and Technical Information

PO Box 62

Oak Ridge, TN 37831

Telephone 865-576-8401

Fax 865-576-5728

E-mail reports@osti.gov

Website http://www.osti.gov/contact.html

This report was prepared as an account of work sponsored by an agency of the United States Government. Neither the United States Government nor any agency thereof, nor any of their employees, makes any warranty, express or implied, or assumes any legal liability or responsibility for the accuracy, completeness, or usefulness of any information, apparatus, product, or process disclosed, or represents that its use would not infringe privately owned rights. Reference herein to any specific commercial product, process, or service by trade name, trademark, manufacturer, or otherwise, does not necessarily constitute or imply its endorsement, recommendation, or favoring by the United States Government or any agency thereof. The views and opinions of authors expressed herein do not necessarily state or reflect those of the United States Government or any agency thereof. 
Nuclear and Radiological Protection Division

\title{
CALCULATION OF HAZARD CATEGORY 2/3 THRESHOLD QUANTITIES USING CONTEMPORARY DOSIMETRIC DATA
}

\author{
William C. Walker
}

Date Published: November 2017

Prepared by OAK RIDGE NATIONAL LABORATORY

Oak Ridge, TN 37831-6283

managed by

UT-BATTELLE, LLC

for the

US DEPARTMENT OF ENERGY

under contract DE-AC05-00OR22725 
(This page intentionally blank) 


\section{CONTENTS}

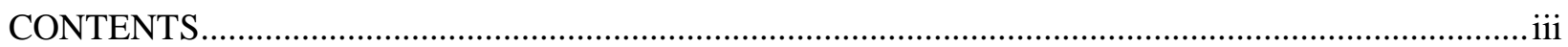

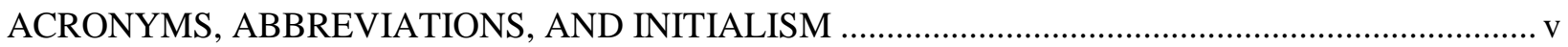

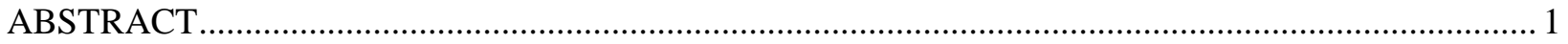

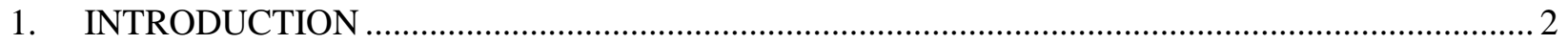

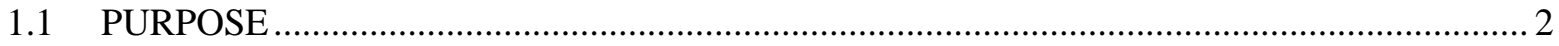

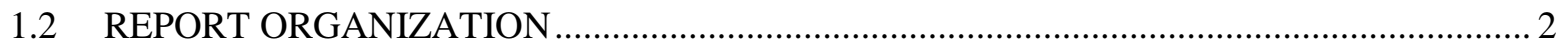

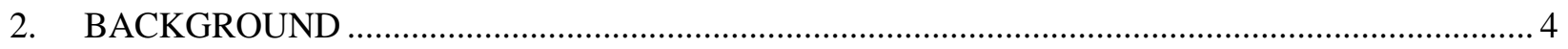

2.1 ORIGIN OF THE EXISTING HAZARD CATEGORIZATION THRESHOLD

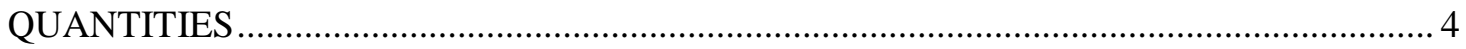

2.2 AVAILABILITY OF UPDATED DOSIMETRIC DATA …......................................... 4

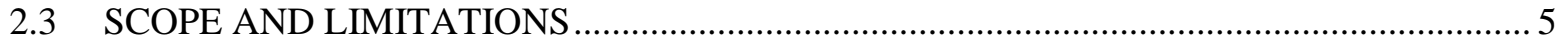

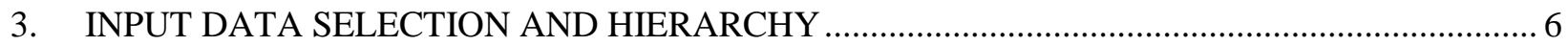

3.1 DEFINING THE DOSE COEFFICIENT INPUT DATA PUBLICATIONS .......................... 6

3.1.1 Dose Coefficients Utilized by DOE-STD-1027-92 for Calculating HC-2 and

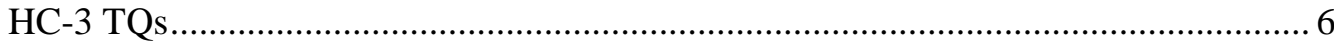

3.1.2 Selection of Contemporary Dose Coefficient Publications ......................................... 7

3.1.3 Hierarchy of Selected Contemporary Dose Coefficient Publications ............................ 9

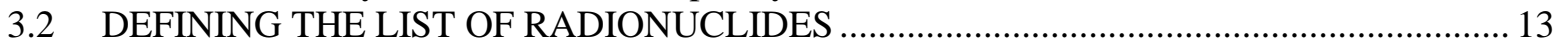

3.2.1 Developing the Master List of Radionuclides......................................................... 13

3.2.2 Reconciliation of Radionuclide Nomenclature Differences ..................................... 13

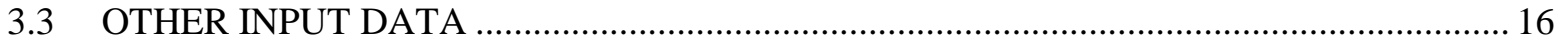

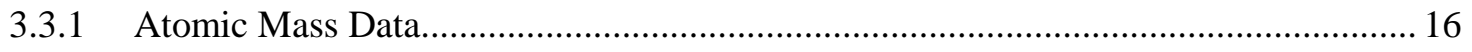

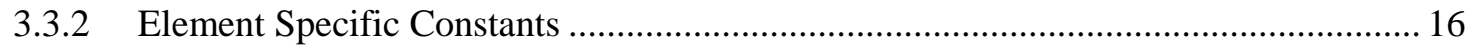

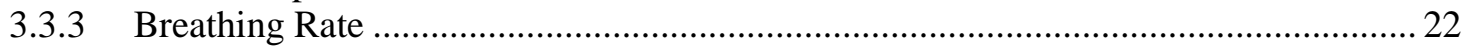

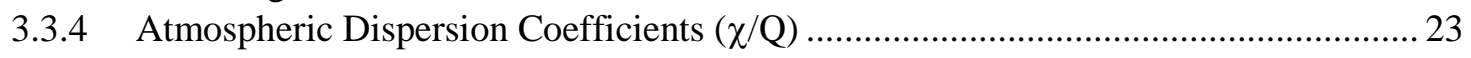

3.3.5 Miscellaneous Inputs and Conversion Factors................................................................ 26

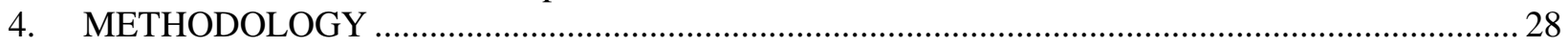

4.1 HAZARD CATEGORY 2 THRESHOLD QUANTITIES METHODOLOGY ...................... 28

4.1.1 HC-2 Threshold Quantity Exposure Pathway Model ............................................... 28

4.2 HAZARD CATEGORY 3 THRESHOLD QUANTITIES METHODOLOGY ..................... 29

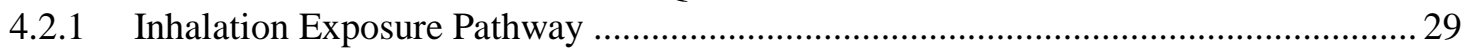

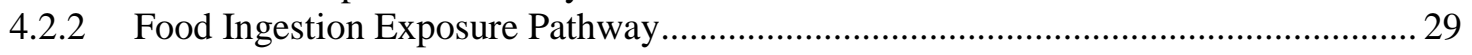

4.2.3 Ground Water Ingestion Exposure Pathway .......................................................... 31

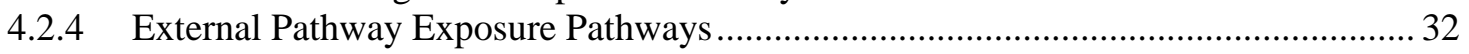

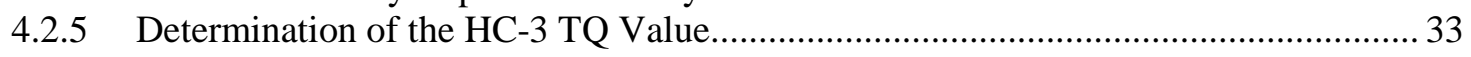

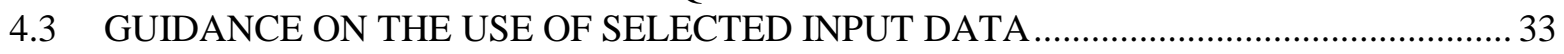

4.3.1 HC-2 Inhalation Dose Coefficient Selection Criteria ................................................ 33

4.3.2 HC-3 Inhalation, Air Immersion, Ingestion Dose Coefficient Selection Criteria........ 37

4.3.3 Direct Exposure Pathway TQ Calculation Considerations ....................................... 39

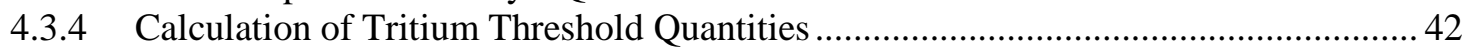

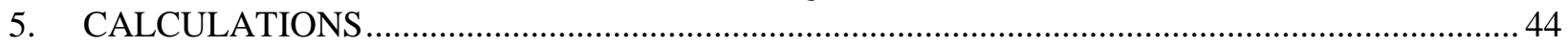

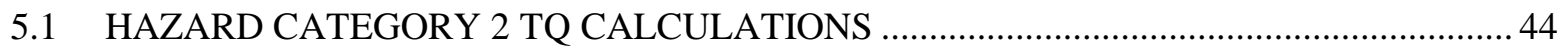

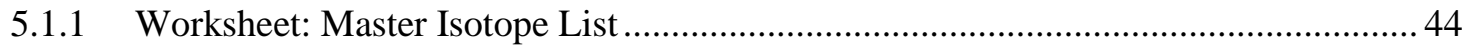

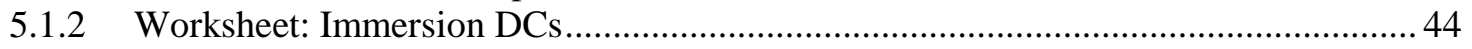

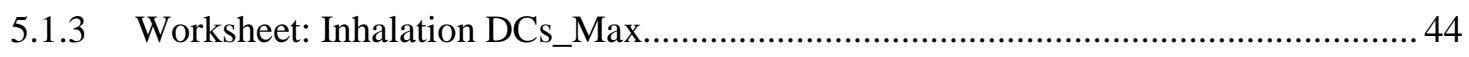

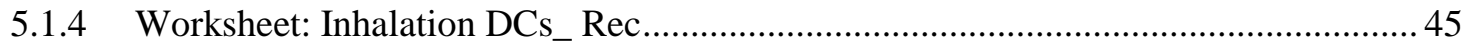




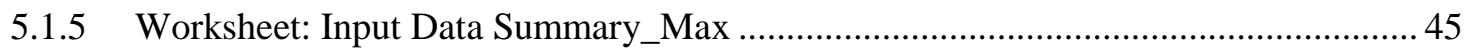

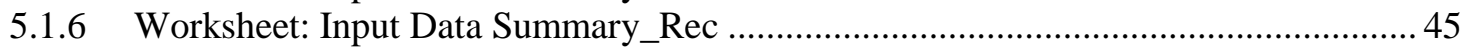

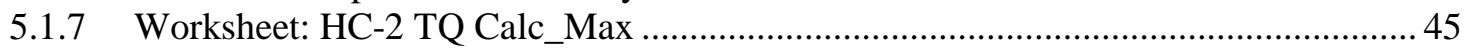

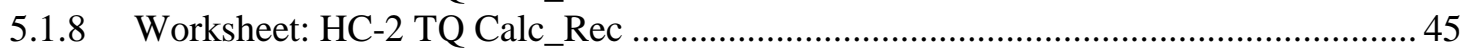

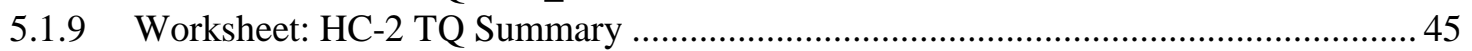

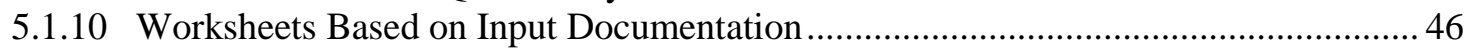

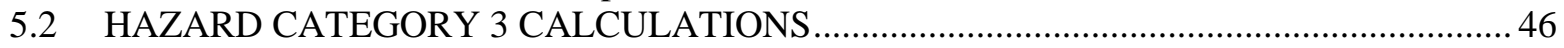

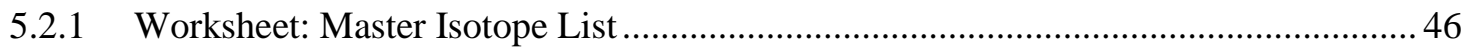

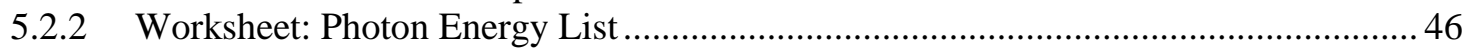

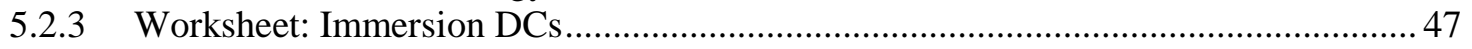

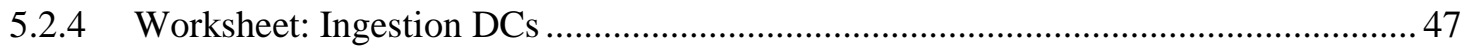

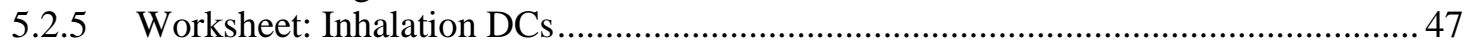

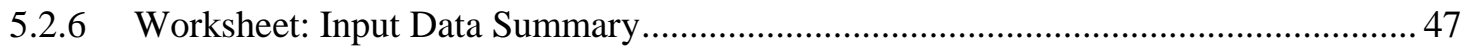

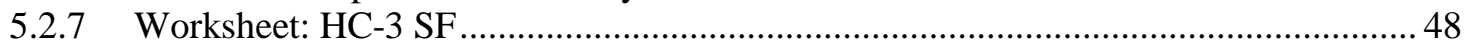

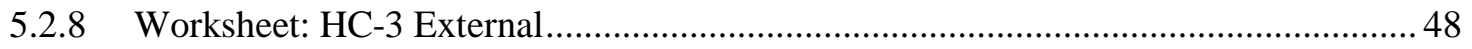

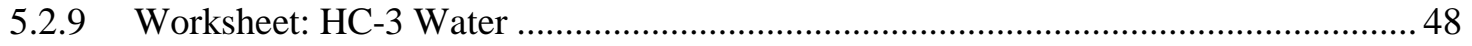

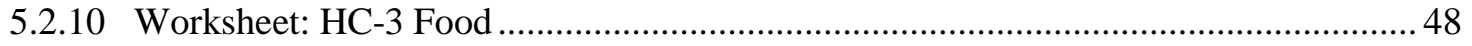

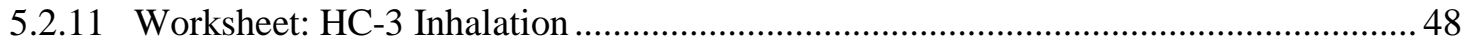

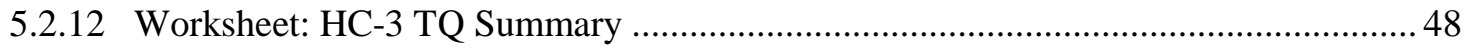

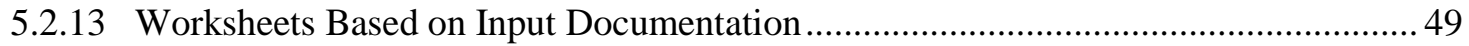

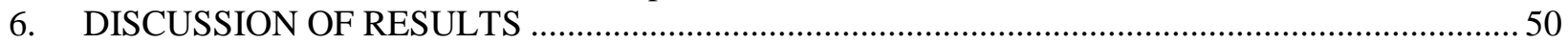

6.1 EXPANSION OF THE SET OF RADIONUCLIDES WITH HC-2 AND HC-3 TQS ............ 50

6.2 RADIONUCLIDES WITHOUT A CALCULATED TQ VALUE …................................... 50

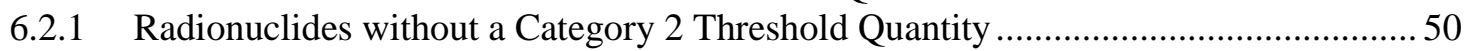

6.2.2 Radionuclides without a Category 3 Threshold Quantity .......................................... 51

6.3 ASSESSMENT OF EXCLUSION OF NEUTRON DOSE EXPOSURE PATHWAY ........... 52

6.4 COMPARISON TO NNSA CALCULATED UPDATED TQS …........................................... 54

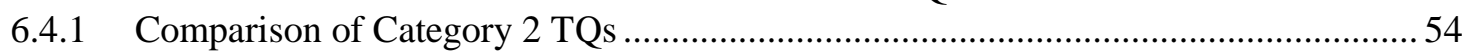

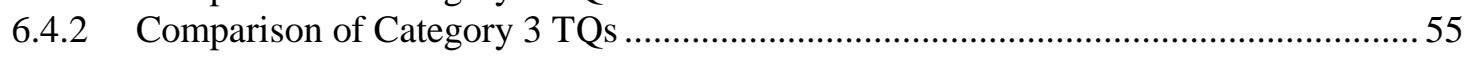

6.5 COMPENSATION FOR EXCEL CALCULATION PRECISION ISSUES ........................ 56

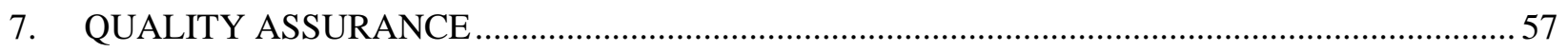

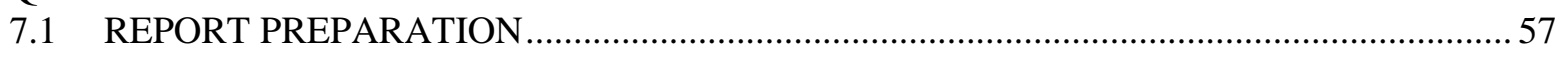

7.1.1 Development of Final Report and Supporting Calculations ...................................... 57

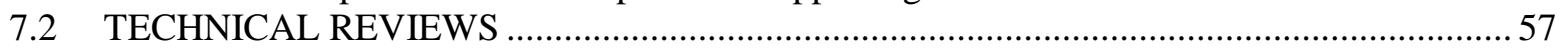

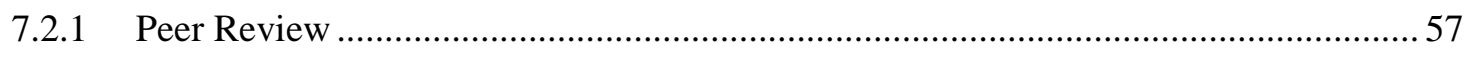

7.2.2 Department of Energy Independent Technical Review ........................................ 58

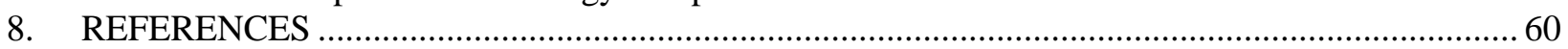

APPENDIX A. CALCULATION OF HAZARD CATEGORY 2 THRESHOLD QUANTITIES USING MAXIMUM DOSE COEFFICIENTS .....................................................................

APPENDIX B. CALCULATION OF HAZARD CATEGORY 2 THRESHOLD QUANTITIES USING RECOMMENDED DOSE COEFFICIENTS ................................................................ B-3

APPENDIX C. CALCULATION OF HAZARD CATEGORY 3 THRESHOLD QUANTITIES .......... C-3

APPENDIX D. SELECTION OF MASTER RADIONUCLIDE LIST ……....................................... D-3

APPENDIX E. PRECISION LIMITATIONS FOR THE CALCULATION OF THE EFFECTIVE

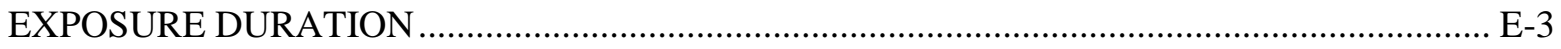




\section{ACRONYMS, ABBREVIATIONS, AND INITIALISM}

ALI

AMAD

AMDC

BR

CEDE

CFR

CSDE

CTA

DAC

DC

DOE

ENSDF

EPA

FGR

HC

IAEA

ICRP

IMP

IOP

JAERI

MIRD

MS

NNDC

NNSA
Annual Limit for Intake

Average Mean Aerodynamic Diameter

Atomic Mass Data Center

Breathing Rate

Committed Effective Dose Equivalent

Code of Federal Regulations

Cloud Shine Dose Equivalent

Central Technical Authority

Derived Airborne Concentration

Dose Coefficient

U. S. Department of Energy

Evaluated Nuclear Structure Data File

U. S. Environmental Protection Agency

Federal Guidance Report

Hazard Category

International Atomic Energy Agency

International Commission on Radiological Protection

Institute of Modern Physics

Internal Operating Procedure

Japan Atomic Energy Research Institute

Medical Internal Radiation Dose

Microsoft

National Nuclear Data Center

National Nuclear Security Administration 


$\begin{array}{ll}\text { NRC } & \text { U. S. Nuclear Regulatory Commission } \\ \text { NTD } & \text { Nuclear Transformation Data } \\ \text { ORNL } & \text { Oak Ridge National Laboratory } \\ \text { RF } & \text { Release Fraction } \\ \text { RV } & \text { Release Value } \\ \text { SFF } & \text { Spontaneous Fission Factor } \\ \text { SI } & \text { International System of Units } \\ \text { TFG } & \text { Tritium Focus Group } \\ \text { TQ } & \text { Threshold Quantity }\end{array}$




\begin{abstract}
The purpose of this report is to describe the methodology and selection of input data utilized to calculate updated Hazard Category 2 and Hazard Category 3 Threshold Quantities (TQs) using contemporary dosimetric information. The calculation of the updated TQs will be considered for use in the revision to the Department of Energy (DOE) Technical Standard (STD-) 1027-92 Change Notice (CN)-1, "Hazard Categorization and Accident Analysis Techniques for Compliance with DOE Order 5480.23, Nuclear Safety Analysis Reports.”

The updated TQs documented in this report complement an effort previously undertaken by the National Nuclear Security Administration (NNSA), which in 2014 issued revised Supplemental Guidance documenting the calculation of updated TQs for approximately 100 radionuclides listed in DOE-STD-1027-92, CN-1. The calculations documented in this report complement the NNSA effort by expanding the set of radionuclides to more than 1,250 radionuclides with a published TQ.

The development of this report was sponsored by the Department of Energy's Office of Nuclear Safety (AU-30) within the Associate Under Secretary for Environment, Health, Safety, and Security organization.
\end{abstract}




\section{INTRODUCTION}

\subsection{PURPOSE}

The purpose of this report is to document the technical basis, methodologies, input document, and supporting calculations utilized in the calculation of updated Hazard Category (HC)-2 and HC-3 Threshold Quantities (TQs) for possible inclusion in a revision to DOE-STD-1027.

\subsection{REPORT ORGANIZATION}

This report is organized as follows:

- $\quad$ Section 2: Background

This section discusses the use of HC-2 and HC-3 TQs and the regulatory allowances specific to the calculation of updated TQs.

- $\quad$ Section 3: Input Data Selection and Hierarchy

This section discusses the selection of contemporary dosimetric data (e.g., inhalation dose coefficients, ingestion dose coefficients, air-immersion dose coefficients, and nuclear transformation data). Since multiple data sources are used in calculation of updated HC-2 and HC-3 TQs, this section outlines the organizational hierarchy to be employed for consistent selection of input data. This section also discusses the resolution of radionuclide nomenclature issues identified amongst the various input documents.

- Section 4: Methodology

This section outlines the specific methodology and equations to be used for the calculation of the HC-2 and HC-3 TQs. This section also discusses issues arising from the selection of suitable inhalation dose coefficients when more than one value is provided for a specific radionuclide. Finally, this section will discuss the applicability of assumptions made in the development of the methodology for the point source exposure pathway (i.e., Direct Exposure).

- $\quad$ Section 5: Discussion of Results

This section discusses the results of the calculation of the updated HC-2 and HC-3 TQs.

- $\quad$ Section 6: Quality Assurance

This section discusses the quality assurance practices used throughout the preparation of this report and the calculation of the updated TQs.

- $\quad$ Appendix A: Calculation of Hazard Category 2 Threshold Quantities Using Maximum Dose Coefficients

This appendix provides a data table documenting the selected maximum inhalation and airimmersion dose coefficients and the selected nuclear transformation data. A second data table is provided documenting the calculated HC-2 TQs using the selected input data. 
- Appendix B: Calculation of Hazard Category 2 Threshold Quantities Using Recommended Dose $\underline{\text { Coefficients }}$

This appendix provides a data table documenting the selected recommended inhalation dose coefficients, the selected maximum air-immersion dose coefficients, and the selected nuclear transformation data. A second data table is provided documenting the calculated HC-2 TQs using the selected input data. The second data table also includes the HC-2 TQs calculated by the NNSA for comparative purposes.

- $\quad$ Appendix C: Calculation of Hazard Category 3 Threshold Quantities

This appendix provides a data table documenting the selected maximum inhalation, ingestion, and air-immersion dose coefficients as well as the selected nuclear transformation data. A second data table is provided documenting the calculated HC-3 TQs using the selected input data. The second data table also includes the HC-3 TQs calculated by the NNSA for comparative purposes

- $\quad$ Appendix D: Selection of Master Radionuclide List

This appendix provides a data table documenting the compilation of a master list of radionuclides based on the various input documentations used for the calculation of the HC-2 and HC-3 TQs.

- Appendix E: Precision Limitations for the Calculation of the Effective Exposure Duration

This appendix documents the precision limitations encountered using an Excel Spreadsheet to calculate the effective exposure duration used for the following HC-3 exposure pathways:

(i) water ingestion and (ii) direct exposure. This appendix also discusses how the precision issue was resolved to ensure an accurate calculation of the affected exposure pathways. 


\section{BACKGROUND}

\subsection{ORIGIN OF THE EXISTING HAZARD CATEGORIZATION THRESHOLD QUANTITIES}

The U. S. Department of Energy (DOE) categorizes nuclear facilities in accordance with the requirements specified in DOE-STD-1027-92, Change Notice 1, "Hazard Categorization and Accident Analysis Techniques for Compliance with DOE Order 5480.23, Nuclear Safety Analysis Reports.” (1) The standard requires an assessment of the facility radiological and fissionable material inventories when determining the facility categorization.

When the categorization of the facility is conducted solely on the basis of its radiological inventory, the standard prescribes the use of Hazard Category (HC) specific Threshold Quantities (TQs). The determination of the categorization is conducted through the use of the following equation:

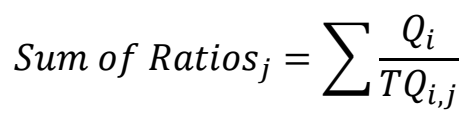

Where;

$$
\begin{array}{ll}
\mathrm{Q}_{\mathrm{i}} & \text { = Quantity of radionuclide “i” [Ci or gm] } \\
\mathrm{TQ}_{\mathrm{i}} & =\text { Threshold Quantity of Radionuclide “i” for Hazard Category “j” [Ci or gm] }
\end{array}
$$

In accordance with the requirement of DOE-STD-1027-92, facilities or facility segments where there are combinations of radioactive materials should be designated as Category 2 or 3 if the sum of the ratios of the quantity of each material to the Category 2 or 3 thresholds exceeds one. DOE-STD-1027-92 and its associated LANL Fact Sheets (2) (3) provide the current set of HC-2 and HC-3 TQs for conducting the sum of ratio calculations.

\subsection{AVAILABILITY OF UPDATED DOSIMETRIC DATA}

The derivation of the HC-2 and HC-3 TQs is associated with available dosimetric data prior to the issuance of DOE-STD-1027. The dosimetric data included inhalation dose coefficients (DCs), ingestion DCs, and nuclear transformation data (NTD) (e.g., half-life and average photon energy data). Since the initial publication of DOE-STD-1027 in 1992, updated dosimetric data has become available.

In 2011 (revised and reissued in 2014), the NNSA issued a supplemental guidance to DOE-STD-1027, outlining specific guidance associated with the calculation of updated HC-2 and HC-3 TQs using the updated dosimetry. (4) The NNSA justified the use of new DCs in recent Publications by the International Commission on Radiological Protection (ICRP); specifically ICRP Pub-68 "Dose Coefficients for Intakes of Radionuclides by Workers," (5) and ICRP Pub-72 "Age-dependent Doses to the Members of the Public from Intake of Radionuclides - Part 5 Compilation of Ingestion and Inhalation Coefficients.” (6) 
Since the NNSA effort to establish ground rules for the use of new DCs, the availability of DCs has expanded with the issuance of the following reports:

- ICRP Pub-119, “Compendium of Dose Coefficients based on ICRP Publication 60.” (7)

- DOE-STD-1196-2011, “Derived Concentration Technical Standard.” (8)

Additionally, a report by the Japan Atomic Energy Research Institute (JAERI) was issued in 2002 which derived DCs for radionuclides not listed in the ICRP publications. (9) Finally, the ICRP updated the radionuclide specific NTD with the issuance of Pub-107 "Nuclear Decay Data for Dosimetric Calculations.” (10)

\subsection{SCOPE AND LIMITATIONS}

This report will calculate updated HC-2 and HC-3 TQs using updated dosimetry data. The NNSA Supplemental Guidance (4) to DOE-STD-1027-92 outlines the guidelines for calculating updated TQs. Specifically, the NNSA states:

"When implementing this [Supplemental Directive] SD G, for radionuclides not listed in Table 1 of Attachment 2, the threshold values should be calculated in accordance with Attachment 4 of this SD G."*

Given that the use of HC-2 and HC-3 TQs are within the context of compliance with DOE regulatory requirements, the NNSA supplemental guidance will be adhered to with respect to the allowed use of new DCs within the existing methodology expectations. Any noted issues with the methodology will be discussed and recommendations provided for consideration when a revision to the methodology occurs.

Although the guidelines in the NNSA Supplemental Guidance (4) are specifically associated with the calculation of radionuclides not listed in the NNSA Supplemental Guidance, the radionuclides in the NNSA Supplemental Guidance will be included in this calculation. The recalculated HC-2 and HC-3 TQs will then be compared to the TQs previously published in the NNSA Supplemental Guidance as a verification of an accurate implementation of the methodology.

Thus, this report will apply the guidelines in the NNSA Supplemental Guidance (4) (i) to calculate the updated TQs of radionuclides not listed in the NNSA Supplemental Guidance, and (ii) to recalculate the TQs of those radionuclides listed in the NNSA Supplemental Guidance.

NNSA SD 1027 (11-28-11), pg AT2-1, Note 


\section{INPUT DATA SELECTION AND HIERARCHY}

\subsection{DEFINING THE DOSE COEFFICIENT INPUT DATA PUBLICATIONS}

In ascertaining the acceptability of contemporary sources of the DCs needed, a review of the pedigree of the DCs previously utilized by the DOE for establishing HC-2 and HC-3 TQs is performed. This review establishes the requirements used to identify and justify the use of contemporary DC data sources.

\subsubsection{Dose Coefficients Utilized by DOE-STD-1027-92 for Calculating HC-2 and HC-3 TQs}

The DCs utilized for the derivation of the HC-2 and HC-3 TQs published in DOE-STD-1027-92 (1) and the supporting LANL Fact Sheets (2) (3) are listed in Table 3.1. Upon inspection, it is noted that multiple publications were utilized for obtaining the various DCs. The use of multiple publications is attributed to the fact that the HC-2 TQ is based on a dose to a member of the public whereas the HC-3 TQ is based on a dose to a worker. Similarly, the publications utilized for calculating HC-3 TQs contain DCs derived using dosimetric models specific to workers.

Table 3.1 - Origin of Nuclear Transformation Data and the Associated Dose Coefficients Used For Calculating HC-2/3 TQs in DOE-STD-1027-92

\begin{tabular}{|c|c|c|c|}
\hline \multicolumn{4}{|c|}{ Coefticients Used For Calculatıng HC-2/3 TQS in DUE-STD-1027-92 } \\
\hline & $\begin{array}{l}\text { Dose } \\
\text { Coefficient }\end{array}$ & $\begin{array}{l}\text { Dose Coefficient } \\
\text { Publication }\end{array}$ & $\begin{array}{l}\text { Supporting Nuclear } \\
\text { Transformation Data } \\
\text { (NTD) }\end{array}$ \\
\hline \multirow{2}{*}{ Ư⿱乛龰 } & Air Immersion DC & $\begin{array}{l}\text { DOE/EH-0070 } \\
(1988)(11)\end{array}$ & $\begin{array}{l}\text { DOE/TIC-11026 } \\
(1981)(12)\end{array}$ \\
\hline & Inhalation DC & $\begin{array}{l}\text { DOE/EH-0071 } \\
(1988)(13)\end{array}$ & \multirow{4}{*}{$\begin{array}{l}\text { ICRP Pub-38 } \\
(1983)(14)\end{array}$} \\
\hline \multirow{4}{*}{$\underbrace{n}_{1}$} & Inhalation DC & \multirow{3}{*}{$\begin{array}{l}\text { ICRP Pub-30 } \\
(1978)(15)\end{array}$} & \\
\hline & Ingestion DC & & \\
\hline & Air Immersion DC & & \\
\hline & Avg. Photon Energy & $* *$ & $* *$ \\
\hline
\end{tabular}

The various DCs were derived by the respective publication via the use of accepted dosimetric models (ingestion, inhalation, and air immersion) and the use of published nuclear transformation data (NTD). The NTD publications listed in Table 3.1 provide information associated with the radionuclide specific radioactive decay characteristics (e.g., half-life, decay modes, and decay energy/intensity). The NTD is based on data files obtained from the Evaluated Nuclear Structure Data File (ENSDF) system. The ENSDF serves as a principal source of data for nuclear structure research, nuclear spectroscopy applications, medical internal radiation dose (MIRD), and publications such as Nuclear Data Sheets and the Table of Isotopes. The ENSDF is maintained by the National Nuclear Data Center (NNDC) by the Brookhaven National Laboratory. 


\subsubsection{Selection of Contemporary Dose Coefficient Publications}

Since the publication of the HC-2 and HC-3 TQs in DOE-STD-1027-92, the DC data source publications have become superseded by subsequent issuance of similar publications. Specifically:

- The NTD information in DOE/TIC-11026 has been superseded by more recent publications from the International Commission on Radiological Protection (ICRP), specifically ICRP Pub-38 (1983) and it successor, ICRP Pub-107 (2008). (10) These ICRP Publications utilize NTD from contemporaneous ENSDF data files.

- The ingestion and inhalation DCs contained within DOE/EH-0071 have been superseded in their usage by subsequent ICRP publications; ICRP Pub-72 (6) and its successor document, ICRP Pub-119. (7)

- The external DCs (e.g., air immersion) contained within DOE/EH-0070 have been superseded by a subsequent EPA publication, Federal Guidance Report No. 12. (17)

The relationship of these various publications is diagrammed in Figure 3.1. A key observation to be noted in Figure 3.1 is that the underlying NTD used in the derivation of the various ingestion, inhalation, and air immersion DCs in FGR-12 and ICRP Pub-119 originate from ICRP Pub-38. As such, the continued use of ICRP Pub-38 is necessary, even though the ICRP has superseded Pub-38 with the issuance of Pub- 107.

Although Pub-38 will still be used in conjunction with the DCs from FGR-12 and Pub-119, it is noted that Pub-107 expanded the radionuclide NTD to 1,252 radionuclides (as compared to the 825 radionuclides with NTD in Pub-38). In recognition of the expanded radionuclide data set in Pub-107, the radionuclides unique to Pub-107 (i.e., not part of the published data in Pub-38) are also assessed in this report for the calculation of HC-2 and HC-3 TQs. DOE-STD-1196-2011 (8) has been identified as a contemporaneous publication with inhalation and air immersion DCs based on Pub-107. It is noted however that DOE-STD-1196-2011 specifically states that the published DCs are computed in a manner similar to ICRP Pub-72 and Federal Guidance Report 13. (18) Since ICRP Pub-72 is specific to public dose calculations, then DOE-STD-1196-2011 will be restricted in use for only HC-2 TQ calculations.

Finally, in 2002, the Japan Atomic Energy Research Institute (JAERI) published a report which provided ingestion and inhalation DCs for radionuclides not addressed by ICRP Pub-68 nor by ICRP Pub-72. (9) The JAERI report noted that the inhalation and ingestion DCs were calculated using tissue and radiation weighting factors recommended in ICRP Pub-60, the human respiratory tract model of ICRP Pub-66, and the biokinetic models of ICRP Pubs-56, 67, 69, and 71. Also, effective dose rates for inert gases (air immersion DCs) were calculated using the methodology described in ICRP Pub-30 and FGR-12. Since the JAERI publication followed the methods of similar ICRP Publications, the DCs are acceptable for use for calculating HC-2 and HC-3 TQs. NOTE: The ICRP Publications only calculate ingestion and inhalation DCs for radionuclides with a half-life equal to or greater than 10 minutes. The JAERI report contains ingestion and inhalation DCs for radionuclides which include some with half-lives less than 10 minutes. To maintain consistency with the ICRP convention, ingestion and inhalation DCs will only be used for radionuclides with half-lives equal to or greater than 10 minutes.

The relationship of the various contemporary DC input documents is illustrated in Figure 3.1. 


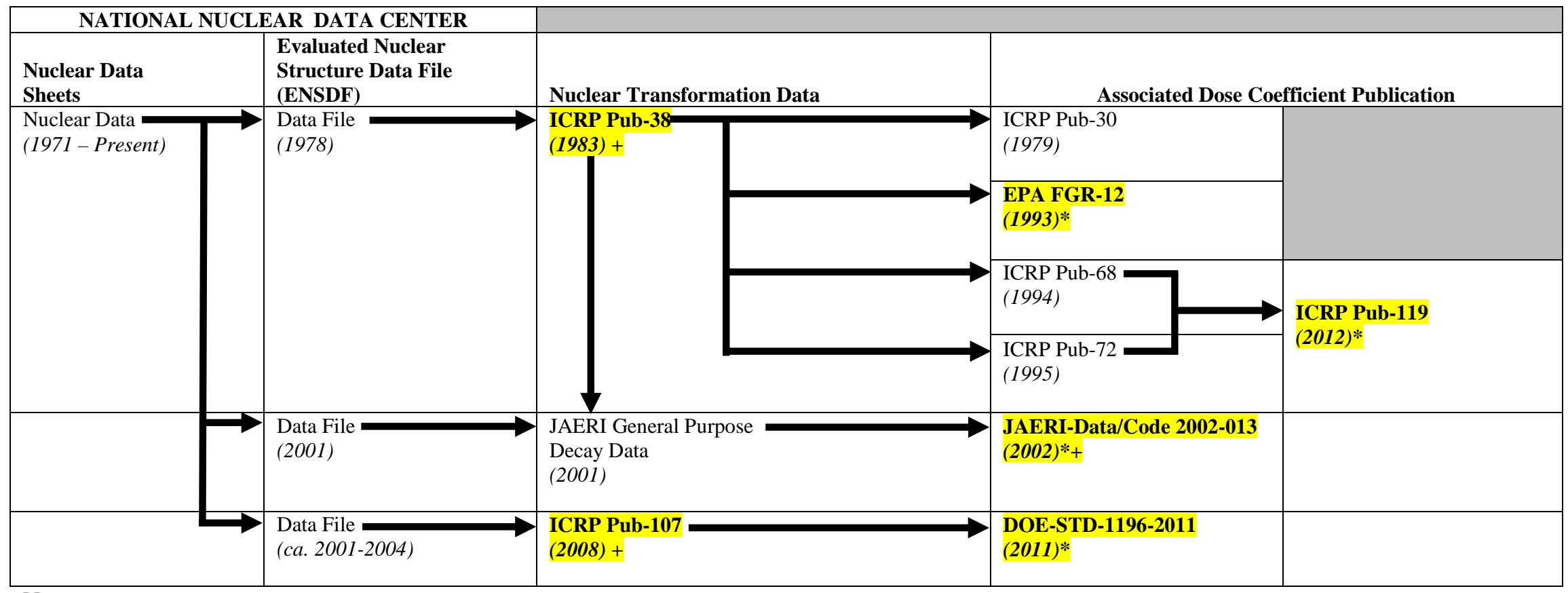

Notes:

* Publications containing ingestion, inhalation, and/or air immersion DCs selected for use in calculating updated HC-2 and HC-3 TQs in this report.

+ Publications containing NTD selected for use of average photon energy/intensity data and for calculating specific activity (using published half-life data).

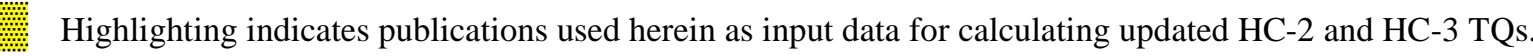

Figure 3.1 - Relationship of Dose Coefficient Publications 
A summary of the selected publications containing contemporary DCs used for the calculation of HC-2 and HC-3 TQs is listed in Table 3.2.

\begin{tabular}{|c|c|c|c|}
\hline \multicolumn{4}{|c|}{$\begin{array}{c}\text { Table } 3.2 \text { - Selection of Contemporary Dose Coefficient Publications } \\
\text { for Calculating HC-2 and HC-3 TQs }\end{array}$} \\
\hline \multirow[b]{2}{*}{ Dose Coefficient Category } & \multirow[b]{2}{*}{ Selected Publication } & \multicolumn{2}{|c|}{ Applicability } \\
\hline & & $\begin{array}{c}\text { HC-2 TQ } \\
\text { Calculation }\end{array}$ & $\begin{array}{l}\text { HC-3 TQ } \\
\text { Calculation }\end{array}$ \\
\hline \multirow{3}{*}{ Inhalation DCs } & ICRP Pub-119 & $\mathbf{X}$ & $\mathbf{X}$ \\
\hline & DOE-STD-1196-2011 & $\mathbf{X}$ & \\
\hline & JAERI-Data/Code 2002-013 & $\mathbf{X}$ & $\mathbf{X}$ \\
\hline \multirow{3}{*}{ Ingestion DCs } & ICRP Pub-119 & & $\mathbf{X}$ \\
\hline & DOE-STD-1196-2011 & & \\
\hline & JAERI-Data/Code 2002-013 & & $\mathbf{X}$ \\
\hline \multirow{3}{*}{ Air Immersion DCs } & ICRP Pub-119 & & $\mathbf{X}$ \\
\hline & FGR-12 & $\mathbf{X}$ & \\
\hline & DOE-STD-1196-2011 & $\mathbf{X}$ & \\
\hline \multirow{2}{*}{$\begin{array}{l}\text { Avg. Photon Energy/Intensity } \\
\text { Data }\end{array}$} & ICRP Pub-38 & & $\mathbf{X}$ \\
\hline & ICRP Pub-107 & & $\mathbf{X}$ \\
\hline
\end{tabular}

\subsubsection{Hierarchy of Selected Contemporary Dose Coefficient Publications}

\subsubsection{Family of Data Concept}

As noted in Table 3.2, multiple publications containing contemporary DCs were selected for use in calculating the HC-2 and HC-3 TQs. The selection of multiple publications was in recognition that no single data source contains all DCs for the scope of radionuclides involved. Accordingly, a hierarchical ranking of the input data is required to ensure a proper calculation of the HC-2 and HC-3 TQs.

For the task at hand, a high level grouping of the information can be made based on the "family of data" concept. As noted in Figure 3.1, the contemporary DC publications can be grouped in accordance with the NTD used in derivation of the ingestion, inhalation and air immersion DCs. Under this "family of data" concept, the NTD and the selected DCs are used together for computing a TQ value.

\section{ICRP Pub-38 Family of Data}

Under the "family of data" concept, the highest ranking data set is assigned to ICRP Pub-38 and its family of publications. The ICRP Pub-38 family ranking is based on the observation that the majority of the available inhalation and ingestion dose coefficients for both worker dose assessments and for members of the public dose assessments currently reside within ICRP Pub-119. The publications assigned to the ICRP Pub-38 family of data include the following:

o ICRP Pub-38

o ICRP Pub-119

o FGR-12 


\section{ICRP Pub-107 Family of Data}

The second highest ranking data set is assigned to ICRP Pub-107 and its family of publications. The ICRP Pub-107 family ranking is based on the observation that ICRP Pub107 provides a substantial increase in the overall radionuclide data set. Whereas ICRP Pub-38 provides NTD for 838 radionuclides, ICRP Pub-107 expands the available data set to 1252 radionuclides (an additional 414 radionuclides). The publications assigned to the ICRP Pub-107 family of data include the following:

o ICRP Pub-107

o DOE-STD-1196-2011

\section{JAERI-Data/Code 2002-013 Family of Data}

The third highest ranking data set is assigned to JAERI-Data/Code 2002-013. This designation is based on the observation that the JAERI data is considered to be a minor supplemental source of information outside of the ICRP publications. The publications assigned to the JAERI-Data/Code 2002-013 family of data include the following:

o JAERI-Data/Code 2002-013

Under this "family of data" concept, the NTD and the DCs are all selected from the same family of data, if possible. If an input element is not available from within the family of data, then the next lower "family of data" level is searched for the necessary data element. This search continues until all lower family of data levels has been exhausted.

\subsubsection{Summary of Dose Coefficient Selection Order}

Based on the "family of data" hierarchy concept, the DC publications can be ranked for their use for the calculation of HC-2 and HC-3 TQs. Table 3.3 lists the relevant DC publications in accordance with the DC data type it is associated with for the calculation of HC-2 TQs. Similarly, Table 3.4 lists the relevant DC publications in accordance with the DC data type it is associated with for the calculation of HC-3 TQs. 
Table 3.3 - Hierarchical Organization of Contemporary Dose Coefficient Publications for HC-2 TQ Calculations

\begin{tabular}{|c|c|c|c|}
\hline Data Type & $\begin{array}{l}\text { Selection } \\
\text { Order }\end{array}$ & Publication & Relevant Dose Coefficient Table \\
\hline \multirow{5}{*}{$\begin{array}{l}\text { Inhalation } \\
\text { DCs }\end{array}$} & \multirow{2}{*}{1} & \multirow{2}{*}{ ICRP Pub-119 } & $\begin{array}{ll}\text { Annex G: } & \text { Effective Dose Coefficients for Inhalation of Radionuclides for } \\
& \text { Members of the Public }\end{array}$ \\
\hline & & & $\begin{array}{c}\text { Annex H: Dose Coefficients for Inhalation of Soluble or Reactive Gases and } \\
\text { Vapours For Members of the Public }\end{array}$ \\
\hline & 2 & DOE-STD-1196-2011 & Table A-2: Effective Dose Coefficients from Inhaled Air \\
\hline & \multirow[b]{2}{*}{3} & \multirow[b]{2}{*}{ JAERI-Data/Code 2002-013 } & $\begin{array}{l}\text { Table 5: Effective Dose Coefficients for Members of the Public-Inhalation } \\
\text { Dose Coefficients, e }(\tau) \text {, to age } 70 y\left(\mathrm{~Sv} B q^{-1}\right)\end{array}$ \\
\hline & & & $\begin{array}{l}\text { Table 7: Effective Dose Coefficients for Members of the Public - Inhalation } \\
\text { Dose Coefficients, e }(\tau) \text {, to age } 70 \text { y }\left(S v B q^{-1}\right) \text { for Soluble or } \\
\text { Reactive Gases and Vapours (Class } S R-1 \text { and SR-2) }\end{array}$ \\
\hline \multirow{2}{*}{$\begin{array}{l}\text { Air } \\
\text { Immersion } \\
\text { DCs }\end{array}$} & 1 & FGR-12 & $\begin{array}{l}\text { Table A.1: Summary Information on the Nuclear Transformation of the } \\
\text { Radionuclides }\end{array}$ \\
\hline & 2 & DOE-STD-1196-2011 & Table A-3: Effective Dose Coefficients from Air Submersion \\
\hline \multirow{4}{*}{$\begin{array}{l}\text { Half-Life } \\
\text { Data }\end{array}$} & 1 & ICRP Pub-38 & Entire Report \\
\hline & 2 & ICRP Pub-107 & Table A.1: Properties of the Radionuclides: ICRP-07 Collection \\
\hline & \multirow{2}{*}{3} & \multirow{2}{*}{ JAERI-Data/Code 2002-013 } & $\begin{array}{l}\text { Table 1: Radionuclides Included in Dose Coefficient Database (Ingestion } \\
\text { and Inhalation of Particulates) }\end{array}$ \\
\hline & & & $\begin{array}{l}\text { Table 2: } \quad \begin{array}{l}\text { Radionuclides Included in Dose Coefficient Database (Inert } \\
\text { Gases) }\end{array}\end{array}$ \\
\hline
\end{tabular}


Table 3.4 - Hierarchical Organization of Contemporary Dose Coefficient Publications for HC-3 TQ Calculations

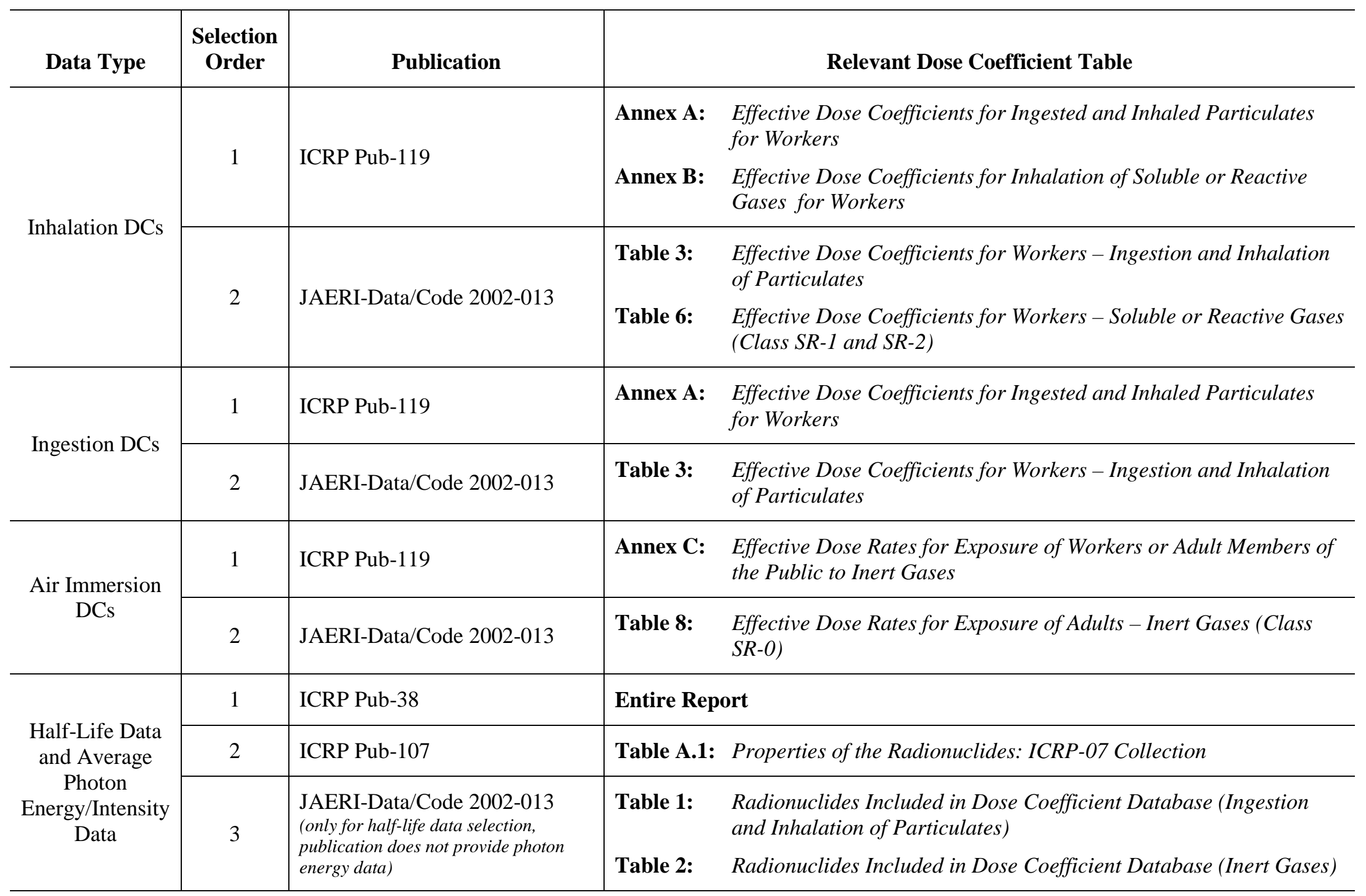




\subsection{DEFINING THE LIST OF RADIONUCLIDES}

The contemporary dosimetry publications selected for use define the set of radionuclides for which HC-2 and HC-3 TQs will be calculated.

\subsubsection{Developing the Master List of Radionuclides}

The following NTD publications were utilized in constructing a master list of radionuclides:

1. ICRP Pub-38

2. ICRP Pub-107

3. JAERI-Data/Code 2002-013

The order of selection was based on the hierarchical "family of data" as previously discussed.

\subsubsection{Reconciliation of Radionuclide Nomenclature Differences}

The use of various NTD publications and their derivative publication that span various agencies (ICRP, DOE, EPA, JAERI) has resulted in noted differences in radionuclide nomenclature. Specifically:

- The treatment of isomer states (i.e., a radionuclide with two different half-lives) has been addressed by the use of the "l" and "s" suffix to denote long and short.

- There are several instances where updated NTD in ICRP Pub-107 has changed a radionuclide identified in ICRP Pub-38 as an isomer to a meta-stable state. An example of this is the radionuclide Eu-150 which was identified in ICRP Pub-38 as existing in an isomeric state (Eu-150a with $\mathrm{t}_{1 / 2}=12.62 \mathrm{~h}$ and Eu-150b with $\left.\mathrm{t}_{1 / 2}=34.2 \mathrm{y}\right){ }^{\dagger}{ }^{\dagger}$ In comparison, ICRP Pub-107 incorporated revised NTD which no longer designated Eu-150 as an isomer, but as a single decay scheme (Eu-150 with $\left.\mathrm{t}_{1 / 2}=36.9 \mathrm{y}\right)$, with a companion metastable state $\left(\mathrm{Eu}-150 \mathrm{~m}\right.$ with $\mathrm{t}_{1 / 2}=12.8$ h). When such nomenclature differences were encountered, the "family of data" hierarchy was used to resolve the nomenclature discrepancy. As such, since ICRP Pub-38 is higher in the “family of data” hierarchy, the name was resolved and established as Eu-150l and Eu-150s.

- Several other instances were noted where ground state and meta-stable state radionuclide definitions differed amongst the publications. An example of this would be Ta-180, which ICRP Pub-38 identifies as having both a ground state (Ta-180 with $\left.\mathrm{t}_{1 / 2}=1 \mathrm{E}+13 \mathrm{y}\right)$ and meta-stable state $\left(\right.$ Ta-180m with $\left.\mathrm{t}_{1 / 2}=8.1 \mathrm{~h}\right)$. The updated NTD in ICRP Pub-107 no longer includes the meta-stable state and identifies Ta-180 has having the previous meta-stable state half-life $\left(\mathrm{t}_{1 / 2}\right.$ $=8.152 \mathrm{~h}$ ). As before, the "family of data" hierarchy was used to resolve the nomenclature discrepancy. As such, since ICRP Pub-38 is higher in the "family of data" hierarchy, the name was resolved and established as Ta-180 and Ta-180m.

A summary of the nomenclature resolution is presented in the form of a crosswalk in Table 3.5. A complete listing of the master radionuclide list is documented in Appendix D of this report.

$\dagger \quad$ The isomeric suffix designators "a” and "b" are unique to the electronic data file containing ICRP Pub-38 NTD. FGR-12 includes the use of the isomeric designators "a" and "b" in both the hard bound copy and electronic data file. Similarly, " $m$ " and " $n$ " suffix designators are universally used to indicate isomers for meta-state radionuclides. 
Table 3.5 - Radionuclide Nomenclature Crosswalk

\begin{tabular}{|c|c|c|c|c|c|c|c|c|c|c|c|c|c|c|c|}
\hline \multirow{3}{*}{$\begin{array}{l}\text { MASTER } \\
\text { LIST } \\
\text { Eu-1501 }\end{array}$} & \multicolumn{3}{|c|}{ DOE-STD-1196 } & \multicolumn{3}{|c|}{ FGR-12 } & \multicolumn{3}{|c|}{ ICRP-38 } & \multicolumn{3}{|c|}{ ICRP-107 } & \multicolumn{3}{|c|}{ JAERI } \\
\hline & \multirow{2}{*}{$\begin{array}{l}\text { Nuclide } \\
\text { Eu-150 }\end{array}$} & \multicolumn{2}{|c|}{$\begin{array}{c}\text { Half-life } \\
\text { (Units) }\end{array}$} & \multirow{2}{*}{$\begin{array}{l}\text { Nuclide } \\
\text { Eu-150b }\end{array}$} & \multicolumn{2}{|c|}{$\begin{array}{c}\text { Half-life } \\
\text { (Units) }\end{array}$} & \multirow{2}{*}{$\begin{array}{l}\text { Nuclide } \\
\text { Eu-150b }\end{array}$} & \multicolumn{2}{|c|}{$\begin{array}{c}\text { Half-life } \\
\text { (Units) }\end{array}$} & \multirow{2}{*}{$\begin{array}{l}\text { Nuclide } \\
\text { Eu-150 }\end{array}$} & \multicolumn{2}{|c|}{$\begin{array}{c}\text { Half-life } \\
\text { (Units) }\end{array}$} & \multirow[t]{2}{*}{ Nuclide } & \multicolumn{2}{|c|}{$\begin{array}{c}\text { Half-life } \\
\text { (Units) }\end{array}$} \\
\hline & & 36.9 & $\mathrm{y}$ & & 34.2 & $\mathrm{y}$ & & 34.2 & $\mathrm{y}$ & & 36.9 & $\mathrm{y}$ & & & \\
\hline Eu-150s & Eu-150m & 12.8 & $\mathrm{~h}$ & Eu-150a & 12.62 & $\mathrm{~h}$ & Eu-150a & 12.62 & $\mathrm{~h}$ & Eu-150m & 12.8 & $\mathrm{~h}$ & & & \\
\hline Eu-152ml & Eu-152m & 9.3116 & $\mathrm{~h}$ & Eu-152m & 9.32 & $\mathrm{~h}$ & Eu-152m & 9.32 & $\mathrm{~h}$ & Eu-152m & 9.3116 & $\mathrm{~h}$ & & & \\
\hline Eu-152ms & Eu-152n & 96 & $\mathrm{~m}$ & & & & & & & Eu-152n & 96 & $\mathrm{~m}$ & Eu-152n & 96 & \\
\hline In-110l & In-110 & 4.9 & $\mathrm{~h}$ & In-110b & 4.9 & $\mathrm{~h}$ & In-110b & 4.9 & $\mathrm{~h}$ & In-110 & 4.9 & $\mathrm{~h}$ & & & \\
\hline In-110s & In-110m & 69.1 & $\mathrm{~m}$ & In-110a & 69.1 & $\mathrm{~m}$ & In-110a & 69.1 & $\mathrm{~m}$ & In-110m & 69.1 & $\mathrm{~m}$ & & & \\
\hline Ir-186l & Ir-186 & 16.64 & $\mathrm{~h}$ & Ir-186a & 15.8 & $\mathrm{~h}$ & Ir-186a & 15.8 & $\mathrm{~h}$ & Ir-186 & 16.64 & $\mathrm{~h}$ & & & \\
\hline Ir-186s & $\mathrm{Ir}-186 \mathrm{~m}$ & 1.92 & $\mathrm{~h}$ & Ir-186b & 1.75 & $\mathrm{~h}$ & $\mathrm{Ir}-186 \mathrm{~b}$ & 1.75 & $\mathrm{~h}$ & Ir-186m & 1.92 & $\mathrm{~h}$ & & & \\
\hline Ir-190ms & $\mathrm{Ir}-190 \mathrm{~m}$ & 1.12 & $\mathrm{~h}$ & $\mathrm{Ir}-190 \mathrm{~m}$ & 1.2 & $\mathrm{~h}$ & $\mathrm{Ir}-190 \mathrm{~m}$ & 1.2 & $\mathrm{~h}$ & Ir-190m & 1.12 & $\mathrm{~h}$ & & & \\
\hline Ir-190ml & Ir-190n & 3.087 & $\mathrm{~h}$ & Ir-190n & 3.1 & $\mathrm{~h}$ & Ir-190n & 3.1 & $\mathrm{~h}$ & Ir-190n & 3.087 & $\mathrm{~h}$ & & & \\
\hline Ir-192ms & $\mathrm{Ir}-192 \mathrm{~m}$ & 1.45 & $\mathrm{~m}$ & & & & & & & $\mathrm{Ir}-192 \mathrm{~m}$ & 1.45 & $\mathrm{~m}$ & & & \\
\hline Ir-192ml & Ir-192n & 241 & $\mathrm{y}$ & Ir-192m & 241 & $\mathrm{y}$ & Ir-192m & 241 & $\mathrm{y}$ & Ir-192n & 241 & $\mathrm{y}$ & & & \\
\hline Nb-89l & Nb-89 & 2.03 & $\mathrm{~h}$ & $\mathrm{Nb}-89 \mathrm{~b}$ & 122 & $\mathrm{~m}$ & Nb-89b & 122 & $\mathrm{~m}$ & Nb-89 & 2.03 & $\mathrm{~h}$ & & & \\
\hline Nb-89s & $\mathrm{Nb}-89 \mathrm{~m}$ & 66 & $\mathrm{~m}$ & Nb-89a & 66 & $\mathrm{~m}$ & Nb-89a & 66 & $\mathrm{~m}$ & $\mathrm{Nb}-89 \mathrm{~m}$ & 66 & $\mathrm{~m}$ & & & \\
\hline Nb-98 & Nb-98m & 51.3 & $\mathrm{~m}$ & Nb-98 & 51.5 & $\mathrm{~m}$ & Nb-98 & 51.5 & $\mathrm{~m}$ & Nb-98m & 51.3 & $\mathrm{~m}$ & & & \\
\hline Np-236l & Np-236 & 154000 & $\mathrm{y}$ & Np-236a & 115000 & $y$ & Np-236a & 115000 & $\mathrm{y}$ & Np-236 & 154000 & $y$ & & & \\
\hline $\mathrm{Np}-236 \mathrm{~s}$ & $\mathrm{~Np}-236 \mathrm{~m}$ & 22.5 & $\mathrm{~h}$ & $\mathrm{~Np}-236 \mathrm{~b}$ & 22.5 & $\mathrm{~h}$ & Np-236b & 22.5 & $\mathrm{~h}$ & $\mathrm{~Np}-236 \mathrm{~m}$ & 22.5 & $\mathrm{~h}$ & & & \\
\hline Re-182l & Re-182 & 64 & $\mathrm{~h}$ & Re-182b & 64 & $\mathrm{~h}$ & Re-182b & 64 & $\mathrm{~h}$ & Re-182 & 64 & $\mathrm{~h}$ & & & \\
\hline Re-182s & Re-182m & 12.7 & $\mathrm{~h}$ & Re-182a & 12.7 & $\mathrm{~h}$ & Re-182a & 12.7 & $\mathrm{~h}$ & Re-182m & 12.7 & $\mathrm{~h}$ & & & \\
\hline Rh-102 & Rh-102m & 3.742 & $\mathrm{y}$ & Rh-102 & 2.9 & $\mathrm{y}$ & Rh-102 & 2.9 & $y$ & Rh-102m & 3.742 & $\mathrm{y}$ & & & \\
\hline Rh-102m & Rh-102 & 207 & $\mathrm{~d}$ & Rh-102m & 207 & $\mathrm{~d}$ & Rh-102m & 207 & $\mathrm{~d}$ & Rh-102 & 207 & $\mathrm{~d}$ & & & \\
\hline Sb-120s & Sb-120 & 15.89 & $\mathrm{~m}$ & Sb-120a & 15.89 & $\mathrm{~m}$ & Sb-120a & 15.89 & $\mathrm{~m}$ & Sb-120 & 15.89 & $\mathrm{~m}$ & & & \\
\hline Sb-120l & Sb-120m & 5.76 & $\mathrm{~d}$ & Sb-120b & 5.76 & $\mathrm{~d}$ & Sb-120b & 5.76 & $\mathrm{~d}$ & Sb-120m & 5.76 & $\mathrm{~d}$ & & & \\
\hline Sb-124ms & Sb-124m & 93 & $\mathrm{~s}$ & Sb-124m & 93 & $\mathrm{~s}$ & Sb-124m & 93 & $\mathrm{~s}$ & Sb-124m & 93 & $\mathrm{~S}$ & Sb-124m & 1.55 & \\
\hline Sb-124ml & Sb-124n & 20.2 & $\mathrm{~m}$ & Sb-124n & 20.2 & $\mathrm{~m}$ & Sb-124n & 20.2 & $\mathrm{~m}$ & Sb-124n & 20.2 & $\mathrm{~m}$ & & & \\
\hline Sb-128l & Sb-128 & 9.01 & $\mathrm{~h}$ & Sb-128b & 9.01 & $\mathrm{~h}$ & Sb-128b & 9.01 & $\mathrm{~h}$ & Sb-128 & 9.01 & $\mathrm{~h}$ & & & \\
\hline Sb-128s & Sb-128m & 10.4 & $\mathrm{~m}$ & Sb-128a & 10.4 & $\mathrm{~m}$ & Sb-128a & 10.4 & $\mathrm{~m}$ & Sb-128m & 10.4 & $\mathrm{~m}$ & & & \\
\hline
\end{tabular}




\begin{tabular}{|c|c|c|c|c|c|c|c|c|c|c|c|}
\hline \multicolumn{12}{|c|}{ Table 3.5 - Radionuclide Nomenclature Crosswalk (continued) } \\
\hline & \multicolumn{3}{|c|}{ DOE-STD-1196 } & \multicolumn{2}{|c|}{ FGR-12 } & \multicolumn{2}{|c|}{ ICRP-38 } & \multicolumn{2}{|c|}{ ICRP-107 } & \multicolumn{2}{|c|}{ JAERI } \\
\hline $\begin{array}{l}\text { MASTER } \\
\text { LIST }\end{array}$ & Nuclide & $\begin{array}{r}\text { Half-lif } \\
\text { (Units) }\end{array}$ & & Nuclide & $\begin{array}{c}\text { Half-life } \\
\text { (Units) }\end{array}$ & Nuclide & $\begin{array}{c}\text { Half-life } \\
\text { (Units) }\end{array}$ & Nuclide & $\begin{array}{c}\text { Half-life } \\
\text { (Units) }\end{array}$ & Nuclide & $\begin{array}{c}\text { Half-life } \\
\text { (Units) }\end{array}$ \\
\hline Ta-178s & Тa-178 & 9.31 & $\mathrm{~m}$ & Та-178a & $9.31 \mathrm{~m}$ & Та-178a & $9.31 \mathrm{~m}$ & Ta-178 & $9.31 \mathrm{~m}$ & Ta-178 & $9.31 \mathrm{~m}$ \\
\hline Ta-178l & Ta-178m & 2.36 & $\mathrm{~h}$ & Ta-178b & 2.2 & Ta-178b & $2.2 \mathrm{~h}$ & Ta-178m & $2.36 \mathrm{~h}$ & & \\
\hline Та-180 & & & & Та-180 & $1 \mathrm{E}+13$ & Та-180 & $1 \mathrm{E}+13 \mathrm{y}$ & & & & \\
\hline Ta-180m & Та-180 & 8.152 & $\mathrm{~h}$ & Ta-180m & 8.1 & Ta-180m & $8.1 \mathrm{~h}$ & Та-180 & $8.152 \mathrm{~h}$ & & \\
\hline Tb-156ml & Tb-156m & 24.4 & $\mathrm{~h}$ & Tb-156m & 24.4 & Tb-156m & $24.4 \mathrm{~h}$ & Tb-156m & $24.4 \mathrm{~h}$ & & \\
\hline Tb-156ms & Tb-156n & 5.3 & $\mathrm{~h}$ & Tb-156n & 5 & Tb-156n & $5 \mathrm{~h}$ & Tb-156n & 5.3 & & \\
\hline
\end{tabular}




\subsection{OTHER INPUT DATA}

\subsubsection{Atomic Mass Data}

Using the methodology described in Section 4 of this report will allow for the calculation of HC-2 and HC-3 TQs in terms of activity (e.g., curies). Through the use of a radionuclide specific activity value, the calculated TQs can be equivalently expressed in terms of mass (e.g., grams). In order to calculate a radionuclide specific activity value, the atomic mass of the associated radionuclide is required.

The Atomic Mass Data Center (AMDC) maintains the official isotope mass tables. The AMDC is hosted by the Institute of Modern Physics (IMP), Chinese Academy of Sciences, Lanzhou, China, with a mirror site maintained by the International Atomic Energy Agency (IAEA). The latest version of the atomic mass data is dated March 1, 2017. (19) The data file contains atomic mass values for atomic mass entities ranging from $\mathrm{A}=0$ to 295 .

\subsubsection{Element Specific Constants}

\subsubsection{HC-2 Element Specific Constants}

The calculation of HC-2 TQs requires the identification of element specific release fractions. The release fraction (RF) is a numerical representation of the fractional amount of the facility radioactive inventory which is lofted into an airborne plume. The RFs used for the calculation of HC-2 TQs is specified in accordance with Attachment 1 (page A-8) of DOE-STD-1027-92 CN-1, (1) which states:

\begin{tabular}{|c|c|c|}
\hline & Element Class & $\begin{array}{c}\text { Assigned Airborne } \\
\text { Release Fraction } \\
\text { (RF) } \\
\end{array}$ \\
\hline 1. & $\begin{array}{l}\text { Gases } \\
\text { (such as tritium, krypton, xenon, argon, radon, chlorine, etc.) }\end{array}$ & 1.0 \\
\hline 2. & $\begin{array}{l}\text { Highly volatile/combustible } \\
\text { (phosphorus, sulfur, potassium, iodine, sodium, bromine) }\end{array}$ & 0.5 \\
\hline 3. & $\begin{array}{l}\text { Semi-volatile } \\
\text { (selenium, mercury, cesium, polonium, tellurium, ruthenium, carbon) }\end{array}$ & $1 \mathrm{E}-2$ \\
\hline 4. & $\begin{array}{l}\text { Solid/Powder/Liquid } \\
\text { (All materials not listed above) }\end{array}$ & $1 \mathrm{E}-3$ \\
\hline
\end{tabular}

\subsubsection{HC-3 Element Specific Constants}

The calculation of HC-3 TQs requires the identification of three element specific constants; (i) airborne release fraction (for the inhalation and food ingestion exposure pathways), (ii) the sorption coefficient (for the water ingestion exposure pathway, and (iii) the soil to plant concentration factor (for the food ingestion exposure pathway). 
These element specific constants were obtained from the following tables in the EPA Technical Background Document (16) and are listed in Table 3.7:

- Exhibit A-1: Inhalation Release Fractions

- Exhibit B-2: Sorption Coefficient Values

- Exhibit C-1: Soil To Plant Concentration Factors

The element specific constants in Table 3.7 have not been altered and represent the published values contained within the EPA Technical Background Document. For several elements, the EPA Technical Background Document did not provide for an element specific constant. These instances are identified in the table with the symbol "--," and the associated pathway was not evaluated for the element. A justification for this disposition is explained as follows:

- Boron: The EPA Technical Background Document did not provide any element specific constants for boron. No radionuclides associated with boron are on the master radionuclide list (Appendix D, Table D.1). Therefore, no element specific constants are required for boron.

- Carbon: As noted in Appendix E of the EPA Technical Background Document, carbon radionuclides were only evaluated for the inhalation, water ingestion, and direct exposure pathways. $^{\ddagger}$ This report follows the same convention as established in the EPA Technical Background Document and only evaluates the inhalation, water ingestion, and direct exposure pathways for carbon radionuclides.

- Helium: The EPA Technical Background Document did not provide an element specific airborne release fraction (RF) nor an element specific sorption coefficient $\left(K_{d}\right)$ for helium. No radionuclides associated with helium are on the master radionuclide list (Appendix D, Table D.1). Therefore, no element specific constants are required for helium.

- Hydrogen: As noted in Appendix E of the EPA Technical Background Document, the lone hydrogen radionuclide (i.e, H-3) was only evaluated for the inhalation and water ingestion pathways. ${ }^{\S}$ This report follows the same convention as established in the EPA Technical Background Document and only evaluates the inhalation and water ingestion pathways for hydrogen radionuclides.

- Lithium: The EPA Technical Background Document did not provide any element specific constants for lithium. No radionuclides associated with the element lithium are on the master radionuclide list (Appendix D, Table D.1). Therefore, no element specific constants are required for lithium.

$\ddagger$ The direct exposure pathway was only evaluated in the EPA Technical Background Document when there was a photon emission component associated with the decay of the radionuclide. As such, the EPA Technical Background Document evaluated the direct exposure pathway for C-11 since it had a photon emission component, whereas C-14 was not evaluated for the direct exposure pathway since there was no photon emission component associated with the its decay.

$\S$ The direct exposure pathway was not evaluated in the EPA Technical Background Document since there was no photon emission component associated with the decay of $\mathrm{H}-3$. The updated input documentation used in this report similarly notes there is no photon emission component associated with the decay of H-3. 
- Neon: The EPA Technical Background Document did not provide any element specific constants for neon. There are two radionuclides associated with neon on the master radionuclide list (Appendix D, Table D.1); Ne-19 $\left(\mathrm{t}_{1 / 2}=17.22 \mathrm{~s}\right)$ and Ne-24 $\left(\mathrm{t}_{1 / 2}=3.38 \mathrm{~m}\right)$. Since these radionuclides each have a half-life less than 10 minutes, the inhalation and ingestion pathways are not evaluated (Refer to section 3.1.2 for a discussion of the 10 minute half-life evaluation threshold). Therefore, no element specific constants are required for neon.

- Nitrogen: The EPA Technical Background Document did not provide any element specific constants for nitrogen. There are two radionuclides associated with nitrogen on the master radionuclide list (Appendix D, Table D.1); $\mathrm{N}-13\left(\mathrm{t}_{1 / 2}=9.965 \mathrm{~m}\right)$ and $\mathrm{N}-16\left(\mathrm{t}_{1 / 2}=7.13 \mathrm{~s}\right)$. Since these radionuclides each have a half-life less than 10 minutes, the inhalation and ingestion pathways are not evaluated (Refer to section 3.1.2 for a discussion of the 10 minute half-life evaluation threshold). Therefore, no element specific constants are required for nitrogen.

- Oxygen: The EPA Technical Background Document did not provide any element specific constants for oxygen. There are three radionuclides associated with oxygen on the master radionuclide list (Appendix D, Table D.1); O-14 ( $\left.\mathrm{t}_{1 / 2}=70.599 \mathrm{~s}\right), \mathrm{O}-15\left(\mathrm{t}_{1 / 2}=122.24 \mathrm{~s}\right)$ and O-19 $\left(\mathrm{t}_{1 / 2}=26.91 \mathrm{~s}\right)$. Since these radionuclides each have a half-life less than 10 minutes, the inhalation and ingestion pathways are not evaluated (Refer to section 3.1.2 for a discussion of the 10 minute half-life evaluation threshold). Therefore, no element specific constants are required for oxygen. 


\begin{tabular}{|c|c|c|c|c|}
\hline \multicolumn{5}{|c|}{$\begin{array}{c}\text { Table 3.7 - Elemental Data Used In The Calculation of Hazard Category } 3 \text { Threshold } \\
\text { Quantities }\end{array}$} \\
\hline Symbol & Name & $\begin{array}{c}\text { Airborne Release } \\
\text { Fraction }^{* *} \\
\text { (RF) }\end{array}$ & $\begin{array}{c}\text { Sorption } \\
\text { Coefficient }^{\dagger+} \\
\left(\mathbf{K}_{\mathbf{d}}\right)\end{array}$ & $\begin{array}{l}\text { Soil to Plant } \\
\text { Concentration } \\
\text { Factor } \\
\text { (B\# } \\
\left(\mathbf{B}_{\mathbf{v}}\right)\end{array}$ \\
\hline Ac & Actinium & 0.001 & 1000 & 0.0035 \\
\hline $\mathrm{Al}$ & Aluminum & 0.01 & 0 & 0.004 \\
\hline $\mathrm{Am}$ & Americium & 0.001 & 1000 & 0.0055 \\
\hline $\mathrm{Sb}$ & Antimony & 0.01 & 1 & 0.2 \\
\hline $\mathrm{Ar}$ & Argon & 1.0 & 0 & 0 \\
\hline As & Arsenic & 0.01 & 0 & 0.04 \\
\hline $\mathrm{At}$ & Astatine & 0.001 & 0 & 1 \\
\hline $\mathrm{Ba}$ & Barium & 0.01 & 100 & 0.15 \\
\hline $\mathrm{Bk}$ & Berkelium & 0.001 & 700 & 0.001 \\
\hline $\mathrm{Be}$ & Beryllium & 0.01 & 75 & 0.01 \\
\hline $\mathrm{Bi}$ & Bismuth & 0.01 & 10 & 0.035 \\
\hline $\mathrm{B}$ & Boron & -- & -- & -- \\
\hline $\mathrm{Br}$ & Bromine & 0.01 & 0 & 1.5 \\
\hline $\mathrm{Cd}$ & Cadmium & 0.01 & $50-100$ & 0.55 \\
\hline $\mathrm{Ca}$ & Calcium & 0.01 & 15 & 3.5 \\
\hline $\mathrm{Cf}$ & Californium & 0.001 & 0 & 0.001 \\
\hline $\mathrm{C}$ & Carbon & 0.5 & 0 & -- \\
\hline $\mathrm{Ce}$ & Cerium & 0.01 & 2000 & 0.01 \\
\hline Cs & Cesium & 0.01 & 2000 & 0.08 \\
\hline $\mathrm{Cl}$ & Chlorine & 0.01 & 0 & 70 \\
\hline $\mathrm{Cr}$ & Chromium & 0.01 & 0 & 0.0075 \\
\hline Co & Cobalt & 0.001 & 2000 & 0.02 \\
\hline $\mathrm{Cu}$ & Copper & 0.01 & 0 & 0.4 \\
\hline $\mathrm{Cm}$ & Curium & 0.001 & 500 & 0.00085 \\
\hline Dy & Dysprosium & 0.01 & 0 & 0.01 \\
\hline Es & Einsteinium & 0.001 & 0 & 0.001 \\
\hline $\mathrm{Er}$ & Erbium & 0.01 & 0 & 0.01 \\
\hline $\mathrm{Eu}$ & Europium & 0.01 & 500 & 0.01 \\
\hline $\mathrm{Fm}$ & Fermium & 0.001 & 0 & 0.001 \\
\hline $\mathrm{F}$ & Fluorine & 0.01 & 0 & 0.06 \\
\hline $\mathrm{Fr}$ & Francium & 0.01 & 200 & 0.03 \\
\hline $\mathrm{Gd}$ & Gadolinium & 0.01 & $500-1000$ & 0.01 \\
\hline $\mathrm{Ga}$ & Gallium & 0.01 & 0 & 0.004 \\
\hline $\mathrm{Ge}$ & Germanium & 0.01 & 0 & 0.4 \\
\hline $\mathrm{Au}$ & Gold & 0.01 & 0 & 0.4 \\
\hline $\mathrm{Hf}$ & Hafnium & 0.01 & 0 & 0.0035 \\
\hline
\end{tabular}

\footnotetext{
** From Exhibit A-1 of the EPA Technical Background Document, 102RQ-RN-5-13

it From Exhibit B-2 of the EPA Technical Background Document, 102RQ-RN-5-13

抹 From Exhibit C-1 of the EPA Technical Background Document, 102RQ-RN-5-13
} 


\begin{tabular}{|c|c|c|c|c|}
\hline Symbol & Name & $\begin{array}{l}\text { Airborne Release } \\
\text { Fraction } \\
\text { (RF) }\end{array}$ & $\begin{array}{c}\text { Sorption } \\
\text { Coefficient } \\
\left(\mathbf{K}_{\mathbf{d}}\right)\end{array}$ & $\begin{array}{l}\text { Soil to Plant } \\
\text { Concentration } \\
\text { Factor } \\
\left(\mathbf{B}_{\mathrm{v}}\right)\end{array}$ \\
\hline $\mathrm{He}$ & Helium & -- & -- & 0.01 \\
\hline Ho & Holmium & 0.01 & 600 & 0.01 \\
\hline $\mathrm{H}$ & Hydrogen & 0.5 & 0 & -- \\
\hline In & Indium & 0.01 & 0 & 0.004 \\
\hline $\mathrm{I}$ & Iodine & 0.5 & 3 & 0.15 \\
\hline $\mathrm{Ir}$ & Iridium & 0.001 & 0 & 0.055 \\
\hline $\mathrm{Fe}$ & Iron & 0.01 & 150 & 0.004 \\
\hline $\mathrm{Kr}$ & Krypton & 1.0 & 0 & 0 \\
\hline $\mathrm{La}$ & Lanthanum & 0.01 & 0 & 0.01 \\
\hline $\mathrm{Pb}$ & Lead & 0.01 & 4000 & 0.045 \\
\hline $\mathrm{Li}$ & Lithium & -- & -- & -- \\
\hline $\mathrm{Lu}$ & Lutetium & 0.01 & 0 & 0.01 \\
\hline $\mathrm{Mg}$ & Magnesium & 0.01 & 0 & 1 \\
\hline $\mathrm{Mn}$ & Manganese & 0.01 & 0 & 0.25 \\
\hline Md & Mendelevium & 0.001 & 0 & 0.001 \\
\hline $\mathrm{Hg}$ & Mercury & 0.01 & 0 & 0.9 \\
\hline Mo & Molybdenum & 0.01 & 5 & 0.25 \\
\hline $\mathrm{Nd}$ & Neodymium & 0.01 & 500 & 0.01 \\
\hline $\mathrm{Ne}$ & Neon & -- & -- & -- \\
\hline $\mathrm{Np}$ & Neptunium & 0.001 & 10 & 0.1 \\
\hline $\mathrm{Ni}$ & Nickel & 0.01 & 100 & 0.06 \\
\hline $\mathrm{Nb}$ & Niobium & 0.01 & 2000 & 0.02 \\
\hline $\mathrm{N}$ & Nitrogen & -- & -- & -- \\
\hline Os & Osmium & 0.01 & 0 & 0.015 \\
\hline $\mathrm{O}$ & Oxygen & -- & -- & -- \\
\hline $\mathrm{Pd}$ & Palladium & 0.01 & $50-100$ & 0.15 \\
\hline $\mathrm{P}$ & Phosphorus & 0.5 & 0 & 3.5 \\
\hline $\mathrm{Pt}$ & Platinum & 0.01 & 0 & 0.095 \\
\hline $\mathrm{Pu}$ & Plutonium & 0.001 & $100-10000$ & 0.00045 \\
\hline Po & Polonium & 0.01 & 25 & 0.0025 \\
\hline $\mathrm{K}$ & Potassium & 0.01 & 35 & 1 \\
\hline $\operatorname{Pr}$ & Praseodymium & 0.01 & $500-1000$ & 0.01 \\
\hline $\mathrm{Pm}$ & Promethium & 0.01 & $1000-10000$ & 0.01 \\
\hline $\mathrm{Pa}$ & Protactinium & 0.001 & 4000 & 0.0025 \\
\hline $\mathrm{Ra}$ & Radium & 0.001 & $100-10000$ & 0.015 \\
\hline $\mathrm{Rn}$ & Radon & 1.0 & 0 & 0 \\
\hline $\operatorname{Re}$ & Rhenium & 0.01 & 0 & 1.5 \\
\hline $\mathrm{Rh}$ & Rhodium & 0.01 & 0 & 0.15 \\
\hline $\mathrm{Rb}$ & Rubidium & 0.01 & 500 & 0.15 \\
\hline $\mathrm{Ru}$ & Ruthenium & 0.01 & $0-500$ & 0.075 \\
\hline Sm & Samarium & 0.01 & $500-1000$ & 0.01 \\
\hline Sc & Scandium & 0.01 & 0 & 0.006 \\
\hline Se & Selenium & 0.01 & 10 & 0.025 \\
\hline
\end{tabular}




\begin{tabular}{|c|c|c|c|c|}
\hline Symbol & Name & $\begin{array}{c}\text { Airborne Release } \\
\text { Fraction } \\
\text { (RF) }\end{array}$ & $\begin{array}{c}\text { Sorption } \\
\text { Coefficient } \\
\left(\mathbf{K}_{\mathrm{d}}\right) \\
\end{array}$ & $\begin{array}{l}\text { Soil to Plant } \\
\text { Concentration } \\
\text { Factor } \\
\left(B_{\mathrm{v}}\right) \\
\end{array}$ \\
\hline $\mathrm{Si}$ & Silicon & 0.01 & 0 & 0.35 \\
\hline $\mathrm{Ag}$ & Silver & 0.01 & $50-100$ & 0.4 \\
\hline $\mathrm{Na}$ & Sodium & 0.01 & 10 & 0.075 \\
\hline $\mathrm{Sr}$ & Strontium & 0.01 & 100 & 2.5 \\
\hline $\mathrm{S}$ & Sulfur & 0.5 & 0 & 1.5 \\
\hline $\mathrm{Ta}$ & Tantalum & 0.001 & 0 & 0.01 \\
\hline Tc & Technetium & 0.01 & 0 & 9.5 \\
\hline $\mathrm{Te}$ & Tellurium & 0.01 & $50-100$ & 0.025 \\
\hline $\mathrm{Tb}$ & Terbium & 0.01 & $500-1000$ & 0.01 \\
\hline $\mathrm{Tl}$ & Thallium & 0.01 & 2 & 0.004 \\
\hline Th & Thorium & 0.001 & $1000-10000$ & 0.00085 \\
\hline $\mathrm{Tm}$ & Thulium & 0.01 & 0 & 0.01 \\
\hline Sn & Tin & 0.01 & $50-100$ & 0.03 \\
\hline $\mathrm{Ti}$ & Titanium & 0.01 & 0 & 0.0055 \\
\hline $\mathrm{W}$ & Tungsten & 0.01 & 0 & 0.045 \\
\hline $\mathrm{U}$ & Uranium & 0.001 & $1-50$ & 0.0085 \\
\hline $\mathrm{V}$ & Vanadium & 0.01 & 0 & 0.0055 \\
\hline $\mathrm{Xe}$ & Xenon & 1.0 & 0 & 0 \\
\hline $\mathrm{Yb}$ & Ytterbium & 0.01 & 0 & 0.01 \\
\hline $\mathrm{Y}$ & Yttrium & 0.01 & 500 & 0.015 \\
\hline $\mathrm{Zn}$ & Zinc & 0.01 & 0 & 1.5 \\
\hline $\mathrm{Zr}$ & Zirconium & 0.01 & 2000 & 0.002 \\
\hline
\end{tabular}

Bk-249, Bk-250, Es-254m RF based on NUREG-1140 release fraction for "any other beta-gamma emitter." 


\subsubsection{Breathing Rate}

\subsubsection{Breathing Rates Used By DOE-STD-1027-92}

For Hazard Category 2, DOE-STD-1027-92 specifies a breathing rate, also referred to as the respiration rate, of $3.5 \times 10^{-4} \mathrm{~m}^{3} / \mathrm{s},(=21 \mathrm{~L} / \mathrm{min})$ a value which is assumed equal to the standard value for an active man. A literature review indicates this recommended breathing rate is approximately the same value specified by ICRP Pub-23 (20) for an adult male engaged in light-activity (=20 L/min). ${ }^{\S \S}$

For Hazard Category 3, DOE-STD-1027-92 uses a breathing rate of $2.3 \times 10^{7} \mathrm{~cm}^{3} /$ day $\left(=2.64 \times 10^{-4} \mathrm{~m}^{3} / \mathrm{s}\right)$ as specified in Appendix A of the EPA Technical Background Document. (16) A literature review indicates this recommended breathing rate is equal to the time-averaged breathing rates for an adult male engaged in 8-hr of working (light-activity), 8-hr of non-occupational activity, and 8-hr of resting as specified by ICRP Pub-23. (20) ${ }^{* * *}$

\subsubsection{Updated Breathing Rate}

In 1994, the ICRP updated the human respiratory tract model with the issuance of Pub-66, Human Respiratory Tract Model for Radiological Protection. (21) Pub-66 defined light-work for workers on the following basis: 2.5-hr sitting (with an inhalation rate of $0.54 \mathrm{~m}^{3} / \mathrm{hr}$ ), and 5.5-hr of light exercise (with an inhalation rate of $1.5 \mathrm{~m}^{3} / \mathrm{hr}$ ). ${ }^{+\dagger}$ This definition of light-work results in a recommended time-averaged breathing rate of $3.3333 \times 10^{-4} \mathrm{~m}^{3} / \mathrm{s}$.

$$
B R_{\text {Light-Activity }}=\frac{\left(0.54 \frac{\mathrm{m}^{3}}{\mathrm{hr}} * 2.5 \mathrm{hr}\right)+\left(1.5 \frac{\mathrm{m}^{3}}{\mathrm{hr}} * 5.5 \mathrm{hr}\right)}{2.5 \mathrm{hr}+5.5 \mathrm{hr}}=1.2 \frac{\mathrm{m}^{3}}{\mathrm{hr}}=3.3 \times 10^{-4} \frac{\mathrm{m}^{3}}{\mathrm{~s}}
$$

\subsubsection{Justification for Use of Updated Breathing Rate}

The primary justification for the use of an updated breathing rate is based on the derivation of the DCs in Pub-68 and Pub-72 reliance on the revised human respiratory tract model as defined in Pub-66. As noted in a Supplemental Guidance (4) to DOE-STD-1027-92, the National Nuclear Security Administration (NNSA) noted that the Central Technical Authority (CTA) issued a memorandum regarding the clarification of dose calculation parameters, specifically for the preparation of safety basis documents for transuranic waste facilities. The NNSA Supplemental Guidance (on page AT4-4) cites the following from the CTA memorandum (emphasis added by the Supplemental Directive in italics):

...the Standard specifies the use of $3.3 \times 10^{-4} \mathrm{~m}^{3} / \mathrm{s}$ as BR [breathing rate] in conjunction with dose conversion factors (DCFs) from International Commission on Radiation Protection (ICRP) Publications 72 and 68. The DCFs in ICRP 72 and 68 are based on a model described in ICRP 66. ICRP 66 provides a range of BRs depending on the age and sex of the person and the type of activity being modeled. The BR specified in the Standard has been called into question because it is not specifically listed in ICRP 66. Since the DCFs in ICRP 72 and 68 are based on the ICRP 66 model, a conclusion was drawn that the BR used in dose calculations must be one of the values explicitly used in ICRP 66.

\footnotetext{
§§ ICRP Pub 23, pg. 347 (Table 120)

${ }^{* * *}$ ICRP Pub 23, pg. 346

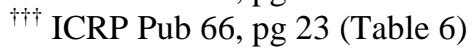


The BR in the Standard represents a weighted average of two BRs in ICRP 66. This average BR is widely used. It is defined and used in ICRP 68 [worker dose coefficients] to represent light work: a combination of 21/2 hours of rest/sitting and 51/2 hours of light exercise, as defined in ICRP 66. This BR is used by DOE in 10 C.F.R. 835, Occupational Radiation Protection, for establishing derived air concentrations for worker protection and in its toolbox modeling codes.

...The DCFs documented in ICRP 72 [public dose coefficients] are not explicitly linked to the $B R s$ identified in ICRP 66. Therefore, using a BR that is within the range specified in ICRP 66 and in conjunction with the DCFs in ICRP 72 is acceptable for a member of the public at a similar activity level. Using this criterion, the BR used in the Standard is within the range of BR values given in ICRP 66 and is reasonable for calculating dose to the public, assuming that the activity level being modeled is the same. That is, the BR specified in DOE-STD-5506 is consistent with that in ICRP 72 for calculating public doses. If a higher activity is likely for a member of the public based on the local conditions at the site boundary, it may then be appropriate to use a higher BR within the range provided in ICRP 66 in the dose calculations.

The NNSA Supplemental Directive concludes that the CTA position supports the use of an updated breathing rate of $3.3333 \times 10^{-4} \mathrm{~m}^{3} / \mathrm{s}$ as an appropriate value to use in conjunction with dose conversion factors pertaining to both the worker (Pub 68) and the public (Pub 72).

This report expands the list of DC publications used for the calculation of HC-2 and HC-3 TQs beyond the NNSA CTA justification associated with the use of Pub-68 and Pub-72. However, as previously discussed, and as illustrated in Figure 3.1, the inhalation DCs derived in DOE-STD-1196-2011 JAERI-Data/Code 2002-013 ${ }^{\S \S \S}$ are computed in a manner consistent with Pub-68 and Pub-72.

Accordingly, the use of the updated breathing rate is within the scope of the justification proffered by the NNSA CTA. Therefore, the HC-2 and HC-3 TQs in this report will utilize the Pub-66 recommended light-work time-averaged breathing rate of $3.3333 \times 10^{-4} \mathrm{~m}^{3} / \mathrm{s}$.

\subsubsection{Atmospheric Dispersion Coefficients ( $\chi / Q)$}

Atmospheric dispersion coefficients $(\chi / \mathrm{Q})$ are utilized to determine the degree by which a passing radioactive plume has dispersed by the time it reaches the downwind location of the analyzed receptor. Since the downwind location of the analyzed receptor differs between the HC-2 and the HC-3 TQ methodologies, the atmospheric dispersion coefficients will be different.

\subsubsection{Hazard Category 2 Atmospheric Dispersion Coefficient}

DOE-STD-1027-92 specifies that for the calculation of HC-2 TQs, the analyzed receptor is located 300 meters downwind from a surface level atmospheric release of radiological material. The release is assumed to disperse in the form of a Gaussian plume. The meteorological conditions of the plume

㧊 DOE-STD-1196-2011, pg 2, “The DCSs of this standard are based on age-specific effective dose coefficients computed in the manner of ICRP Publication $72 \ldots .$.

$\S \S \S$ JAERI-Data/Code 2002-013, pg i, “The dose coefficients were calculated with the computer code DOCAP based on the respiratory tract model and biokinetic model of ICRP. The effective dose rates were calculated by considering both external irradiation from the surrounding cloud and irradiation of the lungs from the gas within them. The calculated results are presented as tables, which are the same forms as those in ICRP Publs. 68 and 72."

${ }^{* * * *}$ FGR-12 is only utilized in this report for air-immersion DCs in support of the calculation of HC-2 TQs. The assessment of the air immersion dose component in the HC-2 TQ calculation does not require the use of a breathing rate. 
dispersion are modeled via a no-bouyancy Pasquill stability class D with a wind speed of $4.5 \mathrm{~m} / \mathrm{s}$. As noted in Figure 3.2, (22) and as stated in DOE-STD-1027-92, these conditions yield a $\chi / \mathrm{Q}$ of approximately $1 \times 10^{-4} \mathrm{~s} / \mathrm{m}^{3}$ when the deposition velocity (for both wet and dry deposition) is assumed to be $1 \mathrm{~cm} / \mathrm{s}$.

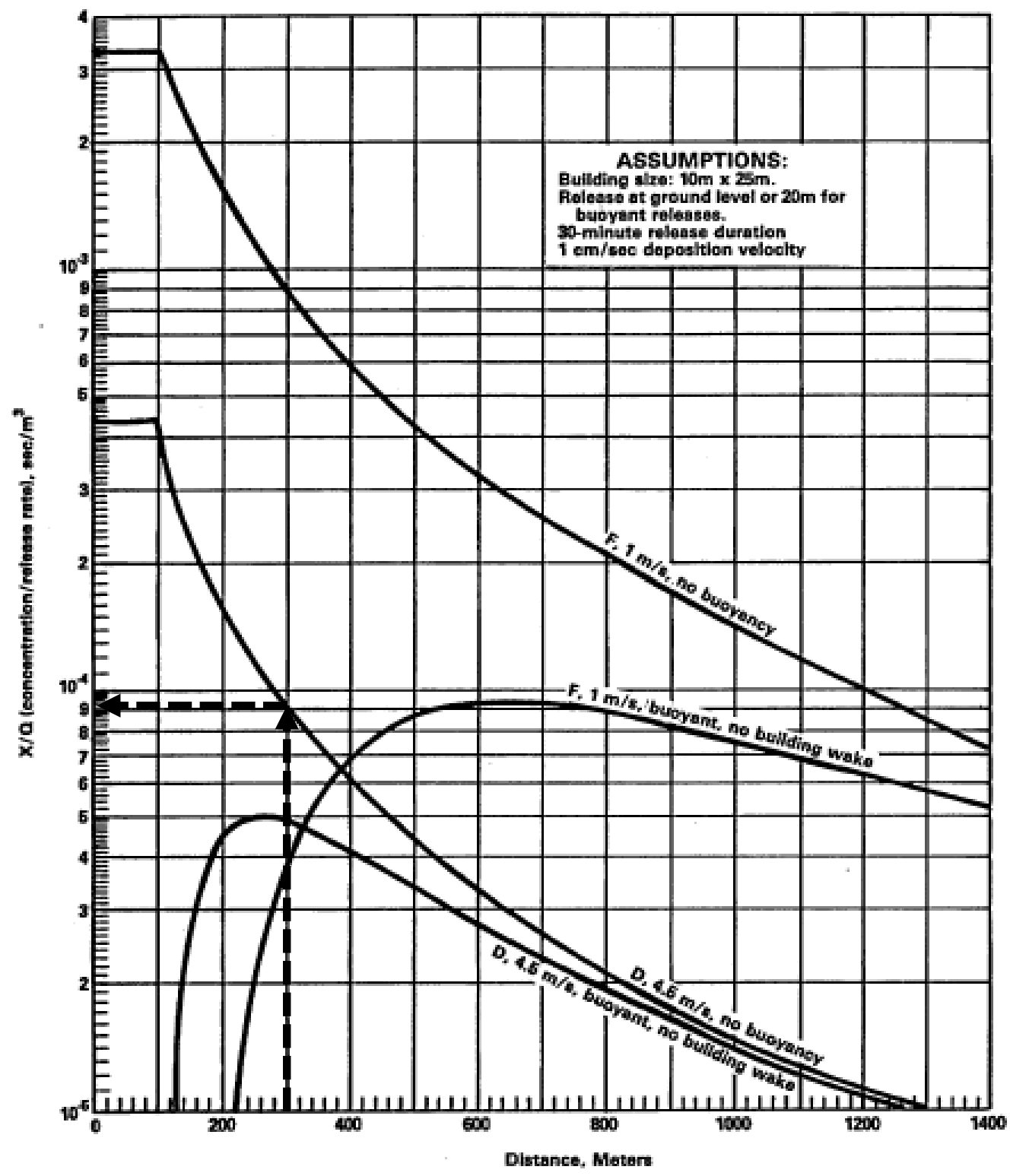

Figure 3.2 - Atmospheric Dispersion Verses Distance (Figure 1 from NUREG-1140)

\subsubsection{Hazard Category 3 Atmospheric Dispersion Coefficient}

DOE-STD-1027-92 defers to the EPA Technical Background Document regarding the specification of the atmospheric dispersion coefficient used for calculating the HC-3 TQs. Per the EPA, the distance from the point source release to the receptor location is assumed to be 30 meters. Additionally, it is assumed that a Gaussian distribution of the released contaminant is established almost immediately within the plume and is fully developed at 30 meters. The meteorological conditions of the plume dispersion are modeled via a 
Pasquill stability class D with a wind speed of $1 \mathrm{~m} / \mathrm{s}$. As noted in Figure 3.3, (16) the EPA concluded that these conditions yield a $\chi / \mathrm{Q}$ of approximately $8.4 \times 10^{-7}$ day $/ \mathrm{m}^{3}\left(=7.2 \times 10^{-2} \mathrm{~s} / \mathrm{m}^{3}\right)$ when the deposition velocity (for both wet and dry deposition) is assumed to be 1,000 meter/day $(=1.16 \mathrm{~cm} / \mathrm{s}) .{ }^{\mathrm{t}+\dagger}$

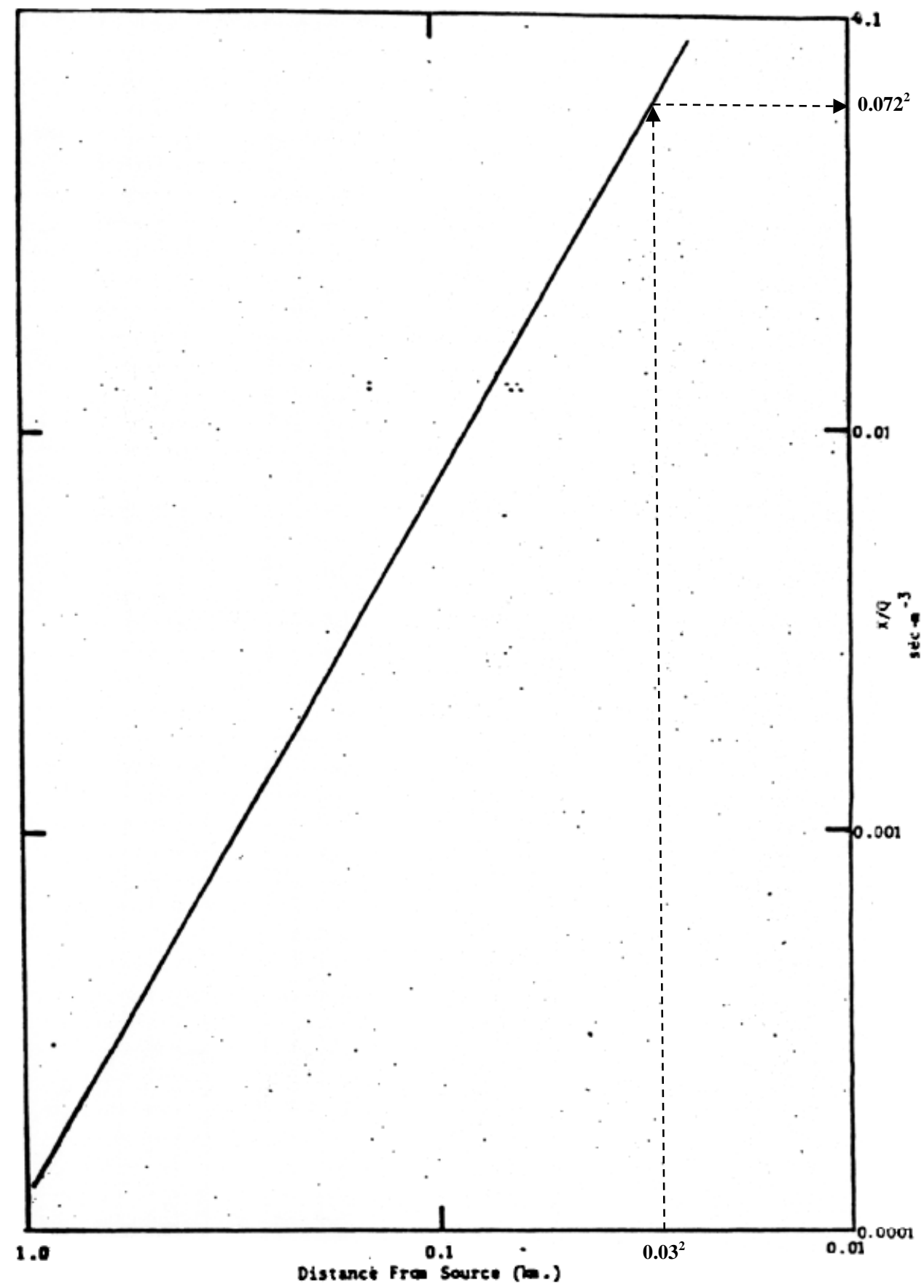

Figure 3.3 - Atmospheric Dispersion Verses Distance (Exhibit A-2 from 102RQ-RN-5-13)

t+†† 102RQ-RN-5-13, pg. C-6 


\subsubsection{Miscellaneous Inputs and Conversion Factors}

\subsubsection{Avogadro's constant}

The calculation of Specific Activity requires the use of Avogadro's constant. DOE-STD-1027-92 does not explicitly state nor imply the value of Avogadro's constant used in calculating specific activity (for converting TQ values from activity to mass). The LANL HC-2 Fact Sheet (2) and the LANL HC-3 Fact Sheet (3) respectively use a value of $6.023 \times 10^{23}$ for Avogadro's constant.

A literature research noted that the Committee on Data for Science and Technology (CODATA) in a 2014 update, specifies a published value of $6.022140857 \times 10^{23} \mathrm{~mol}^{-1}$ (23). A sampling of CODATA publications was conducted to determine if there were any previous changes in the value recommended for Avogadro's constant. The results of the sampling, presented in Table 3.8, note that a truncated version of the constant would result in a value of $6.022 \times 10^{23} \mathrm{~mol}^{-1}$ for all sampled publications going back as far as 1969.

\begin{tabular}{|c|c|c|}
\hline \multicolumn{3}{|c|}{ Table 3.8 - Review of Values for Avogadro's Constant as Recommended by CODATA } \\
\hline CODATA & Publication & Avogadro's Constant \\
\hline 1969 & $\begin{array}{c}\text { Reviews of Modern Physics } \\
\text { Volume 41, No. 3 July 1969 (24) }\end{array}$ & $6.022169 \times 10^{26} \mathrm{kmole}^{-1}$ \\
\hline 1986 & $\begin{array}{c}\text { Reviews of Modern Physics } \\
\text { Volume 59, No. 4 Oct 1987 (25) }\end{array}$ & $6.0221367 \times 10^{23} \mathrm{~mol}^{-1}$ \\
\hline 2002 & $\begin{array}{c}\text { Reviews of Modern Physics } \\
\text { Volume 77, No. 1 Jan 2005 (26) }\end{array}$ & $6.0221415 \times 10^{23} \mathrm{~mol}^{-1}$ \\
\hline 2006 & $\begin{array}{c}\text { Reviews of Modern Physics } \\
\text { Volume 80, No. 2 Apr-June 2008 (27) }\end{array}$ & $6.02214179 \times 10^{23} \mathrm{~mol}^{-1}$ \\
\hline 2014 & $\begin{array}{c}\text { Reviews of Modern Physics } \\
\text { Volume 88, No. 3 July-Sept 2016 (23) }\end{array}$ & $6.022140857 \times 10^{23} \mathrm{~mol}^{-1}$ \\
\hline
\end{tabular}

Based on the literature review of the CODATA recommended values for Avogadro's constant from 1969 to the present, the value used in the TQ calculations will be $6.022 \times 10^{23} \mathrm{~mol}^{-1}$ instead of the value used in the LANL Fact Sheets.

\subsubsection{Conversion Factors}

\section{Dose Equivalent and Activity Conversion Factors}

The contemporary publications selected for use provide inhalation, ingestion, and air immersion DCs utilizing the International System of Units (SI). Specifically, the DCs expressed dose equivalent in seiverts (Sv) and activity in becquerels (Bq). However, the dose equivalent limits specified for the respective HC-2 and HC-3 calculations are expressed in units of roentgen equivalent man (rem). Also, the calculated TQs in terms of activity are expressed as curies (Ci). Accordingly, the following dosimetric conversion factors are utilized in this report:

\begin{tabular}{lc}
\hline Dose Equivalent (28) & $0.01 \frac{\mathrm{Sv}}{\mathrm{rem}}$ \\
\hline Activity (29) & $3.7 \times 10^{10} \frac{\mathrm{Bq}}{\mathrm{Ci}}$ \\
\hline
\end{tabular}




\section{Time Conversion Factors}

Half-life data is obtained from ICRP Pub 38, Pub 107, and JAERI-Data/Code 2002-013. The half-life data is considered to be a NTD, and as such would be formatted in accordance with ENSDF protocols. The NNDC has issued guidance pertaining to the specification requirements for half-life data. Per the ENSDF preparation manual, valid symbols for half-life units are as follows:

- Y, D, H, M, S, MS, US, NS, PS, FS, AS..... for year, day, hour, minute, second(s), $10^{-3} \mathrm{~s}, 10^{-6}$ $\mathrm{s}, 10^{-9} \mathrm{~s}, 10^{-12} \mathrm{~s}, 10^{-15} \mathrm{~s}$, and $10^{-18} \mathrm{~s}$....respectively.

A review of ICRP Pub 38, Pub 107, and JAERI-Data/Code 2002-013 NTD noted the smallest reported half-life was in micro-seconds ( $\mu \mathrm{s}=10^{-6} \mathrm{~s}$ ). As such, the following time conversions in Table 3.9 are used in the calculations.

\begin{tabular}{|c|r|}
\hline \multicolumn{2}{|c|}{$\begin{array}{c}\text { Table 3.9 - Time } \\
\text { Conversion Factors }\end{array}$} \\
\hline Unit & Value (s) \\
\hline $\mathrm{y}$ & 31556952 \\
\hline $\mathrm{d}$ & 86400 \\
\hline $\mathrm{h}$ & 3600 \\
\hline $\mathrm{m}$ & 60 \\
\hline $\mathrm{s}$ & 1 \\
\hline $\mathrm{ms}$ & 0.001 \\
\hline$\mu \mathrm{s}$ & 0.000001 \\
\hline
\end{tabular}

The duration of a year $\left(3.1557 \times 10^{7} \mathrm{~s}\right)$ is based on a mean Gregorian year (365.2425 days per year). ${ }^{\text {抹 }}$

拉执 The current calendar system is established to be the Gregorian calendar, which is based on a cycle of 400 years. Within the 400 year cycle, every $4^{\text {th }}$ year is a leap year (addition of an extra day), except for the $100^{\text {th }}$, $200^{\text {th }}$ and $300^{\text {th }}$ year in the cycle which do not have a leap day. (36) This results in a total of 97 leaps days during the 400 year cycle. Accordingly, a mean Gregorian year is $365 \frac{97}{400}$ days $=365.2425$ days. 


\section{METHODOLOGY}

\subsection{HAZARD CATEGORY 2 THRESHOLD QUANTITIES METHODOLOGY}

\subsubsection{HC-2 Threshold Quantity Exposure Pathway Model}

The derivation of the HC-2 TQs is based on the cumulative dose contribution associated with a person subjected to a passing atmospheric radioactive plume. The specific doses assessed in the model include: (i) the internal dose received as a result of the inhalation of radionuclides contained within the plume, and (ii) external dose received as a result of being submersed in a radioactive gas plume.

Accordingly, the calculation of HC-2 TQs is expressed as follows: (1)

$$
T Q_{H C 2}=\frac{1 \mathrm{rem}}{R F * S A * X / Q *\left(D C_{\text {inhal }} * B R+D C_{\text {sub }}\right)} \quad \text { Eqn. } 1
$$

Where;

$$
\begin{array}{ll}
\mathrm{TQ}_{\mathrm{HC} 2} & =\text { Quantity of material used as threshold (grams) } \\
\mathrm{RF} & =\text { Airborne release fraction of material averaged over an entire facility (unitless) } \\
\mathrm{SA} & =\text { Specific activity of radionuclide released }(\mathrm{Ci} / \mathrm{gm}) \\
\mathrm{X} / \mathrm{Q} & =\text { Meteorological dispersion coefficient }\left(1 \mathrm{E}-4 \mathrm{sec} / \mathrm{m}^{3}\right) \\
\mathrm{DC}_{\text {inhal }} & =\text { Inhalation Dose Coefficient }(\mathrm{Sv} / \mathrm{Bq}) \\
\mathrm{BR} & =\text { Respiration rate }\left(3.3333 \mathrm{E}-4 \mathrm{~m}^{3} / \mathrm{sec}\right) \\
\mathrm{DC}_{\text {sub }} & =\text { Air Immersion Dose Coefficient }\left(\mathrm{Sv} / \mathrm{sec} \text { per } \mathrm{Bq} / \mathrm{m}^{3}\right)
\end{array}
$$

Table 3.3 of this report details the criteria associated with the selection of inhalation DCs and air immersion DCs. The airborne release fractions were selected in as directed per Table 3.6 of this report. The TQ value is converted to grams by dividing the HC-2 TQ (Ci) by the specific activity (Ci/g). The specific activity is calculated using the following equation: (2)

$$
\mathrm{SA}=\frac{\ln (2) * \mathrm{~N}_{\mathrm{A}}}{\mathrm{AW} * t_{1 / 2} *\left(3.1557 \times 10^{7} \frac{S}{y r}\right) *\left(3.7 \times 10^{10} \frac{B q}{C i}\right)} \quad \text { Eqn. } 2
$$

Where; ${ }^{\S \S \S}$

$$
\begin{array}{ll}
\mathrm{SA} & =\text { Specific Activity }(\mathrm{Ci} / \mathrm{g}) \\
\mathrm{N}_{\mathrm{A}} & =\text { Avogadro's Number }\left(6.022 \times 10^{23}\right) \\
\mathrm{AW} & =\text { Atomic Weight (i.e., from the Atomic Mass Data Center) } \\
\mathrm{t}_{1 / 2} & =\text { Half-life }(\mathrm{yr})
\end{array}
$$

§§§ As noted in Section 3.3.5.2 of this report, a time conversion factor of $3.1557 \times 10^{7} \mathrm{sec} / \mathrm{yr}$ is based on a mean Gregorian year. The time conversion value of $3.154 \times 10^{7} \mathrm{sec} / \mathrm{yr}$ as cited in the LANL Fact Sheet, Reference (2), is equivalent to 365 days. This calculation will use the mean Gregorian year length of time (3.1557x $10^{7}$ sec/yr) since it accounts for the 97 leap years in the 400-yr Gregorian calendar cycle. 


\subsection{HAZARD CATEGORY 3 THRESHOLD QUANTITIES METHODOLOGY}

The calculation of HC-3 TQ values is based on the calculation of pathway specific TQs associated with five dose exposure pathways:

- Inhalation

- Ingestion (of food)

- Ingestion (of water)

- $\quad$ Direct Exposure (via point source)

- Air Submersion (in a radioactive cloud)

The limiting exposure pathway is the exposure pathway that results in an effective dose of 10 rem at 30 meters with the smallest amount (i.e., activity) of an isotope.

This methodology is derived from the five exposure pathway methodology presented in the EPA Technical Background Document (16) and modified for a dose exposure of 10 rem (instead of the 0.5 rem dose exposure used in the EPA calculations). The following sections detail the methodologies for calculating pathway specific TQs.

\subsubsection{Inhalation Exposure Pathway}

The inhalation exposure pathway threshold quantity $\left(\mathrm{TQ}_{\mathrm{inh}}\right)$ is expressed as follows (Equation 3):

$$
T Q_{H C 3, \text { inhale }}=\frac{10 \mathrm{rem}}{R *(\chi / Q) * B R * D C_{\text {inhal }}} * \frac{0.01 \frac{\mathrm{Sv}}{\mathrm{rem}}}{3.7 \times 10^{10} \frac{B q}{C i}}
$$

Where;

$$
\begin{array}{ll}
\mathrm{TQ}_{\mathrm{HC} 3, \text { inhale }} & =\text { Inhalation pathway threshold quantity }[\mathrm{Ci}] ; \\
\mathrm{R} & =\text { Airborne release fraction [dimensionless]; } \\
\chi / \mathrm{Q} & =\text { Meteorological dispersion coefficient } 30 \text { meters from ground level release } \\
& {\left[7.2 \times 10^{-2} \mathrm{~s} / \mathrm{m}^{3}\right] ;} \\
\mathrm{BR} & =\text { Breathing Rate }\left[3.3333 \times 10^{-4} \mathrm{~m}^{3} / \mathrm{s}\right] ; \text { and } \\
\mathrm{DC}_{\text {inhal }} & =
\end{array}
$$

Table 3.4 of this report details the criteria associated with the selection of inhalation DCs. The airborne release fractions were selected as directed per Table 3.7 of this report.

\subsubsection{Food Ingestion Exposure Pathway}

The food ingestion exposure pathway threshold quantity ( $\left.\mathrm{TQ}_{\mathrm{HC} 3 \text {,food }}\right)$ is expressed as follows (Equation 4): 


$$
T Q_{H C 3, \text { food }}=\frac{10 \mathrm{rem}}{D F * F C * C T * R * D C_{\text {ingest }}} * \frac{0.01 \frac{\mathrm{Sv}}{\mathrm{rem}}}{3.7 \times 10^{10} \frac{\mathrm{Bq}}{C i}}
$$

Where;

$$
\begin{aligned}
& \mathrm{TQ}_{\mathrm{HC}, \text { food }}=\text { Food ingestion pathway threshold quantity [Ci]; } \\
& \text { DF = Dilution factor accounting for the transfer of radionuclides from air to vegetation } \\
& (\mathrm{Ci} / \mathrm{kg} \text { of vegetables at the point of exposure per curies released to the atmosphere, } \\
& \left.\left[\mathrm{kg}^{-1}\right]\right) \text {; } \\
& \text { FC } \quad=\text { Food (i.e., leafy vegetable) consumption rate of reference man }[0.175 \mathrm{~kg} / \text { day }] \text {; } \\
& \text { CT = Contact Time (effective time over which contaminated vegetables are ingested } \\
& \text { [days]; } \\
& \mathrm{R}=\text { Airborne release fraction [dimensionless]; and } \\
& \mathrm{DC}_{\text {ingest }} \quad=\text { Ingestion dose coefficient }[\mathrm{Sv} / \mathrm{Bq}] \text {. }
\end{aligned}
$$

Table 3.4 of this report details the criteria associated with the selection of ingestion DCs. The airborne release fractions were selected as directed per Table 3.7 of this report. The dilution factor (DF) is calculated in accordance with the following equation:

$$
\mathrm{DF}=1 \times 10^{-4}+\left(3.5 \times 10^{-6} * B_{V}\right) ;\left[\mathrm{kg}^{-1}\right] \quad \text { Eqn. } 5
$$

Where;

$$
\begin{aligned}
\mathrm{B}_{\mathrm{V}} \quad= & \text { Concentration ratio for the transfer of the element to the edible portion of a crop } \\
& \text { from dry soil (dimensionless) }
\end{aligned}
$$

Values for $\mathrm{B}_{\mathrm{V}}$ were taken from Table 3.7 of this report. Values for Contact Time (CT) were determined using the following equation as specified in the EPA Technical Background Document:

$$
\mathrm{CT}=\left(\frac{1-\exp \left(-\left[\left(\lambda_{I}+\lambda_{W}\right) \mathrm{t}_{G}\right]\right)}{\lambda_{I}+\lambda_{W}}\right) ;[\text { days }]
$$

Where;

$$
\begin{array}{ll}
\lambda_{\mathrm{I}} & =\text { Radionuclide decay constant }\left[\text { day }^{-1}\right]=\ln (2) / \mathrm{t}_{1 / 2} ; \\
\mathrm{t}_{1 / 2} & =\text { Radionuclide half-life [days]; } \\
\lambda_{\mathrm{W}} & =\text { Weathering decay constant }\left[\text { day }^{-1}\right]=\ln (2) / 14 \text { days; and } \\
\mathrm{t}_{\mathrm{G}} & =\text { Growing season time }[60 \text { days }] .
\end{array}
$$

Radionuclide half-life data was obtained from the master isotope list in Appendix D of this report (Table D.1). 


\subsubsection{Ground Water Ingestion Exposure Pathway}

The ground water ingestion exposure pathway threshold quantity ( $\mathrm{TQ}_{\mathrm{HC} \text {,water }}$ ) is expressed as follows (Equation 7):

$$
T Q_{H C 3, \text { water }}=\frac{10 \mathrm{rem}}{D F *\left[\frac{1-\exp (-\lambda * C T)}{\lambda}\right] * W C * D C_{\text {ingest }}} * \frac{0.01 \frac{\mathrm{Sv}}{\mathrm{rem}}}{3.7 \times 10^{10} \frac{B q}{C i}} \quad \text { Eqn. } 7
$$

Where;

$$
\begin{array}{ll}
\mathrm{TQ}_{\mathrm{HC}, \text { water }} & =\text { Water ingestion pathway threshold quantity }[\mathrm{Ci}] ; \\
\mathrm{DF} & =\text { Dilution Factor }\left[\mathrm{L}^{-1}\right] ; \\
\lambda & =\text { Radionuclide decay constant }\left[\mathrm{day}^{-1}\right]=\ln (2) / \mathrm{t}_{1 / 2} ; \\
\mathrm{R}_{\mathrm{d}} & =\text { Retardation Factor }[1 \text { day }] ; \\
\mathrm{CT} & =\text { Contact time }[9 \text { days }] ; \\
\mathrm{WC} & =\text { Water consumption of reference man }[2 \mathrm{~L} / \text { day }] ; \text { and } \\
\mathrm{DC}_{\text {ingest }} & =\text { Ingestion dose coefficient }[\mathrm{Sv} / \mathrm{Bq}] .
\end{array}
$$

Table 3.4 of this report details the criteria associated with the selection of ingestion DCs. Radionuclide half-life data was obtained from the master isotope list in Appendix D of this report (Table D.1). The dilution factor (DF) is calculated in accordance with the following equation:

$$
\mathrm{DF}=\left[7.6 \times 10^{-8} * \exp \left(-4.2 * \frac{R_{d}}{t_{\frac{1}{2}}}\right)\right] ;\left[L^{-1}\right]
$$

Where;

$$
\mathrm{R}_{\mathrm{d}} \quad=\text { Retardation Factor (1 day) }
$$

The EPA noted that radioisotopes with a retardation factor greater than one will have a calculated ground water ingestion release value much larger in comparison with the other pathway release values. Accordingly, the EPA did not develop separate ground water ingestion release value equations for situations where the retardation factor is greater than one. As noted by the EPA, when the sorption coefficient, $K_{d}$, is equal to zero, the retardation factor is equal to one. When the sorption coefficient becomes larger, however, the retardation factor increases rapidly. Values for the sorption coefficient were obtained from Table 3.7 of this report. Consistent with the EPA methodology, ground water pathway release values were only calculated for radioisotopes that had a reported sorption coefficient $\left(\mathrm{K}_{\mathrm{d}}\right)$ less than one. 


\subsubsection{External Pathway Exposure Pathways}

The methodology for the determination of release values for external exposure to radiation is dependent upon the form of the radionuclide released. For specific noble gas radioisotopes (argon, krypton, and xenon), the release values are based on exposure due to total body submersion in a radioactive gas cloud. For all other radionuclides, the direct exposure release values are based on the quantity of radioactivity received by an individual exposed to a point source of radiation.

\subsubsection{Direct (a.k.a., Point Source) Exposure Pathway}

Except for noble gases ${ }^{* * * * *}$, the Direct Exposure release value is based on a gamma dose incurred over a 24-hour period from a point source located 30 meters away. The equation for calculating the Direct Exposure pathway threshold quantity is expressed as (Equation 9):

$$
T Q_{\mathrm{HC}, \text { direct }}=\frac{10 \mathrm{rem} * \mathrm{~S}^{2} * C_{\text {gamma }}}{\mathrm{E}_{1} * \mu_{a} *\left(24 \frac{h r}{d a y}\right) *\left(\frac{1-\exp (-\lambda t)}{\lambda}\right) * \exp \left[\left(-100 \frac{\mathrm{cm}}{\mathrm{m}}\right) \mu_{a} S\right]} \quad \text { Eqn. } 9
$$

Where;

$$
\begin{array}{ll}
\mathrm{TQ}_{\mathrm{HC}, \text { direct }}= & \text { Direct exposure pathway threshold quantity }[\mathrm{Ci}] ; \\
\mathrm{S} & =\text { Distance from the point source }[30 \mathrm{~m}] ; \\
\mathrm{C}_{\text {gamma }} & =\text { Equation coefficient }\left[6.41 \times 10^{-5} \frac{\mathrm{Ci}-\mathrm{MeV}-\mathrm{hr}}{r e m-m^{2}-\mathrm{cm}}\right] ; \\
\mathrm{E}_{1} & =\text { Sum of the products of the photon energies and the photon fraction or intensities } \\
& {[\mathrm{MeV}] ;} \\
\mu_{\mathrm{a}} & =\text { Linear energy absorption coefficient for gamma rays in air }\left[3.7 \times 10^{-5} \mathrm{~cm}^{-1}\right] ; \\
\lambda & =\text { Radionuclide decay constant }\left[\text { day }^{-1}\right]=\ln (2) / \mathrm{t}_{1 / 2} ; \\
\mathrm{t} & =\text { Duration of exposure }[1 \text { day }] .
\end{array}
$$

Radionuclide half-life data was obtained from the master isotope list in Appendix D of this report (Table D.1). Table 3.4 of this report details the criteria associated with the selection of the value for the average photon energy.

\subsubsection{Air Immersion Exposure Pathway}

For noble gases (argon, krypton, and xenon), the air immersion release value is based on the dose an individual receives when submerged in a cloud of the radioactive gas. The release value for the air immersion pathway threshold quantity is expressed as (Equation 10):

\footnotetext{
${ }^{* * * * *}$ The ICRP has not published air immersion DCs for radon radionuclides in Pub-119. Accordingly, radon radionuclides are considered only for the $\mathrm{TQ}_{\text {direct }}$ exposure pathway.
} 


$$
T Q_{H C 3, \text { sub }}=\frac{10 \mathrm{rem}}{(\mathrm{\chi} / \mathrm{Q}) *(d a y / 86,400 \mathrm{sec}) * D C_{\text {sub }}} * \frac{0.01 \frac{\mathrm{Sv}}{\mathrm{rem}}}{3.7 \times 10^{10} \frac{B q}{C i}} \quad \text { Eqn. } 10
$$

Where;

$$
\begin{array}{ll}
\mathrm{TQ}_{\mathrm{HC}, \mathrm{sub}}= & \text { Submersion Dose Threshold Quantity }[\mathrm{Ci}] ; \\
\chi / \mathrm{Q} & =\text { Meteorological dispersion coefficient } 30 \text { meters from ground level release } \\
& {\left[7.2 \times 10^{-2} \mathrm{~s} / \mathrm{m}^{3}\right] ; \text { and }} \\
\mathrm{DC}_{\text {sub }} & =
\end{array}
$$

Table 3.4 of this report details the criteria associated with the selection of air immersion DCs.

\subsubsection{Determination of the HC-3 TQ Value}

After calculating the pathway specific TQ values, the HC-3 TQ is determined by selecting the pathway with the lowest calculated activity content that results in a 10-rem dose to the receptor located 30-meters from the facility.

$$
T Q_{H C 3}=\min \left[\begin{array}{c}
T Q_{H C 3, \text { inhale }} \\
T Q_{H C 3, \text { food }} \\
T Q_{H C 3, \text { water }} \\
T Q_{H C 3, \text { direct }} \\
T Q_{H C 3, \text { sub }}
\end{array}\right]
$$

Eqn. 11

\subsection{GUIDANCE ON THE USE OF SELECTED INPUT DATA}

\subsubsection{HC-2 Inhalation Dose Coefficient Selection Criteria}

As noted in Table 3.1, the inhalation DCs used for calculating the HC-2 TQs in DOE-STD-1027-92 were obtained from DOE/EH-0071. (13) The publication provided inhalation DCs for the following lung retention classes: D (daily), W (weekly), and Y (yearly). Per the guidance of DOE/EH-0071, "if the class is unknown, use the largest value.”

As noted in Table 3.2, DOE/EH-0071 is superseded by contemporary publications with updated DCs. The bulk of the inhalation DCs used in the HC-2 TQ calculation will be obtained from ICRP Pub-119 which is a compendium of DCs previously published in Pub-68 (worker) and Pub-72 (public). Table 2 (“Age-Dependent Doses From Intake of Radionuclides”) of Pub-72 (which is not reproduced in Pub-119) describes a modified criterion for the selection of inhalation DCs. Specifically; Table 2 of Pub-72 designates, by element, a recommended default absorption class. The recommended default absorption class was used in the calculation of updated TQs in the NNSA Supplemental Guidance to DOE-STD-1027-92 (4).

In recognition of the differing guidance, this report undertook an effort to calculate the HC-2 TQs using the guidance of Table 2 of ICRP Pub-72 (consistent with the calculated updated TQs in the NNSA Supplemental Guidance) and also calculated the HC-2 TQs using the guidance of DOE/EH-0071 (using the maximum inhalation DC regardless of the recommended inhalation clearance class). 


\subsubsection{Criteria for the Selection of the HC-2 Maximum Inhalation Dose Coefficient}

As observed in Table 3.3, both ICRP Pub-119 and JAERI-Data/Code 2002-013 have more than one table that provide radionuclide specific inhalation DCs. Both the ICRP and the JAERI publication have one table providing DCs (for each analyzed lung clearance class) based on the inhalation of air-borne particulates, and a second table providing inhalation DCs based on the inhalation of gases/vapours. DOESTD-1196-2011 also includes both particulate inhalation DCs, as well as gases/vapours inhalation DCs. However unlike the other publications, the particulate inhalation DCs and the gases/vapours inhalation DCs in DOE-STD-1196-2011 are all listed in a single data table.

The maximum inhalation DC was selected in consideration of both the particulate inhalation DC and the gases/vapours inhalation DC. Typically, the maximum inhalation DC used in the HC-2 TQ calculation was from the inhaled particulate table. However, 48 radionuclides were identified as having the gas/vapor form as the maximum inhalation DC (Table 4.1).

\begin{tabular}{|l|l|l|l|}
\hline \multicolumn{4}{|c|}{$\begin{array}{c}\text { Table 4.1 - Radionuclides With Gas/Vapour as } \\
\text { Maximum Inhalation DC for HC-2 TQ Calculation }\end{array}$} \\
\hline Hg-190 & I-118 & I-131 & Ru-95 \\
\hline Hg-191m & I-119 & I-132 & Ru-97 \\
\hline Hg-192 & I-120 & I-132m & Te-114 \\
\hline Hg-193 & I-120m & I-133 & Te-119m \\
\hline Hg-193m & I-121 & I-134 & Te-121 \\
\hline Hg-194 & I-123 & I-135 & Te-123 \\
\hline Hg-195 & I-124 & Ni-56 & Te-131 \\
\hline Hg-195m & I-125 & Ni-57 & Te-131m \\
\hline Hg-197 & I-126 & Ni-59 & Te-132 \\
\hline Hg-197m & I-128 & Ni-63 & Te-133 \\
\hline Hg-199m & I-129 & Ni-65 & Te-133m \\
\hline Hg-203 & I-130 & Ru-94 & Te-134 \\
\hline
\end{tabular}

The selection of the maximum inhalation DCs and air immersion DCs is documented in Appendix A, Table A.1.

\subsubsection{Criteria for the Selection of the HC-2 Recommended Inhalation Dose Coefficient}

The selection process for using the recommended default absorption class inhalation DCs per Table 2 of ICRP Pub-72 required the following modification to the selection criteria used for identifying the maximum inhalation DC:

- If a radionuclide is not associated with an element identified in Table 2 of ICRP Pub-72 as a candidate for using a recommended default absorption class, then the inhalation DC was selected in the same manner as described for the maximum inhalation DC per Section 4.3.1.1.

- If a radionuclide is associated with an element identified in Table 2 of ICRP Pub-72 as a candidate for using a recommended default absorption class, then only the particulate inhalation DC for the recommended absorption class was used. Table 4.2 summarizes the elements from ICRP Pub-72 that were identified as using a default lung absorption class, as well as those using a maximum lung absorption class. 


\begin{tabular}{|c|c|c|c|}
\hline \multicolumn{4}{|c|}{$\begin{array}{c}\text { Table } 4.2 \text { - Elements With Recommended Default Lung } \\
\text { Absorption Class and Maximum Lung Absorption Class Per } \\
\text { ICRP } 72\end{array}$} \\
\hline Element & Symbol & Max/Rec. & $\begin{array}{c}\text { Rec. Lung } \\
\text { Absorption Type }\end{array}$ \\
\hline Actinium & Ac & Max & \\
\hline Silver & $\mathrm{Ag}$ & Rec & $\mathrm{M}$ \\
\hline Aluminum & $\mathrm{Al}$ & Max & \\
\hline Americium & Am & Rec & $\mathrm{M}$ \\
\hline Arsenic & As & Max & \\
\hline Astatine & $\mathrm{At}$ & Max & \\
\hline Gold & $\mathrm{Au}$ & Max & \\
\hline Barium & $\mathrm{Ba}$ & Rec & $\mathrm{M}$ \\
\hline Beryllium & $\mathrm{Be}$ & Max & \\
\hline Bismuth & $\mathrm{Bi}$ & Max & \\
\hline Berkelium & $\mathrm{Bk}$ & Max & \\
\hline Bromine & $\mathrm{Br}$ & Max & \\
\hline Carbon & $\mathrm{C}$ & Rec & $\mathrm{M}$ \\
\hline Calcium & $\mathrm{Ca}$ & $\operatorname{Rec}$ & $\mathrm{M}$ \\
\hline Cadmium & $\mathrm{Cd}$ & Max & \\
\hline Cerium & $\mathrm{Ce}$ & Rec & $\mathrm{M}$ \\
\hline Californium & $\mathrm{Cf}$ & Max & \\
\hline Chlorine & $\mathrm{Cl}$ & Max & \\
\hline Curium & $\mathrm{Cm}$ & Rec & $\mathrm{M}$ \\
\hline Cobalt & Co & $\operatorname{Rec}$ & $\mathrm{M}$ \\
\hline Chromium & $\mathrm{Cr}$ & Max & \\
\hline Cesium & Cs & Rec & $\mathrm{F}$ \\
\hline Copper & $\mathrm{Cu}$ & Max & \\
\hline Dysprosium & Dy & Max & \\
\hline Erbium & $\mathrm{Er}$ & Max & \\
\hline Einsteinium & Es & Max & \\
\hline Europium & $\mathrm{Eu}$ & Max & \\
\hline Fluorine & $\mathrm{F}$ & Max & \\
\hline Iron & $\mathrm{Fe}$ & $\operatorname{Rec}$ & $\mathrm{M}$ \\
\hline Fermium & $\mathrm{Fm}$ & Max & \\
\hline Francium & $\mathrm{Fr}$ & Max & \\
\hline Gallium & $\mathrm{Ga}$ & Max & \\
\hline Gadolinium & Gd & Max & \\
\hline Germanium & $\mathrm{Ge}$ & Max & \\
\hline
\end{tabular}




\begin{tabular}{|c|c|c|c|}
\hline \multicolumn{4}{|c|}{$\begin{array}{c}\text { Table } 4.2 \text { - Elements With Recommended Default Lung } \\
\text { Absorption Class and Maximum Lung Absorption Class Per } \\
\text { ICRP } 72 \text { (continued) }\end{array}$} \\
\hline Element & Symbol & Max/Rec. & $\begin{array}{c}\text { Rec. Lung } \\
\text { Absorption Type }\end{array}$ \\
\hline Hafnium & Hf & Max & \\
\hline Mercury & $\mathrm{Hg}$ & Max & \\
\hline Holmium & Ho & Max & \\
\hline Iodine & $\mathrm{I}$ & Rec & $\mathrm{F}$ \\
\hline Indium & In & Max & \\
\hline Iridium & $\mathrm{Ir}$ & Max & \\
\hline Potassium & $\mathrm{K}$ & Max & \\
\hline Lanthanum & $\mathrm{La}$ & Max & \\
\hline Lutetium & $\mathrm{Lu}$ & Max & \\
\hline Mendelevium & $\mathrm{Md}$ & Max & \\
\hline Magnesium & $\mathrm{Mg}$ & Max & \\
\hline Manganese & $\mathrm{Mn}$ & Max & \\
\hline Molybdenum & Mo & Rec & $\mathrm{M}$ \\
\hline Sodium & $\mathrm{Na}$ & Max & \\
\hline Niobium & $\mathrm{Nb}$ & $\operatorname{Rec}$ & $\mathrm{M}$ \\
\hline Neodymium & $\mathrm{Nd}$ & Max & \\
\hline Nickel & $\mathrm{Ni}$ & Rec & $\mathrm{M}$ \\
\hline Neptunium & $\mathrm{Np}$ & Rec & $\mathrm{M}$ \\
\hline Osmium & Os & Max & \\
\hline Phosphorus & $\mathrm{P}$ & Max & \\
\hline Protactinium & $\mathrm{Pa}$ & Max & \\
\hline Lead & $\mathrm{Pb}$ & Rec & $\mathrm{M}$ \\
\hline Palladium & $\mathrm{Pd}$ & Max & \\
\hline Promethium & $\mathrm{Pm}$ & Max & \\
\hline Polonium & Po & Rec & $\mathrm{M}$ \\
\hline Praseodymium & $\operatorname{Pr}$ & Max & \\
\hline Platinum & $\mathrm{Pt}$ & Max & \\
\hline Plutonium & $\mathrm{Pu}$ & Rec & $\mathrm{M}$ \\
\hline Radium & $\mathrm{Ra}$ & Rec & $\mathrm{M}$ \\
\hline Rubidium & $\mathrm{Rb}$ & Max & \\
\hline Rhenium & $\operatorname{Re}$ & Max & \\
\hline Rhodium & $\mathrm{Rh}$ & Max & \\
\hline Ruthenium & $\mathrm{Ru}$ & Rec & $\mathrm{M}$ \\
\hline Sulfur & $\mathrm{S}$ & Rec & $\mathrm{M}$ \\
\hline Antimony & $\mathrm{Sb}$ & Rec & $\mathrm{M}$ \\
\hline Scandium & Sc & Max & \\
\hline
\end{tabular}




\begin{tabular}{|c|c|c|c|}
\hline \multicolumn{4}{|c|}{$\begin{array}{c}\text { Table } 4.2 \text { - Elements With Recommended Default Lung } \\
\text { Absorption Class and Maximum Lung Absorption Class Per } \\
\text { ICRP } 72 \text { (continued) }\end{array}$} \\
\hline Element & Symbol & Max/Rec. & $\begin{array}{c}\text { Rec. Lung } \\
\text { Absorption Type }\end{array}$ \\
\hline Selenium & Se & Rec & $\mathrm{F}$ \\
\hline Silicon & $\mathrm{Si}$ & Max & \\
\hline Samarium & $\mathrm{Sm}$ & Max & \\
\hline Tin & Sn & Max & \\
\hline Strontium & $\mathrm{Sr}$ & Rec & $\mathrm{M}$ \\
\hline Tantalum & Ta & Max & \\
\hline Terbium & $\mathrm{Tb}$ & Max & \\
\hline Technetium & Tc & Rec & $\mathrm{M}$ \\
\hline Tellurium & $\mathrm{Te}$ & Rec & M \\
\hline Thorium & Th & Rec & $\mathrm{S}$ \\
\hline Titanium & $\mathrm{Ti}$ & Max & \\
\hline Thallium & $\mathrm{Tl}$ & Max & \\
\hline Thulium & $\mathrm{Tm}$ & Max & \\
\hline Uranium & $\mathrm{U}$ & Rec & $\mathrm{M}$ \\
\hline Vanadium & $\mathrm{V}$ & Max & \\
\hline Tungsten & $\mathrm{W}$ & Max & \\
\hline Yttrium & $\mathrm{Y}$ & Max & \\
\hline Ytterbium & $\mathrm{Yb}$ & Max & \\
\hline Zinc & $\mathrm{Zn}$ & Rec & $\mathrm{M}$ \\
\hline Zirconium & $\mathrm{Zr}$ & Rec & $\mathrm{M}$ \\
\hline Radon & $\mathrm{Rn}$ & Max & \\
\hline Hydrogen & $\mathrm{H}$ & Rec & $\mathrm{M}$ \\
\hline
\end{tabular}

The selection of the recommended inhalation DCs and maximum air immersion DCs is documented in Appendix B, Table B.1.

\subsubsection{HC-3 Inhalation, Air Immersion, Ingestion Dose Coefficient Selection Criteria}

As noted in Table 3.1, the inhalation DCs used for calculating the HC-3 TQs in DOE-STD-1027-92 were obtained from ICRP Pub-30. (15) The publication provided inhalation DCs for the following lung retention classes: D (daily), W (weekly), and Y (yearly). Per the guidance of the EPA Technical Background Document; (16) 
“The inhalation ALIs [Annual Limits for Intake] ${ }^{+1+\dagger \dagger}$ used in the calculations are taken from ICRP's Publication 30 after being converted from becquerels to microcuries. When more than one ALI was given for a particular radionuclide, the lowest value was used for the calculations. This assures that the most hazardous chemical form of a radionuclide is used in the release value calculations.”挷

Also specified in Table 3.1, the ingestion DCs used for calculating the HC-3 TQs in DOE-STD-1027-92 were obtained from ICRP Pub-30. Per the guidance of the EPA Technical Background Document:

The ingestion ALIs used in the calculation are taken from ICRP's Publication 30 after conversion from becquerels to microcuries. Where more than one ALI was given for a particular radionuclide, the lowest value was used in the calculations." $§ \S \S \S$

As noted in Table 3.2, ICRP Pub 30 is superseded by contemporary publications with updated DCs. The bulk of the ingestion and inhalation DCs used in the HC-3 TQ calculation will be obtained from ICRP Pub-119 which is a compendium of DCs previously published in Pub-68 (worker) and Pub-72 (public). Unlike ICRP Pub-72, Pub-68 does not provide for a recommended default lung absorption class.

\subsubsection{Criteria for the Selection of the HC-3 Maximum Inhalation Dose Coefficient and Maximum Air Immersion Dose Coefficient}

As observed in Table 3.4, both ICRP Pub-119 and JAERI-Data/Code 2002-013 have more than one table that provide radionuclide specific inhalation DCs. Both the ICRP and the JAERI publications have one table providing DCs (for each analyzed lung clearance class) based on the inhalation of air-borne particulates, and a second table providing inhalation DCs based on the inhalation of gases/vapours.

The maximum inhalation DC was selected in consideration of both the particulate inhalation DC and the gases/vapours inhalation DC. Typically, the maximum inhalation DC used in the HC-3 TQ calculation was from the inhaled particulate table. However, 51 radionuclides were identified as having the gas/vapor form as the maximum inhalation DC (Table 4.3).

It+1+ It should be noted that the ICRP switched from using Annual Limits for Intake (ALIs) to DCs by the time Pub-68 was published. The ICRP noted in Pub-68 that the ALI for any radionuclide can be obtained by dividing the annual average effective dose limit $(0.02 \mathrm{~Sv})$ by the dose coefficient $e(50)$; which is expressed as follows: $A L I=\frac{0.02}{e(50)}$. Since the ALI is inversely proportional to the e(50) DC, then the larger the DC, the lower the ALI. Therefore, when selecting the lowest ALI per the EPA Technical Background Document, the application to Pub-68 would involve the selection of the maximum DC.

拉抹 102RQ-RN-5-13, pg A-2

§§§§§ 102RQ-RN-5-13, pg B-2 


\begin{tabular}{|l|l|l|}
\hline \multicolumn{3}{|c|}{$\begin{array}{r}\text { Table 4.3 - Radionuclides With Gas/Vapour as } \\
\text { Maximum Inhalation DC for HC-3 TQ Calculation }\end{array}$} \\
\hline C-11 & Hg-203 & I-132 \\
\hline C-14 & Hg-205 & I-132m \\
\hline H-3 & Hg-206 & I-133 \\
\hline Hg-187 & I-118 & I-134 \\
\hline Hg-187m & I-119 & I-135 \\
\hline Hg-188 & I-120 & Ni-56 \\
\hline Hg-190 & I-120m & Ni-59 \\
\hline Hg-191m & I-121 & Ni-63 \\
\hline Hg-192 & I-122 & Ni-65 \\
\hline Hg-193 & I-123 & Te-121 \\
\hline Hg-193m & I-124 & Te-121m \\
\hline Hg-194 & I-125 & Te-123 \\
\hline Hg-195 & I-126 & Te-131 \\
\hline Hg-195m & I-128 & Te-131m \\
\hline Hg-197 & I-129 & Te-132 \\
\hline Hg-197m & I-130 & Te-133 \\
\hline Hg-199m & I-131 & Te-133m \\
\hline
\end{tabular}

The selected maximum inhalation DCs and air immersion DCs are documented in Appendix C, Table C.1.

\subsubsection{Criteria for the Selection of the HC-3 Maximum Ingestion Dose Coefficient}

As observed in Table 3.4, ICRP Pub-119 and JAERI-Data/Code 2002-013 only have one table each that provides radionuclide specific ingestion DCs. As such, following similar guidance used by the EPA Technical Background Document, when more than one ingestion DC was reported for a particular radionuclide, the maximum value was used in the calculations.

The selected maximum ingestion DCs are documented in Appendix C, Table C.1.

\subsubsection{Direct Exposure Pathway TQ Calculation Considerations}

\subsubsection{Assessment of the Exclusion of the Neutron Dose Exposure Pathway}

The direct exposure pathway methodology calculates a TQ assuming the facility inventory behaves like a fixed-point source. The methodology for calculating the direct exposure TQ as outlined in Section 4.2.4.1. of this report assumes the exposure is solely due to photon exposure. Other radiations (alpha, beta, neutron) are not included in the methodology for the following reasons:

- Alpha particles in air will be attenuated before reaching the location of the receptor. Alpha particle energies as high as 8-MeV are reported to have a mean range of approximately 7-cm. 
(30) ${ }^{* * * * * *}$ As such, the assumption of no alpha exposure dose to a receptor located 30-meters away is considered to be valid.

- Similarly, most beta particles in air will be attenuated before reaching the location of the receptor. Beta particle energies as high as 4-MeV are reported to have a maximum range of approximately 800 inches (20.3-m) (30) $)^{t+++t+}$ As such, the assumption of no beta exposure dose to a receptor located 30-meters away is considered to be valid.

- The EPA Technical Background Document evaluated the potential exposure pathway Release Value (RV) associated with an exposure to neutrons resulting from spontaneous fission. The EPA concluded that the calculated neutron exposure $\mathrm{RV}^{\ddagger \neq \neq+\neq \ddagger}$ would be “....uniformly higher than release values calculated on the basis of inhalation......" $\$ \S \S \S \S$ Given this observation, the EPA concluded that the calculation of direct exposure pathways would be solely attributed to photon exposure. The basis for this conclusion is reliant upon use of radionuclide Spontaneous Fission Factors (SFFs) published prior to the issuance of NTD used in this report. To ascertain the validity of this conclusion, updated SFFs from ICRP Pub-107 will be used to calculate TQ values for the neutron exposure pathway. The methodology for calculating the neutron exposure pathway TQs will be as described by the EPA Technical Background Document, adjusted for 10-rem exposure (Equation 12):

$$
T Q_{H C 3, \text { neutron }}=\frac{0.25}{\mathrm{SFF}} * \frac{10 \mathrm{rem}}{0.5 \mathrm{rem}}
$$

Eqn. 12

\subsubsection{Assessment of the Average Photon Intensity Data}

The EPA Technical Background methodology for the direct exposure pathway selected a value for the linear energy absorption coefficient for gamma rays in air of $3.7 \times 10^{-5} \mathrm{~cm}^{-1}$, based on an exposure to a 1 $\mathrm{MeV}$ gamma ray. As noted in Figure 4.1, (31) the linear energy absorption coefficient varies with gamma (a.k.a., x-ray) energy. The EPA justified the selection of the linear energy absorption coefficient value on the following premise:

"The value varies with the energy of the gamma ray involved but is approximately correct $( \pm 15 \%)$ for gamma rays with energies ranging from about $0.07 \mathrm{MeV}$ to about $2 \mathrm{MeV}$. Therefore, recognizing the relatively small error, this single value for $\mu_{\mathrm{a}}$ is used for all radionuclides for the sake of simplicity.”

\footnotetext{
****** The Health Physics and Radiological Health Handbook, 1984, Figure 6.13

†t+†† The Health Physics and Radiological Health Handbook, 1984, Figure 6.11

$\ddagger+\neq+\neq$ The “Release Value” (RV) is defined by the EPA as 0.5 rem to a receptor located 30-meters from a point source. Since the HC-3 TQ is based on 10 rem exposure to a receptor located 30-meters away, the EPA calculated RV is $1 / 20^{\text {th }}$ of a HC-3 TQ.

§§§§§ 102RQ-RN-5-13, pg D-11

102RQ-RN-5-13, pg D-3
} 


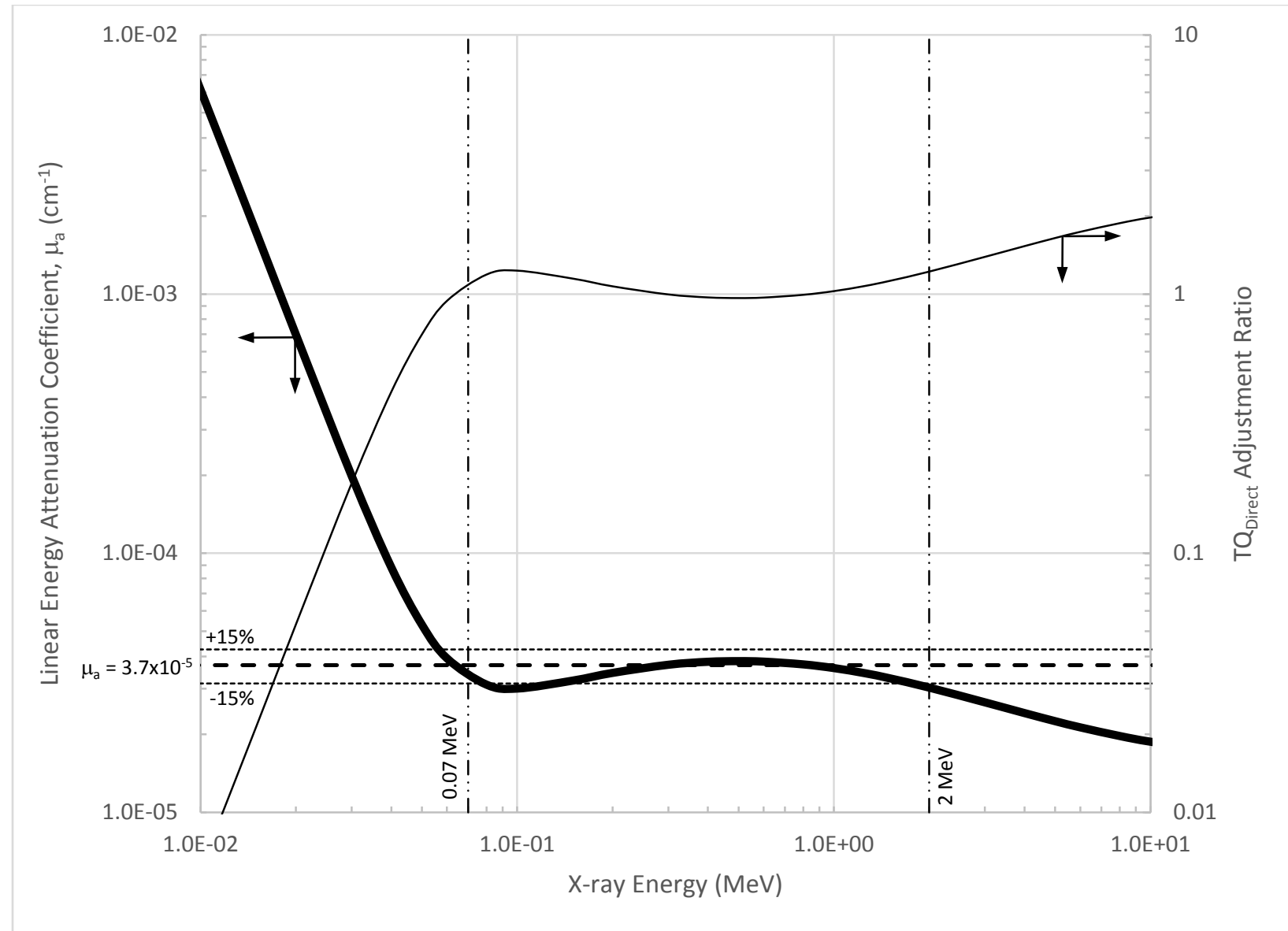

Figure 4.1 - Linear Energy Absorption Coefficient for X-rays in Air (dry)

A review of the NTD that will be used for the calculation of the direct exposure TQ indicates that only $60 \%$ of all radionuclides have an average photon energy within the range of $0.07 \mathrm{MeV}$ to $2 \mathrm{MeV}$

(Table 4.4).

\begin{tabular}{|c|c|c|}
\hline \multicolumn{3}{|c|}{$\begin{array}{l}\text { Table 4.4 - Distribution of Average Photon Intensity Amongst } \\
\text { 1,283 Radionuclides }\end{array}$} \\
\hline $\begin{array}{l}\text { Average Photon Energy } \\
\left(E_{1}\right)\end{array}$ & $\begin{array}{c}\text { No. of } \\
\text { Radionuclides }\end{array}$ & $\%$ \\
\hline $\mathrm{E}_{1}=0 \mathrm{MeV}$ & 96 & $7 \%$ \\
\hline $0.07 \mathrm{MeV}>\mathrm{E}_{1}>0 \mathrm{MeV}$ & 253 & $20 \%$ \\
\hline $2 \mathrm{MeV} \geq \mathrm{E}_{1} \geq 0.07 \mathrm{MeV}$ & 766 & $60 \%$ \\
\hline $\mathrm{E}_{1}>2 \mathrm{MeV}$ & 168 & $13 \%$ \\
\hline
\end{tabular}

Excluding the $7 \%$ of radionuclides with no reported $\mathrm{E}_{1}$ value, this means $33 \%$ of the radionuclide set has an average photon energy value outside of the applicable range associated with the use of the selected value for $\mu_{\mathrm{a}}$. The impact of using a single value for $\mu_{\mathrm{a}}$ can be assessed by comparing the calculated value for $\mathrm{TQ}_{\text {direct }}$ using the linear energy absorption coefficient based on the radionuclide specific average photon energy. Equation 13 represents this concept as a ratio expressed as follows: 


$$
T Q_{\text {direct }} \text { Adjustment Ratio }=\frac{\mathrm{TQ}_{H C 3, \text { direct }}^{\mu_{a} \text { per Fire }}}{\mathrm{TQ}_{H C 3, \text { direct }}^{\mu_{a}=3.7 x 10^{-5}}}=\frac{20728}{\mu_{a} * \exp \left[-\mu_{a}\right]} \quad \text { Eqn. } 13
$$

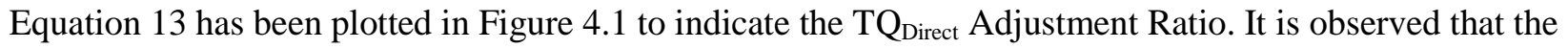
$\mathrm{TQ}_{\text {Direct }}$ Adjustment Ratio does not vary much within the range $0.07 \mathrm{MeV}$ to $2 \mathrm{MeV}$. However, when $\mathrm{E}_{1}>2 \mathrm{MeV}$, the ratio slowly increases to a value of approximately 2 at $10 \mathrm{MeV}$. This indicates that the calculated value for $\mathrm{TQ}_{\text {direct }}$ would be twice that otherwise calculated using the EPA methodology which uses a fixed value for $\mu_{\mathrm{a}}$. Since the EPA methodology would calculate a lower value for TQ $\mathrm{Q}_{\text {direct }}$, the use of the EPA methodology is conservative.

Conversely, as noted in Figure 4.1, the linear energy absorption coefficient rapidly increases for $\mathrm{E}_{1}<0.07 \mathrm{MeV}$. Per Equation 13, this results in the $\mathrm{TQ}_{\text {direct }}$ Adjustment Ratio becoming substantially less than 1 . At an $\mathrm{E}_{1}$ value of $0.02 \mathrm{MeV}$, the $\mathrm{TQ}_{\text {direct }}$ Adjustment Ratio drops to 0.01 . This indicates that the calculated value for $\mathrm{TQ}_{\text {direct }}$ would be $1 \%$ that calculated using the EPA methodology using a fixed value for $\mu_{\mathrm{a}}$. In other words, the EPA methodology is over-predicting the value for $\mathrm{TQ}_{\text {direct }}$ by several orders of magnitude for decreasing values of $\mathrm{E}_{1}$ below $0.07 \mathrm{MeV}$.

Additionally, a footnote in to the Release Value table in Appendix E of the EPA Technical Background Document notes that RVs are not calculated for the direct pathway if the value for $E_{1}$ is less than 0.07 MeV. Specifically, this footnote in the EPA Technical Background Document states;

"No gamma rays are emitted or the gamma rays which are emitted have gamma ray energies of less than $0.07 \mathrm{MeV}$ and are strongly attenuated in air. No release value for the direct exposure pathway was calculated."

As observed in Figure 4.1 and as discussed above, the EPA assertion that the gamma rays are strongly attenuated when $\mathrm{E}_{1}$ is less than $0.07 \mathrm{MeV}$ is an incorrect conclusion.

In light of the results of Equation 13, this report will calculate $\mathrm{TQ}_{\text {direct }}$ values for all radionuclides, even when the value for $E_{1}$ is less than $0.07 \mathrm{MeV}$. Since the scope of the calculations performed are limited to the existing methodology as described in Section 4 of this report, the value for $\mathrm{u}_{\mathrm{a}}$ will be maintained as $3.7 \times 10^{-5} \mathrm{~cm}^{-1}$.

\subsubsection{Calculation of Tritium Threshold Quantities}

As noted in Attachment 1 of DOE-STD-1027-92, the current HC-2 and HC-3 TQ values for tritium are based on the recommendations of the DOE Tritium Focus Group (TFG). During the development of the NNSA Supplemental Guidance to DOE-STD-1027, the NNSA requested the TFG to evaluate the revised HC-2 and HC-3 TQ values and provide a recommendation on the appropriate value to use. The TFG responded to the NNSA in August 2010 with the following:

The position of the TFG is to retain the existing DOE-STD-1027 thresholds for tritium Category 2 and Category 3 nuclear facilities as is. ${ }^{\text {} \neq+\neq ⿰ 扌 \neq}$

+T+t+t+ 102RQ-RN-5-13, pg E-33

拉拉‡ NNSA SD 1027, pg AT4-5 
In June 2013, the NNSA was notified that the TFG had recently met since the publication of the initial issuance of the NNSA Supplemental Guidance to DOE-STD-1027. The TFG voted to continue to endorse the Threshold Quantities as is currently [recommended] while working on new values for recommendation.

While it is recognized that the use of the tritium HC-2 and HC-3 TQs is limited to the thresholds as recommended by the TFG, this report will calculate the HC-2 and HC-3 TQs using the methodology as specified in Sections 4.1 and 4.2, respectively, of this report. The calculation of tritium HC-2 and HC-3 thresholds is provided solely for reference purposes only with the understanding they cannot be used without the review and approval of the TFG. 


\section{CALCULATIONS}

The calculation of the updated HC-2 TQs was performed in MS Excel ${ }^{\circledR}$ file HC2 Updated TQs_ORNL TM-2017 467.xlsx. The calculation of the updated HC-3 TQs was performed in MS Excel ${ }^{\circledR}$ file HC3 Updated TQs_ORNL TM-2017 467.xlsx. These spreadsheets are available upon request to the author of this calculation or through the ORNL Nuclear and Criticality Safety Services group. The following sections explain the organization and purpose of the spreadsheets.

\subsection{HAZARD CATEGORY 2 TQ CALCULATIONS}

\subsubsection{Worksheet: Master Isotope List}

The worksheet Master Isotope List lists the radionuclides and half-life data from the following input documents:

- $\quad$ DOE-STD-1196-2011, CN-1

- FGR-12

- ICRP Pub-38

- ICRP Pub-107

- JAERI-Data/Code 2002-013

This worksheet performs the selection of the half-life based on the "family of data" hierarchy established in Table 3.3 of this report. This worksheet is included as Table D.1 in Appendix D of this report.

\subsubsection{Worksheet: Immersion DCs}

The worksheet Immersion DCs lists the available air immersion DCs from the following input documents:

- FGR-12

- DOE-STD-1196-2011, CN-1

This worksheet performs the selection of the air-immersion DCs based on the "family of data" hierarchy established in Table 3.3 of this report.

\subsubsection{Worksheet: Inhalation DCs_Max}

The worksheet Inhalation DCs_Max lists the available inhalation DCs from the following input documents:

- ICRP Pub-119 (listed as ICRP-72 since the selection is for public inhalation DCs)

- DOE-STD-1196-2011, CN-1

- JAERI-Data/Code 2002-013

The inhalation DC reported from each publication is based on the selection of the radionuclide specific maximum inhalation DCs. The available maximum inhalation DC is based on consideration of particulate inhalation DCs and gases/vapours inhalation DCs (when provided). This worksheet performs the selection of the inhalation DCs based on the "family of data" hierarchy established in Table 3.3 of this report. 


\subsubsection{Worksheet: Inhalation DCs_Rec}

The worksheet Inhalation DCs Rec lists the available inhalation DCs from the following input documents:

- ICRP Pub-119 (listed as ICRP-72 since the selection is for public inhalation DCs)

- DOE-STD-1196-2011, CN-1

- JAERI-Data/Code 2002-013

The inhalation DC reported from each publication is based on the recommended default lung absorption class specified in Table 4.2 of this report. If the radionuclide is associated with an element with a recommended default lung absorption class, then the inhalation DC was reported solely from the particulate inhalation data for the recommended default lung class. If the radionuclide is associated with an element without a recommended default lung absorption class, then the inhalation DC was reported in consideration of particulate inhalation DCs and gases/vapours inhalation DCs (when provided). This worksheet performs the selection of the inhalation DCs based on the "family of data" hierarchy established in Table 3.3 of this report.

\subsubsection{Worksheet: Input Data Summary_Max}

The worksheet Input Data Summary_Max summarizes the selected inhalation and air immersion DCs used for the calculation of the updated HC-2 TQs based on the maximum inhalation DCs. This worksheet is included as Table A.1 in Appendix A of this report.

\subsubsection{Worksheet: Input Data Summary_Rec}

The worksheet Input Data Summary_Rec summarizes the selected inhalation and air immersion DCs used for the calculation of the updated HC-2 TQs based on the recommended default lung absorption class inhalation DCs. This worksheet is included as Table B.1 in Appendix B of this report.

\subsubsection{Worksheet: HC-2 TQ Calc_Max}

The worksheet HC-2 TQ Calc_Max performs the calculation of the radionuclide specific HC-2 TQs using the maximum inhalation and air-immersion DCs. The calculated TQ is based on the inhalation exposure methodology discussed in Section 4.1.1 (Equation 1).

\subsubsection{Worksheet: HC-2 TQ Calc_Rec}

The worksheet HC-2 TQ Calc_Rec performs the of calculation the radionuclide specific HC-2 TQs using the recommended inhalation DCs and the maximum air-immersion DCs. The calculated TQ is based on the inhalation exposure methodology discussed in Section 4.1.1 (Equation 1).

\subsubsection{Worksheet: HC-2 TQ Summary}

The worksheet HC-2 TQ Summary lists the final HC-2 TQs for the following calculations:

- HC-2 TQs calculated using the maximum inhalation and air-immersion DCs.

- HC-2 TQs calculated using the recommended default lung absorption class inhalation DCs and the maximum air-immersion DCs. 
This worksheet also lists the HC-2 TQs from the NNSA Supplemental Guidance document. The HC-2 TQs associated with the maximum inhalation and air-immersion DCs are included as Table A.2 in Appendix A of this report. The HC-2 TQs associated with the recommended default lung absorption class inhalation DCs and the maximum air-immersion DCs are included as Table B.2 in Appendix B of this report. Table B.2 in Appendix B also includes the updated HC-2 TQs from the NNSA Supplemental Guidance.

\subsubsection{Worksheets Based on Input Documentation}

The following worksheets contain the input data used in the calculation of the HC-2 TQs:

- Worksheet: G1_ICRP72 (from ICRP Pub-119)

- Worksheet: H1_ICRP72 (from ICRP Pub-119)

- Worksheet: 1196 Table A-2 (from DOE-STD-1196-2011, CN-1)

- Worksheet: 1196 Table A-3 (from DOE-STD-1196-2011, CN-1)

- Worksheet: JAERI_Table 5 (from JAERI-Data/Code 2002-013)

- Worksheet: JAERI Table 7 (from JAERI-Data/Code 2002-013)

- Worksheet: FGR12 Air Immersion (from FGR-12)

- Worksheet: 1027_RFs (from DOE-STD-1027-92, CN-1)

- Worksheet: Ame2016 (from Atomic Mass Data Center; file mass16.txt)

- Worksheet: ICRP72_RecTable (from ICRP Pub-72)

- Worksheet: NNSA_TQs (from NNSA SD 1027, Admin Change 1)

Manipulations of the input data have been highlighted in orange. Corrections to the input data or alterations to the data selection functions (due to input data formatting issues) have been highlighted in yellow.

There also is a Worksheet: Constants, which contains universally used input constants (i.e., breathing rate, $\chi / \mathrm{Q}$, and Avogadro's Constant), conversion factors (dose and activity), and time conversion factors.

\subsection{HAZARD CATEGORY 3 CALCULATIONS}

\subsubsection{Worksheet: Master Isotope List}

The worksheet Master Isotope List lists the radionuclides and half-life data from the following input documents:

- $\quad$ DOE-STD-1196-2011, CN-1

- FGR-12

- $\quad$ ICRP Pub-38

- ICRP Pub-107

- JAERI-Data/Code 2002-013

This worksheet performs the selection of the half-life based on the "family of data" hierarchy established in Table 3.4 of this report. This worksheet is included as Table D. 1 in Appendix D of this report.

\subsubsection{Worksheet: Photon Energy List}

The worksheet Photon Energy List contains the available radionuclide specific average photon energy values from the following input documents: 
- $\quad$ ICRP Pub-38

- ICRP Pub-107

This worksheet performs the selection of the average photon energy values based on the "family of data" hierarchy established in Table 3.4 of this report.

\subsubsection{Worksheet: Immersion DCs}

The worksheet Immersion DCs lists the available air immersion DCs from the following input documents:

- ICRP Pub-119 (listed as ICRP-68 since the selection is for worker air-immersion DCs)

- JAERI-Data/Code 2002-013

This worksheet performs the selection of the air-immersion DCs based on the "family of data" hierarchy established in Table 3.4 of this report.

\subsubsection{Worksheet: Ingestion DCs}

The worksheet Ingestion DCs lists the available ingestion DCs from the following input documents:

- ICRP Pub-119 (listed as ICRP-68 since the selection is for worker air-immersion DCs)

- JAERI-Data/Code 2002-013

The ingestion DCs reported from each publication are based on the selection of the radionuclide specific maximum ingestion DCs. When more than one ingestion DC is provided for a particular radionuclide, the maximum value is reported. This worksheet performs the selection of the ingestion DCs based on the "family of data" hierarchy established in Table 3.4 of this report.

\subsubsection{Worksheet: Inhalation DCs}

The worksheet Inhalation DCs lists the available inhalation DCs from the following input documents:

- ICRP Pub-119 (listed as ICRP-68 since the selection is for worker air-immersion DCs)

- JAERI-Data/Code 2002-013

The inhalation DCs reported from each publication are based on the selection of the radionuclide specific maximum inhalation DCs. When more than one ingestion DC is provided for a particular radionuclide, the maximum value is reported. The available maximum inhalation DC is based on consideration of particulate inhalation DCs and gases/vapours inhalation DCs (when provided). This worksheet performs the selection of the inhalation DCs based on the "family of data" hierarchy established in Table 3.4 of this report.

\subsubsection{Worksheet: Input Data Summary}

The worksheet Input Data Summary summarizes the selected inhalation, ingestion, and air immersion DCs used for the calculation of the updated HC-3 TQs. This worksheet is included as Table C.1 in Appendix $\mathrm{C}$ of this report. 


\subsubsection{Worksheet: HC-3 SF}

The worksheet HC-3 SF performs the calculation specific to a neutron exposure dose associated with the exposure for radionuclides that spontaneously fission. The calculated pathway TQ is based on the methodology for a neutron emitting source in Section 4.3.3.1 (Equation 12). The results of this calculation are used solely for comparative purposes to validate the original conclusion made by the EPA with respect to disregarding the neutron dose exposure for the external exposure pathway.

\subsubsection{Worksheet: HC-3 External}

The worksheet HC-3 External performs the calculation for the external exposure pathway TQ value. For noble gases (except radon), the calculated pathway TQ is based on the air-immersion methodology in Section 4.2.4.2 (Equation 10). For all other radionuclides, the calculated pathway TQ is based on the point source (i.e., direct exposure) methodology in Section 4.2.4.1 (Equation 9).

\subsubsection{Worksheet: HC-3 Water}

The worksheet HC-3 Water performs the calculation for the water ingestion exposure pathway TQ value. The calculated pathway TQ is based on the ground water ingestion exposure methodology in Section 4.2.3 (Equation 7). The Dilution Factor, used in this pathway TQ calculation, is calculated in accordance with Equation 8.

\subsubsection{Worksheet: HC-3 Food}

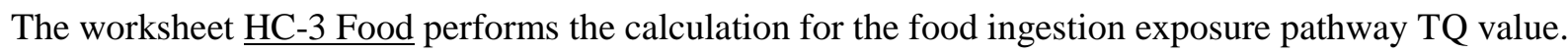
The calculated pathway TQ is based on the food ingestion exposure methodology in Section 4.2.2 (Equation 4). The Dilution Factor, used in this pathway TQ calculation, is calculated in accordance with Equation 5. The Contact Time, also used in this pathway TQ calculation, is calculated in accordance with Equation 6.

\subsubsection{Worksheet: HC-3 Inhalation}

The worksheet HC-3 Inhalation performs the calculation for the inhalation exposure pathway TQ value. The calculated pathway TQ is based on the inhalation exposure methodology in Section 4.2.1 (Equation 3).

\subsubsection{Worksheet: HC-3 TQ Summary}

The worksheet HC-3 TQ Summary lists the following pathway TQs:

- Inhalation exposure pathway TQ.

- Water ingestion exposure pathway TQ.

- Food ingestion exposure pathway TQ.

- Direct exposure (a.k.a., point-source) pathway TQ.

- Air Immersion pathway TQ.

The worksheet selects the final HC-3 TQs in accordance with Equation 11 found in Section 4.2.5. This worksheet also lists the updated HC-3 TQs from the NNSA Supplemental Guidance document. This table is included as Table C.2 in Appendix C of this report. 


\subsubsection{Worksheets Based on Input Documentation}

The following worksheets contain the input data used in the calculation of the HC-3 TQs:

- Worksheet: A1_ICRP68 (from ICRP Pub-119)

- Worksheet: B1_ICRP68 (from ICRP Pub-119)

- Worksheet: C1_ICRP68 (from ICRP Pub-119)

- Worksheet: JAERI_Table 3 (from JAERI-Data/Code 2002-013)

- Worksheet: JAERI Table 6 (from JAERI-Data/Code 2002-013)

- Worksheet: JAERI_Table 8 (from JAERI-Data/Code 2002-013)

- Worksheet: 1027_RFs (from DOE-STD-1027-92, CN-1)

- Worksheet: Ame2016 (from Atomic Mass Data Center; file mass16.txt)

- Worksheet: ICRP07_NDX (from ICRP Pub-107)

- Worksheet: ICRP38_NDX (from ICRP Pub-38)

- Worksheet: NNSA TQs (from NNSA SD 1027, Admin Change 1)

Manipulations of the input data have been highlighted in orange. Corrections to the input data or alterations of the data selection functions (due to input data formatting issues) have been highlighted in yellow.

There also is a Worksheet: Constants, which contains universally used input constants (i.e., breathing rate, $\chi / \mathrm{Q}$, and Avogadro's Constant), pathway specific constants, conversion factors (dose and activity), and time conversion factors. 


\section{DISCUSSION OF RESULTS}

Using the input data as described in Section 3, and the methodology as described in Section 4, updated HC-2 and HC-3 TQs have been calculated for more than 1,200 radionuclides. The updated HC-2 and HC-3 TQs are reported in the following tables:

- Updated HC-2 TQs using maximum inhalation DCs: Table A.2

- $\quad$ Updated HC-2 TQs using recommended inhalation DCs: Table B.2

- $\quad$ Updated HC-3 TQs: Table C.2

\subsection{EXPANSION OF THE SET OF RADIONUCLIDES WITH HC-2 AND HC-3 TQS}

One benefit of this report is the expansion of the available number of radionuclides with a published HC-2 and HC-3 TQ. This report increases the total number of radionuclides with an updated HC-2 TQ to 1,262 and the total number of radionuclides with an updated HC-3 TQ to 1,253 radionuclides. This is a substantial increase in the number of radionuclides with a published TQ as compared to the publications listed in Table 6.1. The increase in the number of radionuclides with a published TQ is attributed to the increase in the available dose coefficients for calculating the TQs.

\begin{tabular}{|l|c|c|}
\hline \multicolumn{3}{|c|}{$\begin{array}{c}\text { Table 6.1 - Number of Radionuclides with Calculated HC-2 and } \\
\text { HC-3 Threshold Quantities }\end{array}$} \\
\hline Publication & HC-2 & HC-3 \\
\hline DOE-STD-1027-92, CN-1 & 94 & 94 \\
\hline LANL Fact Sheets & 97 & 757 \\
\hline NNSA SD 1027 & 98 & 98 \\
\hline ORNL/TM-2017/467 & 1,262 & 1,253 \\
\hline
\end{tabular}

\subsection{RADIONUCLIDES WITHOUT A CALCULATED TQ VALUE}

\subsubsection{Radionuclides without a Category 2 Threshold Quantity}

Of the 1,283 radionuclides on the Master Radionuclide List, 21 radionuclides did not have a calculated updated HC-2 TQ. These radionuclides all have a half-life less than 10 minutes. As noted in Section 3.1.2, inhalation dose coefficients would only be used if the radionuclide had a half-life equal to or greater than 10 minutes. The 10-minute threshold is based on ICRP practices which only provide inhalation dose coefficients for radionuclides with a half-life equal to or greater than 10 minutes.

Additionally, the 21 radionuclides without a calculated updated TQ did not have a published air-immersion coefficient. Even without the use of an available inhalation dose coefficient, a HC-2 TQ can still be calculated solely on the basis of the use of an air-immersion dose coefficient (in fact, 363 radionuclides have an updated HC-2 TQ based solely on the use of an air-immersion dose coefficient).

The 21 radionuclides for which an updated HC-2 TQ was not calculated are listed in Table 6.2. 


\begin{tabular}{|c|c|c|}
\hline \multicolumn{3}{|c|}{ Table 6.2 - Radionuclides Without An } \\
Updated HC-2 TQ \\
\hline At-219 & Hg-188 & Lu-168m \\
\hline Au-188 & Ho-152 & Os-177 \\
\hline Au-189m & Ir-179 & Os-179 \\
\hline Ce-131m & Ir-181 & Pm-138 \\
\hline Er-155 & Lu-164 & Po-212 \\
\hline Hg-187 & Lu-166 & Pt-183 \\
\hline Hg-187m & Lu-166m & Tm-159 \\
\hline
\end{tabular}

\subsubsection{Radionuclides without a Category 3 Threshold Quantity}

Of the 1,283 radionuclides on the Master Radionuclide List, 30 radionuclides did not have a calculated updated HC-3 TQ. These radionuclides all have a half-life less than 10 minutes. As noted in Section 3.1.2, inhalation and ingestion dose coefficients would only be used if the radionuclide had a half-life equal to or greater than 10 minutes. The 10-minute threshold is based on ICRP practices which only provide inhalation and ingestion dose coefficients for radionuclides with a half-life equal to or greater than 10 minutes.

Additionally, the 30 radionuclides without a calculated updated HC-3 TQ did not have a published air-immersion coefficient (for calculating the air-immersion exposure pathway TQ) and did not have a published average photon energy value (for calculating the direct exposure pathway TQ). Even without the use of available inhalation and ingestion dose coefficients, a HC-3 TQ can still be calculated solely on the basis of the use of the external exposure pathway (in fact, 328 radionuclides have an updated HC-3 TQ based solely on an evaluation of the direct exposure pathway TQ and 36 radionuclides have an updated TQ based solely on an evaluation of the air-immersion exposure pathway).

The 30 radionuclides for which an updated HC-3 TQ was not calculated are listed in Table 6.3.

\begin{tabular}{|c|c|c|}
\hline \multicolumn{3}{|c|}{ Table 6.3 - Radionuclides Without An } \\
Updated HC-3 TQ \\
\hline At-219 & Ir-179 & Po-213 \\
\hline Au-188 & Ir-181 & Po-214 \\
\hline Au-189m & Lu-164 & Po-216 \\
\hline Bi-212n & Lu-166 & Po-218 \\
\hline Ce-131m & Lu-166m & Pt-183 \\
\hline Er-155 & Lu-168m & Rn-215 \\
\hline Hg-187 & Os-177 & Rn-216 \\
\hline Hg-187m & Os-179 & Rn-217 \\
\hline Hg-188 & Pm-138 & Tm-159 \\
\hline Ho-152 & Po-212 & U-235m \\
\hline
\end{tabular}




\subsection{ASSESSMENT OF EXCLUSION OF NEUTRON DOSE EXPOSURE PATHWAY}

As noted in Section 4.3.3.1, the methodology employed in the calculation of the direct exposure (i.e., point-source) pathway specified that the neutron dose contribution from radionuclides which spontaneously fission did not need to be considered. The EPA justification for this assertion was based on a comparison of the calculated neutron exposure pathway release value against the inhalation exposure pathway release value. Through this comparative process, the EPA noted that the release value associated with a neutron exposure pathway was always higher than the inhalation exposure pathway release value. Based on this comparison, the EPA concluded the neutron exposure dose pathway would never be the limiting pathway, and as such, could be dropped from further consideration.

With the availability of updated dosimetric data, the original EPA justification can be revisited to determine if it is still applicable. Using the spontaneous fission yield data (i.e., the spontaneous fission factor (SFF)) contained within ICRP Pub-107, the neutron exposure pathway TQ can be calculated using Equation 12, as reported in Table 6.4.

\begin{tabular}{|c|c|c|c|c|}
\hline \multicolumn{5}{|c|}{$\begin{array}{c}\text { Table } 6.4 \text { - Radionuclides Included in ICRP Publication } 107 \text { Which } \\
\text { Decay By Spontaneous Fission }\end{array}$} \\
\hline Isotope & $\begin{array}{l}\text { Spontaneous } \\
\text { Fission } \\
\text { Factor }(10) \\
\end{array}$ & $\begin{array}{c}\text { Neutron } \\
\text { Exposure HC-3 } \\
\text { TQ (Ci) } \\
\end{array}$ & $\begin{array}{c}\text { Inhalation } \\
\text { HC-3 TQ } \\
\text { (Ci) } \\
\end{array}$ & $\begin{array}{c}\text { Inhalation Exposure } \\
\text { TQ > Neutron } \\
\text { Exposure TQ? }\end{array}$ \\
\hline Cf-246 & 2.50E-06 & $2.00 \mathrm{E}+06$ & $2.66 \mathrm{E}+02$ & -- \\
\hline Cf-248 & $2.90 \mathrm{E}-05$ & $1.72 \mathrm{E}+05$ & $1.36 \mathrm{E}+01$ & -- \\
\hline Cf-249 & 5.02E-09 & $9.96 \mathrm{E}+08$ & $1.69 \mathrm{E}+00$ & -- \\
\hline Cf-250 & 7.70E-04 & $6.49 \mathrm{E}+03$ & $3.49 \mathrm{E}+00$ & -- \\
\hline Cf-252 & 3.09E-02 & $1.62 \mathrm{E}+02$ & $6.20 \mathrm{E}+00$ & -- \\
\hline Cf-254 & $9.97 \mathrm{E}-01$ & $5.02 \mathrm{E}+00$ & $3.02 \mathrm{E}+00$ & -- \\
\hline $\mathrm{Cm}-240$ & 3.90E-08 & $1.28 \mathrm{E}+08$ & $3.85 \mathrm{E}+01$ & -- \\
\hline $\mathrm{Cm}-242$ & 6.37E-08 & $7.85 \mathrm{E}+07$ & $2.33 \mathrm{E}+01$ & -- \\
\hline $\mathrm{Cm}-244$ & $1.37 \mathrm{E}-06$ & $3.65 E+06$ & $4.47 \mathrm{E}+00$ & -- \\
\hline Cm-245 & 6.10E-09 & $8.20 \mathrm{E}+08$ & $2.79 \mathrm{E}+00$ & -- \\
\hline $\mathrm{Cm}-246$ & 2.63E-04 & $1.90 \mathrm{E}+04$ & $2.79 \mathrm{E}+00$ & -- \\
\hline $\mathrm{Cm}-248$ & 8.39E-02 & $5.96 \mathrm{E}+01$ & 7.98E-01 & -- \\
\hline Cm-250 & $7.40 \mathrm{E}-01$ & $6.76 \mathrm{E}+00$ & $1.41 \mathrm{E}-01$ & -- \\
\hline Es-253 & $8.90 \mathrm{E}-08$ & $5.62 \mathrm{E}+07$ & $4.47 \mathrm{E}+01$ & -- \\
\hline Es-254 & $3.00 \mathrm{E}-08$ & $1.67 \mathrm{E}+08$ & $1.40 \mathrm{E}+01$ & -- \\
\hline Es-254m & 4.50E-04 & $1.11 \mathrm{E}+04$ & $2.54 \mathrm{E}+01$ & -- \\
\hline Es-255 & 4.50E-05 & $1.11 \mathrm{E}+05$ & $3.10 \mathrm{E}+01$ & -- \\
\hline Fm-252 & $2.30 \mathrm{E}-05$ & $2.17 \mathrm{E}+05$ & $3.72 \mathrm{E}+02$ & -- \\
\hline Fm-254 & 5.92E-04 & $8.45 \mathrm{E}+03$ & $1.45 \mathrm{E}+03$ & -- \\
\hline Fm-255 & 2.30E-07 & $2.17 \mathrm{E}+07$ & $4.30 \mathrm{E}+02$ & -- \\
\hline Fm-256 & $9.19 \mathrm{E}-01$ & $5.44 \mathrm{E}+00$ & $1.60 \mathrm{E}+01$ & No \\
\hline Fm-257 & 2.10E-03 & $2.38 \mathrm{E}+03$ & $1.69 \mathrm{E}+01$ & -- \\
\hline
\end{tabular}




\begin{tabular}{|l|c|c|c|c|}
\hline \multicolumn{5}{|c|}{ Table 6.4 - Radionuclides Included in ICRP Publication 107 Which } \\
Decay By Spontaneous Fission (continued)
\end{tabular}

A review of the results in Table 6.5 notes that only one radionuclide has a calculated neutron exposure pathway TQ value less than the inhalation exposure pathway TQ value. That radionuclide, Fm-256, has a relatively high spontaneous fission yield (0.919), thereby resulting in a calculated neutron exposure pathway TQ of $5.44 \mathrm{Ci}$, which is less than the corresponding inhalation exposure pathway TQ value of 16 Ci.

It is noted that the EPA did not include an adjustment for the half-life of the radionuclides which are undergoing spontaneous fission. This is in contrast to the methodology employed by the EPA in the calculation of the photon exposure pathway where the period of exposure was adjusted to account for the half-life of the radionuclides. As noted by the EPA, the equation used to adjust the exposure time is expressed as follows (Equation 14): ${ }^{\S \S \S \S \S \S \S}$

$$
T=\frac{1-\exp (-\lambda * t)}{\lambda}
$$

Where;

$$
\begin{array}{ll}
\mathrm{T} & =\text { Adjusted exposure duration [day] } \\
\lambda & =\text { Radionuclide decay constant }\left[\text { day }^{-1}\right]=\ln (2) / \mathrm{t}_{1 / 2} \\
\mathrm{t} & =\text { Duration of exposure }[1 \text { day }]
\end{array}
$$

Since Fm-256 has a half-life of 157.6 min (= 0.1094 days), then the adjusted exposure duration is calculated to be:

$$
T=\frac{1-\exp \left(-\frac{\ln (2)}{0.1094} d^{-1} * 1 d\right)}{\frac{\ln (2)}{0.1094} d^{-1}}=0.1576 d
$$

$\S \S \S \S \S \S \S$ In the EPA derivation of the equation, the time unit conversion factor is used to ensure "T" is in units of hours. This conversion is necessitated by the fact that the photon exposure rate was expressed in units of rem/hour. For Equation 12, the calculated TQ is in units of rem for a 1 day unadjusted exposure duration. Therefore, the time conversion factor of $24 \mathrm{hr} /$ day is not needed for the purpose of accounting for the neutron dose adjusted exposure duration. 
As such, the effective exposure duration for Fm-256 is only 0.1576 day, and not the full 1 day assumed in the derivation of Equation 12. Therefore the neutron exposure pathway TQ for Fm-256, when accounting for decay, is $34.5 \mathrm{Ci}$. This adjusted neutron exposure pathway TQ is now greater than the inhalation exposure pathway TQ of $16 \mathrm{Ci}$.

Based on the adjusted neutron exposure pathway for Fm-256, the inhalation exposure continues to be the limiting pathway with respect to the neutron exposure pathway for all analyzed radionuclides with spontaneous fission yield data in ICRP Pub-107. Therefore, the EPA conclusion that the neutron exposure dose pathway would never be the limiting pathway is confirmed to still be an accurate statement.

\subsection{COMPARISON TO NNSA CALCULATED UPDATED TQS}

\subsubsection{Comparison of Category 2 TQs}

The updated HC-2 TQs (in terms of activity, Ci) calculated in this report were within $\pm 5 \%$ for 93 out of 98 radionuclides contained within the NNSA Supplemental Guidance. Two of the five radionuclides that differed by more than $+5 \%$ were $\mathrm{H}-3$ (tritium) and Rn-222. As noted in the NNSA Supplemental Guidance to DOE-STD-1027, the values for H-3 and Rn-222 were not calculated using updated dosimetric data, rather they were left unchanged from the values specified in DOE-STD-1027-92, CN-1. The other three radionuclides are listed Table 6.5.

\begin{tabular}{|c|c|c|c|c|}
\hline \multicolumn{5}{|c|}{ Table 6.5 - Comparison of Updated HC-2 TQs (activity) } \\
\hline Radionuclide & $\begin{array}{l}\text { NNSA SD } 1027 \\
\text { (Ci) }\end{array}$ & $\begin{array}{l}\text { ORNL/TM- } \\
2017 / 467 \text { (Ci) }\end{array}$ & $\Delta$ Activity & $\begin{array}{c}\text { Probable Reason For Difference } \\
\text { (if known) }\end{array}$ \\
\hline Hg-203 & $3.33 E+05$ & $1.15 E+05$ & $-65 \%$ & $\begin{array}{l}\text { NNSA used max particulate } \\
\text { inhalation DC ( } 2.4 \mathrm{E}-09 \mathrm{~Sv} / \mathrm{Bq}) \text {. This } \\
\text { report selected vapor form as max DC } \\
\text { ( } 7.0 \mathrm{E}-09 \mathrm{~Sv} / \mathrm{Bq}) \text {. The relative ratio of } \\
\text { these DCs accounts for the difference. }\end{array}$ \\
\hline Kr-85 & $1.06 \mathrm{E}+07$ & $2.27 \mathrm{E}+07$ & $114 \%$ & $\begin{array}{l}\text { NNSA used ICRP Pub-72 (2.55E-16 } \\
\text { Sv/s per Bq/m³). This report used } \\
\text { FGR-12 }\left(1.19 \mathrm{E}-16 \mathrm{~Sv} / \mathrm{s} \text { per } \mathrm{Bq} / \mathrm{m}^{3}\right) \text {. } \\
\text { The relative ratio of these DCs } \\
\text { accounts for the difference. }\end{array}$ \\
\hline Хe-133 & $1.95 E+06$ & $1.73 E+06$ & $-11 \%$ & 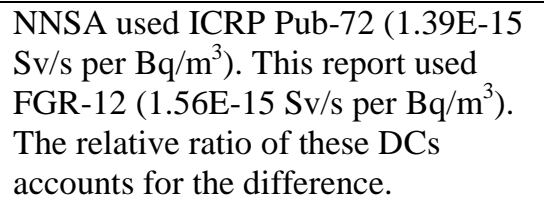 \\
\hline
\end{tabular}

For Hg-203, the difference in the updated HC-2 TQ calculation has been attributed to the data selection criteria in this report which supplemented the inhalation DC data set to include gases/vapours based inhalation DCs. For both Kr-85 and Xe-133, the NNSA updated HC-2 TQs were based on the airimmersion DCs from ICRP Pub-72 whereas this report utilized the air-immersion DCs from FGR-12 for the associated radionuclides.

The updated HC-2 TQs (in terms of mass, g) calculated in this report were within $+5 \%$ for 91 out of 98 radionuclides contained within the NNSA Supplemental Guidance. Similar to the activity comparison, H-3 and Rn-222 have different values since they were not calculated by the NNSA using updated dosimetric data. The other five radionuclides are listed in Table 6.6. 


\begin{tabular}{|c|c|c|c|c|}
\hline \multicolumn{5}{|c|}{ Table 6.6 - Comparison of Updated HC-2 TQs (mass) } \\
\hline Radionuclide & $\begin{array}{l}\text { NNSA SD } 1027 \\
(\mathrm{~g})\end{array}$ & $\begin{array}{l}\text { ORNL/TM- } \\
2017 / 467 \text { (g) }\end{array}$ & $\Delta$ Mass & $\begin{array}{c}\text { Probable Reason for Difference } \\
\text { (if known) }\end{array}$ \\
\hline Am-242m & $2.09 \mathrm{E}+01$ & $2.26 \mathrm{E}+01$ & $8 \%$ & $\begin{array}{l}\text { Suspect difference in the calculated } \\
\text { Specific Activity since the TQ(Ci) values } \\
\text { matched. }\end{array}$ \\
\hline Ge-68 & $8.16 \mathrm{E}+01$ & $8.68 \mathrm{E}+01$ & $6 \%$ & $\begin{array}{l}\text { Suspect difference in the calculated } \\
\text { Specific Activity since the TQ(Ci) values } \\
\text { matched. }\end{array}$ \\
\hline Hg-203 & $2.41 \mathrm{E}+01$ & $8.35 \mathrm{E}+00$ & $-65 \%$ & $\begin{array}{l}\text { NNSA probably used max particulate } \\
\text { inhalation DC ( } 2.4 \mathrm{E}-09 \mathrm{~Sv} / \mathrm{Bq}) \text {. This } \\
\text { report selected vapor form as max DC } \\
\text { ( } 7.0 \mathrm{E}-09 \mathrm{~Sv} / \mathrm{Bq}) \text {. The relative ratio of } \\
\text { these DCs accounts for the difference. }\end{array}$ \\
\hline Kr-85 & $2.70 \mathrm{E}+04$ & $5.78 \mathrm{E}+04$ & $114 \%$ & $\begin{array}{l}\text { NNSA used ICRP Pub-72 (2.55E-16 Sv/s } \\
\left.\text { per } \mathrm{Bq} / \mathrm{m}^{3}\right) \text {. This report used FGR-12 } \\
\left(1.19 \mathrm{E}-16 \mathrm{~Sv} / \mathrm{s} \text { per } \mathrm{Bq} / \mathrm{m}^{3}\right) \text {. The relative } \\
\text { ratio of these DCs accounts for the } \\
\text { difference. }\end{array}$ \\
\hline Хe-133 & $1.04 \mathrm{E}+01$ & $9.25 \mathrm{E}+00$ & $-11 \%$ & 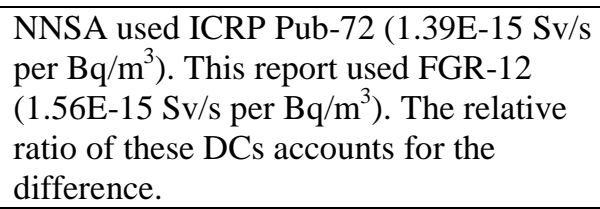 \\
\hline
\end{tabular}

For Am-242m and Ge-68, it is suspected that there are differences in the specific activity values between the NNSA Supplemental Guidance and this report since the activity based TQ value matched. For Hg-203, it appears the NNSA TQ value is based on the maximum particulate inhalation DC whereas this report calculated the TQ using the vapor inhalation DC, which was higher than the particulate inhalation DC. For Kr-85 and Xe-133, the differences in the TQ values are explained by the NNSA most likely using ICRP Pub-72 air-immersion DCs whereas this report selected air-immersion DCs from FGR-12 for these two radionuclides.

\subsubsection{Comparison of Category 3 TQs}

The updated HC-3 TQs (in terms of activity, Ci) calculated in this report were within $\pm 5 \%$ for 96 out of 98 radionuclides contained within the NNSA Supplemental Guidance. The only radionuclides that differed more than $+5 \%$ were $\mathrm{H}-3$ (tritium) and $\mathrm{Rn}-222$. As noted in the NNSA Supplemental Guidance to DOE-STD-1027, the values for H-3 and Rn-222 were not calculated using updated dosimetric data, rather they were left unchanged from the values specified in DOE-STD-1027-92, CN-1.

The updated HC-3 TQs (in terms of mass, g) calculated in this report were within $\pm 5 \%$ for 93 out of 98 radionuclides contained within the NNSA Supplemental Guidance. Similar to the activity comparison, H-3 and Rn-222 have different values since they were not calculated by the NNSA using updated dosimetric data. The other three radionuclides are listed in Table 6.7. 


\begin{tabular}{|c|c|c|c|c|}
\hline \multicolumn{5}{|c|}{ Table 6.7 - Comparison of Updated HC-3 TQs (mass) } \\
\hline Radionuclide & $\begin{array}{l}\text { NNSA SD } 1027 \\
\text { (g) }\end{array}$ & $\begin{array}{l}\text { ORNL/TM- } \\
2017 / 467 \text { (g) }\end{array}$ & $\Delta$ Mass & $\begin{array}{c}\text { Reason for Difference } \\
\text { (if known) }\end{array}$ \\
\hline Am-242m & 3.07E-01 & 3.28E-01 & $7 \%$ & $\begin{array}{l}\text { Suspect difference in the calculated } \\
\text { Specific Activity since the TQ(Ci) } \\
\text { values matched. }\end{array}$ \\
\hline Cd-113 & 8.77E+13 & $9.29 \mathrm{E}+13$ & $6 \%$ & $\begin{array}{l}\text { Suspect difference in the calculated } \\
\text { Specific Activity. The TQ(Ci) values } \\
\text { only differed by } 2 \% \text {. }\end{array}$ \\
\hline Ge-68 & 8.79E-02 & $9.54 \mathrm{E}-02$ & $8 \%$ & $\begin{array}{l}\text { Suspect difference in the calculated } \\
\text { Specific Activity since the TQ(Ci) } \\
\text { values matched. }\end{array}$ \\
\hline
\end{tabular}

For Am-242m and Ge-68, it is suspected that there are differences in the specific activity values between the NNSA Supplemental Guidance and this report since the activity based TQ value matched. Similarly, it is suspected for Cd-113 that there are differences in the specific activity values between the NNSA Supplemental Guidance and this report since the activity based TQ values only differed by $2 \%$.

Finally, a review of the data also noted that the limiting pathway used for the selection of the final HC-3 TQs were a match for 96 of 98 radionuclides, with H-3 and Rn-222 again being the only difference.

\subsection{COMPENSATION FOR EXCEL CALCULATION PRECISION ISSUES}

During the execution of the calculation of the HC-3 TQs for the water ingestion exposure pathway and the direct exposure pathway, calculational stabilities were identified in the calculation of the effective exposure duration. These calculational instabilities have been attributed to the limitations associated with the significant figures retained by MS Excel ${ }^{\circledR}$ to describe a number. Although MS Excel ${ }^{\circledR}$ can display numbers up to 30 decimal places, its precision for a specific number is confined to 15 significant figures.

As described in Appendix E of this report, the instabilities in the calculation of the effective exposure duration were observed to occur for radionuclides with a half-life in excess of $8 \mathrm{E}+12$ days $(\sim 2.19 \mathrm{E}+10 \mathrm{y})$. For radionuclides with increasingly larger half-life values, the calculation instabilities were noted to become worse, resulting in significant deviation from the expected calculated value. To compensate for this issue and preserve calculational accuracy, a conditional statement was used in the MS Excel calculations for the effective exposure duration (also referred to as the Contact Time (CT) by the EPA) for the Water Ingestion pathway and the Direct Exposure pathway.

- If the radionuclide half-life was noted to be less than or equal to1E+12 days, the CT was calculated using Equation E-1.

- Otherwise, if the radionuclide half-life was noted to be greater than $1 \mathrm{E}+12$ days, the CT was set to be equal to the actual exposure duration (9 days for the Water Ingestion pathway and 1 day for the Direct Exposure pathway).

This modification to the CT equation preserved the accuracy of the calculated CT for radionuclides with very large half-life values. Further discussion of this issue can be found in Appendix E of this report. 


\section{QUALITY ASSURANCE}

\subsection{REPORT PREPARATION}

\subsubsection{Development of Final Report and Supporting Calculations}

The development (including the format and content organization), review, and publication of this report was conducted in accordance with the Oak Ridge National Laboratory (ORNL) Standards-Based Management System (SBMS) Subject Area: Publications and Other Scientific Communications, Procedure: Review and Approval of Scientific Communications Internally (32).

The intermediate and final calculations produced in support of the development of this report were conducted in accordance with the ORNL Nuclear and Radiological Protection Division (NRPD) Nuclear and Criticality Safety Services (NCSS) Internal Operation Procedure (IOP): Calculation Production and Control Procedure. (33) The IOP specifies requirements associated with the creation, review, and approval of calculations, computational analyses, and qualitative analyses developed by the Facility and Accelerator Safety staff within the NCSS group.

The development of all calculations and the preparation of the report were conducted by a qualified and experienced analyst with detailed knowledge of the methodologies and assumptions involved in the calculation of the updated HC-2 and HC-3 TQs.

The spreadsheet software application Microsoft (MS) Excel ${ }^{\circledR}$ was exclusively utilized for executing all intermediate and final calculations. As specified in the ORNL SBMS Subject Area: Software Quality Assurance; (34), the use of MS Excel can be exempted from ORNL Software Quality Assurance requirements provided the following stipulation is satisfied:

Software calculations written for use with commercial off-the-shelf software (e.g., Excel spreadsheet applications) which are not intended to be used repeatedly and the results are verified by other methods such as hand calculations, peer review, or an applicable organizational quality assurance process.

For this effort, a peer review process was employed as a verification of the applicability, adequacy, and accuracy of the assumptions, calculations, and conclusions documented in this report.

\subsection{TECHNICAL REVIEWS}

\subsubsection{Peer Review}

A peer review process was utilized for conducting an independent verification of the intermediate and final calculations produced in support of this report. The peer reviewer fulfilled the following roles: (i) perform a verification of the validity and accuracy of the intermediate and final calculations (i.e., the calculation checker), and (ii) served as an independent technical reviewer of the final report. To ensure the review was conducted independently, the peer reviewer did not participate in the development of the calculation spreadsheets and did not participate in the development of the report.

\footnotetext{
${ }^{* * * * * * * *}$ The report format and organization described by the IOP is based on issuance of an internal document. The SBMS Subject Area provides guidance for the release of external publications, such as the case for this report. As noted on page 5 of NCSS-FS-04, R2: "In the case of any inconsistencies with this IOP, the SBMS documents prevail.” Accordingly, the format, organizational content, and approval requirements for this report are based on the guidelines specified in the SBMS Subject Area: Publications and Other Scientific Communications.
} 
In support of the calculation checker role, the peer reviewer performed the following tasks:

- Reviewed and verified input data in the spreadsheets. The review consisted of ensuring the proper input data was utilized as described in Sections 3 and 4 of this report. Additionally, the reviewer conducted a verification sampling of the input data. The verification sampling involved a comparison of randomly selected input data which was then compared to the published reference or electronic data source.

- The peer reviewer verified intermediate and final calculations were accurate by verifying that computation formulas were correct. This verification included $100 \%$ cell verifications to ensure the formulas were correctly copied down their respective columns. Lookup functions and cell formulas which were utilized to import data values from the various worksheets were verified for proper syntax and correct retrieval of the requested data. These lookup functions and cell formulas were checked to ensure the same function / formula was copied correctly to $100 \%$ of the cells down a column.

- Any errors found during the peer review were reported back to the author/calculation developer for required corrections. All corrections were subsequently reviewed by the peer reviewer to ensure an adequate resolution of the identified errors.

In support of the independent technical reviewer role, the peer reviewer performed the following tasks:

- Verified all references cited in the report.

- Verified assumptions in the report have been adequately identified, described, justified, and are reasonable.

- Verified the described input selection organization hierarchy described in Section 3 matched the usage in the spreadsheets.

- Verified the described methodology and associated equations were properly transcribed from the applicable references.

- Provided editorial recommendations in support of ensuring technical clarity of the overall report.

\subsubsection{Department of Energy Independent Technical Review}

An independent technical review of the report was conducted by a DOE-assigned representative in support of the Department of Energy's Office of Nuclear Safety (AU-30), within the Associate Under Secretary for Environment, Health, Safety and Security organization. The DOE-assigned review involved an individual with knowledge of the requirements associated with DOE-STD-1027 and the methodologies and assumptions used in the calculation of the updated HC-2 and HC-3 TQs. The DOE-assigned reviewer did not participate in the development of the report, did not participate in the development of the intermediate and final calculations, and did not collaborate with the assigned peer reviewer. As such, the DOE-assigned representative is considered to be a qualified independent reviewer of the assumptions, assertions, and conclusions made in this report.

The objectives of the DOE Independent Technical Review are summarized as follows: 
- Confirmed the logic, assumptions, and methodology used to expand the list of radioisotopes and to calculate updated HC-2 and HC-3 TQs.

- Confirmed completeness of information presented in the report to support an update to DOE-STD-1027-92.

- Performed a spot check of final results presented in the report.

- Verified the document preparation process reflected adequate quality assurance measures. 


\section{REFERENCES}

1. U. S. Department of Energy. Hazard Categorization and Accidental Analysis Techniques for Compliance With DOE Order 5480.23, Nuclear Safety Analysis Reports. 1997. DOE-STD-102792, Change Notice 1.

2. Los Alamos National Laboratory. Specific Activities and DOE-STD-1027-92 Hazard Category 2 Thresholds. November 1994. LANL Fact Sheet. LA-12846-MS.

3. Los Alamos National Laboratory. Table of DOE-STD-1027-92 Hazard Category 3 Threshold Quantities for the ICRP-30 List of 757 Radionuclides - LANL Fact Sheet. October 2002. Revision 1. LA-12981-MS.

4. National Nuclear Security Administration. Guidance on Using Release Fraction and Modern Dosimetric Information Consistently with DOE STD 1027-92, Hazard Categorization and Accident Analysis Techniques for Compliance with DOE Order 5480.23, Nuclear Safety Analysis Reports, Change Notice No. 1. May 2014. NNSA SD 1027, Admin Change 1.

5. International Commission on Radiological Protection. Dose Coefficients for Intakes of Radionuclides by Workers. Elsevier Ltd., 1994. Publication 68.

6. International Commission on Radiological Protection. Age-dependent Doses to the Members of the Public from Intake of Radionuclides - Part 5 Compilation of Ingestion and Inhalation Coefficients. Elsevier Ltd., 1995. Publication 72.

7. International Commission on Radiological Protection. Compendium of Dose Coefficients based on ICRP Publication 60. Elsevier Ltd., 2012. Publication 119.

8. U. S. Department of Energy. Derived Concentration Technical Standard. 2011. DOE-STD-11962011.

9. Japan Atomic Energy Research Institute. Dose Coeffcients for Radionuclides Produced in High Energy Proton Accelerator Facilities: Coeffciencts for Radionuclides Not Listed in ICRP Publications. May 2002. JAERI-Data/Code 2002-013.

10. International Commission on Radiological Protection. Nuclear Decay Data for Dosimetric Calculations. Elsevier Ltd., 2008. Publication 107.

11. U. S. Department of Energy. External Dose-Rate Conversion Factors for Calculation of Dose to the Public. Office of Environmental Guidance and Compliance. July 1988. DOE/EH-0070.

12. U. S. Department of Energy. Radioactive Decay Data Tables. Office of Scientific and Technical Information. 1981. DOE/TIC-11026.

13. U. S. Department of Energy. Internal Dose Conversion Factors for Calculation of Dose to the Public. July 1988. DOE/EH-0071.

14. International Commission on Radiological Protection. Radionuclide Transformations - Energy and Intensity of Emissions. Pergamon Press Inc., 1983. Publication 38.

15. International Commission on Radiological Protection. Limits for Intakes of Radionuclides by Workers. Pergamon Press Inc., 1979. Publication 30: Part I.

16. ICF Incorporated and C-E Environmental. February 1989. : Environmental Protection Agency, Technical Background Document to Support Final Rule Making Pursuant To Section 102 of the Comprehensive Environmental Response, Compensation, and Liability Act: Radionuclides. 102RQ-RN-5-13. 
17. U. S. Environmental Protection Agency. External Exposure to Radionuclides in Air, Water, and Soil. September 1993. Federal Guidance Report No. 12, EPA-402-R-93-081.

18. U. S. Environmental Protection Agency. Cancer Risk Coefficients for Environmental Exposure to Radionuclides. September 1999. Federal Guidance Report No. 13, EPA-402-R-99-001.

19. International Atomic Energy Agency. Atomic Mass Adjustment. Atomic Mass Data Center. [Online] 2017. https://www-nds.iaea.org/amdc/ame2016/mass16.txt.

20. International Commission on Radiological Protection. Report of the Task Group on Reference Man. Pergamon Press Inc., 1975. Publication 23.

21. International Commission on Radiological Protection. Human Resiratory Tract Model for Radiological Protection. Pergamon Press Inc., 1994. Publication 66.

22. Nuclear Regulatory Commission. A Regulatory Analysis on Emergency Preparedness for Fuel Cycle and Other Radioactive Material Licensees. Office of Nuclear Regulatory Research. January 1988. NUREG-1140.

23. CODATA Recommended Values of the Fundamental Constants: 2014. Mohr, Peter J., Newell, David B. and Taylor, Barry N. Issue No. 3, American Physical Society, 2016, Reviews of Modern Physics, Vol. 88. National Institute of Standards and Technology.

24. Determination of e/h, Using Macroscopic Quantum Phase Coherence in Superconductors: Implications for Quantum Electrodynamics and the Fundamental Physical Constants. Taylor, B. N., Parker, W. H. and Langenberg, D. N. Issue No. 3, The American Physical Society, 1969, Reviews of Modern Physics, Vol. 41.

25. The 1986 Adjustment of the Fundamental Physical Constants. Cohen, E. Richard and Taylor, Barry N. Issue No. 4, The American Physical Society, 1987, Reviews of Modern Physics, Vol. 59.

26. CODATA Recommended Values of the Fundamental Physical Constants: 2002. Mohr, Peter J. and Taylor, Barry N. Issue No. 1, The American Physical Society, Reviews of Modern Physics, Vol. 77.

27. CODATA Recommended Values of the Fundamental Physical Constants: 2006. Mohr, Peter J., Taylor, Barry N. and Newell, David B. Issue No. 2, The American Physical Society, Reviews of Modern Physics, Vol. 80.

28. National Institute of Standards and Technology. Special Publication 11, NIST Guide to the SI, Chapter 5: Units Outside the SI. [Online] August 25, 2016. [Cited: September 14, 2017.] https://www.nist.gov/pml/nist-guide-si-chapter-5-units-outside-si.

29. Bureau International des Poids et Mesures. The International System of Units (SI). 8th Edition. Organisation Intergouvernementale de la Convention du Metre, 2006.

30. Nucleon Lectern Associates. The Health Physics and Radiological Health Handbook. 1984 (7th Printing).

31. National Institute of Standards and Technology. X-ray Mass Attenuation Coeffcients: Air, Dry (near sea level). [Online] https://physics.nist.gov/PhysRefData/XrayMassCoef/ComTab/air.html.

32. Oak Ridge National Laboratory. Review and Approve Scientific Communications Internally. Standards-Based Management System. [Online] UT-Battelle, LLC, July 10, 2017. [Cited: September 19, 2017.] https://sbms.ornl.gov/sbms/SBMSearch/subjarea/publication/pro1.cfm. PROC-3975. 
33. Oak Ridge National Laboratory. Calculation Production and Control Procedure. Nuclear and Criticality Safety Services, Nuclear and Radiological Protection Division. 2013. Internal Operating Procedure. NCSS-FS-04, Revision 2.

34. Oak Ridge National Laboratory. Exhibit: Software Quality Assurance (SQA) Exemptions. Standards-Based Management System. [Online] March 31, 2016. [Cited: September 19, 2017.$]$ https://sbms.ornl.gov/sbms/sbmsearch/subjarea/SQA/ExhibitExemption.cfm. Exhibit-11255.

35. Zwillinger, Daniel, [ed.]. CRC Standard Mathematical Tables and Formulae. 31st Edition. : Chapman and Hall/CRC Press LLC, 2003.

36. Meeus, Jean. Astronomical Algorithms. Second Edition. Richmond, Virginia : Willmann-Bell, Inc., March 2000. 2nd Printing. 


\section{APPENDIX A. CALCULATION OF HAZARD CATEGORY 2 THRESHOLD QUANTITIES USING MAXIMUM DOSE COEFFICIENTS}


(This page is intentionally blank) 


\section{APPENDIX A. CALCULATION OF HAZARD CATEGORY 2 THRESHOLD QUANTITIES USING MAXIMUM DOSE COEFFICIENTS}

This appendix contains the following tables:

- Table A.1: Radionuclide Specific Input Data Used For the Calculation of HC-2 TQs Using Maximum Reported DCs

- Table A.2: HC-2 TQs Using Maximum Inhalation DCs 


\begin{tabular}{|c|c|c|c|c|c|c|c|c|c|c|c|c|c|c|}
\hline \multirow{3}{*}{$\begin{array}{c}\text { MASTER } \\
\text { ISOTOPE } \\
\text { LIST } \\
\text { Ac-223 }\end{array}$} & \multirow{3}{*}{$\begin{array}{c}\begin{array}{c}\text { Atomic } \\
\text { Mass } \\
\text { Nuclide }\end{array} \\
\text { Ac-223 } \\
\end{array}$} & & & \multicolumn{10}{|l|}{ Table A.1 R } & \\
\hline & & \multirow{2}{*}{$\begin{array}{c}\begin{array}{c}\text { Atomic } \\
\text { Mass }\end{array} \\
223.019\end{array}$} & \multirow{2}{*}{$\begin{array}{l}\text { Element } \\
\text { Actinium }\end{array}$} & $\begin{array}{c}\text { RF per } \\
\text { DOE-STD- } \\
1027\end{array}$ & $\begin{array}{c}\text { Half-Life } \\
\text { Reference }\end{array}$ & \multicolumn{2}{|c|}{$\begin{array}{c}\text { Half-Life } \\
\text { (original units) }\end{array}$} & Half Life (s) & $\begin{array}{l}\text { Sp. Act } \\
\text { (Ci/g) }\end{array}$ & $\begin{array}{c}\text { Inhalation DC } \\
\text { Reference }\end{array}$ & $\begin{array}{c}\text { HC-2 } \\
\text { Bounding } \\
\text { Inhalation } \\
\mathrm{DC}(\mathrm{Sv} / \mathrm{Bq})\end{array}$ & $\begin{array}{c}\text { Lung } \\
\text { Absorption } \\
\text { Class/Type }\end{array}$ & \multirow{2}{*}{$\begin{array}{c}\begin{array}{c}\text { Immersion DC } \\
\text { Reference }\end{array} \\
\text { FGR-12 }\end{array}$} & \multirow[t]{2}{*}{$\begin{array}{c}\text { HC-2 } \\
\text { Immersion } \\
\mathrm{DC}(\mathrm{Sv} / \mathrm{s} \\
\left.\text { per } \mathrm{Bq} / \mathrm{m}^{3}\right) \\
2.07 \mathrm{E}-16\end{array}$} \\
\hline & & & & $1.00 \mathrm{E}-03$ & ICRP-38 & $2.20 \mathrm{E}+00$ & $\mathrm{~m}$ & $1.32 \mathrm{E}+02$ & $3.83 \mathrm{E}+08$ & -- & -- & -- & & \\
\hline Ac-224 & Ac-224 & 224.022 & Actinium & $1.00 \mathrm{E}-03$ & ICRP-38 & $2.90 \mathrm{E}+00$ & $\mathrm{~h}$ & $1.04 \mathrm{E}+04$ & $4.82 \mathrm{E}+06$ & ICRP-72 & $1.30 \mathrm{E}-07$ & $\mathrm{~s}$ & FGR-12 & $9.00 \mathrm{E}-15$ \\
\hline Ac-225 & Ac-225 & 225.023 & Actinium & $1.00 \mathrm{E}-03$ & ICRP-38 & $1.00 \mathrm{E}+01$ & $d$ & $8.64 \mathrm{E}+05$ & $5.80 \mathrm{E}+04$ & ICRP-72 & $8.50 \mathrm{E}-06$ & $\mathrm{~s}$ & FGR-12 & $7.21 \mathrm{E}-16$ \\
\hline Ac-226 & Ac-226 & 226.026 & Actinium & $1.00 \mathrm{E}-03$ & ICRP-38 & $2.90 \mathrm{E}+01$ & $\mathrm{~h}$ & $1.04 \mathrm{E}+05$ & $4.78 \mathrm{E}+05$ & ICRP-72 & $1.30 \mathrm{E}-06$ & $\mathrm{~s}$ & FGR-12 & $6.03 E-15$ \\
\hline Ac-227 & Ac-227 & 227.028 & Actinium & $1.00 \mathrm{E}-03$ & ICRP-38 & $2.18 \mathrm{E}+01$ & $y$ & $6.87 \mathrm{E}+08$ & $7.23 \mathrm{E}+01$ & ICRP-72 & $5.50 \mathrm{E}-04$ & $\mathrm{~F}$ & FGR-12 & $5.82 \mathrm{E}-18$ \\
\hline Ac-228 & Ac-228 & 228.031 & Actinium & $1.00 \mathrm{E}-03$ & ICRP-38 & $6.13 \mathrm{E}+00$ & $\mathrm{~h}$ & $2.21 \mathrm{E}+04$ & $2.24 E+06$ & ICRP-72 & $2.50 \mathrm{E}-08$ & $F$ & FGR-12 & $4.78 \mathrm{E}-14$ \\
\hline Ac-229 & Ac-229 & 229.033 & Actinium & $1.00 \mathrm{E}-03$ & JAERI & $6.27 \mathrm{E}+01$ & $\mathrm{~m}$ & $3.76 \mathrm{E}+03$ & $1.31 \mathrm{E}+07$ & JAERI & $3.50 \mathrm{E}-11$ & $M$ & -- & -- \\
\hline Ac-230 & Ac-230 & 230.036 & Actinium & $1.00 \mathrm{E}-03$ & ICRP-107 & $1.22 \mathrm{E}+02$ & $\mathrm{~s}$ & $1.22 \mathrm{E}+02$ & $4.02 E+08$ & -- & -- & -- & DOE-STD-1196 & $2.66 \mathrm{E}-14$ \\
\hline Ac-231 & Ac-231 & 231.038 & Actinium & $1.00 \mathrm{E}-03$ & ICRP-107 & $7.50 \mathrm{E}+00$ & $\mathrm{~m}$ & $4.50 \mathrm{E}+02$ & $1.09 E+08$ & -- & -- & -- & DOE-STD-1196 & $1.84 \mathrm{E}-14$ \\
\hline Ac-232 & Ac-232 & 232.042 & Actinium & $1.00 \mathrm{E}-03$ & ICRP-107 & $1.19 \mathrm{E}+02$ & $\mathrm{~s}$ & $1.19 \mathrm{E}+02$ & $4.09 E+08$ & -- & -- & -- & DOE-STD-1196 & $5.65 \mathrm{E}-14$ \\
\hline Ac-233 & Ac-233 & 233.044 & Actinium & $1.00 \mathrm{E}-03$ & ICRP-107 & $1.45 \mathrm{E}+02$ & $\mathrm{~s}$ & $1.45 \mathrm{E}+02$ & $3.34 \mathrm{E}+08$ & -- & -- & -- & DOE-STD-1196 & $2.29 \mathrm{E}-14$ \\
\hline Ag-100m & $\mathrm{Ag}-100$ & 99.916 & Silver & $1.00 \mathrm{E}-03$ & ICRP-107 & $2.24 \mathrm{E}+00$ & $\mathrm{~m}$ & $1.34 \mathrm{E}+02$ & $8.40 E+08$ & -- & -- & -- & DOE-STD-1196 & $1.33 \mathrm{E}-13$ \\
\hline Ag-101 & Ag-101 & 100.913 & Silver & $1.00 \mathrm{E}-03$ & ICRP-107 & $1.11 \mathrm{E}+01$ & $\mathrm{~m}$ & $6.66 \mathrm{E}+02$ & $1.68 \mathrm{E}+08$ & DOE-STD-1196 & 1.65E-11 & $\mathrm{s}$ & DOE-STD-1196 & 7.19E-14 \\
\hline Ag-102 & Ag-102 & 101.912 & Silver & $1.00 \mathrm{E}-03$ & ICRP-38 & $1.29 \mathrm{E}+01$ & $\mathrm{~m}$ & $7.74 \mathrm{E}+02$ & $1.43 E+08$ & ICRP-72 & $1.80 \mathrm{E}-11$ & $\mathrm{~s}$ & $\begin{array}{l}\text { FGR-12 } \\
\end{array}$ & $1.67 \mathrm{E}-13$ \\
\hline Ag-102m & $\mathrm{Ag}-102$ & 101.912 & Silver & $1.00 \mathrm{E}-03$ & ICRP-107 & $7.70 \mathrm{E}+00$ & $\mathrm{~m}$ & $4.62 \mathrm{E}+02$ & $2.40 \mathrm{E}+08$ & -- & -- & -- & DOE-STD-1196 & $9.72 \mathrm{E}-14$ \\
\hline Ag-103 & Ag-103 & 102.909 & Silver & $1.00 \mathrm{E}-03$ & $\begin{array}{l}\text { ICRP-38 } \\
\end{array}$ & $6.57 \mathrm{E}+01$ & $\mathrm{~m}$ & $3.94 \mathrm{E}+03$ & $2.78 \mathrm{E}+07$ & ICRP-72 & $2.70 \mathrm{E}-11$ & $\mathrm{~s}$ & FGR-12 & $3.68 \mathrm{E}-14$ \\
\hline Ag-104 & $\mathrm{Ag}-104$ & 103.909 & Silver & $1.00 \mathrm{E}-03$ & ICRP-38 & $6.92 \mathrm{E}+01$ & $\mathrm{~m}$ & $4.15 \mathrm{E}+03$ & $2.61 \mathrm{E}+07$ & ICRP-72 & $3.70 \mathrm{E}-11$ & $\mathrm{~s}$ & FGR-12 & $1.32 \mathrm{E}-13$ \\
\hline Ag-104m & $\mathrm{Ag}-104$ & 103.909 & Silver & $1.00 \mathrm{E}-03$ & ICRP-38 & $3.35 \mathrm{E}+01$ & $\mathrm{~m}$ & $2.01 \mathrm{E}+03$ & $5.40 \mathrm{E}+07$ & ICRP-72 & $2.60 \mathrm{E}-11$ & $\mathrm{~s}$ & FGR-12 & $5.82 \mathrm{E}-14$ \\
\hline Ag-105 & Ag-105 & 104.907 & Silver & $1.00 \mathrm{E}-03$ & ICRP-38 & $4.10 \mathrm{E}+01$ & $d$ & $3.54 \mathrm{E}+06$ & $3.04 E+04$ & ICRP-72 & $8.10 \mathrm{E}-10$ & $\mathrm{~s}$ & FGR-12 & $2.45 \mathrm{E}-14$ \\
\hline Ag-105m & $\mathrm{Ag}-105$ & 104.907 & Silver & $1.00 \mathrm{E}-03$ & ICRP-107 & $7.23 \mathrm{E}+00$ & $\mathrm{~m}$ & $4.34 \mathrm{E}+02$ & $2.48 \mathrm{E}+08$ & -- & -- & -- & DOE-STD-1196 & $4.42 \mathrm{E}-17$ \\
\hline Ag-106 & Ag-106 & 105.907 & Silver & $1.00 \mathrm{E}-03$ & ICRP-38 & $2.40 \mathrm{E}+01$ & $\mathrm{~m}$ & $1.44 \mathrm{E}+03$ & $7.41 \mathrm{E}+07$ & ICRP-72 & $1.60 \mathrm{E}-11$ & $\mathrm{~s}$ & FGR-12 & $3.39 \mathrm{E}-14$ \\
\hline Ag-106m & Ag-106 & 105.907 & Silver & $1.00 \mathrm{E}-03$ & ICRP-38 & $8.41 \mathrm{E}+00$ & $d$ & $7.27 E+05$ & $1.47 E+05$ & ICRP-72 & $1.10 \mathrm{E}-09$ & $\mathrm{~F}$ & FGR-12 & $1.38 \mathrm{E}-13$ \\
\hline Ag-108 & Ag-108 & 107.906 & Silver & $1.00 \mathrm{E}-03$ & ICRP-38 & $2.37 \mathrm{E}+00$ & $\mathrm{~m}$ & $1.42 \mathrm{E}+02$ & $7.35 E+08$ & -- & -- & -- & FGR-12 & $9.28 \mathrm{E}-16$ \\
\hline Ag-108m & Ag-108 & 107.906 & Silver & $1.00 \mathrm{E}-03$ & ICRP-38 & $1.27 \mathrm{E}+02$ & $y$ & $4.01 E+09$ & $2.61 \mathrm{E}+01$ & ICRP-72 & $3.70 \mathrm{E}-08$ & $\mathrm{~s}$ & FGR-12 & $7.80 \mathrm{E}-14$ \\
\hline Ag-109m & Ag-109 & 108.905 & Silver & $1.00 \mathrm{E}-03$ & ICRP-38 & $3.96 \mathrm{E}+01$ & $\mathrm{~s}$ & $3.96 \mathrm{E}+01$ & $2.62 E+09$ & -- & -- & -- & FGR-12 & $1.92 \mathrm{E}-16$ \\
\hline Ag-110 & $\mathrm{Ag}-110$ & 109.906 & Silver & $1.00 \mathrm{E}-03$ & ICRP-38 & $2.46 \mathrm{E}+01$ & $\mathrm{~s}$ & $2.46 \mathrm{E}+01$ & $4.17 E+09$ & -- & -- & -- & FGR-12 & $1.78 \mathrm{E}-15$ \\
\hline Ag-110m & $\mathrm{Ag}-110$ & 109.906 & Silver & $1.00 \mathrm{E}-03$ & ICRP-38 & $2.50 \mathrm{E}+02$ & $d$ & $2.16 \mathrm{E}+07$ & $4.75 \mathrm{E}+03$ & ICRP-72 & $1.20 \mathrm{E}-08$ & $\mathrm{~s}$ & FGR-12 & $1.36 \mathrm{E}-13$ \\
\hline Ag-111 & Ag-111 & 110.905 & Silver & $1.00 \mathrm{E}-03$ & ICRP-38 & $7.45 \mathrm{E}+00$ & $d$ & $6.44 E+05$ & $1.58 \mathrm{E}+05$ & ICRP-72 & $1.70 \mathrm{E}-09$ & $\mathrm{~s}$ & FGR-12 & $1.29 \mathrm{E}-15$ \\
\hline Ag-111m & Ag-111 & 110.905 & Silver & $1.00 \mathrm{E}-03$ & ICRP-107 & $6.48 \mathrm{E}+01$ & $\mathrm{~s}$ & $6.48 \mathrm{E}+01$ & $1.57 E+09$ & -- & -- & -- & DOE-STD-1196 & $1.68 \mathrm{E}-16$ \\
\hline Ag-112 & $\mathrm{Ag}-112$ & 111.907 & Silver & $1.00 \mathrm{E}-03$ & ICRP-38 & $3.12 \mathrm{E}+00$ & $\mathrm{~h}$ & $1.12 \mathrm{E}+04$ & $8.98 \mathrm{E}+06$ & ICRP-72 & $1.70 \mathrm{E}-10$ & $\mathrm{~s}$ & FGR-12 & $3.34 \mathrm{E}-14$ \\
\hline Ag-113 & Ag-113 & 112.907 & Silver & $1.00 \mathrm{E}-03$ & ICRP-107 & $5.37 \mathrm{E}+00$ & $\mathrm{~h}$ & $1.93 \mathrm{E}+04$ & $5.17 E+06$ & DOE-STD-1196 & $1.97 \mathrm{E}-10$ & $\mathrm{~s}$ & DOE-STD-1196 & $3.83 \mathrm{E}-15$ \\
\hline Ag-113m & Ag-113 & 112.907 & Silver & $1.00 \mathrm{E}-03$ & ICRP-107 & $6.87 \mathrm{E}+01$ & $\mathrm{~s}$ & $6.87 \mathrm{E}+01$ & $1.45 E+09$ & -- & -- & -- & DOE-STD-1196 & $9.55 \mathrm{E}-15$ \\
\hline Ag-114 & $\mathrm{Ag}-114$ & 113.909 & Silver & $1.00 \mathrm{E}-03$ & ICRP-107 & $4.60 \mathrm{E}+00$ & $\mathrm{~s}$ & $4.60 \mathrm{E}+00$ & $2.15 \mathrm{E}+10$ & -- & -- & -- & DOE-STD-1196 & 1.46E-14 \\
\hline Ag-115 & Ag-115 & 114.909 & Silver & $1.00 \mathrm{E}-03$ & ICRP-38 & $2.00 \mathrm{E}+01$ & $\mathrm{~m}$ & $1.20 \mathrm{E}+03$ & $8.18 \mathrm{E}+07$ & ICRP-72 & $2.90 \mathrm{E}-11$ & $\mathrm{~s}$ & FGR-12 & $3.61 \mathrm{E}-14$ \\
\hline Ag-116 & $\mathrm{Ag}-116$ & 115.911 & Silver & $1.00 \mathrm{E}-03$ & ICRP-107 & $2.68 \mathrm{E}+00$ & $\mathrm{~m}$ & $1.61 \mathrm{E}+02$ & $6.05 E+08$ & -- & -- & -- & DOE-STD-1196 & $1.07 E-13$ \\
\hline Ag-117 & Ag-117 & 116.912 & Silver & $1.00 \mathrm{E}-03$ & ICRP-107 & $7.36 \mathrm{E}+01$ & $\mathrm{~s}$ & $7.36 \mathrm{E}+01$ & $1.31 \mathrm{E}+09$ & -- & -- & -- & DOE-STD-1196 & $6.50 \mathrm{E}-14$ \\
\hline Ag-99 & Ag-99 & 98.918 & $\begin{array}{l}\text { Silver } \\
\end{array}$ & $1.00 \mathrm{E}-03$ & ICRP-107 & $1.24 \mathrm{E}+02$ & $\mathrm{~s}$ & $1.24 \mathrm{E}+02$ & $9.20 \mathrm{E}+08$ & -- & -- & -- & DOE-STD-1196 & $1.08 \mathrm{E}-13$ \\
\hline Al-26 & Al-26 & 25.987 & Aluminum & $1.00 \mathrm{E}-03$ & ICRP-38 & $7.16 \mathrm{E}+05$ & $y$ & $2.26 \mathrm{E}+13$ & $1.92 \mathrm{E}-02$ & ICRP-72 & $2.00 \mathrm{E}-08$ & $M$ & FGR-12 & $1.36 \mathrm{E}-13$ \\
\hline Al-28 & Al-28 & 27.982 & Aluminum & $1.00 \mathrm{E}-03$ & ICRP-38 & $2.24 \mathrm{E}+00$ & $\mathrm{~m}$ & $1.34 \mathrm{E}+02$ & $3.00 \mathrm{E}+09$ & -- & -- & -- & FGR-12 & $9.28 \mathrm{E}-14$ \\
\hline Al-29 & Al-29 & 28.980 & Aluminum & $1.00 \mathrm{E}-03$ & ICRP-107 & $6.56 \mathrm{E}+00$ & $\mathrm{~m}$ & $3.94 \mathrm{E}+02$ & $9.89 E+08$ & -- & -- & -- & DOE-STD-1196 & $6.71 \mathrm{E}-14$ \\
\hline Am-237 & Am-237 & 237.050 & Americium & $1.00 \mathrm{E}-03$ & ICRP-38 & $7.30 \mathrm{E}+01$ & $\mathrm{~m}$ & $4.38 \mathrm{E}+03$ & $1.09 \mathrm{E}+07$ & ICRP-72 & $2.60 \mathrm{E}-11$ & $\mathrm{~s}$ & FGR-12 & $1.70 \mathrm{E}-14$ \\
\hline Am-238 & Am-238 & 238.052 & Americium & $1.00 \mathrm{E}-03$ & ICRP-38 & $9.80 \mathrm{E}+01$ & $\mathrm{~m}$ & $5.88 \mathrm{E}+03$ & $8.06 \mathrm{E}+06$ & ICRP-72 & $1.90 \mathrm{E}-10$ & $\mathrm{~F}$ & FGR-12 & $4.33 \mathrm{E}-14$ \\
\hline Am-239 & Am-239 & 239.053 & Americium & $1.00 \mathrm{E}-03$ & ICRP-38 & $1.19 \mathrm{E}+01$ & $\mathrm{~h}$ & $4.28 \mathrm{E}+04$ & $1.10 \mathrm{E}+06$ & ICRP-72 & $2.40 \mathrm{E}-10$ & $\mathrm{~s}$ & FGR-12 & $1.04 \mathrm{E}-14$ \\
\hline Am-240 & Am-240 & 240.055 & Americium & $1.00 \mathrm{E}-03$ & ICRP-38 & $5.08 \mathrm{E}+01$ & $\mathrm{~h}$ & $1.83 \mathrm{E}+05$ & $2.57 E+05$ & ICRP-72 & $4.30 \mathrm{E}-10$ & $M$ & FGR-12 & $5.00 \mathrm{E}-14$ \\
\hline Am-241 & Am-241 & 241.057 & Americium & $1.00 \mathrm{E}-03$ & ICRP-38 & $4.32 \mathrm{E}+02$ & $y$ & $1.36 \mathrm{E}+10$ & $3.43 \mathrm{E}+00$ & ICRP-72 & $9.60 \mathrm{E}-05$ & $F$ & FGR-12 & $8.18 \mathrm{E}-16$ \\
\hline
\end{tabular}




\begin{tabular}{|c|c|c|c|c|c|c|c|c|c|c|c|c|c|c|}
\hline \multicolumn{15}{|c|}{ Table A.1 Radionuclide Specific Input Data Used For the Calculation of HC-2 TQs Using Maximum Reported DCs (continued) } \\
\hline $\begin{array}{c}\text { MASTER } \\
\text { ISOTOPE } \\
\text { LIST }\end{array}$ & $\begin{array}{l}\text { Atomic } \\
\text { Mass } \\
\text { Nuclide }\end{array}$ & $\begin{array}{l}\text { Atomic } \\
\text { Mass }\end{array}$ & Element & $\begin{array}{c}\text { RF per } \\
\text { DOE-STD- } \\
1027\end{array}$ & $\begin{array}{c}\text { Half-Life } \\
\text { Reference }\end{array}$ & $\begin{array}{r}\text { Half-Li } \\
\text { (original }\end{array}$ & & Half Life (s) & $\begin{array}{l}\text { Sp. Act } \\
\text { (Ci/g) }\end{array}$ & $\begin{array}{l}\text { Inhalation DC } \\
\text { Reference }\end{array}$ & $\begin{array}{c}\text { HC-2 } \\
\text { Bounding } \\
\text { Inhalation } \\
\text { DC (Sv/Bq) }\end{array}$ & $\begin{array}{l}\text { Lung } \\
\text { Absorption } \\
\text { Class/Type }\end{array}$ & $\begin{array}{l}\text { Immersion DC } \\
\text { Reference }\end{array}$ & $\begin{array}{c}\mathrm{HC}-2 \\
\text { Immersion } \\
\mathrm{DC}(\mathrm{Sv} / \mathrm{s} \\
\left.\text { per } \mathrm{Bq} / \mathrm{m}^{3}\right)\end{array}$ \\
\hline Am-242 & Am-242 & 242.060 & Americium & $1.00 \mathrm{E}-03$ & ICRP-38 & $1.60 \mathrm{E}+01$ & $\mathrm{~h}$ & $5.77 \mathrm{E}+04$ & $8.08 \mathrm{E}+05$ & ICRP-72 & $2.00 \mathrm{E}-08$ & $\mathrm{~s}$ & FGR-12 & $6.15 \mathrm{E}-16$ \\
\hline $\mathrm{Am}-242 \mathrm{~m}$ & Am-242 & 242.060 & Americium & $1.00 \mathrm{E}-03$ & ICRP-38 & $1.52 \mathrm{E}+02$ & $\mathrm{y}$ & $4.80 \mathrm{E}+09$ & $9.72 \mathrm{E}+00$ & ICRP-72 & $9.20 \mathrm{E}-05$ & $\mathrm{~F}$ & FGR-12 & $3.17 \mathrm{E}-17$ \\
\hline $\mathrm{Am}-243$ & Am-243 & 243.061 & Americium & $1.00 \mathrm{E}-03$ & ICRP-38 & $7.38 \mathrm{E}+03$ & $y$ & $2.33 \mathrm{E}+11$ & $1.99 \mathrm{E}-01$ & ICRP-72 & $9.60 \mathrm{E}-05$ & $F$ & FGR-12 & $2.18 \mathrm{E}-15$ \\
\hline Am-244 & Am-244 & 244.064 & Americium & $1.00 \mathrm{E}-03$ & ICRP-38 & $1.01 E+01$ & $\mathrm{~h}$ & $3.64 \mathrm{E}+04$ & $1.27 \mathrm{E}+06$ & ICRP-72 & $3.70 \mathrm{E}-09$ & $\mathrm{~F}$ & FGR-12 & $3.85 \mathrm{E}-14$ \\
\hline Am-244m & Am-244 & 244.064 & Americium & $1.00 \mathrm{E}-03$ & ICRP-38 & $2.60 \mathrm{E}+01$ & $\mathrm{~m}$ & $1.56 \mathrm{E}+03$ & $2.96 \mathrm{E}+07$ & ICRP-72 & $1.60 \mathrm{E}-10$ & $\mathrm{~F}$ & FGR-12 & $6.13 \mathrm{E}-17$ \\
\hline Am-245 & Am-245 & 245.066 & Americium & $1.00 \mathrm{E}-03$ & ICRP-38 & $2.05 E+00$ & $\mathrm{~h}$ & $7.38 \mathrm{E}+03$ & $6.24 \mathrm{E}+06$ & ICRP-72 & $5.60 \mathrm{E}-11$ & $\mathrm{~s}$ & FGR-12 & $1.46 \mathrm{E}-15$ \\
\hline Am-246 & Am-246 & 246.070 & Americium & $1.00 \mathrm{E}-03$ & ICRP-38 & $3.90 \mathrm{E}+01$ & $\mathrm{~m}$ & $2.34 \mathrm{E}+03$ & $1.96 \mathrm{E}+07$ & ICRP-72 & $6.90 \mathrm{E}-11$ & $\mathrm{~s}$ & FGR-12 & $3.28 \mathrm{E}-14$ \\
\hline $\mathrm{Am}-246 \mathrm{~m}$ & Am-246 & 246.070 & Americium & $1.00 \mathrm{E}-03$ & ICRP-38 & $2.50 \mathrm{E}+01$ & $\mathrm{~m}$ & $1.50 \mathrm{E}+03$ & $3.06 \mathrm{E}+07$ & ICRP-72 & $2.30 \mathrm{E}-11$ & $\mathrm{~s}$ & $\begin{array}{ll}\text { FGR-12 } \\
\end{array}$ & $5.03 \mathrm{E}-14$ \\
\hline Am-247 & Am-247 & 247.072 & Americium & $1.00 \mathrm{E}-03$ & ICRP-107 & $2.30 \mathrm{E}+01$ & $\mathrm{~m}$ & $1.38 \mathrm{E}+03$ & $3.31 \mathrm{E}+07$ & DOE-STD-1196 & $3.17 \mathrm{E}-11$ & $\mathrm{~s}$ & DOE-STD-1196 & $5.85 \mathrm{E}-15$ \\
\hline Ar-37 & Ar-37 & 36.967 & Argon & $1.00 \mathrm{E}+00$ & ICRP-38 & $3.50 \mathrm{E}+01$ & $d$ & $3.03 \mathrm{E}+06$ & $1.01 \mathrm{E}+05$ & -- & -- & -- & FGR-12 & $1.27 \mathrm{E}-19$ \\
\hline Ar-39 & Ar-39 & 38.964 & Argon & $1.00 E+00$ & ICRP-38 & $2.69 \mathrm{E}+02$ & $y$ & $8.49 \mathrm{E}+09$ & $3.41 \mathrm{E}+01$ & -- & -- & -- & FGR-12 & $9.10 \mathrm{E}-18$ \\
\hline Ar-41 & Ar-41 & 40.965 & Argon & $1.00 \mathrm{E}+00$ & ICRP-38 & $1.83 \mathrm{E}+00$ & $\mathrm{~h}$ & $6.58 \mathrm{E}+03$ & $4.19 \mathrm{E}+07$ & -- & -- & -- & FGR-12 & $6.50 \mathrm{E}-14$ \\
\hline Ar-42 & Ar-42 & 41.963 & Argon & $1.00 \mathrm{E}+00$ & ICRP-107 & $3.29 \mathrm{E}+01$ & $y$ & $1.04 \mathrm{E}+09$ & $2.59 \mathrm{E}+02$ & -- & -- & -- & DOE-STD-1196 & $1.26 \mathrm{E}-16$ \\
\hline Ar-43 & Ar-43 & 42.966 & Argon & $1.00 E+00$ & ICRP-107 & $5.37 \mathrm{E}+00$ & $\mathrm{~m}$ & $3.22 \mathrm{E}+02$ & $8.15 E+08$ & -- & -- & -- & DOE-STD-1196 & $7.55 \mathrm{E}-14$ \\
\hline Ar-44 & Ar-44 & 43.965 & Argon & $1.00 E+00$ & ICRP-107 & $1.19 \mathrm{E}+01$ & $\mathrm{~m}$ & $7.12 \mathrm{E}+02$ & $3.60 E+08$ & -- & -- & -- & DOE-STD-1196 & $9.46 \mathrm{E}-14$ \\
\hline As-68 & As-68 & 67.937 & Arsenic & $1.00 \mathrm{E}-03$ & ICRP-107 & $1.52 \mathrm{E}+02$ & $\mathrm{~s}$ & $1.52 \mathrm{E}+02$ & $1.10 \mathrm{E}+09$ & -- & -- & -- & DOE-STD-1196 & $1.76 \mathrm{E}-13$ \\
\hline As-69 & As-69 & 68.932 & Arsenic & $1.00 \mathrm{E}-03$ & ICRP-38 & $1.52 \mathrm{E}+01$ & $\mathrm{~m}$ & $9.12 \mathrm{E}+02$ & $1.79 E+08$ & ICRP-72 & $2.10 \mathrm{E}-11$ & $M$ & FGR-12 & $4.89 \mathrm{E}-14$ \\
\hline As-70 & As-70 & 69.931 & Arsenic & $1.00 \mathrm{E}-03$ & ICRP-38 & $5.26 \mathrm{E}+01$ & $\mathrm{~m}$ & $3.16 \mathrm{E}+03$ & $5.11 \mathrm{E}+07$ & ICRP-72 & $6.70 \mathrm{E}-11$ & $M$ & FGR-12 & $2.04 \mathrm{E}-13$ \\
\hline As-71 & As-71 & 70.927 & Arsenic & $1.00 \mathrm{E}-03$ & ICRP-38 & $6.48 \mathrm{E}+01$ & $\mathrm{~h}$ & $2.33 E+05$ & $6.82 \mathrm{E}+05$ & ICRP-72 & $4.00 \mathrm{E}-10$ & $M$ & FGR-12 & $2.74 \mathrm{E}-14$ \\
\hline As-72 & As-72 & 71.927 & Arsenic & $1.00 \mathrm{E}-03$ & ICRP-38 & $2.60 \mathrm{E}+01$ & $\mathrm{~h}$ & $9.36 \mathrm{E}+04$ & $1.68 \mathrm{E}+06$ & ICRP-72 & $9.00 \mathrm{E}-10$ & $M$ & FGR-12 & $8.78 \mathrm{E}-14$ \\
\hline As-73 & As-73 & 72.924 & Arsenic & $1.00 \mathrm{E}-03$ & ICRP-38 & $8.03 \mathrm{E}+01$ & $d$ & $6.94 \mathrm{E}+06$ & $2.23 \mathrm{E}+04$ & ICRP-72 & $1.00 \mathrm{E}-09$ & $M$ & FGR-12 & $1.90 \mathrm{E}-16$ \\
\hline As-74 & As-74 & 73.924 & Arsenic & $1.00 \mathrm{E}-03$ & ICRP-38 & $1.78 \mathrm{E}+01$ & $d$ & $1.53 \mathrm{E}+06$ & $9.95 \mathrm{E}+04$ & ICRP-72 & $2.10 \mathrm{E}-09$ & $M$ & FGR-12 & $3.65 \mathrm{E}-14$ \\
\hline As-76 & As-76 & 75.922 & Arsenic & $1.00 \mathrm{E}-03$ & ICRP-38 & $2.63 \mathrm{E}+01$ & $\mathrm{~h}$ & $9.48 \mathrm{E}+04$ & $1.57 \mathrm{E}+06$ & ICRP-72 & $7.40 \mathrm{E}-10$ & $M$ & FGR-12 & $2.13 \mathrm{E}-14$ \\
\hline As-77 & As-77 & 76.921 & Arsenic & $1.00 \mathrm{E}-03$ & ICRP-38 & $3.88 \mathrm{E}+01$ & $\mathrm{~h}$ & $1.40 \mathrm{E}+05$ & $1.05 E+06$ & ICRP-72 & $3.90 \mathrm{E}-10$ & $M$ & FGR-12 & $4.31 \mathrm{E}-16$ \\
\hline As-78 & As-78 & 77.922 & Arsenic & $1.00 \mathrm{E}-03$ & ICRP-38 & $9.07 \mathrm{E}+01$ & $\mathrm{~m}$ & $5.44 \mathrm{E}+03$ & $2.66 \mathrm{E}+07$ & ICRP-72 & $8.90 \mathrm{E}-11$ & $M$ & FGR-12 & $6.32 \mathrm{E}-14$ \\
\hline As-79 & As-79 & 78.921 & Arsenic & $1.00 \mathrm{E}-03$ & ICRP-107 & $9.01 \mathrm{E}+00$ & $\mathrm{~m}$ & $5.41 \mathrm{E}+02$ & $2.64 \mathrm{E}+08$ & -- & -- & -- & DOE-STD-1196 & $2.25 \mathrm{E}-15$ \\
\hline At-204 & At-204 & 203.987 & Astatine & $1.00 \mathrm{E}-03$ & ICRP-107 & $9.20 \mathrm{E}+00$ & $\mathrm{~m}$ & $5.52 \mathrm{E}+02$ & $1.00 E+08$ & -- & -- & -- & DOE-STD-1196 & $1.04 \mathrm{E}-13$ \\
\hline At-205 & At-205 & 204.986 & Astatine & $1.00 \mathrm{E}-03$ & ICRP-107 & $2.62 \mathrm{E}+01$ & $\mathrm{~m}$ & $1.57 \mathrm{E}+03$ & $3.50 \mathrm{E}+07$ & DOE-STD-1196 & $7.83 \mathrm{E}-10$ & $\mathrm{~s}$ & DOE-STD-1196 & $5.22 \mathrm{E}-14$ \\
\hline At-206 & At-206 & 205.987 & Astatine & $1.00 \mathrm{E}-03$ & ICRP-107 & $3.06 \mathrm{E}+01$ & $\mathrm{~m}$ & $1.84 \mathrm{E}+03$ & $2.98 \mathrm{E}+07$ & DOE-STD-1196 & $2.62 \mathrm{E}-10$ & $\mathrm{~s}$ & DOE-STD-1196 & $1.12 \mathrm{E}-13$ \\
\hline At-207 & At-207 & 206.986 & Astatine & $1.00 \mathrm{E}-03$ & ICRP-38 & $1.80 \mathrm{E}+00$ & $\mathrm{~h}$ & $6.48 \mathrm{E}+03$ & $8.41 \mathrm{E}+06$ & ICRP-72 & $2.30 \mathrm{E}-09$ & $M$ & FGR-12 & $6.52 \mathrm{E}-14$ \\
\hline At-208 & At-208 & 207.987 & Astatine & $1.00 \mathrm{E}-03$ & ICRP-107 & $1.63 \mathrm{E}+00$ & $\mathrm{~h}$ & $5.87 \mathrm{E}+03$ & $9.24 \mathrm{E}+06$ & DOE-STD-1196 & $6.56 \mathrm{E}-10$ & $\mathrm{~s}$ & DOE-STD-1196 & $1.40 \mathrm{E}-13$ \\
\hline At-209 & At-209 & 208.986 & Astatine & $1.00 \mathrm{E}-03$ & ICRP-107 & $5.41 E+00$ & $\mathrm{~h}$ & $1.95 E+04$ & $2.77 E+06$ & DOE-STD-1196 & $3.04 \mathrm{E}-09$ & $\mathrm{~s}$ & DOE-STD-1196 & $1.03 \mathrm{E}-13$ \\
\hline At-210 & At-210 & 209.987 & Astatine & $1.00 \mathrm{E}-03$ & ICRP-107 & $8.10 \mathrm{E}+00$ & $\mathrm{~h}$ & $2.92 \mathrm{E}+04$ & $1.84 \mathrm{E}+06$ & DOE-STD-1196 & $1.17 \mathrm{E}-08$ & $\mathrm{~s}$ & DOE-STD-1196 & $1.40 \mathrm{E}-13$ \\
\hline At-211 & At-211 & 210.987 & Astatine & $1.00 \mathrm{E}-03$ & ICRP-38 & $7.21 E+00$ & $\mathrm{~h}$ & $2.60 \mathrm{E}+04$ & $2.06 \mathrm{E}+06$ & ICRP-72 & $1.10 \mathrm{E}-07$ & $M$ & FGR-12 & $1.59 \mathrm{E}-15$ \\
\hline At-215 & At-215 & 214.999 & Astatine & $1.00 \mathrm{E}-03$ & ICRP-38 & $1.00 \mathrm{E}-01$ & $\mathrm{~ms}$ & $1.00 \mathrm{E}-04$ & $5.25 E+14$ & -- & -- & -- & FGR-12 & $9.22 \mathrm{E}-18$ \\
\hline At-216 & At-216 & 216.002 & Astatine & $1.00 \mathrm{E}-03$ & ICRP-38 & $3.00 \mathrm{E}-01$ & $\mathrm{~ms}$ & $3.00 \mathrm{E}-04$ & $1.74 \mathrm{E}+14$ & -- & -- & -- & FGR-12 & $6.24 \mathrm{E}-17$ \\
\hline At-217 & At-217 & 217.005 & Astatine & $1.00 \mathrm{E}-03$ & ICRP-38 & $3.23 \mathrm{E}-02$ & $\mathrm{~s}$ & $3.23 \mathrm{E}-02$ & $1.61 \mathrm{E}+12$ & -- & -- & -- & FGR-12 & $1.48 \mathrm{E}-17$ \\
\hline At-218 & At-218 & 218.009 & Astatine & $1.00 \mathrm{E}-03$ & ICRP-38 & $2.00 \mathrm{E}+00$ & $\mathrm{~s}$ & $2.00 \mathrm{E}+00$ & $2.59 \mathrm{E}+10$ & -- & -- & -- & FGR-12 & $1.19 \mathrm{E}-16$ \\
\hline At-219 & At-219 & 219.011 & Astatine & $1.00 \mathrm{E}-03$ & ICRP-107 & $5.60 \mathrm{E}+01$ & $\mathrm{~s}$ & $5.60 \mathrm{E}+01$ & $9.20 \mathrm{E}+08$ & -- & -- & -- & -- & -- \\
\hline At-220 & At-220 & 220.015 & Astatine & $1.00 \mathrm{E}-03$ & ICRP-107 & $3.71 E+00$ & $\mathrm{~m}$ & $2.23 \mathrm{E}+02$ & $2.30 \mathrm{E}+08$ & -- & -- & -- & DOE-STD-1196 & $2.09 \mathrm{E}-14$ \\
\hline $\mathrm{Au}-186$ & $\mathrm{Au}-186$ & 185.966 & Gold & $1.00 \mathrm{E}-03$ & ICRP-107 & $1.07 \mathrm{E}+01$ & $\mathrm{~m}$ & $6.42 \mathrm{E}+02$ & $9.45 \mathrm{E}+07$ & DOE-STD-1196 & $2.43 \mathrm{E}-11$ & $\mathrm{~s}$ & DOE-STD-1196 & $6.87 \mathrm{E}-14$ \\
\hline Au-187 & Au-187 & 186.965 & Gold & $1.00 \mathrm{E}-03$ & ICRP-107 & $8.40 E+00$ & $\mathrm{~m}$ & $5.04 \mathrm{E}+02$ & $1.20 \mathrm{E}+08$ & -- & -- & -- & DOE-STD-1196 & $4.96 \mathrm{E}-14$ \\
\hline Au-188 & Au-188 & 187.965 & Gold & $1.00 \mathrm{E}-03$ & JAERI & $8.84 \mathrm{E}+00$ & $\mathrm{~m}$ & $5.30 \mathrm{E}+02$ & $1.13 \mathrm{E}+08$ & -- & -- & -- & -- & -- \\
\hline Au-189m & Au-189 & 188.964 & Gold & $1.00 \mathrm{E}-03$ & JAERI & $4.59 \mathrm{E}+00$ & $\mathrm{~m}$ & $2.75 \mathrm{E}+02$ & $2.17 \mathrm{E}+08$ & -- & -- & -- & -- & -- \\
\hline Au-190 & Au-190 & 189.965 & Gold & $1.00 \mathrm{E}-03$ & ICRP-107 & $4.28 \mathrm{E}+01$ & $\mathrm{~m}$ & $2.57 \mathrm{E}+03$ & $2.31 \mathrm{E}+07$ & DOE-STD-1196 & $2.90 \mathrm{E}-11$ & $\mathrm{~s}$ & DOE-STD-1196 & $1.16 \mathrm{E}-13$ \\
\hline $\mathrm{Au}-191$ & Au-191 & 190.964 & Gold & $1.00 \mathrm{E}-03$ & ICRP-107 & $3.18 \mathrm{E}+00$ & $\mathrm{~h}$ & $1.14 \mathrm{E}+04$ & $5.16 \mathrm{E}+06$ & DOE-STD-1196 & $7.56 \mathrm{E}-11$ & $\mathrm{~s}$ & DOE-STD-1196 & $2.55 \mathrm{E}-14$ \\
\hline
\end{tabular}




\begin{tabular}{|c|c|c|c|c|c|c|c|c|c|c|c|c|c|c|}
\hline \multicolumn{15}{|c|}{ Table A.1 Radionuclide Specific Input Data Used For the Calculation of HC-2 TQs Using Maximum Reported DCs (continued) } \\
\hline $\begin{array}{c}\text { MASTER } \\
\text { ISOTOPE } \\
\text { LIST } \\
\end{array}$ & $\begin{array}{l}\text { Atomic } \\
\text { Mass } \\
\text { Nuclide }\end{array}$ & $\begin{array}{l}\text { Atomic } \\
\text { Mass }\end{array}$ & Element & $\begin{array}{c}\text { RF per } \\
\text { DOE-STD- } \\
1027\end{array}$ & $\begin{array}{l}\text { Half-Life } \\
\text { Reference }\end{array}$ & $\begin{array}{r}\text { Half-Li } \\
\text { (original }\end{array}$ & & Half Life (s) & $\begin{array}{l}\text { Sp. Act } \\
\text { (Ci/g) }\end{array}$ & $\begin{array}{c}\text { Inhalation DC } \\
\text { Reference }\end{array}$ & $\begin{array}{c}\text { HC-2 } \\
\text { Bounding } \\
\text { Inhalation } \\
\text { DC (Sv/Bq) }\end{array}$ & $\begin{array}{l}\text { Lung } \\
\text { Absorption } \\
\text { Class/Type }\end{array}$ & $\begin{array}{c}\text { Immersion DC } \\
\text { Reference }\end{array}$ & $\begin{array}{c}\text { HC-2 } \\
\text { Immersion } \\
\mathrm{DC}(\mathrm{Sv} / \mathrm{s} \\
\left.\text { per } \mathrm{Bq} / \mathrm{m}^{3}\right)\end{array}$ \\
\hline Au-192 & Au-192 & 191.965 & Gold & $1.00 \mathrm{E}-03$ & ICRP-107 & $4.94 \mathrm{E}+00$ & $\mathrm{~h}$ & $1.78 \mathrm{E}+04$ & $3.30 \mathrm{E}+06$ & DOE-STD-1196 & $1.15 \mathrm{E}-10$ & $\mathrm{~s}$ & DOE-STD-1196 & $9.29 \mathrm{E}-14$ \\
\hline Au-193 & Au-193 & 192.964 & Gold & $1.00 \mathrm{E}-03$ & ICRP-38 & $1.77 \mathrm{E}+01$ & $\mathrm{~h}$ & $6.35 \mathrm{E}+04$ & $9.20 \mathrm{E}+05$ & ICRP-72 & $1.20 \mathrm{E}-10$ & $\mathrm{~s}$ & FGR-12 & $6.83 \mathrm{E}-15$ \\
\hline Au-193m & Au-193 & 192.964 & Gold & $1.00 \mathrm{E}-03$ & ICRP-107 & $3.90 \mathrm{E}+00$ & $\mathrm{~s}$ & $3.90 \mathrm{E}+00$ & $1.50 \mathrm{E}+10$ & -- & -- & -- & DOE-STD-1196 & $8.37 \mathrm{E}-15$ \\
\hline Au-194 & Au-194 & 193.965 & Gold & $1.00 \mathrm{E}-03$ & ICRP-38 & $3.95 \mathrm{E}+01$ & $\mathrm{~h}$ & $1.42 \mathrm{E}+05$ & $4.09 \mathrm{E}+05$ & ICRP-72 & $2.40 \mathrm{E}-10$ & $\mathrm{~s}$ & FGR-12 & $5.29 \mathrm{E}-14$ \\
\hline Au-195 & Au-195 & 194.965 & Gold & $1.00 \mathrm{E}-03$ & ICRP-38 & $1.83 \mathrm{E}+02$ & $d$ & $1.58 \mathrm{E}+07$ & $3.66 \mathrm{E}+03$ & ICRP-72 & $1.70 \mathrm{E}-09$ & $\mathrm{~s}$ & FGR-12 & $3.21 \mathrm{E}-15$ \\
\hline Au-195m & Au-195 & 194.965 & Gold & $1.00 \mathrm{E}-03$ & ICRP-38 & $3.05 E+01$ & $\mathrm{~s}$ & $3.05 E+01$ & $1.90 E+09$ & -- & -- & -- & FGR-12 & $9.37 \mathrm{E}-15$ \\
\hline Au-196 & Au-196 & 195.967 & Gold & $1.00 \mathrm{E}-03$ & ICRP-107 & $6.18 \mathrm{E}+00$ & $d$ & $5.34 \mathrm{E}+05$ & $1.08 \mathrm{E}+05$ & DOE-STD-1196 & $3.78 \mathrm{E}-10$ & $M$ & DOE-STD-1196 & $2.02 \mathrm{E}-14$ \\
\hline Au-196m & Au-196 & 195.967 & Gold & $1.00 \mathrm{E}-03$ & ICRP-107 & $9.60 \mathrm{E}+00$ & $\mathrm{~h}$ & $3.46 \mathrm{E}+04$ & $1.67 \mathrm{E}+06$ & DOE-STD-1196 & $5.00 \mathrm{E}-10$ & $\mathrm{~s}$ & DOE-STD-1196 & $9.70 \mathrm{E}-15$ \\
\hline Au-198 & $\mathrm{Au}-198$ & 197.968 & Gold & $1.00 \mathrm{E}-03$ & ICRP-38 & $2.70 E+00$ & $d$ & $2.33 \mathrm{E}+05$ & $2.45 E+05$ & ICRP-72 & $8.60 \mathrm{E}-10$ & $\mathrm{~S}$ & FGR-12 & $1.94 \mathrm{E}-14$ \\
\hline Au-198m & Au-198 & 197.968 & Gold & $1.00 \mathrm{E}-03$ & ICRP-38 & $2.30 \mathrm{E}+00$ & $d$ & $1.99 \mathrm{E}+05$ & $2.87 \mathrm{E}+05$ & ICRP-72 & $2.00 \mathrm{E}-09$ & $\mathrm{~s}$ & FGR-12 & $2.66 \mathrm{E}-14$ \\
\hline Au-199 & Au-199 & 198.969 & Gold & $1.00 \mathrm{E}-03$ & ICRP-38 & $3.14 \mathrm{E}+00$ & $d$ & $2.71 \mathrm{E}+05$ & $2.09 \mathrm{E}+05$ & ICRP-72 & $7.90 \mathrm{E}-10$ & $\mathrm{~s}$ & FGR-12 & $4.08 \mathrm{E}-15$ \\
\hline Au-200 & $\mathrm{Au}-200$ & 199.971 & Gold & $1.00 \mathrm{E}-03$ & ICRP-38 & $4.84 \mathrm{E}+01$ & $\mathrm{~m}$ & $2.90 \mathrm{E}+03$ & $1.94 \mathrm{E}+07$ & ICRP-72 & $3.50 \mathrm{E}-11$ & $\mathrm{~s}$ & FGR-12 & $1.37 \mathrm{E}-14$ \\
\hline Au-200m & Au-200 & 199.971 & Gold & $1.00 \mathrm{E}-03$ & ICRP-38 & $1.87 \mathrm{E}+01$ & $\mathrm{~h}$ & $6.73 \mathrm{E}+04$ & $8.38 \mathrm{E}+05$ & ICRP-72 & $7.20 \mathrm{E}-10$ & $\mathrm{~s}$ & FGR-12 & $1.01 \mathrm{E}-13$ \\
\hline Au-201 & Au-201 & 200.972 & Gold & $1.00 \mathrm{E}-03$ & ICRP-38 & $2.64 \mathrm{E}+01$ & $\mathrm{~m}$ & $1.58 \mathrm{E}+03$ & $3.54 \mathrm{E}+07$ & ICRP-72 & $1.70 \mathrm{E}-11$ & $M$ & FGR-12 & $2.57 \mathrm{E}-15$ \\
\hline $\mathrm{Au}-202$ & $\mathrm{Au}-202$ & 201.974 & Gold & $1.00 \mathrm{E}-03$ & ICRP-107 & $2.88 \mathrm{E}+01$ & $s$ & $2.88 \mathrm{E}+01$ & $1.94 \mathrm{E}+09$ & -- & -- & -- & DOE-STD-1196 & $8.90 \mathrm{E}-15$ \\
\hline Ba-124 & Ba-124 & 123.915 & Barium & $1.00 \mathrm{E}-03$ & ICRP-107 & $1.10 \mathrm{E}+01$ & $\mathrm{~m}$ & $6.60 \mathrm{E}+02$ & $1.38 \mathrm{E}+08$ & DOE-STD-1196 & $2.71 \mathrm{E}-11$ & $\mathrm{~s}$ & DOE-STD-1196 & $2.51 \mathrm{E}-14$ \\
\hline Ba-126 & Ba-126 & 125.911 & Barium & $1.00 \mathrm{E}-03$ & ICRP-38 & $9.65 E+01$ & $\mathrm{~m}$ & $5.79 \mathrm{E}+03$ & $1.55 \mathrm{E}+07$ & ICRP-72 & $1.10 \mathrm{E}-10$ & $\mathrm{~s}$ & FGR-12 & $7.03 \mathrm{E}-15$ \\
\hline Ba-127 & Ba-127 & 126.911 & Barium & $1.00 \mathrm{E}-03$ & ICRP-107 & $1.27 \mathrm{E}+01$ & $\mathrm{~m}$ & $7.62 E+02$ & $1.17 \mathrm{E}+08$ & DOE-STD-1196 & $1.34 \mathrm{E}-11$ & $\mathrm{~s}$ & DOE-STD-1196 & $3.28 \mathrm{E}-14$ \\
\hline Ba-128 & Ba-128 & 127.908 & Barium & $1.00 \mathrm{E}-03$ & ICRP-38 & $2.43 \mathrm{E}+00$ & $d$ & $2.10 \mathrm{E}+05$ & $4.20 \mathrm{E}+05$ & ICRP-72 & $1.40 \mathrm{E}-09$ & $\mathrm{~s}$ & FGR-12 & $2.86 \mathrm{E}-15$ \\
\hline Ba-129 & Ba-129 & 128.909 & Barium & $1.00 \mathrm{E}-03$ & ICRP-107 & $2.23 \mathrm{E}+00$ & $\mathrm{~h}$ & $8.03 \mathrm{E}+03$ & $1.09 \mathrm{E}+07$ & DOE-STD-1196 & $3.44 \mathrm{E}-11$ & $\mathrm{~s}$ & DOE-STD-1196 & $1.44 \mathrm{E}-14$ \\
\hline Ba-129m & Ba-129 & 128.909 & Barium & $1.00 \mathrm{E}-03$ & ICRP-107 & $2.16 \mathrm{E}+00$ & $\mathrm{~h}$ & $7.78 \mathrm{E}+03$ & $1.13 \mathrm{E}+07$ & DOE-STD-1196 & $5.57 \mathrm{E}-11$ & $\mathrm{~s}$ & DOE-STD-1196 & $7.20 \mathrm{E}-14$ \\
\hline Ba-131 & Ba-131 & 130.907 & Barium & $1.00 \mathrm{E}-03$ & ICRP-38 & $1.18 \mathrm{E}+01$ & $d$ & $1.02 \mathrm{E}+06$ & $8.45 E+04$ & ICRP-72 & $8.70 \mathrm{E}-10$ & $\mathrm{~s}$ & FGR-12 & $2.10 \mathrm{E}-14$ \\
\hline Ba-131m & Ba-131 & 130.907 & Barium & $1.00 \mathrm{E}-03$ & ICRP-38 & $1.46 \mathrm{E}+01$ & $\mathrm{~m}$ & $8.76 \mathrm{E}+02$ & $9.84 \mathrm{E}+07$ & ICRP-72 & $7.80 \mathrm{E}-12$ & $\mathrm{~s}$ & FGR-12 & $3.04 \mathrm{E}-15$ \\
\hline Ba-133 & Ba-133 & 132.906 & Barium & $1.00 \mathrm{E}-03$ & ICRP-38 & $1.07 \mathrm{E}+01$ & $y$ & $3.39 \mathrm{E}+08$ & $2.50 \mathrm{E}+02$ & ICRP-72 & $1.00 \mathrm{E}-08$ & $\mathrm{~s}$ & FGR-12 & $1.78 \mathrm{E}-14$ \\
\hline Ba-133m & Ba-133 & 132.906 & Barium & $1.00 \mathrm{E}-03$ & ICRP-38 & $3.89 \mathrm{E}+01$ & $\mathrm{~h}$ & $1.40 \mathrm{E}+05$ & $6.06 \mathrm{E}+05$ & ICRP-72 & $4.60 \mathrm{E}-10$ & $\mathrm{~s}$ & FGR-12 & $2.62 \mathrm{E}-15$ \\
\hline Ba-135m & Ba-135 & 134.906 & Barium & $1.00 \mathrm{E}-03$ & ICRP-38 & $2.87 \mathrm{E}+01$ & $\mathrm{~h}$ & $1.03 \mathrm{E}+05$ & $8.09 \mathrm{E}+05$ & ICRP-72 & $3.60 \mathrm{E}-10$ & $\mathrm{~s}$ & FGR-12 & $2.32 \mathrm{E}-15$ \\
\hline Ba-137m & Ba-137 & 136.906 & Barium & $1.00 \mathrm{E}-03$ & ICRP-38 & $2.55 \mathrm{E}+00$ & $\mathrm{~m}$ & $1.53 \mathrm{E}+02$ & $5.38 \mathrm{E}+08$ & -- & -- & -- & FGR-12 & $2.88 \mathrm{E}-14$ \\
\hline Ba-139 & Ba-139 & 138.909 & Barium & $1.00 \mathrm{E}-03$ & ICRP-38 & $8.27 \mathrm{E}+01$ & $\mathrm{~m}$ & $4.96 \mathrm{E}+03$ & $1.64 \mathrm{E}+07$ & ICRP-72 & $5.90 \mathrm{E}-11$ & $\mathrm{~s}$ & FGR-12 & $2.17 \mathrm{E}-15$ \\
\hline Ba-140 & Ba-140 & 139.911 & Barium & $1.00 \mathrm{E}-03$ & ICRP-38 & $1.27 \mathrm{E}+01$ & $d$ & $1.10 \mathrm{E}+06$ & $7.33 \mathrm{E}+04$ & ICRP-72 & $5.80 \mathrm{E}-09$ & $\mathrm{~s}$ & FGR-12 & $8.58 \mathrm{E}-15$ \\
\hline Ba-141 & Ba-141 & 140.914 & Barium & $1.00 \mathrm{E}-03$ & ICRP-38 & $1.83 \mathrm{E}+01$ & $\mathrm{~m}$ & $1.10 \mathrm{E}+03$ & $7.30 \mathrm{E}+07$ & ICRP-72 & $3.40 \mathrm{E}-11$ & $\mathrm{~s}$ & FGR-12 & $4.16 \mathrm{E}-14$ \\
\hline Ba-142 & Ba-142 & 141.916 & Barium & $1.00 \mathrm{E}-03$ & ICRP-38 & $1.06 \mathrm{E}+01$ & $\mathrm{~m}$ & $6.36 \mathrm{E}+02$ & $1.25 \mathrm{E}+08$ & ICRP-72 & $2.20 \mathrm{E}-11$ & $\mathrm{~s}$ & FGR-12 & $5.15 \mathrm{E}-14$ \\
\hline Be-10 & Be-10 & 10.014 & Beryllium & $1.00 \mathrm{E}-03$ & ICRP-38 & $1.60 E+06$ & $y$ & $5.05 \mathrm{E}+13$ & $2.23 \mathrm{E}-02$ & ICRP-72 & $3.50 \mathrm{E}-08$ & $\mathrm{~s}$ & FGR-12 & $1.12 \mathrm{E}-17$ \\
\hline Be-7 & Be-7 & 7.017 & Beryllium & $1.00 \mathrm{E}-03$ & ICRP-38 & $5.33 \mathrm{E}+01$ & d & $4.61 \mathrm{E}+06$ & $3.49 \mathrm{E}+05$ & ICRP-72 & $5.50 \mathrm{E}-11$ & $\mathrm{~s}$ & FGR-12 & $2.36 \mathrm{E}-15$ \\
\hline Bi-197 & Bi-197 & 196.979 & Bismuth & $1.00 \mathrm{E}-03$ & ICRP-107 & $9.30 \mathrm{E}+00$ & $\mathrm{~m}$ & $5.58 \mathrm{E}+02$ & $1.03 E+08$ & -- & -- & -- & DOE-STD-1196 & $7.85 \mathrm{E}-14$ \\
\hline Bi-200 & Bi-200 & 199.978 & Bismuth & $1.00 \mathrm{E}-03$ & ICRP-38 & $3.64 \mathrm{E}+01$ & $\mathrm{~m}$ & $2.18 \mathrm{E}+03$ & $2.58 \mathrm{E}+07$ & ICRP-72 & $3.30 \mathrm{E}-11$ & $M$ & FGR-12 & $1.16 \mathrm{E}-13$ \\
\hline Bi-201 & Bi-201 & 200.977 & Bismuth & $1.00 \mathrm{E}-03$ & ICRP-38 & $1.08 \mathrm{E}+02$ & $\mathrm{~m}$ & $6.48 \mathrm{E}+03$ & $8.66 \mathrm{E}+06$ & ICRP-72 & $6.60 \mathrm{E}-11$ & $M$ & FGR-12 & $6.51 \mathrm{E}-14$ \\
\hline Bi-202 & Bi-202 & 201.978 & Bismuth & $1.00 \mathrm{E}-03$ & ICRP-38 & $1.67 \mathrm{E}+00$ & $\mathrm{~h}$ & $6.01 \mathrm{E}+03$ & $9.29 \mathrm{E}+06$ & ICRP-72 & $5.50 \mathrm{E}-11$ & $M$ & FGR-12 & $1.33 \mathrm{E}-13$ \\
\hline Bi-203 & Bi-203 & 202.977 & Bismuth & $1.00 \mathrm{E}-03$ & ICRP-38 & $1.18 \mathrm{E}+01$ & $\mathrm{~h}$ & $4.23 \mathrm{E}+04$ & $1.31 \mathrm{E}+06$ & ICRP-72 & $2.60 \mathrm{E}-10$ & $M$ & FGR-12 & $1.20 \mathrm{E}-13$ \\
\hline Bi-204 & Bi-204 & 203.978 & Bismuth & $1.00 \mathrm{E}-03$ & ICRP-38 & $1.12 \mathrm{E}+01$ & $\mathrm{~h}$ & $4.04 \mathrm{E}+04$ & $1.37 \mathrm{E}+06$ & DOE-STD-1196 & $3.81 \mathrm{E}-10$ & $\mathrm{~s}$ & DOE-STD-1196 & $1.35 \mathrm{E}-13$ \\
\hline Bi-205 & Bi-205 & 204.977 & Bismuth & $1.00 \mathrm{E}-03$ & ICRP-38 & $1.53 \mathrm{E}+01$ & $d$ & $1.32 \mathrm{E}+06$ & $4.16 \mathrm{E}+04$ & ICRP-72 & $9.30 \mathrm{E}-10$ & $M$ & FGR-12 & $8.49 \mathrm{E}-14$ \\
\hline Bi-206 & Bi-206 & 205.978 & Bismuth & $1.00 \mathrm{E}-03$ & ICRP-38 & $6.24 \mathrm{E}+00$ & $d$ & $5.39 \mathrm{E}+05$ & $1.02 \mathrm{E}+05$ & ICRP-72 & $1.70 \mathrm{E}-09$ & $M$ & FGR-12 & $1.61 \mathrm{E}-13$ \\
\hline Bi-207 & Bi-207 & 206.978 & Bismuth & $1.00 \mathrm{E}-03$ & ICRP-38 & $3.80 \mathrm{E}+01$ & $y$ & $1.20 \mathrm{E}+09$ & $4.55 \mathrm{E}+01$ & ICRP-72 & $5.60 \mathrm{E}-09$ & $M$ & FGR-12 & $7.54 \mathrm{E}-14$ \\
\hline Bi-208 & Bi-208 & 207.980 & Bismuth & $1.00 \mathrm{E}-03$ & ICRP-107 & $3.68 \mathrm{E}+05$ & $y$ & $1.16 \mathrm{E}+13$ & $4.67 \mathrm{E}-03$ & DOE-STD-1196 & $3.83 \mathrm{E}-08$ & $\mathrm{~s}$ & DOE-STD-1196 & $1.35 \mathrm{E}-13$ \\
\hline Bi-210 & Bi-210 & 209.984 & Bismuth & $1.00 \mathrm{E}-03$ & ICRP-38 & $5.01 \mathrm{E}+00$ & $d$ & $4.33 \mathrm{E}+05$ & $1.24 \mathrm{E}+05$ & ICRP-72 & $9.30 \mathrm{E}-08$ & $M$ & FGR-12 & $3.29 \mathrm{E}-17$ \\
\hline $\mathrm{Bi}-210 \mathrm{~m}$ & Bi-210 & 209.984 & Bismuth & $1.00 \mathrm{E}-03$ & ICRP-38 & $3.00 \mathrm{E}+06$ & $y$ & $9.47 \mathrm{E}+13$ & $5.67 \mathrm{E}-04$ & ICRP-72 & $3.40 \mathrm{E}-06$ & $M$ & FGR-12 & $1.22 \mathrm{E}-14$ \\
\hline Bi-211 & Bi-211 & 210.987 & Bismuth & $1.00 \mathrm{E}-03$ & ICRP-38 & $2.14 \mathrm{E}+00$ & $\mathrm{~m}$ & $1.28 \mathrm{E}+02$ & $4.16 \mathrm{E}+08$ & -- & -- & -- & FGR-12 & $2.22 \mathrm{E}-15$ \\
\hline
\end{tabular}




\begin{tabular}{|c|c|c|c|c|c|c|c|c|c|c|c|c|c|c|}
\hline \multicolumn{15}{|c|}{ Table A.1 Radionuclide Specific Input Data Used For the Calculation of HC-2 TQs Using Maximum Reported DCs (continued) } \\
\hline $\begin{array}{c}\text { MASTER } \\
\text { ISOTOPE } \\
\text { LIST } \\
\end{array}$ & $\begin{array}{l}\text { Atomic } \\
\text { Mass } \\
\text { Nuclide }\end{array}$ & $\begin{array}{l}\text { Atomic } \\
\text { Mass }\end{array}$ & Element & $\begin{array}{c}\text { RF per } \\
\text { DOE-STD- } \\
1027 \\
\end{array}$ & $\begin{array}{c}\text { Half-Life } \\
\text { Reference }\end{array}$ & $\begin{array}{r}\text { Half-L } \\
\text { (original }\end{array}$ & & Half Life (s) & $\begin{array}{l}\text { Sp. Act } \\
\text { (Ci/g) }\end{array}$ & $\begin{array}{l}\text { Inhalation DC } \\
\text { Reference }\end{array}$ & $\begin{array}{c}\text { HC-2 } \\
\text { Bounding } \\
\text { Inhalation } \\
\text { DC (Sv/Bq) }\end{array}$ & $\begin{array}{c}\text { Lung } \\
\text { Absorption } \\
\text { Class/Type } \\
\end{array}$ & $\begin{array}{c}\text { Immersion DC } \\
\text { Reference }\end{array}$ & $\begin{array}{c}\mathrm{HC}-2 \\
\text { Immersion } \\
\mathrm{DC}(\mathrm{Sv} / \mathrm{s} \\
\left.\text { per } \mathrm{Bq} / \mathrm{m}^{3}\right)\end{array}$ \\
\hline Bi-212 & Bi-212 & 211.991 & Bismuth & $1.00 \mathrm{E}-03$ & ICRP-38 & $6.06 \mathrm{E}+01$ & $\mathrm{~m}$ & $3.63 \mathrm{E}+03$ & $1.46 \mathrm{E}+07$ & ICRP-72 & $3.10 \mathrm{E}-08$ & $\mathrm{M}$ & FGR-12 & $9.24 \mathrm{E}-15$ \\
\hline Bi-212n & Bi-212 & 211.991 & Bismuth & $1.00 \mathrm{E}-03$ & ICRP-107 & $7.00 \mathrm{E}+00$ & $\mathrm{~m}$ & $4.20 \mathrm{E}+02$ & $1.27 \mathrm{E}+08$ & -- & -- & -- & DOE-STD-1196 & $3.89 \mathrm{E}-16$ \\
\hline Bi-213 & Bi-213 & 212.994 & Bismuth & $1.00 \mathrm{E}-03$ & ICRP-38 & $4.57 \mathrm{E}+01$ & $\mathrm{~m}$ & $2.74 \mathrm{E}+03$ & $1.93 \mathrm{E}+07$ & ICRP-72 & $3.00 \mathrm{E}-08$ & $M$ & FGR-12 & $6.39 \mathrm{E}-15$ \\
\hline Bi-214 & Bi-214 & 213.999 & Bismuth & $1.00 \mathrm{E}-03$ & ICRP-38 & $1.99 \mathrm{E}+01$ & $\mathrm{~m}$ & $1.19 \mathrm{E}+03$ & $4.42 \mathrm{E}+07$ & ICRP-72 & $1.40 \mathrm{E}-08$ & $\mathrm{M}$ & FGR-12 & $7.65 \mathrm{E}-14$ \\
\hline Bi-215 & Bi-215 & 215.002 & Bismuth & $1.00 \mathrm{E}-03$ & ICRP-107 & $7.60 \mathrm{E}+00$ & $\mathrm{~m}$ & $4.56 \mathrm{E}+02$ & $1.15 \mathrm{E}+08$ & -- & -- & -- & DOE-STD-1196 & $1.18 \mathrm{E}-14$ \\
\hline Bi-216 & Bi-216 & 216.006 & Bismuth & $1.00 \mathrm{E}-03$ & ICRP-107 & $2.17 \mathrm{E}+00$ & $\mathrm{~m}$ & $1.30 \mathrm{E}+02$ & $4.01 E+08$ & -- & -- & -- & DOE-STD-1196 & $3.41 \mathrm{E}-14$ \\
\hline$B k-244$ & Bk-244 & 244.065 & Berkelium & $1.00 \mathrm{E}-03$ & JAERI & $4.35 \mathrm{E}+00$ & $\mathrm{~h}$ & $1.57 \mathrm{E}+04$ & $2.95 \mathrm{E}+06$ & JAERI & $1.00 \mathrm{E}-09$ & $M$ & -- & -- \\
\hline Bk-245 & Bk-245 & 245.066 & Berkelium & $1.00 \mathrm{E}-03$ & ICRP-38 & $4.94 \mathrm{E}+00$ & $d$ & $4.27 \mathrm{E}+05$ & $1.08 \mathrm{E}+05$ & ICRP-72 & $2.10 \mathrm{E}-09$ & $M$ & FGR-12 & $1.04 \mathrm{E}-14$ \\
\hline Bk-246 & $B k-246$ & 246.069 & Berkelium & $1.00 \mathrm{E}-03$ & ICRP-38 & $1.83 \mathrm{E}+00$ & $d$ & $1.58 \mathrm{E}+05$ & $2.90 \mathrm{E}+05$ & ICRP-72 & $3.30 \mathrm{E}-10$ & $M$ & FGR-12 & $4.59 \mathrm{E}-14$ \\
\hline Bk-247 & Bk-247 & 247.070 & Berkelium & $1.00 \mathrm{E}-03$ & ICRP-38 & $1.38 \mathrm{E}+03$ & $y$ & $4.35 \mathrm{E}+10$ & $1.05 E+00$ & ICRP-72 & $6.90 \mathrm{E}-05$ & $M$ & FGR-12 & $4.71 \mathrm{E}-15$ \\
\hline Bk-248m & Bk-248 & 248.073 & Berkelium & $1.00 \mathrm{E}-03$ & ICRP-107 & $2.37 \mathrm{E}+01$ & $\mathrm{~h}$ & $8.53 \mathrm{E}+04$ & $5.33 \mathrm{E}+05$ & DOE-STD-1196 & $2.81 \mathrm{E}-08$ & $F$ & DOE-STD-1196 & $2.28 \mathrm{E}-15$ \\
\hline Bk-249 & Bk-249 & 249.075 & Berkelium & $1.00 \mathrm{E}-03$ & ICRP-38 & $3.20 \mathrm{E}+02$ & $d$ & $2.76 \mathrm{E}+07$ & $1.64 \mathrm{E}+03$ & ICRP-72 & $1.60 \mathrm{E}-07$ & $M$ & FGR-12 & $8.21 \mathrm{E}-20$ \\
\hline Bk-250 & Bk-250 & 250.078 & Berkelium & $1.00 \mathrm{E}-03$ & ICRP-38 & $3.22 \mathrm{E}+00$ & $\mathrm{~h}$ & $1.16 \mathrm{E}+04$ & $3.89 \mathrm{E}+06$ & ICRP-72 & $1.00 \mathrm{E}-09$ & $\mathrm{M}$ & FGR-12 & $4.38 \mathrm{E}-14$ \\
\hline Bk-251 & Bk-251 & 251.081 & Berkelium & $1.00 \mathrm{E}-03$ & ICRP-107 & $5.56 \mathrm{E}+01$ & $\mathrm{~m}$ & $3.34 \mathrm{E}+03$ & $1.35 \mathrm{E}+07$ & DOE-STD-1196 & $5.09 \mathrm{E}-11$ & $M$ & DOE-STD-1196 & $3.56 \mathrm{E}-15$ \\
\hline $\mathrm{Br}-72$ & $\mathrm{Br}-72$ & 71.937 & Bromine & $5.00 \mathrm{E}-01$ & ICRP-107 & $7.86 \mathrm{E}+01$ & $\mathrm{~s}$ & $7.86 \mathrm{E}+01$ & $2.00 \mathrm{E}+09$ & -- & -- & -- & DOE-STD-1196 & $1.41 \mathrm{E}-13$ \\
\hline $\mathrm{Br}-73$ & Br-73 & 72.932 & Bromine & $5.00 \mathrm{E}-01$ & ICRP-107 & $3.40 \mathrm{E}+00$ & $\mathrm{~m}$ & $2.04 \mathrm{E}+02$ & $7.58 \mathrm{E}+08$ & -- & -- & -- & DOE-STD-1196 & $6.52 \mathrm{E}-14$ \\
\hline $\mathrm{Br}-74$ & $\mathrm{Br}-74$ & 73.930 & Bromine & $5.00 \mathrm{E}-01$ & ICRP-38 & $2.53 \mathrm{E}+01$ & $\mathrm{~m}$ & $1.52 \mathrm{E}+03$ & $1.01 \mathrm{E}+08$ & ICRP-72 & $3.80 \mathrm{E}-11$ & $M$ & FGR-12 & $2.38 \mathrm{E}-13$ \\
\hline$B r-74 m$ & $\begin{array}{ll}\mathrm{Br}-74 \\
\end{array}$ & 73.930 & Bromine & $5.00 \mathrm{E}-01$ & ICRP-38 & $4.15 \mathrm{E}+01$ & $\mathrm{~m}$ & $2.49 \mathrm{E}+03$ & $6.13 \mathrm{E}+07$ & ICRP-72 & $6.20 \mathrm{E}-11$ & $M$ & FGR-12 & $2.08 \mathrm{E}-13$ \\
\hline $\mathrm{Br}-75$ & $\mathrm{Br}-75$ & 74.926 & Bromine & $5.00 \mathrm{E}-01$ & ICRP-38 & $9.80 \mathrm{E}+01$ & $\mathrm{~m}$ & $5.88 \mathrm{E}+03$ & $2.56 \mathrm{E}+07$ & ICRP-72 & $5.30 \mathrm{E}-11$ & $M$ & FGR-12 & $5.84 \mathrm{E}-14$ \\
\hline $\mathrm{Br}-76$ & Br-76 & 75.925 & Bromine & $5.00 \mathrm{E}-01$ & ICRP-38 & $1.62 \mathrm{E}+01$ & $\mathrm{~h}$ & $5.83 \mathrm{E}+04$ & $2.55 \mathrm{E}+06$ & ICRP-72 & $4.10 \mathrm{E}-10$ & $M$ & FGR-12 & $1.34 \mathrm{E}-13$ \\
\hline $\mathrm{Br}-76 \mathrm{~m}$ & Br-76 & 75.925 & Bromine & $5.00 \mathrm{E}-01$ & ICRP-107 & $1.31 \mathrm{E}+00$ & $\mathrm{~s}$ & $1.31 \mathrm{E}+00$ & $1.13 \mathrm{E}+11$ & -- & -- & -- & DOE-STD-1196 & $9.66 \mathrm{E}-16$ \\
\hline $\mathrm{Br}-77$ & Br-77 & 76.921 & Bromine & $5.00 \mathrm{E}-01$ & ICRP-38 & $5.60 \mathrm{E}+01$ & $\mathrm{~h}$ & $2.02 E+05$ & $7.27 \mathrm{E}+05$ & ICRP-72 & $8.40 \mathrm{E}-11$ & $M$ & FGR-12 & $1.51 \mathrm{E}-14$ \\
\hline $\mathrm{Br}-77 \mathrm{~m}$ & Br-77 & 76.921 & Bromine & $5.00 \mathrm{E}-01$ & ICRP-107 & $4.28 \mathrm{E}+00$ & $\mathrm{~m}$ & $2.57 \mathrm{E}+02$ & $5.71 \mathrm{E}+08$ & -- & -- & -- & DOE-STD-1196 & $5.96 \mathrm{E}-16$ \\
\hline $\mathrm{Br}-78$ & Br-78 & 77.921 & Bromine & $5.00 \mathrm{E}-01$ & ICRP-107 & $6.46 \mathrm{E}+00$ & $\mathrm{~m}$ & $3.88 \mathrm{E}+02$ & $3.74 \mathrm{E}+08$ & --- & -- & -- & DOE-STD-1196 & $4.69 \mathrm{E}-14$ \\
\hline $\mathrm{Br}-80$ & $\mathrm{Br}-80$ & 79.919 & Bromine & $5.00 \mathrm{E}-01$ & ICRP-38 & $1.74 \mathrm{E}+01$ & $\mathrm{~m}$ & $1.04 \mathrm{E}+03$ & $1.35 \mathrm{E}+08$ & ICRP-72 & $9.40 \mathrm{E}-12$ & $M$ & FGR-12 & $3.85 \mathrm{E}-15$ \\
\hline $\mathrm{Br}-80 \mathrm{~m}$ & $\mathrm{Br}-80$ & 79.919 & Bromine & $5.00 \mathrm{E}-01$ & ICRP-38 & $4.42 \mathrm{E}+00$ & $\mathrm{~h}$ & $1.59 \mathrm{E}+04$ & $8.87 \mathrm{E}+06$ & ICRP-72 & $7.60 \mathrm{E}-11$ & $M$ & FGR-12 & $3.11 \mathrm{E}-16$ \\
\hline $\mathrm{Br}-82$ & $\mathrm{Br}-82$ & 81.917 & Bromine & $5.00 \mathrm{E}-01$ & ICRP-38 & $3.53 \mathrm{E}+01$ & $\mathrm{~h}$ & $1.27 \mathrm{E}+05$ & $1.08 \mathrm{E}+06$ & ICRP-72 & $6.30 \mathrm{E}-10$ & $M$ & FGR-12 & $1.30 \mathrm{E}-13$ \\
\hline $\mathrm{Br}-82 \mathrm{~m}$ & Br-82 & 81.917 & Bromine & $5.00 \mathrm{E}-01$ & ICRP-107 & $6.13 \mathrm{E}+00$ & $\mathrm{~m}$ & $3.68 \mathrm{E}+02$ & $3.74 \mathrm{E}+08$ & -- & -- & -- & DOE-STD-1196 & $1.59 \mathrm{E}-16$ \\
\hline $\mathrm{Br}-83$ & $\mathrm{Br}-83$ & 82.915 & Bromine & $5.00 \mathrm{E}-01$ & ICRP-38 & $2.39 \mathrm{E}+00$ & $\mathrm{~h}$ & $8.60 \mathrm{E}+03$ & $1.58 \mathrm{E}+07$ & ICRP-72 & $4.80 \mathrm{E}-11$ & $M$ & FGR-12 & $3.82 \mathrm{E}-16$ \\
\hline $\mathrm{Br}-84$ & Br-84 & 83.916 & Bromine & $5.00 \mathrm{E}-01$ & ICRP-38 & $3.18 \mathrm{E}+01$ & $\mathrm{~m}$ & $1.91 \mathrm{E}+03$ & $7.05 \mathrm{E}+07$ & ICRP-72 & $3.70 \mathrm{E}-11$ & $M$ & FGR-12 & $9.41 \mathrm{E}-14$ \\
\hline $\mathrm{Br}-84 \mathrm{~m}$ & Br-84 & 83.916 & Bromine & $5.00 \mathrm{E}-01$ & ICRP-107 & $6.00 \mathrm{E}+00$ & $\mathrm{~m}$ & $3.60 \mathrm{E}+02$ & $3.73 \mathrm{E}+08$ & -- & -- & -- & DOE-STD-1196 & $1.31 \mathrm{E}-13$ \\
\hline $\mathrm{Br}-85$ & $\mathrm{Br}-85$ & 84.916 & Bromine & $5.00 \mathrm{E}-01$ & ICRP-107 & $2.90 \mathrm{E}+00$ & $\mathrm{~m}$ & $1.74 \mathrm{E}+02$ & $7.64 \mathrm{E}+08$ & -- & -- & -- & DOE-STD-1196 & $3.98 \mathrm{E}-15$ \\
\hline C-10 & $C-10$ & 10.017 & Carbon & $1.00 \mathrm{E}-02$ & ICRP-107 & $1.93 \mathrm{E}+01$ & $\mathrm{~s}$ & $1.93 \mathrm{E}+01$ & $5.85 \mathrm{E}+10$ & -- & -- & -- & DOE-STD-1196 & $7.90 \mathrm{E}-14$ \\
\hline C-11 & C-11 & 11.011 & Carbon & $1.00 \mathrm{E}-02$ & ICRP-38 & $2.04 \mathrm{E}+01$ & $\mathrm{~m}$ & $1.22 \mathrm{E}+03$ & $8.38 \mathrm{E}+08$ & ICRP-72 & $1.80 \mathrm{E}-11$ & $M$ & FGR-12 & $4.89 \mathrm{E}-14$ \\
\hline C-14 & C-14 & 14.003 & Carbon & $1.00 \mathrm{E}-02$ & ICRP-38 & $5.73 E+03$ & $y$ & $1.81 \mathrm{E}+11$ & $4.46 \mathrm{E}+00$ & ICRP-72 & $5.80 \mathrm{E}-09$ & $\mathrm{~s}$ & FGR-12 & $2.24 \mathrm{E}-19$ \\
\hline Ca-41 & Ca-41 & 40.962 & Calcium & $1.00 \mathrm{E}-03$ & ICRP-38 & $1.40 \mathrm{E}+05$ & $y$ & $4.42 \mathrm{E}+12$ & $6.23 \mathrm{E}-02$ & ICRP-72 & $1.80 \mathrm{E}-10$ & $\mathrm{~s}$ & -- & -- \\
\hline Ca-45 & Ca-45 & 44.956 & Calcium & $1.00 \mathrm{E}-03$ & ICRP-38 & $1.63 \mathrm{E}+02$ & $d$ & $1.41 \mathrm{E}+07$ & $1.78 \mathrm{E}+04$ & ICRP-72 & $3.70 \mathrm{E}-09$ & $\mathrm{~s}$ & FGR-12 & $8.63 \mathrm{E}-19$ \\
\hline Ca-47 & Ca-47 & 46.955 & Calcium & $1.00 \mathrm{E}-03$ & ICRP-38 & $4.53 \mathrm{E}+00$ & d & $3.91 \mathrm{E}+05$ & $6.14 \mathrm{E}+05$ & ICRP-72 & $2.10 \mathrm{E}-09$ & $\mathrm{~s}$ & FGR-12 & $5.36 \mathrm{E}-14$ \\
\hline Ca-49 & Ca-49 & 48.956 & Calcium & $1.00 \mathrm{E}-03$ & ICRP-38 & $8.72 \mathrm{E}+00$ & $\mathrm{~m}$ & $5.23 \mathrm{E}+02$ & $4.41 \mathrm{E}+08$ & -- & -- & -- & FGR-12 & $1.73 \mathrm{E}-13$ \\
\hline Cd-101 & Cd-101 & 100.919 & Cadmium & $1.00 \mathrm{E}-03$ & ICRP-107 & $1.36 \mathrm{E}+00$ & $\mathrm{~m}$ & $8.16 \mathrm{E}+01$ & $1.37 \mathrm{E}+09$ & -- & -- & -- & DOE-STD-1196 & $1.17 \mathrm{E}-13$ \\
\hline $\mathrm{Cd}-102$ & Cd-102 & 101.914 & Cadmium & $1.00 \mathrm{E}-03$ & ICRP-107 & $5.50 \mathrm{E}+00$ & $\mathrm{~m}$ & $3.30 \mathrm{E}+02$ & $3.35 \mathrm{E}+08$ & -- & -- & -- & DOE-STD-1196 & $3.73 \mathrm{E}-14$ \\
\hline Cd-103 & Cd-103 & 102.913 & Cadmium & $1.00 \mathrm{E}-03$ & ICRP-107 & $7.30 \mathrm{E}+00$ & $\mathrm{~m}$ & $4.38 \mathrm{E}+02$ & $2.50 \mathrm{E}+08$ & -- & -- & -- & DOE-STD-1196 & $1.00 \mathrm{E}-13$ \\
\hline Cd-104 & Cd-104 & 103.910 & Cadmium & $1.00 \mathrm{E}-03$ & ICRP-38 & $5.77 \mathrm{E}+01$ & $\mathrm{~m}$ & $3.46 \mathrm{E}+03$ & $3.14 \mathrm{E}+07$ & ICRP-72 & $3.50 \mathrm{E}-11$ & $\mathrm{~s}$ & FGR-12 & $1.14 \mathrm{E}-14$ \\
\hline Cd-105 & Cd-105 & 104.909 & Cadmium & $1.00 \mathrm{E}-03$ & ICRP-107 & $5.55 \mathrm{E}+01$ & $\mathrm{~m}$ & $3.33 \mathrm{E}+03$ & $3.23 \mathrm{E}+07$ & DOE-STD-1196 & $2.86 \mathrm{E}-11$ & $\mathrm{~s}$ & DOE-STD-1196 & $6.13 \mathrm{E}-14$ \\
\hline $\mathrm{Cd}-107$ & $\mathrm{Cd}-107$ & 106.907 & Cadmium & $1.00 \mathrm{E}-03$ & ICRP-38 & $6.49 \mathrm{E}+00$ & $\mathrm{~h}$ & $2.34 \mathrm{E}+04$ & $4.52 \mathrm{E}+06$ & ICRP-72 & $8.30 \mathrm{E}-11$ & $M$ & FGR-12 & $6.02 \mathrm{E}-16$ \\
\hline Cd-109 & Cd-109 & 108.905 & Cadmium & $1.00 \mathrm{E}-03$ & ICRP-38 & $4.64 \mathrm{E}+02$ & $d$ & $4.01 \mathrm{E}+07$ & $2.58 \mathrm{E}+03$ & ICRP-72 & $8.10 \mathrm{E}-09$ & $F$ & FGR-12 & $2.94 \mathrm{E}-16$ \\
\hline
\end{tabular}




\begin{tabular}{|c|c|c|c|c|c|c|c|c|c|c|c|c|c|c|}
\hline \multicolumn{15}{|c|}{ Table A.1 Radionuclide Specific Input Data Used For the Calculation of HC-2 TQs Using Maximum Reported DCs (continued) } \\
\hline $\begin{array}{c}\text { MASTER } \\
\text { ISOTOPE } \\
\text { LIST } \\
\end{array}$ & $\begin{array}{l}\text { Atomic } \\
\text { Mass } \\
\text { Nuclide }\end{array}$ & $\begin{array}{l}\text { Atomic } \\
\text { Mass }\end{array}$ & Element & $\begin{array}{c}\text { RF per } \\
\text { DOE-STD- } \\
1027\end{array}$ & $\begin{array}{l}\text { Half-Life } \\
\text { Reference }\end{array}$ & $\begin{array}{r}\text { Half-Li } \\
\text { (original }\end{array}$ & & Half Life (s) & $\begin{array}{l}\text { Sp. Act } \\
\text { (Ci/g) }\end{array}$ & $\begin{array}{c}\text { Inhalation DC } \\
\text { Reference }\end{array}$ & $\begin{array}{c}\text { HC-2 } \\
\text { Bounding } \\
\text { Inhalation } \\
\text { DC (Sv/Bq) }\end{array}$ & $\begin{array}{l}\text { Lung } \\
\text { Absorption } \\
\text { Class/Type }\end{array}$ & $\begin{array}{c}\text { Immersion DC } \\
\text { Reference }\end{array}$ & $\begin{array}{c}\text { HC-2 } \\
\text { Immersion } \\
\mathrm{DC}(\mathrm{Sv} / \mathrm{s} \\
\left.\text { per } \mathrm{Bq} / \mathrm{m}^{3}\right)\end{array}$ \\
\hline $\mathrm{Cd}-111 \mathrm{~m}$ & Cd-111 & 110.904 & Cadmium & $1.00 \mathrm{E}-03$ & ICRP-107 & $4.85 \mathrm{E}+01$ & $\mathrm{~m}$ & $2.91 \mathrm{E}+03$ & $3.50 \mathrm{E}+07$ & DOE-STD-1196 & $2.66 \mathrm{E}-11$ & $\mathrm{~s}$ & DOE-STD-1196 & $1.20 \mathrm{E}-14$ \\
\hline $\mathrm{Cd}-113$ & Cd-113 & 112.904 & Cadmium & $1.00 \mathrm{E}-03$ & ICRP-38 & $9.30 \mathrm{E}+15$ & y & $2.93 \mathrm{E}+23$ & $3.40 \mathrm{E}-13$ & ICRP-72 & $1.20 \mathrm{E}-07$ & $\mathrm{~F}$ & FGR-12 & $1.45 \mathrm{E}-18$ \\
\hline Cd-113m & Cd-113 & 112.904 & Cadmium & $1.00 \mathrm{E}-03$ & ICRP-38 & $1.36 \mathrm{E}+01$ & $y$ & $4.29 \mathrm{E}+08$ & $2.33 \mathrm{E}+02$ & ICRP-72 & $1.10 \mathrm{E}-07$ & $\mathrm{~F}$ & FGR-12 & $6.94 \mathrm{E}-18$ \\
\hline Cd-115 & Cd-115 & 114.905 & Cadmium & $1.00 \mathrm{E}-03$ & ICRP-38 & $5.35 \mathrm{E}+01$ & $\mathrm{~h}$ & $1.92 \mathrm{E}+05$ & $5.10 \mathrm{E}+05$ & ICRP-72 & $1.10 \mathrm{E}-09$ & $\mathrm{~s}$ & FGR-12 & $1.12 \mathrm{E}-14$ \\
\hline $\mathrm{Cd}-115 \mathrm{~m}$ & Cd-115 & 114.905 & Cadmium & $1.00 \mathrm{E}-03$ & ICRP-38 & $4.46 \mathrm{E}+01$ & $d$ & $3.85 \mathrm{E}+06$ & $2.55 \mathrm{E}+04$ & ICRP-72 & $7.70 \mathrm{E}-09$ & $\mathrm{~s}$ & FGR-12 & $1.17 \mathrm{E}-15$ \\
\hline Cd-117 & $\mathrm{Cd}-117$ & 116.907 & Cadmium & $1.00 \mathrm{E}-03$ & ICRP-38 & $2.49 \mathrm{E}+00$ & $\mathrm{~h}$ & $8.96 \mathrm{E}+03$ & $1.08 \mathrm{E}+07$ & ICRP-72 & $1.70 \mathrm{E}-10$ & $\mathrm{~s}$ & FGR-12 & $5.45 \mathrm{E}-14$ \\
\hline Cd-117m & Cd-117 & 116.907 & Cadmium & $1.00 \mathrm{E}-03$ & ICRP-38 & $3.36 \mathrm{E}+00$ & $\mathrm{~h}$ & $1.21 \mathrm{E}+04$ & $7.98 \mathrm{E}+06$ & ICRP-72 & $2.10 \mathrm{E}-10$ & $\mathrm{~s}$ & FGR-12 & $1.05 \mathrm{E}-13$ \\
\hline Cd-118 & Cd-118 & 117.907 & Cadmium & $1.00 \mathrm{E}-03$ & ICRP-107 & $5.03 \mathrm{E}+01$ & $\mathrm{~m}$ & $3.02 \mathrm{E}+03$ & $3.17 \mathrm{E}+07$ & DOE-STD-1196 & $9.60 \mathrm{E}-11$ & $\mathrm{~s}$ & DOE-STD-1196 & $7.25 \mathrm{E}-17$ \\
\hline Cd-119 & Cd-119 & 118.910 & Cadmium & $1.00 \mathrm{E}-03$ & ICRP-107 & $2.69 \mathrm{E}+00$ & $\mathrm{~m}$ & $1.61 \mathrm{E}+02$ & $5.88 \mathrm{E}+08$ & -- & -- & -- & DOE-STD-1196 & $7.96 \mathrm{E}-14$ \\
\hline Cd-119m & $\mathrm{Cd}-119$ & 118.910 & Cadmium & $1.00 \mathrm{E}-03$ & ICRP-107 & $2.20 \mathrm{E}+00$ & $\mathrm{~m}$ & $1.32 \mathrm{E}+02$ & $7.19 E+08$ & -- & -- & -- & DOE-STD-1196 & $1.11 \mathrm{E}-13$ \\
\hline Ce-130 & Ce-130 & 129.915 & Cerium & $1.00 \mathrm{E}-03$ & ICRP-107 & $2.29 \mathrm{E}+01$ & $\mathrm{~m}$ & $1.37 \mathrm{E}+03$ & $6.32 \mathrm{E}+07$ & DOE-STD-1196 & $4.27 \mathrm{E}-11$ & $\mathrm{~s}$ & DOE-STD-1196 & $2.14 \mathrm{E}-14$ \\
\hline Ce-131 & Ce-131 & 130.914 & Cerium & $1.00 \mathrm{E}-03$ & ICRP-107 & $1.02 \mathrm{E}+01$ & $\mathrm{~m}$ & $6.12 \mathrm{E}+02$ & $1.41 \mathrm{E}+08$ & DOE-STD-1196 & $1.68 \mathrm{E}-11$ & $\mathrm{~s}$ & DOE-STD-1196 & $7.43 \mathrm{E}-14$ \\
\hline Ce-131m & Ce-131 & 130.914 & Cerium & $1.00 \mathrm{E}-03$ & JAERI & $5.00 E+00$ & $\mathrm{~m}$ & $3.00 \mathrm{E}+02$ & $2.87 \mathrm{E}+08$ & -- & -- & -- & -- & -- \\
\hline Ce-132 & Ce-132 & 131.911 & Cerium & $1.00 \mathrm{E}-03$ & ICRP-107 & $3.51 E+00$ & $\mathrm{~h}$ & $1.26 \mathrm{E}+04$ & $6.77 \mathrm{E}+06$ & DOE-STD-1196 & $1.72 \mathrm{E}-10$ & $\mathrm{~s}$ & DOE-STD-1196 & $1.09 \mathrm{E}-14$ \\
\hline Ce-133 & Ce-133 & 132.912 & Cerium & $1.00 \mathrm{E}-03$ & ICRP-107 & $9.70 E+01$ & $\mathrm{~m}$ & $5.82 \mathrm{E}+03$ & $1.46 \mathrm{E}+07$ & DOE-STD-1196 & $6.21 \mathrm{E}-11$ & $\mathrm{~s}$ & DOE-STD-1196 & $2.28 \mathrm{E}-14$ \\
\hline Ce-133m & Ce-133 & 132.912 & Cerium & $1.00 \mathrm{E}-03$ & ICRP-107 & $4.90 \mathrm{E}+00$ & $\mathrm{~h}$ & $1.76 \mathrm{E}+04$ & $4.81 \mathrm{E}+06$ & DOE-STD-1196 & $1.42 \mathrm{E}-10$ & $\mathrm{~s}$ & DOE-STD-1196 & $7.95 \mathrm{E}-14$ \\
\hline Ce-134 & Ce-134 & 133.909 & Cerium & $1.00 \mathrm{E}-03$ & ICRP-38 & $7.20 E+01$ & $\mathrm{~h}$ & $2.59 \mathrm{E}+05$ & $3.25 E+05$ & ICRP-72 & $1.30 \mathrm{E}-09$ & $M$ & FGR-12 & $4.71 \mathrm{E}-16$ \\
\hline Ce-135 & Ce-135 & 134.909 & Cerium & $1.00 \mathrm{E}-03$ & ICRP-38 & $1.76 \mathrm{E}+01$ & $\mathrm{~h}$ & $6.34 \mathrm{E}+04$ & $1.32 \mathrm{E}+06$ & ICRP-72 & $5.00 \mathrm{E}-10$ & $\mathrm{~s}$ & FGR-12 & $8.54 \mathrm{E}-14$ \\
\hline Ce-137 & Ce-137 & 136.908 & Cerium & $1.00 \mathrm{E}-03$ & ICRP-38 & $9.00 \mathrm{E}+00$ & $\mathrm{~h}$ & $3.24 \mathrm{E}+04$ & $2.54 \mathrm{E}+06$ & ICRP-72 & $1.00 \mathrm{E}-11$ & $\mathrm{~s}$ & FGR-12 & $8.81 \mathrm{E}-16$ \\
\hline Ce-137m & Ce-137 & 136.908 & Cerium & $1.00 \mathrm{E}-03$ & ICRP-38 & $3.44 \mathrm{E}+01$ & $\mathrm{~h}$ & $1.24 \mathrm{E}+05$ & $6.65 E+05$ & ICRP-72 & $4.40 \mathrm{E}-10$ & $\mathrm{~s}$ & FGR-12 & $1.96 \mathrm{E}-15$ \\
\hline Ce-139 & Ce-139 & 138.907 & Cerium & $1.00 \mathrm{E}-03$ & ICRP-38 & $1.38 \mathrm{E}+02$ & $d$ & $1.19 \mathrm{E}+07$ & $6.83 \mathrm{E}+03$ & ICRP-72 & $1.90 \mathrm{E}-09$ & $\mathrm{~s}$ & FGR-12 & $6.73 \mathrm{E}-15$ \\
\hline Ce-141 & Ce-141 & 140.908 & Cerium & $1.00 \mathrm{E}-03$ & ICRP-38 & $3.25 \mathrm{E}+01$ & $d$ & $2.81 \mathrm{E}+06$ & $2.85 E+04$ & ICRP-72 & $3.80 \mathrm{E}-09$ & $\mathrm{~s}$ & FGR-12 & $3.43 \mathrm{E}-15$ \\
\hline Ce-143 & Ce-143 & 142.912 & Cerium & $1.00 \mathrm{E}-03$ & ICRP-38 & $3.30 \mathrm{E}+01$ & $\mathrm{~h}$ & $1.19 \mathrm{E}+05$ & $6.64 \mathrm{E}+05$ & ICRP-72 & $8.30 \mathrm{E}-10$ & $\mathrm{~s}$ & FGR-12 & $1.29 \mathrm{E}-14$ \\
\hline Ce-144 & Ce-144 & 143.914 & Cerium & $1.00 \mathrm{E}-03$ & ICRP-38 & $2.84 \mathrm{E}+02$ & $d$ & $2.46 \mathrm{E}+07$ & $3.19 \mathrm{E}+03$ & ICRP-72 & $5.30 \mathrm{E}-08$ & $\mathrm{~s}$ & FGR-12 & $8.53 \mathrm{E}-16$ \\
\hline Ce-145 & Ce-145 & 144.917 & Cerium & $1.00 \mathrm{E}-03$ & ICRP-107 & $3.01 E+00$ & $\mathrm{~m}$ & $1.81 \mathrm{E}+02$ & $4.31 \mathrm{E}+08$ & -- & -- & -- & DOE-STD-1196 & $3.64 \mathrm{E}-14$ \\
\hline Ce-146 & Ce-146 & 145.919 & Cerium & $1.00 \mathrm{E}-03$ & JAERI & $1.35 \mathrm{E}+01$ & $\mathrm{~m}$ & $8.11 \mathrm{E}+02$ & $9.53 \mathrm{E}+07$ & JAERI & $2.60 \mathrm{E}-11$ & $\mathrm{~s}$ & -- & -- \\
\hline Cf-244 & Cf-244 & 244.066 & Californium & $1.00 \mathrm{E}-03$ & ICRP-38 & $1.94 \mathrm{E}+01$ & $\mathrm{~m}$ & $1.16 \mathrm{E}+03$ & $3.97 \mathrm{E}+07$ & ICRP-72 & $1.40 \mathrm{E}-08$ & $M$ & FGR-12 & $6.91 \mathrm{E}-18$ \\
\hline Cf-246 & Cf-246 & 246.069 & Californium & $1.00 \mathrm{E}-03$ & ICRP-38 & $3.57 \mathrm{E}+01$ & $\mathrm{~h}$ & $1.29 \mathrm{E}+05$ & $3.57 \mathrm{E}+05$ & ICRP-72 & $4.50 \mathrm{E}-07$ & $M$ & FGR-12 & $5.48 \mathrm{E}-18$ \\
\hline Cf-247 & Cf-247 & 247.071 & Californium & $1.00 \mathrm{E}-03$ & ICRP-107 & $3.11 E+00$ & $\mathrm{~h}$ & $1.12 \mathrm{E}+04$ & $4.08 \mathrm{E}+06$ & DOE-STD-1196 & $5.22 \mathrm{E}-11$ & $\mathrm{~F}$ & DOE-STD-1196 & $3.58 \mathrm{E}-15$ \\
\hline Cf-248 & Cf-248 & 248.072 & Californium & $1.00 \mathrm{E}-03$ & ICRP-38 & $3.34 \mathrm{E}+02$ & $d$ & $2.88 \mathrm{E}+07$ & $1.58 \mathrm{E}+03$ & ICRP-72 & $8.80 \mathrm{E}-06$ & $M$ & FGR-12 & $4.73 \mathrm{E}-18$ \\
\hline Cf-249 & Cf-249 & 249.075 & Californium & $1.00 \mathrm{E}-03$ & ICRP-38 & $3.51 \mathrm{E}+02$ & $y$ & $1.11 \mathrm{E}+10$ & $4.09 \mathrm{E}+00$ & ICRP-72 & $7.00 \mathrm{E}-05$ & $M$ & FGR-12 & $1.58 \mathrm{E}-14$ \\
\hline Cf- 250 & Cf-250 & 250.076 & Californium & $1.00 \mathrm{E}-03$ & ICRP-38 & $1.31 \mathrm{E}+01$ & $y$ & $4.13 \mathrm{E}+08$ & $1.09 \mathrm{E}+02$ & ICRP-72 & $3.40 \mathrm{E}-05$ & $M$ & FGR-12 & $4.50 \mathrm{E}-18$ \\
\hline Cf-251 & Cf-251 & 251.080 & Californium & $1.00 \mathrm{E}-03$ & ICRP-38 & $8.98 \mathrm{E}+02$ & $y$ & $2.83 \mathrm{E}+10$ & $1.59 \mathrm{E}+00$ & ICRP-72 & $7.10 \mathrm{E}-05$ & $M$ & FGR-12 & $5.58 \mathrm{E}-15$ \\
\hline Cf- 252 & Cf- 252 & 252.082 & Californium & $1.00 \mathrm{E}-03$ & ICRP-38 & $2.64 \mathrm{E}+00$ & $y$ & $8.32 \mathrm{E}+07$ & $5.38 \mathrm{E}+02$ & ICRP-72 & $2.00 \mathrm{E}-05$ & $M$ & FGR-12 & $5.06 \mathrm{E}-18$ \\
\hline Cf-253 & Cf-253 & 253.085 & Californium & $1.00 \mathrm{E}-03$ & ICRP-38 & $1.78 \mathrm{E}+01$ & $d$ & $1.54 \mathrm{E}+06$ & $2.90 \mathrm{E}+04$ & ICRP-72 & $1.30 \mathrm{E}-06$ & $M$ & FGR-12 & $1.08 \mathrm{E}-18$ \\
\hline Cf-254 & Cf-254 & 254.087 & Californium & $1.00 \mathrm{E}-03$ & ICRP-38 & $6.05 E+01$ & $d$ & $5.23 \mathrm{E}+06$ & $8.49 \mathrm{E}+03$ & ICRP-72 & $4.10 \mathrm{E}-05$ & $M$ & FGR-12 & $1.47 \mathrm{E}-20$ \\
\hline Cf-255 & Cf-255 & 255.091 & Californium & $1.00 \mathrm{E}-03$ & ICRP-107 & $8.50 \mathrm{E}+01$ & $\mathrm{~m}$ & $5.10 \mathrm{E}+03$ & $8.67 \mathrm{E}+06$ & DOE-STD-1196 & $7.35 \mathrm{E}-09$ & $\mathrm{~s}$ & DOE-STD-1196 & $1.16 \mathrm{E}-16$ \\
\hline Cf-256 & Cf-256 & 256.093 & Californium & $1.00 \mathrm{E}-03$ & JAERI & $1.23 \mathrm{E}+01$ & $\mathrm{~m}$ & $7.38 \mathrm{E}+02$ & $5.97 \mathrm{E}+07$ & JAERI & $1.90 \mathrm{E}-06$ & $M$ & -- & -- \\
\hline $\mathrm{Cl}-34$ & $\mathrm{Cl}-34$ & 33.974 & Chlorine & $1.00 E+00$ & ICRP-107 & $1.53 \mathrm{E}+00$ & $\mathrm{~s}$ & $1.53 \mathrm{E}+00$ & $2.18 \mathrm{E}+11$ & -- & -- & -- & DOE-STD-1196 & $4.77 \mathrm{E}-14$ \\
\hline $\mathrm{Cl}-34 \mathrm{~m}$ & $\mathrm{Cl}-34$ & 33.974 & Chlorine & $1.00 \mathrm{E}+00$ & ICRP-107 & $3.20 \mathrm{E}+01$ & $\mathrm{~m}$ & $1.92 \mathrm{E}+03$ & $1.73 E+08$ & DOE-STD-1196 & $5.50 \mathrm{E}-11$ & $\mathrm{~s}$ & DOE-STD-1196 & $1.03 \mathrm{E}-13$ \\
\hline $\mathrm{Cl}-36$ & $\mathrm{Cl}-36$ & 35.968 & Chlorine & $1.00 \mathrm{E}+00$ & ICRP-38 & $3.01 E+05$ & $y$ & $9.50 \mathrm{E}+12$ & $3.30 \mathrm{E}-02$ & ICRP-72 & $7.30 \mathrm{E}-09$ & $M$ & FGR-12 & $2.23 \mathrm{E}-17$ \\
\hline $\mathrm{Cl}-38$ & $\mathrm{Cl}-38$ & 37.968 & Chlorine & $1.00 E+00$ & ICRP-38 & $3.72 \mathrm{E}+01$ & $\mathrm{~m}$ & $2.23 \mathrm{E}+03$ & $1.33 \mathrm{E}+08$ & ICRP-72 & $4.50 \mathrm{E}-11$ & $M$ & FGR-12 & $7.87 \mathrm{E}-14$ \\
\hline $\mathrm{Cl}-39$ & $\mathrm{Cl}-39$ & 38.968 & Chlorine & $1.00 E+00$ & ICRP-38 & $5.56 \mathrm{E}+01$ & $\mathrm{~m}$ & $3.34 \mathrm{E}+03$ & $8.68 \mathrm{E}+07$ & ICRP-72 & $4.60 \mathrm{E}-11$ & $M$ & FGR-12 & $7.29 \mathrm{E}-14$ \\
\hline $\mathrm{Cl}-40$ & $\mathrm{Cl}-40$ & 39.970 & Chlorine & $1.00 E+00$ & ICRP-107 & $1.35 E+00$ & $\mathrm{~m}$ & $8.10 \mathrm{E}+01$ & $3.48 \mathrm{E}+09$ & -- & -- & -- & DOE-STD-1196 & $2.09 \mathrm{E}-13$ \\
\hline $\mathrm{Cm}-238$ & $\mathrm{Cm}-238$ & 238.053 & Curium & $1.00 \mathrm{E}-03$ & ICRP-38 & $2.40 \mathrm{E}+00$ & $\mathrm{~h}$ & $8.64 \mathrm{E}+03$ & $5.49 \mathrm{E}+06$ & ICRP-72 & $4.90 \mathrm{E}-09$ & $\mathrm{~s}$ & FGR-12 & $3.25 \mathrm{E}-15$ \\
\hline $\mathrm{Cm}-239$ & $\mathrm{Cm}-239$ & 239.055 & Curium & $1.00 \mathrm{E}-03$ & ICRP-107 & $2.90 \mathrm{E}+00$ & $\mathrm{~h}$ & $1.04 \mathrm{E}+04$ & $4.52 \mathrm{E}+06$ & DOE-STD-1196 & $8.06 \mathrm{E}-11$ & $\mathrm{~s}$ & DOE-STD-1196 & $1.05 \mathrm{E}-14$ \\
\hline
\end{tabular}




\begin{tabular}{|c|c|c|c|c|c|c|c|c|c|c|c|c|c|c|}
\hline \multicolumn{15}{|c|}{ Table A.1 Radionuclide Specific Input Data Used For the Calculation of HC-2 TQs Using Maximum Reported DCs (continued) } \\
\hline $\begin{array}{l}\text { MASTER } \\
\text { ISOTOPE } \\
\text { LIST }\end{array}$ & $\begin{array}{l}\text { Atomic } \\
\text { Mass } \\
\text { Nuclide }\end{array}$ & $\begin{array}{l}\text { Atomic } \\
\text { Mass }\end{array}$ & Element & $\begin{array}{c}\text { RF per } \\
\text { DOE-STD- } \\
1027\end{array}$ & $\begin{array}{c}\text { Half-Life } \\
\text { Reference }\end{array}$ & $\begin{array}{r}\text { Half-Li } \\
\text { (original }\end{array}$ & & Half Life (s) & $\begin{array}{l}\text { Sp. Act } \\
\text { (Ci/g) }\end{array}$ & $\begin{array}{l}\text { Inhalation DC } \\
\text { Reference }\end{array}$ & $\begin{array}{c}\text { HC-2 } \\
\text { Bounding } \\
\text { Inhalation } \\
\text { DC (Sv/Bq) }\end{array}$ & $\begin{array}{l}\text { Lung } \\
\text { Absorption } \\
\text { Class/Type }\end{array}$ & $\begin{array}{l}\text { Immersion DC } \\
\text { Reference }\end{array}$ & $\begin{array}{c}\mathrm{HC}-2 \\
\text { Immersion } \\
\mathrm{DC}(\mathrm{Sv} / \mathrm{s} \\
\left.\text { per } \mathrm{Bq} / \mathrm{m}^{3}\right)\end{array}$ \\
\hline $\mathrm{Cm}-240$ & $\mathrm{Cm}-240$ & 240.056 & Curium & $1.00 \mathrm{E}-03$ & ICRP-38 & $2.70 \mathrm{E}+01$ & d & $2.33 \mathrm{E}+06$ & $2.01 \mathrm{E}+04$ & ICRP-72 & $3.50 \mathrm{E}-06$ & $\mathrm{~s}$ & FGR-12 & $6.00 \mathrm{E}-18$ \\
\hline $\mathrm{Cm}-241$ & $\mathrm{Cm}-241$ & 241.058 & Curium & $1.00 \mathrm{E}-03$ & ICRP-38 & $3.28 \mathrm{E}+01$ & d & $2.83 \mathrm{E}+06$ & $1.65 \mathrm{E}+04$ & ICRP-72 & $3.70 \mathrm{E}-08$ & $M$ & FGR-12 & $2.31 \mathrm{E}-14$ \\
\hline $\mathrm{Cm}-242$ & $\mathrm{Cm}-242$ & 242.059 & Curium & $1.00 \mathrm{E}-03$ & ICRP-38 & $1.63 \mathrm{E}+02$ & $d$ & $1.41 \mathrm{E}+07$ & $3.31 \mathrm{E}+03$ & ICRP-72 & $5.90 \mathrm{E}-06$ & $\mathrm{~s}$ & FGR-12 & $5.69 \mathrm{E}-18$ \\
\hline $\mathrm{Cm}-243$ & $\mathrm{Cm}-243$ & 243.061 & Curium & $1.00 \mathrm{E}-03$ & ICRP-38 & $2.85 \mathrm{E}+01$ & $y$ & $8.99 \mathrm{E}+08$ & $5.16 \mathrm{E}+01$ & ICRP-72 & $6.90 \mathrm{E}-05$ & $\mathrm{~F}$ & FGR-12 & $5.88 \mathrm{E}-15$ \\
\hline $\mathrm{Cm}-244$ & $\mathrm{Cm}-244$ & 244.063 & Curium & $1.00 \mathrm{E}-03$ & ICRP-38 & $1.81 \mathrm{E}+01$ & $y$ & $5.72 \mathrm{E}+08$ & $8.09 \mathrm{E}+01$ & ICRP-72 & $5.70 \mathrm{E}-05$ & $\mathrm{~F}$ & FGR-12 & $4.91 \mathrm{E}-18$ \\
\hline $\mathrm{Cm}-245$ & $\mathrm{Cm}-245$ & 245.065 & Curium & $1.00 \mathrm{E}-03$ & ICRP-38 & $8.50 \mathrm{E}+03$ & $y$ & $2.68 \mathrm{E}+11$ & $1.72 \mathrm{E}-01$ & ICRP-72 & $9.90 \mathrm{E}-05$ & $\mathrm{~F}$ & FGR-12 & $3.96 \mathrm{E}-15$ \\
\hline $\mathrm{Cm}-246$ & $\mathrm{Cm}-246$ & 246.067 & Curium & $1.00 \mathrm{E}-03$ & ICRP-38 & $4.73 E+03$ & $y$ & $1.49 \mathrm{E}+11$ & 3.07E-01 & ICRP-72 & $9.80 \mathrm{E}-05$ & $\mathrm{~F}$ & FGR-12 & $4.46 \mathrm{E}-18$ \\
\hline $\mathrm{Cm}-247$ & $\mathrm{Cm}-247$ & 247.070 & Curium & $1.00 \mathrm{E}-03$ & ICRP-38 & $1.56 \mathrm{E}+07$ & $y$ & $4.92 \mathrm{E}+14$ & $9.28 \mathrm{E}-05$ & ICRP-72 & $9.00 \mathrm{E}-05$ & $\mathrm{~F}$ & FGR-12 & $1.50 \mathrm{E}-14$ \\
\hline $\mathrm{Cm}-248$ & $\mathrm{Cm}-248$ & 248.072 & Curium & $1.00 \mathrm{E}-03$ & ICRP-38 & $3.39 \mathrm{E}+05$ & $y$ & $1.07 \mathrm{E}+13$ & $4.25 \mathrm{E}-03$ & ICRP-72 & $3.60 \mathrm{E}-04$ & $\mathrm{~F}$ & FGR-12 & $3.39 \mathrm{E}-18$ \\
\hline $\mathrm{Cm}-249$ & $\mathrm{Cm}-249$ & 249.076 & Curium & $1.00 \mathrm{E}-03$ & ICRP-38 & $6.42 \mathrm{E}+01$ & $\mathrm{~m}$ & $3.85 \mathrm{E}+03$ & $1.18 \mathrm{E}+07$ & ICRP-72 & $4.00 \mathrm{E}-11$ & $F$ & FGR-12 & $9.36 \mathrm{E}-16$ \\
\hline $\mathrm{Cm}-250$ & $\mathrm{Cm}-250$ & 250.078 & Curium & $1.00 \mathrm{E}-03$ & ICRP-38 & $6.90 \mathrm{E}+03$ & $y$ & $2.18 \mathrm{E}+11$ & $2.07 \mathrm{E}-01$ & ICRP-72 & $2.10 \mathrm{E}-03$ & $\mathrm{~F}$ & DOE-STD-1196 & $6.52 \mathrm{E}-13$ \\
\hline $\mathrm{Cm}-251$ & $\mathrm{Cm}-251$ & 251.082 & Curium & $1.00 \mathrm{E}-03$ & ICRP-107 & $1.68 \mathrm{E}+01$ & $\mathrm{~m}$ & $1.01 \mathrm{E}+03$ & $4.46 \mathrm{E}+07$ & DOE-STD-1196 & $2.79 \mathrm{E}-11$ & $\mathrm{~s}$ & DOE-STD-1196 & $5.20 \mathrm{E}-15$ \\
\hline Co-54m & Co-54 & 53.948 & Cobalt & $1.00 \mathrm{E}-03$ & ICRP-107 & $1.48 \mathrm{E}+00$ & $\mathrm{~m}$ & $8.88 \mathrm{E}+01$ & $2.35 \mathrm{E}+09$ & -- & -- & -- & DOE-STD-1196 & $1.85 \mathrm{E}-13$ \\
\hline Co-55 & Co-55 & 54.942 & Cobalt & $1.00 \mathrm{E}-03$ & ICRP-38 & $1.75 E+01$ & $\mathrm{~h}$ & $6.31 \mathrm{E}+04$ & $3.25 E+06$ & ICRP-72 & $5.30 \mathrm{E}-10$ & $\mathrm{~s}$ & FGR-12 & $9.78 \mathrm{E}-14$ \\
\hline Co-56 & Co-56 & 55.940 & Cobalt & $1.00 \mathrm{E}-03$ & ICRP-38 & $7.88 \mathrm{E}+01$ & $d$ & $6.80 \mathrm{E}+06$ & $2.96 \mathrm{E}+04$ & ICRP-72 & $6.70 \mathrm{E}-09$ & $\mathrm{~s}$ & FGR-12 & $1.83 \mathrm{E}-13$ \\
\hline Co-57 & Co-57 & 56.936 & Cobalt & $1.00 \mathrm{E}-03$ & ICRP-38 & $2.71 E+02$ & $d$ & $2.34 \mathrm{E}+07$ & $8.47 \mathrm{E}+03$ & ICRP-72 & $1.00 \mathrm{E}-09$ & $\mathrm{~s}$ & FGR-12 & $5.61 \mathrm{E}-15$ \\
\hline Co-58 & Co-58 & 57.936 & Cobalt & $1.00 \mathrm{E}-03$ & ICRP-38 & $7.08 \mathrm{E}+01$ & $d$ & $6.12 \mathrm{E}+06$ & $3.18 \mathrm{E}+04$ & ICRP-72 & $2.10 \mathrm{E}-09$ & $\mathrm{~s}$ & FGR-12 & $4.76 \mathrm{E}-14$ \\
\hline Co-58m & Co-58 & 57.936 & Cobalt & $1.00 \mathrm{E}-03$ & ICRP-38 & $9.15 \mathrm{E}+00$ & $\mathrm{~h}$ & $3.29 \mathrm{E}+04$ & $5.91 \mathrm{E}+06$ & ICRP-72 & $1.70 \mathrm{E}-11$ & $\mathrm{~s}$ & FGR-12 & $8.77 \mathrm{E}-20$ \\
\hline Co-60 & Co-60 & 59.934 & Cobalt & $1.00 \mathrm{E}-03$ & ICRP-38 & $5.27 \mathrm{E}+00$ & $y$ & $1.66 \mathrm{E}+08$ & $1.13 \mathrm{E}+03$ & ICRP-72 & $3.10 \mathrm{E}-08$ & $\mathrm{~s}$ & FGR-12 & $1.26 \mathrm{E}-13$ \\
\hline Co-60m & Co-60 & 59.934 & Cobalt & $1.00 \mathrm{E}-03$ & ICRP-38 & $1.05 E+01$ & $\mathrm{~m}$ & $6.28 \mathrm{E}+02$ & $3.00 E+08$ & ICRP-72 & $1.40 \mathrm{E}-12$ & $\mathrm{~s}$ & FGR-12 & $2.17 \mathrm{E}-16$ \\
\hline Co-61 & Co-61 & 60.932 & Cobalt & $1.00 \mathrm{E}-03$ & ICRP-38 & $1.65 E+00$ & $\mathrm{~h}$ & $5.94 \mathrm{E}+03$ & $3.12 \mathrm{E}+07$ & ICRP-72 & $5.10 \mathrm{E}-11$ & $\mathrm{~s}$ & FGR-12 & $3.94 \mathrm{E}-15$ \\
\hline Co-62 & Co-62 & 61.934 & Cobalt & $1.00 \mathrm{E}-03$ & ICRP-107 & $1.50 \mathrm{E}+00$ & $\mathrm{~m}$ & $9.00 \mathrm{E}+01$ & $2.02 \mathrm{E}+09$ & -- & -- & -- & DOE-STD-1196 & $7.92 \mathrm{E}-14$ \\
\hline Co-62m & Co-62 & 61.934 & Cobalt & $1.00 \mathrm{E}-03$ & ICRP-38 & $1.39 \mathrm{E}+01$ & $\mathrm{~m}$ & $8.35 E+02$ & $2.18 \mathrm{E}+08$ & ICRP-72 & $2.10 \mathrm{E}-11$ & $\mathrm{~s}$ & FGR-12 & $1.37 \mathrm{E}-13$ \\
\hline $\mathrm{Cr}-48$ & $\mathrm{Cr}-48$ & 47.954 & Chromium & $1.00 \mathrm{E}-03$ & ICRP-38 & $2.30 \mathrm{E}+01$ & $\mathrm{~h}$ & $8.27 \mathrm{E}+04$ & $2.85 \mathrm{E}+06$ & ICRP-72 & $2.20 \mathrm{E}-10$ & $\mathrm{~s}$ & FGR-12 & $2.06 \mathrm{E}-14$ \\
\hline Cr-49 & $\mathrm{Cr}-49$ & 48.951 & Chromium & $1.00 \mathrm{E}-03$ & ICRP-38 & $4.21 \mathrm{E}+01$ & $\mathrm{~m}$ & $2.53 \mathrm{E}+03$ & $9.13 \mathrm{E}+07$ & ICRP-72 & $3.50 \mathrm{E}-11$ & $\mathrm{~s}$ & FGR-12 & $5.03 \mathrm{E}-14$ \\
\hline Cr-51 & Cr-51 & 50.945 & Chromium & $1.00 \mathrm{E}-03$ & ICRP-38 & $2.77 E+01$ & d & $2.39 \mathrm{E}+06$ & $9.25 \mathrm{E}+04$ & ICRP-72 & $3.70 \mathrm{E}-11$ & $\mathrm{~S}$ & FGR-12 & $1.51 \mathrm{E}-15$ \\
\hline Cr-55 & Cr-55 & 54.941 & Chromium & $1.00 \mathrm{E}-03$ & ICRP-107 & $3.50 E+00$ & $\mathrm{~m}$ & $2.10 E+02$ & $9.79 E+08$ & -- & -- & -- & DOE-STD-1196 & $1.00 \mathrm{E}-15$ \\
\hline Cr-56 & Cr-56 & 55.941 & Chromium & $1.00 \mathrm{E}-03$ & ICRP-107 & $5.94 \mathrm{E}+00$ & $\mathrm{~m}$ & $3.56 \mathrm{E}+02$ & $5.66 \mathrm{E}+08$ & -- & -- & -- & DOE-STD-1196 & $3.47 \mathrm{E}-15$ \\
\hline Cs-121 & Cs-121 & 120.917 & Cesium & $1.00 \mathrm{E}-02$ & ICRP-107 & $1.55 \mathrm{E}+02$ & $\mathrm{~s}$ & $1.55 \mathrm{E}+02$ & $6.02 \mathrm{E}+08$ & -- & -- & -- & DOE-STD-1196 & $5.41 \mathrm{E}-14$ \\
\hline Cs-121m & Cs-121 & 120.917 & Cesium & $1.00 \mathrm{E}-02$ & ICRP-107 & $1.22 \mathrm{E}+02$ & $\mathrm{~s}$ & $1.22 \mathrm{E}+02$ & $7.65 E+08$ & -- & -- & -- & DOE-STD-1196 & $5.38 \mathrm{E}-14$ \\
\hline Cs-123 & Cs-123 & 122.913 & Cesium & $1.00 \mathrm{E}-02$ & ICRP-107 & $5.88 \mathrm{E}+00$ & $\mathrm{~m}$ & $3.53 \mathrm{E}+02$ & $2.60 E+08$ & -- & -- & -- & DOE-STD-1196 & $4.89 \mathrm{E}-14$ \\
\hline Cs-124 & Cs-124 & 123.912 & Cesium & $1.00 \mathrm{E}-02$ & ICRP-107 & $3.08 \mathrm{E}+01$ & $\mathrm{~s}$ & $3.08 \mathrm{E}+01$ & $2.96 \mathrm{E}+09$ & -- & -- & -- & DOE-STD-1196 & $5.43 \mathrm{E}-14$ \\
\hline Cs-125 & Cs-125 & 124.910 & Cesium & $1.00 \mathrm{E}-02$ & ICRP-38 & $4.50 \mathrm{E}+01$ & $\mathrm{~m}$ & $2.70 \mathrm{E}+03$ & $3.35 \mathrm{E}+07$ & ICRP-72 & $2.30 \mathrm{E}-11$ & $\mathrm{~s}$ & FGR-12 & $3.22 \mathrm{E}-14$ \\
\hline Cs-126 & Cs-126 & 125.909 & Cesium & $1.00 \mathrm{E}-02$ & ICRP-38 & $1.64 \mathrm{E}+00$ & $\mathrm{~m}$ & $9.84 \mathrm{E}+01$ & $9.11 \mathrm{E}+08$ & -- & -- & -- & FGR-12 & $5.24 \mathrm{E}-14$ \\
\hline Cs-127 & Cs-127 & 126.907 & Cesium & $1.00 \mathrm{E}-02$ & ICRP-38 & $6.25 \mathrm{E}+00$ & $\mathrm{~h}$ & $2.25 E+04$ & $3.95 \mathrm{E}+06$ & ICRP-72 & $3.80 \mathrm{E}-11$ & $\mathrm{~s}$ & FGR-12 & $1.93 \mathrm{E}-14$ \\
\hline Cs-128 & Cs-128 & 127.908 & Cesium & $1.00 \mathrm{E}-02$ & ICRP-38 & $3.90 \mathrm{E}+00$ & $\mathrm{~m}$ & $2.34 \mathrm{E}+02$ & $3.77 \mathrm{E}+08$ & -- & -- & -- & FGR-12 & $4.32 \mathrm{E}-14$ \\
\hline Cs-129 & Cs-129 & 128.906 & Cesium & $1.00 \mathrm{E}-02$ & ICRP-38 & $3.21 \mathrm{E}+01$ & $\mathrm{~h}$ & $1.15 \mathrm{E}+05$ & $7.58 \mathrm{E}+05$ & ICRP-72 & $7.70 \mathrm{E}-11$ & $\mathrm{~s}$ & FGR-12 & $1.24 \mathrm{E}-14$ \\
\hline Cs-130 & Cs-130 & 129.907 & Cesium & $1.00 \mathrm{E}-02$ & ICRP-38 & $2.99 \mathrm{E}+01$ & $\mathrm{~m}$ & $1.79 \mathrm{E}+03$ & $4.84 \mathrm{E}+07$ & ICRP-72 & $1.40 \mathrm{E}-11$ & $M$ & FGR-12 & $2.45 \mathrm{E}-14$ \\
\hline Cs-130m & Cs-130 & 129.907 & Cesium & $1.00 \mathrm{E}-02$ & ICRP-107 & $3.46 \mathrm{E}+00$ & $\mathrm{~m}$ & $2.08 \mathrm{E}+02$ & $4.18 \mathrm{E}+08$ & -- & -- & -- & DOE-STD-1196 & $2.01 \mathrm{E}-15$ \\
\hline Cs-131 & Cs-131 & 130.905 & Cesium & $1.00 \mathrm{E}-02$ & ICRP-38 & $9.69 \mathrm{E}+00$ & d & $8.37 \mathrm{E}+05$ & $1.03 E+05$ & ICRP-72 & $4.70 \mathrm{E}-11$ & $\mathrm{~s}$ & FGR-12 & $3.28 \mathrm{E}-16$ \\
\hline Cs-132 & Cs-132 & 131.906 & Cesium & $1.00 \mathrm{E}-02$ & ICRP-38 & $6.48 \mathrm{E}+00$ & $d$ & $5.59 \mathrm{E}+05$ & $1.53 \mathrm{E}+05$ & ICRP-72 & $3.00 \mathrm{E}-10$ & $\mathrm{~s}$ & FGR-12 & $3.34 \mathrm{E}-14$ \\
\hline Cs-134 & Cs-134 & 133.907 & Cesium & $1.00 \mathrm{E}-02$ & ICRP-38 & $2.06 \mathrm{E}+00$ & $y$ & $6.51 \mathrm{E}+07$ & $1.29 \mathrm{E}+03$ & ICRP-72 & $2.00 \mathrm{E}-08$ & $\mathrm{~s}$ & FGR-12 & $7.57 \mathrm{E}-14$ \\
\hline Cs-134m & Cs-134 & 133.907 & Cesium & $1.00 \mathrm{E}-02$ & ICRP-38 & $2.90 \mathrm{E}+00$ & $\mathrm{~h}$ & $1.04 \mathrm{E}+04$ & $8.07 \mathrm{E}+06$ & ICRP-72 & $6.00 \mathrm{E}-11$ & $\mathrm{~s}$ & FGR-12 & $9.05 \mathrm{E}-16$ \\
\hline Cs-135 & Cs-135 & 134.906 & Cesium & $1.00 \mathrm{E}-02$ & ICRP-38 & $2.30 \mathrm{E}+06$ & $y$ & $7.26 \mathrm{E}+13$ & $1.15 \mathrm{E}-03$ & ICRP-72 & $8.60 \mathrm{E}-09$ & $\mathrm{~s}$ & FGR-12 & $5.65 \mathrm{E}-19$ \\
\hline Cs-135m & Cs-135 & 134.906 & Cesium & $1.00 \mathrm{E}-02$ & ICRP-38 & $5.30 \mathrm{E}+01$ & $\mathrm{~m}$ & $3.18 \mathrm{E}+03$ & $2.63 \mathrm{E}+07$ & ICRP-72 & $1.60 \mathrm{E}-11$ & $\mathrm{~s}$ & FGR-12 & $7.76 \mathrm{E}-14$ \\
\hline Cs-136 & Cs-136 & 135.907 & Cesium & $1.00 \mathrm{E}-02$ & ICRP-38 & $1.31 \mathrm{E}+01$ & $d$ & $1.13 \mathrm{E}+06$ & $7.33 \mathrm{E}+04$ & ICRP-72 & $2.80 \mathrm{E}-09$ & $\mathrm{~s}$ & FGR-12 & $1.06 \mathrm{E}-13$ \\
\hline
\end{tabular}




\begin{tabular}{|c|c|c|c|c|c|c|c|c|c|c|c|c|c|c|}
\hline \multicolumn{15}{|c|}{ Table A.1 Radionuclide Specific Input Data Used For the Calculation of HC-2 TQs Using Maximum Reported DCs (continued) } \\
\hline $\begin{array}{c}\text { MASTER } \\
\text { ISOTOPE } \\
\text { LIST } \\
\end{array}$ & $\begin{array}{l}\text { Atomic } \\
\text { Mass } \\
\text { Nuclide }\end{array}$ & $\begin{array}{c}\text { Atomic } \\
\text { Mass }\end{array}$ & Element & $\begin{array}{c}\text { RF per } \\
\text { DOE-STD- } \\
1027 \\
\end{array}$ & $\begin{array}{c}\text { Half-Life } \\
\text { Reference }\end{array}$ & $\begin{array}{r}\text { Half-L } \\
\text { (original }\end{array}$ & & Half Life (s) & $\begin{array}{l}\text { Sp. Act } \\
\text { (Ci/g) }\end{array}$ & $\begin{array}{l}\text { Inhalation DC } \\
\text { Reference }\end{array}$ & $\begin{array}{c}\text { HC-2 } \\
\text { Bounding } \\
\text { Inhalation } \\
\text { DC (Sv/Bq) }\end{array}$ & $\begin{array}{c}\text { Lung } \\
\text { Absorption } \\
\text { Class/Type } \\
\end{array}$ & $\begin{array}{c}\text { Immersion DC } \\
\text { Reference }\end{array}$ & $\begin{array}{c}\mathrm{HC}-2 \\
\text { Immersion } \\
\mathrm{DC}(\mathrm{Sv} / \mathrm{s} \\
\left.\text { per } \mathrm{Bq} / \mathrm{m}^{3}\right)\end{array}$ \\
\hline Cs-137 & Cs-137 & 136.907 & Cesium & $1.00 \mathrm{E}-02$ & ICRP-38 & $3.00 \mathrm{E}+01$ & $\mathrm{y}$ & $9.47 \mathrm{E}+08$ & $8.70 \mathrm{E}+01$ & ICRP-72 & $3.90 \mathrm{E}-08$ & $\mathrm{~s}$ & FGR-12 & 7.74E-18 \\
\hline Cs-138 & Cs-138 & 137.911 & Cesium & $1.00 \mathrm{E}-02$ & ICRP-38 & $3.22 \mathrm{E}+01$ & $\mathrm{~m}$ & $1.93 \mathrm{E}+03$ & $4.23 \mathrm{E}+07$ & ICRP-72 & $4.30 \mathrm{E}-11$ & $\mathrm{~s}$ & FGR-12 & $1.21 \mathrm{E}-13$ \\
\hline Cs-138m & Cs-138 & 137.911 & Cesium & $1.00 \mathrm{E}-02$ & ICRP-107 & $2.91 \mathrm{E}+00$ & $\mathrm{~m}$ & $1.75 \mathrm{E}+02$ & $4.69 \mathrm{E}+08$ & -- & -- & -- & DOE-STD-1196 & $1.92 \mathrm{E}-14$ \\
\hline Cs-139 & Cs-139 & 138.913 & Cesium & $1.00 \mathrm{E}-02$ & ICRP-107 & $9.27 \mathrm{E}+00$ & $\mathrm{~m}$ & $5.56 \mathrm{E}+02$ & $1.46 \mathrm{E}+08$ & -- & -- & -- & DOE-STD-1196 & $1.66 \mathrm{E}-14$ \\
\hline Cs-140 & Cs-140 & 139.917 & Cesium & $1.00 \mathrm{E}-02$ & ICRP-107 & $6.37 \mathrm{E}+01$ & $\mathrm{~s}$ & $6.37 \mathrm{E}+01$ & $1.27 \mathrm{E}+09$ & -- & -- & -- & DOE-STD-1196 & $8.89 \mathrm{E}-14$ \\
\hline Cu-57 & Cu-57 & 56.949 & Copper & $1.00 \mathrm{E}-03$ & ICRP-38 & $2.33 \mathrm{E}+02$ & $\mathrm{~ms}$ & $2.33 \mathrm{E}-01$ & $8.50 \mathrm{E}+11$ & -- & -- & -- & DOE-STD-1196 & $5.65 \mathrm{E}-14$ \\
\hline Cu-59 & Cu-59 & 58.939 & Copper & $1.00 \mathrm{E}-03$ & ICRP-107 & $8.15 \mathrm{E}+01$ & $\mathrm{~s}$ & $8.15 \mathrm{E}+01$ & $2.35 \mathrm{E}+09$ & -- & -- & -- & DOE-STD-1196 & $6.68 \mathrm{E}-14$ \\
\hline Cu-60 & $\mathrm{Cu}-60$ & 59.937 & Copper & $1.00 \mathrm{E}-03$ & ICRP-38 & $2.32 \mathrm{E}+01$ & $\mathrm{~m}$ & $1.39 \mathrm{E}+03$ & $1.35 \mathrm{E}+08$ & ICRP-72 & $3.40 \mathrm{E}-11$ & $\mathrm{~s}$ & FGR-12 & $1.98 \mathrm{E}-13$ \\
\hline Cu-61 & Cu-61 & 60.933 & Copper & $1.00 \mathrm{E}-03$ & ICRP-38 & $3.41 \mathrm{E}+00$ & $\mathrm{~h}$ & $1.23 \mathrm{E}+04$ & $1.51 \mathrm{E}+07$ & ICRP-72 & $7.80 \mathrm{E}-11$ & $\mathrm{~s}$ & FGR-12 & $3.99 \mathrm{E}-14$ \\
\hline Cu-62 & Cu-62 & 61.933 & Copper & $1.00 \mathrm{E}-03$ & ICRP-38 & $9.74 \mathrm{E}+00$ & $\mathrm{~m}$ & $5.84 \mathrm{E}+02$ & $3.12 \mathrm{E}+08$ & -- & -- & -- & FGR-12 & $4.86 \mathrm{E}-14$ \\
\hline Cu-64 & Cu-64 & 63.930 & Copper & $1.00 \mathrm{E}-03$ & ICRP-38 & $1.27 \mathrm{E}+01$ & $\mathrm{~h}$ & $4.57 \mathrm{E}+04$ & $3.86 \mathrm{E}+06$ & ICRP-72 & $1.20 \mathrm{E}-10$ & $\mathrm{~s}$ & FGR-12 & $9.10 \mathrm{E}-15$ \\
\hline Cu-66 & Cu-66 & 65.929 & Copper & $1.00 \mathrm{E}-03$ & ICRP-38 & $5.10 \mathrm{E}+00$ & $\mathrm{~m}$ & $3.06 \mathrm{E}+02$ & $5.59 \mathrm{E}+08$ & -- & -- & -- & FGR-12 & $4.46 \mathrm{E}-15$ \\
\hline Cu-67 & Cu-67 & 66.928 & Copper & $1.00 \mathrm{E}-03$ & ICRP-38 & $6.19 \mathrm{E}+01$ & $\mathrm{~h}$ & $2.23 \mathrm{E}+05$ & $7.57 \mathrm{E}+05$ & ICRP-72 & $6.10 \mathrm{E}-10$ & $\mathrm{~s}$ & FGR-12 & $5.41 \mathrm{E}-15$ \\
\hline Cu-69 & Cu-69 & 68.929 & Copper & $1.00 \mathrm{E}-03$ & ICRP-107 & $2.85 \mathrm{E}+00$ & $\mathrm{~m}$ & $1.71 \mathrm{E}+02$ & $9.57 \mathrm{E}+08$ & -- & -- & -- & DOE-STD-1196 & $2.53 \mathrm{E}-14$ \\
\hline Dy-148 & Dy-148 & 147.927 & Dysprosium & $1.00 \mathrm{E}-03$ & ICRP-107 & $3.30 \mathrm{E}+00$ & $\mathrm{~m}$ & $1.98 \mathrm{E}+02$ & $3.85 \mathrm{E}+08$ & -- & -- & -- & DOE-STD-1196 & $3.15 \mathrm{E}-14$ \\
\hline Dy-149 & Dy-149 & 148.927 & Dysprosium & $1.00 \mathrm{E}-03$ & ICRP-107 & $4.20 \mathrm{E}+00$ & $\mathrm{~m}$ & $2.52 E+02$ & $3.01 E+08$ & -- & -- & -- & DOE-STD-1196 & $7.52 \mathrm{E}-14$ \\
\hline Dy-150 & Dy-150 & 149.926 & Dysprosium & $1.00 \mathrm{E}-03$ & ICRP-107 & $7.17 \mathrm{E}+00$ & $\mathrm{~m}$ & $4.30 \mathrm{E}+02$ & $1.75 \mathrm{E}+08$ & -- & -- & -- & DOE-STD-1196 & $1.18 \mathrm{E}-14$ \\
\hline Dy-151 & Dy-151 & 150.926 & Dysprosium & $1.00 \mathrm{E}-03$ & ICRP-107 & $1.79 \mathrm{E}+01$ & $\mathrm{~m}$ & $1.07 \mathrm{E}+03$ & $6.96 \mathrm{E}+07$ & DOE-STD-1196 & $1.51 \mathrm{E}-10$ & $\mathrm{~s}$ & DOE-STD-1196 & $6.28 \mathrm{E}-14$ \\
\hline Dy-152 & Dy-152 & 151.925 & Dysprosium & $1.00 \mathrm{E}-03$ & ICRP-107 & $2.38 \mathrm{E}+00$ & $\mathrm{~h}$ & $8.57 \mathrm{E}+03$ & $8.67 E+06$ & DOE-STD-1196 & $7.46 \mathrm{E}-11$ & $\mathrm{~s}$ & DOE-STD-1196 & $1.18 \mathrm{E}-14$ \\
\hline Dy-153 & Dy-153 & 152.926 & Dysprosium & $1.00 \mathrm{E}-03$ & ICRP-107 & $6.40 \mathrm{E}+00$ & $\mathrm{~h}$ & $2.30 \mathrm{E}+04$ & $3.20 \mathrm{E}+06$ & DOE-STD-1196 & $1.51 \mathrm{E}-10$ & $\mathrm{~s}$ & DOE-STD-1196 & $3.82 \mathrm{E}-14$ \\
\hline Dy-154 & Dy-154 & 153.924 & Dysprosium & $1.00 \mathrm{E}-03$ & ICRP-107 & $3.00 \mathrm{E}+06$ & $y$ & $9.47 \mathrm{E}+13$ & $7.74 \mathrm{E}-04$ & DOE-STD-1196 & $2.74 \mathrm{E}-05$ & $F$ & -- & -- \\
\hline Dy-155 & Dy-155 & 154.926 & Dysprosium & $1.00 \mathrm{E}-03$ & ICRP-38 & $1.00 \mathrm{E}+01$ & $\mathrm{~h}$ & $3.60 \mathrm{E}+04$ & $2.02 \mathrm{E}+06$ & ICRP-72 & $7.70 \mathrm{E}-11$ & $M$ & FGR-12 & $2.77 E-14$ \\
\hline Dy-157 & Dy-157 & 156.925 & Dysprosium & $1.00 \mathrm{E}-03$ & ICRP-38 & $8.10 \mathrm{E}+00$ & $\mathrm{~h}$ & $2.92 \mathrm{E}+04$ & $2.47 \mathrm{E}+06$ & ICRP-72 & $3.00 \mathrm{E}-11$ & $M$ & FGR-12 & $1.63 \mathrm{E}-14$ \\
\hline Dy-159 & Dy-159 & 158.926 & Dysprosium & $1.00 \mathrm{E}-03$ & ICRP-38 & $1.44 \mathrm{E}+02$ & $d$ & $1.25 \mathrm{E}+07$ & $5.69 \mathrm{E}+03$ & ICRP-72 & $3.70 \mathrm{E}-10$ & $M$ & FGR-12 & $1.25 \mathrm{E}-15$ \\
\hline Dy-165 & Dy-165 & 164.932 & Dysprosium & $1.00 \mathrm{E}-03$ & ICRP-38 & $2.33 \mathrm{E}+00$ & $\mathrm{~h}$ & $8.40 \mathrm{E}+03$ & $8.14 \mathrm{E}+06$ & ICRP-72 & $6.00 \mathrm{E}-11$ & $M$ & FGR-12 & $1.20 \mathrm{E}-15$ \\
\hline Dy-165m & Dy-165 & 164.932 & Dysprosium & $1.00 \mathrm{E}-03$ & ICRP-107 & $1.26 \mathrm{E}+00$ & $\mathrm{~m}$ & $7.54 \mathrm{E}+01$ & $9.07 E+08$ & -- & -- & -- & DOE-STD-1196 & $7.08 \mathrm{E}-16$ \\
\hline Dy-166 & Dy-166 & 165.933 & Dysprosium & $1.00 \mathrm{E}-03$ & ICRP-38 & $8.16 \mathrm{E}+01$ & $\mathrm{~h}$ & $2.94 \mathrm{E}+05$ & $2.31 \mathrm{E}+05$ & ICRP-72 & $1.90 \mathrm{E}-09$ & $M$ & FGR-12 & $1.40 \mathrm{E}-15$ \\
\hline Dy-167 & Dy-167 & 166.936 & Dysprosium & $1.00 \mathrm{E}-03$ & ICRP-107 & $6.20 \mathrm{E}+00$ & $\mathrm{~m}$ & $3.72 \mathrm{E}+02$ & $1.82 \mathrm{E}+08$ & -- & -- & -- & DOE-STD-1196 & $2.40 \mathrm{E}-14$ \\
\hline Dy-168 & Dy-168 & 167.937 & Dysprosium & $1.00 \mathrm{E}-03$ & ICRP-107 & $8.70 E+00$ & $\mathrm{~m}$ & $5.22 \mathrm{E}+02$ & $1.29 \mathrm{E}+08$ & -- & -- & -- & DOE-STD-1196 & $1.73 \mathrm{E}-14$ \\
\hline Er-154 & Er-154 & 153.933 & Erbium & $1.00 \mathrm{E}-03$ & ICRP-107 & $3.73 \mathrm{E}+00$ & $\mathrm{~m}$ & $2.24 \mathrm{E}+02$ & $3.27 \mathrm{E}+08$ & -- & -- & -- & DOE-STD-1196 & $2.24 \mathrm{E}-15$ \\
\hline Er-155 & Er-155 & 154.933 & Erbium & $1.00 \mathrm{E}-03$ & JAERI & $5.30 \mathrm{E}+00$ & $\mathrm{~m}$ & $3.18 \mathrm{E}+02$ & $2.29 \mathrm{E}+08$ & -- & -- & -- & -- & -- \\
\hline Er-156 & Er-156 & 155.931 & Erbium & $1.00 \mathrm{E}-03$ & ICRP-107 & $1.95 \mathrm{E}+01$ & $\mathrm{~m}$ & $1.17 \mathrm{E}+03$ & $6.18 \mathrm{E}+07$ & DOE-STD-1196 & $2.38 \mathrm{E}-11$ & $\mathrm{~s}$ & DOE-STD-1196 & $1.72 \mathrm{E}-15$ \\
\hline Er-159 & Er-159 & 158.931 & Erbium & $1.00 \mathrm{E}-03$ & ICRP-107 & $3.60 \mathrm{E}+01$ & $\mathrm{~m}$ & $2.16 \mathrm{E}+03$ & $3.29 \mathrm{E}+07$ & DOE-STD-1196 & $2.07 E-11$ & $\mathrm{~s}$ & DOE-STD-1196 & $4.36 \mathrm{E}-14$ \\
\hline Er-161 & Er-161 & 160.930 & Erbium & $1.00 \mathrm{E}-03$ & ICRP-38 & $3.24 \mathrm{E}+00$ & $\mathrm{~h}$ & $1.17 \mathrm{E}+04$ & $6.01 \mathrm{E}+06$ & ICRP-72 & $4.80 \mathrm{E}-11$ & $M$ & FGR-12 & $4.42 \mathrm{E}-14$ \\
\hline Er-163 & Er-163 & 162.930 & Erbium & $1.00 \mathrm{E}-03$ & ICRP-107 & $7.50 E+01$ & $\mathrm{~m}$ & $4.50 \mathrm{E}+03$ & $1.54 \mathrm{E}+07$ & DOE-STD-1196 & $1.56 \mathrm{E}-12$ & $\mathrm{~s}$ & DOE-STD-1196 & $9.82 \mathrm{E}-16$ \\
\hline Er-165 & Er-165 & 164.931 & Erbium & $1.00 \mathrm{E}-03$ & ICRP-38 & $1.04 \mathrm{E}+01$ & $\mathrm{~h}$ & $3.73 E+04$ & $1.83 \mathrm{E}+06$ & ICRP-72 & $7.90 \mathrm{E}-12$ & $M$ & FGR-12 & $1.11 \mathrm{E}-15$ \\
\hline Er-167m & Er-167 & 166.932 & Erbium & $1.00 \mathrm{E}-03$ & ICRP-38 & $2.28 \mathrm{E}+00$ & $\mathrm{~s}$ & $2.28 \mathrm{E}+00$ & $2.96 \mathrm{E}+10$ & -- & -- & -- & DOE-STD-1196 & $4.03 \mathrm{E}-15$ \\
\hline Er-169 & Er-169 & 168.935 & Erbium & $1.00 \mathrm{E}-03$ & ICRP-38 & $9.30 \mathrm{E}+00$ & $d$ & $8.04 \mathrm{E}+05$ & $8.31 \mathrm{E}+04$ & ICRP-72 & $1.00 \mathrm{E}-09$ & $M$ & FGR-12 & $1.74 \mathrm{E}-18$ \\
\hline Er-171 & Er-171 & 170.938 & Erbium & $1.00 \mathrm{E}-03$ & ICRP-38 & $7.52 \mathrm{E}+00$ & $\mathrm{~h}$ & $2.71 E+04$ & $2.44 \mathrm{E}+06$ & ICRP-72 & $2.20 \mathrm{E}-10$ & $M$ & FGR-12 & $1.78 \mathrm{E}-14$ \\
\hline Er-172 & Er-172 & 171.939 & Erbium & $1.00 \mathrm{E}-03$ & ICRP-38 & $4.93 \mathrm{E}+01$ & $\mathrm{~h}$ & $1.77 \mathrm{E}+05$ & $3.70 \mathrm{E}+05$ & ICRP-72 & $1.10 \mathrm{E}-09$ & $M$ & FGR-12 & $2.47 \mathrm{E}-14$ \\
\hline Er-173 & Er-173 & 172.942 & $\begin{array}{l}\text { Erbium } \\
\end{array}$ & $1.00 \mathrm{E}-03$ & ICRP-107 & $1.43 \mathrm{E}+00$ & $\mathrm{~m}$ & $8.60 \mathrm{E}+01$ & $7.58 \mathrm{E}+08$ & -- & -- & -- & DOE-STD-1196 & $3.73 \mathrm{E}-14$ \\
\hline Es-249 & Es-249 & 249.076 & Einsteinium & $1.00 \mathrm{E}-03$ & ICRP-107 & $1.02 \mathrm{E}+02$ & $\mathrm{~m}$ & $6.13 E+03$ & $7.39 \mathrm{E}+06$ & DOE-STD-1196 & $2.49 \mathrm{E}-10$ & $M$ & DOE-STD-1196 & $1.77 \mathrm{E}-14$ \\
\hline Es-250 & Es-250 & 250.079 & Einsteinium & $1.00 \mathrm{E}-03$ & ICRP-38 & $2.10 \mathrm{E}+00$ & $\mathrm{~h}$ & $7.56 \mathrm{E}+03$ & $5.97 \mathrm{E}+06$ & ICRP-72 & $6.30 \mathrm{E}-10$ & $M$ & FGR-12 & $1.90 \mathrm{E}-14$ \\
\hline Es-250m & Es-250 & 250.079 & Einsteinium & $1.00 \mathrm{E}-03$ & ICRP-107 & $2.22 \mathrm{E}+00$ & $\mathrm{~h}$ & $7.99 \mathrm{E}+03$ & $5.64 \mathrm{E}+06$ & DOE-STD-1196 & $1.59 \mathrm{E}-09$ & $F$ & DOE-STD-1196 & $2.49 \mathrm{E}-14$ \\
\hline Es-251 & Es-251 & 251.080 & Einsteinium & $1.00 \mathrm{E}-03$ & ICRP-38 & $3.30 \mathrm{E}+01$ & $\mathrm{~h}$ & $1.19 \mathrm{E}+05$ & $3.78 \mathrm{E}+05$ & ICRP-72 & $2.10 \mathrm{E}-09$ & $M$ & FGR-12 & $4.13 \mathrm{E}-15$ \\
\hline Es-253 & Es-253 & 253.085 & Einsteinium & $1.00 \mathrm{E}-03$ & ICRP-38 & $2.05 E+01$ & $d$ & $1.77 \mathrm{E}+06$ & $2.52 \mathrm{E}+04$ & ICRP-72 & $2.70 \mathrm{E}-06$ & $M$ & FGR-12 & $1.83 \mathrm{E}-17$ \\
\hline
\end{tabular}




\begin{tabular}{|c|c|c|c|c|c|c|c|c|c|c|c|c|c|c|}
\hline \multicolumn{15}{|c|}{ Table A.1 Radionuclide Specific Input Data Used For the Calculation of HC-2 TQs Using Maximum Reported DCs (continued) } \\
\hline $\begin{array}{c}\text { MASTER } \\
\text { ISOTOPE } \\
\text { LIST }\end{array}$ & $\begin{array}{l}\text { Atomic } \\
\text { Mass } \\
\text { Nuclide }\end{array}$ & $\begin{array}{l}\text { Atomic } \\
\text { Mass }\end{array}$ & Element & $\begin{array}{c}\text { RF per } \\
\text { DOE-STD- } \\
1027\end{array}$ & $\begin{array}{c}\text { Half-Life } \\
\text { Reference }\end{array}$ & $\begin{array}{r}\text { Half-Li } \\
\text { (original }\end{array}$ & & Half Life (s) & $\begin{array}{l}\text { Sp. Act } \\
\text { (Ci/g) }\end{array}$ & $\begin{array}{l}\text { Inhalation DC } \\
\text { Reference }\end{array}$ & $\begin{array}{c}\text { HC-2 } \\
\text { Bounding } \\
\text { Inhalation } \\
\text { DC (Sv/Bq) }\end{array}$ & $\begin{array}{l}\text { Lung } \\
\text { Absorption } \\
\text { Class/Type }\end{array}$ & $\begin{array}{l}\text { Immersion DC } \\
\text { Reference }\end{array}$ & $\begin{array}{c}\mathrm{HC}-2 \\
\text { Immersion } \\
\mathrm{DC}(\mathrm{Sv} / \mathrm{s} \\
\left.\text { per } \mathrm{Bq} / \mathrm{m}^{3}\right)\end{array}$ \\
\hline Es-254 & Es-254 & 254.088 & Einsteinium & $1.00 \mathrm{E}-03$ & ICRP-38 & $2.76 \mathrm{E}+02$ & d & $2.38 \mathrm{E}+07$ & $1.86 \mathrm{E}+03$ & ICRP-72 & $8.60 \mathrm{E}-06$ & $\mathrm{M}$ & FGR-12 & $1.93 \mathrm{E}-16$ \\
\hline Es-254m & Es-254 & 254.088 & Einsteinium & $1.00 \mathrm{E}-03$ & ICRP-38 & $3.93 \mathrm{E}+01$ & $\mathrm{~h}$ & $1.41 \mathrm{E}+05$ & $3.14 \mathrm{E}+05$ & ICRP-72 & $4.70 \mathrm{E}-07$ & $M$ & FGR-12 & $2.25 \mathrm{E}-14$ \\
\hline Es-255 & Es-255 & 255.090 & Einsteinium & $1.00 \mathrm{E}-03$ & ICRP-107 & $3.98 \mathrm{E}+01$ & $d$ & $3.44 \mathrm{E}+06$ & $1.29 \mathrm{E}+04$ & DOE-STD-1196 & $4.95 \mathrm{E}-06$ & $\mathrm{~s}$ & DOE-STD-1196 & $4.95 \mathrm{E}-17$ \\
\hline Es-256 & Es-256 & 256.094 & Einsteinium & $1.00 \mathrm{E}-03$ & ICRP-107 & $2.54 \mathrm{E}+01$ & $\mathrm{~m}$ & $1.52 E+03$ & $2.89 \mathrm{E}+07$ & DOE-STD-1196 & $4.55 \mathrm{E}-08$ & $\mathrm{~s}$ & DOE-STD-1196 & $4.33 \mathrm{E}-16$ \\
\hline Eu-142 & Eu-142 & 141.923 & Europium & $1.00 \mathrm{E}-03$ & ICRP-107 & $2.34 \mathrm{E}+00$ & $\mathrm{~s}$ & $2.34 \mathrm{E}+00$ & $3.40 \mathrm{E}+10$ & -- & -- & -- & DOE-STD-1196 & $5.78 \mathrm{E}-14$ \\
\hline Eu-142m & Eu-142 & 141.923 & Europium & $1.00 \mathrm{E}-03$ & ICRP-107 & $1.22 \mathrm{E}+00$ & $\mathrm{~m}$ & $7.34 \mathrm{E}+01$ & $1.08 \mathrm{E}+09$ & -- & -- & -- & DOE-STD-1196 & $1.58 \mathrm{E}-13$ \\
\hline Eu-143 & Eu-143 & 142.920 & Europium & $1.00 \mathrm{E}-03$ & ICRP-107 & $2.59 \mathrm{E}+00$ & $\mathrm{~m}$ & $1.55 \mathrm{E}+02$ & $5.08 \mathrm{E}+08$ & -- & -- & -- & DOE-STD-1196 & $5.26 \mathrm{E}-14$ \\
\hline Eu-144 & Eu-144 & 143.919 & Europium & $1.00 \mathrm{E}-03$ & ICRP-107 & $1.02 \mathrm{E}+01$ & $s$ & $1.02 \mathrm{E}+01$ & $7.69 \mathrm{E}+09$ & -- & -- & -- & DOE-STD-1196 & $5.18 \mathrm{E}-14$ \\
\hline Eu-145 & Eu-145 & 144.916 & Europium & $1.00 \mathrm{E}-03$ & ICRP-38 & $5.94 \mathrm{E}+00$ & $d$ & $5.13 E+05$ & $1.52 \mathrm{E}+05$ & ICRP-72 & $5.50 \mathrm{E}-10$ & $M$ & FGR-12 & $7.22 \mathrm{E}-14$ \\
\hline Eu-146 & Eu-146 & 145.917 & Europium & $1.00 \mathrm{E}-03$ & ICRP-38 & $4.61 \mathrm{E}+00$ & $d$ & $3.98 \mathrm{E}+05$ & $1.94 \mathrm{E}+05$ & ICRP-72 & $8.00 \mathrm{E}-10$ & $M$ & FGR-12 & $1.23 \mathrm{E}-13$ \\
\hline Eu-147 & Eu-147 & 146.917 & Europium & $1.00 \mathrm{E}-03$ & ICRP-38 & $2.40 \mathrm{E}+01$ & $d$ & $2.07 E+06$ & $3.70 \mathrm{E}+04$ & ICRP-72 & $1.10 \mathrm{E}-09$ & $M$ & FGR-12 & $2.32 \mathrm{E}-14$ \\
\hline Eu-148 & Eu-148 & 147.918 & Europium & $1.00 \mathrm{E}-03$ & ICRP-38 & $5.45 \mathrm{E}+01$ & $d$ & $4.71 \mathrm{E}+06$ & $1.62 \mathrm{E}+04$ & ICRP-72 & $2.60 \mathrm{E}-09$ & $M$ & FGR-12 & $1.06 \mathrm{E}-13$ \\
\hline Eu-149 & Eu-149 & 148.918 & Europium & $1.00 \mathrm{E}-03$ & ICRP-38 & $9.31 \mathrm{E}+01$ & $d$ & $8.04 \mathrm{E}+06$ & $9.42 \mathrm{E}+03$ & ICRP-72 & $2.90 \mathrm{E}-10$ & $M$ & FGR-12 & $2.25 \mathrm{E}-15$ \\
\hline Eu-150l & Eu-150 & 149.920 & Europium & $1.00 \mathrm{E}-03$ & ICRP-38 & $3.42 \mathrm{E}+01$ & $y$ & $1.08 \mathrm{E}+09$ & $6.97 \mathrm{E}+01$ & ICRP-72 & $5.30 \mathrm{E}-08$ & $M$ & FGR-12 & $7.17 \mathrm{E}-14$ \\
\hline Eu-150s & Eu-150 & 149.920 & Europium & $1.00 \mathrm{E}-03$ & ICRP-38 & $1.26 \mathrm{E}+01$ & $\mathrm{~h}$ & $4.54 \mathrm{E}+04$ & $1.66 \mathrm{E}+06$ & ICRP-72 & $1.90 \mathrm{E}-10$ & $M$ & FGR-12 & $2.21 \mathrm{E}-15$ \\
\hline Eu-152 & Eu-152 & 151.922 & Europium & $1.00 \mathrm{E}-03$ & ICRP-38 & $1.33 \mathrm{E}+01$ & $y$ & $4.21 E+08$ & $1.77 \mathrm{E}+02$ & ICRP-72 & $4.20 \mathrm{E}-08$ & $M$ & FGR-12 & $5.65 \mathrm{E}-14$ \\
\hline Eu-152ml & Eu-152 & 151.922 & Europium & $1.00 \mathrm{E}-03$ & ICRP-38 & $9.32 \mathrm{E}+00$ & $\mathrm{~h}$ & $3.36 \mathrm{E}+04$ & $2.21 E+06$ & ICRP-72 & $2.20 \mathrm{E}-10$ & $M$ & FGR-12 & $1.42 \mathrm{E}-14$ \\
\hline Eu-152ms & Eu-152 & 151.922 & Europium & $1.00 \mathrm{E}-03$ & ICRP-107 & $9.60 \mathrm{E}+01$ & $\mathrm{~m}$ & $5.76 \mathrm{E}+03$ & $1.29 \mathrm{E}+07$ & DOE-STD-1196 & $1.01 \mathrm{E}-11$ & $M$ & DOE-STD-1196 & $2.63 \mathrm{E}-15$ \\
\hline Eu-154 & Eu-154 & 153.923 & Europium & $1.00 \mathrm{E}-03$ & ICRP-38 & $8.80 \mathrm{E}+00$ & $y$ & $2.78 \mathrm{E}+08$ & $2.64 \mathrm{E}+02$ & ICRP-72 & $5.30 \mathrm{E}-08$ & $M$ & FGR-12 & $6.14 \mathrm{E}-14$ \\
\hline Eu-154m & Eu-154 & 153.923 & Europium & $1.00 \mathrm{E}-03$ & ICRP-107 & $4.60 \mathrm{E}+01$ & $\mathrm{~m}$ & $2.76 \mathrm{E}+03$ & $2.66 \mathrm{E}+07$ & DOE-STD-1196 & $4.76 \mathrm{E}-12$ & $\mathrm{~s}$ & DOE-STD-1196 & $2.15 \mathrm{E}-15$ \\
\hline Eu-155 & Eu-155 & 154.923 & Europium & $1.00 \mathrm{E}-03$ & ICRP-38 & $4.96 \mathrm{E}+00$ & $y$ & $1.57 \mathrm{E}+08$ & $4.65 E+02$ & ICRP-72 & $6.90 \mathrm{E}-09$ & $M$ & FGR-12 & $2.49 \mathrm{E}-15$ \\
\hline Eu-156 & Eu-156 & 155.925 & Europium & $1.00 \mathrm{E}-03$ & ICRP-38 & $1.52 \mathrm{E}+01$ & $d$ & $1.31 \mathrm{E}+06$ & $5.51 \mathrm{E}+04$ & ICRP-72 & $3.40 \mathrm{E}-09$ & $M$ & FGR-12 & $6.75 \mathrm{E}-14$ \\
\hline Eu-157 & Eu-157 & 156.925 & Europium & $1.00 \mathrm{E}-03$ & ICRP-38 & $1.52 \mathrm{E}+01$ & $\mathrm{~h}$ & $5.45 \mathrm{E}+04$ & $1.32 \mathrm{E}+06$ & ICRP-72 & $2.80 \mathrm{E}-10$ & $M$ & FGR-12 & $1.17 \mathrm{E}-14$ \\
\hline Eu-158 & Eu-158 & 157.928 & Europium & $1.00 \mathrm{E}-03$ & ICRP-38 & $4.59 \mathrm{E}+01$ & $\mathrm{~m}$ & $2.75 E+03$ & $2.59 \mathrm{E}+07$ & ICRP-72 & $4.70 \mathrm{E}-11$ & $M$ & FGR-12 & $5.27 \mathrm{E}-14$ \\
\hline Eu-159 & Eu-159 & 158.929 & Europium & $1.00 \mathrm{E}-03$ & ICRP-107 & $1.81 \mathrm{E}+01$ & $\mathrm{~m}$ & $1.09 \mathrm{E}+03$ & $6.54 \mathrm{E}+07$ & DOE-STD-1196 & $2.85 \mathrm{E}-11$ & $\mathrm{~s}$ & DOE-STD-1196 & $1.32 \mathrm{E}-14$ \\
\hline $\mathrm{F}-17$ & F-17 & 17.002 & Fluorine & $1.00 E+00$ & ICRP-107 & $6.45 \mathrm{E}+01$ & $\mathrm{~s}$ & $6.45 \mathrm{E}+01$ & $1.03 \mathrm{E}+10$ & -- & -- & -- & DOE-STD-1196 & $4.60 \mathrm{E}-14$ \\
\hline F-18 & F-18 & 18.001 & Fluorine & $1.00 \mathrm{E}+00$ & ICRP-38 & $1.10 \mathrm{E}+02$ & $\mathrm{~m}$ & $6.59 \mathrm{E}+03$ & $9.52 \mathrm{E}+07$ & ICRP-72 & $5.90 \mathrm{E}-11$ & $\mathrm{~s}$ & FGR-12 & $4.90 \mathrm{E}-14$ \\
\hline Fe-52 & Fe-52 & 51.948 & Iron & $1.00 \mathrm{E}-03$ & ICRP-38 & $8.28 \mathrm{E}+00$ & $\mathrm{~h}$ & $2.98 \mathrm{E}+04$ & $7.29 \mathrm{E}+06$ & ICRP-72 & $6.30 \mathrm{E}-10$ & $\mathrm{~s}$ & FGR-12 & $3.54 \mathrm{E}-14$ \\
\hline Fe-53 & Fe-53 & 52.945 & Iron & $1.00 \mathrm{E}-03$ & ICRP-107 & $8.51 \mathrm{E}+00$ & $\mathrm{~m}$ & $5.11 E+02$ & $4.17 \mathrm{E}+08$ & -- & -- & -- & DOE-STD-1196 & $5.35 \mathrm{E}-14$ \\
\hline Fe-53m & Fe-53 & 52.945 & Iron & $1.00 \mathrm{E}-03$ & ICRP-107 & $2.53 \mathrm{E}+00$ & $\mathrm{~m}$ & $1.52 \mathrm{E}+02$ & $1.41 \mathrm{E}+09$ & -- & -- & -- & DOE-STD-1196 & $1.44 \mathrm{E}-13$ \\
\hline Fe-55 & Fe-55 & 54.938 & Iron & $1.00 \mathrm{E}-03$ & ICRP-38 & $2.70 E+00$ & $y$ & $8.52 \mathrm{E}+07$ & $2.41 E+03$ & ICRP-72 & 7.70E-10 & $\mathrm{F}$ & DOE-STD-1196 & $6.69 \mathrm{E}-24$ \\
\hline Fe-59 & Fe-59 & 58.935 & Iron & $1.00 \mathrm{E}-03$ & ICRP-38 & $4.45 \mathrm{E}+01$ & d & $3.85 \mathrm{E}+06$ & $4.98 \mathrm{E}+04$ & ICRP-72 & $4.00 \mathrm{E}-09$ & $\mathrm{~s}$ & FGR-12 & $5.97 \mathrm{E}-14$ \\
\hline Fe-60 & Fe-60 & 59.934 & Iron & $1.00 \mathrm{E}-03$ & ICRP-38 & $1.00 \mathrm{E}+05$ & $y$ & $3.16 \mathrm{E}+12$ & $5.96 \mathrm{E}-02$ & ICRP-72 & $2.80 \mathrm{E}-07$ & $\mathrm{~F}$ & FGR-12 & $1.95 \mathrm{E}-19$ \\
\hline Fe-61 & Fe-61 & 60.937 & Iron & $1.00 \mathrm{E}-03$ & ICRP-107 & $5.98 \mathrm{E}+00$ & $\mathrm{~m}$ & $3.59 \mathrm{E}+02$ & $5.16 \mathrm{E}+08$ & -- & -- & -- & DOE-STD-1196 & $6.68 \mathrm{E}-14$ \\
\hline Fe-62 & Fe-62 & 61.937 & Iron & $1.00 \mathrm{E}-03$ & ICRP-107 & $6.80 \mathrm{E}+01$ & $\mathrm{~s}$ & $6.80 \mathrm{E}+01$ & $2.68 \mathrm{E}+09$ & $\begin{array}{ll}-- \\
\end{array}$ & -- & -- & DOE-STD-1196 & $2.32 \mathrm{E}-14$ \\
\hline $\mathrm{Fm}-251$ & $\mathrm{Fm}-251$ & 251.082 & Fermium & $1.00 \mathrm{E}-03$ & ICRP-107 & $5.30 \mathrm{E}+00$ & $\mathrm{~h}$ & $1.91 \mathrm{E}+04$ & $2.35 E+06$ & DOE-STD-1196 & $2.16 \mathrm{E}-09$ & $\mathrm{~s}$ & DOE-STD-1196 & $6.35 \mathrm{E}-15$ \\
\hline Fm-252 & Fm-252 & 252.082 & Fermium & $1.00 \mathrm{E}-03$ & ICRP-38 & $2.27 \mathrm{E}+01$ & $\mathrm{~h}$ & $8.17 \mathrm{E}+04$ & $5.48 \mathrm{E}+05$ & ICRP-72 & $3.20 \mathrm{E}-07$ & $M$ & FGR-12 & $5.03 \mathrm{E}-18$ \\
\hline Fm-253 & Fm-253 & 253.085 & Fermium & $1.00 \mathrm{E}-03$ & ICRP-38 & $3.00 \mathrm{E}+00$ & $d$ & $2.59 \mathrm{E}+05$ & $1.72 \mathrm{E}+05$ & ICRP-72 & $4.00 \mathrm{E}-07$ & $M$ & FGR-12 & $3.53 \mathrm{E}-15$ \\
\hline Fm-254 & Fm-254 & 254.087 & Fermium & $1.00 \mathrm{E}-03$ & ICRP-38 & $3.24 \mathrm{E}+00$ & $\mathrm{~h}$ & $1.17 \mathrm{E}+04$ & $3.81 \mathrm{E}+06$ & ICRP-72 & $6.10 \mathrm{E}-08$ & $M$ & FGR-12 & $6.57 \mathrm{E}-18$ \\
\hline $\mathrm{Fm}-255$ & $\mathrm{Fm}-255$ & 255.090 & Fermium & $1.00 \mathrm{E}-03$ & ICRP-38 & $2.01 E+01$ & $\mathrm{~h}$ & $7.23 E+04$ & $6.12 \mathrm{E}+05$ & ICRP-72 & $2.70 \mathrm{E}-07$ & $M$ & FGR-12 & $1.10 \mathrm{E}-16$ \\
\hline Fm-256 & Fm-256 & 256.092 & Fermium & $1.00 \mathrm{E}-03$ & ICRP-107 & $1.58 \mathrm{E}+02$ & $\mathrm{~m}$ & $9.46 \mathrm{E}+03$ & $4.66 \mathrm{E}+06$ & DOE-STD-1196 & $2.85 \mathrm{E}-07$ & $\mathrm{~s}$ & DOE-STD-1196 & $6.07 E-13$ \\
\hline $\mathrm{Fm}-257$ & Fm-257 & 257.095 & Fermium & $1.00 \mathrm{E}-03$ & ICRP-38 & $1.01 E+02$ & d & $8.68 \mathrm{E}+06$ & $5.05 E+03$ & ICRP-72 & $7.10 \mathrm{E}-06$ & $M$ & FGR-12 & $4.66 \mathrm{E}-15$ \\
\hline $\mathrm{Fr}-212$ & $\mathrm{Fr}-212$ & 211.996 & Francium & $1.00 \mathrm{E}-03$ & ICRP-107 & $2.00 E+01$ & $\mathrm{~m}$ & $1.20 \mathrm{E}+03$ & $4.43 \mathrm{E}+07$ & DOE-STD-1196 & $6.98 \mathrm{E}-09$ & $\mathrm{~s}$ & DOE-STD-1196 & $5.26 \mathrm{E}-14$ \\
\hline Fr-219 & Fr-219 & 219.009 & Francium & $1.00 \mathrm{E}-03$ & ICRP-38 & $2.10 \mathrm{E}+01$ & $\mathrm{~ms}$ & $2.10 \mathrm{E}-02$ & $2.45 E+12$ & -- & -- & -- & FGR-12 & $1.66 \mathrm{E}-16$ \\
\hline Fr-220 & Fr-220 & 220.012 & Francium & $1.00 \mathrm{E}-03$ & ICRP-38 & $2.74 \mathrm{E}+01$ & $\mathrm{~s}$ & $2.74 \mathrm{E}+01$ & $1.87 \mathrm{E}+09$ & -- & -- & -- & FGR-12 & $4.92 \mathrm{E}-16$ \\
\hline Fr-221 & Fr-221 & 221.014 & Francium & $1.00 \mathrm{E}-03$ & ICRP-38 & $4.80 E+00$ & $\mathrm{~m}$ & $2.88 \mathrm{E}+02$ & $1.77 \mathrm{E}+08$ & -- & -- & -- & FGR-12 & $1.46 \mathrm{E}-15$ \\
\hline
\end{tabular}

A-11 


\begin{tabular}{|c|c|c|c|c|c|c|c|c|c|c|c|c|c|c|}
\hline \multicolumn{15}{|c|}{ Table A.1 Radionuclide Specific Input Data Used For the Calculation of HC-2 TQs Using Maximum Reported DCs (continued) } \\
\hline $\begin{array}{c}\text { MASTER } \\
\text { ISOTOPE } \\
\text { LIST }\end{array}$ & $\begin{array}{l}\text { Atomic } \\
\text { Mass } \\
\text { Nuclide }\end{array}$ & $\begin{array}{l}\text { Atomic } \\
\text { Mass }\end{array}$ & Element & $\begin{array}{c}\text { RF per } \\
\text { DOE-STD- } \\
1027\end{array}$ & $\begin{array}{c}\text { Half-Life } \\
\text { Reference }\end{array}$ & $\begin{array}{r}\text { Half-Li } \\
\text { (original }\end{array}$ & & Half Life (s) & $\begin{array}{l}\text { Sp. Act } \\
\text { (Ci/g) }\end{array}$ & $\begin{array}{l}\text { Inhalation DC } \\
\text { Reference }\end{array}$ & $\begin{array}{c}\text { HC-2 } \\
\text { Bounding } \\
\text { Inhalation } \\
\text { DC (Sv/Bq) }\end{array}$ & $\begin{array}{l}\text { Lung } \\
\text { Absorption } \\
\text { Class/Type }\end{array}$ & $\begin{array}{l}\text { Immersion DC } \\
\text { Reference }\end{array}$ & $\begin{array}{c}\mathrm{HC}-2 \\
\text { Immersion } \\
\mathrm{DC}(\mathrm{Sv} / \mathrm{s} \\
\left.\text { per } \mathrm{Bq} / \mathrm{m}^{3}\right)\end{array}$ \\
\hline Fr-222 & Fr-222 & 222.018 & Francium & $1.00 \mathrm{E}-03$ & ICRP-38 & $1.44 \mathrm{E}+01$ & $\mathrm{~m}$ & $8.64 \mathrm{E}+02$ & $5.88 \mathrm{E}+07$ & ICRP-72 & $1.40 \mathrm{E}-08$ & $\mathrm{~F}$ & FGR-12 & $1.17 \mathrm{E}-16$ \\
\hline Fr-223 & Fr-223 & 223.020 & Francium & $1.00 \mathrm{E}-03$ & ICRP-38 & $2.18 \mathrm{E}+01$ & $\mathrm{~m}$ & $1.31 \mathrm{E}+03$ & $3.87 \mathrm{E}+07$ & ICRP-72 & $8.90 \mathrm{E}-10$ & $\mathrm{~F}$ & FGR-12 & $2.29 \mathrm{E}-15$ \\
\hline Fr-224 & Fr-224 & 224.023 & Francium & $1.00 \mathrm{E}-03$ & ICRP-107 & $3.33 \mathrm{E}+00$ & $\mathrm{~m}$ & $2.00 \mathrm{E}+02$ & $2.52 \mathrm{E}+08$ & -- & -- & -- & DOE-STD-1196 & $2.62 \mathrm{E}-14$ \\
\hline Fr-227 & Fr-227 & 227.032 & Francium & $1.00 \mathrm{E}-03$ & ICRP-107 & $2.47 \mathrm{E}+00$ & $\mathrm{~m}$ & $1.48 \mathrm{E}+02$ & $3.35 E+08$ & -- & -- & -- & DOE-STD-1196 & $2.00 \mathrm{E}-14$ \\
\hline Ga-64 & Ga-64 & 63.937 & Gallium & $1.00 \mathrm{E}-03$ & ICRP-107 & $2.63 \mathrm{E}+00$ & $\mathrm{~m}$ & $1.58 \mathrm{E}+02$ & $1.12 \mathrm{E}+09$ & -- & --- & -- & DOE-STD-1196 & $1.64 \mathrm{E}-13$ \\
\hline Ga-65 & Ga-65 & 64.933 & Gallium & $1.00 \mathrm{E}-03$ & ICRP-38 & $1.52 \mathrm{E}+01$ & $\mathrm{~m}$ & $9.12 \mathrm{E}+02$ & $1.91 \mathrm{E}+08$ & ICRP-72 & $1.70 \mathrm{E}-11$ & $M$ & FGR-12 & $5.65 \mathrm{E}-14$ \\
\hline Ga-66 & Ga-66 & 65.932 & Gallium & $1.00 \mathrm{E}-03$ & ICRP-38 & $9.40 \mathrm{E}+00$ & $\mathrm{~h}$ & $3.38 \mathrm{E}+04$ & $5.06 \mathrm{E}+06$ & ICRP-72 & $4.40 \mathrm{E}-10$ & M & FGR-12 & $1.29 \mathrm{E}-13$ \\
\hline Ga-67 & Ga-67 & 66.928 & Gallium & $1.00 \mathrm{E}-03$ & ICRP-38 & $7.83 \mathrm{E}+01$ & $\mathrm{~h}$ & $2.82 \mathrm{E}+05$ & $5.98 \mathrm{E}+05$ & ICRP-72 & $2.40 \mathrm{E}-10$ & $M$ & FGR-12 & $7.20 \mathrm{E}-15$ \\
\hline Ga-68 & Ga-68 & 67.928 & Gallium & $1.00 \mathrm{E}-03$ & ICRP-38 & $6.80 \mathrm{E}+01$ & $\mathrm{~m}$ & $4.08 \mathrm{E}+03$ & $4.07 \mathrm{E}+07$ & ICRP-72 & $4.90 \mathrm{E}-11$ & $M$ & FGR-12 & $4.58 \mathrm{E}-14$ \\
\hline Ga-70 & Ga-70 & 69.926 & Gallium & $1.00 \mathrm{E}-03$ & ICRP-38 & $2.12 \mathrm{E}+01$ & $\mathrm{~m}$ & $1.27 \mathrm{E}+03$ & $1.27 \mathrm{E}+08$ & ICRP-72 & $1.60 \mathrm{E}-11$ & $M$ & FGR-12 & $4.62 \mathrm{E}-16$ \\
\hline Ga-72 & Ga-72 & 71.926 & Gallium & $1.00 \mathrm{E}-03$ & ICRP-38 & $1.41 \mathrm{E}+01$ & $\mathrm{~h}$ & $5.08 \mathrm{E}+04$ & $3.09 \mathrm{E}+06$ & ICRP-72 & $5.30 \mathrm{E}-10$ & $M$ & FGR-12 & $1.39 \mathrm{E}-13$ \\
\hline Ga-73 & Ga-73 & 72.925 & Gallium & $1.00 \mathrm{E}-03$ & ICRP-38 & $4.91 \mathrm{E}+00$ & $\mathrm{~h}$ & $1.77 \mathrm{E}+04$ & $8.75 E+06$ & ICRP-72 & $1.40 \mathrm{E}-10$ & $M$ & FGR-12 & $1.48 \mathrm{E}-14$ \\
\hline Ga-74 & Ga-74 & 73.927 & Gallium & $1.00 \mathrm{E}-03$ & ICRP-107 & $8.12 \mathrm{E}+00$ & $\mathrm{~m}$ & $4.87 \mathrm{E}+02$ & $3.13 \mathrm{E}+08$ & -- & -- & -- & DOE-STD-1196 & $1.55 \mathrm{E}-13$ \\
\hline Gd-142 & Gd-142 & 141.928 & Gadolinium & $1.00 \mathrm{E}-03$ & ICRP-107 & $7.02 \mathrm{E}+01$ & $\mathrm{~s}$ & $7.02 \mathrm{E}+01$ & $1.13 \mathrm{E}+09$ & -- & -- & -- & DOE-STD-1196 & $4.78 \mathrm{E}-14$ \\
\hline Gd-143m & Gd-143 & 142.927 & Gadolinium & $1.00 \mathrm{E}-03$ & ICRP-107 & $1.10 \mathrm{E}+02$ & $\mathrm{~s}$ & $1.10 \mathrm{E}+02$ & $7.18 \mathrm{E}+08$ & -- & -- & -- & DOE-STD-1196 & $9.77 \mathrm{E}-14$ \\
\hline Gd-144 & Gd-144 & 143.923 & Gadolinium & $1.00 \mathrm{E}-03$ & ICRP-107 & $4.47 \mathrm{E}+00$ & $\mathrm{~m}$ & $2.68 \mathrm{E}+02$ & $2.92 \mathrm{E}+08$ & -- & -- & -- & DOE-STD-1196 & $4.25 \mathrm{E}-14$ \\
\hline Gd-145 & Gd-145 & 144.922 & Gadolinium & $1.00 \mathrm{E}-03$ & ICRP-38 & $2.29 \mathrm{E}+01$ & $\mathrm{~m}$ & $1.37 \mathrm{E}+03$ & $5.67 \mathrm{E}+07$ & ICRP-72 & $2.00 \mathrm{E}-11$ & $M$ & FGR-12 & $1.15 \mathrm{E}-13$ \\
\hline Gd-145m & Gd-145 & 144.922 & Gadolinium & $1.00 \mathrm{E}-03$ & ICRP-107 & $8.50 \mathrm{E}+01$ & $\mathrm{~s}$ & $8.50 \mathrm{E}+01$ & $9.16 \mathrm{E}+08$ & -- & -- & -- & DOE-STD-1196 & $3.08 \mathrm{E}-14$ \\
\hline Gd-146 & Gd-146 & 145.918 & Gadolinium & $1.00 \mathrm{E}-03$ & ICRP-38 & $4.83 \mathrm{E}+01$ & $d$ & $4.17 \mathrm{E}+06$ & $1.85 \mathrm{E}+04$ & ICRP-72 & $6.40 \mathrm{E}-09$ & $M$ & FGR-12 & $9.95 \mathrm{E}-15$ \\
\hline Gd-147 & Gd-147 & 146.919 & Gadolinium & $1.00 \mathrm{E}-03$ & ICRP-38 & $3.81 \mathrm{E}+01$ & $\mathrm{~h}$ & $1.37 \mathrm{E}+05$ & $5.60 \mathrm{E}+05$ & ICRP-72 & $4.00 \mathrm{E}-10$ & $M$ & FGR-12 & $6.45 \mathrm{E}-14$ \\
\hline Gd-148 & Gd-148 & 147.918 & Gadolinium & $1.00 \mathrm{E}-03$ & ICRP-38 & $9.30 \mathrm{E}+01$ & $y$ & $2.93 \mathrm{E}+09$ & $2.60 \mathrm{E}+01$ & ICRP-72 & $2.60 \mathrm{E}-05$ & $\mathrm{~F}$ & -- & -- \\
\hline Gd-149 & Gd-149 & 148.919 & Gadolinium & $1.00 \mathrm{E}-03$ & ICRP-38 & $9.40 \mathrm{E}+00$ & $d$ & $8.12 \mathrm{E}+05$ & $9.33 \mathrm{E}+04$ & ICRP-72 & $7.30 \mathrm{E}-10$ & $M$ & FGR-12 & $1.92 \mathrm{E}-14$ \\
\hline Gd-150 & Gd-150 & 149.919 & Gadolinium & $1.00 \mathrm{E}-03$ & ICRP-107 & $1.79 \mathrm{E}+06$ & $y$ & $5.65 \mathrm{E}+13$ & $1.33 \mathrm{E}-03$ & DOE-STD-1196 & $2.56 \mathrm{E}-05$ & $F$ & -- & -- \\
\hline Gd-151 & Gd-151 & 150.920 & Gadolinium & $1.00 \mathrm{E}-03$ & ICRP-38 & $1.20 \mathrm{E}+02$ & $d$ & $1.04 \mathrm{E}+07$ & $7.21 \mathrm{E}+03$ & ICRP-72 & $8.60 \mathrm{E}-10$ & $M$ & FGR-12 & $2.20 \mathrm{E}-15$ \\
\hline Gd-152 & Gd-152 & 151.920 & Gadolinium & $1.00 \mathrm{E}-03$ & ICRP-38 & $1.08 \mathrm{E}+14$ & $y$ & $3.41 \mathrm{E}+21$ & $2.18 \mathrm{E}-11$ & ICRP-72 & $1.90 \mathrm{E}-05$ & $\mathrm{~F}$ & -- & -- \\
\hline Gd-153 & Gd-153 & 152.922 & Gadolinium & $1.00 \mathrm{E}-03$ & ICRP-38 & $2.42 \mathrm{E}+02$ & $d$ & $2.09 \mathrm{E}+07$ & $3.53 \mathrm{E}+03$ & ICRP-72 & $2.10 \mathrm{E}-09$ & $\mathrm{~F}$ & FGR-12 & $3.71 \mathrm{E}-15$ \\
\hline Gd-159 & Gd-159 & 158.926 & Gadolinium & $1.00 \mathrm{E}-03$ & ICRP-38 & $1.86 \mathrm{E}+01$ & $\mathrm{~h}$ & $6.68 \mathrm{E}+04$ & $1.06 \mathrm{E}+06$ & ICRP-72 & $2.70 \mathrm{E}-10$ & $M$ & FGR-12 & $2.21 \mathrm{E}-15$ \\
\hline Gd-162 & Gd-162 & 161.931 & Gadolinium & $1.00 \mathrm{E}-03$ & ICRP-107 & $8.40 \mathrm{E}+00$ & $\mathrm{~m}$ & $5.04 \mathrm{E}+02$ & $1.38 \mathrm{E}+08$ & -- & -- & -- & DOE-STD-1196 & $1.86 \mathrm{E}-14$ \\
\hline Ge-66 & Ge-66 & 65.934 & Germanium & $1.00 \mathrm{E}-03$ & ICRP-38 & $2.27 \mathrm{E}+00$ & $\mathrm{~h}$ & $8.17 \mathrm{E}+03$ & $2.09 \mathrm{E}+07$ & ICRP-72 & $9.10 \mathrm{E}-11$ & $M$ & FGR-12 & $3.25 \mathrm{E}-14$ \\
\hline Ge-67 & Ge-67 & 66.933 & Germanium & $1.00 \mathrm{E}-03$ & ICRP-38 & $1.87 \mathrm{E}+01$ & $\mathrm{~m}$ & $1.12 \mathrm{E}+03$ & $1.50 E+08$ & ICRP-72 & $2.50 \mathrm{E}-11$ & $M$ & FGR-12 & $6.86 \mathrm{E}-14$ \\
\hline Ge-68 & Ge-68 & 67.928 & Germanium & $1.00 \mathrm{E}-03$ & ICRP-38 & $2.88 \mathrm{E}+02$ & $d$ & $2.49 \mathrm{E}+07$ & $6.67 \mathrm{E}+03$ & ICRP-72 & $1.40 \mathrm{E}-08$ & $M$ & FGR-12 & $7.37 \mathrm{E}-20$ \\
\hline Ge-69 & Ge-69 & 68.928 & Germanium & $1.00 \mathrm{E}-03$ & ICRP-38 & $3.91 \mathrm{E}+01$ & $\mathrm{~h}$ & $1.41 \mathrm{E}+05$ & $1.16 \mathrm{E}+06$ & ICRP-72 & $2.90 \mathrm{E}-10$ & $M$ & FGR-12 & $4.27 \mathrm{E}-14$ \\
\hline Ge-71 & Ge-71 & 70.925 & Germanium & $1.00 \mathrm{E}-03$ & ICRP-38 & $1.18 \mathrm{E}+01$ & $d$ & $1.02 \mathrm{E}+06$ & $1.56 \mathrm{E}+05$ & ICRP-72 & $1.10 \mathrm{E}-11$ & $M$ & FGR-12 & $7.47 \mathrm{E}-20$ \\
\hline Ge-75 & Ge-75 & 74.923 & Germanium & $1.00 \mathrm{E}-03$ & ICRP-38 & $8.28 \mathrm{E}+01$ & $\mathrm{~m}$ & $4.97 \mathrm{E}+03$ & $3.03 E+07$ & ICRP-72 & $3.60 \mathrm{E}-11$ & $M$ & FGR-12 & $1.68 \mathrm{E}-15$ \\
\hline Ge-77 & Ge-77 & 76.924 & Germanium & $1.00 \mathrm{E}-03$ & ICRP-38 & $1.13 \mathrm{E}+01$ & $\mathrm{~h}$ & $4.07 E+04$ & $3.61 \mathrm{E}+06$ & ICRP-72 & $3.70 \mathrm{E}-10$ & $M$ & FGR-12 & $5.32 \mathrm{E}-14$ \\
\hline Ge-78 & Ge-78 & 77.923 & Germanium & $1.00 \mathrm{E}-03$ & ICRP-38 & $8.70 \mathrm{E}+01$ & $\mathrm{~m}$ & $5.22 \mathrm{E}+03$ & $2.77 \mathrm{E}+07$ & ICRP-72 & $9.50 \mathrm{E}-11$ & $M$ & FGR-12 & $1.34 \mathrm{E}-14$ \\
\hline $\mathrm{H}-3$ & $\mathrm{H}-3$ & 3.016 & Hydrogen & $1.00 E+00$ & ICRP-38 & $1.24 \mathrm{E}+01$ & $y$ & $3.90 \mathrm{E}+08$ & $9.60 \mathrm{E}+03$ & ICRP-72 & $2.60 \mathrm{E}-10$ & $\mathrm{~s}$ & FGR-12 & $3.31 \mathrm{E}-19$ \\
\hline Hf-167 & Hf-167 & 166.943 & Hafnium & $1.00 \mathrm{E}-03$ & ICRP-107 & $2.05 E+00$ & $\mathrm{~m}$ & $1.23 \mathrm{E}+02$ & $5.49 \mathrm{E}+08$ & -- & -- & -- & DOE-STD-1196 & $2.73 \mathrm{E}-14$ \\
\hline $\mathrm{Hf}-169$ & $\mathrm{Hf}-169$ & 168.941 & Hafnium & $1.00 \mathrm{E}-03$ & ICRP-107 & $3.24 \mathrm{E}+00$ & $\mathrm{~m}$ & $1.94 \mathrm{E}+02$ & $3.44 \mathrm{E}+08$ & -- & -- & -- & DOE-STD-1196 & $2.78 \mathrm{E}-14$ \\
\hline $\mathrm{Hf}-170$ & $\mathrm{Hf}-170$ & 169.940 & Hafnium & $1.00 \mathrm{E}-03$ & ICRP-38 & $1.60 \mathrm{E}+01$ & $\mathrm{~h}$ & $5.76 \mathrm{E}+04$ & $1.15 \mathrm{E}+06$ & ICRP-72 & $3.20 \mathrm{E}-10$ & $M$ & FGR-12 & $2.52 \mathrm{E}-14$ \\
\hline $\mathrm{Hf}-172$ & $\mathrm{Hf}-172$ & 171.939 & Hafnium & $1.00 \mathrm{E}-03$ & ICRP-38 & $1.87 \mathrm{E}+00$ & $y$ & $5.90 \mathrm{E}+07$ & $1.11 \mathrm{E}+03$ & ICRP-72 & $3.20 \mathrm{E}-08$ & $F$ & FGR-12 & $4.06 \mathrm{E}-15$ \\
\hline $\mathrm{Hf}-173$ & $\mathrm{Hf}-173$ & 172.941 & Hafnium & $1.00 \mathrm{E}-03$ & ICRP-38 & $2.40 \mathrm{E}+01$ & $\mathrm{~h}$ & $8.64 \mathrm{E}+04$ & $7.55 E+05$ & ICRP-72 & $1.60 \mathrm{E}-10$ & $M$ & FGR-12 & $1.85 \mathrm{E}-14$ \\
\hline Hf-174 & $\mathrm{Hf}-174$ & 173.940 & Hafnium & $1.00 \mathrm{E}-03$ & ICRP-107 & $2.00 E+15$ & $y$ & $6.31 E+22$ & $1.03 \mathrm{E}-12$ & DOE-STD-1196 & $3.20 \mathrm{E}-05$ & $F$ & -- & -- \\
\hline Hf-175 & Hf-175 & 174.942 & Hafnium & $1.00 \mathrm{E}-03$ & ICRP-38 & $7.00 \mathrm{E}+01$ & $\mathrm{~d}$ & $6.05 E+06$ & $1.07 E+04$ & ICRP-72 & $1.20 \mathrm{E}-09$ & $M$ & FGR-12 & $1.69 \mathrm{E}-14$ \\
\hline $\mathrm{Hf}-177 \mathrm{~m}$ & Hf-177 & 176.943 & Hafnium & $1.00 \mathrm{E}-03$ & ICRP-38 & $5.14 \mathrm{E}+01$ & $\mathrm{~m}$ & $3.08 \mathrm{E}+03$ & $2.07 E+07$ & ICRP-72 & $9.00 \mathrm{E}-11$ & $M$ & FGR-12 & $1.06 \mathrm{E}-13$ \\
\hline $\mathrm{Hf}-178 \mathrm{~m}$ & $\mathrm{Hf}-178$ & 177.944 & Hafnium & $1.00 \mathrm{E}-03$ & ICRP-38 & $3.10 \mathrm{E}+01$ & $y$ & $9.78 \mathrm{E}+08$ & $6.48 \mathrm{E}+01$ & ICRP-72 & $2.60 \mathrm{E}-07$ & $F$ & FGR-12 & $1.12 \mathrm{E}-13$ \\
\hline
\end{tabular}

A-12 


\begin{tabular}{|c|c|c|c|c|c|c|c|c|c|c|c|c|c|c|}
\hline \multicolumn{15}{|c|}{ Table A.1 Radionuclide Specific Input Data Used For the Calculation of HC-2 TQs Using Maximum Reported DCs (continued) } \\
\hline $\begin{array}{c}\text { MASTER } \\
\text { ISOTOPE } \\
\text { LIST }\end{array}$ & $\begin{array}{l}\text { Atomic } \\
\text { Mass } \\
\text { Nuclide }\end{array}$ & $\begin{array}{l}\text { Atomic } \\
\text { Mass }\end{array}$ & Element & $\begin{array}{c}\text { RF per } \\
\text { DOE-STD- } \\
1027\end{array}$ & $\begin{array}{l}\text { Half-Life } \\
\text { Reference }\end{array}$ & $\begin{array}{r}\begin{array}{r}\text { Half-Li } \\
\text { (original }\end{array}\end{array}$ & & Half Life (s) & $\begin{array}{l}\text { Sp. Act } \\
\text { (Ci/g) }\end{array}$ & $\begin{array}{c}\text { Inhalation DC } \\
\text { Reference }\end{array}$ & $\begin{array}{c}\text { HC-2 } \\
\text { Bounding } \\
\text { Inhalation } \\
\text { DC (Sv/Bq) }\end{array}$ & $\begin{array}{l}\text { Lung } \\
\text { Absorption } \\
\text { Class/Type }\end{array}$ & $\begin{array}{l}\text { Immersion DC } \\
\text { Reference }\end{array}$ & $\begin{array}{c}\mathrm{HC}-2 \\
\text { Immersion } \\
\mathrm{DC}(\mathrm{Sv} / \mathrm{s} \\
\left.\text { per } \mathrm{Bq} / \mathrm{m}^{3}\right)\end{array}$ \\
\hline $\mathrm{Hf}-179 \mathrm{~m}$ & Hf-179 & 178.946 & Hafnium & $1.00 \mathrm{E}-03$ & ICRP-38 & $2.51 \mathrm{E}+01$ & d & $2.17 \mathrm{E}+06$ & $2.91 \mathrm{E}+04$ & ICRP-72 & $3.80 \mathrm{E}-09$ & $\mathrm{M}$ & FGR-12 & $4.21 \mathrm{E}-14$ \\
\hline $\mathrm{Hf}-180 \mathrm{~m}$ & $\mathrm{Hf}-180$ & 179.947 & Hafnium & $1.00 \mathrm{E}-03$ & ICRP-38 & $5.50 \mathrm{E}+00$ & $\mathrm{~h}$ & $1.98 \mathrm{E}+04$ & $3.17 \mathrm{E}+06$ & ICRP-72 & $1.30 \mathrm{E}-10$ & $M$ & FGR-12 & $4.74 \mathrm{E}-14$ \\
\hline Hf-181 & $\mathrm{Hf}-181$ & 180.949 & Hafnium & $1.00 \mathrm{E}-03$ & ICRP-38 & $4.24 \mathrm{E}+01$ & $d$ & $3.66 \mathrm{E}+06$ & $1.70 \mathrm{E}+04$ & ICRP-72 & $5.00 \mathrm{E}-09$ & $M$ & FGR-12 & $2.62 \mathrm{E}-14$ \\
\hline $\mathrm{Hf}-182$ & Hf-182 & 181.951 & Hafnium & $1.00 \mathrm{E}-03$ & ICRP-38 & $9.00 E+06$ & $y$ & $2.84 \mathrm{E}+14$ & $2.18 \mathrm{E}-04$ & ICRP-72 & $3.10 \mathrm{E}-07$ & $\mathrm{~F}$ & FGR-12 & $1.14 \mathrm{E}-14$ \\
\hline Hf-182m & Hf-182 & 181.951 & Hafnium & $1.00 \mathrm{E}-03$ & ICRP-38 & $6.15 \mathrm{E}+01$ & $\mathrm{~m}$ & $3.69 \mathrm{E}+03$ & $1.68 \mathrm{E}+07$ & ICRP-72 & $4.60 \mathrm{E}-11$ & $M$ & FGR-12 & $4.43 \mathrm{E}-14$ \\
\hline Hf-183 & $\mathrm{Hf}-183$ & 182.954 & Hafnium & $1.00 \mathrm{E}-03$ & ICRP-38 & $6.40 \mathrm{E}+01$ & $\mathrm{~m}$ & $3.84 \mathrm{E}+03$ & $1.61 \mathrm{E}+07$ & ICRP-72 & $5.70 \mathrm{E}-11$ & $M$ & FGR-12 & $3.63 \mathrm{E}-14$ \\
\hline $\mathrm{Hf}-184$ & $\mathrm{Hf}-184$ & 183.955 & Hafnium & $1.00 \mathrm{E}-03$ & ICRP-38 & $4.12 \mathrm{E}+00$ & $\mathrm{~h}$ & $1.48 \mathrm{E}+04$ & $4.13 \mathrm{E}+06$ & ICRP-72 & $3.30 \mathrm{E}-10$ & $M$ & FGR-12 & $1.14 \mathrm{E}-14$ \\
\hline Hg-187 & Hg-187 & 186.970 & Mercury & $1.00 \mathrm{E}-02$ & JAERI & $2.20 \mathrm{E}+00$ & $\mathrm{~m}$ & $1.32 \mathrm{E}+02$ & $4.57 \mathrm{E}+08$ & -- & -- & -- & -- & -- \\
\hline $\mathrm{Hg}-187 \mathrm{~m}$ & $\mathrm{Hg}-187$ & 186.970 & Mercury & $1.00 \mathrm{E}-02$ & JAERI & $2.40 \mathrm{E}+00$ & $\mathrm{~m}$ & $1.44 \mathrm{E}+02$ & $4.19 \mathrm{E}+08$ & -- & -- & -- & -- & -- \\
\hline $\mathrm{Hg}-188$ & $\mathrm{Hg}-188$ & 187.968 & Mercury & $1.00 \mathrm{E}-02$ & JAERI & $3.25 \mathrm{E}+00$ & $\mathrm{~m}$ & $1.95 \mathrm{E}+02$ & $3.08 \mathrm{E}+08$ & -- & -- & -- & -- & -- \\
\hline $\mathrm{Hg}-190$ & $\mathrm{Hg}-190$ & 189.966 & Mercury & $1.00 \mathrm{E}-02$ & ICRP-107 & $2.00 \mathrm{E}+01$ & $\mathrm{~m}$ & $1.20 \mathrm{E}+03$ & $4.95 \mathrm{E}+07$ & DOE-STD-1196 & $8.33 \mathrm{E}-11$ & $\mathrm{~V}$ & DOE-STD-1196 & $7.74 \mathrm{E}-15$ \\
\hline $\mathrm{Hg}-191 \mathrm{~m}$ & Hg-191 & 190.967 & Mercury & $1.00 \mathrm{E}-02$ & ICRP-107 & $5.08 \mathrm{E}+01$ & $\mathrm{~m}$ & $3.05 E+03$ & $1.94 \mathrm{E}+07$ & DOE-STD-1196 & $3.47 \mathrm{E}-10$ & $\mathrm{~V}$ & DOE-STD-1196 & $6.73 \mathrm{E}-14$ \\
\hline $\mathrm{Hg}-192$ & $\mathrm{Hg}-192$ & 191.966 & Mercury & $1.00 \mathrm{E}-02$ & ICRP-107 & $4.85 \mathrm{E}+00$ & $\mathrm{~h}$ & $1.75 \mathrm{E}+04$ & $3.37 \mathrm{E}+06$ & DOE-STD-1196 & $1.08 \mathrm{E}-09$ & $\mathrm{~V}$ & DOE-STD-1196 & $1.10 \mathrm{E}-14$ \\
\hline $\mathrm{Hg}-193$ & $\mathrm{Hg}-193$ & 192.967 & Mercury & $1.00 \mathrm{E}-02$ & ICRP-38 & $3.50 \mathrm{E}+00$ & $\mathrm{~h}$ & $1.26 \mathrm{E}+04$ & $4.64 \mathrm{E}+06$ & ICRP-72 & $1.10 \mathrm{E}-09$ & $\mathrm{~V}$ & FGR-12 & $8.69 \mathrm{E}-15$ \\
\hline Hg-193m & $\mathrm{Hg}-193$ & 192.967 & Mercury & $1.00 \mathrm{E}-02$ & ICRP-38 & $1.11 E+01$ & $\mathrm{~h}$ & $4.00 \mathrm{E}+04$ & $1.46 \mathrm{E}+06$ & ICRP-72 & $3.10 \mathrm{E}-09$ & $\mathrm{~V}$ & FGR-12 & $5.05 \mathrm{E}-14$ \\
\hline $\mathrm{Hg}-194$ & Hg-194 & 193.965 & Mercury & $1.00 \mathrm{E}-02$ & ICRP-38 & $2.60 \mathrm{E}+02$ & $y$ & $8.20 \mathrm{E}+09$ & $7.09 \mathrm{E}+00$ & ICRP-72 & $4.00 \mathrm{E}-08$ & $\mathrm{~V}$ & FGR-12 & $6.92 \mathrm{E}-19$ \\
\hline Hg-195 & $\mathrm{Hg}-195$ & 194.967 & Mercury & $1.00 \mathrm{E}-02$ & ICRP-38 & $9.90 \mathrm{E}+00$ & $\mathrm{~h}$ & $3.56 \mathrm{E}+04$ & $1.62 E+06$ & ICRP-72 & $1.40 \mathrm{E}-09$ & $\mathrm{~V}$ & FGR-12 & $9.20 \mathrm{E}-15$ \\
\hline $\mathrm{Hg}-195 \mathrm{~m}$ & Hg-195 & 194.967 & Mercury & $1.00 \mathrm{E}-02$ & ICRP-38 & $4.16 \mathrm{E}+01$ & $\mathrm{~h}$ & $1.50 \mathrm{E}+05$ & $3.86 \mathrm{E}+05$ & ICRP-72 & $8.20 \mathrm{E}-09$ & $\mathrm{~V}$ & FGR-12 & $9.63 \mathrm{E}-15$ \\
\hline Hg-197 & $\mathrm{Hg}-197$ & 196.967 & Mercury & $1.00 \mathrm{E}-02$ & ICRP-38 & $6.41 \mathrm{E}+01$ & $\mathrm{~h}$ & $2.31 \mathrm{E}+05$ & $2.48 \mathrm{E}+05$ & ICRP-72 & $4.40 \mathrm{E}-09$ & $\mathrm{~V}$ & FGR-12 & $2.66 \mathrm{E}-15$ \\
\hline $\mathrm{Hg}-197 \mathrm{~m}$ & Hg-197 & 196.967 & Mercury & $1.00 \mathrm{E}-02$ & ICRP-38 & $2.38 \mathrm{E}+01$ & $\mathrm{~h}$ & $8.57 \mathrm{E}+04$ & $6.68 \mathrm{E}+05$ & ICRP-72 & $5.80 \mathrm{E}-09$ & $\mathrm{~V}$ & FGR-12 & $4.05 \mathrm{E}-15$ \\
\hline Hg-199m & $\mathrm{Hg}-199$ & 198.968 & Mercury & $1.00 \mathrm{E}-02$ & ICRP-38 & $4.26 \mathrm{E}+01$ & $\mathrm{~m}$ & $2.56 \mathrm{E}+03$ & $2.22 \mathrm{E}+07$ & ICRP-72 & $1.80 \mathrm{E}-10$ & $\mathrm{v}$ & FGR-12 & $8.36 \mathrm{E}-15$ \\
\hline $\mathrm{Hg}-203$ & $\mathrm{Hg}-203$ & 202.973 & Mercury & $1.00 \mathrm{E}-02$ & ICRP-38 & $4.66 \mathrm{E}+01$ & $d$ & $4.03 E+06$ & $1.38 \mathrm{E}+04$ & ICRP-72 & $7.00 \mathrm{E}-09$ & $\mathrm{~V}$ & FGR-12 & $1.13 \mathrm{E}-14$ \\
\hline $\mathrm{Hg}-205$ & $\mathrm{Hg}-205$ & 204.976 & Mercury & $1.00 \mathrm{E}-02$ & ICRP-107 & $5.20 \mathrm{E}+00$ & $\mathrm{~m}$ & $3.12 \mathrm{E}+02$ & $1.76 \mathrm{E}+08$ & -- & -- & -- & DOE-STD-1196 & $6.19 \mathrm{E}-16$ \\
\hline $\mathrm{Hg}-206$ & Hg-206 & 205.978 & Mercury & $1.00 \mathrm{E}-02$ & ICRP-38 & $8.15 E+00$ & $\mathrm{~m}$ & $4.89 \mathrm{E}+02$ & $1.12 \mathrm{E}+08$ & -- & -- & -- & DOE-STD-1196 & $5.56 \mathrm{E}-15$ \\
\hline $\mathrm{Hg}-207$ & $\mathrm{Hg}-207$ & 206.982 & Mercury & $1.00 \mathrm{E}-02$ & ICRP-107 & $2.90 \mathrm{E}+00$ & $\mathrm{~m}$ & $1.74 \mathrm{E}+02$ & $3.13 E+08$ & -- & -- & -- & DOE-STD-1196 & $1.28 \mathrm{E}-13$ \\
\hline Ho-150 & Ho-150 & 149.933 & Holmium & $1.00 \mathrm{E}-03$ & ICRP-107 & $7.68 \mathrm{E}+01$ & $\mathrm{~s}$ & $7.68 \mathrm{E}+01$ & $9.80 \mathrm{E}+08$ & -- & -- & -- & DOE-STD-1196 & $8.73 \mathrm{E}-14$ \\
\hline Ho-152 & Ho-152 & 151.932 & Holmium & $1.00 \mathrm{E}-03$ & JAERI & $2.70 E+00$ & $\mathrm{~m}$ & $1.62 \mathrm{E}+02$ & $4.59 \mathrm{E}+08$ & -- & -- & -- & -- & -- \\
\hline Ho-153 & Ho-153 & 152.930 & Holmium & $1.00 \mathrm{E}-03$ & ICRP-107 & $2.01 E+00$ & $\mathrm{~m}$ & $1.21 \mathrm{E}+02$ & $6.12 \mathrm{E}+08$ & -- & -- & -- & DOE-STD-1196 & $4.61 \mathrm{E}-14$ \\
\hline Ho-153m & Ho-153 & 152.930 & Holmium & $1.00 \mathrm{E}-03$ & ICRP-107 & $9.30 \mathrm{E}+00$ & $\mathrm{~m}$ & $5.58 \mathrm{E}+02$ & $1.32 \mathrm{E}+08$ & -- & -- & -- & DOE-STD-1196 & $4.72 \mathrm{E}-14$ \\
\hline Ho-154 & Ho-154 & 153.931 & Holmium & $1.00 \mathrm{E}-03$ & ICRP-107 & $1.18 \mathrm{E}+01$ & $\mathrm{~m}$ & $7.06 \mathrm{E}+02$ & $1.04 \mathrm{E}+08$ & DOE-STD-1196 & $1.82 \mathrm{E}-11$ & $\mathrm{~s}$ & DOE-STD-1196 & $8.63 \mathrm{E}-14$ \\
\hline Ho- $154 \mathrm{~m}$ & Ho-154 & 153.931 & Holmium & $1.00 \mathrm{E}-03$ & ICRP-107 & $3.10 E+00$ & $\mathrm{~m}$ & $1.86 \mathrm{E}+02$ & $3.94 \mathrm{E}+08$ & -- & -- & -- & DOE-STD-1196 & $1.09 \mathrm{E}-13$ \\
\hline Ho-155 & Ho-155 & 154.929 & Holmium & $1.00 \mathrm{E}-03$ & ICRP-38 & $4.80 \mathrm{E}+01$ & $\mathrm{~m}$ & $2.88 \mathrm{E}+03$ & $2.53 \mathrm{E}+07$ & ICRP-72 & $2.00 \mathrm{E}-11$ & $M$ & FGR-12 & $1.79 \mathrm{E}-14$ \\
\hline Ho-156 & Ho-156 & 155.930 & Holmium & $1.00 \mathrm{E}-03$ & ICRP-107 & $5.60 \mathrm{E}+01$ & $\mathrm{~m}$ & $3.36 \mathrm{E}+03$ & $2.15 \mathrm{E}+07$ & DOE-STD-1196 & $6.56 \mathrm{E}-11$ & $\mathrm{~s}$ & DOE-STD-1196 & $9.78 \mathrm{E}-14$ \\
\hline Ho-157 & Ho-157 & 156.928 & Holmium & $1.00 \mathrm{E}-03$ & ICRP-38 & $1.26 \mathrm{E}+01$ & $\mathrm{~m}$ & $7.56 \mathrm{E}+02$ & $9.51 \mathrm{E}+07$ & ICRP-72 & $4.20 \mathrm{E}-12$ & $M$ & FGR-12 & $2.24 \mathrm{E}-14$ \\
\hline Ho-158 & Ho-158 & 157.929 & Holmium & $1.00 \mathrm{E}-03$ & JAERI & $1.13 \mathrm{E}+01$ & $\mathrm{~m}$ & $6.78 \mathrm{E}+02$ & $1.05 E+08$ & JAERI & $7.80 \mathrm{E}-12$ & $M$ & -- & -- \\
\hline Ho-159 & Ho-159 & 158.928 & Holmium & $1.00 \mathrm{E}-03$ & ICRP-38 & $3.30 \mathrm{E}+01$ & $\mathrm{~m}$ & $1.98 \mathrm{E}+03$ & $3.59 \mathrm{E}+07$ & ICRP-72 & $6.10 \mathrm{E}-12$ & $M$ & FGR-12 & $1.60 \mathrm{E}-14$ \\
\hline Ho-160 & Ho-160 & 159.929 & Holmium & $1.00 \mathrm{E}-03$ & ICRP-107 & $2.56 \mathrm{E}+01$ & $\mathrm{~m}$ & $1.54 \mathrm{E}+03$ & $4.59 \mathrm{E}+07$ & DOE-STD-1196 & $1.63 \mathrm{E}-11$ & $\mathrm{~s}$ & DOE-STD-1196 & $7.64 \mathrm{E}-14$ \\
\hline Ho-161 & Ho-161 & 160.928 & Holmium & $1.00 \mathrm{E}-03$ & ICRP-38 & $2.50 \mathrm{E}+00$ & $\mathrm{~h}$ & $9.00 \mathrm{E}+03$ & $7.79 \mathrm{E}+06$ & ICRP-72 & $6.00 \mathrm{E}-12$ & $M$ & FGR-12 & $1.73 \mathrm{E}-15$ \\
\hline Ho-162 & Ho-162 & 161.929 & Holmium & $1.00 \mathrm{E}-03$ & ICRP-38 & $1.50 \mathrm{E}+01$ & $\mathrm{~m}$ & $9.00 \mathrm{E}+02$ & $7.74 \mathrm{E}+07$ & ICRP-72 & $2.80 \mathrm{E}-12$ & $M$ & FGR-12 & $7.35 \mathrm{E}-15$ \\
\hline Ho-162m & Ho-162 & 161.929 & Holmium & $1.00 \mathrm{E}-03$ & ICRP-38 & $6.80 \mathrm{E}+01$ & $\mathrm{~m}$ & $4.08 \mathrm{E}+03$ & $1.71 \mathrm{E}+07$ & ICRP-72 & $2.10 \mathrm{E}-11$ & $M$ & FGR-12 & $2.74 \mathrm{E}-14$ \\
\hline Ho-163 & Ho-163 & 162.929 & Holmium & $1.00 \mathrm{E}-03$ & ICRP-107 & $4.57 \mathrm{E}+03$ & $y$ & $1.44 \mathrm{E}+11$ & $4.80 \mathrm{E}-01$ & DOE-STD-1196 & $2.82 \mathrm{E}-10$ & $F$ & -- & -- \\
\hline Ho-164 & Ho-164 & 163.930 & Holmium & $1.00 \mathrm{E}-03$ & ICRP-38 & $2.90 \mathrm{E}+01$ & $\mathrm{~m}$ & $1.74 \mathrm{E}+03$ & $3.96 \mathrm{E}+07$ & ICRP-72 & $8.40 \mathrm{E}-12$ & $M$ & FGR-12 & $9.05 \mathrm{E}-16$ \\
\hline Ho-164m & Ho-164 & 163.930 & Holmium & $1.00 \mathrm{E}-03$ & ICRP-38 & $3.75 E+01$ & $\mathrm{~m}$ & $2.25 E+03$ & $3.06 \mathrm{E}+07$ & ICRP-72 & $1.20 \mathrm{E}-11$ & $M$ & FGR-12 & $1.32 \mathrm{E}-15$ \\
\hline Ho-166 & Ho-166 & 165.932 & Holmium & $1.00 \mathrm{E}-03$ & ICRP-38 & $2.68 \mathrm{E}+01$ & $\mathrm{~h}$ & $9.65 E+04$ & $7.05 E+05$ & ICRP-72 & $6.50 \mathrm{E}-10$ & $M$ & FGR-12 & $1.42 \mathrm{E}-15$ \\
\hline Ho-166m & Ho-166 & 165.932 & Holmium & $1.00 \mathrm{E}-03$ & ICRP-38 & $1.20 \mathrm{E}+03$ & $y$ & $3.79 \mathrm{E}+10$ & $1.80 \mathrm{E}+00$ & ICRP-72 & $1.20 \mathrm{E}-07$ & $M$ & FGR-12 & $8.45 \mathrm{E}-14$ \\
\hline Ho-167 & Ho-167 & 166.933 & Holmium & $1.00 \mathrm{E}-03$ & ICRP-38 & $3.10 \mathrm{E}+00$ & $\mathrm{~h}$ & $1.12 \mathrm{E}+04$ & $6.06 \mathrm{E}+06$ & ICRP-72 & $7.10 \mathrm{E}-11$ & $M$ & FGR-12 & $1.73 \mathrm{E}-14$ \\
\hline
\end{tabular}

A-13 


\begin{tabular}{|c|c|c|c|c|c|c|c|c|c|c|c|c|c|c|}
\hline \multicolumn{15}{|c|}{ Table A.1 Radionuclide Specific Input Data Used For the Calculation of HC-2 TQs Using Maximum Reported DCs (continued) } \\
\hline $\begin{array}{l}\text { MASTER } \\
\text { ISOTOPE } \\
\text { LIST }\end{array}$ & $\begin{array}{l}\text { Atomic } \\
\text { Mass } \\
\text { Nuclide }\end{array}$ & $\begin{array}{l}\text { Atomic } \\
\text { Mass }\end{array}$ & Element & $\begin{array}{c}\text { RF per } \\
\text { DOE-STD- } \\
1027\end{array}$ & $\begin{array}{c}\text { Half-Life } \\
\text { Reference }\end{array}$ & $\begin{array}{r}\text { Half-Li } \\
\text { (original }\end{array}$ & & Half Life (s) & $\begin{array}{l}\text { Sp. Act } \\
\text { (Ci/g) }\end{array}$ & $\begin{array}{l}\text { Inhalation DC } \\
\text { Reference }\end{array}$ & $\begin{array}{c}\text { HC-2 } \\
\text { Bounding } \\
\text { Inhalation } \\
\text { DC (Sv/Bq) }\end{array}$ & $\begin{array}{l}\text { Lung } \\
\text { Absorption } \\
\text { Class/Type }\end{array}$ & $\begin{array}{l}\text { Immersion DC } \\
\text { Reference }\end{array}$ & $\begin{array}{c}\mathrm{HC}-2 \\
\text { Immersion } \\
\mathrm{DC}(\mathrm{Sv} / \mathrm{s} \\
\left.\text { per } \mathrm{Bq} / \mathrm{m}^{3}\right)\end{array}$ \\
\hline Ho-168 & Ho-168 & 167.936 & Holmium & $1.00 \mathrm{E}-03$ & ICRP-107 & $2.99 \mathrm{E}+00$ & $\mathrm{~m}$ & $1.79 \mathrm{E}+02$ & $3.74 \mathrm{E}+08$ & --- & --- & --- & DOE-STD-1196 & $4.06 \mathrm{E}-14$ \\
\hline Ho-168m & Ho-168 & 167.936 & Holmium & $1.00 \mathrm{E}-03$ & ICRP-107 & $1.32 \mathrm{E}+02$ & $\mathrm{~s}$ & $1.32 \mathrm{E}+02$ & $5.09 \mathrm{E}+08$ & -- & -- & -- & DOE-STD-1196 & $1.50 \mathrm{E}-16$ \\
\hline Ho-170 & Ho-170 & 169.940 & Holmium & $1.00 \mathrm{E}-03$ & ICRP-107 & $2.76 \mathrm{E}+00$ & $\mathrm{~m}$ & $1.66 \mathrm{E}+02$ & $4.01 E+08$ & -- & -- & -- & DOE-STD-1196 & 7.83E-14 \\
\hline |-118 & $\mathrm{I}-118$ & 117.913 & lodine & $5.00 \mathrm{E}-01$ & ICRP-107 & $1.37 \mathrm{E}+01$ & $\mathrm{~m}$ & $8.22 \mathrm{E}+02$ & $1.16 \mathrm{E}+08$ & DOE-STD-1196 & $2.03 \mathrm{E}-10$ & $V(g)$ & DOE-STD-1196 & $9.38 \mathrm{E}-14$ \\
\hline I-118m & $\mathrm{I}-118$ & 117.913 & Iodine & $5.00 \mathrm{E}-01$ & ICRP-107 & $8.50 \mathrm{E}+00$ & $\mathrm{~m}$ & $5.10 \mathrm{E}+02$ & $1.88 \mathrm{E}+08$ & -- & -- & -- & DOE-STD-1196 & $1.71 \mathrm{E}-13$ \\
\hline I-119 & I-119 & 118.910 & Iodine & $5.00 \mathrm{E}-01$ & ICRP-107 & $1.91 \mathrm{E}+01$ & $\mathrm{~m}$ & $1.15 \mathrm{E}+03$ & $8.28 \mathrm{E}+07$ & DOE-STD-1196 & $5.66 \mathrm{E}-11$ & $V(g)$ & DOE-STD-1196 & 4.06E-14 \\
\hline I-120 & I-120 & 119.910 & lodine & $5.00 \mathrm{E}-01$ & ICRP-38 & $8.10 \mathrm{E}+01$ & $\mathrm{~m}$ & $4.86 \mathrm{E}+03$ & $1.94 \mathrm{E}+07$ & ICRP-72 & $3.00 \mathrm{E}-10$ & $\mathrm{~V}$ & FGR-12 & $1.38 \mathrm{E}-13$ \\
\hline $\mathrm{I}-120 \mathrm{~m}$ & I-120 & 119.910 & Iodine & $5.00 \mathrm{E}-01$ & ICRP-38 & $5.30 \mathrm{E}+01$ & $\mathrm{~m}$ & $3.18 \mathrm{E}+03$ & $2.96 \mathrm{E}+07$ & ICRP-72 & $1.80 \mathrm{E}-10$ & $\mathrm{~V}$ & FGR-12 & $2.65 \mathrm{E}-13$ \\
\hline $\mid-121$ & I-121 & 120.907 & Iodine & $5.00 \mathrm{E}-01$ & ICRP-38 & $2.12 \mathrm{E}+00$ & $\mathrm{~h}$ & $7.63 E+03$ & $1.22 \mathrm{E}+07$ & ICRP-72 & $8.60 \mathrm{E}-11$ & $\mathrm{~V}$ & FGR-12 & $1.94 \mathrm{E}-14$ \\
\hline $\mid-122$ & I-122 & 121.908 & Iodine & $5.00 \mathrm{E}-01$ & ICRP-38 & $3.62 \mathrm{E}+00$ & $\mathrm{~m}$ & $2.17 \mathrm{E}+02$ & $4.26 \mathrm{E}+08$ & -- & -- & -- & FGR-12 & $4.56 \mathrm{E}-14$ \\
\hline I-123 & I-123 & 122.906 & Iodine & $5.00 \mathrm{E}-01$ & ICRP-38 & $1.32 \mathrm{E}+01$ & $\mathrm{~h}$ & $4.75 E+04$ & $1.93 \mathrm{E}+06$ & ICRP-72 & $2.10 \mathrm{E}-10$ & $\mathrm{~V}$ & FGR-12 & $7.28 \mathrm{E}-15$ \\
\hline I-124 & I-124 & 123.906 & Iodine & $5.00 \mathrm{E}-01$ & ICRP-38 & $4.18 \mathrm{E}+00$ & $d$ & $3.61 \mathrm{E}+05$ & $2.52 \mathrm{E}+05$ & ICRP-72 & $1.20 \mathrm{E}-08$ & $\mathrm{~V}$ & FGR-12 & $5.38 \mathrm{E}-14$ \\
\hline $1-125$ & I-125 & 124.905 & Iodine & $5.00 \mathrm{E}-01$ & ICRP-38 & $6.01 \mathrm{E}+01$ & $d$ & $5.20 \mathrm{E}+06$ & $1.74 \mathrm{E}+04$ & ICRP-72 & $1.40 \mathrm{E}-08$ & $\mathrm{~V}$ & FGR-12 & $5.22 \mathrm{E}-16$ \\
\hline |-126 & I-126 & 125.906 & Iodine & $5.00 \mathrm{E}-01$ & ICRP-38 & $1.30 \mathrm{E}+01$ & $d$ & $1.12 \mathrm{E}+06$ & $7.97 \mathrm{E}+04$ & ICRP-72 & $2.60 \mathrm{E}-08$ & $\mathrm{~V}$ & FGR-12 & $2.15 \mathrm{E}-14$ \\
\hline I-128 & I-128 & 127.906 & Iodine & $5.00 \mathrm{E}-01$ & ICRP-38 & $2.50 \mathrm{E}+01$ & $\mathrm{~m}$ & $1.50 \mathrm{E}+03$ & $5.88 \mathrm{E}+07$ & ICRP-72 & $6.50 \mathrm{E}-11$ & $\mathrm{~V}$ & FGR-12 & $4.16 \mathrm{E}-15$ \\
\hline I-129 & I-129 & 128.905 & Iodine & $5.00 \mathrm{E}-01$ & ICRP-38 & $1.57 \mathrm{E}+07$ & $y$ & $4.95 E+14$ & $1.77 \mathrm{E}-04$ & ICRP-72 & $9.60 \mathrm{E}-08$ & $\mathrm{~V}$ & FGR-12 & $3.80 \mathrm{E}-16$ \\
\hline |-130 & I-130 & 129.907 & Iodine & $5.00 \mathrm{E}-01$ & ICRP-38 & $1.24 \mathrm{E}+01$ & $\mathrm{~h}$ & $4.45 \mathrm{E}+04$ & $1.95 \mathrm{E}+06$ & ICRP-72 & $1.90 \mathrm{E}-09$ & $\mathrm{~V}$ & FGR-12 & $1.04 \mathrm{E}-13$ \\
\hline I-130m & I-130 & 129.907 & Iodine & $5.00 \mathrm{E}-01$ & ICRP-107 & $8.84 \mathrm{E}+00$ & $\mathrm{~m}$ & $5.30 \mathrm{E}+02$ & $1.64 \mathrm{E}+08$ & -- & -- & -- & DOE-STD-1196 & $4.88 \mathrm{E}-15$ \\
\hline $\mid-131$ & I-131 & 130.906 & Iodine & $5.00 \mathrm{E}-01$ & ICRP-38 & $8.04 \mathrm{E}+00$ & $d$ & $6.95 \mathrm{E}+05$ & $1.24 \mathrm{E}+05$ & ICRP-72 & $2.00 \mathrm{E}-08$ & $\mathrm{~V}$ & FGR-12 & $1.82 \mathrm{E}-14$ \\
\hline I-132 & $1-132$ & 131.908 & Iodine & $5.00 \mathrm{E}-01$ & ICRP-38 & $2.30 \mathrm{E}+00$ & $\mathrm{~h}$ & $8.28 \mathrm{E}+03$ & $1.03 E+07$ & ICRP-72 & $3.10 \mathrm{E}-10$ & $\mathrm{~V}$ & FGR-12 & $1.12 \mathrm{E}-13$ \\
\hline $\mathrm{I}-132 \mathrm{~m}$ & I-132 & 131.908 & Iodine & $5.00 \mathrm{E}-01$ & ICRP-38 & $8.36 \mathrm{E}+01$ & $\mathrm{~m}$ & $5.02 \mathrm{E}+03$ & $1.71 \mathrm{E}+07$ & ICRP-72 & $2.70 \mathrm{E}-10$ & $\mathrm{v}$ & FGR-12 & $1.53 \mathrm{E}-14$ \\
\hline I-133 & I-133 & 132.908 & Iodine & $5.00 \mathrm{E}-01$ & ICRP-38 & $2.08 \mathrm{E}+01$ & $\mathrm{~h}$ & $7.49 \mathrm{E}+04$ & $1.13 \mathrm{E}+06$ & ICRP-72 & $4.00 \mathrm{E}-09$ & $\mathrm{~V}$ & FGR-12 & $2.94 \mathrm{E}-14$ \\
\hline I-134 & I-134 & 133.910 & Iodine & $5.00 \mathrm{E}-01$ & ICRP-38 & $5.26 \mathrm{E}+01$ & $\mathrm{~m}$ & $3.16 \mathrm{E}+03$ & $2.67 \mathrm{E}+07$ & ICRP-72 & $1.50 \mathrm{E}-10$ & $\mathrm{~V}$ & FGR-12 & $1.30 \mathrm{E}-13$ \\
\hline I-134m & I-134 & 133.910 & Iodine & $5.00 \mathrm{E}-01$ & ICRP-107 & $3.60 \mathrm{E}+00$ & $\mathrm{~m}$ & $2.16 \mathrm{E}+02$ & $3.90 E+08$ & -- & -- & -- & DOE-STD-1196 & $1.19 \mathrm{E}-14$ \\
\hline I-135 & I-135 & 134.910 & Iodine & $5.00 \mathrm{E}-01$ & ICRP-38 & $6.61 \mathrm{E}+00$ & $\mathrm{~h}$ & $2.38 \mathrm{E}+04$ & $3.51 \mathrm{E}+06$ & ICRP-72 & $9.20 \mathrm{E}-10$ & $\mathrm{~V}$ & FGR-12 & $7.98 \mathrm{E}-14$ \\
\hline In-103 & $\ln -103$ & 102.920 & Indium & $1.00 \mathrm{E}-03$ & ICRP-107 & $6.00 \mathrm{E}+01$ & $\mathrm{~s}$ & $6.00 \mathrm{E}+01$ & $1.83 \mathrm{E}+09$ & -- & -- & -- & DOE-STD-1196 & $1.30 \mathrm{E}-13$ \\
\hline In-105 & $\ln -105$ & 104.915 & Indium & $1.00 \mathrm{E}-03$ & ICRP-107 & $5.07 \mathrm{E}+00$ & $\mathrm{~m}$ & $3.04 \mathrm{E}+02$ & $3.53 \mathrm{E}+08$ & -- & -- & -- & DOE-STD-1196 & $8.97 \mathrm{E}-14$ \\
\hline In-106 & $\ln -106$ & 105.913 & Indium & $1.00 \mathrm{E}-03$ & ICRP-107 & $6.20 \mathrm{E}+00$ & $\mathrm{~m}$ & $3.72 \mathrm{E}+02$ & $2.86 \mathrm{E}+08$ & -- & -- & -- & DOE-STD-1196 & $1.63 \mathrm{E}-13$ \\
\hline In-106m & $\ln -106$ & 105.913 & Indium & $1.00 \mathrm{E}-03$ & ICRP-107 & $5.20 \mathrm{E}+00$ & $\mathrm{~m}$ & $3.12 \mathrm{E}+02$ & $3.41 \mathrm{E}+08$ & -- & -- & -- & DOE-STD-1196 & $1.35 \mathrm{E}-13$ \\
\hline In-107 & $\ln -107$ & 106.910 & Indium & $1.00 \mathrm{E}-03$ & ICRP-107 & $3.24 \mathrm{E}+01$ & $\mathrm{~m}$ & $1.94 \mathrm{E}+03$ & $5.43 \mathrm{E}+07$ & DOE-STD-1196 & $3.06 \mathrm{E}-11$ & $\mathrm{~s}$ & DOE-STD-1196 & $7.18 \mathrm{E}-14$ \\
\hline In-108 & $\ln -108$ & 107.910 & Indium & $1.00 \mathrm{E}-03$ & ICRP-107 & $5.80 \mathrm{E}+01$ & $\mathrm{~m}$ & $3.48 \mathrm{E}+03$ & $3.00 E+07$ & DOE-STD-1196 & $5.71 \mathrm{E}-11$ & $\mathrm{~s}$ & DOE-STD-1196 & $1.81 \mathrm{E}-13$ \\
\hline In-108m & $\ln -108$ & 107.910 & Indium & $1.00 \mathrm{E}-03$ & ICRP-107 & $3.96 \mathrm{E}+01$ & $\mathrm{~m}$ & $2.38 \mathrm{E}+03$ & $4.40 \mathrm{E}+07$ & DOE-STD-1196 & $4.53 \mathrm{E}-11$ & $\mathrm{~s}$ & DOE-STD-1196 & $1.35 \mathrm{E}-13$ \\
\hline $\ln -109$ & In-109 & 108.907 & Indium & $1.00 \mathrm{E}-03$ & ICRP-38 & $4.20 \mathrm{E}+00$ & $\mathrm{~h}$ & $1.51 \mathrm{E}+04$ & $6.85 E+06$ & ICRP-72 & $4.20 \mathrm{E}-11$ & $M$ & FGR-12 & $3.21 \mathrm{E}-14$ \\
\hline In-109m & In-109 & 108.907 & Indium & $1.00 \mathrm{E}-03$ & ICRP-107 & $1.34 \mathrm{E}+00$ & $\mathrm{~m}$ & $8.04 \mathrm{E}+01$ & $1.29 \mathrm{E}+09$ & -- & -- & -- & DOE-STD-1196 & $2.74 \mathrm{E}-14$ \\
\hline |n-110| & $\ln -110$ & 109.907 & Indium & $1.00 \mathrm{E}-03$ & ICRP-38 & $4.90 \mathrm{E}+00$ & $\mathrm{~h}$ & $1.76 \mathrm{E}+04$ & $5.82 \mathrm{E}+06$ & ICRP-72 & $1.30 \mathrm{E}-10$ & $M$ & FGR-12 & $1.49 \mathrm{E}-13$ \\
\hline In-110s & $\ln -110$ & 109.907 & Indium & $1.00 \mathrm{E}-03$ & ICRP-38 & $6.91 \mathrm{E}+01$ & $\mathrm{~m}$ & $4.15 \mathrm{E}+03$ & $2.48 \mathrm{E}+07$ & ICRP-72 & $4.70 \mathrm{E}-11$ & $M$ & FGR-12 & $7.62 \mathrm{E}-14$ \\
\hline In-111 & $\ln -111$ & 110.905 & Indium & $1.00 \mathrm{E}-03$ & ICRP-38 & $2.83 \mathrm{E}+00$ & d & $2.45 \mathrm{E}+05$ & $4.16 \mathrm{E}+05$ & ICRP-72 & $2.30 \mathrm{E}-10$ & $M$ & FGR-12 & $1.86 \mathrm{E}-14$ \\
\hline In-111m & $\ln -111$ & 110.905 & Indium & $1.00 \mathrm{E}-03$ & ICRP-38 & $7.70 E+00$ & $\mathrm{~m}$ & $4.62 \mathrm{E}+02$ & $2.20 \mathrm{E}+08$ & -- & -- & -- & DOE-STD-1196 & $2.10 \mathrm{E}-14$ \\
\hline $\ln -112$ & $\ln -112$ & 111.906 & Indium & $1.00 \mathrm{E}-03$ & ICRP-38 & $1.44 \mathrm{E}+01$ & $\mathrm{~m}$ & $8.64 \mathrm{E}+02$ & $1.17 \mathrm{E}+08$ & ICRP-72 & $7.40 \mathrm{E}-12$ & $M$ & FGR-12 & $1.26 \mathrm{E}-14$ \\
\hline $\ln -112 m$ & $\ln -112$ & 111.906 & Indium & $1.00 \mathrm{E}-03$ & ICRP-107 & $2.06 \mathrm{E}+01$ & $\mathrm{~m}$ & $1.23 \mathrm{E}+03$ & $8.17 \mathrm{E}+07$ & DOE-STD-1196 & $2.60 \mathrm{E}-11$ & $\mathrm{~s}$ & DOE-STD-1196 & $9.86 \mathrm{E}-16$ \\
\hline $\ln -113 m$ & $\ln -113$ & 112.904 & Indium & $1.00 \mathrm{E}-03$ & ICRP-38 & $1.66 \mathrm{E}+00$ & $\mathrm{~h}$ & $5.97 \mathrm{E}+03$ & $1.67 \mathrm{E}+07$ & ICRP-72 & $2.00 \mathrm{E}-11$ & $M$ & FGR-12 & $1.21 \mathrm{E}-14$ \\
\hline In-114 & $\ln -114$ & 113.905 & Indium & $1.00 \mathrm{E}-03$ & ICRP-38 & $7.19 \mathrm{E}+01$ & $\mathrm{~s}$ & $7.19 \mathrm{E}+01$ & $1.38 \mathrm{E}+09$ & -- & -- & -- & FGR-12 & $1.39 \mathrm{E}-16$ \\
\hline In-114m & $\ln -114$ & 113.905 & Indium & $1.00 \mathrm{E}-03$ & ICRP-38 & $4.95 \mathrm{E}+01$ & $d$ & $4.28 \mathrm{E}+06$ & $2.32 \mathrm{E}+04$ & ICRP-72 & $9.30 \mathrm{E}-09$ & $\mathrm{~F}$ & FGR-12 & $4.18 \mathrm{E}-15$ \\
\hline $\ln -115$ & $\ln -115$ & 114.904 & Indium & $1.00 \mathrm{E}-03$ & ICRP-38 & $5.10 \mathrm{E}+15$ & $y$ & $1.61 \mathrm{E}+23$ & $6.10 \mathrm{E}-13$ & ICRP-72 & $3.90 \mathrm{E}-07$ & $F$ & FGR-12 & $4.50 \mathrm{E}-18$ \\
\hline In-115m & $\ln -115$ & 114.904 & Indium & $1.00 \mathrm{E}-03$ & ICRP-38 & $4.49 \mathrm{E}+00$ & $\mathrm{~h}$ & $1.61 \mathrm{E}+04$ & $6.08 \mathrm{E}+06$ & ICRP-72 & $5.90 \mathrm{E}-11$ & $M$ & FGR-12 & $7.39 \mathrm{E}-15$ \\
\hline $\ln -116 m$ & $\ln -116$ & 115.905 & Indium & $1.00 \mathrm{E}-03$ & ICRP-38 & $5.42 \mathrm{E}+01$ & $\mathrm{~m}$ & $3.25 \mathrm{E}+03$ & $3.00 \mathrm{E}+07$ & ICRP-72 & $4.50 \mathrm{E}-11$ & $M$ & FGR-12 & $1.25 \mathrm{E}-13$ \\
\hline
\end{tabular}




\begin{tabular}{|c|c|c|c|c|c|c|c|c|c|c|c|c|c|c|}
\hline \multicolumn{15}{|c|}{ Table A.1 Radionuclide Specific Input Data Used For the Calculation of HC-2 TQs Using Maximum Reported DCs (continued) } \\
\hline $\begin{array}{c}\text { MASTER } \\
\text { ISOTOPE } \\
\text { LIST }\end{array}$ & $\begin{array}{l}\text { Atomic } \\
\text { Mass } \\
\text { Nuclide }\end{array}$ & $\begin{array}{l}\text { Atomic } \\
\text { Mass }\end{array}$ & Element & $\begin{array}{c}\text { RF per } \\
\text { DOE-STD- } \\
1027\end{array}$ & $\begin{array}{l}\text { Half-Life } \\
\text { Reference }\end{array}$ & $\begin{array}{r}\begin{array}{r}\text { Half-Li } \\
\text { (original }\end{array}\end{array}$ & & Half Life (s) & $\begin{array}{l}\text { Sp. Act } \\
\text { (Ci/g) }\end{array}$ & $\begin{array}{c}\text { Inhalation DC } \\
\text { Reference }\end{array}$ & $\begin{array}{c}\text { HC-2 } \\
\text { Bounding } \\
\text { Inhalation } \\
\text { DC (Sv/Bq) }\end{array}$ & $\begin{array}{l}\text { Lung } \\
\text { Absorption } \\
\text { Class/Type }\end{array}$ & $\begin{array}{l}\text { Immersion DC } \\
\text { Reference }\end{array}$ & $\begin{array}{c}\mathrm{HC}-2 \\
\text { Immersion } \\
\mathrm{DC}(\mathrm{Sv} / \mathrm{s} \\
\left.\text { per } \mathrm{Bq} / \mathrm{m}^{3}\right)\end{array}$ \\
\hline In-117 & $\ln -117$ & 116.905 & Indium & $1.00 \mathrm{E}-03$ & ICRP-38 & $4.38 \mathrm{E}+01$ & $\mathrm{~m}$ & $2.63 \mathrm{E}+03$ & $3.67 \mathrm{E}+07$ & ICRP-72 & $2.90 \mathrm{E}-11$ & $\mathrm{M}$ & FGR-12 & $3.31 \mathrm{E}-14$ \\
\hline $\mathrm{In}-117 \mathrm{~m}$ & $\ln -117$ & 116.905 & Indium & $1.00 \mathrm{E}-03$ & ICRP-38 & $1.17 \mathrm{E}+02$ & $\mathrm{~m}$ & $6.99 \mathrm{E}+03$ & $1.38 \mathrm{E}+07$ & ICRP-72 & $7.20 \mathrm{E}-11$ & $M$ & FGR-12 & $4.19 \mathrm{E}-15$ \\
\hline $\ln -118$ & $\ln -118$ & 117.906 & Indium & $1.00 \mathrm{E}-03$ & ICRP-107 & $5.00 E+00$ & $\mathrm{~s}$ & $5.00 \mathrm{E}+00$ & $1.91 \mathrm{E}+10$ & -- & -- & -- & DOE-STD-1196 & $5.72 \mathrm{E}-15$ \\
\hline In-118m & $\ln -118$ & 117.906 & Indium & $1.00 \mathrm{E}-03$ & ICRP-107 & $4.36 \mathrm{E}+00$ & $\mathrm{~m}$ & $2.62 \mathrm{E}+02$ & $3.65 E+08$ & -- & -- & -- & DOE-STD-1196 & $1.31 \mathrm{E}-13$ \\
\hline In-119 & $\ln -119$ & 118.906 & Indium & $1.00 \mathrm{E}-03$ & ICRP-38 & $2.40 \mathrm{E}+00$ & $\mathrm{~m}$ & $1.44 \mathrm{E}+02$ & $6.59 \mathrm{E}+08$ & -- & -- & -- & FGR-12 & $3.74 \mathrm{E}-14$ \\
\hline $\ln -119 m$ & $\ln -119$ & 118.906 & Indium & $1.00 \mathrm{E}-03$ & ICRP-38 & $1.80 \mathrm{E}+01$ & $\mathrm{~m}$ & $1.08 \mathrm{E}+03$ & $8.78 \mathrm{E}+07$ & ICRP-72 & $1.70 \mathrm{E}-11$ & $M$ & FGR-12 & $6.14 \mathrm{E}-16$ \\
\hline In-121 & $\ln -121$ & 120.908 & Indium & $1.00 \mathrm{E}-03$ & ICRP-107 & $2.31 \mathrm{E}+01$ & $\mathrm{~s}$ & $2.31 \mathrm{E}+01$ & $4.04 \mathrm{E}+09$ & -- & -- & -- & DOE-STD-1196 & $4.36 \mathrm{E}-14$ \\
\hline In-121m & $\ln -121$ & 120.908 & Indium & $1.00 \mathrm{E}-03$ & ICRP-107 & $3.88 \mathrm{E}+00$ & $\mathrm{~m}$ & $2.33 \mathrm{E}+02$ & $4.01 E+08$ & -- & -- & -- & DOE-STD-1196 & $3.92 \mathrm{E}-15$ \\
\hline |r-179 & Ir-179 & 178.959 & Iridium & $1.00 \mathrm{E}-03$ & JAERI & $1.32 \mathrm{E}+00$ & $\mathrm{~m}$ & $7.90 \mathrm{E}+01$ & $7.98 \mathrm{E}+08$ & -- & -- & -- & -- & -- \\
\hline $\mid r-180$ & $\mid \mathrm{Ir}-180$ & 179.959 & Iridium & $1.00 \mathrm{E}-03$ & ICRP-107 & $1.50 \mathrm{E}+00$ & $\mathrm{~m}$ & $9.00 \mathrm{E}+01$ & $6.97 \mathrm{E}+08$ & -- & -- & -- & DOE-STD-1196 & $7.24 \mathrm{E}-14$ \\
\hline $\mid r-181$ & $\mid \mathrm{Ir}-181$ & 180.958 & Iridium & $1.00 \mathrm{E}-03$ & JAERI & $4.90 E+00$ & $\mathrm{~m}$ & $2.94 \mathrm{E}+02$ & $2.12 \mathrm{E}+08$ & -- & -- & -- & -- & -- \\
\hline $\mid r-182$ & $\mid \mathrm{Ir}-182$ & 181.958 & Iridium & $1.00 \mathrm{E}-03$ & ICRP-38 & $1.50 \mathrm{E}+01$ & $\mathrm{~m}$ & $9.00 \mathrm{E}+02$ & $6.89 \mathrm{E}+07$ & ICRP-72 & $2.40 \mathrm{E}-11$ & $\mathrm{~s}$ & FGR-12 & $6.50 \mathrm{E}-14$ \\
\hline Ir-183 & Ir-183 & 182.957 & Iridium & $1.00 \mathrm{E}-03$ & ICRP-107 & $5.80 \mathrm{E}+01$ & $\mathrm{~m}$ & $3.48 \mathrm{E}+03$ & $1.77 \mathrm{E}+07$ & DOE-STD-1196 & $4.71 \mathrm{E}-11$ & $\mathrm{~s}$ & DOE-STD-1196 & $5.48 \mathrm{E}-14$ \\
\hline $\mid r-184$ & $\mid \mathrm{Ir}-184$ & 183.957 & Iridium & $1.00 \mathrm{E}-03$ & ICRP-38 & $3.02 \mathrm{E}+00$ & $\mathrm{~h}$ & $1.09 \mathrm{E}+04$ & $5.64 \mathrm{E}+06$ & ICRP-72 & $1.20 \mathrm{E}-10$ & $\mathrm{~s}$ & FGR-12 & $9.38 \mathrm{E}-14$ \\
\hline Ir-185 & Ir-185 & 184.957 & Iridium & $1.00 \mathrm{E}-03$ & ICRP-38 & $1.40 \mathrm{E}+01$ & $\mathrm{~h}$ & $5.04 \mathrm{E}+04$ & $1.21 \mathrm{E}+06$ & ICRP-72 & $1.90 \mathrm{E}-10$ & $\mathrm{~s}$ & FGR-12 & $2.94 \mathrm{E}-14$ \\
\hline$|r-186|$ & Ir-186 & 185.958 & Iridium & $1.00 \mathrm{E}-03$ & ICRP-38 & $1.58 \mathrm{E}+01$ & $\mathrm{~h}$ & $5.69 \mathrm{E}+04$ & $1.07 E+06$ & ICRP-72 & $3.20 \mathrm{E}-10$ & $\mathrm{~s}$ & FGR-12 & $8.06 \mathrm{E}-14$ \\
\hline $\mid r-186 s$ & Ir-186 & 185.958 & Iridium & $1.00 \mathrm{E}-03$ & ICRP-38 & $1.75 E+00$ & $\mathrm{~h}$ & $6.30 \mathrm{E}+03$ & $9.63 \mathrm{E}+06$ & ICRP-72 & $4.40 \mathrm{E}-11$ & $\mathrm{~s}$ & FGR-12 & $4.65 \mathrm{E}-14$ \\
\hline Ir-187 & Ir-187 & 186.958 & Iridium & $1.00 \mathrm{E}-03$ & ICRP-38 & $1.05 E+01$ & $\mathrm{~h}$ & $3.78 \mathrm{E}+04$ & $1.60 E+06$ & ICRP-72 & $7.90 \mathrm{E}-11$ & $\mathrm{~s}$ & FGR-12 & $1.68 \mathrm{E}-14$ \\
\hline $\mid \mathrm{r}-188$ & Ir-188 & 187.959 & Iridium & $1.00 \mathrm{E}-03$ & ICRP-38 & $4.15 \mathrm{E}+01$ & $\mathrm{~h}$ & $1.49 \mathrm{E}+05$ & $4.02 \mathrm{E}+05$ & ICRP-72 & $4.20 \mathrm{E}-10$ & $\mathrm{~s}$ & FGR-12 & $8.01 \mathrm{E}-14$ \\
\hline |r-189 & $\mid r-189$ & 188.959 & Iridium & $1.00 \mathrm{E}-03$ & ICRP-38 & $1.33 \mathrm{E}+01$ & $d$ & $1.15 \mathrm{E}+06$ & $5.20 \mathrm{E}+04$ & ICRP-72 & $6.00 \mathrm{E}-10$ & $\mathrm{~s}$ & FGR-12 & $3.21 \mathrm{E}-15$ \\
\hline $\mid \mathrm{r}-190$ & $\mid \mathrm{Ir}-190$ & 189.961 & Iridium & $1.00 \mathrm{E}-03$ & ICRP-38 & $1.21 \mathrm{E}+01$ & $d$ & $1.05 \mathrm{E}+06$ & $5.68 \mathrm{E}+04$ & ICRP-72 & $2.40 \mathrm{E}-09$ & $\mathrm{~s}$ & FGR-12 & $6.86 \mathrm{E}-14$ \\
\hline Ir-190ms & Ir-190 & 189.961 & Iridium & $1.00 \mathrm{E}-03$ & ICRP-38 & $1.20 \mathrm{E}+00$ & $\mathrm{~h}$ & $4.32 \mathrm{E}+03$ & $1.37 \mathrm{E}+07$ & ICRP-72 & $1.00 \mathrm{E}-11$ & $\mathrm{~s}$ & FGR-12 & $1.27 \mathrm{E}-19$ \\
\hline$|r-190 m|$ & Ir-190 & 189.961 & Iridium & $1.00 \mathrm{E}-03$ & ICRP-38 & $3.10 \mathrm{E}+00$ & $\mathrm{~h}$ & $1.12 \mathrm{E}+04$ & $5.32 \mathrm{E}+06$ & ICRP-72 & $8.30 \mathrm{E}-11$ & $\mathrm{~s}$ & FGR-12 & $7.39 \mathrm{E}-14$ \\
\hline Ir-191m & $\mid r-191$ & 190.961 & Iridium & $1.00 \mathrm{E}-03$ & ICRP-38 & $4.94 \mathrm{E}+00$ & $\mathrm{~s}$ & $4.94 \mathrm{E}+00$ & $1.20 \mathrm{E}+10$ & -- & -- & -- & FGR-12 & $3.02 \mathrm{E}-15$ \\
\hline $\mid \mathrm{r}-192$ & Ir-192 & 191.963 & Iridium & $1.00 \mathrm{E}-03$ & ICRP-38 & $7.40 \mathrm{E}+01$ & $d$ & $6.40 \mathrm{E}+06$ & $9.19 \mathrm{E}+03$ & ICRP-72 & $6.60 \mathrm{E}-09$ & $\mathrm{~s}$ & FGR-12 & $3.91 \mathrm{E}-14$ \\
\hline $\mid r-192 m s$ & |r-192 & 191.963 & Iridium & $1.00 \mathrm{E}-03$ & ICRP-107 & $1.45 E+00$ & $\mathrm{~m}$ & $8.70 \mathrm{E}+01$ & $6.76 \mathrm{E}+08$ & -- & -- & -- & DOE-STD-1196 & $2.68 \mathrm{E}-18$ \\
\hline $\mid \mathrm{r}-192 \mathrm{ml}$ & $\mid r-192$ & 191.963 & Iridium & $1.00 \mathrm{E}-03$ & ICRP-38 & $2.41 E+02$ & $y$ & $7.61 E+09$ & $7.73 E+00$ & ICRP-72 & $3.90 \mathrm{E}-08$ & $\mathrm{~s}$ & FGR-12 & $7.63 \mathrm{E}-15$ \\
\hline Ir-193m & $\begin{array}{ll}r-193 \\
\end{array}$ & 192.963 & Iridium & $1.00 \mathrm{E}-03$ & ICRP-107 & $1.05 E+01$ & $d$ & $9.10 \mathrm{E}+05$ & $6.43 E+04$ & ICRP-72 & $1.30 \mathrm{E}-09$ & $\mathrm{~s}$ & DOE-STD-1196 & $1.04 \mathrm{E}-17$ \\
\hline Ir-194 & Ir-194 & 193.965 & Iridium & $1.00 \mathrm{E}-03$ & ICRP-38 & $1.92 \mathrm{E}+01$ & $\mathrm{~h}$ & $6.89 \mathrm{E}+04$ & $8.44 \mathrm{E}+05$ & ICRP-72 & $5.60 \mathrm{E}-10$ & $\mathrm{~s}$ & FGR-12 & $4.54 \mathrm{E}-15$ \\
\hline Ir-194m & $\mid \mathrm{Ir}-194$ & 193.965 & Iridium & $1.00 \mathrm{E}-03$ & ICRP-38 & $1.71 \mathrm{E}+02$ & $d$ & $1.48 \mathrm{E}+07$ & $3.94 \mathrm{E}+03$ & ICRP-72 & $1.30 \mathrm{E}-08$ & $\mathrm{~s}$ & FGR-12 & $1.12 \mathrm{E}-13$ \\
\hline Ir-195 & Ir-195 & 194.966 & Iridium & $1.00 \mathrm{E}-03$ & ICRP-38 & $2.50 E+00$ & $\mathrm{~h}$ & $9.00 \mathrm{E}+03$ & $6.43 \mathrm{E}+06$ & ICRP-72 & $7.10 \mathrm{E}-11$ & $\mathrm{~s}$ & FGR-12 & $2.32 \mathrm{E}-15$ \\
\hline $\mid r-195 m$ & Ir-195 & 194.966 & Iridium & $1.00 \mathrm{E}-03$ & ICRP-38 & $3.80 \mathrm{E}+00$ & $\mathrm{~h}$ & $1.37 \mathrm{E}+04$ & $4.23 E+06$ & ICRP-72 & $1.70 \mathrm{E}-10$ & $\mathrm{~s}$ & FGR-12 & $1.93 \mathrm{E}-14$ \\
\hline |r-196 & Ir-196 & 195.968 & Iridium & $1.00 \mathrm{E}-03$ & ICRP-107 & $5.20 \mathrm{E}+01$ & $\mathrm{~s}$ & $5.20 \mathrm{E}+01$ & $1.11 \mathrm{E}+09$ & -- & -- & -- & DOE-STD-1196 & $1.16 \mathrm{E}-14$ \\
\hline $\mid r-196 m$ & Ir-196 & 195.968 & Iridium & $1.00 \mathrm{E}-03$ & ICRP-107 & $1.40 \mathrm{E}+00$ & $\mathrm{~h}$ & $5.04 \mathrm{E}+03$ & $1.14 \mathrm{E}+07$ & DOE-STD-1196 & $1.08 \mathrm{E}-10$ & $\mathrm{~s}$ & DOE-STD-1196 & $1.10 \mathrm{E}-13$ \\
\hline $\mathrm{K}-38$ & K-38 & 37.969 & Potassium & $5.00 \mathrm{E}-01$ & ICRP-38 & $7.64 \mathrm{E}+00$ & $\mathrm{~m}$ & $4.58 \mathrm{E}+02$ & $6.49 \mathrm{E}+08$ & -- & -- & -- & FGR-12 & $1.64 \mathrm{E}-13$ \\
\hline K-40 & $\mathrm{K}-40$ & 39.964 & Potassium & $5.00 \mathrm{E}-01$ & ICRP-38 & $1.28 \mathrm{E}+09$ & $y$ & $4.04 \mathrm{E}+16$ & $6.99 \mathrm{E}-06$ & ICRP-72 & $2.10 \mathrm{E}-09$ & $\mathrm{~F}$ & FGR-12 & $8.05 \mathrm{E}-15$ \\
\hline K-42 & K-42 & 41.962 & Potassium & $5.00 \mathrm{E}-01$ & ICRP-38 & $1.24 \mathrm{E}+01$ & $\mathrm{~h}$ & $4.45 \mathrm{E}+04$ & $6.04 \mathrm{E}+06$ & ICRP-72 & $1.20 \mathrm{E}-10$ & $\mathrm{~F}$ & FGR-12 & $1.46 \mathrm{E}-14$ \\
\hline K-43 & K-43 & 42.961 & Potassium & $5.00 \mathrm{E}-01$ & ICRP-38 & $2.26 \mathrm{E}+01$ & $\mathrm{~h}$ & $8.14 \mathrm{E}+04$ & $3.23 \mathrm{E}+06$ & ICRP-72 & $1.40 \mathrm{E}-10$ & $F$ & FGR-12 & 4.67E-14 \\
\hline K-44 & K-44 & 43.962 & Potassium & $5.00 \mathrm{E}-01$ & ICRP-38 & $2.21 \mathrm{E}+01$ & $\mathrm{~m}$ & $1.33 \mathrm{E}+03$ & $1.93 \mathrm{E}+08$ & ICRP-72 & $2.00 \mathrm{E}-11$ & $\mathrm{~F}$ & FGR-12 & $1.19 \mathrm{E}-13$ \\
\hline K-45 & K-45 & 44.961 & Potassium & $5.00 \mathrm{E}-01$ & ICRP-38 & $2.00 \mathrm{E}+01$ & $\mathrm{~m}$ & $1.20 \mathrm{E}+03$ & $2.09 \mathrm{E}+08$ & ICRP-72 & $1.50 \mathrm{E}-11$ & $\mathrm{~F}$ & FGR-12 & $9.67 \mathrm{E}-14$ \\
\hline K-46 & K-46 & 45.962 & Potassium & $5.00 \mathrm{E}-01$ & ICRP-107 & $1.05 E+02$ & $\mathrm{~s}$ & $1.05 \mathrm{E}+02$ & $2.34 \mathrm{E}+09$ & -- & -- & -- & DOE-STD-1196 & $1.47 \mathrm{E}-13$ \\
\hline Kr-74 & Kr-74 & 73.933 & Krypton & $1.00 \mathrm{E}+00$ & ICRP-38 & $1.15 \mathrm{E}+01$ & $\mathrm{~m}$ & $6.90 \mathrm{E}+02$ & $2.21 \mathrm{E}+08$ & -- & -- & -- & FGR-12 & $5.59 \mathrm{E}-14$ \\
\hline Kr-75 & Kr-75 & 74.931 & Krypton & $1.00 E+00$ & ICRP-107 & $4.29 \mathrm{E}+00$ & $\mathrm{~m}$ & $2.57 \mathrm{E}+02$ & $5.85 E+08$ & -- & -- & -- & DOE-STD-1196 & $5.85 \mathrm{E}-14$ \\
\hline Kr-76 & Kr-76 & 75.926 & Krypton & $1.00 E+00$ & ICRP-38 & $1.48 \mathrm{E}+01$ & $\mathrm{~h}$ & $5.33 \mathrm{E}+04$ & $2.79 \mathrm{E}+06$ & -- & -- & -- & FGR-12 & $2.03 \mathrm{E}-14$ \\
\hline Kr-77 & Kr-77 & 76.925 & Krypton & $1.00 E+00$ & ICRP-38 & $7.47 \mathrm{E}+01$ & $\mathrm{~m}$ & $4.48 \mathrm{E}+03$ & $3.27 \mathrm{E}+07$ & -- & -- & -- & FGR-12 & $4.86 \mathrm{E}-14$ \\
\hline Kr-79 & Kr-79 & 78.920 & Krypton & $1.00 E+00$ & ICRP-38 & $3.50 \mathrm{E}+01$ & $\mathrm{~h}$ & $1.26 \mathrm{E}+05$ & $1.13 \mathrm{E}+06$ & -- & -- & -- & FGR-12 & $1.21 \mathrm{E}-14$ \\
\hline
\end{tabular}

A-15 


\begin{tabular}{|c|c|c|c|c|c|c|c|c|c|c|c|c|c|c|}
\hline \multicolumn{15}{|c|}{ Table A.1 Radionuclide Specific Input Data Used For the Calculation of HC-2 TQs Using Maximum Reported DCs (continued) } \\
\hline $\begin{array}{c}\text { MASTER } \\
\text { ISOTOPE } \\
\text { LIST } \\
\end{array}$ & $\begin{array}{l}\text { Atomic } \\
\text { Mass } \\
\text { Nuclide }\end{array}$ & $\begin{array}{l}\text { Atomic } \\
\text { Mass }\end{array}$ & Element & $\begin{array}{c}\text { RF per } \\
\text { DOE-STD- } \\
1027\end{array}$ & $\begin{array}{l}\text { Half-Life } \\
\text { Reference }\end{array}$ & $\begin{array}{r}\text { Half-Li } \\
\text { (original }\end{array}$ & & Half Life (s) & $\begin{array}{l}\text { Sp. Act } \\
\text { (Ci/g) }\end{array}$ & $\begin{array}{c}\text { Inhalation DC } \\
\text { Reference }\end{array}$ & $\begin{array}{c}\text { HC-2 } \\
\text { Bounding } \\
\text { Inhalation } \\
\text { DC (Sv/Bq) }\end{array}$ & $\begin{array}{l}\text { Lung } \\
\text { Absorption } \\
\text { Class/Type }\end{array}$ & $\begin{array}{c}\text { Immersion DC } \\
\text { Reference }\end{array}$ & $\begin{array}{c}\text { HC-2 } \\
\text { Immersion } \\
\mathrm{DC}(\mathrm{Sv} / \mathrm{s} \\
\left.\text { per } \mathrm{Bq} / \mathrm{m}^{3}\right)\end{array}$ \\
\hline Kr-81 & Kr-81 & 80.917 & Krypton & $1.00 \mathrm{E}+00$ & ICRP-38 & $2.10 \mathrm{E}+05$ & y & $6.63 \mathrm{E}+12$ & $2.10 \mathrm{E}-02$ & -- & -- & -- & FGR-12 & $2.67 \mathrm{E}-16$ \\
\hline Kr-81m & Kr-81 & 80.917 & Krypton & $1.00 \mathrm{E}+00$ & ICRP-38 & $1.30 \mathrm{E}+01$ & s & $1.30 \mathrm{E}+01$ & $1.07 \mathrm{E}+10$ & -- & -- & -- & FGR-12 & $6.14 \mathrm{E}-15$ \\
\hline $\mathrm{Kr}-83 \mathrm{~m}$ & Kr-83 & 82.914 & Krypton & $1.00 E+00$ & ICRP-38 & $1.83 \mathrm{E}+00$ & $\mathrm{~h}$ & $6.59 \mathrm{E}+03$ & $2.07 E+07$ & -- & -- & -- & FGR-12 & $1.50 \mathrm{E}-18$ \\
\hline $\mathrm{Kr}-85$ & Kr-85 & 84.913 & Krypton & $1.00 E+00$ & ICRP-38 & $1.07 \mathrm{E}+01$ & $y$ & $3.38 \mathrm{E}+08$ & $3.93 \mathrm{E}+02$ & -- & -- & -- & FGR-12 & $1.19 \mathrm{E}-16$ \\
\hline $\mathrm{Kr}-85 \mathrm{~m}$ & Kr-85 & 84.913 & Krypton & $1.00 E+00$ & ICRP-38 & $4.48 \mathrm{E}+00$ & $\mathrm{~h}$ & $1.61 \mathrm{E}+04$ & $8.24 E+06$ & -- & -- & -- & FGR-12 & $7.48 \mathrm{E}-15$ \\
\hline $\mathrm{Kr}-87$ & Kr-87 & 86.913 & Krypton & $1.00 E+00$ & ICRP-38 & $7.63 \mathrm{E}+01$ & $\mathrm{~m}$ & $4.58 \mathrm{E}+03$ & $2.84 E+07$ & -- & -- & -- & FGR-12 & $4.12 \mathrm{E}-14$ \\
\hline $\mathrm{Kr}-88$ & Kr-88 & 87.914 & Krypton & $1.00 \mathrm{E}+00$ & ICRP-38 & $2.84 \mathrm{E}+00$ & $\mathrm{~h}$ & $1.02 \mathrm{E}+04$ & $1.26 \mathrm{E}+07$ & -- & -- & -- & FGR-12 & $1.02 \mathrm{E}-13$ \\
\hline Kr-89 & Kr-89 & 88.918 & Krypton & $1.00 E+00$ & ICRP-107 & $3.15 \mathrm{E}+00$ & $\mathrm{~m}$ & $1.89 \mathrm{E}+02$ & $6.71 \mathrm{E}+08$ & -- & -- & -- & DOE-STD-1196 & $9.56 \mathrm{E}-14$ \\
\hline La-128 & La-128 & 127.916 & Lanthanum & $1.00 \mathrm{E}-03$ & ICRP-107 & $5.18 \mathrm{E}+00$ & $\mathrm{~m}$ & $3.11 \mathrm{E}+02$ & $2.84 E+08$ & -- & -- & -- & DOE-STD-1196 & $1.30 \mathrm{E}-13$ \\
\hline La-129 & La-129 & 128.913 & Lanthanum & $1.00 \mathrm{E}-03$ & ICRP-107 & $1.16 \mathrm{E}+01$ & $\mathrm{~m}$ & $6.96 \mathrm{E}+02$ & $1.26 \mathrm{E}+08$ & DOE-STD-1196 & $1.48 \mathrm{E}-11$ & $\mathrm{~s}$ & DOE-STD-1196 & $4.11 \mathrm{E}-14$ \\
\hline La-130 & La-130 & 129.912 & Lanthanum & $1.00 \mathrm{E}-03$ & ICRP-107 & $8.70 \mathrm{E}+00$ & $\mathrm{~m}$ & $5.22 \mathrm{E}+02$ & $1.66 \mathrm{E}+08$ & -- & -- & -- & DOE-STD-1196 & $1.03 \mathrm{E}-13$ \\
\hline La-131 & La-131 & 130.910 & Lanthanum & $1.00 \mathrm{E}-03$ & ICRP-38 & $5.90 \mathrm{E}+01$ & $\mathrm{~m}$ & $3.54 \mathrm{E}+03$ & $2.43 \mathrm{E}+07$ & ICRP-72 & $2.30 \mathrm{E}-11$ & $M$ & FGR-12 & $3.14 \mathrm{E}-14$ \\
\hline La-132 & La-132 & 131.910 & Lanthanum & $1.00 \mathrm{E}-03$ & ICRP-38 & $4.80 \mathrm{E}+00$ & $\mathrm{~h}$ & $1.73 \mathrm{E}+04$ & $4.95 \mathrm{E}+06$ & ICRP-72 & $1.60 \mathrm{E}-10$ & $M$ & FGR-12 & $1.00 \mathrm{E}-13$ \\
\hline La-132m & La-132 & 131.910 & Lanthanum & $1.00 \mathrm{E}-03$ & ICRP-107 & $2.43 \mathrm{E}+01$ & $\mathrm{~m}$ & $1.46 \mathrm{E}+03$ & $5.87 E+07$ & DOE-STD-1196 & $2.51 \mathrm{E}-11$ & $\mathrm{~s}$ & DOE-STD-1196 & $2.95 \mathrm{E}-14$ \\
\hline La-133 & La-133 & 132.908 & Lanthanum & $1.00 \mathrm{E}-03$ & ICRP-107 & $3.91 \mathrm{E}+00$ & $\mathrm{~h}$ & $1.41 \mathrm{E}+04$ & $6.03 \mathrm{E}+06$ & DOE-STD-1196 & $2.08 \mathrm{E}-11$ & $\mathrm{~s}$ & DOE-STD-1196 & $6.39 \mathrm{E}-15$ \\
\hline La-134 & La-134 & 133.909 & Lanthanum & $1.00 \mathrm{E}-03$ & ICRP-38 & $6.67 \mathrm{E}+00$ & $\mathrm{~m}$ & $4.00 \mathrm{E}+02$ & $2.11 \mathrm{E}+08$ & -- & -- & -- & FGR-12 & $3.35 \mathrm{E}-14$ \\
\hline La-135 & La-135 & 134.907 & Lanthanum & $1.00 \mathrm{E}-03$ & ICRP-38 & $1.95 \mathrm{E}+01$ & $\mathrm{~h}$ & $7.02 E+04$ & $1.19 E+06$ & ICRP-72 & $1.40 \mathrm{E}-11$ & $M$ & FGR-12 & $9.21 \mathrm{E}-16$ \\
\hline La-136 & La-136 & 135.908 & Lanthanum & $1.00 \mathrm{E}-03$ & ICRP-107 & $9.87 \mathrm{E}+00$ & $\mathrm{~m}$ & $5.92 \mathrm{E}+02$ & $1.40 \mathrm{E}+08$ & -- & -- & -- & DOE-STD-1196 & $1.79 \mathrm{E}-14$ \\
\hline La-137 & La-137 & 136.906 & Lanthanum & $1.00 \mathrm{E}-03$ & ICRP-38 & $6.00 \mathrm{E}+04$ & $y$ & $1.89 \mathrm{E}+12$ & $4.35 \mathrm{E}-02$ & ICRP-72 & $8.70 \mathrm{E}-09$ & $\mathrm{~F}$ & FGR-12 & $4.06 \mathrm{E}-16$ \\
\hline La-138 & La-138 & 137.907 & Lanthanum & $1.00 \mathrm{E}-03$ & ICRP-38 & $1.35 \mathrm{E}+11$ & $y$ & $4.26 \mathrm{E}+18$ & $1.92 \mathrm{E}-08$ & ICRP-72 & $1.50 \mathrm{E}-07$ & $\mathrm{~F}$ & FGR-12 & $6.20 \mathrm{E}-14$ \\
\hline La-140 & La-140 & 139.909 & Lanthanum & $1.00 \mathrm{E}-03$ & ICRP-38 & $4.03 \mathrm{E}+01$ & $\mathrm{~h}$ & $1.45 \mathrm{E}+05$ & $5.56 \mathrm{E}+05$ & ICRP-72 & $1.10 \mathrm{E}-09$ & $M$ & FGR-12 & $1.17 \mathrm{E}-13$ \\
\hline La-141 & La-141 & 140.911 & Lanthanum & $1.00 \mathrm{E}-03$ & ICRP-38 & $3.93 \mathrm{E}+00$ & $\mathrm{~h}$ & $1.41 \mathrm{E}+04$ & $5.66 \mathrm{E}+06$ & ICRP-72 & $1.50 \mathrm{E}-10$ & $M$ & FGR-12 & $2.39 \mathrm{E}-15$ \\
\hline La-142 & La-142 & 141.914 & Lanthanum & $1.00 \mathrm{E}-03$ & ICRP-38 & $9.25 \mathrm{E}+01$ & $\mathrm{~m}$ & $5.55 \mathrm{E}+03$ & $1.43 \mathrm{E}+07$ & ICRP-72 & $8.90 \mathrm{E}-11$ & $M$ & FGR-12 & $1.44 \mathrm{E}-13$ \\
\hline La-143 & La-143 & 142.916 & Lanthanum & $1.00 \mathrm{E}-03$ & ICRP-38 & $1.42 \mathrm{E}+01$ & $\mathrm{~m}$ & $8.54 \mathrm{E}+02$ & $9.25 \mathrm{E}+07$ & ICRP-72 & $2.10 \mathrm{E}-11$ & $M$ & FGR-12 & $5.18 \mathrm{E}-15$ \\
\hline Lu-164 & Lu-164 & 163.941 & Lutetium & $1.00 \mathrm{E}-03$ & JAERI & $3.14 \mathrm{E}+00$ & $\mathrm{~m}$ & $1.88 \mathrm{E}+02$ & $3.65 E+08$ & -- & -- & -- & -- & -- \\
\hline Lu-165 & Lu-165 & 164.939 & Lutetium & $1.00 \mathrm{E}-03$ & ICRP-107 & $1.07 \mathrm{E}+01$ & $\mathrm{~m}$ & $6.44 \mathrm{E}+02$ & $1.06 \mathrm{E}+08$ & DOE-STD-1196 & $1.63 \mathrm{E}-11$ & $\mathrm{~S}$ & DOE-STD-1196 & $5.02 \mathrm{E}-14$ \\
\hline Lu-166 & Lu-166 & 165.940 & Lutetium & $1.00 \mathrm{E}-03$ & JAERI & $2.65 E+00$ & $\mathrm{~m}$ & $1.59 \mathrm{E}+02$ & $4.28 \mathrm{E}+08$ & -- & -- & -- & -- & -- \\
\hline Lu-166m & Lu-166 & 165.940 & Lutetium & $1.00 \mathrm{E}-03$ & JAERI & $1.41 \mathrm{E}+00$ & $\mathrm{~m}$ & $8.46 \mathrm{E}+01$ & $8.04 E+08$ & -- & -- & -- & -- & -- \\
\hline Lu-167 & Lu-167 & 166.938 & Lutetium & $1.00 \mathrm{E}-03$ & ICRP-107 & $5.15 \mathrm{E}+01$ & $\mathrm{~m}$ & $3.09 \mathrm{E}+03$ & $2.19 E+07$ & DOE-STD-1196 & $4.56 \mathrm{E}-11$ & $\mathrm{~s}$ & DOE-STD-1196 & $7.94 \mathrm{E}-14$ \\
\hline Lu-168m & Lu-168 & 167.939 & Lutetium & $1.00 \mathrm{E}-03$ & JAERI & $6.70 \mathrm{E}+00$ & $\mathrm{~m}$ & $4.02 \mathrm{E}+02$ & $1.67 E+08$ & -- & -- & -- & -- & -- \\
\hline Lu-169 & Lu-169 & 168.938 & Lutetium & $1.00 \mathrm{E}-03$ & ICRP-38 & $3.41 \mathrm{E}+01$ & $\mathrm{~h}$ & $1.23 \mathrm{E}+05$ & $5.45 \mathrm{E}+05$ & ICRP-72 & $3.80 \mathrm{E}-10$ & $\mathrm{~s}$ & FGR-12 & $5.09 \mathrm{E}-14$ \\
\hline Lu-169m & Lu-169 & 168.938 & Lutetium & $1.00 \mathrm{E}-03$ & ICRP-107 & $1.60 \mathrm{E}+02$ & $\mathrm{~s}$ & $1.60 \mathrm{E}+02$ & $4.17 E+08$ & -- & -- & -- & DOE-STD-1196 & $2.52 \mathrm{E}-20$ \\
\hline Lu-170 & Lu-170 & 169.938 & Lutetium & $1.00 \mathrm{E}-03$ & ICRP-38 & $2.00 E+00$ & $d$ & $1.73 \mathrm{E}+05$ & $3.84 E+05$ & ICRP-72 & $6.60 \mathrm{E}-10$ & $\mathrm{~s}$ & FGR-12 & $1.28 \mathrm{E}-13$ \\
\hline Lu-171 & Lu-171 & 170.938 & Lutetium & $1.00 \mathrm{E}-03$ & ICRP-38 & $8.22 \mathrm{E}+00$ & $d$ & $7.10 \mathrm{E}+05$ & $9.29 \mathrm{E}+04$ & ICRP-72 & $8.80 \mathrm{E}-10$ & $\mathrm{~s}$ & FGR-12 & $3.25 \mathrm{E}-14$ \\
\hline Lu-171m & Lu-171 & 170.938 & Lutetium & $1.00 \mathrm{E}-03$ & ICRP-107 & $7.90 \mathrm{E}+01$ & $\mathrm{~s}$ & $7.90 \mathrm{E}+01$ & $8.35 \mathrm{E}+08$ & -- & -- & -- & DOE-STD-1196 & $9.86 \mathrm{E}-18$ \\
\hline Lu-172 & Lu-172 & 171.939 & Lutetium & $1.00 \mathrm{E}-03$ & ICRP-38 & $6.70 E+00$ & $d$ & $5.79 \mathrm{E}+05$ & $1.13 \mathrm{E}+05$ & ICRP-72 & $1.60 \mathrm{E}-09$ & $\mathrm{~s}$ & FGR-12 & $9.25 \mathrm{E}-14$ \\
\hline Lu-172m & Lu-172 & 171.939 & Lutetium & $1.00 \mathrm{E}-03$ & ICRP-107 & $3.70 E+00$ & $\mathrm{~m}$ & $2.22 \mathrm{E}+02$ & $2.96 \mathrm{E}+08$ & -- & -- & -- & DOE-STD-1196 & $3.90 \mathrm{E}-20$ \\
\hline Lu-173 & Lu-173 & 172.939 & Lutetium & $1.00 \mathrm{E}-03$ & ICRP-38 & $1.37 \mathrm{E}+00$ & $y$ & $4.32 \mathrm{E}+07$ & $1.51 \mathrm{E}+03$ & ICRP-72 & $2.40 \mathrm{E}-09$ & $\mathrm{~s}$ & FGR-12 & $5.10 \mathrm{E}-15$ \\
\hline Lu-174 & Lu-174 & 173.940 & Lutetium & $1.00 \mathrm{E}-03$ & ICRP-38 & $3.31 \mathrm{E}+00$ & $y$ & $1.04 \mathrm{E}+08$ & $6.21 \mathrm{E}+02$ & ICRP-72 & $4.20 \mathrm{E}-09$ & $M$ & FGR-12 & $5.46 \mathrm{E}-15$ \\
\hline Lu-174m & Lu-174 & 173.940 & Lutetium & $1.00 \mathrm{E}-03$ & ICRP-38 & $1.42 \mathrm{E}+02$ & $d$ & $1.23 \mathrm{E}+07$ & $5.29 \mathrm{E}+03$ & ICRP-72 & $4.20 \mathrm{E}-09$ & $\mathrm{~s}$ & FGR-12 & $2.18 \mathrm{E}-15$ \\
\hline Lu-176 & Lu-176 & 175.943 & Lutetium & $1.00 \mathrm{E}-03$ & ICRP-38 & $3.60 \mathrm{E}+10$ & $y$ & $1.14 \mathrm{E}+18$ & $5.64 \mathrm{E}-08$ & ICRP-72 & $7.00 \mathrm{E}-08$ & $M$ & FGR-12 & $2.32 \mathrm{E}-14$ \\
\hline Lu-176m & Lu-176 & 175.943 & Lutetium & $1.00 \mathrm{E}-03$ & ICRP-38 & $3.68 \mathrm{E}+00$ & $\mathrm{~h}$ & $1.32 \mathrm{E}+04$ & $4.84 E+06$ & ICRP-72 & $1.20 \mathrm{E}-10$ & $\mathrm{~s}$ & FGR-12 & $5.87 \mathrm{E}-16$ \\
\hline Lu-177 & Lu-177 & 176.944 & Lutetium & $1.00 \mathrm{E}-03$ & ICRP-38 & $6.71 \mathrm{E}+00$ & $d$ & $5.80 \mathrm{E}+05$ & $1.10 \mathrm{E}+05$ & ICRP-72 & $1.20 \mathrm{E}-09$ & $\mathrm{~s}$ & FGR-12 & $1.62 \mathrm{E}-15$ \\
\hline Lu-177m & Lu-177 & 176.944 & Lutetium & $1.00 \mathrm{E}-03$ & ICRP-38 & $1.61 \mathrm{E}+02$ & $d$ & $1.39 \mathrm{E}+07$ & $4.59 \mathrm{E}+03$ & ICRP-72 & $1.60 \mathrm{E}-08$ & $\mathrm{~s}$ & FGR-12 & $4.67 \mathrm{E}-14$ \\
\hline Lu-178 & Lu-178 & 177.946 & Lutetium & $1.00 \mathrm{E}-03$ & ICRP-38 & $2.84 \mathrm{E}+01$ & $\mathrm{~m}$ & $1.70 \mathrm{E}+03$ & $3.72 E+07$ & ICRP-72 & $2.60 \mathrm{E}-11$ & $\mathrm{~s}$ & FGR-12 & $7.09 \mathrm{E}-15$ \\
\hline Lu-178m & Lu-178 & 177.946 & Lutetium & $1.00 \mathrm{E}-03$ & ICRP-38 & $2.27 \mathrm{E}+01$ & $\mathrm{~m}$ & $1.36 \mathrm{E}+03$ & $4.65 E+07$ & ICRP-72 & $3.30 \mathrm{E}-11$ & $\mathrm{~s}$ & FGR-12 & $5.23 \mathrm{E}-14$ \\
\hline
\end{tabular}

A-16 


\begin{tabular}{|c|c|c|c|c|c|c|c|c|c|c|c|c|c|c|}
\hline \multicolumn{15}{|c|}{ Table A.1 Radionuclide Specific Input Data Used For the Calculation of HC-2 TQs Using Maximum Reported DCs (continued) } \\
\hline $\begin{array}{c}\text { MASTER } \\
\text { ISOTOPE } \\
\text { LIST } \\
\end{array}$ & $\begin{array}{c}\text { Atomic } \\
\text { Mass } \\
\text { Nuclide } \\
\end{array}$ & $\begin{array}{c}\text { Atomic } \\
\text { Mass }\end{array}$ & Element & $\begin{array}{c}\text { RF per } \\
\text { DOE-STD- } \\
1027 \\
\end{array}$ & $\begin{array}{c}\text { Half-Life } \\
\text { Reference }\end{array}$ & $\begin{array}{r}\text { Half-L } \\
\text { (original }\end{array}$ & & Half Life (s) & $\begin{array}{l}\text { Sp. Act } \\
\text { (Ci/g) }\end{array}$ & $\begin{array}{l}\text { Inhalation DC } \\
\text { Reference }\end{array}$ & $\begin{array}{c}\text { HC-2 } \\
\text { Bounding } \\
\text { Inhalation } \\
\text { DC (Sv/Bq) }\end{array}$ & $\begin{array}{c}\text { Lung } \\
\text { Absorption } \\
\text { Class/Type } \\
\end{array}$ & $\begin{array}{l}\text { Immersion DC } \\
\text { Reference }\end{array}$ & $\begin{array}{c}\mathrm{HC}-2 \\
\text { Immersion } \\
\mathrm{DC}(\mathrm{Sv} / \mathrm{s} \\
\left.\text { per } \mathrm{Bq} / \mathrm{m}^{3}\right)\end{array}$ \\
\hline Lu-179 & Lu-179 & 178.947 & Lutetium & $1.00 \mathrm{E}-03$ & ICRP-38 & $4.59 \mathrm{E}+00$ & $\mathrm{~h}$ & $1.65 \mathrm{E}+04$ & $3.82 \mathrm{E}+06$ & ICRP-72 & $1.20 \mathrm{E}-10$ & $\mathrm{~s}$ & FGR-12 & $1.52 \mathrm{E}-15$ \\
\hline Lu-180 & Lu-180 & 179.950 & Lutetium & $1.00 \mathrm{E}-03$ & ICRP-107 & $5.70 \mathrm{E}+00$ & $\mathrm{~m}$ & $3.42 \mathrm{E}+02$ & $1.83 \mathrm{E}+08$ & -- & -- & -- & DOE-STD-1196 & 7.09E-14 \\
\hline Lu-181 & Lu-181 & 180.952 & Lutetium & $1.00 \mathrm{E}-03$ & ICRP-107 & $3.50 \mathrm{E}+00$ & $\mathrm{~m}$ & $2.10 \mathrm{E}+02$ & $2.97 \mathrm{E}+08$ & -- & --- & -- & DOE-STD-1196 & $2.58 \mathrm{E}-14$ \\
\hline Md-257 & Md-257 & 257.096 & Mendelevium & $1.00 \mathrm{E}-03$ & ICRP-38 & $5.20 \mathrm{E}+00$ & $\mathrm{~h}$ & $1.87 \mathrm{E}+04$ & $2.34 \mathrm{E}+06$ & ICRP-72 & $2.50 \mathrm{E}-08$ & $\mathrm{M}$ & FGR-12 & $5.03 \mathrm{E}-15$ \\
\hline Md-258 & Md-258 & 258.098 & Mendelevium & $1.00 \mathrm{E}-03$ & ICRP-38 & $5.50 \mathrm{E}+01$ & $d$ & $4.75 \mathrm{E}+06$ & $9.20 \mathrm{E}+03$ & ICRP-72 & $5.90 \mathrm{E}-06$ & $M$ & FGR-12 & $5.08 \mathrm{E}-17$ \\
\hline $\mathrm{Mg}-27$ & Mg-27 & 26.984 & Magnesium & $1.00 \mathrm{E}-03$ & ICRP-107 & $9.46 \mathrm{E}+00$ & $\mathrm{~m}$ & $5.67 \mathrm{E}+02$ & $7.37 \mathrm{E}+08$ & -- & -- & -- & DOE-STD-1196 & $4.16 \mathrm{E}-14$ \\
\hline $\mathrm{Mg}-28$ & $M g-28$ & 27.984 & Magnesium & $1.00 \mathrm{E}-03$ & ICRP-38 & $2.09 \mathrm{E}+01$ & $\mathrm{~h}$ & $7.53 \mathrm{E}+04$ & $5.36 \mathrm{E}+06$ & ICRP-72 & $1.20 \mathrm{E}-09$ & $M$ & FGR-12 & $6.79 \mathrm{E}-14$ \\
\hline $\mathrm{Mn}-50 \mathrm{~m}$ & $\mathrm{Mn}-50$ & 49.954 & Manganese & $1.00 \mathrm{E}-03$ & ICRP-107 & $1.75 \mathrm{E}+00$ & $\mathrm{~m}$ & $1.05 \mathrm{E}+02$ & $2.15 \mathrm{E}+09$ & -- & -- & -- & DOE-STD-1196 & $2.18 \mathrm{E}-13$ \\
\hline Mn-51 & Mn-51 & 50.948 & Manganese & $1.00 \mathrm{E}-03$ & ICRP-38 & $4.62 \mathrm{E}+01$ & $\mathrm{~m}$ & $2.77 \mathrm{E}+03$ & $7.99 \mathrm{E}+07$ & ICRP-72 & $4.10 \mathrm{E}-11$ & $M$ & FGR-12 & $4.80 \mathrm{E}-14$ \\
\hline $\mathrm{Mn}-52$ & Mn-52 & 51.946 & Manganese & $1.00 \mathrm{E}-03$ & ICRP-38 & $5.59 \mathrm{E}+00$ & $d$ & $4.83 \mathrm{E}+05$ & $4.50 \mathrm{E}+05$ & ICRP-72 & $1.40 \mathrm{E}-09$ & $M$ & FGR-12 & $1.72 \mathrm{E}-13$ \\
\hline$M n-52 m$ & Mn-52 & 51.946 & Manganese & $1.00 \mathrm{E}-03$ & ICRP-38 & $2.11 \mathrm{E}+01$ & $\mathrm{~m}$ & $1.27 \mathrm{E}+03$ & $1.72 \mathrm{E}+08$ & ICRP-72 & $2.90 \mathrm{E}-11$ & $M$ & FGR-12 & $1.20 \mathrm{E}-13$ \\
\hline$M n-53$ & $M n-53$ & 52.941 & Manganese & $1.00 \mathrm{E}-03$ & ICRP-38 & $3.70 \mathrm{E}+06$ & $y$ & $1.17 \mathrm{E}+14$ & $1.83 \mathrm{E}-03$ & ICRP-72 & $5.40 \mathrm{E}-11$ & $M$ & -- & -- \\
\hline $\mathrm{Mn}-54$ & Mn-54 & 53.940 & Manganese & $1.00 \mathrm{E}-03$ & ICRP-38 & $3.13 \mathrm{E}+02$ & $d$ & $2.70 \mathrm{E}+07$ & $7.75 E+03$ & ICRP-72 & $1.50 \mathrm{E}-09$ & $\mathrm{M}$ & FGR-12 & $4.09 \mathrm{E}-14$ \\
\hline $\mathrm{Mn}-56$ & Mn-56 & 55.939 & Manganese & $1.00 \mathrm{E}-03$ & ICRP-38 & $2.58 \mathrm{E}+00$ & $\mathrm{~h}$ & $9.28 \mathrm{E}+03$ & $2.17 \mathrm{E}+07$ & ICRP-72 & $1.20 \mathrm{E}-10$ & $M$ & FGR-12 & $8.61 \mathrm{E}-14$ \\
\hline $\mathrm{Mn}-57$ & Mn-57 & 56.938 & Manganese & $1.00 \mathrm{E}-03$ & ICRP-107 & $8.54 \mathrm{E}+01$ & $\mathrm{~s}$ & $8.54 \mathrm{E}+01$ & $2.32 \mathrm{E}+09$ & -- & -- & -- & DOE-STD-1196 & $5.30 \mathrm{E}-15$ \\
\hline $\mathrm{Mn}-58 \mathrm{~m}$ & Mn-58 & 57.940 & Manganese & $1.00 \mathrm{E}-03$ & ICRP-107 & $6.52 E+01$ & $\mathrm{~s}$ & $6.52 E+01$ & $2.99 \mathrm{E}+09$ & -- & -- & -- & DOE-STD-1196 & $1.15 \mathrm{E}-13$ \\
\hline Mo-101 & Mo-101 & 100.910 & Molybdenum & $1.00 \mathrm{E}-03$ & ICRP-38 & $1.46 \mathrm{E}+01$ & $\mathrm{~m}$ & $8.77 E+02$ & $1.27 \mathrm{E}+08$ & ICRP-72 & $2.60 \mathrm{E}-11$ & $\mathrm{~s}$ & FGR-12 & $6.87 E-14$ \\
\hline Mo-102 & Mo-102 & 101.910 & Molybdenum & $1.00 \mathrm{E}-03$ & ICRP-107 & $1.13 \mathrm{E}+01$ & $\mathrm{~m}$ & $6.78 \mathrm{E}+02$ & $1.63 \mathrm{E}+08$ & DOE-STD-1196 & $3.00 \mathrm{E}-11$ & $\mathrm{~s}$ & DOE-STD-1196 & $1.02 \mathrm{E}-15$ \\
\hline Mo-89 & Mo-89 & 88.919 & Molybdenum & $1.00 \mathrm{E}-03$ & ICRP-107 & $2.11 \mathrm{E}+00$ & $\mathrm{~m}$ & $1.27 \mathrm{E}+02$ & $1.00 \mathrm{E}+09$ & -- & -- & -- & DOE-STD-1196 & $5.70 \mathrm{E}-14$ \\
\hline Mo-90 & Mo-90 & 89.914 & Molybdenum & $1.00 \mathrm{E}-03$ & ICRP-38 & $5.67 \mathrm{E}+00$ & $\mathrm{~h}$ & $2.04 \mathrm{E}+04$ & $6.15 \mathrm{E}+06$ & ICRP-72 & $3.60 \mathrm{E}-10$ & $\mathrm{~S}$ & FGR-12 & $3.93 \mathrm{E}-14$ \\
\hline Mo-91 & Mo-91 & 90.912 & Molybdenum & $1.00 \mathrm{E}-03$ & ICRP-107 & $1.55 \mathrm{E}+01$ & $\mathrm{~m}$ & $9.29 \mathrm{E}+02$ & $1.34 \mathrm{E}+08$ & DOE-STD-1196 & $2.48 \mathrm{E}-11$ & $\mathrm{~s}$ & DOE-STD-1196 & $4.50 \mathrm{E}-14$ \\
\hline Mo-91m & Mo-91 & 90.912 & Molybdenum & $1.00 \mathrm{E}-03$ & ICRP-107 & $6.46 \mathrm{E}+01$ & $\mathrm{~s}$ & $6.46 \mathrm{E}+01$ & $1.92 \mathrm{E}+09$ & -- & -- & -- & DOE-STD-1196 & $6.45 \mathrm{E}-14$ \\
\hline Mo-93 & Mo-93 & 92.907 & Molybdenum & $1.00 \mathrm{E}-03$ & ICRP-38 & $3.50 \mathrm{E}+03$ & $y$ & $1.10 \mathrm{E}+11$ & $1.10 \mathrm{E}+00$ & ICRP-72 & $2.30 \mathrm{E}-09$ & $\mathrm{~s}$ & FGR-12 & $2.52 \mathrm{E}-17$ \\
\hline Mo-93m & Mo-93 & 92.907 & Molybdenum & $1.00 \mathrm{E}-03$ & ICRP-38 & $6.85 \mathrm{E}+00$ & $\mathrm{~h}$ & $2.47 \mathrm{E}+04$ & $4.92 \mathrm{E}+06$ & ICRP-72 & $1.70 \mathrm{E}-10$ & $\mathrm{~s}$ & FGR-12 & $1.13 \mathrm{E}-13$ \\
\hline Mo-99 & Mo-99 & 98.908 & Molybdenum & $1.00 \mathrm{E}-03$ & ICRP-38 & $6.60 \mathrm{E}+01$ & $\mathrm{~h}$ & $2.38 \mathrm{E}+05$ & $4.80 \mathrm{E}+05$ & ICRP-72 & $9.90 \mathrm{E}-10$ & $\mathrm{~s}$ & FGR-12 & $7.28 \mathrm{E}-15$ \\
\hline $\mathrm{N}-13$ & $\mathrm{~N}-1$ & 1.009 & Nitrogen & $1.00 E+00$ & ICRP-38 & $9.97 \mathrm{E}+00$ & $\mathrm{~m}$ & $5.98 \mathrm{E}+02$ & $1.87 \mathrm{E}+10$ & -- & -- & -- & FGR-12 & $4.90 \mathrm{E}-14$ \\
\hline $\mathrm{N}-16$ & $\mathrm{~N}-16$ & 16.006 & Nitrogen & $1.00 \mathrm{E}+00$ & ICRP-107 & $7.13 E+00$ & $\mathrm{~s}$ & $7.13 \mathrm{E}+00$ & $9.89 \mathrm{E}+10$ & -- & -- & -- & DOE-STD-1196 & $2.59 \mathrm{E}-13$ \\
\hline $\mathrm{Na}-22$ & $\mathrm{Na}-22$ & 21.994 & Sodium & $5.00 \mathrm{E}-01$ & ICRP-38 & $2.60 \mathrm{E}+00$ & $y$ & $8.21 \mathrm{E}+07$ & $6.25 \mathrm{E}+03$ & ICRP-72 & $1.30 \mathrm{E}-09$ & $F$ & FGR-12 & $1.08 \mathrm{E}-13$ \\
\hline $\mathrm{Na}-24$ & $\mathrm{Na}-24$ & 23.991 & Sodium & $5.00 \mathrm{E}-01$ & ICRP-38 & $1.50 E+01$ & $\mathrm{~h}$ & $5.40 \mathrm{E}+04$ & $8.71 E+06$ & ICRP-72 & $2.70 \mathrm{E}-10$ & $\mathrm{~F}$ & FGR-12 & $2.18 \mathrm{E}-13$ \\
\hline $\mathrm{Nb}-87$ & Nb-87 & 86.921 & Niobium & $1.00 \mathrm{E}-03$ & ICRP-107 & $3.75 \mathrm{E}+00$ & $\mathrm{~m}$ & $2.25 \mathrm{E}+02$ & $5.77 \mathrm{E}+08$ & -- & -- & -- & DOE-STD-1196 & $5.54 \mathrm{E}-14$ \\
\hline $\mathrm{Nb}-88$ & $\mathrm{Nb}-88$ & 87.918 & Niobium & $1.00 \mathrm{E}-03$ & ICRP-38 & $1.43 \mathrm{E}+01$ & $\mathrm{~m}$ & $8.58 \mathrm{E}+02$ & $1.50 \mathrm{E}+08$ & ICRP-72 & $2.80 \mathrm{E}-11$ & $\mathrm{~s}$ & FGR-12 & $2.02 \mathrm{E}-13$ \\
\hline $\mathrm{Nb}-88 \mathrm{~m}$ & $\mathrm{Nb}-88$ & 87.918 & Niobium & $1.00 \mathrm{E}-03$ & ICRP-107 & $7.78 \mathrm{E}+00$ & $\mathrm{~m}$ & $4.67 E+02$ & $2.75 E+08$ & -- & -- & -- & DOE-STD-1196 & $1.91 \mathrm{E}-13$ \\
\hline $\mathrm{Nb}-89 \mathrm{I}$ & $\mathrm{Nb}-89$ & 88.913 & Niobium & $1.00 \mathrm{E}-03$ & ICRP-38 & $1.22 \mathrm{E}+02$ & $\mathrm{~m}$ & $7.32 \mathrm{E}+03$ & $1.73 \mathrm{E}+07$ & ICRP-72 & $1.20 \mathrm{E}-10$ & $\mathrm{~s}$ & FGR-12 & $6.98 \mathrm{E}-14$ \\
\hline $\mathrm{Nb}-89 \mathrm{~s}$ & $\mathrm{Nb}-89$ & 88.913 & Niobium & $1.00 \mathrm{E}-03$ & ICRP-38 & $6.60 \mathrm{E}+01$ & $\mathrm{~m}$ & $3.96 \mathrm{E}+03$ & $3.20 \mathrm{E}+07$ & ICRP-72 & $7.10 \mathrm{E}-11$ & $\mathrm{~s}$ & FGR-12 & $9.26 \mathrm{E}-14$ \\
\hline $\mathrm{Nb}-90$ & $\mathrm{Nb}-90$ & 89.911 & Niobium & $1.00 \mathrm{E}-03$ & ICRP-38 & $1.46 \mathrm{E}+01$ & $\mathrm{~h}$ & $5.26 \mathrm{E}+04$ & $2.39 \mathrm{E}+06$ & ICRP-72 & $6.60 \mathrm{E}-10$ & $\mathrm{~s}$ & FGR-12 & $2.17 \mathrm{E}-13$ \\
\hline $\mathrm{Nb}-91$ & $\mathrm{Nb}-91$ & 90.907 & Niobium & $1.00 \mathrm{E}-03$ & ICRP-107 & $6.80 \mathrm{E}+02$ & $y$ & $2.15 \mathrm{E}+10$ & $5.78 \mathrm{E}+00$ & DOE-STD-1196 & $1.97 \mathrm{E}-09$ & $\mathrm{~s}$ & DOE-STD-1196 & $8.45 \mathrm{E}-17$ \\
\hline $\mathrm{Nb}-91 \mathrm{~m}$ & $\mathrm{Nb}-91$ & 90.907 & Niobium & $1.00 \mathrm{E}-03$ & ICRP-107 & $6.09 \mathrm{E}+01$ & $d$ & $5.26 \mathrm{E}+06$ & $2.36 \mathrm{E}+04$ & DOE-STD-1196 & $4.57 \mathrm{E}-09$ & $\mathrm{~s}$ & DOE-STD-1196 & $1.20 \mathrm{E}-15$ \\
\hline $\mathrm{Nb}-92$ & $\mathrm{Nb}-92$ & 91.907 & Niobium & $1.00 \mathrm{E}-03$ & ICRP-107 & $3.47 \mathrm{E}+07$ & $y$ & $1.10 \mathrm{E}+15$ & $1.12 \mathrm{E}-04$ & DOE-STD-1196 & $2.84 \mathrm{E}-08$ & $\mathrm{~s}$ & DOE-STD-1196 & $6.83 \mathrm{E}-14$ \\
\hline $\mathrm{Nb}-92 \mathrm{~m}$ & $\mathrm{Nb}-92$ & 91.907 & Niobium & $1.00 \mathrm{E}-03$ & ICRP-107 & $1.02 \mathrm{E}+01$ & $d$ & $8.77 \mathrm{E}+05$ & $1.40 \mathrm{E}+05$ & DOE-STD-1196 & $5.19 \mathrm{E}-10$ & $\mathrm{~s}$ & DOE-STD-1196 & $4.44 \mathrm{E}-14$ \\
\hline $\mathrm{Nb}-93 \mathrm{~m}$ & $\mathrm{Nb}-93$ & 92.906 & Niobium & $1.00 \mathrm{E}-03$ & ICRP-38 & $1.36 \mathrm{E}+01$ & $y$ & $4.29 \mathrm{E}+08$ & $2.83 \mathrm{E}+02$ & ICRP-72 & $1.80 \mathrm{E}-09$ & $\mathrm{~s}$ & FGR-12 & $4.44 \mathrm{E}-18$ \\
\hline $\mathrm{Nb}-94$ & $\mathrm{Nb}-94$ & 93.907 & Niobium & $1.00 \mathrm{E}-03$ & ICRP-38 & $2.03 \mathrm{E}+04$ & $y$ & $6.41 \mathrm{E}+11$ & $1.88 \mathrm{E}-01$ & ICRP-72 & $4.90 \mathrm{E}-08$ & $\mathrm{~s}$ & FGR-12 & $7.70 \mathrm{E}-14$ \\
\hline $\mathrm{Nb}-94 \mathrm{~m}$ & Nb-94 & 93.907 & Niobium & $1.00 \mathrm{E}-03$ & ICRP-107 & $6.26 \mathrm{E}+00$ & $\mathrm{~m}$ & $3.76 \mathrm{E}+02$ & $3.20 \mathrm{E}+08$ & -- & -- & -- & DOE-STD-1196 & $2.15 \mathrm{E}-16$ \\
\hline $\mathrm{Nb}-95$ & $\mathrm{Nb}-95$ & 94.907 & Niobium & $1.00 \mathrm{E}-03$ & ICRP-38 & $3.52 \mathrm{E}+01$ & $d$ & $3.04 \mathrm{E}+06$ & $3.91 \mathrm{E}+04$ & ICRP-72 & $1.80 \mathrm{E}-09$ & $\mathrm{~s}$ & FGR-12 & $3.74 \mathrm{E}-14$ \\
\hline $\mathrm{Nb}-95 \mathrm{~m}$ & $\mathrm{Nb}-95$ & 94.907 & Niobium & $1.00 \mathrm{E}-03$ & ICRP-38 & $8.66 \mathrm{E}+01$ & $\mathrm{~h}$ & $3.12 \mathrm{E}+05$ & $3.81 \mathrm{E}+05$ & ICRP-72 & $8.80 \mathrm{E}-10$ & $\mathrm{~s}$ & FGR-12 & $2.93 \mathrm{E}-15$ \\
\hline $\mathrm{Nb}-96$ & $\mathrm{Nb}-96$ & 95.908 & Niobium & $1.00 \mathrm{E}-03$ & ICRP-38 & $2.34 \mathrm{E}+01$ & $\mathrm{~h}$ & $8.41 \mathrm{E}+04$ & $1.40 \mathrm{E}+06$ & ICRP-72 & $6.60 \mathrm{E}-10$ & $\mathrm{~s}$ & FGR-12 & $1.21 \mathrm{E}-13$ \\
\hline $\mathrm{Nb}-97$ & $\mathrm{Nb}-97$ & 96.908 & Niobium & $1.00 \mathrm{E}-03$ & ICRP-38 & $7.21 \mathrm{E}+01$ & $\mathrm{~m}$ & $4.33 \mathrm{E}+03$ & $2.69 \mathrm{E}+07$ & ICRP-72 & $4.50 \mathrm{E}-11$ & $\mathrm{~s}$ & FGR-12 & $3.18 \mathrm{E}-14$ \\
\hline
\end{tabular}

A-17 


\begin{tabular}{|c|c|c|c|c|c|c|c|c|c|c|c|c|c|c|}
\hline \multicolumn{15}{|c|}{ Table A.1 Radionuclide Specific Input Data Used For the Calculation of HC-2 TQs Using Maximum Reported DCs (continued) } \\
\hline $\begin{array}{l}\text { MASTER } \\
\text { ISOTOPE } \\
\text { LIST }\end{array}$ & $\begin{array}{l}\text { Atomic } \\
\text { Mass } \\
\text { Nuclide }\end{array}$ & $\begin{array}{l}\text { Atomic } \\
\text { Mass }\end{array}$ & Element & $\begin{array}{c}\text { RF per } \\
\text { DOE-STD- } \\
1027\end{array}$ & $\begin{array}{l}\text { Half-Life } \\
\text { Reference }\end{array}$ & $\begin{array}{r}\text { Half-L } \\
\text { (original }\end{array}$ & & Half Life (s) & $\begin{array}{l}\text { Sp. Act } \\
(\mathrm{Ci} / \mathrm{g})\end{array}$ & $\begin{array}{c}\text { Inhalation DC } \\
\text { Reference }\end{array}$ & $\begin{array}{c}\text { HC-2 } \\
\text { Bounding } \\
\text { Inhalation } \\
\text { DC (Sv/Bq) }\end{array}$ & $\begin{array}{l}\text { Lung } \\
\text { Absorption } \\
\text { Class/Type }\end{array}$ & $\begin{array}{c}\text { Immersion DC } \\
\text { Reference }\end{array}$ & $\begin{array}{c}\text { HC-2 } \\
\text { Immersion } \\
\text { DC }(\mathrm{Sv} / \mathrm{s} \\
\left.\text { per } \mathrm{Bq} / \mathrm{m}^{3}\right)\end{array}$ \\
\hline $\mathrm{Nb}-97 \mathrm{~m}$ & $\mathrm{Nb}-97$ & 96.908 & Niobium & $1.00 \mathrm{E}-03$ & ICRP-38 & $6.00 \mathrm{E}+01$ & $\mathrm{~s}$ & $6.00 \mathrm{E}+01$ & $1.94 \mathrm{E}+09$ & --- & -- & -- & FGR-12 & $3.55 \mathrm{E}-14$ \\
\hline $\mathrm{Nb}-98$ & $\mathrm{Nb}-98$ & 97.910 & Niobium & $1.00 \mathrm{E}-03$ & ICRP-38 & $5.15 \mathrm{E}+01$ & $\mathrm{~m}$ & $3.09 \mathrm{E}+03$ & $3.73 \mathrm{E}+07$ & ICRP-72 & $5.80 \mathrm{E}-11$ & $\mathrm{~s}$ & FGR-12 & $1.21 \mathrm{E}-13$ \\
\hline Nb-99 & $\mathrm{Nb}-99$ & 98.912 & Niobium & $1.00 \mathrm{E}-03$ & ICRP-107 & $1.50 \mathrm{E}+01$ & $\mathrm{~s}$ & $1.50 \mathrm{E}+01$ & $7.60 E+09$ & -- & -- & -- & DOE-STD-1196 & $8.32 \mathrm{E}-15$ \\
\hline $\mathrm{Nb}-99 \mathrm{~m}$ & $\mathrm{Nb}-99$ & 98.912 & Niobium & $1.00 \mathrm{E}-03$ & ICRP-107 & $2.60 E+00$ & $\mathrm{~m}$ & $1.56 \mathrm{E}+02$ & $7.31 \mathrm{E}+08$ & -- & -- & -- & DOE-STD-1196 & $3.86 \mathrm{E}-14$ \\
\hline $\mathrm{Nd}-134$ & $\mathrm{Nd}-134$ & 133.919 & Neodymium & $1.00 \mathrm{E}-03$ & ICRP-107 & $8.50 E+00$ & $\mathrm{~m}$ & $5.10 \mathrm{E}+02$ & $1.65 E+08$ & -- & -- & -- & DOE-STD-1196 & $2.32 \mathrm{E}-14$ \\
\hline $\mathrm{Nd}-135$ & $\mathrm{Nd}-135$ & 134.918 & Neodymium & $1.00 \mathrm{E}-03$ & ICRP-107 & $1.24 \mathrm{E}+01$ & $\mathrm{~m}$ & $7.44 E+02$ & $1.12 \mathrm{E}+08$ & DOE-STD-1196 & $3.18 \mathrm{E}-11$ & $\mathrm{~s}$ & DOE-STD-1196 & $5.63 \mathrm{E}-14$ \\
\hline $\mathrm{Nd}-136$ & $\mathrm{Nd}-136$ & 135.915 & Neodymium & $1.00 \mathrm{E}-03$ & ICRP-38 & $5.07 \mathrm{E}+01$ & $\mathrm{~m}$ & $3.04 \mathrm{E}+03$ & $2.73 E+07$ & ICRP-72 & $5.40 \mathrm{E}-11$ & $\mathrm{~s}$ & FGR-12 & $1.27 \mathrm{E}-14$ \\
\hline Nd-137 & Nd-137 & 136.915 & Neodymium & $1.00 \mathrm{E}-03$ & ICRP-107 & $3.85 \mathrm{E}+01$ & $\mathrm{~m}$ & $2.31 \mathrm{E}+03$ & $3.57 \mathrm{E}+07$ & DOE-STD-1196 & $3.39 \mathrm{E}-11$ & $\mathrm{~s}$ & DOE-STD-1196 & $5.33 \mathrm{E}-14$ \\
\hline Nd-138 & $\mathrm{Nd}-138$ & 137.912 & Neodymium & $1.00 \mathrm{E}-03$ & ICRP-38 & $5.04 \mathrm{E}+00$ & $\mathrm{~h}$ & $1.81 \mathrm{E}+04$ & $4.51 \mathrm{E}+06$ & ICRP-72 & $2.50 \mathrm{E}-10$ & $\mathrm{~S}$ & FGR-12 & $1.27 \mathrm{E}-15$ \\
\hline Nd-139 & $\mathrm{Nd}-139$ & 138.912 & Neodymium & $1.00 \mathrm{E}-03$ & ICRP-38 & $2.97 \mathrm{E}+01$ & $\mathrm{~m}$ & $1.78 \mathrm{E}+03$ & $4.56 \mathrm{E}+07$ & ICRP-72 & $1.00 \mathrm{E}-11$ & $\mathrm{~s}$ & FGR-12 & $1.90 \mathrm{E}-14$ \\
\hline $\mathrm{Nd}-139 \mathrm{~m}$ & Nd-139 & 138.912 & Neodymium & $1.00 \mathrm{E}-03$ & ICRP-38 & $5.50 \mathrm{E}+00$ & $\mathrm{~h}$ & $1.98 \mathrm{E}+04$ & $4.10 \mathrm{E}+06$ & ICRP-72 & $1.50 \mathrm{E}-10$ & $M$ & FGR-12 & $7.63 \mathrm{E}-14$ \\
\hline $\mathrm{Nd}-140$ & $\mathrm{Nd}-140$ & 139.910 & Neodymium & $1.00 \mathrm{E}-03$ & ICRP-107 & $3.37 \mathrm{E}+00$ & $d$ & $2.91 E+05$ & $2.77 E+05$ & DOE-STD-1196 & $1.33 \mathrm{E}-09$ & $\mathrm{~s}$ & DOE-STD-1196 & $4.44 \mathrm{E}-16$ \\
\hline $\mathrm{Nd}-141$ & $\mathrm{Nd}-141$ & 140.910 & Neodymium & $1.00 \mathrm{E}-03$ & ICRP-38 & $2.49 \mathrm{E}+00$ & $\mathrm{~h}$ & $8.96 \mathrm{E}+03$ & $8.93 \mathrm{E}+06$ & ICRP-72 & $5.00 \mathrm{E}-12$ & $\mathrm{~s}$ & FGR-12 & $2.88 \mathrm{E}-15$ \\
\hline $\mathrm{Nd}-141 \mathrm{~m}$ & $\mathrm{Nd}-141$ & 140.910 & Neodymium & $1.00 \mathrm{E}-03$ & ICRP-38 & $6.24 \mathrm{E}+01$ & $\mathrm{~s}$ & $6.24 \mathrm{E}+01$ & $1.28 \mathrm{E}+09$ & -- & -- & -- & FGR-12 & $3.70 \mathrm{E}-14$ \\
\hline Nd-144 & Nd-144 & 143.910 & Neodymium & $1.00 \mathrm{E}-03$ & ICRP-107 & $2.29 \mathrm{E}+15$ & $y$ & $7.23 E+22$ & $1.08 \mathrm{E}-12$ & DOE-STD-1196 & $2.01 \mathrm{E}-05$ & $F$ & -- & -- \\
\hline Nd-147 & Nd-147 & 146.916 & Neodymium & $1.00 \mathrm{E}-03$ & ICRP-38 & $1.10 \mathrm{E}+01$ & $d$ & $9.49 \mathrm{E}+05$ & $8.09 \mathrm{E}+04$ & ICRP-72 & $2.40 \mathrm{E}-09$ & $\mathrm{~s}$ & FGR-12 & $6.19 \mathrm{E}-15$ \\
\hline $\mathrm{Nd}-149$ & $\mathrm{Nd}-149$ & 148.920 & Neodymium & $1.00 \mathrm{E}-03$ & ICRP-38 & $1.73 E+00$ & $\mathrm{~h}$ & $6.23 \mathrm{E}+03$ & $1.22 \mathrm{E}+07$ & ICRP-72 & $8.90 \mathrm{E}-11$ & $\mathrm{~S}$ & FGR-12 & $1.81 \mathrm{E}-14$ \\
\hline Nd-151 & Nd-151 & 150.924 & Neodymium & $1.00 \mathrm{E}-03$ & ICRP-38 & $1.24 \mathrm{E}+01$ & $\mathrm{~m}$ & $7.46 \mathrm{E}+02$ & $1.00 E+08$ & ICRP-72 & $1.70 \mathrm{E}-11$ & $M$ & FGR-12 & $4.48 \mathrm{E}-14$ \\
\hline $\mathrm{Nd}-152$ & $\mathrm{Nd}-152$ & 151.925 & Neodymium & $1.00 \mathrm{E}-03$ & ICRP-107 & $1.14 \mathrm{E}+01$ & $\mathrm{~m}$ & $6.84 \mathrm{E}+02$ & $1.09 \mathrm{E}+08$ & DOE-STD-1196 & $2.80 \mathrm{E}-11$ & $\mathrm{~s}$ & DOE-STD-1196 & $7.29 \mathrm{E}-15$ \\
\hline $\mathrm{Ne}-19$ & Ne-19 & 19.002 & Neon & $1.00 \mathrm{E}+00$ & ICRP-38 & $1.72 \mathrm{E}+01$ & $\mathrm{~s}$ & $1.72 \mathrm{E}+01$ & $3.45 \mathrm{E}+10$ & -- & -- & -- & FGR-12 & $4.92 \mathrm{E}-14$ \\
\hline $\mathrm{Ne}-24$ & $\mathrm{Ne}-24$ & 23.994 & Neon & $1.00 E+00$ & ICRP-107 & $3.38 \mathrm{E}+00$ & $\mathrm{~m}$ & $2.03 E+02$ & $2.32 \mathrm{E}+09$ & -- & -- & -- & DOE-STD-1196 & $2.48 \mathrm{E}-14$ \\
\hline $\mathrm{Ni}-56$ & $\mathrm{Ni}-56$ & 55.942 & Nickel & $1.00 \mathrm{E}-03$ & ICRP-38 & $6.10 E+00$ & $d$ & $5.27 \mathrm{E}+05$ & $3.83 \mathrm{E}+05$ & ICRP-72 & $1.20 \mathrm{E}-09$ & V & FGR-12 & $8.41 \mathrm{E}-14$ \\
\hline $\mathrm{Ni}-57$ & $\mathrm{Ni}-57$ & 56.940 & Nickel & $1.00 \mathrm{E}-03$ & ICRP-38 & $3.61 \mathrm{E}+01$ & $\mathrm{~h}$ & $1.30 \mathrm{E}+05$ & $1.53 \mathrm{E}+06$ & ICRP-72 & $5.60 \mathrm{E}-10$ & $\mathrm{~V}$ & FGR-12 & $9.69 \mathrm{E}-14$ \\
\hline $\mathrm{Ni}-59$ & $\mathrm{Ni}-59$ & 58.934 & Nickel & $1.00 \mathrm{E}-03$ & ICRP-38 & $7.50 E+04$ & $y$ & $2.37 \mathrm{E}+12$ & $8.09 \mathrm{E}-02$ & ICRP-72 & $8.30 \mathrm{E}-10$ & $\mathrm{~V}$ & DOE-STD-1196 & $6.92 \mathrm{E}-19$ \\
\hline $\mathrm{Ni}-63$ & $\mathrm{Ni}-63$ & 62.930 & Nickel & $1.00 \mathrm{E}-03$ & ICRP-38 & $9.60 \mathrm{E}+01$ & $y$ & $3.03 E+09$ & $5.92 \mathrm{E}+01$ & ICRP-72 & $2.00 \mathrm{E}-09$ & V & -- & -- \\
\hline $\mathrm{Ni}-65$ & $\mathrm{Ni}-65$ & 64.930 & Nickel & $1.00 \mathrm{E}-03$ & ICRP-38 & $2.52 \mathrm{E}+00$ & $\mathrm{~h}$ & $9.07 \mathrm{E}+03$ & $1.92 \mathrm{E}+07$ & ICRP-72 & $3.60 \mathrm{E}-10$ & $\mathrm{~V}$ & FGR-12 & $2.79 \mathrm{E}-14$ \\
\hline $\mathrm{Ni}-66$ & $\mathrm{Ni}-66$ & 65.929 & Nickel & $1.00 \mathrm{E}-03$ & ICRP-38 & $5.46 \mathrm{E}+01$ & $\mathrm{~h}$ & $1.97 \mathrm{E}+05$ & $8.71 E+05$ & ICRP-72 & $1.80 \mathrm{E}-09$ & $\mathrm{~s}$ & FGR-12 & $6.16 \mathrm{E}-19$ \\
\hline Np-231 & Np-231 & 231.038 & Neptunium & $1.00 \mathrm{E}-03$ & JAERI & $4.88 \mathrm{E}+01$ & $\mathrm{~m}$ & $2.93 \mathrm{E}+03$ & $1.67 \mathrm{E}+07$ & JAERI & $1.80 \mathrm{E}-09$ & $\mathrm{~s}$ & -- & -- \\
\hline Np-232 & $\mathrm{Np}-232$ & 232.040 & Neptunium & $1.00 \mathrm{E}-03$ & ICRP-38 & $1.47 \mathrm{E}+01$ & $\mathrm{~m}$ & $8.82 \mathrm{E}+02$ & $5.51 \mathrm{E}+07$ & ICRP-72 & $1.20 \mathrm{E}-10$ & $\mathrm{~F}$ & FGR-12 & $5.80 \mathrm{E}-14$ \\
\hline Np-233 & Np-233 & 233.041 & Neptunium & $1.00 \mathrm{E}-03$ & ICRP-38 & $3.62 \mathrm{E}+01$ & $\mathrm{~m}$ & $2.17 \mathrm{E}+03$ & $2.23 \mathrm{E}+07$ & ICRP-72 & $1.70 \mathrm{E}-12$ & $\mathrm{~s}$ & FGR-12 & $3.85 \mathrm{E}-15$ \\
\hline $\mathrm{Np}-234$ & Np-234 & 234.043 & Neptunium & $1.00 \mathrm{E}-03$ & ICRP-38 & $4.40 \mathrm{E}+00$ & d & $3.80 \mathrm{E}+05$ & $1.27 \mathrm{E}+05$ & ICRP-72 & $5.50 \mathrm{E}-10$ & $\mathrm{~s}$ & FGR-12 & $7.26 \mathrm{E}-14$ \\
\hline Np-235 & Np-235 & 235.044 & Neptunium & $1.00 \mathrm{E}-03$ & ICRP-38 & $3.96 \mathrm{E}+02$ & $d$ & $3.42 \mathrm{E}+07$ & $1.40 \mathrm{E}+03$ & ICRP-72 & $6.30 \mathrm{E}-10$ & $\mathrm{~F}$ & FGR-12 & $5.10 \mathrm{E}-17$ \\
\hline Np-236l & $\mathrm{Np}-236$ & 236.047 & Neptunium & $1.00 \mathrm{E}-03$ & ICRP-38 & $1.15 \mathrm{E}+05$ & $y$ & $3.63 \mathrm{E}+12$ & $1.32 \mathrm{E}-02$ & ICRP-72 & $8.00 \mathrm{E}-06$ & $\mathrm{~F}$ & FGR-12 & $5.36 \mathrm{E}-15$ \\
\hline Np-236s & $\mathrm{Np}-236$ & 236.047 & Neptunium & $1.00 \mathrm{E}-03$ & ICRP-38 & $2.25 E+01$ & $\mathrm{~h}$ & $8.10 \mathrm{E}+04$ & $5.90 E+05$ & ICRP-72 & $9.00 \mathrm{E}-09$ & $\mathrm{~F}$ & FGR-12 & $2.14 \mathrm{E}-15$ \\
\hline Np-237 & Np-237 & 237.048 & Neptunium & $1.00 \mathrm{E}-03$ & ICRP-38 & $2.14 \mathrm{E}+06$ & $y$ & $6.75 E+13$ & $7.05 \mathrm{E}-04$ & ICRP-72 & $5.00 \mathrm{E}-05$ & $\mathrm{~F}$ & FGR-12 & $1.03 \mathrm{E}-15$ \\
\hline Np-238 & Np-238 & 238.051 & Neptunium & $1.00 \mathrm{E}-03$ & ICRP-38 & $2.12 \mathrm{E}+00$ & $d$ & $1.83 \mathrm{E}+05$ & $2.59 \mathrm{E}+05$ & ICRP-72 & $3.50 \mathrm{E}-09$ & $\mathrm{~F}$ & FGR-12 & $2.72 \mathrm{E}-14$ \\
\hline Np-239 & Np-239 & 239.053 & Neptunium & $1.00 \mathrm{E}-03$ & ICRP-38 & $2.36 \mathrm{E}+00$ & d & $2.03 E+05$ & $2.32 \mathrm{E}+05$ & ICRP-72 & $1.00 \mathrm{E}-09$ & $\mathrm{~s}$ & FGR-12 & $7.69 \mathrm{E}-15$ \\
\hline $\mathrm{Np}-240$ & $\mathrm{~Np}-240$ & 240.056 & Neptunium & $1.00 \mathrm{E}-03$ & ICRP-38 & $6.50 \mathrm{E}+01$ & $\mathrm{~m}$ & $3.90 \mathrm{E}+03$ & $1.20 \mathrm{E}+07$ & ICRP-72 & $9.00 \mathrm{E}-11$ & $\mathrm{~s}$ & FGR-12 & $6.31 \mathrm{E}-14$ \\
\hline $\mathrm{Np}-240 \mathrm{~m}$ & Np-240 & 240.056 & Neptunium & $1.00 \mathrm{E}-03$ & ICRP-38 & $7.40 \mathrm{E}+00$ & $\mathrm{~m}$ & $4.44 \mathrm{E}+02$ & $1.06 \mathrm{E}+08$ & -- & -- & -- & FGR-12 & $1.62 \mathrm{E}-14$ \\
\hline $\mathrm{Np}-241$ & Np-241 & 241.058 & Neptunium & $1.00 \mathrm{E}-03$ & ICRP-107 & $1.39 \mathrm{E}+01$ & $\mathrm{~m}$ & $8.34 \mathrm{E}+02$ & $5.61 \mathrm{E}+07$ & DOE-STD-1196 & $1.41 \mathrm{E}-11$ & $\mathrm{~s}$ & DOE-STD-1196 & $1.79 \mathrm{E}-15$ \\
\hline $\mathrm{Np}-242$ & $\mathrm{~Np}-242$ & 242.062 & Neptunium & $1.00 \mathrm{E}-03$ & ICRP-107 & $2.20 \mathrm{E}+00$ & $\mathrm{~m}$ & $1.32 \mathrm{E}+02$ & $3.53 \mathrm{E}+08$ & -- & -- & -- & DOE-STD-1196 & $1.33 \mathrm{E}-14$ \\
\hline $\mathrm{Np}-242 \mathrm{~m}$ & Np-242 & 242.062 & Neptunium & $1.00 \mathrm{E}-03$ & ICRP-107 & $5.50 \mathrm{E}+00$ & $\mathrm{~m}$ & $3.30 \mathrm{E}+02$ & $1.41 \mathrm{E}+08$ & -- & -- & -- & DOE-STD-1196 & $4.16 \mathrm{E}-14$ \\
\hline $0-14$ & $0-14$ & 14.009 & Oxygen & $1.00 E+00$ & ICRP-38 & $7.06 \mathrm{E}+01$ & $\mathrm{~s}$ & $7.06 \mathrm{E}+01$ & $1.14 \mathrm{E}+10$ & -- & -- & -- & DOE-STD-1196 & $1.63 \mathrm{E}-13$ \\
\hline $0-15$ & $0-15$ & 15.003 & Oxygen & $1.00 E+00$ & ICRP-38 & $1.22 \mathrm{E}+02$ & $\mathrm{~s}$ & $1.22 \mathrm{E}+02$ & $6.15 \mathrm{E}+09$ & -- & -- & -- & FGR-12 & $4.91 \mathrm{E}-14$ \\
\hline $0-19$ & $0-19$ & 19.004 & Oxygen & $1.00 E+00$ & ICRP-38 & $2.69 \mathrm{E}+01$ & $\mathrm{~s}$ & $2.69 \mathrm{E}+01$ & $2.21 \mathrm{E}+10$ & -- & -- & -- & DOE-STD-1196 & $4.60 \mathrm{E}-14$ \\
\hline Os-177 & Os-177 & 176.955 & Osmium & $1.00 \mathrm{E}-03$ & JAERI & $2.80 \mathrm{E}+00$ & $\mathrm{~m}$ & $1.68 \mathrm{E}+02$ & $3.79 E+08$ & -- & -- & -- & -- & -- \\
\hline
\end{tabular}

A-18 


\begin{tabular}{|c|c|c|c|c|c|c|c|c|c|c|c|c|c|c|}
\hline \multicolumn{15}{|c|}{ Table A.1 Radionuclide Specific Input Data Used For the Calculation of HC-2 TQs Using Maximum Reported DCs (continued) } \\
\hline $\begin{array}{l}\text { MASTER } \\
\text { ISOTOPE } \\
\text { LIST } \\
\end{array}$ & $\begin{array}{l}\text { Atomic } \\
\text { Mass } \\
\text { Nuclide }\end{array}$ & $\begin{array}{c}\text { Atomic } \\
\text { Mass }\end{array}$ & Element & $\begin{array}{c}\text { RF per } \\
\text { DOE-STD- } \\
1027 \\
\end{array}$ & $\begin{array}{c}\text { Half-Life } \\
\text { Reference }\end{array}$ & $\begin{array}{r}\text { Half-Li } \\
\text { (original }\end{array}$ & & Half Life (s) & $\begin{array}{l}\text { Sp. Act } \\
\text { (Ci/g) }\end{array}$ & $\begin{array}{c}\text { Inhalation DC } \\
\text { Reference }\end{array}$ & $\begin{array}{c}\text { HC-2 } \\
\text { Bounding } \\
\text { Inhalation } \\
\text { DC (Sv/Bq) }\end{array}$ & $\begin{array}{c}\text { Lung } \\
\text { Absorption } \\
\text { Class/Type }\end{array}$ & $\begin{array}{c}\text { Immersion DC } \\
\text { Reference }\end{array}$ & $\begin{array}{c}\text { HC-2 } \\
\text { Immersion } \\
\mathrm{DC}(\mathrm{Sv} / \mathrm{s} \\
\left.\text { per } \mathrm{Bq} / \mathrm{m}^{3}\right)\end{array}$ \\
\hline Os-179 & Os-179 & 178.954 & Osmium & $1.00 \mathrm{E}-03$ & JAERI & $6.50 \mathrm{E}+00$ & $\mathrm{~m}$ & $3.90 \mathrm{E}+02$ & $1.62 \mathrm{E}+08$ & --- & --- & -- & -- & -- \\
\hline Os-180 & Os-180 & 179.952 & Osmium & $1.00 \mathrm{E}-03$ & ICRP-38 & $2.20 \mathrm{E}+01$ & $\mathrm{~m}$ & $1.32 \mathrm{E}+03$ & $4.75 \mathrm{E}+07$ & ICRP-72 & $1.50 \mathrm{E}-11$ & $\mathrm{~s}$ & FGR-12 & $2.30 \mathrm{E}-15$ \\
\hline Os-181 & Os-181 & 180.953 & Osmium & $1.00 \mathrm{E}-03$ & ICRP-38 & $1.05 E+02$ & $\mathrm{~m}$ & $6.30 \mathrm{E}+03$ & $9.90 \mathrm{E}+06$ & ICRP-72 & $6.50 \mathrm{E}-11$ & $\mathrm{~s}$ & FGR-12 & $5.94 \mathrm{E}-14$ \\
\hline Os-182 & Os-182 & 181.952 & Osmium & $1.00 \mathrm{E}-03$ & ICRP-38 & $2.20 \mathrm{E}+01$ & $\mathrm{~h}$ & $7.92 \mathrm{E}+04$ & $7.83 E+05$ & ICRP-72 & $3.80 \mathrm{E}-10$ & $\mathrm{~s}$ & FGR-12 & $2.01 \mathrm{E}-14$ \\
\hline Os-183 & Os-183 & 182.953 & Osmium & $1.00 \mathrm{E}-03$ & ICRP-107 & $1.30 \mathrm{E}+01$ & $\mathrm{~h}$ & $4.68 \mathrm{E}+04$ & $1.32 \mathrm{E}+06$ & DOE-STD-1196 & $2.20 \mathrm{E}-10$ & $\mathrm{~s}$ & DOE-STD-1196 & $2.66 \mathrm{E}-14$ \\
\hline Os-183m & Os-183 & 182.953 & Osmium & $1.00 \mathrm{E}-03$ & ICRP-107 & $9.90 \mathrm{E}+00$ & $\mathrm{~h}$ & $3.56 \mathrm{E}+04$ & $1.73 E+06$ & DOE-STD-1196 & $1.74 \mathrm{E}-10$ & $\mathrm{~s}$ & DOE-STD-1196 & $4.63 \mathrm{E}-14$ \\
\hline Os-185 & Os-185 & 184.954 & Osmium & $1.00 \mathrm{E}-03$ & ICRP-38 & $9.40 \mathrm{E}+01$ & $d$ & $8.12 \mathrm{E}+06$ & $7.51 \mathrm{E}+03$ & ICRP-72 & $1.60 \mathrm{E}-09$ & $\mathrm{~s}$ & FGR-12 & $3.43 \mathrm{E}-14$ \\
\hline Os-186 & Os-186 & 185.954 & Osmium & $1.00 \mathrm{E}-03$ & ICRP-107 & $2.00 \mathrm{E}+15$ & $y$ & $6.31 \mathrm{E}+22$ & $9.61 \mathrm{E}-13$ & DOE-STD-1196 & $4.51 \mathrm{E}-06$ & $\mathrm{~s}$ & -- & -- \\
\hline Os-189m & Os-189 & 188.958 & Osmium & $1.00 \mathrm{E}-03$ & ICRP-38 & $6.00 \mathrm{E}+00$ & $\mathrm{~h}$ & $2.16 \mathrm{E}+04$ & $2.76 \mathrm{E}+06$ & ICRP-72 & $5.30 \mathrm{E}-12$ & $\mathrm{~S}$ & FGR-12 & $1.06 \mathrm{E}-19$ \\
\hline Os-190m & Os-190 & 189.958 & Osmium & $1.00 \mathrm{E}-03$ & ICRP-38 & $9.90 \mathrm{E}+00$ & $\mathrm{~m}$ & $5.94 \mathrm{E}+02$ & $1.00 E+08$ & -- & -- & -- & FGR-12 & $7.60 \mathrm{E}-14$ \\
\hline Os-191 & Os-191 & 190.961 & Osmium & $1.00 \mathrm{E}-03$ & ICRP-38 & $1.54 \mathrm{E}+01$ & d & $1.33 \mathrm{E}+06$ & $4.44 \mathrm{E}+04$ & ICRP-72 & $1.90 \mathrm{E}-09$ & $\mathrm{~s}$ & FGR-12 & $3.21 \mathrm{E}-15$ \\
\hline Os-191m & Os-191 & 190.961 & Osmium & $1.00 \mathrm{E}-03$ & ICRP-38 & $1.30 \mathrm{E}+01$ & $\mathrm{~h}$ & $4.69 \mathrm{E}+04$ & $1.26 \mathrm{E}+06$ & ICRP-72 & $1.60 \mathrm{E}-10$ & $\mathrm{~s}$ & FGR-12 & $2.75 \mathrm{E}-16$ \\
\hline Os-193 & Os-193 & 192.964 & Osmium & $1.00 \mathrm{E}-03$ & ICRP-38 & $3.00 \mathrm{E}+01$ & $\mathrm{~h}$ & $1.08 \mathrm{E}+05$ & $5.41 \mathrm{E}+05$ & ICRP-72 & $5.20 \mathrm{E}-10$ & $\mathrm{~s}$ & FGR-12 & $3.40 \mathrm{E}-15$ \\
\hline Os-194 & Os-194 & 193.965 & Osmium & $1.00 \mathrm{E}-03$ & ICRP-38 & $6.00 E+00$ & $y$ & $1.89 \mathrm{E}+08$ & $3.07 \mathrm{E}+02$ & ICRP-72 & $8.50 \mathrm{E}-08$ & $\mathrm{~s}$ & FGR-12 & $2.75 \mathrm{E}-17$ \\
\hline Os-196 & Os-196 & 195.970 & Osmium & $1.00 \mathrm{E}-03$ & ICRP-107 & $3.49 \mathrm{E}+01$ & $\mathrm{~m}$ & $2.09 \mathrm{E}+03$ & $2.75 \mathrm{E}+07$ & DOE-STD-1196 & $6.64 \mathrm{E}-11$ & $\mathrm{~s}$ & DOE-STD-1196 & $3.64 \mathrm{E}-15$ \\
\hline $\mathrm{P}-30$ & P-30 & 29.978 & Phosphorus & $5.00 \mathrm{E}-01$ & ICRP-38 & $2.50 \mathrm{E}+00$ & $\mathrm{~m}$ & $1.50 \mathrm{E}+02$ & $2.51 \mathrm{E}+09$ & -- & -- & -- & FGR-12 & $4.94 \mathrm{E}-14$ \\
\hline P-32 & P-32 & 31.974 & Phosphorus & $5.00 \mathrm{E}-01$ & ICRP-38 & $1.43 \mathrm{E}+01$ & d & $1.23 \mathrm{E}+06$ & $2.86 \mathrm{E}+05$ & ICRP-72 & $3.40 \mathrm{E}-09$ & $M$ & FGR-12 & $9.90 \mathrm{E}-17$ \\
\hline $\mathrm{P}-33$ & $P-33$ & 32.972 & Phosphorus & $5.00 \mathrm{E}-01$ & ICRP-38 & $2.54 \mathrm{E}+01$ & $d$ & $2.19 \mathrm{E}+06$ & $1.56 \mathrm{E}+05$ & ICRP-72 & $1.50 \mathrm{E}-09$ & $M$ & FGR-12 & $8.23 \mathrm{E}-19$ \\
\hline Pa-227 & Pa-227 & 227.029 & Protactinium & $1.00 \mathrm{E}-03$ & ICRP-38 & $3.83 \mathrm{E}+01$ & $\mathrm{~m}$ & $2.30 \mathrm{E}+03$ & $2.16 \mathrm{E}+07$ & ICRP-72 & $8.00 \mathrm{E}-08$ & $\mathrm{~s}$ & FGR-12 & $8.54 \mathrm{E}-16$ \\
\hline Pa-228 & Pa-228 & 228.031 & Protactinium & $1.00 \mathrm{E}-03$ & ICRP-38 & $2.20 \mathrm{E}+01$ & $\mathrm{~h}$ & $7.92 \mathrm{E}+04$ & $6.25 \mathrm{E}+05$ & ICRP-72 & $7.50 \mathrm{E}-08$ & $\mathrm{~s}$ & FGR-12 & $5.54 \mathrm{E}-14$ \\
\hline Pa-229 & Pa-229 & 229.032 & Protactinium & $1.00 \mathrm{E}-03$ & ICRP-107 & $1.50 E+00$ & $d$ & $1.30 \mathrm{E}+05$ & $3.80 \mathrm{E}+05$ & DOE-STD-1196 & $7.78 \mathrm{E}-09$ & $\mathrm{~s}$ & DOE-STD-1196 & $2.33 \mathrm{E}-15$ \\
\hline $\mathrm{Pa}-230$ & Pa-230 & 230.035 & Protactinium & $1.00 \mathrm{E}-03$ & ICRP-38 & $1.74 \mathrm{E}+01$ & $d$ & $1.50 \mathrm{E}+06$ & $3.26 \mathrm{E}+04$ & ICRP-72 & $7.60 \mathrm{E}-07$ & $\mathrm{~s}$ & FGR-12 & $3.13 \mathrm{E}-14$ \\
\hline Pa-231 & Pa-231 & 231.036 & Protactinium & $1.00 \mathrm{E}-03$ & ICRP-38 & $3.28 \mathrm{E}+04$ & $y$ & $1.03 \mathrm{E}+12$ & $4.72 \mathrm{E}-02$ & ICRP-72 & $1.40 \mathrm{E}-04$ & $M$ & FGR-12 & $1.72 \mathrm{E}-15$ \\
\hline Pa-232 & $\mathrm{Pa}-232$ & 232.039 & Protactinium & $1.00 \mathrm{E}-03$ & ICRP-38 & $1.31 \mathrm{E}+00$ & $d$ & $1.13 \mathrm{E}+05$ & $4.30 \mathrm{E}+05$ & ICRP-72 & $1.00 \mathrm{E}-08$ & $M$ & FGR-12 & $4.56 \mathrm{E}-14$ \\
\hline $\mathrm{Pa}-233$ & $\mathrm{~Pa}-233$ & 233.040 & Protactinium & $1.00 \mathrm{E}-03$ & ICRP-38 & $2.70 E+01$ & $d$ & $2.33 E+06$ & $2.08 \mathrm{E}+04$ & ICRP-72 & $3.90 \mathrm{E}-09$ & $\mathrm{~s}$ & FGR-12 & $9.35 \mathrm{E}-15$ \\
\hline Pa-234 & $\mathrm{Pa}-234$ & 234.043 & Protactinium & $1.00 \mathrm{E}-03$ & ICRP-38 & $6.70 E+00$ & $\mathrm{~h}$ & $2.41 \mathrm{E}+04$ & $2.00 E+06$ & ICRP-72 & $4.00 \mathrm{E}-10$ & $\mathrm{~s}$ & FGR-12 & $9.34 \mathrm{E}-14$ \\
\hline $\mathrm{Pa}-234 \mathrm{~m}$ & $\mathrm{~Pa}-234$ & 234.043 & Protactinium & $1.00 \mathrm{E}-03$ & ICRP-38 & $1.17 \mathrm{E}+00$ & $\mathrm{~m}$ & $7.02 \mathrm{E}+01$ & $6.87 \mathrm{E}+08$ & -- & -- & -- & FGR-12 & $7.19 \mathrm{E}-16$ \\
\hline Pa-235 & Pa-235 & 235.045 & Protactinium & $1.00 \mathrm{E}-03$ & ICRP-107 & $2.45 \mathrm{E}+01$ & $\mathrm{~m}$ & $1.47 \mathrm{E}+03$ & $3.27 \mathrm{E}+07$ & DOE-STD-1196 & $1.98 \mathrm{E}-11$ & $\mathrm{~s}$ & DOE-STD-1196 & $3.35 \mathrm{E}-16$ \\
\hline $\mathrm{Pa}-236$ & $\mathrm{~Pa}-236$ & 236.049 & Protactinium & $1.00 \mathrm{E}-03$ & ICRP-107 & $9.10 E+00$ & $\mathrm{~m}$ & $5.46 \mathrm{E}+02$ & $8.75 E+07$ & -- & -- & -- & DOE-STD-1196 & $4.36 \mathrm{E}-14$ \\
\hline Pa-237 & $\mathrm{Pa}-237$ & 237.051 & Protactinium & $1.00 \mathrm{E}-03$ & ICRP-107 & $8.70 \mathrm{E}+00$ & $\mathrm{~m}$ & $5.22 \mathrm{E}+02$ & $9.12 \mathrm{E}+07$ & -- & -- & -- & DOE-STD-1196 & $2.82 \mathrm{E}-14$ \\
\hline $\mathrm{Pb}-194$ & Pb-194 & 193.974 & Lead & $1.00 \mathrm{E}-03$ & ICRP-107 & $1.20 \mathrm{E}+01$ & $\mathrm{~m}$ & $7.20 \mathrm{E}+02$ & $8.08 \mathrm{E}+07$ & DOE-STD-1196 & $1.57 \mathrm{E}-11$ & $\mathrm{~s}$ & DOE-STD-1196 & $4.94 \mathrm{E}-14$ \\
\hline $\mathrm{Pb}-195 \mathrm{~m}$ & Pb-195 & 194.975 & Lead & $1.00 \mathrm{E}-03$ & ICRP-38 & $1.58 \mathrm{E}+01$ & $\mathrm{~m}$ & $9.48 \mathrm{E}+02$ & $6.10 \mathrm{E}+07$ & ICRP-72 & $2.70 \mathrm{E}-11$ & $\mathrm{~s}$ & FGR-12 & $7.68 \mathrm{E}-14$ \\
\hline $\mathrm{Pb}-196$ & Pb-196 & 195.973 & Lead & $1.00 \mathrm{E}-03$ & ICRP-107 & $3.70 \mathrm{E}+01$ & $\mathrm{~m}$ & $2.22 \mathrm{E}+03$ & $2.59 \mathrm{E}+07$ & DOE-STD-1196 & $2.99 \mathrm{E}-11$ & $\mathrm{~s}$ & DOE-STD-1196 & $2.10 \mathrm{E}-14$ \\
\hline Pb-197 & Pb-197 & 196.973 & Lead & $1.00 \mathrm{E}-03$ & ICRP-107 & $8.00 \mathrm{E}+00$ & $\mathrm{~m}$ & $4.80 \mathrm{E}+02$ & $1.19 \mathrm{E}+08$ & -- & -- & -- & DOE-STD-1196 & $7.14 \mathrm{E}-14$ \\
\hline $\mathrm{Pb}-197 \mathrm{~m}$ & Pb-197 & 196.973 & Lead & $1.00 \mathrm{E}-03$ & ICRP-107 & $4.30 \mathrm{E}+01$ & $\mathrm{~m}$ & $2.58 \mathrm{E}+03$ & $2.22 \mathrm{E}+07$ & DOE-STD-1196 & $5.54 \mathrm{E}-11$ & $\mathrm{~s}$ & DOE-STD-1196 & $5.22 \mathrm{E}-14$ \\
\hline $\mathrm{Pb}-198$ & Pb-198 & 197.972 & Lead & $1.00 \mathrm{E}-03$ & ICRP-38 & $2.40 \mathrm{E}+00$ & $\mathrm{~h}$ & $8.64 \mathrm{E}+03$ & $6.60 \mathrm{E}+06$ & ICRP-72 & $7.00 \mathrm{E}-11$ & $\mathrm{~s}$ & FGR-12 & $2.04 \mathrm{E}-14$ \\
\hline Pb-199 & Pb-199 & 198.973 & Lead & $1.00 \mathrm{E}-03$ & ICRP-38 & $9.00 \mathrm{E}+01$ & $\mathrm{~m}$ & $5.40 \mathrm{E}+03$ & $1.05 E+07$ & ICRP-72 & $3.70 \mathrm{E}-11$ & $\mathrm{~s}$ & FGR-12 & $7.31 \mathrm{E}-14$ \\
\hline $\mathrm{Pb}-200$ & $\mathrm{~Pb}-200$ & 199.972 & Lead & $1.00 \mathrm{E}-03$ & ICRP-38 & $2.15 \mathrm{E}+01$ & $\mathrm{~h}$ & $7.74 \mathrm{E}+04$ & $7.29 \mathrm{E}+05$ & ICRP-72 & $3.50 \mathrm{E}-10$ & $\mathrm{~s}$ & FGR-12 & $9.20 \mathrm{E}-15$ \\
\hline $\mathrm{Pb}-201$ & $\mathrm{~Pb}-201$ & 200.973 & Lead & $1.00 \mathrm{E}-03$ & ICRP-38 & $9.40 \mathrm{E}+00$ & $\mathrm{~h}$ & $3.38 \mathrm{E}+04$ & $1.66 \mathrm{E}+06$ & ICRP-72 & $1.20 \mathrm{E}-10$ & $\mathrm{~s}$ & FGR-12 & $3.63 \mathrm{E}-14$ \\
\hline $\mathrm{Pb}-201 \mathrm{~m}$ & $\mathrm{~Pb}-201$ & 200.973 & Lead & $1.00 \mathrm{E}-03$ & ICRP-107 & $6.10 \mathrm{E}+01$ & $\mathrm{~s}$ & $6.10 \mathrm{E}+01$ & $9.20 \mathrm{E}+08$ & -- & -- & -- & DOE-STD-1196 & $1.63 \mathrm{E}-14$ \\
\hline $\mathrm{Pb}-202$ & $\mathrm{~Pb}-202$ & 201.972 & Lead & $1.00 \mathrm{E}-03$ & ICRP-38 & $3.00 E+05$ & $y$ & $9.47 \mathrm{E}+12$ & $5.90 \mathrm{E}-03$ & ICRP-72 & $1.20 \mathrm{E}-08$ & $\mathrm{~s}$ & FGR-12 & $4.52 \mathrm{E}-19$ \\
\hline $\mathrm{Pb}-202 \mathrm{~m}$ & $\mathrm{~Pb}-202$ & 201.972 & Lead & $1.00 \mathrm{E}-03$ & ICRP-38 & $3.62 \mathrm{E}+00$ & $\mathrm{~h}$ & $1.30 \mathrm{E}+04$ & $4.29 \mathrm{E}+06$ & ICRP-72 & $1.00 \mathrm{E}-10$ & $\mathrm{~s}$ & FGR-12 & $9.96 \mathrm{E}-14$ \\
\hline $\mathrm{Pb}-203$ & $\mathrm{~Pb}-203$ & 202.973 & Lead & $1.00 \mathrm{E}-03$ & ICRP-38 & $5.21 \mathrm{E}+01$ & $\mathrm{~h}$ & $1.87 \mathrm{E}+05$ & $2.97 \mathrm{E}+05$ & ICRP-72 & $2.20 \mathrm{E}-10$ & $\mathrm{~s}$ & FGR-12 & $1.44 \mathrm{E}-14$ \\
\hline $\mathrm{Pb}-204 \mathrm{~m}$ & $\mathrm{~Pb}-204$ & 203.973 & Lead & $1.00 \mathrm{E}-03$ & ICRP-38 & $6.72 \mathrm{E}+01$ & $\mathrm{~m}$ & $4.03 \mathrm{E}+03$ & $1.37 \mathrm{E}+07$ & DOE-STD-1196 & $3.54 \mathrm{E}-11$ & $\mathrm{~s}$ & DOE-STD-1196 & $9.45 \mathrm{E}-14$ \\
\hline $\mathrm{Pb}-205$ & $\mathrm{~Pb}-205$ & 204.974 & Lead & $1.00 \mathrm{E}-03$ & ICRP-38 & $1.43 \mathrm{E}+07$ & $y$ & $4.51 \mathrm{E}+14$ & $1.22 \mathrm{E}-04$ & ICRP-72 & $8.50 \mathrm{E}-10$ & $\mathrm{~s}$ & FGR-12 & $5.06 \mathrm{E}-19$ \\
\hline $\mathrm{Pb}-209$ & Pb-209 & 208.981 & Lead & $1.00 \mathrm{E}-03$ & ICRP-38 & $3.25 E+00$ & $\mathrm{~h}$ & $1.17 \mathrm{E}+04$ & $4.61 \mathrm{E}+06$ & ICRP-72 & $6.10 \mathrm{E}-11$ & $\mathrm{~s}$ & FGR-12 & $8.12 \mathrm{E}-18$ \\
\hline
\end{tabular}

A-19 


\begin{tabular}{|c|c|c|c|c|c|c|c|c|c|c|c|c|c|c|}
\hline \multicolumn{15}{|c|}{ Table A.1 Radionuclide Specific Input Data Used For the Calculation of HC-2 TQs Using Maximum Reported DCs (continued) } \\
\hline $\begin{array}{c}\text { MASTER } \\
\text { ISOTOPE } \\
\text { LIST }\end{array}$ & $\begin{array}{l}\text { Atomic } \\
\text { Mass } \\
\text { Nuclide }\end{array}$ & $\begin{array}{l}\text { Atomic } \\
\text { Mass }\end{array}$ & Element & $\begin{array}{c}\text { RF per } \\
\text { DOE-STD- } \\
1027\end{array}$ & $\begin{array}{c}\text { Half-Life } \\
\text { Reference }\end{array}$ & $\begin{array}{r}\text { Half-Li } \\
\text { (original }\end{array}$ & & Half Life (s) & $\begin{array}{l}\text { Sp. Act } \\
\text { (Ci/g) }\end{array}$ & $\begin{array}{l}\text { Inhalation DC } \\
\text { Reference }\end{array}$ & $\begin{array}{c}\text { HC-2 } \\
\text { Bounding } \\
\text { Inhalation } \\
\text { DC (Sv/Bq) }\end{array}$ & $\begin{array}{l}\text { Lung } \\
\text { Absorption } \\
\text { Class/Type }\end{array}$ & $\begin{array}{l}\text { Immersion DC } \\
\text { Reference }\end{array}$ & $\begin{array}{c}\mathrm{HC}-2 \\
\text { Immersion } \\
\mathrm{DC}(\mathrm{Sv} / \mathrm{s} \\
\left.\text { per } \mathrm{Bq} / \mathrm{m}^{3}\right)\end{array}$ \\
\hline $\mathrm{Pb}-210$ & $\mathrm{~Pb}-210$ & 209.984 & Lead & $1.00 \mathrm{E}-03$ & ICRP-38 & $2.23 \mathrm{E}+01$ & y & $7.04 \mathrm{E}+08$ & $7.63 \mathrm{E}+01$ & ICRP-72 & $5.60 \mathrm{E}-06$ & $\mathrm{~s}$ & FGR-12 & $5.64 \mathrm{E}-17$ \\
\hline $\mathrm{Pb}-211$ & $\mathrm{~Pb}-211$ & 210.989 & Lead & $1.00 \mathrm{E}-03$ & ICRP-38 & $3.61 \mathrm{E}+01$ & $\mathrm{~m}$ & $2.17 \mathrm{E}+03$ & $2.47 \mathrm{E}+07$ & ICRP-72 & $1.20 \mathrm{E}-08$ & $\mathrm{~s}$ & FGR-12 & $2.49 \mathrm{E}-15$ \\
\hline $\mathrm{Pb}-212$ & $\mathrm{~Pb}-212$ & 211.992 & Lead & $1.00 \mathrm{E}-03$ & ICRP-38 & $1.06 \mathrm{E}+01$ & $\mathrm{~h}$ & $3.83 \mathrm{E}+04$ & $1.39 \mathrm{E}+06$ & ICRP-72 & $1.90 \mathrm{E}-07$ & $\mathrm{~s}$ & FGR-12 & $6.87 E-15$ \\
\hline $\mathrm{Pb}-214$ & $\mathrm{~Pb}-214$ & 214.000 & Lead & $1.00 \mathrm{E}-03$ & ICRP-38 & $2.68 \mathrm{E}+01$ & $\mathrm{~m}$ & $1.61 E+03$ & $3.28 \mathrm{E}+07$ & ICRP-72 & $1.50 \mathrm{E}-08$ & $\mathrm{~s}$ & FGR-12 & $1.18 \mathrm{E}-14$ \\
\hline $\mathrm{Pd}-100$ & $\mathrm{Pd}-100$ & 99.909 & Palladium & $1.00 \mathrm{E}-03$ & ICRP-38 & $3.63 \mathrm{E}+00$ & $d$ & $3.14 \mathrm{E}+05$ & $3.60 \mathrm{E}+05$ & ICRP-72 & $8.50 \mathrm{E}-10$ & $\mathrm{~s}$ & FGR-12 & $4.65 \mathrm{E}-15$ \\
\hline Pd-101 & Pd-101 & 100.908 & Palladium & $1.00 \mathrm{E}-03$ & ICRP-38 & $8.27 \mathrm{E}+00$ & $\mathrm{~h}$ & $2.98 \mathrm{E}+04$ & $3.76 \mathrm{E}+06$ & ICRP-72 & $6.20 \mathrm{E}-11$ & $\mathrm{~s}$ & FGR-12 & $1.53 \mathrm{E}-14$ \\
\hline $\mathrm{Pd}-103$ & $\mathrm{Pd}-103$ & 102.906 & Palladium & $1.00 \mathrm{E}-03$ & ICRP-38 & $1.70 E+01$ & $d$ & $1.47 \mathrm{E}+06$ & $7.48 \mathrm{E}+04$ & ICRP-72 & $4.50 \mathrm{E}-10$ & $\mathrm{~s}$ & FGR-12 & $7.68 \mathrm{E}-17$ \\
\hline $\mathrm{Pd}-107$ & Pd-107 & 106.905 & Palladium & $1.00 \mathrm{E}-03$ & ICRP-38 & $6.50 \mathrm{E}+06$ & $y$ & $2.05 E+14$ & $5.14 \mathrm{E}-04$ & ICRP-72 & $5.90 \mathrm{E}-10$ & $\mathrm{~s}$ & -- & -- \\
\hline Pd-109 & Pd-109 & 108.906 & Palladium & $1.00 \mathrm{E}-03$ & ICRP-38 & $1.34 \mathrm{E}+01$ & $\mathrm{~h}$ & $4.83 \mathrm{E}+04$ & $2.14 \mathrm{E}+06$ & ICRP-72 & $3.70 \mathrm{E}-10$ & $\mathrm{~s}$ & FGR-12 & $2.51 \mathrm{E}-16$ \\
\hline Pd-109m & Pd-109 & 108.906 & Palladium & $1.00 \mathrm{E}-03$ & ICRP-107 & $4.69 \mathrm{E}+00$ & $\mathrm{~m}$ & $2.81 \mathrm{E}+02$ & $3.68 \mathrm{E}+08$ & -- & -- & -- & DOE-STD-1196 & $4.59 \mathrm{E}-15$ \\
\hline $\mathrm{Pd}-111$ & $\mathrm{Pd}-111$ & 110.908 & Palladium & $1.00 \mathrm{E}-03$ & ICRP-107 & $2.34 \mathrm{E}+01$ & $\mathrm{~m}$ & $1.40 \mathrm{E}+03$ & $7.24 \mathrm{E}+07$ & DOE-STD-1196 & $3.14 \mathrm{E}-11$ & $\mathrm{~s}$ & DOE-STD-1196 & $2.89 \mathrm{E}-15$ \\
\hline $\mathrm{Pd}-112$ & $\mathrm{Pd}-112$ & 111.907 & Palladium & $1.00 \mathrm{E}-03$ & ICRP-107 & $2.10 \mathrm{E}+01$ & $\mathrm{~h}$ & $7.57 E+04$ & $1.33 \mathrm{E}+06$ & DOE-STD-1196 & $1.32 \mathrm{E}-09$ & $\mathrm{~s}$ & DOE-STD-1196 & $2.68 \mathrm{E}-17$ \\
\hline $\mathrm{Pd}-114$ & Pd-114 & 113.910 & Palladium & $1.00 \mathrm{E}-03$ & ICRP-107 & $2.42 \mathrm{E}+00$ & $\mathrm{~m}$ & $1.45 \mathrm{E}+02$ & $6.82 \mathrm{E}+08$ & -- & -- & -- & DOE-STD-1196 & $1.49 \mathrm{E}-15$ \\
\hline Pd-96 & Pd-96 & 95.918 & Palladium & $1.00 \mathrm{E}-03$ & ICRP-107 & $1.22 \mathrm{E}+02$ & $\mathrm{~s}$ & $1.22 \mathrm{E}+02$ & $9.64 \mathrm{E}+08$ & -- & -- & -- & DOE-STD-1196 & $6.51 \mathrm{E}-14$ \\
\hline Pd-97 & Pd-97 & 96.916 & Palladium & $1.00 \mathrm{E}-03$ & ICRP-107 & $3.10 E+00$ & $\mathrm{~m}$ & $1.86 \mathrm{E}+02$ & $6.26 \mathrm{E}+08$ & -- & -- & -- & DOE-STD-1196 & $1.12 \mathrm{E}-13$ \\
\hline Pd-98 & $\begin{array}{l}\mathrm{Pd}-98 \\
\end{array}$ & 97.913 & Palladium & $1.00 \mathrm{E}-03$ & ICRP-107 & $1.77 \mathrm{E}+01$ & $\mathrm{~m}$ & $1.06 \mathrm{E}+03$ & $1.08 \mathrm{E}+08$ & DOE-STD-1196 & $3.35 \mathrm{E}-11$ & $\mathrm{~s}$ & DOE-STD-1196 & $1.76 \mathrm{E}-14$ \\
\hline Pd-99 & Pd-99 & 98.912 & Palladium & $1.00 \mathrm{E}-03$ & ICRP-107 & $2.14 \mathrm{E}+01$ & $\mathrm{~m}$ & $1.28 \mathrm{E}+03$ & $8.88 \mathrm{E}+07$ & DOE-STD-1196 & $2.28 \mathrm{E}-11$ & $\mathrm{~s}$ & DOE-STD-1196 & $5.88 \mathrm{E}-14$ \\
\hline Pm-136 & Pm-136 & 135.924 & Promethium & $1.00 \mathrm{E}-03$ & ICRP-107 & $1.07 \mathrm{E}+02$ & $\mathrm{~s}$ & $1.07 \mathrm{E}+02$ & $7.76 \mathrm{E}+08$ & -- & -- & -- & DOE-STD-1196 & $1.25 \mathrm{E}-13$ \\
\hline $\mathrm{Pm}-137 \mathrm{~m}$ & Pm-137 & 136.920 & Promethium & $1.00 \mathrm{E}-03$ & ICRP-107 & $2.40 \mathrm{E}+00$ & $\mathrm{~m}$ & $1.44 \mathrm{E}+02$ & $5.72 \mathrm{E}+08$ & -- & -- & -- & DOE-STD-1196 & $8.01 \mathrm{E}-14$ \\
\hline Pm-138 & $\mathrm{Pm}-138$ & 137.920 & Promethium & $1.00 \mathrm{E}-03$ & JAERI & $3.24 \mathrm{E}+00$ & $\mathrm{~m}$ & $1.94 \mathrm{E}+02$ & $4.21 \mathrm{E}+08$ & -- & -- & -- & -- & -- \\
\hline Pm-139 & Pm-139 & 138.917 & Promethium & $1.00 \mathrm{E}-03$ & ICRP-107 & $4.15 \mathrm{E}+00$ & $\mathrm{~m}$ & $2.49 \mathrm{E}+02$ & $3.26 \mathrm{E}+08$ & -- & -- & -- & DOE-STD-1196 & $4.29 \mathrm{E}-14$ \\
\hline Pm-140 & $\mathrm{Pm}-140$ & 139.916 & Promethium & $1.00 \mathrm{E}-03$ & ICRP-107 & $9.20 \mathrm{E}+00$ & $\mathrm{~s}$ & $9.20 \mathrm{E}+00$ & $8.76 \mathrm{E}+09$ & -- & -- & -- & DOE-STD-1196 & $4.93 \mathrm{E}-14$ \\
\hline Pm-140m & $\mathrm{Pm}-140$ & 139.916 & Promethium & $1.00 \mathrm{E}-03$ & ICRP-107 & $5.95 \mathrm{E}+00$ & $\mathrm{~m}$ & $3.57 \mathrm{E}+02$ & $2.26 \mathrm{E}+08$ & -- & -- & -- & DOE-STD-1196 & $1.39 \mathrm{E}-13$ \\
\hline Pm-141 & Pm-141 & 140.914 & Promethium & $1.00 \mathrm{E}-03$ & ICRP-38 & $2.09 \mathrm{E}+01$ & $\mathrm{~m}$ & $1.25 \mathrm{E}+03$ & $6.38 \mathrm{E}+07$ & ICRP-72 & $1.50 \mathrm{E}-11$ & $\mathrm{~s}$ & FGR-12 & $3.60 \mathrm{E}-14$ \\
\hline Pm-142 & Pm-142 & 141.913 & Promethium & $1.00 \mathrm{E}-03$ & ICRP-38 & $4.05 E+01$ & $\mathrm{~s}$ & $4.05 \mathrm{E}+01$ & $1.96 \mathrm{E}+09$ & -- & -- & -- & FGR-12 & $4.22 \mathrm{E}-14$ \\
\hline Pm-143 & Pm-143 & 142.911 & Promethium & $1.00 \mathrm{E}-03$ & ICRP-38 & $2.65 E+02$ & $d$ & $2.29 \mathrm{E}+07$ & $3.45 \mathrm{E}+03$ & ICRP-72 & $1.50 \mathrm{E}-09$ & $M$ & FGR-12 & $1.46 \mathrm{E}-14$ \\
\hline Pm-144 & Pm-144 & 143.913 & Promethium & $1.00 \mathrm{E}-03$ & ICRP-38 & $3.63 \mathrm{E}+02$ & $d$ & $3.14 \mathrm{E}+07$ & $2.50 E+03$ & ICRP-72 & $8.20 \mathrm{E}-09$ & $M$ & FGR-12 & $7.48 \mathrm{E}-14$ \\
\hline Pm-145 & Pm-145 & 144.913 & Promethium & $1.00 \mathrm{E}-03$ & ICRP-38 & $1.77 \mathrm{E}+01$ & $y$ & $5.59 \mathrm{E}+08$ & $1.39 \mathrm{E}+02$ & ICRP-72 & $3.60 \mathrm{E}-09$ & $M$ & FGR-12 & $7.09 \mathrm{E}-16$ \\
\hline Pm-146 & Pm-146 & 145.915 & Promethium & $1.00 \mathrm{E}-03$ & ICRP-38 & $2.02 \mathrm{E}+03$ & $d$ & $1.75 \mathrm{E}+08$ & $4.43 \mathrm{E}+02$ & ICRP-72 & $2.10 \mathrm{E}-08$ & $M$ & FGR-12 & $3.59 \mathrm{E}-14$ \\
\hline Pm-147 & Pm-147 & 146.915 & Promethium & $1.00 \mathrm{E}-03$ & ICRP-38 & $2.62 \mathrm{E}+00$ & $y$ & $8.28 \mathrm{E}+07$ & $9.28 \mathrm{E}+02$ & ICRP-72 & $5.00 \mathrm{E}-09$ & $M$ & FGR-12 & $6.93 \mathrm{E}-19$ \\
\hline Pm-148 & Pm-148 & 147.917 & Promethium & $1.00 \mathrm{E}-03$ & ICRP-38 & $5.37 \mathrm{E}+00$ & d & $4.64 \mathrm{E}+05$ & $1.64 \mathrm{E}+05$ & ICRP-72 & $2.20 \mathrm{E}-09$ & $\mathrm{~S}$ & FGR-12 & $2.89 \mathrm{E}-14$ \\
\hline $\mathrm{Pm}-148 \mathrm{~m}$ & Pm-148 & 147.917 & Promethium & $1.00 \mathrm{E}-03$ & ICRP-38 & $4.13 \mathrm{E}+01$ & $d$ & $3.57 \mathrm{E}+06$ & $2.14 \mathrm{E}+04$ & ICRP-72 & $5.70 \mathrm{E}-09$ & $\mathrm{~s}$ & FGR-12 & $9.68 \mathrm{E}-14$ \\
\hline Pm-149 & Pm-149 & 148.918 & Promethium & $1.00 \mathrm{E}-03$ & ICRP-38 & $5.31 \mathrm{E}+01$ & $\mathrm{~h}$ & $1.91 \mathrm{E}+05$ & $3.96 \mathrm{E}+05$ & ICRP-72 & $7.30 \mathrm{E}-10$ & $\mathrm{~s}$ & FGR-12 & $5.41 \mathrm{E}-16$ \\
\hline Pm-150 & Pm-150 & 149.921 & Promethium & $1.00 \mathrm{E}-03$ & ICRP-38 & $2.68 \mathrm{E}+00$ & $\mathrm{~h}$ & $9.65 E+03$ & $7.80 E+06$ & ICRP-72 & $1.30 \mathrm{E}-10$ & $\mathrm{~s}$ & FGR-12 & $7.17 \mathrm{E}-14$ \\
\hline Pm-151 & Pm-151 & 150.921 & Promethium & $1.00 \mathrm{E}-03$ & ICRP-38 & $2.84 \mathrm{E}+01$ & $\mathrm{~h}$ & $1.02 \mathrm{E}+05$ & $7.31 \mathrm{E}+05$ & ICRP-72 & $4.60 \mathrm{E}-10$ & $\mathrm{~s}$ & FGR-12 & $1.51 \mathrm{E}-14$ \\
\hline Pm-152 & Pm-152 & 151.924 & Promethium & $1.00 \mathrm{E}-03$ & ICRP-107 & $4.12 \mathrm{E}+00$ & $\mathrm{~m}$ & $2.47 \mathrm{E}+02$ & $3.00 E+08$ & -- & -- & -- & DOE-STD-1196 & $1.45 \mathrm{E}-14$ \\
\hline Pm-152m & Pm-152 & 151.924 & Promethium & $1.00 \mathrm{E}-03$ & ICRP-107 & $7.52 E+00$ & $\mathrm{~m}$ & $4.51 \mathrm{E}+02$ & $1.65 E+08$ & -- & -- & -- & DOE-STD-1196 & $7.07 E-14$ \\
\hline Pm-153 & Pm-153 & 152.924 & Promethium & $1.00 \mathrm{E}-03$ & ICRP-107 & $5.25 \mathrm{E}+00$ & $\mathrm{~m}$ & $3.15 \mathrm{E}+02$ & $2.34 \mathrm{E}+08$ & -- & -- & -- & DOE-STD-1196 & $3.40 \mathrm{E}-15$ \\
\hline $\mathrm{Pm}-154$ & Pm-154 & 153.926 & Promethium & $1.00 \mathrm{E}-03$ & ICRP-107 & $1.73 \mathrm{E}+00$ & $\mathrm{~m}$ & $1.04 \mathrm{E}+02$ & $7.06 \mathrm{E}+08$ & -- & -- & -- & DOE-STD-1196 & $8.73 E-14$ \\
\hline Pm-154m & Pm-154 & 153.926 & Promethium & $1.00 \mathrm{E}-03$ & ICRP-107 & $2.68 \mathrm{E}+00$ & $\mathrm{~m}$ & $1.61 \mathrm{E}+02$ & $4.56 \mathrm{E}+08$ & -- & -- & -- & DOE-STD-1196 & $8.50 \mathrm{E}-14$ \\
\hline Po-203 & Po-203 & 202.981 & Polonium & $1.00 \mathrm{E}-02$ & ICRP-38 & $3.67 \mathrm{E}+01$ & $\mathrm{~m}$ & $2.20 \mathrm{E}+03$ & $2.52 \mathrm{E}+07$ & ICRP-72 & $3.60 \mathrm{E}-11$ & $\mathrm{~s}$ & FGR-12 & $8.12 \mathrm{E}-14$ \\
\hline Po-204 & Po-204 & 203.980 & $\begin{array}{l}\text { Polonium } \\
\end{array}$ & $1.00 \mathrm{E}-02$ & ICRP-107 & $3.53 \mathrm{E}+00$ & $\mathrm{~h}$ & $1.27 \mathrm{E}+04$ & $4.35 \mathrm{E}+06$ & DOE-STD-1196 & $4.61 \mathrm{E}-10$ & $\mathrm{~s}$ & DOE-STD-1196 & $5.16 \mathrm{E}-14$ \\
\hline Po-205 & Po-205 & 204.981 & Polonium & $1.00 \mathrm{E}-02$ & ICRP-38 & $1.80 \mathrm{E}+00$ & $\mathrm{~h}$ & $6.48 \mathrm{E}+03$ & $8.49 \mathrm{E}+06$ & ICRP-72 & $6.90 \mathrm{E}-11$ & $\mathrm{~s}$ & FGR-12 & $7.80 \mathrm{E}-14$ \\
\hline Po-206 & Po-206 & 205.980 & Polonium & $1.00 \mathrm{E}-02$ & ICRP-107 & $8.80 \mathrm{E}+00$ & $d$ & $7.60 \mathrm{E}+05$ & $7.20 \mathrm{E}+04$ & DOE-STD-1196 & $7.09 \mathrm{E}-08$ & $\mathrm{~s}$ & DOE-STD-1196 & $5.33 \mathrm{E}-14$ \\
\hline Po-207 & Po-207 & 206.982 & $\begin{array}{l}\text { Polonium } \\
\end{array}$ & $1.00 \mathrm{E}-02$ & ICRP-38 & $3.50 \mathrm{E}+02$ & $\mathrm{~m}$ & $2.10 E+04$ & $2.60 \mathrm{E}+06$ & ICRP-72 & $8.20 \mathrm{E}-11$ & $\mathrm{~s}$ & FGR-12 & $6.51 \mathrm{E}-14$ \\
\hline Po-208 & Po-208 & 207.981 & Polonium & $1.00 \mathrm{E}-02$ & ICRP-107 & $2.90 \mathrm{E}+00$ & $y$ & $9.15 \mathrm{E}+07$ & $5.93 \mathrm{E}+02$ & DOE-STD-1196 & $7.42 \mathrm{E}-06$ & $\mathrm{~s}$ & DOE-STD-1196 & $9.36 \mathrm{E}-19$ \\
\hline
\end{tabular}




\begin{tabular}{|c|c|c|c|c|c|c|c|c|c|c|c|c|c|c|}
\hline \multicolumn{15}{|c|}{ Table A.1 Radionuclide Specific Input Data Used For the Calculation of HC-2 TQs Using Maximum Reported DCs (continued) } \\
\hline $\begin{array}{c}\text { MASTER } \\
\text { ISOTOPE } \\
\text { LIST }\end{array}$ & $\begin{array}{l}\text { Atomic } \\
\text { Mass } \\
\text { Nuclide }\end{array}$ & $\begin{array}{l}\text { Atomic } \\
\text { Mass }\end{array}$ & Element & $\begin{array}{c}\text { RF per } \\
\text { DOE-STD- } \\
1027\end{array}$ & $\begin{array}{l}\text { Half-Life } \\
\text { Reference }\end{array}$ & $\begin{array}{r}\text { Half-L } \\
\text { (original }\end{array}$ & & Half Life (s) & $\begin{array}{l}\text { Sp. Act } \\
\text { (Ci/g) }\end{array}$ & $\begin{array}{l}\text { Inhalation DC } \\
\text { Reference }\end{array}$ & $\begin{array}{c}\text { HC-2 } \\
\text { Bounding } \\
\text { Inhalation } \\
\text { DC (Sv/Bq) }\end{array}$ & $\begin{array}{l}\text { Lung } \\
\text { Absorption } \\
\text { Class/Type }\end{array}$ & $\begin{array}{l}\text { Immersion DC } \\
\text { Reference }\end{array}$ & $\begin{array}{c}\text { HC-2 } \\
\text { Immersion } \\
\text { DC }(\mathrm{Sv} / \mathrm{s} \\
\left.\text { per } \mathrm{Bq} / \mathrm{m}^{3}\right)\end{array}$ \\
\hline Po-209 & Po-209 & 208.982 & Polonium & $1.00 \mathrm{E}-02$ & ICRP-38 & $1.02 \mathrm{E}+02$ & y & $3.22 \mathrm{E}+09$ & $1.68 \mathrm{E}+01$ & DOE-STD-1196 & $1.02 \mathrm{E}-05$ & $\mathrm{~s}$ & DOE-STD-1196 & $2.76 \mathrm{E}-16$ \\
\hline Po-210 & Po-210 & 209.983 & Polonium & $1.00 \mathrm{E}-02$ & ICRP-38 & $1.38 \mathrm{E}+02$ & d & $1.20 \mathrm{E}+07$ & $4.49 \mathrm{E}+03$ & ICRP-72 & $4.30 \mathrm{E}-06$ & $\mathrm{~s}$ & FGR-12 & $4.16 \mathrm{E}-19$ \\
\hline Po-211 & Po-211 & 210.987 & Polonium & $1.00 \mathrm{E}-02$ & ICRP-38 & $5.16 \mathrm{E}-01$ & $\mathrm{~s}$ & $5.16 \mathrm{E}-01$ & $1.04 \mathrm{E}+11$ & -- & -- & -- & FGR-12 & $3.81 \mathrm{E}-16$ \\
\hline Po-212 & Po-212 & 211.989 & Polonium & $1.00 \mathrm{E}-02$ & ICRP-38 & $3.05 \mathrm{E}-01$ & us & $3.05 \mathrm{E}-07$ & $1.74 \mathrm{E}+17$ & -- & -- & -- & -- & -- \\
\hline Po-212m & Po-212 & 211.989 & Polonium & $1.00 \mathrm{E}-02$ & ICRP-107 & $4.51 \mathrm{E}+01$ & $\mathrm{~s}$ & $4.51 \mathrm{E}+01$ & $1.18 \mathrm{E}+09$ & -- & -- & -- & DOE-STD-1196 & $3.99 \mathrm{E}-15$ \\
\hline Po-213 & Po-213 & 212.993 & $\begin{array}{l}\text { Polonium } \\
\end{array}$ & $1.00 \mathrm{E}-02$ & ICRP-38 & $4.20 \mathrm{E}+00$ & us & $4.20 \mathrm{E}-06$ & $1.26 \mathrm{E}+16$ & -- & -- & -- & DOE-STD-1196 & $1.71 \mathrm{E}-18$ \\
\hline Po-214 & Po-214 & 213.995 & Polonium & $1.00 \mathrm{E}-02$ & ICRP-38 & $1.64 \mathrm{E}+02$ & us & $1.64 \mathrm{E}-04$ & $3.21 \mathrm{E}+14$ & -- & -- & -- & FGR-12 & $4.08 \mathrm{E}-18$ \\
\hline Po-215 & Po-215 & 214.999 & Polonium & $1.00 \mathrm{E}-02$ & ICRP-38 & $1.78 \mathrm{E}-03$ & $\mathrm{~s}$ & $1.78 \mathrm{E}-03$ & $2.95 \mathrm{E}+13$ & -- & -- & -- & FGR-12 & $8.43 \mathrm{E}-18$ \\
\hline Po-216 & Po-216 & 216.002 & $\begin{array}{l}\text { Polonium } \\
\end{array}$ & $1.00 \mathrm{E}-02$ & ICRP-38 & $1.50 \mathrm{E}-01$ & $\mathrm{~s}$ & $1.50 \mathrm{E}-01$ & $3.48 \mathrm{E}+11$ & -- & -- & -- & FGR-12 & $8.29 \mathrm{E}-19$ \\
\hline Po-218 & Po-218 & 218.009 & Polonium & $1.00 \mathrm{E}-02$ & ICRP-38 & $3.05 E+00$ & $\mathrm{~m}$ & $1.83 \mathrm{E}+02$ & $2.83 \mathrm{E}+08$ & -- & -- & -- & FGR-12 & $4.48 \mathrm{E}-19$ \\
\hline Pr-134 & Pr-134 & 133.916 & Praseodymium & $1.00 \mathrm{E}-03$ & ICRP-107 & $1.10 \mathrm{E}+01$ & $\mathrm{~m}$ & $6.60 \mathrm{E}+02$ & $1.28 \mathrm{E}+08$ & DOE-STD-1196 & $2.52 \mathrm{E}-11$ & $\mathrm{~s}$ & DOE-STD-1196 & $1.44 \mathrm{E}-13$ \\
\hline Pr-134m & Pr-134 & 133.916 & Praseodymium & $1.00 \mathrm{E}-03$ & ICRP-107 & $1.70 \mathrm{E}+01$ & $\mathrm{~m}$ & $1.02 \mathrm{E}+03$ & $8.26 \mathrm{E}+07$ & DOE-STD-1196 & $3.83 \mathrm{E}-11$ & $\mathrm{~s}$ & DOE-STD-1196 & $1.08 \mathrm{E}-13$ \\
\hline Pr-135 & Pr-135 & 134.913 & Praseodymium & $1.00 \mathrm{E}-03$ & ICRP-107 & $2.40 \mathrm{E}+01$ & $\mathrm{~m}$ & $1.44 \mathrm{E}+03$ & $5.81 \mathrm{E}+07$ & DOE-STD-1196 & $2.45 \mathrm{E}-11$ & $\mathrm{~s}$ & DOE-STD-1196 & $3.89 \mathrm{E}-14$ \\
\hline Pr-136 & Pr-136 & 135.913 & Praseodymium & $1.00 \mathrm{E}-03$ & ICRP-38 & $1.31 \mathrm{E}+01$ & $\mathrm{~m}$ & $7.86 \mathrm{E}+02$ & $1.06 \mathrm{E}+08$ & ICRP-72 & $1.40 \mathrm{E}-11$ & $\mathrm{~s}$ & FGR-12 & $1.03 \mathrm{E}-13$ \\
\hline Pr-137 & Pr-137 & 136.911 & Praseodymium & $1.00 \mathrm{E}-03$ & ICRP-38 & $7.66 \mathrm{E}+01$ & $\mathrm{~m}$ & $4.60 \mathrm{E}+03$ & $1.79 \mathrm{E}+07$ & ICRP-72 & $2.10 \mathrm{E}-11$ & $\mathrm{~s}$ & FGR-12 & $2.36 \mathrm{E}-14$ \\
\hline Pr-138 & Pr-138 & 137.911 & Praseodymium & $1.00 \mathrm{E}-03$ & ICRP-38 & $1.45 \mathrm{E}+00$ & $\mathrm{~m}$ & $8.70 \mathrm{E}+01$ & $9.40 \mathrm{E}+08$ & -- & -- & -- & FGR-12 & $3.92 \mathrm{E}-14$ \\
\hline Pr-138m & Pr-138 & 137.911 & Praseodymium & $1.00 \mathrm{E}-03$ & ICRP-38 & $2.10 E+00$ & $\mathrm{~h}$ & $7.56 \mathrm{E}+03$ & $1.08 \mathrm{E}+07$ & ICRP-72 & $7.40 \mathrm{E}-11$ & $\mathrm{~s}$ & FGR-12 & $1.21 \mathrm{E}-13$ \\
\hline Pr-139 & Pr-139 & 138.909 & Praseodymium & $1.00 \mathrm{E}-03$ & ICRP-38 & $4.51 \mathrm{E}+00$ & $\mathrm{~h}$ & $1.62 \mathrm{E}+04$ & $5.00 \mathrm{E}+06$ & ICRP-72 & $2.00 \mathrm{E}-11$ & $\mathrm{~s}$ & FGR-12 & $5.17 \mathrm{E}-15$ \\
\hline Pr-140 & Pr-140 & 139.909 & Praseodymium & $1.00 \mathrm{E}-03$ & ICRP-107 & $3.39 \mathrm{E}+00$ & $\mathrm{~m}$ & $2.03 E+02$ & $3.96 \mathrm{E}+08$ & -- & -- & -- & DOE-STD-1196 & $2.44 \mathrm{E}-14$ \\
\hline Pr-142 & Pr-142 & 141.910 & Praseodymium & $1.00 \mathrm{E}-03$ & ICRP-38 & $1.91 \mathrm{E}+01$ & $\mathrm{~h}$ & $6.89 \mathrm{E}+04$ & $1.15 \mathrm{E}+06$ & ICRP-72 & $5.50 \mathrm{E}-10$ & $\mathrm{~s}$ & FGR-12 & $3.15 \mathrm{E}-15$ \\
\hline Pr-142m & Pr-142 & 141.910 & Praseodymium & $1.00 \mathrm{E}-03$ & ICRP-38 & $1.46 \mathrm{E}+01$ & $\mathrm{~m}$ & $8.76 \mathrm{E}+02$ & $9.08 \mathrm{E}+07$ & ICRP-72 & $7.00 \mathrm{E}-12$ & $\mathrm{~s}$ & -- & -- \\
\hline Pr-143 & Pr-143 & 142.911 & Praseodymium & $1.00 \mathrm{E}-03$ & ICRP-38 & $1.36 \mathrm{E}+01$ & $d$ & $1.17 E+06$ & $6.74 \mathrm{E}+04$ & ICRP-72 & $2.40 \mathrm{E}-09$ & $\mathrm{~s}$ & FGR-12 & $2.10 \mathrm{E}-17$ \\
\hline Pr-144 & Pr-144 & 143.913 & Praseodymium & $1.00 \mathrm{E}-03$ & ICRP-38 & $1.73 \mathrm{E}+01$ & $\mathrm{~m}$ & $1.04 \mathrm{E}+03$ & $7.56 \mathrm{E}+07$ & ICRP-72 & $1.80 \mathrm{E}-11$ & $M$ & FGR-12 & $1.95 \mathrm{E}-15$ \\
\hline Pr-144m & Pr-144 & 143.913 & Praseodymium & $1.00 \mathrm{E}-03$ & ICRP-38 & $7.20 E+00$ & $\mathrm{~m}$ & $4.32 \mathrm{E}+02$ & $1.81 \mathrm{E}+08$ & -- & -- & -- & FGR-12 & $2.79 \mathrm{E}-16$ \\
\hline Pr-145 & Pr-145 & 144.915 & Praseodymium & $1.00 \mathrm{E}-03$ & ICRP-38 & $5.98 \mathrm{E}+00$ & $\mathrm{~h}$ & $2.15 E+04$ & $3.62 \mathrm{E}+06$ & ICRP-72 & $1.70 \mathrm{E}-10$ & $\mathrm{~s}$ & FGR-12 & $7.36 \mathrm{E}-16$ \\
\hline Pr-146 & Pr-146 & 145.918 & Praseodymium & $1.00 \mathrm{E}-03$ & ICRP-107 & $2.42 \mathrm{E}+01$ & $\mathrm{~m}$ & $1.45 \mathrm{E}+03$ & $5.34 \mathrm{E}+07$ & DOE-STD-1196 & $3.58 \mathrm{E}-11$ & $\mathrm{~s}$ & DOE-STD-1196 & $4.92 \mathrm{E}-14$ \\
\hline Pr-147 & Pr-147 & 146.919 & Praseodymium & $1.00 \mathrm{E}-03$ & ICRP-38 & $1.36 \mathrm{E}+01$ & $\mathrm{~m}$ & $8.16 \mathrm{E}+02$ & $9.41 \mathrm{E}+07$ & ICRP-72 & $1.80 \mathrm{E}-11$ & M & FGR-12 & $4.15 \mathrm{E}-14$ \\
\hline Pr-148 & Pr-148 & 147.922 & Praseodymium & $1.00 \mathrm{E}-03$ & ICRP-107 & $2.29 \mathrm{E}+00$ & $\mathrm{~m}$ & $1.37 \mathrm{E}+02$ & $5.55 E+08$ & -- & -- & -- & DOE-STD-1196 & $4.83 \mathrm{E}-14$ \\
\hline Pr-148m & Pr-148 & 147.922 & Praseodymium & $1.00 \mathrm{E}-03$ & ICRP-107 & $2.01 E+00$ & $\mathrm{~m}$ & $1.21 \mathrm{E}+02$ & $6.32 \mathrm{E}+08$ & -- & -- & -- & DOE-STD-1196 & $4.37 \mathrm{E}-14$ \\
\hline Pt-183 & Pt-183 & 182.962 & Platinum & $1.00 \mathrm{E}-03$ & JAERI & $6.50 \mathrm{E}+00$ & $\mathrm{~m}$ & $3.90 \mathrm{E}+02$ & $1.58 \mathrm{E}+08$ & -- & -- & -- & -- & -- \\
\hline Pt-184 & Pt-184 & 183.960 & Platinum & $1.00 \mathrm{E}-03$ & ICRP-107 & $1.73 \mathrm{E}+01$ & $\mathrm{~m}$ & $1.04 \mathrm{E}+03$ & $5.91 \mathrm{E}+07$ & DOE-STD-1196 & $3.06 \mathrm{E}-11$ & $\mathrm{~s}$ & DOE-STD-1196 & $3.02 \mathrm{E}-14$ \\
\hline Pt-186 & Pt-186 & 185.959 & Platinum & $1.00 \mathrm{E}-03$ & ICRP-38 & $2.00 \mathrm{E}+00$ & $\mathrm{~h}$ & $7.20 \mathrm{E}+03$ & $8.43 \mathrm{E}+06$ & ICRP-72 & $3.30 \mathrm{E}-11$ & $\mathrm{~F}$ & FGR-12 & $3.53 \mathrm{E}-14$ \\
\hline Pt-187 & Pt-187 & 186.961 & Platinum & $1.00 \mathrm{E}-03$ & ICRP-107 & $2.35 \mathrm{E}+00$ & $\mathrm{~h}$ & $8.46 \mathrm{E}+03$ & $7.13 E+06$ & DOE-STD-1196 & $8.29 \mathrm{E}-11$ & $\mathrm{~s}$ & DOE-STD-1196 & $2.66 \mathrm{E}-14$ \\
\hline Pt-188 & Pt-188 & 187.959 & Platinum & $1.00 \mathrm{E}-03$ & ICRP-38 & $1.02 \mathrm{E}+01$ & $d$ & $8.81 \mathrm{E}+05$ & $6.81 \mathrm{E}+04$ & ICRP-72 & $4.20 \mathrm{E}-10$ & $\mathrm{~F}$ & FGR-12 & $8.86 \mathrm{E}-15$ \\
\hline Pt-189 & Pt-189 & 188.961 & Platinum & $1.00 \mathrm{E}-03$ & ICRP-38 & $1.09 \mathrm{E}+01$ & $\mathrm{~h}$ & $3.91 \mathrm{E}+04$ & $1.53 \mathrm{E}+06$ & ICRP-72 & $3.80 \mathrm{E}-11$ & $\mathrm{~F}$ & FGR-12 & $1.48 \mathrm{E}-14$ \\
\hline Pt-190 & Pt-190 & 189.960 & Platinum & $1.00 \mathrm{E}-03$ & ICRP-107 & $6.50 \mathrm{E}+11$ & $y$ & $2.05 E+19$ & $2.90 \mathrm{E}-09$ & DOE-STD-1196 & $5.58 \mathrm{E}-06$ & $\mathrm{~s}$ & -- & -- \\
\hline Pt-191 & Pt-191 & 190.962 & Platinum & $1.00 \mathrm{E}-03$ & ICRP-38 & $2.80 \mathrm{E}+00$ & $d$ & $2.42 \mathrm{E}+05$ & $2.44 \mathrm{E}+05$ & ICRP-72 & $1.10 \mathrm{E}-10$ & $F$ & FGR-12 & $1.34 \mathrm{E}-14$ \\
\hline Pt-193 & Pt-193 & 192.963 & Platinum & $1.00 \mathrm{E}-03$ & ICRP-38 & $5.00 \mathrm{E}+01$ & $y$ & $1.58 \mathrm{E}+09$ & $3.71 \mathrm{E}+01$ & ICRP-72 & $2.10 \mathrm{E}-11$ & $F$ & FGR-12 & $3.98 \mathrm{E}-19$ \\
\hline Pt-193m & Pt-193 & 192.963 & Platinum & $1.00 \mathrm{E}-03$ & ICRP-38 & $4.33 \mathrm{E}+00$ & $d$ & $3.74 \mathrm{E}+05$ & $1.56 \mathrm{E}+05$ & ICRP-72 & $1.20 \mathrm{E}-10$ & $\mathrm{~F}$ & FGR-12 & $4.15 \mathrm{E}-16$ \\
\hline Pt-195m & Pt-195 & 194.965 & Platinum & $1.00 \mathrm{E}-03$ & ICRP-38 & $4.02 \mathrm{E}+00$ & $d$ & $3.47 \mathrm{E}+05$ & $1.67 \mathrm{E}+05$ & ICRP-72 & $1.80 \mathrm{E}-10$ & $F$ & FGR-12 & $2.84 \mathrm{E}-15$ \\
\hline Pt-197 & Pt-197 & 196.967 & Platinum & $1.00 \mathrm{E}-03$ & ICRP-38 & $1.83 \mathrm{E}+01$ & $\mathrm{~h}$ & $6.59 \mathrm{E}+04$ & $8.69 \mathrm{E}+05$ & ICRP-72 & $8.50 \mathrm{E}-11$ & $F$ & FGR-12 & $1.01 \mathrm{E}-15$ \\
\hline Pt-197m & Pt-197 & 196.967 & Platinum & $1.00 \mathrm{E}-03$ & ICRP-38 & $9.44 \mathrm{E}+01$ & $\mathrm{~m}$ & $5.66 \mathrm{E}+03$ & $1.01 \mathrm{E}+07$ & ICRP-72 & $2.40 \mathrm{E}-11$ & $F$ & FGR-12 & $3.49 \mathrm{E}-15$ \\
\hline Pt-199 & Pt-199 & 198.971 & Platinum & $1.00 \mathrm{E}-03$ & ICRP-38 & $3.08 \mathrm{E}+01$ & $\mathrm{~m}$ & $1.85 \mathrm{E}+03$ & $3.07 \mathrm{E}+07$ & ICRP-72 & $1.20 \mathrm{E}-11$ & $\mathrm{~F}$ & FGR-12 & $9.73 \mathrm{E}-15$ \\
\hline Pt-200 & Pt-200 & 199.971 & Platinum & $1.00 \mathrm{E}-03$ & ICRP-38 & $1.25 \mathrm{E}+01$ & $\mathrm{~h}$ & $4.50 \mathrm{E}+04$ & $1.25 \mathrm{E}+06$ & ICRP-72 & $2.20 \mathrm{E}-10$ & $\mathrm{~F}$ & FGR-12 & $2.55 \mathrm{E}-15$ \\
\hline Pt-202 & Pt-202 & 201.976 & Platinum & $1.00 \mathrm{E}-03$ & ICRP-107 & $4.40 \mathrm{E}+01$ & $\mathrm{~h}$ & $1.58 \mathrm{E}+05$ & $3.53 \mathrm{E}+05$ & DOE-STD-1196 & $2.61 \mathrm{E}-09$ & $\mathrm{~s}$ & DOE-STD-1196 & $5.03 \mathrm{E}-16$ \\
\hline Pu-232 & Pu-232 & 232.041 & Plutonium & $1.00 \mathrm{E}-03$ & ICRP-107 & $3.37 \mathrm{E}+01$ & $\mathrm{~m}$ & $2.02 \mathrm{E}+03$ & $2.40 \mathrm{E}+07$ & DOE-STD-1196 & $2.59 \mathrm{E}-08$ & $\mathrm{~s}$ & DOE-STD-1196 & $2.31 \mathrm{E}-15$ \\
\hline
\end{tabular}




\begin{tabular}{|c|c|c|c|c|c|c|c|c|c|c|c|c|c|c|}
\hline \multicolumn{15}{|c|}{ Table A.1 Radionuclide Specific Input Data Used For the Calculation of HC-2 TQs Using Maximum Reported DCs (continued) } \\
\hline $\begin{array}{c}\text { MASTER } \\
\text { ISOTOPE } \\
\text { LIST } \\
\end{array}$ & $\begin{array}{l}\text { Atomic } \\
\text { Mass } \\
\text { Nuclide }\end{array}$ & $\begin{array}{l}\text { Atomic } \\
\text { Mass }\end{array}$ & Element & $\begin{array}{c}\text { RF per } \\
\text { DOE-STD- } \\
1027 \\
\end{array}$ & $\begin{array}{c}\text { Half-Life } \\
\text { Reference }\end{array}$ & $\begin{array}{r}\text { Half-L } \\
\text { (original }\end{array}$ & & Half Life (s) & $\begin{array}{l}\text { Sp. Act } \\
\text { (Ci/g) }\end{array}$ & $\begin{array}{l}\text { Inhalation DC } \\
\text { Reference }\end{array}$ & $\begin{array}{c}\text { HC-2 } \\
\text { Bounding } \\
\text { Inhalation } \\
\text { DC (Sv/Bq) }\end{array}$ & $\begin{array}{c}\text { Lung } \\
\text { Absorption } \\
\text { Class/Type } \\
\end{array}$ & $\begin{array}{l}\text { Immersion DC } \\
\text { Reference }\end{array}$ & $\begin{array}{c}\mathrm{HC}-2 \\
\text { Immersion } \\
\mathrm{DC}(\mathrm{Sv} / \mathrm{s} \\
\left.\text { per } \mathrm{Bq} / \mathrm{m}^{3}\right)\end{array}$ \\
\hline Pu-234 & Pu-234 & 234.043 & Plutonium & $1.00 \mathrm{E}-03$ & ICRP-38 & $8.80 \mathrm{E}+00$ & $\mathrm{~h}$ & $3.17 \mathrm{E}+04$ & $1.52 \mathrm{E}+06$ & ICRP-72 & $2.40 \mathrm{E}-08$ & $\mathrm{~s}$ & FGR-12 & $2.85 \mathrm{E}-15$ \\
\hline Pu-235 & Pu-235 & 235.045 & Plutonium & $1.00 \mathrm{E}-03$ & ICRP-38 & $2.53 \mathrm{E}+01$ & $\mathrm{~m}$ & $1.52 \mathrm{E}+03$ & $3.16 \mathrm{E}+07$ & ICRP-72 & $1.50 \mathrm{E}-12$ & $\mathrm{~s}$ & FGR-12 & $3.92 \mathrm{E}-15$ \\
\hline $\begin{array}{l}\text { Pu-236 } \\
\end{array}$ & $\begin{array}{l}\text { Pu-236 } \\
\end{array}$ & 236.046 & Plutonium & $1.00 \mathrm{E}-03$ & ICRP-38 & $2.85 \mathrm{E}+00$ & $y$ & $9.00 \mathrm{E}+07$ & $5.31 \mathrm{E}+02$ & ICRP-72 & $4.00 \mathrm{E}-05$ & $\mathrm{~F}$ & FGR-12 & $6.35 \mathrm{E}-18$ \\
\hline Pu-237 & Pu-237 & 237.048 & Plutonium & $1.00 \mathrm{E}-03$ & ICRP-38 & $4.53 \mathrm{E}+01$ & $d$ & $3.91 \mathrm{E}+06$ & $1.22 \mathrm{E}+04$ & ICRP-72 & $3.90 \mathrm{E}-10$ & $\mathrm{~s}$ & FGR-12 & $2.02 \mathrm{E}-15$ \\
\hline Pu-238 & Pu-238 & 238.050 & Plutonium & $1.00 \mathrm{E}-03$ & ICRP-38 & $8.77 \mathrm{E}+01$ & $y$ & $2.77 \mathrm{E}+09$ & $1.71 \mathrm{E}+01$ & ICRP-72 & $1.10 \mathrm{E}-04$ & $F$ & FGR-12 & $4.88 \mathrm{E}-18$ \\
\hline Pu-239 & Pu-239 & 239.052 & $\begin{array}{l}\text { Plutonium } \\
\end{array}$ & $1.00 \mathrm{E}-03$ & ICRP-38 & $2.41 E+04$ & $y$ & $7.59 \mathrm{E}+11$ & $6.21 \mathrm{E}-02$ & ICRP-72 & $1.20 \mathrm{E}-04$ & $\mathrm{~F}$ & FGR-12 & $4.24 \mathrm{E}-18$ \\
\hline Pu-240 & Pu-240 & 240.054 & Plutonium & $1.00 \mathrm{E}-03$ & ICRP-38 & $6.54 \mathrm{E}+03$ & $y$ & $2.06 \mathrm{E}+11$ & $2.28 \mathrm{E}-01$ & ICRP-72 & $1.20 \mathrm{E}-04$ & $\mathrm{~F}$ & FGR-12 & $4.75 \mathrm{E}-18$ \\
\hline Pu-241 & Pu-241 & 241.057 & Plutonium & $1.00 \mathrm{E}-03$ & ICRP-38 & $1.44 \mathrm{E}+01$ & $y$ & $4.54 \mathrm{E}+08$ & $1.03 \mathrm{E}+02$ & ICRP-72 & $2.30 \mathrm{E}-06$ & $F$ & FGR-12 & $7.25 \mathrm{E}-20$ \\
\hline Pu-242 & Pu-242 & 242.059 & Plutonium & $1.00 \mathrm{E}-03$ & ICRP-38 & $3.76 \mathrm{E}+05$ & $y$ & $1.19 \mathrm{E}+13$ & $3.92 \mathrm{E}-03$ & ICRP-72 & $1.10 \mathrm{E}-04$ & $\mathrm{~F}$ & FGR-12 & $4.01 \mathrm{E}-18$ \\
\hline Pu-243 & Pu-243 & 243.062 & Plutonium & $1.00 \mathrm{E}-03$ & ICRP-38 & $4.96 \mathrm{E}+00$ & $\mathrm{~h}$ & $1.78 \mathrm{E}+04$ & $2.60 E+06$ & ICRP-72 & $8.60 \mathrm{E}-11$ & $\mathrm{~s}$ & FGR-12 & $1.03 \mathrm{E}-15$ \\
\hline Pu-244 & Pu-244 & 244.064 & Plutonium & $1.00 \mathrm{E}-03$ & ICRP-38 & $8.26 \mathrm{E}+07$ & $y$ & $2.61 \mathrm{E}+15$ & $1.77 \mathrm{E}-05$ & ICRP-72 & $1.10 \mathrm{E}-04$ & $F$ & FGR-12 & $2.97 \mathrm{E}-18$ \\
\hline Pu-245 & Pu-245 & 245.068 & Plutonium & $1.00 \mathrm{E}-03$ & ICRP-38 & $1.05 \mathrm{E}+01$ & $\mathrm{~h}$ & $3.78 \mathrm{E}+04$ & $1.22 \mathrm{E}+06$ & ICRP-72 & $4.30 \mathrm{E}-10$ & $\mathrm{~s}$ & FGR-12 & $1.99 \mathrm{E}-14$ \\
\hline Pu-246 & Pu-246 & 246.070 & Plutonium & $1.00 \mathrm{E}-03$ & ICRP-38 & $1.09 \mathrm{E}+01$ & $d$ & $9.37 \mathrm{E}+05$ & $4.89 \mathrm{E}+04$ & ICRP-72 & $8.00 \mathrm{E}-09$ & $\mathrm{~s}$ & FGR-12 & $6.01 \mathrm{E}-15$ \\
\hline Ra-219 & Ra-219 & 219.010 & Radium & $1.00 \mathrm{E}-03$ & ICRP-107 & $1.00 \mathrm{E}+01$ & $\mathrm{~ms}$ & $1.00 \mathrm{E}-02$ & $5.15 \mathrm{E}+12$ & -- & -- & -- & DOE-STD-1196 & $7.37 \mathrm{E}-15$ \\
\hline Ra-220 & Ra-220 & 220.011 & Radium & $1.00 \mathrm{E}-03$ & ICRP-107 & $1.79 \mathrm{E}-02$ & $\mathrm{~s}$ & $1.79 \mathrm{E}-02$ & $2.86 \mathrm{E}+12$ & -- & -- & -- & DOE-STD-1196 & $2.07 E-16$ \\
\hline Ra-221 & Ra-221 & 221.014 & Radium & $1.00 \mathrm{E}-03$ & ICRP-107 & $2.80 \mathrm{E}+01$ & $\mathrm{~s}$ & $2.80 \mathrm{E}+01$ & $1.82 E+09$ & -- & -- & -- & DOE-STD-1196 & $1.47 \mathrm{E}-15$ \\
\hline Ra-222 & Ra-222 & 222.015 & Radium & $1.00 \mathrm{E}-03$ & ICRP-38 & $3.80 \mathrm{E}+01$ & $\mathrm{~s}$ & $3.80 \mathrm{E}+01$ & $1.34 \mathrm{E}+09$ & --- & -- & -- & FGR-12 & $4.39 \mathrm{E}-16$ \\
\hline Ra-223 & Ra-223 & 223.019 & Radium & $1.00 \mathrm{E}-03$ & ICRP-38 & $1.14 \mathrm{E}+01$ & $d$ & $9.88 \mathrm{E}+05$ & $5.12 \mathrm{E}+04$ & ICRP-72 & $8.70 \mathrm{E}-06$ & $\mathrm{~s}$ & FGR-12 & $6.09 \mathrm{E}-15$ \\
\hline Ra-224 & Ra-224 & 224.020 & Radium & $1.00 \mathrm{E}-03$ & ICRP-38 & $3.66 \mathrm{E}+00$ & $d$ & $3.16 \mathrm{E}+05$ & $1.59 \mathrm{E}+05$ & ICRP-72 & $3.40 \mathrm{E}-06$ & $\mathrm{~s}$ & FGR-12 & $4.71 \mathrm{E}-16$ \\
\hline Ra-225 & Ra-225 & 225.024 & Radium & $1.00 \mathrm{E}-03$ & ICRP-38 & $1.48 \mathrm{E}+01$ & $d$ & $1.28 \mathrm{E}+06$ & $3.92 \mathrm{E}+04$ & ICRP-72 & $7.70 \mathrm{E}-06$ & $\mathrm{~S}$ & FGR-12 & $2.79 \mathrm{E}-16$ \\
\hline Ra-226 & Ra-226 & 226.025 & Radium & $1.00 \mathrm{E}-03$ & ICRP-38 & $1.60 \mathrm{E}+03$ & $\mathrm{y}$ & $5.05 \mathrm{E}+10$ & $9.89 \mathrm{E}-01$ & ICRP-72 & $9.50 \mathrm{E}-06$ & $\mathrm{~s}$ & FGR-12 & $3.15 \mathrm{E}-16$ \\
\hline Ra-227 & Ra-227 & 227.029 & Radium & $1.00 \mathrm{E}-03$ & ICRP-38 & $4.22 \mathrm{E}+01$ & $\mathrm{~m}$ & $2.53 \mathrm{E}+03$ & $1.96 \mathrm{E}+07$ & ICRP-72 & $4.60 \mathrm{E}-10$ & $\mathrm{~F}$ & FGR-12 & $7.41 \mathrm{E}-15$ \\
\hline Ra-228 & Ra-228 & 228.031 & Radium & $1.00 \mathrm{E}-03$ & ICRP-38 & $5.75 \mathrm{E}+00$ & $y$ & $1.81 \mathrm{E}+08$ & $2.73 \mathrm{E}+02$ & ICRP-72 & $1.60 \mathrm{E}-05$ & $\mathrm{~s}$ & DOE-STD-1196 & $2.89 \mathrm{E}-18$ \\
\hline Ra-230 & Ra-230 & 230.037 & Radium & $1.00 \mathrm{E}-03$ & ICRP-107 & $9.30 \mathrm{E}+01$ & $\mathrm{~m}$ & $5.58 \mathrm{E}+03$ & $8.79 \mathrm{E}+06$ & DOE-STD-1196 & $1.35 \mathrm{E}-10$ & $\mathrm{~s}$ & DOE-STD-1196 & $3.33 \mathrm{E}-15$ \\
\hline Rb-77 & Rb-77 & 76.930 & Rubidium & $1.00 \mathrm{E}-03$ & ICRP-38 & $3.70 \mathrm{E}+00$ & $\mathrm{~m}$ & $2.22 \mathrm{E}+02$ & $6.61 \mathrm{E}+08$ & -- & -- & -- & DOE-STD-1196 & $7.09 \mathrm{E}-14$ \\
\hline Rb-78 & Rb-78 & 77.928 & Rubidium & $1.00 \mathrm{E}-03$ & ICRP-107 & $1.77 \mathrm{E}+01$ & $\mathrm{~m}$ & $1.06 \mathrm{E}+03$ & $1.37 \mathrm{E}+08$ & DOE-STD-1196 & $3.55 \mathrm{E}-11$ & $\mathrm{~s}$ & DOE-STD-1196 & $2.04 \mathrm{E}-13$ \\
\hline Rb-78m & $\mathrm{Rb}-78$ & 77.928 & Rubidium & $1.00 \mathrm{E}-03$ & ICRP-107 & $5.74 \mathrm{E}+00$ & $\mathrm{~m}$ & $3.44 \mathrm{E}+02$ & $4.20 \mathrm{E}+08$ & -- & -- & -- & DOE-STD-1196 & $1.52 \mathrm{E}-13$ \\
\hline $\mathrm{Rb}-79$ & Rb-79 & 78.924 & Rubidium & $1.00 \mathrm{E}-03$ & ICRP-38 & $2.29 \mathrm{E}+01$ & $\mathrm{~m}$ & $1.37 \mathrm{E}+03$ & $1.04 \mathrm{E}+08$ & ICRP-72 & $1.60 \mathrm{E}-11$ & $F$ & FGR-12 & $6.51 \mathrm{E}-14$ \\
\hline $\mathrm{Rb}-80$ & $\mathrm{Rb}-80$ & 79.923 & Rubidium & $1.00 \mathrm{E}-03$ & ICRP-38 & $3.40 \mathrm{E}+01$ & $\mathrm{~s}$ & $3.40 \mathrm{E}+01$ & $4.15 \mathrm{E}+09$ & -- & -- & -- & FGR-12 & $6.07 \mathrm{E}-14$ \\
\hline $\mathrm{Rb}-81$ & Rb-81 & 80.919 & Rubidium & $1.00 \mathrm{E}-03$ & ICRP-38 & $4.58 \mathrm{E}+00$ & $\mathrm{~h}$ & $1.65 E+04$ & $8.46 \mathrm{E}+06$ & ICRP-72 & $3.40 \mathrm{E}-11$ & $\mathrm{~F}$ & FGR-12 & $2.96 \mathrm{E}-14$ \\
\hline $\mathrm{Rb}-81 \mathrm{~m}$ & $\mathrm{Rb}-81$ & 80.919 & Rubidium & $1.00 \mathrm{E}-03$ & ICRP-38 & $3.20 \mathrm{E}+01$ & $\mathrm{~m}$ & $1.92 \mathrm{E}+03$ & $7.26 \mathrm{E}+07$ & ICRP-72 & $7.00 \mathrm{E}-12$ & $\mathrm{~F}$ & FGR-12 & $1.88 \mathrm{E}-16$ \\
\hline $\mathrm{Rb}-82$ & Rb-82 & 81.918 & Rubidium & $1.00 \mathrm{E}-03$ & ICRP-38 & $1.30 \mathrm{E}+00$ & $\mathrm{~m}$ & $7.80 \mathrm{E}+01$ & $1.77 E+09$ & -- & -- & -- & FGR-12 & $5.30 \mathrm{E}-14$ \\
\hline $\mathrm{Rb}-82 \mathrm{~m}$ & Rb-82 & 81.918 & Rubidium & $1.00 \mathrm{E}-03$ & ICRP-38 & $6.20 \mathrm{E}+00$ & $\mathrm{~h}$ & $2.23 E+04$ & $6.17 \mathrm{E}+06$ & ICRP-72 & $1.10 \mathrm{E}-10$ & $F$ & FGR-12 & $1.43 \mathrm{E}-13$ \\
\hline $\mathrm{Rb}-83$ & $\mathrm{Rb}-83$ & 82.915 & Rubidium & $1.00 \mathrm{E}-03$ & ICRP-38 & $8.62 \mathrm{E}+01$ & d & $7.45 \mathrm{E}+06$ & $1.83 \mathrm{E}+04$ & ICRP-72 & $6.90 \mathrm{E}-10$ & $\mathrm{~F}$ & FGR-12 & $2.39 \mathrm{E}-14$ \\
\hline $\mathrm{Rb}-84$ & Rb-84 & 83.914 & Rubidium & $1.00 \mathrm{E}-03$ & ICRP-38 & $3.28 \mathrm{E}+01$ & $d$ & $2.83 E+06$ & $4.75 E+04$ & ICRP-72 & $1.00 \mathrm{E}-09$ & $\mathrm{~F}$ & FGR-12 & 4.47E-14 \\
\hline $\mathrm{Rb}-84 \mathrm{~m}$ & $\mathrm{Rb}-84$ & 83.914 & Rubidium & $1.00 \mathrm{E}-03$ & ICRP-107 & $2.03 \mathrm{E}+01$ & $\mathrm{~m}$ & $1.22 \mathrm{E}+03$ & $1.11 \mathrm{E}+08$ & DOE-STD-1196 & $1.01 \mathrm{E}-11$ & $\mathrm{~s}$ & DOE-STD-1196 & $1.68 \mathrm{E}-14$ \\
\hline $\mathrm{Rb}-86$ & $\mathrm{Rb}-86$ & 85.911 & Rubidium & $1.00 \mathrm{E}-03$ & $\begin{array}{l}\text { ICRP-38 } \\
\end{array}$ & $1.87 \mathrm{E}+01$ & $d$ & $1.61 \mathrm{E}+06$ & $8.14 \mathrm{E}+04$ & ICRP-72 & $9.30 \mathrm{E}-10$ & $\mathrm{~F}$ & $\begin{array}{l}\text { FGR-12 } \\
\end{array}$ & $4.81 \mathrm{E}-15$ \\
\hline $\mathrm{Rb}-86 \mathrm{~m}$ & Rb-86 & 85.911 & Rubidium & $1.00 \mathrm{E}-03$ & ICRP-107 & $1.02 \mathrm{E}+00$ & $\mathrm{~m}$ & $6.10 \mathrm{E}+01$ & $2.15 \mathrm{E}+09$ & -- & -- & -- & DOE-STD-1196 & $2.44 \mathrm{E}-14$ \\
\hline $\mathrm{Rb}-87$ & Rb-87 & 86.909 & Rubidium & $1.00 \mathrm{E}-03$ & ICRP-38 & $4.70 \mathrm{E}+10$ & $y$ & $1.48 \mathrm{E}+18$ & $8.75 \mathrm{E}-08$ & ICRP-72 & $5.00 \mathrm{E}-10$ & $\mathrm{~F}$ & FGR-12 & $1.82 \mathrm{E}-18$ \\
\hline $\mathrm{Rb}-88$ & Rb-88 & 87.911 & Rubidium & $1.00 \mathrm{E}-03$ & ICRP-38 & $1.78 \mathrm{E}+01$ & $\mathrm{~m}$ & $1.07 \mathrm{E}+03$ & $1.20 \mathrm{E}+08$ & ICRP-72 & $1.60 \mathrm{E}-11$ & $F$ & FGR-12 & $3.36 \mathrm{E}-14$ \\
\hline $\mathrm{Rb}-89$ & $\mathrm{Rb}-89$ & 88.912 & Rubidium & $1.00 \mathrm{E}-03$ & ICRP-38 & $1.52 \mathrm{E}+01$ & $\mathrm{~m}$ & $9.12 \mathrm{E}+02$ & $1.39 \mathrm{E}+08$ & ICRP-72 & $1.40 \mathrm{E}-11$ & $\mathrm{~F}$ & FGR-12 & $1.06 \mathrm{E}-13$ \\
\hline Rb-90 & Rb-90 & 89.915 & Rubidium & $1.00 \mathrm{E}-03$ & ICRP-107 & $1.58 \mathrm{E}+02$ & $\mathrm{~s}$ & $1.58 \mathrm{E}+02$ & $7.94 \mathrm{E}+08$ & -- & -- & -- & DOE-STD-1196 & $1.08 \mathrm{E}-13$ \\
\hline Rb-90m & Rb-90 & 89.915 & Rubidium & $1.00 \mathrm{E}-03$ & ICRP-107 & $2.58 \mathrm{E}+02$ & $s$ & $2.58 \mathrm{E}+02$ & $4.86 \mathrm{E}+08$ & -- & -- & -- & DOE-STD-1196 & $1.63 \mathrm{E}-13$ \\
\hline Re-177 & Re-177 & 176.950 & Rhenium & $1.00 \mathrm{E}-03$ & ICRP-38 & $1.40 \mathrm{E}+01$ & $\mathrm{~m}$ & $8.40 \mathrm{E}+02$ & $7.59 \mathrm{E}+07$ & ICRP-72 & $1.40 \mathrm{E}-11$ & $M$ & FGR-12 & $2.96 \mathrm{E}-14$ \\
\hline Re-178 & Re-178 & 177.951 & Rhenium & $1.00 \mathrm{E}-03$ & ICRP-38 & $1.32 \mathrm{E}+01$ & $\mathrm{~m}$ & $7.92 \mathrm{E}+02$ & $8.00 \mathrm{E}+07$ & ICRP-72 & $1.40 \mathrm{E}-11$ & $M$ & FGR-12 & $6.09 \mathrm{E}-14$ \\
\hline Re-179 & Re-179 & 178.950 & Rhenium & $1.00 \mathrm{E}-03$ & ICRP-107 & $1.95 \mathrm{E}+01$ & $\mathrm{~m}$ & $1.17 \mathrm{E}+03$ & $5.39 \mathrm{E}+07$ & DOE-STD-1196 & $1.32 \mathrm{E}-11$ & $\mathrm{~s}$ & DOE-STD-1196 & $4.90 \mathrm{E}-14$ \\
\hline
\end{tabular}




\begin{tabular}{|c|c|c|c|c|c|c|c|c|c|c|c|c|c|c|}
\hline \multicolumn{15}{|c|}{ Table A.1 Radionuclide Specific Input Data Used For the Calculation of HC-2 TQs Using Maximum Reported DCs (continued) } \\
\hline $\begin{array}{c}\text { MASTER } \\
\text { ISOTOPE } \\
\text { LIST }\end{array}$ & $\begin{array}{l}\text { Atomic } \\
\text { Mass } \\
\text { Nuclide }\end{array}$ & $\begin{array}{l}\text { Atomic } \\
\text { Mass }\end{array}$ & Element & $\begin{array}{c}\text { RF per } \\
\text { DOE-STD- } \\
1027\end{array}$ & $\begin{array}{c}\text { Half-Life } \\
\text { Reference }\end{array}$ & $\begin{array}{r}\text { Half-Li } \\
\text { (original }\end{array}$ & & Half Life (s) & $\begin{array}{l}\text { Sp. Act } \\
\text { (Ci/g) }\end{array}$ & $\begin{array}{l}\text { Inhalation DC } \\
\text { Reference }\end{array}$ & $\begin{array}{c}\text { HC-2 } \\
\text { Bounding } \\
\text { Inhalation } \\
\text { DC (Sv/Bq) }\end{array}$ & $\begin{array}{l}\text { Lung } \\
\text { Absorption } \\
\text { Class/Type }\end{array}$ & $\begin{array}{l}\text { Immersion DC } \\
\text { Reference }\end{array}$ & $\begin{array}{c}\mathrm{HC}-2 \\
\text { Immersion } \\
\mathrm{DC}(\mathrm{Sv} / \mathrm{s} \\
\left.\text { per } \mathrm{Bq} / \mathrm{m}^{3}\right)\end{array}$ \\
\hline Re-180 & Re-180 & 179.951 & Rhenium & $1.00 \mathrm{E}-03$ & ICRP-38 & $2.43 \mathrm{E}+00$ & $\mathrm{~m}$ & $1.46 \mathrm{E}+02$ & $4.30 \mathrm{E}+08$ & --- & --- & --- & FGR-12 & $5.72 \mathrm{E}-14$ \\
\hline Re-181 & Re-181 & 180.950 & Rhenium & $1.00 \mathrm{E}-03$ & ICRP-38 & $2.00 \mathrm{E}+01$ & $\mathrm{~h}$ & $7.20 \mathrm{E}+04$ & $8.66 \mathrm{E}+05$ & ICRP-72 & $2.50 \mathrm{E}-10$ & $M$ & FGR-12 & $3.65 E-14$ \\
\hline Re-182I & Re-182 & 181.951 & Rhenium & $1.00 \mathrm{E}-03$ & ICRP-38 & $6.40 \mathrm{E}+01$ & $\mathrm{~h}$ & $2.30 \mathrm{E}+05$ & $2.69 \mathrm{E}+05$ & ICRP-72 & $1.20 \mathrm{E}-09$ & $M$ & FGR-12 & $9.16 \mathrm{E}-14$ \\
\hline Re-182s & Re-182 & 181.951 & Rhenium & $1.00 \mathrm{E}-03$ & ICRP-38 & $1.27 \mathrm{E}+01$ & $\mathrm{~h}$ & $4.57 \mathrm{E}+04$ & $1.36 \mathrm{E}+06$ & ICRP-72 & $2.00 \mathrm{E}-10$ & $M$ & FGR-12 & $5.78 \mathrm{E}-14$ \\
\hline Re-183 & Re-183 & 182.951 & Rhenium & $1.00 \mathrm{E}-03$ & ICRP-107 & $7.00 \mathrm{E}+01$ & $d$ & $6.05 E+06$ & $1.02 \mathrm{E}+04$ & DOE-STD-1196 & $3.94 \mathrm{E}-09$ & $\mathrm{~s}$ & DOE-STD-1196 & $5.53 \mathrm{E}-15$ \\
\hline Re-184 & Re-184 & 183.953 & Rhenium & $1.00 \mathrm{E}-03$ & ICRP-38 & $3.80 \mathrm{E}+01$ & $d$ & $3.28 \mathrm{E}+06$ & $1.87 \mathrm{E}+04$ & ICRP-72 & $1.90 \mathrm{E}-09$ & $M$ & FGR-12 & $4.29 \mathrm{E}-14$ \\
\hline Re-184m & Re-184 & 183.953 & Rhenium & $1.00 \mathrm{E}-03$ & ICRP-38 & $1.65 E+02$ & $d$ & $1.43 \mathrm{E}+07$ & $4.30 \mathrm{E}+03$ & ICRP-72 & $6.50 \mathrm{E}-09$ & M & FGR-12 & $1.82 \mathrm{E}-14$ \\
\hline Re-186 & Re-186 & 185.955 & Rhenium & $1.00 \mathrm{E}-03$ & ICRP-38 & $9.06 \mathrm{E}+01$ & $\mathrm{~h}$ & $3.26 \mathrm{E}+05$ & $1.86 \mathrm{E}+05$ & ICRP-72 & $1.10 \mathrm{E}-09$ & $M$ & FGR-12 & $9.19 \mathrm{E}-16$ \\
\hline Re-186m & Re-186 & 185.955 & Rhenium & $1.00 \mathrm{E}-03$ & ICRP-38 & $2.00 \mathrm{E}+05$ & $y$ & $6.31 \mathrm{E}+12$ & $9.61 \mathrm{E}-03$ & ICRP-72 & $1.20 \mathrm{E}-08$ & $M$ & FGR-12 & $5.00 \mathrm{E}-16$ \\
\hline Re-187 & Re-187 & 186.956 & Rhenium & $1.00 \mathrm{E}-03$ & ICRP-38 & $5.00 \mathrm{E}+10$ & $y$ & $1.58 \mathrm{E}+18$ & $3.82 \mathrm{E}-08$ & ICRP-72 & $6.30 \mathrm{E}-12$ & $M$ & -- & -- \\
\hline Re-188 & Re-188 & 187.958 & Rhenium & $1.00 \mathrm{E}-03$ & ICRP-38 & $1.70 \mathrm{E}+01$ & $\mathrm{~h}$ & $6.11 \mathrm{E}+04$ & $9.82 E+05$ & ICRP-72 & $5.40 \mathrm{E}-10$ & $M$ & FGR-12 & $2.87 \mathrm{E}-15$ \\
\hline Re-188m & Re-188 & 187.958 & Rhenium & $1.00 \mathrm{E}-03$ & ICRP-38 & $1.86 \mathrm{E}+01$ & $\mathrm{~m}$ & $1.12 \mathrm{E}+03$ & $5.38 \mathrm{E}+07$ & ICRP-72 & $1.30 \mathrm{E}-11$ & $M$ & FGR-12 & $3.02 \mathrm{E}-15$ \\
\hline Re-189 & Re-189 & 188.959 & Rhenium & $1.00 \mathrm{E}-03$ & ICRP-38 & $2.43 \mathrm{E}+01$ & $\mathrm{~h}$ & $8.75 \mathrm{E}+04$ & $6.82 \mathrm{E}+05$ & ICRP-72 & $4.30 \mathrm{E}-10$ & $M$ & $\begin{array}{ll}\text { FGR-12 } \\
\end{array}$ & $3.22 \mathrm{E}-15$ \\
\hline Re-190 & Re-190 & 189.962 & Rhenium & $1.00 \mathrm{E}-03$ & ICRP-107 & $3.10 \mathrm{E}+00$ & $\mathrm{~m}$ & $1.86 \mathrm{E}+02$ & $3.19 \mathrm{E}+08$ & -- & -- & -- & DOE-STD-1196 & $6.04 \mathrm{E}-14$ \\
\hline Re-190m & Re-190 & 189.962 & Rhenium & $1.00 \mathrm{E}-03$ & ICRP-107 & $3.20 \mathrm{E}+00$ & $\mathrm{~h}$ & $1.15 \mathrm{E}+04$ & $5.16 \mathrm{E}+06$ & DOE-STD-1196 & $2.44 \mathrm{E}-10$ & $\mathrm{~s}$ & DOE-STD-1196 & $4.13 \mathrm{E}-14$ \\
\hline Rh-100 & Rh-100 & 99.908 & Rhodium & $1.00 \mathrm{E}-03$ & ICRP-38 & $2.08 \mathrm{E}+01$ & $\mathrm{~h}$ & $7.49 \mathrm{E}+04$ & $1.51 E+06$ & ICRP-72 & $3.50 \mathrm{E}-10$ & $\mathrm{~s}$ & FGR-12 & $1.41 \mathrm{E}-13$ \\
\hline Rh-100m & Rh-100 & 99.908 & Rhodium & $1.00 \mathrm{E}-03$ & ICRP-107 & $4.60 E+00$ & $\mathrm{~m}$ & $2.76 \mathrm{E}+02$ & $4.09 E+08$ & -- & -- & -- & DOE-STD-1196 & $2.03 \mathrm{E}-15$ \\
\hline Rh-101 & Rh-101 & 100.906 & Rhodium & $1.00 \mathrm{E}-03$ & ICRP-38 & $3.20 \mathrm{E}+00$ & $y$ & $1.01 \mathrm{E}+08$ & $1.11 \mathrm{E}+03$ & ICRP-72 & $5.40 \mathrm{E}-09$ & $\mathrm{~s}$ & FGR-12 & $1.21 \mathrm{E}-14$ \\
\hline Rh-101m & Rh-101 & 100.906 & Rhodium & $1.00 \mathrm{E}-03$ & ICRP-38 & $4.34 \mathrm{E}+00$ & $d$ & $3.75 E+05$ & $2.98 \mathrm{E}+05$ & ICRP-72 & $2.10 \mathrm{E}-10$ & $\mathrm{~s}$ & FGR-12 & $1.41 \mathrm{E}-14$ \\
\hline Rh-102 & Rh-102 & 101.907 & Rhodium & $1.00 \mathrm{E}-03$ & ICRP-38 & $2.90 \mathrm{E}+00$ & $y$ & $9.15 E+07$ & $1.21 \mathrm{E}+03$ & ICRP-72 & $1.70 \mathrm{E}-08$ & $\mathrm{~s}$ & FGR-12 & $1.04 \mathrm{E}-13$ \\
\hline Rh-102m & Rh-102 & 101.907 & Rhodium & $1.00 \mathrm{E}-03$ & ICRP-38 & $2.07 \mathrm{E}+02$ & $d$ & $1.79 \mathrm{E}+07$ & $6.19 \mathrm{E}+03$ & ICRP-72 & $7.10 \mathrm{E}-09$ & $\mathrm{~s}$ & FGR-12 & $2.31 \mathrm{E}-14$ \\
\hline Rh-103m & Rh-103 & 102.905 & Rhodium & $1.00 \mathrm{E}-03$ & ICRP-38 & $5.61 \mathrm{E}+01$ & $\mathrm{~m}$ & $3.37 \mathrm{E}+03$ & $3.26 \mathrm{E}+07$ & ICRP-72 & $2.70 \mathrm{E}-12$ & $\mathrm{~s}$ & FGR-12 & $8.80 \mathrm{E}-18$ \\
\hline Rh-104 & Rh-104 & 103.907 & Rhodium & $1.00 \mathrm{E}-03$ & ICRP-107 & $4.23 \mathrm{E}+01$ & $\mathrm{~s}$ & $4.23 \mathrm{E}+01$ & $2.57 \mathrm{E}+09$ & -- & -- & -- & DOE-STD-1196 & $1.40 \mathrm{E}-15$ \\
\hline Rh-104m & Rh-104 & 103.907 & Rhodium & $1.00 \mathrm{E}-03$ & ICRP-107 & $4.34 \mathrm{E}+00$ & $\mathrm{~m}$ & $2.60 \mathrm{E}+02$ & $4.17 \mathrm{E}+08$ & -- & -- & -- & DOE-STD-1196 & $9.14 \mathrm{E}-16$ \\
\hline Rh-105 & Rh-105 & 104.906 & Rhodium & $1.00 \mathrm{E}-03$ & ICRP-38 & $3.54 \mathrm{E}+01$ & $\mathrm{~h}$ & $1.27 \mathrm{E}+05$ & $8.45 E+05$ & ICRP-72 & $3.50 \mathrm{E}-10$ & $\mathrm{~s}$ & FGR-12 & $3.72 \mathrm{E}-15$ \\
\hline Rh-106 & Rh-106 & 105.907 & Rhodium & $1.00 \mathrm{E}-03$ & ICRP-38 & $2.99 \mathrm{E}+01$ & $\mathrm{~s}$ & $2.99 \mathrm{E}+01$ & $3.56 \mathrm{E}+09$ & -- & -- & -- & FGR-12 & $1.04 \mathrm{E}-14$ \\
\hline Rh-106m & Rh-106 & 105.907 & Rhodium & $1.00 \mathrm{E}-03$ & ICRP-38 & $1.32 \mathrm{E}+02$ & $\mathrm{~m}$ & $7.92 \mathrm{E}+03$ & $1.34 \mathrm{E}+07$ & ICRP-72 & $1.10 \mathrm{E}-10$ & $M$ & FGR-12 & $1.44 \mathrm{E}-13$ \\
\hline Rh-107 & Rh-107 & 106.907 & Rhodium & $1.00 \mathrm{E}-03$ & ICRP-38 & $2.17 \mathrm{E}+01$ & $\mathrm{~m}$ & $1.30 \mathrm{E}+03$ & $8.10 \mathrm{E}+07$ & ICRP-72 & $1.70 \mathrm{E}-11$ & $\mathrm{~s}$ & FGR-12 & $1.50 \mathrm{E}-14$ \\
\hline Rh-108 & Rh-108 & 107.909 & Rhodium & $1.00 \mathrm{E}-03$ & ICRP-107 & $1.68 \mathrm{E}+01$ & $\mathrm{~s}$ & $1.68 \mathrm{E}+01$ & $6.22 \mathrm{E}+09$ & -- & -- & -- & DOE-STD-1196 & $1.61 \mathrm{E}-14$ \\
\hline Rh-109 & Rh-109 & 108.909 & Rhodium & $1.00 \mathrm{E}-03$ & ICRP-107 & $8.00 \mathrm{E}+01$ & $\mathrm{~s}$ & $8.00 \mathrm{E}+01$ & $1.29 \mathrm{E}+09$ & -- & -- & -- & DOE-STD-1196 & $1.38 \mathrm{E}-14$ \\
\hline Rh-94 & Rh-94 & 93.922 & Rhodium & $1.00 \mathrm{E}-03$ & ICRP-107 & $7.06 \mathrm{E}+01$ & $\mathrm{~s}$ & $7.06 \mathrm{E}+01$ & $1.70 E+09$ & -- & -- & -- & DOE-STD-1196 & $1.81 \mathrm{E}-13$ \\
\hline Rh-95 & Rh-95 & 94.916 & Rhodium & $1.00 \mathrm{E}-03$ & ICRP-107 & $5.02 \mathrm{E}+00$ & $\mathrm{~m}$ & $3.01 \mathrm{E}+02$ & $3.95 E+08$ & -- & -- & -- & DOE-STD-1196 & $1.21 \mathrm{E}-13$ \\
\hline Rh-95m & Rh-95 & 94.916 & Rhodium & $1.00 \mathrm{E}-03$ & ICRP-107 & $1.96 \mathrm{E}+00$ & $\mathrm{~m}$ & $1.18 \mathrm{E}+02$ & $1.01 \mathrm{E}+09$ & -- & -- & -- & DOE-STD-1196 & $4.29 \mathrm{E}-14$ \\
\hline Rh-96 & Rh-96 & 95.914 & Rhodium & $1.00 \mathrm{E}-03$ & ICRP-107 & $9.90 \mathrm{E}+00$ & $\mathrm{~m}$ & $5.94 \mathrm{E}+02$ & $1.98 \mathrm{E}+08$ & -- & -- & -- & DOE-STD-1196 & $1.81 \mathrm{E}-13$ \\
\hline Rh-96m & Rh-96 & 95.914 & Rhodium & $1.00 \mathrm{E}-03$ & ICRP-107 & $1.51 E+00$ & $\mathrm{~m}$ & $9.06 \mathrm{E}+01$ & $1.30 \mathrm{E}+09$ & -- & -- & -- & DOE-STD-1196 & $6.03 \mathrm{E}-14$ \\
\hline Rh-97 & Rh-97 & 96.911 & Rhodium & $1.00 \mathrm{E}-03$ & ICRP-107 & $3.07 \mathrm{E}+01$ & $\mathrm{~m}$ & $1.84 \mathrm{E}+03$ & $6.32 \mathrm{E}+07$ & DOE-STD-1196 & $2.84 \mathrm{E}-11$ & $\mathrm{~s}$ & DOE-STD-1196 & $6.60 \mathrm{E}-14$ \\
\hline Rh-97m & Rh-97 & 96.911 & Rhodium & $1.00 \mathrm{E}-03$ & ICRP-107 & $4.62 \mathrm{E}+01$ & $\mathrm{~m}$ & $2.77 \mathrm{E}+03$ & $4.20 \mathrm{E}+07$ & DOE-STD-1196 & $3.30 \mathrm{E}-11$ & $\mathrm{~s}$ & DOE-STD-1196 & $1.06 \mathrm{E}-13$ \\
\hline Rh-98 & Rh-98 & 97.911 & Rhodium & $1.00 \mathrm{E}-03$ & ICRP-107 & $8.70 \mathrm{E}+00$ & $\mathrm{~m}$ & $5.22 \mathrm{E}+02$ & $2.21 \mathrm{E}+08$ & -- & -- & -- & DOE-STD-1196 & $8.34 \mathrm{E}-14$ \\
\hline Rh-99 & Rh-99 & 98.908 & Rhodium & $1.00 \mathrm{E}-03$ & ICRP-38 & $1.60 \mathrm{E}+01$ & $d$ & $1.38 \mathrm{E}+06$ & $8.25 E+04$ & ICRP-72 & $8.70 \mathrm{E}-10$ & $\mathrm{~s}$ & FGR-12 & $2.85 \mathrm{E}-14$ \\
\hline Rh-99m & Rh-99 & 98.908 & Rhodium & $1.00 \mathrm{E}-03$ & ICRP-38 & $4.70 E+00$ & $\mathrm{~h}$ & $1.69 \mathrm{E}+04$ & $6.74 \mathrm{E}+06$ & ICRP-72 & $4.00 \mathrm{E}-11$ & $\mathrm{~s}$ & FGR-12 & $3.29 \mathrm{E}-14$ \\
\hline $\mathrm{Rn}-207$ & Rn-207 & 206.991 & Radon & $1.00 \mathrm{E}+00$ & ICRP-107 & $9.25 \mathrm{E}+00$ & $\mathrm{~m}$ & $5.55 \mathrm{E}+02$ & $9.82 \mathrm{E}+07$ & -- & -- & -- & DOE-STD-1196 & $4.41 \mathrm{E}-14$ \\
\hline Rn-209 & Rn-209 & 208.990 & Radon & $1.00 \mathrm{E}+00$ & ICRP-107 & $2.85 E+01$ & $\mathrm{~m}$ & $1.71 \mathrm{E}+03$ & $3.16 \mathrm{E}+07$ & -- & -- & -- & DOE-STD-1196 & $5.47 \mathrm{E}-14$ \\
\hline $\mathrm{Rn}-210$ & $\mathrm{Rn}-210$ & 209.990 & Radon & $1.00 E+00$ & ICRP-107 & $2.40 E+00$ & $\mathrm{~h}$ & $8.64 \mathrm{E}+03$ & $6.22 \mathrm{E}+06$ & -- & -- & -- & DOE-STD-1196 & $1.27 \mathrm{E}-14$ \\
\hline Rn-211 & Rn-211 & 210.991 & Radon & $1.00 \mathrm{E}+00$ & ICRP-107 & $1.46 \mathrm{E}+01$ & $\mathrm{~h}$ & $5.26 \mathrm{E}+04$ & $1.02 E+06$ & -- & -- & -- & DOE-STD-1196 & $8.62 \mathrm{E}-14$ \\
\hline Rn-212 & Rn-212 & 211.991 & Radon & $1.00 E+00$ & ICRP-107 & $2.39 \mathrm{E}+01$ & $\mathrm{~m}$ & $1.43 \mathrm{E}+03$ & $3.71 \mathrm{E}+07$ & -- & -- & -- & DOE-STD-1196 & $1.38 \mathrm{E}-14$ \\
\hline Rn-215 & Rn-215 & 214.999 & Radon & $1.00 E+00$ & ICRP-107 & $2.30 \mathrm{E}+00$ & us & $2.30 \mathrm{E}-06$ & $2.28 \mathrm{E}+16$ & -- & -- & -- & DOE-STD-1196 & $1.90 \mathrm{E}-14$ \\
\hline
\end{tabular}




\begin{tabular}{|c|c|c|c|c|c|c|c|c|c|c|c|c|c|c|}
\hline \multicolumn{15}{|c|}{ Table A.1 Radionuclide Specific Input Data Used For the Calculation of HC-2 TQs Using Maximum Reported DCs (continued) } \\
\hline $\begin{array}{c}\text { MASTER } \\
\text { ISOTOPE } \\
\text { LIST } \\
\end{array}$ & $\begin{array}{l}\text { Atomic } \\
\text { Mass } \\
\text { Nuclide }\end{array}$ & $\begin{array}{l}\text { Atomic } \\
\text { Mass }\end{array}$ & Element & $\begin{array}{c}\text { RF per } \\
\text { DOE-STD- } \\
1027\end{array}$ & $\begin{array}{l}\text { Half-Life } \\
\text { Reference }\end{array}$ & $\begin{array}{r}\text { Half-Li } \\
\text { (original }\end{array}$ & & Half Life (s) & $\begin{array}{l}\text { Sp. Act } \\
\text { (Ci/g) }\end{array}$ & $\begin{array}{c}\text { Inhalation DC } \\
\text { Reference }\end{array}$ & $\begin{array}{c}\text { HC-2 } \\
\text { Bounding } \\
\text { Inhalation } \\
\text { DC (Sv/Bq) }\end{array}$ & $\begin{array}{l}\text { Lung } \\
\text { Absorption } \\
\text { Class/Type }\end{array}$ & $\begin{array}{c}\text { Immersion DC } \\
\text { Reference }\end{array}$ & $\begin{array}{c}\text { HC-2 } \\
\text { Immersion } \\
\mathrm{DC}(\mathrm{Sv} / \mathrm{s} \\
\left.\text { per } \mathrm{Bq} / \mathrm{m}^{3}\right)\end{array}$ \\
\hline $\mathrm{Rn}-216$ & $\mathrm{Rn}-216$ & 216.000 & Radon & $1.00 \mathrm{E}+00$ & ICRP-107 & $4.50 \mathrm{E}-05$ & $\mathrm{~s}$ & $4.50 \mathrm{E}-05$ & $1.16 \mathrm{E}+15$ & -- & -- & -- & DOE-STD-1196 & $1.77 \mathrm{E}-14$ \\
\hline $\mathrm{Rn}-217$ & Rn-217 & 217.004 & Radon & $1.00 \mathrm{E}+00$ & ICRP-107 & $5.40 \mathrm{E}-04$ & $\mathrm{~s}$ & $5.40 \mathrm{E}-04$ & $9.63 \mathrm{E}+13$ & -- & -- & -- & DOE-STD-1196 & $1.70 \mathrm{E}-14$ \\
\hline $\mathrm{Rn}-218$ & Rn-218 & 218.006 & Radon & $1.00 E+00$ & ICRP-38 & $3.50 \mathrm{E}+01$ & $\mathrm{~ms}$ & $3.50 \mathrm{E}-02$ & $1.48 \mathrm{E}+12$ & -- & -- & -- & FGR-12 & $3.65 \mathrm{E}-17$ \\
\hline Rn-219 & Rn-219 & 219.009 & Radon & $1.00 E+00$ & ICRP-38 & $3.96 \mathrm{E}+00$ & $\mathrm{~s}$ & $3.96 \mathrm{E}+00$ & $1.30 \mathrm{E}+10$ & -- & -- & -- & FGR-12 & $2.68 \mathrm{E}-15$ \\
\hline $\mathrm{Rn}-220$ & $\mathrm{Rn}-220$ & 220.011 & Radon & $1.00 E+00$ & ICRP-38 & $5.56 \mathrm{E}+01$ & $\mathrm{~s}$ & $5.56 \mathrm{E}+01$ & $9.22 \mathrm{E}+08$ & -- & -- & -- & FGR-12 & $1.85 \mathrm{E}-17$ \\
\hline $\mathrm{Rn}-222$ & $\mathrm{Rn}-222$ & 222.018 & Radon & $1.00 E+00$ & ICRP-38 & $3.82 \mathrm{E}+00$ & $d$ & $3.30 \mathrm{E}+05$ & $1.54 \mathrm{E}+05$ & -- & -- & -- & FGR-12 & $1.91 \mathrm{E}-17$ \\
\hline $\mathrm{Rn}-223$ & $\mathrm{Rn}-223$ & 223.022 & Radon & $1.00 E+00$ & ICRP-107 & $2.43 \mathrm{E}+01$ & $\mathrm{~m}$ & $1.46 \mathrm{E}+03$ & $3.47 \mathrm{E}+07$ & -- & -- & -- & DOE-STD-1196 & $1.56 \mathrm{E}-14$ \\
\hline Ru-103 & Ru-103 & 102.906 & Ruthenium & $1.00 \mathrm{E}-02$ & ICRP-38 & $3.93 \mathrm{E}+01$ & d & $3.39 \mathrm{E}+06$ & $3.23 \mathrm{E}+04$ & ICRP-72 & $3.00 \mathrm{E}-09$ & $\mathrm{~s}$ & FGR-12 & $2.25 \mathrm{E}-14$ \\
\hline Ru-105 & Ru-105 & 104.908 & Ruthenium & $1.00 \mathrm{E}-02$ & ICRP-38 & $4.44 \mathrm{E}+00$ & $\mathrm{~h}$ & $1.60 \mathrm{E}+04$ & $6.73 E+06$ & ICRP-72 & $1.80 \mathrm{E}-10$ & $\mathrm{~s}$ & FGR-12 & $3.81 \mathrm{E}-14$ \\
\hline Ru-106 & Ru-106 & 105.907 & Ruthenium & $1.00 \mathrm{E}-02$ & ICRP-38 & $3.68 \mathrm{E}+02$ & d & $3.18 \mathrm{E}+07$ & $3.35 \mathrm{E}+03$ & ICRP-72 & $6.60 \mathrm{E}-08$ & $\mathrm{~s}$ & -- & -- \\
\hline Ru-107 & Ru-107 & 106.910 & Ruthenium & $1.00 \mathrm{E}-02$ & ICRP-107 & $3.75 E+00$ & $\mathrm{~m}$ & $2.25 \mathrm{E}+02$ & $4.69 \mathrm{E}+08$ & -- & -- & -- & DOE-STD-1196 & $1.69 \mathrm{E}-14$ \\
\hline Ru-108 & Ru-108 & 107.910 & Ruthenium & $1.00 \mathrm{E}-02$ & ICRP-107 & $4.55 \mathrm{E}+00$ & $\mathrm{~m}$ & $2.73 \mathrm{E}+02$ & $3.83 \mathrm{E}+08$ & -- & -- & -- & DOE-STD-1196 & $2.92 \mathrm{E}-15$ \\
\hline Ru-92 & Ru-92 & 91.920 & Ruthenium & $1.00 \mathrm{E}-02$ & ICRP-107 & $3.65 E+00$ & $\mathrm{~m}$ & $2.19 \mathrm{E}+02$ & $5.60 E+08$ & --- & -- & -- & DOE-STD-1196 & $9.44 \mathrm{E}-14$ \\
\hline Ru-94 & Ru-94 & 93.911 & Ruthenium & $1.00 \mathrm{E}-02$ & ICRP-38 & $5.18 \mathrm{E}+01$ & $\mathrm{~m}$ & $3.11 \mathrm{E}+03$ & $3.87 \mathrm{E}+07$ & ICRP-72 & $5.60 \mathrm{E}-11$ & $\mathrm{~V}$ & FGR-12 & $2.54 \mathrm{E}-14$ \\
\hline Ru-95 & Ru-95 & 94.910 & Ruthenium & $1.00 \mathrm{E}-02$ & ICRP-107 & $1.64 \mathrm{E}+00$ & $\mathrm{~h}$ & $5.91 \mathrm{E}+03$ & $2.01 \mathrm{E}+07$ & DOE-STD-1196 & $5.29 \mathrm{E}-11$ & $\mathrm{~V}$ & DOE-STD-1196 & $5.68 \mathrm{E}-14$ \\
\hline Ru-97 & Ru-97 & 96.908 & Ruthenium & $1.00 \mathrm{E}-02$ & ICRP-38 & $2.90 \mathrm{E}+00$ & $d$ & $2.51 \mathrm{E}+05$ & $4.65 E+05$ & ICRP-72 & $1.20 \mathrm{E}-10$ & $\mathrm{~V}$ & FGR-12 & $1.09 \mathrm{E}-14$ \\
\hline S-35 & S-35 & 34.969 & Sulfur & $5.00 \mathrm{E}-01$ & ICRP-38 & $8.74 \mathrm{E}+01$ & d & $7.55 E+06$ & $4.27 \mathrm{E}+04$ & ICRP-72 & $1.90 \mathrm{E}-09$ & $\mathrm{~s}$ & FGR-12 & $2.43 \mathrm{E}-19$ \\
\hline S-37 & S-37 & 36.971 & Sulfur & $5.00 \mathrm{E}-01$ & ICRP-107 & $5.05 E+00$ & $\mathrm{~m}$ & $3.03 E+02$ & $1.01 \mathrm{E}+09$ & -- & -- & -- & DOE-STD-1196 & $1.54 \mathrm{E}-13$ \\
\hline S-38 & $\mathrm{S}-38$ & 37.971 & $\begin{array}{l}\text { Sulfur } \\
\end{array}$ & $5.00 \mathrm{E}-01$ & ICRP-107 & $1.70 \mathrm{E}+02$ & $\mathrm{~m}$ & $1.02 \mathrm{E}+04$ & $2.91 \mathrm{E}+07$ & DOE-STD-1196 & $3.53 \mathrm{E}-10$ & $\mathrm{~s}$ & DOE-STD-1196 & $8.47 \mathrm{E}-14$ \\
\hline Sb-111 & Sb-111 & 110.913 & Antimony & $1.00 \mathrm{E}-03$ & ICRP-107 & $7.50 \mathrm{E}+01$ & $\mathrm{~s}$ & $7.50 \mathrm{E}+01$ & $1.36 \mathrm{E}+09$ & -- & -- & -- & DOE-STD-1196 & $6.76 \mathrm{E}-14$ \\
\hline Sb-113 & Sb-113 & 112.909 & Antimony & $1.00 \mathrm{E}-03$ & ICRP-107 & $6.67 \mathrm{E}+00$ & $\mathrm{~m}$ & $4.00 \mathrm{E}+02$ & $2.50 E+08$ & -- & -- & -- & DOE-STD-1196 & $5.71 \mathrm{E}-14$ \\
\hline Sb-114 & Sb-114 & 113.909 & Antimony & $1.00 \mathrm{E}-03$ & ICRP-107 & $3.49 \mathrm{E}+00$ & $\mathrm{~m}$ & $2.09 \mathrm{E}+02$ & $4.73 E+08$ & -- & -- & -- & DOE-STD-1196 & $1.27 \mathrm{E}-13$ \\
\hline Sb-115 & Sb-115 & 114.907 & Antimony & $1.00 \mathrm{E}-03$ & ICRP-38 & $3.18 \mathrm{E}+01$ & $\mathrm{~m}$ & $1.91 \mathrm{E}+03$ & $5.15 \mathrm{E}+07$ & ICRP-72 & $1.40 \mathrm{E}-11$ & $\mathrm{~s}$ & FGR-12 & $4.32 \mathrm{E}-14$ \\
\hline Sb-116 & Sb-116 & 115.907 & Antimony & $1.00 \mathrm{E}-03$ & ICRP-38 & $1.58 \mathrm{E}+01$ & $\mathrm{~m}$ & $9.48 \mathrm{E}+02$ & $1.03 E+08$ & ICRP-72 & $1.30 \mathrm{E}-11$ & $M$ & FGR-12 & $1.08 \mathrm{E}-13$ \\
\hline Sb-116m & Sb-116 & 115.907 & Antimony & $1.00 \mathrm{E}-03$ & ICRP-38 & $6.03 E+01$ & $\mathrm{~m}$ & $3.62 E+03$ & $2.69 \mathrm{E}+07$ & ICRP-72 & $4.90 \mathrm{E}-11$ & $\mathrm{~s}$ & FGR-12 & $1.55 \mathrm{E}-13$ \\
\hline Sb-117 & Sb-117 & 116.905 & Antimony & $1.00 \mathrm{E}-03$ & ICRP-38 & $2.80 \mathrm{E}+00$ & $\mathrm{~h}$ & $1.01 \mathrm{E}+04$ & $9.57 \mathrm{E}+06$ & ICRP-72 & $1.70 \mathrm{E}-11$ & $\mathrm{~s}$ & FGR-12 & $7.97 \mathrm{E}-15$ \\
\hline Sb-118 & Sb-118 & 117.906 & Antimony & $1.00 \mathrm{E}-03$ & ICRP-38 & $3.60 \mathrm{E}+00$ & $\mathrm{~m}$ & $2.16 \mathrm{E}+02$ & $4.43 E+08$ & -- & -- & -- & DOE-STD-1196 & $3.65 \mathrm{E}-14$ \\
\hline Sb-118m & Sb-118 & 117.906 & Antimony & $1.00 \mathrm{E}-03$ & ICRP-38 & $5.00 E+00$ & $\mathrm{~h}$ & $1.80 \mathrm{E}+04$ & $5.32 \mathrm{E}+06$ & ICRP-72 & $1.20 \mathrm{E}-10$ & $M$ & FGR-12 & $1.27 \mathrm{E}-13$ \\
\hline Sb-119 & Sb-119 & 118.904 & Antimony & $1.00 \mathrm{E}-03$ & ICRP-38 & $3.81 \mathrm{E}+01$ & $\mathrm{~h}$ & $1.37 \mathrm{E}+05$ & $6.92 \mathrm{E}+05$ & ICRP-72 & $3.60 \mathrm{E}-11$ & $\mathrm{~s}$ & FGR-12 & $2.16 \mathrm{E}-16$ \\
\hline Sb-120s & Sb-120 & 119.905 & Antimony & $1.00 \mathrm{E}-03$ & ICRP-38 & $1.59 \mathrm{E}+01$ & $\mathrm{~m}$ & $9.53 \mathrm{E}+02$ & $9.87 \mathrm{E}+07$ & ICRP-72 & $7.30 \mathrm{E}-12$ & $\mathrm{~s}$ & FGR-12 & $2.13 \mathrm{E}-14$ \\
\hline Sb-120l & Sb-120 & 119.905 & Antimony & $1.00 \mathrm{E}-03$ & ICRP-38 & $5.76 \mathrm{E}+00$ & d & $4.98 \mathrm{E}+05$ & $1.89 \mathrm{E}+05$ & ICRP-72 & $1.10 \mathrm{E}-09$ & $\mathrm{~s}$ & FGR-12 & $1.22 \mathrm{E}-13$ \\
\hline Sb-122 & Sb-122 & 121.905 & Antimony & $1.00 \mathrm{E}-03$ & ICRP-38 & $2.70 E+00$ & $d$ & $2.33 E+05$ & $3.97 \mathrm{E}+05$ & ICRP-72 & $1.10 \mathrm{E}-09$ & $\mathrm{~s}$ & FGR-12 & $2.13 \mathrm{E}-14$ \\
\hline Sb-122m & Sb-122 & 121.905 & Antimony & $1.00 \mathrm{E}-03$ & ICRP-107 & $4.19 \mathrm{E}+00$ & $\mathrm{~m}$ & $2.51 \mathrm{E}+02$ & $3.68 \mathrm{E}+08$ & -- & -- & -- & DOE-STD-1196 & $1.77 \mathrm{E}-15$ \\
\hline Sb-124 & Sb-124 & 123.906 & Antimony & $1.00 \mathrm{E}-03$ & ICRP-38 & $6.02 \mathrm{E}+01$ & $d$ & $5.20 \mathrm{E}+06$ & $1.75 E+04$ & ICRP-72 & $8.60 \mathrm{E}-09$ & $\mathrm{~s}$ & FGR-12 & $9.15 \mathrm{E}-14$ \\
\hline Sb-124ms & Sb-124 & 123.906 & Antimony & $1.00 \mathrm{E}-03$ & ICRP-38 & $9.30 \mathrm{E}+01$ & $\mathrm{~s}$ & $9.30 \mathrm{E}+01$ & $9.79 E+08$ & -- & -- & -- & FGR-12 & $1.70 \mathrm{E}-14$ \\
\hline Sb-124ml & Sb-124 & 123.906 & Antimony & $1.00 \mathrm{E}-03$ & ICRP-38 & $2.02 \mathrm{E}+01$ & $\mathrm{~m}$ & $1.21 \mathrm{E}+03$ & $7.51 \mathrm{E}+07$ & ICRP-72 & $5.90 \mathrm{E}-12$ & $\mathrm{~s}$ & FGR-12 & $6.75 \mathrm{E}-19$ \\
\hline Sb-125 & Sb-125 & 124.905 & Antimony & $1.00 \mathrm{E}-03$ & ICRP-38 & $2.77 E+00$ & $y$ & $8.74 \mathrm{E}+07$ & $1.03 E+03$ & ICRP-72 & $1.20 \mathrm{E}-08$ & $\mathrm{~s}$ & FGR-12 & $2.02 \mathrm{E}-14$ \\
\hline Sb-126 & Sb-126 & 125.907 & Antimony & $1.00 \mathrm{E}-03$ & ICRP-38 & $1.24 \mathrm{E}+01$ & d & $1.07 \mathrm{E}+06$ & $8.36 \mathrm{E}+04$ & ICRP-72 & $3.20 \mathrm{E}-09$ & $\mathrm{~s}$ & FGR-12 & $1.37 \mathrm{E}-13$ \\
\hline Sb-126m & Sb-126 & 125.907 & Antimony & $1.00 \mathrm{E}-03$ & ICRP-38 & $1.90 \mathrm{E}+01$ & $\mathrm{~m}$ & $1.14 \mathrm{E}+03$ & $7.86 \mathrm{E}+07$ & ICRP-72 & $2.00 \mathrm{E}-11$ & $\mathrm{~s}$ & FGR-12 & $7.50 \mathrm{E}-14$ \\
\hline Sb-127 & Sb-127 & 126.907 & Antimony & $1.00 \mathrm{E}-03$ & ICRP-38 & $3.85 \mathrm{E}+00$ & d & $3.33 \mathrm{E}+05$ & $2.67 \mathrm{E}+05$ & ICRP-72 & $1.90 \mathrm{E}-09$ & $\mathrm{~s}$ & FGR-12 & $3.33 \mathrm{E}-14$ \\
\hline Sb-128I & Sb-128 & 127.909 & Antimony & $1.00 \mathrm{E}-03$ & ICRP-38 & $9.01 E+00$ & $\mathrm{~h}$ & $3.24 \mathrm{E}+04$ & $2.72 E+06$ & ICRP-72 & $4.20 \mathrm{E}-10$ & $\mathrm{~s}$ & FGR-12 & $1.51 \mathrm{E}-13$ \\
\hline Sb-128s & Sb-128 & 127.909 & Antimony & $1.00 \mathrm{E}-03$ & ICRP-38 & $1.04 \mathrm{E}+01$ & $\mathrm{~m}$ & $6.24 \mathrm{E}+02$ & $1.41 \mathrm{E}+08$ & ICRP-72 & $1.50 \mathrm{E}-11$ & $\mathrm{~s}$ & FGR-12 & $9.69 \mathrm{E}-14$ \\
\hline Sb-129 & Sb-129 & 128.909 & Antimony & $1.00 \mathrm{E}-03$ & ICRP-38 & $4.32 \mathrm{E}+00$ & $\mathrm{~h}$ & $1.56 \mathrm{E}+04$ & $5.63 \mathrm{E}+06$ & ICRP-72 & $2.50 \mathrm{E}-10$ & $\mathrm{~s}$ & FGR-12 & $7.14 \mathrm{E}-14$ \\
\hline Sb-130 & Sb-130 & 129.912 & Antimony & $1.00 \mathrm{E}-03$ & ICRP-38 & $4.00 \mathrm{E}+01$ & $\mathrm{~m}$ & $2.40 \mathrm{E}+03$ & $3.62 \mathrm{E}+07$ & ICRP-72 & $5.30 \mathrm{E}-11$ & $\mathrm{~s}$ & FGR-12 & $1.60 \mathrm{E}-13$ \\
\hline Sb-130m & Sb-130 & 129.912 & Antimony & $1.00 \mathrm{E}-03$ & ICRP-107 & $6.30 \mathrm{E}+00$ & $\mathrm{~m}$ & $3.78 \mathrm{E}+02$ & $2.30 \mathrm{E}+08$ & -- & -- & -- & DOE-STD-1196 & $1.26 \mathrm{E}-13$ \\
\hline Sb-131 & Sb-131 & 130.912 & Antimony & $1.00 \mathrm{E}-03$ & ICRP-38 & $2.30 \mathrm{E}+01$ & $\mathrm{~m}$ & $1.38 \mathrm{E}+03$ & $6.24 \mathrm{E}+07$ & ICRP-72 & $4.40 \mathrm{E}-11$ & $M$ & FGR-12 & $9.37 \mathrm{E}-14$ \\
\hline
\end{tabular}




\begin{tabular}{|c|c|c|c|c|c|c|c|c|c|c|c|c|c|c|}
\hline \multicolumn{15}{|c|}{ Table A.1 Radionuclide Specific Input Data Used For the Calculation of HC-2 TQs Using Maximum Reported DCs (continued) } \\
\hline $\begin{array}{c}\text { MASTER } \\
\text { ISOTOPE } \\
\text { LIST } \\
\end{array}$ & $\begin{array}{l}\text { Atomic } \\
\text { Mass } \\
\text { Nuclide }\end{array}$ & $\begin{array}{l}\text { Atomic } \\
\text { Mass }\end{array}$ & Element & $\begin{array}{c}\text { RF per } \\
\text { DOE-STD- } \\
1027\end{array}$ & $\begin{array}{l}\text { Half-Life } \\
\text { Reference }\end{array}$ & $\begin{array}{r}\text { Half-Li } \\
\text { (original }\end{array}$ & & Half Life (s) & $\begin{array}{l}\text { Sp. Act } \\
\text { (Ci/g) }\end{array}$ & $\begin{array}{c}\text { Inhalation DC } \\
\text { Reference }\end{array}$ & $\begin{array}{c}\text { HC-2 } \\
\text { Bounding } \\
\text { Inhalation } \\
\text { DC (Sv/Bq) }\end{array}$ & $\begin{array}{l}\text { Lung } \\
\text { Absorption } \\
\text { Class/Type }\end{array}$ & $\begin{array}{c}\text { Immersion DC } \\
\text { Reference }\end{array}$ & $\begin{array}{c}\text { HC-2 } \\
\text { Immersion } \\
\mathrm{DC}(\mathrm{Sv} / \mathrm{s} \\
\left.\text { per } \mathrm{Bq} / \mathrm{m}^{3}\right)\end{array}$ \\
\hline Sb-133 & Sb-133 & 132.915 & Antimony & $1.00 \mathrm{E}-03$ & ICRP-107 & $2.50 \mathrm{E}+00$ & $\mathrm{~m}$ & $1.50 \mathrm{E}+02$ & $5.66 \mathrm{E}+08$ & -- & -- & -- & DOE-STD-1196 & $1.33 \mathrm{E}-13$ \\
\hline Sc- $42 \mathrm{~m}$ & Sc-42 & 41.966 & Scandium & $1.00 \mathrm{E}-03$ & ICRP-107 & $6.20 \mathrm{E}+01$ & $\mathrm{~s}$ & $6.20 \mathrm{E}+01$ & $4.34 \mathrm{E}+09$ & --- & -- & -- & DOE-STD-1196 & $1.98 \mathrm{E}-13$ \\
\hline Sc-43 & Sc-43 & 42.961 & Scandium & $1.00 \mathrm{E}-03$ & ICRP-38 & $3.89 \mathrm{E}+00$ & $\mathrm{~h}$ & $1.40 \mathrm{E}+04$ & $1.87 E+07$ & ICRP-72 & $1.10 \mathrm{E}-10$ & $\mathrm{~s}$ & FGR-12 & $5.26 \mathrm{E}-14$ \\
\hline Sc-44 & Sc-44 & 43.959 & Scandium & $1.00 \mathrm{E}-03$ & ICRP-38 & $3.93 \mathrm{E}+00$ & $\mathrm{~h}$ & $1.41 \mathrm{E}+04$ & $1.82 \mathrm{E}+07$ & ICRP-72 & $1.80 \mathrm{E}-10$ & $\mathrm{~s}$ & FGR-12 & $1.05 \mathrm{E}-13$ \\
\hline Sc-44m & Sc-44 & 43.959 & Scandium & $1.00 \mathrm{E}-03$ & ICRP-38 & $5.86 \mathrm{E}+01$ & $\mathrm{~h}$ & $2.11 E+05$ & $1.22 \mathrm{E}+06$ & ICRP-72 & $1.40 \mathrm{E}-09$ & $\mathrm{~s}$ & FGR-12 & $1.35 \mathrm{E}-14$ \\
\hline Sc-46 & Sc-46 & 45.955 & Scandium & $1.00 \mathrm{E}-03$ & ICRP-38 & $8.38 \mathrm{E}+01$ & $d$ & $7.24 E+06$ & $3.39 \mathrm{E}+04$ & ICRP-72 & $6.80 \mathrm{E}-09$ & $\mathrm{~s}$ & FGR-12 & $9.98 \mathrm{E}-14$ \\
\hline Sc-47 & Sc-47 & 46.952 & Scandium & $1.00 \mathrm{E}-03$ & ICRP-38 & $3.35 E+00$ & $d$ & $2.90 \mathrm{E}+05$ & $8.30 \mathrm{E}+05$ & ICRP-72 & $7.30 \mathrm{E}-10$ & $\mathrm{~s}$ & FGR-12 & $5.14 \mathrm{E}-15$ \\
\hline Sc-48 & Sc-48 & 47.952 & Scandium & $1.00 \mathrm{E}-03$ & ICRP-38 & $4.37 \mathrm{E}+01$ & $\mathrm{~h}$ & $1.57 \mathrm{E}+05$ & $1.50 \mathrm{E}+06$ & ICRP-72 & $1.10 \mathrm{E}-09$ & $\mathrm{~s}$ & FGR-12 & $1.68 \mathrm{E}-13$ \\
\hline Sc-49 & Sc-49 & 48.950 & Scandium & $1.00 \mathrm{E}-03$ & ICRP-38 & $5.74 \mathrm{E}+01$ & $\mathrm{~m}$ & $3.44 \mathrm{E}+03$ & $6.69 \mathrm{E}+07$ & ICRP-72 & $4.00 \mathrm{E}-11$ & $\mathrm{~S}$ & FGR-12 & $1.93 \mathrm{E}-16$ \\
\hline Sc-50 & Sc-50 & 49.952 & Scandium & $1.00 \mathrm{E}-03$ & ICRP-107 & $1.03 E+02$ & $s$ & $1.03 \mathrm{E}+02$ & $2.20 E+09$ & -- & -- & -- & DOE-STD-1196 & $1.54 \mathrm{E}-13$ \\
\hline Se-70 & Se-70 & 69.934 & Selenium & $1.00 \mathrm{E}-02$ & ICRP-38 & $4.10 \mathrm{E}+01$ & $\mathrm{~m}$ & $2.46 \mathrm{E}+03$ & $6.56 E+07$ & ICRP-72 & $7.60 \mathrm{E}-11$ & $\mathrm{~s}$ & FGR-12 & $4.73 \mathrm{E}-14$ \\
\hline Se-71 & Se-71 & 70.932 & Selenium & $1.00 \mathrm{E}-02$ & ICRP-107 & $4.74 \mathrm{E}+00$ & $\mathrm{~m}$ & $2.84 \mathrm{E}+02$ & $5.59 \mathrm{E}+08$ & -- & -- & -- & DOE-STD-1196 & $7.40 \mathrm{E}-14$ \\
\hline Se-72 & Se-72 & 71.927 & Selenium & $1.00 \mathrm{E}-02$ & ICRP-38 & $8.40 \mathrm{E}+00$ & $d$ & $7.26 \mathrm{E}+05$ & $2.16 \mathrm{E}+05$ & DOE-STD-1196 & $4.16 \mathrm{E}-09$ & $\mathrm{~s}$ & DOE-STD-1196 & $6.10 \mathrm{E}-16$ \\
\hline Se-73 & Se-73 & 72.927 & Selenium & $1.00 \mathrm{E}-02$ & ICRP-38 & $7.15 E+00$ & $\mathrm{~h}$ & $2.57 \mathrm{E}+04$ & $6.01 \mathrm{E}+06$ & ICRP-72 & $2.10 \mathrm{E}-10$ & $\mathrm{~s}$ & FGR-12 & $5.16 \mathrm{E}-14$ \\
\hline Se-73m & Se-73 & 72.927 & Selenium & $1.00 \mathrm{E}-02$ & ICRP-38 & $3.90 \mathrm{E}+01$ & $\mathrm{~m}$ & $2.34 \mathrm{E}+03$ & $6.61 \mathrm{E}+07$ & ICRP-72 & $2.20 \mathrm{E}-11$ & $\mathrm{~s}$ & FGR-12 & $1.17 \mathrm{E}-14$ \\
\hline Se-75 & Se-75 & 74.923 & Selenium & $1.00 \mathrm{E}-02$ & ICRP-38 & $1.20 \mathrm{E}+02$ & d & $1.04 \mathrm{E}+07$ & $1.45 \mathrm{E}+04$ & ICRP-72 & $1.30 \mathrm{E}-09$ & $\mathrm{~s}$ & FGR-12 & $1.85 \mathrm{E}-14$ \\
\hline Se-77m & Se-77 & 76.920 & Selenium & $1.00 \mathrm{E}-02$ & ICRP-38 & $1.75 E+01$ & $\mathrm{~s}$ & $1.75 \mathrm{E}+01$ & $8.40 E+09$ & -- & -- & -- & FGR-12 & $4.03 \mathrm{E}-15$ \\
\hline Se-79 & Se-79 & 78.918 & Selenium & $1.00 \mathrm{E}-02$ & ICRP-38 & $6.50 \mathrm{E}+04$ & $y$ & $2.05 E+12$ & $6.97 \mathrm{E}-02$ & ICRP-72 & $6.80 \mathrm{E}-09$ & $\mathrm{~s}$ & FGR-12 & $3.03 \mathrm{E}-19$ \\
\hline Se-79m & Se-79 & 78.918 & Selenium & $1.00 \mathrm{E}-02$ & ICRP-107 & $3.92 \mathrm{E}+00$ & $\mathrm{~m}$ & $2.35 E+02$ & $6.08 \mathrm{E}+08$ & -- & -- & -- & DOE-STD-1196 & $3.60 \mathrm{E}-16$ \\
\hline Se-81 & Se-81 & 80.918 & Selenium & $1.00 \mathrm{E}-02$ & ICRP-38 & $1.85 \mathrm{E}+01$ & $\mathrm{~m}$ & $1.11 \mathrm{E}+03$ & $1.26 \mathrm{E}+08$ & ICRP-72 & $1.50 \mathrm{E}-11$ & $\mathrm{~s}$ & FGR-12 & $5.24 \mathrm{E}-16$ \\
\hline Se-81m & Se-81 & 80.918 & Selenium & $1.00 \mathrm{E}-02$ & ICRP-38 & $5.73 \mathrm{E}+01$ & $\mathrm{~m}$ & $3.44 \mathrm{E}+03$ & $4.06 E+07$ & ICRP-72 & $5.10 \mathrm{E}-11$ & $\mathrm{~s}$ & FGR-12 & $6.18 \mathrm{E}-16$ \\
\hline Se-83 & Se- 83 & 82.919 & Selenium & $1.00 \mathrm{E}-02$ & ICRP-38 & $2.25 \mathrm{E}+01$ & $\mathrm{~m}$ & $1.35 \mathrm{E}+03$ & $1.01 E+08$ & ICRP-72 & $3.40 \mathrm{E}-11$ & $\mathrm{~s}$ & FGR-12 & $1.21 \mathrm{E}-13$ \\
\hline Se-83m & Se-83 & 82.919 & Selenium & $1.00 \mathrm{E}-02$ & ICRP-107 & $7.01 \mathrm{E}+01$ & $\mathrm{~s}$ & $7.01 \mathrm{E}+01$ & $1.94 \mathrm{E}+09$ & -- & -- & -- & DOE-STD-1196 & $4.78 \mathrm{E}-14$ \\
\hline Se-84 & Se- 84 & 83.918 & Selenium & $1.00 \mathrm{E}-02$ & ICRP-107 & $3.10 \mathrm{E}+00$ & $\mathrm{~m}$ & $1.86 \mathrm{E}+02$ & $7.23 E+08$ & -- & -- & -- & DOE-STD-1196 & $1.90 \mathrm{E}-14$ \\
\hline Si-31 & Si-31 & 30.975 & Silicon & $1.00 \mathrm{E}-03$ & ICRP-38 & $1.57 \mathrm{E}+02$ & $\mathrm{~m}$ & $9.44 \mathrm{E}+03$ & $3.86 \mathrm{E}+07$ & ICRP-72 & $7.90 \mathrm{E}-11$ & $\mathrm{~s}$ & FGR-12 & $1.17 \mathrm{E}-16$ \\
\hline Si-32 & Si-32 & 31.974 & Silicon & $1.00 \mathrm{E}-03$ & ICRP-38 & $4.50 \mathrm{E}+02$ & $y$ & $1.42 \mathrm{E}+10$ & $2.48 \mathrm{E}+01$ & ICRP-72 & $1.10 \mathrm{E}-07$ & $\mathrm{~s}$ & FGR-12 & $5.24 \mathrm{E}-19$ \\
\hline Sm-139 & Sm-139 & 138.922 & Samarium & $1.00 \mathrm{E}-03$ & ICRP-107 & $2.57 \mathrm{E}+00$ & $\mathrm{~m}$ & $1.54 \mathrm{E}+02$ & $5.27 \mathrm{E}+08$ & -- & -- & -- & DOE-STD-1196 & $6.64 \mathrm{E}-14$ \\
\hline Sm-140 & Sm-140 & 139.919 & Samarium & $1.00 \mathrm{E}-03$ & ICRP-107 & $1.48 \mathrm{E}+01$ & $\mathrm{~m}$ & $8.89 \mathrm{E}+02$ & $9.07 E+07$ & DOE-STD-1196 & $3.92 \mathrm{E}-11$ & $\mathrm{~s}$ & DOE-STD-1196 & $2.53 \mathrm{E}-14$ \\
\hline Sm-141 & Sm-141 & 140.918 & Samarium & $1.00 \mathrm{E}-03$ & ICRP-38 & $1.02 \mathrm{E}+01$ & $\mathrm{~m}$ & $6.12 \mathrm{E}+02$ & $1.31 E+08$ & ICRP-72 & $1.50 \mathrm{E}-11$ & $M$ & FGR-12 & $6.87 \mathrm{E}-14$ \\
\hline Sm-141m & Sm-141 & 140.918 & Samarium & $1.00 \mathrm{E}-03$ & ICRP-38 & $2.26 \mathrm{E}+01$ & $\mathrm{~m}$ & $1.36 \mathrm{E}+03$ & $5.90 \mathrm{E}+07$ & ICRP-72 & $3.20 \mathrm{E}-11$ & $M$ & FGR-12 & $9.71 \mathrm{E}-14$ \\
\hline Sm-142 & Sm-142 & 141.915 & Samarium & $1.00 \mathrm{E}-03$ & ICRP-38 & $7.25 E+01$ & $\mathrm{~m}$ & $4.35 \mathrm{E}+03$ & $1.83 \mathrm{E}+07$ & ICRP-72 & $7.10 \mathrm{E}-11$ & $M$ & FGR-12 & $3.79 \mathrm{E}-15$ \\
\hline Sm-143 & Sm-143 & 142.915 & Samarium & $1.00 \mathrm{E}-03$ & ICRP-107 & $8.75 E+00$ & $\mathrm{~m}$ & $5.25 \mathrm{E}+02$ & $1.50 \mathrm{E}+08$ & -- & -- & -- & DOE-STD-1196 & $2.36 \mathrm{E}-14$ \\
\hline Sm-143m & Sm-143 & 142.915 & Samarium & $1.00 \mathrm{E}-03$ & ICRP-107 & $6.60 \mathrm{E}+01$ & $\mathrm{~s}$ & $6.60 \mathrm{E}+01$ & $1.20 \mathrm{E}+09$ & -- & -- & -- & DOE-STD-1196 & $3.11 \mathrm{E}-14$ \\
\hline Sm-145 & Sm-145 & 144.913 & Samarium & $1.00 \mathrm{E}-03$ & ICRP-38 & $3.40 \mathrm{E}+02$ & $d$ & $2.94 \mathrm{E}+07$ & $2.65 \mathrm{E}+03$ & ICRP-72 & $1.60 \mathrm{E}-09$ & $M$ & FGR-12 & $1.61 \mathrm{E}-15$ \\
\hline Sm-146 & Sm-146 & 145.913 & Samarium & $1.00 \mathrm{E}-03$ & ICRP-38 & $1.03 E+08$ & $y$ & $3.25 \mathrm{E}+15$ & $2.38 \mathrm{E}-05$ & ICRP-72 & $1.10 \mathrm{E}-05$ & $M$ & -- & -- \\
\hline Sm-147 & Sm-147 & 146.915 & Samarium & $1.00 \mathrm{E}-03$ & ICRP-38 & $1.06 \mathrm{E}+11$ & $y$ & $3.35 \mathrm{E}+18$ & $2.30 \mathrm{E}-08$ & ICRP-72 & $9.60 \mathrm{E}-06$ & $M$ & -- & -- \\
\hline Sm-148 & Sm-148 & 147.915 & Samarium & $1.00 \mathrm{E}-03$ & ICRP-107 & $7.00 E+15$ & $y$ & $2.21 \mathrm{E}+23$ & $3.45 \mathrm{E}-13$ & DOE-STD-1196 & $2.10 \mathrm{E}-05$ & $F$ & -- & -- \\
\hline Sm-151 & Sm-151 & 150.920 & Samarium & $1.00 \mathrm{E}-03$ & ICRP-38 & $9.00 \mathrm{E}+01$ & $y$ & $2.84 \mathrm{E}+09$ & $2.63 \mathrm{E}+01$ & ICRP-72 & $4.00 \mathrm{E}-09$ & $M$ & FGR-12 & $3.61 \mathrm{E}-20$ \\
\hline Sm-153 & Sm-153 & 152.922 & Samarium & $1.00 \mathrm{E}-03$ & ICRP-38 & $4.67 \mathrm{E}+01$ & $\mathrm{~h}$ & $1.68 \mathrm{E}+05$ & $4.39 \mathrm{E}+05$ & ICRP-72 & $6.30 \mathrm{E}-10$ & $M$ & FGR-12 & $2.28 \mathrm{E}-15$ \\
\hline Sm-155 & Sm-155 & 154.925 & Samarium & $1.00 \mathrm{E}-03$ & ICRP-38 & $2.21 E+01$ & $\mathrm{~m}$ & $1.33 \mathrm{E}+03$ & $5.49 E+07$ & ICRP-72 & $1.70 \mathrm{E}-11$ & $M$ & FGR-12 & $4.65 \mathrm{E}-15$ \\
\hline Sm-156 & Sm-156 & 155.926 & Samarium & $1.00 \mathrm{E}-03$ & ICRP-38 & $9.40 \mathrm{E}+00$ & $\mathrm{~h}$ & $3.38 \mathrm{E}+04$ & $2.14 E+06$ & ICRP-72 & $2.20 \mathrm{E}-10$ & $M$ & FGR-12 & $5.43 \mathrm{E}-15$ \\
\hline Sm-157 & Sm-157 & 156.928 & Samarium & $1.00 \mathrm{E}-03$ & ICRP-107 & $8.03 \mathrm{E}+00$ & $\mathrm{~m}$ & $4.82 \mathrm{E}+02$ & $1.49 \mathrm{E}+08$ & -- & -- & -- & DOE-STD-1196 & $1.91 \mathrm{E}-14$ \\
\hline Sn-106 & Sn-106 & 105.917 & Tin & $1.00 \mathrm{E}-03$ & ICRP-107 & $1.92 \mathrm{E}+00$ & $\mathrm{~m}$ & $1.15 \mathrm{E}+02$ & $9.25 \mathrm{E}+08$ & -- & -- & -- & DOE-STD-1196 & $5.40 \mathrm{E}-14$ \\
\hline Sn-108 & Sn-108 & 107.912 & Tin & $1.00 \mathrm{E}-03$ & ICRP-107 & $1.03 \mathrm{E}+01$ & $\mathrm{~m}$ & $6.18 \mathrm{E}+02$ & $1.69 \mathrm{E}+08$ & DOE-STD-1196 & $1.40 \mathrm{E}-11$ & $\mathrm{~s}$ & DOE-STD-1196 & $2.96 \mathrm{E}-14$ \\
\hline Sn-109 & Sn-109 & 108.911 & Tin & $1.00 \mathrm{E}-03$ & ICRP-107 & $1.80 \mathrm{E}+01$ & $\mathrm{~m}$ & $1.08 \mathrm{E}+03$ & $9.59 E+07$ & DOE-STD-1196 & $1.44 \mathrm{E}-11$ & $\mathrm{~s}$ & DOE-STD-1196 & $1.05 \mathrm{E}-13$ \\
\hline Sn-110 & Sn-110 & 109.908 & Tin & $1.00 \mathrm{E}-03$ & ICRP-38 & $4.00 E+00$ & $\mathrm{~h}$ & $1.44 \mathrm{E}+04$ & $7.13 E+06$ & ICRP-72 & $1.60 \mathrm{E}-10$ & $M$ & FGR-12 & $1.37 \mathrm{E}-14$ \\
\hline
\end{tabular}




\begin{tabular}{|c|c|c|c|c|c|c|c|c|c|c|c|c|c|c|}
\hline \multicolumn{15}{|c|}{ Table A.1 Radionuclide Specific Input Data Used For the Calculation of HC-2 TQs Using Maximum Reported DCs (continued) } \\
\hline $\begin{array}{c}\text { MASTER } \\
\text { ISOTOPE } \\
\text { LIST } \\
\end{array}$ & $\begin{array}{l}\text { Atomic } \\
\text { Mass } \\
\text { Nuclide }\end{array}$ & $\begin{array}{l}\text { Atomic } \\
\text { Mass }\end{array}$ & Element & $\begin{array}{c}\text { RF per } \\
\text { DOE-STD- } \\
1027\end{array}$ & $\begin{array}{l}\text { Half-Life } \\
\text { Reference }\end{array}$ & $\begin{array}{r}\text { Half-Li } \\
\text { (original }\end{array}$ & & Half Life (s) & $\begin{array}{l}\text { Sp. Act } \\
\text { (Ci/g) }\end{array}$ & $\begin{array}{c}\text { Inhalation DC } \\
\text { Reference }\end{array}$ & $\begin{array}{c}\text { HC-2 } \\
\text { Bounding } \\
\text { Inhalation } \\
\text { DC (Sv/Bq) }\end{array}$ & $\begin{array}{l}\text { Lung } \\
\text { Absorption } \\
\text { Class/Type }\end{array}$ & $\begin{array}{c}\text { Immersion DC } \\
\text { Reference }\end{array}$ & $\begin{array}{c}\text { HC-2 } \\
\text { Immersion } \\
\mathrm{DC}(\mathrm{Sv} / \mathrm{s} \\
\left.\text { per } \mathrm{Bq} / \mathrm{m}^{3}\right)\end{array}$ \\
\hline Sn-111 & Sn-111 & 110.908 & Tin & $1.00 \mathrm{E}-03$ & ICRP-38 & $3.53 \mathrm{E}+01$ & $\mathrm{~m}$ & $2.12 \mathrm{E}+03$ & $4.80 \mathrm{E}+07$ & ICRP-72 & $1.30 \mathrm{E}-11$ & $\mathrm{M}$ & FGR-12 & $2.45 \mathrm{E}-14$ \\
\hline Sn-113 & Sn-113 & 112.905 & Tin & $1.00 \mathrm{E}-03$ & ICRP-38 & $1.15 \mathrm{E}+02$ & d & $9.94 \mathrm{E}+06$ & $1.00 \mathrm{E}+04$ & ICRP-72 & $2.70 \mathrm{E}-09$ & $M$ & FGR-12 & $3.82 \mathrm{E}-16$ \\
\hline Sn-113m & Sn-113 & 112.905 & Tin & $1.00 \mathrm{E}-03$ & ICRP-107 & $2.14 \mathrm{E}+01$ & $\mathrm{~m}$ & $1.28 \mathrm{E}+03$ & $7.78 \mathrm{E}+07$ & DOE-STD-1196 & $5.19 \mathrm{E}-12$ & $\mathrm{~s}$ & DOE-STD-1196 & $1.02 \mathrm{E}-16$ \\
\hline Sn-117m & Sn-117 & 116.903 & Tin & $1.00 \mathrm{E}-03$ & ICRP-38 & $1.36 \mathrm{E}+01$ & d & $1.18 \mathrm{E}+06$ & $8.21 \mathrm{E}+04$ & ICRP-72 & $2.40 \mathrm{E}-09$ & $M$ & FGR-12 & $6.82 \mathrm{E}-15$ \\
\hline Sn-119m & Sn-119 & 118.903 & Tin & $1.00 \mathrm{E}-03$ & ICRP-38 & $2.93 \mathrm{E}+02$ & $d$ & $2.53 \mathrm{E}+07$ & $3.75 E+03$ & ICRP-72 & $2.20 \mathrm{E}-09$ & $M$ & FGR-12 & $1.01 \mathrm{E}-16$ \\
\hline Sn-121 & Sn-121 & 120.904 & Tin & $1.00 \mathrm{E}-03$ & ICRP-38 & $2.71 E+01$ & $\mathrm{~h}$ & $9.74 \mathrm{E}+04$ & $9.58 \mathrm{E}+05$ & ICRP-72 & $2.30 \mathrm{E}-10$ & $M$ & FGR-12 & $2.37 \mathrm{E}-18$ \\
\hline Sn-121m & Sn-121 & 120.904 & Tin & $1.00 \mathrm{E}-03$ & ICRP-38 & $5.50 \mathrm{E}+01$ & $y$ & $1.74 \mathrm{E}+09$ & $5.38 \mathrm{E}+01$ & ICRP-72 & $4.50 \mathrm{E}-09$ & $M$ & FGR-12 & $6.02 \mathrm{E}-17$ \\
\hline Sn-123 & Sn-123 & 122.906 & Tin & $1.00 \mathrm{E}-03$ & ICRP-38 & $1.29 \mathrm{E}+02$ & $d$ & $1.12 \mathrm{E}+07$ & $8.22 \mathrm{E}+03$ & ICRP-72 & $8.10 \mathrm{E}-09$ & $M$ & FGR-12 & $4.03 E-16$ \\
\hline Sn-123m & Sn-123 & 122.906 & Tin & $1.00 \mathrm{E}-03$ & ICRP-38 & $4.01 \mathrm{E}+01$ & $\mathrm{~m}$ & $2.40 \mathrm{E}+03$ & $3.82 \mathrm{E}+07$ & ICRP-72 & $2.70 \mathrm{E}-11$ & $M$ & FGR-12 & $6.55 \mathrm{E}-15$ \\
\hline Sn-125 & Sn-125 & 124.908 & Tin & $1.00 \mathrm{E}-03$ & ICRP-38 & $9.64 \mathrm{E}+00$ & d & $8.33 \mathrm{E}+05$ & $1.08 \mathrm{E}+05$ & ICRP-72 & $3.10 \mathrm{E}-09$ & $M$ & FGR-12 & $1.58 \mathrm{E}-14$ \\
\hline Sn-125m & Sn-125 & 124.908 & Tin & $1.00 \mathrm{E}-03$ & ICRP-107 & $9.52 \mathrm{E}+00$ & $\mathrm{~m}$ & $5.71 \mathrm{E}+02$ & $1.58 \mathrm{E}+08$ & -- & -- & -- & DOE-STD-1196 & $1.60 \mathrm{E}-14$ \\
\hline Sn-126 & Sn-126 & 125.908 & Tin & $1.00 \mathrm{E}-03$ & ICRP-38 & $1.00 \mathrm{E}+05$ & $y$ & $3.16 \mathrm{E}+12$ & $2.84 \mathrm{E}-02$ & ICRP-72 & $2.80 \mathrm{E}-08$ & $M$ & FGR-12 & $2.11 \mathrm{E}-15$ \\
\hline Sn-127 & Sn-127 & 126.910 & Tin & $1.00 \mathrm{E}-03$ & ICRP-38 & $2.10 \mathrm{E}+00$ & $\mathrm{~h}$ & $7.56 \mathrm{E}+03$ & $1.18 \mathrm{E}+07$ & ICRP-72 & $1.30 \mathrm{E}-10$ & $M$ & FGR-12 & $9.59 \mathrm{E}-14$ \\
\hline Sn-127m & Sn-127 & 126.910 & Tin & $1.00 \mathrm{E}-03$ & ICRP-107 & $4.13 \mathrm{E}+00$ & $\mathrm{~m}$ & $2.48 \mathrm{E}+02$ & $3.59 E+08$ & -- & -- & -- & DOE-STD-1196 & $2.67 \mathrm{E}-14$ \\
\hline Sn-128 & Sn-128 & 127.911 & Tin & $1.00 \mathrm{E}-03$ & ICRP-38 & $5.91 \mathrm{E}+01$ & $\mathrm{~m}$ & $3.55 \mathrm{E}+03$ & $2.49 \mathrm{E}+07$ & ICRP-72 & $9.20 \mathrm{E}-11$ & $M$ & FGR-12 & $3.00 \mathrm{E}-14$ \\
\hline Sn-129 & Sn-129 & 128.913 & Tin & $1.00 \mathrm{E}-03$ & ICRP-107 & $2.23 \mathrm{E}+00$ & $\mathrm{~m}$ & $1.34 \mathrm{E}+02$ & $6.54 E+08$ & -- & -- & -- & DOE-STD-1196 & $4.76 \mathrm{E}-14$ \\
\hline Sn-130 & Sn-130 & 129.914 & Tin & $1.00 \mathrm{E}-03$ & ICRP-107 & $3.72 \mathrm{E}+00$ & $\mathrm{~m}$ & $2.23 \mathrm{E}+02$ & $3.89 E+08$ & -- & -- & -- & DOE-STD-1196 & $4.16 \mathrm{E}-14$ \\
\hline Sn-130m & Sn-130 & 129.914 & Tin & $1.00 \mathrm{E}-03$ & ICRP-107 & $1.70 E+00$ & $\mathrm{~m}$ & $1.02 \mathrm{E}+02$ & $8.51 \mathrm{E}+08$ & -- & -- & -- & DOE-STD-1196 & $4.20 \mathrm{E}-14$ \\
\hline Sr-79 & Sr-79 & 78.930 & Strontium & $1.00 \mathrm{E}-03$ & ICRP-107 & $2.25 E+00$ & $\mathrm{~m}$ & $1.35 \mathrm{E}+02$ & $1.06 \mathrm{E}+09$ & -- & -- & -- & DOE-STD-1196 & $5.39 \mathrm{E}-14$ \\
\hline Sr-80 & Sr-80 & 79.925 & Strontium & $1.00 \mathrm{E}-03$ & ICRP-38 & $1.00 \mathrm{E}+02$ & $\mathrm{~m}$ & $6.00 \mathrm{E}+03$ & $2.35 \mathrm{E}+07$ & ICRP-72 & $1.40 \mathrm{E}-10$ & $\mathrm{~s}$ & FGR-12 & $6.53 \mathrm{E}-18$ \\
\hline Sr-81 & Sr-81 & 80.923 & Strontium & $1.00 \mathrm{E}-03$ & ICRP-38 & $2.55 \mathrm{E}+01$ & $\mathrm{~m}$ & $1.53 \mathrm{E}+03$ & $9.11 \mathrm{E}+07$ & ICRP-72 & $3.70 \mathrm{E}-11$ & $\mathrm{~s}$ & FGR-12 & $6.68 \mathrm{E}-14$ \\
\hline Sr-82 & Sr-82 & 81.918 & Strontium & $1.00 \mathrm{E}-03$ & ICRP-38 & $2.50 \mathrm{E}+01$ & d & $2.16 \mathrm{E}+06$ & $6.38 \mathrm{E}+04$ & ICRP-72 & $1.10 \mathrm{E}-08$ & $\mathrm{~s}$ & FGR-12 & $6.43 \mathrm{E}-18$ \\
\hline Sr-83 & Sr-83 & 82.918 & Strontium & $1.00 \mathrm{E}-03$ & ICRP-38 & $3.24 \mathrm{E}+01$ & $\mathrm{~h}$ & $1.17 \mathrm{E}+05$ & $1.17 E+06$ & ICRP-72 & $3.40 \mathrm{E}-10$ & $\mathrm{~s}$ & FGR-12 & $3.86 \mathrm{E}-14$ \\
\hline Sr-85 & Sr-85 & 84.913 & Strontium & $1.00 \mathrm{E}-03$ & ICRP-38 & $6.48 \mathrm{E}+01$ & $d$ & $5.60 \mathrm{E}+06$ & $2.37 E+04$ & ICRP-72 & $8.10 \mathrm{E}-10$ & $\mathrm{~s}$ & FGR-12 & $2.42 \mathrm{E}-14$ \\
\hline Sr-85m & Sr-85 & 84.913 & Strontium & $1.00 \mathrm{E}-03$ & ICRP-38 & $6.95 \mathrm{E}+01$ & $\mathrm{~m}$ & $4.17 \mathrm{E}+03$ & $3.19 E+07$ & ICRP-72 & $4.30 \mathrm{E}-12$ & $\mathrm{~s}$ & FGR-12 & $1.05 \mathrm{E}-14$ \\
\hline Sr-87m & Sr-87 & 86.909 & Strontium & $1.00 \mathrm{E}-03$ & ICRP-38 & $2.81 \mathrm{E}+00$ & $\mathrm{~h}$ & $1.01 \mathrm{E}+04$ & $1.29 E+07$ & ICRP-72 & $2.10 \mathrm{E}-11$ & $\mathrm{~s}$ & FGR-12 & $1.52 \mathrm{E}-14$ \\
\hline $\mathrm{Sr}-89$ & Sr-89 & 88.907 & Strontium & $1.00 \mathrm{E}-03$ & ICRP-38 & $5.05 \mathrm{E}+01$ & $d$ & $4.36 \mathrm{E}+06$ & $2.91 E+04$ & ICRP-72 & $7.90 \mathrm{E}-09$ & $\mathrm{~s}$ & FGR-12 & $7.73 \mathrm{E}-17$ \\
\hline Sr-90 & Sr-90 & 89.908 & Strontium & $1.00 \mathrm{E}-03$ & ICRP-38 & $2.91 \mathrm{E}+01$ & $y$ & $9.19 \mathrm{E}+08$ & $1.37 E+02$ & ICRP-72 & $1.60 \mathrm{E}-07$ & $\mathrm{~s}$ & FGR-12 & $7.53 \mathrm{E}-18$ \\
\hline Sr-91 & Sr-91 & 90.910 & Strontium & $1.00 \mathrm{E}-03$ & ICRP-38 & $9.50 E+00$ & $\mathrm{~h}$ & $3.42 \mathrm{E}+04$ & $3.63 \mathrm{E}+06$ & ICRP-72 & $4.10 \mathrm{E}-10$ & $\mathrm{~s}$ & FGR-12 & $3.45 \mathrm{E}-14$ \\
\hline Sr-92 & Sr-92 & 91.911 & Strontium & $1.00 \mathrm{E}-03$ & ICRP-38 & $2.71 E+00$ & $\mathrm{~h}$ & $9.76 \mathrm{E}+03$ & $1.26 E+07$ & ICRP-72 & $2.30 \mathrm{E}-10$ & $\mathrm{~s}$ & FGR-12 & $6.79 \mathrm{E}-14$ \\
\hline Sr-93 & Sr-93 & 92.914 & Strontium & $1.00 \mathrm{E}-03$ & ICRP-107 & $7.42 \mathrm{E}+00$ & $\mathrm{~m}$ & $4.45 \mathrm{E}+02$ & $2.73 \mathrm{E}+08$ & -- & -- & -- & DOE-STD-1196 & $1.07 \mathrm{E}-13$ \\
\hline Sr-94 & Sr-94 & 93.915 & Strontium & $1.00 \mathrm{E}-03$ & ICRP-107 & $7.53 \mathrm{E}+01$ & $\mathrm{~s}$ & $7.53 \mathrm{E}+01$ & $1.60 E+09$ & -- & -- & -- & DOE-STD-1196 & $6.92 \mathrm{E}-14$ \\
\hline Ta-170 & Ta-170 & 169.946 & Tantalum & $1.00 \mathrm{E}-03$ & ICRP-107 & $6.76 \mathrm{E}+00$ & $\mathrm{~m}$ & $4.06 \mathrm{E}+02$ & $1.64 \mathrm{E}+08$ & -- & -- & -- & DOE-STD-1196 & $4.88 \mathrm{E}-14$ \\
\hline Ta-172 & Ta-172 & 171.945 & Tantalum & $1.00 \mathrm{E}-03$ & ICRP-38 & $3.68 \mathrm{E}+01$ & $\mathrm{~m}$ & $2.21 \mathrm{E}+03$ & $2.97 E+07$ & ICRP-72 & $3.50 \mathrm{E}-11$ & $\mathrm{~s}$ & FGR-12 & $7.59 \mathrm{E}-14$ \\
\hline Ta-173 & Ta-173 & 172.944 & Tantalum & $1.00 \mathrm{E}-03$ & ICRP-38 & $3.65 E+00$ & $\mathrm{~h}$ & $1.31 \mathrm{E}+04$ & $4.96 \mathrm{E}+06$ & ICRP-72 & $1.10 \mathrm{E}-10$ & $M$ & FGR-12 & $2.75 \mathrm{E}-14$ \\
\hline Ta-174 & Ta-174 & 173.944 & Tantalum & $1.00 \mathrm{E}-03$ & ICRP-38 & $1.20 \mathrm{E}+00$ & $\mathrm{~h}$ & $4.32 \mathrm{E}+03$ & $1.50 \mathrm{E}+07$ & ICRP-72 & $4.30 \mathrm{E}-11$ & $\mathrm{~s}$ & FGR-12 & $2.97 \mathrm{E}-14$ \\
\hline Ta-175 & Ta-175 & 174.944 & Tantalum & $1.00 \mathrm{E}-03$ & ICRP-38 & $1.05 E+01$ & $\mathrm{~h}$ & $3.78 \mathrm{E}+04$ & $1.71 \mathrm{E}+06$ & ICRP-72 & $1.30 \mathrm{E}-10$ & $\mathrm{~s}$ & FGR-12 & $4.55 \mathrm{E}-14$ \\
\hline Ta-176 & Ta-176 & 175.945 & Tantalum & $1.00 \mathrm{E}-03$ & ICRP-38 & $8.08 \mathrm{E}+00$ & $\mathrm{~h}$ & $2.91 \mathrm{E}+04$ & $2.20 \mathrm{E}+06$ & ICRP-72 & $2.00 \mathrm{E}-10$ & $\mathrm{~s}$ & FGR-12 & $1.09 \mathrm{E}-13$ \\
\hline Ta-177 & Ta-177 & 176.944 & Tantalum & $1.00 \mathrm{E}-03$ & ICRP-38 & $5.66 \mathrm{E}+01$ & $\mathrm{~h}$ & $2.04 \mathrm{E}+05$ & $3.13 \mathrm{E}+05$ & ICRP-72 & $1.10 \mathrm{E}-10$ & $\mathrm{~s}$ & FGR-12 & $2.53 \mathrm{E}-15$ \\
\hline Ta-178s & Ta-178 & 177.946 & Tantalum & $1.00 \mathrm{E}-03$ & ICRP-38 & $9.31 \mathrm{E}+00$ & $\mathrm{~m}$ & $5.59 \mathrm{E}+02$ & $1.13 \mathrm{E}+08$ & -- & -- & -- & FGR-12 & $4.61 \mathrm{E}-15$ \\
\hline Ta-1781 & Ta-178 & 177.946 & Tantalum & $1.00 \mathrm{E}-03$ & ICRP-38 & $2.20 \mathrm{E}+00$ & $\mathrm{~h}$ & $7.92 \mathrm{E}+03$ & $8.00 E+06$ & ICRP-72 & $6.80 \mathrm{E}-11$ & $\mathrm{~s}$ & FGR-12 & $4.75 \mathrm{E}-14$ \\
\hline Ta-179 & Ta-179 & 178.946 & Tantalum & $1.00 \mathrm{E}-03$ & ICRP-38 & $6.65 E+02$ & $d$ & $5.74 \mathrm{E}+07$ & $1.10 E+03$ & ICRP-72 & $5.60 \mathrm{E}-10$ & $\mathrm{~s}$ & FGR-12 & $1.09 \mathrm{E}-15$ \\
\hline Ta-180 & Ta-180 & 179.947 & Tantalum & $1.00 \mathrm{E}-03$ & ICRP-38 & $1.00 \mathrm{E}+13$ & $y$ & $3.16 \mathrm{E}+20$ & $1.99 \mathrm{E}-10$ & ICRP-72 & $2.60 \mathrm{E}-08$ & $\mathrm{~s}$ & FGR-12 & $2.59 \mathrm{E}-14$ \\
\hline Ta-180m & Ta-180 & 179.947 & Tantalum & $1.00 \mathrm{E}-03$ & ICRP-38 & $8.10 \mathrm{E}+00$ & $\mathrm{~h}$ & $2.92 \mathrm{E}+04$ & $2.15 \mathrm{E}+06$ & ICRP-72 & $4.40 \mathrm{E}-11$ & $M$ & FGR-12 & $1.71 \mathrm{E}-15$ \\
\hline Ta-182 & Ta-182 & 181.950 & Tantalum & $1.00 \mathrm{E}-03$ & ICRP-38 & $1.15 \mathrm{E}+02$ & d & $9.94 \mathrm{E}+06$ & $6.24 E+03$ & ICRP-72 & $1.00 \mathrm{E}-08$ & $\mathrm{~s}$ & FGR-12 & $6.40 \mathrm{E}-14$ \\
\hline Ta-182m & Ta-182 & 181.950 & Tantalum & $1.00 \mathrm{E}-03$ & ICRP-38 & $1.58 \mathrm{E}+01$ & $\mathrm{~m}$ & $9.50 \mathrm{E}+02$ & $6.52 E+07$ & ICRP-72 & $2.10 \mathrm{E}-11$ & $\mathrm{~s}$ & FGR-12 & $1.11 \mathrm{E}-14$ \\
\hline
\end{tabular}




\begin{tabular}{|c|c|c|c|c|c|c|c|c|c|c|c|c|c|c|}
\hline \multicolumn{15}{|c|}{ Table A.1 Radionuclide Specific Input Data Used For the Calculation of HC-2 TQs Using Maximum Reported DCs (continued) } \\
\hline $\begin{array}{c}\text { MASTER } \\
\text { ISOTOPE } \\
\text { LIST }\end{array}$ & $\begin{array}{l}\text { Atomic } \\
\text { Mass } \\
\text { Nuclide }\end{array}$ & $\begin{array}{l}\text { Atomic } \\
\text { Mass }\end{array}$ & Element & $\begin{array}{c}\text { RF per } \\
\text { DOE-STD- } \\
1027\end{array}$ & $\begin{array}{c}\text { Half-Life } \\
\text { Reference }\end{array}$ & $\begin{array}{r}\text { Half-Li } \\
\text { (original }\end{array}$ & & Half Life (s) & $\begin{array}{l}\text { Sp. Act } \\
\text { (Ci/g) }\end{array}$ & $\begin{array}{l}\text { Inhalation DC } \\
\text { Reference }\end{array}$ & $\begin{array}{c}\text { HC-2 } \\
\text { Bounding } \\
\text { Inhalation } \\
\text { DC (Sv/Bq) }\end{array}$ & $\begin{array}{l}\text { Lung } \\
\text { Absorption } \\
\text { Class/Type }\end{array}$ & $\begin{array}{l}\text { Immersion DC } \\
\text { Reference }\end{array}$ & $\begin{array}{c}\mathrm{HC}-2 \\
\text { Immersion } \\
\mathrm{DC}(\mathrm{Sv} / \mathrm{s} \\
\left.\text { per } \mathrm{Bq} / \mathrm{m}^{3}\right)\end{array}$ \\
\hline Ta-183 & Ta-183 & 182.951 & Tantalum & $1.00 \mathrm{E}-03$ & ICRP-38 & $5.10 \mathrm{E}+00$ & d & $4.41 \mathrm{E}+05$ & $1.40 \mathrm{E}+05$ & ICRP-72 & $2.10 \mathrm{E}-09$ & $\mathrm{~s}$ & FGR-12 & $1.31 \mathrm{E}-14$ \\
\hline Ta-184 & Ta-184 & 183.954 & Tantalum & $1.00 \mathrm{E}-03$ & ICRP-38 & $8.70 \mathrm{E}+00$ & $\mathrm{~h}$ & $3.13 \mathrm{E}+04$ & $1.96 \mathrm{E}+06$ & ICRP-72 & $4.30 \mathrm{E}-10$ & $\mathrm{~s}$ & FGR-12 & $7.80 \mathrm{E}-14$ \\
\hline Ta-185 & Ta-185 & 184.956 & Tantalum & $1.00 \mathrm{E}-03$ & ICRP-38 & $4.90 \mathrm{E}+01$ & $\mathrm{~m}$ & $2.94 \mathrm{E}+03$ & $2.07 \mathrm{E}+07$ & ICRP-72 & $4.80 \mathrm{E}-11$ & $\mathrm{~s}$ & FGR-12 & $8.73 \mathrm{E}-15$ \\
\hline Ta-186 & Ta-186 & 185.959 & Tantalum & $1.00 \mathrm{E}-03$ & ICRP-38 & $1.05 E+01$ & $\mathrm{~m}$ & $6.30 \mathrm{E}+02$ & $9.63 \mathrm{E}+07$ & ICRP-72 & $1.80 \mathrm{E}-11$ & $\mathrm{~s}$ & FGR-12 & $7.53 \mathrm{E}-14$ \\
\hline Tb-146 & Tb-146 & 145.927 & Terbium & $1.00 \mathrm{E}-03$ & ICRP-107 & $2.30 \mathrm{E}+01$ & $\mathrm{~s}$ & $2.30 \mathrm{E}+01$ & $3.36 \mathrm{E}+09$ & -- & -- & -- & DOE-STD-1196 & $1.74 \mathrm{E}-13$ \\
\hline Tb-147 & Tb-147 & 146.924 & Terbium & $1.00 \mathrm{E}-03$ & ICRP-38 & $1.65 \mathrm{E}+00$ & $\mathrm{~h}$ & $5.94 \mathrm{E}+03$ & $1.29 \mathrm{E}+07$ & ICRP-72 & $7.60 \mathrm{E}-11$ & $M$ & FGR-12 & $7.78 \mathrm{E}-14$ \\
\hline Tb-147m & Tb-147 & 146.924 & Terbium & $1.00 \mathrm{E}-03$ & ICRP-107 & $1.87 \mathrm{E}+00$ & $\mathrm{~m}$ & $1.12 \mathrm{E}+02$ & $6.84 \mathrm{E}+08$ & -- & -- & -- & DOE-STD-1196 & $9.11 \mathrm{E}-14$ \\
\hline Tb-148 & Tb-148 & 147.924 & Terbium & $1.00 \mathrm{E}-03$ & ICRP-107 & $6.00 \mathrm{E}+01$ & $\mathrm{~m}$ & $3.60 \mathrm{E}+03$ & $2.12 \mathrm{E}+07$ & DOE-STD-1196 & $8.28 \mathrm{E}-11$ & $M$ & DOE-STD-1196 & $1.11 \mathrm{E}-13$ \\
\hline Tb-148m & Tb-148 & 147.924 & Terbium & $1.00 \mathrm{E}-03$ & ICRP-107 & $2.20 \mathrm{E}+00$ & $\mathrm{~m}$ & $1.32 \mathrm{E}+02$ & $5.78 \mathrm{E}+08$ & -- & -- & -- & DOE-STD-1196 & $1.42 \mathrm{E}-13$ \\
\hline Tb-149 & Tb-149 & 148.923 & Terbium & $1.00 \mathrm{E}-03$ & ICRP-38 & $4.15 \mathrm{E}+00$ & $\mathrm{~h}$ & $1.49 \mathrm{E}+04$ & $5.07 \mathrm{E}+06$ & ICRP-72 & $4.90 \mathrm{E}-09$ & $M$ & FGR-12 & $8.02 \mathrm{E}-14$ \\
\hline Tb-149m & Tb-149 & 148.923 & Terbium & $1.00 \mathrm{E}-03$ & ICRP-107 & $4.16 \mathrm{E}+00$ & $\mathrm{~m}$ & $2.50 \mathrm{E}+02$ & $3.03 E+08$ & -- & -- & -- & DOE-STD-1196 & $6.20 \mathrm{E}-14$ \\
\hline Tb-150 & Tb-150 & 149.924 & Terbium & $1.00 \mathrm{E}-03$ & ICRP-38 & $3.27 \mathrm{E}+00$ & $\mathrm{~h}$ & $1.18 \mathrm{E}+04$ & $6.39 \mathrm{E}+06$ & ICRP-72 & $1.10 \mathrm{E}-10$ & $M$ & FGR-12 & $8.26 \mathrm{E}-14$ \\
\hline Tb-150m & Tb-150 & 149.924 & Terbium & $1.00 \mathrm{E}-03$ & ICRP-107 & $5.80 \mathrm{E}+00$ & $\mathrm{~m}$ & $3.48 \mathrm{E}+02$ & $2.16 \mathrm{E}+08$ & -- & -- & -- & DOE-STD-1196 & $1.12 \mathrm{E}-13$ \\
\hline Tb-151 & Tb-151 & 150.923 & Terbium & $1.00 \mathrm{E}-03$ & ICRP-38 & $1.76 \mathrm{E}+01$ & $\mathrm{~h}$ & $6.34 \mathrm{E}+04$ & $1.18 \mathrm{E}+06$ & ICRP-72 & $2.30 \mathrm{E}-10$ & $M$ & FGR-12 & $4.20 \mathrm{E}-14$ \\
\hline Tb-151m & Tb-151 & 150.923 & Terbium & $1.00 \mathrm{E}-03$ & ICRP-107 & $2.50 \mathrm{E}+01$ & $\mathrm{~s}$ & $2.50 \mathrm{E}+01$ & $2.99 \mathrm{E}+09$ & -- & -- & -- & DOE-STD-1196 & $3.20 \mathrm{E}-15$ \\
\hline Tb-152 & Tb-152 & 151.924 & Terbium & $1.00 \mathrm{E}-03$ & ICRP-107 & $1.75 E+01$ & $\mathrm{~h}$ & $6.30 \mathrm{E}+04$ & $1.18 \mathrm{E}+06$ & DOE-STD-1196 & $3.82 \mathrm{E}-10$ & $\mathrm{~s}$ & DOE-STD-1196 & $6.98 \mathrm{E}-14$ \\
\hline Tb-152m & Tb-152 & 151.924 & Terbium & $1.00 \mathrm{E}-03$ & ICRP-107 & $4.20 E+00$ & $\mathrm{~m}$ & $2.52 \mathrm{E}+02$ & $2.95 E+08$ & -- & -- & -- & DOE-STD-1196 & $3.28 \mathrm{E}-14$ \\
\hline Tb-153 & Tb-153 & 152.923 & Terbium & $1.00 \mathrm{E}-03$ & ICRP-38 & $2.34 \mathrm{E}+00$ & d & $2.02 \mathrm{E}+05$ & $3.65 E+05$ & ICRP-72 & $1.90 \mathrm{E}-10$ & $M$ & FGR-12 & $9.89 \mathrm{E}-15$ \\
\hline Tb-154 & Tb-154 & 153.925 & Terbium & $1.00 \mathrm{E}-03$ & ICRP-38 & $2.14 \mathrm{E}+01$ & $\mathrm{~h}$ & $7.70 \mathrm{E}+04$ & $9.51 \mathrm{E}+05$ & ICRP-72 & $3.60 \mathrm{E}-10$ & $M$ & FGR-12 & $1.21 \mathrm{E}-13$ \\
\hline Tb-155 & Tb-155 & 154.924 & Terbium & $1.00 \mathrm{E}-03$ & ICRP-38 & $5.32 \mathrm{E}+00$ & $d$ & $4.60 \mathrm{E}+05$ & $1.58 \mathrm{E}+05$ & ICRP-72 & $2.20 \mathrm{E}-10$ & $M$ & FGR-12 & $5.56 \mathrm{E}-15$ \\
\hline Tb-156 & Tb-156 & 155.925 & Terbium & $1.00 \mathrm{E}-03$ & ICRP-38 & $5.34 \mathrm{E}+00$ & $d$ & $4.61 \mathrm{E}+05$ & $1.57 \mathrm{E}+05$ & ICRP-72 & $1.20 \mathrm{E}-09$ & $M$ & FGR-12 & $8.94 \mathrm{E}-14$ \\
\hline Tb-156ml & Tb-156 & 155.925 & Terbium & $1.00 \mathrm{E}-03$ & ICRP-38 & $2.44 \mathrm{E}+01$ & $\mathrm{~h}$ & $8.78 \mathrm{E}+04$ & $8.24 \mathrm{E}+05$ & ICRP-72 & $2.10 \mathrm{E}-10$ & $M$ & FGR-12 & $7.75 \mathrm{E}-16$ \\
\hline Tb-156ms & Tb-156 & 155.925 & Terbium & $1.00 \mathrm{E}-03$ & ICRP-38 & $5.00 \mathrm{E}+00$ & $\mathrm{~h}$ & $1.80 \mathrm{E}+04$ & $4.02 E+06$ & ICRP-72 & $9.60 \mathrm{E}-11$ & $M$ & FGR-12 & $1.16 \mathrm{E}-16$ \\
\hline Tb-157 & Tb-157 & 156.924 & Terbium & $1.00 \mathrm{E}-03$ & ICRP-38 & $1.50 \mathrm{E}+02$ & $y$ & $4.73 E+09$ & $1.52 \mathrm{E}+01$ & ICRP-72 & $1.20 \mathrm{E}-09$ & $M$ & FGR-12 & $6.78 \mathrm{E}-17$ \\
\hline Tb-158 & Tb-158 & 157.925 & Terbium & $1.00 \mathrm{E}-03$ & ICRP-38 & $1.50 \mathrm{E}+02$ & $y$ & $4.73 \mathrm{E}+09$ & $1.51 \mathrm{E}+01$ & ICRP-72 & $4.60 \mathrm{E}-08$ & $M$ & FGR-12 & $3.84 \mathrm{E}-14$ \\
\hline Tb-160 & Tb-160 & 159.927 & Terbium & $1.00 \mathrm{E}-03$ & ICRP-38 & $7.23 E+01$ & $d$ & $6.25 \mathrm{E}+06$ & $1.13 E+04$ & ICRP-72 & $7.00 \mathrm{E}-09$ & $M$ & FGR-12 & $5.54 \mathrm{E}-14$ \\
\hline Tb-161 & Tb-161 & 160.928 & Terbium & $1.00 \mathrm{E}-03$ & ICRP-38 & $6.91 \mathrm{E}+00$ & d & $5.97 \mathrm{E}+05$ & $1.17 \mathrm{E}+05$ & ICRP-72 & $1.30 \mathrm{E}-09$ & $M$ & FGR-12 & $1.02 \mathrm{E}-15$ \\
\hline Tb-162 & Tb-162 & 161.929 & Terbium & $1.00 \mathrm{E}-03$ & ICRP-107 & $7.60 E+00$ & $\mathrm{~m}$ & $4.56 \mathrm{E}+02$ & $1.53 \mathrm{E}+08$ & -- & -- & -- & DOE-STD-1196 & $5.04 \mathrm{E}-14$ \\
\hline Tb-163 & Tb-163 & 162.931 & Terbium & $1.00 \mathrm{E}-03$ & ICRP-107 & $1.95 \mathrm{E}+01$ & $\mathrm{~m}$ & $1.17 \mathrm{E}+03$ & $5.92 \mathrm{E}+07$ & DOE-STD-1196 & $2.03 \mathrm{E}-11$ & $\mathrm{~s}$ & DOE-STD-1196 & $3.51 \mathrm{E}-14$ \\
\hline Tb-164 & Tb-164 & 163.933 & Terbium & $1.00 \mathrm{E}-03$ & ICRP-107 & $3.00 E+00$ & $\mathrm{~m}$ & $1.80 \mathrm{E}+02$ & $3.82 \mathrm{E}+08$ & -- & -- & -- & DOE-STD-1196 & $1.14 \mathrm{E}-13$ \\
\hline Tb-165 & Tb-165 & 164.935 & Terbium & $1.00 \mathrm{E}-03$ & ICRP-107 & $2.11 E+00$ & $\mathrm{~m}$ & $1.27 \mathrm{E}+02$ & $5.40 E+08$ & -- & -- & -- & DOE-STD-1196 & $4.04 \mathrm{E}-14$ \\
\hline Tc-101 & Tc-101 & 100.907 & Technetium & $1.00 \mathrm{E}-03$ & ICRP-38 & $1.42 \mathrm{E}+01$ & $\mathrm{~m}$ & $8.52 \mathrm{E}+02$ & $1.31 \mathrm{E}+08$ & ICRP-72 & $1.20 \mathrm{E}-11$ & $M$ & FGR-12 & $1.61 \mathrm{E}-14$ \\
\hline Tc-102 & Tc-102 & 101.909 & Technetium & $1.00 \mathrm{E}-03$ & ICRP-107 & $5.28 \mathrm{E}+00$ & $\mathrm{~s}$ & $5.28 \mathrm{E}+00$ & $2.10 \mathrm{E}+10$ & -- & -- & -- & DOE-STD-1196 & $5.85 \mathrm{E}-15$ \\
\hline Tc-102m & Tc-102 & 101.909 & Technetium & $1.00 \mathrm{E}-03$ & ICRP-107 & $4.35 E+00$ & $\mathrm{~m}$ & $2.61 \mathrm{E}+02$ & $4.24 \mathrm{E}+08$ & -- & -- & -- & DOE-STD-1196 & $1.18 \mathrm{E}-13$ \\
\hline Tc-104 & Tc-104 & 103.911 & Technetium & $1.00 \mathrm{E}-03$ & ICRP-38 & $1.82 \mathrm{E}+01$ & $\mathrm{~m}$ & $1.09 \mathrm{E}+03$ & $9.94 \mathrm{E}+07$ & ICRP-72 & $2.90 \mathrm{E}-11$ & $\mathrm{~s}$ & FGR-12 & $1.01 \mathrm{E}-13$ \\
\hline Tc-105 & Tc-105 & 104.912 & Technetium & $1.00 \mathrm{E}-03$ & ICRP-107 & $7.60 E+00$ & $\mathrm{~m}$ & $4.56 \mathrm{E}+02$ & $2.36 \mathrm{E}+08$ & -- & -- & -- & DOE-STD-1196 & $3.77 \mathrm{E}-14$ \\
\hline Tc-91 & Tc-91 & 90.918 & Technetium & $1.00 \mathrm{E}-03$ & ICRP-107 & $3.14 \mathrm{E}+00$ & $\mathrm{~m}$ & $1.88 \mathrm{E}+02$ & $6.59 \mathrm{E}+08$ & -- & -- & -- & DOE-STD-1196 & $1.20 \mathrm{E}-13$ \\
\hline Tc-91m & Tc-91 & 90.918 & Technetium & $1.00 \mathrm{E}-03$ & ICRP-107 & $3.30 \mathrm{E}+00$ & $\mathrm{~m}$ & $1.98 \mathrm{E}+02$ & $6.27 \mathrm{E}+08$ & -- & -- & -- & DOE-STD-1196 & $6.60 \mathrm{E}-14$ \\
\hline Tc-92 & Tc-92 & 91.915 & Technetium & $1.00 \mathrm{E}-03$ & ICRP-107 & $4.25 E+00$ & $\mathrm{~m}$ & $2.55 E+02$ & $4.81 \mathrm{E}+08$ & -- & -- & -- & DOE-STD-1196 & $1.79 \mathrm{E}-13$ \\
\hline Tc-93 & Tc-93 & 92.910 & Technetium & $1.00 \mathrm{E}-03$ & ICRP-38 & $2.75 E+00$ & $\mathrm{~h}$ & $9.90 \mathrm{E}+03$ & $1.23 \mathrm{E}+07$ & ICRP-72 & $3.50 \mathrm{E}-11$ & $M$ & FGR-12 & $7.38 \mathrm{E}-14$ \\
\hline Tc-93m & Tc-93 & 92.910 & Technetium & $1.00 \mathrm{E}-03$ & ICRP-38 & $4.35 \mathrm{E}+01$ & $\mathrm{~m}$ & $2.61 \mathrm{E}+03$ & $4.65 \mathrm{E}+07$ & ICRP-72 & $1.70 \mathrm{E}-11$ & $M$ & FGR-12 & $3.73 \mathrm{E}-14$ \\
\hline Tc-94 & Tc-94 & 93.910 & Technetium & $1.00 \mathrm{E}-03$ & ICRP-38 & $2.93 \mathrm{E}+02$ & $\mathrm{~m}$ & $1.76 \mathrm{E}+04$ & $6.83 \mathrm{E}+06$ & ICRP-72 & $1.30 \mathrm{E}-10$ & $\mathrm{~s}$ & FGR-12 & $1.30 \mathrm{E}-13$ \\
\hline Tc-94m & TC-94 & 93.910 & Technetium & $1.00 \mathrm{E}-03$ & ICRP-38 & $5.20 \mathrm{E}+01$ & $\mathrm{~m}$ & $3.12 \mathrm{E}+03$ & $3.85 \mathrm{E}+07$ & ICRP-72 & $4.60 \mathrm{E}-11$ & $\mathrm{~s}$ & FGR-12 & $9.18 \mathrm{E}-14$ \\
\hline Tc-95 & TC-95 & 94.908 & Technetium & $1.00 \mathrm{E}-03$ & ICRP-38 & $2.00 \mathrm{E}+01$ & $\mathrm{~h}$ & $7.20 \mathrm{E}+04$ & $1.65 E+06$ & ICRP-72 & $1.10 \mathrm{E}-10$ & $\mathrm{~s}$ & FGR-12 & $3.84 \mathrm{E}-14$ \\
\hline Tc-95m & Tc-95 & 94.908 & Technetium & $1.00 \mathrm{E}-03$ & ICRP-38 & $6.10 \mathrm{E}+01$ & $d$ & $5.27 \mathrm{E}+06$ & $2.26 \mathrm{E}+04$ & ICRP-72 & $1.20 \mathrm{E}-09$ & $\mathrm{~s}$ & FGR-12 & $3.23 \mathrm{E}-14$ \\
\hline Tc-96 & Tc-96 & 95.908 & Technetium & $1.00 \mathrm{E}-03$ & ICRP-38 & $4.28 \mathrm{E}+00$ & $d$ & $3.70 \mathrm{E}+05$ & $3.18 \mathrm{E}+05$ & ICRP-72 & $7.00 \mathrm{E}-10$ & $\mathrm{~s}$ & FGR-12 & $1.22 \mathrm{E}-13$ \\
\hline
\end{tabular}




\begin{tabular}{|c|c|c|c|c|c|c|c|c|c|c|c|c|c|c|}
\hline \multicolumn{15}{|c|}{ Table A.1 Radionuclide Specific Input Data Used For the Calculation of HC-2 TQs Using Maximum Reported DCs (continued) } \\
\hline $\begin{array}{c}\text { MASTER } \\
\text { ISOTOPE } \\
\text { LIST } \\
\end{array}$ & $\begin{array}{l}\text { Atomic } \\
\text { Mass } \\
\text { Nuclide }\end{array}$ & $\begin{array}{c}\text { Atomic } \\
\text { Mass }\end{array}$ & Element & $\begin{array}{c}\text { RF per } \\
\text { DOE-STD- } \\
1027\end{array}$ & $\begin{array}{l}\text { Half-Life } \\
\text { Reference }\end{array}$ & $\begin{array}{r}\text { Half-Li } \\
\text { (original }\end{array}$ & & Half Life (s) & $\begin{array}{l}\text { Sp. Act } \\
\text { (Ci/g) }\end{array}$ & $\begin{array}{c}\text { Inhalation DC } \\
\text { Reference }\end{array}$ & $\begin{array}{c}\text { HC-2 } \\
\text { Bounding } \\
\text { Inhalation } \\
\text { DC (Sv/Bq) }\end{array}$ & $\begin{array}{l}\text { Lung } \\
\text { Absorption } \\
\text { Class/Type }\end{array}$ & $\begin{array}{c}\text { Immersion DC } \\
\text { Reference }\end{array}$ & $\begin{array}{c}\text { HC-2 } \\
\text { Immersion } \\
\mathrm{DC}(\mathrm{Sv} / \mathrm{s} \\
\left.\text { per } \mathrm{Bq} / \mathrm{m}^{3}\right)\end{array}$ \\
\hline Tc-96m & Tc-96 & 95.908 & Technetium & $1.00 \mathrm{E}-03$ & ICRP-38 & $5.15 \mathrm{E}+01$ & $\mathrm{~m}$ & $3.09 \mathrm{E}+03$ & $3.81 \mathrm{E}+07$ & ICRP-72 & $7.50 \mathrm{E}-12$ & $\mathrm{~s}$ & FGR-12 & $2.24 \mathrm{E}-15$ \\
\hline Tc-97 & Tc-97 & 96.906 & Technetium & $1.00 \mathrm{E}-03$ & ICRP-38 & $2.60 \mathrm{E}+06$ & y & $8.20 \mathrm{E}+13$ & $1.42 \mathrm{E}-03$ & ICRP-72 & $1.80 \mathrm{E}-09$ & $\mathrm{~s}$ & FGR-12 & $3.33 \mathrm{E}-17$ \\
\hline Tc-97m & TC-97 & 96.906 & Technetium & $1.00 \mathrm{E}-03$ & ICRP-38 & $8.70 \mathrm{E}+01$ & $d$ & $7.52 \mathrm{E}+06$ & $1.55 \mathrm{E}+04$ & ICRP-72 & $4.10 \mathrm{E}-09$ & $\mathrm{~s}$ & FGR-12 & $4.64 \mathrm{E}-17$ \\
\hline Tc-98 & Tc-98 & 97.907 & Technetium & $1.00 \mathrm{E}-03$ & ICRP-38 & $4.20 E+06$ & $y$ & $1.33 \mathrm{E}+14$ & $8.69 \mathrm{E}-04$ & ICRP-72 & $4.50 \mathrm{E}-08$ & $\mathrm{~s}$ & FGR-12 & $6.86 \mathrm{E}-14$ \\
\hline Tc-99 & Tc-99 & 98.906 & Technetium & $1.00 \mathrm{E}-03$ & ICRP-38 & $2.13 \mathrm{E}+05$ & $y$ & $6.72 \mathrm{E}+12$ & $1.70 \mathrm{E}-02$ & ICRP-72 & $1.30 \mathrm{E}-08$ & $\mathrm{~s}$ & FGR-12 & $1.62 \mathrm{E}-18$ \\
\hline Tc-99m & Tc-99 & 98.906 & Technetium & $1.00 \mathrm{E}-03$ & ICRP-38 & $6.02 \mathrm{E}+00$ & $\mathrm{~h}$ & $2.17 E+04$ & $5.26 \mathrm{E}+06$ & ICRP-72 & $2.00 \mathrm{E}-11$ & $\mathrm{~s}$ & FGR-12 & $5.89 \mathrm{E}-15$ \\
\hline Te-113 & Te-113 & 112.916 & Tellurium & $1.00 \mathrm{E}-02$ & ICRP-107 & $1.70 \mathrm{E}+00$ & $\mathrm{~m}$ & $1.02 \mathrm{E}+02$ & $9.80 \mathrm{E}+08$ & -- & -- & -- & DOE-STD-1196 & $1.05 \mathrm{E}-13$ \\
\hline Te-114 & Te-114 & 113.912 & $\begin{array}{l}\text { Tellurium } \\
\end{array}$ & $1.00 \mathrm{E}-02$ & ICRP-107 & $1.52 \mathrm{E}+01$ & $\mathrm{~m}$ & $9.12 \mathrm{E}+02$ & $1.09 \mathrm{E}+08$ & DOE-STD-1196 & $3.82 \mathrm{E}-11$ & $\mathrm{~V}$ & DOE-STD-1196 & $5.92 \mathrm{E}-14$ \\
\hline Te-115 & Te-115 & 114.912 & Tellurium & $1.00 \mathrm{E}-02$ & ICRP-107 & $5.80 \mathrm{E}+00$ & $\mathrm{~m}$ & $3.48 \mathrm{E}+02$ & $2.82 \mathrm{E}+08$ & -- & -- & -- & DOE-STD-1196 & $1.05 \mathrm{E}-13$ \\
\hline Te-115m & Te-115 & 114.912 & Tellurium & $1.00 \mathrm{E}-02$ & ICRP-107 & $6.70 \mathrm{E}+00$ & $\mathrm{~m}$ & $4.02 \mathrm{E}+02$ & $2.44 \mathrm{E}+08$ & -- & -- & -- & DOE-STD-1196 & $1.22 \mathrm{E}-13$ \\
\hline Te-116 & Te-116 & 115.908 & Tellurium & $1.00 \mathrm{E}-02$ & ICRP-38 & $2.49 \mathrm{E}+00$ & $\mathrm{~h}$ & $8.96 \mathrm{E}+03$ & $1.09 \mathrm{E}+07$ & ICRP-72 & $1.10 \mathrm{E}-10$ & $\mathrm{~s}$ & FGR-12 & $2.29 \mathrm{E}-15$ \\
\hline Te-117 & Te-117 & 116.909 & Tellurium & $1.00 \mathrm{E}-02$ & ICRP-107 & $6.20 \mathrm{E}+01$ & $\mathrm{~m}$ & $3.72 E+03$ & $2.59 \mathrm{E}+07$ & DOE-STD-1196 & $3.91 \mathrm{E}-11$ & $\mathrm{~s}$ & DOE-STD-1196 & $7.25 \mathrm{E}-14$ \\
\hline Te-118 & Te-118 & 117.906 & Tellurium & $1.00 \mathrm{E}-02$ & ICRP-107 & $6.00 \mathrm{E}+00$ & $d$ & $5.18 \mathrm{E}+05$ & $1.85 \mathrm{E}+05$ & DOE-STD-1196 & $2.95 \mathrm{E}-09$ & $\mathrm{~s}$ & DOE-STD-1196 & $1.51 \mathrm{E}-16$ \\
\hline Te-119 & Te-119 & 118.906 & Tellurium & $1.00 \mathrm{E}-02$ & ICRP-107 & $1.61 \mathrm{E}+01$ & $\mathrm{~h}$ & $5.78 \mathrm{E}+04$ & $1.64 \mathrm{E}+06$ & DOE-STD-1196 & $1.32 \mathrm{E}-10$ & $\mathrm{~s}$ & DOE-STD-1196 & $3.43 \mathrm{E}-14$ \\
\hline Te-119m & Te-119 & 118.906 & Tellurium & $1.00 \mathrm{E}-02$ & ICRP-107 & $4.70 E+00$ & $d$ & $4.06 \mathrm{E}+05$ & $2.34 \mathrm{E}+05$ & DOE-STD-1196 & $7.32 \mathrm{E}-10$ & $\mathrm{~V}$ & DOE-STD-1196 & $6.97 \mathrm{E}-14$ \\
\hline Te-121 & Te-121 & 120.905 & Tellurium & $1.00 \mathrm{E}-02$ & ICRP-38 & $1.70 \mathrm{E}+01$ & $d$ & $1.47 \mathrm{E}+06$ & $6.35 \mathrm{E}+04$ & ICRP-72 & $5.10 \mathrm{E}-10$ & $\mathrm{~V}$ & FGR-12 & $2.70 \mathrm{E}-14$ \\
\hline Te-121m & Te-121 & 120.905 & Tellurium & $1.00 \mathrm{E}-02$ & ICRP-38 & $1.54 \mathrm{E}+02$ & $d$ & $1.33 \mathrm{E}+07$ & $7.01 E+03$ & ICRP-72 & $5.70 \mathrm{E}-09$ & $\mathrm{~s}$ & FGR-12 & $9.90 \mathrm{E}-15$ \\
\hline Te-123 & Te-123 & 122.904 & Tellurium & $1.00 \mathrm{E}-02$ & ICRP-38 & $1.00 \mathrm{E}+13$ & $y$ & $3.16 \mathrm{E}+20$ & $2.91 \mathrm{E}-10$ & ICRP-72 & $1.20 \mathrm{E}-08$ & $\mathrm{~V}$ & FGR-12 & $2.15 \mathrm{E}-16$ \\
\hline Te-123m & Te-123 & 122.904 & Tellurium & $1.00 \mathrm{E}-02$ & ICRP-38 & $1.20 \mathrm{E}+02$ & $d$ & $1.03 E+07$ & $8.88 \mathrm{E}+03$ & ICRP-72 & $5.10 \mathrm{E}-09$ & $\mathrm{~s}$ & FGR-12 & $6.51 \mathrm{E}-15$ \\
\hline Te-125m & Te-125 & 124.904 & Tellurium & $1.00 \mathrm{E}-02$ & ICRP-38 & $5.80 \mathrm{E}+01$ & $d$ & $5.01 \mathrm{E}+06$ & $1.80 \mathrm{E}+04$ & ICRP-72 & $4.20 \mathrm{E}-09$ & $\mathrm{~s}$ & FGR-12 & $4.53 \mathrm{E}-16$ \\
\hline Te-127 & Te-127 & 126.905 & Tellurium & $1.00 \mathrm{E}-02$ & ICRP-38 & $9.35 \mathrm{E}+00$ & $\mathrm{~h}$ & $3.37 \mathrm{E}+04$ & $2.64 \mathrm{E}+06$ & ICRP-72 & $1.40 \mathrm{E}-10$ & $\mathrm{~s}$ & FGR-12 & $2.42 \mathrm{E}-16$ \\
\hline Te-127m & Te-127 & 126.905 & Tellurium & $1.00 \mathrm{E}-02$ & ICRP-38 & $1.09 \mathrm{E}+02$ & $d$ & $9.42 \mathrm{E}+06$ & $9.44 \mathrm{E}+03$ & ICRP-72 & $9.80 \mathrm{E}-09$ & $\mathrm{~s}$ & FGR-12 & $1.47 \mathrm{E}-16$ \\
\hline Te-129 & Te-129 & 128.907 & Tellurium & $1.00 \mathrm{E}-02$ & ICRP-38 & $6.96 \mathrm{E}+01$ & $\mathrm{~m}$ & $4.18 \mathrm{E}+03$ & $2.10 \mathrm{E}+07$ & ICRP-72 & $3.90 \mathrm{E}-11$ & $\mathrm{~s}$ & FGR-12 & $2.75 \mathrm{E}-15$ \\
\hline Te-129m & Te-129 & 128.907 & Tellurium & $1.00 \mathrm{E}-02$ & ICRP-38 & $3.36 \mathrm{E}+01$ & $d$ & $2.90 \mathrm{E}+06$ & $3.01 \mathrm{E}+04$ & ICRP-72 & $7.90 \mathrm{E}-09$ & $\mathrm{~s}$ & FGR-12 & $1.55 \mathrm{E}-15$ \\
\hline Te-131 & Te-131 & 130.909 & Tellurium & $1.00 \mathrm{E}-02$ & ICRP-38 & $2.50 \mathrm{E}+01$ & $\mathrm{~m}$ & $1.50 \mathrm{E}+03$ & $5.75 \mathrm{E}+07$ & ICRP-72 & $6.80 \mathrm{E}-11$ & V & FGR-12 & $2.04 \mathrm{E}-14$ \\
\hline Te-131m & Te-131 & 130.909 & Tellurium & $1.00 \mathrm{E}-02$ & ICRP-38 & $3.00 \mathrm{E}+01$ & $\mathrm{~h}$ & $1.08 \mathrm{E}+05$ & $7.98 \mathrm{E}+05$ & ICRP-72 & $2.40 \mathrm{E}-09$ & $\mathrm{~V}$ & FGR-12 & $7.01 \mathrm{E}-14$ \\
\hline Te-132 & Te-132 & 131.909 & Tellurium & $1.00 \mathrm{E}-02$ & ICRP-38 & $7.82 \mathrm{E}+01$ & $\mathrm{~h}$ & $2.82 \mathrm{E}+05$ & $3.04 \mathrm{E}+05$ & ICRP-72 & $5.10 \mathrm{E}-09$ & $\mathrm{~V}$ & FGR-12 & $1.03 E-14$ \\
\hline Te-133 & Te-133 & 132.911 & Tellurium & $1.00 \mathrm{E}-02$ & ICRP-38 & $1.25 \mathrm{E}+01$ & $\mathrm{~m}$ & $7.47 \mathrm{E}+02$ & $1.14 \mathrm{E}+08$ & ICRP-72 & $5.60 \mathrm{E}-11$ & $\mathrm{~V}$ & FGR-12 & $4.60 \mathrm{E}-14$ \\
\hline Te-133m & Te-133 & 132.911 & Tellurium & $1.00 \mathrm{E}-02$ & ICRP-38 & $5.54 \mathrm{E}+01$ & $\mathrm{~m}$ & $3.32 \mathrm{E}+03$ & $2.55 \mathrm{E}+07$ & ICRP-72 & $2.20 \mathrm{E}-10$ & $\mathrm{~V}$ & FGR-12 & $1.14 \mathrm{E}-13$ \\
\hline Te-134 & Te-134 & 133.911 & Tellurium & $1.00 \mathrm{E}-02$ & ICRP-38 & $4.18 \mathrm{E}+01$ & $\mathrm{~m}$ & $2.51 \mathrm{E}+03$ & $3.36 \mathrm{E}+07$ & ICRP-72 & $8.40 \mathrm{E}-11$ & $\mathrm{~V}$ & FGR-12 & $4.24 \mathrm{E}-14$ \\
\hline Th-223 & Th-223 & 223.021 & Thorium & $1.00 \mathrm{E}-03$ & ICRP-107 & $6.00 \mathrm{E}-01$ & $\mathrm{~s}$ & $6.00 \mathrm{E}-01$ & $8.43 \mathrm{E}+10$ & -- & -- & -- & DOE-STD-1196 & $2.78 \mathrm{E}-15$ \\
\hline Th-224 & Th-224 & 224.021 & Thorium & $1.00 \mathrm{E}-03$ & ICRP-107 & $1.05 E+00$ & $\mathrm{~s}$ & $1.05 \mathrm{E}+00$ & $4.80 \mathrm{E}+10$ & -- & -- & -- & DOE-STD-1196 & $9.75 \mathrm{E}-16$ \\
\hline Th-226 & Th-226 & 226.025 & Thorium & $1.00 \mathrm{E}-03$ & ICRP-38 & $3.09 \mathrm{E}+01$ & $\mathrm{~m}$ & $1.85 \mathrm{E}+03$ & $2.69 \mathrm{E}+07$ & ICRP-72 & $6.10 \mathrm{E}-08$ & $\mathrm{~s}$ & FGR-12 & $3.59 \mathrm{E}-16$ \\
\hline Th-227 & Th-227 & 227.028 & Thorium & $1.00 \mathrm{E}-03$ & ICRP-38 & $1.87 \mathrm{E}+01$ & $d$ & $1.62 \mathrm{E}+06$ & $3.07 \mathrm{E}+04$ & ICRP-72 & $1.00 \mathrm{E}-05$ & $\mathrm{~s}$ & FGR-12 & $4.88 \mathrm{E}-15$ \\
\hline Th-228 & Th-228 & 228.029 & Thorium & $1.00 \mathrm{E}-03$ & ICRP-38 & $1.91 \mathrm{E}+00$ & $y$ & $6.04 \mathrm{E}+07$ & $8.19 \mathrm{E}+02$ & ICRP-72 & $4.00 \mathrm{E}-05$ & $\mathrm{~s}$ & FGR-12 & $9.20 \mathrm{E}-17$ \\
\hline Th-229 & Th-229 & 229.032 & Thorium & $1.00 \mathrm{E}-03$ & ICRP-38 & $7.34 \mathrm{E}+03$ & $y$ & $2.32 \mathrm{E}+11$ & $2.13 \mathrm{E}-01$ & ICRP-72 & $2.40 \mathrm{E}-04$ & $\mathrm{~F}$ & FGR-12 & $3.83 \mathrm{E}-15$ \\
\hline Th-230 & Th-230 & 230.033 & Thorium & $1.00 \mathrm{E}-03$ & ICRP-38 & $7.70 E+04$ & $y$ & $2.43 \mathrm{E}+12$ & $2.02 \mathrm{E}-02$ & ICRP-72 & $1.00 \mathrm{E}-04$ & $\mathrm{~F}$ & FGR-12 & $1.74 \mathrm{E}-17$ \\
\hline Th-231 & Th-231 & 231.036 & Thorium & $1.00 \mathrm{E}-03$ & ICRP-38 & $2.55 \mathrm{E}+01$ & $\mathrm{~h}$ & $9.19 \mathrm{E}+04$ & $5.31 \mathrm{E}+05$ & ICRP-72 & $3.30 \mathrm{E}-10$ & $\mathrm{~s}$ & FGR-12 & $5.22 \mathrm{E}-16$ \\
\hline Th-232 & Th-232 & 232.038 & Thorium & $1.00 \mathrm{E}-03$ & ICRP-38 & $1.41 \mathrm{E}+10$ & $y$ & $4.43 \mathrm{E}+17$ & $1.10 \mathrm{E}-07$ & ICRP-72 & $1.10 \mathrm{E}-04$ & $\mathrm{~F}$ & FGR-12 & $8.72 \mathrm{E}-18$ \\
\hline Th-233 & Th-233 & 233.042 & Thorium & $1.00 \mathrm{E}-03$ & ICRP-107 & $2.23 \mathrm{E}+01$ & $\mathrm{~m}$ & $1.34 \mathrm{E}+03$ & $3.62 \mathrm{E}+07$ & DOE-STD-1196 & $2.12 \mathrm{E}-11$ & $\mathrm{~s}$ & DOE-STD-1196 & $1.79 \mathrm{E}-15$ \\
\hline Th-234 & Th-234 & 234.044 & Thorium & $1.00 \mathrm{E}-03$ & ICRP-38 & $2.41 E+01$ & $d$ & $2.08 \mathrm{E}+06$ & $2.31 \mathrm{E}+04$ & ICRP-72 & $7.70 \mathrm{E}-09$ & $\mathrm{~s}$ & FGR-12 & $3.38 \mathrm{E}-16$ \\
\hline Th-235 & Th-235 & 235.047 & Thorium & $1.00 \mathrm{E}-03$ & ICRP-107 & $7.10 \mathrm{E}+00$ & $\mathrm{~m}$ & $4.26 \mathrm{E}+02$ & $1.13 \mathrm{E}+08$ & -- & -- & -- & DOE-STD-1196 & $2.93 \mathrm{E}-15$ \\
\hline Th-236 & Th-236 & 236.050 & Thorium & $1.00 \mathrm{E}-03$ & ICRP-107 & $3.75 E+01$ & $\mathrm{~m}$ & $2.25 \mathrm{E}+03$ & $2.12 \mathrm{E}+07$ & DOE-STD-1196 & $6.71 \mathrm{E}-11$ & $\mathrm{~s}$ & DOE-STD-1196 & $1.68 \mathrm{E}-15$ \\
\hline Ti-44 & Ti-44 & 43.960 & Titanium & $1.00 \mathrm{E}-03$ & ICRP-38 & $4.73 \mathrm{E}+01$ & $y$ & $1.49 \mathrm{E}+09$ & $1.72 \mathrm{E}+02$ & ICRP-72 & $1.20 \mathrm{E}-07$ & $\mathrm{~s}$ & FGR-12 & $5.53 \mathrm{E}-15$ \\
\hline Ti-45 & Ti-45 & 44.958 & Titanium & $1.00 \mathrm{E}-03$ & ICRP-38 & $3.08 \mathrm{E}+00$ & $\mathrm{~h}$ & $1.11 \mathrm{E}+04$ & $2.26 \mathrm{E}+07$ & ICRP-72 & $9.30 \mathrm{E}-11$ & $\mathrm{~s}$ & FGR-12 & $4.18 \mathrm{E}-14$ \\
\hline Ti-51 & Ti-51 & 50.947 & Titanium & $1.00 \mathrm{E}-03$ & ICRP-107 & $5.76 \mathrm{E}+00$ & $\mathrm{~m}$ & $3.46 \mathrm{E}+02$ & $6.41 \mathrm{E}+08$ & -- & -- & -- & DOE-STD-1196 & $1.71 \mathrm{E}-14$ \\
\hline
\end{tabular}




\begin{tabular}{|c|c|c|c|c|c|c|c|c|c|c|c|c|c|c|}
\hline \multicolumn{15}{|c|}{ Table A.1 Radionuclide Specific Input Data Used For the Calculation of HC-2 TQs Using Maximum Reported DCs (continued) } \\
\hline $\begin{array}{c}\text { MASTER } \\
\text { ISOTOPE } \\
\text { LIST } \\
\end{array}$ & $\begin{array}{l}\text { Atomic } \\
\text { Mass } \\
\text { Nuclide }\end{array}$ & $\begin{array}{l}\text { Atomic } \\
\text { Mass }\end{array}$ & Element & $\begin{array}{c}\text { RF per } \\
\text { DOE-STD- } \\
1027\end{array}$ & $\begin{array}{l}\text { Half-Life } \\
\text { Reference }\end{array}$ & $\begin{array}{r}\text { Half-Li } \\
\text { (original }\end{array}$ & & Half Life (s) & $\begin{array}{l}\text { Sp. Act } \\
\text { (Ci/g) }\end{array}$ & $\begin{array}{c}\text { Inhalation DC } \\
\text { Reference }\end{array}$ & $\begin{array}{c}\text { HC-2 } \\
\text { Bounding } \\
\text { Inhalation } \\
\text { DC (Sv/Bq) }\end{array}$ & $\begin{array}{l}\text { Lung } \\
\text { Absorption } \\
\text { Class/Type }\end{array}$ & $\begin{array}{c}\text { Immersion DC } \\
\text { Reference }\end{array}$ & $\begin{array}{c}\text { HC-2 } \\
\text { Immersion } \\
\mathrm{DC}(\mathrm{Sv} / \mathrm{s} \\
\left.\text { per } \mathrm{Bq} / \mathrm{m}^{3}\right)\end{array}$ \\
\hline Ti-52 & Ti-52 & 51.947 & Titanium & $1.00 \mathrm{E}-03$ & ICRP-107 & $1.70 \mathrm{E}+00$ & $\mathrm{~m}$ & $1.02 \mathrm{E}+02$ & $2.13 \mathrm{E}+09$ & -- & -- & -- & DOE-STD-1196 & $5.75 \mathrm{E}-15$ \\
\hline TI-190 & TI-190 & 189.974 & Thallium & $1.00 \mathrm{E}-03$ & ICRP-107 & $2.60 \mathrm{E}+00$ & $\mathrm{~m}$ & $1.56 \mathrm{E}+02$ & $3.81 \mathrm{E}+08$ & -- & -- & -- & DOE-STD-1196 & $5.94 \mathrm{E}-14$ \\
\hline Tl-190m & TI-190 & 189.974 & Thallium & $1.00 \mathrm{E}-03$ & ICRP-107 & $3.70 \mathrm{E}+00$ & $\mathrm{~m}$ & $2.22 \mathrm{E}+02$ & $2.67 \mathrm{E}+08$ & -- & $\begin{array}{ll}- \\
\end{array}$ & -- & DOE-STD-1196 & $1.11 \mathrm{E}-13$ \\
\hline Tl-194 & TI-194 & 193.971 & Thallium & $1.00 \mathrm{E}-03$ & ICRP-38 & $3.30 \mathrm{E}+01$ & $\mathrm{~m}$ & $1.98 \mathrm{E}+03$ & $2.94 \mathrm{E}+07$ & ICRP-72 & $4.40 \mathrm{E}-12$ & $\mathrm{~F}$ & FGR-12 & $3.70 \mathrm{E}-14$ \\
\hline Tl-194m & TI-194 & 193.971 & Thallium & $1.00 \mathrm{E}-03$ & ICRP-38 & $3.28 \mathrm{E}+01$ & $\mathrm{~m}$ & $1.97 \mathrm{E}+03$ & $2.96 \mathrm{E}+07$ & ICRP-72 & $1.90 \mathrm{E}-11$ & $\mathrm{~F}$ & FGR-12 & $1.11 \mathrm{E}-13$ \\
\hline Tl-195 & TI-195 & 194.970 & Thallium & $1.00 \mathrm{E}-03$ & ICRP-38 & $1.16 \mathrm{E}+00$ & $\mathrm{~h}$ & $4.18 \mathrm{E}+03$ & $1.39 \mathrm{E}+07$ & ICRP-72 & $1.50 \mathrm{E}-11$ & $\mathrm{~F}$ & FGR-12 & $6.34 \mathrm{E}-14$ \\
\hline TI-196 & TI-196 & 195.970 & Thallium & $1.00 \mathrm{E}-03$ & ICRP-107 & $1.84 \mathrm{E}+00$ & $\mathrm{~h}$ & $6.62 \mathrm{E}+03$ & $8.69 \mathrm{E}+06$ & DOE-STD-1196 & $4.70 \mathrm{E}-11$ & $\mathrm{~s}$ & DOE-STD-1196 & $8.80 \mathrm{E}-14$ \\
\hline TI-197 & TI-197 & 196.970 & Thallium & $1.00 \mathrm{E}-03$ & ICRP-38 & $2.84 \mathrm{E}+00$ & $\mathrm{~h}$ & $1.02 \mathrm{E}+04$ & $5.60 \mathrm{E}+06$ & ICRP-72 & $1.40 \mathrm{E}-11$ & $\mathrm{~F}$ & FGR-12 & $1.93 \mathrm{E}-14$ \\
\hline TI-198 & TI-198 & 197.970 & Thallium & $1.00 \mathrm{E}-03$ & ICRP-38 & $5.30 \mathrm{E}+00$ & $\mathrm{~h}$ & $1.91 \mathrm{E}+04$ & $2.99 \mathrm{E}+06$ & ICRP-72 & $6.00 \mathrm{E}-11$ & $\mathrm{~F}$ & FGR-12 & $1.01 \mathrm{E}-13$ \\
\hline TI-198m & TI-198 & 197.970 & Thallium & $1.00 \mathrm{E}-03$ & ICRP-38 & $1.87 \mathrm{E}+00$ & $\mathrm{~h}$ & $6.73 \mathrm{E}+03$ & $8.46 \mathrm{E}+06$ & ICRP-72 & $3.70 \mathrm{E}-11$ & $\mathrm{~F}$ & FGR-12 & $5.69 \mathrm{E}-14$ \\
\hline TI-199 & TI-199 & 198.970 & Thallium & $1.00 \mathrm{E}-03$ & ICRP-38 & $7.42 \mathrm{E}+00$ & $\mathrm{~h}$ & $2.67 \mathrm{E}+04$ & $2.12 \mathrm{E}+06$ & ICRP-72 & $1.90 \mathrm{E}-11$ & $\mathrm{~F}$ & FGR-12 & $1.13 \mathrm{E}-14$ \\
\hline TI-200 & TI-200 & 199.971 & Thallium & $1.00 \mathrm{E}-03$ & ICRP-38 & $2.61 \mathrm{E}+01$ & $\mathrm{~h}$ & $9.40 \mathrm{E}+04$ & $6.00 E+05$ & ICRP-72 & $1.30 \mathrm{E}-10$ & $\mathrm{~F}$ & FGR-12 & $6.42 \mathrm{E}-14$ \\
\hline TI-201 & TI-201 & 200.971 & Thallium & $1.00 \mathrm{E}-03$ & ICRP-38 & $3.04 \mathrm{E}+00$ & $d$ & $2.63 \mathrm{E}+05$ & $2.13 \mathrm{E}+05$ & ICRP-72 & $4.40 \mathrm{E}-11$ & $\mathrm{~F}$ & FGR-12 & $3.78 \mathrm{E}-15$ \\
\hline TI-202 & TI-202 & 201.972 & Thallium & $1.00 \mathrm{E}-03$ & ICRP-38 & $1.22 \mathrm{E}+01$ & $d$ & $1.06 \mathrm{E}+06$ & $5.29 \mathrm{E}+04$ & ICRP-72 & $1.90 \mathrm{E}-10$ & $\mathrm{~F}$ & FGR-12 & $2.18 \mathrm{E}-14$ \\
\hline TI-204 & TI-204 & 203.974 & Thallium & $1.00 \mathrm{E}-03$ & ICRP-38 & $3.78 \mathrm{E}+00$ & $y$ & $1.19 \mathrm{E}+08$ & $4.64 \mathrm{E}+02$ & ICRP-72 & $3.90 \mathrm{E}-10$ & $F$ & FGR-12 & $5.59 \mathrm{E}-17$ \\
\hline TI-206 & TI-206 & 205.976 & Thallium & $1.00 \mathrm{E}-03$ & ICRP-38 & $4.20 \mathrm{E}+00$ & $\mathrm{~m}$ & $2.52 \mathrm{E}+02$ & $2.17 \mathrm{E}+08$ & -- & -- & -- & FGR-12 & $6.73 \mathrm{E}-17$ \\
\hline TI-206m & TI-206 & 205.976 & Thallium & $1.00 \mathrm{E}-03$ & ICRP-107 & $3.74 \mathrm{E}+00$ & $\mathrm{~m}$ & $2.24 \mathrm{E}+02$ & $2.44 \mathrm{E}+08$ & -- & -- & -- & DOE-STD-1196 & $1.09 \mathrm{E}-13$ \\
\hline TI-207 & TI-207 & 206.977 & Thallium & $1.00 \mathrm{E}-03$ & ICRP-38 & $4.77 \mathrm{E}+00$ & $\mathrm{~m}$ & $2.86 \mathrm{E}+02$ & $1.90 \mathrm{E}+08$ & -- & -- & -- & FGR-12 & $1.62 \mathrm{E}-16$ \\
\hline TI-208 & TI-208 & 207.982 & Thallium & $1.00 \mathrm{E}-03$ & ICRP-38 & $3.07 E+00$ & $\mathrm{~m}$ & $1.84 \mathrm{E}+02$ & $2.94 \mathrm{E}+08$ & -- & -- & -- & FGR-12 & $1.77 \mathrm{E}-13$ \\
\hline TI-209 & TI-209 & 208.985 & Thallium & $1.00 \mathrm{E}-03$ & ICRP-38 & $2.20 \mathrm{E}+00$ & $\mathrm{~m}$ & $1.32 \mathrm{E}+02$ & $4.09 \mathrm{E}+08$ & -- & -- & -- & FGR-12 & $1.02 \mathrm{E}-13$ \\
\hline TI-210 & TI-210 & 209.990 & Thallium & $1.00 \mathrm{E}-03$ & ICRP-38 & $1.30 \mathrm{E}+00$ & $\mathrm{~m}$ & $7.80 \mathrm{E}+01$ & $6.89 \mathrm{E}+08$ & -- & -- & -- & DOE-STD-1196 & $1.32 \mathrm{E}-13$ \\
\hline Tm-159 & Tm-159 & 158.935 & Thulium & $1.00 \mathrm{E}-03$ & JAERI & $9.13 \mathrm{E}+00$ & $\mathrm{~m}$ & $5.48 \mathrm{E}+02$ & $1.30 E+08$ & -- & -- & -- & -- & -- \\
\hline Tm-161 & Tm-161 & 160.934 & Thulium & $1.00 \mathrm{E}-03$ & ICRP-107 & $3.02 \mathrm{E}+01$ & $\mathrm{~m}$ & $1.81 \mathrm{E}+03$ & $3.87 \mathrm{E}+07$ & DOE-STD-1196 & $3.06 \mathrm{E}-11$ & $\mathrm{~s}$ & DOE-STD-1196 & $5.89 \mathrm{E}-14$ \\
\hline Tm-162 & Tm-162 & 161.934 & Thulium & $1.00 \mathrm{E}-03$ & ICRP-38 & $2.17 \mathrm{E}+01$ & $\mathrm{~m}$ & $1.30 \mathrm{E}+03$ & $5.35 \mathrm{E}+07$ & ICRP-72 & $1.60 \mathrm{E}-11$ & $M$ & FGR-12 & $9.01 \mathrm{E}-14$ \\
\hline Tm-163 & Tm-163 & 162.933 & Thulium & $1.00 \mathrm{E}-03$ & ICRP-107 & $1.81 \mathrm{E}+00$ & $\mathrm{~h}$ & $6.52 \mathrm{E}+03$ & $1.06 \mathrm{E}+07$ & DOE-STD-1196 & $4.53 \mathrm{E}-11$ & $\mathrm{~s}$ & DOE-STD-1196 & $6.03 \mathrm{E}-14$ \\
\hline Tm-164 & $\mathrm{Tm}-164$ & 163.934 & Thulium & $1.00 \mathrm{E}-03$ & ICRP-107 & $2.00 E+00$ & $\mathrm{~m}$ & $1.20 \mathrm{E}+02$ & $5.73 E+08$ & -- & -- & -- & DOE-STD-1196 & $3.57 \mathrm{E}-14$ \\
\hline Tm-165 & Tm-165 & 164.932 & Thulium & $1.00 \mathrm{E}-03$ & ICRP-107 & $3.01 \mathrm{E}+01$ & $\mathrm{~h}$ & $1.08 \mathrm{E}+05$ & $6.32 \mathrm{E}+05$ & DOE-STD-1196 & $2.74 \mathrm{E}-10$ & $\mathrm{~s}$ & DOE-STD-1196 & $2.40 \mathrm{E}-14$ \\
\hline Tm-166 & Tm-166 & 165.934 & Thulium & $1.00 \mathrm{E}-03$ & ICRP-38 & $7.70 E+00$ & $\mathrm{~h}$ & $2.77 E+04$ & $2.45 E+06$ & ICRP-72 & $1.70 \mathrm{E}-10$ & $M$ & FGR-12 & $9.35 \mathrm{E}-14$ \\
\hline Tm-167 & Tm-167 & 166.933 & Thulium & $1.00 \mathrm{E}-03$ & ICRP-38 & $9.24 \mathrm{E}+00$ & $d$ & $7.98 \mathrm{E}+05$ & $8.47 E+04$ & ICRP-72 & $1.10 \mathrm{E}-09$ & $M$ & FGR-12 & $6.06 \mathrm{E}-15$ \\
\hline Tm-168 & Tm-168 & 167.934 & Thulium & $1.00 \mathrm{E}-03$ & ICRP-107 & $9.31 \mathrm{E}+01$ & $\mathrm{~d}$ & $8.04 \mathrm{E}+06$ & $8.35 \mathrm{E}+03$ & DOE-STD-1196 & $5.60 \mathrm{E}-09$ & $\mathrm{~s}$ & DOE-STD-1196 & $5.51 \mathrm{E}-14$ \\
\hline Tm-170 & Tm-170 & 169.936 & Thulium & $1.00 \mathrm{E}-03$ & ICRP-38 & $1.29 \mathrm{E}+02$ & $d$ & $1.11 \mathrm{E}+07$ & $5.97 \mathrm{E}+03$ & ICRP-72 & $7.00 \mathrm{E}-09$ & $M$ & FGR-12 & $2.23 \mathrm{E}-16$ \\
\hline Tm-171 & Tm-171 & 170.936 & Thulium & $1.00 \mathrm{E}-03$ & ICRP-38 & $1.92 \mathrm{E}+00$ & $y$ & $6.06 \mathrm{E}+07$ & $1.09 \mathrm{E}+03$ & ICRP-72 & $1.40 \mathrm{E}-09$ & $M$ & FGR-12 & $2.15 \mathrm{E}-17$ \\
\hline Tm-172 & Tm-172 & 171.938 & Thulium & $1.00 \mathrm{E}-03$ & ICRP-38 & $6.36 \mathrm{E}+01$ & $\mathrm{~h}$ & $2.29 \mathrm{E}+05$ & $2.87 \mathrm{E}+05$ & ICRP-72 & $1.10 \mathrm{E}-09$ & $M$ & FGR-12 & $2.41 \mathrm{E}-14$ \\
\hline Tm-173 & Tm-173 & 172.940 & Thulium & $1.00 \mathrm{E}-03$ & ICRP-38 & $8.24 \mathrm{E}+00$ & $\mathrm{~h}$ & $2.97 \mathrm{E}+04$ & $2.20 \mathrm{E}+06$ & ICRP-72 & $1.80 \mathrm{E}-10$ & $M$ & FGR-12 & $1.85 \mathrm{E}-14$ \\
\hline Tm-174 & Tm-174 & 173.942 & Thulium & $1.00 \mathrm{E}-03$ & ICRP-107 & $5.40 \mathrm{E}+00$ & $\mathrm{~m}$ & $3.24 \mathrm{E}+02$ & $2.00 \mathrm{E}+08$ & -- & -- & -- & DOE-STD-1196 & $8.05 \mathrm{E}-14$ \\
\hline Tm-175 & Tm-175 & 174.944 & Thulium & $1.00 \mathrm{E}-03$ & ICRP-38 & $1.52 \mathrm{E}+01$ & $\mathrm{~m}$ & $9.12 \mathrm{E}+02$ & $7.07 E+07$ & ICRP-72 & $1.80 \mathrm{E}-11$ & M & FGR-12 & $5.13 \mathrm{E}-14$ \\
\hline Tm-176 & Tm-176 & 175.947 & Thulium & $1.00 \mathrm{E}-03$ & ICRP-107 & $1.85 \mathrm{E}+00$ & $\mathrm{~m}$ & $1.11 \mathrm{E}+02$ & $5.78 \mathrm{E}+08$ & -- & -- & -- & DOE-STD-1196 & $9.39 \mathrm{E}-14$ \\
\hline U-227 & U-227 & 227.031 & Uranium & $1.00 \mathrm{E}-03$ & ICRP-107 & $1.10 \mathrm{E}+00$ & $\mathrm{~m}$ & $6.60 \mathrm{E}+01$ & $7.53 \mathrm{E}+08$ & -- & -- & -- & DOE-STD-1196 & $4.85 \mathrm{E}-15$ \\
\hline U-228 & U-228 & 228.031 & Uranium & $1.00 \mathrm{E}-03$ & ICRP-107 & $9.10 \mathrm{E}+00$ & $\mathrm{~m}$ & $5.46 \mathrm{E}+02$ & $9.06 \mathrm{E}+07$ & -- & -- & -- & DOE-STD-1196 & $1.59 \mathrm{E}-16$ \\
\hline U-230 & $\mathrm{U}-230$ & 230.034 & Uranium & $1.00 \mathrm{E}-03$ & ICRP-38 & $2.08 \mathrm{E}+01$ & d & $1.80 \mathrm{E}+06$ & $2.73 E+04$ & ICRP-72 & $1.60 \mathrm{E}-05$ & $\mathrm{~s}$ & FGR-12 & $5.23 \mathrm{E}-17$ \\
\hline U-231 & U-231 & 231.036 & Uranium & $1.00 \mathrm{E}-03$ & ICRP-38 & $4.20 \mathrm{E}+00$ & $d$ & $3.63 \mathrm{E}+05$ & $1.35 E+05$ & ICRP-72 & $5.20 \mathrm{E}-10$ & $\mathrm{~s}$ & FGR-12 & $2.95 \mathrm{E}-15$ \\
\hline $\mathrm{U}-232$ & $\mathrm{U}-232$ & 232.037 & Uranium & $1.00 \mathrm{E}-03$ & ICRP-38 & $7.20 \mathrm{E}+01$ & $y$ & $2.27 \mathrm{E}+09$ & $2.14 \mathrm{E}+01$ & ICRP-72 & $3.70 \mathrm{E}-05$ & $\mathrm{~s}$ & FGR-12 & $1.42 \mathrm{E}-17$ \\
\hline U-233 & U-233 & 233.040 & Uranium & $1.00 \mathrm{E}-03$ & ICRP-38 & $1.59 \mathrm{E}+05$ & $y$ & $5.00 \mathrm{E}+12$ & $9.68 \mathrm{E}-03$ & ICRP-72 & $9.60 \mathrm{E}-06$ & $\mathrm{~s}$ & FGR-12 & $1.63 \mathrm{E}-17$ \\
\hline U-234 & U-234 & 234.041 & Uranium & $1.00 \mathrm{E}-03$ & ICRP-38 & $2.45 E+05$ & $y$ & $7.72 \mathrm{E}+12$ & $6.25 \mathrm{E}-03$ & ICRP-72 & $9.40 \mathrm{E}-06$ & $\mathrm{~s}$ & FGR-12 & $7.63 \mathrm{E}-18$ \\
\hline U-235 & U-235 & 235.044 & Uranium & $1.00 \mathrm{E}-03$ & ICRP-38 & $7.04 \mathrm{E}+08$ & $y$ & $2.22 \mathrm{E}+16$ & $2.16 \mathrm{E}-06$ & ICRP-72 & $8.50 \mathrm{E}-06$ & $\mathrm{~s}$ & FGR-12 & $7.20 \mathrm{E}-15$ \\
\hline U-235m & U-235 & 235.044 & Uranium & $1.00 \mathrm{E}-03$ & ICRP-107 & $2.60 \mathrm{E}+01$ & $\mathrm{~m}$ & $1.56 \mathrm{E}+03$ & $3.08 \mathrm{E}+07$ & DOE-STD-1196 & $9.17 \mathrm{E}-16$ & $\mathrm{~s}$ & -- & -- \\
\hline
\end{tabular}




\begin{tabular}{|c|c|c|c|c|c|c|c|c|c|c|c|c|c|c|}
\hline \multicolumn{15}{|c|}{ Table A.1 Radionuclide Specific Input Data Used For the Calculation of HC-2 TQs Using Maximum Reported DCs (continued) } \\
\hline $\begin{array}{c}\text { MASTER } \\
\text { ISOTOPE } \\
\text { LIST } \\
\end{array}$ & $\begin{array}{l}\text { Atomic } \\
\text { Mass } \\
\text { Nuclide }\end{array}$ & $\begin{array}{c}\text { Atomic } \\
\text { Mass }\end{array}$ & Element & $\begin{array}{c}\text { RF per } \\
\text { DOE-STD- } \\
1027 \\
\end{array}$ & $\begin{array}{c}\text { Half-Life } \\
\text { Reference }\end{array}$ & $\begin{array}{r}\text { Half-L } \\
\text { (original }\end{array}$ & & Half Life (s) & $\begin{array}{l}\text { Sp. Act } \\
\text { (Ci/g) }\end{array}$ & $\begin{array}{l}\text { Inhalation DC } \\
\text { Reference }\end{array}$ & $\begin{array}{c}\text { HC-2 } \\
\text { Bounding } \\
\text { Inhalation } \\
\text { DC (Sv/Bq) }\end{array}$ & $\begin{array}{c}\text { Lung } \\
\text { Absorption } \\
\text { Class/Type } \\
\end{array}$ & $\begin{array}{l}\text { Immersion DC } \\
\text { Reference }\end{array}$ & $\begin{array}{c}\mathrm{HC}-2 \\
\text { Immersion } \\
\mathrm{DC}(\mathrm{Sv} / \mathrm{s} \\
\left.\text { per } \mathrm{Bq} / \mathrm{m}^{3}\right)\end{array}$ \\
\hline $\mathrm{U}-236$ & U-236 & 236.046 & Uranium & $1.00 \mathrm{E}-03$ & ICRP-38 & $2.34 \mathrm{E}+07$ & $\mathrm{y}$ & $7.39 \mathrm{E}+14$ & $6.47 \mathrm{E}-05$ & ICRP-72 & $8.70 \mathrm{E}-06$ & $\mathrm{~s}$ & FGR-12 & $5.01 \mathrm{E}-18$ \\
\hline U-237 & U-237 & 237.049 & Uranium & $1.00 \mathrm{E}-03$ & ICRP-38 & $6.75 \mathrm{E}+00$ & d & $5.83 \mathrm{E}+05$ & $8.16 \mathrm{E}+04$ & ICRP-72 & $1.90 \mathrm{E}-09$ & $\mathrm{~s}$ & FGR-12 & $5.97 \mathrm{E}-15$ \\
\hline $\mathrm{U}-238$ & $\mathrm{U}-238$ & 238.051 & Uranium & $1.00 \mathrm{E}-03$ & ICRP-38 & $4.47 \mathrm{E}+09$ & $y$ & $1.41 \mathrm{E}+17$ & $3.36 \mathrm{E}-07$ & ICRP-72 & $8.00 \mathrm{E}-06$ & $\mathrm{~s}$ & FGR-12 & $3.41 \mathrm{E}-18$ \\
\hline U-239 & U-239 & 239.054 & Uranium & $1.00 \mathrm{E}-03$ & ICRP-38 & $2.35 \mathrm{E}+01$ & $\mathrm{~m}$ & $1.41 \mathrm{E}+03$ & $3.34 \mathrm{E}+07$ & ICRP-72 & $2.40 \mathrm{E}-11$ & $\mathrm{~s}$ & FGR-12 & $2.17 E-15$ \\
\hline$U-240$ & $\mathrm{U}-240$ & 240.057 & Uranium & $1.00 \mathrm{E}-03$ & ICRP-38 & $1.41 \mathrm{E}+01$ & $\mathrm{~h}$ & $5.08 \mathrm{E}+04$ & $9.26 \mathrm{E}+05$ & ICRP-72 & $5.80 \mathrm{E}-10$ & $\mathrm{~s}$ & FGR-12 & $3.93 \mathrm{E}-17$ \\
\hline U-242 & U-242 & 242.063 & Uranium & $1.00 \mathrm{E}-03$ & ICRP-107 & $1.68 \mathrm{E}+01$ & $\mathrm{~m}$ & $1.01 \mathrm{E}+03$ & $4.62 \mathrm{E}+07$ & DOE-STD-1196 & $3.35 \mathrm{E}-11$ & $\mathrm{~s}$ & DOE-STD-1196 & $1.96 \mathrm{E}-15$ \\
\hline V-47 & V-47 & 46.955 & Vanadium & $1.00 \mathrm{E}-03$ & ICRP-38 & $3.26 \mathrm{E}+01$ & $\mathrm{~m}$ & $1.96 \mathrm{E}+03$ & $1.23 E+08$ & ICRP-72 & $2.90 \mathrm{E}-11$ & $M$ & FGR-12 & $4.79 \mathrm{E}-14$ \\
\hline V-48 & $\mathrm{V}-48$ & 47.952 & Vanadium & $1.00 \mathrm{E}-03$ & ICRP-38 & $1.62 \mathrm{E}+01$ & d & $1.40 \mathrm{E}+06$ & $1.68 \mathrm{E}+05$ & ICRP-72 & $2.40 \mathrm{E}-09$ & $M$ & FGR-12 & $1.45 \mathrm{E}-13$ \\
\hline V-49 & V-49 & 48.949 & Vanadium & $1.00 \mathrm{E}-03$ & ICRP-38 & $3.30 \mathrm{E}+02$ & $d$ & $2.85 \mathrm{E}+07$ & $8.08 \mathrm{E}+03$ & ICRP-72 & $3.40 \mathrm{E}-11$ & $M$ & -- & -- \\
\hline V-50 & V-50 & 49.947 & Vanadium & $1.00 \mathrm{E}-03$ & ICRP-107 & $1.50 \mathrm{E}+17$ & $y$ & $4.73 \mathrm{E}+24$ & $4.77 \mathrm{E}-14$ & DOE-STD-1196 & $6.69 \mathrm{E}-08$ & $\mathrm{~F}$ & DOE-STD-1196 & $6.87 E-14$ \\
\hline V-52 & V-52 & 51.945 & Vanadium & $1.00 \mathrm{E}-03$ & ICRP-107 & $3.74 \mathrm{E}+00$ & $\mathrm{~m}$ & $2.25 \mathrm{E}+02$ & $9.67 E+08$ & -- & -- & -- & DOE-STD-1196 & $7.05 \mathrm{E}-14$ \\
\hline V-53 & V-53 & 52.944 & Vanadium & $1.00 \mathrm{E}-03$ & ICRP-107 & $1.61 \mathrm{E}+00$ & $\mathrm{~m}$ & $9.66 \mathrm{E}+01$ & $2.21 \mathrm{E}+09$ & -- & -- & -- & DOE-STD-1196 & $4.93 \mathrm{E}-14$ \\
\hline W-176 & W-176 & 175.946 & Tungsten & $1.00 \mathrm{E}-03$ & ICRP-38 & $2.30 \mathrm{E}+00$ & $\mathrm{~h}$ & $8.28 \mathrm{E}+03$ & $7.74 \mathrm{E}+06$ & ICRP-72 & $4.10 \mathrm{E}-11$ & $\mathrm{~F}$ & FGR-12 & $7.02 \mathrm{E}-15$ \\
\hline W-177 & W-177 & 176.947 & Tungsten & $1.00 \mathrm{E}-03$ & ICRP-38 & $1.35 \mathrm{E}+02$ & $\mathrm{~m}$ & $8.10 \mathrm{E}+03$ & $7.87 \mathrm{E}+06$ & ICRP-72 & $2.40 \mathrm{E}-11$ & $\mathrm{~F}$ & FGR-12 & $4.26 \mathrm{E}-14$ \\
\hline W-178 & W-178 & 177.946 & Tungsten & $1.00 \mathrm{E}-03$ & ICRP-38 & $2.17 \mathrm{E}+01$ & $d$ & $1.87 \mathrm{E}+06$ & $3.38 \mathrm{E}+04$ & ICRP-72 & $7.20 \mathrm{E}-11$ & $\mathrm{~F}$ & FGR-12 & $4.62 \mathrm{E}-16$ \\
\hline W-179 & W-179 & 178.947 & Tungsten & $1.00 \mathrm{E}-03$ & ICRP-38 & $3.75 E+01$ & $\mathrm{~m}$ & $2.25 \mathrm{E}+03$ & $2.80 \mathrm{E}+07$ & ICRP-72 & $9.20 \mathrm{E}-13$ & $\mathrm{~F}$ & FGR-12 & $1.83 \mathrm{E}-15$ \\
\hline W-179m & W-179 & 178.947 & Tungsten & $1.00 \mathrm{E}-03$ & ICRP-107 & $6.40 \mathrm{E}+00$ & $\mathrm{~m}$ & $3.84 \mathrm{E}+02$ & $1.64 \mathrm{E}+08$ & -- & -- & -- & DOE-STD-1196 & $1.99 \mathrm{E}-15$ \\
\hline W-181 & W-181 & 180.948 & Tungsten & $1.00 \mathrm{E}-03$ & ICRP-38 & $1.21 \mathrm{E}+02$ & $d$ & $1.05 \mathrm{E}+07$ & $5.95 \mathrm{E}+03$ & ICRP-72 & $2.70 \mathrm{E}-11$ & $\mathrm{~F}$ & $\begin{array}{l}\text { FGR-12 } \\
\end{array}$ & $1.40 \mathrm{E}-15$ \\
\hline W-185 & W-185 & 184.953 & Tungsten & $1.00 \mathrm{E}-03$ & ICRP-38 & $7.51 \mathrm{E}+01$ & $d$ & $6.49 \mathrm{E}+06$ & $9.40 \mathrm{E}+03$ & ICRP-72 & $1.20 \mathrm{E}-10$ & $\mathrm{~F}$ & FGR-12 & $5.37 \mathrm{E}-18$ \\
\hline W-185m & W-185 & 184.953 & Tungsten & $1.00 \mathrm{E}-03$ & ICRP-107 & $1.60 \mathrm{E}+00$ & $\mathrm{~m}$ & $9.58 \mathrm{E}+01$ & $6.37 \mathrm{E}+08$ & -- & -- & -- & DOE-STD-1196 & $9.33 \mathrm{E}-16$ \\
\hline W-187 & W-187 & 186.957 & Tungsten & $1.00 \mathrm{E}-03$ & ICRP-38 & $2.39 \mathrm{E}+01$ & $\mathrm{~h}$ & $8.60 \mathrm{E}+04$ & $7.01 \mathrm{E}+05$ & ICRP-72 & $1.90 \mathrm{E}-10$ & $\mathrm{~F}$ & FGR-12 & $2.28 \mathrm{E}-14$ \\
\hline W-188 & W-188 & 187.958 & Tungsten & $1.00 \mathrm{E}-03$ & ICRP-38 & $6.94 \mathrm{E}+01$ & d & $6.00 \mathrm{E}+06$ & $1.00 \mathrm{E}+04$ & ICRP-72 & $5.70 \mathrm{E}-10$ & $\mathrm{~F}$ & FGR-12 & $9.04 \mathrm{E}-17$ \\
\hline W-190 & W-190 & 189.963 & Tungsten & $1.00 \mathrm{E}-03$ & ICRP-107 & $3.00 \mathrm{E}+01$ & $\mathrm{~m}$ & $1.80 \mathrm{E}+03$ & $3.30 \mathrm{E}+07$ & DOE-STD-1196 & $8.54 \mathrm{E}-11$ & $\mathrm{~s}$ & DOE-STD-1196 & $5.75 \mathrm{E}-15$ \\
\hline Xe-120 & Xe-120 & 119.912 & Xenon & $1.00 \mathrm{E}+00$ & ICRP-38 & $4.00 \mathrm{E}+01$ & $\mathrm{~m}$ & $2.40 \mathrm{E}+03$ & $3.92 \mathrm{E}+07$ & -- & -- & -- & FGR-12 & $1.94 \mathrm{E}-14$ \\
\hline Xe-121 & Xe-121 & 120.911 & Xenon & $1.00 \mathrm{E}+00$ & ICRP-38 & $4.01 \mathrm{E}+01$ & $\mathrm{~m}$ & $2.41 \mathrm{E}+03$ & $3.88 \mathrm{E}+07$ & -- & -- & -- & FGR-12 & $9.14 \mathrm{E}-14$ \\
\hline Xe-122 & Xe-122 & 121.908 & Xenon & $1.00 E+00$ & ICRP-38 & $2.01 E+01$ & $\mathrm{~h}$ & $7.24 \mathrm{E}+04$ & $1.28 \mathrm{E}+06$ & -- & -- & -- & FGR-12 & $2.46 \mathrm{E}-15$ \\
\hline Xe-123 & Xe-123 & 122.908 & Xenon & $1.00 \mathrm{E}+00$ & ICRP-38 & $2.08 \mathrm{E}+00$ & $\mathrm{~h}$ & $7.49 \mathrm{E}+03$ & $1.23 \mathrm{E}+07$ & -- & -- & -- & FGR-12 & $3.03 \mathrm{E}-14$ \\
\hline Xe-125 & Xe-125 & 124.906 & Xenon & $1.00 \mathrm{E}+00$ & ICRP-38 & $1.70 \mathrm{E}+01$ & $\mathrm{~h}$ & $6.12 \mathrm{E}+04$ & $1.48 \mathrm{E}+06$ & -- & -- & -- & FGR-12 & $1.19 \mathrm{E}-14$ \\
\hline Xe-127 & Xe-127 & 126.905 & Xenon & $1.00 E+00$ & ICRP-38 & $3.64 \mathrm{E}+01$ & $d$ & $3.15 \mathrm{E}+06$ & $2.83 \mathrm{E}+04$ & -- & -- & -- & FGR-12 & $1.25 \mathrm{E}-14$ \\
\hline Xe-127m & Xe-127 & 126.905 & Xenon & $1.00 E+00$ & ICRP-107 & $6.92 \mathrm{E}+01$ & $\mathrm{~s}$ & $6.92 \mathrm{E}+01$ & $1.28 \mathrm{E}+09$ & -- & -- & -- & DOE-STD-1196 & $6.57 \mathrm{E}-15$ \\
\hline Xe-129m & Xe-129 & 128.905 & Xenon & $1.00 \mathrm{E}+00$ & ICRP-38 & $8.00 \mathrm{E}+00$ & $d$ & $6.91 \mathrm{E}+05$ & $1.27 \mathrm{E}+05$ & -- & -- & -- & FGR-12 & $1.06 \mathrm{E}-15$ \\
\hline Xe-131m & Xe-131 & 130.905 & Xenon & $1.00 \mathrm{E}+00$ & ICRP-38 & $1.19 \mathrm{E}+01$ & $d$ & $1.03 E+06$ & $8.38 \mathrm{E}+04$ & -- & -- & -- & FGR-12 & $3.89 \mathrm{E}-16$ \\
\hline Xe-133 & Xe-133 & 132.906 & Xenon & $1.00 \mathrm{E}+00$ & ICRP-38 & $5.25 \mathrm{E}+00$ & $d$ & $4.53 E+05$ & $1.87 \mathrm{E}+05$ & -- & -- & -- & FGR-12 & $1.56 \mathrm{E}-15$ \\
\hline Xe-133m & Xe-133 & 132.906 & Xenon & $1.00 \mathrm{E}+00$ & ICRP-38 & $2.19 \mathrm{E}+00$ & $d$ & $1.89 \mathrm{E}+05$ & $4.49 \mathrm{E}+05$ & -- & -- & -- & FGR-12 & $1.37 \mathrm{E}-15$ \\
\hline Xe-135 & Xe-135 & 134.907 & Xenon & $1.00 \mathrm{E}+00$ & ICRP-38 & $9.09 \mathrm{E}+00$ & $\mathrm{~h}$ & $3.27 \mathrm{E}+04$ & $2.56 \mathrm{E}+06$ & -- & -- & -- & FGR-12 & $1.19 \mathrm{E}-14$ \\
\hline Xe-135m & Xe-135 & 134.907 & Xenon & $1.00 \mathrm{E}+00$ & ICRP-38 & $1.53 \mathrm{E}+01$ & $\mathrm{~m}$ & $9.17 \mathrm{E}+02$ & $9.12 \mathrm{E}+07$ & -- & -- & -- & FGR-12 & $2.04 \mathrm{E}-14$ \\
\hline Xe-137 & Xe-137 & 136.912 & Xenon & $1.00 \mathrm{E}+00$ & ICRP-107 & $3.82 \mathrm{E}+00$ & $\mathrm{~m}$ & $2.29 \mathrm{E}+02$ & $3.60 \mathrm{E}+08$ & -- & -- & -- & DOE-STD-1196 & $1.04 \mathrm{E}-14$ \\
\hline Xe-138 & Xe-138 & 137.914 & Xenon & $1.00 \mathrm{E}+00$ & ICRP-38 & $1.42 \mathrm{E}+01$ & $\mathrm{~m}$ & $8.50 \mathrm{E}+02$ & $9.62 \mathrm{E}+07$ & -- & -- & -- & FGR-12 & $5.77 E-14$ \\
\hline $\mathrm{Y}-81$ & $\mathrm{Y}-81$ & 80.929 & Yttrium & $1.00 \mathrm{E}-03$ & ICRP-107 & $7.04 \mathrm{E}+01$ & $\mathrm{~s}$ & $7.04 \mathrm{E}+01$ & $1.98 \mathrm{E}+09$ & -- & -- & -- & DOE-STD-1196 & $5.35 \mathrm{E}-14$ \\
\hline Y-83 & $Y-83$ & 82.922 & Yttrium & $1.00 \mathrm{E}-03$ & ICRP-107 & $7.08 \mathrm{E}+00$ & $\mathrm{~m}$ & $4.25 \mathrm{E}+02$ & $3.20 \mathrm{E}+08$ & -- & -- & -- & DOE-STD-1196 & $6.16 \mathrm{E}-14$ \\
\hline$Y-83 m$ & $Y-83$ & 82.922 & Yttrium & $1.00 \mathrm{E}-03$ & ICRP-107 & $2.85 \mathrm{E}+00$ & $\mathrm{~m}$ & $1.71 \mathrm{E}+02$ & $7.96 \mathrm{E}+08$ & -- & -- & -- & DOE-STD-1196 & $3.77 \mathrm{E}-14$ \\
\hline $\mathrm{Y}-84 \mathrm{~m}$ & $\mathrm{Y}-84$ & 83.921 & Yttrium & $1.00 \mathrm{E}-03$ & ICRP-107 & $3.95 \mathrm{E}+01$ & $\mathrm{~m}$ & $2.37 \mathrm{E}+03$ & $5.67 \mathrm{E}+07$ & DOE-STD-1196 & $7.50 \mathrm{E}-11$ & $\mathrm{~s}$ & DOE-STD-1196 & $1.84 \mathrm{E}-13$ \\
\hline $\begin{array}{r}\mathrm{Y}-85 \\
\end{array}$ & $Y-85$ & 84.916 & Yttrium & $1.00 \mathrm{E}-03$ & ICRP-107 & $2.68 \mathrm{E}+00$ & $\mathrm{~h}$ & $9.65 \mathrm{E}+03$ & $1.38 \mathrm{E}+07$ & DOE-STD-1196 & $1.16 \mathrm{E}-10$ & $\mathrm{~s}$ & DOE-STD-1196 & $4.85 \mathrm{E}-14$ \\
\hline $\mathrm{Y}-85 \mathrm{~m}$ & $Y-85$ & 84.916 & Yttrium & $1.00 \mathrm{E}-03$ & ICRP-107 & $4.86 \mathrm{E}+00$ & $\mathrm{~h}$ & $1.75 \mathrm{E}+04$ & $7.59 \mathrm{E}+06$ & DOE-STD-1196 & $2.04 \mathrm{E}-10$ & $\mathrm{~s}$ & DOE-STD-1196 & $6.18 \mathrm{E}-14$ \\
\hline Y-86 & $Y-86$ & 85.915 & Yttrium & $1.00 \mathrm{E}-03$ & ICRP-38 & $1.47 \mathrm{E}+01$ & $\mathrm{~h}$ & $5.31 \mathrm{E}+04$ & $2.47 E+06$ & ICRP-72 & $4.70 \mathrm{E}-10$ & $\mathrm{~s}$ & FGR-12 & $1.79 \mathrm{E}-13$ \\
\hline $\mathrm{Y}-86 \mathrm{~m}$ & Y-86 & 85.915 & Yttrium & $1.00 \mathrm{E}-03$ & ICRP-38 & $4.80 \mathrm{E}+01$ & $\mathrm{~m}$ & $2.88 \mathrm{E}+03$ & $4.56 \mathrm{E}+07$ & ICRP-72 & $2.80 \mathrm{E}-11$ & $\mathrm{~S}$ & FGR-12 & $1.06 \mathrm{E}-14$ \\
\hline
\end{tabular}




\begin{tabular}{|c|c|c|c|c|c|c|c|c|c|c|c|c|c|c|}
\hline $\begin{array}{c}\text { MASTER } \\
\text { ISOTOPE } \\
\text { LIST } \\
\end{array}$ & $\begin{array}{l}\text { Atomic } \\
\text { Mass } \\
\text { Nuclide }\end{array}$ & $\begin{array}{c}\text { Atomic } \\
\text { Mass }\end{array}$ & Element & $\begin{array}{c}\text { RF per } \\
\text { DOE-STD- } \\
1027 \\
\end{array}$ & $\begin{array}{c}\text { Half-Life } \\
\text { Reference }\end{array}$ & $\begin{array}{r}\text { Half-Li } \\
\text { (original }\end{array}$ & & Half Life (s) & $\begin{array}{l}\text { Sp. Act } \\
\text { (Ci/g) }\end{array}$ & $\begin{array}{c}\text { Inhalation DC } \\
\text { Reference }\end{array}$ & $\begin{array}{c}\text { HC-2 } \\
\text { Bounding } \\
\text { Inhalation } \\
\text { DC (Sv/Bq) }\end{array}$ & $\begin{array}{c}\text { Lung } \\
\text { Absorption } \\
\text { Class/Type }\end{array}$ & $\begin{array}{c}\text { Immersion DC } \\
\text { Reference }\end{array}$ & $\begin{array}{c}\mathrm{HC}-2 \\
\text { Immersion } \\
\mathrm{DC}(\mathrm{Sv} / \mathrm{s} \\
\left.\text { per } \mathrm{Bq} / \mathrm{m}^{3}\right)\end{array}$ \\
\hline Y-87 & $\mathrm{Y}-87$ & 86.911 & Yttrium & $1.00 \mathrm{E}-03$ & ICRP-38 & $8.03 \mathrm{E}+01$ & $\mathrm{~h}$ & $2.89 \mathrm{E}+05$ & $4.49 \mathrm{E}+05$ & ICRP-72 & $3.90 \mathrm{E}-10$ & $\mathrm{~S}$ & FGR-12 & $2.15 \mathrm{E}-14$ \\
\hline $\mathrm{Y}-87 \mathrm{~m}$ & $Y-87$ & 86.911 & Yttrium & $1.00 \mathrm{E}-03$ & ICRP-107 & $1.34 \mathrm{E}+01$ & $\mathrm{~h}$ & $4.81 \mathrm{E}+04$ & $2.70 \mathrm{E}+06$ & DOE-STD-1196 & $1.66 \mathrm{E}-10$ & $\mathrm{~s}$ & DOE-STD-1196 & $1.35 \mathrm{E}-14$ \\
\hline$Y-88$ & $Y-88$ & 87.910 & Yttrium & $1.00 \mathrm{E}-03$ & ICRP-38 & $1.07 \mathrm{E}+02$ & $d$ & $9.21 \mathrm{E}+06$ & $1.39 \mathrm{E}+04$ & ICRP-72 & $4.40 \mathrm{E}-09$ & $\mathrm{~s}$ & FGR-12 & $1.37 \mathrm{E}-13$ \\
\hline $\mathrm{Y}-89 \mathrm{~m}$ & $Y-89$ & 88.906 & Yttrium & $1.00 \mathrm{E}-03$ & ICRP-107 & $1.57 \mathrm{E}+01$ & $\mathrm{~s}$ & $1.57 \mathrm{E}+01$ & $8.10 \mathrm{E}+09$ & -- & -- & -- & DOE-STD-1196 & $4.16 \mathrm{E}-14$ \\
\hline $\mathrm{Y}-90$ & Y-90 & 89.907 & Yttrium & $1.00 \mathrm{E}-03$ & ICRP-38 & $6.40 \mathrm{E}+01$ & $\mathrm{~h}$ & $2.30 \mathrm{E}+05$ & $5.45 \mathrm{E}+05$ & ICRP-72 & $1.50 \mathrm{E}-09$ & $\mathrm{~s}$ & FGR-12 & $1.90 \mathrm{E}-16$ \\
\hline Y-90m & $Y-90$ & 89.907 & Yttrium & $1.00 \mathrm{E}-03$ & ICRP-38 & $3.19 \mathrm{E}+00$ & $\mathrm{~h}$ & $1.15 \mathrm{E}+04$ & $1.09 \mathrm{E}+07$ & ICRP-72 & $1.00 \mathrm{E}-10$ & $\mathrm{~s}$ & FGR-12 & $3.01 \mathrm{E}-14$ \\
\hline$Y-91$ & Y-91 & 90.907 & Yttrium & $1.00 \mathrm{E}-03$ & ICRP-38 & $5.85 \mathrm{E}+01$ & d & $5.06 \mathrm{E}+06$ & $2.45 \mathrm{E}+04$ & ICRP-72 & $8.90 \mathrm{E}-09$ & $\mathrm{~s}$ & FGR-12 & $2.60 \mathrm{E}-16$ \\
\hline $\mathrm{Y}-91 \mathrm{~m}$ & Y-91 & 90.907 & Yttrium & $1.00 \mathrm{E}-03$ & ICRP-38 & $4.97 \mathrm{E}+01$ & $\mathrm{~m}$ & $2.98 \mathrm{E}+03$ & $4.16 \mathrm{E}+07$ & ICRP-72 & $1.10 \mathrm{E}-11$ & $\mathrm{~s}$ & FGR-12 & $2.55 \mathrm{E}-14$ \\
\hline $\begin{array}{r}\mathrm{Y}-92 \\
\end{array}$ & Y-92 & 91.909 & Yttrium & $1.00 \mathrm{E}-03$ & ICRP-38 & $3.54 \mathrm{E}+00$ & $\mathrm{~h}$ & $1.27 \mathrm{E}+04$ & $9.63 \mathrm{E}+06$ & ICRP-72 & $1.80 \mathrm{E}-10$ & $\mathrm{~s}$ & FGR-12 & $1.30 \mathrm{E}-14$ \\
\hline Y-93 & Y-93 & 92.910 & Yttrium & $1.00 \mathrm{E}-03$ & ICRP-38 & $1.01 \mathrm{E}+01$ & $\mathrm{~h}$ & $3.64 \mathrm{E}+04$ & $3.34 \mathrm{E}+06$ & ICRP-72 & $4.20 \mathrm{E}-10$ & $\mathrm{~s}$ & FGR-12 & $4.80 \mathrm{E}-15$ \\
\hline Y-94 & $Y-94$ & 93.912 & Yttrium & $1.00 \mathrm{E}-03$ & ICRP-38 & $1.91 \mathrm{E}+01$ & $\mathrm{~m}$ & $1.15 \mathrm{E}+03$ & $1.05 \mathrm{E}+08$ & ICRP-72 & $2.80 \mathrm{E}-11$ & $\mathrm{~s}$ & FGR-12 & $5.62 \mathrm{E}-14$ \\
\hline Y-95 & $\mathrm{Y}-95$ & 94.913 & Yttrium & $1.00 \mathrm{E}-03$ & ICRP-38 & $1.07 \mathrm{E}+01$ & $\mathrm{~m}$ & $6.42 \mathrm{E}+02$ & $1.85 \mathrm{E}+08$ & ICRP-72 & $1.60 \mathrm{E}-11$ & $\mathrm{~s}$ & FGR-12 & $4.79 \mathrm{E}-14$ \\
\hline Yb-162 & Yb-162 & 161.936 & Ytterbium & $1.00 \mathrm{E}-03$ & ICRP-38 & $1.89 \mathrm{E}+01$ & $\mathrm{~m}$ & $1.13 \mathrm{E}+03$ & $6.14 \mathrm{E}+07$ & ICRP-72 & $1.40 \mathrm{E}-11$ & $\mathrm{~s}$ & FGR-12 & $5.66 \mathrm{E}-15$ \\
\hline Yb-163 & Yb-163 & 162.936 & Ytterbium & $1.00 \mathrm{E}-03$ & ICRP-107 & $1.11 \mathrm{E}+01$ & $\mathrm{~m}$ & $6.63 \mathrm{E}+02$ & $1.04 \mathrm{E}+08$ & DOE-STD-1196 & $1.02 \mathrm{E}-11$ & $\mathrm{~s}$ & DOE-STD-1196 & $3.29 \mathrm{E}-14$ \\
\hline Yb-164 & $\mathrm{Yb}-164$ & 163.934 & Ytterbium & $1.00 \mathrm{E}-03$ & ICRP-107 & $7.58 \mathrm{E}+01$ & $\mathrm{~m}$ & $4.55 \mathrm{E}+03$ & $1.51 \mathrm{E}+07$ & DOE-STD-1196 & $5.31 \mathrm{E}-11$ & $\mathrm{~s}$ & DOE-STD-1196 & $1.61 \mathrm{E}-15$ \\
\hline Yb-165 & Yb-165 & 164.935 & Ytterbium & $1.00 \mathrm{E}-03$ & ICRP-107 & $9.90 \mathrm{E}+00$ & $\mathrm{~m}$ & $5.94 \mathrm{E}+02$ & $1.15 \mathrm{E}+08$ & -- & -- & -- & DOE-STD-1196 & $1.37 \mathrm{E}-14$ \\
\hline Yb-166 & Yb-166 & 165.934 & Ytterbium & $1.00 \mathrm{E}-03$ & ICRP-38 & $5.67 \mathrm{E}+01$ & $\mathrm{~h}$ & $2.04 \mathrm{E}+05$ & $3.33 \mathrm{E}+05$ & ICRP-72 & $7.70 \mathrm{E}-10$ & $\mathrm{~s}$ & FGR-12 & $2.86 \mathrm{E}-15$ \\
\hline $\begin{array}{l}\mathrm{Yb}-167 \\
\end{array}$ & $\begin{array}{l}b-167 \\
\end{array}$ & 166.935 & Ytterbium & $1.00 \mathrm{E}-03$ & ICRP-38 & $1.75 \mathrm{E}+01$ & $\mathrm{~m}$ & $1.05 \mathrm{E}+03$ & $6.44 \mathrm{E}+07$ & ICRP-72 & $6.90 \mathrm{E}-12$ & $\mathrm{~s}$ & FGR-12 & $1.09 \mathrm{E}-14$ \\
\hline $\begin{array}{l}\mathrm{Yb}-169 \\
\end{array}$ & Yb-169 & 168.935 & Ytterbium & $1.00 \mathrm{E}-03$ & ICRP-38 & $3.20 \mathrm{E}+01$ & $d$ & $2.77 \mathrm{E}+06$ & $2.41 \mathrm{E}+04$ & ICRP-72 & $3.00 \mathrm{E}-09$ & $\mathrm{~s}$ & FGR-12 & $1.29 \mathrm{E}-14$ \\
\hline Yb-175 & Yb-175 & 174.941 & Ytterbium & $1.00 \mathrm{E}-03$ & ICRP-38 & $4.19 \mathrm{E}+00$ & $d$ & $3.62 \mathrm{E}+05$ & $1.78 \mathrm{E}+05$ & ICRP-72 & $7.30 \mathrm{E}-10$ & $\mathrm{~s}$ & FGR-12 & $1.87 \mathrm{E}-15$ \\
\hline Yb-177 & Yb-177 & 176.945 & Ytterbium & $1.00 \mathrm{E}-03$ & ICRP-38 & $1.90 \mathrm{E}+00$ & $\mathrm{~h}$ & $6.84 \mathrm{E}+03$ & $9.32 \mathrm{E}+06$ & ICRP-72 & $6.90 \mathrm{E}-11$ & $\mathrm{~s}$ & FGR-12 & $9.23 \mathrm{E}-15$ \\
\hline Yb-178 & $\mathrm{Yb}-178$ & 177.947 & Ytterbium & $1.00 \mathrm{E}-03$ & ICRP-38 & $7.40 \mathrm{E}+01$ & $\mathrm{~m}$ & $4.44 \mathrm{E}+03$ & $1.43 \mathrm{E}+07$ & ICRP-72 & $7.50 \mathrm{E}-11$ & $\mathrm{~s}$ & FGR-12 & $1.67 \mathrm{E}-15$ \\
\hline Yb-179 & Yb-179 & 178.950 & Ytterbium & $1.00 \mathrm{E}-03$ & ICRP-107 & $8.00 \mathrm{E}+00$ & $\mathrm{~m}$ & $4.80 \mathrm{E}+02$ & $1.31 \mathrm{E}+08$ & -- & -- & -- & DOE-STD-1196 & $4.40 \mathrm{E}-14$ \\
\hline $\mathrm{Zn}-60$ & $\mathrm{Zn}-60$ & 59.942 & Zinc & $1.00 \mathrm{E}-03$ & ICRP-107 & $2.38 \mathrm{E}+00$ & $\mathrm{~m}$ & $1.43 \mathrm{E}+02$ & $1.32 \mathrm{E}+09$ & -- & -- & -- & DOE-STD-1196 & $6.91 \mathrm{E}-14$ \\
\hline$Z n-61$ & $\mathrm{Zn}-61$ & 60.940 & Zinc & $1.00 \mathrm{E}-03$ & ICRP-107 & $8.91 \mathrm{E}+01$ & $\mathrm{~s}$ & $8.91 \mathrm{E}+01$ & $2.08 \mathrm{E}+09$ & -- & -- & -- & DOE-STD-1196 & $7.23 \mathrm{E}-14$ \\
\hline $\mathrm{Zn}-62$ & $\mathrm{Zn}-62$ & 61.934 & Zinc & $1.00 \mathrm{E}-03$ & ICRP-38 & $9.26 \mathrm{E}+00$ & $\mathrm{~h}$ & $3.33 \mathrm{E}+04$ & $5.46 \mathrm{E}+06$ & ICRP-72 & $5.50 \mathrm{E}-10$ & $\mathrm{~s}$ & FGR-12 & 2.07E-14 \\
\hline $2 n-63$ & $2 n-63$ & 62.933 & Zinc & $1.00 \mathrm{E}-03$ & ICRP-38 & $3.81 \mathrm{E}+01$ & $\mathrm{~m}$ & $2.29 \mathrm{E}+03$ & $7.84 \mathrm{E}+07$ & ICRP-72 & $3.70 \mathrm{E}-11$ & $\mathrm{~s}$ & FGR-12 & $5.32 \mathrm{E}-14$ \\
\hline $\mathrm{Zn}-65$ & $\mathrm{Zn}-65$ & 64.929 & Zinc & $1.00 \mathrm{E}-03$ & ICRP-38 & $2.44 \mathrm{E}+02$ & $d$ & $2.11 \mathrm{E}+07$ & $8.25 \mathrm{E}+03$ & ICRP-72 & $2.20 \mathrm{E}-09$ & $\mathrm{~F}$ & FGR-12 & $2.90 \mathrm{E}-14$ \\
\hline $\mathrm{Zn}-69$ & $2 n-69$ & 68.927 & Zinc & $1.00 \mathrm{E}-03$ & ICRP-38 & $5.70 \mathrm{E}+01$ & $\mathrm{~m}$ & $3.42 \mathrm{E}+03$ & $4.79 \mathrm{E}+07$ & ICRP-72 & $2.80 \mathrm{E}-11$ & $\mathrm{~s}$ & FGR-12 & $2.16 \mathrm{E}-17$ \\
\hline $\mathrm{Zn}-69 \mathrm{~m}$ & $2 n-69$ & 68.927 & Zinc & $1.00 \mathrm{E}-03$ & ICRP-38 & $1.38 \mathrm{E}+01$ & $\mathrm{~h}$ & $4.95 \mathrm{E}+04$ & $3.30 \mathrm{E}+06$ & ICRP-72 & $2.70 \mathrm{E}-10$ & $\mathrm{~s}$ & FGR-12 & $1.99 \mathrm{E}-14$ \\
\hline $2 n-71$ & Zn-71 & 70.928 & Zinc & $1.00 \mathrm{E}-03$ & ICRP-107 & $2.45 \mathrm{E}+00$ & $\mathrm{~m}$ & $1.47 \mathrm{E}+02$ & $1.08 \mathrm{E}+09$ & -- & -- & -- & DOE-STD-1196 & $1.52 \mathrm{E}-14$ \\
\hline $\mathrm{Zn-71m}$ & $Z n-71$ & 70.928 & Zinc & $1.00 \mathrm{E}-03$ & ICRP-38 & $3.92 \mathrm{E}+00$ & $\mathrm{~h}$ & $1.41 \mathrm{E}+04$ & $1.13 \mathrm{E}+07$ & ICRP-72 & $1.60 \mathrm{E}-10$ & $\mathrm{~s}$ & FGR-12 & $7.50 \mathrm{E}-14$ \\
\hline $2 n-72$ & $2 n-72$ & 71.927 & Zinc & $1.00 \mathrm{E}-03$ & ICRP-38 & $4.65 \mathrm{E}+01$ & $\mathrm{~h}$ & $1.67 \mathrm{E}+05$ & $9.37 \mathrm{E}+05$ & ICRP-72 & $1.30 \mathrm{E}-09$ & $\mathrm{~s}$ & FGR-12 & $6.90 \mathrm{E}-15$ \\
\hline Zr-85 & Zr-85 & 84.921 & Zirconium & $1.00 \mathrm{E}-03$ & ICRP-107 & $7.86 \mathrm{E}+00$ & $\mathrm{~m}$ & $4.72 \mathrm{E}+02$ & $2.82 \mathrm{E}+08$ & -- & -- & -- & DOE-STD-1196 & $6.74 \mathrm{E}-14$ \\
\hline $\mathrm{Zr}-86$ & Zr-86 & 85.916 & Zirconium & $1.00 \mathrm{E}-03$ & ICRP-38 & $1.65 \mathrm{E}+01$ & $\mathrm{~h}$ & $5.94 \mathrm{E}+04$ & $2.21 \mathrm{E}+06$ & ICRP-72 & $4.30 \mathrm{E}-10$ & $\mathrm{~s}$ & FGR-12 & $1.28 \mathrm{E}-14$ \\
\hline Zr-87 & Zr-87 & 86.915 & Zirconium & $1.00 \mathrm{E}-03$ & ICRP-107 & $1.68 \mathrm{E}+00$ & $\mathrm{~h}$ & $6.05 \mathrm{E}+03$ & $2.15 \mathrm{E}+07$ & DOE-STD-1196 & $1.08 \mathrm{E}-10$ & $\mathrm{~s}$ & DOE-STD-1196 & $4.22 \mathrm{E}-14$ \\
\hline Zr-88 & Zr-88 & 87.910 & Zirconium & $1.00 \mathrm{E}-03$ & ICRP-38 & $8.34 \mathrm{E}+01$ & $d$ & $7.21 \mathrm{E}+06$ & $1.78 \mathrm{E}+04$ & ICRP-72 & $3.60 \mathrm{E}-09$ & $\mathrm{~s}$ & FGR-12 & $1.88 \mathrm{E}-14$ \\
\hline Zr-89 & Zr-89 & 88.909 & Zirconium & $1.00 \mathrm{E}-03$ & ICRP-38 & $7.84 \mathrm{E}+01$ & $\mathrm{~h}$ & $2.82 \mathrm{E}+05$ & $4.49 \mathrm{E}+05$ & ICRP-72 & $5.50 \mathrm{E}-10$ & $\mathrm{~s}$ & FGR-12 & $5.68 \mathrm{E}-14$ \\
\hline Zr-89m & Zr-89 & 88.909 & Zirconium & $1.00 \mathrm{E}-03$ & ICRP-107 & $4.16 \mathrm{E}+00$ & $\mathrm{~m}$ & $2.50 \mathrm{E}+02$ & $5.08 \mathrm{E}+08$ & -- & -- & -- & DOE-STD-1196 & $2.88 \mathrm{E}-14$ \\
\hline Zr-93 & Zr-93 & 92.906 & Zirconium & $1.00 \mathrm{E}-03$ & ICRP-38 & $1.53 \mathrm{E}+06$ & $y$ & $4.83 \mathrm{E}+13$ & $2.51 \mathrm{E}-03$ & ICRP-72 & $2.50 \mathrm{E}-08$ & $\mathrm{~F}$ & DOE-STD-1196 & $6.45 \mathrm{E}-22$ \\
\hline Zr-95 & Zr-95 & 94.908 & Zirconium & $1.00 \mathrm{E}-03$ & ICRP-38 & $6.40 \mathrm{E}+01$ & $d$ & $5.53 \mathrm{E}+06$ & $2.15 \mathrm{E}+04$ & ICRP-72 & $5.90 \mathrm{E}-09$ & $\mathrm{~s}$ & FGR-12 & $3.60 \mathrm{E}-14$ \\
\hline Zr-97 & Zr-97 & 96.911 & Zirconium & $1.00 \mathrm{E}-03$ & ICRP-38 & $1.69 \mathrm{E}+01$ & $\mathrm{~h}$ & $6.08 \mathrm{E}+04$ & $1.91 \mathrm{E}+06$ & ICRP-72 & $9.20 \mathrm{E}-10$ & $\mathrm{M}$ & FGR-12 & $9.02 \mathrm{E}-15$ \\
\hline
\end{tabular}




\begin{tabular}{|c|c|c|}
\hline \multicolumn{3}{|c|}{ Table A.2 HC-2 TQs Using Maximum Inhalation DCs } \\
\hline $\begin{array}{c}\text { MASTER } \\
\text { ISOTOPE LIST }\end{array}$ & Activity (Ci) & Mass (g) \\
\hline Ac- 223 & $1.31 \mathrm{E}+10$ & $3.41 \mathrm{E}+01$ \\
\hline Ac-224 & $6.24 \mathrm{E}+04$ & $1.29 \mathrm{E}-02$ \\
\hline Ac-225 & $9.54 \mathrm{E}+02$ & 1.64E-02 \\
\hline Ac-226 & $6.24 \mathrm{E}+03$ & $1.30 \mathrm{E}-02$ \\
\hline Ac-227 & $1.47 \mathrm{E}+01$ & $2.04 \mathrm{E}-01$ \\
\hline Ac-228 & $3.22 \mathrm{E}+05$ & 1.44E-01 \\
\hline Ac-229 & $2.32 \mathrm{E}+08$ & $1.77 \mathrm{E}+01$ \\
\hline Ac-230 & $1.02 \mathrm{E}+08$ & 2.53E-01 \\
\hline Ac-231 & $1.47 \mathrm{E}+08$ & $1.35 \mathrm{E}+00$ \\
\hline Ac-232 & $4.78 \mathrm{E}+07$ & 1.17E-01 \\
\hline Ac-233 & $1.18 \mathrm{E}+08$ & 3.54E-01 \\
\hline Ag-100m & $2.03 \mathrm{E}+07$ & 2.42E-02 \\
\hline Ag-101 & $3.49 \mathrm{E}+07$ & $2.08 \mathrm{E}-01$ \\
\hline Ag-102 & $1.56 \mathrm{E}+07$ & 1.09E-01 \\
\hline Ag-102m & $2.78 \mathrm{E}+07$ & $1.16 \mathrm{E}-01$ \\
\hline Ag-103 & $5.90 \mathrm{E}+07$ & $2.12 \mathrm{E}+00$ \\
\hline Ag-104 & $1.87 \mathrm{E}+07$ & 7.16E-01 \\
\hline Ag-104m & $4.04 \mathrm{E}+07$ & 7.48E-01 \\
\hline Ag-105 & $9.18 \mathrm{E}+06$ & $3.02 E+02$ \\
\hline Ag-105m & $6.11 \mathrm{E}+10$ & $2.47 \mathrm{E}+02$ \\
\hline Ag-106 & $6.89 \mathrm{E}+07$ & $9.30 \mathrm{E}-01$ \\
\hline Ag-106m & $5.36 \mathrm{E}+06$ & $3.65 E+01$ \\
\hline Ag-108 & $2.91 \mathrm{E}+09$ & $3.96 \mathrm{E}+00$ \\
\hline Ag-108m & $2.18 \mathrm{E}+05$ & $8.35 \mathrm{E}+03$ \\
\hline Ag-109m & $1.41 \mathrm{E}+10$ & $5.38 \mathrm{E}+00$ \\
\hline Ag-110 & $1.52 \mathrm{E}+09$ & $3.64 \mathrm{E}-01$ \\
\hline Ag-110m & $6.53 \mathrm{E}+05$ & $1.37 \mathrm{E}+02$ \\
\hline Ag-111 & $4.76 \mathrm{E}+06$ & $3.01 \mathrm{E}+01$ \\
\hline Ag-111m & $1.61 \mathrm{E}+10$ & $1.02 \mathrm{E}+01$ \\
\hline Ag-112 & $3.00 \mathrm{E}+07$ & $3.34 \mathrm{E}+00$ \\
\hline Ag-113 & $3.89 \mathrm{E}+07$ & $7.52 \mathrm{E}+00$ \\
\hline Ag-113m & $2.83 E+08$ & $1.95 \mathrm{E}-01$ \\
\hline Ag-114 & $1.85 \mathrm{E}+08$ & 8.60E-03 \\
\hline Ag-115 & $5.91 \mathrm{E}+07$ & 7.22E-01 \\
\hline Ag-116 & $2.53 \mathrm{E}+07$ & 4.17E-02 \\
\hline Ag-117 & $4.16 \mathrm{E}+07$ & $3.17 \mathrm{E}-02$ \\
\hline Ag-99 & $2.50 \mathrm{E}+07$ & 2.72E-02 \\
\hline
\end{tabular}




\begin{tabular}{|c|c|c|}
\hline \multicolumn{3}{|c|}{$\begin{array}{l}\text { Table A.2 HC-2 TQs Using Maximum Inhalation DCs } \\
\text { (continued) }\end{array}$} \\
\hline $\begin{array}{c}\text { MASTER } \\
\text { ISOTOPE LIST }\end{array}$ & Activity (Ci) & Mass (g) \\
\hline Al-26 & $3.97 \mathrm{E}+05$ & $2.07 \mathrm{E}+07$ \\
\hline $\mathrm{Al}-28$ & $2.91 \mathrm{E}+07$ & $9.71 \mathrm{E}-03$ \\
\hline Al-29 & $4.03 E+07$ & 4.07E-02 \\
\hline Am-237 & $1.05 E+08$ & $9.69 \mathrm{E}+00$ \\
\hline Am-238 & $2.53 \mathrm{E}+07$ & $3.14 \mathrm{E}+00$ \\
\hline Am-239 & $2.99 \mathrm{E}+07$ & $2.71 \mathrm{E}+01$ \\
\hline Am-240 & $1.40 \mathrm{E}+07$ & $5.44 \mathrm{E}+01$ \\
\hline Am-241 & $8.45 \mathrm{E}+01$ & $2.46 \mathrm{E}+01$ \\
\hline Am-242 & $4.05 E+05$ & $5.02 \mathrm{E}-01$ \\
\hline Am-242m & $8.81 \mathrm{E}+01$ & $9.07 \mathrm{E}+00$ \\
\hline Am-243 & $8.45 \mathrm{E}+01$ & $4.24 \mathrm{E}+02$ \\
\hline Am-244 & $2.13 \mathrm{E}+06$ & $1.67 \mathrm{E}+00$ \\
\hline Am-244m & $5.06 \mathrm{E}+07$ & $1.71 \mathrm{E}+00$ \\
\hline Am-245 & $1.34 \mathrm{E}+08$ & $2.15 \mathrm{E}+01$ \\
\hline Am-246 & $4.84 \mathrm{E}+07$ & $2.47 \mathrm{E}+00$ \\
\hline Am-246m & $4.66 \mathrm{E}+07$ & $1.53 \mathrm{E}+00$ \\
\hline Am-247 & $1.65 \mathrm{E}+08$ & $4.98 \mathrm{E}+00$ \\
\hline Ar-37 & $2.13 \mathrm{E}+10$ & $2.11 \mathrm{E}+05$ \\
\hline Ar-39 & $2.97 E+08$ & $8.71 \mathrm{E}+06$ \\
\hline Ar-41 & $4.16 \mathrm{E}+04$ & 9.93E-04 \\
\hline Ar-42 & $2.15 E+07$ & $8.28 \mathrm{E}+04$ \\
\hline Ar-43 & $3.58 \mathrm{E}+04$ & 4.39E-05 \\
\hline Ar-44 & $2.86 \mathrm{E}+04$ & 7.93E-05 \\
\hline As-68 & $1.54 \mathrm{E}+07$ & $1.40 \mathrm{E}-02$ \\
\hline As-69 & $4.83 \mathrm{E}+07$ & 2.69E-01 \\
\hline As-70 & $1.19 \mathrm{E}+07$ & 2.34E-01 \\
\hline As-71 & $1.68 \mathrm{E}+07$ & $2.47 \mathrm{E}+01$ \\
\hline As-72 & $6.97 \mathrm{E}+06$ & $4.16 \mathrm{E}+00$ \\
\hline As-73 & $8.10 \mathrm{E}+06$ & $3.63 \mathrm{E}+02$ \\
\hline As-74 & $3.67 \mathrm{E}+06$ & $3.69 \mathrm{E}+01$ \\
\hline As-76 & $1.01 \mathrm{E}+07$ & $6.43 \mathrm{E}+00$ \\
\hline As-77 & $2.07 \mathrm{E}+07$ & $1.97 \mathrm{E}+01$ \\
\hline As-78 & $2.91 \mathrm{E}+07$ & $1.09 \mathrm{E}+00$ \\
\hline As-79 & $1.20 \mathrm{E}+09$ & $4.54 \mathrm{E}+00$ \\
\hline At-204 & $2.60 \mathrm{E}+07$ & $2.59 \mathrm{E}-01$ \\
\hline At-205 & $8.63 \mathrm{E}+06$ & $2.46 \mathrm{E}-01$ \\
\hline At-206 & $1.36 \mathrm{E}+07$ & 4.55E-01 \\
\hline At-207 & $3.25 \mathrm{E}+06$ & 3.86E-01 \\
\hline
\end{tabular}




\begin{tabular}{|c|c|c|}
\hline \multicolumn{3}{|c|}{$\begin{array}{l}\text { Table A.2 HC-2 TQs Using Maximum Inhalation DCs } \\
\text { (continued) }\end{array}$} \\
\hline $\begin{array}{c}\text { MASTER } \\
\text { ISOTOPE LIST }\end{array}$ & Activity (Ci) & Mass (g) \\
\hline At-208 & $7.54 \mathrm{E}+06$ & 8.15E-01 \\
\hline At-209 & $2.42 \mathrm{E}+06$ & 8.73E-01 \\
\hline At-210 & $6.69 \mathrm{E}+05$ & 3.63E-01 \\
\hline At-211 & $7.37 \mathrm{E}+04$ & 3.58E-02 \\
\hline At-215 & $2.93 \mathrm{E}+11$ & 5.59E-04 \\
\hline At-216 & $4.33 \mathrm{E}+10$ & 2.49E-04 \\
\hline At-217 & $1.83 \mathrm{E}+11$ & 1.13E-01 \\
\hline At-218 & $2.27 \mathrm{E}+10$ & 8.78E-01 \\
\hline At-220 & $1.29 \mathrm{E}+08$ & 5.61E-01 \\
\hline Au-186 & $3.52 \mathrm{E}+07$ & 3.72E-01 \\
\hline Au-187 & $5.45 \mathrm{E}+07$ & 4.55E-01 \\
\hline Au-190 & $2.15 \mathrm{E}+07$ & 9.30E-01 \\
\hline Au-191 & $5.33 \mathrm{E}+07$ & $1.03 \mathrm{E}+01$ \\
\hline Au-192 & $2.06 \mathrm{E}+07$ & $6.23 E+00$ \\
\hline Au-193 & $5.77 \mathrm{E}+07$ & $6.27 \mathrm{E}+01$ \\
\hline Au-193m & $3.23 E+08$ & 2.15E-02 \\
\hline $\mathrm{Au}-194$ & $2.03 \mathrm{E}+07$ & $4.97 \mathrm{E}+01$ \\
\hline Au-195 & $4.74 \mathrm{E}+06$ & $1.30 \mathrm{E}+03$ \\
\hline $\mathrm{Au}-195 \mathrm{~m}$ & $2.88 \mathrm{E}+08$ & 1.52E-01 \\
\hline Au-196 & $1.85 \mathrm{E}+07$ & $1.72 \mathrm{E}+02$ \\
\hline Au-196m & $1.53 \mathrm{E}+07$ & $9.20 \mathrm{E}+00$ \\
\hline $\mathrm{Au}-198$ & $8.83 \mathrm{E}+06$ & $3.61 \mathrm{E}+01$ \\
\hline Au-198m & $3.90 \mathrm{E}+06$ & $1.36 \mathrm{E}+01$ \\
\hline Au-199 & $1.01 \mathrm{E}+07$ & $4.83 \mathrm{E}+01$ \\
\hline Au-200 & $1.07 \mathrm{E}+08$ & $5.48 \mathrm{E}+00$ \\
\hline $\mathrm{Au}-200 \mathrm{~m}$ & $7.93 \mathrm{E}+06$ & $9.46 \mathrm{E}+00$ \\
\hline Au-201 & $3.28 \mathrm{E}+08$ & $9.26 \mathrm{E}+00$ \\
\hline Au-202 & $3.04 \mathrm{E}+08$ & 1.57E-01 \\
\hline Ва-124 & $7.92 \mathrm{E}+07$ & 5.74E-01 \\
\hline Вa-126 & $6.19 \mathrm{E}+07$ & $4.00 \mathrm{E}+00$ \\
\hline Вa-127 & $7.25 \mathrm{E}+07$ & $6.22 \mathrm{E}-01$ \\
\hline Ва-128 & $5.76 \mathrm{E}+06$ & $1.37 \mathrm{E}+01$ \\
\hline Вa-129 & $1.04 \mathrm{E}+08$ & $9.58 \mathrm{E}+00$ \\
\hline Ba-129m & $2.98 \mathrm{E}+07$ & $2.65 \mathrm{E}+00$ \\
\hline Ва-131 & $8.69 \mathrm{E}+06$ & $1.03 \mathrm{E}+02$ \\
\hline Ba-131m & $4.79 E+08$ & $4.87 \mathrm{E}+00$ \\
\hline Ba-133 & $8.07 \mathrm{E}+05$ & $3.22 \mathrm{E}+03$ \\
\hline Ва-133m & $1.73 \mathrm{E}+07$ & $2.86 \mathrm{E}+01$ \\
\hline
\end{tabular}




\begin{tabular}{|c|c|c|}
\hline \multicolumn{3}{|c|}{$\begin{array}{l}\text { Table A.2 HC-2 TQs Using Maximum Inhalation DCs } \\
\text { (continued) }\end{array}$} \\
\hline $\begin{array}{c}\text { MASTER } \\
\text { ISOTOPE LIST }\end{array}$ & Activity (Ci) & Mass (g) \\
\hline Ва-135m & $2.21 \mathrm{E}+07$ & $2.73 E+01$ \\
\hline Ba-137m & $9.38 \mathrm{E}+07$ & 1.74E-01 \\
\hline Ва-139 & $1.24 \mathrm{E}+08$ & $7.56 \mathrm{E}+00$ \\
\hline Ва-140 & $1.39 \mathrm{E}+06$ & $1.90 \mathrm{E}+01$ \\
\hline Вa-141 & $5.11 \mathrm{E}+07$ & 6.99E-01 \\
\hline Ва-142 & $4.59 \mathrm{E}+07$ & 3.68E-01 \\
\hline Be-10 & $2.32 \mathrm{E}+05$ & $1.04 \mathrm{E}+07$ \\
\hline Be-7 & $1.31 \mathrm{E}+08$ & $3.74 \mathrm{E}+02$ \\
\hline Bi-197 & $3.44 \mathrm{E}+07$ & 3.35E-01 \\
\hline $\mathrm{Bi}-200$ & $2.13 \mathrm{E}+07$ & 8.24E-01 \\
\hline Bi-201 & $3.10 \mathrm{E}+07$ & $3.58 \mathrm{E}+00$ \\
\hline Bi-202 & $1.79 \mathrm{E}+07$ & $1.92 \mathrm{E}+00$ \\
\hline Bi-203 & $1.31 \mathrm{E}+07$ & $9.96 \mathrm{E}+00$ \\
\hline Bi-204 & $1.03 \mathrm{E}+07$ & $7.53 \mathrm{E}+00$ \\
\hline Bi-205 & $6.84 \mathrm{E}+06$ & $1.64 \mathrm{E}+02$ \\
\hline Bi-206 & $3.71 \mathrm{E}+06$ & $3.66 \mathrm{E}+01$ \\
\hline Bi-207 & $1.39 \mathrm{E}+06$ & $3.06 \mathrm{E}+04$ \\
\hline $\mathrm{Bi}-208$ & $2.09 \mathrm{E}+05$ & $4.48 \mathrm{E}+07$ \\
\hline Bi-210 & $8.72 E+04$ & 7.03E-01 \\
\hline $\mathrm{Bi}-210 \mathrm{~m}$ & $2.38 \mathrm{E}+03$ & $4.20 \mathrm{E}+06$ \\
\hline Bi-211 & $1.22 \mathrm{E}+09$ & $2.92 \mathrm{E}+00$ \\
\hline Bi-212 & $2.61 \mathrm{E}+05$ & $1.78 \mathrm{E}-02$ \\
\hline Bi-212n & $6.95 \mathrm{E}+09$ & $5.48 \mathrm{E}+01$ \\
\hline $\mathrm{Bi}-213$ & $2.70 \mathrm{E}+05$ & $1.40 \mathrm{E}-02$ \\
\hline $\mathrm{Bi}-214$ & $5.70 \mathrm{E}+05$ & 1.29E-02 \\
\hline $\mathrm{Bi}-215$ & $2.29 \mathrm{E}+08$ & $1.99 \mathrm{E}+00$ \\
\hline Bi-216 & $7.93 \mathrm{E}+07$ & $1.98 \mathrm{E}-01$ \\
\hline Bk-244 & $8.11 \mathrm{E}+06$ & $2.75 \mathrm{E}+00$ \\
\hline Bk-245 & $3.80 \mathrm{E}+06$ & $3.53 \mathrm{E}+01$ \\
\hline Bk-246 & $1.73 \mathrm{E}+07$ & $5.98 \mathrm{E}+01$ \\
\hline Bk-247 & $1.18 \mathrm{E}+02$ & $1.12 \mathrm{E}+02$ \\
\hline Bk-248m & $2.88 \mathrm{E}+05$ & $5.41 \mathrm{E}-01$ \\
\hline Bk-249 & $5.07 \mathrm{E}+04$ & $3.09 \mathrm{E}+01$ \\
\hline Bk-250 & $7.17 \mathrm{E}+06$ & $1.84 \mathrm{E}+00$ \\
\hline Bk-251 & $1.32 \mathrm{E}+08$ & $9.78 \mathrm{E}+00$ \\
\hline $\mathrm{Br}-72$ & $3.83 \mathrm{E}+04$ & $1.92 \mathrm{E}-05$ \\
\hline Br-73 & $8.29 \mathrm{E}+04$ & 1.09E-04 \\
\hline Br-74 & $2.16 \mathrm{E}+04$ & 2.15E-04 \\
\hline
\end{tabular}




\begin{tabular}{|c|c|c|}
\hline \multicolumn{3}{|c|}{$\begin{array}{l}\text { Table A.2 HC-2 TQs Using Maximum Inhalation DCs } \\
\text { (continued) }\end{array}$} \\
\hline $\begin{array}{c}\text { MASTER } \\
\text { ISOTOPE LIST }\end{array}$ & Activity (Ci) & Mass (g) \\
\hline $\mathrm{Br}-74 \mathrm{~m}$ & $2.36 \mathrm{E}+04$ & $3.86 \mathrm{E}-04$ \\
\hline Br-75 & $7.11 \mathrm{E}+04$ & 2.78E-03 \\
\hline Br-76 & $2.00 \mathrm{E}+04$ & 7.84E-03 \\
\hline Br-76m & $5.60 \mathrm{E}+06$ & 4.93E-05 \\
\hline Br-77 & $1.25 \mathrm{E}+05$ & $1.72 \mathrm{E}-01$ \\
\hline Br-77m & $9.07 \mathrm{E}+06$ & 1.59E-02 \\
\hline Br-78 & $1.15 \mathrm{E}+05$ & 3.09E-04 \\
\hline Br-80 & $7.74 \mathrm{E}+05$ & 5.72E-03 \\
\hline $\mathrm{Br}-80 \mathrm{~m}$ & $2.11 \mathrm{E}+05$ & 2.38E-02 \\
\hline Br-82 & $1.59 \mathrm{E}+04$ & 1.47E-02 \\
\hline Br-82m & $3.40 \mathrm{E}+07$ & $9.08 \mathrm{E}-02$ \\
\hline Br-83 & $3.30 \mathrm{E}+05$ & 2.09E-02 \\
\hline $\mathrm{Br}-84$ & $5.08 \mathrm{E}+04$ & 7.21E-04 \\
\hline $\mathrm{Br}-84 \mathrm{~m}$ & $4.13 \mathrm{E}+04$ & $1.10 \mathrm{E}-04$ \\
\hline $\mathrm{Br}-85$ & $1.36 \mathrm{E}+06$ & $1.78 \mathrm{E}-03$ \\
\hline C-10 & $3.42 \mathrm{E}+06$ & 5.85E-05 \\
\hline C-11 & $4.92 \mathrm{E}+06$ & $5.88 \mathrm{E}-03$ \\
\hline C-14 & $1.40 \mathrm{E}+05$ & $3.14 \mathrm{E}+04$ \\
\hline Ca-41 & $4.50 \mathrm{E}+07$ & $7.23 \mathrm{E}+08$ \\
\hline Ca-45 & $2.19 \mathrm{E}+06$ & $1.23 \mathrm{E}+02$ \\
\hline Ca-47 & $3.59 \mathrm{E}+06$ & $5.84 \mathrm{E}+00$ \\
\hline Са-49 & $1.56 \mathrm{E}+07$ & 3.55E-02 \\
\hline Cd-101 & $2.31 \mathrm{E}+07$ & 1.69E-02 \\
\hline Cd-102 & $7.25 \mathrm{E}+07$ & $2.16 \mathrm{E}-01$ \\
\hline Cd-103 & $2.70 \mathrm{E}+07$ & $1.08 \mathrm{E}-01$ \\
\hline Cd-104 & $1.17 \mathrm{E}+08$ & $3.74 \mathrm{E}+00$ \\
\hline Cd-105 & $3.82 \mathrm{E}+07$ & $1.18 \mathrm{E}+00$ \\
\hline Cd-107 & $9.56 \mathrm{E}+07$ & $2.12 \mathrm{E}+01$ \\
\hline Cd-109 & $1.00 \mathrm{E}+06$ & $3.87 \mathrm{E}+02$ \\
\hline Cd-111m & $1.30 \mathrm{E}+08$ & $3.71 \mathrm{E}+00$ \\
\hline Cd-113 & $6.76 \mathrm{E}+04$ & $1.98 \mathrm{E}+17$ \\
\hline Cd-113m & $7.37 \mathrm{E}+04$ & $3.17 \mathrm{E}+02$ \\
\hline Cd-115 & $7.15 \mathrm{E}+06$ & $1.40 \mathrm{E}+01$ \\
\hline Cd-115m & $1.05 \mathrm{E}+06$ & $4.13 \mathrm{E}+01$ \\
\hline Cd-117 & $2.43 \mathrm{E}+07$ & $2.26 \mathrm{E}+00$ \\
\hline $\mathrm{Cd}-117 \mathrm{~m}$ & $1.54 \mathrm{E}+07$ & $1.94 \mathrm{E}+00$ \\
\hline Cd-118 & $8.43 \mathrm{E}+07$ & $2.66 \mathrm{E}+00$ \\
\hline Cd-119 & $3.40 \mathrm{E}+07$ & 5.78E-02 \\
\hline
\end{tabular}




\begin{tabular}{|c|c|c|}
\hline \multicolumn{3}{|c|}{$\begin{array}{l}\text { Table A.2 HC-2 TQs Using Maximum Inhalation DCs } \\
\text { (continued) }\end{array}$} \\
\hline $\begin{array}{c}\text { MASTER } \\
\text { ISOTOPE LIST }\end{array}$ & Activity (Ci) & Mass (g) \\
\hline Cd-119m & $2.43 \mathrm{E}+07$ & 3.39E-02 \\
\hline Ce-130 & $7.58 \mathrm{E}+07$ & $1.20 \mathrm{E}+00$ \\
\hline Сe-131 & $3.38 \mathrm{E}+07$ & 2.40E-01 \\
\hline Ce-132 & $3.96 \mathrm{E}+07$ & $5.85 \mathrm{E}+00$ \\
\hline Ce-133 & $6.21 \mathrm{E}+07$ & $4.26 \mathrm{E}+00$ \\
\hline Ce-133m & $2.13 \mathrm{E}+07$ & $4.43 \mathrm{E}+00$ \\
\hline Ce-134 & $6.23 \mathrm{E}+06$ & $1.92 \mathrm{E}+01$ \\
\hline Ce-135 & $1.07 \mathrm{E}+07$ & $8.12 \mathrm{E}+00$ \\
\hline Ce-137 & $6.41 \mathrm{E}+08$ & $2.52 \mathrm{E}+02$ \\
\hline Ce-137m & $1.82 \mathrm{E}+07$ & $2.73 E+01$ \\
\hline Сe-139 & $4.22 \mathrm{E}+06$ & $6.18 \mathrm{E}+02$ \\
\hline Ce-141 & $2.13 \mathrm{E}+06$ & $7.46 \mathrm{E}+01$ \\
\hline Сe-143 & $9.33 \mathrm{E}+06$ & $1.40 \mathrm{E}+01$ \\
\hline Ce-144 & $1.53 \mathrm{E}+05$ & $4.79 \mathrm{E}+01$ \\
\hline Ce-145 & $7.43 \mathrm{E}+07$ & $1.72 \mathrm{E}-01$ \\
\hline Ce-146 & $3.12 \mathrm{E}+08$ & $3.27 \mathrm{E}+00$ \\
\hline Cf-244 & $5.79 \mathrm{E}+05$ & $1.46 \mathrm{E}-02$ \\
\hline Cf-246 & $1.80 \mathrm{E}+04$ & 5.05E-02 \\
\hline Cf-247 & $1.29 \mathrm{E}+08$ & $3.16 \mathrm{E}+01$ \\
\hline Cf-248 & $9.21 \mathrm{E}+02$ & 5.84E-01 \\
\hline Cf-249 & $1.16 \mathrm{E}+02$ & $2.83 \mathrm{E}+01$ \\
\hline Cf-250 & $2.38 \mathrm{E}+02$ & $2.18 \mathrm{E}+00$ \\
\hline Cf-251 & $1.14 \mathrm{E}+02$ & $7.20 \mathrm{E}+01$ \\
\hline Cf-252 & $4.05 \mathrm{E}+02$ & 7.54E-01 \\
\hline Cf-253 & $6.24 \mathrm{E}+03$ & 2.15E-01 \\
\hline Cf-254 & $1.98 \mathrm{E}+02$ & 2.33E-02 \\
\hline Cf-255 & $1.10 \mathrm{E}+06$ & $1.27 \mathrm{E}-01$ \\
\hline Cf-256 & $4.27 \mathrm{E}+03$ & 7.15E-05 \\
\hline Cl-34 & $5.67 \mathrm{E}+04$ & 2.60E-07 \\
\hline Cl-34m & $2.23 \mathrm{E}+04$ & 1.29E-04 \\
\hline Cl-36 & $1.11 \mathrm{E}+03$ & $3.36 \mathrm{E}+04$ \\
\hline Cl-38 & $2.88 \mathrm{E}+04$ & 2.17E-04 \\
\hline Cl-39 & $3.06 \mathrm{E}+04$ & 3.53E-04 \\
\hline $\mathrm{Cl}-40$ & $1.29 \mathrm{E}+04$ & 3.71E-06 \\
\hline Cm-238 & $1.65 \mathrm{E}+06$ & 3.01E-01 \\
\hline Cm-239 & $7.23 \mathrm{E}+07$ & $1.60 \mathrm{E}+01$ \\
\hline $\mathrm{Cm}-240$ & $2.32 \mathrm{E}+03$ & $1.15 \mathrm{E}-01$ \\
\hline Cm-241 & $2.19 \mathrm{E}+05$ & $1.32 \mathrm{E}+01$ \\
\hline
\end{tabular}




\begin{tabular}{|c|c|c|}
\hline \multicolumn{3}{|c|}{$\begin{array}{l}\text { Table A.2 HC-2 TQs Using Maximum Inhalation DCs } \\
\text { (continued) }\end{array}$} \\
\hline $\begin{array}{c}\text { MASTER } \\
\text { ISOTOPE LIST }\end{array}$ & Activity (Ci) & Mass (g) \\
\hline Cm-242 & $1.37 \mathrm{E}+03$ & 4.15E-01 \\
\hline Cm-243 & $1.18 \mathrm{E}+02$ & $2.28 \mathrm{E}+00$ \\
\hline Cm-244 & $1.42 \mathrm{E}+02$ & $1.76 \mathrm{E}+00$ \\
\hline Cm-245 & $8.19 \mathrm{E}+01$ & $4.77 \mathrm{E}+02$ \\
\hline Cm-246 & $8.27 \mathrm{E}+01$ & $2.69 \mathrm{E}+02$ \\
\hline Cm-247 & $9.01 \mathrm{E}+01$ & $9.71 \mathrm{E}+05$ \\
\hline Cm-248 & $2.25 \mathrm{E}+01$ & $5.30 \mathrm{E}+03$ \\
\hline Cm-249 & $1.89 \mathrm{E}+08$ & $1.61 \mathrm{E}+01$ \\
\hline $\mathrm{Cm}-250$ & $3.86 \mathrm{E}+00$ & $1.86 \mathrm{E}+01$ \\
\hline Cm-251 & $1.86 \mathrm{E}+08$ & $4.18 \mathrm{E}+00$ \\
\hline Co-54m & $1.46 \mathrm{E}+07$ & $6.20 \mathrm{E}-03$ \\
\hline Co-55 & $9.85 E+06$ & $3.03 \mathrm{E}+00$ \\
\hline Co-56 & $1.12 \mathrm{E}+06$ & $3.77 \mathrm{E}+01$ \\
\hline Co-57 & $7.97 \mathrm{E}+06$ & $9.42 \mathrm{E}+02$ \\
\hline Co-58 & $3.62 E+06$ & $1.14 \mathrm{E}+02$ \\
\hline Co-58m & $4.77 \mathrm{E}+08$ & $8.07 \mathrm{E}+01$ \\
\hline Co-60 & $2.58 \mathrm{E}+05$ & $2.28 \mathrm{E}+02$ \\
\hline Сo-60m & $3.95 \mathrm{E}+09$ & $1.32 \mathrm{E}+01$ \\
\hline Сo-61 & $1.29 \mathrm{E}+08$ & $4.14 \mathrm{E}+00$ \\
\hline Co-62 & $3.41 \mathrm{E}+07$ & 1.69E-02 \\
\hline Co-62m & $1.88 \mathrm{E}+07$ & 8.60E-02 \\
\hline Cr-48 & $2.88 \mathrm{E}+07$ & $1.01 \mathrm{E}+01$ \\
\hline Cr-49 & $4.36 \mathrm{E}+07$ & 4.78E-01 \\
\hline Cr-51 & $1.95 \mathrm{E}+08$ & $2.11 \mathrm{E}+03$ \\
\hline Cr-55 & $2.70 \mathrm{E}+09$ & $2.76 \mathrm{E}+00$ \\
\hline Cr-56 & $7.79 \mathrm{E}+08$ & $1.38 \mathrm{E}+00$ \\
\hline Cs-121 & $5.00 \mathrm{E}+06$ & 8.30E-03 \\
\hline Cs-121m & $5.02 \mathrm{E}+06$ & $6.57 \mathrm{E}-03$ \\
\hline Cs-123 & $5.53 \mathrm{E}+06$ & 2.12E-02 \\
\hline Cs-124 & $4.98 \mathrm{E}+06$ & 1.68E-03 \\
\hline Cs-125 & $6.78 \mathrm{E}+06$ & 2.03E-01 \\
\hline Cs-126 & $5.16 \mathrm{E}+06$ & 5.66E-03 \\
\hline Cs-127 & $8.45 E+06$ & $2.14 \mathrm{E}+00$ \\
\hline Cs-128 & $6.26 \mathrm{E}+06$ & $1.66 \mathrm{E}-02$ \\
\hline Cs-129 & $7.10 \mathrm{E}+06$ & $9.36 \mathrm{E}+00$ \\
\hline Cs-130 & $9.27 \mathrm{E}+06$ & $1.91 \mathrm{E}-01$ \\
\hline Cs-130m & $1.34 \mathrm{E}+08$ & $3.21 \mathrm{E}-01$ \\
\hline Cs-131 & $1.69 \mathrm{E}+07$ & $1.64 \mathrm{E}+02$ \\
\hline
\end{tabular}




\begin{tabular}{|c|c|c|}
\hline \multicolumn{3}{|c|}{$\begin{array}{l}\text { Table A.2 HC-2 TQs Using Maximum Inhalation DCs } \\
\text { (continued) }\end{array}$} \\
\hline $\begin{array}{c}\text { MASTER } \\
\text { ISOTOPE LIST }\end{array}$ & Activity (Ci) & Mass (g) \\
\hline Cs-132 & $2.03 \mathrm{E}+06$ & $1.33 \mathrm{E}+01$ \\
\hline Cs-134 & $4.01 \mathrm{E}+04$ & $3.10 \mathrm{E}+01$ \\
\hline Cs-134m & $1.29 \mathrm{E}+07$ & $1.60 \mathrm{E}+00$ \\
\hline Cs-135 & $9.43 \mathrm{E}+04$ & $8.18 \mathrm{E}+07$ \\
\hline Cs-135m & $3.26 \mathrm{E}+06$ & $1.24 \mathrm{E}-01$ \\
\hline Cs-136 & $2.60 \mathrm{E}+05$ & $3.55 \mathrm{E}+00$ \\
\hline Cs-137 & $2.08 \mathrm{E}+04$ & $2.39 \mathrm{E}+02$ \\
\hline Cs-138 & $2.00 \mathrm{E}+06$ & 4.72E-02 \\
\hline Cs-138m & $1.41 \mathrm{E}+07$ & 3.00E-02 \\
\hline Cs-139 & $1.63 \mathrm{E}+07$ & $1.12 \mathrm{E}-01$ \\
\hline Cs-140 & $3.04 \mathrm{E}+06$ & $2.40 \mathrm{E}-03$ \\
\hline Cu-57 & $4.78 \mathrm{E}+07$ & 5.63E-05 \\
\hline Cu-59 & $4.05 \mathrm{E}+07$ & $1.72 \mathrm{E}-02$ \\
\hline $\mathrm{Cu}-60$ & $1.29 \mathrm{E}+07$ & $9.55 \mathrm{E}-02$ \\
\hline Cu-61 & $4.10 \mathrm{E}+07$ & $2.72 E+00$ \\
\hline $\mathrm{Cu}-62$ & $5.56 \mathrm{E}+07$ & $1.78 \mathrm{E}-01$ \\
\hline $\mathrm{Cu}-64$ & $5.50 \mathrm{E}+07$ & $1.43 \mathrm{E}+01$ \\
\hline Cu-66 & $6.06 \mathrm{E}+08$ & $1.08 \mathrm{E}+00$ \\
\hline Cu-67 & $1.29 \mathrm{E}+07$ & $1.71 \mathrm{E}+01$ \\
\hline Cu-69 & $1.07 \mathrm{E}+08$ & 1.12E-01 \\
\hline Dy-148 & $8.58 \mathrm{E}+07$ & 2.23E-01 \\
\hline Dy-149 & $3.59 \mathrm{E}+07$ & $1.20 \mathrm{E}-01$ \\
\hline Dy-150 & $2.29 \mathrm{E}+08$ & $1.31 \mathrm{E}+00$ \\
\hline Dy-151 & $2.39 \mathrm{E}+07$ & 3.43E-01 \\
\hline Dy-152 & $7.37 \mathrm{E}+07$ & $8.50 \mathrm{E}+00$ \\
\hline Dy-153 & $3.05 E+07$ & $9.53 \mathrm{E}+00$ \\
\hline Dy-154 & $2.96 \mathrm{E}+02$ & $3.82 E+05$ \\
\hline Dy-155 & $5.06 \mathrm{E}+07$ & $2.50 \mathrm{E}+01$ \\
\hline Dy-157 & $1.03 \mathrm{E}+08$ & $4.17 \mathrm{E}+01$ \\
\hline Dy-159 & $2.17 \mathrm{E}+07$ & $3.81 \mathrm{E}+03$ \\
\hline Dy-165 & $1.27 \mathrm{E}+08$ & $1.57 \mathrm{E}+01$ \\
\hline Dy-165m & $3.82 E+09$ & $4.21 \mathrm{E}+00$ \\
\hline Dy-166 & $4.26 \mathrm{E}+06$ & $1.84 \mathrm{E}+01$ \\
\hline Dy-167 & $1.13 \mathrm{E}+08$ & $6.20 \mathrm{E}-01$ \\
\hline Dy-168 & $1.56 \mathrm{E}+08$ & $1.21 \mathrm{E}+00$ \\
\hline Er-154 & $1.21 \mathrm{E}+09$ & $3.68 \mathrm{E}+00$ \\
\hline Er-156 & $2.80 \mathrm{E}+08$ & $4.53 \mathrm{E}+00$ \\
\hline Er-159 & $5.35 \mathrm{E}+07$ & $1.63 \mathrm{E}+00$ \\
\hline
\end{tabular}




\begin{tabular}{|c|c|c|}
\hline \multicolumn{3}{|c|}{$\begin{array}{l}\text { Table A.2 HC-2 TQs Using Maximum Inhalation DCs } \\
\text { (continued) }\end{array}$} \\
\hline $\begin{array}{c}\text { MASTER } \\
\text { ISOTOPE LIST }\end{array}$ & Activity (Ci) & Mass (g) \\
\hline Er-161 & $4.49 \mathrm{E}+07$ & $7.47 \mathrm{E}+00$ \\
\hline Er-163 & $1.80 \mathrm{E}+09$ & $1.17 \mathrm{E}+02$ \\
\hline Er-165 & $7.22 \mathrm{E}+08$ & $3.94 \mathrm{E}+02$ \\
\hline Er-167m & $6.71 \mathrm{E}+08$ & 2.26E-02 \\
\hline Er-169 & $8.11 \mathrm{E}+06$ & $9.76 \mathrm{E}+01$ \\
\hline Er-171 & $2.97 \mathrm{E}+07$ & $1.22 \mathrm{E}+01$ \\
\hline Er-172 & $6.91 \mathrm{E}+06$ & $1.87 \mathrm{E}+01$ \\
\hline Er-173 & $7.25 \mathrm{E}+07$ & $9.56 \mathrm{E}-02$ \\
\hline Es-249 & $2.68 \mathrm{E}+07$ & $3.63 \mathrm{E}+00$ \\
\hline Es-250 & $1.18 \mathrm{E}+07$ & $1.98 \mathrm{E}+00$ \\
\hline Es-250m & $4.87 \mathrm{E}+06$ & 8.63E-01 \\
\hline Es-251 & $3.84 \mathrm{E}+06$ & $1.01 \mathrm{E}+01$ \\
\hline Es-253 & $3.00 \mathrm{E}+03$ & 1.19E-01 \\
\hline Es-254 & $9.43 \mathrm{E}+02$ & 5.06E-01 \\
\hline Es-254m & $1.72 \mathrm{E}+04$ & $5.50 \mathrm{E}-02$ \\
\hline Es-255 & $1.64 \mathrm{E}+03$ & $1.27 \mathrm{E}-01$ \\
\hline Es-256 & $1.78 \mathrm{E}+05$ & $6.16 \mathrm{E}-03$ \\
\hline Eu-142 & $4.68 \mathrm{E}+07$ & 1.38E-03 \\
\hline Eu-142m & $1.71 \mathrm{E}+07$ & $1.58 \mathrm{E}-02$ \\
\hline Eu-143 & $5.14 \mathrm{E}+07$ & 1.01E-01 \\
\hline Eu-144 & $5.22 \mathrm{E}+07$ & 6.79E-03 \\
\hline Eu-145 & $1.06 \mathrm{E}+07$ & $6.97 \mathrm{E}+01$ \\
\hline Eu-146 & $6.94 \mathrm{E}+06$ & $3.57 \mathrm{E}+01$ \\
\hline Eu-147 & $6.93 \mathrm{E}+06$ & $1.87 \mathrm{E}+02$ \\
\hline Eu-148 & $2.78 \mathrm{E}+06$ & $1.72 \mathrm{E}+02$ \\
\hline Eu-149 & $2.73 \mathrm{E}+07$ & $2.90 \mathrm{E}+03$ \\
\hline Eu-150l & $1.52 \mathrm{E}+05$ & $2.19 \mathrm{E}+03$ \\
\hline Eu-150s & $4.12 \mathrm{E}+07$ & $2.49 \mathrm{E}+01$ \\
\hline Eu-152 & $1.92 \mathrm{E}+05$ & $1.09 \mathrm{E}+03$ \\
\hline Eu-152ml & $3.09 \mathrm{E}+07$ & $1.40 \mathrm{E}+01$ \\
\hline Eu-152ms & $4.51 \mathrm{E}+08$ & $3.50 \mathrm{E}+01$ \\
\hline Eu-154 & $1.52 \mathrm{E}+05$ & $5.78 \mathrm{E}+02$ \\
\hline Eu-154m & $7.23 \mathrm{E}+08$ & $2.72 E+01$ \\
\hline Eu-155 & $1.17 \mathrm{E}+06$ & $2.52 \mathrm{E}+03$ \\
\hline Eu-156 & $2.25 \mathrm{E}+06$ & $4.08 \mathrm{E}+01$ \\
\hline Eu-157 & $2.57 \mathrm{E}+07$ & $1.95 \mathrm{E}+01$ \\
\hline Eu-158 & $3.95 \mathrm{E}+07$ & $1.52 \mathrm{E}+00$ \\
\hline Eu-159 & $1.19 \mathrm{E}+08$ & $1.82 \mathrm{E}+00$ \\
\hline
\end{tabular}




\begin{tabular}{|c|c|c|}
\hline \multicolumn{3}{|c|}{$\begin{array}{l}\text { Table A.2 HC-2 TQs Using Maximum Inhalation DCs } \\
\text { (continued) }\end{array}$} \\
\hline $\begin{array}{c}\text { MASTER } \\
\text { ISOTOPE LIST }\end{array}$ & Activity (Ci) & Mass (g) \\
\hline F-17 & $5.88 \mathrm{E}+04$ & 5.71E-06 \\
\hline F-18 & $3.94 \mathrm{E}+04$ & $4.14 \mathrm{E}-04$ \\
\hline Fe-52 & $1.10 \mathrm{E}+07$ & $1.51 \mathrm{E}+00$ \\
\hline $\mathrm{Fe}-53$ & $5.05 \mathrm{E}+07$ & $1.21 \mathrm{E}-01$ \\
\hline Fe-53m & $1.88 \mathrm{E}+07$ & 1.34E-02 \\
\hline Fe-55 & $1.05 \mathrm{E}+07$ & $4.37 \mathrm{E}+03$ \\
\hline $\mathrm{Fe}-59$ & $1.94 \mathrm{E}+06$ & $3.90 \mathrm{E}+01$ \\
\hline Fe-60 & $2.90 \mathrm{E}+04$ & $4.85 \mathrm{E}+05$ \\
\hline Fe-61 & $4.05 E+07$ & 7.84E-02 \\
\hline Fe-62 & $1.16 \mathrm{E}+08$ & 4.35E-02 \\
\hline Fm-251 & $3.72 \mathrm{E}+06$ & $1.58 \mathrm{E}+00$ \\
\hline Fm-252 & $2.53 \mathrm{E}+04$ & 4.63E-02 \\
\hline Fm-253 & $2.03 \mathrm{E}+04$ & 1.18E-01 \\
\hline Fm-254 & $1.33 \mathrm{E}+05$ & 3.49E-02 \\
\hline Fm-255 & $3.00 \mathrm{E}+04$ & 4.91E-02 \\
\hline Fm-256 & $2.83 E+04$ & 6.07E-03 \\
\hline Fm-257 & $1.14 \mathrm{E}+03$ & 2.26E-01 \\
\hline Fr-212 & $1.14 \mathrm{E}+06$ & 2.56E-02 \\
\hline Fr-219 & $1.63 \mathrm{E}+10$ & 6.64E-03 \\
\hline Fr-220 & $5.49 \mathrm{E}+09$ & $2.94 \mathrm{E}+00$ \\
\hline Fr-221 & $1.85 \mathrm{E}+09$ & $1.04 \mathrm{E}+01$ \\
\hline Fr-222 & $5.79 \mathrm{E}+05$ & $9.85 \mathrm{E}-03$ \\
\hline Fr-223 & $9.04 \mathrm{E}+06$ & 2.34E-01 \\
\hline Fr-224 & $1.03 \mathrm{E}+08$ & 4.09E-01 \\
\hline Fr-227 & $1.35 \mathrm{E}+08$ & 4.03E-01 \\
\hline Ga-64 & $1.65 \mathrm{E}+07$ & $1.47 \mathrm{E}-02$ \\
\hline Ga-65 & $4.35 \mathrm{E}+07$ & 2.28E-01 \\
\hline Ga-66 & $9.80 \mathrm{E}+06$ & $1.94 \mathrm{E}+00$ \\
\hline Ga-67 & $3.10 \mathrm{E}+07$ & $5.18 \mathrm{E}+01$ \\
\hline Ga-68 & $4.35 \mathrm{E}+07$ & $1.07 \mathrm{E}+00$ \\
\hline Ga-70 & $4.66 \mathrm{E}+08$ & $3.67 \mathrm{E}+00$ \\
\hline Ga-72 & $8.56 \mathrm{E}+06$ & $2.77 E+00$ \\
\hline Ga-73 & $4.40 \mathrm{E}+07$ & $5.02 \mathrm{E}+00$ \\
\hline Ga-74 & $1.74 \mathrm{E}+07$ & 5.57E-02 \\
\hline Gd-142 & $5.65 \mathrm{E}+07$ & 4.99E-02 \\
\hline Gd-143m & $2.77 \mathrm{E}+07$ & 3.86E-02 \\
\hline Gd-144 & $6.36 \mathrm{E}+07$ & 2.18E-01 \\
\hline Gd-145 & $2.22 \mathrm{E}+07$ & 3.92E-01 \\
\hline
\end{tabular}




\begin{tabular}{|c|c|c|}
\hline \multicolumn{3}{|c|}{$\begin{array}{l}\text { Table A.2 HC-2 TQs Using Maximum Inhalation DCs } \\
\text { (continued) }\end{array}$} \\
\hline $\begin{array}{c}\text { MASTER } \\
\text { ISOTOPE LIST }\end{array}$ & Activity (Ci) & Mass (g) \\
\hline Gd-145m & $8.78 \mathrm{E}+07$ & $9.58 \mathrm{E}-02$ \\
\hline Gd-146 & $1.26 \mathrm{E}+06$ & $6.81 \mathrm{E}+01$ \\
\hline Gd-147 & $1.37 \mathrm{E}+07$ & $2.44 \mathrm{E}+01$ \\
\hline Gd-148 & $3.12 \mathrm{E}+02$ & $1.20 \mathrm{E}+01$ \\
\hline Gd-149 & $1.03 \mathrm{E}+07$ & $1.10 \mathrm{E}+02$ \\
\hline Gd-150 & $3.17 \mathrm{E}+02$ & $2.38 \mathrm{E}+05$ \\
\hline Gd-151 & $9.36 \mathrm{E}+06$ & $1.30 \mathrm{E}+03$ \\
\hline Gd-152 & $4.27 \mathrm{E}+02$ & $1.96 \mathrm{E}+13$ \\
\hline Gd-153 & $3.84 \mathrm{E}+06$ & $1.09 \mathrm{E}+03$ \\
\hline Gd-159 & $2.93 \mathrm{E}+07$ & $2.76 \mathrm{E}+01$ \\
\hline Gd-162 & $1.45 \mathrm{E}+08$ & $1.05 E+00$ \\
\hline Ge-66 & $4.30 \mathrm{E}+07$ & $2.05 \mathrm{E}+00$ \\
\hline Ge-67 & $3.51 \mathrm{E}+07$ & 2.34E-01 \\
\hline Ge-68 & $5.79 \mathrm{E}+05$ & $8.68 \mathrm{E}+01$ \\
\hline Ge-69 & $1.94 \mathrm{E}+07$ & $1.67 \mathrm{E}+01$ \\
\hline Ge-71 & 7.37E+08 & $4.72 E+03$ \\
\hline Ge-75 & $1.98 \mathrm{E}+08$ & $6.52 \mathrm{E}+00$ \\
\hline Ge-77 & $1.53 \mathrm{E}+07$ & $4.25 \mathrm{E}+00$ \\
\hline Ge-78 & $6.00 \mathrm{E}+07$ & $2.16 \mathrm{E}+00$ \\
\hline H-3 & $3.12 \mathrm{E}+04$ & $3.25 \mathrm{E}+00$ \\
\hline Hf-167 & $9.90 \mathrm{E}+07$ & $1.80 \mathrm{E}-01$ \\
\hline Hf-169 & $9.72 E+07$ & 2.83E-01 \\
\hline Hf-170 & $2.05 \mathrm{E}+07$ & $1.78 \mathrm{E}+01$ \\
\hline Hf-172 & $2.53 \mathrm{E}+05$ & $2.28 \mathrm{E}+02$ \\
\hline Hf-173 & $3.76 \mathrm{E}+07$ & $4.98 \mathrm{E}+01$ \\
\hline Hf-174 & $2.53 \mathrm{E}+02$ & $2.47 \mathrm{E}+14$ \\
\hline Hf-175 & $6.48 \mathrm{E}+06$ & $6.08 \mathrm{E}+02$ \\
\hline Hf-177m & $1.99 \mathrm{E}+07$ & 9.61E-01 \\
\hline Hf-178m & $3.11 \mathrm{E}+04$ & $4.81 \mathrm{E}+02$ \\
\hline Hf-179m & $2.07 \mathrm{E}+06$ & $7.10 \mathrm{E}+01$ \\
\hline Hf-180m & $2.98 \mathrm{E}+07$ & $9.41 \mathrm{E}+00$ \\
\hline Hf-181 & $1.60 \mathrm{E}+06$ & $9.38 \mathrm{E}+01$ \\
\hline Hf-182 & $2.62 \mathrm{E}+04$ & $1.20 \mathrm{E}+08$ \\
\hline Hf-182m & $4.53 \mathrm{E}+07$ & $2.70 \mathrm{E}+00$ \\
\hline Hf-183 & $4.89 \mathrm{E}+07$ & $3.04 \mathrm{E}+00$ \\
\hline Hf-184 & $2.23 \mathrm{E}+07$ & $5.38 \mathrm{E}+00$ \\
\hline Hg-190 & $7.61 \mathrm{E}+06$ & $1.54 \mathrm{E}-01$ \\
\hline Hg-191m & $1.48 \mathrm{E}+06$ & 7.62E-02 \\
\hline
\end{tabular}




\begin{tabular}{|c|c|c|}
\hline \multicolumn{3}{|c|}{$\begin{array}{l}\text { Table A.2 HC-2 TQs Using Maximum Inhalation DCs } \\
\text { (continued) }\end{array}$} \\
\hline $\begin{array}{c}\text { MASTER } \\
\text { ISOTOPE LIST }\end{array}$ & Activity (Ci) & Mass (g) \\
\hline Hg-192 & $7.28 \mathrm{E}+05$ & $2.16 \mathrm{E}-01$ \\
\hline Hg-193 & $7.20 \mathrm{E}+05$ & $1.55 \mathrm{E}-01$ \\
\hline Hg-193m & $2.49 \mathrm{E}+05$ & $1.70 \mathrm{E}-01$ \\
\hline Hg-194 & $2.03 \mathrm{E}+04$ & $2.86 \mathrm{E}+03$ \\
\hline Hg-195 & $5.68 \mathrm{E}+05$ & 3.50E-01 \\
\hline Hg-195m & $9.85 \mathrm{E}+04$ & 2.55E-01 \\
\hline Hg-197 & $1.84 \mathrm{E}+05$ & 7.41E-01 \\
\hline Hg-197m & $1.40 \mathrm{E}+05$ & 2.09E-01 \\
\hline Hg-199m & $3.95 \mathrm{E}+06$ & $1.78 \mathrm{E}-01$ \\
\hline Hg-203 & $1.15 \mathrm{E}+05$ & $8.35 \mathrm{E}+00$ \\
\hline Hg-205 & $4.37 \mathrm{E}+08$ & $2.48 \mathrm{E}+00$ \\
\hline Hg-206 & $4.86 \mathrm{E}+07$ & 4.34E-01 \\
\hline Hg-207 & $2.11 \mathrm{E}+06$ & $6.74 \mathrm{E}-03$ \\
\hline Ho-150 & $3.10 \mathrm{E}+07$ & $3.16 \mathrm{E}-02$ \\
\hline Ho-153 & $5.86 \mathrm{E}+07$ & $9.58 \mathrm{E}-02$ \\
\hline Ho-153m & $5.73 \mathrm{E}+07$ & 4.33E-01 \\
\hline Ho-154 & $2.93 \mathrm{E}+07$ & 2.82E-01 \\
\hline Но-154m & $2.48 \mathrm{E}+07$ & $6.29 \mathrm{E}-02$ \\
\hline Ho-155 & $1.10 \mathrm{E}+08$ & $4.35 \mathrm{E}+00$ \\
\hline Но-156 & $2.26 \mathrm{E}+07$ & $1.05 \mathrm{E}+00$ \\
\hline Нo-157 & $1.14 \mathrm{E}+08$ & $1.19 \mathrm{E}+00$ \\
\hline Но-158 & $1.04 \mathrm{E}+09$ & $9.87 \mathrm{E}+00$ \\
\hline Ho-159 & $1.50 \mathrm{E}+08$ & $4.18 \mathrm{E}+00$ \\
\hline Но-160 & $3.30 \mathrm{E}+07$ & 7.19E-01 \\
\hline Ho-161 & $7.25 \mathrm{E}+08$ & $9.30 \mathrm{E}+01$ \\
\hline Ho-162 & $3.26 \mathrm{E}+08$ & $4.21 \mathrm{E}+00$ \\
\hline Ho-162m & $7.86 \mathrm{E}+07$ & $4.60 \mathrm{E}+00$ \\
\hline Ho-163 & $2.88 \mathrm{E}+07$ & $5.99 \mathrm{E}+07$ \\
\hline Но-164 & $7.29 \mathrm{E}+08$ & $1.84 \mathrm{E}+01$ \\
\hline Ho-164m & $5.08 \mathrm{E}+08$ & $1.66 \mathrm{E}+01$ \\
\hline Ho-166 & $1.24 \mathrm{E}+07$ & $1.76 \mathrm{E}+01$ \\
\hline Но-166m & $6.74 \mathrm{E}+04$ & $3.76 \mathrm{E}+04$ \\
\hline Но-167 & $6.60 \mathrm{E}+07$ & $1.09 \mathrm{E}+01$ \\
\hline Ho-168 & $6.66 \mathrm{E}+07$ & $1.78 \mathrm{E}-01$ \\
\hline Но-168m & $1.80 \mathrm{E}+10$ & $3.54 \mathrm{E}+01$ \\
\hline Ho-170 & $3.45 \mathrm{E}+07$ & 8.61E-02 \\
\hline $\mathrm{I}-118$ & $3.35 \mathrm{E}+04$ & $2.88 \mathrm{E}-04$ \\
\hline $\mathrm{I}-118 \mathrm{~m}$ & $3.16 \mathrm{E}+04$ & 1.68E-04 \\
\hline
\end{tabular}




\begin{tabular}{|c|c|c|}
\hline \multicolumn{3}{|c|}{$\begin{array}{l}\text { Table A.2 HC-2 TQs Using Maximum Inhalation DCs } \\
\text { (continued) }\end{array}$} \\
\hline $\begin{array}{c}\text { MASTER } \\
\text { ISOTOPE LIST }\end{array}$ & Activity (Ci) & Mass (g) \\
\hline I-119 & $9.09 \mathrm{E}+04$ & $1.10 \mathrm{E}-03$ \\
\hline $\mathrm{I}-120$ & $2.27 \mathrm{E}+04$ & $1.17 \mathrm{E}-03$ \\
\hline $\mathrm{I}-120 \mathrm{~m}$ & $1.66 \mathrm{E}+04$ & 5.62E-04 \\
\hline I-121 & $1.12 \mathrm{E}+05$ & $9.20 \mathrm{E}-03$ \\
\hline I-122 & $1.19 \mathrm{E}+05$ & 2.78E-04 \\
\hline I-123 & $6.99 \mathrm{E}+04$ & 3.62E-02 \\
\hline I-124 & $1.33 \mathrm{E}+03$ & 5.29E-03 \\
\hline I-125 & $1.16 \mathrm{E}+03$ & 6.66E-02 \\
\hline $\mathrm{I}-126$ & $6.22 \mathrm{E}+02$ & 7.81E-03 \\
\hline I-128 & $2.09 \mathrm{E}+05$ & 3.56E-03 \\
\hline I-129 & $1.69 \mathrm{E}+02$ & $9.56 \mathrm{E}+05$ \\
\hline I-130 & $7.33 \mathrm{E}+03$ & $3.76 \mathrm{E}-03$ \\
\hline $\mathrm{I}-130 \mathrm{~m}$ & $1.11 \mathrm{E}+06$ & 6.77E-03 \\
\hline I-131 & $8.09 \mathrm{E}+02$ & $6.52 \mathrm{E}-03$ \\
\hline $\mathrm{I}-132$ & $2.51 \mathrm{E}+04$ & 2.43E-03 \\
\hline $\mathrm{I}-132 \mathrm{~m}$ & $5.13 \mathrm{E}+04$ & 3.01E-03 \\
\hline I-133 & $3.97 \mathrm{E}+03$ & $3.50 \mathrm{E}-03$ \\
\hline I-134 & $3.00 \mathrm{E}+04$ & 1.12E-03 \\
\hline $\mathrm{I}-134 \mathrm{~m}$ & $4.54 \mathrm{E}+05$ & $1.16 \mathrm{E}-03$ \\
\hline I-135 & $1.40 \mathrm{E}+04$ & 3.98E-03 \\
\hline In-103 & $2.08 \mathrm{E}+07$ & $1.14 \mathrm{E}-02$ \\
\hline In-105 & $3.01 \mathrm{E}+07$ & 8.52E-02 \\
\hline In-106 & $1.66 \mathrm{E}+07$ & 5.79E-02 \\
\hline In-106m & $2.00 \mathrm{E}+07$ & $5.86 \mathrm{E}-02$ \\
\hline In-107 & $3.30 \mathrm{E}+07$ & 6.07E-01 \\
\hline In-108 & $1.35 \mathrm{E}+07$ & 4.50E-01 \\
\hline In-108m & $1.80 \mathrm{E}+07$ & 4.09E-01 \\
\hline In-109 & $5.86 \mathrm{E}+07$ & $8.56 \mathrm{E}+00$ \\
\hline In-109m & $9.86 \mathrm{E}+07$ & 7.66E-02 \\
\hline In-110l & $1.41 \mathrm{E}+07$ & $2.41 \mathrm{E}+00$ \\
\hline In-110s & $2.94 \mathrm{E}+07$ & $1.19 \mathrm{E}+00$ \\
\hline In-111 & $2.84 \mathrm{E}+07$ & $6.82 \mathrm{E}+01$ \\
\hline In-111m & $1.29 \mathrm{E}+08$ & 5.85E-01 \\
\hline In-112 & $1.79 \mathrm{E}+08$ & $1.54 \mathrm{E}+00$ \\
\hline In-112m & $2.80 \mathrm{E}+08$ & $3.43 \mathrm{E}+00$ \\
\hline In-113m & $1.44 \mathrm{E}+08$ & $8.60 \mathrm{E}+00$ \\
\hline In-114 & $1.94 \mathrm{E}+10$ & $1.41 \mathrm{E}+01$ \\
\hline In-114m & $8.71 \mathrm{E}+05$ & $3.76 \mathrm{E}+01$ \\
\hline
\end{tabular}




\begin{tabular}{|c|c|c|}
\hline \multicolumn{3}{|c|}{$\begin{array}{l}\text { Table A.2 HC-2 TQs Using Maximum Inhalation DCs } \\
\text { (continued) }\end{array}$} \\
\hline $\begin{array}{c}\text { MASTER } \\
\text { ISOTOPE LIST }\end{array}$ & Activity (Ci) & Mass (g) \\
\hline In-115 & $2.08 \mathrm{E}+04$ & $3.41 \mathrm{E}+16$ \\
\hline In-115m & $9.99 \mathrm{E}+07$ & $1.64 \mathrm{E}+01$ \\
\hline In-116m & $1.93 \mathrm{E}+07$ & 6.44E-01 \\
\hline In-117 & $6.32 \mathrm{E}+07$ & $1.72 \mathrm{E}+00$ \\
\hline In-117m & $9.59 \mathrm{E}+07$ & $6.94 \mathrm{E}+00$ \\
\hline In-118 & $4.73 \mathrm{E}+08$ & 2.47E-02 \\
\hline In-118m & $2.06 \mathrm{E}+07$ & 5.65E-02 \\
\hline In-119 & $7.23 \mathrm{E}+07$ & $1.10 \mathrm{E}-01$ \\
\hline In-119m & $4.30 \mathrm{E}+08$ & $4.90 \mathrm{E}+00$ \\
\hline In-121 & $6.20 \mathrm{E}+07$ & $1.53 \mathrm{E}-02$ \\
\hline In-121m & $6.89 \mathrm{E}+08$ & $1.72 \mathrm{E}+00$ \\
\hline Ir-180 & $3.73 \mathrm{E}+07$ & 5.36E-02 \\
\hline Ir-182 & $3.70 \mathrm{E}+07$ & 5.37E-01 \\
\hline Ir-183 & $3.83 \mathrm{E}+07$ & $2.16 \mathrm{E}+00$ \\
\hline Ir-184 & $2.02 \mathrm{E}+07$ & $3.58 \mathrm{E}+00$ \\
\hline Ir-185 & $2.91 \mathrm{E}+07$ & $2.41 \mathrm{E}+01$ \\
\hline Ir-186l & $1.44 \mathrm{E}+07$ & $1.35 \mathrm{E}+01$ \\
\hline Ir-186s & $4.42 \mathrm{E}+07$ & $4.59 \mathrm{E}+00$ \\
\hline Ir-187 & $6.27 \mathrm{E}+07$ & $3.93 \mathrm{E}+01$ \\
\hline Ir-188 & $1.23 \mathrm{E}+07$ & $3.06 \mathrm{E}+01$ \\
\hline Ir-189 & $1.33 \mathrm{E}+07$ & $2.56 \mathrm{E}+02$ \\
\hline Ir-190 & $3.11 \mathrm{E}+06$ & $5.48 \mathrm{E}+01$ \\
\hline Ir-190ms & $8.11 \mathrm{E}+08$ & $5.90 \mathrm{E}+01$ \\
\hline Ir-190ml & $2.66 \mathrm{E}+07$ & $5.00 \mathrm{E}+00$ \\
\hline Ir-191m & $8.95 \mathrm{E}+08$ & 7.48E-02 \\
\hline Ir-192 & $1.21 \mathrm{E}+06$ & $1.31 \mathrm{E}+02$ \\
\hline Ir-192ms & $1.01 \mathrm{E}+12$ & $1.49 \mathrm{E}+03$ \\
\hline Ir-192ml & $2.08 \mathrm{E}+05$ & $2.69 \mathrm{E}+04$ \\
\hline Ir-193m & $6.24 \mathrm{E}+06$ & $9.71 \mathrm{E}+01$ \\
\hline Ir-194 & $1.41 \mathrm{E}+07$ & $1.68 \mathrm{E}+01$ \\
\hline Ir-194m & $6.08 \mathrm{E}+05$ & $1.54 \mathrm{E}+02$ \\
\hline Ir-195 & $1.04 \mathrm{E}+08$ & $1.62 \mathrm{E}+01$ \\
\hline Ir-195m & $3.56 \mathrm{E}+07$ & $8.41 \mathrm{E}+00$ \\
\hline Ir-196 & $2.33 \mathrm{E}+08$ & $2.10 \mathrm{E}-01$ \\
\hline Ir-196m & $1.85 \mathrm{E}+07$ & $1.62 \mathrm{E}+00$ \\
\hline K-38 & $3.30 \mathrm{E}+04$ & 5.08E-05 \\
\hline K-40 & $7.63 \mathrm{E}+03$ & $1.09 \mathrm{E}+09$ \\
\hline K-42 & $9.90 \mathrm{E}+04$ & $1.64 \mathrm{E}-02$ \\
\hline
\end{tabular}




\begin{tabular}{|c|c|c|}
\hline \multicolumn{3}{|c|}{$\begin{array}{c}\text { Table A.2 HC-2 TQs Using Maximum Inhalation DCs } \\
\text { (continued) }\end{array}$} \\
\hline $\begin{array}{c}\text { MASTER } \\
\text { ISOTOPE LIST }\end{array}$ & Activity (Ci) & Mass (g) \\
\hline K-43 & $5.79 \mathrm{E}+04$ & 1.79E-02 \\
\hline K-44 & $4.30 \mathrm{E}+04$ & 2.23E-04 \\
\hline K-45 & $5.32 \mathrm{E}+04$ & $2.54 \mathrm{E}-04$ \\
\hline K-46 & $3.68 \mathrm{E}+04$ & 1.57E-05 \\
\hline Kr-74 & $4.83 \mathrm{E}+04$ & 2.19E-04 \\
\hline Kr-75 & $4.62 \mathrm{E}+04$ & 7.90E-05 \\
\hline Kr-76 & $1.33 \mathrm{E}+05$ & 4.77E-02 \\
\hline $\mathrm{Kr}-77$ & $5.56 \mathrm{E}+04$ & 1.70E-03 \\
\hline Kr-79 & $2.23 \mathrm{E}+05$ & $1.97 \mathrm{E}-01$ \\
\hline Kr-81 & $1.01 \mathrm{E}+07$ & $4.81 \mathrm{E}+08$ \\
\hline Kr-81m & $4.40 \mathrm{E}+05$ & 4.10E-05 \\
\hline Kr-83m & $1.80 \mathrm{E}+09$ & $8.72 \mathrm{E}+01$ \\
\hline Kr-85 & $2.27 \mathrm{E}+07$ & $5.78 \mathrm{E}+04$ \\
\hline Kr-85m & $3.61 \mathrm{E}+05$ & 4.39E-02 \\
\hline $\mathrm{Kr}-87$ & $6.56 \mathrm{E}+04$ & 2.31E-03 \\
\hline Kr-88 & $2.65 \mathrm{E}+04$ & 2.11E-03 \\
\hline $\mathrm{Kr}-89$ & $2.83 \mathrm{E}+04$ & 4.21E-05 \\
\hline La-128 & $2.08 \mathrm{E}+07$ & 7.33E-02 \\
\hline La-129 & $5.87 \mathrm{E}+07$ & 4.67E-01 \\
\hline La-130 & $2.62 \mathrm{E}+07$ & $1.58 \mathrm{E}-01$ \\
\hline La-131 & $6.92 \mathrm{E}+07$ & $2.84 \mathrm{E}+00$ \\
\hline La-132 & $1.76 \mathrm{E}+07$ & $3.56 \mathrm{E}+00$ \\
\hline La-132m & $7.14 \mathrm{E}+07$ & $1.22 \mathrm{E}+00$ \\
\hline La-133 & $2.03 \mathrm{E}+08$ & $3.37 \mathrm{E}+01$ \\
\hline La-134 & $8.07 \mathrm{E}+07$ & 3.83E-01 \\
\hline La-135 & $4.84 \mathrm{E}+08$ & $4.06 \mathrm{E}+02$ \\
\hline La-136 & $1.51 \mathrm{E}+08$ & $1.08 \mathrm{E}+00$ \\
\hline La-137 & $9.32 \mathrm{E}+05$ & $2.14 \mathrm{E}+07$ \\
\hline La-138 & $5.40 \mathrm{E}+04$ & $2.81 \mathrm{E}+12$ \\
\hline La-140 & $5.59 \mathrm{E}+06$ & $1.00 \mathrm{E}+01$ \\
\hline La-141 & $5.16 \mathrm{E}+07$ & $9.12 \mathrm{E}+00$ \\
\hline La-142 & $1.56 \mathrm{E}+07$ & $1.09 \mathrm{E}+00$ \\
\hline La-143 & $2.22 \mathrm{E}+08$ & $2.40 \mathrm{E}+00$ \\
\hline Lu-165 & $4.86 \mathrm{E}+07$ & $4.58 \mathrm{E}-01$ \\
\hline Lu-167 & $2.86 \mathrm{E}+07$ & $1.31 \mathrm{E}+00$ \\
\hline Lu-169 & $1.52 \mathrm{E}+07$ & $2.79 \mathrm{E}+01$ \\
\hline Lu-169m & $1.07 \mathrm{E}+14$ & $2.57 \mathrm{E}+05$ \\
\hline Lu-170 & $7.77 \mathrm{E}+06$ & $2.02 \mathrm{E}+01$ \\
\hline
\end{tabular}




\begin{tabular}{|c|c|c|}
\hline \multicolumn{3}{|c|}{$\begin{array}{l}\text { Table A.2 HC-2 TQs Using Maximum Inhalation DCs } \\
\text { (continued) }\end{array}$} \\
\hline $\begin{array}{c}\text { MASTER } \\
\text { ISOTOPE LIST }\end{array}$ & Activity (Ci) & Mass (g) \\
\hline Lu-171 & $8.29 \mathrm{E}+06$ & $8.93 \mathrm{E}+01$ \\
\hline Lu-171m & $2.74 \mathrm{E}+11$ & $3.28 \mathrm{E}+02$ \\
\hline Lu-172 & $4.32 \mathrm{E}+06$ & $3.81 \mathrm{E}+01$ \\
\hline Lu-172m & $6.93 \mathrm{E}+13$ & $2.34 \mathrm{E}+05$ \\
\hline Lu-173 & $3.36 \mathrm{E}+06$ & $2.22 \mathrm{E}+03$ \\
\hline Lu-174 & $1.92 \mathrm{E}+06$ & $3.10 \mathrm{E}+03$ \\
\hline Lu-174m & $1.93 \mathrm{E}+06$ & $3.65 \mathrm{E}+02$ \\
\hline Lu-176 & $1.16 \mathrm{E}+05$ & $2.05 E+12$ \\
\hline Lu-176m & $6.66 \mathrm{E}+07$ & $1.38 \mathrm{E}+01$ \\
\hline Lu-177 & $6.73 \mathrm{E}+06$ & $6.12 \mathrm{E}+01$ \\
\hline Lu-177m & $5.02 \mathrm{E}+05$ & $1.10 \mathrm{E}+02$ \\
\hline Lu-178 & $1.72 \mathrm{E}+08$ & $4.61 \mathrm{E}+00$ \\
\hline Lu-178m & $4.27 \mathrm{E}+07$ & $9.17 \mathrm{E}-01$ \\
\hline Lu-179 & $6.51 \mathrm{E}+07$ & $1.71 \mathrm{E}+01$ \\
\hline Lu-180 & $3.81 \mathrm{E}+07$ & $2.08 \mathrm{E}-01$ \\
\hline Lu-181 & $1.05 \mathrm{E}+08$ & 3.53E-01 \\
\hline Md-257 & $3.24 \mathrm{E}+05$ & 1.38E-01 \\
\hline Md-258 & $1.37 \mathrm{E}+03$ & 1.49E-01 \\
\hline Mg-27 & $6.50 \mathrm{E}+07$ & 8.82E-02 \\
\hline Mg-28 & $5.78 \mathrm{E}+06$ & $1.08 \mathrm{E}+00$ \\
\hline $\mathrm{Mn}-50 \mathrm{~m}$ & $1.24 \mathrm{E}+07$ & $5.76 \mathrm{E}-03$ \\
\hline Mn-51 & $4.38 \mathrm{E}+07$ & 5.49E-01 \\
\hline Mn-52 & $4.23 \mathrm{E}+06$ & $9.41 \mathrm{E}+00$ \\
\hline Mn-52m & $2.08 \mathrm{E}+07$ & $1.22 \mathrm{E}-01$ \\
\hline Mn-53 & $1.50 \mathrm{E}+08$ & $8.23 E+10$ \\
\hline Mn-54 & $5.00 \mathrm{E}+06$ & $6.45 E+02$ \\
\hline Mn-56 & $2.14 \mathrm{E}+07$ & 9.87E-01 \\
\hline Mn-57 & $5.10 \mathrm{E}+08$ & 2.20E-01 \\
\hline Mn-58m & $2.35 \mathrm{E}+07$ & 7.87E-03 \\
\hline Mo-101 & $3.49 \mathrm{E}+07$ & 2.74E-01 \\
\hline Mo-102 & $2.45 \mathrm{E}+08$ & $1.50 \mathrm{E}+00$ \\
\hline Mo-89 & $4.74 \mathrm{E}+07$ & 4.73E-02 \\
\hline Mo-90 & $1.70 \mathrm{E}+07$ & $2.76 \mathrm{E}+00$ \\
\hline Mo-91 & $5.07 \mathrm{E}+07$ & 3.80E-01 \\
\hline Mo-91m & $4.19 \mathrm{E}+07$ & $2.18 \mathrm{E}-02$ \\
\hline Mo-93 & $3.53 \mathrm{E}+06$ & $3.21 \mathrm{E}+06$ \\
\hline Mo-93m & $1.59 \mathrm{E}+07$ & $3.24 \mathrm{E}+00$ \\
\hline Мo-99 & $8.01 \mathrm{E}+06$ & $1.67 \mathrm{E}+01$ \\
\hline
\end{tabular}




\begin{tabular}{|c|c|c|}
\hline \multicolumn{3}{|c|}{$\begin{array}{l}\text { Table A.2 HC-2 TQs Using Maximum Inhalation DCs } \\
\text { (continued) }\end{array}$} \\
\hline $\begin{array}{c}\text { MASTER } \\
\text { ISOTOPE LIST }\end{array}$ & Activity (Ci) & Mass (g) \\
\hline $\mathrm{N}-13$ & $5.52 \mathrm{E}+04$ & 2.95E-06 \\
\hline $\mathrm{N}-16$ & $1.04 \mathrm{E}+04$ & $1.06 \mathrm{E}-07$ \\
\hline $\mathrm{Na}-22$ & $9.99 E+03$ & $1.60 \mathrm{E}+00$ \\
\hline $\mathrm{Na}-24$ & $1.76 \mathrm{E}+04$ & 2.02E-03 \\
\hline Nb-87 & $4.88 \mathrm{E}+07$ & 8.46E-02 \\
\hline Nb-88 & $1.28 \mathrm{E}+07$ & 8.55E-02 \\
\hline Nb-88m & $1.42 \mathrm{E}+07$ & 5.15E-02 \\
\hline Nb-89l & $2.46 \mathrm{E}+07$ & $1.42 \mathrm{E}+00$ \\
\hline Nb-89s & $2.32 \mathrm{E}+07$ & $7.26 \mathrm{E}-01$ \\
\hline Nb-90 & $6.18 \mathrm{E}+06$ & $2.59 \mathrm{E}+00$ \\
\hline Nb-91 & $4.12 \mathrm{E}+06$ & $7.12 \mathrm{E}+05$ \\
\hline Nb-91m & $1.77 \mathrm{E}+06$ & $7.51 \mathrm{E}+01$ \\
\hline Nb-92 & $2.83 \mathrm{E}+05$ & $2.53 \mathrm{E}+09$ \\
\hline $\mathrm{Nb}-92 \mathrm{~m}$ & $1.24 \mathrm{E}+07$ & $8.88 \mathrm{E}+01$ \\
\hline Nb-93m & $4.50 \mathrm{E}+06$ & $1.59 \mathrm{E}+04$ \\
\hline Nb-94 & $1.65 \mathrm{E}+05$ & $8.78 \mathrm{E}+05$ \\
\hline Nb-94m & $1.26 \mathrm{E}+10$ & $3.93 \mathrm{E}+01$ \\
\hline Nb-95 & $4.24 \mathrm{E}+06$ & $1.08 \mathrm{E}+02$ \\
\hline Nb-95m & $9.12 \mathrm{E}+06$ & $2.39 \mathrm{E}+01$ \\
\hline Nb-96 & $7.93 \mathrm{E}+06$ & $5.66 \mathrm{E}+00$ \\
\hline Nb-97 & $5.78 \mathrm{E}+07$ & $2.15 \mathrm{E}+00$ \\
\hline Nb-97m & $7.61 \mathrm{E}+07$ & 3.92E-02 \\
\hline Nb-98 & $1.93 \mathrm{E}+07$ & $5.16 \mathrm{E}-01$ \\
\hline Nb-99 & $3.25 \mathrm{E}+08$ & 4.27E-02 \\
\hline Nb-99m & $7.00 \mathrm{E}+07$ & $9.58 \mathrm{E}-02$ \\
\hline Nd-134 & $1.16 \mathrm{E}+08$ & 7.05E-01 \\
\hline Nd-135 & $4.04 \mathrm{E}+07$ & 3.59E-01 \\
\hline Nd-136 & $8.80 \mathrm{E}+07$ & $3.22 \mathrm{E}+00$ \\
\hline Nd-137 & $4.18 \mathrm{E}+07$ & $1.17 \mathrm{E}+00$ \\
\hline Nd-138 & $3.19 \mathrm{E}+07$ & $7.09 \mathrm{E}+00$ \\
\hline Nd-139 & $1.21 \mathrm{E}+08$ & $2.66 \mathrm{E}+00$ \\
\hline Nd-139m & $2.14 \mathrm{E}+07$ & $5.22 \mathrm{E}+00$ \\
\hline Nd-140 & $6.09 \mathrm{E}+06$ & $2.20 \mathrm{E}+01$ \\
\hline Nd-141 & $5.94 \mathrm{E}+08$ & $6.66 \mathrm{E}+01$ \\
\hline Nd-141m & $7.30 \mathrm{E}+07$ & 5.69E-02 \\
\hline Nd-144 & $4.03 \mathrm{E}+02$ & $3.72 E+14$ \\
\hline Nd-147 & $3.35 \mathrm{E}+06$ & $4.14 \mathrm{E}+01$ \\
\hline Nd-149 & $5.66 \mathrm{E}+07$ & $4.65 \mathrm{E}+00$ \\
\hline
\end{tabular}




\begin{tabular}{|c|c|c|}
\hline \multicolumn{3}{|c|}{$\begin{array}{l}\text { Table A.2 HC-2 TQs Using Maximum Inhalation DCs } \\
\text { (continued) }\end{array}$} \\
\hline $\begin{array}{c}\text { MASTER } \\
\text { ISOTOPE LIST }\end{array}$ & Activity (Ci) & Mass (g) \\
\hline Nd-151 & $5.36 \mathrm{E}+07$ & 5.35E-01 \\
\hline Nd-152 & $1.63 \mathrm{E}+08$ & $1.50 \mathrm{E}+00$ \\
\hline Ne-19 & $5.49 \mathrm{E}+04$ & 1.59E-06 \\
\hline $\mathrm{Ne}-24$ & $1.09 \mathrm{E}+05$ & $4.70 \mathrm{E}-05$ \\
\hline $\mathrm{Ni}-56$ & $5.58 \mathrm{E}+06$ & $1.46 \mathrm{E}+01$ \\
\hline $\mathrm{Ni}-57$ & $9.53 \mathrm{E}+06$ & $6.25 \mathrm{E}+00$ \\
\hline $\mathrm{Ni}-59$ & $9.77 \mathrm{E}+06$ & $1.21 \mathrm{E}+08$ \\
\hline $\mathrm{Ni}-63$ & $4.05 E+06$ & $6.85 \mathrm{E}+04$ \\
\hline $\mathrm{Ni}-65$ & $1.83 \mathrm{E}+07$ & 9.54E-01 \\
\hline $\mathrm{Ni}-66$ & $4.50 \mathrm{E}+06$ & $5.17 \mathrm{E}+00$ \\
\hline Np-231 & $4.50 \mathrm{E}+06$ & 2.70E-01 \\
\hline Np-232 & $2.76 \mathrm{E}+07$ & $5.00 \mathrm{E}-01$ \\
\hline Np-233 & $6.12 \mathrm{E}+08$ & $2.75 E+01$ \\
\hline Np-234 & $1.06 \mathrm{E}+07$ & 8.33E+01 \\
\hline Np-235 & $1.29 \mathrm{E}+07$ & $9.17 \mathrm{E}+03$ \\
\hline Np-236l & $1.01 \mathrm{E}+03$ & $7.70 \mathrm{E}+04$ \\
\hline Np-236s & $9.00 \mathrm{E}+05$ & $1.53 \mathrm{E}+00$ \\
\hline Np-237 & $1.62 \mathrm{E}+02$ & $2.30 \mathrm{E}+05$ \\
\hline Np-238 & $2.26 \mathrm{E}+06$ & $8.74 \mathrm{E}+00$ \\
\hline Np-239 & $7.93 \mathrm{E}+06$ & $3.42 \mathrm{E}+01$ \\
\hline Np-240 & $2.90 \mathrm{E}+07$ & $2.41 \mathrm{E}+00$ \\
\hline Np-240m & $1.67 \mathrm{E}+08$ & $1.58 \mathrm{E}+00$ \\
\hline Np-241 & $4.16 \mathrm{E}+08$ & $7.42 \mathrm{E}+00$ \\
\hline Np-242 & $2.03 \mathrm{E}+08$ & 5.76E-01 \\
\hline $\mathrm{Np}-242 \mathrm{~m}$ & $6.50 \mathrm{E}+07$ & 4.60E-01 \\
\hline O-14 & $1.66 \mathrm{E}+04$ & $1.45 \mathrm{E}-06$ \\
\hline O-15 & $5.50 \mathrm{E}+04$ & 8.95E-06 \\
\hline O-19 & $5.88 \mathrm{E}+04$ & 2.66E-06 \\
\hline Os-180 & $3.70 \mathrm{E}+08$ & $7.80 \mathrm{E}+00$ \\
\hline Os-181 & $3.33 \mathrm{E}+07$ & $3.37 \mathrm{E}+00$ \\
\hline Os-182 & $1.84 \mathrm{E}+07$ & $2.35 \mathrm{E}+01$ \\
\hline Os-183 & $2.70 \mathrm{E}+07$ & $2.05 E+01$ \\
\hline Os-183m & $2.59 \mathrm{E}+07$ & $1.50 \mathrm{E}+01$ \\
\hline Os-185 & $4.76 \mathrm{E}+06$ & $6.34 \mathrm{E}+02$ \\
\hline Os-186 & $1.80 \mathrm{E}+03$ & $1.87 \mathrm{E}+15$ \\
\hline Os-189m & $1.53 \mathrm{E}+09$ & $5.53 \mathrm{E}+02$ \\
\hline Os-190m & $3.56 \mathrm{E}+07$ & 3.56E-01 \\
\hline Os-191 & $4.25 \mathrm{E}+06$ & $9.56 \mathrm{E}+01$ \\
\hline
\end{tabular}




\begin{tabular}{|c|c|c|}
\hline \multicolumn{3}{|c|}{$\begin{array}{c}\text { Table A.2 HC-2 TQs Using Maximum Inhalation DCs } \\
\text { (continued) }\end{array}$} \\
\hline $\begin{array}{c}\text { MASTER } \\
\text { ISOTOPE LIST }\end{array}$ & Activity (Ci) & Mass (g) \\
\hline Os-191m & $5.04 \mathrm{E}+07$ & $4.00 \mathrm{E}+01$ \\
\hline Os-193 & $1.53 \mathrm{E}+07$ & $2.83 E+01$ \\
\hline Os-194 & $9.54 \mathrm{E}+04$ & $3.11 \mathrm{E}+02$ \\
\hline Os-196 & $1.05 E+08$ & $3.81 \mathrm{E}+00$ \\
\hline P-30 & $1.09 \mathrm{E}+05$ & 4.36E-05 \\
\hline P-32 & $4.77 \mathrm{E}+03$ & 1.67E-02 \\
\hline P-33 & $1.08 \mathrm{E}+04$ & 6.93E-02 \\
\hline Рa-227 & $1.01 \mathrm{E}+05$ & 4.69E-03 \\
\hline Рa-228 & $1.08 \mathrm{E}+05$ & 1.73E-01 \\
\hline Ра-229 & $1.04 \mathrm{E}+06$ & $2.74 \mathrm{E}+00$ \\
\hline Рa-230 & $1.07 \mathrm{E}+04$ & 3.27E-01 \\
\hline Рa-231 & $5.79 \mathrm{E}+01$ & $1.23 \mathrm{E}+03$ \\
\hline Рa-232 & $8.00 \mathrm{E}+05$ & $1.86 \mathrm{E}+00$ \\
\hline Рa-233 & $2.06 \mathrm{E}+06$ & $9.95 \mathrm{E}+01$ \\
\hline Рa-234 & $1.19 \mathrm{E}+07$ & $5.96 \mathrm{E}+00$ \\
\hline Pa-234m & $3.76 \mathrm{E}+09$ & $5.47 \mathrm{E}+00$ \\
\hline Рa-235 & $3.90 \mathrm{E}+08$ & $1.19 \mathrm{E}+01$ \\
\hline Ра-236 & $6.20 \mathrm{E}+07$ & 7.08E-01 \\
\hline Рa-237 & $9.58 \mathrm{E}+07$ & $1.05 \mathrm{E}+00$ \\
\hline Pb-194 & $4.95 \mathrm{E}+07$ & 6.12E-01 \\
\hline $\mathrm{Pb}-195 \mathrm{~m}$ & $3.15 \mathrm{E}+07$ & 5.16E-01 \\
\hline Pb-196 & $8.73 E+07$ & $3.37 \mathrm{E}+00$ \\
\hline Pb-197 & $3.79 \mathrm{E}+07$ & 3.17E-01 \\
\hline Pb-197m & $3.82 \mathrm{E}+07$ & $1.72 \mathrm{E}+00$ \\
\hline $\mathrm{Pb}-198$ & $6.18 \mathrm{E}+07$ & $9.37 \mathrm{E}+00$ \\
\hline Pb-199 & $3.16 \mathrm{E}+07$ & $3.01 \mathrm{E}+00$ \\
\hline $\mathrm{Pb}-200$ & $2.15 \mathrm{E}+07$ & $2.95 \mathrm{E}+01$ \\
\hline Pb-201 & $3.54 \mathrm{E}+07$ & $2.14 \mathrm{E}+01$ \\
\hline $\mathrm{Pb}-201 \mathrm{~m}$ & $1.66 \mathrm{E}+08$ & $1.80 \mathrm{E}-01$ \\
\hline Pb-202 & $6.76 \mathrm{E}+05$ & $1.15 \mathrm{E}+08$ \\
\hline Pb-202m & $2.03 \mathrm{E}+07$ & $4.74 \mathrm{E}+00$ \\
\hline Pb-203 & $3.08 \mathrm{E}+07$ & $1.04 \mathrm{E}+02$ \\
\hline $\mathrm{Pb}-204 \mathrm{~m}$ & $2.54 \mathrm{E}+07$ & $1.85 \mathrm{E}+00$ \\
\hline Pb-205 & $9.54 \mathrm{E}+06$ & $7.82 \mathrm{E}+10$ \\
\hline Pb-209 & $1.33 \mathrm{E}+08$ & $2.88 \mathrm{E}+01$ \\
\hline $\mathrm{Pb}-210$ & $1.45 E+03$ & $1.90 \mathrm{E}+01$ \\
\hline $\mathrm{Pb}-211$ & $6.75 \mathrm{E}+05$ & $2.74 \mathrm{E}-02$ \\
\hline $\mathrm{Pb}-212$ & $4.27 \mathrm{E}+04$ & 3.07E-02 \\
\hline
\end{tabular}




\begin{tabular}{|c|c|c|}
\hline \multicolumn{3}{|c|}{$\begin{array}{l}\text { Table A.2 HC-2 TQs Using Maximum Inhalation DCs } \\
\text { (continued) }\end{array}$} \\
\hline $\begin{array}{c}\text { MASTER } \\
\text { ISOTOPE LIST }\end{array}$ & Activity (Ci) & Mass (g) \\
\hline $\mathrm{Pb}-214$ & $5.39 \mathrm{E}+05$ & $1.64 \mathrm{E}-02$ \\
\hline Pd-100 & $9.39 \mathrm{E}+06$ & $2.61 \mathrm{E}+01$ \\
\hline Pd-101 & $7.51 \mathrm{E}+07$ & $2.00 \mathrm{E}+01$ \\
\hline Pd-103 & $1.80 \mathrm{E}+07$ & $2.41 \mathrm{E}+02$ \\
\hline Pd-107 & $1.37 \mathrm{E}+07$ & $2.67 \mathrm{E}+10$ \\
\hline Pd-109 & $2.19 \mathrm{E}+07$ & $1.02 \mathrm{E}+01$ \\
\hline Pd-109m & $5.89 \mathrm{E}+08$ & $1.60 \mathrm{E}+00$ \\
\hline Pd-111 & $2.02 E+08$ & $2.79 \mathrm{E}+00$ \\
\hline Pd-112 & $6.14 \mathrm{E}+06$ & $4.61 \mathrm{E}+00$ \\
\hline Pd-114 & $1.81 \mathrm{E}+09$ & $2.66 \mathrm{E}+00$ \\
\hline Pd-96 & $4.15 \mathrm{E}+07$ & 4.31E-02 \\
\hline Pd-97 & $2.41 \mathrm{E}+07$ & 3.86E-02 \\
\hline Pd-98 & $9.40 \mathrm{E}+07$ & 8.66E-01 \\
\hline Pd-99 & $4.07 \mathrm{E}+07$ & $4.58 \mathrm{E}-01$ \\
\hline $\mathrm{Pm}-136$ & $2.16 \mathrm{E}+07$ & 2.79E-02 \\
\hline Pm-137m & $3.37 \mathrm{E}+07$ & 5.90E-02 \\
\hline Pm-139 & $6.30 \mathrm{E}+07$ & 1.93E-01 \\
\hline Pm-140 & $5.48 \mathrm{E}+07$ & $6.26 \mathrm{E}-03$ \\
\hline Pm-140m & $1.94 \mathrm{E}+07$ & 8.61E-02 \\
\hline Pm-141 & $6.59 \mathrm{E}+07$ & $1.03 \mathrm{E}+00$ \\
\hline Pm-142 & $6.40 \mathrm{E}+07$ & $3.26 \mathrm{E}-02$ \\
\hline Pm-143 & $5.25 \mathrm{E}+06$ & $1.52 \mathrm{E}+03$ \\
\hline Pm-144 & $9.62 \mathrm{E}+05$ & $3.85 \mathrm{E}+02$ \\
\hline Pm-145 & $2.25 \mathrm{E}+06$ & $1.62 \mathrm{E}+04$ \\
\hline Pm-146 & $3.84 \mathrm{E}+05$ & $8.67 \mathrm{E}+02$ \\
\hline $\mathrm{Pm}-147$ & $1.62 \mathrm{E}+06$ & $1.75 E+03$ \\
\hline Pm-148 & $3.55 \mathrm{E}+06$ & $2.16 \mathrm{E}+01$ \\
\hline Pm-148m & $1.35 \mathrm{E}+06$ & $6.33 \mathrm{E}+01$ \\
\hline Pm-149 & $1.11 \mathrm{E}+07$ & $2.80 \mathrm{E}+01$ \\
\hline $\mathrm{Pm}-150$ & $2.35 \mathrm{E}+07$ & $3.01 \mathrm{E}+00$ \\
\hline Pm-151 & $1.60 \mathrm{E}+07$ & $2.19 \mathrm{E}+01$ \\
\hline Pm-152 & $1.86 \mathrm{E}+08$ & $6.20 \mathrm{E}-01$ \\
\hline Pm-152m & $3.82 \mathrm{E}+07$ & 2.32E-01 \\
\hline Pm-153 & $7.95 \mathrm{E}+08$ & $3.39 \mathrm{E}+00$ \\
\hline Pm-154 & $3.10 \mathrm{E}+07$ & 4.38E-02 \\
\hline Pm-154m & $3.18 \mathrm{E}+07$ & 6.98E-02 \\
\hline Po-203 & $2.90 \mathrm{E}+06$ & $1.15 \mathrm{E}-01$ \\
\hline Po-204 & $1.32 \mathrm{E}+06$ & 3.03E-01 \\
\hline
\end{tabular}




\begin{tabular}{|c|c|c|}
\hline \multicolumn{3}{|c|}{$\begin{array}{l}\text { Table A.2 HC-2 TQs Using Maximum Inhalation DCs } \\
\text { (continued) }\end{array}$} \\
\hline $\begin{array}{c}\text { MASTER } \\
\text { ISOTOPE LIST }\end{array}$ & Activity (Ci) & Mass (g) \\
\hline Po-205 & $2.68 \mathrm{E}+06$ & $3.15 \mathrm{E}-01$ \\
\hline Po-206 & $1.14 \mathrm{E}+04$ & $1.58 \mathrm{E}-01$ \\
\hline Рo-207 & $2.92 \mathrm{E}+06$ & $1.13 E+00$ \\
\hline Рo-208 & $1.09 \mathrm{E}+02$ & 1.84E-01 \\
\hline Po-209 & $7.95 \mathrm{E}+01$ & $4.74 \mathrm{E}+00$ \\
\hline Po-210 & $1.89 \mathrm{E}+02$ & 4.20E-02 \\
\hline Po-211 & $7.09 \mathrm{E}+08$ & 6.85E-03 \\
\hline Po-212m & $6.77 \mathrm{E}+07$ & 5.74E-02 \\
\hline Рo-213 & $1.58 \mathrm{E}+11$ & $1.25 \mathrm{E}-05$ \\
\hline Po-214 & $6.62 \mathrm{E}+10$ & $2.06 \mathrm{E}-04$ \\
\hline Po-215 & $3.21 \mathrm{E}+10$ & 1.09E-03 \\
\hline Po-216 & $3.26 \mathrm{E}+11$ & 9.36E-01 \\
\hline Рo-218 & $6.03 \mathrm{E}+11$ & $2.13 \mathrm{E}+03$ \\
\hline Pr-134 & $1.77 \mathrm{E}+07$ & 1.39E-01 \\
\hline Pr-134m & $2.24 \mathrm{E}+07$ & 2.71E-01 \\
\hline Pr-135 & $5.74 \mathrm{E}+07$ & 9.89E-01 \\
\hline Pr-136 & $2.51 \mathrm{E}+07$ & 2.38E-01 \\
\hline Pr-137 & $8.83 \mathrm{E}+07$ & $4.93 \mathrm{E}+00$ \\
\hline Pr-138 & $6.89 \mathrm{E}+07$ & 7.33E-02 \\
\hline Pr-138m & $1.86 \mathrm{E}+07$ & $1.71 \mathrm{E}+00$ \\
\hline Pr-139 & $2.28 \mathrm{E}+08$ & $4.56 \mathrm{E}+01$ \\
\hline Pr-140 & $1.11 \mathrm{E}+08$ & 2.79E-01 \\
\hline Pr-142 & $1.45 \mathrm{E}+07$ & $1.26 \mathrm{E}+01$ \\
\hline Pr-142m & $1.16 \mathrm{E}+09$ & $1.28 \mathrm{E}+01$ \\
\hline Pr-143 & $3.38 \mathrm{E}+06$ & $5.01 \mathrm{E}+01$ \\
\hline Pr-144 & $3.40 \mathrm{E}+08$ & $4.50 \mathrm{E}+00$ \\
\hline Pr-144m & $9.69 \mathrm{E}+09$ & $5.34 \mathrm{E}+01$ \\
\hline Pr-145 & $4.71 \mathrm{E}+07$ & $1.30 \mathrm{E}+01$ \\
\hline Pr-146 & $4.42 \mathrm{E}+07$ & 8.29E-01 \\
\hline Pr-147 & $5.69 \mathrm{E}+07$ & $6.05 \mathrm{E}-01$ \\
\hline Pr-148 & $5.60 \mathrm{E}+07$ & $1.01 \mathrm{E}-01$ \\
\hline Pr-148m & $6.18 \mathrm{E}+07$ & $9.78 \mathrm{E}-02$ \\
\hline Pt-184 & $6.69 \mathrm{E}+07$ & $1.13 \mathrm{E}+00$ \\
\hline Pt-186 & $5.84 \mathrm{E}+07$ & $6.93 \mathrm{E}+00$ \\
\hline Pt-187 & $4.98 \mathrm{E}+07$ & $6.99 \mathrm{E}+00$ \\
\hline Pt-188 & $1.82 \mathrm{E}+07$ & $2.67 \mathrm{E}+02$ \\
\hline Pt-189 & $9.84 \mathrm{E}+07$ & $6.45 \mathrm{E}+01$ \\
\hline Pt-190 & $1.45 \mathrm{E}+03$ & $5.02 \mathrm{E}+11$ \\
\hline
\end{tabular}




\begin{tabular}{|c|c|c|}
\hline \multicolumn{3}{|c|}{$\begin{array}{l}\text { Table A.2 HC-2 TQs Using Maximum Inhalation DCs } \\
\text { (continued) }\end{array}$} \\
\hline $\begin{array}{c}\text { MASTER } \\
\text { ISOTOPE LIST }\end{array}$ & Activity (Ci) & Mass (g) \\
\hline Pt-191 & $5.40 \mathrm{E}+07$ & $2.21 \mathrm{E}+02$ \\
\hline Pt-193 & $3.86 \mathrm{E}+08$ & $1.04 \mathrm{E}+07$ \\
\hline Pt-193m & $6.69 \mathrm{E}+07$ & $4.28 \mathrm{E}+02$ \\
\hline Pt-195m & $4.30 \mathrm{E}+07$ & $2.58 \mathrm{E}+02$ \\
\hline Pt-197 & $9.21 \mathrm{E}+07$ & $1.06 \mathrm{E}+02$ \\
\hline Pt-197m & $2.35 \mathrm{E}+08$ & $2.33 \mathrm{E}+01$ \\
\hline Pt-199 & $1.97 \mathrm{E}+08$ & $6.42 \mathrm{E}+00$ \\
\hline Pt-200 & $3.56 \mathrm{E}+07$ & $2.84 \mathrm{E}+01$ \\
\hline Pt-202 & $3.10 \mathrm{E}+06$ & $8.80 \mathrm{E}+00$ \\
\hline $\mathrm{Pu}-232$ & $3.13 \mathrm{E}+05$ & 1.30E-02 \\
\hline Pu-234 & $3.38 \mathrm{E}+05$ & 2.22E-01 \\
\hline Pu-235 & $6.11 \mathrm{E}+08$ & $1.93 \mathrm{E}+01$ \\
\hline Pu-236 & $2.03 \mathrm{E}+02$ & 3.82E-01 \\
\hline $\mathrm{Pu}-237$ & $2.05 \mathrm{E}+07$ & $1.68 \mathrm{E}+03$ \\
\hline Pu-238 & $7.37 \mathrm{E}+01$ & $4.31 \mathrm{E}+00$ \\
\hline $\mathrm{Pu}-239$ & $6.76 \mathrm{E}+01$ & $1.09 \mathrm{E}+03$ \\
\hline $\mathrm{Pu}-240$ & $6.76 \mathrm{E}+01$ & $2.97 \mathrm{E}+02$ \\
\hline Pu-241 & $3.53 \mathrm{E}+03$ & $3.42 \mathrm{E}+01$ \\
\hline Pu-242 & $7.37 \mathrm{E}+01$ & $1.88 \mathrm{E}+04$ \\
\hline $\mathrm{Pu}-243$ & $9.10 \mathrm{E}+07$ & $3.50 \mathrm{E}+01$ \\
\hline $\mathrm{Pu}-244$ & 7.37E+01 & $4.16 \mathrm{E}+06$ \\
\hline $\mathrm{Pu}-245$ & $1.66 \mathrm{E}+07$ & $1.36 \mathrm{E}+01$ \\
\hline $\mathrm{Pu}-246$ & $1.01 \mathrm{E}+06$ & $2.07 \mathrm{E}+01$ \\
\hline Ra-219 & $3.67 \mathrm{E}+08$ & 7.12E-05 \\
\hline Ra-220 & $1.31 \mathrm{E}+10$ & 4.56E-03 \\
\hline Ra-221 & $1.84 \mathrm{E}+09$ & $1.01 \mathrm{E}+00$ \\
\hline Ra-222 & $6.16 \mathrm{E}+09$ & $4.60 \mathrm{E}+00$ \\
\hline Ra-223 & $9.32 \mathrm{E}+02$ & $1.82 \mathrm{E}-02$ \\
\hline Ra-224 & $2.38 \mathrm{E}+03$ & $1.50 \mathrm{E}-02$ \\
\hline Ra-225 & $1.05 \mathrm{E}+03$ & 2.69E-02 \\
\hline Ra-226 & $8.53 \mathrm{E}+02$ & $8.63 \mathrm{E}+02$ \\
\hline Ra-227 & $1.68 \mathrm{E}+07$ & 8.57E-01 \\
\hline Ra-228 & $5.07 \mathrm{E}+02$ & $1.86 \mathrm{E}+00$ \\
\hline Ra-230 & $5.59 \mathrm{E}+07$ & $6.36 \mathrm{E}+00$ \\
\hline Rb-77 & $3.81 \mathrm{E}+07$ & 5.77E-02 \\
\hline $\mathrm{Rb}-78$ & $1.25 \mathrm{E}+07$ & $9.17 \mathrm{E}-02$ \\
\hline Rb-78m & $1.78 \mathrm{E}+07$ & 4.23E-02 \\
\hline Rb-79 & $3.84 \mathrm{E}+07$ & 3.69E-01 \\
\hline
\end{tabular}




\begin{tabular}{|c|c|c|}
\hline \multicolumn{3}{|c|}{$\begin{array}{l}\text { Table A.2 HC-2 TQs Using Maximum Inhalation DCs } \\
\text { (continued) }\end{array}$} \\
\hline $\begin{array}{c}\text { MASTER } \\
\text { ISOTOPE LIST }\end{array}$ & Activity (Ci) & Mass (g) \\
\hline Rb-80 & $4.45 \mathrm{E}+07$ & 1.07E-02 \\
\hline Rb-81 & $6.60 \mathrm{E}+07$ & $7.81 \mathrm{E}+00$ \\
\hline Rb-81m & $1.07 \mathrm{E}+09$ & $1.48 \mathrm{E}+01$ \\
\hline Rb-82 & $5.10 \mathrm{E}+07$ & 2.89E-02 \\
\hline Rb-82m & $1.50 \mathrm{E}+07$ & $2.44 \mathrm{E}+00$ \\
\hline Rb-83 & $1.06 \mathrm{E}+07$ & $5.83 \mathrm{E}+02$ \\
\hline Rb-84 & $7.15 E+06$ & $1.51 \mathrm{E}+02$ \\
\hline Rb-84m & $1.34 \mathrm{E}+08$ & $1.21 \mathrm{E}+00$ \\
\hline Rb-86 & $8.59 \mathrm{E}+06$ & $1.05 \mathrm{E}+02$ \\
\hline $\mathrm{Rb}-86 \mathrm{~m}$ & $1.11 \mathrm{E}+08$ & 5.15E-02 \\
\hline Rb-87 & $1.62 \mathrm{E}+07$ & $1.85 \mathrm{E}+14$ \\
\hline Rb-88 & $6.94 \mathrm{E}+07$ & $5.78 \mathrm{E}-01$ \\
\hline $\mathrm{Rb}-89$ & $2.44 \mathrm{E}+07$ & $1.76 \mathrm{E}-01$ \\
\hline Rb-90 & $2.50 \mathrm{E}+07$ & 3.15E-02 \\
\hline Rb-90m & $1.66 \mathrm{E}+07$ & 3.41E-02 \\
\hline Re-177 & $7.89 \mathrm{E}+07$ & $1.04 \mathrm{E}+00$ \\
\hline Re-178 & $4.12 \mathrm{E}+07$ & 5.15E-01 \\
\hline Re-179 & $5.06 \mathrm{E}+07$ & 9.39E-01 \\
\hline Re-180 & $4.73 \mathrm{E}+07$ & $1.10 \mathrm{E}-01$ \\
\hline Re-181 & $2.26 \mathrm{E}+07$ & $2.60 \mathrm{E}+01$ \\
\hline Re-182l & $5.50 \mathrm{E}+06$ & $2.04 \mathrm{E}+01$ \\
\hline Re-182s & $2.17 \mathrm{E}+07$ & $1.60 \mathrm{E}+01$ \\
\hline Re-183 & $2.05 E+06$ & $2.01 \mathrm{E}+02$ \\
\hline Re-184 & $4.00 \mathrm{E}+06$ & $2.14 \mathrm{E}+02$ \\
\hline Re-184m & $1.24 \mathrm{E}+06$ & $2.88 \mathrm{E}+02$ \\
\hline Re-186 & $7.35 \mathrm{E}+06$ & $3.95 \mathrm{E}+01$ \\
\hline Re-186m & $6.76 \mathrm{E}+05$ & $7.03 \mathrm{E}+07$ \\
\hline Re-187 & $1.29 \mathrm{E}+09$ & $3.37 \mathrm{E}+16$ \\
\hline Re-188 & $1.48 \mathrm{E}+07$ & $1.51 \mathrm{E}+01$ \\
\hline Re-188m & $3.68 \mathrm{E}+08$ & $6.83 \mathrm{E}+00$ \\
\hline Re-189 & $1.84 \mathrm{E}+07$ & $2.70 \mathrm{E}+01$ \\
\hline Re-190 & $4.47 \mathrm{E}+07$ & $1.40 \mathrm{E}-01$ \\
\hline Re-190m & $2.20 \mathrm{E}+07$ & $4.28 \mathrm{E}+00$ \\
\hline Rh-100 & $1.05 \mathrm{E}+07$ & $6.96 \mathrm{E}+00$ \\
\hline Rh-100m & $1.33 \mathrm{E}+09$ & $3.25 \mathrm{E}+00$ \\
\hline Rh-101 & $1.49 \mathrm{E}+06$ & $1.35 \mathrm{E}+03$ \\
\hline Rh-101m & $3.21 \mathrm{E}+07$ & $1.08 \mathrm{E}+02$ \\
\hline Rh-102 & $4.68 \mathrm{E}+05$ & $3.87 \mathrm{E}+02$ \\
\hline
\end{tabular}




\begin{tabular}{|c|c|c|}
\hline \multicolumn{3}{|c|}{$\begin{array}{l}\text { Table A.2 HC-2 TQs Using Maximum Inhalation DCs } \\
\text { (continued) }\end{array}$} \\
\hline $\begin{array}{c}\text { MASTER } \\
\text { ISOTOPE LIST }\end{array}$ & Activity (Ci) & Mass (g) \\
\hline Rh-102m & $1.13 \mathrm{E}+06$ & $1.83 \mathrm{E}+02$ \\
\hline Rh-103m & $2.97 \mathrm{E}+09$ & $9.13 E+01$ \\
\hline Rh-104 & $1.93 \mathrm{E}+09$ & 7.52E-01 \\
\hline Rh-104m & $2.96 \mathrm{E}+09$ & $7.09 \mathrm{E}+00$ \\
\hline Rh-105 & $2.25 \mathrm{E}+07$ & $2.66 \mathrm{E}+01$ \\
\hline Rh-106 & $2.60 \mathrm{E}+08$ & 7.29E-02 \\
\hline Rh-106m & $1.50 \mathrm{E}+07$ & $1.11 \mathrm{E}+00$ \\
\hline Rh-107 & $1.31 \mathrm{E}+08$ & $1.61 \mathrm{E}+00$ \\
\hline Rh-108 & $1.68 \mathrm{E}+08$ & 2.70E-02 \\
\hline Rh-109 & $1.96 \mathrm{E}+08$ & $1.51 \mathrm{E}-01$ \\
\hline Rh-94 & $1.49 \mathrm{E}+07$ & 8.78E-03 \\
\hline Rh-95 & $2.23 \mathrm{E}+07$ & 5.66E-02 \\
\hline Rh-95m & $6.30 \mathrm{E}+07$ & 6.23E-02 \\
\hline Rh-96 & $1.49 \mathrm{E}+07$ & 7.54E-02 \\
\hline Rh-96m & $4.48 \mathrm{E}+07$ & 3.45E-02 \\
\hline Rh-97 & $3.58 \mathrm{E}+07$ & 5.67E-01 \\
\hline Rh-97m & $2.31 \mathrm{E}+07$ & 5.50E-01 \\
\hline Rh-98 & $3.24 \mathrm{E}+07$ & 1.47E-01 \\
\hline Rh-99 & $8.49 \mathrm{E}+06$ & $1.03 \mathrm{E}+02$ \\
\hline Rh-99m & $5.85 \mathrm{E}+07$ & $8.67 \mathrm{E}+00$ \\
\hline Rn-207 & $6.13 E+04$ & $6.24 \mathrm{E}-04$ \\
\hline Rn-209 & $4.94 \mathrm{E}+04$ & $1.57 \mathrm{E}-03$ \\
\hline Rn-210 & $2.13 \mathrm{E}+05$ & 3.42E-02 \\
\hline Rn-211 & $3.14 \mathrm{E}+04$ & 3.08E-02 \\
\hline Rn-212 & $1.96 \mathrm{E}+05$ & $5.28 \mathrm{E}-03$ \\
\hline Rn-215 & $1.42 \mathrm{E}+05$ & $6.24 \mathrm{E}-12$ \\
\hline Rn-216 & $1.53 \mathrm{E}+05$ & $1.32 \mathrm{E}-10$ \\
\hline Rn-217 & $1.59 \mathrm{E}+05$ & 1.65E-09 \\
\hline Rn-218 & $7.40 \mathrm{E}+07$ & 5.01E-05 \\
\hline Rn-219 & $1.01 \mathrm{E}+06$ & 7.75E-05 \\
\hline Rn-220 & $1.46 \mathrm{E}+08$ & $1.58 \mathrm{E}-01$ \\
\hline Rn-222 & $1.42 \mathrm{E}+08$ & $9.20 \mathrm{E}+02$ \\
\hline $\mathrm{Rn}-223$ & $1.73 E+05$ & 4.99E-03 \\
\hline Ru-103 & $2.64 \mathrm{E}+05$ & $8.18 \mathrm{E}+00$ \\
\hline Ru-105 & $2.76 \mathrm{E}+06$ & $4.10 \mathrm{E}-01$ \\
\hline Ru-106 & $1.23 \mathrm{E}+04$ & $3.67 \mathrm{E}+00$ \\
\hline $\mathrm{Ru}-107$ & $1.60 \mathrm{E}+07$ & $3.41 \mathrm{E}-02$ \\
\hline Ru-108 & $9.26 \mathrm{E}+07$ & $2.42 \mathrm{E}-01$ \\
\hline
\end{tabular}




\begin{tabular}{|c|c|c|}
\hline \multicolumn{3}{|c|}{$\begin{array}{l}\text { Table A.2 HC-2 TQs Using Maximum Inhalation DCs } \\
\text { (continued) }\end{array}$} \\
\hline $\begin{array}{c}\text { MASTER } \\
\text { ISOTOPE LIST }\end{array}$ & Activity (Ci) & Mass (g) \\
\hline Ru-92 & $2.86 \mathrm{E}+06$ & 5.11E-03 \\
\hline Ru-94 & $6.13 E+06$ & 1.59E-01 \\
\hline Ru-95 & $3.63 \mathrm{E}+06$ & 1.81E-01 \\
\hline Ru-97 & $5.31 \mathrm{E}+06$ & $1.14 \mathrm{E}+01$ \\
\hline S-35 & $8.53 \mathrm{E}+03$ & $2.00 \mathrm{E}-01$ \\
\hline S-37 & $3.51 \mathrm{E}+04$ & 3.49E-05 \\
\hline S-38 & $2.67 \mathrm{E}+04$ & 9.19E-04 \\
\hline Sb-111 & $4.00 \mathrm{E}+07$ & 2.95E-02 \\
\hline Sb-113 & $4.73 \mathrm{E}+07$ & $1.90 \mathrm{E}-01$ \\
\hline Sb-114 & $2.13 \mathrm{E}+07$ & $4.50 \mathrm{E}-02$ \\
\hline Sb-115 & $5.65 \mathrm{E}+07$ & $1.10 \mathrm{E}+00$ \\
\hline Sb-116 & $2.41 \mathrm{E}+07$ & 2.34E-01 \\
\hline Sb-116m & $1.58 \mathrm{E}+07$ & $5.86 \mathrm{E}-01$ \\
\hline Sb-117 & $1.98 \mathrm{E}+08$ & $2.07 \mathrm{E}+01$ \\
\hline Sb-118 & $7.40 \mathrm{E}+07$ & $1.67 \mathrm{E}-01$ \\
\hline Sb-118m & $1.62 \mathrm{E}+07$ & $3.04 \mathrm{E}+00$ \\
\hline Sb-119 & $2.21 \mathrm{E}+08$ & $3.20 \mathrm{E}+02$ \\
\hline Sb-120s & $1.14 \mathrm{E}+08$ & $1.15 \mathrm{E}+00$ \\
\hline Sb-120l & $5.53 \mathrm{E}+06$ & $2.93 \mathrm{E}+01$ \\
\hline Sb-122 & $6.97 \mathrm{E}+06$ & $1.76 \mathrm{E}+01$ \\
\hline Sb-122m & $1.53 \mathrm{E}+09$ & $4.15 E+00$ \\
\hline Sb-124 & $9.14 \mathrm{E}+05$ & $5.22 \mathrm{E}+01$ \\
\hline Sb-124ms & $1.59 \mathrm{E}+08$ & $1.62 \mathrm{E}-01$ \\
\hline Sb-124ml & $1.37 \mathrm{E}+09$ & $1.83 \mathrm{E}+01$ \\
\hline Sb-125 & $6.72 \mathrm{E}+05$ & $6.51 \mathrm{E}+02$ \\
\hline Sb-126 & $2.25 \mathrm{E}+06$ & $2.68 \mathrm{E}+01$ \\
\hline Sb-126m & $3.31 \mathrm{E}+07$ & 4.21E-01 \\
\hline Sb-127 & $4.05 E+06$ & $1.52 \mathrm{E}+01$ \\
\hline Sb-128l & $9.29 E+06$ & $3.42 \mathrm{E}+00$ \\
\hline Sb-128s & $2.65 \mathrm{E}+07$ & $1.88 \mathrm{E}-01$ \\
\hline Sb-129 & $1.75 \mathrm{E}+07$ & $3.10 \mathrm{E}+00$ \\
\hline Sb-130 & $1.52 \mathrm{E}+07$ & 4.20E-01 \\
\hline Sb-130m & $2.15 \mathrm{E}+07$ & 9.34E-02 \\
\hline Sb-131 & $2.49 \mathrm{E}+07$ & 3.99E-01 \\
\hline Sb-133 & $2.03 \mathrm{E}+07$ & 3.59E-02 \\
\hline Sc- $42 m$ & $1.37 \mathrm{E}+07$ & $3.15 \mathrm{E}-03$ \\
\hline Sc-43 & $3.03 \mathrm{E}+07$ & $1.62 \mathrm{E}+00$ \\
\hline Sc-44 & $1.64 \mathrm{E}+07$ & 9.02E-01 \\
\hline
\end{tabular}




\begin{tabular}{|c|c|c|}
\hline \multicolumn{3}{|c|}{$\begin{array}{l}\text { Table A.2 HC-2 TQs Using Maximum Inhalation DCs } \\
\text { (continued) }\end{array}$} \\
\hline $\begin{array}{c}\text { MASTER } \\
\text { ISOTOPE LIST }\end{array}$ & Activity (Ci) & Mass (g) \\
\hline Sc- $44 \mathrm{~m}$ & $5.63 \mathrm{E}+06$ & $4.63 \mathrm{E}+00$ \\
\hline Sc-46 & $1.14 \mathrm{E}+06$ & $3.37 \mathrm{E}+01$ \\
\hline Sc-47 & $1.09 \mathrm{E}+07$ & $1.31 \mathrm{E}+01$ \\
\hline Sc-48 & $5.05 \mathrm{E}+06$ & $3.38 \mathrm{E}+00$ \\
\hline Sc-49 & $2.00 \mathrm{E}+08$ & $2.99 \mathrm{E}+00$ \\
\hline Sc-50 & $1.76 \mathrm{E}+07$ & 7.97E-03 \\
\hline Se-70 & $3.72 \mathrm{E}+06$ & 5.67E-02 \\
\hline Se-71 & $3.65 E+06$ & $6.53 \mathrm{E}-03$ \\
\hline Se-72 & $1.95 \mathrm{E}+05$ & $9.01 \mathrm{E}-01$ \\
\hline Se-73 & $2.22 \mathrm{E}+06$ & 3.70E-01 \\
\hline Se-73m & $1.42 \mathrm{E}+07$ & 2.15E-01 \\
\hline Se-75 & $5.98 \mathrm{E}+05$ & $4.11 \mathrm{E}+01$ \\
\hline Se-77m & $6.71 \mathrm{E}+07$ & 7.98E-03 \\
\hline Se-79 & $1.19 \mathrm{E}+05$ & $1.71 \mathrm{E}+06$ \\
\hline Se-79m & $7.51 \mathrm{E}+08$ & $1.24 \mathrm{E}+00$ \\
\hline Se-81 & $4.89 \mathrm{E}+07$ & 3.90E-01 \\
\hline Se-81m & $1.53 \mathrm{E}+07$ & 3.78E-01 \\
\hline Se-83 & $2.04 \mathrm{E}+06$ & 2.03E-02 \\
\hline Se-83m & $5.65 \mathrm{E}+06$ & 2.91E-03 \\
\hline Se-84 & $1.42 \mathrm{E}+07$ & 1.97E-02 \\
\hline Si-31 & $1.02 \mathrm{E}+08$ & $2.65 \mathrm{E}+00$ \\
\hline Si-32 & $7.37 \mathrm{E}+04$ & $2.97 \mathrm{E}+03$ \\
\hline Sm-139 & $4.07 \mathrm{E}+07$ & 7.73E-02 \\
\hline Sm-140 & $7.04 \mathrm{E}+07$ & 7.77E-01 \\
\hline Sm-141 & $3.67 \mathrm{E}+07$ & 2.80E-01 \\
\hline Sm-141m & $2.51 \mathrm{E}+07$ & 4.25E-01 \\
\hline Sm-142 & $9.84 \mathrm{E}+07$ & $5.39 \mathrm{E}+00$ \\
\hline Sm-143 & $1.15 \mathrm{E}+08$ & 7.62E-01 \\
\hline Sm-143m & $8.69 \mathrm{E}+07$ & 7.27E-02 \\
\hline Sm-145 & $5.05 E+06$ & $1.91 \mathrm{E}+03$ \\
\hline Sm-146 & 7.37E+02 & $3.10 \mathrm{E}+07$ \\
\hline Sm-147 & $8.45 E+02$ & $3.68 E+10$ \\
\hline Sm-148 & $3.86 \mathrm{E}+02$ & $1.12 \mathrm{E}+15$ \\
\hline Sm-151 & $2.03 E+06$ & $7.70 \mathrm{E}+04$ \\
\hline Sm-153 & $1.27 \mathrm{E}+07$ & $2.90 \mathrm{E}+01$ \\
\hline Sm-155 & $2.62 E+08$ & $4.77 \mathrm{E}+00$ \\
\hline Sm-156 & $3.43 \mathrm{E}+07$ & $1.60 \mathrm{E}+01$ \\
\hline Sm-157 & $1.42 \mathrm{E}+08$ & $9.48 \mathrm{E}-01$ \\
\hline
\end{tabular}




\begin{tabular}{|c|c|c|}
\hline \multicolumn{3}{|c|}{$\begin{array}{l}\text { Table A.2 HC-2 TQs Using Maximum Inhalation DCs } \\
\text { (continued) }\end{array}$} \\
\hline $\begin{array}{c}\text { MASTER } \\
\text { ISOTOPE LIST }\end{array}$ & Activity (Ci) & Mass (g) \\
\hline Sn-106 & $5.01 \mathrm{E}+07$ & $5.41 \mathrm{E}-02$ \\
\hline Sn-108 & $7.89 \mathrm{E}+07$ & 4.66E-01 \\
\hline Sn-109 & $2.46 \mathrm{E}+07$ & 2.57E-01 \\
\hline Sn-110 & $4.03 \mathrm{E}+07$ & $5.66 \mathrm{E}+00$ \\
\hline Sn-111 & $9.37 \mathrm{E}+07$ & $1.95 \mathrm{E}+00$ \\
\hline Sn-113 & $3.00 \mathrm{E}+06$ & $2.99 \mathrm{E}+02$ \\
\hline Sn-113m & $1.48 \mathrm{E}+09$ & $1.90 \mathrm{E}+01$ \\
\hline Sn-117m & $3.35 \mathrm{E}+06$ & $4.08 \mathrm{E}+01$ \\
\hline Sn-119m & $3.69 \mathrm{E}+06$ & $9.83 \mathrm{E}+02$ \\
\hline Sn-121 & $3.53 \mathrm{E}+07$ & $3.68 \mathrm{E}+01$ \\
\hline Sn-121m & $1.80 \mathrm{E}+06$ & $3.35 E+04$ \\
\hline Sn-123 & $1.00 \mathrm{E}+06$ & $1.22 \mathrm{E}+02$ \\
\hline Sn-123m & $1.74 \mathrm{E}+08$ & $4.55 \mathrm{E}+00$ \\
\hline Sn-125 & $2.58 \mathrm{E}+06$ & $2.38 \mathrm{E}+01$ \\
\hline Sn-125m & $1.69 \mathrm{E}+08$ & $1.07 \mathrm{E}+00$ \\
\hline Sn-126 & $2.90 \mathrm{E}+05$ & $1.02 \mathrm{E}+07$ \\
\hline Sn-127 & $1.94 \mathrm{E}+07$ & $1.65 \mathrm{E}+00$ \\
\hline Sn-127m & $1.01 \mathrm{E}+08$ & 2.82E-01 \\
\hline Sn-128 & $4.46 \mathrm{E}+07$ & $1.79 \mathrm{E}+00$ \\
\hline Sn-129 & $5.68 \mathrm{E}+07$ & 8.68E-02 \\
\hline Sn-130 & $6.50 \mathrm{E}+07$ & 1.67E-01 \\
\hline Sn-130m & $6.44 \mathrm{E}+07$ & 7.56E-02 \\
\hline Sr-79 & $5.01 \mathrm{E}+07$ & 4.74E-02 \\
\hline Sr-80 & $5.79 \mathrm{E}+07$ & $2.46 \mathrm{E}+00$ \\
\hline Sr-81 & $3.42 \mathrm{E}+07$ & 3.75E-01 \\
\hline Sr-82 & $7.37 \mathrm{E}+05$ & $1.16 \mathrm{E}+01$ \\
\hline Sr-83 & $1.78 \mathrm{E}+07$ & $1.53 \mathrm{E}+01$ \\
\hline Sr-85 & $9.19 \mathrm{E}+06$ & $3.87 \mathrm{E}+02$ \\
\hline Sr-85m & $2.26 \mathrm{E}+08$ & $7.11 \mathrm{E}+00$ \\
\hline Sr-87m & $1.22 \mathrm{E}+08$ & $9.47 \mathrm{E}+00$ \\
\hline Sr-89 & $1.03 \mathrm{E}+06$ & $3.53 \mathrm{E}+01$ \\
\hline Sr-90 & $5.07 \mathrm{E}+04$ & $3.71 E+02$ \\
\hline Sr-91 & $1.58 \mathrm{E}+07$ & $4.35 \mathrm{E}+00$ \\
\hline Sr-92 & $1.87 \mathrm{E}+07$ & $1.49 \mathrm{E}+00$ \\
\hline Sr-93 & $2.53 \mathrm{E}+07$ & 9.27E-02 \\
\hline Sr-94 & $3.91 \mathrm{E}+07$ & $2.45 \mathrm{E}-02$ \\
\hline Тa-170 & $5.54 \mathrm{E}+07$ & 3.38E-01 \\
\hline Тa-172 & $3.09 \mathrm{E}+07$ & $1.04 \mathrm{E}+00$ \\
\hline
\end{tabular}




\begin{tabular}{|c|c|c|}
\hline \multicolumn{3}{|c|}{$\begin{array}{l}\text { Table A.2 HC-2 TQs Using Maximum Inhalation DCs } \\
\text { (continued) }\end{array}$} \\
\hline $\begin{array}{c}\text { MASTER } \\
\text { ISOTOPE LIST }\end{array}$ & Activity (Ci) & Mass (g) \\
\hline Та-173 & $4.21 \mathrm{E}+07$ & $8.48 \mathrm{E}+00$ \\
\hline Та-174 & $6.14 \mathrm{E}+07$ & $4.09 \mathrm{E}+00$ \\
\hline Та-175 & $3.04 \mathrm{E}+07$ & $1.78 \mathrm{E}+01$ \\
\hline Та-176 & $1.54 \mathrm{E}+07$ & $6.98 \mathrm{E}+00$ \\
\hline Та-177 & $6.90 \mathrm{E}+07$ & $2.20 \mathrm{E}+02$ \\
\hline Та-178s & $5.86 \mathrm{E}+08$ & $5.17 \mathrm{E}+00$ \\
\hline Та-178l & $3.85 \mathrm{E}+07$ & $4.81 \mathrm{E}+00$ \\
\hline Та-179 & $1.44 \mathrm{E}+07$ & $1.31 \mathrm{E}+04$ \\
\hline Тa-180 & $3.11 \mathrm{E}+05$ & $1.57 \mathrm{E}+15$ \\
\hline Та-180m & $1.65 \mathrm{E}+08$ & $7.68 \mathrm{E}+01$ \\
\hline Та-182 & $7.96 \mathrm{E}+05$ & $1.27 \mathrm{E}+02$ \\
\hline Та-182m & $1.49 \mathrm{E}+08$ & $2.29 \mathrm{E}+00$ \\
\hline Та-183 & $3.79 \mathrm{E}+06$ & $2.71 \mathrm{E}+01$ \\
\hline Та-184 & $1.22 \mathrm{E}+07$ & $6.24 \mathrm{E}+00$ \\
\hline Та-185 & $1.09 \mathrm{E}+08$ & $5.27 \mathrm{E}+00$ \\
\hline Та-186 & $3.32 \mathrm{E}+07$ & $3.45 \mathrm{E}-01$ \\
\hline Tb-146 & $1.55 \mathrm{E}+07$ & 4.62E-03 \\
\hline Tb-147 & $2.62 \mathrm{E}+07$ & $2.03 \mathrm{E}+00$ \\
\hline Tb-147m & $2.97 \mathrm{E}+07$ & $4.34 \mathrm{E}-02$ \\
\hline Tb-148 & $1.95 \mathrm{E}+07$ & $9.20 \mathrm{E}-01$ \\
\hline Tb-148m & $1.90 \mathrm{E}+07$ & 3.29E-02 \\
\hline Tb-149 & $1.58 \mathrm{E}+06$ & 3.11E-01 \\
\hline Tb-149m & $4.36 \mathrm{E}+07$ & $1.44 \mathrm{E}-01$ \\
\hline Tb-150 & $2.27 \mathrm{E}+07$ & $3.55 \mathrm{E}+00$ \\
\hline Tb-150m & $2.41 \mathrm{E}+07$ & $1.12 \mathrm{E}-01$ \\
\hline Tb-151 & $2.28 \mathrm{E}+07$ & $1.93 \mathrm{E}+01$ \\
\hline Tb-151m & $8.45 \mathrm{E}+08$ & 2.82E-01 \\
\hline Tb-152 & $1.37 \mathrm{E}+07$ & $1.16 \mathrm{E}+01$ \\
\hline Tb-152m & $8.24 \mathrm{E}+07$ & 2.80E-01 \\
\hline Tb-153 & $3.69 \mathrm{E}+07$ & $1.01 \mathrm{E}+02$ \\
\hline Tb-154 & $1.12 \mathrm{E}+07$ & $1.18 \mathrm{E}+01$ \\
\hline Tb-155 & $3.43 \mathrm{E}+07$ & $2.16 \mathrm{E}+02$ \\
\hline Tb-156 & $5.52 \mathrm{E}+06$ & $3.52 \mathrm{E}+01$ \\
\hline Tb-156ml & $3.82 \mathrm{E}+07$ & $4.64 \mathrm{E}+01$ \\
\hline Tb-156ms & $8.42 \mathrm{E}+07$ & $2.09 \mathrm{E}+01$ \\
\hline Tb-157 & $6.76 \mathrm{E}+06$ & $4.45 \mathrm{E}+05$ \\
\hline Tb-158 & $1.76 \mathrm{E}+05$ & $1.17 \mathrm{E}+04$ \\
\hline Tb-160 & $1.13 \mathrm{E}+06$ & $1.00 \mathrm{E}+02$ \\
\hline
\end{tabular}




\begin{tabular}{|c|c|c|}
\hline \multicolumn{3}{|c|}{$\begin{array}{l}\text { Table A.2 HC-2 TQs Using Maximum Inhalation DCs } \\
\text { (continued) }\end{array}$} \\
\hline $\begin{array}{c}\text { MASTER } \\
\text { ISOTOPE LIST }\end{array}$ & Activity (Ci) & Mass (g) \\
\hline Tb-161 & $6.22 \mathrm{E}+06$ & $5.30 \mathrm{E}+01$ \\
\hline Tb-162 & $5.36 \mathrm{E}+07$ & 3.51E-01 \\
\hline Tb-163 & $6.46 \mathrm{E}+07$ & $1.09 \mathrm{E}+00$ \\
\hline Tb-164 & $2.37 \mathrm{E}+07$ & $6.20 \mathrm{E}-02$ \\
\hline Tb-165 & $6.69 \mathrm{E}+07$ & $1.24 \mathrm{E}-01$ \\
\hline Tc-101 & $1.34 \mathrm{E}+08$ & $1.02 \mathrm{E}+00$ \\
\hline Тс-102 & $4.62 \mathrm{E}+08$ & 2.20E-02 \\
\hline Tc-102m & $2.29 \mathrm{E}+07$ & $5.40 \mathrm{E}-02$ \\
\hline Tc-104 & $2.44 \mathrm{E}+07$ & $2.46 \mathrm{E}-01$ \\
\hline Tc-105 & $7.17 \mathrm{E}+07$ & 3.04E-01 \\
\hline Тс-91 & $2.25 \mathrm{E}+07$ & $3.42 \mathrm{E}-02$ \\
\hline Tc-91m & $4.10 \mathrm{E}+07$ & 6.53E-02 \\
\hline Тc-92 & $1.51 \mathrm{E}+07$ & 3.14E-02 \\
\hline Tc-93 & $3.16 \mathrm{E}+07$ & $2.58 \mathrm{E}+00$ \\
\hline Tc-93m & $6.29 \mathrm{E}+07$ & $1.35 \mathrm{E}+00$ \\
\hline Тc-94 & $1.56 \mathrm{E}+07$ & $2.28 \mathrm{E}+00$ \\
\hline Tc-94m & $2.52 \mathrm{E}+07$ & $6.55 \mathrm{E}-01$ \\
\hline Тc-95 & $3.60 \mathrm{E}+07$ & $2.18 \mathrm{E}+01$ \\
\hline Tc-95m & $6.25 E+06$ & $2.77 \mathrm{E}+02$ \\
\hline Тc-96 & $7.61 \mathrm{E}+06$ & $2.39 \mathrm{E}+01$ \\
\hline Tc-96m & $5.70 \mathrm{E}+08$ & $1.50 \mathrm{E}+01$ \\
\hline Тс-97 & $4.50 \mathrm{E}+06$ & $3.17 \mathrm{E}+09$ \\
\hline Tc-97m & $1.98 \mathrm{E}+06$ & $1.28 \mathrm{E}+02$ \\
\hline Тс-98 & $1.79 \mathrm{E}+05$ & $2.06 \mathrm{E}+08$ \\
\hline Tc-99 & $6.24 \mathrm{E}+05$ & $3.68 \mathrm{E}+07$ \\
\hline Tc-99m & $2.15 E+08$ & $4.09 \mathrm{E}+01$ \\
\hline Te-113 & $2.57 \mathrm{E}+06$ & 2.63E-03 \\
\hline Тe-114 & $3.76 \mathrm{E}+06$ & 3.46E-02 \\
\hline Te-115 & $2.57 \mathrm{E}+06$ & 9.12E-03 \\
\hline Te-115m & $2.22 \mathrm{E}+06$ & $9.07 \mathrm{E}-03$ \\
\hline Te-116 & $6.94 \mathrm{E}+06$ & 6.39E-01 \\
\hline Te-117 & $3.16 \mathrm{E}+06$ & $1.22 \mathrm{E}-01$ \\
\hline Te-118 & $2.75 \mathrm{E}+05$ & $1.49 \mathrm{E}+00$ \\
\hline Te-119 & $3.45 \mathrm{E}+06$ & $2.10 \mathrm{E}+00$ \\
\hline Te-119m & $8.62 \mathrm{E}+05$ & $3.69 \mathrm{E}+00$ \\
\hline Te-121 & $1.37 \mathrm{E}+06$ & $2.16 \mathrm{E}+01$ \\
\hline Te-121m & $1.42 \mathrm{E}+05$ & $2.02 \mathrm{E}+01$ \\
\hline Тe-123 & $6.76 \mathrm{E}+04$ & $2.32 \mathrm{E}+14$ \\
\hline
\end{tabular}




\begin{tabular}{|c|c|c|}
\hline \multicolumn{3}{|c|}{$\begin{array}{l}\text { Table A.2 HC-2 TQs Using Maximum Inhalation DCs } \\
\text { (continued) }\end{array}$} \\
\hline $\begin{array}{c}\text { MASTER } \\
\text { ISOTOPE LIST }\end{array}$ & Activity (Ci) & Mass (g) \\
\hline Te-123m & $1.58 \mathrm{E}+05$ & $1.78 \mathrm{E}+01$ \\
\hline Te-125m & $1.93 \mathrm{E}+05$ & $1.07 \mathrm{E}+01$ \\
\hline Тe-127 & $5.76 \mathrm{E}+06$ & $2.18 \mathrm{E}+00$ \\
\hline Te-127m & $8.27 \mathrm{E}+04$ & $8.76 \mathrm{E}+00$ \\
\hline Тe-129 & $1.72 \mathrm{E}+07$ & 8.19E-01 \\
\hline Te-129m & $1.03 \mathrm{E}+05$ & $3.40 \mathrm{E}+00$ \\
\hline Тe-131 & $6.28 \mathrm{E}+06$ & 1.09E-01 \\
\hline Te-131m & $3.11 \mathrm{E}+05$ & 3.89E-01 \\
\hline Te-132 & $1.58 \mathrm{E}+05$ & $5.20 \mathrm{E}-01$ \\
\hline Te-133 & $4.18 \mathrm{E}+06$ & 3.68E-02 \\
\hline Te-133m & $1.44 \mathrm{E}+06$ & 5.65E-02 \\
\hline Тe-134 & $3.84 \mathrm{E}+06$ & $1.14 \mathrm{E}-01$ \\
\hline Th-223 & $9.72 \mathrm{E}+08$ & 1.15E-02 \\
\hline Th-224 & $2.77 E+09$ & 5.78E-02 \\
\hline Th-226 & $1.33 \mathrm{E}+05$ & 4.94E-03 \\
\hline Th-227 & $8.11 \mathrm{E}+02$ & 2.64E-02 \\
\hline Th-228 & $2.03 \mathrm{E}+02$ & 2.47E-01 \\
\hline Th-229 & $3.38 \mathrm{E}+01$ & $1.59 \mathrm{E}+02$ \\
\hline Th-230 & $8.11 \mathrm{E}+01$ & $4.02 \mathrm{E}+03$ \\
\hline Th-231 & $2.45 \mathrm{E}+07$ & $4.60 \mathrm{E}+01$ \\
\hline Th-232 & 7.37E+01 & $6.72 E+08$ \\
\hline Th-233 & $3.05 E+08$ & $8.43 E+00$ \\
\hline Th-234 & $1.05 \mathrm{E}+06$ & $4.55 \mathrm{E}+01$ \\
\hline Th-235 & $9.22 \mathrm{E}+08$ & $8.19 \mathrm{E}+00$ \\
\hline Th-236 & $1.12 \mathrm{E}+08$ & $5.29 E+00$ \\
\hline $\mathrm{Ti}-44$ & $6.76 \mathrm{E}+04$ & $3.93 \mathrm{E}+02$ \\
\hline Ti-45 & $3.71 \mathrm{E}+07$ & $1.64 \mathrm{E}+00$ \\
\hline Ti-51 & $1.58 \mathrm{E}+08$ & 2.47E-01 \\
\hline Ti-52 & $4.70 \mathrm{E}+08$ & 2.21E-01 \\
\hline Tl-190 & $4.55 \mathrm{E}+07$ & $1.20 \mathrm{E}-01$ \\
\hline Tl-190m & $2.43 \mathrm{E}+07$ & $9.10 \mathrm{E}-02$ \\
\hline Tl-194 & $7.03 \mathrm{E}+07$ & $2.39 \mathrm{E}+00$ \\
\hline Tl-194m & $2.30 \mathrm{E}+07$ & 7.79E-01 \\
\hline Tl-195 & $3.95 \mathrm{E}+07$ & $2.85 \mathrm{E}+00$ \\
\hline Tl-196 & $2.61 \mathrm{E}+07$ & $3.00 \mathrm{E}+00$ \\
\hline Tl-197 & $1.13 \mathrm{E}+08$ & $2.01 \mathrm{E}+01$ \\
\hline Tl-198 & $2.23 \mathrm{E}+07$ & $7.48 \mathrm{E}+00$ \\
\hline Tl-198m & $3.90 \mathrm{E}+07$ & $4.61 \mathrm{E}+00$ \\
\hline
\end{tabular}




\begin{tabular}{|c|c|c|}
\hline \multicolumn{3}{|c|}{$\begin{array}{l}\text { Table A.2 HC-2 TQs Using Maximum Inhalation DCs } \\
\text { (continued) }\end{array}$} \\
\hline $\begin{array}{c}\text { MASTER } \\
\text { ISOTOPE LIST }\end{array}$ & Activity (Ci) & Mass (g) \\
\hline Tl-199 & $1.53 \mathrm{E}+08$ & $7.22 \mathrm{E}+01$ \\
\hline Tl-200 & $2.51 \mathrm{E}+07$ & $4.19 \mathrm{E}+01$ \\
\hline Tl-201 & $1.47 \mathrm{E}+08$ & $6.86 \mathrm{E}+02$ \\
\hline Tl-202 & $3.17 \mathrm{E}+07$ & $6.01 \mathrm{E}+02$ \\
\hline Tl-204 & $2.08 \mathrm{E}+07$ & $4.48 \mathrm{E}+04$ \\
\hline Tl-206 & $4.02 \mathrm{E}+10$ & $1.85 \mathrm{E}+02$ \\
\hline Tl-206m & $2.48 \mathrm{E}+07$ & 1.02E-01 \\
\hline Tl-207 & $1.67 \mathrm{E}+10$ & $8.76 \mathrm{E}+01$ \\
\hline Tl-208 & $1.53 \mathrm{E}+07$ & 5.19E-02 \\
\hline Tl-209 & $2.65 \mathrm{E}+07$ & $6.48 \mathrm{E}-02$ \\
\hline Tl-210 & $2.05 \mathrm{E}+07$ & 2.97E-02 \\
\hline Tm-161 & $3.91 \mathrm{E}+07$ & $1.01 \mathrm{E}+00$ \\
\hline Tm-162 & $2.83 \mathrm{E}+07$ & 5.29E-01 \\
\hline Tm-163 & $3.58 \mathrm{E}+07$ & $3.37 \mathrm{E}+00$ \\
\hline Tm-164 & $7.57 \mathrm{E}+07$ & 1.32E-01 \\
\hline Tm-165 & $2.34 \mathrm{E}+07$ & $3.71 \mathrm{E}+01$ \\
\hline Tm-166 & $1.80 \mathrm{E}+07$ & $7.34 \mathrm{E}+00$ \\
\hline Tm-167 & $7.25 \mathrm{E}+06$ & $8.57 \mathrm{E}+01$ \\
\hline Tm-168 & $1.41 \mathrm{E}+06$ & $1.68 \mathrm{E}+02$ \\
\hline Tm-170 & $1.16 \mathrm{E}+06$ & $1.94 \mathrm{E}+02$ \\
\hline Tm-171 & $5.79 \mathrm{E}+06$ & $5.32 E+03$ \\
\hline Tm-172 & $6.92 E+06$ & $2.41 \mathrm{E}+01$ \\
\hline Tm-173 & $3.44 \mathrm{E}+07$ & $1.57 \mathrm{E}+01$ \\
\hline Tm-174 & $3.36 \mathrm{E}+07$ & $1.68 \mathrm{E}-01$ \\
\hline Tm-175 & $4.72 \mathrm{E}+07$ & 6.67E-01 \\
\hline Tm-176 & $2.88 \mathrm{E}+07$ & 4.98E-02 \\
\hline $\mathrm{U}-227$ & $5.57 \mathrm{E}+08$ & 7.40E-01 \\
\hline U-228 & $1.70 \mathrm{E}+10$ & $1.88 \mathrm{E}+02$ \\
\hline U-230 & $5.07 \mathrm{E}+02$ & $1.86 \mathrm{E}-02$ \\
\hline U-231 & $1.53 \mathrm{E}+07$ & $1.14 \mathrm{E}+02$ \\
\hline $\mathrm{U}-232$ & $2.19 \mathrm{E}+02$ & $1.02 \mathrm{E}+01$ \\
\hline U-233 & $8.45 E+02$ & $8.73 E+04$ \\
\hline U-234 & $8.63 \mathrm{E}+02$ & $1.38 \mathrm{E}+05$ \\
\hline U-235 & $9.54 \mathrm{E}+02$ & $4.41 \mathrm{E}+08$ \\
\hline U-235m & $8.84 \mathrm{E}+12$ & $2.87 \mathrm{E}+05$ \\
\hline U-236 & $9.32 \mathrm{E}+02$ & $1.44 \mathrm{E}+07$ \\
\hline $\mathrm{U}-237$ & $4.23 \mathrm{E}+06$ & $5.18 \mathrm{E}+01$ \\
\hline U-238 & $1.01 \mathrm{E}+03$ & $3.02 \mathrm{E}+09$ \\
\hline
\end{tabular}




\begin{tabular}{|c|c|c|}
\hline \multicolumn{3}{|c|}{$\begin{array}{l}\text { Table A.2 HC-2 TQs Using Maximum Inhalation DCs } \\
\text { (continued) }\end{array}$} \\
\hline $\begin{array}{c}\text { MASTER } \\
\text { ISOTOPE LIST }\end{array}$ & Activity (Ci) & Mass (g) \\
\hline U-239 & $2.66 \mathrm{E}+08$ & $7.95 \mathrm{E}+00$ \\
\hline U-240 & $1.40 \mathrm{E}+07$ & $1.51 \mathrm{E}+01$ \\
\hline U-242 & $2.06 \mathrm{E}+08$ & $4.45 \mathrm{E}+00$ \\
\hline V-47 & $4.69 \mathrm{E}+07$ & 3.82E-01 \\
\hline V-48 & $2.86 \mathrm{E}+06$ & $1.71 \mathrm{E}+01$ \\
\hline V-49 & $2.38 \mathrm{E}+08$ & $2.95 \mathrm{E}+04$ \\
\hline V-50 & $1.21 \mathrm{E}+05$ & $2.53 \mathrm{E}+18$ \\
\hline V-52 & $3.83 \mathrm{E}+07$ & 3.96E-02 \\
\hline V-53 & $5.48 \mathrm{E}+07$ & 2.49E-02 \\
\hline W-176 & $1.31 \mathrm{E}+08$ & $1.69 \mathrm{E}+01$ \\
\hline W-177 & $5.34 \mathrm{E}+07$ & $6.79 \mathrm{E}+00$ \\
\hline W-178 & $1.10 \mathrm{E}+08$ & $3.27 \mathrm{E}+03$ \\
\hline W-179 & $1.26 \mathrm{E}+09$ & $4.51 \mathrm{E}+01$ \\
\hline W-179m & $1.36 \mathrm{E}+09$ & $8.27 E+00$ \\
\hline W-181 & $2.60 \mathrm{E}+08$ & $4.36 \mathrm{E}+04$ \\
\hline W-185 & $6.76 \mathrm{E}+07$ & $7.19 E+03$ \\
\hline W-185m & $2.90 \mathrm{E}+09$ & $4.55 \mathrm{E}+00$ \\
\hline W-187 & $3.14 \mathrm{E}+07$ & $4.47 \mathrm{E}+01$ \\
\hline W-188 & $1.42 \mathrm{E}+07$ & $1.42 \mathrm{E}+03$ \\
\hline W-190 & $7.90 \mathrm{E}+07$ & $2.39 \mathrm{E}+00$ \\
\hline Xe-120 & $1.39 \mathrm{E}+05$ & 3.55E-03 \\
\hline Хe-121 & $2.96 \mathrm{E}+04$ & 7.63E-04 \\
\hline Xe-122 & $1.10 \mathrm{E}+06$ & 8.59E-01 \\
\hline Хe-123 & $8.92 \mathrm{E}+04$ & $7.28 \mathrm{E}-03$ \\
\hline Xe-125 & $2.27 \mathrm{E}+05$ & $1.54 \mathrm{E}-01$ \\
\hline Хe-127 & $2.16 \mathrm{E}+05$ & $7.65 \mathrm{E}+00$ \\
\hline Xe-127m & $4.11 \mathrm{E}+05$ & $3.20 \mathrm{E}-04$ \\
\hline Хe-129m & $2.55 \mathrm{E}+06$ & $2.01 \mathrm{E}+01$ \\
\hline Xe-131m & $6.95 \mathrm{E}+06$ & $8.29 E+01$ \\
\hline Хe-133 & $1.73 \mathrm{E}+06$ & $9.25 \mathrm{E}+00$ \\
\hline Xe-133m & $1.97 \mathrm{E}+06$ & $4.39 \mathrm{E}+00$ \\
\hline Хe-135 & $2.27 \mathrm{E}+05$ & 8.89E-02 \\
\hline Xe-135m & $1.32 \mathrm{E}+05$ & $1.45 \mathrm{E}-03$ \\
\hline Хe-137 & $2.60 \mathrm{E}+05$ & 7.22E-04 \\
\hline Хе-138 & $4.68 \mathrm{E}+04$ & 4.87E-04 \\
\hline Y-81 & $5.05 \mathrm{E}+07$ & 2.55E-02 \\
\hline Y-83 & $4.39 \mathrm{E}+07$ & $1.37 \mathrm{E}-01$ \\
\hline Y-83m & $7.17 \mathrm{E}+07$ & $9.01 \mathrm{E}-02$ \\
\hline
\end{tabular}




\begin{tabular}{|c|c|c|}
\hline \multicolumn{3}{|c|}{$\begin{array}{l}\text { Table A.2 HC-2 TQs Using Maximum Inhalation DCs } \\
\text { (continued) }\end{array}$} \\
\hline $\begin{array}{c}\text { MASTER } \\
\text { ISOTOPE LIST }\end{array}$ & Activity (Ci) & Mass (g) \\
\hline Y-84m & $1.29 \mathrm{E}+07$ & 2.28E-01 \\
\hline Y-85 & $3.10 \mathrm{E}+07$ & $2.25 \mathrm{E}+00$ \\
\hline Y-85m & $2.08 \mathrm{E}+07$ & $2.74 \mathrm{E}+00$ \\
\hline Y-86 & $8.05 E+06$ & $3.25 \mathrm{E}+00$ \\
\hline Y-86m & $1.36 \mathrm{E}+08$ & $2.97 \mathrm{E}+00$ \\
\hline Y-87 & $1.78 \mathrm{E}+07$ & $3.97 \mathrm{E}+01$ \\
\hline Y-87m & $3.93 \mathrm{E}+07$ & $1.46 \mathrm{E}+01$ \\
\hline Y-88 & $1.69 \mathrm{E}+06$ & $1.21 \mathrm{E}+02$ \\
\hline Y-89m & $6.50 \mathrm{E}+07$ & 8.02E-03 \\
\hline Y-90 & $5.40 \mathrm{E}+06$ & $9.92 \mathrm{E}+00$ \\
\hline Y-90m & $4.26 \mathrm{E}+07$ & $3.90 \mathrm{E}+00$ \\
\hline Y-91 & $9.11 \mathrm{E}+05$ & $3.71 \mathrm{E}+01$ \\
\hline Y-91m & $9.27 \mathrm{E}+07$ & $2.23 \mathrm{E}+00$ \\
\hline Y-92 & $3.70 \mathrm{E}+07$ & $3.84 \mathrm{E}+00$ \\
\hline Y-93 & $1.87 \mathrm{E}+07$ & $5.59 \mathrm{E}+00$ \\
\hline Y-94 & $4.12 \mathrm{E}+07$ & 3.93E-01 \\
\hline Y-95 & $5.08 \mathrm{E}+07$ & 2.74E-01 \\
\hline Yb-162 & $2.62 \mathrm{E}+08$ & $4.26 \mathrm{E}+00$ \\
\hline Yb-163 & $7.45 \mathrm{E}+07$ & 7.13E-01 \\
\hline Yb-164 & $1.40 \mathrm{E}+08$ & $9.25 \mathrm{E}+00$ \\
\hline Yb-165 & $1.97 \mathrm{E}+08$ & $1.71 \mathrm{E}+00$ \\
\hline Yb-166 & $1.04 \mathrm{E}+07$ & $3.13 E+01$ \\
\hline Yb-167 & $2.05 E+08$ & $3.18 \mathrm{E}+00$ \\
\hline Yb-169 & $2.67 \mathrm{E}+06$ & $1.11 \mathrm{E}+02$ \\
\hline Yb-175 & $1.10 \mathrm{E}+07$ & $6.19 \mathrm{E}+01$ \\
\hline Yb-177 & $8.39 \mathrm{E}+07$ & $9.00 \mathrm{E}+00$ \\
\hline Yb-178 & $1.01 \mathrm{E}+08$ & $7.10 \mathrm{E}+00$ \\
\hline Yb-179 & $6.14 \mathrm{E}+07$ & 4.68E-01 \\
\hline $\mathrm{Zn}-60$ & $3.91 \mathrm{E}+07$ & 2.97E-02 \\
\hline Zn-61 & $3.74 \mathrm{E}+07$ & $1.80 \mathrm{E}-02$ \\
\hline Zn-62 & $1.32 \mathrm{E}+07$ & $2.42 \mathrm{E}+00$ \\
\hline Zn-63 & $4.12 \mathrm{E}+07$ & $5.26 \mathrm{E}-01$ \\
\hline Zn-65 & $3.55 \mathrm{E}+06$ & 4.30E+02 \\
\hline Zn-69 & $2.89 \mathrm{E}+08$ & $6.04 \mathrm{E}+00$ \\
\hline Zn-69m & $2.46 \mathrm{E}+07$ & $7.44 \mathrm{E}+00$ \\
\hline Zn-71 & $1.78 \mathrm{E}+08$ & $1.64 \mathrm{E}-01$ \\
\hline Zn-71m & $2.11 \mathrm{E}+07$ & $1.87 \mathrm{E}+00$ \\
\hline Zn-72 & $6.14 \mathrm{E}+06$ & $6.55 \mathrm{E}+00$ \\
\hline
\end{tabular}




\begin{tabular}{|c|c|c|}
\hline \multicolumn{3}{|c|}{ Table A.2 HC-2 TQs Using Maximum Inhalation DCs } \\
(continued) \\
\hline $\begin{array}{c}\text { MASTER } \\
\text { ISOTOPE LIST }\end{array}$ & Activity (Ci) & Mass (g) \\
\hline Zr-85 & $4.01 \mathrm{E}+07$ & $1.42 \mathrm{E}-01$ \\
\hline Zr-86 & $1.73 \mathrm{E}+07$ & $7.83 \mathrm{E}+00$ \\
\hline Zr-87 & $3.46 \mathrm{E}+07$ & $1.61 \mathrm{E}+00$ \\
\hline Zr-88 & $2.22 \mathrm{E}+06$ & $1.25 \mathrm{E}+02$ \\
\hline Zr-89 & $1.13 \mathrm{E}+07$ & $2.50 \mathrm{E}+01$ \\
\hline Zr-89m & $9.38 \mathrm{E}+07$ & $1.85 \mathrm{E}-01$ \\
\hline Zr-93 & $3.24 \mathrm{E}+05$ & $1.29 \mathrm{E}+08$ \\
\hline Zr-95 & $1.35 \mathrm{E}+06$ & $6.28 \mathrm{E}+01$ \\
\hline Zr-97 & $8.56 \mathrm{E}+06$ & $4.47 \mathrm{E}+00$ \\
\hline
\end{tabular}




\section{APPENDIX B. CALCULATION OF HAZARD CATEGORY 2 THRESHOLD QUANTITIES USING RECOMMENDED DOSE COEFFICIENTS}


(This page is intentionally blank)

B-2 


\section{APPENDIX B. CALCULATION OF HAZARD CATEGORY 2 THRESHOLD QUANTITIES USING RECOMMENDED DOSE COEFFICIENTS}

This appendix contains the following tables:

- Table B.1: Radionuclide Specific Input Data Used For the Calculation of HC-2 TQs Using Recommended DCs

- Table B.2: HC-2 TQs Using Recommended Inhalation DCs 


\begin{tabular}{|c|c|c|c|c|c|c|c|c|c|c|c|c|c|c|c|}
\hline $\begin{array}{l}\text { MASTER } \\
\text { ISOTOPE } \\
\text { LIST }\end{array}$ & $\begin{array}{l}\text { Atomic } \\
\text { Mass } \\
\text { Nuclide }\end{array}$ & $\begin{array}{l}\text { Atomic } \\
\text { Mass }\end{array}$ & Element & $\begin{array}{c}\text { RF per } \\
\text { DOE-STD- } \\
1027 \\
\end{array}$ & $\begin{array}{c}\text { Half-Life } \\
\text { Reference }\end{array}$ & $\begin{array}{r}\text { Half-Li } \\
\text { (original L }\end{array}$ & & Half Life (s) & $\begin{array}{l}\text { Sp. Act } \\
(\mathrm{Ci} / \mathrm{g})\end{array}$ & $\begin{array}{c}\text { Inhalation DC } \\
\text { Reference }\end{array}$ & $\begin{array}{c}\mathrm{HC}-2 \\
\text { Inhalation } \\
\mathrm{DC} \\
(\mathrm{Sv} / \mathrm{Bq}) \\
\end{array}$ & & $\begin{array}{l}\text { ung } \\
\text { rption } \\
\text { /Type }\end{array}$ & $\begin{array}{c}\text { Immersion DC } \\
\text { Reference }\end{array}$ & $\begin{array}{c}\text { HC-2 } \\
\text { Immersion } \\
\mathrm{DC}(\mathrm{Sv} / \mathrm{s} \\
\left.\text { per } \mathrm{Bq} / \mathrm{m}^{3}\right)\end{array}$ \\
\hline Ac-223 & Ac-223 & 223.019 & Actinium & $1.00 \mathrm{E}-03$ & ICRP-38 & $2.20 \mathrm{E}+00$ & $\mathrm{~m}$ & $1.32 \mathrm{E}+02$ & $3.83 \mathrm{E}+08$ & -- & -- & -- & -- & FGR-12 & $2.07 \mathrm{E}-16$ \\
\hline Ac-224 & Ac-224 & 224.022 & Actinium & $1.00 \mathrm{E}-03$ & ICRP-38 & $2.90 \mathrm{E}+00$ & $\mathrm{~h}$ & $1.04 \mathrm{E}+04$ & $4.82 \mathrm{E}+06$ & ICRP-72 & $1.30 \mathrm{E}-07$ & $\mathrm{~s}$ & Max & FGR-12 & $9.00 \mathrm{E}-15$ \\
\hline Ac-225 & Ac-225 & 225.023 & Actinium & $1.00 \mathrm{E}-03$ & ICRP-38 & $1.00 \mathrm{E}+01$ & $d$ & $8.64 \mathrm{E}+05$ & $5.80 \mathrm{E}+04$ & ICRP-72 & $8.50 \mathrm{E}-06$ & $\mathrm{~s}$ & Max & FGR-12 & $7.21 \mathrm{E}-16$ \\
\hline Ac-226 & Ac-226 & 226.026 & Actinium & $1.00 \mathrm{E}-03$ & ICRP-38 & $2.90 \mathrm{E}+01$ & $\mathrm{~h}$ & $1.04 \mathrm{E}+05$ & $4.78 \mathrm{E}+05$ & ICRP-72 & $1.30 \mathrm{E}-06$ & $\mathrm{~s}$ & Max & FGR-12 & $6.03 \mathrm{E}-15$ \\
\hline Ac-227 & Ac-227 & 227.028 & Actinium & $1.00 \mathrm{E}-03$ & ICRP-38 & $2.18 \mathrm{E}+01$ & $y$ & $6.87 E+08$ & $7.23 \mathrm{E}+01$ & ICRP-72 & $5.50 \mathrm{E}-04$ & $\mathrm{~F}$ & Max & FGR-12 & $5.82 \mathrm{E}-18$ \\
\hline Ac-228 & Ac-228 & 228.031 & Actinium & $1.00 \mathrm{E}-03$ & ICRP-38 & $6.13 \mathrm{E}+00$ & $\mathrm{~h}$ & $2.21 \mathrm{E}+04$ & $2.24 \mathrm{E}+06$ & ICRP-72 & $2.50 \mathrm{E}-08$ & $F$ & Max & FGR-12 & $4.78 \mathrm{E}-14$ \\
\hline Ac-229 & Ac-229 & 229.033 & Actinium & $1.00 \mathrm{E}-03$ & JAERI & $6.27 \mathrm{E}+01$ & $\mathrm{~m}$ & $3.76 \mathrm{E}+03$ & $1.31 \mathrm{E}+07$ & JAERI & $3.50 \mathrm{E}-11$ & $M$ & Max & -- & -- \\
\hline Ac-230 & Ac-230 & 230.036 & Actinium & $1.00 \mathrm{E}-03$ & ICRP-107 & $1.22 \mathrm{E}+02$ & $\mathrm{~s}$ & $1.22 \mathrm{E}+02$ & $4.02 \mathrm{E}+08$ & -- & -- & -- & -- & DOE-STD-1196 & $2.66 \mathrm{E}-14$ \\
\hline Ac-231 & Ac-231 & 231.038 & Actinium & $1.00 \mathrm{E}-03$ & ICRP-107 & $7.50 \mathrm{E}+00$ & $\mathrm{~m}$ & $4.50 E+02$ & $1.09 \mathrm{E}+08$ & -- & -- & -- & -- & DOE-STD-1196 & $1.84 \mathrm{E}-14$ \\
\hline Ac-232 & Ac-232 & 232.042 & Actinium & $1.00 \mathrm{E}-03$ & ICRP-107 & $1.19 \mathrm{E}+02$ & $\mathrm{~s}$ & $1.19 \mathrm{E}+02$ & $4.09 \mathrm{E}+08$ & -- & -- & -- & -- & DOE-STD-1196 & $5.65 \mathrm{E}-14$ \\
\hline Ac-233 & Ac-233 & 233.044 & Actinium & $1.00 \mathrm{E}-03$ & ICRP-107 & $1.45 \mathrm{E}+02$ & $\mathrm{~s}$ & $1.45 \mathrm{E}+02$ & $3.34 \mathrm{E}+08$ & -- & -- & -- & -- & DOE-STD-1196 & $2.29 \mathrm{E}-14$ \\
\hline Ag-100m & Ag-100 & 99.916 & Silver & $1.00 \mathrm{E}-03$ & ICRP-107 & $2.24 \mathrm{E}+00$ & $\mathrm{~m}$ & $1.34 \mathrm{E}+02$ & $8.40 \mathrm{E}+08$ & -- & -- & -- & -- & DOE-STD-1196 & $1.33 \mathrm{E}-13$ \\
\hline Ag-101 & Ag-101 & 100.913 & Silver & $1.00 \mathrm{E}-03$ & ICRP-107 & $1.11 \mathrm{E}+01$ & $\mathrm{~m}$ & $6.66 \mathrm{E}+02$ & $1.68 \mathrm{E}+08$ & DOE-STD-1196 & $1.60 \mathrm{E}-11$ & $M$ & $\operatorname{Rec}$ & DOE-STD-1196 & $7.19 \mathrm{E}-14$ \\
\hline Ag-102 & Ag-102 & 101.912 & Silver & $1.00 \mathrm{E}-03$ & ICRP-38 & $1.29 \mathrm{E}+01$ & $\mathrm{~m}$ & $7.74 E+02$ & $1.43 \mathrm{E}+08$ & ICRP-72 & $1.70 \mathrm{E}-11$ & $M$ & Rec & FGR-12 & $1.67 \mathrm{E}-13$ \\
\hline Ag-102m & Ag-102 & 101.912 & Silver & $1.00 \mathrm{E}-03$ & ICRP-107 & $7.70 \mathrm{E}+00$ & $\mathrm{~m}$ & $4.62 \mathrm{E}+02$ & $2.40 \mathrm{E}+08$ & -- & -- & -- & -- & DOE-STD-1196 & $9.72 \mathrm{E}-14$ \\
\hline Ag-103 & Ag-103 & 102.909 & Silver & $1.00 \mathrm{E}-03$ & ICRP-38 & $6.57 \mathrm{E}+01$ & $\mathrm{~m}$ & $3.94 \mathrm{E}+03$ & $2.78 \mathrm{E}+07$ & ICRP-72 & $2.60 \mathrm{E}-11$ & $M$ & $\operatorname{Rec}$ & FGR-12 & $3.68 \mathrm{E}-14$ \\
\hline Ag-104 & Ag-104 & 103.909 & Silver & $1.00 \mathrm{E}-03$ & ICRP-38 & $6.92 \mathrm{E}+01$ & $\mathrm{~m}$ & $4.15 \mathrm{E}+03$ & $2.61 \mathrm{E}+07$ & ICRP-72 & $3.60 \mathrm{E}-11$ & $M$ & $\operatorname{Rec}$ & FGR-12 & $1.32 \mathrm{E}-13$ \\
\hline Ag-104m & Ag-104 & 103.909 & Silver & $1.00 \mathrm{E}-03$ & ICRP-38 & $3.35 \mathrm{E}+01$ & $\mathrm{~m}$ & $2.01 \mathrm{E}+03$ & $5.40 \mathrm{E}+07$ & ICRP-72 & $2.50 \mathrm{E}-11$ & $M$ & $\operatorname{Rec}$ & FGR-12 & $5.82 \mathrm{E}-14$ \\
\hline Ag-105 & Ag-105 & 104.907 & Silver & $1.00 \mathrm{E}-03$ & ICRP-38 & $4.10 \mathrm{E}+01$ & d & $3.54 \mathrm{E}+06$ & $3.04 \mathrm{E}+04$ & ICRP-72 & $7.30 \mathrm{E}-10$ & $M$ & $\operatorname{Rec}$ & FGR-12 & $2.45 \mathrm{E}-14$ \\
\hline Ag-105m & Ag-105 & 104.907 & Silver & $1.00 \mathrm{E}-03$ & ICRP-107 & $7.23 \mathrm{E}+00$ & $\mathrm{~m}$ & $4.34 \mathrm{E}+02$ & $2.48 \mathrm{E}+08$ & -- & -- & -- & -- & DOE-STD-1196 & $4.42 \mathrm{E}-17$ \\
\hline Ag-106 & Ag-106 & 105.907 & Silver & $1.00 \mathrm{E}-03$ & ICRP-38 & $2.40 \mathrm{E}+01$ & $\mathrm{~m}$ & $1.44 \mathrm{E}+03$ & $7.41 \mathrm{E}+07$ & ICRP-72 & $1.50 \mathrm{E}-11$ & $M$ & $\operatorname{Rec}$ & FGR-12 & $3.39 \mathrm{E}-14$ \\
\hline Ag-106m & Ag-106 & 105.907 & Silver & $1.00 \mathrm{E}-03$ & ICRP-38 & $8.41 \mathrm{E}+00$ & $d$ & $7.27 E+05$ & $1.47 \mathrm{E}+05$ & ICRP-72 & $1.10 \mathrm{E}-09$ & $M$ & $\operatorname{Rec}$ & FGR-12 & $1.38 \mathrm{E}-13$ \\
\hline Ag-108 & Ag-108 & 107.906 & Silver & $1.00 \mathrm{E}-03$ & ICRP-38 & $2.37 \mathrm{E}+00$ & $\mathrm{~m}$ & $1.42 \mathrm{E}+02$ & $7.35 \mathrm{E}+08$ & -- & -- & -- & -- & FGR-12 & $9.28 \mathrm{E}-16$ \\
\hline Ag-108m & Ag-108 & 107.906 & Silver & $1.00 \mathrm{E}-03$ & ICRP-38 & $1.27 E+02$ & $y$ & $4.01 \mathrm{E}+09$ & $2.61 \mathrm{E}+01$ & ICRP-72 & $7.40 \mathrm{E}-09$ & $M$ & $\operatorname{Rec}$ & FGR-12 & $7.80 \mathrm{E}-14$ \\
\hline Ag-109m & Ag-109 & 108.905 & Silver & $1.00 \mathrm{E}-03$ & ICRP-38 & $3.96 \mathrm{E}+01$ & $\mathrm{~s}$ & $3.96 \mathrm{E}+01$ & $2.62 \mathrm{E}+09$ & -- & -- & -- & -- & FGR-12 & $1.92 \mathrm{E}-16$ \\
\hline Ag-110 & Ag-110 & 109.906 & Silver & $1.00 \mathrm{E}-03$ & ICRP-38 & $2.46 \mathrm{E}+01$ & $\mathrm{~s}$ & $2.46 \mathrm{E}+01$ & $4.17 \mathrm{E}+09$ & -- & -- & -- & -- & FGR-12 & $1.78 \mathrm{E}-15$ \\
\hline $\mathrm{Ag}-110 \mathrm{~m}$ & Ag-110 & 109.906 & Silver & $1.00 \mathrm{E}-03$ & ICRP-38 & $2.50 \mathrm{E}+02$ & $d$ & $2.16 \mathrm{E}+07$ & $4.75 \mathrm{E}+03$ & ICRP-72 & $7.60 \mathrm{E}-09$ & $M$ & $\operatorname{Rec}$ & FGR-12 & $1.36 \mathrm{E}-13$ \\
\hline Ag-111 & Ag-111 & 110.905 & Silver & $1.00 \mathrm{E}-03$ & ICRP-38 & $7.45 E+00$ & $d$ & $6.44 E+05$ & $1.58 \mathrm{E}+05$ & ICRP-72 & $1.50 \mathrm{E}-09$ & $M$ & $\operatorname{Rec}$ & FGR-12 & $1.29 \mathrm{E}-15$ \\
\hline Ag-111m & Ag-111 & 110.905 & Silver & $1.00 \mathrm{E}-03$ & ICRP-107 & $6.48 \mathrm{E}+01$ & $\mathrm{~s}$ & $6.48 \mathrm{E}+01$ & $1.57 \mathrm{E}+09$ & -- & -- & -- & -- & DOE-STD-1196 & $1.68 \mathrm{E}-16$ \\
\hline $\mathrm{Ag}-112$ & Ag-112 & 111.907 & Silver & $1.00 \mathrm{E}-03$ & ICRP-38 & $3.12 \mathrm{E}+00$ & $\mathrm{~h}$ & $1.12 \mathrm{E}+04$ & $8.98 \mathrm{E}+06$ & ICRP-72 & $1.60 \mathrm{E}-10$ & $M$ & $\operatorname{Rec}$ & FGR-12 & $3.34 \mathrm{E}-14$ \\
\hline Ag-113 & $\mathrm{Ag}-113$ & 112.907 & Silver & $1.00 \mathrm{E}-03$ & ICRP-107 & $5.37 \mathrm{E}+00$ & $\mathrm{~h}$ & $1.93 \mathrm{E}+04$ & $5.17 \mathrm{E}+06$ & DOE-STD-1196 & $1.83 \mathrm{E}-10$ & $M$ & Rec & DOE-STD-1196 & $3.83 \mathrm{E}-15$ \\
\hline Ag-113m & Ag-113 & 112.907 & Silver & $1.00 \mathrm{E}-03$ & ICRP-107 & $6.87 \mathrm{E}+01$ & $\mathrm{~s}$ & $6.87 \mathrm{E}+01$ & $1.45 \mathrm{E}+09$ & -- & -- & -- & -- & DOE-STD-1196 & $9.55 \mathrm{E}-15$ \\
\hline Ag-114 & Ag-114 & 113.909 & Silver & $1.00 \mathrm{E}-03$ & ICRP-107 & $4.60 \mathrm{E}+00$ & $\mathrm{~s}$ & $4.60 \mathrm{E}+00$ & $2.15 \mathrm{E}+10$ & -- & -- & -- & -- & DOE-STD-1196 & $1.46 E-14$ \\
\hline Ag-115 & Ag-115 & 114.909 & Silver & $1.00 \mathrm{E}-03$ & ICRP-38 & $2.00 \mathrm{E}+01$ & $\mathrm{~m}$ & $1.20 \mathrm{E}+03$ & $8.18 \mathrm{E}+07$ & ICRP-72 & $2.70 \mathrm{E}-11$ & $M$ & Rec & FGR-12 & $3.61 \mathrm{E}-14$ \\
\hline Ag-116 & Ag-116 & 115.911 & Silver & $1.00 \mathrm{E}-03$ & ICRP-107 & $2.68 \mathrm{E}+00$ & $\mathrm{~m}$ & $1.61 \mathrm{E}+02$ & $6.05 \mathrm{E}+08$ & -- & -- & -- & -- & DOE-STD-1196 & $1.07 \mathrm{E}-13$ \\
\hline Ag-117 & Ag-117 & 116.912 & Silver & $1.00 \mathrm{E}-03$ & ICRP-107 & $7.36 \mathrm{E}+01$ & $\mathrm{~s}$ & $7.36 \mathrm{E}+01$ & $1.31 \mathrm{E}+09$ & -- & -- & -- & -- & DOE-STD-1196 & $6.50 \mathrm{E}-14$ \\
\hline Ag-99 & Ag-99 & 98.918 & Silver & $1.00 \mathrm{E}-03$ & ICRP-107 & $1.24 \mathrm{E}+02$ & $\mathrm{~s}$ & $1.24 \mathrm{E}+02$ & $9.20 \mathrm{E}+08$ & -- & -- & -- & -- & DOE-STD-1196 & $1.08 \mathrm{E}-13$ \\
\hline Al-26 & Al-26 & 25.987 & Aluminum & $1.00 \mathrm{E}-03$ & ICRP-38 & $7.16 \mathrm{E}+05$ & $y$ & $2.26 \mathrm{E}+13$ & $1.92 \mathrm{E}-02$ & ICRP-72 & $2.00 \mathrm{E}-08$ & $M$ & $\operatorname{Max}$ & FGR-12 & $1.36 \mathrm{E}-13$ \\
\hline Al-28 & Al-28 & 27.982 & Aluminum & $1.00 \mathrm{E}-03$ & ICRP-38 & $2.24 \mathrm{E}+00$ & $\mathrm{~m}$ & $1.34 \mathrm{E}+02$ & $3.00 \mathrm{E}+09$ & -- & -- & -- & -- & FGR-12 & $9.28 \mathrm{E}-14$ \\
\hline $\mathrm{Al}-29$ & $\mathrm{Al}-29$ & 28.980 & Aluminum & $1.00 \mathrm{E}-03$ & ICRP-107 & $6.56 \mathrm{E}+00$ & $\mathrm{~m}$ & $3.94 \mathrm{E}+02$ & $9.89 \mathrm{E}+08$ & -- & -- & -- & -- & DOE-STD-1196 & $6.71 \mathrm{E}-14$ \\
\hline Am-237 & Am-237 & 237.050 & Americium & $1.00 \mathrm{E}-03$ & ICRP-38 & $7.30 \mathrm{E}+01$ & $\mathrm{~m}$ & $4.38 \mathrm{E}+03$ & $1.09 \mathrm{E}+07$ & ICRP-72 & $2.50 \mathrm{E}-11$ & $\mathrm{M}$ & Rec & FGR-12 & $1.70 \mathrm{E}-14$ \\
\hline Am-238 & Am-238 & 238.052 & Americium & $1.00 \mathrm{E}-03$ & ICRP-38 & $9.80 \mathrm{E}+01$ & $\mathrm{~m}$ & $5.88 \mathrm{E}+03$ & $8.06 \mathrm{E}+06$ & ICRP-72 & $9.00 \mathrm{E}-11$ & $M$ & $\operatorname{Rec}$ & FGR-12 & $4.33 \mathrm{E}-14$ \\
\hline Am-239 & Am-239 & 239.053 & Americium & $1.00 \mathrm{E}-03$ & ICRP-38 & $1.19 \mathrm{E}+01$ & $\mathrm{~h}$ & $4.28 \mathrm{E}+04$ & $1.10 \mathrm{E}+06$ & ICRP-72 & $2.20 \mathrm{E}-10$ & $M$ & $\operatorname{Rec}$ & FGR-12 & $1.04 \mathrm{E}-14$ \\
\hline Am-240 & Am-240 & 240.055 & Americium & $1.00 \mathrm{E}-03$ & ICRP-38 & $5.08 \mathrm{E}+01$ & $\mathrm{~h}$ & $1.83 \mathrm{E}+05$ & $2.57 \mathrm{E}+05$ & ICRP-72 & $4.30 \mathrm{E}-10$ & $M$ & Rec & FGR-12 & $5.00 \mathrm{E}-14$ \\
\hline
\end{tabular}




\begin{tabular}{|c|c|c|c|c|c|c|c|c|c|c|c|c|c|c|c|}
\hline $\begin{array}{l}\text { MASTER } \\
\text { ISOTOPE } \\
\text { LIST }\end{array}$ & $\begin{array}{l}\text { Atomic } \\
\text { Mass } \\
\text { Nuclide }\end{array}$ & $\begin{array}{l}\text { Atomic } \\
\text { Mass }\end{array}$ & Element & $\begin{array}{c}\text { RF per } \\
\text { DOE-STD- } \\
1027\end{array}$ & $\begin{array}{l}\text { Half-Life } \\
\text { Reference }\end{array}$ & $\begin{array}{r}\text { Half-Li } \\
\text { (original u }\end{array}$ & & Half Life (s) & $\begin{array}{l}\text { Sp. Act } \\
\text { (Ci/g) }\end{array}$ & $\begin{array}{l}\text { Inhalation DC } \\
\text { Reference }\end{array}$ & $\begin{array}{c}\mathrm{HC}-2 \\
\text { Inhalation } \\
\mathrm{DC} \\
(\mathrm{Sv} / \mathrm{Bq})\end{array}$ & & $\begin{array}{l}\text { ng } \\
\text { ption } \\
\text { Type }\end{array}$ & $\begin{array}{l}\text { Immersion DC } \\
\text { Reference }\end{array}$ & $\begin{array}{c}\mathrm{HC}-2 \\
\text { Immersion } \\
\mathrm{DC}(\mathrm{Sv} / \mathrm{s} \\
\left.\text { per } \mathrm{Bq} / \mathrm{m}^{3}\right)\end{array}$ \\
\hline $\mathrm{Am}-241$ & Am-241 & 241.057 & Americium & $1.00 \mathrm{E}-03$ & ICRP-38 & $4.32 E+02$ & $y$ & $1.36 \mathrm{E}+10$ & $3.43 \mathrm{E}+00$ & ICRP-72 & $4.20 \mathrm{E}-05$ & $\mathrm{M}$ & $\operatorname{Rec}$ & FGR-12 & $8.18 \mathrm{E}-16$ \\
\hline Am-242 & Am-242 & 242.060 & Americium & $1.00 \mathrm{E}-03$ & ICRP-38 & $1.60 E+01$ & $\mathrm{~h}$ & $5.77 \mathrm{E}+04$ & $8.08 \mathrm{E}+05$ & ICRP-72 & $1.70 \mathrm{E}-08$ & $\mathrm{M}$ & $\operatorname{Rec}$ & FGR-12 & $6.15 \mathrm{E}-16$ \\
\hline$A m-242 m$ & Am-242 & 242.060 & Americium & $1.00 \mathrm{E}-03$ & ICRP-38 & $1.52 \mathrm{E}+02$ & $y$ & $4.80 \mathrm{E}+09$ & $9.72 \mathrm{E}+00$ & ICRP-72 & $3.70 \mathrm{E}-05$ & $M$ & Rec & FGR-12 & $3.17 \mathrm{E}-17$ \\
\hline$A m-243$ & Am-243 & 243.061 & Americium & $1.00 \mathrm{E}-03$ & ICRP-38 & $7.38 \mathrm{E}+03$ & $y$ & $2.33 \mathrm{E}+11$ & $1.99 \mathrm{E}-01$ & ICRP-72 & $4.10 \mathrm{E}-05$ & $M$ & Rec & FGR-12 & $2.18 \mathrm{E}-15$ \\
\hline Am-244 & Am-244 & 244.064 & Americium & $1.00 \mathrm{E}-03$ & ICRP-38 & $1.01 \mathrm{E}+01$ & $\mathrm{~h}$ & $3.64 \mathrm{E}+04$ & $1.27 \mathrm{E}+06$ & ICRP-72 & $2.00 \mathrm{E}-09$ & $M$ & Rec & FGR-12 & $3.85 \mathrm{E}-14$ \\
\hline$A m-244 m$ & Am-244 & 244.064 & Americium & $1.00 \mathrm{E}-03$ & ICRP-38 & $2.60 \mathrm{E}+01$ & $\mathrm{~m}$ & $1.56 \mathrm{E}+03$ & $2.96 \mathrm{E}+07$ & ICRP-72 & $8.40 \mathrm{E}-11$ & $M$ & $\operatorname{Rec}$ & FGR-12 & $6.13 \mathrm{E}-17$ \\
\hline Am-245 & Am-245 & 245.066 & Americium & $1.00 \mathrm{E}-03$ & ICRP-38 & $2.05 \mathrm{E}+00$ & $\mathrm{~h}$ & $7.38 \mathrm{E}+03$ & $6.24 \mathrm{E}+06$ & ICRP-72 & $5.30 \mathrm{E}-11$ & $M$ & Rec & FGR-12 & $1.46 \mathrm{E}-15$ \\
\hline Am-246 & Am-246 & 246.070 & Americium & $1.00 \mathrm{E}-03$ & ICRP-38 & $3.90 \mathrm{E}+01$ & $\mathrm{~m}$ & $2.34 \mathrm{E}+03$ & $1.96 \mathrm{E}+07$ & ICRP-72 & $6.60 \mathrm{E}-11$ & $M$ & Rec & FGR-12 & $3.28 \mathrm{E}-14$ \\
\hline$A m-246 m$ & Am-246 & 246.070 & Americium & $1.00 \mathrm{E}-03$ & ICRP-38 & $2.50 \mathrm{E}+01$ & $\mathrm{~m}$ & $1.50 \mathrm{E}+03$ & $3.06 \mathrm{E}+07$ & ICRP-72 & $2.20 \mathrm{E}-11$ & $M$ & Rec & FGR-12 & $5.03 \mathrm{E}-14$ \\
\hline Am-247 & Am-247 & 247.072 & Americium & $1.00 \mathrm{E}-03$ & ICRP-107 & $2.30 \mathrm{E}+01$ & $\mathrm{~m}$ & $1.38 \mathrm{E}+03$ & $3.31 \mathrm{E}+07$ & DOE-STD-1196 & $3.02 \mathrm{E}-11$ & $M$ & Rec & DOE-STD-1196 & $5.85 \mathrm{E}-15$ \\
\hline Ar-37 & Ar-37 & 36.967 & Argon & $1.00 \mathrm{E}+00$ & ICRP-38 & $3.50 \mathrm{E}+01$ & $d$ & $3.03 E+06$ & $1.01 \mathrm{E}+05$ & -- & -- & -- & -- & FGR-12 & $1.27 \mathrm{E}-19$ \\
\hline Ar-39 & Ar-39 & 38.964 & Argon & $1.00 \mathrm{E}+00$ & ICRP-38 & $2.69 \mathrm{E}+02$ & $y$ & $8.49 \mathrm{E}+09$ & $3.41 \mathrm{E}+01$ & -- & -- & -- & -- & FGR-12 & $9.10 \mathrm{E}-18$ \\
\hline Ar-41 & Ar-41 & 40.965 & Argon & $1.00 \mathrm{E}+00$ & ICRP-38 & $1.83 \mathrm{E}+00$ & $\mathrm{~h}$ & $6.58 \mathrm{E}+03$ & $4.19 \mathrm{E}+07$ & -- & -- & -- & -- & FGR-12 & $6.50 \mathrm{E}-14$ \\
\hline Ar-42 & Ar-42 & 41.963 & Argon & $1.00 \mathrm{E}+00$ & ICRP-107 & $3.29 \mathrm{E}+01$ & $y$ & $1.04 \mathrm{E}+09$ & $2.59 \mathrm{E}+02$ & -- & -- & -- & -- & DOE-STD-1196 & $1.26 \mathrm{E}-16$ \\
\hline Ar-43 & Ar-43 & 42.966 & Argon & $1.00 \mathrm{E}+00$ & ICRP-107 & $5.37 \mathrm{E}+00$ & $\mathrm{~m}$ & $3.22 \mathrm{E}+02$ & $8.15 \mathrm{E}+08$ & -- & -- & -- & -- & DOE-STD-1196 & $7.55 \mathrm{E}-14$ \\
\hline Ar-44 & Ar-44 & 43.965 & Argon & $1.00 \mathrm{E}+00$ & ICRP-107 & $1.19 \mathrm{E}+01$ & $\mathrm{~m}$ & $7.12 \mathrm{E}+02$ & $3.60 \mathrm{E}+08$ & -- & -- & -- & -- & DOE-STD-1196 & $9.46 \mathrm{E}-14$ \\
\hline As-68 & As-68 & 67.937 & Arsenic & $1.00 \mathrm{E}-03$ & ICRP-107 & $1.52 \mathrm{E}+02$ & $\mathrm{~s}$ & $1.52 \mathrm{E}+02$ & $1.10 \mathrm{E}+09$ & -- & -- & -- & -- & DOE-STD-1196 & $1.76 \mathrm{E}-13$ \\
\hline As-69 & As-69 & 68.932 & Arsenic & $1.00 \mathrm{E}-03$ & ICRP-38 & $1.52 \mathrm{E}+01$ & $\mathrm{~m}$ & $9.12 \mathrm{E}+02$ & $1.79 \mathrm{E}+08$ & ICRP-72 & $2.10 \mathrm{E}-11$ & $M$ & Max & FGR-12 & $4.89 \mathrm{E}-14$ \\
\hline As-70 & As-70 & 69.931 & Arsenic & $1.00 \mathrm{E}-03$ & ICRP-38 & $5.26 \mathrm{E}+01$ & $\mathrm{~m}$ & $3.16 \mathrm{E}+03$ & $5.11 \mathrm{E}+07$ & ICRP-72 & $6.70 \mathrm{E}-11$ & $M$ & Max & FGR-12 & $2.04 \mathrm{E}-13$ \\
\hline As-71 & As-71 & 70.927 & Arsenic & $1.00 \mathrm{E}-03$ & ICRP-38 & $6.48 \mathrm{E}+01$ & $\mathrm{~h}$ & $2.33 \mathrm{E}+05$ & $6.82 \mathrm{E}+05$ & ICRP-72 & $4.00 \mathrm{E}-10$ & $M$ & Max & FGR-12 & $2.74 \mathrm{E}-14$ \\
\hline As-72 & As-72 & 71.927 & Arsenic & $1.00 \mathrm{E}-03$ & ICRP-38 & $2.60 \mathrm{E}+01$ & $\mathrm{~h}$ & $9.36 \mathrm{E}+04$ & $1.68 \mathrm{E}+06$ & ICRP-72 & $9.00 \mathrm{E}-10$ & $M$ & Max & FGR-12 & $8.78 \mathrm{E}-14$ \\
\hline As-73 & As-73 & 72.924 & Arsenic & $1.00 \mathrm{E}-03$ & ICRP-38 & $8.03 \mathrm{E}+01$ & $\mathrm{~d}$ & $6.94 \mathrm{E}+06$ & $2.23 \mathrm{E}+04$ & ICRP-72 & $1.00 \mathrm{E}-09$ & $M$ & Max & FGR-12 & $1.90 \mathrm{E}-16$ \\
\hline As-74 & As-74 & 73.924 & Arsenic & $1.00 \mathrm{E}-03$ & ICRP-38 & $1.78 \mathrm{E}+01$ & $\mathrm{~d}$ & $1.53 \mathrm{E}+06$ & $9.95 \mathrm{E}+04$ & ICRP-72 & $2.10 \mathrm{E}-09$ & $M$ & Max & FGR-12 & $3.65 \mathrm{E}-14$ \\
\hline As-76 & As-76 & 75.922 & Arsenic & $1.00 \mathrm{E}-03$ & ICRP-38 & $2.63 \mathrm{E}+01$ & $\mathrm{~h}$ & $9.48 \mathrm{E}+04$ & $1.57 \mathrm{E}+06$ & ICRP-72 & $7.40 \mathrm{E}-10$ & $\mathrm{M}$ & Max & FGR-12 & $2.13 \mathrm{E}-14$ \\
\hline As-77 & As-77 & 76.921 & Arsenic & $1.00 \mathrm{E}-03$ & ICRP-38 & $3.88 \mathrm{E}+01$ & $\mathrm{~h}$ & $1.40 \mathrm{E}+05$ & $1.05 \mathrm{E}+06$ & ICRP-72 & $3.90 \mathrm{E}-10$ & $M$ & Max & FGR-12 & $4.31 \mathrm{E}-16$ \\
\hline As-78 & As-78 & 77.922 & Arsenic & $1.00 \mathrm{E}-03$ & ICRP-38 & $9.07 \mathrm{E}+01$ & $\mathrm{~m}$ & $5.44 \mathrm{E}+03$ & $2.66 \mathrm{E}+07$ & ICRP-72 & $8.90 \mathrm{E}-11$ & $M$ & Max & FGR-12 & $6.32 \mathrm{E}-14$ \\
\hline As-79 & As-79 & 78.921 & Arsenic & $1.00 \mathrm{E}-03$ & ICRP-107 & $9.01 \mathrm{E}+00$ & $\mathrm{~m}$ & $5.41 \mathrm{E}+02$ & $2.64 \mathrm{E}+08$ & -- & -- & -- & $\begin{array}{ll}- \\
\end{array}$ & DOE-STD-1196 & $2.25 \mathrm{E}-15$ \\
\hline At-204 & At-204 & 203.987 & Astatine & $1.00 \mathrm{E}-03$ & ICRP-107 & $9.20 \mathrm{E}+00$ & $\mathrm{~m}$ & $5.52 \mathrm{E}+02$ & $1.00 \mathrm{E}+08$ & $\begin{array}{l}-- \\
\end{array}$ & -- & -- & -- & DOE-STD-1196 & $1.04 \mathrm{E}-13$ \\
\hline At-205 & At-205 & 204.986 & Astatine & $1.00 \mathrm{E}-03$ & ICRP-107 & $2.62 \mathrm{E}+01$ & $\mathrm{~m}$ & $1.57 \mathrm{E}+03$ & $3.50 \mathrm{E}+07$ & DOE-STD-1196 & $7.83 \mathrm{E}-10$ & $\mathrm{~s}$ & Max & DOE-STD-1196 & $5.22 \mathrm{E}-14$ \\
\hline At-206 & At-206 & 205.987 & Astatine & $1.00 \mathrm{E}-03$ & ICRP-107 & $3.06 \mathrm{E}+01$ & $\mathrm{~m}$ & $1.84 \mathrm{E}+03$ & $2.98 \mathrm{E}+07$ & DOE-STD-1196 & $2.62 \mathrm{E}-10$ & $\mathrm{~s}$ & Max & DOE-STD-1196 & $1.12 \mathrm{E}-13$ \\
\hline At-207 & At-207 & 206.986 & Astatine & $1.00 \mathrm{E}-03$ & ICRP-38 & $1.80 \mathrm{E}+00$ & $\mathrm{~h}$ & $6.48 \mathrm{E}+03$ & $8.41 \mathrm{E}+06$ & ICRP-72 & $2.30 \mathrm{E}-09$ & $\mathrm{M}$ & Max & FGR-12 & $6.52 \mathrm{E}-14$ \\
\hline At-208 & At-208 & 207.987 & Astatine & $1.00 \mathrm{E}-03$ & ICRP-107 & $1.63 \mathrm{E}+00$ & $\mathrm{~h}$ & $5.87 \mathrm{E}+03$ & $9.24 \mathrm{E}+06$ & DOE-STD-1196 & $6.56 \mathrm{E}-10$ & $\mathrm{~s}$ & Max & DOE-STD-1196 & $1.40 \mathrm{E}-13$ \\
\hline At-209 & At-209 & 208.986 & Astatine & $1.00 \mathrm{E}-03$ & ICRP-107 & $5.41 \mathrm{E}+00$ & $\mathrm{~h}$ & $1.95 \mathrm{E}+04$ & $2.77 \mathrm{E}+06$ & DOE-STD-1196 & $3.04 \mathrm{E}-09$ & $\mathrm{~s}$ & Max & DOE-STD-1196 & $1.03 \mathrm{E}-13$ \\
\hline At-210 & At-210 & 209.987 & Astatine & $1.00 \mathrm{E}-03$ & ICRP-107 & $8.10 E+00$ & $\mathrm{~h}$ & $2.92 \mathrm{E}+04$ & $1.84 \mathrm{E}+06$ & DOE-STD-1196 & 1.17E-08 & $\mathrm{s}$ & Max & DOE-STD-1196 & $1.40 \mathrm{E}-13$ \\
\hline At-211 & At-211 & 210.987 & Astatine & $1.00 \mathrm{E}-03$ & ICRP-38 & $7.21 \mathrm{E}+00$ & $\mathrm{~h}$ & $2.60 \mathrm{E}+04$ & $2.06 \mathrm{E}+06$ & ICRP-72 & $1.10 \mathrm{E}-07$ & $\mathrm{M}$ & Max & FGR-12 & $1.59 \mathrm{E}-15$ \\
\hline At-215 & At-215 & 214.999 & Astatine & $1.00 \mathrm{E}-03$ & ICRP-38 & $1.00 \mathrm{E}-01$ & $\mathrm{~ms}$ & $1.00 \mathrm{E}-04$ & $5.25 \mathrm{E}+14$ & -- & -- & -- & -- & FGR-12 & $9.22 \mathrm{E}-18$ \\
\hline At-216 & At-216 & 216.002 & Astatine & $1.00 \mathrm{E}-03$ & ICRP-38 & $3.00 \mathrm{E}-01$ & $\mathrm{~ms}$ & $3.00 \mathrm{E}-04$ & $1.74 \mathrm{E}+14$ & -- & -- & -- & -- & FGR-12 & $6.24 \mathrm{E}-17$ \\
\hline At-217 & At-217 & 217.005 & Astatine & $1.00 \mathrm{E}-03$ & ICRP-38 & $3.23 \mathrm{E}-02$ & $\mathrm{~s}$ & $3.23 \mathrm{E}-02$ & $1.61 \mathrm{E}+12$ & -- & -- & -- & -- & FGR-12 & $1.48 \mathrm{E}-17$ \\
\hline At-218 & At-218 & 218.009 & Astatine & $1.00 \mathrm{E}-03$ & ICRP-38 & $2.00 \mathrm{E}+00$ & $\mathrm{~s}$ & $2.00 \mathrm{E}+00$ & $2.59 \mathrm{E}+10$ & -- & -- & -- & -- & FGR-12 & $1.19 \mathrm{E}-16$ \\
\hline At-219 & At-219 & 219.011 & Astatine & $1.00 \mathrm{E}-03$ & ICRP-107 & $5.60 \mathrm{E}+01$ & $\mathrm{~s}$ & $5.60 \mathrm{E}+01$ & $9.20 \mathrm{E}+08$ & -- & -- & -- & -- & -- & -- \\
\hline At-220 & At-220 & 220.015 & Astatine & $1.00 \mathrm{E}-03$ & ICRP-107 & $3.71 \mathrm{E}+00$ & $\mathrm{~m}$ & $2.23 \mathrm{E}+02$ & $2.30 \mathrm{E}+08$ & -- & -- & -- & -- & DOE-STD-1196 & $2.09 \mathrm{E}-14$ \\
\hline $\mathrm{Au}-186$ & $\mathrm{Au}-186$ & 185.966 & Gold & $1.00 \mathrm{E}-03$ & ICRP-107 & $1.07 \mathrm{E}+01$ & $\mathrm{~m}$ & $6.42 \mathrm{E}+02$ & $9.45 \mathrm{E}+07$ & DOE-STD-1196 & $2.43 \mathrm{E}-11$ & $\mathrm{~s}$ & Max & DOE-STD-1196 & $6.87 \mathrm{E}-14$ \\
\hline $\mathrm{Au}-187$ & $\mathrm{Au}-187$ & 186.965 & Gold & $1.00 \mathrm{E}-03$ & ICRP-107 & $8.40 \mathrm{E}+00$ & $\mathrm{~m}$ & $5.04 \mathrm{E}+02$ & $1.20 \mathrm{E}+08$ & -- & -- & -- & -- & DOE-STD-1196 & $4.96 \mathrm{E}-14$ \\
\hline Au-188 & $\mathrm{Au}-188$ & 187.965 & Gold & $1.00 \mathrm{E}-03$ & JAERI & $8.84 \mathrm{E}+00$ & $\mathrm{~m}$ & $5.30 \mathrm{E}+02$ & $1.13 \mathrm{E}+08$ & -- & -- & -- & -- & -- & -- \\
\hline $\mathrm{Au}-189 \mathrm{~m}$ & Au-189 & 188.964 & Gold & $1.00 \mathrm{E}-03$ & JAERI & $4.59 \mathrm{E}+00$ & $\mathrm{~m}$ & $2.75 \mathrm{E}+02$ & $2.17 \mathrm{E}+08$ & -- & -- & -- & -- & -- & -- \\
\hline Au-190 & $\mathrm{Au}-190$ & 189.965 & Gold & $1.00 \mathrm{E}-03$ & ICRP-107 & $4.28 \mathrm{E}+01$ & $\mathrm{~m}$ & $2.57 \mathrm{E}+03$ & $2.31 \mathrm{E}+07$ & DOE-STD-1196 & $2.90 \mathrm{E}-11$ & $\mathrm{~s}$ & Max & DOE-STD-1196 & $1.16 \mathrm{E}-13$ \\
\hline
\end{tabular}




\begin{tabular}{|c|c|c|c|c|c|c|c|c|c|c|c|c|c|c|c|}
\hline \multicolumn{16}{|c|}{ Table B.1 Radionuclide Specific Input Data Used For the Calculation of HC-2 TQs Using Recommended DCs (continued) } \\
\hline $\begin{array}{l}\text { MASTER } \\
\text { ISOTOPE } \\
\text { LIST }\end{array}$ & $\begin{array}{c}\text { Atomic } \\
\text { Mass } \\
\text { Nuclide }\end{array}$ & $\begin{array}{l}\text { Atomic } \\
\text { Mass }\end{array}$ & Element & $\begin{array}{c}\text { RF per } \\
\text { DOE-STD- } \\
1027\end{array}$ & $\begin{array}{l}\text { Half-Life } \\
\text { Reference }\end{array}$ & \multicolumn{2}{|c|}{$\begin{array}{c}\text { Half-Life } \\
\text { (original units) }\end{array}$} & \multirow{2}{*}{$\begin{array}{c}\text { Half Life (s) } \\
1.14 E+04\end{array}$} & \multirow{2}{*}{$\begin{array}{c}\begin{array}{c}\text { Sp. Act } \\
\text { (Ci/g) }\end{array} \\
5.16 \mathrm{E}+06\end{array}$} & \multirow{2}{*}{$\begin{array}{c}\begin{array}{c}\text { Inhalation DC } \\
\text { Reference }\end{array} \\
\text { DOE-STD-1196 }\end{array}$} & \multirow[t]{2}{*}{$\begin{array}{c}\text { HC-2 } \\
\text { Inhalation } \\
\text { DC } \\
\text { (Sv/Bq) } \\
7.56 E-11 \\
\end{array}$} & \multicolumn{2}{|c|}{$\begin{array}{c}\text { Lung } \\
\text { Absorption } \\
\text { Class/Type }\end{array}$} & \multirow{2}{*}{$\begin{array}{c}\begin{array}{c}\text { Immersion DC } \\
\text { Reference }\end{array} \\
\text { DOE-STD-1196 }\end{array}$} & \multirow{2}{*}{$\begin{array}{c}\text { HC-2 } \\
\begin{array}{c}\text { Immersion } \\
\text { DC (Sv/s }\end{array} \\
\left.\text { per Bq/m } / \mathrm{m}^{3}\right) \\
2.55 \mathrm{E}-14 \\
\end{array}$} \\
\hline Au-191 & Au-191 & 190.964 & Gold & $1.00 \mathrm{E}-03$ & ICRP-107 & $3.18 \mathrm{E}+00$ & $\mathrm{~h}$ & & & & & $\mathrm{~s}$ & Max & & \\
\hline Au-192 & Au-192 & 191.965 & Gold & $1.00 \mathrm{E}-03$ & ICRP-107 & $4.94 \mathrm{E}+00$ & $\mathrm{~h}$ & $1.78 \mathrm{E}+04$ & $3.30 \mathrm{E}+06$ & DOE-STD-1196 & $1.15 \mathrm{E}-10$ & $\mathrm{~s}$ & Max & DOE-STD-1196 & $9.29 \mathrm{E}-14$ \\
\hline Au-193 & Au-193 & 192.964 & Gold & $1.00 \mathrm{E}-03$ & ICRP-38 & $1.77 \mathrm{E}+01$ & $\mathrm{~h}$ & $6.35 \mathrm{E}+04$ & $9.20 \mathrm{E}+05$ & ICRP-72 & $1.20 \mathrm{E}-10$ & $\mathrm{~s}$ & Max & FGR-12 & $6.83 \mathrm{E}-15$ \\
\hline Au-193m & Au-193 & 192.964 & Gold & $1.00 \mathrm{E}-03$ & ICRP-107 & $3.90 \mathrm{E}+00$ & $\mathrm{~s}$ & $3.90 \mathrm{E}+00$ & $1.50 \mathrm{E}+10$ & -- & -- & -- & -- & DOE-STD-1196 & $8.37 \mathrm{E}-15$ \\
\hline Au-194 & Au-194 & 193.965 & Gold & $1.00 \mathrm{E}-03$ & ICRP-38 & $3.95 \mathrm{E}+01$ & $\mathrm{~h}$ & $1.42 \mathrm{E}+05$ & $4.09 \mathrm{E}+05$ & ICRP-72 & $2.40 \mathrm{E}-10$ & $\mathrm{~s}$ & Max & FGR-12 & $5.29 \mathrm{E}-14$ \\
\hline $\mathrm{Au}-195$ & Au-195 & 194.965 & Gold & $1.00 \mathrm{E}-03$ & ICRP-38 & $1.83 \mathrm{E}+02$ & $d$ & $1.58 \mathrm{E}+07$ & $3.66 \mathrm{E}+03$ & ICRP-72 & $1.70 \mathrm{E}-09$ & $\mathrm{~s}$ & Max & FGR-12 & $3.21 \mathrm{E}-15$ \\
\hline Au-195m & Au-195 & 194.965 & Gold & $1.00 \mathrm{E}-03$ & ICRP-38 & $3.05 \mathrm{E}+01$ & $\mathrm{~s}$ & $3.05 \mathrm{E}+01$ & $1.90 \mathrm{E}+09$ & -- & -- & -- & -- & FGR-12 & $9.37 \mathrm{E}-15$ \\
\hline Au-196 & Au-196 & 195.967 & Gold & $1.00 \mathrm{E}-03$ & ICRP-107 & $6.18 \mathrm{E}+00$ & $d$ & $5.34 \mathrm{E}+05$ & $1.08 \mathrm{E}+05$ & DOE-STD-1196 & $3.78 \mathrm{E}-10$ & $M$ & Max & DOE-STD-1196 & $2.02 \mathrm{E}-14$ \\
\hline Au-196m & Au-196 & 195.967 & Gold & $1.00 \mathrm{E}-03$ & ICRP-107 & $9.60 \mathrm{E}+00$ & $\mathrm{~h}$ & $3.46 \mathrm{E}+04$ & $1.67 \mathrm{E}+06$ & DOE-STD-1196 & $5.00 \mathrm{E}-10$ & $\mathrm{~s}$ & Max & DOE-STD-1196 & $9.70 \mathrm{E}-15$ \\
\hline Au-198 & Au-198 & 197.968 & Gold & $1.00 \mathrm{E}-03$ & ICRP-38 & $2.70 E+00$ & $d$ & $2.33 \mathrm{E}+05$ & $2.45 \mathrm{E}+05$ & ICRP-72 & $8.60 \mathrm{E}-10$ & $\mathrm{~s}$ & Max & FGR-12 & $1.94 \mathrm{E}-14$ \\
\hline Au-198m & Au-198 & 197.968 & Gold & $1.00 \mathrm{E}-03$ & ICRP-38 & $2.30 \mathrm{E}+00$ & $d$ & $1.99 \mathrm{E}+05$ & $2.87 \mathrm{E}+05$ & ICRP-72 & $2.00 \mathrm{E}-09$ & $\mathrm{~s}$ & Max & FGR-12 & $2.66 \mathrm{E}-14$ \\
\hline Au-199 & Au-199 & 198.969 & Gold & $1.00 \mathrm{E}-03$ & ICRP-38 & $3.14 \mathrm{E}+00$ & $d$ & $2.71 \mathrm{E}+05$ & $2.09 \mathrm{E}+05$ & ICRP-72 & $7.90 \mathrm{E}-10$ & $\mathrm{~s}$ & Max & FGR-12 & $4.08 \mathrm{E}-15$ \\
\hline $\mathrm{Au}-200$ & Au-200 & 199.971 & Gold & $1.00 \mathrm{E}-03$ & ICRP-38 & $4.84 \mathrm{E}+01$ & $\mathrm{~m}$ & $2.90 \mathrm{E}+03$ & $1.94 \mathrm{E}+07$ & ICRP-72 & $3.50 \mathrm{E}-11$ & $\mathrm{~s}$ & Max & FGR-12 & $1.37 \mathrm{E}-14$ \\
\hline $\mathrm{Au}-200 \mathrm{~m}$ & Au-200 & 199.971 & Gold & $1.00 \mathrm{E}-03$ & ICRP-38 & $1.87 \mathrm{E}+01$ & $\mathrm{~h}$ & $6.73 \mathrm{E}+04$ & $8.38 \mathrm{E}+05$ & ICRP-72 & $7.20 \mathrm{E}-10$ & $\mathrm{~s}$ & Max & FGR-12 & $1.01 \mathrm{E}-13$ \\
\hline Au-201 & Au-201 & 200.972 & Gold & $1.00 \mathrm{E}-03$ & ICRP-38 & $2.64 \mathrm{E}+01$ & $\mathrm{~m}$ & $1.58 \mathrm{E}+03$ & $3.54 \mathrm{E}+07$ & ICRP-72 & $1.70 \mathrm{E}-11$ & $M$ & Max & FGR-12 & $2.57 \mathrm{E}-15$ \\
\hline Au-202 & Au-202 & 201.974 & Gold & $1.00 \mathrm{E}-03$ & ICRP-107 & $2.88 \mathrm{E}+01$ & $\mathrm{~s}$ & $2.88 \mathrm{E}+01$ & $1.94 \mathrm{E}+09$ & -- & -- & -- & -- & DOE-STD-1196 & $8.90 \mathrm{E}-15$ \\
\hline Ba-124 & Ba-124 & 123.915 & Barium & $1.00 \mathrm{E}-03$ & ICRP-107 & $1.10 \mathrm{E}+01$ & $\mathrm{~m}$ & $6.60 \mathrm{E}+02$ & $1.38 \mathrm{E}+08$ & DOE-STD-1196 & $2.63 \mathrm{E}-11$ & $M$ & $\operatorname{Rec}$ & DOE-STD-1196 & $2.51 \mathrm{E}-14$ \\
\hline Ba-126 & Ba-126 & 125.911 & Barium & $1.00 \mathrm{E}-03$ & ICRP-38 & $9.65 \mathrm{E}+01$ & $\mathrm{~m}$ & $5.79 \mathrm{E}+03$ & $1.55 \mathrm{E}+07$ & ICRP-72 & $1.00 \mathrm{E}-10$ & $\mathrm{M}$ & Rec & FGR-12 & $7.03 \mathrm{E}-15$ \\
\hline Ba-127 & Ba-127 & 126.911 & Barium & $1.00 \mathrm{E}-03$ & ICRP-107 & $1.27 \mathrm{E}+01$ & $\mathrm{~m}$ & $7.62 \mathrm{E}+02$ & $1.17 \mathrm{E}+08$ & DOE-STD-1196 & $1.30 \mathrm{E}-11$ & $M$ & $\operatorname{Rec}$ & DOE-STD-1196 & $3.28 \mathrm{E}-14$ \\
\hline Ba-128 & Ba-128 & 127.908 & Barium & $1.00 \mathrm{E}-03$ & ICRP-38 & $2.43 \mathrm{E}+00$ & $d$ & $2.10 \mathrm{E}+05$ & $4.20 \mathrm{E}+05$ & ICRP-72 & $1.30 \mathrm{E}-09$ & $M$ & $\operatorname{Rec}$ & FGR-12 & $2.86 \mathrm{E}-15$ \\
\hline Ba-129 & Ba-129 & 128.909 & Barium & $1.00 \mathrm{E}-03$ & ICRP-107 & $2.23 \mathrm{E}+00$ & $\mathrm{~h}$ & $8.03 E+03$ & $1.09 \mathrm{E}+07$ & DOE-STD-1196 & $3.31 \mathrm{E}-11$ & $M$ & $\operatorname{Rec}$ & DOE-STD-1196 & $1.44 \mathrm{E}-14$ \\
\hline Ba-129m & Ba-129 & 128.909 & Barium & $1.00 \mathrm{E}-03$ & ICRP-107 & $2.16 \mathrm{E}+00$ & $\mathrm{~h}$ & $7.78 \mathrm{E}+03$ & $1.13 \mathrm{E}+07$ & DOE-STD-1196 & $5.44 \mathrm{E}-11$ & $M$ & $\operatorname{Rec}$ & DOE-STD-1196 & $7.20 \mathrm{E}-14$ \\
\hline Ba-131 & Ba-131 & 130.907 & Barium & $1.00 \mathrm{E}-03$ & ICRP-38 & $1.18 \mathrm{E}+01$ & $d$ & $1.02 \mathrm{E}+06$ & $8.45 \mathrm{E}+04$ & ICRP-72 & $7.60 \mathrm{E}-10$ & $M$ & $\operatorname{Rec}$ & FGR-12 & $2.10 \mathrm{E}-14$ \\
\hline Ba-131m & Ba-131 & 130.907 & Barium & $1.00 \mathrm{E}-03$ & ICRP-38 & $1.46 \mathrm{E}+01$ & $\mathrm{~m}$ & $8.76 \mathrm{E}+02$ & $9.84 \mathrm{E}+07$ & ICRP-72 & $7.40 \mathrm{E}-12$ & $M$ & $\operatorname{Rec}$ & FGR-12 & $3.04 \mathrm{E}-15$ \\
\hline Ba-133 & Ba-133 & 132.906 & Barium & $1.00 \mathrm{E}-03$ & ICRP-38 & $1.07 E+01$ & $y$ & $3.39 \mathrm{E}+08$ & $2.50 \mathrm{E}+02$ & ICRP-72 & $3.10 \mathrm{E}-09$ & $M$ & Rec & FGR-12 & $1.78 \mathrm{E}-14$ \\
\hline Ba-133m & Ba-133 & 132.906 & Barium & $1.00 \mathrm{E}-03$ & ICRP-38 & $3.89 \mathrm{E}+01$ & $\mathrm{~h}$ & $1.40 \mathrm{E}+05$ & $6.06 \mathrm{E}+05$ & ICRP-72 & $4.20 \mathrm{E}-10$ & $\mathrm{M}$ & $\operatorname{Rec}$ & FGR-12 & $2.62 \mathrm{E}-15$ \\
\hline Ba-135m & Ba-135 & 134.906 & Barium & $1.00 \mathrm{E}-03$ & ICRP-38 & $2.87 \mathrm{E}+01$ & $\mathrm{~h}$ & $1.03 \mathrm{E}+05$ & $8.09 \mathrm{E}+05$ & ICRP-72 & $3.30 \mathrm{E}-10$ & $M$ & $\operatorname{Rec}$ & FGR-12 & $2.32 \mathrm{E}-15$ \\
\hline Ba-137m & Ba-137 & 136.906 & Barium & $1.00 \mathrm{E}-03$ & ICRP-38 & $2.55 \mathrm{E}+00$ & $\mathrm{~m}$ & $1.53 \mathrm{E}+02$ & $5.38 \mathrm{E}+08$ & -- & -- & -- & -- & FGR-12 & $2.88 \mathrm{E}-14$ \\
\hline Ba-139 & Ba-139 & 138.909 & Barium & $1.00 \mathrm{E}-03$ & ICRP-38 & $8.27 \mathrm{E}+01$ & $\mathrm{~m}$ & $4.96 \mathrm{E}+03$ & $1.64 \mathrm{E}+07$ & ICRP-72 & $5.60 \mathrm{E}-11$ & $M$ & Rec & FGR-12 & $2.17 \mathrm{E}-15$ \\
\hline Ba-140 & Ba-140 & 139.911 & Barium & $1.00 \mathrm{E}-03$ & ICRP-38 & $1.27 \mathrm{E}+01$ & $d$ & $1.10 \mathrm{E}+06$ & $7.33 \mathrm{E}+04$ & ICRP-72 & $5.10 \mathrm{E}-09$ & $M$ & $\operatorname{Rec}$ & FGR-12 & $8.58 \mathrm{E}-15$ \\
\hline Ba-141 & Ba-141 & 140.914 & Barium & $1.00 \mathrm{E}-03$ & ICRP-38 & $1.83 \mathrm{E}+01$ & $\mathrm{~m}$ & $1.10 \mathrm{E}+03$ & $7.30 \mathrm{E}+07$ & ICRP-72 & $3.20 \mathrm{E}-11$ & $M$ & $\operatorname{Rec}$ & FGR-12 & $4.16 \mathrm{E}-14$ \\
\hline Ba-142 & Ba-142 & 141.916 & Barium & $1.00 \mathrm{E}-03$ & ICRP-38 & $1.06 \mathrm{E}+01$ & $\mathrm{~m}$ & $6.36 \mathrm{E}+02$ & $1.25 \mathrm{E}+08$ & ICRP-72 & $2.10 \mathrm{E}-11$ & $M$ & $\operatorname{Rec}$ & FGR-12 & $5.15 \mathrm{E}-14$ \\
\hline Be-10 & Be-10 & 10.014 & Beryllium & $1.00 \mathrm{E}-03$ & ICRP-38 & $1.60 \mathrm{E}+06$ & $y$ & $5.05 \mathrm{E}+13$ & $2.23 \mathrm{E}-02$ & ICRP-72 & $3.50 \mathrm{E}-08$ & $\mathrm{~s}$ & Max & FGR-12 & $1.12 \mathrm{E}-17$ \\
\hline Be-7 & Be-7 & 7.017 & Beryllium & $1.00 \mathrm{E}-03$ & ICRP-38 & $5.33 \mathrm{E}+01$ & $d$ & $4.61 \mathrm{E}+06$ & $3.49 \mathrm{E}+05$ & ICRP-72 & $5.50 \mathrm{E}-11$ & $\mathrm{~s}$ & Max & FGR-12 & $2.36 \mathrm{E}-15$ \\
\hline Bi-197 & Bi-197 & 196.979 & Bismuth & $1.00 \mathrm{E}-03$ & ICRP-107 & $9.30 \mathrm{E}+00$ & $\mathrm{~m}$ & $5.58 \mathrm{E}+02$ & $1.03 E+08$ & -- & -- & -- & -- & DOE-STD-1196 & $7.85 \mathrm{E}-14$ \\
\hline Bi-200 & Bi-200 & 199.978 & Bismuth & $1.00 \mathrm{E}-03$ & ICRP-38 & $3.64 \mathrm{E}+01$ & $\mathrm{~m}$ & $2.18 \mathrm{E}+03$ & $2.58 \mathrm{E}+07$ & ICRP-72 & $3.30 \mathrm{E}-11$ & $M$ & Max & FGR-12 & $1.16 \mathrm{E}-13$ \\
\hline Bi-201 & Bi-201 & 200.977 & Bismuth & $1.00 \mathrm{E}-03$ & ICRP-38 & $1.08 \mathrm{E}+02$ & $\mathrm{~m}$ & $6.48 \mathrm{E}+03$ & $8.66 \mathrm{E}+06$ & ICRP-72 & $6.60 \mathrm{E}-11$ & $M$ & Max & FGR-12 & $6.51 \mathrm{E}-14$ \\
\hline $\mathrm{Bi}-202$ & Bi-202 & 201.978 & Bismuth & $1.00 \mathrm{E}-03$ & ICRP-38 & $1.67 E+00$ & $\mathrm{~h}$ & $6.01 \mathrm{E}+03$ & $9.29 \mathrm{E}+06$ & ICRP-72 & $5.50 \mathrm{E}-11$ & $M$ & Max & FGR-12 & $1.33 \mathrm{E}-13$ \\
\hline Bi-203 & Bi-203 & 202.977 & Bismuth & $1.00 \mathrm{E}-03$ & ICRP-38 & $1.18 \mathrm{E}+01$ & $\mathrm{~h}$ & $4.23 \mathrm{E}+04$ & $1.31 \mathrm{E}+06$ & ICRP-72 & $2.60 \mathrm{E}-10$ & $M$ & Max & FGR-12 & $1.20 \mathrm{E}-13$ \\
\hline Bi-204 & Bi-204 & 203.978 & Bismuth & $1.00 \mathrm{E}-03$ & ICRP-38 & $1.12 \mathrm{E}+01$ & $\mathrm{~h}$ & $4.04 \mathrm{E}+04$ & $1.37 \mathrm{E}+06$ & DOE-STD-1196 & $3.81 \mathrm{E}-10$ & $\mathrm{~s}$ & Max & DOE-STD-1196 & $1.35 \mathrm{E}-13$ \\
\hline Bi-205 & Bi-205 & 204.977 & Bismuth & $1.00 \mathrm{E}-03$ & ICRP-38 & $1.53 \mathrm{E}+01$ & $d$ & $1.32 \mathrm{E}+06$ & $4.16 \mathrm{E}+04$ & ICRP-72 & $9.30 \mathrm{E}-10$ & $\mathrm{M}$ & Max & FGR-12 & $8.49 \mathrm{E}-14$ \\
\hline Bi-206 & Bi-206 & 205.978 & Bismuth & $1.00 \mathrm{E}-03$ & ICRP-38 & $6.24 \mathrm{E}+00$ & $d$ & $5.39 \mathrm{E}+05$ & $1.02 \mathrm{E}+05$ & ICRP-72 & $1.70 \mathrm{E}-09$ & $M$ & Max & FGR-12 & $1.61 \mathrm{E}-13$ \\
\hline Bi-207 & Bi-207 & 206.978 & Bismuth & $1.00 \mathrm{E}-03$ & ICRP-38 & $3.80 \mathrm{E}+01$ & $y$ & $1.20 \mathrm{E}+09$ & $4.55 \mathrm{E}+01$ & ICRP-72 & $5.60 \mathrm{E}-09$ & $M$ & Max & FGR-12 & $7.54 \mathrm{E}-14$ \\
\hline Bi-208 & Bi-208 & 207.980 & Bismuth & $1.00 \mathrm{E}-03$ & ICRP-107 & $3.68 \mathrm{E}+05$ & $y$ & $1.16 \mathrm{E}+13$ & $4.67 \mathrm{E}-03$ & DOE-STD-1196 & $3.83 \mathrm{E}-08$ & $\mathrm{~s}$ & Max & DOE-STD-1196 & $1.35 \mathrm{E}-13$ \\
\hline Bi-210 & Bi-210 & 209.984 & Bismuth & $1.00 \mathrm{E}-03$ & ICRP-38 & $5.01 \mathrm{E}+00$ & $d$ & $4.33 \mathrm{E}+05$ & $1.24 \mathrm{E}+05$ & ICRP-72 & $9.30 \mathrm{E}-08$ & $M$ & Max & FGR-12 & $3.29 \mathrm{E}-17$ \\
\hline Bi-210m & Bi-210 & 209.984 & Bismuth & $1.00 \mathrm{E}-03$ & ICRP-38 & $3.00 \mathrm{E}+06$ & $y$ & $9.47 E+13$ & $5.67 \mathrm{E}-04$ & ICRP-72 & $3.40 \mathrm{E}-06$ & $M$ & Max & FGR-12 & $1.22 \mathrm{E}-14$ \\
\hline
\end{tabular}




\begin{tabular}{|c|c|c|c|c|c|c|c|c|c|c|c|c|c|c|c|}
\hline \multicolumn{16}{|c|}{ Table B.1 Radionuclide Specific Input Data Used For the Calculation of HC-2 TQs Using Recommended DCs (continued) } \\
\hline $\begin{array}{l}\text { MASTER } \\
\text { ISOTOPE } \\
\text { LIST }\end{array}$ & $\begin{array}{c}\text { Atomic } \\
\text { Mass } \\
\text { Nuclide }\end{array}$ & $\begin{array}{l}\text { Atomic } \\
\text { Mass }\end{array}$ & Element & $\begin{array}{c}\text { RF per } \\
\text { DOE-STD- } \\
1027\end{array}$ & $\begin{array}{l}\text { Half-Life } \\
\text { Reference }\end{array}$ & \multicolumn{2}{|c|}{$\begin{array}{l}\text { Half-Life } \\
\text { (original units) }\end{array}$} & \multirow{2}{*}{$\begin{array}{c}\text { Half Life (s) } \\
1.28 \mathrm{E}+02\end{array}$} & \multirow{2}{*}{$\begin{array}{c}\begin{array}{c}\text { Sp. Act } \\
\text { (Ci/g) }\end{array} \\
4.16 \mathrm{E}+08\end{array}$} & \multirow{2}{*}{$\begin{array}{c}\text { Inhalation DC } \\
\text { Reference }\end{array}$} & \multirow[t]{2}{*}{$\begin{array}{c}\text { HC-2 } \\
\text { Inhalation } \\
\text { DC } \\
\text { (Sv/Bq) } \\
--\end{array}$} & \multicolumn{2}{|c|}{$\begin{array}{c}\text { Lung } \\
\text { Absorption } \\
\text { Class/Type }\end{array}$} & \multirow{2}{*}{$\begin{array}{c}\begin{array}{c}\text { Immersion DC } \\
\text { Reference }\end{array} \\
\text { FGR-12 }\end{array}$} & \multirow[t]{2}{*}{ 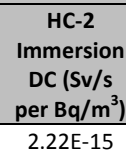 } \\
\hline Bi-211 & Bi-211 & 210.987 & Bismuth & $1.00 \mathrm{E}-03$ & ICRP-38 & $2.14 \mathrm{E}+00$ & $\mathrm{~m}$ & & & & & -- & -- & & \\
\hline Bi-212 & Bi-212 & 211.991 & Bismuth & $1.00 \mathrm{E}-03$ & ICRP-38 & $6.06 \mathrm{E}+01$ & $\mathrm{~m}$ & $3.63 \mathrm{E}+03$ & $1.46 \mathrm{E}+07$ & ICRP-72 & $3.10 \mathrm{E}-08$ & $\mathrm{M}$ & Max & FGR-12 & $9.24 \mathrm{E}-15$ \\
\hline Bi-212n & Bi-212 & 211.991 & Bismuth & $1.00 \mathrm{E}-03$ & ICRP-107 & $7.00 E+00$ & $\mathrm{~m}$ & $4.20 \mathrm{E}+02$ & $1.27 \mathrm{E}+08$ & -- & -- & -- & -- & DOE-STD-1196 & $3.89 \mathrm{E}-16$ \\
\hline Bi-213 & Bi-213 & 212.994 & Bismuth & $1.00 \mathrm{E}-03$ & ICRP-38 & $4.57 \mathrm{E}+01$ & $\mathrm{~m}$ & $2.74 \mathrm{E}+03$ & $1.93 \mathrm{E}+07$ & ICRP-72 & $3.00 \mathrm{E}-08$ & $M$ & Max & FGR-12 & $6.39 \mathrm{E}-15$ \\
\hline Bi-214 & Bi-214 & 213.999 & Bismuth & $1.00 \mathrm{E}-03$ & ICRP-38 & $1.99 \mathrm{E}+01$ & $\mathrm{~m}$ & $1.19 \mathrm{E}+03$ & $4.42 \mathrm{E}+07$ & ICRP-72 & $1.40 \mathrm{E}-08$ & $M$ & Max & FGR-12 & $7.65 \mathrm{E}-14$ \\
\hline Bi-215 & Bi-215 & 215.002 & Bismuth & $1.00 \mathrm{E}-03$ & ICRP-107 & $7.60 E+00$ & $\mathrm{~m}$ & $4.56 \mathrm{E}+02$ & $1.15 \mathrm{E}+08$ & -- & -- & -- & -- & DOE-STD-1196 & $1.18 \mathrm{E}-14$ \\
\hline Bi-216 & Bi-216 & 216.006 & Bismuth & $1.00 \mathrm{E}-03$ & ICRP-107 & $2.17 E+00$ & $\mathrm{~m}$ & $1.30 \mathrm{E}+02$ & $4.01 \mathrm{E}+08$ & -- & -- & -- & -- & DOE-STD-1196 & $3.41 \mathrm{E}-14$ \\
\hline Bk-244 & Bk-244 & 244.065 & Berkelium & $1.00 \mathrm{E}-03$ & JAERI & $4.35 \mathrm{E}+00$ & $\mathrm{~h}$ & $1.57 \mathrm{E}+04$ & $2.95 \mathrm{E}+06$ & JAERI & $1.00 \mathrm{E}-09$ & $M$ & Max & -- & -- \\
\hline Bk-245 & Bk-245 & 245.066 & Berkelium & $1.00 \mathrm{E}-03$ & ICRP-38 & $4.94 \mathrm{E}+00$ & $d$ & $4.27 \mathrm{E}+05$ & $1.08 \mathrm{E}+05$ & ICRP-72 & $2.10 \mathrm{E}-09$ & $M$ & Max & FGR-12 & $1.04 \mathrm{E}-14$ \\
\hline Bk-246 & Bk-246 & 246.069 & Berkelium & $1.00 \mathrm{E}-03$ & ICRP-38 & $1.83 \mathrm{E}+00$ & $d$ & $1.58 \mathrm{E}+05$ & $2.90 E+05$ & ICRP-72 & $3.30 \mathrm{E}-10$ & $M$ & Max & FGR-12 & $4.59 \mathrm{E}-14$ \\
\hline Bk-247 & Bk-247 & 247.070 & Berkelium & $1.00 \mathrm{E}-03$ & ICRP-38 & $1.38 \mathrm{E}+03$ & $y$ & $4.35 \mathrm{E}+10$ & $1.05 \mathrm{E}+00$ & ICRP-72 & $6.90 \mathrm{E}-05$ & $M$ & Max & FGR-12 & $4.71 \mathrm{E}-15$ \\
\hline Bk-248m & Bk-248 & 248.073 & Berkelium & $1.00 \mathrm{E}-03$ & ICRP-107 & $2.37 \mathrm{E}+01$ & $\mathrm{~h}$ & $8.53 \mathrm{E}+04$ & $5.33 \mathrm{E}+05$ & DOE-STD-1196 & $2.81 \mathrm{E}-08$ & $\mathrm{~F}$ & Max & DOE-STD-1196 & $2.28 \mathrm{E}-15$ \\
\hline Bk-249 & Bk-249 & 249.075 & Berkelium & $1.00 \mathrm{E}-03$ & ICRP-38 & $3.20 \mathrm{E}+02$ & $d$ & $2.76 \mathrm{E}+07$ & $1.64 \mathrm{E}+03$ & ICRP-72 & $1.60 \mathrm{E}-07$ & $M$ & Max & FGR-12 & $8.21 \mathrm{E}-20$ \\
\hline Bk-250 & Bk-250 & 250.078 & Berkelium & $1.00 \mathrm{E}-03$ & ICRP-38 & $3.22 \mathrm{E}+00$ & $\mathrm{~h}$ & $1.16 \mathrm{E}+04$ & $3.89 \mathrm{E}+06$ & ICRP-72 & $1.00 \mathrm{E}-09$ & $M$ & Max & FGR-12 & $4.38 \mathrm{E}-14$ \\
\hline Bk-251 & Bk-251 & 251.081 & Berkelium & $1.00 \mathrm{E}-03$ & ICRP-107 & $5.56 \mathrm{E}+01$ & $\mathrm{~m}$ & $3.34 \mathrm{E}+03$ & $1.35 \mathrm{E}+07$ & DOE-STD-1196 & $5.09 \mathrm{E}-11$ & $M$ & Max & DOE-STD-1196 & $3.56 \mathrm{E}-15$ \\
\hline $\mathrm{Br}-72$ & $\mathrm{Br}-72$ & 71.937 & Bromine & $5.00 \mathrm{E}-01$ & ICRP-107 & $7.86 \mathrm{E}+01$ & $\mathrm{~s}$ & $7.86 \mathrm{E}+01$ & $2.00 \mathrm{E}+09$ & -- & -- & -- & -- & DOE-STD-1196 & $1.41 \mathrm{E}-13$ \\
\hline $\mathrm{Br}-73$ & $\mathrm{Br}-73$ & 72.932 & Bromine & $5.00 \mathrm{E}-01$ & ICRP-107 & $3.40 \mathrm{E}+00$ & $\mathrm{~m}$ & $2.04 \mathrm{E}+02$ & $7.58 \mathrm{E}+08$ & -- & -- & -- & -- & DOE-STD-1196 & $6.52 \mathrm{E}-14$ \\
\hline $\mathrm{Br}-74$ & $\mathrm{Br}-74$ & 73.930 & Bromine & $5.00 \mathrm{E}-01$ & ICRP-38 & $2.53 \mathrm{E}+01$ & $\mathrm{~m}$ & $1.52 \mathrm{E}+03$ & $1.01 \mathrm{E}+08$ & ICRP-72 & $3.80 \mathrm{E}-11$ & $M$ & $\operatorname{Max}$ & FGR-12 & $2.38 \mathrm{E}-13$ \\
\hline $\mathrm{Br}-74 \mathrm{~m}$ & $\mathrm{Br}-74$ & 73.930 & Bromine & $5.00 \mathrm{E}-01$ & ICRP-38 & $4.15 \mathrm{E}+01$ & $\mathrm{~m}$ & $2.49 \mathrm{E}+03$ & $6.13 \mathrm{E}+07$ & ICRP-72 & $6.20 \mathrm{E}-11$ & $M$ & Max & FGR-12 & $2.08 \mathrm{E}-13$ \\
\hline $\mathrm{Br}-75$ & $\mathrm{Br}-75$ & 74.926 & Bromine & $5.00 \mathrm{E}-01$ & ICRP-38 & $9.80 \mathrm{E}+01$ & $\mathrm{~m}$ & $5.88 \mathrm{E}+03$ & $2.56 \mathrm{E}+07$ & ICRP-72 & $5.30 \mathrm{E}-11$ & $M$ & Max & FGR-12 & $5.84 \mathrm{E}-14$ \\
\hline $\mathrm{Br}-76$ & $\mathrm{Br}-76$ & 75.925 & Bromine & $5.00 \mathrm{E}-01$ & ICRP-38 & $1.62 \mathrm{E}+01$ & $\mathrm{~h}$ & $5.83 \mathrm{E}+04$ & $2.55 \mathrm{E}+06$ & ICRP-72 & $4.10 \mathrm{E}-10$ & $M$ & Max & FGR-12 & $1.34 \mathrm{E}-13$ \\
\hline $\mathrm{Br}-76 \mathrm{~m}$ & $\mathrm{Br}-76$ & 75.925 & Bromine & $5.00 \mathrm{E}-01$ & ICRP-107 & $1.31 \mathrm{E}+00$ & $\mathrm{~s}$ & $1.31 \mathrm{E}+00$ & $1.13 \mathrm{E}+11$ & -- & -- & -- & -- & DOE-STD-1196 & $9.66 \mathrm{E}-16$ \\
\hline $\mathrm{Br}-77$ & $\mathrm{Br}-77$ & 76.921 & Bromine & $5.00 \mathrm{E}-01$ & ICRP-38 & $5.60 \mathrm{E}+01$ & $\mathrm{~h}$ & $2.02 \mathrm{E}+05$ & $7.27 \mathrm{E}+05$ & ICRP-72 & $8.40 \mathrm{E}-11$ & $M$ & Max & FGR-12 & $1.51 \mathrm{E}-14$ \\
\hline $\mathrm{Br}-77 \mathrm{~m}$ & $\mathrm{Br}-77$ & 76.921 & Bromine & $5.00 \mathrm{E}-01$ & ICRP-107 & $4.28 \mathrm{E}+00$ & $\mathrm{~m}$ & $2.57 \mathrm{E}+02$ & $5.71 \mathrm{E}+08$ & -- & -- & -- & -- & DOE-STD-1196 & $5.96 \mathrm{E}-16$ \\
\hline $\mathrm{Br}-78$ & $\mathrm{Br}-78$ & 77.921 & Bromine & $5.00 \mathrm{E}-01$ & ICRP-107 & $6.46 \mathrm{E}+00$ & $\mathrm{~m}$ & $3.88 \mathrm{E}+02$ & $3.74 \mathrm{E}+08$ & -- & -- & -- & -- & DOE-STD-1196 & $4.69 \mathrm{E}-14$ \\
\hline $\mathrm{Br}-80$ & $\mathrm{Br}-80$ & 79.919 & Bromine & $5.00 \mathrm{E}-01$ & ICRP-38 & $1.74 \mathrm{E}+01$ & $\mathrm{~m}$ & $1.04 \mathrm{E}+03$ & $1.35 \mathrm{E}+08$ & ICRP-72 & $9.40 \mathrm{E}-12$ & $\mathrm{M}$ & Max & FGR-12 & $3.85 \mathrm{E}-15$ \\
\hline $\mathrm{Br}-80 \mathrm{~m}$ & $\mathrm{Br}-80$ & 79.919 & Bromine & $5.00 \mathrm{E}-01$ & ICRP-38 & $4.42 \mathrm{E}+00$ & $\mathrm{~h}$ & $1.59 \mathrm{E}+04$ & $8.87 \mathrm{E}+06$ & ICRP-72 & $7.60 \mathrm{E}-11$ & $M$ & Max & FGR-12 & $3.11 \mathrm{E}-16$ \\
\hline $\mathrm{Br}-82$ & $\mathrm{Br}-82$ & 81.917 & Bromine & $5.00 \mathrm{E}-01$ & ICRP-38 & $3.53 \mathrm{E}+01$ & $\mathrm{~h}$ & $1.27 \mathrm{E}+05$ & $1.08 \mathrm{E}+06$ & ICRP-72 & $6.30 \mathrm{E}-10$ & $M$ & $\operatorname{Max}$ & FGR-12 & $1.30 \mathrm{E}-13$ \\
\hline $\mathrm{Br}-82 \mathrm{~m}$ & $\mathrm{Br}-82$ & 81.917 & Bromine & $5.00 \mathrm{E}-01$ & ICRP-107 & $6.13 \mathrm{E}+00$ & $\mathrm{~m}$ & $3.68 \mathrm{E}+02$ & $3.74 \mathrm{E}+08$ & -- & -- & -- & -- & DOE-STD-1196 & $1.59 \mathrm{E}-16$ \\
\hline $\mathrm{Br}-83$ & $\mathrm{Br}-83$ & 82.915 & Bromine & $5.00 \mathrm{E}-01$ & ICRP-38 & $2.39 \mathrm{E}+00$ & $\mathrm{~h}$ & $8.60 \mathrm{E}+03$ & $1.58 \mathrm{E}+07$ & ICRP-72 & $4.80 \mathrm{E}-11$ & $M$ & Max & FGR-12 & $3.82 \mathrm{E}-16$ \\
\hline $\mathrm{Br}-84$ & $\mathrm{Br}-84$ & 83.916 & Bromine & $5.00 \mathrm{E}-01$ & ICRP-38 & $3.18 \mathrm{E}+01$ & $\mathrm{~m}$ & $1.91 \mathrm{E}+03$ & $7.05 \mathrm{E}+07$ & ICRP-72 & $3.70 \mathrm{E}-11$ & $M$ & Max & FGR-12 & $9.41 \mathrm{E}-14$ \\
\hline $\mathrm{Br}-84 \mathrm{~m}$ & $\mathrm{Br}-84$ & 83.916 & Bromine & $5.00 \mathrm{E}-01$ & ICRP-107 & $6.00 \mathrm{E}+00$ & $\mathrm{~m}$ & $3.60 \mathrm{E}+02$ & $3.73 \mathrm{E}+08$ & -- & -- & -- & -- & DOE-STD-1196 & $1.31 \mathrm{E}-13$ \\
\hline $\mathrm{Br}-85$ & $\mathrm{Br}-85$ & 84.916 & Bromine & $5.00 \mathrm{E}-01$ & ICRP-107 & $2.90 \mathrm{E}+00$ & $\mathrm{~m}$ & $1.74 \mathrm{E}+02$ & $7.64 E+08$ & -- & -- & -- & -- & DOE-STD-1196 & $3.98 \mathrm{E}-15$ \\
\hline C-10 & C-10 & 10.017 & Carbon & $1.00 \mathrm{E}-02$ & ICRP-107 & $1.93 \mathrm{E}+01$ & $\mathrm{~s}$ & $1.93 \mathrm{E}+01$ & $5.85 \mathrm{E}+10$ & -- & -- & -- & -- & DOE-STD-1196 & $7.90 \mathrm{E}-14$ \\
\hline C-11 & C-11 & 11.011 & Carbon & $1.00 \mathrm{E}-02$ & ICRP-38 & $2.04 \mathrm{E}+01$ & $\mathrm{~m}$ & $1.22 \mathrm{E}+03$ & $8.38 \mathrm{E}+08$ & ICRP-72 & $1.80 \mathrm{E}-11$ & $M$ & $\operatorname{Rec}$ & FGR-12 & $4.89 \mathrm{E}-14$ \\
\hline C-14 & C-14 & 14.003 & Carbon & $1.00 \mathrm{E}-02$ & ICRP-38 & $5.73 \mathrm{E}+03$ & $y$ & $1.81 \mathrm{E}+11$ & $4.46 \mathrm{E}+00$ & ICRP-72 & $2.00 \mathrm{E}-09$ & $M$ & $\operatorname{Rec}$ & FGR-12 & $2.24 \mathrm{E}-19$ \\
\hline Ca-41 & Ca-41 & 40.962 & Calcium & $1.00 \mathrm{E}-03$ & ICRP-38 & $1.40 \mathrm{E}+05$ & $y$ & $4.42 \mathrm{E}+12$ & $6.23 \mathrm{E}-02$ & ICRP-72 & $9.50 \mathrm{E}-11$ & $M$ & $\operatorname{Rec}$ & -- & -- \\
\hline Сa-45 & Ca-45 & 44.956 & Calcium & $1.00 \mathrm{E}-03$ & ICRP-38 & $1.63 \mathrm{E}+02$ & $d$ & $1.41 \mathrm{E}+07$ & $1.78 \mathrm{E}+04$ & ICRP-72 & $2.70 \mathrm{E}-09$ & $M$ & $\operatorname{Rec}$ & FGR-12 & $8.63 \mathrm{E}-19$ \\
\hline Ca-47 & Ca-47 & 46.955 & Calcium & $1.00 \mathrm{E}-03$ & ICRP-38 & $4.53 \mathrm{E}+00$ & $d$ & $3.91 \mathrm{E}+05$ & $6.14 \mathrm{E}+05$ & ICRP-72 & $1.90 \mathrm{E}-09$ & $M$ & $\operatorname{Rec}$ & FGR-12 & $5.36 \mathrm{E}-14$ \\
\hline Ca-49 & Ca-49 & 48.956 & Calcium & $1.00 \mathrm{E}-03$ & ICRP-38 & $8.72 \mathrm{E}+00$ & $\mathrm{~m}$ & $5.23 \mathrm{E}+02$ & $4.41 \mathrm{E}+08$ & -- & -- & -- & -- & FGR-12 & $1.73 \mathrm{E}-13$ \\
\hline Cd-101 & Cd-101 & 100.919 & Cadmium & $1.00 \mathrm{E}-03$ & ICRP-107 & $1.36 \mathrm{E}+00$ & $\mathrm{~m}$ & $8.16 \mathrm{E}+01$ & $1.37 E+09$ & -- & -- & -- & -- & DOE-STD-1196 & $1.17 \mathrm{E}-13$ \\
\hline Cd-102 & Cd-102 & 101.914 & Cadmium & $1.00 \mathrm{E}-03$ & ICRP-107 & $5.50 \mathrm{E}+00$ & $\mathrm{~m}$ & $3.30 \mathrm{E}+02$ & $3.35 \mathrm{E}+08$ & -- & -- & -- & -- & DOE-STD-1196 & $3.73 \mathrm{E}-14$ \\
\hline Cd-103 & Cd-103 & 102.913 & Cadmium & $1.00 \mathrm{E}-03$ & ICRP-107 & $7.30 \mathrm{E}+00$ & $\mathrm{~m}$ & $4.38 \mathrm{E}+02$ & $2.50 \mathrm{E}+08$ & -- & -- & -- & -- & DOE-STD-1196 & $1.00 \mathrm{E}-13$ \\
\hline Cd-104 & Cd-104 & 103.910 & Cadmium & $1.00 \mathrm{E}-03$ & ICRP-38 & $5.77 \mathrm{E}+01$ & $\mathrm{~m}$ & $3.46 \mathrm{E}+03$ & $3.14 \mathrm{E}+07$ & ICRP-72 & $3.50 \mathrm{E}-11$ & $\mathrm{~s}$ & Max & FGR-12 & $1.14 \mathrm{E}-14$ \\
\hline Cd-105 & Cd-105 & 104.909 & Cadmium & $1.00 \mathrm{E}-03$ & ICRP-107 & $5.55 \mathrm{E}+01$ & $\mathrm{~m}$ & $3.33 \mathrm{E}+03$ & $3.23 \mathrm{E}+07$ & DOE-STD-1196 & $2.86 \mathrm{E}-11$ & $\mathrm{~s}$ & Max & DOE-STD-1196 & $6.13 \mathrm{E}-14$ \\
\hline Cd-107 & Cd-107 & 106.907 & Cadmium & $1.00 \mathrm{E}-03$ & ICRP-38 & $6.49 \mathrm{E}+00$ & $\mathrm{~h}$ & $2.34 \mathrm{E}+04$ & $4.52 \mathrm{E}+06$ & ICRP-72 & $8.30 \mathrm{E}-11$ & $M$ & Max & FGR-12 & $6.02 \mathrm{E}-16$ \\
\hline
\end{tabular}




\begin{tabular}{|c|c|c|c|c|c|c|c|c|c|c|c|c|c|c|c|}
\hline \multicolumn{16}{|c|}{ Table B.1 Radionuclide Specific Input Data Used For the Calculation of HC-2 TQs Using Recommended DCs (continued) } \\
\hline $\begin{array}{l}\text { MASTER } \\
\text { ISOTOPE } \\
\text { LIST }\end{array}$ & $\begin{array}{c}\text { Atomic } \\
\text { Mass } \\
\text { Nuclide }\end{array}$ & $\begin{array}{l}\text { Atomic } \\
\text { Mass }\end{array}$ & Element & $\begin{array}{c}\text { RF per } \\
\text { DOE-STD- } \\
1027\end{array}$ & $\begin{array}{l}\text { Half-Life } \\
\text { Reference }\end{array}$ & $\begin{array}{r}\text { Half-Li } \\
\text { (original } 4\end{array}$ & & Half Life (s) & $\begin{array}{l}\text { Sp. Act } \\
\text { (Ci/g) }\end{array}$ & $\begin{array}{l}\text { Inhalation DC } \\
\text { Reference }\end{array}$ & $\begin{array}{c}\text { HC-2 } \\
\text { Inhalation } \\
\text { DC } \\
\text { (Sv/Bq) }\end{array}$ & & $\begin{array}{l}\text { ung } \\
\text { rption } \\
\text { /Type }\end{array}$ & $\begin{array}{l}\text { Immersion DC } \\
\text { Reference }\end{array}$ & $\begin{array}{c}\text { HC-2 } \\
\text { Immersion } \\
\text { DC (Sv/s } \\
\left.\text { per } \mathrm{Bq} / \mathrm{m}^{3}\right)\end{array}$ \\
\hline Cd-109 & Cd-109 & 108.905 & Cadmium & $1.00 \mathrm{E}-03$ & ICRP-38 & $4.64 \mathrm{E}+02$ & d & $4.01 \mathrm{E}+07$ & $2.58 \mathrm{E}+03$ & ICRP-72 & $8.10 \mathrm{E}-09$ & $\mathrm{~F}$ & Max & FGR-12 & $2.94 \mathrm{E}-16$ \\
\hline Cd-111m & Cd-111 & 110.904 & Cadmium & $1.00 \mathrm{E}-03$ & ICRP-107 & $4.85 \mathrm{E}+01$ & $\mathrm{~m}$ & $2.91 \mathrm{E}+03$ & $3.50 \mathrm{E}+07$ & DOE-STD-1196 & $2.66 \mathrm{E}-11$ & $\mathrm{~s}$ & Max & DOE-STD-1196 & $1.20 \mathrm{E}-14$ \\
\hline Cd-113 & Cd-113 & 112.904 & Cadmium & $1.00 \mathrm{E}-03$ & ICRP-38 & $9.30 \mathrm{E}+15$ & $y$ & $2.93 \mathrm{E}+23$ & $3.40 \mathrm{E}-13$ & ICRP-72 & $1.20 \mathrm{E}-07$ & $\mathrm{~F}$ & Max & FGR-12 & $1.45 \mathrm{E}-18$ \\
\hline Cd-113m & Cd-113 & 112.904 & Cadmium & $1.00 \mathrm{E}-03$ & ICRP-38 & $1.36 \mathrm{E}+01$ & $y$ & $4.29 \mathrm{E}+08$ & $2.33 \mathrm{E}+02$ & ICRP-72 & $1.10 \mathrm{E}-07$ & $\mathrm{~F}$ & Max & FGR-12 & $6.94 \mathrm{E}-18$ \\
\hline Cd-115 & Cd-115 & 114.905 & Cadmium & $1.00 \mathrm{E}-03$ & ICRP-38 & $5.35 \mathrm{E}+01$ & $\mathrm{~h}$ & $1.92 \mathrm{E}+05$ & $5.10 \mathrm{E}+05$ & ICRP-72 & $1.10 \mathrm{E}-09$ & $\mathrm{~s}$ & Max & FGR-12 & $1.12 \mathrm{E}-14$ \\
\hline Cd-115m & Cd-115 & 114.905 & Cadmium & $1.00 \mathrm{E}-03$ & ICRP-38 & $4.46 \mathrm{E}+01$ & $d$ & $3.85 \mathrm{E}+06$ & $2.55 \mathrm{E}+04$ & ICRP-72 & $7.70 \mathrm{E}-09$ & $\mathrm{~s}$ & Max & FGR-12 & $1.17 \mathrm{E}-15$ \\
\hline Cd-117 & Cd-117 & 116.907 & Cadmium & $1.00 \mathrm{E}-03$ & ICRP-38 & $2.49 \mathrm{E}+00$ & $\mathrm{~h}$ & $8.96 \mathrm{E}+03$ & $1.08 \mathrm{E}+07$ & ICRP-72 & $1.70 \mathrm{E}-10$ & $\mathrm{~s}$ & Max & FGR-12 & $5.45 \mathrm{E}-14$ \\
\hline Cd-117m & Cd-117 & 116.907 & Cadmium & $1.00 \mathrm{E}-03$ & ICRP-38 & $3.36 \mathrm{E}+00$ & $\mathrm{~h}$ & $1.21 \mathrm{E}+04$ & $7.98 \mathrm{E}+06$ & ICRP-72 & $2.10 \mathrm{E}-10$ & $\mathrm{~s}$ & Max & FGR-12 & $1.05 \mathrm{E}-13$ \\
\hline Cd-118 & Cd-118 & 117.907 & Cadmium & $1.00 \mathrm{E}-03$ & ICRP-107 & $5.03 \mathrm{E}+01$ & $\mathrm{~m}$ & $3.02 \mathrm{E}+03$ & $3.17 \mathrm{E}+07$ & DOE-STD-1196 & $9.60 \mathrm{E}-11$ & $\mathrm{~s}$ & Max & DOE-STD-1196 & $7.25 \mathrm{E}-17$ \\
\hline $\mathrm{Cd}-119$ & Cd-119 & 118.910 & Cadmium & $1.00 \mathrm{E}-03$ & ICRP-107 & $2.69 \mathrm{E}+00$ & $\mathrm{~m}$ & $1.61 \mathrm{E}+02$ & $5.88 \mathrm{E}+08$ & -- & -- & -- & -- & DOE-STD-1196 & $7.96 \mathrm{E}-14$ \\
\hline Cd-119m & Cd-119 & 118.910 & Cadmium & $1.00 \mathrm{E}-03$ & ICRP-107 & $2.20 \mathrm{E}+00$ & $\mathrm{~m}$ & $1.32 \mathrm{E}+02$ & $7.19 \mathrm{E}+08$ & -- & -- & -- & -- & DOE-STD-1196 & $1.11 \mathrm{E}-13$ \\
\hline Ce-130 & Ce-130 & 129.915 & Cerium & $1.00 \mathrm{E}-03$ & ICRP-107 & $2.29 \mathrm{E}+01$ & $\mathrm{~m}$ & $1.37 \mathrm{E}+03$ & $6.32 \mathrm{E}+07$ & DOE-STD-1196 & $4.09 \mathrm{E}-11$ & $M$ & $\operatorname{Rec}$ & DOE-STD-1196 & $2.14 \mathrm{E}-14$ \\
\hline Ce-131 & Ce-131 & 130.914 & Cerium & $1.00 \mathrm{E}-03$ & ICRP-107 & $1.02 \mathrm{E}+01$ & $\mathrm{~m}$ & $6.12 \mathrm{E}+02$ & $1.41 \mathrm{E}+08$ & DOE-STD-1196 & $1.62 \mathrm{E}-11$ & $M$ & $\operatorname{Rec}$ & DOE-STD-1196 & $7.43 \mathrm{E}-14$ \\
\hline Ce-131m & Ce-131 & 130.914 & Cerium & $1.00 \mathrm{E}-03$ & JAERI & $5.00 \mathrm{E}+00$ & $\mathrm{~m}$ & $3.00 \mathrm{E}+02$ & $2.87 E+08$ & -- & -- & -- & -- & -- & -- \\
\hline Ce-132 & Ce-132 & 131.911 & Cerium & $1.00 \mathrm{E}-03$ & ICRP-107 & $3.51 \mathrm{E}+00$ & $\mathrm{~h}$ & $1.26 \mathrm{E}+04$ & $6.77 \mathrm{E}+06$ & DOE-STD-1196 & $1.65 \mathrm{E}-10$ & $M$ & $\operatorname{Rec}$ & DOE-STD-1196 & $1.09 \mathrm{E}-14$ \\
\hline Ce-133 & Ce-133 & 132.912 & Cerium & $1.00 \mathrm{E}-03$ & ICRP-107 & $9.70 \mathrm{E}+01$ & $\mathrm{~m}$ & $5.82 \mathrm{E}+03$ & $1.46 \mathrm{E}+07$ & DOE-STD-1196 & $5.90 \mathrm{E}-11$ & $M$ & $\operatorname{Rec}$ & DOE-STD-1196 & $2.28 \mathrm{E}-14$ \\
\hline Ce-133m & Ce-133 & 132.912 & Cerium & $1.00 \mathrm{E}-03$ & ICRP-107 & $4.90 \mathrm{E}+00$ & $\mathrm{~h}$ & $1.76 \mathrm{E}+04$ & $4.81 \mathrm{E}+06$ & DOE-STD-1196 & $1.38 \mathrm{E}-10$ & $M$ & Rec & DOE-STD-1196 & $7.95 \mathrm{E}-14$ \\
\hline Ce-134 & Ce-134 & 133.909 & Cerium & $1.00 \mathrm{E}-03$ & ICRP-38 & $7.20 \mathrm{E}+01$ & $\mathrm{~h}$ & $2.59 \mathrm{E}+05$ & $3.25 \mathrm{E}+05$ & ICRP-72 & $1.30 \mathrm{E}-09$ & $M$ & Rec & FGR-12 & $4.71 \mathrm{E}-16$ \\
\hline Ce-135 & Ce-135 & 134.909 & Cerium & $1.00 \mathrm{E}-03$ & ICRP-38 & $1.76 \mathrm{E}+01$ & $\mathrm{~h}$ & $6.34 \mathrm{E}+04$ & $1.32 \mathrm{E}+06$ & ICRP-72 & $4.80 \mathrm{E}-10$ & $M$ & $\operatorname{Rec}$ & FGR-12 & $8.54 \mathrm{E}-14$ \\
\hline Ce-137 & Ce-137 & 136.908 & Cerium & $1.00 \mathrm{E}-03$ & ICRP-38 & $9.00 \mathrm{E}+00$ & $\mathrm{~h}$ & $3.24 \mathrm{E}+04$ & $2.54 \mathrm{E}+06$ & ICRP-72 & $9.80 \mathrm{E}-12$ & $M$ & $\operatorname{Rec}$ & FGR-12 & $8.81 \mathrm{E}-16$ \\
\hline Ce-137m & Ce-137 & 136.908 & Cerium & $1.00 \mathrm{E}-03$ & ICRP-38 & $3.44 \mathrm{E}+01$ & $\mathrm{~h}$ & $1.24 \mathrm{E}+05$ & $6.65 \mathrm{E}+05$ & ICRP-72 & $4.10 \mathrm{E}-10$ & $M$ & $\operatorname{Rec}$ & FGR-12 & $1.96 \mathrm{E}-15$ \\
\hline Ce-139 & Ce-139 & 138.907 & Cerium & $1.00 \mathrm{E}-03$ & ICRP-38 & $1.38 \mathrm{E}+02$ & $d$ & $1.19 \mathrm{E}+07$ & $6.83 \mathrm{E}+03$ & ICRP-72 & $1.70 \mathrm{E}-09$ & $M$ & $\operatorname{Rec}$ & FGR-12 & $6.73 \mathrm{E}-15$ \\
\hline Ce-141 & Ce-141 & 140.908 & Cerium & $1.00 \mathrm{E}-03$ & ICRP-38 & $3.25 \mathrm{E}+01$ & $d$ & $2.81 \mathrm{E}+06$ & $2.85 \mathrm{E}+04$ & ICRP-72 & $3.20 \mathrm{E}-09$ & $M$ & $\operatorname{Rec}$ & FGR-12 & $3.43 \mathrm{E}-15$ \\
\hline Ce-143 & Ce-143 & 142.912 & Cerium & $1.00 \mathrm{E}-03$ & ICRP-38 & $3.30 \mathrm{E}+01$ & $\mathrm{~h}$ & $1.19 \mathrm{E}+05$ & $6.64 \mathrm{E}+05$ & ICRP-72 & $7.50 \mathrm{E}-10$ & $M$ & $\operatorname{Rec}$ & FGR-12 & $1.29 \mathrm{E}-14$ \\
\hline Ce-144 & Ce-144 & 143.914 & Cerium & $1.00 \mathrm{E}-03$ & ICRP-38 & $2.84 \mathrm{E}+02$ & $d$ & $2.46 \mathrm{E}+07$ & $3.19 \mathrm{E}+03$ & ICRP-72 & $3.60 \mathrm{E}-08$ & $M$ & $\operatorname{Rec}$ & FGR-12 & $8.53 \mathrm{E}-16$ \\
\hline Ce-145 & Ce-145 & 144.917 & Cerium & $1.00 \mathrm{E}-03$ & ICRP-107 & $3.01 \mathrm{E}+00$ & $\mathrm{~m}$ & $1.81 \mathrm{E}+02$ & $4.31 \mathrm{E}+08$ & -- & -- & -- & -- & DOE-STD-1196 & $3.64 \mathrm{E}-14$ \\
\hline Ce-146 & Ce-146 & 145.919 & Cerium & $1.00 \mathrm{E}-03$ & JAERI & $1.35 \mathrm{E}+01$ & $\mathrm{~m}$ & $8.11 \mathrm{E}+02$ & $9.53 \mathrm{E}+07$ & JAERI & $2.50 \mathrm{E}-11$ & $M$ & $\operatorname{Rec}$ & -- & -- \\
\hline Cf-244 & Cf-244 & 244.066 & Californium & $1.00 \mathrm{E}-03$ & ICRP-38 & $1.94 \mathrm{E}+01$ & $\mathrm{~m}$ & $1.16 \mathrm{E}+03$ & $3.97 \mathrm{E}+07$ & ICRP-72 & $1.40 \mathrm{E}-08$ & $M$ & Max & FGR-12 & $6.91 \mathrm{E}-18$ \\
\hline Cf-246 & Cf-246 & 246.069 & Californium & $1.00 \mathrm{E}-03$ & ICRP-38 & $3.57 \mathrm{E}+01$ & $\mathrm{~h}$ & $1.29 \mathrm{E}+05$ & $3.57 \mathrm{E}+05$ & ICRP-72 & $4.50 \mathrm{E}-07$ & $M$ & Max & FGR-12 & $5.48 \mathrm{E}-18$ \\
\hline Cf-247 & Cf-247 & 247.071 & Californium & $1.00 \mathrm{E}-03$ & ICRP-107 & $3.11 \mathrm{E}+00$ & $\mathrm{~h}$ & $1.12 \mathrm{E}+04$ & $4.08 \mathrm{E}+06$ & DOE-STD-1196 & $5.22 \mathrm{E}-11$ & $\mathrm{~F}$ & Max & DOE-STD-1196 & $3.58 \mathrm{E}-15$ \\
\hline Cf-248 & Cf-248 & 248.072 & Californium & $1.00 \mathrm{E}-03$ & ICRP-38 & $3.34 \mathrm{E}+02$ & $d$ & $2.88 \mathrm{E}+07$ & $1.58 \mathrm{E}+03$ & ICRP-72 & $8.80 \mathrm{E}-06$ & $M$ & Max & FGR-12 & $4.73 \mathrm{E}-18$ \\
\hline Cf-249 & Cf-249 & 249.075 & Californium & $1.00 \mathrm{E}-03$ & ICRP-38 & $3.51 \mathrm{E}+02$ & $y$ & $1.11 \mathrm{E}+10$ & $4.09 \mathrm{E}+00$ & ICRP-72 & $7.00 \mathrm{E}-05$ & $M$ & Max & FGR-12 & $1.58 \mathrm{E}-14$ \\
\hline Cf-250 & Cf-250 & 250.076 & Californium & $1.00 \mathrm{E}-03$ & ICRP-38 & $1.31 \mathrm{E}+01$ & $y$ & $4.13 \mathrm{E}+08$ & $1.09 \mathrm{E}+02$ & ICRP-72 & $3.40 \mathrm{E}-05$ & $M$ & Max & FGR-12 & $4.50 \mathrm{E}-18$ \\
\hline Cf-251 & Cf-251 & 251.080 & Californium & $1.00 \mathrm{E}-03$ & ICRP-38 & $8.98 \mathrm{E}+02$ & $y$ & $2.83 \mathrm{E}+10$ & $1.59 \mathrm{E}+00$ & ICRP-72 & $7.10 \mathrm{E}-05$ & $M$ & Max & FGR-12 & $5.58 \mathrm{E}-15$ \\
\hline Cf-252 & Cf-252 & 252.082 & Californium & $1.00 \mathrm{E}-03$ & ICRP-38 & $2.64 \mathrm{E}+00$ & $y$ & $8.32 \mathrm{E}+07$ & $5.38 \mathrm{E}+02$ & ICRP-72 & $2.00 \mathrm{E}-05$ & $M$ & Max & FGR-12 & $5.06 \mathrm{E}-18$ \\
\hline Cf-253 & Cf-253 & 253.085 & Californium & $1.00 \mathrm{E}-03$ & ICRP-38 & $1.78 \mathrm{E}+01$ & $d$ & $1.54 \mathrm{E}+06$ & $2.90 \mathrm{E}+04$ & ICRP-72 & $1.30 \mathrm{E}-06$ & $M$ & Max & FGR-12 & $1.08 \mathrm{E}-18$ \\
\hline Cf-254 & Cf-254 & 254.087 & Californium & $1.00 \mathrm{E}-03$ & ICRP-38 & $6.05 E+01$ & $d$ & $5.23 \mathrm{E}+06$ & $8.49 \mathrm{E}+03$ & ICRP-72 & $4.10 \mathrm{E}-05$ & $M$ & Max & FGR-12 & $1.47 \mathrm{E}-20$ \\
\hline Cf- 255 & Cf-255 & 255.091 & Californium & $1.00 \mathrm{E}-03$ & ICRP-107 & $8.50 \mathrm{E}+01$ & $\mathrm{~m}$ & $5.10 \mathrm{E}+03$ & $8.67 \mathrm{E}+06$ & DOE-STD-1196 & $7.35 \mathrm{E}-09$ & $\mathrm{~s}$ & Max & DOE-STD-1196 & $1.16 \mathrm{E}-16$ \\
\hline Cf-256 & Cf-256 & 256.093 & Californium & $1.00 \mathrm{E}-03$ & JAERI & $1.23 \mathrm{E}+01$ & $\mathrm{~m}$ & $7.38 \mathrm{E}+02$ & $5.97 \mathrm{E}+07$ & JAERI & $1.90 \mathrm{E}-06$ & $M$ & Max & -- & -- \\
\hline $\mathrm{Cl}-34$ & $\mathrm{Cl}-34$ & 33.974 & Chlorine & $1.00 \mathrm{E}+00$ & ICRP-107 & $1.53 \mathrm{E}+00$ & $\mathrm{~s}$ & $1.53 \mathrm{E}+00$ & $2.18 \mathrm{E}+11$ & -- & -- & -- & -- & DOE-STD-1196 & $4.77 \mathrm{E}-14$ \\
\hline $\mathrm{Cl}-34 \mathrm{~m}$ & $\mathrm{Cl}-34$ & 33.974 & Chlorine & $1.00 \mathrm{E}+00$ & ICRP-107 & $3.20 \mathrm{E}+01$ & $\mathrm{~m}$ & $1.92 \mathrm{E}+03$ & $1.73 \mathrm{E}+08$ & DOE-STD-1196 & $5.50 \mathrm{E}-11$ & $\mathrm{~s}$ & Max & DOE-STD-1196 & $1.03 \mathrm{E}-13$ \\
\hline $\mathrm{Cl}-36$ & $\mathrm{Cl}-36$ & 35.968 & Chlorine & $1.00 \mathrm{E}+00$ & ICRP-38 & $3.01 \mathrm{E}+05$ & $y$ & $9.50 \mathrm{E}+12$ & $3.30 \mathrm{E}-02$ & ICRP-72 & $7.30 \mathrm{E}-09$ & $M$ & Max & FGR-12 & $2.23 \mathrm{E}-17$ \\
\hline $\mathrm{Cl}-38$ & $\mathrm{Cl}-38$ & 37.968 & Chlorine & $1.00 \mathrm{E}+00$ & ICRP-38 & $3.72 \mathrm{E}+01$ & $\mathrm{~m}$ & $2.23 \mathrm{E}+03$ & $1.33 \mathrm{E}+08$ & ICRP-72 & $4.50 \mathrm{E}-11$ & $M$ & Max & FGR-12 & $7.87 \mathrm{E}-14$ \\
\hline $\mathrm{Cl}-39$ & $\mathrm{Cl}-39$ & 38.968 & Chlorine & $1.00 \mathrm{E}+00$ & ICRP-38 & $5.56 \mathrm{E}+01$ & $\mathrm{~m}$ & $3.34 \mathrm{E}+03$ & $8.68 \mathrm{E}+07$ & ICRP-72 & $4.60 \mathrm{E}-11$ & $M$ & Max & FGR-12 & $7.29 \mathrm{E}-14$ \\
\hline $\mathrm{Cl}-40$ & $\mathrm{Cl}-40$ & 39.970 & Chlorine & $1.00 E+00$ & ICRP-107 & $1.35 \mathrm{E}+00$ & $\mathrm{~m}$ & $8.10 \mathrm{E}+01$ & $3.48 \mathrm{E}+09$ & -- & -- & -- & -- & DOE-STD-1196 & $2.09 \mathrm{E}-13$ \\
\hline $\mathrm{Cm}-238$ & Cm-238 & 238.053 & Curium & $1.00 \mathrm{E}-03$ & ICRP-38 & $2.40 \mathrm{E}+00$ & $\mathrm{~h}$ & $8.64 \mathrm{E}+03$ & $5.49 \mathrm{E}+06$ & ICRP-72 & $4.50 \mathrm{E}-09$ & $M$ & $\operatorname{Rec}$ & FGR-12 & $3.25 \mathrm{E}-15$ \\
\hline
\end{tabular}




\begin{tabular}{|c|c|c|c|c|c|c|c|c|c|c|c|c|c|c|c|}
\hline \multicolumn{16}{|c|}{ Table B.1 Radionuclide Specific Input Data Used For the Calculation of HC-2 TQs Using Recommended DCs (continued) } \\
\hline $\begin{array}{l}\text { MASTER } \\
\text { ISOTOPE } \\
\text { LIST }\end{array}$ & $\begin{array}{c}\text { Atomic } \\
\text { Mass } \\
\text { Nuclide }\end{array}$ & $\begin{array}{l}\text { Atomic } \\
\text { Mass }\end{array}$ & Element & $\begin{array}{c}\text { RF per } \\
\text { DOE-STD- } \\
1027\end{array}$ & $\begin{array}{l}\text { Half-Life } \\
\text { Reference }\end{array}$ & \multicolumn{2}{|c|}{$\begin{array}{c}\text { Half-Life } \\
\text { (original units) }\end{array}$} & \multirow{2}{*}{$\begin{array}{c}\text { Half Life (s) } \\
1.04 E+04\end{array}$} & \multirow{2}{*}{$\begin{array}{c}\begin{array}{c}\text { Sp. Act } \\
\text { (Ci/g) }\end{array} \\
4.52 \mathrm{E}+06\end{array}$} & \multirow{2}{*}{$\begin{array}{c}\begin{array}{c}\text { Inhalation DC } \\
\text { Reference }\end{array} \\
\text { DOE-STD-1196 }\end{array}$} & \multirow{2}{*}{$\begin{array}{c}\text { HC-2 } \\
\text { Inhalation } \\
\text { DC } \\
\text { (Sv/Bq) } \\
7.59 E-11 \\
\end{array}$} & \multicolumn{2}{|c|}{$\begin{array}{c}\text { Lung } \\
\text { Absorption } \\
\text { Class/Type }\end{array}$} & \multirow{2}{*}{$\begin{array}{c}\text { Immersion DC } \\
\text { Reference } \\
\text { DOE-STD-1196 }\end{array}$} & \multirow{2}{*}{$\begin{array}{c}\text { HC-2 } \\
\text { Immersion } \\
\text { DC (Sv/s } \\
\left.\text { per } \mathrm{Bq} / \mathrm{m}^{3}\right) \\
1.05 \mathrm{E}-14\end{array}$} \\
\hline $\mathrm{Cm}-239$ & $\mathrm{Cm}-239$ & 239.055 & Curium & $1.00 \mathrm{E}-03$ & ICRP-107 & $2.90 \mathrm{E}+00$ & $\mathrm{~h}$ & & & & & $\mathrm{M}$ & Rec & & \\
\hline $\mathrm{Cm}-240$ & $\mathrm{Cm}-240$ & 240.056 & Curium & $1.00 \mathrm{E}-03$ & ICRP-38 & $2.70 \mathrm{E}+01$ & $\mathrm{~d}$ & $2.33 \mathrm{E}+06$ & $2.01 \mathrm{E}+04$ & ICRP-72 & $3.20 \mathrm{E}-06$ & $\mathrm{M}$ & $\operatorname{Rec}$ & FGR-12 & $6.00 \mathrm{E}-18$ \\
\hline $\mathrm{Cm}-241$ & $\mathrm{Cm}-241$ & 241.058 & Curium & $1.00 \mathrm{E}-03$ & ICRP-38 & $3.28 \mathrm{E}+01$ & $d$ & $2.83 \mathrm{E}+06$ & $1.65 \mathrm{E}+04$ & ICRP-72 & $3.70 \mathrm{E}-08$ & $M$ & $\operatorname{Rec}$ & FGR-12 & $2.31 \mathrm{E}-14$ \\
\hline $\mathrm{Cm}-242$ & Cm-242 & 242.059 & Curium & $1.00 \mathrm{E}-03$ & ICRP-38 & $1.63 \mathrm{E}+02$ & $d$ & $1.41 \mathrm{E}+07$ & $3.31 \mathrm{E}+03$ & ICRP-72 & $5.20 \mathrm{E}-06$ & $M$ & $\operatorname{Rec}$ & FGR-12 & $5.69 \mathrm{E}-18$ \\
\hline $\mathrm{Cm}-243$ & Cm-243 & 243.061 & Curium & $1.00 \mathrm{E}-03$ & ICRP-38 & $2.85 \mathrm{E}+01$ & $y$ & $8.99 \mathrm{E}+08$ & $5.16 \mathrm{E}+01$ & ICRP-72 & $3.10 \mathrm{E}-05$ & $M$ & $\operatorname{Rec}$ & FGR-12 & $5.88 \mathrm{E}-15$ \\
\hline $\mathrm{Cm}-244$ & $\mathrm{Cm}-244$ & 244.063 & Curium & $1.00 \mathrm{E}-03$ & ICRP-38 & $1.81 \mathrm{E}+01$ & $y$ & $5.72 \mathrm{E}+08$ & $8.09 \mathrm{E}+01$ & ICRP-72 & $2.70 \mathrm{E}-05$ & $M$ & $\operatorname{Rec}$ & FGR-12 & $4.91 \mathrm{E}-18$ \\
\hline $\mathrm{Cm}-245$ & $\mathrm{Cm}-245$ & 245.065 & Curium & $1.00 \mathrm{E}-03$ & ICRP-38 & $8.50 \mathrm{E}+03$ & $y$ & $2.68 \mathrm{E}+11$ & $1.72 \mathrm{E}-01$ & ICRP-72 & $4.20 \mathrm{E}-05$ & $M$ & $\operatorname{Rec}$ & FGR-12 & $3.96 \mathrm{E}-15$ \\
\hline $\mathrm{Cm}-246$ & $\mathrm{Cm}-246$ & 246.067 & Curium & $1.00 \mathrm{E}-03$ & ICRP-38 & $4.73 \mathrm{E}+03$ & $y$ & $1.49 \mathrm{E}+11$ & $3.07 \mathrm{E}-01$ & ICRP-72 & $4.20 \mathrm{E}-05$ & $M$ & $\operatorname{Rec}$ & FGR-12 & $4.46 \mathrm{E}-18$ \\
\hline $\mathrm{Cm}-247$ & Cm-247 & 247.070 & Curium & $1.00 \mathrm{E}-03$ & ICRP-38 & $1.56 \mathrm{E}+07$ & $y$ & $4.92 \mathrm{E}+14$ & $9.28 \mathrm{E}-05$ & ICRP-72 & $3.90 \mathrm{E}-05$ & $M$ & $\operatorname{Rec}$ & FGR-12 & $1.50 \mathrm{E}-14$ \\
\hline $\mathrm{Cm}-248$ & $\mathrm{Cm}-248$ & 248.072 & Curium & $1.00 \mathrm{E}-03$ & ICRP-38 & $3.39 \mathrm{E}+05$ & $y$ & $1.07 \mathrm{E}+13$ & $4.25 \mathrm{E}-03$ & ICRP-72 & $1.50 \mathrm{E}-04$ & $M$ & $\operatorname{Rec}$ & FGR-12 & $3.39 \mathrm{E}-18$ \\
\hline $\mathrm{Cm}-249$ & $\mathrm{Cm}-249$ & 249.076 & Curium & $1.00 \mathrm{E}-03$ & ICRP-38 & $6.42 \mathrm{E}+01$ & $\mathrm{~m}$ & $3.85 \mathrm{E}+03$ & $1.18 \mathrm{E}+07$ & ICRP-72 & $3.30 \mathrm{E}-11$ & $M$ & $\operatorname{Rec}$ & FGR-12 & $9.36 \mathrm{E}-16$ \\
\hline $\mathrm{Cm}-250$ & $\mathrm{Cm}-250$ & 250.078 & Curium & $1.00 \mathrm{E}-03$ & ICRP-38 & $6.90 \mathrm{E}+03$ & $y$ & $2.18 \mathrm{E}+11$ & $2.07 \mathrm{E}-01$ & ICRP-72 & $8.40 \mathrm{E}-04$ & $M$ & $\operatorname{Rec}$ & DOE-STD-1196 & $6.52 \mathrm{E}-13$ \\
\hline $\mathrm{Cm}-251$ & Cm-251 & 251.082 & Curium & $1.00 \mathrm{E}-03$ & ICRP-107 & $1.68 \mathrm{E}+01$ & $\mathrm{~m}$ & $1.01 \mathrm{E}+03$ & $4.46 \mathrm{E}+07$ & DOE-STD-1196 & $2.74 \mathrm{E}-11$ & $M$ & $\operatorname{Rec}$ & DOE-STD-1196 & $5.20 \mathrm{E}-15$ \\
\hline Co-54m & Co-54 & 53.948 & Cobalt & $1.00 \mathrm{E}-03$ & ICRP-107 & $1.48 \mathrm{E}+00$ & $\mathrm{~m}$ & $8.88 \mathrm{E}+01$ & $2.35 \mathrm{E}+09$ & -- & -- & -- & -- & DOE-STD-1196 & $1.85 \mathrm{E}-13$ \\
\hline Co-55 & Co-55 & 54.942 & Cobalt & $1.00 \mathrm{E}-03$ & ICRP-38 & $1.75 \mathrm{E}+01$ & $\mathrm{~h}$ & $6.31 \mathrm{E}+04$ & $3.25 \mathrm{E}+06$ & ICRP-72 & $5.00 \mathrm{E}-10$ & $M$ & $\operatorname{Rec}$ & FGR-12 & $9.78 \mathrm{E}-14$ \\
\hline Co-56 & Co-56 & 55.940 & Cobalt & $1.00 \mathrm{E}-03$ & ICRP-38 & $7.88 \mathrm{E}+01$ & $d$ & $6.80 \mathrm{E}+06$ & $2.96 \mathrm{E}+04$ & ICRP-72 & $4.80 \mathrm{E}-09$ & $M$ & $\operatorname{Rec}$ & FGR-12 & $1.83 \mathrm{E}-13$ \\
\hline Co-57 & Co-57 & 56.936 & Cobalt & $1.00 \mathrm{E}-03$ & ICRP-38 & $2.71 \mathrm{E}+02$ & $d$ & $2.34 \mathrm{E}+07$ & $8.47 \mathrm{E}+03$ & ICRP-72 & $5.50 \mathrm{E}-10$ & $M$ & $\operatorname{Rec}$ & FGR-12 & $5.61 \mathrm{E}-15$ \\
\hline Co-58 & Co-58 & 57.936 & Cobalt & $1.00 \mathrm{E}-03$ & ICRP-38 & $7.08 \mathrm{E}+01$ & $d$ & $6.12 \mathrm{E}+06$ & $3.18 \mathrm{E}+04$ & ICRP-72 & $1.60 \mathrm{E}-09$ & $M$ & Rec & FGR-12 & $4.76 \mathrm{E}-14$ \\
\hline Co-58m & Co-58 & 57.936 & Cobalt & $1.00 \mathrm{E}-03$ & ICRP-38 & $9.15 \mathrm{E}+00$ & $\mathrm{~h}$ & $3.29 \mathrm{E}+04$ & $5.91 \mathrm{E}+06$ & ICRP-72 & $1.30 \mathrm{E}-11$ & $M$ & $\operatorname{Rec}$ & FGR-12 & $8.77 \mathrm{E}-20$ \\
\hline Co-60 & Co-60 & 59.934 & Cobalt & $1.00 \mathrm{E}-03$ & ICRP-38 & $5.27 \mathrm{E}+00$ & $y$ & $1.66 \mathrm{E}+08$ & $1.13 \mathrm{E}+03$ & ICRP-72 & $1.00 \mathrm{E}-08$ & $M$ & $\operatorname{Rec}$ & FGR-12 & $1.26 \mathrm{E}-13$ \\
\hline Co-60m & Co-60 & 59.934 & Cobalt & $1.00 \mathrm{E}-03$ & ICRP-38 & $1.05 \mathrm{E}+01$ & $\mathrm{~m}$ & $6.28 \mathrm{E}+02$ & $3.00 \mathrm{E}+08$ & ICRP-72 & $1.20 \mathrm{E}-12$ & $M$ & $\operatorname{Rec}$ & FGR-12 & $2.17 \mathrm{E}-16$ \\
\hline Co-61 & Co-61 & 60.932 & Cobalt & $1.00 \mathrm{E}-03$ & ICRP-38 & $1.65 \mathrm{E}+00$ & $\mathrm{~h}$ & $5.94 \mathrm{E}+03$ & $3.12 \mathrm{E}+07$ & ICRP-72 & $4.70 \mathrm{E}-11$ & $M$ & $\operatorname{Rec}$ & FGR-12 & $3.94 \mathrm{E}-15$ \\
\hline Co-62 & Co-62 & 61.934 & Cobalt & $1.00 \mathrm{E}-03$ & ICRP-107 & $1.50 \mathrm{E}+00$ & $m$ & $9.00 \mathrm{E}+01$ & $2.02 \mathrm{E}+09$ & -- & -- & -- & -- & DOE-STD-1196 & $7.92 \mathrm{E}-14$ \\
\hline Co-62m & Co-62 & 61.934 & Cobalt & $1.00 \mathrm{E}-03$ & ICRP-38 & $1.39 \mathrm{E}+01$ & $\mathrm{~m}$ & $8.35 \mathrm{E}+02$ & $2.18 \mathrm{E}+08$ & ICRP-72 & $2.00 \mathrm{E}-11$ & $M$ & $\operatorname{Rec}$ & FGR-12 & $1.37 \mathrm{E}-13$ \\
\hline $\mathrm{Cr}-48$ & $\mathrm{Cr}-48$ & 47.954 & Chromium & $1.00 \mathrm{E}-03$ & ICRP-38 & $2.30 \mathrm{E}+01$ & $\mathrm{~h}$ & $8.27 \mathrm{E}+04$ & $2.85 \mathrm{E}+06$ & ICRP-72 & $2.20 \mathrm{E}-10$ & $\mathrm{~s}$ & Max & FGR-12 & $2.06 \mathrm{E}-14$ \\
\hline Cr-49 & Cr-49 & 48.951 & Chromium & $1.00 \mathrm{E}-03$ & ICRP-38 & $4.21 \mathrm{E}+01$ & $\mathrm{~m}$ & $2.53 E+03$ & $9.13 \mathrm{E}+07$ & ICRP-72 & $3.50 \mathrm{E}-11$ & $\mathrm{~s}$ & Max & FGR-12 & $5.03 \mathrm{E}-14$ \\
\hline Cr-51 & $\mathrm{Cr}-51$ & 50.945 & Chromium & $1.00 \mathrm{E}-03$ & ICRP-38 & $2.77 \mathrm{E}+01$ & $d$ & $2.39 \mathrm{E}+06$ & $9.25 \mathrm{E}+04$ & ICRP-72 & $3.70 \mathrm{E}-11$ & $\mathrm{~s}$ & Max & FGR-12 & $1.51 \mathrm{E}-15$ \\
\hline Cr-55 & $\mathrm{Cr}-55$ & 54.941 & Chromium & $1.00 \mathrm{E}-03$ & ICRP-107 & $3.50 \mathrm{E}+00$ & $\mathrm{~m}$ & $2.10 \mathrm{E}+02$ & $9.79 \mathrm{E}+08$ & -- & -- & -- & -- & DOE-STD-1196 & $1.00 \mathrm{E}-15$ \\
\hline Cr-56 & Cr-56 & 55.941 & Chromium & $1.00 \mathrm{E}-03$ & ICRP-107 & $5.94 \mathrm{E}+00$ & $\mathrm{~m}$ & $3.56 \mathrm{E}+02$ & $5.66 \mathrm{E}+08$ & -- & -- & -- & -- & DOE-STD-1196 & $3.47 \mathrm{E}-15$ \\
\hline Cs-121 & Cs-121 & 120.917 & Cesium & $1.00 \mathrm{E}-02$ & ICRP-107 & $1.55 \mathrm{E}+02$ & $\mathrm{~s}$ & $1.55 \mathrm{E}+02$ & $6.02 \mathrm{E}+08$ & -- & -- & -- & -- & DOE-STD-1196 & $5.41 \mathrm{E}-14$ \\
\hline Cs-121m & Cs-121 & 120.917 & Cesium & $1.00 \mathrm{E}-02$ & ICRP-107 & $1.22 \mathrm{E}+02$ & $\mathrm{~s}$ & $1.22 \mathrm{E}+02$ & $7.65 \mathrm{E}+08$ & -- & -- & -- & -- & DOE-STD-1196 & $5.38 \mathrm{E}-14$ \\
\hline Cs-123 & Cs-123 & 122.913 & Cesium & $1.00 \mathrm{E}-02$ & ICRP-107 & $5.88 \mathrm{E}+00$ & $\mathrm{~m}$ & $3.53 \mathrm{E}+02$ & $2.60 \mathrm{E}+08$ & -- & -- & -- & -- & DOE-STD-1196 & $4.89 \mathrm{E}-14$ \\
\hline Cs-124 & Cs-124 & 123.912 & Cesium & $1.00 \mathrm{E}-02$ & ICRP-107 & $3.08 \mathrm{E}+01$ & $\mathrm{~s}$ & $3.08 \mathrm{E}+01$ & $2.96 \mathrm{E}+09$ & -- & -- & -- & -- & DOE-STD-1196 & $5.43 \mathrm{E}-14$ \\
\hline Cs-125 & Cs-125 & 124.910 & Cesium & $1.00 \mathrm{E}-02$ & ICRP-38 & $4.50 \mathrm{E}+01$ & $\mathrm{~m}$ & $2.70 \mathrm{E}+03$ & $3.35 \mathrm{E}+07$ & ICRP-72 & $1.20 \mathrm{E}-11$ & $F$ & $\operatorname{Rec}$ & FGR-12 & $3.22 \mathrm{E}-14$ \\
\hline Cs-126 & Cs-126 & 125.909 & Cesium & $1.00 \mathrm{E}-02$ & ICRP-38 & $1.64 \mathrm{E}+00$ & $\mathrm{~m}$ & $9.84 \mathrm{E}+01$ & $9.11 \mathrm{E}+08$ & -- & -- & -- & -- & FGR-12 & $5.24 \mathrm{E}-14$ \\
\hline Cs-127 & Cs-127 & 126.907 & Cesium & $1.00 \mathrm{E}-02$ & ICRP-38 & $6.25 \mathrm{E}+00$ & $\mathrm{~h}$ & $2.25 \mathrm{E}+04$ & $3.95 \mathrm{E}+06$ & ICRP-72 & $2.00 \mathrm{E}-11$ & $\mathrm{~F}$ & $\operatorname{Rec}$ & FGR-12 & $1.93 \mathrm{E}-14$ \\
\hline Cs-128 & Cs-128 & 127.908 & Cesium & $1.00 \mathrm{E}-02$ & ICRP-38 & $3.90 \mathrm{E}+00$ & $\mathrm{~m}$ & $2.34 \mathrm{E}+02$ & $3.77 E+08$ & -- & -- & -- & -- & FGR-12 & $4.32 \mathrm{E}-14$ \\
\hline Cs-129 & Cs-129 & 128.906 & Cesium & $1.00 \mathrm{E}-02$ & ICRP-38 & $3.21 \mathrm{E}+01$ & $\mathrm{~h}$ & $1.15 \mathrm{E}+05$ & $7.58 \mathrm{E}+05$ & ICRP-72 & $4.20 \mathrm{E}-11$ & $\mathrm{~F}$ & $\operatorname{Rec}$ & FGR-12 & $1.24 \mathrm{E}-14$ \\
\hline Cs-130 & Cs-130 & 129.907 & Cesium & $1.00 \mathrm{E}-02$ & ICRP-38 & $2.99 \mathrm{E}+01$ & $\mathrm{~m}$ & $1.79 \mathrm{E}+03$ & $4.84 \mathrm{E}+07$ & ICRP-72 & $7.80 \mathrm{E}-12$ & $F$ & $\operatorname{Rec}$ & FGR-12 & $2.45 \mathrm{E}-14$ \\
\hline Cs- $130 \mathrm{~m}$ & Cs-130 & 129.907 & Cesium & $1.00 \mathrm{E}-02$ & ICRP-107 & $3.46 \mathrm{E}+00$ & $\mathrm{~m}$ & $2.08 \mathrm{E}+02$ & $4.18 \mathrm{E}+08$ & -- & -- & -- & -- & DOE-STD-1196 & $2.01 \mathrm{E}-15$ \\
\hline Cs-131 & Cs-131 & 130.905 & Cesium & $1.00 \mathrm{E}-02$ & $\begin{array}{l}\text { ICRP-38 } \\
\end{array}$ & $9.69 \mathrm{E}+00$ & $d$ & $8.37 E+05$ & $1.03 E+05$ & ICRP-72 & $2.70 \mathrm{E}-11$ & $F$ & $\operatorname{Rec}$ & FGR-12 & $3.28 \mathrm{E}-16$ \\
\hline Cs-132 & Cs-132 & 131.906 & Cesium & $1.00 \mathrm{E}-02$ & ICRP-38 & $6.48 \mathrm{E}+00$ & $d$ & $5.59 \mathrm{E}+05$ & $1.53 \mathrm{E}+05$ & ICRP-72 & $2.30 \mathrm{E}-10$ & $\mathrm{~F}$ & $\operatorname{Rec}$ & FGR-12 & $3.34 \mathrm{E}-14$ \\
\hline Cs-134 & Cs-134 & 133.907 & Cesium & $1.00 \mathrm{E}-02$ & ICRP-38 & $2.06 \mathrm{E}+00$ & $y$ & $6.51 \mathrm{E}+07$ & $1.29 \mathrm{E}+03$ & ICRP-72 & $6.60 \mathrm{E}-09$ & $\mathrm{~F}$ & $\operatorname{Rec}$ & FGR-12 & $7.57 \mathrm{E}-14$ \\
\hline Cs-134m & Cs-134 & 133.907 & Cesium & $1.00 \mathrm{E}-02$ & ICRP-38 & $2.90 \mathrm{E}+00$ & $\mathrm{~h}$ & $1.04 \mathrm{E}+04$ & $8.07 \mathrm{E}+06$ & ICRP-72 & $1.40 \mathrm{E}-11$ & $\mathrm{~F}$ & $\operatorname{Rec}$ & FGR-12 & $9.05 \mathrm{E}-16$ \\
\hline Cs-135 & Cs-135 & 134.906 & Cesium & $1.00 \mathrm{E}-02$ & ICRP-38 & $2.30 \mathrm{E}+06$ & $y$ & $7.26 \mathrm{E}+13$ & $1.15 \mathrm{E}-03$ & ICRP-72 & $6.90 \mathrm{E}-10$ & $\mathrm{~F}$ & $\operatorname{Rec}$ & FGR-12 & $5.65 \mathrm{E}-19$ \\
\hline Cs-135m & Cs-135 & 134.906 & Cesium & $1.00 \mathrm{E}-02$ & ICRP-38 & $5.30 \mathrm{E}+01$ & $\mathrm{~m}$ & $3.18 \mathrm{E}+03$ & $2.63 E+07$ & ICRP-72 & $1.20 \mathrm{E}-11$ & $\mathrm{~F}$ & $\operatorname{Rec}$ & FGR-12 & $7.76 \mathrm{E}-14$ \\
\hline
\end{tabular}




\begin{tabular}{|c|c|c|c|c|c|c|c|c|c|c|c|c|c|c|c|}
\hline $\begin{array}{l}\text { MASTER } \\
\text { ISOTOPE } \\
\text { LIST }\end{array}$ & $\begin{array}{c}\text { Atomic } \\
\text { Mass } \\
\text { Nuclide }\end{array}$ & $\begin{array}{c}\text { Atomic } \\
\text { Mass }\end{array}$ & Element & $\begin{array}{c}\text { RF per } \\
\text { DOE-STD- } \\
1027\end{array}$ & $\begin{array}{c}\text { Half-Life } \\
\text { Reference }\end{array}$ & $\begin{array}{r}\text { Half-Li } \\
\text { (original L }\end{array}$ & or th & Half Life (s) & $\begin{array}{l}\text { Sp. Act } \\
(\mathrm{Ci} / \mathrm{g})\end{array}$ & $\begin{array}{c}\text { Inhalation DC } \\
\text { Reference }\end{array}$ & $\begin{array}{c}\text { (continued) } \\
\text { HC-2 } \\
\text { Inhalation } \\
\text { DC } \\
\text { (Sv/Bq) }\end{array}$ & & $\begin{array}{l}\text { ung } \\
\text { rption } \\
\text { /Type }\end{array}$ & $\begin{array}{l}\text { Immersion DC } \\
\text { Reference }\end{array}$ & $\begin{array}{c}\text { HC-2 } \\
\text { Immersion } \\
\mathrm{DC}(\mathrm{Sv} / \mathrm{s} \\
\left.\text { per } \mathrm{Bq} / \mathrm{m}^{3}\right)\end{array}$ \\
\hline Cs-136 & Cs-136 & 135.907 & Cesium & $1.00 E-02$ & ICRP-38 & $1.31 E+01$ & $d$ & $1.13 \mathrm{E}+06$ & $7.33 \mathrm{E}+04$ & ICRP-72 & $1.20 \mathrm{E}-09$ & $\mathrm{~F}$ & $\operatorname{Rec}$ & FGR-12 & 1.06E-13 \\
\hline Cs-137 & Cs-137 & 136.907 & Cesium & $1.00 \mathrm{E}-02$ & ICRP-38 & $3.00 \mathrm{E}+01$ & $y$ & $9.47 \mathrm{E}+08$ & $8.70 \mathrm{E}+01$ & ICRP-72 & $4.60 \mathrm{E}-09$ & $F$ & $\operatorname{Rec}$ & FGR-12 & $7.74 \mathrm{E}-18$ \\
\hline Cs-138 & Cs-138 & 137.911 & Cesium & $1.00 \mathrm{E}-02$ & ICRP-38 & $3.22 \mathrm{E}+01$ & $\mathrm{~m}$ & $1.93 \mathrm{E}+03$ & $4.23 \mathrm{E}+07$ & ICRP-72 & $2.40 \mathrm{E}-11$ & $\mathrm{~F}$ & Rec & FGR-12 & $1.21 \mathrm{E}-13$ \\
\hline Cs-138m & Cs-138 & 137.911 & Cesium & $1.00 \mathrm{E}-02$ & ICRP-107 & $2.91 \mathrm{E}+00$ & $\mathrm{~m}$ & $1.75 \mathrm{E}+02$ & $4.69 \mathrm{E}+08$ & -- & -- & -- & -- & DOE-STD-1196 & $1.92 \mathrm{E}-14$ \\
\hline Cs-139 & Cs-139 & 138.913 & Cesium & $1.00 \mathrm{E}-02$ & ICRP-107 & $9.27 \mathrm{E}+00$ & $\mathrm{~m}$ & $5.56 \mathrm{E}+02$ & $1.46 \mathrm{E}+08$ & -- & -- & -- & -- & DOE-STD-1196 & $1.66 \mathrm{E}-14$ \\
\hline Cs-140 & Cs-140 & 139.917 & Cesium & $1.00 \mathrm{E}-02$ & ICRP-107 & $6.37 \mathrm{E}+01$ & $\mathrm{~s}$ & $6.37 \mathrm{E}+01$ & $1.27 \mathrm{E}+09$ & -- & -- & -- & -- & DOE-STD-1196 & $8.89 \mathrm{E}-14$ \\
\hline Cu-57 & Cu-57 & 56.949 & Copper & $1.00 \mathrm{E}-03$ & ICRP-38 & $2.33 \mathrm{E}+02$ & $\mathrm{~ms}$ & $2.33 \mathrm{E}-01$ & $8.50 \mathrm{E}+11$ & -- & -- & -- & -- & DOE-STD-1196 & $5.65 \mathrm{E}-14$ \\
\hline Cu-59 & Cu-59 & 58.939 & Copper & $1.00 \mathrm{E}-03$ & ICRP-107 & $8.15 \mathrm{E}+01$ & $\mathrm{~s}$ & $8.15 \mathrm{E}+01$ & $2.35 \mathrm{E}+09$ & -- & -- & -- & -- & DOE-STD-1196 & $6.68 \mathrm{E}-14$ \\
\hline Cu-60 & Cu-60 & 59.937 & Copper & $1.00 \mathrm{E}-03$ & $\begin{array}{l}\text { ICRP-38 } \\
\end{array}$ & $2.32 \mathrm{E}+01$ & $\mathrm{~m}$ & $1.39 \mathrm{E}+03$ & $1.35 \mathrm{E}+08$ & ICRP-72 & $3.40 \mathrm{E}-11$ & $\mathrm{~s}$ & Max & FGR-12 & $1.98 \mathrm{E}-13$ \\
\hline Cu-61 & Cu-61 & 60.933 & Copper & $1.00 \mathrm{E}-03$ & ICRP-38 & $3.41 \mathrm{E}+00$ & $\mathrm{~h}$ & $1.23 \mathrm{E}+04$ & $1.51 \mathrm{E}+07$ & ICRP-72 & $7.80 \mathrm{E}-11$ & $\mathrm{~s}$ & Max & FGR-12 & $3.99 \mathrm{E}-14$ \\
\hline Cu-62 & Cu-62 & 61.933 & Copper & $1.00 \mathrm{E}-03$ & ICRP-38 & $9.74 \mathrm{E}+00$ & $\mathrm{~m}$ & $5.84 \mathrm{E}+02$ & $3.12 \mathrm{E}+08$ & -- & -- & -- & -- & FGR-12 & $4.86 \mathrm{E}-14$ \\
\hline Cu-64 & Cu-64 & 63.930 & Copper & $1.00 \mathrm{E}-03$ & ICRP-38 & $1.27 \mathrm{E}+01$ & $\mathrm{~h}$ & $4.57 \mathrm{E}+04$ & $3.86 \mathrm{E}+06$ & ICRP-72 & $1.20 \mathrm{E}-10$ & $\mathrm{~s}$ & Max & FGR-12 & $9.10 \mathrm{E}-15$ \\
\hline Cu-66 & Cu-66 & 65.929 & Copper & $1.00 \mathrm{E}-03$ & ICRP-38 & $5.10 \mathrm{E}+00$ & $\mathrm{~m}$ & $3.06 \mathrm{E}+02$ & $5.59 \mathrm{E}+08$ & -- & -- & -- & -- & FGR-12 & $4.46 \mathrm{E}-15$ \\
\hline Cu-67 & Cu-67 & 66.928 & Copper & $1.00 \mathrm{E}-03$ & ICRP-38 & $6.19 \mathrm{E}+01$ & $\mathrm{~h}$ & $2.23 \mathrm{E}+05$ & $7.57 E+05$ & ICRP-72 & $6.10 \mathrm{E}-10$ & $\mathrm{~s}$ & Max & FGR-12 & $5.41 \mathrm{E}-15$ \\
\hline Cu-69 & Cu-69 & 68.929 & Copper & $1.00 \mathrm{E}-03$ & ICRP-107 & $2.85 \mathrm{E}+00$ & $\mathrm{~m}$ & $1.71 \mathrm{E}+02$ & $9.57 \mathrm{E}+08$ & -- & -- & -- & -- & DOE-STD-1196 & $2.53 \mathrm{E}-14$ \\
\hline Dy-148 & Dy-148 & 147.927 & Dysprosium & $1.00 \mathrm{E}-03$ & ICRP-107 & $3.30 \mathrm{E}+00$ & $\mathrm{~m}$ & $1.98 \mathrm{E}+02$ & $3.85 \mathrm{E}+08$ & -- & -- & -- & -- & DOE-STD-1196 & $3.15 \mathrm{E}-14$ \\
\hline Dy-149 & Dy-149 & 148.927 & Dysprosium & $1.00 \mathrm{E}-03$ & ICRP-107 & $4.20 \mathrm{E}+00$ & $\mathrm{~m}$ & $2.52 \mathrm{E}+02$ & $3.01 \mathrm{E}+08$ & -- & -- & -- & -- & DOE-STD-1196 & $7.52 \mathrm{E}-14$ \\
\hline Dy-150 & Dy-150 & 149.926 & Dysprosium & $1.00 \mathrm{E}-03$ & ICRP-107 & $7.17 E+00$ & $\mathrm{~m}$ & $4.30 \mathrm{E}+02$ & $1.75 \mathrm{E}+08$ & $\begin{array}{ll}-- \\
\end{array}$ & $\begin{array}{ll}-- \\
\end{array}$ & -- & -- & DOE-STD-1196 & $1.18 \mathrm{E}-14$ \\
\hline Dy-151 & Dy-151 & 150.926 & Dysprosium & $1.00 \mathrm{E}-03$ & ICRP-107 & $1.79 \mathrm{E}+01$ & $\mathrm{~m}$ & $1.07 \mathrm{E}+03$ & $6.96 \mathrm{E}+07$ & DOE-STD-1196 & $1.51 \mathrm{E}-10$ & $\mathrm{~s}$ & Max & DOE-STD-1196 & $6.28 \mathrm{E}-14$ \\
\hline Dy-152 & Dy-152 & 151.925 & Dysprosium & $1.00 \mathrm{E}-03$ & ICRP-107 & $2.38 \mathrm{E}+00$ & $\mathrm{~h}$ & $8.57 \mathrm{E}+03$ & $8.67 E+06$ & DOE-STD-1196 & $7.46 \mathrm{E}-11$ & $\mathrm{~s}$ & Max & DOE-STD-1196 & $1.18 \mathrm{E}-14$ \\
\hline Dy-153 & Dy-153 & 152.926 & Dysprosium & $1.00 \mathrm{E}-03$ & ICRP-107 & $6.40 \mathrm{E}+00$ & $\mathrm{~h}$ & $2.30 \mathrm{E}+04$ & $3.20 \mathrm{E}+06$ & DOE-STD-1196 & $1.51 \mathrm{E}-10$ & $\mathrm{~s}$ & Max & DOE-STD-1196 & $3.82 \mathrm{E}-14$ \\
\hline Dy-154 & Dy-154 & 153.924 & Dysprosium & $1.00 \mathrm{E}-03$ & ICRP-107 & $3.00 \mathrm{E}+06$ & $y$ & $9.47 \mathrm{E}+13$ & $7.74 \mathrm{E}-04$ & DOE-STD-1196 & $2.74 \mathrm{E}-05$ & $F$ & Max & -- & -- \\
\hline Dy-155 & Dy-155 & 154.926 & Dysprosium & $1.00 \mathrm{E}-03$ & ICRP-38 & $1.00 \mathrm{E}+01$ & $\mathrm{~h}$ & $3.60 \mathrm{E}+04$ & $2.02 \mathrm{E}+06$ & ICRP-72 & $7.70 \mathrm{E}-11$ & $M$ & Max & FGR-12 & $2.77 \mathrm{E}-14$ \\
\hline Dy-157 & Dy-157 & 156.925 & Dysprosium & $1.00 \mathrm{E}-03$ & ICRP-38 & $8.10 \mathrm{E}+00$ & $\mathrm{~h}$ & $2.92 \mathrm{E}+04$ & $2.47 \mathrm{E}+06$ & ICRP-72 & $3.00 E-11$ & $M$ & Max & FGR-12 & $1.63 \mathrm{E}-14$ \\
\hline Dy-159 & Dy-159 & 158.926 & Dysprosium & $1.00 \mathrm{E}-03$ & ICRP-38 & $1.44 \mathrm{E}+02$ & d & $1.25 \mathrm{E}+07$ & $5.69 \mathrm{E}+03$ & ICRP-72 & $3.70 \mathrm{E}-10$ & $M$ & Max & FGR-12 & $1.25 \mathrm{E}-15$ \\
\hline Dy-165 & Dy-165 & 164.932 & Dysprosium & $1.00 \mathrm{E}-03$ & ICRP-38 & $2.33 \mathrm{E}+00$ & $\mathrm{~h}$ & $8.40 \mathrm{E}+03$ & $8.14 \mathrm{E}+06$ & ICRP-72 & $6.00 \mathrm{E}-11$ & $M$ & Max & FGR-12 & $1.20 \mathrm{E}-15$ \\
\hline Dy-165m & Dy-165 & 164.932 & Dysprosium & $1.00 \mathrm{E}-03$ & ICRP-107 & $1.26 \mathrm{E}+00$ & $\mathrm{~m}$ & $7.54 \mathrm{E}+01$ & $9.07 E+08$ & -- & -- & -- & -- & DOE-STD-1196 & 7.08E-16 \\
\hline Dy-166 & Dy-166 & 165.933 & Dysprosium & $1.00 \mathrm{E}-03$ & ICRP-38 & $8.16 \mathrm{E}+01$ & $\mathrm{~h}$ & $2.94 \mathrm{E}+05$ & $2.31 \mathrm{E}+05$ & ICRP-72 & $1.90 \mathrm{E}-09$ & $M$ & Max & FGR-12 & $1.40 \mathrm{E}-15$ \\
\hline Dy-167 & Dy-167 & 166.936 & Dysprosium & $1.00 \mathrm{E}-03$ & ICRP-107 & $6.20 \mathrm{E}+00$ & $\mathrm{~m}$ & $3.72 \mathrm{E}+02$ & $1.82 \mathrm{E}+08$ & -- & -- & -- & -- & DOE-STD-1196 & $2.40 \mathrm{E}-14$ \\
\hline Dy-168 & Dy-168 & 167.937 & Dysprosium & $1.00 \mathrm{E}-03$ & ICRP-107 & $8.70 \mathrm{E}+00$ & $\mathrm{~m}$ & $5.22 \mathrm{E}+02$ & $1.29 \mathrm{E}+08$ & -- & -- & -- & -- & DOE-STD-1196 & $1.73 \mathrm{E}-14$ \\
\hline Er-154 & Er-154 & 153.933 & Erbium & $1.00 \mathrm{E}-03$ & ICRP-107 & $3.73 \mathrm{E}+00$ & $\mathrm{~m}$ & $2.24 \mathrm{E}+02$ & $3.27 \mathrm{E}+08$ & -- & -- & -- & -- & DOE-STD-1196 & $2.24 \mathrm{E}-15$ \\
\hline Er-155 & Er-155 & 154.933 & Erbium & $1.00 \mathrm{E}-03$ & JAERI & $5.30 \mathrm{E}+00$ & $\mathrm{~m}$ & $3.18 \mathrm{E}+02$ & $2.29 \mathrm{E}+08$ & -- & -- & -- & -- & -- & -- \\
\hline Er-156 & Er-156 & 155.931 & Erbium & $1.00 \mathrm{E}-03$ & ICRP-107 & $1.95 \mathrm{E}+01$ & $\mathrm{~m}$ & $1.17 \mathrm{E}+03$ & $6.18 \mathrm{E}+07$ & DOE-STD-1196 & $2.38 \mathrm{E}-11$ & $\mathrm{~s}$ & Max & DOE-STD-1196 & $1.72 \mathrm{E}-15$ \\
\hline Er-159 & Er-159 & 158.931 & Erbium & $1.00 \mathrm{E}-03$ & ICRP-107 & $3.60 \mathrm{E}+01$ & $\mathrm{~m}$ & $2.16 \mathrm{E}+03$ & $3.29 \mathrm{E}+07$ & DOE-STD-1196 & $2.07 \mathrm{E}-11$ & $\mathrm{~s}$ & Max & DOE-STD-1196 & $4.36 \mathrm{E}-14$ \\
\hline Er-161 & Er-161 & 160.930 & Erbium & $1.00 \mathrm{E}-03$ & ICRP-38 & $3.24 \mathrm{E}+00$ & $\mathrm{~h}$ & $1.17 \mathrm{E}+04$ & $6.01 \mathrm{E}+06$ & ICRP-72 & $4.80 \mathrm{E}-11$ & $M$ & Max & FGR-12 & $4.42 \mathrm{E}-14$ \\
\hline Er-163 & Er-163 & 162.930 & Erbium & $1.00 \mathrm{E}-03$ & ICRP-107 & $7.50 \mathrm{E}+01$ & $\mathrm{~m}$ & $4.50 \mathrm{E}+03$ & $1.54 \mathrm{E}+07$ & DOE-STD-1196 & $1.56 \mathrm{E}-12$ & $\mathrm{~s}$ & Max & DOE-STD-1196 & $9.82 \mathrm{E}-16$ \\
\hline Er-165 & Er-165 & 164.931 & Erbium & $1.00 \mathrm{E}-03$ & ICRP-38 & $1.04 \mathrm{E}+01$ & $h$ & $3.73 E+04$ & $1.83 \mathrm{E}+06$ & ICRP-72 & $7.90 \mathrm{E}-12$ & $M$ & Max & FGR-12 & $1.11 \mathrm{E}-15$ \\
\hline Er-167m & Er-167 & 166.932 & Erbium & $1.00 \mathrm{E}-03$ & ICRP-38 & $2.28 \mathrm{E}+00$ & $\mathrm{~s}$ & $2.28 \mathrm{E}+00$ & $2.96 \mathrm{E}+10$ & -- & -- & -- & -- & DOE-STD-1196 & 4.03E-15 \\
\hline Er-169 & Er-169 & 168.935 & Erbium & $1.00 \mathrm{E}-03$ & ICRP-38 & $9.30 \mathrm{E}+00$ & $d$ & $8.04 \mathrm{E}+05$ & $8.31 \mathrm{E}+04$ & ICRP-72 & 1.00E-09 & $M$ & Max & FGR-12 & $1.74 \mathrm{E}-18$ \\
\hline Er-171 & Er-171 & 170.938 & Erbium & $1.00 \mathrm{E}-03$ & ICRP-38 & $7.52 \mathrm{E}+00$ & $\mathrm{~h}$ & $2.71 \mathrm{E}+04$ & $2.44 \mathrm{E}+06$ & ICRP-72 & $2.20 \mathrm{E}-10$ & $M$ & Max & FGR-12 & $1.78 \mathrm{E}-14$ \\
\hline Er-172 & Er-172 & 171.939 & Erbium & $1.00 \mathrm{E}-03$ & ICRP-38 & $4.93 \mathrm{E}+01$ & $\mathrm{~h}$ & $1.77 \mathrm{E}+05$ & $3.70 \mathrm{E}+05$ & ICRP-72 & $1.10 \mathrm{E}-09$ & $M$ & Max & FGR-12 & $2.47 \mathrm{E}-14$ \\
\hline Er-173 & Er-173 & 172.942 & Erbium & $1.00 \mathrm{E}-03$ & ICRP-107 & $1.43 \mathrm{E}+00$ & $\mathrm{~m}$ & $8.60 \mathrm{E}+01$ & $7.58 \mathrm{E}+08$ & -- & -- & -- & -- & DOE-STD-1196 & $3.73 \mathrm{E}-14$ \\
\hline Es-249 & Es-249 & 249.076 & Einsteinium & $1.00 \mathrm{E}-03$ & ICRP-107 & $1.02 \mathrm{E}+02$ & $\mathrm{~m}$ & $6.13 \mathrm{E}+03$ & $7.39 \mathrm{E}+06$ & DOE-STD-1196 & $2.49 \mathrm{E}-10$ & $M$ & Max & DOE-STD-1196 & $1.77 \mathrm{E}-14$ \\
\hline Es-250 & Es-250 & 250.079 & Einsteinium & $1.00 \mathrm{E}-03$ & ICRP-38 & $2.10 \mathrm{E}+00$ & $\mathrm{~h}$ & $7.56 \mathrm{E}+03$ & $5.97 \mathrm{E}+06$ & ICRP-72 & $6.30 \mathrm{E}-10$ & $M$ & Max & FGR-12 & $1.90 \mathrm{E}-14$ \\
\hline Es-250m & Es-250 & 250.079 & Einsteinium & $1.00 \mathrm{E}-03$ & ICRP-107 & $2.22 \mathrm{E}+00$ & $\mathrm{~h}$ & $7.99 \mathrm{E}+03$ & $5.64 \mathrm{E}+06$ & DOE-STD-1196 & $1.59 \mathrm{E}-09$ & $\mathrm{~F}$ & Max & DOE-STD-1196 & $2.49 \mathrm{E}-14$ \\
\hline Es-251 & Es-251 & 251.080 & Einsteinium & $1.00 \mathrm{E}-03$ & ICRP-38 & $3.30 \mathrm{E}+01$ & $\mathrm{~h}$ & $1.19 E+05$ & $3.78 \mathrm{E}+05$ & ICRP-72 & $2.10 \mathrm{E}-09$ & $M$ & Max & FGR-12 & $4.13 \mathrm{E}-15$ \\
\hline
\end{tabular}




\begin{tabular}{|c|c|c|c|c|c|c|c|c|c|c|c|c|c|c|c|}
\hline \multicolumn{16}{|c|}{ Table B.1 Radionuclide Specific Input Data Used For the Calculation of HC-2 TQs Using Recommended DCs (continued) } \\
\hline $\begin{array}{l}\text { MASTER } \\
\text { ISOTOPE } \\
\text { LIST }\end{array}$ & $\begin{array}{c}\text { Atomic } \\
\text { Mass } \\
\text { Nuclide }\end{array}$ & $\begin{array}{l}\text { Atomic } \\
\text { Mass }\end{array}$ & Element & $\begin{array}{c}\text { RF per } \\
\text { DOE-STD- } \\
1027\end{array}$ & $\begin{array}{l}\text { Half-Life } \\
\text { Reference }\end{array}$ & \multicolumn{2}{|c|}{$\begin{array}{c}\text { Half-Life } \\
\text { (original units) }\end{array}$} & \multirow{2}{*}{$\begin{array}{c}\text { Half Life (s) } \\
1.77 E+06\end{array}$} & \multirow{2}{*}{$\begin{array}{c}\begin{array}{c}\text { Sp. Act } \\
\text { (Ci/g) }\end{array} \\
2.52 \mathrm{E}+04\end{array}$} & \multirow{2}{*}{$\begin{array}{c}\text { Inhalation DC } \\
\text { Reference } \\
\text { ICRP-72 }\end{array}$} & \multirow{2}{*}{$\begin{array}{c}\text { HC-2 } \\
\text { Inhalation } \\
\text { DC } \\
\text { (Sv/Bq) } \\
2.70 E-06 \\
\end{array}$} & \multicolumn{2}{|c|}{$\begin{array}{c}\text { Lung } \\
\text { Absorption } \\
\text { Class/Type }\end{array}$} & \multirow{2}{*}{$\begin{array}{c}\begin{array}{c}\text { Immersion DC } \\
\text { Reference }\end{array} \\
\text { FGR-12 }\end{array}$} & \multirow{2}{*}{ 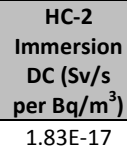 } \\
\hline Es-253 & Es-253 & 253.085 & Einsteinium & $1.00 \mathrm{E}-03$ & ICRP-38 & $2.05 \mathrm{E}+01$ & d & & & & & $\mathrm{M}$ & Max & & \\
\hline Es-254 & Es-254 & 254.088 & Einsteinium & $1.00 \mathrm{E}-03$ & ICRP-38 & $2.76 \mathrm{E}+02$ & $\mathrm{~d}$ & $2.38 \mathrm{E}+07$ & $1.86 \mathrm{E}+03$ & ICRP-72 & $8.60 \mathrm{E}-06$ & $\mathrm{M}$ & Max & FGR-12 & $1.93 \mathrm{E}-16$ \\
\hline Es-254m & Es-254 & 254.088 & Einsteinium & $1.00 \mathrm{E}-03$ & ICRP-38 & $3.93 \mathrm{E}+01$ & $\mathrm{~h}$ & $1.41 \mathrm{E}+05$ & $3.14 \mathrm{E}+05$ & ICRP-72 & $4.70 \mathrm{E}-07$ & $M$ & Max & FGR-12 & $2.25 \mathrm{E}-14$ \\
\hline Es-255 & Es-255 & 255.090 & Einsteinium & $1.00 \mathrm{E}-03$ & ICRP-107 & $3.98 \mathrm{E}+01$ & $d$ & $3.44 \mathrm{E}+06$ & $1.29 \mathrm{E}+04$ & DOE-STD-1196 & $4.95 \mathrm{E}-06$ & $\mathrm{~s}$ & Max & DOE-STD-1196 & $4.95 \mathrm{E}-17$ \\
\hline Es-256 & Es-256 & 256.094 & Einsteinium & $1.00 \mathrm{E}-03$ & ICRP-107 & $2.54 \mathrm{E}+01$ & $\mathrm{~m}$ & $1.52 \mathrm{E}+03$ & $2.89 \mathrm{E}+07$ & DOE-STD-1196 & $4.55 \mathrm{E}-08$ & $\mathrm{~s}$ & Max & DOE-STD-1196 & $4.33 \mathrm{E}-16$ \\
\hline Eu-142 & Eu-142 & 141.923 & Europium & $1.00 \mathrm{E}-03$ & ICRP-107 & $2.34 \mathrm{E}+00$ & $\mathrm{~s}$ & $2.34 \mathrm{E}+00$ & $3.40 \mathrm{E}+10$ & -- & -- & -- & -- & DOE-STD-1196 & $5.78 \mathrm{E}-14$ \\
\hline Eu-142m & Eu-142 & 141.923 & Europium & $1.00 \mathrm{E}-03$ & ICRP-107 & $1.22 \mathrm{E}+00$ & $\mathrm{~m}$ & $7.34 \mathrm{E}+01$ & $1.08 \mathrm{E}+09$ & -- & -- & -- & -- & DOE-STD-1196 & $1.58 \mathrm{E}-13$ \\
\hline Eu-143 & Eu-143 & 142.920 & Europium & $1.00 \mathrm{E}-03$ & ICRP-107 & $2.59 \mathrm{E}+00$ & $\mathrm{~m}$ & $1.55 \mathrm{E}+02$ & $5.08 \mathrm{E}+08$ & -- & -- & -- & -- & DOE-STD-1196 & $5.26 \mathrm{E}-14$ \\
\hline Eu-144 & Eu-144 & 143.919 & Europium & $1.00 \mathrm{E}-03$ & ICRP-107 & $1.02 \mathrm{E}+01$ & $\mathrm{~s}$ & $1.02 \mathrm{E}+01$ & $7.69 \mathrm{E}+09$ & -- & -- & -- & -- & DOE-STD-1196 & $5.18 \mathrm{E}-14$ \\
\hline Eu-145 & Eu-145 & 144.916 & Europium & $1.00 \mathrm{E}-03$ & ICRP-38 & $5.94 \mathrm{E}+00$ & $d$ & $5.13 \mathrm{E}+05$ & $1.52 \mathrm{E}+05$ & ICRP-72 & $5.50 \mathrm{E}-10$ & $M$ & Max & FGR-12 & $7.22 \mathrm{E}-14$ \\
\hline Eu-146 & Eu-146 & 145.917 & Europium & $1.00 \mathrm{E}-03$ & ICRP-38 & $4.61 \mathrm{E}+00$ & $d$ & $3.98 \mathrm{E}+05$ & $1.94 \mathrm{E}+05$ & ICRP-72 & $8.00 \mathrm{E}-10$ & $M$ & Max & FGR-12 & $1.23 \mathrm{E}-13$ \\
\hline Eu-147 & Eu-147 & 146.917 & Europium & $1.00 \mathrm{E}-03$ & ICRP-38 & $2.40 \mathrm{E}+01$ & $d$ & $2.07 \mathrm{E}+06$ & $3.70 \mathrm{E}+04$ & ICRP-72 & $1.10 \mathrm{E}-09$ & $M$ & Max & FGR-12 & $2.32 \mathrm{E}-14$ \\
\hline Eu-148 & Eu-148 & 147.918 & Europium & $1.00 \mathrm{E}-03$ & ICRP-38 & $5.45 \mathrm{E}+01$ & $d$ & $4.71 \mathrm{E}+06$ & $1.62 \mathrm{E}+04$ & ICRP-72 & $2.60 \mathrm{E}-09$ & $M$ & Max & FGR-12 & $1.06 \mathrm{E}-13$ \\
\hline Eu-149 & Eu-149 & 148.918 & Europium & $1.00 \mathrm{E}-03$ & ICRP-38 & $9.31 \mathrm{E}+01$ & $d$ & $8.04 \mathrm{E}+06$ & $9.42 \mathrm{E}+03$ & ICRP-72 & $2.90 \mathrm{E}-10$ & $M$ & Max & FGR-12 & $2.25 \mathrm{E}-15$ \\
\hline Eu-150l & Eu-150 & 149.920 & Europium & $1.00 \mathrm{E}-03$ & ICRP-38 & $3.42 \mathrm{E}+01$ & $y$ & $1.08 \mathrm{E}+09$ & $6.97 \mathrm{E}+01$ & ICRP-72 & $5.30 \mathrm{E}-08$ & $M$ & Max & FGR-12 & 7.17E-14 \\
\hline Eu-150s & Eu-150 & 149.920 & Europium & $1.00 \mathrm{E}-03$ & ICRP-38 & $1.26 \mathrm{E}+01$ & $\mathrm{~h}$ & $4.54 \mathrm{E}+04$ & $1.66 \mathrm{E}+06$ & ICRP-72 & $1.90 \mathrm{E}-10$ & $M$ & Max & FGR-12 & $2.21 \mathrm{E}-15$ \\
\hline Eu-152 & Eu-152 & 151.922 & Europium & $1.00 \mathrm{E}-03$ & ICRP-38 & $1.33 \mathrm{E}+01$ & $y$ & $4.21 \mathrm{E}+08$ & $1.77 \mathrm{E}+02$ & ICRP-72 & $4.20 \mathrm{E}-08$ & $M$ & Max & FGR-12 & $5.65 \mathrm{E}-14$ \\
\hline Eu-152ml & Eu-152 & 151.922 & Europium & $1.00 \mathrm{E}-03$ & ICRP-38 & $9.32 \mathrm{E}+00$ & $\mathrm{~h}$ & $3.36 \mathrm{E}+04$ & $2.21 \mathrm{E}+06$ & ICRP-72 & $2.20 \mathrm{E}-10$ & $\mathrm{M}$ & Max & FGR-12 & $1.42 \mathrm{E}-14$ \\
\hline Eu-152ms & Eu-152 & 151.922 & Europium & $1.00 \mathrm{E}-03$ & ICRP-107 & $9.60 \mathrm{E}+01$ & $\mathrm{~m}$ & $5.76 \mathrm{E}+03$ & $1.29 \mathrm{E}+07$ & DOE-STD-1196 & $1.01 \mathrm{E}-11$ & $M$ & Max & DOE-STD-1196 & $2.63 \mathrm{E}-15$ \\
\hline Eu-154 & Eu-154 & 153.923 & Europium & $1.00 \mathrm{E}-03$ & ICRP-38 & $8.80 \mathrm{E}+00$ & $y$ & $2.78 \mathrm{E}+08$ & $2.64 \mathrm{E}+02$ & ICRP-72 & $5.30 \mathrm{E}-08$ & $M$ & Max & FGR-12 & $6.14 \mathrm{E}-14$ \\
\hline Eu-154m & Eu-154 & 153.923 & Europium & $1.00 \mathrm{E}-03$ & ICRP-107 & $4.60 \mathrm{E}+01$ & $\mathrm{~m}$ & $2.76 \mathrm{E}+03$ & $2.66 \mathrm{E}+07$ & DOE-STD-1196 & $4.76 \mathrm{E}-12$ & $\mathrm{~s}$ & Max & DOE-STD-1196 & $2.15 \mathrm{E}-15$ \\
\hline Eu-155 & Eu-155 & 154.923 & Europium & $1.00 \mathrm{E}-03$ & $\begin{array}{l}\text { ICRP-38 } \\
\end{array}$ & $4.96 \mathrm{E}+00$ & $y$ & $1.57 E+08$ & $4.65 \mathrm{E}+02$ & ICRP-72 & $6.90 \mathrm{E}-09$ & $M$ & Max & FGR-12 & $2.49 \mathrm{E}-15$ \\
\hline Eu-156 & Eu-156 & 155.925 & Europium & $1.00 \mathrm{E}-03$ & ICRP-38 & $1.52 \mathrm{E}+01$ & $d$ & $1.31 \mathrm{E}+06$ & $5.51 \mathrm{E}+04$ & ICRP-72 & $3.40 \mathrm{E}-09$ & $M$ & Max & FGR-12 & $6.75 \mathrm{E}-14$ \\
\hline Eu-157 & Eu-157 & 156.925 & Europium & $1.00 \mathrm{E}-03$ & ICRP-38 & $1.52 \mathrm{E}+01$ & $\mathrm{~h}$ & $5.45 \mathrm{E}+04$ & $1.32 \mathrm{E}+06$ & ICRP-72 & $2.80 \mathrm{E}-10$ & $M$ & Max & FGR-12 & $1.17 \mathrm{E}-14$ \\
\hline Eu-158 & Eu-158 & 157.928 & Europium & $1.00 \mathrm{E}-03$ & ICRP-38 & $4.59 \mathrm{E}+01$ & $\mathrm{~m}$ & $2.75 E+03$ & $2.59 \mathrm{E}+07$ & ICRP-72 & $4.70 \mathrm{E}-11$ & $M$ & Max & FGR-12 & $5.27 \mathrm{E}-14$ \\
\hline Eu-159 & Eu-159 & 158.929 & Europium & $1.00 \mathrm{E}-03$ & ICRP-107 & $1.81 \mathrm{E}+01$ & $\mathrm{~m}$ & $1.09 \mathrm{E}+03$ & $6.54 \mathrm{E}+07$ & DOE-STD-1196 & $2.85 \mathrm{E}-11$ & $\mathrm{~s}$ & Max & DOE-STD-1196 & $1.32 \mathrm{E}-14$ \\
\hline F-17 & F-17 & 17.002 & Fluorine & $1.00 \mathrm{E}+00$ & ICRP-107 & $6.45 \mathrm{E}+01$ & $\mathrm{~s}$ & $6.45 \mathrm{E}+01$ & $1.03 \mathrm{E}+10$ & -- & -- & -- & -- & DOE-STD-1196 & $4.60 \mathrm{E}-14$ \\
\hline F-18 & F-18 & 18.001 & Fluorine & $1.00 \mathrm{E}+00$ & ICRP-38 & $1.10 \mathrm{E}+02$ & $\mathrm{~m}$ & $6.59 \mathrm{E}+03$ & $9.52 \mathrm{E}+07$ & ICRP-72 & $5.90 \mathrm{E}-11$ & $\mathrm{~s}$ & Max & FGR-12 & $4.90 \mathrm{E}-14$ \\
\hline Fe-52 & Fe-52 & 51.948 & Iron & $1.00 \mathrm{E}-03$ & ICRP-38 & $8.28 \mathrm{E}+00$ & $\mathrm{~h}$ & $2.98 \mathrm{E}+04$ & $7.29 \mathrm{E}+06$ & ICRP-72 & $6.00 \mathrm{E}-10$ & $M$ & $\operatorname{Rec}$ & FGR-12 & $3.54 \mathrm{E}-14$ \\
\hline Fe-53 & Fe-53 & 52.945 & Iron & $1.00 \mathrm{E}-03$ & ICRP-107 & $8.51 \mathrm{E}+00$ & $\mathrm{~m}$ & $5.11 \mathrm{E}+02$ & $4.17 \mathrm{E}+08$ & -- & -- & -- & -- & DOE-STD-1196 & $5.35 \mathrm{E}-14$ \\
\hline Fe-53m & Fe-53 & 52.945 & Iron & $1.00 \mathrm{E}-03$ & ICRP-107 & $2.53 \mathrm{E}+00$ & $\mathrm{~m}$ & $1.52 \mathrm{E}+02$ & $1.41 \mathrm{E}+09$ & -- & -- & -- & -- & DOE-STD-1196 & $1.44 \mathrm{E}-13$ \\
\hline Fe-55 & Fe-55 & 54.938 & Iron & $1.00 \mathrm{E}-03$ & ICRP-38 & $2.70 \mathrm{E}+00$ & $y$ & $8.52 \mathrm{E}+07$ & $2.41 \mathrm{E}+03$ & ICRP-72 & $3.80 \mathrm{E}-10$ & $M$ & $\operatorname{Rec}$ & DOE-STD-1196 & $6.69 \mathrm{E}-24$ \\
\hline Fe-59 & Fe-59 & 58.935 & Iron & $1.00 \mathrm{E}-03$ & ICRP-38 & $4.45 \mathrm{E}+01$ & $d$ & $3.85 \mathrm{E}+06$ & $4.98 \mathrm{E}+04$ & ICRP-72 & $3.70 \mathrm{E}-09$ & $M$ & $\operatorname{Rec}$ & FGR-12 & $5.97 \mathrm{E}-14$ \\
\hline Fe-60 & $\mathrm{Fe}-60$ & 59.934 & Iron & $1.00 \mathrm{E}-03$ & ICRP-38 & $1.00 E+05$ & $y$ & $3.16 \mathrm{E}+12$ & $5.96 \mathrm{E}-02$ & ICRP-72 & $1.40 \mathrm{E}-07$ & $M$ & $\operatorname{Rec}$ & FGR-12 & $1.95 \mathrm{E}-19$ \\
\hline Fe-61 & Fe-61 & 60.937 & Iron & $1.00 \mathrm{E}-03$ & ICRP-107 & $5.98 \mathrm{E}+00$ & $\mathrm{~m}$ & $3.59 \mathrm{E}+02$ & $5.16 \mathrm{E}+08$ & -- & -- & -- & -- & DOE-STD-1196 & $6.68 \mathrm{E}-14$ \\
\hline Fe-62 & Fe-62 & 61.937 & Iron & $1.00 \mathrm{E}-03$ & ICRP-107 & $6.80 \mathrm{E}+01$ & $\mathrm{~s}$ & $6.80 \mathrm{E}+01$ & $2.68 \mathrm{E}+09$ & -- & -- & -- & -- & DOE-STD-1196 & $2.32 \mathrm{E}-14$ \\
\hline Fm-251 & Fm-251 & 251.082 & Fermium & $1.00 \mathrm{E}-03$ & ICRP-107 & $5.30 \mathrm{E}+00$ & $\mathrm{~h}$ & $1.91 \mathrm{E}+04$ & $2.35 \mathrm{E}+06$ & DOE-STD-1196 & $2.16 \mathrm{E}-09$ & $\mathrm{~s}$ & Max & DOE-STD-1196 & $6.35 \mathrm{E}-15$ \\
\hline Fm-252 & Fm-252 & 252.082 & Fermium & $1.00 \mathrm{E}-03$ & ICRP-38 & $2.27 \mathrm{E}+01$ & $\mathrm{~h}$ & $8.17 E+04$ & $5.48 \mathrm{E}+05$ & ICRP-72 & $3.20 \mathrm{E}-07$ & $M$ & Max & FGR-12 & $5.03 \mathrm{E}-18$ \\
\hline Fm-253 & Fm-253 & 253.085 & Fermium & $1.00 \mathrm{E}-03$ & ICRP-38 & $3.00 \mathrm{E}+00$ & $d$ & $2.59 \mathrm{E}+05$ & $1.72 \mathrm{E}+05$ & ICRP-72 & $4.00 \mathrm{E}-07$ & $M$ & Max & FGR-12 & $3.53 \mathrm{E}-15$ \\
\hline Fm-254 & Fm-254 & 254.087 & Fermium & $1.00 \mathrm{E}-03$ & ICRP-38 & $3.24 \mathrm{E}+00$ & $\mathrm{~h}$ & $1.17 \mathrm{E}+04$ & $3.81 \mathrm{E}+06$ & ICRP-72 & $6.10 \mathrm{E}-08$ & $M$ & Max & FGR-12 & $6.57 \mathrm{E}-18$ \\
\hline Fm-255 & Fm-255 & 255.090 & Fermium & $1.00 \mathrm{E}-03$ & ICRP-38 & $2.01 \mathrm{E}+01$ & $\mathrm{~h}$ & $7.23 E+04$ & $6.12 \mathrm{E}+05$ & ICRP-72 & $2.70 \mathrm{E}-07$ & $\mathrm{M}$ & Max & FGR-12 & $1.10 \mathrm{E}-16$ \\
\hline Fm-256 & Fm-256 & 256.092 & Fermium & $1.00 \mathrm{E}-03$ & ICRP-107 & $1.58 \mathrm{E}+02$ & $\mathrm{~m}$ & $9.46 \mathrm{E}+03$ & $4.66 \mathrm{E}+06$ & DOE-STD-1196 & $2.85 \mathrm{E}-07$ & $\mathrm{~s}$ & Max & DOE-STD-1196 & $6.07 \mathrm{E}-13$ \\
\hline Fm-257 & Fm-257 & 257.095 & Fermium & $1.00 \mathrm{E}-03$ & ICRP-38 & $1.01 \mathrm{E}+02$ & d & $8.68 \mathrm{E}+06$ & $5.05 \mathrm{E}+03$ & ICRP-72 & $7.10 \mathrm{E}-06$ & $M$ & Max & FGR-12 & $4.66 \mathrm{E}-15$ \\
\hline $\mathrm{Fr}-212$ & Fr-212 & 211.996 & Francium & $1.00 \mathrm{E}-03$ & ICRP-107 & $2.00 \mathrm{E}+01$ & $\mathrm{~m}$ & $1.20 \mathrm{E}+03$ & $4.43 \mathrm{E}+07$ & DOE-STD-1196 & $6.98 \mathrm{E}-09$ & $\mathrm{~s}$ & Max & DOE-STD-1196 & $5.26 \mathrm{E}-14$ \\
\hline Fr-219 & Fr-219 & 219.009 & Francium & $1.00 \mathrm{E}-03$ & ICRP-38 & $2.10 \mathrm{E}+01$ & $\mathrm{~ms}$ & $2.10 \mathrm{E}-02$ & $2.45 \mathrm{E}+12$ & -- & -- & -- & -- & FGR-12 & $1.66 \mathrm{E}-16$ \\
\hline Fr-220 & Fr-220 & 220.012 & Francium & $1.00 \mathrm{E}-03$ & ICRP-38 & $2.74 \mathrm{E}+01$ & $\mathrm{~s}$ & $2.74 \mathrm{E}+01$ & $1.87 \mathrm{E}+09$ & -- & -- & -- & -- & FGR-12 & $4.92 \mathrm{E}-16$ \\
\hline
\end{tabular}

B-11 


\begin{tabular}{|c|c|c|c|c|c|c|c|c|c|c|c|c|c|c|c|}
\hline \multicolumn{16}{|c|}{ Table B.1 Radionuclide Specific Input Data Used For the Calculation of HC-2 TQs Using Recommended DCs (continued) } \\
\hline $\begin{array}{l}\text { MASTER } \\
\text { ISOTOPE } \\
\text { LIST }\end{array}$ & $\begin{array}{l}\text { Atomic } \\
\text { Mass } \\
\text { Nuclide }\end{array}$ & $\begin{array}{l}\text { Atomic } \\
\text { Mass }\end{array}$ & Element & $\begin{array}{c}\text { RF per } \\
\text { DOE-STD- } \\
1027\end{array}$ & $\begin{array}{l}\text { Half-Life } \\
\text { Reference }\end{array}$ & \multicolumn{2}{|c|}{$\begin{array}{c}\text { Half-Life } \\
\text { (original units) }\end{array}$} & \multirow{2}{*}{$\begin{array}{c}\text { Half Life (s) } \\
2.88 \mathrm{E}+02\end{array}$} & \multirow{2}{*}{$\begin{array}{c}\begin{array}{c}\text { Sp. Act } \\
\text { (Ci/g) }\end{array} \\
1.77 \mathrm{E}+08\end{array}$} & \multirow{2}{*}{$\begin{array}{c}\text { Inhalation DC } \\
\text { Reference }\end{array}$} & \multirow[t]{2}{*}{$\begin{array}{c}\text { HC-2 } \\
\text { Inhalation } \\
\text { DC } \\
\text { (Sv/Bq) } \\
--\end{array}$} & \multicolumn{2}{|c|}{$\begin{array}{c}\text { Lung } \\
\text { Absorption } \\
\text { Class/Type }\end{array}$} & \multirow{2}{*}{$\begin{array}{c}\text { Immersion DC } \\
\text { Reference } \\
\text { FGR-12 }\end{array}$} & \multirow[t]{2}{*}{$\begin{array}{c}\text { HC-2 } \\
\text { Immersion } \\
\mathrm{DC}(\mathrm{Sv} / \mathrm{s} \\
\left.\text { per } \mathrm{Bq} / \mathrm{m}^{3}\right)\end{array}$} \\
\hline Fr-221 & Fr-221 & 221.014 & Francium & $1.00 \mathrm{E}-03$ & ICRP-38 & $4.80 \mathrm{E}+00$ & $\mathrm{~m}$ & & & & & -- & -- & & \\
\hline Fr-222 & Fr-222 & 222.018 & Francium & $1.00 \mathrm{E}-03$ & ICRP-38 & $1.44 \mathrm{E}+01$ & $\mathrm{~m}$ & $8.64 \mathrm{E}+02$ & $5.88 \mathrm{E}+07$ & ICRP-72 & 1.40E-08 & $\mathrm{F}$ & Max & FGR-12 & $1.17 \mathrm{E}-16$ \\
\hline Fr-223 & Fr-223 & 223.020 & Francium & $1.00 \mathrm{E}-03$ & ICRP-38 & $2.18 \mathrm{E}+01$ & $\mathrm{~m}$ & $1.31 \mathrm{E}+03$ & $3.87 \mathrm{E}+07$ & ICRP-72 & $8.90 \mathrm{E}-10$ & $\mathrm{~F}$ & Max & FGR-12 & $2.29 \mathrm{E}-15$ \\
\hline Fr-224 & Fr-224 & 224.023 & Francium & $1.00 \mathrm{E}-03$ & ICRP-107 & $3.33 \mathrm{E}+00$ & $\mathrm{~m}$ & $2.00 \mathrm{E}+02$ & $2.52 E+08$ & -- & -- & -- & -- & DOE-STD-1196 & $2.62 \mathrm{E}-14$ \\
\hline Fr-227 & Fr-227 & 227.032 & Francium & $1.00 \mathrm{E}-03$ & ICRP-107 & $2.47 \mathrm{E}+00$ & $\mathrm{~m}$ & $1.48 \mathrm{E}+02$ & $3.35 \mathrm{E}+08$ & -- & -- & -- & -- & DOE-STD-1196 & $2.00 \mathrm{E}-14$ \\
\hline Ga-64 & Ga-64 & 63.937 & Gallium & $1.00 \mathrm{E}-03$ & ICRP-107 & $2.63 E+00$ & $\mathrm{~m}$ & $1.58 \mathrm{E}+02$ & $1.12 \mathrm{E}+09$ & -- & --- & -- & -- & DOE-STD-1196 & $1.64 \mathrm{E}-13$ \\
\hline Ga-65 & Ga-65 & 64.933 & Gallium & $1.00 \mathrm{E}-03$ & ICRP-38 & $1.52 \mathrm{E}+01$ & $\mathrm{~m}$ & $9.12 \mathrm{E}+02$ & $1.91 \mathrm{E}+08$ & ICRP-72 & $1.70 \mathrm{E}-11$ & $M$ & Max & FGR-12 & $5.65 \mathrm{E}-14$ \\
\hline Ga-66 & Ga-66 & 65.932 & Gallium & $1.00 \mathrm{E}-03$ & ICRP-38 & $9.40 \mathrm{E}+00$ & $\mathrm{~h}$ & $3.38 \mathrm{E}+04$ & $5.06 \mathrm{E}+06$ & ICRP-72 & $4.40 \mathrm{E}-10$ & $M$ & Max & FGR-12 & $1.29 \mathrm{E}-13$ \\
\hline Ga-67 & Ga-67 & 66.928 & Gallium & $1.00 \mathrm{E}-03$ & ICRP-38 & $7.83 \mathrm{E}+01$ & $\mathrm{~h}$ & $2.82 \mathrm{E}+05$ & $5.98 \mathrm{E}+05$ & ICRP-72 & $2.40 \mathrm{E}-10$ & $M$ & Max & FGR-12 & $7.20 \mathrm{E}-15$ \\
\hline Ga-68 & Ga-68 & 67.928 & Gallium & $1.00 \mathrm{E}-03$ & ICRP-38 & $6.80 \mathrm{E}+01$ & $\mathrm{~m}$ & $4.08 \mathrm{E}+03$ & $4.07 E+07$ & ICRP-72 & $4.90 \mathrm{E}-11$ & $M$ & Max & FGR-12 & $4.58 \mathrm{E}-14$ \\
\hline Ga-70 & Ga-70 & 69.926 & Gallium & $1.00 \mathrm{E}-03$ & ICRP-38 & $2.12 \mathrm{E}+01$ & $\mathrm{~m}$ & $1.27 \mathrm{E}+03$ & $1.27 \mathrm{E}+08$ & ICRP-72 & $1.60 \mathrm{E}-11$ & $M$ & Max & FGR-12 & $4.62 \mathrm{E}-16$ \\
\hline Ga-72 & Ga-72 & 71.926 & Gallium & $1.00 \mathrm{E}-03$ & ICRP-38 & $1.41 \mathrm{E}+01$ & $\mathrm{~h}$ & $5.08 \mathrm{E}+04$ & $3.09 \mathrm{E}+06$ & ICRP-72 & $5.30 \mathrm{E}-10$ & $M$ & Max & FGR-12 & $1.39 \mathrm{E}-13$ \\
\hline Ga-73 & Ga-73 & 72.925 & Gallium & $1.00 \mathrm{E}-03$ & ICRP-38 & $4.91 \mathrm{E}+00$ & $\mathrm{~h}$ & $1.77 \mathrm{E}+04$ & $8.75 E+06$ & ICRP-72 & 1.40E-10 & $M$ & Max & FGR-12 & $1.48 \mathrm{E}-14$ \\
\hline Ga-74 & Ga-74 & 73.927 & Gallium & $1.00 \mathrm{E}-03$ & ICRP-107 & $8.12 \mathrm{E}+00$ & $\mathrm{~m}$ & $4.87 \mathrm{E}+02$ & $3.13 \mathrm{E}+08$ & -- & -- & -- & -- & DOE-STD-1196 & $1.55 \mathrm{E}-13$ \\
\hline Gd-142 & Gd-142 & 141.928 & Gadolinium & $1.00 \mathrm{E}-03$ & ICRP-107 & $7.02 \mathrm{E}+01$ & $\mathrm{~s}$ & $7.02 \mathrm{E}+01$ & $1.13 \mathrm{E}+09$ & -- & -- & -- & -- & DOE-STD-1196 & $4.78 \mathrm{E}-14$ \\
\hline Gd-143m & Gd-143 & 142.927 & Gadolinium & $1.00 \mathrm{E}-03$ & ICRP-107 & $1.10 \mathrm{E}+02$ & $\mathrm{~s}$ & $1.10 \mathrm{E}+02$ & $7.18 \mathrm{E}+08$ & -- & -- & -- & -- & DOE-STD-1196 & $9.77 \mathrm{E}-14$ \\
\hline Gd-144 & Gd-144 & 143.923 & Gadolinium & $1.00 \mathrm{E}-03$ & ICRP-107 & $4.47 \mathrm{E}+00$ & $\mathrm{~m}$ & $2.68 \mathrm{E}+02$ & $2.92 \mathrm{E}+08$ & -- & -- & -- & -- & DOE-STD-1196 & $4.25 \mathrm{E}-14$ \\
\hline Gd-145 & Gd-145 & 144.922 & Gadolinium & $1.00 \mathrm{E}-03$ & ICRP-38 & $2.29 \mathrm{E}+01$ & $\mathrm{~m}$ & $1.37 \mathrm{E}+03$ & $5.67 \mathrm{E}+07$ & ICRP-72 & $2.00 \mathrm{E}-11$ & $M$ & Max & FGR-12 & $1.15 \mathrm{E}-13$ \\
\hline Gd-145m & Gd-145 & 144.922 & Gadolinium & $1.00 \mathrm{E}-03$ & ICRP-107 & $8.50 \mathrm{E}+01$ & $\mathrm{~s}$ & $8.50 \mathrm{E}+01$ & $9.16 \mathrm{E}+08$ & -- & -- & -- & -- & DOE-STD-1196 & $3.08 \mathrm{E}-14$ \\
\hline Gd-146 & Gd-146 & 145.918 & Gadolinium & $1.00 \mathrm{E}-03$ & ICRP-38 & $4.83 \mathrm{E}+01$ & $d$ & $4.17 \mathrm{E}+06$ & $1.85 \mathrm{E}+04$ & ICRP-72 & $6.40 \mathrm{E}-09$ & $M$ & Max & FGR-12 & $9.95 \mathrm{E}-15$ \\
\hline Gd-147 & Gd-147 & 146.919 & Gadolinium & $1.00 \mathrm{E}-03$ & ICRP-38 & $3.81 \mathrm{E}+01$ & $\mathrm{~h}$ & $1.37 \mathrm{E}+05$ & $5.60 \mathrm{E}+05$ & ICRP-72 & $4.00 \mathrm{E}-10$ & $M$ & Max & FGR-12 & $6.45 \mathrm{E}-14$ \\
\hline Gd-148 & Gd-148 & 147.918 & Gadolinium & $1.00 \mathrm{E}-03$ & ICRP-38 & $9.30 \mathrm{E}+01$ & $y$ & $2.93 \mathrm{E}+09$ & $2.60 \mathrm{E}+01$ & ICRP-72 & $2.60 \mathrm{E}-05$ & $\mathrm{~F}$ & Max & -- & -- \\
\hline Gd-149 & Gd-149 & 148.919 & Gadolinium & $1.00 \mathrm{E}-03$ & ICRP-38 & $9.40 \mathrm{E}+00$ & $d$ & $8.12 \mathrm{E}+05$ & $9.33 \mathrm{E}+04$ & ICRP-72 & $7.30 \mathrm{E}-10$ & $M$ & Max & FGR-12 & $1.92 \mathrm{E}-14$ \\
\hline Gd-150 & Gd-150 & 149.919 & Gadolinium & $1.00 \mathrm{E}-03$ & ICRP-107 & $1.79 \mathrm{E}+06$ & $y$ & $5.65 \mathrm{E}+13$ & $1.33 \mathrm{E}-03$ & DOE-STD-1196 & $2.56 \mathrm{E}-05$ & $\mathrm{~F}$ & Max & -- & -- \\
\hline Gd-151 & Gd-151 & 150.920 & Gadolinium & $1.00 \mathrm{E}-03$ & ICRP-38 & $1.20 \mathrm{E}+02$ & $d$ & $1.04 \mathrm{E}+07$ & $7.21 E+03$ & ICRP-72 & $8.60 \mathrm{E}-10$ & $M$ & Max & FGR-12 & $2.20 \mathrm{E}-15$ \\
\hline Gd-152 & Gd-152 & 151.920 & Gadolinium & $1.00 \mathrm{E}-03$ & ICRP-38 & $1.08 \mathrm{E}+14$ & $y$ & $3.41 \mathrm{E}+21$ & $2.18 \mathrm{E}-11$ & ICRP-72 & $1.90 \mathrm{E}-05$ & $\mathrm{~F}$ & Max & -- & -- \\
\hline Gd-153 & Gd-153 & 152.922 & Gadolinium & $1.00 \mathrm{E}-03$ & ICRP-38 & $2.42 E+02$ & $d$ & $2.09 \mathrm{E}+07$ & $3.53 \mathrm{E}+03$ & ICRP-72 & $2.10 \mathrm{E}-09$ & $\mathrm{~F}$ & Max & FGR-12 & $3.71 \mathrm{E}-15$ \\
\hline Gd-159 & Gd-159 & 158.926 & Gadolinium & $1.00 \mathrm{E}-03$ & ICRP-38 & $1.86 \mathrm{E}+01$ & $\mathrm{~h}$ & $6.68 \mathrm{E}+04$ & $1.06 \mathrm{E}+06$ & ICRP-72 & $2.70 \mathrm{E}-10$ & $M$ & Max & FGR-12 & $2.21 \mathrm{E}-15$ \\
\hline Gd-162 & Gd-162 & 161.931 & Gadolinium & $1.00 \mathrm{E}-03$ & ICRP-107 & $8.40 \mathrm{E}+00$ & $\mathrm{~m}$ & $5.04 \mathrm{E}+02$ & $1.38 \mathrm{E}+08$ & -- & -- & -- & -- & DOE-STD-1196 & $1.86 \mathrm{E}-14$ \\
\hline Ge-66 & Ge-66 & 65.934 & Germanium & $1.00 \mathrm{E}-03$ & ICRP-38 & $2.27 \mathrm{E}+00$ & $\mathrm{~h}$ & $8.17 \mathrm{E}+03$ & $2.09 \mathrm{E}+07$ & ICRP-72 & $9.10 \mathrm{E}-11$ & $M$ & Max & FGR-12 & $3.25 \mathrm{E}-14$ \\
\hline Ge-67 & Ge-67 & 66.933 & Germanium & $1.00 \mathrm{E}-03$ & ICRP-38 & $1.87 \mathrm{E}+01$ & $\mathrm{~m}$ & $1.12 \mathrm{E}+03$ & $1.50 \mathrm{E}+08$ & ICRP-72 & $2.50 \mathrm{E}-11$ & $M$ & Max & FGR-12 & $6.86 \mathrm{E}-14$ \\
\hline Ge-68 & Ge-68 & 67.928 & Germanium & $1.00 \mathrm{E}-03$ & ICRP-38 & $2.88 \mathrm{E}+02$ & $d$ & $2.49 \mathrm{E}+07$ & $6.67 \mathrm{E}+03$ & ICRP-72 & $1.40 \mathrm{E}-08$ & $M$ & Max & FGR-12 & $7.37 \mathrm{E}-20$ \\
\hline Ge-69 & Ge-69 & 68.928 & Germanium & $1.00 \mathrm{E}-03$ & ICRP-38 & $3.91 \mathrm{E}+01$ & $\mathrm{~h}$ & $1.41 \mathrm{E}+05$ & $1.16 \mathrm{E}+06$ & ICRP-72 & $2.90 \mathrm{E}-10$ & $M$ & Max & FGR-12 & $4.27 \mathrm{E}-14$ \\
\hline Ge-71 & Ge-71 & 70.925 & Germanium & $1.00 \mathrm{E}-03$ & ICRP-38 & $1.18 \mathrm{E}+01$ & $d$ & $1.02 \mathrm{E}+06$ & $1.56 \mathrm{E}+05$ & ICRP-72 & $1.10 \mathrm{E}-11$ & $M$ & Max & FGR-12 & $7.47 \mathrm{E}-20$ \\
\hline Ge-75 & Ge-75 & 74.923 & Germanium & $1.00 \mathrm{E}-03$ & ICRP-38 & $8.28 \mathrm{E}+01$ & $\mathrm{~m}$ & $4.97 \mathrm{E}+03$ & $3.03 \mathrm{E}+07$ & ICRP-72 & $3.60 \mathrm{E}-11$ & $M$ & Max & FGR-12 & $1.68 \mathrm{E}-15$ \\
\hline Ge-77 & Ge-77 & 76.924 & Germanium & $1.00 \mathrm{E}-03$ & ICRP-38 & $1.13 \mathrm{E}+01$ & $\mathrm{~h}$ & $4.07 \mathrm{E}+04$ & $3.61 \mathrm{E}+06$ & ICRP-72 & $3.70 \mathrm{E}-10$ & $M$ & Max & FGR-12 & $5.32 \mathrm{E}-14$ \\
\hline Ge-78 & Ge-78 & 77.923 & Germanium & $1.00 \mathrm{E}-03$ & ICRP-38 & $8.70 \mathrm{E}+01$ & $\mathrm{~m}$ & $5.22 \mathrm{E}+03$ & $2.77 E+07$ & ICRP-72 & $9.50 \mathrm{E}-11$ & $M$ & Max & FGR-12 & $1.34 \mathrm{E}-14$ \\
\hline $\mathrm{H}-3$ & $\mathrm{H}-3$ & 3.016 & Hydrogen & $1.00 E+00$ & ICRP-38 & $1.24 \mathrm{E}+01$ & $y$ & $3.90 \mathrm{E}+08$ & $9.60 \mathrm{E}+03$ & ICRP-72 & $4.50 \mathrm{E}-11$ & $M$ & $\operatorname{Rec}$ & FGR-12 & $3.31 \mathrm{E}-19$ \\
\hline Hf-167 & Hf-167 & 166.943 & Hafnium & $1.00 \mathrm{E}-03$ & ICRP-107 & $2.05 E+00$ & $\mathrm{~m}$ & $1.23 \mathrm{E}+02$ & $5.49 \mathrm{E}+08$ & -- & -- & -- & -- & DOE-STD-1196 & $2.73 \mathrm{E}-14$ \\
\hline $\mathrm{Hf}-169$ & Hf-169 & 168.941 & Hafnium & $1.00 \mathrm{E}-03$ & ICRP-107 & $3.24 \mathrm{E}+00$ & $\mathrm{~m}$ & $1.94 \mathrm{E}+02$ & $3.44 \mathrm{E}+08$ & -- & -- & -- & -- & DOE-STD-1196 & $2.78 \mathrm{E}-14$ \\
\hline $\mathrm{Hf}-170$ & $\mathrm{Hf}-170$ & 169.940 & Hafnium & $1.00 \mathrm{E}-03$ & ICRP-38 & $1.60 \mathrm{E}+01$ & $\mathrm{~h}$ & $5.76 \mathrm{E}+04$ & $1.15 \mathrm{E}+06$ & ICRP-72 & $3.20 \mathrm{E}-10$ & $M$ & Max & FGR-12 & $2.52 \mathrm{E}-14$ \\
\hline $\mathrm{Hf}-172$ & $\mathrm{Hf}-172$ & 171.939 & Hafnium & $1.00 \mathrm{E}-03$ & ICRP-38 & $1.87 \mathrm{E}+00$ & $y$ & $5.90 \mathrm{E}+07$ & $1.11 \mathrm{E}+03$ & ICRP-72 & $3.20 \mathrm{E}-08$ & $\mathrm{~F}$ & Max & FGR-12 & 4.06E-15 \\
\hline $\mathrm{Hf}-173$ & $\mathrm{Hf}-173$ & 172.941 & Hafnium & $1.00 \mathrm{E}-03$ & ICRP-38 & $2.40 \mathrm{E}+01$ & $\mathrm{~h}$ & $8.64 \mathrm{E}+04$ & $7.55 \mathrm{E}+05$ & ICRP-72 & $1.60 \mathrm{E}-10$ & $M$ & Max & FGR-12 & $1.85 \mathrm{E}-14$ \\
\hline Hf-174 & $\mathrm{Hf}-174$ & 173.940 & Hafnium & $1.00 \mathrm{E}-03$ & ICRP-107 & $2.00 \mathrm{E}+15$ & $y$ & $6.31 \mathrm{E}+22$ & $1.03 \mathrm{E}-12$ & DOE-STD-1196 & $3.20 \mathrm{E}-05$ & $\mathrm{~F}$ & Max & -- & -- \\
\hline $\mathrm{Hf}-175$ & $\mathrm{Hf}-175$ & 174.942 & Hafnium & $1.00 \mathrm{E}-03$ & ICRP-38 & $7.00 E+01$ & $d$ & $6.05 E+06$ & $1.07 E+04$ & ICRP-72 & $1.20 \mathrm{E}-09$ & $M$ & Max & FGR-12 & $1.69 \mathrm{E}-14$ \\
\hline Hf- $177 \mathrm{~m}$ & Hf-177 & 176.943 & Hafnium & $1.00 \mathrm{E}-03$ & ICRP-38 & $5.14 \mathrm{E}+01$ & $\mathrm{~m}$ & $3.08 \mathrm{E}+03$ & $2.07 \mathrm{E}+07$ & ICRP-72 & $9.00 \mathrm{E}-11$ & $M$ & Max & FGR-12 & $1.06 \mathrm{E}-13$ \\
\hline
\end{tabular}

B-12 


\begin{tabular}{|c|c|c|c|c|c|c|c|c|c|c|c|c|c|c|c|}
\hline \multicolumn{16}{|c|}{ Table B.1 Radionuclide Specific Input Data Used For the Calculation of HC-2 TQs Using Recommended DCs (continued) } \\
\hline $\begin{array}{l}\text { MASTER } \\
\text { ISOTOPE } \\
\text { LIST }\end{array}$ & $\begin{array}{c}\text { Atomic } \\
\text { Mass } \\
\text { Nuclide }\end{array}$ & $\begin{array}{l}\text { Atomic } \\
\text { Mass }\end{array}$ & Element & $\begin{array}{c}\text { RF per } \\
\text { DOE-STD- } \\
1027\end{array}$ & $\begin{array}{l}\text { Half-Life } \\
\text { Reference }\end{array}$ & \multicolumn{2}{|c|}{$\begin{array}{c}\text { Half-Life } \\
\text { (original units) }\end{array}$} & \multirow{2}{*}{$\begin{array}{c}\text { Half Life (s) } \\
9.78 \mathrm{E}+08\end{array}$} & \multirow{2}{*}{$\begin{array}{c}\begin{array}{c}\text { Sp. Act } \\
\text { (Ci/g) }\end{array} \\
6.48 \mathrm{E}+01\end{array}$} & \multirow{2}{*}{$\begin{array}{c}\begin{array}{c}\text { Inhalation DC } \\
\text { Reference }\end{array} \\
\text { ICRP-72 }\end{array}$} & \multirow[t]{2}{*}{$\begin{array}{c}\text { HC-2 } \\
\text { Inhalation } \\
\text { DC } \\
\text { (Sv/Bq) } \\
2.60 \mathrm{E}-07\end{array}$} & \multicolumn{2}{|c|}{$\begin{array}{c}\text { Lung } \\
\text { Absorption } \\
\text { Class/Type }\end{array}$} & \multirow{2}{*}{$\begin{array}{c}\begin{array}{c}\text { Immersion DC } \\
\text { Reference }\end{array} \\
\text { FGR-12 }\end{array}$} & \multirow[t]{2}{*}{ 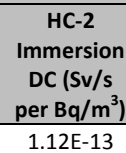 } \\
\hline $\mathrm{Hf}-178 \mathrm{~m}$ & $\mathrm{Hf}-178$ & 177.944 & Hafnium & $1.00 \mathrm{E}-03$ & ICRP-38 & $3.10 \mathrm{E}+01$ & y & & & & & $\mathrm{F}$ & Max & & \\
\hline $\mathrm{Hf}-179 \mathrm{~m}$ & Hf-179 & 178.946 & Hafnium & $1.00 \mathrm{E}-03$ & ICRP-38 & $2.51 \mathrm{E}+01$ & d & $2.17 \mathrm{E}+06$ & $2.91 \mathrm{E}+04$ & ICRP-72 & $3.80 \mathrm{E}-09$ & $\mathrm{M}$ & Max & FGR-12 & $4.21 \mathrm{E}-14$ \\
\hline Hf-180m & Hf-180 & 179.947 & Hafnium & $1.00 \mathrm{E}-03$ & ICRP-38 & $5.50 \mathrm{E}+00$ & $\mathrm{~h}$ & $1.98 \mathrm{E}+04$ & $3.17 \mathrm{E}+06$ & ICRP-72 & $1.30 \mathrm{E}-10$ & $M$ & Max & FGR-12 & $4.74 \mathrm{E}-14$ \\
\hline Hf-181 & Hf-181 & 180.949 & Hafnium & $1.00 \mathrm{E}-03$ & ICRP-38 & $4.24 \mathrm{E}+01$ & $d$ & $3.66 \mathrm{E}+06$ & $1.70 \mathrm{E}+04$ & ICRP-72 & $5.00 \mathrm{E}-09$ & $M$ & Max & FGR-12 & $2.62 \mathrm{E}-14$ \\
\hline Hf-182 & Hf-182 & 181.951 & Hafnium & $1.00 \mathrm{E}-03$ & ICRP-38 & $9.00 \mathrm{E}+06$ & $y$ & $2.84 \mathrm{E}+14$ & $2.18 \mathrm{E}-04$ & ICRP-72 & $3.10 \mathrm{E}-07$ & $\mathrm{~F}$ & Max & FGR-12 & $1.14 \mathrm{E}-14$ \\
\hline Hf- $182 m$ & Hf-182 & 181.951 & Hafnium & $1.00 \mathrm{E}-03$ & ICRP-38 & $6.15 \mathrm{E}+01$ & $\mathrm{~m}$ & $3.69 \mathrm{E}+03$ & $1.68 \mathrm{E}+07$ & ICRP-72 & $4.60 \mathrm{E}-11$ & $M$ & Max & FGR-12 & 4.43E-14 \\
\hline Hf-183 & Hf-183 & 182.954 & Hafnium & $1.00 \mathrm{E}-03$ & ICRP-38 & $6.40 \mathrm{E}+01$ & $\mathrm{~m}$ & $3.84 \mathrm{E}+03$ & $1.61 \mathrm{E}+07$ & ICRP-72 & $5.70 \mathrm{E}-11$ & $M$ & Max & FGR-12 & $3.63 \mathrm{E}-14$ \\
\hline Hf-184 & Hf-184 & 183.955 & Hafnium & $1.00 \mathrm{E}-03$ & ICRP-38 & $4.12 \mathrm{E}+00$ & $\mathrm{~h}$ & $1.48 \mathrm{E}+04$ & $4.13 \mathrm{E}+06$ & ICRP-72 & $3.30 \mathrm{E}-10$ & $M$ & Max & FGR-12 & $1.14 \mathrm{E}-14$ \\
\hline $\mathrm{Hg}-187$ & Hg-187 & 186.970 & Mercury & $1.00 \mathrm{E}-02$ & JAERI & $2.20 \mathrm{E}+00$ & $\mathrm{~m}$ & $1.32 \mathrm{E}+02$ & $4.57 \mathrm{E}+08$ & -- & -- & -- & -- & -- & -- \\
\hline Hg-187m & Hg-187 & 186.970 & Mercury & $1.00 \mathrm{E}-02$ & JAERI & $2.40 \mathrm{E}+00$ & $\mathrm{~m}$ & $1.44 \mathrm{E}+02$ & $4.19 \mathrm{E}+08$ & -- & -- & -- & -- & -- & -- \\
\hline Hg-188 & Hg-188 & 187.968 & Mercury & $1.00 \mathrm{E}-02$ & JAERI & $3.25 \mathrm{E}+00$ & $\mathrm{~m}$ & $1.95 \mathrm{E}+02$ & $3.08 \mathrm{E}+08$ & -- & -- & -- & -- & $\begin{array}{ll}- \\
\end{array}$ & $\begin{array}{ll}- \\
\end{array}$ \\
\hline $\mathrm{Hg}-190$ & Hg-190 & 189.966 & Mercury & $1.00 \mathrm{E}-02$ & ICRP-107 & $2.00 \mathrm{E}+01$ & $\mathrm{~m}$ & $1.20 \mathrm{E}+03$ & $4.95 \mathrm{E}+07$ & DOE-STD-1196 & $8.33 \mathrm{E}-11$ & $\mathrm{v}$ & Max & DOE-STD-1196 & $7.74 \mathrm{E}-15$ \\
\hline Hg-191m & Hg-191 & 190.967 & Mercury & $1.00 \mathrm{E}-02$ & ICRP-107 & $5.08 \mathrm{E}+01$ & $\mathrm{~m}$ & $3.05 E+03$ & $1.94 \mathrm{E}+07$ & DOE-STD-1196 & $3.47 \mathrm{E}-10$ & v & Max & DOE-STD-1196 & $6.73 \mathrm{E}-14$ \\
\hline Hg-192 & Hg-192 & 191.966 & Mercury & $1.00 \mathrm{E}-02$ & ICRP-107 & $4.85 \mathrm{E}+00$ & $\mathrm{~h}$ & $1.75 \mathrm{E}+04$ & $3.37 \mathrm{E}+06$ & DOE-STD-1196 & $1.08 \mathrm{E}-09$ & $\mathrm{v}$ & Max & DOE-STD-1196 & $1.10 \mathrm{E}-14$ \\
\hline Hg-193 & Hg-193 & 192.967 & Mercury & $1.00 \mathrm{E}-02$ & ICRP-38 & $3.50 \mathrm{E}+00$ & $\mathrm{~h}$ & $1.26 \mathrm{E}+04$ & $4.64 \mathrm{E}+06$ & ICRP-72 & $1.10 \mathrm{E}-09$ & $M$ & Max & FGR-12 & $8.69 \mathrm{E}-15$ \\
\hline Hg-193m & Hg-193 & 192.967 & Mercury & $1.00 \mathrm{E}-02$ & ICRP-38 & $1.11 \mathrm{E}+01$ & $\mathrm{~h}$ & $4.00 \mathrm{E}+04$ & $1.46 \mathrm{E}+06$ & ICRP-72 & $3.10 \mathrm{E}-09$ & $M$ & Max & FGR-12 & $5.05 \mathrm{E}-14$ \\
\hline $\mathrm{Hg}-194$ & Hg-194 & 193.965 & Mercury & $1.00 \mathrm{E}-02$ & ICRP-38 & $2.60 \mathrm{E}+02$ & $y$ & $8.20 \mathrm{E}+09$ & $7.09 \mathrm{E}+00$ & ICRP-72 & $4.00 \mathrm{E}-08$ & $\mathrm{~F}$ & Max & FGR-12 & $6.92 \mathrm{E}-19$ \\
\hline Hg-195 & Hg-195 & 194.967 & Mercury & $1.00 \mathrm{E}-02$ & ICRP-38 & $9.90 \mathrm{E}+00$ & $\mathrm{~h}$ & $3.56 \mathrm{E}+04$ & $1.62 \mathrm{E}+06$ & ICRP-72 & $1.40 \mathrm{E}-09$ & $\mathrm{M}$ & Max & FGR-12 & $9.20 \mathrm{E}-15$ \\
\hline Hg-195m & Hg-195 & 194.967 & Mercury & $1.00 \mathrm{E}-02$ & ICRP-38 & $4.16 \mathrm{E}+01$ & $\mathrm{~h}$ & $1.50 \mathrm{E}+05$ & $3.86 \mathrm{E}+05$ & ICRP-72 & $8.20 \mathrm{E}-09$ & $M$ & Max & FGR-12 & $9.63 \mathrm{E}-15$ \\
\hline Hg-197 & Hg-197 & 196.967 & Mercury & $1.00 \mathrm{E}-02$ & ICRP-38 & $6.41 \mathrm{E}+01$ & $\mathrm{~h}$ & $2.31 \mathrm{E}+05$ & $2.48 \mathrm{E}+05$ & ICRP-72 & $4.40 \mathrm{E}-09$ & $M$ & Max & FGR-12 & $2.66 \mathrm{E}-15$ \\
\hline Hg-197m & Hg-197 & 196.967 & Mercury & $1.00 \mathrm{E}-02$ & ICRP-38 & $2.38 \mathrm{E}+01$ & $\mathrm{~h}$ & $8.57 E+04$ & $6.68 \mathrm{E}+05$ & ICRP-72 & $5.80 \mathrm{E}-09$ & $M$ & Max & FGR-12 & $4.05 \mathrm{E}-15$ \\
\hline Hg-199m & Hg-199 & 198.968 & Mercury & $1.00 \mathrm{E}-02$ & ICRP-38 & $4.26 \mathrm{E}+01$ & $\mathrm{~m}$ & $2.56 \mathrm{E}+03$ & $2.22 \mathrm{E}+07$ & ICRP-72 & $1.80 \mathrm{E}-10$ & $M$ & Max & FGR-12 & $8.36 \mathrm{E}-15$ \\
\hline $\mathrm{Hg}-203$ & Hg-203 & 202.973 & Mercury & $1.00 \mathrm{E}-02$ & ICRP-38 & $4.66 \mathrm{E}+01$ & $d$ & $4.03 \mathrm{E}+06$ & $1.38 \mathrm{E}+04$ & ICRP-72 & $7.00 \mathrm{E}-09$ & $M$ & Max & FGR-12 & $1.13 \mathrm{E}-14$ \\
\hline $\mathrm{Hg}-205$ & Hg-205 & 204.976 & Mercury & $1.00 \mathrm{E}-02$ & ICRP-107 & $5.20 \mathrm{E}+00$ & $\mathrm{~m}$ & $3.12 \mathrm{E}+02$ & $1.76 \mathrm{E}+08$ & -- & -- & -- & -- & DOE-STD-1196 & $6.19 \mathrm{E}-16$ \\
\hline $\mathrm{Hg}-206$ & Hg-206 & 205.978 & Mercury & $1.00 \mathrm{E}-02$ & ICRP-38 & $8.15 \mathrm{E}+00$ & $\mathrm{~m}$ & $4.89 \mathrm{E}+02$ & $1.12 \mathrm{E}+08$ & -- & -- & -- & -- & DOE-STD-1196 & $5.56 \mathrm{E}-15$ \\
\hline $\mathrm{Hg}-207$ & Hg-207 & 206.982 & Mercury & $1.00 \mathrm{E}-02$ & ICRP-107 & $2.90 \mathrm{E}+00$ & $\mathrm{~m}$ & $1.74 \mathrm{E}+02$ & $3.13 \mathrm{E}+08$ & -- & -- & -- & -- & DOE-STD-1196 & $1.28 \mathrm{E}-13$ \\
\hline Ho-150 & Ho-150 & 149.933 & Holmium & $1.00 \mathrm{E}-03$ & ICRP-107 & $7.68 \mathrm{E}+01$ & $\mathrm{~s}$ & $7.68 \mathrm{E}+01$ & $9.80 \mathrm{E}+08$ & -- & -- & -- & -- & DOE-STD-1196 & $8.73 \mathrm{E}-14$ \\
\hline Ho-152 & Ho-152 & 151.932 & Holmium & $1.00 \mathrm{E}-03$ & JAERI & $2.70 \mathrm{E}+00$ & $\mathrm{~m}$ & $1.62 \mathrm{E}+02$ & $4.59 \mathrm{E}+08$ & -- & -- & -- & -- & -- & -- \\
\hline Ho-153 & Ho-153 & 152.930 & Holmium & $1.00 \mathrm{E}-03$ & ICRP-107 & $2.01 E+00$ & $\mathrm{~m}$ & $1.21 \mathrm{E}+02$ & $6.12 \mathrm{E}+08$ & -- & -- & -- & -- & DOE-STD-1196 & $4.61 \mathrm{E}-14$ \\
\hline Ho-153m & Ho-153 & 152.930 & Holmium & $1.00 \mathrm{E}-03$ & ICRP-107 & $9.30 \mathrm{E}+00$ & $\mathrm{~m}$ & $5.58 \mathrm{E}+02$ & $1.32 \mathrm{E}+08$ & -- & -- & -- & -- & DOE-STD-1196 & $4.72 \mathrm{E}-14$ \\
\hline Ho-154 & Ho-154 & 153.931 & Holmium & $1.00 \mathrm{E}-03$ & ICRP-107 & $1.18 \mathrm{E}+01$ & $\mathrm{~m}$ & $7.06 \mathrm{E}+02$ & $1.04 \mathrm{E}+08$ & DOE-STD-1196 & $1.82 \mathrm{E}-11$ & $\mathrm{~s}$ & $\operatorname{Max}$ & DOE-STD-1196 & $8.63 \mathrm{E}-14$ \\
\hline Ho-154m & Ho-154 & 153.931 & Holmium & $1.00 \mathrm{E}-03$ & ICRP-107 & $3.10 \mathrm{E}+00$ & $\mathrm{~m}$ & $1.86 \mathrm{E}+02$ & $3.94 \mathrm{E}+08$ & -- & -- & -- & -- & DOE-STD-1196 & $1.09 \mathrm{E}-13$ \\
\hline Ho-155 & Ho-155 & 154.929 & Holmium & $1.00 \mathrm{E}-03$ & ICRP-38 & $4.80 \mathrm{E}+01$ & $\mathrm{~m}$ & $2.88 \mathrm{E}+03$ & $2.53 \mathrm{E}+07$ & ICRP-72 & $2.00 \mathrm{E}-11$ & $M$ & $\operatorname{Max}$ & FGR-12 & $1.79 \mathrm{E}-14$ \\
\hline Ho-156 & Ho-156 & 155.930 & Holmium & $1.00 \mathrm{E}-03$ & ICRP-107 & $5.60 \mathrm{E}+01$ & $\mathrm{~m}$ & $3.36 \mathrm{E}+03$ & $2.15 \mathrm{E}+07$ & DOE-STD-1196 & $6.56 \mathrm{E}-11$ & $\mathrm{~s}$ & Max & DOE-STD-1196 & $9.78 \mathrm{E}-14$ \\
\hline Ho-157 & Ho-157 & 156.928 & Holmium & $1.00 \mathrm{E}-03$ & ICRP-38 & $1.26 \mathrm{E}+01$ & $\mathrm{~m}$ & $7.56 \mathrm{E}+02$ & $9.51 \mathrm{E}+07$ & ICRP-72 & $4.20 \mathrm{E}-12$ & $M$ & $\operatorname{Max}$ & FGR-12 & $2.24 \mathrm{E}-14$ \\
\hline Ho-158 & Ho-158 & 157.929 & Holmium & $1.00 \mathrm{E}-03$ & JAERI & $1.13 \mathrm{E}+01$ & $\mathrm{~m}$ & $6.78 \mathrm{E}+02$ & $1.05 E+08$ & JAERI & $7.80 \mathrm{E}-12$ & $M$ & Max & -- & -- \\
\hline Ho-159 & Ho-159 & 158.928 & Holmium & $1.00 \mathrm{E}-03$ & ICRP-38 & $3.30 \mathrm{E}+01$ & $\mathrm{~m}$ & $1.98 \mathrm{E}+03$ & $3.59 \mathrm{E}+07$ & ICRP-72 & $6.10 \mathrm{E}-12$ & $M$ & Max & FGR-12 & $1.60 \mathrm{E}-14$ \\
\hline Ho-160 & Ho-160 & 159.929 & Holmium & $1.00 \mathrm{E}-03$ & ICRP-107 & $2.56 \mathrm{E}+01$ & $\mathrm{~m}$ & $1.54 \mathrm{E}+03$ & $4.59 \mathrm{E}+07$ & DOE-STD-1196 & 1.63E-11 & $\mathrm{s}$ & Max & DOE-STD-1196 & $7.64 \mathrm{E}-14$ \\
\hline Ho-161 & Ho-161 & 160.928 & Holmium & $1.00 \mathrm{E}-03$ & ICRP-38 & $2.50 \mathrm{E}+00$ & $\mathrm{~h}$ & $9.00 \mathrm{E}+03$ & $7.79 \mathrm{E}+06$ & ICRP-72 & $6.00 \mathrm{E}-12$ & $M$ & Max & FGR-12 & $1.73 \mathrm{E}-15$ \\
\hline Ho-162 & Ho-162 & 161.929 & Holmium & $1.00 \mathrm{E}-03$ & ICRP-38 & $1.50 \mathrm{E}+01$ & $\mathrm{~m}$ & $9.00 \mathrm{E}+02$ & $7.74 \mathrm{E}+07$ & ICRP-72 & $2.80 \mathrm{E}-12$ & $M$ & Max & FGR-12 & $7.35 \mathrm{E}-15$ \\
\hline Ho-162m & Ho-162 & 161.929 & Holmium & $1.00 \mathrm{E}-03$ & ICRP-38 & $6.80 \mathrm{E}+01$ & $\mathrm{~m}$ & $4.08 \mathrm{E}+03$ & $1.71 \mathrm{E}+07$ & ICRP-72 & $2.10 \mathrm{E}-11$ & $\mathrm{M}$ & Max & FGR-12 & $2.74 \mathrm{E}-14$ \\
\hline Ho-163 & Ho-163 & 162.929 & Holmium & $1.00 \mathrm{E}-03$ & ICRP-107 & $4.57 \mathrm{E}+03$ & $y$ & $1.44 \mathrm{E}+11$ & $4.80 \mathrm{E}-01$ & DOE-STD-1196 & $2.82 \mathrm{E}-10$ & $\mathrm{~F}$ & Max & -- & -- \\
\hline Ho-164 & Ho-164 & 163.930 & Holmium & $1.00 \mathrm{E}-03$ & ICRP-38 & $2.90 \mathrm{E}+01$ & $\mathrm{~m}$ & $1.74 \mathrm{E}+03$ & $3.96 \mathrm{E}+07$ & ICRP-72 & $8.40 \mathrm{E}-12$ & $M$ & Max & FGR-12 & $9.05 \mathrm{E}-16$ \\
\hline Ho-164m & Ho-164 & 163.930 & Holmium & $1.00 \mathrm{E}-03$ & ICRP-38 & $3.75 \mathrm{E}+01$ & $\mathrm{~m}$ & $2.25 \mathrm{E}+03$ & $3.06 \mathrm{E}+07$ & ICRP-72 & $1.20 \mathrm{E}-11$ & $M$ & Max & FGR-12 & $1.32 \mathrm{E}-15$ \\
\hline Ho-166 & Ho-166 & 165.932 & Holmium & $1.00 \mathrm{E}-03$ & ICRP-38 & $2.68 \mathrm{E}+01$ & $\mathrm{~h}$ & $9.65 \mathrm{E}+04$ & $7.05 E+05$ & ICRP-72 & $6.50 \mathrm{E}-10$ & $M$ & Max & FGR-12 & $1.42 \mathrm{E}-15$ \\
\hline Ho-166m & Ho-166 & 165.932 & Holmium & $1.00 \mathrm{E}-03$ & ICRP-38 & $1.20 \mathrm{E}+03$ & $y$ & $3.79 \mathrm{E}+10$ & $1.80 \mathrm{E}+00$ & ICRP-72 & $1.20 \mathrm{E}-07$ & $M$ & Max & FGR-12 & $8.45 \mathrm{E}-14$ \\
\hline
\end{tabular}




\begin{tabular}{|c|c|c|c|c|c|c|c|c|c|c|c|c|c|c|c|}
\hline \multicolumn{16}{|c|}{ Table B.1 Radionuclide Specific Input Data Used For the Calculation of HC-2 TQs Using Recommended DCs (continued) } \\
\hline $\begin{array}{l}\text { MASTER } \\
\text { ISOTOPE } \\
\text { LIST }\end{array}$ & $\begin{array}{l}\text { Atomic } \\
\text { Mass } \\
\text { Nuclide }\end{array}$ & $\begin{array}{l}\text { Atomic } \\
\text { Mass }\end{array}$ & Element & $\begin{array}{c}\text { RF per } \\
\text { DOE-STD- } \\
1027\end{array}$ & $\begin{array}{l}\text { Half-Life } \\
\text { Reference }\end{array}$ & \multicolumn{2}{|c|}{$\begin{array}{c}\text { Half-Life } \\
\text { (original units) }\end{array}$} & \multirow{2}{*}{$\begin{array}{c}\text { Half Life (s) } \\
1.12 E+04\end{array}$} & \multirow{2}{*}{$\begin{array}{c}\begin{array}{c}\text { Sp. Act } \\
\text { (Ci/g) }\end{array} \\
6.06 \mathrm{E}+06\end{array}$} & \multirow{2}{*}{$\begin{array}{c}\begin{array}{c}\text { Inhalation DC } \\
\text { Reference }\end{array} \\
\text { ICRP-72 }\end{array}$} & \multirow[t]{2}{*}{$\begin{array}{c}\text { HC-2 } \\
\text { Inhalation } \\
\text { DC } \\
\text { (Sv/Bq) } \\
7.10 E-11 \\
\end{array}$} & \multicolumn{2}{|c|}{$\begin{array}{c}\text { Lung } \\
\text { Absorption } \\
\text { Class/Type }\end{array}$} & \multirow{2}{*}{$\begin{array}{c}\begin{array}{c}\text { Immersion DC } \\
\text { Reference }\end{array} \\
\text { FGR-12 }\end{array}$} & \multirow[t]{2}{*}{$\begin{array}{c}\text { HC-2 } \\
\text { Immersion } \\
\text { DC (Sv/s } \\
\left.\text { per } \mathrm{Bq} / \mathrm{m}^{3}\right) \\
1.73 \mathrm{E}-14\end{array}$} \\
\hline Ho-167 & Ho-167 & 166.933 & Holmium & $1.00 \mathrm{E}-03$ & ICRP-38 & $3.10 \mathrm{E}+00$ & $\mathrm{~h}$ & & & & & $\mathrm{M}$ & Max & & \\
\hline Ho-168 & Ho-168 & 167.936 & Holmium & $1.00 \mathrm{E}-03$ & ICRP-107 & $2.99 \mathrm{E}+00$ & $\mathrm{~m}$ & $1.79 \mathrm{E}+02$ & $3.74 \mathrm{E}+08$ & --- & -- & --- & -- & DOE-STD-1196 & $4.06 \mathrm{E}-14$ \\
\hline Ho-168m & Ho-168 & 167.936 & Holmium & $1.00 \mathrm{E}-03$ & ICRP-107 & $1.32 \mathrm{E}+02$ & $\mathrm{~s}$ & $1.32 \mathrm{E}+02$ & $5.09 \mathrm{E}+08$ & -- & -- & -- & -- & DOE-STD-1196 & $1.50 \mathrm{E}-16$ \\
\hline Ho-170 & Ho-170 & 169.940 & Holmium & $1.00 \mathrm{E}-03$ & ICRP-107 & $2.76 \mathrm{E}+00$ & $\mathrm{~m}$ & $1.66 \mathrm{E}+02$ & $4.01 \mathrm{E}+08$ & -- & -- & -- & -- & DOE-STD-1196 & $7.83 \mathrm{E}-14$ \\
\hline I-118 & I-118 & 117.913 & lodine & $5.00 \mathrm{E}-01$ & ICRP-107 & $1.37 \mathrm{E}+01$ & $\mathrm{~m}$ & $8.22 \mathrm{E}+02$ & $1.16 \mathrm{E}+08$ & DOE-STD-1196 & $6.86 \mathrm{E}-11$ & $\mathrm{~F}$ & $\operatorname{Rec}$ & DOE-STD-1196 & $9.38 \mathrm{E}-14$ \\
\hline $\mathrm{l}-118 \mathrm{~m}$ & I-118 & 117.913 & Iodine & $5.00 \mathrm{E}-01$ & ICRP-107 & $8.50 \mathrm{E}+00$ & $\mathrm{~m}$ & $5.10 \mathrm{E}+02$ & $1.88 \mathrm{E}+08$ & -- & -- & -- & -- & DOE-STD-1196 & $1.71 \mathrm{E}-13$ \\
\hline I-119 & I-119 & 118.910 & Iodine & $5.00 \mathrm{E}-01$ & ICRP-107 & $1.91 \mathrm{E}+01$ & $\mathrm{~m}$ & $1.15 \mathrm{E}+03$ & $8.28 \mathrm{E}+07$ & DOE-STD-1196 & $1.85 \mathrm{E}-11$ & $F$ & $\operatorname{Rec}$ & DOE-STD-1196 & $4.06 \mathrm{E}-14$ \\
\hline I-120 & I-120 & 119.910 & lodine & $5.00 \mathrm{E}-01$ & ICRP-38 & $8.10 \mathrm{E}+01$ & $\mathrm{~m}$ & $4.86 \mathrm{E}+03$ & $1.94 \mathrm{E}+07$ & ICRP-72 & $1.00 \mathrm{E}-10$ & $\mathrm{~F}$ & $\operatorname{Rec}$ & FGR-12 & $1.38 \mathrm{E}-13$ \\
\hline I-120m & $1-120$ & 119.910 & Iodine & $5.00 \mathrm{E}-01$ & ICRP-38 & $5.30 \mathrm{E}+01$ & $\mathrm{~m}$ & $3.18 \mathrm{E}+03$ & $2.96 \mathrm{E}+07$ & ICRP-72 & $8.20 \mathrm{E}-11$ & $\mathrm{~F}$ & $\operatorname{Rec}$ & FGR-12 & $2.65 \mathrm{E}-13$ \\
\hline I-121 & I-121 & 120.907 & lodine & $5.00 \mathrm{E}-01$ & ICRP-38 & $2.12 E+00$ & $\mathrm{~h}$ & $7.63 E+03$ & $1.22 \mathrm{E}+07$ & ICRP-72 & $2.70 \mathrm{E}-11$ & $\mathrm{~F}$ & $\operatorname{Rec}$ & FGR-12 & $1.94 \mathrm{E}-14$ \\
\hline $1-122$ & I-122 & 121.908 & lodine & $5.00 \mathrm{E}-01$ & ICRP-38 & $3.62 \mathrm{E}+00$ & $\mathrm{~m}$ & $2.17 E+02$ & $4.26 \mathrm{E}+08$ & -- & -- & -- & -- & FGR-12 & $4.56 \mathrm{E}-14$ \\
\hline $1-123$ & I-123 & 122.906 & lodine & $5.00 \mathrm{E}-01$ & ICRP-38 & $1.32 \mathrm{E}+01$ & $\mathrm{~h}$ & $4.75 E+04$ & $1.93 \mathrm{E}+06$ & ICRP-72 & $7.40 \mathrm{E}-11$ & $\mathrm{~F}$ & $\operatorname{Rec}$ & FGR-12 & $7.28 \mathrm{E}-15$ \\
\hline I-124 & I-124 & 123.906 & lodine & $5.00 \mathrm{E}-01$ & ICRP-38 & $4.18 \mathrm{E}+00$ & $d$ & $3.61 \mathrm{E}+05$ & $2.52 \mathrm{E}+05$ & ICRP-72 & $4.40 \mathrm{E}-09$ & $\mathrm{~F}$ & $\operatorname{Rec}$ & FGR-12 & $5.38 \mathrm{E}-14$ \\
\hline $1-125$ & $1-125$ & 124.905 & Iodine & $5.00 \mathrm{E}-01$ & ICRP-38 & $6.01 \mathrm{E}+01$ & $d$ & $5.20 \mathrm{E}+06$ & $1.74 \mathrm{E}+04$ & ICRP-72 & $5.10 \mathrm{E}-09$ & $F$ & $\operatorname{Rec}$ & FGR-12 & $5.22 \mathrm{E}-16$ \\
\hline I-126 & I-126 & 125.906 & lodine & $5.00 \mathrm{E}-01$ & ICRP-38 & $1.30 \mathrm{E}+01$ & $d$ & $1.12 \mathrm{E}+06$ & $7.97 \mathrm{E}+04$ & ICRP-72 & $9.80 \mathrm{E}-09$ & $\mathrm{~F}$ & $\operatorname{Rec}$ & FGR-12 & $2.15 \mathrm{E}-14$ \\
\hline $1-128$ & I-128 & 127.906 & lodine & $5.00 \mathrm{E}-01$ & ICRP-38 & $2.50 \mathrm{E}+01$ & $\mathrm{~m}$ & $1.50 \mathrm{E}+03$ & $5.88 \mathrm{E}+07$ & ICRP-72 & $1.30 \mathrm{E}-11$ & $\mathrm{~F}$ & $\operatorname{Rec}$ & FGR-12 & $4.16 \mathrm{E}-15$ \\
\hline $1-129$ & I-129 & 128.905 & lodine & $5.00 \mathrm{E}-01$ & ICRP-38 & $1.57 \mathrm{E}+07$ & $y$ & $4.95 E+14$ & $1.77 \mathrm{E}-04$ & ICRP-72 & $3.60 \mathrm{E}-08$ & $\mathrm{~F}$ & $\operatorname{Rec}$ & FGR-12 & $3.80 \mathrm{E}-16$ \\
\hline $1-130$ & $1-130$ & 129.907 & Iodine & $5.00 \mathrm{E}-01$ & ICRP-38 & $1.24 \mathrm{E}+01$ & $\mathrm{~h}$ & $4.45 \mathrm{E}+04$ & $1.95 \mathrm{E}+06$ & ICRP-72 & $6.70 \mathrm{E}-10$ & $F$ & Rec & FGR-12 & $1.04 \mathrm{E}-13$ \\
\hline $\mathrm{I}-130 \mathrm{~m}$ & I-130 & 129.907 & Iodine & $5.00 \mathrm{E}-01$ & ICRP-107 & $8.84 \mathrm{E}+00$ & $\mathrm{~m}$ & $5.30 \mathrm{E}+02$ & $1.64 \mathrm{E}+08$ & -- & -- & -- & -- & DOE-STD-1196 & $4.88 \mathrm{E}-15$ \\
\hline I-131 & I-131 & 130.906 & lodine & $5.00 \mathrm{E}-01$ & ICRP-38 & $8.04 \mathrm{E}+00$ & d & $6.95 \mathrm{E}+05$ & $1.24 \mathrm{E}+05$ & ICRP-72 & $7.40 \mathrm{E}-09$ & $\mathrm{~F}$ & $\operatorname{Rec}$ & FGR-12 & $1.82 \mathrm{E}-14$ \\
\hline I-132 & I-132 & 131.908 & Iodine & $5.00 \mathrm{E}-01$ & ICRP-38 & $2.30 \mathrm{E}+00$ & $\mathrm{~h}$ & $8.28 \mathrm{E}+03$ & $1.03 \mathrm{E}+07$ & ICRP-72 & $9.40 \mathrm{E}-11$ & $F$ & $\operatorname{Rec}$ & FGR-12 & $1.12 \mathrm{E}-13$ \\
\hline $\mathrm{I}-132 \mathrm{~m}$ & $\mid-132$ & 131.908 & lodine & $5.00 \mathrm{E}-01$ & ICRP-38 & $8.36 \mathrm{E}+01$ & $\mathrm{~m}$ & $5.02 \mathrm{E}+03$ & $1.71 \mathrm{E}+07$ & ICRP-72 & $7.90 \mathrm{E}-11$ & $\mathrm{~F}$ & $\operatorname{Rec}$ & FGR-12 & $1.53 \mathrm{E}-14$ \\
\hline $\mid-133$ & I-133 & 132.908 & Iodine & $5.00 \mathrm{E}-01$ & ICRP-38 & $2.08 \mathrm{E}+01$ & $\mathrm{~h}$ & $7.49 \mathrm{E}+04$ & $1.13 \mathrm{E}+06$ & ICRP-72 & $1.50 \mathrm{E}-09$ & $\mathrm{~F}$ & $\operatorname{Rec}$ & FGR-12 & $2.94 \mathrm{E}-14$ \\
\hline I-134 & I-134 & 133.910 & Iodine & $5.00 \mathrm{E}-01$ & ICRP-38 & $5.26 \mathrm{E}+01$ & $\mathrm{~m}$ & $3.16 \mathrm{E}+03$ & $2.67 \mathrm{E}+07$ & ICRP-72 & $4.50 \mathrm{E}-11$ & $\mathrm{~F}$ & $\operatorname{Rec}$ & FGR-12 & $1.30 \mathrm{E}-13$ \\
\hline I-134m & I-134 & 133.910 & Iodine & $5.00 \mathrm{E}-01$ & ICRP-107 & $3.60 \mathrm{E}+00$ & $\mathrm{~m}$ & $2.16 \mathrm{E}+02$ & $3.90 \mathrm{E}+08$ & -- & -- & -- & -- & DOE-STD-1196 & $1.19 \mathrm{E}-14$ \\
\hline I-135 & I-135 & 134.910 & lodine & $5.00 \mathrm{E}-01$ & ICRP-38 & $6.61 \mathrm{E}+00$ & $\mathrm{~h}$ & $2.38 \mathrm{E}+04$ & $3.51 \mathrm{E}+06$ & ICRP-72 & $3.20 \mathrm{E}-10$ & $F$ & $\operatorname{Rec}$ & FGR-12 & $7.98 \mathrm{E}-14$ \\
\hline $\ln -103$ & $\ln -103$ & 102.920 & Indium & $1.00 \mathrm{E}-03$ & ICRP-107 & $6.00 \mathrm{E}+01$ & $\mathrm{~s}$ & $6.00 \mathrm{E}+01$ & $1.83 \mathrm{E}+09$ & -- & -- & -- & -- & DOE-STD-1196 & $1.30 \mathrm{E}-13$ \\
\hline In-105 & $\ln -105$ & 104.915 & Indium & $1.00 \mathrm{E}-03$ & ICRP-107 & $5.07 \mathrm{E}+00$ & $\mathrm{~m}$ & $3.04 \mathrm{E}+02$ & $3.53 \mathrm{E}+08$ & -- & -- & -- & -- & DOE-STD-1196 & $8.97 \mathrm{E}-14$ \\
\hline $\ln -106$ & $\ln -106$ & 105.913 & Indium & $1.00 \mathrm{E}-03$ & ICRP-107 & $6.20 \mathrm{E}+00$ & $\mathrm{~m}$ & $3.72 \mathrm{E}+02$ & $2.86 \mathrm{E}+08$ & -- & -- & -- & -- & DOE-STD-1196 & $1.63 \mathrm{E}-13$ \\
\hline In-106m & $\ln -106$ & 105.913 & Indium & $1.00 \mathrm{E}-03$ & ICRP-107 & $5.20 \mathrm{E}+00$ & $\mathrm{~m}$ & $3.12 \mathrm{E}+02$ & $3.41 \mathrm{E}+08$ & -- & -- & -- & -- & DOE-STD-1196 & $1.35 \mathrm{E}-13$ \\
\hline In-107 & $\ln -107$ & 106.910 & Indium & $1.00 \mathrm{E}-03$ & ICRP-107 & $3.24 \mathrm{E}+01$ & $\mathrm{~m}$ & $1.94 \mathrm{E}+03$ & $5.43 \mathrm{E}+07$ & DOE-STD-1196 & $3.06 \mathrm{E}-11$ & $\mathrm{~s}$ & Max & DOE-STD-1196 & $7.18 \mathrm{E}-14$ \\
\hline $\ln -108$ & $\ln -108$ & 107.910 & Indium & $1.00 \mathrm{E}-03$ & ICRP-107 & $5.80 \mathrm{E}+01$ & $\mathrm{~m}$ & $3.48 \mathrm{E}+03$ & $3.00 \mathrm{E}+07$ & DOE-STD-1196 & $5.71 \mathrm{E}-11$ & $\mathrm{~s}$ & Max & DOE-STD-1196 & $1.81 \mathrm{E}-13$ \\
\hline In-108m & $\ln -108$ & 107.910 & Indium & $1.00 \mathrm{E}-03$ & ICRP-107 & $3.96 \mathrm{E}+01$ & $\mathrm{~m}$ & $2.38 \mathrm{E}+03$ & $4.40 \mathrm{E}+07$ & DOE-STD-1196 & $4.53 \mathrm{E}-11$ & $\mathrm{~s}$ & Max & DOE-STD-1196 & $1.35 \mathrm{E}-13$ \\
\hline In-109 & $\ln -109$ & 108.907 & Indium & $1.00 \mathrm{E}-03$ & ICRP-38 & $4.20 \mathrm{E}+00$ & $\mathrm{~h}$ & $1.51 \mathrm{E}+04$ & $6.85 \mathrm{E}+06$ & ICRP-72 & $4.20 \mathrm{E}-11$ & $M$ & Max & FGR-12 & $3.21 \mathrm{E}-14$ \\
\hline In-109m & $\ln -109$ & 108.907 & Indium & $1.00 \mathrm{E}-03$ & ICRP-107 & $1.34 \mathrm{E}+00$ & $\mathrm{~m}$ & $8.04 \mathrm{E}+01$ & $1.29 \mathrm{E}+09$ & -- & -- & -- & -- & DOE-STD-1196 & $2.74 \mathrm{E}-14$ \\
\hline$|n-110|$ & $\ln -110$ & 109.907 & Indium & $1.00 \mathrm{E}-03$ & ICRP-38 & $4.90 \mathrm{E}+00$ & $\mathrm{~h}$ & $1.76 \mathrm{E}+04$ & $5.82 \mathrm{E}+06$ & ICRP-72 & $1.30 \mathrm{E}-10$ & $M$ & Max & FGR-12 & $1.49 \mathrm{E}-13$ \\
\hline In-110s & $\ln -110$ & 109.907 & Indium & $1.00 \mathrm{E}-03$ & ICRP-38 & $6.91 \mathrm{E}+01$ & $\mathrm{~m}$ & $4.15 \mathrm{E}+03$ & $2.48 \mathrm{E}+07$ & ICRP-72 & $4.70 \mathrm{E}-11$ & $M$ & Max & FGR-12 & $7.62 \mathrm{E}-14$ \\
\hline $\ln -111$ & $\ln -111$ & 110.905 & Indium & $1.00 \mathrm{E}-03$ & ICRP-38 & $2.83 \mathrm{E}+00$ & $d$ & $2.45 \mathrm{E}+05$ & $4.16 \mathrm{E}+05$ & ICRP-72 & $2.30 \mathrm{E}-10$ & $M$ & Max & FGR-12 & $1.86 \mathrm{E}-14$ \\
\hline In-111m & $\ln -111$ & 110.905 & Indium & $1.00 \mathrm{E}-03$ & ICRP-38 & $7.70 \mathrm{E}+00$ & $\mathrm{~m}$ & $4.62 \mathrm{E}+02$ & $2.20 \mathrm{E}+08$ & -- & -- & -- & $\begin{array}{ll}- \\
\end{array}$ & DOE-STD-1196 & $2.10 \mathrm{E}-14$ \\
\hline $\ln -112$ & $\ln -112$ & 111.906 & Indium & $1.00 \mathrm{E}-03$ & ICRP-38 & $1.44 \mathrm{E}+01$ & $\mathrm{~m}$ & $8.64 \mathrm{E}+02$ & $1.17 \mathrm{E}+08$ & ICRP-72 & $7.40 \mathrm{E}-12$ & $M$ & Max & FGR-12 & $1.26 \mathrm{E}-14$ \\
\hline In-112m & $\ln -112$ & 111.906 & Indium & $1.00 \mathrm{E}-03$ & ICRP-107 & $2.06 \mathrm{E}+01$ & $\mathrm{~m}$ & $1.23 \mathrm{E}+03$ & $8.17 \mathrm{E}+07$ & DOE-STD-1196 & $2.60 \mathrm{E}-11$ & $\mathrm{~s}$ & Max & DOE-STD-1196 & $9.86 \mathrm{E}-16$ \\
\hline In-113m & $\ln -113$ & 112.904 & Indium & $1.00 \mathrm{E}-03$ & ICRP-38 & $1.66 \mathrm{E}+00$ & $\mathrm{~h}$ & $5.97 \mathrm{E}+03$ & $1.67 \mathrm{E}+07$ & ICRP-72 & $2.00 \mathrm{E}-11$ & $M$ & Max & FGR-12 & $1.21 \mathrm{E}-14$ \\
\hline In-114 & $\ln -114$ & 113.905 & Indium & $1.00 \mathrm{E}-03$ & ICRP-38 & $7.19 \mathrm{E}+01$ & $\mathrm{~s}$ & $7.19 \mathrm{E}+01$ & $1.38 \mathrm{E}+09$ & -- & -- & -- & -- & FGR-12 & $1.39 \mathrm{E}-16$ \\
\hline In-114m & $\ln -114$ & 113.905 & Indium & $1.00 \mathrm{E}-03$ & ICRP-38 & $4.95 \mathrm{E}+01$ & $d$ & $4.28 \mathrm{E}+06$ & $2.32 \mathrm{E}+04$ & ICRP-72 & $9.30 \mathrm{E}-09$ & $\mathrm{~F}$ & Max & FGR-12 & $4.18 \mathrm{E}-15$ \\
\hline $\ln -115$ & $\ln -115$ & 114.904 & Indium & $1.00 \mathrm{E}-03$ & ICRP-38 & $5.10 \mathrm{E}+15$ & $y$ & $1.61 \mathrm{E}+23$ & $6.10 \mathrm{E}-13$ & ICRP-72 & $3.90 \mathrm{E}-07$ & $\mathrm{~F}$ & Max & FGR-12 & $4.50 \mathrm{E}-18$ \\
\hline In-115m & $\ln -115$ & 114.904 & Indium & $1.00 \mathrm{E}-03$ & ICRP-38 & $4.49 \mathrm{E}+00$ & $\mathrm{~h}$ & $1.61 \mathrm{E}+04$ & $6.08 \mathrm{E}+06$ & ICRP-72 & $5.90 \mathrm{E}-11$ & $M$ & Max & FGR-12 & $7.39 \mathrm{E}-15$ \\
\hline
\end{tabular}




\begin{tabular}{|c|c|c|c|c|c|c|c|c|c|c|c|c|c|c|c|}
\hline \multicolumn{16}{|c|}{ Table B.1 Radionuclide Specific Input Data Used For the Calculation of HC-2 TQs Using Recommended DCs (continued) } \\
\hline $\begin{array}{l}\text { MASTER } \\
\text { ISOTOPE } \\
\text { LIST }\end{array}$ & $\begin{array}{l}\text { Atomic } \\
\text { Mass } \\
\text { Nuclide }\end{array}$ & $\begin{array}{l}\text { Atomic } \\
\text { Mass }\end{array}$ & Element & $\begin{array}{c}\text { RF per } \\
\text { DOE-STD- } \\
1027\end{array}$ & $\begin{array}{l}\text { Half-Life } \\
\text { Reference }\end{array}$ & $\begin{array}{r}\text { Half-Li } \\
\text { (original u }\end{array}$ & & Half Life (s) & $\begin{array}{l}\text { Sp. Act } \\
\text { (Ci/g) }\end{array}$ & $\begin{array}{c}\text { Inhalation DC } \\
\text { Reference }\end{array}$ & $\begin{array}{c}\text { HC-2 } \\
\text { Inhalation } \\
\text { DC } \\
\text { (Sv/Bq) }\end{array}$ & & $\begin{array}{l}\text { ung } \\
\text { rption } \\
\text { /Type }\end{array}$ & $\begin{array}{l}\text { Immersion DC } \\
\text { Reference }\end{array}$ & $\begin{array}{c}\text { HC-2 } \\
\text { Immersion } \\
\text { DC }(\mathrm{Sv} / \mathrm{s} \\
\left.\text { per } \mathrm{Bq} / \mathrm{m}^{3}\right)\end{array}$ \\
\hline In-116m & $\ln -116$ & 115.905 & Indium & $1.00 \mathrm{E}-03$ & ICRP-38 & $5.42 \mathrm{E}+01$ & $\mathrm{~m}$ & $3.25 \mathrm{E}+03$ & $3.00 \mathrm{E}+07$ & ICRP-72 & 4.50E-11 & $\mathrm{M}$ & Max & FGR-12 & $1.25 \mathrm{E}-13$ \\
\hline $\ln -117$ & $\ln -117$ & 116.905 & Indium & $1.00 \mathrm{E}-03$ & ICRP-38 & $4.38 \mathrm{E}+01$ & $\mathrm{~m}$ & $2.63 \mathrm{E}+03$ & $3.67 \mathrm{E}+07$ & ICRP-72 & $2.90 \mathrm{E}-11$ & $M$ & Max & FGR-12 & $3.31 \mathrm{E}-14$ \\
\hline In-117m & $\ln -117$ & 116.905 & Indium & $1.00 \mathrm{E}-03$ & ICRP-38 & $1.17 \mathrm{E}+02$ & $\mathrm{~m}$ & $6.99 \mathrm{E}+03$ & $1.38 \mathrm{E}+07$ & ICRP-72 & $7.20 \mathrm{E}-11$ & $M$ & Max & FGR-12 & $4.19 \mathrm{E}-15$ \\
\hline $\ln -118$ & $\ln -118$ & 117.906 & Indium & $1.00 \mathrm{E}-03$ & ICRP-107 & $5.00 E+00$ & $\mathrm{~s}$ & $5.00 \mathrm{E}+00$ & $1.91 \mathrm{E}+10$ & -- & -- & -- & -- & DOE-STD-1196 & $5.72 \mathrm{E}-15$ \\
\hline In-118m & $\ln -118$ & 117.906 & Indium & $1.00 \mathrm{E}-03$ & ICRP-107 & $4.36 \mathrm{E}+00$ & $\mathrm{~m}$ & $2.62 \mathrm{E}+02$ & $3.65 E+08$ & -- & -- & -- & -- & DOE-STD-1196 & $1.31 \mathrm{E}-13$ \\
\hline In-119 & In-119 & 118.906 & Indium & $1.00 \mathrm{E}-03$ & ICRP-38 & $2.40 E+00$ & $\mathrm{~m}$ & $1.44 \mathrm{E}+02$ & $6.59 \mathrm{E}+08$ & --- & --- & -- & -- & FGR-12 & $3.74 \mathrm{E}-14$ \\
\hline In-119m & $\ln -119$ & 118.906 & Indium & $1.00 \mathrm{E}-03$ & ICRP-38 & $1.80 \mathrm{E}+01$ & $\mathrm{~m}$ & $1.08 \mathrm{E}+03$ & $8.78 \mathrm{E}+07$ & ICRP-72 & $1.70 \mathrm{E}-11$ & $M$ & Max & FGR-12 & $6.14 \mathrm{E}-16$ \\
\hline In-121 & $\ln -121$ & 120.908 & Indium & $1.00 \mathrm{E}-03$ & ICRP-107 & $2.31 \mathrm{E}+01$ & $\mathrm{~s}$ & $2.31 \mathrm{E}+01$ & $4.04 \mathrm{E}+09$ & -- & -- & -- & -- & DOE-STD-1196 & $4.36 \mathrm{E}-14$ \\
\hline In-121m & $\ln -121$ & 120.908 & Indium & $1.00 \mathrm{E}-03$ & ICRP-107 & $3.88 \mathrm{E}+00$ & $\mathrm{~m}$ & $2.33 \mathrm{E}+02$ & $4.01 E+08$ & -- & -- & -- & -- & DOE-STD-1196 & $3.92 \mathrm{E}-15$ \\
\hline $\mid r-179$ & Ir-179 & 178.959 & Iridium & $1.00 \mathrm{E}-03$ & JAERI & $1.32 \mathrm{E}+00$ & $\mathrm{~m}$ & $7.90 \mathrm{E}+01$ & $7.98 E+08$ & -- & -- & -- & -- & -- & -- \\
\hline $\mid \mathrm{r}-180$ & Ir-180 & 179.959 & Iridium & $1.00 \mathrm{E}-03$ & ICRP-107 & $1.50 \mathrm{E}+00$ & $\mathrm{~m}$ & $9.00 \mathrm{E}+01$ & $6.97 \mathrm{E}+08$ & -- & -- & -- & -- & DOE-STD-1196 & $7.24 \mathrm{E}-14$ \\
\hline $\mid r-181$ & $\mid \mathrm{Ir}-181$ & 180.958 & Iridium & $1.00 \mathrm{E}-03$ & JAERI & $4.90 \mathrm{E}+00$ & $\mathrm{~m}$ & $2.94 \mathrm{E}+02$ & $2.12 \mathrm{E}+08$ & -- & -- & -- & -- & -- & -- \\
\hline $\mid r-182$ & $\mid r-182$ & 181.958 & Iridium & $1.00 \mathrm{E}-03$ & ICRP-38 & $1.50 \mathrm{E}+01$ & $\mathrm{~m}$ & $9.00 \mathrm{E}+02$ & $6.89 \mathrm{E}+07$ & ICRP-72 & $2.40 \mathrm{E}-11$ & $\mathrm{~s}$ & Max & FGR-12 & $6.50 \mathrm{E}-14$ \\
\hline $\mid r-183$ & $\mid r-183$ & 182.957 & Iridium & $1.00 \mathrm{E}-03$ & ICRP-107 & $5.80 \mathrm{E}+01$ & $\mathrm{~m}$ & $3.48 \mathrm{E}+03$ & $1.77 \mathrm{E}+07$ & DOE-STD-1196 & $4.71 \mathrm{E}-11$ & $\mathrm{~s}$ & Max & DOE-STD-1196 & $5.48 \mathrm{E}-14$ \\
\hline $\mid r-184$ & Ir-184 & 183.957 & Iridium & $1.00 \mathrm{E}-03$ & ICRP-38 & $3.02 \mathrm{E}+00$ & $\mathrm{~h}$ & $1.09 \mathrm{E}+04$ & $5.64 \mathrm{E}+06$ & ICRP-72 & $1.20 \mathrm{E}-10$ & $\mathrm{~s}$ & Max & FGR-12 & $9.38 \mathrm{E}-14$ \\
\hline |r-185 & Ir-185 & 184.957 & Iridium & $1.00 \mathrm{E}-03$ & ICRP-38 & $1.40 \mathrm{E}+01$ & $\mathrm{~h}$ & $5.04 \mathrm{E}+04$ & $1.21 \mathrm{E}+06$ & ICRP-72 & $1.90 \mathrm{E}-10$ & $\mathrm{~s}$ & Max & FGR-12 & $2.94 \mathrm{E}-14$ \\
\hline$|r-186|$ & Ir-186 & 185.958 & Iridium & $1.00 \mathrm{E}-03$ & ICRP-38 & $1.58 \mathrm{E}+01$ & $\mathrm{~h}$ & $5.69 \mathrm{E}+04$ & $1.07 E+06$ & ICRP-72 & $3.20 \mathrm{E}-10$ & $\mathrm{~s}$ & Max & FGR-12 & $8.06 \mathrm{E}-14$ \\
\hline $\mid r-186 s$ & Ir-186 & 185.958 & Iridium & $1.00 \mathrm{E}-03$ & ICRP-38 & $1.75 E+00$ & $\mathrm{~h}$ & $6.30 \mathrm{E}+03$ & $9.63 \mathrm{E}+06$ & ICRP-72 & $4.40 \mathrm{E}-11$ & $\mathrm{~s}$ & Max & FGR-12 & $4.65 \mathrm{E}-14$ \\
\hline |r-187 & Ir-187 & 186.958 & Iridium & $1.00 \mathrm{E}-03$ & ICRP-38 & $1.05 E+01$ & $\mathrm{~h}$ & $3.78 \mathrm{E}+04$ & $1.60 E+06$ & ICRP-72 & $7.90 \mathrm{E}-11$ & $\mathrm{~s}$ & Max & FGR-12 & $1.68 \mathrm{E}-14$ \\
\hline $\mid \mathrm{r}-188$ & Ir-188 & 187.959 & Iridium & $1.00 \mathrm{E}-03$ & ICRP-38 & $4.15 \mathrm{E}+01$ & $\mathrm{~h}$ & $1.49 \mathrm{E}+05$ & $4.02 E+05$ & ICRP-72 & $4.20 \mathrm{E}-10$ & $\mathrm{~s}$ & Max & FGR-12 & $8.01 \mathrm{E}-14$ \\
\hline |r-189 & Ir-189 & 188.959 & Iridium & $1.00 \mathrm{E}-03$ & ICRP-38 & $1.33 \mathrm{E}+01$ & $d$ & $1.15 \mathrm{E}+06$ & $5.20 E+04$ & ICRP-72 & $6.00 \mathrm{E}-10$ & $\mathrm{~s}$ & Max & FGR-12 & $3.21 \mathrm{E}-15$ \\
\hline $\mid \mathrm{Ir}-190$ & Ir-190 & 189.961 & Iridium & $1.00 \mathrm{E}-03$ & ICRP-38 & $1.21 \mathrm{E}+01$ & $d$ & $1.05 E+06$ & $5.68 \mathrm{E}+04$ & ICRP-72 & $2.40 \mathrm{E}-09$ & $\mathrm{~s}$ & Max & FGR-12 & $6.86 \mathrm{E}-14$ \\
\hline Ir-190ms & $\mid r-190$ & 189.961 & Iridium & $1.00 \mathrm{E}-03$ & ICRP-38 & $1.20 \mathrm{E}+00$ & $\mathrm{~h}$ & $4.32 \mathrm{E}+03$ & $1.37 \mathrm{E}+07$ & ICRP-72 & $1.00 \mathrm{E}-11$ & $\mathrm{~s}$ & Max & FGR-12 & $1.27 \mathrm{E}-19$ \\
\hline$|r-190 m|$ & Ir-190 & 189.961 & Iridium & $1.00 \mathrm{E}-03$ & ICRP-38 & $3.10 E+00$ & $\mathrm{~h}$ & $1.12 \mathrm{E}+04$ & $5.32 \mathrm{E}+06$ & ICRP-72 & $8.30 \mathrm{E}-11$ & $\mathrm{~s}$ & Max & FGR-12 & $7.39 \mathrm{E}-14$ \\
\hline $\mid r-191 m$ & $\mid \mathrm{Ir}-191$ & 190.961 & Iridium & $1.00 \mathrm{E}-03$ & ICRP-38 & $4.94 \mathrm{E}+00$ & $\mathrm{~s}$ & $4.94 \mathrm{E}+00$ & $1.20 \mathrm{E}+10$ & -- & -- & -- & -- & FGR-12 & $3.02 \mathrm{E}-15$ \\
\hline Ir-192 & Ir-192 & 191.963 & Iridium & $1.00 \mathrm{E}-03$ & ICRP-38 & $7.40 \mathrm{E}+01$ & $d$ & $6.40 \mathrm{E}+06$ & $9.19 \mathrm{E}+03$ & ICRP-72 & $6.60 \mathrm{E}-09$ & $\mathrm{~s}$ & Max & FGR-12 & $3.91 \mathrm{E}-14$ \\
\hline $\mid r-192 m s$ & $\mid \mathrm{r}-192$ & 191.963 & Iridium & $1.00 \mathrm{E}-03$ & ICRP-107 & $1.45 E+00$ & $\mathrm{~m}$ & $8.70 \mathrm{E}+01$ & $6.76 \mathrm{E}+08$ & -- & -- & -- & -- & DOE-STD-1196 & $2.68 \mathrm{E}-18$ \\
\hline $\mid \mathrm{r}-192 \mathrm{ml}$ & $\mid r-192$ & 191.963 & Iridium & $1.00 \mathrm{E}-03$ & ICRP-38 & $2.41 \mathrm{E}+02$ & $y$ & $7.61 E+09$ & $7.73 E+00$ & ICRP-72 & $3.90 \mathrm{E}-08$ & $\mathrm{~s}$ & Max & FGR-12 & $7.63 \mathrm{E}-15$ \\
\hline $\mid \mathrm{r}-193 \mathrm{~m}$ & $\mid \mathrm{Ir}-193$ & 192.963 & Iridium & $1.00 \mathrm{E}-03$ & ICRP-107 & $1.05 E+01$ & $d$ & $9.10 \mathrm{E}+05$ & $6.43 \mathrm{E}+04$ & ICRP-72 & $1.30 \mathrm{E}-09$ & $\mathrm{~s}$ & Max & DOE-STD-1196 & $1.04 \mathrm{E}-17$ \\
\hline Ir-194 & Ir-194 & 193.965 & Iridium & $1.00 \mathrm{E}-03$ & ICRP-38 & $1.92 \mathrm{E}+01$ & $\mathrm{~h}$ & $6.89 \mathrm{E}+04$ & $8.44 \mathrm{E}+05$ & ICRP-72 & $5.60 \mathrm{E}-10$ & $\mathrm{~s}$ & Max & FGR-12 & $4.54 \mathrm{E}-15$ \\
\hline Ir-194m & $\mid \mathrm{Ir}-194$ & 193.965 & Iridium & $1.00 \mathrm{E}-03$ & ICRP-38 & $1.71 \mathrm{E}+02$ & $d$ & $1.48 \mathrm{E}+07$ & $3.94 \mathrm{E}+03$ & ICRP-72 & $1.30 \mathrm{E}-08$ & $\mathrm{~s}$ & Max & FGR-12 & $1.12 \mathrm{E}-13$ \\
\hline $\mid \mathrm{r}-195$ & $\mid \mathrm{Ir}-195$ & 194.966 & Iridium & $1.00 \mathrm{E}-03$ & ICRP-38 & $2.50 \mathrm{E}+00$ & $\mathrm{~h}$ & $9.00 \mathrm{E}+03$ & $6.43 \mathrm{E}+06$ & ICRP-72 & $7.10 \mathrm{E}-11$ & $\mathrm{~s}$ & Max & FGR-12 & $2.32 \mathrm{E}-15$ \\
\hline Ir-195m & Ir-195 & 194.966 & Iridium & $1.00 \mathrm{E}-03$ & ICRP-38 & $3.80 \mathrm{E}+00$ & $\mathrm{~h}$ & $1.37 \mathrm{E}+04$ & $4.23 \mathrm{E}+06$ & ICRP-72 & $1.70 \mathrm{E}-10$ & $\mathrm{~s}$ & Max & FGR-12 & $1.93 \mathrm{E}-14$ \\
\hline |r-196 & Ir-196 & 195.968 & Iridium & $1.00 \mathrm{E}-03$ & ICRP-107 & $5.20 \mathrm{E}+01$ & $\mathrm{~s}$ & $5.20 \mathrm{E}+01$ & $1.11 \mathrm{E}+09$ & -- & -- & -- & -- & DOE-STD-1196 & $1.16 \mathrm{E}-14$ \\
\hline Ir-196m & Ir-196 & 195.968 & Iridium & $1.00 \mathrm{E}-03$ & ICRP-107 & $1.40 \mathrm{E}+00$ & $\mathrm{~h}$ & $5.04 \mathrm{E}+03$ & $1.14 \mathrm{E}+07$ & DOE-STD-1196 & $1.08 \mathrm{E}-10$ & $\mathrm{~s}$ & Max & DOE-STD-1196 & $1.10 \mathrm{E}-13$ \\
\hline $\mathrm{K}-38$ & $\mathrm{~K}-38$ & 37.969 & Potassium & $5.00 \mathrm{E}-01$ & ICRP-38 & $7.64 \mathrm{E}+00$ & $\mathrm{~m}$ & $4.58 \mathrm{E}+02$ & $6.49 \mathrm{E}+08$ & -- & -- & -- & -- & FGR-12 & $1.64 \mathrm{E}-13$ \\
\hline K-40 & K-40 & 39.964 & Potassium & $5.00 \mathrm{E}-01$ & ICRP-38 & $1.28 \mathrm{E}+09$ & $y$ & $4.04 \mathrm{E}+16$ & $6.99 \mathrm{E}-06$ & ICRP-72 & $2.10 \mathrm{E}-09$ & $\mathrm{~F}$ & Max & FGR-12 & $8.05 \mathrm{E}-15$ \\
\hline K-42 & K-42 & 41.962 & Potassium & $5.00 \mathrm{E}-01$ & ICRP-38 & $1.24 \mathrm{E}+01$ & $\mathrm{~h}$ & $4.45 \mathrm{E}+04$ & $6.04 \mathrm{E}+06$ & ICRP-72 & $1.20 \mathrm{E}-10$ & $\mathrm{~F}$ & Max & FGR-12 & 1.46E-14 \\
\hline K-43 & K-43 & 42.961 & Potassium & $5.00 \mathrm{E}-01$ & ICRP-38 & $2.26 \mathrm{E}+01$ & $\mathrm{~h}$ & $8.14 \mathrm{E}+04$ & $3.23 \mathrm{E}+06$ & ICRP-72 & 1.40E-10 & $F$ & Max & FGR-12 & $4.67 \mathrm{E}-14$ \\
\hline K-44 & K-44 & 43.962 & Potassium & $5.00 \mathrm{E}-01$ & ICRP-38 & $2.21 \mathrm{E}+01$ & $\mathrm{~m}$ & $1.33 \mathrm{E}+03$ & $1.93 \mathrm{E}+08$ & ICRP-72 & $2.00 \mathrm{E}-11$ & $\mathrm{~F}$ & Max & FGR-12 & $1.19 \mathrm{E}-13$ \\
\hline K-45 & K-45 & 44.961 & Potassium & $5.00 \mathrm{E}-01$ & ICRP-38 & $2.00 \mathrm{E}+01$ & $m$ & $1.20 \mathrm{E}+03$ & $2.09 \mathrm{E}+08$ & ICRP-72 & $1.50 \mathrm{E}-11$ & $\mathrm{~F}$ & Max & FGR-12 & $9.67 \mathrm{E}-14$ \\
\hline K-46 & $\mathrm{K}-46$ & 45.962 & Potassium & $5.00 \mathrm{E}-01$ & ICRP-107 & $1.05 E+02$ & $\mathrm{~s}$ & $1.05 E+02$ & $2.34 \mathrm{E}+09$ & -- & -- & -- & -- & DOE-STD-1196 & $1.47 \mathrm{E}-13$ \\
\hline Kr-74 & Kr-74 & 73.933 & Krypton & $1.00 E+00$ & ICRP-38 & $1.15 \mathrm{E}+01$ & $\mathrm{~m}$ & $6.90 \mathrm{E}+02$ & $2.21 \mathrm{E}+08$ & -- & -- & -- & -- & FGR-12 & $5.59 \mathrm{E}-14$ \\
\hline Kr-75 & Kr-75 & 74.931 & Krypton & $1.00 \mathrm{E}+00$ & ICRP-107 & $4.29 \mathrm{E}+00$ & $\mathrm{~m}$ & $2.57 \mathrm{E}+02$ & $5.85 \mathrm{E}+08$ & -- & -- & -- & -- & DOE-STD-1196 & $5.85 \mathrm{E}-14$ \\
\hline Kr-76 & Kr-76 & 75.926 & Krypton & $1.00 E+00$ & ICRP-38 & $1.48 \mathrm{E}+01$ & $\mathrm{~h}$ & $5.33 \mathrm{E}+04$ & $2.79 E+06$ & -- & -- & -- & -- & FGR-12 & $2.03 \mathrm{E}-14$ \\
\hline Kr-77 & Kr-77 & 76.925 & Krypton & $1.00 E+00$ & ICRP-38 & $7.47 \mathrm{E}+01$ & $\mathrm{~m}$ & $4.48 \mathrm{E}+03$ & $3.27 \mathrm{E}+07$ & -- & -- & -- & -- & FGR-12 & $4.86 \mathrm{E}-14$ \\
\hline
\end{tabular}




\begin{tabular}{|c|c|c|c|c|c|c|c|c|c|c|c|c|c|c|c|}
\hline \multicolumn{16}{|c|}{ Table B.1 Radionuclide Specific Input Data Used For the Calculation of HC-2 TQs Using Recommended DCs (continued) } \\
\hline $\begin{array}{l}\text { MASTER } \\
\text { ISOTOPE } \\
\text { LIST }\end{array}$ & $\begin{array}{c}\text { Atomic } \\
\text { Mass } \\
\text { Nuclide }\end{array}$ & $\begin{array}{l}\text { Atomic } \\
\text { Mass }\end{array}$ & Element & $\begin{array}{c}\text { RF per } \\
\text { DOE-STD- } \\
1027\end{array}$ & $\begin{array}{l}\text { Half-Life } \\
\text { Reference }\end{array}$ & \multicolumn{2}{|c|}{$\begin{array}{c}\text { Half-Life } \\
\text { (original units) }\end{array}$} & \multirow{2}{*}{$\begin{array}{c}\text { Half Life (s) } \\
1.26 E+05\end{array}$} & \multirow{2}{*}{$\begin{array}{c}\begin{array}{c}\text { Sp. Act } \\
\text { (Ci/g) }\end{array} \\
1.13 \mathrm{E}+06\end{array}$} & \multirow{2}{*}{$\begin{array}{c}\begin{array}{c}\text { Inhalation DC } \\
\text { Reference }\end{array} \\
--\end{array}$} & \multirow[t]{2}{*}{$\begin{array}{c}\text { HC-2 } \\
\text { Inhalation } \\
\text { DC } \\
\text { (Sv/Bq) } \\
--\end{array}$} & \multicolumn{2}{|c|}{$\begin{array}{c}\text { Lung } \\
\text { Absorption } \\
\text { Class/Type }\end{array}$} & \multirow{2}{*}{$\begin{array}{c}\begin{array}{c}\text { Immersion DC } \\
\text { Reference }\end{array} \\
\text { FGR-12 }\end{array}$} & \multirow[t]{2}{*}{$\begin{array}{c}\text { HC-2 } \\
\text { Immersion } \\
\text { DC (Sv/s } \\
\left.\text { per } \mathrm{Bq} / \mathrm{m}^{3}\right) \\
1.21 \mathrm{E}-14\end{array}$} \\
\hline Kr-79 & Kr-79 & 78.920 & Krypton & $1.00 \mathrm{E}+00$ & ICRP-38 & $3.50 \mathrm{E}+01$ & $\mathrm{~h}$ & & & & & -- & -- & & \\
\hline Kr-81 & $\mathrm{Kr}-81$ & 80.917 & Krypton & $1.00 \mathrm{E}+00$ & ICRP-38 & $2.10 \mathrm{E}+05$ & $\mathrm{y}$ & $6.63 \mathrm{E}+12$ & $2.10 \mathrm{E}-02$ & --- & -- & --- & -- & FGR-12 & $2.67 \mathrm{E}-16$ \\
\hline $\mathrm{Kr}-81 \mathrm{~m}$ & $\mathrm{Kr}-81$ & 80.917 & Krypton & $1.00 \mathrm{E}+00$ & ICRP-38 & $1.30 \mathrm{E}+01$ & $\mathrm{~s}$ & $1.30 \mathrm{E}+01$ & $1.07 \mathrm{E}+10$ & -- & -- & -- & -- & FGR-12 & $6.14 \mathrm{E}-15$ \\
\hline $\mathrm{Kr}-83 \mathrm{~m}$ & $\mathrm{Kr}-83$ & 82.914 & Krypton & $1.00 \mathrm{E}+00$ & ICRP-38 & $1.83 \mathrm{E}+00$ & $\mathrm{~h}$ & $6.59 \mathrm{E}+03$ & $2.07 \mathrm{E}+07$ & -- & -- & -- & -- & FGR-12 & $1.50 \mathrm{E}-18$ \\
\hline $\mathrm{Kr}-85$ & $\mathrm{Kr}-85$ & 84.913 & Krypton & $1.00 \mathrm{E}+00$ & ICRP-38 & $1.07 E+01$ & $y$ & $3.38 \mathrm{E}+08$ & $3.93 \mathrm{E}+02$ & -- & -- & -- & -- & FGR-12 & $1.19 \mathrm{E}-16$ \\
\hline $\mathrm{Kr}-85 \mathrm{~m}$ & $\mathrm{Kr}-85$ & 84.913 & Krypton & $1.00 \mathrm{E}+00$ & ICRP-38 & $4.48 \mathrm{E}+00$ & $\mathrm{~h}$ & $1.61 \mathrm{E}+04$ & $8.24 \mathrm{E}+06$ & -- & -- & -- & -- & FGR-12 & $7.48 \mathrm{E}-15$ \\
\hline $\mathrm{Kr}-87$ & $\mathrm{Kr}-87$ & 86.913 & Krypton & $1.00 \mathrm{E}+00$ & ICRP-38 & $7.63 \mathrm{E}+01$ & $\mathrm{~m}$ & $4.58 \mathrm{E}+03$ & $2.84 \mathrm{E}+07$ & -- & -- & -- & -- & FGR-12 & $4.12 \mathrm{E}-14$ \\
\hline Kr-88 & $\mathrm{Kr}-88$ & 87.914 & Krypton & $1.00 \mathrm{E}+00$ & ICRP-38 & $2.84 \mathrm{E}+00$ & $\mathrm{~h}$ & $1.02 \mathrm{E}+04$ & $1.26 \mathrm{E}+07$ & -- & -- & -- & -- & FGR-12 & $1.02 \mathrm{E}-13$ \\
\hline Kr-89 & $\mathrm{Kr}-89$ & 88.918 & Krypton & $1.00 \mathrm{E}+00$ & ICRP-107 & $3.15 \mathrm{E}+00$ & $\mathrm{~m}$ & $1.89 \mathrm{E}+02$ & $6.71 \mathrm{E}+08$ & -- & -- & -- & -- & DOE-STD-1196 & $9.56 \mathrm{E}-14$ \\
\hline La-128 & La-128 & 127.916 & Lanthanum & $1.00 \mathrm{E}-03$ & ICRP-107 & $5.18 \mathrm{E}+00$ & $\mathrm{~m}$ & $3.11 \mathrm{E}+02$ & $2.84 \mathrm{E}+08$ & -- & -- & -- & -- & DOE-STD-1196 & $1.30 \mathrm{E}-13$ \\
\hline La-129 & La-129 & 128.913 & Lanthanum & $1.00 \mathrm{E}-03$ & ICRP-107 & $1.16 \mathrm{E}+01$ & $\mathrm{~m}$ & $6.96 \mathrm{E}+02$ & $1.26 \mathrm{E}+08$ & DOE-STD-1196 & $1.48 \mathrm{E}-11$ & $\mathrm{~s}$ & Max & DOE-STD-1196 & $4.11 \mathrm{E}-14$ \\
\hline La-130 & La-130 & 129.912 & Lanthanum & $1.00 \mathrm{E}-03$ & ICRP-107 & $8.70 E+00$ & $\mathrm{~m}$ & $5.22 \mathrm{E}+02$ & $1.66 \mathrm{E}+08$ & -- & -- & -- & -- & DOE-STD-1196 & $1.03 \mathrm{E}-13$ \\
\hline La-131 & La-131 & 130.910 & Lanthanum & $1.00 \mathrm{E}-03$ & ICRP-38 & $5.90 \mathrm{E}+01$ & $\mathrm{~m}$ & $3.54 \mathrm{E}+03$ & $2.43 \mathrm{E}+07$ & ICRP-72 & $2.30 \mathrm{E}-11$ & $M$ & Max & FGR-12 & $3.14 \mathrm{E}-14$ \\
\hline La-132 & La-132 & 131.910 & Lanthanum & $1.00 \mathrm{E}-03$ & ICRP-38 & $4.80 \mathrm{E}+00$ & $\mathrm{~h}$ & $1.73 \mathrm{E}+04$ & $4.95 \mathrm{E}+06$ & ICRP-72 & $1.60 \mathrm{E}-10$ & $M$ & Max & FGR-12 & $1.00 \mathrm{E}-13$ \\
\hline La-132m & La-132 & 131.910 & Lanthanum & $1.00 \mathrm{E}-03$ & ICRP-107 & $2.43 \mathrm{E}+01$ & $\mathrm{~m}$ & $1.46 \mathrm{E}+03$ & $5.87 \mathrm{E}+07$ & DOE-STD-1196 & $2.51 \mathrm{E}-11$ & $\mathrm{~s}$ & Max & DOE-STD-1196 & $2.95 \mathrm{E}-14$ \\
\hline La-133 & La-133 & 132.908 & Lanthanum & $1.00 \mathrm{E}-03$ & ICRP-107 & $3.91 \mathrm{E}+00$ & $\mathrm{~h}$ & $1.41 \mathrm{E}+04$ & $6.03 \mathrm{E}+06$ & DOE-STD-1196 & $2.08 \mathrm{E}-11$ & $\mathrm{~s}$ & Max & DOE-STD-1196 & $6.39 \mathrm{E}-15$ \\
\hline La-134 & La-134 & 133.909 & Lanthanum & $1.00 \mathrm{E}-03$ & ICRP-38 & $6.67 E+00$ & $\mathrm{~m}$ & $4.00 \mathrm{E}+02$ & $2.11 \mathrm{E}+08$ & -- & -- & -- & -- & FGR-12 & $3.35 \mathrm{E}-14$ \\
\hline La-135 & La-135 & 134.907 & Lanthanum & $1.00 \mathrm{E}-03$ & ICRP-38 & $1.95 \mathrm{E}+01$ & $\mathrm{~h}$ & $7.02 E+04$ & $1.19 \mathrm{E}+06$ & ICRP-72 & 1.40E-11 & $M$ & Max & FGR-12 & $9.21 \mathrm{E}-16$ \\
\hline La-136 & La-136 & 135.908 & Lanthanum & $1.00 \mathrm{E}-03$ & ICRP-107 & $9.87 E+00$ & $\mathrm{~m}$ & $5.92 \mathrm{E}+02$ & $1.40 \mathrm{E}+08$ & -- & -- & -- & -- & DOE-STD-1196 & $1.79 \mathrm{E}-14$ \\
\hline La-137 & La-137 & 136.906 & Lanthanum & $1.00 \mathrm{E}-03$ & ICRP-38 & $6.00 \mathrm{E}+04$ & $y$ & $1.89 \mathrm{E}+12$ & $4.35 \mathrm{E}-02$ & ICRP-72 & $8.70 \mathrm{E}-09$ & $\mathrm{~F}$ & Max & FGR-12 & $4.06 \mathrm{E}-16$ \\
\hline La-138 & La-138 & 137.907 & Lanthanum & $1.00 \mathrm{E}-03$ & ICRP-38 & $1.35 \mathrm{E}+11$ & $y$ & $4.26 \mathrm{E}+18$ & $1.92 \mathrm{E}-08$ & ICRP-72 & $1.50 \mathrm{E}-07$ & $\mathrm{~F}$ & Max & FGR-12 & $6.20 \mathrm{E}-14$ \\
\hline La-140 & La-140 & 139.909 & Lanthanum & $1.00 \mathrm{E}-03$ & ICRP-38 & $4.03 \mathrm{E}+01$ & $\mathrm{~h}$ & $1.45 \mathrm{E}+05$ & $5.56 \mathrm{E}+05$ & ICRP-72 & $1.10 \mathrm{E}-09$ & $M$ & Max & FGR-12 & $1.17 \mathrm{E}-13$ \\
\hline La-141 & La-141 & 140.911 & Lanthanum & $1.00 \mathrm{E}-03$ & ICRP-38 & $3.93 \mathrm{E}+00$ & $\mathrm{~h}$ & $1.41 \mathrm{E}+04$ & $5.66 \mathrm{E}+06$ & ICRP-72 & $1.50 \mathrm{E}-10$ & $M$ & Max & FGR-12 & $2.39 \mathrm{E}-15$ \\
\hline La-142 & La-142 & 141.914 & Lanthanum & $1.00 \mathrm{E}-03$ & ICRP-38 & $9.25 \mathrm{E}+01$ & $\mathrm{~m}$ & $5.55 \mathrm{E}+03$ & $1.43 \mathrm{E}+07$ & ICRP-72 & $8.90 \mathrm{E}-11$ & $M$ & Max & FGR-12 & $1.44 \mathrm{E}-13$ \\
\hline La-143 & La-143 & 142.916 & Lanthanum & $1.00 \mathrm{E}-03$ & ICRP-38 & $1.42 \mathrm{E}+01$ & $\mathrm{~m}$ & $8.54 \mathrm{E}+02$ & $9.25 \mathrm{E}+07$ & ICRP-72 & $2.10 \mathrm{E}-11$ & $M$ & Max & FGR-12 & $5.18 \mathrm{E}-15$ \\
\hline Lu-164 & Lu-164 & 163.941 & Lutetium & $1.00 \mathrm{E}-03$ & JAERI & $3.14 \mathrm{E}+00$ & $\mathrm{~m}$ & $1.88 \mathrm{E}+02$ & $3.65 \mathrm{E}+08$ & -- & -- & -- & -- & -- & -- \\
\hline Lu-165 & Lu-165 & 164.939 & Lutetium & $1.00 \mathrm{E}-03$ & ICRP-107 & $1.07 E+01$ & $\mathrm{~m}$ & $6.44 \mathrm{E}+02$ & $1.06 \mathrm{E}+08$ & DOE-STD-1196 & $1.63 \mathrm{E}-11$ & $\mathrm{~s}$ & Max & DOE-STD-1196 & $5.02 \mathrm{E}-14$ \\
\hline Lu-166 & Lu-166 & 165.940 & Lutetium & $1.00 \mathrm{E}-03$ & JAERI & $2.65 \mathrm{E}+00$ & $\mathrm{~m}$ & $1.59 \mathrm{E}+02$ & $4.28 \mathrm{E}+08$ & -- & -- & -- & -- & -- & -- \\
\hline Lu-166m & Lu-166 & 165.940 & Lutetium & $1.00 \mathrm{E}-03$ & JAERI & $1.41 \mathrm{E}+00$ & $\mathrm{~m}$ & $8.46 \mathrm{E}+01$ & $8.04 \mathrm{E}+08$ & -- & -- & -- & -- & -- & $\begin{array}{l}- \\
\end{array}$ \\
\hline Lu-167 & Lu-167 & 166.938 & Lutetium & $1.00 \mathrm{E}-03$ & ICRP-107 & $5.15 \mathrm{E}+01$ & $\mathrm{~m}$ & $3.09 \mathrm{E}+03$ & $2.19 \mathrm{E}+07$ & DOE-STD-1196 & $4.56 \mathrm{E}-11$ & $\mathrm{~s}$ & Max & DOE-STD-1196 & $7.94 \mathrm{E}-14$ \\
\hline Lu-168m & Lu-168 & 167.939 & Lutetium & $1.00 \mathrm{E}-03$ & JAERI & $6.70 \mathrm{E}+00$ & $\mathrm{~m}$ & $4.02 \mathrm{E}+02$ & $1.67 \mathrm{E}+08$ & -- & -- & -- & -- & -- & -- \\
\hline Lu-169 & Lu-169 & 168.938 & Lutetium & $1.00 \mathrm{E}-03$ & ICRP-38 & $3.41 \mathrm{E}+01$ & $\mathrm{~h}$ & $1.23 \mathrm{E}+05$ & $5.45 \mathrm{E}+05$ & ICRP-72 & $3.80 \mathrm{E}-10$ & $\mathrm{~s}$ & Max & FGR-12 & $5.09 \mathrm{E}-14$ \\
\hline Lu-169m & Lu-169 & 168.938 & Lutetium & $1.00 \mathrm{E}-03$ & ICRP-107 & $1.60 \mathrm{E}+02$ & $\mathrm{~s}$ & $1.60 \mathrm{E}+02$ & $4.17 \mathrm{E}+08$ & -- & -- & -- & -- & DOE-STD-1196 & $2.52 \mathrm{E}-20$ \\
\hline Lu-170 & Lu-170 & 169.938 & Lutetium & $1.00 \mathrm{E}-03$ & ICRP-38 & $2.00 E+00$ & $d$ & $1.73 \mathrm{E}+05$ & $3.84 \mathrm{E}+05$ & ICRP-72 & $6.60 \mathrm{E}-10$ & $\mathrm{~s}$ & Max & FGR-12 & $1.28 \mathrm{E}-13$ \\
\hline Lu-171 & Lu-171 & 170.938 & Lutetium & $1.00 \mathrm{E}-03$ & ICRP-38 & $8.22 \mathrm{E}+00$ & $d$ & $7.10 \mathrm{E}+05$ & $9.29 \mathrm{E}+04$ & ICRP-72 & $8.80 \mathrm{E}-10$ & $\mathrm{~s}$ & Max & FGR-12 & $3.25 \mathrm{E}-14$ \\
\hline Lu-171m & Lu-171 & 170.938 & Lutetium & $1.00 \mathrm{E}-03$ & ICRP-107 & $7.90 \mathrm{E}+01$ & $\mathrm{~s}$ & $7.90 \mathrm{E}+01$ & $8.35 \mathrm{E}+08$ & -- & -- & -- & -- & DOE-STD-1196 & $9.86 \mathrm{E}-18$ \\
\hline Lu-172 & Lu-172 & 171.939 & Lutetium & $1.00 \mathrm{E}-03$ & ICRP-38 & $6.70 \mathrm{E}+00$ & $d$ & $5.79 \mathrm{E}+05$ & $1.13 \mathrm{E}+05$ & ICRP-72 & $1.60 \mathrm{E}-09$ & $\mathrm{~s}$ & Max & FGR-12 & $9.25 \mathrm{E}-14$ \\
\hline Lu-172m & Lu-172 & 171.939 & Lutetium & $1.00 \mathrm{E}-03$ & ICRP-107 & $3.70 \mathrm{E}+00$ & $\mathrm{~m}$ & $2.22 \mathrm{E}+02$ & $2.96 \mathrm{E}+08$ & -- & -- & -- & -- & DOE-STD-1196 & $3.90 \mathrm{E}-20$ \\
\hline Lu-173 & Lu-173 & 172.939 & Lutetium & $1.00 \mathrm{E}-03$ & ICRP-38 & $1.37 E+00$ & $y$ & $4.32 \mathrm{E}+07$ & $1.51 \mathrm{E}+03$ & ICRP-72 & $2.40 \mathrm{E}-09$ & $\mathrm{~s}$ & Max & FGR-12 & $5.10 \mathrm{E}-15$ \\
\hline Lu-174 & Lu-174 & 173.940 & Lutetium & $1.00 \mathrm{E}-03$ & ICRP-38 & $3.31 \mathrm{E}+00$ & $y$ & $1.04 \mathrm{E}+08$ & $6.21 \mathrm{E}+02$ & ICRP-72 & $4.20 \mathrm{E}-09$ & $M$ & Max & FGR-12 & $5.46 \mathrm{E}-15$ \\
\hline Lu-174m & Lu-174 & 173.940 & Lutetium & $1.00 \mathrm{E}-03$ & ICRP-38 & $1.42 \mathrm{E}+02$ & $d$ & $1.23 \mathrm{E}+07$ & $5.29 \mathrm{E}+03$ & ICRP-72 & $4.20 \mathrm{E}-09$ & $\mathrm{~s}$ & Max & FGR-12 & $2.18 \mathrm{E}-15$ \\
\hline Lu-176 & Lu-176 & 175.943 & Lutetium & $1.00 \mathrm{E}-03$ & ICRP-38 & $3.60 \mathrm{E}+10$ & $y$ & $1.14 \mathrm{E}+18$ & $5.64 \mathrm{E}-08$ & ICRP-72 & $7.00 \mathrm{E}-08$ & $M$ & Max & FGR-12 & $2.32 \mathrm{E}-14$ \\
\hline Lu-176m & Lu-176 & 175.943 & Lutetium & $1.00 \mathrm{E}-03$ & ICRP-38 & $3.68 \mathrm{E}+00$ & $\mathrm{~h}$ & $1.32 \mathrm{E}+04$ & $4.84 \mathrm{E}+06$ & ICRP-72 & $1.20 \mathrm{E}-10$ & $\mathrm{~s}$ & Max & FGR-12 & $5.87 \mathrm{E}-16$ \\
\hline Lu-177 & Lu-177 & 176.944 & Lutetium & $1.00 \mathrm{E}-03$ & ICRP-38 & $6.71 \mathrm{E}+00$ & $d$ & $5.80 \mathrm{E}+05$ & $1.10 \mathrm{E}+05$ & ICRP-72 & $1.20 \mathrm{E}-09$ & $\mathrm{~s}$ & Max & FGR-12 & $1.62 \mathrm{E}-15$ \\
\hline Lu-177m & Lu-177 & 176.944 & Lutetium & $1.00 \mathrm{E}-03$ & ICRP-38 & $1.61 \mathrm{E}+02$ & $d$ & $1.39 \mathrm{E}+07$ & $4.59 \mathrm{E}+03$ & ICRP-72 & $1.60 \mathrm{E}-08$ & $\mathrm{~s}$ & Max & FGR-12 & $4.67 \mathrm{E}-14$ \\
\hline Lu-178 & Lu-178 & 177.946 & Lutetium & $1.00 \mathrm{E}-03$ & ICRP-38 & $2.84 \mathrm{E}+01$ & $\mathrm{~m}$ & $1.70 \mathrm{E}+03$ & $3.72 \mathrm{E}+07$ & ICRP-72 & $2.60 \mathrm{E}-11$ & $\mathrm{~s}$ & Max & FGR-12 & $7.09 \mathrm{E}-15$ \\
\hline
\end{tabular}




\begin{tabular}{|c|c|c|c|c|c|c|c|c|c|c|c|c|c|c|c|}
\hline \multicolumn{16}{|c|}{ Table B.1 Radionuclide Specific Input Data Used For the Calculation of HC-2 TQs Using Recommended DCs (continued) } \\
\hline $\begin{array}{l}\text { MASTER } \\
\text { ISOTOPE } \\
\text { LIST }\end{array}$ & $\begin{array}{l}\text { Atomic } \\
\text { Mass } \\
\text { Nuclide }\end{array}$ & $\begin{array}{l}\text { Atomic } \\
\text { Mass }\end{array}$ & Element & $\begin{array}{c}\text { RF per } \\
\text { DOE-STD- } \\
1027\end{array}$ & $\begin{array}{l}\text { Half-Life } \\
\text { Reference }\end{array}$ & $\begin{array}{r}\text { Half-Li } \\
\text { (original L }\end{array}$ & & Half Life (s) & $\begin{array}{l}\text { Sp. Act } \\
\text { (Ci/g) }\end{array}$ & $\begin{array}{c}\text { Inhalation DC } \\
\text { Reference }\end{array}$ & $\begin{array}{c}\text { HC-2 } \\
\text { Inhalation } \\
\text { DC } \\
(\mathrm{Sv} / \mathrm{Bq})\end{array}$ & & $\begin{array}{l}\text { ung } \\
\text { rption } \\
\text { /Type }\end{array}$ & $\begin{array}{l}\text { Immersion DC } \\
\text { Reference }\end{array}$ & $\begin{array}{c}\text { HC-2 } \\
\text { Immersion } \\
\mathrm{DC}(\mathrm{Sv} / \mathrm{s} \\
\left.\text { per } \mathrm{Bq} / \mathrm{m}^{3}\right)\end{array}$ \\
\hline Lu-178m & Lu-178 & 177.946 & Lutetium & $1.00 \mathrm{E}-03$ & ICRP-38 & $2.27 \mathrm{E}+01$ & $\mathrm{~m}$ & $1.36 \mathrm{E}+03$ & $4.65 \mathrm{E}+07$ & ICRP-72 & $3.30 \mathrm{E}-11$ & $\mathrm{~s}$ & Max & FGR-12 & $5.23 \mathrm{E}-14$ \\
\hline Lu-179 & Lu-179 & 178.947 & Lutetium & $1.00 \mathrm{E}-03$ & ICRP-38 & $4.59 \mathrm{E}+00$ & $\mathrm{~h}$ & $1.65 \mathrm{E}+04$ & $3.82 \mathrm{E}+06$ & ICRP-72 & $1.20 \mathrm{E}-10$ & $\mathrm{~s}$ & Max & FGR-12 & $1.52 \mathrm{E}-15$ \\
\hline Lu-180 & Lu-180 & 179.950 & Lutetium & $1.00 \mathrm{E}-03$ & ICRP-107 & $5.70 \mathrm{E}+00$ & $\mathrm{~m}$ & $3.42 \mathrm{E}+02$ & $1.83 \mathrm{E}+08$ & -- & -- & -- & -- & DOE-STD-1196 & $7.09 \mathrm{E}-14$ \\
\hline Lu-181 & Lu-181 & 180.952 & Lutetium & $1.00 \mathrm{E}-03$ & ICRP-107 & $3.50 \mathrm{E}+00$ & $\mathrm{~m}$ & $2.10 \mathrm{E}+02$ & $2.97 \mathrm{E}+08$ & -- & -- & -- & -- & DOE-STD-1196 & $2.58 \mathrm{E}-14$ \\
\hline Md-257 & Md-257 & 257.096 & Mendelevium & $1.00 \mathrm{E}-03$ & ICRP-38 & $5.20 \mathrm{E}+00$ & $\mathrm{~h}$ & $1.87 \mathrm{E}+04$ & $2.34 \mathrm{E}+06$ & ICRP-72 & $2.50 \mathrm{E}-08$ & $M$ & Max & FGR-12 & $5.03 \mathrm{E}-15$ \\
\hline Md-258 & $\mathrm{Md}-258$ & 258.098 & Mendelevium & $1.00 \mathrm{E}-03$ & ICRP-38 & $5.50 \mathrm{E}+01$ & $d$ & $4.75 \mathrm{E}+06$ & $9.20 \mathrm{E}+03$ & ICRP-72 & $5.90 \mathrm{E}-06$ & $\mathrm{M}$ & Max & FGR-12 & $5.08 \mathrm{E}-17$ \\
\hline $\mathrm{Mg}-27$ & $\mathrm{Mg}-27$ & 26.984 & Magnesium & $1.00 \mathrm{E}-03$ & ICRP-107 & $9.46 \mathrm{E}+00$ & $\mathrm{~m}$ & $5.67 \mathrm{E}+02$ & $7.37 E+08$ & -- & -- & -- & -- & DOE-STD-1196 & $4.16 \mathrm{E}-14$ \\
\hline $\mathrm{Mg}-28$ & $\mathrm{Mg}-28$ & 27.984 & Magnesium & $1.00 \mathrm{E}-03$ & ICRP-38 & $2.09 \mathrm{E}+01$ & $\mathrm{~h}$ & $7.53 E+04$ & $5.36 \mathrm{E}+06$ & ICRP-72 & $1.20 \mathrm{E}-09$ & $M$ & Max & FGR-12 & $6.79 \mathrm{E}-14$ \\
\hline $\mathrm{Mn}-50 \mathrm{~m}$ & $M n-50$ & 49.954 & Manganese & $1.00 \mathrm{E}-03$ & ICRP-107 & $1.75 \mathrm{E}+00$ & $\mathrm{~m}$ & $1.05 \mathrm{E}+02$ & $2.15 \mathrm{E}+09$ & -- & -- & -- & -- & DOE-STD-1196 & $2.18 \mathrm{E}-13$ \\
\hline Mn-51 & Mn-51 & 50.948 & Manganese & $1.00 \mathrm{E}-03$ & ICRP-38 & $4.62 E+01$ & $\mathrm{~m}$ & $2.77 \mathrm{E}+03$ & $7.99 \mathrm{E}+07$ & ICRP-72 & $4.10 \mathrm{E}-11$ & $M$ & Max & FGR-12 & $4.80 \mathrm{E}-14$ \\
\hline Mn-52 & $M n-52$ & 51.946 & Manganese & $1.00 \mathrm{E}-03$ & ICRP-38 & $5.59 \mathrm{E}+00$ & d & $4.83 \mathrm{E}+05$ & $4.50 \mathrm{E}+05$ & ICRP-72 & $1.40 \mathrm{E}-09$ & $M$ & Max & FGR-12 & $1.72 \mathrm{E}-13$ \\
\hline$M n-52 m$ & $M n-52$ & 51.946 & Manganese & $1.00 \mathrm{E}-03$ & ICRP-38 & $2.11 \mathrm{E}+01$ & $\mathrm{~m}$ & $1.27 \mathrm{E}+03$ & $1.72 \mathrm{E}+08$ & ICRP-72 & $2.90 \mathrm{E}-11$ & $M$ & Max & FGR-12 & $1.20 \mathrm{E}-13$ \\
\hline Mn-53 & $M n-53$ & 52.941 & Manganese & $1.00 \mathrm{E}-03$ & ICRP-38 & $3.70 \mathrm{E}+06$ & $y$ & $1.17 \mathrm{E}+14$ & $1.83 \mathrm{E}-03$ & ICRP-72 & $5.40 \mathrm{E}-11$ & $M$ & Max & -- & -- \\
\hline Mn-54 & Mn-54 & 53.940 & Manganese & $1.00 \mathrm{E}-03$ & ICRP-38 & $3.13 \mathrm{E}+02$ & $d$ & $2.70 \mathrm{E}+07$ & $7.75 E+03$ & ICRP-72 & $1.50 \mathrm{E}-09$ & $M$ & Max & FGR-12 & $4.09 \mathrm{E}-14$ \\
\hline Mn-56 & Mn-56 & 55.939 & Manganese & $1.00 \mathrm{E}-03$ & ICRP-38 & $2.58 \mathrm{E}+00$ & $\mathrm{~h}$ & $9.28 \mathrm{E}+03$ & $2.17 \mathrm{E}+07$ & ICRP-72 & $1.20 \mathrm{E}-10$ & $M$ & Max & FGR-12 & $8.61 \mathrm{E}-14$ \\
\hline Mn-57 & Mn-57 & 56.938 & Manganese & $1.00 \mathrm{E}-03$ & ICRP-107 & $8.54 \mathrm{E}+01$ & $\mathrm{~s}$ & $8.54 \mathrm{E}+01$ & $2.32 \mathrm{E}+09$ & -- & -- & -- & -- & DOE-STD-1196 & $5.30 \mathrm{E}-15$ \\
\hline Mn-58m & $M n-58$ & 57.940 & Manganese & $1.00 \mathrm{E}-03$ & ICRP-107 & $6.52 \mathrm{E}+01$ & $\mathrm{~s}$ & $6.52 \mathrm{E}+01$ & $2.99 \mathrm{E}+09$ & -- & -- & -- & -- & DOE-STD-1196 & $1.15 \mathrm{E}-13$ \\
\hline Mo-101 & Mo-101 & 100.910 & Molybdenum & $1.00 \mathrm{E}-03$ & ICRP-38 & $1.46 \mathrm{E}+01$ & $\mathrm{~m}$ & $8.77 \mathrm{E}+02$ & $1.27 \mathrm{E}+08$ & ICRP-72 & $2.50 \mathrm{E}-11$ & $M$ & Rec & FGR-12 & $6.87 \mathrm{E}-14$ \\
\hline Mo-102 & Mo-102 & 101.910 & Molybdenum & $1.00 \mathrm{E}-03$ & ICRP-107 & $1.13 \mathrm{E}+01$ & $\mathrm{~m}$ & $6.78 \mathrm{E}+02$ & $1.63 \mathrm{E}+08$ & DOE-STD-1196 & $2.89 \mathrm{E}-11$ & $M$ & $\operatorname{Rec}$ & DOE-STD-1196 & $1.02 \mathrm{E}-15$ \\
\hline Mo-89 & Mo-89 & 88.919 & Molybdenum & $1.00 \mathrm{E}-03$ & ICRP-107 & $2.11 \mathrm{E}+00$ & $\mathrm{~m}$ & $1.27 \mathrm{E}+02$ & $1.00 \mathrm{E}+09$ & -- & -- & -- & -- & DOE-STD-1196 & $5.70 \mathrm{E}-14$ \\
\hline Mo-90 & Mo-90 & 89.914 & Molybdenum & $1.00 \mathrm{E}-03$ & ICRP-38 & $5.67 \mathrm{E}+00$ & $\mathrm{~h}$ & $2.04 \mathrm{E}+04$ & $6.15 \mathrm{E}+06$ & ICRP-72 & $3.40 \mathrm{E}-10$ & $M$ & $\operatorname{Rec}$ & FGR-12 & $3.93 \mathrm{E}-14$ \\
\hline Mo-91 & Mo-91 & 90.912 & Molybdenum & $1.00 \mathrm{E}-03$ & ICRP-107 & $1.55 \mathrm{E}+01$ & $\mathrm{~m}$ & $9.29 \mathrm{E}+02$ & $1.34 \mathrm{E}+08$ & DOE-STD-1196 & $2.39 \mathrm{E}-11$ & $M$ & $\operatorname{Rec}$ & DOE-STD-1196 & $4.50 \mathrm{E}-14$ \\
\hline Mo-91m & Mo-91 & 90.912 & Molybdenum & $1.00 \mathrm{E}-03$ & ICRP-107 & $6.46 \mathrm{E}+01$ & $\mathrm{~s}$ & $6.46 \mathrm{E}+01$ & $1.92 \mathrm{E}+09$ & -- & -- & -- & --- & DOE-STD-1196 & $6.45 \mathrm{E}-14$ \\
\hline Mo-93 & Mo-93 & 92.907 & Molybdenum & $1.00 \mathrm{E}-03$ & ICRP-38 & $3.50 \mathrm{E}+03$ & $y$ & $1.10 \mathrm{E}+11$ & $1.10 \mathrm{E}+00$ & ICRP-72 & $5.90 \mathrm{E}-10$ & $M$ & $\operatorname{Rec}$ & FGR-12 & $2.52 \mathrm{E}-17$ \\
\hline Mo-93m & Mo-93 & 92.907 & Molybdenum & $1.00 \mathrm{E}-03$ & ICRP-38 & $6.85 \mathrm{E}+00$ & $\mathrm{~h}$ & $2.47 \mathrm{E}+04$ & $4.92 \mathrm{E}+06$ & ICRP-72 & $1.60 \mathrm{E}-10$ & $M$ & $\operatorname{Rec}$ & FGR-12 & $1.13 \mathrm{E}-13$ \\
\hline Mo-99 & Mo-99 & 98.908 & Molybdenum & $1.00 \mathrm{E}-03$ & ICRP-38 & $6.60 \mathrm{E}+01$ & $\mathrm{~h}$ & $2.38 \mathrm{E}+05$ & $4.80 \mathrm{E}+05$ & ICRP-72 & $8.90 \mathrm{E}-10$ & $M$ & $\operatorname{Rec}$ & FGR-12 & $7.28 \mathrm{E}-15$ \\
\hline $\mathrm{N}-13$ & $\mathrm{~N}-1$ & 1.009 & Nitrogen & $1.00 \mathrm{E}+00$ & ICRP-38 & $9.97 \mathrm{E}+00$ & $\mathrm{~m}$ & $5.98 \mathrm{E}+02$ & $1.87 \mathrm{E}+10$ & -- & -- & -- & -- & FGR-12 & $4.90 \mathrm{E}-14$ \\
\hline $\mathrm{N}-16$ & $\mathrm{~N}-16$ & 16.006 & Nitrogen & $1.00 \mathrm{E}+00$ & ICRP-107 & $7.13 \mathrm{E}+00$ & $\mathrm{~s}$ & $7.13 \mathrm{E}+00$ & $9.89 \mathrm{E}+10$ & -- & -- & -- & -- & DOE-STD-1196 & $2.59 \mathrm{E}-13$ \\
\hline $\mathrm{Na}-22$ & $\mathrm{Na}-22$ & 21.994 & Sodium & $5.00 \mathrm{E}-01$ & ICRP-38 & $2.60 \mathrm{E}+00$ & $y$ & $8.21 \mathrm{E}+07$ & $6.25 \mathrm{E}+03$ & ICRP-72 & $1.30 \mathrm{E}-09$ & $F$ & Max & FGR-12 & $1.08 \mathrm{E}-13$ \\
\hline $\mathrm{Na}-24$ & $\mathrm{Na}-24$ & 23.991 & Sodium & $5.00 \mathrm{E}-01$ & ICRP-38 & $1.50 \mathrm{E}+01$ & $\mathrm{~h}$ & $5.40 \mathrm{E}+04$ & $8.71 \mathrm{E}+06$ & ICRP-72 & $2.70 \mathrm{E}-10$ & $\mathrm{~F}$ & Max & FGR-12 & $2.18 \mathrm{E}-13$ \\
\hline $\mathrm{Nb}-87$ & Nb-87 & 86.921 & Niobium & $1.00 \mathrm{E}-03$ & ICRP-107 & $3.75 \mathrm{E}+00$ & $\mathrm{~m}$ & $2.25 \mathrm{E}+02$ & $5.77 \mathrm{E}+08$ & -- & -- & -- & -- & DOE-STD-1196 & $5.54 \mathrm{E}-14$ \\
\hline $\mathrm{Nb}-88$ & $\mathrm{Nb}-88$ & 87.918 & Niobium & $1.00 \mathrm{E}-03$ & ICRP-38 & $1.43 \mathrm{E}+01$ & $\mathrm{~m}$ & $8.58 \mathrm{E}+02$ & $1.50 \mathrm{E}+08$ & ICRP-72 & $2.70 \mathrm{E}-11$ & $M$ & $\operatorname{Rec}$ & FGR-12 & $2.02 \mathrm{E}-13$ \\
\hline $\mathrm{Nb}-88 \mathrm{~m}$ & $\mathrm{Nb}-88$ & 87.918 & Niobium & $1.00 \mathrm{E}-03$ & ICRP-107 & $7.78 \mathrm{E}+00$ & $\mathrm{~m}$ & $4.67 \mathrm{E}+02$ & $2.75 E+08$ & -- & -- & -- & -- & DOE-STD-1196 & $1.91 \mathrm{E}-13$ \\
\hline Nb-89| & $\mathrm{Nb}-89$ & 88.913 & Niobium & $1.00 \mathrm{E}-03$ & ICRP-38 & $1.22 \mathrm{E}+02$ & $\mathrm{~m}$ & $7.32 \mathrm{E}+03$ & $1.73 \mathrm{E}+07$ & ICRP-72 & $1.10 \mathrm{E}-10$ & $M$ & $\operatorname{Rec}$ & FGR-12 & $6.98 \mathrm{E}-14$ \\
\hline $\mathrm{Nb}-89 \mathrm{~s}$ & $\mathrm{Nb}-89$ & 88.913 & Niobium & $1.00 \mathrm{E}-03$ & ICRP-38 & $6.60 \mathrm{E}+01$ & $\mathrm{~m}$ & $3.96 \mathrm{E}+03$ & $3.20 \mathrm{E}+07$ & ICRP-72 & $6.80 \mathrm{E}-11$ & $M$ & $\operatorname{Rec}$ & FGR-12 & $9.26 \mathrm{E}-14$ \\
\hline $\mathrm{Nb}-90$ & $\mathrm{Nb}-90$ & 89.911 & Niobium & $1.00 \mathrm{E}-03$ & ICRP-38 & $1.46 \mathrm{E}+01$ & $\mathrm{~h}$ & $5.26 \mathrm{E}+04$ & $2.39 \mathrm{E}+06$ & ICRP-72 & $6.30 \mathrm{E}-10$ & $M$ & $\operatorname{Rec}$ & FGR-12 & $2.17 \mathrm{E}-13$ \\
\hline Nb-91 & $\mathrm{Nb}-91$ & 90.907 & Niobium & $1.00 \mathrm{E}-03$ & ICRP-107 & $6.80 \mathrm{E}+02$ & $y$ & $2.15 \mathrm{E}+10$ & $5.78 \mathrm{E}+00$ & DOE-STD-1196 & $3.03 \mathrm{E}-10$ & $M$ & $\operatorname{Rec}$ & DOE-STD-1196 & $8.45 \mathrm{E}-17$ \\
\hline $\mathrm{Nb}-91 \mathrm{~m}$ & $\mathrm{Nb}-91$ & 90.907 & Niobium & $1.00 \mathrm{E}-03$ & ICRP-107 & $6.09 \mathrm{E}+01$ & $d$ & $5.26 \mathrm{E}+06$ & $2.36 \mathrm{E}+04$ & DOE-STD-1196 & $3.65 \mathrm{E}-09$ & $M$ & $\operatorname{Rec}$ & DOE-STD-1196 & $1.20 \mathrm{E}-15$ \\
\hline $\mathrm{Nb}-92$ & $\mathrm{Nb}-92$ & 91.907 & Niobium & $1.00 \mathrm{E}-03$ & ICRP-107 & $3.47 \mathrm{E}+07$ & $y$ & $1.10 \mathrm{E}+15$ & $1.12 \mathrm{E}-04$ & DOE-STD-1196 & $5.46 \mathrm{E}-09$ & $M$ & $\operatorname{Rec}$ & DOE-STD-1196 & $6.83 \mathrm{E}-14$ \\
\hline $\mathrm{Nb}-92 \mathrm{~m}$ & $\mathrm{Nb}-92$ & 91.907 & Niobium & $1.00 \mathrm{E}-03$ & ICRP-107 & $1.02 \mathrm{E}+01$ & $d$ & $8.77 \mathrm{E}+05$ & $1.40 \mathrm{E}+05$ & DOE-STD-1196 & $5.00 \mathrm{E}-10$ & $M$ & $\operatorname{Rec}$ & DOE-STD-1196 & 4.44E-14 \\
\hline $\mathrm{Nb}-93 \mathrm{~m}$ & $\mathrm{Nb}-93$ & 92.906 & Niobium & $1.00 \mathrm{E}-03$ & ICRP-38 & $1.36 \mathrm{E}+01$ & $y$ & $4.29 \mathrm{E}+08$ & $2.83 \mathrm{E}+02$ & ICRP-72 & $5.10 \mathrm{E}-10$ & $\mathrm{M}$ & $\operatorname{Rec}$ & FGR-12 & $4.44 \mathrm{E}-18$ \\
\hline $\mathrm{Nb}-94$ & $\mathrm{Nb}-94$ & 93.907 & Niobium & $1.00 \mathrm{E}-03$ & ICRP-38 & $2.03 E+04$ & $y$ & $6.41 \mathrm{E}+11$ & $1.88 \mathrm{E}-01$ & ICRP-72 & $1.10 \mathrm{E}-08$ & $M$ & $\operatorname{Rec}$ & FGR-12 & $7.70 \mathrm{E}-14$ \\
\hline $\mathrm{Nb}-94 \mathrm{~m}$ & $\mathrm{Nb}-94$ & 93.907 & Niobium & $1.00 \mathrm{E}-03$ & ICRP-107 & $6.26 \mathrm{E}+00$ & $\mathrm{~m}$ & $3.76 \mathrm{E}+02$ & $3.20 \mathrm{E}+08$ & -- & -- & -- & -- & DOE-STD-1196 & $2.15 \mathrm{E}-16$ \\
\hline $\mathrm{Nb}-95$ & $\mathrm{Nb}-95$ & 94.907 & Niobium & $1.00 \mathrm{E}-03$ & ICRP-38 & $3.52 \mathrm{E}+01$ & $d$ & $3.04 \mathrm{E}+06$ & $3.91 \mathrm{E}+04$ & ICRP-72 & $1.50 \mathrm{E}-09$ & $M$ & $\operatorname{Rec}$ & FGR-12 & $3.74 \mathrm{E}-14$ \\
\hline $\mathrm{Nb}-95 \mathrm{~m}$ & $\mathrm{Nb}-95$ & 94.907 & Niobium & $1.00 \mathrm{E}-03$ & ICRP-38 & $8.66 \mathrm{E}+01$ & $\mathrm{~h}$ & $3.12 \mathrm{E}+05$ & $3.81 \mathrm{E}+05$ & ICRP-72 & $7.90 \mathrm{E}-10$ & $M$ & $\operatorname{Rec}$ & FGR-12 & $2.93 \mathrm{E}-15$ \\
\hline $\mathrm{Nb}-96$ & Nb-96 & 95.908 & Niobium & $1.00 \mathrm{E}-03$ & ICRP-38 & $2.34 \mathrm{E}+01$ & $\mathrm{~h}$ & $8.41 \mathrm{E}+04$ & $1.40 \mathrm{E}+06$ & ICRP-72 & $6.30 \mathrm{E}-10$ & $M$ & $\operatorname{Rec}$ & FGR-12 & $1.21 \mathrm{E}-13$ \\
\hline
\end{tabular}




\begin{tabular}{|c|c|c|c|c|c|c|c|c|c|c|c|c|c|c|c|}
\hline $\begin{array}{l}\text { MASTER } \\
\text { ISOTOPE } \\
\text { LIST }\end{array}$ & $\begin{array}{l}\text { Atomic } \\
\text { Mass } \\
\text { Nuclide }\end{array}$ & $\begin{array}{l}\text { Atomic } \\
\text { Mass }\end{array}$ & Element & $\begin{array}{c}\text { RF per } \\
\text { DOE-STD- } \\
1027\end{array}$ & $\begin{array}{c}\text { Half-Life } \\
\text { Reference }\end{array}$ & $\begin{array}{r}\text { Half-Li } \\
\text { (original u }\end{array}$ & & Half Life (s) & $\begin{array}{l}\text { Sp. Act } \\
\text { (Ci/g) }\end{array}$ & $\begin{array}{l}\text { Inhalation DC } \\
\text { Reference }\end{array}$ & $\begin{array}{c}\mathrm{HC}-2 \\
\text { Inhalation } \\
\mathrm{DC} \\
(\mathrm{Sv} / \mathrm{Bq})\end{array}$ & & $\begin{array}{l}\text { Ing } \\
\text { rption } \\
\text { /Type }\end{array}$ & $\begin{array}{l}\text { Immersion DC } \\
\text { Reference }\end{array}$ & $\begin{array}{c}\mathrm{HC}-2 \\
\text { Immersion } \\
\mathrm{DC}(\mathrm{Sv} / \mathrm{s} \\
\left.\text { per } \mathrm{Bq} / \mathrm{m}^{3}\right)\end{array}$ \\
\hline $\mathrm{Nb}-97$ & $\mathrm{Nb}-97$ & 96.908 & Niobium & $1.00 \mathrm{E}-03$ & ICRP-38 & $7.21 \mathrm{E}+01$ & $\mathrm{~m}$ & $4.33 \mathrm{E}+03$ & $2.69 \mathrm{E}+07$ & ICRP-72 & $4.30 \mathrm{E}-11$ & $M$ & $\operatorname{Rec}$ & FGR-12 & $3.18 \mathrm{E}-14$ \\
\hline $\mathrm{Nb}-97 \mathrm{~m}$ & $\mathrm{Nb}-97$ & 96.908 & Niobium & $1.00 \mathrm{E}-03$ & ICRP-38 & $6.00 \mathrm{E}+01$ & $s$ & $6.00 \mathrm{E}+01$ & $1.94 \mathrm{E}+09$ & -- & -- & -- & -- & FGR-12 & $3.55 \mathrm{E}-14$ \\
\hline $\mathrm{Nb}-98$ & $\mathrm{Nb}-98$ & 97.910 & Niobium & $1.00 \mathrm{E}-03$ & ICRP-38 & $5.15 \mathrm{E}+01$ & $\mathrm{~m}$ & $3.09 \mathrm{E}+03$ & $3.73 \mathrm{E}+07$ & ICRP-72 & $5.60 \mathrm{E}-11$ & $M$ & Rec & FGR-12 & $1.21 \mathrm{E}-13$ \\
\hline Nb-99 & $\mathrm{Nb}-99$ & 98.912 & Niobium & $1.00 \mathrm{E}-03$ & ICRP-107 & $1.50 \mathrm{E}+01$ & $s$ & $1.50 \mathrm{E}+01$ & $7.60 \mathrm{E}+09$ & -- & -- & -- & -- & DOE-STD-1196 & $8.32 \mathrm{E}-15$ \\
\hline $\mathrm{Nb}-99 \mathrm{~m}$ & Nb-99 & 98.912 & Niobium & $1.00 \mathrm{E}-03$ & ICRP-107 & $2.60 \mathrm{E}+00$ & $\mathrm{~m}$ & $1.56 \mathrm{E}+02$ & $7.31 \mathrm{E}+08$ & -- & -- & -- & -- & DOE-STD-1196 & $3.86 \mathrm{E}-14$ \\
\hline $\mathrm{Nd}-134$ & $\mathrm{Nd}-134$ & 133.919 & Neodymium & $1.00 \mathrm{E}-03$ & ICRP-107 & $8.50 \mathrm{E}+00$ & $\mathrm{~m}$ & $5.10 \mathrm{E}+02$ & $1.65 E+08$ & -- & -- & -- & -- & DOE-STD-1196 & $2.32 \mathrm{E}-14$ \\
\hline Nd-135 & $\mathrm{Nd}-135$ & 134.918 & Neodymium & $1.00 \mathrm{E}-03$ & ICRP-107 & $1.24 \mathrm{E}+01$ & $\mathrm{~m}$ & $7.44 \mathrm{E}+02$ & $1.12 \mathrm{E}+08$ & DOE-STD-1196 & $3.18 \mathrm{E}-11$ & $\mathrm{~s}$ & Max & DOE-STD-1196 & $5.63 \mathrm{E}-14$ \\
\hline Nd-136 & $\mathrm{Nd}-136$ & 135.915 & Neodymium & $1.00 \mathrm{E}-03$ & ICRP-38 & $5.07 \mathrm{E}+01$ & $\mathrm{~m}$ & $3.04 \mathrm{E}+03$ & $2.73 \mathrm{E}+07$ & ICRP-72 & $5.40 \mathrm{E}-11$ & $\mathrm{~s}$ & Max & FGR-12 & $1.27 \mathrm{E}-14$ \\
\hline Nd-137 & $\mathrm{Nd}-137$ & 136.915 & Neodymium & $1.00 \mathrm{E}-03$ & ICRP-107 & $3.85 \mathrm{E}+01$ & $\mathrm{~m}$ & $2.31 \mathrm{E}+03$ & $3.57 \mathrm{E}+07$ & DOE-STD-1196 & $3.39 \mathrm{E}-11$ & $\mathrm{~s}$ & Max & DOE-STD-1196 & $5.33 \mathrm{E}-14$ \\
\hline $\mathrm{Nd}-138$ & $\mathrm{Nd}-138$ & 137.912 & Neodymium & $1.00 \mathrm{E}-03$ & ICRP-38 & $5.04 \mathrm{E}+00$ & $\mathrm{~h}$ & $1.81 \mathrm{E}+04$ & $4.51 \mathrm{E}+06$ & ICRP-72 & $2.50 \mathrm{E}-10$ & $\mathrm{~s}$ & Max & FGR-12 & $1.27 \mathrm{E}-15$ \\
\hline Nd-139 & $\mathrm{Nd}-139$ & 138.912 & Neodymium & $1.00 \mathrm{E}-03$ & ICRP-38 & $2.97 \mathrm{E}+01$ & $\mathrm{~m}$ & $1.78 \mathrm{E}+03$ & $4.56 \mathrm{E}+07$ & ICRP-72 & $1.00 \mathrm{E}-11$ & $\mathrm{~s}$ & Max & FGR-12 & $1.90 \mathrm{E}-14$ \\
\hline $\mathrm{Nd}-139 \mathrm{~m}$ & $\mathrm{Nd}-139$ & 138.912 & Neodymium & $1.00 \mathrm{E}-03$ & ICRP-38 & $5.50 \mathrm{E}+00$ & $\mathrm{~h}$ & $1.98 \mathrm{E}+04$ & $4.10 \mathrm{E}+06$ & ICRP-72 & $1.50 \mathrm{E}-10$ & $M$ & Max & $\begin{array}{ll}\text { FGR-12 } \\
\end{array}$ & $7.63 \mathrm{E}-14$ \\
\hline $\mathrm{Nd}-140$ & $\mathrm{Nd}-140$ & 139.910 & Neodymium & $1.00 \mathrm{E}-03$ & ICRP-107 & $3.37 \mathrm{E}+00$ & $d$ & $2.91 \mathrm{E}+05$ & $2.77 \mathrm{E}+05$ & DOE-STD-1196 & $1.33 \mathrm{E}-09$ & $\mathrm{~s}$ & Max & DOE-STD-1196 & 4.44E-16 \\
\hline Nd-141 & $\mathrm{Nd}-141$ & 140.910 & Neodymium & $1.00 \mathrm{E}-03$ & ICRP-38 & $2.49 \mathrm{E}+00$ & $\mathrm{~h}$ & $8.96 \mathrm{E}+03$ & $8.93 \mathrm{E}+06$ & ICRP-72 & $5.00 \mathrm{E}-12$ & $\mathrm{~s}$ & Max & FGR-12 & $2.88 \mathrm{E}-15$ \\
\hline $\mathrm{Nd}-141 \mathrm{~m}$ & $\mathrm{Nd}-141$ & 140.910 & Neodymium & $1.00 \mathrm{E}-03$ & ICRP-38 & $6.24 \mathrm{E}+01$ & $\mathrm{~s}$ & $6.24 \mathrm{E}+01$ & $1.28 \mathrm{E}+09$ & -- & -- & -- & -- & FGR-12 & $3.70 \mathrm{E}-14$ \\
\hline Nd-144 & $\mathrm{Nd}-144$ & 143.910 & Neodymium & $1.00 \mathrm{E}-03$ & ICRP-107 & $2.29 \mathrm{E}+15$ & $y$ & $7.23 \mathrm{E}+22$ & $1.08 \mathrm{E}-12$ & DOE-STD-1196 & $2.01 \mathrm{E}-05$ & $\mathrm{~F}$ & Max & -- & -- \\
\hline Nd-147 & $\mathrm{Nd}-147$ & 146.916 & Neodymium & $1.00 \mathrm{E}-03$ & ICRP-38 & $1.10 \mathrm{E}+01$ & $d$ & $9.49 \mathrm{E}+05$ & $8.09 \mathrm{E}+04$ & ICRP-72 & $2.40 \mathrm{E}-09$ & $\mathrm{~s}$ & Max & FGR-12 & $6.19 \mathrm{E}-15$ \\
\hline Nd-149 & Nd-149 & 148.920 & Neodymium & $1.00 \mathrm{E}-03$ & ICRP-38 & $1.73 E+00$ & $\mathrm{~h}$ & $6.23 \mathrm{E}+03$ & $1.22 \mathrm{E}+07$ & ICRP-72 & $8.90 \mathrm{E}-11$ & $\mathrm{~s}$ & Max & FGR-12 & $1.81 \mathrm{E}-14$ \\
\hline Nd-151 & Nd-151 & 150.924 & Neodymium & $1.00 \mathrm{E}-03$ & ICRP-38 & $1.24 \mathrm{E}+01$ & $\mathrm{~m}$ & $7.46 \mathrm{E}+02$ & $1.00 \mathrm{E}+08$ & ICRP-72 & $1.70 \mathrm{E}-11$ & $M$ & Max & FGR-12 & $4.48 \mathrm{E}-14$ \\
\hline Nd-152 & $\mathrm{Nd}-152$ & 151.925 & Neodymium & $1.00 \mathrm{E}-03$ & ICRP-107 & $1.14 \mathrm{E}+01$ & $\mathrm{~m}$ & $6.84 \mathrm{E}+02$ & $1.09 \mathrm{E}+08$ & DOE-STD-1196 & $2.80 \mathrm{E}-11$ & $\mathrm{~s}$ & Max & DOE-STD-1196 & $7.29 \mathrm{E}-15$ \\
\hline $\mathrm{Ne}-19$ & $\begin{array}{l}\mathrm{Ne}-19 \\
\end{array}$ & 19.002 & Neon & $1.00 \mathrm{E}+00$ & ICRP-38 & $1.72 \mathrm{E}+01$ & $\mathrm{~s}$ & $1.72 \mathrm{E}+01$ & $3.45 \mathrm{E}+10$ & -- & -- & -- & -- & FGR-12 & $4.92 \mathrm{E}-14$ \\
\hline $\mathrm{Ne}-24$ & $\mathrm{Ne}-24$ & 23.994 & Neon & $1.00 \mathrm{E}+00$ & ICRP-107 & $3.38 \mathrm{E}+00$ & $\mathrm{~m}$ & $2.03 E+02$ & $2.32 \mathrm{E}+09$ & -- & -- & -- & -- & DOE-STD-1196 & $2.48 \mathrm{E}-14$ \\
\hline Ni-56 & $\mathrm{Ni}-56$ & 55.942 & Nickel & $1.00 \mathrm{E}-03$ & ICRP-38 & $6.10 \mathrm{E}+00$ & d & $5.27 \mathrm{E}+05$ & $3.83 \mathrm{E}+05$ & ICRP-72 & $8.70 \mathrm{E}-10$ & $M$ & Rec & FGR-12 & $8.41 \mathrm{E}-14$ \\
\hline Ni-57 & $\mathrm{Ni}-57$ & 56.940 & Nickel & $1.00 \mathrm{E}-03$ & ICRP-38 & $3.61 \mathrm{E}+01$ & $\mathrm{~h}$ & $1.30 \mathrm{E}+05$ & $1.53 \mathrm{E}+06$ & ICRP-72 & $5.00 \mathrm{E}-10$ & $M$ & $\operatorname{Rec}$ & FGR-12 & $9.69 \mathrm{E}-14$ \\
\hline $\mathrm{Ni}-59$ & $\mathrm{Ni}-59$ & 58.934 & Nickel & $1.00 \mathrm{E}-03$ & ICRP-38 & $7.50 E+04$ & $y$ & $2.37 \mathrm{E}+12$ & $8.09 \mathrm{E}-02$ & ICRP-72 & $1.30 \mathrm{E}-10$ & $M$ & $\operatorname{Rec}$ & DOE-STD-1196 & $6.92 \mathrm{E}-19$ \\
\hline $\mathrm{Ni}-63$ & $\mathrm{Ni}-63$ & 62.930 & Nickel & $1.00 \mathrm{E}-03$ & ICRP-38 & $9.60 \mathrm{E}+01$ & $y$ & $3.03 E+09$ & $5.92 \mathrm{E}+01$ & ICRP-72 & $4.80 \mathrm{E}-10$ & $M$ & Rec & -- & -- \\
\hline $\mathrm{Ni}-65$ & $\mathrm{Ni}-65$ & 64.930 & Nickel & $1.00 \mathrm{E}-03$ & ICRP-38 & $2.52 \mathrm{E}+00$ & $\mathrm{~h}$ & $9.07 \mathrm{E}+03$ & $1.92 \mathrm{E}+07$ & ICRP-72 & $8.50 \mathrm{E}-11$ & $M$ & Rec & FGR-12 & $2.79 \mathrm{E}-14$ \\
\hline Ni-66 & $\mathrm{Ni}-66$ & 65.929 & Nickel & $1.00 \mathrm{E}-03$ & ICRP-38 & $5.46 \mathrm{E}+01$ & $\mathrm{~h}$ & $1.97 \mathrm{E}+05$ & $8.71 E+05$ & ICRP-72 & $1.60 \mathrm{E}-09$ & $M$ & Rec & FGR-12 & $6.16 \mathrm{E}-19$ \\
\hline Np-231 & Np-231 & 231.038 & Neptunium & $1.00 \mathrm{E}-03$ & JAERI & $4.88 \mathrm{E}+01$ & $\mathrm{~m}$ & $2.93 \mathrm{E}+03$ & $1.67 \mathrm{E}+07$ & JAERI & $1.70 \mathrm{E}-09$ & $M$ & Rec & -- & -- \\
\hline $\mathrm{Np}-232$ & $\mathrm{~Np}-232$ & 232.040 & Neptunium & $1.00 \mathrm{E}-03$ & ICRP-38 & $1.47 \mathrm{E}+01$ & $\mathrm{~m}$ & $8.82 \mathrm{E}+02$ & $5.51 \mathrm{E}+07$ & ICRP-72 & $5.00 \mathrm{E}-11$ & $M$ & $\operatorname{Rec}$ & FGR-12 & $5.80 \mathrm{E}-14$ \\
\hline Np-233 & Np-233 & 233.041 & Neptunium & $1.00 \mathrm{E}-03$ & ICRP-38 & $3.62 \mathrm{E}+01$ & $\mathrm{~m}$ & $2.17 \mathrm{E}+03$ & $2.23 \mathrm{E}+07$ & ICRP-72 & $1.60 \mathrm{E}-12$ & $M$ & $\operatorname{Rec}$ & FGR-12 & $3.85 \mathrm{E}-15$ \\
\hline Np-234 & Np-234 & 234.043 & Neptunium & $1.00 \mathrm{E}-03$ & ICRP-38 & $4.40 \mathrm{E}+00$ & $d$ & $3.80 \mathrm{E}+05$ & $1.27 \mathrm{E}+05$ & ICRP-72 & $5.30 \mathrm{E}-10$ & $M$ & Rec & FGR-12 & $7.26 \mathrm{E}-14$ \\
\hline Np-235 & $\mathrm{Np}-235$ & 235.044 & Neptunium & $1.00 \mathrm{E}-03$ & ICRP-38 & $3.96 \mathrm{E}+02$ & $d$ & $3.42 \mathrm{E}+07$ & $1.40 \mathrm{E}+03$ & ICRP-72 & $4.20 \mathrm{E}-10$ & $M$ & $\operatorname{Rec}$ & FGR-12 & $5.10 \mathrm{E}-17$ \\
\hline Np-236l & $\mathrm{Np}-236$ & 236.047 & Neptunium & $1.00 \mathrm{E}-03$ & ICRP-38 & $1.15 E+05$ & $y$ & $3.63 \mathrm{E}+12$ & $1.32 \mathrm{E}-02$ & ICRP-72 & $3.20 \mathrm{E}-06$ & $M$ & Rec & FGR-12 & $5.36 \mathrm{E}-15$ \\
\hline $\mathrm{Np}-236 \mathrm{~s}$ & $\mathrm{~Np}-236$ & 236.047 & Neptunium & $1.00 \mathrm{E}-03$ & ICRP-38 & $2.25 \mathrm{E}+01$ & $\mathrm{~h}$ & $8.10 \mathrm{E}+04$ & $5.90 \mathrm{E}+05$ & ICRP-72 & $5.30 \mathrm{E}-09$ & $M$ & Rec & FGR-12 & $2.14 \mathrm{E}-15$ \\
\hline Np-237 & Np-237 & 237.048 & Neptunium & $1.00 \mathrm{E}-03$ & ICRP-38 & $2.14 \mathrm{E}+06$ & $y$ & $6.75 \mathrm{E}+13$ & $7.05 \mathrm{E}-04$ & ICRP-72 & $2.30 \mathrm{E}-05$ & $M$ & $\operatorname{Rec}$ & FGR-12 & $1.03 \mathrm{E}-15$ \\
\hline Np-238 & $\mathrm{Np}-238$ & 238.051 & Neptunium & $1.00 \mathrm{E}-03$ & ICRP-38 & $2.12 \mathrm{E}+00$ & $d$ & $1.83 \mathrm{E}+05$ & $2.59 \mathrm{E}+05$ & ICRP-72 & $2.10 \mathrm{E}-09$ & $M$ & $\operatorname{Rec}$ & FGR-12 & $2.72 \mathrm{E}-14$ \\
\hline Np-239 & $\mathrm{Np}-239$ & 239.053 & Neptunium & $1.00 \mathrm{E}-03$ & ICRP-38 & $2.36 \mathrm{E}+00$ & $d$ & $2.03 \mathrm{E}+05$ & $2.32 \mathrm{E}+05$ & ICRP-72 & $9.30 \mathrm{E}-10$ & $\mathrm{M}$ & $\operatorname{Rec}$ & FGR-12 & 7.69E-15 \\
\hline $\mathrm{Np}-240$ & $\mathrm{~Np}-240$ & 240.056 & Neptunium & $1.00 \mathrm{E}-03$ & ICRP-38 & $6.50 \mathrm{E}+01$ & $\mathrm{~m}$ & $3.90 \mathrm{E}+03$ & $1.20 \mathrm{E}+07$ & ICRP-72 & $8.50 \mathrm{E}-11$ & $M$ & Rec & FGR-12 & $6.31 \mathrm{E}-14$ \\
\hline $\mathrm{Np}-240 \mathrm{~m}$ & $\mathrm{~Np}-240$ & 240.056 & Neptunium & $1.00 \mathrm{E}-03$ & ICRP-38 & $7.40 \mathrm{E}+00$ & $\mathrm{~m}$ & $4.44 \mathrm{E}+02$ & $1.06 \mathrm{E}+08$ & -- & -- & -- & -- & FGR-12 & $1.62 \mathrm{E}-14$ \\
\hline $\mathrm{Np}-241$ & Np-241 & 241.058 & Neptunium & $1.00 \mathrm{E}-03$ & ICRP-107 & $1.39 \mathrm{E}+01$ & $\mathrm{~m}$ & $8.34 \mathrm{E}+02$ & $5.61 \mathrm{E}+07$ & DOE-STD-1196 & $1.40 \mathrm{E}-11$ & $M$ & $\operatorname{Rec}$ & DOE-STD-1196 & $1.79 \mathrm{E}-15$ \\
\hline Np-242 & $\mathrm{Np}-242$ & 242.062 & Neptunium & $1.00 \mathrm{E}-03$ & ICRP-107 & $2.20 \mathrm{E}+00$ & $\mathrm{~m}$ & $1.32 \mathrm{E}+02$ & $3.53 \mathrm{E}+08$ & -- & -- & -- & -- & DOE-STD-1196 & $1.33 \mathrm{E}-14$ \\
\hline $\mathrm{Np}-242 \mathrm{~m}$ & $\mathrm{~Np}-242$ & 242.062 & Neptunium & $1.00 \mathrm{E}-03$ & ICRP-107 & $5.50 \mathrm{E}+00$ & $\mathrm{~m}$ & $3.30 \mathrm{E}+02$ & $1.41 \mathrm{E}+08$ & -- & -- & -- & -- & DOE-STD-1196 & $4.16 \mathrm{E}-14$ \\
\hline $0-14$ & $0-14$ & 14.009 & Oxygen & $1.00 \mathrm{E}+00$ & ICRP-38 & $7.06 \mathrm{E}+01$ & $\mathrm{~s}$ & $7.06 \mathrm{E}+01$ & $1.14 \mathrm{E}+10$ & -- & -- & -- & -- & DOE-STD-1196 & $1.63 \mathrm{E}-13$ \\
\hline $0-15$ & $0-15$ & 15.003 & Oxygen & $1.00 E+00$ & ICRP-38 & $1.22 \mathrm{E}+02$ & $\mathrm{~s}$ & $1.22 \mathrm{E}+02$ & $6.15 \mathrm{E}+09$ & -- & -- & -- & -- & FGR-12 & $4.91 \mathrm{E}-14$ \\
\hline $0-19$ & $0-19$ & 19.004 & Oxygen & $1.00 \mathrm{E}+00$ & ICRP-38 & $2.69 \mathrm{E}+01$ & $\mathrm{~s}$ & $2.69 \mathrm{E}+01$ & $2.21 \mathrm{E}+10$ & -- & -- & -- & -- & DOE-STD-1196 & $4.60 \mathrm{E}-14$ \\
\hline
\end{tabular}




\begin{tabular}{|c|c|c|c|c|c|c|c|c|c|c|c|c|c|c|c|}
\hline \multirow{2}{*}{$\begin{array}{l}\text { MASTER } \\
\text { ISOTOPE } \\
\text { LIST } \\
\text { Os-177 } \\
\end{array}$} & \multirow{2}{*}{$\begin{array}{c}\begin{array}{c}\text { Atomic } \\
\text { Mass } \\
\text { Nuclide }\end{array} \\
\text { Os-177 } \\
\end{array}$} & \multirow{2}{*}{$\begin{array}{c}\begin{array}{c}\text { Atomic } \\
\text { Mass }\end{array} \\
176.955\end{array}$} & \multirow{2}{*}{$\begin{array}{l}\text { Element } \\
\text { Osmium }\end{array}$} & \multirow{2}{*}{$\begin{array}{c}\text { RF per } \\
\text { DOE-STD- } \\
1027 \\
1.00 \mathrm{E}-03\end{array}$} & \multirow{2}{*}{$\begin{array}{c}\begin{array}{c}\text { Half-Life } \\
\text { Reference }\end{array} \\
\text { JAERI }\end{array}$} & \multicolumn{2}{|c|}{$\begin{array}{c}\text { Half-Life } \\
\text { (original units) }\end{array}$} & \multirow{2}{*}{$\begin{array}{c}\text { Half Life }(s) \\
1.68 \mathrm{E}+02\end{array}$} & \multirow{2}{*}{$\begin{array}{c}\begin{array}{c}\text { Sp. Act } \\
\text { (Ci/g) }\end{array} \\
3.79 \mathrm{E}+08 \\
\end{array}$} & \multirow{2}{*}{$\begin{array}{c}\text { Inhalation DC } \\
\text { Reference } \\
--\end{array}$} & \multirow[t]{2}{*}{$\begin{array}{c}\text { HC-2 } \\
\text { Inhalation } \\
\text { DC } \\
\text { (Sv/Bq) } \\
-- \\
\end{array}$} & \multicolumn{2}{|c|}{$\begin{array}{c}\text { Lung } \\
\text { Absorption } \\
\text { Class/Type }\end{array}$} & \multirow{2}{*}{$\begin{array}{c}\text { Immersion DC } \\
\text { Reference } \\
-- \\
\end{array}$} & \multirow[t]{2}{*}{$\begin{array}{c}\text { HC-2 } \\
\text { Immersion } \\
\mathrm{DC}(\mathrm{Sv} / \mathrm{s} \\
\left.\text { per } \mathrm{Bq} / \mathrm{m}^{3}\right) \\
--\end{array}$} \\
\hline & & & & & & $2.80 \mathrm{E}+00$ & $\mathrm{~m}$ & & & & & -- & -- & & \\
\hline Os-179 & Os-179 & 178.954 & Osmium & $1.00 \mathrm{E}-03$ & JAERI & $6.50 \mathrm{E}+00$ & $\mathrm{~m}$ & $3.90 \mathrm{E}+02$ & $1.62 \mathrm{E}+08$ & -- & -- & -- & -- & -- & -- \\
\hline Os-180 & Os-180 & 179.952 & Osmium & $1.00 \mathrm{E}-03$ & ICRP-38 & $2.20 \mathrm{E}+01$ & $\mathrm{~m}$ & $1.32 \mathrm{E}+03$ & $4.75 \mathrm{E}+07$ & ICRP-72 & $1.50 \mathrm{E}-11$ & $\mathrm{~s}$ & Max & FGR-12 & $2.30 \mathrm{E}-15$ \\
\hline Os-181 & Os-181 & 180.953 & Osmium & $1.00 \mathrm{E}-03$ & ICRP-38 & $1.05 \mathrm{E}+02$ & $\mathrm{~m}$ & $6.30 \mathrm{E}+03$ & $9.90 \mathrm{E}+06$ & ICRP-72 & $6.50 \mathrm{E}-11$ & $\mathrm{~s}$ & Max & FGR-12 & $5.94 \mathrm{E}-14$ \\
\hline Os-182 & Os-182 & 181.952 & Osmium & $1.00 \mathrm{E}-03$ & ICRP-38 & $2.20 \mathrm{E}+01$ & $\mathrm{~h}$ & $7.92 \mathrm{E}+04$ & $7.83 \mathrm{E}+05$ & ICRP-72 & $3.80 \mathrm{E}-10$ & $\mathrm{~s}$ & Max & FGR-12 & $2.01 \mathrm{E}-14$ \\
\hline Os-183 & Os-183 & 182.953 & Osmium & $1.00 \mathrm{E}-03$ & ICRP-107 & $1.30 \mathrm{E}+01$ & $\mathrm{~h}$ & $4.68 \mathrm{E}+04$ & $1.32 \mathrm{E}+06$ & DOE-STD-1196 & $2.20 \mathrm{E}-10$ & $\mathrm{~s}$ & Max & DOE-STD-1196 & $2.66 \mathrm{E}-14$ \\
\hline Os-183m & Os-183 & 182.953 & Osmium & $1.00 \mathrm{E}-03$ & ICRP-107 & $9.90 \mathrm{E}+00$ & $\mathrm{~h}$ & $3.56 \mathrm{E}+04$ & $1.73 \mathrm{E}+06$ & DOE-STD-1196 & $1.74 \mathrm{E}-10$ & $\mathrm{~s}$ & Max & DOE-STD-1196 & $4.63 \mathrm{E}-14$ \\
\hline Os-185 & Os-185 & 184.954 & Osmium & $1.00 \mathrm{E}-03$ & ICRP-38 & $9.40 \mathrm{E}+01$ & $d$ & $8.12 E+06$ & $7.51 \mathrm{E}+03$ & ICRP-72 & $1.60 \mathrm{E}-09$ & $\mathrm{~s}$ & Max & FGR-12 & $3.43 \mathrm{E}-14$ \\
\hline Os-186 & Os-186 & 185.954 & Osmium & $1.00 \mathrm{E}-03$ & ICRP-107 & $2.00 \mathrm{E}+15$ & $y$ & $6.31 \mathrm{E}+22$ & $9.61 \mathrm{E}-13$ & DOE-STD-1196 & $4.51 \mathrm{E}-06$ & $\mathrm{~s}$ & Max & -- & -- \\
\hline Os-189m & Os-189 & 188.958 & Osmium & $1.00 \mathrm{E}-03$ & ICRP-38 & $6.00 \mathrm{E}+00$ & $\mathrm{~h}$ & $2.16 \mathrm{E}+04$ & $2.76 \mathrm{E}+06$ & ICRP-72 & $5.30 \mathrm{E}-12$ & $\mathrm{~s}$ & Max & FGR-12 & $1.06 \mathrm{E}-19$ \\
\hline Os-190m & Os-190 & 189.958 & Osmium & $1.00 \mathrm{E}-03$ & ICRP-38 & $9.90 \mathrm{E}+00$ & $\mathrm{~m}$ & $5.94 \mathrm{E}+02$ & $1.00 \mathrm{E}+08$ & -- & -- & -- & -- & FGR-12 & $7.60 \mathrm{E}-14$ \\
\hline Os-191 & Os-191 & 190.961 & Osmium & $1.00 \mathrm{E}-03$ & ICRP-38 & $1.54 \mathrm{E}+01$ & $d$ & $1.33 \mathrm{E}+06$ & $4.44 \mathrm{E}+04$ & ICRP-72 & $1.90 \mathrm{E}-09$ & $\mathrm{~s}$ & Max & FGR-12 & $3.21 \mathrm{E}-15$ \\
\hline Os-191m & Os-191 & 190.961 & Osmium & $1.00 \mathrm{E}-03$ & ICRP-38 & $1.30 \mathrm{E}+01$ & $\mathrm{~h}$ & $4.69 \mathrm{E}+04$ & $1.26 \mathrm{E}+06$ & ICRP-72 & $1.60 \mathrm{E}-10$ & $\mathrm{~s}$ & Max & FGR-12 & $2.75 \mathrm{E}-16$ \\
\hline Os-193 & Os-193 & 192.964 & Osmium & $1.00 \mathrm{E}-03$ & ICRP-38 & $3.00 \mathrm{E}+01$ & $\mathrm{~h}$ & $1.08 \mathrm{E}+05$ & $5.41 \mathrm{E}+05$ & ICRP-72 & $5.20 \mathrm{E}-10$ & $S$ & Max & FGR-12 & $3.40 \mathrm{E}-15$ \\
\hline Os-194 & Os-194 & 193.965 & Osmium & $1.00 \mathrm{E}-03$ & ICRP-38 & $6.00 E+00$ & $y$ & $1.89 \mathrm{E}+08$ & $3.07 E+02$ & ICRP-72 & $8.50 \mathrm{E}-08$ & $\mathrm{~s}$ & Max & FGR-12 & $2.75 \mathrm{E}-17$ \\
\hline Os-196 & Os-196 & 195.970 & Osmium & $1.00 \mathrm{E}-03$ & ICRP-107 & $3.49 \mathrm{E}+01$ & $\mathrm{~m}$ & $2.09 \mathrm{E}+03$ & $2.75 \mathrm{E}+07$ & DOE-STD-1196 & $6.64 \mathrm{E}-11$ & $\mathrm{~s}$ & Max & DOE-STD-1196 & $3.64 \mathrm{E}-15$ \\
\hline $\mathrm{P}-30$ & $\mathrm{P}-30$ & 29.978 & Phosphorus & $5.00 \mathrm{E}-01$ & ICRP-38 & $2.50 \mathrm{E}+00$ & $\mathrm{~m}$ & $1.50 \mathrm{E}+02$ & $2.51 \mathrm{E}+09$ & -- & -- & -- & -- & FGR-12 & $4.94 \mathrm{E}-14$ \\
\hline $\mathrm{P}-32$ & P-32 & 31.974 & Phosphorus & $5.00 \mathrm{E}-01$ & ICRP-38 & $1.43 \mathrm{E}+01$ & $d$ & $1.23 \mathrm{E}+06$ & $2.86 \mathrm{E}+05$ & ICRP-72 & $3.40 \mathrm{E}-09$ & $\mathrm{M}$ & Max & FGR-12 & $9.90 \mathrm{E}-17$ \\
\hline P-33 & $\mathrm{P}-33$ & 32.972 & Phosphorus & $5.00 \mathrm{E}-01$ & ICRP-38 & $2.54 \mathrm{E}+01$ & $d$ & $2.19 \mathrm{E}+06$ & $1.56 \mathrm{E}+05$ & ICRP-72 & $1.50 \mathrm{E}-09$ & $M$ & Max & FGR-12 & $8.23 \mathrm{E}-19$ \\
\hline Pa-227 & Pa-227 & 227.029 & Protactinium & $1.00 \mathrm{E}-03$ & ICRP-38 & $3.83 \mathrm{E}+01$ & $\mathrm{~m}$ & $2.30 \mathrm{E}+03$ & $2.16 \mathrm{E}+07$ & ICRP-72 & $8.00 \mathrm{E}-08$ & $\mathrm{~s}$ & Max & FGR-12 & $8.54 \mathrm{E}-16$ \\
\hline Pa-228 & Pa-228 & 228.031 & Protactinium & $1.00 \mathrm{E}-03$ & ICRP-38 & $2.20 \mathrm{E}+01$ & $\mathrm{~h}$ & $7.92 \mathrm{E}+04$ & $6.25 \mathrm{E}+05$ & ICRP-72 & $7.50 \mathrm{E}-08$ & $\mathrm{~s}$ & Max & FGR-12 & $5.54 \mathrm{E}-14$ \\
\hline Pa-229 & Pa-229 & 229.032 & Protactinium & $1.00 \mathrm{E}-03$ & ICRP-107 & $1.50 \mathrm{E}+00$ & $d$ & $1.30 \mathrm{E}+05$ & $3.80 \mathrm{E}+05$ & DOE-STD-1196 & $7.78 \mathrm{E}-09$ & $\mathrm{~s}$ & Max & DOE-STD-1196 & $2.33 \mathrm{E}-15$ \\
\hline Pa-230 & Pa-230 & 230.035 & Protactinium & $1.00 \mathrm{E}-03$ & ICRP-38 & $1.74 \mathrm{E}+01$ & $d$ & $1.50 \mathrm{E}+06$ & $3.26 \mathrm{E}+04$ & ICRP-72 & $7.60 \mathrm{E}-07$ & $\mathrm{~s}$ & Max & FGR-12 & $3.13 \mathrm{E}-14$ \\
\hline Pa-231 & Pa-231 & 231.036 & Protactinium & $1.00 \mathrm{E}-03$ & ICRP-38 & $3.28 \mathrm{E}+04$ & $y$ & $1.03 \mathrm{E}+12$ & $4.72 \mathrm{E}-02$ & ICRP-72 & $1.40 \mathrm{E}-04$ & $M$ & Max & FGR-12 & $1.72 \mathrm{E}-15$ \\
\hline Pa-232 & Pa-232 & 232.039 & Protactinium & $1.00 \mathrm{E}-03$ & ICRP-38 & $1.31 \mathrm{E}+00$ & $d$ & $1.13 \mathrm{E}+05$ & $4.30 \mathrm{E}+05$ & ICRP-72 & $1.00 \mathrm{E}-08$ & $M$ & Max & FGR-12 & $4.56 \mathrm{E}-14$ \\
\hline Pa-233 & Pa-233 & 233.040 & Protactinium & $1.00 \mathrm{E}-03$ & ICRP-38 & $2.70 \mathrm{E}+01$ & $d$ & $2.33 \mathrm{E}+06$ & $2.08 \mathrm{E}+04$ & ICRP-72 & $3.90 \mathrm{E}-09$ & $\mathrm{~s}$ & Max & FGR-12 & $9.35 \mathrm{E}-15$ \\
\hline Pa-234 & Pa-234 & 234.043 & Protactinium & $1.00 \mathrm{E}-03$ & ICRP-38 & $6.70 \mathrm{E}+00$ & $\mathrm{~h}$ & $2.41 \mathrm{E}+04$ & $2.00 \mathrm{E}+06$ & ICRP-72 & $4.00 \mathrm{E}-10$ & $\mathrm{~s}$ & Max & FGR-12 & $9.34 \mathrm{E}-14$ \\
\hline $\mathrm{Pa}-234 \mathrm{~m}$ & Pa-234 & 234.043 & Protactinium & $1.00 \mathrm{E}-03$ & ICRP-38 & $1.17 \mathrm{E}+00$ & $\mathrm{~m}$ & $7.02 \mathrm{E}+01$ & $6.87 \mathrm{E}+08$ & -- & -- & -- & -- & FGR-12 & $7.19 \mathrm{E}-16$ \\
\hline Pa-235 & Pa-235 & 235.045 & Protactinium & $1.00 \mathrm{E}-03$ & ICRP-107 & $2.45 \mathrm{E}+01$ & $\mathrm{~m}$ & $1.47 \mathrm{E}+03$ & $3.27 \mathrm{E}+07$ & DOE-STD-1196 & $1.98 \mathrm{E}-11$ & $\mathrm{~s}$ & Max & DOE-STD-1196 & $3.35 \mathrm{E}-16$ \\
\hline Pa-236 & Pa-236 & 236.049 & Protactinium & $1.00 \mathrm{E}-03$ & ICRP-107 & $9.10 \mathrm{E}+00$ & $\mathrm{~m}$ & $5.46 \mathrm{E}+02$ & $8.75 \mathrm{E}+07$ & -- & -- & -- & -- & DOE-STD-1196 & $4.36 \mathrm{E}-14$ \\
\hline Pa-237 & Pa-237 & 237.051 & Protactinium & $1.00 \mathrm{E}-03$ & ICRP-107 & $8.70 \mathrm{E}+00$ & $\mathrm{~m}$ & $5.22 \mathrm{E}+02$ & $9.12 \mathrm{E}+07$ & -- & -- & -- & -- & DOE-STD-1196 & $2.82 \mathrm{E}-14$ \\
\hline Pb-194 & Pb-194 & 193.974 & Lead & $1.00 \mathrm{E}-03$ & ICRP-107 & $1.20 \mathrm{E}+01$ & $\mathrm{~m}$ & $7.20 \mathrm{E}+02$ & $8.08 \mathrm{E}+07$ & DOE-STD-1196 & $1.51 \mathrm{E}-11$ & $M$ & $\operatorname{Rec}$ & DOE-STD-1196 & $4.94 \mathrm{E}-14$ \\
\hline $\mathrm{Pb}-195 \mathrm{~m}$ & $\mathrm{~Pb}-195$ & 194.975 & Lead & $1.00 \mathrm{E}-03$ & ICRP-38 & $1.58 \mathrm{E}+01$ & $\mathrm{~m}$ & $9.48 \mathrm{E}+02$ & $6.10 \mathrm{E}+07$ & ICRP-72 & $2.50 \mathrm{E}-11$ & $M$ & $\operatorname{Rec}$ & FGR-12 & $7.68 \mathrm{E}-14$ \\
\hline Pb-196 & Pb-196 & 195.973 & Lead & $1.00 \mathrm{E}-03$ & ICRP-107 & $3.70 \mathrm{E}+01$ & $\mathrm{~m}$ & $2.22 \mathrm{E}+03$ & $2.59 \mathrm{E}+07$ & DOE-STD-1196 & $2.88 \mathrm{E}-11$ & $M$ & $\operatorname{Rec}$ & DOE-STD-1196 & $2.10 \mathrm{E}-14$ \\
\hline $\mathrm{Pb}-197$ & $\mathrm{~Pb}-197$ & 196.973 & Lead & $1.00 \mathrm{E}-03$ & ICRP-107 & $8.00 \mathrm{E}+00$ & $\mathrm{~m}$ & $4.80 \mathrm{E}+02$ & $1.19 \mathrm{E}+08$ & -- & -- & -- & -- & DOE-STD-1196 & 7.14E-14 \\
\hline Pb-197m & Pb-197 & 196.973 & Lead & $1.00 \mathrm{E}-03$ & ICRP-107 & $4.30 \mathrm{E}+01$ & $\mathrm{~m}$ & $2.58 \mathrm{E}+03$ & $2.22 \mathrm{E}+07$ & DOE-STD-1196 & $5.27 \mathrm{E}-11$ & $M$ & $\operatorname{Rec}$ & DOE-STD-1196 & $5.22 \mathrm{E}-14$ \\
\hline Pb-198 & Pb-198 & 197.972 & Lead & $1.00 \mathrm{E}-03$ & ICRP-38 & $2.40 \mathrm{E}+00$ & $\mathrm{~h}$ & $8.64 \mathrm{E}+03$ & $6.60 \mathrm{E}+06$ & ICRP-72 & $6.60 \mathrm{E}-11$ & $M$ & $\operatorname{Rec}$ & FGR-12 & $2.04 \mathrm{E}-14$ \\
\hline Pb-199 & Pb-199 & 198.973 & Lead & $1.00 \mathrm{E}-03$ & ICRP-38 & $9.00 \mathrm{E}+01$ & $\mathrm{~m}$ & $5.40 \mathrm{E}+03$ & $1.05 \mathrm{E}+07$ & ICRP-72 & $3.60 E-11$ & $\mathrm{M}$ & $\operatorname{Rec}$ & FGR-12 & 7.31E-14 \\
\hline $\mathrm{Pb}-200$ & $\mathrm{~Pb}-200$ & 199.972 & Lead & $1.00 \mathrm{E}-03$ & ICRP-38 & $2.15 \mathrm{E}+01$ & $\mathrm{~h}$ & $7.74 \mathrm{E}+04$ & $7.29 \mathrm{E}+05$ & ICRP-72 & $3.30 \mathrm{E}-10$ & $M$ & Rec & FGR-12 & $9.20 \mathrm{E}-15$ \\
\hline $\mathrm{Pb}-201$ & $\mathrm{~Pb}-201$ & 200.973 & Lead & $1.00 \mathrm{E}-03$ & ICRP-38 & $9.40 \mathrm{E}+00$ & $\mathrm{~h}$ & $3.38 \mathrm{E}+04$ & $1.66 \mathrm{E}+06$ & ICRP-72 & $1.10 \mathrm{E}-10$ & $M$ & $\operatorname{Rec}$ & FGR-12 & $3.63 \mathrm{E}-14$ \\
\hline $\mathrm{Pb}-201 \mathrm{~m}$ & $\mathrm{~Pb}-201$ & 200.973 & Lead & $1.00 \mathrm{E}-03$ & ICRP-107 & $6.10 \mathrm{E}+01$ & $\mathrm{~s}$ & $6.10 \mathrm{E}+01$ & $9.20 \mathrm{E}+08$ & -- & -- & -- & -- & DOE-STD-1196 & $1.63 \mathrm{E}-14$ \\
\hline $\mathrm{Pb}-202$ & $\mathrm{~Pb}-202$ & 201.972 & Lead & $1.00 \mathrm{E}-03$ & ICRP-38 & $3.00 \mathrm{E}+05$ & $y$ & $9.47 \mathrm{E}+12$ & $5.90 \mathrm{E}-03$ & ICRP-72 & $6.30 \mathrm{E}-09$ & $M$ & $\operatorname{Rec}$ & FGR-12 & $4.52 \mathrm{E}-19$ \\
\hline $\mathrm{Pb}-202 \mathrm{~m}$ & $\mathrm{~Pb}-202$ & 201.972 & Lead & $1.00 \mathrm{E}-03$ & ICRP-38 & $3.62 \mathrm{E}+00$ & $\mathrm{~h}$ & $1.30 \mathrm{E}+04$ & $4.29 \mathrm{E}+06$ & ICRP-72 & $9.50 \mathrm{E}-11$ & $M$ & $\operatorname{Rec}$ & FGR-12 & $9.96 \mathrm{E}-14$ \\
\hline Pb-203 & $\mathrm{Pb}-203$ & 202.973 & Lead & $1.00 \mathrm{E}-03$ & ICRP-38 & $5.21 \mathrm{E}+01$ & $\mathrm{~h}$ & $1.87 \mathrm{E}+05$ & $2.97 \mathrm{E}+05$ & ICRP-72 & $2.00 \mathrm{E}-10$ & $M$ & $\operatorname{Rec}$ & FGR-12 & $1.44 \mathrm{E}-14$ \\
\hline $\mathrm{Pb}-204 \mathrm{~m}$ & $\mathrm{~Pb}-204$ & 203.973 & Lead & $1.00 \mathrm{E}-03$ & ICRP-38 & $6.72 E+01$ & $\mathrm{~m}$ & $4.03 E+03$ & $1.37 \mathrm{E}+07$ & DOE-STD-1196 & $3.44 \mathrm{E}-11$ & $M$ & $\operatorname{Rec}$ & DOE-STD-1196 & $9.45 \mathrm{E}-14$ \\
\hline Pb-205 & $P b-205$ & 204.974 & Lead & $1.00 \mathrm{E}-03$ & ICRP-38 & $1.43 \mathrm{E}+07$ & $y$ & $4.51 \mathrm{E}+14$ & $1.22 \mathrm{E}-04$ & ICRP-72 & $2.50 \mathrm{E}-10$ & $M$ & $\operatorname{Rec}$ & FGR-12 & $5.06 \mathrm{E}-19$ \\
\hline
\end{tabular}




\begin{tabular}{|c|c|c|c|c|c|c|c|c|c|c|c|c|c|c|c|}
\hline \multicolumn{16}{|c|}{ Table B.1 Radionuclide Specific Input Data Used For the Calculation of HC-2 TQs Using Recommended DCs (continued) } \\
\hline $\begin{array}{l}\text { MASTER } \\
\text { ISOTOPE } \\
\text { LIST }\end{array}$ & $\begin{array}{l}\text { Atomic } \\
\text { Mass } \\
\text { Nuclide }\end{array}$ & $\begin{array}{c}\text { Atomic } \\
\text { Mass }\end{array}$ & Element & $\begin{array}{c}\text { RF per } \\
\text { DOE-STD- } \\
1027\end{array}$ & $\begin{array}{c}\text { Half-Life } \\
\text { Reference }\end{array}$ & \multicolumn{2}{|c|}{$\begin{array}{c}\text { Half-Life } \\
\text { (original units) }\end{array}$} & \multirow{2}{*}{$\begin{array}{c}\text { Half Life (s) } \\
1.17 \mathrm{E}+04\end{array}$} & \multirow{2}{*}{$\begin{array}{c}\begin{array}{c}\text { Sp. Act } \\
\text { (Ci/g) }\end{array} \\
4.61 \mathrm{E}+06 \\
\end{array}$} & \multirow{2}{*}{$\begin{array}{c}\begin{array}{c}\text { Inhalation DC } \\
\text { Reference }\end{array} \\
\text { ICRP-72 }\end{array}$} & \multirow[t]{2}{*}{$\begin{array}{c}\text { HC-2 } \\
\text { Inhalation } \\
\text { DC } \\
\text { (Sv/Bq) } \\
5.60 \mathrm{E}-11 \\
\end{array}$} & \multicolumn{2}{|c|}{$\begin{array}{c}\text { Lung } \\
\text { Absorption } \\
\text { Class/Type }\end{array}$} & \multirow{2}{*}{$\begin{array}{c}\text { Immersion DC } \\
\text { Reference } \\
\text { FGR-12 }\end{array}$} & \multirow[t]{2}{*}{$\begin{array}{c}\text { HC-2 } \\
\begin{array}{c}\text { Immersion } \\
\text { DC }(\mathrm{Sv} / \mathrm{s}\end{array} \\
\left.\text { per Bq/m } / \mathrm{m}^{3}\right) \\
8.12 \mathrm{E}-18 \\
\end{array}$} \\
\hline $\mathrm{Pb}-209$ & $\mathrm{~Pb}-209$ & 208.981 & Lead & $1.00 \mathrm{E}-03$ & ICRP-38 & $3.25 \mathrm{E}+00$ & $\mathrm{~h}$ & & & & & $\mathrm{M}$ & $\operatorname{Rec}$ & & \\
\hline $\mathrm{Pb}-210$ & $\mathrm{~Pb}-210$ & 209.984 & Lead & $1.00 \mathrm{E}-03$ & ICRP-38 & $2.23 \mathrm{E}+01$ & $\mathrm{y}$ & $7.04 \mathrm{E}+08$ & $7.63 \mathrm{E}+01$ & ICRP-72 & $1.10 \mathrm{E}-06$ & $\mathrm{M}$ & Rec & FGR-12 & $5.64 \mathrm{E}-17$ \\
\hline $\mathrm{Pb}-211$ & $\mathrm{~Pb}-211$ & 210.989 & Lead & $1.00 \mathrm{E}-03$ & ICRP-38 & $3.61 \mathrm{E}+01$ & $\mathrm{~m}$ & $2.17 E+03$ & $2.47 \mathrm{E}+07$ & ICRP-72 & $1.10 \mathrm{E}-08$ & $M$ & Rec & FGR-12 & $2.49 \mathrm{E}-15$ \\
\hline $\mathrm{Pb}-212$ & $\mathrm{~Pb}-212$ & 211.992 & Lead & $1.00 \mathrm{E}-03$ & ICRP-38 & $1.06 \mathrm{E}+01$ & $\mathrm{~h}$ & $3.83 \mathrm{E}+04$ & $1.39 \mathrm{E}+06$ & ICRP-72 & $1.70 \mathrm{E}-07$ & $M$ & $\operatorname{Rec}$ & FGR-12 & $6.87 \mathrm{E}-15$ \\
\hline $\mathrm{Pb}-214$ & $\mathrm{~Pb}-214$ & 214.000 & Lead & $1.00 \mathrm{E}-03$ & ICRP-38 & $2.68 \mathrm{E}+01$ & $\mathrm{~m}$ & $1.61 \mathrm{E}+03$ & $3.28 \mathrm{E}+07$ & ICRP-72 & $1.40 \mathrm{E}-08$ & $M$ & $\operatorname{Rec}$ & FGR-12 & $1.18 \mathrm{E}-14$ \\
\hline $\mathrm{Pd}-100$ & $\mathrm{Pd}-100$ & 99.909 & Palladium & $1.00 \mathrm{E}-03$ & ICRP-38 & $3.63 \mathrm{E}+00$ & $d$ & $3.14 \mathrm{E}+05$ & $3.60 \mathrm{E}+05$ & ICRP-72 & $8.50 \mathrm{E}-10$ & $\mathrm{~s}$ & Max & FGR-12 & $4.65 \mathrm{E}-15$ \\
\hline $\mathrm{Pd}-101$ & Pd-101 & 100.908 & $\begin{array}{l}\text { Palladium } \\
\end{array}$ & $1.00 \mathrm{E}-03$ & ICRP-38 & $8.27 \mathrm{E}+00$ & $\mathrm{~h}$ & $2.98 \mathrm{E}+04$ & $3.76 \mathrm{E}+06$ & ICRP-72 & $6.20 \mathrm{E}-11$ & $\mathrm{~s}$ & Max & FGR-12 & $1.53 \mathrm{E}-14$ \\
\hline $\mathrm{Pd}-103$ & $\mathrm{Pd}-103$ & 102.906 & Palladium & $1.00 \mathrm{E}-03$ & ICRP-38 & $1.70 \mathrm{E}+01$ & $d$ & $1.47 \mathrm{E}+06$ & $7.48 \mathrm{E}+04$ & ICRP-72 & $4.50 \mathrm{E}-10$ & $\mathrm{~s}$ & Max & FGR-12 & $7.68 \mathrm{E}-17$ \\
\hline Pd-107 & Pd-107 & 106.905 & Palladium & $1.00 \mathrm{E}-03$ & ICRP-38 & $6.50 \mathrm{E}+06$ & $y$ & $2.05 E+14$ & $5.14 \mathrm{E}-04$ & ICRP-72 & $5.90 \mathrm{E}-10$ & $\mathrm{~s}$ & Max & -- & -- \\
\hline Pd-109 & $\mathrm{Pd}-109$ & 108.906 & Palladium & $1.00 \mathrm{E}-03$ & ICRP-38 & $1.34 \mathrm{E}+01$ & $\mathrm{~h}$ & $4.83 \mathrm{E}+04$ & $2.14 \mathrm{E}+06$ & ICRP-72 & $3.70 \mathrm{E}-10$ & $\mathrm{~s}$ & Max & FGR-12 & $2.51 \mathrm{E}-16$ \\
\hline Pd-109m & Pd-109 & 108.906 & $\begin{array}{l}\text { Palladium } \\
\end{array}$ & $1.00 \mathrm{E}-03$ & ICRP-107 & $4.69 \mathrm{E}+00$ & $\mathrm{~m}$ & $2.81 \mathrm{E}+02$ & $3.68 \mathrm{E}+08$ & -- & -- & -- & -- & DOE-STD-1196 & $4.59 \mathrm{E}-15$ \\
\hline Pd-111 & $\mathrm{Pd}-1111$ & 110.908 & Palladium & $1.00 \mathrm{E}-03$ & ICRP-107 & $2.34 \mathrm{E}+01$ & $\mathrm{~m}$ & $1.40 \mathrm{E}+03$ & $7.24 \mathrm{E}+07$ & DOE-STD-1196 & $3.14 \mathrm{E}-11$ & $\mathrm{~s}$ & Max & DOE-STD-1196 & $2.89 \mathrm{E}-15$ \\
\hline $\mathrm{Pd}-112$ & $\mathrm{Pd}-112$ & 111.907 & Palladium & $1.00 \mathrm{E}-03$ & ICRP-107 & $2.10 \mathrm{E}+01$ & $\mathrm{~h}$ & $7.57 \mathrm{E}+04$ & $1.33 \mathrm{E}+06$ & DOE-STD-1196 & $1.32 \mathrm{E}-09$ & $\mathrm{~s}$ & Max & DOE-STD-1196 & $2.68 \mathrm{E}-17$ \\
\hline $\mathrm{Pd}-114$ & $\mathrm{Pd}-114$ & 113.910 & Palladium & $1.00 \mathrm{E}-03$ & ICRP-107 & $2.42 \mathrm{E}+00$ & $\mathrm{~m}$ & $1.45 \mathrm{E}+02$ & $6.82 \mathrm{E}+08$ & -- & -- & -- & -- & DOE-STD-1196 & $1.49 \mathrm{E}-15$ \\
\hline $\mathrm{Pd}-96$ & Pd-96 & 95.918 & Palladium & $1.00 \mathrm{E}-03$ & ICRP-107 & $1.22 \mathrm{E}+02$ & $\mathrm{~s}$ & $1.22 \mathrm{E}+02$ & $9.64 \mathrm{E}+08$ & -- & -- & -- & -- & DOE-STD-1196 & $6.51 \mathrm{E}-14$ \\
\hline Pd-97 & $\mathrm{Pd}-97$ & 96.916 & $\begin{array}{l}\text { Palladium } \\
\end{array}$ & $1.00 \mathrm{E}-03$ & ICRP-107 & $3.10 \mathrm{E}+00$ & $\mathrm{~m}$ & $1.86 \mathrm{E}+02$ & $6.26 \mathrm{E}+08$ & -- & -- & -- & -- & DOE-STD-1196 & $1.12 \mathrm{E}-13$ \\
\hline Pd-98 & $\mathrm{Pd}-98$ & 97.913 & Palladium & $1.00 \mathrm{E}-03$ & ICRP-107 & $1.77 \mathrm{E}+01$ & $\mathrm{~m}$ & $1.06 \mathrm{E}+03$ & $1.08 \mathrm{E}+08$ & DOE-STD-1196 & $3.35 \mathrm{E}-11$ & $\mathrm{~s}$ & Max & DOE-STD-1196 & $1.76 \mathrm{E}-14$ \\
\hline $\begin{array}{l}\mathrm{Pd}-99 \\
\end{array}$ & Pd-99 & 98.912 & Palladium & $1.00 \mathrm{E}-03$ & ICRP-107 & $2.14 \mathrm{E}+01$ & $\mathrm{~m}$ & $1.28 \mathrm{E}+03$ & $8.88 \mathrm{E}+07$ & DOE-STD-1196 & $2.28 \mathrm{E}-11$ & $\mathrm{~s}$ & Max & DOE-STD-1196 & $5.88 \mathrm{E}-14$ \\
\hline Pm-136 & Pm-136 & 135.924 & Promethium & $1.00 \mathrm{E}-03$ & ICRP-107 & $1.07 \mathrm{E}+02$ & $\mathrm{~s}$ & $1.07 \mathrm{E}+02$ & $7.76 \mathrm{E}+08$ & -- & -- & -- & -- & DOE-STD-1196 & $1.25 \mathrm{E}-13$ \\
\hline$P m-137 m$ & Pm-137 & 136.920 & Promethium & $1.00 \mathrm{E}-03$ & ICRP-107 & $2.40 \mathrm{E}+00$ & $\mathrm{~m}$ & $1.44 \mathrm{E}+02$ & $5.72 \mathrm{E}+08$ & -- & -- & -- & -- & DOE-STD-1196 & $8.01 \mathrm{E}-14$ \\
\hline $\mathrm{Pm}-138$ & Pm-138 & 137.920 & Promethium & $1.00 \mathrm{E}-03$ & JAERI & $3.24 \mathrm{E}+00$ & $\mathrm{~m}$ & $1.94 \mathrm{E}+02$ & $4.21 \mathrm{E}+08$ & -- & -- & -- & -- & -- & -- \\
\hline Pm-139 & Pm-139 & 138.917 & Promethium & $1.00 \mathrm{E}-03$ & ICRP-107 & $4.15 \mathrm{E}+00$ & $\mathrm{~m}$ & $2.49 \mathrm{E}+02$ & $3.26 \mathrm{E}+08$ & -- & -- & -- & -- & DOE-STD-1196 & $4.29 \mathrm{E}-14$ \\
\hline Pm-140 & Pm-140 & 139.916 & Promethium & $1.00 \mathrm{E}-03$ & ICRP-107 & $9.20 \mathrm{E}+00$ & $\mathrm{~s}$ & $9.20 \mathrm{E}+00$ & $8.76 \mathrm{E}+09$ & -- & -- & -- & -- & DOE-STD-1196 & $4.93 \mathrm{E}-14$ \\
\hline $\mathrm{Pm}-140 \mathrm{~m}$ & Pm-140 & 139.916 & Promethium & $1.00 \mathrm{E}-03$ & ICRP-107 & $5.95 \mathrm{E}+00$ & $\mathrm{~m}$ & $3.57 \mathrm{E}+02$ & $2.26 \mathrm{E}+08$ & -- & -- & -- & -- & DOE-STD-1196 & $1.39 \mathrm{E}-13$ \\
\hline $\mathrm{Pm}-141$ & Pm-141 & 140.914 & Promethium & $1.00 \mathrm{E}-03$ & ICRP-38 & $2.09 \mathrm{E}+01$ & $\mathrm{~m}$ & $1.25 \mathrm{E}+03$ & $6.38 \mathrm{E}+07$ & ICRP-72 & $1.50 \mathrm{E}-11$ & $\mathrm{~s}$ & Max & FGR-12 & $3.60 \mathrm{E}-14$ \\
\hline Pm-142 & Pm-142 & 141.913 & Promethium & $1.00 \mathrm{E}-03$ & ICRP-38 & $4.05 \mathrm{E}+01$ & $s$ & $4.05 \mathrm{E}+01$ & $1.96 \mathrm{E}+09$ & -- & -- & -- & -- & FGR-12 & $4.22 \mathrm{E}-14$ \\
\hline Pm-143 & Pm-143 & 142.911 & Promethium & $1.00 \mathrm{E}-03$ & ICRP-38 & $2.65 \mathrm{E}+02$ & $d$ & $2.29 \mathrm{E}+07$ & $3.45 \mathrm{E}+03$ & ICRP-72 & $1.50 \mathrm{E}-09$ & $M$ & Max & FGR-12 & $1.46 \mathrm{E}-14$ \\
\hline $\mathrm{Pm}-144$ & Pm-144 & 143.913 & Promethium & $1.00 \mathrm{E}-03$ & ICRP-38 & $3.63 \mathrm{E}+02$ & $d$ & $3.14 \mathrm{E}+07$ & $2.50 \mathrm{E}+03$ & ICRP-72 & $8.20 \mathrm{E}-09$ & $M$ & Max & FGR-12 & $7.48 \mathrm{E}-14$ \\
\hline Pm-145 & Pm-145 & 144.913 & Promethium & $1.00 \mathrm{E}-03$ & ICRP-38 & $1.77 \mathrm{E}+01$ & $y$ & $5.59 \mathrm{E}+08$ & $1.39 \mathrm{E}+02$ & ICRP-72 & $3.60 \mathrm{E}-09$ & $M$ & Max & FGR-12 & $7.09 \mathrm{E}-16$ \\
\hline Pm-146 & Pm-146 & 145.915 & Promethium & $1.00 \mathrm{E}-03$ & ICRP-38 & $2.02 E+03$ & $d$ & $1.75 \mathrm{E}+08$ & $4.43 \mathrm{E}+02$ & ICRP-72 & $2.10 \mathrm{E}-08$ & $M$ & Max & FGR-12 & $3.59 \mathrm{E}-14$ \\
\hline Pm-147 & Pm-147 & 146.915 & Promethium & $1.00 \mathrm{E}-03$ & ICRP-38 & $2.62 \mathrm{E}+00$ & $y$ & $8.28 \mathrm{E}+07$ & $9.28 \mathrm{E}+02$ & ICRP-72 & 5.00E-09 & $M$ & Max & FGR-12 & $6.93 \mathrm{E}-19$ \\
\hline Pm-148 & Pm-148 & 147.917 & Promethium & $1.00 \mathrm{E}-03$ & ICRP-38 & $5.37 \mathrm{E}+00$ & $d$ & $4.64 \mathrm{E}+05$ & $1.64 \mathrm{E}+05$ & ICRP-72 & $2.20 \mathrm{E}-09$ & $\mathrm{~s}$ & Max & FGR-12 & $2.89 \mathrm{E}-14$ \\
\hline $\mathrm{Pm}-148 \mathrm{~m}$ & Pm-148 & 147.917 & Promethium & $1.00 \mathrm{E}-03$ & ICRP-38 & $4.13 \mathrm{E}+01$ & $d$ & $3.57 \mathrm{E}+06$ & $2.14 \mathrm{E}+04$ & ICRP-72 & $5.70 \mathrm{E}-09$ & $\mathrm{~s}$ & Max & FGR-12 & $9.68 \mathrm{E}-14$ \\
\hline Pm-149 & Pm-149 & 148.918 & Promethium & $1.00 \mathrm{E}-03$ & ICRP-38 & $5.31 \mathrm{E}+01$ & $\mathrm{~h}$ & $1.91 \mathrm{E}+05$ & $3.96 \mathrm{E}+05$ & ICRP-72 & $7.30 \mathrm{E}-10$ & $\mathrm{~s}$ & Max & FGR-12 & $5.41 \mathrm{E}-16$ \\
\hline $\mathrm{Pm}-150$ & Pm-150 & 149.921 & Promethium & $1.00 \mathrm{E}-03$ & ICRP-38 & $2.68 \mathrm{E}+00$ & $\mathrm{~h}$ & $9.65 \mathrm{E}+03$ & $7.80 \mathrm{E}+06$ & ICRP-72 & $1.30 \mathrm{E}-10$ & $\mathrm{~s}$ & Max & FGR-12 & $7.17 E-14$ \\
\hline Pm-151 & Pm-151 & 150.921 & Promethium & $1.00 \mathrm{E}-03$ & ICRP-38 & $2.84 \mathrm{E}+01$ & $\mathrm{~h}$ & $1.02 \mathrm{E}+05$ & $7.31 \mathrm{E}+05$ & ICRP-72 & $4.60 \mathrm{E}-10$ & $\mathrm{~s}$ & Max & FGR-12 & $1.51 \mathrm{E}-14$ \\
\hline Pm-152 & Pm-152 & 151.924 & Promethium & $1.00 \mathrm{E}-03$ & ICRP-107 & $4.12 \mathrm{E}+00$ & $\mathrm{~m}$ & $2.47 \mathrm{E}+02$ & $3.00 \mathrm{E}+08$ & -- & -- & -- & -- & DOE-STD-1196 & $1.45 \mathrm{E}-14$ \\
\hline $\mathrm{Pm}-152 \mathrm{~m}$ & Pm-152 & 151.924 & Promethium & $1.00 \mathrm{E}-03$ & ICRP-107 & $7.52 \mathrm{E}+00$ & $\mathrm{~m}$ & $4.51 \mathrm{E}+02$ & $1.65 \mathrm{E}+08$ & -- & -- & -- & -- & DOE-STD-1196 & $7.07 E-14$ \\
\hline Pm-153 & Pm-153 & 152.924 & Promethium & $1.00 \mathrm{E}-03$ & ICRP-107 & $5.25 \mathrm{E}+00$ & $\mathrm{~m}$ & $3.15 \mathrm{E}+02$ & $2.34 \mathrm{E}+08$ & -- & -- & -- & -- & DOE-STD-1196 & $3.40 \mathrm{E}-15$ \\
\hline Pm-154 & Pm-154 & 153.926 & Promethium & $1.00 \mathrm{E}-03$ & ICRP-107 & $1.73 \mathrm{E}+00$ & $\mathrm{~m}$ & $1.04 \mathrm{E}+02$ & $7.06 \mathrm{E}+08$ & -- & -- & -- & -- & DOE-STD-1196 & $8.73 \mathrm{E}-14$ \\
\hline Pm-154m & Pm-154 & 153.926 & Promethium & $1.00 \mathrm{E}-03$ & ICRP-107 & $2.68 \mathrm{E}+00$ & $\mathrm{~m}$ & $1.61 \mathrm{E}+02$ & $4.56 \mathrm{E}+08$ & -- & -- & -- & -- & DOE-STD-1196 & $8.50 \mathrm{E}-14$ \\
\hline Po-203 & Po-203 & 202.981 & Polonium & $1.00 \mathrm{E}-02$ & ICRP-38 & $3.67 \mathrm{E}+01$ & $\mathrm{~m}$ & $2.20 \mathrm{E}+03$ & $2.52 \mathrm{E}+07$ & $\begin{array}{l}\text { ICRP-72 } \\
\end{array}$ & $3.50 \mathrm{E}-11$ & $M$ & Rec & FGR-12 & $8.12 \mathrm{E}-14$ \\
\hline Po-204 & Po-204 & 203.980 & Polonium & $1.00 \mathrm{E}-02$ & ICRP-107 & $3.53 \mathrm{E}+00$ & $\mathrm{~h}$ & $1.27 \mathrm{E}+04$ & $4.35 \mathrm{E}+06$ & DOE-STD-1196 & $4.24 \mathrm{E}-10$ & $M$ & $\operatorname{Rec}$ & DOE-STD-1196 & $5.16 \mathrm{E}-14$ \\
\hline Po-205 & Po-205 & 204.981 & Polonium & $1.00 \mathrm{E}-02$ & ICRP-38 & $1.80 \mathrm{E}+00$ & $\mathrm{~h}$ & $6.48 \mathrm{E}+03$ & $8.49 \mathrm{E}+06$ & ICRP-72 & $6.50 \mathrm{E}-11$ & $M$ & $\operatorname{Rec}$ & FGR-12 & $7.80 \mathrm{E}-14$ \\
\hline Po-206 & Po-206 & 205.980 & Polonium & $1.00 \mathrm{E}-02$ & ICRP-107 & $8.80 \mathrm{E}+00$ & $d$ & $7.60 \mathrm{E}+05$ & $7.20 \mathrm{E}+04$ & DOE-STD-1196 & $6.27 \mathrm{E}-08$ & $M$ & Rec & DOE-STD-1196 & $5.33 \mathrm{E}-14$ \\
\hline Po-207 & Po-207 & 206.982 & Polonium & $1.00 \mathrm{E}-02$ & ICRP-38 & $3.50 \mathrm{E}+02$ & $\mathrm{~m}$ & $2.10 E+04$ & $2.60 \mathrm{E}+06$ & ICRP-72 & $7.80 \mathrm{E}-11$ & $M$ & Rec & FGR-12 & $6.51 \mathrm{E}-14$ \\
\hline
\end{tabular}




\begin{tabular}{|c|c|c|c|c|c|c|c|c|c|c|c|c|c|c|c|}
\hline $\begin{array}{l}\text { MASTER } \\
\text { ISOTOPE } \\
\text { LIST }\end{array}$ & $\begin{array}{l}\text { Atomic } \\
\text { Mass } \\
\text { Nuclide }\end{array}$ & $\begin{array}{l}\text { Atomic } \\
\text { Mass }\end{array}$ & Element & $\begin{array}{c}\text { RF per } \\
\text { DOE-STD- } \\
1027\end{array}$ & $\begin{array}{c}\text { Half-Life } \\
\text { Reference }\end{array}$ & $\begin{array}{r}\text { Half-Li } \\
\text { (original u }\end{array}$ & & Half Life (s) & $\begin{array}{l}\text { Sp. Act } \\
\text { (Ci/g) }\end{array}$ & $\begin{array}{l}\text { Inhalation DC } \\
\text { Reference }\end{array}$ & $\begin{array}{c}\mathrm{HC}-2 \\
\text { Inhalation } \\
\mathrm{DC} \\
(\mathrm{Sv} / \mathrm{Bq})\end{array}$ & & $\begin{array}{l}\text { ng } \\
\text { ption } \\
\text { Type }\end{array}$ & $\begin{array}{l}\text { Immersion DC } \\
\text { Reference }\end{array}$ & $\begin{array}{c}\mathrm{HC}-2 \\
\text { Immersion } \\
\mathrm{DC}(\mathrm{Sv} / \mathrm{s} \\
\left.\text { per } \mathrm{Bq} / \mathrm{m}^{3}\right)\end{array}$ \\
\hline Po-208 & Po-208 & 207.981 & Polonium & $1.00 \mathrm{E}-02$ & ICRP-107 & $2.90 \mathrm{E}+00$ & $y$ & $9.15 \mathrm{E}+07$ & $5.93 \mathrm{E}+02$ & DOE-STD-1196 & $4.16 \mathrm{E}-06$ & $\mathrm{M}$ & $\operatorname{Rec}$ & DOE-STD-1196 & 9.36E-19 \\
\hline Po-209 & Po-209 & 208.982 & Polonium & $1.00 \mathrm{E}-02$ & ICRP-38 & $1.02 E+02$ & $\mathrm{y}$ & $3.22 E+09$ & $1.68 \mathrm{E}+01$ & DOE-STD-1196 & $4.02 \mathrm{E}-06$ & $\mathrm{M}$ & $\operatorname{Rec}$ & DOE-STD-1196 & $2.76 \mathrm{E}-16$ \\
\hline Po-210 & Po-210 & 209.983 & Polonium & $1.00 \mathrm{E}-02$ & ICRP-38 & $1.38 \mathrm{E}+02$ & d & $1.20 \mathrm{E}+07$ & $4.49 \mathrm{E}+03$ & ICRP-72 & $3.30 \mathrm{E}-06$ & $M$ & Rec & FGR-12 & 4.16E-19 \\
\hline Po-211 & Po-211 & 210.987 & Polonium & $1.00 \mathrm{E}-02$ & ICRP-38 & $5.16 \mathrm{E}-01$ & $s$ & $5.16 \mathrm{E}-01$ & $1.04 \mathrm{E}+11$ & -- & -- & -- & -- & FGR-12 & $3.81 \mathrm{E}-16$ \\
\hline Po-212 & Po-212 & 211.989 & Polonium & $1.00 \mathrm{E}-02$ & ICRP-38 & $3.05 \mathrm{E}-01$ & us & $3.05 \mathrm{E}-07$ & $1.74 \mathrm{E}+17$ & -- & -- & -- & -- & -- & -- \\
\hline Po-212m & Po-212 & 211.989 & Polonium & $1.00 \mathrm{E}-02$ & ICRP-107 & $4.51 \mathrm{E}+01$ & $\mathrm{~s}$ & $4.51 \mathrm{E}+01$ & $1.18 \mathrm{E}+09$ & -- & -- & -- & -- & DOE-STD-1196 & $3.99 \mathrm{E}-15$ \\
\hline Po-213 & Po-213 & 212.993 & Polonium & $1.00 \mathrm{E}-02$ & ICRP-38 & $4.20 \mathrm{E}+00$ & us & $4.20 \mathrm{E}-06$ & $1.26 \mathrm{E}+16$ & -- & -- & -- & -- & DOE-STD-1196 & $1.71 \mathrm{E}-18$ \\
\hline Po-214 & Po-214 & 213.995 & Polonium & $1.00 \mathrm{E}-02$ & ICRP-38 & $1.64 \mathrm{E}+02$ & us & $1.64 \mathrm{E}-04$ & $3.21 \mathrm{E}+14$ & -- & -- & -- & -- & FGR-12 & $4.08 \mathrm{E}-18$ \\
\hline Po-215 & Po-215 & 214.999 & Polonium & $1.00 \mathrm{E}-02$ & ICRP-38 & $1.78 \mathrm{E}-03$ & $\mathrm{~s}$ & $1.78 \mathrm{E}-03$ & $2.95 \mathrm{E}+13$ & -- & -- & -- & -- & FGR-12 & $8.43 \mathrm{E}-18$ \\
\hline Po-216 & Po-216 & 216.002 & Polonium & $1.00 \mathrm{E}-02$ & ICRP-38 & $1.50 \mathrm{E}-01$ & $\mathrm{~s}$ & $1.50 \mathrm{E}-01$ & $3.48 \mathrm{E}+11$ & -- & -- & -- & -- & FGR-12 & $8.29 \mathrm{E}-19$ \\
\hline Po-218 & Po-218 & 218.009 & $\begin{array}{l}\text { Polonium } \\
\end{array}$ & $1.00 \mathrm{E}-02$ & ICRP-38 & $3.05 E+00$ & $\mathrm{~m}$ & $1.83 \mathrm{E}+02$ & $2.83 \mathrm{E}+08$ & -- & -- & -- & -- & FGR-12 & $4.48 \mathrm{E}-19$ \\
\hline Pr-134 & Pr-134 & 133.916 & Praseodymium & $1.00 \mathrm{E}-03$ & ICRP-107 & $1.10 \mathrm{E}+01$ & $\mathrm{~m}$ & $6.60 \mathrm{E}+02$ & $1.28 \mathrm{E}+08$ & DOE-STD-1196 & $2.52 \mathrm{E}-11$ & $\mathrm{~s}$ & Max & DOE-STD-1196 & $1.44 \mathrm{E}-13$ \\
\hline Pr-134m & Pr-134 & 133.916 & Praseodymium & $1.00 \mathrm{E}-03$ & ICRP-107 & $1.70 \mathrm{E}+01$ & $\mathrm{~m}$ & $1.02 \mathrm{E}+03$ & $8.26 \mathrm{E}+07$ & DOE-STD-1196 & 3.83E-11 & $\mathrm{s}$ & Max & DOE-STD-1196 & $1.08 \mathrm{E}-13$ \\
\hline Pr-135 & Pr-135 & 134.913 & Praseodymium & $1.00 \mathrm{E}-03$ & ICRP-107 & $2.40 \mathrm{E}+01$ & $\mathrm{~m}$ & $1.44 \mathrm{E}+03$ & $5.81 \mathrm{E}+07$ & DOE-STD-1196 & $2.45 \mathrm{E}-11$ & $\mathrm{~s}$ & Max & DOE-STD-1196 & $3.89 \mathrm{E}-14$ \\
\hline Pr-136 & Pr-136 & 135.913 & Praseodymium & $1.00 \mathrm{E}-03$ & ICRP-38 & $1.31 \mathrm{E}+01$ & $\mathrm{~m}$ & $7.86 \mathrm{E}+02$ & $1.06 \mathrm{E}+08$ & ICRP-72 & 1.40E-11 & $\mathrm{s}$ & Max & FGR-12 & $1.03 \mathrm{E}-13$ \\
\hline Pr-137 & Pr-137 & 136.911 & Praseodymium & $1.00 \mathrm{E}-03$ & ICRP-38 & $7.66 \mathrm{E}+01$ & $\mathrm{~m}$ & $4.60 \mathrm{E}+03$ & $1.79 \mathrm{E}+07$ & ICRP-72 & $2.10 \mathrm{E}-11$ & $\mathrm{~s}$ & Max & FGR-12 & $2.36 \mathrm{E}-14$ \\
\hline Pr-138 & Pr-138 & 137.911 & Praseodymium & $1.00 \mathrm{E}-03$ & ICRP-38 & $1.45 \mathrm{E}+00$ & $\mathrm{~m}$ & $8.70 \mathrm{E}+01$ & $9.40 \mathrm{E}+08$ & -- & -- & -- & -- & FGR-12 & $3.92 \mathrm{E}-14$ \\
\hline Pr-138m & $\begin{array}{ll}\text { Pr-138 } \\
\end{array}$ & 137.911 & Praseodymium & $1.00 \mathrm{E}-03$ & ICRP-38 & $2.10 \mathrm{E}+00$ & $\mathrm{~h}$ & $7.56 \mathrm{E}+03$ & $1.08 \mathrm{E}+07$ & ICRP-72 & $7.40 \mathrm{E}-11$ & $\mathrm{~s}$ & Max & FGR-12 & $1.21 \mathrm{E}-13$ \\
\hline Pr-139 & Pr-139 & 138.909 & Praseodymium & $1.00 \mathrm{E}-03$ & ICRP-38 & $4.51 \mathrm{E}+00$ & $\mathrm{~h}$ & $1.62 \mathrm{E}+04$ & $5.00 \mathrm{E}+06$ & ICRP-72 & $2.00 \mathrm{E}-11$ & $\mathrm{~s}$ & Max & FGR-12 & $5.17 \mathrm{E}-15$ \\
\hline $\begin{array}{ll}\mathrm{Pr}-140 \\
\end{array}$ & Pr-140 & 139.909 & Praseodymium & $1.00 \mathrm{E}-03$ & ICRP-107 & $3.39 \mathrm{E}+00$ & $\mathrm{~m}$ & $2.03 \mathrm{E}+02$ & $3.96 \mathrm{E}+08$ & -- & -- & -- & -- & DOE-STD-1196 & $2.44 \mathrm{E}-14$ \\
\hline Pr-142 & Pr-142 & 141.910 & Praseodymium & $1.00 \mathrm{E}-03$ & ICRP-38 & $1.91 \mathrm{E}+01$ & $\mathrm{~h}$ & $6.89 \mathrm{E}+04$ & $1.15 \mathrm{E}+06$ & ICRP-72 & $5.50 \mathrm{E}-10$ & $\mathrm{~s}$ & Max & FGR-12 & $3.15 \mathrm{E}-15$ \\
\hline Pr-142m & Pr-142 & 141.910 & Praseodymium & $1.00 \mathrm{E}-03$ & ICRP-38 & $1.46 \mathrm{E}+01$ & $\mathrm{~m}$ & $8.76 \mathrm{E}+02$ & $9.08 \mathrm{E}+07$ & ICRP-72 & $7.00 \mathrm{E}-12$ & $\mathrm{~s}$ & Max & -- & -- \\
\hline Pr-143 & Pr-143 & 142.911 & Praseodymium & $1.00 \mathrm{E}-03$ & ICRP-38 & $1.36 \mathrm{E}+01$ & d & $1.17 E+06$ & $6.74 \mathrm{E}+04$ & ICRP-72 & $2.40 \mathrm{E}-09$ & $\mathrm{~s}$ & Max & FGR-12 & $2.10 \mathrm{E}-17$ \\
\hline Pr-144 & Pr-144 & 143.913 & Praseodymium & $1.00 \mathrm{E}-03$ & ICRP-38 & $1.73 \mathrm{E}+01$ & $\mathrm{~m}$ & $1.04 \mathrm{E}+03$ & $7.56 \mathrm{E}+07$ & ICRP-72 & $1.80 \mathrm{E}-11$ & $\mathrm{M}$ & Max & FGR-12 & $1.95 \mathrm{E}-15$ \\
\hline Pr-144m & Pr-144 & 143.913 & Praseodymium & $1.00 \mathrm{E}-03$ & ICRP-38 & $7.20 E+00$ & $\mathrm{~m}$ & $4.32 \mathrm{E}+02$ & $1.81 \mathrm{E}+08$ & -- & -- & -- & -- & FGR-12 & $2.79 \mathrm{E}-16$ \\
\hline Pr-145 & Pr-145 & 144.915 & Praseodymium & $1.00 \mathrm{E}-03$ & ICRP-38 & $5.98 \mathrm{E}+00$ & $\mathrm{~h}$ & $2.15 \mathrm{E}+04$ & $3.62 \mathrm{E}+06$ & ICRP-72 & $1.70 \mathrm{E}-10$ & $\mathrm{~s}$ & Max & FGR-12 & $7.36 \mathrm{E}-16$ \\
\hline Pr-146 & Pr-146 & 145.918 & Praseodymium & $1.00 \mathrm{E}-03$ & ICRP-107 & $2.42 \mathrm{E}+01$ & $\mathrm{~m}$ & $1.45 \mathrm{E}+03$ & $5.34 \mathrm{E}+07$ & DOE-STD-1196 & $3.58 \mathrm{E}-11$ & $\mathrm{~s}$ & Max & DOE-STD-1196 & $4.92 \mathrm{E}-14$ \\
\hline Pr-147 & Pr-147 & 146.919 & Praseodymium & $1.00 \mathrm{E}-03$ & ICRP-38 & $1.36 \mathrm{E}+01$ & $\mathrm{~m}$ & $8.16 \mathrm{E}+02$ & $9.41 \mathrm{E}+07$ & ICRP-72 & $1.80 \mathrm{E}-11$ & $M$ & Max & FGR-12 & $4.15 \mathrm{E}-14$ \\
\hline $\begin{array}{ll}\text { Pr-148 } \\
\end{array}$ & Pr-148 & 147.922 & Praseodymium & $1.00 \mathrm{E}-03$ & ICRP-107 & $2.29 \mathrm{E}+00$ & $\mathrm{~m}$ & $1.37 \mathrm{E}+02$ & $5.55 \mathrm{E}+08$ & -- & -- & -- & $\begin{array}{l}- \\
\end{array}$ & DOE-STD-1196 & $4.83 \mathrm{E}-14$ \\
\hline Pr-148m & Pr-148 & 147.922 & Praseodymium & $1.00 \mathrm{E}-03$ & ICRP-107 & $2.01 \mathrm{E}+00$ & $\mathrm{~m}$ & $1.21 \mathrm{E}+02$ & $6.32 \mathrm{E}+08$ & -- & -- & -- & -- & DOE-STD-1196 & $4.37 \mathrm{E}-14$ \\
\hline Pt-183 & Pt-183 & 182.962 & Platinum & $1.00 \mathrm{E}-03$ & JAERI & $6.50 \mathrm{E}+00$ & $\mathrm{~m}$ & $3.90 \mathrm{E}+02$ & $1.58 \mathrm{E}+08$ & -- & -- & -- & -- & -- & -- \\
\hline Pt-184 & Pt-184 & 183.960 & Platinum & $1.00 \mathrm{E}-03$ & ICRP-107 & $1.73 \mathrm{E}+01$ & $\mathrm{~m}$ & $1.04 \mathrm{E}+03$ & $5.91 \mathrm{E}+07$ & DOE-STD-1196 & $3.06 \mathrm{E}-11$ & $\mathrm{~s}$ & Max & DOE-STD-1196 & $3.02 \mathrm{E}-14$ \\
\hline Pt-186 & Pt-186 & 185.959 & Platinum & $1.00 \mathrm{E}-03$ & ICRP-38 & $2.00 \mathrm{E}+00$ & $\mathrm{~h}$ & $7.20 \mathrm{E}+03$ & $8.43 \mathrm{E}+06$ & ICRP-72 & $3.30 \mathrm{E}-11$ & $\mathrm{~F}$ & Max & FGR-12 & $3.53 \mathrm{E}-14$ \\
\hline Pt-187 & Pt-187 & 186.961 & Platinum & $1.00 \mathrm{E}-03$ & ICRP-107 & $2.35 E+00$ & $\mathrm{~h}$ & $8.46 \mathrm{E}+03$ & $7.13 \mathrm{E}+06$ & DOE-STD-1196 & $8.29 \mathrm{E}-11$ & $\mathrm{~S}$ & Max & DOE-STD-1196 & $2.66 \mathrm{E}-14$ \\
\hline Pt-188 & Pt-188 & 187.959 & Platinum & $1.00 \mathrm{E}-03$ & ICRP-38 & $1.02 \mathrm{E}+01$ & $d$ & $8.81 \mathrm{E}+05$ & $6.81 \mathrm{E}+04$ & ICRP-72 & $4.20 \mathrm{E}-10$ & $\mathrm{~F}$ & Max & FGR-12 & $8.86 \mathrm{E}-15$ \\
\hline Pt-189 & Pt-189 & 188.961 & Platinum & $1.00 \mathrm{E}-03$ & ICRP-38 & $1.09 \mathrm{E}+01$ & $\mathrm{~h}$ & $3.91 \mathrm{E}+04$ & $1.53 \mathrm{E}+06$ & ICRP-72 & $3.80 \mathrm{E}-11$ & $\mathrm{~F}$ & Max & FGR-12 & $1.48 \mathrm{E}-14$ \\
\hline Pt-190 & Pt-190 & 189.960 & Platinum & $1.00 \mathrm{E}-03$ & ICRP-107 & $6.50 \mathrm{E}+11$ & $y$ & $2.05 \mathrm{E}+19$ & $2.90 \mathrm{E}-09$ & DOE-STD-1196 & $5.58 \mathrm{E}-06$ & $\mathrm{~s}$ & Max & -- & -- \\
\hline Pt-191 & Pt-191 & 190.962 & Platinum & $1.00 \mathrm{E}-03$ & ICRP-38 & $2.80 \mathrm{E}+00$ & d & $2.42 \mathrm{E}+05$ & $2.44 \mathrm{E}+05$ & ICRP-72 & $1.10 \mathrm{E}-10$ & $\mathrm{~F}$ & Max & FGR-12 & $1.34 \mathrm{E}-14$ \\
\hline Pt-193 & Pt-193 & 192.963 & Platinum & $1.00 \mathrm{E}-03$ & ICRP-38 & $5.00 \mathrm{E}+01$ & $y$ & $1.58 \mathrm{E}+09$ & $3.71 \mathrm{E}+01$ & ICRP-72 & $2.10 \mathrm{E}-11$ & $F$ & Max & FGR-12 & $3.98 \mathrm{E}-19$ \\
\hline Pt-193m & Pt-193 & 192.963 & Platinum & $1.00 \mathrm{E}-03$ & ICRP-38 & $4.33 \mathrm{E}+00$ & $d$ & $3.74 \mathrm{E}+05$ & $1.56 \mathrm{E}+05$ & ICRP-72 & $1.20 \mathrm{E}-10$ & $\mathrm{~F}$ & Max & FGR-12 & $4.15 \mathrm{E}-16$ \\
\hline Pt-195m & Pt-195 & 194.965 & Platinum & $1.00 \mathrm{E}-03$ & ICRP-38 & $4.02 E+00$ & $d$ & $3.47 \mathrm{E}+05$ & $1.67 \mathrm{E}+05$ & ICRP-72 & $1.80 \mathrm{E}-10$ & $\mathrm{~F}$ & Max & FGR-12 & $2.84 \mathrm{E}-15$ \\
\hline Pt-197 & Pt-197 & 196.967 & Platinum & $1.00 \mathrm{E}-03$ & ICRP-38 & $1.83 \mathrm{E}+01$ & $\mathrm{~h}$ & $6.59 \mathrm{E}+04$ & $8.69 \mathrm{E}+05$ & ICRP-72 & $8.50 \mathrm{E}-11$ & $\mathrm{~F}$ & Max & FGR-12 & $1.01 \mathrm{E}-15$ \\
\hline Pt-197m & Pt-197 & 196.967 & Platinum & $1.00 \mathrm{E}-03$ & ICRP-38 & $9.44 \mathrm{E}+01$ & $\mathrm{~m}$ & $5.66 \mathrm{E}+03$ & $1.01 \mathrm{E}+07$ & ICRP-72 & $2.40 \mathrm{E}-11$ & $\mathrm{~F}$ & Max & FGR-12 & $3.49 \mathrm{E}-15$ \\
\hline Pt-199 & Pt-199 & 198.971 & Platinum & $1.00 \mathrm{E}-03$ & ICRP-38 & $3.08 \mathrm{E}+01$ & $\mathrm{~m}$ & $1.85 \mathrm{E}+03$ & $3.07 \mathrm{E}+07$ & ICRP-72 & $1.20 \mathrm{E}-11$ & $\mathrm{~F}$ & Max & FGR-12 & $9.73 \mathrm{E}-15$ \\
\hline Pt-200 & Pt-200 & 199.971 & Platinum & $1.00 \mathrm{E}-03$ & ICRP-38 & $1.25 \mathrm{E}+01$ & $\mathrm{~h}$ & $4.50 \mathrm{E}+04$ & $1.25 \mathrm{E}+06$ & ICRP-72 & $2.20 \mathrm{E}-10$ & $\mathrm{~F}$ & Max & FGR-12 & $2.55 \mathrm{E}-15$ \\
\hline Pt-202 & Pt-202 & 201.976 & Platinum & $1.00 \mathrm{E}-03$ & ICRP-107 & $4.40 \mathrm{E}+01$ & $\mathrm{~h}$ & $1.58 \mathrm{E}+05$ & $3.53 \mathrm{E}+05$ & DOE-STD-1196 & $2.61 \mathrm{E}-09$ & $\mathrm{~s}$ & Max & DOE-STD-1196 & $5.03 \mathrm{E}-16$ \\
\hline
\end{tabular}




\begin{tabular}{|c|c|c|c|c|c|c|c|c|c|c|c|c|c|c|c|}
\hline \multicolumn{16}{|c|}{ Table B.1 Radionuclide Specific Input Data Used For the Calculation of HC-2 TQs Using Recommended DCs (continued) } \\
\hline $\begin{array}{l}\text { MASTER } \\
\text { ISOTOPE } \\
\text { LIST }\end{array}$ & $\begin{array}{c}\text { Atomic } \\
\text { Mass } \\
\text { Nuclide }\end{array}$ & $\begin{array}{l}\text { Atomic } \\
\text { Mass }\end{array}$ & Element & $\begin{array}{c}\text { RF per } \\
\text { DOE-STD- } \\
1027\end{array}$ & $\begin{array}{l}\text { Half-Life } \\
\text { Reference }\end{array}$ & \multicolumn{2}{|c|}{$\begin{array}{c}\text { Half-Life } \\
\text { (original units) }\end{array}$} & \multirow{2}{*}{$\begin{array}{c}\text { Half Life (s) } \\
2.02 E+03\end{array}$} & \multirow{2}{*}{$\begin{array}{c}\begin{array}{c}\text { Sp. Act } \\
\text { (Ci/g) }\end{array} \\
2.40 \mathrm{E}+07\end{array}$} & \multirow{2}{*}{$\begin{array}{c}\begin{array}{l}\text { Inhalation DC } \\
\text { Reference }\end{array} \\
\text { DOE-STD-1196 }\end{array}$} & \multirow[t]{2}{*}{$\begin{array}{c}\text { HC-2 } \\
\text { Inhalation } \\
\text { DC } \\
\text { (Sv/Bq) } \\
2.44 E-08 \\
\end{array}$} & \multicolumn{2}{|c|}{$\begin{array}{c}\text { Lung } \\
\text { Absorption } \\
\text { Class/Type }\end{array}$} & \multirow{2}{*}{$\begin{array}{c}\text { Immersion DC } \\
\text { Reference } \\
\text { DOE-STD-1196 }\end{array}$} & \multirow[t]{2}{*}{ 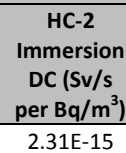 } \\
\hline Pu-232 & Pu-232 & 232.041 & Plutonium & $1.00 \mathrm{E}-03$ & ICRP-107 & $3.37 \mathrm{E}+01$ & $\mathrm{~m}$ & & & & & $\mathrm{M}$ & Rec & & \\
\hline $\mathrm{Pu}-234$ & Pu-234 & 234.043 & Plutonium & $1.00 \mathrm{E}-03$ & ICRP-38 & $8.80 \mathrm{E}+00$ & $\mathrm{~h}$ & $3.17 \mathrm{E}+04$ & $1.52 \mathrm{E}+06$ & ICRP-72 & $2.10 \mathrm{E}-08$ & $\mathrm{M}$ & Rec & FGR-12 & $2.85 \mathrm{E}-15$ \\
\hline Pu-235 & Pu-235 & 235.045 & Plutonium & $1.00 \mathrm{E}-03$ & ICRP-38 & $2.53 \mathrm{E}+01$ & $\mathrm{~m}$ & $1.52 \mathrm{E}+03$ & $3.16 \mathrm{E}+07$ & ICRP-72 & 1.40E-12 & $M$ & $\operatorname{Rec}$ & FGR-12 & $3.92 \mathrm{E}-15$ \\
\hline Pu-236 & Pu-236 & 236.046 & Plutonium & $1.00 \mathrm{E}-03$ & ICRP-38 & $2.85 \mathrm{E}+00$ & $y$ & $9.00 \mathrm{E}+07$ & $5.31 \mathrm{E}+02$ & ICRP-72 & $2.00 \mathrm{E}-05$ & $M$ & $\operatorname{Rec}$ & FGR-12 & $6.35 \mathrm{E}-18$ \\
\hline Pu-237 & Pu-237 & 237.048 & Plutonium & $1.00 \mathrm{E}-03$ & ICRP-38 & $4.53 \mathrm{E}+01$ & $d$ & $3.91 \mathrm{E}+06$ & $1.22 \mathrm{E}+04$ & ICRP-72 & $3.50 \mathrm{E}-10$ & $M$ & $\operatorname{Rec}$ & FGR-12 & $2.02 \mathrm{E}-15$ \\
\hline Pu-238 & Pu-238 & 238.050 & Plutonium & $1.00 \mathrm{E}-03$ & ICRP-38 & $8.77 \mathrm{E}+01$ & $y$ & $2.77 \mathrm{E}+09$ & $1.71 \mathrm{E}+01$ & ICRP-72 & $4.60 \mathrm{E}-05$ & $M$ & $\operatorname{Rec}$ & FGR-12 & $4.88 \mathrm{E}-18$ \\
\hline Pu-239 & Pu-239 & 239.052 & Plutonium & $1.00 \mathrm{E}-03$ & ICRP-38 & $2.41 \mathrm{E}+04$ & $y$ & $7.59 \mathrm{E}+11$ & $6.21 \mathrm{E}-02$ & ICRP-72 & $5.00 \mathrm{E}-05$ & $M$ & $\operatorname{Rec}$ & FGR-12 & $4.24 \mathrm{E}-18$ \\
\hline Pu-240 & Pu-240 & 240.054 & Plutonium & $1.00 \mathrm{E}-03$ & ICRP-38 & $6.54 \mathrm{E}+03$ & $y$ & $2.06 \mathrm{E}+11$ & $2.28 \mathrm{E}-01$ & ICRP-72 & $5.00 \mathrm{E}-05$ & $M$ & $\operatorname{Rec}$ & FGR-12 & $4.75 \mathrm{E}-18$ \\
\hline Pu-241 & Pu-241 & 241.057 & Plutonium & $1.00 \mathrm{E}-03$ & ICRP-38 & $1.44 \mathrm{E}+01$ & $y$ & $4.54 \mathrm{E}+08$ & $1.03 \mathrm{E}+02$ & ICRP-72 & $9.00 \mathrm{E}-07$ & $M$ & $\operatorname{Rec}$ & FGR-12 & $7.25 \mathrm{E}-20$ \\
\hline Pu-242 & Pu-242 & 242.059 & Plutonium & $1.00 \mathrm{E}-03$ & ICRP-38 & $3.76 \mathrm{E}+05$ & $y$ & $1.19 \mathrm{E}+13$ & $3.92 \mathrm{E}-03$ & ICRP-72 & $4.80 \mathrm{E}-05$ & $M$ & $\operatorname{Rec}$ & FGR-12 & $4.01 \mathrm{E}-18$ \\
\hline Pu-243 & Pu-243 & 243.062 & Plutonium & $1.00 \mathrm{E}-03$ & ICRP-38 & $4.96 \mathrm{E}+00$ & $h$ & $1.78 \mathrm{E}+04$ & $2.60 \mathrm{E}+06$ & ICRP-72 & $8.30 \mathrm{E}-11$ & $M$ & $\operatorname{Rec}$ & FGR-12 & $1.03 \mathrm{E}-15$ \\
\hline Pu-244 & Pu-244 & 244.064 & Plutonium & $1.00 \mathrm{E}-03$ & ICRP-38 & $8.26 \mathrm{E}+07$ & $y$ & $2.61 \mathrm{E}+15$ & $1.77 \mathrm{E}-05$ & ICRP-72 & $4.70 \mathrm{E}-05$ & $M$ & $\operatorname{Rec}$ & FGR-12 & $2.97 \mathrm{E}-18$ \\
\hline Pu-245 & Pu-245 & 245.068 & Plutonium & $1.00 \mathrm{E}-03$ & ICRP-38 & $1.05 \mathrm{E}+01$ & $\mathrm{~h}$ & $3.78 \mathrm{E}+04$ & $1.22 \mathrm{E}+06$ & ICRP-72 & $4.00 \mathrm{E}-10$ & $M$ & $\operatorname{Rec}$ & FGR-12 & $1.99 \mathrm{E}-14$ \\
\hline Pu-246 & Pu-246 & 246.070 & Plutonium & $1.00 \mathrm{E}-03$ & ICRP-38 & $1.09 \mathrm{E}+01$ & d & $9.37 \mathrm{E}+05$ & $4.89 \mathrm{E}+04$ & ICRP-72 & $7.40 \mathrm{E}-09$ & $M$ & $\operatorname{Rec}$ & FGR-12 & $6.01 \mathrm{E}-15$ \\
\hline Ra-219 & Ra-219 & 219.010 & Radium & $1.00 \mathrm{E}-03$ & ICRP-107 & $1.00 \mathrm{E}+01$ & $\mathrm{~ms}$ & $1.00 \mathrm{E}-02$ & $5.15 \mathrm{E}+12$ & -- & -- & -- & -- & DOE-STD-1196 & $7.37 E-15$ \\
\hline Ra-220 & Ra-220 & 220.011 & Radium & $1.00 \mathrm{E}-03$ & ICRP-107 & $1.79 \mathrm{E}-02$ & $\mathrm{~s}$ & $1.79 \mathrm{E}-02$ & $2.86 \mathrm{E}+12$ & -- & -- & -- & -- & DOE-STD-1196 & $2.07 \mathrm{E}-16$ \\
\hline Ra-221 & Ra-221 & 221.014 & Radium & $1.00 \mathrm{E}-03$ & ICRP-107 & $2.80 \mathrm{E}+01$ & $\mathrm{~s}$ & $2.80 \mathrm{E}+01$ & $1.82 \mathrm{E}+09$ & -- & -- & -- & -- & DOE-STD-1196 & $1.47 \mathrm{E}-15$ \\
\hline Ra-222 & Ra-222 & 222.015 & Radium & $1.00 \mathrm{E}-03$ & ICRP-38 & $3.80 \mathrm{E}+01$ & $\mathrm{~s}$ & $3.80 \mathrm{E}+01$ & $1.34 \mathrm{E}+09$ & -- & -- & -- & -- & FGR-12 & $4.39 \mathrm{E}-16$ \\
\hline Ra-223 & Ra-223 & 223.019 & Radium & $1.00 \mathrm{E}-03$ & ICRP-38 & $1.14 \mathrm{E}+01$ & $d$ & $9.88 \mathrm{E}+05$ & $5.12 \mathrm{E}+04$ & ICRP-72 & $7.40 \mathrm{E}-06$ & $M$ & $\operatorname{Rec}$ & FGR-12 & $6.09 \mathrm{E}-15$ \\
\hline Ra-224 & Ra-224 & 224.020 & Radium & $1.00 \mathrm{E}-03$ & ICRP-38 & $3.66 \mathrm{E}+00$ & $d$ & $3.16 \mathrm{E}+05$ & $1.59 \mathrm{E}+05$ & ICRP-72 & $3.00 \mathrm{E}-06$ & $M$ & $\operatorname{Rec}$ & FGR-12 & $4.71 \mathrm{E}-16$ \\
\hline Ra-225 & Ra-225 & 225.024 & Radium & $1.00 \mathrm{E}-03$ & ICRP-38 & $1.48 \mathrm{E}+01$ & $d$ & $1.28 \mathrm{E}+06$ & $3.92 \mathrm{E}+04$ & ICRP-72 & $6.30 \mathrm{E}-06$ & $M$ & $\operatorname{Rec}$ & FGR-12 & $2.79 \mathrm{E}-16$ \\
\hline Ra-226 & Ra-226 & 226.025 & Radium & $1.00 \mathrm{E}-03$ & ICRP-38 & $1.60 \mathrm{E}+03$ & $y$ & $5.05 \mathrm{E}+10$ & $9.89 \mathrm{E}-01$ & ICRP-72 & $3.50 \mathrm{E}-06$ & $M$ & $\operatorname{Rec}$ & FGR-12 & $3.15 \mathrm{E}-16$ \\
\hline Ra-227 & Ra-227 & 227.029 & Radium & $1.00 \mathrm{E}-03$ & ICRP-38 & $4.22 \mathrm{E}+01$ & $\mathrm{~m}$ & $2.53 \mathrm{E}+03$ & $1.96 \mathrm{E}+07$ & ICRP-72 & $2.80 \mathrm{E}-10$ & $M$ & $\operatorname{Rec}$ & FGR-12 & $7.41 \mathrm{E}-15$ \\
\hline Ra-228 & Ra-228 & 228.031 & Radium & $1.00 \mathrm{E}-03$ & ICRP-38 & $5.75 \mathrm{E}+00$ & $y$ & $1.81 \mathrm{E}+08$ & $2.73 \mathrm{E}+02$ & ICRP-72 & $2.60 \mathrm{E}-06$ & $M$ & $\operatorname{Rec}$ & DOE-STD-1196 & $2.89 \mathrm{E}-18$ \\
\hline Ra-230 & Ra-230 & 230.037 & Radium & $1.00 \mathrm{E}-03$ & ICRP-107 & $9.30 \mathrm{E}+01$ & $\mathrm{~m}$ & $5.58 \mathrm{E}+03$ & $8.79 \mathrm{E}+06$ & DOE-STD-1196 & $1.29 \mathrm{E}-10$ & $M$ & $\operatorname{Rec}$ & DOE-STD-1196 & $3.33 \mathrm{E}-15$ \\
\hline $\mathrm{Rb}-77$ & Rb-77 & 76.930 & Rubidium & $1.00 \mathrm{E}-03$ & ICRP-38 & $3.70 \mathrm{E}+00$ & $\mathrm{~m}$ & $2.22 \mathrm{E}+02$ & $6.61 \mathrm{E}+08$ & -- & -- & -- & -- & DOE-STD-1196 & $7.09 \mathrm{E}-14$ \\
\hline Rb-78 & Rb-78 & 77.928 & Rubidium & $1.00 \mathrm{E}-03$ & ICRP-107 & $1.77 \mathrm{E}+01$ & $\mathrm{~m}$ & $1.06 \mathrm{E}+03$ & $1.37 \mathrm{E}+08$ & DOE-STD-1196 & $3.55 \mathrm{E}-11$ & $\mathrm{~s}$ & Max & DOE-STD-1196 & $2.04 \mathrm{E}-13$ \\
\hline $\mathrm{Rb}-78 \mathrm{~m}$ & Rb-78 & 77.928 & Rubidium & $1.00 \mathrm{E}-03$ & ICRP-107 & $5.74 \mathrm{E}+00$ & $\mathrm{~m}$ & $3.44 \mathrm{E}+02$ & $4.20 \mathrm{E}+08$ & -- & -- & -- & -- & DOE-STD-1196 & $1.52 \mathrm{E}-13$ \\
\hline Rb-79 & Rb-79 & 78.924 & Rubidium & $1.00 \mathrm{E}-03$ & $\begin{array}{l}\text { ICRP-38 } \\
\end{array}$ & $2.29 \mathrm{E}+01$ & $\mathrm{~m}$ & $1.37 E+03$ & $1.04 \mathrm{E}+08$ & ICRP-72 & $1.60 \mathrm{E}-11$ & $\mathrm{~F}$ & Max & FGR-12 & $6.51 \mathrm{E}-14$ \\
\hline Rb-80 & $\mathrm{Rb}-80$ & 79.923 & Rubidium & $1.00 \mathrm{E}-03$ & ICRP-38 & $3.40 \mathrm{E}+01$ & $\mathrm{~s}$ & $3.40 \mathrm{E}+01$ & $4.15 \mathrm{E}+09$ & -- & -- & -- & -- & FGR-12 & $6.07 E-14$ \\
\hline $\mathrm{Rb}-81$ & $\mathrm{Rb}-81$ & 80.919 & Rubidium & $1.00 \mathrm{E}-03$ & ICRP-38 & $4.58 \mathrm{E}+00$ & $\mathrm{~h}$ & $1.65 \mathrm{E}+04$ & $8.46 \mathrm{E}+06$ & ICRP-72 & $3.40 \mathrm{E}-11$ & $\mathrm{~F}$ & Max & FGR-12 & $2.96 \mathrm{E}-14$ \\
\hline Rb-81m & $\mathrm{Rb}-81$ & 80.919 & Rubidium & $1.00 \mathrm{E}-03$ & ICRP-38 & $3.20 \mathrm{E}+01$ & $\mathrm{~m}$ & $1.92 \mathrm{E}+03$ & $7.26 \mathrm{E}+07$ & ICRP-72 & $7.00 \mathrm{E}-12$ & $\mathrm{~F}$ & Max & FGR-12 & $1.88 \mathrm{E}-16$ \\
\hline $\mathrm{Rb}-82$ & $\mathrm{Rb}-82$ & 81.918 & Rubidium & $1.00 \mathrm{E}-03$ & ICRP-38 & $1.30 \mathrm{E}+00$ & $\mathrm{~m}$ & $7.80 \mathrm{E}+01$ & $1.77 \mathrm{E}+09$ & -- & -- & -- & -- & FGR-12 & $5.30 \mathrm{E}-14$ \\
\hline$R b-82 m$ & Rb-82 & 81.918 & Rubidium & $1.00 \mathrm{E}-03$ & ICRP-38 & $6.20 \mathrm{E}+00$ & $\mathrm{~h}$ & $2.23 \mathrm{E}+04$ & $6.17 \mathrm{E}+06$ & ICRP-72 & $1.10 \mathrm{E}-10$ & $\mathrm{~F}$ & Max & FGR-12 & $1.43 \mathrm{E}-13$ \\
\hline $\mathrm{Rb}-83$ & $\mathrm{Rb}-83$ & 82.915 & Rubidium & $1.00 \mathrm{E}-03$ & ICRP-38 & $8.62 \mathrm{E}+01$ & $d$ & $7.45 \mathrm{E}+06$ & $1.83 \mathrm{E}+04$ & ICRP-72 & $6.90 \mathrm{E}-10$ & $\mathrm{~F}$ & Max & FGR-12 & $2.39 \mathrm{E}-14$ \\
\hline $\mathrm{Rb}-84$ & $\mathrm{Rb}-84$ & 83.914 & Rubidium & $1.00 \mathrm{E}-03$ & ICRP-38 & $3.28 \mathrm{E}+01$ & $d$ & $2.83 \mathrm{E}+06$ & $4.75 \mathrm{E}+04$ & ICRP-72 & $1.00 \mathrm{E}-09$ & $\mathrm{~F}$ & Max & FGR-12 & $4.47 \mathrm{E}-14$ \\
\hline $\mathrm{Rb}-84 \mathrm{~m}$ & $\mathrm{Rb}-84$ & 83.914 & Rubidium & $1.00 \mathrm{E}-03$ & ICRP-107 & $2.03 \mathrm{E}+01$ & $\mathrm{~m}$ & $1.22 \mathrm{E}+03$ & $1.11 \mathrm{E}+08$ & DOE-STD-1196 & $1.01 \mathrm{E}-11$ & $\mathrm{~s}$ & Max & DOE-STD-1196 & $1.68 \mathrm{E}-14$ \\
\hline $\mathrm{Rb}-86$ & $\mathrm{Rb}-86$ & 85.911 & Rubidium & $1.00 \mathrm{E}-03$ & ICRP-38 & $1.87 \mathrm{E}+01$ & $d$ & $1.61 \mathrm{E}+06$ & $8.14 \mathrm{E}+04$ & ICRP-72 & $9.30 \mathrm{E}-10$ & $\mathrm{~F}$ & Max & FGR-12 & $4.81 \mathrm{E}-15$ \\
\hline $\mathrm{Rb}-86 \mathrm{~m}$ & $\mathrm{Rb}-86$ & 85.911 & Rubidium & $1.00 \mathrm{E}-03$ & ICRP-107 & $1.02 \mathrm{E}+00$ & $\mathrm{~m}$ & $6.10 \mathrm{E}+01$ & $2.15 \mathrm{E}+09$ & -- & -- & -- & $\begin{array}{ll}- \\
\end{array}$ & DOE-STD-1196 & $2.44 \mathrm{E}-14$ \\
\hline $\mathrm{Rb}-87$ & $\mathrm{Rb}-87$ & 86.909 & Rubidium & $1.00 \mathrm{E}-03$ & ICRP-38 & $4.70 \mathrm{E}+10$ & $y$ & $1.48 \mathrm{E}+18$ & $8.75 \mathrm{E}-08$ & ICRP-72 & $5.00 \mathrm{E}-10$ & $\mathrm{~F}$ & Max & FGR-12 & $1.82 \mathrm{E}-18$ \\
\hline Rb-88 & Rb-88 & 87.911 & Rubidium & $1.00 \mathrm{E}-03$ & ICRP-38 & $1.78 \mathrm{E}+01$ & $\mathrm{~m}$ & $1.07 E+03$ & $1.20 \mathrm{E}+08$ & ICRP-72 & $1.60 \mathrm{E}-11$ & $F$ & Max & FGR-12 & $3.36 \mathrm{E}-14$ \\
\hline $\mathrm{Rb}-89$ & $\mathrm{Rb}-89$ & 88.912 & Rubidium & $1.00 \mathrm{E}-03$ & ICRP-38 & $1.52 \mathrm{E}+01$ & $\mathrm{~m}$ & $9.12 \mathrm{E}+02$ & $1.39 \mathrm{E}+08$ & ICRP-72 & 1.40E-11 & $\mathrm{F}$ & Max & FGR-12 & 1.06E-13 \\
\hline Rb-90 & Rb-90 & 89.915 & Rubidium & $1.00 \mathrm{E}-03$ & ICRP-107 & $1.58 \mathrm{E}+02$ & $\mathrm{~s}$ & $1.58 \mathrm{E}+02$ & $7.94 \mathrm{E}+08$ & -- & -- & -- & -- & DOE-STD-1196 & $1.08 \mathrm{E}-13$ \\
\hline Rb-90m & $\mathrm{Rb}-90$ & 89.915 & Rubidium & $1.00 \mathrm{E}-03$ & ICRP-107 & $2.58 \mathrm{E}+02$ & $\mathrm{~s}$ & $2.58 \mathrm{E}+02$ & $4.86 \mathrm{E}+08$ & -- & -- & -- & -- & DOE-STD-1196 & $1.63 \mathrm{E}-13$ \\
\hline Re-177 & Re-177 & 176.950 & Rhenium & $1.00 \mathrm{E}-03$ & ICRP-38 & $1.40 \mathrm{E}+01$ & $\mathrm{~m}$ & $8.40 \mathrm{E}+02$ & $7.59 \mathrm{E}+07$ & ICRP-72 & $1.40 \mathrm{E}-11$ & $M$ & Max & FGR-12 & $2.96 \mathrm{E}-14$ \\
\hline Re-178 & Re-178 & 177.951 & Rhenium & $1.00 \mathrm{E}-03$ & ICRP-38 & $1.32 \mathrm{E}+01$ & $\mathrm{~m}$ & $7.92 \mathrm{E}+02$ & $8.00 \mathrm{E}+07$ & ICRP-72 & $1.40 \mathrm{E}-11$ & $M$ & Max & FGR-12 & $6.09 \mathrm{E}-14$ \\
\hline
\end{tabular}




\begin{tabular}{|c|c|c|c|c|c|c|c|c|c|c|c|c|c|c|c|}
\hline $\begin{array}{l}\text { MASTER } \\
\text { ISOTOPE } \\
\text { LIST }\end{array}$ & $\begin{array}{c}\text { Atomic } \\
\text { Mass } \\
\text { Nuclide }\end{array}$ & $\begin{array}{c}\text { Atomic } \\
\text { Mass }\end{array}$ & Element & $\begin{array}{c}\text { RF per } \\
\text { DOE-STD- } \\
1027\end{array}$ & $\begin{array}{c}\text { Half-Life } \\
\text { Reference }\end{array}$ & $\begin{array}{r}\text { Half-Li } \\
\text { (original L }\end{array}$ & or th & Calculation of & $\begin{array}{l}\text { Sp. Act } \\
(\mathrm{Ci} / \mathrm{g})\end{array}$ & $\begin{array}{c}\text { Inhalation DC } \\
\text { Reference }\end{array}$ & $\begin{array}{c}\text { continued) } \\
\text { HC-2 } \\
\text { Inhalation } \\
\text { DC } \\
\text { (Sv/Bq) }\end{array}$ & & $\begin{array}{l}\text { ung } \\
\text { rption } \\
\text { /Type }\end{array}$ & $\begin{array}{l}\text { Immersion DC } \\
\text { Reference }\end{array}$ & $\begin{array}{c}\text { HC-2 } \\
\text { Immersion } \\
\mathrm{DC}(\mathrm{Sv} / \mathrm{s} \\
\left.\text { per } \mathrm{Bq} / \mathrm{m}^{3}\right)\end{array}$ \\
\hline Re-179 & Re-179 & 178.950 & Rhenium & $1.00 E-03$ & ICRP-107 & $1.95 \mathrm{E}+01$ & $\mathrm{~m}$ & $1.17 \mathrm{E}+03$ & $5.39 \mathrm{E}+07$ & DOE-STD-1196 & $1.32 \mathrm{E}-11$ & $\mathrm{~s}$ & Max & DOE-STD-1196 & 4.90E-14 \\
\hline Re-180 & Re-180 & 179.951 & Rhenium & $1.00 \mathrm{E}-03$ & ICRP-38 & $2.43 \mathrm{E}+00$ & $\mathrm{~m}$ & $1.46 \mathrm{E}+02$ & $4.30 \mathrm{E}+08$ & -- & -- & -- & -- & FGR-12 & $5.72 \mathrm{E}-14$ \\
\hline Re-181 & Re-181 & 180.950 & Rhenium & $1.00 \mathrm{E}-03$ & ICRP-38 & $2.00 \mathrm{E}+01$ & $\mathrm{~h}$ & $7.20 \mathrm{E}+04$ & $8.66 \mathrm{E}+05$ & ICRP-72 & $2.50 \mathrm{E}-10$ & $M$ & Max & FGR-12 & $3.65 \mathrm{E}-14$ \\
\hline Re-182I & Re-182 & 181.951 & Rhenium & $1.00 \mathrm{E}-03$ & ICRP-38 & $6.40 \mathrm{E}+01$ & $\mathrm{~h}$ & $2.30 \mathrm{E}+05$ & $2.69 \mathrm{E}+05$ & ICRP-72 & $1.20 \mathrm{E}-09$ & $M$ & Max & FGR-12 & $9.16 \mathrm{E}-14$ \\
\hline Re-182s & Re-182 & 181.951 & Rhenium & $1.00 \mathrm{E}-03$ & ICRP-38 & $1.27 \mathrm{E}+01$ & $\mathrm{~h}$ & $4.57 \mathrm{E}+04$ & $1.36 \mathrm{E}+06$ & ICRP-72 & $2.00 \mathrm{E}-10$ & $M$ & Max & FGR-12 & $5.78 \mathrm{E}-14$ \\
\hline Re-183 & Re-183 & 182.951 & Rhenium & $1.00 \mathrm{E}-03$ & ICRP-107 & $7.00 \mathrm{E}+01$ & $d$ & $6.05 \mathrm{E}+06$ & $1.02 \mathrm{E}+04$ & DOE-STD-1196 & $3.94 \mathrm{E}-09$ & $\mathrm{~s}$ & Max & DOE-STD-1196 & $5.53 \mathrm{E}-15$ \\
\hline Re-184 & Re-184 & 183.953 & Rhenium & $1.00 \mathrm{E}-03$ & ICRP-38 & $3.80 \mathrm{E}+01$ & $d$ & $3.28 \mathrm{E}+06$ & $1.87 \mathrm{E}+04$ & ICRP-72 & 1.90E-09 & $M$ & Max & FGR-12 & $4.29 \mathrm{E}-14$ \\
\hline Re- $184 \mathrm{~m}$ & Re-184 & 183.953 & Rhenium & $1.00 \mathrm{E}-03$ & ICRP-38 & $1.65 E+02$ & $d$ & $1.43 \mathrm{E}+07$ & $4.30 \mathrm{E}+03$ & ICRP-72 & $6.50 \mathrm{E}-09$ & $M$ & Max & FGR-12 & $1.82 \mathrm{E}-14$ \\
\hline Re-186 & Re-186 & 185.955 & Rhenium & $1.00 \mathrm{E}-03$ & ICRP-38 & $9.06 \mathrm{E}+01$ & $\mathrm{~h}$ & $3.26 \mathrm{E}+05$ & $1.86 \mathrm{E}+05$ & ICRP-72 & $1.10 \mathrm{E}-09$ & $M$ & Max & FGR-12 & 9.19E-16 \\
\hline Re-186m & Re-186 & 185.955 & Rhenium & $1.00 \mathrm{E}-03$ & ICRP-38 & $2.00 \mathrm{E}+05$ & $y$ & $6.31 \mathrm{E}+12$ & $9.61 \mathrm{E}-03$ & ICRP-72 & $1.20 \mathrm{E}-08$ & $M$ & Max & FGR-12 & $5.00 \mathrm{E}-16$ \\
\hline Re-187 & Re-187 & 186.956 & Rhenium & $1.00 \mathrm{E}-03$ & ICRP-38 & $5.00 \mathrm{E}+10$ & $y$ & $1.58 \mathrm{E}+18$ & $3.82 \mathrm{E}-08$ & ICRP-72 & $6.30 \mathrm{E}-12$ & $M$ & Max & -- & -- \\
\hline Re-188 & Re-188 & 187.958 & Rhenium & $1.00 \mathrm{E}-03$ & ICRP-38 & $1.70 \mathrm{E}+01$ & $\mathrm{~h}$ & $6.11 \mathrm{E}+04$ & $9.82 \mathrm{E}+05$ & ICRP-72 & $5.40 \mathrm{E}-10$ & $M$ & Max & FGR-12 & $2.87 \mathrm{E}-15$ \\
\hline Re-188m & Re-188 & 187.958 & Rhenium & $1.00 \mathrm{E}-03$ & ICRP-38 & $1.86 \mathrm{E}+01$ & $\mathrm{~m}$ & $1.12 \mathrm{E}+03$ & $5.38 \mathrm{E}+07$ & ICRP-72 & $1.30 \mathrm{E}-11$ & $M$ & Max & FGR-12 & $3.02 \mathrm{E}-15$ \\
\hline Re-189 & Re-189 & 188.959 & Rhenium & $1.00 \mathrm{E}-03$ & $\begin{array}{l}\text { ICRP-38 } \\
\end{array}$ & $2.43 \mathrm{E}+01$ & $\mathrm{~h}$ & $8.75 \mathrm{E}+04$ & $6.82 \mathrm{E}+05$ & ICRP-72 & $4.30 \mathrm{E}-10$ & $M$ & Max & FGR-12 & $3.22 \mathrm{E}-15$ \\
\hline Re-190 & Re-190 & 189.962 & Rhenium & $1.00 \mathrm{E}-03$ & ICRP-107 & $3.10 \mathrm{E}+00$ & $\mathrm{~m}$ & $1.86 \mathrm{E}+02$ & $3.19 \mathrm{E}+08$ & -- & -- & -- & -- & DOE-STD-1196 & $6.04 \mathrm{E}-14$ \\
\hline Re-190m & Re-190 & 189.962 & Rhenium & $1.00 \mathrm{E}-03$ & ICRP-107 & $3.20 \mathrm{E}+00$ & $\mathrm{~h}$ & $1.15 \mathrm{E}+04$ & $5.16 \mathrm{E}+06$ & DOE-STD-1196 & $2.44 \mathrm{E}-10$ & $\mathrm{~s}$ & Max & DOE-STD-1196 & 4.13E-14 \\
\hline Rh-100 & Rh-100 & 99.908 & Rhodium & $1.00 \mathrm{E}-03$ & ICRP-38 & $2.08 \mathrm{E}+01$ & $\mathrm{~h}$ & $7.49 \mathrm{E}+04$ & $1.51 \mathrm{E}+06$ & ICRP-72 & $3.50 \mathrm{E}-10$ & $\mathrm{~s}$ & Max & FGR-12 & $1.41 \mathrm{E}-13$ \\
\hline Rh-100m & Rh-100 & 99.908 & Rhodium & $1.00 \mathrm{E}-03$ & ICRP-107 & $4.60 \mathrm{E}+00$ & $\mathrm{~m}$ & $2.76 \mathrm{E}+02$ & $4.09 \mathrm{E}+08$ & -- & -- & -- & -- & DOE-STD-1196 & $2.03 \mathrm{E}-15$ \\
\hline Rh-101 & Rh-101 & 100.906 & Rhodium & $1.00 \mathrm{E}-03$ & $\begin{array}{l}\text { ICRP-38 } \\
\end{array}$ & $3.20 \mathrm{E}+00$ & $y$ & $1.01 \mathrm{E}+08$ & $1.11 \mathrm{E}+03$ & ICRP-72 & 5.40E-09 & $\mathrm{s}$ & Max & FGR-12 & $1.21 \mathrm{E}-14$ \\
\hline Rh-101m & Rh-101 & 100.906 & Rhodium & $1.00 \mathrm{E}-03$ & ICRP-38 & $4.34 \mathrm{E}+00$ & $d$ & $3.75 \mathrm{E}+05$ & $2.98 \mathrm{E}+05$ & ICRP-72 & $2.10 \mathrm{E}-10$ & $\mathrm{~s}$ & Max & FGR-12 & $1.41 \mathrm{E}-14$ \\
\hline Rh-102 & Rh-102 & 101.907 & Rhodium & $1.00 \mathrm{E}-03$ & ICRP-38 & $2.90 \mathrm{E}+00$ & $y$ & $9.15 \mathrm{E}+07$ & $1.21 \mathrm{E}+03$ & ICRP-72 & $1.70 \mathrm{E}-08$ & $\mathrm{~s}$ & Max & FGR-12 & $1.04 \mathrm{E}-13$ \\
\hline Rh-102m & Rh-102 & 101.907 & Rhodium & $1.00 \mathrm{E}-03$ & ICRP-38 & $2.07 E+02$ & $d$ & $1.79 \mathrm{E}+07$ & $6.19 \mathrm{E}+03$ & ICRP-72 & $7.10 \mathrm{E}-09$ & $\mathrm{~s}$ & Max & FGR-12 & $2.31 \mathrm{E}-14$ \\
\hline Rh-103m & Rh-103 & 102.905 & Rhodium & $1.00 \mathrm{E}-03$ & ICRP-38 & $5.61 \mathrm{E}+01$ & $\mathrm{~m}$ & $3.37 \mathrm{E}+03$ & $3.26 \mathrm{E}+07$ & ICRP-72 & $2.70 \mathrm{E}-12$ & $\mathrm{~s}$ & Max & FGR-12 & $8.80 \mathrm{E}-18$ \\
\hline Rh-104 & Rh-104 & 103.907 & Rhodium & $1.00 \mathrm{E}-03$ & ICRP-107 & $4.23 \mathrm{E}+01$ & $\mathrm{~s}$ & $4.23 \mathrm{E}+01$ & $2.57 \mathrm{E}+09$ & -- & -- & -- & -- & DOE-STD-1196 & $1.40 \mathrm{E}-15$ \\
\hline Rh-104m & Rh-104 & 103.907 & Rhodium & $1.00 \mathrm{E}-03$ & ICRP-107 & $4.34 \mathrm{E}+00$ & $\mathrm{~m}$ & $2.60 \mathrm{E}+02$ & $4.17 \mathrm{E}+08$ & -- & -- & -- & -- & DOE-STD-1196 & $9.14 \mathrm{E}-16$ \\
\hline Rh-105 & Rh-105 & 104.906 & Rhodium & $1.00 \mathrm{E}-03$ & ICRP-38 & $3.54 \mathrm{E}+01$ & $\mathrm{~h}$ & $1.27 E+05$ & $8.45 \mathrm{E}+05$ & ICRP-72 & $3.50 \mathrm{E}-10$ & $\mathrm{~s}$ & Max & FGR-12 & $3.72 \mathrm{E}-15$ \\
\hline Rh-106 & Rh-106 & 105.907 & Rhodium & $1.00 \mathrm{E}-03$ & ICRP-38 & $2.99 \mathrm{E}+01$ & $\mathrm{~s}$ & $2.99 \mathrm{E}+01$ & $3.56 \mathrm{E}+09$ & -- & -- & -- & -- & FGR-12 & $1.04 \mathrm{E}-14$ \\
\hline Rh-106m & Rh-106 & 105.907 & Rhodium & $1.00 \mathrm{E}-03$ & ICRP-38 & $1.32 \mathrm{E}+02$ & $\mathrm{~m}$ & $7.92 \mathrm{E}+03$ & $1.34 \mathrm{E}+07$ & ICRP-72 & $1.10 \mathrm{E}-10$ & $M$ & $\operatorname{Max}$ & FGR-12 & $1.44 \mathrm{E}-13$ \\
\hline Rh-107 & Rh-107 & 106.907 & Rhodium & $1.00 \mathrm{E}-03$ & ICRP-38 & $2.17 E+01$ & $\mathrm{~m}$ & $1.30 \mathrm{E}+03$ & $8.10 \mathrm{E}+07$ & ICRP-72 & $1.70 \mathrm{E}-11$ & $\mathrm{~s}$ & Max & FGR-12 & $1.50 \mathrm{E}-14$ \\
\hline Rh-108 & Rh-108 & 107.909 & Rhodium & $1.00 \mathrm{E}-03$ & ICRP-107 & $1.68 \mathrm{E}+01$ & $s$ & $1.68 \mathrm{E}+01$ & $6.22 \mathrm{E}+09$ & -- & -- & -- & -- & DOE-STD-1196 & $1.61 \mathrm{E}-14$ \\
\hline Rh-109 & Rh-109 & 108.909 & Rhodium & $1.00 \mathrm{E}-03$ & ICRP-107 & $8.00 \mathrm{E}+01$ & $\mathrm{~s}$ & $8.00 \mathrm{E}+01$ & $1.29 \mathrm{E}+09$ & -- & -- & -- & -- & DOE-STD-1196 & $1.38 \mathrm{E}-14$ \\
\hline Rh-94 & Rh-94 & 93.922 & Rhodium & $1.00 \mathrm{E}-03$ & $\begin{array}{ll}\text { ICRP-107 } \\
\end{array}$ & $7.06 \mathrm{E}+01$ & $\mathrm{~s}$ & $7.06 \mathrm{E}+01$ & $1.70 \mathrm{E}+09$ & -- & -- & -- & -- & DOE-STD-1196 & $1.81 \mathrm{E}-13$ \\
\hline Rh-95 & $\mathrm{Rh}-95$ & 94.916 & Rhodium & $1.00 \mathrm{E}-03$ & ICRP-107 & $5.02 \mathrm{E}+00$ & $\mathrm{~m}$ & $3.01 \mathrm{E}+02$ & $3.95 \mathrm{E}+08$ & -- & -- & -- & -- & DOE-STD-1196 & $1.21 \mathrm{E}-13$ \\
\hline Rh-95m & Rh-95 & 94.916 & Rhodium & $1.00 \mathrm{E}-03$ & ICRP-107 & $1.96 \mathrm{E}+00$ & $\mathrm{~m}$ & $1.18 \mathrm{E}+02$ & $1.01 \mathrm{E}+09$ & -- & -- & -- & -- & DOE-STD-1196 & $4.29 \mathrm{E}-14$ \\
\hline Rh-96 & Rh-96 & 95.914 & Rhodium & $1.00 \mathrm{E}-03$ & ICRP-107 & $9.90 \mathrm{E}+00$ & $\mathrm{~m}$ & $5.94 \mathrm{E}+02$ & $1.98 \mathrm{E}+08$ & -- & -- & -- & -- & DOE-STD-1196 & $1.81 \mathrm{E}-13$ \\
\hline Rh-96m & Rh-96 & 95.914 & Rhodium & $1.00 \mathrm{E}-03$ & ICRP-107 & $1.51 \mathrm{E}+00$ & $\mathrm{~m}$ & $9.06 \mathrm{E}+01$ & $1.30 \mathrm{E}+09$ & -- & -- & -- & -- & DOE-STD-1196 & $6.03 \mathrm{E}-14$ \\
\hline Rh-97 & Rh-97 & 96.911 & Rhodium & $1.00 \mathrm{E}-03$ & ICRP-107 & $3.07 E+01$ & $\mathrm{~m}$ & $1.84 \mathrm{E}+03$ & $6.32 \mathrm{E}+07$ & DOE-STD-1196 & $2.84 \mathrm{E}-11$ & $\mathrm{~s}$ & Max & DOE-STD-1196 & $6.60 \mathrm{E}-14$ \\
\hline Rh-97m & Rh-97 & 96.911 & Rhodium & $1.00 \mathrm{E}-03$ & ICRP-107 & $4.62 \mathrm{E}+01$ & $\mathrm{~m}$ & $2.77 \mathrm{E}+03$ & $4.20 \mathrm{E}+07$ & DOE-STD-1196 & $3.30 \mathrm{E}-11$ & $\mathrm{~s}$ & Max & DOE-STD-1196 & $1.06 \mathrm{E}-13$ \\
\hline Rh-98 & Rh-98 & 97.911 & Rhodium & $1.00 \mathrm{E}-03$ & ICRP-107 & $8.70 \mathrm{E}+00$ & $\mathrm{~m}$ & $5.22 \mathrm{E}+02$ & $2.21 \mathrm{E}+08$ & -- & -- & -- & -- & DOE-STD-1196 & $8.34 \mathrm{E}-14$ \\
\hline Rh-99 & Rh-99 & 98.908 & Rhodium & $1.00 \mathrm{E}-03$ & ICRP-38 & $1.60 \mathrm{E}+01$ & $d$ & $1.38 \mathrm{E}+06$ & $8.25 \mathrm{E}+04$ & ICRP-72 & $8.70 \mathrm{E}-10$ & $\mathrm{~s}$ & Max & FGR-12 & $2.85 \mathrm{E}-14$ \\
\hline Rh-99m & Rh-99 & 98.908 & Rhodium & $1.00 \mathrm{E}-03$ & ICRP-38 & $4.70 \mathrm{E}+00$ & $\mathrm{~h}$ & $1.69 \mathrm{E}+04$ & $6.74 \mathrm{E}+06$ & ICRP-72 & $4.00 \mathrm{E}-11$ & $\mathrm{~s}$ & Max & FGR-12 & $3.29 \mathrm{E}-14$ \\
\hline Rn-207 & Rn-207 & 206.991 & Radon & $1.00 \mathrm{E}+00$ & ICRP-107 & $9.25 \mathrm{E}+00$ & $\mathrm{~m}$ & $5.55 \mathrm{E}+02$ & $9.82 \mathrm{E}+07$ & -- & -- & -- & -- & DOE-STD-1196 & $4.41 \mathrm{E}-14$ \\
\hline Rn-209 & Rn-209 & 208.990 & Radon & $1.00 \mathrm{E}+00$ & ICRP-107 & $2.85 \mathrm{E}+01$ & $\mathrm{~m}$ & $1.71 \mathrm{E}+03$ & $3.16 \mathrm{E}+07$ & -- & -- & -- & -- & DOE-STD-1196 & $5.47 \mathrm{E}-14$ \\
\hline $\mathrm{Rn}-210$ & $\mathrm{Rn}-210$ & 209.990 & Radon & $1.00 \mathrm{E}+00$ & ICRP-107 & $2.40 \mathrm{E}+00$ & $\mathrm{~h}$ & $8.64 \mathrm{E}+03$ & $6.22 \mathrm{E}+06$ & -- & -- & -- & -- & DOE-STD-1196 & $1.27 \mathrm{E}-14$ \\
\hline Rn-211 & Rn-211 & 210.991 & Radon & $1.00 \mathrm{E}+00$ & ICRP-107 & $1.46 \mathrm{E}+01$ & $\mathrm{~h}$ & $5.26 \mathrm{E}+04$ & $1.02 \mathrm{E}+06$ & -- & -- & -- & -- & DOE-STD-1196 & $8.62 \mathrm{E}-14$ \\
\hline Rn-212 & Rn-212 & 211.991 & Radon & $1.00 \mathrm{E}+00$ & ICRP-107 & $2.39 \mathrm{E}+01$ & $\mathrm{~m}$ & $1.43 \mathrm{E}+03$ & $3.71 \mathrm{E}+07$ & -- & -- & -- & -- & DOE-STD-1196 & $1.38 \mathrm{E}-14$ \\
\hline
\end{tabular}




\begin{tabular}{|c|c|c|c|c|c|c|c|c|c|c|c|c|c|c|c|}
\hline $\begin{array}{l}\text { MASTER } \\
\text { ISOTOPE } \\
\text { LIST }\end{array}$ & $\begin{array}{c}\text { Atomic } \\
\text { Mass } \\
\text { Nuclide }\end{array}$ & $\begin{array}{l}\text { Atomic } \\
\text { Mass }\end{array}$ & Element & $\begin{array}{c}\text { RF per } \\
\text { DOE-STD- } \\
1027\end{array}$ & $\begin{array}{c}\text { Half-Life } \\
\text { Reference }\end{array}$ & $\begin{array}{r}\text { Half-Li } \\
\text { (original L }\end{array}$ & or th & Half Life (s) & $\begin{array}{l}\text { Sp. Act } \\
(\mathrm{Ci} / \mathrm{g})\end{array}$ & $\begin{array}{l}\text { Inhalation DC } \\
\text { Reference }\end{array}$ & $\begin{array}{c}\text { (continued) } \\
\text { HC-2 } \\
\text { Inhalation } \\
\text { DC } \\
\text { (Sv/Bq) }\end{array}$ & & $\begin{array}{l}\text { ng } \\
\text { ption } \\
\text { Type }\end{array}$ & $\begin{array}{l}\text { Immersion DC } \\
\text { Reference }\end{array}$ & $\begin{array}{c}\text { HC-2 } \\
\text { Immersion } \\
\mathrm{DC}(\mathrm{Sv} / \mathrm{s} \\
\left.\text { per } \mathrm{Bq} / \mathrm{m}^{3}\right)\end{array}$ \\
\hline Rn-215 & $\mathrm{Rn}-215$ & 214.999 & Radon & $1.00 \mathrm{E}+00$ & ICRP-107 & $2.30 \mathrm{E}+00$ & us & $2.30 \mathrm{E}-06$ & $2.28 \mathrm{E}+16$ & -- & -- & -- & -- & DOE-STD-1196 & $1.90 \mathrm{E}-14$ \\
\hline Rn-216 & Rn-216 & 216.000 & Radon & $1.00 \mathrm{E}+00$ & ICRP-107 & $4.50 \mathrm{E}-05$ & $\mathrm{~s}$ & $4.50 \mathrm{E}-05$ & $1.16 \mathrm{E}+15$ & -- & -- & -- & -- & DOE-STD-1196 & $1.77 \mathrm{E}-14$ \\
\hline Rn-217 & $\mathrm{Rn}-217$ & 217.004 & Radon & $1.00 \mathrm{E}+00$ & ICRP-107 & $5.40 \mathrm{E}-04$ & $\mathrm{~s}$ & $5.40 \mathrm{E}-04$ & $9.63 \mathrm{E}+13$ & -- & -- & -- & -- & DOE-STD-1196 & $1.70 \mathrm{E}-14$ \\
\hline Rn-218 & $\mathrm{Rn}-218$ & 218.006 & Radon & $1.00 \mathrm{E}+00$ & ICRP-38 & $3.50 \mathrm{E}+01$ & $\mathrm{~ms}$ & $3.50 \mathrm{E}-02$ & $1.48 \mathrm{E}+12$ & -- & -- & -- & -- & FGR-12 & $3.65 \mathrm{E}-17$ \\
\hline Rn-219 & Rn-219 & 219.009 & Radon & $1.00 \mathrm{E}+00$ & ICRP-38 & $3.96 \mathrm{E}+00$ & $\mathrm{~s}$ & $3.96 \mathrm{E}+00$ & $1.30 \mathrm{E}+10$ & -- & -- & -- & -- & FGR-12 & $2.68 \mathrm{E}-15$ \\
\hline Rn-220 & $\mathrm{Rn}-220$ & 220.011 & Radon & $1.00 \mathrm{E}+00$ & ICRP-38 & $5.56 \mathrm{E}+01$ & $\mathrm{~s}$ & $5.56 \mathrm{E}+01$ & $9.22 \mathrm{E}+08$ & -- & -- & -- & -- & FGR-12 & $1.85 \mathrm{E}-17$ \\
\hline Rn-222 & $\mathrm{Rn}-222$ & 222.018 & Radon & $1.00 \mathrm{E}+00$ & ICRP-38 & $3.82 \mathrm{E}+00$ & $d$ & $3.30 \mathrm{E}+05$ & $1.54 \mathrm{E}+05$ & -- & -- & -- & -- & FGR-12 & $1.91 \mathrm{E}-17$ \\
\hline $\mathrm{Rn}-223$ & $\mathrm{Rn}-223$ & 223.022 & Radon & $1.00 E+00$ & ICRP-107 & $2.43 E+01$ & $\mathrm{~m}$ & $1.46 \mathrm{E}+03$ & $3.47 \mathrm{E}+07$ & -- & -- & -- & -- & DOE-STD-1196 & $1.56 \mathrm{E}-14$ \\
\hline Ru-103 & Ru-103 & 102.906 & Ruthenium & $1.00 \mathrm{E}-02$ & $\begin{array}{l}\text { ICRP-38 } \\
\end{array}$ & $3.93 \mathrm{E}+01$ & $d$ & $3.39 \mathrm{E}+06$ & $3.23 \mathrm{E}+04$ & ICRP-72 & $2.40 \mathrm{E}-09$ & $M$ & $\operatorname{Rec}$ & FGR-12 & $2.25 \mathrm{E}-14$ \\
\hline Ru-105 & Ru-105 & 104.908 & Ruthenium & $1.00 \mathrm{E}-02$ & ICRP-38 & $4.44 \mathrm{E}+00$ & $\mathrm{~h}$ & $1.60 \mathrm{E}+04$ & $6.73 \mathrm{E}+06$ & ICRP-72 & $1.70 \mathrm{E}-10$ & $M$ & $\operatorname{Rec}$ & FGR-12 & $3.81 \mathrm{E}-14$ \\
\hline Ru-106 & Ru-106 & 105.907 & Ruthenium & $1.00 \mathrm{E}-02$ & ICRP-38 & $3.68 \mathrm{E}+02$ & $d$ & $3.18 \mathrm{E}+07$ & $3.35 \mathrm{E}+03$ & ICRP-72 & $2.80 \mathrm{E}-08$ & $M$ & $\operatorname{Rec}$ & -- & -- \\
\hline Ru-107 & Ru-107 & 106.910 & Ruthenium & $1.00 \mathrm{E}-02$ & ICRP-107 & $3.75 \mathrm{E}+00$ & $\mathrm{~m}$ & $2.25 \mathrm{E}+02$ & $4.69 \mathrm{E}+08$ & -- & -- & -- & -- & DOE-STD-1196 & $1.69 \mathrm{E}-14$ \\
\hline Ru-108 & Ru-108 & 107.910 & Ruthenium & $1.00 \mathrm{E}-02$ & ICRP-107 & $4.55 \mathrm{E}+00$ & $\mathrm{~m}$ & $2.73 E+02$ & $3.83 \mathrm{E}+08$ & -- & -- & -- & -- & DOE-STD-1196 & $2.92 \mathrm{E}-15$ \\
\hline Ru-92 & Ru-92 & 91.920 & Ruthenium & $1.00 \mathrm{E}-02$ & ICRP-107 & $3.65 \mathrm{E}+00$ & $\mathrm{~m}$ & $2.19 \mathrm{E}+02$ & $5.60 \mathrm{E}+08$ & -- & -- & -- & -- & DOE-STD-1196 & $9.44 \mathrm{E}-14$ \\
\hline Ru-94 & Ru-94 & 93.911 & Ruthenium & $1.00 \mathrm{E}-02$ & ICRP-38 & $5.18 \mathrm{E}+01$ & $\mathrm{~m}$ & $3.11 \mathrm{E}+03$ & $3.87 \mathrm{E}+07$ & ICRP-72 & $4.20 \mathrm{E}-11$ & $M$ & $\operatorname{Rec}$ & FGR-12 & $2.54 \mathrm{E}-14$ \\
\hline Ru-95 & Ru-95 & 94.910 & Ruthenium & $1.00 \mathrm{E}-02$ & ICRP-107 & $1.64 \mathrm{E}+00$ & $\mathrm{~h}$ & $5.91 \mathrm{E}+03$ & $2.01 \mathrm{E}+07$ & DOE-STD-1196 & $4.29 \mathrm{E}-11$ & $M$ & $\operatorname{Rec}$ & DOE-STD-1196 & $5.68 \mathrm{E}-14$ \\
\hline Ru-97 & Ru-97 & 96.908 & Ruthenium & $1.00 \mathrm{E}-02$ & ICRP-38 & $2.90 \mathrm{E}+00$ & $d$ & $2.51 \mathrm{E}+05$ & $4.65 \mathrm{E}+05$ & ICRP-72 & $1.00 \mathrm{E}-10$ & $M$ & $\operatorname{Rec}$ & FGR-12 & $1.09 \mathrm{E}-14$ \\
\hline S-35 & S-35 & 34.969 & Sulfur & $5.00 \mathrm{E}-01$ & ICRP-38 & $8.74 \mathrm{E}+01$ & d & $7.55 \mathrm{E}+06$ & $4.27 \mathrm{E}+04$ & ICRP-72 & $1.40 \mathrm{E}-09$ & $M$ & $\operatorname{Rec}$ & FGR-12 & $2.43 \mathrm{E}-19$ \\
\hline S-37 & S-37 & 36.971 & Sulfur & $5.00 \mathrm{E}-01$ & ICRP-107 & $5.05 E+00$ & $\mathrm{~m}$ & $3.03 \mathrm{E}+02$ & $1.01 \mathrm{E}+09$ & -- & -- & -- & -- & DOE-STD-1196 & $1.54 \mathrm{E}-13$ \\
\hline S-38 & $\mathrm{S}-38$ & 37.971 & Sulfur & $5.00 \mathrm{E}-01$ & ICRP-107 & $1.70 \mathrm{E}+02$ & $\mathrm{~m}$ & $1.02 \mathrm{E}+04$ & $2.91 \mathrm{E}+07$ & DOE-STD-1196 & $3.33 \mathrm{E}-10$ & $M$ & $\operatorname{Rec}$ & DOE-STD-1196 & $8.47 \mathrm{E}-14$ \\
\hline Sb-111 & Sb-111 & 110.913 & Antimony & $1.00 \mathrm{E}-03$ & ICRP-107 & $7.50 \mathrm{E}+01$ & $\mathrm{~s}$ & $7.50 \mathrm{E}+01$ & $1.36 \mathrm{E}+09$ & -- & -- & -- & -- & DOE-STD-1196 & $6.76 \mathrm{E}-14$ \\
\hline Sb-113 & Sb-113 & 112.909 & Antimony & $1.00 \mathrm{E}-03$ & ICRP-107 & $6.67 \mathrm{E}+00$ & $\mathrm{~m}$ & $4.00 \mathrm{E}+02$ & $2.50 \mathrm{E}+08$ & -- & -- & -- & -- & DOE-STD-1196 & $5.71 \mathrm{E}-14$ \\
\hline Sb-114 & Sb-114 & 113.909 & Antimony & $1.00 \mathrm{E}-03$ & ICRP-107 & $3.49 \mathrm{E}+00$ & $\mathrm{~m}$ & $2.09 E+02$ & $4.73 \mathrm{E}+08$ & -- & -- & -- & -- & DOE-STD-1196 & $1.27 \mathrm{E}-13$ \\
\hline Sb-115 & Sb-115 & 114.907 & Antimony & $1.00 \mathrm{E}-03$ & ICRP-38 & $3.18 \mathrm{E}+01$ & $\mathrm{~m}$ & $1.91 \mathrm{E}+03$ & $5.15 \mathrm{E}+07$ & ICRP-72 & $1.30 \mathrm{E}-11$ & $M$ & $\operatorname{Rec}$ & FGR-12 & $4.32 \mathrm{E}-14$ \\
\hline Sb-116 & Sb-116 & 115.907 & Antimony & $1.00 \mathrm{E}-03$ & ICRP-38 & $1.58 \mathrm{E}+01$ & $\mathrm{~m}$ & $9.48 \mathrm{E}+02$ & $1.03 \mathrm{E}+08$ & ICRP-72 & $1.30 \mathrm{E}-11$ & $M$ & $\operatorname{Rec}$ & FGR-12 & $1.08 \mathrm{E}-13$ \\
\hline Sb-116m & Sb-116 & 115.907 & Antimony & $1.00 \mathrm{E}-03$ & ICRP-38 & $6.03 \mathrm{E}+01$ & $\mathrm{~m}$ & $3.62 \mathrm{E}+03$ & $2.69 \mathrm{E}+07$ & ICRP-72 & $4.70 \mathrm{E}-11$ & $M$ & $\operatorname{Rec}$ & FGR-12 & $1.55 \mathrm{E}-13$ \\
\hline Sb-117 & Sb-117 & 116.905 & Antimony & $1.00 \mathrm{E}-03$ & ICRP-38 & $2.80 \mathrm{E}+00$ & $\mathrm{~h}$ & $1.01 \mathrm{E}+04$ & $9.57 \mathrm{E}+06$ & ICRP-72 & $1.60 \mathrm{E}-11$ & $M$ & $\operatorname{Rec}$ & FGR-12 & $7.97 \mathrm{E}-15$ \\
\hline Sb-118 & Sb-118 & 117.906 & Antimony & $1.00 \mathrm{E}-03$ & ICRP-38 & $3.60 \mathrm{E}+00$ & $\mathrm{~m}$ & $2.16 \mathrm{E}+02$ & $4.43 \mathrm{E}+08$ & -- & -- & -- & -- & DOE-STD-1196 & $3.65 \mathrm{E}-14$ \\
\hline Sb-118m & Sb-118 & 117.906 & Antimony & $1.00 \mathrm{E}-03$ & ICRP-38 & $5.00 \mathrm{E}+00$ & $\mathrm{~h}$ & $1.80 \mathrm{E}+04$ & $5.32 \mathrm{E}+06$ & ICRP-72 & $1.20 \mathrm{E}-10$ & $M$ & $\operatorname{Rec}$ & FGR-12 & $1.27 \mathrm{E}-13$ \\
\hline Sb-119 & Sb-119 & 118.904 & Antimony & $1.00 \mathrm{E}-03$ & ICRP-38 & $3.81 \mathrm{E}+01$ & $\mathrm{~h}$ & $1.37 \mathrm{E}+05$ & $6.92 \mathrm{E}+05$ & ICRP-72 & $3.50 \mathrm{E}-11$ & $M$ & $\operatorname{Rec}$ & FGR-12 & $2.16 \mathrm{E}-16$ \\
\hline Sb-120s & Sb-120 & 119.905 & Antimony & $1.00 \mathrm{E}-03$ & ICRP-38 & $1.59 \mathrm{E}+01$ & $\mathrm{~m}$ & $9.53 \mathrm{E}+02$ & $9.87 \mathrm{E}+07$ & ICRP-72 & $7.00 \mathrm{E}-12$ & $M$ & $\operatorname{Rec}$ & FGR-12 & $2.13 \mathrm{E}-14$ \\
\hline Sb-120l & Sb-120 & 119.905 & Antimony & $1.00 \mathrm{E}-03$ & ICRP-38 & $5.76 \mathrm{E}+00$ & $d$ & $4.98 \mathrm{E}+05$ & $1.89 \mathrm{E}+05$ & ICRP-72 & $1.00 \mathrm{E}-09$ & $M$ & $\operatorname{Rec}$ & FGR-12 & $1.22 \mathrm{E}-13$ \\
\hline Sb-122 & Sb-122 & 121.905 & Antimony & $1.00 \mathrm{E}-03$ & ICRP-38 & $2.70 \mathrm{E}+00$ & d & $2.33 \mathrm{E}+05$ & $3.97 \mathrm{E}+05$ & ICRP-72 & $1.00 \mathrm{E}-09$ & $M$ & Rec & FGR-12 & $2.13 \mathrm{E}-14$ \\
\hline Sb-122m & Sb-122 & 121.905 & Antimony & $1.00 \mathrm{E}-03$ & ICRP-107 & $4.19 \mathrm{E}+00$ & $\mathrm{~m}$ & $2.51 \mathrm{E}+02$ & $3.68 \mathrm{E}+08$ & -- & -- & -- & -- & DOE-STD-1196 & $1.77 \mathrm{E}-15$ \\
\hline Sb-124 & Sb-124 & 123.906 & Antimony & $1.00 \mathrm{E}-03$ & ICRP-38 & $6.02 E+01$ & d & $5.20 \mathrm{E}+06$ & $1.75 \mathrm{E}+04$ & ICRP-72 & $6.40 \mathrm{E}-09$ & $M$ & $\operatorname{Rec}$ & FGR-12 & $9.15 \mathrm{E}-14$ \\
\hline Sb-124ms & Sb-124 & 123.906 & Antimony & $1.00 \mathrm{E}-03$ & ICRP-38 & $9.30 \mathrm{E}+01$ & $\mathrm{~s}$ & $9.30 \mathrm{E}+01$ & $9.79 \mathrm{E}+08$ & -- & -- & -- & -- & FGR-12 & $1.70 \mathrm{E}-14$ \\
\hline Sb-124ml & Sb-124 & 123.906 & Antimony & $1.00 \mathrm{E}-03$ & ICRP-38 & $2.02 \mathrm{E}+01$ & $\mathrm{~m}$ & $1.21 \mathrm{E}+03$ & $7.51 \mathrm{E}+07$ & ICRP-72 & $5.40 \mathrm{E}-12$ & $M$ & $\operatorname{Rec}$ & FGR-12 & $6.75 \mathrm{E}-19$ \\
\hline Sb-125 & Sb-125 & 124.905 & Antimony & $1.00 \mathrm{E}-03$ & ICRP-38 & $2.77 \mathrm{E}+00$ & $y$ & $8.74 \mathrm{E}+07$ & $1.03 \mathrm{E}+03$ & ICRP-72 & $4.80 \mathrm{E}-09$ & $M$ & $\operatorname{Rec}$ & FGR-12 & $2.02 \mathrm{E}-14$ \\
\hline Sb-126 & Sb-126 & 125.907 & Antimony & $1.00 \mathrm{E}-03$ & ICRP-38 & $1.24 \mathrm{E}+01$ & d & $1.07 \mathrm{E}+06$ & $8.36 \mathrm{E}+04$ & ICRP-72 & $2.80 \mathrm{E}-09$ & $M$ & $\operatorname{Rec}$ & FGR-12 & $1.37 \mathrm{E}-13$ \\
\hline Sb-126m & Sb-126 & 125.907 & Antimony & $1.00 \mathrm{E}-03$ & ICRP-38 & $1.90 \mathrm{E}+01$ & $\mathrm{~m}$ & $1.14 \mathrm{E}+03$ & $7.86 \mathrm{E}+07$ & ICRP-72 & $1.90 \mathrm{E}-11$ & $M$ & $\operatorname{Rec}$ & FGR-12 & $7.50 \mathrm{E}-14$ \\
\hline Sb-127 & Sb-127 & 126.907 & Antimony & $1.00 \mathrm{E}-03$ & ICRP-38 & $3.85 \mathrm{E}+00$ & $d$ & $3.33 \mathrm{E}+05$ & $2.67 \mathrm{E}+05$ & ICRP-72 & $1.70 \mathrm{E}-09$ & $M$ & $\operatorname{Rec}$ & FGR-12 & $3.33 \mathrm{E}-14$ \\
\hline Sb-128l & Sb-128 & 127.909 & Antimony & $1.00 \mathrm{E}-03$ & ICRP-38 & $9.01 \mathrm{E}+00$ & $\mathrm{~h}$ & $3.24 \mathrm{E}+04$ & $2.72 \mathrm{E}+06$ & ICRP-72 & $4.00 \mathrm{E}-10$ & $M$ & $\operatorname{Rec}$ & FGR-12 & $1.51 \mathrm{E}-13$ \\
\hline Sb-128s & Sb-128 & 127.909 & Antimony & $1.00 \mathrm{E}-03$ & ICRP-38 & $1.04 \mathrm{E}+01$ & $\mathrm{~m}$ & $6.24 E+02$ & $1.41 \mathrm{E}+08$ & ICRP-72 & $1.40 \mathrm{E}-11$ & $M$ & $\operatorname{Rec}$ & FGR-12 & $9.69 \mathrm{E}-14$ \\
\hline Sb-129 & Sb-129 & 128.909 & Antimony & $1.00 \mathrm{E}-03$ & ICRP-38 & $4.32 \mathrm{E}+00$ & $\mathrm{~h}$ & $1.56 \mathrm{E}+04$ & $5.63 \mathrm{E}+06$ & ICRP-72 & $2.30 \mathrm{E}-10$ & $M$ & Rec & FGR-12 & $7.14 \mathrm{E}-14$ \\
\hline Sb-130 & Sb-130 & 129.912 & Antimony & $1.00 \mathrm{E}-03$ & ICRP-38 & $4.00 \mathrm{E}+01$ & $\mathrm{~m}$ & $2.40 \mathrm{E}+03$ & $3.62 \mathrm{E}+07$ & ICRP-72 & $5.10 \mathrm{E}-11$ & $M$ & $\operatorname{Rec}$ & FGR-12 & $1.60 \mathrm{E}-13$ \\
\hline Sb-130m & Sb-130 & 129.912 & Antimony & $1.00 \mathrm{E}-03$ & ICRP-107 & $6.30 \mathrm{E}+00$ & $\mathrm{~m}$ & $3.78 \mathrm{E}+02$ & $2.30 \mathrm{E}+08$ & -- & -- & -- & -- & DOE-STD-1196 & $1.26 \mathrm{E}-13$ \\
\hline
\end{tabular}




\begin{tabular}{|c|c|c|c|c|c|c|c|c|c|c|c|c|c|c|c|}
\hline $\begin{array}{l}\text { MASTER } \\
\text { ISOTOPE } \\
\text { LIST }\end{array}$ & $\begin{array}{l}\text { Atomic } \\
\text { Mass } \\
\text { Nuclide }\end{array}$ & $\begin{array}{l}\text { Atomic } \\
\text { Mass }\end{array}$ & Element & $\begin{array}{c}\text { RF per } \\
\text { DOE-STD- } \\
1027\end{array}$ & $\begin{array}{c}\text { Half-Life } \\
\text { Reference }\end{array}$ & $\begin{array}{r}\text { Half-Li } \\
\text { (original L }\end{array}$ & or th & Half Life (s) & $\begin{array}{l}\text { Sp. Act } \\
(\mathrm{Ci} / \mathrm{g})\end{array}$ & $\begin{array}{l}\text { Inhalation DC } \\
\text { Reference }\end{array}$ & $\begin{array}{c}\text { continued) } \\
\text { HC-2 } \\
\text { Inhalation } \\
\text { DC } \\
\text { (Sv/Bq) }\end{array}$ & & $\begin{array}{l}\text { ung } \\
\text { rption } \\
\text { /Type }\end{array}$ & $\begin{array}{l}\text { Immersion DC } \\
\text { Reference }\end{array}$ & $\begin{array}{c}\text { HC-2 } \\
\text { Immersion } \\
\mathrm{DC}(\mathrm{Sv} / \mathrm{s} \\
\left.\text { per } \mathrm{Bq} / \mathrm{m}^{3}\right)\end{array}$ \\
\hline Sb-131 & Sb-131 & 130.912 & Antimony & $1.00 \mathrm{E}-03$ & ICRP-38 & $2.30 \mathrm{E}+01$ & $\mathrm{~m}$ & $1.38 \mathrm{E}+03$ & $6.24 \mathrm{E}+07$ & ICRP-72 & $4.40 \mathrm{E}-11$ & $M$ & $\operatorname{Rec}$ & FGR-12 & 9.37E-14 \\
\hline Sb-133 & Sb-133 & 132.915 & Antimony & $1.00 \mathrm{E}-03$ & ICRP-107 & $2.50 \mathrm{E}+00$ & $\mathrm{~m}$ & $1.50 \mathrm{E}+02$ & $5.66 \mathrm{E}+08$ & -- & -- & -- & -- & DOE-STD-1196 & $1.33 \mathrm{E}-13$ \\
\hline Sc $-42 \mathrm{~m}$ & Sc-42 & 41.966 & Scandium & $1.00 \mathrm{E}-03$ & ICRP-107 & $6.20 \mathrm{E}+01$ & $\mathrm{~s}$ & $6.20 \mathrm{E}+01$ & $4.34 \mathrm{E}+09$ & -- & -- & -- & -- & DOE-STD-1196 & $1.98 \mathrm{E}-13$ \\
\hline Sc-43 & Sc-43 & 42.961 & Scandium & $1.00 \mathrm{E}-03$ & ICRP-38 & $3.89 \mathrm{E}+00$ & $\mathrm{~h}$ & $1.40 \mathrm{E}+04$ & $1.87 \mathrm{E}+07$ & ICRP-72 & $1.10 \mathrm{E}-10$ & $\mathrm{~s}$ & Max & FGR-12 & $5.26 \mathrm{E}-14$ \\
\hline Sc-44 & Sc-44 & 43.959 & Scandium & $1.00 \mathrm{E}-03$ & ICRP-38 & $3.93 \mathrm{E}+00$ & $\mathrm{~h}$ & $1.41 \mathrm{E}+04$ & $1.82 \mathrm{E}+07$ & ICRP-72 & $1.80 \mathrm{E}-10$ & $\mathrm{~s}$ & Max & FGR-12 & $1.05 \mathrm{E}-13$ \\
\hline Sc- $44 m$ & Sc-44 & 43.959 & Scandium & $1.00 \mathrm{E}-03$ & ICRP-38 & $5.86 \mathrm{E}+01$ & $\mathrm{~h}$ & $2.11 \mathrm{E}+05$ & $1.22 \mathrm{E}+06$ & ICRP-72 & $1.40 \mathrm{E}-09$ & $\mathrm{~s}$ & Max & FGR-12 & $1.35 \mathrm{E}-14$ \\
\hline Sc-46 & Sc-46 & 45.955 & Scandium & $1.00 \mathrm{E}-03$ & ICRP-38 & $8.38 \mathrm{E}+01$ & $d$ & $7.24 \mathrm{E}+06$ & $3.39 \mathrm{E}+04$ & ICRP-72 & $6.80 \mathrm{E}-09$ & $\mathrm{~s}$ & Max & FGR-12 & $9.98 \mathrm{E}-14$ \\
\hline Sc-47 & Sc-47 & 46.952 & Scandium & $1.00 \mathrm{E}-03$ & ICRP-38 & $3.35 E+00$ & $d$ & $2.90 E+05$ & $8.30 \mathrm{E}+05$ & ICRP-72 & $7.30 \mathrm{E}-10$ & $\mathrm{~s}$ & Max & FGR-12 & $5.14 \mathrm{E}-15$ \\
\hline Sc-48 & Sc-48 & 47.952 & Scandium & $1.00 \mathrm{E}-03$ & ICRP-38 & $4.37 \mathrm{E}+01$ & $\mathrm{~h}$ & $1.57 E+05$ & $1.50 \mathrm{E}+06$ & ICRP-72 & $1.10 \mathrm{E}-09$ & $\mathrm{~s}$ & Max & FGR-12 & $1.68 \mathrm{E}-13$ \\
\hline Sc-49 & Sc-49 & 48.950 & Scandium & $1.00 \mathrm{E}-03$ & ICRP-38 & $5.74 \mathrm{E}+01$ & $\mathrm{~m}$ & $3.44 \mathrm{E}+03$ & $6.69 \mathrm{E}+07$ & ICRP-72 & $4.00 E-11$ & $\mathrm{~s}$ & Max & FGR-12 & $1.93 \mathrm{E}-16$ \\
\hline Sc-50 & Sc-50 & 49.952 & Scandium & $1.00 \mathrm{E}-03$ & ICRP-107 & $1.03 \mathrm{E}+02$ & $\mathrm{~s}$ & $1.03 \mathrm{E}+02$ & $2.20 \mathrm{E}+09$ & -- & -- & -- & -- & DOE-STD-1196 & $1.54 \mathrm{E}-13$ \\
\hline Se-70 & Se-70 & 69.934 & Selenium & $1.00 \mathrm{E}-02$ & ICRP-38 & $4.10 \mathrm{E}+01$ & $\mathrm{~m}$ & $2.46 \mathrm{E}+03$ & $6.56 \mathrm{E}+07$ & ICRP-72 & $4.20 \mathrm{E}-11$ & $F$ & $\operatorname{Rec}$ & FGR-12 & $4.73 \mathrm{E}-14$ \\
\hline Se-71 & Se-71 & 70.932 & Selenium & $1.00 \mathrm{E}-02$ & ICRP-107 & $4.74 \mathrm{E}+00$ & $\mathrm{~m}$ & $2.84 \mathrm{E}+02$ & $5.59 \mathrm{E}+08$ & -- & -- & -- & -- & DOE-STD-1196 & $7.40 \mathrm{E}-14$ \\
\hline Se-72 & Se-72 & 71.927 & Selenium & $1.00 \mathrm{E}-02$ & ICRP-38 & $8.40 \mathrm{E}+00$ & $d$ & $7.26 \mathrm{E}+05$ & $2.16 \mathrm{E}+05$ & DOE-STD-1196 & $2.84 \mathrm{E}-09$ & $\mathrm{~F}$ & Rec & DOE-STD-1196 & $6.10 \mathrm{E}-16$ \\
\hline Se-73 & Se-73 & 72.927 & Selenium & $1.00 \mathrm{E}-02$ & ICRP-38 & $7.15 \mathrm{E}+00$ & $\mathrm{~h}$ & $2.57 \mathrm{E}+04$ & $6.01 \mathrm{E}+06$ & ICRP-72 & $8.00 \mathrm{E}-11$ & $\mathrm{~F}$ & $\operatorname{Rec}$ & FGR-12 & $5.16 \mathrm{E}-14$ \\
\hline Se-73m & Se-73 & 72.927 & Selenium & $1.00 \mathrm{E}-02$ & ICRP-38 & $3.90 \mathrm{E}+01$ & $\mathrm{~m}$ & $2.34 \mathrm{E}+03$ & $6.61 \mathrm{E}+07$ & ICRP-72 & $9.20 \mathrm{E}-12$ & $F$ & $\operatorname{Rec}$ & FGR-12 & $1.17 \mathrm{E}-14$ \\
\hline Se-75 & Se-75 & 74.923 & Selenium & $1.00 \mathrm{E}-02$ & ICRP-38 & $1.20 \mathrm{E}+02$ & $d$ & $1.04 \mathrm{E}+07$ & $1.45 \mathrm{E}+04$ & ICRP-72 & $1.00 \mathrm{E}-09$ & $F$ & $\operatorname{Rec}$ & FGR-12 & $1.85 \mathrm{E}-14$ \\
\hline Se-77m & Se-77 & 76.920 & Selenium & $1.00 \mathrm{E}-02$ & ICRP-38 & $1.75 \mathrm{E}+01$ & $\mathrm{~s}$ & $1.75 \mathrm{E}+01$ & $8.40 \mathrm{E}+09$ & -- & -- & -- & -- & FGR-12 & $4.03 \mathrm{E}-15$ \\
\hline Se-79 & Se-79 & 78.918 & Selenium & $1.00 \mathrm{E}-02$ & ICRP-38 & $6.50 \mathrm{E}+04$ & $y$ & $2.05 \mathrm{E}+12$ & $6.97 \mathrm{E}-02$ & ICRP-72 & $1.10 \mathrm{E}-09$ & $\mathrm{~F}$ & $\operatorname{Rec}$ & FGR-12 & $3.03 \mathrm{E}-19$ \\
\hline Se-79m & Se-79 & 78.918 & Selenium & $1.00 \mathrm{E}-02$ & ICRP-107 & $3.92 \mathrm{E}+00$ & $\mathrm{~m}$ & $2.35 \mathrm{E}+02$ & $6.08 \mathrm{E}+08$ & -- & -- & -- & -- & DOE-STD-1196 & $3.60 \mathrm{E}-16$ \\
\hline Se-81 & Se-81 & 80.918 & Selenium & $1.00 \mathrm{E}-02$ & ICRP-38 & $1.85 \mathrm{E}+01$ & $\mathrm{~m}$ & $1.11 \mathrm{E}+03$ & $1.26 \mathrm{E}+08$ & ICRP-72 & $8.00 \mathrm{E}-12$ & $\mathrm{~F}$ & $\operatorname{Rec}$ & FGR-12 & $5.24 \mathrm{E}-16$ \\
\hline Se-81m & Se-81 & 80.918 & Selenium & $1.00 \mathrm{E}-02$ & ICRP-38 & $5.73 \mathrm{E}+01$ & $\mathrm{~m}$ & $3.44 \mathrm{E}+03$ & $4.06 \mathrm{E}+07$ & ICRP-72 & $1.60 \mathrm{E}-11$ & $\mathrm{~F}$ & $\operatorname{Rec}$ & FGR-12 & $6.18 \mathrm{E}-16$ \\
\hline Se-83 & Se-83 & 82.919 & Selenium & $1.00 \mathrm{E}-02$ & ICRP-38 & $2.25 \mathrm{E}+01$ & $\mathrm{~m}$ & $1.35 \mathrm{E}+03$ & $1.01 \mathrm{E}+08$ & ICRP-72 & $1.80 \mathrm{E}-11$ & $\mathrm{~F}$ & $\operatorname{Rec}$ & FGR-12 & $1.21 \mathrm{E}-13$ \\
\hline Se-83m & Se-83 & 82.919 & Selenium & $1.00 \mathrm{E}-02$ & ICRP-107 & $7.01 \mathrm{E}+01$ & $\mathrm{~s}$ & $7.01 \mathrm{E}+01$ & $1.94 \mathrm{E}+09$ & -- & -- & -- & -- & DOE-STD-1196 & $4.78 \mathrm{E}-14$ \\
\hline Se-84 & Se-84 & 83.918 & Selenium & $1.00 \mathrm{E}-02$ & ICRP-107 & $3.10 \mathrm{E}+00$ & $\mathrm{~m}$ & $1.86 \mathrm{E}+02$ & $7.23 \mathrm{E}+08$ & -- & -- & -- & -- & DOE-STD-1196 & $1.90 \mathrm{E}-14$ \\
\hline Si-31 & Si-31 & 30.975 & Silicon & $1.00 \mathrm{E}-03$ & ICRP-38 & $1.57 \mathrm{E}+02$ & $\mathrm{~m}$ & $9.44 E+03$ & $3.86 \mathrm{E}+07$ & ICRP-72 & $7.90 \mathrm{E}-11$ & $\mathrm{~s}$ & Max & FGR-12 & $1.17 \mathrm{E}-16$ \\
\hline Si-32 & $\mathrm{Si}-32$ & 31.974 & Silicon & $1.00 \mathrm{E}-03$ & ICRP-38 & $4.50 \mathrm{E}+02$ & $y$ & $1.42 \mathrm{E}+10$ & $2.48 \mathrm{E}+01$ & ICRP-72 & $1.10 \mathrm{E}-07$ & $\mathrm{~s}$ & Max & FGR-12 & $5.24 \mathrm{E}-19$ \\
\hline Sm-139 & Sm-139 & 138.922 & Samarium & $1.00 \mathrm{E}-03$ & ICRP-107 & $2.57 \mathrm{E}+00$ & $\mathrm{~m}$ & $1.54 \mathrm{E}+02$ & $5.27 \mathrm{E}+08$ & -- & -- & -- & -- & DOE-STD-1196 & $6.64 \mathrm{E}-14$ \\
\hline Sm-140 & Sm-140 & 139.919 & Samarium & $1.00 \mathrm{E}-03$ & ICRP-107 & $1.48 \mathrm{E}+01$ & $\mathrm{~m}$ & $8.89 \mathrm{E}+02$ & $9.07 \mathrm{E}+07$ & DOE-STD-1196 & $3.92 \mathrm{E}-11$ & $\mathrm{~s}$ & Max & DOE-STD-1196 & $2.53 \mathrm{E}-14$ \\
\hline Sm-141 & Sm-141 & 140.918 & Samarium & $1.00 \mathrm{E}-03$ & ICRP-38 & $1.02 \mathrm{E}+01$ & $\mathrm{~m}$ & $6.12 \mathrm{E}+02$ & $1.31 \mathrm{E}+08$ & ICRP-72 & $1.50 \mathrm{E}-11$ & $M$ & Max & FGR-12 & $6.87 \mathrm{E}-14$ \\
\hline Sm-141m & Sm-141 & 140.918 & Samarium & $1.00 \mathrm{E}-03$ & ICRP-38 & $2.26 \mathrm{E}+01$ & $\mathrm{~m}$ & $1.36 \mathrm{E}+03$ & $5.90 \mathrm{E}+07$ & ICRP-72 & $3.20 \mathrm{E}-11$ & $M$ & Max & FGR-12 & $9.71 \mathrm{E}-14$ \\
\hline Sm-142 & Sm-142 & 141.915 & Samarium & $1.00 \mathrm{E}-03$ & ICRP-38 & $7.25 E+01$ & $\mathrm{~m}$ & $4.35 \mathrm{E}+03$ & $1.83 \mathrm{E}+07$ & ICRP-72 & $7.10 \mathrm{E}-11$ & $M$ & Max & FGR-12 & $3.79 \mathrm{E}-15$ \\
\hline Sm-143 & Sm-143 & 142.915 & Samarium & $1.00 \mathrm{E}-03$ & ICRP-107 & $8.75 \mathrm{E}+00$ & $\mathrm{~m}$ & $5.25 \mathrm{E}+02$ & $1.50 \mathrm{E}+08$ & -- & -- & -- & -- & DOE-STD-1196 & $2.36 \mathrm{E}-14$ \\
\hline Sm-143m & Sm-143 & 142.915 & Samarium & $1.00 \mathrm{E}-03$ & ICRP-107 & $6.60 \mathrm{E}+01$ & $\mathrm{~s}$ & $6.60 \mathrm{E}+01$ & $1.20 \mathrm{E}+09$ & -- & -- & -- & -- & DOE-STD-1196 & $3.11 \mathrm{E}-14$ \\
\hline Sm-145 & Sm-145 & 144.913 & Samarium & $1.00 \mathrm{E}-03$ & ICRP-38 & $3.40 \mathrm{E}+02$ & $d$ & $2.94 \mathrm{E}+07$ & $2.65 \mathrm{E}+03$ & ICRP-72 & $1.60 \mathrm{E}-09$ & $M$ & $\operatorname{Max}$ & FGR-12 & $1.61 \mathrm{E}-15$ \\
\hline Sm-146 & Sm-146 & 145.913 & Samarium & $1.00 \mathrm{E}-03$ & ICRP-38 & $1.03 E+08$ & $y$ & $3.25 \mathrm{E}+15$ & $2.38 \mathrm{E}-05$ & ICRP-72 & $1.10 \mathrm{E}-05$ & $M$ & Max & -- & -- \\
\hline Sm-147 & Sm-147 & 146.915 & Samarium & $1.00 \mathrm{E}-03$ & ICRP-38 & $1.06 \mathrm{E}+11$ & $y$ & $3.35 \mathrm{E}+18$ & $2.30 \mathrm{E}-08$ & ICRP-72 & $9.60 \mathrm{E}-06$ & $M$ & Max & -- & -- \\
\hline Sm-148 & Sm-148 & 147.915 & Samarium & $1.00 \mathrm{E}-03$ & ICRP-107 & $7.00 \mathrm{E}+15$ & $y$ & $2.21 \mathrm{E}+23$ & $3.45 \mathrm{E}-13$ & DOE-STD-1196 & $2.10 \mathrm{E}-05$ & $\mathrm{~F}$ & $\operatorname{Max}$ & -- & -- \\
\hline Sm-151 & Sm-151 & 150.920 & Samarium & $1.00 \mathrm{E}-03$ & ICRP-38 & $9.00 \mathrm{E}+01$ & $y$ & $2.84 \mathrm{E}+09$ & $2.63 \mathrm{E}+01$ & ICRP-72 & $4.00 \mathrm{E}-09$ & $M$ & Max & FGR-12 & $3.61 \mathrm{E}-20$ \\
\hline Sm-153 & Sm-153 & 152.922 & Samarium & $1.00 \mathrm{E}-03$ & ICRP-38 & $4.67 \mathrm{E}+01$ & $\mathrm{~h}$ & $1.68 \mathrm{E}+05$ & $4.39 \mathrm{E}+05$ & ICRP-72 & $6.30 \mathrm{E}-10$ & $M$ & Max & FGR-12 & $2.28 \mathrm{E}-15$ \\
\hline Sm-155 & Sm-155 & 154.925 & Samarium & $1.00 \mathrm{E}-03$ & ICRP-38 & $2.21 \mathrm{E}+01$ & $\mathrm{~m}$ & $1.33 \mathrm{E}+03$ & $5.49 \mathrm{E}+07$ & ICRP-72 & $1.70 \mathrm{E}-11$ & $M$ & Max & FGR-12 & $4.65 \mathrm{E}-15$ \\
\hline Sm-156 & Sm-156 & 155.926 & Samarium & $1.00 \mathrm{E}-03$ & ICRP-38 & $9.40 \mathrm{E}+00$ & $\mathrm{~h}$ & $3.38 \mathrm{E}+04$ & $2.14 \mathrm{E}+06$ & ICRP-72 & $2.20 \mathrm{E}-10$ & $M$ & Max & FGR-12 & $5.43 \mathrm{E}-15$ \\
\hline Sm-157 & Sm-157 & 156.928 & Samarium & $1.00 \mathrm{E}-03$ & ICRP-107 & $8.03 E+00$ & $\mathrm{~m}$ & $4.82 \mathrm{E}+02$ & $1.49 \mathrm{E}+08$ & -- & -- & -- & -- & DOE-STD-1196 & $1.91 \mathrm{E}-14$ \\
\hline Sn-106 & Sn-106 & 105.917 & Tin & $1.00 \mathrm{E}-03$ & ICRP-107 & $1.92 \mathrm{E}+00$ & $\mathrm{~m}$ & $1.15 \mathrm{E}+02$ & $9.25 \mathrm{E}+08$ & -- & -- & -- & -- & DOE-STD-1196 & $5.40 \mathrm{E}-14$ \\
\hline Sn-108 & Sn-108 & 107.912 & Tin & $1.00 \mathrm{E}-03$ & ICRP-107 & $1.03 \mathrm{E}+01$ & $\mathrm{~m}$ & $6.18 \mathrm{E}+02$ & $1.69 \mathrm{E}+08$ & DOE-STD-1196 & $1.40 \mathrm{E}-11$ & $\mathrm{~s}$ & Max & DOE-STD-1196 & $2.96 \mathrm{E}-14$ \\
\hline Sn-109 & Sn-109 & 108.911 & Tin & $1.00 \mathrm{E}-03$ & ICRP-107 & $1.80 \mathrm{E}+01$ & $\mathrm{~m}$ & $1.08 \mathrm{E}+03$ & $9.59 \mathrm{E}+07$ & DOE-STD-1196 & $1.44 \mathrm{E}-11$ & $\mathrm{~s}$ & Max & DOE-STD-1196 & $1.05 \mathrm{E}-13$ \\
\hline
\end{tabular}




\begin{tabular}{|c|c|c|c|c|c|c|c|c|c|c|c|c|c|c|c|}
\hline \multicolumn{16}{|c|}{ Table B.1 Radionuclide Specific Input Data Used For the Calculation of HC-2 TQs Using Recommended DCs (continued) } \\
\hline $\begin{array}{l}\text { MASTER } \\
\text { ISOTOPE } \\
\text { LIST }\end{array}$ & $\begin{array}{c}\text { Atomic } \\
\text { Mass } \\
\text { Nuclide }\end{array}$ & $\begin{array}{l}\text { Atomic } \\
\text { Mass }\end{array}$ & Element & $\begin{array}{c}\text { RF per } \\
\text { DOE-STD- } \\
1027\end{array}$ & $\begin{array}{l}\text { Half-Life } \\
\text { Reference }\end{array}$ & $\begin{array}{r}\text { Half-Li } \\
\text { (original } 4\end{array}$ & & Half Life (s) & $\begin{array}{l}\text { Sp. Act } \\
\text { (Ci/g) }\end{array}$ & $\begin{array}{c}\text { Inhalation DC } \\
\text { Reference }\end{array}$ & $\begin{array}{c}\text { HC-2 } \\
\text { Inhalation } \\
\text { DC } \\
\text { (Sv/Bq) }\end{array}$ & & $\begin{array}{l}\text { ung } \\
\text { rption } \\
\text { /Type }\end{array}$ & $\begin{array}{c}\text { Immersion DC } \\
\text { Reference }\end{array}$ & $\begin{array}{c}\text { HC-2 } \\
\text { Immersion } \\
\mathrm{DC}(\mathrm{Sv} / \mathrm{s} \\
\left.\text { per } \mathrm{Bq} / \mathrm{m}^{3}\right)\end{array}$ \\
\hline Sn-110 & Sn-110 & 109.908 & Tin & $1.00 \mathrm{E}-03$ & ICRP-38 & $4.00 \mathrm{E}+00$ & $\mathrm{~h}$ & $1.44 \mathrm{E}+04$ & $7.13 \mathrm{E}+06$ & ICRP-72 & $1.60 \mathrm{E}-10$ & $\mathrm{M}$ & Max & FGR-12 & $1.37 \mathrm{E}-14$ \\
\hline Sn-111 & Sn-111 & 110.908 & Tin & $1.00 \mathrm{E}-03$ & ICRP-38 & $3.53 \mathrm{E}+01$ & $\mathrm{~m}$ & $2.12 \mathrm{E}+03$ & $4.80 \mathrm{E}+07$ & ICRP-72 & $1.30 \mathrm{E}-11$ & $M$ & Max & FGR-12 & $2.45 \mathrm{E}-14$ \\
\hline Sn-113 & Sn-113 & 112.905 & Tin & $1.00 \mathrm{E}-03$ & ICRP-38 & $1.15 \mathrm{E}+02$ & $d$ & $9.94 \mathrm{E}+06$ & $1.00 \mathrm{E}+04$ & ICRP-72 & $2.70 \mathrm{E}-09$ & $M$ & Max & FGR-12 & $3.82 \mathrm{E}-16$ \\
\hline Sn-113m & Sn-113 & 112.905 & Tin & $1.00 \mathrm{E}-03$ & ICRP-107 & $2.14 \mathrm{E}+01$ & $\mathrm{~m}$ & $1.28 \mathrm{E}+03$ & $7.78 \mathrm{E}+07$ & DOE-STD-1196 & $5.19 \mathrm{E}-12$ & $\mathrm{~s}$ & Max & DOE-STD-1196 & $1.02 \mathrm{E}-16$ \\
\hline Sn-117m & Sn-117 & 116.903 & Tin & $1.00 \mathrm{E}-03$ & ICRP-38 & $1.36 \mathrm{E}+01$ & $d$ & $1.18 \mathrm{E}+06$ & $8.21 \mathrm{E}+04$ & ICRP-72 & $2.40 \mathrm{E}-09$ & $M$ & Max & FGR-12 & $6.82 \mathrm{E}-15$ \\
\hline Sn-119m & Sn-119 & 118.903 & Tin & $1.00 \mathrm{E}-03$ & ICRP-38 & $2.93 \mathrm{E}+02$ & $d$ & $2.53 \mathrm{E}+07$ & $3.75 \mathrm{E}+03$ & ICRP-72 & $2.20 \mathrm{E}-09$ & $M$ & Max & FGR-12 & $1.01 \mathrm{E}-16$ \\
\hline Sn-121 & Sn-121 & 120.904 & Tin & $1.00 \mathrm{E}-03$ & ICRP-38 & $2.71 \mathrm{E}+01$ & $\mathrm{~h}$ & $9.74 \mathrm{E}+04$ & $9.58 \mathrm{E}+05$ & ICRP-72 & $2.30 \mathrm{E}-10$ & $M$ & Max & FGR-12 & $2.37 \mathrm{E}-18$ \\
\hline Sn-121m & Sn-121 & 120.904 & Tin & $1.00 \mathrm{E}-03$ & ICRP-38 & $5.50 \mathrm{E}+01$ & $y$ & $1.74 \mathrm{E}+09$ & $5.38 \mathrm{E}+01$ & ICRP-72 & $4.50 \mathrm{E}-09$ & $M$ & Max & FGR-12 & $6.02 \mathrm{E}-17$ \\
\hline Sn-123 & Sn-123 & 122.906 & Tin & $1.00 \mathrm{E}-03$ & ICRP-38 & $1.29 \mathrm{E}+02$ & $d$ & $1.12 \mathrm{E}+07$ & $8.22 \mathrm{E}+03$ & ICRP-72 & $8.10 \mathrm{E}-09$ & $M$ & Max & FGR-12 & $4.03 \mathrm{E}-16$ \\
\hline Sn-123m & Sn-123 & 122.906 & Tin & $1.00 \mathrm{E}-03$ & ICRP-38 & $4.01 \mathrm{E}+01$ & $\mathrm{~m}$ & $2.40 \mathrm{E}+03$ & $3.82 \mathrm{E}+07$ & ICRP-72 & $2.70 \mathrm{E}-11$ & $M$ & Max & FGR-12 & $6.55 \mathrm{E}-15$ \\
\hline Sn-125 & Sn-125 & 124.908 & Tin & $1.00 \mathrm{E}-03$ & ICRP-38 & $9.64 \mathrm{E}+00$ & $d$ & $8.33 \mathrm{E}+05$ & $1.08 \mathrm{E}+05$ & ICRP-72 & $3.10 \mathrm{E}-09$ & $M$ & Max & FGR-12 & $1.58 \mathrm{E}-14$ \\
\hline Sn-125m & Sn-125 & 124.908 & Tin & $1.00 \mathrm{E}-03$ & ICRP-107 & $9.52 \mathrm{E}+00$ & $\mathrm{~m}$ & $5.71 \mathrm{E}+02$ & $1.58 \mathrm{E}+08$ & -- & -- & -- & -- & DOE-STD-1196 & $1.60 \mathrm{E}-14$ \\
\hline Sn-126 & Sn-126 & 125.908 & Tin & $1.00 \mathrm{E}-03$ & ICRP-38 & $1.00 E+05$ & $y$ & $3.16 \mathrm{E}+12$ & $2.84 \mathrm{E}-02$ & ICRP-72 & $2.80 \mathrm{E}-08$ & $M$ & Max & FGR-12 & $2.11 \mathrm{E}-15$ \\
\hline Sn-127 & Sn-127 & 126.910 & Tin & $1.00 \mathrm{E}-03$ & ICRP-38 & $2.10 \mathrm{E}+00$ & $\mathrm{~h}$ & $7.56 E+03$ & $1.18 \mathrm{E}+07$ & ICRP-72 & $1.30 \mathrm{E}-10$ & $M$ & Max & FGR-12 & $9.59 \mathrm{E}-14$ \\
\hline Sn-127m & Sn-127 & 126.910 & Tin & $1.00 \mathrm{E}-03$ & ICRP-107 & $4.13 \mathrm{E}+00$ & $\mathrm{~m}$ & $2.48 \mathrm{E}+02$ & $3.59 \mathrm{E}+08$ & -- & -- & -- & -- & DOE-STD-1196 & $2.67 \mathrm{E}-14$ \\
\hline Sn-128 & Sn-128 & 127.911 & Tin & $1.00 \mathrm{E}-03$ & ICRP-38 & $5.91 \mathrm{E}+01$ & $\mathrm{~m}$ & $3.55 \mathrm{E}+03$ & $2.49 \mathrm{E}+07$ & ICRP-72 & $9.20 \mathrm{E}-11$ & $M$ & Max & FGR-12 & $3.00 \mathrm{E}-14$ \\
\hline Sn-129 & Sn-129 & 128.913 & Tin & $1.00 \mathrm{E}-03$ & ICRP-107 & $2.23 \mathrm{E}+00$ & $\mathrm{~m}$ & $1.34 \mathrm{E}+02$ & $6.54 \mathrm{E}+08$ & -- & -- & -- & -- & DOE-STD-1196 & $4.76 \mathrm{E}-14$ \\
\hline Sn-130 & Sn-130 & 129.914 & Tin & $1.00 \mathrm{E}-03$ & ICRP-107 & $3.72 \mathrm{E}+00$ & $\mathrm{~m}$ & $2.23 \mathrm{E}+02$ & $3.89 \mathrm{E}+08$ & -- & -- & -- & -- & DOE-STD-1196 & $4.16 \mathrm{E}-14$ \\
\hline Sn-130m & Sn-130 & 129.914 & Tin & $1.00 \mathrm{E}-03$ & ICRP-107 & $1.70 \mathrm{E}+00$ & $\mathrm{~m}$ & $1.02 \mathrm{E}+02$ & $8.51 \mathrm{E}+08$ & -- & -- & -- & -- & DOE-STD-1196 & $4.20 \mathrm{E}-14$ \\
\hline Sr-79 & Sr-79 & 78.930 & Strontium & $1.00 \mathrm{E}-03$ & ICRP-107 & $2.25 \mathrm{E}+00$ & $\mathrm{~m}$ & $1.35 \mathrm{E}+02$ & $1.06 \mathrm{E}+09$ & -- & -- & -- & -- & DOE-STD-1196 & $5.39 \mathrm{E}-14$ \\
\hline Sr-80 & Sr-80 & 79.925 & Strontium & $1.00 \mathrm{E}-03$ & ICRP-38 & $1.00 E+02$ & $\mathrm{~m}$ & $6.00 \mathrm{E}+03$ & $2.35 \mathrm{E}+07$ & ICRP-72 & $1.30 \mathrm{E}-10$ & $M$ & $\operatorname{Rec}$ & FGR-12 & $6.53 \mathrm{E}-18$ \\
\hline Sr-81 & $\mathrm{Sr}-81$ & 80.923 & Strontium & $1.00 \mathrm{E}-03$ & ICRP-38 & $2.55 \mathrm{E}+01$ & $\mathrm{~m}$ & $1.53 \mathrm{E}+03$ & $9.11 \mathrm{E}+07$ & ICRP-72 & $3.50 \mathrm{E}-11$ & $M$ & $\operatorname{Rec}$ & FGR-12 & $6.68 \mathrm{E}-14$ \\
\hline Sr-82 & Sr-82 & 81.918 & Strontium & $1.00 \mathrm{E}-03$ & ICRP-38 & $2.50 \mathrm{E}+01$ & $d$ & $2.16 \mathrm{E}+06$ & $6.38 \mathrm{E}+04$ & ICRP-72 & $8.90 \mathrm{E}-09$ & $M$ & $\operatorname{Rec}$ & FGR-12 & $6.43 \mathrm{E}-18$ \\
\hline Sr-83 & $\mathrm{Sr}-83$ & 82.918 & Strontium & $1.00 \mathrm{E}-03$ & ICRP-38 & $3.24 \mathrm{E}+01$ & $\mathrm{~h}$ & $1.17 \mathrm{E}+05$ & $1.17 \mathrm{E}+06$ & ICRP-72 & $3.10 \mathrm{E}-10$ & $M$ & $\operatorname{Rec}$ & FGR-12 & $3.86 \mathrm{E}-14$ \\
\hline Sr-85 & Sr-85 & 84.913 & Strontium & $1.00 \mathrm{E}-03$ & ICRP-38 & $6.48 \mathrm{E}+01$ & $d$ & $5.60 \mathrm{E}+06$ & $2.37 \mathrm{E}+04$ & ICRP-72 & $6.40 \mathrm{E}-10$ & $M$ & $\operatorname{Rec}$ & FGR-12 & $2.42 \mathrm{E}-14$ \\
\hline Sr-85m & Sr-85 & 84.913 & Strontium & $1.00 \mathrm{E}-03$ & ICRP-38 & $6.95 \mathrm{E}+01$ & $\mathrm{~m}$ & $4.17 \mathrm{E}+03$ & $3.19 \mathrm{E}+07$ & ICRP-72 & $4.10 \mathrm{E}-12$ & $\mathrm{M}$ & $\operatorname{Rec}$ & FGR-12 & $1.05 \mathrm{E}-14$ \\
\hline Sr-87m & Sr-87 & 86.909 & Strontium & $1.00 \mathrm{E}-03$ & ICRP-38 & $2.81 \mathrm{E}+00$ & $\mathrm{~h}$ & $1.01 \mathrm{E}+04$ & $1.29 \mathrm{E}+07$ & ICRP-72 & $2.00 \mathrm{E}-11$ & $M$ & $\operatorname{Rec}$ & FGR-12 & $1.52 \mathrm{E}-14$ \\
\hline Sr-89 & $\mathrm{Sr}-89$ & 88.907 & Strontium & $1.00 \mathrm{E}-03$ & ICRP-38 & $5.05 \mathrm{E}+01$ & $d$ & $4.36 \mathrm{E}+06$ & $2.91 \mathrm{E}+04$ & ICRP-72 & $6.10 \mathrm{E}-09$ & $M$ & $\operatorname{Rec}$ & FGR-12 & $7.73 \mathrm{E}-17$ \\
\hline Sr-90 & Sr-90 & 89.908 & Strontium & $1.00 \mathrm{E}-03$ & ICRP-38 & $2.91 \mathrm{E}+01$ & $y$ & $9.19 \mathrm{E}+08$ & $1.37 \mathrm{E}+02$ & ICRP-72 & $3.60 \mathrm{E}-08$ & $M$ & Rec & FGR-12 & $7.53 \mathrm{E}-18$ \\
\hline Sr-91 & Sr-91 & 90.910 & Strontium & $1.00 \mathrm{E}-03$ & ICRP-38 & $9.50 \mathrm{E}+00$ & $\mathrm{~h}$ & $3.42 \mathrm{E}+04$ & $3.63 \mathrm{E}+06$ & ICRP-72 & $3.70 \mathrm{E}-10$ & $M$ & $\operatorname{Rec}$ & FGR-12 & $3.45 \mathrm{E}-14$ \\
\hline Sr-92 & Sr-92 & 91.911 & Strontium & $1.00 \mathrm{E}-03$ & ICRP-38 & $2.71 \mathrm{E}+00$ & $\mathrm{~h}$ & $9.76 \mathrm{E}+03$ & $1.26 \mathrm{E}+07$ & ICRP-72 & $2.10 \mathrm{E}-10$ & $M$ & $\operatorname{Rec}$ & FGR-12 & $6.79 \mathrm{E}-14$ \\
\hline Sr-93 & Sr-93 & 92.914 & Strontium & $1.00 \mathrm{E}-03$ & ICRP-107 & $7.42 \mathrm{E}+00$ & $\mathrm{~m}$ & $4.45 \mathrm{E}+02$ & $2.73 \mathrm{E}+08$ & -- & -- & -- & -- & DOE-STD-1196 & $1.07 \mathrm{E}-13$ \\
\hline Sr-94 & Sr-94 & 93.915 & Strontium & $1.00 \mathrm{E}-03$ & ICRP-107 & $7.53 \mathrm{E}+01$ & $\mathrm{~s}$ & $7.53 \mathrm{E}+01$ & $1.60 \mathrm{E}+09$ & -- & -- & -- & -- & DOE-STD-1196 & $6.92 \mathrm{E}-14$ \\
\hline Ta-170 & Ta-170 & 169.946 & Tantalum & $1.00 \mathrm{E}-03$ & ICRP-107 & $6.76 \mathrm{E}+00$ & $\mathrm{~m}$ & $4.06 \mathrm{E}+02$ & $1.64 \mathrm{E}+08$ & -- & -- & -- & -- & DOE-STD-1196 & $4.88 \mathrm{E}-14$ \\
\hline Ta-172 & Ta-172 & 171.945 & Tantalum & $1.00 \mathrm{E}-03$ & ICRP-38 & $3.68 \mathrm{E}+01$ & $\mathrm{~m}$ & $2.21 \mathrm{E}+03$ & $2.97 \mathrm{E}+07$ & ICRP-72 & $3.50 \mathrm{E}-11$ & $\mathrm{~s}$ & Max & FGR-12 & $7.59 \mathrm{E}-14$ \\
\hline Ta-173 & Ta-173 & 172.944 & Tantalum & $1.00 \mathrm{E}-03$ & ICRP-38 & $3.65 \mathrm{E}+00$ & $\mathrm{~h}$ & $1.31 \mathrm{E}+04$ & $4.96 \mathrm{E}+06$ & ICRP-72 & $1.10 \mathrm{E}-10$ & $M$ & Max & FGR-12 & $2.75 \mathrm{E}-14$ \\
\hline Ta-174 & Ta-174 & 173.944 & Tantalum & $1.00 \mathrm{E}-03$ & ICRP-38 & $1.20 \mathrm{E}+00$ & $\mathrm{~h}$ & $4.32 \mathrm{E}+03$ & $1.50 \mathrm{E}+07$ & ICRP-72 & $4.30 \mathrm{E}-11$ & $\mathrm{~s}$ & Max & FGR-12 & $2.97 \mathrm{E}-14$ \\
\hline Ta-175 & Ta-175 & 174.944 & Tantalum & $1.00 \mathrm{E}-03$ & ICRP-38 & $1.05 \mathrm{E}+01$ & $\mathrm{~h}$ & $3.78 \mathrm{E}+04$ & $1.71 \mathrm{E}+06$ & ICRP-72 & $1.30 \mathrm{E}-10$ & $\mathrm{~s}$ & Max & FGR-12 & $4.55 \mathrm{E}-14$ \\
\hline Ta-176 & Ta-176 & 175.945 & Tantalum & $1.00 \mathrm{E}-03$ & ICRP-38 & $8.08 \mathrm{E}+00$ & $\mathrm{~h}$ & $2.91 \mathrm{E}+04$ & $2.20 \mathrm{E}+06$ & ICRP-72 & $2.00 \mathrm{E}-10$ & $\mathrm{~s}$ & Max & FGR-12 & $1.09 \mathrm{E}-13$ \\
\hline Ta-177 & Ta-177 & 176.944 & Tantalum & $1.00 \mathrm{E}-03$ & ICRP-38 & $5.66 \mathrm{E}+01$ & $\mathrm{~h}$ & $2.04 \mathrm{E}+05$ & $3.13 \mathrm{E}+05$ & ICRP-72 & $1.10 \mathrm{E}-10$ & $\mathrm{~s}$ & Max & FGR-12 & $2.53 \mathrm{E}-15$ \\
\hline Ta-178s & Ta-178 & 177.946 & Tantalum & $1.00 \mathrm{E}-03$ & ICRP-38 & $9.31 \mathrm{E}+00$ & $\mathrm{~m}$ & $5.59 \mathrm{E}+02$ & $1.13 \mathrm{E}+08$ & -- & -- & -- & -- & FGR-12 & $4.61 \mathrm{E}-15$ \\
\hline Ta-1781 & Ta-178 & 177.946 & Tantalum & $1.00 \mathrm{E}-03$ & ICRP-38 & $2.20 E+00$ & $\mathrm{~h}$ & $7.92 \mathrm{E}+03$ & $8.00 \mathrm{E}+06$ & ICRP-72 & $6.80 \mathrm{E}-11$ & $\mathrm{~s}$ & Max & FGR-12 & $4.75 \mathrm{E}-14$ \\
\hline Ta-179 & Ta-179 & 178.946 & Tantalum & $1.00 \mathrm{E}-03$ & ICRP-38 & $6.65 \mathrm{E}+02$ & $d$ & $5.74 \mathrm{E}+07$ & $1.10 \mathrm{E}+03$ & ICRP-72 & $5.60 \mathrm{E}-10$ & $\mathrm{~s}$ & Max & FGR-12 & $1.09 \mathrm{E}-15$ \\
\hline Ta-180 & Ta-180 & 179.947 & Tantalum & $1.00 \mathrm{E}-03$ & ICRP-38 & $1.00 \mathrm{E}+13$ & $y$ & $3.16 \mathrm{E}+20$ & $1.99 \mathrm{E}-10$ & ICRP-72 & $2.60 \mathrm{E}-08$ & $\mathrm{~s}$ & Max & FGR-12 & $2.59 \mathrm{E}-14$ \\
\hline Ta-180m & Ta-180 & 179.947 & Tantalum & $1.00 \mathrm{E}-03$ & ICRP-38 & $8.10 \mathrm{E}+00$ & $\mathrm{~h}$ & $2.92 \mathrm{E}+04$ & $2.15 \mathrm{E}+06$ & ICRP-72 & $4.40 \mathrm{E}-11$ & $M$ & Max & FGR-12 & $1.71 \mathrm{E}-15$ \\
\hline Ta-182 & Ta-182 & 181.950 & Tantalum & $1.00 \mathrm{E}-03$ & ICRP-38 & $1.15 \mathrm{E}+02$ & $d$ & $9.94 \mathrm{E}+06$ & $6.24 \mathrm{E}+03$ & ICRP-72 & $1.00 \mathrm{E}-08$ & $\mathrm{~s}$ & Max & FGR-12 & $6.40 \mathrm{E}-14$ \\
\hline
\end{tabular}




\begin{tabular}{|c|c|c|c|c|c|c|c|c|c|c|c|c|c|c|c|}
\hline \multicolumn{16}{|c|}{ Table B.1 Radionuclide Specific Input Data Used For the Calculation of HC-2 TQs Using Recommended DCs (continued) } \\
\hline $\begin{array}{l}\text { MASTER } \\
\text { ISOTOPE } \\
\text { LIST }\end{array}$ & $\begin{array}{c}\text { Atomic } \\
\text { Mass } \\
\text { Nuclide }\end{array}$ & $\begin{array}{l}\text { Atomic } \\
\text { Mass }\end{array}$ & Element & $\begin{array}{c}\text { RF per } \\
\text { DOE-STD- } \\
1027\end{array}$ & $\begin{array}{c}\text { Half-Life } \\
\text { Reference }\end{array}$ & \multicolumn{2}{|c|}{$\begin{array}{c}\text { Half-Life } \\
\text { (original units) }\end{array}$} & \multirow{2}{*}{$\begin{array}{c}\text { Half Life (s) } \\
9.50 \mathrm{E}+02\end{array}$} & \multirow{2}{*}{$\begin{array}{c}\begin{array}{c}\text { Sp. Act } \\
\text { (Ci/g) }\end{array} \\
6.52 \mathrm{E}+07\end{array}$} & \multirow{2}{*}{$\begin{array}{c}\text { Inhalation DC } \\
\text { Reference } \\
\text { ICRP-72 }\end{array}$} & \multirow[t]{2}{*}{$\begin{array}{c}\text { HC-2 } \\
\text { Inhalation } \\
\text { DC } \\
\text { (Sv/Bq) } \\
2.10 E-11 \\
\end{array}$} & \multicolumn{2}{|c|}{$\begin{array}{c}\text { Lung } \\
\text { Absorption } \\
\text { Class/Type }\end{array}$} & \multirow{2}{*}{$\begin{array}{c}\text { Immersion DC } \\
\text { Reference } \\
\text { FGR-12 } \\
\end{array}$} & \multirow[t]{2}{*}{$\begin{array}{c}\text { HC-2 } \\
\text { Immersion } \\
\mathrm{DC}(\mathrm{Sv} / \mathrm{s} \\
\left.\text { per } \mathrm{Bq} / \mathrm{m}^{3}\right) \\
1.11 \mathrm{E}-14\end{array}$} \\
\hline Ta-182m & Ta-182 & 181.950 & Tantalum & $1.00 \mathrm{E}-03$ & ICRP-38 & $1.58 \mathrm{E}+01$ & $\mathrm{~m}$ & & & & & $\mathrm{~s}$ & Max & & \\
\hline Ta-183 & Ta-183 & 182.951 & Tantalum & $1.00 \mathrm{E}-03$ & ICRP-38 & $5.10 \mathrm{E}+00$ & d & $4.41 \mathrm{E}+05$ & $1.40 \mathrm{E}+05$ & ICRP-72 & $2.10 \mathrm{E}-09$ & $\mathrm{~s}$ & Max & FGR-12 & $1.31 \mathrm{E}-14$ \\
\hline Ta-184 & Ta-184 & 183.954 & Tantalum & $1.00 \mathrm{E}-03$ & ICRP-38 & $8.70 \mathrm{E}+00$ & $\mathrm{~h}$ & $3.13 \mathrm{E}+04$ & $1.96 \mathrm{E}+06$ & ICRP-72 & $4.30 \mathrm{E}-10$ & $\mathrm{~s}$ & Max & FGR-12 & $7.80 \mathrm{E}-14$ \\
\hline Ta-185 & Ta-185 & 184.956 & Tantalum & $1.00 \mathrm{E}-03$ & ICRP-38 & $4.90 \mathrm{E}+01$ & $\mathrm{~m}$ & $2.94 \mathrm{E}+03$ & $2.07 E+07$ & ICRP-72 & $4.80 \mathrm{E}-11$ & $\mathrm{~s}$ & Max & FGR-12 & $8.73 \mathrm{E}-15$ \\
\hline Ta-186 & Ta-186 & 185.959 & Tantalum & $1.00 \mathrm{E}-03$ & ICRP-38 & $1.05 \mathrm{E}+01$ & $\mathrm{~m}$ & $6.30 \mathrm{E}+02$ & $9.63 \mathrm{E}+07$ & ICRP-72 & $1.80 \mathrm{E}-11$ & $\mathrm{~s}$ & Max & FGR-12 & $7.53 \mathrm{E}-14$ \\
\hline Tb-146 & Tb-146 & 145.927 & Terbium & $1.00 \mathrm{E}-03$ & ICRP-107 & $2.30 \mathrm{E}+01$ & $\mathrm{~s}$ & $2.30 \mathrm{E}+01$ & $3.36 \mathrm{E}+09$ & -- & -- & -- & -- & DOE-STD-1196 & $1.74 \mathrm{E}-13$ \\
\hline Tb-147 & Tb-147 & 146.924 & Terbium & $1.00 \mathrm{E}-03$ & ICRP-38 & $1.65 \mathrm{E}+00$ & $\mathrm{~h}$ & $5.94 \mathrm{E}+03$ & $1.29 \mathrm{E}+07$ & ICRP-72 & $7.60 \mathrm{E}-11$ & $M$ & Max & FGR-12 & $7.78 \mathrm{E}-14$ \\
\hline Tb-147m & Tb-147 & 146.924 & Terbium & $1.00 \mathrm{E}-03$ & ICRP-107 & $1.87 \mathrm{E}+00$ & $\mathrm{~m}$ & $1.12 \mathrm{E}+02$ & $6.84 \mathrm{E}+08$ & -- & -- & -- & -- & DOE-STD-1196 & $9.11 \mathrm{E}-14$ \\
\hline Tb-148 & Tb-148 & 147.924 & Terbium & $1.00 \mathrm{E}-03$ & ICRP-107 & $6.00 \mathrm{E}+01$ & $m$ & $3.60 \mathrm{E}+03$ & $2.12 \mathrm{E}+07$ & DOE-STD-1196 & $8.28 \mathrm{E}-11$ & $M$ & Max & DOE-STD-1196 & $1.11 \mathrm{E}-13$ \\
\hline Tb-148m & Tb-148 & 147.924 & Terbium & $1.00 \mathrm{E}-03$ & ICRP-107 & $2.20 \mathrm{E}+00$ & $\mathrm{~m}$ & $1.32 \mathrm{E}+02$ & $5.78 \mathrm{E}+08$ & -- & -- & -- & -- & DOE-STD-1196 & $1.42 \mathrm{E}-13$ \\
\hline Tb-149 & Tb-149 & 148.923 & Terbium & $1.00 \mathrm{E}-03$ & ICRP-38 & $4.15 \mathrm{E}+00$ & $\mathrm{~h}$ & $1.49 \mathrm{E}+04$ & $5.07 E+06$ & ICRP-72 & $4.90 \mathrm{E}-09$ & $M$ & Max & FGR-12 & $8.02 \mathrm{E}-14$ \\
\hline Tb-149m & Tb-149 & 148.923 & Terbium & $1.00 \mathrm{E}-03$ & ICRP-107 & $4.16 \mathrm{E}+00$ & $\mathrm{~m}$ & $2.50 \mathrm{E}+02$ & $3.03 \mathrm{E}+08$ & -- & -- & -- & -- & DOE-STD-1196 & $6.20 \mathrm{E}-14$ \\
\hline Tb-150 & Tb-150 & 149.924 & Terbium & $1.00 \mathrm{E}-03$ & ICRP-38 & $3.27 E+00$ & $\mathrm{~h}$ & $1.18 \mathrm{E}+04$ & $6.39 \mathrm{E}+06$ & ICRP-72 & $1.10 \mathrm{E}-10$ & $M$ & Max & FGR-12 & $8.26 \mathrm{E}-14$ \\
\hline Tb-150m & Tb-150 & 149.924 & Terbium & $1.00 \mathrm{E}-03$ & ICRP-107 & $5.80 \mathrm{E}+00$ & $\mathrm{~m}$ & $3.48 \mathrm{E}+02$ & $2.16 \mathrm{E}+08$ & -- & -- & -- & -- & DOE-STD-1196 & $1.12 \mathrm{E}-13$ \\
\hline Tb-151 & Tb-151 & 150.923 & Terbium & $1.00 \mathrm{E}-03$ & ICRP-38 & $1.76 \mathrm{E}+01$ & $h$ & $6.34 \mathrm{E}+04$ & $1.18 \mathrm{E}+06$ & ICRP-72 & $2.30 \mathrm{E}-10$ & $M$ & Max & FGR-12 & $4.20 \mathrm{E}-14$ \\
\hline Tb-151m & Tb-151 & 150.923 & Terbium & $1.00 \mathrm{E}-03$ & $\begin{array}{l}\text { ICRP-107 } \\
\end{array}$ & $2.50 \mathrm{E}+01$ & $\mathrm{~s}$ & $2.50 \mathrm{E}+01$ & $2.99 \mathrm{E}+09$ & -- & -- & -- & -- & DOE-STD-1196 & $3.20 \mathrm{E}-15$ \\
\hline Tb-152 & Tb-152 & 151.924 & Terbium & $1.00 \mathrm{E}-03$ & $\begin{array}{l}\text { ICRP-107 } \\
\end{array}$ & $1.75 \mathrm{E}+01$ & $\mathrm{~h}$ & $6.30 \mathrm{E}+04$ & $1.18 \mathrm{E}+06$ & DOE-STD-1196 & $3.82 \mathrm{E}-10$ & $\mathrm{~s}$ & Max & DOE-STD-1196 & $6.98 \mathrm{E}-14$ \\
\hline Tb-152m & Tb-152 & 151.924 & Terbium & $1.00 \mathrm{E}-03$ & $\begin{array}{l}\text { ICRP-107 } \\
\end{array}$ & $4.20 \mathrm{E}+00$ & $\mathrm{~m}$ & $2.52 \mathrm{E}+02$ & $2.95 \mathrm{E}+08$ & -- & -- & -- & -- & DOE-STD-1196 & $3.28 \mathrm{E}-14$ \\
\hline Tb-153 & Tb-153 & 152.923 & Terbium & $1.00 \mathrm{E}-03$ & $\begin{array}{l}\text { ICRP-38 } \\
\end{array}$ & $2.34 \mathrm{E}+00$ & $d$ & $2.02 \mathrm{E}+05$ & $3.65 \mathrm{E}+05$ & ICRP-72 & $1.90 \mathrm{E}-10$ & $M$ & Max & FGR-12 & $9.89 \mathrm{E}-15$ \\
\hline Tb-154 & Tb-154 & 153.925 & Terbium & $1.00 \mathrm{E}-03$ & ICRP-38 & $2.14 \mathrm{E}+01$ & $\mathrm{~h}$ & $7.70 \mathrm{E}+04$ & $9.51 \mathrm{E}+05$ & ICRP-72 & $3.60 \mathrm{E}-10$ & $M$ & Max & FGR-12 & $1.21 \mathrm{E}-13$ \\
\hline Tb-155 & Tb-155 & 154.924 & Terbium & $1.00 \mathrm{E}-03$ & ICRP-38 & $5.32 \mathrm{E}+00$ & $d$ & $4.60 \mathrm{E}+05$ & $1.58 \mathrm{E}+05$ & ICRP-72 & $2.20 \mathrm{E}-10$ & $M$ & Max & FGR-12 & $5.56 \mathrm{E}-15$ \\
\hline Tb-156 & Tb-156 & 155.925 & Terbium & $1.00 \mathrm{E}-03$ & ICRP-38 & $5.34 \mathrm{E}+00$ & $d$ & $4.61 \mathrm{E}+05$ & $1.57 \mathrm{E}+05$ & ICRP-72 & $1.20 \mathrm{E}-09$ & $M$ & Max & FGR-12 & $8.94 \mathrm{E}-14$ \\
\hline Tb-156ml & Tb-156 & 155.925 & Terbium & $1.00 \mathrm{E}-03$ & ICRP-38 & $2.44 \mathrm{E}+01$ & $\mathrm{~h}$ & $8.78 \mathrm{E}+04$ & $8.24 \mathrm{E}+05$ & ICRP-72 & $2.10 \mathrm{E}-10$ & $M$ & Max & FGR-12 & $7.75 \mathrm{E}-16$ \\
\hline Tb-156ms & Tb-156 & 155.925 & Terbium & $1.00 \mathrm{E}-03$ & ICRP-38 & $5.00 \mathrm{E}+00$ & $\mathrm{~h}$ & $1.80 \mathrm{E}+04$ & $4.02 \mathrm{E}+06$ & ICRP-72 & $9.60 \mathrm{E}-11$ & $M$ & Max & FGR-12 & $1.16 \mathrm{E}-16$ \\
\hline Tb-157 & Tb-157 & 156.924 & Terbium & $1.00 \mathrm{E}-03$ & ICRP-38 & $1.50 \mathrm{E}+02$ & $y$ & $4.73 E+09$ & $1.52 \mathrm{E}+01$ & ICRP-72 & $1.20 \mathrm{E}-09$ & $M$ & Max & FGR-12 & $6.78 \mathrm{E}-17$ \\
\hline Tb-158 & Tb-158 & 157.925 & Terbium & $1.00 \mathrm{E}-03$ & ICRP-38 & $1.50 \mathrm{E}+02$ & $y$ & $4.73 \mathrm{E}+09$ & $1.51 \mathrm{E}+01$ & ICRP-72 & $4.60 \mathrm{E}-08$ & $M$ & Max & FGR-12 & $3.84 \mathrm{E}-14$ \\
\hline Tb-160 & Tb-160 & 159.927 & Terbium & $1.00 \mathrm{E}-03$ & ICRP-38 & $7.23 \mathrm{E}+01$ & $d$ & $6.25 \mathrm{E}+06$ & $1.13 \mathrm{E}+04$ & ICRP-72 & $7.00 \mathrm{E}-09$ & $M$ & Max & FGR-12 & $5.54 \mathrm{E}-14$ \\
\hline Tb-161 & Tb-161 & 160.928 & Terbium & $1.00 \mathrm{E}-03$ & ICRP-38 & $6.91 \mathrm{E}+00$ & $d$ & $5.97 \mathrm{E}+05$ & $1.17 E+05$ & ICRP-72 & $1.30 \mathrm{E}-09$ & $M$ & Max & FGR-12 & $1.02 \mathrm{E}-15$ \\
\hline Tb-162 & Tb-162 & 161.929 & Terbium & $1.00 \mathrm{E}-03$ & ICRP-107 & $7.60 \mathrm{E}+00$ & $\mathrm{~m}$ & $4.56 \mathrm{E}+02$ & $1.53 \mathrm{E}+08$ & -- & -- & -- & -- & DOE-STD-1196 & $5.04 \mathrm{E}-14$ \\
\hline Tb-163 & Tb-163 & 162.931 & Terbium & $1.00 \mathrm{E}-03$ & ICRP-107 & $1.95 \mathrm{E}+01$ & $\mathrm{~m}$ & $1.17 \mathrm{E}+03$ & $5.92 \mathrm{E}+07$ & DOE-STD-1196 & $2.03 \mathrm{E}-11$ & $\mathrm{~s}$ & Max & DOE-STD-1196 & $3.51 \mathrm{E}-14$ \\
\hline Tb-164 & Tb-164 & 163.933 & Terbium & $1.00 \mathrm{E}-03$ & ICRP-107 & $3.00 \mathrm{E}+00$ & $\mathrm{~m}$ & $1.80 \mathrm{E}+02$ & $3.82 \mathrm{E}+08$ & -- & -- & -- & -- & DOE-STD-1196 & $1.14 \mathrm{E}-13$ \\
\hline Tb-165 & Tb-165 & 164.935 & Terbium & $1.00 \mathrm{E}-03$ & $\begin{array}{ll}\text { ICRP-107 } \\
\end{array}$ & $2.11 \mathrm{E}+00$ & $\mathrm{~m}$ & $1.27 \mathrm{E}+02$ & $5.40 \mathrm{E}+08$ & -- & -- & -- & -- & DOE-STD-1196 & $4.04 \mathrm{E}-14$ \\
\hline Tc-101 & Tc-101 & 100.907 & Technetium & $1.00 \mathrm{E}-03$ & ICRP-38 & $1.42 \mathrm{E}+01$ & $\mathrm{~m}$ & $8.52 \mathrm{E}+02$ & $1.31 \mathrm{E}+08$ & ICRP-72 & $1.20 \mathrm{E}-11$ & $M$ & $\operatorname{Rec}$ & FGR-12 & $1.61 \mathrm{E}-14$ \\
\hline Tc-102 & Tc-102 & 101.909 & Technetium & $1.00 \mathrm{E}-03$ & $\begin{array}{l}\text { ICRP-107 } \\
\end{array}$ & $5.28 \mathrm{E}+00$ & $\mathrm{~s}$ & $5.28 \mathrm{E}+00$ & $2.10 \mathrm{E}+10$ & -- & -- & -- & -- & DOE-STD-1196 & $5.85 \mathrm{E}-15$ \\
\hline Tc-102m & Tc-102 & 101.909 & Technetium & $1.00 \mathrm{E}-03$ & ICRP-107 & $4.35 \mathrm{E}+00$ & $\mathrm{~m}$ & $2.61 \mathrm{E}+02$ & $4.24 \mathrm{E}+08$ & -- & -- & -- & -- & DOE-STD-1196 & $1.18 \mathrm{E}-13$ \\
\hline Tc-104 & Tc-104 & 103.911 & Technetium & $1.00 \mathrm{E}-03$ & ICRP-38 & $1.82 \mathrm{E}+01$ & $\mathrm{~m}$ & $1.09 \mathrm{E}+03$ & $9.94 \mathrm{E}+07$ & ICRP-72 & $2.80 \mathrm{E}-11$ & $M$ & $\operatorname{Rec}$ & FGR-12 & $1.01 \mathrm{E}-13$ \\
\hline Tc-105 & Tc-105 & 104.912 & Technetium & $1.00 \mathrm{E}-03$ & ICRP-107 & $7.60 \mathrm{E}+00$ & $\mathrm{~m}$ & $4.56 \mathrm{E}+02$ & $2.36 \mathrm{E}+08$ & -- & -- & -- & -- & DOE-STD-1196 & $3.77 \mathrm{E}-14$ \\
\hline Tc-91 & Tc-91 & 90.918 & Technetium & $1.00 \mathrm{E}-03$ & ICRP-107 & $3.14 \mathrm{E}+00$ & $\mathrm{~m}$ & $1.88 \mathrm{E}+02$ & $6.59 \mathrm{E}+08$ & -- & -- & -- & -- & DOE-STD-1196 & $1.20 \mathrm{E}-13$ \\
\hline Tc-91m & Tc-91 & 90.918 & Technetium & $1.00 \mathrm{E}-03$ & $\begin{array}{l}\text { ICRP-107 } \\
\end{array}$ & $3.30 \mathrm{E}+00$ & $\mathrm{~m}$ & $1.98 \mathrm{E}+02$ & $6.27 \mathrm{E}+08$ & -- & -- & -- & -- & DOE-STD-1196 & $6.60 \mathrm{E}-14$ \\
\hline Tc-92 & TC-92 & 91.915 & Technetium & $1.00 \mathrm{E}-03$ & ICRP-107 & $4.25 \mathrm{E}+00$ & $\mathrm{~m}$ & $2.55 \mathrm{E}+02$ & $4.81 \mathrm{E}+08$ & -- & -- & -- & -- & DOE-STD-1196 & $1.79 \mathrm{E}-13$ \\
\hline Tc-93 & Tc-93 & 92.910 & Technetium & $1.00 \mathrm{E}-03$ & ICRP-38 & $2.75 E+00$ & $\mathrm{~h}$ & $9.90 \mathrm{E}+03$ & $1.23 \mathrm{E}+07$ & ICRP-72 & $3.50 \mathrm{E}-11$ & $M$ & $\operatorname{Rec}$ & FGR-12 & $7.38 \mathrm{E}-14$ \\
\hline Tc-93m & Tc-93 & 92.910 & Technetium & $1.00 \mathrm{E}-03$ & ICRP-38 & $4.35 \mathrm{E}+01$ & $\mathrm{~m}$ & $2.61 \mathrm{E}+03$ & $4.65 \mathrm{E}+07$ & ICRP-72 & $1.70 \mathrm{E}-11$ & $M$ & Rec & FGR-12 & $3.73 \mathrm{E}-14$ \\
\hline TC-94 & TC-94 & 93.910 & Technetium & $1.00 \mathrm{E}-03$ & ICRP-38 & $2.93 \mathrm{E}+02$ & $\mathrm{~m}$ & $1.76 \mathrm{E}+04$ & $6.83 \mathrm{E}+06$ & ICRP-72 & $1.20 \mathrm{E}-10$ & $M$ & $\operatorname{Rec}$ & FGR-12 & $1.30 \mathrm{E}-13$ \\
\hline Tc-94m & TC-94 & 93.910 & Technetium & $1.00 \mathrm{E}-03$ & ICRP-38 & $5.20 \mathrm{E}+01$ & $\mathrm{~m}$ & $3.12 \mathrm{E}+03$ & $3.85 \mathrm{E}+07$ & ICRP-72 & $4.50 \mathrm{E}-11$ & $M$ & $\operatorname{Rec}$ & FGR-12 & $9.18 \mathrm{E}-14$ \\
\hline Tc-95 & TC-95 & 94.908 & Technetium & $1.00 \mathrm{E}-03$ & ICRP-38 & $2.00 \mathrm{E}+01$ & $\mathrm{~h}$ & $7.20 \mathrm{E}+04$ & $1.65 \mathrm{E}+06$ & ICRP-72 & $1.00 \mathrm{E}-10$ & $M$ & Rec & FGR-12 & $3.84 \mathrm{E}-14$ \\
\hline Tc-95m & Tc-95 & 94.908 & Technetium & $1.00 \mathrm{E}-03$ & ICRP-38 & $6.10 \mathrm{E}+01$ & $d$ & $5.27 E+06$ & $2.26 \mathrm{E}+04$ & ICRP-72 & $8.80 \mathrm{E}-10$ & $M$ & $\operatorname{Rec}$ & FGR-12 & $3.23 \mathrm{E}-14$ \\
\hline
\end{tabular}




\begin{tabular}{|c|c|c|c|c|c|c|c|c|c|c|c|c|c|c|c|}
\hline \multicolumn{16}{|c|}{ Table B.1 Radionuclide Specific Input Data Used For the Calculation of HC-2 TQs Using Recommended DCs (continued) } \\
\hline $\begin{array}{l}\text { MASTER } \\
\text { ISOTOPE } \\
\text { LIST }\end{array}$ & $\begin{array}{c}\text { Atomic } \\
\text { Mass } \\
\text { Nuclide }\end{array}$ & $\begin{array}{l}\text { Atomic } \\
\text { Mass }\end{array}$ & Element & $\begin{array}{c}\text { RF per } \\
\text { DOE-STD- } \\
1027\end{array}$ & $\begin{array}{l}\text { Half-Life } \\
\text { Reference }\end{array}$ & $\begin{array}{r}\text { Half-L } \\
\text { (original }\end{array}$ & & Half Life (s) & $\begin{array}{l}\text { Sp. Act } \\
\text { (Ci/g) }\end{array}$ & $\begin{array}{l}\text { Inhalation DC } \\
\text { Reference }\end{array}$ & $\begin{array}{c}\text { HC-2 } \\
\text { Inhalation } \\
\mathrm{DC} \\
(\mathrm{Sv} / \mathrm{Bq})\end{array}$ & & $\begin{array}{l}\text { ung } \\
\text { rption } \\
\text { /Type }\end{array}$ & $\begin{array}{c}\text { Immersion DC } \\
\text { Reference }\end{array}$ & $\begin{array}{c}\text { HC-2 } \\
\text { Immersion } \\
\mathrm{DC}(\mathrm{Sv} / \mathrm{s} \\
\left.\text { per } \mathrm{Bq} / \mathrm{m}^{3}\right)\end{array}$ \\
\hline Tc-96 & Tc-96 & 95.908 & Technetium & $1.00 \mathrm{E}-03$ & ICRP-38 & $4.28 \mathrm{E}+00$ & d & $3.70 \mathrm{E}+05$ & $3.18 \mathrm{E}+05$ & ICRP-72 & $6.80 \mathrm{E}-10$ & $\mathrm{M}$ & Rec & FGR-12 & $1.22 \mathrm{E}-13$ \\
\hline Tc-96m & Tc-96 & 95.908 & Technetium & $1.00 \mathrm{E}-03$ & ICRP-38 & $5.15 \mathrm{E}+01$ & $\mathrm{~m}$ & $3.09 \mathrm{E}+03$ & $3.81 \mathrm{E}+07$ & ICRP-72 & $7.40 \mathrm{E}-12$ & $M$ & Rec & FGR-12 & $2.24 \mathrm{E}-15$ \\
\hline Tc-97 & TC-97 & 96.906 & Technetium & $1.00 \mathrm{E}-03$ & ICRP-38 & $2.60 \mathrm{E}+06$ & $y$ & $8.20 \mathrm{E}+13$ & $1.42 \mathrm{E}-03$ & ICRP-72 & $2.20 \mathrm{E}-10$ & $M$ & $\operatorname{Rec}$ & FGR-12 & $3.33 \mathrm{E}-17$ \\
\hline Tc-97m & TC-97 & 96.906 & Technetium & $1.00 \mathrm{E}-03$ & ICRP-38 & $8.70 \mathrm{E}+01$ & $d$ & $7.52 \mathrm{E}+06$ & $1.55 \mathrm{E}+04$ & ICRP-72 & $3.20 \mathrm{E}-09$ & $M$ & $\operatorname{Rec}$ & FGR-12 & $4.64 \mathrm{E}-17$ \\
\hline Tc-98 & TC-98 & 97.907 & Technetium & $1.00 \mathrm{E}-03$ & ICRP-38 & $4.20 \mathrm{E}+06$ & $y$ & $1.33 \mathrm{E}+14$ & $8.69 \mathrm{E}-04$ & ICRP-72 & $8.30 \mathrm{E}-09$ & $M$ & $\operatorname{Rec}$ & FGR-12 & $6.86 \mathrm{E}-14$ \\
\hline TC-99 & TC-99 & 98.906 & Technetium & $1.00 \mathrm{E}-03$ & ICRP-38 & $2.13 \mathrm{E}+05$ & $y$ & $6.72 \mathrm{E}+12$ & $1.70 \mathrm{E}-02$ & ICRP-72 & 4.00E-09 & $M$ & $\operatorname{Rec}$ & FGR-12 & $1.62 \mathrm{E}-18$ \\
\hline Tc-99m & Tc-99 & 98.906 & Technetium & $1.00 \mathrm{E}-03$ & ICRP-38 & $6.02 \mathrm{E}+00$ & $\mathrm{~h}$ & $2.17 E+04$ & $5.26 \mathrm{E}+06$ & ICRP-72 & $1.90 \mathrm{E}-11$ & $M$ & $\operatorname{Rec}$ & FGR-12 & $5.89 \mathrm{E}-15$ \\
\hline Te-113 & Te-113 & 112.916 & Tellurium & $1.00 \mathrm{E}-02$ & ICRP-107 & $1.70 \mathrm{E}+00$ & $\mathrm{~m}$ & $1.02 \mathrm{E}+02$ & $9.80 \mathrm{E}+08$ & -- & -- & -- & -- & DOE-STD-1196 & $1.05 \mathrm{E}-13$ \\
\hline Te-114 & Te-114 & 113.912 & Tellurium & $1.00 \mathrm{E}-02$ & ICRP-107 & $1.52 \mathrm{E}+01$ & $\mathrm{~m}$ & $9.12 \mathrm{E}+02$ & $1.09 \mathrm{E}+08$ & DOE-STD-1196 & $3.34 \mathrm{E}-11$ & $M$ & $\operatorname{Rec}$ & DOE-STD-1196 & $5.92 \mathrm{E}-14$ \\
\hline Te-115 & Te-115 & 114.912 & Tellurium & $1.00 \mathrm{E}-02$ & ICRP-107 & $5.80 \mathrm{E}+00$ & $\mathrm{~m}$ & $3.48 \mathrm{E}+02$ & $2.82 E+08$ & -- & -- & -- & -- & DOE-STD-1196 & $1.05 \mathrm{E}-13$ \\
\hline Te-115m & Te-115 & 114.912 & Tellurium & $1.00 \mathrm{E}-02$ & ICRP-107 & $6.70 E+00$ & $\mathrm{~m}$ & $4.02 \mathrm{E}+02$ & $2.44 \mathrm{E}+08$ & -- & -- & -- & -- & DOE-STD-1196 & $1.22 \mathrm{E}-13$ \\
\hline Te-116 & Te-116 & 115.908 & Tellurium & $1.00 \mathrm{E}-02$ & ICRP-38 & $2.49 \mathrm{E}+00$ & $\mathrm{~h}$ & $8.96 \mathrm{E}+03$ & $1.09 \mathrm{E}+07$ & ICRP-72 & $1.00 \mathrm{E}-10$ & $M$ & $\operatorname{Rec}$ & FGR-12 & $2.29 \mathrm{E}-15$ \\
\hline Te-117 & Te-117 & 116.909 & Tellurium & $1.00 \mathrm{E}-02$ & ICRP-107 & $6.20 \mathrm{E}+01$ & $\mathrm{~m}$ & $3.72 \mathrm{E}+03$ & $2.59 \mathrm{E}+07$ & DOE-STD-1196 & $3.76 \mathrm{E}-11$ & $M$ & $\operatorname{Rec}$ & DOE-STD-1196 & $7.25 \mathrm{E}-14$ \\
\hline Te-118 & Te-118 & 117.906 & Tellurium & $1.00 \mathrm{E}-02$ & ICRP-107 & $6.00 E+00$ & $d$ & $5.18 \mathrm{E}+05$ & $1.85 \mathrm{E}+05$ & DOE-STD-1196 & $2.68 \mathrm{E}-09$ & $M$ & $\operatorname{Rec}$ & DOE-STD-1196 & $1.51 \mathrm{E}-16$ \\
\hline Te-119 & Te-119 & 118.906 & Tellurium & $1.00 \mathrm{E}-02$ & ICRP-107 & $1.61 \mathrm{E}+01$ & $\mathrm{~h}$ & $5.78 \mathrm{E}+04$ & $1.64 \mathrm{E}+06$ & DOE-STD-1196 & $1.26 \mathrm{E}-10$ & $M$ & $\operatorname{Rec}$ & DOE-STD-1196 & $3.43 \mathrm{E}-14$ \\
\hline Te-119m & Te-119 & 118.906 & $\begin{array}{l}\text { Tellurium } \\
\end{array}$ & $1.00 \mathrm{E}-02$ & ICRP-107 & $4.70 E+00$ & $d$ & $4.06 \mathrm{E}+05$ & $2.34 \mathrm{E}+05$ & DOE-STD-1196 & $6.12 \mathrm{E}-10$ & $M$ & $\operatorname{Rec}$ & DOE-STD-1196 & $6.97 \mathrm{E}-14$ \\
\hline Te-121 & Te-121 & 120.905 & Tellurium & $1.00 \mathrm{E}-02$ & ICRP-38 & $1.70 \mathrm{E}+01$ & $d$ & $1.47 \mathrm{E}+06$ & $6.35 \mathrm{E}+04$ & ICRP-72 & $3.80 \mathrm{E}-10$ & $M$ & $\operatorname{Rec}$ & FGR-12 & $2.70 \mathrm{E}-14$ \\
\hline Te-121m & Te-121 & 120.905 & Tellurium & $1.00 \mathrm{E}-02$ & ICRP-38 & $1.54 \mathrm{E}+02$ & $d$ & $1.33 \mathrm{E}+07$ & $7.01 E+03$ & ICRP-72 & $4.20 \mathrm{E}-09$ & $\mathrm{M}$ & Rec & FGR-12 & $9.90 \mathrm{E}-15$ \\
\hline Te-123 & Te-123 & 122.904 & Tellurium & $1.00 \mathrm{E}-02$ & ICRP-38 & $1.00 \mathrm{E}+13$ & $y$ & $3.16 \mathrm{E}+20$ & $2.91 \mathrm{E}-10$ & ICRP-72 & $1.90 \mathrm{E}-09$ & $M$ & $\operatorname{Rec}$ & FGR-12 & $2.15 \mathrm{E}-16$ \\
\hline Te-123m & Te-123 & 122.904 & Tellurium & $1.00 \mathrm{E}-02$ & ICRP-38 & $1.20 \mathrm{E}+02$ & $d$ & $1.03 \mathrm{E}+07$ & $8.88 \mathrm{E}+03$ & ICRP-72 & $4.00 \mathrm{E}-09$ & $M$ & $\operatorname{Rec}$ & FGR-12 & $6.51 \mathrm{E}-15$ \\
\hline Te-125m & Te-125 & 124.904 & Tellurium & $1.00 \mathrm{E}-02$ & ICRP-38 & $5.80 \mathrm{E}+01$ & $d$ & $5.01 \mathrm{E}+06$ & $1.80 \mathrm{E}+04$ & ICRP-72 & $3.40 \mathrm{E}-09$ & $M$ & $\operatorname{Rec}$ & FGR-12 & $4.53 \mathrm{E}-16$ \\
\hline Te-127 & Te-127 & 126.905 & Tellurium & $1.00 \mathrm{E}-02$ & ICRP-38 & $9.35 \mathrm{E}+00$ & $\mathrm{~h}$ & $3.37 \mathrm{E}+04$ & $2.64 \mathrm{E}+06$ & ICRP-72 & $1.30 \mathrm{E}-10$ & $M$ & $\operatorname{Rec}$ & FGR-12 & $2.42 \mathrm{E}-16$ \\
\hline Te-127m & Te-127 & 126.905 & Tellurium & $1.00 \mathrm{E}-02$ & ICRP-38 & $1.09 \mathrm{E}+02$ & $d$ & $9.42 \mathrm{E}+06$ & $9.44 \mathrm{E}+03$ & ICRP-72 & $7.40 \mathrm{E}-09$ & $M$ & $\operatorname{Rec}$ & FGR-12 & $1.47 \mathrm{E}-16$ \\
\hline Te-129 & Te-129 & 128.907 & Tellurium & $1.00 \mathrm{E}-02$ & ICRP-38 & $6.96 \mathrm{E}+01$ & $\mathrm{~m}$ & $4.18 \mathrm{E}+03$ & $2.10 \mathrm{E}+07$ & ICRP-72 & $3.70 \mathrm{E}-11$ & $M$ & $\operatorname{Rec}$ & FGR-12 & $2.75 \mathrm{E}-15$ \\
\hline Te-129m & Te-129 & 128.907 & Tellurium & $1.00 \mathrm{E}-02$ & ICRP-38 & $3.36 \mathrm{E}+01$ & $d$ & $2.90 \mathrm{E}+06$ & $3.01 \mathrm{E}+04$ & ICRP-72 & $6.60 \mathrm{E}-09$ & $M$ & $\operatorname{Rec}$ & FGR-12 & $1.55 \mathrm{E}-15$ \\
\hline Te-131 & Te-131 & 130.909 & Tellurium & $1.00 \mathrm{E}-02$ & ICRP-38 & $2.50 \mathrm{E}+01$ & $\mathrm{~m}$ & $1.50 \mathrm{E}+03$ & $5.75 \mathrm{E}+07$ & ICRP-72 & $2.80 \mathrm{E}-11$ & $\mathrm{M}$ & $\operatorname{Rec}$ & FGR-12 & $2.04 \mathrm{E}-14$ \\
\hline Te-131m & Te-131 & 130.909 & Tellurium & $1.00 \mathrm{E}-02$ & ICRP-38 & $3.00 \mathrm{E}+01$ & $\mathrm{~h}$ & $1.08 \mathrm{E}+05$ & $7.98 \mathrm{E}+05$ & ICRP-72 & $9.40 \mathrm{E}-10$ & $M$ & $\operatorname{Rec}$ & FGR-12 & $7.01 \mathrm{E}-14$ \\
\hline Te-132 & Te-132 & 131.909 & Tellurium & $1.00 \mathrm{E}-02$ & ICRP-38 & $7.82 \mathrm{E}+01$ & $\mathrm{~h}$ & $2.82 \mathrm{E}+05$ & $3.04 \mathrm{E}+05$ & ICRP-72 & $2.00 \mathrm{E}-09$ & $M$ & $\operatorname{Rec}$ & FGR-12 & $1.03 \mathrm{E}-14$ \\
\hline Te-133 & Te-133 & 132.911 & Tellurium & $1.00 \mathrm{E}-02$ & ICRP-38 & $1.25 \mathrm{E}+01$ & $\mathrm{~m}$ & $7.47 E+02$ & $1.14 \mathrm{E}+08$ & ICRP-72 & $2.00 \mathrm{E}-11$ & $M$ & $\operatorname{Rec}$ & FGR-12 & $4.60 \mathrm{E}-14$ \\
\hline Te-133m & Te-133 & 132.911 & Tellurium & $1.00 \mathrm{E}-02$ & ICRP-38 & $5.54 \mathrm{E}+01$ & $\mathrm{~m}$ & $3.32 \mathrm{E}+03$ & $2.55 \mathrm{E}+07$ & ICRP-72 & $8.70 \mathrm{E}-11$ & $M$ & $\operatorname{Rec}$ & FGR-12 & $1.14 \mathrm{E}-13$ \\
\hline Te-134 & Te-134 & 133.911 & Tellurium & $1.00 \mathrm{E}-02$ & ICRP-38 & $4.18 \mathrm{E}+01$ & $\mathrm{~m}$ & $2.51 \mathrm{E}+03$ & $3.36 \mathrm{E}+07$ & ICRP-72 & $6.60 \mathrm{E}-11$ & $M$ & $\operatorname{Rec}$ & FGR-12 & $4.24 \mathrm{E}-14$ \\
\hline Th-223 & Th-223 & 223.021 & Thorium & $1.00 \mathrm{E}-03$ & ICRP-107 & $6.00 \mathrm{E}-01$ & $\mathrm{~s}$ & $6.00 \mathrm{E}-01$ & $8.43 \mathrm{E}+10$ & -- & -- & -- & -- & DOE-STD-1196 & $2.78 \mathrm{E}-15$ \\
\hline Th-224 & Th-224 & 224.021 & Thorium & $1.00 \mathrm{E}-03$ & ICRP-107 & $1.05 \mathrm{E}+00$ & $\mathrm{~s}$ & $1.05 \mathrm{E}+00$ & $4.80 \mathrm{E}+10$ & -- & -- & -- & -- & DOE-STD-1196 & $9.75 \mathrm{E}-16$ \\
\hline Th-226 & Th-226 & 226.025 & Thorium & $1.00 \mathrm{E}-03$ & ICRP-38 & $3.09 \mathrm{E}+01$ & $\mathrm{~m}$ & $1.85 \mathrm{E}+03$ & $2.69 \mathrm{E}+07$ & ICRP-72 & $6.10 \mathrm{E}-08$ & $\mathrm{~s}$ & $\operatorname{Rec}$ & FGR-12 & $3.59 \mathrm{E}-16$ \\
\hline Th-227 & Th-227 & 227.028 & Thorium & $1.00 \mathrm{E}-03$ & ICRP-38 & $1.87 E+01$ & $d$ & $1.62 \mathrm{E}+06$ & $3.07 \mathrm{E}+04$ & ICRP-72 & $1.00 \mathrm{E}-05$ & $\mathrm{~s}$ & $\operatorname{Rec}$ & FGR-12 & $4.88 \mathrm{E}-15$ \\
\hline Th-228 & Th-228 & 228.029 & Thorium & $1.00 \mathrm{E}-03$ & ICRP-38 & $1.91 \mathrm{E}+00$ & $y$ & $6.04 \mathrm{E}+07$ & $8.19 \mathrm{E}+02$ & ICRP-72 & $4.00 \mathrm{E}-05$ & $\mathrm{~s}$ & $\operatorname{Rec}$ & FGR-12 & $9.20 \mathrm{E}-17$ \\
\hline Th-229 & Th-229 & 229.032 & Thorium & $1.00 \mathrm{E}-03$ & ICRP-38 & $7.34 \mathrm{E}+03$ & $y$ & $2.32 \mathrm{E}+11$ & $2.13 \mathrm{E}-01$ & ICRP-72 & $7.10 \mathrm{E}-05$ & $\mathrm{~s}$ & $\operatorname{Rec}$ & FGR-12 & $3.83 \mathrm{E}-15$ \\
\hline Th-230 & Th-230 & 230.033 & Thorium & $1.00 \mathrm{E}-03$ & ICRP-38 & $7.70 \mathrm{E}+04$ & $y$ & $2.43 \mathrm{E}+12$ & $2.02 \mathrm{E}-02$ & ICRP-72 & 1.40E-05 & $\mathrm{s}$ & $\operatorname{Rec}$ & FGR-12 & $1.74 \mathrm{E}-17$ \\
\hline Th-231 & Th-231 & 231.036 & Thorium & $1.00 \mathrm{E}-03$ & ICRP-38 & $2.55 \mathrm{E}+01$ & $\mathrm{~h}$ & $9.19 \mathrm{E}+04$ & $5.31 \mathrm{E}+05$ & ICRP-72 & $3.30 \mathrm{E}-10$ & $\mathrm{~s}$ & $\operatorname{Rec}$ & FGR-12 & $5.22 \mathrm{E}-16$ \\
\hline Th-232 & Th-232 & 232.038 & Thorium & $1.00 \mathrm{E}-03$ & ICRP-38 & $1.41 \mathrm{E}+10$ & $y$ & $4.43 \mathrm{E}+17$ & $1.10 \mathrm{E}-07$ & ICRP-72 & $2.50 \mathrm{E}-05$ & $\mathrm{~s}$ & $\operatorname{Rec}$ & FGR-12 & $8.72 \mathrm{E}-18$ \\
\hline Th-233 & Th-233 & 233.042 & Thorium & $1.00 \mathrm{E}-03$ & ICRP-107 & $2.23 \mathrm{E}+01$ & $m$ & $1.34 \mathrm{E}+03$ & $3.62 \mathrm{E}+07$ & DOE-STD-1196 & $2.12 \mathrm{E}-11$ & $\mathrm{~s}$ & $\operatorname{Rec}$ & DOE-STD-1196 & $1.79 \mathrm{E}-15$ \\
\hline Th-234 & Th-234 & 234.044 & Thorium & $1.00 \mathrm{E}-03$ & ICRP-38 & $2.41 \mathrm{E}+01$ & $d$ & $2.08 \mathrm{E}+06$ & $2.31 \mathrm{E}+04$ & ICRP-72 & $7.70 \mathrm{E}-09$ & $\mathrm{~s}$ & $\operatorname{Rec}$ & FGR-12 & $3.38 \mathrm{E}-16$ \\
\hline Th-235 & Th-235 & 235.047 & Thorium & $1.00 \mathrm{E}-03$ & ICRP-107 & $7.10 E+00$ & $\mathrm{~m}$ & $4.26 \mathrm{E}+02$ & $1.13 \mathrm{E}+08$ & -- & -- & -- & -- & DOE-STD-1196 & $2.93 \mathrm{E}-15$ \\
\hline Th-236 & Th-236 & 236.050 & Thorium & $1.00 \mathrm{E}-03$ & ICRP-107 & $3.75 E+01$ & $\mathrm{~m}$ & $2.25 \mathrm{E}+03$ & $2.12 \mathrm{E}+07$ & DOE-STD-1196 & $6.71 \mathrm{E}-11$ & $\mathrm{~s}$ & $\operatorname{Rec}$ & DOE-STD-1196 & $1.68 \mathrm{E}-15$ \\
\hline Ti-44 & Ti-44 & 43.960 & Titanium & $1.00 \mathrm{E}-03$ & ICRP-38 & $4.73 \mathrm{E}+01$ & $y$ & $1.49 \mathrm{E}+09$ & $1.72 \mathrm{E}+02$ & ICRP-72 & $1.20 \mathrm{E}-07$ & $\mathrm{~s}$ & Max & FGR-12 & $5.53 \mathrm{E}-15$ \\
\hline Ti-45 & Ti-45 & 44.958 & Titanium & $1.00 \mathrm{E}-03$ & ICRP-38 & $3.08 \mathrm{E}+00$ & $\mathrm{~h}$ & $1.11 \mathrm{E}+04$ & $2.26 \mathrm{E}+07$ & ICRP-72 & $9.30 \mathrm{E}-11$ & $\mathrm{~s}$ & Max & FGR-12 & $4.18 \mathrm{E}-14$ \\
\hline
\end{tabular}




\begin{tabular}{|c|c|c|c|c|c|c|c|c|c|c|c|c|c|c|c|}
\hline \multicolumn{16}{|c|}{ Table B.1 Radionuclide Specific Input Data Used For the Calculation of HC-2 TQs Using Recommended DCs (continued) } \\
\hline $\begin{array}{l}\text { MASTER } \\
\text { ISOTOPE } \\
\text { LIST }\end{array}$ & $\begin{array}{l}\text { Atomic } \\
\text { Mass } \\
\text { Nuclide }\end{array}$ & $\begin{array}{l}\text { Atomic } \\
\text { Mass }\end{array}$ & Element & $\begin{array}{c}\text { RF per } \\
\text { DOE-STD- } \\
1027\end{array}$ & $\begin{array}{l}\text { Half-Life } \\
\text { Reference }\end{array}$ & $\begin{array}{r}\text { Half-Li } \\
\text { (original } 4\end{array}$ & & Half Life (s) & $\begin{array}{l}\text { Sp. Act } \\
\text { (Ci/g) }\end{array}$ & $\begin{array}{l}\text { Inhalation DC } \\
\text { Reference }\end{array}$ & $\begin{array}{c}\text { HC-2 } \\
\text { Inhalation } \\
\mathrm{DC} \\
(\mathrm{Sv} / \mathrm{Bq})\end{array}$ & & $\begin{array}{l}\text { ung } \\
\text { rption } \\
\text { /Type }\end{array}$ & $\begin{array}{c}\text { Immersion DC } \\
\text { Reference }\end{array}$ & $\begin{array}{c}\text { HC-2 } \\
\text { Immersion } \\
\mathrm{DC}(\mathrm{Sv} / \mathrm{s} \\
\left.\text { per } \mathrm{Bq} / \mathrm{m}^{3}\right)\end{array}$ \\
\hline Ti-51 & Ti-51 & 50.947 & Titanium & $1.00 \mathrm{E}-03$ & ICRP-107 & $5.76 \mathrm{E}+00$ & $\mathrm{~m}$ & $3.46 \mathrm{E}+02$ & $6.41 \mathrm{E}+08$ & -- & -- & -- & -- & DOE-STD-1196 & $1.71 \mathrm{E}-14$ \\
\hline Ti-52 & Ti-52 & 51.947 & Titanium & $1.00 \mathrm{E}-03$ & ICRP-107 & $1.70 \mathrm{E}+00$ & $\mathrm{~m}$ & $1.02 \mathrm{E}+02$ & $2.13 \mathrm{E}+09$ & -- & -- & -- & -- & DOE-STD-1196 & $5.75 \mathrm{E}-15$ \\
\hline Tl-190 & TI-190 & 189.974 & Thallium & $1.00 \mathrm{E}-03$ & ICRP-107 & $2.60 E+00$ & $\mathrm{~m}$ & $1.56 \mathrm{E}+02$ & $3.81 \mathrm{E}+08$ & -- & -- & -- & -- & DOE-STD-1196 & $5.94 \mathrm{E}-14$ \\
\hline Tl-190m & TI-190 & 189.974 & Thallium & $1.00 \mathrm{E}-03$ & ICRP-107 & $3.70 \mathrm{E}+00$ & $\mathrm{~m}$ & $2.22 \mathrm{E}+02$ & $2.67 \mathrm{E}+08$ & -- & -- & -- & -- & DOE-STD-1196 & $1.11 \mathrm{E}-13$ \\
\hline Tl-194 & Tl-194 & 193.971 & Thallium & $1.00 \mathrm{E}-03$ & ICRP-38 & $3.30 \mathrm{E}+01$ & $\mathrm{~m}$ & $1.98 \mathrm{E}+03$ & $2.94 \mathrm{E}+07$ & ICRP-72 & $4.40 \mathrm{E}-12$ & $\mathrm{~F}$ & Max & FGR-12 & $3.70 \mathrm{E}-14$ \\
\hline Tl-194m & TI-194 & 193.971 & Thallium & $1.00 \mathrm{E}-03$ & ICRP-38 & $3.28 \mathrm{E}+01$ & $\mathrm{~m}$ & $1.97 \mathrm{E}+03$ & $2.96 \mathrm{E}+07$ & ICRP-72 & $1.90 \mathrm{E}-11$ & $\mathrm{~F}$ & Max & FGR-12 & $1.11 \mathrm{E}-13$ \\
\hline Tl-195 & TI-195 & 194.970 & Thallium & $1.00 \mathrm{E}-03$ & ICRP-38 & $1.16 \mathrm{E}+00$ & $\mathrm{~h}$ & $4.18 \mathrm{E}+03$ & $1.39 \mathrm{E}+07$ & ICRP-72 & $1.50 \mathrm{E}-11$ & $F$ & Max & FGR-12 & $6.34 \mathrm{E}-14$ \\
\hline TI-196 & TI-196 & 195.970 & Thallium & $1.00 \mathrm{E}-03$ & ICRP-107 & $1.84 \mathrm{E}+00$ & $\mathrm{~h}$ & $6.62 \mathrm{E}+03$ & $8.69 \mathrm{E}+06$ & DOE-STD-1196 & $4.70 \mathrm{E}-11$ & $\mathrm{~s}$ & Max & DOE-STD-1196 & $8.80 \mathrm{E}-14$ \\
\hline TI-197 & TI-197 & 196.970 & Thallium & $1.00 \mathrm{E}-03$ & ICRP-38 & $2.84 \mathrm{E}+00$ & $\mathrm{~h}$ & $1.02 \mathrm{E}+04$ & $5.60 \mathrm{E}+06$ & ICRP-72 & $1.40 \mathrm{E}-11$ & $\mathrm{~F}$ & Max & FGR-12 & $1.93 \mathrm{E}-14$ \\
\hline TI-198 & TI-198 & 197.970 & Thallium & $1.00 \mathrm{E}-03$ & ICRP-38 & $5.30 \mathrm{E}+00$ & $\mathrm{~h}$ & $1.91 \mathrm{E}+04$ & $2.99 \mathrm{E}+06$ & ICRP-72 & $6.00 \mathrm{E}-11$ & $\mathrm{~F}$ & Max & FGR-12 & $1.01 \mathrm{E}-13$ \\
\hline Tl-198m & TT-198 & 197.970 & Thallium & $1.00 \mathrm{E}-03$ & ICRP-38 & $1.87 \mathrm{E}+00$ & $\mathrm{~h}$ & $6.73 \mathrm{E}+03$ & $8.46 \mathrm{E}+06$ & ICRP-72 & $3.70 \mathrm{E}-11$ & $\mathrm{~F}$ & Max & FGR-12 & $5.69 \mathrm{E}-14$ \\
\hline Tl-199 & Tl-199 & 198.970 & Thallium & $1.00 \mathrm{E}-03$ & ICRP-38 & $7.42 \mathrm{E}+00$ & $\mathrm{~h}$ & $2.67 \mathrm{E}+04$ & $2.12 \mathrm{E}+06$ & ICRP-72 & $1.90 \mathrm{E}-11$ & $\mathrm{~F}$ & Max & FGR-12 & $1.13 \mathrm{E}-14$ \\
\hline TI-200 & TI-200 & 199.971 & Thallium & $1.00 \mathrm{E}-03$ & ICRP-38 & $2.61 E+01$ & $\mathrm{~h}$ & $9.40 \mathrm{E}+04$ & $6.00 \mathrm{E}+05$ & ICRP-72 & $1.30 \mathrm{E}-10$ & $\mathrm{~F}$ & Max & FGR-12 & $6.42 \mathrm{E}-14$ \\
\hline TI-201 & TI-201 & 200.971 & Thallium & $1.00 \mathrm{E}-03$ & ICRP-38 & $3.04 \mathrm{E}+00$ & $d$ & $2.63 \mathrm{E}+05$ & $2.13 \mathrm{E}+05$ & ICRP-72 & $4.40 \mathrm{E}-11$ & $F$ & Max & FGR-12 & $3.78 \mathrm{E}-15$ \\
\hline TI-202 & TT-202 & 201.972 & Thallium & $1.00 \mathrm{E}-03$ & ICRP-38 & $1.22 \mathrm{E}+01$ & $d$ & $1.06 \mathrm{E}+06$ & $5.29 \mathrm{E}+04$ & ICRP-72 & $1.90 \mathrm{E}-10$ & $\mathrm{~F}$ & Max & FGR-12 & $2.18 \mathrm{E}-14$ \\
\hline TI-204 & TT-204 & 203.974 & Thallium & $1.00 \mathrm{E}-03$ & ICRP-38 & $3.78 \mathrm{E}+00$ & $y$ & $1.19 \mathrm{E}+08$ & $4.64 \mathrm{E}+02$ & ICRP-72 & $3.90 \mathrm{E}-10$ & $\mathrm{~F}$ & Max & FGR-12 & $5.59 \mathrm{E}-17$ \\
\hline TI-206 & TT-206 & 205.976 & Thallium & $1.00 \mathrm{E}-03$ & ICRP-38 & $4.20 \mathrm{E}+00$ & $\mathrm{~m}$ & $2.52 E+02$ & $2.17 \mathrm{E}+08$ & -- & -- & -- & -- & FGR-12 & $6.73 \mathrm{E}-17$ \\
\hline TI-206m & TT-206 & 205.976 & Thallium & $1.00 \mathrm{E}-03$ & ICRP-107 & $3.74 \mathrm{E}+00$ & $\mathrm{~m}$ & $2.24 \mathrm{E}+02$ & $2.44 \mathrm{E}+08$ & -- & -- & -- & -- & DOE-STD-1196 & $1.09 \mathrm{E}-13$ \\
\hline Tl-207 & Tl-207 & 206.977 & Thallium & $1.00 \mathrm{E}-03$ & ICRP-38 & $4.77 \mathrm{E}+00$ & $\mathrm{~m}$ & $2.86 \mathrm{E}+02$ & $1.90 \mathrm{E}+08$ & -- & -- & -- & -- & FGR-12 & $1.62 \mathrm{E}-16$ \\
\hline TI-208 & TT-208 & 207.982 & Thallium & $1.00 \mathrm{E}-03$ & ICRP-38 & $3.07 \mathrm{E}+00$ & $\mathrm{~m}$ & $1.84 \mathrm{E}+02$ & $2.94 \mathrm{E}+08$ & -- & -- & -- & -- & FGR-12 & $1.77 \mathrm{E}-13$ \\
\hline TI-209 & Tl-209 & 208.985 & Thallium & $1.00 \mathrm{E}-03$ & ICRP-38 & $2.20 \mathrm{E}+00$ & $\mathrm{~m}$ & $1.32 \mathrm{E}+02$ & $4.09 \mathrm{E}+08$ & -- & -- & -- & -- & FGR-12 & $1.02 \mathrm{E}-13$ \\
\hline TI-210 & TI-210 & 209.990 & Thallium & $1.00 \mathrm{E}-03$ & ICRP-38 & $1.30 \mathrm{E}+00$ & $\mathrm{~m}$ & $7.80 \mathrm{E}+01$ & $6.89 \mathrm{E}+08$ & -- & -- & -- & -- & DOE-STD-1196 & $1.32 \mathrm{E}-13$ \\
\hline Tm-159 & Tm-159 & 158.935 & Thulium & $1.00 \mathrm{E}-03$ & JAERI & $9.13 \mathrm{E}+00$ & $\mathrm{~m}$ & $5.48 \mathrm{E}+02$ & $1.30 \mathrm{E}+08$ & -- & -- & -- & -- & -- & -- \\
\hline Tm-161 & Tm-161 & 160.934 & Thulium & $1.00 \mathrm{E}-03$ & ICRP-107 & $3.02 \mathrm{E}+01$ & $\mathrm{~m}$ & $1.81 \mathrm{E}+03$ & $3.87 \mathrm{E}+07$ & DOE-STD-1196 & $3.06 \mathrm{E}-11$ & $\mathrm{~s}$ & Max & DOE-STD-1196 & $5.89 \mathrm{E}-14$ \\
\hline Tm-162 & Tm-162 & 161.934 & Thulium & $1.00 \mathrm{E}-03$ & ICRP-38 & $2.17 \mathrm{E}+01$ & $\mathrm{~m}$ & $1.30 \mathrm{E}+03$ & $5.35 \mathrm{E}+07$ & ICRP-72 & $1.60 \mathrm{E}-11$ & $M$ & Max & FGR-12 & $9.01 \mathrm{E}-14$ \\
\hline $\mathrm{Tm}-163$ & $\mathrm{Tm}-163$ & 162.933 & Thulium & $1.00 \mathrm{E}-03$ & ICRP-107 & $1.81 \mathrm{E}+00$ & $\mathrm{~h}$ & $6.52 \mathrm{E}+03$ & $1.06 \mathrm{E}+07$ & DOE-STD-1196 & $4.53 \mathrm{E}-11$ & $\mathrm{~s}$ & Max & DOE-STD-1196 & $6.03 \mathrm{E}-14$ \\
\hline Tm-164 & Tm-164 & 163.934 & Thulium & $1.00 \mathrm{E}-03$ & ICRP-107 & $2.00 \mathrm{E}+00$ & $\mathrm{~m}$ & $1.20 \mathrm{E}+02$ & $5.73 \mathrm{E}+08$ & -- & -- & -- & -- & DOE-STD-1196 & $3.57 \mathrm{E}-14$ \\
\hline Tm-165 & Tm-165 & 164.932 & Thulium & $1.00 \mathrm{E}-03$ & ICRP-107 & $3.01 \mathrm{E}+01$ & $\mathrm{~h}$ & $1.08 \mathrm{E}+05$ & $6.32 \mathrm{E}+05$ & DOE-STD-1196 & $2.74 \mathrm{E}-10$ & $\mathrm{~s}$ & Max & DOE-STD-1196 & $2.40 \mathrm{E}-14$ \\
\hline Tm-166 & $\mathrm{Tm}-166$ & 165.934 & Thulium & $1.00 \mathrm{E}-03$ & $\begin{array}{l}\text { ICRP-38 } \\
\end{array}$ & $7.70 \mathrm{E}+00$ & $\mathrm{~h}$ & $2.77 E+04$ & $2.45 \mathrm{E}+06$ & ICRP-72 & $1.70 \mathrm{E}-10$ & $M$ & Max & FGR-12 & $9.35 \mathrm{E}-14$ \\
\hline Tm-167 & $\mathrm{Tm}-167$ & 166.933 & Thulium & $1.00 \mathrm{E}-03$ & ICRP-38 & $9.24 \mathrm{E}+00$ & $d$ & $7.98 \mathrm{E}+05$ & $8.47 \mathrm{E}+04$ & ICRP-72 & $1.10 \mathrm{E}-09$ & $M$ & Max & FGR-12 & $6.06 \mathrm{E}-15$ \\
\hline $\mathrm{Tm}-168$ & $\mathrm{Tm}-168$ & 167.934 & Thulium & $1.00 \mathrm{E}-03$ & ICRP-107 & $9.31 \mathrm{E}+01$ & $d$ & $8.04 \mathrm{E}+06$ & $8.35 \mathrm{E}+03$ & DOE-STD-1196 & $5.60 \mathrm{E}-09$ & $\mathrm{~s}$ & Max & DOE-STD-1196 & $5.51 \mathrm{E}-14$ \\
\hline Tm-170 & Tm-170 & 169.936 & Thulium & $1.00 \mathrm{E}-03$ & ICRP-38 & $1.29 \mathrm{E}+02$ & $d$ & $1.11 \mathrm{E}+07$ & $5.97 \mathrm{E}+03$ & ICRP-72 & $7.00 \mathrm{E}-09$ & $M$ & Max & FGR-12 & $2.23 \mathrm{E}-16$ \\
\hline Tm-171 & Tm-171 & 170.936 & Thulium & $1.00 \mathrm{E}-03$ & ICRP-38 & $1.92 \mathrm{E}+00$ & $y$ & $6.06 \mathrm{E}+07$ & $1.09 \mathrm{E}+03$ & ICRP-72 & $1.40 \mathrm{E}-09$ & $M$ & Max & FGR-12 & $2.15 \mathrm{E}-17$ \\
\hline Tm-172 & Tm-172 & 171.938 & Thulium & $1.00 \mathrm{E}-03$ & ICRP-38 & $6.36 \mathrm{E}+01$ & $\mathrm{~h}$ & $2.29 \mathrm{E}+05$ & $2.87 E+05$ & ICRP-72 & $1.10 \mathrm{E}-09$ & $M$ & Max & FGR-12 & $2.41 \mathrm{E}-14$ \\
\hline Tm-173 & Tm-173 & 172.940 & Thulium & $1.00 \mathrm{E}-03$ & ICRP-38 & $8.24 \mathrm{E}+00$ & $\mathrm{~h}$ & $2.97 \mathrm{E}+04$ & $2.20 \mathrm{E}+06$ & ICRP-72 & $1.80 \mathrm{E}-10$ & $M$ & Max & FGR-12 & $1.85 \mathrm{E}-14$ \\
\hline Tm-174 & Tm-174 & 173.942 & Thulium & $1.00 \mathrm{E}-03$ & ICRP-107 & $5.40 \mathrm{E}+00$ & $\mathrm{~m}$ & $3.24 \mathrm{E}+02$ & $2.00 E+08$ & -- & -- & -- & -- & DOE-STD-1196 & $8.05 \mathrm{E}-14$ \\
\hline Tm-175 & Tm-175 & 174.944 & Thulium & $1.00 \mathrm{E}-03$ & ICRP-38 & $1.52 \mathrm{E}+01$ & $\mathrm{~m}$ & $9.12 \mathrm{E}+02$ & $7.07 E+07$ & ICRP-72 & $1.80 \mathrm{E}-11$ & $M$ & Max & FGR-12 & $5.13 \mathrm{E}-14$ \\
\hline Tm-176 & Tm-176 & 175.947 & Thulium & $1.00 \mathrm{E}-03$ & ICRP-107 & $1.85 \mathrm{E}+00$ & $\mathrm{~m}$ & $1.11 \mathrm{E}+02$ & $5.78 \mathrm{E}+08$ & -- & -- & -- & -- & DOE-STD-1196 & $9.39 \mathrm{E}-14$ \\
\hline U-227 & U-227 & 227.031 & Uranium & $1.00 \mathrm{E}-03$ & ICRP-107 & $1.10 \mathrm{E}+00$ & $\mathrm{~m}$ & $6.60 \mathrm{E}+01$ & $7.53 \mathrm{E}+08$ & -- & -- & -- & -- & DOE-STD-1196 & $4.85 \mathrm{E}-15$ \\
\hline U-228 & U-228 & 228.031 & Uranium & $1.00 \mathrm{E}-03$ & ICRP-107 & $9.10 \mathrm{E}+00$ & $\mathrm{~m}$ & $5.46 \mathrm{E}+02$ & $9.06 \mathrm{E}+07$ & -- & -- & -- & -- & DOE-STD-1196 & $1.59 \mathrm{E}-16$ \\
\hline$U-230$ & $U-230$ & 230.034 & Uranium & $1.00 \mathrm{E}-03$ & $\begin{array}{l}\text { ICRP-38 } \\
\end{array}$ & $2.08 \mathrm{E}+01$ & $d$ & $1.80 \mathrm{E}+06$ & $2.73 \mathrm{E}+04$ & ICRP-72 & $1.30 \mathrm{E}-05$ & $\mathrm{M}$ & $\operatorname{Rec}$ & FGR-12 & $5.23 \mathrm{E}-17$ \\
\hline U-231 & $U-231$ & 231.036 & Uranium & $1.00 \mathrm{E}-03$ & ICRP-38 & $4.20 \mathrm{E}+00$ & $d$ & $3.63 \mathrm{E}+05$ & $1.35 \mathrm{E}+05$ & ICRP-72 & $4.70 \mathrm{E}-10$ & $M$ & $\operatorname{Rec}$ & FGR-12 & $2.95 \mathrm{E}-15$ \\
\hline U-232 & $U-232$ & 232.037 & Uranium & $1.00 \mathrm{E}-03$ & ICRP-38 & $7.20 \mathrm{E}+01$ & $y$ & $2.27 \mathrm{E}+09$ & $2.14 \mathrm{E}+01$ & ICRP-72 & $7.80 \mathrm{E}-06$ & $M$ & $\operatorname{Rec}$ & FGR-12 & $1.42 \mathrm{E}-17$ \\
\hline U-233 & $U-233$ & 233.040 & Uranium & $1.00 \mathrm{E}-03$ & ICRP-38 & $1.59 \mathrm{E}+05$ & $y$ & $5.00 \mathrm{E}+12$ & $9.68 \mathrm{E}-03$ & ICRP-72 & $3.60 \mathrm{E}-06$ & $M$ & $\operatorname{Rec}$ & FGR-12 & $1.63 \mathrm{E}-17$ \\
\hline U-234 & U-234 & 234.041 & Uranium & $1.00 \mathrm{E}-03$ & ICRP-38 & $2.45 E+05$ & $y$ & $7.72 \mathrm{E}+12$ & $6.25 \mathrm{E}-03$ & ICRP-72 & $3.50 \mathrm{E}-06$ & $M$ & $\operatorname{Rec}$ & FGR-12 & $7.63 \mathrm{E}-18$ \\
\hline U-235 & U-235 & 235.044 & Uranium & $1.00 \mathrm{E}-03$ & ICRP-38 & $7.04 E+08$ & $y$ & $2.22 \mathrm{E}+16$ & $2.16 \mathrm{E}-06$ & ICRP-72 & $3.10 \mathrm{E}-06$ & $M$ & $\operatorname{Rec}$ & FGR-12 & $7.20 \mathrm{E}-15$ \\
\hline
\end{tabular}




\begin{tabular}{|c|c|c|c|c|c|c|c|c|c|c|c|c|c|c|c|}
\hline \multicolumn{16}{|c|}{ Table B.1 Radionuclide Specific Input Data Used For the Calculation of HC-2 TQs Using Recommended DCs (continued) } \\
\hline $\begin{array}{l}\text { MASTER } \\
\text { ISOTOPE } \\
\text { LIST }\end{array}$ & $\begin{array}{c}\text { Atomic } \\
\text { Mass } \\
\text { Nuclide }\end{array}$ & $\begin{array}{l}\text { Atomic } \\
\text { Mass }\end{array}$ & Element & $\begin{array}{c}\text { RF per } \\
\text { DOE-STD- } \\
1027\end{array}$ & $\begin{array}{l}\text { Half-Life } \\
\text { Reference }\end{array}$ & \multicolumn{2}{|c|}{$\begin{array}{c}\text { Half-Life } \\
\text { (original units) }\end{array}$} & \multirow{2}{*}{$\begin{array}{c}\text { Half Life (s) } \\
1.56 \mathrm{E}+03\end{array}$} & \multirow{2}{*}{$\begin{array}{c}\begin{array}{c}\text { Sp. Act } \\
\text { (Ci/g) }\end{array} \\
3.08 \mathrm{E}+07\end{array}$} & \multirow{2}{*}{$\begin{array}{c}\begin{array}{c}\text { Inhalation DC } \\
\text { Reference }\end{array} \\
\text { DOE-STD-1196 }\end{array}$} & \multirow[t]{2}{*}{$\begin{array}{c}\text { HC-2 } \\
\text { Inhalation } \\
\text { DC } \\
\text { (Sv/Bq) } \\
9.01 \mathrm{E}-16\end{array}$} & \multicolumn{2}{|c|}{$\begin{array}{c}\text { Lung } \\
\text { Absorption } \\
\text { Class/Type }\end{array}$} & \multirow{2}{*}{$\begin{array}{c}\begin{array}{c}\text { Immersion DC } \\
\text { Reference }\end{array} \\
---\end{array}$} & \multirow[t]{2}{*}{$\begin{array}{c}\text { HC-2 } \\
\text { Immersion } \\
\mathrm{DC}(\mathrm{Sv} / \mathrm{s} \\
\left.\text { per } \mathrm{Bq} / \mathrm{m}^{3}\right) \\
---\end{array}$} \\
\hline $\mathrm{U}-235 \mathrm{~m}$ & U-235 & 235.044 & Uranium & $1.00 \mathrm{E}-03$ & ICRP-107 & $2.60 \mathrm{E}+01$ & $\mathrm{~m}$ & & & & & $\mathrm{M}$ & Rec & & \\
\hline $\mathrm{U}-236$ & $\mathrm{U}-236$ & 236.046 & Uranium & $1.00 \mathrm{E}-03$ & ICRP-38 & $2.34 \mathrm{E}+07$ & $\mathrm{y}$ & $7.39 \mathrm{E}+14$ & $6.47 \mathrm{E}-05$ & ICRP-72 & $3.20 \mathrm{E}-06$ & $\mathrm{M}$ & $\operatorname{Rec}$ & FGR-12 & $5.01 \mathrm{E}-18$ \\
\hline U-237 & $\mathrm{U}-237$ & 237.049 & Uranium & $1.00 \mathrm{E}-03$ & ICRP-38 & $6.75 \mathrm{E}+00$ & $d$ & $5.83 \mathrm{E}+05$ & $8.16 \mathrm{E}+04$ & ICRP-72 & $1.70 \mathrm{E}-09$ & $M$ & $\operatorname{Rec}$ & FGR-12 & $5.97 \mathrm{E}-15$ \\
\hline U-238 & U-238 & 238.051 & Uranium & $1.00 \mathrm{E}-03$ & ICRP-38 & $4.47 \mathrm{E}+09$ & $y$ & $1.41 \mathrm{E}+17$ & $3.36 \mathrm{E}-07$ & ICRP-72 & $2.90 \mathrm{E}-06$ & $M$ & $\operatorname{Rec}$ & FGR-12 & $3.41 \mathrm{E}-18$ \\
\hline U-239 & U-239 & 239.054 & Uranium & $1.00 \mathrm{E}-03$ & ICRP-38 & $2.35 \mathrm{E}+01$ & $\mathrm{~m}$ & $1.41 \mathrm{E}+03$ & $3.34 \mathrm{E}+07$ & ICRP-72 & $2.20 \mathrm{E}-11$ & $M$ & $\operatorname{Rec}$ & FGR-12 & $2.17 \mathrm{E}-15$ \\
\hline U-240 & U-240 & 240.057 & Uranium & $1.00 \mathrm{E}-03$ & ICRP-38 & $1.41 \mathrm{E}+01$ & $\mathrm{~h}$ & $5.08 \mathrm{E}+04$ & $9.26 \mathrm{E}+05$ & ICRP-72 & $5.30 \mathrm{E}-10$ & $M$ & $\operatorname{Rec}$ & FGR-12 & $3.93 \mathrm{E}-17$ \\
\hline U-242 & U-242 & 242.063 & Uranium & $1.00 \mathrm{E}-03$ & ICRP-107 & $1.68 \mathrm{E}+01$ & $\mathrm{~m}$ & $1.01 \mathrm{E}+03$ & $4.62 E+07$ & DOE-STD-1196 & $3.21 \mathrm{E}-11$ & $M$ & $\operatorname{Rec}$ & DOE-STD-1196 & $1.96 \mathrm{E}-15$ \\
\hline V-47 & V-47 & 46.955 & Vanadium & $1.00 \mathrm{E}-03$ & ICRP-38 & $3.26 \mathrm{E}+01$ & $\mathrm{~m}$ & $1.96 \mathrm{E}+03$ & $1.23 \mathrm{E}+08$ & ICRP-72 & $2.90 \mathrm{E}-11$ & $M$ & Max & FGR-12 & $4.79 \mathrm{E}-14$ \\
\hline V-48 & $V-48$ & 47.952 & Vanadium & $1.00 \mathrm{E}-03$ & ICRP-38 & $1.62 \mathrm{E}+01$ & $d$ & $1.40 \mathrm{E}+06$ & $1.68 \mathrm{E}+05$ & ICRP-72 & $2.40 \mathrm{E}-09$ & $M$ & Max & FGR-12 & $1.45 \mathrm{E}-13$ \\
\hline V-49 & $V-49$ & 48.949 & Vanadium & $1.00 \mathrm{E}-03$ & ICRP-38 & $3.30 \mathrm{E}+02$ & $d$ & $2.85 \mathrm{E}+07$ & $8.08 \mathrm{E}+03$ & ICRP-72 & $3.40 \mathrm{E}-11$ & $M$ & Max & -- & -- \\
\hline V-50 & $V-50$ & 49.947 & $\begin{array}{l}\text { Vanadium } \\
\end{array}$ & $1.00 \mathrm{E}-03$ & ICRP-107 & $1.50 \mathrm{E}+17$ & $y$ & $4.73 \mathrm{E}+24$ & $4.77 \mathrm{E}-14$ & DOE-STD-1196 & $6.69 \mathrm{E}-08$ & $\mathrm{~F}$ & Max & DOE-STD-1196 & $6.87 \mathrm{E}-14$ \\
\hline V-52 & $V-52$ & 51.945 & Vanadium & $1.00 \mathrm{E}-03$ & ICRP-107 & $3.74 \mathrm{E}+00$ & $\mathrm{~m}$ & $2.25 \mathrm{E}+02$ & $9.67 E+08$ & -- & -- & -- & -- & DOE-STD-1196 & $7.05 \mathrm{E}-14$ \\
\hline V-53 & V-53 & 52.944 & Vanadium & $1.00 \mathrm{E}-03$ & ICRP-107 & $1.61 \mathrm{E}+00$ & $\mathrm{~m}$ & $9.66 \mathrm{E}+01$ & $2.21 \mathrm{E}+09$ & -- & -- & -- & -- & DOE-STD-1196 & $4.93 \mathrm{E}-14$ \\
\hline W-176 & W-176 & 175.946 & Tungsten & $1.00 \mathrm{E}-03$ & ICRP-38 & $2.30 \mathrm{E}+00$ & $\mathrm{~h}$ & $8.28 \mathrm{E}+03$ & $7.74 E+06$ & ICRP-72 & $4.10 \mathrm{E}-11$ & $\mathrm{~F}$ & Max & FGR-12 & $7.02 \mathrm{E}-15$ \\
\hline W-177 & W-177 & 176.947 & Tungsten & $1.00 \mathrm{E}-03$ & ICRP-38 & $1.35 \mathrm{E}+02$ & $\mathrm{~m}$ & $8.10 \mathrm{E}+03$ & $7.87 E+06$ & ICRP-72 & $2.40 \mathrm{E}-11$ & $\mathrm{~F}$ & Max & FGR-12 & $4.26 \mathrm{E}-14$ \\
\hline W-178 & W-178 & 177.946 & Tungsten & $1.00 \mathrm{E}-03$ & ICRP-38 & $2.17 \mathrm{E}+01$ & $d$ & $1.87 E+06$ & $3.38 \mathrm{E}+04$ & ICRP-72 & $7.20 \mathrm{E}-11$ & $\mathrm{~F}$ & Max & FGR-12 & $4.62 \mathrm{E}-16$ \\
\hline W-179 & W-179 & 178.947 & Tungsten & $1.00 \mathrm{E}-03$ & ICRP-38 & $3.75 \mathrm{E}+01$ & $\mathrm{~m}$ & $2.25 \mathrm{E}+03$ & $2.80 E+07$ & ICRP-72 & $9.20 \mathrm{E}-13$ & $\mathrm{~F}$ & Max & FGR-12 & $1.83 \mathrm{E}-15$ \\
\hline W-179m & W-179 & 178.947 & Tungsten & $1.00 \mathrm{E}-03$ & ICRP-107 & $6.40 \mathrm{E}+00$ & $\mathrm{~m}$ & $3.84 \mathrm{E}+02$ & $1.64 \mathrm{E}+08$ & -- & -- & -- & -- & DOE-STD-1196 & $1.99 \mathrm{E}-15$ \\
\hline W-181 & W-181 & 180.948 & Tungsten & $1.00 \mathrm{E}-03$ & ICRP-38 & $1.21 \mathrm{E}+02$ & $d$ & $1.05 E+07$ & $5.95 \mathrm{E}+03$ & ICRP-72 & $2.70 \mathrm{E}-11$ & $\mathrm{~F}$ & Max & FGR-12 & $1.40 \mathrm{E}-15$ \\
\hline W-185 & W-185 & 184.953 & Tungsten & $1.00 \mathrm{E}-03$ & ICRP-38 & $7.51 \mathrm{E}+01$ & $d$ & $6.49 \mathrm{E}+06$ & $9.40 \mathrm{E}+03$ & ICRP-72 & $1.20 \mathrm{E}-10$ & $\mathrm{~F}$ & Max & FGR-12 & $5.37 \mathrm{E}-18$ \\
\hline W-185m & W-185 & 184.953 & Tungsten & $1.00 \mathrm{E}-03$ & ICRP-107 & $1.60 \mathrm{E}+00$ & $\mathrm{~m}$ & $9.58 \mathrm{E}+01$ & $6.37 E+08$ & -- & -- & -- & -- & DOE-STD-1196 & $9.33 \mathrm{E}-16$ \\
\hline W-187 & W-187 & 186.957 & Tungsten & $1.00 \mathrm{E}-03$ & ICRP-38 & $2.39 \mathrm{E}+01$ & $\mathrm{~h}$ & $8.60 \mathrm{E}+04$ & $7.01 E+05$ & ICRP-72 & $1.90 \mathrm{E}-10$ & $\mathrm{~F}$ & Max & FGR-12 & $2.28 \mathrm{E}-14$ \\
\hline W-188 & W-188 & 187.958 & Tungsten & $1.00 \mathrm{E}-03$ & ICRP-38 & $6.94 \mathrm{E}+01$ & $d$ & $6.00 \mathrm{E}+06$ & $1.00 E+04$ & ICRP-72 & $5.70 \mathrm{E}-10$ & $\mathrm{~F}$ & Max & FGR-12 & $9.04 \mathrm{E}-17$ \\
\hline W-190 & W-190 & 189.963 & Tungsten & $1.00 \mathrm{E}-03$ & ICRP-107 & $3.00 \mathrm{E}+01$ & $\mathrm{~m}$ & $1.80 \mathrm{E}+03$ & $3.30 \mathrm{E}+07$ & DOE-STD-1196 & $8.54 \mathrm{E}-11$ & $\mathrm{~s}$ & Max & DOE-STD-1196 & $5.75 \mathrm{E}-15$ \\
\hline $\mathrm{Xe}-120$ & Xe-120 & 119.912 & Xenon & $1.00 \mathrm{E}+00$ & ICRP-38 & $4.00 \mathrm{E}+01$ & $\mathrm{~m}$ & $2.40 \mathrm{E}+03$ & $3.92 E+07$ & -- & -- & -- & -- & FGR-12 & $1.94 \mathrm{E}-14$ \\
\hline Xe-121 & Xe-121 & 120.911 & Xenon & $1.00 \mathrm{E}+00$ & ICRP-38 & $4.01 \mathrm{E}+01$ & $\mathrm{~m}$ & $2.41 \mathrm{E}+03$ & $3.88 \mathrm{E}+07$ & -- & -- & -- & -- & FGR-12 & $9.14 \mathrm{E}-14$ \\
\hline $\mathrm{Xe}-122$ & Xe-122 & 121.908 & Xenon & $1.00 \mathrm{E}+00$ & ICRP-38 & $2.01 \mathrm{E}+01$ & $\mathrm{~h}$ & $7.24 E+04$ & $1.28 \mathrm{E}+06$ & -- & -- & -- & -- & FGR-12 & $2.46 \mathrm{E}-15$ \\
\hline Xe-123 & Xe-123 & 122.908 & Xenon & $1.00 \mathrm{E}+00$ & ICRP-38 & $2.08 \mathrm{E}+00$ & $\mathrm{~h}$ & $7.49 \mathrm{E}+03$ & $1.23 \mathrm{E}+07$ & -- & -- & -- & -- & FGR-12 & $3.03 \mathrm{E}-14$ \\
\hline Xe-125 & Xe-125 & 124.906 & Xenon & $1.00 \mathrm{E}+00$ & ICRP-38 & $1.70 \mathrm{E}+01$ & $\mathrm{~h}$ & $6.12 \mathrm{E}+04$ & $1.48 \mathrm{E}+06$ & -- & -- & -- & -- & FGR-12 & $1.19 \mathrm{E}-14$ \\
\hline Xe-127 & Xe-127 & 126.905 & Xenon & $1.00 \mathrm{E}+00$ & ICRP-38 & $3.64 \mathrm{E}+01$ & $d$ & $3.15 \mathrm{E}+06$ & $2.83 \mathrm{E}+04$ & -- & -- & -- & -- & FGR-12 & $1.25 \mathrm{E}-14$ \\
\hline Xe-127m & Xe-127 & 126.905 & Xenon & $1.00 \mathrm{E}+00$ & ICRP-107 & $6.92 \mathrm{E}+01$ & $\mathrm{~s}$ & $6.92 \mathrm{E}+01$ & $1.28 \mathrm{E}+09$ & -- & -- & -- & -- & DOE-STD-1196 & $6.57 \mathrm{E}-15$ \\
\hline Xe-129m & Xe-129 & 128.905 & Xenon & $1.00 \mathrm{E}+00$ & ICRP-38 & $8.00 \mathrm{E}+00$ & $d$ & $6.91 \mathrm{E}+05$ & $1.27 E+05$ & -- & -- & -- & -- & FGR-12 & $1.06 \mathrm{E}-15$ \\
\hline Xe-131m & Xe-131 & 130.905 & Xenon & $1.00 \mathrm{E}+00$ & ICRP-38 & $1.19 \mathrm{E}+01$ & $d$ & $1.03 \mathrm{E}+06$ & $8.38 \mathrm{E}+04$ & -- & -- & -- & -- & FGR-12 & $3.89 \mathrm{E}-16$ \\
\hline Xe-133 & Xe-133 & 132.906 & Xenon & $1.00 \mathrm{E}+00$ & ICRP-38 & $5.25 \mathrm{E}+00$ & $d$ & $4.53 \mathrm{E}+05$ & $1.87 E+05$ & -- & -- & -- & -- & FGR-12 & $1.56 \mathrm{E}-15$ \\
\hline Xe-133m & Xe-133 & 132.906 & Xenon & $1.00 \mathrm{E}+00$ & ICRP-38 & $2.19 \mathrm{E}+00$ & $d$ & $1.89 \mathrm{E}+05$ & $4.49 \mathrm{E}+05$ & -- & -- & -- & -- & FGR-12 & $1.37 \mathrm{E}-15$ \\
\hline Xe-135 & Xe-135 & 134.907 & Xenon & $1.00 \mathrm{E}+00$ & ICRP-38 & $9.09 \mathrm{E}+00$ & $\mathrm{~h}$ & $3.27 \mathrm{E}+04$ & $2.56 E+06$ & -- & -- & -- & -- & FGR-12 & $1.19 \mathrm{E}-14$ \\
\hline Xe-135m & Xe-135 & 134.907 & Xenon & $1.00 \mathrm{E}+00$ & ICRP-38 & $1.53 \mathrm{E}+01$ & $\mathrm{~m}$ & $9.17 E+02$ & $9.12 \mathrm{E}+07$ & -- & -- & -- & -- & FGR-12 & $2.04 \mathrm{E}-14$ \\
\hline Xe-137 & Xe-137 & 136.912 & Xenon & $1.00 \mathrm{E}+00$ & ICRP-107 & $3.82 \mathrm{E}+00$ & $\mathrm{~m}$ & $2.29 \mathrm{E}+02$ & $3.60 E+08$ & -- & -- & -- & -- & DOE-STD-1196 & $1.04 \mathrm{E}-14$ \\
\hline Xe-138 & Xe-138 & 137.914 & Xenon & $1.00 \mathrm{E}+00$ & ICRP-38 & $1.42 \mathrm{E}+01$ & $\mathrm{~m}$ & $8.50 \mathrm{E}+02$ & $9.62 E+07$ & -- & -- & -- & -- & FGR-12 & $5.77 \mathrm{E}-14$ \\
\hline $\mathrm{Y}-81$ & $\mathrm{Y}-81$ & 80.929 & Yttrium & $1.00 \mathrm{E}-03$ & ICRP-107 & $7.04 E+01$ & $\mathrm{~s}$ & $7.04 \mathrm{E}+01$ & $1.98 \mathrm{E}+09$ & -- & -- & -- & -- & DOE-STD-1196 & $5.35 \mathrm{E}-14$ \\
\hline Y-83 & $Y-83$ & 82.922 & Yttrium & $1.00 \mathrm{E}-03$ & ICRP-107 & $7.08 \mathrm{E}+00$ & $\mathrm{~m}$ & $4.25 \mathrm{E}+02$ & $3.20 E+08$ & -- & -- & -- & -- & DOE-STD-1196 & $6.16 \mathrm{E}-14$ \\
\hline $\mathrm{Y}-83 \mathrm{~m}$ & $Y-83$ & 82.922 & Yttrium & $1.00 \mathrm{E}-03$ & ICRP-107 & $2.85 \mathrm{E}+00$ & $\mathrm{~m}$ & $1.71 \mathrm{E}+02$ & $7.96 \mathrm{E}+08$ & -- & -- & -- & -- & DOE-STD-1196 & $3.77 \mathrm{E}-14$ \\
\hline $\mathrm{Y}-84 \mathrm{~m}$ & $Y-84$ & 83.921 & Yttrium & $1.00 \mathrm{E}-03$ & ICRP-107 & $3.95 \mathrm{E}+01$ & $\mathrm{~m}$ & $2.37 E+03$ & $5.67 E+07$ & DOE-STD-1196 & $7.50 \mathrm{E}-11$ & $\mathrm{~s}$ & Max & DOE-STD-1196 & $1.84 \mathrm{E}-13$ \\
\hline$Y-85$ & $Y-85$ & 84.916 & Yttrium & $1.00 \mathrm{E}-03$ & ICRP-107 & $2.68 \mathrm{E}+00$ & $\mathrm{~h}$ & $9.65 \mathrm{E}+03$ & $1.38 \mathrm{E}+07$ & DOE-STD-1196 & $1.16 \mathrm{E}-10$ & $\mathrm{~s}$ & Max & DOE-STD-1196 & $4.85 \mathrm{E}-14$ \\
\hline$Y-85 m$ & $Y-85$ & 84.916 & Yttrium & $1.00 \mathrm{E}-03$ & ICRP-107 & $4.86 \mathrm{E}+00$ & $\mathrm{~h}$ & $1.75 \mathrm{E}+04$ & $7.59 E+06$ & DOE-STD-1196 & $2.04 \mathrm{E}-10$ & $\mathrm{~s}$ & Max & DOE-STD-1196 & $6.18 \mathrm{E}-14$ \\
\hline Y-86 & $Y-86$ & 85.915 & Yttrium & $1.00 \mathrm{E}-03$ & ICRP-38 & $1.47 \mathrm{E}+01$ & $\mathrm{~h}$ & $5.31 \mathrm{E}+04$ & $2.47 E+06$ & ICRP-72 & $4.70 \mathrm{E}-10$ & $\mathrm{~s}$ & Max & FGR-12 & $1.79 \mathrm{E}-13$ \\
\hline
\end{tabular}




\begin{tabular}{|c|c|c|c|c|c|c|c|c|c|c|c|c|c|c|c|}
\hline $\mathrm{Y}-86 \mathrm{~m}$ & $\mathrm{Y}-86$ & 85.915 & Yttrium & $1.00 \mathrm{E}-03$ & ICRP-38 & $4.80 \mathrm{E}+01$ & $\mathrm{~m}$ & $2.88 \mathrm{E}+03$ & $4.56 \mathrm{E}+07$ & ICRP-72 & $2.80 \mathrm{E}-11$ & $\mathrm{~s}$ & Max & FGR-12 & 1.06E-14 \\
\hline $\begin{array}{l}-87 \\
\end{array}$ & Y-87 & 86.911 & Yttrium & $1.00 \mathrm{E}-03$ & ICRP-38 & $8.03 \mathrm{E}+01$ & $\mathrm{~h}$ & $2.89 \mathrm{E}+05$ & $4.49 \mathrm{E}+05$ & ICRP-72 & $3.90 \mathrm{E}-10$ & $\mathrm{~s}$ & Max & FGR-12 & $2.15 \mathrm{E}-14$ \\
\hline$Y-87 m$ & Y-87 & 86.911 & Yttrium & $1.00 \mathrm{E}-03$ & ICRP-107 & $1.34 \mathrm{E}+01$ & $\mathrm{~h}$ & $4.81 \mathrm{E}+04$ & $2.70 \mathrm{E}+06$ & DOE-STD-1196 & $1.66 \mathrm{E}-10$ & $\mathrm{~s}$ & Max & DOE-STD-1196 & $1.35 \mathrm{E}-14$ \\
\hline$Y-88$ & Y-88 & 87.910 & Yttrium & $1.00 \mathrm{E}-03$ & ICRP-38 & $1.07 \mathrm{E}+02$ & d & $9.21 \mathrm{E}+06$ & $1.39 \mathrm{E}+04$ & ICRP-72 & $4.40 \mathrm{E}-09$ & $\mathrm{~s}$ & Max & FGR-12 & $1.37 \mathrm{E}-13$ \\
\hline $\mathrm{Y}-89 \mathrm{~m}$ & $Y-89$ & 88.906 & Yttrium & $1.00 \mathrm{E}-03$ & ICRP-107 & $1.57 \mathrm{E}+01$ & $\mathrm{~s}$ & $1.57 \mathrm{E}+01$ & $8.10 \mathrm{E}+09$ & -- & -- & -- & -- & DOE-STD-1196 & $4.16 \mathrm{E}-14$ \\
\hline Y-90 & $Y-90$ & 89.907 & Yttrium & $1.00 \mathrm{E}-03$ & ICRP-38 & $6.40 \mathrm{E}+01$ & $\mathrm{~h}$ & $2.30 \mathrm{E}+05$ & $5.45 \mathrm{E}+05$ & ICRP-72 & $1.50 \mathrm{E}-09$ & $\mathrm{~s}$ & Max & FGR-12 & $1.90 \mathrm{E}-16$ \\
\hline Y-90m & Y-90 & 89.907 & Yttrium & $1.00 \mathrm{E}-03$ & ICRP-38 & $3.19 \mathrm{E}+00$ & $\mathrm{~h}$ & $1.15 E+04$ & $1.09 \mathrm{E}+07$ & ICRP-72 & $1.00 \mathrm{E}-10$ & $\mathrm{~s}$ & Max & FGR-12 & $3.01 \mathrm{E}-14$ \\
\hline Y-91 & Y-91 & 90.907 & Yttrium & $1.00 \mathrm{E}-03$ & ICRP-38 & $5.85 \mathrm{E}+01$ & $d$ & $5.06 \mathrm{E}+06$ & $2.45 \mathrm{E}+04$ & ICRP-72 & $8.90 \mathrm{E}-09$ & $\mathrm{~s}$ & Max & FGR-12 & $2.60 \mathrm{E}-16$ \\
\hline Y-91m & Y-91 & 90.907 & Yttrium & $1.00 \mathrm{E}-03$ & ICRP-38 & $4.97 \mathrm{E}+01$ & $\mathrm{~m}$ & $2.98 \mathrm{E}+03$ & $4.16 \mathrm{E}+07$ & ICRP-72 & $1.10 \mathrm{E}-11$ & $\mathrm{~s}$ & Max & FGR-12 & $2.55 \mathrm{E}-14$ \\
\hline Y-92 & $Y-92$ & 91.909 & Yttrium & $1.00 \mathrm{E}-03$ & ICRP-38 & $3.54 \mathrm{E}+00$ & $\mathrm{~h}$ & $1.27 \mathrm{E}+04$ & $9.63 E+06$ & ICRP-72 & $1.80 \mathrm{E}-10$ & $\mathrm{~s}$ & Max & FGR-12 & $1.30 \mathrm{E}-14$ \\
\hline Y-93 & $\mathrm{Y}-93$ & 92.910 & Yttrium & $1.00 \mathrm{E}-03$ & ICRP-38 & $1.01 \mathrm{E}+01$ & $\mathrm{~h}$ & $3.64 \mathrm{E}+04$ & $3.34 \mathrm{E}+06$ & ICRP-72 & $4.20 \mathrm{E}-10$ & $\mathrm{~s}$ & Max & FGR-12 & $4.80 \mathrm{E}-15$ \\
\hline Y-94 & $Y-94$ & 93.912 & Yttrium & $1.00 \mathrm{E}-03$ & ICRP-38 & $1.91 \mathrm{E}+01$ & $\mathrm{~m}$ & $1.15 \mathrm{E}+03$ & $1.05 \mathrm{E}+08$ & ICRP-72 & $2.80 \mathrm{E}-11$ & $\mathrm{~s}$ & Max & FGR-12 & $5.62 \mathrm{E}-14$ \\
\hline $\begin{array}{l}\mathrm{Y}-95 \\
\end{array}$ & $\mathrm{Y}-95$ & 94.913 & Yttrium & $1.00 \mathrm{E}-03$ & ICRP-38 & $1.07 \mathrm{E}+01$ & $\mathrm{~m}$ & $6.42 \mathrm{E}+02$ & $1.85 \mathrm{E}+08$ & ICRP-72 & $1.60 \mathrm{E}-11$ & $\mathrm{~s}$ & Max & FGR-12 & 4.79E-14 \\
\hline Yb-162 & Yb-162 & 161.936 & Ytterbium & $1.00 \mathrm{E}-03$ & ICRP-38 & $1.89 \mathrm{E}+01$ & $\mathrm{~m}$ & $1.13 \mathrm{E}+03$ & $6.14 \mathrm{E}+07$ & ICRP-72 & $1.40 \mathrm{E}-11$ & $\mathrm{~s}$ & Max & FGR-12 & $5.66 \mathrm{E}-15$ \\
\hline Yb-163 & Yb-163 & 162.936 & Ytterbium & $1.00 \mathrm{E}-03$ & ICRP-107 & $1.11 \mathrm{E}+01$ & $\mathrm{~m}$ & $6.63 \mathrm{E}+02$ & $1.04 \mathrm{E}+08$ & DOE-STD-1196 & $1.02 \mathrm{E}-11$ & $\mathrm{~s}$ & Max & DOE-STD-1196 & $3.29 \mathrm{E}-14$ \\
\hline Yb-164 & Yb-164 & 163.934 & Ytterbium & $1.00 \mathrm{E}-03$ & ICRP-107 & $7.58 \mathrm{E}+01$ & $\mathrm{~m}$ & $4.55 \mathrm{E}+03$ & $1.51 \mathrm{E}+07$ & DOE-STD-1196 & $5.31 \mathrm{E}-11$ & $\mathrm{~s}$ & Max & DOE-STD-1196 & $1.61 \mathrm{E}-15$ \\
\hline Yb-165 & Yb-165 & 164.935 & Ytterbium & $1.00 \mathrm{E}-03$ & ICRP-107 & $9.90 \mathrm{E}+00$ & $\mathrm{~m}$ & $5.94 \mathrm{E}+02$ & $1.15 \mathrm{E}+08$ & -- & -- & -- & -- & DOE-STD-1196 & $1.37 \mathrm{E}-14$ \\
\hline Yb-166 & $\mathrm{Yb}-166$ & 165.934 & Ytterbium & $1.00 \mathrm{E}-03$ & ICRP-38 & $5.67 \mathrm{E}+01$ & $\mathrm{~h}$ & $2.04 \mathrm{E}+05$ & $3.33 \mathrm{E}+05$ & ICRP-72 & $7.70 \mathrm{E}-10$ & $\mathrm{~s}$ & Max & FGR-12 & $2.86 \mathrm{E}-15$ \\
\hline Yb-167 & Yb-167 & 166.935 & Ytterbium & $1.00 \mathrm{E}-03$ & ICRP-38 & $1.75 \mathrm{E}+01$ & $\mathrm{~m}$ & $1.05 \mathrm{E}+03$ & $6.44 \mathrm{E}+07$ & ICRP-72 & $6.90 \mathrm{E}-12$ & $\mathrm{~s}$ & Max & FGR-12 & $1.09 \mathrm{E}-14$ \\
\hline Yb-169 & Yb-169 & 168.935 & Ytterbium & $1.00 \mathrm{E}-03$ & ICRP-38 & $3.20 \mathrm{E}+01$ & $d$ & $2.77 \mathrm{E}+06$ & $2.41 \mathrm{E}+04$ & ICRP-72 & $3.00 \mathrm{E}-09$ & $\mathrm{~s}$ & Max & FGR-12 & $1.29 \mathrm{E}-14$ \\
\hline Yb-175 & $\mathrm{Yb}-175$ & 174.941 & Ytterbium & $1.00 \mathrm{E}-03$ & ICRP-38 & $4.19 \mathrm{E}+00$ & $\mathrm{~d}$ & $3.62 \mathrm{E}+05$ & $1.78 \mathrm{E}+05$ & ICRP-72 & $7.30 \mathrm{E}-10$ & $\mathrm{~s}$ & Max & FGR-12 & $1.87 \mathrm{E}-15$ \\
\hline Yb-177 & Yb-177 & 176.945 & Ytterbium & $1.00 \mathrm{E}-03$ & ICRP-38 & $1.90 \mathrm{E}+00$ & $\mathrm{~h}$ & $6.84 \mathrm{E}+03$ & $9.32 \mathrm{E}+06$ & ICRP-72 & $6.90 \mathrm{E}-11$ & $\mathrm{~s}$ & Max & FGR-12 & $9.23 \mathrm{E}-15$ \\
\hline Yb-178 & $\mathrm{Yb}-178$ & 177.947 & Ytterbium & $1.00 \mathrm{E}-03$ & ICRP-38 & $7.40 \mathrm{E}+01$ & $\mathrm{~m}$ & $4.44 \mathrm{E}+03$ & $1.43 \mathrm{E}+07$ & ICRP-72 & $7.50 \mathrm{E}-11$ & $\mathrm{~s}$ & Max & FGR-12 & $1.67 \mathrm{E}-15$ \\
\hline Yb-179 & Yb-179 & 178.950 & Ytterbium & $1.00 \mathrm{E}-03$ & ICRP-107 & $8.00 E+00$ & $\mathrm{~m}$ & $4.80 \mathrm{E}+02$ & $1.31 \mathrm{E}+08$ & -- & -- & -- & -- & DOE-STD-1196 & $4.40 \mathrm{E}-14$ \\
\hline $\mathrm{Zn}-60$ & $2 n-60$ & 59.942 & Zinc & $1.00 \mathrm{E}-03$ & ICRP-107 & $2.38 \mathrm{E}+00$ & $\mathrm{~m}$ & $1.43 \mathrm{E}+02$ & $1.32 \mathrm{E}+09$ & -- & -- & -- & -- & DOE-STD-1196 & $6.91 \mathrm{E}-14$ \\
\hline $\mathrm{Zn}-61$ & Zn-61 & 60.940 & Zinc & $1.00 \mathrm{E}-03$ & ICRP-107 & $8.91 \mathrm{E}+01$ & $\mathrm{~s}$ & $8.91 \mathrm{E}+01$ & $2.08 \mathrm{E}+09$ & -- & $\begin{array}{ll}-- \\
\end{array}$ & -- & -- & DOE-STD-1196 & $7.23 \mathrm{E}-14$ \\
\hline$Z n-62$ & Zn-62 & 61.934 & Zinc & $1.00 \mathrm{E}-03$ & ICRP-38 & $9.26 \mathrm{E}+00$ & $\mathrm{~h}$ & $3.33 \mathrm{E}+04$ & $5.46 \mathrm{E}+06$ & ICRP-72 & $5.00 \mathrm{E}-10$ & $M$ & Rec & FGR-12 & $2.07 \mathrm{E}-14$ \\
\hline$Z n-63$ & Zn-63 & 62.933 & Zinc & $1.00 \mathrm{E}-03$ & ICRP-38 & $3.81 \mathrm{E}+01$ & $\mathrm{~m}$ & $2.29 \mathrm{E}+03$ & $7.84 \mathrm{E}+07$ & ICRP-72 & $3.50 \mathrm{E}-11$ & $M$ & Rec & FGR-12 & $5.32 \mathrm{E}-14$ \\
\hline $\mathrm{Zn}-65$ & Zn-65 & 64.929 & Zinc & $1.00 \mathrm{E}-03$ & ICRP-38 & $2.44 \mathrm{E}+02$ & $d$ & $2.11 \mathrm{E}+07$ & $8.25 \mathrm{E}+03$ & ICRP-72 & $1.60 \mathrm{E}-09$ & $M$ & Rec & FGR-12 & $2.90 \mathrm{E}-14$ \\
\hline $\mathrm{Zn}-69$ & Zn-69 & 68.927 & Zinc & $1.00 \mathrm{E}-03$ & ICRP-38 & $5.70 \mathrm{E}+01$ & $\mathrm{~m}$ & $3.42 \mathrm{E}+03$ & $4.79 \mathrm{E}+07$ & ICRP-72 & $2.60 \mathrm{E}-11$ & $M$ & Rec & FGR-12 & $2.16 \mathrm{E}-17$ \\
\hline $\mathrm{Zn}-69 m$ & $2 n-69$ & 68.927 & Zinc & $1.00 \mathrm{E}-03$ & ICRP-38 & $1.38 \mathrm{E}+01$ & $\mathrm{~h}$ & $4.95 \mathrm{E}+04$ & $3.30 \mathrm{E}+06$ & ICRP-72 & $2.40 \mathrm{E}-10$ & $M$ & Rec & FGR-12 & $1.99 \mathrm{E}-14$ \\
\hline $\mathrm{Zn-71}$ & Zn-71 & 70.928 & Zinc & $1.00 \mathrm{E}-03$ & ICRP-107 & $2.45 E+00$ & $\mathrm{~m}$ & $1.47 \mathrm{E}+02$ & $1.08 \mathrm{E}+09$ & -- & -- & -- & -- & DOE-STD-1196 & $1.52 \mathrm{E}-14$ \\
\hline $\mathrm{Zn}-71 m$ & $2 n-71$ & 70.928 & Zinc & $1.00 \mathrm{E}-03$ & ICRP-38 & $3.92 \mathrm{E}+00$ & $\mathrm{~h}$ & $1.41 \mathrm{E}+04$ & $1.13 E+07$ & ICRP-72 & $1.50 \mathrm{E}-10$ & $M$ & Rec & FGR-12 & $7.50 \mathrm{E}-14$ \\
\hline $\mathrm{Zn}-72$ & Zn-72 & 71.927 & Zinc & $1.00 \mathrm{E}-03$ & ICRP-38 & $4.65 \mathrm{E}+01$ & $\mathrm{~h}$ & $1.67 E+05$ & $9.37 \mathrm{E}+05$ & ICRP-72 & $1.20 \mathrm{E}-09$ & $M$ & $\operatorname{Rec}$ & FGR-12 & $6.90 \mathrm{E}-15$ \\
\hline $\mathrm{Zr}-85$ & Zr-85 & 84.921 & Zirconium & $1.00 \mathrm{E}-03$ & ICRP-107 & $7.86 \mathrm{E}+00$ & $\mathrm{~m}$ & $4.72 E+02$ & $2.82 \mathrm{E}+08$ & -- & -- & -- & -- & DOE-STD-1196 & $6.74 \mathrm{E}-14$ \\
\hline $\mathrm{Zr}-86$ & Zr-86 & 85.916 & Zirconium & $1.00 \mathrm{E}-03$ & ICRP-38 & $1.65 \mathrm{E}+01$ & $\mathrm{~h}$ & $5.94 \mathrm{E}+04$ & $2.21 \mathrm{E}+06$ & ICRP-72 & $4.20 \mathrm{E}-10$ & $M$ & $\operatorname{Rec}$ & FGR-12 & $1.28 \mathrm{E}-14$ \\
\hline $\mathrm{Zr}-87$ & Zr-87 & 86.915 & Zirconium & $1.00 \mathrm{E}-03$ & ICRP-107 & $1.68 \mathrm{E}+00$ & $\mathrm{~h}$ & $6.05 E+03$ & $2.15 \mathrm{E}+07$ & DOE-STD-1196 & $1.02 \mathrm{E}-10$ & $M$ & $\operatorname{Rec}$ & DOE-STD-1196 & $4.22 \mathrm{E}-14$ \\
\hline $\mathrm{Zr}-88$ & Zr-88 & 87.910 & Zirconium & $1.00 \mathrm{E}-03$ & ICRP-38 & $8.34 \mathrm{E}+01$ & $d$ & $7.21 \mathrm{E}+06$ & $1.78 \mathrm{E}+04$ & ICRP-72 & $2.60 \mathrm{E}-09$ & $M$ & Rec & FGR-12 & $1.88 \mathrm{E}-14$ \\
\hline $\mathrm{Zr}-89$ & Zr-89 & 88.909 & Zirconium & $1.00 \mathrm{E}-03$ & ICRP-38 & $7.84 \mathrm{E}+01$ & $\mathrm{~h}$ & $2.82 \mathrm{E}+05$ & $4.49 \mathrm{E}+05$ & ICRP-72 & $5.20 \mathrm{E}-10$ & $M$ & Rec & FGR-12 & $5.68 \mathrm{E}-14$ \\
\hline $\mathrm{Zr}-89 \mathrm{~m}$ & Zr-89 & 88.909 & Zirconium & $1.00 \mathrm{E}-03$ & ICRP-107 & $4.16 \mathrm{E}+00$ & $\mathrm{~m}$ & $2.50 \mathrm{E}+02$ & $5.08 \mathrm{E}+08$ & -- & -- & -- & -- & DOE-STD-1196 & $2.88 \mathrm{E}-14$ \\
\hline Zr-93 & Zr-93 & 92.906 & Zirconium & $1.00 \mathrm{E}-03$ & ICRP-38 & $1.53 \mathrm{E}+06$ & $y$ & $4.83 \mathrm{E}+13$ & $2.51 \mathrm{E}-03$ & ICRP-72 & $1.00 \mathrm{E}-08$ & $M$ & $\operatorname{Rec}$ & DOE-STD-1196 & $6.45 \mathrm{E}-22$ \\
\hline $\mathrm{Zr}-95$ & Zr-95 & 94.908 & Zirconium & $1.00 \mathrm{E}-03$ & ICRP-38 & $6.40 \mathrm{E}+01$ & d & $5.53 \mathrm{E}+06$ & $2.15 \mathrm{E}+04$ & ICRP-72 & $4.80 \mathrm{E}-09$ & $M$ & Rec & FGR-12 & $3.60 \mathrm{E}-14$ \\
\hline Zr-97 & Zr-97 & 96.911 & Zirconium & $1.00 \mathrm{E}-03$ & ICRP-38 & $1.69 \mathrm{E}+01$ & $\mathrm{~h}$ & $6.08 \mathrm{E}+04$ & $1.91 \mathrm{E}+06$ & ICRP-72 & $9.20 \mathrm{E}-10$ & $M$ & $\operatorname{Rec}$ & FGR-12 & $9.02 \mathrm{E}-15$ \\
\hline
\end{tabular}




\begin{tabular}{|c|c|c|c|c|}
\hline \multicolumn{5}{|c|}{ Table B.2 HC-2 TQs Using Recommended Inhalation DCs } \\
\hline \multirow{2}{*}{$\begin{array}{c}\text { MASTER } \\
\text { ISOTOPE LIST }\end{array}$} & \multicolumn{2}{|c|}{ NNSA SD 1027} & \multicolumn{2}{|c|}{ Final Threshold Quantity } \\
\hline & Act (Ci) & Mass (g) & Act (Ci) & Mass (g) \\
\hline Ac-223 & & & $1.31 \mathrm{E}+10$ & $3.41 \mathrm{E}+01$ \\
\hline Ac-224 & & & $6.24 \mathrm{E}+04$ & $1.29 \mathrm{E}-02$ \\
\hline Ac-225 & $9.54 \mathrm{E}+02$ & $1.64 \mathrm{E}-02$ & $9.54 \mathrm{E}+02$ & $1.64 \mathrm{E}-02$ \\
\hline Ac-226 & & & $6.24 \mathrm{E}+03$ & $1.30 \mathrm{E}-02$ \\
\hline Ac-227 & $1.47 \mathrm{E}+01$ & 2.04E-01 & $1.47 \mathrm{E}+01$ & $2.04 \mathrm{E}-01$ \\
\hline Ac-228 & & & $3.22 \mathrm{E}+05$ & $1.44 \mathrm{E}-01$ \\
\hline Ac-229 & & & $2.32 \mathrm{E}+08$ & $1.77 \mathrm{E}+01$ \\
\hline Ac-230 & & & $1.02 \mathrm{E}+08$ & 2.53E-01 \\
\hline Ac-231 & & & $1.47 \mathrm{E}+08$ & $1.35 \mathrm{E}+00$ \\
\hline Ac-232 & & & $4.78 \mathrm{E}+07$ & $1.17 \mathrm{E}-01$ \\
\hline Ac-233 & & & $1.18 \mathrm{E}+08$ & $3.54 \mathrm{E}-01$ \\
\hline Ag-100m & & & $2.03 \mathrm{E}+07$ & 2.42E-02 \\
\hline Ag-101 & & & $3.50 \mathrm{E}+07$ & $2.08 \mathrm{E}-01$ \\
\hline Ag-102 & & & $1.57 \mathrm{E}+07$ & $1.09 \mathrm{E}-01$ \\
\hline Ag-102m & & & $2.78 \mathrm{E}+07$ & $1.16 \mathrm{E}-01$ \\
\hline Ag-103 & & & $5.94 \mathrm{E}+07$ & $2.14 \mathrm{E}+00$ \\
\hline Ag-104 & & & $1.88 \mathrm{E}+07$ & $7.18 \mathrm{E}-01$ \\
\hline Ag-104m & & & $4.06 \mathrm{E}+07$ & 7.52E-01 \\
\hline Ag-105 & & & $1.01 \mathrm{E}+07$ & $3.32 \mathrm{E}+02$ \\
\hline Ag-105m & & & $6.11 \mathrm{E}+10$ & $2.47 \mathrm{E}+02$ \\
\hline Ag-106 & & & $6.95 \mathrm{E}+07$ & $9.38 \mathrm{E}-01$ \\
\hline Ag-106m & & & $5.36 \mathrm{E}+06$ & $3.65 \mathrm{E}+01$ \\
\hline Ag-108 & & & $2.91 \mathrm{E}+09$ & $3.96 \mathrm{E}+00$ \\
\hline Ag-108m & & & $1.06 \mathrm{E}+06$ & $4.07 \mathrm{E}+04$ \\
\hline Ag-109m & & & $1.41 \mathrm{E}+10$ & $5.38 \mathrm{E}+00$ \\
\hline Ag-110 & & & $1.52 \mathrm{E}+09$ & 3.64E-01 \\
\hline Ag-110m & $1.01 \mathrm{E}+06$ & $2.13 \mathrm{E}+02$ & $1.01 \mathrm{E}+06$ & $2.13 \mathrm{E}+02$ \\
\hline Ag-111 & & & $5.39 \mathrm{E}+06$ & $3.41 \mathrm{E}+01$ \\
\hline Ag-111m & & & $1.61 \mathrm{E}+10$ & $1.02 \mathrm{E}+01$ \\
\hline Ag-112 & & & $3.12 \mathrm{E}+07$ & $3.47 \mathrm{E}+00$ \\
\hline Ag-113 & & & $4.17 \mathrm{E}+07$ & $8.07 \mathrm{E}+00$ \\
\hline Ag-113m & & & $2.83 \mathrm{E}+08$ & 1.95E-01 \\
\hline Ag-114 & & & $1.85 \mathrm{E}+08$ & $8.60 \mathrm{E}-03$ \\
\hline Ag-115 & & & $5.99 \mathrm{E}+07$ & 7.32E-01 \\
\hline Ag-116 & & & $2.53 \mathrm{E}+07$ & 4.17E-02 \\
\hline Ag-117 & & & $4.16 \mathrm{E}+07$ & $3.17 \mathrm{E}-02$ \\
\hline Ag-99 & & & $2.50 \mathrm{E}+07$ & $2.72 \mathrm{E}-02$ \\
\hline
\end{tabular}




\begin{tabular}{|c|c|c|c|c|}
\hline \multicolumn{5}{|c|}{ Table B.2 HC-2 TQs Using Recommended Inhalation DCs (continued) } \\
\hline \multirow{2}{*}{$\begin{array}{c}\text { MASTER } \\
\text { ISOTOPE LIST }\end{array}$} & \multicolumn{2}{|c|}{ NNSA SD 1027} & \multicolumn{2}{|c|}{ Final Threshold Quantity } \\
\hline & Act (Ci) & Mass (g) & Act (Ci) & Mass (g) \\
\hline Al-26 & & & $3.97 \mathrm{E}+05$ & $2.07 \mathrm{E}+07$ \\
\hline $\mathrm{Al}-28$ & & & $2.91 \mathrm{E}+07$ & $9.71 \mathrm{E}-03$ \\
\hline $\mathrm{Al}-29$ & & & $4.03 \mathrm{E}+07$ & 4.07E-02 \\
\hline Am-237 & & & $1.07 \mathrm{E}+08$ & $9.82 \mathrm{E}+00$ \\
\hline Am-238 & & & $3.69 \mathrm{E}+07$ & $4.57 \mathrm{E}+00$ \\
\hline Am-239 & & & $3.23 \mathrm{E}+07$ & $2.93 \mathrm{E}+01$ \\
\hline Am-240 & & & $1.40 \mathrm{E}+07$ & $5.44 \mathrm{E}+01$ \\
\hline Am-241 & $1.93 \mathrm{E}+02$ & $5.63 \mathrm{E}+01$ & $1.93 \mathrm{E}+02$ & $5.63 \mathrm{E}+01$ \\
\hline Am-242 & & & $4.77 \mathrm{E}+05$ & $5.90 \mathrm{E}-01$ \\
\hline Am-242m & $2.19 \mathrm{E}+02$ & $2.09 \mathrm{E}+01$ & $2.19 \mathrm{E}+02$ & $2.26 \mathrm{E}+01$ \\
\hline Am-243 & $1.98 \mathrm{E}+02$ & $9.90 \mathrm{E}+02$ & $1.98 \mathrm{E}+02$ & $9.92 \mathrm{E}+02$ \\
\hline Am-244 & & & $3.83 \mathrm{E}+06$ & $3.01 \mathrm{E}+00$ \\
\hline Am-244m & & & $9.63 \mathrm{E}+07$ & $3.25 \mathrm{E}+00$ \\
\hline Am-245 & & & $1.41 \mathrm{E}+08$ & $2.27 \mathrm{E}+01$ \\
\hline Am-246 & & & $4.93 \mathrm{E}+07$ & $2.52 E+00$ \\
\hline Am-246m & & & $4.69 \mathrm{E}+07$ & $1.53 \mathrm{E}+00$ \\
\hline Am-247 & & & $1.70 \mathrm{E}+08$ & $5.13 \mathrm{E}+00$ \\
\hline Ar-37 & & & $2.13 \mathrm{E}+10$ & $2.11 \mathrm{E}+05$ \\
\hline Ar-39 & & & $2.97 \mathrm{E}+08$ & $8.71 \mathrm{E}+06$ \\
\hline Ar-41 & & & $4.16 \mathrm{E}+04$ & 9.93E-04 \\
\hline Ar-42 & & & $2.15 \mathrm{E}+07$ & $8.28 E+04$ \\
\hline Ar-43 & & & $3.58 \mathrm{E}+04$ & 4.39E-05 \\
\hline Ar-44 & & & $2.86 \mathrm{E}+04$ & 7.93E-05 \\
\hline As-68 & & & $1.54 \mathrm{E}+07$ & $1.40 \mathrm{E}-02$ \\
\hline As-69 & & & $4.83 \mathrm{E}+07$ & $2.69 \mathrm{E}-01$ \\
\hline As-70 & & & $1.19 \mathrm{E}+07$ & $2.34 \mathrm{E}-01$ \\
\hline As-71 & & & $1.68 \mathrm{E}+07$ & $2.47 \mathrm{E}+01$ \\
\hline As-72 & & & $6.97 \mathrm{E}+06$ & $4.16 \mathrm{E}+00$ \\
\hline As-73 & & & $8.10 \mathrm{E}+06$ & $3.63 \mathrm{E}+02$ \\
\hline As-74 & & & $3.67 \mathrm{E}+06$ & $3.69 \mathrm{E}+01$ \\
\hline As-76 & & & $1.01 \mathrm{E}+07$ & $6.43 \mathrm{E}+00$ \\
\hline As-77 & & & $2.07 \mathrm{E}+07$ & $1.97 \mathrm{E}+01$ \\
\hline As-78 & & & $2.91 \mathrm{E}+07$ & $1.09 \mathrm{E}+00$ \\
\hline As-79 & & & $1.20 \mathrm{E}+09$ & $4.54 \mathrm{E}+00$ \\
\hline At-204 & & & $2.60 \mathrm{E}+07$ & $2.59 \mathrm{E}-01$ \\
\hline At-205 & & & $8.63 \mathrm{E}+06$ & $2.46 \mathrm{E}-01$ \\
\hline At-206 & & & $1.36 \mathrm{E}+07$ & $4.55 \mathrm{E}-01$ \\
\hline At-207 & & & $3.25 E+06$ & 3.86E-01 \\
\hline
\end{tabular}




\begin{tabular}{|c|c|c|c|c|}
\hline \multicolumn{5}{|c|}{ Table B.2 HC-2 TQs Using Recommended Inhalation DCs (continued) } \\
\hline \multirow{2}{*}{$\begin{array}{c}\text { MASTER } \\
\text { ISOTOPE LIST }\end{array}$} & \multicolumn{2}{|c|}{ NNSA SD 1027} & \multicolumn{2}{|c|}{ Final Threshold Quantity } \\
\hline & Act (Ci) & Mass (g) & Act (Ci) & Mass (g) \\
\hline At-208 & & & $7.54 \mathrm{E}+06$ & 8.15E-01 \\
\hline At-209 & & & $2.42 \mathrm{E}+06$ & 8.73E-01 \\
\hline At-210 & & & $6.69 \mathrm{E}+05$ & 3.63E-01 \\
\hline At-211 & & & $7.37 \mathrm{E}+04$ & $3.58 \mathrm{E}-02$ \\
\hline At-215 & & & $2.93 \mathrm{E}+11$ & 5.59E-04 \\
\hline At-216 & & & $4.33 \mathrm{E}+10$ & $2.49 \mathrm{E}-04$ \\
\hline At-217 & & & $1.83 \mathrm{E}+11$ & 1.13E-01 \\
\hline At-218 & & & $2.27 \mathrm{E}+10$ & $8.78 \mathrm{E}-01$ \\
\hline At-220 & & & $1.29 \mathrm{E}+08$ & 5.61E-01 \\
\hline Au-186 & & & $3.52 \mathrm{E}+07$ & 3.72E-01 \\
\hline $\mathrm{Au}-187$ & & & $5.45 \mathrm{E}+07$ & 4.55E-01 \\
\hline Au-190 & & & $2.15 \mathrm{E}+07$ & $9.30 \mathrm{E}-01$ \\
\hline $\mathrm{Au}-191$ & & & $5.33 \mathrm{E}+07$ & $1.03 \mathrm{E}+01$ \\
\hline Au-192 & & & $2.06 \mathrm{E}+07$ & $6.23 E+00$ \\
\hline Au-193 & & & $5.77 \mathrm{E}+07$ & $6.27 \mathrm{E}+01$ \\
\hline $\mathrm{Au}-193 \mathrm{~m}$ & & & $3.23 E+08$ & 2.15E-02 \\
\hline Au-194 & & & $2.03 \mathrm{E}+07$ & $4.97 \mathrm{E}+01$ \\
\hline Au-195 & & & $4.74 \mathrm{E}+06$ & $1.30 \mathrm{E}+03$ \\
\hline Au-195m & & & $2.88 \mathrm{E}+08$ & 1.52E-01 \\
\hline $\mathrm{Au}-196$ & & & $1.85 \mathrm{E}+07$ & $1.72 \mathrm{E}+02$ \\
\hline $\mathrm{Au}-196 \mathrm{~m}$ & & & $1.53 \mathrm{E}+07$ & $9.20 \mathrm{E}+00$ \\
\hline Au-198 & $8.83 E+06$ & $3.61 \mathrm{E}+01$ & $8.83 E+06$ & $3.61 \mathrm{E}+01$ \\
\hline $\mathrm{Au}-198 \mathrm{~m}$ & & & $3.90 \mathrm{E}+06$ & $1.36 \mathrm{E}+01$ \\
\hline Au-199 & & & $1.01 \mathrm{E}+07$ & $4.83 \mathrm{E}+01$ \\
\hline Au-200 & & & $1.07 \mathrm{E}+08$ & $5.48 \mathrm{E}+00$ \\
\hline $\mathrm{Au}-200 \mathrm{~m}$ & & & $7.93 \mathrm{E}+06$ & $9.46 \mathrm{E}+00$ \\
\hline Au-201 & & & $3.28 \mathrm{E}+08$ & $9.26 \mathrm{E}+00$ \\
\hline $\mathrm{Au}-202$ & & & $3.04 \mathrm{E}+08$ & $1.57 \mathrm{E}-01$ \\
\hline Вa-124 & & & $7.98 \mathrm{E}+07$ & 5.79E-01 \\
\hline Ba-126 & & & $6.70 \mathrm{E}+07$ & $4.33 \mathrm{E}+00$ \\
\hline Вa-127 & & & $7.28 \mathrm{E}+07$ & $6.24 \mathrm{E}-01$ \\
\hline Ba-128 & & & $6.20 \mathrm{E}+06$ & $1.47 \mathrm{E}+01$ \\
\hline Ba-129 & & & $1.06 \mathrm{E}+08$ & $9.75 \mathrm{E}+00$ \\
\hline Ba-129m & & & $3.00 \mathrm{E}+07$ & $2.66 \mathrm{E}+00$ \\
\hline Ва-131 & & & $9.85 \mathrm{E}+06$ & $1.17 \mathrm{E}+02$ \\
\hline Вa-131m & & & $4.91 \mathrm{E}+08$ & $4.99 \mathrm{E}+00$ \\
\hline Ba-133 & $2.57 \mathrm{E}+06$ & $1.01 \mathrm{E}+04$ & $2.57 \mathrm{E}+06$ & $1.03 \mathrm{E}+04$ \\
\hline Вa-133m & & & $1.90 \mathrm{E}+07$ & $3.13 \mathrm{E}+01$ \\
\hline
\end{tabular}




\begin{tabular}{|c|c|c|c|c|}
\hline \multicolumn{5}{|c|}{ Table B.2 HC-2 TQs Using Recommended Inhalation DCs (continued) } \\
\hline \multirow{2}{*}{$\begin{array}{c}\text { MASTER } \\
\text { ISOTOPE LIST }\end{array}$} & \multicolumn{2}{|c|}{ NNSA SD 1027} & \multicolumn{2}{|c|}{ Final Threshold Quantity } \\
\hline & Act (Ci) & Mass (g) & Act (Ci) & Mass (g) \\
\hline Ba-135m & & & $2.41 \mathrm{E}+07$ & $2.97 \mathrm{E}+01$ \\
\hline Ba-137m & & & $9.38 \mathrm{E}+07$ & $1.74 \mathrm{E}-01$ \\
\hline Ba-139 & & & $1.30 \mathrm{E}+08$ & $7.92 \mathrm{E}+00$ \\
\hline Ba-140 & $1.58 \mathrm{E}+06$ & $2.16 \mathrm{E}+01$ & $1.58 \mathrm{E}+06$ & $2.16 \mathrm{E}+01$ \\
\hline Ba-141 & & & $5.17 \mathrm{E}+07$ & 7.08E-01 \\
\hline Ba-142 & & & $4.62 \mathrm{E}+07$ & $3.70 \mathrm{E}-01$ \\
\hline Be-10 & & & $2.32 \mathrm{E}+05$ & $1.04 \mathrm{E}+07$ \\
\hline Be-7 & & & $1.31 \mathrm{E}+08$ & $3.74 \mathrm{E}+02$ \\
\hline Bi-197 & & & $3.44 \mathrm{E}+07$ & 3.35E-01 \\
\hline $\mathrm{Bi}-200$ & & & $2.13 \mathrm{E}+07$ & $8.24 \mathrm{E}-01$ \\
\hline $\mathrm{Bi}-201$ & & & $3.10 \mathrm{E}+07$ & $3.58 \mathrm{E}+00$ \\
\hline Bi-202 & & & $1.79 \mathrm{E}+07$ & $1.92 \mathrm{E}+00$ \\
\hline $\mathrm{Bi}-203$ & & & $1.31 \mathrm{E}+07$ & $9.96 \mathrm{E}+00$ \\
\hline Bi-204 & & & $1.03 \mathrm{E}+07$ & $7.53 \mathrm{E}+00$ \\
\hline $\mathrm{Bi}-205$ & & & $6.84 \mathrm{E}+06$ & $1.64 \mathrm{E}+02$ \\
\hline Bi-206 & & & $3.71 \mathrm{E}+06$ & $3.66 \mathrm{E}+01$ \\
\hline $\mathrm{Bi}-207$ & $1.39 \mathrm{E}+06$ & $3.06 \mathrm{E}+04$ & $1.39 \mathrm{E}+06$ & $3.06 \mathrm{E}+04$ \\
\hline $\mathrm{Bi}-208$ & & & $2.09 \mathrm{E}+05$ & $4.48 \mathrm{E}+07$ \\
\hline $\mathrm{Bi}-210$ & $8.72 \mathrm{E}+04$ & 7.01E-01 & $8.72 \mathrm{E}+04$ & 7.03E-01 \\
\hline Bi-210m & & & $2.38 \mathrm{E}+03$ & $4.20 \mathrm{E}+06$ \\
\hline $\mathrm{Bi}-211$ & & & $1.22 \mathrm{E}+09$ & $2.92 \mathrm{E}+00$ \\
\hline $\mathrm{Bi}-212$ & & & $2.61 \mathrm{E}+05$ & $1.78 \mathrm{E}-02$ \\
\hline Bi-212n & & & $6.95 \mathrm{E}+09$ & $5.48 \mathrm{E}+01$ \\
\hline $\mathrm{Bi}-213$ & & & $2.70 \mathrm{E}+05$ & $1.40 \mathrm{E}-02$ \\
\hline $\mathrm{Bi}-214$ & & & $5.70 \mathrm{E}+05$ & $1.29 \mathrm{E}-02$ \\
\hline $\mathrm{Bi}-215$ & & & $2.29 \mathrm{E}+08$ & $1.99 \mathrm{E}+00$ \\
\hline Bi-216 & & & $7.93 \mathrm{E}+07$ & $1.98 \mathrm{E}-01$ \\
\hline Bk-244 & & & $8.11 \mathrm{E}+06$ & $2.75 \mathrm{E}+00$ \\
\hline Bk-245 & & & $3.80 \mathrm{E}+06$ & $3.53 \mathrm{E}+01$ \\
\hline Bk-246 & & & $1.73 \mathrm{E}+07$ & $5.98 \mathrm{E}+01$ \\
\hline Bk-247 & & & $1.18 \mathrm{E}+02$ & $1.12 \mathrm{E}+02$ \\
\hline Bk-248m & & & $2.88 \mathrm{E}+05$ & $5.41 \mathrm{E}-01$ \\
\hline Bk-249 & & & $5.07 \mathrm{E}+04$ & $3.09 \mathrm{E}+01$ \\
\hline Bk-250 & & & $7.17 \mathrm{E}+06$ & $1.84 \mathrm{E}+00$ \\
\hline Bk-251 & & & $1.32 \mathrm{E}+08$ & $9.78 \mathrm{E}+00$ \\
\hline $\mathrm{Br}-72$ & & & $3.83 \mathrm{E}+04$ & $1.92 \mathrm{E}-05$ \\
\hline Br-73 & & & $8.29 E+04$ & $1.09 \mathrm{E}-04$ \\
\hline Br-74 & & & $2.16 \mathrm{E}+04$ & $2.15 \mathrm{E}-04$ \\
\hline
\end{tabular}




\begin{tabular}{|c|c|c|c|c|}
\hline \multicolumn{5}{|c|}{ Table B.2 HC-2 TQs Using Recommended Inhalation DCs (continued) } \\
\hline \multirow{2}{*}{$\begin{array}{c}\text { MASTER } \\
\text { ISOTOPE LIST }\end{array}$} & \multicolumn{2}{|c|}{ NNSA SD 1027} & \multicolumn{2}{|c|}{ Final Threshold Quantity } \\
\hline & Act (Ci) & Mass (g) & Act (Ci) & Mass (g) \\
\hline $\mathrm{Br}-74 \mathrm{~m}$ & & & $2.36 \mathrm{E}+04$ & 3.86E-04 \\
\hline $\mathrm{Br}-75$ & & & $7.11 \mathrm{E}+04$ & $2.78 \mathrm{E}-03$ \\
\hline $\mathrm{Br}-76$ & & & $2.00 \mathrm{E}+04$ & 7.84E-03 \\
\hline Br-76m & & & $5.60 \mathrm{E}+06$ & 4.93E-05 \\
\hline $\mathrm{Br}-77$ & & & $1.25 \mathrm{E}+05$ & $1.72 \mathrm{E}-01$ \\
\hline Br-77m & & & $9.07 \mathrm{E}+06$ & $1.59 \mathrm{E}-02$ \\
\hline $\mathrm{Br}-78$ & & & $1.15 \mathrm{E}+05$ & 3.09E-04 \\
\hline $\mathrm{Br}-80$ & & & $7.74 \mathrm{E}+05$ & 5.72E-03 \\
\hline Br-80m & & & $2.11 \mathrm{E}+05$ & $2.38 \mathrm{E}-02$ \\
\hline $\mathrm{Br}-82$ & & & $1.59 \mathrm{E}+04$ & $1.47 \mathrm{E}-02$ \\
\hline Br-82m & & & $3.40 \mathrm{E}+07$ & $9.08 \mathrm{E}-02$ \\
\hline $\mathrm{Br}-83$ & & & $3.30 \mathrm{E}+05$ & $2.09 \mathrm{E}-02$ \\
\hline $\mathrm{Br}-84$ & & & $5.08 \mathrm{E}+04$ & $7.21 \mathrm{E}-04$ \\
\hline Br-84m & & & $4.13 \mathrm{E}+04$ & $1.10 \mathrm{E}-04$ \\
\hline $\mathrm{Br}-85$ & & & $1.36 \mathrm{E}+06$ & $1.78 \mathrm{E}-03$ \\
\hline C-10 & & & $3.42 \mathrm{E}+06$ & 5.85E-05 \\
\hline $\mathrm{C}-11$ & & & $4.92 \mathrm{E}+06$ & $5.88 \mathrm{E}-03$ \\
\hline $\mathrm{C}-14$ & $4.05 \mathrm{E}+05$ & $9.07 \mathrm{E}+04$ & $4.05 E+05$ & $9.10 \mathrm{E}+04$ \\
\hline Ca-41 & & & $8.53 \mathrm{E}+07$ & $1.37 \mathrm{E}+09$ \\
\hline Ca-45 & $3.00 \mathrm{E}+06$ & $1.68 \mathrm{E}+02$ & $3.00 \mathrm{E}+06$ & $1.69 \mathrm{E}+02$ \\
\hline Ca-47 & $3.93 \mathrm{E}+06$ & $6.42 \mathrm{E}+00$ & $3.93 \mathrm{E}+06$ & $6.41 \mathrm{E}+00$ \\
\hline Ca-49 & & & $1.56 \mathrm{E}+07$ & 3.55E-02 \\
\hline Cd-101 & & & $2.31 \mathrm{E}+07$ & $1.69 \mathrm{E}-02$ \\
\hline Cd-102 & & & $7.25 \mathrm{E}+07$ & $2.16 \mathrm{E}-01$ \\
\hline Cd-103 & & & $2.70 \mathrm{E}+07$ & $1.08 \mathrm{E}-01$ \\
\hline Cd-104 & & & $1.17 \mathrm{E}+08$ & $3.74 \mathrm{E}+00$ \\
\hline Cd-105 & & & $3.82 \mathrm{E}+07$ & $1.18 \mathrm{E}+00$ \\
\hline Cd-107 & & & $9.56 \mathrm{E}+07$ & $2.12 \mathrm{E}+01$ \\
\hline Cd-109 & $1.00 \mathrm{E}+06$ & $3.86 \mathrm{E}+02$ & $1.00 \mathrm{E}+06$ & $3.87 \mathrm{E}+02$ \\
\hline Cd-111m & & & $1.30 \mathrm{E}+08$ & $3.71 \mathrm{E}+00$ \\
\hline Cd-113 & $6.76 \mathrm{E}+04$ & $1.92 \mathrm{E}+17$ & $6.76 \mathrm{E}+04$ & $1.98 \mathrm{E}+17$ \\
\hline Cd-113m & & & $7.37 \mathrm{E}+04$ & $3.17 \mathrm{E}+02$ \\
\hline Cd-115 & & & $7.15 E+06$ & $1.40 \mathrm{E}+01$ \\
\hline Cd-115m & & & $1.05 \mathrm{E}+06$ & $4.13 \mathrm{E}+01$ \\
\hline Cd-117 & & & $2.43 \mathrm{E}+07$ & $2.26 \mathrm{E}+00$ \\
\hline Cd-117m & & & $1.54 \mathrm{E}+07$ & $1.94 \mathrm{E}+00$ \\
\hline Cd-118 & & & $8.43 \mathrm{E}+07$ & $2.66 \mathrm{E}+00$ \\
\hline Cd-119 & & & $3.40 \mathrm{E}+07$ & $5.78 \mathrm{E}-02$ \\
\hline
\end{tabular}




\begin{tabular}{|c|c|c|c|c|}
\hline \multicolumn{5}{|c|}{ Table B.2 HC-2 TQs Using Recommended Inhalation DCs (continued) } \\
\hline \multirow{2}{*}{$\begin{array}{c}\text { MASTER } \\
\text { ISOTOPE LIST }\end{array}$} & \multicolumn{2}{|c|}{ NNSA SD 1027} & \multicolumn{2}{|c|}{ Final Threshold Quantity } \\
\hline & Act (Ci) & Mass (g) & Act (Ci) & Mass (g) \\
\hline Cd-119m & & & $2.43 \mathrm{E}+07$ & 3.39E-02 \\
\hline Ce-130 & & & $7.71 \mathrm{E}+07$ & $1.22 \mathrm{E}+00$ \\
\hline Ce-131 & & & $3.39 \mathrm{E}+07$ & $2.41 \mathrm{E}-01$ \\
\hline Ce-132 & & & $4.10 \mathrm{E}+07$ & $6.06 \mathrm{E}+00$ \\
\hline Ce-133 & & & $6.36 \mathrm{E}+07$ & $4.36 \mathrm{E}+00$ \\
\hline Ce-133m & & & $2.15 \mathrm{E}+07$ & $4.48 \mathrm{E}+00$ \\
\hline Ce-134 & & & $6.23 \mathrm{E}+06$ & $1.92 \mathrm{E}+01$ \\
\hline Ce-135 & & & $1.10 \mathrm{E}+07$ & $8.34 \mathrm{E}+00$ \\
\hline Ce-137 & & & $6.52 \mathrm{E}+08$ & $2.56 \mathrm{E}+02$ \\
\hline Ce-137m & & & $1.95 \mathrm{E}+07$ & $2.93 \mathrm{E}+01$ \\
\hline Ce-139 & & & $4.71 \mathrm{E}+06$ & $6.90 \mathrm{E}+02$ \\
\hline Ce-141 & $2.53 \mathrm{E}+06$ & $8.86 \mathrm{E}+01$ & $2.53 \mathrm{E}+06$ & $8.86 \mathrm{E}+01$ \\
\hline Ce-143 & & & $1.03 \mathrm{E}+07$ & $1.55 \mathrm{E}+01$ \\
\hline Ce-144 & $2.25 \mathrm{E}+05$ & $7.06 \mathrm{E}+01$ & $2.25 \mathrm{E}+05$ & $7.06 \mathrm{E}+01$ \\
\hline Ce-145 & & & $7.43 \mathrm{E}+07$ & $1.72 \mathrm{E}-01$ \\
\hline Ce-146 & & & $3.24 \mathrm{E}+08$ & $3.40 \mathrm{E}+00$ \\
\hline Cf-244 & & & $5.79 \mathrm{E}+05$ & $1.46 \mathrm{E}-02$ \\
\hline Cf-246 & & & $1.80 \mathrm{E}+04$ & 5.05E-02 \\
\hline Cf-247 & & & $1.29 \mathrm{E}+08$ & $3.16 \mathrm{E}+01$ \\
\hline Cf-248 & & & $9.21 \mathrm{E}+02$ & $5.84 \mathrm{E}-01$ \\
\hline Cf-249 & & & $1.16 \mathrm{E}+02$ & $2.83 E+01$ \\
\hline Cf-250 & & & $2.38 \mathrm{E}+02$ & $2.18 \mathrm{E}+00$ \\
\hline Cf-251 & & & $1.14 \mathrm{E}+02$ & $7.20 \mathrm{E}+01$ \\
\hline Cf-252 & $4.05 E+02$ & 7.56E-01 & $4.05 E+02$ & 7.54E-01 \\
\hline Cf-253 & & & $6.24 \mathrm{E}+03$ & $2.15 \mathrm{E}-01$ \\
\hline Cf-254 & & & $1.98 \mathrm{E}+02$ & $2.33 \mathrm{E}-02$ \\
\hline Cf-255 & & & $1.10 \mathrm{E}+06$ & $1.27 \mathrm{E}-01$ \\
\hline Cf-256 & & & $4.27 \mathrm{E}+03$ & 7.15E-05 \\
\hline Cl-34 & & & $5.67 \mathrm{E}+04$ & $2.60 \mathrm{E}-07$ \\
\hline $\mathrm{Cl}-34 \mathrm{~m}$ & & & $2.23 \mathrm{E}+04$ & $1.29 \mathrm{E}-04$ \\
\hline Cl-36 & $1.11 \mathrm{E}+03$ & $3.36 \mathrm{E}+04$ & $1.11 \mathrm{E}+03$ & $3.36 \mathrm{E}+04$ \\
\hline Cl-38 & & & $2.88 \mathrm{E}+04$ & $2.17 \mathrm{E}-04$ \\
\hline Cl-39 & & & $3.06 \mathrm{E}+04$ & 3.53E-04 \\
\hline Cl-40 & & & $1.29 \mathrm{E}+04$ & $3.71 \mathrm{E}-06$ \\
\hline Cm-238 & & & $1.80 \mathrm{E}+06$ & $3.28 \mathrm{E}-01$ \\
\hline Cm-239 & & & $7.55 \mathrm{E}+07$ & $1.67 \mathrm{E}+01$ \\
\hline $\mathrm{Cm}-240$ & & & $2.53 \mathrm{E}+03$ & $1.26 \mathrm{E}-01$ \\
\hline $\mathrm{Cm}-241$ & & & $2.19 \mathrm{E}+05$ & $1.32 \mathrm{E}+01$ \\
\hline
\end{tabular}




\begin{tabular}{|c|c|c|c|c|}
\hline \multicolumn{5}{|c|}{ Table B.2 HC-2 TQs Using Recommended Inhalation DCs (continued) } \\
\hline \multirow{2}{*}{$\begin{array}{c}\text { MASTER } \\
\text { ISOTOPE LIST }\end{array}$} & \multicolumn{2}{|c|}{ NNSA SD 1027} & \multicolumn{2}{|c|}{ Final Threshold Quantity } \\
\hline & Act (Ci) & Mass (g) & Act (Ci) & Mass (g) \\
\hline $\mathrm{Cm}-242$ & $1.56 \mathrm{E}+03$ & 4.71E-01 & $1.56 \mathrm{E}+03$ & 4.71E-01 \\
\hline $\mathrm{Cm}-243$ & & & $2.62 \mathrm{E}+02$ & $5.07 \mathrm{E}+00$ \\
\hline $\mathrm{Cm}-244$ & & & $3.00 \mathrm{E}+02$ & $3.71 \mathrm{E}+00$ \\
\hline $\mathrm{Cm}-245$ & $1.93 \mathrm{E}+02$ & $1.12 \mathrm{E}+03$ & $1.93 \mathrm{E}+02$ & $1.12 \mathrm{E}+03$ \\
\hline $\mathrm{Cm}-246$ & & & $1.93 \mathrm{E}+02$ & $6.29 \mathrm{E}+02$ \\
\hline $\mathrm{Cm}-247$ & & & $2.08 \mathrm{E}+02$ & $2.24 \mathrm{E}+06$ \\
\hline $\mathrm{Cm}-248$ & & & $5.41 \mathrm{E}+01$ & $1.27 \mathrm{E}+04$ \\
\hline Cm-249 & & & $2.26 \mathrm{E}+08$ & $1.92 \mathrm{E}+01$ \\
\hline $\mathrm{Cm}-250$ & & & $9.65 \mathrm{E}+00$ & $4.66 \mathrm{E}+01$ \\
\hline Cm-251 & & & $1.89 \mathrm{E}+08$ & $4.23 \mathrm{E}+00$ \\
\hline Co-54m & & & $1.46 \mathrm{E}+07$ & $6.20 \mathrm{E}-03$ \\
\hline Co-55 & & & $1.02 \mathrm{E}+07$ & $3.14 \mathrm{E}+00$ \\
\hline Co-56 & & & $1.52 \mathrm{E}+06$ & $5.11 \mathrm{E}+01$ \\
\hline Co-57 & & & $1.43 \mathrm{E}+07$ & $1.69 \mathrm{E}+03$ \\
\hline Co-58 & & & $4.65 \mathrm{E}+06$ & $1.46 \mathrm{E}+02$ \\
\hline Co-58m & & & $6.24 \mathrm{E}+08$ & $1.06 \mathrm{E}+02$ \\
\hline Co-60 & $7.81 \mathrm{E}+05$ & $6.90 \mathrm{E}+02$ & $7.81 \mathrm{E}+05$ & $6.90 \mathrm{E}+02$ \\
\hline Co-60m & & & $4.38 \mathrm{E}+09$ & $1.46 \mathrm{E}+01$ \\
\hline Co-61 & & & $1.38 \mathrm{E}+08$ & $4.42 \mathrm{E}+00$ \\
\hline Co-62 & & & $3.41 \mathrm{E}+07$ & $1.69 \mathrm{E}-02$ \\
\hline Co-62m & & & $1.88 \mathrm{E}+07$ & 8.62E-02 \\
\hline Cr-48 & & & $2.88 \mathrm{E}+07$ & $1.01 \mathrm{E}+01$ \\
\hline Cr-49 & & & $4.36 \mathrm{E}+07$ & $4.78 \mathrm{E}-01$ \\
\hline Cr-51 & $1.95 \mathrm{E}+08$ & $2.11 \mathrm{E}+03$ & $1.95 \mathrm{E}+08$ & $2.11 \mathrm{E}+03$ \\
\hline Cr-55 & & & $2.70 \mathrm{E}+09$ & $2.76 \mathrm{E}+00$ \\
\hline Cr-56 & & & $7.79 \mathrm{E}+08$ & $1.38 \mathrm{E}+00$ \\
\hline Cs-121 & & & $5.00 \mathrm{E}+06$ & $8.30 \mathrm{E}-03$ \\
\hline Cs-121m & & & $5.02 \mathrm{E}+06$ & $6.57 \mathrm{E}-03$ \\
\hline Cs-123 & & & $5.53 \mathrm{E}+06$ & $2.12 \mathrm{E}-02$ \\
\hline Cs-124 & & & $4.98 \mathrm{E}+06$ & $1.68 \mathrm{E}-03$ \\
\hline Cs-125 & & & $7.47 \mathrm{E}+06$ & $2.23 \mathrm{E}-01$ \\
\hline Cs-126 & & & $5.16 \mathrm{E}+06$ & $5.66 \mathrm{E}-03$ \\
\hline Cs-127 & & & $1.04 \mathrm{E}+07$ & $2.63 \mathrm{E}+00$ \\
\hline Cs-128 & & & $6.26 \mathrm{E}+06$ & $1.66 \mathrm{E}-02$ \\
\hline Cs-129 & & & $1.02 \mathrm{E}+07$ & $1.35 \mathrm{E}+01$ \\
\hline Cs-130 & & & $9.97 \mathrm{E}+06$ & $2.06 \mathrm{E}-01$ \\
\hline Cs-130m & & & $1.34 \mathrm{E}+08$ & $3.21 \mathrm{E}-01$ \\
\hline Cs-131 & & & $2.90 \mathrm{E}+07$ & $2.81 \mathrm{E}+02$ \\
\hline
\end{tabular}




\begin{tabular}{|c|c|c|c|c|}
\hline \multicolumn{5}{|c|}{ Table B.2 HC-2 TQs Using Recommended Inhalation DCs (continued) } \\
\hline \multirow{2}{*}{$\begin{array}{c}\text { MASTER } \\
\text { ISOTOPE LIST }\end{array}$} & \multicolumn{2}{|c|}{ NNSA SD 1027} & \multicolumn{2}{|c|}{ Final Threshold Quantity } \\
\hline & Act (Ci) & Mass (g) & Act (Ci) & Mass (g) \\
\hline Cs-132 & & & $2.46 \mathrm{E}+06$ & $1.61 \mathrm{E}+01$ \\
\hline Cs-134 & $1.19 \mathrm{E}+05$ & $9.18 \mathrm{E}+01$ & $1.19 \mathrm{E}+05$ & $9.17 \mathrm{E}+01$ \\
\hline Cs-134m & & & $4.85 \mathrm{E}+07$ & $6.01 \mathrm{E}+00$ \\
\hline Cs-135 & & & $1.18 \mathrm{E}+06$ & $1.02 \mathrm{E}+09$ \\
\hline Cs-135m & & & $3.31 \mathrm{E}+06$ & $1.26 \mathrm{E}-01$ \\
\hline Cs-136 & & & $5.34 \mathrm{E}+05$ & $7.28 \mathrm{E}+00$ \\
\hline Cs-137 & $1.76 \mathrm{E}+05$ & $2.03 \mathrm{E}+03$ & $1.76 \mathrm{E}+05$ & $2.03 \mathrm{E}+03$ \\
\hline Cs-138 & & & $2.10 \mathrm{E}+06$ & $4.95 \mathrm{E}-02$ \\
\hline Cs-138m & & & $1.41 \mathrm{E}+07$ & $3.00 \mathrm{E}-02$ \\
\hline Cs-139 & & & $1.63 \mathrm{E}+07$ & $1.12 \mathrm{E}-01$ \\
\hline Cs-140 & & & $3.04 \mathrm{E}+06$ & $2.40 \mathrm{E}-03$ \\
\hline Cu-57 & & & $4.78 \mathrm{E}+07$ & 5.63E-05 \\
\hline Cu-59 & & & $4.05 \mathrm{E}+07$ & $1.72 \mathrm{E}-02$ \\
\hline $\mathrm{Cu}-60$ & & & $1.29 \mathrm{E}+07$ & $9.55 \mathrm{E}-02$ \\
\hline $\mathrm{Cu}-61$ & & & $4.10 \mathrm{E}+07$ & $2.72 \mathrm{E}+00$ \\
\hline Cu-62 & & & $5.56 \mathrm{E}+07$ & $1.78 \mathrm{E}-01$ \\
\hline $\mathrm{Cu}-64$ & & & $5.50 \mathrm{E}+07$ & $1.43 \mathrm{E}+01$ \\
\hline Cu-66 & & & $6.06 \mathrm{E}+08$ & $1.08 \mathrm{E}+00$ \\
\hline Cu-67 & & & $1.29 \mathrm{E}+07$ & $1.71 \mathrm{E}+01$ \\
\hline Cu-69 & & & $1.07 \mathrm{E}+08$ & $1.12 \mathrm{E}-01$ \\
\hline Dy-148 & & & $8.58 \mathrm{E}+07$ & $2.23 \mathrm{E}-01$ \\
\hline Dy-149 & & & $3.59 \mathrm{E}+07$ & $1.20 \mathrm{E}-01$ \\
\hline Dy-150 & & & $2.29 \mathrm{E}+08$ & $1.31 \mathrm{E}+00$ \\
\hline Dy-151 & & & $2.39 \mathrm{E}+07$ & 3.43E-01 \\
\hline Dy-152 & & & $7.37 \mathrm{E}+07$ & $8.50 \mathrm{E}+00$ \\
\hline Dy-153 & & & $3.05 \mathrm{E}+07$ & $9.53 \mathrm{E}+00$ \\
\hline Dy-154 & & & $2.96 \mathrm{E}+02$ & $3.82 \mathrm{E}+05$ \\
\hline Dy-155 & & & $5.06 \mathrm{E}+07$ & $2.50 \mathrm{E}+01$ \\
\hline Dy-157 & & & $1.03 E+08$ & $4.17 \mathrm{E}+01$ \\
\hline Dy-159 & & & $2.17 \mathrm{E}+07$ & $3.81 \mathrm{E}+03$ \\
\hline Dy-165 & & & $1.27 \mathrm{E}+08$ & $1.57 \mathrm{E}+01$ \\
\hline Dy-165m & & & $3.82 \mathrm{E}+09$ & $4.21 \mathrm{E}+00$ \\
\hline Dy-166 & & & $4.26 \mathrm{E}+06$ & $1.84 \mathrm{E}+01$ \\
\hline Dy-167 & & & $1.13 \mathrm{E}+08$ & $6.20 \mathrm{E}-01$ \\
\hline Dy-168 & & & $1.56 \mathrm{E}+08$ & $1.21 \mathrm{E}+00$ \\
\hline Er-154 & & & $1.21 \mathrm{E}+09$ & $3.68 \mathrm{E}+00$ \\
\hline Er-156 & & & $2.80 \mathrm{E}+08$ & $4.53 \mathrm{E}+00$ \\
\hline Er-159 & & & $5.35 \mathrm{E}+07$ & $1.63 \mathrm{E}+00$ \\
\hline
\end{tabular}




\begin{tabular}{|c|c|c|c|c|}
\hline \multicolumn{5}{|c|}{ Table B.2 HC-2 TQs Using Recommended Inhalation DCs (continued) } \\
\hline \multirow{2}{*}{$\begin{array}{c}\text { MASTER } \\
\text { ISOTOPE LIST }\end{array}$} & \multicolumn{2}{|c|}{ NNSA SD 1027} & \multicolumn{2}{|c|}{ Final Threshold Quantity } \\
\hline & Act (Ci) & Mass (g) & Act (Ci) & Mass (g) \\
\hline Er-161 & & & $4.49 \mathrm{E}+07$ & $7.47 \mathrm{E}+00$ \\
\hline Er-163 & & & $1.80 \mathrm{E}+09$ & $1.17 \mathrm{E}+02$ \\
\hline Er-165 & & & $7.22 \mathrm{E}+08$ & $3.94 \mathrm{E}+02$ \\
\hline Er-167m & & & $6.71 \mathrm{E}+08$ & $2.26 \mathrm{E}-02$ \\
\hline Er-169 & & & $8.11 \mathrm{E}+06$ & $9.76 \mathrm{E}+01$ \\
\hline Er-171 & & & $2.97 \mathrm{E}+07$ & $1.22 \mathrm{E}+01$ \\
\hline Er-172 & & & $6.91 \mathrm{E}+06$ & $1.87 \mathrm{E}+01$ \\
\hline Er-173 & & & $7.25 \mathrm{E}+07$ & $9.56 \mathrm{E}-02$ \\
\hline Es-249 & & & $2.68 \mathrm{E}+07$ & $3.63 \mathrm{E}+00$ \\
\hline Es-250 & & & $1.18 \mathrm{E}+07$ & $1.98 \mathrm{E}+00$ \\
\hline Es-250m & & & $4.87 \mathrm{E}+06$ & 8.63E-01 \\
\hline Es-251 & & & $3.84 \mathrm{E}+06$ & $1.01 \mathrm{E}+01$ \\
\hline Es-253 & & & $3.00 \mathrm{E}+03$ & 1.19E-01 \\
\hline Es-254 & & & $9.43 \mathrm{E}+02$ & $5.06 \mathrm{E}-01$ \\
\hline Es-254m & & & $1.72 \mathrm{E}+04$ & $5.50 \mathrm{E}-02$ \\
\hline Es-255 & & & $1.64 \mathrm{E}+03$ & $1.27 \mathrm{E}-01$ \\
\hline Es-256 & & & $1.78 \mathrm{E}+05$ & $6.16 \mathrm{E}-03$ \\
\hline Eu-142 & & & $4.68 \mathrm{E}+07$ & $1.38 \mathrm{E}-03$ \\
\hline Eu-142m & & & $1.71 \mathrm{E}+07$ & $1.58 \mathrm{E}-02$ \\
\hline Eu-143 & & & $5.14 \mathrm{E}+07$ & $1.01 \mathrm{E}-01$ \\
\hline Eu-144 & & & $5.22 \mathrm{E}+07$ & $6.79 \mathrm{E}-03$ \\
\hline Eu-145 & & & $1.06 \mathrm{E}+07$ & $6.97 \mathrm{E}+01$ \\
\hline Eu-146 & & & $6.94 \mathrm{E}+06$ & $3.57 \mathrm{E}+01$ \\
\hline Eu-147 & & & $6.93 \mathrm{E}+06$ & $1.87 \mathrm{E}+02$ \\
\hline Eu-148 & & & $2.78 \mathrm{E}+06$ & $1.72 \mathrm{E}+02$ \\
\hline Eu-149 & & & $2.73 \mathrm{E}+07$ & $2.90 \mathrm{E}+03$ \\
\hline Eu-150l & & & $1.52 \mathrm{E}+05$ & $2.19 \mathrm{E}+03$ \\
\hline Eu-150s & & & $4.12 \mathrm{E}+07$ & $2.49 \mathrm{E}+01$ \\
\hline Eu-152 & $1.92 \mathrm{E}+05$ & $1.11 \mathrm{E}+03$ & $1.92 \mathrm{E}+05$ & $1.09 \mathrm{E}+03$ \\
\hline Eu-152ml & & & $3.09 \mathrm{E}+07$ & $1.40 \mathrm{E}+01$ \\
\hline Eu-152ms & & & $4.51 \mathrm{E}+08$ & $3.50 \mathrm{E}+01$ \\
\hline Eu-154 & $1.52 \mathrm{E}+05$ & $5.64 \mathrm{E}+02$ & $1.52 \mathrm{E}+05$ & $5.78 \mathrm{E}+02$ \\
\hline Eu-154m & & & $7.23 \mathrm{E}+08$ & $2.72 \mathrm{E}+01$ \\
\hline Eu-155 & $1.17 \mathrm{E}+06$ & $2.41 \mathrm{E}+03$ & $1.17 \mathrm{E}+06$ & $2.52 \mathrm{E}+03$ \\
\hline Eu-156 & & & $2.25 E+06$ & $4.08 \mathrm{E}+01$ \\
\hline Eu-157 & & & $2.57 \mathrm{E}+07$ & $1.95 \mathrm{E}+01$ \\
\hline Eu-158 & & & $3.95 \mathrm{E}+07$ & $1.52 \mathrm{E}+00$ \\
\hline Eu-159 & & & $1.19 \mathrm{E}+08$ & $1.82 \mathrm{E}+00$ \\
\hline
\end{tabular}




\begin{tabular}{|c|c|c|c|c|}
\hline \multicolumn{5}{|c|}{ Table B.2 HC-2 TQs Using Recommended Inhalation DCs (continued) } \\
\hline \multirow{2}{*}{$\begin{array}{c}\text { MASTER } \\
\text { ISOTOPE LIST }\end{array}$} & \multicolumn{2}{|c|}{ NNSA SD 1027} & \multicolumn{2}{|c|}{ Final Threshold Quantity } \\
\hline & Act (Ci) & Mass (g) & Act (Ci) & Mass (g) \\
\hline F-17 & & & $5.88 \mathrm{E}+04$ & $5.71 \mathrm{E}-06$ \\
\hline F-18 & & & $3.94 \mathrm{E}+04$ & $4.14 \mathrm{E}-04$ \\
\hline Fe-52 & & & $1.15 \mathrm{E}+07$ & $1.57 \mathrm{E}+00$ \\
\hline Fe-53 & & & $5.05 \mathrm{E}+07$ & $1.21 \mathrm{E}-01$ \\
\hline Fe-53m & & & $1.88 \mathrm{E}+07$ & $1.34 \mathrm{E}-02$ \\
\hline Fe-55 & $2.13 \mathrm{E}+07$ & $8.95 \mathrm{E}+03$ & $2.13 \mathrm{E}+07$ & $8.85 \mathrm{E}+03$ \\
\hline Fe-59 & $2.09 \mathrm{E}+06$ & $4.20 \mathrm{E}+01$ & $2.09 \mathrm{E}+06$ & $4.20 \mathrm{E}+01$ \\
\hline Fe-60 & & & $5.79 \mathrm{E}+04$ & $9.71 \mathrm{E}+05$ \\
\hline Fe-61 & & & $4.05 \mathrm{E}+07$ & 7.84E-02 \\
\hline Fe-62 & & & $1.16 \mathrm{E}+08$ & 4.35E-02 \\
\hline Fm-251 & & & $3.72 \mathrm{E}+06$ & $1.58 \mathrm{E}+00$ \\
\hline Fm-252 & & & $2.53 \mathrm{E}+04$ & 4.63E-02 \\
\hline Fm-253 & & & $2.03 \mathrm{E}+04$ & $1.18 \mathrm{E}-01$ \\
\hline Fm-254 & & & $1.33 \mathrm{E}+05$ & $3.49 \mathrm{E}-02$ \\
\hline Fm-255 & & & $3.00 \mathrm{E}+04$ & $4.91 \mathrm{E}-02$ \\
\hline Fm-256 & & & $2.83 \mathrm{E}+04$ & 6.07E-03 \\
\hline Fm-257 & & & $1.14 \mathrm{E}+03$ & $2.26 \mathrm{E}-01$ \\
\hline Fr-212 & & & $1.14 \mathrm{E}+06$ & $2.56 \mathrm{E}-02$ \\
\hline Fr-219 & & & $1.63 \mathrm{E}+10$ & $6.64 \mathrm{E}-03$ \\
\hline Fr-220 & & & $5.49 \mathrm{E}+09$ & $2.94 \mathrm{E}+00$ \\
\hline Fr-221 & & & $1.85 \mathrm{E}+09$ & $1.04 \mathrm{E}+01$ \\
\hline Fr-222 & & & $5.79 \mathrm{E}+05$ & $9.85 \mathrm{E}-03$ \\
\hline Fr-223 & & & $9.04 \mathrm{E}+06$ & $2.34 \mathrm{E}-01$ \\
\hline Fr-224 & & & $1.03 \mathrm{E}+08$ & 4.09E-01 \\
\hline Fr-227 & & & $1.35 \mathrm{E}+08$ & 4.03E-01 \\
\hline Ga-64 & & & $1.65 \mathrm{E}+07$ & $1.47 \mathrm{E}-02$ \\
\hline Ga-65 & & & $4.35 \mathrm{E}+07$ & $2.28 \mathrm{E}-01$ \\
\hline Ga-66 & & & $9.80 \mathrm{E}+06$ & $1.94 \mathrm{E}+00$ \\
\hline Ga-67 & & & $3.10 \mathrm{E}+07$ & $5.18 \mathrm{E}+01$ \\
\hline Ga-68 & & & $4.35 \mathrm{E}+07$ & $1.07 \mathrm{E}+00$ \\
\hline Ga-70 & & & $4.66 \mathrm{E}+08$ & $3.67 \mathrm{E}+00$ \\
\hline Ga-72 & & & $8.56 \mathrm{E}+06$ & $2.77 \mathrm{E}+00$ \\
\hline Ga-73 & & & $4.40 \mathrm{E}+07$ & $5.02 \mathrm{E}+00$ \\
\hline Ga-74 & & & $1.74 \mathrm{E}+07$ & $5.57 \mathrm{E}-02$ \\
\hline Gd-142 & & & $5.65 \mathrm{E}+07$ & 4.99E-02 \\
\hline Gd-143m & & & $2.77 \mathrm{E}+07$ & $3.86 \mathrm{E}-02$ \\
\hline Gd-144 & & & $6.36 \mathrm{E}+07$ & $2.18 \mathrm{E}-01$ \\
\hline Gd-145 & & & $2.22 \mathrm{E}+07$ & 3.92E-01 \\
\hline
\end{tabular}




\begin{tabular}{|c|c|c|c|c|}
\hline \multicolumn{5}{|c|}{ Table B.2 HC-2 TQs Using Recommended Inhalation DCs (continued) } \\
\hline \multirow{2}{*}{$\begin{array}{c}\text { MASTER } \\
\text { ISOTOPE LIST }\end{array}$} & \multicolumn{2}{|c|}{ NNSA SD 1027} & \multicolumn{2}{|c|}{ Final Threshold Quantity } \\
\hline & Act (Ci) & Mass (g) & Act (Ci) & Mass (g) \\
\hline Gd-145m & & & $8.78 \mathrm{E}+07$ & 9.58E-02 \\
\hline Gd-146 & & & $1.26 \mathrm{E}+06$ & $6.81 \mathrm{E}+01$ \\
\hline Gd-147 & & & $1.37 \mathrm{E}+07$ & $2.44 \mathrm{E}+01$ \\
\hline Gd-148 & & & $3.12 \mathrm{E}+02$ & $1.20 \mathrm{E}+01$ \\
\hline Gd-149 & & & $1.03 \mathrm{E}+07$ & $1.10 \mathrm{E}+02$ \\
\hline Gd-150 & & & $3.17 \mathrm{E}+02$ & $2.38 \mathrm{E}+05$ \\
\hline Gd-151 & & & $9.36 \mathrm{E}+06$ & $1.30 \mathrm{E}+03$ \\
\hline Gd-152 & & & $4.27 \mathrm{E}+02$ & $1.96 \mathrm{E}+13$ \\
\hline Gd-153 & $3.84 \mathrm{E}+06$ & $1.09 \mathrm{E}+03$ & $3.84 \mathrm{E}+06$ & $1.09 \mathrm{E}+03$ \\
\hline Gd-159 & & & $2.93 \mathrm{E}+07$ & $2.76 \mathrm{E}+01$ \\
\hline Gd-162 & & & $1.45 \mathrm{E}+08$ & $1.05 \mathrm{E}+00$ \\
\hline Ge-66 & & & $4.30 \mathrm{E}+07$ & $2.05 \mathrm{E}+00$ \\
\hline Ge-67 & & & $3.51 \mathrm{E}+07$ & $2.34 \mathrm{E}-01$ \\
\hline Ge-68 & $5.79 \mathrm{E}+05$ & $8.16 \mathrm{E}+01$ & $5.79 \mathrm{E}+05$ & $8.68 \mathrm{E}+01$ \\
\hline Ge-69 & & & $1.94 \mathrm{E}+07$ & $1.67 \mathrm{E}+01$ \\
\hline Ge-71 & & & $7.37 \mathrm{E}+08$ & $4.72 \mathrm{E}+03$ \\
\hline Ge-75 & & & $1.98 \mathrm{E}+08$ & $6.52 \mathrm{E}+00$ \\
\hline Ge-77 & & & $1.53 \mathrm{E}+07$ & $4.25 \mathrm{E}+00$ \\
\hline Ge-78 & & & $6.00 \mathrm{E}+07$ & $2.16 \mathrm{E}+00$ \\
\hline $\mathrm{H}-3$ & $3.00 \mathrm{E}+05$ & $3.00 \mathrm{E}+01$ & $1.80 \mathrm{E}+05$ & $1.88 \mathrm{E}+01$ \\
\hline Hf-167 & & & $9.90 \mathrm{E}+07$ & $1.80 \mathrm{E}-01$ \\
\hline Hf-169 & & & $9.72 \mathrm{E}+07$ & 2.83E-01 \\
\hline Hf-170 & & & $2.05 \mathrm{E}+07$ & $1.78 \mathrm{E}+01$ \\
\hline Hf-172 & & & $2.53 \mathrm{E}+05$ & $2.28 \mathrm{E}+02$ \\
\hline Hf-173 & & & $3.76 \mathrm{E}+07$ & $4.98 \mathrm{E}+01$ \\
\hline Hf-174 & & & $2.53 \mathrm{E}+02$ & $2.47 \mathrm{E}+14$ \\
\hline Hf-175 & & & $6.48 \mathrm{E}+06$ & $6.08 \mathrm{E}+02$ \\
\hline Hf-177m & & & $1.99 \mathrm{E}+07$ & $9.61 \mathrm{E}-01$ \\
\hline Hf-178m & & & $3.11 \mathrm{E}+04$ & $4.81 \mathrm{E}+02$ \\
\hline Hf-179m & & & $2.07 \mathrm{E}+06$ & $7.10 \mathrm{E}+01$ \\
\hline Hf-180m & & & $2.98 \mathrm{E}+07$ & $9.41 \mathrm{E}+00$ \\
\hline Hf-181 & $1.60 \mathrm{E}+06$ & $9.38 \mathrm{E}+01$ & $1.60 \mathrm{E}+06$ & $9.38 \mathrm{E}+01$ \\
\hline Hf-182 & & & $2.62 E+04$ & $1.20 \mathrm{E}+08$ \\
\hline Hf-182m & & & $4.53 \mathrm{E}+07$ & $2.70 \mathrm{E}+00$ \\
\hline Hf-183 & & & $4.89 \mathrm{E}+07$ & $3.04 \mathrm{E}+00$ \\
\hline Hf-184 & & & $2.23 \mathrm{E}+07$ & $5.38 \mathrm{E}+00$ \\
\hline Hg-190 & & & $7.61 \mathrm{E}+06$ & $1.54 \mathrm{E}-01$ \\
\hline Hg-191m & & & $1.48 \mathrm{E}+06$ & 7.62E-02 \\
\hline
\end{tabular}




\begin{tabular}{|c|c|c|c|c|}
\hline \multicolumn{5}{|c|}{ Table B.2 HC-2 TQs Using Recommended Inhalation DCs (continued) } \\
\hline \multirow{2}{*}{$\begin{array}{c}\text { MASTER } \\
\text { ISOTOPE LIST }\end{array}$} & \multicolumn{2}{|c|}{ NNSA SD 1027} & \multicolumn{2}{|c|}{ Final Threshold Quantity } \\
\hline & Act (Ci) & Mass (g) & Act (Ci) & Mass (g) \\
\hline Hg-192 & & & $7.28 \mathrm{E}+05$ & 2.16E-01 \\
\hline Hg-193 & & & $7.20 \mathrm{E}+05$ & $1.55 \mathrm{E}-01$ \\
\hline Hg-193m & & & $2.49 \mathrm{E}+05$ & $1.70 \mathrm{E}-01$ \\
\hline Hg-194 & & & $2.03 \mathrm{E}+04$ & $2.86 \mathrm{E}+03$ \\
\hline Hg-195 & & & $5.68 \mathrm{E}+05$ & 3.50E-01 \\
\hline Hg-195m & & & $9.85 \mathrm{E}+04$ & $2.55 \mathrm{E}-01$ \\
\hline Hg-197 & & & $1.84 \mathrm{E}+05$ & $7.41 \mathrm{E}-01$ \\
\hline Hg-197m & & & $1.40 \mathrm{E}+05$ & $2.09 \mathrm{E}-01$ \\
\hline Hg-199m & & & $3.95 \mathrm{E}+06$ & $1.78 \mathrm{E}-01$ \\
\hline Hg-203 & $3.33 \mathrm{E}+05$ & $2.41 \mathrm{E}+01$ & $1.15 \mathrm{E}+05$ & $8.35 \mathrm{E}+00$ \\
\hline Hg-205 & & & $4.37 \mathrm{E}+08$ & $2.48 \mathrm{E}+00$ \\
\hline Hg-206 & & & $4.86 \mathrm{E}+07$ & $4.34 \mathrm{E}-01$ \\
\hline Hg-207 & & & $2.11 \mathrm{E}+06$ & $6.74 \mathrm{E}-03$ \\
\hline Ho-150 & & & $3.10 \mathrm{E}+07$ & $3.16 \mathrm{E}-02$ \\
\hline Ho-153 & & & $5.86 \mathrm{E}+07$ & $9.58 \mathrm{E}-02$ \\
\hline Ho-153m & & & $5.73 \mathrm{E}+07$ & 4.33E-01 \\
\hline Ho-154 & & & $2.93 \mathrm{E}+07$ & $2.82 \mathrm{E}-01$ \\
\hline Ho-154m & & & $2.48 \mathrm{E}+07$ & $6.29 \mathrm{E}-02$ \\
\hline Ho-155 & & & $1.10 \mathrm{E}+08$ & $4.35 \mathrm{E}+00$ \\
\hline Ho-156 & & & $2.26 \mathrm{E}+07$ & $1.05 \mathrm{E}+00$ \\
\hline Ho-157 & & & $1.14 \mathrm{E}+08$ & $1.19 \mathrm{E}+00$ \\
\hline Ho-158 & & & $1.04 \mathrm{E}+09$ & $9.87 \mathrm{E}+00$ \\
\hline Ho-159 & & & $1.50 \mathrm{E}+08$ & $4.18 \mathrm{E}+00$ \\
\hline Ho-160 & & & $3.30 \mathrm{E}+07$ & 7.19E-01 \\
\hline Ho-161 & & & $7.25 \mathrm{E}+08$ & $9.30 \mathrm{E}+01$ \\
\hline Ho-162 & & & $3.26 \mathrm{E}+08$ & $4.21 \mathrm{E}+00$ \\
\hline Нo-162m & & & $7.86 \mathrm{E}+07$ & $4.60 \mathrm{E}+00$ \\
\hline Но-163 & & & $2.88 \mathrm{E}+07$ & $5.99 \mathrm{E}+07$ \\
\hline Ho-164 & & & $7.29 \mathrm{E}+08$ & $1.84 \mathrm{E}+01$ \\
\hline Ho-164m & & & $5.08 \mathrm{E}+08$ & $1.66 \mathrm{E}+01$ \\
\hline Но-166 & & & $1.24 \mathrm{E}+07$ & $1.76 \mathrm{E}+01$ \\
\hline Ho-166m & $6.74 \mathrm{E}+04$ & $3.75 \mathrm{E}+04$ & $6.74 \mathrm{E}+04$ & $3.76 \mathrm{E}+04$ \\
\hline Но-167 & & & $6.60 \mathrm{E}+07$ & $1.09 \mathrm{E}+01$ \\
\hline Но-168 & & & $6.66 \mathrm{E}+07$ & $1.78 \mathrm{E}-01$ \\
\hline Нo-168m & & & $1.80 \mathrm{E}+10$ & $3.54 \mathrm{E}+01$ \\
\hline Но-170 & & & $3.45 \mathrm{E}+07$ & $8.61 \mathrm{E}-02$ \\
\hline $\mathrm{I}-118$ & & & $4.63 \mathrm{E}+04$ & $3.98 \mathrm{E}-04$ \\
\hline $\mathrm{I}-118 \mathrm{~m}$ & & & $3.16 \mathrm{E}+04$ & $1.68 \mathrm{E}-04$ \\
\hline
\end{tabular}




\begin{tabular}{|c|c|c|c|c|}
\hline \multicolumn{5}{|c|}{ Table B.2 HC-2 TQs Using Recommended Inhalation DCs (continued) } \\
\hline \multirow{2}{*}{$\begin{array}{c}\text { MASTER } \\
\text { ISOTOPE LIST }\end{array}$} & \multicolumn{2}{|c|}{ NNSA SD 1027} & \multicolumn{2}{|c|}{ Final Threshold Quantity } \\
\hline & Act (Ci) & Mass (g) & Act (Ci) & Mass (g) \\
\hline $\mathrm{I}-119$ & & & $1.16 \mathrm{E}+05$ & 1.40E-03 \\
\hline $\mathrm{I}-120$ & & & $3.15 \mathrm{E}+04$ & $1.63 \mathrm{E}-03$ \\
\hline $\mathrm{I}-120 \mathrm{~m}$ & & & $1.85 \mathrm{E}+04$ & $6.25 \mathrm{E}-04$ \\
\hline $\mathrm{I}-121$ & & & $1.90 \mathrm{E}+05$ & $1.56 \mathrm{E}-02$ \\
\hline $\mathrm{I}-122$ & & & $1.19 \mathrm{E}+05$ & $2.78 \mathrm{E}-04$ \\
\hline $\mathrm{I}-123$ & & & $1.69 \mathrm{E}+05$ & $8.76 \mathrm{E}-02$ \\
\hline $\mathrm{I}-124$ & & & $3.56 \mathrm{E}+03$ & $1.41 \mathrm{E}-02$ \\
\hline $\mathrm{I}-125$ & $3.18 \mathrm{E}+03$ & $1.81 \mathrm{E}-01$ & $3.18 \mathrm{E}+03$ & $1.83 \mathrm{E}-01$ \\
\hline $\mathrm{I}-126$ & & & $1.64 \mathrm{E}+03$ & $2.06 \mathrm{E}-02$ \\
\hline $\mathrm{I}-128$ & & & $6.36 \mathrm{E}+05$ & $1.08 \mathrm{E}-02$ \\
\hline I-129 & & & $4.50 \mathrm{E}+02$ & $2.55 E+06$ \\
\hline I-130 & & & $1.65 \mathrm{E}+04$ & $8.46 \mathrm{E}-03$ \\
\hline $\mathrm{I}-130 \mathrm{~m}$ & & & $1.11 \mathrm{E}+06$ & 6.77E-03 \\
\hline I-131 & $2.18 \mathrm{E}+03$ & $1.75 \mathrm{E}-02$ & $2.18 \mathrm{E}+03$ & $1.75 \mathrm{E}-02$ \\
\hline I-132 & & & $3.77 \mathrm{E}+04$ & $3.65 \mathrm{E}-03$ \\
\hline $\mathrm{I}-132 \mathrm{~m}$ & & & $1.30 \mathrm{E}+05$ & 7.61E-03 \\
\hline $\mathrm{I}-133$ & & & $1.02 \mathrm{E}+04$ & $9.01 \mathrm{E}-03$ \\
\hline $\mathrm{I}-134$ & & & $3.73 E+04$ & $1.40 \mathrm{E}-03$ \\
\hline I-134m & & & $4.54 \mathrm{E}+05$ & $1.16 \mathrm{E}-03$ \\
\hline $\mathrm{I}-135$ & & & $2.90 \mathrm{E}+04$ & $8.25 \mathrm{E}-03$ \\
\hline In-103 & & & $2.08 \mathrm{E}+07$ & $1.14 \mathrm{E}-02$ \\
\hline In-105 & & & $3.01 \mathrm{E}+07$ & 8.52E-02 \\
\hline In-106 & & & $1.66 \mathrm{E}+07$ & 5.79E-02 \\
\hline In-106m & & & $2.00 \mathrm{E}+07$ & $5.86 \mathrm{E}-02$ \\
\hline In-107 & & & $3.30 \mathrm{E}+07$ & $6.07 \mathrm{E}-01$ \\
\hline In-108 & & & $1.35 \mathrm{E}+07$ & $4.50 \mathrm{E}-01$ \\
\hline In-108m & & & $1.80 \mathrm{E}+07$ & 4.09E-01 \\
\hline In-109 & & & $5.86 \mathrm{E}+07$ & $8.56 \mathrm{E}+00$ \\
\hline In-109m & & & $9.86 \mathrm{E}+07$ & $7.66 \mathrm{E}-02$ \\
\hline In-110l & & & $1.41 \mathrm{E}+07$ & $2.41 \mathrm{E}+00$ \\
\hline In-110s & & & $2.94 \mathrm{E}+07$ & $1.19 \mathrm{E}+00$ \\
\hline In-111 & & & $2.84 \mathrm{E}+07$ & $6.82 \mathrm{E}+01$ \\
\hline In-111m & & & $1.29 \mathrm{E}+08$ & 5.85E-01 \\
\hline In-112 & & & $1.79 \mathrm{E}+08$ & $1.54 \mathrm{E}+00$ \\
\hline In-112m & & & $2.80 \mathrm{E}+08$ & $3.43 \mathrm{E}+00$ \\
\hline In-113m & & & $1.44 \mathrm{E}+08$ & $8.60 \mathrm{E}+00$ \\
\hline In-114 & & & $1.94 \mathrm{E}+10$ & $1.41 \mathrm{E}+01$ \\
\hline In-114m & $8.71 \mathrm{E}+05$ & $3.76 \mathrm{E}+01$ & $8.71 \mathrm{E}+05$ & $3.76 \mathrm{E}+01$ \\
\hline
\end{tabular}




\begin{tabular}{|c|c|c|c|c|}
\hline \multicolumn{5}{|c|}{ Table B.2 HC-2 TQs Using Recommended Inhalation DCs (continued) } \\
\hline \multirow{2}{*}{$\begin{array}{c}\text { MASTER } \\
\text { ISOTOPE LIST }\end{array}$} & \multicolumn{2}{|c|}{ NNSA SD 1027} & \multicolumn{2}{|c|}{ Final Threshold Quantity } \\
\hline & Act (Ci) & Mass (g) & Act (Ci) & Mass (g) \\
\hline In-115 & & & $2.08 \mathrm{E}+04$ & $3.41 \mathrm{E}+16$ \\
\hline In-115m & & & $9.99 \mathrm{E}+07$ & $1.64 \mathrm{E}+01$ \\
\hline In-116m & & & $1.93 \mathrm{E}+07$ & $6.44 \mathrm{E}-01$ \\
\hline In-117 & & & $6.32 \mathrm{E}+07$ & $1.72 \mathrm{E}+00$ \\
\hline In-117m & & & $9.59 \mathrm{E}+07$ & $6.94 \mathrm{E}+00$ \\
\hline In-118 & & & $4.73 \mathrm{E}+08$ & $2.47 \mathrm{E}-02$ \\
\hline In-118m & & & $2.06 \mathrm{E}+07$ & $5.65 \mathrm{E}-02$ \\
\hline In-119 & & & $7.23 \mathrm{E}+07$ & $1.10 \mathrm{E}-01$ \\
\hline In-119m & & & $4.30 \mathrm{E}+08$ & $4.90 \mathrm{E}+00$ \\
\hline In-121 & & & $6.20 \mathrm{E}+07$ & $1.53 \mathrm{E}-02$ \\
\hline In-121m & & & $6.89 \mathrm{E}+08$ & $1.72 \mathrm{E}+00$ \\
\hline Ir-180 & & & $3.73 \mathrm{E}+07$ & $5.36 \mathrm{E}-02$ \\
\hline Ir-182 & & & $3.70 \mathrm{E}+07$ & 5.37E-01 \\
\hline Ir-183 & & & $3.83 \mathrm{E}+07$ & $2.16 \mathrm{E}+00$ \\
\hline Ir-184 & & & $2.02 E+07$ & $3.58 \mathrm{E}+00$ \\
\hline Ir-185 & & & $2.91 \mathrm{E}+07$ & $2.41 \mathrm{E}+01$ \\
\hline Ir-186l & & & $1.44 \mathrm{E}+07$ & $1.35 \mathrm{E}+01$ \\
\hline Ir-186s & & & $4.42 \mathrm{E}+07$ & $4.59 \mathrm{E}+00$ \\
\hline Ir-187 & & & $6.27 \mathrm{E}+07$ & $3.93 \mathrm{E}+01$ \\
\hline Ir-188 & & & $1.23 \mathrm{E}+07$ & $3.06 \mathrm{E}+01$ \\
\hline Ir-189 & & & $1.33 \mathrm{E}+07$ & $2.56 \mathrm{E}+02$ \\
\hline Ir-190 & & & $3.11 \mathrm{E}+06$ & $5.48 \mathrm{E}+01$ \\
\hline Ir-190ms & & & $8.11 \mathrm{E}+08$ & $5.90 \mathrm{E}+01$ \\
\hline Ir-190ml & & & $2.66 \mathrm{E}+07$ & $5.00 \mathrm{E}+00$ \\
\hline Ir-191m & & & $8.95 \mathrm{E}+08$ & $7.48 \mathrm{E}-02$ \\
\hline Ir-192 & $1.21 \mathrm{E}+06$ & $1.31 \mathrm{E}+02$ & $1.21 \mathrm{E}+06$ & $1.31 \mathrm{E}+02$ \\
\hline Ir-192ms & & & $1.01 \mathrm{E}+12$ & $1.49 \mathrm{E}+03$ \\
\hline Ir-192ml & & & $2.08 \mathrm{E}+05$ & $2.69 \mathrm{E}+04$ \\
\hline Ir-193m & & & $6.24 \mathrm{E}+06$ & $9.71 \mathrm{E}+01$ \\
\hline Ir-194 & & & $1.41 \mathrm{E}+07$ & $1.68 \mathrm{E}+01$ \\
\hline Ir-194m & & & $6.08 \mathrm{E}+05$ & $1.54 \mathrm{E}+02$ \\
\hline Ir-195 & & & $1.04 \mathrm{E}+08$ & $1.62 \mathrm{E}+01$ \\
\hline Ir-195m & & & $3.56 \mathrm{E}+07$ & $8.41 \mathrm{E}+00$ \\
\hline Ir-196 & & & $2.33 \mathrm{E}+08$ & $2.10 \mathrm{E}-01$ \\
\hline Ir-196m & & & $1.85 \mathrm{E}+07$ & $1.62 \mathrm{E}+00$ \\
\hline K-38 & & & $3.30 \mathrm{E}+04$ & $5.08 \mathrm{E}-05$ \\
\hline $\mathrm{K}-40$ & $7.63 \mathrm{E}+03$ & $1.10 \mathrm{E}+09$ & $7.63 \mathrm{E}+03$ & $1.09 \mathrm{E}+09$ \\
\hline K-42 & & & $9.90 \mathrm{E}+04$ & $1.64 \mathrm{E}-02$ \\
\hline
\end{tabular}




\begin{tabular}{|c|c|c|c|c|}
\hline \multicolumn{5}{|c|}{ Table B.2 HC-2 TQs Using Recommended Inhalation DCs (continued) } \\
\hline \multirow{2}{*}{$\begin{array}{c}\text { MASTER } \\
\text { ISOTOPE LIST }\end{array}$} & \multicolumn{2}{|c|}{ NNSA SD 1027} & \multicolumn{2}{|c|}{ Final Threshold Quantity } \\
\hline & Act (Ci) & Mass (g) & Act (Ci) & Mass (g) \\
\hline K-43 & & & $5.79 \mathrm{E}+04$ & 1.79E-02 \\
\hline K-44 & & & $4.30 \mathrm{E}+04$ & $2.23 \mathrm{E}-04$ \\
\hline K-45 & & & $5.32 \mathrm{E}+04$ & $2.54 \mathrm{E}-04$ \\
\hline K-46 & & & $3.68 \mathrm{E}+04$ & $1.57 \mathrm{E}-05$ \\
\hline Kr-74 & & & $4.83 \mathrm{E}+04$ & $2.19 \mathrm{E}-04$ \\
\hline Kr-75 & & & $4.62 \mathrm{E}+04$ & $7.90 \mathrm{E}-05$ \\
\hline Kr-76 & & & $1.33 \mathrm{E}+05$ & 4.77E-02 \\
\hline Kr-77 & & & $5.56 \mathrm{E}+04$ & $1.70 \mathrm{E}-03$ \\
\hline Kr-79 & & & $2.23 \mathrm{E}+05$ & $1.97 \mathrm{E}-01$ \\
\hline $\mathrm{Kr}-81$ & & & $1.01 \mathrm{E}+07$ & $4.81 \mathrm{E}+08$ \\
\hline Kr-81m & & & $4.40 \mathrm{E}+05$ & $4.10 \mathrm{E}-05$ \\
\hline Kr-83m & & & $1.80 \mathrm{E}+09$ & $8.72 \mathrm{E}+01$ \\
\hline Kr-85 & $1.06 \mathrm{E}+07$ & $2.70 \mathrm{E}+04$ & $2.27 \mathrm{E}+07$ & $5.78 \mathrm{E}+04$ \\
\hline Kr-85m & & & $3.61 \mathrm{E}+05$ & 4.39E-02 \\
\hline Kr-87 & & & $6.56 \mathrm{E}+04$ & $2.31 \mathrm{E}-03$ \\
\hline Kr-88 & & & $2.65 E+04$ & $2.11 \mathrm{E}-03$ \\
\hline Kr-89 & & & $2.83 \mathrm{E}+04$ & $4.21 \mathrm{E}-05$ \\
\hline La-128 & & & $2.08 \mathrm{E}+07$ & 7.33E-02 \\
\hline La-129 & & & $5.87 \mathrm{E}+07$ & 4.67E-01 \\
\hline La-130 & & & $2.62 \mathrm{E}+07$ & $1.58 \mathrm{E}-01$ \\
\hline La-131 & & & $6.92 \mathrm{E}+07$ & $2.84 \mathrm{E}+00$ \\
\hline La-132 & & & $1.76 \mathrm{E}+07$ & $3.56 \mathrm{E}+00$ \\
\hline La-132m & & & $7.14 \mathrm{E}+07$ & $1.22 \mathrm{E}+00$ \\
\hline La-133 & & & $2.03 \mathrm{E}+08$ & $3.37 \mathrm{E}+01$ \\
\hline La-134 & & & $8.07 \mathrm{E}+07$ & 3.83E-01 \\
\hline La-135 & & & $4.84 \mathrm{E}+08$ & $4.06 \mathrm{E}+02$ \\
\hline La-136 & & & $1.51 \mathrm{E}+08$ & $1.08 \mathrm{E}+00$ \\
\hline La-137 & & & $9.32 E+05$ & $2.14 \mathrm{E}+07$ \\
\hline La-138 & & & $5.40 \mathrm{E}+04$ & $2.81 \mathrm{E}+12$ \\
\hline La-140 & & & $5.59 \mathrm{E}+06$ & $1.00 \mathrm{E}+01$ \\
\hline La-141 & & & $5.16 \mathrm{E}+07$ & $9.12 \mathrm{E}+00$ \\
\hline La-142 & & & $1.56 \mathrm{E}+07$ & $1.09 \mathrm{E}+00$ \\
\hline La-143 & & & $2.22 \mathrm{E}+08$ & $2.40 \mathrm{E}+00$ \\
\hline Lu-165 & & & $4.86 \mathrm{E}+07$ & $4.58 \mathrm{E}-01$ \\
\hline Lu-167 & & & $2.86 \mathrm{E}+07$ & $1.31 \mathrm{E}+00$ \\
\hline Lu-169 & & & $1.52 \mathrm{E}+07$ & $2.79 \mathrm{E}+01$ \\
\hline Lu-169m & & & $1.07 \mathrm{E}+14$ & $2.57 \mathrm{E}+05$ \\
\hline Lu-170 & & & $7.77 \mathrm{E}+06$ & $2.02 \mathrm{E}+01$ \\
\hline
\end{tabular}




\begin{tabular}{|c|c|c|c|c|}
\hline \multicolumn{5}{|c|}{ Table B.2 HC-2 TQs Using Recommended Inhalation DCs (continued) } \\
\hline \multirow{2}{*}{$\begin{array}{c}\text { MASTER } \\
\text { ISOTOPE LIST }\end{array}$} & \multicolumn{2}{|c|}{ NNSA SD 1027} & \multicolumn{2}{|c|}{ Final Threshold Quantity } \\
\hline & Act (Ci) & Mass (g) & Act (Ci) & Mass (g) \\
\hline Lu-171 & & & $8.29 \mathrm{E}+06$ & $8.93 E+01$ \\
\hline Lu-171m & & & $2.74 \mathrm{E}+11$ & $3.28 \mathrm{E}+02$ \\
\hline Lu-172 & & & $4.32 \mathrm{E}+06$ & $3.81 \mathrm{E}+01$ \\
\hline Lu-172m & & & $6.93 \mathrm{E}+13$ & $2.34 \mathrm{E}+05$ \\
\hline Lu-173 & & & $3.36 \mathrm{E}+06$ & $2.22 \mathrm{E}+03$ \\
\hline Lu-174 & & & $1.92 \mathrm{E}+06$ & $3.10 \mathrm{E}+03$ \\
\hline Lu-174m & & & $1.93 \mathrm{E}+06$ & $3.65 \mathrm{E}+02$ \\
\hline Lu-176 & & & $1.16 \mathrm{E}+05$ & $2.05 E+12$ \\
\hline Lu-176m & & & $6.66 \mathrm{E}+07$ & $1.38 \mathrm{E}+01$ \\
\hline Lu-177 & & & $6.73 \mathrm{E}+06$ & $6.12 \mathrm{E}+01$ \\
\hline Lu-177m & & & $5.02 \mathrm{E}+05$ & $1.10 \mathrm{E}+02$ \\
\hline Lu-178 & & & $1.72 \mathrm{E}+08$ & $4.61 \mathrm{E}+00$ \\
\hline Lu-178m & & & $4.27 \mathrm{E}+07$ & 9.17E-01 \\
\hline Lu-179 & & & $6.51 \mathrm{E}+07$ & $1.71 \mathrm{E}+01$ \\
\hline Lu-180 & & & $3.81 \mathrm{E}+07$ & $2.08 \mathrm{E}-01$ \\
\hline Lu-181 & & & $1.05 \mathrm{E}+08$ & 3.53E-01 \\
\hline Md-257 & & & $3.24 \mathrm{E}+05$ & $1.38 \mathrm{E}-01$ \\
\hline Md-258 & & & $1.37 \mathrm{E}+03$ & 1.49E-01 \\
\hline Mg-27 & & & $6.50 \mathrm{E}+07$ & $8.82 \mathrm{E}-02$ \\
\hline Mg-28 & & & $5.78 \mathrm{E}+06$ & $1.08 \mathrm{E}+00$ \\
\hline Mn-50m & & & $1.24 \mathrm{E}+07$ & $5.76 \mathrm{E}-03$ \\
\hline Mn-51 & & & $4.38 \mathrm{E}+07$ & 5.49E-01 \\
\hline Mn-52 & $4.23 \mathrm{E}+06$ & $9.41 \mathrm{E}+00$ & $4.23 \mathrm{E}+06$ & $9.41 \mathrm{E}+00$ \\
\hline Mn-52m & & & $2.08 \mathrm{E}+07$ & $1.22 \mathrm{E}-01$ \\
\hline Mn-53 & & & $1.50 \mathrm{E}+08$ & $8.23 \mathrm{E}+10$ \\
\hline Mn-54 & & & $5.00 \mathrm{E}+06$ & $6.45 \mathrm{E}+02$ \\
\hline Mn-56 & & & $2.14 \mathrm{E}+07$ & 9.87E-01 \\
\hline Mn-57 & & & $5.10 \mathrm{E}+08$ & $2.20 \mathrm{E}-01$ \\
\hline Mn-58m & & & $2.35 E+07$ & 7.87E-03 \\
\hline Mo-101 & & & $3.51 \mathrm{E}+07$ & $2.75 \mathrm{E}-01$ \\
\hline Mo-102 & & & $2.54 \mathrm{E}+08$ & $1.55 \mathrm{E}+00$ \\
\hline Mo-89 & & & $4.74 \mathrm{E}+07$ & 4.73E-02 \\
\hline Mo-90 & & & $1.77 \mathrm{E}+07$ & $2.88 \mathrm{E}+00$ \\
\hline Mo-91 & & & $5.10 \mathrm{E}+07$ & 3.82E-01 \\
\hline Mo-91m & & & $4.19 \mathrm{E}+07$ & $2.18 \mathrm{E}-02$ \\
\hline Mo-93 & & & $1.37 \mathrm{E}+07$ & $1.25 \mathrm{E}+07$ \\
\hline Mo-93m & & & $1.62 \mathrm{E}+07$ & $3.30 \mathrm{E}+00$ \\
\hline Мo-99 & $8.89 \mathrm{E}+06$ & $1.85 \mathrm{E}+01$ & $8.89 \mathrm{E}+06$ & $1.85 \mathrm{E}+01$ \\
\hline
\end{tabular}




\begin{tabular}{|c|c|c|c|c|}
\hline \multicolumn{5}{|c|}{ Table B.2 HC-2 TQs Using Recommended Inhalation DCs (continued) } \\
\hline \multirow{2}{*}{$\begin{array}{c}\text { MASTER } \\
\text { ISOTOPE LIST }\end{array}$} & \multicolumn{2}{|c|}{ NNSA SD 1027} & \multicolumn{2}{|c|}{ Final Threshold Quantity } \\
\hline & Act (Ci) & Mass (g) & Act (Ci) & Mass (g) \\
\hline $\mathrm{N}-13$ & & & $5.52 \mathrm{E}+04$ & 2.95E-06 \\
\hline $\mathrm{N}-16$ & & & $1.04 \mathrm{E}+04$ & $1.06 \mathrm{E}-07$ \\
\hline $\mathrm{Na}-22$ & $9.99 \mathrm{E}+03$ & $1.60 \mathrm{E}+00$ & $9.99 \mathrm{E}+03$ & $1.60 \mathrm{E}+00$ \\
\hline $\mathrm{Na}-24$ & & & $1.76 \mathrm{E}+04$ & $2.02 \mathrm{E}-03$ \\
\hline Nb-87 & & & $4.88 \mathrm{E}+07$ & $8.46 \mathrm{E}-02$ \\
\hline Nb-88 & & & $1.28 \mathrm{E}+07$ & $8.56 \mathrm{E}-02$ \\
\hline $\mathrm{Nb}-88 \mathrm{~m}$ & & & $1.42 \mathrm{E}+07$ & $5.15 \mathrm{E}-02$ \\
\hline Nb-89l & & & $2.54 \mathrm{E}+07$ & $1.46 \mathrm{E}+00$ \\
\hline Nb-89s & & & $2.34 \mathrm{E}+07$ & 7.32E-01 \\
\hline Nb-90 & & & $6.33 \mathrm{E}+06$ & $2.65 \mathrm{E}+00$ \\
\hline Nb-91 & & & $2.67 \mathrm{E}+07$ & $4.62 E+06$ \\
\hline Nb-91m & & & $2.22 \mathrm{E}+06$ & $9.40 \mathrm{E}+01$ \\
\hline Nb-92 & & & $1.43 \mathrm{E}+06$ & $1.28 \mathrm{E}+10$ \\
\hline $\mathrm{Nb}-92 \mathrm{~m}$ & & & $1.28 \mathrm{E}+07$ & $9.15 \mathrm{E}+01$ \\
\hline Nb-93m & & & $1.59 \mathrm{E}+07$ & $5.62 E+04$ \\
\hline Nb-94 & $7.22 \mathrm{E}+05$ & $3.79 \mathrm{E}+06$ & $7.22 \mathrm{E}+05$ & $3.85 \mathrm{E}+06$ \\
\hline Nb-94m & & & $1.26 \mathrm{E}+10$ & $3.93 \mathrm{E}+01$ \\
\hline Nb-95 & & & $5.03 \mathrm{E}+06$ & $1.28 \mathrm{E}+02$ \\
\hline Nb-95m & & & $1.02 \mathrm{E}+07$ & $2.66 \mathrm{E}+01$ \\
\hline Nb-96 & & & $8.17 \mathrm{E}+06$ & $5.84 \mathrm{E}+00$ \\
\hline Nb-97 & & & $5.86 \mathrm{E}+07$ & $2.18 \mathrm{E}+00$ \\
\hline $\mathrm{Nb}-97 \mathrm{~m}$ & & & $7.61 \mathrm{E}+07$ & 3.92E-02 \\
\hline Nb-98 & & & $1.94 \mathrm{E}+07$ & 5.19E-01 \\
\hline Nb-99 & & & $3.25 \mathrm{E}+08$ & 4.27E-02 \\
\hline Nb-99m & & & $7.00 \mathrm{E}+07$ & $9.58 \mathrm{E}-02$ \\
\hline Nd-134 & & & $1.16 \mathrm{E}+08$ & 7.05E-01 \\
\hline Nd-135 & & & $4.04 \mathrm{E}+07$ & 3.59E-01 \\
\hline Nd-136 & & & $8.80 \mathrm{E}+07$ & $3.22 \mathrm{E}+00$ \\
\hline Nd-137 & & & $4.18 \mathrm{E}+07$ & $1.17 \mathrm{E}+00$ \\
\hline Nd-138 & & & $3.19 \mathrm{E}+07$ & $7.09 \mathrm{E}+00$ \\
\hline Nd-139 & & & $1.21 \mathrm{E}+08$ & $2.66 \mathrm{E}+00$ \\
\hline Nd-139m & & & $2.14 \mathrm{E}+07$ & $5.22 \mathrm{E}+00$ \\
\hline Nd-140 & & & $6.09 \mathrm{E}+06$ & $2.20 \mathrm{E}+01$ \\
\hline Nd-141 & & & $5.94 \mathrm{E}+08$ & $6.66 \mathrm{E}+01$ \\
\hline Nd-141m & & & $7.30 \mathrm{E}+07$ & 5.69E-02 \\
\hline Nd-144 & & & $4.03 \mathrm{E}+02$ & $3.72 \mathrm{E}+14$ \\
\hline Nd-147 & & & $3.35 \mathrm{E}+06$ & $4.14 \mathrm{E}+01$ \\
\hline Nd-149 & & & $5.66 \mathrm{E}+07$ & $4.65 \mathrm{E}+00$ \\
\hline
\end{tabular}




\begin{tabular}{|c|c|c|c|c|}
\hline \multicolumn{5}{|c|}{ Table B.2 HC-2 TQs Using Recommended Inhalation DCs (continued) } \\
\hline \multirow{2}{*}{$\begin{array}{c}\text { MASTER } \\
\text { ISOTOPE LIST }\end{array}$} & \multicolumn{2}{|c|}{ NNSA SD 1027} & \multicolumn{2}{|c|}{ Final Threshold Quantity } \\
\hline & Act (Ci) & Mass (g) & Act (Ci) & Mass (g) \\
\hline Nd-151 & & & $5.36 \mathrm{E}+07$ & 5.35E-01 \\
\hline Nd-152 & & & $1.63 \mathrm{E}+08$ & $1.50 \mathrm{E}+00$ \\
\hline Ne-19 & & & $5.49 \mathrm{E}+04$ & 1.59E-06 \\
\hline $\mathrm{Ne}-24$ & & & $1.09 \mathrm{E}+05$ & 4.70E-05 \\
\hline $\mathrm{Ni}-56$ & & & $7.22 \mathrm{E}+06$ & $1.89 \mathrm{E}+01$ \\
\hline $\mathrm{Ni}-57$ & & & $1.03 \mathrm{E}+07$ & $6.72 \mathrm{E}+00$ \\
\hline $\mathrm{Ni}-59$ & & & $6.24 \mathrm{E}+07$ & $7.71 \mathrm{E}+08$ \\
\hline $\mathrm{Ni}-63$ & $1.69 \mathrm{E}+07$ & $2.97 \mathrm{E}+05$ & $1.69 \mathrm{E}+07$ & $2.85 \mathrm{E}+05$ \\
\hline $\mathrm{Ni}-65$ & & & $4.81 \mathrm{E}+07$ & $2.51 \mathrm{E}+00$ \\
\hline $\mathrm{Ni}-66$ & & & $5.07 \mathrm{E}+06$ & $5.82 \mathrm{E}+00$ \\
\hline Np-231 & & & $4.77 \mathrm{E}+06$ & $2.86 \mathrm{E}-01$ \\
\hline Np-232 & & & $3.62 \mathrm{E}+07$ & $6.57 \mathrm{E}-01$ \\
\hline Np-233 & & & $6.17 \mathrm{E}+08$ & $2.77 \mathrm{E}+01$ \\
\hline Np-234 & & & $1.08 \mathrm{E}+07$ & $8.55 \mathrm{E}+01$ \\
\hline Np-235 & & & $1.93 \mathrm{E}+07$ & $1.38 \mathrm{E}+04$ \\
\hline Np-236l & & & $2.53 \mathrm{E}+03$ & $1.92 \mathrm{E}+05$ \\
\hline Np-236s & & & $1.53 \mathrm{E}+06$ & $2.59 \mathrm{E}+00$ \\
\hline Np-237 & $3.53 \mathrm{E}+02$ & $5.00 \mathrm{E}+05$ & $3.53 \mathrm{E}+02$ & $5.00 \mathrm{E}+05$ \\
\hline Np-238 & $3.72 \mathrm{E}+06$ & $1.43 \mathrm{E}+01$ & $3.72 \mathrm{E}+06$ & $1.43 \mathrm{E}+01$ \\
\hline Np-239 & & & $8.51 \mathrm{E}+06$ & $3.67 \mathrm{E}+01$ \\
\hline Np-240 & & & $2.96 \mathrm{E}+07$ & $2.45 \mathrm{E}+00$ \\
\hline Np-240m & & & $1.67 \mathrm{E}+08$ & $1.58 \mathrm{E}+00$ \\
\hline $\mathrm{Np}-241$ & & & $4.19 \mathrm{E}+08$ & $7.46 \mathrm{E}+00$ \\
\hline Np-242 & & & $2.03 \mathrm{E}+08$ & $5.76 \mathrm{E}-01$ \\
\hline Np-242m & & & $6.50 \mathrm{E}+07$ & $4.60 \mathrm{E}-01$ \\
\hline O-14 & & & $1.66 \mathrm{E}+04$ & $1.45 \mathrm{E}-06$ \\
\hline O-15 & & & $5.50 \mathrm{E}+04$ & $8.95 \mathrm{E}-06$ \\
\hline O-19 & & & $5.88 \mathrm{E}+04$ & $2.66 \mathrm{E}-06$ \\
\hline Os-180 & & & $3.70 \mathrm{E}+08$ & $7.80 \mathrm{E}+00$ \\
\hline Os-181 & & & $3.33 \mathrm{E}+07$ & $3.37 \mathrm{E}+00$ \\
\hline Os-182 & & & $1.84 \mathrm{E}+07$ & $2.35 \mathrm{E}+01$ \\
\hline Os-183 & & & $2.70 \mathrm{E}+07$ & $2.05 \mathrm{E}+01$ \\
\hline Os-183m & & & $2.59 \mathrm{E}+07$ & $1.50 \mathrm{E}+01$ \\
\hline Os-185 & & & $4.76 \mathrm{E}+06$ & $6.34 \mathrm{E}+02$ \\
\hline Os-186 & & & $1.80 \mathrm{E}+03$ & $1.87 \mathrm{E}+15$ \\
\hline Os-189m & & & $1.53 \mathrm{E}+09$ & $5.53 \mathrm{E}+02$ \\
\hline Os-190m & & & $3.56 \mathrm{E}+07$ & 3.56E-01 \\
\hline Os-191 & & & $4.25 \mathrm{E}+06$ & $9.56 \mathrm{E}+01$ \\
\hline
\end{tabular}




\begin{tabular}{|c|c|c|c|c|}
\hline \multicolumn{5}{|c|}{ Table B.2 HC-2 TQs Using Recommended Inhalation DCs (continued) } \\
\hline \multirow{2}{*}{$\begin{array}{c}\text { MASTER } \\
\text { ISOTOPE LIST }\end{array}$} & \multicolumn{2}{|c|}{ NNSA SD 1027} & \multicolumn{2}{|c|}{ Final Threshold Quantity } \\
\hline & Act (Ci) & Mass (g) & Act (Ci) & Mass (g) \\
\hline Os-191m & & & $5.04 \mathrm{E}+07$ & $4.00 \mathrm{E}+01$ \\
\hline Os-193 & & & $1.53 \mathrm{E}+07$ & $2.83 \mathrm{E}+01$ \\
\hline Os-194 & & & $9.54 \mathrm{E}+04$ & $3.11 \mathrm{E}+02$ \\
\hline Os-196 & & & $1.05 \mathrm{E}+08$ & $3.81 \mathrm{E}+00$ \\
\hline $\mathrm{P}-30$ & & & $1.09 \mathrm{E}+05$ & 4.36E-05 \\
\hline $\mathrm{P}-32$ & $4.77 \mathrm{E}+03$ & 1.67E-02 & $4.77 \mathrm{E}+03$ & $1.67 \mathrm{E}-02$ \\
\hline $\mathrm{P}-33$ & $1.08 \mathrm{E}+04$ & 6.91E-02 & $1.08 \mathrm{E}+04$ & 6.93E-02 \\
\hline Рa-227 & & & $1.01 \mathrm{E}+05$ & $4.69 \mathrm{E}-03$ \\
\hline Рa-228 & & & $1.08 \mathrm{E}+05$ & $1.73 \mathrm{E}-01$ \\
\hline Рa-229 & & & $1.04 \mathrm{E}+06$ & $2.74 \mathrm{E}+00$ \\
\hline Pa-230 & & & $1.07 \mathrm{E}+04$ & 3.27E-01 \\
\hline Pa-231 & & & $5.79 \mathrm{E}+01$ & $1.23 \mathrm{E}+03$ \\
\hline Pa-232 & & & $8.00 \mathrm{E}+05$ & $1.86 \mathrm{E}+00$ \\
\hline Рa-233 & & & $2.06 \mathrm{E}+06$ & $9.95 \mathrm{E}+01$ \\
\hline Рa-234 & & & $1.19 \mathrm{E}+07$ & $5.96 \mathrm{E}+00$ \\
\hline Pa-234m & & & $3.76 \mathrm{E}+09$ & $5.47 \mathrm{E}+00$ \\
\hline Pa-235 & & & $3.90 \mathrm{E}+08$ & $1.19 \mathrm{E}+01$ \\
\hline $\mathrm{Pa}-236$ & & & $6.20 \mathrm{E}+07$ & $7.08 \mathrm{E}-01$ \\
\hline Pa-237 & & & $9.58 \mathrm{E}+07$ & $1.05 \mathrm{E}+00$ \\
\hline $\mathrm{Pb}-194$ & & & $4.97 \mathrm{E}+07$ & $6.15 \mathrm{E}-01$ \\
\hline $\mathrm{Pb}-195 \mathrm{~m}$ & & & $3.17 \mathrm{E}+07$ & $5.20 \mathrm{E}-01$ \\
\hline $\mathrm{Pb}-196$ & & & $8.83 \mathrm{E}+07$ & $3.41 \mathrm{E}+00$ \\
\hline $\mathrm{Pb}-197$ & & & $3.79 \mathrm{E}+07$ & 3.17E-01 \\
\hline $\mathrm{Pb}-197 \mathrm{~m}$ & & & $3.87 \mathrm{E}+07$ & $1.75 \mathrm{E}+00$ \\
\hline Pb-198 & & & $6.37 \mathrm{E}+07$ & $9.66 \mathrm{E}+00$ \\
\hline $\mathrm{Pb}-199$ & & & $3.18 \mathrm{E}+07$ & $3.02 \mathrm{E}+00$ \\
\hline $\mathrm{Pb}-200$ & & & $2.27 \mathrm{E}+07$ & $3.11 \mathrm{E}+01$ \\
\hline $\mathrm{Pb}-201$ & & & $3.70 \mathrm{E}+07$ & $2.23 \mathrm{E}+01$ \\
\hline $\mathrm{Pb}-201 \mathrm{~m}$ & & & $1.66 \mathrm{E}+08$ & $1.80 \mathrm{E}-01$ \\
\hline Pb-202 & & & $1.29 \mathrm{E}+06$ & $2.18 \mathrm{E}+08$ \\
\hline $\mathrm{Pb}-202 \mathrm{~m}$ & & & $2.06 \mathrm{E}+07$ & $4.80 \mathrm{E}+00$ \\
\hline Pb-203 & & & $3.33 \mathrm{E}+07$ & $1.12 \mathrm{E}+02$ \\
\hline $\mathrm{Pb}-204 \mathrm{~m}$ & & & $2.55 \mathrm{E}+07$ & $1.86 \mathrm{E}+00$ \\
\hline $\mathrm{Pb}-205$ & & & $3.24 \mathrm{E}+07$ & $2.66 \mathrm{E}+11$ \\
\hline $\mathrm{Pb}-209$ & & & $1.45 \mathrm{E}+08$ & $3.14 \mathrm{E}+01$ \\
\hline $\mathrm{Pb}-210$ & $7.37 \mathrm{E}+03$ & $9.78 \mathrm{E}+01$ & $7.37 \mathrm{E}+03$ & $9.66 \mathrm{E}+01$ \\
\hline $\mathrm{Pb}-211$ & & & $7.37 \mathrm{E}+05$ & $2.98 \mathrm{E}-02$ \\
\hline $\mathrm{Pb}-212$ & & & $4.77 \mathrm{E}+04$ & 3.43E-02 \\
\hline
\end{tabular}




\begin{tabular}{|c|c|c|c|c|}
\hline \multicolumn{5}{|c|}{ Table B.2 HC-2 TQs Using Recommended Inhalation DCs (continued) } \\
\hline \multirow{2}{*}{$\begin{array}{c}\text { MASTER } \\
\text { ISOTOPE LIST }\end{array}$} & \multicolumn{2}{|c|}{ NNSA SD 1027} & \multicolumn{2}{|c|}{ Final Threshold Quantity } \\
\hline & Act (Ci) & Mass (g) & Act (Ci) & Mass (g) \\
\hline $\mathrm{Pb}-214$ & & & $5.78 \mathrm{E}+05$ & 1.76E-02 \\
\hline Pd-100 & & & $9.39 \mathrm{E}+06$ & $2.61 \mathrm{E}+01$ \\
\hline Pd-101 & & & $7.51 \mathrm{E}+07$ & $2.00 \mathrm{E}+01$ \\
\hline Pd-103 & & & $1.80 \mathrm{E}+07$ & $2.41 \mathrm{E}+02$ \\
\hline Pd-107 & & & $1.37 \mathrm{E}+07$ & $2.67 \mathrm{E}+10$ \\
\hline Pd-109 & & & $2.19 \mathrm{E}+07$ & $1.02 \mathrm{E}+01$ \\
\hline Pd-109m & & & $5.89 \mathrm{E}+08$ & $1.60 \mathrm{E}+00$ \\
\hline Pd-111 & & & $2.02 \mathrm{E}+08$ & $2.79 \mathrm{E}+00$ \\
\hline Pd-112 & & & $6.14 \mathrm{E}+06$ & $4.61 \mathrm{E}+00$ \\
\hline Pd-114 & & & $1.81 \mathrm{E}+09$ & $2.66 \mathrm{E}+00$ \\
\hline Pd-96 & & & $4.15 \mathrm{E}+07$ & 4.31E-02 \\
\hline Pd-97 & & & $2.41 \mathrm{E}+07$ & $3.86 \mathrm{E}-02$ \\
\hline Pd-98 & & & $9.40 \mathrm{E}+07$ & 8.66E-01 \\
\hline Pd-99 & & & $4.07 \mathrm{E}+07$ & $4.58 \mathrm{E}-01$ \\
\hline Pm-136 & & & $2.16 \mathrm{E}+07$ & $2.79 \mathrm{E}-02$ \\
\hline Pm-137m & & & $3.37 \mathrm{E}+07$ & $5.90 \mathrm{E}-02$ \\
\hline Pm-139 & & & $6.30 \mathrm{E}+07$ & $1.93 \mathrm{E}-01$ \\
\hline Pm-140 & & & $5.48 \mathrm{E}+07$ & $6.26 \mathrm{E}-03$ \\
\hline Pm-140m & & & $1.94 \mathrm{E}+07$ & $8.61 \mathrm{E}-02$ \\
\hline Pm-141 & & & $6.59 \mathrm{E}+07$ & $1.03 \mathrm{E}+00$ \\
\hline Pm-142 & & & $6.40 \mathrm{E}+07$ & $3.26 \mathrm{E}-02$ \\
\hline Pm-143 & & & $5.25 \mathrm{E}+06$ & $1.52 \mathrm{E}+03$ \\
\hline $\mathrm{Pm}-144$ & & & $9.62 \mathrm{E}+05$ & $3.85 \mathrm{E}+02$ \\
\hline Pm-145 & $2.25 \mathrm{E}+06$ & $1.61 \mathrm{E}+04$ & $2.25 E+06$ & $1.62 \mathrm{E}+04$ \\
\hline Pm-146 & & & $3.84 \mathrm{E}+05$ & $8.67 \mathrm{E}+02$ \\
\hline Pm-147 & $1.62 \mathrm{E}+06$ & $1.75 \mathrm{E}+03$ & $1.62 \mathrm{E}+06$ & $1.75 \mathrm{E}+03$ \\
\hline Pm-148 & & & $3.55 \mathrm{E}+06$ & $2.16 \mathrm{E}+01$ \\
\hline Pm-148m & & & $1.35 \mathrm{E}+06$ & $6.33 \mathrm{E}+01$ \\
\hline Pm-149 & & & $1.11 \mathrm{E}+07$ & $2.80 \mathrm{E}+01$ \\
\hline Pm-150 & & & $2.35 \mathrm{E}+07$ & $3.01 \mathrm{E}+00$ \\
\hline Pm-151 & & & $1.60 \mathrm{E}+07$ & $2.19 \mathrm{E}+01$ \\
\hline Pm-152 & & & $1.86 \mathrm{E}+08$ & $6.20 \mathrm{E}-01$ \\
\hline Pm-152m & & & $3.82 \mathrm{E}+07$ & 2.32E-01 \\
\hline Pm-153 & & & $7.95 \mathrm{E}+08$ & $3.39 \mathrm{E}+00$ \\
\hline Pm-154 & & & $3.10 \mathrm{E}+07$ & 4.38E-02 \\
\hline Pm-154m & & & $3.18 \mathrm{E}+07$ & $6.98 \mathrm{E}-02$ \\
\hline Po-203 & & & $2.91 \mathrm{E}+06$ & $1.15 \mathrm{E}-01$ \\
\hline Po-204 & & & $1.40 \mathrm{E}+06$ & $3.22 \mathrm{E}-01$ \\
\hline
\end{tabular}




\begin{tabular}{|c|c|c|c|c|}
\hline \multicolumn{5}{|c|}{ Table B.2 HC-2 TQs Using Recommended Inhalation DCs (continued) } \\
\hline \multirow{2}{*}{$\begin{array}{c}\text { MASTER } \\
\text { ISOTOPE LIST }\end{array}$} & \multicolumn{2}{|c|}{ NNSA SD 1027} & \multicolumn{2}{|c|}{ Final Threshold Quantity } \\
\hline & Act (Ci) & Mass (g) & Act (Ci) & Mass (g) \\
\hline Po-205 & & & $2.71 \mathrm{E}+06$ & 3.19E-01 \\
\hline Po-206 & & & $1.29 \mathrm{E}+04$ & $1.79 \mathrm{E}-01$ \\
\hline Po-207 & & & $2.97 \mathrm{E}+06$ & $1.14 \mathrm{E}+00$ \\
\hline Po-208 & & & $1.95 \mathrm{E}+02$ & $3.29 \mathrm{E}-01$ \\
\hline Po-209 & & & $2.02 \mathrm{E}+02$ & $1.20 \mathrm{E}+01$ \\
\hline Po-210 & $2.46 \mathrm{E}+02$ & 5.47E-02 & $2.46 \mathrm{E}+02$ & $5.47 \mathrm{E}-02$ \\
\hline Po-211 & & & $7.09 \mathrm{E}+08$ & $6.85 \mathrm{E}-03$ \\
\hline Po-212m & & & $6.77 \mathrm{E}+07$ & $5.74 \mathrm{E}-02$ \\
\hline Po-213 & & & $1.58 \mathrm{E}+11$ & $1.25 \mathrm{E}-05$ \\
\hline Po-214 & & & $6.62 \mathrm{E}+10$ & $2.06 \mathrm{E}-04$ \\
\hline Po-215 & & & $3.21 \mathrm{E}+10$ & $1.09 \mathrm{E}-03$ \\
\hline Po-216 & & & $3.26 \mathrm{E}+11$ & $9.36 \mathrm{E}-01$ \\
\hline Po-218 & & & $6.03 \mathrm{E}+11$ & $2.13 \mathrm{E}+03$ \\
\hline Pr-134 & & & $1.77 \mathrm{E}+07$ & $1.39 \mathrm{E}-01$ \\
\hline Pr-134m & & & $2.24 \mathrm{E}+07$ & $2.71 \mathrm{E}-01$ \\
\hline Pr-135 & & & $5.74 \mathrm{E}+07$ & 9.89E-01 \\
\hline Pr-136 & & & $2.51 \mathrm{E}+07$ & $2.38 \mathrm{E}-01$ \\
\hline Pr-137 & & & $8.83 \mathrm{E}+07$ & $4.93 \mathrm{E}+00$ \\
\hline Pr-138 & & & $6.89 \mathrm{E}+07$ & 7.33E-02 \\
\hline Pr-138m & & & $1.86 \mathrm{E}+07$ & $1.71 \mathrm{E}+00$ \\
\hline Pr-139 & & & $2.28 \mathrm{E}+08$ & $4.56 \mathrm{E}+01$ \\
\hline Pr-140 & & & $1.11 \mathrm{E}+08$ & 2.79E-01 \\
\hline Pr-142 & & & $1.45 \mathrm{E}+07$ & $1.26 \mathrm{E}+01$ \\
\hline Pr-142m & & & $1.16 \mathrm{E}+09$ & $1.28 \mathrm{E}+01$ \\
\hline Pr-143 & & & $3.38 \mathrm{E}+06$ & $5.01 \mathrm{E}+01$ \\
\hline Pr-144 & & & $3.40 \mathrm{E}+08$ & $4.50 \mathrm{E}+00$ \\
\hline Pr-144m & & & $9.69 \mathrm{E}+09$ & $5.34 \mathrm{E}+01$ \\
\hline Pr-145 & & & $4.71 \mathrm{E}+07$ & $1.30 \mathrm{E}+01$ \\
\hline Pr-146 & & & $4.42 \mathrm{E}+07$ & $8.29 \mathrm{E}-01$ \\
\hline Pr-147 & & & $5.69 \mathrm{E}+07$ & $6.05 \mathrm{E}-01$ \\
\hline Pr-148 & & & $5.60 \mathrm{E}+07$ & $1.01 \mathrm{E}-01$ \\
\hline Pr-148m & & & $6.18 \mathrm{E}+07$ & $9.78 \mathrm{E}-02$ \\
\hline Pt-184 & & & $6.69 \mathrm{E}+07$ & $1.13 \mathrm{E}+00$ \\
\hline Pt-186 & & & $5.84 \mathrm{E}+07$ & $6.93 \mathrm{E}+00$ \\
\hline Pt-187 & & & $4.98 \mathrm{E}+07$ & $6.99 \mathrm{E}+00$ \\
\hline Pt-188 & & & $1.82 \mathrm{E}+07$ & $2.67 \mathrm{E}+02$ \\
\hline Pt-189 & & & $9.84 \mathrm{E}+07$ & $6.45 \mathrm{E}+01$ \\
\hline Pt-190 & & & $1.45 \mathrm{E}+03$ & $5.02 \mathrm{E}+11$ \\
\hline
\end{tabular}




\begin{tabular}{|c|c|c|c|c|}
\hline \multicolumn{5}{|c|}{ Table B.2 HC-2 TQs Using Recommended Inhalation DCs (continued) } \\
\hline \multirow{2}{*}{$\begin{array}{c}\text { MASTER } \\
\text { ISOTOPE LIST }\end{array}$} & \multicolumn{2}{|c|}{ NNSA SD 1027} & \multicolumn{2}{|c|}{ Final Threshold Quantity } \\
\hline & Act (Ci) & Mass (g) & Act (Ci) & Mass (g) \\
\hline Pt-191 & & & $5.40 \mathrm{E}+07$ & $2.21 \mathrm{E}+02$ \\
\hline Pt-193 & & & $3.86 \mathrm{E}+08$ & $1.04 \mathrm{E}+07$ \\
\hline Pt-193m & & & $6.69 \mathrm{E}+07$ & $4.28 \mathrm{E}+02$ \\
\hline Pt-195m & & & $4.30 \mathrm{E}+07$ & $2.58 \mathrm{E}+02$ \\
\hline Pt-197 & & & $9.21 \mathrm{E}+07$ & $1.06 \mathrm{E}+02$ \\
\hline Pt-197m & & & $2.35 \mathrm{E}+08$ & $2.33 \mathrm{E}+01$ \\
\hline Pt-199 & & & $1.97 \mathrm{E}+08$ & $6.42 \mathrm{E}+00$ \\
\hline Pt-200 & & & $3.56 \mathrm{E}+07$ & $2.84 \mathrm{E}+01$ \\
\hline Pt-202 & & & $3.10 \mathrm{E}+06$ & $8.80 \mathrm{E}+00$ \\
\hline $\mathrm{Pu}-232$ & & & $3.32 \mathrm{E}+05$ & $1.38 \mathrm{E}-02$ \\
\hline $\mathrm{Pu}-234$ & & & $3.86 \mathrm{E}+05$ & $2.54 \mathrm{E}-01$ \\
\hline $\mathrm{Pu}-235$ & & & $6.16 \mathrm{E}+08$ & $1.95 \mathrm{E}+01$ \\
\hline $\mathrm{Pu}-236$ & & & $4.05 \mathrm{E}+02$ & 7.63E-01 \\
\hline $\mathrm{Pu}-237$ & & & $2.28 \mathrm{E}+07$ & $1.87 \mathrm{E}+03$ \\
\hline $\mathrm{Pu}-238$ & $1.76 \mathrm{E}+02$ & $1.03 \mathrm{E}+01$ & $1.76 \mathrm{E}+02$ & $1.03 \mathrm{E}+01$ \\
\hline $\mathrm{Pu}-239$ & $1.62 \mathrm{E}+02$ & $2.61 \mathrm{E}+03$ & $1.62 \mathrm{E}+02$ & $2.61 \mathrm{E}+03$ \\
\hline $\mathrm{Pu}-240$ & $1.62 \mathrm{E}+02$ & $7.14 \mathrm{E}+02$ & $1.62 \mathrm{E}+02$ & $7.12 \mathrm{E}+02$ \\
\hline $\mathrm{Pu}-241$ & $9.01 \mathrm{E}+03$ & $8.74 \mathrm{E}+01$ & $9.01 \mathrm{E}+03$ & $8.75 \mathrm{E}+01$ \\
\hline $\mathrm{Pu}-242$ & $1.69 \mathrm{E}+02$ & $4.29 \mathrm{E}+04$ & $1.69 \mathrm{E}+02$ & $4.30 \mathrm{E}+04$ \\
\hline $\mathrm{Pu}-243$ & & & $9.42 \mathrm{E}+07$ & $3.62 \mathrm{E}+01$ \\
\hline $\mathrm{Pu}-244$ & & & $1.73 \mathrm{E}+02$ & $9.73 \mathrm{E}+06$ \\
\hline $\mathrm{Pu}-245$ & & & $1.76 \mathrm{E}+07$ & $1.45 \mathrm{E}+01$ \\
\hline $\mathrm{Pu}-246$ & & & $1.09 \mathrm{E}+06$ & $2.23 \mathrm{E}+01$ \\
\hline Ra-219 & & & $3.67 \mathrm{E}+08$ & 7.12E-05 \\
\hline Ra-220 & & & $1.31 \mathrm{E}+10$ & $4.56 \mathrm{E}-03$ \\
\hline Ra-221 & & & $1.84 \mathrm{E}+09$ & $1.01 \mathrm{E}+00$ \\
\hline Ra-222 & & & $6.16 \mathrm{E}+09$ & $4.60 \mathrm{E}+00$ \\
\hline Ra-223 & $1.10 \mathrm{E}+03$ & $2.14 \mathrm{E}-02$ & $1.10 \mathrm{E}+03$ & $2.14 \mathrm{E}-02$ \\
\hline Ra-224 & $2.70 \mathrm{E}+03$ & $1.70 \mathrm{E}-02$ & $2.70 \mathrm{E}+03$ & $1.70 \mathrm{E}-02$ \\
\hline Ra-225 & $1.29 \mathrm{E}+03$ & 3.30E-02 & $1.29 \mathrm{E}+03$ & $3.28 \mathrm{E}-02$ \\
\hline Ra-226 & & & $2.32 E+03$ & $2.34 \mathrm{E}+03$ \\
\hline Ra-227 & & & $2.68 \mathrm{E}+07$ & $1.37 \mathrm{E}+00$ \\
\hline Ra-228 & & & $3.12 \mathrm{E}+03$ & $1.14 \mathrm{E}+01$ \\
\hline Ra-230 & & & $5.83 \mathrm{E}+07$ & $6.64 \mathrm{E}+00$ \\
\hline Rb-77 & & & $3.81 \mathrm{E}+07$ & 5.77E-02 \\
\hline $\mathrm{Rb}-78$ & & & $1.25 \mathrm{E}+07$ & $9.17 \mathrm{E}-02$ \\
\hline Rb-78m & & & $1.78 \mathrm{E}+07$ & $4.23 \mathrm{E}-02$ \\
\hline Rb-79 & & & $3.84 \mathrm{E}+07$ & 3.69E-01 \\
\hline
\end{tabular}




\begin{tabular}{|c|c|c|c|c|}
\hline \multicolumn{5}{|c|}{ Table B.2 HC-2 TQs Using Recommended Inhalation DCs (continued) } \\
\hline \multirow{2}{*}{$\begin{array}{c}\text { MASTER } \\
\text { ISOTOPE LIST }\end{array}$} & \multicolumn{2}{|c|}{ NNSA SD 1027} & \multicolumn{2}{|c|}{ Final Threshold Quantity } \\
\hline & Act $(\mathbf{C i})$ & Mass (g) & Act (Ci) & Mass (g) \\
\hline $\mathrm{Rb}-80$ & & & $4.45 \mathrm{E}+07$ & 1.07E-02 \\
\hline $\mathrm{Rb}-81$ & & & $6.60 \mathrm{E}+07$ & $7.81 \mathrm{E}+00$ \\
\hline Rb-81m & & & $1.07 \mathrm{E}+09$ & $1.48 \mathrm{E}+01$ \\
\hline Rb-82 & & & $5.10 \mathrm{E}+07$ & $2.89 \mathrm{E}-02$ \\
\hline Rb-82m & & & $1.50 \mathrm{E}+07$ & $2.44 \mathrm{E}+00$ \\
\hline Rb-83 & & & $1.06 \mathrm{E}+07$ & $5.83 \mathrm{E}+02$ \\
\hline $\mathrm{Rb}-84$ & & & $7.15 \mathrm{E}+06$ & $1.51 \mathrm{E}+02$ \\
\hline Rb-84m & & & $1.34 \mathrm{E}+08$ & $1.21 \mathrm{E}+00$ \\
\hline Rb-86 & & & $8.59 \mathrm{E}+06$ & $1.05 \mathrm{E}+02$ \\
\hline Rb-86m & & & $1.11 \mathrm{E}+08$ & $5.15 \mathrm{E}-02$ \\
\hline Rb-87 & & & $1.62 \mathrm{E}+07$ & $1.85 \mathrm{E}+14$ \\
\hline Rb-88 & & & $6.94 \mathrm{E}+07$ & $5.78 \mathrm{E}-01$ \\
\hline Rb-89 & & & $2.44 \mathrm{E}+07$ & $1.76 \mathrm{E}-01$ \\
\hline Rb-90 & & & $2.50 \mathrm{E}+07$ & $3.15 \mathrm{E}-02$ \\
\hline Rb-90m & & & $1.66 \mathrm{E}+07$ & $3.41 \mathrm{E}-02$ \\
\hline Re-177 & & & $7.89 \mathrm{E}+07$ & $1.04 \mathrm{E}+00$ \\
\hline Re-178 & & & $4.12 \mathrm{E}+07$ & $5.15 \mathrm{E}-01$ \\
\hline Re-179 & & & $5.06 \mathrm{E}+07$ & 9.39E-01 \\
\hline Re-180 & & & $4.73 \mathrm{E}+07$ & $1.10 \mathrm{E}-01$ \\
\hline Re-181 & & & $2.26 \mathrm{E}+07$ & $2.60 \mathrm{E}+01$ \\
\hline Re-182l & & & $5.50 \mathrm{E}+06$ & $2.04 \mathrm{E}+01$ \\
\hline Re-182s & & & $2.17 \mathrm{E}+07$ & $1.60 \mathrm{E}+01$ \\
\hline Re-183 & & & $2.05 \mathrm{E}+06$ & $2.01 \mathrm{E}+02$ \\
\hline Re-184 & & & $4.00 \mathrm{E}+06$ & $2.14 \mathrm{E}+02$ \\
\hline Re-184m & & & $1.24 \mathrm{E}+06$ & $2.88 \mathrm{E}+02$ \\
\hline Re-186 & & & $7.35 \mathrm{E}+06$ & $3.95 \mathrm{E}+01$ \\
\hline Re-186m & & & $6.76 \mathrm{E}+05$ & $7.03 \mathrm{E}+07$ \\
\hline Re-187 & & & $1.29 \mathrm{E}+09$ & $3.37 \mathrm{E}+16$ \\
\hline Re-188 & & & $1.48 \mathrm{E}+07$ & $1.51 \mathrm{E}+01$ \\
\hline Re-188m & & & $3.68 \mathrm{E}+08$ & $6.83 \mathrm{E}+00$ \\
\hline Re-189 & & & $1.84 \mathrm{E}+07$ & $2.70 \mathrm{E}+01$ \\
\hline Re-190 & & & $4.47 \mathrm{E}+07$ & $1.40 \mathrm{E}-01$ \\
\hline Re-190m & & & $2.20 \mathrm{E}+07$ & $4.28 \mathrm{E}+00$ \\
\hline Rh-100 & & & $1.05 \mathrm{E}+07$ & $6.96 \mathrm{E}+00$ \\
\hline Rh-100m & & & $1.33 \mathrm{E}+09$ & $3.25 \mathrm{E}+00$ \\
\hline Rh-101 & & & $1.49 \mathrm{E}+06$ & $1.35 \mathrm{E}+03$ \\
\hline Rh-101m & & & $3.21 \mathrm{E}+07$ & $1.08 \mathrm{E}+02$ \\
\hline Rh-102 & & & $4.68 \mathrm{E}+05$ & $3.87 \mathrm{E}+02$ \\
\hline
\end{tabular}




\begin{tabular}{|c|c|c|c|c|}
\hline \multicolumn{5}{|c|}{ Table B.2 HC-2 TQs Using Recommended Inhalation DCs (continued) } \\
\hline \multirow{2}{*}{$\begin{array}{c}\text { MASTER } \\
\text { ISOTOPE LIST }\end{array}$} & \multicolumn{2}{|c|}{ NNSA SD 1027} & \multicolumn{2}{|c|}{ Final Threshold Quantity } \\
\hline & Act (Ci) & Mass (g) & Act (Ci) & Mass (g) \\
\hline Rh-102m & & & $1.13 \mathrm{E}+06$ & $1.83 \mathrm{E}+02$ \\
\hline Rh-103m & & & $2.97 \mathrm{E}+09$ & $9.13 \mathrm{E}+01$ \\
\hline Rh-104 & & & $1.93 \mathrm{E}+09$ & 7.52E-01 \\
\hline Rh-104m & & & $2.96 \mathrm{E}+09$ & $7.09 \mathrm{E}+00$ \\
\hline Rh-105 & & & $2.25 \mathrm{E}+07$ & $2.66 \mathrm{E}+01$ \\
\hline Rh-106 & & & $2.60 \mathrm{E}+08$ & $7.29 \mathrm{E}-02$ \\
\hline Rh-106m & & & $1.50 \mathrm{E}+07$ & $1.11 \mathrm{E}+00$ \\
\hline Rh-107 & & & $1.31 \mathrm{E}+08$ & $1.61 \mathrm{E}+00$ \\
\hline Rh-108 & & & $1.68 \mathrm{E}+08$ & $2.70 \mathrm{E}-02$ \\
\hline Rh-109 & & & $1.96 \mathrm{E}+08$ & $1.51 \mathrm{E}-01$ \\
\hline Rh-94 & & & $1.49 \mathrm{E}+07$ & 8.78E-03 \\
\hline Rh-95 & & & $2.23 \mathrm{E}+07$ & $5.66 \mathrm{E}-02$ \\
\hline Rh-95m & & & $6.30 \mathrm{E}+07$ & $6.23 \mathrm{E}-02$ \\
\hline Rh-96 & & & $1.49 \mathrm{E}+07$ & $7.54 \mathrm{E}-02$ \\
\hline Rh-96m & & & $4.48 \mathrm{E}+07$ & 3.45E-02 \\
\hline Rh-97 & & & $3.58 \mathrm{E}+07$ & 5.67E-01 \\
\hline Rh-97m & & & $2.31 \mathrm{E}+07$ & $5.50 \mathrm{E}-01$ \\
\hline Rh-98 & & & $3.24 \mathrm{E}+07$ & 1.47E-01 \\
\hline Rh-99 & & & $8.49 \mathrm{E}+06$ & $1.03 \mathrm{E}+02$ \\
\hline Rh-99m & & & $5.85 \mathrm{E}+07$ & $8.67 \mathrm{E}+00$ \\
\hline Rn-207 & & & $6.13 \mathrm{E}+04$ & $6.24 \mathrm{E}-04$ \\
\hline Rn-209 & & & $4.94 \mathrm{E}+04$ & $1.57 \mathrm{E}-03$ \\
\hline Rn-210 & & & $2.13 \mathrm{E}+05$ & $3.42 \mathrm{E}-02$ \\
\hline Rn-211 & & & $3.14 \mathrm{E}+04$ & $3.08 \mathrm{E}-02$ \\
\hline Rn-212 & & & $1.96 \mathrm{E}+05$ & $5.28 \mathrm{E}-03$ \\
\hline Rn-215 & & & $1.42 \mathrm{E}+05$ & $6.24 \mathrm{E}-12$ \\
\hline Rn-216 & & & $1.53 \mathrm{E}+05$ & $1.32 \mathrm{E}-10$ \\
\hline Rn-217 & & & $1.59 \mathrm{E}+05$ & $1.65 \mathrm{E}-09$ \\
\hline Rn-218 & & & $7.40 \mathrm{E}+07$ & 5.01E-05 \\
\hline Rn-219 & & & $1.01 \mathrm{E}+06$ & 7.75E-05 \\
\hline Rn-220 & & & $1.46 \mathrm{E}+08$ & $1.58 \mathrm{E}-01$ \\
\hline Rn-222 & $1.60 \mathrm{E}+08$ & $1.10 \mathrm{E}+03$ & $1.42 \mathrm{E}+08$ & $9.20 \mathrm{E}+02$ \\
\hline Rn-223 & & & $1.73 \mathrm{E}+05$ & 4.99E-03 \\
\hline $\mathrm{Ru}-103$ & & & $3.29 \mathrm{E}+05$ & $1.02 \mathrm{E}+01$ \\
\hline $\mathrm{Ru}-105$ & & & $2.85 E+06$ & 4.24E-01 \\
\hline Ru-106 & $2.90 \mathrm{E}+04$ & $8.74 \mathrm{E}+00$ & $2.90 \mathrm{E}+04$ & $8.65 \mathrm{E}+00$ \\
\hline $\mathrm{Ru}-107$ & & & $1.60 \mathrm{E}+07$ & $3.41 \mathrm{E}-02$ \\
\hline Ru-108 & & & $9.26 \mathrm{E}+07$ & $2.42 \mathrm{E}-01$ \\
\hline
\end{tabular}




\begin{tabular}{|c|c|c|c|c|}
\hline \multicolumn{5}{|c|}{ Table B.2 HC-2 TQs Using Recommended Inhalation DCs (continued) } \\
\hline \multirow{2}{*}{$\begin{array}{c}\text { MASTER } \\
\text { ISOTOPE LIST }\end{array}$} & \multicolumn{2}{|c|}{ NNSA SD 1027} & \multicolumn{2}{|c|}{ Final Threshold Quantity } \\
\hline & Act (Ci) & Mass (g) & Act (Ci) & Mass (g) \\
\hline Ru-92 & & & $2.86 \mathrm{E}+06$ & $5.11 \mathrm{E}-03$ \\
\hline Ru-94 & & & $6.86 \mathrm{E}+06$ & $1.77 \mathrm{E}-01$ \\
\hline Ru-95 & & & $3.80 \mathrm{E}+06$ & 1.89E-01 \\
\hline Ru-97 & & & $6.11 \mathrm{E}+06$ & $1.32 \mathrm{E}+01$ \\
\hline S-35 & $1.16 \mathrm{E}+04$ & 2.70E-01 & $1.16 \mathrm{E}+04$ & $2.71 \mathrm{E}-01$ \\
\hline S-37 & & & $3.51 \mathrm{E}+04$ & $3.49 \mathrm{E}-05$ \\
\hline S-38 & & & $2.76 \mathrm{E}+04$ & $9.50 \mathrm{E}-04$ \\
\hline Sb-111 & & & $4.00 \mathrm{E}+07$ & $2.95 \mathrm{E}-02$ \\
\hline Sb-113 & & & $4.73 \mathrm{E}+07$ & $1.90 \mathrm{E}-01$ \\
\hline Sb-114 & & & $2.13 \mathrm{E}+07$ & $4.50 \mathrm{E}-02$ \\
\hline Sb-115 & & & $5.69 \mathrm{E}+07$ & $1.10 \mathrm{E}+00$ \\
\hline Sb-116 & & & $2.41 \mathrm{E}+07$ & $2.34 \mathrm{E}-01$ \\
\hline Sb-116m & & & $1.58 \mathrm{E}+07$ & $5.89 \mathrm{E}-01$ \\
\hline Sb-117 & & & $2.03 E+08$ & $2.12 \mathrm{E}+01$ \\
\hline Sb-118 & & & $7.40 \mathrm{E}+07$ & $1.67 \mathrm{E}-01$ \\
\hline Sb-118m & & & $1.62 \mathrm{E}+07$ & $3.04 \mathrm{E}+00$ \\
\hline Sb-119 & & & $2.27 \mathrm{E}+08$ & $3.29 \mathrm{E}+02$ \\
\hline Sb-120s & & & $1.14 \mathrm{E}+08$ & $1.16 \mathrm{E}+00$ \\
\hline Sb-120l & & & $5.94 \mathrm{E}+06$ & $3.14 \mathrm{E}+01$ \\
\hline Sb-122 & & & $7.62 \mathrm{E}+06$ & $1.92 \mathrm{E}+01$ \\
\hline Sb-122m & & & $1.53 \mathrm{E}+09$ & $4.15 E+00$ \\
\hline Sb-124 & $1.21 \mathrm{E}+06$ & $6.94 \mathrm{E}+01$ & $1.21 \mathrm{E}+06$ & $6.94 \mathrm{E}+01$ \\
\hline Sb-124ms & & & $1.59 \mathrm{E}+08$ & $1.62 \mathrm{E}-01$ \\
\hline Sb-124ml & & & $1.50 \mathrm{E}+09$ & $2.00 \mathrm{E}+01$ \\
\hline Sb-125 & & & $1.67 \mathrm{E}+06$ & $1.61 \mathrm{E}+03$ \\
\hline Sb-126 & $2.53 \mathrm{E}+06$ & $3.02 \mathrm{E}+01$ & $2.53 \mathrm{E}+06$ & $3.02 \mathrm{E}+01$ \\
\hline Sb-126m & & & $3.32 \mathrm{E}+07$ & 4.23E-01 \\
\hline Sb-127 & & & $4.50 \mathrm{E}+06$ & $1.69 \mathrm{E}+01$ \\
\hline Sb-128l & & & $9.51 \mathrm{E}+06$ & $3.50 \mathrm{E}+00$ \\
\hline Sb-128s & & & $2.66 \mathrm{E}+07$ & $1.88 \mathrm{E}-01$ \\
\hline Sb-129 & & & $1.83 \mathrm{E}+07$ & $3.24 \mathrm{E}+00$ \\
\hline Sb-130 & & & $1.53 \mathrm{E}+07$ & $4.22 \mathrm{E}-01$ \\
\hline Sb-130m & & & $2.15 E+07$ & $9.34 \mathrm{E}-02$ \\
\hline Sb-131 & & & $2.49 \mathrm{E}+07$ & 3.99E-01 \\
\hline Sb-133 & & & $2.03 \mathrm{E}+07$ & 3.59E-02 \\
\hline Sc-42m & & & $1.37 \mathrm{E}+07$ & $3.15 \mathrm{E}-03$ \\
\hline Sc-43 & & & $3.03 E+07$ & $1.62 \mathrm{E}+00$ \\
\hline Sc-44 & & & $1.64 \mathrm{E}+07$ & $9.02 \mathrm{E}-01$ \\
\hline
\end{tabular}




\begin{tabular}{|c|c|c|c|c|}
\hline \multicolumn{5}{|c|}{ Table B.2 HC-2 TQs Using Recommended Inhalation DCs (continued) } \\
\hline \multirow{2}{*}{$\begin{array}{c}\text { MASTER } \\
\text { ISOTOPE LIST }\end{array}$} & \multicolumn{2}{|c|}{ NNSA SD 1027} & \multicolumn{2}{|c|}{ Final Threshold Quantity } \\
\hline & Act (Ci) & Mass (g) & Act (Ci) & Mass (g) \\
\hline Sc- $-44 \mathrm{~m}$ & & & $5.63 \mathrm{E}+06$ & $4.63 \mathrm{E}+00$ \\
\hline Sc-46 & $1.14 \mathrm{E}+06$ & $3.37 \mathrm{E}+01$ & $1.14 \mathrm{E}+06$ & $3.37 \mathrm{E}+01$ \\
\hline Sc-47 & & & $1.09 \mathrm{E}+07$ & $1.31 \mathrm{E}+01$ \\
\hline Sc-48 & & & $5.05 \mathrm{E}+06$ & $3.38 \mathrm{E}+00$ \\
\hline Sc-49 & & & $2.00 \mathrm{E}+08$ & $2.99 \mathrm{E}+00$ \\
\hline Sc-50 & & & $1.76 \mathrm{E}+07$ & 7.97E-03 \\
\hline Se-70 & & & $4.41 \mathrm{E}+06$ & $6.72 \mathrm{E}-02$ \\
\hline Se-71 & & & $3.65 \mathrm{E}+06$ & $6.53 \mathrm{E}-03$ \\
\hline Se-72 & & & $2.85 E+05$ & $1.32 \mathrm{E}+00$ \\
\hline Se-73 & & & $3.45 \mathrm{E}+06$ & $5.75 \mathrm{E}-01$ \\
\hline Se-73m & & & $1.83 \mathrm{E}+07$ & 2.77E-01 \\
\hline Se-75 & $7.68 \mathrm{E}+05$ & $5.28 \mathrm{E}+01$ & $7.68 \mathrm{E}+05$ & $5.28 \mathrm{E}+01$ \\
\hline Se-77m & & & $6.71 \mathrm{E}+07$ & $7.98 \mathrm{E}-03$ \\
\hline Se-79 & & & $7.37 \mathrm{E}+05$ & $1.06 \mathrm{E}+07$ \\
\hline Se-79m & & & $7.51 \mathrm{E}+08$ & $1.24 \mathrm{E}+00$ \\
\hline Se-81 & & & $8.47 \mathrm{E}+07$ & 6.74E-01 \\
\hline Se-81m & & & $4.54 \mathrm{E}+07$ & $1.12 \mathrm{E}+00$ \\
\hline Se-83 & & & $2.13 E+06$ & $2.11 \mathrm{E}-02$ \\
\hline Se-83m & & & $5.65 \mathrm{E}+06$ & $2.91 \mathrm{E}-03$ \\
\hline Se-84 & & & $1.42 \mathrm{E}+07$ & $1.97 \mathrm{E}-02$ \\
\hline Si-31 & & & $1.02 \mathrm{E}+08$ & $2.65 \mathrm{E}+00$ \\
\hline Si-32 & & & $7.37 \mathrm{E}+04$ & $2.97 \mathrm{E}+03$ \\
\hline Sm-139 & & & $4.07 \mathrm{E}+07$ & 7.73E-02 \\
\hline Sm-140 & & & $7.04 \mathrm{E}+07$ & 7.77E-01 \\
\hline Sm-141 & & & $3.67 \mathrm{E}+07$ & $2.80 \mathrm{E}-01$ \\
\hline Sm-141m & & & $2.51 \mathrm{E}+07$ & $4.25 \mathrm{E}-01$ \\
\hline Sm-142 & & & $9.84 \mathrm{E}+07$ & $5.39 \mathrm{E}+00$ \\
\hline Sm-143 & & & $1.15 \mathrm{E}+08$ & 7.62E-01 \\
\hline Sm-143m & & & $8.69 \mathrm{E}+07$ & 7.27E-02 \\
\hline Sm-145 & & & $5.05 \mathrm{E}+06$ & $1.91 \mathrm{E}+03$ \\
\hline Sm-146 & & & $7.37 \mathrm{E}+02$ & $3.10 \mathrm{E}+07$ \\
\hline Sm-147 & & & $8.45 \mathrm{E}+02$ & $3.68 \mathrm{E}+10$ \\
\hline Sm-148 & & & $3.86 \mathrm{E}+02$ & $1.12 \mathrm{E}+15$ \\
\hline Sm-151 & $2.03 \mathrm{E}+06$ & $7.70 \mathrm{E}+04$ & $2.03 \mathrm{E}+06$ & $7.70 \mathrm{E}+04$ \\
\hline Sm-153 & & & $1.27 \mathrm{E}+07$ & $2.90 \mathrm{E}+01$ \\
\hline Sm-155 & & & $2.62 \mathrm{E}+08$ & $4.77 \mathrm{E}+00$ \\
\hline Sm-156 & & & $3.43 \mathrm{E}+07$ & $1.60 \mathrm{E}+01$ \\
\hline Sm-157 & & & $1.42 \mathrm{E}+08$ & $9.48 \mathrm{E}-01$ \\
\hline
\end{tabular}




\begin{tabular}{|c|c|c|c|c|}
\hline \multicolumn{5}{|c|}{ Table B.2 HC-2 TQs Using Recommended Inhalation DCs (continued) } \\
\hline \multirow{2}{*}{$\begin{array}{c}\text { MASTER } \\
\text { ISOTOPE LIST }\end{array}$} & \multicolumn{2}{|c|}{ NNSA SD 1027} & \multicolumn{2}{|c|}{ Final Threshold Quantity } \\
\hline & Act (Ci) & Mass (g) & Act (Ci) & Mass (g) \\
\hline Sn-106 & & & $5.01 \mathrm{E}+07$ & $5.41 \mathrm{E}-02$ \\
\hline Sn-108 & & & $7.89 \mathrm{E}+07$ & $4.66 \mathrm{E}-01$ \\
\hline Sn-109 & & & $2.46 \mathrm{E}+07$ & 2.57E-01 \\
\hline Sn-110 & & & $4.03 \mathrm{E}+07$ & $5.66 \mathrm{E}+00$ \\
\hline Sn-111 & & & $9.37 \mathrm{E}+07$ & $1.95 \mathrm{E}+00$ \\
\hline Sn-113 & $3.00 \mathrm{E}+06$ & $2.99 \mathrm{E}+02$ & $3.00 \mathrm{E}+06$ & $2.99 \mathrm{E}+02$ \\
\hline Sn-113m & & & $1.48 \mathrm{E}+09$ & $1.90 \mathrm{E}+01$ \\
\hline Sn-117m & & & $3.35 \mathrm{E}+06$ & $4.08 \mathrm{E}+01$ \\
\hline Sn-119m & & & $3.69 \mathrm{E}+06$ & $9.83 \mathrm{E}+02$ \\
\hline Sn-121 & & & $3.53 \mathrm{E}+07$ & $3.68 \mathrm{E}+01$ \\
\hline Sn-121m & & & $1.80 \mathrm{E}+06$ & $3.35 E+04$ \\
\hline Sn-123 & $1.00 \mathrm{E}+06$ & $1.22 \mathrm{E}+02$ & $1.00 \mathrm{E}+06$ & $1.22 \mathrm{E}+02$ \\
\hline Sn-123m & & & $1.74 \mathrm{E}+08$ & $4.55 \mathrm{E}+00$ \\
\hline Sn-125 & & & $2.58 \mathrm{E}+06$ & $2.38 \mathrm{E}+01$ \\
\hline Sn-125m & & & $1.69 \mathrm{E}+08$ & $1.07 E+00$ \\
\hline Sn-126 & $2.90 \mathrm{E}+05$ & $1.02 \mathrm{E}+07$ & $2.90 \mathrm{E}+05$ & $1.02 \mathrm{E}+07$ \\
\hline Sn-127 & & & $1.94 \mathrm{E}+07$ & $1.65 \mathrm{E}+00$ \\
\hline Sn-127m & & & $1.01 \mathrm{E}+08$ & $2.82 \mathrm{E}-01$ \\
\hline Sn-128 & & & $4.46 \mathrm{E}+07$ & $1.79 \mathrm{E}+00$ \\
\hline Sn-129 & & & $5.68 \mathrm{E}+07$ & $8.68 \mathrm{E}-02$ \\
\hline Sn-130 & & & $6.50 \mathrm{E}+07$ & $1.67 \mathrm{E}-01$ \\
\hline Sn-130m & & & $6.44 \mathrm{E}+07$ & 7.56E-02 \\
\hline Sr-79 & & & $5.01 \mathrm{E}+07$ & $4.74 \mathrm{E}-02$ \\
\hline Sr-80 & & & $6.24 \mathrm{E}+07$ & $2.65 \mathrm{E}+00$ \\
\hline Sr-81 & & & $3.44 \mathrm{E}+07$ & 3.78E-01 \\
\hline Sr-82 & & & $9.11 \mathrm{E}+05$ & $1.43 \mathrm{E}+01$ \\
\hline Sr-83 & & & $1.90 \mathrm{E}+07$ & $1.63 \mathrm{E}+01$ \\
\hline Sr-85 & & & $1.14 \mathrm{E}+07$ & $4.80 \mathrm{E}+02$ \\
\hline Sr-85m & & & $2.28 \mathrm{E}+08$ & $7.15 \mathrm{E}+00$ \\
\hline Sr-87m & & & $1.24 \mathrm{E}+08$ & $9.62 \mathrm{E}+00$ \\
\hline Sr-89 & $1.33 \mathrm{E}+06$ & $4.57 \mathrm{E}+01$ & $1.33 \mathrm{E}+06$ & $4.57 \mathrm{E}+01$ \\
\hline Sr-90 & $2.25 \mathrm{E}+05$ & $1.63 \mathrm{E}+03$ & $2.25 \mathrm{E}+05$ & $1.65 \mathrm{E}+03$ \\
\hline Sr-91 & & & $1.71 \mathrm{E}+07$ & $4.72 \mathrm{E}+00$ \\
\hline Sr-92 & & & $1.96 \mathrm{E}+07$ & $1.56 \mathrm{E}+00$ \\
\hline Sr-93 & & & $2.53 \mathrm{E}+07$ & $9.27 \mathrm{E}-02$ \\
\hline Sr-94 & & & $3.91 \mathrm{E}+07$ & $2.45 \mathrm{E}-02$ \\
\hline Тa-170 & & & $5.54 \mathrm{E}+07$ & 3.38E-01 \\
\hline Та-172 & & & $3.09 \mathrm{E}+07$ & $1.04 \mathrm{E}+00$ \\
\hline
\end{tabular}




\begin{tabular}{|c|c|c|c|c|}
\hline \multicolumn{5}{|c|}{ Table B.2 HC-2 TQs Using Recommended Inhalation DCs (continued) } \\
\hline \multirow{2}{*}{$\begin{array}{c}\text { MASTER } \\
\text { ISOTOPE LIST }\end{array}$} & \multicolumn{2}{|c|}{ NNSA SD 1027} & \multicolumn{2}{|c|}{ Final Threshold Quantity } \\
\hline & Act (Ci) & Mass (g) & Act (Ci) & Mass (g) \\
\hline Тa-173 & & & $4.21 \mathrm{E}+07$ & $8.48 \mathrm{E}+00$ \\
\hline Тa-174 & & & $6.14 \mathrm{E}+07$ & $4.09 \mathrm{E}+00$ \\
\hline Ta-175 & & & $3.04 \mathrm{E}+07$ & $1.78 \mathrm{E}+01$ \\
\hline Ta-176 & & & $1.54 \mathrm{E}+07$ & $6.98 \mathrm{E}+00$ \\
\hline Ta-177 & & & $6.90 \mathrm{E}+07$ & $2.20 \mathrm{E}+02$ \\
\hline Ta-178s & & & $5.86 \mathrm{E}+08$ & $5.17 \mathrm{E}+00$ \\
\hline Ta-1781 & & & $3.85 \mathrm{E}+07$ & $4.81 \mathrm{E}+00$ \\
\hline Ta-179 & & & $1.44 \mathrm{E}+07$ & $1.31 \mathrm{E}+04$ \\
\hline Та-180 & & & $3.11 \mathrm{E}+05$ & $1.57 \mathrm{E}+15$ \\
\hline Ta-180m & & & $1.65 \mathrm{E}+08$ & $7.68 \mathrm{E}+01$ \\
\hline Та-182 & & & $7.96 \mathrm{E}+05$ & $1.27 \mathrm{E}+02$ \\
\hline Ta-182m & & & $1.49 \mathrm{E}+08$ & $2.29 \mathrm{E}+00$ \\
\hline Та-183 & & & $3.79 \mathrm{E}+06$ & $2.71 \mathrm{E}+01$ \\
\hline Та-184 & & & $1.22 \mathrm{E}+07$ & $6.24 \mathrm{E}+00$ \\
\hline Та-185 & & & $1.09 \mathrm{E}+08$ & $5.27 \mathrm{E}+00$ \\
\hline Та-186 & & & $3.32 \mathrm{E}+07$ & 3.45E-01 \\
\hline Tb-146 & & & $1.55 \mathrm{E}+07$ & $4.62 \mathrm{E}-03$ \\
\hline Tb-147 & & & $2.62 \mathrm{E}+07$ & $2.03 \mathrm{E}+00$ \\
\hline Tb-147m & & & $2.97 \mathrm{E}+07$ & 4.34E-02 \\
\hline Tb-148 & & & $1.95 \mathrm{E}+07$ & $9.20 \mathrm{E}-01$ \\
\hline Tb-148m & & & $1.90 \mathrm{E}+07$ & $3.29 \mathrm{E}-02$ \\
\hline Tb-149 & & & $1.58 \mathrm{E}+06$ & 3.11E-01 \\
\hline Tb-149m & & & $4.36 \mathrm{E}+07$ & $1.44 \mathrm{E}-01$ \\
\hline Tb-150 & & & $2.27 \mathrm{E}+07$ & $3.55 \mathrm{E}+00$ \\
\hline Tb-150m & & & $2.41 \mathrm{E}+07$ & $1.12 \mathrm{E}-01$ \\
\hline Tb-151 & & & $2.28 \mathrm{E}+07$ & $1.93 \mathrm{E}+01$ \\
\hline Tb-151m & & & $8.45 \mathrm{E}+08$ & $2.82 \mathrm{E}-01$ \\
\hline Tb-152 & & & $1.37 \mathrm{E}+07$ & $1.16 \mathrm{E}+01$ \\
\hline Tb-152m & & & $8.24 \mathrm{E}+07$ & $2.80 \mathrm{E}-01$ \\
\hline Tb-153 & & & $3.69 \mathrm{E}+07$ & $1.01 \mathrm{E}+02$ \\
\hline Tb-154 & & & $1.12 \mathrm{E}+07$ & $1.18 \mathrm{E}+01$ \\
\hline Tb-155 & & & $3.43 \mathrm{E}+07$ & $2.16 \mathrm{E}+02$ \\
\hline Tb-156 & & & $5.52 \mathrm{E}+06$ & $3.52 \mathrm{E}+01$ \\
\hline Tb-156ml & & & $3.82 \mathrm{E}+07$ & $4.64 \mathrm{E}+01$ \\
\hline Tb-156ms & & & $8.42 \mathrm{E}+07$ & $2.09 \mathrm{E}+01$ \\
\hline Tb-157 & & & $6.76 \mathrm{E}+06$ & $4.45 \mathrm{E}+05$ \\
\hline Tb-158 & & & $1.76 \mathrm{E}+05$ & $1.17 \mathrm{E}+04$ \\
\hline Tb-160 & $1.13 \mathrm{E}+06$ & $1.00 \mathrm{E}+02$ & $1.13 \mathrm{E}+06$ & $1.00 \mathrm{E}+02$ \\
\hline
\end{tabular}




\begin{tabular}{|c|c|c|c|c|}
\hline \multicolumn{5}{|c|}{ Table B.2 HC-2 TQs Using Recommended Inhalation DCs (continued) } \\
\hline \multirow{2}{*}{$\begin{array}{c}\text { MASTER } \\
\text { ISOTOPE LIST }\end{array}$} & \multicolumn{2}{|c|}{ NNSA SD 1027} & \multicolumn{2}{|c|}{ Final Threshold Quantity } \\
\hline & Act (Ci) & Mass (g) & Act (Ci) & Mass (g) \\
\hline Tb-161 & & & $6.22 \mathrm{E}+06$ & $5.30 \mathrm{E}+01$ \\
\hline Tb-162 & & & $5.36 \mathrm{E}+07$ & $3.51 \mathrm{E}-01$ \\
\hline Tb-163 & & & $6.46 \mathrm{E}+07$ & $1.09 \mathrm{E}+00$ \\
\hline Tb-164 & & & $2.37 \mathrm{E}+07$ & $6.20 \mathrm{E}-02$ \\
\hline Tb-165 & & & $6.69 \mathrm{E}+07$ & $1.24 \mathrm{E}-01$ \\
\hline Tc-101 & & & $1.34 \mathrm{E}+08$ & $1.02 \mathrm{E}+00$ \\
\hline Tc-102 & & & $4.62 \mathrm{E}+08$ & $2.20 \mathrm{E}-02$ \\
\hline Tc-102m & & & $2.29 \mathrm{E}+07$ & $5.40 \mathrm{E}-02$ \\
\hline Tc-104 & & & $2.45 \mathrm{E}+07$ & $2.46 \mathrm{E}-01$ \\
\hline Tc-105 & & & $7.17 \mathrm{E}+07$ & 3.04E-01 \\
\hline Tc-91 & & & $2.25 \mathrm{E}+07$ & 3.42E-02 \\
\hline Tc-91m & & & $4.10 \mathrm{E}+07$ & $6.53 \mathrm{E}-02$ \\
\hline Tc-92 & & & $1.51 \mathrm{E}+07$ & $3.14 \mathrm{E}-02$ \\
\hline Tc-93 & & & $3.16 \mathrm{E}+07$ & $2.58 \mathrm{E}+00$ \\
\hline Tc-93m & & & $6.29 \mathrm{E}+07$ & $1.35 \mathrm{E}+00$ \\
\hline Tc-94 & & & $1.59 \mathrm{E}+07$ & $2.33 \mathrm{E}+00$ \\
\hline Tc-94m & & & $2.53 \mathrm{E}+07$ & $6.57 \mathrm{E}-01$ \\
\hline Tc-95 & & & $3.77 \mathrm{E}+07$ & $2.28 \mathrm{E}+01$ \\
\hline Tc-95m & & & $8.30 \mathrm{E}+06$ & $3.68 \mathrm{E}+02$ \\
\hline Tc-96 & & & $7.75 \mathrm{E}+06$ & $2.44 \mathrm{E}+01$ \\
\hline Tc-96m & & & $5.74 \mathrm{E}+08$ & $1.51 \mathrm{E}+01$ \\
\hline Tc-97 & & & $3.68 \mathrm{E}+07$ & $2.60 \mathrm{E}+10$ \\
\hline Tc-97m & & & $2.53 \mathrm{E}+06$ & $1.64 \mathrm{E}+02$ \\
\hline Tc-98 & & & $9.53 \mathrm{E}+05$ & $1.10 \mathrm{E}+09$ \\
\hline Tc-99 & $2.03 \mathrm{E}+06$ & $1.19 \mathrm{E}+08$ & $2.03 \mathrm{E}+06$ & $1.19 \mathrm{E}+08$ \\
\hline Tc-99m & & & $2.21 \mathrm{E}+08$ & $4.20 \mathrm{E}+01$ \\
\hline Te-113 & & & $2.57 \mathrm{E}+06$ & $2.63 \mathrm{E}-03$ \\
\hline Te-114 & & & $3.84 \mathrm{E}+06$ & $3.54 \mathrm{E}-02$ \\
\hline Te-115 & & & $2.57 \mathrm{E}+06$ & 9.12E-03 \\
\hline Te-115m & & & $2.22 \mathrm{E}+06$ & $9.07 \mathrm{E}-03$ \\
\hline Te-116 & & & $7.59 \mathrm{E}+06$ & 6.99E-01 \\
\hline Te-117 & & & $3.18 \mathrm{E}+06$ & $1.23 \mathrm{E}-01$ \\
\hline Te-118 & & & $3.02 E+05$ & $1.64 \mathrm{E}+00$ \\
\hline Te-119 & & & $3.54 \mathrm{E}+06$ & $2.16 \mathrm{E}+00$ \\
\hline Te-119m & & & $9.87 \mathrm{E}+05$ & $4.23 \mathrm{E}+00$ \\
\hline Te-121 & & & $1.76 \mathrm{E}+06$ & $2.77 \mathrm{E}+01$ \\
\hline Te-121m & & & $1.92 \mathrm{E}+05$ & $2.73 \mathrm{E}+01$ \\
\hline Te-123 & & & $4.27 \mathrm{E}+05$ & $1.47 \mathrm{E}+15$ \\
\hline
\end{tabular}




\begin{tabular}{|c|c|c|c|c|}
\hline \multicolumn{5}{|c|}{ Table B.2 HC-2 TQs Using Recommended Inhalation DCs (continued) } \\
\hline \multirow{2}{*}{$\begin{array}{c}\text { MASTER } \\
\text { ISOTOPE LIST }\end{array}$} & \multicolumn{2}{|c|}{ NNSA SD 1027} & \multicolumn{2}{|c|}{ Final Threshold Quantity } \\
\hline & Act (Ci) & Mass (g) & Act (Ci) & Mass (g) \\
\hline Te-123m & & & $2.02 \mathrm{E}+05$ & $2.27 \mathrm{E}+01$ \\
\hline Te-125m & & & $2.38 \mathrm{E}+05$ & $1.32 \mathrm{E}+01$ \\
\hline Te-127 & & & $6.20 \mathrm{E}+06$ & $2.35 \mathrm{E}+00$ \\
\hline Te-127m & $1.10 \mathrm{E}+05$ & $1.16 \mathrm{E}+01$ & $1.10 \mathrm{E}+05$ & $1.16 \mathrm{E}+01$ \\
\hline Te-129 & & & $1.79 \mathrm{E}+07$ & 8.55E-01 \\
\hline Te-129m & $1.23 \mathrm{E}+05$ & $4.07 \mathrm{E}+00$ & $1.23 \mathrm{E}+05$ & $4.07 \mathrm{E}+00$ \\
\hline Te-131 & & & $9.09 \mathrm{E}+06$ & $1.58 \mathrm{E}-01$ \\
\hline Te-131m & & & $7.05 \mathrm{E}+05$ & 8.83E-01 \\
\hline Te-132 & & & $3.99 \mathrm{E}+05$ & $1.31 \mathrm{E}+00$ \\
\hline Te-133 & & & $5.13 \mathrm{E}+06$ & $4.52 \mathrm{E}-02$ \\
\hline Te-133m & & & $1.89 \mathrm{E}+06$ & $7.40 \mathrm{E}-02$ \\
\hline Te-134 & & & $4.20 \mathrm{E}+06$ & $1.25 \mathrm{E}-01$ \\
\hline Th-223 & & & $9.72 \mathrm{E}+08$ & $1.15 \mathrm{E}-02$ \\
\hline Th-224 & & & $2.77 \mathrm{E}+09$ & $5.78 \mathrm{E}-02$ \\
\hline Th-226 & & & $1.33 \mathrm{E}+05$ & $4.94 \mathrm{E}-03$ \\
\hline Th-227 & & & $8.11 \mathrm{E}+02$ & $2.64 \mathrm{E}-02$ \\
\hline Th-228 & $2.03 \mathrm{E}+02$ & 2.47E-01 & $2.03 \mathrm{E}+02$ & $2.47 \mathrm{E}-01$ \\
\hline Th-229 & & & $1.14 \mathrm{E}+02$ & $5.37 \mathrm{E}+02$ \\
\hline Th-230 & $5.79 \mathrm{E}+02$ & $2.81 \mathrm{E}+04$ & $5.79 \mathrm{E}+02$ & $2.87 \mathrm{E}+04$ \\
\hline Th-231 & & & $2.45 \mathrm{E}+07$ & $4.60 \mathrm{E}+01$ \\
\hline Th-232 & $3.24 \mathrm{E}+02$ & $2.96 \mathrm{E}+09$ & $3.24 \mathrm{E}+02$ & $2.96 \mathrm{E}+09$ \\
\hline Th-233 & & & $3.05 \mathrm{E}+08$ & $8.43 \mathrm{E}+00$ \\
\hline Th-234 & & & $1.05 \mathrm{E}+06$ & $4.55 \mathrm{E}+01$ \\
\hline Th-235 & & & $9.22 \mathrm{E}+08$ & $8.19 \mathrm{E}+00$ \\
\hline Th-236 & & & $1.12 \mathrm{E}+08$ & $5.29 \mathrm{E}+00$ \\
\hline $\mathrm{Ti}-44$ & $6.76 \mathrm{E}+04$ & $3.93 \mathrm{E}+02$ & $6.76 \mathrm{E}+04$ & $3.93 \mathrm{E}+02$ \\
\hline Ti-45 & & & $3.71 \mathrm{E}+07$ & $1.64 \mathrm{E}+00$ \\
\hline Ti-51 & & & $1.58 \mathrm{E}+08$ & $2.47 \mathrm{E}-01$ \\
\hline Ti-52 & & & $4.70 \mathrm{E}+08$ & $2.21 \mathrm{E}-01$ \\
\hline Tl-190 & & & $4.55 \mathrm{E}+07$ & $1.20 \mathrm{E}-01$ \\
\hline $\mathrm{Tl}-190 \mathrm{~m}$ & & & $2.43 \mathrm{E}+07$ & $9.10 \mathrm{E}-02$ \\
\hline Tl-194 & & & $7.03 \mathrm{E}+07$ & $2.39 \mathrm{E}+00$ \\
\hline Tl-194m & & & $2.30 \mathrm{E}+07$ & 7.79E-01 \\
\hline Tl-195 & & & $3.95 \mathrm{E}+07$ & $2.85 \mathrm{E}+00$ \\
\hline Tl-196 & & & $2.61 \mathrm{E}+07$ & $3.00 \mathrm{E}+00$ \\
\hline Tl-197 & & & $1.13 \mathrm{E}+08$ & $2.01 \mathrm{E}+01$ \\
\hline Tl-198 & & & $2.23 \mathrm{E}+07$ & $7.48 \mathrm{E}+00$ \\
\hline Tl-198m & & & $3.90 \mathrm{E}+07$ & $4.61 \mathrm{E}+00$ \\
\hline
\end{tabular}




\begin{tabular}{|c|c|c|c|c|}
\hline \multicolumn{5}{|c|}{ Table B.2 HC-2 TQs Using Recommended Inhalation DCs (continued) } \\
\hline \multirow{2}{*}{$\begin{array}{c}\text { MASTER } \\
\text { ISOTOPE LIST }\end{array}$} & \multicolumn{2}{|c|}{ NNSA SD 1027} & \multicolumn{2}{|c|}{ Final Threshold Quantity } \\
\hline & Act (Ci) & Mass (g) & Act (Ci) & Mass (g) \\
\hline Tl-199 & & & $1.53 \mathrm{E}+08$ & $7.22 \mathrm{E}+01$ \\
\hline Tl-200 & & & $2.51 \mathrm{E}+07$ & $4.19 \mathrm{E}+01$ \\
\hline Tl-201 & & & $1.47 \mathrm{E}+08$ & $6.86 \mathrm{E}+02$ \\
\hline Tl-202 & & & $3.17 \mathrm{E}+07$ & $6.01 \mathrm{E}+02$ \\
\hline Tl-204 & & & $2.08 \mathrm{E}+07$ & $4.48 \mathrm{E}+04$ \\
\hline Tl-206 & & & $4.02 \mathrm{E}+10$ & $1.85 \mathrm{E}+02$ \\
\hline Tl-206m & & & $2.48 \mathrm{E}+07$ & $1.02 \mathrm{E}-01$ \\
\hline Tl-207 & & & $1.67 \mathrm{E}+10$ & $8.76 \mathrm{E}+01$ \\
\hline Tl-208 & & & $1.53 \mathrm{E}+07$ & 5.19E-02 \\
\hline Tl-209 & & & $2.65 \mathrm{E}+07$ & $6.48 \mathrm{E}-02$ \\
\hline Tl-210 & & & $2.05 \mathrm{E}+07$ & $2.97 \mathrm{E}-02$ \\
\hline Tm-161 & & & $3.91 \mathrm{E}+07$ & $1.01 \mathrm{E}+00$ \\
\hline Tm-162 & & & $2.83 \mathrm{E}+07$ & $5.29 \mathrm{E}-01$ \\
\hline Tm-163 & & & $3.58 \mathrm{E}+07$ & $3.37 \mathrm{E}+00$ \\
\hline Tm-164 & & & $7.57 \mathrm{E}+07$ & $1.32 \mathrm{E}-01$ \\
\hline Tm-165 & & & $2.34 \mathrm{E}+07$ & $3.71 \mathrm{E}+01$ \\
\hline Tm-166 & & & $1.80 \mathrm{E}+07$ & $7.34 \mathrm{E}+00$ \\
\hline Tm-167 & & & $7.25 \mathrm{E}+06$ & $8.57 \mathrm{E}+01$ \\
\hline Tm-168 & & & $1.41 \mathrm{E}+06$ & $1.68 \mathrm{E}+02$ \\
\hline Tm-170 & $1.16 \mathrm{E}+06$ & $1.94 \mathrm{E}+02$ & $1.16 \mathrm{E}+06$ & $1.94 \mathrm{E}+02$ \\
\hline Tm-171 & & & $5.79 \mathrm{E}+06$ & $5.32 \mathrm{E}+03$ \\
\hline Tm-172 & & & $6.92 \mathrm{E}+06$ & $2.41 \mathrm{E}+01$ \\
\hline Tm-173 & & & $3.44 \mathrm{E}+07$ & $1.57 \mathrm{E}+01$ \\
\hline Tm-174 & & & $3.36 \mathrm{E}+07$ & $1.68 \mathrm{E}-01$ \\
\hline Tm-175 & & & $4.72 \mathrm{E}+07$ & 6.67E-01 \\
\hline Tm-176 & & & $2.88 \mathrm{E}+07$ & $4.98 \mathrm{E}-02$ \\
\hline U-227 & & & $5.57 \mathrm{E}+08$ & $7.40 \mathrm{E}-01$ \\
\hline U-228 & & & $1.70 \mathrm{E}+10$ & $1.88 \mathrm{E}+02$ \\
\hline U-230 & & & $6.24 \mathrm{E}+02$ & $2.29 \mathrm{E}-02$ \\
\hline U-231 & & & $1.69 \mathrm{E}+07$ & $1.26 \mathrm{E}+02$ \\
\hline U-232 & $1.04 \mathrm{E}+03$ & $4.71 \mathrm{E}+01$ & $1.04 \mathrm{E}+03$ & $4.86 \mathrm{E}+01$ \\
\hline U-233 & $2.25 \mathrm{E}+03$ & $2.34 \mathrm{E}+05$ & $2.25 \mathrm{E}+03$ & $2.33 \mathrm{E}+05$ \\
\hline U-234 & $2.32 \mathrm{E}+03$ & $3.73 E+05$ & $2.32 \mathrm{E}+03$ & $3.71 \mathrm{E}+05$ \\
\hline U-235 & $2.62 \mathrm{E}+03$ & $1.21 \mathrm{E}+09$ & $2.62 \mathrm{E}+03$ & $1.21 \mathrm{E}+09$ \\
\hline U-235m & & & $9.00 \mathrm{E}+12$ & $2.92 \mathrm{E}+05$ \\
\hline U-236 & $2.53 \mathrm{E}+03$ & $3.92 \mathrm{E}+07$ & $2.53 \mathrm{E}+03$ & $3.92 \mathrm{E}+07$ \\
\hline U-237 & & & $4.72 \mathrm{E}+06$ & $5.78 \mathrm{E}+01$ \\
\hline U-238 & $2.80 \mathrm{E}+03$ & $8.32 \mathrm{E}+09$ & $2.80 \mathrm{E}+03$ & $8.32 E+09$ \\
\hline
\end{tabular}




\begin{tabular}{|c|c|c|c|c|}
\hline \multicolumn{5}{|c|}{ Table B.2 HC-2 TQs Using Recommended Inhalation DCs (continued) } \\
\hline \multirow{2}{*}{$\begin{array}{c}\text { MASTER } \\
\text { ISOTOPE LIST }\end{array}$} & \multicolumn{2}{|c|}{ NNSA SD 1027} & \multicolumn{2}{|c|}{ Final Threshold Quantity } \\
\hline & Act (Ci) & Mass (g) & Act (Ci) & Mass (g) \\
\hline U-239 & & & $2.84 \mathrm{E}+08$ & $8.51 E+00$ \\
\hline $\mathrm{U}-240$ & & & $1.53 \mathrm{E}+07$ & $1.65 \mathrm{E}+01$ \\
\hline $\mathrm{U}-242$ & & & $2.13 \mathrm{E}+08$ & $4.62 \mathrm{E}+00$ \\
\hline V-47 & & & $4.69 \mathrm{E}+07$ & $3.82 \mathrm{E}-01$ \\
\hline $\mathrm{V}-48$ & $2.86 \mathrm{E}+06$ & $1.68 \mathrm{E}+01$ & $2.86 \mathrm{E}+06$ & $1.71 \mathrm{E}+01$ \\
\hline V-49 & & & $2.38 \mathrm{E}+08$ & $2.95 \mathrm{E}+04$ \\
\hline V-50 & & & $1.21 \mathrm{E}+05$ & $2.53 \mathrm{E}+18$ \\
\hline V-52 & & & $3.83 \mathrm{E}+07$ & $3.96 \mathrm{E}-02$ \\
\hline V-53 & & & $5.48 \mathrm{E}+07$ & $2.49 \mathrm{E}-02$ \\
\hline W-176 & & & $1.31 \mathrm{E}+08$ & $1.69 \mathrm{E}+01$ \\
\hline W-177 & & & $5.34 \mathrm{E}+07$ & $6.79 \mathrm{E}+00$ \\
\hline W-178 & & & $1.10 \mathrm{E}+08$ & $3.27 \mathrm{E}+03$ \\
\hline W-179 & & & $1.26 \mathrm{E}+09$ & $4.51 \mathrm{E}+01$ \\
\hline $\mathrm{W}-179 \mathrm{~m}$ & & & $1.36 \mathrm{E}+09$ & $8.27 \mathrm{E}+00$ \\
\hline W-181 & & & $2.60 \mathrm{E}+08$ & $4.36 \mathrm{E}+04$ \\
\hline W-185 & & & $6.76 \mathrm{E}+07$ & $7.19 \mathrm{E}+03$ \\
\hline $\mathrm{W}-185 \mathrm{~m}$ & & & $2.90 \mathrm{E}+09$ & $4.55 \mathrm{E}+00$ \\
\hline W-187 & & & $3.14 \mathrm{E}+07$ & $4.47 \mathrm{E}+01$ \\
\hline W-188 & & & $1.42 \mathrm{E}+07$ & $1.42 \mathrm{E}+03$ \\
\hline W-190 & & & $7.90 \mathrm{E}+07$ & $2.39 \mathrm{E}+00$ \\
\hline Xe-120 & & & $1.39 \mathrm{E}+05$ & $3.55 \mathrm{E}-03$ \\
\hline Xe-121 & & & $2.96 \mathrm{E}+04$ & 7.63E-04 \\
\hline Xe-122 & & & $1.10 \mathrm{E}+06$ & $8.59 \mathrm{E}-01$ \\
\hline Xe-123 & & & $8.92 \mathrm{E}+04$ & $7.28 \mathrm{E}-03$ \\
\hline Xe-125 & & & $2.27 \mathrm{E}+05$ & $1.54 \mathrm{E}-01$ \\
\hline Хe-127 & & & $2.16 \mathrm{E}+05$ & $7.65 \mathrm{E}+00$ \\
\hline Xe-127m & & & $4.11 \mathrm{E}+05$ & $3.20 \mathrm{E}-04$ \\
\hline Xe-129m & & & $2.55 \mathrm{E}+06$ & $2.01 \mathrm{E}+01$ \\
\hline Xe-131m & & & $6.95 \mathrm{E}+06$ & $8.29 \mathrm{E}+01$ \\
\hline Хe-133 & $1.95 \mathrm{E}+06$ & $1.04 \mathrm{E}+01$ & $1.73 \mathrm{E}+06$ & $9.25 \mathrm{E}+00$ \\
\hline Xe-133m & & & $1.97 \mathrm{E}+06$ & $4.39 \mathrm{E}+00$ \\
\hline Xe-135 & & & $2.27 \mathrm{E}+05$ & $8.89 \mathrm{E}-02$ \\
\hline Xe-135m & & & $1.32 \mathrm{E}+05$ & $1.45 \mathrm{E}-03$ \\
\hline Xe-137 & & & $2.60 \mathrm{E}+05$ & $7.22 \mathrm{E}-04$ \\
\hline Хe-138 & & & $4.68 \mathrm{E}+04$ & 4.87E-04 \\
\hline Y-81 & & & $5.05 \mathrm{E}+07$ & $2.55 \mathrm{E}-02$ \\
\hline Y-83 & & & $4.39 \mathrm{E}+07$ & $1.37 \mathrm{E}-01$ \\
\hline Y-83m & & & $7.17 \mathrm{E}+07$ & $9.01 \mathrm{E}-02$ \\
\hline
\end{tabular}




\begin{tabular}{|c|c|c|c|c|}
\hline \multicolumn{5}{|c|}{ Table B.2 HC-2 TQs Using Recommended Inhalation DCs (continued) } \\
\hline \multirow{2}{*}{$\begin{array}{c}\text { MASTER } \\
\text { ISOTOPE LIST }\end{array}$} & \multicolumn{2}{|c|}{ NNSA SD 1027} & \multicolumn{2}{|c|}{ Final Threshold Quantity } \\
\hline & Act (Ci) & Mass (g) & Act (Ci) & Mass (g) \\
\hline Y-84m & & & $1.29 \mathrm{E}+07$ & 2.28E-01 \\
\hline Y-85 & & & $3.10 \mathrm{E}+07$ & $2.25 \mathrm{E}+00$ \\
\hline Y-85m & & & $2.08 \mathrm{E}+07$ & $2.74 \mathrm{E}+00$ \\
\hline Y-86 & & & $8.05 \mathrm{E}+06$ & $3.25 \mathrm{E}+00$ \\
\hline Y-86m & & & $1.36 \mathrm{E}+08$ & $2.97 \mathrm{E}+00$ \\
\hline Y-87 & & & $1.78 \mathrm{E}+07$ & $3.97 \mathrm{E}+01$ \\
\hline Y-87m & & & $3.93 \mathrm{E}+07$ & $1.46 \mathrm{E}+01$ \\
\hline $\mathrm{Y}-88$ & & & $1.69 \mathrm{E}+06$ & $1.21 \mathrm{E}+02$ \\
\hline Y-89m & & & $6.50 \mathrm{E}+07$ & 8.02E-03 \\
\hline Y-90 & & & $5.40 \mathrm{E}+06$ & $9.92 \mathrm{E}+00$ \\
\hline Y-90m & & & $4.26 \mathrm{E}+07$ & $3.90 \mathrm{E}+00$ \\
\hline $\mathrm{Y}-91$ & $9.11 \mathrm{E}+05$ & $3.71 \mathrm{E}+01$ & $9.11 \mathrm{E}+05$ & $3.71 \mathrm{E}+01$ \\
\hline Y-91m & & & $9.27 \mathrm{E}+07$ & $2.23 \mathrm{E}+00$ \\
\hline Y-92 & & & $3.70 \mathrm{E}+07$ & $3.84 \mathrm{E}+00$ \\
\hline Y-93 & & & $1.87 \mathrm{E}+07$ & $5.59 \mathrm{E}+00$ \\
\hline Y-94 & & & $4.12 \mathrm{E}+07$ & 3.93E-01 \\
\hline Y-95 & & & $5.08 \mathrm{E}+07$ & $2.74 \mathrm{E}-01$ \\
\hline Yb-162 & & & $2.62 \mathrm{E}+08$ & $4.26 \mathrm{E}+00$ \\
\hline Yb-163 & & & $7.45 \mathrm{E}+07$ & $7.13 \mathrm{E}-01$ \\
\hline Yb-164 & & & $1.40 \mathrm{E}+08$ & $9.25 \mathrm{E}+00$ \\
\hline Yb-165 & & & $1.97 \mathrm{E}+08$ & $1.71 \mathrm{E}+00$ \\
\hline Yb-166 & & & $1.04 \mathrm{E}+07$ & $3.13 \mathrm{E}+01$ \\
\hline Yb-167 & & & $2.05 E+08$ & $3.18 \mathrm{E}+00$ \\
\hline Yb-169 & & & $2.67 \mathrm{E}+06$ & $1.11 \mathrm{E}+02$ \\
\hline Yb-175 & & & $1.10 \mathrm{E}+07$ & $6.19 \mathrm{E}+01$ \\
\hline Yb-177 & & & $8.39 \mathrm{E}+07$ & $9.00 \mathrm{E}+00$ \\
\hline Yb-178 & & & $1.01 \mathrm{E}+08$ & $7.10 \mathrm{E}+00$ \\
\hline Yb-179 & & & $6.14 \mathrm{E}+07$ & $4.68 \mathrm{E}-01$ \\
\hline Zn-60 & & & $3.91 \mathrm{E}+07$ & $2.97 \mathrm{E}-02$ \\
\hline Zn-61 & & & $3.74 \mathrm{E}+07$ & $1.80 \mathrm{E}-02$ \\
\hline Zn-62 & & & $1.44 \mathrm{E}+07$ & $2.64 \mathrm{E}+00$ \\
\hline Zn-63 & & & $4.17 \mathrm{E}+07$ & $5.31 \mathrm{E}-01$ \\
\hline Zn-65 & $4.81 \mathrm{E}+06$ & $5.83 \mathrm{E}+02$ & $4.81 \mathrm{E}+06$ & $5.83 \mathrm{E}+02$ \\
\hline Zn-69 & & & $3.11 \mathrm{E}+08$ & $6.50 \mathrm{E}+00$ \\
\hline Zn-69m & & & $2.71 \mathrm{E}+07$ & $8.19 \mathrm{E}+00$ \\
\hline Zn-71 & & & $1.78 \mathrm{E}+08$ & $1.64 \mathrm{E}-01$ \\
\hline Zn-71m & & & $2.16 \mathrm{E}+07$ & $1.92 \mathrm{E}+00$ \\
\hline Zn-72 & & & $6.64 \mathrm{E}+06$ & $7.09 \mathrm{E}+00$ \\
\hline
\end{tabular}




\begin{tabular}{|c|c|c|c|c|}
\hline \multicolumn{2}{|c}{ Table B.2 HC-2 TQs Using Recommended Inhalation DCs (continued) } \\
\hline $\begin{array}{c}\text { MASTER } \\
\text { ISOTOPE LIST }\end{array}$ & \multicolumn{2}{|c|}{ NNSA SD 1027 } & \multicolumn{2}{c|}{ Final Threshold Quantity } \\
\cline { 2 - 5 } & Act (Ci) & Mass (g) & Act (Ci) & Mass (g) \\
\hline Zr-85 & & & $4.01 \mathrm{E}+07$ & $1.42 \mathrm{E}-01$ \\
\hline Zr-86 & & & $1.77 \mathrm{E}+07$ & $8.00 \mathrm{E}+00$ \\
\hline Zr-87 & & & $3.55 \mathrm{E}+07$ & $1.65 \mathrm{E}+00$ \\
\hline Zr-88 & & & $3.05 \mathrm{E}+06$ & $1.71 \mathrm{E}+02$ \\
\hline Zr-89 & & & $1.17 \mathrm{E}+07$ & $2.61 \mathrm{E}+01$ \\
\hline Zr-89m & & $3.16 \mathrm{E}+08$ & $8.11 \mathrm{E}+05$ & $3.22 \mathrm{E}+08$ \\
\hline Zr-93 & $8.11 \mathrm{E}+05$ & $7.69 \mathrm{E}+01$ & $1.65 \mathrm{E}+06$ & $7.68 \mathrm{E}+01$ \\
\hline Zr-95 & $1.65 \mathrm{E}+06$ & & $8.56 \mathrm{E}+06$ & $4.47 \mathrm{E}+00$ \\
\hline Zr-97 & & &
\end{tabular}




\section{APPENDIX C. CALCULATION OF HAZARD CATEGORY 3}

THRESHOLD QUANTITIES 
(This page is intentionally blank) 


\section{APPENDIX C. CALCULATION OF HAZARD CATEGORY 3 THRESHOLD QUANTITIES}

This appendix contains the following tables:

- Table C.1: Radionuclide Specific Input Data Used For the Calculation of HC-3 TQs Using Maximum Reported DCs

- Table C.2: Summary of Exposure Pathway TQs and Selection of Final HC-3 TQs 


\begin{tabular}{|c|c|c|c|c|c|c|c|c|c|c|c|c|c|c|c|c|c|c|}
\hline \multicolumn{19}{|c|}{ Table C.1 Radionuclide Specific Input Data Used For the Calculation of HC-3 TQs Using Maximum Reported DCs } \\
\hline $\begin{array}{l}\text { MASTER } \\
\text { ISOTOPE } \\
\text { LIST }\end{array}$ & $\begin{array}{l}\text { Atomic } \\
\text { Mass } \\
\text { Nuclide }\end{array}$ & $\begin{array}{l}\text { Atomic } \\
\text { Mass }\end{array}$ & Element & $\begin{array}{c}\text { EPA } \\
\text { RF }\end{array}$ & $\begin{array}{c}\text { EPA } \\
\text { Conc } \\
\text { Factors } \\
\left(B_{v}\right) \\
\end{array}$ & $\begin{array}{c}\text { EPA } \\
\text { Sorption } \\
\text { Coefficient } \\
\left(K_{d}\right)\end{array}$ & $\begin{array}{c}\text { Half-Life } \\
\text { Reference }\end{array}$ & $\begin{array}{r}\begin{array}{r}\text { Half-Lif } \\
\text { (original u }\end{array} \\
\text { (oring }\end{array}$ & & $\begin{array}{l}\text { Half Life } \\
\text { (s) }\end{array}$ & $\begin{array}{l}\text { Sp. Act } \\
\text { (Ci/g) }\end{array}$ & $\begin{array}{l}\text { Inhalation } \\
\text { DC } \\
\text { Reference }\end{array}$ & $\begin{array}{c}\text { HC-3 } \\
\text { Bounding } \\
\text { Inhalation } \\
\text { DC } \\
\text { (Sv/Bq) } \\
\end{array}$ & $\begin{array}{l}\text { Lung } \\
\text { Absorption } \\
\text { Class/Type }\end{array}$ & $\begin{array}{l}\text { Ingestion } \\
\text { DC } \\
\text { Reference } \\
\end{array}$ & $\begin{array}{c}\mathrm{HC}-3 \\
\text { Bounding } \\
\text { Ingestion } \\
\mathrm{DC} \\
\text { (Sv/Bq) } \\
\end{array}$ & $\begin{array}{l}\text { Immersion } \\
\text { DC } \\
\text { Reference }\end{array}$ & $\begin{array}{c}\text { HC-3 } \\
\text { Immersion } \\
\mathrm{DC}(\mathrm{Sv} / \mathrm{d} \\
\left.\operatorname{per} \mathrm{Bq} / \mathrm{m}^{3}\right)\end{array}$ \\
\hline Ac-223 & Ac-223 & 223.019 & Actinium & 0.001 & 0.0035 & 1000 & ICRP-38 & $2.20 \mathrm{E}+00$ & $\mathrm{~m}$ & $1.32 \mathrm{E}+02$ & $3.83 \mathrm{E}+08$ & -- & --- & -- & -- & -- & -- & --- \\
\hline Ac-224 & Ac-224 & 224.022 & Actinium & 0.001 & 0.0035 & 1000 & ICRP-38 & $2.90 \mathrm{E}+00$ & $\mathrm{~h}$ & $1.04 \mathrm{E}+04$ & $4.82 E+06$ & ICRP-68 & $1.2 \mathrm{E}-07$ & $\mathrm{~s}$ & ICRP-68 & $7.0 \mathrm{E}-10$ & -- & -- \\
\hline Ac-225 & Ac-225 & 225.023 & Actinium & 0.001 & 0.0035 & 1000 & ICRP-38 & $1.00 \mathrm{E}+01$ & $d$ & $8.64 \mathrm{E}+05$ & $5.80 \mathrm{E}+04$ & ICRP-68 & $7.9 \mathrm{E}-06$ & $\mathrm{~s}$ & $\begin{array}{l}\text { ICRP-68 } \\
\end{array}$ & $2.4 \mathrm{E}-08$ & -- & -- \\
\hline Ac-226 & Ac-226 & 226.026 & Actinium & 0.001 & 0.0035 & 1000 & ICRP-38 & $2.90 \mathrm{E}+01$ & $\mathrm{~h}$ & $1.04 \mathrm{E}+05$ & $4.78 \mathrm{E}+05$ & ICRP-68 & $1.2 \mathrm{E}-06$ & $\mathrm{~s}$ & ICRP-68 & 1.0E-08 & -- & -- \\
\hline Ac-227 & Ac-227 & 227.028 & Actinium & 0.001 & 0.0035 & 1000 & ICRP-38 & $2.18 \mathrm{E}+01$ & $y$ & $6.87 \mathrm{E}+08$ & $7.23 \mathrm{E}+01$ & ICRP-68 & $6.3 \mathrm{E}-04$ & $F$ & ICRP-68 & $1.1 \mathrm{E}-06$ & -- & -- \\
\hline Ac-228 & Ac-228 & 228.031 & Actinium & 0.001 & 0.0035 & 1000 & ICRP-38 & $6.13 \mathrm{E}+00$ & $\mathrm{~h}$ & $2.21 \mathrm{E}+04$ & $2.24 \mathrm{E}+06$ & ICRP-68 & $2.9 \mathrm{E}-08$ & $\mathrm{~F}$ & ICRP-68 & $4.3 \mathrm{E}-10$ & -- & -- \\
\hline Ac-229 & Ac-229 & 229.033 & Actinium & 0.001 & 0.0035 & 1000 & JAERI & $6.27 \mathrm{E}+01$ & $m$ & $3.76 \mathrm{E}+03$ & $1.31 \mathrm{E}+07$ & JAERI & $5.4 \mathrm{E}-11$ & $\mathrm{~s}$ & JAERI & $4.4 \mathrm{E}-11$ & -- & -- \\
\hline Ac-230 & Ac-230 & 230.036 & Actinium & 0.001 & 0.0035 & 1000 & ICRP-107 & $1.22 \mathrm{E}+02$ & $\mathrm{~s}$ & $1.22 \mathrm{E}+02$ & $4.02 E+08$ & -- & -- & -- & --- & -- & -- & -- \\
\hline Ac-231 & Ac-231 & 231.038 & Actinium & 0.001 & 0.0035 & 1000 & ICRP-107 & $7.50 \mathrm{E}+00$ & $\mathrm{~m}$ & $4.50 \mathrm{E}+02$ & $1.09 \mathrm{E}+08$ & -- & -- & -- & -- & -- & -- & -- \\
\hline Ac-232 & Ac-232 & 232.042 & Actinium & 0.001 & 0.0035 & 1000 & ICRP-107 & $1.19 \mathrm{E}+02$ & $\mathrm{~s}$ & $1.19 E+02$ & $4.09 \mathrm{E}+08$ & -- & -- & -- & -- & -- & -- & -- \\
\hline Ac-233 & Ac-233 & 233.044 & Actinium & 0.001 & 0.0035 & 1000 & ICRP-107 & $1.45 \mathrm{E}+02$ & $\mathrm{~s}$ & $1.45 \mathrm{E}+02$ & $3.34 \mathrm{E}+08$ & -- & -- & -- & -- & -- & -- & -- \\
\hline Ag-100m & Ag-100 & 99.916 & $\begin{array}{l}\text { Silver } \\
\end{array}$ & 0.01 & 0.4 & $50-100$ & ICRP-107 & $2.24 \mathrm{E}+00$ & $\mathrm{~m}$ & $1.34 \mathrm{E}+02$ & $8.40 \mathrm{E}+08$ & -- & -- & -- & $\begin{array}{ll}- \\
\end{array}$ & -- & -- & -- \\
\hline Ag-101 & Ag-101 & 100.913 & Silver & 0.01 & 0.4 & $50-100$ & ICRP-107 & $1.11 \mathrm{E}+01$ & $m$ & $6.66 \mathrm{E}+02$ & $1.68 \mathrm{E}+08$ & JAERI & $2.4 \mathrm{E}-11$ & $M$ & JAERI & $3.2 \mathrm{E}-11$ & -- & -- \\
\hline $\mathrm{Ag}-102$ & $\mathrm{Ag}-102$ & 101.912 & Silver & 0.01 & 0.4 & $50-100$ & ICRP-38 & $1.29 \mathrm{E}+01$ & $\mathrm{~m}$ & $7.74 \mathrm{E}+02$ & $1.43 E+08$ & ICRP-68 & $3.2 \mathrm{E}-11$ & $M$ & ICRP-68 & 4.0E-11 & -- & -- \\
\hline $\mathrm{Ag}-102 \mathrm{~m}$ & Ag-102 & 101.912 & Silver & 0.01 & 0.4 & $50-100$ & ICRP-107 & $7.70 \mathrm{E}+00$ & $\mathrm{~m}$ & $4.62 E+02$ & $2.40 E+08$ & -- & -- & -- & -- & -- & -- & -- \\
\hline Ag-103 & Ag-103 & 102.909 & Silver & 0.01 & 0.4 & $50-100$ & ICRP-38 & $6.57 \mathrm{E}+01$ & $\mathrm{~m}$ & $3.94 \mathrm{E}+03$ & $2.78 \mathrm{E}+07$ & ICRP-68 & $4.5 \mathrm{E}-11$ & $\mathrm{~s}$ & ICRP-68 & $4.3 \mathrm{E}-11$ & -- & -- \\
\hline Ag-104 & Ag-104 & 103.909 & Silver & 0.01 & 0.4 & $50-100$ & ICRP-38 & $6.92 \mathrm{E}+01$ & $\mathrm{~m}$ & $4.15 \mathrm{E}+03$ & $2.61 \mathrm{E}+07$ & ICRP-68 & $7.1 \mathrm{E}-11$ & $\mathrm{~s}$ & ICRP-68 & $6.0 \mathrm{E}-11$ & -- & -- \\
\hline $\mathrm{Ag}-104 \mathrm{~m}$ & Ag-104 & 103.909 & Silver & 0.01 & 0.4 & $50-100$ & ICRP-38 & $3.35 \mathrm{E}+01$ & $\mathrm{~m}$ & $2.01 \mathrm{E}+03$ & $5.40 \mathrm{E}+07$ & ICRP-68 & $4.5 \mathrm{E}-11$ & $\mathrm{~s}$ & ICRP-68 & $5.4 \mathrm{E}-11$ & -- & -- \\
\hline Ag-105 & Ag-105 & 104.907 & Silver & 0.01 & 0.4 & $50-100$ & ICRP-38 & $4.10 \mathrm{E}+01$ & $d$ & $3.54 \mathrm{E}+06$ & $3.04 \mathrm{E}+04$ & ICRP-68 & $8.0 \mathrm{E}-10$ & $\mathrm{~F}$ & ICRP-68 & $4.7 \mathrm{E}-10$ & -- & -- \\
\hline Ag-105m & Ag-105 & 104.907 & Silver & 0.01 & 0.4 & $50-100$ & ICRP-107 & $7.23 E+00$ & $\mathrm{~m}$ & $4.34 \mathrm{E}+02$ & $2.48 \mathrm{E}+08$ & -- & -- & -- & -- & -- & -- & -- \\
\hline $\mathrm{Ag}-106$ & $\mathrm{Ag}-106$ & 105.907 & Silver & 0.01 & 0.4 & $50-100$ & ICRP-38 & $2.40 \mathrm{E}+01$ & $\mathrm{~m}$ & $1.44 \mathrm{E}+03$ & $7.41 \mathrm{E}+07$ & ICRP-68 & $2.7 \mathrm{E}-11$ & $\mathrm{~s}$ & ICRP-68 & $3.2 \mathrm{E}-11$ & -- & -- \\
\hline Ag-106m & Ag-106 & 105.907 & Silver & 0.01 & 0.4 & $50-100$ & ICRP-38 & $8.41 \mathrm{E}+00$ & $d$ & $7.27 E+05$ & $1.47 \mathrm{E}+05$ & ICRP-68 & $1.6 \mathrm{E}-09$ & $\mathrm{~F}$ & ICRP-68 & $1.5 \mathrm{E}-09$ & -- & -- \\
\hline Ag-108 & Ag-108 & 107.906 & Silver & 0.01 & 0.4 & $50-100$ & ICRP-38 & $2.37 E+00$ & $\mathrm{~m}$ & $1.42 \mathrm{E}+02$ & $7.35 \mathrm{E}+08$ & -- & -- & -- & -- & -- & -- & -- \\
\hline $\mathrm{Ag}-108 \mathrm{~m}$ & Ag-108 & 107.906 & Silver & 0.01 & 0.4 & $50-100$ & ICRP-38 & $1.27 \mathrm{E}+02$ & $y$ & $4.01 \mathrm{E}+09$ & $2.61 \mathrm{E}+01$ & ICRP-68 & $3.5 \mathrm{E}-08$ & $\mathrm{~s}$ & ICRP-68 & $2.3 \mathrm{E}-09$ & -- & -- \\
\hline Ag-109m & Ag-109 & 108.905 & Silver & 0.01 & 0.4 & $50-100$ & ICRP-38 & $3.96 \mathrm{E}+01$ & $\mathrm{~s}$ & $3.96 \mathrm{E}+01$ & $2.62 E+09$ & -- & -- & -- & -- & -- & -- & -- \\
\hline Ag-110 & Ag-110 & 109.906 & Silver & 0.01 & 0.4 & $50-100$ & ICRP-38 & $2.46 \mathrm{E}+01$ & $\mathrm{~s}$ & $2.46 \mathrm{E}+01$ & $4.17 E+09$ & -- & -- & -- & -- & --- & -- & -- \\
\hline $\mathrm{Ag}-110 \mathrm{~m}$ & Ag-110 & 109.906 & Silver & 0.01 & 0.4 & $50-100$ & ICRP-38 & $2.50 \mathrm{E}+02$ & $d$ & $2.16 \mathrm{E}+07$ & $4.75 \mathrm{E}+03$ & ICRP-68 & $1.2 \mathrm{E}-08$ & $\mathrm{~s}$ & $\begin{array}{l}\text { ICRP-68 } \\
\end{array}$ & $2.8 \mathrm{E}-09$ & -- & -- \\
\hline Ag-111 & Ag-111 & 110.905 & Silver & 0.01 & 0.4 & $50-100$ & ICRP-38 & $7.45 \mathrm{E}+00$ & d & $6.44 \mathrm{E}+05$ & $1.58 \mathrm{E}+05$ & ICRP-68 & $1.7 \mathrm{E}-09$ & $\mathrm{~s}$ & ICRP-68 & $1.3 \mathrm{E}-09$ & -- & -- \\
\hline $\mathrm{Ag}-111 \mathrm{~m}$ & Ag-111 & 110.905 & Silver & 0.01 & 0.4 & $50-100$ & ICRP-107 & $6.48 \mathrm{E}+01$ & $\mathrm{~s}$ & $6.48 \mathrm{E}+01$ & $1.57 E+09$ & -- & -- & -- & -- & -- & -- & -- \\
\hline Ag-112 & Ag-112 & 111.907 & Silver & 0.01 & 0.4 & $50-100$ & ICRP-38 & $3.12 \mathrm{E}+00$ & $\mathrm{~h}$ & $1.12 \mathrm{E}+04$ & $8.98 \mathrm{E}+06$ & ICRP-68 & $2.6 \mathrm{E}-10$ & $\mathrm{~s}$ & ICRP-68 & $4.3 \mathrm{E}-10$ & -- & -- \\
\hline Ag-113 & Ag-113 & 112.907 & Silver & 0.01 & 0.4 & $50-100$ & ICRP-107 & $5.37 \mathrm{E}+00$ & $\mathrm{~h}$ & $1.93 \mathrm{E}+04$ & $5.17 \mathrm{E}+06$ & JAERI & $2.5 \mathrm{E}-10$ & $\mathrm{~s}$ & JAERI & $4.1 \mathrm{E}-10$ & -- & -- \\
\hline Ag-113m & Ag-113 & 112.907 & Silver & 0.01 & 0.4 & $50-100$ & ICRP-107 & $6.87 \mathrm{E}+01$ & $\mathrm{~s}$ & $6.87 E+01$ & $1.45 \mathrm{E}+09$ & $\begin{array}{l}-- \\
\end{array}$ & -- & -- & $\begin{array}{ll}- \\
\end{array}$ & -- & -- & -- \\
\hline Ag-114 & Ag-114 & 113.909 & Silver & 0.01 & 0.4 & $50-100$ & ICRP-107 & $4.60 E+00$ & $\mathrm{~s}$ & $4.60 E+00$ & $2.15 \mathrm{E}+10$ & -- & -- & -- & -- & -- & -- & -- \\
\hline $\mathrm{Ag}-115$ & Ag-115 & 114.909 & Silver & 0.01 & 0.4 & $50-100$ & ICRP-38 & $2.00 \mathrm{E}+01$ & $\mathrm{~m}$ & $1.20 \mathrm{E}+03$ & $8.18 \mathrm{E}+07$ & ICRP-68 & $4.4 \mathrm{E}-11$ & $\mathrm{~s}$ & ICRP-68 & $6.0 \mathrm{E}-11$ & -- & -- \\
\hline Ag-116 & Ag-116 & 115.911 & Silver & 0.01 & 0.4 & $50-100$ & ICRP-107 & $2.68 \mathrm{E}+00$ & $\mathrm{~m}$ & $1.61 E+02$ & $6.05 \mathrm{E}+08$ & -- & -- & -- & -- & -- & -- & -- \\
\hline $\mathrm{Ag}-117$ & Ag-117 & 116.912 & Silver & 0.01 & 0.4 & $50-100$ & ICRP-107 & $7.36 \mathrm{E}+01$ & $\mathrm{~s}$ & $7.36 \mathrm{E}+01$ & $1.31 \mathrm{E}+09$ & -- & -- & -- & -- & -- & -- & -- \\
\hline Ag-99 & Ag-99 & 98.918 & Silver & 0.01 & 0.4 & $50-100$ & ICRP-107 & $1.24 \mathrm{E}+02$ & $\mathrm{~s}$ & $1.24 \mathrm{E}+02$ & $9.20 \mathrm{E}+08$ & -- & -- & -- & -- & -- & -- & -- \\
\hline Al-26 & Al-26 & 25.987 & Aluminum & 0.01 & 0.004 & 0 & ICRP-38 & $7.16 \mathrm{E}+05$ & $y$ & $2.26 \mathrm{E}+13$ & $1.92 \mathrm{E}-02$ & ICRP-68 & $1.8 \mathrm{E}-08$ & $M$ & ICRP-68 & $3.5 \mathrm{E}-09$ & -- & -- \\
\hline Al-28 & Al-28 & 27.982 & Aluminum & 0.01 & 0.004 & 0 & ICRP-38 & $2.24 \mathrm{E}+00$ & $\mathrm{~m}$ & $1.34 \mathrm{E}+02$ & $3.00 \mathrm{E}+09$ & -- & -- & -- & -- & -- & -- & -- \\
\hline Al-29 & Al-29 & 28.980 & Aluminum & 0.01 & 0.004 & 0 & ICRP-107 & $6.56 \mathrm{E}+00$ & $m$ & $3.94 \mathrm{E}+02$ & $9.89 \mathrm{E}+08$ & -- & -- & -- & -- & -- & -- & -- \\
\hline Am-237 & Am-237 & 237.050 & Americium & 0.001 & 0.0055 & 1000 & ICRP-38 & $7.30 \mathrm{E}+01$ & $\mathrm{~m}$ & $4.38 \mathrm{E}+03$ & $1.09 \mathrm{E}+07$ & ICRP-68 & $3.6 \mathrm{E}-11$ & $M$ & ICRP-68 & $1.8 \mathrm{E}-11$ & -- & -- \\
\hline Am-238 & Am-238 & 238.052 & Americium & 0.001 & 0.0055 & 1000 & ICRP-38 & $9.80 \mathrm{E}+01$ & $\mathrm{~m}$ & $5.88 \mathrm{E}+03$ & $8.06 \mathrm{E}+06$ & ICRP-68 & $8.5 \mathrm{E}-11$ & $M$ & ICRP-68 & $3.2 \mathrm{E}-11$ & -- & -- \\
\hline Am-239 & Am-239 & 239.053 & Americium & 0.001 & 0.0055 & 1000 & ICRP-38 & $1.19 \mathrm{E}+01$ & $\mathrm{~h}$ & $4.28 \mathrm{E}+04$ & $1.10 \mathrm{E}+06$ & ICRP-68 & $2.9 \mathrm{E}-10$ & $M$ & ICRP-68 & $2.4 \mathrm{E}-10$ & -- & -- \\
\hline Am-240 & Am-240 & 240.055 & Americium & 0.001 & 0.0055 & 1000 & ICRP-38 & $5.08 \mathrm{E}+01$ & $\mathrm{~h}$ & $1.83 \mathrm{E}+05$ & $2.57 \mathrm{E}+05$ & ICRP-68 & $5.9 \mathrm{E}-10$ & $M$ & ICRP-68 & $5.8 \mathrm{E}-10$ & -- & -- \\
\hline
\end{tabular}




\begin{tabular}{|c|c|c|c|c|c|c|c|c|c|c|c|c|c|c|c|c|c|c|}
\hline \multirow{3}{*}{$\begin{array}{l}\text { MASTER } \\
\text { ISOTOPE } \\
\text { LIST } \\
\text { Am-241 } \\
\end{array}$} & \multirow{3}{*}{$\begin{array}{c}\begin{array}{c}\text { Atomic } \\
\text { Mass } \\
\text { Nuclide }\end{array} \\
\text { Am-241 } \\
\end{array}$} & \multirow{3}{*}{$\begin{array}{c}\begin{array}{c}\text { Atomic } \\
\text { Mass }\end{array} \\
241.057 \\
\end{array}$} & \multirow{3}{*}{$\begin{array}{c}\text { Element } \\
\text { Americium }\end{array}$} & & \multicolumn{14}{|c|}{ Table C.1 Radionuclide Specific Input Dat } \\
\hline & & & & \multirow{2}{*}{$\begin{array}{c}\text { EPA } \\
\text { RF } \\
0.001\end{array}$} & $\begin{array}{c}\text { EPA } \\
\text { Conc } \\
\text { Factors } \\
\left(B_{v}\right) \\
\end{array}$ & $\begin{array}{c}\text { EPA } \\
\text { Sorption } \\
\text { Coefficient } \\
\left(K_{d}\right)\end{array}$ & $\begin{array}{c}\text { Half-Life } \\
\text { Reference }\end{array}$ & $\begin{array}{r}\text { Half-Lit } \\
\text { (original u }\end{array}$ & & $\begin{array}{l}\text { Half Life } \\
\text { (s) }\end{array}$ & $\begin{array}{l}\text { Sp. Act } \\
\text { (Ci/g) }\end{array}$ & $\begin{array}{l}\text { Inhalation } \\
\text { DC } \\
\text { Reference }\end{array}$ & $\begin{array}{c}\text { HC-3 } \\
\text { Bounding } \\
\text { Inhalation } \\
\text { DC } \\
\text { (Sv/Bq) } \\
\end{array}$ & $\begin{array}{c}\text { Lung } \\
\text { Absorption } \\
\text { Class/Type }\end{array}$ & $\begin{array}{l}\text { Ingestion } \\
\text { DC } \\
\text { Reference }\end{array}$ & $\begin{array}{c}\text { HC-3 } \\
\text { Bounding } \\
\text { Ingestion } \\
\text { DC } \\
\text { (Sv/Bq) } \\
\end{array}$ & $\begin{array}{c}\text { Immersion } \\
\text { DC } \\
\text { Reference }\end{array}$ & $\begin{array}{c}\mathrm{HC}-3 \\
\text { Immersion } \\
\mathrm{DC}(\mathrm{Sv} / \mathrm{d} \\
\left.\operatorname{per} \mathrm{Bq} / \mathrm{m}^{3}\right)\end{array}$ \\
\hline & & & & & 0.0055 & 1000 & ICRP-38 & $4.32 \mathrm{E}+02$ & $\mathrm{y}$ & $1.36 \mathrm{E}+10$ & $3.43 \mathrm{E}+00$ & ICRP-68 & $3.9 \mathrm{E}-05$ & $\mathrm{M}$ & ICRP-68 & $2.0 \mathrm{E}-07$ & -- & -- \\
\hline Am-242 & Am-242 & 242.060 & Americium & 0.001 & 0.0055 & 1000 & ICRP-38 & $1.60 \mathrm{E}+01$ & $\mathrm{~h}$ & $5.77 \mathrm{E}+04$ & $8.08 \mathrm{E}+05$ & ICRP-68 & $1.6 \mathrm{E}-08$ & $\mathrm{M}$ & ICRP-68 & $3.0 \mathrm{E}-10$ & -- & -- \\
\hline$A m-242 m$ & Am-242 & 242.060 & Americium & 0.001 & 0.0055 & 1000 & ICRP-38 & $1.52 \mathrm{E}+02$ & $\mathrm{y}$ & $4.80 \mathrm{E}+09$ & $9.72 \mathrm{E}+00$ & ICRP-68 & $3.5 \mathrm{E}-05$ & $\mathrm{M}$ & ICRP-68 & $1.9 \mathrm{E}-07$ & -- & -- \\
\hline $\mathrm{Am}-243$ & $\mathrm{Am}-243$ & 243.061 & Americium & 0.001 & 0.0055 & 1000 & ICRP-38 & $7.38 \mathrm{E}+03$ & $\mathrm{y}$ & $2.33 \mathrm{E}+11$ & $1.99 \mathrm{E}-01$ & ICRP-68 & $3.9 \mathrm{E}-05$ & $\mathrm{M}$ & ICRP-68 & $2.0 \mathrm{E}-07$ & -- & -- \\
\hline Am-244 & Am-244 & 244.064 & Americium & 0.001 & 0.0055 & 1000 & ICRP-38 & $1.01 \mathrm{E}+01$ & $\mathrm{~h}$ & $3.64 \mathrm{E}+04$ & $1.27 \mathrm{E}+06$ & ICRP-68 & $1.9 \mathrm{E}-09$ & $M$ & ICRP-68 & $4.6 \mathrm{E}-10$ & -- & -- \\
\hline Am-244m & Am-244 & 244.064 & Americium & 0.001 & 0.0055 & 1000 & ICRP-38 & $2.60 \mathrm{E}+01$ & $\mathrm{~m}$ & $1.56 \mathrm{E}+03$ & $2.96 \mathrm{E}+07$ & ICRP-68 & $7.9 \mathrm{E}-11$ & $M$ & ICRP-68 & $2.9 \mathrm{E}-11$ & -- & -- \\
\hline Am-245 & Am-245 & 245.066 & Americium & 0.001 & 0.0055 & 1000 & ICRP-38 & $2.05 \mathrm{E}+00$ & $\mathrm{~h}$ & $7.38 \mathrm{E}+03$ & $6.24 \mathrm{E}+06$ & ICRP-68 & $7.6 \mathrm{E}-11$ & $M$ & ICRP-68 & $6.2 \mathrm{E}-11$ & -- & -- \\
\hline Am-246 & Am-246 & 246.070 & Americium & 0.001 & 0.0055 & 1000 & ICRP-38 & $3.90 \mathrm{E}+01$ & $\mathrm{~m}$ & $2.34 \mathrm{E}+03$ & $1.96 \mathrm{E}+07$ & ICRP-68 & $1.1 \mathrm{E}-10$ & $M$ & ICRP-68 & $5.8 \mathrm{E}-11$ & -- & -- \\
\hline $\mathrm{Am}-246 \mathrm{~m}$ & Am-246 & 246.070 & Americium & 0.001 & 0.0055 & 1000 & ICRP-38 & $2.50 \mathrm{E}+01$ & $\mathrm{~m}$ & $1.50 \mathrm{E}+03$ & $3.06 \mathrm{E}+07$ & ICRP-68 & $3.8 \mathrm{E}-11$ & $M$ & ICRP-68 & $3.4 \mathrm{E}-11$ & -- & -- \\
\hline Am-247 & Am-247 & 247.072 & Americium & 0.001 & 0.0055 & 1000 & ICRP-107 & $2.30 \mathrm{E}+01$ & $\mathrm{~m}$ & $1.38 \mathrm{E}+03$ & $3.31 \mathrm{E}+07$ & JAERI & $4.4 \mathrm{E}-11$ & $M$ & JAERI & $3.1 \mathrm{E}-11$ & -- & -- \\
\hline Ar-37 & Ar-37 & 36.967 & Argon & 1 & 0 & 0 & ICRP-38 & $3.50 \mathrm{E}+01$ & $d$ & $3.03 \mathrm{E}+06$ & $1.01 \mathrm{E}+05$ & -- & -- & -- & -- & -- & ICRP-68 & $4.10 \mathrm{E}-15$ \\
\hline Ar-39 & Ar-39 & 38.964 & Argon & 1 & 0 & 0 & ICRP-38 & $2.69 \mathrm{E}+02$ & $y$ & $8.49 \mathrm{E}+09$ & $3.41 \mathrm{E}+01$ & -- & -- & -- & -- & -- & ICRP-68 & 1.10E-11 \\
\hline Ar-41 & Ar-41 & 40.965 & Argon & 1 & 0 & 0 & ICRP-38 & $1.83 \mathrm{E}+00$ & $\mathrm{~h}$ & $6.58 \mathrm{E}+03$ & $4.19 \mathrm{E}+07$ & -- & -- & -- & -- & -- & ICRP-68 & $5.30 \mathrm{E}-09$ \\
\hline Ar-42 & Ar-42 & 41.963 & Argon & 1 & 0 & 0 & ICRP-107 & $3.29 \mathrm{E}+01$ & $y$ & $1.04 \mathrm{E}+09$ & $2.59 \mathrm{E}+02$ & -- & -- & -- & -- & -- & JAERI & $1.30 \mathrm{E}-11$ \\
\hline Ar-43 & Ar-43 & 42.966 & Argon & 1 & 0 & 0 & ICRP-107 & $5.37 \mathrm{E}+00$ & $\mathrm{~m}$ & $3.22 \mathrm{E}+02$ & $8.15 \mathrm{E}+08$ & -- & -- & -- & -- & -- & -- & -- \\
\hline Ar-44 & Ar-44 & 43.965 & Argon & 1 & 0 & 0 & ICRP-107 & $1.19 \mathrm{E}+01$ & $\mathrm{~m}$ & $7.12 \mathrm{E}+02$ & $3.60 \mathrm{E}+08$ & -- & -- & -- & -- & -- & JAERI & $8.10 \mathrm{E}-09$ \\
\hline As-68 & As-68 & 67.937 & Arsenic & 0.01 & 0.04 & 0 & ICRP-107 & $1.52 \mathrm{E}+02$ & $\mathrm{~s}$ & $1.52 \mathrm{E}+02$ & $1.10 \mathrm{E}+09$ & -- & -- & -- & -- & -- & -- & -- \\
\hline As-69 & As-69 & 68.932 & Arsenic & 0.01 & 0.04 & 0 & ICRP-38 & $1.52 \mathrm{E}+01$ & $\mathrm{~m}$ & $9.12 \mathrm{E}+02$ & $1.79 \mathrm{E}+08$ & ICRP-68 & $3.5 \mathrm{E}-11$ & $M$ & ICRP-68 & $5.7 \mathrm{E}-11$ & -- & -- \\
\hline As-70 & As-70 & 69.931 & Arsenic & 0.01 & 0.04 & 0 & ICRP-38 & $5.26 \mathrm{E}+01$ & $\mathrm{~m}$ & $3.16 \mathrm{E}+03$ & $5.11 \mathrm{E}+07$ & ICRP-68 & $1.2 \mathrm{E}-10$ & $M$ & ICRP-68 & $1.3 \mathrm{E}-10$ & -- & -- \\
\hline As-71 & As-71 & 70.927 & Arsenic & 0.01 & 0.04 & 0 & ICRP-38 & $6.48 \mathrm{E}+01$ & $\mathrm{~h}$ & $2.33 \mathrm{E}+05$ & $6.82 \mathrm{E}+05$ & ICRP-68 & $5.0 \mathrm{E}-10$ & $M$ & ICRP-68 & $4.6 \mathrm{E}-10$ & -- & -- \\
\hline As-72 & As-72 & 71.927 & Arsenic & 0.01 & 0.04 & 0 & ICRP-38 & $2.60 \mathrm{E}+01$ & $\mathrm{~h}$ & $9.36 \mathrm{E}+04$ & $1.68 \mathrm{E}+06$ & ICRP-68 & $1.3 \mathrm{E}-09$ & $M$ & ICRP-68 & $1.8 \mathrm{E}-09$ & -- & -- \\
\hline As-73 & As-73 & 72.924 & Arsenic & 0.01 & 0.04 & 0 & ICRP-38 & $8.03 \mathrm{E}+01$ & $d$ & $6.94 \mathrm{E}+06$ & $2.23 \mathrm{E}+04$ & ICRP-68 & $9.3 \mathrm{E}-10$ & $M$ & ICRP-68 & $2.6 \mathrm{E}-10$ & -- & -- \\
\hline As-74 & As-74 & 73.924 & Arsenic & 0.01 & 0.04 & 0 & ICRP-38 & $1.78 \mathrm{E}+01$ & $d$ & $1.53 \mathrm{E}+06$ & $9.95 \mathrm{E}+04$ & ICRP-68 & $2.1 \mathrm{E}-09$ & $M$ & ICRP-68 & $1.3 \mathrm{E}-09$ & -- & -- \\
\hline As-76 & As-76 & 75.922 & Arsenic & 0.01 & 0.04 & 0 & ICRP-38 & $2.63 \mathrm{E}+01$ & $\mathrm{~h}$ & $9.48 \mathrm{E}+04$ & $1.57 \mathrm{E}+06$ & ICRP-68 & $9.2 \mathrm{E}-10$ & $M$ & ICRP-68 & $1.6 \mathrm{E}-09$ & -- & -- \\
\hline As-77 & As-77 & 76.921 & Arsenic & 0.01 & 0.04 & 0 & ICRP-38 & $3.88 \mathrm{E}+01$ & $\mathrm{~h}$ & $1.40 \mathrm{E}+05$ & $1.05 \mathrm{E}+06$ & ICRP-68 & $4.2 \mathrm{E}-10$ & $M$ & ICRP-68 & $4.0 \mathrm{E}-10$ & -- & -- \\
\hline As-78 & As-78 & 77.922 & Arsenic & 0.01 & 0.04 & 0 & ICRP-38 & $9.07 E+01$ & $\mathrm{~m}$ & $5.44 \mathrm{E}+03$ & $2.66 \mathrm{E}+07$ & ICRP-68 & $1.4 \mathrm{E}-10$ & $M$ & ICRP-68 & $2.1 \mathrm{E}-10$ & -- & -- \\
\hline As-79 & As-79 & 78.921 & Arsenic & 0.01 & 0.04 & 0 & ICRP-107 & $9.01 \mathrm{E}+00$ & $\mathrm{~m}$ & $5.41 \mathrm{E}+02$ & $2.64 \mathrm{E}+08$ & -- & -- & -- & -- & -- & -- & -- \\
\hline At-204 & At-204 & 203.987 & Astatine & 0.001 & 1 & 0 & ICRP-107 & $9.20 \mathrm{E}+00$ & $\mathrm{~m}$ & $5.52 \mathrm{E}+02$ & $1.00 \mathrm{E}+08$ & -- & -- & -- & -- & -- & -- & -- \\
\hline At-205 & At-205 & 204.986 & Astatine & 0.001 & 1 & 0 & ICRP-107 & $2.62 \mathrm{E}+01$ & $\mathrm{~m}$ & $1.57 \mathrm{E}+03$ & $3.50 \mathrm{E}+07$ & JAERI & $6.7 \mathrm{E}-10$ & $\mathrm{M}$ & JAERI & $7.7 \mathrm{E}-11$ & -- & -- \\
\hline At-206 & At-206 & 205.987 & Astatine & 0.001 & 1 & 0 & ICRP-107 & $3.06 \mathrm{E}+01$ & $\mathrm{~m}$ & $1.84 \mathrm{E}+03$ & $2.98 \mathrm{E}+07$ & -- & -- & -- & -- & -- & -- & -- \\
\hline At-207 & At-207 & 206.986 & Astatine & 0.001 & 1 & 0 & ICRP-38 & $1.80 \mathrm{E}+00$ & $\mathrm{~h}$ & $6.48 \mathrm{E}+03$ & $8.41 \mathrm{E}+06$ & ICRP-68 & $2.1 \mathrm{E}-09$ & $M$ & ICRP-68 & $2.3 \mathrm{E}-10$ & -- & -- \\
\hline At-208 & At-208 & 207.987 & Astatine & 0.001 & 1 & 0 & ICRP-107 & $1.63 \mathrm{E}+00$ & $\mathrm{~h}$ & $5.87 \mathrm{E}+03$ & $9.24 \mathrm{E}+06$ & JAERI & $3.8 \mathrm{E}-10$ & $M$ & JAERI & $9.4 \mathrm{E}-11$ & -- & -- \\
\hline At-209 & At-209 & 208.986 & Astatine & 0.001 & 1 & 0 & ICRP-107 & $5.41 \mathrm{E}+00$ & $\mathrm{~h}$ & $1.95 \mathrm{E}+04$ & $2.77 E+06$ & JAERI & $2.8 \mathrm{E}-09$ & $M$ & JAERI & $1.9 \mathrm{E}-09$ & -- & -- \\
\hline At-210 & At-210 & 209.987 & Astatine & 0.001 & 1 & 0 & ICRP-107 & $8.10 \mathrm{E}+00$ & $\mathrm{~h}$ & $2.92 \mathrm{E}+04$ & $1.84 \mathrm{E}+06$ & JAERI & $9.8 \mathrm{E}-09$ & $M$ & JAERI & $9.8 \mathrm{E}-09$ & -- & -- \\
\hline At-211 & At-211 & 210.987 & Astatine & 0.001 & 1 & 0 & ICRP-38 & $7.21 \mathrm{E}+00$ & $\mathrm{~h}$ & $2.60 \mathrm{E}+04$ & $2.06 \mathrm{E}+06$ & ICRP-68 & $1.1 \mathrm{E}-07$ & $M$ & ICRP-68 & $1.1 \mathrm{E}-08$ & -- & -- \\
\hline At-215 & At-215 & 214.999 & Astatine & 0.001 & 1 & 0 & ICRP-38 & $1.00 \mathrm{E}-01$ & $\mathrm{~ms}$ & $1.00 \mathrm{E}-04$ & $5.25 \mathrm{E}+14$ & -- & -- & -- & -- & -- & -- & -- \\
\hline At-216 & At-216 & 216.002 & Astatine & 0.001 & 1 & 0 & ICRP-38 & $3.00 \mathrm{E}-01$ & $\mathrm{~ms}$ & $3.00 \mathrm{E}-04$ & $1.74 \mathrm{E}+14$ & -- & -- & -- & -- & -- & -- & -- \\
\hline At-217 & At-217 & 217.005 & Astatine & 0.001 & 1 & 0 & ICRP-38 & $3.23 \mathrm{E}-02$ & $\mathrm{~s}$ & $3.23 \mathrm{E}-02$ & $1.61 \mathrm{E}+12$ & -- & -- & -- & -- & -- & -- & -- \\
\hline At-218 & At-218 & 218.009 & Astatine & 0.001 & 1 & 0 & ICRP-38 & $2.00 \mathrm{E}+00$ & $\mathrm{~s}$ & $2.00 \mathrm{E}+00$ & $2.59 \mathrm{E}+10$ & -- & -- & -- & -- & -- & -- & -- \\
\hline At-219 & At-219 & 219.011 & Astatine & 0.001 & 1 & 0 & ICRP-107 & $5.60 \mathrm{E}+01$ & $\mathrm{~s}$ & $5.60 \mathrm{E}+01$ & $9.20 \mathrm{E}+08$ & -- & -- & -- & -- & -- & -- & -- \\
\hline At-220 & At-220 & 220.015 & Astatine & 0.001 & 1 & 0 & ICRP-107 & $3.71 \mathrm{E}+00$ & $\mathrm{~m}$ & $2.23 \mathrm{E}+02$ & $2.30 \mathrm{E}+08$ & -- & -- & -- & -- & -- & -- & -- \\
\hline $\mathrm{Au}-186$ & Au-186 & 185.966 & Gold & 0.01 & 0.4 & 0 & ICRP-107 & $1.07 \mathrm{E}+01$ & $\mathrm{~m}$ & $6.42 \mathrm{E}+02$ & $9.45 \mathrm{E}+07$ & JAERI & $3.5 \mathrm{E}-11$ & $\mathrm{~s}$ & JAERI & $4.6 \mathrm{E}-11$ & -- & -- \\
\hline Au-187 & Au-187 & 186.965 & Gold & 0.01 & 0.4 & 0 & ICRP-107 & $8.40 \mathrm{E}+00$ & $\mathrm{~m}$ & $5.04 \mathrm{E}+02$ & $1.20 \mathrm{E}+08$ & -- & -- & -- & -- & -- & -- & -- \\
\hline $\mathrm{Au}-188$ & Au-188 & 187.965 & Gold & 0.01 & 0.4 & 0 & JAERI & $8.84 \mathrm{E}+00$ & $\mathrm{~m}$ & $5.30 \mathrm{E}+02$ & $1.13 \mathrm{E}+08$ & -- & -- & -- & -- & -- & -- & -- \\
\hline Au-189m & Au-189 & 188.964 & Gold & 0.01 & 0.4 & 0 & JAERI & $4.59 \mathrm{E}+00$ & $\mathrm{~m}$ & $2.75 \mathrm{E}+02$ & $2.17 E+08$ & -- & -- & -- & -- & -- & -- & -- \\
\hline
\end{tabular}




\begin{tabular}{|c|c|c|c|c|c|c|c|c|c|c|c|c|c|c|c|c|c|c|}
\hline \multirow{3}{*}{$\begin{array}{l}\text { MASTER } \\
\text { ISOTOPE } \\
\text { LIST } \\
\text { Au-190 } \\
\end{array}$} & \multirow{3}{*}{$\begin{array}{c}\begin{array}{c}\text { Atomic } \\
\text { Mass } \\
\text { Nuclide }\end{array} \\
\text { Au-190 }\end{array}$} & \multirow{3}{*}{$\begin{array}{c}\begin{array}{c}\text { Atomic } \\
\text { Mass }\end{array} \\
189.965\end{array}$} & & & \multicolumn{14}{|c|}{ Table C.1 Radionuclide Spe } \\
\hline & & & \multirow{2}{*}{$\begin{array}{c}\text { Element } \\
\text { Gold }\end{array}$} & \multirow{2}{*}{$\begin{array}{c}\text { EPA } \\
\text { RF } \\
0.01\end{array}$} & $\begin{array}{c}\text { EPA } \\
\text { Conc } \\
\text { Factors } \\
\left(B_{v}\right) \\
\end{array}$ & $\begin{array}{c}\text { EPA } \\
\text { Sorption } \\
\text { Coefficient } \\
\left(K_{d}\right)\end{array}$ & $\begin{array}{c}\text { Half-Life } \\
\text { Reference }\end{array}$ & $\begin{array}{r}\text { Half-Lif } \\
\text { (original u }\end{array}$ & & $\begin{array}{l}\text { Half Life } \\
\text { (s) }\end{array}$ & $\begin{array}{l}\text { Sp. Act } \\
\text { (Ci/g) }\end{array}$ & $\begin{array}{c}\text { Inhalation } \\
\text { DC } \\
\text { Reference }\end{array}$ & $\begin{array}{c}\text { HC-3 } \\
\text { Bounding } \\
\text { Inhalation } \\
\text { DC } \\
\text { (Sv/Bq) } \\
\end{array}$ & $\begin{array}{c}\text { Lung } \\
\text { Absorption } \\
\text { Class/Type }\end{array}$ & $\begin{array}{l}\text { Ingestion } \\
\text { DC } \\
\text { Reference }\end{array}$ & $\begin{array}{c}\text { HC-3 } \\
\text { Bounding } \\
\text { Ingestion } \\
\text { DC } \\
\text { (Sv/Bq) } \\
\end{array}$ & $\begin{array}{c}\text { Immersion } \\
\text { DC } \\
\text { Reference }\end{array}$ & $\begin{array}{c}\mathrm{HC}-3 \\
\text { Immersion } \\
\mathrm{DC}(\mathrm{Sv} / \mathrm{d} \\
\left.\operatorname{per} \mathrm{Bq} / \mathrm{m}^{3}\right)\end{array}$ \\
\hline & & & & & 0.4 & 0 & ICRP-107 & $4.28 \mathrm{E}+01$ & $\mathrm{~m}$ & $2.57 \mathrm{E}+03$ & $2.31 \mathrm{E}+07$ & JAERI & $4.6 \mathrm{E}-11$ & $\mathrm{~s}$ & JAERI & $4.7 \mathrm{E}-11$ & -- & -- \\
\hline $\mathrm{Au}-191$ & Au-191 & 190.964 & Gold & 0.01 & 0.4 & 0 & ICRP-107 & $3.18 \mathrm{E}+00$ & $\mathrm{~h}$ & $1.14 \mathrm{E}+04$ & $5.16 \mathrm{E}+06$ & JAERI & $9.7 \mathrm{E}-11$ & $\mathrm{~s}$ & JAERI & $7.7 \mathrm{E}-11$ & -- & -- \\
\hline $\mathrm{Au}-192$ & Au-192 & 191.965 & Gold & 0.01 & 0.4 & 0 & ICRP-107 & $4.94 \mathrm{E}+00$ & $\mathrm{~h}$ & $1.78 \mathrm{E}+04$ & $3.30 \mathrm{E}+06$ & JAERI & $1.7 \mathrm{E}-10$ & $\mathrm{M}$ & JAERI & $1.8 \mathrm{E}-10$ & -- & -- \\
\hline Au-193 & $\mathrm{Au}-193$ & 192.964 & Gold & 0.01 & 0.4 & 0 & ICRP-38 & $1.77 \mathrm{E}+01$ & $\mathrm{~h}$ & $6.35 \mathrm{E}+04$ & $9.20 \mathrm{E}+05$ & ICRP-68 & $1.6 \mathrm{E}-10$ & $\mathrm{~s}$ & ICRP-68 & $1.3 \mathrm{E}-10$ & -- & -- \\
\hline $\mathrm{Au}-193 \mathrm{~m}$ & $\mathrm{Au}-193$ & 192.964 & Gold & 0.01 & 0.4 & 0 & ICRP-107 & $3.90 \mathrm{E}+00$ & $\mathrm{~s}$ & $3.90 \mathrm{E}+00$ & $1.50 \mathrm{E}+10$ & -- & -- & -- & -- & -- & -- & -- \\
\hline Au-194 & $\mathrm{Au}-194$ & 193.965 & Gold & 0.01 & 0.4 & 0 & ICRP-38 & $3.95 \mathrm{E}+01$ & $\mathrm{~h}$ & $1.42 \mathrm{E}+05$ & $4.09 \mathrm{E}+05$ & ICRP-68 & $3.8 \mathrm{E}-10$ & $\mathrm{~s}$ & ICRP-68 & $4.2 \mathrm{E}-10$ & -- & -- \\
\hline $\mathrm{Au}-195$ & $\mathrm{Au}-195$ & 194.965 & Gold & 0.01 & 0.4 & 0 & ICRP-38 & $1.83 \mathrm{E}+02$ & $\mathrm{~d}$ & $1.58 \mathrm{E}+07$ & $3.66 \mathrm{E}+03$ & ICRP-68 & $1.6 \mathrm{E}-09$ & $\mathrm{~s}$ & ICRP-68 & $2.5 \mathrm{E}-10$ & -- & -- \\
\hline Au-195m & $\mathrm{Au}-195$ & 194.965 & Gold & 0.01 & 0.4 & 0 & ICRP-38 & $3.05 \mathrm{E}+01$ & $\mathrm{~s}$ & $3.05 \mathrm{E}+01$ & $1.90 \mathrm{E}+09$ & -- & -- & -- & -- & -- & -- & -- \\
\hline Au-196 & $\mathrm{Au}-196$ & 195.967 & Gold & 0.01 & 0.4 & 0 & ICRP-107 & $6.18 \mathrm{E}+00$ & $d$ & $5.34 \mathrm{E}+05$ & $1.08 \mathrm{E}+05$ & JAERI & $7.3 \mathrm{E}-10$ & $\mathrm{~s}$ & JAERI & $5.3 \mathrm{E}-10$ & -- & -- \\
\hline Au-196m & Au-196 & 195.967 & Gold & 0.01 & 0.4 & 0 & ICRP-107 & $9.60 \mathrm{E}+00$ & $\mathrm{~h}$ & $3.46 \mathrm{E}+04$ & $1.67 \mathrm{E}+06$ & JAERI & $6.1 \mathrm{E}-10$ & $\mathrm{~s}$ & JAERI & $4.1 \mathrm{E}-10$ & -- & -- \\
\hline Au-198 & Au-198 & 197.968 & Gold & 0.01 & 0.4 & 0 & ICRP-38 & $2.70 \mathrm{E}+00$ & $d$ & $2.33 \mathrm{E}+05$ & $2.45 \mathrm{E}+05$ & ICRP-68 & $1.1 \mathrm{E}-09$ & $\mathrm{~s}$ & ICRP-68 & $1.0 \mathrm{E}-09$ & -- & -- \\
\hline Au-198m & $\mathrm{Au}-198$ & 197.968 & Gold & 0.01 & 0.4 & 0 & ICRP-38 & $2.30 \mathrm{E}+00$ & $d$ & $1.99 \mathrm{E}+05$ & $2.87 \mathrm{E}+05$ & ICRP-68 & $2.0 \mathrm{E}-09$ & $M$ & ICRP-68 & $1.3 \mathrm{E}-09$ & -- & -- \\
\hline Au-199 & Au-199 & 198.969 & Gold & 0.01 & 0.4 & 0 & ICRP-38 & $3.14 \mathrm{E}+00$ & $d$ & $2.71 \mathrm{E}+05$ & $2.09 \mathrm{E}+05$ & $\begin{array}{l}\text { ICRP-68 } \\
\end{array}$ & $7.6 \mathrm{E}-10$ & $\mathrm{~s}$ & ICRP-68 & $4.4 \mathrm{E}-10$ & -- & -- \\
\hline $\mathrm{Au}-200$ & $\mathrm{Au}-200$ & 199.971 & Gold & 0.01 & 0.4 & 0 & ICRP-38 & $4.84 \mathrm{E}+01$ & $\mathrm{~m}$ & $2.90 \mathrm{E}+03$ & $1.94 \mathrm{E}+07$ & ICRP-68 & $5.6 \mathrm{E}-11$ & $\mathrm{~s}$ & ICRP-68 & $6.8 \mathrm{E}-11$ & -- & -- \\
\hline Au-200m & $\mathrm{Au}-200$ & 199.971 & Gold & 0.01 & 0.4 & 0 & ICRP-38 & $1.87 \mathrm{E}+01$ & $\mathrm{~h}$ & $6.73 \mathrm{E}+04$ & $8.38 \mathrm{E}+05$ & ICRP-68 & $1.0 \mathrm{E}-09$ & $\mathrm{~s}$ & ICRP-68 & $1.1 \mathrm{E}-09$ & -- & -- \\
\hline Au-201 & Au-201 & 200.972 & Gold & 0.01 & 0.4 & 0 & ICRP-38 & $2.64 \mathrm{E}+01$ & $\mathrm{~m}$ & $1.58 \mathrm{E}+03$ & $3.54 \mathrm{E}+07$ & ICRP-68 & $2.9 \mathrm{E}-11$ & $\mathrm{~s}$ & ICRP-68 & $2.4 \mathrm{E}-11$ & -- & -- \\
\hline $\mathrm{Au}-202$ & $\mathrm{Au}-202$ & 201.974 & Gold & 0.01 & 0.4 & 0 & ICRP-107 & $2.88 \mathrm{E}+01$ & $\mathrm{~s}$ & $2.88 \mathrm{E}+01$ & $1.94 \mathrm{E}+09$ & -- & -- & -- & -- & -- & -- & -- \\
\hline Ba-124 & Ba-124 & 123.915 & Barium & 0.01 & 0.15 & 100 & ICRP-107 & $1.10 \mathrm{E}+01$ & $\mathrm{~m}$ & $6.60 \mathrm{E}+02$ & $1.38 \mathrm{E}+08$ & JAERI & $2.8 \mathrm{E}-11$ & $F$ & JAERI & $6.9 \mathrm{E}-11$ & -- & -- \\
\hline Ba-126 & Ba-126 & 125.911 & Barium & 0.01 & 0.15 & 100 & ICRP-38 & $9.65 \mathrm{E}+01$ & $\mathrm{~m}$ & $5.79 \mathrm{E}+03$ & $1.55 \mathrm{E}+07$ & ICRP-68 & $1.2 \mathrm{E}-10$ & $\mathrm{~F}$ & ICRP-68 & $2.6 \mathrm{E}-10$ & -- & -- \\
\hline Ba-127 & Ba-127 & 126.911 & Barium & 0.01 & 0.15 & 100 & ICRP-107 & $1.27 \mathrm{E}+01$ & $\mathrm{~m}$ & $7.62 \mathrm{E}+02$ & $1.17 E+08$ & JAERI & $1.4 \mathrm{E}-11$ & $\mathrm{~F}$ & JAERI & $2.5 \mathrm{E}-11$ & -- & -- \\
\hline Ba-128 & Ba-128 & 127.908 & Barium & 0.01 & 0.15 & 100 & ICRP-38 & $2.43 \mathrm{E}+00$ & $d$ & $2.10 E+05$ & $4.20 \mathrm{E}+05$ & ICRP-68 & $1.3 \mathrm{E}-09$ & $\mathrm{~F}$ & ICRP-68 & $2.7 \mathrm{E}-09$ & -- & -- \\
\hline Ba-129 & Ba-129 & 128.909 & Barium & 0.01 & 0.15 & 100 & ICRP-107 & $2.23 \mathrm{E}+00$ & $\mathrm{~h}$ & $8.03 E+03$ & $1.09 \mathrm{E}+07$ & JAERI & $3.6 \mathrm{E}-11$ & $F$ & JAERI & $5.2 \mathrm{E}-11$ & -- & -- \\
\hline Ba-129m & Ba-129 & 128.909 & Barium & 0.01 & 0.15 & 100 & ICRP-107 & $2.16 \mathrm{E}+00$ & $h$ & $7.78 \mathrm{E}+03$ & $1.13 \mathrm{E}+07$ & JAERI & $7.6 \mathrm{E}-11$ & $\mathrm{~F}$ & JAERI & $7.8 \mathrm{E}-11$ & -- & -- \\
\hline Ba-131 & Ba-131 & 130.907 & Barium & 0.01 & 0.15 & 100 & ICRP-38 & $1.18 \mathrm{E}+01$ & $d$ & $1.02 \mathrm{E}+06$ & $8.45 \mathrm{E}+04$ & ICRP-68 & $3.5 \mathrm{E}-10$ & $\mathrm{~F}$ & ICRP-68 & $4.5 \mathrm{E}-10$ & -- & -- \\
\hline Ba-131m & Ba-131 & 130.907 & Barium & 0.01 & 0.15 & 100 & ICRP-38 & $1.46 \mathrm{E}+01$ & $\mathrm{~m}$ & $8.76 \mathrm{E}+02$ & $9.84 \mathrm{E}+07$ & ICRP-68 & $6.4 \mathrm{E}-12$ & $\mathrm{~F}$ & ICRP-68 & $4.9 \mathrm{E}-12$ & -- & -- \\
\hline Ba-133 & Ba-133 & 132.906 & Barium & 0.01 & 0.15 & 100 & ICRP-38 & $1.07 E+01$ & $y$ & $3.39 \mathrm{E}+08$ & $2.50 \mathrm{E}+02$ & ICRP-68 & $1.8 \mathrm{E}-09$ & $\mathrm{~F}$ & ICRP-68 & $1.0 \mathrm{E}-09$ & -- & -- \\
\hline Ba-133m & Ba-133 & 132.906 & Barium & 0.01 & 0.15 & 100 & ICRP-38 & $3.89 \mathrm{E}+01$ & $\mathrm{~h}$ & $1.40 \mathrm{E}+05$ & $6.06 \mathrm{E}+05$ & ICRP-68 & $2.8 \mathrm{E}-10$ & $\mathrm{~F}$ & ICRP-68 & $5.5 \mathrm{E}-10$ & -- & -- \\
\hline Ba-135m & Ba-135 & 134.906 & Barium & 0.01 & 0.15 & 100 & ICRP-38 & $2.87 \mathrm{E}+01$ & $\mathrm{~h}$ & $1.03 \mathrm{E}+05$ & $8.09 \mathrm{E}+05$ & ICRP-68 & $2.3 \mathrm{E}-10$ & $\mathrm{~F}$ & ICRP-68 & $4.5 \mathrm{E}-10$ & -- & -- \\
\hline Ba-137m & Ba-137 & 136.906 & Barium & 0.01 & 0.15 & 100 & ICRP-38 & $2.55 \mathrm{E}+00$ & $\mathrm{~m}$ & $1.53 \mathrm{E}+02$ & $5.38 \mathrm{E}+08$ & -- & -- & -- & -- & -- & -- & -- \\
\hline Ba-139 & Ba-139 & 138.909 & Barium & 0.01 & 0.15 & 100 & ICRP-38 & $8.27 \mathrm{E}+01$ & $\mathrm{~m}$ & $4.96 \mathrm{E}+03$ & $1.64 \mathrm{E}+07$ & ICRP-68 & $5.5 \mathrm{E}-11$ & $F$ & ICRP-68 & $1.2 \mathrm{E}-10$ & -- & -- \\
\hline Ba-140 & Ba-140 & 139.911 & Barium & 0.01 & 0.15 & 100 & ICRP-38 & $1.27 \mathrm{E}+01$ & $d$ & $1.10 \mathrm{E}+06$ & $7.33 \mathrm{E}+04$ & ICRP-68 & $1.6 \mathrm{E}-09$ & $\mathrm{~F}$ & ICRP-68 & $2.5 \mathrm{E}-09$ & -- & -- \\
\hline Ba-141 & Ba-141 & 140.914 & Barium & 0.01 & 0.15 & 100 & ICRP-38 & $1.83 \mathrm{E}+01$ & $\mathrm{~m}$ & $1.10 \mathrm{E}+03$ & $7.30 \mathrm{E}+07$ & ICRP-68 & $3.5 \mathrm{E}-11$ & $F$ & ICRP-68 & $7.0 \mathrm{E}-11$ & -- & -- \\
\hline Ba-142 & Ba-142 & 141.916 & Barium & 0.01 & 0.15 & 100 & ICRP-38 & $1.06 \mathrm{E}+01$ & $\mathrm{~m}$ & $6.36 \mathrm{E}+02$ & $1.25 \mathrm{E}+08$ & ICRP-68 & $2.7 \mathrm{E}-11$ & $\mathrm{~F}$ & ICRP-68 & $3.5 \mathrm{E}-11$ & -- & -- \\
\hline Be-10 & Be-10 & 10.014 & Beryllium & 0.01 & 0.01 & 75 & ICRP-38 & $1.60 \mathrm{E}+06$ & $y$ & $5.05 \mathrm{E}+13$ & $2.23 E-02$ & ICRP-68 & $3.2 \mathrm{E}-08$ & $\mathrm{~s}$ & ICRP-68 & $1.1 \mathrm{E}-09$ & -- & -- \\
\hline $\mathrm{Be}-7$ & Be-7 & 7.017 & Beryllium & 0.01 & 0.01 & 75 & ICRP-38 & $5.33 \mathrm{E}+01$ & $d$ & $4.61 \mathrm{E}+06$ & $3.49 \mathrm{E}+05$ & ICRP-68 & $5.2 \mathrm{E}-11$ & $\mathrm{~s}$ & ICRP-68 & $2.8 \mathrm{E}-11$ & -- & -- \\
\hline Bi-197 & Bi-197 & 196.979 & Bismuth & 0.01 & 0.035 & 10 & ICRP-107 & $9.30 \mathrm{E}+00$ & $\mathrm{~m}$ & $5.58 \mathrm{E}+02$ & $1.03 \mathrm{E}+08$ & -- & -- & -- & -- & -- & -- & -- \\
\hline Bi-200 & Bi-200 & 199.978 & Bismuth & 0.01 & 0.035 & 10 & ICRP-38 & $3.64 \mathrm{E}+01$ & $\mathrm{~m}$ & $2.18 \mathrm{E}+03$ & $2.58 \mathrm{E}+07$ & ICRP-68 & $5.6 \mathrm{E}-11$ & $M$ & ICRP-68 & $5.1 \mathrm{E}-11$ & -- & -- \\
\hline Bi-201 & Bi-201 & 200.977 & Bismuth & 0.01 & 0.035 & 10 & ICRP-38 & $1.08 \mathrm{E}+02$ & $\mathrm{~m}$ & $6.48 \mathrm{E}+03$ & $8.66 \mathrm{E}+06$ & ICRP-68 & $1.1 \mathrm{E}-10$ & $\mathrm{M}$ & ICRP-68 & $1.2 \mathrm{E}-10$ & -- & -- \\
\hline Bi-202 & $\mathrm{Bi}-202$ & 201.978 & Bismuth & 0.01 & 0.035 & 10 & ICRP-38 & $1.67 \mathrm{E}+00$ & $\mathrm{~h}$ & $6.01 \mathrm{E}+03$ & $9.29 \mathrm{E}+06$ & ICRP-68 & $1.0 \mathrm{E}-10$ & $M$ & ICRP-68 & $8.9 \mathrm{E}-11$ & -- & -- \\
\hline Bi-203 & Bi-203 & 202.977 & Bismuth & 0.01 & 0.035 & 10 & ICRP-38 & $1.18 \mathrm{E}+01$ & $\mathrm{~h}$ & $4.23 \mathrm{E}+04$ & $1.31 \mathrm{E}+06$ & ICRP-68 & $4.5 \mathrm{E}-10$ & $M$ & ICRP-68 & $4.8 \mathrm{E}-10$ & -- & -- \\
\hline Bi-204 & Bi-204 & 203.978 & Bismuth & 0.01 & 0.035 & 10 & ICRP-38 & $1.12 \mathrm{E}+01$ & $\mathrm{~h}$ & $4.04 \mathrm{E}+04$ & $1.37 \mathrm{E}+06$ & JAERI & $5.8 \mathrm{E}-10$ & $\mathrm{M}$ & JAERI & $6.1 \mathrm{E}-10$ & -- & -- \\
\hline Bi-205 & Bi-205 & 204.977 & Bismuth & 0.01 & 0.035 & 10 & ICRP-38 & $1.53 \mathrm{E}+01$ & $d$ & $1.32 \mathrm{E}+06$ & $4.16 \mathrm{E}+04$ & ICRP-68 & $1.0 \mathrm{E}-09$ & $\mathrm{M}$ & ICRP-68 & $9.0 \mathrm{E}-10$ & -- & -- \\
\hline Bi-206 & Bi-206 & 205.978 & Bismuth & 0.01 & 0.035 & 10 & ICRP-38 & $6.24 \mathrm{E}+00$ & $d$ & $5.39 \mathrm{E}+05$ & $1.02 \mathrm{E}+05$ & ICRP-68 & $2.1 \mathrm{E}-09$ & $M$ & ICRP-68 & $1.9 \mathrm{E}-09$ & -- & -- \\
\hline Bi-207 & Bi-207 & 206.978 & Bismuth & 0.01 & 0.035 & 10 & ICRP-38 & $3.80 \mathrm{E}+01$ & $y$ & $1.20 \mathrm{E}+09$ & $4.55 \mathrm{E}+01$ & ICRP-68 & $5.2 \mathrm{E}-09$ & $M$ & ICRP-68 & $1.3 \mathrm{E}-09$ & -- & -- \\
\hline Bi-208 & Bi-208 & 207.980 & Bismuth & 0.01 & 0.035 & 10 & ICRP-107 & $3.68 \mathrm{E}+05$ & $y$ & $1.16 \mathrm{E}+13$ & $4.67 \mathrm{E}-03$ & JAERI & $4.4 \mathrm{E}-09$ & $M$ & JAERI & $1.2 \mathrm{E}-09$ & -- & -- \\
\hline
\end{tabular}




\begin{tabular}{|c|c|c|c|c|c|c|c|c|c|c|c|c|c|c|c|c|c|c|}
\hline \multirow{3}{*}{$\begin{array}{l}\text { MASTER } \\
\text { ISOTOPE } \\
\text { LIST } \\
\text { Bi-210 }\end{array}$} & \multirow{3}{*}{$\begin{array}{c}\begin{array}{c}\text { Atomic } \\
\text { Mass } \\
\text { Nuclide }\end{array} \\
\text { Bi-210 }\end{array}$} & & & & \multicolumn{14}{|c|}{ Table C.1 Radionuclide Specific Input Data Used For the Calculation of HC-3 TQs Using Maximum Reported DCs (continued) } \\
\hline & & \multirow{2}{*}{$\begin{array}{c}\begin{array}{c}\text { Atomic } \\
\text { Mass }\end{array} \\
209.984\end{array}$} & \multirow{2}{*}{$\begin{array}{l}\text { Element } \\
\text { Bismuth }\end{array}$} & \multirow{2}{*}{$\begin{array}{c}\text { EPA } \\
\text { RF } \\
0.01\end{array}$} & $\begin{array}{c}\text { EPA } \\
\text { Conc } \\
\text { Factors } \\
\left(B_{v}\right) \\
\end{array}$ & $\begin{array}{c}\text { EPA } \\
\text { Sorption } \\
\text { Coefficient } \\
\left(K_{d}\right)\end{array}$ & $\begin{array}{c}\text { Half-Life } \\
\text { Reference }\end{array}$ & $\begin{array}{r}\text { Half-Lit } \\
\text { (original u }\end{array}$ & & $\begin{array}{l}\text { Half Life } \\
\text { (s) }\end{array}$ & $\begin{array}{l}\text { Sp. Act } \\
\text { (Ci/g) }\end{array}$ & $\begin{array}{c}\text { Inhalation } \\
\text { DC } \\
\text { Reference }\end{array}$ & $\begin{array}{c}\text { HC-3 } \\
\text { Bounding } \\
\text { Inhalation } \\
\text { DC } \\
\text { (Sv/Bq) } \\
\end{array}$ & $\begin{array}{c}\text { Lung } \\
\text { Absorption } \\
\text { Class/Type }\end{array}$ & $\begin{array}{l}\text { Ingestion } \\
\text { DC } \\
\text { Reference }\end{array}$ & $\begin{array}{c}\text { HC-3 } \\
\text { Bounding } \\
\text { Ingestion } \\
\text { DC } \\
\text { (Sv/Bq) } \\
\end{array}$ & $\begin{array}{c}\text { Immersion } \\
\text { DC } \\
\text { Reference }\end{array}$ & $\begin{array}{c}\mathrm{HC}-3 \\
\text { Immersion } \\
\mathrm{DC}(\mathrm{Sv} / \mathrm{d} \\
\left.\operatorname{per} \mathrm{Bq} / \mathrm{m}^{3}\right)\end{array}$ \\
\hline & & & & & 0.035 & 10 & ICRP-38 & $5.01 \mathrm{E}+00$ & $\mathrm{~d}$ & $4.33 \mathrm{E}+05$ & $1.24 \mathrm{E}+05$ & ICRP-68 & $8.4 \mathrm{E}-08$ & $\mathrm{M}$ & ICRP-68 & $1.3 \mathrm{E}-09$ & -- & -- \\
\hline Bi-210m & Bi-210 & 209.984 & Bismuth & 0.01 & 0.035 & 10 & ICRP-38 & $3.00 \mathrm{E}+06$ & $\mathrm{y}$ & $9.47 \mathrm{E}+13$ & $5.67 \mathrm{E}-04$ & ICRP-68 & $3.1 \mathrm{E}-06$ & $M$ & ICRP-68 & $1.5 \mathrm{E}-08$ & -- & -- \\
\hline Bi-211 & Bi-211 & 210.987 & Bismuth & 0.01 & 0.035 & 10 & ICRP-38 & $2.14 \mathrm{E}+00$ & $\mathrm{~m}$ & $1.28 \mathrm{E}+02$ & $4.16 \mathrm{E}+08$ & -- & -- & -- & -- & -- & -- & -- \\
\hline Bi-212 & Bi-212 & 211.991 & Bismuth & 0.01 & 0.035 & 10 & ICRP-38 & $6.06 \mathrm{E}+01$ & $\mathrm{~m}$ & $3.63 \mathrm{E}+03$ & $1.46 \mathrm{E}+07$ & ICRP-68 & $3.9 \mathrm{E}-08$ & $M$ & ICRP-68 & $2.6 \mathrm{E}-10$ & -- & -- \\
\hline Bi-212n & Bi-212 & 211.991 & Bismuth & 0.01 & 0.035 & 10 & ICRP-107 & $7.00 \mathrm{E}+00$ & $\mathrm{~m}$ & $4.20 \mathrm{E}+02$ & $1.27 E+08$ & -- & -- & -- & -- & -- & -- & -- \\
\hline Bi-213 & Bi-213 & 212.994 & Bismuth & 0.01 & 0.035 & 10 & ICRP-38 & $4.57 \mathrm{E}+01$ & $\mathrm{~m}$ & $2.74 \mathrm{E}+03$ & $1.93 \mathrm{E}+07$ & ICRP-68 & $4.1 \mathrm{E}-08$ & $\mathrm{M}$ & ICRP-68 & $2.0 \mathrm{E}-10$ & -- & -- \\
\hline Bi-214 & Bi-214 & 213.999 & Bismuth & 0.01 & 0.035 & 10 & ICRP-38 & $1.99 \mathrm{E}+01$ & $\mathrm{~m}$ & $1.19 \mathrm{E}+03$ & $4.42 \mathrm{E}+07$ & ICRP-68 & $2.1 \mathrm{E}-08$ & $M$ & $\begin{array}{l}\text { ICRP-68 } \\
\end{array}$ & $1.1 \mathrm{E}-10$ & -- & -- \\
\hline Bi-215 & Bi-215 & 215.002 & Bismuth & 0.01 & 0.035 & 10 & ICRP-107 & $7.60 \mathrm{E}+00$ & $\mathrm{~m}$ & $4.56 \mathrm{E}+02$ & $1.15 \mathrm{E}+08$ & -- & -- & -- & -- & -- & -- & -- \\
\hline Bi-216 & Bi-216 & 216.006 & Bismuth & 0.01 & 0.035 & 10 & ICRP-107 & $2.17 \mathrm{E}+00$ & $\mathrm{~m}$ & $1.30 \mathrm{E}+02$ & $4.01 \mathrm{E}+08$ & -- & -- & -- & -- & -- & -- & -- \\
\hline Bk-244 & Bk-244 & 244.065 & Berkelium & 0.001 & 0.001 & 700 & JAERI & $4.35 \mathrm{E}+00$ & $\mathrm{~h}$ & $1.57 \mathrm{E}+04$ & $2.95 \mathrm{E}+06$ & JAERI & $1.0 \mathrm{E}-09$ & $\mathrm{M}$ & JAERI & $6.9 \mathrm{E}-10$ & -- & -- \\
\hline Bk-245 & Bk-245 & 245.066 & Berkelium & 0.001 & 0.001 & 700 & ICRP-38 & $4.94 \mathrm{E}+00$ & $d$ & $4.27 \mathrm{E}+05$ & $1.08 \mathrm{E}+05$ & ICRP-68 & $2.0 \mathrm{E}-09$ & $M$ & ICRP-68 & $5.7 \mathrm{E}-10$ & -- & -- \\
\hline Bk-246 & Bk-246 & 246.069 & Berkelium & 0.001 & 0.001 & 700 & ICRP-38 & $1.83 \mathrm{E}+00$ & $d$ & $1.58 \mathrm{E}+05$ & $2.90 \mathrm{E}+05$ & ICRP-68 & $4.6 \mathrm{E}-10$ & $M$ & ICRP-68 & $4.8 \mathrm{E}-10$ & -- & -- \\
\hline Bk-247 & Bk-247 & 247.070 & Berkelium & 0.001 & 0.001 & 700 & ICRP-38 & $1.38 \mathrm{E}+03$ & $y$ & $4.35 \mathrm{E}+10$ & $1.05 \mathrm{E}+00$ & $\begin{array}{l}\text { ICRP-68 } \\
\end{array}$ & $6.5 \mathrm{E}-05$ & $\mathrm{M}$ & ICRP-68 & $3.5 \mathrm{E}-07$ & -- & -- \\
\hline Bk-248m & Bk-248 & 248.073 & Berkelium & 0.001 & 0.001 & 700 & ICRP-107 & $2.37 \mathrm{E}+01$ & $\mathrm{~h}$ & $8.53 \mathrm{E}+04$ & $5.33 \mathrm{E}+05$ & JAERI & $1.5 \mathrm{E}-08$ & $\mathrm{M}$ & JAERI & $3.3 \mathrm{E}-09$ & -- & -- \\
\hline Bk-249 & Bk-249 & 249.075 & Berkelium & 0.01 & 0.001 & 700 & ICRP-38 & $3.20 \mathrm{E}+02$ & $d$ & $2.76 \mathrm{E}+07$ & $1.64 \mathrm{E}+03$ & ICRP-68 & $1.5 \mathrm{E}-07$ & $M$ & ICRP-68 & $9.7 \mathrm{E}-10$ & -- & -- \\
\hline$B k-250$ & Bk-250 & 250.078 & Berkelium & 0.01 & 0.001 & 700 & ICRP-38 & $3.22 \mathrm{E}+00$ & $\mathrm{~h}$ & $1.16 \mathrm{E}+04$ & $3.89 \mathrm{E}+06$ & ICRP-68 & $9.6 \mathrm{E}-10$ & $M$ & ICRP-68 & $1.4 \mathrm{E}-10$ & -- & -- \\
\hline Bk-251 & Bk-251 & 251.081 & Berkelium & 0.001 & 0.001 & 700 & ICRP-107 & $5.56 \mathrm{E}+01$ & $\mathrm{~m}$ & $3.34 \mathrm{E}+03$ & $1.35 \mathrm{E}+07$ & JAERI & $6.4 \mathrm{E}-11$ & $M$ & JAERI & $4.4 \mathrm{E}-11$ & -- & -- \\
\hline $\mathrm{Br}-72$ & Br-72 & 71.937 & Bromine & 0.01 & 1.5 & 0 & ICRP-107 & $7.86 \mathrm{E}+01$ & $\mathrm{~s}$ & $7.86 \mathrm{E}+01$ & $2.00 E+09$ & -- & -- & -- & -- & -- & -- & -- \\
\hline $\mathrm{Br}-73$ & Br-73 & 72.932 & Bromine & 0.01 & 1.5 & 0 & ICRP-107 & $3.40 \mathrm{E}+00$ & $\mathrm{~m}$ & $2.04 \mathrm{E}+02$ & $7.58 \mathrm{E}+08$ & -- & -- & -- & -- & -- & -- & -- \\
\hline $\mathrm{Br}-74$ & Br-74 & 73.930 & Bromine & 0.01 & 1.5 & 0 & ICRP-38 & $2.53 \mathrm{E}+01$ & $\mathrm{~m}$ & $1.52 \mathrm{E}+03$ & $1.01 \mathrm{E}+08$ & ICRP-68 & $6.8 \mathrm{E}-11$ & $M$ & ICRP-68 & $8.4 \mathrm{E}-11$ & -- & -- \\
\hline $\mathrm{Br}-74 \mathrm{~m}$ & Br-74 & 73.930 & Bromine & 0.01 & 1.5 & 0 & ICRP-38 & $4.15 \mathrm{E}+01$ & $\mathrm{~m}$ & $2.49 \mathrm{E}+03$ & $6.13 \mathrm{E}+07$ & ICRP-68 & $1.1 \mathrm{E}-10$ & $M$ & ICRP-68 & $1.4 \mathrm{E}-10$ & -- & -- \\
\hline $\mathrm{Br}-75$ & $\mathrm{Br}-75$ & 74.926 & Bromine & 0.01 & 1.5 & 0 & ICRP-38 & $9.80 \mathrm{E}+01$ & $\mathrm{~m}$ & $5.88 \mathrm{E}+03$ & $2.56 \mathrm{E}+07$ & ICRP-68 & $8.5 \mathrm{E}-11$ & $M$ & ICRP-68 & $7.9 \mathrm{E}-11$ & -- & -- \\
\hline Br-76 & Br-76 & 75.925 & Bromine & 0.01 & 1.5 & 0 & ICRP-38 & $1.62 \mathrm{E}+01$ & $\mathrm{~h}$ & $5.83 \mathrm{E}+04$ & $2.55 \mathrm{E}+06$ & ICRP-68 & $5.8 \mathrm{E}-10$ & $M$ & ICRP-68 & $4.6 \mathrm{E}-10$ & -- & -- \\
\hline $\mathrm{Br}-76 \mathrm{~m}$ & $\mathrm{Br}-76$ & 75.925 & Bromine & 0.01 & 1.5 & 0 & ICRP-107 & $1.31 \mathrm{E}+00$ & $\mathrm{~s}$ & $1.31 \mathrm{E}+00$ & $1.13 \mathrm{E}+11$ & -- & -- & -- & -- & -- & -- & -- \\
\hline $\mathrm{Br}-77$ & Br-77 & 76.921 & Bromine & 0.01 & 1.5 & 0 & ICRP-38 & $5.60 \mathrm{E}+01$ & $\mathrm{~h}$ & $2.02 \mathrm{E}+05$ & $7.27 E+05$ & ICRP-68 & $1.3 \mathrm{E}-10$ & $M$ & ICRP-68 & $9.6 \mathrm{E}-11$ & -- & -- \\
\hline $\mathrm{Br}-77 \mathrm{~m}$ & $\mathrm{Br}-77$ & 76.921 & Bromine & 0.01 & 1.5 & 0 & ICRP-107 & $4.28 \mathrm{E}+00$ & $\mathrm{~m}$ & $2.57 \mathrm{E}+02$ & $5.71 \mathrm{E}+08$ & -- & -- & -- & -- & -- & -- & -- \\
\hline $\mathrm{Br}-78$ & Br-78 & 77.921 & Bromine & 0.01 & 1.5 & 0 & $\begin{array}{l}\text { ICRP-107 } \\
\end{array}$ & $6.46 \mathrm{E}+00$ & $\mathrm{~m}$ & $3.88 \mathrm{E}+02$ & $3.74 \mathrm{E}+08$ & -- & -- & -- & $\begin{array}{l}- \\
\end{array}$ & -- & -- & -- \\
\hline $\mathrm{Br}-80$ & $\mathrm{Br}-80$ & 79.919 & Bromine & 0.01 & 1.5 & 0 & ICRP-38 & $1.74 \mathrm{E}+01$ & $\mathrm{~m}$ & $1.04 \mathrm{E}+03$ & $1.35 \mathrm{E}+08$ & ICRP-68 & $1.7 \mathrm{E}-11$ & $M$ & ICRP-68 & $3.1 \mathrm{E}-11$ & -- & -- \\
\hline $\mathrm{Br}-80 \mathrm{~m}$ & $\mathrm{Br}-80$ & 79.919 & Bromine & 0.01 & 1.5 & 0 & ICRP-38 & $4.42 \mathrm{E}+00$ & $\mathrm{~h}$ & $1.59 \mathrm{E}+04$ & $8.87 E+06$ & ICRP-68 & $1.0 \mathrm{E}-10$ & $\mathrm{M}$ & ICRP-68 & $1.1 \mathrm{E}-10$ & -- & -- \\
\hline $\mathrm{Br}-82$ & $\mathrm{Br}-82$ & 81.917 & Bromine & 0.01 & 1.5 & 0 & ICRP-38 & $3.53 \mathrm{E}+01$ & $\mathrm{~h}$ & $1.27 \mathrm{E}+05$ & $1.08 \mathrm{E}+06$ & ICRP-68 & $8.8 \mathrm{E}-10$ & $M$ & ICRP-68 & $5.4 \mathrm{E}-10$ & -- & -- \\
\hline $\mathrm{Br}-82 \mathrm{~m}$ & $\mathrm{Br}-82$ & 81.917 & Bromine & 0.01 & 1.5 & 0 & ICRP-107 & $6.13 \mathrm{E}+00$ & $\mathrm{~m}$ & $3.68 \mathrm{E}+02$ & $3.74 \mathrm{E}+08$ & -- & -- & -- & -- & -- & -- & -- \\
\hline $\mathrm{Br}-83$ & $\mathrm{Br}-83$ & 82.915 & Bromine & 0.01 & 1.5 & 0 & ICRP-38 & $2.39 \mathrm{E}+00$ & $\mathrm{~h}$ & $8.60 \mathrm{E}+03$ & $1.58 \mathrm{E}+07$ & ICRP-68 & $6.7 \mathrm{E}-11$ & $M$ & ICRP-68 & $4.3 \mathrm{E}-11$ & -- & -- \\
\hline $\mathrm{Br}-84$ & $\mathrm{Br}-84$ & 83.916 & Bromine & 0.01 & 1.5 & 0 & ICRP-38 & $3.18 \mathrm{E}+01$ & $\mathrm{~m}$ & $1.91 \mathrm{E}+03$ & $7.05 \mathrm{E}+07$ & ICRP-68 & $6.2 \mathrm{E}-11$ & $M$ & ICRP-68 & $8.8 \mathrm{E}-11$ & -- & -- \\
\hline $\mathrm{Br}-84 \mathrm{~m}$ & $\mathrm{Br}-84$ & 83.916 & Bromine & 0.01 & 1.5 & 0 & ICRP-107 & $6.00 \mathrm{E}+00$ & $\mathrm{~m}$ & $3.60 \mathrm{E}+02$ & $3.73 \mathrm{E}+08$ & -- & -- & -- & -- & -- & -- & -- \\
\hline $\mathrm{Br}-85$ & Br-85 & 84.916 & Bromine & 0.01 & 1.5 & 0 & ICRP-107 & $2.90 \mathrm{E}+00$ & $\mathrm{~m}$ & $1.74 \mathrm{E}+02$ & $7.64 E+08$ & -- & -- & -- & -- & -- & -- & -- \\
\hline C-10 & $\mathrm{C}-10$ & 10.017 & Carbon & 0.5 & -- & 0 & ICRP-107 & $1.93 \mathrm{E}+01$ & $\mathrm{~s}$ & $1.93 \mathrm{E}+01$ & $5.85 \mathrm{E}+10$ & -- & -- & -- & -- & -- & -- & -- \\
\hline C-11 & $C-11$ & 11.011 & Carbon & 0.5 & -- & 0 & ICRP-38 & $2.04 \mathrm{E}+01$ & $\mathrm{~m}$ & $1.22 \mathrm{E}+03$ & $8.38 \mathrm{E}+08$ & ICRP-68 & $3.2 \mathrm{E}-12$ & $\begin{array}{l}\text { Organic } \\
\text { gasses/ } \\
\text { vapours }\end{array}$ & ICRP-68 & $2.4 \mathrm{E}-11$ & -- & -- \\
\hline C-14 & C-14 & 14.003 & Carbon & 0.5 & -- & 0 & ICRP-38 & $5.73 E+03$ & $y$ & $1.81 \mathrm{E}+11$ & $4.46 \mathrm{E}+00$ & ICRP-68 & $5.8 \mathrm{E}-10$ & $\begin{array}{l}\text { Organic } \\
\text { gasses/ } \\
\text { vapours }\end{array}$ & ICRP-68 & $5.8 \mathrm{E}-10$ & -- & -- \\
\hline Ca-41 & Ca-41 & 40.962 & Calcium & 0.01 & 3.5 & 15 & ICRP-38 & $1.40 \mathrm{E}+05$ & $y$ & $4.42 \mathrm{E}+12$ & $6.23 \mathrm{E}-02$ & ICRP-68 & $1.9 \mathrm{E}-10$ & $M$ & ICRP-68 & $2.9 \mathrm{E}-10$ & -- & -- \\
\hline Ca-45 & Ca-45 & 44.956 & Calcium & 0.01 & 3.5 & 15 & ICRP-38 & $1.63 \mathrm{E}+02$ & $d$ & $1.41 \mathrm{E}+07$ & $1.78 \mathrm{E}+04$ & ICRP-68 & $2.7 \mathrm{E}-09$ & $\mathrm{M}$ & ICRP-68 & $7.6 \mathrm{E}-10$ & -- & -- \\
\hline Ca-47 & Ca-47 & 46.955 & Calcium & 0.01 & 3.5 & 15 & ICRP-38 & $4.53 \mathrm{E}+00$ & $d$ & $3.91 \mathrm{E}+05$ & $6.14 \mathrm{E}+05$ & ICRP-68 & $2.1 \mathrm{E}-09$ & $M$ & ICRP-68 & $1.6 \mathrm{E}-09$ & -- & -- \\
\hline Ca-49 & Ca-49 & 48.956 & Calcium & 0.01 & 3.5 & 15 & ICRP-38 & $8.72 \mathrm{E}+00$ & $\mathrm{~m}$ & $5.23 \mathrm{E}+02$ & $4.41 \mathrm{E}+08$ & -- & -- & -- & -- & -- & -- & -- \\
\hline
\end{tabular}




\begin{tabular}{|c|c|c|c|c|c|c|c|c|c|c|c|c|c|c|c|c|c|c|}
\hline \multirow{3}{*}{$\begin{array}{l}\text { MASTER } \\
\text { ISOTOPE } \\
\text { LIST } \\
\text { Cd-101 }\end{array}$} & \multirow{3}{*}{$\begin{array}{c}\begin{array}{c}\text { Atomic } \\
\text { Mass } \\
\text { Nuclide }\end{array} \\
\text { Cd-101 }\end{array}$} & \multirow{3}{*}{$\begin{array}{c}\begin{array}{c}\text { Atomic } \\
\text { Mass }\end{array} \\
100.919\end{array}$} & & & able C.1 F & dionuclide $\mathrm{Sp}$ & ic Input Da & Ised For & & & $2 \mathrm{sus}$ & imum $R$ & & nued) & & & & \\
\hline & & & \multirow{2}{*}{$\begin{array}{l}\text { Element } \\
\text { Cadmium }\end{array}$} & \multirow{2}{*}{$\begin{array}{c}\text { EPA } \\
\text { RF } \\
0.01\end{array}$} & \multirow[t]{2}{*}{$\begin{array}{c}\text { EPA } \\
\text { Conc } \\
\text { Factors } \\
\left(\mathbf{B}_{\mathrm{v}}\right) \\
0.55\end{array}$} & \multirow[t]{2}{*}{$\begin{array}{c}\text { EPA } \\
\begin{array}{c}\text { Sorption } \\
\text { Coefficient } \\
\left(K_{d}\right)\end{array} \\
50-100\end{array}$} & \multirow{2}{*}{$\begin{array}{c}\begin{array}{c}\text { Half-Life } \\
\text { Reference }\end{array} \\
\text { ICRP-107 }\end{array}$} & \multicolumn{2}{|c|}{$\begin{array}{c}\text { Half-Life } \\
\text { (original units) }\end{array}$} & \multirow{2}{*}{$\begin{array}{c}\begin{array}{c}\text { Half Life } \\
\text { (s) }\end{array} \\
8.16 \mathrm{E}+01\end{array}$} & \multirow{2}{*}{$\begin{array}{c}\begin{array}{c}\text { Sp. Act } \\
\text { (Ci/g) }\end{array} \\
1.37 \mathrm{E}+09\end{array}$} & \multirow[t]{2}{*}{$\begin{array}{c}\begin{array}{c}\text { Inhalation } \\
\text { DC } \\
\text { Reference }\end{array} \\
--\end{array}$} & \multirow[t]{2}{*}{$\begin{array}{c}\text { HC-3 } \\
\text { Bounding } \\
\text { Inhalation } \\
\text { DC } \\
\text { (Sv/Bq) } \\
--\end{array}$} & \multirow[t]{2}{*}{$\begin{array}{c}\text { Lung } \\
\text { Absorption } \\
\text { Class/Type }\end{array}$} & \multirow[t]{2}{*}{$\begin{array}{c}\begin{array}{c}\text { Ingestion } \\
\text { DC } \\
\text { Reference }\end{array} \\
--\end{array}$} & \multirow[t]{2}{*}{$\begin{array}{c}\text { HC-3 } \\
\text { Bounding } \\
\text { Ingestion } \\
\text { DC } \\
\text { (Sv/Bq) } \\
--\end{array}$} & $\begin{array}{c}\text { Immersion } \\
\text { DC } \\
\text { Reference }\end{array}$ & $\begin{array}{c}\text { HC-3 } \\
\text { Immersion } \\
\mathrm{DC}(\mathrm{Sv} / \mathrm{d} \\
\left.\text { per } \mathrm{Bq} / \mathrm{m}^{3}\right)\end{array}$ \\
\hline & & & & & & & & $1.36 \mathrm{E}+00$ & $\mathrm{~m}$ & & & & & & & & -- & -- \\
\hline $\mathrm{Cd}-102$ & $\mathrm{Cd}-102$ & 101.914 & Cadmium & 0.01 & 0.55 & $50-100$ & ICRP-107 & $5.50 \mathrm{E}+00$ & $\mathrm{~m}$ & $3.30 E+02$ & $3.35 \mathrm{E}+08$ & -- & -- & -- & -- & -- & -- & -- \\
\hline Cd-103 & Cd-103 & 102.913 & Cadmium & 0.01 & 0.55 & $50-100$ & ICRP-107 & $7.30 \mathrm{E}+00$ & $\mathrm{~m}$ & $4.38 \mathrm{E}+02$ & $2.50 \mathrm{E}+08$ & -- & -- & -- & -- & -- & -- & -- \\
\hline Cd-104 & Cd-104 & 103.910 & Cadmium & 0.01 & 0.55 & $50-100$ & ICRP-38 & $5.77 \mathrm{E}+01$ & $\mathrm{~m}$ & $3.46 E+03$ & $3.14 \mathrm{E}+07$ & ICRP-68 & $6.3 \mathrm{E}-11$ & $\mathrm{~s}$ & ICRP-68 & $5.8 \mathrm{E}-11$ & -- & -- \\
\hline Cd-105 & $\mathrm{Cd}-105$ & 104.909 & Cadmium & 0.01 & 0.55 & $50-100$ & ICRP-107 & $5.55 \mathrm{E}+01$ & $\mathrm{~m}$ & $3.33 \mathrm{E}+03$ & $3.23 \mathrm{E}+07$ & JAERI & $4.2 \mathrm{E}-11$ & $\mathrm{~s}$ & JAERI & $4.4 \mathrm{E}-11$ & -- & -- \\
\hline Cd-107 & $\mathrm{Cd}-107$ & 106.907 & Cadmium & 0.01 & 0.55 & $50-100$ & ICRP-38 & $6.49 \mathrm{E}+00$ & $\mathrm{~h}$ & $2.34 E+04$ & $4.52 \mathrm{E}+06$ & ICRP-68 & $1.1 \mathrm{E}-10$ & $\mathrm{~s}$ & ICRP-68 & $6.2 \mathrm{E}-11$ & -- & -- \\
\hline Cd-109 & Cd-109 & 108.905 & Cadmium & 0.01 & 0.55 & $50-100$ & ICRP-38 & $4.64 \mathrm{E}+02$ & $d$ & $4.01 \mathrm{E}+07$ & $2.58 \mathrm{E}+03$ & ICRP-68 & $9.6 \mathrm{E}-09$ & $F$ & ICRP-68 & $2.0 \mathrm{E}-09$ & -- & -- \\
\hline Cd-111m & Cd-111 & 110.904 & Cadmium & 0.01 & 0.55 & $50-100$ & ICRP-107 & $4.85 \mathrm{E}+01$ & $\mathrm{~m}$ & $2.91 E+03$ & $3.50 \mathrm{E}+07$ & JAERI & $3.8 \mathrm{E}-11$ & $\mathrm{~s}$ & JAERI & $1.4 \mathrm{E}-11$ & -- & -- \\
\hline Cd-113 & Cd-113 & 112.904 & Cadmium & 0.01 & 0.55 & $50-100$ & ICRP-38 & $9.30 \mathrm{E}+15$ & $y$ & $2.93 E+23$ & $3.40 \mathrm{E}-13$ & ICRP-68 & $1.4 \mathrm{E}-07$ & $\mathrm{~F}$ & ICRP-68 & $2.5 \mathrm{E}-08$ & -- & -- \\
\hline Cd-113m & $\mathrm{Cd}-113$ & 112.904 & Cadmium & 0.01 & 0.55 & $50-100$ & ICRP-38 & $1.36 \mathrm{E}+01$ & $y$ & $4.29 E+08$ & $2.33 \mathrm{E}+02$ & ICRP-68 & $1.3 \mathrm{E}-07$ & $\mathrm{~F}$ & ICRP-68 & $2.3 \mathrm{E}-08$ & -- & -- \\
\hline Cd-115 & Cd-115 & 114.905 & Cadmium & 0.01 & 0.55 & $50-100$ & ICRP-38 & $5.35 \mathrm{E}+01$ & $\mathrm{~h}$ & $1.92 E+05$ & $5.10 \mathrm{E}+05$ & ICRP-68 & $1.3 \mathrm{E}-09$ & $\mathrm{~s}$ & ICRP-68 & $1.4 \mathrm{E}-09$ & -- & -- \\
\hline Cd-115m & Cd-115 & 114.905 & Cadmium & 0.01 & 0.55 & $50-100$ & ICRP-38 & $4.46 \mathrm{E}+01$ & $d$ & $3.85 \mathrm{E}+06$ & $2.55 \mathrm{E}+04$ & ICRP-68 & $7.3 \mathrm{E}-09$ & $\mathrm{~s}$ & ICRP-68 & $3.3 \mathrm{E}-09$ & -- & -- \\
\hline Cd-117 & Cd-117 & 116.907 & Cadmium & 0.01 & 0.55 & $50-100$ & ICRP-38 & $2.49 \mathrm{E}+00$ & $\mathrm{~h}$ & $8.96 \mathrm{E}+03$ & $1.08 \mathrm{E}+07$ & ICRP-68 & $2.5 \mathrm{E}-10$ & $\mathrm{~s}$ & ICRP-68 & $2.8 \mathrm{E}-10$ & -- & -- \\
\hline Cd-117m & Cd-117 & 116.907 & Cadmium & 0.01 & 0.55 & $50-100$ & ICRP-38 & $3.36 \mathrm{E}+00$ & $\mathrm{~h}$ & $1.21 \mathrm{E}+04$ & $7.98 \mathrm{E}+06$ & ICRP-68 & $3.2 \mathrm{E}-10$ & $\mathrm{~s}$ & ICRP-68 & $2.8 \mathrm{E}-10$ & -- & -- \\
\hline Cd-118 & $\mathrm{Cd}-118$ & 117.907 & Cadmium & 0.01 & 0.55 & $50-100$ & ICRP-107 & $5.03 \mathrm{E}+01$ & $\mathrm{~m}$ & $3.02 \mathrm{E}+03$ & $3.17 \mathrm{E}+07$ & JAERI & $1.2 \mathrm{E}-10$ & $\mathrm{M}$ & JAERI & $1.9 \mathrm{E}-10$ & -- & -- \\
\hline Cd-119 & Cd-119 & 118.910 & Cadmium & 0.01 & 0.55 & $50-100$ & ICRP-107 & $2.69 \mathrm{E}+00$ & $\mathrm{~m}$ & $1.61 E+02$ & $5.88 \mathrm{E}+08$ & -- & -- & -- & -- & -- & -- & -- \\
\hline Cd-119m & Cd-119 & 118.910 & Cadmium & 0.01 & 0.55 & $50-100$ & ICRP-107 & $2.20 \mathrm{E}+00$ & $\mathrm{~m}$ & $1.32 \mathrm{E}+02$ & $7.19 \mathrm{E}+08$ & -- & -- & -- & -- & -- & -- & -- \\
\hline Ce-130 & $\mathrm{Ce}-130$ & 129.915 & Cerium & 0.01 & 0.01 & 2000 & ICRP-107 & $2.29 \mathrm{E}+01$ & $\mathrm{~m}$ & $1.37 E+03$ & $6.32 \mathrm{E}+07$ & JAERI & $6.1 \mathrm{E}-11$ & $\mathrm{~s}$ & JAERI & $7.2 \mathrm{E}-11$ & -- & -- \\
\hline Ce-131 & Ce-131 & 130.914 & Cerium & 0.01 & 0.01 & 2000 & ICRP-107 & $1.02 \mathrm{E}+01$ & $\mathrm{~m}$ & $6.12 \mathrm{E}+02$ & $1.41 \mathrm{E}+08$ & JAERI & $2.4 \mathrm{E}-11$ & $M$ & JAERI & $2.8 \mathrm{E}-11$ & -- & -- \\
\hline Ce-131m & Ce-131 & 130.914 & Cerium & 0.01 & 0.01 & 2000 & JAERI & $5.00 \mathrm{E}+00$ & $\mathrm{~m}$ & $3.00 E+02$ & $2.87 \mathrm{E}+08$ & -- & -- & -- & -- & -- & -- & -- \\
\hline Ce-132 & Ce-132 & 131.911 & Cerium & 0.01 & 0.01 & 2000 & ICRP-107 & $3.51 \mathrm{E}+00$ & $\mathrm{~h}$ & $1.26 E+04$ & $6.77 \mathrm{E}+06$ & JAERI & $2.3 \mathrm{E}-10$ & $M$ & JAERI & $3.4 \mathrm{E}-10$ & -- & -- \\
\hline Ce-133 & Ce-133 & 132.912 & Cerium & 0.01 & 0.01 & 2000 & ICRP-107 & $9.70 \mathrm{E}+01$ & $\mathrm{~m}$ & $5.82 E+03$ & $1.46 \mathrm{E}+07$ & JAERI & $7.9 \mathrm{E}-11$ & $\mathrm{~s}$ & JAERI & $9.1 \mathrm{E}-11$ & -- & -- \\
\hline Ce-133m & Ce-133 & 132.912 & Cerium & 0.01 & 0.01 & 2000 & ICRP-107 & $4.90 \mathrm{E}+00$ & $h$ & $1.76 \mathrm{E}+04$ & $4.81 \mathrm{E}+06$ & JAERI & $2.2 \mathrm{E}-10$ & $\mathrm{~s}$ & JAERI & $2.3 \mathrm{E}-10$ & -- & -- \\
\hline Ce-134 & Ce-134 & 133.909 & Cerium & 0.01 & 0.01 & 2000 & ICRP-38 & $7.20 \mathrm{E}+01$ & $\mathrm{~h}$ & $2.59 \mathrm{E}+05$ & $3.25 \mathrm{E}+05$ & ICRP-68 & $1.6 \mathrm{E}-09$ & $\mathrm{~s}$ & ICRP-68 & $2.5 \mathrm{E}-09$ & -- & -- \\
\hline Ce-135 & Ce-135 & 134.909 & Cerium & 0.01 & 0.01 & 2000 & ICRP-38 & $1.76 \mathrm{E}+01$ & $\mathrm{~h}$ & $6.34 \mathrm{E}+04$ & $1.32 \mathrm{E}+06$ & ICRP-68 & $7.6 \mathrm{E}-10$ & $\mathrm{~s}$ & ICRP-68 & $7.9 \mathrm{E}-10$ & -- & -- \\
\hline Ce-137 & Ce-137 & 136.908 & Cerium & 0.01 & 0.01 & 2000 & ICRP-38 & $9.00 \mathrm{E}+00$ & $\mathrm{~h}$ & $3.24 E+04$ & $2.54 \mathrm{E}+06$ & ICRP-68 & $1.9 \mathrm{E}-11$ & $\mathrm{~s}$ & ICRP-68 & $2.5 \mathrm{E}-11$ & -- & -- \\
\hline Ce-137m & Ce-137 & 136.908 & Cerium & 0.01 & 0.01 & 2000 & ICRP-38 & $3.44 \mathrm{E}+01$ & $\mathrm{~h}$ & $1.24 E+05$ & $6.65 \mathrm{E}+05$ & ICRP-68 & $5.9 \mathrm{E}-10$ & $\mathrm{~s}$ & ICRP-68 & $5.4 \mathrm{E}-10$ & -- & -- \\
\hline Ce-139 & Ce-139 & 138.907 & Cerium & 0.01 & 0.01 & 2000 & ICRP-38 & $1.38 \mathrm{E}+02$ & $d$ & $1.19 \mathrm{E}+07$ & $6.83 \mathrm{E}+03$ & ICRP-68 & $1.8 \mathrm{E}-09$ & $\mathrm{~s}$ & ICRP-68 & $2.6 \mathrm{E}-10$ & -- & -- \\
\hline Ce-141 & Ce-141 & 140.908 & Cerium & 0.01 & 0.01 & 2000 & ICRP-38 & $3.25 \mathrm{E}+01$ & $d$ & $2.81 E+06$ & $2.85 \mathrm{E}+04$ & ICRP-68 & $3.6 \mathrm{E}-09$ & $\mathrm{~s}$ & ICRP-68 & $7.1 \mathrm{E}-10$ & -- & -- \\
\hline Ce-143 & Ce-143 & 142.912 & Cerium & 0.01 & 0.01 & 2000 & ICRP-38 & $3.30 \mathrm{E}+01$ & $\mathrm{~h}$ & $1.19 E+05$ & $6.64 \mathrm{E}+05$ & ICRP-68 & $1.0 \mathrm{E}-09$ & $\mathrm{~s}$ & ICRP-68 & $1.1 \mathrm{E}-09$ & -- & -- \\
\hline Ce-144 & Ce-144 & 143.914 & Cerium & 0.01 & 0.01 & 2000 & ICRP-38 & $2.84 \mathrm{E}+02$ & $d$ & $2.46 \mathrm{E}+07$ & $3.19 \mathrm{E}+03$ & ICRP-68 & $4.9 \mathrm{E}-08$ & $\mathrm{~s}$ & ICRP-68 & $5.2 \mathrm{E}-09$ & -- & -- \\
\hline Ce-145 & Ce-145 & 144.917 & Cerium & 0.01 & 0.01 & 2000 & ICRP-107 & $3.01 \mathrm{E}+00$ & $\mathrm{~m}$ & $1.81 \mathrm{E}+02$ & $4.31 \mathrm{E}+08$ & -- & -- & -- & -- & -- & -- & -- \\
\hline Ce-146 & Ce-146 & 145.919 & Cerium & 0.01 & 0.01 & 2000 & JAERI & $1.35 \mathrm{E}+01$ & $\mathrm{~m}$ & $8.11 \mathrm{E}+02$ & $9.53 \mathrm{E}+07$ & JAERI & $4.4 \mathrm{E}-11$ & $\mathrm{~s}$ & JAERI & $4.7 \mathrm{E}-11$ & -- & -- \\
\hline Cf-244 & Cf-244 & 244.066 & Californium & 0.001 & 0.001 & 0 & ICRP-38 & $1.94 \mathrm{E}+01$ & $\mathrm{~m}$ & $1.16 \mathrm{E}+03$ & $3.97 \mathrm{E}+07$ & ICRP-68 & $1.8 \mathrm{E}-08$ & $M$ & ICRP-68 & $7.0 \mathrm{E}-11$ & -- & -- \\
\hline Cf-246 & Cf-246 & 246.069 & Californium & 0.001 & 0.001 & 0 & ICRP-38 & $3.57 \mathrm{E}+01$ & $\mathrm{~h}$ & $1.29 \mathrm{E}+05$ & $3.57 \mathrm{E}+05$ & ICRP-68 & $4.2 \mathrm{E}-07$ & $M$ & ICRP-68 & $3.3 \mathrm{E}-09$ & -- & -- \\
\hline Cf-247 & Cf-247 & 247.071 & Californium & 0.001 & 0.001 & 0 & ICRP-107 & $3.11 \mathrm{E}+00$ & $\mathrm{~h}$ & $1.12 E+04$ & $4.08 \mathrm{E}+06$ & JAERI & $4.9 \mathrm{E}-11$ & $M$ & JAERI & $2.9 \mathrm{E}-11$ & -- & -- \\
\hline Cf-248 & Cf-248 & 248.072 & Californium & 0.001 & 0.001 & 0 & ICRP-38 & $3.34 \mathrm{E}+02$ & $d$ & $2.88 \mathrm{E}+07$ & $1.58 \mathrm{E}+03$ & ICRP-68 & $8.2 \mathrm{E}-06$ & $M$ & ICRP-68 & $2.8 \mathrm{E}-08$ & -- & -- \\
\hline Cf-249 & Cf-249 & 249.075 & Californium & 0.001 & 0.001 & 0 & ICRP-38 & $3.51 \mathrm{E}+02$ & $y$ & $1.11 \mathrm{E}+10$ & $4.09 \mathrm{E}+00$ & ICRP-68 & $6.6 \mathrm{E}-05$ & $M$ & ICRP-68 & $3.5 \mathrm{E}-07$ & -- & -- \\
\hline Cf-250 & Cf- 250 & 250.076 & Californium & 0.001 & 0.001 & 0 & ICRP-38 & $1.31 \mathrm{E}+01$ & $y$ & $4.13 \mathrm{E}+08$ & $1.09 \mathrm{E}+02$ & ICRP-68 & $3.2 \mathrm{E}-05$ & $M$ & ICRP-68 & $1.6 \mathrm{E}-07$ & -- & -- \\
\hline Cf-251 & Cf-251 & 251.080 & Californium & 0.001 & 0.001 & 0 & ICRP-38 & $8.98 \mathrm{E}+02$ & $y$ & $2.83 E+10$ & $1.59 \mathrm{E}+00$ & ICRP-68 & $6.7 \mathrm{E}-05$ & $M$ & ICRP-68 & $3.6 \mathrm{E}-07$ & -- & -- \\
\hline $\mathrm{Cf}-252$ & $\mathrm{Cf}-252$ & 252.082 & Californium & 0.001 & 0.001 & 0 & ICRP-38 & $2.64 \mathrm{E}+00$ & $y$ & $8.32 E+07$ & $5.38 \mathrm{E}+02$ & ICRP-68 & $1.8 \mathrm{E}-05$ & $M$ & ICRP-68 & $9.0 \mathrm{E}-08$ & -- & -- \\
\hline Cf-253 & Cf-253 & 253.085 & Californium & 0.001 & 0.001 & 0 & ICRP-38 & $1.78 \mathrm{E}+01$ & $d$ & $1.54 \mathrm{E}+06$ & $2.90 \mathrm{E}+04$ & ICRP-68 & $1.2 \mathrm{E}-06$ & $\mathrm{M}$ & ICRP-68 & $1.4 \mathrm{E}-09$ & -- & -- \\
\hline Cf-254 & Cf-254 & 254.087 & Californium & 0.001 & 0.001 & 0 & ICRP-38 & $6.05 \mathrm{E}+01$ & $d$ & $5.23 E+06$ & $8.49 \mathrm{E}+03$ & ICRP-68 & $3.7 \mathrm{E}-05$ & $M$ & ICRP-68 & $4.0 \mathrm{E}-07$ & -- & -- \\
\hline Cf-255 & Cf-255 & 255.091 & Californium & 0.001 & 0.001 & 0 & ICRP-107 & $8.50 \mathrm{E}+01$ & $\mathrm{~m}$ & $5.10 \mathrm{E}+03$ & $8.67 \mathrm{E}+06$ & JAERI & $5.4 \mathrm{E}-09$ & $\mathrm{M}$ & JAERI & $4.0 \mathrm{E}-11$ & -- & -- \\
\hline Cf-256 & Cf-256 & 256.093 & Californium & 0.001 & 0.001 & 0 & JAERI & $1.23 \mathrm{E}+01$ & $\mathrm{~m}$ & $7.38 \mathrm{E}+02$ & $5.97 \mathrm{E}+07$ & JAERI & $4.0 \mathrm{E}-06$ & $M$ & JAERI & $3.3 \mathrm{E}-09$ & -- & -- \\
\hline
\end{tabular}




\begin{tabular}{|c|c|c|c|c|c|c|c|c|c|c|c|c|c|c|c|c|c|c|}
\hline \multirow{3}{*}{$\begin{array}{l}\text { MASTER } \\
\text { ISOTOPE } \\
\text { LIST } \\
\text { Cl-34 }\end{array}$} & \multirow{3}{*}{$\begin{array}{c}\begin{array}{c}\text { Atomic } \\
\text { Mass } \\
\text { Nuclide }\end{array} \\
\text { Cl-34 } \\
\end{array}$} & & & & \multicolumn{14}{|c|}{ Table C.1 Radionuclide Spe } \\
\hline & & \multirow{2}{*}{$\begin{array}{l}\begin{array}{c}\text { Atomic } \\
\text { Mass }\end{array} \\
33.974\end{array}$} & \multirow{2}{*}{$\begin{array}{l}\text { Element } \\
\text { Chlorine }\end{array}$} & \multirow{2}{*}{$\begin{array}{c}\text { EPA } \\
\text { RF } \\
0.01\end{array}$} & $\begin{array}{c}\text { EPA } \\
\text { Conc } \\
\text { Factors } \\
\left(B_{v}\right) \\
\end{array}$ & $\begin{array}{c}\text { EPA } \\
\text { Sorption } \\
\text { Coefficient } \\
\left(K_{d}\right)\end{array}$ & $\begin{array}{c}\text { Half-Life } \\
\text { Reference }\end{array}$ & $\begin{array}{r}\text { Half-Lit } \\
\text { (original u }\end{array}$ & & $\begin{array}{l}\text { Half Life } \\
\text { (s) }\end{array}$ & $\begin{array}{l}\text { Sp. Act } \\
\text { (Ci/g) }\end{array}$ & $\begin{array}{l}\text { Inhalation } \\
\text { DC } \\
\text { Reference }\end{array}$ & $\begin{array}{c}\text { HC-3 } \\
\text { Bounding } \\
\text { Inhalation } \\
\text { DC } \\
\text { (Sv/Bq) } \\
\end{array}$ & $\begin{array}{c}\text { Lung } \\
\text { Absorption } \\
\text { Class/Type }\end{array}$ & $\begin{array}{l}\text { Ingestion } \\
\text { DC } \\
\text { Reference }\end{array}$ & $\begin{array}{c}\text { HC-3 } \\
\text { Bounding } \\
\text { Ingestion } \\
\text { DC } \\
\text { (Sv/Bq) } \\
\end{array}$ & $\begin{array}{c}\text { Immersion } \\
\text { DC } \\
\text { Reference }\end{array}$ & $\begin{array}{c}\mathrm{HC}-3 \\
\text { Immersion } \\
\mathrm{DC}(\mathrm{Sv} / \mathrm{d} \\
\left.\operatorname{per} \mathrm{Bq} / \mathrm{m}^{3}\right)\end{array}$ \\
\hline & & & & & 70 & 0 & ICRP-107 & $1.53 \mathrm{E}+00$ & $\mathrm{~s}$ & $1.53 \mathrm{E}+00$ & $2.18 \mathrm{E}+11$ & -- & -- & -- & -- & -- & -- & -- \\
\hline $\mathrm{Cl}-34 \mathrm{~m}$ & $\mathrm{Cl}-34$ & 33.974 & Chlorine & 0.01 & 70 & 0 & ICRP-107 & $3.20 \mathrm{E}+01$ & $\mathrm{~m}$ & $1.92 \mathrm{E}+03$ & $1.73 \mathrm{E}+08$ & JAERI & $7.5 \mathrm{E}-11$ & $\mathrm{M}$ & JAERI & $1.0 \mathrm{E}-10$ & -- & -- \\
\hline $\mathrm{Cl}-36$ & $\mathrm{Cl}-36$ & 35.968 & Chlorine & 0.01 & 70 & 0 & ICRP-38 & $3.01 \mathrm{E}+05$ & $y$ & $9.50 \mathrm{E}+12$ & $3.30 \mathrm{E}-02$ & ICRP-68 & $6.9 \mathrm{E}-09$ & $\mathrm{M}$ & ICRP-68 & $9.3 \mathrm{E}-10$ & -- & -- \\
\hline $\mathrm{Cl}-38$ & $\mathrm{Cl}-38$ & 37.968 & Chlorine & 0.01 & 70 & 0 & ICRP-38 & $3.72 \mathrm{E}+01$ & $\mathrm{~m}$ & $2.23 \mathrm{E}+03$ & $1.33 \mathrm{E}+08$ & ICRP-68 & $7.3 \mathrm{E}-11$ & $\mathrm{M}$ & ICRP-68 & $1.2 \mathrm{E}-10$ & -- & -- \\
\hline $\mathrm{Cl}-39$ & $\mathrm{Cl}-39$ & 38.968 & Chlorine & 0.01 & 70 & 0 & ICRP-38 & $5.56 \mathrm{E}+01$ & $\mathrm{~m}$ & $3.34 \mathrm{E}+03$ & $8.68 \mathrm{E}+07$ & ICRP-68 & $7.6 \mathrm{E}-11$ & $\mathrm{M}$ & ICRP-68 & $8.5 \mathrm{E}-11$ & -- & -- \\
\hline $\mathrm{Cl}-40$ & $\mathrm{Cl}-40$ & 39.970 & Chlorine & 0.01 & 70 & 0 & ICRP-107 & $1.35 \mathrm{E}+00$ & $\mathrm{~m}$ & $8.10 \mathrm{E}+01$ & $3.48 \mathrm{E}+09$ & -- & -- & -- & -- & -- & -- & -- \\
\hline $\mathrm{Cm}-238$ & $\mathrm{Cm}-238$ & 238.053 & Curium & 0.001 & 0.00085 & 500 & ICRP-38 & $2.40 \mathrm{E}+00$ & $\mathrm{~h}$ & $8.64 \mathrm{E}+03$ & $5.49 \mathrm{E}+06$ & ICRP-68 & $4.8 \mathrm{E}-09$ & $\mathrm{M}$ & ICRP-68 & $8.0 \mathrm{E}-11$ & -- & -- \\
\hline $\mathrm{Cm}-239$ & Cm-239 & 239.055 & Curium & 0.001 & 0.00085 & 500 & ICRP-107 & $2.90 \mathrm{E}+00$ & $\mathrm{~h}$ & $1.04 \mathrm{E}+04$ & $4.52 \mathrm{E}+06$ & JAERI & $8.8 \mathrm{E}-11$ & $M$ & JAERI & $8.2 \mathrm{E}-11$ & -- & -- \\
\hline $\mathrm{Cm}-240$ & $\mathrm{Cm}-240$ & 240.056 & Curium & 0.001 & 0.00085 & 500 & $\begin{array}{l}\text { ICRP-38 } \\
\end{array}$ & $2.70 \mathrm{E}+01$ & $d$ & $2.33 \mathrm{E}+06$ & $2.01 \mathrm{E}+04$ & ICRP-68 & $2.9 \mathrm{E}-06$ & $M$ & ICRP-68 & $7.6 \mathrm{E}-09$ & -- & -- \\
\hline $\mathrm{Cm}-241$ & $\mathrm{Cm}-241$ & 241.058 & Curium & 0.001 & 0.00085 & 500 & ICRP-38 & $3.28 \mathrm{E}+01$ & $d$ & $2.83 \mathrm{E}+06$ & $1.65 \mathrm{E}+04$ & ICRP-68 & $3.4 \mathrm{E}-08$ & $M$ & ICRP-68 & $9.1 \mathrm{E}-10$ & -- & -- \\
\hline $\mathrm{Cm}-242$ & $\mathrm{Cm}-242$ & 242.059 & Curium & 0.001 & 0.00085 & 500 & ICRP-38 & $1.63 \mathrm{E}+02$ & $d$ & $1.41 \mathrm{E}+07$ & $3.31 \mathrm{E}+03$ & ICRP-68 & $4.8 \mathrm{E}-06$ & $M$ & ICRP-68 & $1.2 \mathrm{E}-08$ & -- & -- \\
\hline $\mathrm{Cm}-243$ & $\mathrm{Cm}-243$ & 243.061 & Curium & 0.001 & 0.00085 & 500 & ICRP-38 & $2.85 \mathrm{E}+01$ & $y$ & $8.99 \mathrm{E}+08$ & $5.16 \mathrm{E}+01$ & ICRP-68 & $2.9 \mathrm{E}-05$ & $M$ & ICRP-68 & $1.5 \mathrm{E}-07$ & -- & -- \\
\hline $\mathrm{Cm}-244$ & $\mathrm{Cm}-244$ & 244.063 & Curium & 0.001 & 0.00085 & 500 & ICRP-38 & $1.81 \mathrm{E}+01$ & $y$ & $5.72 \mathrm{E}+08$ & $8.09 \mathrm{E}+01$ & $\begin{array}{l}\text { ICRP-68 } \\
\end{array}$ & $2.5 \mathrm{E}-05$ & $M$ & ICRP-68 & $1.2 \mathrm{E}-07$ & -- & -- \\
\hline $\mathrm{Cm}-245$ & $\mathrm{Cm}-245$ & 245.065 & Curium & 0.001 & 0.00085 & 500 & ICRP-38 & $8.50 \mathrm{E}+03$ & $y$ & $2.68 \mathrm{E}+11$ & $1.72 \mathrm{E}-01$ & ICRP-68 & $4.0 \mathrm{E}-05$ & $M$ & ICRP-68 & $2.1 \mathrm{E}-07$ & -- & -- \\
\hline $\mathrm{Cm}-246$ & $\mathrm{Cm}-246$ & 246.067 & Curium & 0.001 & 0.00085 & 500 & ICRP-38 & $4.73 \mathrm{E}+03$ & $y$ & $1.49 \mathrm{E}+11$ & $3.07 \mathrm{E}-01$ & ICRP-68 & $4.0 \mathrm{E}-05$ & $M$ & ICRP-68 & $2.1 \mathrm{E}-07$ & -- & -- \\
\hline $\mathrm{Cm}-247$ & $\mathrm{Cm}-247$ & 247.070 & Curium & 0.001 & 0.00085 & 500 & ICRP-38 & $1.56 \mathrm{E}+07$ & $y$ & $4.92 \mathrm{E}+14$ & $9.28 \mathrm{E}-05$ & ICRP-68 & $3.6 \mathrm{E}-05$ & $M$ & ICRP-68 & $1.9 \mathrm{E}-07$ & -- & -- \\
\hline $\mathrm{Cm}-248$ & $\mathrm{Cm}-248$ & 248.072 & Curium & 0.001 & 0.00085 & 500 & ICRP-38 & $3.39 \mathrm{E}+05$ & $y$ & $1.07 \mathrm{E}+13$ & $4.25 \mathrm{E}-03$ & ICRP-68 & $1.4 \mathrm{E}-04$ & $\mathrm{M}$ & ICRP-68 & $7.7 \mathrm{E}-07$ & -- & -- \\
\hline $\mathrm{Cm}-249$ & $\mathrm{Cm}-249$ & 249.076 & Curium & 0.001 & 0.00085 & 500 & ICRP-38 & $6.42 \mathrm{E}+01$ & $\mathrm{~m}$ & $3.85 \mathrm{E}+03$ & $1.18 \mathrm{E}+07$ & ICRP-68 & $5.1 \mathrm{E}-11$ & $M$ & ICRP-68 & $3.1 \mathrm{E}-11$ & -- & -- \\
\hline $\mathrm{Cm}-250$ & $\mathrm{Cm}-250$ & 250.078 & Curium & 0.001 & 0.00085 & 500 & ICRP-38 & $6.90 \mathrm{E}+03$ & $y$ & $2.18 \mathrm{E}+11$ & $2.07 E-01$ & ICRP-68 & $7.9 \mathrm{E}-04$ & $M$ & ICRP-68 & $4.4 \mathrm{E}-06$ & -- & -- \\
\hline $\mathrm{Cm}-251$ & Cm-251 & 251.082 & Curium & 0.001 & 0.00085 & 500 & ICRP-107 & $1.68 \mathrm{E}+01$ & $\mathrm{~m}$ & $1.01 \mathrm{E}+03$ & $4.46 \mathrm{E}+07$ & -- & -- & -- & -- & -- & -- & -- \\
\hline Co-54m & Co-54 & 53.948 & Cobalt & 0.001 & 0.02 & 2000 & ICRP-107 & $1.48 \mathrm{E}+00$ & $\mathrm{~m}$ & $8.88 \mathrm{E}+01$ & $2.35 \mathrm{E}+09$ & -- & -- & -- & -- & -- & -- & -- \\
\hline Co-55 & Co-55 & 54.942 & Cobalt & 0.001 & 0.02 & 2000 & ICRP-38 & $1.75 \mathrm{E}+01$ & $\mathrm{~h}$ & $6.31 \mathrm{E}+04$ & $3.25 \mathrm{E}+06$ & ICRP-68 & $8.3 \mathrm{E}-10$ & $\mathrm{~s}$ & ICRP-68 & $1.1 \mathrm{E}-09$ & -- & -- \\
\hline Co-56 & Co-56 & 55.940 & Cobalt & 0.001 & 0.02 & 2000 & ICRP-38 & $7.88 \mathrm{E}+01$ & $d$ & $6.80 \mathrm{E}+06$ & $2.96 \mathrm{E}+04$ & ICRP-68 & $6.3 \mathrm{E}-09$ & $\mathrm{~s}$ & ICRP-68 & $2.5 \mathrm{E}-09$ & -- & -- \\
\hline Co-57 & Co-57 & 56.936 & Cobalt & 0.001 & 0.02 & 2000 & ICRP-38 & $2.71 \mathrm{E}+02$ & $d$ & $2.34 \mathrm{E}+07$ & $8.47 E+03$ & ICRP-68 & $9.4 \mathrm{E}-10$ & $\mathrm{~s}$ & ICRP-68 & $2.1 \mathrm{E}-10$ & -- & -- \\
\hline Co-58 & Co-58 & 57.936 & Cobalt & 0.001 & 0.02 & 2000 & ICRP-38 & $7.08 \mathrm{E}+01$ & $d$ & $6.12 \mathrm{E}+06$ & $3.18 \mathrm{E}+04$ & ICRP-68 & $2.0 \mathrm{E}-09$ & $\mathrm{~s}$ & ICRP-68 & $7.4 \mathrm{E}-10$ & -- & -- \\
\hline Co-58m & Co-58 & 57.936 & Cobalt & 0.001 & 0.02 & 2000 & ICRP-38 & $9.15 \mathrm{E}+00$ & $\mathrm{~h}$ & $3.29 \mathrm{E}+04$ & $5.91 \mathrm{E}+06$ & ICRP-68 & $1.7 \mathrm{E}-11$ & $\mathrm{~s}$ & ICRP-68 & $2.4 \mathrm{E}-11$ & -- & -- \\
\hline Co-60 & Co-60 & 59.934 & Cobalt & 0.001 & 0.02 & 2000 & ICRP-38 & $5.27 \mathrm{E}+00$ & $y$ & $1.66 \mathrm{E}+08$ & $1.13 \mathrm{E}+03$ & ICRP-68 & $2.9 \mathrm{E}-08$ & $\mathrm{~s}$ & ICRP-68 & $3.4 \mathrm{E}-09$ & -- & -- \\
\hline Co-60m & Co-60 & 59.934 & Cobalt & 0.001 & 0.02 & 2000 & ICRP-38 & $1.05 \mathrm{E}+01$ & $\mathrm{~m}$ & $6.28 \mathrm{E}+02$ & $3.00 \mathrm{E}+08$ & ICRP-68 & $1.3 \mathrm{E}-12$ & $\mathrm{~s}$ & ICRP-68 & 1.7E-12 & -- & -- \\
\hline Co-61 & Co-61 & 60.932 & Cobalt & 0.001 & 0.02 & 2000 & ICRP-38 & $1.65 \mathrm{E}+00$ & $\mathrm{~h}$ & $5.94 \mathrm{E}+03$ & $3.12 \mathrm{E}+07$ & ICRP-68 & $7.5 \mathrm{E}-11$ & $\mathrm{~s}$ & ICRP-68 & $7.4 \mathrm{E}-11$ & -- & -- \\
\hline Co-62 & Co-62 & 61.934 & Cobalt & 0.001 & 0.02 & 2000 & ICRP-107 & $1.50 \mathrm{E}+00$ & $\mathrm{~m}$ & $9.00 \mathrm{E}+01$ & $2.02 \mathrm{E}+09$ & -- & -- & -- & -- & -- & -- & -- \\
\hline Co-62m & Co-62 & 61.934 & Cobalt & 0.001 & 0.02 & 2000 & ICRP-38 & $1.39 \mathrm{E}+01$ & $\mathrm{~m}$ & $8.35 \mathrm{E}+02$ & $2.18 \mathrm{E}+08$ & ICRP-68 & 3.7E-11 & $\mathrm{s}$ & ICRP-68 & 4.7E-11 & -- & -- \\
\hline $\mathrm{Cr}-48$ & $\mathrm{Cr}-48$ & 47.954 & Chromium & 0.01 & 0.0075 & 0 & ICRP-38 & $2.30 \mathrm{E}+01$ & $\mathrm{~h}$ & $8.27 \mathrm{E}+04$ & $2.85 \mathrm{E}+06$ & ICRP-68 & $2.5 \mathrm{E}-10$ & $\mathrm{~s}$ & ICRP-68 & $2.0 \mathrm{E}-10$ & -- & -- \\
\hline Cr-49 & $\mathrm{Cr}-49$ & 48.951 & Chromium & 0.01 & 0.0075 & 0 & ICRP-38 & $4.21 \mathrm{E}+01$ & $\mathrm{~m}$ & $2.53 \mathrm{E}+03$ & $9.13 \mathrm{E}+07$ & ICRP-68 & $5.9 \mathrm{E}-11$ & $\mathrm{~s}$ & ICRP-68 & $6.1 \mathrm{E}-11$ & -- & -- \\
\hline Cr-51 & Cr-51 & 50.945 & Chromium & 0.01 & 0.0075 & 0 & ICRP-38 & $2.77 \mathrm{E}+01$ & $d$ & $2.39 \mathrm{E}+06$ & $9.25 \mathrm{E}+04$ & ICRP-68 & $3.6 \mathrm{E}-11$ & $\mathrm{~s}$ & ICRP-68 & $3.8 \mathrm{E}-11$ & -- & -- \\
\hline Cr-55 & Cr-55 & 54.941 & Chromium & 0.01 & 0.0075 & 0 & ICRP-107 & $3.50 \mathrm{E}+00$ & $\mathrm{~m}$ & $2.10 \mathrm{E}+02$ & $9.79 E+08$ & -- & -- & -- & -- & -- & -- & -- \\
\hline Cr-56 & Cr-56 & 55.941 & Chromium & 0.01 & 0.0075 & 0 & ICRP-107 & $5.94 \mathrm{E}+00$ & $\mathrm{~m}$ & $3.56 \mathrm{E}+02$ & $5.66 \mathrm{E}+08$ & -- & -- & -- & -- & -- & -- & -- \\
\hline Cs-121 & Cs-121 & 120.917 & Cesium & 0.01 & 0.08 & 2000 & ICRP-107 & $1.55 \mathrm{E}+02$ & $\mathrm{~s}$ & $1.55 \mathrm{E}+02$ & $6.02 \mathrm{E}+08$ & -- & -- & -- & -- & -- & -- & -- \\
\hline Cs-121m & Cs-121 & 120.917 & Cesium & 0.01 & 0.08 & 2000 & ICRP-107 & $1.22 \mathrm{E}+02$ & $\mathrm{~s}$ & $1.22 \mathrm{E}+02$ & $7.65 \mathrm{E}+08$ & -- & -- & -- & -- & -- & -- & -- \\
\hline Cs-123 & Cs-123 & 122.913 & Cesium & 0.01 & 0.08 & 2000 & ICRP-107 & $5.88 \mathrm{E}+00$ & $\mathrm{~m}$ & $3.53 \mathrm{E}+02$ & $2.60 \mathrm{E}+08$ & -- & -- & -- & -- & -- & -- & -- \\
\hline Cs-124 & Cs-124 & 123.912 & Cesium & 0.01 & 0.08 & 2000 & ICRP-107 & $3.08 \mathrm{E}+01$ & $\mathrm{~s}$ & $3.08 \mathrm{E}+01$ & $2.96 \mathrm{E}+09$ & -- & -- & -- & -- & -- & -- & -- \\
\hline Cs-125 & Cs-125 & 124.910 & Cesium & 0.01 & 0.08 & 2000 & ICRP-38 & $4.50 \mathrm{E}+01$ & $\mathrm{~m}$ & $2.70 \mathrm{E}+03$ & $3.35 \mathrm{E}+07$ & ICRP-68 & $2.3 \mathrm{E}-11$ & $F$ & ICRP-68 & $3.5 \mathrm{E}-11$ & -- & -- \\
\hline Cs-126 & Cs-126 & 125.909 & Cesium & 0.01 & 0.08 & 2000 & ICRP-38 & $1.64 \mathrm{E}+00$ & $\mathrm{~m}$ & $9.84 \mathrm{E}+01$ & $9.11 \mathrm{E}+08$ & -- & -- & -- & -- & -- & -- & -- \\
\hline Cs-127 & Cs-127 & 126.907 & Cesium & 0.01 & 0.08 & 2000 & ICRP-38 & $6.25 \mathrm{E}+00$ & $\mathrm{~h}$ & $2.25 \mathrm{E}+04$ & $3.95 \mathrm{E}+06$ & ICRP-68 & $4.0 \mathrm{E}-11$ & $\mathrm{~F}$ & ICRP-68 & $2.4 \mathrm{E}-11$ & -- & -- \\
\hline Cs-128 & Cs-128 & 127.908 & Cesium & 0.01 & 0.08 & 2000 & ICRP-38 & $3.90 \mathrm{E}+00$ & $\mathrm{~m}$ & $2.34 \mathrm{E}+02$ & $3.77 \mathrm{E}+08$ & -- & -- & -- & -- & -- & -- & -- \\
\hline Cs-129 & Cs-129 & 128.906 & Cesium & 0.01 & 0.08 & 2000 & ICRP-38 & $3.21 \mathrm{E}+01$ & $\mathrm{~h}$ & $1.15 \mathrm{E}+05$ & $7.58 \mathrm{E}+05$ & ICRP-68 & $8.1 \mathrm{E}-11$ & $F$ & ICRP-68 & $6.0 \mathrm{E}-11$ & -- & -- \\
\hline
\end{tabular}




\begin{tabular}{|c|c|c|c|c|c|c|c|c|c|c|c|c|c|c|c|c|c|c|}
\hline \multirow{3}{*}{$\begin{array}{l}\text { MASTER } \\
\text { ISOTOPE } \\
\text { LIST } \\
\text { CS-130 }\end{array}$} & \multirow{3}{*}{$\begin{array}{c}\begin{array}{c}\text { Atomic } \\
\text { Mass } \\
\text { Nuclide }\end{array} \\
\text { Cs-130 }\end{array}$} & & & & able C.1 F & dionuclide Sp & fic Input Da & Jsed For th & ialc & tion of $\mathrm{H}$ & 2 s Using & ximum Rep & & ued) & & & & \\
\hline & & \multirow{2}{*}{$\begin{array}{c}\begin{array}{c}\text { Atomic } \\
\text { Mass }\end{array} \\
129.907\end{array}$} & \multirow{2}{*}{$\begin{array}{l}\text { Element } \\
\text { Cesium }\end{array}$} & \multirow{2}{*}{$\begin{array}{c}\text { EPA } \\
\text { RF } \\
0.01\end{array}$} & \multirow[t]{2}{*}{$\begin{array}{c}\text { EPA } \\
\text { Conc } \\
\text { Factors } \\
\left(\mathbf{B}_{\mathrm{v}}\right) \\
0.08 \\
\end{array}$} & \multirow[t]{2}{*}{$\begin{array}{c}\text { EPA } \\
\begin{array}{c}\text { Sorption } \\
\text { Coefficient } \\
\left(K_{d}\right)\end{array} \\
2000 \\
\end{array}$} & \multirow{2}{*}{$\begin{array}{c}\begin{array}{c}\text { Half-Life } \\
\text { Reference }\end{array} \\
\text { ICRP-38 } \\
\end{array}$} & \multicolumn{2}{|c|}{$\begin{array}{c}\text { Half-Life } \\
\text { (original units) }\end{array}$} & \multirow{2}{*}{$\begin{array}{c}\begin{array}{c}\text { Half Life } \\
\text { (s) }\end{array} \\
1.79 \mathrm{E}+03\end{array}$} & \multirow{2}{*}{$\begin{array}{c}\begin{array}{c}\text { Sp. Act } \\
\text { (Ci/g) }\end{array} \\
4.84 \mathrm{E}+07\end{array}$} & \multirow{2}{*}{$\begin{array}{c}\text { Inhalation } \\
\text { DC } \\
\text { Reference }\end{array}$} & \multirow[t]{2}{*}{$\begin{array}{c}\text { HC-3 } \\
\text { Bounding } \\
\text { Inhalation } \\
\text { DC } \\
(\mathrm{Sv} / \mathrm{Bq}) \\
1.5 \mathrm{E}-11\end{array}$} & \multirow{2}{*}{$\begin{array}{c}\begin{array}{c}\text { Lung } \\
\text { Absorption } \\
\text { Class/Type }\end{array} \\
\text { F }\end{array}$} & \multirow[t]{2}{*}{$\begin{array}{c}\text { Ingestion } \\
\text { DC } \\
\text { Reference }\end{array}$} & \multirow[t]{2}{*}{$\begin{array}{c}\text { HC-3 } \\
\text { Bounding } \\
\text { Ingestion } \\
\text { DC } \\
(\mathrm{Sv} / \mathrm{Bq}) \\
2.8 \mathrm{E}-11\end{array}$} & $\begin{array}{c}\text { Immersion } \\
\text { DC } \\
\text { Reference }\end{array}$ & $\begin{array}{c}\mathrm{HC}-3 \\
\text { Immersion } \\
\mathrm{DC}(\mathrm{Sv} / \mathrm{d} \\
\left.\operatorname{per} \mathrm{Bq} / \mathrm{m}^{3}\right)\end{array}$ \\
\hline & & & & & & & & $2.99 \mathrm{E}+01$ & $\mathrm{~m}$ & & & & & & & & & -- \\
\hline Cs-130m & Cs-130 & 129.907 & Cesium & 0.01 & 0.08 & 2000 & $\begin{array}{l}\text { ICRP-107 } \\
\end{array}$ & $3.46 \mathrm{E}+00$ & $\mathrm{~m}$ & $2.08 \mathrm{E}+02$ & $4.18 \mathrm{E}+08$ & -- & -- & -- & -- & -- & -- & -- \\
\hline Cs-131 & Cs-131 & 130.905 & Cesium & 0.01 & 0.08 & 2000 & ICRP-38 & $9.69 \mathrm{E}+00$ & $d$ & $8.37 E+05$ & $1.03 E+05$ & ICRP-68 & $4.5 \mathrm{E}-11$ & $F$ & ICRP-68 & $5.8 \mathrm{E}-11$ & -- & -- \\
\hline Cs-132 & Cs-132 & 131.906 & Cesium & 0.01 & 0.08 & 2000 & ICRP-38 & $6.48 \mathrm{E}+00$ & $d$ & $5.59 \mathrm{E}+05$ & $1.53 \mathrm{E}+05$ & ICRP-68 & $3.8 \mathrm{E}-10$ & $F$ & ICRP-68 & $5.0 \mathrm{E}-10$ & -- & -- \\
\hline Cs-134 & Cs-134 & 133.907 & Cesium & 0.01 & 0.08 & 2000 & ICRP-38 & $2.06 \mathrm{E}+00$ & $y$ & $6.51 \mathrm{E}+07$ & $1.29 \mathrm{E}+03$ & ICRP-68 & $9.6 \mathrm{E}-09$ & $F$ & ICRP-68 & $1.9 \mathrm{E}-08$ & -- & -- \\
\hline Cs-134m & Cs-134 & 133.907 & Cesium & 0.01 & 0.08 & 2000 & ICRP-38 & $2.90 \mathrm{E}+00$ & $\mathrm{~h}$ & $1.04 \mathrm{E}+04$ & $8.07 E+06$ & ICRP-68 & $2.6 \mathrm{E}-11$ & $\mathrm{~F}$ & ICRP-68 & $2.0 \mathrm{E}-11$ & -- & -- \\
\hline Cs-135 & Cs-135 & 134.906 & Cesium & 0.01 & 0.08 & 2000 & ICRP-38 & $2.30 \mathrm{E}+06$ & $y$ & $7.26 \mathrm{E}+13$ & $1.15 \mathrm{E}-03$ & ICRP-68 & $9.9 \mathrm{E}-10$ & $F$ & ICRP-68 & $2.0 \mathrm{E}-09$ & -- & -- \\
\hline Cs-135m & Cs-135 & 134.906 & Cesium & 0.01 & 0.08 & 2000 & ICRP-38 & $5.30 \mathrm{E}+01$ & $\mathrm{~m}$ & $3.18 \mathrm{E}+03$ & $2.63 \mathrm{E}+07$ & ICRP-68 & $2.4 \mathrm{E}-11$ & $\mathrm{~F}$ & ICRP-68 & $1.9 \mathrm{E}-11$ & -- & -- \\
\hline Cs-136 & Cs-136 & 135.907 & Cesium & 0.01 & 0.08 & 2000 & ICRP-38 & $1.31 \mathrm{E}+01$ & $d$ & $1.13 \mathrm{E}+06$ & $7.33 \mathrm{E}+04$ & ICRP-68 & $1.9 \mathrm{E}-09$ & $\mathrm{~F}$ & ICRP-68 & $3.0 \mathrm{E}-09$ & -- & -- \\
\hline Cs-137 & Cs-137 & 136.907 & Cesium & 0.01 & 0.08 & 2000 & ICRP-38 & $3.00 \mathrm{E}+01$ & $y$ & $9.47 E+08$ & $8.70 \mathrm{E}+01$ & ICRP-68 & $6.7 \mathrm{E}-09$ & $F$ & ICRP-68 & $1.3 \mathrm{E}-08$ & -- & -- \\
\hline Cs-138 & Cs-138 & 137.911 & Cesium & 0.01 & 0.08 & 2000 & ICRP-38 & $3.22 \mathrm{E}+01$ & $\mathrm{~m}$ & $1.93 \mathrm{E}+03$ & $4.23 \mathrm{E}+07$ & ICRP-68 & $4.6 \mathrm{E}-11$ & $\mathrm{~F}$ & ICRP-68 & $9.2 \mathrm{E}-11$ & -- & -- \\
\hline Cs-138m & Cs-138 & 137.911 & Cesium & 0.01 & 0.08 & 2000 & ICRP-107 & $2.91 \mathrm{E}+00$ & $\mathrm{~m}$ & $1.75 \mathrm{E}+02$ & $4.69 \mathrm{E}+08$ & -- & -- & -- & -- & -- & -- & -- \\
\hline Cs-139 & Cs-139 & 138.913 & Cesium & 0.01 & 0.08 & 2000 & ICRP-107 & $9.27 E+00$ & $\mathrm{~m}$ & $5.56 \mathrm{E}+02$ & $1.46 \mathrm{E}+08$ & -- & -- & -- & -- & -- & -- & -- \\
\hline Cs-140 & Cs-140 & 139.917 & Cesium & 0.01 & 0.08 & 2000 & ICRP-107 & $6.37 \mathrm{E}+01$ & $\mathrm{~s}$ & $6.37 \mathrm{E}+01$ & $1.27 \mathrm{E}+09$ & -- & -- & -- & -- & -- & -- & -- \\
\hline Cu-57 & Cu-57 & 56.949 & Copper & 0.01 & 0.4 & 0 & ICRP-38 & $2.33 \mathrm{E}+02$ & $\mathrm{~ms}$ & $2.33 \mathrm{E}-01$ & $8.50 \mathrm{E}+11$ & -- & -- & -- & -- & -- & -- & -- \\
\hline Cu-59 & Cu-59 & 58.939 & Copper & 0.01 & 0.4 & 0 & ICRP-107 & $8.15 \mathrm{E}+01$ & $\mathrm{~s}$ & $8.15 \mathrm{E}+01$ & $2.35 \mathrm{E}+09$ & -- & -- & -- & -- & -- & -- & -- \\
\hline $\mathrm{Cu}-60$ & Cu-60 & 59.937 & Copper & 0.01 & 0.4 & 0 & ICRP-38 & $2.32 \mathrm{E}+01$ & $\mathrm{~m}$ & $1.39 \mathrm{E}+03$ & $1.35 \mathrm{E}+08$ & ICRP-68 & $6.2 \mathrm{E}-11$ & $\mathrm{~s}$ & ICRP-68 & $7.0 \mathrm{E}-11$ & -- & -- \\
\hline Cu-61 & Cu-61 & 60.933 & Copper & 0.01 & 0.4 & 0 & ICRP-38 & $3.41 \mathrm{E}+00$ & $\mathrm{~h}$ & $1.23 \mathrm{E}+04$ & $1.51 \mathrm{E}+07$ & ICRP-68 & $1.2 \mathrm{E}-10$ & $M$ & ICRP-68 & $1.2 \mathrm{E}-10$ & -- & -- \\
\hline Cu-62 & Cu-62 & 61.933 & Copper & 0.01 & 0.4 & 0 & ICRP-38 & $9.74 \mathrm{E}+00$ & $\mathrm{~m}$ & $5.84 \mathrm{E}+02$ & $3.12 \mathrm{E}+08$ & -- & -- & -- & -- & -- & -- & -- \\
\hline Cu-64 & Cu-64 & 63.930 & Copper & 0.01 & 0.4 & 0 & ICRP-38 & $1.27 \mathrm{E}+01$ & $\mathrm{~h}$ & $4.57 \mathrm{E}+04$ & $3.86 \mathrm{E}+06$ & ICRP-68 & $1.5 \mathrm{E}-10$ & $M$ & ICRP-68 & $1.2 \mathrm{E}-10$ & -- & -- \\
\hline Cu-66 & Cu-66 & 65.929 & Copper & 0.01 & 0.4 & 0 & ICRP-38 & $5.10 \mathrm{E}+00$ & $\mathrm{~m}$ & $3.06 \mathrm{E}+02$ & $5.59 \mathrm{E}+08$ & -- & -- & -- & -- & -- & -- & -- \\
\hline Cu-67 & Cu-67 & 66.928 & Copper & 0.01 & 0.4 & 0 & ICRP-38 & $6.19 \mathrm{E}+01$ & $\mathrm{~h}$ & $2.23 \mathrm{E}+05$ & $7.57 \mathrm{E}+05$ & ICRP-68 & $5.8 \mathrm{E}-10$ & $\mathrm{~s}$ & ICRP-68 & $3.4 \mathrm{E}-10$ & -- & -- \\
\hline Cu-69 & Cu-69 & 68.929 & Copper & 0.01 & 0.4 & 0 & ICRP-107 & $2.85 \mathrm{E}+00$ & $\mathrm{~m}$ & $1.71 \mathrm{E}+02$ & $9.57 E+08$ & -- & -- & -- & -- & -- & -- & -- \\
\hline Dy-148 & Dy-148 & 147.927 & Dysprosium & 0.01 & 0.01 & 0 & ICRP-107 & $3.30 \mathrm{E}+00$ & $\mathrm{~m}$ & $1.98 \mathrm{E}+02$ & $3.85 \mathrm{E}+08$ & -- & -- & -- & -- & -- & -- & -- \\
\hline Dy-149 & Dy-149 & 148.927 & Dysprosium & 0.01 & 0.01 & 0 & ICRP-107 & $4.20 \mathrm{E}+00$ & $\mathrm{~m}$ & $2.52 \mathrm{E}+02$ & $3.01 \mathrm{E}+08$ & -- & -- & -- & -- & -- & -- & -- \\
\hline Dy-150 & Dy-150 & 149.926 & Dysprosium & 0.01 & 0.01 & 0 & ICRP-107 & $7.17 \mathrm{E}+00$ & $\mathrm{~m}$ & $4.30 \mathrm{E}+02$ & $1.75 \mathrm{E}+08$ & -- & -- & -- & -- & -- & -- & -- \\
\hline Dy-151 & Dy-151 & 150.926 & Dysprosium & 0.01 & 0.01 & 0 & ICRP-107 & $1.79 \mathrm{E}+01$ & $\mathrm{~m}$ & $1.07 E+03$ & $6.96 \mathrm{E}+07$ & JAERI & $1.2 \mathrm{E}-10$ & $M$ & JAERI & $2.0 \mathrm{E}-11$ & -- & -- \\
\hline Dy-152 & Dy-152 & 151.925 & Dysprosium & 0.01 & 0.01 & 0 & ICRP-107 & $2.38 \mathrm{E}+00$ & $\mathrm{~h}$ & $8.57 \mathrm{E}+03$ & $8.67 \mathrm{E}+06$ & JAERI & $8.8 \mathrm{E}-11$ & $M$ & JAERI & $1.1 \mathrm{E}-10$ & -- & -- \\
\hline Dy-153 & Dy-153 & 152.926 & Dysprosium & 0.01 & 0.01 & 0 & ICRP-107 & $6.40 \mathrm{E}+00$ & $\mathrm{~h}$ & $2.30 \mathrm{E}+04$ & $3.20 \mathrm{E}+06$ & JAERI & $1.8 \mathrm{E}-10$ & $\mathrm{M}$ & JAERI & $1.9 \mathrm{E}-10$ & -- & -- \\
\hline Dy-154 & Dy-154 & 153.924 & Dysprosium & 0.01 & 0.01 & 0 & ICRP-107 & $3.00 \mathrm{E}+06$ & $y$ & $9.47 \mathrm{E}+13$ & $7.74 \mathrm{E}-04$ & JAERI & $1.1 \mathrm{E}-05$ & $M$ & JAERI & $5.6 \mathrm{E}-08$ & -- & -- \\
\hline Dy-155 & Dy-155 & 154.926 & Dysprosium & 0.01 & 0.01 & 0 & ICRP-38 & $1.00 \mathrm{E}+01$ & $h$ & $3.60 \mathrm{E}+04$ & $2.02 \mathrm{E}+06$ & ICRP-68 & $1.2 \mathrm{E}-10$ & $M$ & ICRP-68 & $1.3 \mathrm{E}-10$ & -- & -- \\
\hline Dy-157 & Dy-157 & 156.925 & Dysprosium & 0.01 & 0.01 & 0 & ICRP-38 & $8.10 \mathrm{E}+00$ & $\mathrm{~h}$ & $2.92 \mathrm{E}+04$ & $2.47 E+06$ & ICRP-68 & $5.5 \mathrm{E}-11$ & $M$ & ICRP-68 & $6.1 \mathrm{E}-11$ & -- & -- \\
\hline Dy-159 & Dy-159 & 158.926 & Dysprosium & 0.01 & 0.01 & 0 & ICRP-38 & $1.44 \mathrm{E}+02$ & $d$ & $1.25 \mathrm{E}+07$ & $5.69 \mathrm{E}+03$ & ICRP-68 & $3.5 \mathrm{E}-10$ & $M$ & ICRP-68 & $1.0 \mathrm{E}-10$ & -- & -- \\
\hline Dy-165 & Dy-165 & 164.932 & Dysprosium & 0.01 & 0.01 & 0 & ICRP-38 & $2.33 \mathrm{E}+00$ & $\mathrm{~h}$ & $8.40 \mathrm{E}+03$ & $8.14 \mathrm{E}+06$ & ICRP-68 & $8.7 E-11$ & $M$ & ICRP-68 & $1.1 \mathrm{E}-10$ & -- & -- \\
\hline Dy-165m & Dy-165 & 164.932 & Dysprosium & 0.01 & 0.01 & 0 & ICRP-107 & $1.26 \mathrm{E}+00$ & $\mathrm{~m}$ & $7.54 \mathrm{E}+01$ & $9.07 E+08$ & -- & -- & -- & -- & -- & -- & -- \\
\hline Dy-166 & Dy-166 & 165.933 & Dysprosium & 0.01 & 0.01 & 0 & $\begin{array}{l}\text { ICRP-38 } \\
\end{array}$ & $8.16 \mathrm{E}+01$ & $\mathrm{~h}$ & $2.94 \mathrm{E}+05$ & $2.31 \mathrm{E}+05$ & ICRP-68 & $1.8 \mathrm{E}-09$ & $M$ & ICRP-68 & $1.6 \mathrm{E}-09$ & -- & -- \\
\hline Dy-167 & Dy-167 & 166.936 & Dysprosium & 0.01 & 0.01 & 0 & ICRP-107 & $6.20 \mathrm{E}+00$ & $\mathrm{~m}$ & $3.72 \mathrm{E}+02$ & $1.82 \mathrm{E}+08$ & -- & -- & -- & -- & -- & -- & -- \\
\hline Dy-168 & Dy-168 & 167.937 & Dysprosium & 0.01 & 0.01 & 0 & ICRP-107 & $8.70 \mathrm{E}+00$ & $\mathrm{~m}$ & $5.22 \mathrm{E}+02$ & $1.29 \mathrm{E}+08$ & -- & -- & -- & -- & -- & -- & -- \\
\hline Er-154 & Er-154 & 153.933 & Erbium & 0.01 & 0.01 & 0 & ICRP-107 & $3.73 \mathrm{E}+00$ & $\mathrm{~m}$ & $2.24 \mathrm{E}+02$ & $3.27 \mathrm{E}+08$ & -- & -- & -- & -- & -- & -- & -- \\
\hline Er-155 & Er-155 & 154.933 & Erbium & 0.01 & 0.01 & 0 & JAERI & $5.30 \mathrm{E}+00$ & $\mathrm{~m}$ & $3.18 \mathrm{E}+02$ & $2.29 \mathrm{E}+08$ & -- & -- & -- & -- & -- & -- & -- \\
\hline Er-156 & Er-156 & 155.931 & Erbium & 0.01 & 0.01 & 0 & ICRP-107 & $1.95 \mathrm{E}+01$ & $\mathrm{~m}$ & $1.17 \mathrm{E}+03$ & $6.18 \mathrm{E}+07$ & JAERI & $3.0 \mathrm{E}-11$ & $\mathrm{M}$ & JAERI & $3.8 \mathrm{E}-11$ & -- & -- \\
\hline Er-159 & Er-159 & 158.931 & Erbium & 0.01 & 0.01 & 0 & ICRP-107 & $3.60 \mathrm{E}+01$ & $\mathrm{~m}$ & $2.16 \mathrm{E}+03$ & $3.29 \mathrm{E}+07$ & JAERI & $3.0 \mathrm{E}-11$ & $\mathrm{M}$ & JAERI & $2.4 \mathrm{E}-11$ & -- & -- \\
\hline Er-161 & Er-161 & 160.930 & Erbium & 0.01 & 0.01 & 0 & ICRP-38 & $3.24 \mathrm{E}+00$ & $\mathrm{~h}$ & $1.17 \mathrm{E}+04$ & $6.01 \mathrm{E}+06$ & ICRP-68 & $8.5 \mathrm{E}-11$ & $M$ & ICRP-68 & $8.0 \mathrm{E}-11$ & -- & -- \\
\hline Er-163 & Er-163 & 162.930 & Erbium & 0.01 & 0.01 & 0 & ICRP-107 & $7.50 \mathrm{E}+01$ & $\mathrm{~m}$ & $4.50 \mathrm{E}+03$ & $1.54 \mathrm{E}+07$ & JAERI & $2.2 \mathrm{E}-12$ & $M$ & JAERI & $2.7 \mathrm{E}-12$ & -- & -- \\
\hline Er-165 & Er-165 & 164.931 & Erbium & 0.01 & 0.01 & 0 & ICRP-38 & $1.04 \mathrm{E}+01$ & $\mathrm{~h}$ & $3.73 \mathrm{E}+04$ & $1.83 \mathrm{E}+06$ & ICRP-68 & $1.4 \mathrm{E}-11$ & $M$ & ICRP-68 & $1.9 \mathrm{E}-11$ & -- & -- \\
\hline
\end{tabular}




\begin{tabular}{|c|c|c|c|c|c|c|c|c|c|c|c|c|c|c|c|c|c|c|}
\hline \multirow{3}{*}{$\begin{array}{l}\text { MASTER } \\
\text { ISOTOPE } \\
\text { LIST } \\
\text { Er-167m }\end{array}$} & \multirow{3}{*}{$\begin{array}{c}\begin{array}{c}\text { Atomic } \\
\text { Mass } \\
\text { Nuclide }\end{array} \\
\text { Er-167 } \\
\end{array}$} & \multirow{3}{*}{$\begin{array}{c}\begin{array}{c}\text { Atomic } \\
\text { Mass }\end{array} \\
166.932\end{array}$} & \multirow{3}{*}{$\begin{array}{c}\text { Element } \\
\text { Erbium }\end{array}$} & \multirow{3}{*}{$\begin{array}{c}\text { EPA } \\
\text { RF } \\
0.01\end{array}$} & \multicolumn{14}{|c|}{ Table C.1 Radionuclide Specific Input Data Used For the Calculation of HC-3 TQs Using Maximum Reported DCs (continued) } \\
\hline & & & & & $\begin{array}{c}\text { EPA } \\
\text { Conc } \\
\text { Factors } \\
\left(B_{v}\right) \\
\end{array}$ & $\begin{array}{c}\text { EPA } \\
\text { Sorption } \\
\text { Coefficient } \\
\left(K_{d}\right)\end{array}$ & $\begin{array}{c}\text { Half-Life } \\
\text { Reference }\end{array}$ & \multicolumn{2}{|c|}{$\begin{array}{c}\begin{array}{c}\text { Half-Life } \\
\text { (original units) }\end{array}\end{array}$} & \multirow{2}{*}{$\begin{array}{c}\text { Half Life } \\
\text { (s) } \\
2.28 \mathrm{E}+00\end{array}$} & \multirow{2}{*}{$\begin{array}{c}\begin{array}{c}\text { Sp. Act } \\
\text { (Ci/g) }\end{array} \\
2.96 \mathrm{E}+10\end{array}$} & \multirow[t]{2}{*}{$\begin{array}{c}\begin{array}{c}\text { Inhalation } \\
\text { DC } \\
\text { Reference }\end{array} \\
--\end{array}$} & \multirow[t]{2}{*}{$\begin{array}{c}\text { HC-3 } \\
\text { Bounding } \\
\text { Inhalation } \\
\mathrm{DC} \\
(\mathrm{Sv} / \mathrm{Bq}) \\
--\end{array}$} & \multirow[t]{2}{*}{$\begin{array}{c}\begin{array}{c}\text { Lung } \\
\text { Absorption } \\
\text { Class/Type }\end{array} \\
--\end{array}$} & \multirow[t]{2}{*}{$\begin{array}{c}\begin{array}{c}\text { Ingestion } \\
\text { DC } \\
\text { Reference }\end{array} \\
--\end{array}$} & \multirow[t]{2}{*}{$\begin{array}{c}\text { HC-3 } \\
\text { Bounding } \\
\text { Ingestion } \\
\text { DC } \\
\text { (Sv/Bq) } \\
--\end{array}$} & \multirow[t]{2}{*}{$\begin{array}{c}\text { Immersion } \\
\text { DC } \\
\text { Reference }\end{array}$} & \multirow[t]{2}{*}{$\begin{array}{c}\mathrm{HC}-3 \\
\text { Immersion } \\
\mathrm{DC}(\mathrm{Sv} / \mathrm{d} \\
\text { per } \mathrm{Bq} / \mathrm{m}^{3}\end{array}$} \\
\hline & & & & & 0.01 & 0 & ICRP-38 & $2.28 \mathrm{E}+00$ & $\mathrm{~s}$ & & & & & & & & & \\
\hline Er-169 & Er-169 & 168.935 & Erbium & 0.01 & 0.01 & 0 & ICRP-38 & $9.30 \mathrm{E}+00$ & d & $8.04 \mathrm{E}+05$ & $8.31 \mathrm{E}+04$ & ICRP-68 & $9.8 \mathrm{E}-10$ & $\mathrm{M}$ & ICRP-68 & $3.7 \mathrm{E}-10$ & -- & -- \\
\hline Er-171 & Er-171 & 170.938 & Erbium & 0.01 & 0.01 & 0 & ICRP-38 & $7.52 \mathrm{E}+00$ & $\mathrm{~h}$ & $2.71 \mathrm{E}+04$ & $2.44 \mathrm{E}+06$ & ICRP-68 & $3.0 \mathrm{E}-10$ & $\mathrm{M}$ & ICRP-68 & $3.6 \mathrm{E}-10$ & -- & -- \\
\hline Er-172 & Er-172 & 171.939 & Erbium & 0.01 & 0.01 & 0 & ICRP-38 & $4.93 \mathrm{E}+01$ & $\mathrm{~h}$ & $1.77 E+05$ & $3.70 \mathrm{E}+05$ & ICRP-68 & $1.2 \mathrm{E}-09$ & $M$ & ICRP-68 & $1.0 \mathrm{E}-09$ & -- & -- \\
\hline Er-173 & Er-173 & 172.942 & Erbium & 0.01 & 0.01 & 0 & ICRP-107 & $1.43 \mathrm{E}+00$ & $\mathrm{~m}$ & $8.60 E+01$ & $7.58 \mathrm{E}+08$ & -- & -- & -- & -- & -- & -- & -- \\
\hline Es-249 & Es-249 & 249.076 & Einsteinium & 0.001 & 0.001 & 0 & ICRP-107 & $1.02 \mathrm{E}+02$ & $\mathrm{~m}$ & $6.13 \mathrm{E}+03$ & $7.39 \mathrm{E}+06$ & JAERI & $2.7 \mathrm{E}-10$ & $M$ & JAERI & $2.2 \mathrm{E}-11$ & -- & -- \\
\hline Es-250 & Es-250 & 250.079 & Einsteinium & 0.001 & 0.001 & 0 & ICRP-38 & $2.10 \mathrm{E}+00$ & $\mathrm{~h}$ & $7.56 \mathrm{E}+03$ & $5.97 \mathrm{E}+06$ & ICRP-68 & $5.9 \mathrm{E}-10$ & $\mathrm{M}$ & ICRP-68 & $2.1 \mathrm{E}-11$ & -- & -- \\
\hline Es-250m & Es-250 & 250.079 & Einsteinium & 0.001 & 0.001 & 0 & ICRP-107 & $2.22 \mathrm{E}+00$ & $\mathrm{~h}$ & $7.99 \mathrm{E}+03$ & $5.64 \mathrm{E}+06$ & -- & -- & -- & -- & -- & -- & -- \\
\hline Es-251 & Es-251 & 251.080 & Einsteinium & 0.001 & 0.001 & 0 & ICRP-38 & $3.30 \mathrm{E}+01$ & $\mathrm{~h}$ & $1.19 \mathrm{E}+05$ & $3.78 \mathrm{E}+05$ & ICRP-68 & $2.0 \mathrm{E}-09$ & $M$ & ICRP-68 & $1.7 \mathrm{E}-10$ & -- & -- \\
\hline Es-253 & Es-253 & 253.085 & Einsteinium & 0.001 & 0.001 & 0 & ICRP-38 & $2.05 \mathrm{E}+01$ & $d$ & $1.77 E+06$ & $2.52 \mathrm{E}+04$ & ICRP-68 & $2.5 \mathrm{E}-06$ & $M$ & ICRP-68 & $6.1 \mathrm{E}-09$ & -- & -- \\
\hline Es-254 & Es-254 & 254.088 & Einsteinium & 0.001 & 0.001 & 0 & ICRP-38 & $2.76 \mathrm{E}+02$ & $d$ & $2.38 \mathrm{E}+07$ & $1.86 \mathrm{E}+03$ & ICRP-68 & $8.0 \mathrm{E}-06$ & $M$ & ICRP-68 & $2.8 \mathrm{E}-08$ & -- & -- \\
\hline Es-254m & Es-254 & 254.088 & Einsteinium & 0.01 & 0.001 & 0 & ICRP-38 & $3.93 \mathrm{E}+01$ & $\mathrm{~h}$ & $1.41 \mathrm{E}+05$ & $3.14 \mathrm{E}+05$ & ICRP-68 & $4.4 \mathrm{E}-07$ & $M$ & ICRP-68 & $4.2 \mathrm{E}-09$ & -- & -- \\
\hline Es-255 & Es-255 & 255.090 & Einsteinium & 0.001 & 0.001 & 0 & ICRP-107 & $3.98 \mathrm{E}+01$ & $d$ & $3.44 E+06$ & $1.29 \mathrm{E}+04$ & JAERI & $3.6 \mathrm{E}-06$ & $M$ & JAERI & $6.0 \mathrm{E}-09$ & -- & -- \\
\hline Es-256 & Es-256 & 256.094 & Einsteinium & 0.001 & 0.001 & 0 & ICRP-107 & $2.54 \mathrm{E}+01$ & $\mathrm{~m}$ & $1.52 \mathrm{E}+03$ & $2.89 \mathrm{E}+07$ & JAERI & $3.4 \mathrm{E}-07$ & $\mathrm{M}$ & JAERI & $4.1 \mathrm{E}-09$ & -- & -- \\
\hline Eu-142 & Eu-142 & 141.923 & Europium & 0.01 & 0.01 & 500 & ICRP-107 & $2.34 \mathrm{E}+00$ & $\mathrm{~s}$ & $2.34 \mathrm{E}+00$ & $3.40 \mathrm{E}+10$ & -- & -- & -- & -- & -- & -- & -- \\
\hline Eu-142m & Eu-142 & 141.923 & Europium & 0.01 & 0.01 & 500 & ICRP-107 & $1.22 \mathrm{E}+00$ & $\mathrm{~m}$ & $7.34 \mathrm{E}+01$ & $1.08 \mathrm{E}+09$ & -- & -- & -- & -- & -- & -- & -- \\
\hline Eu-143 & Eu-143 & 142.920 & Europium & 0.01 & 0.01 & 500 & ICRP-107 & $2.59 \mathrm{E}+00$ & $\mathrm{~m}$ & $1.55 \mathrm{E}+02$ & $5.08 \mathrm{E}+08$ & -- & -- & -- & -- & -- & -- & -- \\
\hline Eu-144 & Eu-144 & 143.919 & Europium & 0.01 & 0.01 & 500 & ICRP-107 & $1.02 \mathrm{E}+01$ & $\mathrm{~s}$ & $1.02 E+01$ & $7.69 \mathrm{E}+09$ & -- & -- & -- & -- & -- & -- & -- \\
\hline Eu-145 & Eu-145 & 144.916 & Europium & 0.01 & 0.01 & 500 & ICRP-38 & $5.94 \mathrm{E}+00$ & $d$ & $5.13 \mathrm{E}+05$ & $1.52 \mathrm{E}+05$ & ICRP-68 & $7.3 \mathrm{E}-10$ & $M$ & ICRP-68 & $7.5 \mathrm{E}-10$ & -- & -- \\
\hline Eu-146 & Eu-146 & 145.917 & Europium & 0.01 & 0.01 & 500 & ICRP-38 & $4.61 \mathrm{E}+00$ & $d$ & $3.98 \mathrm{E}+05$ & $1.94 \mathrm{E}+05$ & ICRP-68 & $1.2 \mathrm{E}-09$ & $M$ & ICRP-68 & $1.3 \mathrm{E}-09$ & -- & -- \\
\hline Eu-147 & Eu-147 & 146.917 & Europium & 0.01 & 0.01 & 500 & ICRP-38 & $2.40 \mathrm{E}+01$ & $d$ & $2.07 E+06$ & $3.70 \mathrm{E}+04$ & ICRP-68 & $1.0 \mathrm{E}-09$ & $M$ & ICRP-68 & 4.4E-10 & -- & -- \\
\hline Eu-148 & Eu-148 & 147.918 & Europium & 0.01 & 0.01 & 500 & ICRP-38 & $5.45 \mathrm{E}+01$ & $d$ & $4.71 E+06$ & $1.62 \mathrm{E}+04$ & ICRP-68 & $2.7 \mathrm{E}-09$ & $M$ & ICRP-68 & $1.3 \mathrm{E}-09$ & -- & -- \\
\hline Eu-149 & Eu-149 & 148.918 & Europium & 0.01 & 0.01 & 500 & ICRP-38 & $9.31 \mathrm{E}+01$ & d & $8.04 E+06$ & $9.42 \mathrm{E}+03$ & ICRP-68 & $2.7 \mathrm{E}-10$ & $M$ & ICRP-68 & $1.0 \mathrm{E}-10$ & -- & -- \\
\hline Eu-150l & Eu-150 & 149.920 & Europium & 0.01 & 0.01 & 500 & ICRP-38 & $3.42 \mathrm{E}+01$ & $y$ & $1.08 \mathrm{E}+09$ & $6.97 \mathrm{E}+01$ & ICRP-68 & $5.0 \mathrm{E}-08$ & $M$ & ICRP-68 & $1.3 \mathrm{E}-09$ & -- & -- \\
\hline Eu-150s & Eu-150 & 149.920 & Europium & 0.01 & 0.01 & 500 & ICRP-38 & $1.26 \mathrm{E}+01$ & $\mathrm{~h}$ & $4.54 E+04$ & $1.66 \mathrm{E}+06$ & ICRP-68 & $2.8 \mathrm{E}-10$ & $M$ & ICRP-68 & $3.8 \mathrm{E}-10$ & -- & -- \\
\hline Eu-152 & Eu-152 & 151.922 & Europium & 0.01 & 0.01 & 500 & ICRP-38 & $1.33 \mathrm{E}+01$ & $y$ & $4.21 E+08$ & $1.77 \mathrm{E}+02$ & ICRP-68 & $3.9 \mathrm{E}-08$ & $M$ & ICRP-68 & $1.4 \mathrm{E}-09$ & -- & -- \\
\hline Eu-152ml & Eu-152 & 151.922 & Europium & 0.01 & 0.01 & 500 & ICRP-38 & $9.32 \mathrm{E}+00$ & $\mathrm{~h}$ & $3.36 \mathrm{E}+04$ & $2.21 \mathrm{E}+06$ & $\begin{array}{l}\text { ICRP-68 } \\
\end{array}$ & $3.2 \mathrm{E}-10$ & $\mathrm{M}$ & ICRP-68 & $5.0 \mathrm{E}-10$ & -- & -- \\
\hline Eu-152ms & Eu-152 & 151.922 & Europium & 0.01 & 0.01 & 500 & ICRP-107 & $9.60 \mathrm{E}+01$ & $\mathrm{~m}$ & $5.76 \mathrm{E}+03$ & $1.29 \mathrm{E}+07$ & JAERI & $1.1 \mathrm{E}-11$ & $M$ & JAERI & $1.4 \mathrm{E}-11$ & -- & -- \\
\hline Eu-154 & Eu-154 & 153.923 & Europium & 0.01 & 0.01 & 500 & $\begin{array}{l}\text { ICRP-38 } \\
\end{array}$ & $8.80 \mathrm{E}+00$ & $y$ & $2.78 \mathrm{E}+08$ & $2.64 \mathrm{E}+02$ & ICRP-68 & $5.0 \mathrm{E}-08$ & $M$ & ICRP-68 & $2.0 \mathrm{E}-09$ & -- & -- \\
\hline Eu-154m & Eu-154 & 153.923 & Europium & 0.01 & 0.01 & 500 & ICRP-107 & $4.60 \mathrm{E}+01$ & $\mathrm{~m}$ & $2.76 \mathrm{E}+03$ & $2.66 \mathrm{E}+07$ & JAERI & $5.8 \mathrm{E}-12$ & $\mathrm{M}$ & JAERI & $9.1 \mathrm{E}-12$ & -- & -- \\
\hline Eu-155 & Eu-155 & 154.923 & Europium & 0.01 & 0.01 & 500 & ICRP-38 & $4.96 \mathrm{E}+00$ & $y$ & $1.57 E+08$ & $4.65 \mathrm{E}+02$ & ICRP-68 & $6.5 \mathrm{E}-09$ & $M$ & ICRP-68 & $3.2 \mathrm{E}-10$ & -- & -- \\
\hline Eu-156 & Eu-156 & 155.925 & Europium & 0.01 & 0.01 & 500 & ICRP-38 & $1.52 \mathrm{E}+01$ & $d$ & $1.31 \mathrm{E}+06$ & $5.51 \mathrm{E}+04$ & ICRP-68 & $3.3 \mathrm{E}-09$ & $M$ & ICRP-68 & $2.2 \mathrm{E}-09$ & -- & -- \\
\hline Eu-157 & Eu-157 & 156.925 & Europium & 0.01 & 0.01 & 500 & ICRP-38 & $1.52 \mathrm{E}+01$ & $\mathrm{~h}$ & $5.45 E+04$ & $1.32 \mathrm{E}+06$ & ICRP-68 & $4.4 \mathrm{E}-10$ & $M$ & ICRP-68 & $6.0 \mathrm{E}-10$ & -- & -- \\
\hline Eu-158 & Eu-158 & 157.928 & Europium & 0.01 & 0.01 & 500 & ICRP-38 & $4.59 \mathrm{E}+01$ & $\mathrm{~m}$ & $2.75 \mathrm{E}+03$ & $2.59 \mathrm{E}+07$ & ICRP-68 & $7.5 \mathrm{E}-11$ & $M$ & ICRP-68 & $9.4 \mathrm{E}-11$ & -- & -- \\
\hline Eu-159 & Eu-159 & 158.929 & Europium & 0.01 & 0.01 & 500 & ICRP-107 & $1.81 \mathrm{E}+01$ & $\mathrm{~m}$ & $1.09 \mathrm{E}+03$ & $6.54 \mathrm{E}+07$ & JAERI & $3.6 \mathrm{E}-11$ & $M$ & JAERI & $4.9 \mathrm{E}-11$ & -- & -- \\
\hline F-17 & F-17 & 17.002 & $\begin{array}{l}\text { Fluorine } \\
\end{array}$ & 0.01 & 0.06 & 0 & ICRP-107 & $6.45 \mathrm{E}+01$ & $\mathrm{~s}$ & $6.45 \mathrm{E}+01$ & $1.03 \mathrm{E}+10$ & -- & -- & -- & -- & -- & -- & -- \\
\hline F-18 & F-18 & 18.001 & Fluorine & 0.01 & 0.06 & 0 & ICRP-38 & $1.10 \mathrm{E}+02$ & $\mathrm{~m}$ & $6.59 \mathrm{E}+03$ & $9.52 \mathrm{E}+07$ & ICRP-68 & $9.3 \mathrm{E}-11$ & $\mathrm{~s}$ & ICRP-68 & $4.9 \mathrm{E}-11$ & -- & -- \\
\hline Fe-52 & Fe-52 & 51.948 & Iron & 0.01 & 0.004 & 150 & ICRP-38 & $8.28 \mathrm{E}+00$ & $\mathrm{~h}$ & $2.98 \mathrm{E}+04$ & $7.29 E+06$ & ICRP-68 & $9.5 \mathrm{E}-10$ & $M$ & ICRP-68 & $1.4 \mathrm{E}-09$ & -- & -- \\
\hline Fe-53 & $\mathrm{Fe}-53$ & 52.945 & Iron & 0.01 & 0.004 & 150 & ICRP-107 & $8.51 E+00$ & $\mathrm{~m}$ & $5.11 \mathrm{E}+02$ & $4.17 \mathrm{E}+08$ & -- & -- & -- & -- & -- & -- & -- \\
\hline Fe-53m & Fe-53 & 52.945 & Iron & 0.01 & 0.004 & 150 & ICRP-107 & $2.53 \mathrm{E}+00$ & $\mathrm{~m}$ & $1.52 E+02$ & $1.41 \mathrm{E}+09$ & -- & -- & -- & -- & -- & -- & -- \\
\hline Fe-55 & Fe-55 & 54.938 & Iron & 0.01 & 0.004 & 150 & ICRP-38 & $2.70 \mathrm{E}+00$ & $y$ & $8.52 E+07$ & $2.41 \mathrm{E}+03$ & ICRP-68 & $9.2 \mathrm{E}-10$ & $\mathrm{~F}$ & ICRP-68 & $3.3 \mathrm{E}-10$ & -- & -- \\
\hline Fe-59 & $\mathrm{Fe}-59$ & 58.935 & Iron & 0.01 & 0.004 & 150 & ICRP-38 & $4.45 \mathrm{E}+01$ & $d$ & $3.85 \mathrm{E}+06$ & $4.98 \mathrm{E}+04$ & ICRP-68 & $3.5 \mathrm{E}-09$ & $M$ & ICRP-68 & $1.8 \mathrm{E}-09$ & -- & -- \\
\hline Fe-60 & Fe-60 & 59.934 & Iron & 0.01 & 0.004 & 150 & ICRP-38 & $1.00 \mathrm{E}+05$ & $y$ & $3.16 \mathrm{E}+12$ & $5.96 \mathrm{E}-02$ & ICRP-68 & $3.3 \mathrm{E}-07$ & $F$ & ICRP-68 & $1.1 \mathrm{E}-07$ & -- & -- \\
\hline Fe-61 & Fe-61 & 60.937 & Iron & 0.01 & 0.004 & 150 & ICRP-107 & $5.98 \mathrm{E}+00$ & $\mathrm{~m}$ & $3.59 \mathrm{E}+02$ & $5.16 \mathrm{E}+08$ & -- & -- & -- & -- & -- & -- & -- \\
\hline Fe-62 & Fe-62 & 61.937 & Iron & 0.01 & 0.004 & 150 & ICRP-107 & $6.80 \mathrm{E}+01$ & $\mathrm{~s}$ & $6.80 \mathrm{E}+01$ & $2.68 \mathrm{E}+09$ & -- & -- & -- & -- & -- & -- & -- \\
\hline
\end{tabular}




\begin{tabular}{|c|c|c|c|c|c|c|c|c|c|c|c|c|c|c|c|c|c|c|}
\hline \multirow{3}{*}{$\begin{array}{l}\text { MASTER } \\
\text { ISOTOPE } \\
\text { LIST } \\
\text { Fm-251 }\end{array}$} & \multirow{3}{*}{$\begin{array}{c}\begin{array}{c}\text { Atomic } \\
\text { Mass } \\
\text { Nuclide }\end{array} \\
\text { Fm-251 }\end{array}$} & & & & \multicolumn{14}{|c|}{ Table C.1 Radionuclide Spe } \\
\hline & & \multirow{2}{*}{$\begin{array}{c}\begin{array}{c}\text { Atomic } \\
\text { Mass }\end{array} \\
251.082\end{array}$} & \multirow{2}{*}{$\begin{array}{l}\text { Element } \\
\text { Fermium }\end{array}$} & \multirow{2}{*}{$\begin{array}{c}\text { EPA } \\
\text { RF } \\
0.001\end{array}$} & $\begin{array}{c}\text { EPA } \\
\text { Conc } \\
\text { Factors } \\
\left(B_{v}\right) \\
\end{array}$ & $\begin{array}{c}\text { EPA } \\
\text { Sorption } \\
\text { Coefficient } \\
\left(K_{d}\right)\end{array}$ & $\begin{array}{c}\text { Half-Life } \\
\text { Reference }\end{array}$ & $\begin{array}{r}\text { Half-Lif } \\
\text { (original u }\end{array}$ & & $\begin{array}{l}\text { Half Life } \\
\text { (s) }\end{array}$ & $\begin{array}{l}\text { Sp. Act } \\
\text { (Ci/g) }\end{array}$ & $\begin{array}{c}\text { Inhalation } \\
\text { DC } \\
\text { Reference }\end{array}$ & $\begin{array}{c}\text { HC-3 } \\
\text { Bounding } \\
\text { Inhalation } \\
\text { DC } \\
\text { (Sv/Bq) } \\
\end{array}$ & $\begin{array}{c}\text { Lung } \\
\text { Absorption } \\
\text { Class/Type }\end{array}$ & $\begin{array}{l}\text { Ingestion } \\
\text { DC } \\
\text { Reference }\end{array}$ & $\begin{array}{c}\text { HC-3 } \\
\text { Bounding } \\
\text { Ingestion } \\
\text { DC } \\
\text { (Sv/Bq) } \\
\end{array}$ & $\begin{array}{c}\text { Immersion } \\
\text { DC } \\
\text { Reference }\end{array}$ & $\begin{array}{c}\mathrm{HC}-3 \\
\text { Immersion } \\
\mathrm{DC}(\mathrm{Sv} / \mathrm{d} \\
\left.\operatorname{per} \mathrm{Bq} / \mathrm{m}^{3}\right)\end{array}$ \\
\hline & & & & & 0.001 & 0 & ICRP-107 & $5.30 \mathrm{E}+00$ & $\mathrm{~h}$ & $1.91 \mathrm{E}+04$ & $2.35 \mathrm{E}+06$ & JAERI & $1.8 \mathrm{E}-09$ & $M$ & JAERI & $7.2 \mathrm{E}-11$ & -- & -- \\
\hline Fm-252 & $\mathrm{Fm}-252$ & 252.082 & Fermium & 0.001 & 0.001 & 0 & ICRP-38 & $2.27 \mathrm{E}+01$ & $\mathrm{~h}$ & $8.17 E+04$ & $5.48 \mathrm{E}+05$ & ICRP-68 & $3.0 \mathrm{E}-07$ & $\mathrm{M}$ & ICRP-68 & $2.7 \mathrm{E}-09$ & -- & -- \\
\hline Fm-253 & Fm-253 & 253.085 & Fermium & 0.001 & 0.001 & 0 & ICRP-38 & $3.00 \mathrm{E}+00$ & $\mathrm{~d}$ & $2.59 \mathrm{E}+05$ & $1.72 \mathrm{E}+05$ & ICRP-68 & $3.7 \mathrm{E}-07$ & $\mathrm{M}$ & ICRP-68 & $9.1 \mathrm{E}-10$ & -- & -- \\
\hline Fm-254 & $\mathrm{Fm}-254$ & 254.087 & Fermium & 0.001 & 0.001 & 0 & ICRP-38 & $3.24 \mathrm{E}+00$ & $\mathrm{~h}$ & $1.17 E+04$ & $3.81 \mathrm{E}+06$ & ICRP-68 & $7.7 \mathrm{E}-08$ & $\mathrm{M}$ & ICRP-68 & $4.4 \mathrm{E}-10$ & -- & -- \\
\hline Fm-255 & Fm-255 & 255.090 & Fermium & 0.001 & 0.001 & 0 & ICRP-38 & $2.01 \mathrm{E}+01$ & $\mathrm{~h}$ & $7.23 \mathrm{E}+04$ & $6.12 \mathrm{E}+05$ & ICRP-68 & $2.6 \mathrm{E}-07$ & $\mathrm{M}$ & ICRP-68 & $2.5 \mathrm{E}-09$ & -- & -- \\
\hline Fm-256 & Fm-256 & 256.092 & Fermium & 0.001 & 0.001 & 0 & ICRP-107 & $1.58 \mathrm{E}+02$ & $\mathrm{~m}$ & $9.46 \mathrm{E}+03$ & $4.66 \mathrm{E}+06$ & JAERI & $7.0 \mathrm{E}-06$ & $\mathrm{M}$ & JAERI & $2.6 \mathrm{E}-08$ & -- & -- \\
\hline Fm-257 & Fm-257 & 257.095 & Fermium & 0.001 & 0.001 & 0 & ICRP-38 & $1.01 \mathrm{E}+02$ & d & $8.68 \mathrm{E}+06$ & $5.05 \mathrm{E}+03$ & ICRP-68 & $6.6 \mathrm{E}-06$ & $\mathrm{M}$ & ICRP-68 & $1.5 \mathrm{E}-08$ & -- & -- \\
\hline Fr-212 & Fr-212 & 211.996 & Francium & 0.01 & 0.03 & 200 & ICRP-107 & $2.00 \mathrm{E}+01$ & $\mathrm{~m}$ & $1.20 \mathrm{E}+03$ & $4.43 \mathrm{E}+07$ & JAERI & $2.8 \mathrm{E}-09$ & $\mathrm{~F}$ & JAERI & $7.1 \mathrm{E}-10$ & -- & -- \\
\hline Fr-219 & Fr-219 & 219.009 & Francium & 0.01 & 0.03 & 200 & $\begin{array}{l}\text { ICRP-38 } \\
\end{array}$ & $2.10 \mathrm{E}+01$ & $\mathrm{~ms}$ & $2.10 \mathrm{E}-02$ & $2.45 \mathrm{E}+12$ & -- & -- & -- & -- & -- & -- & -- \\
\hline Fr-220 & Fr-220 & 220.012 & Francium & 0.01 & 0.03 & 200 & ICRP-38 & $2.74 \mathrm{E}+01$ & $\mathrm{~s}$ & $2.74 \mathrm{E}+01$ & $1.87 \mathrm{E}+09$ & -- & -- & -- & -- & -- & -- & -- \\
\hline Fr-221 & Fr-221 & 221.014 & Francium & 0.01 & 0.03 & 200 & ICRP-38 & $4.80 \mathrm{E}+00$ & $\mathrm{~m}$ & $2.88 \mathrm{E}+02$ & $1.77 E+08$ & -- & -- & -- & -- & -- & -- & -- \\
\hline Fr-222 & Fr-222 & 222.018 & Francium & 0.01 & 0.03 & 200 & ICRP-38 & $1.44 \mathrm{E}+01$ & $\mathrm{~m}$ & $8.64 \mathrm{E}+02$ & $5.88 \mathrm{E}+07$ & ICRP-68 & $2.1 \mathrm{E}-08$ & $\mathrm{~F}$ & ICRP-68 & $7.1 \mathrm{E}-10$ & -- & -- \\
\hline Fr-223 & Fr-223 & 223.020 & Francium & 0.01 & 0.03 & 200 & ICRP-38 & $2.18 \mathrm{E}+01$ & $\mathrm{~m}$ & $1.31 \mathrm{E}+03$ & $3.87 \mathrm{E}+07$ & $\begin{array}{l}\text { ICRP-68 } \\
\end{array}$ & $1.3 \mathrm{E}-09$ & $F$ & ICRP-68 & $2.3 \mathrm{E}-09$ & -- & -- \\
\hline Fr-224 & Fr-224 & 224.023 & Francium & 0.01 & 0.03 & 200 & ICRP-107 & $3.33 \mathrm{E}+00$ & $\mathrm{~m}$ & $2.00 \mathrm{E}+02$ & $2.52 \mathrm{E}+08$ & -- & -- & -- & -- & -- & -- & -- \\
\hline Fr-227 & Fr-227 & 227.032 & Francium & 0.01 & 0.03 & 200 & ICRP-107 & $2.47 \mathrm{E}+00$ & $\mathrm{~m}$ & $1.48 \mathrm{E}+02$ & $3.35 \mathrm{E}+08$ & -- & -- & -- & -- & -- & -- & -- \\
\hline Ga-64 & Ga-64 & 63.937 & Gallium & 0.01 & 0.004 & 0 & ICRP-107 & $2.63 \mathrm{E}+00$ & $\mathrm{~m}$ & $1.58 \mathrm{E}+02$ & $1.12 \mathrm{E}+09$ & -- & -- & -- & -- & -- & -- & -- \\
\hline Ga-65 & Ga-65 & 64.933 & Gallium & 0.01 & 0.004 & 0 & ICRP-38 & $1.52 \mathrm{E}+01$ & $\mathrm{~m}$ & $9.12 \mathrm{E}+02$ & $1.91 \mathrm{E}+08$ & ICRP-68 & $2.9 \mathrm{E}-11$ & $\mathrm{M}$ & ICRP-68 & $3.7 \mathrm{E}-11$ & -- & -- \\
\hline Ga-66 & Ga-66 & 65.932 & Gallium & 0.01 & 0.004 & 0 & ICRP-38 & $9.40 \mathrm{E}+00$ & $\mathrm{~h}$ & $3.38 \mathrm{E}+04$ & $5.06 \mathrm{E}+06$ & ICRP-68 & $7.1 \mathrm{E}-10$ & $M$ & ICRP-68 & $1.2 \mathrm{E}-09$ & -- & -- \\
\hline Ga-67 & Ga-67 & 66.928 & Gallium & 0.01 & 0.004 & 0 & ICRP-38 & $7.83 \mathrm{E}+01$ & $h$ & $2.82 \mathrm{E}+05$ & $5.98 \mathrm{E}+05$ & ICRP-68 & $2.8 \mathrm{E}-10$ & $M$ & ICRP-68 & $1.9 \mathrm{E}-10$ & -- & -- \\
\hline Ga-68 & Ga-68 & 67.928 & Gallium & 0.01 & 0.004 & 0 & ICRP-38 & $6.80 \mathrm{E}+01$ & $\mathrm{~m}$ & $4.08 \mathrm{E}+03$ & $4.07 E+07$ & ICRP-68 & $8.1 \mathrm{E}-11$ & $M$ & ICRP-68 & $1.0 \mathrm{E}-10$ & -- & -- \\
\hline Ga-70 & Ga-70 & 69.926 & Gallium & 0.01 & 0.004 & 0 & ICRP-38 & $2.12 \mathrm{E}+01$ & $\mathrm{~m}$ & $1.27 \mathrm{E}+03$ & $1.27 \mathrm{E}+08$ & ICRP-68 & $2.6 \mathrm{E}-11$ & $M$ & ICRP-68 & $3.1 \mathrm{E}-11$ & -- & -- \\
\hline Ga-72 & Ga-72 & 71.926 & Gallium & 0.01 & 0.004 & 0 & ICRP-38 & $1.41 \mathrm{E}+01$ & $\mathrm{~h}$ & $5.08 \mathrm{E}+04$ & $3.09 \mathrm{E}+06$ & ICRP-68 & $8.4 \mathrm{E}-10$ & $M$ & ICRP-68 & $1.1 \mathrm{E}-09$ & -- & -- \\
\hline Ga-73 & Ga-73 & 72.925 & Gallium & 0.01 & 0.004 & 0 & ICRP-38 & $4.91 \mathrm{E}+00$ & $h$ & $1.77 E+04$ & $8.75 \mathrm{E}+06$ & ICRP-68 & $2.0 \mathrm{E}-10$ & $M$ & ICRP-68 & $2.6 \mathrm{E}-10$ & -- & -- \\
\hline Ga-74 & Ga-74 & 73.927 & $\begin{array}{l}\text { Gallium } \\
\end{array}$ & 0.01 & 0.004 & 0 & ICRP-107 & $8.12 \mathrm{E}+00$ & $\mathrm{~m}$ & $4.87 \mathrm{E}+02$ & $3.13 \mathrm{E}+08$ & -- & -- & -- & -- & -- & -- & -- \\
\hline Gd-142 & Gd-142 & 141.928 & Gadolinium & 0.01 & 0.01 & $500-1000$ & ICRP-107 & $7.02 \mathrm{E}+01$ & $\mathrm{~s}$ & $7.02 \mathrm{E}+01$ & $1.13 \mathrm{E}+09$ & -- & -- & -- & -- & -- & -- & -- \\
\hline Gd-143m & Gd-143 & 142.927 & Gadolinium & 0.01 & 0.01 & $500-1000$ & ICRP-107 & $1.10 \mathrm{E}+02$ & $\mathrm{~s}$ & $1.10 \mathrm{E}+02$ & $7.18 \mathrm{E}+08$ & -- & -- & -- & -- & -- & -- & -- \\
\hline Gd-144 & Gd-144 & 143.923 & Gadolinium & 0.01 & 0.01 & $500-1000$ & ICRP-107 & $4.47 \mathrm{E}+00$ & $\mathrm{~m}$ & $2.68 \mathrm{E}+02$ & $2.92 \mathrm{E}+08$ & -- & -- & -- & -- & -- & -- & -- \\
\hline Gd-145 & Gd-145 & 144.922 & Gadolinium & 0.01 & 0.01 & $500-1000$ & ICRP-38 & $2.29 \mathrm{E}+01$ & $\mathrm{~m}$ & $1.37 \mathrm{E}+03$ & $5.67 \mathrm{E}+07$ & ICRP-68 & 3.5E-11 & $M$ & ICRP-68 & $4.4 \mathrm{E}-11$ & -- & -- \\
\hline Gd-145m & Gd-145 & 144.922 & Gadolinium & 0.01 & 0.01 & $500-1000$ & ICRP-107 & $8.50 \mathrm{E}+01$ & $\mathrm{~s}$ & $8.50 \mathrm{E}+01$ & $9.16 \mathrm{E}+08$ & -- & -- & -- & -- & -- & -- & -- \\
\hline Gd-146 & Gd-146 & 145.918 & Gadolinium & 0.01 & 0.01 & $500-1000$ & ICRP-38 & $4.83 \mathrm{E}+01$ & $d$ & $4.17 \mathrm{E}+06$ & $1.85 \mathrm{E}+04$ & ICRP-68 & $6.0 \mathrm{E}-09$ & $M$ & ICRP-68 & $9.6 \mathrm{E}-10$ & -- & -- \\
\hline Gd-147 & Gd-147 & 146.919 & Gadolinium & 0.01 & 0.01 & $500-1000$ & ICRP-38 & $3.81 \mathrm{E}+01$ & $h$ & $1.37 \mathrm{E}+05$ & $5.60 \mathrm{E}+05$ & ICRP-68 & $5.9 \mathrm{E}-10$ & $M$ & ICRP-68 & $6.1 \mathrm{E}-10$ & -- & -- \\
\hline Gd-148 & Gd-148 & 147.918 & Gadolinium & 0.01 & 0.01 & $500-1000$ & ICRP-38 & $9.30 \mathrm{E}+01$ & $y$ & $2.93 \mathrm{E}+09$ & $2.60 \mathrm{E}+01$ & ICRP-68 & $3.0 \mathrm{E}-05$ & $\mathrm{~F}$ & ICRP-68 & $5.5 \mathrm{E}-08$ & -- & -- \\
\hline Gd-149 & Gd-149 & 148.919 & Gadolinium & 0.01 & 0.01 & $500-1000$ & ICRP-38 & $9.40 \mathrm{E}+00$ & $d$ & $8.12 \mathrm{E}+05$ & $9.33 \mathrm{E}+04$ & ICRP-68 & $7.9 \mathrm{E}-10$ & $M$ & ICRP-68 & $4.5 \mathrm{E}-10$ & -- & -- \\
\hline Gd-150 & Gd-150 & 149.919 & Gadolinium & 0.01 & 0.01 & $500-1000$ & ICRP-107 & $1.79 \mathrm{E}+06$ & $y$ & $5.65 \mathrm{E}+13$ & $1.33 \mathrm{E}-03$ & JAERI & $2.8 \mathrm{E}-05$ & $F$ & JAERI & $5.2 \mathrm{E}-08$ & -- & -- \\
\hline Gd-151 & Gd-151 & 150.920 & Gadolinium & 0.01 & 0.01 & $500-1000$ & ICRP-38 & $1.20 \mathrm{E}+02$ & d & $1.04 \mathrm{E}+07$ & $7.21 \mathrm{E}+03$ & ICRP-68 & $9.3 \mathrm{E}-10$ & $\mathrm{~F}$ & ICRP-68 & $2.0 \mathrm{E}-10$ & -- & -- \\
\hline Gd-152 & Gd-152 & 151.920 & Gadolinium & 0.01 & 0.01 & $500-1000$ & ICRP-38 & $1.08 \mathrm{E}+14$ & $y$ & $3.41 \mathrm{E}+21$ & $2.18 \mathrm{E}-11$ & ICRP-68 & $2.2 \mathrm{E}-05$ & $\mathrm{~F}$ & ICRP-68 & $4.1 \mathrm{E}-08$ & -- & -- \\
\hline Gd-153 & Gd-153 & 152.922 & Gadolinium & 0.01 & 0.01 & $500-1000$ & ICRP-38 & $2.42 \mathrm{E}+02$ & $d$ & $2.09 \mathrm{E}+07$ & $3.53 \mathrm{E}+03$ & ICRP-68 & $2.5 \mathrm{E}-09$ & $F$ & ICRP-68 & $2.7 \mathrm{E}-10$ & -- & -- \\
\hline Gd-159 & Gd-159 & 158.926 & Gadolinium & 0.01 & 0.01 & $500-1000$ & ICRP-38 & $1.86 \mathrm{E}+01$ & $\mathrm{~h}$ & $6.68 \mathrm{E}+04$ & $1.06 \mathrm{E}+06$ & ICRP-68 & $3.9 \mathrm{E}-10$ & $M$ & ICRP-68 & $4.9 \mathrm{E}-10$ & -- & -- \\
\hline Gd-162 & Gd-162 & 161.931 & Gadolinium & 0.01 & 0.01 & $500-1000$ & ICRP-107 & $8.40 \mathrm{E}+00$ & $\mathrm{~m}$ & $5.04 \mathrm{E}+02$ & $1.38 \mathrm{E}+08$ & -- & -- & -- & -- & -- & -- & -- \\
\hline Ge-66 & Ge-66 & 65.934 & Germanium & 0.01 & 0.4 & 0 & ICRP-38 & $2.27 E+00$ & $\mathrm{~h}$ & $8.17 E+03$ & $2.09 \mathrm{E}+07$ & ICRP-68 & $1.3 \mathrm{E}-10$ & $M$ & ICRP-68 & $1.0 \mathrm{E}-10$ & -- & -- \\
\hline Ge-67 & Ge-67 & 66.933 & Germanium & 0.01 & 0.4 & 0 & ICRP-38 & $1.87 \mathrm{E}+01$ & $\mathrm{~m}$ & $1.12 \mathrm{E}+03$ & $1.50 \mathrm{E}+08$ & ICRP-68 & $4.2 \mathrm{E}-11$ & $\mathrm{M}$ & ICRP-68 & $6.5 \mathrm{E}-11$ & -- & -- \\
\hline Ge-68 & Ge-68 & 67.928 & Germanium & 0.01 & 0.4 & 0 & ICRP-38 & $2.88 \mathrm{E}+02$ & $\mathrm{~d}$ & $2.49 \mathrm{E}+07$ & $6.67 \mathrm{E}+03$ & ICRP-68 & $1.3 \mathrm{E}-08$ & $\mathrm{M}$ & ICRP-68 & $1.3 \mathrm{E}-09$ & -- & -- \\
\hline Ge-69 & Ge-69 & 68.928 & Germanium & 0.01 & 0.4 & 0 & ICRP-38 & $3.91 \mathrm{E}+01$ & $\mathrm{~h}$ & $1.41 \mathrm{E}+05$ & $1.16 \mathrm{E}+06$ & ICRP-68 & $3.7 \mathrm{E}-10$ & $M$ & ICRP-68 & $2.4 \mathrm{E}-10$ & -- & -- \\
\hline Ge-71 & Ge-71 & 70.925 & Germanium & 0.01 & 0.4 & 0 & ICRP-38 & $1.18 \mathrm{E}+01$ & $d$ & $1.02 \mathrm{E}+06$ & $1.56 \mathrm{E}+05$ & ICRP-68 & $1.1 \mathrm{E}-11$ & $M$ & ICRP-68 & $1.2 \mathrm{E}-11$ & -- & -- \\
\hline Ge-75 & Ge-75 & 74.923 & Germanium & 0.01 & 0.4 & 0 & ICRP-38 & $8.28 \mathrm{E}+01$ & $\mathrm{~m}$ & $4.97 E+03$ & $3.03 \mathrm{E}+07$ & ICRP-68 & $5.4 \mathrm{E}-11$ & $M$ & ICRP-68 & $4.6 \mathrm{E}-11$ & -- & -- \\
\hline
\end{tabular}




\begin{tabular}{|c|c|c|c|c|c|c|c|c|c|c|c|c|c|c|c|c|c|c|}
\hline \multirow{3}{*}{$\begin{array}{l}\text { MASTER } \\
\text { ISOTOPE } \\
\text { LIST } \\
\text { Ge-77 } \\
\end{array}$} & \multirow{3}{*}{$\begin{array}{c}\begin{array}{c}\text { Atomic } \\
\text { Mass } \\
\text { Nuclide }\end{array} \\
\text { Ge-77 } \\
\end{array}$} & \multirow{3}{*}{$\begin{array}{c}\begin{array}{c}\text { Atomic } \\
\text { Mass }\end{array} \\
76.924 \\
\end{array}$} & & & able C.1 F & dionuclide $\mathrm{Sp}$ & fic Input Da & Jsed For & als & ion of $\mathrm{t}$ & Qs Usir & ximum $\mathbf{R e}$ & (1) & nued) & & & & \\
\hline & & & \multirow{2}{*}{$\begin{array}{c}\text { Element } \\
\text { Germanium }\end{array}$} & \multirow{2}{*}{$\begin{array}{c}\text { EPA } \\
\text { RF } \\
0.01\end{array}$} & \multirow[t]{2}{*}{$\begin{array}{c}\text { EPA } \\
\text { Conc } \\
\text { Factors } \\
\left(B_{v}\right) \\
0.4\end{array}$} & \multirow[t]{2}{*}{$\begin{array}{c}\text { EPA } \\
\begin{array}{c}\text { Sorption } \\
\text { Coefficient } \\
\left(K_{d}\right)\end{array} \\
0\end{array}$} & \multirow{2}{*}{$\begin{array}{c}\begin{array}{c}\text { Half-Life } \\
\text { Reference }\end{array} \\
\text { ICRP-38 }\end{array}$} & \multicolumn{2}{|c|}{$\begin{array}{c}\text { Half-Life } \\
\text { (original units) }\end{array}$} & \multirow{2}{*}{$\begin{array}{c}\begin{array}{c}\text { Half Life } \\
\text { (s) }\end{array} \\
4.07 E+04\end{array}$} & \multirow{2}{*}{$\begin{array}{c}\begin{array}{c}\text { Sp. Act } \\
\text { (Ci/g) }\end{array} \\
3.61 \mathrm{E}+06\end{array}$} & \multirow[t]{2}{*}{$\begin{array}{c}\begin{array}{c}\text { Inhalation } \\
\text { DC } \\
\text { Reference }\end{array} \\
\text { ICRP-68 }\end{array}$} & \multirow[t]{2}{*}{$\begin{array}{c}\text { HC-3 } \\
\text { Bounding } \\
\text { Inhalation } \\
\text { DC } \\
\text { (Sv/Bq) } \\
4.5 \mathrm{E}-10\end{array}$} & \multirow[t]{2}{*}{$\begin{array}{c}\text { Lung } \\
\text { Absorption } \\
\text { Class/Type }\end{array}$} & \multirow[t]{2}{*}{$\begin{array}{c}\text { Ingestion } \\
\text { DC } \\
\text { Reference }\end{array}$} & \multirow[t]{2}{*}{$\begin{array}{c}\text { HC-3 } \\
\text { Bounding } \\
\text { Ingestion } \\
\text { DC } \\
(\mathrm{Sv} / \mathrm{Bq}) \\
3.3 \mathrm{E}-10\end{array}$} & $\begin{array}{c}\text { Immersion } \\
\text { DC } \\
\text { Reference }\end{array}$ & $\begin{array}{c}\text { HC-3 } \\
\text { Immersion } \\
\mathrm{DC}(\mathrm{Sv} / \mathrm{d} \\
\left.\text { per } \mathrm{Bq} / \mathrm{m}^{3}\right)\end{array}$ \\
\hline & & & & & & & & $1.13 \mathrm{E}+01$ & $\mathrm{~h}$ & & & & & & & & -- & $\begin{array}{c}-1 \\
--\end{array}$ \\
\hline Ge-78 & Ge-78 & 77.923 & Germanium & 0.01 & 0.4 & 0 & ICRP-38 & $8.70 \mathrm{E}+01$ & $\mathrm{~m}$ & $5.22 \mathrm{E}+03$ & $2.77 E+07$ & ICRP-68 & $1.4 \mathrm{E}-10$ & $M$ & ICRP-68 & $1.2 \mathrm{E}-10$ & -- & -- \\
\hline $\mathrm{H}-3$ & $\mathrm{H}-3$ & 3.016 & Hydrogen & 0.5 & -- & 0 & ICRP-38 & $1.24 \mathrm{E}+01$ & $y$ & $3.90 E+08$ & $9.60 \mathrm{E}+03$ & ICRP-68 & $4.1 \mathrm{E}-11$ & OBT & ICRP-68 & $4.2 \mathrm{E}-11$ & -- & -- \\
\hline Hf-167 & Hf-167 & 166.943 & Hafnium & 0.01 & 0.0035 & 0 & ICRP-107 & $2.05 \mathrm{E}+00$ & $\mathrm{~m}$ & $1.23 \mathrm{E}+02$ & $5.49 \mathrm{E}+08$ & -- & -- & -- & -- & -- & -- & -- \\
\hline Hf-169 & Hf-169 & 168.941 & Hafnium & 0.01 & 0.0035 & 0 & ICRP-107 & $3.24 \mathrm{E}+00$ & $\mathrm{~m}$ & $1.94 \mathrm{E}+02$ & $3.44 \mathrm{E}+08$ & -- & -- & -- & -- & -- & -- & -- \\
\hline $\mathrm{Hf}-170$ & $\mathrm{Hf}-170$ & 169.940 & Hafnium & 0.01 & 0.0035 & 0 & ICRP-38 & $1.60 \mathrm{E}+01$ & $\mathrm{~h}$ & $5.76 \mathrm{E}+04$ & $1.15 \mathrm{E}+06$ & ICRP-68 & $4.3 \mathrm{E}-10$ & $M$ & ICRP-68 & $4.8 \mathrm{E}-10$ & -- & -- \\
\hline Hf-172 & Hf-172 & 171.939 & Hafnium & 0.01 & 0.0035 & 0 & ICRP-38 & $1.87 \mathrm{E}+00$ & $y$ & $5.90 \mathrm{E}+07$ & $1.11 \mathrm{E}+03$ & ICRP-68 & $3.7 \mathrm{E}-08$ & $F$ & ICRP-68 & $1.0 \mathrm{E}-09$ & -- & -- \\
\hline Hf-173 & Hf-173 & 172.941 & Hafnium & 0.01 & 0.0035 & 0 & ICRP-38 & $2.40 \mathrm{E}+01$ & $\mathrm{~h}$ & $8.64 E+04$ & $7.55 \mathrm{E}+05$ & ICRP-68 & $2.2 \mathrm{E}-10$ & $M$ & ICRP-68 & $2.3 \mathrm{E}-10$ & -- & -- \\
\hline Hf-174 & Hf-174 & 173.940 & Hafnium & 0.01 & 0.0035 & 0 & ICRP-107 & $2.00 \mathrm{E}+15$ & $y$ & $6.31 E+22$ & $1.03 E-12$ & JAERI & $3.6 \mathrm{E}-05$ & $\mathrm{~F}$ & JAERI & $2.5 \mathrm{E}-07$ & -- & -- \\
\hline Hf-175 & $\mathrm{Hf}-175$ & 174.942 & Hafnium & 0.01 & 0.0035 & 0 & ICRP-38 & $7.00 \mathrm{E}+01$ & $d$ & $6.05 E+06$ & $1.07 E+04$ & ICRP-68 & $1.1 \mathrm{E}-09$ & $M$ & ICRP-68 & $4.1 \mathrm{E}-10$ & -- & -- \\
\hline $\mathrm{Hf}-177 \mathrm{~m}$ & Hf-177 & 176.943 & Hafnium & 0.01 & 0.0035 & 0 & ICRP-38 & $5.14 \mathrm{E}+01$ & $\mathrm{~m}$ & $3.08 E+03$ & $2.07 \mathrm{E}+07$ & ICRP-68 & $1.5 \mathrm{E}-10$ & $M$ & ICRP-68 & $8.1 \mathrm{E}-11$ & -- & -- \\
\hline $\mathrm{Hf}-178 \mathrm{~m}$ & Hf-178 & 177.944 & Hafnium & 0.01 & 0.0035 & 0 & ICRP-38 & $3.10 \mathrm{E}+01$ & $y$ & $9.78 \mathrm{E}+08$ & $6.48 \mathrm{E}+01$ & ICRP-68 & $3.1 \mathrm{E}-07$ & $\mathrm{~F}$ & ICRP-68 & $4.7 \mathrm{E}-09$ & -- & -- \\
\hline Hf-179m & Hf-179 & 178.946 & Hafnium & 0.01 & 0.0035 & 0 & ICRP-38 & $2.51 \mathrm{E}+01$ & $d$ & $2.17 E+06$ & $2.91 \mathrm{E}+04$ & ICRP-68 & $3.6 \mathrm{E}-09$ & $M$ & ICRP-68 & $1.2 \mathrm{E}-09$ & -- & -- \\
\hline $\mathrm{Hf}-180 \mathrm{~m}$ & Hf-180 & 179.947 & Hafnium & 0.01 & 0.0035 & 0 & ICRP-38 & $5.50 \mathrm{E}+00$ & $\mathrm{~h}$ & $1.98 \mathrm{E}+04$ & $3.17 \mathrm{E}+06$ & ICRP-68 & $2.0 \mathrm{E}-10$ & $M$ & ICRP-68 & $1.7 \mathrm{E}-10$ & -- & -- \\
\hline Hf-181 & $\mathrm{Hf}-181$ & 180.949 & Hafnium & 0.01 & 0.0035 & 0 & ICRP-38 & $4.24 \mathrm{E}+01$ & $d$ & $3.66 \mathrm{E}+06$ & $1.70 \mathrm{E}+04$ & ICRP-68 & $4.7 \mathrm{E}-09$ & $M$ & ICRP-68 & $1.1 \mathrm{E}-09$ & -- & -- \\
\hline Hf-182 & Hf-182 & 181.951 & Hafnium & 0.01 & 0.0035 & 0 & ICRP-38 & $9.00 \mathrm{E}+06$ & $y$ & $2.84 E+14$ & $2.18 \mathrm{E}-04$ & ICRP-68 & $3.6 \mathrm{E}-07$ & $\mathrm{~F}$ & ICRP-68 & $3.0 \mathrm{E}-09$ & -- & -- \\
\hline Hf- $182 m$ & Hf-182 & 181.951 & Hafnium & 0.01 & 0.0035 & 0 & ICRP-38 & $6.15 \mathrm{E}+01$ & $\mathrm{~m}$ & $3.69 \mathrm{E}+03$ & $1.68 \mathrm{E}+07$ & ICRP-68 & $7.1 \mathrm{E}-11$ & $M$ & ICRP-68 & $4.2 \mathrm{E}-11$ & -- & -- \\
\hline Hf-183 & Hf-183 & 182.954 & Hafnium & 0.01 & 0.0035 & 0 & ICRP-38 & $6.40 \mathrm{E}+01$ & $\mathrm{~m}$ & $3.84 \mathrm{E}+03$ & $1.61 \mathrm{E}+07$ & ICRP-68 & $8.3 \mathrm{E}-11$ & $M$ & ICRP-68 & $7.3 \mathrm{E}-11$ & -- & -- \\
\hline Hf-184 & Hf-184 & 183.955 & Hafnium & 0.01 & 0.0035 & 0 & ICRP-38 & $4.12 \mathrm{E}+00$ & $\mathrm{~h}$ & $1.48 \mathrm{E}+04$ & $4.13 \mathrm{E}+06$ & ICRP-68 & $4.5 \mathrm{E}-10$ & $M$ & ICRP-68 & $5.2 \mathrm{E}-10$ & -- & -- \\
\hline Hg-187 & Hg-187 & 186.970 & Mercury & 0.01 & 0.9 & 0 & JAERI & $2.20 \mathrm{E}+00$ & $\mathrm{~m}$ & $1.32 \mathrm{E}+02$ & $4.57 \mathrm{E}+08$ & -- & -- & -- & -- & -- & -- & -- \\
\hline Hg-187m & Hg-187 & 186.970 & Mercury & 0.01 & 0.9 & 0 & JAERI & $2.40 \mathrm{E}+00$ & $\mathrm{~m}$ & $1.44 \mathrm{E}+02$ & $4.19 \mathrm{E}+08$ & -- & -- & -- & -- & -- & -- & -- \\
\hline Hg-188 & $\mathrm{Hg}-188$ & 187.968 & Mercury & 0.01 & 0.9 & 0 & JAERI & $3.25 \mathrm{E}+00$ & $\mathrm{~m}$ & $1.95 \mathrm{E}+02$ & $3.08 \mathrm{E}+08$ & -- & -- & -- & -- & -- & -- & -- \\
\hline Hg-190 & $\mathrm{Hg}-190$ & 189.966 & Mercury & 0.01 & 0.9 & 0 & ICRP-107 & $2.00 \mathrm{E}+01$ & $\mathrm{~m}$ & $1.20 \mathrm{E}+03$ & $4.95 \mathrm{E}+07$ & JAERI & $9.6 \mathrm{E}-11$ & Vap & JAERI & $2.5 \mathrm{E}-11$ & -- & -- \\
\hline Hg-191m & $\mathrm{Hg}-191$ & 190.967 & Mercury & 0.01 & 0.9 & 0 & ICRP-107 & $5.08 \mathrm{E}+01$ & $\mathrm{~m}$ & $3.05 E+03$ & $1.94 \mathrm{E}+07$ & JAERI & $3.2 \mathrm{E}-10$ & Vap & JAERI & $5.5 \mathrm{E}-11$ & -- & -- \\
\hline Hg-192 & $\mathrm{Hg}-192$ & 191.966 & Mercury & 0.01 & 0.9 & 0 & ICRP-107 & $4.85 \mathrm{E}+00$ & $\mathrm{~h}$ & $1.75 E+04$ & $3.37 \mathrm{E}+06$ & JAERI & $1.0 \mathrm{E}-09$ & Vap & JAERI & $2.3 \mathrm{E}-10$ & -- & -- \\
\hline Hg-193 & $\mathrm{Hg}-193$ & 192.967 & Mercury & 0.01 & 0.9 & 0 & ICRP-38 & $3.50 \mathrm{E}+00$ & $\mathrm{~h}$ & $1.26 \mathrm{E}+04$ & $4.64 \mathrm{E}+06$ & ICRP-68 & $1.1 \mathrm{E}-09$ & 0 & ICRP-68 & $8.2 \mathrm{E}-11$ & -- & -- \\
\hline Hg-193m & $\mathrm{Hg}-193$ & 192.967 & Mercury & 0.01 & 0.9 & 0 & ICRP-38 & $1.11 \mathrm{E}+01$ & $\mathrm{~h}$ & $4.00 E+04$ & $1.46 \mathrm{E}+06$ & ICRP-68 & $3.1 \mathrm{E}-09$ & 0 & ICRP-68 & $4.0 \mathrm{E}-10$ & -- & -- \\
\hline Hg-194 & $\mathrm{Hg}-194$ & 193.965 & Mercury & 0.01 & 0.9 & 0 & ICRP-38 & $2.60 \mathrm{E}+02$ & $y$ & $8.20 \mathrm{E}+09$ & $7.09 \mathrm{E}+00$ & ICRP-68 & $4.0 \mathrm{E}-08$ & 0 & ICRP-68 & $5.1 \mathrm{E}-08$ & -- & -- \\
\hline $\mathrm{Hg}-195$ & $\mathrm{Hg}-195$ & 194.967 & Mercury & 0.01 & 0.9 & 0 & ICRP-38 & $9.90 \mathrm{E}+00$ & $\mathrm{~h}$ & $3.56 \mathrm{E}+04$ & $1.62 \mathrm{E}+06$ & ICRP-68 & $1.4 \mathrm{E}-09$ & 0 & ICRP-68 & $9.7 \mathrm{E}-11$ & -- & -- \\
\hline $\mathrm{Hg}-195 \mathrm{~m}$ & Hg-195 & 194.967 & Mercury & 0.01 & 0.9 & 0 & ICRP-38 & $4.16 \mathrm{E}+01$ & $\mathrm{~h}$ & $1.50 E+05$ & $3.86 \mathrm{E}+05$ & ICRP-68 & $8.2 \mathrm{E}-09$ & 0 & ICRP-68 & $5.6 \mathrm{E}-10$ & -- & -- \\
\hline Hg-197 & $\mathrm{Hg}-197$ & 196.967 & Mercury & 0.01 & 0.9 & 0 & ICRP-38 & $6.41 \mathrm{E}+01$ & $h$ & $2.31 \mathrm{E}+05$ & $2.48 \mathrm{E}+05$ & ICRP-68 & $4.4 \mathrm{E}-09$ & 0 & ICRP-68 & $2.3 \mathrm{E}-10$ & -- & -- \\
\hline Hg-197m & $\mathrm{Hg}-197$ & 196.967 & Mercury & 0.01 & 0.9 & 0 & ICRP-38 & $2.38 \mathrm{E}+01$ & $\mathrm{~h}$ & $8.57 E+04$ & $6.68 \mathrm{E}+05$ & ICRP-68 & $5.8 \mathrm{E}-09$ & 0 & ICRP-68 & $4.7 \mathrm{E}-10$ & -- & -- \\
\hline Hg-199m & Hg-199 & 198.968 & Mercury & 0.01 & 0.9 & 0 & ICRP-38 & $4.26 \mathrm{E}+01$ & $\mathrm{~m}$ & $2.56 E+03$ & $2.22 \mathrm{E}+07$ & ICRP-68 & $1.8 \mathrm{E}-10$ & 0 & ICRP-68 & $3.1 \mathrm{E}-11$ & -- & -- \\
\hline Hg-203 & $\mathrm{Hg}-203$ & 202.973 & Mercury & 0.01 & 0.9 & 0 & ICRP-38 & $4.66 \mathrm{E}+01$ & $d$ & $4.03 E+06$ & $1.38 \mathrm{E}+04$ & ICRP-68 & $7.0 \mathrm{E}-09$ & 0 & ICRP-68 & $1.9 \mathrm{E}-09$ & -- & -- \\
\hline $\mathrm{Hg}-205$ & $\mathrm{Hg}-205$ & 204.976 & Mercury & 0.01 & 0.9 & 0 & ICRP-107 & $5.20 \mathrm{E}+00$ & $\mathrm{~m}$ & $3.12 \mathrm{E}+02$ & $1.76 \mathrm{E}+08$ & -- & -- & -- & -- & -- & -- & -- \\
\hline $\mathrm{Hg}-206$ & $\mathrm{Hg}-206$ & 205.978 & Mercury & 0.01 & 0.9 & 0 & ICRP-38 & $8.15 \mathrm{E}+00$ & $\mathrm{~m}$ & $4.89 \mathrm{E}+02$ & $1.12 \mathrm{E}+08$ & -- & -- & -- & -- & -- & -- & -- \\
\hline $\mathrm{Hg}-207$ & $\mathrm{Hg}-207$ & 206.982 & Mercury & 0.01 & 0.9 & 0 & ICRP-107 & $2.90 \mathrm{E}+00$ & $\mathrm{~m}$ & $1.74 E+02$ & $3.13 \mathrm{E}+08$ & -- & -- & -- & -- & -- & -- & -- \\
\hline Ho-150 & Ho-150 & 149.933 & Holmium & 0.01 & 0.01 & 600 & ICRP-107 & $7.68 \mathrm{E}+01$ & $\mathrm{~s}$ & $7.68 \mathrm{E}+01$ & $9.80 \mathrm{E}+08$ & -- & -- & -- & -- & -- & -- & -- \\
\hline Ho-152 & Ho-152 & 151.932 & Holmium & 0.01 & 0.01 & 600 & JAERI & $2.70 \mathrm{E}+00$ & $\mathrm{~m}$ & $1.62 \mathrm{E}+02$ & $4.59 \mathrm{E}+08$ & -- & -- & -- & -- & -- & -- & -- \\
\hline Ho-153 & Ho-153 & 152.930 & Holmium & 0.01 & 0.01 & 600 & ICRP-107 & $2.01 \mathrm{E}+00$ & $\mathrm{~m}$ & $1.21 \mathrm{E}+02$ & $6.12 \mathrm{E}+08$ & -- & -- & -- & -- & -- & -- & -- \\
\hline Ho-153m & Ho-153 & 152.930 & Holmium & 0.01 & 0.01 & 600 & ICRP-107 & $9.30 \mathrm{E}+00$ & $\mathrm{~m}$ & $5.58 \mathrm{E}+02$ & $1.32 \mathrm{E}+08$ & -- & -- & -- & -- & -- & -- & -- \\
\hline Ho-154 & Ho-154 & 153.931 & Holmium & 0.01 & 0.01 & 600 & ICRP-107 & $1.18 \mathrm{E}+01$ & $\mathrm{~m}$ & $7.06 \mathrm{E}+02$ & $1.04 \mathrm{E}+08$ & JAERI & $2.7 \mathrm{E}-11$ & $\mathrm{M}$ & JAERI & $4.1 \mathrm{E}-11$ & -- & -- \\
\hline Ho- $154 \mathrm{~m}$ & Ho-154 & 153.931 & Holmium & 0.01 & 0.01 & 600 & ICRP-107 & $3.10 \mathrm{E}+00$ & $\mathrm{~m}$ & $1.86 \mathrm{E}+02$ & $3.94 E+08$ & -- & -- & -- & -- & -- & -- & -- \\
\hline Ho-155 & Ho-155 & 154.929 & Holmium & 0.01 & 0.01 & 600 & ICRP-38 & $4.80 \mathrm{E}+01$ & $\mathrm{~m}$ & $2.88 \mathrm{E}+03$ & $2.53 \mathrm{E}+07$ & ICRP-68 & $3.2 \mathrm{E}-11$ & $M$ & ICRP-68 & $3.7 \mathrm{E}-11$ & -- & -- \\
\hline Ho-156 & Ho-156 & 155.930 & Holmium & 0.01 & 0.01 & 600 & ICRP-107 & $5.60 \mathrm{E}+01$ & $\mathrm{~m}$ & $3.36 \mathrm{E}+03$ & $2.15 \mathrm{E}+07$ & JAERI & $9.1 \mathrm{E}-11$ & $M$ & JAERI & $9.0 \mathrm{E}-12$ & -- & -- \\
\hline
\end{tabular}




\begin{tabular}{|c|c|c|c|c|c|c|c|c|c|c|c|c|c|c|c|c|c|c|}
\hline \multicolumn{19}{|c|}{ Table C.1 Radionuclide Specific Input Data Used For the Calculation of HC-3 TQs Using Maximum Reported DCs (continued) } \\
\hline $\begin{array}{l}\text { MASTER } \\
\text { ISOTOPE } \\
\text { LIST }\end{array}$ & $\begin{array}{c}\text { Atomic } \\
\text { Mass } \\
\text { Nuclide }\end{array}$ & $\begin{array}{l}\text { Atomic } \\
\text { Mass }\end{array}$ & Element & $\begin{array}{c}\text { EPA } \\
\text { RF }\end{array}$ & $\begin{array}{c}\text { EPA } \\
\text { Conc } \\
\text { Factors } \\
\left(B_{v}\right) \\
\end{array}$ & $\begin{array}{c}\text { EPA } \\
\text { Sorption } \\
\text { Coefficient } \\
\left(K_{d}\right)\end{array}$ & $\begin{array}{c}\text { Half-Life } \\
\text { Reference }\end{array}$ & $\begin{array}{r}\text { Half-Lif } \\
\text { (original u }\end{array}$ & & $\begin{array}{l}\text { Half Life } \\
\text { (s) }\end{array}$ & $\begin{array}{l}\text { Sp. Act } \\
\text { (Ci/g) }\end{array}$ & $\begin{array}{l}\text { Inhalation } \\
\text { DC } \\
\text { Reference }\end{array}$ & $\begin{array}{c}\text { HC-3 } \\
\text { Bounding } \\
\text { Inhalation } \\
\text { DC } \\
\text { (Sv/Bq) } \\
\end{array}$ & $\begin{array}{c}\text { Lung } \\
\text { Absorption } \\
\text { Class/Type }\end{array}$ & $\begin{array}{l}\text { Ingestion } \\
\text { DC } \\
\text { Reference }\end{array}$ & $\begin{array}{c}\text { HC-3 } \\
\text { Bounding } \\
\text { Ingestion } \\
\text { DC } \\
\text { (Sv/Bq) } \\
\end{array}$ & $\begin{array}{l}\text { Immersion } \\
\text { DC } \\
\text { Reference }\end{array}$ & $\begin{array}{c}\mathrm{HC}-3 \\
\text { Immersion } \\
\mathrm{DC}(\mathrm{Sv} / \mathrm{d} \\
\left.\text { per } \mathrm{Bq} / \mathrm{m}^{3}\right)\end{array}$ \\
\hline Ho-157 & Ho-157 & 156.928 & Holmium & 0.01 & 0.01 & 600 & ICRP-38 & $1.26 \mathrm{E}+01$ & $\mathrm{~m}$ & $7.56 \mathrm{E}+02$ & $9.51 \mathrm{E}+07$ & ICRP-68 & $7.6 \mathrm{E}-12$ & $\mathrm{M}$ & ICRP-68 & $6.5 \mathrm{E}-12$ & -- & -- \\
\hline Ho-158 & Ho-158 & 157.929 & Holmium & 0.01 & 0.01 & 600 & JAERI & $1.13 \mathrm{E}+01$ & $\mathrm{~m}$ & $6.78 \mathrm{E}+02$ & $1.05 E+08$ & JAERI & $1.4 \mathrm{E}-11$ & $\mathrm{M}$ & JAERI & $8.9 \mathrm{E}-12$ & -- & -- \\
\hline Ho-159 & Ho-159 & 158.928 & Holmium & 0.01 & 0.01 & 600 & ICRP-38 & $3.30 \mathrm{E}+01$ & $\mathrm{~m}$ & $1.98 \mathrm{E}+03$ & $3.59 \mathrm{E}+07$ & ICRP-68 & $1.0 \mathrm{E}-11$ & $M$ & ICRP-68 & $7.9 \mathrm{E}-12$ & -- & -- \\
\hline Ho-160 & Ho-160 & 159.929 & Holmium & 0.01 & 0.01 & 600 & ICRP-107 & $2.56 \mathrm{E}+01$ & $\mathrm{~m}$ & $1.54 \mathrm{E}+03$ & $4.59 \mathrm{E}+07$ & JAERI & $2.4 \mathrm{E}-11$ & $M$ & JAERI & $1.7 \mathrm{E}-11$ & -- & -- \\
\hline Ho-161 & Ho-161 & 160.928 & Holmium & 0.01 & 0.01 & 600 & ICRP-38 & $2.50 \mathrm{E}+00$ & $\mathrm{~h}$ & $9.00 \mathrm{E}+03$ & $7.79 \mathrm{E}+06$ & ICRP-68 & $1.0 \mathrm{E}-11$ & $M$ & ICRP-68 & $1.3 \mathrm{E}-11$ & -- & -- \\
\hline Ho-162 & Ho-162 & 161.929 & Holmium & 0.01 & 0.01 & 600 & ICRP-38 & $1.50 \mathrm{E}+01$ & $\mathrm{~m}$ & $9.00 \mathrm{E}+02$ & $7.74 \mathrm{E}+07$ & ICRP-68 & $4.5 \mathrm{E}-12$ & $\mathrm{M}$ & ICRP-68 & $3.3 \mathrm{E}-12$ & -- & -- \\
\hline Ho-162m & Ho-162 & 161.929 & Holmium & 0.01 & 0.01 & 600 & ICRP-38 & $6.80 \mathrm{E}+01$ & $\mathrm{~m}$ & $4.08 \mathrm{E}+03$ & $1.71 \mathrm{E}+07$ & ICRP-68 & $3.3 \mathrm{E}-11$ & $M$ & ICRP-68 & $2.6 \mathrm{E}-11$ & -- & -- \\
\hline Ho-163 & Ho-163 & 162.929 & Holmium & 0.01 & 0.01 & 600 & ICRP-107 & $4.57 \mathrm{E}+03$ & $y$ & $1.44 \mathrm{E}+11$ & $4.80 \mathrm{E}-01$ & JAERI & $2.4 \mathrm{E}-10$ & $M$ & JAERI & $6.8 \mathrm{E}-12$ & -- & -- \\
\hline Ho-164 & Ho-164 & 163.930 & Holmium & 0.01 & 0.01 & 600 & $\begin{array}{l}\text { ICRP-38 } \\
\end{array}$ & $2.90 \mathrm{E}+01$ & $\mathrm{~m}$ & $1.74 E+03$ & $3.96 \mathrm{E}+07$ & ICRP-68 & $1.3 \mathrm{E}-11$ & $M$ & ICRP-68 & $9.5 \mathrm{E}-12$ & -- & -- \\
\hline Ho-164m & Ho-164 & 163.930 & Holmium & 0.01 & 0.01 & 600 & ICRP-38 & $3.75 \mathrm{E}+01$ & $\mathrm{~m}$ & $2.25 \mathrm{E}+03$ & $3.06 \mathrm{E}+07$ & ICRP-68 & $1.6 \mathrm{E}-11$ & $\mathrm{M}$ & ICRP-68 & $1.6 \mathrm{E}-11$ & -- & -- \\
\hline Ho-166 & Ho-166 & 165.932 & Holmium & 0.01 & 0.01 & 600 & ICRP-38 & $2.68 \mathrm{E}+01$ & $\mathrm{~h}$ & $9.65 \mathrm{E}+04$ & $7.05 E+05$ & ICRP-68 & $8.3 \mathrm{E}-10$ & $M$ & ICRP-68 & $1.4 \mathrm{E}-09$ & -- & -- \\
\hline Ho-166m & Ho-166 & 165.932 & Holmium & 0.01 & 0.01 & 600 & ICRP-38 & $1.20 \mathrm{E}+03$ & $y$ & $3.79 E+10$ & $1.80 \mathrm{E}+00$ & ICRP-68 & $1.1 \mathrm{E}-07$ & $M$ & ICRP-68 & $2.0 \mathrm{E}-09$ & -- & -- \\
\hline Ho-167 & Ho-167 & 166.933 & Holmium & 0.01 & 0.01 & 600 & ICRP-38 & $3.10 \mathrm{E}+00$ & $\mathrm{~h}$ & $1.12 \mathrm{E}+04$ & $6.06 \mathrm{E}+06$ & $\begin{array}{l}\text { ICRP-68 } \\
\end{array}$ & $1.0 \mathrm{E}-10$ & $\mathrm{M}$ & ICRP-68 & $8.3 \mathrm{E}-11$ & -- & -- \\
\hline Ho-168 & Ho-168 & 167.936 & Holmium & 0.01 & 0.01 & 600 & ICRP-107 & $2.99 \mathrm{E}+00$ & $\mathrm{~m}$ & $1.79 \mathrm{E}+02$ & $3.74 \mathrm{E}+08$ & -- & -- & -- & -- & -- & -- & -- \\
\hline Ho-168m & Ho-168 & 167.936 & Holmium & 0.01 & 0.01 & 600 & ICRP-107 & $1.32 \mathrm{E}+02$ & $\mathrm{~s}$ & $1.32 \mathrm{E}+02$ & $5.09 \mathrm{E}+08$ & -- & -- & -- & -- & -- & -- & -- \\
\hline Ho-170 & Ho- 170 & 169.940 & Holmium & 0.01 & 0.01 & 600 & ICRP-107 & $2.76 \mathrm{E}+00$ & $\mathrm{~m}$ & $1.66 \mathrm{E}+02$ & $4.01 \mathrm{E}+08$ & -- & -- & -- & -- & -- & -- & -- \\
\hline I-118 & $\mathrm{I}-118$ & 117.913 & lodine & 0.5 & 0.15 & 3 & ICRP-107 & $1.37 \mathrm{E}+01$ & $\mathrm{~m}$ & $8.22 \mathrm{E}+02$ & $1.16 \mathrm{E}+08$ & JAERI & $1.7 \mathrm{E}-10$ & Vap & JAERI & $2.0 \mathrm{E}-10$ & -- & -- \\
\hline $\mathrm{I}-118 \mathrm{~m}$ & I-118 & 117.913 & Iodine & 0.5 & 0.15 & 3 & ICRP-107 & $8.50 \mathrm{E}+00$ & $\mathrm{~m}$ & $5.10 \mathrm{E}+02$ & $1.88 \mathrm{E}+08$ & -- & -- & -- & -- & -- & -- & -- \\
\hline I-119 & I-119 & 118.910 & lodine & 0.5 & 0.15 & 3 & ICRP-107 & $1.91 \mathrm{E}+01$ & $\mathrm{~m}$ & $1.15 \mathrm{E}+03$ & $8.28 \mathrm{E}+07$ & JAERI & $5.6 \mathrm{E}-11$ & Vap & JAERI & $4.6 \mathrm{E}-11$ & -- & -- \\
\hline $1-120$ & I-120 & 119.910 & Iodine & 0.5 & 0.15 & 3 & ICRP-38 & $8.10 \mathrm{E}+01$ & $\mathrm{~m}$ & $4.86 \mathrm{E}+03$ & $1.94 \mathrm{E}+07$ & ICRP-68 & $3.0 \mathrm{E}-10$ & 12 & $\begin{array}{l}\text { ICRP-68 } \\
\end{array}$ & $3.4 \mathrm{E}-10$ & -- & -- \\
\hline $\mathrm{I}-120 \mathrm{~m}$ & I-120 & 119.910 & lodine & 0.5 & 0.15 & 3 & ICRP-38 & $5.30 \mathrm{E}+01$ & $\mathrm{~m}$ & $3.18 \mathrm{E}+03$ & $2.96 \mathrm{E}+07$ & ICRP-68 & $1.8 \mathrm{E}-10$ & 12 & ICRP-68 & $2.1 \mathrm{E}-10$ & -- & -- \\
\hline $\mathrm{I}-121$ & I-121 & 120.907 & lodine & 0.5 & 0.15 & 3 & ICRP-38 & $2.12 \mathrm{E}+00$ & $\mathrm{~h}$ & $7.63 \mathrm{E}+03$ & $1.22 \mathrm{E}+07$ & ICRP-68 & $8.6 \mathrm{E}-11$ & 12 & ICRP-68 & $8.2 \mathrm{E}-11$ & -- & -- \\
\hline $1-122$ & I-122 & 121.908 & Iodine & 0.5 & 0.15 & 3 & ICRP-38 & $3.62 \mathrm{E}+00$ & $\mathrm{~m}$ & $2.17 E+02$ & $4.26 \mathrm{E}+08$ & -- & -- & -- & -- & -- & -- & -- \\
\hline I-123 & I-123 & 122.906 & lodine & 0.5 & 0.15 & 3 & ICRP-38 & $1.32 \mathrm{E}+01$ & $\mathrm{~h}$ & $4.75 \mathrm{E}+04$ & $1.93 \mathrm{E}+06$ & ICRP-68 & $2.1 \mathrm{E}-10$ & 12 & ICRP-68 & $2.1 \mathrm{E}-10$ & -- & -- \\
\hline $1-124$ & I-124 & 123.906 & Iodine & 0.5 & 0.15 & 3 & ICRP-38 & $4.18 \mathrm{E}+00$ & $d$ & $3.61 \mathrm{E}+05$ & $2.52 \mathrm{E}+05$ & ICRP-68 & $1.2 \mathrm{E}-08$ & 12 & ICRP-68 & $1.3 \mathrm{E}-08$ & -- & -- \\
\hline $1-125$ & I-125 & 124.905 & lodine & 0.5 & 0.15 & 3 & ICRP-38 & $6.01 \mathrm{E}+01$ & $d$ & $5.20 \mathrm{E}+06$ & $1.74 \mathrm{E}+04$ & ICRP-68 & $1.4 \mathrm{E}-08$ & 12 & ICRP-68 & $1.5 \mathrm{E}-08$ & -- & -- \\
\hline $1-126$ & I-126 & 125.906 & Iodine & 0.5 & 0.15 & 3 & ICRP-38 & $1.30 \mathrm{E}+01$ & $d$ & $1.12 \mathrm{E}+06$ & $7.97 \mathrm{E}+04$ & ICRP-68 & $2.6 \mathrm{E}-08$ & 12 & ICRP-68 & $2.9 \mathrm{E}-08$ & -- & -- \\
\hline $1-128$ & I-128 & 127.906 & Iodine & 0.5 & 0.15 & 3 & ICRP-38 & $2.50 \mathrm{E}+01$ & $\mathrm{~m}$ & $1.50 \mathrm{E}+03$ & $5.88 \mathrm{E}+07$ & ICRP-68 & $6.5 \mathrm{E}-11$ & 12 & ICRP-68 & $4.6 \mathrm{E}-11$ & -- & -- \\
\hline $1-129$ & I-129 & 128.905 & Iodine & 0.5 & 0.15 & 3 & ICRP-38 & $1.57 \mathrm{E}+07$ & $y$ & $4.95 \mathrm{E}+14$ & $1.77 \mathrm{E}-04$ & ICRP-68 & $9.6 \mathrm{E}-08$ & 12 & ICRP-68 & $1.1 \mathrm{E}-07$ & -- & -- \\
\hline $1-130$ & I-130 & 129.907 & Iodine & 0.5 & 0.15 & 3 & ICRP-38 & $1.24 \mathrm{E}+01$ & $\mathrm{~h}$ & $4.45 \mathrm{E}+04$ & $1.95 \mathrm{E}+06$ & ICRP-68 & $1.9 \mathrm{E}-09$ & 12 & ICRP-68 & $2.0 \mathrm{E}-09$ & -- & -- \\
\hline $\mathrm{I}-130 \mathrm{~m}$ & I-130 & 129.907 & Iodine & 0.5 & 0.15 & 3 & ICRP-107 & $8.84 \mathrm{E}+00$ & $\mathrm{~m}$ & $5.30 \mathrm{E}+02$ & $1.64 \mathrm{E}+08$ & -- & -- & -- & -- & -- & -- & -- \\
\hline I-131 & I-131 & 130.906 & Iodine & 0.5 & 0.15 & 3 & ICRP-38 & $8.04 \mathrm{E}+00$ & $d$ & $6.95 E+05$ & $1.24 \mathrm{E}+05$ & ICRP-68 & $2.0 \mathrm{E}-08$ & 12 & ICRP-68 & $2.2 \mathrm{E}-08$ & -- & -- \\
\hline $1-132$ & I-132 & 131.908 & lodine & 0.5 & 0.15 & 3 & ICRP-38 & $2.30 \mathrm{E}+00$ & $\mathrm{~h}$ & $8.28 \mathrm{E}+03$ & $1.03 E+07$ & ICRP-68 & $3.1 \mathrm{E}-10$ & 12 & ICRP-68 & $2.9 \mathrm{E}-10$ & -- & -- \\
\hline I-132m & I-132 & 131.908 & Iodine & 0.5 & 0.15 & 3 & ICRP-38 & $8.36 \mathrm{E}+01$ & $\mathrm{~m}$ & $5.02 \mathrm{E}+03$ & $1.71 \mathrm{E}+07$ & ICRP-68 & $2.7 \mathrm{E}-10$ & 12 & ICRP-68 & $2.2 \mathrm{E}-10$ & -- & -- \\
\hline I-133 & I-133 & 132.908 & Iodine & 0.5 & 0.15 & 3 & ICRP-38 & $2.08 \mathrm{E}+01$ & $\mathrm{~h}$ & $7.49 E+04$ & $1.13 \mathrm{E}+06$ & ICRP-68 & $4.0 \mathrm{E}-09$ & 12 & ICRP-68 & $4.3 \mathrm{E}-09$ & -- & -- \\
\hline I-134 & I-134 & 133.910 & Iodine & 0.5 & 0.15 & 3 & ICRP-38 & $5.26 \mathrm{E}+01$ & $\mathrm{~m}$ & $3.16 \mathrm{E}+03$ & $2.67 E+07$ & ICRP-68 & $1.5 \mathrm{E}-10$ & 12 & ICRP-68 & $1.1 \mathrm{E}-10$ & -- & -- \\
\hline I-134m & I-134 & 133.910 & Iodine & 0.5 & 0.15 & 3 & ICRP-107 & $3.60 \mathrm{E}+00$ & $\mathrm{~m}$ & $2.16 \mathrm{E}+02$ & $3.90 \mathrm{E}+08$ & -- & -- & -- & -- & -- & -- & -- \\
\hline $\mathrm{I}-135$ & I-135 & 134.910 & lodine & 0.5 & 0.15 & 3 & ICRP-38 & $6.61 \mathrm{E}+00$ & $\mathrm{~h}$ & $2.38 E+04$ & $3.51 \mathrm{E}+06$ & ICRP-68 & $9.2 \mathrm{E}-10$ & 12 & ICRP-68 & $9.3 \mathrm{E}-10$ & -- & -- \\
\hline $\ln -103$ & $\ln -103$ & 102.920 & Indium & 0.01 & 0.004 & 0 & ICRP-107 & $6.00 \mathrm{E}+01$ & $\mathrm{~s}$ & $6.00 \mathrm{E}+01$ & $1.83 \mathrm{E}+09$ & -- & -- & -- & -- & -- & -- & -- \\
\hline $\ln -105$ & $\ln -105$ & 104.915 & Indium & 0.01 & 0.004 & 0 & ICRP-107 & $5.07 \mathrm{E}+00$ & $\mathrm{~m}$ & $3.04 \mathrm{E}+02$ & $3.53 \mathrm{E}+08$ & -- & -- & -- & -- & -- & -- & -- \\
\hline $\ln -106$ & $\ln -106$ & 105.913 & Indium & 0.01 & 0.004 & 0 & ICRP-107 & $6.20 \mathrm{E}+00$ & $\mathrm{~m}$ & $3.72 \mathrm{E}+02$ & $2.86 \mathrm{E}+08$ & -- & -- & -- & -- & -- & -- & -- \\
\hline $\mathrm{In}-106 \mathrm{~m}$ & $\ln -106$ & 105.913 & Indium & 0.01 & 0.004 & 0 & ICRP-107 & $5.20 \mathrm{E}+00$ & $\mathrm{~m}$ & $3.12 \mathrm{E}+02$ & $3.41 \mathrm{E}+08$ & -- & -- & -- & -- & -- & -- & -- \\
\hline In-107 & $\ln -107$ & 106.910 & Indium & 0.01 & 0.004 & 0 & ICRP-107 & $3.24 \mathrm{E}+01$ & $\mathrm{~m}$ & $1.94 \mathrm{E}+03$ & $5.43 \mathrm{E}+07$ & JAERI & $4.1 \mathrm{E}-11$ & $M$ & JAERI & $4.1 \mathrm{E}-11$ & -- & -- \\
\hline $\ln -108$ & $\ln -108$ & 107.910 & Indium & 0.01 & 0.004 & 0 & ICRP-107 & $5.80 \mathrm{E}+01$ & $\mathrm{~m}$ & $3.48 \mathrm{E}+03$ & $3.00 \mathrm{E}+07$ & JAERI & $9.2 \mathrm{E}-11$ & $M$ & JAERI & $8.3 \mathrm{E}-11$ & -- & -- \\
\hline In-108m & $\ln -108$ & 107.910 & Indium & 0.01 & 0.004 & 0 & ICRP-107 & $3.96 \mathrm{E}+01$ & $\mathrm{~m}$ & $2.38 \mathrm{E}+03$ & $4.40 \mathrm{E}+07$ & JAERI & $6.5 \mathrm{E}-11$ & $M$ & JAERI & $8.5 \mathrm{E}-11$ & -- & -- \\
\hline
\end{tabular}




\begin{tabular}{|c|c|c|c|c|c|c|c|c|c|c|c|c|c|c|c|c|c|c|}
\hline \multirow{3}{*}{$\begin{array}{l}\text { MASTER } \\
\text { ISOTOPE } \\
\text { LIST } \\
\text { In-109 }\end{array}$} & \multirow{3}{*}{$\begin{array}{c}\begin{array}{c}\text { Atomic } \\
\text { Mass } \\
\text { Nuclide }\end{array} \\
\text { In-109 }\end{array}$} & \multirow{3}{*}{$\begin{array}{c}\begin{array}{c}\text { Atomic } \\
\text { Mass }\end{array} \\
108.907\end{array}$} & & & Table C.1 & dionuclide Sp & fic Input Da & Jsed For th & & tion of $\mathrm{H}$ & 2 s Usin & ximum Rep & & ued) & & & & \\
\hline & & & \multirow{2}{*}{$\frac{\text { Element }}{\text { Indium }}$} & \multirow{2}{*}{$\begin{array}{c}\text { EPA } \\
\text { RF } \\
0.01\end{array}$} & \multirow[t]{2}{*}{$\begin{array}{c}\text { EPA } \\
\text { Conc } \\
\text { Factors } \\
\left(\mathbf{B}_{\mathrm{v}}\right) \\
0.004 \\
\end{array}$} & \multirow[t]{2}{*}{$\begin{array}{c}\text { EPA } \\
\begin{array}{c}\text { Sorption } \\
\text { Coefficient } \\
\left(K_{d}\right)\end{array} \\
0\end{array}$} & \multirow{2}{*}{$\begin{array}{c}\begin{array}{c}\text { Half-Life } \\
\text { Reference }\end{array} \\
\text { ICRP-38 } \\
\end{array}$} & \multicolumn{2}{|c|}{$\begin{array}{c}\text { Half-Life } \\
\text { (original units) }\end{array}$} & \multirow{2}{*}{$\begin{array}{c}\begin{array}{c}\text { Half Life } \\
\text { (s) }\end{array} \\
1.51 \mathrm{E}+04 \\
\end{array}$} & \multirow{2}{*}{$\begin{array}{c}\begin{array}{c}\text { Sp. Act } \\
\text { (Ci/g) }\end{array} \\
6.85 \mathrm{E}+06\end{array}$} & \multirow[t]{2}{*}{$\begin{array}{c}\text { Inhalation } \\
\text { DC } \\
\text { Reference }\end{array}$} & \multirow[t]{2}{*}{$\begin{array}{c}\text { HC-3 } \\
\text { Bounding } \\
\text { Inhalation } \\
\text { DC } \\
\text { (Sv/Bq) } \\
7.3 \mathrm{E}-11 \\
\end{array}$} & \multirow{2}{*}{$\begin{array}{c}\begin{array}{c}\text { Lung } \\
\text { Absorption } \\
\text { Class/Type }\end{array} \\
\mathrm{M}\end{array}$} & \multirow[t]{2}{*}{$\begin{array}{c}\text { Ingestion } \\
\text { DC } \\
\text { Reference }\end{array}$} & \multirow[t]{2}{*}{$\begin{array}{c}\text { HC-3 } \\
\text { Bounding } \\
\text { Ingestion } \\
\text { DC } \\
(\mathrm{Sv} / \mathrm{Bq}) \\
6.6 \mathrm{E}-11\end{array}$} & $\begin{array}{c}\text { Immersion } \\
\text { DC } \\
\text { Reference }\end{array}$ & $\begin{array}{c}\mathrm{HC}-3 \\
\text { Immersion } \\
\mathrm{DC}(\mathrm{Sv} / \mathrm{d} \\
\left.\operatorname{per} \mathrm{Bq} / \mathrm{m}^{3}\right)\end{array}$ \\
\hline & & & & & & & & $4.20 \mathrm{E}+00$ & $\mathrm{~h}$ & & & & & & & & & -- \\
\hline In-109m & $\ln -109$ & 108.907 & Indium & 0.01 & 0.004 & 0 & $\begin{array}{l}\text { ICRP-107 } \\
\end{array}$ & $1.34 \mathrm{E}+00$ & $\mathrm{~m}$ & $8.04 \mathrm{E}+01$ & $1.29 \mathrm{E}+09$ & -- & -- & -- & -- & -- & -- & -- \\
\hline$|n-110|$ & $\ln -110$ & 109.907 & Indium & 0.01 & 0.004 & 0 & ICRP-38 & $4.90 \mathrm{E}+00$ & $\mathrm{~h}$ & $1.76 \mathrm{E}+04$ & $5.82 \mathrm{E}+06$ & ICRP-68 & $2.5 \mathrm{E}-10$ & $M$ & ICRP-68 & $2.4 \mathrm{E}-10$ & -- & -- \\
\hline $\ln -110 \mathrm{~s}$ & $\ln -110$ & 109.907 & Indium & 0.01 & 0.004 & 0 & ICRP-38 & $6.91 \mathrm{E}+01$ & $\mathrm{~m}$ & $4.15 \mathrm{E}+03$ & $2.48 \mathrm{E}+07$ & ICRP-68 & $8.1 \mathrm{E}-11$ & $M$ & ICRP-68 & $1.0 \mathrm{E}-10$ & -- & -- \\
\hline $\ln -111$ & $\ln -111$ & 110.905 & Indium & 0.01 & 0.004 & 0 & ICRP-38 & $2.83 \mathrm{E}+00$ & d & $2.45 \mathrm{E}+05$ & $4.16 \mathrm{E}+05$ & ICRP-68 & $3.1 \mathrm{E}-10$ & $M$ & ICRP-68 & $2.9 \mathrm{E}-10$ & -- & -- \\
\hline $\ln -111 \mathrm{~m}$ & $\ln -111$ & 110.905 & Indium & 0.01 & 0.004 & 0 & ICRP-38 & $7.70 \mathrm{E}+00$ & $\mathrm{~m}$ & $4.62 \mathrm{E}+02$ & $2.20 \mathrm{E}+08$ & -- & -- & -- & -- & -- & -- & -- \\
\hline $\ln -112$ & $\ln -112$ & 111.906 & Indium & 0.01 & 0.004 & 0 & ICRP-38 & $1.44 \mathrm{E}+01$ & $\mathrm{~m}$ & $8.64 \mathrm{E}+02$ & $1.17 \mathrm{E}+08$ & ICRP-68 & $1.3 \mathrm{E}-11$ & $M$ & ICRP-68 & $1.0 \mathrm{E}-11$ & -- & -- \\
\hline $\ln -112 m$ & $\ln -112$ & 111.906 & Indium & 0.01 & 0.004 & 0 & ICRP-107 & $2.06 \mathrm{E}+01$ & $\mathrm{~m}$ & $1.23 \mathrm{E}+03$ & $8.17 E+07$ & JAERI & $3.6 \mathrm{E}-11$ & $M$ & JAERI & $1.7 \mathrm{E}-11$ & -- & -- \\
\hline $\ln -113 m$ & $\ln -113$ & 112.904 & Indium & 0.01 & 0.004 & 0 & $\begin{array}{l}\text { ICRP-38 } \\
\end{array}$ & $1.66 \mathrm{E}+00$ & $\mathrm{~h}$ & $5.97 E+03$ & $1.67 \mathrm{E}+07$ & ICRP-68 & $3.2 \mathrm{E}-11$ & $M$ & ICRP-68 & $2.8 \mathrm{E}-11$ & -- & -- \\
\hline $\ln -114$ & $\ln -114$ & 113.905 & Indium & 0.01 & 0.004 & 0 & ICRP-38 & $7.19 \mathrm{E}+01$ & $\mathrm{~s}$ & $7.19 \mathrm{E}+01$ & $1.38 \mathrm{E}+09$ & -- & -- & -- & -- & -- & -- & -- \\
\hline $\ln -114 m$ & $\ln -114$ & 113.905 & Indium & 0.01 & 0.004 & 0 & ICRP-38 & $4.95 \mathrm{E}+01$ & $d$ & $4.28 \mathrm{E}+06$ & $2.32 \mathrm{E}+04$ & ICRP-68 & $1.1 \mathrm{E}-08$ & $\mathrm{~F}$ & ICRP-68 & $4.1 \mathrm{E}-09$ & -- & -- \\
\hline $\ln -115$ & $\ln -115$ & 114.904 & Indium & 0.01 & 0.004 & 0 & ICRP-38 & $5.10 \mathrm{E}+15$ & $y$ & $1.61 \mathrm{E}+23$ & $6.10 \mathrm{E}-13$ & ICRP-68 & $4.5 \mathrm{E}-07$ & $\mathrm{~F}$ & ICRP-68 & $3.2 \mathrm{E}-08$ & -- & -- \\
\hline In-115m & $\ln -115$ & 114.904 & Indium & 0.01 & 0.004 & 0 & ICRP-38 & $4.49 \mathrm{E}+00$ & $\mathrm{~h}$ & $1.61 \mathrm{E}+04$ & $6.08 \mathrm{E}+06$ & $\begin{array}{l}\text { ICRP-68 } \\
\end{array}$ & $8.7 E-11$ & $M$ & ICRP-68 & $8.6 \mathrm{E}-11$ & -- & -- \\
\hline $\mathrm{In}-116 \mathrm{~m}$ & $\ln -116$ & 115.905 & Indium & 0.01 & 0.004 & 0 & ICRP-38 & $5.42 \mathrm{E}+01$ & $\mathrm{~m}$ & $3.25 \mathrm{E}+03$ & $3.00 \mathrm{E}+07$ & ICRP-68 & $8.0 \mathrm{E}-11$ & $M$ & ICRP-68 & $6.4 \mathrm{E}-11$ & -- & -- \\
\hline $\ln -117$ & $\ln -117$ & 116.905 & Indium & 0.01 & 0.004 & 0 & ICRP-38 & $4.38 \mathrm{E}+01$ & $\mathrm{~m}$ & $2.63 \mathrm{E}+03$ & $3.67 \mathrm{E}+07$ & ICRP-68 & $4.8 \mathrm{E}-11$ & $M$ & ICRP-68 & $3.1 \mathrm{E}-11$ & -- & -- \\
\hline $\ln -117 m$ & $\ln -117$ & 116.905 & Indium & 0.01 & 0.004 & 0 & ICRP-38 & $1.17 \mathrm{E}+02$ & $\mathrm{~m}$ & $6.99 \mathrm{E}+03$ & $1.38 \mathrm{E}+07$ & ICRP-68 & $1.1 \mathrm{E}-10$ & $M$ & ICRP-68 & $1.2 \mathrm{E}-10$ & -- & -- \\
\hline $\ln -118$ & $\ln -118$ & 117.906 & Indium & 0.01 & 0.004 & 0 & ICRP-107 & $5.00 \mathrm{E}+00$ & $\mathrm{~s}$ & $5.00 \mathrm{E}+00$ & $1.91 \mathrm{E}+10$ & -- & -- & -- & -- & -- & -- & -- \\
\hline In-118m & $\ln -118$ & 117.906 & Indium & 0.01 & 0.004 & 0 & ICRP-107 & $4.36 \mathrm{E}+00$ & $\mathrm{~m}$ & $2.62 \mathrm{E}+02$ & $3.65 \mathrm{E}+08$ & -- & -- & -- & -- & -- & -- & -- \\
\hline $\ln -119$ & $\ln -119$ & 118.906 & Indium & 0.01 & 0.004 & 0 & ICRP-38 & $2.40 \mathrm{E}+00$ & $\mathrm{~m}$ & $1.44 \mathrm{E}+02$ & $6.59 \mathrm{E}+08$ & -- & -- & -- & -- & -- & -- & -- \\
\hline In-119m & $\ln -119$ & 118.906 & Indium & 0.01 & 0.004 & 0 & ICRP-38 & $1.80 \mathrm{E}+01$ & $\mathrm{~m}$ & $1.08 \mathrm{E}+03$ & $8.78 \mathrm{E}+07$ & ICRP-68 & $2.9 \mathrm{E}-11$ & $M$ & ICRP-68 & $4.7 \mathrm{E}-11$ & -- & -- \\
\hline In-121 & $\ln -121$ & 120.908 & Indium & 0.01 & 0.004 & 0 & ICRP-107 & $2.31 \mathrm{E}+01$ & $\mathrm{~s}$ & $2.31 \mathrm{E}+01$ & $4.04 \mathrm{E}+09$ & -- & -- & -- & -- & -- & -- & -- \\
\hline In-121m & $\ln -121$ & 120.908 & Indium & 0.01 & 0.004 & 0 & ICRP-107 & $3.88 \mathrm{E}+00$ & $\mathrm{~m}$ & $2.33 \mathrm{E}+02$ & $4.01 \mathrm{E}+08$ & -- & -- & -- & -- & -- & -- & -- \\
\hline Ir-179 & Ir-179 & 178.959 & Iridium & 0.001 & 0.055 & 0 & JAERI & $1.32 \mathrm{E}+00$ & $\mathrm{~m}$ & $7.90 \mathrm{E}+01$ & $7.98 \mathrm{E}+08$ & -- & -- & -- & -- & -- & -- & -- \\
\hline $\mid r-180$ & Ir-180 & 179.959 & Iridium & 0.001 & 0.055 & 0 & ICRP-107 & $1.50 \mathrm{E}+00$ & $\mathrm{~m}$ & $9.00 \mathrm{E}+01$ & $6.97 E+08$ & -- & -- & -- & -- & -- & -- & -- \\
\hline $\mid r-181$ & $\mid \mathrm{Ir}-181$ & 180.958 & Iridium & 0.001 & 0.055 & 0 & JAERI & $4.90 \mathrm{E}+00$ & $\mathrm{~m}$ & $2.94 \mathrm{E}+02$ & $2.12 \mathrm{E}+08$ & -- & -- & -- & -- & -- & -- & -- \\
\hline Ir-182 & $\mid r-182$ & 181.958 & Iridium & 0.001 & 0.055 & 0 & ICRP-38 & $1.50 \mathrm{E}+01$ & $\mathrm{~m}$ & $9.00 \mathrm{E}+02$ & $6.89 \mathrm{E}+07$ & ICRP-68 & $4.0 \mathrm{E}-11$ & $\mathrm{~s}$ & ICRP-68 & $4.8 \mathrm{E}-11$ & -- & -- \\
\hline Ir-183 & Ir-183 & 182.957 & Iridium & 0.001 & 0.055 & 0 & ICRP-107 & $5.80 \mathrm{E}+01$ & $\mathrm{~m}$ & $3.48 \mathrm{E}+03$ & $1.77 \mathrm{E}+07$ & JAERI & $6.4 \mathrm{E}-11$ & $\mathrm{~s}$ & JAERI & $5.5 \mathrm{E}-11$ & -- & -- \\
\hline Ir-184 & Ir-184 & 183.957 & Iridium & 0.001 & 0.055 & 0 & ICRP-38 & $3.02 \mathrm{E}+00$ & $\mathrm{~h}$ & $1.09 \mathrm{E}+04$ & $5.64 \mathrm{E}+06$ & ICRP-68 & $1.9 \mathrm{E}-10$ & $\mathrm{~s}$ & ICRP-68 & $1.7 \mathrm{E}-10$ & -- & -- \\
\hline Ir-185 & Ir-185 & 184.957 & Iridium & 0.001 & 0.055 & 0 & ICRP-38 & $1.40 \mathrm{E}+01$ & $\mathrm{~h}$ & $5.04 \mathrm{E}+04$ & $1.21 \mathrm{E}+06$ & ICRP-68 & $2.6 \mathrm{E}-10$ & $\mathrm{~s}$ & ICRP-68 & $2.6 \mathrm{E}-10$ & -- & -- \\
\hline$|r-186|$ & Ir-186 & 185.958 & Iridium & 0.001 & 0.055 & 0 & ICRP-38 & $1.58 \mathrm{E}+01$ & $\mathrm{~h}$ & $5.69 \mathrm{E}+04$ & $1.07 E+06$ & ICRP-68 & $5.0 \mathrm{E}-10$ & $\mathrm{~s}$ & ICRP-68 & $4.9 \mathrm{E}-10$ & -- & -- \\
\hline Ir-186s & Ir-186 & 185.958 & Iridium & 0.001 & 0.055 & 0 & ICRP-38 & $1.75 \mathrm{E}+00$ & $h$ & $6.30 \mathrm{E}+03$ & $9.63 \mathrm{E}+06$ & ICRP-68 & $7.1 \mathrm{E}-11$ & $\mathrm{~s}$ & ICRP-68 & $6.1 \mathrm{E}-11$ & -- & -- \\
\hline Ir-187 & Ir-187 & 186.958 & Iridium & 0.001 & 0.055 & 0 & ICRP-38 & $1.05 \mathrm{E}+01$ & $\mathrm{~h}$ & $3.78 \mathrm{E}+04$ & $1.60 \mathrm{E}+06$ & ICRP-68 & $1.2 \mathrm{E}-10$ & $\mathrm{~s}$ & ICRP-68 & $1.2 \mathrm{E}-10$ & -- & -- \\
\hline Ir-188 & $\mid r-188$ & 187.959 & Iridium & 0.001 & 0.055 & 0 & ICRP-38 & $4.15 \mathrm{E}+01$ & $\mathrm{~h}$ & $1.49 \mathrm{E}+05$ & $4.02 \mathrm{E}+05$ & ICRP-68 & $6.2 \mathrm{E}-10$ & $\mathrm{~s}$ & ICRP-68 & $6.3 \mathrm{E}-10$ & -- & -- \\
\hline Ir-189 & Ir-189 & 188.959 & Iridium & 0.001 & 0.055 & 0 & ICRP-38 & $1.33 \mathrm{E}+01$ & $d$ & $1.15 \mathrm{E}+06$ & $5.20 \mathrm{E}+04$ & ICRP-68 & $5.5 \mathrm{E}-10$ & $\mathrm{~s}$ & ICRP-68 & $2.4 \mathrm{E}-10$ & -- & -- \\
\hline $\mid \mathrm{Ir}-190$ & $\mid \mathrm{Ir}-190$ & 189.961 & Iridium & 0.001 & 0.055 & 0 & ICRP-38 & $1.21 \mathrm{E}+01$ & $d$ & $1.05 E+06$ & $5.68 \mathrm{E}+04$ & ICRP-68 & $2.5 \mathrm{E}-09$ & $\mathrm{~s}$ & ICRP-68 & $1.2 \mathrm{E}-09$ & -- & -- \\
\hline $\mathrm{Ir}-190 \mathrm{~ms}$ & Ir-190 & 189.961 & Iridium & 0.001 & 0.055 & 0 & ICRP-38 & $1.20 \mathrm{E}+00$ & $\mathrm{~h}$ & $4.32 \mathrm{E}+03$ & $1.37 \mathrm{E}+07$ & ICRP-68 & $1.1 \mathrm{E}-11$ & $\mathrm{~s}$ & ICRP-68 & $8.0 \mathrm{E}-12$ & -- & -- \\
\hline $\mid \mathrm{r}-190 \mathrm{ml}$ & Ir-190 & 189.961 & Iridium & 0.001 & 0.055 & 0 & ICRP-38 & $3.10 \mathrm{E}+00$ & $\mathrm{~h}$ & $1.12 \mathrm{E}+04$ & $5.32 \mathrm{E}+06$ & ICRP-68 & $1.4 \mathrm{E}-10$ & $M$ & ICRP-68 & $1.2 \mathrm{E}-10$ & -- & -- \\
\hline Ir-191m & $\mid r-191$ & 190.961 & Iridium & 0.001 & 0.055 & 0 & ICRP-38 & $4.94 \mathrm{E}+00$ & $\mathrm{~s}$ & $4.94 \mathrm{E}+00$ & $1.20 \mathrm{E}+10$ & -- & -- & -- & -- & -- & -- & -- \\
\hline Ir-192 & $\mid r-192$ & 191.963 & Iridium & 0.001 & 0.055 & 0 & ICRP-38 & $7.40 \mathrm{E}+01$ & $d$ & $6.40 \mathrm{E}+06$ & $9.19 \mathrm{E}+03$ & ICRP-68 & $6.2 \mathrm{E}-09$ & $\mathrm{~s}$ & ICRP-68 & $1.4 \mathrm{E}-09$ & -- & -- \\
\hline Ir-192ms & $\mid \mathrm{Ir}-192$ & 191.963 & Iridium & 0.001 & 0.055 & 0 & ICRP-107 & $1.45 \mathrm{E}+00$ & $\mathrm{~m}$ & $8.70 \mathrm{E}+01$ & $6.76 \mathrm{E}+08$ & -- & -- & -- & -- & -- & -- & -- \\
\hline $\mid \mathrm{r}-192 \mathrm{ml}$ & $\mid r-192$ & 191.963 & Iridium & 0.001 & 0.055 & 0 & ICRP-38 & $2.41 \mathrm{E}+02$ & $y$ & $7.61 \mathrm{E}+09$ & $7.73 \mathrm{E}+00$ & ICRP-68 & $3.6 \mathrm{E}-08$ & $\mathrm{~s}$ & ICRP-68 & $3.1 \mathrm{E}-10$ & -- & -- \\
\hline Ir-193m & Ir-193 & 192.963 & Iridium & 0.001 & 0.055 & 0 & ICRP-107 & $1.05 \mathrm{E}+01$ & $d$ & $9.10 \mathrm{E}+05$ & $6.43 \mathrm{E}+04$ & ICRP-68 & $1.2 \mathrm{E}-09$ & $\mathrm{~s}$ & ICRP-68 & $2.7 \mathrm{E}-10$ & -- & -- \\
\hline Ir-194 & $\mid \mathrm{Ir}-194$ & 193.965 & Iridium & 0.001 & 0.055 & 0 & ICRP-38 & $1.92 \mathrm{E}+01$ & $\mathrm{~h}$ & $6.89 \mathrm{E}+04$ & $8.44 \mathrm{E}+05$ & ICRP-68 & $7.5 \mathrm{E}-10$ & $\mathrm{~s}$ & ICRP-68 & $1.3 \mathrm{E}-09$ & -- & -- \\
\hline Ir-194m & Ir-194 & 193.965 & Iridium & 0.001 & 0.055 & 0 & ICRP-38 & $1.71 \mathrm{E}+02$ & $d$ & $1.48 \mathrm{E}+07$ & $3.94 \mathrm{E}+03$ & ICRP-68 & $1.2 \mathrm{E}-08$ & $\mathrm{~s}$ & ICRP-68 & $2.1 \mathrm{E}-09$ & -- & -- \\
\hline Ir-195 & Ir-195 & 194.966 & Iridium & 0.001 & 0.055 & 0 & ICRP-38 & $2.50 \mathrm{E}+00$ & $\mathrm{~h}$ & $9.00 \mathrm{E}+03$ & $6.43 \mathrm{E}+06$ & ICRP-68 & $1.0 \mathrm{E}-10$ & $\mathrm{~s}$ & ICRP-68 & $1.0 \mathrm{E}-10$ & -- & -- \\
\hline
\end{tabular}




\begin{tabular}{|c|c|c|c|c|c|c|c|c|c|c|c|c|c|c|c|c|c|c|}
\hline \multirow{3}{*}{$\begin{array}{l}\text { MASTER } \\
\text { ISOTOPE } \\
\text { LIST } \\
\text { Ir-195m }\end{array}$} & \multirow{3}{*}{$\begin{array}{c}\begin{array}{c}\text { Atomic } \\
\text { Mass } \\
\text { Nuclide }\end{array} \\
\text { Ir-195 }\end{array}$} & & & & \multicolumn{14}{|c|}{ Table C.1 Radionuclide Specific Input Data Used For the } \\
\hline & & \multirow{2}{*}{$\begin{array}{c}\begin{array}{c}\text { Atomic } \\
\text { Mass }\end{array} \\
194.966\end{array}$} & \multirow{2}{*}{$\begin{array}{l}\text { Element } \\
\text { Iridium }\end{array}$} & \multirow{2}{*}{$\begin{array}{c}\text { EPA } \\
\text { RF } \\
0.001\end{array}$} & $\begin{array}{c}\text { EPA } \\
\text { Conc } \\
\text { Factors } \\
\left(B_{v}\right) \\
\end{array}$ & $\begin{array}{c}\text { EPA } \\
\text { Sorption } \\
\text { Coefficient } \\
\left(K_{d}\right)\end{array}$ & $\begin{array}{c}\text { Half-Life } \\
\text { Reference }\end{array}$ & $\begin{array}{r}\text { Half-Lif } \\
\text { (original u }\end{array}$ & & $\begin{array}{l}\text { Half Life } \\
\text { (s) }\end{array}$ & $\begin{array}{l}\text { Sp. Act } \\
\text { (Ci/g) }\end{array}$ & $\begin{array}{l}\text { Inhalation } \\
\text { DC } \\
\text { Reference }\end{array}$ & $\begin{array}{c}\text { HC-3 } \\
\text { Bounding } \\
\text { Inhalation } \\
\text { DC } \\
\text { (Sv/Bq) } \\
\end{array}$ & $\begin{array}{c}\text { Lung } \\
\text { Absorption } \\
\text { Class/Type }\end{array}$ & $\begin{array}{l}\text { Ingestion } \\
\text { DC } \\
\text { Reference }\end{array}$ & $\begin{array}{c}\text { HC-3 } \\
\text { Bounding } \\
\text { Ingestion } \\
\text { DC } \\
\text { (Sv/Bq) } \\
\end{array}$ & $\begin{array}{c}\text { Immersion } \\
\text { DC } \\
\text { Reference }\end{array}$ & $\begin{array}{c}\mathrm{HC}-3 \\
\text { Immersion } \\
\mathrm{DC}(\mathrm{Sv} / \mathrm{d} \\
\left.\operatorname{per} \mathrm{Bq} / \mathrm{m}^{3}\right)\end{array}$ \\
\hline & & & & & 0.055 & 0 & ICRP-38 & $3.80 \mathrm{E}+00$ & $\mathrm{~h}$ & $1.37 E+04$ & $4.23 \mathrm{E}+06$ & ICRP-68 & $2.4 \mathrm{E}-10$ & $\mathrm{~s}$ & ICRP-68 & $2.1 \mathrm{E}-10$ & -- & -- \\
\hline Ir-196 & Ir-196 & 195.968 & Iridium & 0.001 & 0.055 & 0 & ICRP-107 & $5.20 \mathrm{E}+01$ & $\mathrm{~s}$ & $5.20 \mathrm{E}+01$ & $1.11 \mathrm{E}+09$ & -- & -- & -- & -- & -- & -- & -- \\
\hline Ir-196m & Ir-196 & 195.968 & Iridium & 0.001 & 0.055 & 0 & ICRP-107 & $1.40 \mathrm{E}+00$ & $\mathrm{~h}$ & $5.04 \mathrm{E}+03$ & $1.14 \mathrm{E}+07$ & JAERI & $1.6 \mathrm{E}-10$ & $\mathrm{~s}$ & JAERI & $1.3 \mathrm{E}-10$ & -- & -- \\
\hline $\mathrm{K}-38$ & $\mathrm{~K}-38$ & 37.969 & Potassium & 0.01 & 1 & 35 & ICRP-38 & $7.64 \mathrm{E}+00$ & $\mathrm{~m}$ & $4.58 \mathrm{E}+02$ & $6.49 \mathrm{E}+08$ & -- & -- & -- & -- & -- & -- & -- \\
\hline K-40 & K-40 & 39.964 & $\begin{array}{l}\text { Potassium } \\
\end{array}$ & 0.01 & 1 & 35 & ICRP-38 & $1.28 \mathrm{E}+09$ & $y$ & $4.04 \mathrm{E}+16$ & $6.99 \mathrm{E}-06$ & ICRP-68 & $3.0 \mathrm{E}-09$ & $F$ & ICRP-68 & $6.2 \mathrm{E}-09$ & -- & -- \\
\hline K-42 & $\mathrm{K}-42$ & 41.962 & Potassium & 0.01 & 1 & 35 & ICRP-38 & $1.24 \mathrm{E}+01$ & $\mathrm{~h}$ & $4.45 \mathrm{E}+04$ & $6.04 \mathrm{E}+06$ & ICRP-68 & $2.0 \mathrm{E}-10$ & $F$ & ICRP-68 & $4.3 \mathrm{E}-10$ & -- & -- \\
\hline K-43 & K-43 & 42.961 & Potassium & 0.01 & 1 & 35 & ICRP-38 & $2.26 \mathrm{E}+01$ & $\mathrm{~h}$ & $8.14 \mathrm{E}+04$ & $3.23 \mathrm{E}+06$ & ICRP-68 & $2.6 \mathrm{E}-10$ & $F$ & ICRP-68 & $2.5 \mathrm{E}-10$ & -- & -- \\
\hline K-44 & $\mathrm{K}-44$ & 43.962 & Potassium & 0.01 & 1 & 35 & ICRP-38 & $2.21 \mathrm{E}+01$ & $\mathrm{~m}$ & $1.33 \mathrm{E}+03$ & $1.93 \mathrm{E}+08$ & ICRP-68 & $3.7 E-11$ & $\mathrm{~F}$ & ICRP-68 & $8.4 \mathrm{E}-11$ & -- & -- \\
\hline K-45 & K-45 & 44.961 & Potassium & 0.01 & 1 & 35 & ICRP-38 & $2.00 \mathrm{E}+01$ & $\mathrm{~m}$ & $1.20 \mathrm{E}+03$ & $2.09 \mathrm{E}+08$ & ICRP-68 & $2.8 \mathrm{E}-11$ & $\mathrm{~F}$ & ICRP-68 & $5.4 \mathrm{E}-11$ & -- & -- \\
\hline K-46 & K-46 & 45.962 & Potassium & 0.01 & 1 & 35 & ICRP-107 & $1.05 \mathrm{E}+02$ & $\mathrm{~s}$ & $1.05 E+02$ & $2.34 \mathrm{E}+09$ & -- & -- & -- & -- & -- & -- & -- \\
\hline Kr-74 & Kr-74 & 73.933 & Krypton & 1 & 0 & 0 & ICRP-38 & $1.15 \mathrm{E}+01$ & $\mathrm{~m}$ & $6.90 \mathrm{E}+02$ & $2.21 \mathrm{E}+08$ & -- & -- & -- & -- & -- & ICRP-68 & 4.50E-09 \\
\hline Kr-75 & $\mathrm{Kr}-75$ & 74.931 & Krypton & 1 & 0 & 0 & ICRP-107 & $4.29 \mathrm{E}+00$ & $\mathrm{~m}$ & $2.57 E+02$ & $5.85 \mathrm{E}+08$ & -- & -- & -- & -- & -- & JAERI & $5.10 \mathrm{E}-09$ \\
\hline Kr-76 & $\mathrm{Kr}-76$ & 75.926 & Krypton & 1 & 0 & 0 & ICRP-38 & $1.48 \mathrm{E}+01$ & $\mathrm{~h}$ & $5.33 \mathrm{E}+04$ & $2.79 \mathrm{E}+06$ & -- & -- & -- & -- & -- & ICRP-68 & $1.60 \mathrm{E}-09$ \\
\hline Kr-77 & $\mathrm{Kr}-77$ & 76.925 & Krypton & 1 & 0 & 0 & ICRP-38 & $7.47 \mathrm{E}+01$ & $\mathrm{~m}$ & $4.48 \mathrm{E}+03$ & $3.27 \mathrm{E}+07$ & -- & -- & -- & -- & -- & ICRP-68 & $3.90 \mathrm{E}-09$ \\
\hline Kr-79 & Kr-79 & 78.920 & Krypton & 1 & 0 & 0 & ICRP-38 & $3.50 \mathrm{E}+01$ & $\mathrm{~h}$ & $1.26 \mathrm{E}+05$ & $1.13 \mathrm{E}+06$ & -- & -- & -- & -- & -- & ICRP-68 & $9.70 \mathrm{E}-10$ \\
\hline Kr-81 & $\mathrm{Kr}-81$ & 80.917 & Krypton & 1 & 0 & 0 & ICRP-38 & $2.10 \mathrm{E}+05$ & $y$ & $6.63 \mathrm{E}+12$ & $2.10 \mathrm{E}-02$ & -- & -- & -- & -- & -- & ICRP-68 & $2.10 \mathrm{E}-11$ \\
\hline $\mathrm{Kr}-81 \mathrm{~m}$ & $\mathrm{Kr}-81$ & 80.917 & Krypton & 1 & 0 & 0 & ICRP-38 & $1.30 \mathrm{E}+01$ & $\mathrm{~s}$ & $1.30 \mathrm{E}+01$ & $1.07 \mathrm{E}+10$ & -- & -- & -- & -- & -- & ICRP-68 & $4.80 \mathrm{E}-10$ \\
\hline $\mathrm{Kr}-83 \mathrm{~m}$ & $\mathrm{Kr}-83$ & 82.914 & Krypton & 1 & 0 & 0 & ICRP-38 & $1.83 \mathrm{E}+00$ & $\mathrm{~h}$ & $6.59 \mathrm{E}+03$ & $2.07 E+07$ & -- & -- & -- & -- & -- & ICRP-68 & $2.10 \mathrm{E}-13$ \\
\hline $\mathrm{Kr}-85$ & $\mathrm{Kr}-85$ & 84.913 & Krypton & 1 & 0 & 0 & ICRP-38 & $1.07 \mathrm{E}+01$ & $y$ & $3.38 E+08$ & $3.93 \mathrm{E}+02$ & -- & -- & -- & -- & -- & ICRP-68 & $2.20 \mathrm{E}-11$ \\
\hline $\mathrm{Kr}-85 \mathrm{~m}$ & $\mathrm{Kr}-85$ & 84.913 & Krypton & 1 & 0 & 0 & ICRP-38 & $4.48 \mathrm{E}+00$ & $\mathrm{~h}$ & $1.61 E+04$ & $8.24 \mathrm{E}+06$ & -- & -- & -- & -- & -- & ICRP-68 & $5.90 \mathrm{E}-10$ \\
\hline $\mathrm{Kr}-87$ & $\mathrm{Kr}-87$ & 86.913 & Krypton & 1 & 0 & 0 & ICRP-38 & $7.63 E+01$ & $\mathrm{~m}$ & $4.58 \mathrm{E}+03$ & $2.84 \mathrm{E}+07$ & -- & -- & -- & -- & -- & ICRP-68 & $3.40 \mathrm{E}-09$ \\
\hline Kr-88 & $\mathrm{Kr}-88$ & 87.914 & Krypton & 1 & 0 & 0 & ICRP-38 & $2.84 \mathrm{E}+00$ & $\mathrm{~h}$ & $1.02 E+04$ & $1.26 \mathrm{E}+07$ & -- & -- & -- & -- & -- & ICRP-68 & $8.40 \mathrm{E}-09$ \\
\hline Kr-89 & $\mathrm{Kr}-89$ & 88.918 & Krypton & 1 & 0 & 0 & ICRP-107 & $3.15 \mathrm{E}+00$ & $\mathrm{~m}$ & $1.89 \mathrm{E}+02$ & $6.71 \mathrm{E}+08$ & -- & -- & -- & -- & -- & JAERI & $8.30 \mathrm{E}-09$ \\
\hline La-128 & La-128 & 127.916 & Lanthanum & 0.01 & 0.01 & 0 & ICRP-107 & $5.18 \mathrm{E}+00$ & $\mathrm{~m}$ & $3.11 \mathrm{E}+02$ & $2.84 \mathrm{E}+08$ & -- & -- & -- & -- & -- & -- & -- \\
\hline La-129 & La-129 & 128.913 & Lanthanum & 0.01 & 0.01 & 0 & ICRP-107 & $1.16 \mathrm{E}+01$ & $\mathrm{~m}$ & $6.96 \mathrm{E}+02$ & $1.26 \mathrm{E}+08$ & JAERI & $2.1 \mathrm{E}-11$ & $M$ & JAERI & $2.7 \mathrm{E}-11$ & -- & -- \\
\hline La-130 & La-130 & 129.912 & Lanthanum & 0.01 & 0.01 & 0 & ICRP-107 & $8.70 \mathrm{E}+00$ & $\mathrm{~m}$ & $5.22 \mathrm{E}+02$ & $1.66 \mathrm{E}+08$ & -- & -- & -- & -- & -- & -- & -- \\
\hline La-131 & La-131 & 130.910 & Lanthanum & 0.01 & 0.01 & 0 & ICRP-38 & $5.90 \mathrm{E}+01$ & $\mathrm{~m}$ & $3.54 E+03$ & $2.43 \mathrm{E}+07$ & ICRP-68 & $3.6 \mathrm{E}-11$ & $M$ & ICRP-68 & $3.5 \mathrm{E}-11$ & -- & -- \\
\hline La-132 & La-132 & 131.910 & Lanthanum & 0.01 & 0.01 & 0 & ICRP-38 & $4.80 \mathrm{E}+00$ & $\mathrm{~h}$ & $1.73 E+04$ & $4.95 \mathrm{E}+06$ & ICRP-68 & $2.8 \mathrm{E}-10$ & $M$ & ICRP-68 & $3.9 \mathrm{E}-10$ & -- & -- \\
\hline La-132m & La-132 & 131.910 & Lanthanum & 0.01 & 0.01 & 0 & ICRP-107 & $2.43 \mathrm{E}+01$ & $\mathrm{~m}$ & $1.46 \mathrm{E}+03$ & $5.87 \mathrm{E}+07$ & JAERI & $3.4 \mathrm{E}-11$ & $\mathrm{M}$ & JAERI & $3.8 \mathrm{E}-11$ & -- & -- \\
\hline La-133 & La-133 & 132.908 & Lanthanum & 0.01 & 0.01 & 0 & ICRP-107 & $3.91 \mathrm{E}+00$ & $\mathrm{~h}$ & $1.41 \mathrm{E}+04$ & $6.03 \mathrm{E}+06$ & JAERI & $3.7 \mathrm{E}-11$ & $M$ & JAERI & $4.6 \mathrm{E}-11$ & -- & -- \\
\hline La-134 & La-134 & 133.909 & Lanthanum & 0.01 & 0.01 & 0 & ICRP-38 & $6.67 \mathrm{E}+00$ & $\mathrm{~m}$ & $4.00 \mathrm{E}+02$ & $2.11 \mathrm{E}+08$ & -- & -- & -- & -- & -- & -- & -- \\
\hline La-135 & La-135 & 134.907 & Lanthanum & 0.01 & 0.01 & 0 & ICRP-38 & $1.95 \mathrm{E}+01$ & $\mathrm{~h}$ & $7.02 E+04$ & $1.19 \mathrm{E}+06$ & ICRP-68 & $2.5 \mathrm{E}-11$ & $M$ & ICRP-68 & $3.0 \mathrm{E}-11$ & -- & -- \\
\hline La-136 & La-136 & 135.908 & Lanthanum & 0.01 & 0.01 & 0 & ICRP-107 & $9.87 \mathrm{E}+00$ & $\mathrm{~m}$ & $5.92 E+02$ & $1.40 \mathrm{E}+08$ & -- & -- & -- & -- & -- & -- & -- \\
\hline La-137 & La-137 & 136.906 & Lanthanum & 0.01 & 0.01 & 0 & ICRP-38 & $6.00 \mathrm{E}+04$ & $y$ & $1.89 \mathrm{E}+12$ & $4.35 \mathrm{E}-02$ & ICRP-68 & $1.0 \mathrm{E}-08$ & $F$ & ICRP-68 & $8.1 \mathrm{E}-11$ & -- & -- \\
\hline La-138 & La-138 & 137.907 & Lanthanum & 0.01 & 0.01 & 0 & ICRP-38 & $1.35 \mathrm{E}+11$ & $y$ & $4.26 \mathrm{E}+18$ & $1.92 \mathrm{E}-08$ & ICRP-68 & $1.8 \mathrm{E}-07$ & $\mathrm{~F}$ & ICRP-68 & $1.1 \mathrm{E}-09$ & -- & -- \\
\hline La-140 & La-140 & 139.909 & Lanthanum & 0.01 & 0.01 & 0 & ICRP-38 & $4.03 \mathrm{E}+01$ & $\mathrm{~h}$ & $1.45 \mathrm{E}+05$ & $5.56 \mathrm{E}+05$ & ICRP-68 & $1.5 \mathrm{E}-09$ & $M$ & ICRP-68 & $2.0 \mathrm{E}-09$ & -- & -- \\
\hline La-141 & La-141 & 140.911 & Lanthanum & 0.01 & 0.01 & 0 & ICRP-38 & $3.93 \mathrm{E}+00$ & $\mathrm{~h}$ & $1.41 \mathrm{E}+04$ & $5.66 \mathrm{E}+06$ & ICRP-68 & $2.2 \mathrm{E}-10$ & $M$ & ICRP-68 & $3.6 \mathrm{E}-10$ & -- & -- \\
\hline La-142 & La-142 & 141.914 & Lanthanum & 0.01 & 0.01 & 0 & ICRP-38 & $9.25 \mathrm{E}+01$ & $\mathrm{~m}$ & $5.55 \mathrm{E}+03$ & $1.43 \mathrm{E}+07$ & ICRP-68 & $1.5 \mathrm{E}-10$ & $M$ & ICRP-68 & $1.8 \mathrm{E}-10$ & -- & -- \\
\hline La-143 & La-143 & 142.916 & Lanthanum & 0.01 & 0.01 & 0 & ICRP-38 & $1.42 \mathrm{E}+01$ & $\mathrm{~m}$ & $8.54 \mathrm{E}+02$ & $9.25 \mathrm{E}+07$ & ICRP-68 & $3.3 \mathrm{E}-11$ & $M$ & ICRP-68 & $5.6 \mathrm{E}-11$ & -- & -- \\
\hline Lu-164 & Lu-164 & 163.941 & Lutetium & 0.01 & 0.01 & 0 & JAERI & $3.14 \mathrm{E}+00$ & $\mathrm{~m}$ & $1.88 \mathrm{E}+02$ & $3.65 \mathrm{E}+08$ & -- & -- & -- & -- & -- & -- & -- \\
\hline Lu-165 & Lu-165 & 164.939 & Lutetium & 0.01 & 0.01 & 0 & ICRP-107 & $1.07 \mathrm{E}+01$ & $\mathrm{~m}$ & $6.44 E+02$ & $1.06 \mathrm{E}+08$ & JAERI & $2.3 \mathrm{E}-11$ & $\mathrm{~s}$ & JAERI & $2.3 \mathrm{E}-11$ & -- & -- \\
\hline Lu-166 & Lu-166 & 165.940 & Lutetium & 0.01 & 0.01 & 0 & JAERI & $2.65 \mathrm{E}+00$ & $\mathrm{~m}$ & $1.59 \mathrm{E}+02$ & $4.28 \mathrm{E}+08$ & -- & -- & -- & -- & -- & -- & -- \\
\hline Lu-166m & Lu-166 & 165.940 & Lutetium & 0.01 & 0.01 & 0 & JAERI & $1.41 \mathrm{E}+00$ & $\mathrm{~m}$ & $8.46 \mathrm{E}+01$ & $8.04 E+08$ & -- & -- & -- & -- & -- & -- & -- \\
\hline Lu-167 & Lu-167 & 166.938 & Lutetium & 0.01 & 0.01 & 0 & ICRP-107 & $5.15 \mathrm{E}+01$ & $\mathrm{~m}$ & $3.09 \mathrm{E}+03$ & $2.19 \mathrm{E}+07$ & JAERI & $6.0 \mathrm{E}-11$ & $\mathrm{~s}$ & JAERI & $5.0 \mathrm{E}-11$ & -- & -- \\
\hline Lu-168m & Lu-168 & 167.939 & Lutetium & 0.01 & 0.01 & 0 & JAERI & $6.70 \mathrm{E}+00$ & $\mathrm{~m}$ & $4.02 \mathrm{E}+02$ & $1.67 E+08$ & -- & -- & -- & -- & -- & -- & -- \\
\hline
\end{tabular}

C-16 


\begin{tabular}{|c|c|c|c|c|c|c|c|c|c|c|c|c|c|c|c|c|c|c|}
\hline \multirow{3}{*}{$\begin{array}{l}\text { MASTER } \\
\text { ISOTOPE } \\
\text { LIST } \\
\text { LU-169 }\end{array}$} & \multirow{3}{*}{$\begin{array}{c}\begin{array}{c}\text { Atomic } \\
\text { Mass } \\
\text { Nuclide }\end{array} \\
\text { Lu-169 }\end{array}$} & \multirow{3}{*}{$\begin{array}{c}\begin{array}{c}\text { Atomic } \\
\text { Mass }\end{array} \\
168.938\end{array}$} & \multirow{3}{*}{$\begin{array}{l}\text { Element } \\
\text { Lutetium }\end{array}$} & \multirow{3}{*}{$\begin{array}{c}\text { EPA } \\
\text { RF } \\
0.01\end{array}$} & \multicolumn{14}{|c|}{ Table C.1 Radionuclide Specific Input Data Used For the Calculation of HC-3 TQs Using Maximum Reported DCs (continued) } \\
\hline & & & & & $\begin{array}{c}\text { EPA } \\
\text { Conc } \\
\text { Factors } \\
\left(B_{v}\right) \\
\end{array}$ & $\begin{array}{c}\text { EPA } \\
\text { Sorption } \\
\text { Coefficient } \\
\left(K_{d}\right)\end{array}$ & $\begin{array}{c}\text { Half-Life } \\
\text { Reference }\end{array}$ & \multicolumn{2}{|c|}{$\begin{array}{c}\begin{array}{c}\text { Half-Life } \\
\text { (original units) }\end{array}\end{array}$} & \multirow{2}{*}{$\begin{array}{c}\begin{array}{c}\text { Half Life } \\
\text { (s) }\end{array} \\
1.23 E+05\end{array}$} & \multirow{2}{*}{$\begin{array}{c}\begin{array}{c}\text { Sp. Act } \\
\text { (Ci/g) }\end{array} \\
5.45 \mathrm{E}+05\end{array}$} & \multirow[t]{2}{*}{$\begin{array}{c}\text { Inhalation } \\
\text { DC } \\
\text { Reference }\end{array}$} & \multirow[t]{2}{*}{$\begin{array}{c}\text { HC-3 } \\
\text { Bounding } \\
\text { Inhalation } \\
\text { DC } \\
(\mathrm{Sv} / \mathrm{Bq}) \\
4.9 \mathrm{E}-10\end{array}$} & \multirow[t]{2}{*}{$\begin{array}{c}\begin{array}{c}\text { Lung } \\
\text { Absorption } \\
\text { Class/Type }\end{array} \\
\text { S }\end{array}$} & \multirow[t]{2}{*}{$\begin{array}{c}\text { Ingestion } \\
\text { DC } \\
\text { Reference }\end{array}$} & \multirow[t]{2}{*}{$\begin{array}{c}\text { HC-3 } \\
\text { Bounding } \\
\text { Ingestion } \\
\text { DC } \\
\text { (Sv/Bq) } \\
4.6 \mathrm{E}-10 \\
\end{array}$} & \multirow[t]{2}{*}{$\begin{array}{c}\text { Immersion } \\
\text { DC } \\
\text { Reference }\end{array}$} & \multirow[t]{2}{*}{$\begin{array}{c}\mathrm{HC}-3 \\
\text { Immersion } \\
\mathrm{DC}(\mathrm{Sv} / \mathrm{d} \\
\text { per } \mathrm{Bq} / \mathrm{m}^{3}\end{array}$} \\
\hline & & & & & 0.01 & 0 & ICRP-38 & $3.41 \mathrm{E}+01$ & $\mathrm{~h}$ & & & & & & & & & \\
\hline Lu-169m & Lu-169 & 168.938 & Lutetium & 0.01 & 0.01 & 0 & ICRP-107 & $1.60 \mathrm{E}+02$ & $\mathrm{~s}$ & $1.60 \mathrm{E}+02$ & $4.17 \mathrm{E}+08$ & --- & -- & -- & -- & -- & -- & -- \\
\hline Lu-170 & Lu-170 & 169.938 & Lutetium & 0.01 & 0.01 & 0 & $\begin{array}{l}\text { ICRP-38 } \\
\end{array}$ & $2.00 \mathrm{E}+00$ & $d$ & $1.73 \mathrm{E}+05$ & $3.84 \mathrm{E}+05$ & ICRP-68 & $9.5 \mathrm{E}-10$ & $\mathrm{~s}$ & ICRP-68 & $9.9 \mathrm{E}-10$ & -- & -- \\
\hline Lu-171 & Lu-171 & 170.938 & Lutetium & 0.01 & 0.01 & 0 & ICRP-38 & $8.22 \mathrm{E}+00$ & $d$ & $7.10 E+05$ & $9.29 \mathrm{E}+04$ & ICRP-68 & $9.3 \mathrm{E}-10$ & $\mathrm{~s}$ & ICRP-68 & $6.7 \mathrm{E}-10$ & -- & -- \\
\hline Lu-171m & Lu-171 & 170.938 & Lutetium & 0.01 & 0.01 & 0 & ICRP-107 & $7.90 \mathrm{E}+01$ & $\mathrm{~s}$ & $7.90 \mathrm{E}+01$ & $8.35 \mathrm{E}+08$ & -- & -- & -- & -- & -- & -- & -- \\
\hline Lu-172 & Lu-172 & 171.939 & Lutetium & 0.01 & 0.01 & 0 & ICRP-38 & $6.70 \mathrm{E}+00$ & $d$ & $5.79 E+05$ & $1.13 \mathrm{E}+05$ & ICRP-68 & $1.8 \mathrm{E}-09$ & $\mathrm{~s}$ & ICRP-68 & $1.3 \mathrm{E}-09$ & -- & -- \\
\hline Lu-172m & Lu-172 & 171.939 & Lutetium & 0.01 & 0.01 & 0 & ICRP-107 & $3.70 \mathrm{E}+00$ & $\mathrm{~m}$ & $2.22 \mathrm{E}+02$ & $2.96 \mathrm{E}+08$ & -- & -- & -- & -- & -- & -- & -- \\
\hline Lu-173 & Lu-173 & 172.939 & Lutetium & 0.01 & 0.01 & 0 & ICRP-38 & $1.37 \mathrm{E}+00$ & $y$ & $4.32 \mathrm{E}+07$ & $1.51 \mathrm{E}+03$ & ICRP-68 & $2.3 \mathrm{E}-09$ & $\mathrm{~s}$ & ICRP-68 & $2.6 \mathrm{E}-10$ & -- & -- \\
\hline Lu-174 & Lu-174 & 173.940 & Lutetium & 0.01 & 0.01 & 0 & ICRP-38 & $3.31 \mathrm{E}+00$ & $y$ & $1.04 E+08$ & $6.21 \mathrm{E}+02$ & ICRP-68 & $4.0 \mathrm{E}-09$ & $M$ & ICRP-68 & $2.7 \mathrm{E}-10$ & -- & -- \\
\hline Lu-174m & Lu-174 & 173.940 & Lutetium & 0.01 & 0.01 & 0 & ICRP-38 & $1.42 \mathrm{E}+02$ & $d$ & $1.23 \mathrm{E}+07$ & $5.29 \mathrm{E}+03$ & ICRP-68 & $3.8 \mathrm{E}-09$ & $\mathrm{~s}$ & ICRP-68 & $5.3 \mathrm{E}-10$ & -- & -- \\
\hline Lu-176 & Lu-176 & 175.943 & Lutetium & 0.01 & 0.01 & 0 & ICRP-38 & $3.60 \mathrm{E}+10$ & $y$ & $1.14 \mathrm{E}+18$ & $5.64 \mathrm{E}-08$ & ICRP-68 & $6.6 \mathrm{E}-08$ & $M$ & ICRP-68 & $1.8 \mathrm{E}-09$ & -- & -- \\
\hline Lu-176m & Lu-176 & 175.943 & Lutetium & 0.01 & 0.01 & 0 & ICRP-38 & $3.68 \mathrm{E}+00$ & $\mathrm{~h}$ & $1.32 E+04$ & $4.84 \mathrm{E}+06$ & ICRP-68 & $1.6 \mathrm{E}-10$ & $\mathrm{~s}$ & ICRP-68 & $1.7 \mathrm{E}-10$ & -- & -- \\
\hline Lu-177 & Lu-177 & 176.944 & Lutetium & 0.01 & 0.01 & 0 & ICRP-38 & $6.71 \mathrm{E}+00$ & $d$ & $5.80 E+05$ & $1.10 \mathrm{E}+05$ & ICRP-68 & $1.1 \mathrm{E}-09$ & $\mathrm{~s}$ & ICRP-68 & $5.3 \mathrm{E}-10$ & -- & -- \\
\hline Lu-177m & Lu-177 & 176.944 & Lutetium & 0.01 & 0.01 & 0 & ICRP-38 & $1.61 \mathrm{E}+02$ & $d$ & $1.39 \mathrm{E}+07$ & $4.59 \mathrm{E}+03$ & ICRP-68 & $1.5 \mathrm{E}-08$ & $\mathrm{~s}$ & ICRP-68 & $1.7 \mathrm{E}-09$ & -- & -- \\
\hline Lu-178 & Lu-178 & 177.946 & Lutetium & 0.01 & 0.01 & 0 & ICRP-38 & $2.84 \mathrm{E}+01$ & $\mathrm{~m}$ & $1.70 \mathrm{E}+03$ & $3.72 \mathrm{E}+07$ & ICRP-68 & $4.1 \mathrm{E}-11$ & $\mathrm{~s}$ & ICRP-68 & 4.7E-11 & -- & -- \\
\hline Lu-178m & Lu-178 & 177.946 & Lutetium & 0.01 & 0.01 & 0 & ICRP-38 & $2.27 \mathrm{E}+01$ & $\mathrm{~m}$ & $1.36 \mathrm{E}+03$ & $4.65 \mathrm{E}+07$ & ICRP-68 & $5.6 \mathrm{E}-11$ & $\mathrm{~s}$ & ICRP-68 & $3.8 \mathrm{E}-11$ & -- & -- \\
\hline Lu-179 & Lu-179 & 178.947 & Lutetium & 0.01 & 0.01 & 0 & ICRP-38 & $4.59 \mathrm{E}+00$ & $\mathrm{~h}$ & $1.65 \mathrm{E}+04$ & $3.82 \mathrm{E}+06$ & ICRP-68 & $1.6 \mathrm{E}-10$ & $M$ & ICRP-68 & $2.1 \mathrm{E}-10$ & -- & -- \\
\hline Lu-180 & Lu-180 & 179.950 & Lutetium & 0.01 & 0.01 & 0 & ICRP-107 & $5.70 \mathrm{E}+00$ & $\mathrm{~m}$ & $3.42 E+02$ & $1.83 \mathrm{E}+08$ & -- & -- & -- & -- & -- & -- & -- \\
\hline Lu-181 & Lu-181 & 180.952 & Lutetium & 0.01 & 0.01 & 0 & ICRP-107 & $3.50 \mathrm{E}+00$ & $\mathrm{~m}$ & $2.10 E+02$ & $2.97 E+08$ & -- & -- & -- & -- & -- & -- & -- \\
\hline Md-257 & Md-257 & 257.096 & Mendelevium & 0.001 & 0.001 & 0 & ICRP-38 & $5.20 \mathrm{E}+00$ & $\mathrm{~h}$ & $1.87 \mathrm{E}+04$ & $2.34 \mathrm{E}+06$ & ICRP-68 & $2.3 \mathrm{E}-08$ & $M$ & ICRP-68 & $1.2 \mathrm{E}-10$ & -- & -- \\
\hline Md-258 & Md-258 & 258.098 & Mendelevium & 0.001 & 0.001 & 0 & ICRP-38 & $5.50 \mathrm{E}+01$ & $d$ & $4.75 E+06$ & $9.20 \mathrm{E}+03$ & ICRP-68 & $5.5 \mathrm{E}-06$ & $M$ & ICRP-68 & $1.3 \mathrm{E}-08$ & -- & -- \\
\hline $\mathrm{Mg}-27$ & $\mathrm{Mg}-27$ & 26.984 & Magnesium & 0.01 & 1 & 0 & ICRP-107 & $9.46 \mathrm{E}+00$ & $\mathrm{~m}$ & $5.67 E+02$ & $7.37 E+08$ & -- & -- & -- & -- & -- & -- & -- \\
\hline $\mathrm{Mg}-28$ & $\mathrm{Mg}-28$ & 27.984 & Magnesium & 0.01 & 1 & 0 & ICRP-38 & $2.09 \mathrm{E}+01$ & $\mathrm{~h}$ & $7.53 E+04$ & $5.36 \mathrm{E}+06$ & ICRP-68 & $1.7 \mathrm{E}-09$ & $M$ & ICRP-68 & $2.2 \mathrm{E}-09$ & -- & -- \\
\hline Mn-50m & $\mathrm{Mn}-50$ & 49.954 & Manganese & 0.01 & 0.25 & 0 & ICRP-107 & $1.75 \mathrm{E}+00$ & $\mathrm{~m}$ & $1.05 E+02$ & $2.15 \mathrm{E}+09$ & -- & -- & -- & -- & -- & -- & -- \\
\hline Mn-51 & $M n-51$ & 50.948 & Manganese & 0.01 & 0.25 & 0 & ICRP-38 & $4.62 \mathrm{E}+01$ & $\mathrm{~m}$ & $2.77 E+03$ & $7.99 \mathrm{E}+07$ & ICRP-68 & $6.8 \mathrm{E}-11$ & $M$ & ICRP-68 & $9.3 \mathrm{E}-11$ & -- & -- \\
\hline $\mathrm{Mn}-52$ & $M n-52$ & 51.946 & Manganese & 0.01 & 0.25 & 0 & ICRP-38 & $5.59 \mathrm{E}+00$ & $d$ & $4.83 \mathrm{E}+05$ & $4.50 \mathrm{E}+05$ & ICRP-68 & $1.8 \mathrm{E}-09$ & $M$ & ICRP-68 & $1.8 \mathrm{E}-09$ & -- & -- \\
\hline Mn-52m & $M n-52$ & 51.946 & Manganese & 0.01 & 0.25 & 0 & ICRP-38 & $2.11 \mathrm{E}+01$ & $\mathrm{~m}$ & $1.27 E+03$ & $1.72 \mathrm{E}+08$ & $\begin{array}{l}\text { ICRP-68 } \\
\end{array}$ & $5.0 \mathrm{E}-11$ & $\mathrm{M}$ & ICRP-68 & $6.9 \mathrm{E}-11$ & -- & -- \\
\hline Mn-53 & Mn-53 & 52.941 & Manganese & 0.01 & 0.25 & 0 & ICRP-38 & $3.70 \mathrm{E}+06$ & $y$ & $1.17 E+14$ & $1.83 \mathrm{E}-03$ & ICRP-68 & $5.2 \mathrm{E}-11$ & $M$ & ICRP-68 & $3.0 \mathrm{E}-11$ & -- & -- \\
\hline $\mathrm{Mn}-54$ & Mn-54 & 53.940 & Manganese & 0.01 & 0.25 & 0 & ICRP-38 & $3.13 \mathrm{E}+02$ & $d$ & $2.70 \mathrm{E}+07$ & $7.75 \mathrm{E}+03$ & ICRP-68 & $1.5 \mathrm{E}-09$ & $M$ & ICRP-68 & $7.1 \mathrm{E}-10$ & -- & -- \\
\hline$M n-56$ & Mn-56 & 55.939 & Manganese & 0.01 & 0.25 & 0 & ICRP-38 & $2.58 \mathrm{E}+00$ & $\mathrm{~h}$ & $9.28 \mathrm{E}+03$ & $2.17 E+07$ & ICRP-68 & $2.0 \mathrm{E}-10$ & $\mathrm{M}$ & ICRP-68 & $2.5 \mathrm{E}-10$ & -- & -- \\
\hline Mn-57 & Mn-57 & 56.938 & Manganese & 0.01 & 0.25 & 0 & ICRP-107 & $8.54 \mathrm{E}+01$ & $\mathrm{~s}$ & $8.54 \mathrm{E}+01$ & $2.32 \mathrm{E}+09$ & -- & -- & -- & -- & -- & -- & -- \\
\hline Mn-58m & Mn-58 & 57.940 & Manganese & 0.01 & 0.25 & 0 & ICRP-107 & $6.52 \mathrm{E}+01$ & $s$ & $6.52 \mathrm{E}+01$ & $2.99 \mathrm{E}+09$ & -- & -- & -- & -- & -- & -- & -- \\
\hline Mo-101 & Mo-101 & 100.910 & Molybdenum & 0.01 & 0.25 & 5 & ICRP-38 & $1.46 \mathrm{E}+01$ & $\mathrm{~m}$ & $8.77 E+02$ & $1.27 \mathrm{E}+08$ & ICRP-68 & $4.5 \mathrm{E}-11$ & $\mathrm{~s}$ & ICRP-68 & $4.2 \mathrm{E}-11$ & -- & -- \\
\hline Mo-102 & Mo-102 & 101.910 & Molybdenum & 0.01 & 0.25 & 5 & ICRP-107 & $1.13 \mathrm{E}+01$ & $\mathrm{~m}$ & $6.78 \mathrm{E}+02$ & $1.63 \mathrm{E}+08$ & JAERI & $4.2 \mathrm{E}-11$ & $\mathrm{~s}$ & JAERI & $6.9 \mathrm{E}-11$ & -- & -- \\
\hline Mo-89 & Mo-89 & 88.919 & Molybdenum & 0.01 & 0.25 & 5 & ICRP-107 & $2.11 \mathrm{E}+00$ & $\mathrm{~m}$ & $1.27 E+02$ & $1.00 \mathrm{E}+09$ & -- & -- & -- & -- & -- & -- & -- \\
\hline Mo-90 & Mo-90 & 89.914 & Molybdenum & 0.01 & 0.25 & 5 & ICRP-38 & $5.67 \mathrm{E}+00$ & $\mathrm{~h}$ & $2.04 E+04$ & $6.15 \mathrm{E}+06$ & ICRP-68 & $5.6 \mathrm{E}-10$ & $\mathrm{~s}$ & ICRP-68 & $6.2 \mathrm{E}-10$ & -- & -- \\
\hline Mo-91 & Mo-91 & 90.912 & Molybdenum & 0.01 & 0.25 & 5 & ICRP-107 & $1.55 \mathrm{E}+01$ & $\mathrm{~m}$ & $9.29 \mathrm{E}+02$ & $1.34 \mathrm{E}+08$ & JAERI & $3.5 \mathrm{E}-11$ & $\mathrm{~s}$ & JAERI & $6.1 \mathrm{E}-11$ & -- & -- \\
\hline Mo-91m & Mo-91 & 90.912 & Molybdenum & 0.01 & 0.25 & 5 & ICRP-107 & $6.46 \mathrm{E}+01$ & $\mathrm{~s}$ & $6.46 \mathrm{E}+01$ & $1.92 \mathrm{E}+09$ & -- & -- & -- & -- & -- & -- & -- \\
\hline Mo-93 & Mo-93 & 92.907 & Molybdenum & 0.01 & 0.25 & 5 & ICRP-38 & $3.50 \mathrm{E}+03$ & $y$ & $1.10 \mathrm{E}+11$ & $1.10 \mathrm{E}+00$ & ICRP-68 & $2.2 \mathrm{E}-09$ & $\mathrm{~s}$ & ICRP-68 & $2.6 \mathrm{E}-09$ & -- & -- \\
\hline Mo-93m & Mo-93 & 92.907 & Molybdenum & 0.01 & 0.25 & 5 & ICRP-38 & $6.85 \mathrm{E}+00$ & $\mathrm{~h}$ & $2.47 E+04$ & $4.92 \mathrm{E}+06$ & ICRP-68 & $3.0 \mathrm{E}-10$ & $\mathrm{~s}$ & ICRP-68 & $2.8 \mathrm{E}-10$ & -- & -- \\
\hline Mo-99 & Mo-99 & 98.908 & Molybdenum & 0.01 & 0.25 & 5 & ICRP-38 & $6.60 \mathrm{E}+01$ & $\mathrm{~h}$ & $2.38 \mathrm{E}+05$ & $4.80 \mathrm{E}+05$ & ICRP-68 & $1.1 \mathrm{E}-09$ & $\mathrm{~s}$ & ICRP-68 & $1.2 \mathrm{E}-09$ & -- & -- \\
\hline $\mathrm{N}-13$ & $\mathrm{~N}-13$ & 13.006 & Nitrogen & -- & -- & -- & ICRP-38 & $9.97 E+00$ & $\mathrm{~m}$ & $5.98 \mathrm{E}+02$ & $1.45 \mathrm{E}+09$ & -- & -- & -- & -- & -- & JAERI & $4.00 \mathrm{E}-09$ \\
\hline $\mathrm{N}-16$ & $\mathrm{~N}-16$ & 16.006 & Nitrogen & -- & -- & -- & ICRP-107 & $7.13 \mathrm{E}+00$ & $\mathrm{~s}$ & $7.13 \mathrm{E}+00$ & $9.89 \mathrm{E}+10$ & -- & -- & -- & -- & -- & -- & -- \\
\hline $\mathrm{Na}-22$ & $\mathrm{Na}-22$ & 21.994 & Sodium & 0.01 & 0.075 & 10 & ICRP-38 & $2.60 \mathrm{E}+00$ & $y$ & $8.21 \mathrm{E}+07$ & $6.25 \mathrm{E}+03$ & ICRP-68 & $2.0 \mathrm{E}-09$ & $\mathrm{~F}$ & ICRP-68 & $3.2 \mathrm{E}-09$ & -- & -- \\
\hline $\mathrm{Na}-24$ & $\mathrm{Na}-24$ & 23.991 & Sodium & 0.01 & 0.075 & 10 & ICRP-38 & $1.50 \mathrm{E}+01$ & $\mathrm{~h}$ & $5.40 E+04$ & $8.71 \mathrm{E}+06$ & ICRP-68 & $5.3 \mathrm{E}-10$ & $\mathrm{~F}$ & ICRP-68 & $4.3 \mathrm{E}-10$ & -- & -- \\
\hline
\end{tabular}




\begin{tabular}{|c|c|c|c|c|c|c|c|c|c|c|c|c|c|c|c|c|c|c|}
\hline \multirow{3}{*}{$\begin{array}{l}\text { MASTER } \\
\text { ISOTOPE } \\
\text { LIST } \\
\mathrm{Nb}-87\end{array}$} & \multirow{3}{*}{$\begin{array}{c}\begin{array}{c}\text { Atomic } \\
\text { Mass } \\
\text { Nuclide }\end{array} \\
\mathrm{Nb}-87\end{array}$} & \multirow{3}{*}{$\begin{array}{l}\begin{array}{c}\text { Atomic } \\
\text { Mass }\end{array} \\
86.921\end{array}$} & & & \multicolumn{14}{|c|}{ Table C.1 Radionuclide Spe } \\
\hline & & & \multirow{2}{*}{$\begin{array}{l}\text { Element } \\
\text { Niobium }\end{array}$} & \multirow{2}{*}{$\begin{array}{c}\text { EPA } \\
\text { RF } \\
0.01\end{array}$} & $\begin{array}{c}\text { EPA } \\
\text { Conc } \\
\text { Factors } \\
\left(B_{v}\right) \\
\end{array}$ & $\begin{array}{c}\text { EPA } \\
\text { Sorption } \\
\text { Coefficient } \\
\left(K_{d}\right)\end{array}$ & $\begin{array}{c}\text { Half-Life } \\
\text { Reference }\end{array}$ & $\begin{array}{r}\text { Half-Lif } \\
\text { (original u }\end{array}$ & & $\begin{array}{l}\text { Half Life } \\
\text { (s) }\end{array}$ & $\begin{array}{l}\text { Sp. Act } \\
\text { (Ci/g) }\end{array}$ & $\begin{array}{l}\text { Inhalation } \\
\text { DC } \\
\text { Reference }\end{array}$ & $\begin{array}{c}\text { HC-3 } \\
\text { Bounding } \\
\text { Inhalation } \\
\text { DC } \\
\text { (Sv/Bq) } \\
\end{array}$ & $\begin{array}{c}\text { Lung } \\
\text { Absorption } \\
\text { Class/Type }\end{array}$ & $\begin{array}{l}\text { Ingestion } \\
\text { DC } \\
\text { Reference }\end{array}$ & $\begin{array}{c}\text { HC-3 } \\
\text { Bounding } \\
\text { Ingestion } \\
\text { DC } \\
\text { (Sv/Bq) } \\
\end{array}$ & $\begin{array}{c}\text { Immersion } \\
\text { DC } \\
\text { Reference }\end{array}$ & $\begin{array}{c}\mathrm{HC}-3 \\
\text { Immersion } \\
\mathrm{DC}(\mathrm{Sv} / \mathrm{d} \\
\left.\operatorname{per} \mathrm{Bq} / \mathrm{m}^{3}\right)\end{array}$ \\
\hline & & & & & 0.02 & 2000 & ICRP-107 & $3.75 \mathrm{E}+00$ & $\mathrm{~m}$ & $2.25 \mathrm{E}+02$ & $5.77 \mathrm{E}+08$ & -- & -- & -- & -- & -- & -- & -- \\
\hline $\mathrm{Nb}-88$ & $\mathrm{Nb}-88$ & 87.918 & Niobium & 0.01 & 0.02 & 2000 & ICRP-38 & $1.43 \mathrm{E}+01$ & $\mathrm{~m}$ & $8.58 \mathrm{E}+02$ & $1.50 \mathrm{E}+08$ & ICRP-68 & $5.0 \mathrm{E}-11$ & $\mathrm{~s}$ & ICRP-68 & $6.3 \mathrm{E}-11$ & -- & -- \\
\hline $\mathrm{Nb}-88 \mathrm{~m}$ & $\mathrm{Nb}-88$ & 87.918 & Niobium & 0.01 & 0.02 & 2000 & ICRP-107 & $7.78 \mathrm{E}+00$ & $\mathrm{~m}$ & $4.67 \mathrm{E}+02$ & $2.75 \mathrm{E}+08$ & -- & -- & -- & -- & -- & -- & -- \\
\hline Nb-89I & $\mathrm{Nb}-89$ & 88.913 & Niobium & 0.01 & 0.02 & 2000 & ICRP-38 & $1.22 \mathrm{E}+02$ & $\mathrm{~m}$ & $7.32 \mathrm{E}+03$ & $1.73 \mathrm{E}+07$ & ICRP-68 & $1.9 \mathrm{E}-10$ & $\mathrm{~s}$ & ICRP-68 & $3.0 \mathrm{E}-10$ & -- & -- \\
\hline $\mathrm{Nb}-89 \mathrm{~s}$ & $\mathrm{Nb}-89$ & 88.913 & Niobium & 0.01 & 0.02 & 2000 & ICRP-38 & $6.60 \mathrm{E}+01$ & $\mathrm{~m}$ & $3.96 \mathrm{E}+03$ & $3.20 \mathrm{E}+07$ & ICRP-68 & $1.2 \mathrm{E}-10$ & $\mathrm{~s}$ & ICRP-68 & $1.4 \mathrm{E}-10$ & -- & -- \\
\hline $\mathrm{Nb}-90$ & $\mathrm{Nb}-90$ & 89.911 & Niobium & 0.01 & 0.02 & 2000 & ICRP-38 & $1.46 \mathrm{E}+01$ & $\mathrm{~h}$ & $5.26 \mathrm{E}+04$ & $2.39 \mathrm{E}+06$ & ICRP-68 & $1.1 \mathrm{E}-09$ & $\mathrm{~s}$ & ICRP-68 & $1.2 \mathrm{E}-09$ & -- & -- \\
\hline Nb-91 & $\mathrm{Nb}-91$ & 90.907 & Niobium & 0.01 & 0.02 & 2000 & ICRP-107 & $6.80 \mathrm{E}+02$ & $y$ & $2.15 \mathrm{E}+10$ & $5.78 \mathrm{E}+00$ & JAERI & $1.7 \mathrm{E}-09$ & $\mathrm{~s}$ & JAERI & $4.6 \mathrm{E}-11$ & -- & -- \\
\hline $\mathrm{Nb}-91 \mathrm{~m}$ & $\mathrm{Nb}-91$ & 90.907 & Niobium & 0.01 & 0.02 & 2000 & ICRP-107 & $6.09 \mathrm{E}+01$ & $d$ & $5.26 \mathrm{E}+06$ & $2.36 \mathrm{E}+04$ & JAERI & $3.9 \mathrm{E}-09$ & $\mathrm{~s}$ & JAERI & $4.1 \mathrm{E}-10$ & -- & -- \\
\hline Nb-92 & $\mathrm{Nb}-92$ & 91.907 & Niobium & 0.01 & 0.02 & 2000 & ICRP-107 & $3.47 \mathrm{E}+07$ & $y$ & $1.10 \mathrm{E}+15$ & $1.12 \mathrm{E}-04$ & JAERI & $2.5 \mathrm{E}-08$ & $\mathrm{~s}$ & JAERI & $1.0 \mathrm{E}-09$ & -- & -- \\
\hline$N b-92 m$ & $\mathrm{Nb}-92$ & 91.907 & Niobium & 0.01 & 0.02 & 2000 & ICRP-107 & $1.02 \mathrm{E}+01$ & d & $8.77 \mathrm{E}+05$ & $1.40 \mathrm{E}+05$ & JAERI & $5.4 \mathrm{E}-10$ & $M$ & JAERI & $5.0 \mathrm{E}-10$ & -- & -- \\
\hline $\mathrm{Nb}-93 \mathrm{~m}$ & $\mathrm{Nb}-93$ & 92.906 & Niobium & 0.01 & 0.02 & 2000 & ICRP-38 & $1.36 \mathrm{E}+01$ & $y$ & $4.29 \mathrm{E}+08$ & $2.83 \mathrm{E}+02$ & ICRP-68 & $1.6 \mathrm{E}-09$ & $\mathrm{~s}$ & ICRP-68 & $1.2 \mathrm{E}-10$ & -- & -- \\
\hline $\mathrm{Nb}-94$ & $\mathrm{Nb}-94$ & 93.907 & Niobium & 0.01 & 0.02 & 2000 & ICRP-38 & $2.03 \mathrm{E}+04$ & $y$ & $6.41 \mathrm{E}+11$ & 1.88E-01 & ICRP-68 & $4.5 \mathrm{E}-08$ & $\mathrm{~s}$ & ICRP-68 & $1.7 \mathrm{E}-09$ & -- & -- \\
\hline $\mathrm{Nb}-94 \mathrm{~m}$ & $\mathrm{Nb}-94$ & 93.907 & Niobium & 0.01 & 0.02 & 2000 & ICRP-107 & $6.26 \mathrm{E}+00$ & $\mathrm{~m}$ & $3.76 \mathrm{E}+02$ & $3.20 \mathrm{E}+08$ & -- & -- & -- & -- & -- & -- & -- \\
\hline $\mathrm{Nb}-95$ & $\mathrm{Nb}-95$ & 94.907 & Niobium & 0.01 & 0.02 & 2000 & ICRP-38 & $3.52 \mathrm{E}+01$ & $d$ & $3.04 \mathrm{E}+06$ & $3.91 \mathrm{E}+04$ & ICRP-68 & $1.6 \mathrm{E}-09$ & $\mathrm{~s}$ & ICRP-68 & $5.8 \mathrm{E}-10$ & -- & -- \\
\hline $\mathrm{Nb}-95 \mathrm{~m}$ & $\mathrm{Nb}-95$ & 94.907 & Niobium & 0.01 & 0.02 & 2000 & ICRP-38 & $8.66 \mathrm{E}+01$ & $\mathrm{~h}$ & $3.12 \mathrm{E}+05$ & $3.81 \mathrm{E}+05$ & ICRP-68 & $8.5 \mathrm{E}-10$ & $\mathrm{~s}$ & ICRP-68 & $5.6 \mathrm{E}-10$ & -- & -- \\
\hline $\mathrm{Nb}-96$ & $\mathrm{Nb}-96$ & 95.908 & Niobium & 0.01 & 0.02 & 2000 & ICRP-38 & $2.34 \mathrm{E}+01$ & $\mathrm{~h}$ & $8.41 \mathrm{E}+04$ & $1.40 \mathrm{E}+06$ & ICRP-68 & $1.0 \mathrm{E}-09$ & $\mathrm{~s}$ & ICRP-68 & $1.1 \mathrm{E}-09$ & -- & -- \\
\hline $\mathrm{Nb}-97$ & $\mathrm{Nb}-97$ & 96.908 & Niobium & 0.01 & 0.02 & 2000 & ICRP-38 & $7.21 \mathrm{E}+01$ & $\mathrm{~m}$ & $4.33 \mathrm{E}+03$ & $2.69 \mathrm{E}+07$ & ICRP-68 & $7.2 \mathrm{E}-11$ & $\mathrm{~s}$ & ICRP-68 & $6.8 \mathrm{E}-11$ & -- & -- \\
\hline $\mathrm{Nb}-97 \mathrm{~m}$ & $\mathrm{Nb}-97$ & 96.908 & Niobium & 0.01 & 0.02 & 2000 & ICRP-38 & $6.00 \mathrm{E}+01$ & $\mathrm{~s}$ & $6.00 \mathrm{E}+01$ & $1.94 \mathrm{E}+09$ & -- & -- & -- & -- & -- & -- & -- \\
\hline $\mathrm{Nb}-98$ & $\mathrm{Nb}-98$ & 97.910 & Niobium & 0.01 & 0.02 & 2000 & ICRP-38 & $5.15 \mathrm{E}+01$ & $\mathrm{~m}$ & $3.09 \mathrm{E}+03$ & $3.73 \mathrm{E}+07$ & ICRP-68 & $9.9 \mathrm{E}-11$ & $\mathrm{~s}$ & ICRP-68 & $1.1 \mathrm{E}-10$ & -- & -- \\
\hline $\mathrm{Nb}-99$ & $\mathrm{Nb}-99$ & 98.912 & Niobium & 0.01 & 0.02 & 2000 & ICRP-107 & $1.50 \mathrm{E}+01$ & $\mathrm{~s}$ & $1.50 \mathrm{E}+01$ & $7.60 \mathrm{E}+09$ & -- & -- & -- & -- & -- & -- & -- \\
\hline $\mathrm{Nb}-99 \mathrm{~m}$ & $\mathrm{Nb}-99$ & 98.912 & Niobium & 0.01 & 0.02 & 2000 & ICRP-107 & $2.60 \mathrm{E}+00$ & $\mathrm{~m}$ & $1.56 \mathrm{E}+02$ & $7.31 \mathrm{E}+08$ & -- & -- & -- & -- & -- & -- & -- \\
\hline $\mathrm{Nd}-134$ & Nd-134 & 133.919 & Neodymium & 0.01 & 0.01 & 500 & ICRP-107 & $8.50 \mathrm{E}+00$ & $\mathrm{~m}$ & $5.10 \mathrm{E}+02$ & $1.65 \mathrm{E}+08$ & -- & -- & -- & -- & -- & -- & -- \\
\hline $\mathrm{Nd}-135$ & $\mathrm{Nd}-135$ & 134.918 & Neodymium & 0.01 & 0.01 & 500 & ICRP-107 & $1.24 \mathrm{E}+01$ & $\mathrm{~m}$ & $7.44 \mathrm{E}+02$ & $1.12 \mathrm{E}+08$ & JAERI & $4.5 \mathrm{E}-11$ & $\mathrm{~s}$ & JAERI & $6.0 \mathrm{E}-11$ & -- & -- \\
\hline $\mathrm{Nd}-136$ & $\mathrm{Nd}-136$ & 135.915 & Neodymium & 0.01 & 0.01 & 500 & ICRP-38 & $5.07 \mathrm{E}+01$ & $\mathrm{~m}$ & $3.04 \mathrm{E}+03$ & $2.73 \mathrm{E}+07$ & ICRP-68 & $8.9 \mathrm{E}-11$ & $\mathrm{~s}$ & ICRP-68 & $9.9 \mathrm{E}-11$ & -- & -- \\
\hline $\mathrm{Nd}-137$ & Nd-137 & 136.915 & Neodymium & 0.01 & 0.01 & 500 & ICRP-107 & $3.85 \mathrm{E}+01$ & $\mathrm{~m}$ & $2.31 \mathrm{E}+03$ & $3.57 \mathrm{E}+07$ & JAERI & $5.0 \mathrm{E}-11$ & $\mathrm{~s}$ & JAERI & $5.9 \mathrm{E}-11$ & -- & -- \\
\hline $\mathrm{Nd}-138$ & $\mathrm{Nd}-138$ & 137.912 & Neodymium & 0.01 & 0.01 & 500 & ICRP-38 & $5.04 \mathrm{E}+00$ & $\mathrm{~h}$ & $1.81 \mathrm{E}+04$ & $4.51 \mathrm{E}+06$ & ICRP-68 & $3.8 \mathrm{E}-10$ & $\mathrm{~s}$ & ICRP-68 & $6.4 \mathrm{E}-10$ & -- & -- \\
\hline Nd-139 & $\mathrm{Nd}-139$ & 138.912 & Neodymium & 0.01 & 0.01 & 500 & ICRP-38 & $2.97 \mathrm{E}+01$ & $\mathrm{~m}$ & $1.78 \mathrm{E}+03$ & $4.56 \mathrm{E}+07$ & ICRP-68 & $1.7 \mathrm{E}-11$ & $M$ & ICRP-68 & $2.0 \mathrm{E}-11$ & -- & -- \\
\hline $\mathrm{Nd}-139 \mathrm{~m}$ & $\mathrm{Nd}-139$ & 138.912 & Neodymium & 0.01 & 0.01 & 500 & ICRP-38 & $5.50 \mathrm{E}+00$ & $\mathrm{~h}$ & $1.98 \mathrm{E}+04$ & $4.10 \mathrm{E}+06$ & ICRP-68 & $2.5 \mathrm{E}-10$ & $M$ & ICRP-68 & $2.5 \mathrm{E}-10$ & -- & -- \\
\hline Nd-140 & $\mathrm{Nd}-140$ & 139.910 & Neodymium & 0.01 & 0.01 & 500 & ICRP-107 & $3.37 \mathrm{E}+00$ & $d$ & $2.91 \mathrm{E}+05$ & $2.77 E+05$ & JAERI & $1.3 \mathrm{E}-09$ & $\mathrm{M}$ & JAERI & $2.0 \mathrm{E}-09$ & -- & -- \\
\hline Nd-141 & $\mathrm{Nd}-141$ & 140.910 & Neodymium & 0.01 & 0.01 & 500 & ICRP-38 & $2.49 \mathrm{E}+00$ & $\mathrm{~h}$ & $8.96 \mathrm{E}+03$ & $8.93 \mathrm{E}+06$ & ICRP-68 & $8.8 \mathrm{E}-12$ & $\mathrm{~s}$ & ICRP-68 & $8.3 \mathrm{E}-12$ & -- & -- \\
\hline $\mathrm{Nd}-141 \mathrm{~m}$ & $\mathrm{Nd}-141$ & 140.910 & Neodymium & 0.01 & 0.01 & 500 & ICRP-38 & $6.24 \mathrm{E}+01$ & $\mathrm{~s}$ & $6.24 \mathrm{E}+01$ & $1.28 \mathrm{E}+09$ & -- & -- & -- & -- & -- & -- & -- \\
\hline $\mathrm{Nd}-144$ & $\mathrm{Nd}-144$ & 143.910 & Neodymium & 0.01 & 0.01 & 500 & ICRP-107 & $2.29 \mathrm{E}+15$ & $y$ & $7.23 \mathrm{E}+22$ & $1.08 \mathrm{E}-12$ & JAERI & $7.4 \mathrm{E}-06$ & $M$ & JAERI & $4.1 \mathrm{E}-08$ & -- & -- \\
\hline $\mathrm{Nd}-147$ & $\mathrm{Nd}-147$ & 146.916 & Neodymium & 0.01 & 0.01 & 500 & ICRP-38 & $1.10 \mathrm{E}+01$ & $d$ & $9.49 \mathrm{E}+05$ & $8.09 \mathrm{E}+04$ & ICRP-68 & $2.3 \mathrm{E}-09$ & $\mathrm{~s}$ & ICRP-68 & $1.1 \mathrm{E}-09$ & -- & -- \\
\hline $\mathrm{Nd}-149$ & $\mathrm{Nd}-149$ & 148.920 & Neodymium & 0.01 & 0.01 & 500 & ICRP-38 & $1.73 \mathrm{E}+00$ & $\mathrm{~h}$ & $6.23 \mathrm{E}+03$ & $1.22 \mathrm{E}+07$ & ICRP-68 & $1.3 \mathrm{E}-10$ & $\mathrm{~s}$ & ICRP-68 & $1.2 \mathrm{E}-10$ & -- & -- \\
\hline Nd-151 & Nd-151 & 150.924 & Neodymium & 0.01 & 0.01 & 500 & ICRP-38 & $1.24 \mathrm{E}+01$ & $\mathrm{~m}$ & $7.46 \mathrm{E}+02$ & $1.00 E+08$ & ICRP-68 & $2.9 \mathrm{E}-11$ & $\mathrm{~s}$ & ICRP-68 & $3.0 \mathrm{E}-11$ & -- & -- \\
\hline $\mathrm{Nd}-152$ & $\mathrm{Nd}-152$ & 151.925 & Neodymium & 0.01 & 0.01 & 500 & ICRP-107 & $1.14 \mathrm{E}+01$ & $\mathrm{~m}$ & $6.84 \mathrm{E}+02$ & $1.09 \mathrm{E}+08$ & JAERI & $4.0 \mathrm{E}-11$ & $\mathrm{~s}$ & JAERI & $4.9 \mathrm{E}-11$ & -- & -- \\
\hline $\mathrm{Ne}-19$ & $\mathrm{Ne}-19$ & 19.002 & Neon & -- & -- & -- & ICRP-38 & $1.72 \mathrm{E}+01$ & $\mathrm{~s}$ & $1.72 \mathrm{E}+01$ & $3.45 \mathrm{E}+10$ & -- & -- & -- & -- & -- & -- & -- \\
\hline $\mathrm{Ne}-24$ & $\mathrm{Ne}-24$ & 23.994 & Neon & -- & -- & -- & ICRP-107 & $3.38 \mathrm{E}+00$ & $\mathrm{~m}$ & $2.03 \mathrm{E}+02$ & $2.32 \mathrm{E}+09$ & -- & -- & -- & -- & -- & -- & -- \\
\hline $\mathrm{Ni}-56$ & $\mathrm{Ni}-56$ & 55.942 & Nickel & 0.01 & 0.06 & 100 & ICRP-38 & $6.10 \mathrm{E}+00$ & $d$ & $5.27 \mathrm{E}+05$ & $3.83 \mathrm{E}+05$ & ICRP-68 & $1.2 \mathrm{E}-09$ & 0 & ICRP-68 & $8.6 \mathrm{E}-10$ & -- & -- \\
\hline Ni-57 & $\mathrm{Ni}-57$ & 56.940 & Nickel & 0.01 & 0.06 & 100 & ICRP-38 & $3.61 \mathrm{E}+01$ & $\mathrm{~h}$ & $1.30 \mathrm{E}+05$ & $1.53 \mathrm{E}+06$ & ICRP-68 & $7.6 \mathrm{E}-10$ & $M$ & ICRP-68 & $8.7 E-10$ & -- & -- \\
\hline $\mathrm{Ni}-59$ & $\mathrm{Ni}-59$ & 58.934 & Nickel & 0.01 & 0.06 & 100 & ICRP-38 & $7.50 \mathrm{E}+04$ & $y$ & $2.37 E+12$ & $8.09 \mathrm{E}-02$ & ICRP-68 & $8.3 \mathrm{E}-10$ & 0 & ICRP-68 & $6.3 \mathrm{E}-11$ & -- & -- \\
\hline $\mathrm{Ni}-63$ & $\mathrm{Ni}-63$ & 62.930 & Nickel & 0.01 & 0.06 & 100 & ICRP-38 & $9.60 \mathrm{E}+01$ & $y$ & $3.03 \mathrm{E}+09$ & $5.92 \mathrm{E}+01$ & ICRP-68 & $2.0 \mathrm{E}-09$ & 0 & ICRP-68 & $1.5 \mathrm{E}-10$ & -- & -- \\
\hline $\mathrm{Ni}-65$ & $\mathrm{Ni}-65$ & 64.930 & Nickel & 0.01 & 0.06 & 100 & ICRP-38 & $2.52 \mathrm{E}+00$ & $\mathrm{~h}$ & $9.07 \mathrm{E}+03$ & $1.92 \mathrm{E}+07$ & ICRP-68 & $3.6 \mathrm{E}-10$ & 0 & ICRP-68 & $1.8 \mathrm{E}-10$ & -- & -- \\
\hline $\mathrm{Ni}-66$ & $\mathrm{Ni}-66$ & 65.929 & Nickel & 0.01 & 0.06 & 100 & ICRP-38 & $5.46 \mathrm{E}+01$ & $\mathrm{~h}$ & $1.97 \mathrm{E}+05$ & $8.71 \mathrm{E}+05$ & ICRP-68 & $1.9 \mathrm{E}-09$ & $M$ & ICRP-68 & $3.0 \mathrm{E}-09$ & -- & -- \\
\hline Np-231 & Np-231 & 231.038 & Neptunium & 0.001 & 0.1 & 10 & JAERI & $4.88 \mathrm{E}+01$ & $\mathrm{~m}$ & $2.93 E+03$ & $1.67 \mathrm{E}+07$ & JAERI & $1.7 \mathrm{E}-09$ & $M$ & JAERI & $2.9 \mathrm{E}-11$ & -- & -- \\
\hline
\end{tabular}




\begin{tabular}{|c|c|c|c|c|c|c|c|c|c|c|c|c|c|c|c|c|c|c|}
\hline \multirow{3}{*}{$\begin{array}{l}\text { MASTER } \\
\text { ISOTOPE } \\
\text { LIST } \\
\text { Np-232 }\end{array}$} & \multirow{3}{*}{$\begin{array}{c}\begin{array}{c}\text { Atomic } \\
\text { Mass } \\
\text { Nuclide }\end{array} \\
\text { Np-232 }\end{array}$} & & & & \multicolumn{14}{|c|}{ Table C.1 Radionuclide Spe } \\
\hline & & \multirow{2}{*}{$\begin{array}{c}\begin{array}{c}\text { Atomic } \\
\text { Mass }\end{array} \\
232.040\end{array}$} & \multirow{2}{*}{$\begin{array}{c}\text { Element } \\
\text { Neptunium }\end{array}$} & \multirow{2}{*}{$\begin{array}{c}\text { EPA } \\
\text { RF } \\
0.001\end{array}$} & $\begin{array}{c}\text { EPA } \\
\text { Conc } \\
\text { Factors } \\
\left(B_{v}\right) \\
\end{array}$ & $\begin{array}{c}\text { EPA } \\
\text { Sorption } \\
\text { Coefficient } \\
\left(K_{d}\right)\end{array}$ & $\begin{array}{c}\text { Half-Life } \\
\text { Reference }\end{array}$ & $\begin{array}{r}\text { Half-Lit } \\
\text { (original u }\end{array}$ & & $\begin{array}{l}\text { Half Life } \\
\text { (s) }\end{array}$ & $\begin{array}{l}\text { Sp. Act } \\
\text { (Ci/g) }\end{array}$ & $\begin{array}{l}\text { Inhalation } \\
\text { DC } \\
\text { Reference }\end{array}$ & $\begin{array}{c}\text { HC-3 } \\
\text { Bounding } \\
\text { Inhalation } \\
\text { DC } \\
\text { (Sv/Bq) } \\
\end{array}$ & $\begin{array}{c}\text { Lung } \\
\text { Absorption } \\
\text { Class/Type }\end{array}$ & $\begin{array}{l}\text { Ingestion } \\
\text { DC } \\
\text { Reference }\end{array}$ & $\begin{array}{c}\text { HC-3 } \\
\text { Bounding } \\
\text { Ingestion } \\
\text { DC } \\
\text { (Sv/Bq) } \\
\end{array}$ & $\begin{array}{c}\text { Immersion } \\
\text { DC } \\
\text { Reference }\end{array}$ & $\begin{array}{c}\mathrm{HC}-3 \\
\text { Immersion } \\
\mathrm{DC}(\mathrm{Sv} / \mathrm{d} \\
\left.\operatorname{per} \mathrm{Bq} / \mathrm{m}^{3}\right)\end{array}$ \\
\hline & & & & & 0.1 & 10 & ICRP-38 & $1.47 \mathrm{E}+01$ & $\mathrm{~m}$ & $8.82 E+02$ & $5.51 \mathrm{E}+07$ & ICRP-68 & 4.7E-11 & $\mathrm{M}$ & ICRP-68 & $9.7 \mathrm{E}-12$ & -- & -- \\
\hline $\mathrm{Np}-233$ & $\mathrm{~Np}-233$ & 233.041 & Neptunium & 0.001 & 0.1 & 10 & ICRP-38 & $3.62 \mathrm{E}+01$ & $\mathrm{~m}$ & $2.17 \mathrm{E}+03$ & $2.23 \mathrm{E}+07$ & ICRP-68 & $3.0 \mathrm{E}-12$ & $\mathrm{M}$ & ICRP-68 & $2.2 \mathrm{E}-12$ & -- & -- \\
\hline $\mathrm{Np}-234$ & $\mathrm{~Np}-234$ & 234.043 & Neptunium & 0.001 & 0.1 & 10 & ICRP-38 & $4.40 \mathrm{E}+00$ & $d$ & $3.80 \mathrm{E}+05$ & $1.27 \mathrm{E}+05$ & ICRP-68 & $7.3 \mathrm{E}-10$ & $\mathrm{M}$ & ICRP-68 & $8.1 \mathrm{E}-10$ & -- & -- \\
\hline Np-235 & $\mathrm{Np}-235$ & 235.044 & Neptunium & 0.001 & 0.1 & 10 & ICRP-38 & $3.96 \mathrm{E}+02$ & $d$ & $3.42 \mathrm{E}+07$ & $1.40 \mathrm{E}+03$ & ICRP-68 & $4.0 \mathrm{E}-10$ & $\mathrm{M}$ & ICRP-68 & $5.3 \mathrm{E}-11$ & -- & -- \\
\hline Np-236I & $\mathrm{Np}-236$ & 236.047 & Neptunium & 0.001 & 0.1 & 10 & ICRP-38 & $1.15 \mathrm{E}+05$ & $y$ & $3.63 \mathrm{E}+12$ & $1.32 \mathrm{E}-02$ & ICRP-68 & $3.0 \mathrm{E}-06$ & $\mathrm{M}$ & ICRP-68 & $1.7 \mathrm{E}-08$ & -- & -- \\
\hline Np-236s & $\mathrm{Np}-236$ & 236.047 & Neptunium & 0.001 & 0.1 & 10 & ICRP-38 & $2.25 \mathrm{E}+01$ & $\mathrm{~h}$ & $8.10 \mathrm{E}+04$ & $5.90 \mathrm{E}+05$ & ICRP-68 & $5.0 \mathrm{E}-09$ & $\mathrm{M}$ & ICRP-68 & $1.9 \mathrm{E}-10$ & -- & -- \\
\hline Np-237 & $\mathrm{Np}-237$ & 237.048 & Neptunium & 0.001 & 0.1 & 10 & ICRP-38 & $2.14 \mathrm{E}+06$ & $y$ & $6.75 \mathrm{E}+13$ & $7.05 \mathrm{E}-04$ & ICRP-68 & $2.1 \mathrm{E}-05$ & $\mathrm{M}$ & ICRP-68 & $1.1 \mathrm{E}-07$ & -- & -- \\
\hline Np-238 & Np-238 & 238.051 & Neptunium & 0.001 & 0.1 & 10 & ICRP-38 & $2.12 \mathrm{E}+00$ & $d$ & $1.83 \mathrm{E}+05$ & $2.59 \mathrm{E}+05$ & ICRP-68 & $2.0 \mathrm{E}-09$ & $M$ & ICRP-68 & $9.1 \mathrm{E}-10$ & -- & -- \\
\hline Np-239 & Np-239 & 239.053 & Neptunium & 0.001 & 0.1 & 10 & ICRP-38 & $2.36 \mathrm{E}+00$ & $d$ & $2.03 E+05$ & $2.32 \mathrm{E}+05$ & ICRP-68 & $1.1 \mathrm{E}-09$ & $M$ & ICRP-68 & $8.0 \mathrm{E}-10$ & -- & -- \\
\hline $\mathrm{Np}-240$ & $\mathrm{~Np}-240$ & 240.056 & Neptunium & 0.001 & 0.1 & 10 & ICRP-38 & $6.50 \mathrm{E}+01$ & $\mathrm{~m}$ & $3.90 \mathrm{E}+03$ & $1.20 \mathrm{E}+07$ & ICRP-68 & $1.3 \mathrm{E}-10$ & $M$ & ICRP-68 & $8.2 \mathrm{E}-11$ & -- & -- \\
\hline $\mathrm{Np}-240 \mathrm{~m}$ & $\mathrm{~Np}-240$ & 240.056 & Neptunium & 0.001 & 0.1 & 10 & ICRP-38 & $7.40 \mathrm{E}+00$ & $\mathrm{~m}$ & $4.44 \mathrm{E}+02$ & $1.06 \mathrm{E}+08$ & -- & -- & -- & -- & -- & -- & -- \\
\hline Np-241 & Np-241 & 241.058 & Neptunium & 0.001 & 0.1 & 10 & ICRP-107 & $1.39 \mathrm{E}+01$ & $\mathrm{~m}$ & $8.34 \mathrm{E}+02$ & $5.61 \mathrm{E}+07$ & JAERI & $2.2 \mathrm{E}-11$ & $M$ & JAERI & $1.8 \mathrm{E}-11$ & -- & -- \\
\hline Np-242 & $\mathrm{Np}-242$ & 242.062 & Neptunium & 0.001 & 0.1 & 10 & ICRP-107 & $2.20 \mathrm{E}+00$ & $\mathrm{~m}$ & $1.32 \mathrm{E}+02$ & $3.53 \mathrm{E}+08$ & -- & -- & -- & -- & -- & -- & -- \\
\hline $\mathrm{Np}-242 \mathrm{~m}$ & $\mathrm{~Np}-242$ & 242.062 & Neptunium & 0.001 & 0.1 & 10 & ICRP-107 & $5.50 \mathrm{E}+00$ & $\mathrm{~m}$ & $3.30 \mathrm{E}+02$ & $1.41 \mathrm{E}+08$ & -- & -- & -- & -- & -- & -- & -- \\
\hline $0-14$ & $0-14$ & 14.009 & Oxygen & -- & -- & -- & ICRP-38 & $7.06 \mathrm{E}+01$ & $\mathrm{~s}$ & $7.06 \mathrm{E}+01$ & $1.14 \mathrm{E}+10$ & -- & -- & -- & -- & -- & JAERI & $1.40 \mathrm{E}-08$ \\
\hline $0-15$ & $0-15$ & 15.003 & Oxygen & -- & -- & -- & ICRP-38 & $1.22 \mathrm{E}+02$ & $\mathrm{~s}$ & $1.22 \mathrm{E}+02$ & $6.15 \mathrm{E}+09$ & -- & -- & -- & -- & -- & JAERI & $4.00 \mathrm{E}-09$ \\
\hline $0-19$ & $0-19$ & 19.004 & Oxygen & --- & -- & -- & ICRP-38 & $2.69 \mathrm{E}+01$ & $\mathrm{~s}$ & $2.69 \mathrm{E}+01$ & $2.21 \mathrm{E}+10$ & -- & -- & -- & -- & -- & -- & -- \\
\hline Os-177 & Os-177 & 176.955 & Osmium & 0.01 & 0.015 & 0 & JAERI & $2.80 \mathrm{E}+00$ & $\mathrm{~m}$ & $1.68 \mathrm{E}+02$ & $3.79 \mathrm{E}+08$ & -- & -- & -- & -- & -- & -- & -- \\
\hline Os-179 & Os-179 & 178.954 & Osmium & 0.01 & 0.015 & 0 & JAERI & $6.50 \mathrm{E}+00$ & $\mathrm{~m}$ & $3.90 \mathrm{E}+02$ & $1.62 \mathrm{E}+08$ & -- & -- & -- & -- & -- & -- & -- \\
\hline Os-180 & Os-180 & 179.952 & Osmium & 0.01 & 0.015 & 0 & ICRP-38 & $2.20 \mathrm{E}+01$ & $\mathrm{~m}$ & $1.32 \mathrm{E}+03$ & $4.75 \mathrm{E}+07$ & ICRP-68 & $2.5 \mathrm{E}-11$ & $\mathrm{~s}$ & ICRP-68 & $1.7 \mathrm{E}-11$ & -- & -- \\
\hline Os-181 & Os-181 & 180.953 & Osmium & 0.01 & 0.015 & 0 & ICRP-38 & $1.05 \mathrm{E}+02$ & $\mathrm{~m}$ & $6.30 \mathrm{E}+03$ & $9.90 \mathrm{E}+06$ & ICRP-68 & $1.0 \mathrm{E}-10$ & $\mathrm{~s}$ & ICRP-68 & $8.9 \mathrm{E}-11$ & -- & -- \\
\hline Os-182 & Os-182 & 181.952 & Osmium & 0.01 & 0.015 & 0 & ICRP-38 & $2.20 \mathrm{E}+01$ & $\mathrm{~h}$ & $7.92 \mathrm{E}+04$ & $7.83 \mathrm{E}+05$ & ICRP-68 & $5.2 \mathrm{E}-10$ & $\mathrm{~s}$ & ICRP-68 & $5.6 \mathrm{E}-10$ & -- & -- \\
\hline Os-183 & Os-183 & 182.953 & Osmium & 0.01 & 0.015 & 0 & ICRP-107 & $1.30 \mathrm{E}+01$ & $h$ & $4.68 \mathrm{E}+04$ & $1.32 \mathrm{E}+06$ & JAERI & $2.5 \mathrm{E}-10$ & $\mathrm{~s}$ & JAERI & $2.3 \mathrm{E}-10$ & -- & -- \\
\hline Os-183m & Os-183 & 182.953 & Osmium & 0.01 & 0.015 & 0 & ICRP-107 & $9.90 \mathrm{E}+00$ & $\mathrm{~h}$ & $3.56 \mathrm{E}+04$ & $1.73 \mathrm{E}+06$ & JAERI & $2.2 \mathrm{E}-10$ & $M$ & JAERI & $2.1 \mathrm{E}-10$ & -- & -- \\
\hline Os-185 & Os-185 & 184.954 & Osmium & 0.01 & 0.015 & 0 & ICRP-38 & $9.40 \mathrm{E}+01$ & $d$ & $8.12 \mathrm{E}+06$ & $7.51 \mathrm{E}+03$ & ICRP-68 & $1.5 \mathrm{E}-09$ & $\mathrm{~s}$ & ICRP-68 & $5.1 \mathrm{E}-10$ & -- & -- \\
\hline Os-186 & Os-186 & 185.954 & Osmium & 0.01 & 0.015 & 0 & ICRP-107 & $2.00 \mathrm{E}+15$ & $y$ & $6.31 \mathrm{E}+22$ & $9.61 \mathrm{E}-13$ & JAERI & $3.8 \mathrm{E}-06$ & $\mathrm{~s}$ & JAERI & $3.2 \mathrm{E}-08$ & -- & -- \\
\hline Os-189m & Os-189 & 188.958 & Osmium & 0.01 & 0.015 & 0 & ICRP-38 & $6.00 \mathrm{E}+00$ & $\mathrm{~h}$ & $2.16 \mathrm{E}+04$ & $2.76 \mathrm{E}+06$ & ICRP-68 & $7.9 \mathrm{E}-12$ & $\mathrm{~s}$ & ICRP-68 & $1.8 \mathrm{E}-11$ & -- & -- \\
\hline Os-190m & Os-190 & 189.958 & Osmium & 0.01 & 0.015 & 0 & ICRP-38 & $9.90 \mathrm{E}+00$ & $\mathrm{~m}$ & $5.94 \mathrm{E}+02$ & $1.00 \mathrm{E}+08$ & -- & -- & -- & -- & -- & -- & -- \\
\hline Os-191 & Os-191 & 190.961 & Osmium & 0.01 & 0.015 & 0 & ICRP-38 & $1.54 \mathrm{E}+01$ & $d$ & $1.33 \mathrm{E}+06$ & $4.44 \mathrm{E}+04$ & ICRP-68 & $1.8 \mathrm{E}-09$ & $\mathrm{~s}$ & ICRP-68 & $5.7 \mathrm{E}-10$ & -- & -- \\
\hline Os-191m & Os-191 & 190.961 & Osmium & 0.01 & 0.015 & 0 & ICRP-38 & $1.30 \mathrm{E}+01$ & $\mathrm{~h}$ & $4.69 \mathrm{E}+04$ & $1.26 \mathrm{E}+06$ & ICRP-68 & $1.5 \mathrm{E}-10$ & $\mathrm{~s}$ & ICRP-68 & $9.6 \mathrm{E}-11$ & -- & -- \\
\hline Os-193 & Os-193 & 192.964 & Osmium & 0.01 & 0.015 & 0 & ICRP-38 & $3.00 \mathrm{E}+01$ & $h$ & $1.08 \mathrm{E}+05$ & $5.41 \mathrm{E}+05$ & ICRP-68 & $6.8 \mathrm{E}-10$ & $\mathrm{~s}$ & ICRP-68 & $8.1 \mathrm{E}-10$ & -- & -- \\
\hline Os-194 & Os-194 & 193.965 & Osmium & 0.01 & 0.015 & 0 & ICRP-38 & $6.00 \mathrm{E}+00$ & $y$ & $1.89 \mathrm{E}+08$ & $3.07 E+02$ & ICRP-68 & $7.9 \mathrm{E}-08$ & $\mathrm{~s}$ & ICRP-68 & $2.4 \mathrm{E}-09$ & -- & -- \\
\hline Os-196 & Os-196 & 195.970 & Osmium & 0.01 & 0.015 & 0 & ICRP-107 & $3.49 \mathrm{E}+01$ & $\mathrm{~m}$ & $2.09 \mathrm{E}+03$ & $2.75 \mathrm{E}+07$ & JAERI & $9.1 \mathrm{E}-11$ & $\mathrm{~s}$ & JAERI & $1.2 \mathrm{E}-10$ & -- & -- \\
\hline $\mathrm{P}-30$ & P-30 & 29.978 & Phosphorus & 0.5 & 3.5 & 0 & ICRP-38 & $2.50 \mathrm{E}+00$ & $\mathrm{~m}$ & $1.50 \mathrm{E}+02$ & $2.51 \mathrm{E}+09$ & -- & -- & -- & -- & -- & -- & -- \\
\hline P-32 & P-32 & 31.974 & Phosphorus & 0.5 & 3.5 & 0 & ICRP-38 & $1.43 \mathrm{E}+01$ & $d$ & $1.23 \mathrm{E}+06$ & $2.86 \mathrm{E}+05$ & ICRP-68 & $3.2 \mathrm{E}-09$ & $M$ & ICRP-68 & $2.4 \mathrm{E}-09$ & -- & -- \\
\hline $\mathrm{P}-33$ & P-33 & 32.972 & Phosphorus & $\begin{array}{l}0.5 \\
\end{array}$ & 3.5 & 0 & ICRP-38 & $2.54 \mathrm{E}+01$ & $d$ & $2.19 \mathrm{E}+06$ & $1.56 \mathrm{E}+05$ & ICRP-68 & $1.4 \mathrm{E}-09$ & $M$ & ICRP-68 & $2.4 \mathrm{E}-10$ & -- & -- \\
\hline Pa-227 & Pa-227 & 227.029 & Protactinium & 0.001 & 0.0025 & 4000 & ICRP-38 & $3.83 \mathrm{E}+01$ & $\mathrm{~m}$ & $2.30 \mathrm{E}+03$ & $2.16 \mathrm{E}+07$ & ICRP-68 & $9.7 \mathrm{E}-08$ & $\mathrm{~s}$ & ICRP-68 & $4.5 \mathrm{E}-10$ & -- & -- \\
\hline Pa-228 & Pa-228 & 228.031 & Protactinium & 0.001 & 0.0025 & 4000 & ICRP-38 & $2.20 \mathrm{E}+01$ & $\mathrm{~h}$ & $7.92 \mathrm{E}+04$ & $6.25 \mathrm{E}+05$ & ICRP-68 & $6.9 \mathrm{E}-08$ & $\mathrm{~s}$ & ICRP-68 & $7.8 \mathrm{E}-10$ & -- & -- \\
\hline Pa-229 & Pa-229 & 229.032 & Protactinium & 0.001 & 0.0025 & 4000 & ICRP-107 & $1.50 \mathrm{E}+00$ & $d$ & $1.30 \mathrm{E}+05$ & $3.80 \mathrm{E}+05$ & JAERI & $6.7 \mathrm{E}-09$ & $\mathrm{~s}$ & JAERI & $2.2 \mathrm{E}-10$ & -- & -- \\
\hline $\mathrm{Pa}-230$ & Pa-230 & 230.035 & Protactinium & 0.001 & 0.0025 & 4000 & ICRP-38 & $1.74 \mathrm{E}+01$ & $d$ & $1.50 \mathrm{E}+06$ & $3.26 \mathrm{E}+04$ & ICRP-68 & $7.1 \mathrm{E}-07$ & $\mathrm{~s}$ & ICRP-68 & $9.2 \mathrm{E}-10$ & -- & -- \\
\hline $\mathrm{Pa}-231$ & Pa-231 & 231.036 & Protactinium & 0.001 & 0.0025 & 4000 & ICRP-38 & $3.28 \mathrm{E}+04$ & $y$ & $1.03 \mathrm{E}+12$ & $4.72 \mathrm{E}-02$ & ICRP-68 & $1.3 \mathrm{E}-04$ & $\mathrm{M}$ & ICRP-68 & $7.1 \mathrm{E}-07$ & -- & -- \\
\hline Pa-232 & $\mathrm{Pa}-232$ & 232.039 & Protactinium & 0.001 & 0.0025 & 4000 & ICRP-38 & $1.31 \mathrm{E}+00$ & $d$ & $1.13 \mathrm{E}+05$ & $4.30 \mathrm{E}+05$ & ICRP-68 & $9.5 \mathrm{E}-09$ & $\mathrm{M}$ & ICRP-68 & $7.2 \mathrm{E}-10$ & -- & -- \\
\hline $\mathrm{Pa}-233$ & Pa-233 & 233.040 & Protactinium & 0.001 & 0.0025 & 4000 & ICRP-38 & $2.70 \mathrm{E}+01$ & $d$ & $2.33 \mathrm{E}+06$ & $2.08 \mathrm{E}+04$ & ICRP-68 & $3.7 \mathrm{E}-09$ & $\mathrm{~s}$ & ICRP-68 & $8.7 \mathrm{E}-10$ & -- & -- \\
\hline Pa-234 & $\mathrm{Pa}-234$ & 234.043 & Protactinium & 0.001 & 0.0025 & 4000 & ICRP-38 & $6.70 \mathrm{E}+00$ & $\mathrm{~h}$ & $2.41 \mathrm{E}+04$ & $2.00 E+06$ & ICRP-68 & $5.8 \mathrm{E}-10$ & $\mathrm{~s}$ & ICRP-68 & $5.1 \mathrm{E}-10$ & -- & -- \\
\hline Pa-234m & Pa-234 & 234.043 & Protactinium & 0.001 & 0.0025 & 4000 & ICRP-38 & $1.17 \mathrm{E}+00$ & $\mathrm{~m}$ & $7.02 \mathrm{E}+01$ & $6.87 \mathrm{E}+08$ & -- & -- & -- & -- & -- & -- & -- \\
\hline
\end{tabular}




\begin{tabular}{|c|c|c|c|c|c|c|c|c|c|c|c|c|c|c|c|c|c|c|}
\hline \multirow{3}{*}{$\begin{array}{l}\text { MASTER } \\
\text { ISOTOPE } \\
\text { LIST } \\
\text { Pa-235 }\end{array}$} & \multirow{3}{*}{$\begin{array}{c}\begin{array}{c}\text { Atomic } \\
\text { Mass } \\
\text { Nuclide }\end{array} \\
\text { Pa-235 }\end{array}$} & & & & \multicolumn{14}{|c|}{ Table C.1 Radionuclide Specific Input Data Used For the Calculation of HC-3 TQs Using Maximum Reported DCs (continued) } \\
\hline & & \multirow{2}{*}{$\begin{array}{c}\begin{array}{c}\text { Atomic } \\
\text { Mass }\end{array} \\
235.045\end{array}$} & \multirow{2}{*}{$\begin{array}{c}\text { Element } \\
\text { Protactinium }\end{array}$} & \multirow{2}{*}{$\begin{array}{c}\text { EPA } \\
\text { RF } \\
0.001\end{array}$} & $\begin{array}{c}\text { EPA } \\
\text { Conc } \\
\text { Factors } \\
\left(B_{v}\right) \\
\end{array}$ & $\begin{array}{c}\text { EPA } \\
\text { Sorption } \\
\text { Coefficient } \\
\left(K_{d}\right)\end{array}$ & $\begin{array}{c}\text { Half-Life } \\
\text { Reference }\end{array}$ & $\begin{array}{r}\text { Half-Lit } \\
\text { (original u }\end{array}$ & & $\begin{array}{l}\text { Half Life } \\
\text { (s) }\end{array}$ & $\begin{array}{l}\text { Sp. Act } \\
\text { (Ci/g) }\end{array}$ & $\begin{array}{l}\text { Inhalation } \\
\text { DC } \\
\text { Reference }\end{array}$ & $\begin{array}{c}\text { HC-3 } \\
\text { Bounding } \\
\text { Inhalation } \\
\text { DC } \\
\text { (Sv/Bq) } \\
\end{array}$ & $\begin{array}{c}\text { Lung } \\
\text { Absorption } \\
\text { Class/Type }\end{array}$ & $\begin{array}{l}\text { Ingestion } \\
\text { DC } \\
\text { Reference }\end{array}$ & $\begin{array}{c}\text { HC-3 } \\
\text { Bounding } \\
\text { Ingestion } \\
\text { DC } \\
\text { (Sv/Bq) } \\
\end{array}$ & $\begin{array}{c}\text { Immersion } \\
\text { DC } \\
\text { Reference }\end{array}$ & $\begin{array}{c}\mathrm{HC}-3 \\
\text { Immersion } \\
\mathrm{DC}(\mathrm{Sv} / \mathrm{d} \\
\left.\operatorname{per} \mathrm{Bq} / \mathrm{m}^{3}\right)\end{array}$ \\
\hline & & & & & 0.0025 & 4000 & ICRP-107 & $2.45 \mathrm{E}+01$ & $\mathrm{~m}$ & $1.47 \mathrm{E}+03$ & $3.27 \mathrm{E}+07$ & -- & -- & -- & -- & -- & -- & -- \\
\hline $\mathrm{Pa}-236$ & Pa-236 & 236.049 & Protactinium & 0.001 & 0.0025 & 4000 & ICRP-107 & $9.10 \mathrm{E}+00$ & $\mathrm{~m}$ & $5.46 \mathrm{E}+02$ & $8.75 \mathrm{E}+07$ & -- & -- & -- & -- & -- & -- & -- \\
\hline Pa-237 & Pa-237 & 237.051 & Protactinium & 0.001 & 0.0025 & 4000 & ICRP-107 & $8.70 \mathrm{E}+00$ & $\mathrm{~m}$ & $5.22 \mathrm{E}+02$ & $9.12 \mathrm{E}+07$ & -- & -- & -- & -- & -- & -- & -- \\
\hline Pb-194 & $\mathrm{Pb}-194$ & $\begin{array}{l}193.974 \\
\end{array}$ & Lead & 0.01 & 0.045 & 4000 & ICRP-107 & $1.20 \mathrm{E}+01$ & $\mathrm{~m}$ & $7.20 \mathrm{E}+02$ & $8.08 \mathrm{E}+07$ & JAERI & $1.5 \mathrm{E}-11$ & $F$ & JAERI & $2.0 \mathrm{E}-11$ & -- & -- \\
\hline $\mathrm{Pb}-195 \mathrm{~m}$ & $\mathrm{~Pb}-195$ & 194.975 & Lead & 0.01 & 0.045 & 4000 & ICRP-38 & $1.58 \mathrm{E}+01$ & $\mathrm{~m}$ & $9.48 \mathrm{E}+02$ & $6.10 \mathrm{E}+07$ & ICRP-68 & $3.0 \mathrm{E}-11$ & $\mathrm{~F}$ & ICRP-68 & $2.9 \mathrm{E}-11$ & -- & -- \\
\hline Pb-196 & $\mathrm{Pb}-196$ & 195.973 & Lead & 0.01 & 0.045 & 4000 & ICRP-107 & $3.70 \mathrm{E}+01$ & $\mathrm{~m}$ & $2.22 \mathrm{E}+03$ & $2.59 \mathrm{E}+07$ & JAERI & $3.1 \mathrm{E}-11$ & $\mathrm{~F}$ & JAERI & $2.8 \mathrm{E}-11$ & -- & -- \\
\hline Pb-197 & $\mathrm{Pb}-197$ & 196.973 & Lead & 0.01 & 0.045 & 4000 & ICRP-107 & $8.00 \mathrm{E}+00$ & $\mathrm{~m}$ & $4.80 \mathrm{E}+02$ & $1.19 \mathrm{E}+08$ & -- & -- & -- & -- & -- & -- & -- \\
\hline $\mathrm{Pb}-197 \mathrm{~m}$ & $\mathrm{~Pb}-197$ & 196.973 & Lead & 0.01 & 0.045 & 4000 & ICRP-107 & $4.30 \mathrm{E}+01$ & $\mathrm{~m}$ & $2.58 \mathrm{E}+03$ & $2.22 \mathrm{E}+07$ & JAERI & $4.6 \mathrm{E}-11$ & $\mathrm{~F}$ & JAERI & $4.5 \mathrm{E}-11$ & -- & -- \\
\hline $\mathrm{Pb}-198$ & $\mathrm{~Pb}-198$ & 197.972 & Lead & 0.01 & 0.045 & 4000 & $\begin{array}{l}\text { ICRP-38 } \\
\end{array}$ & $2.40 \mathrm{E}+00$ & $\mathrm{~h}$ & $8.64 \mathrm{E}+03$ & $6.60 \mathrm{E}+06$ & ICRP-68 & $8.7 \mathrm{E}-11$ & $\mathrm{~F}$ & ICRP-68 & $1.0 \mathrm{E}-10$ & -- & -- \\
\hline Pb-199 & $\mathrm{Pb}-199$ & 198.973 & Lead & 0.01 & 0.045 & 4000 & ICRP-38 & $9.00 \mathrm{E}+01$ & $\mathrm{~m}$ & $5.40 \mathrm{E}+03$ & $1.05 \mathrm{E}+07$ & ICRP-68 & $4.8 \mathrm{E}-11$ & $F$ & ICRP-68 & $5.4 \mathrm{E}-11$ & -- & -- \\
\hline $\mathrm{Pb}-200$ & $\mathrm{~Pb}-200$ & 199.972 & Lead & 0.01 & 0.045 & 4000 & ICRP-38 & $2.15 \mathrm{E}+01$ & $\mathrm{~h}$ & $7.74 \mathrm{E}+04$ & $7.29 \mathrm{E}+05$ & ICRP-68 & $2.6 \mathrm{E}-10$ & $\mathrm{~F}$ & ICRP-68 & $4.0 \mathrm{E}-10$ & -- & -- \\
\hline $\begin{array}{l}\mathrm{Pb}-201 \\
\end{array}$ & $\mathrm{~Pb}-201$ & 200.973 & Lead & 0.01 & 0.045 & 4000 & ICRP-38 & $9.40 \mathrm{E}+00$ & $\mathrm{~h}$ & $3.38 \mathrm{E}+04$ & $1.66 \mathrm{E}+06$ & ICRP-68 & $1.2 \mathrm{E}-10$ & $\mathrm{~F}$ & ICRP-68 & $1.6 \mathrm{E}-10$ & -- & -- \\
\hline $\mathrm{Pb}-201 \mathrm{~m}$ & $\mathrm{~Pb}-201$ & 200.973 & Lead & 0.01 & 0.045 & 4000 & ICRP-107 & $6.10 \mathrm{E}+01$ & $\mathrm{~s}$ & $6.10 \mathrm{E}+01$ & $9.20 \mathrm{E}+08$ & -- & -- & -- & -- & -- & -- & -- \\
\hline $\mathrm{Pb}-202$ & $\mathrm{~Pb}-202$ & 201.972 & Lead & 0.01 & 0.045 & 4000 & ICRP-38 & $3.00 \mathrm{E}+05$ & $y$ & $9.47 \mathrm{E}+12$ & $5.90 \mathrm{E}-03$ & ICRP-68 & $1.4 \mathrm{E}-08$ & $\mathrm{~F}$ & ICRP-68 & $8.7 \mathrm{E}-09$ & -- & -- \\
\hline $\mathrm{Pb}-202 \mathrm{~m}$ & $\mathrm{~Pb}-202$ & 201.972 & Lead & 0.01 & 0.045 & 4000 & ICRP-38 & $3.62 \mathrm{E}+00$ & $\mathrm{~h}$ & $1.30 \mathrm{E}+04$ & $4.29 \mathrm{E}+06$ & ICRP-68 & $1.2 \mathrm{E}-10$ & $F$ & ICRP-68 & $1.3 \mathrm{E}-10$ & -- & -- \\
\hline $\mathrm{Pb}-203$ & $\mathrm{~Pb}-203$ & 202.973 & Lead & 0.01 & 0.045 & 4000 & ICRP-38 & $5.21 \mathrm{E}+01$ & $\mathrm{~h}$ & $1.87 \mathrm{E}+05$ & $2.97 E+05$ & ICRP-68 & $1.6 \mathrm{E}-10$ & $\mathrm{~F}$ & ICRP-68 & $2.4 \mathrm{E}-10$ & -- & -- \\
\hline $\mathrm{Pb}-204 \mathrm{~m}$ & $\mathrm{~Pb}-204$ & 203.973 & Lead & 0.01 & 0.045 & 4000 & ICRP-38 & $6.72 \mathrm{E}+01$ & $\mathrm{~m}$ & $4.03 \mathrm{E}+03$ & $1.37 \mathrm{E}+07$ & JAERI & $4.4 \mathrm{E}-11$ & $\mathrm{~F}$ & JAERI & $5.0 \mathrm{E}-11$ & -- & -- \\
\hline $\mathrm{Pb}-205$ & $\mathrm{~Pb}-205$ & 204.974 & Lead & 0.01 & 0.045 & 4000 & ICRP-38 & $1.43 \mathrm{E}+07$ & $y$ & $4.51 \mathrm{E}+14$ & $1.22 \mathrm{E}-04$ & ICRP-68 & $4.1 \mathrm{E}-10$ & $F$ & ICRP-68 & $2.8 \mathrm{E}-10$ & -- & -- \\
\hline $\mathrm{Pb}-209$ & $\mathrm{~Pb}-209$ & 208.981 & Lead & 0.01 & 0.045 & 4000 & ICRP-38 & $3.25 \mathrm{E}+00$ & $\mathrm{~h}$ & $1.17 \mathrm{E}+04$ & $4.61 \mathrm{E}+06$ & ICRP-68 & $3.2 \mathrm{E}-11$ & $\mathrm{~F}$ & ICRP-68 & $5.7 \mathrm{E}-11$ & -- & -- \\
\hline $\mathrm{Pb}-210$ & $\mathrm{~Pb}-210$ & 209.984 & Lead & 0.01 & 0.045 & 4000 & ICRP-38 & $2.23 \mathrm{E}+01$ & $y$ & $7.04 \mathrm{E}+08$ & $7.63 \mathrm{E}+01$ & ICRP-68 & $1.1 \mathrm{E}-06$ & $\mathrm{~F}$ & ICRP-68 & $6.8 \mathrm{E}-07$ & -- & -- \\
\hline $\mathrm{Pb}-211$ & $\mathrm{~Pb}-211$ & 210.989 & Lead & 0.01 & 0.045 & 4000 & ICRP-38 & $3.61 \mathrm{E}+01$ & $\mathrm{~m}$ & $2.17 E+03$ & $2.47 E+07$ & ICRP-68 & $5.6 \mathrm{E}-09$ & $\mathrm{~F}$ & ICRP-68 & $1.8 \mathrm{E}-10$ & -- & -- \\
\hline $\mathrm{Pb}-212$ & $\mathrm{~Pb}-212$ & 211.992 & Lead & 0.01 & 0.045 & 4000 & ICRP-38 & $1.06 \mathrm{E}+01$ & $\mathrm{~h}$ & $3.83 \mathrm{E}+04$ & $1.39 \mathrm{E}+06$ & ICRP-68 & $3.3 \mathrm{E}-08$ & $\mathrm{~F}$ & ICRP-68 & $5.9 \mathrm{E}-09$ & -- & -- \\
\hline $\mathrm{Pb}-214$ & $\mathrm{~Pb}-214$ & 214.000 & Lead & 0.01 & 0.045 & 4000 & ICRP-38 & $2.68 \mathrm{E}+01$ & $\mathrm{~m}$ & $1.61 \mathrm{E}+03$ & $3.28 \mathrm{E}+07$ & ICRP-68 & $4.8 \mathrm{E}-09$ & $\mathrm{~F}$ & ICRP-68 & $1.4 \mathrm{E}-10$ & -- & -- \\
\hline $\mathrm{Pd}-100$ & $\mathrm{Pd}-100$ & 99.909 & Palladium & 0.01 & 0.15 & $50-100$ & ICRP-38 & $3.63 \mathrm{E}+00$ & d & $3.14 \mathrm{E}+05$ & $3.60 \mathrm{E}+05$ & ICRP-68 & $9.7 \mathrm{E}-10$ & $\mathrm{~s}$ & ICRP-68 & $9.4 \mathrm{E}-10$ & -- & -- \\
\hline Pd-101 & Pd-101 & 100.908 & Palladium & 0.01 & 0.15 & $50-100$ & ICRP-38 & $8.27 \mathrm{E}+00$ & $\mathrm{~h}$ & $2.98 \mathrm{E}+04$ & $3.76 \mathrm{E}+06$ & ICRP-68 & $1.0 \mathrm{E}-10$ & $\mathrm{~s}$ & ICRP-68 & $9.4 \mathrm{E}-11$ & -- & -- \\
\hline $\mathrm{Pd}-103$ & Pd-103 & 102.906 & Palladium & 0.01 & 0.15 & $50-100$ & ICRP-38 & $1.70 \mathrm{E}+01$ & $d$ & $1.47 E+06$ & $7.48 \mathrm{E}+04$ & ICRP-68 & $4.0 \mathrm{E}-10$ & $\mathrm{~s}$ & ICRP-68 & $1.9 \mathrm{E}-10$ & -- & -- \\
\hline $\mathrm{Pd}-107$ & Pd-107 & 106.905 & Palladium & 0.01 & 0.15 & $50-100$ & ICRP-38 & $6.50 \mathrm{E}+06$ & $y$ & $2.05 E+14$ & $5.14 \mathrm{E}-04$ & ICRP-68 & $5.5 \mathrm{E}-10$ & $\mathrm{~s}$ & ICRP-68 & $3.7 \mathrm{E}-11$ & -- & -- \\
\hline $\mathrm{Pd}-109$ & Pd-109 & 108.906 & Palladium & 0.01 & 0.15 & $50-100$ & ICRP-38 & $1.34 \mathrm{E}+01$ & $\mathrm{~h}$ & $4.83 \mathrm{E}+04$ & $2.14 \mathrm{E}+06$ & ICRP-68 & $5.0 \mathrm{E}-10$ & $\mathrm{~s}$ & ICRP-68 & $5.5 \mathrm{E}-10$ & -- & -- \\
\hline Pd-109m & Pd-109 & 108.906 & Palladium & 0.01 & 0.15 & $50-100$ & ICRP-107 & $4.69 \mathrm{E}+00$ & $\mathrm{~m}$ & $2.81 \mathrm{E}+02$ & $3.68 \mathrm{E}+08$ & -- & -- & -- & -- & -- & -- & -- \\
\hline Pd-111 & Pd-111 & 110.908 & Palladium & 0.01 & 0.15 & $50-100$ & $\begin{array}{l}\text { ICRP-107 } \\
\end{array}$ & $2.34 \mathrm{E}+01$ & $\mathrm{~m}$ & $1.40 \mathrm{E}+03$ & $7.24 \mathrm{E}+07$ & JAERI & $3.9 \mathrm{E}-11$ & $\mathrm{~s}$ & JAERI & $5.0 \mathrm{E}-11$ & -- & -- \\
\hline $\mathrm{Pd}-112$ & $\mathrm{Pd}-112$ & 111.907 & Palladium & 0.01 & 0.15 & $50-100$ & ICRP-107 & $2.10 \mathrm{E}+01$ & $\mathrm{~h}$ & $7.57 E+04$ & $1.33 \mathrm{E}+06$ & JAERI & $1.5 \mathrm{E}-09$ & $\mathrm{~s}$ & JAERI & $2.6 \mathrm{E}-09$ & -- & -- \\
\hline Pd-114 & $\mathrm{Pd}-114$ & 113.910 & Palladium & 0.01 & 0.15 & $50-100$ & ICRP-107 & $2.42 \mathrm{E}+00$ & $\mathrm{~m}$ & $1.45 \mathrm{E}+02$ & $6.82 \mathrm{E}+08$ & -- & -- & -- & -- & -- & -- & -- \\
\hline Pd-96 & Pd-96 & 95.918 & Palladium & 0.01 & 0.15 & $50-100$ & ICRP-107 & $1.22 \mathrm{E}+02$ & $\mathrm{~s}$ & $1.22 \mathrm{E}+02$ & $9.64 \mathrm{E}+08$ & -- & -- & -- & -- & -- & -- & -- \\
\hline Pd-97 & Pd-97 & 96.916 & $\begin{array}{l}\text { Palladium } \\
\end{array}$ & 0.01 & 0.15 & $50-100$ & ICRP-107 & $3.10 \mathrm{E}+00$ & $\mathrm{~m}$ & $1.86 \mathrm{E}+02$ & $6.26 \mathrm{E}+08$ & -- & -- & -- & -- & -- & -- & -- \\
\hline Pd-98 & Pd-98 & 97.913 & Palladium & 0.01 & 0.15 & $50-100$ & ICRP-107 & $1.77 \mathrm{E}+01$ & $\mathrm{~m}$ & $1.06 \mathrm{E}+03$ & $1.08 \mathrm{E}+08$ & JAERI & $4.7 \mathrm{E}-11$ & $\mathrm{~s}$ & JAERI & $6.3 \mathrm{E}-11$ & -- & -- \\
\hline Pd-99 & Pd-99 & 98.912 & $\begin{array}{l}\text { Palladium } \\
\end{array}$ & 0.01 & 0.15 & $50-100$ & ICRP-107 & $2.14 \mathrm{E}+01$ & $\mathrm{~m}$ & $1.28 \mathrm{E}+03$ & $8.88 \mathrm{E}+07$ & JAERI & $3.4 \mathrm{E}-11$ & $\mathrm{~s}$ & JAERI & $3.6 \mathrm{E}-11$ & -- & -- \\
\hline Pm-136 & Pm-136 & 135.924 & Promethium & 0.01 & 0.01 & $1000-10000$ & ICRP-107 & $1.07 E+02$ & $\mathrm{~s}$ & $1.07 E+02$ & $7.76 \mathrm{E}+08$ & -- & -- & -- & -- & -- & -- & -- \\
\hline $\mathrm{Pm}-137 \mathrm{~m}$ & Pm-137 & 136.920 & Promethium & 0.01 & 0.01 & $1000-10000$ & ICRP-107 & $2.40 \mathrm{E}+00$ & $\mathrm{~m}$ & $1.44 \mathrm{E}+02$ & $5.72 \mathrm{E}+08$ & -- & -- & -- & -- & -- & -- & -- \\
\hline Pm-138 & Pm-138 & 137.920 & Promethium & 0.01 & 0.01 & $1000-10000$ & JAERI & $3.24 \mathrm{E}+00$ & $\mathrm{~m}$ & $1.94 \mathrm{E}+02$ & $4.21 \mathrm{E}+08$ & -- & -- & -- & -- & -- & -- & -- \\
\hline Pm-139 & Pm-139 & 138.917 & Promethium & 0.01 & 0.01 & $1000-10000$ & ICRP-107 & $4.15 \mathrm{E}+00$ & $\mathrm{~m}$ & $2.49 \mathrm{E}+02$ & $3.26 \mathrm{E}+08$ & -- & -- & -- & -- & -- & -- & -- \\
\hline $\begin{array}{l}\mathrm{Pm}-140 \\
\end{array}$ & Pm-140 & 139.916 & Promethium & 0.01 & 0.01 & $1000-10000$ & ICRP-107 & $9.20 \mathrm{E}+00$ & $\mathrm{~s}$ & $9.20 \mathrm{E}+00$ & $8.76 \mathrm{E}+09$ & -- & -- & -- & -- & -- & -- & -- \\
\hline Pm-140m & Pm-140 & 139.916 & Promethium & 0.01 & 0.01 & $1000-10000$ & ICRP-107 & $5.95 \mathrm{E}+00$ & $\mathrm{~m}$ & $3.57 \mathrm{E}+02$ & $2.26 \mathrm{E}+08$ & -- & -- & -- & -- & -- & -- & -- \\
\hline Pm-141 & Pm-141 & 140.914 & Promethium & 0.01 & 0.01 & $1000-10000$ & ICRP-38 & $2.09 \mathrm{E}+01$ & $\mathrm{~m}$ & $1.25 \mathrm{E}+03$ & $6.38 \mathrm{E}+07$ & ICRP-68 & $2.5 \mathrm{E}-11$ & $\mathrm{~s}$ & ICRP-68 & $3.6 \mathrm{E}-11$ & -- & -- \\
\hline Pm-142 & Pm-142 & 141.913 & Promethium & 0.01 & 0.01 & $1000-10000$ & ICRP-38 & $4.05 \mathrm{E}+01$ & $\mathrm{~s}$ & $4.05 \mathrm{E}+01$ & $1.96 \mathrm{E}+09$ & -- & -- & -- & -- & -- & -- & -- \\
\hline Pm-143 & Pm-143 & 142.911 & Promethium & 0.01 & 0.01 & $1000-10000$ & ICRP-38 & $2.65 \mathrm{E}+02$ & $d$ & $2.29 \mathrm{E}+07$ & $3.45 \mathrm{E}+03$ & ICRP-68 & $1.4 \mathrm{E}-09$ & $M$ & ICRP-68 & $2.3 \mathrm{E}-10$ & -- & -- \\
\hline
\end{tabular}




\begin{tabular}{|c|c|c|c|c|c|c|c|c|c|c|c|c|c|c|c|c|c|c|}
\hline \multirow{3}{*}{$\begin{array}{l}\text { MASTER } \\
\text { ISOTOPE } \\
\text { LIST } \\
\text { Pm-144 }\end{array}$} & \multirow{3}{*}{$\begin{array}{c}\begin{array}{c}\text { Atomic } \\
\text { Mass } \\
\text { Nuclide }\end{array} \\
\text { Pm-144 } \\
\end{array}$} & \multirow{3}{*}{$\begin{array}{c}\begin{array}{c}\text { Atomic } \\
\text { Mass }\end{array} \\
143.913\end{array}$} & \multirow{3}{*}{$\begin{array}{c}\text { Element } \\
\text { Promethium }\end{array}$} & \multirow{3}{*}{$\begin{array}{c}\text { EPA } \\
\text { RF } \\
0.01\end{array}$} & \multicolumn{14}{|c|}{ Table C.1 Radionuclide Specific Input Data Used For the Calculation of HC-3 TQs Using Maximum Reported DCs (continued) } \\
\hline & & & & & $\begin{array}{c}\text { EPA } \\
\text { Conc } \\
\text { Factors } \\
\left(B_{v}\right) \\
\end{array}$ & $\begin{array}{c}\text { EPA } \\
\text { Sorption } \\
\text { Coefficient } \\
\left(K_{d}\right)\end{array}$ & $\begin{array}{c}\text { Half-Life } \\
\text { Reference }\end{array}$ & \multicolumn{2}{|c|}{$\begin{array}{c}\begin{array}{c}\text { Half-Life } \\
\text { (original units) }\end{array}\end{array}$} & \multirow{2}{*}{$\begin{array}{c}\begin{array}{c}\text { Half Life } \\
\text { (s) }\end{array} \\
3.14 E+07\end{array}$} & \multirow{2}{*}{$\begin{array}{c}\text { Sp. Act } \\
\text { (Ci/g) } \\
2.50 \mathrm{E}+03\end{array}$} & \multirow[t]{2}{*}{$\begin{array}{c}\text { Inhalation } \\
\text { DC } \\
\text { Reference }\end{array}$} & \multirow[t]{2}{*}{$\begin{array}{c}\text { HC-3 } \\
\text { Bounding } \\
\text { Inhalation } \\
\text { DC } \\
(\mathrm{Sv} / \mathrm{Bq}) \\
7.8 \mathrm{E}-09\end{array}$} & \multirow[t]{2}{*}{$\begin{array}{c}\begin{array}{c}\text { Lung } \\
\text { Absorption } \\
\text { Class/Type }\end{array} \\
\text { M }\end{array}$} & \multirow[t]{2}{*}{$\begin{array}{c}\text { Ingestion } \\
\text { DC } \\
\text { Reference }\end{array}$} & \multirow[t]{2}{*}{$\begin{array}{c}\text { HC-3 } \\
\text { Bounding } \\
\text { Ingestion } \\
\text { DC } \\
\text { (Sv/Bq) } \\
9.7 \mathrm{E}-10 \\
\end{array}$} & \multirow[t]{2}{*}{$\begin{array}{c}\text { Immersion } \\
\text { DC } \\
\text { Reference }\end{array}$} & \multirow[t]{2}{*}{$\begin{array}{c}\mathrm{HC}-3 \\
\text { Immersion } \\
\mathrm{DC}(\mathrm{Sv} / \mathrm{d} \\
\text { per } \mathrm{Bq} / \mathrm{m}^{3}\end{array}$} \\
\hline & & & & & 0.01 & $1000-10000$ & ICRP-38 & $3.63 \mathrm{E}+02$ & $\mathrm{~d}$ & & & & & & & & & \\
\hline Pm-145 & Pm-145 & 144.913 & Promethium & 0.01 & 0.01 & $1000-10000$ & ICRP-38 & $1.77 \mathrm{E}+01$ & $\mathrm{y}$ & $5.59 \mathrm{E}+08$ & $1.39 \mathrm{E}+02$ & ICRP-68 & $3.4 \mathrm{E}-09$ & $\mathrm{M}$ & ICRP-68 & $1.1 \mathrm{E}-10$ & -- & -- \\
\hline $\mathrm{Pm}-146$ & Pm-146 & 145.915 & Promethium & 0.01 & 0.01 & $1000-10000$ & ICRP-38 & $2.02 \mathrm{E}+03$ & $d$ & $1.75 E+08$ & $4.43 \mathrm{E}+02$ & ICRP-68 & $1.9 \mathrm{E}-08$ & $M$ & ICRP-68 & $9.0 \mathrm{E}-10$ & -- & -- \\
\hline Pm-147 & Pm-147 & 146.915 & Promethium & 0.01 & 0.01 & $1000-10000$ & ICRP-38 & $2.62 \mathrm{E}+00$ & $y$ & $8.28 \mathrm{E}+07$ & $9.28 \mathrm{E}+02$ & ICRP-68 & $4.7 \mathrm{E}-09$ & $M$ & ICRP-68 & $2.6 \mathrm{E}-10$ & -- & -- \\
\hline Pm-148 & Pm-148 & 147.917 & Promethium & 0.01 & 0.01 & $1000-10000$ & ICRP-38 & $5.37 \mathrm{E}+00$ & $d$ & $4.64 \mathrm{E}+05$ & $1.64 \mathrm{E}+05$ & ICRP-68 & $2.2 \mathrm{E}-09$ & $\mathrm{~s}$ & ICRP-68 & $2.7 \mathrm{E}-09$ & -- & -- \\
\hline Pm-148m & Pm-148 & 147.917 & Promethium & 0.01 & 0.01 & $1000-10000$ & ICRP-38 & $4.13 \mathrm{E}+01$ & $d$ & $3.57 E+06$ & $2.14 E+04$ & ICRP-68 & $5.4 \mathrm{E}-09$ & $\mathrm{~s}$ & ICRP-68 & $1.8 \mathrm{E}-09$ & -- & -- \\
\hline Pm-149 & Pm-149 & 148.918 & Promethium & 0.01 & 0.01 & $1000-10000$ & ICRP-38 & $5.31 \mathrm{E}+01$ & $\mathrm{~h}$ & $1.91 \mathrm{E}+05$ & $3.96 \mathrm{E}+05$ & ICRP-68 & $8.2 \mathrm{E}-10$ & $\mathrm{~s}$ & ICRP-68 & $9.9 \mathrm{E}-10$ & -- & -- \\
\hline Pm-150 & Pm-150 & 149.921 & Promethium & 0.01 & 0.01 & $1000-10000$ & ICRP-38 & $2.68 \mathrm{E}+00$ & $\mathrm{~h}$ & $9.65 \mathrm{E}+03$ & $7.80 \mathrm{E}+06$ & ICRP-68 & $2.1 \mathrm{E}-10$ & $\mathrm{~s}$ & ICRP-68 & $2.6 \mathrm{E}-10$ & -- & -- \\
\hline Pm-151 & Pm-151 & 150.921 & Promethium & 0.01 & 0.01 & $1000-10000$ & ICRP-38 & $2.84 \mathrm{E}+01$ & $\mathrm{~h}$ & $1.02 E+05$ & $7.31 \mathrm{E}+05$ & ICRP-68 & $6.4 \mathrm{E}-10$ & $\mathrm{~s}$ & ICRP-68 & $7.3 \mathrm{E}-10$ & -- & -- \\
\hline $\mathrm{Pm}-152$ & Pm-152 & 151.924 & Promethium & 0.01 & 0.01 & $1000-10000$ & ICRP-107 & $4.12 \mathrm{E}+00$ & $\mathrm{~m}$ & $2.47 E+02$ & $3.00 \mathrm{E}+08$ & -- & -- & -- & -- & -- & -- & -- \\
\hline Pm-152m & Pm-152 & 151.924 & Promethium & 0.01 & 0.01 & $1000-10000$ & ICRP-107 & $7.52 \mathrm{E}+00$ & $\mathrm{~m}$ & $4.51 \mathrm{E}+02$ & $1.65 \mathrm{E}+08$ & -- & -- & -- & -- & -- & -- & -- \\
\hline Pm-153 & Pm-153 & 152.924 & Promethium & 0.01 & 0.01 & $1000-10000$ & ICRP-107 & $5.25 \mathrm{E}+00$ & $\mathrm{~m}$ & $3.15 \mathrm{E}+02$ & $2.34 \mathrm{E}+08$ & -- & -- & -- & -- & -- & -- & -- \\
\hline Pm-154 & Pm-154 & 153.926 & Promethium & 0.01 & 0.01 & $1000-10000$ & ICRP-107 & $1.73 \mathrm{E}+00$ & $\mathrm{~m}$ & $1.04 E+02$ & $7.06 \mathrm{E}+08$ & -- & -- & -- & -- & -- & -- & -- \\
\hline Pm-154m & Pm-154 & 153.926 & Promethium & 0.01 & 0.01 & $1000-10000$ & ICRP-107 & $2.68 \mathrm{E}+00$ & $\mathrm{~m}$ & $1.61 \mathrm{E}+02$ & $4.56 \mathrm{E}+08$ & -- & -- & -- & -- & -- & -- & -- \\
\hline Po-203 & Po-203 & 202.981 & Polonium & 0.01 & 0.0025 & 25 & ICRP-38 & $3.67 \mathrm{E}+01$ & $\mathrm{~m}$ & $2.20 \mathrm{E}+03$ & $2.52 \mathrm{E}+07$ & ICRP-68 & $6.1 \mathrm{E}-11$ & $\mathrm{M}$ & ICRP-68 & $5.2 \mathrm{E}-11$ & -- & -- \\
\hline Po-204 & Po-204 & 203.980 & $\begin{array}{l}\text { Polonium } \\
\end{array}$ & 0.01 & 0.0025 & 25 & ICRP-107 & $3.53 \mathrm{E}+00$ & $\mathrm{~h}$ & $1.27 E+04$ & $4.35 \mathrm{E}+06$ & JAERI & $3.8 \mathrm{E}-10$ & $\mathrm{M}$ & JAERI & $3.1 \mathrm{E}-10$ & -- & -- \\
\hline Po-205 & Po-205 & 204.981 & Polonium & 0.01 & 0.0025 & 25 & ICRP-38 & $1.80 \mathrm{E}+00$ & $\mathrm{~h}$ & $6.48 \mathrm{E}+03$ & $8.49 \mathrm{E}+06$ & ICRP-68 & $8.9 \mathrm{E}-11$ & $M$ & ICRP-68 & $5.9 \mathrm{E}-11$ & -- & -- \\
\hline Po-206 & Po-206 & 205.980 & Polonium & 0.01 & 0.0025 & 25 & ICRP-107 & $8.80 \mathrm{E}+00$ & $d$ & $7.60 E+05$ & $7.20 \mathrm{E}+04$ & JAERI & $5.2 \mathrm{E}-08$ & $M$ & JAERI & $4.6 \mathrm{E}-09$ & -- & -- \\
\hline Po-207 & Po-207 & 206.982 & $\begin{array}{l}\text { Polonium } \\
\end{array}$ & 0.01 & 0.0025 & 25 & ICRP-38 & $3.50 \mathrm{E}+02$ & $\mathrm{~m}$ & $2.10 E+04$ & $2.60 \mathrm{E}+06$ & ICRP-68 & $1.5 \mathrm{E}-10$ & $M$ & ICRP-68 & $1.4 \mathrm{E}-10$ & -- & -- \\
\hline Po-208 & Po-208 & 207.981 & Polonium & 0.01 & 0.0025 & 25 & ICRP-107 & $2.90 \mathrm{E}+00$ & $y$ & $9.15 \mathrm{E}+07$ & $5.93 \mathrm{E}+02$ & JAERI & $3.5 \mathrm{E}-06$ & $M$ & JAERI & $3.1 \mathrm{E}-07$ & -- & -- \\
\hline Po-209 & Po-209 & 208.982 & Polonium & 0.01 & 0.0025 & 25 & ICRP-38 & $1.02 \mathrm{E}+02$ & $y$ & $3.22 \mathrm{E}+09$ & $1.68 \mathrm{E}+01$ & JAERI & $3.4 \mathrm{E}-06$ & $M$ & JAERI & $3.0 \mathrm{E}-07$ & -- & -- \\
\hline Po-210 & Po-210 & 209.983 & Polonium & 0.01 & 0.0025 & 25 & ICRP-38 & $1.38 \mathrm{E}+02$ & $d$ & $1.20 E+07$ & $4.49 \mathrm{E}+03$ & ICRP-68 & $3.0 \mathrm{E}-06$ & $M$ & ICRP-68 & $2.4 \mathrm{E}-07$ & -- & -- \\
\hline Po-211 & Po-211 & 210.987 & Polonium & 0.01 & 0.0025 & 25 & ICRP-38 & $5.16 \mathrm{E}-01$ & $\mathrm{~s}$ & $5.16 \mathrm{E}-01$ & $1.04 \mathrm{E}+11$ & -- & -- & -- & -- & -- & -- & -- \\
\hline Po-212 & Po-212 & 211.989 & Polonium & 0.01 & 0.0025 & 25 & ICRP-38 & $3.05 \mathrm{E}-01$ & us & $3.05 \mathrm{E}-07$ & $1.74 \mathrm{E}+17$ & -- & -- & -- & -- & -- & -- & -- \\
\hline Po-212m & Po-212 & 211.989 & $\begin{array}{l}\text { Polonium } \\
\end{array}$ & 0.01 & 0.0025 & 25 & ICRP-107 & $4.51 \mathrm{E}+01$ & $\mathrm{~s}$ & $4.51 E+01$ & $1.18 \mathrm{E}+09$ & -- & -- & -- & -- & -- & -- & -- \\
\hline Po-213 & Po-213 & 212.993 & Polonium & 0.01 & 0.0025 & 25 & ICRP-38 & $4.20 \mathrm{E}+00$ & us & $4.20 \mathrm{E}-06$ & $1.26 \mathrm{E}+16$ & -- & -- & -- & -- & -- & -- & -- \\
\hline Po-214 & Po-214 & 213.995 & Polonium & 0.01 & 0.0025 & 25 & ICRP-38 & $1.64 \mathrm{E}+02$ & us & $1.64 \mathrm{E}-04$ & $3.21 \mathrm{E}+14$ & -- & -- & -- & -- & -- & -- & -- \\
\hline Po-215 & Po-215 & 214.999 & Polonium & 0.01 & 0.0025 & 25 & ICRP-38 & $1.78 \mathrm{E}-03$ & $\mathrm{~s}$ & $1.78 \mathrm{E}-03$ & $2.95 \mathrm{E}+13$ & -- & -- & -- & -- & -- & -- & -- \\
\hline Po-216 & Po-216 & 216.002 & $\begin{array}{l}\text { Polonium } \\
\end{array}$ & 0.01 & 0.0025 & 25 & ICRP-38 & $1.50 \mathrm{E}-01$ & $\mathrm{~s}$ & $1.50 \mathrm{E}-01$ & $3.48 \mathrm{E}+11$ & -- & -- & -- & -- & -- & -- & -- \\
\hline Po-218 & Po-218 & 218.009 & Polonium & 0.01 & 0.0025 & 25 & ICRP-38 & $3.05 \mathrm{E}+00$ & $\mathrm{~m}$ & $1.83 \mathrm{E}+02$ & $2.83 E+08$ & -- & -- & -- & -- & -- & -- & -- \\
\hline Pr-134 & Pr-134 & 133.916 & Praseodymium & 0.01 & 0.01 & $500-1000$ & ICRP-107 & $1.10 \mathrm{E}+01$ & $\mathrm{~m}$ & $6.60 \mathrm{E}+02$ & $1.28 \mathrm{E}+08$ & JAERI & $5.0 \mathrm{E}-11$ & $\mathrm{~s}$ & JAERI & $8.1 \mathrm{E}-11$ & -- & -- \\
\hline Pr-134m & Pr-134 & 133.916 & Praseodymium & 0.01 & 0.01 & $500-1000$ & ICRP-107 & $1.70 \mathrm{E}+01$ & $\mathrm{~m}$ & $1.02 \mathrm{E}+03$ & $8.26 \mathrm{E}+07$ & JAERI & $3.4 \mathrm{E}-11$ & $\mathrm{~s}$ & JAERI & $4.6 \mathrm{E}-11$ & -- & -- \\
\hline Pr-135 & Pr-135 & 134.913 & Praseodymium & 0.01 & 0.01 & $500-1000$ & ICRP-107 & $2.40 \mathrm{E}+01$ & $\mathrm{~m}$ & $1.44 \mathrm{E}+03$ & $5.81 \mathrm{E}+07$ & JAERI & $3.6 \mathrm{E}-11$ & $\mathrm{~s}$ & JAERI & $4.6 \mathrm{E}-11$ & -- & -- \\
\hline Pr-136 & Pr-136 & 135.913 & Praseodymium & 0.01 & 0.01 & $500-1000$ & ICRP-38 & $1.31 \mathrm{E}+01$ & $\mathrm{~m}$ & $7.86 \mathrm{E}+02$ & $1.06 \mathrm{E}+08$ & ICRP-68 & $2.5 \mathrm{E}-11$ & $\mathrm{~s}$ & ICRP-68 & $3.3 \mathrm{E}-11$ & -- & -- \\
\hline Pr-137 & Pr-137 & 136.911 & Praseodymium & 0.01 & 0.01 & $500-1000$ & ICRP-38 & $7.66 \mathrm{E}+01$ & $\mathrm{~m}$ & $4.60 \mathrm{E}+03$ & $1.79 \mathrm{E}+07$ & ICRP-68 & $3.5 \mathrm{E}-11$ & $\mathrm{~s}$ & ICRP-68 & $4.0 \mathrm{E}-11$ & -- & -- \\
\hline Pr-138 & Pr-138 & 137.911 & Praseodymium & 0.01 & 0.01 & $500-1000$ & ICRP-38 & $1.45 \mathrm{E}+00$ & $\mathrm{~m}$ & $8.70 E+01$ & $9.40 \mathrm{E}+08$ & -- & -- & -- & -- & -- & -- & -- \\
\hline Pr-138m & Pr-138 & 137.911 & Praseodymium & 0.01 & 0.01 & $500-1000$ & ICRP-38 & $2.10 \mathrm{E}+00$ & $\mathrm{~h}$ & $7.56 \mathrm{E}+03$ & $1.08 \mathrm{E}+07$ & ICRP-68 & $1.3 \mathrm{E}-10$ & $M$ & ICRP-68 & $1.3 \mathrm{E}-10$ & -- & -- \\
\hline Pr-139 & Pr-139 & 138.909 & Praseodymium & 0.01 & 0.01 & $500-1000$ & ICRP-38 & $4.51 \mathrm{E}+00$ & $\mathrm{~h}$ & $1.62 E+04$ & $5.00 \mathrm{E}+06$ & ICRP-68 & $3.0 \mathrm{E}-11$ & $\mathrm{~s}$ & ICRP-68 & $3.1 \mathrm{E}-11$ & -- & -- \\
\hline Pr-140 & Pr-140 & 139.909 & Praseodymium & 0.01 & 0.01 & $500-1000$ & ICRP-107 & $3.39 \mathrm{E}+00$ & $\mathrm{~m}$ & $2.03 E+02$ & $3.96 \mathrm{E}+08$ & -- & -- & -- & -- & -- & -- & -- \\
\hline Pr-142 & Pr-142 & 141.910 & Praseodymium & 0.01 & 0.01 & $500-1000$ & ICRP-38 & $1.91 \mathrm{E}+01$ & $\mathrm{~h}$ & $6.89 \mathrm{E}+04$ & $1.15 \mathrm{E}+06$ & ICRP-68 & $7.4 \mathrm{E}-10$ & $\mathrm{~s}$ & ICRP-68 & $1.3 \mathrm{E}-09$ & -- & -- \\
\hline Pr-142m & Pr-142 & 141.910 & Praseodymium & 0.01 & 0.01 & $500-1000$ & ICRP-38 & $1.46 \mathrm{E}+01$ & $\mathrm{~m}$ & $8.76 \mathrm{E}+02$ & $9.08 \mathrm{E}+07$ & ICRP-68 & $9.4 \mathrm{E}-12$ & $\mathrm{~s}$ & ICRP-68 & $1.7 \mathrm{E}-11$ & -- & -- \\
\hline Pr-143 & Pr-143 & 142.911 & Praseodymium & 0.01 & 0.01 & $500-1000$ & ICRP-38 & $1.36 \mathrm{E}+01$ & d & $1.17 E+06$ & $6.74 \mathrm{E}+04$ & ICRP-68 & $2.3 \mathrm{E}-09$ & $\mathrm{~s}$ & ICRP-68 & $1.2 \mathrm{E}-09$ & -- & -- \\
\hline Pr-144 & Pr-144 & 143.913 & Praseodymium & 0.01 & 0.01 & $500-1000$ & ICRP-38 & $1.73 \mathrm{E}+01$ & $\mathrm{~m}$ & $1.04 \mathrm{E}+03$ & $7.56 \mathrm{E}+07$ & ICRP-68 & $3.0 \mathrm{E}-11$ & $\mathrm{~s}$ & ICRP-68 & $5.0 \mathrm{E}-11$ & -- & -- \\
\hline Pr-144m & Pr-144 & 143.913 & Praseodymium & 0.01 & 0.01 & $500-1000$ & ICRP-38 & $7.20 \mathrm{E}+00$ & $\mathrm{~m}$ & $4.32 \mathrm{E}+02$ & $1.81 \mathrm{E}+08$ & -- & -- & -- & -- & -- & -- & -- \\
\hline Pr-145 & Pr-145 & 144.915 & Praseodymium & 0.01 & 0.01 & $500-1000$ & ICRP-38 & $5.98 \mathrm{E}+00$ & $\mathrm{~h}$ & $2.15 E+04$ & $3.62 \mathrm{E}+06$ & ICRP-68 & $2.6 \mathrm{E}-10$ & $\mathrm{~s}$ & ICRP-68 & $3.9 \mathrm{E}-10$ & -- & -- \\
\hline
\end{tabular}




\begin{tabular}{|c|c|c|c|c|c|c|c|c|c|c|c|c|c|c|c|c|c|c|}
\hline \multirow{3}{*}{$\begin{array}{l}\text { MASTER } \\
\text { ISOTOPE } \\
\text { LIST } \\
\text { Pr-146 }\end{array}$} & \multirow{3}{*}{$\begin{array}{c}\begin{array}{c}\text { Atomic } \\
\text { Mass } \\
\text { Nuclide }\end{array} \\
\text { Pr-146 }\end{array}$} & & \multirow{3}{*}{$\begin{array}{c}\text { Element } \\
\text { Praseodymium }\end{array}$} & & \multicolumn{14}{|c|}{ Table C.1 Radionuclide S } \\
\hline & & \multirow{2}{*}{$\begin{array}{c}\begin{array}{c}\text { Atomic } \\
\text { Mass }\end{array} \\
145.918\end{array}$} & & \multirow{2}{*}{$\begin{array}{c}\text { EPA } \\
\text { RF } \\
0.01\end{array}$} & $\begin{array}{c}\text { EPA } \\
\text { Conc } \\
\text { Factors } \\
\left(B_{v}\right) \\
\end{array}$ & $\begin{array}{c}\text { EPA } \\
\text { Sorption } \\
\text { Coefficient } \\
\left(K_{d}\right)\end{array}$ & $\begin{array}{c}\text { Half-Life } \\
\text { Reference }\end{array}$ & $\begin{array}{r}\text { Half-Lif } \\
\text { (original u }\end{array}$ & & $\begin{array}{l}\text { Half Life } \\
\text { (s) }\end{array}$ & $\begin{array}{l}\text { Sp. Act } \\
\text { (Ci/g) }\end{array}$ & $\begin{array}{c}\text { Inhalation } \\
\text { DC } \\
\text { Reference }\end{array}$ & $\begin{array}{c}\text { HC-3 } \\
\text { Bounding } \\
\text { Inhalation } \\
\text { DC } \\
\text { (Sv/Bq) } \\
\end{array}$ & $\begin{array}{c}\text { Lung } \\
\text { Absorption } \\
\text { Class/Type }\end{array}$ & $\begin{array}{l}\text { Ingestion } \\
\text { DC } \\
\text { Reference }\end{array}$ & $\begin{array}{c}\text { HC-3 } \\
\text { Bounding } \\
\text { Ingestion } \\
\mathrm{DC} \\
\text { (Sv/Bq) } \\
\end{array}$ & $\begin{array}{c}\text { Immersion } \\
\text { DC } \\
\text { Reference }\end{array}$ & $\begin{array}{c}\mathrm{HC}-3 \\
\text { Immersion } \\
\mathrm{DC}(\mathrm{Sv} / \mathrm{d} \\
\left.\operatorname{per} \mathrm{Bq} / \mathrm{m}^{3}\right)\end{array}$ \\
\hline & & & & & 0.01 & $500-1000$ & ICRP-107 & $2.42 \mathrm{E}+01$ & $\mathrm{~m}$ & $1.45 \mathrm{E}+03$ & $5.34 \mathrm{E}+07$ & JAERI & $4.9 \mathrm{E}-11$ & $\mathrm{~s}$ & JAERI & $7.8 \mathrm{E}-11$ & -- & -- \\
\hline Pr-147 & Pr-147 & 146.919 & Praseodymium & 0.01 & 0.01 & $500-1000$ & ICRP-38 & $1.36 \mathrm{E}+01$ & $\mathrm{~m}$ & $8.16 \mathrm{E}+02$ & $9.41 \mathrm{E}+07$ & ICRP-68 & $3.0 \mathrm{E}-11$ & $\mathrm{~s}$ & ICRP-68 & $3.3 \mathrm{E}-11$ & -- & -- \\
\hline Pr-148 & Pr-148 & 147.922 & Praseodymium & 0.01 & 0.01 & $500-1000$ & ICRP-107 & $2.29 \mathrm{E}+00$ & $\mathrm{~m}$ & $1.37 E+02$ & $5.55 \mathrm{E}+08$ & -- & -- & -- & -- & -- & -- & -- \\
\hline Pr-148m & Pr-148 & 147.922 & Praseodymium & 0.01 & 0.01 & $500-1000$ & ICRP-107 & $2.01 \mathrm{E}+00$ & $\mathrm{~m}$ & $1.21 \mathrm{E}+02$ & $6.32 \mathrm{E}+08$ & -- & -- & -- & -- & -- & -- & -- \\
\hline Pt-183 & Pt-183 & 182.962 & Platinum & 0.01 & 0.095 & 0 & JAERI & $6.50 \mathrm{E}+00$ & $\mathrm{~m}$ & $3.90 \mathrm{E}+02$ & $1.58 \mathrm{E}+08$ & -- & -- & -- & -- & -- & -- & -- \\
\hline Pt-184 & Pt-184 & 183.960 & Platinum & 0.01 & 0.095 & 0 & ICRP-107 & $1.73 \mathrm{E}+01$ & $\mathrm{~m}$ & $1.04 \mathrm{E}+03$ & $5.91 \mathrm{E}+07$ & JAERI & $2.6 \mathrm{E}-11$ & $\mathrm{~F}$ & JAERI & $3.0 \mathrm{E}-11$ & -- & -- \\
\hline Pt-186 & Pt-186 & 185.959 & Platinum & 0.01 & 0.095 & 0 & ICRP-38 & $2.00 \mathrm{E}+00$ & $\mathrm{~h}$ & $7.20 \mathrm{E}+03$ & $8.43 \mathrm{E}+06$ & ICRP-68 & $6.6 \mathrm{E}-11$ & $\mathrm{~F}$ & ICRP-68 & $9.3 \mathrm{E}-11$ & -- & -- \\
\hline Pt-187 & Pt-187 & 186.961 & Platinum & 0.01 & 0.095 & 0 & ICRP-107 & $2.35 \mathrm{E}+00$ & $\mathrm{~h}$ & $8.46 \mathrm{E}+03$ & $7.13 \mathrm{E}+06$ & JAERI & $6.0 \mathrm{E}-11$ & $\mathrm{~F}$ & JAERI & $8.8 \mathrm{E}-11$ & -- & -- \\
\hline Pt-188 & Pt-188 & 187.959 & Platinum & 0.01 & 0.095 & 0 & $\begin{array}{l}\text { ICRP-38 } \\
\end{array}$ & $1.02 \mathrm{E}+01$ & $d$ & $8.81 \mathrm{E}+05$ & $6.81 \mathrm{E}+04$ & ICRP-68 & $6.3 \mathrm{E}-10$ & $\mathrm{~F}$ & ICRP-68 & $7.6 \mathrm{E}-10$ & -- & -- \\
\hline Pt-189 & Pt-189 & 188.961 & Platinum & 0.01 & 0.095 & 0 & ICRP-38 & $1.09 \mathrm{E}+01$ & $\mathrm{~h}$ & $3.91 \mathrm{E}+04$ & $1.53 \mathrm{E}+06$ & ICRP-68 & $7.3 \mathrm{E}-11$ & $F$ & ICRP-68 & $1.2 \mathrm{E}-10$ & -- & -- \\
\hline Pt-190 & Pt-190 & 189.960 & Platinum & 0.01 & 0.095 & 0 & ICRP-107 & $6.50 \mathrm{E}+11$ & $y$ & $2.05 \mathrm{E}+19$ & $2.90 \mathrm{E}-09$ & JAERI & $1.3 \mathrm{E}-07$ & $\mathrm{~F}$ & JAERI & $6.8 \mathrm{E}-09$ & -- & -- \\
\hline Pt-191 & Pt-191 & 190.962 & Platinum & 0.01 & 0.095 & 0 & $\begin{array}{l}\text { ICRP-38 } \\
\end{array}$ & $2.80 \mathrm{E}+00$ & $d$ & $2.42 \mathrm{E}+05$ & $2.44 \mathrm{E}+05$ & ICRP-68 & $1.9 \mathrm{E}-10$ & $\mathrm{~F}$ & ICRP-68 & $3.4 \mathrm{E}-10$ & -- & -- \\
\hline Pt-193 & Pt-193 & 192.963 & Platinum & 0.01 & 0.095 & 0 & ICRP-38 & $5.00 \mathrm{E}+01$ & $y$ & $1.58 \mathrm{E}+09$ & $3.71 \mathrm{E}+01$ & $\begin{array}{l}\text { ICRP-68 } \\
\end{array}$ & $2.7 \mathrm{E}-11$ & $F$ & ICRP-68 & $3.1 \mathrm{E}-11$ & -- & -- \\
\hline Pt-193m & Pt-193 & 192.963 & Platinum & 0.01 & 0.095 & 0 & ICRP-38 & $4.33 \mathrm{E}+00$ & $d$ & $3.74 \mathrm{E}+05$ & $1.56 \mathrm{E}+05$ & ICRP-68 & $2.1 \mathrm{E}-10$ & $\mathrm{~F}$ & ICRP-68 & $4.5 E-10$ & -- & -- \\
\hline Pt-195m & Pt-195 & 194.965 & Platinum & 0.01 & 0.095 & 0 & ICRP-38 & $4.02 \mathrm{E}+00$ & $d$ & $3.47 \mathrm{E}+05$ & $1.67 E+05$ & ICRP-68 & $3.1 \mathrm{E}-10$ & $F$ & ICRP-68 & $6.3 \mathrm{E}-10$ & -- & -- \\
\hline Pt-197 & Pt-197 & 196.967 & Platinum & 0.01 & 0.095 & 0 & ICRP-38 & $1.83 \mathrm{E}+01$ & $\mathrm{~h}$ & $6.59 \mathrm{E}+04$ & $8.69 \mathrm{E}+05$ & ICRP-68 & $1.6 \mathrm{E}-10$ & $\mathrm{~F}$ & ICRP-68 & $4.0 \mathrm{E}-10$ & -- & -- \\
\hline Pt-197m & Pt-197 & 196.967 & Platinum & 0.01 & 0.095 & 0 & ICRP-38 & $9.44 \mathrm{E}+01$ & $\mathrm{~m}$ & $5.66 \mathrm{E}+03$ & $1.01 \mathrm{E}+07$ & ICRP-68 & $4.3 \mathrm{E}-11$ & $\mathrm{~F}$ & ICRP-68 & $8.4 \mathrm{E}-11$ & -- & -- \\
\hline Pt-199 & Pt-199 & 198.971 & Platinum & 0.01 & 0.095 & 0 & ICRP-38 & $3.08 \mathrm{E}+01$ & $\mathrm{~m}$ & $1.85 \mathrm{E}+03$ & $3.07 \mathrm{E}+07$ & ICRP-68 & $2.2 \mathrm{E}-11$ & $F$ & ICRP-68 & $3.9 \mathrm{E}-11$ & -- & -- \\
\hline Pt-200 & Pt-200 & 199.971 & Platinum & 0.01 & 0.095 & 0 & ICRP-38 & $1.25 \mathrm{E}+01$ & $\mathrm{~h}$ & $4.50 \mathrm{E}+04$ & $1.25 \mathrm{E}+06$ & ICRP-68 & $4.0 \mathrm{E}-10$ & $\mathrm{~F}$ & ICRP-68 & $1.2 \mathrm{E}-09$ & -- & -- \\
\hline Pt-202 & Pt-202 & 201.976 & $\begin{array}{l}\text { Platinum } \\
\end{array}$ & 0.01 & 0.095 & 0 & ICRP-107 & $4.40 \mathrm{E}+01$ & $\mathrm{~h}$ & $1.58 \mathrm{E}+05$ & $3.53 \mathrm{E}+05$ & JAERI & $1.4 \mathrm{E}-09$ & $\mathrm{~F}$ & JAERI & $4.5 \mathrm{E}-09$ & -- & -- \\
\hline Pu-232 & Pu-232 & 232.041 & Plutonium & 0.001 & 0.00045 & $100-10000$ & ICRP-107 & $3.37 \mathrm{E}+01$ & $\mathrm{~m}$ & $2.02 E+03$ & $2.40 \mathrm{E}+07$ & JAERI & $2.5 \mathrm{E}-08$ & $\mathrm{~s}$ & JAERI & $2.9 \mathrm{E}-10$ & -- & -- \\
\hline Pu-234 & Pu-234 & 234.043 & Plutonium & 0.001 & 0.00045 & $100-10000$ & ICRP-38 & $8.80 \mathrm{E}+00$ & $\mathrm{~h}$ & $3.17 E+04$ & $1.52 \mathrm{E}+06$ & ICRP-68 & $2.2 \mathrm{E}-08$ & $\mathrm{~s}$ & ICRP-68 & $1.6 \mathrm{E}-10$ & -- & -- \\
\hline Pu-235 & Pu-235 & 235.045 & Plutonium & 0.001 & 0.00045 & $100-10000$ & ICRP-38 & $2.53 \mathrm{E}+01$ & $\mathrm{~m}$ & $1.52 \mathrm{E}+03$ & $3.16 \mathrm{E}+07$ & ICRP-68 & $2.6 \mathrm{E}-12$ & $\mathrm{~s}$ & ICRP-68 & $2.1 \mathrm{E}-12$ & -- & -- \\
\hline Pu-236 & Pu-236 & 236.046 & Plutonium & 0.001 & 0.00045 & $100-10000$ & ICRP-38 & $2.85 \mathrm{E}+00$ & $y$ & $9.00 \mathrm{E}+07$ & $5.31 \mathrm{E}+02$ & ICRP-68 & $1.8 \mathrm{E}-05$ & $M$ & ICRP-68 & $8.6 \mathrm{E}-08$ & -- & -- \\
\hline Pu-237 & Pu-237 & 237.048 & Plutonium & 0.001 & 0.00045 & $100-10000$ & ICRP-38 & $4.53 \mathrm{E}+01$ & $d$ & $3.91 \mathrm{E}+06$ & $1.22 \mathrm{E}+04$ & ICRP-68 & $3.6 \mathrm{E}-10$ & $\mathrm{~s}$ & ICRP-68 & $1.0 \mathrm{E}-10$ & -- & -- \\
\hline Pu-238 & Pu-238 & 238.050 & Plutonium & 0.001 & 0.00045 & $100-10000$ & ICRP-38 & $8.77 \mathrm{E}+01$ & $y$ & $2.77 \mathrm{E}+09$ & $1.71 \mathrm{E}+01$ & ICRP-68 & $4.3 \mathrm{E}-05$ & $M$ & ICRP-68 & $2.3 \mathrm{E}-07$ & -- & -- \\
\hline Pu-239 & Pu-239 & 239.052 & Plutonium & 0.001 & 0.00045 & $100-10000$ & ICRP-38 & $2.41 \mathrm{E}+04$ & $y$ & $7.59 \mathrm{E}+11$ & $6.21 \mathrm{E}-02$ & ICRP-68 & $4.7 \mathrm{E}-05$ & $M$ & ICRP-68 & $2.5 \mathrm{E}-07$ & -- & -- \\
\hline Pu-240 & Pu-240 & 240.054 & Plutonium & 0.001 & 0.00045 & $100-10000$ & ICRP-38 & $6.54 \mathrm{E}+03$ & $y$ & $2.06 \mathrm{E}+11$ & $2.28 \mathrm{E}-01$ & ICRP-68 & 4.7E-05 & $M$ & ICRP-68 & $2.5 \mathrm{E}-07$ & -- & -- \\
\hline Pu-241 & Pu-241 & 241.057 & $\begin{array}{l}\text { Plutonium } \\
\end{array}$ & 0.001 & 0.00045 & $100-10000$ & ICRP-38 & $1.44 \mathrm{E}+01$ & $y$ & $4.54 \mathrm{E}+08$ & $1.03 \mathrm{E}+02$ & ICRP-68 & $8.5 \mathrm{E}-07$ & $\mathrm{M}$ & ICRP-68 & $4.7 \mathrm{E}-09$ & -- & -- \\
\hline Pu-242 & Pu-242 & 242.059 & Plutonium & 0.001 & 0.00045 & $100-10000$ & ICRP-38 & $3.76 \mathrm{E}+05$ & $y$ & $1.19 \mathrm{E}+13$ & $3.92 \mathrm{E}-03$ & ICRP-68 & $4.4 \mathrm{E}-05$ & $M$ & ICRP-68 & $2.4 \mathrm{E}-07$ & -- & -- \\
\hline Pu-243 & Pu-243 & 243.062 & Plutonium & 0.001 & 0.00045 & $100-10000$ & ICRP-38 & $4.96 \mathrm{E}+00$ & $h$ & $1.78 \mathrm{E}+04$ & $2.60 \mathrm{E}+06$ & ICRP-68 & $1.1 \mathrm{E}-10$ & $M$ & ICRP-68 & $8.5 \mathrm{E}-11$ & -- & -- \\
\hline Pu-244 & Pu-244 & 244.064 & Plutonium & 0.001 & 0.00045 & $100-10000$ & ICRP-38 & $8.26 \mathrm{E}+07$ & $y$ & $2.61 \mathrm{E}+15$ & $1.77 \mathrm{E}-05$ & ICRP-68 & $4.4 \mathrm{E}-05$ & $M$ & ICRP-68 & $2.4 \mathrm{E}-07$ & -- & -- \\
\hline Pu-245 & Pu-245 & 245.068 & Plutonium & 0.001 & 0.00045 & $100-10000$ & ICRP-38 & $1.05 \mathrm{E}+01$ & $\mathrm{~h}$ & $3.78 \mathrm{E}+04$ & $1.22 \mathrm{E}+06$ & ICRP-68 & $6.5 \mathrm{E}-10$ & $\mathrm{~s}$ & ICRP-68 & $7.2 \mathrm{E}-10$ & -- & -- \\
\hline Pu-246 & Pu-246 & 246.070 & $\begin{array}{l}\text { Plutonium } \\
\end{array}$ & 0.001 & 0.00045 & $100-10000$ & ICRP-38 & $1.09 \mathrm{E}+01$ & $d$ & $9.37 \mathrm{E}+05$ & $4.89 \mathrm{E}+04$ & ICRP-68 & $7.6 \mathrm{E}-09$ & $\mathrm{~s}$ & ICRP-68 & $3.3 \mathrm{E}-09$ & -- & -- \\
\hline Ra-219 & Ra-219 & 219.010 & Radium & 0.001 & 0.015 & $100-10000$ & ICRP-107 & $1.00 \mathrm{E}+01$ & $\mathrm{~ms}$ & $1.00 \mathrm{E}-02$ & $5.15 \mathrm{E}+12$ & -- & -- & -- & -- & -- & -- & -- \\
\hline Ra-220 & Ra-220 & 220.011 & Radium & 0.001 & 0.015 & $100-10000$ & ICRP-107 & $1.79 E-02$ & $\mathrm{~s}$ & $1.79 \mathrm{E}-02$ & $2.86 \mathrm{E}+12$ & -- & -- & -- & -- & -- & -- & -- \\
\hline Ra-221 & Ra-221 & 221.014 & Radium & 0.001 & 0.015 & $100-10000$ & ICRP-107 & $2.80 \mathrm{E}+01$ & $\mathrm{~s}$ & $2.80 \mathrm{E}+01$ & $1.82 \mathrm{E}+09$ & -- & -- & -- & -- & -- & -- & -- \\
\hline Ra-222 & Ra-222 & 222.015 & Radium & 0.001 & 0.015 & $100-10000$ & ICRP-38 & $3.80 \mathrm{E}+01$ & $\mathrm{~s}$ & $3.80 \mathrm{E}+01$ & $1.34 \mathrm{E}+09$ & -- & -- & -- & -- & -- & -- & -- \\
\hline Ra-223 & Ra-223 & 223.019 & Radium & 0.001 & 0.015 & $100-10000$ & ICRP-38 & $1.14 \mathrm{E}+01$ & $d$ & $9.88 \mathrm{E}+05$ & $5.12 \mathrm{E}+04$ & ICRP-68 & $6.9 \mathrm{E}-06$ & $M$ & ICRP-68 & $1.0 \mathrm{E}-07$ & -- & -- \\
\hline Ra-224 & Ra-224 & 224.020 & Radium & 0.001 & 0.015 & $100-10000$ & ICRP-38 & $3.66 \mathrm{E}+00$ & $d$ & $3.16 \mathrm{E}+05$ & $1.59 \mathrm{E}+05$ & ICRP-68 & $2.9 \mathrm{E}-06$ & $M$ & ICRP-68 & $6.5 \mathrm{E}-08$ & -- & -- \\
\hline Ra-225 & Ra-225 & 225.024 & Radium & 0.001 & 0.015 & $100-10000$ & ICRP-38 & $1.48 \mathrm{E}+01$ & $d$ & $1.28 \mathrm{E}+06$ & $3.92 \mathrm{E}+04$ & ICRP-68 & $5.8 \mathrm{E}-06$ & $\mathrm{M}$ & ICRP-68 & $9.5 \mathrm{E}-08$ & -- & -- \\
\hline Ra-226 & Ra-226 & 226.025 & Radium & 0.001 & 0.015 & $100-10000$ & ICRP-38 & $1.60 \mathrm{E}+03$ & $y$ & $5.05 \mathrm{E}+10$ & 9.89E-01 & ICRP-68 & $3.2 \mathrm{E}-06$ & $\mathrm{M}$ & ICRP-68 & $2.8 \mathrm{E}-07$ & -- & -- \\
\hline Ra-227 & Ra-227 & 227.029 & Radium & 0.001 & 0.015 & $100-10000$ & ICRP-38 & $4.22 \mathrm{E}+01$ & $\mathrm{~m}$ & $2.53 \mathrm{E}+03$ & $1.96 \mathrm{E}+07$ & ICRP-68 & $2.8 \mathrm{E}-10$ & $M$ & ICRP-68 & $8.4 \mathrm{E}-11$ & -- & -- \\
\hline Ra-228 & Ra-228 & 228.031 & Radium & 0.001 & 0.015 & $100-10000$ & ICRP-38 & $5.75 \mathrm{E}+00$ & $y$ & $1.81 \mathrm{E}+08$ & $2.73 \mathrm{E}+02$ & ICRP-68 & $2.6 \mathrm{E}-06$ & $M$ & ICRP-68 & $6.7 \mathrm{E}-07$ & -- & -- \\
\hline Ra-230 & Ra-230 & 230.037 & Radium & 0.001 & 0.015 & $100-10000$ & ICRP-107 & $9.30 \mathrm{E}+01$ & $\mathrm{~m}$ & $5.58 \mathrm{E}+03$ & $8.79 \mathrm{E}+06$ & JAERI & $1.7 \mathrm{E}-10$ & $M$ & JAERI & $1.9 \mathrm{E}-10$ & -- & -- \\
\hline
\end{tabular}




\begin{tabular}{|c|c|c|c|c|c|c|c|c|c|c|c|c|c|c|c|c|c|c|}
\hline \multirow{3}{*}{$\begin{array}{l}\text { MASTER } \\
\text { ISOTOPE } \\
\text { LIST } \\
\text { Rb-77 }\end{array}$} & \multirow{3}{*}{$\begin{array}{c}\begin{array}{c}\text { Atomic } \\
\text { Mass } \\
\text { Nuclide }\end{array} \\
\text { Rb-77 } \\
\end{array}$} & & & & \multicolumn{14}{|c|}{ Table C.1 Radionuclide Spe } \\
\hline & & \multirow{2}{*}{$\begin{array}{l}\begin{array}{c}\text { Atomic } \\
\text { Mass }\end{array} \\
76.930\end{array}$} & \multirow{2}{*}{$\begin{array}{l}\text { Element } \\
\text { Rubidium }\end{array}$} & \multirow{2}{*}{$\begin{array}{c}\text { EPA } \\
\text { RF } \\
0.01\end{array}$} & $\begin{array}{c}\text { EPA } \\
\text { Conc } \\
\text { Factors } \\
\left(B_{v}\right) \\
\end{array}$ & $\begin{array}{c}\text { EPA } \\
\text { Sorption } \\
\text { Coefficient } \\
\left(K_{d}\right)\end{array}$ & $\begin{array}{c}\text { Half-Life } \\
\text { Reference }\end{array}$ & $\begin{array}{r}\text { Half-Lif } \\
\text { (original u }\end{array}$ & & $\begin{array}{l}\text { Half Life } \\
\text { (s) }\end{array}$ & $\begin{array}{l}\text { Sp. Act } \\
\text { (Ci/g) }\end{array}$ & $\begin{array}{l}\text { Inhalation } \\
\text { DC } \\
\text { Reference }\end{array}$ & $\begin{array}{c}\text { HC-3 } \\
\text { Bounding } \\
\text { Inhalation } \\
\text { DC } \\
\text { (Sv/Bq) } \\
\end{array}$ & $\begin{array}{c}\text { Lung } \\
\text { Absorption } \\
\text { Class/Type }\end{array}$ & $\begin{array}{l}\text { Ingestion } \\
\text { DC } \\
\text { Reference }\end{array}$ & $\begin{array}{c}\text { HC-3 } \\
\text { Bounding } \\
\text { Ingestion } \\
\text { DC } \\
\text { (Sv/Bq) } \\
\end{array}$ & $\begin{array}{c}\text { Immersion } \\
\text { DC } \\
\text { Reference }\end{array}$ & $\begin{array}{c}\mathrm{HC}-3 \\
\text { Immersion } \\
\mathrm{DC}(\mathrm{Sv} / \mathrm{d} \\
\left.\operatorname{per} \mathrm{Bq} / \mathrm{m}^{3}\right)\end{array}$ \\
\hline & & & & & 0.15 & 500 & ICRP-38 & $3.70 \mathrm{E}+00$ & $\mathrm{~m}$ & $2.22 E+02$ & $6.61 \mathrm{E}+08$ & -- & -- & -- & -- & -- & -- & -- \\
\hline Rb-78 & Rb-78 & 77.928 & Rubidium & 0.01 & 0.15 & 500 & ICRP-107 & $1.77 \mathrm{E}+01$ & $\mathrm{~m}$ & $1.06 \mathrm{E}+03$ & $1.37 E+08$ & JAERI & $3.7 \mathrm{E}-11$ & $\mathrm{~F}$ & JAERI & $7.0 \mathrm{E}-11$ & -- & -- \\
\hline $\mathrm{Rb}-78 \mathrm{~m}$ & $\mathrm{Rb}-78$ & 77.928 & Rubidium & 0.01 & 0.15 & 500 & ICRP-107 & $5.74 \mathrm{E}+00$ & $\mathrm{~m}$ & $3.44 \mathrm{E}+02$ & $4.20 \mathrm{E}+08$ & -- & -- & -- & -- & -- & -- & -- \\
\hline $\mathrm{Rb}-79$ & $\mathrm{Rb}-79$ & 78.924 & Rubidium & 0.01 & 0.15 & 500 & ICRP-38 & $2.29 \mathrm{E}+01$ & $\mathrm{~m}$ & $1.37 \mathrm{E}+03$ & $1.04 \mathrm{E}+08$ & ICRP-68 & $3.0 \mathrm{E}-11$ & $\mathrm{~F}$ & ICRP-68 & $5.0 \mathrm{E}-11$ & -- & -- \\
\hline $\mathrm{Rb}-80$ & $\mathrm{Rb}-80$ & 79.923 & Rubidium & 0.01 & 0.15 & 500 & ICRP-38 & $3.40 \mathrm{E}+01$ & $\mathrm{~s}$ & $3.40 \mathrm{E}+01$ & $4.15 \mathrm{E}+09$ & -- & -- & -- & -- & -- & -- & -- \\
\hline $\mathrm{Rb}-81$ & $\mathrm{Rb}-81$ & 80.919 & Rubidium & 0.01 & 0.15 & 500 & ICRP-38 & $4.58 \mathrm{E}+00$ & $\mathrm{~h}$ & $1.65 \mathrm{E}+04$ & $8.46 \mathrm{E}+06$ & ICRP-68 & $6.8 \mathrm{E}-11$ & $\mathrm{~F}$ & ICRP-68 & $5.4 \mathrm{E}-11$ & -- & -- \\
\hline $\mathrm{Rb}-81 \mathrm{~m}$ & $\mathrm{Rb}-81$ & 80.919 & Rubidium & 0.01 & 0.15 & 500 & ICRP-38 & $3.20 \mathrm{E}+01$ & $\mathrm{~m}$ & $1.92 \mathrm{E}+03$ & $7.26 \mathrm{E}+07$ & ICRP-68 & $1.3 \mathrm{E}-11$ & $\mathrm{~F}$ & ICRP-68 & $9.7 \mathrm{E}-12$ & -- & -- \\
\hline $\mathrm{Rb}-82$ & $\mathrm{Rb}-82$ & 81.918 & Rubidium & 0.01 & 0.15 & 500 & ICRP-38 & $1.30 \mathrm{E}+00$ & $\mathrm{~m}$ & $7.80 \mathrm{E}+01$ & $1.77 E+09$ & -- & -- & -- & -- & -- & -- & -- \\
\hline $\mathrm{Rb}-82 \mathrm{~m}$ & $\mathrm{Rb}-82$ & 81.918 & Rubidium & 0.01 & 0.15 & 500 & ICRP-38 & $6.20 \mathrm{E}+00$ & $\mathrm{~h}$ & $2.23 \mathrm{E}+04$ & $6.17 E+06$ & ICRP-68 & $2.2 \mathrm{E}-10$ & $\mathrm{~F}$ & ICRP-68 & $1.3 \mathrm{E}-10$ & -- & -- \\
\hline $\mathrm{Rb}-83$ & $\mathrm{Rb}-83$ & 82.915 & Rubidium & 0.01 & 0.15 & 500 & ICRP-38 & $8.62 \mathrm{E}+01$ & $d$ & $7.45 \mathrm{E}+06$ & $1.83 \mathrm{E}+04$ & ICRP-68 & $1.0 \mathrm{E}-09$ & $F$ & ICRP-68 & $1.9 \mathrm{E}-09$ & -- & -- \\
\hline $\mathrm{Rb}-84$ & $\mathrm{Rb}-84$ & 83.914 & Rubidium & 0.01 & 0.15 & 500 & ICRP-38 & $3.28 \mathrm{E}+01$ & $d$ & $2.83 \mathrm{E}+06$ & $4.75 \mathrm{E}+04$ & ICRP-68 & $1.5 \mathrm{E}-09$ & $\mathrm{~F}$ & ICRP-68 & $2.8 \mathrm{E}-09$ & -- & -- \\
\hline $\mathrm{Rb}-84 \mathrm{~m}$ & $\mathrm{Rb}-84$ & 83.914 & Rubidium & 0.01 & 0.15 & 500 & ICRP-107 & $2.03 \mathrm{E}+01$ & $\mathrm{~m}$ & $1.22 \mathrm{E}+03$ & $1.11 \mathrm{E}+08$ & JAERI & $8.9 \mathrm{E}-12$ & $\mathrm{~F}$ & JAERI & $7.1 \mathrm{E}-12$ & -- & -- \\
\hline $\mathrm{Rb}-86$ & Rb-86 & 85.911 & Rubidium & 0.01 & 0.15 & 500 & ICRP-38 & $1.87 \mathrm{E}+01$ & $d$ & $1.61 \mathrm{E}+06$ & $8.14 \mathrm{E}+04$ & $\begin{array}{l}\text { ICRP-68 } \\
\end{array}$ & $1.3 \mathrm{E}-09$ & $F$ & ICRP-68 & $2.8 \mathrm{E}-09$ & -- & -- \\
\hline $\mathrm{Rb}-86 \mathrm{~m}$ & $\mathrm{Rb}-86$ & 85.911 & Rubidium & 0.01 & 0.15 & 500 & ICRP-107 & $1.02 \mathrm{E}+00$ & $\mathrm{~m}$ & $6.10 \mathrm{E}+01$ & $2.15 \mathrm{E}+09$ & -- & -- & -- & -- & -- & -- & -- \\
\hline $\mathrm{Rb}-87$ & $\mathrm{Rb}-87$ & 86.909 & Rubidium & 0.01 & 0.15 & 500 & ICRP-38 & $4.70 \mathrm{E}+10$ & $y$ & $1.48 \mathrm{E}+18$ & $8.75 \mathrm{E}-08$ & ICRP-68 & $7.6 \mathrm{E}-10$ & $F$ & ICRP-68 & $1.5 \mathrm{E}-09$ & -- & -- \\
\hline $\mathrm{Rb}-88$ & $\mathrm{Rb}-88$ & 87.911 & Rubidium & 0.01 & 0.15 & 500 & ICRP-38 & $1.78 \mathrm{E}+01$ & $\mathrm{~m}$ & $1.07 \mathrm{E}+03$ & $1.20 \mathrm{E}+08$ & ICRP-68 & $2.8 \mathrm{E}-11$ & $\mathrm{~F}$ & ICRP-68 & $9.0 \mathrm{E}-11$ & -- & -- \\
\hline $\mathrm{Rb}-89$ & $\mathrm{Rb}-89$ & 88.912 & Rubidium & 0.01 & 0.15 & 500 & ICRP-38 & $1.52 \mathrm{E}+01$ & $\mathrm{~m}$ & $9.12 \mathrm{E}+02$ & $1.39 \mathrm{E}+08$ & ICRP-68 & $2.5 \mathrm{E}-11$ & $\mathrm{~F}$ & ICRP-68 & $4.7 \mathrm{E}-11$ & -- & -- \\
\hline Rb-90 & $\mathrm{Rb}-90$ & 89.915 & Rubidium & 0.01 & 0.15 & 500 & ICRP-107 & $1.58 \mathrm{E}+02$ & $\mathrm{~s}$ & $1.58 \mathrm{E}+02$ & $7.94 \mathrm{E}+08$ & -- & -- & -- & -- & -- & -- & -- \\
\hline $\mathrm{Rb}-90 \mathrm{~m}$ & $\mathrm{Rb}-90$ & 89.915 & Rubidium & 0.01 & 0.15 & 500 & ICRP-107 & $2.58 \mathrm{E}+02$ & $\mathrm{~s}$ & $2.58 \mathrm{E}+02$ & $4.86 \mathrm{E}+08$ & -- & -- & -- & -- & -- & -- & -- \\
\hline Re-177 & Re-177 & 176.950 & Rhenium & 0.01 & 1.5 & 0 & ICRP-38 & $1.40 \mathrm{E}+01$ & $\mathrm{~m}$ & $8.40 \mathrm{E}+02$ & $7.59 \mathrm{E}+07$ & ICRP-68 & $2.2 \mathrm{E}-11$ & $M$ & ICRP-68 & $2.2 \mathrm{E}-11$ & -- & -- \\
\hline Re-178 & Re-178 & 177.951 & Rhenium & 0.01 & 1.5 & 0 & ICRP-38 & $1.32 \mathrm{E}+01$ & $\mathrm{~m}$ & $7.92 E+02$ & $8.00 \mathrm{E}+07$ & ICRP-68 & $2.4 \mathrm{E}-11$ & $M$ & ICRP-68 & $2.5 \mathrm{E}-11$ & -- & -- \\
\hline Re-179 & Re-179 & 178.950 & Rhenium & 0.01 & 1.5 & 0 & ICRP-107 & $1.95 \mathrm{E}+01$ & $\mathrm{~m}$ & $1.17 \mathrm{E}+03$ & $5.39 \mathrm{E}+07$ & JAERI & $2.1 \mathrm{E}-11$ & $M$ & JAERI & $1.6 \mathrm{E}-11$ & -- & -- \\
\hline Re-180 & Re-180 & 179.951 & Rhenium & 0.01 & 1.5 & 0 & ICRP-38 & $2.43 \mathrm{E}+00$ & $\mathrm{~m}$ & $1.46 \mathrm{E}+02$ & $4.30 \mathrm{E}+08$ & -- & -- & -- & -- & -- & -- & -- \\
\hline Re-181 & Re-181 & 180.950 & Rhenium & 0.01 & 1.5 & 0 & ICRP-38 & $2.00 \mathrm{E}+01$ & $\mathrm{~h}$ & $7.20 \mathrm{E}+04$ & $8.66 \mathrm{E}+05$ & ICRP-68 & $3.7 \mathrm{E}-10$ & $M$ & ICRP-68 & $4.2 \mathrm{E}-10$ & -- & -- \\
\hline Re-182I & Re-182 & 181.951 & Rhenium & 0.01 & 1.5 & 0 & ICRP-38 & $6.40 \mathrm{E}+01$ & $\mathrm{~h}$ & $2.30 \mathrm{E}+05$ & $2.69 \mathrm{E}+05$ & ICRP-68 & $1.7 \mathrm{E}-09$ & $M$ & ICRP-68 & $1.4 \mathrm{E}-09$ & -- & -- \\
\hline Re-182s & Re-182 & 181.951 & Rhenium & 0.01 & 1.5 & 0 & ICRP-38 & $1.27 \mathrm{E}+01$ & $\mathrm{~h}$ & $4.57 \mathrm{E}+04$ & $1.36 \mathrm{E}+06$ & ICRP-68 & $3.0 \mathrm{E}-10$ & $M$ & ICRP-68 & $2.7 \mathrm{E}-10$ & -- & -- \\
\hline Re-183 & Re-183 & 182.951 & Rhenium & 0.01 & 1.5 & 0 & ICRP-107 & $7.00 \mathrm{E}+01$ & $d$ & $6.05 \mathrm{E}+06$ & $1.02 \mathrm{E}+04$ & JAERI & $2.6 \mathrm{E}-09$ & $M$ & JAERI & $9.5 \mathrm{E}-10$ & -- & -- \\
\hline Re-184 & Re-184 & 183.953 & Rhenium & 0.01 & 1.5 & 0 & ICRP-38 & $3.80 \mathrm{E}+01$ & $d$ & $3.28 \mathrm{E}+06$ & $1.87 \mathrm{E}+04$ & ICRP-68 & $1.8 \mathrm{E}-09$ & $M$ & ICRP-68 & $1.0 \mathrm{E}-09$ & -- & -- \\
\hline Re-184m & Re-184 & 183.953 & Rhenium & 0.01 & 1.5 & 0 & ICRP-38 & $1.65 \mathrm{E}+02$ & $d$ & $1.43 \mathrm{E}+07$ & $4.30 \mathrm{E}+03$ & ICRP-68 & $6.1 \mathrm{E}-09$ & $\mathrm{M}$ & ICRP-68 & $1.5 \mathrm{E}-09$ & -- & -- \\
\hline Re-186 & Re-186 & 185.955 & Rhenium & 0.01 & 1.5 & 0 & ICRP-38 & $9.06 \mathrm{E}+01$ & $\mathrm{~h}$ & $3.26 \mathrm{E}+05$ & $1.86 \mathrm{E}+05$ & ICRP-68 & $1.2 \mathrm{E}-09$ & $M$ & ICRP-68 & $1.5 \mathrm{E}-09$ & -- & -- \\
\hline Re-186m & Re-186 & 185.955 & Rhenium & 0.01 & 1.5 & 0 & ICRP-38 & $2.00 \mathrm{E}+05$ & $y$ & $6.31 \mathrm{E}+12$ & $9.61 \mathrm{E}-03$ & ICRP-68 & $1.1 \mathrm{E}-08$ & $M$ & ICRP-68 & $2.2 \mathrm{E}-09$ & -- & -- \\
\hline Re-187 & Re-187 & 186.956 & Rhenium & 0.01 & 1.5 & 0 & ICRP-38 & $5.00 \mathrm{E}+10$ & $y$ & $1.58 \mathrm{E}+18$ & $3.82 \mathrm{E}-08$ & ICRP-68 & $6.0 \mathrm{E}-12$ & $M$ & ICRP-68 & $5.1 \mathrm{E}-12$ & -- & -- \\
\hline Re-188 & Re-188 & 187.958 & Rhenium & 0.01 & 1.5 & 0 & ICRP-38 & $1.70 \mathrm{E}+01$ & $\mathrm{~h}$ & $6.11 \mathrm{E}+04$ & $9.82 \mathrm{E}+05$ & ICRP-68 & $7.4 \mathrm{E}-10$ & $M$ & ICRP-68 & $1.4 \mathrm{E}-09$ & -- & -- \\
\hline Re-188m & Re-188 & 187.958 & Rhenium & 0.01 & 1.5 & 0 & ICRP-38 & $1.86 \mathrm{E}+01$ & $\mathrm{~m}$ & $1.12 \mathrm{E}+03$ & $5.38 \mathrm{E}+07$ & ICRP-68 & $2.0 \mathrm{E}-11$ & $M$ & ICRP-68 & $3.0 \mathrm{E}-11$ & -- & -- \\
\hline Re-189 & Re-189 & 188.959 & Rhenium & 0.01 & 1.5 & 0 & ICRP-38 & $2.43 \mathrm{E}+01$ & $\mathrm{~h}$ & $8.75 \mathrm{E}+04$ & $6.82 E+05$ & ICRP-68 & $6.0 \mathrm{E}-10$ & $M$ & ICRP-68 & $7.8 \mathrm{E}-10$ & -- & -- \\
\hline Re-190 & Re-190 & 189.962 & Rhenium & 0.01 & 1.5 & 0 & ICRP-107 & $3.10 \mathrm{E}+00$ & $\mathrm{~m}$ & $1.86 \mathrm{E}+02$ & $3.19 \mathrm{E}+08$ & -- & -- & -- & -- & -- & -- & -- \\
\hline Re-190m & Re-190 & 189.962 & Rhenium & 0.01 & 1.5 & 0 & ICRP-107 & $3.20 \mathrm{E}+00$ & $\mathrm{~h}$ & $1.15 \mathrm{E}+04$ & $5.16 \mathrm{E}+06$ & -- & -- & -- & -- & -- & -- & -- \\
\hline Rh-100 & Rh-100 & 99.908 & Rhodium & 0.01 & 0.15 & 0 & ICRP-38 & $2.08 \mathrm{E}+01$ & $\mathrm{~h}$ & $7.49 \mathrm{E}+04$ & $1.51 \mathrm{E}+06$ & ICRP-68 & $6.3 \mathrm{E}-10$ & $\mathrm{~s}$ & ICRP-68 & $7.1 \mathrm{E}-10$ & -- & -- \\
\hline Rh-100m & Rh-100 & 99.908 & Rhodium & 0.01 & 0.15 & 0 & ICRP-107 & $4.60 \mathrm{E}+00$ & $\mathrm{~m}$ & $2.76 \mathrm{E}+02$ & $4.09 \mathrm{E}+08$ & -- & -- & -- & -- & -- & -- & -- \\
\hline Rh-101 & Rh-101 & 100.906 & Rhodium & 0.01 & 0.15 & 0 & ICRP-38 & $3.20 \mathrm{E}+00$ & $y$ & $1.01 \mathrm{E}+08$ & $1.11 \mathrm{E}+03$ & ICRP-68 & $5.0 \mathrm{E}-09$ & $\mathrm{~s}$ & ICRP-68 & $5.5 \mathrm{E}-10$ & -- & -- \\
\hline Rh-101m & Rh-101 & 100.906 & Rhodium & 0.01 & 0.15 & 0 & ICRP-38 & $4.34 \mathrm{E}+00$ & $d$ & $3.75 \mathrm{E}+05$ & $2.98 \mathrm{E}+05$ & ICRP-68 & $2.7 \mathrm{E}-10$ & $\mathrm{~s}$ & ICRP-68 & $2.2 \mathrm{E}-10$ & -- & -- \\
\hline Rh-102 & Rh-102 & 101.907 & Rhodium & 0.01 & 0.15 & 0 & ICRP-38 & $2.90 \mathrm{E}+00$ & $y$ & $9.15 \mathrm{E}+07$ & $1.21 \mathrm{E}+03$ & ICRP-68 & $1.6 \mathrm{E}-08$ & $\mathrm{~s}$ & ICRP-68 & $2.6 \mathrm{E}-09$ & -- & -- \\
\hline Rh-102m & Rh-102 & 101.907 & Rhodium & 0.01 & 0.15 & 0 & ICRP-38 & $2.07 \mathrm{E}+02$ & $d$ & $1.79 \mathrm{E}+07$ & $6.19 \mathrm{E}+03$ & ICRP-68 & $6.7 \mathrm{E}-09$ & $\mathrm{~s}$ & ICRP-68 & $1.2 \mathrm{E}-09$ & -- & -- \\
\hline Rh-103m & Rh-103 & 102.905 & Rhodium & 0.01 & 0.15 & 0 & ICRP-38 & $5.61 \mathrm{E}+01$ & $\mathrm{~m}$ & $3.37 \mathrm{E}+03$ & $3.26 \mathrm{E}+07$ & ICRP-68 & $2.5 \mathrm{E}-12$ & $\mathrm{~s}$ & ICRP-68 & $3.8 \mathrm{E}-12$ & -- & -- \\
\hline Rh-104 & Rh-104 & 103.907 & Rhodium & 0.01 & 0.15 & 0 & ICRP-107 & $4.23 \mathrm{E}+01$ & $\mathrm{~s}$ & $4.23 \mathrm{E}+01$ & $2.57 E+09$ & -- & -- & -- & -- & -- & -- & -- \\
\hline
\end{tabular}




\begin{tabular}{|c|c|c|c|c|c|c|c|c|c|c|c|c|c|c|c|c|c|c|}
\hline \multirow{3}{*}{$\begin{array}{l}\text { MASTER } \\
\text { ISOTOPE } \\
\text { LIST } \\
\text { Rh-104m }\end{array}$} & \multirow{3}{*}{$\begin{array}{c}\begin{array}{c}\text { Atomic } \\
\text { Mass } \\
\text { Nuclide }\end{array} \\
\text { Rh-104 }\end{array}$} & \multirow{3}{*}{$\begin{array}{c}\begin{array}{c}\text { Atomic } \\
\text { Mass }\end{array} \\
103.907\end{array}$} & & & \multicolumn{14}{|c|}{ Table C.1 Radionuclide Specific Input Date } \\
\hline & & & \multirow{2}{*}{$\begin{array}{l}\text { Element } \\
\text { Rhodium }\end{array}$} & \multirow{2}{*}{$\begin{array}{c}\text { EPA } \\
\text { RF } \\
0.01\end{array}$} & $\begin{array}{c}\text { EPA } \\
\text { Conc } \\
\text { Factors } \\
\left(B_{v}\right) \\
\end{array}$ & $\begin{array}{c}\text { EPA } \\
\text { Sorption } \\
\text { Coefficient } \\
\left(K_{d}\right)\end{array}$ & $\begin{array}{c}\text { Half-Life } \\
\text { Reference }\end{array}$ & $\begin{array}{r}\text { Half-Lit } \\
\text { (original u }\end{array}$ & & $\begin{array}{l}\text { Half Life } \\
\text { (s) }\end{array}$ & $\begin{array}{l}\text { Sp. Act } \\
\text { (Ci/g) }\end{array}$ & $\begin{array}{l}\text { Inhalation } \\
\text { DC } \\
\text { Reference }\end{array}$ & $\begin{array}{c}\text { HC-3 } \\
\text { Bounding } \\
\text { Inhalation } \\
\text { DC } \\
\text { (Sv/Bq) } \\
\end{array}$ & $\begin{array}{c}\text { Lung } \\
\text { Absorption } \\
\text { Class/Type }\end{array}$ & $\begin{array}{l}\text { Ingestion } \\
\text { DC } \\
\text { Reference }\end{array}$ & $\begin{array}{c}\text { HC-3 } \\
\text { Bounding } \\
\text { Ingestion } \\
\text { DC } \\
\text { (Sv/Bq) } \\
\end{array}$ & $\begin{array}{c}\text { Immersion } \\
\text { DC } \\
\text { Reference }\end{array}$ & $\begin{array}{c}\mathrm{HC}-3 \\
\text { Immersion } \\
\mathrm{DC}(\mathrm{Sv} / \mathrm{d} \\
\left.\operatorname{per} \mathrm{Bq} / \mathrm{m}^{3}\right)\end{array}$ \\
\hline & & & & & 0.15 & 0 & ICRP-107 & $4.34 \mathrm{E}+00$ & $\mathrm{~m}$ & $2.60 E+02$ & $4.17 \mathrm{E}+08$ & -- & -- & -- & -- & -- & -- & -- \\
\hline Rh-105 & Rh-105 & 104.906 & Rhodium & 0.01 & 0.15 & 0 & ICRP-38 & $3.54 \mathrm{E}+01$ & $\mathrm{~h}$ & $1.27 \mathrm{E}+05$ & $8.45 \mathrm{E}+05$ & ICRP-68 & $4.4 \mathrm{E}-10$ & $\mathrm{~s}$ & ICRP-68 & $3.7 \mathrm{E}-10$ & -- & -- \\
\hline Rh-106 & Rh-106 & 105.907 & Rhodium & 0.01 & 0.15 & 0 & ICRP-38 & $2.99 \mathrm{E}+01$ & $\mathrm{~s}$ & $2.99 \mathrm{E}+01$ & $3.56 \mathrm{E}+09$ & -- & -- & -- & -- & -- & -- & -- \\
\hline Rh-106m & Rh-106 & 105.907 & Rhodium & 0.01 & 0.15 & 0 & ICRP-38 & $1.32 \mathrm{E}+02$ & $\mathrm{~m}$ & $7.92 \mathrm{E}+03$ & $1.34 \mathrm{E}+07$ & ICRP-68 & $1.9 \mathrm{E}-10$ & $\mathrm{~s}$ & ICRP-68 & $1.6 \mathrm{E}-10$ & -- & -- \\
\hline Rh-107 & Rh-107 & 106.907 & Rhodium & 0.01 & 0.15 & 0 & ICRP-38 & $2.17 \mathrm{E}+01$ & $\mathrm{~m}$ & $1.30 \mathrm{E}+03$ & $8.10 \mathrm{E}+07$ & ICRP-68 & $2.8 \mathrm{E}-11$ & $\mathrm{~s}$ & ICRP-68 & $2.4 \mathrm{E}-11$ & -- & -- \\
\hline Rh-108 & Rh-108 & 107.909 & Rhodium & 0.01 & 0.15 & 0 & ICRP-107 & $1.68 \mathrm{E}+01$ & $\mathrm{~s}$ & $1.68 \mathrm{E}+01$ & $6.22 \mathrm{E}+09$ & -- & -- & -- & -- & -- & -- & -- \\
\hline Rh-109 & Rh-109 & 108.909 & Rhodium & 0.01 & 0.15 & 0 & ICRP-107 & $8.00 \mathrm{E}+01$ & $\mathrm{~s}$ & $8.00 \mathrm{E}+01$ & $1.29 \mathrm{E}+09$ & -- & -- & -- & -- & -- & -- & -- \\
\hline Rh-94 & Rh-94 & 93.922 & Rhodium & 0.01 & 0.15 & 0 & ICRP-107 & $7.06 \mathrm{E}+01$ & $\mathrm{~s}$ & $7.06 \mathrm{E}+01$ & $1.70 \mathrm{E}+09$ & -- & -- & -- & -- & -- & -- & -- \\
\hline Rh-95 & Rh-95 & 94.916 & Rhodium & 0.01 & 0.15 & 0 & ICRP-107 & $5.02 \mathrm{E}+00$ & $\mathrm{~m}$ & $3.01 \mathrm{E}+02$ & $3.95 \mathrm{E}+08$ & -- & -- & -- & -- & -- & -- & -- \\
\hline Rh-95m & Rh-95 & 94.916 & Rhodium & 0.01 & 0.15 & 0 & ICRP-107 & $1.96 \mathrm{E}+00$ & $\mathrm{~m}$ & $1.18 \mathrm{E}+02$ & $1.01 \mathrm{E}+09$ & -- & -- & -- & -- & -- & -- & -- \\
\hline Rh-96 & Rh-96 & 95.914 & Rhodium & 0.01 & 0.15 & 0 & ICRP-107 & $9.90 \mathrm{E}+00$ & $\mathrm{~m}$ & $5.94 \mathrm{E}+02$ & $1.98 \mathrm{E}+08$ & -- & -- & -- & -- & -- & -- & -- \\
\hline Rh-96m & Rh-96 & 95.914 & Rhodium & 0.01 & 0.15 & 0 & ICRP-107 & $1.51 \mathrm{E}+00$ & $\mathrm{~m}$ & $9.06 \mathrm{E}+01$ & $1.30 \mathrm{E}+09$ & --- & -- & -- & -- & -- & -- & -- \\
\hline Rh-97 & Rh-97 & 96.911 & Rhodium & 0.01 & 0.15 & 0 & ICRP-107 & $3.07 E+01$ & $\mathrm{~m}$ & $1.84 \mathrm{E}+03$ & $6.32 \mathrm{E}+07$ & JAERI & $4.2 \mathrm{E}-11$ & $\mathrm{~s}$ & JAERI & $4.9 \mathrm{E}-11$ & -- & -- \\
\hline Rh-97m & Rh-97 & 96.911 & Rhodium & 0.01 & 0.15 & 0 & ICRP-107 & $4.62 \mathrm{E}+01$ & $\mathrm{~m}$ & $2.77 \mathrm{E}+03$ & $4.20 \mathrm{E}+07$ & JAERI & $5.0 \mathrm{E}-11$ & $\mathrm{~s}$ & JAERI & $4.8 \mathrm{E}-11$ & -- & -- \\
\hline Rh-98 & Rh-98 & 97.911 & Rhodium & 0.01 & 0.15 & 0 & ICRP-107 & $8.70 \mathrm{E}+00$ & $\mathrm{~m}$ & $5.22 \mathrm{E}+02$ & $2.21 \mathrm{E}+08$ & -- & -- & -- & -- & -- & -- & -- \\
\hline Rh-99 & Rh-99 & 98.908 & Rhodium & 0.01 & 0.15 & 0 & ICRP-38 & $1.60 \mathrm{E}+01$ & $d$ & $1.38 \mathrm{E}+06$ & $8.25 \mathrm{E}+04$ & ICRP-68 & $8.9 \mathrm{E}-10$ & $\mathrm{~s}$ & ICRP-68 & $5.1 \mathrm{E}-10$ & -- & -- \\
\hline Rh-99m & Rh-99 & 98.908 & Rhodium & 0.01 & 0.15 & 0 & ICRP-38 & $4.70 \mathrm{E}+00$ & $\mathrm{~h}$ & $1.69 \mathrm{E}+04$ & $6.74 \mathrm{E}+06$ & ICRP-68 & $7.3 \mathrm{E}-11$ & $\mathrm{~s}$ & ICRP-68 & $6.6 \mathrm{E}-11$ & -- & -- \\
\hline Rn-207 & Rn-207 & 206.991 & Radon & 1 & 0 & 0 & ICRP-107 & $9.25 \mathrm{E}+00$ & $\mathrm{~m}$ & $5.55 \mathrm{E}+02$ & $9.82 \mathrm{E}+07$ & -- & -- & -- & -- & -- & -- & -- \\
\hline Rn-209 & Rn-209 & 208.990 & Radon & 1 & 0 & 0 & ICRP-107 & $2.85 \mathrm{E}+01$ & $\mathrm{~m}$ & $1.71 \mathrm{E}+03$ & $3.16 \mathrm{E}+07$ & -- & -- & -- & -- & -- & -- & -- \\
\hline $\mathrm{Rn}-210$ & Rn-210 & 209.990 & Radon & 1 & 0 & 0 & ICRP-107 & $2.40 \mathrm{E}+00$ & $\mathrm{~h}$ & $8.64 \mathrm{E}+03$ & $6.22 \mathrm{E}+06$ & -- & -- & -- & -- & -- & -- & -- \\
\hline Rn-211 & Rn-211 & 210.991 & Radon & 1 & 0 & 0 & ICRP-107 & $1.46 \mathrm{E}+01$ & $\mathrm{~h}$ & $5.26 \mathrm{E}+04$ & $1.02 \mathrm{E}+06$ & -- & -- & -- & -- & -- & -- & -- \\
\hline Rn-212 & Rn-212 & 211.991 & Radon & 1 & 0 & 0 & ICRP-107 & $2.39 \mathrm{E}+01$ & $\mathrm{~m}$ & $1.43 \mathrm{E}+03$ & $3.71 \mathrm{E}+07$ & -- & -- & -- & -- & -- & -- & -- \\
\hline Rn-215 & Rn-215 & 214.999 & Radon & 1 & 0 & 0 & ICRP-107 & $2.30 \mathrm{E}+00$ & us & $2.30 \mathrm{E}-06$ & $2.28 \mathrm{E}+16$ & -- & -- & -- & -- & -- & -- & -- \\
\hline Rn-216 & $\mathrm{Rn}-216$ & 216.000 & Radon & 1 & 0 & 0 & ICRP-107 & $4.50 \mathrm{E}-05$ & $\mathrm{~s}$ & $4.50 \mathrm{E}-05$ & $1.16 \mathrm{E}+15$ & -- & -- & -- & -- & -- & -- & -- \\
\hline Rn-217 & Rn-217 & 217.004 & Radon & 1 & 0 & 0 & ICRP-107 & $5.40 \mathrm{E}-04$ & $\mathrm{~s}$ & $5.40 \mathrm{E}-04$ & $9.63 \mathrm{E}+13$ & -- & -- & -- & -- & -- & -- & -- \\
\hline $\mathrm{Rn}-218$ & Rn-218 & 218.006 & Radon & 1 & 0 & 0 & ICRP-38 & $3.50 \mathrm{E}+01$ & $\mathrm{~ms}$ & $3.50 \mathrm{E}-02$ & $1.48 \mathrm{E}+12$ & -- & -- & -- & -- & -- & -- & -- \\
\hline Rn-219 & Rn-219 & 219.009 & Radon & 1 & 0 & 0 & ICRP-38 & $3.96 \mathrm{E}+00$ & $\mathrm{~s}$ & $3.96 \mathrm{E}+00$ & $1.30 \mathrm{E}+10$ & -- & -- & -- & -- & -- & -- & -- \\
\hline $\mathrm{Rn}-220$ & Rn-220 & 220.011 & Radon & 1 & 0 & 0 & ICRP-38 & $5.56 \mathrm{E}+01$ & $\mathrm{~s}$ & $5.56 \mathrm{E}+01$ & $9.22 \mathrm{E}+08$ & -- & -- & -- & -- & -- & -- & -- \\
\hline Rn-222 & Rn-222 & 222.018 & Radon & 1 & 0 & 0 & ICRP-38 & $3.82 \mathrm{E}+00$ & $d$ & $3.30 \mathrm{E}+05$ & $1.54 \mathrm{E}+05$ & -- & -- & -- & -- & -- & -- & -- \\
\hline Rn-223 & Rn-223 & 223.022 & Radon & 1 & 0 & 0 & ICRP-107 & $2.43 \mathrm{E}+01$ & $\mathrm{~m}$ & $1.46 \mathrm{E}+03$ & $3.47 \mathrm{E}+07$ & -- & -- & -- & -- & -- & -- & -- \\
\hline Ru-103 & Ru-103 & 102.906 & Ruthenium & 0.01 & 0.075 & $0-500$ & ICRP-38 & $3.93 \mathrm{E}+01$ & $d$ & $3.39 \mathrm{E}+06$ & $3.23 \mathrm{E}+04$ & ICRP-68 & $2.8 \mathrm{E}-09$ & $\mathrm{~s}$ & ICRP-68 & $7.3 \mathrm{E}-10$ & -- & -- \\
\hline Ru-105 & Ru-105 & 104.908 & Ruthenium & 0.01 & 0.075 & $0-500$ & ICRP-38 & $4.44 \mathrm{E}+00$ & $\mathrm{~h}$ & $1.60 \mathrm{E}+04$ & $6.73 \mathrm{E}+06$ & ICRP-68 & $2.5 \mathrm{E}-10$ & $\mathrm{~s}$ & ICRP-68 & $2.6 \mathrm{E}-10$ & -- & -- \\
\hline Ru-106 & Ru-106 & 105.907 & Ruthenium & 0.01 & 0.075 & $0-500$ & ICRP-38 & $3.68 \mathrm{E}+02$ & $d$ & $3.18 \mathrm{E}+07$ & $3.35 \mathrm{E}+03$ & ICRP-68 & $6.2 \mathrm{E}-08$ & $\mathrm{~s}$ & ICRP-68 & $7.0 \mathrm{E}-09$ & -- & -- \\
\hline Ru-107 & Ru-107 & 106.910 & Ruthenium & 0.01 & 0.075 & $0-500$ & ICRP-107 & $3.75 \mathrm{E}+00$ & $\mathrm{~m}$ & $2.25 \mathrm{E}+02$ & $4.69 \mathrm{E}+08$ & -- & -- & -- & -- & -- & -- & -- \\
\hline Ru-108 & Ru-108 & 107.910 & Ruthenium & 0.01 & 0.075 & $0-500$ & ICRP-107 & $4.55 \mathrm{E}+00$ & $\mathrm{~m}$ & $2.73 \mathrm{E}+02$ & $3.83 \mathrm{E}+08$ & -- & -- & -- & -- & -- & -- & -- \\
\hline Ru-92 & Ru-92 & 91.920 & Ruthenium & 0.01 & 0.075 & $0-500$ & ICRP-107 & $3.65 \mathrm{E}+00$ & $\mathrm{~m}$ & $2.19 \mathrm{E}+02$ & $5.60 \mathrm{E}+08$ & -- & -- & -- & -- & -- & -- & -- \\
\hline Ru-94 & Ru-94 & 93.911 & Ruthenium & 0.01 & 0.075 & $0-500$ & ICRP-38 & $5.18 \mathrm{E}+01$ & $\mathrm{~m}$ & $3.11 \mathrm{E}+03$ & $3.87 \mathrm{E}+07$ & ICRP-68 & $7.4 \mathrm{E}-11$ & $\mathrm{~s}$ & ICRP-68 & $9.4 \mathrm{E}-11$ & -- & -- \\
\hline Ru-95 & Ru-95 & 94.910 & Ruthenium & 0.01 & 0.075 & $0-500$ & ICRP-107 & $1.64 \mathrm{E}+00$ & $\mathrm{~h}$ & $5.91 \mathrm{E}+03$ & $2.01 \mathrm{E}+07$ & JAERI & $6.7 \mathrm{E}-11$ & $\mathrm{~s}$ & JAERI & $6.3 \mathrm{E}-11$ & -- & -- \\
\hline Ru-97 & Ru-97 & 96.908 & Ruthenium & 0.01 & 0.075 & $0-500$ & ICRP-38 & $2.90 \mathrm{E}+00$ & $d$ & $2.51 \mathrm{E}+05$ & $4.65 \mathrm{E}+05$ & ICRP-68 & $1.6 \mathrm{E}-10$ & $M$ & ICRP-68 & $1.5 \mathrm{E}-10$ & -- & -- \\
\hline S-35 & S-35 & 34.969 & Sulfur & 0.5 & 1.5 & 0 & ICRP-38 & $8.74 \mathrm{E}+01$ & $d$ & $7.55 \mathrm{E}+06$ & $4.27 \mathrm{E}+04$ & ICRP-68 & $1.3 \mathrm{E}-09$ & $\mathrm{M}$ & ICRP-68 & $7.7 \mathrm{E}-10$ & -- & -- \\
\hline S-37 & S-37 & 36.971 & Sulfur & 0.5 & 1.5 & 0 & ICRP-107 & $5.05 \mathrm{E}+00$ & $\mathrm{~m}$ & $3.03 \mathrm{E}+02$ & $1.01 \mathrm{E}+09$ & -- & -- & -- & -- & -- & -- & -- \\
\hline$S-38$ & $\mathrm{~S}-38$ & 37.971 & Sulfur & 0.5 & 1.5 & 0 & ICRP-107 & $1.70 \mathrm{E}+02$ & $\mathrm{~m}$ & $1.02 \mathrm{E}+04$ & $2.91 \mathrm{E}+07$ & JAERI & $3.6 \mathrm{E}-10$ & $\mathrm{M}$ & JAERI & $6.4 \mathrm{E}-10$ & -- & -- \\
\hline Sb-111 & Sb-111 & 110.913 & Antimony & 0.01 & 0.2 & 1 & ICRP-107 & $7.50 \mathrm{E}+01$ & $\mathrm{~s}$ & $7.50 \mathrm{E}+01$ & $1.36 \mathrm{E}+09$ & -- & -- & -- & -- & -- & -- & -- \\
\hline Sb-113 & Sb-113 & 112.909 & Antimony & 0.01 & 0.2 & 1 & ICRP-107 & $6.67 \mathrm{E}+00$ & $\mathrm{~m}$ & $4.00 E+02$ & $2.50 \mathrm{E}+08$ & -- & -- & -- & -- & -- & -- & -- \\
\hline Sb-114 & Sb-114 & 113.909 & Antimony & 0.01 & 0.2 & 1 & ICRP-107 & $3.49 \mathrm{E}+00$ & $\mathrm{~m}$ & $2.09 \mathrm{E}+02$ & $4.73 \mathrm{E}+08$ & -- & -- & -- & -- & -- & -- & -- \\
\hline
\end{tabular}




\begin{tabular}{|c|c|c|c|c|c|c|c|c|c|c|c|c|c|c|c|c|c|c|}
\hline \multicolumn{19}{|c|}{ Table C.1 Radionuclide Specific Input Data Used For the Calculation of HC-3 TQs Using Maximum Reported DCs (continued) } \\
\hline $\begin{array}{l}\text { MASTER } \\
\text { ISOTOPE } \\
\text { LIST }\end{array}$ & $\begin{array}{c}\text { Atomic } \\
\text { Mass } \\
\text { Nuclide }\end{array}$ & $\begin{array}{l}\text { Atomic } \\
\text { Mass }\end{array}$ & Element & $\begin{array}{c}\text { EPA } \\
\text { RF }\end{array}$ & $\begin{array}{c}\text { EPA } \\
\text { Conc } \\
\text { Factors } \\
\left(B_{v}\right) \\
\end{array}$ & $\begin{array}{c}\text { EPA } \\
\text { Sorption } \\
\text { Coefficient } \\
\left(K_{d}\right)\end{array}$ & $\begin{array}{c}\text { Half-Life } \\
\text { Reference }\end{array}$ & $\begin{array}{r}\text { Half-Lif } \\
\text { (original u }\end{array}$ & & $\begin{array}{l}\text { Half Life } \\
\text { (s) }\end{array}$ & $\begin{array}{l}\text { Sp. Act } \\
\text { (Ci/g) }\end{array}$ & $\begin{array}{l}\text { Inhalation } \\
\text { DC } \\
\text { Reference }\end{array}$ & $\begin{array}{c}\text { HC-3 } \\
\text { Bounding } \\
\text { Inhalation } \\
\text { DC } \\
\text { (Sv/Bq) } \\
\end{array}$ & $\begin{array}{c}\text { Lung } \\
\text { Absorption } \\
\text { Class/Type }\end{array}$ & $\begin{array}{l}\text { Ingestion } \\
\text { DC } \\
\text { Reference }\end{array}$ & $\begin{array}{c}\text { HC-3 } \\
\text { Bounding } \\
\text { Ingestion } \\
\text { DC } \\
\text { (Sv/Bq) } \\
\end{array}$ & $\begin{array}{l}\text { Immersion } \\
\text { DC } \\
\text { Reference }\end{array}$ & $\begin{array}{c}\mathrm{HC}-3 \\
\text { Immersion } \\
\mathrm{DC}(\mathrm{Sv} / \mathrm{d} \\
\left.\text { per } \mathrm{Bq} / \mathrm{m}^{3}\right)\end{array}$ \\
\hline Sb-115 & Sb-115 & 114.907 & Antimony & 0.01 & 0.2 & 1 & ICRP-38 & $3.18 \mathrm{E}+01$ & $\mathrm{~m}$ & $1.91 \mathrm{E}+03$ & $5.15 \mathrm{E}+07$ & ICRP-68 & $2.3 \mathrm{E}-11$ & $\mathrm{M}$ & ICRP-68 & $2.4 \mathrm{E}-11$ & -- & -- \\
\hline Sb-116 & Sb-116 & 115.907 & Antimony & 0.01 & 0.2 & 1 & ICRP-38 & $1.58 \mathrm{E}+01$ & $\mathrm{~m}$ & $9.48 \mathrm{E}+02$ & $1.03 E+08$ & ICRP-68 & $2.3 \mathrm{E}-11$ & $\mathrm{M}$ & ICRP-68 & $2.6 \mathrm{E}-11$ & -- & -- \\
\hline Sb-116m & Sb-116 & 115.907 & Antimony & 0.01 & 0.2 & 1 & ICRP-38 & $6.03 \mathrm{E}+01$ & $\mathrm{~m}$ & $3.62 E+03$ & $2.69 \mathrm{E}+07$ & ICRP-68 & $8.5 \mathrm{E}-11$ & $M$ & ICRP-68 & $6.7 \mathrm{E}-11$ & -- & -- \\
\hline Sb-117 & Sb-117 & 116.905 & Antimony & 0.01 & 0.2 & 1 & ICRP-38 & $2.80 \mathrm{E}+00$ & $\mathrm{~h}$ & $1.01 \mathrm{E}+04$ & $9.57 \mathrm{E}+06$ & ICRP-68 & $2.7 \mathrm{E}-11$ & $M$ & ICRP-68 & $1.8 \mathrm{E}-11$ & -- & -- \\
\hline Sb-118 & Sb-118 & 117.906 & Antimony & 0.01 & 0.2 & 1 & ICRP-38 & $3.60 \mathrm{E}+00$ & $\mathrm{~m}$ & $2.16 \mathrm{E}+02$ & $4.43 \mathrm{E}+08$ & -- & -- & -- & -- & -- & -- & -- \\
\hline Sb-118m & Sb-118 & 117.906 & Antimony & 0.01 & 0.2 & 1 & ICRP-38 & $5.00 \mathrm{E}+00$ & $\mathrm{~h}$ & $1.80 \mathrm{E}+04$ & $5.32 \mathrm{E}+06$ & ICRP-68 & $2.3 \mathrm{E}-10$ & $M$ & ICRP-68 & $2.1 \mathrm{E}-10$ & -- & -- \\
\hline Sb-119 & Sb-119 & 118.904 & Antimony & 0.01 & 0.2 & 1 & ICRP-38 & $3.81 \mathrm{E}+01$ & $\mathrm{~h}$ & $1.37 E+05$ & $6.92 \mathrm{E}+05$ & ICRP-68 & $5.9 \mathrm{E}-11$ & $M$ & ICRP-68 & $8.1 \mathrm{E}-11$ & -- & -- \\
\hline$S b-120 s$ & Sb-120 & 119.905 & Antimony & 0.01 & 0.2 & 1 & ICRP-38 & $1.59 \mathrm{E}+01$ & $\mathrm{~m}$ & $9.53 \mathrm{E}+02$ & $9.87 \mathrm{E}+07$ & ICRP-68 & $1.2 \mathrm{E}-11$ & $M$ & ICRP-68 & $1.4 \mathrm{E}-11$ & -- & -- \\
\hline Sb-120l & Sb-120 & 119.905 & Antimony & 0.01 & 0.2 & 1 & ICRP-38 & $5.76 \mathrm{E}+00$ & $d$ & $4.98 \mathrm{E}+05$ & $1.89 \mathrm{E}+05$ & ICRP-68 & $1.3 \mathrm{E}-09$ & $M$ & ICRP-68 & $1.2 \mathrm{E}-09$ & -- & -- \\
\hline Sb-122 & Sb-122 & 121.905 & Antimony & 0.01 & 0.2 & 1 & ICRP-38 & $2.70 \mathrm{E}+00$ & $d$ & $2.33 E+05$ & $3.97 \mathrm{E}+05$ & ICRP-68 & $1.2 \mathrm{E}-09$ & $M$ & ICRP-68 & $1.7 \mathrm{E}-09$ & -- & -- \\
\hline Sb-122m & Sb-122 & 121.905 & Antimony & 0.01 & 0.2 & 1 & ICRP-107 & $4.19 \mathrm{E}+00$ & $\mathrm{~m}$ & $2.51 \mathrm{E}+02$ & $3.68 \mathrm{E}+08$ & -- & -- & -- & -- & -- & -- & -- \\
\hline Sb-124 & Sb-124 & 123.906 & Antimony & 0.01 & 0.2 & 1 & $\begin{array}{l}\text { ICRP-38 } \\
\end{array}$ & $6.02 \mathrm{E}+01$ & $d$ & $5.20 \mathrm{E}+06$ & $1.75 \mathrm{E}+04$ & ICRP-68 & $6.1 \mathrm{E}-09$ & $M$ & ICRP-68 & $2.5 \mathrm{E}-09$ & -- & -- \\
\hline Sb-124ms & Sb-124 & 123.906 & Antimony & 0.01 & 0.2 & 1 & ICRP-38 & $9.30 \mathrm{E}+01$ & $\mathrm{~s}$ & $9.30 \mathrm{E}+01$ & $9.79 \mathrm{E}+08$ & -- & -- & -- & -- & -- & -- & -- \\
\hline Sb-124ml & Sb-124 & 123.906 & Antimony & 0.01 & 0.2 & 1 & ICRP-38 & $2.02 \mathrm{E}+01$ & $\mathrm{~m}$ & $1.21 \mathrm{E}+03$ & $7.51 \mathrm{E}+07$ & ICRP-68 & $8.3 \mathrm{E}-12$ & $\mathrm{M}$ & ICRP-68 & $8.0 \mathrm{E}-12$ & -- & -- \\
\hline Sb-125 & Sb-125 & 124.905 & Antimony & 0.01 & 0.2 & 1 & ICRP-38 & $2.77 \mathrm{E}+00$ & $y$ & $8.74 \mathrm{E}+07$ & $1.03 \mathrm{E}+03$ & ICRP-68 & $4.5 \mathrm{E}-09$ & $M$ & ICRP-68 & $1.1 \mathrm{E}-09$ & -- & -- \\
\hline Sb-126 & Sb-126 & 125.907 & Antimony & 0.01 & 0.2 & 1 & ICRP-38 & $1.24 \mathrm{E}+01$ & $d$ & $1.07 E+06$ & $8.36 \mathrm{E}+04$ & ICRP-68 & $3.2 \mathrm{E}-09$ & $M$ & ICRP-68 & $2.4 \mathrm{E}-09$ & -- & -- \\
\hline Sb-126m & Sb-126 & 125.907 & Antimony & 0.01 & 0.2 & 1 & ICRP-38 & $1.90 \mathrm{E}+01$ & $\mathrm{~m}$ & $1.14 \mathrm{E}+03$ & $7.86 \mathrm{E}+07$ & ICRP-68 & $3.3 \mathrm{E}-11$ & $M$ & ICRP-68 & $3.6 \mathrm{E}-11$ & -- & -- \\
\hline Sb-127 & Sb-127 & 126.907 & Antimony & 0.01 & 0.2 & 1 & ICRP-38 & $3.85 \mathrm{E}+00$ & $d$ & $3.33 \mathrm{E}+05$ & $2.67 \mathrm{E}+05$ & ICRP-68 & $1.7 \mathrm{E}-09$ & $M$ & ICRP-68 & $1.7 \mathrm{E}-09$ & -- & -- \\
\hline Sb-128l & Sb-128 & 127.909 & Antimony & 0.01 & 0.2 & 1 & ICRP-38 & $9.01 \mathrm{E}+00$ & $h$ & $3.24 \mathrm{E}+04$ & $2.72 \mathrm{E}+06$ & ICRP-68 & $6.7 \mathrm{E}-10$ & $M$ & ICRP-68 & $7.6 \mathrm{E}-10$ & -- & -- \\
\hline$S b-128 s$ & Sb-128 & 127.909 & Antimony & 0.01 & 0.2 & 1 & ICRP-38 & $1.04 \mathrm{E}+01$ & $\mathrm{~m}$ & $6.24 E+02$ & $1.41 \mathrm{E}+08$ & ICRP-68 & $2.6 \mathrm{E}-11$ & $M$ & ICRP-68 & $3.3 \mathrm{E}-11$ & -- & -- \\
\hline Sb-129 & Sb-129 & 128.909 & Antimony & 0.01 & 0.2 & 1 & ICRP-38 & $4.32 \mathrm{E}+00$ & $\mathrm{~h}$ & $1.56 \mathrm{E}+04$ & $5.63 \mathrm{E}+06$ & ICRP-68 & $3.5 \mathrm{E}-10$ & $M$ & ICRP-68 & $4.2 \mathrm{E}-10$ & -- & -- \\
\hline Sb-130 & Sb-130 & 129.912 & Antimony & 0.01 & 0.2 & 1 & ICRP-38 & $4.00 \mathrm{E}+01$ & $\mathrm{~m}$ & $2.40 \mathrm{E}+03$ & $3.62 \mathrm{E}+07$ & ICRP-68 & $9.1 \mathrm{E}-11$ & $M$ & ICRP-68 & $9.1 \mathrm{E}-11$ & -- & -- \\
\hline Sb-130m & Sb-130 & 129.912 & Antimony & 0.01 & 0.2 & 1 & ICRP-107 & $6.30 \mathrm{E}+00$ & $\mathrm{~m}$ & $3.78 \mathrm{E}+02$ & $2.30 \mathrm{E}+08$ & -- & -- & -- & -- & -- & -- & -- \\
\hline Sb-131 & Sb-131 & 130.912 & Antimony & 0.01 & 0.2 & 1 & ICRP-38 & $2.30 \mathrm{E}+01$ & $\mathrm{~m}$ & $1.38 \mathrm{E}+03$ & $6.24 \mathrm{E}+07$ & ICRP-68 & $8.3 \mathrm{E}-11$ & $M$ & ICRP-68 & $1.0 \mathrm{E}-10$ & -- & -- \\
\hline Sb-133 & Sb-133 & 132.915 & Antimony & 0.01 & 0.2 & 1 & ICRP-107 & $2.50 \mathrm{E}+00$ & $\mathrm{~m}$ & $1.50 \mathrm{E}+02$ & $5.66 \mathrm{E}+08$ & -- & -- & -- & -- & -- & -- & -- \\
\hline Sc- $42 \mathrm{~m}$ & Sc-42 & 41.966 & Scandium & 0.01 & 0.006 & 0 & ICRP-107 & $6.20 \mathrm{E}+01$ & $\mathrm{~s}$ & $6.20 \mathrm{E}+01$ & $4.34 \mathrm{E}+09$ & -- & -- & -- & -- & -- & -- & -- \\
\hline Sc-43 & Sc-43 & 42.961 & Scandium & 0.01 & 0.006 & 0 & ICRP-38 & $3.89 \mathrm{E}+00$ & $\mathrm{~h}$ & $1.40 \mathrm{E}+04$ & $1.87 \mathrm{E}+07$ & ICRP-68 & $1.8 \mathrm{E}-10$ & $\mathrm{~s}$ & ICRP-68 & $1.9 \mathrm{E}-10$ & -- & -- \\
\hline Sc-44 & Sc-44 & 43.959 & Scandium & 0.01 & 0.006 & 0 & ICRP-38 & $3.93 \mathrm{E}+00$ & $\mathrm{~h}$ & $1.41 \mathrm{E}+04$ & $1.82 \mathrm{E}+07$ & ICRP-68 & $3.0 \mathrm{E}-10$ & $\mathrm{~s}$ & ICRP-68 & $3.5 \mathrm{E}-10$ & -- & -- \\
\hline Sc-44m & Sc-44 & 43.959 & Scandium & 0.01 & 0.006 & 0 & ICRP-38 & $5.86 \mathrm{E}+01$ & $\mathrm{~h}$ & $2.11 \mathrm{E}+05$ & $1.22 \mathrm{E}+06$ & ICRP-68 & $2.0 \mathrm{E}-09$ & $\mathrm{~s}$ & ICRP-68 & $2.4 \mathrm{E}-09$ & -- & -- \\
\hline Sc-46 & Sc-46 & 45.955 & Scandium & 0.01 & 0.006 & 0 & ICRP-38 & $8.38 \mathrm{E}+01$ & $d$ & $7.24 E+06$ & $3.39 \mathrm{E}+04$ & ICRP-68 & $6.4 \mathrm{E}-09$ & $\mathrm{~s}$ & ICRP-68 & $1.5 \mathrm{E}-09$ & -- & -- \\
\hline Sc-47 & Sc-47 & 46.952 & Scandium & 0.01 & 0.006 & 0 & ICRP-38 & $3.35 \mathrm{E}+00$ & $d$ & $2.90 \mathrm{E}+05$ & $8.30 \mathrm{E}+05$ & ICRP-68 & $7.3 \mathrm{E}-10$ & $\mathrm{~s}$ & ICRP-68 & $5.4 \mathrm{E}-10$ & -- & -- \\
\hline Sc-48 & Sc-48 & 47.952 & Scandium & 0.01 & 0.006 & 0 & ICRP-38 & $4.37 \mathrm{E}+01$ & $\mathrm{~h}$ & $1.57 E+05$ & $1.50 \mathrm{E}+06$ & ICRP-68 & $1.6 \mathrm{E}-09$ & $\mathrm{~s}$ & ICRP-68 & $1.7 \mathrm{E}-09$ & -- & -- \\
\hline Sc-49 & Sc-49 & 48.950 & Scandium & 0.01 & 0.006 & 0 & ICRP-38 & $5.74 \mathrm{E}+01$ & $\mathrm{~m}$ & $3.44 \mathrm{E}+03$ & $6.69 \mathrm{E}+07$ & ICRP-68 & $6.1 \mathrm{E}-11$ & $\mathrm{~s}$ & ICRP-68 & $8.2 \mathrm{E}-11$ & -- & -- \\
\hline Sc-50 & Sc-50 & 49.952 & Scandium & 0.01 & 0.006 & 0 & ICRP-107 & $1.03 \mathrm{E}+02$ & $\mathrm{~s}$ & $1.03 E+02$ & $2.20 \mathrm{E}+09$ & -- & -- & -- & -- & -- & -- & -- \\
\hline Se-70 & Se-70 & 69.934 & Selenium & 0.01 & 0.025 & 10 & ICRP-38 & $4.10 \mathrm{E}+01$ & $\mathrm{~m}$ & $2.46 \mathrm{E}+03$ & $6.56 \mathrm{E}+07$ & ICRP-68 & $1.2 \mathrm{E}-10$ & $M$ & ICRP-68 & $1.4 \mathrm{E}-10$ & -- & -- \\
\hline Se-71 & $\begin{array}{l}\text { Se-71 } \\
\end{array}$ & 70.932 & Selenium & 0.01 & 0.025 & 10 & ICRP-107 & $4.74 \mathrm{E}+00$ & $\mathrm{~m}$ & $2.84 \mathrm{E}+02$ & $5.59 \mathrm{E}+08$ & -- & -- & -- & -- & -- & -- & -- \\
\hline Se-72 & Se-72 & 71.927 & Selenium & 0.01 & 0.025 & 10 & ICRP-38 & $8.40 \mathrm{E}+00$ & $d$ & $7.26 \mathrm{E}+05$ & $2.16 \mathrm{E}+05$ & JAERI & $3.9 \mathrm{E}-09$ & $\mathrm{M}$ & JAERI & $5.1 \mathrm{E}-09$ & -- & -- \\
\hline Se-73 & Se-73 & 72.927 & Selenium & 0.01 & 0.025 & 10 & ICRP-38 & $7.15 \mathrm{E}+00$ & $\mathrm{~h}$ & $2.57 E+04$ & $6.01 \mathrm{E}+06$ & ICRP-68 & $2.4 \mathrm{E}-10$ & $M$ & ICRP-68 & $3.9 \mathrm{E}-10$ & -- & -- \\
\hline Se-73m & Se-73 & 72.927 & Selenium & 0.01 & 0.025 & 10 & ICRP-38 & $3.90 \mathrm{E}+01$ & $\mathrm{~m}$ & $2.34 \mathrm{E}+03$ & $6.61 \mathrm{E}+07$ & ICRP-68 & $2.7 \mathrm{E}-11$ & $M$ & ICRP-68 & $4.1 \mathrm{E}-11$ & -- & -- \\
\hline Se-75 & Se-75 & 74.923 & Selenium & 0.01 & 0.025 & 10 & ICRP-38 & $1.20 \mathrm{E}+02$ & $d$ & $1.04 \mathrm{E}+07$ & $1.45 \mathrm{E}+04$ & ICRP-68 & $1.7 \mathrm{E}-09$ & $\mathrm{M}$ & ICRP-68 & $2.6 \mathrm{E}-09$ & -- & -- \\
\hline Se-77m & Se-77 & 76.920 & Selenium & 0.01 & 0.025 & 10 & ICRP-38 & $1.75 \mathrm{E}+01$ & $\mathrm{~s}$ & $1.75 \mathrm{E}+01$ & $8.40 \mathrm{E}+09$ & -- & -- & -- & -- & -- & -- & -- \\
\hline Se-79 & Se-79 & 78.918 & Selenium & 0.01 & 0.025 & 10 & ICRP-38 & $6.50 \mathrm{E}+04$ & $y$ & $2.05 \mathrm{E}+12$ & $6.97 \mathrm{E}-02$ & ICRP-68 & $3.1 \mathrm{E}-09$ & $M$ & ICRP-68 & $2.9 \mathrm{E}-09$ & -- & -- \\
\hline Se-79m & Se-79 & 78.918 & Selenium & 0.01 & 0.025 & 10 & ICRP-107 & $3.92 \mathrm{E}+00$ & $\mathrm{~m}$ & $2.35 \mathrm{E}+02$ & $6.08 \mathrm{E}+08$ & -- & -- & -- & -- & -- & -- & -- \\
\hline Se-81 & Se-81 & 80.918 & Selenium & 0.01 & 0.025 & 10 & ICRP-38 & $1.85 \mathrm{E}+01$ & $\mathrm{~m}$ & $1.11 \mathrm{E}+03$ & $1.26 \mathrm{E}+08$ & ICRP-68 & $2.4 \mathrm{E}-11$ & $M$ & ICRP-68 & $2.7 \mathrm{E}-11$ & -- & -- \\
\hline Se-81m & $\begin{array}{ll}\text { Se- } 81 \\
\end{array}$ & 80.918 & Selenium & 0.01 & 0.025 & 10 & ICRP-38 & $5.73 \mathrm{E}+01$ & $\mathrm{~m}$ & $3.44 E+03$ & $4.06 \mathrm{E}+07$ & ICRP-68 & $6.8 \mathrm{E}-11$ & $M$ & ICRP-68 & $5.9 \mathrm{E}-11$ & -- & -- \\
\hline
\end{tabular}




\begin{tabular}{|c|c|c|c|c|c|c|c|c|c|c|c|c|c|c|c|c|c|c|}
\hline \multirow{3}{*}{$\begin{array}{l}\text { MASTER } \\
\text { ISOTOPE } \\
\text { LIST } \\
\text { Se- } 83 \\
\end{array}$} & \multirow{3}{*}{$\begin{array}{c}\begin{array}{c}\text { Atomic } \\
\text { Mass } \\
\text { Nuclide }\end{array} \\
\text { Se- } 83 \\
\end{array}$} & \multirow{3}{*}{$\begin{array}{l}\begin{array}{c}\text { Atomic } \\
\text { Mass }\end{array} \\
82.919\end{array}$} & & & \multicolumn{14}{|c|}{ Table C.1 Radionuclide Specific Input Dat } \\
\hline & & & \multirow{2}{*}{$\begin{array}{l}\text { Element } \\
\text { Selenium }\end{array}$} & \multirow{2}{*}{$\begin{array}{c}\text { EPA } \\
\text { RF } \\
0.01\end{array}$} & $\begin{array}{c}\text { EPA } \\
\text { Conc } \\
\text { Factors } \\
\left(B_{v}\right) \\
\end{array}$ & $\begin{array}{c}\text { EPA } \\
\text { Sorption } \\
\text { Coefficient } \\
\left(K_{d}\right)\end{array}$ & $\begin{array}{c}\text { Half-Life } \\
\text { Reference }\end{array}$ & $\begin{array}{r}\text { Half-Lit } \\
\text { (original u }\end{array}$ & & $\begin{array}{l}\text { Half Life } \\
\text { (s) }\end{array}$ & $\begin{array}{l}\text { Sp. Act } \\
\text { (Ci/g) }\end{array}$ & $\begin{array}{c}\text { Inhalation } \\
\text { DC } \\
\text { Reference }\end{array}$ & $\begin{array}{c}\text { HC-3 } \\
\text { Bounding } \\
\text { Inhalation } \\
\text { DC } \\
\text { (Sv/Bq) } \\
\end{array}$ & $\begin{array}{c}\text { Lung } \\
\text { Absorption } \\
\text { Class/Type }\end{array}$ & $\begin{array}{l}\text { Ingestion } \\
\text { DC } \\
\text { Reference }\end{array}$ & $\begin{array}{c}\text { HC-3 } \\
\text { Bounding } \\
\text { Ingestion } \\
\text { DC } \\
\text { (Sv/Bq) } \\
\end{array}$ & $\begin{array}{c}\text { Immersion } \\
\text { DC } \\
\text { Reference }\end{array}$ & $\begin{array}{c}\mathrm{HC}-3 \\
\text { Immersion } \\
\mathrm{DC}(\mathrm{Sv} / \mathrm{d} \\
\left.\operatorname{per} \mathrm{Bq} / \mathrm{m}^{3}\right)\end{array}$ \\
\hline & & & & & 0.025 & 10 & ICRP-38 & $2.25 \mathrm{E}+01$ & $\mathrm{~m}$ & $1.35 \mathrm{E}+03$ & $1.01 \mathrm{E}+08$ & ICRP-68 & $5.3 \mathrm{E}-11$ & $\mathrm{M}$ & ICRP-68 & $5.1 \mathrm{E}-11$ & -- & -- \\
\hline Se-83m & Se- 83 & 82.919 & Selenium & 0.01 & 0.025 & 10 & ICRP-107 & $7.01 \mathrm{E}+01$ & $\mathrm{~s}$ & $7.01 \mathrm{E}+01$ & $1.94 \mathrm{E}+09$ & -- & -- & -- & -- & -- & -- & -- \\
\hline Se-84 & Se- 84 & 83.918 & Selenium & 0.01 & 0.025 & 10 & ICRP-107 & $3.10 \mathrm{E}+00$ & $\mathrm{~m}$ & $1.86 \mathrm{E}+02$ & $7.23 \mathrm{E}+08$ & -- & -- & -- & -- & -- & -- & -- \\
\hline Si-31 & Si-31 & 30.975 & Silicon & 0.01 & 0.35 & 0 & ICRP-38 & $1.57 \mathrm{E}+02$ & $\mathrm{~m}$ & $9.44 \mathrm{E}+03$ & $3.86 \mathrm{E}+07$ & ICRP-68 & $1.1 \mathrm{E}-10$ & $\mathrm{M}$ & ICRP-68 & $1.6 \mathrm{E}-10$ & -- & -- \\
\hline Si-32 & Si-32 & 31.974 & Silicon & 0.01 & 0.35 & 0 & ICRP-38 & $4.50 \mathrm{E}+02$ & $\mathrm{y}$ & $1.42 \mathrm{E}+10$ & $2.48 \mathrm{E}+01$ & ICRP-68 & $1.1 \mathrm{E}-07$ & $\mathrm{~s}$ & ICRP-68 & $5.6 \mathrm{E}-10$ & -- & -- \\
\hline Sm-139 & Sm-139 & 138.922 & Samarium & 0.01 & 0.01 & $500-1000$ & ICRP-107 & $2.57 \mathrm{E}+00$ & $\mathrm{~m}$ & $1.54 \mathrm{E}+02$ & $5.27 \mathrm{E}+08$ & -- & -- & -- & -- & -- & -- & -- \\
\hline Sm-140 & Sm-140 & 139.919 & Samarium & 0.01 & 0.01 & $500-1000$ & ICRP-107 & $1.48 \mathrm{E}+01$ & $\mathrm{~m}$ & $8.89 \mathrm{E}+02$ & $9.07 \mathrm{E}+07$ & JAERI & $5.2 \mathrm{E}-11$ & $M$ & JAERI & $9.8 \mathrm{E}-11$ & -- & -- \\
\hline Sm-141 & Sm-141 & 140.918 & Samarium & 0.01 & 0.01 & $500-1000$ & ICRP-38 & $1.02 \mathrm{E}+01$ & $\mathrm{~m}$ & $6.12 \mathrm{E}+02$ & $1.31 \mathrm{E}+08$ & ICRP-68 & $2.7 \mathrm{E}-11$ & $M$ & ICRP-68 & $3.9 \mathrm{E}-11$ & -- & -- \\
\hline Sm-141m & Sm-141 & 140.918 & Samarium & 0.01 & 0.01 & $500-1000$ & ICRP-38 & $2.26 \mathrm{E}+01$ & $\mathrm{~m}$ & $1.36 \mathrm{E}+03$ & $5.90 \mathrm{E}+07$ & ICRP-68 & $5.6 \mathrm{E}-11$ & $M$ & ICRP-68 & $6.5 \mathrm{E}-11$ & -- & -- \\
\hline Sm-142 & Sm-142 & 141.915 & Samarium & 0.01 & 0.01 & $500-1000$ & ICRP-38 & $7.25 \mathrm{E}+01$ & $\mathrm{~m}$ & $4.35 \mathrm{E}+03$ & $1.83 \mathrm{E}+07$ & ICRP-68 & $1.1 \mathrm{E}-10$ & $M$ & ICRP-68 & $1.9 \mathrm{E}-10$ & -- & -- \\
\hline Sm-143 & Sm-143 & 142.915 & Samarium & 0.01 & 0.01 & $500-1000$ & ICRP-107 & $8.75 \mathrm{E}+00$ & $\mathrm{~m}$ & $5.25 \mathrm{E}+02$ & $1.50 \mathrm{E}+08$ & -- & -- & -- & -- & -- & -- & -- \\
\hline Sm-143m & Sm-143 & 142.915 & Samarium & 0.01 & 0.01 & $500-1000$ & ICRP-107 & $6.60 \mathrm{E}+01$ & $\mathrm{~s}$ & $6.60 \mathrm{E}+01$ & $1.20 \mathrm{E}+09$ & -- & -- & -- & -- & -- & -- & -- \\
\hline Sm-145 & Sm-145 & 144.913 & Samarium & 0.01 & 0.01 & $500-1000$ & ICRP-38 & $3.40 \mathrm{E}+02$ & $\mathrm{~d}$ & $2.94 \mathrm{E}+07$ & $2.65 \mathrm{E}+03$ & $\begin{array}{l}\text { ICRP-68 } \\
\end{array}$ & $1.5 \mathrm{E}-09$ & $M$ & ICRP-68 & $2.1 \mathrm{E}-10$ & -- & -- \\
\hline Sm-146 & Sm-146 & 145.913 & Samarium & 0.01 & 0.01 & $500-1000$ & ICRP-38 & $1.03 E+08$ & $y$ & $3.25 \mathrm{E}+15$ & $2.38 \mathrm{E}-05$ & ICRP-68 & $9.9 \mathrm{E}-06$ & $M$ & ICRP-68 & $5.4 \mathrm{E}-08$ & -- & -- \\
\hline Sm-147 & Sm-147 & 146.915 & Samarium & 0.01 & 0.01 & $500-1000$ & ICRP-38 & $1.06 \mathrm{E}+11$ & $y$ & $3.35 \mathrm{E}+18$ & $2.30 \mathrm{E}-08$ & ICRP-68 & $8.9 \mathrm{E}-06$ & $M$ & ICRP-68 & $4.9 \mathrm{E}-08$ & -- & -- \\
\hline Sm-148 & Sm-148 & 147.915 & Samarium & 0.01 & 0.01 & $500-1000$ & ICRP-107 & $7.00 \mathrm{E}+15$ & $y$ & $2.21 \mathrm{E}+23$ & $3.45 E-13$ & JAERI & $7.7 \mathrm{E}-06$ & $M$ & JAERI & $4.3 \mathrm{E}-08$ & -- & -- \\
\hline Sm-151 & Sm-151 & 150.920 & Samarium & 0.01 & 0.01 & $500-1000$ & ICRP-38 & $9.00 \mathrm{E}+01$ & $y$ & $2.84 \mathrm{E}+09$ & $2.63 \mathrm{E}+01$ & ICRP-68 & $3.7 \mathrm{E}-09$ & $\mathrm{M}$ & ICRP-68 & $9.8 \mathrm{E}-11$ & -- & -- \\
\hline Sm-153 & Sm-153 & 152.922 & Samarium & 0.01 & 0.01 & $500-1000$ & ICRP-38 & $4.67 \mathrm{E}+01$ & $\mathrm{~h}$ & $1.68 \mathrm{E}+05$ & $4.39 \mathrm{E}+05$ & ICRP-68 & $6.8 \mathrm{E}-10$ & $M$ & ICRP-68 & $7.4 \mathrm{E}-10$ & -- & -- \\
\hline Sm-155 & Sm-155 & 154.925 & Samarium & 0.01 & 0.01 & $500-1000$ & ICRP-38 & $2.21 \mathrm{E}+01$ & $\mathrm{~m}$ & $1.33 \mathrm{E}+03$ & $5.49 \mathrm{E}+07$ & ICRP-68 & $2.8 \mathrm{E}-11$ & $M$ & ICRP-68 & $2.9 \mathrm{E}-11$ & -- & -- \\
\hline Sm-156 & Sm-156 & 155.926 & Samarium & 0.01 & 0.01 & $500-1000$ & ICRP-38 & $9.40 \mathrm{E}+00$ & $\mathrm{~h}$ & $3.38 \mathrm{E}+04$ & $2.14 \mathrm{E}+06$ & ICRP-68 & $2.8 \mathrm{E}-10$ & $M$ & ICRP-68 & $2.5 \mathrm{E}-10$ & -- & -- \\
\hline Sm-157 & Sm-157 & 156.928 & Samarium & 0.01 & 0.01 & $500-1000$ & ICRP-107 & $8.03 E+00$ & $\mathrm{~m}$ & $4.82 \mathrm{E}+02$ & $1.49 \mathrm{E}+08$ & -- & -- & -- & -- & -- & -- & -- \\
\hline Sn-106 & Sn-106 & 105.917 & Tin & 0.01 & 0.03 & $50-100$ & ICRP-107 & $1.92 \mathrm{E}+00$ & $\mathrm{~m}$ & $1.15 \mathrm{E}+02$ & $9.25 \mathrm{E}+08$ & -- & -- & -- & -- & -- & -- & -- \\
\hline Sn-108 & Sn-108 & 107.912 & Tin & 0.01 & 0.03 & $50-100$ & ICRP-107 & $1.03 \mathrm{E}+01$ & $\mathrm{~m}$ & $6.18 \mathrm{E}+02$ & $1.69 \mathrm{E}+08$ & JAERI & $2.0 \mathrm{E}-11$ & $M$ & JAERI & $2.3 \mathrm{E}-11$ & -- & -- \\
\hline Sn-109 & Sn-109 & 108.911 & Tin & 0.01 & 0.03 & $50-100$ & ICRP-107 & $1.80 \mathrm{E}+01$ & $\mathrm{~m}$ & $1.08 \mathrm{E}+03$ & $9.59 \mathrm{E}+07$ & JAERI & $2.2 \mathrm{E}-11$ & $M$ & JAERI & $1.9 \mathrm{E}-11$ & -- & -- \\
\hline Sn-110 & Sn-110 & 109.908 & Tin & 0.01 & 0.03 & $50-100$ & ICRP-38 & $4.00 \mathrm{E}+00$ & $\mathrm{~h}$ & $1.44 \mathrm{E}+04$ & $7.13 \mathrm{E}+06$ & ICRP-68 & $2.6 \mathrm{E}-10$ & $M$ & ICRP-68 & $3.5 \mathrm{E}-10$ & -- & -- \\
\hline Sn-111 & Sn-111 & 110.908 & Tin & 0.01 & 0.03 & $50-100$ & ICRP-38 & $3.53 \mathrm{E}+01$ & $\mathrm{~m}$ & $2.12 \mathrm{E}+03$ & $4.80 \mathrm{E}+07$ & ICRP-68 & $2.2 \mathrm{E}-11$ & $M$ & ICRP-68 & $2.3 \mathrm{E}-11$ & -- & -- \\
\hline Sn-113 & Sn-113 & 112.905 & Tin & 0.01 & 0.03 & $50-100$ & ICRP-38 & $1.15 \mathrm{E}+02$ & $d$ & $9.94 \mathrm{E}+06$ & $1.00 \mathrm{E}+04$ & ICRP-68 & $2.5 \mathrm{E}-09$ & $M$ & ICRP-68 & $7.3 \mathrm{E}-10$ & -- & -- \\
\hline Sn-113m & Sn-113 & 112.905 & Tin & 0.01 & 0.03 & $50-100$ & ICRP-107 & $2.14 \mathrm{E}+01$ & $\mathrm{~m}$ & $1.28 \mathrm{E}+03$ & $7.78 \mathrm{E}+07$ & JAERI & $5.3 \mathrm{E}-12$ & $M$ & JAERI & $3.3 \mathrm{E}-12$ & -- & -- \\
\hline Sn-117m & Sn-117 & 116.903 & Tin & 0.01 & 0.03 & $50-100$ & ICRP-38 & $1.36 \mathrm{E}+01$ & $d$ & $1.18 \mathrm{E}+06$ & $8.21 \mathrm{E}+04$ & ICRP-68 & $2.3 \mathrm{E}-09$ & $\mathrm{M}$ & ICRP-68 & $7.1 \mathrm{E}-10$ & -- & -- \\
\hline Sn-119m & Sn-119 & 118.903 & Tin & 0.01 & 0.03 & $50-100$ & ICRP-38 & $2.93 \mathrm{E}+02$ & $d$ & $2.53 \mathrm{E}+07$ & $3.75 \mathrm{E}+03$ & ICRP-68 & $2.0 \mathrm{E}-09$ & $M$ & ICRP-68 & $3.4 \mathrm{E}-10$ & -- & -- \\
\hline Sn-121 & Sn-121 & 120.904 & Tin & 0.01 & 0.03 & $50-100$ & ICRP-38 & $2.71 \mathrm{E}+01$ & $h$ & $9.74 \mathrm{E}+04$ & $9.58 \mathrm{E}+05$ & ICRP-68 & $2.8 \mathrm{E}-10$ & $M$ & ICRP-68 & $2.3 \mathrm{E}-10$ & -- & -- \\
\hline Sn-121m & Sn-121 & 120.904 & Tin & 0.01 & 0.03 & $50-100$ & ICRP-38 & $5.50 \mathrm{E}+01$ & $y$ & $1.74 \mathrm{E}+09$ & $5.38 \mathrm{E}+01$ & ICRP-68 & $4.2 \mathrm{E}-09$ & $M$ & ICRP-68 & $3.8 \mathrm{E}-10$ & -- & -- \\
\hline Sn-123 & Sn-123 & 122.906 & Tin & 0.01 & 0.03 & $50-100$ & ICRP-38 & $1.29 \mathrm{E}+02$ & $d$ & $1.12 \mathrm{E}+07$ & $8.22 \mathrm{E}+03$ & ICRP-68 & $7.7 \mathrm{E}-09$ & $M$ & ICRP-68 & $2.1 \mathrm{E}-09$ & -- & -- \\
\hline Sn-123m & Sn-123 & 122.906 & Tin & 0.01 & 0.03 & $50-100$ & ICRP-38 & $4.01 \mathrm{E}+01$ & $\mathrm{~m}$ & $2.40 \mathrm{E}+03$ & $3.82 \mathrm{E}+07$ & ICRP-68 & $4.4 \mathrm{E}-11$ & $M$ & ICRP-68 & $3.8 \mathrm{E}-11$ & -- & -- \\
\hline Sn-125 & Sn-125 & 124.908 & Tin & 0.01 & 0.03 & $50-100$ & ICRP-38 & $9.64 \mathrm{E}+00$ & $d$ & $8.33 \mathrm{E}+05$ & $1.08 \mathrm{E}+05$ & ICRP-68 & $3.0 \mathrm{E}-09$ & $M$ & ICRP-68 & $3.1 \mathrm{E}-09$ & -- & -- \\
\hline Sn-125m & Sn-125 & 124.908 & Tin & 0.01 & 0.03 & $50-100$ & ICRP-107 & $9.52 \mathrm{E}+00$ & $\mathrm{~m}$ & $5.71 \mathrm{E}+02$ & $1.58 \mathrm{E}+08$ & -- & -- & -- & -- & -- & -- & -- \\
\hline Sn-126 & Sn-126 & 125.908 & Tin & 0.01 & 0.03 & $50-100$ & ICRP-38 & $1.00 \mathrm{E}+05$ & $y$ & $3.16 \mathrm{E}+12$ & $2.84 \mathrm{E}-02$ & ICRP-68 & $2.7 \mathrm{E}-08$ & $M$ & ICRP-68 & $4.7 \mathrm{E}-09$ & -- & -- \\
\hline Sn-127 & Sn-127 & 126.910 & Tin & 0.01 & 0.03 & $50-100$ & ICRP-38 & $2.10 \mathrm{E}+00$ & $\mathrm{~h}$ & $7.56 \mathrm{E}+03$ & $1.18 \mathrm{E}+07$ & ICRP-68 & $2.0 \mathrm{E}-10$ & $\mathrm{M}$ & ICRP-68 & $2.0 \mathrm{E}-10$ & -- & -- \\
\hline Sn-127m & Sn-127 & 126.910 & Tin & 0.01 & 0.03 & $50-100$ & ICRP-107 & $4.13 \mathrm{E}+00$ & $\mathrm{~m}$ & $2.48 \mathrm{E}+02$ & $3.59 \mathrm{E}+08$ & -- & -- & -- & -- & -- & -- & -- \\
\hline Sn-128 & Sn-128 & 127.911 & Tin & 0.01 & 0.03 & $50-100$ & ICRP-38 & $5.91 \mathrm{E}+01$ & $\mathrm{~m}$ & $3.55 \mathrm{E}+03$ & $2.49 \mathrm{E}+07$ & ICRP-68 & $1.5 \mathrm{E}-10$ & $M$ & ICRP-68 & $1.5 \mathrm{E}-10$ & -- & -- \\
\hline Sn-129 & Sn-129 & 128.913 & Tin & 0.01 & 0.03 & $50-100$ & ICRP-107 & $2.23 \mathrm{E}+00$ & $\mathrm{~m}$ & $1.34 \mathrm{E}+02$ & $6.54 \mathrm{E}+08$ & -- & -- & -- & -- & -- & -- & -- \\
\hline Sn-130 & Sn-130 & 129.914 & Tin & 0.01 & 0.03 & $50-100$ & ICRP-107 & $3.72 \mathrm{E}+00$ & $\mathrm{~m}$ & $2.23 \mathrm{E}+02$ & $3.89 \mathrm{E}+08$ & -- & -- & -- & -- & -- & -- & -- \\
\hline Sn-130m & Sn-130 & 129.914 & Tin & 0.01 & 0.03 & $50-100$ & ICRP-107 & $1.70 \mathrm{E}+00$ & $\mathrm{~m}$ & $1.02 \mathrm{E}+02$ & $8.51 \mathrm{E}+08$ & -- & -- & -- & -- & -- & -- & -- \\
\hline Sr-79 & Sr-79 & 78.930 & Strontium & 0.01 & 2.5 & 100 & ICRP-107 & $2.25 \mathrm{E}+00$ & $\mathrm{~m}$ & $1.35 \mathrm{E}+02$ & $1.06 \mathrm{E}+09$ & -- & -- & -- & -- & -- & -- & -- \\
\hline Sr-80 & Sr-80 & 79.925 & Strontium & 0.01 & 2.5 & 100 & ICRP-38 & $1.00 \mathrm{E}+02$ & $\mathrm{~m}$ & $6.00 \mathrm{E}+03$ & $2.35 \mathrm{E}+07$ & ICRP-68 & $2.1 \mathrm{E}-10$ & $\mathrm{~s}$ & ICRP-68 & $3.5 \mathrm{E}-10$ & -- & -- \\
\hline
\end{tabular}




\begin{tabular}{|c|c|c|c|c|c|c|c|c|c|c|c|c|c|c|c|c|c|c|}
\hline \multirow{3}{*}{$\begin{array}{l}\text { MASTER } \\
\text { ISOTOPE } \\
\text { LIST } \\
\text { Sr-81 } \\
\end{array}$} & \multirow{3}{*}{$\begin{array}{c}\begin{array}{c}\text { Atomic } \\
\text { Mass } \\
\text { Nuclide }\end{array} \\
\text { Sr-81 }\end{array}$} & \multirow{3}{*}{$\begin{array}{l}\begin{array}{c}\text { Atomic } \\
\text { Mass }\end{array} \\
80.923\end{array}$} & & & \multicolumn{14}{|c|}{ Table C.1 Radionuclide Specific Input Dat } \\
\hline & & & \multirow{2}{*}{$\begin{array}{c}\text { Element } \\
\text { Strontium }\end{array}$} & \multirow{2}{*}{$\begin{array}{c}\text { EPA } \\
\text { RF } \\
0.01\end{array}$} & $\begin{array}{c}\text { EPA } \\
\text { Conc } \\
\text { Factors } \\
\left(B_{v}\right) \\
\end{array}$ & $\begin{array}{c}\text { EPA } \\
\text { Sorption } \\
\text { Coefficient } \\
\left(K_{d}\right)\end{array}$ & $\begin{array}{c}\text { Half-Life } \\
\text { Reference }\end{array}$ & $\begin{array}{r}\text { Half-Lit } \\
\text { (original u }\end{array}$ & & $\begin{array}{l}\text { Half Life } \\
\text { (s) }\end{array}$ & $\begin{array}{l}\text { Sp. Act } \\
\text { (Ci/g) }\end{array}$ & $\begin{array}{l}\text { Inhalation } \\
\text { DC } \\
\text { Reference }\end{array}$ & $\begin{array}{c}\text { HC-3 } \\
\text { Bounding } \\
\text { Inhalation } \\
\text { DC } \\
\text { (Sv/Bq) } \\
\end{array}$ & $\begin{array}{c}\text { Lung } \\
\text { Absorption } \\
\text { Class/Type }\end{array}$ & $\begin{array}{l}\text { Ingestion } \\
\text { DC } \\
\text { Reference }\end{array}$ & $\begin{array}{c}\text { HC-3 } \\
\text { Bounding } \\
\text { Ingestion } \\
\text { DC } \\
\text { (Sv/Bq) } \\
\end{array}$ & $\begin{array}{c}\text { Immersion } \\
\text { DC } \\
\text { Reference }\end{array}$ & $\begin{array}{c}\mathrm{HC}-3 \\
\text { Immersion } \\
\mathrm{DC}(\mathrm{Sv} / \mathrm{d} \\
\left.\operatorname{per} \mathrm{Bq} / \mathrm{m}^{3}\right)\end{array}$ \\
\hline & & & & & 2.5 & 100 & ICRP-38 & $2.55 \mathrm{E}+01$ & $\mathrm{~m}$ & $1.53 \mathrm{E}+03$ & $9.11 \mathrm{E}+07$ & ICRP-68 & $6.1 \mathrm{E}-11$ & $\mathrm{~s}$ & ICRP-68 & $7.8 \mathrm{E}-11$ & -- & -- \\
\hline Sr-82 & Sr-82 & 81.918 & Strontium & 0.01 & 2.5 & 100 & ICRP-38 & $2.50 \mathrm{E}+01$ & d & $2.16 \mathrm{E}+06$ & $6.38 \mathrm{E}+04$ & ICRP-68 & $1.0 \mathrm{E}-08$ & $\mathrm{~s}$ & ICRP-68 & $6.1 \mathrm{E}-09$ & -- & -- \\
\hline Sr-83 & Sr-83 & 82.918 & Strontium & 0.01 & 2.5 & 100 & ICRP-38 & $3.24 \mathrm{E}+01$ & $\mathrm{~h}$ & $1.17 \mathrm{E}+05$ & $1.17 \mathrm{E}+06$ & ICRP-68 & $4.9 \mathrm{E}-10$ & $\mathrm{~s}$ & ICRP-68 & $5.8 \mathrm{E}-10$ & -- & -- \\
\hline Sr-85 & Sr-85 & 84.913 & Strontium & 0.01 & 2.5 & 100 & ICRP-38 & $6.48 \mathrm{E}+01$ & $d$ & $5.60 \mathrm{E}+06$ & $2.37 \mathrm{E}+04$ & ICRP-68 & $7.7 \mathrm{E}-10$ & $\mathrm{~s}$ & ICRP-68 & $5.6 \mathrm{E}-10$ & -- & -- \\
\hline Sr-85m & Sr-85 & 84.913 & Strontium & 0.01 & 2.5 & 100 & ICRP-38 & $6.95 \mathrm{E}+01$ & $\mathrm{~m}$ & $4.17 \mathrm{E}+03$ & $3.19 \mathrm{E}+07$ & ICRP-68 & $7.4 \mathrm{E}-12$ & $\mathrm{~s}$ & ICRP-68 & $6.1 \mathrm{E}-12$ & -- & -- \\
\hline Sr-87m & Sr-87 & 86.909 & Strontium & 0.01 & 2.5 & 100 & ICRP-38 & $2.81 \mathrm{E}+00$ & $\mathrm{~h}$ & $1.01 \mathrm{E}+04$ & $1.29 \mathrm{E}+07$ & ICRP-68 & $3.5 \mathrm{E}-11$ & $\mathrm{~s}$ & ICRP-68 & $3.3 \mathrm{E}-11$ & -- & -- \\
\hline Sr-89 & Sr-89 & 88.907 & Strontium & 0.01 & 2.5 & 100 & ICRP-38 & $5.05 \mathrm{E}+01$ & $d$ & $4.36 \mathrm{E}+06$ & $2.91 \mathrm{E}+04$ & ICRP-68 & $7.5 \mathrm{E}-09$ & $\mathrm{~s}$ & ICRP-68 & $2.6 \mathrm{E}-09$ & -- & -- \\
\hline Sr-90 & Sr-90 & 89.908 & Strontium & 0.01 & 2.5 & 100 & ICRP-38 & $2.91 \mathrm{E}+01$ & $y$ & $9.19 \mathrm{E}+08$ & $1.37 \mathrm{E}+02$ & ICRP-68 & $1.5 \mathrm{E}-07$ & $\mathrm{~s}$ & ICRP-68 & $2.8 \mathrm{E}-08$ & -- & -- \\
\hline Sr-91 & Sr-91 & 90.910 & Strontium & 0.01 & 2.5 & 100 & ICRP-38 & $9.50 \mathrm{E}+00$ & $\mathrm{~h}$ & $3.42 \mathrm{E}+04$ & $3.63 \mathrm{E}+06$ & ICRP-68 & $5.7 \mathrm{E}-10$ & $\mathrm{~s}$ & ICRP-68 & $7.6 \mathrm{E}-10$ & -- & -- \\
\hline Sr-92 & Sr-92 & 91.911 & Strontium & 0.01 & 2.5 & 100 & ICRP-38 & $2.71 \mathrm{E}+00$ & $\mathrm{~h}$ & $9.76 \mathrm{E}+03$ & $1.26 \mathrm{E}+07$ & ICRP-68 & $3.4 \mathrm{E}-10$ & $\mathrm{~s}$ & ICRP-68 & $4.9 \mathrm{E}-10$ & -- & -- \\
\hline Sr-93 & Sr-93 & 92.914 & Strontium & 0.01 & 2.5 & 100 & ICRP-107 & $7.42 \mathrm{E}+00$ & $\mathrm{~m}$ & $4.45 E+02$ & $2.73 \mathrm{E}+08$ & -- & -- & -- & -- & -- & -- & -- \\
\hline Sr-94 & Sr-94 & 93.915 & Strontium & 0.01 & 2.5 & 100 & ICRP-107 & $7.53 \mathrm{E}+01$ & $\mathrm{~s}$ & $7.53 \mathrm{E}+01$ & $1.60 \mathrm{E}+09$ & -- & -- & -- & -- & -- & -- & -- \\
\hline Ta-170 & Ta-170 & 169.946 & Tantalum & 0.001 & 0.01 & 0 & ICRP-107 & $6.76 \mathrm{E}+00$ & $\mathrm{~m}$ & $4.06 \mathrm{E}+02$ & $1.64 \mathrm{E}+08$ & -- & -- & -- & -- & -- & -- & -- \\
\hline Ta-172 & Ta-172 & 171.945 & Tantalum & 0.001 & 0.01 & 0 & ICRP-38 & $3.68 \mathrm{E}+01$ & $\mathrm{~m}$ & $2.21 \mathrm{E}+03$ & $2.97 \mathrm{E}+07$ & ICRP-68 & $5.7 \mathrm{E}-11$ & $\mathrm{~s}$ & ICRP-68 & $5.3 \mathrm{E}-11$ & -- & -- \\
\hline Ta-173 & Ta-173 & 172.944 & $\begin{array}{l}\text { Tantalum } \\
\end{array}$ & 0.001 & 0.01 & 0 & ICRP-38 & $3.65 \mathrm{E}+00$ & $\mathrm{~h}$ & $1.31 \mathrm{E}+04$ & $4.96 \mathrm{E}+06$ & ICRP-68 & $1.6 \mathrm{E}-10$ & $M$ & ICRP-68 & $1.9 \mathrm{E}-10$ & -- & -- \\
\hline Ta-174 & Ta-174 & 173.944 & Tantalum & 0.001 & 0.01 & 0 & ICRP-38 & $1.20 \mathrm{E}+00$ & $\mathrm{~h}$ & $4.32 \mathrm{E}+03$ & $1.50 \mathrm{E}+07$ & ICRP-68 & $6.6 \mathrm{E}-11$ & $\mathrm{~s}$ & ICRP-68 & $5.7 \mathrm{E}-11$ & -- & -- \\
\hline Ta-175 & Ta-175 & 174.944 & Tantalum & 0.001 & 0.01 & 0 & ICRP-38 & $1.05 \mathrm{E}+01$ & $\mathrm{~h}$ & $3.78 \mathrm{E}+04$ & $1.71 \mathrm{E}+06$ & ICRP-68 & $2.0 \mathrm{E}-10$ & $\mathrm{M}$ & ICRP-68 & $2.1 \mathrm{E}-10$ & -- & -- \\
\hline Ta-176 & Ta-176 & 175.945 & Tantalum & 0.001 & 0.01 & 0 & ICRP-38 & $8.08 \mathrm{E}+00$ & $\mathrm{~h}$ & $2.91 \mathrm{E}+04$ & $2.20 \mathrm{E}+06$ & ICRP-68 & $3.3 \mathrm{E}-10$ & $\mathrm{~s}$ & ICRP-68 & $3.1 \mathrm{E}-10$ & -- & -- \\
\hline Ta-177 & Ta-177 & 176.944 & Tantalum & 0.001 & 0.01 & 0 & ICRP-38 & $5.66 \mathrm{E}+01$ & $\mathrm{~h}$ & $2.04 \mathrm{E}+05$ & $3.13 \mathrm{E}+05$ & ICRP-68 & $1.3 \mathrm{E}-10$ & $\mathrm{~s}$ & ICRP-68 & $1.1 \mathrm{E}-10$ & -- & -- \\
\hline Ta-178s & Ta-178 & 177.946 & Tantalum & 0.001 & 0.01 & 0 & ICRP-38 & $9.31 \mathrm{E}+00$ & $\mathrm{~m}$ & $5.59 \mathrm{E}+02$ & $1.13 \mathrm{E}+08$ & -- & -- & -- & -- & -- & -- & -- \\
\hline Ta-178I & Ta-178 & 177.946 & Tantalum & 0.001 & 0.01 & 0 & ICRP-38 & $2.20 \mathrm{E}+00$ & $\mathrm{~h}$ & $7.92 E+03$ & $8.00 \mathrm{E}+06$ & ICRP-68 & $1.1 \mathrm{E}-10$ & $\mathrm{~s}$ & ICRP-68 & $7.8 \mathrm{E}-11$ & -- & -- \\
\hline Ta-179 & Ta-179 & 178.946 & Tantalum & 0.001 & 0.01 & 0 & ICRP-38 & $6.65 \mathrm{E}+02$ & $d$ & $5.74 \mathrm{E}+07$ & $1.10 \mathrm{E}+03$ & ICRP-68 & $5.2 \mathrm{E}-10$ & $\mathrm{~s}$ & ICRP-68 & $6.5 \mathrm{E}-11$ & -- & -- \\
\hline Ta-180 & Ta-180 & 179.947 & Tantalum & 0.001 & 0.01 & 0 & ICRP-38 & $1.00 \mathrm{E}+13$ & $y$ & $3.16 \mathrm{E}+20$ & $1.99 \mathrm{E}-10$ & ICRP-68 & $2.4 \mathrm{E}-08$ & $\mathrm{~s}$ & ICRP-68 & $8.4 \mathrm{E}-10$ & -- & -- \\
\hline Ta-180m & Ta-180 & 179.947 & Tantalum & 0.001 & 0.01 & 0 & ICRP-38 & $8.10 \mathrm{E}+00$ & $\mathrm{~h}$ & $2.92 E+04$ & $2.15 \mathrm{E}+06$ & ICRP-68 & $6.2 \mathrm{E}-11$ & $\mathrm{~s}$ & ICRP-68 & $5.4 \mathrm{E}-11$ & -- & -- \\
\hline Ta-182 & Ta-182 & 181.950 & Tantalum & 0.001 & 0.01 & 0 & ICRP-38 & $1.15 \mathrm{E}+02$ & $d$ & $9.94 \mathrm{E}+06$ & $6.24 \mathrm{E}+03$ & ICRP-68 & $9.7 \mathrm{E}-09$ & $\mathrm{~s}$ & ICRP-68 & $1.5 \mathrm{E}-09$ & -- & -- \\
\hline Ta-182m & Ta-182 & 181.950 & Tantalum & 0.001 & 0.01 & 0 & ICRP-38 & $1.58 \mathrm{E}+01$ & $\mathrm{~m}$ & $9.50 \mathrm{E}+02$ & $6.52 \mathrm{E}+07$ & ICRP-68 & $3.6 \mathrm{E}-11$ & $\mathrm{~s}$ & ICRP-68 & $1.2 \mathrm{E}-11$ & -- & -- \\
\hline Ta-183 & Ta-183 & 182.951 & Tantalum & 0.001 & 0.01 & 0 & ICRP-38 & $5.10 \mathrm{E}+00$ & d & $4.41 \mathrm{E}+05$ & $1.40 \mathrm{E}+05$ & ICRP-68 & $2.0 \mathrm{E}-09$ & $\mathrm{~s}$ & ICRP-68 & $1.3 \mathrm{E}-09$ & -- & -- \\
\hline Ta-184 & Тa-184 & 183.954 & Tantalum & 0.001 & 0.01 & 0 & ICRP-38 & $8.70 \mathrm{E}+00$ & $\mathrm{~h}$ & $3.13 E+04$ & $1.96 \mathrm{E}+06$ & ICRP-68 & $6.3 \mathrm{E}-10$ & $\mathrm{~s}$ & ICRP-68 & $6.8 \mathrm{E}-10$ & -- & -- \\
\hline Ta-185 & Ta-185 & 184.956 & Tantalum & 0.001 & 0.01 & 0 & ICRP-38 & $4.90 \mathrm{E}+01$ & $\mathrm{~m}$ & $2.94 \mathrm{E}+03$ & $2.07 E+07$ & ICRP-68 & $7.2 \mathrm{E}-11$ & $\mathrm{~s}$ & ICRP-68 & $6.8 \mathrm{E}-11$ & -- & -- \\
\hline Ta-186 & Ta-186 & 185.959 & Tantalum & 0.001 & 0.01 & 0 & ICRP-38 & $1.05 \mathrm{E}+01$ & $\mathrm{~m}$ & $6.30 \mathrm{E}+02$ & $9.63 \mathrm{E}+07$ & ICRP-68 & $3.1 \mathrm{E}-11$ & $\mathrm{~s}$ & ICRP-68 & $3.3 \mathrm{E}-11$ & -- & -- \\
\hline Tb-146 & Tb-146 & 145.927 & Terbium & 0.01 & 0.01 & $500-1000$ & ICRP-107 & $2.30 \mathrm{E}+01$ & $\mathrm{~s}$ & $2.30 \mathrm{E}+01$ & $3.36 \mathrm{E}+09$ & -- & -- & -- & -- & -- & -- & -- \\
\hline Tb-147 & Tb-147 & 146.924 & Terbium & 0.01 & 0.01 & $500-1000$ & ICRP-38 & $1.65 \mathrm{E}+00$ & $\mathrm{~h}$ & $5.94 \mathrm{E}+03$ & $1.29 \mathrm{E}+07$ & ICRP-68 & $1.2 \mathrm{E}-10$ & $M$ & ICRP-68 & $1.6 \mathrm{E}-10$ & -- & -- \\
\hline Tb-147m & Tb-147 & 146.924 & Terbium & 0.01 & 0.01 & $500-1000$ & ICRP-107 & $1.87 \mathrm{E}+00$ & $\mathrm{~m}$ & $1.12 \mathrm{E}+02$ & $6.84 \mathrm{E}+08$ & -- & -- & -- & -- & -- & -- & -- \\
\hline Tb-148 & Tb-148 & 147.924 & Terbium & 0.01 & 0.01 & $500-1000$ & ICRP-107 & $6.00 \mathrm{E}+01$ & $\mathrm{~m}$ & $3.60 \mathrm{E}+03$ & $2.12 \mathrm{E}+07$ & JAERI & $1.0 \mathrm{E}-10$ & $M$ & JAERI & $1.3 \mathrm{E}-10$ & -- & -- \\
\hline Tb-148m & Tb-148 & 147.924 & Terbium & 0.01 & 0.01 & $500-1000$ & ICRP-107 & $2.20 \mathrm{E}+00$ & $\mathrm{~m}$ & $1.32 \mathrm{E}+02$ & $5.78 \mathrm{E}+08$ & -- & -- & -- & -- & -- & -- & -- \\
\hline Tb-149 & Tb-149 & 148.923 & Terbium & 0.01 & 0.01 & $500-1000$ & $\begin{array}{l}\text { ICRP-38 } \\
\end{array}$ & $4.15 \mathrm{E}+00$ & $\mathrm{~h}$ & $1.49 \mathrm{E}+04$ & $5.07 \mathrm{E}+06$ & ICRP-68 & $4.3 \mathrm{E}-09$ & $M$ & ICRP-68 & $2.5 \mathrm{E}-10$ & -- & -- \\
\hline Tb-149m & Tb-149 & 148.923 & Terbium & 0.01 & 0.01 & $500-1000$ & ICRP-107 & $4.16 \mathrm{E}+00$ & $\mathrm{~m}$ & $2.50 \mathrm{E}+02$ & $3.03 E+08$ & -- & -- & -- & -- & -- & -- & -- \\
\hline Tb-150 & Tb-150 & 149.924 & Terbium & 0.01 & 0.01 & $500-1000$ & ICRP-38 & $3.27 \mathrm{E}+00$ & $\mathrm{~h}$ & $1.18 \mathrm{E}+04$ & $6.39 \mathrm{E}+06$ & ICRP-68 & $1.8 \mathrm{E}-10$ & $\mathrm{M}$ & ICRP-68 & $2.5 \mathrm{E}-10$ & -- & -- \\
\hline Tb-150m & Tb-150 & 149.924 & Terbium & 0.01 & 0.01 & $500-1000$ & ICRP-107 & $5.80 \mathrm{E}+00$ & $\mathrm{~m}$ & $3.48 \mathrm{E}+02$ & $2.16 \mathrm{E}+08$ & -- & -- & -- & -- & -- & -- & -- \\
\hline Tb-151 & Tb-151 & 150.923 & Terbium & 0.01 & 0.01 & $500-1000$ & ICRP-38 & $1.76 \mathrm{E}+01$ & $\mathrm{~h}$ & $6.34 \mathrm{E}+04$ & $1.18 \mathrm{E}+06$ & ICRP-68 & $3.3 \mathrm{E}-10$ & $M$ & ICRP-68 & $3.4 \mathrm{E}-10$ & -- & -- \\
\hline Tb-151m & Tb-151 & 150.923 & Terbium & 0.01 & $\begin{array}{ll}0.01 \\
\end{array}$ & $500-1000$ & ICRP-107 & $2.50 \mathrm{E}+01$ & $\mathrm{~s}$ & $2.50 \mathrm{E}+01$ & $2.99 \mathrm{E}+09$ & -- & -- & -- & -- & -- & -- & -- \\
\hline Tb-152 & Tb-152 & 151.924 & Terbium & 0.01 & 0.01 & $500-1000$ & ICRP-107 & $1.75 \mathrm{E}+01$ & $\mathrm{~h}$ & $6.30 \mathrm{E}+04$ & $1.18 \mathrm{E}+06$ & JAERI & $5.0 \mathrm{E}-10$ & $M$ & JAERI & $7.1 \mathrm{E}-10$ & -- & -- \\
\hline Tb-152m & Tb-152 & 151.924 & Terbium & 0.01 & 0.01 & $500-1000$ & ICRP-107 & $4.20 \mathrm{E}+00$ & $\mathrm{~m}$ & $2.52 \mathrm{E}+02$ & $2.95 \mathrm{E}+08$ & -- & -- & -- & -- & -- & -- & -- \\
\hline Tb-153 & Tb-153 & 152.923 & Terbium & 0.01 & 0.01 & $500-1000$ & ICRP-38 & $2.34 \mathrm{E}+00$ & $d$ & $2.02 \mathrm{E}+05$ & $3.65 \mathrm{E}+05$ & ICRP-68 & $2.4 \mathrm{E}-10$ & $M$ & ICRP-68 & $2.5 \mathrm{E}-10$ & -- & -- \\
\hline Tb-154 & Tb-154 & 153.925 & Terbium & 0.01 & 0.01 & $500-1000$ & ICRP-38 & $2.14 \mathrm{E}+01$ & $\mathrm{~h}$ & $7.70 E+04$ & $9.51 \mathrm{E}+05$ & ICRP-68 & $6.0 \mathrm{E}-10$ & $M$ & ICRP-68 & $6.5 \mathrm{E}-10$ & -- & -- \\
\hline
\end{tabular}




\begin{tabular}{|c|c|c|c|c|c|c|c|c|c|c|c|c|c|c|c|c|c|c|}
\hline \multirow{3}{*}{$\begin{array}{l}\text { MASTER } \\
\text { ISOTOPE } \\
\text { LIST } \\
\text { Tb-155 }\end{array}$} & \multirow{3}{*}{$\begin{array}{c}\begin{array}{c}\text { Atomic } \\
\text { Mass } \\
\text { Nuclide }\end{array} \\
\text { Tb-155 }\end{array}$} & & & & \multicolumn{14}{|c|}{ Table C.1 Radionuclide Spe } \\
\hline & & \multirow{2}{*}{$\begin{array}{c}\begin{array}{c}\text { Atomic } \\
\text { Mass }\end{array} \\
154.924\end{array}$} & \multirow{2}{*}{$\begin{array}{l}\text { Element } \\
\text { Terbium }\end{array}$} & \multirow{2}{*}{$\begin{array}{c}\text { EPA } \\
\text { RF } \\
0.01\end{array}$} & $\begin{array}{c}\text { EPA } \\
\text { Conc } \\
\text { Factors } \\
\left(B_{v}\right) \\
\end{array}$ & $\begin{array}{c}\text { EPA } \\
\text { Sorption } \\
\text { Coefficient } \\
\left(K_{d}\right)\end{array}$ & $\begin{array}{c}\text { Half-Life } \\
\text { Reference }\end{array}$ & $\begin{array}{r}\text { Half-Lit } \\
\text { (original u }\end{array}$ & & $\begin{array}{l}\text { Half Life } \\
\text { (s) }\end{array}$ & $\begin{array}{l}\text { Sp. Act } \\
\text { (Ci/g) }\end{array}$ & $\begin{array}{l}\text { Inhalation } \\
\text { DC } \\
\text { Reference }\end{array}$ & $\begin{array}{c}\text { HC-3 } \\
\text { Bounding } \\
\text { Inhalation } \\
\text { DC } \\
\text { (Sv/Bq) } \\
\end{array}$ & $\begin{array}{c}\text { Lung } \\
\text { Absorption } \\
\text { Class/Type }\end{array}$ & $\begin{array}{l}\text { Ingestion } \\
\text { DC } \\
\text { Reference }\end{array}$ & $\begin{array}{c}\text { HC-3 } \\
\text { Bounding } \\
\text { Ingestion } \\
\text { DC } \\
\text { (Sv/Bq) } \\
\end{array}$ & $\begin{array}{c}\text { Immersion } \\
\text { DC } \\
\text { Reference }\end{array}$ & $\begin{array}{c}\mathrm{HC}-3 \\
\text { Immersion } \\
\mathrm{DC}(\mathrm{Sv} / \mathrm{d} \\
\left.\operatorname{per} \mathrm{Bq} / \mathrm{m}^{3}\right)\end{array}$ \\
\hline & & & & & 0.01 & $500-1000$ & ICRP-38 & $5.32 \mathrm{E}+00$ & $\mathrm{~d}$ & $4.60 E+05$ & $1.58 \mathrm{E}+05$ & ICRP-68 & $2.5 \mathrm{E}-10$ & $\mathrm{M}$ & ICRP-68 & $2.1 \mathrm{E}-10$ & -- & -- \\
\hline Tb-156 & Tb-156 & 155.925 & Terbium & 0.01 & 0.01 & $500-1000$ & ICRP-38 & $5.34 \mathrm{E}+00$ & $\mathrm{~d}$ & $4.61 \mathrm{E}+05$ & $1.57 \mathrm{E}+05$ & ICRP-68 & $1.4 \mathrm{E}-09$ & $\mathrm{M}$ & ICRP-68 & $1.2 \mathrm{E}-09$ & -- & -- \\
\hline Tb-156ml & Tb-156 & 155.925 & Terbium & 0.01 & 0.01 & $500-1000$ & ICRP-38 & $2.44 \mathrm{E}+01$ & $\mathrm{~h}$ & $8.78 \mathrm{E}+04$ & $8.24 \mathrm{E}+05$ & ICRP-68 & $2.3 \mathrm{E}-10$ & $\mathrm{M}$ & ICRP-68 & $1.7 \mathrm{E}-10$ & -- & -- \\
\hline Tb-156ms & Tb-156 & 155.925 & Terbium & 0.01 & 0.01 & $500-1000$ & ICRP-38 & $5.00 \mathrm{E}+00$ & $\mathrm{~h}$ & $1.80 \mathrm{E}+04$ & $4.02 \mathrm{E}+06$ & ICRP-68 & $1.3 \mathrm{E}-10$ & $\mathrm{M}$ & ICRP-68 & $8.1 \mathrm{E}-11$ & -- & -- \\
\hline Tb-157 & Tb-157 & 156.924 & Terbium & 0.01 & 0.01 & $500-1000$ & ICRP-38 & $1.50 \mathrm{E}+02$ & $y$ & $4.73 \mathrm{E}+09$ & $1.52 \mathrm{E}+01$ & ICRP-68 & $1.1 \mathrm{E}-09$ & $\mathrm{M}$ & ICRP-68 & $3.4 \mathrm{E}-11$ & -- & -- \\
\hline Tb-158 & Tb-158 & 157.925 & Terbium & 0.01 & 0.01 & $500-1000$ & ICRP-38 & $1.50 \mathrm{E}+02$ & $y$ & $4.73 \mathrm{E}+09$ & $1.51 \mathrm{E}+01$ & ICRP-68 & $4.3 \mathrm{E}-08$ & $M$ & ICRP-68 & $1.1 \mathrm{E}-09$ & -- & -- \\
\hline Tb-160 & Tb-160 & 159.927 & Terbium & 0.01 & 0.01 & $500-1000$ & ICRP-38 & $7.23 \mathrm{E}+01$ & $\mathrm{~d}$ & $6.25 \mathrm{E}+06$ & $1.13 \mathrm{E}+04$ & ICRP-68 & $6.6 \mathrm{E}-09$ & $\mathrm{M}$ & ICRP-68 & $1.6 \mathrm{E}-09$ & -- & -- \\
\hline Tb-161 & Tb-161 & 160.928 & Terbium & 0.01 & 0.01 & $500-1000$ & ICRP-38 & $6.91 \mathrm{E}+00$ & $d$ & $5.97 \mathrm{E}+05$ & $1.17 E+05$ & ICRP-68 & $1.2 \mathrm{E}-09$ & $M$ & ICRP-68 & $7.2 \mathrm{E}-10$ & -- & -- \\
\hline Tb-162 & Tb-162 & 161.929 & Terbium & 0.01 & 0.01 & $500-1000$ & ICRP-107 & $7.60 \mathrm{E}+00$ & $\mathrm{~m}$ & $4.56 \mathrm{E}+02$ & $1.53 \mathrm{E}+08$ & -- & -- & -- & -- & -- & -- & -- \\
\hline Tb-163 & Tb-163 & 162.931 & Terbium & 0.01 & 0.01 & $500-1000$ & ICRP-107 & $1.95 \mathrm{E}+01$ & $\mathrm{~m}$ & $1.17 \mathrm{E}+03$ & $5.92 \mathrm{E}+07$ & JAERI & $2.9 \mathrm{E}-11$ & $M$ & JAERI & $2.2 \mathrm{E}-11$ & -- & -- \\
\hline Tb-164 & Tb-164 & 163.933 & Terbium & 0.01 & 0.01 & $500-1000$ & ICRP-107 & $3.00 \mathrm{E}+00$ & $\mathrm{~m}$ & $1.80 \mathrm{E}+02$ & $3.82 \mathrm{E}+08$ & -- & -- & -- & -- & -- & -- & -- \\
\hline Tb-165 & Tb-165 & 164.935 & Terbium & 0.01 & 0.01 & $500-1000$ & ICRP-107 & $2.11 \mathrm{E}+00$ & $\mathrm{~m}$ & $1.27 \mathrm{E}+02$ & $5.40 \mathrm{E}+08$ & -- & -- & -- & -- & -- & -- & -- \\
\hline Tc-101 & Tc-101 & 100.907 & Technetium & 0.01 & 9.5 & 0 & ICRP-38 & $1.42 \mathrm{E}+01$ & $\mathrm{~m}$ & $8.52 \mathrm{E}+02$ & $1.31 \mathrm{E}+08$ & $\begin{array}{l}\text { ICRP-68 } \\
\end{array}$ & $2.1 \mathrm{E}-11$ & $M$ & ICRP-68 & $1.9 \mathrm{E}-11$ & -- & -- \\
\hline Tc-102 & Tc-102 & 101.909 & Technetium & 0.01 & 9.5 & 0 & ICRP-107 & $5.28 \mathrm{E}+00$ & $\mathrm{~s}$ & $5.28 \mathrm{E}+00$ & $2.10 \mathrm{E}+10$ & -- & -- & -- & -- & -- & -- & -- \\
\hline Tc-102m & Tc-102 & 101.909 & Technetium & 0.01 & 9.5 & 0 & ICRP-107 & $4.35 \mathrm{E}+00$ & $\mathrm{~m}$ & $2.61 \mathrm{E}+02$ & $4.24 \mathrm{E}+08$ & -- & -- & -- & -- & -- & -- & -- \\
\hline Tc-104 & Tc-104 & 103.911 & Technetium & 0.01 & 9.5 & 0 & ICRP-38 & $1.82 \mathrm{E}+01$ & $\mathrm{~m}$ & $1.09 \mathrm{E}+03$ & $9.94 \mathrm{E}+07$ & ICRP-68 & $4.8 \mathrm{E}-11$ & $M$ & ICRP-68 & $8.1 \mathrm{E}-11$ & -- & -- \\
\hline Tc-105 & Tc-105 & 104.912 & Technetium & 0.01 & 9.5 & 0 & ICRP-107 & $7.60 \mathrm{E}+00$ & $\mathrm{~m}$ & $4.56 \mathrm{E}+02$ & $2.36 \mathrm{E}+08$ & -- & -- & -- & -- & -- & -- & -- \\
\hline Tc-91 & TC-91 & 90.918 & Technetium & 0.01 & 9.5 & 0 & ICRP-107 & $3.14 \mathrm{E}+00$ & $\mathrm{~m}$ & $1.88 \mathrm{E}+02$ & $6.59 \mathrm{E}+08$ & -- & -- & -- & -- & -- & -- & -- \\
\hline Tc-91m & TC-91 & 90.918 & Technetium & 0.01 & 9.5 & 0 & ICRP-107 & $3.30 \mathrm{E}+00$ & $\mathrm{~m}$ & $1.98 \mathrm{E}+02$ & $6.27 \mathrm{E}+08$ & -- & -- & -- & -- & -- & -- & -- \\
\hline Tc-92 & Tc-92 & 91.915 & Technetium & 0.01 & 9.5 & 0 & ICRP-107 & $4.25 \mathrm{E}+00$ & $\mathrm{~m}$ & $2.55 \mathrm{E}+02$ & $4.81 \mathrm{E}+08$ & -- & -- & -- & -- & -- & -- & -- \\
\hline Tc-93 & Tc-93 & 92.910 & Technetium & 0.01 & 9.5 & 0 & ICRP-38 & $2.75 \mathrm{E}+00$ & $\mathrm{~h}$ & $9.90 \mathrm{E}+03$ & $1.23 \mathrm{E}+07$ & ICRP-68 & $6.5 \mathrm{E}-11$ & $M$ & ICRP-68 & $4.9 \mathrm{E}-11$ & -- & -- \\
\hline Tc-93m & Tc-93 & 92.910 & Technetium & 0.01 & 9.5 & 0 & ICRP-38 & $4.35 \mathrm{E}+01$ & $\mathrm{~m}$ & $2.61 \mathrm{E}+03$ & $4.65 \mathrm{E}+07$ & ICRP-68 & $3.1 \mathrm{E}-11$ & $M$ & ICRP-68 & $2.4 \mathrm{E}-11$ & -- & -- \\
\hline Tc-94 & Tc-94 & 93.910 & Technetium & 0.01 & 9.5 & 0 & ICRP-38 & $2.93 \mathrm{E}+02$ & $\mathrm{~m}$ & $1.76 \mathrm{E}+04$ & $6.83 \mathrm{E}+06$ & ICRP-68 & $2.2 \mathrm{E}-10$ & $M$ & ICRP-68 & $1.8 \mathrm{E}-10$ & -- & -- \\
\hline Tc-94m & Tc-94 & 93.910 & Technetium & 0.01 & 9.5 & 0 & ICRP-38 & $5.20 \mathrm{E}+01$ & $\mathrm{~m}$ & $3.12 \mathrm{E}+03$ & $3.85 \mathrm{E}+07$ & ICRP-68 & $8.0 \mathrm{E}-11$ & $M$ & ICRP-68 & $1.1 \mathrm{E}-10$ & -- & -- \\
\hline Tc-95 & TC-95 & 94.908 & Technetium & 0.01 & 9.5 & 0 & ICRP-38 & $2.00 \mathrm{E}+01$ & $\mathrm{~h}$ & $7.20 \mathrm{E}+04$ & $1.65 \mathrm{E}+06$ & ICRP-68 & $1.8 \mathrm{E}-10$ & $\mathrm{~F}$ & ICRP-68 & $1.6 \mathrm{E}-10$ & -- & -- \\
\hline Tc-95m & Tc-95 & 94.908 & Technetium & 0.01 & 9.5 & 0 & ICRP-38 & $6.10 \mathrm{E}+01$ & $d$ & $5.27 \mathrm{E}+06$ & $2.26 \mathrm{E}+04$ & ICRP-68 & $8.7 E-10$ & $M$ & ICRP-68 & $6.2 \mathrm{E}-10$ & -- & -- \\
\hline Tc-96 & Tc-96 & 95.908 & Technetium & 0.01 & 9.5 & 0 & ICRP-38 & $4.28 \mathrm{E}+00$ & $d$ & $3.70 \mathrm{E}+05$ & $3.18 \mathrm{E}+05$ & ICRP-68 & $1.0 \mathrm{E}-09$ & $M$ & ICRP-68 & $1.1 \mathrm{E}-09$ & -- & -- \\
\hline Tc-96m & Tc-96 & 95.908 & Technetium & 0.01 & 9.5 & 0 & ICRP-38 & $5.15 \mathrm{E}+01$ & $\mathrm{~m}$ & $3.09 \mathrm{E}+03$ & $3.81 \mathrm{E}+07$ & ICRP-68 & $1.1 \mathrm{E}-11$ & $\mathrm{~F}$ & ICRP-68 & $1.3 \mathrm{E}-11$ & -- & -- \\
\hline TC-97 & TC-97 & 96.906 & Technetium & 0.01 & 9.5 & 0 & ICRP-38 & $2.60 \mathrm{E}+06$ & $y$ & $8.20 \mathrm{E}+13$ & $1.42 \mathrm{E}-03$ & ICRP-68 & $2.1 \mathrm{E}-10$ & $\mathrm{M}$ & ICRP-68 & $8.3 \mathrm{E}-11$ & -- & -- \\
\hline Tc-97m & TC-97 & 96.906 & Technetium & 0.01 & 9.5 & 0 & ICRP-38 & $8.70 \mathrm{E}+01$ & $d$ & $7.52 \mathrm{E}+06$ & $1.55 \mathrm{E}+04$ & ICRP-68 & $3.1 \mathrm{E}-09$ & $M$ & ICRP-68 & $6.6 \mathrm{E}-10$ & -- & -- \\
\hline Tc-98 & Tc-98 & 97.907 & Technetium & 0.01 & 9.5 & 0 & ICRP-38 & $4.20 \mathrm{E}+06$ & $y$ & $1.33 \mathrm{E}+14$ & $8.69 \mathrm{E}-04$ & ICRP-68 & $8.1 \mathrm{E}-09$ & $M$ & ICRP-68 & $2.3 \mathrm{E}-09$ & -- & -- \\
\hline Tc-99 & Tc-99 & 98.906 & Technetium & 0.01 & 9.5 & 0 & ICRP-38 & $2.13 \mathrm{E}+05$ & $y$ & $6.72 \mathrm{E}+12$ & $1.70 \mathrm{E}-02$ & ICRP-68 & $3.9 \mathrm{E}-09$ & $M$ & ICRP-68 & $7.8 \mathrm{E}-10$ & -- & -- \\
\hline Tc-99m & TC-99 & 98.906 & Technetium & 0.01 & 9.5 & 0 & ICRP-38 & $6.02 \mathrm{E}+00$ & $\mathrm{~h}$ & $2.17 \mathrm{E}+04$ & $5.26 \mathrm{E}+06$ & ICRP-68 & $2.9 \mathrm{E}-11$ & $M$ & ICRP-68 & $2.2 \mathrm{E}-11$ & -- & -- \\
\hline Te-113 & Te-113 & 112.916 & Tellurium & 0.01 & 0.025 & $50-100$ & ICRP-107 & $1.70 \mathrm{E}+00$ & $\mathrm{~m}$ & $1.02 \mathrm{E}+02$ & $9.80 \mathrm{E}+08$ & -- & -- & -- & -- & -- & -- & -- \\
\hline Te-114 & Te-114 & 113.912 & Tellurium & 0.01 & 0.025 & $50-100$ & ICRP-107 & $1.52 \mathrm{E}+01$ & $\mathrm{~m}$ & $9.12 \mathrm{E}+02$ & $1.09 \mathrm{E}+08$ & -- & -- & -- & -- & -- & -- & -- \\
\hline Te-115 & Te-115 & 114.912 & $\begin{array}{l}\text { Tellurium } \\
\end{array}$ & 0.01 & 0.025 & $50-100$ & ICRP-107 & $5.80 \mathrm{E}+00$ & $\mathrm{~m}$ & $3.48 \mathrm{E}+02$ & $2.82 \mathrm{E}+08$ & -- & -- & -- & -- & -- & -- & -- \\
\hline Te-115m & Te-115 & 114.912 & Tellurium & 0.01 & 0.025 & $50-100$ & ICRP-107 & $6.70 \mathrm{E}+00$ & $\mathrm{~m}$ & $4.02 \mathrm{E}+02$ & $2.44 E+08$ & -- & -- & -- & -- & -- & -- & -- \\
\hline Te-116 & Te-116 & 115.908 & Tellurium & 0.01 & 0.025 & $50-100$ & ICRP-38 & $2.49 \mathrm{E}+00$ & $\mathrm{~h}$ & $8.96 \mathrm{E}+03$ & $1.09 \mathrm{E}+07$ & ICRP-68 & $1.7 \mathrm{E}-10$ & $\mathrm{M}$ & ICRP-68 & $1.7 \mathrm{E}-10$ & -- & -- \\
\hline Te-117 & Te-117 & 116.909 & $\begin{array}{l}\text { Tellurium } \\
\end{array}$ & 0.01 & 0.025 & $50-100$ & ICRP-107 & $6.20 \mathrm{E}+01$ & $\mathrm{~m}$ & $3.72 \mathrm{E}+03$ & $2.59 \mathrm{E}+07$ & JAERI & $5.5 \mathrm{E}-11$ & $M$ & JAERI & $5.4 \mathrm{E}-11$ & -- & -- \\
\hline Te-118 & Te-118 & 117.906 & Tellurium & 0.01 & 0.025 & $50-100$ & ICRP-107 & $6.00 \mathrm{E}+00$ & $d$ & $5.18 \mathrm{E}+05$ & $1.85 \mathrm{E}+05$ & JAERI & $2.3 \mathrm{E}-09$ & $M$ & JAERI & $3.0 \mathrm{E}-09$ & -- & -- \\
\hline Te-119 & Te-119 & 118.906 & Tellurium & 0.01 & 0.025 & $50-100$ & ICRP-107 & $1.61 \mathrm{E}+01$ & $\mathrm{~h}$ & $5.78 \mathrm{E}+04$ & $1.64 \mathrm{E}+06$ & JAERI & $1.8 \mathrm{E}-10$ & $\mathrm{M}$ & JAERI & $1.8 \mathrm{E}-10$ & -- & -- \\
\hline Te-119m & Te-119 & 118.906 & Tellurium & 0.01 & 0.025 & $50-100$ & ICRP-107 & $4.70 \mathrm{E}+00$ & $d$ & $4.06 \mathrm{E}+05$ & $2.34 \mathrm{E}+05$ & JAERI & $7.3 \mathrm{E}-10$ & $\mathrm{M}$ & JAERI & $7.3 \mathrm{E}-10$ & -- & -- \\
\hline Te-121 & Te-121 & 120.905 & Tellurium & 0.01 & 0.025 & $50-100$ & ICRP-38 & $1.70 \mathrm{E}+01$ & $d$ & $1.47 \mathrm{E}+06$ & $6.35 \mathrm{E}+04$ & ICRP-68 & $5.1 \mathrm{E}-10$ & 0 & ICRP-68 & $4.3 \mathrm{E}-10$ & -- & -- \\
\hline Te-121m & Te-121 & 120.905 & Tellurium & 0.01 & 0.025 & $50-100$ & ICRP-38 & $1.54 \mathrm{E}+02$ & $d$ & $1.33 \mathrm{E}+07$ & $7.01 E+03$ & ICRP-68 & $5.5 \mathrm{E}-09$ & 0 & ICRP-68 & $2.3 \mathrm{E}-09$ & -- & -- \\
\hline Te-123 & Te-123 & 122.904 & Tellurium & 0.01 & 0.025 & $50-100$ & ICRP-38 & $1.00 \mathrm{E}+13$ & $y$ & $3.16 \mathrm{E}+20$ & $2.91 \mathrm{E}-10$ & ICRP-68 & $1.2 \mathrm{E}-08$ & 0 & ICRP-68 & $4.4 \mathrm{E}-09$ & -- & -- \\
\hline
\end{tabular}




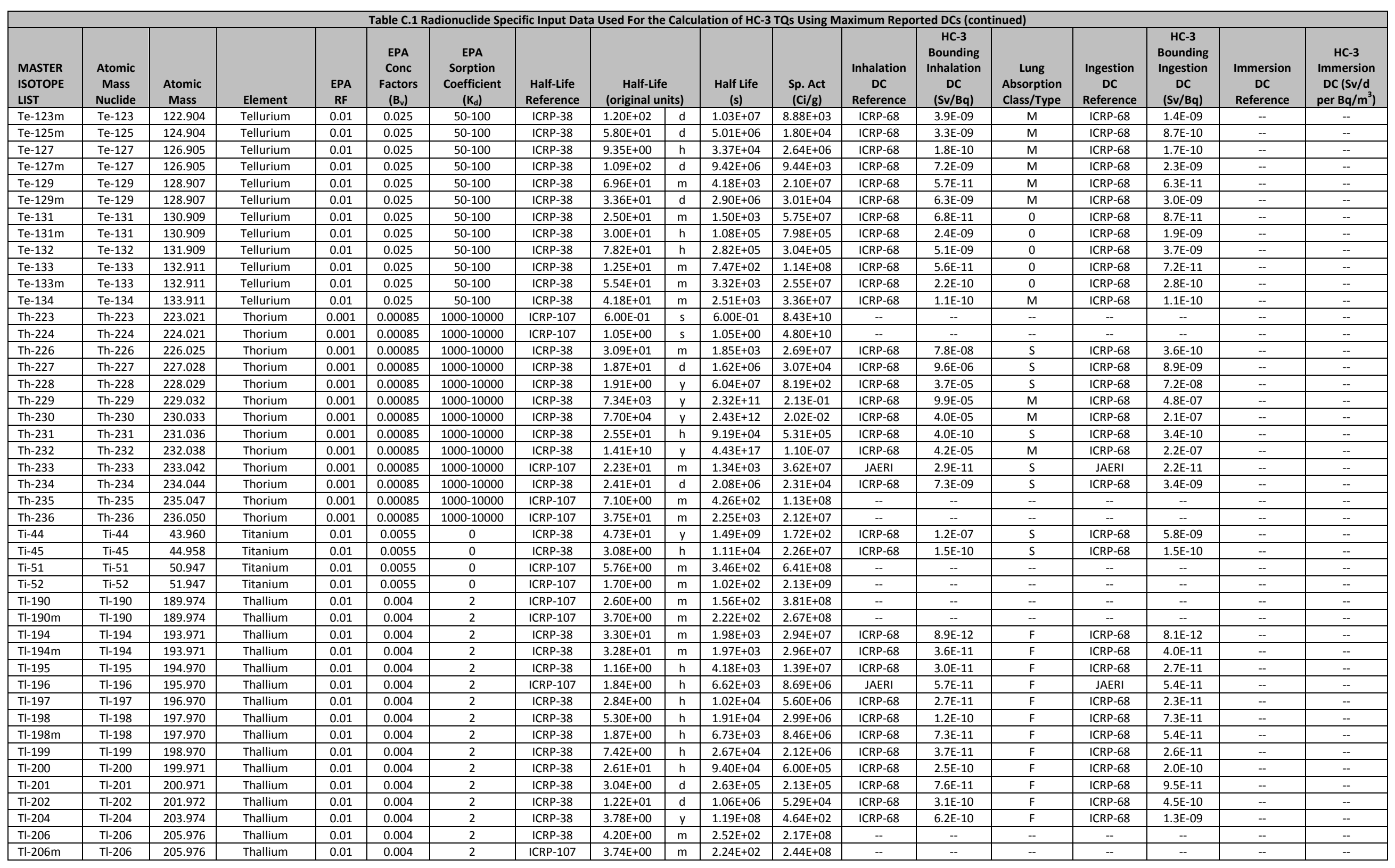




\begin{tabular}{|c|c|c|c|c|c|c|c|c|c|c|c|c|c|c|c|c|c|c|}
\hline \multirow{3}{*}{$\begin{array}{l}\text { MASTER } \\
\text { ISOTOPE } \\
\text { LIST } \\
\text { TI-207 }\end{array}$} & \multirow{3}{*}{$\begin{array}{c}\begin{array}{c}\text { Atomic } \\
\text { Mass } \\
\text { Nuclide }\end{array} \\
\text { Tl-207 }\end{array}$} & \multirow{3}{*}{$\begin{array}{c}\begin{array}{c}\text { Atomic } \\
\text { Mass }\end{array} \\
206.977\end{array}$} & & & \multicolumn{14}{|c|}{ Table C.1 Radionuclide Specific Input Dat } \\
\hline & & & \multirow{2}{*}{$\begin{array}{l}\text { Element } \\
\text { Thallium }\end{array}$} & \multirow{2}{*}{$\begin{array}{c}\text { EPA } \\
\text { RF } \\
0.01\end{array}$} & $\begin{array}{c}\text { EPA } \\
\text { Conc } \\
\text { Factors } \\
\left(B_{v}\right) \\
\end{array}$ & $\begin{array}{c}\text { EPA } \\
\text { Sorption } \\
\text { Coefficient } \\
\left(K_{d}\right)\end{array}$ & $\begin{array}{c}\text { Half-Life } \\
\text { Reference }\end{array}$ & $\begin{array}{r}\text { Half-Lit } \\
\text { (original u }\end{array}$ & & $\begin{array}{l}\text { Half Life } \\
\text { (s) }\end{array}$ & $\begin{array}{l}\text { Sp. Act } \\
\text { (Ci/g) }\end{array}$ & $\begin{array}{l}\text { Inhalation } \\
\text { DC } \\
\text { Reference }\end{array}$ & $\begin{array}{c}\text { HC-3 } \\
\text { Bounding } \\
\text { Inhalation } \\
\text { DC } \\
\text { (Sv/Bq) } \\
\end{array}$ & $\begin{array}{c}\text { Lung } \\
\text { Absorption } \\
\text { Class/Type }\end{array}$ & $\begin{array}{l}\text { Ingestion } \\
\text { DC } \\
\text { Reference }\end{array}$ & $\begin{array}{c}\text { HC-3 } \\
\text { Bounding } \\
\text { Ingestion } \\
\text { DC } \\
\text { (Sv/Bq) } \\
\end{array}$ & $\begin{array}{c}\text { Immersion } \\
\text { DC } \\
\text { Reference }\end{array}$ & $\begin{array}{c}\mathrm{HC}-3 \\
\text { Immersion } \\
\mathrm{DC}(\mathrm{Sv} / \mathrm{d} \\
\left.\operatorname{per} \mathrm{Bq} / \mathrm{m}^{3}\right)\end{array}$ \\
\hline & & & & & 0.004 & 2 & ICRP-38 & $4.77 \mathrm{E}+00$ & $\mathrm{~m}$ & $2.86 \mathrm{E}+02$ & $1.90 \mathrm{E}+08$ & -- & -- & -- & -- & -- & -- & -- \\
\hline TI-208 & TI-208 & 207.982 & Thallium & 0.01 & 0.004 & 2 & ICRP-38 & $3.07 \mathrm{E}+00$ & $\mathrm{~m}$ & $1.84 \mathrm{E}+02$ & $2.94 \mathrm{E}+08$ & -- & -- & -- & -- & -- & -- & -- \\
\hline TI-209 & TT-209 & 208.985 & Thallium & 0.01 & 0.004 & 2 & ICRP-38 & $2.20 \mathrm{E}+00$ & $\mathrm{~m}$ & $1.32 \mathrm{E}+02$ & $4.09 \mathrm{E}+08$ & -- & -- & -- & -- & -- & -- & -- \\
\hline $\mathrm{TI}-210$ & $\mathrm{TI}-210$ & 209.990 & Thallium & 0.01 & 0.004 & 2 & ICRP-38 & $1.30 \mathrm{E}+00$ & $\mathrm{~m}$ & $7.80 \mathrm{E}+01$ & $6.89 \mathrm{E}+08$ & -- & -- & -- & -- & -- & -- & -- \\
\hline Tm-159 & Tm-159 & 158.935 & Thulium & 0.01 & 0.01 & 0 & JAERI & $9.13 \mathrm{E}+00$ & $\mathrm{~m}$ & $5.48 \mathrm{E}+02$ & $1.30 \mathrm{E}+08$ & -- & -- & -- & -- & -- & -- & -- \\
\hline Tm-161 & Tm-161 & 160.934 & Thulium & 0.01 & 0.01 & 0 & ICRP-107 & $3.02 \mathrm{E}+01$ & $\mathrm{~m}$ & $1.81 \mathrm{E}+03$ & $3.87 \mathrm{E}+07$ & -- & -- & -- & -- & -- & -- & -- \\
\hline Tm-162 & Tm-162 & 161.934 & Thulium & 0.01 & 0.01 & 0 & ICRP-38 & $2.17 \mathrm{E}+01$ & $\mathrm{~m}$ & $1.30 \mathrm{E}+03$ & $5.35 \mathrm{E}+07$ & ICRP-68 & $2.7 \mathrm{E}-11$ & $M$ & ICRP-68 & $2.9 \mathrm{E}-11$ & -- & -- \\
\hline Tm-163 & Tm-163 & 162.933 & Thulium & 0.01 & 0.01 & 0 & ICRP-107 & $1.81 \mathrm{E}+00$ & $\mathrm{~h}$ & $6.52 \mathrm{E}+03$ & $1.06 \mathrm{E}+07$ & JAERI & $6.3 \mathrm{E}-11$ & $M$ & JAERI & $5.6 \mathrm{E}-11$ & -- & -- \\
\hline Tm-164 & Tm-164 & 163.934 & Thulium & 0.01 & 0.01 & 0 & ICRP-107 & $2.00 \mathrm{E}+00$ & $\mathrm{~m}$ & $1.20 \mathrm{E}+02$ & $5.73 \mathrm{E}+08$ & -- & -- & -- & -- & -- & -- & -- \\
\hline Tm-165 & $\mathrm{Tm}-165$ & 164.932 & Thulium & 0.01 & 0.01 & 0 & ICRP-107 & $3.01 \mathrm{E}+01$ & $\mathrm{~h}$ & $1.08 \mathrm{E}+05$ & $6.32 \mathrm{E}+05$ & JAERI & $3.1 \mathrm{E}-10$ & $M$ & JAERI & $3.6 \mathrm{E}-10$ & -- & -- \\
\hline Tm-166 & Tm-166 & 165.934 & Thulium & 0.01 & 0.01 & 0 & ICRP-38 & $7.70 \mathrm{E}+00$ & $\mathrm{~h}$ & $2.77 E+04$ & $2.45 \mathrm{E}+06$ & ICRP-68 & $2.8 \mathrm{E}-10$ & $M$ & ICRP-68 & $2.8 \mathrm{E}-10$ & -- & -- \\
\hline Tm-167 & $\mathrm{Tm}-167$ & 166.933 & Thulium & 0.01 & 0.01 & 0 & ICRP-38 & $9.24 \mathrm{E}+00$ & $d$ & $7.98 \mathrm{E}+05$ & $8.47 E+04$ & ICRP-68 & $1.1 \mathrm{E}-09$ & $M$ & ICRP-68 & $5.6 \mathrm{E}-10$ & -- & -- \\
\hline $\mathrm{Tm}-168$ & $\mathrm{Tm}-168$ & 167.934 & Thulium & 0.01 & 0.01 & 0 & ICRP-107 & $9.31 \mathrm{E}+01$ & $\mathrm{~d}$ & $8.04 \mathrm{E}+06$ & $8.35 \mathrm{E}+03$ & JAERI & $4.3 \mathrm{E}-09$ & $M$ & JAERI & $1.0 \mathrm{E}-09$ & -- & -- \\
\hline $\mathrm{Tm}-170$ & Tm-170 & 169.936 & Thulium & 0.01 & 0.01 & 0 & ICRP-38 & $1.29 \mathrm{E}+02$ & $d$ & $1.11 \mathrm{E}+07$ & $5.97 \mathrm{E}+03$ & ICRP-68 & $6.6 \mathrm{E}-09$ & $M$ & ICRP-68 & $1.3 \mathrm{E}-09$ & -- & -- \\
\hline Tm-171 & Tm-171 & 170.936 & Thulium & 0.01 & 0.01 & 0 & ICRP-38 & $1.92 \mathrm{E}+00$ & $y$ & $6.06 \mathrm{E}+07$ & $1.09 \mathrm{E}+03$ & ICRP-68 & $1.3 \mathrm{E}-09$ & $M$ & ICRP-68 & $1.1 \mathrm{E}-10$ & -- & -- \\
\hline Tm-172 & Tm-172 & 171.938 & Thulium & 0.01 & 0.01 & 0 & ICRP-38 & $6.36 \mathrm{E}+01$ & $\mathrm{~h}$ & $2.29 \mathrm{E}+05$ & $2.87 E+05$ & ICRP-68 & $1.4 \mathrm{E}-09$ & $M$ & ICRP-68 & $1.7 \mathrm{E}-09$ & -- & -- \\
\hline Tm-173 & $\mathrm{Tm}-173$ & 172.940 & Thulium & 0.01 & 0.01 & 0 & ICRP-38 & $8.24 \mathrm{E}+00$ & $\mathrm{~h}$ & $2.97 \mathrm{E}+04$ & $2.20 \mathrm{E}+06$ & ICRP-68 & $2.6 \mathrm{E}-10$ & $\mathrm{M}$ & ICRP-68 & $3.1 \mathrm{E}-10$ & -- & -- \\
\hline Tm-174 & Tm-174 & 173.942 & Thulium & 0.01 & 0.01 & 0 & ICRP-107 & $5.40 \mathrm{E}+00$ & $\mathrm{~m}$ & $3.24 \mathrm{E}+02$ & $2.00 \mathrm{E}+08$ & -- & -- & -- & -- & -- & -- & -- \\
\hline Tm-175 & Tm-175 & 174.944 & Thulium & 0.01 & 0.01 & 0 & ICRP-38 & $1.52 \mathrm{E}+01$ & $\mathrm{~m}$ & $9.12 \mathrm{E}+02$ & $7.07 E+07$ & ICRP-68 & $3.1 \mathrm{E}-11$ & $M$ & ICRP-68 & $2.7 \mathrm{E}-11$ & -- & -- \\
\hline Tm-176 & Tm-176 & 175.947 & Thulium & 0.01 & 0.01 & 0 & ICRP-107 & $1.85 \mathrm{E}+00$ & $\mathrm{~m}$ & $1.11 \mathrm{E}+02$ & $5.78 \mathrm{E}+08$ & -- & -- & -- & -- & -- & -- & -- \\
\hline U-227 & U-227 & 227.031 & Uranium & 0.001 & 0.0085 & $1-50$ & ICRP-107 & $1.10 \mathrm{E}+00$ & $\mathrm{~m}$ & $6.60 \mathrm{E}+01$ & $7.53 \mathrm{E}+08$ & -- & -- & -- & -- & -- & -- & -- \\
\hline $\mathrm{U}-228$ & $\mathrm{U}-228$ & 228.031 & Uranium & 0.001 & 0.0085 & $1-50$ & ICRP-107 & $9.10 \mathrm{E}+00$ & $\mathrm{~m}$ & $5.46 \mathrm{E}+02$ & $9.06 \mathrm{E}+07$ & -- & -- & -- & -- & -- & -- & -- \\
\hline U-230 & $U-230$ & 230.034 & Uranium & 0.001 & 0.0085 & $1-50$ & ICRP-38 & $2.08 \mathrm{E}+01$ & $d$ & $1.80 \mathrm{E}+06$ & $2.73 \mathrm{E}+04$ & ICRP-68 & $1.5 \mathrm{E}-05$ & $\mathrm{~s}$ & ICRP-68 & $5.5 \mathrm{E}-08$ & -- & -- \\
\hline U-231 & U-231 & 231.036 & Uranium & 0.001 & 0.0085 & $1-50$ & ICRP-38 & $4.20 \mathrm{E}+00$ & $d$ & $3.63 \mathrm{E}+05$ & $1.35 \mathrm{E}+05$ & ICRP-68 & $4.9 \mathrm{E}-10$ & $\mathrm{~s}$ & ICRP-68 & $2.8 \mathrm{E}-10$ & -- & -- \\
\hline U-232 & $\mathrm{U}-232$ & 232.037 & Uranium & 0.001 & 0.0085 & $1-50$ & ICRP-38 & $7.20 \mathrm{E}+01$ & $y$ & $2.27 E+09$ & $2.14 \mathrm{E}+01$ & ICRP-68 & $3.5 E-05$ & $\mathrm{~s}$ & ICRP-68 & $3.3 \mathrm{E}-07$ & -- & -- \\
\hline U-233 & $\mathrm{U}-233$ & 233.040 & Uranium & 0.001 & 0.0085 & $1-50$ & ICRP-38 & $1.59 \mathrm{E}+05$ & $y$ & $5.00 \mathrm{E}+12$ & $9.68 \mathrm{E}-03$ & ICRP-68 & $8.7 \mathrm{E}-06$ & $\mathrm{~s}$ & ICRP-68 & $5.0 \mathrm{E}-08$ & -- & -- \\
\hline U-234 & $\mathrm{U}-234$ & 234.041 & Uranium & 0.001 & 0.0085 & $1-50$ & ICRP-38 & $2.45 \mathrm{E}+05$ & $y$ & $7.72 \mathrm{E}+12$ & $6.25 \mathrm{E}-03$ & ICRP-68 & $8.5 \mathrm{E}-06$ & $\mathrm{~s}$ & ICRP-68 & $4.9 \mathrm{E}-08$ & -- & -- \\
\hline $\mathrm{U}-235$ & $\mathrm{U}-235$ & 235.044 & Uranium & 0.001 & 0.0085 & $1-50$ & ICRP-38 & $7.04 \mathrm{E}+08$ & $y$ & $2.22 \mathrm{E}+16$ & $2.16 \mathrm{E}-06$ & ICRP-68 & $7.7 \mathrm{E}-06$ & $\mathrm{~s}$ & ICRP-68 & $4.6 \mathrm{E}-08$ & -- & -- \\
\hline$U-235 m$ & $U-235$ & 235.044 & Uranium & 0.001 & 0.0085 & $1-50$ & ICRP-107 & $2.60 \mathrm{E}+01$ & $\mathrm{~m}$ & $1.56 \mathrm{E}+03$ & $3.08 \mathrm{E}+07$ & -- & -- & -- & -- & -- & -- & -- \\
\hline $\mathrm{U}-236$ & U-236 & 236.046 & Uranium & 0.001 & 0.0085 & $1-50$ & ICRP-38 & $2.34 \mathrm{E}+07$ & $y$ & $7.39 \mathrm{E}+14$ & $6.47 \mathrm{E}-05$ & ICRP-68 & $7.9 \mathrm{E}-06$ & $\mathrm{~s}$ & ICRP-68 & $4.6 \mathrm{E}-08$ & -- & -- \\
\hline U-237 & U-237 & 237.049 & Uranium & 0.001 & 0.0085 & $1-50$ & ICRP-38 & $6.75 \mathrm{E}+00$ & $d$ & $5.83 \mathrm{E}+05$ & $8.16 \mathrm{E}+04$ & ICRP-68 & $1.8 \mathrm{E}-09$ & $\mathrm{~s}$ & ICRP-68 & $7.7 \mathrm{E}-10$ & -- & -- \\
\hline U-238 & $\mathrm{U}-238$ & 238.051 & Uranium & 0.001 & 0.0085 & $1-50$ & ICRP-38 & $4.47 \mathrm{E}+09$ & $y$ & $1.41 \mathrm{E}+17$ & $3.36 \mathrm{E}-07$ & ICRP-68 & $7.3 \mathrm{E}-06$ & $\mathrm{~s}$ & ICRP-68 & $4.4 \mathrm{E}-08$ & -- & -- \\
\hline U-239 & U-239 & 239.054 & Uranium & 0.001 & 0.0085 & $1-50$ & ICRP-38 & $2.35 \mathrm{E}+01$ & $\mathrm{~m}$ & $1.41 \mathrm{E}+03$ & $3.34 \mathrm{E}+07$ & ICRP-68 & $3.5 \mathrm{E}-11$ & $\mathrm{~s}$ & ICRP-68 & $2.8 \mathrm{E}-11$ & -- & -- \\
\hline $\mathrm{U}-240$ & $\mathrm{U}-240$ & 240.057 & Uranium & 0.001 & 0.0085 & $1-50$ & ICRP-38 & $1.41 \mathrm{E}+01$ & $\mathrm{~h}$ & $5.08 \mathrm{E}+04$ & $9.26 \mathrm{E}+05$ & ICRP-68 & $8.4 \mathrm{E}-10$ & $\mathrm{~s}$ & ICRP-68 & $1.1 \mathrm{E}-09$ & -- & -- \\
\hline U-242 & U-242 & 242.063 & Uranium & 0.001 & 0.0085 & $1-50$ & ICRP-107 & $1.68 \mathrm{E}+01$ & $\mathrm{~m}$ & $1.01 \mathrm{E}+03$ & $4.62 \mathrm{E}+07$ & -- & -- & -- & -- & -- & -- & -- \\
\hline V-47 & $\mathrm{V}-47$ & 46.955 & Vanadium & 0.01 & 0.0055 & 0 & $\begin{array}{l}\text { ICRP-38 } \\
\end{array}$ & $3.26 \mathrm{E}+01$ & $\mathrm{~m}$ & $1.96 \mathrm{E}+03$ & $1.23 \mathrm{E}+08$ & ICRP-68 & $5.0 \mathrm{E}-11$ & $M$ & ICRP-68 & $6.3 \mathrm{E}-11$ & -- & -- \\
\hline $\mathrm{V}-48$ & $V-48$ & 47.952 & Vanadium & 0.01 & 0.0055 & 0 & ICRP-38 & $1.62 \mathrm{E}+01$ & $d$ & $1.40 \mathrm{E}+06$ & $1.68 \mathrm{E}+05$ & ICRP-68 & $2.7 \mathrm{E}-09$ & $M$ & ICRP-68 & $2.0 \mathrm{E}-09$ & -- & -- \\
\hline V-49 & V-49 & 48.949 & Vanadium & 0.01 & 0.0055 & 0 & ICRP-38 & $3.30 \mathrm{E}+02$ & $d$ & $2.85 \mathrm{E}+07$ & $8.08 \mathrm{E}+03$ & ICRP-68 & $3.2 \mathrm{E}-11$ & $\mathrm{M}$ & ICRP-68 & $1.8 \mathrm{E}-11$ & -- & -- \\
\hline $\mathrm{V}-50$ & V-50 & 49.947 & Vanadium & 0.01 & 0.0055 & 0 & ICRP-107 & $1.50 \mathrm{E}+17$ & $y$ & $4.73 \mathrm{E}+24$ & $4.77 \mathrm{E}-14$ & JAERI & $9.9 \mathrm{E}-08$ & $F$ & JAERI & $4.2 \mathrm{E}-09$ & -- & -- \\
\hline$V-52$ & $V-52$ & 51.945 & Vanadium & 0.01 & 0.0055 & 0 & ICRP-107 & $3.74 \mathrm{E}+00$ & $\mathrm{~m}$ & $2.25 \mathrm{E}+02$ & $9.67 \mathrm{E}+08$ & -- & -- & -- & -- & -- & -- & -- \\
\hline V-53 & $\mathrm{V}-53$ & 52.944 & Vanadium & 0.01 & 0.0055 & 0 & ICRP-107 & $1.61 \mathrm{E}+00$ & $\mathrm{~m}$ & $9.66 \mathrm{E}+01$ & $2.21 \mathrm{E}+09$ & -- & -- & -- & -- & -- & -- & -- \\
\hline W-176 & W-176 & 175.946 & Tungsten & 0.01 & 0.045 & 0 & ICRP-38 & $2.30 \mathrm{E}+00$ & $\mathrm{~h}$ & $8.28 \mathrm{E}+03$ & $7.74 \mathrm{E}+06$ & ICRP-68 & $7.6 \mathrm{E}-11$ & $\mathrm{~F}$ & ICRP-68 & $1.1 \mathrm{E}-10$ & -- & -- \\
\hline W-177 & W-177 & 176.947 & Tungsten & 0.01 & 0.045 & 0 & ICRP-38 & $1.35 \mathrm{E}+02$ & $\mathrm{~m}$ & $8.10 \mathrm{E}+03$ & $7.87 \mathrm{E}+06$ & ICRP-68 & $4.6 \mathrm{E}-11$ & $\mathrm{~F}$ & ICRP-68 & $6.1 \mathrm{E}-11$ & -- & -- \\
\hline W-178 & W-178 & 177.946 & Tungsten & 0.01 & 0.045 & 0 & ICRP-38 & $2.17 \mathrm{E}+01$ & $d$ & $1.87 \mathrm{E}+06$ & $3.38 \mathrm{E}+04$ & ICRP-68 & $1.2 \mathrm{E}-10$ & $\mathrm{~F}$ & ICRP-68 & $2.5 \mathrm{E}-10$ & -- & -- \\
\hline W-179 & W-179 & 178.947 & Tungsten & 0.01 & 0.045 & 0 & ICRP-38 & $3.75 \mathrm{E}+01$ & $\mathrm{~m}$ & $2.25 \mathrm{E}+03$ & $2.80 \mathrm{E}+07$ & ICRP-68 & $1.8 \mathrm{E}-12$ & $F$ & ICRP-68 & $3.3 \mathrm{E}-12$ & -- & -- \\
\hline
\end{tabular}




\begin{tabular}{|c|c|c|c|c|c|c|c|c|c|c|c|c|c|c|c|c|c|c|}
\hline \multirow{3}{*}{$\begin{array}{l}\text { MASTER } \\
\text { ISOTOPE } \\
\text { LIST } \\
\text { W-179m }\end{array}$} & \multirow{3}{*}{$\begin{array}{c}\begin{array}{c}\text { Atomic } \\
\text { Mass } \\
\text { Nuclide }\end{array} \\
\text { W-179 } \\
\end{array}$} & \multirow{3}{*}{$\begin{array}{c}\begin{array}{c}\text { Atomic } \\
\text { Mass }\end{array} \\
178.947\end{array}$} & \multirow{3}{*}{$\begin{array}{l}\text { Element } \\
\text { Tungsten }\end{array}$} & & \multicolumn{14}{|c|}{ Table C.1 Radionuclide Specific Input Dat } \\
\hline & & & & \multirow{2}{*}{$\begin{array}{c}\text { EPA } \\
\text { RF } \\
0.01\end{array}$} & $\begin{array}{c}\text { EPA } \\
\text { Conc } \\
\text { Factors } \\
\left(B_{v}\right) \\
\end{array}$ & $\begin{array}{c}\text { EPA } \\
\text { Sorption } \\
\text { Coefficient } \\
\left(K_{d}\right)\end{array}$ & $\begin{array}{c}\text { Half-Life } \\
\text { Reference }\end{array}$ & $\begin{array}{r}\text { Half-Lif } \\
\text { (original u }\end{array}$ & & $\begin{array}{l}\text { Half Life } \\
\text { (s) }\end{array}$ & $\begin{array}{l}\text { Sp. Act } \\
\text { (Ci/g) }\end{array}$ & $\begin{array}{l}\text { Inhalation } \\
\text { DC } \\
\text { Reference }\end{array}$ & $\begin{array}{c}\text { HC-3 } \\
\text { Bounding } \\
\text { Inhalation } \\
\text { DC } \\
\text { (Sv/Bq) } \\
\end{array}$ & $\begin{array}{c}\text { Lung } \\
\text { Absorption } \\
\text { Class/Type }\end{array}$ & $\begin{array}{l}\text { Ingestion } \\
\text { DC } \\
\text { Reference }\end{array}$ & $\begin{array}{c}\text { HC-3 } \\
\text { Bounding } \\
\text { Ingestion } \\
\text { DC } \\
\text { (Sv/Bq) } \\
\end{array}$ & $\begin{array}{c}\text { Immersion } \\
\text { DC } \\
\text { Reference }\end{array}$ & $\begin{array}{c}\mathrm{HC}-3 \\
\text { Immersion } \\
\mathrm{DC}(\mathrm{Sv} / \mathrm{d} \\
\left.\text { per } \mathrm{Bq} / \mathrm{m}^{3}\right)\end{array}$ \\
\hline & & & & & 0.045 & 0 & ICRP-107 & $6.40 \mathrm{E}+00$ & $\mathrm{~m}$ & $3.84 \mathrm{E}+02$ & $1.64 \mathrm{E}+08$ & -- & -- & -- & -- & -- & -- & -- \\
\hline W-181 & W-181 & 180.948 & Tungsten & 0.01 & 0.045 & 0 & ICRP-38 & $1.21 \mathrm{E}+02$ & d & $1.05 \mathrm{E}+07$ & $5.95 \mathrm{E}+03$ & ICRP-68 & $4.3 \mathrm{E}-11$ & $\mathrm{~F}$ & ICRP-68 & $8.2 \mathrm{E}-11$ & -- & -- \\
\hline W-185 & W-185 & 184.953 & Tungsten & 0.01 & 0.045 & 0 & ICRP-38 & $7.51 \mathrm{E}+01$ & d & $6.49 \mathrm{E}+06$ & $9.40 \mathrm{E}+03$ & ICRP-68 & $2.2 \mathrm{E}-10$ & $\mathrm{~F}$ & ICRP-68 & $5.0 \mathrm{E}-10$ & -- & -- \\
\hline $\mathrm{W}-185 \mathrm{~m}$ & W-185 & 184.953 & Tungsten & 0.01 & 0.045 & 0 & ICRP-107 & $1.60 \mathrm{E}+00$ & $\mathrm{~m}$ & $9.58 \mathrm{E}+01$ & $6.37 \mathrm{E}+08$ & -- & -- & -- & -- & -- & -- & -- \\
\hline W-187 & W-187 & 186.957 & Tungsten & 0.01 & 0.045 & 0 & ICRP-38 & $2.39 \mathrm{E}+01$ & $\mathrm{~h}$ & $8.60 \mathrm{E}+04$ & $7.01 \mathrm{E}+05$ & ICRP-68 & $3.3 \mathrm{E}-10$ & $\mathrm{~F}$ & ICRP-68 & $7.1 \mathrm{E}-10$ & -- & -- \\
\hline W-188 & W-188 & 187.958 & Tungsten & 0.01 & 0.045 & 0 & ICRP-38 & $6.94 \mathrm{E}+01$ & $d$ & $6.00 \mathrm{E}+06$ & $1.00 \mathrm{E}+04$ & ICRP-68 & $8.4 \mathrm{E}-10$ & $\mathrm{~F}$ & ICRP-68 & $2.3 \mathrm{E}-09$ & -- & -- \\
\hline W-190 & W-190 & 189.963 & Tungsten & 0.01 & 0.045 & 0 & ICRP-107 & $3.00 \mathrm{E}+01$ & $\mathrm{~m}$ & $1.80 \mathrm{E}+03$ & $3.30 \mathrm{E}+07$ & JAERI & $6.6 \mathrm{E}-11$ & $F$ & JAERI & $8.5 \mathrm{E}-11$ & --- & $\begin{array}{ll}- \\
\end{array}$ \\
\hline $\mathrm{Xe}-120$ & Xe-120 & 119.912 & Xenon & 1 & 0 & 0 & ICRP-38 & $4.00 \mathrm{E}+01$ & $\mathrm{~m}$ & $2.40 \mathrm{E}+03$ & $3.92 \mathrm{E}+07$ & -- & -- & -- & -- & -- & ICRP-68 & 1.50E-09 \\
\hline Xe-121 & Xe-121 & 120.911 & Xenon & 1 & 0 & 0 & ICRP-38 & $4.01 \mathrm{E}+01$ & $\mathrm{~m}$ & $2.41 \mathrm{E}+03$ & $3.88 \mathrm{E}+07$ & -- & -- & -- & -- & -- & ICRP-68 & $7.50 \mathrm{E}-09$ \\
\hline $\mathrm{Xe}-122$ & $\mathrm{Xe}-122$ & 121.908 & Xenon & 1 & 0 & 0 & ICRP-38 & $2.01 \mathrm{E}+01$ & $\mathrm{~h}$ & $7.24 E+04$ & $1.28 \mathrm{E}+06$ & -- & -- & -- & -- & -- & ICRP-68 & $1.90 \mathrm{E}-10$ \\
\hline Xe-123 & Xe-123 & 122.908 & Xenon & 1 & 0 & 0 & ICRP-38 & $2.08 \mathrm{E}+00$ & $\mathrm{~h}$ & $7.49 \mathrm{E}+03$ & $1.23 \mathrm{E}+07$ & -- & -- & -- & -- & -- & ICRP-68 & $2.40 \mathrm{E}-09$ \\
\hline Xe-125 & Xe-125 & 124.906 & Xenon & 1 & 0 & 0 & ICRP-38 & $1.70 \mathrm{E}+01$ & $\mathrm{~h}$ & $6.12 \mathrm{E}+04$ & $1.48 \mathrm{E}+06$ & -- & -- & -- & -- & -- & ICRP-68 & $9.30 \mathrm{E}-10$ \\
\hline Xe-127 & Xe-127 & 126.905 & Xenon & 1 & 0 & 0 & ICRP-38 & $3.64 \mathrm{E}+01$ & $d$ & $3.15 \mathrm{E}+06$ & $2.83 \mathrm{E}+04$ & -- & -- & -- & -- & -- & ICRP-68 & $9.70 \mathrm{E}-10$ \\
\hline Xe-127m & Xe-127 & 126.905 & Xenon & 1 & 0 & 0 & ICRP-107 & $6.92 \mathrm{E}+01$ & $\mathrm{~s}$ & $6.92 \mathrm{E}+01$ & $1.28 \mathrm{E}+09$ & -- & -- & -- & -- & -- & JAERI & $6.00 \mathrm{E}-10$ \\
\hline Xe-129m & Xe-129 & 128.905 & Xenon & 1 & 0 & 0 & ICRP-38 & $8.00 \mathrm{E}+00$ & $d$ & $6.91 \mathrm{E}+05$ & $1.27 \mathrm{E}+05$ & -- & -- & -- & -- & -- & ICRP-68 & $8.10 \mathrm{E}-11$ \\
\hline Xe-131m & Xe-131 & 130.905 & Xenon & 1 & 0 & 0 & ICRP-38 & $1.19 \mathrm{E}+01$ & $d$ & $1.03 E+06$ & $8.38 \mathrm{E}+04$ & -- & -- & -- & -- & -- & ICRP-68 & $3.20 \mathrm{E}-11$ \\
\hline Xe-133 & Xe-133 & 132.906 & Xenon & 1 & 0 & 0 & ICRP-38 & $5.25 \mathrm{E}+00$ & $d$ & $4.53 \mathrm{E}+05$ & $1.87 \mathrm{E}+05$ & -- & -- & -- & -- & -- & ICRP-68 & $1.20 \mathrm{E}-10$ \\
\hline Xe-133m & Xe-133 & 132.906 & Xenon & 1 & 0 & 0 & ICRP-38 & $2.19 \mathrm{E}+00$ & $d$ & $1.89 \mathrm{E}+05$ & $4.49 \mathrm{E}+05$ & -- & -- & -- & -- & -- & ICRP-68 & $1.10 \mathrm{E}-10$ \\
\hline Xe-135 & Xe-135 & 134.907 & Xenon & 1 & 0 & 0 & ICRP-38 & $9.09 \mathrm{E}+00$ & $\mathrm{~h}$ & $3.27 E+04$ & $2.56 \mathrm{E}+06$ & -- & -- & -- & -- & -- & ICRP-68 & $9.60 \mathrm{E}-10$ \\
\hline Xe-135m & Xe-135 & 134.907 & Xenon & 1 & 0 & 0 & ICRP-38 & $1.53 \mathrm{E}+01$ & $\mathrm{~m}$ & $9.17 E+02$ & $9.12 \mathrm{E}+07$ & -- & -- & -- & -- & -- & ICRP-68 & 1.60E-09 \\
\hline Xe-137 & Xe-137 & 136.912 & Xenon & 1 & 0 & 0 & ICRP-107 & $3.82 \mathrm{E}+00$ & $\mathrm{~m}$ & $2.29 E+02$ & $3.60 \mathrm{E}+08$ & -- & -- & -- & -- & -- & JAERI & $9.40 \mathrm{E}-10$ \\
\hline Xe-138 & Xe-138 & 137.914 & Xenon & 1 & 0 & 0 & ICRP-38 & $1.42 \mathrm{E}+01$ & $\mathrm{~m}$ & $8.50 \mathrm{E}+02$ & $9.62 \mathrm{E}+07$ & -- & -- & -- & -- & -- & ICRP-68 & $4.70 \mathrm{E}-09$ \\
\hline$Y-81$ & $\mathrm{Y}-81$ & 80.929 & Yttrium & 0.01 & 0.015 & 500 & ICRP-107 & $7.04 \mathrm{E}+01$ & $\mathrm{~s}$ & $7.04 \mathrm{E}+01$ & $1.98 \mathrm{E}+09$ & -- & -- & -- & -- & -- & -- & -- \\
\hline$Y-83$ & $\mathrm{Y}-83$ & 82.922 & Yttrium & 0.01 & 0.015 & 500 & ICRP-107 & $7.08 \mathrm{E}+00$ & $\mathrm{~m}$ & $4.25 E+02$ & $3.20 \mathrm{E}+08$ & -- & -- & -- & -- & -- & -- & -- \\
\hline $\mathrm{Y}-83 \mathrm{~m}$ & $Y-83$ & 82.922 & Yttrium & 0.01 & 0.015 & 500 & ICRP-107 & $2.85 \mathrm{E}+00$ & $\mathrm{~m}$ & $1.71 \mathrm{E}+02$ & $7.96 \mathrm{E}+08$ & -- & -- & -- & -- & -- & -- & -- \\
\hline$Y-84 m$ & $Y-84$ & 83.921 & Yttrium & 0.01 & 0.015 & 500 & ICRP-107 & $3.95 \mathrm{E}+01$ & $\mathrm{~m}$ & $2.37 E+03$ & $5.67 \mathrm{E}+07$ & JAERI & $1.0 \mathrm{E}-10$ & $M$ & JAERI & $1.2 \mathrm{E}-10$ & -- & -- \\
\hline$Y-85$ & $Y-85$ & 84.916 & Yttrium & 0.01 & 0.015 & 500 & ICRP-107 & $2.68 \mathrm{E}+00$ & $\mathrm{~h}$ & $9.65 \mathrm{E}+03$ & $1.38 \mathrm{E}+07$ & JAERI & $1.6 \mathrm{E}-10$ & $\mathrm{~s}$ & JAERI & $1.9 \mathrm{E}-10$ & -- & -- \\
\hline$Y-85 m$ & $Y-85$ & 84.916 & Yttrium & 0.01 & 0.015 & 500 & ICRP-107 & $4.86 \mathrm{E}+00$ & $\mathrm{~h}$ & $1.75 \mathrm{E}+04$ & $7.59 \mathrm{E}+06$ & JAERI & $2.7 \mathrm{E}-10$ & $\mathrm{~s}$ & JAERI & $3.8 \mathrm{E}-10$ & -- & -- \\
\hline$Y-86$ & $Y-86$ & 85.915 & Yttrium & 0.01 & 0.015 & 500 & ICRP-38 & $1.47 \mathrm{E}+01$ & $\mathrm{~h}$ & $5.31 \mathrm{E}+04$ & $2.47 \mathrm{E}+06$ & ICRP-68 & $8.1 \mathrm{E}-10$ & $\mathrm{~s}$ & ICRP-68 & $9.6 \mathrm{E}-10$ & -- & -- \\
\hline$Y-86 m$ & $Y-86$ & 85.915 & Yttrium & 0.01 & 0.015 & 500 & ICRP-38 & $4.80 \mathrm{E}+01$ & $\mathrm{~m}$ & $2.88 \mathrm{E}+03$ & $4.56 \mathrm{E}+07$ & ICRP-68 & $4.9 \mathrm{E}-11$ & $\mathrm{~s}$ & ICRP-68 & $5.6 \mathrm{E}-11$ & -- & -- \\
\hline$Y-87$ & $Y-87$ & 86.911 & Yttrium & 0.01 & 0.015 & 500 & ICRP-38 & $8.03 \mathrm{E}+01$ & $\mathrm{~h}$ & $2.89 \mathrm{E}+05$ & $4.49 \mathrm{E}+05$ & ICRP-68 & $5.3 \mathrm{E}-10$ & $\mathrm{~s}$ & ICRP-68 & $5.5 \mathrm{E}-10$ & -- & -- \\
\hline$Y-87 m$ & $Y-87$ & 86.911 & Yttrium & 0.01 & 0.015 & 500 & ICRP-107 & $1.34 \mathrm{E}+01$ & $\mathrm{~h}$ & $4.81 E+04$ & $2.70 \mathrm{E}+06$ & JAERI & $2.0 \mathrm{E}-10$ & $\mathrm{~s}$ & JAERI & $2.2 \mathrm{E}-10$ & -- & -- \\
\hline$Y-88$ & $Y-88$ & 87.910 & Yttrium & 0.01 & 0.015 & 500 & ICRP-38 & $1.07 \mathrm{E}+02$ & $d$ & $9.21 \mathrm{E}+06$ & $1.39 \mathrm{E}+04$ & ICRP-68 & $4.1 \mathrm{E}-09$ & $\mathrm{~s}$ & ICRP-68 & $1.3 \mathrm{E}-09$ & -- & -- \\
\hline $\mathrm{Y}-89 \mathrm{~m}$ & $Y-89$ & 88.906 & Yttrium & 0.01 & 0.015 & 500 & ICRP-107 & $1.57 \mathrm{E}+01$ & $\mathrm{~s}$ & $1.57 \mathrm{E}+01$ & $8.10 \mathrm{E}+09$ & -- & -- & -- & -- & -- & -- & -- \\
\hline$Y-90$ & $Y-90$ & 89.907 & Yttrium & 0.01 & 0.015 & 500 & ICRP-38 & $6.40 \mathrm{E}+01$ & $\mathrm{~h}$ & $2.30 \mathrm{E}+05$ & $5.45 \mathrm{E}+05$ & ICRP-68 & $1.7 \mathrm{E}-09$ & $\mathrm{~s}$ & ICRP-68 & $2.7 \mathrm{E}-09$ & -- & -- \\
\hline $\mathrm{Y}-90 \mathrm{~m}$ & $Y-90$ & 89.907 & Yttrium & 0.01 & 0.015 & 500 & ICRP-38 & $3.19 \mathrm{E}+00$ & $\mathrm{~h}$ & $1.15 E+04$ & $1.09 \mathrm{E}+07$ & ICRP-68 & $1.3 \mathrm{E}-10$ & $M$ & ICRP-68 & $1.7 \mathrm{E}-10$ & -- & -- \\
\hline Y-91 & Y-91 & 90.907 & Yttrium & 0.01 & 0.015 & 500 & ICRP-38 & $5.85 \mathrm{E}+01$ & $d$ & $5.06 \mathrm{E}+06$ & $2.45 \mathrm{E}+04$ & ICRP-68 & $8.4 \mathrm{E}-09$ & $\mathrm{~s}$ & ICRP-68 & $2.4 \mathrm{E}-09$ & -- & -- \\
\hline Y-91m & $Y-91$ & 90.907 & Yttrium & 0.01 & 0.015 & 500 & ICRP-38 & $4.97 \mathrm{E}+01$ & $\mathrm{~m}$ & $2.98 E+03$ & $4.16 \mathrm{E}+07$ & ICRP-68 & $1.5 \mathrm{E}-11$ & $\mathrm{~s}$ & ICRP-68 & $1.1 \mathrm{E}-11$ & -- & -- \\
\hline Y-92 & $Y-92$ & 91.909 & Yttrium & 0.01 & 0.015 & 500 & ICRP-38 & $3.54 \mathrm{E}+00$ & $\mathrm{~h}$ & $1.27 E+04$ & $9.63 \mathrm{E}+06$ & ICRP-68 & $2.8 \mathrm{E}-10$ & $\mathrm{~s}$ & ICRP-68 & $4.9 \mathrm{E}-10$ & -- & -- \\
\hline Y-93 & $Y-93$ & 92.910 & Yttrium & 0.01 & 0.015 & 500 & ICRP-38 & $1.01 \mathrm{E}+01$ & $\mathrm{~h}$ & $3.64 E+04$ & $3.34 \mathrm{E}+06$ & ICRP-68 & $6.0 \mathrm{E}-10$ & $\mathrm{~s}$ & ICRP-68 & $1.2 \mathrm{E}-09$ & -- & -- \\
\hline$Y-94$ & $Y-94$ & 93.912 & Yttrium & 0.01 & 0.015 & 500 & ICRP-38 & $1.91 \mathrm{E}+01$ & $\mathrm{~m}$ & $1.15 \mathrm{E}+03$ & $1.05 \mathrm{E}+08$ & ICRP-68 & $4.6 \mathrm{E}-11$ & $\mathrm{~s}$ & ICRP-68 & $8.1 \mathrm{E}-11$ & -- & -- \\
\hline Y-95 & $\mathrm{Y}-95$ & 94.913 & Yttrium & 0.01 & 0.015 & 500 & ICRP-38 & $1.07 \mathrm{E}+01$ & $\mathrm{~m}$ & $6.42 \mathrm{E}+02$ & $1.85 \mathrm{E}+08$ & ICRP-68 & $2.6 \mathrm{E}-11$ & $\mathrm{~s}$ & ICRP-68 & $4.6 \mathrm{E}-11$ & -- & -- \\
\hline Yb-162 & Yb-162 & 161.936 & Ytterbium & 0.01 & 0.01 & 0 & ICRP-38 & $1.89 \mathrm{E}+01$ & $\mathrm{~m}$ & $1.13 \mathrm{E}+03$ & $6.14 \mathrm{E}+07$ & ICRP-68 & $2.3 \mathrm{E}-11$ & $\mathrm{~s}$ & ICRP-68 & $2.3 \mathrm{E}-11$ & -- & -- \\
\hline Yb-163 & Yb-163 & 162.936 & Ytterbium & 0.01 & 0.01 & 0 & ICRP-107 & $1.11 \mathrm{E}+01$ & $\mathrm{~m}$ & $6.63 \mathrm{E}+02$ & $1.04 \mathrm{E}+08$ & JAERI & $1.5 \mathrm{E}-11$ & $\mathrm{~s}$ & JAERI & $1.6 \mathrm{E}-11$ & -- & -- \\
\hline $\mathrm{Yb}-164$ & $\mathrm{Yb}-164$ & 163.934 & Ytterbium & 0.01 & 0.01 & 0 & ICRP-107 & $7.58 \mathrm{E}+01$ & $\mathrm{~m}$ & $4.55 \mathrm{E}+03$ & $1.51 \mathrm{E}+07$ & JAERI & $6.7 \mathrm{E}-11$ & $\mathrm{~s}$ & JAERI & $9.1 \mathrm{E}-11$ & -- & -- \\
\hline
\end{tabular}




\begin{tabular}{|c|c|c|c|c|c|c|c|c|c|c|c|c|c|c|c|c|c|c|}
\hline $\begin{array}{l}\text { MASTER } \\
\text { ISOTOPE } \\
\text { LIST }\end{array}$ & $\begin{array}{c}\text { Atomic } \\
\text { Mass } \\
\text { Nuclide }\end{array}$ & $\begin{array}{l}\text { Atomic } \\
\text { Mass }\end{array}$ & Element & $\begin{array}{c}\text { EPA } \\
\text { RF }\end{array}$ & $\begin{array}{c}\text { EPA } \\
\text { Conc } \\
\text { Factors } \\
\left(B_{v}\right)\end{array}$ & $\begin{array}{c}\text { EPA } \\
\text { Sorption } \\
\text { Coefficient } \\
\left(K_{d}\right)\end{array}$ & $\begin{array}{c}\text { Half-Life } \\
\text { Reference }\end{array}$ & $\begin{array}{r}\text { Half-Lit } \\
\text { (original u }\end{array}$ & & $\begin{array}{l}\text { Half Life } \\
\text { (s) }\end{array}$ & $\begin{array}{l}\text { Sp. Act } \\
(\mathrm{Ci} / \mathrm{g})\end{array}$ & $\begin{array}{l}\text { Inhalation } \\
\text { DC } \\
\text { Reference }\end{array}$ & $\begin{array}{c}\text { HC-3 } \\
\text { Bounding } \\
\text { Inhalation } \\
\text { DC } \\
\text { (Sv/Bq) }\end{array}$ & $\begin{array}{l}\text { Lung } \\
\text { Absorption } \\
\text { Class/Type }\end{array}$ & $\begin{array}{l}\text { Ingestion } \\
\text { DC } \\
\text { Reference }\end{array}$ & $\begin{array}{c}\text { HC-3 } \\
\text { Bounding } \\
\text { Ingestion } \\
\text { DC } \\
\text { (Sv/Bq) }\end{array}$ & $\begin{array}{l}\text { Immersion } \\
\text { DC } \\
\text { Reference }\end{array}$ & $\begin{array}{c}\text { HC-3 } \\
\text { Immersion } \\
\mathrm{DC}(\mathrm{Sv} / \mathrm{d} \\
\left.\text { per } \mathrm{Bq} / \mathrm{m}^{3}\right)\end{array}$ \\
\hline Yb-165 & Yb-165 & 164.935 & Ytterbium & 0.01 & 0.01 & 0 & ICRP-107 & $9.90 \mathrm{E}+00$ & $\mathrm{~m}$ & $5.94 \mathrm{E}+02$ & $1.15 \mathrm{E}+08$ & -- & -- & -- & -- & -- & -- & -- \\
\hline $\mathrm{Yb}-166$ & Yb-166 & 165.934 & Ytterbium & 0.01 & 0.01 & 0 & ICRP-38 & $5.67 \mathrm{E}+01$ & $\mathrm{~h}$ & $2.04 \mathrm{E}+05$ & $3.33 \mathrm{E}+05$ & ICRP-68 & $9.5 \mathrm{E}-10$ & $\mathrm{~s}$ & ICRP-68 & $9.5 \mathrm{E}-10$ & -- & -- \\
\hline $\mathrm{Yb}-167$ & Yb-167 & 166.935 & Ytterbium & 0.01 & 0.01 & 0 & ICRP-38 & $1.75 \mathrm{E}+01$ & $\mathrm{~m}$ & $1.05 \mathrm{E}+03$ & $6.44 \mathrm{E}+07$ & ICRP-68 & $9.5 \mathrm{E}-12$ & $\mathrm{~s}$ & ICRP-68 & $6.7 \mathrm{E}-12$ & -- & -- \\
\hline Yb-169 & Yb-169 & 168.935 & Ytterbium & 0.01 & 0.01 & 0 & ICRP-38 & $3.20 \mathrm{E}+01$ & $d$ & $2.77 E+06$ & $2.41 \mathrm{E}+04$ & ICRP-68 & $2.8 \mathrm{E}-09$ & $\mathrm{~s}$ & ICRP-68 & $7.1 \mathrm{E}-10$ & -- & -- \\
\hline $\mathrm{Yb}-175$ & Yb-175 & 174.941 & Ytterbium & 0.01 & 0.01 & 0 & ICRP-38 & $4.19 \mathrm{E}+00$ & d & $3.62 \mathrm{E}+05$ & $1.78 \mathrm{E}+05$ & ICRP-68 & $7.0 \mathrm{E}-10$ & $\mathrm{~s}$ & ICRP-68 & $4.4 \mathrm{E}-10$ & -- & -- \\
\hline $\mathrm{Yb}-177$ & Yb-177 & 176.945 & Ytterbium & 0.01 & 0.01 & 0 & ICRP-38 & $1.90 \mathrm{E}+00$ & $\mathrm{~h}$ & $6.84 \mathrm{E}+03$ & $9.32 \mathrm{E}+06$ & ICRP-68 & $9.4 \mathrm{E}-11$ & $\mathrm{~s}$ & ICRP-68 & $9.7 \mathrm{E}-11$ & -- & -- \\
\hline Yb-178 & Yb-178 & 177.947 & Ytterbium & 0.01 & 0.01 & 0 & ICRP-38 & $7.40 \mathrm{E}+01$ & $\mathrm{~m}$ & $4.44 \mathrm{E}+03$ & $1.43 \mathrm{E}+07$ & ICRP-68 & $1.1 \mathrm{E}-10$ & $\mathrm{~s}$ & ICRP-68 & $1.2 \mathrm{E}-10$ & -- & -- \\
\hline $\mathrm{Yb}-179$ & Yb-179 & 178.950 & Ytterbium & 0.01 & 0.01 & 0 & ICRP-107 & $8.00 E+00$ & $\mathrm{~m}$ & $4.80 \mathrm{E}+02$ & $1.31 \mathrm{E}+08$ & -- & -- & -- & -- & -- & -- & -- \\
\hline $\mathrm{Zn}-60$ & $\mathrm{Zn}-60$ & 59.942 & Zinc & 0.01 & 1.5 & 0 & ICRP-107 & $2.38 \mathrm{E}+00$ & $\mathrm{~m}$ & $1.43 \mathrm{E}+02$ & $1.32 \mathrm{E}+09$ & -- & -- & -- & -- & -- & -- & -- \\
\hline Zn-61 & $Z n-61$ & 60.940 & Zinc & 0.01 & 1.5 & 0 & ICRP-107 & $8.91 \mathrm{E}+01$ & $\mathrm{~s}$ & $8.91 \mathrm{E}+01$ & $2.08 \mathrm{E}+09$ & -- & -- & -- & -- & -- & -- & -- \\
\hline Zn-62 & Zn-62 & 61.934 & Zinc & 0.01 & 1.5 & 0 & ICRP-38 & $9.26 \mathrm{E}+00$ & $\mathrm{~h}$ & $3.33 \mathrm{E}+04$ & $5.46 \mathrm{E}+06$ & ICRP-68 & $6.6 \mathrm{E}-10$ & $\mathrm{~s}$ & ICRP-68 & $9.4 \mathrm{E}-10$ & -- & -- \\
\hline Zn-63 & $Z n-63$ & 62.933 & Zinc & 0.01 & 1.5 & 0 & ICRP-38 & $3.81 \mathrm{E}+01$ & $\mathrm{~m}$ & $2.29 E+03$ & $7.84 E+07$ & ICRP-68 & $6.1 \mathrm{E}-11$ & $\mathrm{~s}$ & ICRP-68 & $7.9 \mathrm{E}-11$ & -- & -- \\
\hline Zn-65 & $Z n-65$ & 64.929 & Zinc & 0.01 & 1.5 & 0 & ICRP-38 & $2.44 \mathrm{E}+02$ & $d$ & $2.11 \mathrm{E}+07$ & $8.25 \mathrm{E}+03$ & ICRP-68 & $2.9 \mathrm{E}-09$ & $\mathrm{~s}$ & ICRP-68 & $3.9 \mathrm{E}-09$ & -- & -- \\
\hline$Z n-69$ & $Z n-69$ & 68.927 & Zinc & 0.01 & 1.5 & 0 & ICRP-38 & $5.70 \mathrm{E}+01$ & $\mathrm{~m}$ & $3.42 E+03$ & $4.79 E+07$ & ICRP-68 & $4.3 \mathrm{E}-11$ & $\mathrm{~s}$ & ICRP-68 & $3.1 \mathrm{E}-11$ & -- & -- \\
\hline Zn-69m & $2 n-69$ & 68.927 & Zinc & 0.01 & 1.5 & 0 & ICRP-38 & $1.38 \mathrm{E}+01$ & $\mathrm{~h}$ & $4.95 E+04$ & $3.30 \mathrm{E}+06$ & ICRP-68 & $3.3 \mathrm{E}-10$ & $\mathrm{~s}$ & ICRP-68 & $3.3 \mathrm{E}-10$ & -- & -- \\
\hline$Z n-71$ & Zn-71 & 70.928 & Zinc & 0.01 & 1.5 & 0 & ICRP-107 & $2.45 \mathrm{E}+00$ & $\mathrm{~m}$ & $1.47 E+02$ & $1.08 \mathrm{E}+09$ & -- & -- & -- & -- & -- & -- & -- \\
\hline Zn-71m & $2 n-71$ & 70.928 & Zinc & 0.01 & 1.5 & 0 & ICRP-38 & $3.92 \mathrm{E}+00$ & $\mathrm{~h}$ & $1.41 E+04$ & $1.13 \mathrm{E}+07$ & ICRP-68 & $2.4 \mathrm{E}-10$ & $\mathrm{~s}$ & ICRP-68 & $2.4 \mathrm{E}-10$ & -- & -- \\
\hline $\mathrm{Zn}-72$ & Zn-72 & 71.927 & Zinc & 0.01 & 1.5 & 0 & ICRP-38 & $4.65 \mathrm{E}+01$ & $\mathrm{~h}$ & $1.67 E+05$ & $9.37 E+05$ & ICRP-68 & $1.5 \mathrm{E}-09$ & $\mathrm{~s}$ & ICRP-68 & $1.4 \mathrm{E}-09$ & -- & -- \\
\hline Zr-85 & Zr-85 & 84.921 & Zirconium & 0.01 & 0.002 & 2000 & ICRP-107 & $7.86 \mathrm{E}+00$ & $\mathrm{~m}$ & $4.72 \mathrm{E}+02$ & $2.82 E+08$ & -- & -- & -- & -- & -- & -- & -- \\
\hline Zr-86 & Zr-86 & 85.916 & Zirconium & 0.01 & 0.002 & 2000 & ICRP-38 & $1.65 \mathrm{E}+01$ & $\mathrm{~h}$ & $5.94 E+04$ & $2.21 \mathrm{E}+06$ & ICRP-68 & $7.0 \mathrm{E}-10$ & $\mathrm{~s}$ & ICRP-68 & $8.6 \mathrm{E}-10$ & -- & -- \\
\hline Zr-87 & Zr-87 & 86.915 & Zirconium & 0.01 & 0.002 & 2000 & ICRP-107 & $1.68 \mathrm{E}+00$ & $\mathrm{~h}$ & $6.05 E+03$ & $2.15 E+07$ & JAERI & $1.4 \mathrm{E}-10$ & $M$ & JAERI & $2.0 \mathrm{E}-10$ & -- & -- \\
\hline Zr-88 & Zr-88 & 87.910 & Zirconium & 0.01 & 0.002 & 2000 & ICRP-38 & $8.34 \mathrm{E}+01$ & $d$ & $7.21 E+06$ & $1.78 \mathrm{E}+04$ & ICRP-68 & $4.1 \mathrm{E}-09$ & $\mathrm{~F}$ & ICRP-68 & $3.3 \mathrm{E}-10$ & -- & -- \\
\hline Zr-89 & Zr-89 & 88.909 & Zirconium & 0.01 & 0.002 & 2000 & ICRP-38 & $7.84 \mathrm{E}+01$ & $\mathrm{~h}$ & $2.82 E+05$ & $4.49 \mathrm{E}+05$ & ICRP-68 & $7.5 \mathrm{E}-10$ & $\mathrm{~s}$ & ICRP-68 & $7.9 \mathrm{E}-10$ & -- & -- \\
\hline $\mathrm{Zr}-89 \mathrm{~m}$ & Zr-89 & 88.909 & Zirconium & 0.01 & 0.002 & 2000 & ICRP-107 & $4.16 \mathrm{E}+00$ & $\mathrm{~m}$ & $2.50 \mathrm{E}+02$ & $5.08 \mathrm{E}+08$ & -- & -- & -- & -- & -- & -- & -- \\
\hline Zr-93 & Zr-93 & 92.906 & Zirconium & 0.01 & 0.002 & 2000 & ICRP-38 & $1.53 \mathrm{E}+06$ & $y$ & $4.83 \mathrm{E}+13$ & $2.51 \mathrm{E}-03$ & ICRP-68 & $2.9 \mathrm{E}-08$ & $\mathrm{~F}$ & ICRP-68 & $2.8 \mathrm{E}-10$ & -- & -- \\
\hline Zr-95 & Zr-95 & 94.908 & Zirconium & 0.01 & 0.002 & 2000 & ICRP-38 & $6.40 \mathrm{E}+01$ & $d$ & $5.53 \mathrm{E}+06$ & $2.15 \mathrm{E}+04$ & ICRP-68 & $5.5 \mathrm{E}-09$ & $\mathrm{~s}$ & ICRP-68 & $8.8 \mathrm{E}-10$ & -- & -- \\
\hline Zr-97 & Zr-97 & 96.911 & Zirconium & 0.01 & 0.002 & 2000 & ICRP-38 & $1.69 \mathrm{E}+01$ & $\mathrm{~h}$ & $6.08 \mathrm{E}+04$ & $1.91 \mathrm{E}+06$ & ICRP-68 & $1.4 \mathrm{E}-09$ & $\mathrm{~s}$ & ICRP-68 & $2.1 \mathrm{E}-09$ & -- & -- \\
\hline
\end{tabular}


Table C.2 Summary of Exposure Pathway TQs and Selection of Final HC-3 TQs

\begin{tabular}{|c|c|c|c|c|c|c|c|c|c|c|c|c|}
\hline \multirow{2}{*}{$\begin{array}{c}\text { MASTER } \\
\text { ISOTOPE } \\
\text { LIST }\end{array}$} & \multicolumn{3}{|c|}{ from NNSA SD G 1027} & \multicolumn{5}{|c|}{ Calculated Pathway Specific TQ Values } & \multicolumn{4}{|c|}{ Selected Final Threshold Quantities } \\
\hline & Act (Ci) & Mass (g) & $\begin{array}{l}\text { Limiting } \\
\text { Pathway }\end{array}$ & $\begin{array}{c}\mathrm{TQ}_{\text {inhale }} \\
\text { (Ci) }\end{array}$ & $\begin{array}{l}\text { TQ }_{\text {water }} \\
\text { (Ci) }\end{array}$ & $\mathrm{TQ}_{\text {food }}(\mathrm{Ci})$ & $\begin{array}{l}\mathrm{TQ}_{\text {direct }} \\
\text { (Ci) }\end{array}$ & $\begin{array}{l}\mathrm{TQ}_{\text {sub }} \\
(\mathrm{Ci})\end{array}$ & Act $(\mathrm{Ci})$ & Mass (g) & $\begin{array}{l}\text { Sp. Act. } \\
\text { (Ci/g) }\end{array}$ & $\begin{array}{l}\text { Limiting } \\
\text { Pathway }\end{array}$ \\
\hline Ac-223 & & & & -- & -- & -- & $5.31 \mathrm{E}+07$ & -- & $5.31 \mathrm{E}+07$ & $1.39 \mathrm{E}-01$ & $3.83 \mathrm{E}+08$ & Direct \\
\hline Ac-224 & & & & $9.31 \mathrm{E}+02$ & -- & $1.28 \mathrm{E}+06$ & $2.09 \mathrm{E}+04$ & -- & $9.31 \mathrm{E}+02$ & $1.93 \mathrm{E}-04$ & $4.82 \mathrm{E}+06$ & Inh. \\
\hline Ac-225 & $1.43 \mathrm{E}+01$ & $2.46 \mathrm{E}-04$ & $\mathrm{i}$ & $1.41 \mathrm{E}+01$ & -- & $7.65 \mathrm{E}+02$ & $4.17 \mathrm{E}+04$ & -- & $1.41 \mathrm{E}+01$ & $2.44 \mathrm{E}-04$ & $5.80 \mathrm{E}+04$ & Inh. \\
\hline Ac-226 & & & & $9.31 \mathrm{E}+01$ & -- & $9.62 \mathrm{E}+03$ & $7.34 \mathrm{E}+03$ & -- & $9.31 \mathrm{E}+01$ & $1.95 \mathrm{E}-04$ & $4.78 \mathrm{E}+05$ & Inh. \\
\hline Ac-227 & $1.78 \mathrm{E}-01$ & $2.45 \mathrm{E}-03$ & $\mathrm{i}$ & 1.77E-01 & -- & $7.34 \mathrm{E}+00$ & $3.63 E+06$ & -- & 1.77E-01 & $2.45 \mathrm{E}-03$ & $7.23 E+01$ & Inh. \\
\hline Ac-228 & & & & $3.85 \mathrm{E}+03$ & -- & $9.92 \mathrm{E}+05$ & $2.17 \mathrm{E}+03$ & -- & $2.17 \mathrm{E}+03$ & 9.69E-04 & $2.24 \mathrm{E}+06$ & Direct \\
\hline Ac-229 & & & & $2.07 E+06$ & -- & $5.60 \mathrm{E}+07$ & -- & -- & $2.07 \mathrm{E}+06$ & $1.58 \mathrm{E}-01$ & $1.31 \mathrm{E}+07$ & Inh. \\
\hline Ac-230 & & & & -- & -- & -- & $6.55 \mathrm{E}+05$ & -- & $6.55 \mathrm{E}+05$ & $1.63 \mathrm{E}-03$ & $4.02 E+08$ & Direct \\
\hline Ac-231 & & & & -- & -- & -- & $2.31 \mathrm{E}+05$ & -- & $2.31 \mathrm{E}+05$ & $2.13 \mathrm{E}-03$ & $1.09 \mathrm{E}+08$ & Direct \\
\hline Ac-232 & & & & -- & -- & -- & $3.17 \mathrm{E}+05$ & -- & $3.17 E+05$ & $7.76 \mathrm{E}-04$ & $4.09 \mathrm{E}+08$ & Direct \\
\hline Ac-233 & & & & -- & -- & -- & $6.01 \mathrm{E}+05$ & -- & $6.01 \mathrm{E}+05$ & $1.80 \mathrm{E}-03$ & $3.34 \mathrm{E}+08$ & Direct \\
\hline $\mathrm{Ag}-100 \mathrm{~m}$ & & & & -- & -- & -- & $1.15 \mathrm{E}+05$ & -- & $1.15 \mathrm{E}+05$ & $1.36 \mathrm{E}-04$ & $8.40 \mathrm{E}+08$ & Direct \\
\hline $\mathrm{Ag}-101$ & & & & $4.65 \mathrm{E}+05$ & -- & $4.28 \mathrm{E}+07$ & $4.16 \mathrm{E}+04$ & -- & $4.16 \mathrm{E}+04$ & $2.48 \mathrm{E}-04$ & $1.68 \mathrm{E}+08$ & Direct \\
\hline Ag-102 & & & & $3.49 \mathrm{E}+05$ & -- & $2.95 \mathrm{E}+07$ & $1.68 \mathrm{E}+04$ & -- & $1.68 \mathrm{E}+04$ & 1.17E-04 & $1.43 \mathrm{E}+08$ & Direct \\
\hline $\mathrm{Ag}-102 \mathrm{~m}$ & & & & -- & -- & -- & $4.72 \mathrm{E}+04$ & -- & $4.72 \mathrm{E}+04$ & $1.97 \mathrm{E}-04$ & $2.40 \mathrm{E}+08$ & Direct \\
\hline Ag-103 & & & & $2.48 \mathrm{E}+05$ & -- & $5.40 \mathrm{E}+06$ & $1.44 \mathrm{E}+04$ & -- & $1.44 \mathrm{E}+04$ & $5.18 \mathrm{E}-04$ & $2.78 \mathrm{E}+07$ & Direct \\
\hline Ag-104 & & & & $1.57 \mathrm{E}+05$ & -- & $3.67 \mathrm{E}+06$ & $3.90 \mathrm{E}+03$ & -- & $3.90 \mathrm{E}+03$ & $1.49 \mathrm{E}-04$ & $2.61 \mathrm{E}+07$ & Direct \\
\hline Ag-104m & & & & $2.48 \mathrm{E}+05$ & -- & $8.42 E+06$ & $1.84 \mathrm{E}+04$ & -- & $1.84 \mathrm{E}+04$ & $3.41 \mathrm{E}-04$ & $5.40 \mathrm{E}+07$ & Direct \\
\hline Ag-105 & & & & $1.40 \mathrm{E}+04$ & -- & $2.19 \mathrm{E}+03$ & $1.39 \mathrm{E}+03$ & -- & $1.39 \mathrm{E}+03$ & 4.59E-02 & $3.04 \mathrm{E}+04$ & Direct \\
\hline $\mathrm{Ag}-105 \mathrm{~m}$ & & & & -- & -- & -- & $7.95 \mathrm{E}+07$ & -- & $7.95 \mathrm{E}+07$ & $3.21 \mathrm{E}-01$ & $2.48 \mathrm{E}+08$ & Direct \\
\hline $\mathrm{Ag}-106$ & & & & $4.14 \mathrm{E}+05$ & -- & $1.99 \mathrm{E}+07$ & $4.25 \mathrm{E}+04$ & -- & $4.25 \mathrm{E}+04$ & 5.74E-04 & $7.41 \mathrm{E}+07$ & Direct \\
\hline Ag-106m & & & & $6.98 \mathrm{E}+03$ & -- & $1.34 \mathrm{E}+03$ & $2.68 \mathrm{E}+02$ & -- & $2.68 \mathrm{E}+02$ & $1.83 \mathrm{E}-03$ & $1.47 \mathrm{E}+05$ & Direct \\
\hline Ag-108 & & & & -- & -- & -- & $1.72 \mathrm{E}+07$ & -- & $1.72 \mathrm{E}+07$ & $2.34 \mathrm{E}-02$ & $7.35 \mathrm{E}+08$ & Direct \\
\hline Ag-108m & & & & $3.19 \mathrm{E}+02$ & -- & $3.46 \mathrm{E}+02$ & $4.46 \mathrm{E}+02$ & -- & $3.19 \mathrm{E}+02$ & $1.22 \mathrm{E}+01$ & $2.61 \mathrm{E}+01$ & Inh. \\
\hline Ag-109m & & & & -- & -- & -- & $9.89 \mathrm{E}+07$ & -- & $9.89 \mathrm{E}+07$ & $3.78 \mathrm{E}-02$ & $2.62 E+09$ & Direct \\
\hline $\mathrm{Ag}-110$ & & & & -- & -- & -- & $5.78 \mathrm{E}+07$ & -- & $5.78 \mathrm{E}+07$ & $1.38 \mathrm{E}-02$ & $4.17 \mathrm{E}+09$ & Direct \\
\hline $\mathrm{Ag}-110 \mathrm{~m}$ & $2.63 \mathrm{E}+02$ & $5.54 \mathrm{E}-02$ & de & $9.31 \mathrm{E}+02$ & -- & $2.97 \mathrm{E}+02$ & $2.64 \mathrm{E}+02$ & -- & $2.64 \mathrm{E}+02$ & $5.56 \mathrm{E}-02$ & $4.75 \mathrm{E}+03$ & Direct \\
\hline Ag-111 & & & & $6.57 \mathrm{E}+03$ & -- & $1.67 \mathrm{E}+03$ & $2.89 \mathrm{E}+04$ & -- & $1.67 \mathrm{E}+03$ & $1.06 \mathrm{E}-02$ & $1.58 \mathrm{E}+05$ & Food \\
\hline
\end{tabular}

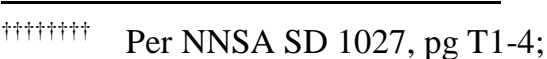

de - direct exposure from a point source

i - inhalation

ing - ingestion

sub - submersion in a radioactive cloud 
Table C.2 Summary of Exposure Pathway TQs and Selection of Final HC-3 TQs (continued)

\begin{tabular}{|c|c|c|c|c|c|c|c|c|c|c|c|c|}
\hline \multirow{2}{*}{$\begin{array}{c}\text { MASTER } \\
\text { ISOTOPE } \\
\text { LIST } \\
\end{array}$} & \multicolumn{3}{|c|}{ from NNSA SD G 1027} & \multicolumn{5}{|c|}{ Calculated Pathway Specific TQ Values } & \multicolumn{4}{|c|}{ Selected Final Threshold Quantities } \\
\hline & Act (Ci) & Mass (g) & $\begin{array}{l}\text { Limiting } \\
\text { Pathway }\end{array}$ & $\begin{array}{c}\text { TQ }_{\text {inhale }} \\
\text { (Ci) }\end{array}$ & $\begin{array}{c}\text { TQ }_{\text {water }} \\
\text { (Ci) }\end{array}$ & $\mathrm{TQ}_{\text {food }}(\mathrm{Ci})$ & $\begin{array}{c}\mathrm{TQ}_{\text {direct }} \\
(\mathrm{Ci}) \\
\end{array}$ & $\mathrm{TQ}_{\text {sub }}(\mathrm{Ci})$ & Act (Ci) & Mass (g) & $\begin{array}{c}\text { Sp. Act. } \\
\text { (Ci/g) }\end{array}$ & $\begin{array}{l}\text { Limiting } \\
\text { Pathway }\end{array}$ \\
\hline Ag-111m & & & & -- & -- & -- & $8.54 \mathrm{E}+07$ & -- & $8.54 \mathrm{E}+07$ & $5.44 \mathrm{E}-02$ & $1.57 \mathrm{E}+09$ & Direct \\
\hline Ag-112 & & & & $4.30 \mathrm{E}+04$ & -- & $1.91 \mathrm{E}+05$ & $5.92 \mathrm{E}+03$ & -- & $5.92 \mathrm{E}+03$ & $6.59 \mathrm{E}-04$ & $8.98 \mathrm{E}+06$ & Direct \\
\hline $\mathrm{Ag}-113$ & & & & $4.47 \mathrm{E}+04$ & -- & $1.17 \mathrm{E}+05$ & $3.27 \mathrm{E}+04$ & -- & $3.27 \mathrm{E}+04$ & $6.33 \mathrm{E}-03$ & $5.17 \mathrm{E}+06$ & Direct \\
\hline $\mathrm{Ag}-113 \mathrm{~m}$ & & & & -- & -- & -- & $2.97 \mathrm{E}+06$ & -- & $2.97 \mathrm{E}+06$ & $2.04 \mathrm{E}-03$ & $1.45 \mathrm{E}+09$ & Direct \\
\hline $\mathrm{Ag}-114$ & & & & -- & -- & -- & $3.65 \mathrm{E}+07$ & -- & $3.65 E+07$ & $1.70 \mathrm{E}-03$ & $2.15 \mathrm{E}+10$ & Direct \\
\hline $\mathrm{Ag}-115$ & & & & $2.54 \mathrm{E}+05$ & -- & $1.27 \mathrm{E}+07$ & $5.13 E+04$ & -- & $5.13 \mathrm{E}+04$ & $6.26 \mathrm{E}-04$ & $8.18 \mathrm{E}+07$ & Direct \\
\hline Ag-116 & & & & -- & -- & -- & $1.26 \mathrm{E}+05$ & -- & $1.26 \mathrm{E}+05$ & $2.08 \mathrm{E}-04$ & $6.05 \mathrm{E}+08$ & Direct \\
\hline Ag-117 & & & & -- & -- & -- & $4.53 \mathrm{E}+05$ & -- & $4.53 \mathrm{E}+05$ & $3.46 \mathrm{E}-04$ & $1.31 \mathrm{E}+09$ & Direct \\
\hline Ag-99 & & & & -- & -- & -- & $1.52 \mathrm{E}+05$ & -- & $1.52 \mathrm{E}+05$ & $1.65 \mathrm{E}-04$ & $9.20 \mathrm{E}+08$ & Direct \\
\hline Al-26 & & & & $6.20 \mathrm{E}+02$ & $5.64 \mathrm{E}+02$ & $2.30 \mathrm{E}+02$ & $2.71 \mathrm{E}+02$ & -- & $2.30 \mathrm{E}+02$ & $1.20 \mathrm{E}+04$ & $1.92 \mathrm{E}-02$ & Food \\
\hline Al-28 & & & & -- & -- & -- & $1.82 \mathrm{E}+05$ & -- & $1.82 \mathrm{E}+05$ & $6.06 \mathrm{E}-05$ & $3.00 \mathrm{E}+09$ & Direct \\
\hline Al-29 & & & & -- & -- & -- & $8.01 \mathrm{E}+04$ & -- & $8.01 E+04$ & $8.10 \mathrm{E}-05$ & $9.89 \mathrm{E}+08$ & Direct \\
\hline Am-237 & & & & $3.10 \mathrm{E}+06$ & -- & $1.18 \mathrm{E}+08$ & $2.69 \mathrm{E}+04$ & -- & $2.69 E+04$ & $2.47 \mathrm{E}-03$ & $1.09 \mathrm{E}+07$ & Direct \\
\hline Am-238 & & & & $1.31 \mathrm{E}+06$ & -- & $4.94 \mathrm{E}+07$ & $8.30 \mathrm{E}+03$ & -- & $8.30 \mathrm{E}+03$ & $1.03 \mathrm{E}-03$ & $8.06 \mathrm{E}+06$ & Direct \\
\hline Am-239 & & & & $3.85 \mathrm{E}+05$ & -- & $9.31 \mathrm{E}+05$ & $5.63 \mathrm{E}+03$ & -- & $5.63 \mathrm{E}+03$ & $5.11 \mathrm{E}-03$ & $1.10 \mathrm{E}+06$ & Direct \\
\hline Am-240 & & & & $1.89 \mathrm{E}+05$ & -- & $1.00 \mathrm{E}+05$ & $8.28 \mathrm{E}+02$ & -- & $8.28 \mathrm{E}+02$ & $3.22 \mathrm{E}-03$ & $2.57 \mathrm{E}+05$ & Direct \\
\hline Am-241 & $2.89 \mathrm{E}+00$ & $8.42 \mathrm{E}-01$ & $\mathrm{i}$ & $2.86 \mathrm{E}+00$ & -- & $4.03 \mathrm{E}+01$ & $2.23 E+04$ & -- & $2.86 \mathrm{E}+00$ & 8.35E-01 & $3.43 E+00$ & Inh. \\
\hline Am-242 & & & & $6.98 \mathrm{E}+03$ & -- & $5.60 \mathrm{E}+05$ & $6.38 \mathrm{E}+04$ & -- & $6.98 \mathrm{E}+03$ & $8.64 \mathrm{E}-03$ & $8.08 \mathrm{E}+05$ & Inh. \\
\hline Am-242m & $3.22 \mathrm{E}+00$ & 3.07E-01 & $\mathrm{i}$ & $3.19 \mathrm{E}+00$ & -- & $4.24 \mathrm{E}+01$ & $1.42 \mathrm{E}+05$ & -- & $3.19 \mathrm{E}+00$ & $3.28 \mathrm{E}-01$ & $9.72 \mathrm{E}+00$ & Inh. \\
\hline Am-243 & $2.89 \mathrm{E}+00$ & $1.45 \mathrm{E}+01$ & $\mathrm{i}$ & $2.86 \mathrm{E}+00$ & -- & $4.03 \mathrm{E}+01$ & $1.30 \mathrm{E}+04$ & -- & $2.86 \mathrm{E}+00$ & $1.44 \mathrm{E}+01$ & $1.99 \mathrm{E}-01$ & Inh. \\
\hline Am-244 & & & & $5.88 \mathrm{E}+04$ & -- & $5.70 \mathrm{E}+05$ & $1.83 \mathrm{E}+03$ & -- & $1.83 \mathrm{E}+03$ & $1.44 \mathrm{E}-03$ & $1.27 \mathrm{E}+06$ & Direct \\
\hline Am-244m & & & & $1.41 \mathrm{E}+06$ & -- & $2.05 \mathrm{E}+08$ & $1.86 \mathrm{E}+07$ & -- & $1.41 \mathrm{E}+06$ & 4.77E-02 & $2.96 \mathrm{E}+07$ & Inh. \\
\hline Am-245 & & & & $1.47 \mathrm{E}+06$ & -- & $2.03 \mathrm{E}+07$ & $1.82 \mathrm{E}+05$ & -- & $1.82 \mathrm{E}+05$ & $2.92 \mathrm{E}-02$ & $6.24 \mathrm{E}+06$ & Direct \\
\hline Am-246 & & & & $1.02 \mathrm{E}+06$ & -- & $6.83 \mathrm{E}+07$ & $2.66 \mathrm{E}+04$ & -- & $2.66 \mathrm{E}+04$ & $1.36 \mathrm{E}-03$ & $1.96 \mathrm{E}+07$ & Direct \\
\hline Am-246m & & & & $2.94 \mathrm{E}+06$ & -- & $1.82 \mathrm{E}+08$ & $2.85 \mathrm{E}+04$ & -- & $2.85 \mathrm{E}+04$ & $9.31 \mathrm{E}-04$ & $3.06 \mathrm{E}+07$ & Direct \\
\hline Am-247 & & & & $2.54 \mathrm{E}+06$ & -- & $2.16 \mathrm{E}+08$ & $2.34 \mathrm{E}+05$ & -- & $2.34 \mathrm{E}+05$ & 7.06E-03 & $3.31 \mathrm{E}+07$ & Direct \\
\hline Ar-37 & & & & -- & -- & -- & -- & $7.84 \mathrm{E}+08$ & $7.84 \mathrm{E}+08$ & $7.78 \mathrm{E}+03$ & $1.01 \mathrm{E}+05$ & Sub \\
\hline Ar-39 & & & & -- & -- & -- & -- & $2.92 \mathrm{E}+05$ & $2.92 \mathrm{E}+05$ & $8.57 \mathrm{E}+03$ & $3.41 \mathrm{E}+01$ & Sub \\
\hline Ar-41 & & & & -- & -- & -- & -- & $6.07 \mathrm{E}+02$ & $6.07 E+02$ & $1.45 \mathrm{E}-05$ & $4.19 \mathrm{E}+07$ & Sub \\
\hline Ar-42 & & & & -- & -- & -- & -- & $2.47 \mathrm{E}+05$ & $2.47 \mathrm{E}+05$ & $9.55 \mathrm{E}+02$ & $2.59 \mathrm{E}+02$ & Sub \\
\hline Ar-43 & & & & -- & -- & -- & $8.79 \mathrm{E}+04$ & -- & $8.79 \mathrm{E}+04$ & $1.08 \mathrm{E}-04$ & $8.15 \mathrm{E}+08$ & Direct \\
\hline Ar-44 & & & & -- & -- & -- & -- & $3.97 \mathrm{E}+02$ & $3.97 \mathrm{E}+02$ & $1.10 \mathrm{E}-06$ & $3.60 \mathrm{E}+08$ & Sub \\
\hline As-68 & & & & -- & -- & -- & $7.73 E+04$ & -- & $7.73 E+04$ & $7.05 \mathrm{E}-05$ & $1.10 \mathrm{E}+09$ & Direct \\
\hline As-69 & & & & $3.19 \mathrm{E}+05$ & $1.30 \mathrm{E}+180$ & $1.78 \mathrm{E}+07$ & $4.71 \mathrm{E}+04$ & -- & $4.71 \mathrm{E}+04$ & $2.62 \mathrm{E}-04$ & $1.79 \mathrm{E}+08$ & Direct \\
\hline As-70 & & & & $9.31 \mathrm{E}+04$ & $2.24 \mathrm{E}+56$ & $2.26 \mathrm{E}+06$ & $3.36 \mathrm{E}+03$ & -- & $3.36 \mathrm{E}+03$ & $6.58 \mathrm{E}-05$ & $5.11 \mathrm{E}+07$ & Direct \\
\hline
\end{tabular}


Table C.2 Summary of Exposure Pathway TQs and Selection of Final HC-3 TQs (continued)

\begin{tabular}{|c|c|c|c|c|c|c|c|c|c|c|c|c|}
\hline \multirow[b]{2}{*}{$\begin{array}{c}\text { MASTER } \\
\text { ISOTOPE } \\
\text { LIST } \\
\end{array}$} & \multicolumn{3}{|c|}{ from NNSA SD G 1027} & \multicolumn{5}{|c|}{ Calculated Pathway Specific TQ Values } & \multicolumn{4}{|c|}{ Selected Final Threshold Quantities } \\
\hline & Act $(\mathrm{Ci})$ & Mass (g) & $\begin{array}{l}\text { Limiting } \\
\text { Pathway }\end{array}$ & $\begin{array}{c}\text { TQ }_{\text {inhale }} \\
(\mathrm{Ci})\end{array}$ & $\begin{array}{c}\text { TQ }_{\text {water }} \\
\text { (Ci) }\end{array}$ & $\mathrm{TQ}_{\text {food }}(\mathrm{Ci})$ & $\begin{array}{c}T_{\text {direct }} \\
\text { (Ci) }\end{array}$ & $\mathrm{TQ}_{\text {sub }}(\mathrm{Ci})$ & Act (Ci) & Mass (g) & $\begin{array}{c}\text { Sp. Act. } \\
\text { (Ci/g) }\end{array}$ & $\begin{array}{l}\text { Limiting } \\
\text { Pathway }\end{array}$ \\
\hline As-71 & & & & $2.23 E+04$ & $5.22 \mathrm{E}+04$ & $1.03 \mathrm{E}+04$ & $1.43 \mathrm{E}+03$ & -- & $1.43 \mathrm{E}+03$ & $2.10 \mathrm{E}-03$ & $6.82 \mathrm{E}+05$ & Direct \\
\hline As-72 & & & & $8.59 \mathrm{E}+03$ & $3.06 \mathrm{E}+05$ & $5.91 \mathrm{E}+03$ & $5.48 \mathrm{E}+02$ & -- & $5.48 \mathrm{E}+02$ & $3.27 \mathrm{E}-04$ & $1.68 \mathrm{E}+06$ & Direct \\
\hline As-73 & & & & $1.20 \mathrm{E}+04$ & $8.32 \mathrm{E}+03$ & $3.56 \mathrm{E}+03$ & $4.56 \mathrm{E}+04$ & -- & $3.56 \mathrm{E}+03$ & $1.60 \mathrm{E}-01$ & $2.23 \mathrm{E}+04$ & Food \\
\hline As-74 & & & & $5.32 \mathrm{E}+03$ & $2.28 \mathrm{E}+03$ & $1.06 \mathrm{E}+03$ & $9.76 \mathrm{E}+02$ & -- & $9.76 \mathrm{E}+02$ & $9.81 \mathrm{E}-03$ & $9.95 \mathrm{E}+04$ & Direct \\
\hline As-76 & & & & $1.21 \mathrm{E}+04$ & $3.25 \mathrm{E}+05$ & $6.57 \mathrm{E}+03$ & $2.28 \mathrm{E}+03$ & -- & $2.28 \mathrm{E}+03$ & $1.45 \mathrm{E}-03$ & $1.57 \mathrm{E}+06$ & Direct \\
\hline As-77 & & & & $2.66 \mathrm{E}+04$ & $2.62 \mathrm{E}+05$ & $1.84 \mathrm{E}+04$ & $1.03 \mathrm{E}+05$ & -- & $1.84 \mathrm{E}+04$ & $1.76 \mathrm{E}-02$ & $1.05 \mathrm{E}+06$ & Food \\
\hline As-78 & & & & $7.98 \mathrm{E}+04$ & $8.49 \mathrm{E}+34$ & $8.12 \mathrm{E}+05$ & $6.38 \mathrm{E}+03$ & -- & $6.38 \mathrm{E}+03$ & $2.40 \mathrm{E}-04$ & $2.66 \mathrm{E}+07$ & Direct \\
\hline As-79 & & & & -- & -- & -- & $2.38 \mathrm{E}+06$ & -- & $2.38 \mathrm{E}+06$ & $9.00 \mathrm{E}-03$ & $2.64 \mathrm{E}+08$ & Direct \\
\hline At-204 & & & & -- & -- & -- & $3.39 E+04$ & -- & $3.39 E+04$ & $3.38 \mathrm{E}-04$ & $1.00 \mathrm{E}+08$ & Direct \\
\hline At-205 & & & & $1.67 \mathrm{E}+05$ & $1.57 \mathrm{E}+107$ & $7.39 \mathrm{E}+07$ & $2.42 \mathrm{E}+04$ & -- & $2.42 \mathrm{E}+04$ & $6.90 \mathrm{E}-04$ & $3.50 \mathrm{E}+07$ & Direct \\
\hline At-206 & & & & -- & -- & -- & $9.55 \mathrm{E}+03$ & -- & $9.55 \mathrm{E}+03$ & $3.20 \mathrm{E}-04$ & $2.98 \mathrm{E}+07$ & Direct \\
\hline At-207 & & & & $5.32 \mathrm{E}+04$ & $1.49 \mathrm{E}+30$ & $6.03 E+06$ & $5.07 \mathrm{E}+03$ & -- & $5.07 \mathrm{E}+03$ & $6.02 \mathrm{E}-04$ & $8.41 \mathrm{E}+06$ & Direct \\
\hline At-208 & & & & $2.94 \mathrm{E}+05$ & $1.39 \mathrm{E}+33$ & $1.63 \mathrm{E}+07$ & $2.44 \mathrm{E}+03$ & -- & $2.44 \mathrm{E}+03$ & $2.64 \mathrm{E}-04$ & $9.24 \mathrm{E}+06$ & Direct \\
\hline At-209 & & & & $3.99 \mathrm{E}+04$ & $3.56 \mathrm{E}+12$ & $2.45 \mathrm{E}+05$ & $1.02 \mathrm{E}+03$ & -- & $1.02 \mathrm{E}+03$ & $3.70 \mathrm{E}-04$ & $2.77 \mathrm{E}+06$ & Direct \\
\hline At-210 & & & & $1.14 \mathrm{E}+04$ & $9.46 \mathrm{E}+08$ & $3.20 \mathrm{E}+04$ & $5.77 \mathrm{E}+02$ & -- & $5.77 \mathrm{E}+02$ & $3.13 E-04$ & $1.84 \mathrm{E}+06$ & Direct \\
\hline At-211 & & & & $1.02 \mathrm{E}+03$ & $4.36 \mathrm{E}+09$ & $3.20 \mathrm{E}+04$ & $4.76 \mathrm{E}+04$ & -- & $1.02 \mathrm{E}+03$ & $4.93 \mathrm{E}-04$ & $2.06 \mathrm{E}+06$ & Inh. \\
\hline At-215 & & & & -- & -- & -- & $4.35 \mathrm{E}+15$ & -- & $4.35 \mathrm{E}+15$ & $8.29 \mathrm{E}+00$ & $5.25 \mathrm{E}+14$ & Direct \\
\hline At-216 & & & & -- & -- & -- & $9.66 \mathrm{E}+13$ & -- & $9.66 \mathrm{E}+13$ & $5.55 \mathrm{E}-01$ & $1.74 \mathrm{E}+14$ & Direct \\
\hline At-217 & & & & -- & -- & -- & $4.49 \mathrm{E}+12$ & -- & $4.49 \mathrm{E}+12$ & $2.79 \mathrm{E}+00$ & $1.61 \mathrm{E}+12$ & Direct \\
\hline At-218 & & & & -- & -- & -- & $3.24 \mathrm{E}+09$ & -- & $3.24 \mathrm{E}+09$ & $1.25 \mathrm{E}-01$ & $2.59 \mathrm{E}+10$ & Direct \\
\hline At-219 & & & & -- & -- & -- & -- & -- & -- & -- & $9.20 \mathrm{E}+08$ & -- \\
\hline At-220 & & & & -- & -- & -- & $4.34 \mathrm{E}+05$ & -- & $4.34 \mathrm{E}+05$ & $1.89 \mathrm{E}-03$ & $2.30 \mathrm{E}+08$ & Direct \\
\hline Au-186 & & & & $3.19 E+05$ & $1.08 \mathrm{E}+253$ & $3.09 \mathrm{E}+07$ & $4.52 E+04$ & -- & $4.52 E+04$ & $4.78 \mathrm{E}-04$ & $9.45 \mathrm{E}+07$ & Direct \\
\hline Au-187 & & & & -- & -- & -- & $8.10 \mathrm{E}+04$ & -- & $8.10 E+04$ & $6.76 \mathrm{E}-04$ & $1.20 \mathrm{E}+08$ & Direct \\
\hline Au-188 & & & & -- & -- & -- & -- & -- & -- & -- & $1.13 \mathrm{E}+08$ & -- \\
\hline Au-189m & & & & -- & -- & -- & -- & -- & -- & -- & $2.17 E+08$ & -- \\
\hline Au-190 & & & & $2.43 \mathrm{E}+05$ & $2.07 E+68$ & $7.57 \mathrm{E}+06$ & $7.11 \mathrm{E}+03$ & -- & $7.11 E+03$ & 3.07E-04 & $2.31 \mathrm{E}+07$ & Direct \\
\hline Au-191 & & & & $1.15 \mathrm{E}+05$ & $7.05 \mathrm{E}+19$ & $1.04 \mathrm{E}+06$ & $6.42 \mathrm{E}+03$ & -- & $6.42 \mathrm{E}+03$ & $1.24 \mathrm{E}-03$ & $5.16 \mathrm{E}+06$ & Direct \\
\hline Au-192 & & & & $6.57 E+04$ & $2.42 \mathrm{E}+14$ & $2.89 \mathrm{E}+05$ & $1.31 \mathrm{E}+03$ & -- & $1.31 \mathrm{E}+03$ & $3.95 \mathrm{E}-04$ & $3.30 \mathrm{E}+06$ & Direct \\
\hline Au-193 & & & & $6.98 \mathrm{E}+04$ & $3.90 \mathrm{E}+07$ & $1.16 \mathrm{E}+05$ & $7.03 E+03$ & -- & $7.03 E+03$ & $7.64 \mathrm{E}-03$ & $9.20 \mathrm{E}+05$ & Direct \\
\hline Au-193m & & & & -- & -- & -- & $5.63 \mathrm{E}+07$ & -- & $5.63 \mathrm{E}+07$ & $3.76 \mathrm{E}-03$ & $1.50 \mathrm{E}+10$ & Direct \\
\hline Au-194 & & & & $2.94 \mathrm{E}+04$ & $2.34 \mathrm{E}+05$ & $1.71 \mathrm{E}+04$ & $8.34 \mathrm{E}+02$ & -- & $8.34 \mathrm{E}+02$ & $2.04 \mathrm{E}-03$ & $4.09 \mathrm{E}+05$ & Direct \\
\hline Au-195 & & & & $6.98 \mathrm{E}+03$ & $8.22 \mathrm{E}+03$ & $3.39 \mathrm{E}+03$ & $8.60 \mathrm{E}+03$ & -- & $3.39 E+03$ & $9.25 \mathrm{E}-01$ & $3.66 \mathrm{E}+03$ & Food \\
\hline Au-195m & & & & -- & -- & -- & $7.08 \mathrm{E}+06$ & -- & $7.08 \mathrm{E}+06$ & $3.73 \mathrm{E}-03$ & $1.90 \mathrm{E}+09$ & Direct \\
\hline Au-196 & & & & $1.53 \mathrm{E}+04$ & $1.17 \mathrm{E}+04$ & $4.64 \mathrm{E}+03$ & $1.62 \mathrm{E}+03$ & -- & $1.62 \mathrm{E}+03$ & $1.50 \mathrm{E}-02$ & $1.08 \mathrm{E}+05$ & Direct \\
\hline
\end{tabular}


Table C.2 Summary of Exposure Pathway TQs and Selection of Final HC-3 TQs (continued)

\begin{tabular}{|c|c|c|c|c|c|c|c|c|c|c|c|c|}
\hline \multirow[b]{2}{*}{$\begin{array}{c}\text { MASTER } \\
\text { ISOTOPE } \\
\text { LIST } \\
\end{array}$} & \multicolumn{3}{|c|}{ from NNSA SD G 1027} & \multicolumn{5}{|c|}{ Calculated Pathway Specific TQ Values } & \multicolumn{4}{|c|}{ Selected Final Threshold Quantities } \\
\hline & Act (Ci) & Mass (g) & $\begin{array}{l}\text { Limiting } \\
\text { Pathway }\end{array}$ & $\begin{array}{c}\text { TQ }_{\text {inhale }} \\
\text { (Ci) }\end{array}$ & $\begin{array}{c}\text { TQ }_{\text {water }} \\
\text { (Ci) }\end{array}$ & $\mathrm{TQ}_{\text {food }}(\mathrm{Ci})$ & $\begin{array}{c}T_{\text {direct }} \\
\text { (Ci) }\end{array}$ & $\mathrm{TQ}_{\text {sub }}(\mathrm{Ci})$ & Act (Ci) & Mass (g) & $\begin{array}{l}\text { Sp. Act. } \\
\text { (Ci/g) }\end{array}$ & $\begin{array}{l}\text { Limiting } \\
\text { Pathway }\end{array}$ \\
\hline Au-196m & & & & $1.83 \mathrm{E}+04$ & $2.73 \mathrm{E}+09$ & $6.62 \mathrm{E}+04$ & $6.18 \mathrm{E}+03$ & -- & $6.18 \mathrm{E}+03$ & $3.71 \mathrm{E}-03$ & $1.67 \mathrm{E}+06$ & Direct \\
\hline Au-198 & $2.03 \mathrm{E}+03$ & $8.30 \mathrm{E}-03$ & de & $1.02 \mathrm{E}+04$ & $2.41 \mathrm{E}+04$ & $4.67 \mathrm{E}+03$ & $2.03 \mathrm{E}+03$ & -- & $2.03 \mathrm{E}+03$ & $8.31 \mathrm{E}-03$ & $2.45 \mathrm{E}+05$ & Direct \\
\hline Au-198m & & & & $5.58 \mathrm{E}+03$ & $2.74 \mathrm{E}+04$ & $4.11 \mathrm{E}+03$ & $1.46 \mathrm{E}+03$ & -- & $1.46 \mathrm{E}+03$ & $5.08 \mathrm{E}-03$ & $2.87 \mathrm{E}+05$ & Direct \\
\hline Au-199 & & & & $1.47 \mathrm{E}+04$ & $3.94 \mathrm{E}+04$ & $9.36 \mathrm{E}+03$ & $9.11 \mathrm{E}+03$ & -- & $9.11 \mathrm{E}+03$ & $4.36 \mathrm{E}-02$ & $2.09 \mathrm{E}+05$ & Direct \\
\hline $\mathrm{Au}-200$ & & & & $1.99 \mathrm{E}+05$ & $1.00 \mathrm{E}+61$ & $4.63 \mathrm{E}+06$ & $5.50 \mathrm{E}+04$ & -- & $5.50 \mathrm{E}+04$ & $2.83 \mathrm{E}-03$ & $1.94 \mathrm{E}+07$ & Direct \\
\hline Au-200m & & & & $1.12 \mathrm{E}+04$ & $3.15 E+06$ & $1.30 \mathrm{E}+04$ & $5.25 \mathrm{E}+02$ & -- & $5.25 \mathrm{E}+02$ & $6.27 \mathrm{E}-04$ & $8.38 \mathrm{E}+05$ & Direct \\
\hline Au-201 & & & & $3.85 \mathrm{E}+05$ & $8.71 E+106$ & $2.40 \mathrm{E}+07$ & $5.14 \mathrm{E}+05$ & -- & $3.85 \mathrm{E}+05$ & 1.09E-02 & $3.54 \mathrm{E}+07$ & Inh. \\
\hline Au-202 & & & & -- & -- & -- & $8.77 E+06$ & -- & $8.77 \mathrm{E}+06$ & $4.52 \mathrm{E}-03$ & $1.94 \mathrm{E}+09$ & Direct \\
\hline Ba-124 & & & & $3.99 \mathrm{E}+05$ & -- & $2.02 \mathrm{E}+07$ & $1.15 \mathrm{E}+05$ & -- & $1.15 \mathrm{E}+05$ & 8.34E-04 & $1.38 \mathrm{E}+08$ & Direct \\
\hline Ba-126 & & & & $9.31 \mathrm{E}+04$ & -- & $6.14 \mathrm{E}+05$ & $4.60 \mathrm{E}+04$ & -- & $4.60 \mathrm{E}+04$ & $2.98 \mathrm{E}-03$ & $1.55 \mathrm{E}+07$ & Direct \\
\hline Ba-127 & & & & $7.98 \mathrm{E}+05$ & -- & $4.83 \mathrm{E}+07$ & $7.83 \mathrm{E}+04$ & -- & $7.83 E+04$ & $6.72 \mathrm{E}-04$ & $1.17 \mathrm{E}+08$ & Direct \\
\hline Ba-128 & & & & $8.59 \mathrm{E}+03$ & -- & $1.90 \mathrm{E}+03$ & $1.10 \mathrm{E}+04$ & -- & $1.90 \mathrm{E}+03$ & $4.53 \mathrm{E}-03$ & $4.20 \mathrm{E}+05$ & Food \\
\hline Ba-129 & & & & $3.10 \mathrm{E}+05$ & -- & $2.22 \mathrm{E}+06$ & $1.63 \mathrm{E}+04$ & -- & $1.63 \mathrm{E}+04$ & $1.49 \mathrm{E}-03$ & $1.09 \mathrm{E}+07$ & Direct \\
\hline Ba-129m & & & & $1.47 \mathrm{E}+05$ & -- & $1.53 \mathrm{E}+06$ & $3.53 E+03$ & -- & $3.53 E+03$ & $3.14 \mathrm{E}-04$ & $1.13 \mathrm{E}+07$ & Direct \\
\hline Ba-131 & & & & $3.19 E+04$ & -- & $3.70 \mathrm{E}+03$ & $1.63 \mathrm{E}+03$ & -- & $1.63 E+03$ & 1.93E-02 & $8.45 E+04$ & Direct \\
\hline Ba-131m & & & & $1.75 \mathrm{E}+06$ & -- & $2.15 \mathrm{E}+08$ & $6.48 \mathrm{E}+05$ & -- & $6.48 \mathrm{E}+05$ & $6.59 \mathrm{E}-03$ & $9.84 \mathrm{E}+07$ & Direct \\
\hline Ba-133 & $7.85 \mathrm{E}+02$ & $3.07 E+00$ & ing & $6.20 \mathrm{E}+03$ & -- & $8.04 \mathrm{E}+02$ & $1.81 \mathrm{E}+03$ & -- & $8.04 \mathrm{E}+02$ & $3.21 \mathrm{E}+00$ & $2.50 \mathrm{E}+02$ & Food \\
\hline Ba-133m & & & & $3.99 \mathrm{E}+04$ & -- & $1.33 \mathrm{E}+04$ & $1.34 \mathrm{E}+04$ & -- & $1.33 \mathrm{E}+04$ & $2.20 \mathrm{E}-02$ & $6.06 \mathrm{E}+05$ & Food \\
\hline Ba-135m & & & & $4.86 \mathrm{E}+04$ & -- & $2.15 \mathrm{E}+04$ & $1.59 \mathrm{E}+04$ & -- & $1.59 \mathrm{E}+04$ & 1.97E-02 & $8.09 \mathrm{E}+05$ & Direct \\
\hline Ba-137m & & & & -- & -- & -- & $4.76 \mathrm{E}+05$ & -- & $4.76 \mathrm{E}+05$ & $8.84 \mathrm{E}-04$ & $5.38 \mathrm{E}+08$ & Direct \\
\hline Ba-139 & & & & $2.03 E+05$ & -- & $1.55 \mathrm{E}+06$ & $2.05 \mathrm{E}+05$ & -- & $2.03 E+05$ & $1.24 \mathrm{E}-02$ & $1.64 \mathrm{E}+07$ & Inh. \\
\hline Ba-140 & $6.44 \mathrm{E}+02$ & 8.80E-03 & ing & $6.98 \mathrm{E}+03$ & -- & $6.40 \mathrm{E}+02$ & $4.08 \mathrm{E}+03$ & -- & $6.40 \mathrm{E}+02$ & $8.73 \mathrm{E}-03$ & $7.33 \mathrm{E}+04$ & Food \\
\hline Ba-141 & & & & $3.19 E+05$ & -- & $1.20 \mathrm{E}+07$ & $4.69 E+04$ & -- & $4.69 E+04$ & $6.43 \mathrm{E}-04$ & $7.30 \mathrm{E}+07$ & Direct \\
\hline Ba-142 & & & & $4.14 \mathrm{E}+05$ & -- & $4.14 \mathrm{E}+07$ & $6.53 E+04$ & -- & $6.53 E+04$ & $5.22 \mathrm{E}-04$ & $1.25 \mathrm{E}+08$ & Direct \\
\hline $\mathrm{Be}-10$ & & & & $3.49 \mathrm{E}+02$ & -- & $7.32 \mathrm{E}+02$ & -- & -- & $3.49 E+02$ & $1.56 \mathrm{E}+04$ & $2.23 \mathrm{E}-02$ & Inh. \\
\hline $\mathrm{Be}-7$ & & & & $2.15 \mathrm{E}+05$ & -- & $3.53 \mathrm{E}+04$ & $1.48 \mathrm{E}+04$ & -- & $1.48 \mathrm{E}+04$ & $4.25 \mathrm{E}-02$ & $3.49 \mathrm{E}+05$ & Direct \\
\hline Bi-197 & & & & -- & -- & -- & $4.59 \mathrm{E}+04$ & -- & $4.59 \mathrm{E}+04$ & $4.47 \mathrm{E}-04$ & $1.03 \mathrm{E}+08$ & Direct \\
\hline $\mathrm{Bi}-200$ & & & & $1.99 \mathrm{E}+05$ & -- & $8.31 \mathrm{E}+06$ & $8.32 \mathrm{E}+03$ & -- & $8.32 \mathrm{E}+03$ & $3.22 \mathrm{E}-04$ & $2.58 \mathrm{E}+07$ & Direct \\
\hline Bi-201 & & & & $1.02 \mathrm{E}+05$ & -- & $1.19 \mathrm{E}+06$ & $5.01 \mathrm{E}+03$ & -- & $5.01 \mathrm{E}+03$ & $5.79 \mathrm{E}-04$ & $8.66 \mathrm{E}+06$ & Direct \\
\hline Bi-202 & & & & $1.12 \mathrm{E}+05$ & -- & $1.74 \mathrm{E}+06$ & $2.67 \mathrm{E}+03$ & -- & $2.67 E+03$ & $2.87 \mathrm{E}-04$ & $9.29 \mathrm{E}+06$ & Direct \\
\hline Bi-203 & & & & $2.48 \mathrm{E}+04$ & -- & $4.70 \mathrm{E}+04$ & $5.69 \mathrm{E}+02$ & -- & $5.69 \mathrm{E}+02$ & $4.33 \mathrm{E}-04$ & $1.31 \mathrm{E}+06$ & Direct \\
\hline Bi-204 & & & & $1.93 \mathrm{E}+04$ & -- & $3.87 \mathrm{E}+04$ & $4.54 \mathrm{E}+02$ & -- & $4.54 \mathrm{E}+02$ & $3.32 \mathrm{E}-04$ & $1.37 \mathrm{E}+06$ & Direct \\
\hline Bi-205 & & & & $1.12 \mathrm{E}+04$ & -- & $1.63 \mathrm{E}+03$ & $4.39 \mathrm{E}+02$ & -- & $4.39 E+02$ & $1.06 \mathrm{E}-02$ & $4.16 \mathrm{E}+04$ & Direct \\
\hline Bi-206 & & & & $5.32 \mathrm{E}+03$ & -- & $1.30 \mathrm{E}+03$ & $2.34 \mathrm{E}+02$ & -- & $2.34 \mathrm{E}+02$ & $2.30 \mathrm{E}-03$ & $1.02 \mathrm{E}+05$ & Direct \\
\hline $\mathrm{Bi}-207$ & $4.71 \mathrm{E}+02$ & $1.04 \mathrm{E}+01$ & de & $2.15 \mathrm{E}+03$ & -- & $6.20 \mathrm{E}+02$ & $4.71 \mathrm{E}+02$ & -- & $4.71 E+02$ & $1.04 \mathrm{E}+01$ & $4.55 \mathrm{E}+01$ & Direct \\
\hline
\end{tabular}


Table C.2 Summary of Exposure Pathway TQs and Selection of Final HC-3 TQs (continued)

\begin{tabular}{|c|c|c|c|c|c|c|c|c|c|c|c|c|}
\hline \multirow{2}{*}{$\begin{array}{c}\text { MASTER } \\
\text { ISOTOPE } \\
\text { LIST } \\
\end{array}$} & \multicolumn{3}{|c|}{ from NNSA SD G 1027} & \multicolumn{5}{|c|}{ Calculated Pathway Specific TQ Values } & \multicolumn{4}{|c|}{ Selected Final Threshold Quantities } \\
\hline & Act (Ci) & Mass (g) & $\begin{array}{l}\text { Limiting } \\
\text { Pathway }\end{array}$ & $\begin{array}{c}\mathrm{TQ}_{\text {inhale }} \\
(\mathrm{Ci}) \\
\end{array}$ & $\begin{array}{c}\text { TQ }_{\text {water }} \\
\text { (Ci) }\end{array}$ & $\mathrm{TQ}_{\text {food }}(\mathrm{Ci})$ & $\begin{array}{c}\mathrm{TQ}_{\text {direct }} \\
(\mathrm{Ci}) \\
\end{array}$ & $\mathrm{TQ}_{\text {sub }}(\mathrm{Ci})$ & Act (Ci) & Mass (g) & $\begin{array}{c}\text { Sp. Act. } \\
\text { (Ci/g) }\end{array}$ & $\begin{array}{l}\text { Limiting } \\
\text { Pathway }\end{array}$ \\
\hline $\mathrm{Bi}-208$ & & & & $2.54 \mathrm{E}+03$ & -- & $6.71 \mathrm{E}+02$ & $2.74 \mathrm{E}+02$ & -- & $2.74 \mathrm{E}+02$ & $5.87 \mathrm{E}+04$ & 4.67E-03 & Direct \\
\hline $\mathrm{Bi}-210$ & $1.34 \mathrm{E}+02$ & $1.08 \mathrm{E}-03$ & $\mathrm{i}$ & $1.33 \mathrm{E}+02$ & -- & $2.23 \mathrm{E}+03$ & -- & -- & $1.33 \mathrm{E}+02$ & $1.07 \mathrm{E}-03$ & $1.24 \mathrm{E}+05$ & Inh. \\
\hline $\mathrm{Bi}-210 \mathrm{~m}$ & & & & $3.60 \mathrm{E}+00$ & -- & $5.37 \mathrm{E}+01$ & $2.83 \mathrm{E}+03$ & -- & $3.60 \mathrm{E}+00$ & $6.35 \mathrm{E}+03$ & $5.67 \mathrm{E}-04$ & Inh. \\
\hline $\mathrm{Bi}-211$ & & & & -- & -- & -- & $7.25 \mathrm{E}+06$ & -- & $7.25 \mathrm{E}+06$ & $1.74 \mathrm{E}-02$ & $4.16 \mathrm{E}+08$ & Direct \\
\hline $\mathrm{Bi}-212$ & & & & $2.86 \mathrm{E}+02$ & -- & $9.81 \mathrm{E}+05$ & $6.45 \mathrm{E}+04$ & -- & $2.86 \mathrm{E}+02$ & $1.95 \mathrm{E}-05$ & $1.46 \mathrm{E}+07$ & Inh. \\
\hline $\mathrm{Bi}-212 \mathrm{n}$ & & & & -- & -- & -- & -- & -- & -- & -- & $1.27 \mathrm{E}+08$ & -- \\
\hline Bi-213 & & & & $2.72 \mathrm{E}+02$ & -- & $1.69 \mathrm{E}+06$ & $1.19 \mathrm{E}+05$ & -- & $2.72 E+02$ & $1.41 \mathrm{E}-05$ & $1.93 \mathrm{E}+07$ & Inh. \\
\hline $\mathrm{Bi}-214$ & & & & $5.32 \mathrm{E}+02$ & -- & $7.04 \mathrm{E}+06$ & $2.41 \mathrm{E}+04$ & -- & $5.32 \mathrm{E}+02$ & $1.20 \mathrm{E}-05$ & $4.42 \mathrm{E}+07$ & Inh. \\
\hline $\mathrm{Bi}-215$ & & & & -- & -- & -- & $3.76 \mathrm{E}+05$ & -- & $3.76 \mathrm{E}+05$ & $3.27 \mathrm{E}-03$ & $1.15 \mathrm{E}+08$ & Direct \\
\hline $\mathrm{Bi}-216$ & & & & -- & -- & -- & $4.52 \mathrm{E}+05$ & -- & $4.52 \mathrm{E}+05$ & $1.13 \mathrm{E}-03$ & $4.01 \mathrm{E}+08$ & Direct \\
\hline Bk-244 & & & & $1.12 \mathrm{E}+05$ & -- & $8.67 \mathrm{E}+05$ & -- & -- & $1.12 \mathrm{E}+05$ & $3.78 \mathrm{E}-02$ & $2.95 \mathrm{E}+06$ & Inh. \\
\hline Bk-245 & & & & $5.58 \mathrm{E}+04$ & -- & $5.14 \mathrm{E}+04$ & $3.32 \mathrm{E}+03$ & -- & $3.32 E+03$ & $3.08 \mathrm{E}-02$ & $1.08 \mathrm{E}+05$ & Direct \\
\hline Bk-246 & & & & $2.43 \mathrm{E}+05$ & -- & $1.38 \mathrm{E}+05$ & $9.17 \mathrm{E}+02$ & -- & $9.17 \mathrm{E}+02$ & $3.16 \mathrm{E}-03$ & $2.90 \mathrm{E}+05$ & Direct \\
\hline Bk-247 & & & & $1.72 \mathrm{E}+00$ & -- & $2.30 \mathrm{E}+01$ & $6.89 \mathrm{E}+03$ & -- & $1.72 \mathrm{E}+00$ & $1.64 \mathrm{E}+00$ & $1.05 \mathrm{E}+00$ & Inh. \\
\hline Bk-248m & & & & $7.45 \mathrm{E}+03$ & -- & $3.52 \mathrm{E}+04$ & $1.81 \mathrm{E}+04$ & -- & $7.45 \mathrm{E}+03$ & $1.40 \mathrm{E}-02$ & $5.33 \mathrm{E}+05$ & Inh. \\
\hline Bk-249 & & & & $7.45 \mathrm{E}+01$ & -- & $8.62 \mathrm{E}+02$ & -- & -- & $7.45 \mathrm{E}+01$ & $4.54 \mathrm{E}-02$ & $1.64 \mathrm{E}+03$ & Inh. \\
\hline Bk-250 & & & & $1.16 \mathrm{E}+04$ & -- & $5.75 \mathrm{E}+05$ & $4.25 \mathrm{E}+03$ & -- & $4.25 \mathrm{E}+03$ & 1.09E-03 & $3.89 \mathrm{E}+06$ & Direct \\
\hline Bk-251 & & & & $1.75 \mathrm{E}+06$ & -- & $6.32 \mathrm{E}+07$ & $1.42 \mathrm{E}+05$ & -- & $1.42 \mathrm{E}+05$ & $1.06 \mathrm{E}-02$ & $1.35 \mathrm{E}+07$ & Direct \\
\hline $\mathrm{Br}-72$ & & & & -- & -- & -- & $1.87 \mathrm{E}+05$ & -- & $1.87 \mathrm{E}+05$ & $9.36 \mathrm{E}-05$ & $2.00 \mathrm{E}+09$ & Direct \\
\hline $\mathrm{Br}-73$ & & & & -- & -- & -- & $1.49 \mathrm{E}+05$ & -- & $1.49 \mathrm{E}+05$ & $1.96 \mathrm{E}-04$ & $7.58 \mathrm{E}+08$ & Direct \\
\hline $\mathrm{Br}-74$ & & & & $1.64 \mathrm{E}+05$ & $5.50 \mathrm{E}+110$ & $6.90 \mathrm{E}+06$ & $6.30 \mathrm{E}+03$ & -- & $6.30 \mathrm{E}+03$ & $6.26 \mathrm{E}-05$ & $1.01 \mathrm{E}+08$ & Direct \\
\hline $\mathrm{Br}-74 \mathrm{~m}$ & & & & $1.02 \mathrm{E}+05$ & $5.98 \mathrm{E}+69$ & $2.53 \mathrm{E}+06$ & $4.28 \mathrm{E}+03$ & -- & $4.28 \mathrm{E}+03$ & $6.98 \mathrm{E}-05$ & $6.13 \mathrm{E}+07$ & Direct \\
\hline $\mathrm{Br}-75$ & & & & $1.31 \mathrm{E}+05$ & $1.45 \mathrm{E}+33$ & $1.90 \mathrm{E}+06$ & $6.08 \mathrm{E}+03$ & -- & $6.08 \mathrm{E}+03$ & $2.38 \mathrm{E}-04$ & $2.56 \mathrm{E}+07$ & Direct \\
\hline $\mathrm{Br}-76$ & & & & $1.93 \mathrm{E}+04$ & $2.00 \mathrm{E}+07$ & $3.43 E+04$ & $4.41 \mathrm{E}+02$ & -- & $4.41 E+02$ & 1.73E-04 & $2.55 \mathrm{E}+06$ & Direct \\
\hline $\mathrm{Br}-76 \mathrm{~m}$ & & & & -- & -- & -- & $7.67 \mathrm{E}+08$ & -- & $7.67 \mathrm{E}+08$ & $6.76 \mathrm{E}-03$ & $1.13 \mathrm{E}+11$ & Direct \\
\hline $\mathrm{Br}-77$ & & & & $8.59 \mathrm{E}+04$ & $3.58 \mathrm{E}+05$ & $5.30 \mathrm{E}+04$ & $2.62 \mathrm{E}+03$ & -- & $2.62 \mathrm{E}+03$ & $3.60 \mathrm{E}-03$ & $7.27 \mathrm{E}+05$ & Direct \\
\hline $\mathrm{Br}-77 \mathrm{~m}$ & & & & -- & -- & -- & $8.61 \mathrm{E}+06$ & -- & $8.61 \mathrm{E}+06$ & $1.51 \mathrm{E}-02$ & $5.71 \mathrm{E}+08$ & Direct \\
\hline $\mathrm{Br}-78$ & & & & -- & -- & -- & $1.09 \mathrm{E}+05$ & -- & $1.09 \mathrm{E}+05$ & $2.91 \mathrm{E}-04$ & $3.74 \mathrm{E}+08$ & Direct \\
\hline $\mathrm{Br}-80$ & & & & $6.57 \mathrm{E}+05$ & $2.96 \mathrm{E}+158$ & $2.72 \mathrm{E}+07$ & $5.23 E+05$ & -- & $5.23 E+05$ & $3.87 \mathrm{E}-03$ & $1.35 \mathrm{E}+08$ & Direct \\
\hline $\mathrm{Br}-80 \mathrm{~m}$ & & & & $1.12 \mathrm{E}+05$ & $4.88 \mathrm{E}+15$ & $5.09 \mathrm{E}+05$ & $1.17 \mathrm{E}+05$ & -- & $1.12 \mathrm{E}+05$ & $1.26 \mathrm{E}-02$ & $8.87 \mathrm{E}+06$ & Inh. \\
\hline $\mathrm{Br}-82$ & & & & $1.27 \mathrm{E}+04$ & $2.74 \mathrm{E}+05$ & $1.42 \mathrm{E}+04$ & $3.45 \mathrm{E}+02$ & -- & $3.45 \mathrm{E}+02$ & $3.18 \mathrm{E}-04$ & $1.08 \mathrm{E}+06$ & Direct \\
\hline $\mathrm{Br}-82 \mathrm{~m}$ & & & & -- & -- & -- & $1.46 \mathrm{E}+07$ & -- & $1.46 \mathrm{E}+07$ & 3.90E-02 & $3.74 \mathrm{E}+08$ & Direct \\
\hline $\mathrm{Br}-83$ & & & & $1.67 \mathrm{E}+05$ & $5.97 \mathrm{E}+24$ & $2.39 \mathrm{E}+06$ & $6.74 \mathrm{E}+05$ & -- & $1.67 \mathrm{E}+05$ & $1.05 \mathrm{E}-02$ & $1.58 \mathrm{E}+07$ & Inh. \\
\hline $\mathrm{Br}-84$ & & & & $1.80 \mathrm{E}+05$ & $2.51 \mathrm{E}+89$ & $5.24 \mathrm{E}+06$ & $1.27 \mathrm{E}+04$ & -- & $1.27 \mathrm{E}+04$ & $1.81 \mathrm{E}-04$ & $7.05 \mathrm{E}+07$ & Direct \\
\hline $\mathrm{Br}-84 \mathrm{~m}$ & & & & -- & -- & -- & $4.36 \mathrm{E}+04$ & -- & $4.36 \mathrm{E}+04$ & 1.17E-04 & $3.73 E+08$ & Direct \\
\hline
\end{tabular}


Table C.2 Summary of Exposure Pathway TQs and Selection of Final HC-3 TQs (continued)

\begin{tabular}{|c|c|c|c|c|c|c|c|c|c|c|c|c|}
\hline \multirow[b]{2}{*}{$\begin{array}{c}\text { MASTER } \\
\text { ISOTOPE } \\
\text { LIST } \\
\end{array}$} & \multicolumn{3}{|c|}{ from NNSA SD G 1027} & \multicolumn{5}{|c|}{ Calculated Pathway Specific TQ Values } & \multicolumn{4}{|c|}{ Selected Final Threshold Quantities } \\
\hline & Act $(\mathrm{Ci})$ & Mass (g) & $\begin{array}{l}\text { Limiting } \\
\text { Pathway }\end{array}$ & $\begin{array}{c}\text { TQ }_{\text {inhale }} \\
(\mathrm{Ci})\end{array}$ & $\begin{array}{c}\text { TQ }_{\text {water }} \\
\text { (Ci) }\end{array}$ & $\mathrm{TQ}_{\text {food }}(\mathrm{Ci})$ & $\begin{array}{c}\mathrm{TQ}_{\text {direct }} \\
\text { (Ci) }\end{array}$ & $\mathrm{TQ}_{\text {sub }}(\mathrm{Ci})$ & Act (Ci) & Mass (g) & $\begin{array}{c}\text { Sp. Act. } \\
\text { (Ci/g) }\end{array}$ & $\begin{array}{l}\text { Limiting } \\
\text { Pathway }\end{array}$ \\
\hline $\mathrm{Br}-85$ & & & & -- & -- & -- & $3.78 \mathrm{E}+06$ & -- & $3.78 \mathrm{E}+06$ & $4.96 \mathrm{E}-03$ & $7.64 \mathrm{E}+08$ & Direct \\
\hline $\mathrm{C}-10$ & & & & -- & -- & -- & $1.29 \mathrm{E}+06$ & -- & $1.29 \mathrm{E}+06$ & $2.21 \mathrm{E}-05$ & $5.85 \mathrm{E}+10$ & Direct \\
\hline C-11 & & & & $6.98 \mathrm{E}+04$ & $2.76 \mathrm{E}+136$ & -- & $3.49 \mathrm{E}+04$ & -- & $3.49 \mathrm{E}+04$ & $4.16 \mathrm{E}-05$ & $8.38 \mathrm{E}+08$ & Direct \\
\hline C-14 & $3.88 \mathrm{E}+02$ & $8.69 \mathrm{E}+01$ & $\mathrm{i}$ & $3.85 \mathrm{E}+02$ & $3.41 \mathrm{E}+03$ & -- & -- & -- & $3.85 \mathrm{E}+02$ & $8.64 \mathrm{E}+01$ & $4.46 \mathrm{E}+00$ & Inh. \\
\hline Ca-41 & & & & $5.88 \mathrm{E}+04$ & -- & $2.48 \mathrm{E}+03$ & $1.81 \mathrm{E}+06$ & -- & $2.48 \mathrm{E}+03$ & $3.97 \mathrm{E}+04$ & $6.23 \mathrm{E}-02$ & Food \\
\hline Ca-45 & $9.94 \mathrm{E}+02$ & $5.57 \mathrm{E}-02$ & ing & $4.14 \mathrm{E}+03$ & -- & $1.01 \mathrm{E}+03$ & -- & -- & $1.01 \mathrm{E}+03$ & $5.69 \mathrm{E}-02$ & $1.78 \mathrm{E}+04$ & Food \\
\hline Ca-47 & $7.37 \mathrm{E}+02$ & $1.20 \mathrm{E}-03$ & de & $5.32 \mathrm{E}+03$ & -- & $1.74 \mathrm{E}+03$ & $7.37 \mathrm{E}+02$ & -- & $7.37 \mathrm{E}+02$ & $1.20 \mathrm{E}-03$ & $6.14 \mathrm{E}+05$ & Direct \\
\hline Ca-49 & & & & -- & -- & -- & $2.63 \mathrm{E}+04$ & -- & $2.63 \mathrm{E}+04$ & 5.96E-05 & $4.41 \mathrm{E}+08$ & Direct \\
\hline Cd-101 & & & & -- & -- & -- & $2.14 \mathrm{E}+05$ & -- & $2.14 \mathrm{E}+05$ & $1.56 \mathrm{E}-04$ & $1.37 \mathrm{E}+09$ & Direct \\
\hline Cd-102 & & & & -- & -- & -- & $1.57 \mathrm{E}+05$ & -- & $1.57 \mathrm{E}+05$ & $4.69 \mathrm{E}-04$ & $3.35 \mathrm{E}+08$ & Direct \\
\hline Cd-103 & & & & -- & -- & -- & $4.75 E+04$ & -- & $4.75 \mathrm{E}+04$ & $1.90 \mathrm{E}-04$ & $2.50 \mathrm{E}+08$ & Direct \\
\hline Cd-104 & & & & $1.77 \mathrm{E}+05$ & -- & $4.53 \mathrm{E}+06$ & $4.86 \mathrm{E}+04$ & -- & $4.86 \mathrm{E}+04$ & $1.55 \mathrm{E}-03$ & $3.14 \mathrm{E}+07$ & Direct \\
\hline Cd-105 & & & & $2.66 \mathrm{E}+05$ & -- & $6.21 \mathrm{E}+06$ & $1.00 \mathrm{E}+04$ & -- & $1.00 \mathrm{E}+04$ & $3.11 \mathrm{E}-04$ & $3.23 \mathrm{E}+07$ & Direct \\
\hline Cd-107 & & & & $1.02 \mathrm{E}+05$ & -- & $6.39 \mathrm{E}+05$ & $5.93 E+04$ & -- & $5.93 \mathrm{E}+04$ & $1.31 \mathrm{E}-02$ & $4.52 \mathrm{E}+06$ & Direct \\
\hline Cd-109 & $3.96 \mathrm{E}+02$ & $1.53 \mathrm{E}-01$ & ing & $1.16 \mathrm{E}+03$ & -- & $4.05 \mathrm{E}+02$ & $2.76 \mathrm{E}+04$ & -- & $4.05 \mathrm{E}+02$ & 1.57E-01 & $2.58 \mathrm{E}+03$ & Food \\
\hline Cd-111m & & & & $2.94 \mathrm{E}+05$ & -- & $2.23 \mathrm{E}+07$ & $5.26 \mathrm{E}+04$ & -- & $5.26 \mathrm{E}+04$ & $1.50 \mathrm{E}-03$ & $3.50 \mathrm{E}+07$ & Direct \\
\hline Cd-113 & $3.09 \mathrm{E}+01$ & 8.77E+13 & ing & $7.98 \mathrm{E}+01$ & -- & $3.16 \mathrm{E}+01$ & -- & -- & $3.16 \mathrm{E}+01$ & $9.29 \mathrm{E}+13$ & $3.40 \mathrm{E}-13$ & Food \\
\hline $\mathrm{Cd}-113 \mathrm{~m}$ & & & & $8.59 \mathrm{E}+01$ & -- & $3.45 \mathrm{E}+01$ & -- & -- & $3.45 \mathrm{E}+01$ & $1.48 \mathrm{E}-01$ & $2.33 \mathrm{E}+02$ & Food \\
\hline Cd-115 & & & & $8.59 \mathrm{E}+03$ & -- & $3.90 \mathrm{E}+03$ & $3.63 E+03$ & -- & $3.63 E+03$ & 7.11E-03 & $5.10 \mathrm{E}+05$ & Direct \\
\hline Cd-115m & & & & $1.53 \mathrm{E}+03$ & -- & $3.05 \mathrm{E}+02$ & $3.34 \mathrm{E}+04$ & -- & $3.05 E+02$ & $1.20 \mathrm{E}-02$ & $2.55 \mathrm{E}+04$ & Food \\
\hline $\mathrm{Cd}-117$ & & & & $4.47 E+04$ & -- & $3.64 \mathrm{E}+05$ & $4.47 \mathrm{E}+03$ & -- & $4.47 \mathrm{E}+03$ & $4.15 \mathrm{E}-04$ & $1.08 \mathrm{E}+07$ & Direct \\
\hline Cd-117m & & & & $3.49 E+04$ & -- & $2.71 \mathrm{E}+05$ & $1.77 \mathrm{E}+03$ & -- & $1.77 \mathrm{E}+03$ & $2.22 \mathrm{E}-04$ & $7.98 \mathrm{E}+06$ & Direct \\
\hline Cd-118 & & & & $9.31 \mathrm{E}+04$ & -- & $1.59 \mathrm{E}+06$ & -- & -- & $9.31 \mathrm{E}+04$ & $2.94 \mathrm{E}-03$ & $3.17 \mathrm{E}+07$ & Inh. \\
\hline Cd-119 & & & & -- & -- & -- & $1.64 \mathrm{E}+05$ & -- & $1.64 \mathrm{E}+05$ & $2.80 \mathrm{E}-04$ & $5.88 \mathrm{E}+08$ & Direct \\
\hline $\mathrm{Cd}-119 \mathrm{~m}$ & & & & -- & -- & -- & $1.43 \mathrm{E}+05$ & -- & $1.43 \mathrm{E}+05$ & $1.99 \mathrm{E}-04$ & $7.19 \mathrm{E}+08$ & Direct \\
\hline Ce-130 & & & & $1.83 \mathrm{E}+05$ & -- & $9.36 \mathrm{E}+06$ & $6.32 \mathrm{E}+04$ & -- & $6.32 E+04$ & $1.00 \mathrm{E}-03$ & $6.32 \mathrm{E}+07$ & Direct \\
\hline Ce-131 & & & & $4.65 \mathrm{E}+05$ & -- & $5.40 \mathrm{E}+07$ & $4.37 \mathrm{E}+04$ & -- & $4.37 \mathrm{E}+04$ & $3.10 \mathrm{E}-04$ & $1.41 \mathrm{E}+08$ & Direct \\
\hline Ce-131m & & & & -- & -- & -- & -- & -- & -- & -- & $2.87 \mathrm{E}+08$ & -- \\
\hline Ce-132 & & & & $4.86 \mathrm{E}+04$ & -- & $2.17 E+05$ & $1.27 \mathrm{E}+04$ & -- & $1.27 \mathrm{E}+04$ & $1.88 \mathrm{E}-03$ & $6.77 \mathrm{E}+06$ & Direct \\
\hline Ce-133 & & & & $1.41 \mathrm{E}+05$ & -- & $1.75 \mathrm{E}+06$ & $1.38 \mathrm{E}+04$ & -- & $1.38 \mathrm{E}+04$ & $9.43 \mathrm{E}-04$ & $1.46 \mathrm{E}+07$ & Direct \\
\hline Ce-133m & & & & $5.08 \mathrm{E}+04$ & -- & $2.31 \mathrm{E}+05$ & $1.47 \mathrm{E}+03$ & -- & $1.47 \mathrm{E}+03$ & 3.05E-04 & $4.81 \mathrm{E}+06$ & Direct \\
\hline Ce-134 & & & & $6.98 \mathrm{E}+03$ & -- & $1.73 \mathrm{E}+03$ & $3.10 \mathrm{E}+04$ & -- & $1.73 E+03$ & $5.33 \mathrm{E}-03$ & $3.25 \mathrm{E}+05$ & Food \\
\hline Ce-135 & & & & $1.47 \mathrm{E}+04$ & -- & $1.94 \mathrm{E}+04$ & $6.32 \mathrm{E}+02$ & -- & $6.32 \mathrm{E}+02$ & 4.79E-04 & $1.32 \mathrm{E}+06$ & Direct \\
\hline Ce-137 & & & & $5.88 \mathrm{E}+05$ & -- & $1.17 \mathrm{E}+06$ & $4.49 E+04$ & -- & $4.49 E+04$ & $1.76 \mathrm{E}-02$ & $2.54 \mathrm{E}+06$ & Direct \\
\hline Ce-137m & & & & $1.89 \mathrm{E}+04$ & -- & $1.52 \mathrm{E}+04$ & $1.73 E+04$ & -- & $1.52 \mathrm{E}+04$ & $2.29 \mathrm{E}-02$ & $6.65 \mathrm{E}+05$ & Food \\
\hline
\end{tabular}


Table C.2 Summary of Exposure Pathway TQs and Selection of Final HC-3 TQs (continued)

\begin{tabular}{|c|c|c|c|c|c|c|c|c|c|c|c|c|}
\hline \multirow[b]{2}{*}{$\begin{array}{c}\text { MASTER } \\
\text { ISOTOPE } \\
\text { LIST } \\
\end{array}$} & \multicolumn{3}{|c|}{ from NNSA SD G 1027} & \multicolumn{5}{|c|}{ Calculated Pathway Specific TQ Values } & \multicolumn{4}{|c|}{ Selected Final Threshold Quantities } \\
\hline & Act $(\mathrm{Ci})$ & Mass (g) & $\begin{array}{l}\text { Limiting } \\
\text { Pathway }\end{array}$ & $\begin{array}{c}\text { TQ }_{\text {inhale }} \\
(\mathrm{Ci})\end{array}$ & $\begin{array}{c}\text { TQ }_{\text {water }} \\
\text { (Ci) }\end{array}$ & $\mathrm{TQ}_{\text {food }}(\mathrm{Ci})$ & $\begin{array}{c}T_{\text {direct }} \\
\text { (Ci) }\end{array}$ & $\mathrm{TQ}_{\text {sub }}(\mathrm{Ci})$ & Act (Ci) & Mass (g) & $\begin{array}{c}\text { Sp. Act. } \\
\text { (Ci/g) }\end{array}$ & $\begin{array}{l}\text { Limiting } \\
\text { Pathway }\end{array}$ \\
\hline Ce-139 & & & & $6.20 \mathrm{E}+03$ & -- & $3.37 \mathrm{E}+03$ & $4.56 \mathrm{E}+03$ & -- & $3.37 E+03$ & $4.93 \mathrm{E}-01$ & $6.83 \mathrm{E}+03$ & Food \\
\hline Ce-141 & $1.54 \mathrm{E}+03$ & $5.41 \mathrm{E}-02$ & ing & $3.10 \mathrm{E}+03$ & -- & $1.56 \mathrm{E}+03$ & $9.63 \mathrm{E}+03$ & -- & $1.56 \mathrm{E}+03$ & $5.48 \mathrm{E}-02$ & $2.85 \mathrm{E}+04$ & Food \\
\hline Ce-143 & & & & $1.12 \mathrm{E}+04$ & -- & $7.77 \mathrm{E}+03$ & $3.27 \mathrm{E}+03$ & -- & $3.27 \mathrm{E}+03$ & 4.93E-03 & $6.64 \mathrm{E}+05$ & Direct \\
\hline Ce-144 & $1.58 \mathrm{E}+02$ & $4.95 \mathrm{E}-02$ & ing & $2.28 \mathrm{E}+02$ & -- & $1.61 \mathrm{E}+02$ & $3.51 \mathrm{E}+04$ & -- & $1.61 \mathrm{E}+02$ & $5.06 \mathrm{E}-02$ & $3.19 \mathrm{E}+03$ & Food \\
\hline Ce-145 & & & & -- & -- & -- & $2.96 \mathrm{E}+05$ & -- & $2.96 \mathrm{E}+05$ & $6.86 \mathrm{E}-04$ & $4.31 \mathrm{E}+08$ & Direct \\
\hline Ce-146 & & & & $2.54 \mathrm{E}+05$ & -- & $2.43 \mathrm{E}+07$ & -- & -- & $2.54 \mathrm{E}+05$ & $2.66 \mathrm{E}-03$ & $9.53 \mathrm{E}+07$ & Inh. \\
\hline Cf-244 & & & & $6.20 \mathrm{E}+03$ & $3.23 E+142$ & $1.14 \mathrm{E}+08$ & $1.97 \mathrm{E}+07$ & -- & $6.20 \mathrm{E}+03$ & $1.56 \mathrm{E}-04$ & $3.97 \mathrm{E}+07$ & Inh. \\
\hline Cf-246 & & & & $2.66 \mathrm{E}+02$ & $4.29 \mathrm{E}+04$ & $2.41 E+04$ & $6.99 \mathrm{E}+05$ & -- & $2.66 \mathrm{E}+02$ & $7.45 \mathrm{E}-04$ & $3.57 \mathrm{E}+05$ & Inh. \\
\hline Cf-247 & & & & $2.28 \mathrm{E}+06$ & $3.91 \mathrm{E}+20$ & $2.87 \mathrm{E}+07$ & $3.72 E+04$ & -- & $3.72 \mathrm{E}+04$ & $9.11 \mathrm{E}-03$ & $4.08 \mathrm{E}+06$ & Direct \\
\hline Cf-248 & & & & $1.36 \mathrm{E}+01$ & $7.21 \mathrm{E}+01$ & $2.98 \mathrm{E}+02$ & $5.59 \mathrm{E}+05$ & -- & $1.36 \mathrm{E}+01$ & $8.63 \mathrm{E}-03$ & $1.58 \mathrm{E}+03$ & Inh. \\
\hline Cf-249 & & & & $1.69 \mathrm{E}+00$ & $5.65 \mathrm{E}+00$ & $2.30 \mathrm{E}+01$ & $2.17 E+03$ & -- & $1.69 \mathrm{E}+00$ & $4.13 \mathrm{E}-01$ & $4.09 \mathrm{E}+00$ & Inh. \\
\hline Cf-250 & & & & $3.49 \mathrm{E}+00$ & $1.24 \mathrm{E}+01$ & $5.05 \mathrm{E}+01$ & $6.05 \mathrm{E}+05$ & -- & $3.49 \mathrm{E}+00$ & $3.19 \mathrm{E}-02$ & $1.09 \mathrm{E}+02$ & Inh. \\
\hline Cf-251 & & & & $1.67 \mathrm{E}+00$ & $5.49 \mathrm{E}+00$ & $2.24 \mathrm{E}+01$ & $5.51 \mathrm{E}+03$ & -- & $1.67 \mathrm{E}+00$ & $1.05 \mathrm{E}+00$ & $1.59 \mathrm{E}+00$ & Inh. \\
\hline Cf-252 & $6.26 \mathrm{E}+00$ & $1.17 \mathrm{E}-02$ & $\mathrm{i}$ & $6.20 \mathrm{E}+00$ & $2.21 \mathrm{E}+01$ & $9.06 \mathrm{E}+01$ & $6.05 \mathrm{E}+05$ & -- & $6.20 \mathrm{E}+00$ & $1.15 \mathrm{E}-02$ & $5.38 \mathrm{E}+02$ & Inh. \\
\hline Cf-253 & & & & $9.31 \mathrm{E}+01$ & $2.12 \mathrm{E}+03$ & $9.80 \mathrm{E}+03$ & -- & -- & $9.31 \mathrm{E}+01$ & $3.21 \mathrm{E}-03$ & $2.90 \mathrm{E}+04$ & Inh. \\
\hline Cf-254 & & & & $3.02 \mathrm{E}+00$ & $5.57 \mathrm{E}+00$ & $2.42 \mathrm{E}+01$ & -- & -- & $3.02 \mathrm{E}+00$ & $3.55 \mathrm{E}-04$ & $8.49 E+03$ & Inh. \\
\hline Cf-255 & & & & $2.07 E+04$ & $4.16 \mathrm{E}+37$ & $4.55 \mathrm{E}+07$ & -- & -- & $2.07 E+04$ & $2.39 \mathrm{E}-03$ & $8.67 E+06$ & Inh. \\
\hline Cf-256 & & & & $2.79 \mathrm{E}+01$ & $1.54 \mathrm{E}+219$ & $3.80 \mathrm{E}+06$ & -- & -- & $2.79 \mathrm{E}+01$ & $4.68 \mathrm{E}-07$ & $5.97 \mathrm{E}+07$ & Inh. \\
\hline $\mathrm{Cl}-34$ & & & & -- & -- & -- & $2.79 \mathrm{E}+07$ & -- & $2.79 \mathrm{E}+07$ & $1.28 \mathrm{E}-04$ & $2.18 \mathrm{E}+11$ & Direct \\
\hline $\mathrm{Cl}-34 \mathrm{~m}$ & & & & $1.49 \mathrm{E}+05$ & $6.69 \mathrm{E}+88$ & $1.40 \mathrm{E}+06$ & $1.07 E+04$ & -- & $1.07 \mathrm{E}+04$ & $6.20 \mathrm{E}-05$ & $1.73 \mathrm{E}+08$ & Direct \\
\hline $\mathrm{Cl}-36$ & $2.49 \mathrm{E}+02$ & $7.55 \mathrm{E}+03$ & ing & $1.62 \mathrm{E}+03$ & $2.12 \mathrm{E}+03$ & $2.51 \mathrm{E}+02$ & $7.26 \mathrm{E}+06$ & -- & $2.51 \mathrm{E}+02$ & $7.61 \mathrm{E}+03$ & $3.30 \mathrm{E}-02$ & Food \\
\hline $\mathrm{Cl}-38$ & & & & $1.53 \mathrm{E}+05$ & $1.54 \mathrm{E}+77$ & $1.00 \mathrm{E}+06$ & $1.31 \mathrm{E}+04$ & -- & $1.31 \mathrm{E}+04$ & 9.83E-05 & $1.33 \mathrm{E}+08$ & Direct \\
\hline $\mathrm{Cl}-39$ & & & & $1.47 \mathrm{E}+05$ & $6.54 \mathrm{E}+53$ & $9.48 \mathrm{E}+05$ & $9.06 \mathrm{E}+03$ & -- & $9.06 \mathrm{E}+03$ & $1.04 \mathrm{E}-04$ & $8.68 \mathrm{E}+07$ & Direct \\
\hline $\mathrm{Cl}-40$ & & & & -- & -- & -- & $1.31 \mathrm{E}+05$ & -- & $1.31 \mathrm{E}+05$ & $3.77 \mathrm{E}-05$ & $3.48 \mathrm{E}+09$ & Direct \\
\hline $\mathrm{Cm}-238$ & & & & $2.33 \mathrm{E}+04$ & -- & $1.35 \mathrm{E}+07$ & $6.53 \mathrm{E}+04$ & -- & $2.33 E+04$ & $4.24 \mathrm{E}-03$ & $5.49 \mathrm{E}+06$ & Inh. \\
\hline $\mathrm{Cm}-239$ & & & & $1.27 \mathrm{E}+06$ & -- & $1.09 \mathrm{E}+07$ & $1.61 \mathrm{E}+04$ & -- & $1.61 \mathrm{E}+04$ & $3.56 \mathrm{E}-03$ & $4.52 E+06$ & Direct \\
\hline $\mathrm{Cm}-240$ & & & & $3.85 \mathrm{E}+01$ & -- & $1.54 \mathrm{E}+03$ & $3.68 \mathrm{E}+05$ & -- & $3.85 \mathrm{E}+01$ & $1.91 \mathrm{E}-03$ & $2.01 \mathrm{E}+04$ & Inh. \\
\hline $\mathrm{Cm}-241$ & & & & $3.28 \mathrm{E}+03$ & -- & $1.22 \mathrm{E}+04$ & $1.46 \mathrm{E}+03$ & -- & $1.46 \mathrm{E}+03$ & $8.86 \mathrm{E}-02$ & $1.65 \mathrm{E}+04$ & Direct \\
\hline $\mathrm{Cm}-242$ & $2.35 \mathrm{E}+01$ & $7.08 \mathrm{E}-03$ & $\mathrm{i}$ & $2.33 \mathrm{E}+01$ & -- & $7.21 \mathrm{E}+02$ & $4.04 \mathrm{E}+05$ & -- & $2.33 \mathrm{E}+01$ & $7.02 \mathrm{E}-03$ & $3.31 \mathrm{E}+03$ & Inh. \\
\hline $\mathrm{Cm}-243$ & & & & $3.85 \mathrm{E}+00$ & -- & $5.38 \mathrm{E}+01$ & $5.41 E+03$ & -- & $3.85 \mathrm{E}+00$ & $7.46 \mathrm{E}-02$ & $5.16 \mathrm{E}+01$ & Inh. \\
\hline $\mathrm{Cm}-244$ & & & & $4.47 \mathrm{E}+00$ & -- & $6.73 \mathrm{E}+01$ & $4.54 \mathrm{E}+05$ & -- & $4.47 \mathrm{E}+00$ & $5.52 \mathrm{E}-02$ & $8.09 \mathrm{E}+01$ & Inh. \\
\hline $\mathrm{Cm}-245$ & $2.82 \mathrm{E}+00$ & $1.64 \mathrm{E}+01$ & $i$ & $2.79 \mathrm{E}+00$ & -- & $3.84 \mathrm{E}+01$ & $7.59 \mathrm{E}+03$ & -- & $2.79 E+00$ & $1.63 \mathrm{E}+01$ & $1.72 \mathrm{E}-01$ & Inh. \\
\hline $\mathrm{Cm}-246$ & & & & $2.79 \mathrm{E}+00$ & -- & $3.84 \mathrm{E}+01$ & $4.84 \mathrm{E}+05$ & -- & $2.79 E+00$ & $9.09 \mathrm{E}+00$ & 3.07E-01 & Inh. \\
\hline $\mathrm{Cm}-247$ & & & & $3.10 \mathrm{E}+00$ & -- & $4.24 \mathrm{E}+01$ & $2.30 \mathrm{E}+03$ & -- & $3.10 \mathrm{E}+00$ & $3.34 \mathrm{E}+04$ & $9.28 \mathrm{E}-05$ & Inh. \\
\hline $\mathrm{Cm}-248$ & & & & 7.98E-01 & -- & $1.05 \mathrm{E}+01$ & $6.60 \mathrm{E}+05$ & -- & 7.98E-01 & $1.88 \mathrm{E}+02$ & $4.25 \mathrm{E}-03$ & Inh. \\
\hline
\end{tabular}


Table C.2 Summary of Exposure Pathway TQs and Selection of Final HC-3 TQs (continued)

\begin{tabular}{|c|c|c|c|c|c|c|c|c|c|c|c|c|}
\hline \multirow[b]{2}{*}{$\begin{array}{c}\text { MASTER } \\
\text { ISOTOPE } \\
\text { LIST } \\
\end{array}$} & \multicolumn{3}{|c|}{ from NNSA SD G 1027} & \multicolumn{5}{|c|}{ Calculated Pathway Specific TQ Values } & \multicolumn{4}{|c|}{ Selected Final Threshold Quantities } \\
\hline & Act (Ci) & Mass (g) & $\begin{array}{l}\text { Limiting } \\
\text { Pathway }\end{array}$ & $\begin{array}{c}\mathrm{TQ}_{\text {inhale }} \\
(\mathrm{Ci})\end{array}$ & $\begin{array}{l}\text { TQ }_{\text {water }} \\
\text { (Ci) }\end{array}$ & $T_{\text {food }}(C i)$ & $\begin{array}{c}T_{\text {direct }} \\
\text { (Ci) }\end{array}$ & $\mathrm{TQ}_{\text {sub }}(\mathrm{Ci})$ & Act $(\mathrm{Ci})$ & Mass (g) & $\begin{array}{l}\text { Sp. Act. } \\
\text { (Ci/g) }\end{array}$ & $\begin{array}{l}\text { Limiting } \\
\text { Pathway }\end{array}$ \\
\hline $\mathrm{Cm}-249$ & & & & $2.19 E+06$ & -- & $7.78 \mathrm{E}+07$ & $5.91 \mathrm{E}+05$ & -- & $5.91 \mathrm{E}+05$ & $5.03 \mathrm{E}-02$ & $1.18 \mathrm{E}+07$ & Direct \\
\hline $\mathrm{Cm}-250$ & & & & $1.41 \mathrm{E}-01$ & -- & $1.83 \mathrm{E}+00$ & -- & -- & $1.41 \mathrm{E}-01$ & $6.82 \mathrm{E}-01$ & 2.07E-01 & Inh. \\
\hline $\mathrm{Cm}-251$ & & & & -- & -- & -- & $3.88 \mathrm{E}+05$ & -- & $3.88 \mathrm{E}+05$ & $8.70 \mathrm{E}-03$ & $4.46 \mathrm{E}+07$ & Direct \\
\hline Co-54m & & & & -- & -- & -- & $1.25 \mathrm{E}+05$ & -- & $1.25 \mathrm{E}+05$ & 5.29E-05 & $2.35 \mathrm{E}+09$ & Direct \\
\hline Co-55 & & & & $1.35 \mathrm{E}+05$ & -- & $1.40 \mathrm{E}+05$ & $5.64 \mathrm{E}+02$ & -- & $5.64 \mathrm{E}+02$ & $1.73 \mathrm{E}-04$ & $3.25 \mathrm{E}+06$ & Direct \\
\hline Co-56 & & & & $1.77 \mathrm{E}+04$ & -- & $3.71 E+03$ & $2.04 \mathrm{E}+02$ & -- & $2.04 \mathrm{E}+02$ & 6.87E-03 & $2.96 \mathrm{E}+04$ & Direct \\
\hline Co-57 & & & & $1.19 \mathrm{E}+05$ & -- & $4.00 \mathrm{E}+04$ & $5.81 \mathrm{E}+03$ & -- & $5.81 \mathrm{E}+03$ & $6.86 \mathrm{E}-01$ & $8.47 E+03$ & Direct \\
\hline Co-58 & & & & $5.58 \mathrm{E}+04$ & -- & $1.27 \mathrm{E}+04$ & $7.48 \mathrm{E}+02$ & -- & $7.48 \mathrm{E}+02$ & $2.35 \mathrm{E}-02$ & $3.18 \mathrm{E}+04$ & Direct \\
\hline Co-58m & & & & $6.57 E+06$ & -- & $1.20 \mathrm{E}+07$ & $7.88 \mathrm{E}+05$ & -- & $7.88 \mathrm{E}+05$ & $1.33 \mathrm{E}-01$ & $5.91 \mathrm{E}+06$ & Direct \\
\hline Co-60 & $2.90 \mathrm{E}+02$ & $2.56 \mathrm{E}-01$ & de & $3.85 \mathrm{E}+03$ & -- & $2.38 \mathrm{E}+03$ & $2.90 \mathrm{E}+02$ & -- & $2.90 \mathrm{E}+02$ & $2.56 \mathrm{E}-01$ & $1.13 \mathrm{E}+03$ & Direct \\
\hline Co-60m & & & & $8.59 E+07$ & -- & $8.66 \mathrm{E}+09$ & $1.02 E+07$ & -- & $1.02 \mathrm{E}+07$ & $3.40 \mathrm{E}-02$ & $3.00 \mathrm{E}+08$ & Direct \\
\hline Co-61 & & & & $1.49 \mathrm{E}+06$ & -- & $2.11 \mathrm{E}+07$ & $8.08 \mathrm{E}+04$ & -- & $8.08 \mathrm{E}+04$ & $2.59 \mathrm{E}-03$ & $3.12 \mathrm{E}+07$ & Direct \\
\hline Co-62 & & & & -- & -- & -- & $3.01 E+05$ & -- & $3.01 \mathrm{E}+05$ & $1.49 \mathrm{E}-04$ & $2.02 E+09$ & Direct \\
\hline Co-62m & & & & $3.02 \mathrm{E}+06$ & -- & $2.36 \mathrm{E}+08$ & $1.93 \mathrm{E}+04$ & -- & $1.93 \mathrm{E}+04$ & $8.85 \mathrm{E}-05$ & $2.18 \mathrm{E}+08$ & Direct \\
\hline $\mathrm{Cr}-48$ & & & & $4.47 E+04$ & $5.20 \mathrm{E}+06$ & $5.98 \mathrm{E}+04$ & $2.34 \mathrm{E}+03$ & -- & $2.34 \mathrm{E}+03$ & $8.23 \mathrm{E}-04$ & $2.85 \mathrm{E}+06$ & Direct \\
\hline $\mathrm{Cr}-49$ & & & & $1.89 \mathrm{E}+05$ & $1.76 \mathrm{E}+69$ & $6.01 E+06$ & $1.63 E+04$ & -- & $1.63 E+04$ & $1.79 \mathrm{E}-04$ & $9.13 E+07$ & Direct \\
\hline Cr-51 & $2.26 \mathrm{E}+04$ & $2.44 \mathrm{E}-01$ & de & $3.10 \mathrm{E}+05$ & $6.76 \mathrm{E}+04$ & $3.06 \mathrm{E}+04$ & $2.26 \mathrm{E}+04$ & -- & $2.26 \mathrm{E}+04$ & $2.44 \mathrm{E}-01$ & $9.25 \mathrm{E}+04$ & Direct \\
\hline Cr-55 & & & & -- & -- & -- & $3.09 E+08$ & -- & $3.09 E+08$ & $3.16 \mathrm{E}-01$ & $9.79 \mathrm{E}+08$ & Direct \\
\hline Cr-56 & & & & -- & -- & -- & $1.33 \mathrm{E}+06$ & -- & $1.33 \mathrm{E}+06$ & $2.35 \mathrm{E}-03$ & $5.66 \mathrm{E}+08$ & Direct \\
\hline Cs-121 & & & & -- & -- & -- & $2.39 \mathrm{E}+05$ & -- & $2.39 \mathrm{E}+05$ & $3.97 \mathrm{E}-04$ & $6.02 \mathrm{E}+08$ & Direct \\
\hline Cs-121m & & & & -- & -- & -- & $3.01 \mathrm{E}+05$ & -- & $3.01 \mathrm{E}+05$ & $3.94 \mathrm{E}-04$ & $7.65 \mathrm{E}+08$ & Direct \\
\hline Cs-123 & & & & -- & -- & -- & $1.13 E+05$ & -- & $1.13 \mathrm{E}+05$ & $4.36 \mathrm{E}-04$ & $2.60 \mathrm{E}+08$ & Direct \\
\hline Cs-124 & & & & -- & -- & -- & $1.22 \mathrm{E}+06$ & -- & $1.22 \mathrm{E}+06$ & $4.11 \mathrm{E}-04$ & $2.96 \mathrm{E}+09$ & Direct \\
\hline Cs-125 & & & & $4.86 \mathrm{E}+05$ & -- & $9.78 \mathrm{E}+06$ & $2.37 E+04$ & -- & $2.37 E+04$ & 7.10E-04 & $3.35 \mathrm{E}+07$ & Direct \\
\hline Cs-126 & & & & -- & -- & -- & $4.07 \mathrm{E}+05$ & -- & $4.07 E+05$ & 4.47E-04 & $9.11 \mathrm{E}+08$ & Direct \\
\hline Cs-127 & & & & $2.79 E+05$ & -- & $1.74 \mathrm{E}+06$ & $4.95 \mathrm{E}+03$ & -- & $4.95 \mathrm{E}+03$ & $1.25 \mathrm{E}-03$ & $3.95 \mathrm{E}+06$ & Direct \\
\hline Cs-128 & & & & -- & -- & -- & $2.06 \mathrm{E}+05$ & -- & $2.06 \mathrm{E}+05$ & 5.47E-04 & $3.77 \mathrm{E}+08$ & Direct \\
\hline Cs-129 & & & & $1.38 \mathrm{E}+05$ & -- & $1.46 \mathrm{E}+05$ & $3.31 \mathrm{E}+03$ & -- & $3.31 \mathrm{E}+03$ & $4.36 \mathrm{E}-03$ & $7.58 \mathrm{E}+05$ & Direct \\
\hline Cs-130 & & & & $7.45 \mathrm{E}+05$ & -- & $1.84 \mathrm{E}+07$ & $4.69 \mathrm{E}+04$ & -- & $4.69 \mathrm{E}+04$ & $9.69 \mathrm{E}-04$ & $4.84 \mathrm{E}+07$ & Direct \\
\hline Cs-130m & & & & -- & -- & -- & $2.84 \mathrm{E}+06$ & -- & $2.84 \mathrm{E}+06$ & $6.79 \mathrm{E}-03$ & $4.18 \mathrm{E}+08$ & Direct \\
\hline Cs-131 & & & & $2.48 \mathrm{E}+05$ & -- & $3.22 \mathrm{E}+04$ & $3.30 \mathrm{E}+04$ & -- & $3.22 \mathrm{E}+04$ & $3.12 \mathrm{E}-01$ & $1.03 E+05$ & Food \\
\hline Cs-132 & & & & $2.94 \mathrm{E}+04$ & -- & $4.82 \mathrm{E}+03$ & $1.09 \mathrm{E}+03$ & -- & $1.09 \mathrm{E}+03$ & $7.10 \mathrm{E}-03$ & $1.53 \mathrm{E}+05$ & Direct \\
\hline Cs-134 & $4.20 \mathrm{E}+01$ & $3.24 \mathrm{E}-02$ & ing & $1.16 \mathrm{E}+03$ & -- & $4.30 \mathrm{E}+01$ & $4.67 \mathrm{E}+02$ & -- & $4.30 \mathrm{E}+01$ & $3.32 \mathrm{E}-02$ & $1.29 E+03$ & Food \\
\hline Cs-134m & & & & $4.30 \mathrm{E}+05$ & -- & $4.46 \mathrm{E}+06$ & $1.56 \mathrm{E}+05$ & -- & $1.56 \mathrm{E}+05$ & $1.94 \mathrm{E}-02$ & $8.07 E+06$ & Direct \\
\hline Cs-135 & & & & $1.13 E+04$ & -- & $4.02 E+02$ & -- & -- & $4.02 E+02$ & $3.49 E+05$ & $1.15 \mathrm{E}-03$ & Food \\
\hline
\end{tabular}


Table C.2 Summary of Exposure Pathway TQs and Selection of Final HC-3 TQs (continued)

\begin{tabular}{|c|c|c|c|c|c|c|c|c|c|c|c|c|}
\hline \multirow{2}{*}{$\begin{array}{c}\text { MASTER } \\
\text { ISOTOPE } \\
\text { LIST }\end{array}$} & \multicolumn{3}{|c|}{ from NNSA SD G 1027} & \multicolumn{5}{|c|}{ Calculated Pathway Specific TQ Values } & \multicolumn{4}{|c|}{ Selected Final Threshold Quantities } \\
\hline & Act (Ci) & Mass (g) & $\begin{array}{l}\text { Limiting } \\
\text { Pathway }\end{array}$ & $\begin{array}{c}\text { TQ }_{\text {inhale }} \\
\text { (Ci) }\end{array}$ & $\begin{array}{l}\text { TQ }_{\text {water }} \\
\text { (Ci) }\end{array}$ & $\mathrm{TQ}_{\text {food }}(\mathrm{Ci})$ & $\begin{array}{c}\text { TQ }_{\text {direct }} \\
\text { (Ci) }\end{array}$ & $\mathrm{TQ}_{\text {sub }}(\mathrm{Ci})$ & Act (Ci) & Mass (g) & $\begin{array}{l}\text { Sp. Act. } \\
\text { (Ci/g) }\end{array}$ & $\begin{array}{l}\text { Limiting } \\
\text { Pathway }\end{array}$ \\
\hline Cs-135m & & & & $4.65 \mathrm{E}+05$ & -- & $1.53 \mathrm{E}+07$ & $8.62 E+03$ & -- & $8.62 \mathrm{E}+03$ & $3.28 \mathrm{E}-04$ & $2.63 \mathrm{E}+07$ & Direct \\
\hline Cs-136 & & & & $5.88 \mathrm{E}+03$ & -- & $5.27 E+02$ & $3.44 \mathrm{E}+02$ & -- & $3.44 \mathrm{E}+02$ & 4.69E-03 & $7.33 \mathrm{E}+04$ & Direct \\
\hline Cs-137 & $6.04 \mathrm{E}+01$ & $6.95 \mathrm{E}-01$ & ing & $1.67 \mathrm{E}+03$ & -- & $6.19 \mathrm{E}+01$ & -- & -- & $6.19 \mathrm{E}+01$ & $7.11 \mathrm{E}-01$ & $8.70 \mathrm{E}+01$ & Food \\
\hline Cs-138 & & & & $2.43 \mathrm{E}+05$ & -- & $5.20 \mathrm{E}+06$ & $9.53 \mathrm{E}+03$ & -- & $9.53 \mathrm{E}+03$ & $2.25 \mathrm{E}-04$ & $4.23 \mathrm{E}+07$ & Direct \\
\hline Cs-138m & & & & -- & -- & -- & $6.00 \mathrm{E}+05$ & -- & $6.00 \mathrm{E}+05$ & $1.28 \mathrm{E}-03$ & $4.69 \mathrm{E}+08$ & Direct \\
\hline Cs-139 & & & & -- & -- & -- & $2.58 \mathrm{E}+05$ & -- & $2.58 \mathrm{E}+05$ & $1.77 \mathrm{E}-03$ & $1.46 \mathrm{E}+08$ & Direct \\
\hline Cs-140 & & & & -- & -- & -- & $3.86 \mathrm{E}+05$ & -- & $3.86 \mathrm{E}+05$ & $3.05 \mathrm{E}-04$ & 1.27E+09 & Direct \\
\hline Cu-57 & & & & -- & -- & -- & $1.76 \mathrm{E}+08$ & -- & $1.76 \mathrm{E}+08$ & $2.06 \mathrm{E}-04$ & $8.50 \mathrm{E}+11$ & Direct \\
\hline Cu-59 & & & & -- & -- & -- & $3.69 \mathrm{E}+05$ & -- & $3.69 \mathrm{E}+05$ & $1.57 \mathrm{E}-04$ & $2.35 \mathrm{E}+09$ & Direct \\
\hline Cu-60 & & & & $1.80 \mathrm{E}+05$ & $1.80 \mathrm{E}+120$ & $9.37 \mathrm{E}+06$ & $8.01 \mathrm{E}+03$ & -- & $8.01 \mathrm{E}+03$ & $5.93 \mathrm{E}-05$ & $1.35 \mathrm{E}+08$ & Direct \\
\hline Cu-61 & & & & $9.31 \mathrm{E}+04$ & $5.07 \mathrm{E}+18$ & $6.26 \mathrm{E}+05$ & $4.31 \mathrm{E}+03$ & -- & $4.31 \mathrm{E}+03$ & $2.86 \mathrm{E}-04$ & $1.51 \mathrm{E}+07$ & Direct \\
\hline Cu-62 & & & & -- & -- & -- & $7.39 E+04$ & -- & $7.39 E+04$ & $2.37 \mathrm{E}-04$ & $3.12 \mathrm{E}+08$ & Direct \\
\hline Cu-64 & & & & $7.45 \mathrm{E}+04$ & $5.43 E+08$ & $1.73 \mathrm{E}+05$ & $6.83 E+03$ & -- & $6.83 E+03$ & $1.77 \mathrm{E}-03$ & $3.86 \mathrm{E}+06$ & Direct \\
\hline Cu-66 & & & & -- & -- & -- & $1.68 \mathrm{E}+06$ & -- & $1.68 \mathrm{E}+06$ & $3.00 \mathrm{E}-03$ & $5.59 \mathrm{E}+08$ & Direct \\
\hline Cu-67 & & & & $1.93 \mathrm{E}+04$ & $7.87 \mathrm{E}+04$ & $1.43 \mathrm{E}+04$ & $7.18 \mathrm{E}+03$ & -- & $7.18 \mathrm{E}+03$ & $9.49 \mathrm{E}-03$ & $7.57 \mathrm{E}+05$ & Direct \\
\hline Cu-69 & & & & -- & -- & -- & $4.81 \mathrm{E}+05$ & -- & $4.81 \mathrm{E}+05$ & $5.03 \mathrm{E}-04$ & $9.57 \mathrm{E}+08$ & Direct \\
\hline Dy-148 & & & & -- & -- & -- & $3.06 \mathrm{E}+05$ & -- & $3.06 \mathrm{E}+05$ & 7.95E-04 & $3.85 \mathrm{E}+08$ & Direct \\
\hline Dy-149 & & & & -- & -- & -- & $1.06 \mathrm{E}+05$ & -- & $1.06 \mathrm{E}+05$ & $3.54 \mathrm{E}-04$ & $3.01 \mathrm{E}+08$ & Direct \\
\hline Dy-150 & & & & -- & -- & -- & $3.63 \mathrm{E}+05$ & -- & $3.63 E+05$ & $2.07 \mathrm{E}-03$ & $1.75 \mathrm{E}+08$ & Direct \\
\hline Dy-151 & & & & $9.31 \mathrm{E}+04$ & $2.71 \mathrm{E}+154$ & $4.31 \mathrm{E}+07$ & $2.95 \mathrm{E}+04$ & -- & $2.95 \mathrm{E}+04$ & $4.24 \mathrm{E}-04$ & $6.96 \mathrm{E}+07$ & Direct \\
\hline Dy-152 & & & & $1.27 \mathrm{E}+05$ & $2.80 \mathrm{E}+24$ & $9.88 \mathrm{E}+05$ & $1.77 \mathrm{E}+04$ & -- & $1.77 \mathrm{E}+04$ & $2.04 \mathrm{E}-03$ & $8.67 \mathrm{E}+06$ & Direct \\
\hline Dy-153 & & & & $6.20 \mathrm{E}+04$ & $1.68 \mathrm{E}+12$ & $2.15 \mathrm{E}+05$ & $2.33 \mathrm{E}+03$ & -- & $2.33 E+03$ & $7.28 \mathrm{E}-04$ & $3.20 \mathrm{E}+06$ & Direct \\
\hline Dy-154 & & & & $1.02 \mathrm{E}+00$ & $3.53 \mathrm{E}+01$ & $1.44 \mathrm{E}+01$ & -- & -- & $1.02 \mathrm{E}+00$ & $1.31 \mathrm{E}+03$ & 7.74E-04 & Inh. \\
\hline Dy-155 & & & & $9.31 \mathrm{E}+04$ & $5.43 \mathrm{E}+09$ & $2.03 E+05$ & $2.56 \mathrm{E}+03$ & -- & $2.56 \mathrm{E}+03$ & $1.26 \mathrm{E}-03$ & $2.02 \mathrm{E}+06$ & Direct \\
\hline Dy-157 & & & & $2.03 E+05$ & $1.52 \mathrm{E}+11$ & $5.32 \mathrm{E}+05$ & $4.80 \mathrm{E}+03$ & -- & $4.80 \mathrm{E}+03$ & $1.95 \mathrm{E}-03$ & $2.47 \mathrm{E}+06$ & Direct \\
\hline Dy-159 & & & & $3.19 \mathrm{E}+04$ & $2.08 \mathrm{E}+04$ & $8.72 \mathrm{E}+03$ & $1.62 \mathrm{E}+04$ & -- & $8.72 \mathrm{E}+03$ & $1.53 \mathrm{E}+00$ & $5.69 \mathrm{E}+03$ & Food \\
\hline Dy-165 & & & & $1.28 \mathrm{E}+05$ & $6.57 E+24$ & $1.01 \mathrm{E}+06$ & $1.99 \mathrm{E}+05$ & -- & $1.28 \mathrm{E}+05$ & $1.58 \mathrm{E}-02$ & $8.14 \mathrm{E}+06$ & Inh. \\
\hline Dy-165m & & & & -- & -- & -- & $3.01 \mathrm{E}+07$ & -- & $3.01 \mathrm{E}+07$ & $3.32 \mathrm{E}-02$ & $9.07 E+08$ & Direct \\
\hline Dy-166 & & & & $6.20 \mathrm{E}+03$ & $9.27 \mathrm{E}+03$ & $2.44 \mathrm{E}+03$ & $2.00 \mathrm{E}+04$ & -- & $2.44 \mathrm{E}+03$ & $1.06 \mathrm{E}-02$ & $2.31 \mathrm{E}+05$ & Food \\
\hline Dy-167 & & & & -- & -- & -- & $2.19 \mathrm{E}+05$ & -- & $2.19 E+05$ & $1.21 \mathrm{E}-03$ & $1.82 \mathrm{E}+08$ & Direct \\
\hline Dy-168 & & & & -- & -- & -- & $2.11 \mathrm{E}+05$ & -- & $2.11 E+05$ & $1.64 \mathrm{E}-03$ & $1.29 \mathrm{E}+08$ & Direct \\
\hline Er-154 & & & & -- & -- & -- & $2.56 \mathrm{E}+06$ & -- & $2.56 \mathrm{E}+06$ & $7.81 \mathrm{E}-03$ & $3.27 \mathrm{E}+08$ & Direct \\
\hline Er-155 & & & & -- & -- & -- & -- & -- & -- & -- & $2.29 \mathrm{E}+08$ & -- \\
\hline Er-156 & & & & $3.72 \mathrm{E}+05$ & $1.20 \mathrm{E}+142$ & $2.08 \mathrm{E}+07$ & $5.48 \mathrm{E}+05$ & -- & $3.72 E+05$ & $6.02 \mathrm{E}-03$ & $6.18 \mathrm{E}+07$ & Inh. \\
\hline Er-159 & & & & $3.72 E+05$ & $1.88 \mathrm{E}+80$ & $1.79 \mathrm{E}+07$ & $2.09 E+04$ & -- & $2.09 E+04$ & $6.36 \mathrm{E}-04$ & $3.29 E+07$ & Direct \\
\hline
\end{tabular}


Table C.2 Summary of Exposure Pathway TQs and Selection of Final HC-3 TQs (continued)

\begin{tabular}{|c|c|c|c|c|c|c|c|c|c|c|c|c|}
\hline \multirow[b]{2}{*}{$\begin{array}{c}\text { MASTER } \\
\text { ISOTOPE } \\
\text { LIST } \\
\end{array}$} & \multicolumn{3}{|c|}{ from NNSA SD G 1027} & \multicolumn{5}{|c|}{ Calculated Pathway Specific TQ Values } & \multicolumn{4}{|c|}{ Selected Final Threshold Quantities } \\
\hline & Act $(\mathrm{Ci})$ & Mass (g) & $\begin{array}{l}\text { Limiting } \\
\text { Pathway }\end{array}$ & $\begin{array}{c}\text { TQ }_{\text {inhale }} \\
(\mathrm{Ci})\end{array}$ & $\begin{array}{c}\mathrm{TQ}_{\text {water }} \\
\text { (Ci) }\end{array}$ & $\mathrm{TQ}_{\text {food }}(\mathrm{Ci})$ & $\begin{array}{c}T_{\text {direct }} \\
\text { (Ci) }\end{array}$ & $\mathrm{TQ}_{\text {sub }}(\mathrm{Ci})$ & Act (Ci) & Mass (g) & $\begin{array}{c}\text { Sp. Act. } \\
\text { (Ci/g) }\end{array}$ & $\begin{array}{l}\text { Limiting } \\
\text { Pathway }\end{array}$ \\
\hline Er-161 & & & & $1.31 \mathrm{E}+05$ & $3.70 \mathrm{E}+19$ & $1.00 \mathrm{E}+06$ & $4.10 \mathrm{E}+03$ & -- & $4.10 E+03$ & $6.82 \mathrm{E}-04$ & $6.01 \mathrm{E}+06$ & Direct \\
\hline Er-163 & & & & $5.08 \mathrm{E}+06$ & $9.21 \mathrm{E}+42$ & $7.64 \mathrm{E}+07$ & $2.40 \mathrm{E}+05$ & -- & $2.40 \mathrm{E}+05$ & $1.56 \mathrm{E}-02$ & $1.54 \mathrm{E}+07$ & Direct \\
\hline Er-165 & & & & $7.98 \mathrm{E}+05$ & $2.53 \mathrm{E}+10$ & $1.34 \mathrm{E}+06$ & $3.88 \mathrm{E}+04$ & -- & $3.88 \mathrm{E}+04$ & $2.11 \mathrm{E}-02$ & $1.83 \mathrm{E}+06$ & Direct \\
\hline Er-167m & & & & -- & -- & -- & $1.97 \mathrm{E}+08$ & -- & $1.97 \mathrm{E}+08$ & $6.65 \mathrm{E}-03$ & $2.96 \mathrm{E}+10$ & Direct \\
\hline Er-169 & & & & $1.14 \mathrm{E}+04$ & $1.15 \mathrm{E}+04$ & $5.18 \mathrm{E}+03$ & -- & -- & $5.18 \mathrm{E}+03$ & $6.23 \mathrm{E}-02$ & $8.31 E+04$ & Food \\
\hline Er-171 & & & & $3.72 \mathrm{E}+04$ & $7.24 \mathrm{E}+10$ & $9.70 \mathrm{E}+04$ & $4.73 E+03$ & -- & $4.73 \mathrm{E}+03$ & $1.94 \mathrm{E}-03$ & $2.44 \mathrm{E}+06$ & Direct \\
\hline Er-172 & & & & $9.31 \mathrm{E}+03$ & $4.87 E+04$ & $5.97 \mathrm{E}+03$ & $1.64 \mathrm{E}+03$ & -- & $1.64 \mathrm{E}+03$ & $4.43 \mathrm{E}-03$ & $3.70 \mathrm{E}+05$ & Direct \\
\hline Er-173 & & & & -- & -- & -- & $6.08 \mathrm{E}+05$ & -- & $6.08 \mathrm{E}+05$ & $8.02 \mathrm{E}-04$ & $7.58 \mathrm{E}+08$ & Direct \\
\hline Es-249 & & & & $4.14 \mathrm{E}+05$ & $3.96 \mathrm{E}+32$ & $6.89 \mathrm{E}+07$ & $1.72 \mathrm{E}+04$ & -- & $1.72 \mathrm{E}+04$ & $2.32 \mathrm{E}-03$ & $7.39 \mathrm{E}+06$ & Direct \\
\hline Es-250 & & & & $1.89 \mathrm{E}+05$ & $4.71 E+27$ & $5.86 \mathrm{E}+07$ & $1.45 \mathrm{E}+04$ & -- & $1.45 \mathrm{E}+04$ & $2.43 \mathrm{E}-03$ & $5.97 \mathrm{E}+06$ & Direct \\
\hline Es-250m & & & & -- & -- & -- & $9.81 \mathrm{E}+03$ & -- & $9.81 \mathrm{E}+03$ & $1.74 \mathrm{E}-03$ & $5.64 \mathrm{E}+06$ & Direct \\
\hline Es-251 & & & & $5.58 \mathrm{E}+04$ & $1.13 \mathrm{E}+06$ & $5.03 \mathrm{E}+05$ & $9.39 \mathrm{E}+03$ & -- & $9.39 E+03$ & $2.48 \mathrm{E}-02$ & $3.78 \mathrm{E}+05$ & Direct \\
\hline Es-253 & & & & $4.47 \mathrm{E}+01$ & $4.61 \mathrm{E}+02$ & $2.13 \mathrm{E}+03$ & $6.71 \mathrm{E}+05$ & -- & $4.47 \mathrm{E}+01$ & $1.77 \mathrm{E}-03$ & $2.52 \mathrm{E}+04$ & Inh. \\
\hline Es-254 & & & & $1.40 \mathrm{E}+01$ & $7.25 \mathrm{E}+01$ & $3.00 \mathrm{E}+02$ & $3.81 \mathrm{E}+04$ & -- & $1.40 \mathrm{E}+01$ & $7.49 \mathrm{E}-03$ & $1.86 \mathrm{E}+03$ & Inh. \\
\hline Es-254m & & & & $2.54 \mathrm{E}+01$ & $2.38 \mathrm{E}+04$ & $1.74 \mathrm{E}+03$ & $1.90 \mathrm{E}+03$ & -- & $2.54 \mathrm{E}+01$ & 8.09E-05 & $3.14 \mathrm{E}+05$ & Inh. \\
\hline Es-255 & & & & $3.10 \mathrm{E}+01$ & $3.95 \mathrm{E}+02$ & $1.75 \mathrm{E}+03$ & $1.08 \mathrm{E}+06$ & -- & $3.10 \mathrm{E}+01$ & $2.41 \mathrm{E}-03$ & $1.29 \mathrm{E}+04$ & Inh. \\
\hline Es-256 & & & & $3.28 \mathrm{E}+02$ & $4.38 \mathrm{E}+108$ & $1.48 \mathrm{E}+06$ & $9.06 \mathrm{E}+06$ & -- & $3.28 \mathrm{E}+02$ & $1.14 \mathrm{E}-05$ & $2.89 \mathrm{E}+07$ & Inh. \\
\hline Eu-142 & & & & -- & -- & -- & $1.55 \mathrm{E}+07$ & -- & $1.55 \mathrm{E}+07$ & 4.57E-04 & $3.40 \mathrm{E}+10$ & Direct \\
\hline Eu-142m & & & & -- & -- & -- & $1.73 \mathrm{E}+05$ & -- & $1.73 \mathrm{E}+05$ & $1.59 \mathrm{E}-04$ & $1.08 \mathrm{E}+09$ & Direct \\
\hline Eu-143 & & & & -- & -- & -- & $2.49 \mathrm{E}+05$ & -- & $2.49 \mathrm{E}+05$ & $4.91 \mathrm{E}-04$ & $5.08 \mathrm{E}+08$ & Direct \\
\hline Eu-144 & & & & -- & -- & -- & $3.90 \mathrm{E}+06$ & -- & $3.90 \mathrm{E}+06$ & $5.08 \mathrm{E}-04$ & $7.69 \mathrm{E}+09$ & Direct \\
\hline Eu-145 & & & & $1.53 \mathrm{E}+04$ & -- & $3.42 \mathrm{E}+03$ & $5.28 \mathrm{E}+02$ & -- & $5.28 \mathrm{E}+02$ & $3.48 \mathrm{E}-03$ & $1.52 \mathrm{E}+05$ & Direct \\
\hline Eu-146 & & & & $9.31 \mathrm{E}+03$ & -- & $2.37 \mathrm{E}+03$ & $3.12 \mathrm{E}+02$ & -- & $3.12 \mathrm{E}+02$ & $1.61 \mathrm{E}-03$ & $1.94 \mathrm{E}+05$ & Direct \\
\hline Eu-147 & & & & $1.12 \mathrm{E}+04$ & -- & $2.78 \mathrm{E}+03$ & $1.48 \mathrm{E}+03$ & -- & $1.48 \mathrm{E}+03$ & $4.00 \mathrm{E}-02$ & $3.70 \mathrm{E}+04$ & Direct \\
\hline Eu-148 & & & & $4.14 \mathrm{E}+03$ & -- & $7.57 \mathrm{E}+02$ & $3.36 \mathrm{E}+02$ & -- & $3.36 \mathrm{E}+02$ & $2.07 \mathrm{E}-02$ & $1.62 \mathrm{E}+04$ & Direct \\
\hline Eu-149 & & & & $4.14 \mathrm{E}+04$ & -- & $9.09 \mathrm{E}+03$ & $1.15 \mathrm{E}+04$ & -- & $9.09 \mathrm{E}+03$ & $9.65 \mathrm{E}-01$ & $9.42 \mathrm{E}+03$ & Food \\
\hline Eu-150l & & & & $2.23 \mathrm{E}+02$ & -- & $6.20 \mathrm{E}+02$ & $4.85 \mathrm{E}+02$ & -- & $2.23 \mathrm{E}+02$ & $3.20 \mathrm{E}+00$ & $6.97 \mathrm{E}+01$ & Inh. \\
\hline Eu-150s & & & & $3.99 \mathrm{E}+04$ & -- & $5.56 \mathrm{E}+04$ & $2.79 \mathrm{E}+04$ & -- & $2.79 E+04$ & 1.69E-02 & $1.66 \mathrm{E}+06$ & Direct \\
\hline Eu-152 & $2.89 \mathrm{E}+02$ & $1.66 \mathrm{E}+00$ & $\mathrm{i}$ & $2.86 \mathrm{E}+02$ & -- & $5.77 \mathrm{E}+02$ & $6.29 \mathrm{E}+02$ & -- & $2.86 \mathrm{E}+02$ & $1.62 \mathrm{E}+00$ & $1.77 \mathrm{E}+02$ & Inh. \\
\hline Eu-152ml & & & & $3.49 \mathrm{E}+04$ & -- & $5.66 \mathrm{E}+04$ & $5.31 \mathrm{E}+03$ & -- & $5.31 \mathrm{E}+03$ & $2.40 \mathrm{E}-03$ & $2.21 \mathrm{E}+06$ & Direct \\
\hline Eu-152ms & & & & $1.02 \mathrm{E}+06$ & -- & $1.15 \mathrm{E}+07$ & $1.00 \mathrm{E}+05$ & -- & $1.00 \mathrm{E}+05$ & $7.78 \mathrm{E}-03$ & $1.29 \mathrm{E}+07$ & Direct \\
\hline Eu-154 & $2.25 \mathrm{E}+02$ & $8.33 \mathrm{E}-01$ & $\mathrm{i}$ & $2.23 \mathrm{E}+02$ & -- & $4.04 \mathrm{E}+02$ & $5.85 \mathrm{E}+02$ & -- & $2.23 E+02$ & $8.46 \mathrm{E}-01$ & $2.64 \mathrm{E}+02$ & Inh. \\
\hline Eu-154m & & & & $1.93 \mathrm{E}+06$ & -- & $3.69 \mathrm{E}+07$ & $2.23 \mathrm{E}+05$ & -- & $2.23 \mathrm{E}+05$ & $8.40 \mathrm{E}-03$ & $2.66 \mathrm{E}+07$ & Direct \\
\hline Eu-155 & $1.73 \mathrm{E}+03$ & $3.56 \mathrm{E}+00$ & $\mathrm{i}$ & $1.72 \mathrm{E}+03$ & -- & $2.53 \mathrm{E}+03$ & $1.20 \mathrm{E}+04$ & -- & $1.72 \mathrm{E}+03$ & $3.69 \mathrm{E}+00$ & $4.65 \mathrm{E}+02$ & Inh. \\
\hline Eu-156 & & & & $3.38 \mathrm{E}+03$ & -- & $6.70 \mathrm{E}+02$ & $5.59 \mathrm{E}+02$ & -- & $5.59 \mathrm{E}+02$ & $1.01 \mathrm{E}-02$ & $5.51 \mathrm{E}+04$ & Direct \\
\hline
\end{tabular}


Table C.2 Summary of Exposure Pathway TQs and Selection of Final HC-3 TQs (continued)

\begin{tabular}{|c|c|c|c|c|c|c|c|c|c|c|c|c|}
\hline \multirow{2}{*}{$\begin{array}{c}\text { MASTER } \\
\text { ISOTOPE } \\
\text { LIST } \\
\end{array}$} & \multicolumn{3}{|c|}{ from NNSA SD G 1027} & \multicolumn{5}{|c|}{ Calculated Pathway Specific TQ Values } & \multicolumn{4}{|c|}{ Selected Final Threshold Quantities } \\
\hline & Act (Ci) & Mass (g) & $\begin{array}{l}\text { Limiting } \\
\text { Pathway }\end{array}$ & $\begin{array}{c}\mathrm{TQ}_{\text {inhale }} \\
(\mathrm{Ci}) \\
\end{array}$ & $\begin{array}{c}\text { TQ }_{\text {water }} \\
\text { (Ci) }\end{array}$ & $\mathrm{TQ}_{\text {food }}(\mathrm{Ci})$ & $\begin{array}{c}\mathrm{TQ}_{\text {direct }} \\
(\mathrm{Ci}) \\
\end{array}$ & $\mathrm{TQ}_{\text {sub }}(\mathrm{Ci})$ & Act (Ci) & Mass (g) & $\begin{array}{c}\text { Sp. Act. } \\
\text { (Ci/g) }\end{array}$ & $\begin{array}{l}\text { Limiting } \\
\text { Pathway }\end{array}$ \\
\hline Eu-157 & & & & $2.54 \mathrm{E}+04$ & -- & $2.95 \mathrm{E}+04$ & $4.57 \mathrm{E}+03$ & -- & $4.57 \mathrm{E}+03$ & $3.46 \mathrm{E}-03$ & $1.32 \mathrm{E}+06$ & Direct \\
\hline Eu-158 & & & & $1.49 \mathrm{E}+05$ & -- & $3.58 \mathrm{E}+06$ & $1.49 \mathrm{E}+04$ & -- & $1.49 \mathrm{E}+04$ & $5.76 \mathrm{E}-04$ & $2.59 \mathrm{E}+07$ & Direct \\
\hline Eu-159 & & & & $3.10 \mathrm{E}+05$ & -- & $1.74 \mathrm{E}+07$ & $1.32 \mathrm{E}+05$ & -- & $1.32 \mathrm{E}+05$ & $2.02 \mathrm{E}-03$ & $6.54 \mathrm{E}+07$ & Direct \\
\hline $\mathrm{F}-17$ & & & & -- & -- & -- & $6.60 \mathrm{E}+05$ & -- & $6.60 \mathrm{E}+05$ & $6.42 \mathrm{E}-05$ & $1.03 \mathrm{E}+10$ & Direct \\
\hline F-18 & & & & $1.20 \mathrm{E}+05$ & $2.80 \mathrm{E}+30$ & $2.88 \mathrm{E}+06$ & $6.46 \mathrm{E}+03$ & -- & $6.46 \mathrm{E}+03$ & $6.79 \mathrm{E}-05$ & $9.52 \mathrm{E}+07$ & Direct \\
\hline Fe-52 & & & & $1.18 \mathrm{E}+04$ & -- & $2.27 \mathrm{E}+04$ & $2.28 \mathrm{E}+03$ & -- & $2.28 \mathrm{E}+03$ & $3.12 \mathrm{E}-04$ & $7.29 \mathrm{E}+06$ & Direct \\
\hline Fe-53 & & & & -- & -- & -- & $7.23 E+04$ & -- & $7.23 E+04$ & 1.73E-04 & $4.17 \mathrm{E}+08$ & Direct \\
\hline $\mathrm{Fe}-53 \mathrm{~m}$ & & & & -- & -- & -- & $9.39 \mathrm{E}+04$ & -- & $9.39 \mathrm{E}+04$ & $6.68 \mathrm{E}-05$ & $1.41 \mathrm{E}+09$ & Direct \\
\hline Fe-55 & $2.41 \mathrm{E}+03$ & $1.01 \mathrm{E}+00$ & ing & $1.21 \mathrm{E}+04$ & -- & $2.47 \mathrm{E}+03$ & $4.54 \mathrm{E}+05$ & -- & $2.47 \mathrm{E}+03$ & $1.03 \mathrm{E}+00$ & $2.41 \mathrm{E}+03$ & Food \\
\hline Fe-59 & $5.61 \mathrm{E}+02$ & $1.13 \mathrm{E}-02$ & ing & $3.19 \mathrm{E}+03$ & -- & $5.70 \mathrm{E}+02$ & $6.15 \mathrm{E}+02$ & -- & $5.70 \mathrm{E}+02$ & $1.15 \mathrm{E}-02$ & $4.98 \mathrm{E}+04$ & Food \\
\hline Fe-60 & & & & $3.38 \mathrm{E}+01$ & -- & $7.33 \mathrm{E}+00$ & -- & -- & $7.33 \mathrm{E}+00$ & $1.23 \mathrm{E}+02$ & $5.96 \mathrm{E}-02$ & Food \\
\hline Fe-61 & & & & -- & -- & -- & $8.71 E+04$ & -- & $8.71 E+04$ & 1.69E-04 & $5.16 \mathrm{E}+08$ & Direct \\
\hline Fe-62 & & & & -- & -- & -- & $1.26 \mathrm{E}+06$ & -- & $1.26 \mathrm{E}+06$ & $4.72 \mathrm{E}-04$ & $2.68 \mathrm{E}+09$ & Direct \\
\hline Fm-251 & & & & $6.20 \mathrm{E}+04$ & $1.41 \mathrm{E}+14$ & $6.84 \mathrm{E}+06$ & $1.50 \mathrm{E}+04$ & -- & $1.50 \mathrm{E}+04$ & $6.37 \mathrm{E}-03$ & $2.35 \mathrm{E}+06$ & Direct \\
\hline Fm-252 & & & & $3.72 \mathrm{E}+02$ & $4.10 \mathrm{E}+05$ & $4.47 \mathrm{E}+04$ & $9.31 \mathrm{E}+05$ & -- & $3.72 \mathrm{E}+02$ & $6.80 \mathrm{E}-04$ & $5.48 \mathrm{E}+05$ & Inh. \\
\hline $\mathrm{Fm}-253$ & & & & $3.02 \mathrm{E}+02$ & $2.09 \mathrm{E}+04$ & $4.76 \mathrm{E}+04$ & $9.81 \mathrm{E}+03$ & -- & $3.02 \mathrm{E}+02$ & $1.76 \mathrm{E}-03$ & $1.72 \mathrm{E}+05$ & Inh. \\
\hline Fm-254 & & & & $1.45 \mathrm{E}+03$ & $6.74 \mathrm{E}+18$ & $1.82 \mathrm{E}+06$ & $3.12 \mathrm{E}+06$ & -- & $1.45 \mathrm{E}+03$ & $3.81 \mathrm{E}-04$ & $3.81 \mathrm{E}+06$ & Inh. \\
\hline Fm-255 & & & & $4.30 \mathrm{E}+02$ & $8.95 \mathrm{E}+05$ & $5.43 E+04$ & $7.85 \mathrm{E}+04$ & -- & $4.30 \mathrm{E}+02$ & $7.02 \mathrm{E}-04$ & $6.12 \mathrm{E}+05$ & Inh. \\
\hline Fm-256 & & & & $1.60 \mathrm{E}+01$ & $2.01 \mathrm{E}+20$ & $3.79 \mathrm{E}+04$ & $3.70 \mathrm{E}+02$ & -- & $1.60 \mathrm{E}+01$ & $3.42 \mathrm{E}-06$ & $4.66 \mathrm{E}+06$ & Inh. \\
\hline Fm-257 & & & & $1.69 \mathrm{E}+01$ & $1.42 \mathrm{E}+02$ & $6.01 \mathrm{E}+02$ & $6.57 \mathrm{E}+03$ & -- & $1.69 \mathrm{E}+01$ & 3.35E-03 & $5.05 \mathrm{E}+03$ & Inh. \\
\hline Fr-212 & & & & $3.99 \mathrm{E}+03$ & -- & $1.09 \mathrm{E}+06$ & $3.17 \mathrm{E}+04$ & -- & $3.99 \mathrm{E}+03$ & $8.99 \mathrm{E}-05$ & $4.43 \mathrm{E}+07$ & Inh. \\
\hline Fr-219 & & & & -- & -- & -- & $6.09 \mathrm{E}+11$ & -- & $6.09 \mathrm{E}+11$ & $2.48 \mathrm{E}-01$ & $2.45 \mathrm{E}+12$ & Direct \\
\hline $\mathrm{Fr}-220$ & & & & -- & -- & -- & $1.31 \mathrm{E}+08$ & -- & $1.31 \mathrm{E}+08$ & 7.01E-02 & $1.87 \mathrm{E}+09$ & Direct \\
\hline Fr-221 & & & & -- & -- & -- & $4.87 E+06$ & -- & $4.87 E+06$ & $2.75 \mathrm{E}-02$ & $1.77 \mathrm{E}+08$ & Direct \\
\hline Fr-222 & & & & $5.32 \mathrm{E}+02$ & -- & $1.51 \mathrm{E}+06$ & -- & -- & $5.32 \mathrm{E}+02$ & $9.04 \mathrm{E}-06$ & $5.88 \mathrm{E}+07$ & Inh. \\
\hline Fr-223 & & & & $8.59 \mathrm{E}+03$ & -- & $3.07 \mathrm{E}+05$ & $5.60 \mathrm{E}+05$ & -- & $8.59 \mathrm{E}+03$ & $2.22 \mathrm{E}-04$ & $3.87 \mathrm{E}+07$ & Inh. \\
\hline Fr-224 & & & & -- & -- & -- & $3.94 \mathrm{E}+05$ & -- & $3.94 E+05$ & $1.56 \mathrm{E}-03$ & $2.52 \mathrm{E}+08$ & Direct \\
\hline Fr-227 & & & & -- & -- & -- & $6.52 \mathrm{E}+05$ & -- & $6.52 \mathrm{E}+05$ & $1.94 \mathrm{E}-03$ & $3.35 \mathrm{E}+08$ & Direct \\
\hline Ga-64 & & & & -- & -- & -- & $8.18 \mathrm{E}+04$ & -- & $8.18 \mathrm{E}+04$ & 7.31E-05 & $1.12 \mathrm{E}+09$ & Direct \\
\hline Ga-65 & & & & $3.85 \mathrm{E}+05$ & $2.01 \mathrm{E}+180$ & $2.74 \mathrm{E}+07$ & $4.05 \mathrm{E}+04$ & -- & $4.05 E+04$ & $2.13 \mathrm{E}-04$ & $1.91 \mathrm{E}+08$ & Direct \\
\hline Ga-66 & & & & $1.57 \mathrm{E}+04$ & $1.19 \mathrm{E}+09$ & $2.34 \mathrm{E}+04$ & $6.26 \mathrm{E}+02$ & -- & $6.26 \mathrm{E}+02$ & $1.24 \mathrm{E}-04$ & $5.06 \mathrm{E}+06$ & Direct \\
\hline Ga-67 & & & & $3.99 \mathrm{E}+04$ & $8.46 \mathrm{E}+04$ & $2.13 \mathrm{E}+04$ & $5.10 \mathrm{E}+03$ & -- & $5.10 \mathrm{E}+03$ & $8.52 \mathrm{E}-03$ & $5.98 \mathrm{E}+05$ & Direct \\
\hline Ga-68 & & & & $1.38 \mathrm{E}+05$ & $1.10 \mathrm{E}+45$ & $2.27 \mathrm{E}+06$ & $1.12 \mathrm{E}+04$ & -- & $1.12 \mathrm{E}+04$ & $2.75 \mathrm{E}-04$ & $4.07 \mathrm{E}+07$ & Direct \\
\hline Ga-70 & & & & $4.30 \mathrm{E}+05$ & $4.19 \mathrm{E}+131$ & $2.35 \mathrm{E}+07$ & $4.57 \mathrm{E}+06$ & -- & $4.30 \mathrm{E}+05$ & $3.38 \mathrm{E}-03$ & $1.27 \mathrm{E}+08$ & Inh. \\
\hline Ga-72 & & & & $1.33 \mathrm{E}+04$ & $2.43 \mathrm{E}+07$ & $1.73 \mathrm{E}+04$ & $4.56 \mathrm{E}+02$ & -- & $4.56 \mathrm{E}+02$ & $1.48 \mathrm{E}-04$ & $3.09 \mathrm{E}+06$ & Direct \\
\hline
\end{tabular}


Table C.2 Summary of Exposure Pathway TQs and Selection of Final HC-3 TQs (continued)

\begin{tabular}{|c|c|c|c|c|c|c|c|c|c|c|c|c|}
\hline \multirow[b]{2}{*}{$\begin{array}{c}\text { MASTER } \\
\text { ISOTOPE } \\
\text { LIST } \\
\end{array}$} & \multicolumn{3}{|c|}{ from NNSA SD G 1027} & \multicolumn{5}{|c|}{ Calculated Pathway Specific TQ Values } & \multicolumn{4}{|c|}{ Selected Final Threshold Quantities } \\
\hline & Act $(\mathrm{Ci})$ & Mass (g) & $\begin{array}{l}\text { Limiting } \\
\text { Pathway }\end{array}$ & $\begin{array}{c}\text { TQ }_{\text {inhale }} \\
(\mathrm{Ci})\end{array}$ & $\begin{array}{c}\text { TQ }_{\text {water }} \\
\text { (Ci) }\end{array}$ & $\mathrm{TQ}_{\text {food }}(\mathrm{Ci})$ & $\begin{array}{c}T_{\text {direct }} \\
\text { (Ci) }\end{array}$ & $\mathrm{TQ}_{\text {sub }}(\mathrm{Ci})$ & Act (Ci) & Mass (g) & $\begin{array}{c}\text { Sp. Act. } \\
\text { (Ci/g) }\end{array}$ & $\begin{array}{l}\text { Limiting } \\
\text { Pathway }\end{array}$ \\
\hline Ga-73 & & & & $5.58 \mathrm{E}+04$ & $1.91 \mathrm{E}+14$ & $2.04 \mathrm{E}+05$ & $8.07 E+03$ & -- & $8.07 E+03$ & $9.22 \mathrm{E}-04$ & $8.75 \mathrm{E}+06$ & Direct \\
\hline Ga-74 & & & & -- & -- & -- & $2.83 \mathrm{E}+04$ & -- & $2.83 \mathrm{E}+04$ & 9.03E-05 & $3.13 \mathrm{E}+08$ & Direct \\
\hline Gd-142 & & & & -- & -- & -- & $5.94 \mathrm{E}+05$ & -- & $5.94 \mathrm{E}+05$ & $5.24 \mathrm{E}-04$ & $1.13 \mathrm{E}+09$ & Direct \\
\hline Gd-143m & & & & -- & -- & -- & $1.86 \mathrm{E}+05$ & -- & $1.86 \mathrm{E}+05$ & $2.60 \mathrm{E}-04$ & $7.18 \mathrm{E}+08$ & Direct \\
\hline Gd-144 & & & & -- & -- & -- & $1.78 \mathrm{E}+05$ & -- & $1.78 \mathrm{E}+05$ & $6.10 \mathrm{E}-04$ & $2.92 \mathrm{E}+08$ & Direct \\
\hline Gd-145 & & & & $3.19 E+05$ & -- & $1.53 \mathrm{E}+07$ & $1.40 \mathrm{E}+04$ & -- & $1.40 \mathrm{E}+04$ & $2.47 \mathrm{E}-04$ & $5.67 \mathrm{E}+07$ & Direct \\
\hline Gd-145m & & & & -- & -- & -- & $7.51 \mathrm{E}+05$ & -- & $7.51 \mathrm{E}+05$ & $8.20 \mathrm{E}-04$ & $9.16 \mathrm{E}+08$ & Direct \\
\hline Gd-146 & & & & $1.86 \mathrm{E}+03$ & -- & $1.05 \mathrm{E}+03$ & $2.92 \mathrm{E}+03$ & -- & $1.05 E+03$ & $5.67 \mathrm{E}-02$ & $1.85 \mathrm{E}+04$ & Food \\
\hline Gd-147 & & & & $1.89 \mathrm{E}+04$ & -- & $1.23 \mathrm{E}+04$ & $6.70 \mathrm{E}+02$ & -- & $6.70 \mathrm{E}+02$ & $1.20 \mathrm{E}-03$ & $5.60 \mathrm{E}+05$ & Direct \\
\hline Gd-148 & & & & $3.72 \mathrm{E}-01$ & -- & $1.47 \mathrm{E}+01$ & -- & -- & $3.72 \mathrm{E}-01$ & $1.43 \mathrm{E}-02$ & $2.60 \mathrm{E}+01$ & Inh. \\
\hline Gd-149 & & & & $1.41 \mathrm{E}+04$ & -- & $4.23 \mathrm{E}+03$ & $1.79 \mathrm{E}+03$ & -- & $1.79 \mathrm{E}+03$ & $1.92 \mathrm{E}-02$ & $9.33 \mathrm{E}+04$ & Direct \\
\hline Gd-150 & & & & 3.99E-01 & -- & $1.55 \mathrm{E}+01$ & -- & -- & 3.99E-01 & $2.99 \mathrm{E}+02$ & $1.33 \mathrm{E}-03$ & Inh. \\
\hline Gd-151 & & & & $1.20 \mathrm{E}+04$ & -- & $4.43 \mathrm{E}+03$ & $1.14 \mathrm{E}+04$ & -- & $4.43 E+03$ & $6.14 \mathrm{E}-01$ & $7.21 \mathrm{E}+03$ & Food \\
\hline Gd-152 & & & & $5.08 \mathrm{E}-01$ & -- & $1.97 \mathrm{E}+01$ & -- & -- & $5.08 \mathrm{E}-01$ & $2.33 \mathrm{E}+10$ & $2.18 \mathrm{E}-11$ & Inh. \\
\hline Gd-153 & $3.06 \mathrm{E}+03$ & 8.66E-01 & ing & $4.47 \mathrm{E}+03$ & -- & $3.13 \mathrm{E}+03$ & $6.89 \mathrm{E}+03$ & -- & $3.13 E+03$ & 8.87E-01 & $3.53 E+03$ & Food \\
\hline Gd-159 & & & & $2.86 \mathrm{E}+04$ & -- & $2.98 \mathrm{E}+04$ & $2.20 \mathrm{E}+04$ & -- & $2.20 \mathrm{E}+04$ & $2.07 \mathrm{E}-02$ & $1.06 \mathrm{E}+06$ & Direct \\
\hline Gd-162 & & & & -- & -- & -- & $2.07 E+05$ & -- & $2.07 E+05$ & $1.50 \mathrm{E}-03$ & $1.38 \mathrm{E}+08$ & Direct \\
\hline Ge-66 & & & & $8.59 \mathrm{E}+04$ & $2.51 \mathrm{E}+25$ & $1.12 \mathrm{E}+06$ & $7.75 \mathrm{E}+03$ & -- & $7.75 \mathrm{E}+03$ & $3.70 \mathrm{E}-04$ & $2.09 \mathrm{E}+07$ & Direct \\
\hline Ge-67 & & & & $2.66 \mathrm{E}+05$ & $4.22 \mathrm{E}+147$ & $1.25 \mathrm{E}+07$ & $2.76 \mathrm{E}+04$ & -- & $2.76 \mathrm{E}+04$ & $1.83 \mathrm{E}-04$ & $1.50 \mathrm{E}+08$ & Direct \\
\hline Ge-68 & $6.24 \mathrm{E}+02$ & 8.79E-02 & ing & $8.59 \mathrm{E}+02$ & $1.56 \mathrm{E}+03$ & $6.37 \mathrm{E}+02$ & $1.77 \mathrm{E}+05$ & -- & $6.37 \mathrm{E}+02$ & $9.54 \mathrm{E}-02$ & $6.67 \mathrm{E}+03$ & Food \\
\hline Ge-69 & & & & $3.02 \mathrm{E}+04$ & $4.26 \mathrm{E}+05$ & $3.02 \mathrm{E}+04$ & $1.02 \mathrm{E}+03$ & -- & $1.02 \mathrm{E}+03$ & $8.77 \mathrm{E}-04$ & $1.16 \mathrm{E}+06$ & Direct \\
\hline Ge-71 & & & & $1.02 \mathrm{E}+06$ & $3.03 E+05$ & $1.38 \mathrm{E}+05$ & $1.82 \mathrm{E}+05$ & -- & $1.38 \mathrm{E}+05$ & $8.82 \mathrm{E}-01$ & $1.56 \mathrm{E}+05$ & Food \\
\hline Ge-75 & & & & $2.07 E+05$ & $2.50 \mathrm{E}+38$ & $4.01 \mathrm{E}+06$ & $2.57 \mathrm{E}+05$ & -- & $2.07 \mathrm{E}+05$ & $6.82 \mathrm{E}-03$ & $3.03 E+07$ & Inh. \\
\hline Ge-77 & & & & $2.48 \mathrm{E}+04$ & $5.94 \mathrm{E}+08$ & $7.02 E+04$ & $1.28 \mathrm{E}+03$ & -- & $1.28 \mathrm{E}+03$ & $3.54 \mathrm{E}-04$ & $3.61 \mathrm{E}+06$ & Direct \\
\hline Ge-78 & & & & $7.98 \mathrm{E}+04$ & $2.64 \mathrm{E}+36$ & $1.46 \mathrm{E}+06$ & $3.00 \mathrm{E}+04$ & -- & $3.00 \mathrm{E}+04$ & $1.08 \mathrm{E}-03$ & $2.77 \mathrm{E}+07$ & Direct \\
\hline $\mathrm{H}-3$ & $1.60 \mathrm{E}+04$ & $1.60 \mathrm{E}+00$ & $* *$ & $5.45 \mathrm{E}+03$ & $4.71 \mathrm{E}+04$ & -- & -- & -- & $5.45 \mathrm{E}+03$ & $5.68 \mathrm{E}-01$ & $9.60 \mathrm{E}+03$ & Inh. \\
\hline Hf-167 & & & & -- & -- & -- & $5.73 E+05$ & -- & $5.73 E+05$ & $1.04 \mathrm{E}-03$ & $5.49 E+08$ & Direct \\
\hline Hf-169 & & & & -- & -- & -- & $3.49 \mathrm{E}+05$ & -- & $3.49 E+05$ & $1.01 \mathrm{E}-03$ & $3.44 \mathrm{E}+08$ & Direct \\
\hline $\mathrm{Hf}-170$ & & & & $2.60 \mathrm{E}+04$ & $2.09 \mathrm{E}+07$ & $3.50 \mathrm{E}+04$ & $2.13 E+03$ & -- & $2.13 E+03$ & $1.85 \mathrm{E}-03$ & $1.15 \mathrm{E}+06$ & Direct \\
\hline $\mathrm{Hf}-172$ & & & & $3.02 \mathrm{E}+02$ & $2.00 \mathrm{E}+03$ & $8.20 \mathrm{E}+02$ & $6.17 \mathrm{E}+03$ & -- & $3.02 E+02$ & $2.71 \mathrm{E}-01$ & $1.11 \mathrm{E}+03$ & Inh. \\
\hline $\mathrm{Hf}-173$ & & & & $5.08 \mathrm{E}+04$ & $3.58 \mathrm{E}+06$ & $4.99 \mathrm{E}+04$ & $2.47 \mathrm{E}+03$ & -- & $2.47 \mathrm{E}+03$ & $3.27 \mathrm{E}-03$ & $7.55 \mathrm{E}+05$ & Direct \\
\hline $\mathrm{Hf}-174$ & & & & $3.10 \mathrm{E}-01$ & $7.90 \mathrm{E}+00$ & $3.22 \mathrm{E}+00$ & -- & -- & $3.10 \mathrm{E}-01$ & $3.02 \mathrm{E}+11$ & $1.03 \mathrm{E}-12$ & Inh. \\
\hline Hf-175 & & & & $1.02 \mathrm{E}+04$ & $5.35 \mathrm{E}+03$ & $2.30 \mathrm{E}+03$ & $1.98 \mathrm{E}+03$ & -- & $1.98 \mathrm{E}+03$ & $1.85 \mathrm{E}-01$ & $1.07 \mathrm{E}+04$ & Direct \\
\hline $\mathrm{Hf}-177 \mathrm{~m}$ & & & & $7.45 \mathrm{E}+04$ & $5.38 \mathrm{E}+57$ & $3.71 \mathrm{E}+06$ & $6.26 \mathrm{E}+03$ & -- & $6.26 \mathrm{E}+03$ & $3.03 \mathrm{E}-04$ & $2.07 \mathrm{E}+07$ & Direct \\
\hline $\mathrm{Hf}-178 \mathrm{~m}$ & & & & $3.60 \mathrm{E}+01$ & $4.21 \mathrm{E}+02$ & $1.72 \mathrm{E}+02$ & $3.08 \mathrm{E}+02$ & -- & $3.60 \mathrm{E}+01$ & $5.56 \mathrm{E}-01$ & $6.48 \mathrm{E}+01$ & Inh. \\
\hline
\end{tabular}


Table C.2 Summary of Exposure Pathway TQs and Selection of Final HC-3 TQs (continued)

\begin{tabular}{|c|c|c|c|c|c|c|c|c|c|c|c|c|}
\hline \multirow{2}{*}{$\begin{array}{c}\text { MASTER } \\
\text { ISOTOPE } \\
\text { LIST } \\
\end{array}$} & \multicolumn{3}{|c|}{ from NNSA SD G 1027} & \multicolumn{5}{|c|}{ Calculated Pathway Specific TQ Values } & \multicolumn{4}{|c|}{ Selected Final Threshold Quantities } \\
\hline & Act (Ci) & Mass (g) & $\begin{array}{l}\text { Limiting } \\
\text { Pathway }\end{array}$ & $\begin{array}{c}\mathrm{TQ}_{\text {inhale }} \\
(\mathrm{Ci}) \\
\end{array}$ & $\begin{array}{c}\mathrm{TQ}_{\text {water }} \\
\text { (Ci) }\end{array}$ & $\mathrm{TQ}_{\text {food }}(\mathrm{Ci})$ & $\begin{array}{c}\mathrm{TQ}_{\text {direct }} \\
(\mathrm{Ci}) \\
\end{array}$ & $\mathrm{TQ}_{\text {sub }}(\mathrm{Ci})$ & Act (Ci) & Mass (g) & $\begin{array}{c}\text { Sp. Act. } \\
\text { (Ci/g) }\end{array}$ & $\begin{array}{l}\text { Limiting } \\
\text { Pathway }\end{array}$ \\
\hline $\mathrm{Hf}-179 \mathrm{~m}$ & & & & $3.10 \mathrm{E}+03$ & $2.20 \mathrm{E}+03$ & $1.00 \mathrm{E}+03$ & $8.17 \mathrm{E}+02$ & -- & $8.17 \mathrm{E}+02$ & $2.81 \mathrm{E}-02$ & $2.91 \mathrm{E}+04$ & Direct \\
\hline $\mathrm{Hf}-180 \mathrm{~m}$ & & & & $5.58 \mathrm{E}+04$ & $2.88 \mathrm{E}+13$ & $2.79 \mathrm{E}+05$ & $2.29 \mathrm{E}+03$ & -- & $2.29 \mathrm{E}+03$ & $7.23 \mathrm{E}-04$ & $3.17 \mathrm{E}+06$ & Direct \\
\hline $\mathrm{Hf}-181$ & $9.28 \mathrm{E}+02$ & $5.45 \mathrm{E}-02$ & ing & $2.38 \mathrm{E}+03$ & $2.13 \mathrm{E}+03$ & $9.43 \mathrm{E}+02$ & $1.32 \mathrm{E}+03$ & -- & $9.43 \mathrm{E}+02$ & $5.54 \mathrm{E}-02$ & $1.70 \mathrm{E}+04$ & Food \\
\hline $\mathrm{Hf}-182$ & & & & $3.10 \mathrm{E}+01$ & $6.59 \mathrm{E}+02$ & $2.69 \mathrm{E}+02$ & $3.03 \mathrm{E}+03$ & -- & $3.10 \mathrm{E}+01$ & $1.42 \mathrm{E}+05$ & $2.18 \mathrm{E}-04$ & Inh. \\
\hline $\mathrm{Hf}-182 \mathrm{~m}$ & & & & $1.57 \mathrm{E}+05$ & $3.52 \mathrm{E}+49$ & $5.99 \mathrm{E}+06$ & $1.26 \mathrm{E}+04$ & -- & $1.26 \mathrm{E}+04$ & $7.52 \mathrm{E}-04$ & $1.68 \mathrm{E}+07$ & Direct \\
\hline Hf-183 & & & & $1.35 \mathrm{E}+05$ & $4.17 \mathrm{E}+47$ & $3.31 \mathrm{E}+06$ & $1.51 \mathrm{E}+04$ & -- & $1.51 \mathrm{E}+04$ & $9.38 \mathrm{E}-04$ & $1.61 \mathrm{E}+07$ & Direct \\
\hline Hf-184 & & & & $2.48 \mathrm{E}+04$ & $5.83 \mathrm{E}+15$ & $1.21 \mathrm{E}+05$ & $1.19 \mathrm{E}+04$ & -- & $1.19 \mathrm{E}+04$ & $2.88 \mathrm{E}-03$ & $4.13 E+06$ & Direct \\
\hline Hg-187 & & & & -- & -- & -- & -- & -- & -- & -- & $4.57 \mathrm{E}+08$ & -- \\
\hline $\mathrm{Hg}-187 \mathrm{~m}$ & & & & -- & -- & -- & -- & -- & -- & -- & $4.19 \mathrm{E}+08$ & -- \\
\hline $\mathrm{Hg}-188$ & & & & -- & -- & -- & -- & -- & -- & -- & $3.08 \mathrm{E}+08$ & -- \\
\hline $\mathrm{Hg}-190$ & & & & $1.16 \mathrm{E}+05$ & $7.60 \mathrm{E}+138$ & $2.99 \mathrm{E}+07$ & $1.80 \mathrm{E}+05$ & -- & $1.16 \mathrm{E}+05$ & $2.35 \mathrm{E}-03$ & $4.95 \mathrm{E}+07$ & Inh. \\
\hline $\mathrm{Hg}-191 \mathrm{~m}$ & & & & $3.49 \mathrm{E}+04$ & $3.22 \mathrm{E}+58$ & $5.36 \mathrm{E}+06$ & $9.58 \mathrm{E}+03$ & -- & $9.58 \mathrm{E}+03$ & $4.94 \mathrm{E}-04$ & $1.94 \mathrm{E}+07$ & Direct \\
\hline Hg-192 & & & & $1.12 \mathrm{E}+04$ & $2.82 \mathrm{E}+14$ & $2.27 \mathrm{E}+05$ & $9.36 \mathrm{E}+03$ & -- & $9.36 \mathrm{E}+03$ & $2.78 \mathrm{E}-03$ & $3.37 \mathrm{E}+06$ & Direct \\
\hline $\mathrm{Hg}-193$ & & & & $1.02 \mathrm{E}+04$ & $3.32 \mathrm{E}+18$ & $8.77 \mathrm{E}+05$ & $1.72 \mathrm{E}+04$ & -- & $1.02 \mathrm{E}+04$ & $2.19 \mathrm{E}-03$ & $4.64 \mathrm{E}+06$ & Inh. \\
\hline $\mathrm{Hg}-193 \mathrm{~m}$ & & & & $3.60 \mathrm{E}+03$ & $5.85 \mathrm{E}+08$ & $5.80 \mathrm{E}+04$ & $1.34 \mathrm{E}+03$ & -- & $1.34 \mathrm{E}+03$ & $9.16 \mathrm{E}-04$ & $1.46 \mathrm{E}+06$ & Direct \\
\hline $\mathrm{Hg}-194$ & & & & $2.79 \mathrm{E}+02$ & $3.87 \mathrm{E}+01$ & $1.53 \mathrm{E}+01$ & $2.69 \mathrm{E}+05$ & -- & $1.53 \mathrm{E}+01$ & $2.16 \mathrm{E}+00$ & $7.09 \mathrm{E}+00$ & Food \\
\hline $\mathrm{Hg}-195$ & & & & $7.98 \mathrm{E}+03$ & $8.14 \mathrm{E}+09$ & $2.67 \mathrm{E}+05$ & $7.36 \mathrm{E}+03$ & -- & $7.36 \mathrm{E}+03$ & $4.53 \mathrm{E}-03$ & $1.62 \mathrm{E}+06$ & Direct \\
\hline $\mathrm{Hg}-195 \mathrm{~m}$ & & & & $1.36 \mathrm{E}+03$ & $1.47 \mathrm{E}+05$ & $1.20 \mathrm{E}+04$ & $4.12 \mathrm{E}+03$ & -- & $1.36 \mathrm{E}+03$ & $3.53 \mathrm{E}-03$ & $3.86 \mathrm{E}+05$ & Inh. \\
\hline $\mathrm{Hg}-197$ & & & & $2.54 \mathrm{E}+03$ & $1.07 \mathrm{E}+05$ & $2.01 \mathrm{E}+04$ & $1.18 \mathrm{E}+04$ & -- & $2.54 \mathrm{E}+03$ & $1.02 \mathrm{E}-02$ & $2.48 \mathrm{E}+05$ & Inh. \\
\hline $\mathrm{Hg}-197 \mathrm{~m}$ & & & & $1.93 \mathrm{E}+03$ & $1.83 \mathrm{E}+06$ & $2.38 \mathrm{E}+04$ & $1.07 \mathrm{E}+04$ & -- & $1.93 \mathrm{E}+03$ & $2.88 \mathrm{E}-03$ & $6.68 \mathrm{E}+05$ & Inh. \\
\hline $\mathrm{Hg}-199 \mathrm{~m}$ & & & & $6.20 \mathrm{E}+04$ & $6.11 \mathrm{E}+68$ & $1.13 \mathrm{E}+07$ & $9.14 \mathrm{E}+04$ & -- & $6.20 \mathrm{E}+04$ & $2.80 \mathrm{E}-03$ & $2.22 \mathrm{E}+07$ & Inh. \\
\hline $\mathrm{Hg}-203$ & $5.06 \mathrm{E}+02$ & $3.67 \mathrm{E}-02$ & ing & $1.60 \mathrm{E}+03$ & $1.22 \mathrm{E}+03$ & $5.18 \mathrm{E}+02$ & $3.07 E+03$ & -- & $5.18 \mathrm{E}+02$ & $3.75 \mathrm{E}-02$ & $1.38 \mathrm{E}+04$ & Food \\
\hline $\mathrm{Hg}-205$ & & & & -- & -- & -- & $2.61 \mathrm{E}+07$ & -- & $2.61 \mathrm{E}+07$ & $1.48 \mathrm{E}-01$ & $1.76 \mathrm{E}+08$ & Direct \\
\hline $\mathrm{Hg}-206$ & & & & -- & -- & -- & $8.41 \mathrm{E}+05$ & -- & $8.41 \mathrm{E}+05$ & 7.51E-03 & $1.12 \mathrm{E}+08$ & Direct \\
\hline $\mathrm{Hg}-207$ & & & & -- & -- & -- & $9.39 \mathrm{E}+04$ & -- & $9.39 \mathrm{E}+04$ & $3.00 \mathrm{E}-04$ & $3.13 \mathrm{E}+08$ & Direct \\
\hline Ho-150 & & & & -- & -- & -- & $3.00 \mathrm{E}+05$ & -- & $3.00 \mathrm{E}+05$ & $3.07 \mathrm{E}-04$ & $9.80 \mathrm{E}+08$ & Direct \\
\hline Ho-152 & & & & -- & -- & -- & -- & -- & -- & -- & $4.59 \mathrm{E}+08$ & -- \\
\hline Ho-153 & & & & -- & -- & -- & $3.51 \mathrm{E}+05$ & -- & $3.51 \mathrm{E}+05$ & $5.73 \mathrm{E}-04$ & $6.12 \mathrm{E}+08$ & Direct \\
\hline Ho-153m & & & & -- & -- & -- & $7.34 \mathrm{E}+04$ & -- & $7.34 \mathrm{E}+04$ & $5.55 \mathrm{E}-04$ & $1.32 \mathrm{E}+08$ & Direct \\
\hline Ho-154 & & & & $4.14 \mathrm{E}+05$ & -- & $3.20 \mathrm{E}+07$ & $3.27 \mathrm{E}+04$ & -- & $3.27 \mathrm{E}+04$ & $3.15 \mathrm{E}-04$ & $1.04 \mathrm{E}+08$ & Direct \\
\hline Ho-154m & & & & -- & -- & -- & $9.59 \mathrm{E}+04$ & -- & $9.59 \mathrm{E}+04$ & $2.43 \mathrm{E}-04$ & $3.94 \mathrm{E}+08$ & Direct \\
\hline Ho-155 & & & & $3.49 \mathrm{E}+05$ & -- & $8.70 \mathrm{E}+06$ & $3.90 \mathrm{E}+04$ & -- & $3.90 \mathrm{E}+04$ & $1.54 \mathrm{E}-03$ & $2.53 \mathrm{E}+07$ & Direct \\
\hline Ho-156 & & & & $1.23 \mathrm{E}+05$ & -- & $3.07 \mathrm{E}+07$ & $6.14 \mathrm{E}+03$ & -- & $6.14 \mathrm{E}+03$ & $2.85 \mathrm{E}-04$ & $2.15 \mathrm{E}+07$ & Direct \\
\hline Ho-157 & & & & $1.47 \mathrm{E}+06$ & -- & $1.88 \mathrm{E}+08$ & $1.17 \mathrm{E}+05$ & -- & $1.17 \mathrm{E}+05$ & $1.23 \mathrm{E}-03$ & $9.51 \mathrm{E}+07$ & Direct \\
\hline Ho-158 & & & & $7.98 \mathrm{E}+05$ & -- & $1.53 \mathrm{E}+08$ & -- & -- & $7.98 \mathrm{E}+05$ & 7.57E-03 & $1.05 \mathrm{E}+08$ & Inh. \\
\hline
\end{tabular}


Table C.2 Summary of Exposure Pathway TQs and Selection of Final HC-3 TQs (continued)

\begin{tabular}{|c|c|c|c|c|c|c|c|c|c|c|c|c|}
\hline \multirow[b]{2}{*}{$\begin{array}{c}\text { MASTER } \\
\text { ISOTOPE } \\
\text { LIST } \\
\end{array}$} & \multicolumn{3}{|c|}{ from NNSA SD G 1027} & \multicolumn{5}{|c|}{ Calculated Pathway Specific TQ Values } & \multicolumn{4}{|c|}{ Selected Final Threshold Quantities } \\
\hline & Act $(\mathrm{Ci})$ & Mass (g) & $\begin{array}{l}\text { Limiting } \\
\text { Pathway }\end{array}$ & $\begin{array}{c}\text { TQ }_{\text {inhale }} \\
(\mathrm{Ci})\end{array}$ & $\begin{array}{c}\text { TQ }_{\text {water }} \\
\text { (Ci) }\end{array}$ & $\mathrm{TQ}_{\text {food }}(\mathrm{Ci})$ & $\begin{array}{c}T_{\text {direct }} \\
\text { (Ci) }\end{array}$ & $\mathrm{TQ}_{\text {sub }}(\mathrm{Ci})$ & Act (Ci) & Mass (g) & $\begin{array}{c}\text { Sp. Act. } \\
\text { (Ci/g) }\end{array}$ & $\begin{array}{l}\text { Limiting } \\
\text { Pathway }\end{array}$ \\
\hline Ho-159 & & & & $1.12 \mathrm{E}+06$ & -- & $5.92 \mathrm{E}+07$ & $6.00 \mathrm{E}+04$ & -- & $6.00 E+04$ & $1.67 \mathrm{E}-03$ & $3.59 \mathrm{E}+07$ & Direct \\
\hline Ho-160 & & & & $4.65 \mathrm{E}+05$ & -- & $3.55 \mathrm{E}+07$ & $1.67 \mathrm{E}+04$ & -- & $1.67 \mathrm{E}+04$ & $3.64 \mathrm{E}-04$ & $4.59 \mathrm{E}+07$ & Direct \\
\hline Ho-161 & & & & $1.12 \mathrm{E}+06$ & -- & $7.96 \mathrm{E}+06$ & $7.86 \mathrm{E}+04$ & -- & $7.86 \mathrm{E}+04$ & $1.01 \mathrm{E}-02$ & $7.79 \mathrm{E}+06$ & Direct \\
\hline Ho-162 & & & & $2.48 \mathrm{E}+06$ & -- & $3.12 \mathrm{E}+08$ & $2.88 \mathrm{E}+05$ & -- & $2.88 \mathrm{E}+05$ & $3.72 \mathrm{E}-03$ & $7.74 \mathrm{E}+07$ & Direct \\
\hline Ho-162m & & & & $3.38 \mathrm{E}+05$ & -- & $8.75 E+06$ & $1.85 \mathrm{E}+04$ & -- & $1.85 \mathrm{E}+04$ & $1.08 \mathrm{E}-03$ & $1.71 \mathrm{E}+07$ & Direct \\
\hline Ho-163 & & & & $4.65 \mathrm{E}+04$ & -- & $1.18 \mathrm{E}+05$ & $1.21 \mathrm{E}+07$ & -- & $4.65 \mathrm{E}+04$ & $9.69 \mathrm{E}+04$ & $4.80 \mathrm{E}-01$ & Inh. \\
\hline Ho-164 & & & & $8.59 \mathrm{E}+05$ & -- & $5.60 \mathrm{E}+07$ & $8.44 \mathrm{E}+05$ & -- & $8.44 \mathrm{E}+05$ & $2.13 \mathrm{E}-02$ & $3.96 \mathrm{E}+07$ & Direct \\
\hline Ho-164m & & & & $6.98 \mathrm{E}+05$ & -- & $2.57 \mathrm{E}+07$ & $4.09 \mathrm{E}+05$ & -- & $4.09 \mathrm{E}+05$ & $1.34 \mathrm{E}-02$ & $3.06 \mathrm{E}+07$ & Direct \\
\hline Ho-166 & & & & $1.35 \mathrm{E}+04$ & -- & $7.39 \mathrm{E}+03$ & $3.36 \mathrm{E}+04$ & -- & $7.39 \mathrm{E}+03$ & $1.05 \mathrm{E}-02$ & $7.05 \mathrm{E}+05$ & Food \\
\hline Ho-166m & $1.02 \mathrm{E}+02$ & $5.70 \mathrm{E}+01$ & $\mathrm{i}$ & $1.02 \mathrm{E}+02$ & -- & $4.03 E+02$ & $4.16 \mathrm{E}+02$ & -- & $1.02 \mathrm{E}+02$ & $5.66 \mathrm{E}+01$ & $1.80 \mathrm{E}+00$ & Inh. \\
\hline Ho-167 & & & & $1.12 \mathrm{E}+05$ & -- & $1.01 \mathrm{E}+06$ & $1.07 E+04$ & -- & $1.07 \mathrm{E}+04$ & 1.77E-03 & $6.06 \mathrm{E}+06$ & Direct \\
\hline Ho-168 & & & & -- & -- & -- & $2.77 \mathrm{E}+05$ & -- & $2.77 \mathrm{E}+05$ & 7.39E-04 & $3.74 \mathrm{E}+08$ & Direct \\
\hline Ho-168m & & & & -- & -- & -- & $4.53 \mathrm{E}+07$ & -- & $4.53 \mathrm{E}+07$ & $8.90 \mathrm{E}-02$ & $5.09 \mathrm{E}+08$ & Direct \\
\hline Ho-170 & & & & -- & -- & -- & $1.54 \mathrm{E}+05$ & -- & $1.54 \mathrm{E}+05$ & $3.84 \mathrm{E}-04$ & $4.01 \mathrm{E}+08$ & Direct \\
\hline $\mathrm{I}-118$ & & & & $1.31 \mathrm{E}+03$ & -- & $1.12 \mathrm{E}+05$ & $2.62 E+04$ & -- & $1.31 \mathrm{E}+03$ & 1.13E-05 & $1.16 \mathrm{E}+08$ & Inh. \\
\hline $\mathrm{I}-118 \mathrm{~m}$ & & & & -- & -- & -- & $2.27 E+04$ & -- & $2.27 \mathrm{E}+04$ & $1.21 \mathrm{E}-04$ & $1.88 \mathrm{E}+08$ & Direct \\
\hline $\mathrm{I}-119$ & & & & $3.99 \mathrm{E}+03$ & -- & $3.49 \mathrm{E}+05$ & $4.16 \mathrm{E}+04$ & -- & $3.99 E+03$ & $4.82 \mathrm{E}-05$ & $8.28 \mathrm{E}+07$ & Inh. \\
\hline $\mathrm{I}-120$ & & & & $7.45 \mathrm{E}+02$ & -- & $1.12 \mathrm{E}+04$ & $3.28 \mathrm{E}+03$ & -- & $7.45 \mathrm{E}+02$ & $3.85 \mathrm{E}-05$ & $1.94 \mathrm{E}+07$ & Inh. \\
\hline $\mathrm{I}-120 \mathrm{~m}$ & & & & $1.24 \mathrm{E}+03$ & -- & $2.76 \mathrm{E}+04$ & $2.58 \mathrm{E}+03$ & -- & $1.24 \mathrm{E}+03$ & 4.19E-05 & $2.96 \mathrm{E}+07$ & Inh. \\
\hline $\mathrm{I}-121$ & & & & $2.60 \mathrm{E}+03$ & -- & $2.96 \mathrm{E}+04$ & $1.36 \mathrm{E}+04$ & -- & $2.60 E+03$ & $2.12 \mathrm{E}-04$ & $1.22 \mathrm{E}+07$ & Inh. \\
\hline $\mathrm{I}-122$ & & & & -- & -- & -- & $2.12 \mathrm{E}+05$ & -- & $2.12 E+05$ & 4.97E-04 & $4.26 \mathrm{E}+08$ & Direct \\
\hline $\mathrm{I}-123$ & & & & $1.06 \mathrm{E}+03$ & -- & $1.92 \mathrm{E}+03$ & $7.44 \mathrm{E}+03$ & -- & $1.06 \mathrm{E}+03$ & 5.51E-04 & $1.93 \mathrm{E}+06$ & Inh. \\
\hline $\mathrm{I}-124$ & & & & $1.86 \mathrm{E}+01$ & -- & $5.09 \mathrm{E}+00$ & $7.17 \mathrm{E}+02$ & -- & $5.09 \mathrm{E}+00$ & $2.02 \mathrm{E}-05$ & $2.52 \mathrm{E}+05$ & Food \\
\hline $\mathrm{I}-125$ & $1.26 \mathrm{E}+00$ & 7.17E-05 & ing & $1.60 \mathrm{E}+01$ & -- & $1.28 \mathrm{E}+00$ & $1.74 \mathrm{E}+04$ & -- & $1.28 \mathrm{E}+00$ & $7.38 \mathrm{E}-05$ & $1.74 \mathrm{E}+04$ & Food \\
\hline $\mathrm{I}-126$ & & & & $8.59 \mathrm{E}+00$ & -- & $1.09 \mathrm{E}+00$ & $1.64 \mathrm{E}+03$ & -- & $1.09 \mathrm{E}+00$ & 1.37E-05 & $7.97 \mathrm{E}+04$ & Food \\
\hline $\mathrm{I}-128$ & & & & $3.44 \mathrm{E}+03$ & -- & $2.67 E+05$ & $3.41 \mathrm{E}+05$ & -- & $3.44 \mathrm{E}+03$ & 5.84E-05 & $5.88 \mathrm{E}+07$ & Inh. \\
\hline $\mathrm{I}-129$ & & & & $2.33 \mathrm{E}+00$ & -- & $1.46 \mathrm{E}-01$ & $2.95 \mathrm{E}+04$ & -- & $1.46 \mathrm{E}-01$ & $8.25 \mathrm{E}+02$ & 1.77E-04 & Food \\
\hline $\mathrm{I}-130$ & & & & $1.18 \mathrm{E}+02$ & -- & $2.14 \mathrm{E}+02$ & $6.18 \mathrm{E}+02$ & -- & $1.18 \mathrm{E}+02$ & $6.02 \mathrm{E}-05$ & $1.95 \mathrm{E}+06$ & Inh. \\
\hline $\mathrm{I}-130 \mathrm{~m}$ & & & & -- & -- & -- & $7.47 \mathrm{E}+05$ & -- & $7.47 E+05$ & $4.57 \mathrm{E}-03$ & $1.64 \mathrm{E}+08$ & Direct \\
\hline $\mathrm{I}-131$ & $1.93 \mathrm{E}+00$ & $1.56 \mathrm{E}-05$ & ing & $1.12 \mathrm{E}+01$ & -- & $1.90 \mathrm{E}+00$ & $1.99 \mathrm{E}+03$ & -- & $1.90 \mathrm{E}+00$ & $1.53 \mathrm{E}-05$ & $1.24 \mathrm{E}+05$ & Food \\
\hline $\mathrm{I}-132$ & & & & $7.21 \mathrm{E}+02$ & -- & $7.72 \mathrm{E}+03$ & $2.30 \mathrm{E}+03$ & -- & $7.21 \mathrm{E}+02$ & $6.98 \mathrm{E}-05$ & $1.03 \mathrm{E}+07$ & Inh. \\
\hline $\mathrm{I}-132 \mathrm{~m}$ & & & & $8.27 E+02$ & -- & $1.67 \mathrm{E}+04$ & $2.69 \mathrm{E}+04$ & -- & $8.27 \mathrm{E}+02$ & $4.85 \mathrm{E}-05$ & $1.71 \mathrm{E}+07$ & Inh. \\
\hline $\mathrm{I}-133$ & & & & $5.58 \mathrm{E}+01$ & -- & $6.07 \mathrm{E}+01$ & $1.74 \mathrm{E}+03$ & -- & $5.58 \mathrm{E}+01$ & $4.93 \mathrm{E}-05$ & $1.13 \mathrm{E}+06$ & Inh. \\
\hline $\mathrm{I}-134$ & & & & $1.49 \mathrm{E}+03$ & -- & $5.31 \mathrm{E}+04$ & $5.25 \mathrm{E}+03$ & -- & $1.49 E+03$ & $5.58 \mathrm{E}-05$ & $2.67 \mathrm{E}+07$ & Inh. \\
\hline $\mathrm{I}-134 \mathrm{~m}$ & & & & -- & -- & -- & $6.97 E+05$ & -- & $6.97 \mathrm{E}+05$ & 1.79E-03 & $3.90 \mathrm{E}+08$ & Direct \\
\hline
\end{tabular}


Table C.2 Summary of Exposure Pathway TQs and Selection of Final HC-3 TQs (continued)

\begin{tabular}{|c|c|c|c|c|c|c|c|c|c|c|c|c|}
\hline \multirow[b]{2}{*}{$\begin{array}{c}\text { MASTER } \\
\text { ISOTOPE } \\
\text { LIST } \\
\end{array}$} & \multicolumn{3}{|c|}{ from NNSA SD G 1027} & \multicolumn{5}{|c|}{ Calculated Pathway Specific TQ Values } & \multicolumn{4}{|c|}{ Selected Final Threshold Quantities } \\
\hline & Act $(\mathrm{Ci})$ & Mass (g) & $\begin{array}{l}\text { Limiting } \\
\text { Pathway }\end{array}$ & $\begin{array}{c}\text { TQ }_{\text {inhale }} \\
(\mathrm{Ci})\end{array}$ & $\begin{array}{c}\text { TQ }_{\text {water }} \\
\text { (Ci) }\end{array}$ & $\mathrm{TQ}_{\text {food }}(\mathrm{Ci})$ & $\begin{array}{c}\mathrm{TQ}_{\text {direct }} \\
\text { (Ci) }\end{array}$ & $\mathrm{TQ}_{\text {sub }}(\mathrm{Ci})$ & Act (Ci) & Mass (g) & $\begin{array}{c}\text { Sp. Act. } \\
\text { (Ci/g) }\end{array}$ & $\begin{array}{l}\text { Limiting } \\
\text { Pathway }\end{array}$ \\
\hline $\mathrm{I}-135$ & & & & $2.43 E+02$ & -- & $8.48 \mathrm{E}+02$ & $1.26 \mathrm{E}+03$ & -- & $2.43 E+02$ & $6.91 \mathrm{E}-05$ & $3.51 \mathrm{E}+06$ & Inh. \\
\hline $\ln -103$ & & & & -- & -- & -- & $2.63 \mathrm{E}+05$ & -- & $2.63 \mathrm{E}+05$ & $1.44 \mathrm{E}-04$ & $1.83 \mathrm{E}+09$ & Direct \\
\hline $\ln -105$ & & & & -- & -- & -- & $7.40 \mathrm{E}+04$ & -- & $7.40 \mathrm{E}+04$ & $2.09 \mathrm{E}-04$ & $3.53 \mathrm{E}+08$ & Direct \\
\hline $\ln -106$ & & & & -- & -- & -- & $3.29 \mathrm{E}+04$ & -- & $3.29 \mathrm{E}+04$ & $1.15 \mathrm{E}-04$ & $2.86 \mathrm{E}+08$ & Direct \\
\hline In-106m & & & & -- & -- & -- & $4.93 \mathrm{E}+04$ & -- & $4.93 E+04$ & $1.45 \mathrm{E}-04$ & $3.41 \mathrm{E}+08$ & Direct \\
\hline In-107 & & & & $2.72 \mathrm{E}+05$ & $1.56 \mathrm{E}+88$ & $1.16 \mathrm{E}+07$ & $1.46 \mathrm{E}+04$ & -- & $1.46 \mathrm{E}+04$ & $2.69 \mathrm{E}-04$ & $5.43 \mathrm{E}+07$ & Direct \\
\hline $\ln -108$ & & & & $1.21 \mathrm{E}+05$ & $7.13 \mathrm{E}+51$ & $3.21 \mathrm{E}+06$ & $3.19 E+03$ & -- & $3.19 E+03$ & $1.06 \mathrm{E}-04$ & $3.00 \mathrm{E}+07$ & Direct \\
\hline In-108m & & & & $1.72 \mathrm{E}+05$ & $1.12 \mathrm{E}+73$ & $4.59 \mathrm{E}+06$ & $6.62 \mathrm{E}+03$ & -- & $6.62 \mathrm{E}+03$ & 1.50E-04 & $4.40 \mathrm{E}+07$ & Direct \\
\hline In-109 & & & & $1.53 \mathrm{E}+05$ & $2.83 \mathrm{E}+16$ & $9.38 \mathrm{E}+05$ & $4.36 \mathrm{E}+03$ & -- & $4.36 \mathrm{E}+03$ & $6.36 \mathrm{E}-04$ & $6.85 \mathrm{E}+06$ & Direct \\
\hline In-109m & & & & -- & -- & -- & $8.89 \mathrm{E}+05$ & -- & $8.89 E+05$ & $6.90 \mathrm{E}-04$ & $1.29 \mathrm{E}+09$ & Direct \\
\hline In-110| & & & & $4.47 \mathrm{E}+04$ & $2.16 \mathrm{E}+14$ & $2.22 \mathrm{E}+05$ & $8.36 \mathrm{E}+02$ & -- & $8.36 \mathrm{E}+02$ & $1.44 \mathrm{E}-04$ & $5.82 \mathrm{E}+06$ & Direct \\
\hline $\ln -110 \mathrm{~s}$ & & & & $1.38 \mathrm{E}+05$ & $2.64 \mathrm{E}+44$ & $2.24 \mathrm{E}+06$ & $6.74 \mathrm{E}+03$ & -- & $6.74 \mathrm{E}+03$ & $2.72 \mathrm{E}-04$ & $2.48 \mathrm{E}+07$ & Direct \\
\hline In-111 & & & & $3.60 \mathrm{E}+04$ & $7.45 \mathrm{E}+04$ & $1.57 \mathrm{E}+04$ & $2.02 \mathrm{E}+03$ & -- & $2.02 E+03$ & $4.85 \mathrm{E}-03$ & $4.16 \mathrm{E}+05$ & Direct \\
\hline In-111m & & & & -- & -- & -- & $2.00 \mathrm{E}+05$ & -- & $2.00 \mathrm{E}+05$ & $9.10 \mathrm{E}-04$ & $2.20 \mathrm{E}+08$ & Direct \\
\hline In-112 & & & & $8.59 \mathrm{E}+05$ & $3.12 \mathrm{E}+190$ & $1.07 \mathrm{E}+08$ & $1.88 \mathrm{E}+05$ & -- & $1.88 \mathrm{E}+05$ & $1.61 \mathrm{E}-03$ & $1.17 \mathrm{E}+08$ & Direct \\
\hline In-112m & & & & $3.10 \mathrm{E}+05$ & $2.88 \mathrm{E}+135$ & $4.41 \mathrm{E}+07$ & $1.01 \mathrm{E}+06$ & -- & $3.10 \mathrm{E}+05$ & $3.80 \mathrm{E}-03$ & $8.17 \mathrm{E}+07$ & Inh. \\
\hline In-113m & & & & $3.49 \mathrm{E}+05$ & $1.61 \mathrm{E}+33$ & $5.56 \mathrm{E}+06$ & $2.83 \mathrm{E}+04$ & -- & $2.83 E+04$ & $1.69 \mathrm{E}-03$ & $1.67 \mathrm{E}+07$ & Direct \\
\hline In-114 & & & & -- & -- & -- & $2.24 \mathrm{E}+08$ & -- & $2.24 \mathrm{E}+08$ & $1.63 \mathrm{E}-01$ & $1.38 \mathrm{E}+09$ & Direct \\
\hline In-114m & $2.40 \mathrm{E}+02$ & $1.04 \mathrm{E}-02$ & ing & $1.02 \mathrm{E}+03$ & $5.58 \mathrm{E}+02$ & $2.45 \mathrm{E}+02$ & $7.74 \mathrm{E}+03$ & -- & $2.45 \mathrm{E}+02$ & $1.06 \mathrm{E}-02$ & $2.32 \mathrm{E}+04$ & Food \\
\hline In-115 & & & & $2.48 \mathrm{E}+01$ & $6.17 \mathrm{E}+01$ & $2.52 \mathrm{E}+01$ & -- & -- & $2.48 \mathrm{E}+01$ & $4.07 \mathrm{E}+13$ & $6.10 \mathrm{E}-13$ & Inh. \\
\hline In-115m & & & & $1.28 \mathrm{E}+05$ & $4.40 \mathrm{E}+15$ & $6.75 \mathrm{E}+05$ & $1.71 \mathrm{E}+04$ & -- & $1.71 \mathrm{E}+04$ & $2.82 \mathrm{E}-03$ & $6.08 \mathrm{E}+06$ & Direct \\
\hline In-116m & & & & $1.40 \mathrm{E}+05$ & $1.64 \mathrm{E}+55$ & $4.46 \mathrm{E}+06$ & $5.41 \mathrm{E}+03$ & -- & $5.41 E+03$ & $1.81 \mathrm{E}-04$ & $3.00 \mathrm{E}+07$ & Direct \\
\hline In-117 & & & & $2.33 \mathrm{E}+05$ & $1.22 \mathrm{E}+67$ & $1.14 \mathrm{E}+07$ & $2.39 \mathrm{E}+04$ & -- & $2.39 E+04$ & $6.51 \mathrm{E}-04$ & $3.67 \mathrm{E}+07$ & Direct \\
\hline In-117m & & & & $1.02 \mathrm{E}+05$ & $4.46 \mathrm{E}+28$ & $1.11 \mathrm{E}+06$ & $6.84 \mathrm{E}+04$ & -- & $6.84 \mathrm{E}+04$ & $4.96 \mathrm{E}-03$ & $1.38 \mathrm{E}+07$ & Direct \\
\hline In-118 & & & & -- & -- & -- & $1.12 \mathrm{E}+08$ & -- & $1.12 \mathrm{E}+08$ & $5.84 \mathrm{E}-03$ & $1.91 \mathrm{E}+10$ & Direct \\
\hline In-118m & & & & -- & -- & -- & $5.98 \mathrm{E}+04$ & -- & $5.98 \mathrm{E}+04$ & $1.64 \mathrm{E}-04$ & $3.65 \mathrm{E}+08$ & Direct \\
\hline $\ln -119$ & & & & -- & -- & -- & $3.93 E+05$ & -- & $3.93 E+05$ & $5.96 \mathrm{E}-04$ & $6.59 \mathrm{E}+08$ & Direct \\
\hline In-119m & & & & $3.85 \mathrm{E}+05$ & $1.76 \mathrm{E}+153$ & $1.82 \mathrm{E}+07$ & $3.59 \mathrm{E}+06$ & -- & $3.85 \mathrm{E}+05$ & $4.38 \mathrm{E}-03$ & $8.78 \mathrm{E}+07$ & Inh. \\
\hline In-121 & & & & -- & -- & -- & $2.03 E+06$ & -- & $2.03 E+06$ & $5.03 \mathrm{E}-04$ & $4.04 \mathrm{E}+09$ & Direct \\
\hline In-121m & & & & -- & -- & -- & $2.94 \mathrm{E}+06$ & -- & $2.94 \mathrm{E}+06$ & $7.34 \mathrm{E}-03$ & $4.01 \mathrm{E}+08$ & Direct \\
\hline Ir-179 & & & & -- & -- & -- & -- & -- & -- & -- & $7.98 \mathrm{E}+08$ & -- \\
\hline Ir-180 & & & & -- & -- & -- & $3.03 E+05$ & -- & $3.03 E+05$ & $4.35 \mathrm{E}-04$ & $6.97 \mathrm{E}+08$ & Direct \\
\hline Ir-181 & & & & -- & -- & -- & -- & -- & -- & -- & $2.12 \mathrm{E}+08$ & -- \\
\hline Ir-182 & & & & $2.79 E+06$ & $3.16 \mathrm{E}+182$ & $2.14 \mathrm{E}+08$ & $3.61 E+04$ & -- & $3.61 E+04$ & $5.23 \mathrm{E}-04$ & $6.89 \mathrm{E}+07$ & Direct \\
\hline Ir-183 & & & & $1.75 \mathrm{E}+06$ & $1.08 \mathrm{E}+52$ & $4.84 \mathrm{E}+07$ & $1.05 \mathrm{E}+04$ & -- & $1.05 \mathrm{E}+04$ & $5.93 \mathrm{E}-04$ & $1.77 \mathrm{E}+07$ & Direct \\
\hline
\end{tabular}


Table C.2 Summary of Exposure Pathway TQs and Selection of Final HC-3 TQs (continued)

\begin{tabular}{|c|c|c|c|c|c|c|c|c|c|c|c|c|}
\hline \multirow[b]{2}{*}{$\begin{array}{c}\text { MASTER } \\
\text { ISOTOPE } \\
\text { LIST } \\
\end{array}$} & \multicolumn{3}{|c|}{ from NNSA SD G 1027} & \multicolumn{5}{|c|}{ Calculated Pathway Specific TQ Values } & \multicolumn{4}{|c|}{ Selected Final Threshold Quantities } \\
\hline & Act $(\mathrm{Ci})$ & Mass (g) & $\begin{array}{l}\text { Limiting } \\
\text { Pathway }\end{array}$ & $\begin{array}{c}\text { TQ }_{\text {inhale }} \\
(\mathrm{Ci})\end{array}$ & $\begin{array}{c}\mathrm{TQ}_{\text {water }} \\
\text { (Ci) }\end{array}$ & $\mathrm{TQ}_{\text {food }}(\mathrm{Ci})$ & $\begin{array}{c}T_{\text {direct }} \\
\text { (Ci) }\end{array}$ & $\mathrm{TQ}_{\text {sub }}(\mathrm{Ci})$ & Act (Ci) & Mass (g) & $\begin{array}{c}\text { Sp. Act. } \\
\text { (Ci/g) }\end{array}$ & $\begin{array}{l}\text { Limiting } \\
\text { Pathway }\end{array}$ \\
\hline Ir-184 & & & & $5.88 \mathrm{E}+05$ & $1.80 \mathrm{E}+20$ & $5.04 \mathrm{E}+06$ & $2.10 \mathrm{E}+03$ & -- & $2.10 E+03$ & $3.73 \mathrm{E}-04$ & $5.64 \mathrm{E}+06$ & Direct \\
\hline Ir-185 & & & & $4.30 \mathrm{E}+05$ & $1.09 \mathrm{E}+08$ & $7.34 \mathrm{E}+05$ & $2.07 \mathrm{E}+03$ & -- & $2.07 \mathrm{E}+03$ & $1.71 \mathrm{E}-03$ & $1.21 \mathrm{E}+06$ & Direct \\
\hline$|r-186|$ & & & & $2.23 \mathrm{E}+05$ & $2.25 \mathrm{E}+07$ & $3.47 \mathrm{E}+05$ & $7.15 \mathrm{E}+02$ & -- & $7.15 \mathrm{E}+02$ & $6.71 \mathrm{E}-04$ & $1.07 \mathrm{E}+06$ & Direct \\
\hline $\mid \mathrm{Ir}-186 \mathrm{~s}$ & & & & $1.57 \mathrm{E}+06$ & $2.87 \mathrm{E}+31$ & $2.41 \mathrm{E}+07$ & $7.16 \mathrm{E}+03$ & -- & $7.16 \mathrm{E}+03$ & $7.44 \mathrm{E}-04$ & $9.63 \mathrm{E}+06$ & Direct \\
\hline Ir-187 & & & & $9.31 \mathrm{E}+05$ & $3.47 E+09$ & $2.10 \mathrm{E}+06$ & $3.98 \mathrm{E}+03$ & -- & $3.98 \mathrm{E}+03$ & $2.50 \mathrm{E}-03$ & $1.60 \mathrm{E}+06$ & Direct \\
\hline Ir-188 & & & & $1.80 \mathrm{E}+05$ & $1.32 \mathrm{E}+05$ & $1.10 \mathrm{E}+05$ & $5.56 \mathrm{E}+02$ & -- & $5.56 \mathrm{E}+02$ & $1.38 \mathrm{E}-03$ & $4.02 \mathrm{E}+05$ & Direct \\
\hline Ir-189 & & & & $2.03 E+05$ & $1.41 \mathrm{E}+04$ & $6.54 \mathrm{E}+04$ & $9.16 \mathrm{E}+03$ & -- & $9.16 \mathrm{E}+03$ & $1.76 \mathrm{E}-01$ & $5.20 \mathrm{E}+04$ & Direct \\
\hline Ir-190 & & & & $4.47 \mathrm{E}+04$ & $2.98 \mathrm{E}+03$ & $1.37 \mathrm{E}+04$ & $5.18 \mathrm{E}+02$ & -- & $5.18 \mathrm{E}+02$ & $9.11 \mathrm{E}-03$ & $5.68 \mathrm{E}+04$ & Direct \\
\hline Ir-190ms & & & & $1.02 \mathrm{E}+07$ & $9.32 \mathrm{E}+43$ & $2.68 \mathrm{E}+08$ & $5.30 \mathrm{E}+06$ & -- & $5.30 \mathrm{E}+06$ & $3.85 \mathrm{E}-01$ & $1.37 \mathrm{E}+07$ & Direct \\
\hline $\mid r-190 \mathrm{ml}$ & & & & $7.98 \mathrm{E}+05$ & $1.05 E+20$ & $6.96 \mathrm{E}+06$ & $2.52 \mathrm{E}+03$ & -- & $2.52 E+03$ & $4.73 \mathrm{E}-04$ & $5.32 \mathrm{E}+06$ & Direct \\
\hline Ir-191m & & & & -- & -- & -- & $1.18 \mathrm{E}+08$ & -- & $1.18 \mathrm{E}+08$ & $9.85 \mathrm{E}-03$ & $1.20 \mathrm{E}+10$ & Direct \\
\hline Ir-192 & $8.82 \mathrm{E}+02$ & $9.57 \mathrm{E}-02$ & de & $1.80 \mathrm{E}+04$ & $1.56 \mathrm{E}+03$ & $6.68 \mathrm{E}+03$ & $8.92 \mathrm{E}+02$ & -- & $8.92 E+02$ & $9.70 \mathrm{E}-02$ & $9.19 \mathrm{E}+03$ & Direct \\
\hline Ir-192ms & & & & -- & -- & -- & $1.71 \mathrm{E}+08$ & -- & $1.71 \mathrm{E}+08$ & $2.53 \mathrm{E}-01$ & $6.76 \mathrm{E}+08$ & Direct \\
\hline Ir-192ml & & & & $3.10 \mathrm{E}+03$ & $6.37 \mathrm{E}+03$ & $2.60 \mathrm{E}+04$ & $4.51 \mathrm{E}+03$ & -- & $3.10 \mathrm{E}+03$ & $4.01 \mathrm{E}+02$ & $7.73 \mathrm{E}+00$ & Inh. \\
\hline Ir-193m & & & & $9.31 \mathrm{E}+04$ & $1.45 \mathrm{E}+04$ & $6.59 \mathrm{E}+04$ & $2.82 \mathrm{E}+05$ & -- & $1.45 \mathrm{E}+04$ & $2.25 \mathrm{E}-01$ & $6.43 E+04$ & Water \\
\hline Ir-194 & & & & $1.49 \mathrm{E}+05$ & $2.30 \mathrm{E}+06$ & $1.09 \mathrm{E}+05$ & $1.20 \mathrm{E}+04$ & -- & $1.20 \mathrm{E}+04$ & $1.43 \mathrm{E}-02$ & $8.44 \mathrm{E}+05$ & Direct \\
\hline Ir-194m & & & & $9.31 \mathrm{E}+03$ & $9.82 \mathrm{E}+02$ & $4.10 \mathrm{E}+03$ & $3.11 \mathrm{E}+02$ & -- & $3.11 E+02$ & 7.91E-02 & $3.94 \mathrm{E}+03$ & Direct \\
\hline Ir-195 & & & & $1.12 \mathrm{E}+06$ & $3.84 \mathrm{E}+23$ & $1.03 \mathrm{E}+07$ & $8.27 \mathrm{E}+04$ & -- & $8.27 \mathrm{E}+04$ & $1.29 \mathrm{E}-02$ & $6.43 \mathrm{E}+06$ & Direct \\
\hline Ir-195m & & & & $4.65 \mathrm{E}+05$ & $1.23 \mathrm{E}+17$ & $3.25 \mathrm{E}+06$ & $7.45 \mathrm{E}+03$ & -- & $7.45 \mathrm{E}+03$ & $1.76 \mathrm{E}-03$ & $4.23 \mathrm{E}+06$ & Direct \\
\hline Ir-196 & & & & -- & -- & -- & $3.60 \mathrm{E}+06$ & -- & $3.60 \mathrm{E}+06$ & $3.25 \mathrm{E}-03$ & $1.11 \mathrm{E}+09$ & Direct \\
\hline Ir-196m & & & & $6.98 \mathrm{E}+05$ & $3.02 \mathrm{E}+37$ & $1.41 \mathrm{E}+07$ & $3.50 \mathrm{E}+03$ & -- & $3.50 \mathrm{E}+03$ & $3.06 \mathrm{E}-04$ & $1.14 \mathrm{E}+07$ & Direct \\
\hline $\mathrm{K}-38$ & & & & -- & -- & -- & $2.98 \mathrm{E}+04$ & -- & $2.98 \mathrm{E}+04$ & $4.59 \mathrm{E}-05$ & $6.49 E+08$ & Direct \\
\hline $\mathrm{K}-40$ & $1.23 \mathrm{E}+02$ & $1.77 \mathrm{E}+07$ & ing & $3.72 \mathrm{E}+03$ & -- & $1.26 \mathrm{E}+02$ & $4.64 \mathrm{E}+03$ & -- & $1.26 \mathrm{E}+02$ & $1.80 \mathrm{E}+07$ & $6.99 \mathrm{E}-06$ & Food \\
\hline $\mathrm{K}-42$ & & & & $5.58 \mathrm{E}+04$ & -- & $4.84 \mathrm{E}+04$ & $4.79 \mathrm{E}+03$ & -- & $4.79 E+03$ & 7.92E-04 & $6.04 \mathrm{E}+06$ & Direct \\
\hline $\mathrm{K}-43$ & & & & $4.30 \mathrm{E}+04$ & -- & $4.69 \mathrm{E}+04$ & $1.06 \mathrm{E}+03$ & -- & $1.06 \mathrm{E}+03$ & $3.28 \mathrm{E}-04$ & $3.23 \mathrm{E}+06$ & Direct \\
\hline $\mathrm{K}-44$ & & & & $3.02 \mathrm{E}+05$ & -- & $8.02 \mathrm{E}+06$ & $1.44 \mathrm{E}+04$ & -- & $1.44 \mathrm{E}+04$ & 7.47E-05 & $1.93 \mathrm{E}+08$ & Direct \\
\hline $\mathrm{K}-45$ & & & & $3.99 \mathrm{E}+05$ & -- & $1.38 \mathrm{E}+07$ & $1.94 \mathrm{E}+04$ & -- & $1.94 \mathrm{E}+04$ & $9.28 \mathrm{E}-05$ & $2.09 E+08$ & Direct \\
\hline $\mathrm{K}-46$ & & & & -- & -- & -- & $1.44 \mathrm{E}+05$ & -- & $1.44 \mathrm{E}+05$ & $6.17 \mathrm{E}-05$ & $2.34 \mathrm{E}+09$ & Direct \\
\hline $\mathrm{Kr}-74$ & & & & -- & -- & -- & -- & $7.15 \mathrm{E}+02$ & $7.15 E+02$ & $3.23 \mathrm{E}-06$ & $2.21 \mathrm{E}+08$ & Sub \\
\hline Kr-75 & & & & -- & -- & -- & -- & $6.31 \mathrm{E}+02$ & $6.31 E+02$ & $1.08 \mathrm{E}-06$ & $5.85 \mathrm{E}+08$ & Sub \\
\hline $\mathrm{Kr}-76$ & & & & -- & -- & -- & -- & $2.01 \mathrm{E}+03$ & $2.01 \mathrm{E}+03$ & $7.21 \mathrm{E}-04$ & $2.79 \mathrm{E}+06$ & Sub \\
\hline Kr-77 & & & & -- & -- & -- & -- & $8.25 \mathrm{E}+02$ & $8.25 E+02$ & $2.52 \mathrm{E}-05$ & $3.27 \mathrm{E}+07$ & Sub \\
\hline Kr-79 & & & & -- & -- & -- & -- & $3.32 \mathrm{E}+03$ & $3.32 \mathrm{E}+03$ & $2.93 \mathrm{E}-03$ & $1.13 \mathrm{E}+06$ & Sub \\
\hline $\mathrm{Kr}-81$ & & & & -- & -- & -- & -- & $1.53 \mathrm{E}+05$ & $1.53 \mathrm{E}+05$ & $7.28 \mathrm{E}+06$ & $2.10 \mathrm{E}-02$ & Sub \\
\hline $\mathrm{Kr}-81 \mathrm{~m}$ & & & & -- & -- & -- & -- & $6.70 \mathrm{E}+03$ & $6.70 \mathrm{E}+03$ & $6.25 \mathrm{E}-07$ & $1.07 \mathrm{E}+10$ & Sub \\
\hline
\end{tabular}


Table C.2 Summary of Exposure Pathway TQs and Selection of Final HC-3 TQs (continued)

\begin{tabular}{|c|c|c|c|c|c|c|c|c|c|c|c|c|}
\hline \multirow[b]{2}{*}{$\begin{array}{c}\text { MASTER } \\
\text { ISOTOPE } \\
\text { LIST } \\
\end{array}$} & \multicolumn{3}{|c|}{ from NNSA SD G 1027} & \multicolumn{5}{|c|}{ Calculated Pathway Specific TQ Values } & \multicolumn{4}{|c|}{ Selected Final Threshold Quantities } \\
\hline & Act $(\mathrm{Ci})$ & Mass (g) & $\begin{array}{l}\text { Limiting } \\
\text { Pathway }\end{array}$ & $\begin{array}{c}\text { TQ }_{\text {inhale }} \\
\text { (Ci) }\end{array}$ & $\begin{array}{c}\text { TQ }_{\text {water }} \\
\text { (Ci) }\end{array}$ & $\mathrm{TQ}_{\text {food }}(\mathrm{Ci})$ & $\begin{array}{c}T_{\text {direct }} \\
\text { (Ci) }\end{array}$ & $\mathrm{TQ}_{\text {sub }}(\mathrm{Ci})$ & Act (Ci) & Mass (g) & $\begin{array}{c}\text { Sp. Act. } \\
\text { (Ci/g) }\end{array}$ & $\begin{array}{l}\text { Limiting } \\
\text { Pathway }\end{array}$ \\
\hline $\mathrm{Kr}-83 \mathrm{~m}$ & & & & -- & -- & -- & -- & $1.53 \mathrm{E}+07$ & $1.53 \mathrm{E}+07$ & $7.42 \mathrm{E}-01$ & $2.07 \mathrm{E}+07$ & Sub \\
\hline $\mathrm{Kr}-85$ & $1.46 \mathrm{E}+05$ & $3.71 \mathrm{E}+02$ & sub & -- & -- & -- & -- & $1.46 \mathrm{E}+05$ & $1.46 \mathrm{E}+05$ & $3.72 \mathrm{E}+02$ & $3.93 \mathrm{E}+02$ & Sub \\
\hline $\mathrm{Kr}-85 \mathrm{~m}$ & & & & -- & -- & -- & -- & $5.45 \mathrm{E}+03$ & $5.45 \mathrm{E}+03$ & $6.62 \mathrm{E}-04$ & $8.24 \mathrm{E}+06$ & Sub \\
\hline $\mathrm{Kr}-87$ & & & & -- & -- & -- & -- & $9.46 \mathrm{E}+02$ & $9.46 \mathrm{E}+02$ & $3.34 \mathrm{E}-05$ & $2.84 \mathrm{E}+07$ & Sub \\
\hline $\mathrm{Kr}-88$ & & & & -- & -- & -- & -- & $3.83 \mathrm{E}+02$ & $3.83 \mathrm{E}+02$ & $3.05 \mathrm{E}-05$ & $1.26 \mathrm{E}+07$ & Sub \\
\hline $\mathrm{Kr}-89$ & & & & -- & -- & -- & -- & $3.88 \mathrm{E}+02$ & $3.88 \mathrm{E}+02$ & 5.77E-07 & $6.71 \mathrm{E}+08$ & Sub \\
\hline La-128 & & & & -- & -- & -- & $4.95 \mathrm{E}+04$ & -- & $4.95 \mathrm{E}+04$ & 1.74E-04 & $2.84 \mathrm{E}+08$ & Direct \\
\hline La-129 & & & & $5.32 \mathrm{E}+05$ & $1.53 \mathrm{E}+234$ & $4.92 \mathrm{E}+07$ & $6.78 \mathrm{E}+04$ & -- & $6.78 \mathrm{E}+04$ & $5.39 \mathrm{E}-04$ & $1.26 \mathrm{E}+08$ & Direct \\
\hline La-130 & & & & -- & -- & -- & $3.73 E+04$ & -- & $3.73 E+04$ & $2.24 \mathrm{E}-04$ & $1.66 \mathrm{E}+08$ & Direct \\
\hline La-131 & & & & $3.10 \mathrm{E}+05$ & $2.84 \mathrm{E}+51$ & $7.48 \mathrm{E}+06$ & $1.83 \mathrm{E}+04$ & -- & $1.83 \mathrm{E}+04$ & $7.52 \mathrm{E}-04$ & $2.43 \mathrm{E}+07$ & Direct \\
\hline La-132 & & & & $3.99 E+04$ & $2.08 \mathrm{E}+14$ & $1.39 \mathrm{E}+05$ & $1.29 \mathrm{E}+03$ & -- & $1.29 E+03$ & $2.61 \mathrm{E}-04$ & $4.95 \mathrm{E}+06$ & Direct \\
\hline La-132m & & & & $3.28 \mathrm{E}+05$ & $2.37 \mathrm{E}+115$ & $1.67 \mathrm{E}+07$ & $4.45 \mathrm{E}+04$ & -- & $4.45 \mathrm{E}+04$ & $7.58 \mathrm{E}-04$ & $5.87 \mathrm{E}+07$ & Direct \\
\hline La-133 & & & & $3.02 \mathrm{E}+05$ & $2.55 \mathrm{E}+17$ & $1.44 \mathrm{E}+06$ & $1.95 \mathrm{E}+04$ & -- & $1.95 \mathrm{E}+04$ & $3.24 \mathrm{E}-03$ & $6.03 E+06$ & Direct \\
\hline La-134 & & & & -- & -- & -- & $1.56 \mathrm{E}+05$ & -- & $1.56 \mathrm{E}+05$ & $7.40 \mathrm{E}-04$ & $2.11 \mathrm{E}+08$ & Direct \\
\hline La-135 & & & & $4.47 \mathrm{E}+05$ & $8.89 \mathrm{E}+07$ & $4.65 \mathrm{E}+05$ & $3.02 E+04$ & -- & $3.02 \mathrm{E}+04$ & $2.54 \mathrm{E}-02$ & $1.19 \mathrm{E}+06$ & Direct \\
\hline La-136 & & & & -- & -- & -- & $1.80 \mathrm{E}+05$ & -- & $1.80 \mathrm{E}+05$ & $1.29 \mathrm{E}-03$ & $1.40 \mathrm{E}+08$ & Direct \\
\hline La-137 & & & & $1.12 \mathrm{E}+03$ & $2.44 \mathrm{E}+04$ & $9.95 \mathrm{E}+03$ & $3.00 \mathrm{E}+04$ & -- & $1.12 \mathrm{E}+03$ & $2.57 E+04$ & $4.35 \mathrm{E}-02$ & Inh. \\
\hline La-138 & & & & $6.20 \mathrm{E}+01$ & $1.80 \mathrm{E}+03$ & $7.32 \mathrm{E}+02$ & $5.87 \mathrm{E}+02$ & -- & $6.20 \mathrm{E}+01$ & $3.23 \mathrm{E}+09$ & $1.92 \mathrm{E}-08$ & Inh. \\
\hline La-140 & & & & $7.45 \mathrm{E}+03$ & $4.60 \mathrm{E}+04$ & $3.57 \mathrm{E}+03$ & $3.83 E+02$ & -- & $3.83 \mathrm{E}+02$ & $6.88 \mathrm{E}-04$ & $5.56 \mathrm{E}+05$ & Direct \\
\hline La-141 & & & & $5.08 \mathrm{E}+04$ & $2.88 \mathrm{E}+16$ & $1.84 \mathrm{E}+05$ & $7.27 E+04$ & -- & $5.08 \mathrm{E}+04$ & $8.97 \mathrm{E}-03$ & $5.66 \mathrm{E}+06$ & Inh. \\
\hline La-142 & & & & $7.45 \mathrm{E}+04$ & $2.65 \mathrm{E}+34$ & $9.30 \mathrm{E}+05$ & $2.85 \mathrm{E}+03$ & -- & $2.85 \mathrm{E}+03$ & $1.99 \mathrm{E}-04$ & $1.43 \mathrm{E}+07$ & Direct \\
\hline La-143 & & & & $3.38 \mathrm{E}+05$ & $8.52 \mathrm{E}+191$ & $1.94 \mathrm{E}+07$ & $5.42 \mathrm{E}+05$ & -- & $3.38 \mathrm{E}+05$ & $3.66 \mathrm{E}-03$ & $9.25 \mathrm{E}+07$ & Inh. \\
\hline Lu-164 & & & & -- & -- & -- & -- & -- & -- & -- & $3.65 E+08$ & -- \\
\hline Lu-165 & & & & $4.86 \mathrm{E}+05$ & $2.63 E+252$ & $6.24 \mathrm{E}+07$ & $6.08 \mathrm{E}+04$ & -- & $6.08 \mathrm{E}+04$ & $5.73 \mathrm{E}-04$ & $1.06 \mathrm{E}+08$ & Direct \\
\hline Lu-166 & & & & -- & -- & -- & -- & -- & -- & -- & $4.28 \mathrm{E}+08$ & -- \\
\hline Lu-166m & & & & -- & -- & -- & -- & -- & -- & -- & $8.04 \mathrm{E}+08$ & -- \\
\hline Lu-167 & & & & $1.86 \mathrm{E}+05$ & $6.93 \mathrm{E}+57$ & $6.00 \mathrm{E}+06$ & $8.32 \mathrm{E}+03$ & -- & $8.32 \mathrm{E}+03$ & $3.80 \mathrm{E}-04$ & $2.19 E+07$ & Direct \\
\hline Lu-168m & & & & -- & -- & -- & -- & -- & -- & -- & $1.67 \mathrm{E}+08$ & -- \\
\hline Lu-169 & & & & $2.28 \mathrm{E}+04$ & $3.69 \mathrm{E}+05$ & $1.81 \mathrm{E}+04$ & $8.82 \mathrm{E}+02$ & -- & $8.82 \mathrm{E}+02$ & 1.62E-03 & $5.45 \mathrm{E}+05$ & Direct \\
\hline Lu-169m & & & & -- & -- & -- & $1.62 \mathrm{E}+08$ & -- & $1.62 \mathrm{E}+08$ & $3.88 \mathrm{E}-01$ & $4.17 E+08$ & Direct \\
\hline Lu-170 & & & & $1.18 \mathrm{E}+04$ & $5.32 \mathrm{E}+04$ & $6.18 \mathrm{E}+03$ & $3.46 \mathrm{E}+02$ & -- & $3.46 \mathrm{E}+02$ & $9.00 \mathrm{E}-04$ & $3.84 \mathrm{E}+05$ & Direct \\
\hline Lu-171 & & & & $1.20 \mathrm{E}+04$ & $7.01 \mathrm{E}+03$ & $3.08 \mathrm{E}+03$ & $1.09 \mathrm{E}+03$ & -- & $1.09 \mathrm{E}+03$ & 1.17E-02 & $9.29 \mathrm{E}+04$ & Direct \\
\hline Lu-171m & & & & -- & -- & -- & $2.65 \mathrm{E}+08$ & -- & $2.65 \mathrm{E}+08$ & 3.17E-01 & $8.35 E+08$ & Direct \\
\hline Lu-172 & & & & $6.20 \mathrm{E}+03$ & $4.37 \mathrm{E}+03$ & $1.82 \mathrm{E}+03$ & $4.05 \mathrm{E}+02$ & -- & $4.05 \mathrm{E}+02$ & 3.57E-03 & $1.13 \mathrm{E}+05$ & Direct \\
\hline Lu-172m & & & & -- & -- & -- & $1.30 \mathrm{E}+08$ & -- & $1.30 \mathrm{E}+08$ & 4.39E-01 & $2.96 \mathrm{E}+08$ & Direct \\
\hline
\end{tabular}


Table C.2 Summary of Exposure Pathway TQs and Selection of Final HC-3 TQs (continued)

\begin{tabular}{|c|c|c|c|c|c|c|c|c|c|c|c|c|}
\hline \multirow[b]{2}{*}{$\begin{array}{c}\text { MASTER } \\
\text { ISOTOPE } \\
\text { LIST } \\
\end{array}$} & \multicolumn{3}{|c|}{ from NNSA SD G 1027} & \multicolumn{5}{|c|}{ Calculated Pathway Specific TQ Values } & \multicolumn{4}{|c|}{ Selected Final Threshold Quantities } \\
\hline & Act $(\mathrm{Ci})$ & Mass (g) & $\begin{array}{l}\text { Limiting } \\
\text { Pathway }\end{array}$ & $\begin{array}{c}\text { TQ }_{\text {inhale }} \\
(\mathrm{Ci})\end{array}$ & $\begin{array}{c}\text { TQ }_{\text {water }} \\
\text { (Ci) }\end{array}$ & $\mathrm{TQ}_{\text {food }}(\mathrm{Ci})$ & $\begin{array}{c}T_{\text {direct }} \\
\text { (Ci) }\end{array}$ & $\mathrm{TQ}_{\text {sub }}(\mathrm{Ci})$ & Act (Ci) & Mass (g) & $\begin{array}{c}\text { Sp. Act. } \\
\text { (Ci/g) }\end{array}$ & $\begin{array}{l}\text { Limiting } \\
\text { Pathway }\end{array}$ \\
\hline Lu-173 & & & & $4.86 \mathrm{E}+03$ & $7.71 \mathrm{E}+03$ & $3.17 \mathrm{E}+03$ & $5.61 \mathrm{E}+03$ & -- & $3.17 \mathrm{E}+03$ & $2.10 \mathrm{E}+00$ & $1.51 \mathrm{E}+03$ & Food \\
\hline Lu-174 & & & & $2.79 \mathrm{E}+03$ & $7.36 \mathrm{E}+03$ & $3.01 \mathrm{E}+03$ & $5.78 \mathrm{E}+03$ & -- & $2.79 \mathrm{E}+03$ & $4.50 \mathrm{E}+00$ & $6.21 \mathrm{E}+02$ & Inh. \\
\hline Lu-174m & & & & $2.94 \mathrm{E}+03$ & $3.92 \mathrm{E}+03$ & $1.65 \mathrm{E}+03$ & $1.15 \mathrm{E}+04$ & -- & $1.65 \mathrm{E}+03$ & $3.12 \mathrm{E}-01$ & $5.29 \mathrm{E}+03$ & Food \\
\hline Lu-176 & & & & $1.69 \mathrm{E}+02$ & $1.10 \mathrm{E}+03$ & $4.48 \mathrm{E}+02$ & $1.48 \mathrm{E}+03$ & -- & $1.69 \mathrm{E}+02$ & $3.00 \mathrm{E}+09$ & $5.64 \mathrm{E}-08$ & Inh. \\
\hline Lu-176m & & & & $6.98 \mathrm{E}+04$ & $3.72 \mathrm{E}+17$ & $4.15 \mathrm{E}+05$ & $2.32 \mathrm{E}+05$ & -- & $6.98 \mathrm{E}+04$ & $1.44 \mathrm{E}-02$ & $4.84 \mathrm{E}+06$ & Inh. \\
\hline Lu-177 & & & & $1.02 \mathrm{E}+04$ & $1.07 \mathrm{E}+04$ & $4.45 \mathrm{E}+03$ & $2.18 \mathrm{E}+04$ & -- & $4.45 \mathrm{E}+03$ & $4.05 \mathrm{E}-02$ & $1.10 \mathrm{E}+05$ & Food \\
\hline Lu-177m & & & & $7.45 \mathrm{E}+02$ & $1.22 \mathrm{E}+03$ & $5.09 \mathrm{E}+02$ & $7.25 \mathrm{E}+02$ & -- & $5.09 \mathrm{E}+02$ & 1.11E-01 & $4.59 \mathrm{E}+03$ & Food \\
\hline Lu-178 & & & & $2.72 \mathrm{E}+05$ & $4.07 \mathrm{E}+99$ & $1.16 \mathrm{E}+07$ & $1.82 \mathrm{E}+05$ & -- & $1.82 \mathrm{E}+05$ & $4.91 \mathrm{E}-03$ & $3.72 \mathrm{E}+07$ & Direct \\
\hline Lu-178m & & & & $1.99 \mathrm{E}+05$ & $1.05 \mathrm{E}+123$ & $1.79 \mathrm{E}+07$ & $2.88 \mathrm{E}+04$ & -- & $2.88 \mathrm{E}+04$ & $6.19 \mathrm{E}-04$ & $4.65 \mathrm{E}+07$ & Direct \\
\hline Lu-179 & & & & $6.98 \mathrm{E}+04$ & $1.06 \mathrm{E}+15$ & $2.70 \mathrm{E}+05$ & $8.75 E+04$ & -- & $6.98 \mathrm{E}+04$ & $1.83 \mathrm{E}-02$ & $3.82 \mathrm{E}+06$ & Inh. \\
\hline Lu-180 & & & & -- & -- & -- & $8.39 E+04$ & -- & $8.39 E+04$ & $4.58 \mathrm{E}-04$ & $1.83 \mathrm{E}+08$ & Direct \\
\hline Lu-181 & & & & -- & -- & -- & $3.61 \mathrm{E}+05$ & -- & $3.61 \mathrm{E}+05$ & $1.21 \mathrm{E}-03$ & $2.97 \mathrm{E}+08$ & Direct \\
\hline Md-257 & & & & $4.86 \mathrm{E}+03$ & $1.24 \mathrm{E}+14$ & $4.18 \mathrm{E}+06$ & $2.13 E+04$ & -- & $4.86 \mathrm{E}+03$ & $2.07 \mathrm{E}-03$ & $2.34 \mathrm{E}+06$ & Inh. \\
\hline Md-258 & & & & $2.03 \mathrm{E}+01$ & $1.74 \mathrm{E}+02$ & $7.56 \mathrm{E}+02$ & $1.18 \mathrm{E}+05$ & -- & $2.03 \mathrm{E}+01$ & $2.21 \mathrm{E}-03$ & $9.20 \mathrm{E}+03$ & Inh. \\
\hline $\mathrm{Mg}-27$ & & & & -- & -- & -- & $8.60 E+04$ & -- & $8.60 E+04$ & 1.17E-04 & $7.37 E+08$ & Direct \\
\hline $\mathrm{Mg}-28$ & & & & $6.57 \mathrm{E}+03$ & $7.98 \mathrm{E}+05$ & $5.73 \mathrm{E}+03$ & $7.68 \mathrm{E}+02$ & -- & $7.68 \mathrm{E}+02$ & 1.43E-04 & $5.36 \mathrm{E}+06$ & Direct \\
\hline $\mathrm{Mn}-50 \mathrm{~m}$ & & & & -- & -- & -- & $8.92 E+04$ & -- & $8.92 \mathrm{E}+04$ & $4.15 \mathrm{E}-05$ & $2.15 E+09$ & Direct \\
\hline $\mathrm{Mn}-51$ & & & & $1.64 \mathrm{E}+05$ & $2.95 \mathrm{E}+63$ & $3.56 \mathrm{E}+06$ & $1.57 \mathrm{E}+04$ & -- & $1.57 \mathrm{E}+04$ & 1.97E-04 & $7.99 \mathrm{E}+07$ & Direct \\
\hline $\mathrm{Mn}-52$ & $2.23 \mathrm{E}+02$ & 4.97E-04 & de & $6.20 \mathrm{E}+03$ & $3.86 \mathrm{E}+03$ & $1.48 \mathrm{E}+03$ & $2.23 \mathrm{E}+02$ & -- & $2.23 \mathrm{E}+02$ & 4.97E-04 & $4.50 \mathrm{E}+05$ & Direct \\
\hline$M n-52 m$ & & & & $2.23 \mathrm{E}+05$ & $3.72 \mathrm{E}+131$ & $1.05 \mathrm{E}+07$ & $1.43 \mathrm{E}+04$ & -- & $1.43 \mathrm{E}+04$ & $8.31 \mathrm{E}-05$ & $1.72 \mathrm{E}+08$ & Direct \\
\hline $\mathrm{Mn}-53$ & & & & $2.15 \mathrm{E}+05$ & $6.59 \mathrm{E}+04$ & $2.66 \mathrm{E}+04$ & $5.58 \mathrm{E}+05$ & -- & $2.66 \mathrm{E}+04$ & $1.46 \mathrm{E}+07$ & $1.83 \mathrm{E}-03$ & Food \\
\hline Mn-54 & & & & $7.45 E+03$ & $2.85 \mathrm{E}+03$ & $1.17 \mathrm{E}+03$ & $8.69 E+02$ & -- & $8.69 \mathrm{E}+02$ & $1.12 \mathrm{E}-01$ & $7.75 \mathrm{E}+03$ & Direct \\
\hline $\mathrm{Mn}-56$ & & & & $5.58 \mathrm{E}+04$ & $4.36 \mathrm{E}+22$ & $3.98 \mathrm{E}+05$ & $2.77 \mathrm{E}+03$ & -- & $2.77 \mathrm{E}+03$ & $1.28 \mathrm{E}-04$ & $2.17 \mathrm{E}+07$ & Direct \\
\hline $\mathrm{Mn}-57$ & & & & -- & -- & -- & $5.11 \mathrm{E}+06$ & -- & $5.11 \mathrm{E}+06$ & $2.20 \mathrm{E}-03$ & $2.32 \mathrm{E}+09$ & Direct \\
\hline $\mathrm{Mn}-58 \mathrm{~m}$ & & & & -- & -- & -- & $2.80 \mathrm{E}+05$ & -- & $2.80 \mathrm{E}+05$ & $9.36 \mathrm{E}-05$ & $2.99 \mathrm{E}+09$ & Direct \\
\hline Mo-101 & & & & $2.48 \mathrm{E}+05$ & -- & $2.49 \mathrm{E}+07$ & $3.62 \mathrm{E}+04$ & -- & $3.62 E+04$ & $2.84 \mathrm{E}-04$ & $1.27 \mathrm{E}+08$ & Direct \\
\hline Mo-102 & & & & $2.66 \mathrm{E}+05$ & -- & $1.96 \mathrm{E}+07$ & $3.46 \mathrm{E}+06$ & -- & $2.66 \mathrm{E}+05$ & $1.63 \mathrm{E}-03$ & $1.63 \mathrm{E}+08$ & Inh. \\
\hline Mo-89 & & & & -- & -- & -- & $2.82 \mathrm{E}+05$ & -- & $2.82 \mathrm{E}+05$ & $2.82 \mathrm{E}-04$ & $1.00 \mathrm{E}+09$ & Direct \\
\hline Mo-90 & & & & $1.99 \mathrm{E}+04$ & -- & $7.37 \mathrm{E}+04$ & $2.72 \mathrm{E}+03$ & -- & $2.72 \mathrm{E}+03$ & $4.42 \mathrm{E}-04$ & $6.15 \mathrm{E}+06$ & Direct \\
\hline Mo-91 & & & & $3.19 E+05$ & -- & $1.62 \mathrm{E}+07$ & $4.79 E+04$ & -- & $4.79 E+04$ & $3.58 \mathrm{E}-04$ & $1.34 \mathrm{E}+08$ & Direct \\
\hline Mo-91m & & & & -- & -- & -- & $4.86 \mathrm{E}+05$ & -- & $4.86 \mathrm{E}+05$ & $2.53 \mathrm{E}-04$ & $1.92 \mathrm{E}+09$ & Direct \\
\hline Mo-93 & & & & $5.08 \mathrm{E}+03$ & -- & $3.07 \mathrm{E}+02$ & $6.85 \mathrm{E}+04$ & -- & $3.07 \mathrm{E}+02$ & $2.80 \mathrm{E}+02$ & $1.10 \mathrm{E}+00$ & Food \\
\hline Mo-93m & & & & $3.72 \mathrm{E}+04$ & -- & $1.35 \mathrm{E}+05$ & $8.59 E+02$ & -- & $8.59 E+02$ & $1.74 \mathrm{E}-04$ & $4.92 \mathrm{E}+06$ & Direct \\
\hline Mo-99 & $3.76 \mathrm{E}+03$ & 7.84E-03 & ing & $1.02 \mathrm{E}+04$ & -- & $3.85 \mathrm{E}+03$ & $5.48 \mathrm{E}+03$ & -- & $3.85 \mathrm{E}+03$ & $8.01 \mathrm{E}-03$ & $4.80 \mathrm{E}+05$ & Food \\
\hline $\mathrm{N}-13$ & & & & -- & -- & -- & -- & $8.04 \mathrm{E}+02$ & $8.04 \mathrm{E}+02$ & 5.54E-07 & $1.45 \mathrm{E}+09$ & Sub \\
\hline
\end{tabular}


Table C.2 Summary of Exposure Pathway TQs and Selection of Final HC-3 TQs (continued)

\begin{tabular}{|c|c|c|c|c|c|c|c|c|c|c|c|c|}
\hline \multirow[b]{2}{*}{$\begin{array}{c}\text { MASTER } \\
\text { ISOTOPE } \\
\text { LIST } \\
\end{array}$} & \multicolumn{3}{|c|}{ from NNSA SD G 1027} & \multicolumn{5}{|c|}{ Calculated Pathway Specific TQ Values } & \multicolumn{4}{|c|}{ Selected Final Threshold Quantities } \\
\hline & Act $(\mathrm{Ci})$ & Mass (g) & $\begin{array}{l}\text { Limiting } \\
\text { Pathway }\end{array}$ & $\begin{array}{c}\text { TQ }_{\text {inhale }} \\
(\mathrm{Ci})\end{array}$ & $\begin{array}{c}\text { TQ }_{\text {water }} \\
\text { (Ci) }\end{array}$ & $\mathrm{TQ}_{\text {food }}(\mathrm{Ci})$ & $\begin{array}{c}\mathrm{TQ}_{\text {direct }} \\
\text { (Ci) }\end{array}$ & $\mathrm{TQ}_{\text {sub }}(\mathrm{Ci})$ & Act (Ci) & Mass (g) & $\begin{array}{c}\text { Sp. Act. } \\
\text { (Ci/g) }\end{array}$ & $\begin{array}{l}\text { Limiting } \\
\text { Pathway }\end{array}$ \\
\hline $\mathrm{N}-16$ & & & & -- & -- & -- & $1.36 \mathrm{E}+06$ & -- & $1.36 \mathrm{E}+06$ & $1.37 \mathrm{E}-05$ & $9.89 \mathrm{E}+10$ & Direct \\
\hline $\mathrm{Na}-22$ & $2.48 \mathrm{E}+02$ & $3.98 \mathrm{E}-02$ & ing & $5.58 \mathrm{E}+03$ & -- & $2.54 \mathrm{E}+02$ & $3.31 \mathrm{E}+02$ & -- & $2.54 \mathrm{E}+02$ & 4.07E-02 & $6.25 \mathrm{E}+03$ & Food \\
\hline $\mathrm{Na}-24$ & & & & $2.11 \mathrm{E}+04$ & -- & $4.15 \mathrm{E}+04$ & $2.92 \mathrm{E}+02$ & -- & $2.92 \mathrm{E}+02$ & $3.35 \mathrm{E}-05$ & $8.71 \mathrm{E}+06$ & Direct \\
\hline $\mathrm{Nb}-87$ & & & & -- & -- & -- & $1.58 \mathrm{E}+05$ & -- & $1.58 \mathrm{E}+05$ & $2.75 \mathrm{E}-04$ & $5.77 \mathrm{E}+08$ & Direct \\
\hline $\mathrm{Nb}-88$ & & & & $2.23 \mathrm{E}+05$ & -- & $1.71 \mathrm{E}+07$ & $1.23 \mathrm{E}+04$ & -- & $1.23 \mathrm{E}+04$ & $8.21 \mathrm{E}-05$ & $1.50 \mathrm{E}+08$ & Direct \\
\hline $\mathrm{Nb}-88 \mathrm{~m}$ & & & & -- & -- & -- & $2.27 \mathrm{E}+04$ & -- & $2.27 \mathrm{E}+04$ & $8.25 \mathrm{E}-05$ & $2.75 \mathrm{E}+08$ & Direct \\
\hline Nb-89I & & & & $5.88 \mathrm{E}+04$ & -- & $4.23 \mathrm{E}+05$ & $4.27 \mathrm{E}+03$ & -- & $4.27 E+03$ & $2.46 \mathrm{E}-04$ & $1.73 \mathrm{E}+07$ & Direct \\
\hline $\mathrm{Nb}-89 \mathrm{~s}$ & & & & $9.31 \mathrm{E}+04$ & -- & $1.67 \mathrm{E}+06$ & $5.70 \mathrm{E}+03$ & -- & $5.70 \mathrm{E}+03$ & $1.78 \mathrm{E}-04$ & $3.20 \mathrm{E}+07$ & Direct \\
\hline $\mathrm{Nb}-90$ & & & & $1.02 \mathrm{E}+04$ & -- & $1.53 \mathrm{E}+04$ & $2.88 \mathrm{E}+02$ & -- & $2.88 \mathrm{E}+02$ & $1.21 \mathrm{E}-04$ & $2.39 \mathrm{E}+06$ & Direct \\
\hline Nb-91 & & & & $6.57 E+03$ & -- & $1.75 \mathrm{E}+04$ & $6.15 E+04$ & -- & $6.57 \mathrm{E}+03$ & $1.14 \mathrm{E}+03$ & $5.78 \mathrm{E}+00$ & Inh. \\
\hline $\mathrm{Nb}-91 \mathrm{~m}$ & & & & $2.86 \mathrm{E}+03$ & -- & $2.35 \mathrm{E}+03$ & $2.15 E+04$ & -- & $2.35 \mathrm{E}+03$ & 9.97E-02 & $2.36 \mathrm{E}+04$ & Food \\
\hline $\mathrm{Nb}-92$ & & & & $4.47 \mathrm{E}+02$ & -- & $8.05 \mathrm{E}+02$ & $4.82 \mathrm{E}+02$ & -- & $4.47 \mathrm{E}+02$ & $3.99 \mathrm{E}+06$ & 1.12E-04 & Inh. \\
\hline $\mathrm{Nb}-92 \mathrm{~m}$ & & & & $2.07 \mathrm{E}+04$ & -- & $3.64 \mathrm{E}+03$ & $7.75 \mathrm{E}+02$ & -- & $7.75 \mathrm{E}+02$ & $5.54 \mathrm{E}-03$ & $1.40 \mathrm{E}+05$ & Direct \\
\hline $\mathrm{Nb}-93 \mathrm{~m}$ & & & & $6.98 \mathrm{E}+03$ & -- & $6.73 \mathrm{E}+03$ & $3.82 \mathrm{E}+05$ & -- & $6.73 \mathrm{E}+03$ & $2.38 \mathrm{E}+01$ & $2.83 \mathrm{E}+02$ & Food \\
\hline $\mathrm{Nb}-94$ & $2.49 \mathrm{E}+02$ & $1.33 \mathrm{E}+03$ & $i$ & $2.48 \mathrm{E}+02$ & -- & $4.74 \mathrm{E}+02$ & $4.61 \mathrm{E}+02$ & -- & $2.48 \mathrm{E}+02$ & $1.32 \mathrm{E}+03$ & $1.88 \mathrm{E}-01$ & Inh. \\
\hline $\mathrm{Nb}-94 \mathrm{~m}$ & & & & -- & -- & -- & $9.91 \mathrm{E}+06$ & -- & $9.91 E+06$ & $3.10 \mathrm{E}-02$ & $3.20 E+08$ & Direct \\
\hline $\mathrm{Nb}-95$ & & & & $6.98 \mathrm{E}+03$ & -- & $1.87 \mathrm{E}+03$ & $9.57 \mathrm{E}+02$ & -- & $9.57 \mathrm{E}+02$ & $2.45 \mathrm{E}-02$ & $3.91 \mathrm{E}+04$ & Direct \\
\hline $\mathrm{Nb}-95 \mathrm{~m}$ & & & & $1.31 \mathrm{E}+04$ & -- & $6.66 \mathrm{E}+03$ & $1.17 \mathrm{E}+04$ & -- & $6.66 \mathrm{E}+03$ & $1.75 \mathrm{E}-02$ & $3.81 \mathrm{E}+05$ & Food \\
\hline $\mathrm{Nb}-96$ & & & & $1.12 \mathrm{E}+04$ & -- & $1.07 \mathrm{E}+04$ & $4.11 \mathrm{E}+02$ & -- & $4.11 \mathrm{E}+02$ & $2.93 \mathrm{E}-04$ & $1.40 \mathrm{E}+06$ & Direct \\
\hline $\mathrm{Nb}-97$ & & & & $1.55 \mathrm{E}+05$ & -- & $3.15 \mathrm{E}+06$ & $1.53 \mathrm{E}+04$ & -- & $1.53 \mathrm{E}+04$ & $5.70 \mathrm{E}-04$ & $2.69 \mathrm{E}+07$ & Direct \\
\hline $\mathrm{Nb}-97 \mathrm{~m}$ & & & & -- & -- & -- & $9.95 \mathrm{E}+05$ & -- & $9.95 \mathrm{E}+05$ & $5.13 \mathrm{E}-04$ & $1.94 \mathrm{E}+09$ & Direct \\
\hline $\mathrm{Nb}-98$ & & & & $1.13 \mathrm{E}+05$ & -- & $2.73 \mathrm{E}+06$ & $5.80 \mathrm{E}+03$ & -- & $5.80 \mathrm{E}+03$ & $1.56 \mathrm{E}-04$ & $3.73 \mathrm{E}+07$ & Direct \\
\hline $\mathrm{Nb}-99$ & & & & -- & -- & -- & $1.66 \mathrm{E}+07$ & -- & $1.66 \mathrm{E}+07$ & $2.19 \mathrm{E}-03$ & $7.60 \mathrm{E}+09$ & Direct \\
\hline $\mathrm{Nb}-99 \mathrm{~m}$ & & & & -- & -- & -- & $3.68 \mathrm{E}+05$ & -- & $3.68 \mathrm{E}+05$ & $5.04 \mathrm{E}-04$ & $7.31 \mathrm{E}+08$ & Direct \\
\hline $\mathrm{Nd}-134$ & & & & -- & -- & -- & $1.57 \mathrm{E}+05$ & -- & $1.57 \mathrm{E}+05$ & $9.52 \mathrm{E}-04$ & $1.65 \mathrm{E}+08$ & Direct \\
\hline $\mathrm{Nd}-135$ & & & & $2.48 \mathrm{E}+05$ & -- & $2.07 \mathrm{E}+07$ & $4.63 \mathrm{E}+04$ & -- & $4.63 E+04$ & $4.12 \mathrm{E}-04$ & $1.12 \mathrm{E}+08$ & Direct \\
\hline $\mathrm{Nd}-136$ & & & & $1.25 \mathrm{E}+05$ & -- & $3.08 \mathrm{E}+06$ & $4.88 \mathrm{E}+04$ & -- & $4.88 \mathrm{E}+04$ & $1.79 \mathrm{E}-03$ & $2.73 \mathrm{E}+07$ & Direct \\
\hline $\mathrm{Nd}-137$ & & & & $2.23 \mathrm{E}+05$ & -- & $6.80 \mathrm{E}+06$ & $1.60 \mathrm{E}+04$ & -- & $1.60 \mathrm{E}+04$ & $4.48 \mathrm{E}-04$ & $3.57 \mathrm{E}+07$ & Direct \\
\hline $\mathrm{Nd}-138$ & & & & $2.94 \mathrm{E}+04$ & -- & $8.08 \mathrm{E}+04$ & $5.77 \mathrm{E}+04$ & -- & $2.94 \mathrm{E}+04$ & $6.52 \mathrm{E}-03$ & $4.51 \mathrm{E}+06$ & Inh. \\
\hline $\mathrm{Nd}-139$ & & & & $6.57 \mathrm{E}+05$ & -- & $2.60 \mathrm{E}+07$ & $6.02 \mathrm{E}+04$ & -- & $6.02 E+04$ & $1.32 \mathrm{E}-03$ & $4.56 \mathrm{E}+07$ & Direct \\
\hline $\mathrm{Nd}-139 \mathrm{~m}$ & & & & $4.47 \mathrm{E}+04$ & -- & $1.90 \mathrm{E}+05$ & $1.47 \mathrm{E}+03$ & -- & $1.47 \mathrm{E}+03$ & $3.58 \mathrm{E}-04$ & $4.10 \mathrm{E}+06$ & Direct \\
\hline $\mathrm{Nd}-140$ & & & & $8.59 \mathrm{E}+03$ & -- & $1.97 \mathrm{E}+03$ & $2.79 E+04$ & -- & $1.97 \mathrm{E}+03$ & $7.11 \mathrm{E}-03$ & $2.77 \mathrm{E}+05$ & Food \\
\hline $\mathrm{Nd}-141$ & & & & $1.27 \mathrm{E}+06$ & -- & $1.25 \mathrm{E}+07$ & $6.45 \mathrm{E}+04$ & -- & $6.45 \mathrm{E}+04$ & $7.22 \mathrm{E}-03$ & $8.93 E+06$ & Direct \\
\hline $\mathrm{Nd}-141 \mathrm{~m}$ & & & & -- & -- & -- & $9.17 \mathrm{E}+05$ & -- & $9.17 \mathrm{E}+05$ & 7.15E-04 & $1.28 \mathrm{E}+09$ & Direct \\
\hline $\mathrm{Nd}-144$ & & & & $1.51 \mathrm{E}+00$ & -- & $1.97 \mathrm{E}+01$ & -- & -- & $1.51 \mathrm{E}+00$ & $1.39 \mathrm{E}+12$ & $1.08 \mathrm{E}-12$ & Inh. \\
\hline
\end{tabular}


Table C.2 Summary of Exposure Pathway TQs and Selection of Final HC-3 TQs (continued)

\begin{tabular}{|c|c|c|c|c|c|c|c|c|c|c|c|c|}
\hline \multirow[b]{2}{*}{$\begin{array}{c}\text { MASTER } \\
\text { ISOTOPE } \\
\text { LIST } \\
\end{array}$} & \multicolumn{3}{|c|}{ from NNSA SD G 1027} & \multicolumn{5}{|c|}{ Calculated Pathway Specific TQ Values } & \multicolumn{4}{|c|}{ Selected Final Threshold Quantities } \\
\hline & Act $(\mathrm{Ci})$ & Mass (g) & $\begin{array}{l}\text { Limiting } \\
\text { Pathway }\end{array}$ & $\begin{array}{c}\text { TQ }_{\text {inhale }} \\
(\mathrm{Ci})\end{array}$ & $\begin{array}{c}\text { TQ }_{\text {water }} \\
\text { (Ci) }\end{array}$ & $\mathrm{TQ}_{\text {food }}(\mathrm{Ci})$ & $\begin{array}{c}T_{\text {direct }} \\
\text { (Ci) }\end{array}$ & $\mathrm{TQ}_{\text {sub }}(\mathrm{Ci})$ & Act (Ci) & Mass (g) & $\begin{array}{c}\text { Sp. Act. } \\
\text { (Ci/g) }\end{array}$ & $\begin{array}{l}\text { Limiting } \\
\text { Pathway }\end{array}$ \\
\hline $\mathrm{Nd}-147$ & & & & $4.86 \mathrm{E}+03$ & -- & $1.58 \mathrm{E}+03$ & $5.34 \mathrm{E}+03$ & -- & $1.58 \mathrm{E}+03$ & $1.96 \mathrm{E}-02$ & $8.09 E+04$ & Food \\
\hline $\mathrm{Nd}-149$ & & & & $8.59 \mathrm{E}+04$ & -- & $1.24 \mathrm{E}+06$ & $1.82 \mathrm{E}+04$ & -- & $1.82 \mathrm{E}+04$ & $1.49 \mathrm{E}-03$ & $1.22 \mathrm{E}+07$ & Direct \\
\hline $\mathrm{Nd}-151$ & & & & $3.85 \mathrm{E}+05$ & -- & $4.13 \mathrm{E}+07$ & $6.36 \mathrm{E}+04$ & -- & $6.36 \mathrm{E}+04$ & $6.35 \mathrm{E}-04$ & $1.00 \mathrm{E}+08$ & Direct \\
\hline Nd-152 & & & & $2.79 \mathrm{E}+05$ & -- & $2.76 \mathrm{E}+07$ & $3.87 E+05$ & -- & $2.79 \mathrm{E}+05$ & $2.57 \mathrm{E}-03$ & $1.09 \mathrm{E}+08$ & Inh. \\
\hline $\mathrm{Ne}-19$ & & & & -- & -- & -- & $2.47 \mathrm{E}+06$ & -- & $2.47 \mathrm{E}+06$ & $7.16 \mathrm{E}-05$ & $3.45 \mathrm{E}+10$ & Direct \\
\hline $\mathrm{Ne}-24$ & & & & -- & -- & -- & $3.96 \mathrm{E}+05$ & -- & $3.96 \mathrm{E}+05$ & 1.71E-04 & $2.32 \mathrm{E}+09$ & Direct \\
\hline $\mathrm{Ni}-56$ & & & & $9.31 \mathrm{E}+03$ & -- & $2.92 \mathrm{E}+03$ & $4.46 \mathrm{E}+02$ & -- & $4.46 \mathrm{E}+02$ & 1.17E-03 & $3.83 \mathrm{E}+05$ & Direct \\
\hline $\mathrm{Ni}-57$ & & & & $1.47 \mathrm{E}+04$ & -- & $9.04 \mathrm{E}+03$ & $4.71 E+02$ & -- & $4.71 E+02$ & 3.09E-04 & $1.53 \mathrm{E}+06$ & Direct \\
\hline $\mathrm{Ni}-59$ & & & & $1.35 \mathrm{E}+04$ & -- & $1.28 \mathrm{E}+04$ & $3.02 \mathrm{E}+05$ & -- & $1.28 \mathrm{E}+04$ & $1.58 \mathrm{E}+05$ & 8.09E-02 & Food \\
\hline $\mathrm{Ni}-63$ & $5.24 \mathrm{E}+03$ & $9.21 \mathrm{E}+01$ & ing & $5.58 \mathrm{E}+03$ & -- & $5.36 \mathrm{E}+03$ & -- & -- & $5.36 \mathrm{E}+03$ & $9.06 \mathrm{E}+01$ & $5.92 \mathrm{E}+01$ & Food \\
\hline $\mathrm{Ni}-65$ & & & & $3.10 E+04$ & -- & $5.69 \mathrm{E}+05$ & $8.75 E+03$ & -- & $8.75 E+03$ & 4.57E-04 & $1.92 \mathrm{E}+07$ & Direct \\
\hline $\mathrm{Ni}-66$ & & & & $5.88 \mathrm{E}+03$ & -- & $1.82 \mathrm{E}+03$ & -- & -- & $1.82 \mathrm{E}+03$ & $2.09 \mathrm{E}-03$ & $8.71 \mathrm{E}+05$ & Food \\
\hline $\mathrm{Np}-231$ & & & & $6.57 \mathrm{E}+04$ & -- & $1.09 \mathrm{E}+08$ & -- & -- & $6.57 \mathrm{E}+04$ & $3.94 \mathrm{E}-03$ & $1.67 \mathrm{E}+07$ & Inh. \\
\hline Np-232 & & & & $2.38 \mathrm{E}+06$ & -- & $1.08 \mathrm{E}+09$ & $4.10 \mathrm{E}+04$ & -- & $4.10 \mathrm{E}+04$ & $7.43 \mathrm{E}-04$ & $5.51 \mathrm{E}+07$ & Direct \\
\hline $\mathrm{Np}-233$ & & & & $3.72 \mathrm{E}+07$ & -- & $1.93 \mathrm{E}+09$ & $2.20 \mathrm{E}+05$ & -- & $2.20 \mathrm{E}+05$ & $9.89 \mathrm{E}-03$ & $2.23 \mathrm{E}+07$ & Direct \\
\hline $\mathrm{Np}-234$ & & & & $1.53 \mathrm{E}+05$ & -- & $3.93 \mathrm{E}+04$ & $5.44 \mathrm{E}+02$ & -- & $5.44 \mathrm{E}+02$ & $4.29 \mathrm{E}-03$ & $1.27 \mathrm{E}+05$ & Direct \\
\hline $\mathrm{Np}-235$ & & & & $2.79 \mathrm{E}+05$ & -- & $1.56 \mathrm{E}+05$ & $1.02 \mathrm{E}+05$ & -- & $1.02 \mathrm{E}+05$ & $7.30 \mathrm{E}+01$ & $1.40 \mathrm{E}+03$ & Direct \\
\hline $\mathrm{Np}-236 \mathrm{I}$ & & & & $3.72 \mathrm{E}+01$ & -- & $4.72 \mathrm{E}+02$ & $5.33 E+03$ & -- & $3.72 \mathrm{E}+01$ & $2.83 \mathrm{E}+03$ & $1.32 \mathrm{E}-02$ & Inh. \\
\hline $\mathrm{Np}-236 \mathrm{~s}$ & & & & $2.23 \mathrm{E}+04$ & -- & $6.39 \mathrm{E}+05$ & $2.03 E+04$ & -- & $2.03 E+04$ & $3.43 \mathrm{E}-02$ & $5.90 \mathrm{E}+05$ & Direct \\
\hline $\mathrm{Np}-237$ & $5.36 \mathrm{E}+00$ & $7.60 \mathrm{E}+03$ & $\mathrm{i}$ & $5.32 \mathrm{E}+00$ & -- & $7.30 \mathrm{E}+01$ & $2.10 \mathrm{E}+04$ & -- & $5.32 \mathrm{E}+00$ & $7.55 \mathrm{E}+03$ & 7.05E-04 & Inh. \\
\hline $\mathrm{Np}-238$ & $1.54 \mathrm{E}+03$ & $5.93 \mathrm{E}-03$ & de & $5.58 \mathrm{E}+04$ & -- & $6.37 E+04$ & $1.54 \mathrm{E}+03$ & -- & $1.54 \mathrm{E}+03$ & 5.94E-03 & $2.59 \mathrm{E}+05$ & Direct \\
\hline $\mathrm{Np}-239$ & & & & $1.02 \mathrm{E}+05$ & -- & $6.61 \mathrm{E}+04$ & $4.84 \mathrm{E}+03$ & -- & $4.84 \mathrm{E}+03$ & $2.09 \mathrm{E}-02$ & $2.32 \mathrm{E}+05$ & Direct \\
\hline $\mathrm{Np}-240$ & & & & $8.59 E+05$ & -- & $2.89 \mathrm{E}+07$ & $8.49 \mathrm{E}+03$ & -- & $8.49 E+03$ & 7.04E-04 & $1.20 \mathrm{E}+07$ & Direct \\
\hline $\mathrm{Np}-240 \mathrm{~m}$ & & & & -- & -- & -- & $2.90 \mathrm{E}+05$ & -- & $2.90 \mathrm{E}+05$ & $2.74 \mathrm{E}-03$ & $1.06 \mathrm{E}+08$ & Direct \\
\hline $\mathrm{Np}-241$ & & & & $5.08 \mathrm{E}+06$ & -- & $6.14 \mathrm{E}+08$ & $1.32 \mathrm{E}+06$ & -- & $1.32 \mathrm{E}+06$ & $2.35 \mathrm{E}-02$ & $5.61 \mathrm{E}+07$ & Direct \\
\hline $\mathrm{Np}-242$ & & & & -- & -- & -- & $1.24 \mathrm{E}+06$ & -- & $1.24 \mathrm{E}+06$ & $3.51 \mathrm{E}-03$ & $3.53 \mathrm{E}+08$ & Direct \\
\hline $\mathrm{Np}-242 \mathrm{~m}$ & & & & -- & -- & -- & $1.43 \mathrm{E}+05$ & -- & $1.43 \mathrm{E}+05$ & $1.01 \mathrm{E}-03$ & $1.41 \mathrm{E}+08$ & Direct \\
\hline $0-14$ & & & & -- & -- & -- & -- & $2.30 \mathrm{E}+02$ & $2.30 \mathrm{E}+02$ & $2.01 \mathrm{E}-08$ & $1.14 \mathrm{E}+10$ & Sub \\
\hline $0-15$ & & & & -- & -- & -- & -- & $8.04 \mathrm{E}+02$ & $8.04 \mathrm{E}+02$ & $1.31 \mathrm{E}-07$ & $6.15 \mathrm{E}+09$ & Sub \\
\hline $0-19$ & & & & -- & -- & -- & $1.69 \mathrm{E}+06$ & -- & $1.69 \mathrm{E}+06$ & $7.65 \mathrm{E}-05$ & $2.21 \mathrm{E}+10$ & Direct \\
\hline Os-177 & & & & -- & -- & -- & -- & -- & -- & -- & $3.79 \mathrm{E}+08$ & -- \\
\hline Os-179 & & & & -- & -- & -- & -- & -- & -- & -- & $1.62 \mathrm{E}+08$ & -- \\
\hline Os-180 & & & & $4.47 \mathrm{E}+05$ & $1.17 \mathrm{E}+127$ & $4.12 \mathrm{E}+07$ & $5.11 \mathrm{E}+05$ & -- & $4.47 \mathrm{E}+05$ & $9.41 \mathrm{E}-03$ & $4.75 \mathrm{E}+07$ & Inh. \\
\hline Os-181 & & & & $1.12 \mathrm{E}+05$ & $1.97 \mathrm{E}+31$ & $1.66 \mathrm{E}+06$ & $5.65 \mathrm{E}+03$ & -- & $5.65 \mathrm{E}+03$ & $5.71 \mathrm{E}-04$ & $9.90 \mathrm{E}+06$ & Direct \\
\hline Os-182 & & & & $2.15 \mathrm{E}+04$ & $2.35 \mathrm{E}+06$ & $2.22 \mathrm{E}+04$ & $2.38 \mathrm{E}+03$ & -- & $2.38 \mathrm{E}+03$ & 3.04E-03 & $7.83 E+05$ & Direct \\
\hline
\end{tabular}


Table C.2 Summary of Exposure Pathway TQs and Selection of Final HC-3 TQs (continued)

\begin{tabular}{|c|c|c|c|c|c|c|c|c|c|c|c|c|}
\hline \multirow{2}{*}{$\begin{array}{c}\text { MASTER } \\
\text { ISOTOPE } \\
\text { LIST } \\
\end{array}$} & \multicolumn{3}{|c|}{ from NNSA SD G 1027} & \multicolumn{5}{|c|}{ Calculated Pathway Specific TQ Values } & \multicolumn{4}{|c|}{ Selected Final Threshold Quantities } \\
\hline & Act (Ci) & Mass (g) & $\begin{array}{l}\text { Limiting } \\
\text { Pathway }\end{array}$ & $\begin{array}{c}\mathrm{TQ}_{\text {inhale }} \\
(\mathrm{Ci}) \\
\end{array}$ & $\begin{array}{c}\mathrm{TQ}_{\text {water }} \\
\text { (Ci) }\end{array}$ & $\mathrm{TQ}_{\text {food }}(\mathrm{Ci})$ & $\begin{array}{c}\mathrm{TQ}_{\text {direct }} \\
(\mathrm{Ci}) \\
\end{array}$ & $\mathrm{TQ}_{\text {sub }}(\mathrm{Ci})$ & Act (Ci) & Mass (g) & $\begin{array}{c}\text { Sp. Act. } \\
\text { (Ci/g) }\end{array}$ & $\begin{array}{l}\text { Limiting } \\
\text { Pathway }\end{array}$ \\
\hline Os-183 & & & & $4.47 \mathrm{E}+04$ & $2.31 \mathrm{E}+08$ & $8.92 \mathrm{E}+04$ & $2.05 \mathrm{E}+03$ & -- & $2.05 \mathrm{E}+03$ & $1.55 \mathrm{E}-03$ & $1.32 \mathrm{E}+06$ & Direct \\
\hline Os-183m & & & & $5.08 \mathrm{E}+04$ & $3.76 \mathrm{E}+09$ & $1.27 \mathrm{E}+05$ & $1.49 \mathrm{E}+03$ & -- & $1.49 \mathrm{E}+03$ & $8.61 \mathrm{E}-04$ & $1.73 \mathrm{E}+06$ & Direct \\
\hline Os-185 & & & & $7.45 \mathrm{E}+03$ & $4.19 \mathrm{E}+03$ & $1.78 \mathrm{E}+03$ & $1.01 \mathrm{E}+03$ & -- & $1.01 \mathrm{E}+03$ & $1.35 \mathrm{E}-01$ & $7.51 \mathrm{E}+03$ & Direct \\
\hline Os-186 & & & & $2.94 \mathrm{E}+00$ & $6.17 \mathrm{E}+01$ & $2.52 \mathrm{E}+01$ & -- & -- & $2.94 \mathrm{E}+00$ & $3.06 \mathrm{E}+12$ & $9.61 \mathrm{E}-13$ & Inh. \\
\hline Os-189m & & & & $1.41 \mathrm{E}+06$ & $5.42 \mathrm{E}+13$ & $2.42 \mathrm{E}+06$ & $1.19 \mathrm{E}+06$ & -- & $1.19 \mathrm{E}+06$ & $4.32 \mathrm{E}-01$ & $2.76 \mathrm{E}+06$ & Direct \\
\hline Os-190m & & & & -- & -- & -- & $4.61 \mathrm{E}+04$ & -- & $4.61 \mathrm{E}+04$ & $4.61 \mathrm{E}-04$ & $1.00 \mathrm{E}+08$ & Direct \\
\hline Os-191 & & & & $6.20 \mathrm{E}+03$ & $5.54 \mathrm{E}+03$ & $2.57 \mathrm{E}+03$ & $9.34 \mathrm{E}+03$ & -- & $2.57 \mathrm{E}+03$ & $5.78 \mathrm{E}-02$ & $4.44 \mathrm{E}+04$ & Food \\
\hline Os-191m & & & & $7.45 \mathrm{E}+04$ & $5.41 \mathrm{E}+08$ & $2.13 \mathrm{E}+05$ & $1.41 \mathrm{E}+05$ & -- & $7.45 \mathrm{E}+04$ & 5.91E-02 & $1.26 \mathrm{E}+06$ & Inh. \\
\hline Os-193 & & & & $1.64 \mathrm{E}+04$ & $3.53 \mathrm{E}+05$ & $1.15 \mathrm{E}+04$ & $1.29 \mathrm{E}+04$ & -- & $1.15 \mathrm{E}+04$ & $2.13 \mathrm{E}-02$ & $5.41 \mathrm{E}+05$ & Food \\
\hline Os-194 & & & & $1.41 \mathrm{E}+02$ & $8.26 \mathrm{E}+02$ & $3.37 \mathrm{E}+02$ & $4.27 \mathrm{E}+05$ & -- & $1.41 \mathrm{E}+02$ & $4.60 \mathrm{E}-01$ & $3.07 \mathrm{E}+02$ & Inh. \\
\hline Os-196 & & & & $1.23 \mathrm{E}+05$ & $7.73 \mathrm{E}+81$ & $3.69 \mathrm{E}+06$ & $2.58 \mathrm{E}+05$ & -- & $1.23 \mathrm{E}+05$ & $4.46 \mathrm{E}-03$ & $2.75 \mathrm{E}+07$ & Inh. \\
\hline $\mathrm{P}-30$ & & & & -- & -- & -- & $2.84 \mathrm{E}+05$ & -- & $2.84 \mathrm{E}+05$ & 1.13E-04 & $2.51 \mathrm{E}+09$ & Direct \\
\hline $\mathrm{P}-32$ & $1.13 \mathrm{E}+01$ & 3.95E-05 & ing & $6.98 \mathrm{E}+01$ & $1.36 \mathrm{E}+03$ & $1.13 \mathrm{E}+01$ & -- & -- & $1.13 \mathrm{E}+01$ & $3.94 \mathrm{E}-05$ & $2.86 \mathrm{E}+05$ & Food \\
\hline $\mathrm{P}-33$ & $8.84 \mathrm{E}+01$ & $5.64 \mathrm{E}-04$ & ing & $1.60 \mathrm{E}+02$ & $1.10 \mathrm{E}+04$ & $8.89 \mathrm{E}+01$ & -- & -- & $8.89 \mathrm{E}+01$ & $5.70 \mathrm{E}-04$ & $1.56 \mathrm{E}+05$ & Food \\
\hline $\mathrm{Pa}-227$ & & & & $1.15 \mathrm{E}+03$ & -- & $8.96 \mathrm{E}+06$ & $8.52 \mathrm{E}+05$ & -- & $1.15 \mathrm{E}+03$ & $5.32 \mathrm{E}-05$ & $2.16 \mathrm{E}+07$ & Inh. \\
\hline $\mathrm{Pa}-228$ & & & & $1.62 \mathrm{E}+03$ & -- & $1.60 \mathrm{E}+05$ & $9.06 \mathrm{E}+02$ & -- & $9.06 \mathrm{E}+02$ & $1.45 \mathrm{E}-03$ & $6.25 \mathrm{E}+05$ & Direct \\
\hline Pa-229 & & & & $1.67 \mathrm{E}+04$ & -- & $3.59 \mathrm{E}+05$ & $1.36 \mathrm{E}+04$ & -- & $1.36 \mathrm{E}+04$ & $3.58 \mathrm{E}-02$ & $3.80 \mathrm{E}+05$ & Direct \\
\hline Pa-230 & & & & $1.57 \mathrm{E}+02$ & -- & $1.51 \mathrm{E}+04$ & $1.14 \mathrm{E}+03$ & -- & $1.57 \mathrm{E}+02$ & $4.82 \mathrm{E}-03$ & $3.26 \mathrm{E}+04$ & Inh. \\
\hline $\mathrm{Pa}-231$ & & & & $8.59 \mathrm{E}-01$ & -- & $1.14 \mathrm{E}+01$ & $1.51 \mathrm{E}+04$ & -- & $8.59 \mathrm{E}-01$ & $1.82 \mathrm{E}+01$ & $4.72 \mathrm{E}-02$ & Inh. \\
\hline $\mathrm{Pa}-232$ & & & & $1.18 \mathrm{E}+04$ & -- & $1.24 \mathrm{E}+05$ & $9.96 \mathrm{E}+02$ & -- & $9.96 \mathrm{E}+02$ & $2.32 \mathrm{E}-03$ & $4.30 \mathrm{E}+05$ & Direct \\
\hline $\mathrm{Pa}-233$ & & & & $3.02 \mathrm{E}+04$ & -- & $1.35 \mathrm{E}+04$ & $3.60 \mathrm{E}+03$ & -- & $3.60 \mathrm{E}+03$ & $1.74 \mathrm{E}-01$ & $2.08 \mathrm{E}+04$ & Direct \\
\hline Pa-234 & & & & $1.93 \mathrm{E}+05$ & -- & $7.67 \mathrm{E}+05$ & $1.02 \mathrm{E}+03$ & -- & $1.02 \mathrm{E}+03$ & $5.13 \mathrm{E}-04$ & $2.00 \mathrm{E}+06$ & Direct \\
\hline Pa-234m & & & & -- & -- & -- & $5.39 \mathrm{E}+07$ & -- & $5.39 \mathrm{E}+07$ & 7.84E-02 & $6.87 \mathrm{E}+08$ & Direct \\
\hline $\mathrm{Pa}-235$ & & & & -- & -- & -- & $3.74 \mathrm{E}+07$ & -- & $3.74 \mathrm{E}+07$ & $1.15 \mathrm{E}+00$ & $3.27 \mathrm{E}+07$ & Direct \\
\hline Pa-236 & & & & -- & -- & -- & $8.70 \mathrm{E}+04$ & -- & $8.70 \mathrm{E}+04$ & $9.94 \mathrm{E}-04$ & $8.75 \mathrm{E}+07$ & Direct \\
\hline Pa-237 & & & & -- & -- & -- & $1.37 \mathrm{E}+05$ & -- & $1.37 \mathrm{E}+05$ & $1.50 \mathrm{E}-03$ & $9.12 \mathrm{E}+07$ & Direct \\
\hline Pb-194 & & & & $7.45 \mathrm{E}+05$ & -- & $6.42 \mathrm{E}+07$ & $5.57 \mathrm{E}+04$ & -- & $5.57 \mathrm{E}+04$ & $6.90 \mathrm{E}-04$ & $8.08 \mathrm{E}+07$ & Direct \\
\hline $\mathrm{Pb}-195 \mathrm{~m}$ & & & & $3.72 \mathrm{E}+05$ & -- & $3.36 \mathrm{E}+07$ & $2.87 E+04$ & -- & $2.87 E+04$ & $4.70 \mathrm{E}-04$ & $6.10 \mathrm{E}+07$ & Direct \\
\hline Pb-196 & & & & $3.60 \mathrm{E}+05$ & -- & $1.49 \mathrm{E}+07$ & $3.96 \mathrm{E}+04$ & -- & $3.96 \mathrm{E}+04$ & $1.53 \mathrm{E}-03$ & $2.59 \mathrm{E}+07$ & Direct \\
\hline Pb-197 & & & & -- & -- & -- & $5.91 \mathrm{E}+04$ & -- & $5.91 \mathrm{E}+04$ & $4.95 \mathrm{E}-04$ & $1.19 \mathrm{E}+08$ & Direct \\
\hline $\mathrm{Pb}-197 \mathrm{~m}$ & & & & $2.43 \mathrm{E}+05$ & -- & $7.97 \mathrm{E}+06$ & $1.44 \mathrm{E}+04$ & -- & $1.44 \mathrm{E}+04$ & $6.47 \mathrm{E}-04$ & $2.22 \mathrm{E}+07$ & Direct \\
\hline $\mathrm{Pb}-198$ & & & & $1.28 \mathrm{E}+05$ & -- & $1.08 \mathrm{E}+06$ & $1.15 \mathrm{E}+04$ & -- & $1.15 \mathrm{E}+04$ & $1.74 \mathrm{E}-03$ & $6.60 \mathrm{E}+06$ & Direct \\
\hline Pb-199 & & & & $2.33 E+05$ & -- & $3.18 \mathrm{E}+06$ & $5.45 E+03$ & -- & $5.45 \mathrm{E}+03$ & $5.19 \mathrm{E}-04$ & $1.05 \mathrm{E}+07$ & Direct \\
\hline $\mathrm{Pb}-200$ & & & & $4.30 \mathrm{E}+04$ & -- & $3.17 \mathrm{E}+04$ & $4.99 \mathrm{E}+03$ & -- & $4.99 \mathrm{E}+03$ & $6.85 \mathrm{E}-03$ & $7.29 \mathrm{E}+05$ & Direct \\
\hline $\mathrm{Pb}-201$ & & & & $9.31 \mathrm{E}+04$ & -- & $1.75 \mathrm{E}+05$ & $2.04 \mathrm{E}+03$ & -- & $2.04 \mathrm{E}+03$ & $1.23 \mathrm{E}-03$ & $1.66 \mathrm{E}+06$ & Direct \\
\hline
\end{tabular}


Table C.2 Summary of Exposure Pathway TQs and Selection of Final HC-3 TQs (continued)

\begin{tabular}{|c|c|c|c|c|c|c|c|c|c|c|c|c|}
\hline \multirow[b]{2}{*}{$\begin{array}{c}\text { MASTER } \\
\text { ISOTOPE } \\
\text { LIST } \\
\end{array}$} & \multicolumn{3}{|c|}{ from NNSA SD G 1027} & \multicolumn{5}{|c|}{ Calculated Pathway Specific TQ Values } & \multicolumn{4}{|c|}{ Selected Final Threshold Quantities } \\
\hline & Act $(\mathrm{Ci})$ & Mass (g) & $\begin{array}{l}\text { Limiting } \\
\text { Pathway }\end{array}$ & $\begin{array}{c}\text { TQ }_{\text {inhale }} \\
(\mathrm{Ci})\end{array}$ & $\begin{array}{c}\text { TQ }_{\text {water }} \\
\text { (Ci) }\end{array}$ & $\mathrm{TQ}_{\text {food }}(\mathrm{Ci})$ & $\begin{array}{c}\mathrm{TQ}_{\text {direct }} \\
\text { (Ci) }\end{array}$ & $\mathrm{TQ}_{\text {sub }}(\mathrm{Ci})$ & Act (Ci) & Mass (g) & $\begin{array}{c}\text { Sp. Act. } \\
\text { (Ci/g) }\end{array}$ & $\begin{array}{l}\text { Limiting } \\
\text { Pathway }\end{array}$ \\
\hline $\mathrm{Pb}-201 \mathrm{~m}$ & & & & -- & -- & -- & $1.95 \mathrm{E}+06$ & -- & $1.95 \mathrm{E}+06$ & $2.12 \mathrm{E}-03$ & $9.20 \mathrm{E}+08$ & Direct \\
\hline $\mathrm{Pb}-202$ & & & & $7.98 \mathrm{E}+02$ & -- & $9.25 \mathrm{E}+01$ & $3.46 \mathrm{E}+05$ & -- & $9.25 \mathrm{E}+01$ & $1.57 \mathrm{E}+04$ & $5.90 \mathrm{E}-03$ & Food \\
\hline $\mathrm{Pb}-202 \mathrm{~m}$ & & & & $9.31 \mathrm{E}+04$ & -- & $5.51 \mathrm{E}+05$ & $1.65 \mathrm{E}+03$ & -- & $1.65 \mathrm{E}+03$ & $3.85 \mathrm{E}-04$ & $4.29 \mathrm{E}+06$ & Direct \\
\hline $\mathrm{Pb}-203$ & & & & $6.98 \mathrm{E}+04$ & -- & $2.37 E+04$ & $2.72 \mathrm{E}+03$ & -- & $2.72 \mathrm{E}+03$ & $9.17 \mathrm{E}-03$ & $2.97 \mathrm{E}+05$ & Direct \\
\hline $\mathrm{Pb}-204 \mathrm{~m}$ & & & & $2.54 \mathrm{E}+05$ & -- & $4.60 \mathrm{E}+06$ & $5.12 \mathrm{E}+03$ & -- & $5.12 E+03$ & $3.73 \mathrm{E}-04$ & $1.37 \mathrm{E}+07$ & Direct \\
\hline $\mathrm{Pb}-205$ & & & & $2.72 \mathrm{E}+04$ & -- & $2.87 \mathrm{E}+03$ & $3.16 \mathrm{E}+05$ & -- & $2.87 \mathrm{E}+03$ & $2.36 \mathrm{E}+07$ & $1.22 \mathrm{E}-04$ & Food \\
\hline Pb-209 & & & & $3.49 \mathrm{E}+05$ & -- & $1.40 \mathrm{E}+06$ & -- & -- & $3.49 \mathrm{E}+05$ & 7.57E-02 & $4.61 \mathrm{E}+06$ & Inh. \\
\hline $\mathrm{Pb}-210$ & $1.16 \mathrm{E}+00$ & $1.53 \mathrm{E}-02$ & ing & $1.02 \mathrm{E}+01$ & -- & $1.19 \mathrm{E}+00$ & $1.51 \mathrm{E}+05$ & -- & $1.19 \mathrm{E}+00$ & $1.55 \mathrm{E}-02$ & $7.63 \mathrm{E}+01$ & Food \\
\hline $\mathrm{Pb}-211$ & & & & $1.99 \mathrm{E}+03$ & -- & $2.37 E+06$ & $3.97 \mathrm{E}+05$ & -- & $1.99 \mathrm{E}+03$ & $8.08 \mathrm{E}-05$ & $2.47 \mathrm{E}+07$ & Inh. \\
\hline $\mathrm{Pb}-212$ & & & & $3.38 \mathrm{E}+02$ & -- & $4.22 \mathrm{E}+03$ & $9.68 \mathrm{E}+03$ & -- & $3.38 \mathrm{E}+02$ & $2.44 \mathrm{E}-04$ & $1.39 \mathrm{E}+06$ & Inh. \\
\hline $\mathrm{Pb}-214$ & & & & $2.33 \mathrm{E}+03$ & -- & $4.11 \mathrm{E}+06$ & $1.08 \mathrm{E}+05$ & -- & $2.33 \mathrm{E}+03$ & $7.10 \mathrm{E}-05$ & $3.28 \mathrm{E}+07$ & Inh. \\
\hline Pd-100 & & & & $1.15 \mathrm{E}+04$ & -- & $3.93 \mathrm{E}+03$ & $6.19 E+03$ & -- & $3.93 E+03$ & 1.09E-02 & $3.60 \mathrm{E}+05$ & Food \\
\hline Pd-101 & & & & $1.12 \mathrm{E}+05$ & -- & $3.37 \mathrm{E}+05$ & $5.00 \mathrm{E}+03$ & -- & $5.00 \mathrm{E}+03$ & $1.33 \mathrm{E}-03$ & $3.76 \mathrm{E}+06$ & Direct \\
\hline Pd-103 & & & & $2.79 E+04$ & -- & $7.34 \mathrm{E}+03$ & $5.14 \mathrm{E}+04$ & -- & $7.34 \mathrm{E}+03$ & $9.81 \mathrm{E}-02$ & $7.48 \mathrm{E}+04$ & Food \\
\hline Pd-107 & & & & $2.03 E+04$ & -- & $2.17 E+04$ & -- & -- & $2.03 E+04$ & $3.95 \mathrm{E}+07$ & $5.14 \mathrm{E}-04$ & Inh. \\
\hline Pd-109 & & & & $2.23 E+04$ & -- & $3.60 \mathrm{E}+04$ & $1.08 \mathrm{E}+05$ & -- & $2.23 E+04$ & $1.04 \mathrm{E}-02$ & $2.14 \mathrm{E}+06$ & Inh. \\
\hline Pd-109m & & & & -- & -- & -- & $1.39 \mathrm{E}+06$ & -- & $1.39 \mathrm{E}+06$ & $3.77 \mathrm{E}-03$ & $3.68 \mathrm{E}+08$ & Direct \\
\hline $\mathrm{Pd}-111$ & & & & $2.86 \mathrm{E}+05$ & -- & $1.31 \mathrm{E}+07$ & $6.48 \mathrm{E}+05$ & -- & $2.86 \mathrm{E}+05$ & $3.95 \mathrm{E}-03$ & $7.24 \mathrm{E}+07$ & Inh. \\
\hline Pd-112 & & & & $7.45 \mathrm{E}+03$ & -- & $4.97 \mathrm{E}+03$ & $2.07 \mathrm{E}+05$ & -- & $4.97 \mathrm{E}+03$ & $3.73 \mathrm{E}-03$ & $1.33 \mathrm{E}+06$ & Food \\
\hline $\mathrm{Pd}-114$ & & & & -- & -- & -- & $1.16 \mathrm{E}+07$ & -- & $1.16 \mathrm{E}+07$ & $1.70 \mathrm{E}-02$ & $6.82 \mathrm{E}+08$ & Direct \\
\hline Pd-96 & & & & -- & -- & -- & $2.47 \mathrm{E}+05$ & -- & $2.47 E+05$ & $2.57 \mathrm{E}-04$ & $9.64 \mathrm{E}+08$ & Direct \\
\hline Pd-97 & & & & -- & -- & -- & $9.82 \mathrm{E}+04$ & -- & $9.82 \mathrm{E}+04$ & 1.57E-04 & $6.26 \mathrm{E}+08$ & Direct \\
\hline Pd-98 & & & & $2.38 \mathrm{E}+05$ & -- & $1.38 \mathrm{E}+07$ & $9.85 \mathrm{E}+04$ & -- & $9.85 \mathrm{E}+04$ & $9.08 \mathrm{E}-04$ & $1.08 \mathrm{E}+08$ & Direct \\
\hline Pd-99 & & & & $3.28 \mathrm{E}+05$ & -- & $1.99 \mathrm{E}+07$ & $2.64 \mathrm{E}+04$ & -- & $2.64 E+04$ & $2.97 \mathrm{E}-04$ & $8.88 \mathrm{E}+07$ & Direct \\
\hline Pm-136 & & & & -- & -- & -- & $1.49 \mathrm{E}+05$ & -- & $1.49 \mathrm{E}+05$ & $1.93 \mathrm{E}-04$ & $7.76 \mathrm{E}+08$ & Direct \\
\hline Pm-137m & & & & -- & -- & -- & $1.69 \mathrm{E}+05$ & -- & $1.69 E+05$ & $2.96 \mathrm{E}-04$ & $5.72 \mathrm{E}+08$ & Direct \\
\hline Pm-138 & & & & -- & -- & -- & -- & -- & -- & -- & $4.21 \mathrm{E}+08$ & -- \\
\hline Pm-139 & & & & -- & -- & -- & $1.86 \mathrm{E}+05$ & -- & $1.86 \mathrm{E}+05$ & $5.70 \mathrm{E}-04$ & $3.26 \mathrm{E}+08$ & Direct \\
\hline Pm-140 & & & & -- & -- & -- & $4.50 \mathrm{E}+06$ & -- & $4.50 \mathrm{E}+06$ & 5.13E-04 & $8.76 \mathrm{E}+09$ & Direct \\
\hline Pm-140m & & & & -- & -- & -- & $4.02 \mathrm{E}+04$ & -- & $4.02 \mathrm{E}+04$ & $1.78 \mathrm{E}-04$ & $2.26 \mathrm{E}+08$ & Direct \\
\hline Pm-141 & & & & $4.47 \mathrm{E}+05$ & -- & $2.05 \mathrm{E}+07$ & $4.66 \mathrm{E}+04$ & -- & $4.66 \mathrm{E}+04$ & $7.30 \mathrm{E}-04$ & $6.38 \mathrm{E}+07$ & Direct \\
\hline Pm-142 & & & & -- & -- & -- & $1.24 \mathrm{E}+06$ & -- & $1.24 \mathrm{E}+06$ & $6.30 \mathrm{E}-04$ & $1.96 \mathrm{E}+09$ & Direct \\
\hline Pm-143 & & & & $7.98 \mathrm{E}+03$ & -- & $3.66 \mathrm{E}+03$ & $2.30 \mathrm{E}+03$ & -- & $2.30 \mathrm{E}+03$ & $6.68 \mathrm{E}-01$ & $3.45 \mathrm{E}+03$ & Direct \\
\hline Pm-144 & & & & $1.43 \mathrm{E}+03$ & -- & $8.58 \mathrm{E}+02$ & $4.65 \mathrm{E}+02$ & -- & $4.65 E+02$ & $1.86 \mathrm{E}-01$ & $2.50 \mathrm{E}+03$ & Direct \\
\hline Pm-145 & $3.31 E+03$ & $2.37 \mathrm{E}+01$ & i & $3.28 \mathrm{E}+03$ & -- & $7.34 \mathrm{E}+03$ & $2.34 \mathrm{E}+04$ & -- & $3.28 \mathrm{E}+03$ & $2.36 \mathrm{E}+01$ & $1.39 \mathrm{E}+02$ & Inh. \\
\hline
\end{tabular}


Table C.2 Summary of Exposure Pathway TQs and Selection of Final HC-3 TQs (continued)

\begin{tabular}{|c|c|c|c|c|c|c|c|c|c|c|c|c|}
\hline \multirow[b]{2}{*}{$\begin{array}{c}\text { MASTER } \\
\text { ISOTOPE } \\
\text { LIST } \\
\end{array}$} & \multicolumn{3}{|c|}{ from NNSA SD G 1027} & \multicolumn{5}{|c|}{ Calculated Pathway Specific TQ Values } & \multicolumn{4}{|c|}{ Selected Final Threshold Quantities } \\
\hline & Act (Ci) & Mass (g) & $\begin{array}{l}\text { Limiting } \\
\text { Pathway }\end{array}$ & $\begin{array}{c}\text { TQ }_{\text {inhale }} \\
\text { (Ci) }\end{array}$ & $\begin{array}{c}\text { TQ }_{\text {water }} \\
\text { (Ci) }\end{array}$ & $\mathrm{TQ}_{\text {food }}(\mathrm{Ci})$ & $\begin{array}{c}\text { TQ }_{\text {direct }} \\
\text { (Ci) }\end{array}$ & $\mathrm{TQ}_{\text {sub }}(\mathrm{Ci})$ & Act (Ci) & Mass (g) & $\begin{array}{l}\text { Sp. Act. } \\
\text { (Ci/g) }\end{array}$ & $\begin{array}{l}\text { Limiting } \\
\text { Pathway }\end{array}$ \\
\hline $\mathrm{Pm}-146$ & & & & $5.88 \mathrm{E}+02$ & -- & $9.00 \mathrm{E}+02$ & $9.64 \mathrm{E}+02$ & -- & $5.88 \mathrm{E}+02$ & $1.33 \mathrm{E}+00$ & $4.43 E+02$ & Inh. \\
\hline Pm-147 & $2.40 \mathrm{E}+03$ & $2.58 \mathrm{E}+00$ & $\mathrm{i}$ & $2.38 \mathrm{E}+03$ & -- & $3.14 \mathrm{E}+03$ & -- & -- & $2.38 \mathrm{E}+03$ & $2.56 \mathrm{E}+00$ & $9.28 \mathrm{E}+02$ & Inh. \\
\hline Pm-148 & & & & $5.08 \mathrm{E}+03$ & -- & $1.02 \mathrm{E}+03$ & $1.35 \mathrm{E}+03$ & -- & $1.02 \mathrm{E}+03$ & $6.21 \mathrm{E}-03$ & $1.64 \mathrm{E}+05$ & Food \\
\hline Pm-148m & & & & $2.07 \mathrm{E}+03$ & -- & $5.79 \mathrm{E}+02$ & $3.66 \mathrm{E}+02$ & -- & $3.66 \mathrm{E}+02$ & $1.71 \mathrm{E}-02$ & $2.14 \mathrm{E}+04$ & Direct \\
\hline Pm-149 & & & & $1.36 \mathrm{E}+04$ & -- & $5.66 \mathrm{E}+03$ & $7.98 \mathrm{E}+04$ & -- & $5.66 \mathrm{E}+03$ & $1.43 \mathrm{E}-02$ & $3.96 \mathrm{E}+05$ & Food \\
\hline Pm-150 & & & & $5.32 \mathrm{E}+04$ & -- & $3.72 \mathrm{E}+05$ & $3.15 E+03$ & -- & $3.15 E+03$ & $4.04 \mathrm{E}-04$ & $7.80 \mathrm{E}+06$ & Direct \\
\hline Pm-151 & & & & $1.75 \mathrm{E}+04$ & -- & $1.34 \mathrm{E}+04$ & $2.99 \mathrm{E}+03$ & -- & $2.99 \mathrm{E}+03$ & 4.09E-03 & $7.31 \mathrm{E}+05$ & Direct \\
\hline Pm-152 & & & & -- & -- & -- & $6.14 \mathrm{E}+05$ & -- & $6.14 \mathrm{E}+05$ & $2.04 \mathrm{E}-03$ & $3.00 \mathrm{E}+08$ & Direct \\
\hline Pm-152m & & & & -- & -- & -- & $6.34 \mathrm{E}+04$ & -- & $6.34 \mathrm{E}+04$ & $3.85 \mathrm{E}-04$ & $1.65 \mathrm{E}+08$ & Direct \\
\hline Pm-153 & & & & -- & -- & -- & $1.81 \mathrm{E}+06$ & -- & $1.81 \mathrm{E}+06$ & $7.71 \mathrm{E}-03$ & $2.34 \mathrm{E}+08$ & Direct \\
\hline Pm-154 & & & & -- & -- & -- & $2.34 \mathrm{E}+05$ & -- & $2.34 \mathrm{E}+05$ & $3.31 \mathrm{E}-04$ & $7.06 \mathrm{E}+08$ & Direct \\
\hline Pm-154m & & & & -- & -- & -- & $1.50 \mathrm{E}+05$ & -- & $1.50 \mathrm{E}+05$ & 3.30E-04 & $4.56 \mathrm{E}+08$ & Direct \\
\hline Po-203 & & & & $1.83 \mathrm{E}+05$ & -- & $8.09 \mathrm{E}+06$ & $1.20 \mathrm{E}+04$ & -- & $1.20 \mathrm{E}+04$ & $4.76 \mathrm{E}-04$ & $2.52 \mathrm{E}+07$ & Direct \\
\hline Po-204 & & & & $2.94 \mathrm{E}+04$ & -- & $2.37 \mathrm{E}+05$ & $2.96 \mathrm{E}+03$ & -- & $2.96 \mathrm{E}+03$ & $6.81 \mathrm{E}-04$ & $4.35 \mathrm{E}+06$ & Direct \\
\hline Po-205 & & & & $1.25 \mathrm{E}+05$ & -- & $2.43 \mathrm{E}+06$ & $4.24 \mathrm{E}+03$ & -- & $4.24 \mathrm{E}+03$ & $5.00 \mathrm{E}-04$ & $8.49 \mathrm{E}+06$ & Direct \\
\hline Po-206 & & & & $2.15 \mathrm{E}+02$ & -- & $4.31 \mathrm{E}+02$ & $6.33 E+02$ & -- & $2.15 \mathrm{E}+02$ & $2.98 \mathrm{E}-03$ & $7.20 \mathrm{E}+04$ & Inh. \\
\hline Po-207 & & & & $7.45 \mathrm{E}+04$ & -- & $3.20 \mathrm{E}+05$ & $1.65 \mathrm{E}+03$ & -- & $1.65 \mathrm{E}+03$ & $6.36 \mathrm{E}-04$ & $2.60 \mathrm{E}+06$ & Direct \\
\hline Po-208 & & & & $3.19 \mathrm{E}+00$ & -- & $2.63 \mathrm{E}+00$ & $3.63 \mathrm{E}+07$ & -- & $2.63 \mathrm{E}+00$ & $4.43 \mathrm{E}-03$ & $5.93 \mathrm{E}+02$ & Food \\
\hline Po-209 & & & & $3.28 \mathrm{E}+00$ & -- & $2.69 \mathrm{E}+00$ & $2.34 \mathrm{E}+05$ & -- & $2.69 \mathrm{E}+00$ & $1.60 \mathrm{E}-01$ & $1.68 \mathrm{E}+01$ & Food \\
\hline Po-210 & $3.57 \mathrm{E}+00$ & 7.94E-04 & ing & $3.72 \mathrm{E}+00$ & -- & $3.65 \mathrm{E}+00$ & -- & -- & $3.65 \mathrm{E}+00$ & $8.11 \mathrm{E}-04$ & $4.49 \mathrm{E}+03$ & Food \\
\hline Po-211 & & & & -- & -- & -- & $1.08 \mathrm{E}+10$ & -- & $1.08 \mathrm{E}+10$ & $1.04 \mathrm{E}-01$ & $1.04 \mathrm{E}+11$ & Direct \\
\hline Po-212 & & & & -- & -- & -- & -- & -- & -- & -- & $1.74 \mathrm{E}+17$ & -- \\
\hline Po-212m & & & & -- & -- & -- & $1.22 \mathrm{E}+07$ & -- & $1.22 \mathrm{E}+07$ & $1.03 \mathrm{E}-02$ & $1.18 \mathrm{E}+09$ & Direct \\
\hline Po-213 & & & & -- & -- & -- & -- & -- & -- & -- & $1.26 \mathrm{E}+16$ & -- \\
\hline Po-214 & & & & -- & -- & -- & -- & -- & -- & -- & $3.21 \mathrm{E}+14$ & -- \\
\hline Po-215 & & & & -- & -- & -- & $2.44 \mathrm{E}+14$ & -- & $2.44 \mathrm{E}+14$ & $8.29 \mathrm{E}+00$ & $2.95 \mathrm{E}+13$ & Direct \\
\hline Po-216 & & & & -- & -- & -- & -- & -- & -- & -- & $3.48 \mathrm{E}+11$ & -- \\
\hline Po-218 & & & & -- & -- & -- & -- & -- & -- & -- & $2.83 E+08$ & -- \\
\hline Pr-134 & & & & $2.23 E+05$ & -- & $1.73 \mathrm{E}+07$ & $2.09 \mathrm{E}+04$ & -- & $2.09 \mathrm{E}+04$ & 1.64E-04 & $1.28 \mathrm{E}+08$ & Direct \\
\hline Pr-134m & & & & $3.28 \mathrm{E}+05$ & -- & $1.97 \mathrm{E}+07$ & $1.85 \mathrm{E}+04$ & -- & $1.85 \mathrm{E}+04$ & $2.23 \mathrm{E}-04$ & $8.26 \mathrm{E}+07$ & Direct \\
\hline Pr-135 & & & & $3.10 \mathrm{E}+05$ & -- & $1.40 \mathrm{E}+07$ & $3.45 \mathrm{E}+04$ & -- & $3.45 \mathrm{E}+04$ & $5.95 \mathrm{E}-04$ & $5.81 \mathrm{E}+07$ & Direct \\
\hline Pr-136 & & & & $4.47 \mathrm{E}+05$ & -- & $3.57 \mathrm{E}+07$ & $2.63 E+04$ & -- & $2.63 \mathrm{E}+04$ & $2.49 \mathrm{E}-04$ & $1.06 \mathrm{E}+08$ & Direct \\
\hline Pr-137 & & & & $3.19 \mathrm{E}+05$ & -- & $5.05 \mathrm{E}+06$ & $1.89 \mathrm{E}+04$ & -- & $1.89 \mathrm{E}+04$ & $1.05 \mathrm{E}-03$ & $1.79 \mathrm{E}+07$ & Direct \\
\hline Pr-138 & & & & -- & -- & -- & $6.14 \mathrm{E}+05$ & -- & $6.14 \mathrm{E}+05$ & $6.54 \mathrm{E}-04$ & $9.40 \mathrm{E}+08$ & Direct \\
\hline $\operatorname{Pr}-138 \mathrm{~m}$ & & & & $8.59 E+04$ & -- & $9.47 E+05$ & $2.32 \mathrm{E}+03$ & -- & $2.32 \mathrm{E}+03$ & $2.15 \mathrm{E}-04$ & $1.08 \mathrm{E}+07$ & Direct \\
\hline
\end{tabular}


Table C.2 Summary of Exposure Pathway TQs and Selection of Final HC-3 TQs (continued)

\begin{tabular}{|c|c|c|c|c|c|c|c|c|c|c|c|c|}
\hline \multirow[b]{2}{*}{$\begin{array}{c}\text { MASTER } \\
\text { ISOTOPE } \\
\text { LIST } \\
\end{array}$} & \multicolumn{3}{|c|}{ from NNSA SD G 1027} & \multicolumn{5}{|c|}{ Calculated Pathway Specific TQ Values } & \multicolumn{4}{|c|}{ Selected Final Threshold Quantities } \\
\hline & Act $(\mathrm{Ci})$ & Mass (g) & $\begin{array}{l}\text { Limiting } \\
\text { Pathway }\end{array}$ & $\begin{array}{c}\text { TQ }_{\text {inhale }} \\
(\mathrm{Ci})\end{array}$ & $\begin{array}{c}\text { TQ }_{\text {water }} \\
\text { (Ci) }\end{array}$ & $\mathrm{TQ}_{\text {food }}(\mathrm{Ci})$ & $\begin{array}{c}\mathrm{TQ}_{\text {direct }} \\
\text { (Ci) }\end{array}$ & $\mathrm{TQ}_{\text {sub }}(\mathrm{Ci})$ & Act (Ci) & Mass (g) & $\begin{array}{c}\text { Sp. Act. } \\
\text { (Ci/g) }\end{array}$ & $\begin{array}{l}\text { Limiting } \\
\text { Pathway }\end{array}$ \\
\hline Pr-139 & & & & $3.72 \mathrm{E}+05$ & -- & $1.86 \mathrm{E}+06$ & $2.25 \mathrm{E}+04$ & -- & $2.25 \mathrm{E}+04$ & 4.49E-03 & $5.00 \mathrm{E}+06$ & Direct \\
\hline Pr-140 & & & & -- & -- & -- & $3.91 \mathrm{E}+05$ & -- & $3.91 \mathrm{E}+05$ & $9.86 \mathrm{E}-04$ & $3.96 \mathrm{E}+08$ & Direct \\
\hline $\operatorname{Pr}-142$ & & & & $1.51 \mathrm{E}+04$ & -- & $1.09 \mathrm{E}+04$ & $1.86 \mathrm{E}+04$ & -- & $1.09 \mathrm{E}+04$ & $9.46 \mathrm{E}-03$ & $1.15 \mathrm{E}+06$ & Food \\
\hline Pr-142m & & & & $1.19 \mathrm{E}+06$ & -- & $6.21 \mathrm{E}+07$ & -- & -- & $1.19 \mathrm{E}+06$ & $1.31 \mathrm{E}-02$ & $9.08 \mathrm{E}+07$ & Inh. \\
\hline Pr-143 & & & & $4.86 \mathrm{E}+03$ & -- & $1.30 \mathrm{E}+03$ & -- & -- & $1.30 \mathrm{E}+03$ & 1.93E-02 & $6.74 \mathrm{E}+04$ & Food \\
\hline Pr-144 & & & & $3.72 \mathrm{E}+05$ & -- & $1.79 \mathrm{E}+07$ & $1.32 \mathrm{E}+06$ & -- & $3.72 \mathrm{E}+05$ & $4.92 \mathrm{E}-03$ & $7.56 \mathrm{E}+07$ & Inh. \\
\hline Pr-144m & & & & -- & -- & -- & $7.99 \mathrm{E}+06$ & -- & $7.99 \mathrm{E}+06$ & $4.40 \mathrm{E}-02$ & $1.81 \mathrm{E}+08$ & Direct \\
\hline Pr-145 & & & & $4.30 E+04$ & -- & $1.12 \mathrm{E}+05$ & $1.64 \mathrm{E}+05$ & -- & $4.30 E+04$ & 1.19E-02 & $3.62 E+06$ & Inh. \\
\hline Pr-146 & & & & $2.28 \mathrm{E}+05$ & -- & $8.19 \mathrm{E}+06$ & $2.97 E+04$ & -- & $2.97 \mathrm{E}+04$ & $5.56 \mathrm{E}-04$ & $5.34 \mathrm{E}+07$ & Direct \\
\hline Pr-147 & & & & $3.72 \mathrm{E}+05$ & -- & $3.44 \mathrm{E}+07$ & $6.17 E+04$ & -- & $6.17 \mathrm{E}+04$ & $6.56 \mathrm{E}-04$ & $9.41 \mathrm{E}+07$ & Direct \\
\hline Pr-148 & & & & -- & -- & -- & $3.19 \mathrm{E}+05$ & -- & $3.19 E+05$ & $5.76 \mathrm{E}-04$ & $5.55 \mathrm{E}+08$ & Direct \\
\hline Pr-148m & & & & -- & -- & -- & $3.86 \mathrm{E}+05$ & -- & $3.86 \mathrm{E}+05$ & $6.10 \mathrm{E}-04$ & $6.32 \mathrm{E}+08$ & Direct \\
\hline Pt-183 & & & & -- & -- & -- & -- & -- & -- & -- & $1.58 \mathrm{E}+08$ & -- \\
\hline Pt-184 & & & & $4.30 \mathrm{E}+05$ & $2.30 \mathrm{E}+159$ & $2.96 \mathrm{E}+07$ & $5.76 \mathrm{E}+04$ & -- & $5.76 \mathrm{E}+04$ & $9.76 \mathrm{E}-04$ & $5.91 \mathrm{E}+07$ & Direct \\
\hline Pt-186 & & & & $1.69 \mathrm{E}+05$ & $1.23 \mathrm{E}+28$ & $1.38 \mathrm{E}+06$ & $8.16 E+03$ & -- & $8.16 E+03$ & $9.69 \mathrm{E}-04$ & $8.43 E+06$ & Direct \\
\hline Pt-187 & & & & $1.86 \mathrm{E}+05$ & $6.08 \mathrm{E}+24$ & $1.25 \mathrm{E}+06$ & $8.32 E+03$ & -- & $8.32 E+03$ & 1.17E-03 & $7.13 E+06$ & Direct \\
\hline Pt-188 & & & & $1.77 \mathrm{E}+04$ & $5.25 \mathrm{E}+03$ & $2.38 \mathrm{E}+03$ & $3.72 \mathrm{E}+03$ & -- & $2.38 \mathrm{E}+03$ & $3.50 \mathrm{E}-02$ & $6.81 \mathrm{E}+04$ & Food \\
\hline Pt-189 & & & & $1.53 \mathrm{E}+05$ & $2.41 \mathrm{E}+09$ & $2.03 E+05$ & $4.36 \mathrm{E}+03$ & -- & $4.36 \mathrm{E}+03$ & $2.86 \mathrm{E}-03$ & $1.53 \mathrm{E}+06$ & Direct \\
\hline Pt-190 & & & & $8.59 \mathrm{E}+01$ & $2.91 \mathrm{E}+02$ & $1.18 \mathrm{E}+02$ & -- & -- & $8.59 \mathrm{E}+01$ & $2.97 \mathrm{E}+10$ & 2.90E-09 & Inh. \\
\hline Pt-191 & & & & $5.88 \mathrm{E}+04$ & $6.50 \mathrm{E}+04$ & $1.34 \mathrm{E}+04$ & $2.69 \mathrm{E}+03$ & -- & $2.69 \mathrm{E}+03$ & $1.10 \mathrm{E}-02$ & $2.44 \mathrm{E}+05$ & Direct \\
\hline Pt-193 & & & & $4.14 \mathrm{E}+05$ & $6.38 \mathrm{E}+04$ & $2.59 \mathrm{E}+04$ & $3.46 \mathrm{E}+05$ & -- & $2.59 \mathrm{E}+04$ & $7.00 \mathrm{E}+02$ & $3.71 \mathrm{E}+01$ & Food \\
\hline Pt-193m & & & & $5.32 E+04$ & $2.19 \mathrm{E}+04$ & $7.17 E+03$ & $6.14 \mathrm{E}+04$ & -- & $7.17 E+03$ & $4.59 \mathrm{E}-02$ & $1.56 \mathrm{E}+05$ & Food \\
\hline Pt-195m & & & & $3.60 \mathrm{E}+04$ & $1.76 \mathrm{E}+04$ & $5.42 \mathrm{E}+03$ & $1.03 \mathrm{E}+04$ & -- & $5.42 \mathrm{E}+03$ & $3.25 \mathrm{E}-02$ & $1.67 \mathrm{E}+05$ & Food \\
\hline Pt-197 & & & & $6.98 \mathrm{E}+04$ & $9.97 \mathrm{E}+06$ & $3.69 \mathrm{E}+04$ & $4.42 \mathrm{E}+04$ & -- & $3.69 E+04$ & $4.24 \mathrm{E}-02$ & $8.69 \mathrm{E}+05$ & Food \\
\hline Pt-197m & & & & $2.60 \mathrm{E}+05$ & $1.49 \mathrm{E}+34$ & $1.95 \mathrm{E}+06$ & $9.20 \mathrm{E}+04$ & -- & $9.20 \mathrm{E}+04$ & $9.10 \mathrm{E}-03$ & $1.01 \mathrm{E}+07$ & Direct \\
\hline Pt-199 & & & & $5.08 \mathrm{E}+05$ & $2.81 \mathrm{E}+92$ & $1.28 \mathrm{E}+07$ & $1.17 \mathrm{E}+05$ & -- & $1.17 \mathrm{E}+05$ & $3.80 \mathrm{E}-03$ & $3.07 \mathrm{E}+07$ & Direct \\
\hline Pt-200 & & & & $2.79 \mathrm{E}+04$ & $6.27 \mathrm{E}+07$ & $1.77 \mathrm{E}+04$ & $2.17 E+04$ & -- & $1.77 \mathrm{E}+04$ & $1.41 \mathrm{E}-02$ & $1.25 \mathrm{E}+06$ & Food \\
\hline Pt-202 & & & & $7.98 \mathrm{E}+03$ & $1.53 \mathrm{E}+04$ & $1.46 \mathrm{E}+03$ & -- & -- & $1.46 \mathrm{E}+03$ & $4.15 \mathrm{E}-03$ & $3.53 \mathrm{E}+05$ & Food \\
\hline Pu-232 & & & & $4.47 \mathrm{E}+03$ & -- & $1.58 \mathrm{E}+07$ & $3.39 \mathrm{E}+05$ & -- & $4.47 \mathrm{E}+03$ & $1.86 \mathrm{E}-04$ & $2.40 \mathrm{E}+07$ & Inh. \\
\hline $\mathrm{Pu}-234$ & & & & $5.08 \mathrm{E}+03$ & -- & $1.87 \mathrm{E}+06$ & $2.36 \mathrm{E}+04$ & -- & $5.08 \mathrm{E}+03$ & $3.34 \mathrm{E}-03$ & $1.52 \mathrm{E}+06$ & Inh. \\
\hline Pu-235 & & & & $4.30 \mathrm{E}+07$ & -- & $2.91 \mathrm{E}+09$ & $3.02 \mathrm{E}+05$ & -- & $3.02 \mathrm{E}+05$ & $9.56 \mathrm{E}-03$ & $3.16 \mathrm{E}+07$ & Direct \\
\hline Pu-236 & & & & $6.20 \mathrm{E}+00$ & -- & $9.48 \mathrm{E}+01$ & $3.63 E+05$ & -- & $6.20 \mathrm{E}+00$ & $1.17 \mathrm{E}-02$ & $5.31 \mathrm{E}+02$ & Inh. \\
\hline Pu-237 & & & & $3.10 \mathrm{E}+05$ & -- & $1.02 \mathrm{E}+05$ & $1.40 \mathrm{E}+04$ & -- & $1.40 \mathrm{E}+04$ & $1.15 \mathrm{E}+00$ & $1.22 \mathrm{E}+04$ & Direct \\
\hline Pu-238 & $2.62 \mathrm{E}+00$ & $1.53 \mathrm{E}-01$ & $\mathrm{i}$ & $2.60 \mathrm{E}+00$ & -- & $3.51 \mathrm{E}+01$ & $4.03 E+05$ & -- & $2.60 E+00$ & $1.52 \mathrm{E}-01$ & $1.71 \mathrm{E}+01$ & Inh. \\
\hline Pu-239 & $2.40 \mathrm{E}+00$ & $3.86 \mathrm{E}+01$ & i & $2.38 \mathrm{E}+00$ & -- & $3.22 \mathrm{E}+01$ & $9.07 E+05$ & -- & $2.38 \mathrm{E}+00$ & $3.82 \mathrm{E}+01$ & $6.21 \mathrm{E}-02$ & Inh. \\
\hline
\end{tabular}


Table C.2 Summary of Exposure Pathway TQs and Selection of Final HC-3 TQs (continued)

\begin{tabular}{|c|c|c|c|c|c|c|c|c|c|c|c|c|}
\hline \multirow[b]{2}{*}{$\begin{array}{c}\text { MASTER } \\
\text { ISOTOPE } \\
\text { LIST } \\
\end{array}$} & \multicolumn{3}{|c|}{ from NNSA SD G 1027} & \multicolumn{5}{|c|}{ Calculated Pathway Specific TQ Values } & \multicolumn{4}{|c|}{ Selected Final Threshold Quantities } \\
\hline & Act $(\mathrm{Ci})$ & Mass (g) & $\begin{array}{l}\text { Limiting } \\
\text { Pathway }\end{array}$ & $\begin{array}{c}\text { TQ }_{\text {inhale }} \\
(\mathrm{Ci})\end{array}$ & $\begin{array}{c}\text { TQ }_{\text {water }} \\
\text { (Ci) }\end{array}$ & $\mathrm{TQ}_{\text {food }}(\mathrm{Ci})$ & $\begin{array}{c}T_{\text {direct }} \\
\text { (Ci) }\end{array}$ & $\mathrm{TQ}_{\text {sub }}(\mathrm{Ci})$ & Act (Ci) & Mass (g) & $\begin{array}{c}\text { Sp. Act. } \\
\text { (Ci/g) }\end{array}$ & $\begin{array}{l}\text { Limiting } \\
\text { Pathway }\end{array}$ \\
\hline $\mathrm{Pu}-240$ & $2.40 \mathrm{E}+00$ & $1.05 \mathrm{E}+01$ & $\mathrm{i}$ & $2.38 \mathrm{E}+00$ & -- & $3.22 \mathrm{E}+01$ & $4.27 \mathrm{E}+05$ & -- & $2.38 \mathrm{E}+00$ & $1.04 \mathrm{E}+01$ & $2.28 \mathrm{E}-01$ & Inh. \\
\hline Pu-241 & $1.32 \mathrm{E}+02$ & $1.29 \mathrm{E}+00$ & $\mathrm{i}$ & $1.31 \mathrm{E}+02$ & -- & $1.72 \mathrm{E}+03$ & -- & -- & $1.31 \mathrm{E}+02$ & $1.28 \mathrm{E}+00$ & $1.03 \mathrm{E}+02$ & Inh. \\
\hline Pu-242 & $2.56 \mathrm{E}+00$ & $6.49 \mathrm{E}+02$ & $\mathrm{i}$ & $2.54 \mathrm{E}+00$ & -- & $3.36 \mathrm{E}+01$ & $5.19 \mathrm{E}+05$ & -- & $2.54 \mathrm{E}+00$ & $6.47 \mathrm{E}+02$ & $3.92 \mathrm{E}-03$ & Inh. \\
\hline Pu-243 & & & & $1.02 \mathrm{E}+06$ & -- & $6.19 \mathrm{E}+06$ & $9.86 \mathrm{E}+04$ & -- & $9.86 \mathrm{E}+04$ & $3.79 \mathrm{E}-02$ & $2.60 \mathrm{E}+06$ & Direct \\
\hline Pu-244 & & & & $2.54 \mathrm{E}+00$ & -- & $3.36 \mathrm{E}+01$ & $6.05 \mathrm{E}+05$ & -- & $2.54 \mathrm{E}+00$ & $1.43 \mathrm{E}+05$ & 1.77E-05 & Inh. \\
\hline Pu-245 & & & & $1.72 \mathrm{E}+05$ & -- & $3.50 \mathrm{E}+05$ & $3.47 \mathrm{E}+03$ & -- & $3.47 \mathrm{E}+03$ & $2.85 \mathrm{E}-03$ & $1.22 \mathrm{E}+06$ & Direct \\
\hline Pu-246 & & & & $1.47 \mathrm{E}+04$ & -- & $5.31 \mathrm{E}+03$ & $5.34 \mathrm{E}+03$ & -- & $5.31 \mathrm{E}+03$ & $1.09 \mathrm{E}-01$ & $4.89 \mathrm{E}+04$ & Food \\
\hline Ra-219 & & & & -- & -- & -- & $2.56 \mathrm{E}+10$ & -- & $2.56 \mathrm{E}+10$ & $4.96 \mathrm{E}-03$ & $5.15 \mathrm{E}+12$ & Direct \\
\hline Ra-220 & & & & -- & -- & -- & $5.20 \mathrm{E}+11$ & -- & $5.20 \mathrm{E}+11$ & $1.82 \mathrm{E}-01$ & $2.86 \mathrm{E}+12$ & Direct \\
\hline Ra-221 & & & & -- & -- & -- & $3.98 \mathrm{E}+07$ & -- & $3.98 \mathrm{E}+07$ & $2.18 \mathrm{E}-02$ & $1.82 \mathrm{E}+09$ & Direct \\
\hline Ra-222 & & & & -- & -- & -- & $1.26 \mathrm{E}+08$ & -- & $1.26 \mathrm{E}+08$ & $9.40 \mathrm{E}-02$ & $1.34 \mathrm{E}+09$ & Direct \\
\hline Ra-223 & $1.63 \mathrm{E}+01$ & 3.19E-04 & $\mathrm{i}$ & $1.62 \mathrm{E}+01$ & -- & $1.70 \mathrm{E}+02$ & $5.58 \mathrm{E}+03$ & -- & $1.62 \mathrm{E}+01$ & $3.16 \mathrm{E}-04$ & $5.12 \mathrm{E}+04$ & Inh. \\
\hline Ra-224 & $3.88 \mathrm{E}+01$ & $2.44 \mathrm{E}-04$ & $\mathrm{i}$ & $3.85 \mathrm{E}+01$ & -- & $5.67 \mathrm{E}+02$ & $8.05 \mathrm{E}+04$ & -- & $3.85 \mathrm{E}+01$ & $2.42 \mathrm{E}-04$ & $1.59 \mathrm{E}+05$ & Inh. \\
\hline Ra-225 & $1.94 \mathrm{E}+01$ & 4.99E-04 & $\mathrm{i}$ & $1.93 \mathrm{E}+01$ & -- & $1.57 \mathrm{E}+02$ & $5.46 \mathrm{E}+04$ & -- & $1.93 \mathrm{E}+01$ & $4.91 \mathrm{E}-04$ & $3.92 \mathrm{E}+04$ & Inh. \\
\hline Ra-226 & & & & $3.49 \mathrm{E}+01$ & -- & $2.88 \mathrm{E}+01$ & $1.08 \mathrm{E}+05$ & -- & $2.88 \mathrm{E}+01$ & $2.91 \mathrm{E}+01$ & $9.89 \mathrm{E}-01$ & Food \\
\hline Ra-227 & & & & $3.99 \mathrm{E}+05$ & -- & $4.36 \mathrm{E}+07$ & $1.03 \mathrm{E}+05$ & -- & $1.03 E+05$ & $5.25 \mathrm{E}-03$ & $1.96 \mathrm{E}+07$ & Direct \\
\hline Ra-228 & & & & $4.30 \mathrm{E}+01$ & -- & $1.21 \mathrm{E}+01$ & -- & -- & $1.21 \mathrm{E}+01$ & 4.43E-02 & $2.73 \mathrm{E}+02$ & Food \\
\hline Ra-230 & & & & $6.57 \mathrm{E}+05$ & -- & $8.76 \mathrm{E}+06$ & $9.81 \mathrm{E}+04$ & -- & $9.81 \mathrm{E}+04$ & $1.12 \mathrm{E}-02$ & $8.79 \mathrm{E}+06$ & Direct \\
\hline Rb-77 & & & & -- & -- & -- & $1.07 \mathrm{E}+05$ & -- & $1.07 \mathrm{E}+05$ & $1.62 \mathrm{E}-04$ & $6.61 \mathrm{E}+08$ & Direct \\
\hline $\mathrm{Rb}-78$ & & & & $3.02 \mathrm{E}+05$ & -- & $1.24 \mathrm{E}+07$ & $1.00 \mathrm{E}+04$ & -- & $1.00 \mathrm{E}+04$ & 7.34E-05 & $1.37 \mathrm{E}+08$ & Direct \\
\hline $\mathrm{Rb}-78 \mathrm{~m}$ & & & & -- & -- & -- & $3.93 \mathrm{E}+04$ & -- & $3.93 E+04$ & $9.34 \mathrm{E}-05$ & $4.20 \mathrm{E}+08$ & Direct \\
\hline Rb-79 & & & & $3.72 \mathrm{E}+05$ & -- & $1.34 \mathrm{E}+07$ & $2.33 E+04$ & -- & $2.33 E+04$ & $2.24 \mathrm{E}-04$ & $1.04 \mathrm{E}+08$ & Direct \\
\hline $\mathrm{Rb}-80$ & & & & -- & -- & -- & $1.03 \mathrm{E}+06$ & -- & $1.03 \mathrm{E}+06$ & $2.47 \mathrm{E}-04$ & $4.15 \mathrm{E}+09$ & Direct \\
\hline $\mathrm{Rb}-81$ & & & & $1.64 \mathrm{E}+05$ & -- & $1.05 \mathrm{E}+06$ & $4.35 \mathrm{E}+03$ & -- & $4.35 \mathrm{E}+03$ & $5.14 \mathrm{E}-04$ & $8.46 \mathrm{E}+06$ & Direct \\
\hline $\mathrm{Rb}-81 \mathrm{~m}$ & & & & $8.59 \mathrm{E}+05$ & -- & $4.95 \mathrm{E}+07$ & $2.26 \mathrm{E}+06$ & -- & $8.59 E+05$ & $1.18 \mathrm{E}-02$ & $7.26 \mathrm{E}+07$ & Inh. \\
\hline $\mathrm{Rb}-82$ & & & & -- & -- & -- & $5.10 \mathrm{E}+05$ & -- & $5.10 \mathrm{E}+05$ & $2.89 \mathrm{E}-04$ & $1.77 \mathrm{E}+09$ & Direct \\
\hline $\mathrm{Rb}-82 \mathrm{~m}$ & & & & $5.08 \mathrm{E}+04$ & -- & $3.23 \mathrm{E}+05$ & $7.18 \mathrm{E}+02$ & -- & $7.18 \mathrm{E}+02$ & $1.16 \mathrm{E}-04$ & $6.17 \mathrm{E}+06$ & Direct \\
\hline $\mathrm{Rb}-83$ & & & & $1.12 \mathrm{E}+04$ & -- & $4.81 \mathrm{E}+02$ & $1.44 \mathrm{E}+03$ & -- & $4.81 \mathrm{E}+02$ & $2.63 \mathrm{E}-02$ & $1.83 \mathrm{E}+04$ & Food \\
\hline $\mathrm{Rb}-84$ & & & & $7.45 \mathrm{E}+03$ & -- & $3.93 \mathrm{E}+02$ & $7.99 \mathrm{E}+02$ & -- & $3.93 E+02$ & $8.28 \mathrm{E}-03$ & $4.75 \mathrm{E}+04$ & Food \\
\hline $\mathrm{Rb}-84 \mathrm{~m}$ & & & & $1.25 \mathrm{E}+06$ & -- & $1.07 \mathrm{E}+08$ & $9.34 \mathrm{E}+04$ & -- & $9.34 \mathrm{E}+04$ & $8.44 \mathrm{E}-04$ & $1.11 \mathrm{E}+08$ & Direct \\
\hline $\mathrm{Rb}-86$ & & & & $8.59 \mathrm{E}+03$ & -- & $4.78 \mathrm{E}+02$ & $7.83 \mathrm{E}+03$ & -- & $4.78 \mathrm{E}+02$ & $5.87 \mathrm{E}-03$ & $8.14 \mathrm{E}+04$ & Food \\
\hline $\mathrm{Rb}-86 \mathrm{~m}$ & & & & -- & -- & -- & $1.30 \mathrm{E}+06$ & -- & $1.30 \mathrm{E}+06$ & $6.06 \mathrm{E}-04$ & $2.15 \mathrm{E}+09$ & Direct \\
\hline $\mathrm{Rb}-87$ & & & & $1.47 \mathrm{E}+04$ & -- & $5.35 \mathrm{E}+02$ & -- & -- & $5.35 \mathrm{E}+02$ & $6.11 \mathrm{E}+09$ & $8.75 \mathrm{E}-08$ & Food \\
\hline $\mathrm{Rb}-88$ & & & & $3.99 \mathrm{E}+05$ & -- & $9.58 \mathrm{E}+06$ & $6.48 \mathrm{E}+04$ & -- & $6.48 \mathrm{E}+04$ & $5.39 \mathrm{E}-04$ & $1.20 \mathrm{E}+08$ & Direct \\
\hline $\mathrm{Rb}-89$ & & & & $4.47 E+05$ & -- & $2.15 \mathrm{E}+07$ & $2.30 E+04$ & -- & $2.30 E+04$ & $1.65 \mathrm{E}-04$ & $1.39 \mathrm{E}+08$ & Direct \\
\hline
\end{tabular}


Table C.2 Summary of Exposure Pathway TQs and Selection of Final HC-3 TQs (continued)

\begin{tabular}{|c|c|c|c|c|c|c|c|c|c|c|c|c|}
\hline \multirow[b]{2}{*}{$\begin{array}{c}\text { MASTER } \\
\text { ISOTOPE } \\
\text { LIST } \\
\end{array}$} & \multicolumn{3}{|c|}{ from NNSA SD G 1027} & \multicolumn{5}{|c|}{ Calculated Pathway Specific TQ Values } & \multicolumn{4}{|c|}{ Selected Final Threshold Quantities } \\
\hline & Act $(\mathrm{Ci})$ & Mass (g) & $\begin{array}{l}\text { Limiting } \\
\text { Pathway }\end{array}$ & $\begin{array}{c}\text { TQ }_{\text {inhale }} \\
(\mathrm{Ci})\end{array}$ & $\begin{array}{c}\text { TQ }_{\text {water }} \\
\text { (Ci) }\end{array}$ & $\mathrm{TQ}_{\text {food }}(\mathrm{Ci})$ & $\begin{array}{c}T_{\text {direct }} \\
\text { (Ci) }\end{array}$ & $\mathrm{TQ}_{\text {sub }}(\mathrm{Ci})$ & Act (Ci) & Mass (g) & $\begin{array}{c}\text { Sp. Act. } \\
\text { (Ci/g) }\end{array}$ & $\begin{array}{l}\text { Limiting } \\
\text { Pathway }\end{array}$ \\
\hline $\mathrm{Rb}-90$ & & & & -- & -- & -- & $1.36 \mathrm{E}+05$ & -- & $1.36 \mathrm{E}+05$ & $1.71 \mathrm{E}-04$ & $7.94 \mathrm{E}+08$ & Direct \\
\hline $\mathrm{Rb}-90 \mathrm{~m}$ & & & & -- & -- & -- & $5.20 \mathrm{E}+04$ & -- & $5.20 \mathrm{E}+04$ & $1.07 \mathrm{E}-04$ & $4.86 \mathrm{E}+08$ & Direct \\
\hline Re-177 & & & & $5.08 \mathrm{E}+05$ & $2.38 \mathrm{E}+195$ & $4.76 \mathrm{E}+07$ & $8.34 \mathrm{E}+04$ & -- & $8.34 \mathrm{E}+04$ & $1.10 \mathrm{E}-03$ & $7.59 \mathrm{E}+07$ & Direct \\
\hline Re-178 & & & & $4.65 \mathrm{E}+05$ & $5.21 E+206$ & $4.44 \mathrm{E}+07$ & $4.51 \mathrm{E}+04$ & -- & $4.51 \mathrm{E}+04$ & $5.63 \mathrm{E}-04$ & $8.00 \mathrm{E}+07$ & Direct \\
\hline Re-179 & & & & $5.32 \mathrm{E}+05$ & $2.84 \mathrm{E}+142$ & $4.70 \mathrm{E}+07$ & $3.43 \mathrm{E}+04$ & -- & $3.43 E+04$ & $6.36 \mathrm{E}-04$ & $5.39 \mathrm{E}+07$ & Direct \\
\hline Re-180 & & & & -- & -- & -- & $2.52 \mathrm{E}+05$ & -- & $2.52 \mathrm{E}+05$ & $5.86 \mathrm{E}-04$ & $4.30 \mathrm{E}+08$ & Direct \\
\hline Re-181 & & & & $3.02 E+04$ & $5.44 \mathrm{E}+06$ & $3.08 \mathrm{E}+04$ & $1.39 \mathrm{E}+03$ & -- & $1.39 \mathrm{E}+03$ & $1.60 \mathrm{E}-03$ & $8.66 \mathrm{E}+05$ & Direct \\
\hline Re-182I & & & & $6.57 E+03$ & $1.76 \mathrm{E}+04$ & $3.24 \mathrm{E}+03$ & $4.37 E+02$ & -- & $4.37 E+02$ & $1.62 \mathrm{E}-03$ & $2.69 \mathrm{E}+05$ & Direct \\
\hline $\operatorname{Re}-182 \mathrm{~s}$ & & & & $3.72 \mathrm{E}+04$ & $2.41 \mathrm{E}+08$ & $7.39 \mathrm{E}+04$ & $1.10 \mathrm{E}+03$ & -- & $1.10 \mathrm{E}+03$ & $8.14 \mathrm{E}-04$ & $1.36 \mathrm{E}+06$ & Direct \\
\hline Re-183 & & & & $4.30 \mathrm{E}+03$ & $2.31 \mathrm{E}+03$ & $9.44 \mathrm{E}+02$ & $4.63 E+03$ & -- & $9.44 \mathrm{E}+02$ & $9.26 \mathrm{E}-02$ & $1.02 \mathrm{E}+04$ & Food \\
\hline Re-184 & & & & $6.20 \mathrm{E}+03$ & $2.39 \mathrm{E}+03$ & $1.01 \mathrm{E}+03$ & $8.22 \mathrm{E}+02$ & -- & $8.22 \mathrm{E}+02$ & $4.40 \mathrm{E}-02$ & $1.87 \mathrm{E}+04$ & Direct \\
\hline Re-184m & & & & $1.83 \mathrm{E}+03$ & $1.38 \mathrm{E}+03$ & $5.47 \mathrm{E}+02$ & $1.87 \mathrm{E}+03$ & -- & $5.47 \mathrm{E}+02$ & 1.27E-01 & $4.30 \mathrm{E}+03$ & Food \\
\hline Re-186 & & & & $9.31 \mathrm{E}+03$ & $8.18 \mathrm{E}+03$ & $2.28 \mathrm{E}+03$ & $3.88 \mathrm{E}+04$ & -- & $2.28 \mathrm{E}+03$ & $1.23 \mathrm{E}-02$ & $1.86 \mathrm{E}+05$ & Food \\
\hline Re-186m & & & & $1.02 \mathrm{E}+03$ & $8.98 \mathrm{E}+02$ & $3.48 \mathrm{E}+02$ & $3.78 \mathrm{E}+04$ & -- & $3.48 \mathrm{E}+02$ & $3.62 \mathrm{E}+04$ & $9.61 \mathrm{E}-03$ & Food \\
\hline Re-187 & & & & $1.86 \mathrm{E}+06$ & $3.87 E+05$ & $1.50 \mathrm{E}+05$ & -- & -- & $1.50 \mathrm{E}+05$ & $3.93 \mathrm{E}+12$ & $3.82 \mathrm{E}-08$ & Food \\
\hline Re-188 & & & & $1.51 \mathrm{E}+04$ & $4.71 \mathrm{E}+06$ & $1.08 \mathrm{E}+04$ & $1.98 \mathrm{E}+04$ & -- & $1.08 \mathrm{E}+04$ & 1.10E-02 & $9.82 \mathrm{E}+05$ & Food \\
\hline Re-188m & & & & $5.58 \mathrm{E}+05$ & $5.23 E+148$ & $2.63 \mathrm{E}+07$ & $4.86 \mathrm{E}+05$ & -- & $4.86 \mathrm{E}+05$ & $9.03 \mathrm{E}-03$ & $5.38 \mathrm{E}+07$ & Direct \\
\hline Re-189 & & & & $1.86 \mathrm{E}+04$ & $9.90 \mathrm{E}+05$ & $1.38 \mathrm{E}+04$ & $1.45 \mathrm{E}+04$ & -- & $1.38 \mathrm{E}+04$ & $2.02 \mathrm{E}-02$ & $6.82 \mathrm{E}+05$ & Food \\
\hline Re-190 & & & & -- & -- & -- & $1.75 \mathrm{E}+05$ & -- & $1.75 \mathrm{E}+05$ & $5.47 \mathrm{E}-04$ & $3.19 \mathrm{E}+08$ & Direct \\
\hline Re-190m & & & & -- & -- & -- & $4.10 \mathrm{E}+03$ & -- & $4.10 \mathrm{E}+03$ & $7.95 \mathrm{E}-04$ & $5.16 \mathrm{E}+06$ & Direct \\
\hline Rh-100 & & & & $1.77 \mathrm{E}+04$ & $2.55 \mathrm{E}+06$ & $1.84 \mathrm{E}+04$ & $3.81 \mathrm{E}+02$ & -- & $3.81 \mathrm{E}+02$ & $2.53 \mathrm{E}-04$ & $1.51 \mathrm{E}+06$ & Direct \\
\hline Rh-100m & & & & -- & -- & -- & $2.47 E+06$ & -- & $2.47 E+06$ & $6.04 \mathrm{E}-03$ & $4.09 E+08$ & Direct \\
\hline Rh-101 & & & & $2.23 \mathrm{E}+03$ & $3.61 \mathrm{E}+03$ & $1.47 \mathrm{E}+03$ & $2.70 \mathrm{E}+03$ & -- & $1.47 \mathrm{E}+03$ & $1.33 \mathrm{E}+00$ & $1.11 \mathrm{E}+03$ & Food \\
\hline Rh-101m & & & & $4.14 \mathrm{E}+04$ & $4.46 \mathrm{E}+04$ & $1.46 \mathrm{E}+04$ & $2.56 \mathrm{E}+03$ & -- & $2.56 \mathrm{E}+03$ & $8.59 \mathrm{E}-03$ & $2.98 \mathrm{E}+05$ & Direct \\
\hline Rh-102 & & & & $6.98 \mathrm{E}+02$ & $7.65 \mathrm{E}+02$ & $3.12 \mathrm{E}+02$ & $3.39 \mathrm{E}+02$ & -- & $3.12 \mathrm{E}+02$ & $2.58 \mathrm{E}-01$ & $1.21 \mathrm{E}+03$ & Food \\
\hline Rh-102m & & & & $1.67 \mathrm{E}+03$ & $1.71 \mathrm{E}+03$ & $7.06 \mathrm{E}+02$ & $1.50 \mathrm{E}+03$ & -- & $7.06 \mathrm{E}+02$ & $1.14 \mathrm{E}-01$ & $6.19 \mathrm{E}+03$ & Food \\
\hline Rh-103m & & & & $4.47 \mathrm{E}+06$ & $5.29 \mathrm{E}+54$ & $7.21 \mathrm{E}+07$ & $7.59 \mathrm{E}+06$ & -- & $4.47 \mathrm{E}+06$ & $1.37 \mathrm{E}-01$ & $3.26 \mathrm{E}+07$ & Inh. \\
\hline Rh-104 & & & & -- & -- & -- & $8.28 \mathrm{E}+07$ & -- & $8.28 \mathrm{E}+07$ & $3.22 \mathrm{E}-02$ & $2.57 \mathrm{E}+09$ & Direct \\
\hline Rh-104m & & & & -- & -- & -- & $3.79 \mathrm{E}+06$ & -- & $3.79 \mathrm{E}+06$ & $9.08 \mathrm{E}-03$ & $4.17 \mathrm{E}+08$ & Direct \\
\hline Rh-105 & & & & $2.54 \mathrm{E}+04$ & $3.97 \mathrm{E}+05$ & $2.16 \mathrm{E}+04$ & $1.17 \mathrm{E}+04$ & -- & $1.17 \mathrm{E}+04$ & 1.39E-02 & $8.45 \mathrm{E}+05$ & Direct \\
\hline Rh-106 & & & & -- & -- & -- & $7.10 \mathrm{E}+06$ & -- & $7.10 \mathrm{E}+06$ & 1.99E-03 & $3.56 \mathrm{E}+09$ & Direct \\
\hline Rh-106m & & & & $5.88 \mathrm{E}+04$ & $6.65 \mathrm{E}+25$ & $7.31 \mathrm{E}+05$ & $1.88 \mathrm{E}+03$ & -- & $1.88 \mathrm{E}+03$ & $1.40 \mathrm{E}-04$ & $1.34 \mathrm{E}+07$ & Direct \\
\hline Rh-107 & & & & $3.99 \mathrm{E}+05$ & $3.75 \mathrm{E}+128$ & $2.95 \mathrm{E}+07$ & $1.07 \mathrm{E}+05$ & -- & $1.07 \mathrm{E}+05$ & $1.32 \mathrm{E}-03$ & $8.10 \mathrm{E}+07$ & Direct \\
\hline Rh-108 & & & & -- & -- & -- & $8.16 \mathrm{E}+06$ & -- & $8.16 \mathrm{E}+06$ & $1.31 \mathrm{E}-03$ & $6.22 \mathrm{E}+09$ & Direct \\
\hline Rh-109 & & & & -- & -- & -- & $1.81 \mathrm{E}+06$ & -- & $1.81 \mathrm{E}+06$ & $1.40 \mathrm{E}-03$ & $1.29 \mathrm{E}+09$ & Direct \\
\hline
\end{tabular}


Table C.2 Summary of Exposure Pathway TQs and Selection of Final HC-3 TQs (continued)

\begin{tabular}{|c|c|c|c|c|c|c|c|c|c|c|c|c|}
\hline \multirow[b]{2}{*}{$\begin{array}{c}\text { MASTER } \\
\text { ISOTOPE } \\
\text { LIST } \\
\end{array}$} & \multicolumn{3}{|c|}{ from NNSA SD G 1027} & \multicolumn{5}{|c|}{ Calculated Pathway Specific TQ Values } & \multicolumn{4}{|c|}{ Selected Final Threshold Quantities } \\
\hline & Act (Ci) & Mass (g) & $\begin{array}{l}\text { Limiting } \\
\text { Pathway }\end{array}$ & $\begin{array}{c}\text { TQ }_{\text {inhale }} \\
\text { (Ci) }\end{array}$ & $\begin{array}{c}\text { TQ }_{\text {water }} \\
\text { (Ci) }\end{array}$ & $\mathrm{TQ}_{\text {food }}(\mathrm{Ci})$ & $\begin{array}{c}T_{\text {direct }} \\
\text { (Ci) }\end{array}$ & $\mathrm{TQ}_{\text {sub }}(\mathrm{Ci})$ & Act (Ci) & Mass (g) & $\begin{array}{c}\text { Sp. Act. } \\
\text { (Ci/g) }\end{array}$ & $\begin{array}{l}\text { Limiting } \\
\text { Pathway }\end{array}$ \\
\hline Rh-94 & & & & -- & -- & -- & $1.64 \mathrm{E}+05$ & -- & $1.64 \mathrm{E}+05$ & $9.62 \mathrm{E}-05$ & $1.70 \mathrm{E}+09$ & Direct \\
\hline Rh-95 & & & & -- & -- & -- & $5.64 \mathrm{E}+04$ & -- & $5.64 \mathrm{E}+04$ & $1.43 \mathrm{E}-04$ & $3.95 \mathrm{E}+08$ & Direct \\
\hline Rh-95m & & & & -- & -- & -- & $4.12 \mathrm{E}+05$ & -- & $4.12 \mathrm{E}+05$ & $4.08 \mathrm{E}-04$ & $1.01 \mathrm{E}+09$ & Direct \\
\hline Rh-96 & & & & -- & -- & -- & $1.86 \mathrm{E}+04$ & -- & $1.86 \mathrm{E}+04$ & $9.41 \mathrm{E}-05$ & $1.98 \mathrm{E}+08$ & Direct \\
\hline Rh-96m & & & & -- & -- & -- & $3.74 \mathrm{E}+05$ & -- & $3.74 \mathrm{E}+05$ & $2.88 \mathrm{E}-04$ & $1.30 \mathrm{E}+09$ & Direct \\
\hline Rh-97 & & & & $2.66 \mathrm{E}+05$ & $4.26 \mathrm{E}+92$ & $1.02 \mathrm{E}+07$ & $1.63 \mathrm{E}+04$ & -- & $1.63 \mathrm{E}+04$ & $2.58 \mathrm{E}-04$ & $6.32 \mathrm{E}+07$ & Direct \\
\hline Rh-97m & & & & $2.23 \mathrm{E}+05$ & $5.71 \mathrm{E}+63$ & $6.93 \mathrm{E}+06$ & $7.12 \mathrm{E}+03$ & -- & $7.12 E+03$ & $1.70 \mathrm{E}-04$ & $4.20 \mathrm{E}+07$ & Direct \\
\hline Rh-98 & & & & -- & -- & -- & $4.60 \mathrm{E}+04$ & -- & $4.60 \mathrm{E}+04$ & $2.08 \mathrm{E}-04$ & $2.21 \mathrm{E}+08$ & Direct \\
\hline Rh-99 & & & & $1.25 \mathrm{E}+04$ & $6.08 \mathrm{E}+03$ & $2.81 \mathrm{E}+03$ & $1.22 \mathrm{E}+03$ & -- & $1.22 \mathrm{E}+03$ & $1.48 \mathrm{E}-02$ & $8.25 \mathrm{E}+04$ & Direct \\
\hline Rh-99m & & & & $1.53 \mathrm{E}+05$ & $1.97 \mathrm{E}+15$ & $8.35 \mathrm{E}+05$ & $3.86 \mathrm{E}+03$ & -- & $3.86 \mathrm{E}+03$ & $5.73 \mathrm{E}-04$ & $6.74 \mathrm{E}+06$ & Direct \\
\hline $\mathrm{Rn}-207$ & & & & -- & -- & -- & $7.95 E+04$ & -- & $7.95 \mathrm{E}+04$ & $8.10 E-04$ & $9.82 \mathrm{E}+07$ & Direct \\
\hline Rn-209 & & & & -- & -- & -- & $2.13 E+04$ & -- & $2.13 E+04$ & $6.74 \mathrm{E}-04$ & $3.16 \mathrm{E}+07$ & Direct \\
\hline Rn-210 & & & & -- & -- & -- & $8.26 \mathrm{E}+04$ & -- & $8.26 \mathrm{E}+04$ & $1.33 \mathrm{E}-02$ & $6.22 \mathrm{E}+06$ & Direct \\
\hline Rn-211 & & & & -- & -- & -- & $6.50 \mathrm{E}+02$ & -- & $6.50 \mathrm{E}+02$ & $6.38 \mathrm{E}-04$ & $1.02 \mathrm{E}+06$ & Direct \\
\hline $\mathrm{Rn}-212$ & & & & -- & -- & -- & $9.19 \mathrm{E}+07$ & -- & $9.19 \mathrm{E}+07$ & $2.48 \mathrm{E}+00$ & $3.71 \mathrm{E}+07$ & Direct \\
\hline Rn-215 & & & & -- & -- & -- & -- & -- & -- & -- & $2.28 \mathrm{E}+16$ & -- \\
\hline $\mathrm{Rn}-216$ & & & & -- & -- & -- & -- & -- & -- & -- & $1.16 \mathrm{E}+15$ & -- \\
\hline Rn-217 & & & & -- & -- & -- & -- & -- & -- & -- & $9.63 \mathrm{E}+13$ & -- \\
\hline Rn-218 & & & & -- & -- & -- & $1.77 \mathrm{E}+12$ & -- & $1.77 \mathrm{E}+12$ & $1.20 \mathrm{E}+00$ & $1.48 \mathrm{E}+12$ & Direct \\
\hline Rn-219 & & & & -- & -- & -- & $1.96 \mathrm{E}+08$ & -- & $1.96 \mathrm{E}+08$ & $1.50 \mathrm{E}-02$ & $1.30 \mathrm{E}+10$ & Direct \\
\hline Rn-220 & & & & -- & -- & -- & $2.61 \mathrm{E}+09$ & -- & $2.61 \mathrm{E}+09$ & $2.83 \mathrm{E}+00$ & $9.22 \mathrm{E}+08$ & Direct \\
\hline Rn-222 & $1.00 \mathrm{E}+01$ & $6.50 \mathrm{E}-05$ & $\mathrm{i}$ & -- & -- & -- & $2.65 \mathrm{E}+06$ & -- & $2.65 E+06$ & $1.72 \mathrm{E}+01$ & $1.54 \mathrm{E}+05$ & Direct \\
\hline Rn-223 & & & & -- & -- & -- & $8.66 \mathrm{E}+04$ & -- & $8.66 \mathrm{E}+04$ & $2.50 \mathrm{E}-03$ & $3.47 \mathrm{E}+07$ & Direct \\
\hline Ru-103 & & & & $3.99 \mathrm{E}+03$ & -- & $1.44 \mathrm{E}+03$ & $1.56 \mathrm{E}+03$ & -- & $1.44 \mathrm{E}+03$ & 4.47E-02 & $3.23 E+04$ & Food \\
\hline Ru-105 & & & & $4.47 \mathrm{E}+04$ & -- & $2.25 \mathrm{E}+05$ & $3.55 \mathrm{E}+03$ & -- & $3.55 \mathrm{E}+03$ & $5.28 \mathrm{E}-04$ & $6.73 \mathrm{E}+06$ & Direct \\
\hline Ru-106 & $1.15 \mathrm{E}+02$ & 3.49E-02 & ing & $1.80 \mathrm{E}+02$ & -- & $1.19 \mathrm{E}+02$ & -- & -- & $1.19 \mathrm{E}+02$ & $3.54 \mathrm{E}-02$ & $3.35 \mathrm{E}+03$ & Food \\
\hline Ru-107 & & & & -- & -- & -- & $5.60 \mathrm{E}+05$ & -- & $5.60 \mathrm{E}+05$ & $1.19 \mathrm{E}-03$ & $4.69 \mathrm{E}+08$ & Direct \\
\hline Ru-108 & & & & -- & -- & -- & $2.54 \mathrm{E}+06$ & -- & $2.54 \mathrm{E}+06$ & $6.65 \mathrm{E}-03$ & $3.83 \mathrm{E}+08$ & Direct \\
\hline Ru-92 & & & & -- & -- & -- & $9.52 \mathrm{E}+04$ & -- & $9.52 E+04$ & 1.70E-04 & $5.60 \mathrm{E}+08$ & Direct \\
\hline Ru-94 & & & & $1.51 \mathrm{E}+05$ & -- & $3.17 \mathrm{E}+06$ & $2.62 \mathrm{E}+04$ & -- & $2.62 E+04$ & $6.77 \mathrm{E}-04$ & $3.87 \mathrm{E}+07$ & Direct \\
\hline Ru-95 & & & & $1.67 \mathrm{E}+05$ & -- & $2.49 \mathrm{E}+06$ & $5.92 \mathrm{E}+03$ & -- & $5.92 \mathrm{E}+03$ & $2.95 \mathrm{E}-04$ & $2.01 \mathrm{E}+07$ & Direct \\
\hline Ru-97 & & & & $6.98 \mathrm{E}+04$ & -- & $2.96 \mathrm{E}+04$ & $3.40 \mathrm{E}+03$ & -- & $3.40 \mathrm{E}+03$ & $7.32 \mathrm{E}-03$ & $4.65 \mathrm{E}+05$ & Direct \\
\hline S-35 & $2.21 \mathrm{E}+01$ & $5.18 \mathrm{E}-04$ & ing & $1.72 \mathrm{E}+02$ & $2.79 E+03$ & $2.26 \mathrm{E}+01$ & -- & -- & $2.26 \mathrm{E}+01$ & $5.30 \mathrm{E}-04$ & $4.27 \mathrm{E}+04$ & Food \\
\hline S-37 & & & & -- & -- & -- & $4.90 \mathrm{E}+04$ & -- & $4.90 E+04$ & $4.86 \mathrm{E}-05$ & $1.01 \mathrm{E}+09$ & Direct \\
\hline $\mathrm{S}-38$ & & & & $6.20 \mathrm{E}+02$ & $4.32 \mathrm{E}+20$ & $2.71 E+03$ & $2.52 E+03$ & -- & $6.20 E+02$ & 2.13E-05 & $2.91 \mathrm{E}+07$ & Inh. \\
\hline
\end{tabular}


Table C.2 Summary of Exposure Pathway TQs and Selection of Final HC-3 TQs (continued)

\begin{tabular}{|c|c|c|c|c|c|c|c|c|c|c|c|c|}
\hline \multirow[b]{2}{*}{$\begin{array}{c}\text { MASTER } \\
\text { ISOTOPE } \\
\text { LIST } \\
\end{array}$} & \multicolumn{3}{|c|}{ from NNSA SD G 1027} & \multicolumn{5}{|c|}{ Calculated Pathway Specific TQ Values } & \multicolumn{4}{|c|}{ Selected Final Threshold Quantities } \\
\hline & Act $(\mathrm{Ci})$ & Mass (g) & $\begin{array}{l}\text { Limiting } \\
\text { Pathway }\end{array}$ & $\begin{array}{c}\text { TQ }_{\text {inhale }} \\
(\mathrm{Ci})\end{array}$ & $\begin{array}{c}\text { TQ }_{\text {water }} \\
\text { (Ci) }\end{array}$ & $\mathrm{TQ}_{\text {food }}(\mathrm{Ci})$ & $\begin{array}{c}T_{\text {direct }} \\
\text { (Ci) }\end{array}$ & $\mathrm{TQ}_{\text {sub }}(\mathrm{Ci})$ & Act (Ci) & Mass (g) & $\begin{array}{c}\text { Sp. Act. } \\
\text { (Ci/g) }\end{array}$ & $\begin{array}{l}\text { Limiting } \\
\text { Pathway }\end{array}$ \\
\hline Sb-111 & & & & -- & -- & -- & $3.90 \mathrm{E}+05$ & -- & $3.90 \mathrm{E}+05$ & $2.88 \mathrm{E}-04$ & $1.36 \mathrm{E}+09$ & Direct \\
\hline Sb-113 & & & & -- & -- & -- & $8.57 \mathrm{E}+04$ & -- & $8.57 \mathrm{E}+04$ & $3.43 \mathrm{E}-04$ & $2.50 \mathrm{E}+08$ & Direct \\
\hline Sb-114 & & & & -- & -- & -- & $7.72 \mathrm{E}+04$ & -- & $7.72 \mathrm{E}+04$ & $1.63 \mathrm{E}-04$ & $4.73 \mathrm{E}+08$ & Direct \\
\hline Sb-115 & & & & $4.86 \mathrm{E}+05$ & -- & $2.01 \mathrm{E}+07$ & $2.51 \mathrm{E}+04$ & -- & $2.51 \mathrm{E}+04$ & $4.87 \mathrm{E}-04$ & $5.15 \mathrm{E}+07$ & Direct \\
\hline Sb-116 & & & & $4.86 \mathrm{E}+05$ & -- & $3.73 \mathrm{E}+07$ & $2.13 \mathrm{E}+04$ & -- & $2.13 E+04$ & 2.07E-04 & $1.03 E+08$ & Direct \\
\hline Sb-116m & & & & $1.31 \mathrm{E}+05$ & -- & $3.80 \mathrm{E}+06$ & $3.82 \mathrm{E}+03$ & -- & $3.82 \mathrm{E}+03$ & $1.42 \mathrm{E}-04$ & $2.69 \mathrm{E}+07$ & Direct \\
\hline Sb-117 & & & & $4.14 \mathrm{E}+05$ & -- & $5.10 \mathrm{E}+06$ & $2.34 \mathrm{E}+04$ & -- & $2.34 \mathrm{E}+04$ & $2.45 \mathrm{E}-03$ & $9.57 \mathrm{E}+06$ & Direct \\
\hline Sb-118 & & & & -- & -- & -- & $2.48 \mathrm{E}+05$ & -- & $2.48 \mathrm{E}+05$ & 5.60E-04 & $4.43 \mathrm{E}+08$ & Direct \\
\hline Sb-118m & & & & $4.86 \mathrm{E}+04$ & -- & $2.47 E+05$ & $9.69 \mathrm{E}+02$ & -- & $9.69 \mathrm{E}+02$ & $1.82 \mathrm{E}-04$ & $5.32 \mathrm{E}+06$ & Direct \\
\hline Sb-119 & & & & $1.89 \mathrm{E}+05$ & -- & $9.20 \mathrm{E}+04$ & $3.88 \mathrm{E}+04$ & -- & $3.88 \mathrm{E}+04$ & $5.61 \mathrm{E}-02$ & $6.92 \mathrm{E}+05$ & Direct \\
\hline Sb-120s & & & & $9.31 \mathrm{E}+05$ & -- & $6.89 \mathrm{E}+07$ & $1.01 \mathrm{E}+05$ & -- & $1.01 \mathrm{E}+05$ & $1.02 \mathrm{E}-03$ & $9.87 \mathrm{E}+07$ & Direct \\
\hline Sb-120l & & & & $8.59 \mathrm{E}+03$ & -- & $2.17 E+03$ & $3.12 \mathrm{E}+02$ & -- & $3.12 \mathrm{E}+02$ & $1.65 \mathrm{E}-03$ & $1.89 \mathrm{E}+05$ & Direct \\
\hline Sb-122 & & & & $9.31 \mathrm{E}+03$ & -- & $2.76 \mathrm{E}+03$ & $1.87 \mathrm{E}+03$ & -- & $1.87 \mathrm{E}+03$ & 4.70E-03 & $3.97 \mathrm{E}+05$ & Direct \\
\hline $\mathrm{Sb}-122 \mathrm{~m}$ & & & & -- & -- & -- & $2.44 \mathrm{E}+06$ & -- & $2.44 \mathrm{E}+06$ & $6.64 \mathrm{E}-03$ & $3.68 \mathrm{E}+08$ & Direct \\
\hline Sb-124 & $3.77 \mathrm{E}+02$ & $2.16 \mathrm{E}-02$ & ing & $1.83 \mathrm{E}+03$ & -- & $3.84 \mathrm{E}+02$ & $4.02 E+02$ & -- & $3.84 \mathrm{E}+02$ & $2.20 \mathrm{E}-02$ & $1.75 \mathrm{E}+04$ & Food \\
\hline $\mathrm{Sb}-124 \mathrm{~ms}$ & & & & -- & -- & -- & $1.33 \mathrm{E}+06$ & -- & $1.33 \mathrm{E}+06$ & $1.36 \mathrm{E}-03$ & $9.79 E+08$ & Direct \\
\hline Sb-124ml & & & & $1.35 \mathrm{E}+06$ & -- & $9.48 \mathrm{E}+07$ & $1.79 \mathrm{E}+08$ & -- & $1.35 \mathrm{E}+06$ & 1.79E-02 & $7.51 \mathrm{E}+07$ & Inh. \\
\hline Sb-125 & & & & $2.48 \mathrm{E}+03$ & -- & $7.36 \mathrm{E}+02$ & $1.69 \mathrm{E}+03$ & -- & $7.36 \mathrm{E}+02$ & $7.12 \mathrm{E}-01$ & $1.03 \mathrm{E}+03$ & Food \\
\hline Sb-126 & $2.64 \mathrm{E}+02$ & $3.15 \mathrm{E}-03$ & de & $3.49 \mathrm{E}+03$ & -- & $6.75 \mathrm{E}+02$ & $2.63 E+02$ & -- & $2.63 \mathrm{E}+02$ & 3.15E-03 & $8.36 \mathrm{E}+04$ & Direct \\
\hline Sb-126m & & & & $3.38 \mathrm{E}+05$ & -- & $2.24 \mathrm{E}+07$ & $2.46 \mathrm{E}+04$ & -- & $2.46 \mathrm{E}+04$ & $3.13 \mathrm{E}-04$ & $7.86 \mathrm{E}+07$ & Direct \\
\hline Sb-127 & & & & $6.57 E+03$ & -- & $2.07 E+03$ & $1.15 \mathrm{E}+03$ & -- & $1.15 \mathrm{E}+03$ & $4.31 \mathrm{E}-03$ & $2.67 \mathrm{E}+05$ & Direct \\
\hline Sb-128I & & & & $1.67 E+04$ & -- & $3.83 \mathrm{E}+04$ & $5.15 \mathrm{E}+02$ & -- & $5.15 \mathrm{E}+02$ & $1.89 \mathrm{E}-04$ & $2.72 \mathrm{E}+06$ & Direct \\
\hline Sb-128s & & & & $4.30 \mathrm{E}+05$ & -- & $4.46 \mathrm{E}+07$ & $3.51 \mathrm{E}+04$ & -- & $3.51 \mathrm{E}+04$ & $2.48 \mathrm{E}-04$ & $1.41 \mathrm{E}+08$ & Direct \\
\hline Sb-129 & & & & $3.19 E+04$ & -- & $1.42 \mathrm{E}+05$ & $1.99 \mathrm{E}+03$ & -- & $1.99 \mathrm{E}+03$ & 3.53E-04 & $5.63 \mathrm{E}+06$ & Direct \\
\hline Sb-130 & & & & $1.23 \mathrm{E}+05$ & -- & $4.21 \mathrm{E}+06$ & $5.55 \mathrm{E}+03$ & -- & $5.55 \mathrm{E}+03$ & $1.53 \mathrm{E}-04$ & $3.62 \mathrm{E}+07$ & Direct \\
\hline Sb-130m & & & & -- & -- & -- & $4.25 \mathrm{E}+04$ & -- & $4.25 E+04$ & $1.85 \mathrm{E}-04$ & $2.30 \mathrm{E}+08$ & Direct \\
\hline Sb-131 & & & & $1.35 \mathrm{E}+05$ & -- & $6.66 \mathrm{E}+06$ & $1.69 \mathrm{E}+04$ & -- & $1.69 \mathrm{E}+04$ & $2.71 \mathrm{E}-04$ & $6.24 \mathrm{E}+07$ & Direct \\
\hline Sb-133 & & & & -- & -- & -- & $1.06 \mathrm{E}+05$ & -- & $1.06 \mathrm{E}+05$ & $1.87 \mathrm{E}-04$ & $5.66 \mathrm{E}+08$ & Direct \\
\hline Sc- $42 \mathrm{~m}$ & & & & -- & -- & -- & $1.67 \mathrm{E}+05$ & -- & $1.67 \mathrm{E}+05$ & $3.85 \mathrm{E}-05$ & $4.34 \mathrm{E}+09$ & Direct \\
\hline Sc-43 & & & & $6.20 \mathrm{E}+04$ & $7.13 \mathrm{E}+16$ & $3.51 \mathrm{E}+05$ & $2.87 \mathrm{E}+03$ & -- & $2.87 \mathrm{E}+03$ & $1.53 \mathrm{E}-04$ & $1.87 \mathrm{E}+07$ & Direct \\
\hline Sc-44 & & & & $3.72 \mathrm{E}+04$ & $3.02 \mathrm{E}+16$ & $1.89 \mathrm{E}+05$ & $1.46 \mathrm{E}+03$ & -- & $1.46 \mathrm{E}+03$ & 8.04E-05 & $1.82 \mathrm{E}+07$ & Direct \\
\hline Sc- $44 m$ & & & & $5.58 \mathrm{E}+03$ & $1.27 \mathrm{E}+04$ & $2.14 \mathrm{E}+03$ & $2.98 \mathrm{E}+03$ & -- & $2.14 \mathrm{E}+03$ & $1.76 \mathrm{E}-03$ & $1.22 \mathrm{E}+06$ & Food \\
\hline Sc-46 & $3.63 E+02$ & 1.07E-02 & de & $1.75 \mathrm{E}+03$ & $1.44 \mathrm{E}+03$ & $6.14 \mathrm{E}+02$ & $3.63 \mathrm{E}+02$ & -- & $3.63 \mathrm{E}+02$ & 1.07E-02 & $3.39 E+04$ & Direct \\
\hline Sc-47 & & & & $1.53 \mathrm{E}+04$ & $2.82 \mathrm{E}+04$ & $7.33 \mathrm{E}+03$ & $7.42 \mathrm{E}+03$ & -- & $7.33 \mathrm{E}+03$ & $8.83 \mathrm{E}-03$ & $8.30 E+05$ & Food \\
\hline Sc-48 & & & & $6.98 \mathrm{E}+03$ & $4.13 E+04$ & $3.91 \mathrm{E}+03$ & $2.61 E+02$ & -- & $2.61 \mathrm{E}+02$ & $1.74 \mathrm{E}-04$ & $1.50 \mathrm{E}+06$ & Direct \\
\hline
\end{tabular}


Table C.2 Summary of Exposure Pathway TQs and Selection of Final HC-3 TQs (continued)

\begin{tabular}{|c|c|c|c|c|c|c|c|c|c|c|c|c|}
\hline \multirow[b]{2}{*}{$\begin{array}{c}\text { MASTER } \\
\text { ISOTOPE } \\
\text { LIST } \\
\end{array}$} & \multicolumn{3}{|c|}{ from NNSA SD G 1027} & \multicolumn{5}{|c|}{ Calculated Pathway Specific TQ Values } & \multicolumn{4}{|c|}{ Selected Final Threshold Quantities } \\
\hline & Act $(\mathrm{Ci})$ & Mass (g) & $\begin{array}{l}\text { Limiting } \\
\text { Pathway }\end{array}$ & $\begin{array}{c}\text { TQ }_{\text {inhale }} \\
(\mathrm{Ci})\end{array}$ & $\begin{array}{c}\mathrm{TQ}_{\text {water }} \\
\text { (Ci) }\end{array}$ & $\mathrm{TQ}_{\text {food }}(\mathrm{Ci})$ & $\begin{array}{c}\mathrm{TQ}_{\text {direct }} \\
\text { (Ci) }\end{array}$ & $\mathrm{TQ}_{\text {sub }}(\mathrm{Ci})$ & Act (Ci) & Mass (g) & $\begin{array}{c}\text { Sp. Act. } \\
\text { (Ci/g) }\end{array}$ & $\begin{array}{l}\text { Limiting } \\
\text { Pathway }\end{array}$ \\
\hline Sc-49 & & & & $1.83 \mathrm{E}+05$ & $2.17 \mathrm{E}+52$ & $3.28 \mathrm{E}+06$ & $1.26 \mathrm{E}+07$ & -- & $1.83 \mathrm{E}+05$ & $2.74 \mathrm{E}-03$ & $6.69 \mathrm{E}+07$ & Inh. \\
\hline Sc-50 & & & & -- & -- & -- & $1.32 \mathrm{E}+05$ & -- & $1.32 \mathrm{E}+05$ & $6.01 \mathrm{E}-05$ & $2.20 \mathrm{E}+09$ & Direct \\
\hline Se-70 & & & & $9.31 \mathrm{E}+04$ & -- & $2.69 \mathrm{E}+06$ & $1.77 \mathrm{E}+04$ & -- & $1.77 \mathrm{E}+04$ & $2.70 \mathrm{E}-04$ & $6.56 \mathrm{E}+07$ & Direct \\
\hline Se-71 & & & & -- & -- & -- & $9.52 \mathrm{E}+04$ & -- & $9.52 \mathrm{E}+04$ & $1.70 \mathrm{E}-04$ & $5.59 \mathrm{E}+08$ & Direct \\
\hline Se-72 & & & & $2.86 \mathrm{E}+03$ & -- & $4.00 \mathrm{E}+02$ & $2.20 \mathrm{E}+04$ & -- & $4.00 E+02$ & $1.85 \mathrm{E}-03$ & $2.16 \mathrm{E}+05$ & Food \\
\hline Se-73 & & & & $4.65 \mathrm{E}+04$ & -- & $9.40 \mathrm{E}+04$ & $1.72 \mathrm{E}+03$ & -- & $1.72 \mathrm{E}+03$ & $2.86 \mathrm{E}-04$ & $6.01 \mathrm{E}+06$ & Direct \\
\hline Se-73m & & & & $4.14 \mathrm{E}+05$ & -- & $9.65 \mathrm{E}+06$ & $7.62 E+04$ & -- & $7.62 \mathrm{E}+04$ & 1.15E-03 & $6.61 \mathrm{E}+07$ & Direct \\
\hline Se-75 & $3.33 \mathrm{E}+02$ & $2.29 \mathrm{E}-02$ & ing & $6.57 \mathrm{E}+03$ & -- & $3.41 \mathrm{E}+02$ & $1.85 \mathrm{E}+03$ & -- & $3.41 E+02$ & $2.34 \mathrm{E}-02$ & $1.45 \mathrm{E}+04$ & Food \\
\hline Se-77m & & & & -- & -- & -- & $2.85 \mathrm{E}+07$ & -- & $2.85 \mathrm{E}+07$ & 3.39E-03 & $8.40 \mathrm{E}+09$ & Direct \\
\hline Se-79 & & & & $3.60 \mathrm{E}+03$ & -- & $2.78 \mathrm{E}+02$ & -- & -- & $2.78 \mathrm{E}+02$ & $3.98 \mathrm{E}+03$ & $6.97 \mathrm{E}-02$ & Food \\
\hline Se-79m & & & & -- & -- & -- & $1.34 \mathrm{E}+07$ & -- & $1.34 \mathrm{E}+07$ & $2.21 \mathrm{E}-02$ & $6.08 \mathrm{E}+08$ & Direct \\
\hline Se-81 & & & & $4.65 \mathrm{E}+05$ & -- & $3.09 \mathrm{E}+07$ & $4.26 \mathrm{E}+06$ & -- & $4.65 E+05$ & $3.70 \mathrm{E}-03$ & $1.26 \mathrm{E}+08$ & Inh. \\
\hline Se-81m & & & & $1.64 \mathrm{E}+05$ & -- & $4.57 \mathrm{E}+06$ & $6.99 \mathrm{E}+05$ & -- & $1.64 \mathrm{E}+05$ & $4.05 \mathrm{E}-03$ & $4.06 \mathrm{E}+07$ & Inh. \\
\hline Se-83 & & & & $2.11 \mathrm{E}+05$ & -- & $1.34 \mathrm{E}+07$ & $1.33 \mathrm{E}+04$ & -- & $1.33 \mathrm{E}+04$ & $1.32 \mathrm{E}-04$ & $1.01 \mathrm{E}+08$ & Direct \\
\hline Se-83m & & & & -- & -- & -- & $6.30 \mathrm{E}+05$ & -- & $6.30 \mathrm{E}+05$ & $3.24 \mathrm{E}-04$ & $1.94 \mathrm{E}+09$ & Direct \\
\hline Se-84 & & & & -- & -- & -- & $5.56 \mathrm{E}+05$ & -- & $5.56 \mathrm{E}+05$ & 7.70E-04 & $7.23 \mathrm{E}+08$ & Direct \\
\hline Si-31 & & & & $1.02 \mathrm{E}+05$ & $3.52 E+22$ & $6.10 \mathrm{E}+05$ & $5.77 \mathrm{E}+06$ & -- & $1.02 \mathrm{E}+05$ & $2.63 \mathrm{E}-03$ & $3.86 \mathrm{E}+07$ & Inh. \\
\hline Si-32 & & & & $1.02 \mathrm{E}+02$ & $3.53 E+03$ & $1.42 \mathrm{E}+03$ & -- & -- & $1.02 \mathrm{E}+02$ & $4.09 \mathrm{E}+00$ & $2.48 \mathrm{E}+01$ & Inh. \\
\hline Sm-139 & & & & -- & -- & -- & $1.94 \mathrm{E}+05$ & -- & $1.94 \mathrm{E}+05$ & $3.68 \mathrm{E}-04$ & $5.27 \mathrm{E}+08$ & Direct \\
\hline Sm-140 & & & & $2.15 \mathrm{E}+05$ & -- & $1.06 \mathrm{E}+07$ & $8.61 \mathrm{E}+04$ & -- & $8.61 E+04$ & $9.50 \mathrm{E}-04$ & $9.07 \mathrm{E}+07$ & Direct \\
\hline Sm-141 & & & & $4.14 \mathrm{E}+05$ & -- & $3.88 \mathrm{E}+07$ & $5.06 \mathrm{E}+04$ & -- & $5.06 \mathrm{E}+04$ & 3.87E-04 & $1.31 \mathrm{E}+08$ & Direct \\
\hline Sm-141m & & & & $1.99 \mathrm{E}+05$ & -- & $1.05 \mathrm{E}+07$ & $1.62 \mathrm{E}+04$ & -- & $1.62 \mathrm{E}+04$ & $2.74 \mathrm{E}-04$ & $5.90 \mathrm{E}+07$ & Direct \\
\hline Sm-142 & & & & $1.02 \mathrm{E}+05$ & -- & $1.12 \mathrm{E}+06$ & $1.06 \mathrm{E}+05$ & -- & $1.02 \mathrm{E}+05$ & $5.56 \mathrm{E}-03$ & $1.83 \mathrm{E}+07$ & Inh. \\
\hline Sm-143 & & & & -- & -- & -- & $1.57 \mathrm{E}+05$ & -- & $1.57 \mathrm{E}+05$ & $1.04 \mathrm{E}-03$ & $1.50 \mathrm{E}+08$ & Direct \\
\hline Sm-143m & & & & -- & -- & -- & $9.62 \mathrm{E}+05$ & -- & $9.62 \mathrm{E}+05$ & $8.05 \mathrm{E}-04$ & $1.20 \mathrm{E}+09$ & Direct \\
\hline Sm-145 & & & & $7.45 \mathrm{E}+03$ & -- & $3.97 \mathrm{E}+03$ & $1.11 \mathrm{E}+04$ & -- & $3.97 \mathrm{E}+03$ & $1.50 \mathrm{E}+00$ & $2.65 \mathrm{E}+03$ & Food \\
\hline Sm-146 & & & & $1.13 \mathrm{E}+00$ & -- & $1.49 \mathrm{E}+01$ & -- & -- & $1.13 \mathrm{E}+00$ & $4.74 \mathrm{E}+04$ & $2.38 \mathrm{E}-05$ & Inh. \\
\hline Sm-147 & & & & $1.25 \mathrm{E}+00$ & -- & $1.64 \mathrm{E}+01$ & -- & -- & $1.25 \mathrm{E}+00$ & $5.47 \mathrm{E}+07$ & $2.30 \mathrm{E}-08$ & Inh. \\
\hline Sm-148 & & & & $1.45 \mathrm{E}+00$ & -- & $1.87 \mathrm{E}+01$ & -- & -- & $1.45 \mathrm{E}+00$ & $4.20 \mathrm{E}+12$ & $3.45 \mathrm{E}-13$ & Inh. \\
\hline Sm-151 & $3.04 \mathrm{E}+03$ & $1.16 \mathrm{E}+02$ & $\mathrm{i}$ & $3.02 \mathrm{E}+03$ & -- & $8.22 \mathrm{E}+03$ & -- & -- & $3.02 E+03$ & $1.15 \mathrm{E}+02$ & $2.63 \mathrm{E}+01$ & Inh. \\
\hline Sm-153 & & & & $1.64 \mathrm{E}+04$ & -- & $8.46 \mathrm{E}+03$ & $1.39 \mathrm{E}+04$ & -- & $8.46 \mathrm{E}+03$ & $1.93 \mathrm{E}-02$ & $4.39 \mathrm{E}+05$ & Food \\
\hline Sm-155 & & & & $3.99 \mathrm{E}+05$ & -- & $2.41 \mathrm{E}+07$ & $3.18 \mathrm{E}+05$ & -- & $3.18 \mathrm{E}+05$ & 5.79E-03 & $5.49 \mathrm{E}+07$ & Direct \\
\hline Sm-156 & & & & $3.99 \mathrm{E}+04$ & -- & $1.12 \mathrm{E}+05$ & $1.28 \mathrm{E}+04$ & -- & $1.28 \mathrm{E}+04$ & $6.00 \mathrm{E}-03$ & $2.14 \mathrm{E}+06$ & Direct \\
\hline Sm-157 & & & & -- & -- & -- & $2.18 \mathrm{E}+05$ & -- & $2.18 \mathrm{E}+05$ & $1.46 \mathrm{E}-03$ & $1.49 \mathrm{E}+08$ & Direct \\
\hline Sn-106 & & & & -- & -- & -- & $3.12 E+05$ & -- & $3.12 \mathrm{E}+05$ & $3.38 \mathrm{E}-04$ & $9.25 \mathrm{E}+08$ & Direct \\
\hline
\end{tabular}


Table C.2 Summary of Exposure Pathway TQs and Selection of Final HC-3 TQs (continued)

\begin{tabular}{|c|c|c|c|c|c|c|c|c|c|c|c|c|}
\hline \multirow[b]{2}{*}{$\begin{array}{c}\text { MASTER } \\
\text { ISOTOPE } \\
\text { LIST } \\
\end{array}$} & \multicolumn{3}{|c|}{ from NNSA SD G 1027} & \multicolumn{5}{|c|}{ Calculated Pathway Specific TQ Values } & \multicolumn{4}{|c|}{ Selected Final Threshold Quantities } \\
\hline & Act (Ci) & Mass (g) & $\begin{array}{l}\text { Limiting } \\
\text { Pathway }\end{array}$ & $\begin{array}{c}\text { TQ }_{\text {inhale }} \\
\text { (Ci) }\end{array}$ & $\begin{array}{c}\text { TQ }_{\text {water }} \\
\text { (Ci) }\end{array}$ & $\mathrm{TQ}_{\text {food }}(\mathrm{Ci})$ & $\begin{array}{c}T_{\text {direct }} \\
\text { (Ci) }\end{array}$ & $\mathrm{TQ}_{\text {sub }}(\mathrm{Ci})$ & Act (Ci) & Mass (g) & $\begin{array}{l}\text { Sp. Act. } \\
\text { (Ci/g) }\end{array}$ & $\begin{array}{l}\text { Limiting } \\
\text { Pathway }\end{array}$ \\
\hline Sn-108 & & & & $5.58 \mathrm{E}+05$ & -- & $6.50 \mathrm{E}+07$ & $1.03 \mathrm{E}+05$ & -- & $1.03 \mathrm{E}+05$ & 6.07E-04 & $1.69 \mathrm{E}+08$ & Direct \\
\hline Sn-109 & & & & $5.08 \mathrm{E}+05$ & -- & $4.51 \mathrm{E}+07$ & $1.82 \mathrm{E}+04$ & -- & $1.82 \mathrm{E}+04$ & $1.90 \mathrm{E}-04$ & $9.59 \mathrm{E}+07$ & Direct \\
\hline Sn-110 & & & & $4.30 \mathrm{E}+04$ & -- & $1.86 \mathrm{E}+05$ & $1.02 \mathrm{E}+04$ & -- & $1.02 \mathrm{E}+04$ & $1.43 \mathrm{E}-03$ & $7.13 E+06$ & Direct \\
\hline Sn-111 & & & & $5.08 \mathrm{E}+05$ & -- & $1.90 \mathrm{E}+07$ & $4.03 E+04$ & -- & $4.03 E+04$ & $8.39 \mathrm{E}-04$ & $4.80 \mathrm{E}+07$ & Direct \\
\hline Sn-113 & $1.19 \mathrm{E}+03$ & $1.19 \mathrm{E}-01$ & ing & $4.47 \mathrm{E}+03$ & -- & $1.22 \mathrm{E}+03$ & $3.19 \mathrm{E}+04$ & -- & $1.22 \mathrm{E}+03$ & $1.21 \mathrm{E}-01$ & $1.00 \mathrm{E}+04$ & Food \\
\hline Sn-113m & & & & $2.11 E+06$ & -- & $2.18 \mathrm{E}+08$ & $2.48 \mathrm{E}+06$ & -- & $2.11 \mathrm{E}+06$ & $2.71 \mathrm{E}-02$ & $7.78 \mathrm{E}+07$ & Inh. \\
\hline Sn-117m & & & & $4.86 \mathrm{E}+03$ & -- & $2.19 \mathrm{E}+03$ & $4.72 \mathrm{E}+03$ & -- & $2.19 E+03$ & $2.67 \mathrm{E}-02$ & $8.21 \mathrm{E}+04$ & Food \\
\hline Sn-119m & & & & $5.58 \mathrm{E}+03$ & -- & $2.46 \mathrm{E}+03$ & $6.38 \mathrm{E}+04$ & -- & $2.46 \mathrm{E}+03$ & 6.57E-01 & $3.75 \mathrm{E}+03$ & Food \\
\hline Sn-121 & & & & $3.99 \mathrm{E}+04$ & -- & $4.46 \mathrm{E}+04$ & -- & -- & $3.99 \mathrm{E}+04$ & $4.16 \mathrm{E}-02$ & $9.58 \mathrm{E}+05$ & Inh. \\
\hline Sn-121m & & & & $2.66 \mathrm{E}+03$ & -- & $2.12 \mathrm{E}+03$ & $1.48 \mathrm{E}+05$ & -- & $2.12 \mathrm{E}+03$ & $3.94 \mathrm{E}+01$ & $5.38 \mathrm{E}+01$ & Food \\
\hline Sn-123 & $4.10 \mathrm{E}+02$ & $4.98 \mathrm{E}-02$ & ing & $1.45 \mathrm{E}+03$ & -- & $4.19 \mathrm{E}+02$ & $1.07 \mathrm{E}+05$ & -- & $4.19 \mathrm{E}+02$ & $5.09 \mathrm{E}-02$ & $8.22 \mathrm{E}+03$ & Food \\
\hline Sn-123m & & & & $2.54 \mathrm{E}+05$ & -- & $1.01 \mathrm{E}+07$ & $1.30 \mathrm{E}+05$ & -- & $1.30 \mathrm{E}+05$ & $3.40 \mathrm{E}-03$ & $3.82 \mathrm{E}+07$ & Direct \\
\hline Sn-125 & & & & $3.72 \mathrm{E}+03$ & -- & $6.05 \mathrm{E}+02$ & $2.41 \mathrm{E}+03$ & -- & $6.05 E+02$ & $5.58 \mathrm{E}-03$ & $1.08 \mathrm{E}+05$ & Food \\
\hline Sn-125m & & & & -- & -- & -- & $2.20 \mathrm{E}+05$ & -- & $2.20 \mathrm{E}+05$ & $1.39 \mathrm{E}-03$ & $1.58 \mathrm{E}+08$ & Direct \\
\hline Sn-126 & $1.67 \mathrm{E}+02$ & $5.89 \mathrm{E}+03$ & ing & $4.14 \mathrm{E}+02$ & -- & $1.71 \mathrm{E}+02$ & $1.28 \mathrm{E}+04$ & -- & $1.71 \mathrm{E}+02$ & $6.03 \mathrm{E}+03$ & $2.84 \mathrm{E}-02$ & Food \\
\hline Sn-127 & & & & $5.58 \mathrm{E}+04$ & -- & $6.15 \mathrm{E}+05$ & $3.01 \mathrm{E}+03$ & -- & $3.01 E+03$ & $2.56 \mathrm{E}-04$ & $1.18 \mathrm{E}+07$ & Direct \\
\hline Sn-127m & & & & -- & -- & -- & $3.09 \mathrm{E}+05$ & -- & $3.09 E+05$ & $8.60 \mathrm{E}-04$ & $3.59 \mathrm{E}+08$ & Direct \\
\hline Sn-128 & & & & $7.45 \mathrm{E}+04$ & -- & $1.74 \mathrm{E}+06$ & $1.84 \mathrm{E}+04$ & -- & $1.84 \mathrm{E}+04$ & $7.40 \mathrm{E}-04$ & $2.49 \mathrm{E}+07$ & Direct \\
\hline Sn-129 & & & & -- & -- & -- & $3.22 \mathrm{E}+05$ & -- & $3.22 \mathrm{E}+05$ & $4.93 \mathrm{E}-04$ & $6.54 \mathrm{E}+08$ & Direct \\
\hline Sn-130 & & & & -- & -- & -- & $2.08 \mathrm{E}+05$ & -- & $2.08 \mathrm{E}+05$ & 5.34E-04 & $3.89 \mathrm{E}+08$ & Direct \\
\hline Sn-130m & & & & -- & -- & -- & $4.81 \mathrm{E}+05$ & -- & $4.81 \mathrm{E}+05$ & $5.65 \mathrm{E}-04$ & $8.51 \mathrm{E}+08$ & Direct \\
\hline Sr-79 & & & & -- & -- & -- & $2.73 \mathrm{E}+05$ & -- & $2.73 E+05$ & $2.58 \mathrm{E}-04$ & $1.06 \mathrm{E}+09$ & Direct \\
\hline Sr-80 & & & & $5.32 E+04$ & -- & $4.07 E+05$ & $9.06 \mathrm{E}+05$ & -- & $5.32 E+04$ & $2.26 \mathrm{E}-03$ & $2.35 \mathrm{E}+07$ & Inh. \\
\hline Sr-81 & & & & $1.83 \mathrm{E}+05$ & -- & $7.14 \mathrm{E}+06$ & $2.05 E+04$ & -- & $2.05 E+04$ & $2.25 \mathrm{E}-04$ & $9.11 \mathrm{E}+07$ & Direct \\
\hline Sr-82 & & & & $1.12 \mathrm{E}+03$ & -- & $1.82 \mathrm{E}+02$ & $9.44 \mathrm{E}+04$ & -- & $1.82 \mathrm{E}+02$ & $2.85 \mathrm{E}-03$ & $6.38 \mathrm{E}+04$ & Food \\
\hline Sr-83 & & & & $2.28 \mathrm{E}+04$ & -- & $1.38 \mathrm{E}+04$ & $1.16 \mathrm{E}+03$ & -- & $1.16 \mathrm{E}+03$ & 9.93E-04 & $1.17 \mathrm{E}+06$ & Direct \\
\hline $\mathrm{Sr}-85$ & & & & $1.45 \mathrm{E}+04$ & -- & $1.57 \mathrm{E}+03$ & $1.43 \mathrm{E}+03$ & -- & $1.43 \mathrm{E}+03$ & $6.01 \mathrm{E}-02$ & $2.37 E+04$ & Direct \\
\hline Sr-85m & & & & $1.51 \mathrm{E}+06$ & -- & $3.36 \mathrm{E}+07$ & $4.75 \mathrm{E}+04$ & -- & $4.75 \mathrm{E}+04$ & $1.49 \mathrm{E}-03$ & $3.19 E+07$ & Direct \\
\hline Sr-87m & & & & $3.19 \mathrm{E}+05$ & -- & $2.57 \mathrm{E}+06$ & $1.35 \mathrm{E}+04$ & -- & $1.35 \mathrm{E}+04$ & $1.05 \mathrm{E}-03$ & $1.29 \mathrm{E}+07$ & Direct \\
\hline Sr-89 & $3.48 \mathrm{E}+02$ & $1.20 \mathrm{E}-02$ & ing & $1.49 \mathrm{E}+03$ & -- & $3.53 \mathrm{E}+02$ & -- & -- & $3.53 E+02$ & $1.22 \mathrm{E}-02$ & $2.91 \mathrm{E}+04$ & Food \\
\hline Sr-90 & $2.59 \mathrm{E}+01$ & $1.87 \mathrm{E}-01$ & ing & $7.45 \mathrm{E}+01$ & -- & $2.65 \mathrm{E}+01$ & -- & -- & $2.65 \mathrm{E}+01$ & $1.94 \mathrm{E}-01$ & $1.37 \mathrm{E}+02$ & Food \\
\hline Sr-91 & & & & $1.96 \mathrm{E}+04$ & -- & $3.36 \mathrm{E}+04$ & $2.21 \mathrm{E}+03$ & -- & $2.21 \mathrm{E}+03$ & $6.08 \mathrm{E}-04$ & $3.63 \mathrm{E}+06$ & Direct \\
\hline Sr-92 & & & & $3.28 \mathrm{E}+04$ & -- & $1.79 \mathrm{E}+05$ & $3.34 \mathrm{E}+03$ & -- & $3.34 \mathrm{E}+03$ & $2.65 \mathrm{E}-04$ & $1.26 \mathrm{E}+07$ & Direct \\
\hline Sr-93 & & & & -- & -- & -- & $4.31 E+04$ & -- & $4.31 \mathrm{E}+04$ & $1.58 \mathrm{E}-04$ & $2.73 \mathrm{E}+08$ & Direct \\
\hline Sr-94 & & & & -- & -- & -- & $4.05 E+05$ & -- & $4.05 E+05$ & $2.54 \mathrm{E}-04$ & $1.60 \mathrm{E}+09$ & Direct \\
\hline
\end{tabular}


Table C.2 Summary of Exposure Pathway TQs and Selection of Final HC-3 TQs (continued)

\begin{tabular}{|c|c|c|c|c|c|c|c|c|c|c|c|c|}
\hline \multirow[b]{2}{*}{$\begin{array}{c}\text { MASTER } \\
\text { ISOTOPE } \\
\text { LIST } \\
\end{array}$} & \multicolumn{3}{|c|}{ from NNSA SD G 1027} & \multicolumn{5}{|c|}{ Calculated Pathway Specific TQ Values } & \multicolumn{4}{|c|}{ Selected Final Threshold Quantities } \\
\hline & Act (Ci) & Mass (g) & $\begin{array}{l}\text { Limiting } \\
\text { Pathway }\end{array}$ & $\begin{array}{c}\text { TQ }_{\text {inhale }} \\
\text { (Ci) }\end{array}$ & $\begin{array}{c}\text { TQ }_{\text {water }} \\
\text { (Ci) }\end{array}$ & $\mathrm{TQ}_{\text {food }}(\mathrm{Ci})$ & $\begin{array}{c}\mathrm{TQ}_{\text {direct }} \\
(\mathrm{Ci}) \\
\end{array}$ & $\mathrm{TQ}_{\text {sub }}(\mathrm{Ci})$ & Act (Ci) & Mass (g) & $\begin{array}{c}\text { Sp. Act. } \\
\text { (Ci/g) }\end{array}$ & $\begin{array}{l}\text { Limiting } \\
\text { Pathway }\end{array}$ \\
\hline Ta-170 & & & & -- & -- & -- & $1.01 \mathrm{E}+05$ & -- & $1.01 \mathrm{E}+05$ & $6.18 \mathrm{E}-04$ & $1.64 \mathrm{E}+08$ & Direct \\
\hline Ta-172 & & & & $1.96 \mathrm{E}+06$ & $2.16 \mathrm{E}+78$ & $7.92 \mathrm{E}+07$ & $1.27 \mathrm{E}+04$ & -- & $1.27 \mathrm{E}+04$ & $4.28 \mathrm{E}-04$ & $2.97 \mathrm{E}+07$ & Direct \\
\hline Ta-173 & & & & $6.98 \mathrm{E}+05$ & $4.20 \mathrm{E}+17$ & $3.74 \mathrm{E}+06$ & $5.72 \mathrm{E}+03$ & -- & $5.72 \mathrm{E}+03$ & $1.15 \mathrm{E}-03$ & $4.96 \mathrm{E}+06$ & Direct \\
\hline Ta-174 & & & & $1.69 \mathrm{E}+06$ & $1.31 \mathrm{E}+43$ & $3.77 \mathrm{E}+07$ & $1.60 \mathrm{E}+04$ & -- & $1.60 \mathrm{E}+04$ & $1.07 \mathrm{E}-03$ & $1.50 \mathrm{E}+07$ & Direct \\
\hline Ta-175 & & & & $5.58 \mathrm{E}+05$ & $1.98 \mathrm{E}+09$ & $1.20 \mathrm{E}+06$ & $1.55 \mathrm{E}+03$ & -- & $1.55 \mathrm{E}+03$ & $9.09 \mathrm{E}-04$ & $1.71 \mathrm{E}+06$ & Direct \\
\hline Ta-176 & & & & $3.38 \mathrm{E}+05$ & $3.09 \mathrm{E}+10$ & $1.05 \mathrm{E}+06$ & $7.99 \mathrm{E}+02$ & -- & $7.99 \mathrm{E}+02$ & $3.62 \mathrm{E}-04$ & $2.20 \mathrm{E}+06$ & Direct \\
\hline Тa-177 & & & & $8.59 \mathrm{E}+05$ & $3.04 \mathrm{E}+05$ & $4.82 \mathrm{E}+05$ & $1.25 \mathrm{E}+04$ & -- & $1.25 \mathrm{E}+04$ & 3.99E-02 & $3.13 E+05$ & Direct \\
\hline Ta-178s & & & & -- & -- & -- & $7.17 \mathrm{E}+05$ & -- & $7.17 \mathrm{E}+05$ & 6.31E-03 & $1.13 \mathrm{E}+08$ & Direct \\
\hline Ta-178I & & & & $1.02 \mathrm{E}+06$ & $1.36 \mathrm{E}+26$ & $1.51 \mathrm{E}+07$ & $5.37 \mathrm{E}+03$ & -- & $5.37 \mathrm{E}+03$ & $6.70 \mathrm{E}-04$ & $8.00 \mathrm{E}+06$ & Direct \\
\hline Ta-179 & & & & $2.15 \mathrm{E}+05$ & $3.07 E+04$ & $1.26 \mathrm{E}+05$ & $2.24 \mathrm{E}+04$ & -- & $2.24 \mathrm{E}+04$ & $2.04 \mathrm{E}+01$ & $1.10 \mathrm{E}+03$ & Direct \\
\hline Та-180 & & & & $4.65 \mathrm{E}+03$ & $2.35 \mathrm{E}+03$ & $9.59 \mathrm{E}+03$ & $1.30 \mathrm{E}+03$ & -- & $1.30 \mathrm{E}+03$ & $6.53 \mathrm{E}+12$ & $1.99 \mathrm{E}-10$ & Direct \\
\hline Ta-180m & & & & $1.80 \mathrm{E}+06$ & $1.72 \mathrm{E}+11$ & $6.01 \mathrm{E}+06$ & $3.53 E+04$ & -- & $3.53 E+04$ & 1.64E-02 & $2.15 \mathrm{E}+06$ & Direct \\
\hline Ta-182 & & & & $1.15 \mathrm{E}+04$ & $1.40 \mathrm{E}+03$ & $5.93 \mathrm{E}+03$ & $5.63 \mathrm{E}+02$ & -- & $5.63 E+02$ & $9.02 \mathrm{E}-02$ & $6.24 \mathrm{E}+03$ & Direct \\
\hline Ta-182m & & & & $3.10 \mathrm{E}+06$ & $6.19 \mathrm{E}+173$ & $8.11 \mathrm{E}+08$ & $1.82 \mathrm{E}+05$ & -- & $1.82 \mathrm{E}+05$ & $2.79 \mathrm{E}-03$ & $6.52 \mathrm{E}+07$ & Direct \\
\hline Тa-183 & & & & $5.58 \mathrm{E}+04$ & $6.00 \mathrm{E}+03$ & $2.20 \mathrm{E}+04$ & $2.65 \mathrm{E}+03$ & -- & $2.65 \mathrm{E}+03$ & $1.89 \mathrm{E}-02$ & $1.40 \mathrm{E}+05$ & Direct \\
\hline Ta-184 & & & & $1.77 \mathrm{E}+05$ & $5.38 \mathrm{E}+09$ & $4.45 \mathrm{E}+05$ & $1.01 \mathrm{E}+03$ & -- & $1.01 E+03$ & $5.16 \mathrm{E}-04$ & $1.96 \mathrm{E}+06$ & Direct \\
\hline Ta-185 & & & & $1.55 \mathrm{E}+06$ & $2.14 \mathrm{E}+60$ & $4.64 \mathrm{E}+07$ & $7.67 E+04$ & -- & $7.67 E+04$ & $3.70 \mathrm{E}-03$ & $2.07 \mathrm{E}+07$ & Direct \\
\hline Ta-186 & & & & $3.60 \mathrm{E}+06$ & $7.30 \mathrm{E}+257$ & $4.45 \mathrm{E}+08$ & $4.42 \mathrm{E}+04$ & -- & $4.42 \mathrm{E}+04$ & $4.59 \mathrm{E}-04$ & $9.63 \mathrm{E}+07$ & Direct \\
\hline Tb-146 & & & & -- & -- & -- & $5.21 \mathrm{E}+05$ & -- & $5.21 \mathrm{E}+05$ & $1.55 \mathrm{E}-04$ & $3.36 \mathrm{E}+09$ & Direct \\
\hline Tb-147 & & & & $9.31 \mathrm{E}+04$ & -- & $9.78 \mathrm{E}+05$ & $4.60 \mathrm{E}+03$ & -- & $4.60 \mathrm{E}+03$ & $3.56 \mathrm{E}-04$ & $1.29 \mathrm{E}+07$ & Direct \\
\hline Tb-147m & & & & -- & -- & -- & $2.03 \mathrm{E}+05$ & -- & $2.03 E+05$ & $2.96 \mathrm{E}-04$ & $6.84 \mathrm{E}+08$ & Direct \\
\hline Tb-148 & & & & $1.12 \mathrm{E}+05$ & -- & $1.98 \mathrm{E}+06$ & $5.12 \mathrm{E}+03$ & -- & $5.12 \mathrm{E}+03$ & $2.42 \mathrm{E}-04$ & $2.12 \mathrm{E}+07$ & Direct \\
\hline Tb-148m & & & & -- & -- & -- & $1.05 E+05$ & -- & $1.05 \mathrm{E}+05$ & $1.82 \mathrm{E}-04$ & $5.78 \mathrm{E}+08$ & Direct \\
\hline Tb-149 & & & & $2.60 \mathrm{E}+03$ & -- & $2.51 \mathrm{E}+05$ & $1.84 \mathrm{E}+03$ & -- & $1.84 \mathrm{E}+03$ & $3.62 \mathrm{E}-04$ & $5.07 \mathrm{E}+06$ & Direct \\
\hline Tb-149m & & & & -- & -- & -- & $1.27 \mathrm{E}+05$ & -- & $1.27 \mathrm{E}+05$ & 4.17E-04 & $3.03 E+08$ & Direct \\
\hline Tb-150 & & & & $6.20 \mathrm{E}+04$ & -- & $3.17 \mathrm{E}+05$ & $2.21 \mathrm{E}+03$ & -- & $2.21 \mathrm{E}+03$ & $3.46 \mathrm{E}-04$ & $6.39 \mathrm{E}+06$ & Direct \\
\hline Tb-150m & & & & -- & -- & -- & $4.96 \mathrm{E}+04$ & -- & $4.96 \mathrm{E}+04$ & $2.29 \mathrm{E}-04$ & $2.16 \mathrm{E}+08$ & Direct \\
\hline Tb-151 & & & & $3.38 \mathrm{E}+04$ & -- & $4.52 \mathrm{E}+04$ & $1.26 \mathrm{E}+03$ & -- & $1.26 \mathrm{E}+03$ & 1.07E-03 & $1.18 \mathrm{E}+06$ & Direct \\
\hline Tb-151m & & & & -- & -- & -- & $2.15 \mathrm{E}+07$ & -- & $2.15 \mathrm{E}+07$ & $7.20 \mathrm{E}-03$ & $2.99 \mathrm{E}+09$ & Direct \\
\hline Tb-152 & & & & $2.23 \mathrm{E}+04$ & -- & $2.17 \mathrm{E}+04$ & $7.53 \mathrm{E}+02$ & -- & $7.53 E+02$ & $6.39 \mathrm{E}-04$ & $1.18 \mathrm{E}+06$ & Direct \\
\hline Tb-152m & & & & -- & -- & -- & $2.26 \mathrm{E}+05$ & -- & $2.26 \mathrm{E}+05$ & $7.68 \mathrm{E}-04$ & $2.95 \mathrm{E}+08$ & Direct \\
\hline Tb-153 & & & & $4.65 \mathrm{E}+04$ & -- & $2.14 \mathrm{E}+04$ & $3.66 \mathrm{E}+03$ & -- & $3.66 \mathrm{E}+03$ & $1.00 \mathrm{E}-02$ & $3.65 \mathrm{E}+05$ & Direct \\
\hline Tb-154 & & & & $1.86 \mathrm{E}+04$ & -- & $1.96 \mathrm{E}+04$ & $4.44 \mathrm{E}+02$ & -- & $4.44 \mathrm{E}+02$ & 4.67E-04 & $9.51 \mathrm{E}+05$ & Direct \\
\hline Tb-155 & & & & $4.47 \mathrm{E}+04$ & -- & $1.32 \mathrm{E}+04$ & $5.54 \mathrm{E}+03$ & -- & $5.54 \mathrm{E}+03$ & $3.50 \mathrm{E}-02$ & $1.58 \mathrm{E}+05$ & Direct \\
\hline Tb-156 & & & & $7.98 \mathrm{E}+03$ & -- & $2.31 E+03$ & $4.24 \mathrm{E}+02$ & -- & $4.24 \mathrm{E}+02$ & $2.70 \mathrm{E}-03$ & $1.57 \mathrm{E}+05$ & Direct \\
\hline
\end{tabular}


Table C.2 Summary of Exposure Pathway TQs and Selection of Final HC-3 TQs (continued)

\begin{tabular}{|c|c|c|c|c|c|c|c|c|c|c|c|c|}
\hline \multirow{2}{*}{$\begin{array}{c}\text { MASTER } \\
\text { ISOTOPE } \\
\text { LIST } \\
\end{array}$} & \multicolumn{3}{|c|}{ from NNSA SD G 1027} & \multicolumn{5}{|c|}{ Calculated Pathway Specific TQ Values } & \multicolumn{4}{|c|}{ Selected Final Threshold Quantities } \\
\hline & Act (Ci) & Mass (g) & $\begin{array}{l}\text { Limiting } \\
\text { Pathway }\end{array}$ & $\begin{array}{c}\mathrm{TQ}_{\text {inhale }} \\
(\mathrm{Ci}) \\
\end{array}$ & $\begin{array}{c}\text { TQ }_{\text {water }} \\
\text { (Ci) }\end{array}$ & $\mathrm{TQ}_{\text {food }}(\mathrm{Ci})$ & $\begin{array}{c}\mathrm{TQ}_{\text {direct }} \\
(\mathrm{Ci}) \\
\end{array}$ & $\mathrm{TQ}_{\text {sub }}(\mathrm{Ci})$ & Act (Ci) & Mass (g) & $\begin{array}{c}\text { Sp. Act. } \\
\text { (Ci/g) }\end{array}$ & $\begin{array}{l}\text { Limiting } \\
\text { Pathway }\end{array}$ \\
\hline $\mathrm{Tb}-156 \mathrm{ml}$ & & & & $4.86 \mathrm{E}+04$ & -- & $6.64 \mathrm{E}+04$ & $3.96 \mathrm{E}+04$ & -- & $3.96 \mathrm{E}+04$ & $4.80 \mathrm{E}-02$ & $8.24 \mathrm{E}+05$ & Direct \\
\hline Tb-156ms & & & & $8.59 \mathrm{E}+04$ & -- & $6.44 \mathrm{E}+05$ & $5.69 \mathrm{E}+05$ & -- & $8.59 E+04$ & $2.14 \mathrm{E}-02$ & $4.02 \mathrm{E}+06$ & Inh. \\
\hline Tb-157 & & & & $1.02 \mathrm{E}+04$ & -- & $2.37 \mathrm{E}+04$ & $2.20 \mathrm{E}+05$ & -- & $1.02 \mathrm{E}+04$ & $6.69 \mathrm{E}+02$ & $1.52 \mathrm{E}+01$ & Inh. \\
\hline Tb-158 & & & & $2.60 \mathrm{E}+02$ & -- & $7.33 \mathrm{E}+02$ & $9.10 \mathrm{E}+02$ & -- & $2.60 \mathrm{E}+02$ & $1.72 \mathrm{E}+01$ & $1.51 \mathrm{E}+01$ & Inh. \\
\hline Tb-160 & $5.76 \mathrm{E}+02$ & $5.10 \mathrm{E}-02$ & ing & $1.69 \mathrm{E}+03$ & -- & $5.87 \mathrm{E}+02$ & $6.49 \mathrm{E}+02$ & -- & $5.87 \mathrm{E}+02$ & $5.20 \mathrm{E}-02$ & $1.13 \mathrm{E}+04$ & Food \\
\hline Tb-161 & & & & $9.31 \mathrm{E}+03$ & -- & $3.21 \mathrm{E}+03$ & $2.17 E+04$ & -- & $3.21 \mathrm{E}+03$ & $2.74 \mathrm{E}-02$ & $1.17 \mathrm{E}+05$ & Food \\
\hline Tb-162 & & & & -- & -- & -- & $8.62 E+04$ & -- & $8.62 E+04$ & $5.64 \mathrm{E}-04$ & $1.53 \mathrm{E}+08$ & Direct \\
\hline Tb-163 & & & & $3.85 \mathrm{E}+05$ & -- & $3.60 \mathrm{E}+07$ & $4.71 E+04$ & -- & $4.71 E+04$ & $7.96 \mathrm{E}-04$ & $5.92 \mathrm{E}+07$ & Direct \\
\hline Tb-164 & & & & -- & -- & -- & $9.88 \mathrm{E}+04$ & -- & $9.88 \mathrm{E}+04$ & $2.58 \mathrm{E}-04$ & $3.82 \mathrm{E}+08$ & Direct \\
\hline Tb-165 & & & & -- & -- & -- & $4.11 \mathrm{E}+05$ & -- & $4.11 \mathrm{E}+05$ & $7.60 \mathrm{E}-04$ & $5.40 \mathrm{E}+08$ & Direct \\
\hline Tc-101 & & & & $5.32 \mathrm{E}+05$ & $6.18 \mathrm{E}+192$ & $4.29 \mathrm{E}+07$ & $1.53 \mathrm{E}+05$ & -- & $1.53 \mathrm{E}+05$ & $1.16 \mathrm{E}-03$ & $1.31 \mathrm{E}+08$ & Direct \\
\hline Tc-102 & & & & -- & -- & -- & $1.02 \mathrm{E}+08$ & -- & $1.02 \mathrm{E}+08$ & $4.86 \mathrm{E}-03$ & $2.10 \mathrm{E}+10$ & Direct \\
\hline Tc-102m & & & & -- & -- & -- & $6.73 \mathrm{E}+04$ & -- & $6.73 E+04$ & $1.59 \mathrm{E}-04$ & $4.24 \mathrm{E}+08$ & Direct \\
\hline Tc-104 & & & & $2.33 \mathrm{E}+05$ & $2.51 \mathrm{E}+151$ & $7.85 \mathrm{E}+06$ & $2.01 \mathrm{E}+04$ & -- & $2.01 \mathrm{E}+04$ & $2.02 \mathrm{E}-04$ & $9.94 \mathrm{E}+07$ & Direct \\
\hline Tc-105 & & & & -- & -- & -- & $1.19 \mathrm{E}+05$ & -- & $1.19 \mathrm{E}+05$ & 5.07E-04 & $2.36 \mathrm{E}+08$ & Direct \\
\hline Tc-91 & & & & -- & -- & -- & $9.29 \mathrm{E}+04$ & -- & $9.29 \mathrm{E}+04$ & $1.41 \mathrm{E}-04$ & $6.59 \mathrm{E}+08$ & Direct \\
\hline Tc-91m & & & & -- & -- & -- & $1.54 \mathrm{E}+05$ & -- & $1.54 \mathrm{E}+05$ & $2.46 \mathrm{E}-04$ & $6.27 \mathrm{E}+08$ & Direct \\
\hline Tc-92 & & & & -- & -- & -- & $4.45 \mathrm{E}+04$ & -- & $4.45 \mathrm{E}+04$ & $9.25 \mathrm{E}-05$ & $4.81 \mathrm{E}+08$ & Direct \\
\hline Tc-93 & & & & $1.72 \mathrm{E}+05$ & $1.82 \mathrm{E}+22$ & $1.44 \mathrm{E}+06$ & $3.02 \mathrm{E}+03$ & -- & $3.02 \mathrm{E}+03$ & $2.46 \mathrm{E}-04$ & $1.23 \mathrm{E}+07$ & Direct \\
\hline Tc-93m & & & & $3.60 \mathrm{E}+05$ & $4.10 \mathrm{E}+67$ & $1.11 \mathrm{E}+07$ & $2.30 \mathrm{E}+04$ & -- & $2.30 \mathrm{E}+04$ & $4.94 \mathrm{E}-04$ & $4.65 \mathrm{E}+07$ & Direct \\
\hline Tc-94 & & & & $5.08 \mathrm{E}+04$ & $3.10 \mathrm{E}+14$ & $2.23 \mathrm{E}+05$ & $9.58 \mathrm{E}+02$ & -- & $9.58 \mathrm{E}+02$ & $1.40 \mathrm{E}-04$ & $6.83 \mathrm{E}+06$ & Direct \\
\hline Tc-94m & & & & $1.40 \mathrm{E}+05$ & $1.01 \mathrm{E}+57$ & $2.03 E+06$ & $7.50 \mathrm{E}+03$ & -- & $7.50 \mathrm{E}+03$ & $1.95 \mathrm{E}-04$ & $3.85 \mathrm{E}+07$ & Direct \\
\hline Tc-95 & & & & $6.20 \mathrm{E}+04$ & $1.43 \mathrm{E}+07$ & $6.38 \mathrm{E}+04$ & $1.34 \mathrm{E}+03$ & -- & $1.34 \mathrm{E}+03$ & $8.13 \mathrm{E}-04$ & $1.65 \mathrm{E}+06$ & Direct \\
\hline Tc-95m & & & & $1.28 \mathrm{E}+04$ & $3.59 \mathrm{E}+03$ & $1.17 \mathrm{E}+03$ & $1.08 \mathrm{E}+03$ & -- & $1.08 \mathrm{E}+03$ & $4.80 \mathrm{E}-02$ & $2.26 \mathrm{E}+04$ & Direct \\
\hline Tc-96 & & & & $1.12 \mathrm{E}+04$ & $9.10 \mathrm{E}+03$ & $2.23 \mathrm{E}+03$ & $3.14 \mathrm{E}+02$ & -- & $3.14 \mathrm{E}+02$ & 9.87E-04 & $3.18 \mathrm{E}+05$ & Direct \\
\hline Tc-96m & & & & $1.02 \mathrm{E}+06$ & $2.66 \mathrm{E}+58$ & $1.73 \mathrm{E}+07$ & $2.73 \mathrm{E}+05$ & -- & $2.73 E+05$ & $7.18 \mathrm{E}-03$ & $3.81 \mathrm{E}+07$ & Direct \\
\hline Tc-97 & & & & $5.32 \mathrm{E}+04$ & $2.38 \mathrm{E}+04$ & $7.29 \mathrm{E}+03$ & $6.42 \mathrm{E}+04$ & -- & $7.29 E+03$ & $5.14 \mathrm{E}+06$ & $1.42 \mathrm{E}-03$ & Food \\
\hline Tc-97m & & & & $3.60 \mathrm{E}+03$ & $3.26 \mathrm{E}+03$ & $1.04 \mathrm{E}+03$ & $7.67 \mathrm{E}+04$ & -- & $1.04 \mathrm{E}+03$ & $6.73 \mathrm{E}-02$ & $1.55 \mathrm{E}+04$ & Food \\
\hline Tc-98 & & & & $1.38 \mathrm{E}+03$ & $8.59 \mathrm{E}+02$ & $2.63 \mathrm{E}+02$ & $5.14 \mathrm{E}+02$ & -- & $2.63 \mathrm{E}+02$ & $3.02 \mathrm{E}+05$ & $8.69 \mathrm{E}-04$ & Food \\
\hline Tc-99 & $7.61 \mathrm{E}+02$ & $4.48 \mathrm{E}+04$ & ing & $2.86 \mathrm{E}+03$ & $2.53 \mathrm{E}+03$ & $7.75 \mathrm{E}+02$ & -- & -- & $7.75 \mathrm{E}+02$ & $4.57 \mathrm{E}+04$ & 1.70E-02 & Food \\
\hline Tc-99m & & & & $3.85 \mathrm{E}+05$ & $4.18 \mathrm{E}+13$ & $1.48 \mathrm{E}+06$ & $1.70 \mathrm{E}+04$ & -- & $1.70 \mathrm{E}+04$ & $3.22 \mathrm{E}-03$ & $5.26 \mathrm{E}+06$ & Direct \\
\hline Te-113 & & & & -- & -- & -- & $1.92 \mathrm{E}+05$ & -- & $1.92 \mathrm{E}+05$ & $1.96 \mathrm{E}-04$ & $9.80 \mathrm{E}+08$ & Direct \\
\hline Te-114 & & & & -- & -- & -- & $3.72 \mathrm{E}+04$ & -- & $3.72 \mathrm{E}+04$ & $3.43 \mathrm{E}-04$ & $1.09 \mathrm{E}+08$ & Direct \\
\hline Te-115 & & & & -- & -- & -- & $5.56 \mathrm{E}+04$ & -- & $5.56 \mathrm{E}+04$ & 1.97E-04 & $2.82 \mathrm{E}+08$ & Direct \\
\hline Te-115m & & & & -- & -- & -- & $4.15 \mathrm{E}+04$ & -- & $4.15 E+04$ & 1.70E-04 & $2.44 \mathrm{E}+08$ & Direct \\
\hline
\end{tabular}


Table C.2 Summary of Exposure Pathway TQs and Selection of Final HC-3 TQs (continued)

\begin{tabular}{|c|c|c|c|c|c|c|c|c|c|c|c|c|}
\hline \multirow{2}{*}{$\begin{array}{c}\text { MASTER } \\
\text { ISOTOPE } \\
\text { LIST } \\
\end{array}$} & \multicolumn{3}{|c|}{ from NNSA SD G 1027} & \multicolumn{5}{|c|}{ Calculated Pathway Specific TQ Values } & \multicolumn{4}{|c|}{ Selected Final Threshold Quantities } \\
\hline & Act (Ci) & Mass (g) & $\begin{array}{l}\text { Limiting } \\
\text { Pathway }\end{array}$ & $\begin{array}{c}\mathrm{TQ}_{\text {inhale }} \\
(\mathrm{Ci}) \\
\end{array}$ & $\begin{array}{c}\text { TQ }_{\text {water }} \\
\text { (Ci) }\end{array}$ & $\mathrm{TQ}_{\text {food }}(\mathrm{Ci})$ & $\begin{array}{c}\mathrm{TQ}_{\text {direct }} \\
(\mathrm{Ci}) \\
\end{array}$ & $\mathrm{TQ}_{\text {sub }}(\mathrm{Ci})$ & Act (Ci) & Mass (g) & $\begin{array}{c}\text { Sp. Act. } \\
\text { (Ci/g) }\end{array}$ & $\begin{array}{l}\text { Limiting } \\
\text { Pathway }\end{array}$ \\
\hline Te-116 & & & & $6.57 \mathrm{E}+04$ & -- & $6.11 \mathrm{E}+05$ & $6.65 \mathrm{E}+04$ & -- & $6.57 \mathrm{E}+04$ & $6.05 \mathrm{E}-03$ & $1.09 \mathrm{E}+07$ & Inh. \\
\hline Te-117 & & & & $2.03 \mathrm{E}+05$ & -- & $4.61 \mathrm{E}+06$ & $7.54 \mathrm{E}+03$ & -- & $7.54 \mathrm{E}+03$ & $2.91 \mathrm{E}-04$ & $2.59 \mathrm{E}+07$ & Direct \\
\hline Te-118 & & & & $4.86 \mathrm{E}+03$ & -- & $8.49 \mathrm{E}+02$ & $3.86 \mathrm{E}+04$ & -- & $8.49 \mathrm{E}+02$ & $4.60 \mathrm{E}-03$ & $1.85 \mathrm{E}+05$ & Food \\
\hline Te-119 & & & & $6.20 \mathrm{E}+04$ & -- & $9.31 \mathrm{E}+04$ & $1.52 \mathrm{E}+03$ & -- & $1.52 \mathrm{E}+03$ & $9.25 \mathrm{E}-04$ & $1.64 \mathrm{E}+06$ & Direct \\
\hline Te-119m & & & & $1.53 \mathrm{E}+04$ & -- & $4.16 \mathrm{E}+03$ & $5.18 \mathrm{E}+02$ & -- & $5.18 \mathrm{E}+02$ & $2.22 \mathrm{E}-03$ & $2.34 \mathrm{E}+05$ & Direct \\
\hline Te-121 & & & & $2.19 \mathrm{E}+04$ & -- & $3.25 \mathrm{E}+03$ & $1.28 \mathrm{E}+03$ & -- & $1.28 \mathrm{E}+03$ & $2.02 \mathrm{E}-02$ & $6.35 \mathrm{E}+04$ & Direct \\
\hline Te-121m & & & & $2.03 E+03$ & -- & $3.77 \mathrm{E}+02$ & $3.36 \mathrm{E}+03$ & -- & $3.77 \mathrm{E}+02$ & $5.38 \mathrm{E}-02$ & $7.01 \mathrm{E}+03$ & Food \\
\hline Te-123 & & & & $9.31 \mathrm{E}+02$ & -- & $1.83 \mathrm{E}+02$ & $3.68 \mathrm{E}+04$ & -- & $1.83 \mathrm{E}+02$ & $6.29 \mathrm{E}+11$ & $2.91 \mathrm{E}-10$ & Food \\
\hline Te-123m & & & & $2.86 \mathrm{E}+03$ & -- & $6.32 \mathrm{E}+02$ & $4.92 \mathrm{E}+03$ & -- & $6.32 \mathrm{E}+02$ & 7.13E-02 & $8.88 \mathrm{E}+03$ & Food \\
\hline Te-125m & & & & $3.38 \mathrm{E}+03$ & -- & $1.12 \mathrm{E}+03$ & $2.06 \mathrm{E}+04$ & -- & $1.12 \mathrm{E}+03$ & $6.20 \mathrm{E}-02$ & $1.80 \mathrm{E}+04$ & Food \\
\hline Te-127 & & & & $6.20 \mathrm{E}+04$ & -- & $1.66 \mathrm{E}+05$ & $3.24 \mathrm{E}+05$ & -- & $6.20 \mathrm{E}+04$ & $2.35 \mathrm{E}-02$ & $2.64 \mathrm{E}+06$ & Inh. \\
\hline Te-127m & $3.80 \mathrm{E}+02$ & 4.03E-02 & ing & $1.55 \mathrm{E}+03$ & -- & $3.88 \mathrm{E}+02$ & $6.50 \mathrm{E}+04$ & -- & $3.88 \mathrm{E}+02$ & $4.11 \mathrm{E}-02$ & $9.44 \mathrm{E}+03$ & Food \\
\hline Te-129 & & & & $1.96 \mathrm{E}+05$ & -- & $3.52 \mathrm{E}+06$ & $1.75 \mathrm{E}+05$ & -- & $1.75 \mathrm{E}+05$ & $8.36 \mathrm{E}-03$ & $2.10 \mathrm{E}+07$ & Direct \\
\hline Te-129m & $3.61 \mathrm{E}+02$ & $1.20 \mathrm{E}-02$ & ing & $1.77 \mathrm{E}+03$ & -- & $3.66 \mathrm{E}+02$ & $1.95 \mathrm{E}+04$ & -- & $3.66 \mathrm{E}+02$ & $1.21 \mathrm{E}-02$ & $3.01 \mathrm{E}+04$ & Food \\
\hline Te-131 & & & & $1.64 \mathrm{E}+05$ & -- & $7.09 \mathrm{E}+06$ & $6.89 \mathrm{E}+04$ & -- & $6.89 E+04$ & $1.20 \mathrm{E}-03$ & $5.75 \mathrm{E}+07$ & Direct \\
\hline Te-131m & & & & $4.65 E+03$ & -- & $4.91 \mathrm{E}+03$ & $6.64 \mathrm{E}+02$ & -- & $6.64 \mathrm{E}+02$ & $8.32 \mathrm{E}-04$ & $7.98 \mathrm{E}+05$ & Direct \\
\hline Te-132 & & & & $2.19 \mathrm{E}+03$ & -- & $1.09 \mathrm{E}+03$ & $3.45 \mathrm{E}+03$ & -- & $1.09 \mathrm{E}+03$ & $3.60 \mathrm{E}-03$ & $3.04 \mathrm{E}+05$ & Food \\
\hline Te-133 & & & & $1.99 \mathrm{E}+05$ & -- & $1.72 \mathrm{E}+07$ & $6.27 \mathrm{E}+04$ & -- & $6.27 E+04$ & $5.51 \mathrm{E}-04$ & $1.14 \mathrm{E}+08$ & Direct \\
\hline Te-133m & & & & $5.08 \mathrm{E}+04$ & -- & $9.96 \mathrm{E}+05$ & $5.66 \mathrm{E}+03$ & -- & $5.66 \mathrm{E}+03$ & $2.21 \mathrm{E}-04$ & $2.55 \mathrm{E}+07$ & Direct \\
\hline Te-134 & & & & $1.02 \mathrm{E}+05$ & -- & $3.36 \mathrm{E}+06$ & $1.96 \mathrm{E}+04$ & -- & $1.96 \mathrm{E}+04$ & $5.83 \mathrm{E}-04$ & $3.36 \mathrm{E}+07$ & Direct \\
\hline Th-223 & & & & -- & -- & -- & $9.61 \mathrm{E}+08$ & -- & $9.61 \mathrm{E}+08$ & $1.14 \mathrm{E}-02$ & $8.43 \mathrm{E}+10$ & Direct \\
\hline Th-224 & & & & -- & -- & -- & $1.79 \mathrm{E}+09$ & -- & $1.79 \mathrm{E}+09$ & $3.72 \mathrm{E}-02$ & $4.80 \mathrm{E}+10$ & Direct \\
\hline Th-226 & & & & $1.43 \mathrm{E}+03$ & -- & $1.39 \mathrm{E}+07$ & $2.70 \mathrm{E}+06$ & -- & $1.43 \mathrm{E}+03$ & $5.32 \mathrm{E}-05$ & $2.69 \mathrm{E}+07$ & Inh. \\
\hline Th-227 & & & & $1.16 \mathrm{E}+01$ & -- & $1.51 \mathrm{E}+03$ & $6.72 \mathrm{E}+03$ & -- & $1.16 \mathrm{E}+01$ & $3.79 \mathrm{E}-04$ & $3.07 E+04$ & Inh. \\
\hline Th-228 & $2.89 \mathrm{E}+00$ & $3.52 \mathrm{E}-03$ & $\mathrm{i}$ & $3.02 \mathrm{E}+00$ & -- & $1.14 \mathrm{E}+02$ & $2.27 \mathrm{E}+05$ & -- & $3.02 \mathrm{E}+00$ & $3.68 \mathrm{E}-03$ & $8.19 \mathrm{E}+02$ & Inh. \\
\hline Th-229 & & & & $1.13 \mathrm{E}+00$ & -- & $1.68 \mathrm{E}+01$ & $7.58 \mathrm{E}+03$ & -- & $1.13 \mathrm{E}+00$ & $5.30 \mathrm{E}+00$ & $2.13 \mathrm{E}-01$ & Inh. \\
\hline Th-230 & $2.82 \mathrm{E}+00$ & $1.36 \mathrm{E}+02$ & $\mathrm{i}$ & $2.79 \mathrm{E}+00$ & -- & $3.84 \mathrm{E}+01$ & $4.84 \mathrm{E}+05$ & -- & $2.79 \mathrm{E}+00$ & $1.38 \mathrm{E}+02$ & $2.02 \mathrm{E}-02$ & Inh. \\
\hline Th-231 & & & & $2.79 \mathrm{E}+05$ & -- & $3.19 \mathrm{E}+05$ & $3.86 \mathrm{E}+04$ & -- & $3.86 \mathrm{E}+04$ & $7.26 \mathrm{E}-02$ & $5.31 \mathrm{E}+05$ & Direct \\
\hline Th-232 & $2.68 \mathrm{E}+00$ & $2.44 \mathrm{E}+07$ & $\mathrm{i}$ & $2.66 \mathrm{E}+00$ & -- & $3.66 \mathrm{E}+01$ & $5.58 \mathrm{E}+05$ & -- & $2.66 \mathrm{E}+00$ & $2.42 \mathrm{E}+07$ & $1.10 \mathrm{E}-07$ & Inh. \\
\hline Th-233 & & & & $3.85 \mathrm{E}+06$ & -- & $3.15 \mathrm{E}+08$ & $8.67 \mathrm{E}+05$ & -- & $8.67 E+05$ & $2.40 \mathrm{E}-02$ & $3.62 \mathrm{E}+07$ & Direct \\
\hline Th-234 & & & & $1.53 \mathrm{E}+04$ & -- & $3.59 \mathrm{E}+03$ & $7.92 \mathrm{E}+04$ & -- & $3.59 \mathrm{E}+03$ & $1.55 \mathrm{E}-01$ & $2.31 \mathrm{E}+04$ & Food \\
\hline Th-235 & & & & -- & -- & -- & $1.90 \mathrm{E}+06$ & -- & $1.90 \mathrm{E}+06$ & $1.69 \mathrm{E}-02$ & $1.13 \mathrm{E}+08$ & Direct \\
\hline Th-236 & & & & -- & -- & -- & $5.58 \mathrm{E}+05$ & -- & $5.58 \mathrm{E}+05$ & $2.63 \mathrm{E}-02$ & $2.12 \mathrm{E}+07$ & Direct \\
\hline $\mathrm{Ti}-44$ & $9.38 \mathrm{E}+01$ & $5.46 \mathrm{E}-01$ & $\mathrm{i}$ & $9.31 \mathrm{E}+01$ & $3.41 \mathrm{E}+02$ & $1.39 \mathrm{E}+02$ & $5.39 \mathrm{E}+03$ & -- & $9.31 \mathrm{E}+01$ & $5.41 \mathrm{E}-01$ & $1.72 \mathrm{E}+02$ & Inh. \\
\hline Ti-45 & & & & $7.45 \mathrm{E}+04$ & $1.05 \mathrm{E}+20$ & $5.61 \mathrm{E}+05$ & $4.53 \mathrm{E}+03$ & -- & $4.53 E+03$ & $2.00 \mathrm{E}-04$ & $2.26 \mathrm{E}+07$ & Direct \\
\hline
\end{tabular}


Table C.2 Summary of Exposure Pathway TQs and Selection of Final HC-3 TQs (continued)

\begin{tabular}{|c|c|c|c|c|c|c|c|c|c|c|c|c|}
\hline \multirow[b]{2}{*}{$\begin{array}{c}\text { MASTER } \\
\text { ISOTOPE } \\
\text { LIST } \\
\end{array}$} & \multicolumn{3}{|c|}{ from NNSA SD G 1027} & \multicolumn{5}{|c|}{ Calculated Pathway Specific TQ Values } & \multicolumn{4}{|c|}{ Selected Final Threshold Quantities } \\
\hline & Act (Ci) & Mass (g) & $\begin{array}{l}\text { Limiting } \\
\text { Pathway }\end{array}$ & $\begin{array}{c}\text { TQ }_{\text {inhale }} \\
(\mathrm{Ci})\end{array}$ & $\begin{array}{c}\text { TQ }_{\text {water }} \\
\text { (Ci) }\end{array}$ & $\mathrm{TQ}_{\text {food }}(\mathrm{Ci})$ & $\begin{array}{c}T_{\text {direct }} \\
\text { (Ci) }\end{array}$ & $\mathrm{TQ}_{\text {sub }}(\mathrm{Ci})$ & Act (Ci) & Mass (g) & $\begin{array}{c}\text { Sp. Act. } \\
\text { (Ci/g) }\end{array}$ & $\begin{array}{l}\text { Limiting } \\
\text { Pathway }\end{array}$ \\
\hline Ti-51 & & & & -- & -- & -- & $3.41 \mathrm{E}+05$ & -- & $3.41 \mathrm{E}+05$ & $5.32 \mathrm{E}-04$ & $6.41 \mathrm{E}+08$ & Direct \\
\hline Ti-52 & & & & -- & -- & -- & $3.32 \mathrm{E}+06$ & -- & $3.32 \mathrm{E}+06$ & $1.56 \mathrm{E}-03$ & $2.13 \mathrm{E}+09$ & Direct \\
\hline TI-190 & & & & -- & -- & -- & $2.16 \mathrm{E}+05$ & -- & $2.16 \mathrm{E}+05$ & $5.67 \mathrm{E}-04$ & $3.81 \mathrm{E}+08$ & Direct \\
\hline $\mathrm{TI}-190 \mathrm{~m}$ & & & & -- & -- & -- & $7.98 \mathrm{E}+04$ & -- & $7.98 \mathrm{E}+04$ & $2.98 \mathrm{E}-04$ & $2.67 \mathrm{E}+08$ & Direct \\
\hline TI-194 & & & & $1.25 \mathrm{E}+06$ & -- & $5.78 \mathrm{E}+07$ & $2.82 \mathrm{E}+04$ & -- & $2.82 \mathrm{E}+04$ & $9.59 E-04$ & $2.94 \mathrm{E}+07$ & Direct \\
\hline TI-194m & & & & $3.10 \mathrm{E}+05$ & -- & $1.18 \mathrm{E}+07$ & $9.53 \mathrm{E}+03$ & -- & $9.53 \mathrm{E}+03$ & $3.22 \mathrm{E}-04$ & $2.96 \mathrm{E}+07$ & Direct \\
\hline TI-195 & & & & $3.72 \mathrm{E}+05$ & -- & $8.23 E+06$ & $8.19 E+03$ & -- & $8.19 E+03$ & $5.91 \mathrm{E}-04$ & $1.39 \mathrm{E}+07$ & Direct \\
\hline TI-196 & & & & $1.96 \mathrm{E}+05$ & -- & $2.60 \mathrm{E}+06$ & $3.50 \mathrm{E}+03$ & -- & $3.50 \mathrm{E}+03$ & $4.03 \mathrm{E}-04$ & $8.69 \mathrm{E}+06$ & Direct \\
\hline TI-197 & & & & $4.14 \mathrm{E}+05$ & -- & $3.97 E+06$ & $1.04 \mathrm{E}+04$ & -- & $1.04 \mathrm{E}+04$ & $1.86 \mathrm{E}-03$ & $5.60 \mathrm{E}+06$ & Direct \\
\hline TI-198 & & & & $9.31 \mathrm{E}+04$ & -- & $6.74 \mathrm{E}+05$ & $1.19 \mathrm{E}+03$ & -- & $1.19 \mathrm{E}+03$ & $3.98 \mathrm{E}-04$ & $2.99 \mathrm{E}+06$ & Direct \\
\hline Tl-198m & & & & $1.53 \mathrm{E}+05$ & -- & $2.56 \mathrm{E}+06$ & $5.40 \mathrm{E}+03$ & -- & $5.40 \mathrm{E}+03$ & $6.38 \mathrm{E}-04$ & $8.46 \mathrm{E}+06$ & Direct \\
\hline Tl-199 & & & & $3.02 \mathrm{E}+05$ & -- & $1.36 \mathrm{E}+06$ & $7.31 \mathrm{E}+03$ & -- & $7.31 \mathrm{E}+03$ & $3.45 \mathrm{E}-03$ & $2.12 \mathrm{E}+06$ & Direct \\
\hline TI-200 & & & & $4.47 \mathrm{E}+04$ & -- & $5.30 \mathrm{E}+04$ & $7.49 \mathrm{E}+02$ & -- & $7.49 E+02$ & $1.25 \mathrm{E}-03$ & $6.00 \mathrm{E}+05$ & Direct \\
\hline TI-201 & & & & $1.47 \mathrm{E}+05$ & -- & $4.51 \mathrm{E}+04$ & $8.69 \mathrm{E}+03$ & -- & $8.69 E+03$ & 4.07E-02 & $2.13 \mathrm{E}+05$ & Direct \\
\hline TI-202 & & & & $3.60 \mathrm{E}+04$ & -- & $3.65 \mathrm{E}+03$ & $1.60 \mathrm{E}+03$ & -- & $1.60 \mathrm{E}+03$ & $3.02 \mathrm{E}-02$ & $5.29 \mathrm{E}+04$ & Direct \\
\hline TI-204 & & & & $1.80 \mathrm{E}+04$ & -- & $6.25 \mathrm{E}+02$ & $6.60 \mathrm{E}+05$ & -- & $6.25 \mathrm{E}+02$ & $1.35 \mathrm{E}+00$ & $4.64 \mathrm{E}+02$ & Food \\
\hline TI-206 & & & & -- & -- & -- & $1.73 \mathrm{E}+09$ & -- & $1.73 E+09$ & $7.94 \mathrm{E}+00$ & $2.17 E+08$ & Direct \\
\hline TI-206m & & & & -- & -- & -- & $8.01 \mathrm{E}+04$ & -- & $8.01 E+04$ & $3.28 \mathrm{E}-04$ & $2.44 \mathrm{E}+08$ & Direct \\
\hline TI-207 & & & & -- & -- & -- & $6.90 \mathrm{E}+07$ & -- & $6.90 \mathrm{E}+07$ & $3.63 \mathrm{E}-01$ & $1.90 \mathrm{E}+08$ & Direct \\
\hline TI-208 & & & & -- & -- & -- & $6.99 \mathrm{E}+04$ & -- & $6.99 E+04$ & $2.38 \mathrm{E}-04$ & $2.94 \mathrm{E}+08$ & Direct \\
\hline TI-209 & & & & -- & -- & -- & $1.62 \mathrm{E}+05$ & -- & $1.62 \mathrm{E}+05$ & $3.96 \mathrm{E}-04$ & $4.09 \mathrm{E}+08$ & Direct \\
\hline TI-210 & & & & -- & -- & -- & $2.04 \mathrm{E}+05$ & -- & $2.04 \mathrm{E}+05$ & $2.96 \mathrm{E}-04$ & $6.89 \mathrm{E}+08$ & Direct \\
\hline Tm-159 & & & & -- & -- & -- & -- & -- & -- & -- & $1.30 \mathrm{E}+08$ & -- \\
\hline $\mathrm{Tm}-161$ & & & & -- & -- & -- & $1.85 \mathrm{E}+04$ & -- & $1.85 \mathrm{E}+04$ & 4.77E-04 & $3.87 \mathrm{E}+07$ & Direct \\
\hline $\mathrm{Tm}-162$ & & & & $4.14 \mathrm{E}+05$ & $3.11 \mathrm{E}+128$ & $2.45 \mathrm{E}+07$ & $1.88 \mathrm{E}+04$ & -- & $1.88 \mathrm{E}+04$ & $3.50 \mathrm{E}-04$ & $5.35 \mathrm{E}+07$ & Direct \\
\hline $\mathrm{Tm}-163$ & & & & $1.77 \mathrm{E}+05$ & $4.48 \mathrm{E}+30$ & $2.55 \mathrm{E}+06$ & $5.06 \mathrm{E}+03$ & -- & $5.06 \mathrm{E}+03$ & $4.76 \mathrm{E}-04$ & $1.06 \mathrm{E}+07$ & Direct \\
\hline Tm-164 & & & & -- & -- & -- & $4.67 \mathrm{E}+05$ & -- & $4.67 \mathrm{E}+05$ & $8.14 \mathrm{E}-04$ & $5.73 E+08$ & Direct \\
\hline Tm-165 & & & & $3.60 \mathrm{E}+04$ & $7.87 \mathrm{E}+05$ & $2.59 \mathrm{E}+04$ & $1.68 \mathrm{E}+03$ & -- & $1.68 \mathrm{E}+03$ & $2.66 \mathrm{E}-03$ & $6.32 \mathrm{E}+05$ & Direct \\
\hline $\mathrm{Tm}-166$ & & & & $3.99 \mathrm{E}+04$ & $6.65 \mathrm{E}+10$ & $1.22 \mathrm{E}+05$ & $9.48 \mathrm{E}+02$ & -- & $9.48 \mathrm{E}+02$ & $3.86 \mathrm{E}-04$ & $2.45 \mathrm{E}+06$ & Direct \\
\hline $\mathrm{Tm}-167$ & & & & $1.02 \mathrm{E}+04$ & $7.64 \mathrm{E}+03$ & $3.44 \mathrm{E}+03$ & $5.18 \mathrm{E}+03$ & -- & $3.44 \mathrm{E}+03$ & $4.06 \mathrm{E}-02$ & $8.47 \mathrm{E}+04$ & Food \\
\hline $\mathrm{Tm}-168$ & & & & $2.60 \mathrm{E}+03$ & $2.14 \mathrm{E}+03$ & $9.09 \mathrm{E}+02$ & $5.86 \mathrm{E}+02$ & -- & $5.86 \mathrm{E}+02$ & $7.02 \mathrm{E}-02$ & $8.35 \mathrm{E}+03$ & Direct \\
\hline $\mathrm{Tm}-170$ & $6.63 \mathrm{E}+02$ & $1.11 \mathrm{E}-01$ & ing & $1.69 \mathrm{E}+03$ & $1.61 \mathrm{E}+03$ & $6.77 \mathrm{E}+02$ & $1.35 \mathrm{E}+05$ & -- & $6.77 \mathrm{E}+02$ & $1.13 \mathrm{E}-01$ & $5.97 \mathrm{E}+03$ & Food \\
\hline Tm-171 & & & & $8.59 E+03$ & $1.81 \mathrm{E}+04$ & $7.45 \mathrm{E}+03$ & $1.21 \mathrm{E}+06$ & -- & $7.45 E+03$ & $6.84 \mathrm{E}+00$ & $1.09 \mathrm{E}+03$ & Food \\
\hline Tm-172 & & & & $7.98 \mathrm{E}+03$ & $1.47 \mathrm{E}+04$ & $2.83 \mathrm{E}+03$ & $1.73 \mathrm{E}+03$ & -- & $1.73 E+03$ & $6.03 \mathrm{E}-03$ & $2.87 E+05$ & Direct \\
\hline Tm-173 & & & & $4.30 \mathrm{E}+04$ & $2.38 \mathrm{E}+10$ & $1.03 E+05$ & $4.35 E+03$ & -- & $4.35 E+03$ & $1.98 \mathrm{E}-03$ & $2.20 \mathrm{E}+06$ & Direct \\
\hline
\end{tabular}


Table C.2 Summary of Exposure Pathway TQs and Selection of Final HC-3 TQs (continued)

\begin{tabular}{|c|c|c|c|c|c|c|c|c|c|c|c|c|}
\hline \multirow[b]{2}{*}{$\begin{array}{c}\text { MASTER } \\
\text { ISOTOPE } \\
\text { LIST } \\
\end{array}$} & \multicolumn{3}{|c|}{ from NNSA SD G 1027} & \multicolumn{5}{|c|}{ Calculated Pathway Specific TQ Values } & \multicolumn{4}{|c|}{ Selected Final Threshold Quantities } \\
\hline & Act (Ci) & Mass (g) & $\begin{array}{l}\text { Limiting } \\
\text { Pathway }\end{array}$ & $\begin{array}{c}\text { TQ }_{\text {inhale }} \\
\text { (Ci) }\end{array}$ & $\begin{array}{l}\text { TQ }_{\text {water }} \\
\text { (Ci) }\end{array}$ & $\mathrm{TQ}_{\text {food }}(\mathrm{Ci})$ & $\begin{array}{c}T_{\text {direct }} \\
\text { (Ci) }\end{array}$ & $\mathrm{TQ}_{\text {sub }}(\mathrm{Ci})$ & Act (Ci) & Mass (g) & $\begin{array}{l}\text { Sp. Act. } \\
\text { (Ci/g) }\end{array}$ & $\begin{array}{l}\text { Limiting } \\
\text { Pathway }\end{array}$ \\
\hline $\mathrm{Tm}-174$ & & & & -- & -- & -- & $7.54 \mathrm{E}+04$ & -- & $7.54 \mathrm{E}+04$ & $3.77 \mathrm{E}-04$ & $2.00 \mathrm{E}+08$ & Direct \\
\hline $\mathrm{Tm}-175$ & & & & $3.60 \mathrm{E}+05$ & $2.75 \mathrm{E}+180$ & $3.76 \mathrm{E}+07$ & $4.53 \mathrm{E}+04$ & -- & $4.53 \mathrm{E}+04$ & $6.40 \mathrm{E}-04$ & $7.07 \mathrm{E}+07$ & Direct \\
\hline $\mathrm{Tm}-176$ & & & & -- & -- & -- & $1.99 \mathrm{E}+05$ & -- & $1.99 \mathrm{E}+05$ & $3.44 \mathrm{E}-04$ & $5.78 \mathrm{E}+08$ & Direct \\
\hline U-227 & & & & -- & -- & -- & $5.50 \mathrm{E}+06$ & -- & $5.50 \mathrm{E}+06$ & $7.30 \mathrm{E}-03$ & $7.53 \mathrm{E}+08$ & Direct \\
\hline $\mathrm{U}-228$ & & & & -- & -- & -- & $1.43 \mathrm{E}+07$ & -- & $1.43 \mathrm{E}+07$ & $1.57 \mathrm{E}-01$ & $9.06 \mathrm{E}+07$ & Direct \\
\hline$U-230$ & & & & $7.45 E+00$ & -- & $2.34 \mathrm{E}+02$ & $2.55 \mathrm{E}+05$ & -- & $7.45 \mathrm{E}+00$ & $2.73 \mathrm{E}-04$ & $2.73 E+04$ & Inh. \\
\hline U-231 & & & & $2.28 \mathrm{E}+05$ & -- & $1.18 \mathrm{E}+05$ & $9.60 \mathrm{E}+03$ & -- & $9.60 \mathrm{E}+03$ & 7.14E-02 & $1.35 \mathrm{E}+05$ & Direct \\
\hline U-232 & $3.21 \mathrm{E}+00$ & $1.49 \mathrm{E}-01$ & $\mathrm{i}$ & $3.19 \mathrm{E}+00$ & -- & $2.44 \mathrm{E}+01$ & $3.46 \mathrm{E}+05$ & -- & $3.19 \mathrm{E}+00$ & 1.49E-01 & $2.14 \mathrm{E}+01$ & Inh. \\
\hline U-233 & $1.29 \mathrm{E}+01$ & $1.34 \mathrm{E}+03$ & $\mathrm{i}$ & $1.28 \mathrm{E}+01$ & -- & $1.61 \mathrm{E}+02$ & $5.58 \mathrm{E}+05$ & -- & $1.28 \mathrm{E}+01$ & $1.33 \mathrm{E}+03$ & $9.68 \mathrm{E}-03$ & Inh. \\
\hline$U-234$ & $1.32 \mathrm{E}+01$ & $2.13 \mathrm{E}+03$ & $\mathrm{i}$ & $1.31 \mathrm{E}+01$ & -- & $1.64 \mathrm{E}+02$ & $4.27 E+05$ & -- & $1.31 \mathrm{E}+01$ & $2.10 \mathrm{E}+03$ & $6.25 \mathrm{E}-03$ & Inh. \\
\hline U-235 & $1.46 \mathrm{E}+01$ & $6.76 \mathrm{E}+06$ & $i$ & $1.45 \mathrm{E}+01$ & -- & $1.75 \mathrm{E}+02$ & $4.66 \mathrm{E}+03$ & -- & $1.45 \mathrm{E}+01$ & $6.71 E+06$ & $2.16 \mathrm{E}-06$ & Inh. \\
\hline $\mathrm{U}-235 \mathrm{~m}$ & & & & -- & -- & -- & -- & -- & -- & -- & $3.08 \mathrm{E}+07$ & -- \\
\hline U-236 & $1.43 \mathrm{E}+01$ & $2.20 \mathrm{E}+05$ & $\mathrm{i}$ & $1.41 \mathrm{E}+01$ & -- & $1.75 \mathrm{E}+02$ & $4.84 \mathrm{E}+05$ & -- & $1.41 \mathrm{E}+01$ & $2.19 \mathrm{E}+05$ & $6.47 \mathrm{E}-05$ & Inh. \\
\hline U-237 & & & & $6.20 \mathrm{E}+04$ & -- & $3.05 E+04$ & $5.35 \mathrm{E}+03$ & -- & $5.35 \mathrm{E}+03$ & $6.55 \mathrm{E}-02$ & $8.16 \mathrm{E}+04$ & Direct \\
\hline U-238 & $1.54 \mathrm{E}+01$ & $4.59 \mathrm{E}+07$ & $i$ & $1.53 \mathrm{E}+01$ & -- & $1.83 \mathrm{E}+02$ & $5.58 \mathrm{E}+05$ & -- & $1.53 \mathrm{E}+01$ & $4.55 \mathrm{E}+07$ & $3.36 \mathrm{E}-07$ & Inh. \\
\hline U-239 & & & & $3.19 E+06$ & -- & $2.34 \mathrm{E}+08$ & $5.85 E+05$ & -- & $5.85 \mathrm{E}+05$ & $1.75 \mathrm{E}-02$ & $3.34 \mathrm{E}+07$ & Direct \\
\hline $\mathrm{U}-240$ & & & & $1.33 \mathrm{E}+05$ & -- & $1.73 \mathrm{E}+05$ & $1.63 \mathrm{E}+05$ & -- & $1.33 \mathrm{E}+05$ & $1.44 \mathrm{E}-01$ & $9.26 \mathrm{E}+05$ & Inh. \\
\hline U-242 & & & & -- & -- & -- & $1.04 \mathrm{E}+06$ & -- & $1.04 \mathrm{E}+06$ & $2.26 \mathrm{E}-02$ & $4.62 \mathrm{E}+07$ & Direct \\
\hline V-47 & & & & $2.23 \mathrm{E}+05$ & $3.22 \mathrm{E}+87$ & $7.52 \mathrm{E}+06$ & $2.23 E+04$ & -- & $2.23 E+04$ & $1.82 \mathrm{E}-04$ & $1.23 \mathrm{E}+08$ & Direct \\
\hline V-48 & $2.54 \mathrm{E}+02$ & $1.49 \mathrm{E}-03$ & de & $4.14 \mathrm{E}+03$ & $1.54 \mathrm{E}+03$ & $7.15 \mathrm{E}+02$ & $2.54 \mathrm{E}+02$ & -- & $2.54 \mathrm{E}+02$ & $1.52 \mathrm{E}-03$ & $1.68 \mathrm{E}+05$ & Direct \\
\hline $\mathrm{V}-49$ & & & & $3.49 E+05$ & $1.12 \mathrm{E}+05$ & $4.64 \mathrm{E}+04$ & $8.07 E+05$ & -- & $4.64 \mathrm{E}+04$ & $5.74 \mathrm{E}+00$ & $8.08 \mathrm{E}+03$ & Food \\
\hline V-50 & & & & $1.13 \mathrm{E}+02$ & $4.70 \mathrm{E}+02$ & $1.92 \mathrm{E}+02$ & $5.10 \mathrm{E}+02$ & -- & $1.13 \mathrm{E}+02$ & $2.36 \mathrm{E}+15$ & $4.77 \mathrm{E}-14$ & Inh. \\
\hline$V-52$ & & & & -- & -- & -- & $1.34 \mathrm{E}+05$ & -- & $1.34 \mathrm{E}+05$ & $1.39 \mathrm{E}-04$ & $9.67 \mathrm{E}+08$ & Direct \\
\hline V-53 & & & & -- & -- & -- & $4.34 \mathrm{E}+05$ & -- & $4.34 \mathrm{E}+05$ & 1.97E-04 & $2.21 \mathrm{E}+09$ & Direct \\
\hline W-176 & & & & $1.47 \mathrm{E}+05$ & $1.26 \mathrm{E}+25$ & $1.02 \mathrm{E}+06$ & $2.96 \mathrm{E}+04$ & -- & $2.96 \mathrm{E}+04$ & $3.83 \mathrm{E}-03$ & $7.74 \mathrm{E}+06$ & Direct \\
\hline W-177 & & & & $2.43 \mathrm{E}+05$ & $6.16 \mathrm{E}+25$ & $1.88 \mathrm{E}+06$ & $5.95 \mathrm{E}+03$ & -- & $5.95 \mathrm{E}+03$ & $7.56 \mathrm{E}-04$ & $7.87 \mathrm{E}+06$ & Direct \\
\hline W-178 & & & & $9.31 \mathrm{E}+04$ & $1.10 \mathrm{E}+04$ & $5.06 \mathrm{E}+03$ & $5.12 \mathrm{E}+04$ & -- & $5.06 \mathrm{E}+03$ & $1.50 \mathrm{E}-01$ & $3.38 \mathrm{E}+04$ & Food \\
\hline W-179 & & & & $6.20 \mathrm{E}+06$ & $1.58 \mathrm{E}+78$ & $1.25 \mathrm{E}+08$ & $3.23 \mathrm{E}+05$ & -- & $3.23 E+05$ & $1.15 \mathrm{E}-02$ & $2.80 \mathrm{E}+07$ & Direct \\
\hline W-179m & & & & -- & -- & -- & $2.02 \mathrm{E}+06$ & -- & $2.02 E+06$ & $1.23 \mathrm{E}-02$ & $1.64 \mathrm{E}+08$ & Direct \\
\hline W-181 & & & & $2.60 \mathrm{E}+05$ & $2.56 \mathrm{E}+04$ & $1.08 \mathrm{E}+04$ & $1.80 \mathrm{E}+04$ & -- & $1.08 \mathrm{E}+04$ & $1.81 \mathrm{E}+00$ & $5.95 \mathrm{E}+03$ & Food \\
\hline W-185 & & & & $5.08 \mathrm{E}+04$ & $4.35 \mathrm{E}+03$ & $1.87 \mathrm{E}+03$ & -- & -- & $1.87 \mathrm{E}+03$ & 1.99E-01 & $9.40 \mathrm{E}+03$ & Food \\
\hline W-185m & & & & -- & -- & -- & $1.58 \mathrm{E}+07$ & -- & $1.58 \mathrm{E}+07$ & $2.48 \mathrm{E}-02$ & $6.37 \mathrm{E}+08$ & Direct \\
\hline W-187 & & & & $3.38 \mathrm{E}+04$ & $1.19 \mathrm{E}+06$ & $1.62 \mathrm{E}+04$ & $2.10 \mathrm{E}+03$ & -- & $2.10 \mathrm{E}+03$ & $2.99 \mathrm{E}-03$ & $7.01 \mathrm{E}+05$ & Direct \\
\hline W-188 & & & & $1.33 \mathrm{E}+04$ & $9.54 \mathrm{E}+02$ & $4.10 \mathrm{E}+02$ & $3.84 \mathrm{E}+05$ & -- & $4.10 \mathrm{E}+02$ & $4.10 \mathrm{E}-02$ & $1.00 \mathrm{E}+04$ & Food \\
\hline W-190 & & & & $1.69 \mathrm{E}+05$ & $2.49 \mathrm{E}+94$ & $6.04 \mathrm{E}+06$ & $1.60 \mathrm{E}+05$ & -- & $1.60 \mathrm{E}+05$ & 4.84E-03 & $3.30 \mathrm{E}+07$ & Direct \\
\hline
\end{tabular}


Table C.2 Summary of Exposure Pathway TQs and Selection of Final HC-3 TQs (continued)

\begin{tabular}{|c|c|c|c|c|c|c|c|c|c|c|c|c|}
\hline \multirow[b]{2}{*}{$\begin{array}{c}\text { MASTER } \\
\text { ISOTOPE } \\
\text { LIST } \\
\end{array}$} & \multicolumn{3}{|c|}{ from NNSA SD G 1027} & \multicolumn{5}{|c|}{ Calculated Pathway Specific TQ Values } & \multicolumn{4}{|c|}{ Selected Final Threshold Quantities } \\
\hline & Act (Ci) & Mass (g) & $\begin{array}{l}\text { Limiting } \\
\text { Pathway }\end{array}$ & $\begin{array}{c}\text { TQ }_{\text {inhale }} \\
\text { (Ci) }\end{array}$ & $\begin{array}{c}\text { TQ }_{\text {water }} \\
\text { (Ci) }\end{array}$ & $\mathrm{TQ}_{\text {food }}(\mathrm{Ci})$ & $\begin{array}{c}\text { TQ }_{\text {direct }} \\
\text { (Ci) }\end{array}$ & $\mathrm{TQ}_{\text {sub }}(\mathrm{Ci})$ & Act (Ci) & Mass (g) & $\begin{array}{l}\text { Sp. Act. } \\
\text { (Ci/g) }\end{array}$ & $\begin{array}{l}\text { Limiting } \\
\text { Pathway }\end{array}$ \\
\hline $\mathrm{Xe}-120$ & & & & -- & -- & -- & -- & $2.14 \mathrm{E}+03$ & $2.14 \mathrm{E}+03$ & $5.47 \mathrm{E}-05$ & $3.92 \mathrm{E}+07$ & Sub \\
\hline Xe-121 & & & & -- & -- & -- & -- & $4.29 E+02$ & $4.29 \mathrm{E}+02$ & $1.11 \mathrm{E}-05$ & $3.88 \mathrm{E}+07$ & Sub \\
\hline Xe-122 & & & & -- & -- & -- & -- & $1.69 \mathrm{E}+04$ & $1.69 \mathrm{E}+04$ & $1.32 \mathrm{E}-02$ & $1.28 \mathrm{E}+06$ & Sub \\
\hline Xe-123 & & & & -- & -- & -- & -- & $1.34 \mathrm{E}+03$ & $1.34 \mathrm{E}+03$ & $1.09 \mathrm{E}-04$ & $1.23 \mathrm{E}+07$ & Sub \\
\hline Xe-125 & & & & -- & -- & -- & -- & $3.46 \mathrm{E}+03$ & $3.46 \mathrm{E}+03$ & $2.34 \mathrm{E}-03$ & $1.48 \mathrm{E}+06$ & Sub \\
\hline Xe-127 & & & & -- & -- & -- & -- & $3.32 \mathrm{E}+03$ & $3.32 \mathrm{E}+03$ & $1.17 \mathrm{E}-01$ & $2.83 E+04$ & Sub \\
\hline Xe-127m & & & & -- & -- & -- & -- & $5.36 \mathrm{E}+03$ & $5.36 \mathrm{E}+03$ & 4.17E-06 & $1.28 \mathrm{E}+09$ & Sub \\
\hline Xe-129m & & & & -- & -- & -- & -- & $3.97 E+04$ & $3.97 E+04$ & 3.14E-01 & $1.27 \mathrm{E}+05$ & Sub \\
\hline Xe-131m & & & & -- & -- & -- & -- & $1.01 \mathrm{E}+05$ & $1.01 \mathrm{E}+05$ & $1.20 \mathrm{E}+00$ & $8.38 \mathrm{E}+04$ & Sub \\
\hline Xe-133 & $2.67 \mathrm{E}+04$ & $1.43 \mathrm{E}-01$ & sub & -- & -- & -- & -- & $2.68 \mathrm{E}+04$ & $2.68 \mathrm{E}+04$ & $1.43 \mathrm{E}-01$ & $1.87 \mathrm{E}+05$ & Sub \\
\hline Xe-133m & & & & -- & -- & -- & -- & $2.92 \mathrm{E}+04$ & $2.92 \mathrm{E}+04$ & $6.51 \mathrm{E}-02$ & $4.49 \mathrm{E}+05$ & Sub \\
\hline Xe-135 & & & & -- & -- & -- & -- & $3.35 \mathrm{E}+03$ & $3.35 \mathrm{E}+03$ & $1.31 \mathrm{E}-03$ & $2.56 \mathrm{E}+06$ & Sub \\
\hline Xe-135m & & & & -- & -- & -- & -- & $2.01 \mathrm{E}+03$ & $2.01 \mathrm{E}+03$ & $2.21 \mathrm{E}-05$ & $9.12 \mathrm{E}+07$ & Sub \\
\hline Xe-137 & & & & -- & -- & -- & -- & $3.42 \mathrm{E}+03$ & $3.42 \mathrm{E}+03$ & $9.51 \mathrm{E}-06$ & $3.60 \mathrm{E}+08$ & Sub \\
\hline Xe-138 & & & & -- & -- & -- & -- & $6.84 \mathrm{E}+02$ & $6.84 \mathrm{E}+02$ & 7.11E-06 & $9.62 \mathrm{E}+07$ & Sub \\
\hline $\mathrm{Y}-81$ & & & & -- & -- & -- & $5.28 \mathrm{E}+05$ & -- & $5.28 \mathrm{E}+05$ & 2.67E-04 & $1.98 \mathrm{E}+09$ & Direct \\
\hline $\mathrm{Y}-83$ & & & & -- & -- & -- & $7.60 \mathrm{E}+04$ & -- & $7.60 E+04$ & $2.37 \mathrm{E}-04$ & $3.20 \mathrm{E}+08$ & Direct \\
\hline $\mathrm{Y}-83 \mathrm{~m}$ & & & & -- & -- & -- & $3.04 \mathrm{E}+05$ & -- & $3.04 \mathrm{E}+05$ & $3.82 \mathrm{E}-04$ & $7.96 \mathrm{E}+08$ & Direct \\
\hline $\mathrm{Y}-84 \mathrm{~m}$ & & & & $1.12 \mathrm{E}+05$ & -- & $3.26 \mathrm{E}+06$ & $4.61 \mathrm{E}+03$ & -- & $4.61 E+03$ & $8.14 \mathrm{E}-05$ & $5.67 \mathrm{E}+07$ & Direct \\
\hline $\mathrm{Y}-85$ & & & & $6.98 \mathrm{E}+04$ & -- & $5.08 \mathrm{E}+05$ & $4.18 \mathrm{E}+03$ & -- & $4.18 \mathrm{E}+03$ & $3.04 \mathrm{E}-04$ & $1.38 \mathrm{E}+07$ & Direct \\
\hline $\mathrm{Y}-85 \mathrm{~m}$ & & & & $4.14 \mathrm{E}+04$ & -- & $1.41 \mathrm{E}+05$ & $1.94 \mathrm{E}+03$ & -- & $1.94 \mathrm{E}+03$ & $2.55 \mathrm{E}-04$ & $7.59 \mathrm{E}+06$ & Direct \\
\hline $\mathrm{Y}-86$ & & & & $1.38 \mathrm{E}+04$ & -- & $1.89 \mathrm{E}+04$ & $3.37 \mathrm{E}+02$ & -- & $3.37 \mathrm{E}+02$ & $1.36 \mathrm{E}-04$ & $2.47 E+06$ & Direct \\
\hline Y-86m & & & & $2.28 \mathrm{E}+05$ & -- & $5.75 \mathrm{E}+06$ & $6.85 E+04$ & -- & $6.85 \mathrm{E}+04$ & $1.50 \mathrm{E}-03$ & $4.56 \mathrm{E}+07$ & Direct \\
\hline Y-87 & & & & $2.11 \mathrm{E}+04$ & -- & $7.20 \mathrm{E}+03$ & $1.76 \mathrm{E}+03$ & -- & $1.76 \mathrm{E}+03$ & $3.91 \mathrm{E}-03$ & $4.49 E+05$ & Direct \\
\hline $\mathrm{Y}-87 \mathrm{~m}$ & & & & $5.58 \mathrm{E}+04$ & -- & $9.08 \mathrm{E}+04$ & $4.13 E+03$ & -- & $4.13 E+03$ & $1.53 \mathrm{E}-03$ & $2.70 \mathrm{E}+06$ & Direct \\
\hline $\mathrm{Y}-88$ & & & & $2.72 \mathrm{E}+03$ & -- & $6.89 \mathrm{E}+02$ & $2.71 \mathrm{E}+02$ & -- & $2.71 \mathrm{E}+02$ & $1.94 \mathrm{E}-02$ & $1.39 \mathrm{E}+04$ & Direct \\
\hline Y-89m & & & & -- & -- & -- & $3.08 \mathrm{E}+06$ & -- & $3.08 \mathrm{E}+06$ & $3.80 \mathrm{E}-04$ & $8.10 E+09$ & Direct \\
\hline $\mathrm{Y}-90$ & & & & $6.57 \mathrm{E}+03$ & -- & $1.77 \mathrm{E}+03$ & -- & -- & $1.77 \mathrm{E}+03$ & $3.25 \mathrm{E}-03$ & $5.45 \mathrm{E}+05$ & Food \\
\hline $\mathrm{Y}-90 \mathrm{~m}$ & & & & $8.59 \mathrm{E}+04$ & -- & $4.78 \mathrm{E}+05$ & $6.05 \mathrm{E}+03$ & -- & $6.05 \mathrm{E}+03$ & $5.54 \mathrm{E}-04$ & $1.09 \mathrm{E}+07$ & Direct \\
\hline $\mathrm{Y}-91$ & $3.98 \mathrm{E}+02$ & $1.62 \mathrm{E}-02$ & ing & $1.33 \mathrm{E}+03$ & -- & $4.05 \mathrm{E}+02$ & $2.03 E+05$ & -- & $4.05 E+02$ & 1.65E-02 & $2.45 \mathrm{E}+04$ & Food \\
\hline $\mathrm{Y}-91 \mathrm{~m}$ & & & & $7.45 \mathrm{E}+05$ & -- & $2.82 \mathrm{E}+07$ & $2.75 \mathrm{E}+04$ & -- & $2.75 \mathrm{E}+04$ & $6.61 \mathrm{E}-04$ & $4.16 \mathrm{E}+07$ & Direct \\
\hline Y-92 & & & & $3.99 \mathrm{E}+04$ & -- & $1.50 \mathrm{E}+05$ & $1.37 \mathrm{E}+04$ & -- & $1.37 \mathrm{E}+04$ & $1.42 \mathrm{E}-03$ & $9.63 \mathrm{E}+06$ & Direct \\
\hline Y-93 & & & & $1.86 \mathrm{E}+04$ & -- & $2.18 \mathrm{E}+04$ & $1.67 \mathrm{E}+04$ & -- & $1.67 E+04$ & 4.99E-03 & $3.34 \mathrm{E}+06$ & Direct \\
\hline Y-94 & & & & $2.43 \mathrm{E}+05$ & -- & $9.97 \mathrm{E}+06$ & $3.42 \mathrm{E}+04$ & -- & $3.42 \mathrm{E}+04$ & $3.26 \mathrm{E}-04$ & $1.05 \mathrm{E}+08$ & Direct \\
\hline Y-95 & & & & $4.30 \mathrm{E}+05$ & -- & $3.13 E+07$ & $7.58 \mathrm{E}+04$ & -- & $7.58 \mathrm{E}+04$ & 4.09E-04 & $1.85 \mathrm{E}+08$ & Direct \\
\hline
\end{tabular}


Table C.2 Summary of Exposure Pathway TQs and Selection of Final HC-3 TQs (continued)

\begin{tabular}{|c|c|c|c|c|c|c|c|c|c|c|c|c|}
\hline \multirow[b]{2}{*}{$\begin{array}{c}\text { MASTER } \\
\text { ISOTOPE } \\
\text { LIST } \\
\end{array}$} & \multicolumn{3}{|c|}{ from NNSA SD G 1027} & \multicolumn{5}{|c|}{ Calculated Pathway Specific TQ Values } & \multicolumn{4}{|c|}{ Selected Final Threshold Quantities } \\
\hline & Act $(\mathrm{Ci})$ & Mass (g) & $\begin{array}{l}\text { Limiting } \\
\text { Pathway }\end{array}$ & $\begin{array}{c}\mathrm{TQ}_{\text {inhale }} \\
(\mathrm{Ci})\end{array}$ & $\begin{array}{c}\text { TQ }_{\text {water }} \\
\text { (Ci) }\end{array}$ & $\mathrm{TQ}_{\text {food }}(\mathrm{Ci})$ & $\begin{array}{c}\text { TQ }_{\text {direct }} \\
\text { (Ci) }\end{array}$ & $T_{Q_{\text {sub }}}(\mathrm{Ci})$ & Act (Ci) & Mass (g) & $\begin{array}{c}\text { Sp. Act. } \\
\text { (Ci/g) }\end{array}$ & $\begin{array}{l}\text { Limiting } \\
\text { Pathway }\end{array}$ \\
\hline Yb-162 & & & & $4.86 \mathrm{E}+05$ & $3.85 E+146$ & $3.55 \mathrm{E}+07$ & $2.81 \mathrm{E}+05$ & -- & $2.81 \mathrm{E}+05$ & $4.57 \mathrm{E}-03$ & $6.14 \mathrm{E}+07$ & Direct \\
\hline Yb-163 & & & & $7.45 \mathrm{E}+05$ & $5.06 \mathrm{E}+245$ & $8.72 \mathrm{E}+07$ & $9.01 \mathrm{E}+04$ & -- & $9.01 \mathrm{E}+04$ & $8.63 \mathrm{E}-04$ & $1.04 \mathrm{E}+08$ & Direct \\
\hline Yb-164 & & & & $1.67 \mathrm{E}+05$ & $1.15 \mathrm{E}+41$ & $2.24 \mathrm{E}+06$ & $1.76 \mathrm{E}+05$ & -- & $1.67 \mathrm{E}+05$ & $1.10 \mathrm{E}-02$ & $1.51 \mathrm{E}+07$ & Inh. \\
\hline Yb-165 & & & & -- & -- & -- & $2.16 \mathrm{E}+05$ & -- & $2.16 \mathrm{E}+05$ & $1.87 \mathrm{E}-03$ & $1.15 \mathrm{E}+08$ & Direct \\
\hline Yb-166 & & & & $1.18 \mathrm{E}+04$ & $3.50 \mathrm{E}+04$ & $5.57 \mathrm{E}+03$ & $9.75 \mathrm{E}+03$ & -- & $5.57 \mathrm{E}+03$ & 1.67E-02 & $3.33 \mathrm{E}+05$ & Food \\
\hline Yb-167 & & & & $1.18 \mathrm{E}+06$ & $1.87 \mathrm{E}+158$ & $1.32 \mathrm{E}+08$ & $1.55 \mathrm{E}+05$ & -- & $1.55 \mathrm{E}+05$ & $2.41 \mathrm{E}-03$ & $6.44 \mathrm{E}+07$ & Direct \\
\hline Yb-169 & & & & $3.99 \mathrm{E}+03$ & $3.49 \mathrm{E}+03$ & $1.57 \mathrm{E}+03$ & $2.37 E+03$ & -- & $1.57 \mathrm{E}+03$ & $6.50 \mathrm{E}-02$ & $2.41 \mathrm{E}+04$ & Food \\
\hline Yb-175 & & & & $1.60 \mathrm{E}+04$ & $2.35 \mathrm{E}+04$ & $7.54 \mathrm{E}+03$ & $1.99 \mathrm{E}+04$ & -- & $7.54 \mathrm{E}+03$ & $4.23 \mathrm{E}-02$ & $1.78 \mathrm{E}+05$ & Food \\
\hline Yb-177 & & & & $1.19 \mathrm{E}+05$ & $1.76 \mathrm{E}+29$ & $1.40 \mathrm{E}+06$ & $3.39 E+04$ & -- & $3.39 \mathrm{E}+04$ & $3.64 \mathrm{E}-03$ & $9.32 \mathrm{E}+06$ & Direct \\
\hline Yb-178 & & & & $1.02 \mathrm{E}+05$ & $6.24 \mathrm{E}+41$ & $1.74 \mathrm{E}+06$ & $2.81 \mathrm{E}+05$ & -- & $1.02 \mathrm{E}+05$ & 7.11E-03 & $1.43 \mathrm{E}+07$ & Inh. \\
\hline Yb-179 & & & & -- & -- & -- & $9.30 \mathrm{E}+04$ & -- & $9.30 \mathrm{E}+04$ & $7.08 \mathrm{E}-04$ & $1.31 \mathrm{E}+08$ & Direct \\
\hline $\mathrm{Zn}-60$ & & & & -- & -- & -- & $1.99 \mathrm{E}+05$ & -- & $1.99 \mathrm{E}+05$ & $1.51 \mathrm{E}-04$ & $1.32 \mathrm{E}+09$ & Direct \\
\hline Zn-61 & & & & -- & -- & -- & $3.18 \mathrm{E}+05$ & -- & $3.18 \mathrm{E}+05$ & $1.53 \mathrm{E}-04$ & $2.08 \mathrm{E}+09$ & Direct \\
\hline Zn-62 & & & & $1.69 \mathrm{E}+04$ & $1.81 \mathrm{E}+09$ & $2.88 \mathrm{E}+04$ & $3.56 \mathrm{E}+03$ & -- & $3.56 \mathrm{E}+03$ & $6.52 \mathrm{E}-04$ & $5.46 \mathrm{E}+06$ & Direct \\
\hline Zn-63 & & & & $1.83 \mathrm{E}+05$ & $5.14 \mathrm{E}+75$ & $4.88 \mathrm{E}+06$ & $1.73 E+04$ & -- & $1.73 \mathrm{E}+04$ & $2.21 \mathrm{E}-04$ & $7.84 \mathrm{E}+07$ & Direct \\
\hline Zn-65 & $2.01 \mathrm{E}+02$ & $2.44 \mathrm{E}-02$ & ing & $3.85 \mathrm{E}+03$ & $5.22 \mathrm{E}+02$ & $2.06 \mathrm{E}+02$ & $1.24 \mathrm{E}+03$ & -- & $2.06 \mathrm{E}+02$ & $2.50 \mathrm{E}-02$ & $8.25 \mathrm{E}+03$ & Food \\
\hline Zn-69 & & & & $2.60 \mathrm{E}+05$ & $1.21 \mathrm{E}+53$ & $8.31 \mathrm{E}+06$ & -- & -- & $2.60 \mathrm{E}+05$ & $5.43 \mathrm{E}-03$ & $4.79 \mathrm{E}+07$ & Inh. \\
\hline Zn-69m & & & & $3.38 \mathrm{E}+04$ & $9.89 \mathrm{E}+07$ & $5.60 \mathrm{E}+04$ & $3.00 \mathrm{E}+03$ & -- & $3.00 \mathrm{E}+03$ & $9.09 \mathrm{E}-04$ & $3.30 \mathrm{E}+06$ & Direct \\
\hline Zn-71 & & & & -- & -- & -- & $9.39 \mathrm{E}+05$ & -- & $9.39 \mathrm{E}+05$ & $8.68 \mathrm{E}-04$ & $1.08 \mathrm{E}+09$ & Direct \\
\hline Zn-71m & & & & $4.65 \mathrm{E}+04$ & $4.62 \mathrm{E}+16$ & $2.62 \mathrm{E}+05$ & $2.01 \mathrm{E}+03$ & -- & $2.01 \mathrm{E}+03$ & $1.79 \mathrm{E}-04$ & $1.13 \mathrm{E}+07$ & Direct \\
\hline $\mathrm{Zn}-72$ & & & & $7.45 \mathrm{E}+03$ & $4.14 \mathrm{E}+04$ & $4.27 \mathrm{E}+03$ & $5.68 \mathrm{E}+03$ & -- & $4.27 \mathrm{E}+03$ & $4.56 \mathrm{E}-03$ & $9.37 \mathrm{E}+05$ & Food \\
\hline Zr-85 & & & & -- & -- & -- & $6.26 \mathrm{E}+04$ & -- & $6.26 \mathrm{E}+04$ & $2.22 \mathrm{E}-04$ & $2.82 \mathrm{E}+08$ & Direct \\
\hline Zr-86 & & & & $1.60 \mathrm{E}+04$ & -- & $1.90 \mathrm{E}+04$ & $4.01 \mathrm{E}+03$ & -- & $4.01 \mathrm{E}+03$ & $1.81 \mathrm{E}-03$ & $2.21 \mathrm{E}+06$ & Direct \\
\hline Zr-87 & & & & $7.98 \mathrm{E}+04$ & -- & $7.68 \mathrm{E}+05$ & $7.75 \mathrm{E}+03$ & -- & $7.75 \mathrm{E}+03$ & $3.61 \mathrm{E}-04$ & $2.15 \mathrm{E}+07$ & Direct \\
\hline Zr-88 & & & & $2.72 \mathrm{E}+03$ & -- & $2.79 \mathrm{E}+03$ & $1.81 \mathrm{E}+03$ & -- & $1.81 \mathrm{E}+03$ & $1.02 \mathrm{E}-01$ & $1.78 \mathrm{E}+04$ & Direct \\
\hline Zr-89 & & & & $1.49 \mathrm{E}+04$ & -- & $5.11 \mathrm{E}+03$ & $6.92 \mathrm{E}+02$ & -- & $6.92 \mathrm{E}+02$ & $1.54 \mathrm{E}-03$ & $4.49 \mathrm{E}+05$ & Direct \\
\hline Zr-89m & & & & -- & -- & -- & $2.74 \mathrm{E}+05$ & -- & $2.74 \mathrm{E}+05$ & $5.40 \mathrm{E}-04$ & $5.08 \mathrm{E}+08$ & Direct \\
\hline Zr-93 & $3.88 \mathrm{E}+02$ & $1.51 \mathrm{E}+05$ & $\mathrm{i}$ & $3.85 \mathrm{E}+02$ & -- & $2.88 \mathrm{E}+03$ & -- & -- & $3.85 \mathrm{E}+02$ & $1.53 \mathrm{E}+05$ & $2.51 \mathrm{E}-03$ & Inh. \\
\hline Zr-95 & $9.93 \mathrm{E}+02$ & $4.62 \mathrm{E}-02$ & de & $2.03 E+03$ & -- & $1.09 \mathrm{E}+03$ & $9.88 \mathrm{E}+02$ & -- & $9.88 \mathrm{E}+02$ & 4.59E-02 & $2.15 \mathrm{E}+04$ & Direct \\
\hline Zr-97 & & & & $7.98 \mathrm{E}+03$ & -- & $7.60 \mathrm{E}+03$ & $6.36 \mathrm{E}+03$ & -- & $6.36 \mathrm{E}+03$ & 3.33E-03 & $1.91 \mathrm{E}+06$ & Direct \\
\hline
\end{tabular}


APPENDIX D. SELECTION OF MASTER RADIONUCLIDE LIST 
(This page is intentionally blank) 


\section{APPENDIX D. SELECTION OF MASTER RADIONUCLIDE LIST}

This appendix contains the following tables:

- Table D.1: Selection of Radionuclides For Calculating HC-2 and HC-3 TQs 


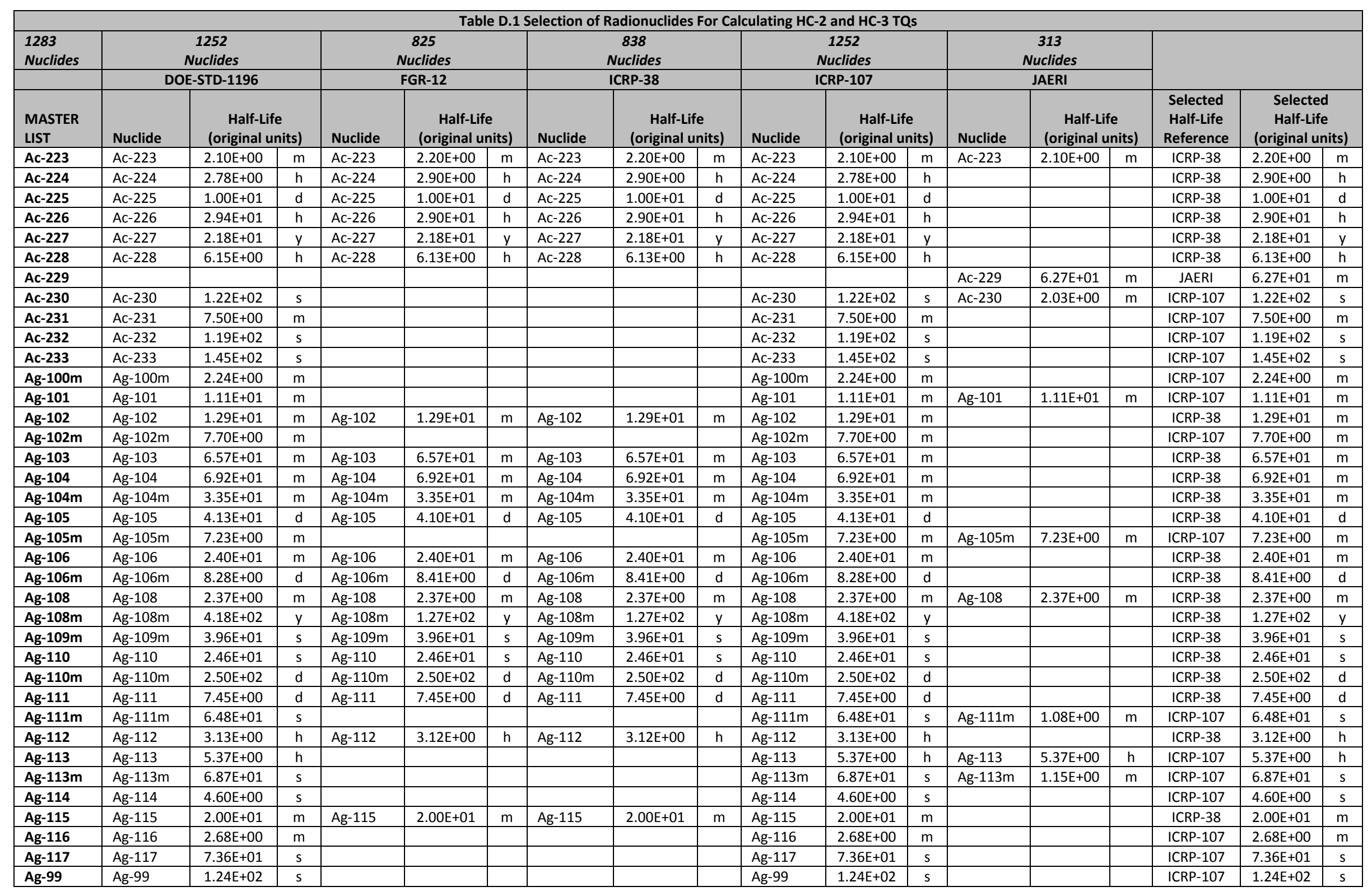




\begin{tabular}{|c|c|c|c|c|c|c|c|c|c|c|c|c|c|c|c|c|c|c|}
\hline \multicolumn{19}{|c|}{ Table D.1 Selection of Radionuclides For Calculating HC-2 and HC-3 TQs (continued) } \\
\hline \multirow{3}{*}{$\begin{array}{l}\text { MASTER } \\
\text { LIST } \\
\text { Al-26 }\end{array}$} & \multicolumn{3}{|c|}{ DOE-STD-1196 } & \multicolumn{3}{|c|}{ FGR-12 } & \multicolumn{3}{|c|}{ ICRP-38 } & \multicolumn{3}{|c|}{ ICRP-107 } & \multicolumn{3}{|c|}{ JAERI } & \multirow{3}{*}{$\begin{array}{c}\begin{array}{c}\text { Selected } \\
\text { Half-Life } \\
\text { Reference }\end{array} \\
\text { ICRP-38 }\end{array}$} & & \\
\hline & \multirow{2}{*}{$\begin{array}{l}\text { Nuclide } \\
\text { Al-26 }\end{array}$} & \multicolumn{2}{|c|}{$\begin{array}{c}\text { Half-Life } \\
\text { (original units) }\end{array}$} & \multirow{2}{*}{$\begin{array}{l}\text { Nuclide } \\
\text { Al-26 }\end{array}$} & \multicolumn{2}{|c|}{$\begin{array}{c}\text { Half-Life } \\
\text { (original units) }\end{array}$} & \multirow{2}{*}{$\begin{array}{l}\text { Nuclide } \\
\text { Al-26 }\end{array}$} & \multicolumn{2}{|c|}{$\begin{array}{c}\text { Half-Life } \\
\text { (original units) }\end{array}$} & \multirow{2}{*}{$\begin{array}{l}\text { Nuclide } \\
\text { Al-26 }\end{array}$} & \multicolumn{2}{|c|}{$\begin{array}{c}\text { Half-Life } \\
\text { (original units) } \\
\end{array}$} & \multirow[t]{2}{*}{ Nuclide } & $\begin{array}{r}\text { Half-Lif } \\
\text { (original u }\end{array}$ & its) & & \multicolumn{2}{|c|}{$\begin{array}{c}\text { Selected } \\
\text { Half-Life } \\
\text { (original units) }\end{array}$} \\
\hline & & $7.17 E+05$ & $y$ & & $7.16 \mathrm{E}+05$ & $y$ & & $7.16 \mathrm{E}+05$ & $y$ & & $7.17 \mathrm{E}+05$ & $y$ & & & & & $7.16 \mathrm{E}+05$ & $y$ \\
\hline Al-28 & $\mathrm{Al}-28$ & $2.24 \mathrm{E}+00$ & $\mathrm{~m}$ & $\mathrm{Al}-28$ & $2.24 \mathrm{E}+00$ & $\mathrm{~m}$ & Al-28 & $2.24 \mathrm{E}+00$ & $\mathrm{~m}$ & $\mathrm{Al}-28$ & $2.24 \mathrm{E}+00$ & $\mathrm{~m}$ & Al-28 & $2.24 \mathrm{E}+00$ & $\mathrm{~m}$ & ICRP-38 & $2.24 \mathrm{E}+00$ & $\mathrm{~m}$ \\
\hline Al-29 & Al-29 & $6.56 \mathrm{E}+00$ & $\mathrm{~m}$ & & & & & & & Al-29 & $6.56 \mathrm{E}+00$ & $\mathrm{~m}$ & Al-29 & $6.56 \mathrm{E}+00$ & $\mathrm{~m}$ & ICRP-107 & $6.56 \mathrm{E}+00$ & $\mathrm{~m}$ \\
\hline Am-237 & Am-237 & $7.30 \mathrm{E}+01$ & $\mathrm{~m}$ & Am-237 & $7.30 \mathrm{E}+01$ & $\mathrm{~m}$ & Am-237 & $7.30 \mathrm{E}+01$ & $\mathrm{~m}$ & Am-237 & $7.30 \mathrm{E}+01$ & $\mathrm{~m}$ & & & & ICRP-38 & $7.30 \mathrm{E}+01$ & $\mathrm{~m}$ \\
\hline Am-238 & Am-238 & $9.80 \mathrm{E}+01$ & $\mathrm{~m}$ & Am-238 & $9.80 \mathrm{E}+01$ & $m$ & Am-238 & $9.80 \mathrm{E}+01$ & $\mathrm{~m}$ & Am-238 & $9.80 \mathrm{E}+01$ & $\mathrm{~m}$ & & & & ICRP-38 & $9.80 \mathrm{E}+01$ & $\mathrm{~m}$ \\
\hline Am-239 & Am-239 & $1.19 \mathrm{E}+01$ & $\mathrm{~h}$ & Am-239 & $1.19 \mathrm{E}+01$ & $\mathrm{~h}$ & Am-239 & $1.19 \mathrm{E}+01$ & $\mathrm{~h}$ & Am-239 & $1.19 \mathrm{E}+01$ & $\mathrm{~h}$ & & & & ICRP-38 & $1.19 \mathrm{E}+01$ & $\mathrm{~h}$ \\
\hline Am-240 & Am-240 & $5.08 \mathrm{E}+01$ & $\mathrm{~h}$ & Am-240 & $5.08 \mathrm{E}+01$ & $\mathrm{~h}$ & Am-240 & $5.08 \mathrm{E}+01$ & $\mathrm{~h}$ & Am-240 & $5.08 \mathrm{E}+01$ & $\mathrm{~h}$ & & & & ICRP-38 & $5.08 \mathrm{E}+01$ & $\mathrm{~h}$ \\
\hline Am-241 & Am-241 & $4.32 \mathrm{E}+02$ & $y$ & Am-241 & $4.32 \mathrm{E}+02$ & $y$ & Am-241 & $4.32 \mathrm{E}+02$ & $y$ & Am-241 & $4.32 \mathrm{E}+02$ & $y$ & & & & ICRP-38 & $4.32 \mathrm{E}+02$ & $y$ \\
\hline Am-242 & $\mathrm{Am}-242$ & $1.60 \mathrm{E}+01$ & $\mathrm{~h}$ & $A m-242$ & $1.60 \mathrm{E}+01$ & $\mathrm{~h}$ & Am-242 & $1.60 \mathrm{E}+01$ & $\mathrm{~h}$ & Am-242 & $1.60 \mathrm{E}+01$ & $\mathrm{~h}$ & & & & ICRP-38 & $1.60 \mathrm{E}+01$ & $\mathrm{~h}$ \\
\hline Am-242m & $A m-242 m$ & $1.41 \mathrm{E}+02$ & $y$ & $A m-242 m$ & $1.52 \mathrm{E}+02$ & $y$ & $A m-242 m$ & $1.52 \mathrm{E}+02$ & $y$ & $A m-242 m$ & $1.41 \mathrm{E}+02$ & $y$ & & & & ICRP-38 & $1.52 \mathrm{E}+02$ & $y$ \\
\hline Am-243 & $\mathrm{Am}-243$ & $7.37 \mathrm{E}+03$ & $y$ & $\mathrm{Am}-243$ & $7.38 \mathrm{E}+03$ & $y$ & Am-243 & $7.38 \mathrm{E}+03$ & $y$ & Am-243 & $7.37 \mathrm{E}+03$ & $y$ & & & & ICRP-38 & $7.38 \mathrm{E}+03$ & $y$ \\
\hline Am-244 & Am-244 & $1.01 \mathrm{E}+01$ & $\mathrm{~h}$ & $\mathrm{Am}-244$ & $1.01 \mathrm{E}+01$ & $\mathrm{~h}$ & Am-244 & $1.01 \mathrm{E}+01$ & $\mathrm{~h}$ & Am-244 & $1.01 \mathrm{E}+01$ & $\mathrm{~h}$ & & & & ICRP-38 & $1.01 \mathrm{E}+01$ & $\mathrm{~h}$ \\
\hline Am-244m & Am-244m & $2.60 \mathrm{E}+01$ & $\mathrm{~m}$ & Am-244m & $2.60 \mathrm{E}+01$ & $m$ & Am-244m & $2.60 \mathrm{E}+01$ & $\mathrm{~m}$ & Am-244m & $2.60 \mathrm{E}+01$ & $\mathrm{~m}$ & & & & ICRP-38 & $2.60 \mathrm{E}+01$ & $\mathrm{~m}$ \\
\hline Am-245 & Am-245 & $2.05 \mathrm{E}+00$ & $\mathrm{~h}$ & Am-245 & $2.05 \mathrm{E}+00$ & $\mathrm{~h}$ & Am-245 & $2.05 \mathrm{E}+00$ & $\mathrm{~h}$ & Am-245 & $2.05 \mathrm{E}+00$ & $\mathrm{~h}$ & & & & ICRP-38 & $2.05 \mathrm{E}+00$ & $\mathrm{~h}$ \\
\hline Am-246 & Am-246 & $3.90 \mathrm{E}+01$ & $\mathrm{~m}$ & Am-246 & $3.90 \mathrm{E}+01$ & $m$ & Am-246 & $3.90 \mathrm{E}+01$ & $m$ & Am-246 & $3.90 \mathrm{E}+01$ & $m$ & & & & ICRP-38 & $3.90 \mathrm{E}+01$ & $m$ \\
\hline Am-246m & $\mathrm{Am}-246 \mathrm{~m}$ & $2.50 \mathrm{E}+01$ & $\mathrm{~m}$ & $\mathrm{Am}-246 \mathrm{~m}$ & $2.50 \mathrm{E}+01$ & $\mathrm{~m}$ & Am-246m & $2.50 \mathrm{E}+01$ & $m$ & Am-246m & $2.50 \mathrm{E}+01$ & $\mathrm{~m}$ & & & & ICRP-38 & $2.50 \mathrm{E}+01$ & $\mathrm{~m}$ \\
\hline Am-247 & $\mathrm{Am}-247$ & $2.30 \mathrm{E}+01$ & $\mathrm{~m}$ & & & & & & & Am-247 & $2.30 \mathrm{E}+01$ & $\mathrm{~m}$ & Am-247 & $2.30 \mathrm{E}+01$ & $\mathrm{~m}$ & ICRP-107 & $2.30 \mathrm{E}+01$ & $\mathrm{~m}$ \\
\hline Ar-37 & Ar-37 & $3.50 \mathrm{E}+01$ & $d$ & Ar-37 & $3.50 \mathrm{E}+01$ & $d$ & Ar-37 & $3.50 \mathrm{E}+01$ & $d$ & Ar-37 & $3.50 \mathrm{E}+01$ & $\mathrm{~d}$ & & & & ICRP-38 & $3.50 \mathrm{E}+01$ & $d$ \\
\hline Ar-39 & Ar-39 & $2.69 \mathrm{E}+02$ & $y$ & Ar-39 & $2.69 \mathrm{E}+02$ & $y$ & Ar-39 & $2.69 \mathrm{E}+02$ & $y$ & Ar-39 & $2.69 \mathrm{E}+02$ & $y$ & & & & ICRP-38 & $2.69 \mathrm{E}+02$ & $y$ \\
\hline Ar-41 & Ar-41 & $1.10 \mathrm{E}+02$ & $\mathrm{~m}$ & Ar-41 & $1.83 \mathrm{E}+00$ & $\mathrm{~h}$ & $A r-41$ & $1.83 \mathrm{E}+00$ & $\mathrm{~h}$ & Ar-41 & $1.10 \mathrm{E}+02$ & $m$ & & & & ICRP-38 & $1.83 \mathrm{E}+00$ & $\mathrm{~h}$ \\
\hline Ar-42 & Ar-42 & $3.29 \mathrm{E}+01$ & $y$ & & & & & & & Ar-42 & $3.29 \mathrm{E}+01$ & $\mathrm{y}$ & Ar-42 & $3.29 \mathrm{E}+01$ & $y$ & ICRP-107 & $3.29 \mathrm{E}+01$ & $y$ \\
\hline Ar-43 & Ar-43 & $5.37 \mathrm{E}+00$ & $\mathrm{~m}$ & & & & & & & Ar-43 & $5.37 \mathrm{E}+00$ & $\mathrm{~m}$ & & & & ICRP-107 & $5.37 \mathrm{E}+00$ & $\mathrm{~m}$ \\
\hline Ar-44 & Ar-44 & $1.19 \mathrm{E}+01$ & $m$ & & & & & & & Ar-44 & $1.19 \mathrm{E}+01$ & $m$ & Ar-44 & $1.19 \mathrm{E}+01$ & $\mathrm{~m}$ & ICRP-107 & $1.19 \mathrm{E}+01$ & $\mathrm{~m}$ \\
\hline As-68 & As-68 & $1.52 \mathrm{E}+02$ & $\mathrm{~s}$ & & & & & & & As-68 & $1.52 \mathrm{E}+02$ & $s$ & As-68 & $2.53 \mathrm{E}+00$ & $m$ & ICRP-107 & $1.52 \mathrm{E}+02$ & $\mathrm{~s}$ \\
\hline As-69 & As-69 & $1.52 \mathrm{E}+01$ & $m$ & As-69 & $1.52 \mathrm{E}+01$ & $\mathrm{~m}$ & As-69 & $1.52 \mathrm{E}+01$ & $m$ & As-69 & $1.52 \mathrm{E}+01$ & $m$ & & & & ICRP-38 & $1.52 \mathrm{E}+01$ & $\mathrm{~m}$ \\
\hline As-70 & As-70 & $5.26 \mathrm{E}+01$ & $m$ & As-70 & $5.26 \mathrm{E}+01$ & $m$ & As-70 & $5.26 \mathrm{E}+01$ & $\mathrm{~m}$ & As-70 & $5.26 \mathrm{E}+01$ & $\mathrm{~m}$ & & & & ICRP-38 & $5.26 \mathrm{E}+01$ & $\mathrm{~m}$ \\
\hline As-71 & As-71 & $6.53 \mathrm{E}+01$ & $\mathrm{~h}$ & As-71 & $6.48 \mathrm{E}+01$ & $\mathrm{~h}$ & As-71 & $6.48 \mathrm{E}+01$ & $\mathrm{~h}$ & As-71 & $6.53 \mathrm{E}+01$ & $\mathrm{~h}$ & & & & ICRP-38 & $6.48 \mathrm{E}+01$ & $\mathrm{~h}$ \\
\hline As-72 & As-72 & $2.60 \mathrm{E}+01$ & $\mathrm{~h}$ & As-72 & $2.60 \mathrm{E}+01$ & $\mathrm{~h}$ & As-72 & $2.60 \mathrm{E}+01$ & $\mathrm{~h}$ & As-72 & $2.60 \mathrm{E}+01$ & $\mathrm{~h}$ & & & & ICRP-38 & $2.60 \mathrm{E}+01$ & $\mathrm{~h}$ \\
\hline As-73 & As-73 & $8.03 E+01$ & $d$ & As-73 & $8.03 E+01$ & $d$ & As-73 & $8.03 E+01$ & $d$ & As-73 & $8.03 E+01$ & $d$ & & & & ICRP-38 & $8.03 E+01$ & $d$ \\
\hline As-74 & As-74 & $1.78 \mathrm{E}+01$ & $d$ & As-74 & $1.78 \mathrm{E}+01$ & d & As-74 & $1.78 \mathrm{E}+01$ & $d$ & As-74 & $1.78 \mathrm{E}+01$ & $d$ & & & & ICRP-38 & $1.78 \mathrm{E}+01$ & $d$ \\
\hline As-76 & As-76 & $1.08 \mathrm{E}+00$ & $d$ & As-76 & $2.63 \mathrm{E}+01$ & $\mathrm{~h}$ & As-76 & $2.63 \mathrm{E}+01$ & $\mathrm{~h}$ & As-76 & $1.08 \mathrm{E}+00$ & $d$ & & & & ICRP-38 & $2.63 \mathrm{E}+01$ & $\mathrm{~h}$ \\
\hline As-77 & As-77 & $3.88 \mathrm{E}+01$ & $\mathrm{~h}$ & As-77 & $3.88 \mathrm{E}+01$ & $\mathrm{~h}$ & As-77 & $3.88 \mathrm{E}+01$ & $\mathrm{~h}$ & As-77 & $3.88 \mathrm{E}+01$ & $\mathrm{~h}$ & & & & ICRP-38 & $3.88 \mathrm{E}+01$ & $\mathrm{~h}$ \\
\hline As-78 & As-78 & $9.07 \mathrm{E}+01$ & $\mathrm{~m}$ & As-78 & $9.07 \mathrm{E}+01$ & $\mathrm{~m}$ & As-78 & $9.07 \mathrm{E}+01$ & $\mathrm{~m}$ & As-78 & $9.07 \mathrm{E}+01$ & $\mathrm{~m}$ & & & & ICRP-38 & $9.07 \mathrm{E}+01$ & $\mathrm{~m}$ \\
\hline As-79 & As-79 & $9.01 \mathrm{E}+00$ & $m$ & & & & & & & As-79 & $9.01 \mathrm{E}+00$ & $m$ & As-79 & $9.01 \mathrm{E}+00$ & $m$ & ICRP-107 & $9.01 \mathrm{E}+00$ & $\mathrm{~m}$ \\
\hline At-204 & At-204 & $9.20 \mathrm{E}+00$ & $\mathrm{~m}$ & & & & & & & At-204 & $9.20 \mathrm{E}+00$ & $m$ & & & & ICRP-107 & $9.20 \mathrm{E}+00$ & $\mathrm{~m}$ \\
\hline At-205 & At-205 & $2.62 \mathrm{E}+01$ & $\mathrm{~m}$ & & & & & & & At-205 & $2.62 \mathrm{E}+01$ & $m$ & At-205 & $2.62 \mathrm{E}+01$ & $m$ & ICRP-107 & $2.62 \mathrm{E}+01$ & $m$ \\
\hline At-206 & At-206 & $3.06 \mathrm{E}+01$ & $\mathrm{~m}$ & & & & & & & At-206 & $3.06 \mathrm{E}+01$ & $\mathrm{~m}$ & & & & ICRP-107 & $3.06 \mathrm{E}+01$ & $\mathrm{~m}$ \\
\hline At-207 & At-207 & $1.80 \mathrm{E}+00$ & $\mathrm{~h}$ & At-207 & $1.80 \mathrm{E}+00$ & $\mathrm{~h}$ & At-207 & $1.80 \mathrm{E}+00$ & $\mathrm{~h}$ & At-207 & $1.80 \mathrm{E}+00$ & $\mathrm{~h}$ & & & & ICRP-38 & $1.80 \mathrm{E}+00$ & $\mathrm{~h}$ \\
\hline At-208 & At-208 & $1.63 \mathrm{E}+00$ & $\mathrm{~h}$ & & & & & & & At-208 & $1.63 \mathrm{E}+00$ & $\mathrm{~h}$ & At-208 & $1.63 \mathrm{E}+00$ & $\mathrm{~h}$ & ICRP-107 & $1.63 \mathrm{E}+00$ & $\mathrm{~h}$ \\
\hline At-209 & At-209 & $5.41 \mathrm{E}+00$ & $\mathrm{~h}$ & & & & & & & At-209 & $5.41 \mathrm{E}+00$ & $\mathrm{~h}$ & At-209 & $5.41 \mathrm{E}+00$ & $\mathrm{~h}$ & ICRP-107 & $5.41 \mathrm{E}+00$ & $\mathrm{~h}$ \\
\hline
\end{tabular}




\begin{tabular}{|c|c|c|c|c|c|c|c|c|c|c|c|c|c|c|c|c|c|c|}
\hline \multirow{3}{*}{$\begin{array}{l}\text { MASTER } \\
\text { LIST } \\
\text { At-210 }\end{array}$} & \multicolumn{3}{|c|}{ DOE-STD-1196 } & \multicolumn{3}{|c|}{ FGR-12 } & \multicolumn{3}{|c|}{ ICRP-38 } & \multicolumn{3}{|c|}{ ICRP-107 } & \multicolumn{3}{|c|}{ JAERI } & \multirow{3}{*}{$\begin{array}{c}\begin{array}{c}\text { Selected } \\
\text { Half-Life } \\
\text { Reference }\end{array} \\
\text { ICRP-107 }\end{array}$} & \multirow{2}{*}{\multicolumn{2}{|c|}{$\begin{array}{c}\text { Selected } \\
\text { Half-Life } \\
\text { (original units) }\end{array}$}} \\
\hline & \multirow{2}{*}{$\begin{array}{l}\text { Nuclide } \\
\text { At-210 }\end{array}$} & \multicolumn{2}{|c|}{$\begin{array}{c}\text { Half-Life } \\
\text { (original units) }\end{array}$} & \multirow[t]{2}{*}{ Nuclide } & \multicolumn{2}{|c|}{$\begin{array}{c}\text { Half-Life } \\
\text { (original units) }\end{array}$} & \multirow[t]{2}{*}{ Nuclide } & \multicolumn{2}{|c|}{$\begin{array}{c}\text { Half-Life } \\
\text { (original units) }\end{array}$} & \multirow{2}{*}{$\begin{array}{l}\text { Nuclide } \\
\text { At-210 }\end{array}$} & \multicolumn{2}{|c|}{$\begin{array}{c}\text { Half-Life } \\
\text { (original units) }\end{array}$} & \multirow{2}{*}{$\begin{array}{l}\text { Nuclide } \\
\text { At-210 }\end{array}$} & \multicolumn{2}{|c|}{$\begin{array}{c}\text { Half-Life } \\
\text { (original units) }\end{array}$} & & & \\
\hline & & $8.10 \mathrm{E}+00$ & $\mathrm{~h}$ & & & & & & & & $8.10 \mathrm{E}+00$ & $\mathrm{~h}$ & & $8.10 \mathrm{E}+00$ & $\mathrm{~h}$ & & $8.10 \mathrm{E}+00$ & $\mathrm{~h}$ \\
\hline At-211 & At-211 & $7.21 \mathrm{E}+00$ & $\mathrm{~h}$ & At-211 & $7.21 \mathrm{E}+00$ & $\mathrm{~h}$ & At-211 & $7.21 \mathrm{E}+00$ & $\mathrm{~h}$ & At-211 & $7.21 \mathrm{E}+00$ & $\mathrm{~h}$ & & & & ICRP-38 & $7.21 \mathrm{E}+00$ & $\mathrm{~h}$ \\
\hline At-215 & At-215 & $1.00 \mathrm{E}-04$ & $\mathrm{~s}$ & At-215 & $1.00 \mathrm{E}-01$ & $\mathrm{~ms}$ & At-215 & $1.00 \mathrm{E}-01$ & $\mathrm{~ms}$ & At-215 & $1.00 \mathrm{E}-04$ & $\mathrm{~s}$ & & & & ICRP-38 & $1.00 \mathrm{E}-01$ & $\mathrm{~ms}$ \\
\hline At-216 & At-216 & $3.00 \mathrm{E}-04$ & $\mathrm{~s}$ & At-216 & $3.00 \mathrm{E}-01$ & $\mathrm{~ms}$ & At-216 & $3.00 \mathrm{E}-01$ & $\mathrm{~ms}$ & At-216 & $3.00 \mathrm{E}-04$ & $\mathrm{~s}$ & & & & ICRP-38 & $3.00 \mathrm{E}-01$ & $\mathrm{~ms}$ \\
\hline At-217 & At-217 & $3.23 \mathrm{E}-02$ & $\mathrm{~s}$ & At-217 & $3.23 \mathrm{E}-02$ & $\mathrm{~s}$ & At-217 & $3.23 \mathrm{E}-02$ & $\mathrm{~s}$ & At-217 & $3.23 \mathrm{E}-02$ & $\mathrm{~s}$ & & & & ICRP-38 & $3.23 \mathrm{E}-02$ & $\mathrm{~s}$ \\
\hline At-218 & At-218 & $1.50 \mathrm{E}+00$ & $\mathrm{~s}$ & At-218 & $2.00 \mathrm{E}+00$ & $\mathrm{~s}$ & At-218 & $2.00 \mathrm{E}+00$ & $\mathrm{~s}$ & At-218 & $1.50 \mathrm{E}+00$ & $\mathrm{~s}$ & & & & ICRP-38 & $2.00 \mathrm{E}+00$ & $\mathrm{~s}$ \\
\hline At-219 & At-219 & $5.60 \mathrm{E}+01$ & $\mathrm{~s}$ & & & & & & & At-219 & $5.60 \mathrm{E}+01$ & $\mathrm{~s}$ & & & & ICRP-107 & $5.60 \mathrm{E}+01$ & $\mathrm{~s}$ \\
\hline At-220 & At-220 & $3.71 E+00$ & $\mathrm{~m}$ & & & & & & & At-220 & $3.71 E+00$ & $\mathrm{~m}$ & & & & ICRP-107 & $3.71 \mathrm{E}+00$ & $\mathrm{~m}$ \\
\hline Au-186 & $\mathrm{Au}-186$ & $1.07 \mathrm{E}+01$ & $\mathrm{~m}$ & & & & & & & Au-186 & $1.07 \mathrm{E}+01$ & $\mathrm{~m}$ & Au-186 & $1.07 \mathrm{E}+01$ & $\mathrm{~m}$ & ICRP-107 & $1.07 \mathrm{E}+01$ & $\mathrm{~m}$ \\
\hline Au-187 & $\mathrm{Au}-187$ & $8.40 \mathrm{E}+00$ & $\mathrm{~m}$ & & & & & & & $\mathrm{Au}-187$ & $8.40 \mathrm{E}+00$ & $\mathrm{~m}$ & Au-187 & $8.40 \mathrm{E}+00$ & $\mathrm{~m}$ & ICRP-107 & $8.40 \mathrm{E}+00$ & $\mathrm{~m}$ \\
\hline Au-188 & & & & & & & & & & & & & $A u-188$ & $8.84 \mathrm{E}+00$ & $\mathrm{~m}$ & JAERI & $8.84 \mathrm{E}+00$ & $\mathrm{~m}$ \\
\hline Au-189m & & & & & & & & & & & & & Au-189m & $4.59 \mathrm{E}+00$ & $\mathrm{~m}$ & JAERI & $4.59 \mathrm{E}+00$ & $\mathrm{~m}$ \\
\hline Au-190 & $\mathrm{Au}-190$ & $4.28 \mathrm{E}+01$ & $\mathrm{~m}$ & & & & & & & $\mathrm{Au}-190$ & $4.28 \mathrm{E}+01$ & $\mathrm{~m}$ & Au-190 & $4.28 \mathrm{E}+01$ & $\mathrm{~m}$ & ICRP-107 & $4.28 \mathrm{E}+01$ & $\mathrm{~m}$ \\
\hline Au-191 & $\mathrm{Au}-191$ & $3.18 \mathrm{E}+00$ & $\mathrm{~h}$ & & & & & & & Au-191 & $3.18 \mathrm{E}+00$ & $\mathrm{~h}$ & Au-191 & $3.18 \mathrm{E}+00$ & $\mathrm{~h}$ & ICRP-107 & $3.18 \mathrm{E}+00$ & $\mathrm{~h}$ \\
\hline Au-192 & $\mathrm{Au}-192$ & $4.94 \mathrm{E}+00$ & $\mathrm{~h}$ & & & & & & & $\mathrm{Au}-192$ & $4.94 \mathrm{E}+00$ & $\mathrm{~h}$ & Au-192 & $4.94 \mathrm{E}+00$ & $\mathrm{~h}$ & ICRP-107 & $4.94 \mathrm{E}+00$ & $\mathrm{~h}$ \\
\hline Au-193 & Au-193 & $1.77 \mathrm{E}+01$ & $\mathrm{~h}$ & $\mathrm{Au}-193$ & $1.77 \mathrm{E}+01$ & $\mathrm{~h}$ & Au-193 & $1.77 \mathrm{E}+01$ & $\mathrm{~h}$ & Au-193 & $1.77 \mathrm{E}+01$ & $\mathrm{~h}$ & & & & ICRP-38 & $1.77 \mathrm{E}+01$ & $\mathrm{~h}$ \\
\hline Au-193m & Au-193m & $3.90 \mathrm{E}+00$ & $\mathrm{~s}$ & & & & & & & Au-193m & $3.90 \mathrm{E}+00$ & $\mathrm{~s}$ & & & & ICRP-107 & $3.90 \mathrm{E}+00$ & $\mathrm{~s}$ \\
\hline Au-194 & Au-194 & $3.80 \mathrm{E}+01$ & $\mathrm{~h}$ & $\mathrm{Au}-194$ & $3.95 \mathrm{E}+01$ & $\mathrm{~h}$ & Au-194 & $3.95 \mathrm{E}+01$ & $\mathrm{~h}$ & Au-194 & $3.80 \mathrm{E}+01$ & $\mathrm{~h}$ & & & & ICRP-38 & $3.95 \mathrm{E}+01$ & $\mathrm{~h}$ \\
\hline Au-195 & Au-195 & $1.86 \mathrm{E}+02$ & $d$ & Au-195 & $1.83 \mathrm{E}+02$ & $d$ & Au-195 & $1.83 \mathrm{E}+02$ & $d$ & Au-195 & $1.86 \mathrm{E}+02$ & $d$ & & & & ICRP-38 & $1.83 \mathrm{E}+02$ & $d$ \\
\hline Au-195m & Au-195m & $3.05 \mathrm{E}+01$ & $\mathrm{~s}$ & $\mathrm{Au}-195 \mathrm{~m}$ & $3.05 \mathrm{E}+01$ & $\mathrm{~s}$ & Au-195m & $3.05 \mathrm{E}+01$ & $s$ & Au-195m & $3.05 \mathrm{E}+01$ & $s$ & & & & ICRP-38 & $3.05 \mathrm{E}+01$ & $s$ \\
\hline Au-196 & Au-196 & $6.18 \mathrm{E}+00$ & $d$ & & & & & & & Au-196 & $6.18 \mathrm{E}+00$ & $d$ & $\mathrm{Au}-196$ & $6.18 \mathrm{E}+00$ & $d$ & ICRP-107 & $6.18 \mathrm{E}+00$ & $d$ \\
\hline Au-196m & Au-196m & $9.60 \mathrm{E}+00$ & $\mathrm{~h}$ & & & & & & & Au-196m & $9.60 \mathrm{E}+00$ & $\mathrm{~h}$ & Au-196m & $9.60 \mathrm{E}+00$ & $\mathrm{~h}$ & ICRP-107 & $9.60 \mathrm{E}+00$ & $\mathrm{~h}$ \\
\hline Au-198 & Au-198 & $2.70 \mathrm{E}+00$ & $d$ & Au-198 & $2.70 \mathrm{E}+00$ & $d$ & $\mathrm{Au}-198$ & $2.70 \mathrm{E}+00$ & $d$ & Au-198 & $2.70 \mathrm{E}+00$ & $d$ & & & & ICRP-38 & $2.70 \mathrm{E}+00$ & $d$ \\
\hline Au-198m & Au-198m & $2.27 \mathrm{E}+00$ & $d$ & $\mathrm{Au}-198 \mathrm{~m}$ & $2.30 \mathrm{E}+00$ & $d$ & Au-198m & $2.30 \mathrm{E}+00$ & $d$ & Au-198m & $2.27 \mathrm{E}+00$ & $d$ & & & & ICRP-38 & $2.30 \mathrm{E}+00$ & $d$ \\
\hline Au-199 & Au-199 & $3.14 \mathrm{E}+00$ & $d$ & Au-199 & $3.14 \mathrm{E}+00$ & $d$ & Au-199 & $3.14 \mathrm{E}+00$ & $d$ & Au-199 & $3.14 \mathrm{E}+00$ & $d$ & & & & ICRP-38 & $3.14 \mathrm{E}+00$ & $d$ \\
\hline Au-200 & $\mathrm{Au}-200$ & $4.84 \mathrm{E}+01$ & $\mathrm{~m}$ & $\mathrm{Au}-200$ & $4.84 \mathrm{E}+01$ & $\mathrm{~m}$ & $\mathrm{Au}-200$ & $4.84 \mathrm{E}+01$ & $\mathrm{~m}$ & $\mathrm{Au}-200$ & $4.84 \mathrm{E}+01$ & $\mathrm{~m}$ & & & & ICRP-38 & $4.84 \mathrm{E}+01$ & $\mathrm{~m}$ \\
\hline Au-200m & Au-200m & $1.87 \mathrm{E}+01$ & $\mathrm{~h}$ & $\mathrm{Au}-200 \mathrm{~m}$ & $1.87 \mathrm{E}+01$ & $\mathrm{~h}$ & $\mathrm{Au}-200 \mathrm{~m}$ & $1.87 \mathrm{E}+01$ & $\mathrm{~h}$ & $\mathrm{Au}-200 \mathrm{~m}$ & $1.87 \mathrm{E}+01$ & $\mathrm{~h}$ & & & & ICRP-38 & $1.87 \mathrm{E}+01$ & $\mathrm{~h}$ \\
\hline Au-201 & $\mathrm{Au}-201$ & $2.60 \mathrm{E}+01$ & $\mathrm{~m}$ & $\mathrm{Au}-201$ & $2.64 \mathrm{E}+01$ & $\mathrm{~m}$ & $\mathrm{Au}-201$ & $2.64 \mathrm{E}+01$ & $\mathrm{~m}$ & $\mathrm{Au}-201$ & $2.60 \mathrm{E}+01$ & $\mathrm{~m}$ & & & & ICRP-38 & $2.64 \mathrm{E}+01$ & $\mathrm{~m}$ \\
\hline Au-202 & $\mathrm{Au}-202$ & $2.88 \mathrm{E}+01$ & $\mathrm{~s}$ & & & & & & & Au-202 & $2.88 \mathrm{E}+01$ & $\mathrm{~s}$ & & & & ICRP-107 & $2.88 \mathrm{E}+01$ & $\mathrm{~s}$ \\
\hline Ba-124 & Ba-124 & $1.10 \mathrm{E}+01$ & $\mathrm{~m}$ & & & & & & & Ba-124 & $1.10 \mathrm{E}+01$ & $\mathrm{~m}$ & Ba-124 & $1.10 \mathrm{E}+01$ & $\mathrm{~m}$ & ICRP-107 & $1.10 \mathrm{E}+01$ & $\mathrm{~m}$ \\
\hline Ba-126 & Ba-126 & $1.00 \mathrm{E}+02$ & $\mathrm{~m}$ & Ba-126 & $9.65 \mathrm{E}+01$ & $\mathrm{~m}$ & Ba-126 & $9.65 \mathrm{E}+01$ & $\mathrm{~m}$ & Ba-126 & $1.00 \mathrm{E}+02$ & $m$ & & & & ICRP-38 & $9.65 \mathrm{E}+01$ & $\mathrm{~m}$ \\
\hline Ba-127 & Ba-127 & $1.27 \mathrm{E}+01$ & $\mathrm{~m}$ & & & & & & & Ba-127 & $1.27 \mathrm{E}+01$ & $m$ & Ba-127 & $1.27 \mathrm{E}+01$ & $\mathrm{~m}$ & ICRP-107 & $1.27 \mathrm{E}+01$ & $\mathrm{~m}$ \\
\hline Ba-128 & Ba-128 & $2.43 \mathrm{E}+00$ & $d$ & Ba-128 & $2.43 \mathrm{E}+00$ & $d$ & Ba-128 & $2.43 \mathrm{E}+00$ & $d$ & Ba-128 & $2.43 \mathrm{E}+00$ & $d$ & & & & ICRP-38 & $2.43 \mathrm{E}+00$ & $d$ \\
\hline Ba-129 & Ba-129 & $2.23 \mathrm{E}+00$ & $\mathrm{~h}$ & & & & & & & Ba-129 & $2.23 \mathrm{E}+00$ & $\mathrm{~h}$ & Ba-129 & $2.23 \mathrm{E}+00$ & $\mathrm{~h}$ & ICRP-107 & $2.23 \mathrm{E}+00$ & $\mathrm{~h}$ \\
\hline Ba-129m & Ba-129m & $2.16 \mathrm{E}+00$ & $\mathrm{~h}$ & & & & & & & Ba-129m & $2.16 \mathrm{E}+00$ & $\mathrm{~h}$ & Ba-129m & $2.16 \mathrm{E}+00$ & $\mathrm{~h}$ & ICRP-107 & $2.16 \mathrm{E}+00$ & $\mathrm{~h}$ \\
\hline Ba-131 & Ba-131 & $1.15 \mathrm{E}+01$ & d & Ва-131 & $1.18 \mathrm{E}+01$ & $d$ & Ba-131 & $1.18 \mathrm{E}+01$ & $d$ & Ba-131 & $1.15 \mathrm{E}+01$ & $d$ & & & & ICRP-38 & $1.18 \mathrm{E}+01$ & $d$ \\
\hline Ba-131m & Ba-131m & $1.46 \mathrm{E}+01$ & $\mathrm{~m}$ & Ba-131m & $1.46 \mathrm{E}+01$ & $m$ & Ba-131m & $1.46 \mathrm{E}+01$ & $\mathrm{~m}$ & Ba-131m & $1.46 \mathrm{E}+01$ & $\mathrm{~m}$ & & & & ICRP-38 & $1.46 \mathrm{E}+01$ & $\mathrm{~m}$ \\
\hline Ba-133 & Ba-133 & $1.05 \mathrm{E}+01$ & $y$ & Ba-133 & $1.07 \mathrm{E}+01$ & $y$ & Ba-133 & $1.07 \mathrm{E}+01$ & $y$ & Ba-133 & $1.05 \mathrm{E}+01$ & $y$ & & & & ICRP-38 & $1.07 \mathrm{E}+01$ & $y$ \\
\hline Ba-133m & Ba-133m & $3.89 \mathrm{E}+01$ & $\mathrm{~h}$ & Ba-133m & $3.89 \mathrm{E}+01$ & $\mathrm{~h}$ & Ba-133m & $3.89 \mathrm{E}+01$ & $\mathrm{~h}$ & Ba-133m & $3.89 \mathrm{E}+01$ & $\mathrm{~h}$ & & & & ICRP-38 & $3.89 \mathrm{E}+01$ & $\mathrm{~h}$ \\
\hline Ba-135m & Ba-135m & $2.87 \mathrm{E}+01$ & $\mathrm{~h}$ & Ba-135m & $2.87 \mathrm{E}+01$ & $\mathrm{~h}$ & $\mathrm{Ba}-135 \mathrm{~m}$ & $2.87 E+01$ & $\mathrm{~h}$ & Ba-135m & $2.87 E+01$ & $\mathrm{~h}$ & & & & ICRP-38 & $2.87 \mathrm{E}+01$ & $\mathrm{~h}$ \\
\hline
\end{tabular}




\begin{tabular}{|c|c|c|c|c|c|c|c|c|c|c|c|c|c|c|c|c|c|c|}
\hline \multicolumn{19}{|c|}{ Table D.1 Selection of Radionuclides For Calculating HC-2 and HC-3 TQs (continued) } \\
\hline \multirow{3}{*}{$\begin{array}{l}\text { MASTER } \\
\text { LIST } \\
\text { Ba-137m }\end{array}$} & \multicolumn{3}{|c|}{ DOE-STD-1196 } & \multicolumn{3}{|c|}{ FGR-12 } & \multicolumn{3}{|c|}{ ICRP-38 } & \multicolumn{3}{|c|}{ ICRP-107 } & \multicolumn{3}{|c|}{ JAERI } & \multirow{3}{*}{$\begin{array}{c}\begin{array}{c}\text { Selected } \\
\text { Half-Life } \\
\text { Reference }\end{array} \\
\text { ICRP-38 }\end{array}$} & & \\
\hline & \multirow{2}{*}{$\begin{array}{l}\text { Nuclide } \\
\text { Ba-137m }\end{array}$} & \multicolumn{2}{|c|}{$\begin{array}{c}\text { Half-Life } \\
\text { (original units) }\end{array}$} & \multirow{2}{*}{$\begin{array}{l}\text { Nuclide } \\
\text { Ba-137m }\end{array}$} & \multicolumn{2}{|c|}{$\begin{array}{c}\text { Half-Life } \\
\text { (original units) }\end{array}$} & \multirow{2}{*}{$\frac{\text { Nuclide }}{\text { Ba-137m }}$} & \multicolumn{2}{|c|}{$\begin{array}{c}\text { Half-Life } \\
\text { (original units) }\end{array}$} & \multirow{2}{*}{$\begin{array}{l}\text { Nuclide } \\
\text { Ba-137m }\end{array}$} & \multicolumn{2}{|c|}{$\begin{array}{c}\text { Half-Life } \\
\text { (original units) }\end{array}$} & \multirow{2}{*}{$\begin{array}{l}\text { Nuclide } \\
\text { Ba-137m }\end{array}$} & $\begin{array}{r}\text { Half-Li } \\
\text { (original }\end{array}$ & its) & & \multicolumn{2}{|c|}{$\begin{array}{c}\text { Selected } \\
\text { Half-Life } \\
\text { (original units) }\end{array}$} \\
\hline & & $2.55 E+00$ & $\mathrm{~m}$ & & $2.55 \mathrm{E}+00$ & $m$ & & $2.55 \mathrm{E}+00$ & $\mathrm{~m}$ & & $2.55 \mathrm{E}+00$ & $\mathrm{~m}$ & & $2.55 E+00$ & $\mathrm{~m}$ & & $2.55 E+00$ & $\mathrm{~m}$ \\
\hline Ba-139 & Ba-139 & $8.31 \mathrm{E}+01$ & $\mathrm{~m}$ & Ba-139 & $8.27 \mathrm{E}+01$ & $\mathrm{~m}$ & Ba-139 & $8.27 \mathrm{E}+01$ & $\mathrm{~m}$ & Ba-139 & $8.31 \mathrm{E}+01$ & $\mathrm{~m}$ & & & & ICRP-38 & $8.27 \mathrm{E}+01$ & $\mathrm{~m}$ \\
\hline Ba-140 & Ba-140 & $1.28 \mathrm{E}+01$ & $d$ & Ba-140 & $1.27 \mathrm{E}+01$ & $\mathrm{~d}$ & Ba-140 & $1.27 \mathrm{E}+01$ & $d$ & Ba-140 & $1.28 \mathrm{E}+01$ & $d$ & & & & ICRP-38 & $1.27 \mathrm{E}+01$ & $d$ \\
\hline Ba-141 & Ba-141 & $1.83 \mathrm{E}+01$ & $\mathrm{~m}$ & Ba-141 & $1.83 \mathrm{E}+01$ & $\mathrm{~m}$ & Ba-141 & $1.83 \mathrm{E}+01$ & $\mathrm{~m}$ & Ba-141 & $1.83 \mathrm{E}+01$ & $\mathrm{~m}$ & & & & ICRP-38 & $1.83 \mathrm{E}+01$ & $\mathrm{~m}$ \\
\hline Ba-142 & Ba-142 & $1.06 \mathrm{E}+01$ & $\mathrm{~m}$ & Ba-142 & $1.06 \mathrm{E}+01$ & $m$ & Ba-142 & $1.06 \mathrm{E}+01$ & $\mathrm{~m}$ & Ba-142 & $1.06 \mathrm{E}+01$ & $\mathrm{~m}$ & & & & ICRP-38 & $1.06 \mathrm{E}+01$ & $\mathrm{~m}$ \\
\hline Be-10 & Be-10 & $1.51 \mathrm{E}+06$ & $\mathrm{y}$ & Be-10 & $1.60 \mathrm{E}+06$ & $\mathrm{y}$ & Be-10 & $1.60 \mathrm{E}+06$ & $\mathrm{y}$ & Be-10 & $1.51 \mathrm{E}+06$ & $\mathrm{y}$ & & & & ICRP-38 & $1.60 \mathrm{E}+06$ & y \\
\hline Be-7 & Be-7 & $5.32 \mathrm{E}+01$ & $\mathrm{~d}$ & Be-7 & $5.33 \mathrm{E}+01$ & d & Be-7 & $5.33 \mathrm{E}+01$ & d & Be-7 & $5.32 \mathrm{E}+01$ & $d$ & & & & ICRP-38 & $5.33 \mathrm{E}+01$ & $d$ \\
\hline Bi-197 & Bi-197 & $9.30 \mathrm{E}+00$ & $\mathrm{~m}$ & & & & & & & Bi-197 & $9.30 \mathrm{E}+00$ & $\mathrm{~m}$ & Bi-197 & $9.33 \mathrm{E}+00$ & $\mathrm{~m}$ & ICRP-107 & $9.30 \mathrm{E}+00$ & $m$ \\
\hline $\mathrm{Bi}-200$ & $\mathrm{Bi}-200$ & $3.64 \mathrm{E}+01$ & $\mathrm{~m}$ & $\mathrm{Bi}-200$ & $3.64 \mathrm{E}+01$ & $\mathrm{~m}$ & $\mathrm{Bi}-200$ & $3.64 \mathrm{E}+01$ & $\mathrm{~m}$ & $\mathrm{Bi}-200$ & $3.64 \mathrm{E}+01$ & $\mathrm{~m}$ & & & & ICRP-38 & $3.64 \mathrm{E}+01$ & $\mathrm{~m}$ \\
\hline $\mathrm{Bi}-201$ & $\mathrm{Bi}-201$ & $1.08 \mathrm{E}+02$ & $\mathrm{~m}$ & $\mathrm{Bi}-201$ & $1.08 \mathrm{E}+02$ & $\mathrm{~m}$ & $\mathrm{Bi}-201$ & $1.08 \mathrm{E}+02$ & $\mathrm{~m}$ & $\mathrm{Bi}-201$ & $1.08 \mathrm{E}+02$ & $\mathrm{~m}$ & & & & ICRP-38 & $1.08 \mathrm{E}+02$ & $\mathrm{~m}$ \\
\hline Bi-202 & $\mathrm{Bi}-202$ & $1.72 \mathrm{E}+00$ & $\mathrm{~h}$ & $\mathrm{Bi}-202$ & $1.67 \mathrm{E}+00$ & $\mathrm{~h}$ & $\mathrm{Bi}-202$ & $1.67 \mathrm{E}+00$ & $\mathrm{~h}$ & $\mathrm{Bi}-202$ & $1.72 \mathrm{E}+00$ & $\mathrm{~h}$ & & & & ICRP-38 & $1.67 \mathrm{E}+00$ & $\mathrm{~h}$ \\
\hline Bi-203 & $\mathrm{Bi}-203$ & $1.18 \mathrm{E}+01$ & $\mathrm{~h}$ & $\mathrm{Bi}-203$ & $1.18 \mathrm{E}+01$ & $\mathrm{~h}$ & $\mathrm{Bi}-203$ & $1.18 \mathrm{E}+01$ & $\mathrm{~h}$ & $\mathrm{Bi}-203$ & $1.18 \mathrm{E}+01$ & $\mathrm{~h}$ & & & & ICRP-38 & $1.18 \mathrm{E}+01$ & $\mathrm{~h}$ \\
\hline Bi-204 & $\mathrm{Bi}-204$ & $1.12 \mathrm{E}+01$ & $\mathrm{~h}$ & & & & $\mathrm{Bi}-204$ & $1.12 \mathrm{E}+01$ & $\mathrm{~h}$ & $\mathrm{Bi}-204$ & $1.12 \mathrm{E}+01$ & $\mathrm{~h}$ & $\mathrm{Bi}-204$ & $1.12 \mathrm{E}+01$ & $\mathrm{~h}$ & ICRP-38 & $1.12 \mathrm{E}+01$ & $\mathrm{~h}$ \\
\hline Bi-205 & $\mathrm{Bi}-205$ & $1.53 \mathrm{E}+01$ & $d$ & Bi-205 & $1.53 \mathrm{E}+01$ & $d$ & $\mathrm{Bi}-205$ & $1.53 \mathrm{E}+01$ & $d$ & $\mathrm{Bi}-205$ & $1.53 \mathrm{E}+01$ & $d$ & & & & ICRP-38 & $1.53 \mathrm{E}+01$ & $d$ \\
\hline Bi-206 & $\mathrm{Bi}-206$ & $6.24 \mathrm{E}+00$ & $d$ & $\mathrm{Bi}-206$ & $6.24 \mathrm{E}+00$ & $\mathrm{~d}$ & $\mathrm{Bi}-206$ & $6.24 \mathrm{E}+00$ & $d$ & $\mathrm{Bi}-206$ & $6.24 \mathrm{E}+00$ & $d$ & & & & ICRP-38 & $6.24 \mathrm{E}+00$ & $d$ \\
\hline Bi-207 & $\mathrm{Bi}-207$ & $3.29 \mathrm{E}+01$ & $y$ & $\mathrm{Bi}-207$ & $3.80 \mathrm{E}+01$ & $y$ & $\mathrm{Bi}-207$ & $3.80 \mathrm{E}+01$ & $y$ & $\mathrm{Bi}-207$ & $3.29 \mathrm{E}+01$ & $y$ & & & & ICRP-38 & $3.80 \mathrm{E}+01$ & $y$ \\
\hline Bi-208 & $\mathrm{Bi}-208$ & $3.68 \mathrm{E}+05$ & $y$ & & & & & & & $\mathrm{Bi}-208$ & $3.68 \mathrm{E}+05$ & $y$ & Bi-208 & $3.68 \mathrm{E}+05$ & $y$ & ICRP-107 & $3.68 \mathrm{E}+05$ & $y$ \\
\hline Bi-210 & Bi-210 & $5.01 \mathrm{E}+00$ & $d$ & Bi-210 & $5.01 \mathrm{E}+00$ & $d$ & Bi-210 & $5.01 \mathrm{E}+00$ & $d$ & Bi-210 & $5.01 \mathrm{E}+00$ & $d$ & & & & ICRP-38 & $5.01 \mathrm{E}+00$ & $d$ \\
\hline Bi-210m & $\mathrm{Bi}-210 \mathrm{~m}$ & $3.04 \mathrm{E}+06$ & $y$ & $\mathrm{Bi}-210 \mathrm{~m}$ & $3.00 \mathrm{E}+06$ & $y$ & $\mathrm{Bi}-210 \mathrm{~m}$ & $3.00 \mathrm{E}+06$ & $y$ & $\mathrm{Bi}-210 \mathrm{~m}$ & $3.04 \mathrm{E}+06$ & $y$ & & & & ICRP-38 & $3.00 E+06$ & $y$ \\
\hline Bi-211 & Bi-211 & $2.14 \mathrm{E}+00$ & $\mathrm{~m}$ & Bi-211 & $2.14 \mathrm{E}+00$ & $\mathrm{~m}$ & Bi-211 & $2.14 \mathrm{E}+00$ & $m$ & Bi-211 & $2.14 \mathrm{E}+00$ & $m$ & Bi-211 & $2.14 E+00$ & $\mathrm{~m}$ & ICRP-38 & $2.14 \mathrm{E}+00$ & $\mathrm{~m}$ \\
\hline Bi-212 & $\mathrm{Bi}-212$ & $6.06 \mathrm{E}+01$ & $\mathrm{~m}$ & $\mathrm{Bi}-212$ & $6.06 \mathrm{E}+01$ & $m$ & $\mathrm{Bi}-212$ & $6.06 \mathrm{E}+01$ & $m$ & $\mathrm{Bi}-212$ & $6.06 \mathrm{E}+01$ & $m$ & & & & ICRP-38 & $6.06 \mathrm{E}+01$ & $\mathrm{~m}$ \\
\hline Bi-212n & $B i-212 n$ & $7.00 \mathrm{E}+00$ & $\mathrm{~m}$ & & & & & & & Bi-212n & $7.00 \mathrm{E}+00$ & $\mathrm{~m}$ & & & & ICRP-107 & $7.00 \mathrm{E}+00$ & $\mathrm{~m}$ \\
\hline Bi-213 & Bi-213 & $4.56 \mathrm{E}+01$ & $m$ & $\mathrm{Bi}-213$ & $4.57 \mathrm{E}+01$ & $\mathrm{~m}$ & $\mathrm{Bi}-213$ & $4.57 \mathrm{E}+01$ & $\mathrm{~m}$ & Bi-213 & $4.56 \mathrm{E}+01$ & $m$ & & & & ICRP-38 & $4.57 \mathrm{E}+01$ & $\mathrm{~m}$ \\
\hline Bi-214 & $\mathrm{Bi}-214$ & $1.99 \mathrm{E}+01$ & $m$ & $\mathrm{Bi}-214$ & $1.99 \mathrm{E}+01$ & $m$ & $\mathrm{Bi}-214$ & $1.99 \mathrm{E}+01$ & $m$ & $\mathrm{Bi}-214$ & $1.99 \mathrm{E}+01$ & $m$ & & & & ICRP-38 & $1.99 \mathrm{E}+01$ & $\mathrm{~m}$ \\
\hline Bi-215 & $\mathrm{Bi}-215$ & $7.60 \mathrm{E}+00$ & $m$ & & & & & & & $\mathrm{Bi}-215$ & $7.60 \mathrm{E}+00$ & $m$ & $\mathrm{Bi}-215$ & $7.60 \mathrm{E}+00$ & $\mathrm{~m}$ & ICRP-107 & $7.60 \mathrm{E}+00$ & $\mathrm{~m}$ \\
\hline Bi-216 & $\mathrm{Bi}-216$ & $2.17 \mathrm{E}+00$ & $\mathrm{~m}$ & & & & & & & $\mathrm{Bi}-216$ & $2.17 E+00$ & $m$ & & & & ICRP-107 & $2.17 \mathrm{E}+00$ & $\mathrm{~m}$ \\
\hline Bk-244 & & & & & & & & & & & & & Bk-244 & $4.35 \mathrm{E}+00$ & $\mathrm{~h}$ & JAERI & $4.35 \mathrm{E}+00$ & $\mathrm{~h}$ \\
\hline Bk-245 & Bk-245 & $4.94 \mathrm{E}+00$ & $d$ & Bk-245 & $4.94 \mathrm{E}+00$ & $d$ & Bk-245 & $4.94 \mathrm{E}+00$ & $d$ & Bk-245 & $4.94 \mathrm{E}+00$ & $d$ & & & & ICRP-38 & $4.94 \mathrm{E}+00$ & $d$ \\
\hline Bk-246 & Bk-246 & $1.80 \mathrm{E}+00$ & $d$ & Bk-246 & $1.83 \mathrm{E}+00$ & $\mathrm{~d}$ & Bk-246 & $1.83 \mathrm{E}+00$ & $d$ & Bk-246 & $1.80 \mathrm{E}+00$ & $d$ & & & & ICRP-38 & $1.83 \mathrm{E}+00$ & $d$ \\
\hline Bk-247 & Bk-247 & $1.38 \mathrm{E}+03$ & $y$ & Bk-247 & $1.38 \mathrm{E}+03$ & $y$ & Bk-247 & $1.38 \mathrm{E}+03$ & $y$ & Bk-247 & $1.38 \mathrm{E}+03$ & $y$ & & & & ICRP-38 & $1.38 \mathrm{E}+03$ & $y$ \\
\hline Bk-248m & Bk-248m & $2.37 \mathrm{E}+01$ & $\mathrm{~h}$ & & & & & & & Bk-248m & $2.37 \mathrm{E}+01$ & $\mathrm{~h}$ & Bk-248m & $2.37 \mathrm{E}+01$ & $\mathrm{~h}$ & ICRP-107 & $2.37 \mathrm{E}+01$ & $\mathrm{~h}$ \\
\hline Bk-249 & Bk-249 & $3.30 \mathrm{E}+02$ & $d$ & Bk-249 & $3.20 \mathrm{E}+02$ & $d$ & Bk-249 & $3.20 \mathrm{E}+02$ & $d$ & Bk-249 & $3.30 \mathrm{E}+02$ & $d$ & & & & ICRP-38 & $3.20 \mathrm{E}+02$ & $d$ \\
\hline Bk-250 & Bk-250 & $3.21 \mathrm{E}+00$ & $\mathrm{~h}$ & Bk-250 & $3.22 \mathrm{E}+00$ & $\mathrm{~h}$ & Bk-250 & $3.22 \mathrm{E}+00$ & $\mathrm{~h}$ & Bk-250 & $3.21 \mathrm{E}+00$ & $\mathrm{~h}$ & & & & ICRP-38 & $3.22 \mathrm{E}+00$ & $\mathrm{~h}$ \\
\hline Bk-251 & Bk-251 & $5.56 \mathrm{E}+01$ & $m$ & & & & & & & Bk-251 & $5.56 \mathrm{E}+01$ & $m$ & Bk-251 & $5.56 \mathrm{E}+01$ & $m$ & ICRP-107 & $5.56 \mathrm{E}+01$ & $\mathrm{~m}$ \\
\hline $\mathrm{Br}-72$ & $\mathrm{Br}-72$ & $7.86 \mathrm{E}+01$ & $\mathrm{~s}$ & & & & & & & $\mathrm{Br}-72$ & $7.86 \mathrm{E}+01$ & $\mathrm{~s}$ & & & & ICRP-107 & $7.86 \mathrm{E}+01$ & $\mathrm{~s}$ \\
\hline $\mathrm{Br}-73$ & $\mathrm{Br}-73$ & $3.40 \mathrm{E}+00$ & $\mathrm{~m}$ & & & & & & & $\mathrm{Br}-73$ & $3.40 \mathrm{E}+00$ & $m$ & & & & ICRP-107 & $3.40 \mathrm{E}+00$ & $m$ \\
\hline $\mathrm{Br}-74$ & $\mathrm{Br}-74$ & $2.54 \mathrm{E}+01$ & $\mathrm{~m}$ & $\mathrm{Br}-74$ & $2.53 \mathrm{E}+01$ & $m$ & $\mathrm{Br}-74$ & $2.53 \mathrm{E}+01$ & $\mathrm{~m}$ & $\mathrm{Br}-74$ & $2.54 \mathrm{E}+01$ & $\mathrm{~m}$ & & & & ICRP-38 & $2.53 \mathrm{E}+01$ & $\mathrm{~m}$ \\
\hline $\mathrm{Br}-74 \mathrm{~m}$ & $\mathrm{Br}-74 \mathrm{~m}$ & $4.60 \mathrm{E}+01$ & $\mathrm{~m}$ & $\mathrm{Br}-74 \mathrm{~m}$ & $4.15 \mathrm{E}+01$ & $\mathrm{~m}$ & $\mathrm{Br}-74 \mathrm{~m}$ & $4.15 \mathrm{E}+01$ & $\mathrm{~m}$ & $\mathrm{Br}-74 \mathrm{~m}$ & $4.60 \mathrm{E}+01$ & $\mathrm{~m}$ & & & & ICRP-38 & $4.15 \mathrm{E}+01$ & $\mathrm{~m}$ \\
\hline $\mathrm{Br}-75$ & $\mathrm{Br}-75$ & $9.67 \mathrm{E}+01$ & $\mathrm{~m}$ & $\mathrm{Br}-75$ & $9.80 \mathrm{E}+01$ & $\mathrm{~m}$ & $\mathrm{Br}-75$ & $9.80 \mathrm{E}+01$ & $\mathrm{~m}$ & $\mathrm{Br}-75$ & $9.67 \mathrm{E}+01$ & $\mathrm{~m}$ & & & & ICRP-38 & $9.80 \mathrm{E}+01$ & $\mathrm{~m}$ \\
\hline $\mathrm{Br}-76$ & $\mathrm{Br}-76$ & $1.62 \mathrm{E}+01$ & $\mathrm{~h}$ & $\mathrm{Br}-76$ & $1.62 \mathrm{E}+01$ & $\mathrm{~h}$ & $\mathrm{Br}-76$ & $1.62 \mathrm{E}+01$ & $\mathrm{~h}$ & $\mathrm{Br}-76$ & $1.62 \mathrm{E}+01$ & $\mathrm{~h}$ & & & & ICRP-38 & $1.62 \mathrm{E}+01$ & $\mathrm{~h}$ \\
\hline
\end{tabular}




\begin{tabular}{|c|c|c|c|c|c|c|c|c|c|c|c|c|c|c|c|c|c|c|}
\hline \multirow{3}{*}{$\begin{array}{l}\text { MASTER } \\
\text { LIST } \\
\mathrm{Br}-76 \mathrm{~m}\end{array}$} & \multicolumn{3}{|c|}{ DOE-STD-1196 } & \multicolumn{3}{|c|}{ FGR-12 } & \multicolumn{3}{|c|}{ ICRP-38 } & \multicolumn{3}{|c|}{ ICRP-107 } & \multicolumn{3}{|c|}{ JAERI } & \multirow{3}{*}{$\begin{array}{c}\begin{array}{c}\text { Selected } \\
\text { Half-Life } \\
\text { Reference }\end{array} \\
\text { ICRP-107 }\end{array}$} & \multirow{2}{*}{\multicolumn{2}{|c|}{$\begin{array}{c}\text { Selected } \\
\text { Half-Life } \\
\text { (original units) }\end{array}$}} \\
\hline & \multirow{2}{*}{$\frac{\text { Nuclide }}{\text { Br-76m }}$} & \multicolumn{2}{|c|}{$\begin{array}{c}\text { Half-Life } \\
\text { (original units) }\end{array}$} & \multirow[t]{2}{*}{ Nuclide } & \multicolumn{2}{|c|}{$\begin{array}{c}\text { Half-Life } \\
\text { (original units) }\end{array}$} & \multirow[t]{2}{*}{ Nuclide } & \multicolumn{2}{|c|}{$\begin{array}{c}\text { Half-Life } \\
\text { (original units) }\end{array}$} & \multirow{2}{*}{$\frac{\text { Nuclide }}{\text { Br-76m }}$} & \multicolumn{2}{|c|}{$\begin{array}{c}\text { Half-Life } \\
\text { (original units) }\end{array}$} & \multirow[t]{2}{*}{ Nuclide } & \multicolumn{2}{|c|}{$\begin{array}{c}\text { Half-Life } \\
\text { (original units) }\end{array}$} & & & \\
\hline & & $1.31 \mathrm{E}+00$ & $\mathrm{~s}$ & & & & & & & & $1.31 \mathrm{E}+00$ & $\mathrm{~s}$ & & & & & $1.31 \mathrm{E}+00$ & $\mathrm{~s}$ \\
\hline Br-77 & $\mathrm{Br}-77$ & $5.70 \mathrm{E}+01$ & $\mathrm{~h}$ & $\mathrm{Br}-77$ & $5.60 \mathrm{E}+01$ & $\mathrm{~h}$ & $\mathrm{Br}-77$ & $5.60 \mathrm{E}+01$ & $\mathrm{~h}$ & Br-77 & $5.70 \mathrm{E}+01$ & $\mathrm{~h}$ & & & & ICRP-38 & $5.60 \mathrm{E}+01$ & $\mathrm{~h}$ \\
\hline $\mathrm{Br}-77 \mathrm{~m}$ & $\mathrm{Br}-77 \mathrm{~m}$ & $4.28 \mathrm{E}+00$ & $\mathrm{~m}$ & & & & & & & $\mathrm{Br}-77 \mathrm{~m}$ & $4.28 \mathrm{E}+00$ & $\mathrm{~m}$ & $\mathrm{Br}-77 \mathrm{~m}$ & $4.28 \mathrm{E}+00$ & $\mathrm{~m}$ & ICRP-107 & $4.28 \mathrm{E}+00$ & $\mathrm{~m}$ \\
\hline Br-78 & $\mathrm{Br}-78$ & $6.46 \mathrm{E}+00$ & $\mathrm{~m}$ & & & & & & & $\mathrm{Br}-78$ & $6.46 \mathrm{E}+00$ & $\mathrm{~m}$ & $\mathrm{Br}-78$ & $6.46 \mathrm{E}+00$ & $\mathrm{~m}$ & ICRP-107 & $6.46 \mathrm{E}+00$ & $\mathrm{~m}$ \\
\hline $\mathrm{Br}-80$ & $\mathrm{Br}-80$ & $1.77 \mathrm{E}+01$ & $\mathrm{~m}$ & $\mathrm{Br}-80$ & $1.74 \mathrm{E}+01$ & $\mathrm{~m}$ & $\mathrm{Br}-80$ & $1.74 \mathrm{E}+01$ & $\mathrm{~m}$ & $\mathrm{Br}-80$ & $1.77 \mathrm{E}+01$ & $\mathrm{~m}$ & & & & ICRP-38 & $1.74 \mathrm{E}+01$ & $\mathrm{~m}$ \\
\hline $\mathrm{Br}-80 \mathrm{~m}$ & $\mathrm{Br}-80 \mathrm{~m}$ & $4.42 \mathrm{E}+00$ & $\mathrm{~h}$ & $\mathrm{Br}-80 \mathrm{~m}$ & $4.42 \mathrm{E}+00$ & $\mathrm{~h}$ & $\mathrm{Br}-80 \mathrm{~m}$ & $4.42 \mathrm{E}+00$ & $\mathrm{~h}$ & $\mathrm{Br}-80 \mathrm{~m}$ & $4.42 \mathrm{E}+00$ & $\mathrm{~h}$ & & & & ICRP-38 & $4.42 \mathrm{E}+00$ & $\mathrm{~h}$ \\
\hline $\mathrm{Br}-82$ & $\mathrm{Br}-82$ & $3.53 \mathrm{E}+01$ & $\mathrm{~h}$ & $\mathrm{Br}-82$ & $3.53 \mathrm{E}+01$ & $\mathrm{~h}$ & $\mathrm{Br}-82$ & $3.53 \mathrm{E}+01$ & $\mathrm{~h}$ & $\mathrm{Br}-82$ & $3.53 \mathrm{E}+01$ & $\mathrm{~h}$ & & & & ICRP-38 & $3.53 \mathrm{E}+01$ & $\mathrm{~h}$ \\
\hline $\mathrm{Br}-82 \mathrm{~m}$ & $\mathrm{Br}-82 \mathrm{~m}$ & $6.13 \mathrm{E}+00$ & $\mathrm{~m}$ & & & & & & & $\mathrm{Br}-82 \mathrm{~m}$ & $6.13 E+00$ & $\mathrm{~m}$ & $\mathrm{Br}-82 \mathrm{~m}$ & $6.13 \mathrm{E}+00$ & $\mathrm{~m}$ & ICRP-107 & $6.13 E+00$ & $\mathrm{~m}$ \\
\hline $\mathrm{Br}-83$ & $\mathrm{Br}-83$ & $2.40 \mathrm{E}+00$ & $\mathrm{~h}$ & $\mathrm{Br}-83$ & $2.39 \mathrm{E}+00$ & $\mathrm{~h}$ & $\mathrm{Br}-83$ & $2.39 \mathrm{E}+00$ & $\mathrm{~h}$ & $\mathrm{Br}-83$ & $2.40 \mathrm{E}+00$ & $\mathrm{~h}$ & & & & ICRP-38 & $2.39 \mathrm{E}+00$ & $\mathrm{~h}$ \\
\hline $\mathrm{Br}-84$ & $\mathrm{Br}-84$ & $3.18 \mathrm{E}+01$ & $\mathrm{~m}$ & $\mathrm{Br}-84$ & $3.18 \mathrm{E}+01$ & $\mathrm{~m}$ & $\mathrm{Br}-84$ & $3.18 \mathrm{E}+01$ & $\mathrm{~m}$ & $\mathrm{Br}-84$ & $3.18 \mathrm{E}+01$ & $\mathrm{~m}$ & & & & ICRP-38 & $3.18 \mathrm{E}+01$ & $\mathrm{~m}$ \\
\hline $\mathrm{Br}-84 \mathrm{~m}$ & $\mathrm{Br}-84 \mathrm{~m}$ & $6.00 \mathrm{E}+00$ & $\mathrm{~m}$ & & & & & & & $\mathrm{Br}-84 \mathrm{~m}$ & $6.00 \mathrm{E}+00$ & $\mathrm{~m}$ & $\mathrm{Br}-84 \mathrm{~m}$ & $6.00 \mathrm{E}+00$ & $\mathrm{~m}$ & ICRP-107 & $6.00 \mathrm{E}+00$ & $\mathrm{~m}$ \\
\hline $\mathrm{Br}-85$ & $\mathrm{Br}-85$ & $2.90 \mathrm{E}+00$ & $\mathrm{~m}$ & & & & & & & $\mathrm{Br}-85$ & $2.90 \mathrm{E}+00$ & $\mathrm{~m}$ & & & & ICRP-107 & $2.90 \mathrm{E}+00$ & $\mathrm{~m}$ \\
\hline C-10 & C-10 & $1.93 \mathrm{E}+01$ & $\mathrm{~s}$ & & & & & & & $\mathrm{C}-10$ & $1.93 \mathrm{E}+01$ & $s$ & & & & ICRP-107 & $1.93 \mathrm{E}+01$ & $\mathrm{~s}$ \\
\hline C-11 & $\mathrm{C}-11$ & $2.04 \mathrm{E}+01$ & $m$ & C-11 & $2.04 \mathrm{E}+01$ & $\mathrm{~m}$ & C-11 & $2.04 \mathrm{E}+01$ & $\mathrm{~m}$ & $\mathrm{C}-11$ & $2.04 \mathrm{E}+01$ & $m$ & & & & ICRP-38 & $2.04 \mathrm{E}+01$ & $\mathrm{~m}$ \\
\hline C-14 & C-14 & $5.70 \mathrm{E}+03$ & $y$ & $\mathrm{C}-14$ & $5.73 E+03$ & $y$ & C-14 & $5.73 \mathrm{E}+03$ & $y$ & C-14 & $5.70 \mathrm{E}+03$ & $y$ & & & & ICRP-38 & $5.73 \mathrm{E}+03$ & $\mathrm{y}$ \\
\hline Ca-41 & $\mathrm{Ca}-41$ & $1.02 \mathrm{E}+05$ & $y$ & Ca-41 & $1.40 \mathrm{E}+05$ & $y$ & Ca-41 & $1.40 \mathrm{E}+05$ & $y$ & $\mathrm{Ca}-41$ & $1.02 \mathrm{E}+05$ & $y$ & & & & ICRP-38 & $1.40 \mathrm{E}+05$ & $y$ \\
\hline Ca-45 & Ca-45 & $1.63 \mathrm{E}+02$ & d & Ca-45 & $1.63 \mathrm{E}+02$ & d & Ca-45 & $1.63 \mathrm{E}+02$ & d & Ca-45 & $1.63 \mathrm{E}+02$ & d & & & & ICRP-38 & $1.63 \mathrm{E}+02$ & d \\
\hline Ca-47 & Ca-47 & $4.54 \mathrm{E}+00$ & $d$ & Ca-47 & $4.53 \mathrm{E}+00$ & $d$ & Ca-47 & $4.53 \mathrm{E}+00$ & $d$ & $\mathrm{Ca}-47$ & $4.54 \mathrm{E}+00$ & $d$ & & & & ICRP-38 & $4.53 \mathrm{E}+00$ & $\mathrm{~d}$ \\
\hline Ca-49 & Ca-49 & $8.72 \mathrm{E}+00$ & $\mathrm{~m}$ & Ca-49 & $8.72 \mathrm{E}+00$ & $\mathrm{~m}$ & Ca-49 & $8.72 \mathrm{E}+00$ & $\mathrm{~m}$ & $\mathrm{Ca}-49$ & $8.72 \mathrm{E}+00$ & $\mathrm{~m}$ & Сa-49 & $8.72 \mathrm{E}+00$ & $\mathrm{~m}$ & ICRP-38 & $8.72 \mathrm{E}+00$ & $\mathrm{~m}$ \\
\hline Cd-101 & Cd-101 & $1.36 \mathrm{E}+00$ & $\mathrm{~m}$ & & & & & & & Cd-101 & $1.36 \mathrm{E}+00$ & $m$ & & & & ICRP-107 & $1.36 \mathrm{E}+00$ & $\mathrm{~m}$ \\
\hline Cd-102 & Cd-102 & $5.50 \mathrm{E}+00$ & $\mathrm{~m}$ & & & & & & & Cd-102 & $5.50 \mathrm{E}+00$ & $m$ & & & & ICRP-107 & $5.50 \mathrm{E}+00$ & $\mathrm{~m}$ \\
\hline Cd-103 & Cd-103 & $7.30 \mathrm{E}+00$ & $\mathrm{~m}$ & & & & & & & Cd-103 & $7.30 \mathrm{E}+00$ & $\mathrm{~m}$ & & & & ICRP-107 & $7.30 \mathrm{E}+00$ & $\mathrm{~m}$ \\
\hline Cd-104 & Cd-104 & $5.77 \mathrm{E}+01$ & $m$ & Cd-104 & $5.77 \mathrm{E}+01$ & $\mathrm{~m}$ & Cd-104 & $5.77 \mathrm{E}+01$ & $\mathrm{~m}$ & Cd-104 & $5.77 \mathrm{E}+01$ & $m$ & & & & ICRP-38 & $5.77 \mathrm{E}+01$ & $\mathrm{~m}$ \\
\hline Cd-105 & Cd-105 & $5.55 \mathrm{E}+01$ & $m$ & & & & & & & Cd-105 & $5.55 \mathrm{E}+01$ & $m$ & Cd-105 & $5.55 \mathrm{E}+01$ & $\mathrm{~m}$ & ICRP-107 & $5.55 \mathrm{E}+01$ & $\mathrm{~m}$ \\
\hline Cd-107 & Cd-107 & $6.50 \mathrm{E}+00$ & $\mathrm{~h}$ & Cd-107 & $6.49 \mathrm{E}+00$ & $\mathrm{~h}$ & Cd-107 & $6.49 \mathrm{E}+00$ & $\mathrm{~h}$ & Cd-107 & $6.50 \mathrm{E}+00$ & $\mathrm{~h}$ & & & & ICRP-38 & $6.49 \mathrm{E}+00$ & $\mathrm{~h}$ \\
\hline Cd-109 & Cd-109 & $4.61 \mathrm{E}+02$ & $d$ & Cd-109 & $4.64 \mathrm{E}+02$ & $\mathrm{~d}$ & Cd-109 & $4.64 \mathrm{E}+02$ & $d$ & Cd-109 & $4.61 E+02$ & $d$ & & & & ICRP-38 & $4.64 \mathrm{E}+02$ & $\mathrm{~d}$ \\
\hline Cd-111m & $C d-111 m$ & $4.85 \mathrm{E}+01$ & $\mathrm{~m}$ & & & & & & & $C d-111 m$ & $4.85 \mathrm{E}+01$ & $\mathrm{~m}$ & $C d-111 m$ & $4.85 E+01$ & $\mathrm{~m}$ & ICRP-107 & $4.85 \mathrm{E}+01$ & $\mathrm{~m}$ \\
\hline Cd-113 & Cd-113 & $7.70 \mathrm{E}+15$ & $y$ & Cd-113 & $9.30 \mathrm{E}+15$ & $\mathrm{y}$ & $\mathrm{Cd}-113$ & $9.30 \mathrm{E}+15$ & $y$ & Cd-113 & $7.70 \mathrm{E}+15$ & $y$ & & & & ICRP-38 & $9.30 \mathrm{E}+15$ & $\mathrm{y}$ \\
\hline Cd-113m & Cd-113m & $1.41 \mathrm{E}+01$ & $y$ & Cd-113m & $1.36 \mathrm{E}+01$ & $y$ & Cd-113m & $1.36 \mathrm{E}+01$ & $y$ & Cd-113m & $1.41 \mathrm{E}+01$ & $y$ & Cd-113m & $1.41 \mathrm{E}+01$ & $y$ & ICRP-38 & $1.36 \mathrm{E}+01$ & $y$ \\
\hline Cd-115 & Cd-115 & $5.35 \mathrm{E}+01$ & $\mathrm{~h}$ & Cd-115 & $5.35 \mathrm{E}+01$ & $\mathrm{~h}$ & Cd-115 & $5.35 \mathrm{E}+01$ & $\mathrm{~h}$ & Cd-115 & $5.35 \mathrm{E}+01$ & $\mathrm{~h}$ & & & & ICRP-38 & $5.35 \mathrm{E}+01$ & $\mathrm{~h}$ \\
\hline Cd-115m & Cd-115m & $4.46 \mathrm{E}+01$ & $d$ & Cd-115m & $4.46 \mathrm{E}+01$ & $d$ & Cd-115m & $4.46 \mathrm{E}+01$ & $d$ & Cd-115m & $4.46 \mathrm{E}+01$ & $d$ & & & & ICRP-38 & $4.46 \mathrm{E}+01$ & d \\
\hline Cd-117 & Cd-117 & $2.49 \mathrm{E}+00$ & $\mathrm{~h}$ & Cd-117 & $2.49 \mathrm{E}+00$ & $\mathrm{~h}$ & Cd-117 & $2.49 \mathrm{E}+00$ & $\mathrm{~h}$ & Cd-117 & $2.49 \mathrm{E}+00$ & $\mathrm{~h}$ & & & & ICRP-38 & $2.49 \mathrm{E}+00$ & $\mathrm{~h}$ \\
\hline Cd-117m & Cd-117m & $3.36 \mathrm{E}+00$ & $\mathrm{~h}$ & Cd-117m & $3.36 \mathrm{E}+00$ & $\mathrm{~h}$ & $\mathrm{Cd}-117 \mathrm{~m}$ & $3.36 \mathrm{E}+00$ & $\mathrm{~h}$ & Cd-117m & $3.36 \mathrm{E}+00$ & $\mathrm{~h}$ & & & & ICRP-38 & $3.36 \mathrm{E}+00$ & $\mathrm{~h}$ \\
\hline Cd-118 & Cd-118 & $5.03 E+01$ & $m$ & & & & & & & Cd-118 & $5.03 \mathrm{E}+01$ & $m$ & Cd-118 & $5.03 \mathrm{E}+01$ & $\mathrm{~m}$ & ICRP-107 & $5.03 \mathrm{E}+01$ & $\mathrm{~m}$ \\
\hline Cd-119 & Cd-119 & $2.69 \mathrm{E}+00$ & $\mathrm{~m}$ & & & & & & & Cd-119 & $2.69 \mathrm{E}+00$ & $\mathrm{~m}$ & & & & ICRP-107 & $2.69 \mathrm{E}+00$ & $\mathrm{~m}$ \\
\hline Cd-119m & Cd-119m & $2.20 \mathrm{E}+00$ & $\mathrm{~m}$ & & & & & & & Cd-119m & $2.20 \mathrm{E}+00$ & $m$ & & & & ICRP-107 & $2.20 \mathrm{E}+00$ & $\mathrm{~m}$ \\
\hline Ce-130 & Ce-130 & $2.29 \mathrm{E}+01$ & $\mathrm{~m}$ & & & & & & & Ce-130 & $2.29 \mathrm{E}+01$ & $\mathrm{~m}$ & Ce-130 & $2.29 \mathrm{E}+01$ & $\mathrm{~m}$ & ICRP-107 & $2.29 \mathrm{E}+01$ & $\mathrm{~m}$ \\
\hline Ce-131 & Ce-131 & $1.02 \mathrm{E}+01$ & $\mathrm{~m}$ & & & & & & & Ce-131 & $1.02 \mathrm{E}+01$ & $\mathrm{~m}$ & Ce-131 & $1.02 \mathrm{E}+01$ & $\mathrm{~m}$ & ICRP-107 & $1.02 \mathrm{E}+01$ & $\mathrm{~m}$ \\
\hline Ce-131m & & & & & & & & & & & & & Ce-131m & $5.00 \mathrm{E}+00$ & $\mathrm{~m}$ & JAERI & $5.00 \mathrm{E}+00$ & $\mathrm{~m}$ \\
\hline Ce-132 & Ce-132 & $3.51 E+00$ & $\mathrm{~h}$ & & & & & & & Ce-132 & $3.51 \mathrm{E}+00$ & $\mathrm{~h}$ & Ce-132 & $3.51 \mathrm{E}+00$ & $\mathrm{~h}$ & ICRP-107 & $3.51 \mathrm{E}+00$ & $\mathrm{~h}$ \\
\hline
\end{tabular}




\begin{tabular}{|c|c|c|c|c|c|c|c|c|c|c|c|c|c|c|c|c|c|c|}
\hline \multirow{3}{*}{$\begin{array}{l}\text { MASTER } \\
\text { LIST } \\
\text { Ce-133 }\end{array}$} & \multicolumn{3}{|c|}{ DOE-STD-1196 } & \multicolumn{3}{|c|}{ FGR-12 } & \multicolumn{3}{|c|}{ ICRP-38 } & \multicolumn{3}{|c|}{ ICRP-107 } & \multicolumn{3}{|c|}{ JAERI } & \multirow{3}{*}{$\begin{array}{c}\begin{array}{c}\text { Selected } \\
\text { Half-Life } \\
\text { Reference }\end{array} \\
\text { ICRP-107 }\end{array}$} & \multirow{2}{*}{\multicolumn{2}{|c|}{$\begin{array}{c}\text { Selected } \\
\text { Half-Life } \\
\text { (original units) }\end{array}$}} \\
\hline & \multirow{2}{*}{$\begin{array}{l}\text { Nuclide } \\
\text { Ce-133 }\end{array}$} & \multicolumn{2}{|c|}{$\begin{array}{c}\text { Half-Life } \\
\text { (original units) }\end{array}$} & \multirow[t]{2}{*}{ Nuclide } & \multicolumn{2}{|c|}{$\begin{array}{c}\text { Half-Life } \\
\text { (original units) }\end{array}$} & \multirow[t]{2}{*}{ Nuclide } & \multicolumn{2}{|c|}{$\begin{array}{c}\text { Half-Life } \\
\text { (original units) }\end{array}$} & \multirow{2}{*}{$\begin{array}{l}\text { Nuclide } \\
\text { Ce-133 }\end{array}$} & \multicolumn{2}{|c|}{$\begin{array}{c}\text { Half-Life } \\
\text { (original units) }\end{array}$} & \multirow{2}{*}{$\begin{array}{l}\text { Nuclide } \\
\text { Ce-133 }\end{array}$} & \multicolumn{2}{|c|}{$\begin{array}{c}\text { Half-Life } \\
\text { (original units) }\end{array}$} & & & \\
\hline & & $9.70 E+01$ & $\mathrm{~m}$ & & & & & & & & $9.70 \mathrm{E}+01$ & $\mathrm{~m}$ & & $9.70 \mathrm{E}+01$ & $\mathrm{~m}$ & & $9.70 \mathrm{E}+01$ & $\mathrm{~m}$ \\
\hline Ce-133m & Ce-133m & $4.90 \mathrm{E}+00$ & $\mathrm{~h}$ & & & & & & & Ce-133m & $4.90 \mathrm{E}+00$ & $\mathrm{~h}$ & Ce-133m & $4.90 \mathrm{E}+00$ & $\mathrm{~h}$ & ICRP-107 & $4.90 \mathrm{E}+00$ & $\mathrm{~h}$ \\
\hline Ce-134 & Ce-134 & $3.16 \mathrm{E}+00$ & d & Ce-134 & $7.20 \mathrm{E}+01$ & $\mathrm{~h}$ & Ce-134 & $7.20 \mathrm{E}+01$ & $\mathrm{~h}$ & Ce-134 & $3.16 \mathrm{E}+00$ & d & & & & ICRP-38 & $7.20 \mathrm{E}+01$ & $\mathrm{~h}$ \\
\hline Ce-135 & Ce-135 & $1.77 \mathrm{E}+01$ & $\mathrm{~h}$ & Ce-135 & $1.76 \mathrm{E}+01$ & $\mathrm{~h}$ & Ce-135 & $1.76 \mathrm{E}+01$ & $\mathrm{~h}$ & Ce-135 & $1.77 \mathrm{E}+01$ & $\mathrm{~h}$ & & & & ICRP-38 & $1.76 \mathrm{E}+01$ & $\mathrm{~h}$ \\
\hline Ce-137 & Ce-137 & $9.00 \mathrm{E}+00$ & $\mathrm{~h}$ & Ce-137 & $9.00 \mathrm{E}+00$ & $\mathrm{~h}$ & Ce-137 & $9.00 \mathrm{E}+00$ & $\mathrm{~h}$ & Ce-137 & $9.00 \mathrm{E}+00$ & $\mathrm{~h}$ & & & & ICRP-38 & $9.00 \mathrm{E}+00$ & $\mathrm{~h}$ \\
\hline Ce-137m & Ce-137m & $3.44 \mathrm{E}+01$ & $\mathrm{~h}$ & Ce-137m & $3.44 \mathrm{E}+01$ & $\mathrm{~h}$ & Ce-137m & $3.44 \mathrm{E}+01$ & $\mathrm{~h}$ & Ce-137m & $3.44 \mathrm{E}+01$ & $\mathrm{~h}$ & & & & ICRP-38 & $3.44 \mathrm{E}+01$ & $\mathrm{~h}$ \\
\hline Ce-139 & Ce-139 & $1.38 \mathrm{E}+02$ & $d$ & Ce-139 & $1.38 \mathrm{E}+02$ & $\mathrm{~d}$ & Ce-139 & $1.38 \mathrm{E}+02$ & d & Ce-139 & $1.38 \mathrm{E}+02$ & d & & & & ICRP-38 & $1.38 \mathrm{E}+02$ & $\mathrm{~d}$ \\
\hline Ce-141 & Ce-141 & $3.25 \mathrm{E}+01$ & $d$ & Ce-141 & $3.25 \mathrm{E}+01$ & $\mathrm{~d}$ & Ce-141 & $3.25 \mathrm{E}+01$ & $d$ & Ce-141 & $3.25 E+01$ & $d$ & & & & ICRP-38 & $3.25 \mathrm{E}+01$ & d \\
\hline Ce-143 & Ce-143 & $3.30 \mathrm{E}+01$ & $\mathrm{~h}$ & Ce-143 & $3.30 \mathrm{E}+01$ & $\mathrm{~h}$ & Ce-143 & $3.30 \mathrm{E}+01$ & $\mathrm{~h}$ & Ce-143 & $3.30 \mathrm{E}+01$ & $\mathrm{~h}$ & & & & ICRP-38 & $3.30 \mathrm{E}+01$ & $\mathrm{~h}$ \\
\hline Ce-144 & Ce-144 & $2.85 \mathrm{E}+02$ & $d$ & Ce-144 & $2.84 \mathrm{E}+02$ & $d$ & Ce-144 & $2.84 \mathrm{E}+02$ & $d$ & Ce-144 & $2.85 \mathrm{E}+02$ & $d$ & & & & ICRP-38 & $2.84 \mathrm{E}+02$ & d \\
\hline Ce-145 & Ce-145 & $3.01 \mathrm{E}+00$ & $\mathrm{~m}$ & & & & & & & Ce-145 & $3.01 \mathrm{E}+00$ & $\mathrm{~m}$ & & & & ICRP-107 & $3.01 \mathrm{E}+00$ & $\mathrm{~m}$ \\
\hline Ce-146 & & & & & & & & & & & & & Ce-146 & $1.35 \mathrm{E}+01$ & $m$ & JAERI & $1.35 \mathrm{E}+01$ & $\mathrm{~m}$ \\
\hline Cf-244 & Cf-244 & $1.94 \mathrm{E}+01$ & $\mathrm{~m}$ & Cf-244 & $1.94 \mathrm{E}+01$ & $\mathrm{~m}$ & Cf-244 & $1.94 \mathrm{E}+01$ & $\mathrm{~m}$ & Cf-244 & $1.94 \mathrm{E}+01$ & $\mathrm{~m}$ & & & & ICRP-38 & $1.94 \mathrm{E}+01$ & $\mathrm{~m}$ \\
\hline Cf-246 & Cf-246 & $3.57 E+01$ & $\mathrm{~h}$ & Cf-246 & $3.57 E+01$ & $\mathrm{~h}$ & Cf-246 & $3.57 \mathrm{E}+01$ & $\mathrm{~h}$ & Cf-246 & $3.57 \mathrm{E}+01$ & $\mathrm{~h}$ & & & & ICRP-38 & $3.57 \mathrm{E}+01$ & $\mathrm{~h}$ \\
\hline Cf-247 & Cf-247 & $3.11 \mathrm{E}+00$ & $\mathrm{~h}$ & & & & & & & Cf-247 & $3.11 \mathrm{E}+00$ & $\mathrm{~h}$ & Cf-247 & $3.11 \mathrm{E}+00$ & $\mathrm{~h}$ & ICRP-107 & $3.11 \mathrm{E}+00$ & $\mathrm{~h}$ \\
\hline Cf-248 & Cf- 248 & $3.34 \mathrm{E}+02$ & $d$ & Cf-248 & $3.34 \mathrm{E}+02$ & $d$ & Cf-248 & $3.34 \mathrm{E}+02$ & $d$ & Cf- 248 & $3.34 \mathrm{E}+02$ & $d$ & & & & ICRP-38 & $3.34 \mathrm{E}+02$ & d \\
\hline Cf-249 & Cf-249 & $3.51 \mathrm{E}+02$ & $y$ & Cf-249 & $3.51 \mathrm{E}+02$ & $y$ & Cf-249 & $3.51 \mathrm{E}+02$ & $y$ & Cf-249 & $3.51 \mathrm{E}+02$ & $y$ & & & & ICRP-38 & $3.51 \mathrm{E}+02$ & $y$ \\
\hline Cf-250 & Cf- 250 & $1.31 \mathrm{E}+01$ & $y$ & Cf- 250 & $1.31 \mathrm{E}+01$ & $y$ & Cf- 250 & $1.31 \mathrm{E}+01$ & $y$ & Cf- 250 & $1.31 \mathrm{E}+01$ & $y$ & & & & ICRP-38 & $1.31 \mathrm{E}+01$ & $y$ \\
\hline Cf-251 & Cf- 251 & $9.00 \mathrm{E}+02$ & $y$ & Cf-251 & $8.98 \mathrm{E}+02$ & $y$ & Cf-251 & $8.98 \mathrm{E}+02$ & $y$ & Cf- 251 & $9.00 \mathrm{E}+02$ & $y$ & & & & ICRP-38 & $8.98 \mathrm{E}+02$ & $y$ \\
\hline Cf-252 & Cf-252 & $2.65 \mathrm{E}+00$ & $y$ & Cf-252 & $2.64 \mathrm{E}+00$ & $y$ & Cf- 252 & $2.64 \mathrm{E}+00$ & $y$ & Cf-252 & $2.65 \mathrm{E}+00$ & $y$ & & & & ICRP-38 & $2.64 \mathrm{E}+00$ & $y$ \\
\hline Cf-253 & Cf-253 & $1.78 \mathrm{E}+01$ & $d$ & Cf- 253 & $1.78 \mathrm{E}+01$ & $d$ & Cf- 253 & $1.78 \mathrm{E}+01$ & $d$ & Cf-253 & $1.78 \mathrm{E}+01$ & $d$ & & & & ICRP-38 & $1.78 \mathrm{E}+01$ & $\frac{1}{d}$ \\
\hline Cf-254 & Cf-254 & $6.05 \mathrm{E}+01$ & $d$ & Cf-254 & $6.05 E+01$ & $d$ & Cf-254 & $6.05 E+01$ & $d$ & Cf-254 & $6.05 \mathrm{E}+01$ & $d$ & & & & ICRP-38 & $6.05 \mathrm{E}+01$ & d \\
\hline Cf-255 & Cf-255 & $8.50 \mathrm{E}+01$ & $m$ & & & & & & & Cf-255 & $8.50 \mathrm{E}+01$ & $m$ & Cf- 255 & $8.50 \mathrm{E}+01$ & $\mathrm{~m}$ & ICRP-107 & $8.50 \mathrm{E}+01$ & $\mathrm{~m}$ \\
\hline Cf-256 & & & & & & & & & & & & & Cf-256 & $1.23 \mathrm{E}+01$ & $m$ & JAERI & $1.23 \mathrm{E}+01$ & $\mathrm{~m}$ \\
\hline $\mathrm{Cl}-34$ & $\mathrm{Cl}-34$ & $1.53 \mathrm{E}+00$ & $\mathrm{~s}$ & & & & & & & $\mathrm{Cl}-34$ & $1.53 \mathrm{E}+00$ & $\mathrm{~s}$ & & & & ICRP-107 & $1.53 \mathrm{E}+00$ & $\mathrm{~s}$ \\
\hline Cl-34m & $\mathrm{Cl}-34 \mathrm{~m}$ & $3.20 \mathrm{E}+01$ & $\mathrm{~m}$ & & & & & & & $\mathrm{Cl}-34 \mathrm{~m}$ & $3.20 \mathrm{E}+01$ & $\mathrm{~m}$ & $\mathrm{Cl}-34 \mathrm{~m}$ & $3.20 \mathrm{E}+01$ & $\mathrm{~m}$ & ICRP-107 & $3.20 \mathrm{E}+01$ & $\mathrm{~m}$ \\
\hline $\mathrm{Cl}-36$ & $\mathrm{Cl}-36$ & $3.01 \mathrm{E}+05$ & $y$ & $\mathrm{Cl}-36$ & $3.01 \mathrm{E}+05$ & $y$ & $\mathrm{Cl}-36$ & $3.01 \mathrm{E}+05$ & $y$ & $\mathrm{Cl}-36$ & $3.01 \mathrm{E}+05$ & $y$ & & & & ICRP-38 & $3.01 \mathrm{E}+05$ & $\mathrm{y}$ \\
\hline $\mathrm{Cl}-38$ & $\mathrm{Cl}-38$ & $3.72 \mathrm{E}+01$ & $\mathrm{~m}$ & $\mathrm{Cl}-38$ & $3.72 \mathrm{E}+01$ & $\mathrm{~m}$ & $\mathrm{Cl}-38$ & $3.72 \mathrm{E}+01$ & $\mathrm{~m}$ & $\mathrm{Cl}-38$ & $3.72 \mathrm{E}+01$ & $\mathrm{~m}$ & & & & ICRP-38 & $3.72 \mathrm{E}+01$ & $\mathrm{~m}$ \\
\hline $\mathrm{Cl}-39$ & $\mathrm{Cl}-39$ & $5.56 \mathrm{E}+01$ & $m$ & $\mathrm{Cl}-39$ & $5.56 \mathrm{E}+01$ & $m$ & $\mathrm{Cl}-39$ & $5.56 \mathrm{E}+01$ & $\mathrm{~m}$ & $\mathrm{Cl}-39$ & $5.56 \mathrm{E}+01$ & $\mathrm{~m}$ & & & & ICRP-38 & $5.56 \mathrm{E}+01$ & $\mathrm{~m}$ \\
\hline $\mathrm{Cl}-40$ & $\mathrm{Cl}-40$ & $1.35 \mathrm{E}+00$ & $\mathrm{~m}$ & & & & & & & $\mathrm{Cl}-40$ & $1.35 \mathrm{E}+00$ & $\mathrm{~m}$ & $\mathrm{Cl}-40$ & $1.35 \mathrm{E}+00$ & $\mathrm{~m}$ & ICRP-107 & $1.35 \mathrm{E}+00$ & $\mathrm{~m}$ \\
\hline Cm-238 & $\mathrm{Cm}-238$ & $2.40 \mathrm{E}+00$ & $\mathrm{~h}$ & $\mathrm{Cm}-238$ & $2.40 \mathrm{E}+00$ & $\mathrm{~h}$ & $\mathrm{Cm}-238$ & $2.40 \mathrm{E}+00$ & $\mathrm{~h}$ & $\mathrm{Cm}-238$ & $2.40 \mathrm{E}+00$ & $\mathrm{~h}$ & & & & ICRP-38 & $2.40 \mathrm{E}+00$ & $\mathrm{~h}$ \\
\hline $\mathrm{Cm}-239$ & $\mathrm{Cm}-239$ & $2.90 \mathrm{E}+00$ & $\mathrm{~h}$ & & & & & & & $\mathrm{Cm}-239$ & $2.90 \mathrm{E}+00$ & $\mathrm{~h}$ & Cm-239 & $2.90 \mathrm{E}+00$ & $\mathrm{~h}$ & ICRP-107 & $2.90 \mathrm{E}+00$ & $\mathrm{~h}$ \\
\hline $\mathrm{Cm}-240$ & $\mathrm{Cm}-240$ & $2.70 \mathrm{E}+01$ & $d$ & $\mathrm{Cm}-240$ & $2.70 \mathrm{E}+01$ & $d$ & $\mathrm{Cm}-240$ & $2.70 \mathrm{E}+01$ & $d$ & $\mathrm{Cm}-240$ & $2.70 \mathrm{E}+01$ & $d$ & & & & ICRP-38 & $2.70 \mathrm{E}+01$ & d \\
\hline $\mathrm{Cm}-241$ & $\mathrm{Cm}-241$ & $3.28 \mathrm{E}+01$ & $d$ & $\mathrm{Cm}-241$ & $3.28 \mathrm{E}+01$ & $\mathrm{~d}$ & $\mathrm{Cm}-241$ & $3.28 \mathrm{E}+01$ & $d$ & $\mathrm{Cm}-241$ & $3.28 \mathrm{E}+01$ & $d$ & & & & ICRP-38 & $3.28 \mathrm{E}+01$ & $\mathrm{~d}$ \\
\hline $\mathrm{Cm}-242$ & $\mathrm{Cm}-242$ & $1.63 E+02$ & $d$ & $\mathrm{Cm}-242$ & $1.63 \mathrm{E}+02$ & $d$ & $\mathrm{Cm}-242$ & $1.63 \mathrm{E}+02$ & $d$ & $\mathrm{Cm}-242$ & $1.63 \mathrm{E}+02$ & $\mathrm{~d}$ & & & & ICRP-38 & $1.63 E+02$ & $\bar{d}$ \\
\hline $\mathrm{Cm}-243$ & $\mathrm{Cm}-243$ & $2.91 \mathrm{E}+01$ & $y$ & $\mathrm{Cm}-243$ & $2.85 \mathrm{E}+01$ & $y$ & $\mathrm{Cm}-243$ & $2.85 \mathrm{E}+01$ & $y$ & $\mathrm{Cm}-243$ & $2.91 \mathrm{E}+01$ & $y$ & & & & ICRP-38 & $2.85 \mathrm{E}+01$ & $y$ \\
\hline $\mathrm{Cm}-244$ & $\mathrm{Cm}-244$ & $1.81 \mathrm{E}+01$ & $y$ & $\mathrm{Cm}-244$ & $1.81 \mathrm{E}+01$ & $y$ & $\mathrm{Cm}-244$ & $1.81 \mathrm{E}+01$ & $y$ & $\mathrm{Cm}-244$ & $1.81 \mathrm{E}+01$ & $y$ & & & & ICRP-38 & $1.81 \mathrm{E}+01$ & $y$ \\
\hline $\mathrm{Cm}-245$ & $\mathrm{Cm}-245$ & $8.50 E+03$ & $y$ & $\mathrm{Cm}-245$ & $8.50 E+03$ & $y$ & $\mathrm{Cm}-245$ & $8.50 \mathrm{E}+03$ & $y$ & $\mathrm{Cm}-245$ & $8.50 \mathrm{E}+03$ & $y$ & & & & ICRP-38 & $8.50 \mathrm{E}+03$ & $y$ \\
\hline $\mathrm{Cm}-246$ & $\mathrm{Cm}-246$ & $4.76 \mathrm{E}+03$ & $y$ & $\mathrm{Cm}-246$ & $4.73 E+03$ & $y$ & $\mathrm{Cm}-246$ & $4.73 \mathrm{E}+03$ & $y$ & $\mathrm{Cm}-246$ & $4.76 \mathrm{E}+03$ & $y$ & & & & ICRP-38 & $4.73 E+03$ & $y$ \\
\hline $\mathrm{Cm}-247$ & $\mathrm{Cm}-247$ & $1.56 \mathrm{E}+07$ & $y$ & $\mathrm{Cm}-247$ & $1.56 \mathrm{E}+07$ & $y$ & $\mathrm{Cm}-247$ & $1.56 \mathrm{E}+07$ & $y$ & $\mathrm{Cm}-247$ & $1.56 \mathrm{E}+07$ & $y$ & & & & ICRP-38 & $1.56 \mathrm{E}+07$ & $y$ \\
\hline
\end{tabular}




\begin{tabular}{|c|c|c|c|c|c|c|c|c|c|c|c|c|c|c|c|c|c|c|}
\hline \multicolumn{19}{|c|}{ Table D.1 Selection of Radionuclides For Calculating HC-2 and HC-3 TQs (continued) } \\
\hline \multirow{3}{*}{$\begin{array}{l}\text { MASTER } \\
\text { LIST } \\
\mathrm{Cm}-248\end{array}$} & \multicolumn{3}{|c|}{ DOE-STD-1196 } & \multicolumn{3}{|c|}{ FGR-12 } & \multicolumn{3}{|c|}{ ICRP-38 } & \multicolumn{3}{|c|}{ ICRP-107 } & \multicolumn{3}{|c|}{ JAERI } & \multirow{3}{*}{$\begin{array}{c}\begin{array}{c}\text { Selected } \\
\text { Half-Life } \\
\text { Reference }\end{array} \\
\text { ICRP-38 }\end{array}$} & \multirow{2}{*}{\multicolumn{2}{|c|}{$\begin{array}{c}\text { Selected } \\
\text { Half-Life } \\
\text { (original units) }\end{array}$}} \\
\hline & \multirow{2}{*}{$\begin{array}{l}\text { Nuclide } \\
\mathrm{Cm}-248\end{array}$} & \multicolumn{2}{|c|}{$\begin{array}{c}\text { Half-Life } \\
\text { (original units) }\end{array}$} & \multirow{2}{*}{$\begin{array}{l}\text { Nuclide } \\
\mathrm{Cm}-248\end{array}$} & \multicolumn{2}{|c|}{$\begin{array}{c}\text { Half-Life } \\
\text { (original units) }\end{array}$} & \multirow{2}{*}{$\begin{array}{l}\text { Nuclide } \\
\mathrm{Cm}-248\end{array}$} & \multicolumn{2}{|c|}{$\begin{array}{c}\text { Half-Life } \\
\text { (original units) }\end{array}$} & \multirow{2}{*}{$\frac{\text { Nuclide }}{\mathrm{Cm}-248}$} & \multicolumn{2}{|c|}{$\begin{array}{c}\text { Half-Life } \\
\text { (original units) }\end{array}$} & \multirow[t]{2}{*}{ Nuclide } & $\begin{array}{r}\text { Half-Lif } \\
\text { (original u }\end{array}$ & its) & & & \\
\hline & & $3.48 E+05$ & $y$ & & $3.39 E+05$ & $y$ & & $3.39 \mathrm{E}+05$ & $y$ & & $3.48 \mathrm{E}+05$ & $y$ & & & & & $3.39 E+05$ & $y$ \\
\hline $\mathrm{Cm}-249$ & Cm-249 & $6.42 \mathrm{E}+01$ & $\mathrm{~m}$ & $\mathrm{Cm}-249$ & $6.42 \mathrm{E}+01$ & $\mathrm{~m}$ & $\mathrm{Cm}-249$ & $6.42 \mathrm{E}+01$ & $\mathrm{~m}$ & $\mathrm{Cm}-249$ & $6.42 \mathrm{E}+01$ & $\mathrm{~m}$ & & & & ICRP-38 & $6.42 \mathrm{E}+01$ & $\mathrm{~m}$ \\
\hline $\mathrm{Cm}-250$ & $\mathrm{Cm}-250$ & $8.30 \mathrm{E}+03$ & $\mathrm{y}$ & $\mathrm{Cm}-250$ & $6.90 \mathrm{E}+03$ & $y$ & $\mathrm{Cm}-250$ & $6.90 \mathrm{E}+03$ & $y$ & $\mathrm{Cm}-250$ & $8.30 \mathrm{E}+03$ & $y$ & & & & ICRP-38 & $6.90 \mathrm{E}+03$ & $y$ \\
\hline $\mathrm{Cm}-251$ & $\mathrm{Cm}-251$ & $1.68 \mathrm{E}+01$ & $\mathrm{~m}$ & & & & & & & $\mathrm{Cm}-251$ & $1.68 \mathrm{E}+01$ & $\mathrm{~m}$ & & & & ICRP-107 & $1.68 \mathrm{E}+01$ & $\mathrm{~m}$ \\
\hline Co-54m & Co-54m & $1.48 \mathrm{E}+00$ & $\mathrm{~m}$ & & & & & & & Co-54m & $1.48 \mathrm{E}+00$ & $\mathrm{~m}$ & & & & ICRP-107 & $1.48 \mathrm{E}+00$ & $\mathrm{~m}$ \\
\hline Co-55 & Co-55 & $1.75 \mathrm{E}+01$ & $\mathrm{~h}$ & Co-55 & $1.75 \mathrm{E}+01$ & $\mathrm{~h}$ & Co-55 & $1.75 \mathrm{E}+01$ & $\mathrm{~h}$ & Co-55 & $1.75 \mathrm{E}+01$ & $\mathrm{~h}$ & & & & ICRP-38 & $1.75 \mathrm{E}+01$ & $\mathrm{~h}$ \\
\hline Co-56 & Co-56 & $7.72 \mathrm{E}+01$ & d & Co-56 & $7.88 \mathrm{E}+01$ & $\mathrm{~d}$ & Co-56 & $7.88 \mathrm{E}+01$ & d & Co-56 & $7.72 \mathrm{E}+01$ & $d$ & & & & ICRP-38 & $7.88 \mathrm{E}+01$ & $\mathrm{~d}$ \\
\hline Co-57 & Co-57 & $2.72 \mathrm{E}+02$ & $d$ & Co-57 & $2.71 \mathrm{E}+02$ & $\mathrm{~d}$ & Co-57 & $2.71 \mathrm{E}+02$ & $d$ & Co-57 & $2.72 \mathrm{E}+02$ & $d$ & & & & ICRP-38 & $2.71 \mathrm{E}+02$ & $d$ \\
\hline Co-58 & Co-58 & $7.09 \mathrm{E}+01$ & $d$ & Co-58 & $7.08 \mathrm{E}+01$ & $\mathrm{~d}$ & Co-58 & $7.08 \mathrm{E}+01$ & $d$ & Co-58 & $7.09 \mathrm{E}+01$ & $d$ & & & & ICRP-38 & $7.08 \mathrm{E}+01$ & $d$ \\
\hline Co-58m & Co-58m & $9.04 \mathrm{E}+00$ & $\mathrm{~h}$ & Co-58m & $9.15 E+00$ & $\mathrm{~h}$ & Co-58m & $9.15 \mathrm{E}+00$ & $\mathrm{~h}$ & Co-58m & $9.04 \mathrm{E}+00$ & $\mathrm{~h}$ & & & & ICRP-38 & $9.15 \mathrm{E}+00$ & $\mathrm{~h}$ \\
\hline Co-60 & Co-60 & $5.27 \mathrm{E}+00$ & $y$ & Co-60 & $5.27 \mathrm{E}+00$ & $y$ & Co-60 & $5.27 \mathrm{E}+00$ & $y$ & Co-60 & $5.27 \mathrm{E}+00$ & $y$ & & & & ICRP-38 & $5.27 \mathrm{E}+00$ & $y$ \\
\hline Co-60m & Co-60m & $1.05 \mathrm{E}+01$ & $\mathrm{~m}$ & Co-60m & $1.05 \mathrm{E}+01$ & $\mathrm{~m}$ & Co-60m & $1.05 \mathrm{E}+01$ & $m$ & Co-60m & $1.05 \mathrm{E}+01$ & $m$ & & & & ICRP-38 & $1.05 \mathrm{E}+01$ & $\mathrm{~m}$ \\
\hline Co-61 & Co-61 & $1.65 \mathrm{E}+00$ & $\mathrm{~h}$ & Co-61 & $1.65 \mathrm{E}+00$ & $\mathrm{~h}$ & Co-61 & $1.65 \mathrm{E}+00$ & $\mathrm{~h}$ & Co-61 & $1.65 \mathrm{E}+00$ & $\mathrm{~h}$ & & & & ICRP-38 & $1.65 \mathrm{E}+00$ & $\mathrm{~h}$ \\
\hline Co-62 & Co-62 & $1.50 \mathrm{E}+00$ & $m$ & & & & & & & Co-62 & $1.50 \mathrm{E}+00$ & $m$ & Co-62 & $1.50 \mathrm{E}+00$ & $m$ & ICRP-107 & $1.50 \mathrm{E}+00$ & $\mathrm{~m}$ \\
\hline Co-62m & Co-62m & $1.39 \mathrm{E}+01$ & $\mathrm{~m}$ & Co-62m & $1.39 \mathrm{E}+01$ & $m$ & Co-62m & $1.39 \mathrm{E}+01$ & $\mathrm{~m}$ & Co-62m & $1.39 \mathrm{E}+01$ & $m$ & & & & ICRP-38 & $1.39 \mathrm{E}+01$ & $m$ \\
\hline Cr-48 & $\mathrm{Cr}-48$ & $2.16 \mathrm{E}+01$ & $\mathrm{~h}$ & $\mathrm{Cr}-48$ & $2.30 \mathrm{E}+01$ & $\mathrm{~h}$ & $\mathrm{Cr}-48$ & $2.30 \mathrm{E}+01$ & $\mathrm{~h}$ & $\mathrm{Cr}-48$ & $2.16 \mathrm{E}+01$ & $\mathrm{~h}$ & & & & ICRP-38 & $2.30 \mathrm{E}+01$ & $\mathrm{~h}$ \\
\hline Cr-49 & Cr-49 & $4.23 \mathrm{E}+01$ & $\mathrm{~m}$ & Cr-49 & $4.21 \mathrm{E}+01$ & $\mathrm{~m}$ & $\mathrm{Cr}-49$ & $4.21 \mathrm{E}+01$ & $\mathrm{~m}$ & $\mathrm{Cr}-49$ & $4.23 \mathrm{E}+01$ & $\mathrm{~m}$ & & & & ICRP-38 & $4.21 \mathrm{E}+01$ & $\mathrm{~m}$ \\
\hline Cr-51 & Cr-51 & $2.77 E+01$ & $d$ & Cr-51 & $2.77 E+01$ & $d$ & Cr-51 & $2.77 \mathrm{E}+01$ & $d$ & $\mathrm{Cr}-51$ & $2.77 \mathrm{E}+01$ & $\mathrm{~d}$ & & & & ICRP-38 & $2.77 \mathrm{E}+01$ & $d$ \\
\hline Cr-55 & Cr-55 & $3.50 \mathrm{E}+00$ & $\mathrm{~m}$ & & & & & & & $\mathrm{Cr}-55$ & $3.50 \mathrm{E}+00$ & $\mathrm{~m}$ & Cr-55 & $3.50 \mathrm{E}+00$ & $\mathrm{~m}$ & ICRP-107 & $3.50 \mathrm{E}+00$ & $\mathrm{~m}$ \\
\hline Cr-56 & $\mathrm{Cr}-56$ & $5.94 \mathrm{E}+00$ & $\mathrm{~m}$ & & & & & & & $\mathrm{Cr}-56$ & $5.94 \mathrm{E}+00$ & $m$ & $\mathrm{Cr}-56$ & $5.94 \mathrm{E}+00$ & $m$ & ICRP-107 & $5.94 \mathrm{E}+00$ & $\mathrm{~m}$ \\
\hline Cs-121 & Cs-121 & $1.55 \mathrm{E}+02$ & $\mathrm{~s}$ & & & & & & & Cs-121 & $1.55 \mathrm{E}+02$ & $\mathrm{~s}$ & & & & ICRP-107 & $1.55 \mathrm{E}+02$ & $s$ \\
\hline Cs-121m & Cs-121m & $1.22 \mathrm{E}+02$ & $\mathrm{~s}$ & & & & & & & Cs-121m & $1.22 \mathrm{E}+02$ & $\mathrm{~s}$ & & & & ICRP-107 & $1.22 \mathrm{E}+02$ & $\mathrm{~s}$ \\
\hline Cs-123 & Cs-123 & $5.88 \mathrm{E}+00$ & $m$ & & & & & & & Cs-123 & $5.88 \mathrm{E}+00$ & $m$ & & & & ICRP-107 & $5.88 \mathrm{E}+00$ & $\mathrm{~m}$ \\
\hline Cs-124 & Cs-124 & $3.08 \mathrm{E}+01$ & $\mathrm{~s}$ & & & & & & & Cs-124 & $3.08 \mathrm{E}+01$ & $s$ & & & & ICRP-107 & $3.08 \mathrm{E}+01$ & $\mathrm{~s}$ \\
\hline Cs-125 & Cs-125 & $4.50 \mathrm{E}+01$ & $m$ & Cs-125 & $4.50 \mathrm{E}+01$ & $m$ & Cs-125 & $4.50 \mathrm{E}+01$ & $m$ & Cs-125 & $4.50 \mathrm{E}+01$ & $m$ & & & & ICRP-38 & $4.50 \mathrm{E}+01$ & $\mathrm{~m}$ \\
\hline Cs-126 & Cs-126 & $1.64 \mathrm{E}+00$ & $m$ & Cs-126 & $1.64 \mathrm{E}+00$ & $m$ & Cs-126 & $1.64 \mathrm{E}+00$ & $\mathrm{~m}$ & Cs-126 & $1.64 \mathrm{E}+00$ & $\mathrm{~m}$ & Cs-126 & $1.64 \mathrm{E}+00$ & $\mathrm{~m}$ & ICRP-38 & $1.64 \mathrm{E}+00$ & $\mathrm{~m}$ \\
\hline Cs-127 & Cs-127 & $6.25 \mathrm{E}+00$ & $\mathrm{~h}$ & Cs-127 & $6.25 \mathrm{E}+00$ & $\mathrm{~h}$ & Cs-127 & $6.25 \mathrm{E}+00$ & $\mathrm{~h}$ & Cs-127 & $6.25 \mathrm{E}+00$ & $\mathrm{~h}$ & & & & ICRP-38 & $6.25 \mathrm{E}+00$ & $\mathrm{~h}$ \\
\hline Cs-128 & Cs-128 & $3.64 \mathrm{E}+00$ & $\mathrm{~m}$ & Cs-128 & $3.90 \mathrm{E}+00$ & $m$ & Cs-128 & $3.90 \mathrm{E}+00$ & $\mathrm{~m}$ & Cs-128 & $3.64 \mathrm{E}+00$ & $\mathrm{~m}$ & Cs-128 & $3.62 \mathrm{E}+00$ & $\mathrm{~m}$ & ICRP-38 & $3.90 \mathrm{E}+00$ & $\mathrm{~m}$ \\
\hline Cs-129 & Cs-129 & $3.21 \mathrm{E}+01$ & $\mathrm{~h}$ & Cs-129 & $3.21 \mathrm{E}+01$ & $\mathrm{~h}$ & Cs-129 & $3.21 \mathrm{E}+01$ & $\mathrm{~h}$ & Cs-129 & $3.21 \mathrm{E}+01$ & $\mathrm{~h}$ & & & & ICRP-38 & $3.21 \mathrm{E}+01$ & $\mathrm{~h}$ \\
\hline Cs-130 & Cs-130 & $2.92 \mathrm{E}+01$ & $\mathrm{~m}$ & Cs-130 & $2.99 \mathrm{E}+01$ & $\mathrm{~m}$ & Cs-130 & $2.99 \mathrm{E}+01$ & $\mathrm{~m}$ & Cs-130 & $2.92 \mathrm{E}+01$ & $\mathrm{~m}$ & & & & ICRP-38 & $2.99 \mathrm{E}+01$ & $\mathrm{~m}$ \\
\hline Cs-130m & Cs-130m & $3.46 \mathrm{E}+00$ & $\mathrm{~m}$ & & & & & & & Cs-130m & $3.46 \mathrm{E}+00$ & $m$ & Cs- $130 \mathrm{~m}$ & $3.46 \mathrm{E}+00$ & $\mathrm{~m}$ & ICRP-107 & $3.46 \mathrm{E}+00$ & $\mathrm{~m}$ \\
\hline Cs-131 & Cs-131 & $9.69 \mathrm{E}+00$ & $\mathrm{~d}$ & Cs-131 & $9.69 \mathrm{E}+00$ & $d$ & Cs-131 & $9.69 \mathrm{E}+00$ & $d$ & Cs-131 & $9.69 \mathrm{E}+00$ & $d$ & & & & ICRP-38 & $9.69 \mathrm{E}+00$ & $d$ \\
\hline Cs-132 & Cs-132 & $6.48 \mathrm{E}+00$ & $d$ & Cs-132 & $6.48 \mathrm{E}+00$ & $d$ & Cs-132 & $6.48 \mathrm{E}+00$ & $d$ & Cs-132 & $6.48 \mathrm{E}+00$ & $d$ & & & & ICRP-38 & $6.48 \mathrm{E}+00$ & $d$ \\
\hline Cs-134 & Cs-134 & $2.06 \mathrm{E}+00$ & $y$ & Cs-134 & $2.06 \mathrm{E}+00$ & $y$ & Cs-134 & $2.06 \mathrm{E}+00$ & $y$ & Cs-134 & $2.06 \mathrm{E}+00$ & $y$ & & & & ICRP-38 & $2.06 \mathrm{E}+00$ & $y$ \\
\hline Cs-134m & Cs-134m & $2.90 \mathrm{E}+00$ & $\mathrm{~h}$ & Cs-134m & $2.90 \mathrm{E}+00$ & $\mathrm{~h}$ & Cs-134m & $2.90 \mathrm{E}+00$ & $\mathrm{~h}$ & Cs-134m & $2.90 \mathrm{E}+00$ & $\mathrm{~h}$ & & & & ICRP-38 & $2.90 \mathrm{E}+00$ & $\mathrm{~h}$ \\
\hline Cs-135 & Cs-135 & $2.30 \mathrm{E}+06$ & $y$ & Cs-135 & $2.30 \mathrm{E}+06$ & $y$ & Cs-135 & $2.30 \mathrm{E}+06$ & $y$ & Cs-135 & $2.30 \mathrm{E}+06$ & $y$ & & & & ICRP-38 & $2.30 \mathrm{E}+06$ & $y$ \\
\hline Cs-135m & Cs-135m & $5.30 \mathrm{E}+01$ & $\mathrm{~m}$ & Cs-135m & $5.30 \mathrm{E}+01$ & $\mathrm{~m}$ & Cs-135m & $5.30 \mathrm{E}+01$ & $m$ & Cs-135m & $5.30 \mathrm{E}+01$ & $\mathrm{~m}$ & & & & ICRP-38 & $5.30 \mathrm{E}+01$ & $\mathrm{~m}$ \\
\hline Cs-136 & Cs-136 & $1.32 \mathrm{E}+01$ & $d$ & Cs-136 & $1.31 \mathrm{E}+01$ & $d$ & Cs-136 & $1.31 \mathrm{E}+01$ & $d$ & Cs-136 & $1.32 \mathrm{E}+01$ & $d$ & & & & ICRP-38 & $1.31 \mathrm{E}+01$ & $d$ \\
\hline Cs-137 & Cs-137 & $3.02 \mathrm{E}+01$ & $y$ & Cs-137 & $3.00 \mathrm{E}+01$ & $y$ & Cs-137 & $3.00 \mathrm{E}+01$ & $y$ & Cs-137 & $3.02 \mathrm{E}+01$ & $y$ & & & & ICRP-38 & $3.00 \mathrm{E}+01$ & $y$ \\
\hline Cs-138 & Cs-138 & $3.34 \mathrm{E}+01$ & $\mathrm{~m}$ & Cs-138 & $3.22 \mathrm{E}+01$ & $m$ & Cs-138 & $3.22 \mathrm{E}+01$ & $\mathrm{~m}$ & Cs-138 & $3.34 \mathrm{E}+01$ & $m$ & & & & ICRP-38 & $3.22 \mathrm{E}+01$ & $\mathrm{~m}$ \\
\hline
\end{tabular}

D-10 


\begin{tabular}{|c|c|c|c|c|c|c|c|c|c|c|c|c|c|c|c|c|c|c|}
\hline \multicolumn{19}{|c|}{ Table D.1 Selection of Radionuclides For Calculating HC-2 and HC-3 TQs (continued) } \\
\hline \multirow{3}{*}{$\begin{array}{l}\text { MASTER } \\
\text { LIST } \\
\text { Cs-138m }\end{array}$} & \multicolumn{3}{|c|}{ DOE-STD-1196 } & \multicolumn{3}{|c|}{ FGR-12 } & \multicolumn{3}{|c|}{ ICRP-38 } & \multicolumn{3}{|c|}{ ICRP-107 } & \multicolumn{3}{|c|}{ JAERI } & \multirow{3}{*}{$\begin{array}{c}\begin{array}{c}\text { Selected } \\
\text { Half-Life } \\
\text { Reference }\end{array} \\
\text { ICRP-107 }\end{array}$} & & \\
\hline & \multirow{2}{*}{$\begin{array}{l}\text { Nuclide } \\
\text { Cs-138m }\end{array}$} & \multicolumn{2}{|c|}{$\begin{array}{c}\text { Half-Life } \\
\text { (original units) }\end{array}$} & \multirow[t]{2}{*}{ Nuclide } & \multicolumn{2}{|c|}{$\begin{array}{c}\text { Half-Life } \\
\text { (original units) }\end{array}$} & \multirow[t]{2}{*}{ Nuclide } & \multicolumn{2}{|c|}{$\begin{array}{c}\text { Half-Life } \\
\text { (original units) }\end{array}$} & \multirow{2}{*}{$\begin{array}{l}\text { Nuclide } \\
\text { Cs-138m }\end{array}$} & \multicolumn{2}{|c|}{$\begin{array}{c}\text { Half-Life } \\
\text { (original units) }\end{array}$} & \multirow[t]{2}{*}{ Nuclide } & $\begin{array}{r}\text { Half-Lif } \\
\text { (original u }\end{array}$ & its) & & \multicolumn{2}{|c|}{$\begin{array}{c}\text { Selected } \\
\text { Half-Life } \\
\text { (original units) }\end{array}$} \\
\hline & & $2.91 E+00$ & $\mathrm{~m}$ & & & & & & & & $2.91 \mathrm{E}+00$ & $\mathrm{~m}$ & & & & & $2.91 \mathrm{E}+00$ & $\mathrm{~m}$ \\
\hline Cs-139 & Cs-139 & $9.27 \mathrm{E}+00$ & $\mathrm{~m}$ & & & & & & & Cs-139 & $9.27 \mathrm{E}+00$ & $\mathrm{~m}$ & Cs-139 & $9.27 \mathrm{E}+00$ & $\mathrm{~m}$ & ICRP-107 & $9.27 \mathrm{E}+00$ & $\mathrm{~m}$ \\
\hline Cs-140 & Cs-140 & $6.37 \mathrm{E}+01$ & $\mathrm{~s}$ & & & & & & & Cs-140 & $6.37 \mathrm{E}+01$ & $\mathrm{~s}$ & & & & ICRP-107 & $6.37 \mathrm{E}+01$ & $\mathrm{~s}$ \\
\hline Cu-57 & Cu-57 & $1.96 \mathrm{E}-01$ & $\mathrm{~s}$ & & & & Cu-57 & $2.33 \mathrm{E}+02$ & $\mathrm{~ms}$ & Cu-57 & $1.96 \mathrm{E}-01$ & $\mathrm{~s}$ & & & & ICRP-38 & $2.33 \mathrm{E}+02$ & $\mathrm{~ms}$ \\
\hline Cu-59 & Cu-59 & $8.15 \mathrm{E}+01$ & $\mathrm{~s}$ & & & & & & & Cu-59 & $8.15 \mathrm{E}+01$ & $\mathrm{~s}$ & & & & ICRP-107 & $8.15 \mathrm{E}+01$ & $\mathrm{~s}$ \\
\hline Cu-60 & Cu-60 & $2.37 \mathrm{E}+01$ & $\mathrm{~m}$ & Cu-60 & $2.32 \mathrm{E}+01$ & $\mathrm{~m}$ & Cu-60 & $2.32 \mathrm{E}+01$ & $\mathrm{~m}$ & Cu-60 & $2.37 \mathrm{E}+01$ & $\mathrm{~m}$ & & & & ICRP-38 & $2.32 \mathrm{E}+01$ & $\mathrm{~m}$ \\
\hline Cu-61 & Cu-61 & $3.33 \mathrm{E}+00$ & $\mathrm{~h}$ & Cu-61 & $3.41 \mathrm{E}+00$ & $\mathrm{~h}$ & Cu-61 & $3.41 \mathrm{E}+00$ & $\mathrm{~h}$ & Cu-61 & $3.33 \mathrm{E}+00$ & $\mathrm{~h}$ & & & & ICRP-38 & $3.41 \mathrm{E}+00$ & $\mathrm{~h}$ \\
\hline $\mathrm{Cu}-62$ & Cu-62 & $9.67 \mathrm{E}+00$ & $\mathrm{~m}$ & Cu-62 & $9.74 \mathrm{E}+00$ & $m$ & Cu-62 & $9.74 \mathrm{E}+00$ & $m$ & Cu-62 & $9.67 \mathrm{E}+00$ & $\mathrm{~m}$ & Cu-62 & $9.74 \mathrm{E}+00$ & $\mathrm{~m}$ & ICRP-38 & $9.74 \mathrm{E}+00$ & $m$ \\
\hline Cu-64 & Cu-64 & $1.27 \mathrm{E}+01$ & $\mathrm{~h}$ & Cu-64 & $1.27 \mathrm{E}+01$ & $\mathrm{~h}$ & Cu-64 & $1.27 \mathrm{E}+01$ & $\mathrm{~h}$ & Cu-64 & $1.27 \mathrm{E}+01$ & $\mathrm{~h}$ & & & & ICRP-38 & $1.27 \mathrm{E}+01$ & $\mathrm{~h}$ \\
\hline Cu-66 & Cu-66 & $5.12 \mathrm{E}+00$ & $\mathrm{~m}$ & Cu-66 & $5.10 \mathrm{E}+00$ & $\mathrm{~m}$ & Cu-66 & $5.10 \mathrm{E}+00$ & $\mathrm{~m}$ & Cu-66 & $5.12 \mathrm{E}+00$ & $\mathrm{~m}$ & Cu-66 & $5.09 \mathrm{E}+00$ & $\mathrm{~m}$ & ICRP-38 & $5.10 \mathrm{E}+00$ & $\mathrm{~m}$ \\
\hline Cu-67 & Cu-67 & $6.18 \mathrm{E}+01$ & $\mathrm{~h}$ & Cu-67 & $6.19 \mathrm{E}+01$ & $\mathrm{~h}$ & Cu-67 & $6.19 \mathrm{E}+01$ & $\mathrm{~h}$ & Cu-67 & $6.18 \mathrm{E}+01$ & $\mathrm{~h}$ & & & & ICRP-38 & $6.19 \mathrm{E}+01$ & $\mathrm{~h}$ \\
\hline Cu-69 & Cu-69 & $2.85 \mathrm{E}+00$ & $\mathrm{~m}$ & & & & & & & Cu-69 & $2.85 \mathrm{E}+00$ & $m$ & & & & ICRP-107 & $2.85 \mathrm{E}+00$ & $\mathrm{~m}$ \\
\hline Dy-148 & Dy-148 & $3.30 \mathrm{E}+00$ & $\mathrm{~m}$ & & & & & & & Dy-148 & $3.30 \mathrm{E}+00$ & $m$ & & & & ICRP-107 & $3.30 \mathrm{E}+00$ & $\mathrm{~m}$ \\
\hline Dy-149 & Dy-149 & $4.20 \mathrm{E}+00$ & $m$ & & & & & & & Dy-149 & $4.20 \mathrm{E}+00$ & $m$ & Dy-149 & $4.20 \mathrm{E}+00$ & $m$ & ICRP-107 & $4.20 \mathrm{E}+00$ & $\mathrm{~m}$ \\
\hline Dy-150 & Dy-150 & $7.17 \mathrm{E}+00$ & $\mathrm{~m}$ & & & & & & & Dy-150 & $7.17 \mathrm{E}+00$ & $m$ & Dy-150 & $7.17 \mathrm{E}+00$ & $m$ & ICRP-107 & $7.17 \mathrm{E}+00$ & $m$ \\
\hline Dy-151 & Dy-151 & $1.79 \mathrm{E}+01$ & $\mathrm{~m}$ & & & & & & & Dy-151 & $1.79 \mathrm{E}+01$ & $\mathrm{~m}$ & Dy-151 & $1.79 \mathrm{E}+01$ & $m$ & ICRP-107 & $1.79 \mathrm{E}+01$ & $\mathrm{~m}$ \\
\hline Dy-152 & Dy-152 & $2.38 \mathrm{E}+00$ & $\mathrm{~h}$ & & & & & & & Dy-152 & $2.38 \mathrm{E}+00$ & $\mathrm{~h}$ & Dy-152 & $2.38 \mathrm{E}+00$ & $\mathrm{~h}$ & ICRP-107 & $2.38 \mathrm{E}+00$ & $\mathrm{~h}$ \\
\hline Dy-153 & Dy-153 & $6.40 \mathrm{E}+00$ & $\mathrm{~h}$ & & & & & & & Dy-153 & $6.40 \mathrm{E}+00$ & $\mathrm{~h}$ & Dy-153 & $6.40 \mathrm{E}+00$ & $\mathrm{~h}$ & ICRP-107 & $6.40 \mathrm{E}+00$ & $\mathrm{~h}$ \\
\hline Dy-154 & Dy-154 & $3.00 \mathrm{E}+06$ & $y$ & & & & & & & Dy-154 & $3.00 \mathrm{E}+06$ & $y$ & Dy-154 & $3.00 \mathrm{E}+06$ & $y$ & ICRP-107 & $3.00 E+06$ & $y$ \\
\hline Dy-155 & Dy-155 & $9.90 \mathrm{E}+00$ & $\mathrm{~h}$ & Dy-155 & $1.00 \mathrm{E}+01$ & $\mathrm{~h}$ & Dy-155 & $1.00 \mathrm{E}+01$ & $\mathrm{~h}$ & Dy-155 & $9.90 \mathrm{E}+00$ & $\mathrm{~h}$ & & & & ICRP-38 & $1.00 \mathrm{E}+01$ & $\mathrm{~h}$ \\
\hline Dy-157 & Dy-157 & $8.14 \mathrm{E}+00$ & $\mathrm{~h}$ & Dy-157 & $8.10 \mathrm{E}+00$ & $\mathrm{~h}$ & Dy-157 & $8.10 \mathrm{E}+00$ & $\mathrm{~h}$ & Dy-157 & $8.14 \mathrm{E}+00$ & $\mathrm{~h}$ & & & & ICRP-38 & $8.10 \mathrm{E}+00$ & $\mathrm{~h}$ \\
\hline Dy-159 & Dy-159 & $1.44 \mathrm{E}+02$ & $d$ & Dy-159 & $1.44 \mathrm{E}+02$ & $d$ & Dy-159 & $1.44 \mathrm{E}+02$ & $d$ & Dy-159 & $1.44 \mathrm{E}+02$ & $d$ & & & & ICRP-38 & $1.44 \mathrm{E}+02$ & $d$ \\
\hline Dy-165 & Dy-165 & $2.33 \mathrm{E}+00$ & $\mathrm{~h}$ & Dy-165 & $2.33 \mathrm{E}+00$ & $\mathrm{~h}$ & Dy-165 & $2.33 \mathrm{E}+00$ & $\mathrm{~h}$ & Dy-165 & $2.33 \mathrm{E}+00$ & $\mathrm{~h}$ & & & & ICRP-38 & $2.33 \mathrm{E}+00$ & $\mathrm{~h}$ \\
\hline Dy-165m & Dy-165m & $1.26 \mathrm{E}+00$ & $m$ & & & & & & & Dy-165m & $1.26 \mathrm{E}+00$ & $m$ & Dy-165m & $1.26 \mathrm{E}+00$ & $m$ & ICRP-107 & $1.26 \mathrm{E}+00$ & $\mathrm{~m}$ \\
\hline Dy-166 & Dy-166 & $8.16 \mathrm{E}+01$ & $\mathrm{~h}$ & Dy-166 & $8.16 \mathrm{E}+01$ & $\mathrm{~h}$ & Dy-166 & $8.16 \mathrm{E}+01$ & $\mathrm{~h}$ & Dy-166 & $8.16 \mathrm{E}+01$ & $\mathrm{~h}$ & & & & ICRP-38 & $8.16 \mathrm{E}+01$ & $\mathrm{~h}$ \\
\hline Dy-167 & Dy-167 & $6.20 \mathrm{E}+00$ & $\mathrm{~m}$ & & & & & & & Dy-167 & $6.20 \mathrm{E}+00$ & $m$ & Dy-167 & $6.20 \mathrm{E}+00$ & $\mathrm{~m}$ & ICRP-107 & $6.20 \mathrm{E}+00$ & $m$ \\
\hline Dy-168 & Dy-168 & $8.70 \mathrm{E}+00$ & $\mathrm{~m}$ & & & & & & & Dy-168 & $8.70 \mathrm{E}+00$ & $\mathrm{~m}$ & Dy-168 & $8.70 \mathrm{E}+00$ & $m$ & ICRP-107 & $8.70 \mathrm{E}+00$ & $\mathrm{~m}$ \\
\hline Er-154 & Er-154 & $3.73 \mathrm{E}+00$ & $\mathrm{~m}$ & & & & & & & Er-154 & $3.73 \mathrm{E}+00$ & $\mathrm{~m}$ & & & & ICRP-107 & $3.73 \mathrm{E}+00$ & $\mathrm{~m}$ \\
\hline Er-155 & & & & & & & & & & & & & Er-155 & $5.30 \mathrm{E}+00$ & $\mathrm{~m}$ & JAERI & $5.30 \mathrm{E}+00$ & $\mathrm{~m}$ \\
\hline Er-156 & Er-156 & $1.95 \mathrm{E}+01$ & $\mathrm{~m}$ & & & & & & & Er-156 & $1.95 \mathrm{E}+01$ & $\mathrm{~m}$ & Er-156 & $1.95 \mathrm{E}+01$ & $\mathrm{~m}$ & ICRP-107 & $1.95 \mathrm{E}+01$ & $\mathrm{~m}$ \\
\hline Er-159 & Er-159 & $3.60 \mathrm{E}+01$ & $\mathrm{~m}$ & & & & & & & Er-159 & $3.60 \mathrm{E}+01$ & $m$ & Er-159 & $3.60 \mathrm{E}+01$ & $m$ & ICRP-107 & $3.60 \mathrm{E}+01$ & $\mathrm{~m}$ \\
\hline Er-161 & Er-161 & $3.21 \mathrm{E}+00$ & $\mathrm{~h}$ & Er-161 & $3.24 \mathrm{E}+00$ & $\mathrm{~h}$ & Er-161 & $3.24 \mathrm{E}+00$ & $\mathrm{~h}$ & Er-161 & $3.21 \mathrm{E}+00$ & $\mathrm{~h}$ & & & & ICRP-38 & $3.24 \mathrm{E}+00$ & $\mathrm{~h}$ \\
\hline Er-163 & Er-163 & $7.50 \mathrm{E}+01$ & $\mathrm{~m}$ & & & & & & & Er-163 & $7.50 \mathrm{E}+01$ & $\mathrm{~m}$ & Er-163 & $7.50 \mathrm{E}+01$ & $\mathrm{~m}$ & ICRP-107 & $7.50 \mathrm{E}+01$ & $\mathrm{~m}$ \\
\hline Er-165 & Er-165 & $1.04 \mathrm{E}+01$ & $\mathrm{~h}$ & Er-165 & $1.04 \mathrm{E}+01$ & $\mathrm{~h}$ & Er-165 & $1.04 \mathrm{E}+01$ & $\mathrm{~h}$ & Er-165 & $1.04 \mathrm{E}+01$ & $\mathrm{~h}$ & & & & ICRP-38 & $1.04 \mathrm{E}+01$ & $\mathrm{~h}$ \\
\hline Er-167m & Er-167m & $2.27 E+00$ & $\mathrm{~s}$ & & & & Er-167m & $2.28 \mathrm{E}+00$ & $s$ & Er-167m & $2.27 \mathrm{E}+00$ & $s$ & & & & ICRP-38 & $2.28 \mathrm{E}+00$ & $\mathrm{~s}$ \\
\hline Er-169 & Er-169 & $9.40 \mathrm{E}+00$ & $d$ & Er-169 & $9.30 \mathrm{E}+00$ & $\mathrm{~d}$ & Er-169 & $9.30 \mathrm{E}+00$ & $d$ & Er-169 & $9.40 \mathrm{E}+00$ & $d$ & & & & ICRP-38 & $9.30 \mathrm{E}+00$ & $d$ \\
\hline Er-171 & Er-171 & $7.52 \mathrm{E}+00$ & $\mathrm{~h}$ & Er-171 & $7.52 \mathrm{E}+00$ & $\mathrm{~h}$ & Er-171 & $7.52 \mathrm{E}+00$ & $\mathrm{~h}$ & Er-171 & $7.52 \mathrm{E}+00$ & $\mathrm{~h}$ & & & & ICRP-38 & $7.52 \mathrm{E}+00$ & $\mathrm{~h}$ \\
\hline Er-172 & Er-172 & $4.93 \mathrm{E}+01$ & $\mathrm{~h}$ & Er-172 & $4.93 \mathrm{E}+01$ & $\mathrm{~h}$ & Er-172 & $4.93 \mathrm{E}+01$ & $\mathrm{~h}$ & Er-172 & $4.93 \mathrm{E}+01$ & $\mathrm{~h}$ & & & & ICRP-38 & $4.93 \mathrm{E}+01$ & $\mathrm{~h}$ \\
\hline Er-173 & Er-173 & $1.43 \mathrm{E}+00$ & $\mathrm{~m}$ & & & & & & & Er-173 & $1.43 \mathrm{E}+00$ & $\mathrm{~m}$ & & & & ICRP-107 & $1.43 \mathrm{E}+00$ & $\mathrm{~m}$ \\
\hline Es-249 & Es-249 & $1.02 \mathrm{E}+02$ & $m$ & & & & & & & Es-249 & $1.02 \mathrm{E}+02$ & $m$ & Es-249 & $1.02 \mathrm{E}+02$ & $\mathrm{~m}$ & ICRP-107 & $1.02 \mathrm{E}+02$ & $\mathrm{~m}$ \\
\hline
\end{tabular}

D-11 


\begin{tabular}{|c|c|c|c|c|c|c|c|c|c|c|c|c|c|c|c|c|c|c|}
\hline \multicolumn{19}{|c|}{ Table D.1 Selection of Radionuclides For Calculating HC-2 and HC-3 TQs (continued) } \\
\hline \multirow{3}{*}{$\begin{array}{l}\text { MASTER } \\
\text { LIST } \\
\text { Es-250 }\end{array}$} & \multicolumn{3}{|c|}{ DOE-STD-1196 } & \multicolumn{3}{|c|}{ FGR-12 } & \multicolumn{3}{|c|}{ ICRP-38 } & \multicolumn{3}{|c|}{ ICRP-107 } & \multicolumn{3}{|c|}{ JAERI } & \multirow{3}{*}{$\begin{array}{c}\begin{array}{c}\text { Selected } \\
\text { Half-Life } \\
\text { Reference }\end{array} \\
\text { ICRP-38 }\end{array}$} & \multirow{2}{*}{\multicolumn{2}{|c|}{$\begin{array}{c}\text { Selected } \\
\text { Half-Life } \\
\text { (original units) }\end{array}$}} \\
\hline & \multirow{2}{*}{$\begin{array}{l}\text { Nuclide } \\
\text { Es-250 }\end{array}$} & \multicolumn{2}{|c|}{$\begin{array}{c}\text { Half-Life } \\
\text { (original units) }\end{array}$} & \multirow{2}{*}{$\begin{array}{l}\text { Nuclide } \\
\text { Es-250 }\end{array}$} & \multicolumn{2}{|c|}{$\begin{array}{c}\text { Half-Life } \\
\text { (original units) }\end{array}$} & \multirow{2}{*}{$\begin{array}{l}\text { Nuclide } \\
\text { Es-250 }\end{array}$} & \multicolumn{2}{|c|}{$\begin{array}{c}\text { Half-Life } \\
\text { (original units) }\end{array}$} & \multirow{2}{*}{$\begin{array}{l}\text { Nuclide } \\
\text { Es-250 }\end{array}$} & \multicolumn{2}{|c|}{$\begin{array}{c}\text { Half-Life } \\
\text { (original units) }\end{array}$} & \multirow[t]{2}{*}{ Nuclide } & $\begin{array}{r}\text { Half-Lif } \\
\text { (original u }\end{array}$ & its) & & & \\
\hline & & $8.60 E+00$ & $\mathrm{~h}$ & & $2.10 \mathrm{E}+00$ & $\mathrm{~h}$ & & $2.10 \mathrm{E}+00$ & $\mathrm{~h}$ & & $8.60 \mathrm{E}+00$ & $\mathrm{~h}$ & & & & & $2.10 E+00$ & $\mathrm{~h}$ \\
\hline Es-250m & Es-250m & $2.22 \mathrm{E}+00$ & $\mathrm{~h}$ & & & & & & & Es-250m & $2.22 \mathrm{E}+00$ & $\mathrm{~h}$ & & & & ICRP-107 & $2.22 \mathrm{E}+00$ & $\mathrm{~h}$ \\
\hline Es-251 & Es-251 & $3.30 \mathrm{E}+01$ & $\mathrm{~h}$ & Es-251 & $3.30 \mathrm{E}+01$ & $\mathrm{~h}$ & Es-251 & $3.30 \mathrm{E}+01$ & $\mathrm{~h}$ & Es-251 & $3.30 \mathrm{E}+01$ & $\mathrm{~h}$ & & & & ICRP-38 & $3.30 \mathrm{E}+01$ & $\mathrm{~h}$ \\
\hline Es-253 & Es-253 & $2.05 \mathrm{E}+01$ & $d$ & Es-253 & $2.05 \mathrm{E}+01$ & $d$ & Es-253 & $2.05 \mathrm{E}+01$ & $d$ & Es-253 & $2.05 \mathrm{E}+01$ & $d$ & & & & ICRP-38 & $2.05 \mathrm{E}+01$ & $d$ \\
\hline Es-254 & Es-254 & $2.76 \mathrm{E}+02$ & $d$ & Es-254 & $2.76 \mathrm{E}+02$ & $d$ & Es-254 & $2.76 \mathrm{E}+02$ & $d$ & Es-254 & $2.76 \mathrm{E}+02$ & $d$ & & & & ICRP-38 & $2.76 \mathrm{E}+02$ & $d$ \\
\hline Es-254m & Es-254m & $3.93 \mathrm{E}+01$ & $\mathrm{~h}$ & Es-254m & $3.93 \mathrm{E}+01$ & $\mathrm{~h}$ & Es-254m & $3.93 \mathrm{E}+01$ & $\mathrm{~h}$ & Es-254m & $3.93 \mathrm{E}+01$ & $\mathrm{~h}$ & & & & ICRP-38 & $3.93 \mathrm{E}+01$ & $\mathrm{~h}$ \\
\hline Es-255 & Es-255 & $3.98 \mathrm{E}+01$ & $\mathrm{~d}$ & & & & & & & Es-255 & $3.98 \mathrm{E}+01$ & $d$ & Es-255 & $3.98 \mathrm{E}+01$ & $d$ & ICRP-107 & $3.98 \mathrm{E}+01$ & $d$ \\
\hline Es-256 & Es-256 & $2.54 \mathrm{E}+01$ & $\mathrm{~m}$ & & & & & & & Es-256 & $2.54 \mathrm{E}+01$ & $\mathrm{~m}$ & Es-256 & $2.54 \mathrm{E}+01$ & $m$ & ICRP-107 & $2.54 \mathrm{E}+01$ & $m$ \\
\hline Eu-142 & Eu-142 & $2.34 \mathrm{E}+00$ & $\mathrm{~s}$ & & & & & & & Eu-142 & $2.34 \mathrm{E}+00$ & $\mathrm{~s}$ & & & & ICRP-107 & $2.34 \mathrm{E}+00$ & $\mathrm{~s}$ \\
\hline Eu-142m & Eu-142m & $1.22 \mathrm{E}+00$ & $\mathrm{~m}$ & & & & & & & Eu-142m & $1.22 \mathrm{E}+00$ & $\mathrm{~m}$ & & & & ICRP-107 & $1.22 \mathrm{E}+00$ & $\mathrm{~m}$ \\
\hline Eu-143 & Eu-143 & $2.59 \mathrm{E}+00$ & $\mathrm{~m}$ & & & & & & & Eu-143 & $2.59 \mathrm{E}+00$ & $m$ & Eu-143 & $2.59 \mathrm{E}+00$ & $\mathrm{~m}$ & ICRP-107 & $2.59 \mathrm{E}+00$ & $\mathrm{~m}$ \\
\hline Eu-144 & Eu-144 & $1.02 \mathrm{E}+01$ & $\mathrm{~s}$ & & & & & & & Eu-144 & $1.02 \mathrm{E}+01$ & $\mathrm{~s}$ & & & & ICRP-107 & $1.02 \mathrm{E}+01$ & $s$ \\
\hline Eu-145 & Eu-145 & $5.93 \mathrm{E}+00$ & $d$ & Eu-145 & $5.94 \mathrm{E}+00$ & $d$ & Eu-145 & $5.94 \mathrm{E}+00$ & $d$ & Eu-145 & $5.93 \mathrm{E}+00$ & $d$ & & & & ICRP-38 & $5.94 \mathrm{E}+00$ & $\mathrm{~d}$ \\
\hline Eu-146 & Eu-146 & $4.61 E+00$ & $d$ & Eu-146 & $4.61 \mathrm{E}+00$ & $\mathrm{~d}$ & Eu-146 & $4.61 \mathrm{E}+00$ & $d$ & Eu-146 & $4.61 \mathrm{E}+00$ & $d$ & & & & ICRP-38 & $4.61 \mathrm{E}+00$ & $d$ \\
\hline Eu-147 & Eu-147 & $2.41 \mathrm{E}+01$ & $d$ & Eu-147 & $2.40 \mathrm{E}+01$ & $\mathrm{~d}$ & Eu-147 & $2.40 \mathrm{E}+01$ & $d$ & Eu-147 & $2.41 \mathrm{E}+01$ & $d$ & & & & ICRP-38 & $2.40 \mathrm{E}+01$ & $d$ \\
\hline Eu-148 & Eu-148 & $5.45 \mathrm{E}+01$ & $d$ & Eu-148 & $5.45 \mathrm{E}+01$ & $d$ & Eu-148 & $5.45 \mathrm{E}+01$ & $d$ & Eu-148 & $5.45 \mathrm{E}+01$ & $d$ & & & & ICRP-38 & $5.45 \mathrm{E}+01$ & $d$ \\
\hline Eu-149 & Eu-149 & $9.31 \mathrm{E}+01$ & $d$ & Eu-149 & $9.31 \mathrm{E}+01$ & $d$ & Eu-149 & $9.31 \mathrm{E}+01$ & $d$ & Eu-149 & $9.31 \mathrm{E}+01$ & $d$ & & & & ICRP-38 & $9.31 \mathrm{E}+01$ & $d$ \\
\hline Eu-150l & Eu-150 & $3.69 \mathrm{E}+01$ & $y$ & Eu-150b & $3.42 \mathrm{E}+01$ & $y$ & Eu-150b & $3.42 \mathrm{E}+01$ & $y$ & Eu-150 & $3.69 \mathrm{E}+01$ & $y$ & & & & ICRP-38 & $3.42 \mathrm{E}+01$ & $y$ \\
\hline Eu-150s & Eu-150m & $1.28 \mathrm{E}+01$ & $\mathrm{~h}$ & Eu-150a & $1.26 \mathrm{E}+01$ & $\mathrm{~h}$ & Eu-150a & $1.26 \mathrm{E}+01$ & $\mathrm{~h}$ & Eu-150m & $1.28 \mathrm{E}+01$ & $\mathrm{~h}$ & & & & ICRP-38 & $1.26 \mathrm{E}+01$ & $\mathrm{~h}$ \\
\hline Eu-152 & Eu-152 & $1.35 \mathrm{E}+01$ & $y$ & Eu-152 & $1.33 \mathrm{E}+01$ & $y$ & Eu-152 & $1.33 \mathrm{E}+01$ & $y$ & Eu-152 & $1.35 \mathrm{E}+01$ & $y$ & & & & ICRP-38 & $1.33 \mathrm{E}+01$ & $y$ \\
\hline Eu-152ml & Eu-152m & $9.31 \mathrm{E}+00$ & $\mathrm{~h}$ & Eu-152m & $9.32 \mathrm{E}+00$ & $\mathrm{~h}$ & Eu-152m & $9.32 \mathrm{E}+00$ & $\mathrm{~h}$ & Eu-152m & $9.31 \mathrm{E}+00$ & $\mathrm{~h}$ & & & & ICRP-38 & $9.32 \mathrm{E}+00$ & $\mathrm{~h}$ \\
\hline Eu-152ms & Eu-152n & $9.60 \mathrm{E}+01$ & $\mathrm{~m}$ & & & & & & & Eu-152n & $9.60 \mathrm{E}+01$ & $\mathrm{~m}$ & Eu-152n & $9.60 \mathrm{E}+01$ & $\mathrm{~m}$ & ICRP-107 & $9.60 \mathrm{E}+01$ & $\mathrm{~m}$ \\
\hline Eu-154 & Eu-154 & $8.59 \mathrm{E}+00$ & $y$ & Eu-154 & $8.80 \mathrm{E}+00$ & $y$ & Eu-154 & $8.80 \mathrm{E}+00$ & $y$ & Eu-154 & $8.59 \mathrm{E}+00$ & $y$ & & & & ICRP-38 & $8.80 \mathrm{E}+00$ & $y$ \\
\hline Eu-154m & Eu-154m & $4.60 \mathrm{E}+01$ & $\mathrm{~m}$ & & & & & & & Eu-154m & $4.60 \mathrm{E}+01$ & $m$ & Eu-154m & $4.60 \mathrm{E}+01$ & $m$ & ICRP-107 & $4.60 \mathrm{E}+01$ & $\mathrm{~m}$ \\
\hline Eu-155 & Eu-155 & $4.76 \mathrm{E}+00$ & $y$ & Eu-155 & $4.96 \mathrm{E}+00$ & $y$ & Eu-155 & $4.96 \mathrm{E}+00$ & $y$ & Eu-155 & $4.76 \mathrm{E}+00$ & $y$ & & & & ICRP-38 & $4.96 \mathrm{E}+00$ & $y$ \\
\hline Eu-156 & Eu-156 & $1.52 \mathrm{E}+01$ & $d$ & Eu-156 & $1.52 \mathrm{E}+01$ & $d$ & Eu-156 & $1.52 \mathrm{E}+01$ & $d$ & Eu-156 & $1.52 \mathrm{E}+01$ & $d$ & & & & ICRP-38 & $1.52 \mathrm{E}+01$ & $d$ \\
\hline Eu-157 & Eu-157 & $1.52 \mathrm{E}+01$ & $\mathrm{~h}$ & Eu-157 & $1.52 \mathrm{E}+01$ & $\mathrm{~h}$ & Eu-157 & $1.52 \mathrm{E}+01$ & $\mathrm{~h}$ & Eu-157 & $1.52 \mathrm{E}+01$ & $\mathrm{~h}$ & & & & ICRP-38 & $1.52 \mathrm{E}+01$ & $\mathrm{~h}$ \\
\hline Eu-158 & Eu-158 & $4.59 \mathrm{E}+01$ & $\mathrm{~m}$ & Eu-158 & $4.59 \mathrm{E}+01$ & $\mathrm{~m}$ & Eu-158 & $4.59 \mathrm{E}+01$ & $\mathrm{~m}$ & Eu-158 & $4.59 \mathrm{E}+01$ & $\mathrm{~m}$ & & & & ICRP-38 & $4.59 \mathrm{E}+01$ & $\mathrm{~m}$ \\
\hline Eu-159 & Eu-159 & $1.81 \mathrm{E}+01$ & $\mathrm{~m}$ & & & & & & & Eu-159 & $1.81 \mathrm{E}+01$ & $\mathrm{~m}$ & Eu-159 & $1.81 \mathrm{E}+01$ & $\mathrm{~m}$ & ICRP-107 & $1.81 \mathrm{E}+01$ & $\mathrm{~m}$ \\
\hline F-17 & F-17 & $6.45 \mathrm{E}+01$ & $\mathrm{~s}$ & & & & & & & $\mathrm{~F}-17$ & $6.45 \mathrm{E}+01$ & $\mathrm{~s}$ & & & & ICRP-107 & $6.45 \mathrm{E}+01$ & $\mathrm{~s}$ \\
\hline F-18 & $F-18$ & $1.10 \mathrm{E}+02$ & $\mathrm{~m}$ & F-18 & $1.10 \mathrm{E}+02$ & $\mathrm{~m}$ & F-18 & $1.10 \mathrm{E}+02$ & $\mathrm{~m}$ & $F-18$ & $1.10 \mathrm{E}+02$ & $m$ & & & & ICRP-38 & $1.10 \mathrm{E}+02$ & $\mathrm{~m}$ \\
\hline Fe-52 & Fe-52 & $8.28 \mathrm{E}+00$ & $\mathrm{~h}$ & Fe-52 & $8.28 \mathrm{E}+00$ & $\mathrm{~h}$ & Fe-52 & $8.28 \mathrm{E}+00$ & $\mathrm{~h}$ & Fe-52 & $8.28 \mathrm{E}+00$ & $\mathrm{~h}$ & & & & ICRP-38 & $8.28 \mathrm{E}+00$ & $\mathrm{~h}$ \\
\hline Fe-53 & Fe-53 & $8.51 \mathrm{E}+00$ & $\mathrm{~m}$ & & & & & & & Fe-53 & $8.51 \mathrm{E}+00$ & $\mathrm{~m}$ & Fe-53 & $8.51 \mathrm{E}+00$ & $\mathrm{~m}$ & ICRP-107 & $8.51 \mathrm{E}+00$ & $\mathrm{~m}$ \\
\hline Fe-53m & Fe- $53 \mathrm{~m}$ & $2.53 \mathrm{E}+00$ & $\mathrm{~m}$ & & & & & & & Fe-53m & $2.53 \mathrm{E}+00$ & $\mathrm{~m}$ & & & & ICRP-107 & $2.53 \mathrm{E}+00$ & $\mathrm{~m}$ \\
\hline Fe-55 & Fe-55 & $2.74 \mathrm{E}+00$ & $y$ & Fe-55 & $2.70 \mathrm{E}+00$ & $y$ & Fe-55 & $2.70 \mathrm{E}+00$ & $y$ & Fe-55 & $2.74 \mathrm{E}+00$ & $\mathrm{y}$ & & & & ICRP-38 & $2.70 \mathrm{E}+00$ & $y$ \\
\hline Fe-59 & Fe-59 & $4.45 \mathrm{E}+01$ & $d$ & Fe-59 & $4.45 \mathrm{E}+01$ & $d$ & Fe-59 & $4.45 \mathrm{E}+01$ & $d$ & Fe-59 & $4.45 \mathrm{E}+01$ & $d$ & & & & ICRP-38 & $4.45 \mathrm{E}+01$ & $d$ \\
\hline Fe-60 & Fe-60 & $1.50 \mathrm{E}+06$ & $y$ & $\mathrm{Fe}-60$ & $1.00 \mathrm{E}+05$ & $y$ & Fe-60 & $1.00 \mathrm{E}+05$ & $y$ & Fe-60 & $1.50 \mathrm{E}+06$ & $y$ & & & & ICRP-38 & $1.00 \mathrm{E}+05$ & $y$ \\
\hline Fe-61 & Fe-61 & $5.98 \mathrm{E}+00$ & $\mathrm{~m}$ & & & & & & & Fe-61 & $5.98 \mathrm{E}+00$ & $\mathrm{~m}$ & & & & ICRP-107 & $5.98 \mathrm{E}+00$ & $\mathrm{~m}$ \\
\hline Fe-62 & Fe-62 & $6.80 \mathrm{E}+01$ & $\mathrm{~s}$ & & & & & & & Fe-62 & $6.80 \mathrm{E}+01$ & $\mathrm{~s}$ & & & & ICRP-107 & $6.80 \mathrm{E}+01$ & $\mathrm{~s}$ \\
\hline Fm-251 & Fm-251 & $5.30 \mathrm{E}+00$ & $\mathrm{~h}$ & & & & & & & Fm-251 & $5.30 \mathrm{E}+00$ & $\mathrm{~h}$ & Fm-251 & $5.30 \mathrm{E}+00$ & $\mathrm{~h}$ & ICRP-107 & $5.30 \mathrm{E}+00$ & $\mathrm{~h}$ \\
\hline
\end{tabular}

D-12 


\begin{tabular}{|c|c|c|c|c|c|c|c|c|c|c|c|c|c|c|c|c|c|c|}
\hline \multicolumn{19}{|c|}{ Table D.1 Selection of Radionuclides For Calculating HC-2 and HC-3 TQs (continued) } \\
\hline \multirow{3}{*}{$\begin{array}{l}\text { MASTER } \\
\text { LIST } \\
\text { Fm-252 }\end{array}$} & \multicolumn{3}{|c|}{ DOE-STD-1196 } & \multicolumn{3}{|c|}{ FGR-12 } & \multicolumn{3}{|c|}{ ICRP-38 } & \multicolumn{3}{|c|}{ ICRP-107 } & \multicolumn{3}{|c|}{ JAERI } & \multirow{3}{*}{$\begin{array}{c}\begin{array}{c}\text { Selected } \\
\text { Half-Life } \\
\text { Reference }\end{array} \\
\text { ICRP-38 }\end{array}$} & \multirow{2}{*}{\multicolumn{2}{|c|}{$\begin{array}{c}\text { Selected } \\
\text { Half-Life } \\
\text { (original units) }\end{array}$}} \\
\hline & \multirow{2}{*}{$\begin{array}{l}\text { Nuclide } \\
\text { Fm-252 }\end{array}$} & \multicolumn{2}{|c|}{$\begin{array}{c}\text { Half-Life } \\
\text { (original units) }\end{array}$} & \multirow{2}{*}{$\begin{array}{l}\text { Nuclide } \\
\text { Fm-252 }\end{array}$} & \multicolumn{2}{|c|}{$\begin{array}{c}\text { Half-Life } \\
\text { (original units) }\end{array}$} & \multirow{2}{*}{$\begin{array}{l}\text { Nuclide } \\
\text { Fm-252 }\end{array}$} & \multicolumn{2}{|c|}{$\begin{array}{c}\text { Half-Life } \\
\text { (original units) }\end{array}$} & \multirow{2}{*}{$\begin{array}{l}\text { Nuclide } \\
\text { Fm-252 }\end{array}$} & \multicolumn{2}{|c|}{$\begin{array}{c}\text { Half-Life } \\
\text { (original units) }\end{array}$} & \multirow[t]{2}{*}{ Nuclide } & $\begin{array}{r}\text { Half-Lif } \\
\text { (original u }\end{array}$ & its) & & & \\
\hline & & $2.54 \mathrm{E}+01$ & $\mathrm{~h}$ & & $2.27 \mathrm{E}+01$ & $\mathrm{~h}$ & & $2.27 \mathrm{E}+01$ & $\mathrm{~h}$ & & $2.54 \mathrm{E}+01$ & $\mathrm{~h}$ & & & & & $2.27 \mathrm{E}+01$ & $\mathrm{~h}$ \\
\hline Fm-253 & Fm-253 & $3.00 \mathrm{E}+00$ & $d$ & Fm-253 & $3.00 \mathrm{E}+00$ & $d$ & Fm-253 & $3.00 \mathrm{E}+00$ & d & $\mathrm{Fm}-253$ & $3.00 \mathrm{E}+00$ & d & & & & ICRP-38 & $3.00 \mathrm{E}+00$ & d \\
\hline Fm-254 & Fm-254 & $3.24 \mathrm{E}+00$ & $\mathrm{~h}$ & Fm-254 & $3.24 \mathrm{E}+00$ & $\mathrm{~h}$ & Fm-254 & $3.24 \mathrm{E}+00$ & $\mathrm{~h}$ & Fm-254 & $3.24 \mathrm{E}+00$ & $\mathrm{~h}$ & & & & ICRP-38 & $3.24 \mathrm{E}+00$ & $\mathrm{~h}$ \\
\hline Fm-255 & Fm-255 & $2.01 \mathrm{E}+01$ & $\mathrm{~h}$ & Fm-255 & $2.01 \mathrm{E}+01$ & $\mathrm{~h}$ & Fm-255 & $2.01 \mathrm{E}+01$ & $\mathrm{~h}$ & Fm-255 & $2.01 \mathrm{E}+01$ & $\mathrm{~h}$ & & & & ICRP-38 & $2.01 \mathrm{E}+01$ & $\mathrm{~h}$ \\
\hline Fm-256 & Fm-256 & $1.58 \mathrm{E}+02$ & $\mathrm{~m}$ & & & & & & & Fm-256 & $1.58 \mathrm{E}+02$ & $\mathrm{~m}$ & Fm-256 & $1.58 \mathrm{E}+02$ & $\mathrm{~m}$ & ICRP-107 & $1.58 \mathrm{E}+02$ & $\mathrm{~m}$ \\
\hline Fm-257 & Fm-257 & $1.01 \mathrm{E}+02$ & $\mathrm{~d}$ & Fm-257 & $1.01 \mathrm{E}+02$ & $d$ & Fm-257 & $1.01 \mathrm{E}+02$ & $d$ & Fm-257 & $1.01 \mathrm{E}+02$ & $\mathrm{~d}$ & & & & ICRP-38 & $1.01 \mathrm{E}+02$ & d \\
\hline Fr-212 & Fr-212 & $2.00 \mathrm{E}+01$ & $m$ & & & & & & & Fr-212 & $2.00 \mathrm{E}+01$ & $\mathrm{~m}$ & Fr-212 & $2.00 \mathrm{E}+01$ & $\mathrm{~m}$ & ICRP-107 & $2.00 \mathrm{E}+01$ & $\mathrm{~m}$ \\
\hline Fr-219 & Fr-219 & $2.00 \mathrm{E}-02$ & $\mathrm{~s}$ & Fr-219 & $2.10 \mathrm{E}+01$ & $\mathrm{~ms}$ & Fr-219 & $2.10 \mathrm{E}+01$ & $\mathrm{~ms}$ & Fr-219 & $2.00 \mathrm{E}-02$ & $\mathrm{~s}$ & & & & ICRP-38 & $2.10 \mathrm{E}+01$ & $\mathrm{~ms}$ \\
\hline Fr-220 & Fr-220 & $2.74 \mathrm{E}+01$ & $\mathrm{~s}$ & Fr-220 & $2.74 \mathrm{E}+01$ & $\mathrm{~s}$ & Fr-220 & $2.74 \mathrm{E}+01$ & $\mathrm{~s}$ & Fr-220 & $2.74 \mathrm{E}+01$ & $\mathrm{~s}$ & & & & ICRP-38 & $2.74 \mathrm{E}+01$ & $\mathrm{~s}$ \\
\hline Fr-221 & Fr-221 & $4.90 \mathrm{E}+00$ & $\mathrm{~m}$ & Fr-221 & $4.80 \mathrm{E}+00$ & $\mathrm{~m}$ & Fr-221 & $4.80 \mathrm{E}+00$ & $\mathrm{~m}$ & Fr-221 & $4.90 \mathrm{E}+00$ & $\mathrm{~m}$ & Fr-221 & $4.90 \mathrm{E}+00$ & $\mathrm{~m}$ & ICRP-38 & $4.80 \mathrm{E}+00$ & $\mathrm{~m}$ \\
\hline Fr-222 & Fr-222 & $1.42 \mathrm{E}+01$ & $\mathrm{~m}$ & Fr-222 & $1.44 \mathrm{E}+01$ & $\mathrm{~m}$ & Fr-222 & $1.44 \mathrm{E}+01$ & $\mathrm{~m}$ & Fr-222 & $1.42 \mathrm{E}+01$ & $m$ & & & & ICRP-38 & $1.44 \mathrm{E}+01$ & $\mathrm{~m}$ \\
\hline Fr-223 & $\mathrm{Fr}-223$ & $2.20 \mathrm{E}+01$ & $\mathrm{~m}$ & Fr-223 & $2.18 \mathrm{E}+01$ & $m$ & Fr-223 & $2.18 \mathrm{E}+01$ & $m$ & Fr-223 & $2.20 \mathrm{E}+01$ & $m$ & & & & ICRP-38 & $2.18 \mathrm{E}+01$ & $\mathrm{~m}$ \\
\hline Fr-224 & Fr-224 & $3.33 \mathrm{E}+00$ & $\mathrm{~m}$ & & & & & & & Fr-224 & $3.33 \mathrm{E}+00$ & $m$ & & & & ICRP-107 & $3.33 \mathrm{E}+00$ & $\mathrm{~m}$ \\
\hline Fr-227 & Fr-227 & $2.47 E+00$ & $m$ & & & & & & & Fr-227 & $2.47 \mathrm{E}+00$ & $m$ & & & & ICRP-107 & $2.47 \mathrm{E}+00$ & $\mathrm{~m}$ \\
\hline Ga-64 & Ga-64 & $2.63 \mathrm{E}+00$ & $\mathrm{~m}$ & & & & & & & Ga-64 & $2.63 \mathrm{E}+00$ & $m$ & & & & ICRP-107 & $2.63 \mathrm{E}+00$ & $m$ \\
\hline Ga-65 & Ga-65 & $1.52 \mathrm{E}+01$ & $\mathrm{~m}$ & Ga-65 & $1.52 \mathrm{E}+01$ & $m$ & Ga-65 & $1.52 \mathrm{E}+01$ & $\mathrm{~m}$ & Ga-65 & $1.52 \mathrm{E}+01$ & $\mathrm{~m}$ & & & & ICRP-38 & $1.52 \mathrm{E}+01$ & $\mathrm{~m}$ \\
\hline Ga-66 & Ga-66 & $9.49 \mathrm{E}+00$ & $\mathrm{~h}$ & Ga-66 & $9.40 \mathrm{E}+00$ & $\mathrm{~h}$ & Ga-66 & $9.40 \mathrm{E}+00$ & $\mathrm{~h}$ & Ga-66 & $9.49 \mathrm{E}+00$ & $\mathrm{~h}$ & & & & ICRP-38 & $9.40 \mathrm{E}+00$ & $\mathrm{~h}$ \\
\hline Ga-67 & Ga-67 & $3.26 \mathrm{E}+00$ & $d$ & Ga-67 & $7.83 \mathrm{E}+01$ & $\mathrm{~h}$ & Ga-67 & $7.83 \mathrm{E}+01$ & $\mathrm{~h}$ & Ga-67 & $3.26 \mathrm{E}+00$ & $\mathrm{~d}$ & & & & ICRP-38 & $7.83 \mathrm{E}+01$ & $\mathrm{~h}$ \\
\hline Ga-68 & Ga-68 & $6.77 \mathrm{E}+01$ & $\mathrm{~m}$ & Ga-68 & $6.80 \mathrm{E}+01$ & $\mathrm{~m}$ & Ga-68 & $6.80 \mathrm{E}+01$ & $\mathrm{~m}$ & Ga-68 & $6.77 \mathrm{E}+01$ & $\mathrm{~m}$ & & & & ICRP-38 & $6.80 \mathrm{E}+01$ & $\mathrm{~m}$ \\
\hline Ga-70 & Ga-70 & $2.11 \mathrm{E}+01$ & $\mathrm{~m}$ & Ga-70 & $2.12 \mathrm{E}+01$ & $m$ & Ga-70 & $2.12 \mathrm{E}+01$ & $m$ & Ga-70 & $2.11 \mathrm{E}+01$ & $m$ & & & & ICRP-38 & $2.12 \mathrm{E}+01$ & $\mathrm{~m}$ \\
\hline Ga-72 & Ga-72 & $1.41 \mathrm{E}+01$ & $\mathrm{~h}$ & Ga-72 & $1.41 \mathrm{E}+01$ & $\mathrm{~h}$ & Ga-72 & $1.41 \mathrm{E}+01$ & $\mathrm{~h}$ & Ga-72 & $1.41 \mathrm{E}+01$ & $\mathrm{~h}$ & & & & ICRP-38 & $1.41 \mathrm{E}+01$ & $\mathrm{~h}$ \\
\hline Ga-73 & Ga-73 & $4.86 \mathrm{E}+00$ & $\mathrm{~h}$ & Ga-73 & $4.91 \mathrm{E}+00$ & $\mathrm{~h}$ & Ga-73 & $4.91 \mathrm{E}+00$ & $\mathrm{~h}$ & Ga-73 & $4.86 \mathrm{E}+00$ & $\mathrm{~h}$ & & & & ICRP-38 & $4.91 \mathrm{E}+00$ & $\mathrm{~h}$ \\
\hline Ga-74 & Ga-74 & $8.12 \mathrm{E}+00$ & $\mathrm{~m}$ & & & & & & & Ga-74 & $8.12 \mathrm{E}+00$ & $\mathrm{~m}$ & & & & ICRP-107 & $8.12 \mathrm{E}+00$ & $\mathrm{~m}$ \\
\hline Gd-142 & Gd-142 & $7.02 \mathrm{E}+01$ & $\mathrm{~s}$ & & & & & & & Gd-142 & $7.02 \mathrm{E}+01$ & $\mathrm{~s}$ & & & & ICRP-107 & $7.02 \mathrm{E}+01$ & $\mathrm{~s}$ \\
\hline Gd-143m & Gd-143m & $1.10 \mathrm{E}+02$ & $\mathrm{~s}$ & & & & & & & Gd-143m & $1.10 \mathrm{E}+02$ & $s$ & & & & ICRP-107 & $1.10 \mathrm{E}+02$ & $\mathrm{~s}$ \\
\hline Gd-144 & Gd-144 & $4.47 \mathrm{E}+00$ & $\mathrm{~m}$ & & & & & & & Gd-144 & $4.47 \mathrm{E}+00$ & $m$ & & & & ICRP-107 & $4.47 \mathrm{E}+00$ & $m$ \\
\hline Gd-145 & Gd-145 & $2.30 \mathrm{E}+01$ & $\mathrm{~m}$ & Gd-145 & $2.29 \mathrm{E}+01$ & $\mathrm{~m}$ & Gd-145 & $2.29 \mathrm{E}+01$ & $m$ & Gd-145 & $2.30 \mathrm{E}+01$ & $\mathrm{~m}$ & & & & ICRP-38 & $2.29 \mathrm{E}+01$ & $\mathrm{~m}$ \\
\hline Gd-145m & Gd-145m & $8.50 \mathrm{E}+01$ & $\mathrm{~s}$ & & & & & & & Gd-145m & $8.50 \mathrm{E}+01$ & $\mathrm{~s}$ & Gd-145m & $1.42 \mathrm{E}+00$ & $\mathrm{~m}$ & ICRP-107 & $8.50 \mathrm{E}+01$ & $\mathrm{~s}$ \\
\hline Gd-146 & Gd-146 & $4.83 \mathrm{E}+01$ & $d$ & Gd-146 & $4.83 \mathrm{E}+01$ & $d$ & Gd-146 & $4.83 \mathrm{E}+01$ & $d$ & Gd-146 & $4.83 \mathrm{E}+01$ & $d$ & & & & ICRP-38 & $4.83 \mathrm{E}+01$ & $d$ \\
\hline Gd-147 & Gd-147 & $3.81 E+01$ & $\mathrm{~h}$ & Gd-147 & $3.81 \mathrm{E}+01$ & $\mathrm{~h}$ & Gd-147 & $3.81 \mathrm{E}+01$ & $\mathrm{~h}$ & Gd-147 & $3.81 \mathrm{E}+01$ & $\mathrm{~h}$ & & & & ICRP-38 & $3.81 \mathrm{E}+01$ & $\mathrm{~h}$ \\
\hline Gd-148 & Gd-148 & $7.46 \mathrm{E}+01$ & $y$ & Gd-148 & $9.30 \mathrm{E}+01$ & $y$ & Gd-148 & $9.30 \mathrm{E}+01$ & $y$ & Gd-148 & $7.46 \mathrm{E}+01$ & $y$ & & & & ICRP-38 & $9.30 \mathrm{E}+01$ & $y$ \\
\hline Gd-149 & Gd-149 & $9.28 \mathrm{E}+00$ & $d$ & Gd-149 & $9.40 \mathrm{E}+00$ & $d$ & Gd-149 & $9.40 \mathrm{E}+00$ & $d$ & Gd-149 & $9.28 \mathrm{E}+00$ & $d$ & & & & ICRP-38 & $9.40 \mathrm{E}+00$ & $d$ \\
\hline Gd-150 & Gd-150 & $1.79 \mathrm{E}+06$ & $y$ & & & & & & & Gd-150 & $1.79 \mathrm{E}+06$ & $y$ & Gd-150 & $1.79 \mathrm{E}+06$ & $y$ & ICRP-107 & $1.79 \mathrm{E}+06$ & $y$ \\
\hline Gd-151 & Gd-151 & $1.24 \mathrm{E}+02$ & $d$ & Gd-151 & $1.20 \mathrm{E}+02$ & $d$ & Gd-151 & $1.20 \mathrm{E}+02$ & $d$ & Gd-151 & $1.24 \mathrm{E}+02$ & $d$ & & & & ICRP-38 & $1.20 \mathrm{E}+02$ & $d$ \\
\hline Gd-152 & Gd-152 & $1.08 \mathrm{E}+14$ & $y$ & Gd-152 & $1.08 \mathrm{E}+14$ & $y$ & Gd-152 & $1.08 \mathrm{E}+14$ & $y$ & Gd-152 & $1.08 \mathrm{E}+14$ & $y$ & & & & ICRP-38 & $1.08 \mathrm{E}+14$ & $y$ \\
\hline Gd-153 & Gd-153 & $2.40 \mathrm{E}+02$ & $d$ & Gd-153 & $2.42 \mathrm{E}+02$ & $d$ & Gd-153 & $2.42 \mathrm{E}+02$ & $d$ & Gd-153 & $2.40 \mathrm{E}+02$ & $d$ & & & & ICRP-38 & $2.42 \mathrm{E}+02$ & $d$ \\
\hline Gd-159 & Gd-159 & $1.85 \mathrm{E}+01$ & $\mathrm{~h}$ & Gd-159 & $1.86 \mathrm{E}+01$ & $\mathrm{~h}$ & Gd-159 & $1.86 \mathrm{E}+01$ & $\mathrm{~h}$ & Gd-159 & $1.85 \mathrm{E}+01$ & $\mathrm{~h}$ & & & & ICRP-38 & $1.86 \mathrm{E}+01$ & $\mathrm{~h}$ \\
\hline Gd-162 & Gd-162 & $8.40 \mathrm{E}+00$ & $\mathrm{~m}$ & & & & & & & Gd-162 & $8.40 \mathrm{E}+00$ & $\mathrm{~m}$ & & & & ICRP-107 & $8.40 \mathrm{E}+00$ & $\mathrm{~m}$ \\
\hline Ge-66 & Ge-66 & $2.26 \mathrm{E}+00$ & $\mathrm{~h}$ & Ge-66 & $2.27 \mathrm{E}+00$ & $\mathrm{~h}$ & Ge-66 & $2.27 \mathrm{E}+00$ & $\mathrm{~h}$ & Ge-66 & $2.26 \mathrm{E}+00$ & $\mathrm{~h}$ & & & & ICRP-38 & $2.27 \mathrm{E}+00$ & $\mathrm{~h}$ \\
\hline Ge-67 & Ge-67 & $1.89 \mathrm{E}+01$ & $m$ & Ge-67 & $1.87 \mathrm{E}+01$ & $m$ & Ge-67 & $1.87 \mathrm{E}+01$ & $m$ & Ge-67 & $1.89 \mathrm{E}+01$ & $m$ & & & & ICRP-38 & $1.87 \mathrm{E}+01$ & $\mathrm{~m}$ \\
\hline
\end{tabular}

D-13 


\begin{tabular}{|c|c|c|c|c|c|c|c|c|c|c|c|c|c|c|c|c|c|c|}
\hline \multicolumn{19}{|c|}{ Table D.1 Selection of Radionuclides For Calculating HC-2 and HC-3 TQs (continued) } \\
\hline \multirow{3}{*}{$\begin{array}{l}\text { MASTER } \\
\text { LIST } \\
\text { Ge-68 }\end{array}$} & \multicolumn{3}{|c|}{ DOE-STD-1196 } & \multicolumn{3}{|c|}{ FGR-12 } & \multicolumn{3}{|c|}{ ICRP-38 } & \multicolumn{3}{|c|}{ ICRP-107 } & \multicolumn{3}{|c|}{ JAERI } & \multirow{3}{*}{$\begin{array}{c}\begin{array}{c}\text { Selected } \\
\text { Half-Life } \\
\text { Reference }\end{array} \\
\text { ICRP-38 }\end{array}$} & \multirow{2}{*}{\multicolumn{2}{|c|}{$\begin{array}{c}\text { Selected } \\
\text { Half-Life } \\
\text { (original units) }\end{array}$}} \\
\hline & \multirow{2}{*}{$\begin{array}{l}\text { Nuclide } \\
\text { Ge-68 }\end{array}$} & \multicolumn{2}{|c|}{$\begin{array}{c}\text { Half-Life } \\
\text { (original units) }\end{array}$} & \multirow{2}{*}{$\begin{array}{l}\text { Nuclide } \\
\text { Ge-68 }\end{array}$} & \multicolumn{2}{|c|}{$\begin{array}{c}\text { Half-Life } \\
\text { (original units) }\end{array}$} & \multirow{2}{*}{$\begin{array}{l}\text { Nuclide } \\
\text { Ge-68 }\end{array}$} & \multicolumn{2}{|c|}{$\begin{array}{c}\text { Half-Life } \\
\text { (original units) }\end{array}$} & \multirow{2}{*}{$\begin{array}{l}\text { Nuclide } \\
\text { Ge-68 }\end{array}$} & \multicolumn{2}{|c|}{$\begin{array}{c}\text { Half-Life } \\
\text { (original units) } \\
\end{array}$} & \multirow[t]{2}{*}{ Nuclide } & $\begin{array}{r}\text { Half-Lif } \\
\text { (original u }\end{array}$ & its) & & & \\
\hline & & $2.71 E+02$ & $\mathrm{~d}$ & & $2.88 \mathrm{E}+02$ & $d$ & & $2.88 \mathrm{E}+02$ & $d$ & & $2.71 \mathrm{E}+02$ & $d$ & & & & & $2.88 \mathrm{E}+02$ & $d$ \\
\hline Ge-69 & Ge-69 & $3.91 \mathrm{E}+01$ & $\mathrm{~h}$ & Ge-69 & $3.91 \mathrm{E}+01$ & $\mathrm{~h}$ & Ge-69 & $3.91 \mathrm{E}+01$ & $\mathrm{~h}$ & Ge-69 & $3.91 \mathrm{E}+01$ & $\mathrm{~h}$ & & & & ICRP-38 & $3.91 \mathrm{E}+01$ & $\mathrm{~h}$ \\
\hline Ge-71 & Ge-71 & $1.14 \mathrm{E}+01$ & $d$ & Ge-71 & $1.18 \mathrm{E}+01$ & $d$ & Ge-71 & $1.18 \mathrm{E}+01$ & $d$ & Ge-71 & $1.14 \mathrm{E}+01$ & $d$ & & & & ICRP-38 & $1.18 \mathrm{E}+01$ & $d$ \\
\hline Ge-75 & Ge-75 & $8.28 \mathrm{E}+01$ & $\mathrm{~m}$ & Ge-75 & $8.28 \mathrm{E}+01$ & $\mathrm{~m}$ & Ge-75 & $8.28 \mathrm{E}+01$ & $\mathrm{~m}$ & Ge-75 & $8.28 \mathrm{E}+01$ & $\mathrm{~m}$ & & & & ICRP-38 & $8.28 \mathrm{E}+01$ & $\mathrm{~m}$ \\
\hline Ge-77 & Ge-77 & $1.13 \mathrm{E}+01$ & $\mathrm{~h}$ & Ge-77 & $1.13 \mathrm{E}+01$ & $\mathrm{~h}$ & Ge-77 & $1.13 \mathrm{E}+01$ & $\mathrm{~h}$ & Ge-77 & $1.13 \mathrm{E}+01$ & $\mathrm{~h}$ & & & & ICRP-38 & $1.13 \mathrm{E}+01$ & $\mathrm{~h}$ \\
\hline Ge-78 & Ge-78 & $8.80 \mathrm{E}+01$ & $\mathrm{~m}$ & Ge-78 & $8.70 \mathrm{E}+01$ & $\mathrm{~m}$ & Ge-78 & $8.70 \mathrm{E}+01$ & $\mathrm{~m}$ & Ge-78 & $8.80 \mathrm{E}+01$ & $\mathrm{~m}$ & & & & ICRP-38 & $8.70 \mathrm{E}+01$ & $\mathrm{~m}$ \\
\hline $\mathrm{H}-3$ & $\mathrm{H}-3$ & $1.23 \mathrm{E}+01$ & $\mathrm{y}$ & $\mathrm{H}-3$ & $1.24 \mathrm{E}+01$ & $y$ & $\mathrm{H}-3$ & $1.24 \mathrm{E}+01$ & $y$ & $\mathrm{H}-3$ & $1.23 \mathrm{E}+01$ & $y$ & & & & ICRP-38 & $1.24 \mathrm{E}+01$ & $y$ \\
\hline Hf-167 & Hf-167 & $2.05 \mathrm{E}+00$ & $\mathrm{~m}$ & & & & & & & Hf-167 & $2.05 E+00$ & $\mathrm{~m}$ & Hf-167 & $2.05 \mathrm{E}+00$ & $\mathrm{~m}$ & ICRP-107 & $2.05 E+00$ & $\mathrm{~m}$ \\
\hline Hf-169 & Hf-169 & $3.24 \mathrm{E}+00$ & $\mathrm{~m}$ & & & & & & & Hf-169 & $3.24 \mathrm{E}+00$ & $\mathrm{~m}$ & Hf-169 & $3.24 \mathrm{E}+00$ & $\mathrm{~m}$ & ICRP-107 & $3.24 \mathrm{E}+00$ & $\mathrm{~m}$ \\
\hline Hf-170 & $\mathrm{Hf}-170$ & $1.60 \mathrm{E}+01$ & $\mathrm{~h}$ & Hf-170 & $1.60 \mathrm{E}+01$ & $\mathrm{~h}$ & Hf-170 & $1.60 \mathrm{E}+01$ & $\mathrm{~h}$ & Hf-170 & $1.60 \mathrm{E}+01$ & $\mathrm{~h}$ & & & & ICRP-38 & $1.60 \mathrm{E}+01$ & $\mathrm{~h}$ \\
\hline Hf-172 & $\mathrm{Hf}-172$ & $1.87 \mathrm{E}+00$ & $y$ & $\mathrm{Hf}-172$ & $1.87 \mathrm{E}+00$ & $y$ & $\mathrm{Hf}-172$ & $1.87 \mathrm{E}+00$ & $y$ & $\mathrm{Hf}-172$ & $1.87 \mathrm{E}+00$ & $y$ & & & & ICRP-38 & $1.87 \mathrm{E}+00$ & $y$ \\
\hline Hf-173 & $\mathrm{Hf}-173$ & $2.36 \mathrm{E}+01$ & $\mathrm{~h}$ & $\mathrm{Hf}-173$ & $2.40 \mathrm{E}+01$ & $\mathrm{~h}$ & $\mathrm{Hf}-173$ & $2.40 \mathrm{E}+01$ & $\mathrm{~h}$ & $\mathrm{Hf}-173$ & $2.36 \mathrm{E}+01$ & $\mathrm{~h}$ & & & & ICRP-38 & $2.40 \mathrm{E}+01$ & $\mathrm{~h}$ \\
\hline Hf-174 & $\mathrm{Hf}-174$ & $2.00 \mathrm{E}+15$ & $y$ & & & & & & & $\mathrm{Hf}-174$ & $2.00 \mathrm{E}+15$ & $y$ & Hf-174 & $2.00 \mathrm{E}+15$ & $y$ & ICRP-107 & $2.00 \mathrm{E}+15$ & $y$ \\
\hline Hf-175 & $\mathrm{Hf}-175$ & $7.00 \mathrm{E}+01$ & $d$ & Hf-175 & $7.00 \mathrm{E}+01$ & $d$ & Hf-175 & $7.00 \mathrm{E}+01$ & $d$ & $\mathrm{Hf}-175$ & $7.00 \mathrm{E}+01$ & $d$ & & & & ICRP-38 & $7.00 \mathrm{E}+01$ & d \\
\hline Hf-177m & $\mathrm{Hf}-177 \mathrm{~m}$ & $5.14 \mathrm{E}+01$ & $\mathrm{~m}$ & Hf-177m & $5.14 \mathrm{E}+01$ & $m$ & Hf-177m & $5.14 \mathrm{E}+01$ & $m$ & $\mathrm{Hf}-177 \mathrm{~m}$ & $5.14 \mathrm{E}+01$ & $m$ & & & & ICRP-38 & $5.14 \mathrm{E}+01$ & $\mathrm{~m}$ \\
\hline Hf-178m & $\mathrm{Hf}-178 \mathrm{~m}$ & $3.10 \mathrm{E}+01$ & $y$ & $\mathrm{Hf}-178 \mathrm{~m}$ & $3.10 \mathrm{E}+01$ & $y$ & $\mathrm{Hf}-178 \mathrm{~m}$ & $3.10 \mathrm{E}+01$ & $y$ & $\mathrm{Hf}-178 \mathrm{~m}$ & $3.10 \mathrm{E}+01$ & $y$ & & & & ICRP-38 & $3.10 \mathrm{E}+01$ & $y$ \\
\hline Hf-179m & $\mathrm{Hf}-179 \mathrm{~m}$ & $2.51 \mathrm{E}+01$ & $d$ & $\mathrm{Hf}-179 \mathrm{~m}$ & $2.51 \mathrm{E}+01$ & $d$ & Hf-179m & $2.51 \mathrm{E}+01$ & $d$ & $\mathrm{Hf}-179 \mathrm{~m}$ & $2.51 \mathrm{E}+01$ & $d$ & & & & ICRP-38 & $2.51 \mathrm{E}+01$ & $d$ \\
\hline Hf-180m & $\mathrm{Hf}-180 \mathrm{~m}$ & $5.50 \mathrm{E}+00$ & $\mathrm{~h}$ & $\mathrm{Hf}-180 \mathrm{~m}$ & $5.50 \mathrm{E}+00$ & $\mathrm{~h}$ & $\mathrm{Hf}-180 \mathrm{~m}$ & $5.50 \mathrm{E}+00$ & $\mathrm{~h}$ & $\mathrm{Hf}-180 \mathrm{~m}$ & $5.50 \mathrm{E}+00$ & $\mathrm{~h}$ & & & & ICRP-38 & $5.50 \mathrm{E}+00$ & $\mathrm{~h}$ \\
\hline Hf-181 & $\mathrm{Hf}-181$ & $4.24 \mathrm{E}+01$ & $d$ & Hf-181 & $4.24 \mathrm{E}+01$ & $d$ & Hf-181 & $4.24 \mathrm{E}+01$ & $d$ & $\mathrm{Hf}-181$ & $4.24 \mathrm{E}+01$ & $d$ & & & & ICRP-38 & $4.24 \mathrm{E}+01$ & $d$ \\
\hline Hf-182 & $\mathrm{Hf}-182$ & $9.00 \mathrm{E}+06$ & $y$ & Hf-182 & $9.00 \mathrm{E}+06$ & $y$ & Hf-182 & $9.00 \mathrm{E}+06$ & $y$ & Hf-182 & $9.00 \mathrm{E}+06$ & $y$ & & & & ICRP-38 & $9.00 \mathrm{E}+06$ & $y$ \\
\hline Hf-182m & $\mathrm{Hf}-182 \mathrm{~m}$ & $6.15 \mathrm{E}+01$ & $\mathrm{~m}$ & Hf-182m & $6.15 \mathrm{E}+01$ & $\mathrm{~m}$ & Hf-182m & $6.15 \mathrm{E}+01$ & $\mathrm{~m}$ & $\mathrm{Hf}-182 \mathrm{~m}$ & $6.15 \mathrm{E}+01$ & $m$ & & & & ICRP-38 & $6.15 \mathrm{E}+01$ & $\mathrm{~m}$ \\
\hline Hf-183 & $\mathrm{Hf}-183$ & $1.07 \mathrm{E}+00$ & $\mathrm{~h}$ & $\mathrm{Hf}-183$ & $6.40 \mathrm{E}+01$ & $\mathrm{~m}$ & $\mathrm{Hf}-183$ & $6.40 \mathrm{E}+01$ & $\mathrm{~m}$ & $\mathrm{Hf}-183$ & $1.07 \mathrm{E}+00$ & $\mathrm{~h}$ & & & & ICRP-38 & $6.40 \mathrm{E}+01$ & $\mathrm{~m}$ \\
\hline Hf-184 & Hf-184 & $4.12 \mathrm{E}+00$ & $\mathrm{~h}$ & Hf-184 & $4.12 \mathrm{E}+00$ & $\mathrm{~h}$ & Hf-184 & $4.12 \mathrm{E}+00$ & $\mathrm{~h}$ & Hf-184 & $4.12 \mathrm{E}+00$ & $\mathrm{~h}$ & & & & ICRP-38 & $4.12 \mathrm{E}+00$ & $\mathrm{~h}$ \\
\hline Hg-187 & & & & & & & & & & & & & Hg-187 & $2.20 \mathrm{E}+00$ & $m$ & JAERI & $2.20 \mathrm{E}+00$ & $\mathrm{~m}$ \\
\hline $\mathrm{Hg}-187 \mathrm{~m}$ & & & & & & & & & & & & & $\mathrm{Hg}-187 \mathrm{~m}$ & $2.40 \mathrm{E}+00$ & $m$ & JAERI & $2.40 \mathrm{E}+00$ & $\mathrm{~m}$ \\
\hline Hg-188 & & & & & & & & & & & & & $\mathrm{Hg}-188$ & $3.25 \mathrm{E}+00$ & $\mathrm{~m}$ & JAERI & $3.25 \mathrm{E}+00$ & $\mathrm{~m}$ \\
\hline $\mathrm{Hg}-190$ & Hg-190 & $2.00 \mathrm{E}+01$ & $\mathrm{~m}$ & & & & & & & Hg-190 & $2.00 \mathrm{E}+01$ & $\mathrm{~m}$ & $\mathrm{Hg}-190$ & $2.00 \mathrm{E}+01$ & $m$ & ICRP-107 & $2.00 \mathrm{E}+01$ & $\mathrm{~m}$ \\
\hline Hg-191m & $\mathrm{Hg}-191 \mathrm{~m}$ & $5.08 \mathrm{E}+01$ & $\mathrm{~m}$ & & & & & & & $\mathrm{Hg}-191 \mathrm{~m}$ & $5.08 \mathrm{E}+01$ & $\mathrm{~m}$ & $\mathrm{Hg}-191 \mathrm{~m}$ & $5.08 \mathrm{E}+01$ & $\mathrm{~m}$ & ICRP-107 & $5.08 \mathrm{E}+01$ & $\mathrm{~m}$ \\
\hline Hg-192 & $\mathrm{Hg}-192$ & $4.85 \mathrm{E}+00$ & $\mathrm{~h}$ & & & & & & & $\mathrm{Hg}-192$ & $4.85 \mathrm{E}+00$ & $\mathrm{~h}$ & $\mathrm{Hg}-192$ & $4.85 \mathrm{E}+00$ & $\mathrm{~h}$ & ICRP-107 & $4.85 \mathrm{E}+00$ & $\mathrm{~h}$ \\
\hline $\mathrm{Hg}-193$ & $\mathrm{Hg}-193$ & $3.80 \mathrm{E}+00$ & $\mathrm{~h}$ & Hg-193 & $3.50 \mathrm{E}+00$ & $\mathrm{~h}$ & Hg-193 & $3.50 \mathrm{E}+00$ & $\mathrm{~h}$ & $\mathrm{Hg}-193$ & $3.80 \mathrm{E}+00$ & $\mathrm{~h}$ & & & & ICRP-38 & $3.50 \mathrm{E}+00$ & $\mathrm{~h}$ \\
\hline Hg-193m & $\mathrm{Hg}-193 \mathrm{~m}$ & $1.18 \mathrm{E}+01$ & $\mathrm{~h}$ & $\mathrm{Hg}-193 \mathrm{~m}$ & $1.11 \mathrm{E}+01$ & $\mathrm{~h}$ & $\mathrm{Hg}-193 \mathrm{~m}$ & $1.11 \mathrm{E}+01$ & $\mathrm{~h}$ & $\mathrm{Hg}-193 \mathrm{~m}$ & $1.18 \mathrm{E}+01$ & $\mathrm{~h}$ & & & & ICRP-38 & $1.11 \mathrm{E}+01$ & $\mathrm{~h}$ \\
\hline $\mathrm{Hg}-194$ & $\mathrm{Hg}-194$ & $4.40 \mathrm{E}+02$ & $y$ & $\mathrm{Hg}-194$ & $2.60 \mathrm{E}+02$ & $y$ & $\mathrm{Hg}-194$ & $2.60 \mathrm{E}+02$ & $y$ & $\mathrm{Hg}-194$ & $4.40 \mathrm{E}+02$ & $y$ & & & & ICRP-38 & $2.60 \mathrm{E}+02$ & $y$ \\
\hline Hg-195 & Hg-195 & $1.05 \mathrm{E}+01$ & $\mathrm{~h}$ & $\mathrm{Hg}-195$ & $9.90 \mathrm{E}+00$ & $\mathrm{~h}$ & $\mathrm{Hg}-195$ & $9.90 \mathrm{E}+00$ & $\mathrm{~h}$ & Hg-195 & $1.05 \mathrm{E}+01$ & $\mathrm{~h}$ & & & & ICRP-38 & $9.90 \mathrm{E}+00$ & $\mathrm{~h}$ \\
\hline Hg-195m & $\mathrm{Hg}-195 \mathrm{~m}$ & $4.16 \mathrm{E}+01$ & $\mathrm{~h}$ & $\mathrm{Hg}-195 \mathrm{~m}$ & $4.16 \mathrm{E}+01$ & $\mathrm{~h}$ & $\mathrm{Hg}-195 \mathrm{~m}$ & $4.16 \mathrm{E}+01$ & $\mathrm{~h}$ & $\mathrm{Hg}-195 \mathrm{~m}$ & $4.16 \mathrm{E}+01$ & $\mathrm{~h}$ & & & & ICRP-38 & $4.16 \mathrm{E}+01$ & $\mathrm{~h}$ \\
\hline Hg-197 & $\mathrm{Hg}-197$ & $6.49 \mathrm{E}+01$ & $\mathrm{~h}$ & $\mathrm{Hg}-197$ & $6.41 \mathrm{E}+01$ & $\mathrm{~h}$ & $\mathrm{Hg}-197$ & $6.41 \mathrm{E}+01$ & $\mathrm{~h}$ & $\mathrm{Hg}-197$ & $6.49 \mathrm{E}+01$ & $\mathrm{~h}$ & & & & ICRP-38 & $6.41 \mathrm{E}+01$ & $\mathrm{~h}$ \\
\hline $\mathrm{Hg}-197 \mathrm{~m}$ & $\mathrm{Hg}-197 \mathrm{~m}$ & $2.38 \mathrm{E}+01$ & $\mathrm{~h}$ & $\mathrm{Hg}-197 \mathrm{~m}$ & $2.38 \mathrm{E}+01$ & $\mathrm{~h}$ & $\mathrm{Hg}-197 \mathrm{~m}$ & $2.38 \mathrm{E}+01$ & $\mathrm{~h}$ & $\mathrm{Hg}-197 \mathrm{~m}$ & $2.38 \mathrm{E}+01$ & $\mathrm{~h}$ & & & & ICRP-38 & $2.38 \mathrm{E}+01$ & $\mathrm{~h}$ \\
\hline $\mathrm{Hg}-199 \mathrm{~m}$ & Hg-199m & $4.27 \mathrm{E}+01$ & $\mathrm{~m}$ & Hg-199m & $4.26 \mathrm{E}+01$ & $\mathrm{~m}$ & $\mathrm{Hg}-199 \mathrm{~m}$ & $4.26 \mathrm{E}+01$ & $\mathrm{~m}$ & Hg-199m & $4.27 \mathrm{E}+01$ & $\mathrm{~m}$ & & & & ICRP-38 & $4.26 \mathrm{E}+01$ & $\mathrm{~m}$ \\
\hline $\mathrm{Hg}-203$ & $\mathrm{Hg}-203$ & $4.66 \mathrm{E}+01$ & $d$ & $\mathrm{Hg}-203$ & $4.66 \mathrm{E}+01$ & $d$ & $\mathrm{Hg}-203$ & $4.66 \mathrm{E}+01$ & $d$ & $\mathrm{Hg}-203$ & $4.66 \mathrm{E}+01$ & $d$ & & & & ICRP-38 & $4.66 \mathrm{E}+01$ & $d$ \\
\hline Hg-205 & $\mathrm{Hg}-205$ & $5.20 \mathrm{E}+00$ & $\mathrm{~m}$ & & & & & & & $\mathrm{Hg}-205$ & $5.20 \mathrm{E}+00$ & $\mathrm{~m}$ & $\mathrm{Hg}-205$ & $5.20 \mathrm{E}+00$ & $\mathrm{~m}$ & ICRP-107 & $5.20 \mathrm{E}+00$ & $\mathrm{~m}$ \\
\hline Hg-206 & $\mathrm{Hg}-206$ & $8.15 \mathrm{E}+00$ & $m$ & & & & Hg-206 & $8.15 \mathrm{E}+00$ & $\mathrm{~m}$ & $\mathrm{Hg}-206$ & $8.15 \mathrm{E}+00$ & $m$ & $\mathrm{Hg}-206$ & $8.15 E+00$ & $m$ & ICRP-38 & $8.15 \mathrm{E}+00$ & $\mathrm{~m}$ \\
\hline
\end{tabular}

D-14 


\begin{tabular}{|c|c|c|c|c|c|c|c|c|c|c|c|c|c|c|c|c|c|c|}
\hline \multicolumn{19}{|c|}{ Table D.1 Selection of Radionuclides For Calculating HC-2 and HC-3 TQs (continued) } \\
\hline \multirow{3}{*}{$\begin{array}{l}\text { MASTER } \\
\text { LIST } \\
\mathrm{Hg}-207\end{array}$} & \multicolumn{3}{|c|}{ DOE-STD-1196 } & \multicolumn{3}{|c|}{ FGR-12 } & \multicolumn{3}{|c|}{ ICRP-38 } & \multicolumn{3}{|c|}{ ICRP-107 } & \multicolumn{3}{|c|}{ JAERI } & \multirow{3}{*}{$\begin{array}{c}\begin{array}{c}\text { Selected } \\
\text { Half-Life } \\
\text { Reference }\end{array} \\
\text { ICRP-107 }\end{array}$} & & \\
\hline & \multirow{2}{*}{$\begin{array}{l}\text { Nuclide } \\
\text { Hg-207 }\end{array}$} & \multicolumn{2}{|c|}{$\begin{array}{c}\text { Half-Life } \\
\text { (original units) }\end{array}$} & \multirow[t]{2}{*}{ Nuclide } & \multicolumn{2}{|c|}{$\begin{array}{c}\text { Half-Life } \\
\text { (original units) }\end{array}$} & \multirow[t]{2}{*}{ Nuclide } & \multicolumn{2}{|c|}{$\begin{array}{c}\text { Half-Life } \\
\text { (original units) }\end{array}$} & \multirow{2}{*}{$\begin{array}{l}\text { Nuclide } \\
\text { Hg-207 }\end{array}$} & \multicolumn{2}{|c|}{$\begin{array}{c}\text { Half-Life } \\
\text { (original units) } \\
\end{array}$} & \multirow[t]{2}{*}{ Nuclide } & $\begin{array}{r}\text { Half-Lif } \\
\text { (original u }\end{array}$ & its) & & \multicolumn{2}{|c|}{$\begin{array}{c}\text { Selected } \\
\text { Half-Life } \\
\text { (original units) }\end{array}$} \\
\hline & & $2.90 \mathrm{E}+00$ & $\mathrm{~m}$ & & & & & & & & $2.90 \mathrm{E}+00$ & $\mathrm{~m}$ & & & & & $2.90 \mathrm{E}+00$ & $\mathrm{~m}$ \\
\hline Ho-150 & Ho-150 & $7.68 \mathrm{E}+01$ & $\mathrm{~s}$ & & & & & & & Ho-150 & $7.68 \mathrm{E}+01$ & $\mathrm{~s}$ & & & & ICRP-107 & $7.68 \mathrm{E}+01$ & $\mathrm{~s}$ \\
\hline Ho-152 & & & & & & & & & & & & & Ho-152 & $2.70 \mathrm{E}+00$ & $\mathrm{~m}$ & JAERI & $2.70 \mathrm{E}+00$ & $\mathrm{~m}$ \\
\hline Ho-153 & Ho-153 & $2.01 \mathrm{E}+00$ & $\mathrm{~m}$ & & & & & & & Ho-153 & $2.01 \mathrm{E}+00$ & $\mathrm{~m}$ & Ho-153 & $2.02 \mathrm{E}+00$ & $\mathrm{~m}$ & ICRP-107 & $2.01 \mathrm{E}+00$ & $\mathrm{~m}$ \\
\hline Ho-153m & Ho- $153 \mathrm{~m}$ & $9.30 \mathrm{E}+00$ & $\mathrm{~m}$ & & & & & & & Ho-153m & $9.30 \mathrm{E}+00$ & $\mathrm{~m}$ & Ho- $153 m$ & $9.30 \mathrm{E}+00$ & $\mathrm{~m}$ & ICRP-107 & $9.30 \mathrm{E}+00$ & $\mathrm{~m}$ \\
\hline Ho-154 & Ho-154 & $1.18 \mathrm{E}+01$ & $\mathrm{~m}$ & & & & & & & Ho-154 & $1.18 \mathrm{E}+01$ & $\mathrm{~m}$ & Ho-154 & $1.18 \mathrm{E}+01$ & $m$ & ICRP-107 & $1.18 \mathrm{E}+01$ & $\mathrm{~m}$ \\
\hline Ho-154m & Ho-154m & $3.10 \mathrm{E}+00$ & $m$ & & & & & & & Ho-154m & $3.10 \mathrm{E}+00$ & $\mathrm{~m}$ & Ho-154m & $3.10 \mathrm{E}+00$ & $\mathrm{~m}$ & ICRP-107 & $3.10 \mathrm{E}+00$ & $\mathrm{~m}$ \\
\hline Ho-155 & Ho-155 & $4.80 \mathrm{E}+01$ & $\mathrm{~m}$ & Ho-155 & $4.80 \mathrm{E}+01$ & $\mathrm{~m}$ & Ho-155 & $4.80 \mathrm{E}+01$ & $\mathrm{~m}$ & Ho-155 & $4.80 \mathrm{E}+01$ & $\mathrm{~m}$ & & & & ICRP-38 & $4.80 \mathrm{E}+01$ & $m$ \\
\hline Ho-156 & Ho-156 & $5.60 \mathrm{E}+01$ & $\mathrm{~m}$ & & & & & & & Ho-156 & $5.60 \mathrm{E}+01$ & $\mathrm{~m}$ & Ho-156 & $5.60 \mathrm{E}+01$ & $\mathrm{~m}$ & ICRP-107 & $5.60 \mathrm{E}+01$ & $\mathrm{~m}$ \\
\hline Ho-157 & Ho-157 & $1.26 \mathrm{E}+01$ & $\mathrm{~m}$ & Ho-157 & $1.26 \mathrm{E}+01$ & $\mathrm{~m}$ & Ho-157 & $1.26 \mathrm{E}+01$ & $\mathrm{~m}$ & Ho-157 & $1.26 \mathrm{E}+01$ & $\mathrm{~m}$ & & & & ICRP-38 & $1.26 \mathrm{E}+01$ & $\mathrm{~m}$ \\
\hline Ho-158 & & & & & & & & & & & & & Ho-158 & $1.13 \mathrm{E}+01$ & $\mathrm{~m}$ & JAERI & $1.13 \mathrm{E}+01$ & $\mathrm{~m}$ \\
\hline Ho-159 & Ho-159 & $3.31 \mathrm{E}+01$ & $\mathrm{~m}$ & Ho-159 & $3.30 \mathrm{E}+01$ & $\mathrm{~m}$ & Ho-159 & $3.30 \mathrm{E}+01$ & $\mathrm{~m}$ & Ho-159 & $3.31 \mathrm{E}+01$ & $\mathrm{~m}$ & & & & ICRP-38 & $3.30 \mathrm{E}+01$ & $\mathrm{~m}$ \\
\hline Ho-160 & Ho-160 & $2.56 \mathrm{E}+01$ & $\mathrm{~m}$ & & & & & & & Ho-160 & $2.56 \mathrm{E}+01$ & $\mathrm{~m}$ & Ho-160 & $2.56 \mathrm{E}+01$ & $\mathrm{~m}$ & ICRP-107 & $2.56 \mathrm{E}+01$ & $\mathrm{~m}$ \\
\hline Ho-161 & Ho-161 & $2.48 \mathrm{E}+00$ & $\mathrm{~h}$ & Ho-161 & $2.50 \mathrm{E}+00$ & $\mathrm{~h}$ & Ho-161 & $2.50 \mathrm{E}+00$ & $\mathrm{~h}$ & Ho-161 & $2.48 \mathrm{E}+00$ & $\mathrm{~h}$ & & & & ICRP-38 & $2.50 \mathrm{E}+00$ & $\mathrm{~h}$ \\
\hline Ho-162 & Ho-162 & $1.50 \mathrm{E}+01$ & $\mathrm{~m}$ & Ho-162 & $1.50 \mathrm{E}+01$ & $m$ & Ho-162 & $1.50 \mathrm{E}+01$ & $m$ & Ho-162 & $1.50 \mathrm{E}+01$ & $m$ & & & & ICRP-38 & $1.50 \mathrm{E}+01$ & $m$ \\
\hline Ho-162m & Ho-162m & $6.70 \mathrm{E}+01$ & $\mathrm{~m}$ & Ho-162m & $6.80 \mathrm{E}+01$ & $m$ & Ho-162m & $6.80 \mathrm{E}+01$ & $\mathrm{~m}$ & Ho-162m & $6.70 \mathrm{E}+01$ & $\mathrm{~m}$ & & & & ICRP-38 & $6.80 \mathrm{E}+01$ & $\mathrm{~m}$ \\
\hline Ho-163 & Ho-163 & $4.57 \mathrm{E}+03$ & $y$ & & & & & & & Ho-163 & $4.57 \mathrm{E}+03$ & $y$ & Ho-163 & $4.57 \mathrm{E}+03$ & $y$ & ICRP-107 & $4.57 \mathrm{E}+03$ & $y$ \\
\hline Ho-164 & Ho-164 & $2.90 \mathrm{E}+01$ & $\mathrm{~m}$ & Ho-164 & $2.90 \mathrm{E}+01$ & $\mathrm{~m}$ & Ho-164 & $2.90 \mathrm{E}+01$ & $\mathrm{~m}$ & Ho-164 & $2.90 \mathrm{E}+01$ & $\mathrm{~m}$ & & & & ICRP-38 & $2.90 \mathrm{E}+01$ & $\mathrm{~m}$ \\
\hline Ho-164m & Ho-164m & $3.80 \mathrm{E}+01$ & $\mathrm{~m}$ & Ho-164m & $3.75 \mathrm{E}+01$ & $\mathrm{~m}$ & Ho-164m & $3.75 \mathrm{E}+01$ & $\mathrm{~m}$ & Ho-164m & $3.80 \mathrm{E}+01$ & $\mathrm{~m}$ & & & & ICRP-38 & $3.75 \mathrm{E}+01$ & $\mathrm{~m}$ \\
\hline Ho-166 & Ho-166 & $2.68 \mathrm{E}+01$ & $\mathrm{~h}$ & Ho-166 & $2.68 \mathrm{E}+01$ & $\mathrm{~h}$ & Ho-166 & $2.68 \mathrm{E}+01$ & $\mathrm{~h}$ & Ho-166 & $2.68 \mathrm{E}+01$ & $\mathrm{~h}$ & & & & ICRP-38 & $2.68 \mathrm{E}+01$ & $\mathrm{~h}$ \\
\hline Ho-166m & Ho-166m & $1.20 \mathrm{E}+03$ & $y$ & Ho-166m & $1.20 \mathrm{E}+03$ & $y$ & Ho-166m & $1.20 \mathrm{E}+03$ & $y$ & Ho-166m & $1.20 \mathrm{E}+03$ & $y$ & & & & ICRP-38 & $1.20 \mathrm{E}+03$ & $y$ \\
\hline Ho-167 & Ho-167 & $3.10 \mathrm{E}+00$ & $\mathrm{~h}$ & Ho-167 & $3.10 \mathrm{E}+00$ & $\mathrm{~h}$ & Ho-167 & $3.10 \mathrm{E}+00$ & $\mathrm{~h}$ & Ho-167 & $3.10 \mathrm{E}+00$ & $\mathrm{~h}$ & & & & ICRP-38 & $3.10 \mathrm{E}+00$ & $\mathrm{~h}$ \\
\hline Ho-168 & Ho-168 & $2.99 \mathrm{E}+00$ & $m$ & & & & & & & Ho-168 & $2.99 \mathrm{E}+00$ & $m$ & Ho-168 & $2.99 \mathrm{E}+00$ & $\mathrm{~m}$ & ICRP-107 & $2.99 \mathrm{E}+00$ & $\mathrm{~m}$ \\
\hline Ho-168m & Ho-168m & $1.32 \mathrm{E}+02$ & $\mathrm{~s}$ & & & & & & & Ho-168m & $1.32 \mathrm{E}+02$ & $s$ & Ho-168m & $2.20 \mathrm{E}+00$ & $m$ & ICRP-107 & $1.32 \mathrm{E}+02$ & $\mathrm{~s}$ \\
\hline Ho-170 & Ho-170 & $2.76 \mathrm{E}+00$ & $m$ & & & & & & & Ho-170 & $2.76 \mathrm{E}+00$ & $m$ & Ho-170 & $2.76 \mathrm{E}+00$ & $m$ & ICRP-107 & $2.76 \mathrm{E}+00$ & $\mathrm{~m}$ \\
\hline I-118 & $\mathrm{I}-118$ & $1.37 \mathrm{E}+01$ & $\mathrm{~m}$ & & & & & & & $\mathrm{I}-118$ & $1.37 \mathrm{E}+01$ & $m$ & $\mathrm{I}-118$ & $1.37 \mathrm{E}+01$ & $m$ & ICRP-107 & $1.37 \mathrm{E}+01$ & $m$ \\
\hline I-118m & $\mathrm{I}-118 \mathrm{~m}$ & $8.50 \mathrm{E}+00$ & $\mathrm{~m}$ & & & & & & & $\mathrm{I}-118 \mathrm{~m}$ & $8.50 \mathrm{E}+00$ & $\mathrm{~m}$ & & & & ICRP-107 & $8.50 \mathrm{E}+00$ & $\mathrm{~m}$ \\
\hline I-119 & $\mathrm{I}-119$ & $1.91 \mathrm{E}+01$ & $\mathrm{~m}$ & & & & & & & $\mathrm{I}-119$ & $1.91 \mathrm{E}+01$ & $\mathrm{~m}$ & I-119 & $1.91 \mathrm{E}+01$ & $\mathrm{~m}$ & ICRP-107 & $1.91 \mathrm{E}+01$ & $\mathrm{~m}$ \\
\hline I-120 & $\mathrm{I}-120$ & $8.16 \mathrm{E}+01$ & $\mathrm{~m}$ & $\mid-120$ & $8.10 \mathrm{E}+01$ & $m$ & $\mathrm{I}-120$ & $8.10 \mathrm{E}+01$ & $\mathrm{~m}$ & $\mathrm{I}-120$ & $8.16 \mathrm{E}+01$ & $\mathrm{~m}$ & & & & ICRP-38 & $8.10 E+01$ & $\mathrm{~m}$ \\
\hline $\mathrm{I}-120 \mathrm{~m}$ & $\mathrm{I}-120 \mathrm{~m}$ & $5.30 \mathrm{E}+01$ & $\mathrm{~m}$ & $\mathrm{I}-120 \mathrm{~m}$ & $5.30 \mathrm{E}+01$ & $\mathrm{~m}$ & $\mathrm{I}-120 \mathrm{~m}$ & $5.30 \mathrm{E}+01$ & $\mathrm{~m}$ & $\mathrm{I}-120 \mathrm{~m}$ & $5.30 \mathrm{E}+01$ & $\mathrm{~m}$ & & & & ICRP-38 & $5.30 \mathrm{E}+01$ & $\mathrm{~m}$ \\
\hline |-121 & $\mathrm{I}-121$ & $2.12 \mathrm{E}+00$ & $\mathrm{~h}$ & I-121 & $2.12 \mathrm{E}+00$ & $\mathrm{~h}$ & $\mathrm{I}-121$ & $2.12 \mathrm{E}+00$ & $\mathrm{~h}$ & $\mathrm{I}-121$ & $2.12 \mathrm{E}+00$ & $\mathrm{~h}$ & & & & ICRP-38 & $2.12 \mathrm{E}+00$ & $\mathrm{~h}$ \\
\hline I-122 & $1-122$ & $3.63 \mathrm{E}+00$ & $\mathrm{~m}$ & $1-122$ & $3.62 \mathrm{E}+00$ & $m$ & $1-122$ & $3.62 \mathrm{E}+00$ & $m$ & $1-122$ & $3.63 \mathrm{E}+00$ & $m$ & $1-122$ & $3.63 \mathrm{E}+00$ & $\mathrm{~m}$ & ICRP-38 & $3.62 \mathrm{E}+00$ & $\mathrm{~m}$ \\
\hline I-123 & I-123 & $1.33 \mathrm{E}+01$ & $\mathrm{~h}$ & I-123 & $1.32 \mathrm{E}+01$ & $\mathrm{~h}$ & $1-123$ & $1.32 \mathrm{E}+01$ & $\mathrm{~h}$ & I-123 & $1.33 \mathrm{E}+01$ & $\mathrm{~h}$ & & & & ICRP-38 & $1.32 \mathrm{E}+01$ & $\mathrm{~h}$ \\
\hline I-124 & I-124 & $4.18 \mathrm{E}+00$ & $d$ & I-124 & $4.18 \mathrm{E}+00$ & $d$ & I-124 & $4.18 \mathrm{E}+00$ & $d$ & I-124 & $4.18 \mathrm{E}+00$ & $d$ & & & & ICRP-38 & $4.18 \mathrm{E}+00$ & $d$ \\
\hline $1-125$ & I-125 & $5.94 \mathrm{E}+01$ & $d$ & I-125 & $6.01 \mathrm{E}+01$ & $d$ & $1-125$ & $6.01 \mathrm{E}+01$ & $d$ & I-125 & $5.94 \mathrm{E}+01$ & $d$ & & & & ICRP-38 & $6.01 \mathrm{E}+01$ & $d$ \\
\hline I-126 & I-126 & $1.29 \mathrm{E}+01$ & $d$ & I-126 & $1.30 \mathrm{E}+01$ & $\mathrm{~d}$ & $1-126$ & $1.30 \mathrm{E}+01$ & $\mathrm{~d}$ & $1-126$ & $1.29 \mathrm{E}+01$ & $d$ & & & & ICRP-38 & $1.30 \mathrm{E}+01$ & $d$ \\
\hline I-128 & $1-128$ & $2.50 \mathrm{E}+01$ & $\mathrm{~m}$ & $1-128$ & $2.50 \mathrm{E}+01$ & $m$ & $1-128$ & $2.50 \mathrm{E}+01$ & $\mathrm{~m}$ & $1-128$ & $2.50 \mathrm{E}+01$ & $m$ & & & & ICRP-38 & $2.50 \mathrm{E}+01$ & $\mathrm{~m}$ \\
\hline $1-129$ & $1-129$ & $1.57 \mathrm{E}+07$ & $y$ & $1-129$ & $1.57 \mathrm{E}+07$ & $y$ & $1-129$ & $1.57 \mathrm{E}+07$ & $y$ & $1-129$ & $1.57 \mathrm{E}+07$ & $y$ & & & & ICRP-38 & $1.57 \mathrm{E}+07$ & $y$ \\
\hline I-130 & I-130 & $1.24 \mathrm{E}+01$ & $\mathrm{~h}$ & I-130 & $1.24 \mathrm{E}+01$ & $\mathrm{~h}$ & $1-130$ & $1.24 \mathrm{E}+01$ & $\mathrm{~h}$ & I-130 & $1.24 \mathrm{E}+01$ & $\mathrm{~h}$ & & & & ICRP-38 & $1.24 \mathrm{E}+01$ & $\mathrm{~h}$ \\
\hline $1-130 m$ & $\mathrm{I}-130 \mathrm{~m}$ & $8.84 \mathrm{E}+00$ & $m$ & & & & & & & $\mathrm{I}-130 \mathrm{~m}$ & $8.84 \mathrm{E}+00$ & $m$ & & & & ICRP-107 & $8.84 \mathrm{E}+00$ & $\mathrm{~m}$ \\
\hline
\end{tabular}

D-15 


\begin{tabular}{|c|c|c|c|c|c|c|c|c|c|c|c|c|c|c|c|c|c|c|}
\hline \multicolumn{19}{|c|}{ Table D.1 Selection of Radionuclides For Calculating HC-2 and HC-3 TQs (continued) } \\
\hline \multirow{3}{*}{$\begin{array}{l}\text { MASTER } \\
\text { LIST } \\
\text { I-131 }\end{array}$} & \multicolumn{3}{|c|}{ DOE-STD-1196 } & \multicolumn{3}{|c|}{ FGR-12 } & \multicolumn{3}{|c|}{ ICRP-38 } & \multicolumn{3}{|c|}{ ICRP-107 } & \multicolumn{3}{|c|}{ JAERI } & \multirow{3}{*}{$\begin{array}{c}\begin{array}{c}\text { Selected } \\
\text { Half-Life } \\
\text { Reference }\end{array} \\
\text { ICRP-38 }\end{array}$} & \multirow{2}{*}{\multicolumn{2}{|c|}{$\begin{array}{c}\text { Selected } \\
\text { Half-Life } \\
\text { (original units) }\end{array}$}} \\
\hline & \multirow{2}{*}{$\begin{array}{l}\text { Nuclide } \\
\text { I-131 }\end{array}$} & \multicolumn{2}{|c|}{$\begin{array}{c}\text { Half-Life } \\
\text { (original units) }\end{array}$} & \multirow{2}{*}{$\begin{array}{l}\text { Nuclide } \\
\text { I-131 }\end{array}$} & \multicolumn{2}{|c|}{$\begin{array}{c}\text { Half-Life } \\
\text { (original units) }\end{array}$} & \multirow{2}{*}{$\begin{array}{l}\text { Nuclide } \\
1-131\end{array}$} & \multicolumn{2}{|c|}{$\begin{array}{c}\text { Half-Life } \\
\text { (original units) }\end{array}$} & \multirow{2}{*}{$\begin{array}{l}\text { Nuclide } \\
\text { I-131 }\end{array}$} & \multicolumn{2}{|c|}{$\begin{array}{c}\text { Half-Life } \\
\text { (original units) }\end{array}$} & \multirow[t]{2}{*}{ Nuclide } & $\begin{array}{r}\text { Half-Li } \\
\text { (original }\end{array}$ & its) & & & \\
\hline & & $8.02 E+00$ & $\mathrm{~d}$ & & $8.04 E+00$ & $\mathrm{~d}$ & & $8.04 \mathrm{E}+00$ & $d$ & & $8.02 \mathrm{E}+00$ & $d$ & & & & & $8.04 E+00$ & $d$ \\
\hline $1-132$ & $1-132$ & $2.30 \mathrm{E}+00$ & $\mathrm{~h}$ & $\mathrm{I}-132$ & $2.30 \mathrm{E}+00$ & $\mathrm{~h}$ & $1-132$ & $2.30 \mathrm{E}+00$ & $\mathrm{~h}$ & $1-132$ & $2.30 \mathrm{E}+00$ & $\mathrm{~h}$ & & & & ICRP-38 & $2.30 \mathrm{E}+00$ & $\mathrm{~h}$ \\
\hline $1-132 m$ & $\mathrm{I}-132 \mathrm{~m}$ & $1.39 \mathrm{E}+00$ & $\mathrm{~h}$ & $\mathrm{I}-132 \mathrm{~m}$ & $8.36 \mathrm{E}+01$ & $\mathrm{~m}$ & $\mathrm{I}-132 \mathrm{~m}$ & $8.36 \mathrm{E}+01$ & $\mathrm{~m}$ & $\mathrm{I}-132 \mathrm{~m}$ & $1.39 \mathrm{E}+00$ & $\mathrm{~h}$ & & & & ICRP-38 & $8.36 \mathrm{E}+01$ & $\mathrm{~m}$ \\
\hline I-133 & $1-133$ & $2.08 \mathrm{E}+01$ & $h$ & I-133 & $2.08 \mathrm{E}+01$ & $\mathrm{~h}$ & I-133 & $2.08 \mathrm{E}+01$ & $\mathrm{~h}$ & $1-133$ & $2.08 \mathrm{E}+01$ & $\mathrm{~h}$ & & & & ICRP-38 & $2.08 \mathrm{E}+01$ & $h$ \\
\hline $1-134$ & I-134 & $5.25 \mathrm{E}+01$ & $\mathrm{~m}$ & $\mid-134$ & $5.26 \mathrm{E}+01$ & $\mathrm{~m}$ & $\mid-134$ & $5.26 \mathrm{E}+01$ & $\mathrm{~m}$ & $\mathrm{I}-134$ & $5.25 \mathrm{E}+01$ & $\mathrm{~m}$ & & & & ICRP-38 & $5.26 \mathrm{E}+01$ & $\mathrm{~m}$ \\
\hline $\mathrm{I}-134 \mathrm{~m}$ & $\mathrm{I}-134 \mathrm{~m}$ & $3.60 \mathrm{E}+00$ & $\mathrm{~m}$ & & & & & & & $\mathrm{I}-134 \mathrm{~m}$ & $3.60 \mathrm{E}+00$ & $\mathrm{~m}$ & & & & ICRP-107 & $3.60 \mathrm{E}+00$ & $\mathrm{~m}$ \\
\hline I-135 & I-135 & $6.57 E+00$ & $\mathrm{~h}$ & I-135 & $6.61 \mathrm{E}+00$ & $\mathrm{~h}$ & I-135 & $6.61 \mathrm{E}+00$ & $\mathrm{~h}$ & I-135 & $6.57 \mathrm{E}+00$ & $\mathrm{~h}$ & & & & ICRP-38 & $6.61 \mathrm{E}+00$ & $\mathrm{~h}$ \\
\hline In-103 & In-103 & $6.00 \mathrm{E}+01$ & $\mathrm{~s}$ & & & & & & & In-103 & $6.00 \mathrm{E}+01$ & $\mathrm{~s}$ & & & & ICRP-107 & $6.00 \mathrm{E}+01$ & $\mathrm{~s}$ \\
\hline In-105 & In-105 & $5.07 E+00$ & $\mathrm{~m}$ & & & & & & & $\ln -105$ & $5.07 \mathrm{E}+00$ & $\mathrm{~m}$ & & & & ICRP-107 & $5.07 \mathrm{E}+00$ & $\mathrm{~m}$ \\
\hline In-106 & In-106 & $6.20 \mathrm{E}+00$ & $\mathrm{~m}$ & & & & & & & $\ln -106$ & $6.20 \mathrm{E}+00$ & $\mathrm{~m}$ & & & & ICRP-107 & $6.20 \mathrm{E}+00$ & $\mathrm{~m}$ \\
\hline In-106m & In-106m & $5.20 \mathrm{E}+00$ & $\mathrm{~m}$ & & & & & & & In-106m & $5.20 \mathrm{E}+00$ & $m$ & & & & ICRP-107 & $5.20 \mathrm{E}+00$ & $\mathrm{~m}$ \\
\hline $\ln -107$ & $\ln -107$ & $3.24 \mathrm{E}+01$ & $\mathrm{~m}$ & & & & & & & In-107 & $3.24 \mathrm{E}+01$ & $m$ & $\ln -107$ & $3.24 \mathrm{E}+01$ & $\mathrm{~m}$ & ICRP-107 & $3.24 \mathrm{E}+01$ & $\mathrm{~m}$ \\
\hline In-108 & $\ln -108$ & $5.80 \mathrm{E}+01$ & $\mathrm{~m}$ & & & & & & & $\ln -108$ & $5.80 \mathrm{E}+01$ & $m$ & $\ln -108$ & $5.80 \mathrm{E}+01$ & $m$ & ICRP-107 & $5.80 \mathrm{E}+01$ & $\mathrm{~m}$ \\
\hline In-108m & In-108m & $3.96 \mathrm{E}+01$ & $m$ & & & & & & & In-108m & $3.96 \mathrm{E}+01$ & $m$ & In-108m & $3.96 \mathrm{E}+01$ & $m$ & ICRP-107 & $3.96 \mathrm{E}+01$ & $\mathrm{~m}$ \\
\hline $\ln -109$ & $\ln -109$ & $4.20 \mathrm{E}+00$ & $\mathrm{~h}$ & In-109 & $4.20 \mathrm{E}+00$ & $\mathrm{~h}$ & $\ln -109$ & $4.20 \mathrm{E}+00$ & $\mathrm{~h}$ & $\ln -109$ & $4.20 \mathrm{E}+00$ & $\mathrm{~h}$ & & & & ICRP-38 & $4.20 \mathrm{E}+00$ & $\mathrm{~h}$ \\
\hline In-109m & In-109m & $1.34 \mathrm{E}+00$ & $\mathrm{~m}$ & & & & & & & In-109m & $1.34 \mathrm{E}+00$ & $\mathrm{~m}$ & In-109m & $1.34 \mathrm{E}+00$ & $\mathrm{~m}$ & ICRP-107 & $1.34 \mathrm{E}+00$ & $\mathrm{~m}$ \\
\hline In-110| & $\ln -110$ & $4.90 \mathrm{E}+00$ & $\mathrm{~h}$ & In-110b & $4.90 \mathrm{E}+00$ & $\mathrm{~h}$ & In-110b & $4.90 \mathrm{E}+00$ & $\mathrm{~h}$ & $\ln -110$ & $4.90 \mathrm{E}+00$ & $\mathrm{~h}$ & & & & ICRP-38 & $4.90 \mathrm{E}+00$ & $\mathrm{~h}$ \\
\hline In-110s & In-110m & $6.91 \mathrm{E}+01$ & $m$ & In-110a & $6.91 \mathrm{E}+01$ & $\mathrm{~m}$ & In-110a & $6.91 \mathrm{E}+01$ & $\mathrm{~m}$ & In-110m & $6.91 \mathrm{E}+01$ & $\mathrm{~m}$ & & & & ICRP-38 & $6.91 \mathrm{E}+01$ & $\mathrm{~m}$ \\
\hline In-111 & $\ln -111$ & $2.80 \mathrm{E}+00$ & $d$ & $\ln -111$ & $2.83 E+00$ & $d$ & $\ln -111$ & $2.83 \mathrm{E}+00$ & $d$ & $\ln -111$ & $2.80 \mathrm{E}+00$ & $d$ & & & & ICRP-38 & $2.83 \mathrm{E}+00$ & $d$ \\
\hline In-111m & In-111m & $7.70 \mathrm{E}+00$ & $\mathrm{~m}$ & & & & In-111m & $7.70 \mathrm{E}+00$ & $m$ & In-111m & $7.70 \mathrm{E}+00$ & $m$ & In-111m & $7.70 \mathrm{E}+00$ & $\mathrm{~m}$ & ICRP-38 & $7.70 \mathrm{E}+00$ & $\mathrm{~m}$ \\
\hline In-112 & $\ln -112$ & $1.50 \mathrm{E}+01$ & $\mathrm{~m}$ & $\ln -112$ & $1.44 \mathrm{E}+01$ & $\mathrm{~m}$ & $\ln -112$ & $1.44 \mathrm{E}+01$ & $m$ & $\ln -112$ & $1.50 \mathrm{E}+01$ & $m$ & & & & ICRP-38 & $1.44 \mathrm{E}+01$ & $\mathrm{~m}$ \\
\hline In-112m & In-112m & $2.06 \mathrm{E}+01$ & $\mathrm{~m}$ & & & & & & & $\ln -112 \mathrm{~m}$ & $2.06 \mathrm{E}+01$ & $\mathrm{~m}$ & In-112m & $2.06 \mathrm{E}+01$ & $\mathrm{~m}$ & ICRP-107 & $2.06 \mathrm{E}+01$ & $\mathrm{~m}$ \\
\hline In-113m & In-113m & $1.66 \mathrm{E}+00$ & $\mathrm{~h}$ & In-113m & $1.66 \mathrm{E}+00$ & $\mathrm{~h}$ & In-113m & $1.66 \mathrm{E}+00$ & $\mathrm{~h}$ & In-113m & $1.66 \mathrm{E}+00$ & $\mathrm{~h}$ & & & & ICRP-38 & $1.66 \mathrm{E}+00$ & $\mathrm{~h}$ \\
\hline In-114 & $\ln -114$ & $7.19 \mathrm{E}+01$ & $s$ & $\ln -114$ & $7.19 \mathrm{E}+01$ & $s$ & $\ln -114$ & $7.19 \mathrm{E}+01$ & $s$ & $\ln -114$ & $7.19 \mathrm{E}+01$ & $\mathrm{~s}$ & $\ln -114$ & $1.20 \mathrm{E}+00$ & $m$ & ICRP-38 & $7.19 \mathrm{E}+01$ & $\mathrm{~s}$ \\
\hline In-114m & In-114m & $4.95 \mathrm{E}+01$ & $d$ & In-114m & $4.95 \mathrm{E}+01$ & $d$ & In-114m & $4.95 \mathrm{E}+01$ & $d$ & In-114m & $4.95 \mathrm{E}+01$ & $d$ & & & & ICRP-38 & $4.95 \mathrm{E}+01$ & $d$ \\
\hline In-115 & $\ln -115$ & $4.41 \mathrm{E}+14$ & $y$ & $\ln -115$ & $5.10 \mathrm{E}+15$ & $y$ & $\ln -115$ & $5.10 \mathrm{E}+15$ & $y$ & $\ln -115$ & $4.41 \mathrm{E}+14$ & $y$ & & & & ICRP-38 & $5.10 \mathrm{E}+15$ & $y$ \\
\hline In-115m & In-115m & $4.49 \mathrm{E}+00$ & $\mathrm{~h}$ & In-115m & $4.49 \mathrm{E}+00$ & $\mathrm{~h}$ & In-115m & $4.49 \mathrm{E}+00$ & $\mathrm{~h}$ & In-115m & $4.49 \mathrm{E}+00$ & $\mathrm{~h}$ & & & & ICRP-38 & $4.49 \mathrm{E}+00$ & $\mathrm{~h}$ \\
\hline In-116m & In-116m & $5.44 \mathrm{E}+01$ & $\mathrm{~m}$ & $\ln -116 m$ & $5.42 \mathrm{E}+01$ & $\mathrm{~m}$ & $\ln -116 m$ & $5.42 \mathrm{E}+01$ & $\mathrm{~m}$ & In-116m & $5.44 \mathrm{E}+01$ & $\mathrm{~m}$ & & & & ICRP-38 & $5.42 \mathrm{E}+01$ & $\mathrm{~m}$ \\
\hline In-117 & $\ln -117$ & $4.32 \mathrm{E}+01$ & $\mathrm{~m}$ & $\ln -117$ & $4.38 \mathrm{E}+01$ & $m$ & $\ln -117$ & $4.38 \mathrm{E}+01$ & $\mathrm{~m}$ & In-117 & $4.32 \mathrm{E}+01$ & $\mathrm{~m}$ & & & & ICRP-38 & $4.38 \mathrm{E}+01$ & $\mathrm{~m}$ \\
\hline In-117m & In-117m & $1.16 \mathrm{E}+02$ & $\mathrm{~m}$ & In-117m & $1.17 E+02$ & $\mathrm{~m}$ & In-117m & $1.17 \mathrm{E}+02$ & $\mathrm{~m}$ & In-117m & $1.16 \mathrm{E}+02$ & $\mathrm{~m}$ & & & & ICRP-38 & $1.17 \mathrm{E}+02$ & $\mathrm{~m}$ \\
\hline In-118 & $\ln -118$ & $5.00 \mathrm{E}+00$ & $\mathrm{~s}$ & & & & & & & $\ln -118$ & $5.00 \mathrm{E}+00$ & $\mathrm{~s}$ & & & & ICRP-107 & $5.00 \mathrm{E}+00$ & $s$ \\
\hline In-118m & In-118m & $4.36 \mathrm{E}+00$ & $\mathrm{~m}$ & & & & & & & In-118m & $4.36 \mathrm{E}+00$ & $m$ & In-118m & $4.36 \mathrm{E}+00$ & $\mathrm{~m}$ & ICRP-107 & $4.36 \mathrm{E}+00$ & $\mathrm{~m}$ \\
\hline In-119 & $\ln -119$ & $2.40 \mathrm{E}+00$ & $M$ & In-119 & $2.40 \mathrm{E}+00$ & $\mathrm{~m}$ & $\ln -119$ & $2.40 \mathrm{E}+00$ & $\mathrm{~m}$ & In-119 & $2.40 \mathrm{E}+00$ & $\mathrm{~m}$ & $\ln -119$ & $2.40 \mathrm{E}+00$ & $m$ & ICRP-38 & $2.40 \mathrm{E}+00$ & $\mathrm{~m}$ \\
\hline In-119m & In-119m & $1.80 \mathrm{E}+01$ & $m$ & In-119m & $1.80 \mathrm{E}+01$ & $m$ & In-119m & $1.80 \mathrm{E}+01$ & $m$ & In-119m & $1.80 \mathrm{E}+01$ & $m$ & & & & ICRP-38 & $1.80 \mathrm{E}+01$ & $\mathrm{~m}$ \\
\hline In-121 & $\ln -121$ & $2.31 E+01$ & $\mathrm{~S}$ & & & & & & & $\ln -121$ & $2.31 \mathrm{E}+01$ & $\mathrm{~s}$ & & & & ICRP-107 & $2.31 \mathrm{E}+01$ & $\mathrm{~s}$ \\
\hline In-121m & In-121m & $3.88 \mathrm{E}+00$ & $M$ & & & & & & & In-121m & $3.88 \mathrm{E}+00$ & $m$ & & & & ICRP-107 & $3.88 \mathrm{E}+00$ & $m$ \\
\hline Ir-179 & & & & & & & & & & & & & $\mid \mathrm{r}-179$ & $1.32 \mathrm{E}+00$ & $\mathrm{~m}$ & JAERI & $1.32 \mathrm{E}+00$ & $\mathrm{~m}$ \\
\hline Ir-180 & $\mid r-180$ & $1.50 \mathrm{E}+00$ & $\mathrm{~m}$ & & & & & & & $\mid r-180$ & $1.50 \mathrm{E}+00$ & $\mathrm{~m}$ & $\mid r-180$ & $1.50 \mathrm{E}+00$ & $\mathrm{~m}$ & ICRP-107 & $1.50 \mathrm{E}+00$ & $\mathrm{~m}$ \\
\hline Ir-181 & & & & & & & & & & & & & $\mid r-181$ & $4.90 \mathrm{E}+00$ & $\mathrm{~m}$ & JAERI & $4.90 \mathrm{E}+00$ & $\mathrm{~m}$ \\
\hline Ir-182 & $\mid r-182$ & $1.50 \mathrm{E}+01$ & $\mathrm{~m}$ & $\mid r-182$ & $1.50 \mathrm{E}+01$ & $\mathrm{~m}$ & $\mid r-182$ & $1.50 \mathrm{E}+01$ & $\mathrm{~m}$ & $\mid r-182$ & $1.50 \mathrm{E}+01$ & $\mathrm{~m}$ & & & & ICRP-38 & $1.50 \mathrm{E}+01$ & $\mathrm{~m}$ \\
\hline
\end{tabular}

D-16 


\begin{tabular}{|c|c|c|c|c|c|c|c|c|c|c|c|c|c|c|c|c|c|c|}
\hline \multicolumn{19}{|c|}{ Table D.1 Selection of Radionuclides For Calculating HC-2 and HC-3 TQs (continued) } \\
\hline \multirow{3}{*}{$\begin{array}{l}\text { MASTER } \\
\text { LIST } \\
\text { Ir-183 }\end{array}$} & \multicolumn{3}{|c|}{ DOE-STD-1196 } & \multicolumn{3}{|c|}{ FGR-12 } & \multicolumn{3}{|c|}{ ICRP-38 } & \multicolumn{3}{|c|}{ ICRP-107 } & \multicolumn{3}{|c|}{ JAERI } & \multirow{3}{*}{$\begin{array}{c}\begin{array}{c}\text { Selected } \\
\text { Half-Life } \\
\text { Reference }\end{array} \\
\text { ICRP-107 }\end{array}$} & & \\
\hline & \multirow{2}{*}{$\begin{array}{l}\text { Nuclide } \\
\text { Ir-183 }\end{array}$} & \multicolumn{2}{|c|}{$\begin{array}{c}\text { Half-Life } \\
\text { (original units) }\end{array}$} & \multirow[t]{2}{*}{ Nuclide } & \multicolumn{2}{|c|}{$\begin{array}{c}\text { Half-Life } \\
\text { (original units) }\end{array}$} & \multirow[t]{2}{*}{ Nuclide } & \multicolumn{2}{|c|}{$\begin{array}{c}\text { Half-Life } \\
\text { (original units) }\end{array}$} & \multirow{2}{*}{$\begin{array}{l}\text { Nuclide } \\
\text { Ir-183 }\end{array}$} & \multicolumn{2}{|c|}{$\begin{array}{c}\text { Half-Life } \\
\text { (original units) }\end{array}$} & \multirow{2}{*}{$\begin{array}{l}\text { Nuclide } \\
\text { Ir-183 }\end{array}$} & $\begin{array}{r}\text { Half-Lif } \\
\text { (original u }\end{array}$ & its) & & \multicolumn{2}{|c|}{$\begin{array}{c}\text { Selected } \\
\text { Half-Life } \\
\text { (original units) }\end{array}$} \\
\hline & & $5.80 \mathrm{E}+01$ & $\mathrm{~m}$ & & & & & & & & $5.80 \mathrm{E}+01$ & $\mathrm{~m}$ & & $5.80 \mathrm{E}+01$ & $\mathrm{~m}$ & & $5.80 \mathrm{E}+01$ & $\mathrm{~m}$ \\
\hline Ir-184 & Ir-184 & $3.09 \mathrm{E}+00$ & $\mathrm{~h}$ & Ir-184 & $3.02 \mathrm{E}+00$ & $\mathrm{~h}$ & Ir-184 & $3.02 \mathrm{E}+00$ & $\mathrm{~h}$ & Ir-184 & $3.09 \mathrm{E}+00$ & $\mathrm{~h}$ & & & & ICRP-38 & $3.02 \mathrm{E}+00$ & $\mathrm{~h}$ \\
\hline Ir-185 & $\operatorname{lr}-185$ & $1.44 \mathrm{E}+01$ & $\mathrm{~h}$ & Ir-185 & $1.40 \mathrm{E}+01$ & $\mathrm{~h}$ & Ir-185 & $1.40 \mathrm{E}+01$ & $\mathrm{~h}$ & |r-185 & $1.44 \mathrm{E}+01$ & $\mathrm{~h}$ & & & & ICRP-38 & $1.40 \mathrm{E}+01$ & $\mathrm{~h}$ \\
\hline Ir-186| & $\operatorname{lr}-186$ & $1.66 \mathrm{E}+01$ & $\mathrm{~h}$ & Ir-186a & $1.58 \mathrm{E}+01$ & $\mathrm{~h}$ & Ir-186a & $1.58 \mathrm{E}+01$ & $\mathrm{~h}$ & Ir-186 & $1.66 \mathrm{E}+01$ & $\mathrm{~h}$ & & & & ICRP-38 & $1.58 \mathrm{E}+01$ & $\mathrm{~h}$ \\
\hline Ir-186s & Ir-186m & $1.92 \mathrm{E}+00$ & $\mathrm{~h}$ & Ir-186b & $1.75 \mathrm{E}+00$ & $\mathrm{~h}$ & Ir-186b & $1.75 \mathrm{E}+00$ & $\mathrm{~h}$ & Ir-186m & $1.92 \mathrm{E}+00$ & $\mathrm{~h}$ & & & & ICRP-38 & $1.75 \mathrm{E}+00$ & $\mathrm{~h}$ \\
\hline Ir-187 & Ir-187 & $1.05 \mathrm{E}+01$ & $\mathrm{~h}$ & Ir-187 & $1.05 \mathrm{E}+01$ & $\mathrm{~h}$ & Ir-187 & $1.05 \mathrm{E}+01$ & $\mathrm{~h}$ & Ir-187 & $1.05 \mathrm{E}+01$ & $\mathrm{~h}$ & & & & ICRP-38 & $1.05 \mathrm{E}+01$ & $\mathrm{~h}$ \\
\hline Ir-188 & Ir-188 & $4.15 \mathrm{E}+01$ & $\mathrm{~h}$ & Ir-188 & $4.15 \mathrm{E}+01$ & $\mathrm{~h}$ & Ir-188 & $4.15 \mathrm{E}+01$ & $\mathrm{~h}$ & Ir-188 & $4.15 \mathrm{E}+01$ & $\mathrm{~h}$ & & & & ICRP-38 & $4.15 \mathrm{E}+01$ & $\mathrm{~h}$ \\
\hline Ir-189 & Ir-189 & $1.32 \mathrm{E}+01$ & $d$ & Ir-189 & $1.33 \mathrm{E}+01$ & $\mathrm{~d}$ & Ir-189 & $1.33 \mathrm{E}+01$ & $d$ & $\mid r-189$ & $1.32 \mathrm{E}+01$ & $d$ & & & & ICRP-38 & $1.33 \mathrm{E}+01$ & $d$ \\
\hline Ir-190 & Ir-190 & $1.18 \mathrm{E}+01$ & $d$ & Ir-190 & $1.21 \mathrm{E}+01$ & $\mathrm{~d}$ & Ir-190 & $1.21 \mathrm{E}+01$ & $d$ & Ir-190 & $1.18 \mathrm{E}+01$ & $d$ & & & & ICRP-38 & $1.21 \mathrm{E}+01$ & $d$ \\
\hline Ir-190ms & Ir-190m & $1.12 \mathrm{E}+00$ & $\mathrm{~h}$ & Ir-190m & $1.20 \mathrm{E}+00$ & $\mathrm{~h}$ & Ir-190m & $1.20 \mathrm{E}+00$ & $\mathrm{~h}$ & Ir-190m & $1.12 \mathrm{E}+00$ & $\mathrm{~h}$ & & & & ICRP-38 & $1.20 \mathrm{E}+00$ & $\mathrm{~h}$ \\
\hline Ir-190ml & Ir-190n & $3.09 \mathrm{E}+00$ & $\mathrm{~h}$ & Ir-190n & $3.10 \mathrm{E}+00$ & $\mathrm{~h}$ & Ir-190n & $3.10 \mathrm{E}+00$ & $\mathrm{~h}$ & Ir-190n & $3.09 \mathrm{E}+00$ & $\mathrm{~h}$ & & & & ICRP-38 & $3.10 \mathrm{E}+00$ & $\mathrm{~h}$ \\
\hline Ir-191m & Ir-191m & $4.94 \mathrm{E}+00$ & $\mathrm{~s}$ & Ir-191m & $4.94 \mathrm{E}+00$ & $\mathrm{~s}$ & Ir-191m & $4.94 \mathrm{E}+00$ & $s$ & Ir-191m & $4.94 \mathrm{E}+00$ & $s$ & & & & ICRP-38 & $4.94 \mathrm{E}+00$ & $s$ \\
\hline Ir-192 & Ir-192 & $7.38 \mathrm{E}+01$ & $d$ & Ir-192 & $7.40 \mathrm{E}+01$ & $d$ & Ir-192 & $7.40 \mathrm{E}+01$ & $d$ & Ir-192 & $7.38 \mathrm{E}+01$ & $d$ & & & & ICRP-38 & $7.40 \mathrm{E}+01$ & $d$ \\
\hline Ir-192ms & Ir-192m & $1.45 \mathrm{E}+00$ & $m$ & & & & & & & Ir-192m & $1.45 \mathrm{E}+00$ & $m$ & & & & ICRP-107 & $1.45 \mathrm{E}+00$ & $\mathrm{~m}$ \\
\hline Ir-192ml & Ir-192n & $2.41 \mathrm{E}+02$ & $y$ & Ir-192m & $2.41 \mathrm{E}+02$ & $y$ & Ir-192m & $2.41 E+02$ & $y$ & Ir-192n & $2.41 \mathrm{E}+02$ & $y$ & & & & ICRP-38 & $2.41 \mathrm{E}+02$ & $y$ \\
\hline Ir-193m & Ir-193m & $1.05 \mathrm{E}+01$ & $d$ & & & & & & & Ir-193m & $1.05 \mathrm{E}+01$ & $d$ & & & & ICRP-107 & $1.05 \mathrm{E}+01$ & d \\
\hline Ir-194 & Ir-194 & $1.93 \mathrm{E}+01$ & $\mathrm{~h}$ & Ir-194 & $1.92 \mathrm{E}+01$ & $\mathrm{~h}$ & Ir-194 & $1.92 \mathrm{E}+01$ & $\mathrm{~h}$ & Ir-194 & $1.93 \mathrm{E}+01$ & $\mathrm{~h}$ & & & & ICRP-38 & $1.92 \mathrm{E}+01$ & $\mathrm{~h}$ \\
\hline Ir-194m & Ir-194m & $1.71 \mathrm{E}+02$ & $d$ & Ir-194m & $1.71 \mathrm{E}+02$ & $d$ & Ir-194m & $1.71 \mathrm{E}+02$ & $d$ & Ir-194m & $1.71 \mathrm{E}+02$ & $d$ & & & & ICRP-38 & $1.71 \mathrm{E}+02$ & $d$ \\
\hline Ir-195 & Ir-195 & $2.50 \mathrm{E}+00$ & $\mathrm{~h}$ & Ir-195 & $2.50 \mathrm{E}+00$ & $\mathrm{~h}$ & Ir-195 & $2.50 \mathrm{E}+00$ & $\mathrm{~h}$ & Ir-195 & $2.50 \mathrm{E}+00$ & $\mathrm{~h}$ & & & & ICRP-38 & $2.50 \mathrm{E}+00$ & $\mathrm{~h}$ \\
\hline Ir-195m & Ir-195m & $3.80 \mathrm{E}+00$ & $\mathrm{~h}$ & Ir-195m & $3.80 \mathrm{E}+00$ & $\mathrm{~h}$ & Ir-195m & $3.80 \mathrm{E}+00$ & $\mathrm{~h}$ & Ir-195m & $3.80 \mathrm{E}+00$ & $\mathrm{~h}$ & & & & ICRP-38 & $3.80 \mathrm{E}+00$ & $\mathrm{~h}$ \\
\hline Ir-196 & Ir-196 & $5.20 \mathrm{E}+01$ & $\mathrm{~s}$ & & & & & & & Ir-196 & $5.20 \mathrm{E}+01$ & $s$ & & & & ICRP-107 & $5.20 \mathrm{E}+01$ & $s$ \\
\hline Ir-196m & Ir-196m & $1.40 \mathrm{E}+00$ & $\mathrm{~h}$ & & & & & & & Ir-196m & $1.40 \mathrm{E}+00$ & $\mathrm{~h}$ & Ir-196m & $1.40 \mathrm{E}+00$ & $\mathrm{~h}$ & ICRP-107 & $1.40 \mathrm{E}+00$ & $\mathrm{~h}$ \\
\hline K-38 & K-38 & $7.64 \mathrm{E}+00$ & $m$ & $\mathrm{~K}-38$ & $7.64 \mathrm{E}+00$ & $\mathrm{~m}$ & $\mathrm{~K}-38$ & $7.64 \mathrm{E}+00$ & $m$ & K-38 & $7.64 \mathrm{E}+00$ & $m$ & $\mathrm{~K}-38$ & $7.64 \mathrm{E}+00$ & $m$ & ICRP-38 & $7.64 \mathrm{E}+00$ & $\mathrm{~m}$ \\
\hline K-40 & $\mathrm{K}-40$ & $1.25 \mathrm{E}+09$ & $y$ & $\mathrm{~K}-40$ & $1.28 \mathrm{E}+09$ & $y$ & $\mathrm{~K}-40$ & $1.28 \mathrm{E}+09$ & $y$ & $\mathrm{~K}-40$ & $1.25 \mathrm{E}+09$ & $y$ & & & & ICRP-38 & $1.28 \mathrm{E}+09$ & $y$ \\
\hline K-42 & $\mathrm{K}-42$ & $1.24 \mathrm{E}+01$ & $\mathrm{~h}$ & $\mathrm{~K}-42$ & $1.24 \mathrm{E}+01$ & $\mathrm{~h}$ & $\mathrm{~K}-42$ & $1.24 \mathrm{E}+01$ & $\mathrm{~h}$ & $\mathrm{~K}-42$ & $1.24 \mathrm{E}+01$ & $\mathrm{~h}$ & & & & ICRP-38 & $1.24 \mathrm{E}+01$ & $\mathrm{~h}$ \\
\hline K-43 & $\mathrm{K}-43$ & $2.23 \mathrm{E}+01$ & $\mathrm{~h}$ & K-43 & $2.26 \mathrm{E}+01$ & $\mathrm{~h}$ & K-43 & $2.26 \mathrm{E}+01$ & $\mathrm{~h}$ & $\mathrm{~K}-43$ & $2.23 \mathrm{E}+01$ & $\mathrm{~h}$ & & & & ICRP-38 & $2.26 \mathrm{E}+01$ & $\mathrm{~h}$ \\
\hline K-44 & $\mathrm{K}-44$ & $2.21 \mathrm{E}+01$ & $\mathrm{~m}$ & $\mathrm{~K}-44$ & $2.21 \mathrm{E}+01$ & $\mathrm{~m}$ & $\mathrm{~K}-44$ & $2.21 \mathrm{E}+01$ & $\mathrm{~m}$ & $\mathrm{~K}-44$ & $2.21 \mathrm{E}+01$ & $\mathrm{~m}$ & & & & ICRP-38 & $2.21 \mathrm{E}+01$ & $\mathrm{~m}$ \\
\hline K-45 & $\mathrm{K}-45$ & $1.73 \mathrm{E}+01$ & $\mathrm{~m}$ & K-45 & $2.00 \mathrm{E}+01$ & $\mathrm{~m}$ & K-45 & $2.00 \mathrm{E}+01$ & $\mathrm{~m}$ & $\mathrm{~K}-45$ & $1.73 \mathrm{E}+01$ & $\mathrm{~m}$ & & & & ICRP-38 & $2.00 \mathrm{E}+01$ & $\mathrm{~m}$ \\
\hline K-46 & K-46 & $1.05 E+02$ & $\mathrm{~s}$ & & & & & & & K-46 & $1.05 \mathrm{E}+02$ & $\mathrm{~s}$ & & & & ICRP-107 & $1.05 E+02$ & $\mathrm{~s}$ \\
\hline Kr-74 & $\mathrm{Kr}-74$ & $1.15 \mathrm{E}+01$ & $\mathrm{~m}$ & Kr-74 & $1.15 \mathrm{E}+01$ & $\mathrm{~m}$ & Kr-74 & $1.15 \mathrm{E}+01$ & $\mathrm{~m}$ & $\mathrm{Kr}-74$ & $1.15 \mathrm{E}+01$ & $\mathrm{~m}$ & & & & ICRP-38 & $1.15 \mathrm{E}+01$ & $\mathrm{~m}$ \\
\hline Kr-75 & $\mathrm{Kr}-75$ & $4.29 \mathrm{E}+00$ & $\mathrm{~m}$ & & & & & & & $\mathrm{Kr}-75$ & $4.29 \mathrm{E}+00$ & $m$ & Kr-75 & $4.29 \mathrm{E}+00$ & $\mathrm{~m}$ & ICRP-107 & $4.29 \mathrm{E}+00$ & $\mathrm{~m}$ \\
\hline Kr-76 & $\mathrm{Kr}-76$ & $1.48 \mathrm{E}+01$ & $\mathrm{~h}$ & Kr-76 & $1.48 \mathrm{E}+01$ & $\mathrm{~h}$ & Kr-76 & $1.48 \mathrm{E}+01$ & $\mathrm{~h}$ & $\mathrm{Kr}-76$ & $1.48 \mathrm{E}+01$ & $\mathrm{~h}$ & & & & ICRP-38 & $1.48 \mathrm{E}+01$ & $\mathrm{~h}$ \\
\hline Kr-77 & Kr-77 & $7.44 \mathrm{E}+01$ & $\mathrm{~m}$ & $\mathrm{Kr}-77$ & $7.47 \mathrm{E}+01$ & $\mathrm{~m}$ & $\mathrm{Kr}-77$ & $7.47 \mathrm{E}+01$ & $\mathrm{~m}$ & $\mathrm{Kr}-77$ & $7.44 \mathrm{E}+01$ & $\mathrm{~m}$ & & & & ICRP-38 & $7.47 \mathrm{E}+01$ & $\mathrm{~m}$ \\
\hline Kr-79 & $\mathrm{Kr}-79$ & $3.50 \mathrm{E}+01$ & $\mathrm{~h}$ & $\mathrm{Kr}-79$ & $3.50 \mathrm{E}+01$ & $\mathrm{~h}$ & $\mathrm{Kr}-79$ & $3.50 \mathrm{E}+01$ & $\mathrm{~h}$ & $\mathrm{Kr}-79$ & $3.50 \mathrm{E}+01$ & $\mathrm{~h}$ & & & & ICRP-38 & $3.50 \mathrm{E}+01$ & $\mathrm{~h}$ \\
\hline Kr-81 & $\mathrm{Kr}-81$ & $2.29 \mathrm{E}+05$ & $y$ & $\mathrm{Kr}-81$ & $2.10 \mathrm{E}+05$ & $y$ & $\mathrm{Kr}-81$ & $2.10 \mathrm{E}+05$ & $y$ & $\mathrm{Kr}-81$ & $2.29 \mathrm{E}+05$ & $y$ & & & & ICRP-38 & $2.10 \mathrm{E}+05$ & $y$ \\
\hline Kr-81m & $\mathrm{Kr}-81 \mathrm{~m}$ & $1.31 \mathrm{E}+01$ & $\mathrm{~s}$ & $\mathrm{Kr}-81 \mathrm{~m}$ & $1.30 \mathrm{E}+01$ & $\mathrm{~s}$ & $\mathrm{Kr}-81 \mathrm{~m}$ & $1.30 \mathrm{E}+01$ & $s$ & $\mathrm{Kr}-81 \mathrm{~m}$ & $1.31 \mathrm{E}+01$ & $s$ & & & & ICRP-38 & $1.30 \mathrm{E}+01$ & $\mathrm{~s}$ \\
\hline Kr-83m & $\mathrm{Kr}-83 \mathrm{~m}$ & $1.83 \mathrm{E}+00$ & $\mathrm{~h}$ & $\mathrm{Kr}-83 \mathrm{~m}$ & $1.83 \mathrm{E}+00$ & $\mathrm{~h}$ & $\mathrm{Kr}-83 \mathrm{~m}$ & $1.83 \mathrm{E}+00$ & $\mathrm{~h}$ & $\mathrm{Kr}-83 \mathrm{~m}$ & $1.83 \mathrm{E}+00$ & $\mathrm{~h}$ & & & & ICRP-38 & $1.83 \mathrm{E}+00$ & $\mathrm{~h}$ \\
\hline Kr-85 & $\mathrm{Kr}-85$ & $1.08 \mathrm{E}+01$ & $y$ & $\mathrm{Kr}-85$ & $1.07 \mathrm{E}+01$ & $y$ & $\mathrm{Kr}-85$ & $1.07 \mathrm{E}+01$ & $y$ & $\mathrm{Kr}-85$ & $1.08 \mathrm{E}+01$ & $y$ & & & & ICRP-38 & $1.07 \mathrm{E}+01$ & $y$ \\
\hline Kr-85m & $\mathrm{Kr}-85 \mathrm{~m}$ & $4.48 \mathrm{E}+00$ & $\mathrm{~h}$ & $\mathrm{Kr}-85 \mathrm{~m}$ & $4.48 \mathrm{E}+00$ & $\mathrm{~h}$ & $\mathrm{Kr}-85 \mathrm{~m}$ & $4.48 \mathrm{E}+00$ & $\mathrm{~h}$ & $\mathrm{Kr}-85 \mathrm{~m}$ & $4.48 \mathrm{E}+00$ & $\mathrm{~h}$ & & & & ICRP-38 & $4.48 \mathrm{E}+00$ & $\mathrm{~h}$ \\
\hline Kr-87 & Kr-87 & $7.63 \mathrm{E}+01$ & $m$ & Kr-87 & $7.63 \mathrm{E}+01$ & $m$ & Kr-87 & $7.63 \mathrm{E}+01$ & $m$ & Kr-87 & $7.63 \mathrm{E}+01$ & $m$ & & & & ICRP-38 & $7.63 \mathrm{E}+01$ & $\mathrm{~m}$ \\
\hline
\end{tabular}

D-17 


\begin{tabular}{|c|c|c|c|c|c|c|c|c|c|c|c|c|c|c|c|c|c|c|}
\hline \multicolumn{19}{|c|}{ Table D.1 Selection of Radionuclides For Calculating HC-2 and HC-3 TQs (continued) } \\
\hline \multirow{3}{*}{$\begin{array}{l}\text { MASTER } \\
\text { LIST } \\
\mathrm{Kr}-88\end{array}$} & \multicolumn{3}{|c|}{ DOE-STD-1196 } & \multicolumn{3}{|c|}{ FGR-12 } & \multicolumn{3}{|c|}{ ICRP-38 } & \multicolumn{3}{|c|}{ ICRP-107 } & \multicolumn{3}{|c|}{ JAERI } & \multirow{3}{*}{$\begin{array}{c}\begin{array}{c}\text { Selected } \\
\text { Half-Life } \\
\text { Reference }\end{array} \\
\text { ICRP-38 }\end{array}$} & & \\
\hline & \multirow{2}{*}{$\begin{array}{l}\text { Nuclide } \\
\text { Kr-88 }\end{array}$} & \multicolumn{2}{|c|}{$\begin{array}{c}\text { Half-Life } \\
\text { (original units) }\end{array}$} & \multirow{2}{*}{$\begin{array}{l}\text { Nuclide } \\
\mathrm{Kr}-88\end{array}$} & \multicolumn{2}{|c|}{$\begin{array}{c}\text { Half-Life } \\
\text { (original units) }\end{array}$} & \multirow{2}{*}{$\begin{array}{l}\text { Nuclide } \\
\mathrm{Kr}-88\end{array}$} & \multicolumn{2}{|c|}{$\begin{array}{c}\text { Half-Life } \\
\text { (original units) }\end{array}$} & \multirow{2}{*}{$\begin{array}{l}\text { Nuclide } \\
\mathrm{Kr}-88\end{array}$} & \multicolumn{2}{|c|}{$\begin{array}{c}\text { Half-Life } \\
\text { (original units) }\end{array}$} & \multirow[t]{2}{*}{ Nuclide } & $\begin{array}{r}\text { Half-Li } \\
\text { (original }\end{array}$ & its) & & \multicolumn{2}{|c|}{$\begin{array}{c}\text { Selected } \\
\text { Half-Life } \\
\text { (original units) }\end{array}$} \\
\hline & & $2.84 \mathrm{E}+00$ & $\mathrm{~h}$ & & $2.84 \mathrm{E}+00$ & $\mathrm{~h}$ & & $2.84 \mathrm{E}+00$ & $\mathrm{~h}$ & & $2.84 \mathrm{E}+00$ & $\mathrm{~h}$ & & & & & $2.84 \mathrm{E}+00$ & $\mathrm{~h}$ \\
\hline Kr-89 & Kr-89 & $3.15 \mathrm{E}+00$ & $\mathrm{~m}$ & & & & & & & Kr-89 & $3.15 \mathrm{E}+00$ & $\mathrm{~m}$ & $\mathrm{Kr}-89$ & $3.15 \mathrm{E}+00$ & $\mathrm{~m}$ & ICRP-107 & $3.15 \mathrm{E}+00$ & $\mathrm{~m}$ \\
\hline La-128 & La-128 & $5.18 \mathrm{E}+00$ & $\mathrm{~m}$ & & & & & & & La-128 & $5.18 \mathrm{E}+00$ & $\mathrm{~m}$ & & & & ICRP-107 & $5.18 \mathrm{E}+00$ & $\mathrm{~m}$ \\
\hline La-129 & La-129 & $1.16 \mathrm{E}+01$ & $\mathrm{~m}$ & & & & & & & La-129 & $1.16 \mathrm{E}+01$ & $\mathrm{~m}$ & La-129 & $1.16 \mathrm{E}+01$ & $\mathrm{~m}$ & ICRP-107 & $1.16 \mathrm{E}+01$ & $\mathrm{~m}$ \\
\hline La-130 & La-130 & $8.70 \mathrm{E}+00$ & $\mathrm{~m}$ & & & & & & & La-130 & $8.70 \mathrm{E}+00$ & $\mathrm{~m}$ & La-130 & $8.70 \mathrm{E}+00$ & $\mathrm{~m}$ & ICRP-107 & $8.70 \mathrm{E}+00$ & $\mathrm{~m}$ \\
\hline La-131 & La-131 & $5.90 \mathrm{E}+01$ & $\mathrm{~m}$ & La-131 & $5.90 \mathrm{E}+01$ & $\mathrm{~m}$ & La-131 & $5.90 \mathrm{E}+01$ & $\mathrm{~m}$ & La-131 & $5.90 \mathrm{E}+01$ & $\mathrm{~m}$ & & & & ICRP-38 & $5.90 \mathrm{E}+01$ & $\mathrm{~m}$ \\
\hline La-132 & La-132 & $4.80 \mathrm{E}+00$ & $\mathrm{~h}$ & La-132 & $4.80 \mathrm{E}+00$ & $\mathrm{~h}$ & La-132 & $4.80 \mathrm{E}+00$ & $\mathrm{~h}$ & La-132 & $4.80 \mathrm{E}+00$ & $\mathrm{~h}$ & & & & ICRP-38 & $4.80 \mathrm{E}+00$ & $\mathrm{~h}$ \\
\hline La-132m & La-132m & $2.43 \mathrm{E}+01$ & $\mathrm{~m}$ & & & & & & & La-132m & $2.43 E+01$ & $\mathrm{~m}$ & La-132m & $2.43 \mathrm{E}+01$ & $\mathrm{~m}$ & ICRP-107 & $2.43 \mathrm{E}+01$ & $m$ \\
\hline La-133 & La-133 & $3.91 \mathrm{E}+00$ & $\mathrm{~h}$ & & & & & & & La-133 & $3.91 \mathrm{E}+00$ & $\mathrm{~h}$ & La-133 & $3.91 \mathrm{E}+00$ & $\mathrm{~h}$ & ICRP-107 & $3.91 \mathrm{E}+00$ & $\mathrm{~h}$ \\
\hline La-134 & La-134 & $6.45 \mathrm{E}+00$ & $\mathrm{~m}$ & La-134 & $6.67 E+00$ & $\mathrm{~m}$ & La-134 & $6.67 \mathrm{E}+00$ & $\mathrm{~m}$ & La-134 & $6.45 \mathrm{E}+00$ & $\mathrm{~m}$ & La-134 & $6.45 E+00$ & $\mathrm{~m}$ & ICRP-38 & $6.67 \mathrm{E}+00$ & $\mathrm{~m}$ \\
\hline La-135 & La-135 & $1.95 \mathrm{E}+01$ & $\mathrm{~h}$ & La-135 & $1.95 \mathrm{E}+01$ & $\mathrm{~h}$ & La-135 & $1.95 \mathrm{E}+01$ & $\mathrm{~h}$ & La-135 & $1.95 \mathrm{E}+01$ & $\mathrm{~h}$ & & & & ICRP-38 & $1.95 \mathrm{E}+01$ & $\mathrm{~h}$ \\
\hline La-136 & La-136 & $9.87 \mathrm{E}+00$ & $\mathrm{~m}$ & & & & & & & La-136 & $9.87 \mathrm{E}+00$ & $m$ & La-136 & $9.87 \mathrm{E}+00$ & $\mathrm{~m}$ & ICRP-107 & $9.87 \mathrm{E}+00$ & $\mathrm{~m}$ \\
\hline La-137 & La-137 & $6.00 \mathrm{E}+04$ & $y$ & La-137 & $6.00 \mathrm{E}+04$ & $y$ & La-137 & $6.00 \mathrm{E}+04$ & $y$ & La-137 & $6.00 \mathrm{E}+04$ & $y$ & & & & ICRP-38 & $6.00 \mathrm{E}+04$ & $y$ \\
\hline La-138 & La-138 & $1.02 \mathrm{E}+11$ & $y$ & La-138 & $1.35 \mathrm{E}+11$ & $y$ & La-138 & $1.35 \mathrm{E}+11$ & $y$ & La-138 & $1.02 \mathrm{E}+11$ & $y$ & & & & ICRP-38 & $1.35 \mathrm{E}+11$ & $y$ \\
\hline La-140 & La-140 & $1.68 \mathrm{E}+00$ & $d$ & La-140 & $4.03 \mathrm{E}+01$ & $\mathrm{~h}$ & La-140 & $4.03 \mathrm{E}+01$ & $\mathrm{~h}$ & La-140 & $1.68 \mathrm{E}+00$ & $d$ & & & & ICRP-38 & $4.03 E+01$ & $\mathrm{~h}$ \\
\hline La-141 & La-141 & $3.92 \mathrm{E}+00$ & $\mathrm{~h}$ & La-141 & $3.93 \mathrm{E}+00$ & $\mathrm{~h}$ & La-141 & $3.93 \mathrm{E}+00$ & $\mathrm{~h}$ & La-141 & $3.92 \mathrm{E}+00$ & $\mathrm{~h}$ & & & & ICRP-38 & $3.93 \mathrm{E}+00$ & $\mathrm{~h}$ \\
\hline La-142 & La-142 & $9.11 \mathrm{E}+01$ & $\mathrm{~m}$ & La-142 & $9.25 \mathrm{E}+01$ & $\mathrm{~m}$ & La-142 & $9.25 \mathrm{E}+01$ & $\mathrm{~m}$ & La-142 & $9.11 \mathrm{E}+01$ & $\mathrm{~m}$ & & & & ICRP-38 & $9.25 \mathrm{E}+01$ & $\mathrm{~m}$ \\
\hline La-143 & La-143 & $1.42 \mathrm{E}+01$ & $m$ & La-143 & $1.42 \mathrm{E}+01$ & $m$ & La-143 & $1.42 \mathrm{E}+01$ & $\mathrm{~m}$ & La-143 & $1.42 \mathrm{E}+01$ & $\mathrm{~m}$ & & & & ICRP-38 & $1.42 \mathrm{E}+01$ & $m$ \\
\hline Lu-164 & & & & & & & & & & & & & Lu-164 & $3.14 \mathrm{E}+00$ & $\mathrm{~m}$ & JAERI & $3.14 \mathrm{E}+00$ & $\mathrm{~m}$ \\
\hline Lu-165 & Lu-165 & $1.07 \mathrm{E}+01$ & $\mathrm{~m}$ & & & & & & & Lu-165 & $1.07 \mathrm{E}+01$ & $\mathrm{~m}$ & Lu-165 & $1.07 \mathrm{E}+01$ & $m$ & ICRP-107 & $1.07 \mathrm{E}+01$ & $\mathrm{~m}$ \\
\hline Lu-166 & & & & & & & & & & & & & Lu-166 & $2.65 \mathrm{E}+00$ & $\mathrm{~m}$ & JAERI & $2.65 \mathrm{E}+00$ & $\mathrm{~m}$ \\
\hline Lu-166m & & & & & & & & & & & & & Lu-166m & $1.41 \mathrm{E}+00$ & $\mathrm{~m}$ & JAERI & $1.41 \mathrm{E}+00$ & $\mathrm{~m}$ \\
\hline Lu-167 & Lu-167 & $5.15 \mathrm{E}+01$ & $\mathrm{~m}$ & & & & & & & Lu-167 & $5.15 \mathrm{E}+01$ & $\mathrm{~m}$ & Lu-167 & $5.15 \mathrm{E}+01$ & $\mathrm{~m}$ & ICRP-107 & $5.15 \mathrm{E}+01$ & $\mathrm{~m}$ \\
\hline Lu-168m & & & & & & & & & & & & & Lu-168m & $6.70 \mathrm{E}+00$ & $m$ & JAERI & $6.70 \mathrm{E}+00$ & $\mathrm{~m}$ \\
\hline Lu-169 & Lu-169 & $3.41 \mathrm{E}+01$ & $\mathrm{~h}$ & Lu-169 & $3.41 \mathrm{E}+01$ & $\mathrm{~h}$ & Lu-169 & $3.41 \mathrm{E}+01$ & $\mathrm{~h}$ & Lu-169 & $3.41 \mathrm{E}+01$ & $\mathrm{~h}$ & & & & ICRP-38 & $3.41 \mathrm{E}+01$ & $\mathrm{~h}$ \\
\hline Lu-169m & Lu-169m & $1.60 \mathrm{E}+02$ & $\mathrm{~s}$ & & & & & & & Lu-169m & $1.60 \mathrm{E}+02$ & $\mathrm{~s}$ & Lu-169m & $2.67 \mathrm{E}+00$ & $\mathrm{~m}$ & ICRP-107 & $1.60 \mathrm{E}+02$ & $\mathrm{~s}$ \\
\hline Lu-170 & Lu-170 & $2.01 \mathrm{E}+00$ & $d$ & Lu-170 & $2.00 \mathrm{E}+00$ & $d$ & Lu-170 & $2.00 \mathrm{E}+00$ & d & Lu-170 & $2.01 \mathrm{E}+00$ & d & & & & ICRP-38 & $2.00 \mathrm{E}+00$ & $d$ \\
\hline Lu-171 & Lu-171 & $8.24 \mathrm{E}+00$ & $d$ & Lu-171 & $8.22 \mathrm{E}+00$ & $d$ & Lu-171 & $8.22 \mathrm{E}+00$ & $d$ & Lu-171 & $8.24 \mathrm{E}+00$ & $d$ & & & & ICRP-38 & $8.22 \mathrm{E}+00$ & $d$ \\
\hline Lu-171m & Lu-171m & $7.90 \mathrm{E}+01$ & $\mathrm{~s}$ & & & & & & & Lu-171m & $7.90 \mathrm{E}+01$ & $\mathrm{~s}$ & Lu-171m & $1.32 \mathrm{E}+00$ & $\mathrm{~m}$ & ICRP-107 & $7.90 \mathrm{E}+01$ & $\mathrm{~s}$ \\
\hline Lu-172 & Lu-172 & $6.70 \mathrm{E}+00$ & $d$ & Lu-172 & $6.70 E+00$ & $d$ & Lu-172 & $6.70 \mathrm{E}+00$ & $d$ & Lu-172 & $6.70 \mathrm{E}+00$ & $d$ & & & & ICRP-38 & $6.70 \mathrm{E}+00$ & $d$ \\
\hline Lu-172m & Lu-172m & $3.70 \mathrm{E}+00$ & $\mathrm{~m}$ & & & & & & & Lu-172m & $3.70 \mathrm{E}+00$ & $m$ & Lu-172m & $3.70 \mathrm{E}+00$ & $\mathrm{~m}$ & ICRP-107 & $3.70 \mathrm{E}+00$ & $\mathrm{~m}$ \\
\hline Lu-173 & Lu-173 & $1.37 \mathrm{E}+00$ & $y$ & Lu-173 & $1.37 \mathrm{E}+00$ & $y$ & Lu-173 & $1.37 \mathrm{E}+00$ & $y$ & Lu-173 & $1.37 \mathrm{E}+00$ & $y$ & & & & ICRP-38 & $1.37 \mathrm{E}+00$ & $y$ \\
\hline Lu-174 & Lu-174 & $3.31 \mathrm{E}+00$ & $y$ & Lu-174 & $3.31 \mathrm{E}+00$ & $y$ & Lu-174 & $3.31 \mathrm{E}+00$ & $y$ & Lu-174 & $3.31 \mathrm{E}+00$ & $y$ & & & & ICRP-38 & $3.31 \mathrm{E}+00$ & $y$ \\
\hline Lu-174m & Lu-174m & $1.42 \mathrm{E}+02$ & $d$ & Lu-174m & $1.42 \mathrm{E}+02$ & $d$ & Lu-174m & $1.42 \mathrm{E}+02$ & $d$ & Lu-174m & $1.42 \mathrm{E}+02$ & $d$ & & & & ICRP-38 & $1.42 \mathrm{E}+02$ & $d$ \\
\hline Lu-176 & Lu-176 & $3.85 \mathrm{E}+10$ & $y$ & Lu-176 & $3.60 \mathrm{E}+10$ & $y$ & Lu-176 & $3.60 \mathrm{E}+10$ & $y$ & Lu-176 & $3.85 \mathrm{E}+10$ & $y$ & & & & ICRP-38 & $3.60 \mathrm{E}+10$ & $y$ \\
\hline Lu-176m & Lu-176m & $3.64 \mathrm{E}+00$ & $\mathrm{~h}$ & Lu-176m & $3.68 \mathrm{E}+00$ & $\mathrm{~h}$ & Lu-176m & $3.68 \mathrm{E}+00$ & $\mathrm{~h}$ & Lu-176m & $3.64 \mathrm{E}+00$ & $\mathrm{~h}$ & & & & ICRP-38 & $3.68 \mathrm{E}+00$ & $\mathrm{~h}$ \\
\hline Lu-177 & Lu-177 & $6.65 \mathrm{E}+00$ & $d$ & Lu-177 & $6.71 \mathrm{E}+00$ & $\mathrm{~d}$ & Lu-177 & $6.71 \mathrm{E}+00$ & $d$ & Lu-177 & $6.65 \mathrm{E}+00$ & $d$ & & & & ICRP-38 & $6.71 \mathrm{E}+00$ & $d$ \\
\hline Lu-177m & Lu-177m & $1.60 \mathrm{E}+02$ & $d$ & Lu-177m & $1.61 \mathrm{E}+02$ & $d$ & Lu-177m & $1.61 \mathrm{E}+02$ & $d$ & Lu-177m & $1.60 \mathrm{E}+02$ & $d$ & & & & ICRP-38 & $1.61 \mathrm{E}+02$ & $d$ \\
\hline Lu-178 & Lu-178 & $2.84 \mathrm{E}+01$ & $\mathrm{~m}$ & Lu-178 & $2.84 \mathrm{E}+01$ & $\mathrm{~m}$ & Lu-178 & $2.84 \mathrm{E}+01$ & $\mathrm{~m}$ & Lu-178 & $2.84 \mathrm{E}+01$ & $\mathrm{~m}$ & & & & ICRP-38 & $2.84 \mathrm{E}+01$ & $\mathrm{~m}$ \\
\hline Lu-178m & Lu-178m & $2.31 \mathrm{E}+01$ & $m$ & Lu-178m & $2.27 \mathrm{E}+01$ & $m$ & Lu-178m & $2.27 \mathrm{E}+01$ & $m$ & Lu-178m & $2.31 \mathrm{E}+01$ & $m$ & & & & ICRP-38 & $2.27 \mathrm{E}+01$ & $\mathrm{~m}$ \\
\hline
\end{tabular}

D-18 


\begin{tabular}{|c|c|c|c|c|c|c|c|c|c|c|c|c|c|c|c|c|c|c|}
\hline \multirow{3}{*}{$\begin{array}{l}\text { MASTER } \\
\text { LIST } \\
\text { Lu-179 }\end{array}$} & \multicolumn{3}{|c|}{ DOE-STD-1196 } & \multicolumn{3}{|c|}{ FGR-12 } & \multicolumn{3}{|c|}{ ICRP-38 } & \multicolumn{3}{|c|}{ ICRP-107 } & \multicolumn{3}{|c|}{ JAERI } & \multirow{3}{*}{$\begin{array}{c}\begin{array}{c}\text { Selected } \\
\text { Half-Life } \\
\text { Reference }\end{array} \\
\text { ICRP-38 }\end{array}$} & \multirow{2}{*}{\multicolumn{2}{|c|}{$\begin{array}{c}\text { Selected } \\
\text { Half-Life } \\
\text { (original units) }\end{array}$}} \\
\hline & \multirow{2}{*}{$\begin{array}{l}\text { Nuclide } \\
\text { Lu-179 }\end{array}$} & \multicolumn{2}{|c|}{$\begin{array}{c}\text { Half-Life } \\
\text { (original units) }\end{array}$} & \multirow{2}{*}{$\begin{array}{l}\text { Nuclide } \\
\text { Lu-179 }\end{array}$} & \multicolumn{2}{|c|}{$\begin{array}{c}\text { Half-Life } \\
\text { (original units) }\end{array}$} & \multirow{2}{*}{$\begin{array}{l}\text { Nuclide } \\
\text { Lu-179 }\end{array}$} & \multicolumn{2}{|c|}{$\begin{array}{c}\text { Half-Life } \\
\text { (original units) }\end{array}$} & \multirow{2}{*}{$\begin{array}{l}\text { Nuclide } \\
\text { Lu-179 }\end{array}$} & \multicolumn{2}{|c|}{$\begin{array}{c}\text { Half-Life } \\
\text { (original units) }\end{array}$} & \multirow[t]{2}{*}{ Nuclide } & \multicolumn{2}{|c|}{$\begin{array}{c}\text { Half-Life } \\
\text { (original units) }\end{array}$} & & & \\
\hline & & $4.59 \mathrm{E}+00$ & $\mathrm{~h}$ & & $4.59 E+00$ & $\mathrm{~h}$ & & $4.59 \mathrm{E}+00$ & $\mathrm{~h}$ & & $4.59 \mathrm{E}+00$ & $\mathrm{~h}$ & & & & & $4.59 \mathrm{E}+00$ & $\mathrm{~h}$ \\
\hline Lu-180 & Lu-180 & $5.70 \mathrm{E}+00$ & $\mathrm{~m}$ & & & & & & & Lu-180 & $5.70 \mathrm{E}+00$ & $\mathrm{~m}$ & & & & ICRP-107 & $5.70 \mathrm{E}+00$ & $\mathrm{~m}$ \\
\hline Lu-181 & Lu-181 & $3.50 \mathrm{E}+00$ & $\mathrm{~m}$ & & & & & & & Lu-181 & $3.50 \mathrm{E}+00$ & $\mathrm{~m}$ & & & & ICRP-107 & $3.50 \mathrm{E}+00$ & $\mathrm{~m}$ \\
\hline Md-257 & & & & Md-257 & $5.20 \mathrm{E}+00$ & $\mathrm{~h}$ & Md-257 & $5.20 \mathrm{E}+00$ & $\mathrm{~h}$ & & & & & & & ICRP-38 & $5.20 \mathrm{E}+00$ & $\mathrm{~h}$ \\
\hline Md-258 & & & & Md-258 & $5.50 \mathrm{E}+01$ & $d$ & Md-258 & $5.50 \mathrm{E}+01$ & $d$ & & & & & & & ICRP-38 & $5.50 \mathrm{E}+01$ & $\mathrm{~d}$ \\
\hline Mg-27 & $M g-27$ & $9.46 \mathrm{E}+00$ & $\mathrm{~m}$ & & & & & & & $M g-27$ & $9.46 \mathrm{E}+00$ & $\mathrm{~m}$ & $M g-27$ & $9.46 \mathrm{E}+00$ & $\mathrm{~m}$ & ICRP-107 & $9.46 \mathrm{E}+00$ & $\mathrm{~m}$ \\
\hline $\mathrm{Mg}-28$ & $\mathrm{Mg}-28$ & $2.09 \mathrm{E}+01$ & $\mathrm{~h}$ & Mg-28 & $2.09 \mathrm{E}+01$ & $\mathrm{~h}$ & $M g-28$ & $2.09 \mathrm{E}+01$ & $\mathrm{~h}$ & $\mathrm{Mg}-28$ & $2.09 \mathrm{E}+01$ & $\mathrm{~h}$ & & & & ICRP-38 & $2.09 \mathrm{E}+01$ & $\mathrm{~h}$ \\
\hline $\mathrm{Mn}-50 \mathrm{~m}$ & $\mathrm{Mn}-50 \mathrm{~m}$ & $1.75 \mathrm{E}+00$ & $\mathrm{~m}$ & & & & & & & $\mathrm{Mn}-50 \mathrm{~m}$ & $1.75 \mathrm{E}+00$ & $\mathrm{~m}$ & & & & ICRP-107 & $1.75 \mathrm{E}+00$ & $\mathrm{~m}$ \\
\hline $\mathrm{Mn}-51$ & $M n-51$ & $4.62 \mathrm{E}+01$ & $\mathrm{~m}$ & Mn-51 & $4.62 \mathrm{E}+01$ & $\mathrm{~m}$ & Mn-51 & $4.62 \mathrm{E}+01$ & $\mathrm{~m}$ & $M n-51$ & $4.62 \mathrm{E}+01$ & $\mathrm{~m}$ & & & & ICRP-38 & $4.62 \mathrm{E}+01$ & $\mathrm{~m}$ \\
\hline Mn-52 & $\mathrm{Mn}-52$ & $5.59 \mathrm{E}+00$ & $d$ & $\mathrm{Mn}-52$ & $5.59 \mathrm{E}+00$ & $d$ & $\mathrm{Mn}-52$ & $5.59 \mathrm{E}+00$ & $d$ & $M n-52$ & $5.59 \mathrm{E}+00$ & $d$ & & & & ICRP-38 & $5.59 \mathrm{E}+00$ & $\mathrm{~d}$ \\
\hline$M n-52 m$ & $M n-52 m$ & $2.11 \mathrm{E}+01$ & $\mathrm{~m}$ & $M n-52 m$ & $2.11 \mathrm{E}+01$ & $\mathrm{~m}$ & $M n-52 m$ & $2.11 \mathrm{E}+01$ & $\mathrm{~m}$ & $M n-52 m$ & $2.11 \mathrm{E}+01$ & $\mathrm{~m}$ & & & & ICRP-38 & $2.11 \mathrm{E}+01$ & $\mathrm{~m}$ \\
\hline Mn-53 & $\mathrm{Mn}-53$ & $3.70 \mathrm{E}+06$ & $\mathrm{y}$ & $M n-53$ & $3.70 \mathrm{E}+06$ & $y$ & $M n-53$ & $3.70 \mathrm{E}+06$ & $y$ & $\mathrm{Mn}-53$ & $3.70 \mathrm{E}+06$ & $\mathrm{y}$ & & & & ICRP-38 & $3.70 \mathrm{E}+06$ & $\mathrm{y}$ \\
\hline Mn-54 & Mn-54 & $3.12 \mathrm{E}+02$ & d & $\mathrm{Mn}-54$ & $3.13 \mathrm{E}+02$ & d & $\mathrm{Mn}-54$ & $3.13 \mathrm{E}+02$ & $d$ & Mn-54 & $3.12 \mathrm{E}+02$ & $d$ & & & & ICRP-38 & $3.13 \mathrm{E}+02$ & d \\
\hline Mn-56 & Mn-56 & $2.58 \mathrm{E}+00$ & $\mathrm{~h}$ & Mn-56 & $2.58 \mathrm{E}+00$ & $\mathrm{~h}$ & Mn-56 & $2.58 \mathrm{E}+00$ & $\mathrm{~h}$ & Mn-56 & $2.58 \mathrm{E}+00$ & $\mathrm{~h}$ & & & & ICRP-38 & $2.58 \mathrm{E}+00$ & $\mathrm{~h}$ \\
\hline Mn-57 & $\mathrm{Mn}-57$ & $8.54 \mathrm{E}+01$ & $\mathrm{~s}$ & & & & & & & $\mathrm{Mn}-57$ & $8.54 \mathrm{E}+01$ & $s$ & $M n-57$ & $1.42 \mathrm{E}+00$ & $\mathrm{~m}$ & ICRP-107 & $8.54 \mathrm{E}+01$ & $s$ \\
\hline $\mathrm{Mn}-58 \mathrm{~m}$ & $M n-58 m$ & $6.52 \mathrm{E}+01$ & $s$ & & & & & & & $M n-58 m$ & $6.52 \mathrm{E}+01$ & $s$ & $M n-58 m$ & $1.09 \mathrm{E}+00$ & $m$ & ICRP-107 & $6.52 \mathrm{E}+01$ & $s$ \\
\hline Mo-101 & Mo-101 & $1.46 \mathrm{E}+01$ & $\mathrm{~m}$ & Mo-101 & $1.46 \mathrm{E}+01$ & $\mathrm{~m}$ & Mo-101 & $1.46 \mathrm{E}+01$ & $\mathrm{~m}$ & Mo-101 & $1.46 \mathrm{E}+01$ & $\mathrm{~m}$ & & & & ICRP-38 & $1.46 \mathrm{E}+01$ & $\mathrm{~m}$ \\
\hline Mo-102 & Mo-102 & $1.13 \mathrm{E}+01$ & $m$ & & & & & & & Mo-102 & $1.13 \mathrm{E}+01$ & $\mathrm{~m}$ & Mo-102 & $1.13 \mathrm{E}+01$ & $\mathrm{~m}$ & ICRP-107 & $1.13 \mathrm{E}+01$ & $\mathrm{~m}$ \\
\hline Mo-89 & Mo-89 & $2.11 E+00$ & $\mathrm{~m}$ & & & & & & & Mo-89 & $2.11 \mathrm{E}+00$ & $\mathrm{~m}$ & & & & ICRP-107 & $2.11 E+00$ & $\mathrm{~m}$ \\
\hline Mo-90 & Mo-90 & $5.56 \mathrm{E}+00$ & $\mathrm{~h}$ & Mo-90 & $5.67 \mathrm{E}+00$ & $\mathrm{~h}$ & Mo-90 & $5.67 \mathrm{E}+00$ & $\mathrm{~h}$ & Mo-90 & $5.56 \mathrm{E}+00$ & $\mathrm{~h}$ & & & & ICRP-38 & $5.67 \mathrm{E}+00$ & $\mathrm{~h}$ \\
\hline Mo-91 & Mo-91 & $1.55 \mathrm{E}+01$ & $\mathrm{~m}$ & & & & & & & Mo-91 & $1.55 \mathrm{E}+01$ & $m$ & Mo-91 & $1.55 \mathrm{E}+01$ & $\mathrm{~m}$ & ICRP-107 & $1.55 \mathrm{E}+01$ & $\mathrm{~m}$ \\
\hline Mo-91m & Mo-91m & $6.46 \mathrm{E}+01$ & $\mathrm{~s}$ & & & & & & & Mo-91m & $6.46 \mathrm{E}+01$ & $\mathrm{~s}$ & & & & ICRP-107 & $6.46 \mathrm{E}+01$ & $\mathrm{~s}$ \\
\hline Mo-93 & Mo-93 & $4.00 \mathrm{E}+03$ & $y$ & Mo-93 & $3.50 \mathrm{E}+03$ & $\mathrm{y}$ & Mo-93 & $3.50 \mathrm{E}+03$ & $y$ & Mo-93 & $4.00 \mathrm{E}+03$ & $y$ & & & & ICRP-38 & $3.50 \mathrm{E}+03$ & $y$ \\
\hline Mo-93m & Mo-93m & $6.85 \mathrm{E}+00$ & $\mathrm{~h}$ & Mo-93m & $6.85 \mathrm{E}+00$ & $\mathrm{~h}$ & Mo-93m & $6.85 \mathrm{E}+00$ & $\mathrm{~h}$ & Mo-93m & $6.85 \mathrm{E}+00$ & $\mathrm{~h}$ & & & & ICRP-38 & $6.85 \mathrm{E}+00$ & $\mathrm{~h}$ \\
\hline Mo-99 & Mo-99 & $6.59 \mathrm{E}+01$ & $\mathrm{~h}$ & Mo-99 & $6.60 \mathrm{E}+01$ & $\mathrm{~h}$ & Mo-99 & $6.60 \mathrm{E}+01$ & $\mathrm{~h}$ & Mo-99 & $6.59 \mathrm{E}+01$ & $\mathrm{~h}$ & & & & ICRP-38 & $6.60 \mathrm{E}+01$ & $\mathrm{~h}$ \\
\hline $\mathrm{N}-13$ & $\mathrm{~N}-13$ & $9.97 \mathrm{E}+00$ & $\mathrm{~m}$ & $\mathrm{~N}-13$ & $9.97 \mathrm{E}+00$ & $\mathrm{~m}$ & $\mathrm{~N}-13$ & $9.97 \mathrm{E}+00$ & $\mathrm{~m}$ & $\mathrm{~N}-13$ & $9.97 \mathrm{E}+00$ & $\mathrm{~m}$ & $\mathrm{~N}-13$ & $9.97 \mathrm{E}+00$ & $\mathrm{~m}$ & ICRP-38 & $9.97 \mathrm{E}+00$ & $\mathrm{~m}$ \\
\hline $\mathrm{N}-16$ & $\mathrm{~N}-16$ & $7.13 \mathrm{E}+00$ & $\mathrm{~s}$ & & & & & & & $\mathrm{~N}-16$ & $7.13 \mathrm{E}+00$ & $\mathrm{~s}$ & & & & ICRP-107 & $7.13 \mathrm{E}+00$ & $\mathrm{~s}$ \\
\hline Na-22 & $\mathrm{Na}-22$ & $2.60 \mathrm{E}+00$ & $y$ & $\mathrm{Na}-22$ & $2.60 \mathrm{E}+00$ & $\mathrm{y}$ & $\mathrm{Na}-22$ & $2.60 \mathrm{E}+00$ & $\mathrm{y}$ & $\mathrm{Na}-22$ & $2.60 \mathrm{E}+00$ & $y$ & & & & ICRP-38 & $2.60 \mathrm{E}+00$ & $y$ \\
\hline $\mathrm{Na}-24$ & $\mathrm{Na}-24$ & $1.50 \mathrm{E}+01$ & $\mathrm{~h}$ & $\mathrm{Na}-24$ & $1.50 \mathrm{E}+01$ & $\mathrm{~h}$ & $\mathrm{Na}-24$ & $1.50 \mathrm{E}+01$ & $\mathrm{~h}$ & $\mathrm{Na}-24$ & $1.50 \mathrm{E}+01$ & $\mathrm{~h}$ & & & & ICRP-38 & $1.50 \mathrm{E}+01$ & $\mathrm{~h}$ \\
\hline Nb-87 & $\mathrm{Nb}-87$ & $3.75 \mathrm{E}+00$ & $\mathrm{~m}$ & & & & & & & $\mathrm{Nb}-87$ & $3.75 \mathrm{E}+00$ & $\mathrm{~m}$ & & & & ICRP-107 & $3.75 E+00$ & $\mathrm{~m}$ \\
\hline $\mathrm{Nb}-88$ & $\mathrm{Nb}-88$ & $1.45 \mathrm{E}+01$ & $\mathrm{~m}$ & $\mathrm{Nb}-88$ & $1.43 \mathrm{E}+01$ & $\mathrm{~m}$ & $\mathrm{Nb}-88$ & $1.43 \mathrm{E}+01$ & $\mathrm{~m}$ & $\mathrm{Nb}-88$ & $1.45 \mathrm{E}+01$ & $\mathrm{~m}$ & & & & ICRP-38 & $1.43 \mathrm{E}+01$ & $\mathrm{~m}$ \\
\hline $\mathrm{Nb}-88 \mathrm{~m}$ & $\mathrm{Nb}-88 \mathrm{~m}$ & $7.78 \mathrm{E}+00$ & $\mathrm{~m}$ & & & & & & & $\mathrm{Nb}-88 \mathrm{~m}$ & $7.78 \mathrm{E}+00$ & $\mathrm{~m}$ & $\mathrm{Nb}-88 \mathrm{~m}$ & $7.80 \mathrm{E}+00$ & $m$ & ICRP-107 & $7.78 \mathrm{E}+00$ & $\mathrm{~m}$ \\
\hline Nb-89I & $\mathrm{Nb}-89$ & $2.03 \mathrm{E}+00$ & $\mathrm{~h}$ & $\mathrm{Nb}-89 \mathrm{~b}$ & $1.22 \mathrm{E}+02$ & $\mathrm{~m}$ & $\mathrm{Nb}-89 \mathrm{~b}$ & $1.22 \mathrm{E}+02$ & $\mathrm{~m}$ & $\mathrm{Nb}-89$ & $2.03 \mathrm{E}+00$ & $\mathrm{~h}$ & & & & ICRP-38 & $1.22 \mathrm{E}+02$ & $\mathrm{~m}$ \\
\hline $\mathrm{Nb}-89 \mathrm{~s}$ & $\mathrm{Nb}-89 \mathrm{~m}$ & $6.60 \mathrm{E}+01$ & $m$ & $\mathrm{Nb}-89 \mathrm{a}$ & $6.60 \mathrm{E}+01$ & $\mathrm{~m}$ & $\mathrm{Nb}-89 \mathrm{a}$ & $6.60 \mathrm{E}+01$ & $m$ & $\mathrm{Nb}-89 \mathrm{~m}$ & $6.60 \mathrm{E}+01$ & $m$ & & & & ICRP-38 & $6.60 \mathrm{E}+01$ & $\mathrm{~m}$ \\
\hline $\mathrm{Nb}-90$ & $\mathrm{Nb}-90$ & $1.46 \mathrm{E}+01$ & $\mathrm{~h}$ & $\mathrm{Nb}-90$ & $1.46 \mathrm{E}+01$ & $\mathrm{~h}$ & $\mathrm{Nb}-90$ & $1.46 \mathrm{E}+01$ & $\mathrm{~h}$ & $\mathrm{Nb}-90$ & $1.46 \mathrm{E}+01$ & $\mathrm{~h}$ & & & & ICRP-38 & $1.46 \mathrm{E}+01$ & $\mathrm{~h}$ \\
\hline $\mathrm{Nb}-91$ & $\mathrm{Nb}-91$ & $6.80 \mathrm{E}+02$ & $y$ & & & & & & & $\mathrm{Nb}-91$ & $6.80 \mathrm{E}+02$ & $y$ & $\mathrm{Nb}-91$ & $6.80 \mathrm{E}+02$ & $y$ & ICRP-107 & $6.80 \mathrm{E}+02$ & $y$ \\
\hline $\mathrm{Nb}-91 \mathrm{~m}$ & $\mathrm{Nb}-91 \mathrm{~m}$ & $6.09 \mathrm{E}+01$ & $d$ & & & & & & & $\mathrm{Nb}-91 \mathrm{~m}$ & $6.09 \mathrm{E}+01$ & $d$ & $\mathrm{Nb}-91 \mathrm{~m}$ & $6.09 \mathrm{E}+01$ & $d$ & ICRP-107 & $6.09 \mathrm{E}+01$ & d \\
\hline $\mathrm{Nb}-92$ & $\mathrm{Nb}-92$ & $3.47 E+07$ & $y$ & & & & & & & $\mathrm{Nb}-92$ & $3.47 \mathrm{E}+07$ & $y$ & $\mathrm{Nb}-92$ & $3.47 E+07$ & $y$ & ICRP-107 & $3.47 \mathrm{E}+07$ & $y$ \\
\hline $\mathrm{Nb}-92 \mathrm{~m}$ & $\mathrm{Nb}-92 \mathrm{~m}$ & $1.02 \mathrm{E}+01$ & d & & & & & & & $\mathrm{Nb}-92 \mathrm{~m}$ & $1.02 \mathrm{E}+01$ & d & $\mathrm{Nb}-92 \mathrm{~m}$ & $1.02 \mathrm{E}+01$ & d & ICRP-107 & $1.02 \mathrm{E}+01$ & d \\
\hline Nb-93m & $\mathrm{Nb}-93 \mathrm{~m}$ & $1.61 \mathrm{E}+01$ & $y$ & $\mathrm{Nb}-93 \mathrm{~m}$ & $1.36 \mathrm{E}+01$ & $\mathrm{y}$ & $\mathrm{Nb}-93 \mathrm{~m}$ & $1.36 \mathrm{E}+01$ & $y$ & $\mathrm{Nb}-93 \mathrm{~m}$ & $1.61 \mathrm{E}+01$ & $y$ & & & & ICRP-38 & $1.36 \mathrm{E}+01$ & $\mathrm{y}$ \\
\hline
\end{tabular}

D-19 


\begin{tabular}{|c|c|c|c|c|c|c|c|c|c|c|c|c|c|c|c|c|c|c|}
\hline \multirow{3}{*}{$\begin{array}{l}\text { MASTER } \\
\text { LIST } \\
\mathrm{Nb}-94\end{array}$} & \multicolumn{3}{|c|}{ DOE-STD-1196 } & \multicolumn{3}{|c|}{ FGR-12 } & \multicolumn{3}{|c|}{ ICRP-38 } & \multicolumn{3}{|c|}{ ICRP-107 } & \multicolumn{3}{|c|}{ JAERI } & \multirow{3}{*}{$\begin{array}{c}\begin{array}{c}\text { Selected } \\
\text { Half-Life } \\
\text { Reference }\end{array} \\
\text { ICRP-38 }\end{array}$} & \multirow{2}{*}{\multicolumn{2}{|c|}{$\begin{array}{c}\text { Selected } \\
\text { Half-Life } \\
\text { (original units) }\end{array}$}} \\
\hline & \multirow{2}{*}{$\begin{array}{l}\text { Nuclide } \\
\mathrm{Nb}-94\end{array}$} & \multicolumn{2}{|c|}{$\begin{array}{c}\text { Half-Life } \\
\text { (original units) }\end{array}$} & \multirow{2}{*}{$\begin{array}{l}\text { Nuclide } \\
\mathrm{Nb}-94\end{array}$} & \multicolumn{2}{|c|}{$\begin{array}{c}\text { Half-Life } \\
\text { (original units) }\end{array}$} & \multirow{2}{*}{$\begin{array}{l}\text { Nuclide } \\
\text { Nb-94 }\end{array}$} & \multicolumn{2}{|c|}{$\begin{array}{c}\text { Half-Life } \\
\text { (original units) }\end{array}$} & \multirow{2}{*}{$\begin{array}{l}\text { Nuclide } \\
\text { Nb-94 }\end{array}$} & \multicolumn{2}{|c|}{$\begin{array}{c}\text { Half-Life } \\
\text { (original units) }\end{array}$} & \multirow[t]{2}{*}{ Nuclide } & \multicolumn{2}{|c|}{$\begin{array}{c}\text { Half-Life } \\
\text { (original units) }\end{array}$} & & & \\
\hline & & $2.03 E+04$ & $y$ & & $2.03 E+04$ & $y$ & & $2.03 E+04$ & $y$ & & $2.03 E+04$ & $y$ & & & & & $2.03 E+04$ & $y$ \\
\hline $\mathrm{Nb}-94 \mathrm{~m}$ & $\mathrm{Nb}-94 \mathrm{~m}$ & $6.26 \mathrm{E}+00$ & $\mathrm{~m}$ & & & & & & & $\mathrm{Nb}-94 \mathrm{~m}$ & $6.26 \mathrm{E}+00$ & $\mathrm{~m}$ & $\mathrm{Nb}-94 \mathrm{~m}$ & $6.26 \mathrm{E}+00$ & $\mathrm{~m}$ & ICRP-107 & $6.26 \mathrm{E}+00$ & $\mathrm{~m}$ \\
\hline $\mathrm{Nb}-95$ & $\mathrm{Nb}-95$ & $3.50 \mathrm{E}+01$ & $\mathrm{~d}$ & $\mathrm{Nb}-95$ & $3.52 \mathrm{E}+01$ & d & $\mathrm{Nb}-95$ & $3.52 \mathrm{E}+01$ & d & $\mathrm{Nb}-95$ & $3.50 \mathrm{E}+01$ & d & & & & ICRP-38 & $3.52 \mathrm{E}+01$ & $\mathrm{~d}$ \\
\hline $\mathrm{Nb}-95 \mathrm{~m}$ & $\mathrm{Nb}-95 \mathrm{~m}$ & $3.61 \mathrm{E}+00$ & $d$ & $\mathrm{Nb}-95 \mathrm{~m}$ & $8.66 \mathrm{E}+01$ & $\mathrm{~h}$ & $\mathrm{Nb}-95 \mathrm{~m}$ & $8.66 \mathrm{E}+01$ & $\mathrm{~h}$ & $\mathrm{Nb}-95 \mathrm{~m}$ & $3.61 \mathrm{E}+00$ & $d$ & & & & ICRP-38 & $8.66 \mathrm{E}+01$ & $\mathrm{~h}$ \\
\hline $\mathrm{Nb}-96$ & $\mathrm{Nb}-96$ & $2.34 \mathrm{E}+01$ & $\mathrm{~h}$ & $\mathrm{Nb}-96$ & $2.34 \mathrm{E}+01$ & $\mathrm{~h}$ & $\mathrm{Nb}-96$ & $2.34 \mathrm{E}+01$ & $\mathrm{~h}$ & $\mathrm{Nb}-96$ & $2.34 \mathrm{E}+01$ & $\mathrm{~h}$ & & & & ICRP-38 & $2.34 \mathrm{E}+01$ & $\mathrm{~h}$ \\
\hline Nb-97 & $\mathrm{Nb}-97$ & $7.21 \mathrm{E}+01$ & $\mathrm{~m}$ & $\mathrm{Nb}-97$ & $7.21 \mathrm{E}+01$ & $\mathrm{~m}$ & $\mathrm{Nb}-97$ & $7.21 \mathrm{E}+01$ & $\mathrm{~m}$ & $\mathrm{Nb}-97$ & $7.21 \mathrm{E}+01$ & $m$ & & & & ICRP-38 & $7.21 \mathrm{E}+01$ & $\mathrm{~m}$ \\
\hline $\mathrm{Nb}-97 \mathrm{~m}$ & & & & $\mathrm{Nb}-97 \mathrm{~m}$ & $6.00 \mathrm{E}+01$ & $\mathrm{~s}$ & Nb-97m & $6.00 \mathrm{E}+01$ & $\mathrm{~s}$ & & & & & & & ICRP-38 & $6.00 \mathrm{E}+01$ & $\mathrm{~s}$ \\
\hline Nb-98 & $\mathrm{Nb}-98 \mathrm{~m}$ & $5.13 \mathrm{E}+01$ & $\mathrm{~m}$ & $\mathrm{Nb}-98$ & $5.15 E+01$ & $m$ & $\mathrm{Nb}-98$ & $5.15 E+01$ & $m$ & $\mathrm{Nb}-98 \mathrm{~m}$ & $5.13 \mathrm{E}+01$ & $\mathrm{~m}$ & & & & ICRP-38 & $5.15 E+01$ & $m$ \\
\hline Nb-99 & $\mathrm{Nb}-99$ & $1.50 \mathrm{E}+01$ & $\mathrm{~s}$ & & & & & & & $\mathrm{Nb}-99$ & $1.50 \mathrm{E}+01$ & $\mathrm{~s}$ & & & & ICRP-107 & $1.50 \mathrm{E}+01$ & $\mathrm{~s}$ \\
\hline $\mathrm{Nb}-99 \mathrm{~m}$ & $\mathrm{Nb}-99 \mathrm{~m}$ & $2.60 \mathrm{E}+00$ & $\mathrm{~m}$ & & & & & & & $\mathrm{Nb}-99 \mathrm{~m}$ & $2.60 \mathrm{E}+00$ & $\mathrm{~m}$ & & & & ICRP-107 & $2.60 \mathrm{E}+00$ & $\mathrm{~m}$ \\
\hline Nd-134 & $\mathrm{Nd}-134$ & $8.50 \mathrm{E}+00$ & $\mathrm{~m}$ & & & & & & & Nd-134 & $8.50 \mathrm{E}+00$ & $\mathrm{~m}$ & & & & ICRP-107 & $8.50 \mathrm{E}+00$ & $\mathrm{~m}$ \\
\hline Nd-135 & $\mathrm{Nd}-135$ & $1.24 \mathrm{E}+01$ & $\mathrm{~m}$ & & & & & & & $\mathrm{Nd}-135$ & $1.24 \mathrm{E}+01$ & $m$ & $\mathrm{Nd}-135$ & $1.24 \mathrm{E}+01$ & $\mathrm{~m}$ & ICRP-107 & $1.24 \mathrm{E}+01$ & $\mathrm{~m}$ \\
\hline Nd-136 & Nd-136 & $5.07 E+01$ & $\mathrm{~m}$ & $\mathrm{Nd}-136$ & $5.07 \mathrm{E}+01$ & $\mathrm{~m}$ & Nd-136 & $5.07 \mathrm{E}+01$ & $\mathrm{~m}$ & Nd-136 & $5.07 \mathrm{E}+01$ & $\mathrm{~m}$ & & & & ICRP-38 & $5.07 \mathrm{E}+01$ & $\mathrm{~m}$ \\
\hline Nd-137 & $\mathrm{Nd}-137$ & $3.85 \mathrm{E}+01$ & $m$ & & & & & & & Nd-137 & $3.85 \mathrm{E}+01$ & $m$ & $\mathrm{Nd}-137$ & $3.85 \mathrm{E}+01$ & $\mathrm{~m}$ & ICRP-107 & $3.85 \mathrm{E}+01$ & $m$ \\
\hline Nd-138 & $\mathrm{Nd}-138$ & $5.04 \mathrm{E}+00$ & $\mathrm{~h}$ & $\mathrm{Nd}-138$ & $5.04 \mathrm{E}+00$ & $\mathrm{~h}$ & $\mathrm{Nd}-138$ & $5.04 \mathrm{E}+00$ & $\mathrm{~h}$ & $\mathrm{Nd}-138$ & $5.04 \mathrm{E}+00$ & $\mathrm{~h}$ & & & & ICRP-38 & $5.04 \mathrm{E}+00$ & $\mathrm{~h}$ \\
\hline Nd-139 & $\mathrm{Nd}-139$ & $2.97 \mathrm{E}+01$ & $\mathrm{~m}$ & $\mathrm{Nd}-139$ & $2.97 \mathrm{E}+01$ & $\mathrm{~m}$ & $\mathrm{Nd}-139$ & $2.97 \mathrm{E}+01$ & $\mathrm{~m}$ & $\mathrm{Nd}-139$ & $2.97 \mathrm{E}+01$ & $\mathrm{~m}$ & & & & ICRP-38 & $2.97 \mathrm{E}+01$ & $\mathrm{~m}$ \\
\hline $\mathrm{Nd}-139 \mathrm{~m}$ & $\mathrm{Nd}-139 \mathrm{~m}$ & $5.50 \mathrm{E}+00$ & $\mathrm{~h}$ & $\mathrm{Nd}-139 \mathrm{~m}$ & $5.50 \mathrm{E}+00$ & $\mathrm{~h}$ & Nd-139m & $5.50 \mathrm{E}+00$ & $\mathrm{~h}$ & $\mathrm{Nd}-139 \mathrm{~m}$ & $5.50 \mathrm{E}+00$ & $\mathrm{~h}$ & & & & ICRP-38 & $5.50 \mathrm{E}+00$ & $\mathrm{~h}$ \\
\hline Nd-140 & $\mathrm{Nd}-140$ & $3.37 E+00$ & $d$ & & & & & & & $\mathrm{Nd}-140$ & $3.37 \mathrm{E}+00$ & $d$ & $\mathrm{Nd}-140$ & $3.37 \mathrm{E}+00$ & $d$ & ICRP-107 & $3.37 \mathrm{E}+00$ & $\mathrm{~d}$ \\
\hline Nd-141 & $\mathrm{Nd}-141$ & $2.49 \mathrm{E}+00$ & $\mathrm{~h}$ & $\mathrm{Nd}-141$ & $2.49 \mathrm{E}+00$ & $\mathrm{~h}$ & Nd-141 & $2.49 \mathrm{E}+00$ & $\mathrm{~h}$ & $\mathrm{Nd}-141$ & $2.49 \mathrm{E}+00$ & $\mathrm{~h}$ & & & & ICRP-38 & $2.49 \mathrm{E}+00$ & $\mathrm{~h}$ \\
\hline $\mathrm{Nd}-141 \mathrm{~m}$ & $\mathrm{Nd}-141 \mathrm{~m}$ & $6.20 \mathrm{E}+01$ & $\mathrm{~s}$ & $\mathrm{Nd}-141 \mathrm{~m}$ & $6.24 \mathrm{E}+01$ & $\mathrm{~s}$ & $\mathrm{Nd}-141 \mathrm{~m}$ & $6.24 \mathrm{E}+01$ & $s$ & $\mathrm{Nd}-141 \mathrm{~m}$ & $6.20 \mathrm{E}+01$ & $s$ & $\mathrm{Nd}-141 \mathrm{~m}$ & $1.03 \mathrm{E}+00$ & $\mathrm{~m}$ & ICRP-38 & $6.24 \mathrm{E}+01$ & $\mathrm{~s}$ \\
\hline Nd-144 & $\mathrm{Nd}-144$ & $2.29 \mathrm{E}+15$ & $y$ & & & & & & & $\mathrm{Nd}-144$ & $2.29 \mathrm{E}+15$ & $y$ & $\mathrm{Nd}-144$ & $2.29 \mathrm{E}+15$ & $y$ & ICRP-107 & $2.29 \mathrm{E}+15$ & $y$ \\
\hline Nd-147 & $\mathrm{Nd}-147$ & $1.10 \mathrm{E}+01$ & $d$ & $\mathrm{Nd}-147$ & $1.10 \mathrm{E}+01$ & $d$ & $\mathrm{Nd}-147$ & $1.10 \mathrm{E}+01$ & $d$ & $\mathrm{Nd}-147$ & $1.10 \mathrm{E}+01$ & $d$ & & & & ICRP-38 & $1.10 \mathrm{E}+01$ & $d$ \\
\hline Nd-149 & Nd-149 & $1.73 \mathrm{E}+00$ & $\mathrm{~h}$ & Nd-149 & $1.73 \mathrm{E}+00$ & $\mathrm{~h}$ & Nd-149 & $1.73 \mathrm{E}+00$ & $\mathrm{~h}$ & Nd-149 & $1.73 \mathrm{E}+00$ & $\mathrm{~h}$ & & & & ICRP-38 & $1.73 \mathrm{E}+00$ & $\mathrm{~h}$ \\
\hline Nd-151 & Nd-151 & $1.24 \mathrm{E}+01$ & $m$ & Nd-151 & $1.24 \mathrm{E}+01$ & $m$ & Nd-151 & $1.24 \mathrm{E}+01$ & $m$ & Nd-151 & $1.24 \mathrm{E}+01$ & $m$ & & & & ICRP-38 & $1.24 \mathrm{E}+01$ & $m$ \\
\hline Nd-152 & Nd-152 & $1.14 \mathrm{E}+01$ & $m$ & & & & & & & Nd-152 & $1.14 \mathrm{E}+01$ & $m$ & $\mathrm{Nd}-152$ & $1.14 \mathrm{E}+01$ & $\mathrm{~m}$ & ICRP-107 & $1.14 \mathrm{E}+01$ & $m$ \\
\hline $\mathrm{Ne}-19$ & $\mathrm{Ne}-19$ & $1.72 \mathrm{E}+01$ & $\mathrm{~s}$ & $\mathrm{Ne}-19$ & $1.72 \mathrm{E}+01$ & $\mathrm{~s}$ & $\mathrm{Ne}-19$ & $1.72 \mathrm{E}+01$ & $\mathrm{~s}$ & $\mathrm{Ne}-19$ & $1.72 \mathrm{E}+01$ & $\mathrm{~s}$ & & & & ICRP-38 & $1.72 \mathrm{E}+01$ & $\mathrm{~s}$ \\
\hline $\mathrm{Ne}-24$ & $\mathrm{Ne}-24$ & $3.38 \mathrm{E}+00$ & $\mathrm{~m}$ & & & & & & & $\mathrm{Ne}-24$ & $3.38 \mathrm{E}+00$ & $\mathrm{~m}$ & & & & ICRP-107 & $3.38 \mathrm{E}+00$ & $\mathrm{~m}$ \\
\hline Ni-56 & $\mathrm{Ni}-56$ & $6.08 \mathrm{E}+00$ & $d$ & $\mathrm{Ni}-56$ & $6.10 \mathrm{E}+00$ & $d$ & $\mathrm{Ni}-56$ & $6.10 \mathrm{E}+00$ & $d$ & $\mathrm{Ni}-56$ & $6.08 \mathrm{E}+00$ & $d$ & & & & ICRP-38 & $6.10 \mathrm{E}+00$ & $\mathrm{~d}$ \\
\hline Ni-57 & $\mathrm{Ni}-57$ & $3.56 \mathrm{E}+01$ & $\mathrm{~h}$ & $\mathrm{Ni}-57$ & $3.61 \mathrm{E}+01$ & $\mathrm{~h}$ & $\mathrm{Ni}-57$ & $3.61 \mathrm{E}+01$ & $\mathrm{~h}$ & $\mathrm{Ni}-57$ & $3.56 \mathrm{E}+01$ & $\mathrm{~h}$ & & & & ICRP-38 & $3.61 \mathrm{E}+01$ & $\mathrm{~h}$ \\
\hline Ni-59 & $\mathrm{Ni}-59$ & $1.01 \mathrm{E}+05$ & $y$ & $\mathrm{Ni}-59$ & $7.50 \mathrm{E}+04$ & $y$ & $\mathrm{Ni}-59$ & $7.50 \mathrm{E}+04$ & $y$ & $\mathrm{Ni}-59$ & $1.01 \mathrm{E}+05$ & $y$ & & & & ICRP-38 & $7.50 \mathrm{E}+04$ & $y$ \\
\hline Ni-63 & $\mathrm{Ni}-63$ & $1.00 \mathrm{E}+02$ & $y$ & $\mathrm{Ni}-63$ & $9.60 \mathrm{E}+01$ & $y$ & $\mathrm{Ni}-63$ & $9.60 \mathrm{E}+01$ & $y$ & $\mathrm{Ni}-63$ & $1.00 \mathrm{E}+02$ & $y$ & & & & ICRP-38 & $9.60 \mathrm{E}+01$ & $y$ \\
\hline Ni-65 & $\mathrm{Ni}-65$ & $2.52 \mathrm{E}+00$ & $\mathrm{~h}$ & $\mathrm{Ni}-65$ & $2.52 \mathrm{E}+00$ & $\mathrm{~h}$ & $\mathrm{Ni}-65$ & $2.52 \mathrm{E}+00$ & $\mathrm{~h}$ & $\mathrm{Ni}-65$ & $2.52 \mathrm{E}+00$ & $\mathrm{~h}$ & & & & ICRP-38 & $2.52 \mathrm{E}+00$ & $\mathrm{~h}$ \\
\hline Ni-66 & $\mathrm{Ni}-66$ & $5.46 \mathrm{E}+01$ & $\mathrm{~h}$ & $\mathrm{Ni}-66$ & $5.46 \mathrm{E}+01$ & $\mathrm{~h}$ & $\mathrm{Ni}-66$ & $5.46 \mathrm{E}+01$ & $\mathrm{~h}$ & $\mathrm{Ni}-66$ & $5.46 \mathrm{E}+01$ & $\mathrm{~h}$ & & & & ICRP-38 & $5.46 \mathrm{E}+01$ & $\mathrm{~h}$ \\
\hline Np-231 & & & & & & & & & & & & & Np-231 & $4.88 \mathrm{E}+01$ & $\mathrm{~m}$ & JAERI & $4.88 \mathrm{E}+01$ & $\mathrm{~m}$ \\
\hline Np-232 & Np-232 & $1.47 \mathrm{E}+01$ & $\mathrm{~m}$ & $\mathrm{~Np}-232$ & $1.47 \mathrm{E}+01$ & $m$ & Np-232 & $1.47 \mathrm{E}+01$ & $m$ & Np-232 & $1.47 \mathrm{E}+01$ & $m$ & & & & ICRP-38 & $1.47 \mathrm{E}+01$ & $\mathrm{~m}$ \\
\hline Np-233 & $\mathrm{Np}-233$ & $3.62 E+01$ & $\mathrm{~m}$ & $\mathrm{~Np}-233$ & $3.62 \mathrm{E}+01$ & $m$ & Np-233 & $3.62 \mathrm{E}+01$ & $\mathrm{~m}$ & Np-233 & $3.62 \mathrm{E}+01$ & $\mathrm{~m}$ & & & & ICRP-38 & $3.62 \mathrm{E}+01$ & $\mathrm{~m}$ \\
\hline Np-234 & Np-234 & $4.40 \mathrm{E}+00$ & $d$ & $\mathrm{~Np}-234$ & $4.40 \mathrm{E}+00$ & $d$ & $\mathrm{~Np}-234$ & $4.40 \mathrm{E}+00$ & $d$ & Np-234 & $4.40 \mathrm{E}+00$ & $d$ & & & & ICRP-38 & $4.40 \mathrm{E}+00$ & $d$ \\
\hline Np-235 & $\mathrm{Np}-235$ & $3.96 \mathrm{E}+02$ & $d$ & Np-235 & $3.96 \mathrm{E}+02$ & $d$ & Np-235 & $3.96 \mathrm{E}+02$ & $d$ & $\mathrm{~Np}-235$ & $3.96 \mathrm{E}+02$ & $d$ & & & & ICRP-38 & $3.96 \mathrm{E}+02$ & d \\
\hline Np-236I & $\mathrm{Np}-236$ & $1.54 \mathrm{E}+05$ & $y$ & $\mathrm{~Np}-236 \mathrm{a}$ & $1.15 \mathrm{E}+05$ & $y$ & $\mathrm{~Np}-236 \mathrm{a}$ & $1.15 \mathrm{E}+05$ & $y$ & $\mathrm{~Np}-236$ & $1.54 \mathrm{E}+05$ & $y$ & & & & ICRP-38 & $1.15 \mathrm{E}+05$ & $y$ \\
\hline Np-236s & $\mathrm{Np}-236 \mathrm{~m}$ & $2.25 \mathrm{E}+01$ & $\mathrm{~h}$ & $\mathrm{~Np}-236 \mathrm{~b}$ & $2.25 \mathrm{E}+01$ & $\mathrm{~h}$ & $\mathrm{~Np}-236 \mathrm{~b}$ & $2.25 \mathrm{E}+01$ & $\mathrm{~h}$ & $\mathrm{~Np}-236 \mathrm{~m}$ & $2.25 \mathrm{E}+01$ & $\mathrm{~h}$ & & & & ICRP-38 & $2.25 \mathrm{E}+01$ & $\mathrm{~h}$ \\
\hline
\end{tabular}

D-20 


\begin{tabular}{|c|c|c|c|c|c|c|c|c|c|c|c|c|c|c|c|c|c|c|}
\hline \multirow{3}{*}{$\begin{array}{l}\text { MASTER } \\
\text { LIST } \\
\text { Np-237 }\end{array}$} & \multicolumn{3}{|c|}{ DOE-STD-1196 } & \multicolumn{3}{|c|}{ FGR-12 } & \multicolumn{3}{|c|}{ ICRP-38 } & \multicolumn{3}{|c|}{ ICRP-107 } & \multicolumn{3}{|c|}{ JAERI } & \multirow{3}{*}{$\begin{array}{c}\begin{array}{c}\text { Selected } \\
\text { Half-Life } \\
\text { Reference }\end{array} \\
\text { ICRP-38 }\end{array}$} & \multirow{2}{*}{\multicolumn{2}{|c|}{$\begin{array}{c}\text { Selected } \\
\text { Half-Life } \\
\text { (original units) }\end{array}$}} \\
\hline & \multirow{2}{*}{$\begin{array}{l}\text { Nuclide } \\
\text { Np-237 }\end{array}$} & \multicolumn{2}{|c|}{$\begin{array}{c}\text { Half-Life } \\
\text { (original units) }\end{array}$} & \multirow{2}{*}{$\begin{array}{l}\text { Nuclide } \\
\text { Np-237 }\end{array}$} & \multicolumn{2}{|c|}{$\begin{array}{c}\text { Half-Life } \\
\text { (original units) }\end{array}$} & \multirow{2}{*}{$\begin{array}{l}\text { Nuclide } \\
\text { Np-237 }\end{array}$} & \multicolumn{2}{|c|}{$\begin{array}{c}\text { Half-Life } \\
\text { (original units) }\end{array}$} & \multirow{2}{*}{$\begin{array}{l}\text { Nuclide } \\
\text { Np-237 }\end{array}$} & \multicolumn{2}{|c|}{$\begin{array}{c}\text { Half-Life } \\
\text { (original units) }\end{array}$} & \multirow[t]{2}{*}{ Nuclide } & \multicolumn{2}{|c|}{$\begin{array}{c}\text { Half-Life } \\
\text { (original units) }\end{array}$} & & & \\
\hline & & $2.14 \mathrm{E}+06$ & $y$ & & $2.14 E+06$ & $y$ & & $2.14 \mathrm{E}+06$ & $y$ & & $2.14 \mathrm{E}+06$ & $y$ & & & & & $2.14 \mathrm{E}+06$ & $y$ \\
\hline Np-238 & $\mathrm{Np}-238$ & $2.12 \mathrm{E}+00$ & $d$ & $\mathrm{~Np}-238$ & $2.12 \mathrm{E}+00$ & $d$ & $\mathrm{~Np}-238$ & $2.12 \mathrm{E}+00$ & d & Np-238 & $2.12 \mathrm{E}+00$ & d & & & & ICRP-38 & $2.12 \mathrm{E}+00$ & d \\
\hline Np-239 & Np-239 & $2.36 \mathrm{E}+00$ & $d$ & Np-239 & $2.36 \mathrm{E}+00$ & $d$ & $\mathrm{~Np}-239$ & $2.36 \mathrm{E}+00$ & $d$ & Np-239 & $2.36 \mathrm{E}+00$ & $d$ & & & & ICRP-38 & $2.36 \mathrm{E}+00$ & d \\
\hline Np-240 & $\mathrm{Np}-240$ & $6.19 \mathrm{E}+01$ & $\mathrm{~m}$ & $\mathrm{~Np}-240$ & $6.50 \mathrm{E}+01$ & $\mathrm{~m}$ & $\mathrm{~Np}-240$ & $6.50 \mathrm{E}+01$ & $\mathrm{~m}$ & $\mathrm{~Np}-240$ & $6.19 \mathrm{E}+01$ & $\mathrm{~m}$ & & & & ICRP-38 & $6.50 \mathrm{E}+01$ & $\mathrm{~m}$ \\
\hline $\mathrm{Np}-240 \mathrm{~m}$ & $\mathrm{~Np}-240 \mathrm{~m}$ & $7.22 \mathrm{E}+00$ & $\mathrm{~m}$ & $\mathrm{~Np}-240 \mathrm{~m}$ & $7.40 \mathrm{E}+00$ & $m$ & $\mathrm{~Np}-240 \mathrm{~m}$ & $7.40 \mathrm{E}+00$ & $\mathrm{~m}$ & $\mathrm{~Np}-240 \mathrm{~m}$ & $7.22 \mathrm{E}+00$ & $\mathrm{~m}$ & $\mathrm{~Np}-240 \mathrm{~m}$ & $7.22 \mathrm{E}+00$ & $\mathrm{~m}$ & ICRP-38 & $7.40 \mathrm{E}+00$ & $\mathrm{~m}$ \\
\hline Np-241 & $\mathrm{Np}-241$ & $1.39 \mathrm{E}+01$ & $\mathrm{~m}$ & & & & & & & $\mathrm{~Np}-241$ & $1.39 \mathrm{E}+01$ & $\mathrm{~m}$ & $\mathrm{~Np}-241$ & $1.39 \mathrm{E}+01$ & $m$ & ICRP-107 & $1.39 \mathrm{E}+01$ & $\mathrm{~m}$ \\
\hline $\mathrm{Np}-242$ & $\mathrm{~Np}-242$ & $2.20 \mathrm{E}+00$ & $\mathrm{~m}$ & & & & & & & $\mathrm{~Np}-242$ & $2.20 \mathrm{E}+00$ & $\mathrm{~m}$ & & & & ICRP-107 & $2.20 \mathrm{E}+00$ & $\mathrm{~m}$ \\
\hline Np-242m & $\mathrm{Np}-242 \mathrm{~m}$ & $5.50 \mathrm{E}+00$ & $\mathrm{~m}$ & & & & & & & $\mathrm{~Np}-242 \mathrm{~m}$ & $5.50 \mathrm{E}+00$ & $\mathrm{~m}$ & & & & ICRP-107 & $5.50 \mathrm{E}+00$ & $\mathrm{~m}$ \\
\hline $0-14$ & $0-14$ & $7.06 \mathrm{E}+01$ & $\mathrm{~s}$ & & & & $0-14$ & $7.06 \mathrm{E}+01$ & $\mathrm{~s}$ & $0-14$ & $7.06 \mathrm{E}+01$ & $\mathrm{~s}$ & $0-14$ & $1.18 \mathrm{E}+00$ & $\mathrm{~m}$ & ICRP-38 & $7.06 \mathrm{E}+01$ & $\mathrm{~s}$ \\
\hline $0-15$ & $0-15$ & $1.22 \mathrm{E}+02$ & $\mathrm{~s}$ & $0-15$ & $1.22 \mathrm{E}+02$ & $\mathrm{~s}$ & $0-15$ & $1.22 \mathrm{E}+02$ & $\mathrm{~s}$ & $0-15$ & $1.22 \mathrm{E}+02$ & $\mathrm{~s}$ & $0-15$ & $2.04 \mathrm{E}+00$ & $\mathrm{~m}$ & ICRP-38 & $1.22 \mathrm{E}+02$ & $\mathrm{~s}$ \\
\hline $0-19$ & $0-19$ & $2.65 \mathrm{E}+01$ & $\mathrm{~s}$ & & & & $0-19$ & $2.69 \mathrm{E}+01$ & $\mathrm{~s}$ & $0-19$ & $2.65 \mathrm{E}+01$ & $\mathrm{~s}$ & & & & ICRP-38 & $2.69 \mathrm{E}+01$ & $\mathrm{~s}$ \\
\hline Os-177 & & & & & & & & & & & & & Os-177 & $2.80 \mathrm{E}+00$ & $\mathrm{~m}$ & JAERI & $2.80 \mathrm{E}+00$ & $\mathrm{~m}$ \\
\hline Os-179 & & & & & & & & & & & & & Os-179 & $6.50 \mathrm{E}+00$ & $\mathrm{~m}$ & JAERI & $6.50 \mathrm{E}+00$ & $\mathrm{~m}$ \\
\hline Os-180 & Os-180 & $2.15 \mathrm{E}+01$ & $\mathrm{~m}$ & Os-180 & $2.20 \mathrm{E}+01$ & $\mathrm{~m}$ & Os-180 & $2.20 \mathrm{E}+01$ & $\mathrm{~m}$ & Os-180 & $2.15 \mathrm{E}+01$ & $\mathrm{~m}$ & & & & ICRP-38 & $2.20 \mathrm{E}+01$ & $\mathrm{~m}$ \\
\hline Os-181 & Os-181 & $1.05 \mathrm{E}+02$ & $\mathrm{~m}$ & Os-181 & $1.05 \mathrm{E}+02$ & $m$ & Os-181 & $1.05 \mathrm{E}+02$ & $m$ & Os-181 & $1.05 \mathrm{E}+02$ & $\mathrm{~m}$ & & & & ICRP-38 & $1.05 E+02$ & $\mathrm{~m}$ \\
\hline Os-182 & Os-182 & $2.21 \mathrm{E}+01$ & $\mathrm{~h}$ & Os-182 & $2.20 \mathrm{E}+01$ & $\mathrm{~h}$ & Os-182 & $2.20 \mathrm{E}+01$ & $\mathrm{~h}$ & Os-182 & $2.21 \mathrm{E}+01$ & $\mathrm{~h}$ & & & & ICRP-38 & $2.20 \mathrm{E}+01$ & $\mathrm{~h}$ \\
\hline Os-183 & Os-183 & $1.30 \mathrm{E}+01$ & $\mathrm{~h}$ & & & & & & & Os-183 & $1.30 \mathrm{E}+01$ & $\mathrm{~h}$ & Os-183 & $1.30 \mathrm{E}+01$ & $\mathrm{~h}$ & ICRP-107 & $1.30 \mathrm{E}+01$ & $\mathrm{~h}$ \\
\hline Os-183m & Os-183m & $9.90 \mathrm{E}+00$ & $\mathrm{~h}$ & & & & & & & Os-183m & $9.90 \mathrm{E}+00$ & $\mathrm{~h}$ & Os-183m & $9.90 \mathrm{E}+00$ & $\mathrm{~h}$ & ICRP-107 & $9.90 \mathrm{E}+00$ & $\mathrm{~h}$ \\
\hline Os-185 & Os-185 & $9.36 \mathrm{E}+01$ & $d$ & Os-185 & $9.40 \mathrm{E}+01$ & $d$ & Os-185 & $9.40 \mathrm{E}+01$ & $d$ & Os-185 & $9.36 \mathrm{E}+01$ & $d$ & & & & ICRP-38 & $9.40 \mathrm{E}+01$ & $\mathrm{~d}$ \\
\hline Os-186 & Os-186 & $2.00 \mathrm{E}+15$ & $y$ & & & & & & & Os-186 & $2.00 \mathrm{E}+15$ & $y$ & Os-186 & $2.00 \mathrm{E}+15$ & $y$ & ICRP-107 & $2.00 \mathrm{E}+15$ & $y$ \\
\hline Os-189m & Os-189m & $5.80 \mathrm{E}+00$ & $\mathrm{~h}$ & Os-189m & $6.00 \mathrm{E}+00$ & $\mathrm{~h}$ & Os-189m & $6.00 \mathrm{E}+00$ & $\mathrm{~h}$ & Os-189m & $5.80 \mathrm{E}+00$ & $\mathrm{~h}$ & & & & ICRP-38 & $6.00 \mathrm{E}+00$ & $\mathrm{~h}$ \\
\hline Os-190m & Os-190m & $9.90 \mathrm{E}+00$ & $\mathrm{~m}$ & Os-190m & $9.90 \mathrm{E}+00$ & $\mathrm{~m}$ & Os-190m & $9.90 \mathrm{E}+00$ & $\mathrm{~m}$ & Os-190m & $9.90 \mathrm{E}+00$ & $\mathrm{~m}$ & Os-190m & $9.90 \mathrm{E}+00$ & $\mathrm{~m}$ & ICRP-38 & $9.90 \mathrm{E}+00$ & $\mathrm{~m}$ \\
\hline Os-191 & Os-191 & $1.54 \mathrm{E}+01$ & $d$ & Os-191 & $1.54 \mathrm{E}+01$ & $d$ & Os-191 & $1.54 \mathrm{E}+01$ & $d$ & Os-191 & $1.54 \mathrm{E}+01$ & $d$ & & & & ICRP-38 & $1.54 \mathrm{E}+01$ & $d$ \\
\hline Os-191m & Os-191m & $1.31 \mathrm{E}+01$ & $\mathrm{~h}$ & Os-191m & $1.30 \mathrm{E}+01$ & $\mathrm{~h}$ & Os-191m & $1.30 \mathrm{E}+01$ & $\mathrm{~h}$ & Os-191m & $1.31 \mathrm{E}+01$ & $\mathrm{~h}$ & & & & ICRP-38 & $1.30 \mathrm{E}+01$ & $\mathrm{~h}$ \\
\hline Os-193 & Os-193 & $3.01 E+01$ & $\mathrm{~h}$ & Os-193 & $3.00 \mathrm{E}+01$ & $\mathrm{~h}$ & Os-193 & $3.00 \mathrm{E}+01$ & $\mathrm{~h}$ & Os-193 & $3.01 \mathrm{E}+01$ & $\mathrm{~h}$ & & & & ICRP-38 & $3.00 \mathrm{E}+01$ & $\mathrm{~h}$ \\
\hline Os-194 & Os-194 & $6.00 \mathrm{E}+00$ & $y$ & Os-194 & $6.00 \mathrm{E}+00$ & $y$ & Os-194 & $6.00 \mathrm{E}+00$ & $y$ & Os-194 & $6.00 \mathrm{E}+00$ & $y$ & & & & ICRP-38 & $6.00 \mathrm{E}+00$ & $y$ \\
\hline Os-196 & Os-196 & $3.49 \mathrm{E}+01$ & $\mathrm{~m}$ & & & & & & & Os-196 & $3.49 \mathrm{E}+01$ & $\mathrm{~m}$ & Os-196 & $3.49 \mathrm{E}+01$ & $\mathrm{~m}$ & ICRP-107 & $3.49 \mathrm{E}+01$ & $\mathrm{~m}$ \\
\hline P-30 & $\mathrm{P}-30$ & $2.50 \mathrm{E}+00$ & $\mathrm{~m}$ & $\mathrm{P}-30$ & $2.50 \mathrm{E}+00$ & $\mathrm{~m}$ & $\mathrm{P}-30$ & $2.50 \mathrm{E}+00$ & $\mathrm{~m}$ & $\mathrm{P}-30$ & $2.50 \mathrm{E}+00$ & $\mathrm{~m}$ & $\mathrm{P}-30$ & $2.50 \mathrm{E}+00$ & $\mathrm{~m}$ & ICRP-38 & $2.50 \mathrm{E}+00$ & $\mathrm{~m}$ \\
\hline P-32 & $\mathrm{P}-32$ & $1.43 \mathrm{E}+01$ & $d$ & $P-32$ & $1.43 \mathrm{E}+01$ & $\mathrm{~d}$ & $P-32$ & $1.43 \mathrm{E}+01$ & $d$ & $P-32$ & $1.43 \mathrm{E}+01$ & $\mathrm{~d}$ & & & & ICRP-38 & $1.43 \mathrm{E}+01$ & $\mathrm{~d}$ \\
\hline P-33 & P-33 & $2.53 \mathrm{E}+01$ & $d$ & P-33 & $2.54 \mathrm{E}+01$ & $d$ & P-33 & $2.54 \mathrm{E}+01$ & $d$ & P-33 & $2.53 \mathrm{E}+01$ & $d$ & & & & ICRP-38 & $2.54 \mathrm{E}+01$ & $d$ \\
\hline $\mathrm{Pa}-227$ & Pa-227 & $3.83 \mathrm{E}+01$ & $\mathrm{~m}$ & $\mathrm{~Pa}-227$ & $3.83 \mathrm{E}+01$ & $\mathrm{~m}$ & Pa-227 & $3.83 \mathrm{E}+01$ & $m$ & Pa-227 & $3.83 \mathrm{E}+01$ & $m$ & & & & ICRP-38 & $3.83 \mathrm{E}+01$ & $\mathrm{~m}$ \\
\hline $\mathrm{Pa}-228$ & Pa-228 & $2.20 \mathrm{E}+01$ & $\mathrm{~h}$ & Pa-228 & $2.20 \mathrm{E}+01$ & $\mathrm{~h}$ & Pa-228 & $2.20 \mathrm{E}+01$ & $\mathrm{~h}$ & Pa-228 & $2.20 \mathrm{E}+01$ & $\mathrm{~h}$ & & & & ICRP-38 & $2.20 \mathrm{E}+01$ & $\mathrm{~h}$ \\
\hline $\mathrm{Pa}-229$ & Pa-229 & $1.50 \mathrm{E}+00$ & $d$ & & & & & & & Pa-229 & $1.50 \mathrm{E}+00$ & $d$ & Pa-229 & $1.50 \mathrm{E}+00$ & $d$ & ICRP-107 & $1.50 \mathrm{E}+00$ & $d$ \\
\hline $\mathrm{Pa}-230$ & Pa-230 & $1.74 \mathrm{E}+01$ & $d$ & Pa-230 & $1.74 \mathrm{E}+01$ & $d$ & Pa-230 & $1.74 \mathrm{E}+01$ & $d$ & Pa-230 & $1.74 \mathrm{E}+01$ & $d$ & & & & ICRP-38 & $1.74 \mathrm{E}+01$ & $\mathrm{~d}$ \\
\hline Pa-231 & Pa-231 & $3.28 \mathrm{E}+04$ & $y$ & Pa-231 & $3.28 \mathrm{E}+04$ & $y$ & Pa-231 & $3.28 \mathrm{E}+04$ & $y$ & Pa-231 & $3.28 \mathrm{E}+04$ & $y$ & & & & ICRP-38 & $3.28 \mathrm{E}+04$ & $y$ \\
\hline $\mathrm{Pa}-232$ & Pa-232 & $1.31 \mathrm{E}+00$ & $d$ & $\mathrm{~Pa}-232$ & $1.31 \mathrm{E}+00$ & $d$ & Pa-232 & $1.31 \mathrm{E}+00$ & $d$ & $\mathrm{~Pa}-232$ & $1.31 \mathrm{E}+00$ & $d$ & & & & ICRP-38 & $1.31 \mathrm{E}+00$ & $d$ \\
\hline $\mathrm{Pa}-233$ & Pa-233 & $2.70 \mathrm{E}+01$ & $d$ & Pa-233 & $2.70 \mathrm{E}+01$ & $d$ & Pa-233 & $2.70 \mathrm{E}+01$ & $d$ & Pa-233 & $2.70 \mathrm{E}+01$ & $\mathrm{~d}$ & & & & ICRP-38 & $2.70 \mathrm{E}+01$ & $\mathrm{~d}$ \\
\hline $\mathrm{Pa}-234$ & $\mathrm{~Pa}-234$ & $6.70 \mathrm{E}+00$ & $\mathrm{~h}$ & $\mathrm{~Pa}-234$ & $6.70 \mathrm{E}+00$ & $\mathrm{~h}$ & $\mathrm{~Pa}-234$ & $6.70 \mathrm{E}+00$ & $\mathrm{~h}$ & $\mathrm{~Pa}-234$ & $6.70 \mathrm{E}+00$ & $\mathrm{~h}$ & & & & ICRP-38 & $6.70 \mathrm{E}+00$ & $\mathrm{~h}$ \\
\hline Pa-234m & Pa-234m & $1.17 E+00$ & $\mathrm{~m}$ & $\mathrm{~Pa}-234 \mathrm{~m}$ & $1.17 E+00$ & $\mathrm{~m}$ & Pa-234m & $1.17 \mathrm{E}+00$ & $\mathrm{~m}$ & $\mathrm{~Pa}-234 \mathrm{~m}$ & $1.17 \mathrm{E}+00$ & $\mathrm{~m}$ & Pa-234m & $1.17 \mathrm{E}+00$ & $\mathrm{~m}$ & ICRP-38 & $1.17 \mathrm{E}+00$ & $\mathrm{~m}$ \\
\hline Pa-235 & $\mathrm{Pa}-235$ & $2.45 \mathrm{E}+01$ & $m$ & & & & & & & $\mathrm{~Pa}-235$ & $2.45 \mathrm{E}+01$ & $m$ & & & & ICRP-107 & $2.45 \mathrm{E}+01$ & $m$ \\
\hline
\end{tabular}

D-21 


\begin{tabular}{|c|c|c|c|c|c|c|c|c|c|c|c|c|c|c|c|c|c|c|}
\hline \multirow{3}{*}{$\begin{array}{l}\text { MASTER } \\
\text { LIST } \\
\text { Pa-236 }\end{array}$} & \multicolumn{3}{|c|}{ DOE-STD-1196 } & \multicolumn{3}{|c|}{ FGR-12 } & \multicolumn{3}{|c|}{ ICRP-38 } & \multicolumn{3}{|c|}{ ICRP-107 } & \multicolumn{3}{|c|}{ JAERI } & \multirow{3}{*}{$\begin{array}{c}\begin{array}{c}\text { Selected } \\
\text { Half-Life } \\
\text { Reference }\end{array} \\
\text { ICRP-107 }\end{array}$} & \multirow{2}{*}{\multicolumn{2}{|c|}{$\begin{array}{c}\text { Selected } \\
\text { Half-Life } \\
\text { (original units) }\end{array}$}} \\
\hline & \multirow{2}{*}{$\begin{array}{l}\text { Nuclide } \\
\text { Pa-236 }\end{array}$} & \multicolumn{2}{|c|}{$\begin{array}{c}\text { Half-Life } \\
\text { (original units) }\end{array}$} & \multirow[t]{2}{*}{ Nuclide } & \multicolumn{2}{|c|}{$\begin{array}{c}\text { Half-Life } \\
\text { (original units) }\end{array}$} & \multirow[t]{2}{*}{ Nuclide } & \multicolumn{2}{|c|}{$\begin{array}{c}\text { Half-Life } \\
\text { (original units) }\end{array}$} & \multirow{2}{*}{$\begin{array}{l}\text { Nuclide } \\
\text { Pa-236 }\end{array}$} & \multicolumn{2}{|c|}{$\begin{array}{c}\text { Half-Life } \\
\text { (original units) }\end{array}$} & \multirow{2}{*}{$\begin{array}{l}\text { Nuclide } \\
\text { Pa-236 }\end{array}$} & \multicolumn{2}{|c|}{$\begin{array}{c}\text { Half-Life } \\
\text { (original units) }\end{array}$} & & & \\
\hline & & $9.10 E+00$ & $\mathrm{~m}$ & & & & & & & & $9.10 \mathrm{E}+00$ & $\mathrm{~m}$ & & $9.10 \mathrm{E}+00$ & $\mathrm{~m}$ & & $9.10 E+00$ & $\mathrm{~m}$ \\
\hline Pa-237 & Pa-237 & $8.70 \mathrm{E}+00$ & $\mathrm{~m}$ & & & & & & & Pa-237 & $8.70 \mathrm{E}+00$ & $\mathrm{~m}$ & & & & ICRP-107 & $8.70 \mathrm{E}+00$ & $\mathrm{~m}$ \\
\hline Pb-194 & Pb-194 & $1.20 \mathrm{E}+01$ & $\mathrm{~m}$ & & & & & & & Pb-194 & $1.20 \mathrm{E}+01$ & $\mathrm{~m}$ & $\mathrm{~Pb}-194$ & $1.20 \mathrm{E}+01$ & $\mathrm{~m}$ & ICRP-107 & $1.20 \mathrm{E}+01$ & $\mathrm{~m}$ \\
\hline Pb-195m & $\mathrm{Pb}-195 \mathrm{~m}$ & $1.50 \mathrm{E}+01$ & $\mathrm{~m}$ & Pb-195m & $1.58 \mathrm{E}+01$ & $\mathrm{~m}$ & $\mathrm{~Pb}-195 \mathrm{~m}$ & $1.58 \mathrm{E}+01$ & $\mathrm{~m}$ & $\mathrm{~Pb}-195 \mathrm{~m}$ & $1.50 \mathrm{E}+01$ & $\mathrm{~m}$ & & & & ICRP-38 & $1.58 \mathrm{E}+01$ & $\mathrm{~m}$ \\
\hline Pb-196 & Pb-196 & $3.70 \mathrm{E}+01$ & $\mathrm{~m}$ & & & & & & & Pb-196 & $3.70 \mathrm{E}+01$ & $\mathrm{~m}$ & Pb-196 & $3.70 \mathrm{E}+01$ & $\mathrm{~m}$ & ICRP-107 & $3.70 \mathrm{E}+01$ & $\mathrm{~m}$ \\
\hline $\mathrm{Pb}-197$ & Pb-197 & $8.00 \mathrm{E}+00$ & $\mathrm{~m}$ & & & & & & & $\mathrm{~Pb}-197$ & $8.00 \mathrm{E}+00$ & $\mathrm{~m}$ & $\mathrm{~Pb}-197$ & $8.00 \mathrm{E}+00$ & $\mathrm{~m}$ & ICRP-107 & $8.00 \mathrm{E}+00$ & $\mathrm{~m}$ \\
\hline Pb-197m & $\mathrm{Pb}-197 \mathrm{~m}$ & $4.30 \mathrm{E}+01$ & $m$ & & & & & & & Pb-197m & $4.30 \mathrm{E}+01$ & $\mathrm{~m}$ & $\mathrm{~Pb}-197 \mathrm{~m}$ & $4.30 \mathrm{E}+01$ & $\mathrm{~m}$ & ICRP-107 & $4.30 \mathrm{E}+01$ & $\mathrm{~m}$ \\
\hline Pb-198 & $\mathrm{Pb}-198$ & $2.40 \mathrm{E}+00$ & $\mathrm{~h}$ & Pb-198 & $2.40 \mathrm{E}+00$ & $\mathrm{~h}$ & Pb-198 & $2.40 \mathrm{E}+00$ & $\mathrm{~h}$ & Pb-198 & $2.40 \mathrm{E}+00$ & $\mathrm{~h}$ & & & & ICRP-38 & $2.40 \mathrm{E}+00$ & $\mathrm{~h}$ \\
\hline Pb-199 & Pb-199 & $9.00 \mathrm{E}+01$ & $\mathrm{~m}$ & Pb-199 & $9.00 \mathrm{E}+01$ & $\mathrm{~m}$ & Pb-199 & $9.00 \mathrm{E}+01$ & $m$ & Pb-199 & $9.00 \mathrm{E}+01$ & $m$ & & & & ICRP-38 & $9.00 \mathrm{E}+01$ & $\mathrm{~m}$ \\
\hline $\mathrm{Pb}-200$ & $\mathrm{~Pb}-200$ & $2.15 \mathrm{E}+01$ & $\mathrm{~h}$ & $\mathrm{~Pb}-200$ & $2.15 \mathrm{E}+01$ & $\mathrm{~h}$ & $\mathrm{~Pb}-200$ & $2.15 \mathrm{E}+01$ & $\mathrm{~h}$ & $\mathrm{~Pb}-200$ & $2.15 E+01$ & $\mathrm{~h}$ & & & & ICRP-38 & $2.15 \mathrm{E}+01$ & $\mathrm{~h}$ \\
\hline Pb-201 & $\mathrm{Pb}-201$ & $9.33 \mathrm{E}+00$ & $\mathrm{~h}$ & $\mathrm{~Pb}-201$ & $9.40 \mathrm{E}+00$ & $\mathrm{~h}$ & $\mathrm{~Pb}-201$ & $9.40 \mathrm{E}+00$ & $\mathrm{~h}$ & $\mathrm{~Pb}-201$ & $9.33 \mathrm{E}+00$ & $\mathrm{~h}$ & & & & ICRP-38 & $9.40 \mathrm{E}+00$ & $\mathrm{~h}$ \\
\hline Pb-201m & $\mathrm{Pb}-201 \mathrm{~m}$ & $6.10 \mathrm{E}+01$ & $\mathrm{~s}$ & & & & & & & Pb-201m & $6.10 \mathrm{E}+01$ & $\mathrm{~s}$ & & & & ICRP-107 & $6.10 \mathrm{E}+01$ & $\mathrm{~s}$ \\
\hline Pb-202 & Pb-202 & $5.25 \mathrm{E}+04$ & $y$ & $\mathrm{~Pb}-202$ & $3.00 \mathrm{E}+05$ & $y$ & Pb-202 & $3.00 \mathrm{E}+05$ & $y$ & Pb-202 & $5.25 E+04$ & $\mathrm{y}$ & & & & ICRP-38 & $3.00 \mathrm{E}+05$ & $y$ \\
\hline Pb-202m & Pb-202m & $3.53 E+00$ & $\mathrm{~h}$ & $\mathrm{~Pb}-202 \mathrm{~m}$ & $3.62 \mathrm{E}+00$ & $\mathrm{~h}$ & $\mathrm{~Pb}-202 \mathrm{~m}$ & $3.62 \mathrm{E}+00$ & $\mathrm{~h}$ & Pb-202m & $3.53 \mathrm{E}+00$ & $\mathrm{~h}$ & & & & ICRP-38 & $3.62 \mathrm{E}+00$ & $\mathrm{~h}$ \\
\hline Pb-203 & Pb-203 & $5.19 \mathrm{E}+01$ & $\mathrm{~h}$ & $\mathrm{~Pb}-203$ & $5.21 \mathrm{E}+01$ & $\mathrm{~h}$ & $\mathrm{~Pb}-203$ & $5.21 \mathrm{E}+01$ & $\mathrm{~h}$ & Pb-203 & $5.19 \mathrm{E}+01$ & $\mathrm{~h}$ & & & & ICRP-38 & $5.21 \mathrm{E}+01$ & $\mathrm{~h}$ \\
\hline Pb-204m & $\mathrm{Pb}-204 \mathrm{~m}$ & $6.72 \mathrm{E}+01$ & $\mathrm{~m}$ & & & & $\mathrm{~Pb}-204 \mathrm{~m}$ & $6.72 \mathrm{E}+01$ & $\mathrm{~m}$ & $\mathrm{~Pb}-204 \mathrm{~m}$ & $6.72 \mathrm{E}+01$ & $\mathrm{~m}$ & $\mathrm{~Pb}-204 \mathrm{~m}$ & $6.72 \mathrm{E}+01$ & $\mathrm{~m}$ & ICRP-38 & $6.72 \mathrm{E}+01$ & $\mathrm{~m}$ \\
\hline Pb-205 & $\mathrm{Pb}-205$ & $1.53 \mathrm{E}+07$ & $y$ & Pb-205 & $1.43 \mathrm{E}+07$ & $\mathrm{y}$ & Pb-205 & $1.43 \mathrm{E}+07$ & $y$ & $\mathrm{~Pb}-205$ & $1.53 \mathrm{E}+07$ & $y$ & & & & ICRP-38 & $1.43 \mathrm{E}+07$ & $\mathrm{y}$ \\
\hline Pb-209 & Pb-209 & $3.25 \mathrm{E}+00$ & $\mathrm{~h}$ & Pb-209 & $3.25 \mathrm{E}+00$ & $\mathrm{~h}$ & Pb-209 & $3.25 \mathrm{E}+00$ & $\mathrm{~h}$ & $\mathrm{~Pb}-209$ & $3.25 \mathrm{E}+00$ & $\mathrm{~h}$ & & & & ICRP-38 & $3.25 \mathrm{E}+00$ & $\mathrm{~h}$ \\
\hline $\mathrm{Pb}-210$ & $\mathrm{~Pb}-210$ & $2.22 \mathrm{E}+01$ & $y$ & $\mathrm{~Pb}-210$ & $2.23 \mathrm{E}+01$ & $y$ & $\mathrm{~Pb}-210$ & $2.23 \mathrm{E}+01$ & $y$ & $\mathrm{~Pb}-210$ & $2.22 \mathrm{E}+01$ & $y$ & & & & ICRP-38 & $2.23 \mathrm{E}+01$ & $y$ \\
\hline Pb-211 & $\mathrm{Pb}-211$ & $3.61 \mathrm{E}+01$ & $\mathrm{~m}$ & $\mathrm{~Pb}-211$ & $3.61 \mathrm{E}+01$ & $\mathrm{~m}$ & $\mathrm{~Pb}-211$ & $3.61 \mathrm{E}+01$ & $m$ & $\mathrm{~Pb}-211$ & $3.61 \mathrm{E}+01$ & $m$ & & & & ICRP-38 & $3.61 \mathrm{E}+01$ & $\mathrm{~m}$ \\
\hline Pb-212 & $\mathrm{Pb}-212$ & $1.06 \mathrm{E}+01$ & $\mathrm{~h}$ & $\mathrm{~Pb}-212$ & $1.06 \mathrm{E}+01$ & $\mathrm{~h}$ & $\mathrm{~Pb}-212$ & $1.06 \mathrm{E}+01$ & $\mathrm{~h}$ & $\mathrm{~Pb}-212$ & $1.06 \mathrm{E}+01$ & $\mathrm{~h}$ & & & & ICRP-38 & $1.06 \mathrm{E}+01$ & $\mathrm{~h}$ \\
\hline Pb-214 & $\mathrm{Pb}-214$ & $2.68 \mathrm{E}+01$ & $\mathrm{~m}$ & $\mathrm{~Pb}-214$ & $2.68 \mathrm{E}+01$ & $\mathrm{~m}$ & $\mathrm{~Pb}-214$ & $2.68 \mathrm{E}+01$ & $\mathrm{~m}$ & $\mathrm{~Pb}-214$ & $2.68 \mathrm{E}+01$ & $\mathrm{~m}$ & & & & ICRP-38 & $2.68 \mathrm{E}+01$ & $\mathrm{~m}$ \\
\hline Pd-100 & Pd-100 & $3.63 \mathrm{E}+00$ & $d$ & Pd-100 & $3.63 E+00$ & $d$ & Pd-100 & $3.63 \mathrm{E}+00$ & $d$ & Pd-100 & $3.63 \mathrm{E}+00$ & $d$ & & & & ICRP-38 & $3.63 \mathrm{E}+00$ & $\mathrm{~d}$ \\
\hline Pd-101 & Pd-101 & $8.47 \mathrm{E}+00$ & $\mathrm{~h}$ & Pd-101 & $8.27 \mathrm{E}+00$ & $\mathrm{~h}$ & Pd-101 & $8.27 \mathrm{E}+00$ & $\mathrm{~h}$ & Pd-101 & $8.47 \mathrm{E}+00$ & $\mathrm{~h}$ & & & & ICRP-38 & $8.27 E+00$ & $\mathrm{~h}$ \\
\hline Pd-103 & Pd-103 & $1.70 \mathrm{E}+01$ & $d$ & Pd-103 & $1.70 \mathrm{E}+01$ & $d$ & Pd-103 & $1.70 \mathrm{E}+01$ & $d$ & Pd-103 & $1.70 \mathrm{E}+01$ & $d$ & & & & ICRP-38 & $1.70 \mathrm{E}+01$ & $\mathrm{~d}$ \\
\hline Pd-107 & $\mathrm{Pd}-107$ & $6.50 \mathrm{E}+06$ & $\mathrm{y}$ & Pd-107 & $6.50 \mathrm{E}+06$ & $\mathrm{y}$ & Pd-107 & $6.50 \mathrm{E}+06$ & $\mathrm{y}$ & Pd-107 & $6.50 \mathrm{E}+06$ & $y$ & & & & ICRP-38 & $6.50 \mathrm{E}+06$ & $\mathrm{y}$ \\
\hline Pd-109 & Pd-109 & $1.37 \mathrm{E}+01$ & $\mathrm{~h}$ & Pd-109 & $1.34 \mathrm{E}+01$ & $\mathrm{~h}$ & Pd-109 & $1.34 \mathrm{E}+01$ & $\mathrm{~h}$ & Pd-109 & $1.37 \mathrm{E}+01$ & $\mathrm{~h}$ & & & & ICRP-38 & $1.34 \mathrm{E}+01$ & $\mathrm{~h}$ \\
\hline Pd-109m & Pd-109m & $4.69 \mathrm{E}+00$ & $\mathrm{~m}$ & & & & & & & Pd-109m & $4.69 \mathrm{E}+00$ & $\mathrm{~m}$ & Pd-109m & $4.69 \mathrm{E}+00$ & $\mathrm{~m}$ & ICRP-107 & $4.69 \mathrm{E}+00$ & $\mathrm{~m}$ \\
\hline Pd-111 & $\mathrm{Pd}-111$ & $2.34 \mathrm{E}+01$ & $m$ & & & & & & & $\mathrm{Pd}-111$ & $2.34 \mathrm{E}+01$ & $\mathrm{~m}$ & Pd-111 & $2.34 \mathrm{E}+01$ & $m$ & ICRP-107 & $2.34 \mathrm{E}+01$ & $\mathrm{~m}$ \\
\hline Pd-112 & Pd-112 & $2.10 \mathrm{E}+01$ & $\mathrm{~h}$ & & & & & & & $\mathrm{Pd}-112$ & $2.10 \mathrm{E}+01$ & $\mathrm{~h}$ & Pd-112 & $2.10 \mathrm{E}+01$ & $\mathrm{~h}$ & ICRP-107 & $2.10 \mathrm{E}+01$ & $\mathrm{~h}$ \\
\hline Pd-114 & Pd-114 & $2.42 \mathrm{E}+00$ & $\mathrm{~m}$ & & & & & & & Pd-114 & $2.42 \mathrm{E}+00$ & $m$ & & & & ICRP-107 & $2.42 \mathrm{E}+00$ & $\mathrm{~m}$ \\
\hline Pd-96 & Pd-96 & $1.22 \mathrm{E}+02$ & $\mathrm{~s}$ & & & & & & & Pd-96 & $1.22 \mathrm{E}+02$ & $\mathrm{~s}$ & & & & ICRP-107 & $1.22 \mathrm{E}+02$ & $\mathrm{~s}$ \\
\hline Pd-97 & Pd-97 & $3.10 \mathrm{E}+00$ & $\mathrm{~m}$ & & & & & & & Pd-97 & $3.10 \mathrm{E}+00$ & $\mathrm{~m}$ & & & & ICRP-107 & $3.10 \mathrm{E}+00$ & $\mathrm{~m}$ \\
\hline Pd-98 & Pd-98 & $1.77 \mathrm{E}+01$ & $m$ & & & & & & & Pd-98 & $1.77 \mathrm{E}+01$ & $m$ & Pd-98 & $1.77 \mathrm{E}+01$ & $\mathrm{~m}$ & ICRP-107 & $1.77 \mathrm{E}+01$ & $\mathrm{~m}$ \\
\hline Pd-99 & Pd-99 & $2.14 \mathrm{E}+01$ & $\mathrm{~m}$ & & & & & & & Pd-99 & $2.14 \mathrm{E}+01$ & $\mathrm{~m}$ & Pd-99 & $2.14 \mathrm{E}+01$ & $\mathrm{~m}$ & ICRP-107 & $2.14 \mathrm{E}+01$ & $\mathrm{~m}$ \\
\hline Pm-136 & Pm-136 & $1.07 \mathrm{E}+02$ & $\mathrm{~s}$ & & & & & & & Pm-136 & $1.07 \mathrm{E}+02$ & $\mathrm{~s}$ & & & & ICRP-107 & $1.07 \mathrm{E}+02$ & $\mathrm{~s}$ \\
\hline Pm-137m & $\mathrm{Pm}-137 \mathrm{~m}$ & $2.40 \mathrm{E}+00$ & $m$ & & & & & & & Pm-137m & $2.40 \mathrm{E}+00$ & $\mathrm{~m}$ & & & & ICRP-107 & $2.40 \mathrm{E}+00$ & $\mathrm{~m}$ \\
\hline Pm-138 & & & & & & & & & & & & & $\mathrm{Pm}-138$ & $3.24 \mathrm{E}+00$ & $\mathrm{~m}$ & JAERI & $3.24 \mathrm{E}+00$ & $\mathrm{~m}$ \\
\hline Pm-139 & Pm-139 & $4.15 \mathrm{E}+00$ & $\mathrm{~m}$ & & & & & & & Pm-139 & $4.15 \mathrm{E}+00$ & $\mathrm{~m}$ & Pm-139 & $4.15 E+00$ & $\mathrm{~m}$ & ICRP-107 & $4.15 \mathrm{E}+00$ & $\mathrm{~m}$ \\
\hline Pm-140 & Pm-140 & $9.20 \mathrm{E}+00$ & $\mathrm{~s}$ & & & & & & & Pm-140 & $9.20 \mathrm{E}+00$ & $\mathrm{~s}$ & & & & ICRP-107 & $9.20 \mathrm{E}+00$ & $\mathrm{~s}$ \\
\hline
\end{tabular}

D-22 


\begin{tabular}{|c|c|c|c|c|c|c|c|c|c|c|c|c|c|c|c|c|c|c|}
\hline \multicolumn{19}{|c|}{ Table D.1 Selection of Radionuclides For Calculating HC-2 and HC-3 TQs (continued) } \\
\hline \multirow{3}{*}{$\begin{array}{l}\text { MASTER } \\
\text { LIST } \\
\text { Pm-140m }\end{array}$} & \multicolumn{3}{|c|}{ DOE-STD-1196 } & \multicolumn{3}{|c|}{ FGR-12 } & \multicolumn{3}{|c|}{ ICRP-38 } & \multicolumn{3}{|c|}{ ICRP-107 } & \multicolumn{3}{|c|}{ JAERI } & \multirow{3}{*}{$\begin{array}{c}\begin{array}{c}\text { Selected } \\
\text { Half-Life } \\
\text { Reference }\end{array} \\
\text { ICRP-107 }\end{array}$} & & \\
\hline & \multirow{2}{*}{$\begin{array}{l}\text { Nuclide } \\
\text { Pm-140m }\end{array}$} & \multicolumn{2}{|c|}{$\begin{array}{c}\text { Half-Life } \\
\text { (original units) }\end{array}$} & \multirow[t]{2}{*}{ Nuclide } & \multicolumn{2}{|c|}{$\begin{array}{c}\text { Half-Life } \\
\text { (original units) }\end{array}$} & \multirow[t]{2}{*}{ Nuclide } & \multicolumn{2}{|c|}{$\begin{array}{c}\text { Half-Life } \\
\text { (original units) }\end{array}$} & \multirow{2}{*}{$\begin{array}{l}\text { Nuclide } \\
\text { Pm-140m }\end{array}$} & \multicolumn{2}{|c|}{$\begin{array}{c}\text { Half-Life } \\
\text { (original units) } \\
\end{array}$} & \multirow{2}{*}{$\begin{array}{l}\text { Nuclide } \\
\text { Pm-140m }\end{array}$} & $\begin{array}{r}\text { Half-Lif } \\
\text { (original u }\end{array}$ & its) & & \multicolumn{2}{|c|}{$\begin{array}{c}\text { Selected } \\
\text { Half-Life } \\
\text { (original units) }\end{array}$} \\
\hline & & $5.95 E+00$ & $\mathrm{~m}$ & & & & & & & & $5.95 \mathrm{E}+00$ & $\mathrm{~m}$ & & $5.95 \mathrm{E}+00$ & $\mathrm{~m}$ & & $5.95 E+00$ & $\mathrm{~m}$ \\
\hline Pm-141 & Pm-141 & $2.09 \mathrm{E}+01$ & $\mathrm{~m}$ & Pm-141 & $2.09 \mathrm{E}+01$ & $\mathrm{~m}$ & Pm-141 & $2.09 \mathrm{E}+01$ & $\mathrm{~m}$ & Pm-141 & $2.09 \mathrm{E}+01$ & $\mathrm{~m}$ & & & & ICRP-38 & $2.09 \mathrm{E}+01$ & $\mathrm{~m}$ \\
\hline Pm-142 & Pm-142 & $4.05 \mathrm{E}+01$ & $s$ & Pm-142 & $4.05 \mathrm{E}+01$ & $\mathrm{~s}$ & Pm-142 & $4.05 \mathrm{E}+01$ & $\mathrm{~s}$ & Pm-142 & $4.05 \mathrm{E}+01$ & $\mathrm{~s}$ & & & & ICRP-38 & $4.05 \mathrm{E}+01$ & $\mathrm{~s}$ \\
\hline Pm-143 & Pm-143 & $2.65 \mathrm{E}+02$ & $d$ & Pm-143 & $2.65 \mathrm{E}+02$ & $d$ & Pm-143 & $2.65 \mathrm{E}+02$ & $d$ & Pm-143 & $2.65 \mathrm{E}+02$ & $d$ & & & & ICRP-38 & $2.65 \mathrm{E}+02$ & $d$ \\
\hline Pm-144 & Pm-144 & $3.63 \mathrm{E}+02$ & $d$ & Pm-144 & $3.63 \mathrm{E}+02$ & $d$ & Pm-144 & $3.63 \mathrm{E}+02$ & $d$ & Pm-144 & $3.63 \mathrm{E}+02$ & $d$ & & & & ICRP-38 & $3.63 \mathrm{E}+02$ & $d$ \\
\hline Pm-145 & Pm-145 & $1.77 \mathrm{E}+01$ & y & Pm-145 & $1.77 \mathrm{E}+01$ & y & Pm-145 & $1.77 \mathrm{E}+01$ & $y$ & Pm-145 & $1.77 \mathrm{E}+01$ & $y$ & & & & ICRP-38 & $1.77 \mathrm{E}+01$ & $y$ \\
\hline Pm-146 & Pm-146 & $5.53 \mathrm{E}+00$ & $y$ & Pm-146 & $2.02 \mathrm{E}+03$ & d & Pm-146 & $2.02 \mathrm{E}+03$ & d & Pm-146 & $5.53 \mathrm{E}+00$ & $y$ & & & & ICRP-38 & $2.02 \mathrm{E}+03$ & d \\
\hline Pm-147 & Pm-147 & $2.62 \mathrm{E}+00$ & $y$ & Pm-147 & $2.62 \mathrm{E}+00$ & $y$ & Pm-147 & $2.62 \mathrm{E}+00$ & $y$ & Pm-147 & $2.62 \mathrm{E}+00$ & $y$ & & & & ICRP-38 & $2.62 \mathrm{E}+00$ & $y$ \\
\hline Pm-148 & $\mathrm{Pm}-148$ & $5.37 E+00$ & $d$ & Pm-148 & $5.37 \mathrm{E}+00$ & d & Pm-148 & $5.37 \mathrm{E}+00$ & $d$ & Pm-148 & $5.37 \mathrm{E}+00$ & $d$ & & & & ICRP-38 & $5.37 \mathrm{E}+00$ & $d$ \\
\hline Pm-148m & Pm-148m & $4.13 E+01$ & $d$ & Pm-148m & $4.13 E+01$ & d & Pm-148m & $4.13 \mathrm{E}+01$ & $d$ & Pm-148m & $4.13 \mathrm{E}+01$ & $d$ & & & & ICRP-38 & $4.13 \mathrm{E}+01$ & $d$ \\
\hline Pm-149 & Pm-149 & $5.31 \mathrm{E}+01$ & $\mathrm{~h}$ & Pm-149 & $5.31 \mathrm{E}+01$ & $\mathrm{~h}$ & Pm-149 & $5.31 \mathrm{E}+01$ & $\mathrm{~h}$ & Pm-149 & $5.31 \mathrm{E}+01$ & $\mathrm{~h}$ & & & & ICRP-38 & $5.31 \mathrm{E}+01$ & $\mathrm{~h}$ \\
\hline Pm-150 & Pm-150 & $2.68 \mathrm{E}+00$ & $\mathrm{~h}$ & Pm-150 & $2.68 \mathrm{E}+00$ & $\mathrm{~h}$ & $\mathrm{Pm}-150$ & $2.68 \mathrm{E}+00$ & $\mathrm{~h}$ & $\mathrm{Pm}-150$ & $2.68 \mathrm{E}+00$ & $\mathrm{~h}$ & & & & ICRP-38 & $2.68 \mathrm{E}+00$ & $\mathrm{~h}$ \\
\hline Pm-151 & Pm-151 & $2.84 \mathrm{E}+01$ & $\mathrm{~h}$ & Pm-151 & $2.84 \mathrm{E}+01$ & $\mathrm{~h}$ & Pm-151 & $2.84 \mathrm{E}+01$ & $\mathrm{~h}$ & Pm-151 & $2.84 \mathrm{E}+01$ & $\mathrm{~h}$ & & & & ICRP-38 & $2.84 \mathrm{E}+01$ & $\mathrm{~h}$ \\
\hline Pm-152 & Pm-152 & $4.12 \mathrm{E}+00$ & $m$ & & & & & & & Pm-152 & $4.12 \mathrm{E}+00$ & $m$ & Pm-152 & $4.12 \mathrm{E}+00$ & $m$ & ICRP-107 & $4.12 \mathrm{E}+00$ & $\mathrm{~m}$ \\
\hline Pm-152m & Pm-152m & $7.52 \mathrm{E}+00$ & $\mathrm{~m}$ & & & & & & & Pm-152m & $7.52 \mathrm{E}+00$ & $m$ & & & & ICRP-107 & $7.52 \mathrm{E}+00$ & $m$ \\
\hline Pm-153 & Pm-153 & $5.25 \mathrm{E}+00$ & $\mathrm{~m}$ & & & & & & & Pm-153 & $5.25 \mathrm{E}+00$ & $\mathrm{~m}$ & & & & ICRP-107 & $5.25 \mathrm{E}+00$ & $\mathrm{~m}$ \\
\hline Pm-154 & Pm-154 & $1.73 \mathrm{E}+00$ & $\mathrm{~m}$ & & & & & & & Pm-154 & $1.73 \mathrm{E}+00$ & $\mathrm{~m}$ & & & & ICRP-107 & $1.73 \mathrm{E}+00$ & $\mathrm{~m}$ \\
\hline Pm-154m & Pm-154m & $2.68 \mathrm{E}+00$ & $m$ & & & & & & & Pm-154m & $2.68 \mathrm{E}+00$ & $\mathrm{~m}$ & & & & ICRP-107 & $2.68 \mathrm{E}+00$ & $m$ \\
\hline Po-203 & Po-203 & $3.67 E+01$ & $\mathrm{~m}$ & Po-203 & $3.67 \mathrm{E}+01$ & $\mathrm{~m}$ & Po-203 & $3.67 \mathrm{E}+01$ & $\mathrm{~m}$ & Po-203 & $3.67 \mathrm{E}+01$ & $\mathrm{~m}$ & & & & ICRP-38 & $3.67 \mathrm{E}+01$ & $\mathrm{~m}$ \\
\hline Po-204 & Po-204 & $3.53 \mathrm{E}+00$ & $\mathrm{~h}$ & & & & & & & Po-204 & $3.53 \mathrm{E}+00$ & $\mathrm{~h}$ & Po-204 & $3.53 \mathrm{E}+00$ & $\mathrm{~h}$ & ICRP-107 & $3.53 \mathrm{E}+00$ & $\mathrm{~h}$ \\
\hline Po-205 & Po-205 & $1.66 \mathrm{E}+00$ & $\mathrm{~h}$ & Po-205 & $1.80 \mathrm{E}+00$ & $\mathrm{~h}$ & Po-205 & $1.80 \mathrm{E}+00$ & $\mathrm{~h}$ & Po-205 & $1.66 \mathrm{E}+00$ & $\mathrm{~h}$ & & & & ICRP-38 & $1.80 \mathrm{E}+00$ & $\mathrm{~h}$ \\
\hline Po-206 & Po-206 & $8.80 \mathrm{E}+00$ & $d$ & & & & & & & Po-206 & $8.80 \mathrm{E}+00$ & $d$ & Po-206 & $8.80 \mathrm{E}+00$ & $d$ & ICRP-107 & $8.80 \mathrm{E}+00$ & $d$ \\
\hline Po-207 & Po-207 & $5.80 \mathrm{E}+00$ & $\mathrm{~h}$ & Po-207 & $3.50 \mathrm{E}+02$ & $\mathrm{~m}$ & Po-207 & $3.50 \mathrm{E}+02$ & $m$ & Po-207 & $5.80 \mathrm{E}+00$ & $\mathrm{~h}$ & & & & ICRP-38 & $3.50 \mathrm{E}+02$ & $\mathrm{~m}$ \\
\hline Po-208 & Po-208 & $2.90 \mathrm{E}+00$ & $y$ & & & & & & & Po-208 & $2.90 \mathrm{E}+00$ & $y$ & Po-208 & $2.90 \mathrm{E}+00$ & $y$ & ICRP-107 & $2.90 \mathrm{E}+00$ & $y$ \\
\hline Po-209 & Po-209 & $1.02 \mathrm{E}+02$ & $y$ & & & & Po-209 & $1.02 \mathrm{E}+02$ & $y$ & Po-209 & $1.02 \mathrm{E}+02$ & $y$ & Po-209 & $1.02 \mathrm{E}+02$ & $y$ & ICRP-38 & $1.02 \mathrm{E}+02$ & $y$ \\
\hline Po-210 & Po-210 & $1.38 \mathrm{E}+02$ & $d$ & Po-210 & $1.38 \mathrm{E}+02$ & $d$ & Po-210 & $1.38 \mathrm{E}+02$ & $d$ & Po-210 & $1.38 \mathrm{E}+02$ & $d$ & & & & ICRP-38 & $1.38 \mathrm{E}+02$ & $d$ \\
\hline Po-211 & Po-211 & $5.16 \mathrm{E}-01$ & $\mathrm{~s}$ & Po-211 & $5.16 \mathrm{E}-01$ & $\mathrm{~s}$ & Po-211 & $5.16 \mathrm{E}-01$ & $\mathrm{~s}$ & Po-211 & $5.16 \mathrm{E}-01$ & $\mathrm{~s}$ & & & & ICRP-38 & $5.16 \mathrm{E}-01$ & $\mathrm{~s}$ \\
\hline Po-212 & Po-212 & $2.99 \mathrm{E}-07$ & $\mathrm{~s}$ & Po-212 & $3.05 \mathrm{E}-01$ & us & Po-212 & $3.05 \mathrm{E}-01$ & us & Po-212 & $2.99 \mathrm{E}-07$ & $\mathrm{~s}$ & & & & ICRP-38 & $3.05 \mathrm{E}-01$ & us \\
\hline Po-212m & Po-212m & $4.51 \mathrm{E}+01$ & $\mathrm{~s}$ & & & & & & & Po-212m & $4.51 \mathrm{E}+01$ & $\mathrm{~s}$ & & & & ICRP-107 & $4.51 \mathrm{E}+01$ & $\mathrm{~s}$ \\
\hline Po-213 & Po-213 & $4.20 \mathrm{E}-06$ & $\mathrm{~s}$ & Po-213 & $4.20 \mathrm{E}+00$ & us & Po-213 & $4.20 \mathrm{E}+00$ & us & Po-213 & $4.20 \mathrm{E}-06$ & $\mathrm{~s}$ & & & & ICRP-38 & $4.20 \mathrm{E}+00$ & us \\
\hline Po-214 & Po-214 & 1.64E-04 & $s$ & Po-214 & $1.64 \mathrm{E}+02$ & us & Po-214 & $1.64 \mathrm{E}+02$ & us & Po-214 & 1.64E-04 & $\mathrm{s}$ & & & & ICRP-38 & $1.64 \mathrm{E}+02$ & us \\
\hline Po-215 & Po-215 & $1.78 \mathrm{E}-03$ & $\mathrm{~s}$ & Po-215 & $1.78 \mathrm{E}-03$ & $\mathrm{~s}$ & Po-215 & $1.78 \mathrm{E}-03$ & $\mathrm{~s}$ & Po-215 & $1.78 \mathrm{E}-03$ & $\mathrm{~s}$ & & & & ICRP-38 & $1.78 \mathrm{E}-03$ & $s$ \\
\hline Po-216 & Po-216 & $1.45 \mathrm{E}-01$ & $\mathrm{~s}$ & Po-216 & $1.50 \mathrm{E}-01$ & $\mathrm{~s}$ & Po-216 & $1.50 \mathrm{E}-01$ & $\mathrm{~s}$ & Po-216 & $1.45 \mathrm{E}-01$ & $\mathrm{~s}$ & & & & ICRP-38 & $1.50 \mathrm{E}-01$ & $\mathrm{~s}$ \\
\hline Po-218 & Po-218 & $3.10 \mathrm{E}+00$ & $m$ & Po-218 & $3.05 E+00$ & $m$ & Po-218 & $3.05 \mathrm{E}+00$ & $m$ & Po-218 & $3.10 \mathrm{E}+00$ & $m$ & Po-218 & $3.10 \mathrm{E}+00$ & $m$ & ICRP-38 & $3.05 \mathrm{E}+00$ & $\mathrm{~m}$ \\
\hline Pr-134 & Pr-134 & $1.10 \mathrm{E}+01$ & $\mathrm{~m}$ & & & & & & & Pr-134 & $1.10 \mathrm{E}+01$ & $m$ & Pr-134 & $1.70 \mathrm{E}+01$ & $m$ & ICRP-107 & $1.10 \mathrm{E}+01$ & $\mathrm{~m}$ \\
\hline Pr-134m & Pr-134m & $1.70 \mathrm{E}+01$ & $\mathrm{~m}$ & & & & & & & Pr-134m & $1.70 \mathrm{E}+01$ & $m$ & $\operatorname{Pr}-134 \mathrm{~m}$ & $1.10 \mathrm{E}+01$ & $m$ & ICRP-107 & $1.70 \mathrm{E}+01$ & $m$ \\
\hline Pr-135 & Pr-135 & $2.40 \mathrm{E}+01$ & $\mathrm{~m}$ & & & & & & & Pr-135 & $2.40 \mathrm{E}+01$ & $\mathrm{~m}$ & $\operatorname{Pr}-135$ & $2.40 \mathrm{E}+01$ & $m$ & ICRP-107 & $2.40 \mathrm{E}+01$ & $\mathrm{~m}$ \\
\hline Pr-136 & $\operatorname{Pr}-136$ & $1.31 \mathrm{E}+01$ & $\mathrm{~m}$ & Pr-136 & $1.31 \mathrm{E}+01$ & $\mathrm{~m}$ & Pr-136 & $1.31 \mathrm{E}+01$ & $\mathrm{~m}$ & Pr-136 & $1.31 \mathrm{E}+01$ & $\mathrm{~m}$ & & & & ICRP-38 & $1.31 \mathrm{E}+01$ & $\mathrm{~m}$ \\
\hline Pr-137 & Pr-137 & $1.28 \mathrm{E}+00$ & $\mathrm{~h}$ & Pr-137 & $7.66 \mathrm{E}+01$ & $\mathrm{~m}$ & Pr-137 & $7.66 \mathrm{E}+01$ & $\mathrm{~m}$ & Pr-137 & $1.28 \mathrm{E}+00$ & $\mathrm{~h}$ & & & & ICRP-38 & $7.66 \mathrm{E}+01$ & $\mathrm{~m}$ \\
\hline Pr-138 & Pr-138 & $1.45 \mathrm{E}+00$ & $m$ & Pr-138 & $1.45 \mathrm{E}+00$ & $m$ & Pr-138 & $1.45 \mathrm{E}+00$ & $m$ & Pr-138 & $1.45 \mathrm{E}+00$ & $m$ & Pr-138 & $1.45 \mathrm{E}+00$ & $\mathrm{~m}$ & ICRP-38 & $1.45 \mathrm{E}+00$ & $\mathrm{~m}$ \\
\hline
\end{tabular}

D-23 


\begin{tabular}{|c|c|c|c|c|c|c|c|c|c|c|c|c|c|c|c|c|c|c|}
\hline \multicolumn{19}{|c|}{ Table D.1 Selection of Radionuclides For Calculating HC-2 and HC-3 TQs (continued) } \\
\hline \multirow{3}{*}{$\begin{array}{l}\text { MASTER } \\
\text { LIST } \\
\text { Pr-138m }\end{array}$} & \multicolumn{3}{|c|}{ DOE-STD-1196 } & \multicolumn{3}{|c|}{ FGR-12 } & \multicolumn{3}{|c|}{ ICRP-38 } & \multicolumn{3}{|c|}{ ICRP-107 } & \multicolumn{3}{|c|}{ JAERI } & \multirow{3}{*}{$\begin{array}{c}\begin{array}{c}\text { Selected } \\
\text { Half-Life } \\
\text { Reference }\end{array} \\
\text { ICRP-38 }\end{array}$} & & \\
\hline & \multirow{2}{*}{$\begin{array}{l}\text { Nuclide } \\
\text { Pr-138m }\end{array}$} & \multicolumn{2}{|c|}{$\begin{array}{c}\text { Half-Life } \\
\text { (original units) }\end{array}$} & \multirow{2}{*}{$\frac{\text { Nuclide }}{\text { Pr-138m }}$} & \multicolumn{2}{|c|}{$\begin{array}{c}\text { Half-Life } \\
\text { (original units) }\end{array}$} & \multirow{2}{*}{$\frac{\text { Nuclide }}{\text { Pr-138m }}$} & \multicolumn{2}{|c|}{$\begin{array}{c}\text { Half-Life } \\
\text { (original units) }\end{array}$} & \multirow{2}{*}{$\frac{\text { Nuclide }}{\text { Pr-138m }}$} & \multicolumn{2}{|c|}{$\begin{array}{c}\text { Half-Life } \\
\text { (original units) }\end{array}$} & \multirow[t]{2}{*}{ Nuclide } & $\begin{array}{r}\text { Half-Li } \\
\text { (original }\end{array}$ & its) & & \multicolumn{2}{|c|}{$\begin{array}{c}\text { Selected } \\
\text { Half-Life } \\
\text { (original units) }\end{array}$} \\
\hline & & $2.12 E+00$ & $\mathrm{~h}$ & & $2.10 \mathrm{E}+00$ & $\mathrm{~h}$ & & $2.10 \mathrm{E}+00$ & $\mathrm{~h}$ & & $2.12 \mathrm{E}+00$ & $\mathrm{~h}$ & & & & & $2.10 E+00$ & $\mathrm{~h}$ \\
\hline Pr-139 & Pr-139 & $4.41 \mathrm{E}+00$ & $\mathrm{~h}$ & Pr-139 & $4.51 E+00$ & $\mathrm{~h}$ & Pr-139 & $4.51 \mathrm{E}+00$ & $\mathrm{~h}$ & Pr-139 & $4.41 \mathrm{E}+00$ & $\mathrm{~h}$ & & & & ICRP-38 & $4.51 \mathrm{E}+00$ & $\mathrm{~h}$ \\
\hline Pr-140 & Pr-140 & $3.39 \mathrm{E}+00$ & $\mathrm{~m}$ & & & & & & & Pr-140 & $3.39 \mathrm{E}+00$ & $\mathrm{~m}$ & Pr-140 & $3.39 \mathrm{E}+00$ & $\mathrm{~m}$ & ICRP-107 & $3.39 \mathrm{E}+00$ & $\mathrm{~m}$ \\
\hline Pr-142 & Pr-142 & $1.91 \mathrm{E}+01$ & $\mathrm{~h}$ & Pr-142 & $1.91 \mathrm{E}+01$ & $\mathrm{~h}$ & Pr-142 & $1.91 \mathrm{E}+01$ & $\mathrm{~h}$ & Pr-142 & $1.91 \mathrm{E}+01$ & $\mathrm{~h}$ & & & & ICRP-38 & $1.91 \mathrm{E}+01$ & $\mathrm{~h}$ \\
\hline Pr-142m & Pr-142m & $1.46 \mathrm{E}+01$ & $\mathrm{~m}$ & Pr-142m & $1.46 \mathrm{E}+01$ & $m$ & Pr-142m & $1.46 \mathrm{E}+01$ & $\mathrm{~m}$ & Pr-142m & $1.46 \mathrm{E}+01$ & $\mathrm{~m}$ & & & & ICRP-38 & $1.46 \mathrm{E}+01$ & $\mathrm{~m}$ \\
\hline Pr-143 & Pr-143 & $1.36 \mathrm{E}+01$ & $\mathrm{~d}$ & Pr-143 & $1.36 \mathrm{E}+01$ & $\mathrm{~d}$ & Pr-143 & $1.36 \mathrm{E}+01$ & $\mathrm{~d}$ & Pr-143 & $1.36 \mathrm{E}+01$ & $d$ & & & & ICRP-38 & $1.36 \mathrm{E}+01$ & $d$ \\
\hline Pr-144 & Pr-144 & $1.73 \mathrm{E}+01$ & $\mathrm{~m}$ & Pr-144 & $1.73 \mathrm{E}+01$ & $m$ & Pr-144 & $1.73 \mathrm{E}+01$ & $\mathrm{~m}$ & Pr-144 & $1.73 \mathrm{E}+01$ & $\mathrm{~m}$ & & & & ICRP-38 & $1.73 \mathrm{E}+01$ & $\mathrm{~m}$ \\
\hline Pr-144m & Pr-144m & $7.20 \mathrm{E}+00$ & $\mathrm{~m}$ & Pr-144m & $7.20 \mathrm{E}+00$ & $m$ & Pr-144m & $7.20 \mathrm{E}+00$ & $m$ & Pr-144m & $7.20 \mathrm{E}+00$ & $\mathrm{~m}$ & Pr-144m & $7.20 \mathrm{E}+00$ & $\mathrm{~m}$ & ICRP-38 & $7.20 \mathrm{E}+00$ & $m$ \\
\hline Pr-145 & Pr-145 & $5.98 \mathrm{E}+00$ & $\mathrm{~h}$ & Pr-145 & $5.98 \mathrm{E}+00$ & $\mathrm{~h}$ & Pr-145 & $5.98 \mathrm{E}+00$ & $\mathrm{~h}$ & Pr-145 & $5.98 \mathrm{E}+00$ & $\mathrm{~h}$ & & & & ICRP-38 & $5.98 \mathrm{E}+00$ & $\mathrm{~h}$ \\
\hline Pr-146 & Pr-146 & $2.42 \mathrm{E}+01$ & $\mathrm{~m}$ & & & & & & & Pr-146 & $2.42 \mathrm{E}+01$ & $\mathrm{~m}$ & Pr-146 & $2.42 \mathrm{E}+01$ & $\mathrm{~m}$ & ICRP-107 & $2.42 \mathrm{E}+01$ & $\mathrm{~m}$ \\
\hline Pr-147 & Pr-147 & $1.34 \mathrm{E}+01$ & $\mathrm{~m}$ & Pr-147 & $1.36 \mathrm{E}+01$ & $\mathrm{~m}$ & Pr-147 & $1.36 \mathrm{E}+01$ & $\mathrm{~m}$ & Pr-147 & $1.34 \mathrm{E}+01$ & $m$ & & & & ICRP-38 & $1.36 \mathrm{E}+01$ & $\mathrm{~m}$ \\
\hline Pr-148 & Pr-148 & $2.29 \mathrm{E}+00$ & $\mathrm{~m}$ & & & & & & & Pr-148 & $2.29 \mathrm{E}+00$ & $m$ & & & & ICRP-107 & $2.29 \mathrm{E}+00$ & $\mathrm{~m}$ \\
\hline Pr-148m & Pr-148m & $2.01 \mathrm{E}+00$ & $\mathrm{~m}$ & & & & & & & Pr-148m & $2.01 \mathrm{E}+00$ & $m$ & & & & ICRP-107 & $2.01 \mathrm{E}+00$ & $\mathrm{~m}$ \\
\hline Pt-183 & & & & & & & & & & & & & Pt-183 & $6.50 \mathrm{E}+00$ & $m$ & JAERI & $6.50 \mathrm{E}+00$ & $\mathrm{~m}$ \\
\hline Pt-184 & Pt-184 & $1.73 E+01$ & $m$ & & & & & & & Pt-184 & $1.73 \mathrm{E}+01$ & $m$ & Pt-184 & $1.73 \mathrm{E}+01$ & $\mathrm{~m}$ & ICRP-107 & $1.73 E+01$ & $\mathrm{~m}$ \\
\hline Pt-186 & Pt-186 & $2.08 \mathrm{E}+00$ & $\mathrm{~h}$ & Pt-186 & $2.00 \mathrm{E}+00$ & $\mathrm{~h}$ & Pt-186 & $2.00 \mathrm{E}+00$ & $\mathrm{~h}$ & Pt-186 & $2.08 \mathrm{E}+00$ & $\mathrm{~h}$ & & & & ICRP-38 & $2.00 \mathrm{E}+00$ & $\mathrm{~h}$ \\
\hline Pt-187 & Pt-187 & $2.35 \mathrm{E}+00$ & $\mathrm{~h}$ & & & & & & & Pt-187 & $2.35 \mathrm{E}+00$ & $\mathrm{~h}$ & Pt-187 & $2.35 \mathrm{E}+00$ & $\mathrm{~h}$ & ICRP-107 & $2.35 \mathrm{E}+00$ & $\mathrm{~h}$ \\
\hline Pt-188 & Pt-188 & $1.02 \mathrm{E}+01$ & $d$ & Pt-188 & $1.02 \mathrm{E}+01$ & $d$ & Pt-188 & $1.02 \mathrm{E}+01$ & $d$ & Pt-188 & $1.02 \mathrm{E}+01$ & $d$ & & & & ICRP-38 & $1.02 \mathrm{E}+01$ & $d$ \\
\hline Pt-189 & Pt-189 & $1.09 \mathrm{E}+01$ & $\mathrm{~h}$ & Pt-189 & $1.09 \mathrm{E}+01$ & $\mathrm{~h}$ & Pt-189 & $1.09 \mathrm{E}+01$ & $\mathrm{~h}$ & Pt-189 & $1.09 \mathrm{E}+01$ & $\mathrm{~h}$ & & & & ICRP-38 & $1.09 \mathrm{E}+01$ & $\mathrm{~h}$ \\
\hline Pt-190 & Pt-190 & $6.50 \mathrm{E}+11$ & $y$ & & & & & & & Pt-190 & $6.50 \mathrm{E}+11$ & $y$ & Pt-190 & $6.50 \mathrm{E}+11$ & $y$ & ICRP-107 & $6.50 \mathrm{E}+11$ & $y$ \\
\hline Pt-191 & Pt-191 & $2.80 \mathrm{E}+00$ & $d$ & Pt-191 & $2.80 \mathrm{E}+00$ & $d$ & Pt-191 & $2.80 \mathrm{E}+00$ & $d$ & Pt-191 & $2.80 \mathrm{E}+00$ & $d$ & & & & ICRP-38 & $2.80 \mathrm{E}+00$ & $d$ \\
\hline Pt-193 & Pt-193 & $5.00 \mathrm{E}+01$ & $y$ & Pt-193 & $5.00 \mathrm{E}+01$ & $y$ & Pt-193 & $5.00 \mathrm{E}+01$ & $y$ & Pt-193 & $5.00 \mathrm{E}+01$ & $y$ & & & & ICRP-38 & $5.00 \mathrm{E}+01$ & $y$ \\
\hline Pt-193m & Pt-193m & $4.33 \mathrm{E}+00$ & $d$ & Pt-193m & $4.33 \mathrm{E}+00$ & $d$ & Pt-193m & $4.33 \mathrm{E}+00$ & $d$ & Pt-193m & $4.33 \mathrm{E}+00$ & $d$ & & & & ICRP-38 & $4.33 E+00$ & $d$ \\
\hline Pt-195m & Pt-195m & $4.02 \mathrm{E}+00$ & $d$ & Pt-195m & $4.02 \mathrm{E}+00$ & $\mathrm{~d}$ & Pt-195m & $4.02 \mathrm{E}+00$ & $d$ & Pt-195m & $4.02 \mathrm{E}+00$ & $d$ & & & & ICRP-38 & $4.02 \mathrm{E}+00$ & $d$ \\
\hline Pt-197 & Pt-197 & $1.99 \mathrm{E}+01$ & $\mathrm{~h}$ & Pt-197 & $1.83 \mathrm{E}+01$ & $\mathrm{~h}$ & Pt-197 & $1.83 \mathrm{E}+01$ & $\mathrm{~h}$ & Pt-197 & $1.99 \mathrm{E}+01$ & $\mathrm{~h}$ & & & & ICRP-38 & $1.83 \mathrm{E}+01$ & $\mathrm{~h}$ \\
\hline Pt-197m & Pt-197m & $9.54 \mathrm{E}+01$ & $\mathrm{~m}$ & Pt-197m & $9.44 \mathrm{E}+01$ & $m$ & Pt-197m & $9.44 \mathrm{E}+01$ & $m$ & Pt-197m & $9.54 \mathrm{E}+01$ & $m$ & & & & ICRP-38 & $9.44 \mathrm{E}+01$ & $m$ \\
\hline Pt-199 & Pt-199 & $3.08 \mathrm{E}+01$ & $\mathrm{~m}$ & Pt-199 & $3.08 \mathrm{E}+01$ & $\mathrm{~m}$ & Pt-199 & $3.08 \mathrm{E}+01$ & $m$ & Pt-199 & $3.08 \mathrm{E}+01$ & $\mathrm{~m}$ & & & & ICRP-38 & $3.08 \mathrm{E}+01$ & $\mathrm{~m}$ \\
\hline Pt-200 & Pt-200 & $1.25 \mathrm{E}+01$ & $\mathrm{~h}$ & Pt-200 & $1.25 \mathrm{E}+01$ & $\mathrm{~h}$ & Pt-200 & $1.25 \mathrm{E}+01$ & $\mathrm{~h}$ & Pt-200 & $1.25 \mathrm{E}+01$ & $\mathrm{~h}$ & & & & ICRP-38 & $1.25 \mathrm{E}+01$ & $\mathrm{~h}$ \\
\hline Pt-202 & Pt-202 & $4.40 \mathrm{E}+01$ & $\mathrm{~h}$ & & & & & & & Pt-202 & $4.40 \mathrm{E}+01$ & $\mathrm{~h}$ & Pt-202 & $4.40 \mathrm{E}+01$ & $\mathrm{~h}$ & ICRP-107 & $4.40 \mathrm{E}+01$ & $\mathrm{~h}$ \\
\hline Pu-232 & Pu-232 & $3.37 E+01$ & $\mathrm{~m}$ & & & & & & & Pu-232 & $3.37 \mathrm{E}+01$ & $\mathrm{~m}$ & Pu-232 & $3.41 \mathrm{E}+01$ & $\mathrm{~m}$ & ICRP-107 & $3.37 E+01$ & $\mathrm{~m}$ \\
\hline Pu-234 & Pu-234 & $8.80 \mathrm{E}+00$ & $\mathrm{~h}$ & Pu-234 & $8.80 \mathrm{E}+00$ & $\mathrm{~h}$ & Pu-234 & $8.80 \mathrm{E}+00$ & $\mathrm{~h}$ & $\mathrm{Pu}-234$ & $8.80 \mathrm{E}+00$ & $\mathrm{~h}$ & & & & ICRP-38 & $8.80 \mathrm{E}+00$ & $\mathrm{~h}$ \\
\hline Pu-235 & Pu-235 & $2.53 \mathrm{E}+01$ & $\mathrm{~m}$ & Pu-235 & $2.53 \mathrm{E}+01$ & $\mathrm{~m}$ & Pu-235 & $2.53 \mathrm{E}+01$ & $m$ & Pu-235 & $2.53 \mathrm{E}+01$ & $m$ & & & & ICRP-38 & $2.53 \mathrm{E}+01$ & $\mathrm{~m}$ \\
\hline Pu-236 & Pu-236 & $2.86 \mathrm{E}+00$ & $\mathrm{y}$ & Pu-236 & $2.85 \mathrm{E}+00$ & $\mathrm{y}$ & Pu-236 & $2.85 \mathrm{E}+00$ & $y$ & $\mathrm{Pu}-236$ & $2.86 \mathrm{E}+00$ & $y$ & & & & ICRP-38 & $2.85 \mathrm{E}+00$ & $y$ \\
\hline Pu-237 & Pu-237 & $4.52 \mathrm{E}+01$ & $d$ & Pu-237 & $4.53 \mathrm{E}+01$ & $d$ & Pu-237 & $4.53 \mathrm{E}+01$ & $d$ & Pu-237 & $4.52 \mathrm{E}+01$ & $d$ & & & & ICRP-38 & $4.53 \mathrm{E}+01$ & $d$ \\
\hline Pu-238 & Pu-238 & $8.77 E+01$ & $y$ & Pu-238 & $8.77 E+01$ & $y$ & Pu-238 & $8.77 \mathrm{E}+01$ & $y$ & Pu-238 & $8.77 \mathrm{E}+01$ & $y$ & & & & ICRP-38 & $8.77 \mathrm{E}+01$ & $y$ \\
\hline Pu-239 & Pu-239 & $2.41 \mathrm{E}+04$ & $y$ & Pu-239 & $2.41 \mathrm{E}+04$ & $y$ & Pu-239 & $2.41 \mathrm{E}+04$ & $y$ & Pu-239 & $2.41 \mathrm{E}+04$ & $y$ & & & & ICRP-38 & $2.41 \mathrm{E}+04$ & $y$ \\
\hline Pu-240 & $\mathrm{Pu}-240$ & $6.56 \mathrm{E}+03$ & $y$ & $\mathrm{Pu}-240$ & $6.54 \mathrm{E}+03$ & $y$ & $\mathrm{Pu}-240$ & $6.54 \mathrm{E}+03$ & $y$ & $\mathrm{Pu}-240$ & $6.56 \mathrm{E}+03$ & $y$ & & & & ICRP-38 & $6.54 \mathrm{E}+03$ & $y$ \\
\hline Pu-241 & $\mathrm{Pu}-241$ & $1.44 \mathrm{E}+01$ & $y$ & $\mathrm{Pu}-241$ & $1.44 \mathrm{E}+01$ & $y$ & $\mathrm{Pu}-241$ & $1.44 \mathrm{E}+01$ & $y$ & $\mathrm{Pu}-241$ & $1.44 \mathrm{E}+01$ & $y$ & & & & ICRP-38 & $1.44 \mathrm{E}+01$ & $y$ \\
\hline Pu-242 & Pu-242 & $3.75 E+05$ & $y$ & $\mathrm{Pu}-242$ & $3.76 \mathrm{E}+05$ & $y$ & Pu-242 & $3.76 \mathrm{E}+05$ & $y$ & Pu-242 & $3.75 E+05$ & $y$ & & & & ICRP-38 & $3.76 \mathrm{E}+05$ & $y$ \\
\hline Pu-243 & Pu-243 & $4.96 \mathrm{E}+00$ & $\mathrm{~h}$ & Pu-243 & $4.96 \mathrm{E}+00$ & $\mathrm{~h}$ & Pu-243 & $4.96 \mathrm{E}+00$ & $\mathrm{~h}$ & Pu-243 & $4.96 \mathrm{E}+00$ & $\mathrm{~h}$ & & & & ICRP-38 & $4.96 \mathrm{E}+00$ & $\mathrm{~h}$ \\
\hline
\end{tabular}

D-24 


\begin{tabular}{|c|c|c|c|c|c|c|c|c|c|c|c|c|c|c|c|c|c|c|}
\hline \multicolumn{19}{|c|}{ Table D.1 Selection of Radionuclides For Calculating HC-2 and HC-3 TQs (continued) } \\
\hline \multirow{3}{*}{$\begin{array}{l}\text { MASTER } \\
\text { LIST } \\
\text { Pu-244 } \\
\end{array}$} & \multicolumn{3}{|c|}{ DOE-STD-1196 } & \multicolumn{3}{|c|}{ FGR-12 } & \multicolumn{3}{|c|}{ ICRP-38 } & \multicolumn{3}{|c|}{ ICRP-107 } & \multicolumn{3}{|c|}{ JAERI } & \multirow{3}{*}{$\begin{array}{c}\begin{array}{c}\text { Selected } \\
\text { Half-Life } \\
\text { Reference }\end{array} \\
\text { ICRP-38 }\end{array}$} & & \\
\hline & \multirow{2}{*}{$\begin{array}{l}\text { Nuclide } \\
\text { Pu-244 }\end{array}$} & \multicolumn{2}{|c|}{$\begin{array}{c}\text { Half-Life } \\
\text { (original units) }\end{array}$} & \multirow{2}{*}{$\begin{array}{l}\text { Nuclide } \\
\text { Pu-244 }\end{array}$} & \multicolumn{2}{|c|}{$\begin{array}{c}\text { Half-Life } \\
\text { (original units) }\end{array}$} & \multirow{2}{*}{$\begin{array}{l}\text { Nuclide } \\
\text { Pu-244 }\end{array}$} & \multicolumn{2}{|c|}{$\begin{array}{c}\text { Half-Life } \\
\text { (original units) }\end{array}$} & \multirow{2}{*}{$\begin{array}{l}\text { Nuclide } \\
\text { Pu-244 }\end{array}$} & \multicolumn{2}{|c|}{$\begin{array}{c}\text { Half-Life } \\
\text { (original units) }\end{array}$} & \multirow[t]{2}{*}{ Nuclide } & $\begin{array}{r}\text { Half-Li } \\
\text { (original }\end{array}$ & its) & & \multicolumn{2}{|c|}{$\begin{array}{c}\text { Selected } \\
\text { Half-Life } \\
\text { (original units) }\end{array}$} \\
\hline & & $8.00 E+07$ & $y$ & & $8.26 \mathrm{E}+07$ & $y$ & & $8.26 \mathrm{E}+07$ & $y$ & & $8.00 \mathrm{E}+07$ & $y$ & & & & & $8.26 \mathrm{E}+07$ & $y$ \\
\hline Pu-245 & Pu-245 & $1.05 \mathrm{E}+01$ & $\mathrm{~h}$ & Pu-245 & $1.05 \mathrm{E}+01$ & $\mathrm{~h}$ & Pu-245 & $1.05 \mathrm{E}+01$ & $\mathrm{~h}$ & Pu-245 & $1.05 \mathrm{E}+01$ & $\mathrm{~h}$ & & & & ICRP-38 & $1.05 \mathrm{E}+01$ & $\mathrm{~h}$ \\
\hline Pu-246 & Pu-246 & $1.08 \mathrm{E}+01$ & $d$ & Pu-246 & $1.09 \mathrm{E}+01$ & $d$ & Pu-246 & $1.09 \mathrm{E}+01$ & $d$ & Pu-246 & $1.08 \mathrm{E}+01$ & $\mathrm{~d}$ & & & & ICRP-38 & $1.09 \mathrm{E}+01$ & $d$ \\
\hline Ra-219 & Ra-219 & $1.00 \mathrm{E}+01$ & $\mathrm{~ms}$ & & & & & & & Ra-219 & $1.00 \mathrm{E}+01$ & $\mathrm{~ms}$ & & & & ICRP-107 & $1.00 \mathrm{E}+01$ & $\mathrm{~ms}$ \\
\hline Ra-220 & Ra-220 & $1.79 \mathrm{E}-02$ & $\mathrm{~s}$ & & & & & & & Ra-220 & $1.79 \mathrm{E}-02$ & $\mathrm{~s}$ & & & & ICRP-107 & $1.79 \mathrm{E}-02$ & $\mathrm{~s}$ \\
\hline Ra-221 & Ra-221 & $2.80 \mathrm{E}+01$ & $\mathrm{~s}$ & & & & & & & Ra-221 & $2.80 \mathrm{E}+01$ & $\mathrm{~s}$ & & & & ICRP-107 & $2.80 \mathrm{E}+01$ & $\mathrm{~s}$ \\
\hline Ra-222 & Ra-222 & $3.80 \mathrm{E}+01$ & $\mathrm{~s}$ & Ra-222 & $3.80 \mathrm{E}+01$ & $\mathrm{~s}$ & Ra-222 & $3.80 \mathrm{E}+01$ & $\mathrm{~s}$ & Ra-222 & $3.80 \mathrm{E}+01$ & $\mathrm{~s}$ & & & & ICRP-38 & $3.80 \mathrm{E}+01$ & $\mathrm{~s}$ \\
\hline Ra-223 & Ra-223 & $1.14 \mathrm{E}+01$ & $d$ & Ra-223 & $1.14 \mathrm{E}+01$ & $\mathrm{~d}$ & Ra-223 & $1.14 \mathrm{E}+01$ & $d$ & Ra-223 & $1.14 \mathrm{E}+01$ & $d$ & & & & ICRP-38 & $1.14 \mathrm{E}+01$ & $d$ \\
\hline Ra-224 & Ra-224 & $3.66 \mathrm{E}+00$ & $d$ & Ra-224 & $3.66 \mathrm{E}+00$ & $\mathrm{~d}$ & Ra-224 & $3.66 \mathrm{E}+00$ & $d$ & Ra-224 & $3.66 \mathrm{E}+00$ & $d$ & & & & ICRP-38 & $3.66 \mathrm{E}+00$ & $d$ \\
\hline Ra-225 & Ra-225 & $1.49 \mathrm{E}+01$ & $d$ & Ra-225 & $1.48 \mathrm{E}+01$ & $d$ & Ra-225 & $1.48 \mathrm{E}+01$ & $d$ & Ra-225 & $1.49 \mathrm{E}+01$ & $d$ & & & & ICRP-38 & $1.48 \mathrm{E}+01$ & $d$ \\
\hline Ra-226 & Ra-226 & $1.60 \mathrm{E}+03$ & $y$ & Ra-226 & $1.60 \mathrm{E}+03$ & $y$ & Ra-226 & $1.60 \mathrm{E}+03$ & $y$ & Ra-226 & $1.60 \mathrm{E}+03$ & $y$ & & & & ICRP-38 & $1.60 \mathrm{E}+03$ & $y$ \\
\hline Ra-227 & Ra-227 & $4.22 \mathrm{E}+01$ & $\mathrm{~m}$ & Ra-227 & $4.22 \mathrm{E}+01$ & $\mathrm{~m}$ & Ra-227 & $4.22 \mathrm{E}+01$ & $m$ & Ra-227 & $4.22 \mathrm{E}+01$ & $m$ & & & & ICRP-38 & $4.22 \mathrm{E}+01$ & $\mathrm{~m}$ \\
\hline Ra-228 & Ra-228 & $5.75 \mathrm{E}+00$ & $y$ & Ra-228 & $5.75 \mathrm{E}+00$ & $y$ & Ra-228 & $5.75 \mathrm{E}+00$ & $y$ & Ra-228 & $5.75 \mathrm{E}+00$ & $y$ & & & & ICRP-38 & $5.75 \mathrm{E}+00$ & $y$ \\
\hline Ra-230 & Ra-230 & $9.30 \mathrm{E}+01$ & $\mathrm{~m}$ & & & & & & & Ra-230 & $9.30 \mathrm{E}+01$ & $\mathrm{~m}$ & Ra-230 & $9.30 \mathrm{E}+01$ & $m$ & ICRP-107 & $9.30 \mathrm{E}+01$ & $\mathrm{~m}$ \\
\hline Rb-77 & $\mathrm{Rb}-77$ & $3.77 \mathrm{E}+00$ & $m$ & & & & Rb-77 & $3.70 \mathrm{E}+00$ & $\mathrm{~m}$ & $\mathrm{Rb}-77$ & $3.77 \mathrm{E}+00$ & $m$ & $\mathrm{Rb}-77$ & $3.77 \mathrm{E}+00$ & $m$ & ICRP-38 & $3.70 \mathrm{E}+00$ & $m$ \\
\hline $\mathrm{Rb}-78$ & $\mathrm{Rb}-78$ & $1.77 \mathrm{E}+01$ & $\mathrm{~m}$ & & & & & & & $\mathrm{Rb}-78$ & $1.77 \mathrm{E}+01$ & $\mathrm{~m}$ & $\mathrm{Rb}-78$ & $1.77 \mathrm{E}+01$ & $m$ & ICRP-107 & $1.77 \mathrm{E}+01$ & $\mathrm{~m}$ \\
\hline Rb-78m & $\mathrm{Rb}-78 \mathrm{~m}$ & $5.74 \mathrm{E}+00$ & $m$ & & & & & & & $\mathrm{Rb}-78 \mathrm{~m}$ & $5.74 \mathrm{E}+00$ & $\mathrm{~m}$ & & & & ICRP-107 & $5.74 \mathrm{E}+00$ & $\mathrm{~m}$ \\
\hline Rb-79 & $\mathrm{Rb}-79$ & $2.29 \mathrm{E}+01$ & $m$ & Rb-79 & $2.29 \mathrm{E}+01$ & $\mathrm{~m}$ & Rb-79 & $2.29 \mathrm{E}+01$ & $\mathrm{~m}$ & Rb-79 & $2.29 \mathrm{E}+01$ & $\mathrm{~m}$ & & & & ICRP-38 & $2.29 \mathrm{E}+01$ & $m$ \\
\hline Rb-80 & $\mathrm{Rb}-80$ & $3.34 \mathrm{E}+01$ & $\mathrm{~s}$ & $\mathrm{Rb}-80$ & $3.40 \mathrm{E}+01$ & $\mathrm{~s}$ & $\mathrm{Rb}-80$ & $3.40 \mathrm{E}+01$ & $\mathrm{~s}$ & $\mathrm{Rb}-80$ & $3.34 \mathrm{E}+01$ & $\mathrm{~s}$ & & & & ICRP-38 & $3.40 \mathrm{E}+01$ & $\mathrm{~s}$ \\
\hline $\mathrm{Rb}-81$ & $\mathrm{Rb}-81$ & $4.58 \mathrm{E}+00$ & $\mathrm{~h}$ & $\mathrm{Rb}-81$ & $4.58 \mathrm{E}+00$ & $\mathrm{~h}$ & $\mathrm{Rb}-81$ & $4.58 \mathrm{E}+00$ & $\mathrm{~h}$ & $\mathrm{Rb}-81$ & $4.58 \mathrm{E}+00$ & $\mathrm{~h}$ & & & & ICRP-38 & $4.58 \mathrm{E}+00$ & $\mathrm{~h}$ \\
\hline Rb-81m & $\mathrm{Rb}-81 \mathrm{~m}$ & $3.05 \mathrm{E}+01$ & $\mathrm{~m}$ & Rb-81m & $3.20 \mathrm{E}+01$ & $\mathrm{~m}$ & Rb-81m & $3.20 \mathrm{E}+01$ & $m$ & $\mathrm{Rb}-81 \mathrm{~m}$ & $3.05 \mathrm{E}+01$ & $m$ & & & & ICRP-38 & $3.20 \mathrm{E}+01$ & $\mathrm{~m}$ \\
\hline Rb-82 & $\mathrm{Rb}-82$ & $1.27 \mathrm{E}+00$ & $\mathrm{~m}$ & $\mathrm{Rb}-82$ & $1.30 \mathrm{E}+00$ & $\mathrm{~m}$ & $\mathrm{Rb}-82$ & $1.30 \mathrm{E}+00$ & $\mathrm{~m}$ & $\mathrm{Rb}-82$ & $1.27 \mathrm{E}+00$ & $\mathrm{~m}$ & $\mathrm{Rb}-82$ & $1.27 \mathrm{E}+00$ & $\mathrm{~m}$ & ICRP-38 & $1.30 \mathrm{E}+00$ & $\mathrm{~m}$ \\
\hline Rb-82m & $\mathrm{Rb}-82 \mathrm{~m}$ & $6.47 E+00$ & $\mathrm{~h}$ & Rb-82m & $6.20 \mathrm{E}+00$ & $\mathrm{~h}$ & Rb-82m & $6.20 \mathrm{E}+00$ & $\mathrm{~h}$ & Rb-82m & $6.47 \mathrm{E}+00$ & $\mathrm{~h}$ & & & & ICRP-38 & $6.20 \mathrm{E}+00$ & $\mathrm{~h}$ \\
\hline Rb-83 & $\mathrm{Rb}-83$ & $8.62 \mathrm{E}+01$ & $d$ & $\mathrm{Rb}-83$ & $8.62 \mathrm{E}+01$ & $d$ & Rb-83 & $8.62 \mathrm{E}+01$ & $d$ & $\mathrm{Rb}-83$ & $8.62 \mathrm{E}+01$ & $d$ & & & & ICRP-38 & $8.62 \mathrm{E}+01$ & $d$ \\
\hline Rb-84 & $\mathrm{Rb}-84$ & $3.28 \mathrm{E}+01$ & $d$ & $\mathrm{Rb}-84$ & $3.28 \mathrm{E}+01$ & $d$ & $\mathrm{Rb}-84$ & $3.28 \mathrm{E}+01$ & $d$ & $\mathrm{Rb}-84$ & $3.28 \mathrm{E}+01$ & $d$ & & & & ICRP-38 & $3.28 \mathrm{E}+01$ & d \\
\hline $\mathrm{Rb}-84 \mathrm{~m}$ & $\mathrm{Rb}-84 \mathrm{~m}$ & $2.03 \mathrm{E}+01$ & $\mathrm{~m}$ & & & & & & & $\mathrm{Rb}-84 \mathrm{~m}$ & $2.03 \mathrm{E}+01$ & $\mathrm{~m}$ & $\mathrm{Rb}-84 \mathrm{~m}$ & $2.03 E+01$ & $\mathrm{~m}$ & ICRP-107 & $2.03 \mathrm{E}+01$ & $\mathrm{~m}$ \\
\hline Rb-86 & $\mathrm{Rb}-86$ & $1.86 \mathrm{E}+01$ & $d$ & $\mathrm{Rb}-86$ & $1.87 \mathrm{E}+01$ & $d$ & $\mathrm{Rb}-86$ & $1.87 \mathrm{E}+01$ & $d$ & $\mathrm{Rb}-86$ & $1.86 \mathrm{E}+01$ & $d$ & & & & ICRP-38 & $1.87 \mathrm{E}+01$ & $\mathrm{~d}$ \\
\hline Rb-86m & $\mathrm{Rb}-86 \mathrm{~m}$ & $1.02 \mathrm{E}+00$ & $\mathrm{~m}$ & & & & & & & Rb-86m & $1.02 \mathrm{E}+00$ & $\mathrm{~m}$ & Rb-86m & $1.02 \mathrm{E}+00$ & $\mathrm{~m}$ & ICRP-107 & $1.02 \mathrm{E}+00$ & $\mathrm{~m}$ \\
\hline Rb-87 & Rb-87 & $4.92 \mathrm{E}+10$ & $y$ & Rb-87 & $4.70 \mathrm{E}+10$ & $y$ & Rb-87 & $4.70 \mathrm{E}+10$ & $y$ & Rb-87 & $4.92 \mathrm{E}+10$ & $y$ & & & & ICRP-38 & $4.70 \mathrm{E}+10$ & $y$ \\
\hline Rb-88 & $\mathrm{Rb}-88$ & $1.78 \mathrm{E}+01$ & $\mathrm{~m}$ & $\mathrm{Rb}-88$ & $1.78 \mathrm{E}+01$ & $\mathrm{~m}$ & $\mathrm{Rb}-88$ & $1.78 \mathrm{E}+01$ & $\mathrm{~m}$ & $\mathrm{Rb}-88$ & $1.78 \mathrm{E}+01$ & $\mathrm{~m}$ & & & & ICRP-38 & $1.78 \mathrm{E}+01$ & $\mathrm{~m}$ \\
\hline Rb-89 & $\mathrm{Rb}-89$ & $1.52 \mathrm{E}+01$ & $\mathrm{~m}$ & $\mathrm{Rb}-89$ & $1.52 \mathrm{E}+01$ & $\mathrm{~m}$ & $\mathrm{Rb}-89$ & $1.52 \mathrm{E}+01$ & $\mathrm{~m}$ & $\mathrm{Rb}-89$ & $1.52 \mathrm{E}+01$ & $m$ & & & & ICRP-38 & $1.52 \mathrm{E}+01$ & $\mathrm{~m}$ \\
\hline Rb-90 & $\mathrm{Rb}-90$ & $1.58 \mathrm{E}+02$ & $\mathrm{~s}$ & & & & & & & $\mathrm{Rb}-90$ & $1.58 \mathrm{E}+02$ & $\mathrm{~s}$ & Rb-90 & $2.63 \mathrm{E}+00$ & $\mathrm{~m}$ & ICRP-107 & $1.58 \mathrm{E}+02$ & $s$ \\
\hline Rb-90m & $\mathrm{Rb}-90 \mathrm{~m}$ & $2.58 \mathrm{E}+02$ & $\mathrm{~s}$ & & & & & & & Rb-90m & $2.58 \mathrm{E}+02$ & $\mathrm{~s}$ & Rb-90m & $4.30 \mathrm{E}+00$ & $m$ & ICRP-107 & $2.58 \mathrm{E}+02$ & $\mathrm{~s}$ \\
\hline Re-177 & & & & Re-177 & $1.40 \mathrm{E}+01$ & $\mathrm{~m}$ & Re-177 & $1.40 \mathrm{E}+01$ & $m$ & & & & & & & ICRP-38 & $1.40 \mathrm{E}+01$ & $\mathrm{~m}$ \\
\hline Re-178 & Re-178 & $1.32 \mathrm{E}+01$ & $\mathrm{~m}$ & Re-178 & $1.32 \mathrm{E}+01$ & $m$ & Re-178 & $1.32 \mathrm{E}+01$ & $m$ & Re-178 & $1.32 \mathrm{E}+01$ & $m$ & & & & ICRP-38 & $1.32 \mathrm{E}+01$ & $\mathrm{~m}$ \\
\hline Re-179 & Re-179 & $1.95 \mathrm{E}+01$ & $m$ & & & & & & & Re-179 & $1.95 \mathrm{E}+01$ & $m$ & Re-179 & $1.95 \mathrm{E}+01$ & $m$ & ICRP-107 & $1.95 \mathrm{E}+01$ & $m$ \\
\hline Re-180 & Re-180 & $2.44 \mathrm{E}+00$ & $\mathrm{~m}$ & Re-180 & $2.43 \mathrm{E}+00$ & $\mathrm{~m}$ & Re-180 & $2.43 \mathrm{E}+00$ & $\mathrm{~m}$ & Re-180 & $2.44 \mathrm{E}+00$ & $\mathrm{~m}$ & Re-180 & $2.44 \mathrm{E}+00$ & $m$ & ICRP-38 & $2.43 \mathrm{E}+00$ & $\mathrm{~m}$ \\
\hline Re-181 & Re-181 & $1.99 \mathrm{E}+01$ & $\mathrm{~h}$ & Re-181 & $2.00 \mathrm{E}+01$ & $\mathrm{~h}$ & Re-181 & $2.00 \mathrm{E}+01$ & $\mathrm{~h}$ & Re-181 & $1.99 \mathrm{E}+01$ & $\mathrm{~h}$ & & & & ICRP-38 & $2.00 \mathrm{E}+01$ & $\mathrm{~h}$ \\
\hline Re-182I & Re-182 & $6.40 \mathrm{E}+01$ & $\mathrm{~h}$ & Re-182b & $6.40 \mathrm{E}+01$ & $\mathrm{~h}$ & Re-182b & $6.40 \mathrm{E}+01$ & $\mathrm{~h}$ & Re-182 & $6.40 \mathrm{E}+01$ & $\mathrm{~h}$ & & & & ICRP-38 & $6.40 \mathrm{E}+01$ & $\mathrm{~h}$ \\
\hline Re-182s & $\operatorname{Re}-182 \mathrm{~m}$ & $1.27 \mathrm{E}+01$ & $\mathrm{~h}$ & Re-182a & $1.27 \mathrm{E}+01$ & $\mathrm{~h}$ & Re-182a & $1.27 \mathrm{E}+01$ & $\mathrm{~h}$ & Re-182m & $1.27 \mathrm{E}+01$ & $\mathrm{~h}$ & & & & ICRP-38 & $1.27 \mathrm{E}+01$ & $\mathrm{~h}$ \\
\hline
\end{tabular}

D-25 


\begin{tabular}{|c|c|c|c|c|c|c|c|c|c|c|c|c|c|c|c|c|c|c|}
\hline \multicolumn{19}{|c|}{ Table D.1 Selection of Radionuclides For Calculating HC-2 and HC-3 TQs (continued) } \\
\hline & \multicolumn{3}{|c|}{ DOE-STD-1196 } & \multicolumn{3}{|c|}{ FGR-12 } & \multicolumn{3}{|c|}{ ICRP-38 } & \multicolumn{3}{|c|}{ ICRP-107 } & \multicolumn{3}{|c|}{ JAERI } & \multirow{3}{*}{$\begin{array}{c}\begin{array}{c}\text { Selected } \\
\text { Half-Life } \\
\text { Reference }\end{array} \\
\text { ICRP-107 }\end{array}$} & \multirow{2}{*}{\multicolumn{2}{|c|}{$\begin{array}{c}\text { Selected } \\
\text { Half-Life } \\
\text { (original units) }\end{array}$}} \\
\hline $\begin{array}{l}\text { MASTER } \\
\text { LIST }\end{array}$ & \multirow{2}{*}{$\begin{array}{l}\text { Nuclide } \\
\text { Re-183 }\end{array}$} & \multicolumn{2}{|c|}{$\begin{array}{c}\text { Half-Life } \\
\text { (original units) }\end{array}$} & \multirow[t]{2}{*}{ Nuclide } & \multicolumn{2}{|c|}{$\begin{array}{c}\text { Half-Life } \\
\text { (original units) }\end{array}$} & \multirow[t]{2}{*}{ Nuclide } & \multicolumn{2}{|c|}{$\begin{array}{c}\text { Half-Life } \\
\text { (original units) }\end{array}$} & \multirow{2}{*}{$\begin{array}{l}\text { Nuclide } \\
\text { Re-183 }\end{array}$} & \multicolumn{2}{|c|}{$\begin{array}{c}\text { Half-Life } \\
\text { (original units) }\end{array}$} & \multirow{2}{*}{$\begin{array}{l}\text { Nuclide } \\
\text { Re-183 }\end{array}$} & \multicolumn{2}{|c|}{$\begin{array}{c}\text { Half-Life } \\
\text { (original units) }\end{array}$} & & & \\
\hline Re-183 & & $7.00 \mathrm{E}+01$ & $\mathrm{~d}$ & & & & & & & & $7.00 \mathrm{E}+01$ & $d$ & & $7.00 \mathrm{E}+01$ & $\mathrm{~d}$ & & $7.00 \mathrm{E}+01$ & $\mathrm{~d}$ \\
\hline Re-184 & Re-184 & $3.80 \mathrm{E}+01$ & d & Re-184 & $3.80 \mathrm{E}+01$ & d & Re-184 & $3.80 \mathrm{E}+01$ & d & Re-184 & $3.80 \mathrm{E}+01$ & d & & & & ICRP-38 & $3.80 \mathrm{E}+01$ & $\mathrm{~d}$ \\
\hline Re-184m & Re-184m & $1.69 \mathrm{E}+02$ & $d$ & Re-184m & $1.65 \mathrm{E}+02$ & $\mathrm{~d}$ & Re-184m & $1.65 \mathrm{E}+02$ & $\mathrm{~d}$ & Re-184m & $1.69 \mathrm{E}+02$ & d & & & & ICRP-38 & $1.65 \mathrm{E}+02$ & $\mathrm{~d}$ \\
\hline Re-186 & Re-186 & $3.72 \mathrm{E}+00$ & $d$ & Re-186 & $9.06 \mathrm{E}+01$ & $\mathrm{~h}$ & Re-186 & $9.06 \mathrm{E}+01$ & $\mathrm{~h}$ & Re-186 & $3.72 \mathrm{E}+00$ & d & & & & ICRP-38 & $9.06 \mathrm{E}+01$ & $\mathrm{~h}$ \\
\hline Re-186m & Re-186m & $2.00 \mathrm{E}+05$ & $\mathrm{y}$ & Re-186m & $2.00 \mathrm{E}+05$ & $\mathrm{y}$ & Re-186m & $2.00 \mathrm{E}+05$ & $\mathrm{y}$ & Re-186m & $2.00 \mathrm{E}+05$ & $\mathrm{y}$ & & & & ICRP-38 & $2.00 \mathrm{E}+05$ & $\mathrm{y}$ \\
\hline Re-187 & Re-187 & $4.12 \mathrm{E}+10$ & $\mathrm{y}$ & Re-187 & $5.00 \mathrm{E}+10$ & $\mathrm{y}$ & Re-187 & $5.00 \mathrm{E}+10$ & y & Re-187 & $4.12 \mathrm{E}+10$ & y & & & & ICRP-38 & $5.00 \mathrm{E}+10$ & $\mathrm{y}$ \\
\hline Re-188 & Re-188 & $1.70 \mathrm{E}+01$ & $\mathrm{~h}$ & Re-188 & $1.70 \mathrm{E}+01$ & $\mathrm{~h}$ & Re-188 & $1.70 \mathrm{E}+01$ & $\mathrm{~h}$ & Re-188 & $1.70 \mathrm{E}+01$ & $\mathrm{~h}$ & & & & ICRP-38 & $1.70 \mathrm{E}+01$ & $\mathrm{~h}$ \\
\hline Re-188m & Re-188m & $1.86 \mathrm{E}+01$ & $\mathrm{~m}$ & Re-188m & $1.86 \mathrm{E}+01$ & $\mathrm{~m}$ & Re-188m & $1.86 \mathrm{E}+01$ & $\mathrm{~m}$ & Re-188m & $1.86 \mathrm{E}+01$ & $\mathrm{~m}$ & & & & ICRP-38 & $1.86 \mathrm{E}+01$ & $\mathrm{~m}$ \\
\hline Re-189 & Re-189 & $2.43 \mathrm{E}+01$ & $\mathrm{~h}$ & Re-189 & $2.43 \mathrm{E}+01$ & $\mathrm{~h}$ & Re-189 & $2.43 \mathrm{E}+01$ & $\mathrm{~h}$ & Re-189 & $2.43 E+01$ & $\mathrm{~h}$ & & & & ICRP-38 & $2.43 \mathrm{E}+01$ & $\mathrm{~h}$ \\
\hline Re-190 & Re-190 & $3.10 \mathrm{E}+00$ & $\mathrm{~m}$ & & & & & & & Re-190 & $3.10 \mathrm{E}+00$ & $\mathrm{~m}$ & Re-190 & $3.10 \mathrm{E}+00$ & $\mathrm{~m}$ & ICRP-107 & $3.10 \mathrm{E}+00$ & $\mathrm{~m}$ \\
\hline Re-190m & Re-190m & $3.20 \mathrm{E}+00$ & $\mathrm{~h}$ & & & & & & & Re-190m & $3.20 \mathrm{E}+00$ & $\mathrm{~h}$ & & & & ICRP-107 & $3.20 E+00$ & $\mathrm{~h}$ \\
\hline Rh-100 & Rh-100 & $2.08 \mathrm{E}+01$ & $\mathrm{~h}$ & Rh-100 & $2.08 \mathrm{E}+01$ & $\mathrm{~h}$ & Rh-100 & $2.08 \mathrm{E}+01$ & $\mathrm{~h}$ & Rh-100 & $2.08 \mathrm{E}+01$ & $\mathrm{~h}$ & & & & ICRP-38 & $2.08 \mathrm{E}+01$ & $\mathrm{~h}$ \\
\hline Rh-100m & Rh-100m & $4.60 \mathrm{E}+00$ & $\mathrm{~m}$ & & & & & & & Rh-100m & $4.60 \mathrm{E}+00$ & $\mathrm{~m}$ & Rh-100m & $4.60 \mathrm{E}+00$ & $\mathrm{~m}$ & ICRP-107 & $4.60 \mathrm{E}+00$ & $\mathrm{~m}$ \\
\hline Rh-101 & Rh-101 & $3.30 \mathrm{E}+00$ & $y$ & Rh-101 & $3.20 \mathrm{E}+00$ & $y$ & Rh-101 & $3.20 \mathrm{E}+00$ & $y$ & Rh-101 & $3.30 \mathrm{E}+00$ & $\mathrm{y}$ & & & & ICRP-38 & $3.20 \mathrm{E}+00$ & $\mathrm{y}$ \\
\hline Rh-101m & Rh-101m & $4.34 \mathrm{E}+00$ & d & Rh-101m & $4.34 \mathrm{E}+00$ & d & Rh-101m & $4.34 \mathrm{E}+00$ & $d$ & Rh-101m & $4.34 \mathrm{E}+00$ & $d$ & & & & ICRP-38 & $4.34 \mathrm{E}+00$ & $\mathrm{~d}$ \\
\hline Rh-102 & Rh-102m & $3.74 \mathrm{E}+00$ & $y$ & Rh-102 & $2.90 \mathrm{E}+00$ & $y$ & Rh-102 & $2.90 \mathrm{E}+00$ & $\mathrm{y}$ & Rh-102m & $3.74 \mathrm{E}+00$ & $\mathrm{y}$ & & & & ICRP-38 & $2.90 \mathrm{E}+00$ & $\mathrm{y}$ \\
\hline Rh-102m & Rh-102 & $2.07 \mathrm{E}+02$ & $d$ & Rh-102m & $2.07 \mathrm{E}+02$ & $d$ & Rh-102m & $2.07 \mathrm{E}+02$ & $d$ & Rh-102 & $2.07 \mathrm{E}+02$ & $d$ & & & & ICRP-38 & $2.07 \mathrm{E}+02$ & $\mathrm{~d}$ \\
\hline Rh-103m & Rh-103m & $5.61 \mathrm{E}+01$ & $\mathrm{~m}$ & Rh-103m & $5.61 \mathrm{E}+01$ & $\mathrm{~m}$ & Rh-103m & $5.61 \mathrm{E}+01$ & $\mathrm{~m}$ & Rh-103m & $5.61 \mathrm{E}+01$ & $\mathrm{~m}$ & & & & ICRP-38 & $5.61 \mathrm{E}+01$ & $\mathrm{~m}$ \\
\hline Rh-104 & Rh-104 & $4.23 \mathrm{E}+01$ & $\mathrm{~s}$ & & & & & & & Rh-104 & $4.23 E+01$ & $\mathrm{~s}$ & & & & ICRP-107 & $4.23 \mathrm{E}+01$ & $\mathrm{~s}$ \\
\hline Rh-104m & Rh-104m & $4.34 \mathrm{E}+00$ & $\mathrm{~m}$ & & & & & & & Rh-104m & $4.34 \mathrm{E}+00$ & $\mathrm{~m}$ & & & & ICRP-107 & $4.34 \mathrm{E}+00$ & $\mathrm{~m}$ \\
\hline Rh-105 & Rh-105 & $3.54 \mathrm{E}+01$ & $\mathrm{~h}$ & Rh-105 & $3.54 \mathrm{E}+01$ & $\mathrm{~h}$ & Rh-105 & $3.54 \mathrm{E}+01$ & $\mathrm{~h}$ & Rh-105 & $3.54 \mathrm{E}+01$ & $\mathrm{~h}$ & & & & ICRP-38 & $3.54 \mathrm{E}+01$ & $\mathrm{~h}$ \\
\hline Rh-106 & Rh-106 & $2.98 \mathrm{E}+01$ & $\mathrm{~s}$ & Rh-106 & $2.99 \mathrm{E}+01$ & $\mathrm{~s}$ & Rh-106 & $2.99 \mathrm{E}+01$ & $\mathrm{~s}$ & Rh-106 & $2.98 \mathrm{E}+01$ & $\mathrm{~s}$ & & & & ICRP-38 & $2.99 \mathrm{E}+01$ & $\mathrm{~s}$ \\
\hline Rh-106m & Rh-106m & $1.31 \mathrm{E}+02$ & $\mathrm{~m}$ & Rh-106m & $1.32 \mathrm{E}+02$ & $\mathrm{~m}$ & Rh-106m & $1.32 \mathrm{E}+02$ & $\mathrm{~m}$ & Rh-106m & $1.31 \mathrm{E}+02$ & $\mathrm{~m}$ & & & & ICRP-38 & $1.32 \mathrm{E}+02$ & $\mathrm{~m}$ \\
\hline Rh-107 & Rh-107 & $2.17 \mathrm{E}+01$ & $\mathrm{~m}$ & Rh-107 & $2.17 \mathrm{E}+01$ & $\mathrm{~m}$ & Rh-107 & $2.17 \mathrm{E}+01$ & $\mathrm{~m}$ & Rh-107 & $2.17 E+01$ & $\mathrm{~m}$ & & & & ICRP-38 & $2.17 \mathrm{E}+01$ & $\mathrm{~m}$ \\
\hline Rh-108 & Rh-108 & $1.68 \mathrm{E}+01$ & $\mathrm{~s}$ & & & & & & & Rh-108 & $1.68 \mathrm{E}+01$ & $\mathrm{~s}$ & & & & ICRP-107 & $1.68 \mathrm{E}+01$ & $\mathrm{~s}$ \\
\hline Rh-109 & Rh-109 & $8.00 \mathrm{E}+01$ & $\mathrm{~s}$ & & & & & & & Rh-109 & $8.00 \mathrm{E}+01$ & $\mathrm{~s}$ & & & & ICRP-107 & $8.00 \mathrm{E}+01$ & $\mathrm{~s}$ \\
\hline Rh-94 & Rh-94 & $7.06 \mathrm{E}+01$ & $\mathrm{~s}$ & & & & & & & Rh-94 & $7.06 \mathrm{E}+01$ & $\mathrm{~s}$ & & & & ICRP-107 & $7.06 \mathrm{E}+01$ & $\mathrm{~s}$ \\
\hline Rh-95 & Rh-95 & $5.02 \mathrm{E}+00$ & $\mathrm{~m}$ & & & & & & & Rh-95 & $5.02 \mathrm{E}+00$ & $\mathrm{~m}$ & & & & ICRP-107 & $5.02 \mathrm{E}+00$ & $\mathrm{~m}$ \\
\hline Rh-95m & Rh-95m & $1.96 \mathrm{E}+00$ & $\mathrm{~m}$ & & & & & & & Rh-95m & $1.96 \mathrm{E}+00$ & $\mathrm{~m}$ & & & & ICRP-107 & $1.96 \mathrm{E}+00$ & $\mathrm{~m}$ \\
\hline Rh-96 & Rh-96 & $9.90 \mathrm{E}+00$ & $\mathrm{~m}$ & & & & & & & Rh-96 & $9.90 \mathrm{E}+00$ & $\mathrm{~m}$ & & & & ICRP-107 & $9.90 \mathrm{E}+00$ & $\mathrm{~m}$ \\
\hline Rh-96m & Rh-96m & $1.51 \mathrm{E}+00$ & $\mathrm{~m}$ & & & & & & & Rh-96m & $1.51 \mathrm{E}+00$ & $\mathrm{~m}$ & & & & ICRP-107 & $1.51 \mathrm{E}+00$ & $\mathrm{~m}$ \\
\hline Rh-97 & Rh-97 & $3.07 E+01$ & $\mathrm{~m}$ & & & & & & & Rh-97 & $3.07 \mathrm{E}+01$ & $\mathrm{~m}$ & Rh-97 & $3.07 \mathrm{E}+01$ & $\mathrm{~m}$ & ICRP-107 & $3.07 \mathrm{E}+01$ & $\mathrm{~m}$ \\
\hline Rh-97m & Rh-97m & $4.62 \mathrm{E}+01$ & $\mathrm{~m}$ & & & & & & & Rh-97m & $4.62 \mathrm{E}+01$ & $\mathrm{~m}$ & Rh-97m & $4.62 \mathrm{E}+01$ & $\mathrm{~m}$ & ICRP-107 & $4.62 \mathrm{E}+01$ & $\mathrm{~m}$ \\
\hline Rh-98 & Rh-98 & $8.70 \mathrm{E}+00$ & $\mathrm{~m}$ & & & & & & & Rh-98 & $8.70 \mathrm{E}+00$ & $\mathrm{~m}$ & Rh-98 & $8.70 \mathrm{E}+00$ & $\mathrm{~m}$ & ICRP-107 & $8.70 \mathrm{E}+00$ & $\mathrm{~m}$ \\
\hline Rh-99 & Rh-99 & $1.61 \mathrm{E}+01$ & $d$ & Rh-99 & $1.60 \mathrm{E}+01$ & $d$ & Rh-99 & $1.60 \mathrm{E}+01$ & $d$ & Rh-99 & $1.61 \mathrm{E}+01$ & $\mathrm{~d}$ & & & & ICRP-38 & $1.60 \mathrm{E}+01$ & $\mathrm{~d}$ \\
\hline Rh-99m & Rh-99m & $4.70 \mathrm{E}+00$ & $\mathrm{~h}$ & Rh-99m & $4.70 \mathrm{E}+00$ & $\mathrm{~h}$ & Rh-99m & $4.70 \mathrm{E}+00$ & $\mathrm{~h}$ & Rh-99m & $4.70 \mathrm{E}+00$ & $\mathrm{~h}$ & & & & ICRP-38 & $4.70 \mathrm{E}+00$ & $\mathrm{~h}$ \\
\hline Rn-207 & Rn-207 & $9.25 \mathrm{E}+00$ & $\mathrm{~m}$ & & & & & & & Rn-207 & $9.25 \mathrm{E}+00$ & $\mathrm{~m}$ & & & & ICRP-107 & $9.25 \mathrm{E}+00$ & $\mathrm{~m}$ \\
\hline Rn-209 & Rn-209 & $2.85 \mathrm{E}+01$ & $\mathrm{~m}$ & & & & & & & Rn-209 & $2.85 \mathrm{E}+01$ & $\mathrm{~m}$ & & & & ICRP-107 & $2.85 \mathrm{E}+01$ & $\mathrm{~m}$ \\
\hline Rn-210 & $\mathrm{Rn}-210$ & $2.40 \mathrm{E}+00$ & $\mathrm{~h}$ & & & & & & & $\mathrm{Rn}-210$ & $2.40 \mathrm{E}+00$ & $\mathrm{~h}$ & & & & ICRP-107 & $2.40 \mathrm{E}+00$ & $\mathrm{~h}$ \\
\hline Rn-211 & Rn-211 & $1.46 \mathrm{E}+01$ & $\mathrm{~h}$ & & & & & & & Rn-211 & $1.46 \mathrm{E}+01$ & $\mathrm{~h}$ & & & & ICRP-107 & $1.46 \mathrm{E}+01$ & $\mathrm{~h}$ \\
\hline
\end{tabular}

D-26 


\begin{tabular}{|c|c|c|c|c|c|c|c|c|c|c|c|c|c|c|c|c|c|c|}
\hline \multicolumn{19}{|c|}{ Table D.1 Selection of Radionuclides For Calculating HC-2 and HC-3 TQs (continued) } \\
\hline \multirow{3}{*}{$\begin{array}{l}\text { MASTER } \\
\text { LIST } \\
\text { Rn-212 }\end{array}$} & \multicolumn{3}{|c|}{ DOE-STD-1196 } & \multicolumn{3}{|c|}{ FGR-12 } & \multicolumn{3}{|c|}{ ICRP-38 } & \multicolumn{3}{|c|}{ ICRP-107 } & \multicolumn{3}{|c|}{ JAERI } & \multirow{3}{*}{$\begin{array}{c}\begin{array}{c}\text { Selected } \\
\text { Half-Life } \\
\text { Reference }\end{array} \\
\text { ICRP-107 }\end{array}$} & & \\
\hline & \multirow{2}{*}{$\begin{array}{l}\text { Nuclide } \\
\text { Rn-212 }\end{array}$} & \multicolumn{2}{|c|}{$\begin{array}{c}\text { Half-Life } \\
\text { (original units) }\end{array}$} & \multirow{2}{*}{ Nuclide } & \multicolumn{2}{|c|}{$\begin{array}{c}\text { Half-Life } \\
\text { (original units) }\end{array}$} & \multirow[t]{2}{*}{ Nuclide } & \multicolumn{2}{|c|}{$\begin{array}{c}\text { Half-Life } \\
\text { (original units) } \\
\end{array}$} & \multirow{2}{*}{$\begin{array}{l}\text { Nuclide } \\
\text { Rn-212 }\end{array}$} & \multicolumn{2}{|c|}{$\begin{array}{c}\text { Half-Life } \\
\text { (original units) } \\
\end{array}$} & \multirow[t]{2}{*}{ Nuclide } & $\begin{array}{r}\text { Half-Lif } \\
\text { (original u }\end{array}$ & its) & & \multicolumn{2}{|c|}{$\begin{array}{c}\text { Selected } \\
\text { Half-Life } \\
\text { (original units) }\end{array}$} \\
\hline & & $2.39 \mathrm{E}+01$ & $\mathrm{~m}$ & & & & & & & & $2.39 \mathrm{E}+01$ & $\mathrm{~m}$ & & & & & $2.39 \mathrm{E}+01$ & $\mathrm{~m}$ \\
\hline Rn-215 & Rn-215 & $2.30 \mathrm{E}+00$ & us & & & & & & & Rn-215 & $2.30 \mathrm{E}+00$ & us & & & & ICRP-107 & $2.30 \mathrm{E}+00$ & us \\
\hline Rn-216 & Rn-216 & $4.50 \mathrm{E}-05$ & $\mathrm{~s}$ & & & & & & & Rn-216 & $4.50 \mathrm{E}-05$ & $\mathrm{~s}$ & & & & ICRP-107 & $4.50 \mathrm{E}-05$ & $\mathrm{~s}$ \\
\hline Rn-217 & Rn-217 & $5.40 \mathrm{E}-04$ & $\mathrm{~s}$ & & & & & & & Rn-217 & $5.40 \mathrm{E}-04$ & $\mathrm{~s}$ & & & & ICRP-107 & $5.40 \mathrm{E}-04$ & $\mathrm{~s}$ \\
\hline Rn-218 & $\mathrm{Rn}-218$ & $3.50 \mathrm{E}-02$ & $\mathrm{~s}$ & Rn-218 & $3.50 \mathrm{E}+01$ & $\mathrm{~ms}$ & Rn-218 & $3.50 \mathrm{E}+01$ & $\mathrm{~ms}$ & Rn-218 & $3.50 \mathrm{E}-02$ & $\mathrm{~s}$ & & & & ICRP-38 & $3.50 \mathrm{E}+01$ & $\mathrm{~ms}$ \\
\hline Rn-219 & Rn-219 & $3.96 \mathrm{E}+00$ & $\mathrm{~s}$ & Rn-219 & $3.96 \mathrm{E}+00$ & $\mathrm{~s}$ & Rn-219 & $3.96 \mathrm{E}+00$ & $\mathrm{~s}$ & Rn-219 & $3.96 \mathrm{E}+00$ & $\mathrm{~s}$ & & & & ICRP-38 & $3.96 \mathrm{E}+00$ & $\mathrm{~s}$ \\
\hline Rn-220 & $\mathrm{Rn}-220$ & $5.56 \mathrm{E}+01$ & $\mathrm{~s}$ & Rn-220 & $5.56 \mathrm{E}+01$ & $s$ & Rn-220 & $5.56 \mathrm{E}+01$ & $\mathrm{~s}$ & Rn-220 & $5.56 \mathrm{E}+01$ & $\mathrm{~s}$ & & & & ICRP-38 & $5.56 \mathrm{E}+01$ & $\mathrm{~s}$ \\
\hline Rn-222 & Rn-222 & $3.82 \mathrm{E}+00$ & $d$ & $\mathrm{Rn}-222$ & $3.82 \mathrm{E}+00$ & $\mathrm{~d}$ & Rn-222 & $3.82 \mathrm{E}+00$ & $d$ & Rn-222 & $3.82 \mathrm{E}+00$ & $d$ & & & & ICRP-38 & $3.82 \mathrm{E}+00$ & $d$ \\
\hline Rn-223 & Rn-223 & $2.43 \mathrm{E}+01$ & $\mathrm{~m}$ & & & & & & & Rn-223 & $2.43 \mathrm{E}+01$ & $\mathrm{~m}$ & & & & ICRP-107 & $2.43 \mathrm{E}+01$ & $\mathrm{~m}$ \\
\hline Ru-103 & Ru-103 & $3.93 E+01$ & $d$ & Ru-103 & $3.93 E+01$ & $d$ & Ru-103 & $3.93 \mathrm{E}+01$ & $d$ & Ru-103 & $3.93 \mathrm{E}+01$ & $d$ & & & & ICRP-38 & $3.93 \mathrm{E}+01$ & $d$ \\
\hline Ru-105 & Ru-105 & $4.44 \mathrm{E}+00$ & $\mathrm{~h}$ & Ru-105 & $4.44 \mathrm{E}+00$ & $\mathrm{~h}$ & Ru-105 & $4.44 \mathrm{E}+00$ & $\mathrm{~h}$ & Ru-105 & $4.44 \mathrm{E}+00$ & $\mathrm{~h}$ & & & & ICRP-38 & $4.44 \mathrm{E}+00$ & $\mathrm{~h}$ \\
\hline Ru-106 & Ru-106 & $3.74 \mathrm{E}+02$ & $\mathrm{~d}$ & Ru-106 & $3.68 \mathrm{E}+02$ & $\mathrm{~d}$ & Ru-106 & $3.68 \mathrm{E}+02$ & $d$ & Ru-106 & $3.74 \mathrm{E}+02$ & $d$ & & & & ICRP-38 & $3.68 \mathrm{E}+02$ & $d$ \\
\hline Ru-107 & Ru-107 & $3.75 \mathrm{E}+00$ & $m$ & & & & & & & Ru-107 & $3.75 \mathrm{E}+00$ & $m$ & & & & ICRP-107 & $3.75 \mathrm{E}+00$ & $\mathrm{~m}$ \\
\hline Ru-108 & Ru-108 & $4.55 \mathrm{E}+00$ & $m$ & & & & & & & Ru-108 & $4.55 \mathrm{E}+00$ & $m$ & & & & ICRP-107 & $4.55 \mathrm{E}+00$ & $\mathrm{~m}$ \\
\hline Ru-92 & Ru-92 & $3.65 \mathrm{E}+00$ & $\mathrm{~m}$ & & & & & & & Ru-92 & $3.65 \mathrm{E}+00$ & $m$ & & & & ICRP-107 & $3.65 \mathrm{E}+00$ & $m$ \\
\hline Ru-94 & Ru-94 & $5.18 \mathrm{E}+01$ & $\mathrm{~m}$ & Ru-94 & $5.18 \mathrm{E}+01$ & $m$ & Ru-94 & $5.18 \mathrm{E}+01$ & $\mathrm{~m}$ & Ru-94 & $5.18 \mathrm{E}+01$ & $\mathrm{~m}$ & & & & ICRP-38 & $5.18 \mathrm{E}+01$ & $\mathrm{~m}$ \\
\hline Ru-95 & Ru-95 & $1.64 \mathrm{E}+00$ & $\mathrm{~h}$ & & & & & & & Ru-95 & $1.64 \mathrm{E}+00$ & $\mathrm{~h}$ & Ru-95 & $1.64 \mathrm{E}+00$ & $\mathrm{~h}$ & ICRP-107 & $1.64 \mathrm{E}+00$ & $\mathrm{~h}$ \\
\hline Ru-97 & Ru-97 & $2.90 \mathrm{E}+00$ & $d$ & Ru-97 & $2.90 \mathrm{E}+00$ & $d$ & Ru-97 & $2.90 \mathrm{E}+00$ & $d$ & Ru-97 & $2.90 \mathrm{E}+00$ & $d$ & & & & ICRP-38 & $2.90 \mathrm{E}+00$ & $d$ \\
\hline S-35 & S-35 & $8.75 \mathrm{E}+01$ & $d$ & S-35 & $8.74 \mathrm{E}+01$ & $d$ & S-35 & $8.74 \mathrm{E}+01$ & $d$ & S-35 & $8.75 \mathrm{E}+01$ & $d$ & & & & ICRP-38 & $8.74 \mathrm{E}+01$ & $d$ \\
\hline S-37 & S-37 & $5.05 \mathrm{E}+00$ & $\mathrm{~m}$ & & & & & & & S-37 & $5.05 \mathrm{E}+00$ & $m$ & S-37 & $5.05 \mathrm{E}+00$ & $\mathrm{~m}$ & ICRP-107 & $5.05 \mathrm{E}+00$ & $m$ \\
\hline S-38 & $\mathrm{S}-38$ & $1.70 \mathrm{E}+02$ & $\mathrm{~m}$ & & & & & & & S-38 & $1.70 \mathrm{E}+02$ & $m$ & $S-38$ & $1.70 \mathrm{E}+02$ & $m$ & ICRP-107 & $1.70 \mathrm{E}+02$ & $\mathrm{~m}$ \\
\hline Sb-111 & Sb-111 & $7.50 \mathrm{E}+01$ & $\mathrm{~s}$ & & & & & & & Sb-111 & $7.50 \mathrm{E}+01$ & $\mathrm{~s}$ & & & & ICRP-107 & $7.50 \mathrm{E}+01$ & $\mathrm{~s}$ \\
\hline Sb-113 & Sb-113 & $6.67 \mathrm{E}+00$ & $\mathrm{~m}$ & & & & & & & Sb-113 & $6.67 \mathrm{E}+00$ & $\mathrm{~m}$ & & & & ICRP-107 & $6.67 \mathrm{E}+00$ & $\mathrm{~m}$ \\
\hline Sb-114 & Sb-114 & $3.49 \mathrm{E}+00$ & $m$ & & & & & & & Sb-114 & $3.49 \mathrm{E}+00$ & $m$ & Sb-114 & $3.49 \mathrm{E}+00$ & $m$ & ICRP-107 & $3.49 \mathrm{E}+00$ & $\mathrm{~m}$ \\
\hline Sb-115 & Sb-115 & $3.21 E+01$ & $m$ & Sb-115 & $3.18 \mathrm{E}+01$ & $m$ & Sb-115 & $3.18 \mathrm{E}+01$ & $m$ & Sb-115 & $3.21 \mathrm{E}+01$ & $m$ & & & & ICRP-38 & $3.18 \mathrm{E}+01$ & $\mathrm{~m}$ \\
\hline Sb-116 & Sb-116 & $1.58 \mathrm{E}+01$ & $\mathrm{~m}$ & Sb-116 & $1.58 \mathrm{E}+01$ & $m$ & Sb-116 & $1.58 \mathrm{E}+01$ & $m$ & Sb-116 & $1.58 \mathrm{E}+01$ & $m$ & & & & ICRP-38 & $1.58 \mathrm{E}+01$ & $m$ \\
\hline Sb-116m & Sb-116m & $6.03 E+01$ & $\mathrm{~m}$ & Sb-116m & $6.03 \mathrm{E}+01$ & $\mathrm{~m}$ & Sb-116m & $6.03 \mathrm{E}+01$ & $m$ & Sb-116m & $6.03 \mathrm{E}+01$ & $\mathrm{~m}$ & & & & ICRP-38 & $6.03 \mathrm{E}+01$ & $\mathrm{~m}$ \\
\hline Sb-117 & Sb-117 & $2.80 \mathrm{E}+00$ & $\mathrm{~h}$ & Sb-117 & $2.80 \mathrm{E}+00$ & $\mathrm{~h}$ & Sb-117 & $2.80 \mathrm{E}+00$ & $\mathrm{~h}$ & Sb-117 & $2.80 \mathrm{E}+00$ & $\mathrm{~h}$ & & & & ICRP-38 & $2.80 \mathrm{E}+00$ & $\mathrm{~h}$ \\
\hline Sb-118 & Sb-118 & $3.60 \mathrm{E}+00$ & $\mathrm{~m}$ & & & & Sb-118 & $3.60 \mathrm{E}+00$ & $\mathrm{~m}$ & Sb-118 & $3.60 \mathrm{E}+00$ & $\mathrm{~m}$ & Sb-118 & $3.60 \mathrm{E}+00$ & $\mathrm{~m}$ & ICRP-38 & $3.60 \mathrm{E}+00$ & $\mathrm{~m}$ \\
\hline Sb-118m & Sb-118m & $5.00 \mathrm{E}+00$ & $\mathrm{~h}$ & Sb-118m & $5.00 \mathrm{E}+00$ & $\mathrm{~h}$ & Sb-118m & $5.00 \mathrm{E}+00$ & $\mathrm{~h}$ & Sb-118m & $5.00 \mathrm{E}+00$ & $\mathrm{~h}$ & & & & ICRP-38 & $5.00 \mathrm{E}+00$ & $\mathrm{~h}$ \\
\hline Sb-119 & Sb-119 & $3.82 \mathrm{E}+01$ & $\mathrm{~h}$ & Sb-119 & $3.81 \mathrm{E}+01$ & $\mathrm{~h}$ & Sb-119 & $3.81 \mathrm{E}+01$ & $\mathrm{~h}$ & Sb-119 & $3.82 \mathrm{E}+01$ & $\mathrm{~h}$ & & & & ICRP-38 & $3.81 \mathrm{E}+01$ & $\mathrm{~h}$ \\
\hline Sb-120s & Sb-120 & $1.59 \mathrm{E}+01$ & $\mathrm{~m}$ & Sb-120a & $1.59 \mathrm{E}+01$ & $m$ & Sb-120a & $1.59 \mathrm{E}+01$ & $m$ & Sb-120 & $1.59 \mathrm{E}+01$ & $m$ & & & & ICRP-38 & $1.59 \mathrm{E}+01$ & $\mathrm{~m}$ \\
\hline Sb-120I & Sb-120m & $5.76 \mathrm{E}+00$ & $\mathrm{~d}$ & Sb-120b & $5.76 \mathrm{E}+00$ & $d$ & Sb-120b & $5.76 \mathrm{E}+00$ & $d$ & Sb-120m & $5.76 \mathrm{E}+00$ & $d$ & & & & ICRP-38 & $5.76 \mathrm{E}+00$ & $d$ \\
\hline Sb-122 & Sb-122 & $2.72 \mathrm{E}+00$ & $d$ & Sb-122 & $2.70 \mathrm{E}+00$ & $d$ & Sb-122 & $2.70 \mathrm{E}+00$ & $d$ & Sb-122 & $2.72 \mathrm{E}+00$ & $d$ & & & & ICRP-38 & $2.70 \mathrm{E}+00$ & d \\
\hline Sb-122m & Sb-122m & $4.19 \mathrm{E}+00$ & $\mathrm{~m}$ & & & & & & & Sb-122m & $4.19 \mathrm{E}+00$ & $m$ & Sb-122m & $4.19 \mathrm{E}+00$ & $m$ & ICRP-107 & $4.19 \mathrm{E}+00$ & $m$ \\
\hline Sb-124 & Sb-124 & $6.02 \mathrm{E}+01$ & $d$ & Sb-124 & $6.02 \mathrm{E}+01$ & $d$ & Sb-124 & $6.02 \mathrm{E}+01$ & $d$ & Sb-124 & $6.02 \mathrm{E}+01$ & $d$ & & & & ICRP-38 & $6.02 \mathrm{E}+01$ & d \\
\hline $\mathrm{Sb}-124 \mathrm{~ms}$ & Sb-124m & $9.30 \mathrm{E}+01$ & $\mathrm{~s}$ & Sb-124m & $9.30 \mathrm{E}+01$ & $s$ & Sb-124m & $9.30 \mathrm{E}+01$ & $\mathrm{~s}$ & Sb-124m & $9.30 \mathrm{E}+01$ & $\mathrm{~s}$ & Sb-124m & $1.55 \mathrm{E}+00$ & $\mathrm{~m}$ & ICRP-38 & $9.30 \mathrm{E}+01$ & $\mathrm{~s}$ \\
\hline Sb-124ml & Sb-124n & $2.02 \mathrm{E}+01$ & $\mathrm{~m}$ & Sb-124n & $2.02 \mathrm{E}+01$ & $\mathrm{~m}$ & Sb-124n & $2.02 \mathrm{E}+01$ & $\mathrm{~m}$ & Sb-124n & $2.02 \mathrm{E}+01$ & $\mathrm{~m}$ & & & & ICRP-38 & $2.02 E+01$ & $\mathrm{~m}$ \\
\hline Sb-125 & Sb-125 & $2.76 \mathrm{E}+00$ & $y$ & Sb-125 & $2.77 \mathrm{E}+00$ & $y$ & Sb-125 & $2.77 \mathrm{E}+00$ & $y$ & Sb-125 & $2.76 \mathrm{E}+00$ & $y$ & & & & ICRP-38 & $2.77 \mathrm{E}+00$ & $y$ \\
\hline Sb-126 & Sb-126 & $1.24 \mathrm{E}+01$ & $d$ & Sb-126 & $1.24 \mathrm{E}+01$ & $d$ & Sb-126 & $1.24 \mathrm{E}+01$ & $d$ & Sb-126 & $1.24 \mathrm{E}+01$ & $d$ & & & & ICRP-38 & $1.24 \mathrm{E}+01$ & $d$ \\
\hline
\end{tabular}

D-27 


\begin{tabular}{|c|c|c|c|c|c|c|c|c|c|c|c|c|c|c|c|c|c|c|}
\hline \multirow{3}{*}{$\begin{array}{l}\text { MASTER } \\
\text { LIST } \\
\text { Sb-126m }\end{array}$} & \multicolumn{3}{|c|}{ DOE-STD-1196 } & \multicolumn{3}{|c|}{ FGR-12 } & \multicolumn{3}{|c|}{ ICRP-38 } & \multicolumn{3}{|c|}{ ICRP-107 } & \multicolumn{3}{|c|}{ JAERI } & \multirow{3}{*}{$\begin{array}{c}\begin{array}{c}\text { Selected } \\
\text { Half-Life } \\
\text { Reference }\end{array} \\
\text { ICRP-38 }\end{array}$} & \multirow{2}{*}{\multicolumn{2}{|c|}{$\begin{array}{c}\text { Selected } \\
\text { Half-Life } \\
\text { (original units) }\end{array}$}} \\
\hline & \multirow{2}{*}{$\begin{array}{l}\text { Nuclide } \\
\text { Sb-126m }\end{array}$} & \multicolumn{2}{|c|}{$\begin{array}{c}\text { Half-Life } \\
\text { (original units) }\end{array}$} & \multirow{2}{*}{$\begin{array}{l}\text { Nuclide } \\
\text { Sb-126m }\end{array}$} & \multicolumn{2}{|c|}{$\begin{array}{c}\text { Half-Life } \\
\text { (original units) }\end{array}$} & \multirow{2}{*}{$\begin{array}{l}\text { Nuclide } \\
\text { Sb-126m }\end{array}$} & \multicolumn{2}{|c|}{$\begin{array}{c}\text { Half-Life } \\
\text { (original units) }\end{array}$} & \multirow{2}{*}{$\begin{array}{l}\text { Nuclide } \\
\text { Sb-126m }\end{array}$} & \multicolumn{2}{|c|}{$\begin{array}{c}\text { Half-Life } \\
\text { (original units) }\end{array}$} & \multirow[t]{2}{*}{ Nuclide } & \multicolumn{2}{|c|}{$\begin{array}{c}\text { Half-Life } \\
\text { (original units) }\end{array}$} & & & \\
\hline & & $1.92 \mathrm{E}+01$ & $\mathrm{~m}$ & & $1.90 \mathrm{E}+01$ & $\mathrm{~m}$ & & $1.90 \mathrm{E}+01$ & $\mathrm{~m}$ & & $1.92 \mathrm{E}+01$ & $\mathrm{~m}$ & & & & & $1.90 \mathrm{E}+01$ & $\mathrm{~m}$ \\
\hline Sb-127 & Sb-127 & $3.85 \mathrm{E}+00$ & d & Sb-127 & $3.85 \mathrm{E}+00$ & $d$ & Sb-127 & $3.85 \mathrm{E}+00$ & d & Sb-127 & $3.85 \mathrm{E}+00$ & d & & & & ICRP-38 & $3.85 \mathrm{E}+00$ & $d$ \\
\hline Sb-128I & Sb-128 & $9.01 \mathrm{E}+00$ & $h$ & Sb-128b & $9.01 \mathrm{E}+00$ & $\mathrm{~h}$ & Sb-128b & $9.01 \mathrm{E}+00$ & $\mathrm{~h}$ & Sb-128 & $9.01 \mathrm{E}+00$ & $\mathrm{~h}$ & & & & ICRP-38 & $9.01 \mathrm{E}+00$ & $\mathrm{~h}$ \\
\hline Sb-128s & Sb-128m & $1.04 \mathrm{E}+01$ & $\mathrm{~m}$ & Sb-128a & $1.04 \mathrm{E}+01$ & $\mathrm{~m}$ & Sb-128a & $1.04 \mathrm{E}+01$ & $\mathrm{~m}$ & Sb-128m & $1.04 \mathrm{E}+01$ & $\mathrm{~m}$ & & & & ICRP-38 & $1.04 \mathrm{E}+01$ & $\mathrm{~m}$ \\
\hline Sb-129 & Sb-129 & $4.40 \mathrm{E}+00$ & $\mathrm{~h}$ & Sb-129 & $4.32 \mathrm{E}+00$ & $\mathrm{~h}$ & Sb-129 & $4.32 \mathrm{E}+00$ & $\mathrm{~h}$ & Sb-129 & $4.40 \mathrm{E}+00$ & $\mathrm{~h}$ & & & & ICRP-38 & $4.32 \mathrm{E}+00$ & $\mathrm{~h}$ \\
\hline Sb-130 & Sb-130 & $3.95 \mathrm{E}+01$ & $\mathrm{~m}$ & Sb-130 & $4.00 \mathrm{E}+01$ & $m$ & Sb-130 & $4.00 \mathrm{E}+01$ & $\mathrm{~m}$ & Sb-130 & $3.95 \mathrm{E}+01$ & $\mathrm{~m}$ & & & & ICRP-38 & $4.00 \mathrm{E}+01$ & $\mathrm{~m}$ \\
\hline Sb-130m & Sb-130m & $6.30 \mathrm{E}+00$ & $m$ & & & & & & & Sb-130m & $6.30 \mathrm{E}+00$ & $\mathrm{~m}$ & & & & ICRP-107 & $6.30 \mathrm{E}+00$ & $\mathrm{~m}$ \\
\hline Sb-131 & Sb-131 & $2.30 \mathrm{E}+01$ & $\mathrm{~m}$ & Sb-131 & $2.30 \mathrm{E}+01$ & $\mathrm{~m}$ & Sb-131 & $2.30 \mathrm{E}+01$ & $\mathrm{~m}$ & Sb-131 & $2.30 \mathrm{E}+01$ & $\mathrm{~m}$ & & & & ICRP-38 & $2.30 \mathrm{E}+01$ & $m$ \\
\hline Sb-133 & Sb-133 & $2.50 \mathrm{E}+00$ & $\mathrm{~m}$ & & & & & & & Sb-133 & $2.50 \mathrm{E}+00$ & $\mathrm{~m}$ & & & & ICRP-107 & $2.50 \mathrm{E}+00$ & $m$ \\
\hline Sc-42m & Sc- $42 m$ & $6.20 \mathrm{E}+01$ & $\mathrm{~s}$ & & & & & & & Sc- $42 m$ & $6.20 \mathrm{E}+01$ & $\mathrm{~s}$ & & & & ICRP-107 & $6.20 \mathrm{E}+01$ & $\mathrm{~s}$ \\
\hline Sc-43 & Sc-43 & $3.89 \mathrm{E}+00$ & $\mathrm{~h}$ & Sc-43 & $3.89 \mathrm{E}+00$ & $\mathrm{~h}$ & Sc-43 & $3.89 \mathrm{E}+00$ & $\mathrm{~h}$ & Sc-43 & $3.89 \mathrm{E}+00$ & $\mathrm{~h}$ & & & & ICRP-38 & $3.89 \mathrm{E}+00$ & $\mathrm{~h}$ \\
\hline Sc-44 & Sc-44 & $3.97 \mathrm{E}+00$ & $\mathrm{~h}$ & Sc-44 & $3.93 \mathrm{E}+00$ & $\mathrm{~h}$ & Sc-44 & $3.93 \mathrm{E}+00$ & $\mathrm{~h}$ & Sc-44 & $3.97 \mathrm{E}+00$ & $\mathrm{~h}$ & & & & ICRP-38 & $3.93 \mathrm{E}+00$ & $\mathrm{~h}$ \\
\hline Sc-44m & Sc- $44 m$ & $5.86 \mathrm{E}+01$ & $\mathrm{~h}$ & Sc- $44 m$ & $5.86 \mathrm{E}+01$ & $\mathrm{~h}$ & Sc- $44 m$ & $5.86 \mathrm{E}+01$ & $\mathrm{~h}$ & Sc- $44 m$ & $5.86 \mathrm{E}+01$ & $\mathrm{~h}$ & & & & ICRP-38 & $5.86 \mathrm{E}+01$ & $\mathrm{~h}$ \\
\hline Sc-46 & Sc-46 & $8.38 \mathrm{E}+01$ & $d$ & Sc-46 & $8.38 \mathrm{E}+01$ & $\mathrm{~d}$ & Sc-46 & $8.38 \mathrm{E}+01$ & $d$ & Sc-46 & $8.38 \mathrm{E}+01$ & $d$ & & & & ICRP-38 & $8.38 \mathrm{E}+01$ & $\mathrm{~d}$ \\
\hline Sc-47 & Sc-47 & $3.35 E+00$ & $d$ & Sc-47 & $3.35 \mathrm{E}+00$ & $d$ & Sc-47 & $3.35 \mathrm{E}+00$ & $d$ & Sc-47 & $3.35 \mathrm{E}+00$ & $d$ & & & & ICRP-38 & $3.35 E+00$ & $d$ \\
\hline Sc-48 & Sc-48 & $4.37 \mathrm{E}+01$ & $\mathrm{~h}$ & Sc-48 & $4.37 \mathrm{E}+01$ & $\mathrm{~h}$ & Sc-48 & $4.37 \mathrm{E}+01$ & $\mathrm{~h}$ & Sc-48 & $4.37 \mathrm{E}+01$ & $\mathrm{~h}$ & & & & ICRP-38 & $4.37 \mathrm{E}+01$ & $\mathrm{~h}$ \\
\hline Sc-49 & Sc-49 & $5.72 \mathrm{E}+01$ & $\mathrm{~m}$ & Sc-49 & $5.74 \mathrm{E}+01$ & $\mathrm{~m}$ & Sc-49 & $5.74 \mathrm{E}+01$ & $\mathrm{~m}$ & Sc-49 & $5.72 \mathrm{E}+01$ & $\mathrm{~m}$ & & & & ICRP-38 & $5.74 \mathrm{E}+01$ & $\mathrm{~m}$ \\
\hline Sc-50 & Sc-50 & $1.03 \mathrm{E}+02$ & $\mathrm{~s}$ & & & & & & & Sc-50 & $1.03 \mathrm{E}+02$ & $\mathrm{~s}$ & & & & ICRP-107 & $1.03 \mathrm{E}+02$ & $\mathrm{~s}$ \\
\hline Se-70 & Se-70 & $4.11 E+01$ & $\mathrm{~m}$ & Se-70 & $4.10 \mathrm{E}+01$ & $\mathrm{~m}$ & Se-70 & 4.10E+01 & $\mathrm{m}$ & Se-70 & $4.11 \mathrm{E}+01$ & $\mathrm{~m}$ & & & & ICRP-38 & $4.10 \mathrm{E}+01$ & $\mathrm{~m}$ \\
\hline Se-71 & Se-71 & $4.74 \mathrm{E}+00$ & $\mathrm{~m}$ & & & & & & & Se-71 & $4.74 \mathrm{E}+00$ & $m$ & Se-71 & $4.74 \mathrm{E}+00$ & $\mathrm{~m}$ & ICRP-107 & $4.74 \mathrm{E}+00$ & $m$ \\
\hline Se-72 & Se-72 & $8.40 \mathrm{E}+00$ & $\mathrm{~d}$ & & & & Se-72 & $8.40 \mathrm{E}+00$ & $d$ & Se-72 & $8.40 \mathrm{E}+00$ & $d$ & Se-72 & $8.40 \mathrm{E}+00$ & $\mathrm{~d}$ & ICRP-38 & $8.40 \mathrm{E}+00$ & $d$ \\
\hline Se-73 & Se-73 & $7.15 \mathrm{E}+00$ & $\mathrm{~h}$ & Se-73 & $7.15 \mathrm{E}+00$ & $\mathrm{~h}$ & Se-73 & $7.15 \mathrm{E}+00$ & $\mathrm{~h}$ & Se-73 & $7.15 \mathrm{E}+00$ & $\mathrm{~h}$ & & & & ICRP-38 & $7.15 \mathrm{E}+00$ & $\mathrm{~h}$ \\
\hline Se-73m & Se-73m & $3.98 \mathrm{E}+01$ & $m$ & Se-73m & $3.90 \mathrm{E}+01$ & $\mathrm{~m}$ & Se-73m & $3.90 \mathrm{E}+01$ & $m$ & Se-73m & $3.98 \mathrm{E}+01$ & $m$ & & & & ICRP-38 & $3.90 \mathrm{E}+01$ & $\mathrm{~m}$ \\
\hline Se-75 & Se-75 & $1.20 \mathrm{E}+02$ & $d$ & Se-75 & $1.20 \mathrm{E}+02$ & $\mathrm{~d}$ & Se-75 & $1.20 \mathrm{E}+02$ & $\mathrm{~d}$ & Se-75 & $1.20 \mathrm{E}+02$ & $\mathrm{~d}$ & & & & ICRP-38 & $1.20 \mathrm{E}+02$ & $\mathrm{~d}$ \\
\hline Se-77m & Se-77m & $1.74 \mathrm{E}+01$ & $\mathrm{~s}$ & Se-77m & $1.75 \mathrm{E}+01$ & $s$ & Se-77m & $1.75 \mathrm{E}+01$ & $s$ & Se-77m & $1.74 \mathrm{E}+01$ & $s$ & & & & ICRP-38 & $1.75 \mathrm{E}+01$ & $\mathrm{~s}$ \\
\hline Se-79 & Se-79 & $2.95 \mathrm{E}+05$ & $y$ & Se-79 & $6.50 \mathrm{E}+04$ & $y$ & Se-79 & $6.50 \mathrm{E}+04$ & $y$ & Se-79 & $2.95 \mathrm{E}+05$ & $y$ & & & & ICRP-38 & $6.50 \mathrm{E}+04$ & $y$ \\
\hline Se-79m & Se-79m & $3.92 \mathrm{E}+00$ & $\mathrm{~m}$ & & & & & & & Se-79m & $3.92 \mathrm{E}+00$ & $\mathrm{~m}$ & Se-79m & $3.92 \mathrm{E}+00$ & $\mathrm{~m}$ & ICRP-107 & $3.92 \mathrm{E}+00$ & $\mathrm{~m}$ \\
\hline Se-81 & Se-81 & $1.85 \mathrm{E}+01$ & $\mathrm{~m}$ & Se-81 & $1.85 \mathrm{E}+01$ & $\mathrm{~m}$ & Se-81 & $1.85 \mathrm{E}+01$ & $\mathrm{~m}$ & Se-81 & $1.85 \mathrm{E}+01$ & $\mathrm{~m}$ & & & & ICRP-38 & $1.85 \mathrm{E}+01$ & $m$ \\
\hline Se-81m & Se-81m & $5.73 E+01$ & $m$ & Se-81m & $5.73 E+01$ & $\mathrm{~m}$ & Se-81m & $5.73 \mathrm{E}+01$ & $\mathrm{~m}$ & Se-81m & $5.73 \mathrm{E}+01$ & $\mathrm{~m}$ & & & & ICRP-38 & $5.73 \mathrm{E}+01$ & $m$ \\
\hline Se-83 & Se-83 & $2.23 \mathrm{E}+01$ & $\mathrm{~m}$ & Se-83 & $2.25 \mathrm{E}+01$ & $\mathrm{~m}$ & Se-83 & $2.25 \mathrm{E}+01$ & $\mathrm{~m}$ & Se-83 & $2.23 \mathrm{E}+01$ & $\mathrm{~m}$ & & & & ICRP-38 & $2.25 \mathrm{E}+01$ & $\mathrm{~m}$ \\
\hline Se-83m & Se-83m & $7.01 \mathrm{E}+01$ & $\mathrm{~s}$ & & & & & & & Se-83m & $7.01 \mathrm{E}+01$ & $\mathrm{~s}$ & & & & ICRP-107 & $7.01 \mathrm{E}+01$ & $s$ \\
\hline Se-84 & Se- 84 & $3.10 \mathrm{E}+00$ & $\mathrm{~m}$ & & & & & & & Se- 84 & $3.10 \mathrm{E}+00$ & $m$ & & & & ICRP-107 & $3.10 \mathrm{E}+00$ & $\mathrm{~m}$ \\
\hline Si-31 & Si-31 & $1.57 \mathrm{E}+02$ & $\mathrm{~m}$ & Si-31 & $1.57 \mathrm{E}+02$ & $\mathrm{~m}$ & Si-31 & $1.57 \mathrm{E}+02$ & $\mathrm{~m}$ & Si-31 & $1.57 \mathrm{E}+02$ & $\mathrm{~m}$ & & & & ICRP-38 & $1.57 \mathrm{E}+02$ & $\mathrm{~m}$ \\
\hline Si-32 & Si-32 & $1.32 \mathrm{E}+02$ & $y$ & Si-32 & $4.50 \mathrm{E}+02$ & $y$ & Si-32 & $4.50 \mathrm{E}+02$ & $y$ & Si-32 & $1.32 \mathrm{E}+02$ & $y$ & & & & ICRP-38 & $4.50 \mathrm{E}+02$ & $y$ \\
\hline Sm-139 & Sm-139 & $2.57 E+00$ & $\mathrm{~m}$ & & & & & & & Sm-139 & $2.57 \mathrm{E}+00$ & $m$ & & & & ICRP-107 & $2.57 \mathrm{E}+00$ & $\mathrm{~m}$ \\
\hline Sm-140 & Sm-140 & $1.48 \mathrm{E}+01$ & $\mathrm{~m}$ & & & & & & & Sm-140 & $1.48 \mathrm{E}+01$ & $m$ & Sm-140 & $1.48 \mathrm{E}+01$ & $\mathrm{~m}$ & ICRP-107 & $1.48 \mathrm{E}+01$ & $m$ \\
\hline Sm-141 & Sm-141 & $1.02 \mathrm{E}+01$ & $\mathrm{~m}$ & Sm-141 & $1.02 \mathrm{E}+01$ & $\mathrm{~m}$ & Sm-141 & $1.02 \mathrm{E}+01$ & $\mathrm{~m}$ & Sm-141 & $1.02 \mathrm{E}+01$ & $\mathrm{~m}$ & & & & ICRP-38 & $1.02 \mathrm{E}+01$ & $\mathrm{~m}$ \\
\hline Sm-141m & Sm-141m & $2.26 \mathrm{E}+01$ & $\mathrm{~m}$ & Sm-141m & $2.26 \mathrm{E}+01$ & $\mathrm{~m}$ & Sm-141m & $2.26 \mathrm{E}+01$ & $\mathrm{~m}$ & Sm-141m & $2.26 \mathrm{E}+01$ & $\mathrm{~m}$ & & & & ICRP-38 & $2.26 \mathrm{E}+01$ & $\mathrm{~m}$ \\
\hline Sm-142 & Sm-142 & $7.25 \mathrm{E}+01$ & $\mathrm{~m}$ & Sm-142 & $7.25 \mathrm{E}+01$ & $\mathrm{~m}$ & Sm-142 & $7.25 \mathrm{E}+01$ & $\mathrm{~m}$ & Sm-142 & $7.25 \mathrm{E}+01$ & $\mathrm{~m}$ & & & & ICRP-38 & $7.25 \mathrm{E}+01$ & $\mathrm{~m}$ \\
\hline Sm-143 & Sm-143 & $8.75 \mathrm{E}+00$ & $m$ & & & & & & & Sm-143 & $8.75 \mathrm{E}+00$ & $m$ & Sm-143 & $8.83 \mathrm{E}+00$ & $\mathrm{~m}$ & ICRP-107 & $8.75 \mathrm{E}+00$ & $m$ \\
\hline
\end{tabular}

D-28 


\begin{tabular}{|c|c|c|c|c|c|c|c|c|c|c|c|c|c|c|c|c|c|c|}
\hline \multirow{3}{*}{$\begin{array}{l}\text { MASTER } \\
\text { LIST } \\
\text { Sm-143m }\end{array}$} & \multicolumn{3}{|c|}{ DOE-STD-1196 } & \multicolumn{3}{|c|}{ FGR-12 } & \multicolumn{3}{|c|}{ ICRP-38 } & \multicolumn{3}{|c|}{ ICRP-107 } & \multicolumn{3}{|c|}{ JAERI } & \multirow{3}{*}{$\begin{array}{c}\begin{array}{c}\text { Selected } \\
\text { Half-Life } \\
\text { Reference }\end{array} \\
\text { ICRP-107 }\end{array}$} & \multirow{2}{*}{\multicolumn{2}{|c|}{$\begin{array}{c}\text { Selected } \\
\text { Half-Life } \\
\text { (original units) }\end{array}$}} \\
\hline & \multirow{2}{*}{$\begin{array}{l}\text { Nuclide } \\
\text { Sm-143m }\end{array}$} & \multicolumn{2}{|c|}{$\begin{array}{c}\text { Half-Life } \\
\text { (original units) }\end{array}$} & \multirow[t]{2}{*}{ Nuclide } & \multicolumn{2}{|c|}{$\begin{array}{c}\text { Half-Life } \\
\text { (original units) }\end{array}$} & \multirow[t]{2}{*}{ Nuclide } & \multicolumn{2}{|c|}{$\begin{array}{c}\text { Half-Life } \\
\text { (original units) }\end{array}$} & \multirow{2}{*}{$\begin{array}{l}\text { Nuclide } \\
\text { Sm-143m }\end{array}$} & \multicolumn{2}{|c|}{$\begin{array}{c}\text { Half-Life } \\
\text { (original units) }\end{array}$} & \multirow{2}{*}{$\begin{array}{l}\text { Nuclide } \\
\text { Sm-143m }\end{array}$} & \multicolumn{2}{|c|}{$\begin{array}{c}\text { Half-Life } \\
\text { (original units) }\end{array}$} & & & \\
\hline & & $6.60 E+01$ & $\mathrm{~s}$ & & & & & & & & $6.60 \mathrm{E}+01$ & $\mathrm{~s}$ & & $1.10 \mathrm{E}+00$ & $\mathrm{~m}$ & & $6.60 \mathrm{E}+01$ & $\mathrm{~s}$ \\
\hline Sm-145 & Sm-145 & $3.40 \mathrm{E}+02$ & d & Sm-145 & $3.40 \mathrm{E}+02$ & d & Sm-145 & $3.40 \mathrm{E}+02$ & d & Sm-145 & $3.40 \mathrm{E}+02$ & d & & & & ICRP-38 & $3.40 \mathrm{E}+02$ & d \\
\hline Sm-146 & Sm-146 & $1.03 \mathrm{E}+08$ & $y$ & Sm-146 & $1.03 \mathrm{E}+08$ & $y$ & Sm-146 & $1.03 E+08$ & $y$ & Sm-146 & $1.03 \mathrm{E}+08$ & $y$ & & & & ICRP-38 & $1.03 \mathrm{E}+08$ & $y$ \\
\hline Sm-147 & Sm-147 & $1.06 \mathrm{E}+11$ & $y$ & Sm-147 & $1.06 \mathrm{E}+11$ & $y$ & Sm-147 & $1.06 \mathrm{E}+11$ & $y$ & Sm-147 & $1.06 \mathrm{E}+11$ & $y$ & & & & ICRP-38 & $1.06 \mathrm{E}+11$ & $y$ \\
\hline Sm-148 & Sm-148 & $7.00 \mathrm{E}+15$ & $y$ & & & & & & & Sm-148 & $7.00 \mathrm{E}+15$ & $y$ & Sm-148 & $7.00 \mathrm{E}+15$ & $y$ & ICRP-107 & $7.00 \mathrm{E}+15$ & $y$ \\
\hline Sm-151 & Sm-151 & $9.00 \mathrm{E}+01$ & $y$ & Sm-151 & $9.00 \mathrm{E}+01$ & $\mathrm{y}$ & Sm-151 & $9.00 \mathrm{E}+01$ & $\mathrm{y}$ & Sm-151 & $9.00 \mathrm{E}+01$ & $y$ & & & & ICRP-38 & $9.00 \mathrm{E}+01$ & $y$ \\
\hline Sm-153 & Sm-153 & $4.65 \mathrm{E}+01$ & $\mathrm{~h}$ & Sm-153 & $4.67 \mathrm{E}+01$ & $\mathrm{~h}$ & Sm-153 & $4.67 \mathrm{E}+01$ & $\mathrm{~h}$ & Sm-153 & $4.65 \mathrm{E}+01$ & $\mathrm{~h}$ & & & & ICRP-38 & $4.67 \mathrm{E}+01$ & $\mathrm{~h}$ \\
\hline Sm-155 & Sm-155 & $2.23 \mathrm{E}+01$ & $\mathrm{~m}$ & Sm-155 & $2.21 \mathrm{E}+01$ & $m$ & Sm-155 & $2.21 \mathrm{E}+01$ & $m$ & Sm-155 & $2.23 \mathrm{E}+01$ & $m$ & & & & ICRP-38 & $2.21 \mathrm{E}+01$ & $m$ \\
\hline Sm-156 & Sm-156 & $9.40 \mathrm{E}+00$ & $\mathrm{~h}$ & Sm-156 & $9.40 \mathrm{E}+00$ & $\mathrm{~h}$ & Sm-156 & $9.40 \mathrm{E}+00$ & $\mathrm{~h}$ & Sm-156 & $9.40 \mathrm{E}+00$ & $\mathrm{~h}$ & & & & ICRP-38 & $9.40 \mathrm{E}+00$ & $\mathrm{~h}$ \\
\hline Sm-157 & Sm-157 & $8.03 E+00$ & $\mathrm{~m}$ & & & & & & & Sm-157 & $8.03 E+00$ & $\mathrm{~m}$ & & & & ICRP-107 & $8.03 E+00$ & $\mathrm{~m}$ \\
\hline Sn-106 & Sn-106 & $1.92 \mathrm{E}+00$ & $\mathrm{~m}$ & & & & & & & Sn-106 & $1.92 \mathrm{E}+00$ & $\mathrm{~m}$ & & & & ICRP-107 & $1.92 \mathrm{E}+00$ & $\mathrm{~m}$ \\
\hline Sn-108 & Sn-108 & $1.03 \mathrm{E}+01$ & $\mathrm{~m}$ & & & & & & & Sn-108 & $1.03 \mathrm{E}+01$ & $m$ & Sn-108 & $1.03 \mathrm{E}+01$ & $\mathrm{~m}$ & ICRP-107 & $1.03 \mathrm{E}+01$ & $\mathrm{~m}$ \\
\hline Sn-109 & Sn-109 & $1.80 \mathrm{E}+01$ & $\mathrm{~m}$ & & & & & & & Sn-109 & $1.80 \mathrm{E}+01$ & $\mathrm{~m}$ & Sn-109 & $1.80 \mathrm{E}+01$ & $\mathrm{~m}$ & ICRP-107 & $1.80 \mathrm{E}+01$ & $\mathrm{~m}$ \\
\hline Sn-110 & Sn-110 & $4.11 E+00$ & $\mathrm{~h}$ & Sn-110 & $4.00 \mathrm{E}+00$ & $\mathrm{~h}$ & Sn-110 & $4.00 \mathrm{E}+00$ & $\mathrm{~h}$ & Sn-110 & $4.11 \mathrm{E}+00$ & $\mathrm{~h}$ & & & & ICRP-38 & $4.00 \mathrm{E}+00$ & $\mathrm{~h}$ \\
\hline Sn-111 & Sn-111 & $3.53 \mathrm{E}+01$ & $\mathrm{~m}$ & Sn-111 & $3.53 \mathrm{E}+01$ & $m$ & Sn-111 & $3.53 \mathrm{E}+01$ & $m$ & Sn-111 & $3.53 \mathrm{E}+01$ & $m$ & & & & ICRP-38 & $3.53 \mathrm{E}+01$ & $m$ \\
\hline Sn-113 & Sn-113 & $1.15 \mathrm{E}+02$ & $d$ & Sn-113 & $1.15 \mathrm{E}+02$ & $\mathrm{~d}$ & Sn-113 & $1.15 \mathrm{E}+02$ & $d$ & Sn-113 & $1.15 \mathrm{E}+02$ & $d$ & & & & ICRP-38 & $1.15 \mathrm{E}+02$ & $d$ \\
\hline Sn-113m & Sn-113m & $2.14 \mathrm{E}+01$ & $\mathrm{~m}$ & & & & & & & Sn-113m & $2.14 \mathrm{E}+01$ & $\mathrm{~m}$ & Sn-113m & $2.14 \mathrm{E}+01$ & $\mathrm{~m}$ & ICRP-107 & $2.14 \mathrm{E}+01$ & $\mathrm{~m}$ \\
\hline Sn-117m & Sn-117m & $1.38 \mathrm{E}+01$ & $d$ & Sn-117m & $1.36 \mathrm{E}+01$ & $\mathrm{~d}$ & Sn-117m & $1.36 \mathrm{E}+01$ & $d$ & Sn-117m & $1.38 \mathrm{E}+01$ & $\mathrm{~d}$ & & & & ICRP-38 & $1.36 \mathrm{E}+01$ & $\mathrm{~d}$ \\
\hline Sn-119m & Sn-119m & $2.93 \mathrm{E}+02$ & $d$ & Sn-119m & $2.93 E+02$ & $d$ & Sn-119m & $2.93 \mathrm{E}+02$ & $d$ & Sn-119m & $2.93 \mathrm{E}+02$ & $d$ & & & & ICRP-38 & $2.93 \mathrm{E}+02$ & d \\
\hline Sn-121 & Sn-121 & $2.70 \mathrm{E}+01$ & $\mathrm{~h}$ & Sn-121 & $2.71 \mathrm{E}+01$ & $\mathrm{~h}$ & Sn-121 & $2.71 \mathrm{E}+01$ & $\mathrm{~h}$ & Sn-121 & $2.70 \mathrm{E}+01$ & $\mathrm{~h}$ & & & & ICRP-38 & $2.71 \mathrm{E}+01$ & $\mathrm{~h}$ \\
\hline Sn-121m & Sn-121m & $4.39 \mathrm{E}+01$ & $y$ & Sn-121m & $5.50 \mathrm{E}+01$ & $y$ & Sn-121m & $5.50 \mathrm{E}+01$ & $y$ & Sn-121m & $4.39 \mathrm{E}+01$ & $y$ & & & & ICRP-38 & $5.50 \mathrm{E}+01$ & $y$ \\
\hline Sn-123 & Sn-123 & $1.29 \mathrm{E}+02$ & $d$ & Sn-123 & $1.29 \mathrm{E}+02$ & $d$ & Sn-123 & $1.29 \mathrm{E}+02$ & $d$ & Sn-123 & $1.29 \mathrm{E}+02$ & d & & & & ICRP-38 & $1.29 \mathrm{E}+02$ & $d$ \\
\hline Sn-123m & Sn-123m & $4.01 \mathrm{E}+01$ & $m$ & Sn-123m & $4.01 \mathrm{E}+01$ & $\mathrm{~m}$ & Sn-123m & $4.01 \mathrm{E}+01$ & $m$ & Sn-123m & $4.01 \mathrm{E}+01$ & $m$ & & & & ICRP-38 & $4.01 \mathrm{E}+01$ & $\mathrm{~m}$ \\
\hline Sn-125 & Sn-125 & $9.64 \mathrm{E}+00$ & $d$ & Sn-125 & $9.64 \mathrm{E}+00$ & $\mathrm{~d}$ & Sn-125 & $9.64 \mathrm{E}+00$ & $d$ & Sn-125 & $9.64 \mathrm{E}+00$ & $\mathrm{~d}$ & & & & ICRP-38 & $9.64 \mathrm{E}+00$ & $\mathrm{~d}$ \\
\hline Sn-125m & Sn-125m & $9.52 \mathrm{E}+00$ & $m$ & & & & & & & Sn-125m & $9.52 \mathrm{E}+00$ & $m$ & Sn-125m & $9.52 \mathrm{E}+00$ & $\mathrm{~m}$ & ICRP-107 & $9.52 \mathrm{E}+00$ & $\mathrm{~m}$ \\
\hline Sn-126 & Sn-126 & $2.30 \mathrm{E}+05$ & $y$ & Sn-126 & $1.00 \mathrm{E}+05$ & $\mathrm{y}$ & Sn-126 & $1.00 \mathrm{E}+05$ & $\mathrm{y}$ & Sn-126 & $2.30 \mathrm{E}+05$ & $y$ & & & & ICRP-38 & $1.00 \mathrm{E}+05$ & $y$ \\
\hline Sn-127 & Sn-127 & $2.10 \mathrm{E}+00$ & $\mathrm{~h}$ & Sn-127 & $2.10 \mathrm{E}+00$ & $\mathrm{~h}$ & Sn-127 & $2.10 \mathrm{E}+00$ & $\mathrm{~h}$ & Sn-127 & $2.10 \mathrm{E}+00$ & $\mathrm{~h}$ & & & & ICRP-38 & $2.10 \mathrm{E}+00$ & $\mathrm{~h}$ \\
\hline Sn-127m & Sn-127m & $4.13 \mathrm{E}+00$ & $\mathrm{~m}$ & & & & & & & Sn-127m & $4.13 \mathrm{E}+00$ & $\mathrm{~m}$ & & & & ICRP-107 & $4.13 \mathrm{E}+00$ & $m$ \\
\hline Sn-128 & Sn-128 & $5.91 \mathrm{E}+01$ & $m$ & Sn-128 & $5.91 \mathrm{E}+01$ & $\mathrm{~m}$ & Sn-128 & $5.91 \mathrm{E}+01$ & $\mathrm{~m}$ & Sn-128 & $5.91 \mathrm{E}+01$ & $\mathrm{~m}$ & & & & ICRP-38 & $5.91 \mathrm{E}+01$ & $m$ \\
\hline Sn-129 & Sn-129 & $2.23 \mathrm{E}+00$ & $\mathrm{~m}$ & & & & & & & Sn-129 & $2.23 \mathrm{E}+00$ & $\mathrm{~m}$ & & & & ICRP-107 & $2.23 \mathrm{E}+00$ & $\mathrm{~m}$ \\
\hline Sn-130 & Sn-130 & $3.72 \mathrm{E}+00$ & $\mathrm{~m}$ & & & & & & & Sn-130 & $3.72 \mathrm{E}+00$ & $m$ & & & & ICRP-107 & $3.72 \mathrm{E}+00$ & $\mathrm{~m}$ \\
\hline Sn-130m & Sn-130m & $1.70 \mathrm{E}+00$ & $\mathrm{~m}$ & & & & & & & Sn-130m & $1.70 \mathrm{E}+00$ & $m$ & & & & ICRP-107 & $1.70 \mathrm{E}+00$ & $\mathrm{~m}$ \\
\hline Sr-79 & Sr-79 & $2.25 \mathrm{E}+00$ & $\mathrm{~m}$ & & & & & & & Sr-79 & $2.25 \mathrm{E}+00$ & $\mathrm{~m}$ & & & & ICRP-107 & $2.25 \mathrm{E}+00$ & $\mathrm{~m}$ \\
\hline Sr-80 & Sr-80 & $1.06 \mathrm{E}+02$ & $m$ & Sr-80 & $1.00 \mathrm{E}+02$ & $\mathrm{~m}$ & Sr-80 & $1.00 \mathrm{E}+02$ & $m$ & Sr-80 & $1.06 \mathrm{E}+02$ & $m$ & & & & ICRP-38 & $1.00 \mathrm{E}+02$ & $\mathrm{~m}$ \\
\hline Sr-81 & Sr-81 & $2.23 \mathrm{E}+01$ & $\mathrm{~m}$ & Sr-81 & $2.55 \mathrm{E}+01$ & $m$ & Sr-81 & $2.55 \mathrm{E}+01$ & $m$ & Sr-81 & $2.23 \mathrm{E}+01$ & $m$ & & & & ICRP-38 & $2.55 \mathrm{E}+01$ & $\mathrm{~m}$ \\
\hline Sr-82 & Sr-82 & $2.54 \mathrm{E}+01$ & $d$ & Sr-82 & $2.50 \mathrm{E}+01$ & $d$ & Sr-82 & $2.50 \mathrm{E}+01$ & $\mathrm{~d}$ & Sr-82 & $2.54 \mathrm{E}+01$ & $d$ & & & & ICRP-38 & $2.50 \mathrm{E}+01$ & $d$ \\
\hline Sr-83 & Sr-83 & $3.24 \mathrm{E}+01$ & $\mathrm{~h}$ & Sr-83 & $3.24 \mathrm{E}+01$ & $\mathrm{~h}$ & Sr-83 & $3.24 \mathrm{E}+01$ & $\mathrm{~h}$ & Sr-83 & $3.24 \mathrm{E}+01$ & $\mathrm{~h}$ & & & & ICRP-38 & $3.24 \mathrm{E}+01$ & $\mathrm{~h}$ \\
\hline Sr-85 & Sr-85 & $6.48 \mathrm{E}+01$ & $d$ & Sr-85 & $6.48 \mathrm{E}+01$ & $d$ & Sr-85 & $6.48 \mathrm{E}+01$ & $d$ & Sr-85 & $6.48 \mathrm{E}+01$ & $d$ & & & & ICRP-38 & $6.48 \mathrm{E}+01$ & d \\
\hline Sr-85m & Sr-85m & $6.76 \mathrm{E}+01$ & $\mathrm{~m}$ & Sr-85m & $6.95 \mathrm{E}+01$ & $\mathrm{~m}$ & Sr-85m & $6.95 \mathrm{E}+01$ & $\mathrm{~m}$ & Sr-85m & $6.76 \mathrm{E}+01$ & $\mathrm{~m}$ & & & & ICRP-38 & $6.95 \mathrm{E}+01$ & $\mathrm{~m}$ \\
\hline Sr-87m & Sr-87m & $2.82 \mathrm{E}+00$ & $\mathrm{~h}$ & Sr-87m & $2.81 \mathrm{E}+00$ & $\mathrm{~h}$ & Sr-87m & $2.81 \mathrm{E}+00$ & $\mathrm{~h}$ & Sr-87m & $2.82 \mathrm{E}+00$ & $\mathrm{~h}$ & & & & ICRP-38 & $2.81 \mathrm{E}+00$ & $\mathrm{~h}$ \\
\hline
\end{tabular}

D-29 


\begin{tabular}{|c|c|c|c|c|c|c|c|c|c|c|c|c|c|c|c|c|c|c|}
\hline \multicolumn{19}{|c|}{ Table D.1 Selection of Radionuclides For Calculating HC-2 and HC-3 TQs (continued) } \\
\hline \multirow{3}{*}{$\begin{array}{l}\text { MASTER } \\
\text { LIST } \\
\text { Sr-89 }\end{array}$} & \multicolumn{3}{|c|}{ DOE-STD-1196 } & \multicolumn{3}{|c|}{ FGR-12 } & \multicolumn{3}{|c|}{ ICRP-38 } & \multicolumn{3}{|c|}{ ICRP-107 } & \multicolumn{3}{|c|}{ JAERI } & \multirow{3}{*}{$\begin{array}{c}\begin{array}{c}\text { Selected } \\
\text { Half-Life } \\
\text { Reference }\end{array} \\
\text { ICRP-38 }\end{array}$} & \multirow{2}{*}{\multicolumn{2}{|c|}{$\begin{array}{c}\text { Selected } \\
\text { Half-Life } \\
\text { (original units) }\end{array}$}} \\
\hline & \multirow{2}{*}{$\begin{array}{l}\text { Nuclide } \\
\text { Sr-89 }\end{array}$} & \multicolumn{2}{|c|}{$\begin{array}{c}\text { Half-Life } \\
\text { (original units) }\end{array}$} & \multirow{2}{*}{$\begin{array}{l}\text { Nuclide } \\
\text { Sr-89 }\end{array}$} & \multicolumn{2}{|c|}{$\begin{array}{c}\text { Half-Life } \\
\text { (original units) }\end{array}$} & \multirow{2}{*}{$\begin{array}{l}\text { Nuclide } \\
\text { Sr-89 }\end{array}$} & \multicolumn{2}{|c|}{$\begin{array}{c}\text { Half-Life } \\
\text { (original units) }\end{array}$} & \multirow{2}{*}{$\begin{array}{l}\text { Nuclide } \\
\text { Sr-89 }\end{array}$} & \multicolumn{2}{|c|}{$\begin{array}{c}\text { Half-Life } \\
\text { (original units) }\end{array}$} & \multirow[t]{2}{*}{ Nuclide } & $\begin{array}{r}\text { Half-Li } \\
\text { (original }\end{array}$ & its) & & & \\
\hline & & $5.05 E+01$ & $\mathrm{~d}$ & & $5.05 E+01$ & $d$ & & $5.05 \mathrm{E}+01$ & $d$ & & $5.05 \mathrm{E}+01$ & $d$ & & & & & $5.05 \mathrm{E}+01$ & $d$ \\
\hline Sr-90 & Sr-90 & $2.88 \mathrm{E}+01$ & $\mathrm{y}$ & Sr-90 & $2.91 \mathrm{E}+01$ & $\mathrm{y}$ & Sr-90 & $2.91 \mathrm{E}+01$ & $\mathrm{y}$ & Sr-90 & $2.88 \mathrm{E}+01$ & $\mathrm{y}$ & & & & ICRP-38 & $2.91 \mathrm{E}+01$ & $\mathrm{y}$ \\
\hline Sr-91 & Sr-91 & $9.63 \mathrm{E}+00$ & $\mathrm{~h}$ & Sr-91 & $9.50 \mathrm{E}+00$ & $\mathrm{~h}$ & Sr-91 & $9.50 \mathrm{E}+00$ & $\mathrm{~h}$ & Sr-91 & $9.63 \mathrm{E}+00$ & $\mathrm{~h}$ & & & & ICRP-38 & $9.50 \mathrm{E}+00$ & $\mathrm{~h}$ \\
\hline Sr-92 & Sr-92 & $2.66 \mathrm{E}+00$ & $\mathrm{~h}$ & Sr-92 & $2.71 \mathrm{E}+00$ & $\mathrm{~h}$ & Sr-92 & $2.71 \mathrm{E}+00$ & $\mathrm{~h}$ & Sr-92 & $2.66 \mathrm{E}+00$ & $\mathrm{~h}$ & & & & ICRP-38 & $2.71 \mathrm{E}+00$ & $\mathrm{~h}$ \\
\hline Sr-93 & Sr-93 & $7.42 \mathrm{E}+00$ & $\mathrm{~m}$ & & & & & & & Sr-93 & $7.42 \mathrm{E}+00$ & $\mathrm{~m}$ & & & & ICRP-107 & $7.42 \mathrm{E}+00$ & $\mathrm{~m}$ \\
\hline Sr-94 & Sr-94 & $7.53 \mathrm{E}+01$ & $\mathrm{~s}$ & & & & & & & Sr-94 & $7.53 \mathrm{E}+01$ & $\mathrm{~s}$ & & & & ICRP-107 & $7.53 \mathrm{E}+01$ & $\mathrm{~s}$ \\
\hline Ta-170 & Ta-170 & $6.76 \mathrm{E}+00$ & $\mathrm{~m}$ & & & & & & & Ta-170 & $6.76 \mathrm{E}+00$ & $\mathrm{~m}$ & Ta-170 & $6.76 \mathrm{E}+00$ & $\mathrm{~m}$ & ICRP-107 & $6.76 \mathrm{E}+00$ & $\mathrm{~m}$ \\
\hline Ta-172 & Ta-172 & $3.68 \mathrm{E}+01$ & $\mathrm{~m}$ & Ta-172 & $3.68 \mathrm{E}+01$ & $\mathrm{~m}$ & Ta-172 & $3.68 \mathrm{E}+01$ & $\mathrm{~m}$ & Ta-172 & $3.68 \mathrm{E}+01$ & $\mathrm{~m}$ & & & & ICRP-38 & $3.68 \mathrm{E}+01$ & $m$ \\
\hline Ta-173 & Ta-173 & $3.14 \mathrm{E}+00$ & $\mathrm{~h}$ & Ta-173 & $3.65 \mathrm{E}+00$ & $\mathrm{~h}$ & Ta-173 & $3.65 \mathrm{E}+00$ & $\mathrm{~h}$ & Ta-173 & $3.14 \mathrm{E}+00$ & $\mathrm{~h}$ & & & & ICRP-38 & $3.65 \mathrm{E}+00$ & $\mathrm{~h}$ \\
\hline Ta-174 & Ta-174 & $1.14 \mathrm{E}+00$ & $\mathrm{~h}$ & Ta-174 & $1.20 \mathrm{E}+00$ & $\mathrm{~h}$ & Ta-174 & $1.20 \mathrm{E}+00$ & $\mathrm{~h}$ & Ta-174 & $1.14 \mathrm{E}+00$ & $\mathrm{~h}$ & & & & ICRP-38 & $1.20 \mathrm{E}+00$ & $\mathrm{~h}$ \\
\hline Ta-175 & Ta-175 & $1.05 \mathrm{E}+01$ & $\mathrm{~h}$ & Ta-175 & $1.05 \mathrm{E}+01$ & $\mathrm{~h}$ & Ta-175 & $1.05 \mathrm{E}+01$ & $\mathrm{~h}$ & Ta-175 & $1.05 \mathrm{E}+01$ & $\mathrm{~h}$ & & & & ICRP-38 & $1.05 \mathrm{E}+01$ & $\mathrm{~h}$ \\
\hline Ta-176 & Ta-176 & $8.09 \mathrm{E}+00$ & $\mathrm{~h}$ & Ta-176 & $8.08 \mathrm{E}+00$ & $\mathrm{~h}$ & Ta-176 & $8.08 \mathrm{E}+00$ & $\mathrm{~h}$ & Ta-176 & $8.09 \mathrm{E}+00$ & $\mathrm{~h}$ & & & & ICRP-38 & $8.08 \mathrm{E}+00$ & $\mathrm{~h}$ \\
\hline Ta-177 & Ta-177 & $5.66 \mathrm{E}+01$ & $\mathrm{~h}$ & Ta-177 & $5.66 \mathrm{E}+01$ & $\mathrm{~h}$ & Ta-177 & $5.66 \mathrm{E}+01$ & $\mathrm{~h}$ & Ta-177 & $5.66 \mathrm{E}+01$ & $\mathrm{~h}$ & & & & ICRP-38 & $5.66 \mathrm{E}+01$ & $\mathrm{~h}$ \\
\hline Ta-178s & Ta-178 & $9.31 \mathrm{E}+00$ & $m$ & Ta-178a & $9.31 \mathrm{E}+00$ & $m$ & Ta-178a & $9.31 \mathrm{E}+00$ & $m$ & Ta-178 & $9.31 \mathrm{E}+00$ & $m$ & Ta-178 & $9.31 \mathrm{E}+00$ & $m$ & ICRP-38 & $9.31 \mathrm{E}+00$ & $\mathrm{~m}$ \\
\hline Ta-178I & Ta-178m & $2.36 \mathrm{E}+00$ & $\mathrm{~h}$ & Ta-178b & $2.20 \mathrm{E}+00$ & $\mathrm{~h}$ & Ta-178b & $2.20 \mathrm{E}+00$ & $\mathrm{~h}$ & Ta-178m & $2.36 \mathrm{E}+00$ & $\mathrm{~h}$ & & & & ICRP-38 & $2.20 \mathrm{E}+00$ & $\mathrm{~h}$ \\
\hline Ta-179 & Тa-179 & $1.82 \mathrm{E}+00$ & $y$ & Ta-179 & $6.65 \mathrm{E}+02$ & $d$ & Ta-179 & $6.65 \mathrm{E}+02$ & $d$ & Ta-179 & $1.82 \mathrm{E}+00$ & $y$ & & & & ICRP-38 & $6.65 \mathrm{E}+02$ & $d$ \\
\hline Ta-180 & & & & Ta-180 & $1.00 \mathrm{E}+13$ & $y$ & Ta-180 & $1.00 \mathrm{E}+13$ & $y$ & & & & & & & ICRP-38 & $1.00 \mathrm{E}+13$ & $y$ \\
\hline Ta-180m & Ta-180 & $8.15 \mathrm{E}+00$ & $\mathrm{~h}$ & Ta-180m & $8.10 \mathrm{E}+00$ & $\mathrm{~h}$ & Ta-180m & $8.10 \mathrm{E}+00$ & $\mathrm{~h}$ & Ta-180 & $8.15 E+00$ & $\mathrm{~h}$ & & & & ICRP-38 & $8.10 \mathrm{E}+00$ & $\mathrm{~h}$ \\
\hline Ta-182 & Ta-182 & $1.14 \mathrm{E}+02$ & $d$ & Ta-182 & $1.15 \mathrm{E}+02$ & $d$ & Ta-182 & $1.15 \mathrm{E}+02$ & $d$ & Ta-182 & $1.14 \mathrm{E}+02$ & $d$ & & & & ICRP-38 & $1.15 \mathrm{E}+02$ & $d$ \\
\hline Ta-182m & Ta-182m & $1.58 \mathrm{E}+01$ & $\mathrm{~m}$ & Ta-182m & $1.58 \mathrm{E}+01$ & $m$ & Ta-182m & $1.58 \mathrm{E}+01$ & $m$ & Ta-182m & $1.58 \mathrm{E}+01$ & $m$ & & & & ICRP-38 & $1.58 \mathrm{E}+01$ & $\mathrm{~m}$ \\
\hline Ta-183 & Ta-183 & $5.10 \mathrm{E}+00$ & $\mathrm{~d}$ & Ta-183 & $5.10 \mathrm{E}+00$ & $d$ & Ta-183 & $5.10 \mathrm{E}+00$ & $d$ & Ta-183 & $5.10 \mathrm{E}+00$ & $\mathrm{~d}$ & & & & ICRP-38 & $5.10 \mathrm{E}+00$ & $d$ \\
\hline Ta-184 & Ta-184 & $8.70 \mathrm{E}+00$ & $\mathrm{~h}$ & Ta-184 & $8.70 \mathrm{E}+00$ & $\mathrm{~h}$ & Ta-184 & $8.70 \mathrm{E}+00$ & $\mathrm{~h}$ & Ta-184 & $8.70 \mathrm{E}+00$ & $\mathrm{~h}$ & & & & ICRP-38 & $8.70 \mathrm{E}+00$ & $\mathrm{~h}$ \\
\hline Ta-185 & Ta-185 & $4.94 \mathrm{E}+01$ & $m$ & Ta-185 & $4.90 \mathrm{E}+01$ & $\mathrm{~m}$ & Ta-185 & $4.90 \mathrm{E}+01$ & $m$ & Ta-185 & $4.94 \mathrm{E}+01$ & $m$ & & & & ICRP-38 & $4.90 \mathrm{E}+01$ & $\mathrm{~m}$ \\
\hline Ta-186 & Ta-186 & $1.05 \mathrm{E}+01$ & $m$ & Ta-186 & $1.05 \mathrm{E}+01$ & $m$ & Ta-186 & $1.05 \mathrm{E}+01$ & $m$ & Ta-186 & $1.05 \mathrm{E}+01$ & $m$ & & & & ICRP-38 & $1.05 \mathrm{E}+01$ & $\mathrm{~m}$ \\
\hline Tb-146 & Tb-146 & $2.30 \mathrm{E}+01$ & $\mathrm{~s}$ & & & & & & & Tb-146 & $2.30 \mathrm{E}+01$ & $\mathrm{~s}$ & & & & ICRP-107 & $2.30 \mathrm{E}+01$ & $\mathrm{~s}$ \\
\hline Tb-147 & Tb-147 & $1.64 \mathrm{E}+00$ & $\mathrm{~h}$ & Tb-147 & $1.65 \mathrm{E}+00$ & $\mathrm{~h}$ & Tb-147 & $1.65 \mathrm{E}+00$ & $\mathrm{~h}$ & Tb-147 & $1.64 \mathrm{E}+00$ & $\mathrm{~h}$ & & & & ICRP-38 & $1.65 \mathrm{E}+00$ & $\mathrm{~h}$ \\
\hline Tb-147m & Tb-147m & $1.87 \mathrm{E}+00$ & $\mathrm{~m}$ & & & & & & & Tb-147m & $1.87 \mathrm{E}+00$ & $\mathrm{~m}$ & Tb-147m & $1.87 \mathrm{E}+00$ & $\mathrm{~m}$ & ICRP-107 & $1.87 \mathrm{E}+00$ & $\mathrm{~m}$ \\
\hline Tb-148 & Tb-148 & $6.00 \mathrm{E}+01$ & $\mathrm{~m}$ & & & & & & & Tb-148 & $6.00 \mathrm{E}+01$ & $\mathrm{~m}$ & Tb-148 & $6.00 \mathrm{E}+01$ & $\mathrm{~m}$ & ICRP-107 & $6.00 \mathrm{E}+01$ & $\mathrm{~m}$ \\
\hline Tb-148m & Tb-148m & $2.20 \mathrm{E}+00$ & $\mathrm{~m}$ & & & & & & & Tb-148m & $2.20 \mathrm{E}+00$ & $\mathrm{~m}$ & Tb-148m & $2.20 \mathrm{E}+00$ & $\mathrm{~m}$ & ICRP-107 & $2.20 \mathrm{E}+00$ & $\mathrm{~m}$ \\
\hline Tb-149 & Tb-149 & $4.12 \mathrm{E}+00$ & $\mathrm{~h}$ & Tb-149 & $4.15 \mathrm{E}+00$ & $\mathrm{~h}$ & Tb-149 & $4.15 E+00$ & $\mathrm{~h}$ & Tb-149 & $4.12 \mathrm{E}+00$ & $\mathrm{~h}$ & & & & ICRP-38 & $4.15 E+00$ & $\mathrm{~h}$ \\
\hline Tb-149m & Tb-149m & $4.16 \mathrm{E}+00$ & $\mathrm{~m}$ & & & & & & & Tb-149m & $4.16 \mathrm{E}+00$ & $m$ & Tb-149m & $4.16 \mathrm{E}+00$ & $\mathrm{~m}$ & ICRP-107 & $4.16 \mathrm{E}+00$ & $\mathrm{~m}$ \\
\hline Tb-150 & Tb-150 & $3.48 \mathrm{E}+00$ & $\mathrm{~h}$ & Tb-150 & $3.27 \mathrm{E}+00$ & $\mathrm{~h}$ & Tb-150 & $3.27 \mathrm{E}+00$ & $\mathrm{~h}$ & Tb-150 & $3.48 \mathrm{E}+00$ & $\mathrm{~h}$ & & & & ICRP-38 & $3.27 \mathrm{E}+00$ & $\mathrm{~h}$ \\
\hline Tb-150m & Tb-150m & $5.80 \mathrm{E}+00$ & $\mathrm{~m}$ & & & & & & & Tb-150m & $5.80 \mathrm{E}+00$ & $\mathrm{~m}$ & Tb-150m & $5.80 \mathrm{E}+00$ & $\mathrm{~m}$ & ICRP-107 & $5.80 \mathrm{E}+00$ & $\mathrm{~m}$ \\
\hline Tb-151 & Tb-151 & $1.76 \mathrm{E}+01$ & $\mathrm{~h}$ & Tb-151 & $1.76 \mathrm{E}+01$ & $\mathrm{~h}$ & Tb-151 & $1.76 \mathrm{E}+01$ & $\mathrm{~h}$ & Tb-151 & $1.76 \mathrm{E}+01$ & $\mathrm{~h}$ & & & & ICRP-38 & $1.76 \mathrm{E}+01$ & $\mathrm{~h}$ \\
\hline Tb-151m & Tb-151m & $2.50 \mathrm{E}+01$ & $\mathrm{~s}$ & & & & & & & Tb-151m & $2.50 \mathrm{E}+01$ & $s$ & & & & ICRP-107 & $2.50 \mathrm{E}+01$ & $\mathrm{~s}$ \\
\hline Tb-152 & Tb-152 & $1.75 \mathrm{E}+01$ & $\mathrm{~h}$ & & & & & & & Tb-152 & $1.75 \mathrm{E}+01$ & $\mathrm{~h}$ & Tb-152 & $1.75 \mathrm{E}+01$ & $\mathrm{~h}$ & ICRP-107 & $1.75 \mathrm{E}+01$ & $\mathrm{~h}$ \\
\hline Tb-152m & Tb-152m & $4.20 \mathrm{E}+00$ & $\mathrm{~m}$ & & & & & & & Tb-152m & $4.20 \mathrm{E}+00$ & $\mathrm{~m}$ & Tb-152m & $4.20 \mathrm{E}+00$ & $\mathrm{~m}$ & ICRP-107 & $4.20 \mathrm{E}+00$ & $\mathrm{~m}$ \\
\hline Tb-153 & Tb-153 & $2.34 \mathrm{E}+00$ & $d$ & Tb-153 & $2.34 \mathrm{E}+00$ & $d$ & Tb-153 & $2.34 \mathrm{E}+00$ & $d$ & Tb-153 & $2.34 \mathrm{E}+00$ & $d$ & & & & ICRP-38 & $2.34 \mathrm{E}+00$ & $d$ \\
\hline Tb-154 & Tb-154 & $2.15 \mathrm{E}+01$ & $\mathrm{~h}$ & Tb-154 & $2.14 \mathrm{E}+01$ & $\mathrm{~h}$ & Tb-154 & $2.14 \mathrm{E}+01$ & $\mathrm{~h}$ & Tb-154 & $2.15 \mathrm{E}+01$ & $\mathrm{~h}$ & & & & ICRP-38 & $2.14 \mathrm{E}+01$ & $\mathrm{~h}$ \\
\hline Tb-155 & Tb-155 & $5.32 \mathrm{E}+00$ & $d$ & Tb-155 & $5.32 \mathrm{E}+00$ & $d$ & Tb-155 & $5.32 \mathrm{E}+00$ & $d$ & Tb-155 & $5.32 \mathrm{E}+00$ & $d$ & & & & ICRP-38 & $5.32 \mathrm{E}+00$ & $d$ \\
\hline
\end{tabular}

D-30 


\begin{tabular}{|c|c|c|c|c|c|c|c|c|c|c|c|c|c|c|c|c|c|c|}
\hline \multicolumn{19}{|c|}{ Table D.1 Selection of Radionuclides For Calculating HC-2 and HC-3 TQs (continued) } \\
\hline \multirow{3}{*}{$\begin{array}{l}\text { MASTER } \\
\text { LIST } \\
\text { Tb-156 }\end{array}$} & \multicolumn{3}{|c|}{ DOE-STD-1196 } & \multicolumn{3}{|c|}{ FGR-12 } & \multicolumn{3}{|c|}{ ICRP-38 } & \multicolumn{3}{|c|}{ ICRP-107 } & \multicolumn{3}{|c|}{ JAERI } & \multirow{3}{*}{$\begin{array}{c}\begin{array}{c}\text { Selected } \\
\text { Half-Life } \\
\text { Reference }\end{array} \\
\text { ICRP-38 }\end{array}$} & \multirow{2}{*}{\multicolumn{2}{|c|}{$\begin{array}{c}\text { Selected } \\
\text { Half-Life } \\
\text { (original units) }\end{array}$}} \\
\hline & \multirow{2}{*}{$\begin{array}{l}\text { Nuclide } \\
\text { Tb-156 }\end{array}$} & \multicolumn{2}{|c|}{$\begin{array}{c}\text { Half-Life } \\
\text { (original units) }\end{array}$} & \multirow{2}{*}{$\begin{array}{l}\text { Nuclide } \\
\text { Tb-156 }\end{array}$} & \multicolumn{2}{|c|}{$\begin{array}{c}\text { Half-Life } \\
\text { (original units) }\end{array}$} & \multirow{2}{*}{$\begin{array}{l}\text { Nuclide } \\
\text { Tb-156 }\end{array}$} & \multicolumn{2}{|c|}{$\begin{array}{c}\text { Half-Life } \\
\text { (original units) }\end{array}$} & \multirow{2}{*}{$\begin{array}{l}\text { Nuclide } \\
\text { Tb-156 }\end{array}$} & \multicolumn{2}{|c|}{$\begin{array}{c}\text { Half-Life } \\
\text { (original units) }\end{array}$} & \multirow[t]{2}{*}{ Nuclide } & $\begin{array}{r}\text { Half-Lif } \\
\text { (original u }\end{array}$ & its) & & & \\
\hline & & $5.35 E+00$ & $\mathrm{~d}$ & & $5.34 \mathrm{E}+00$ & $d$ & & $5.34 \mathrm{E}+00$ & $d$ & & $5.35 \mathrm{E}+00$ & $d$ & & & & & $5.34 \mathrm{E}+00$ & $d$ \\
\hline Tb-156ml & Tb-156m & $2.44 \mathrm{E}+01$ & $\mathrm{~h}$ & Tb-156m & $2.44 \mathrm{E}+01$ & $\mathrm{~h}$ & Tb-156m & $2.44 \mathrm{E}+01$ & $\mathrm{~h}$ & Tb-156m & $2.44 \mathrm{E}+01$ & $\mathrm{~h}$ & & & & ICRP-38 & $2.44 \mathrm{E}+01$ & $\mathrm{~h}$ \\
\hline Tb-156ms & Tb-156n & $5.30 \mathrm{E}+00$ & $\mathrm{~h}$ & Tb-156n & $5.00 \mathrm{E}+00$ & $\mathrm{~h}$ & Tb-156n & $5.00 \mathrm{E}+00$ & $\mathrm{~h}$ & Tb-156n & $5.30 \mathrm{E}+00$ & $\mathrm{~h}$ & & & & ICRP-38 & $5.00 \mathrm{E}+00$ & $\mathrm{~h}$ \\
\hline Tb-157 & Tb-157 & $7.10 \mathrm{E}+01$ & $y$ & Tb-157 & $1.50 \mathrm{E}+02$ & $y$ & Tb-157 & $1.50 \mathrm{E}+02$ & $y$ & Tb-157 & $7.10 \mathrm{E}+01$ & $y$ & & & & ICRP-38 & $1.50 \mathrm{E}+02$ & $y$ \\
\hline Tb-158 & Tb-158 & $1.80 \mathrm{E}+02$ & $y$ & Tb-158 & $1.50 \mathrm{E}+02$ & $y$ & Tb-158 & $1.50 \mathrm{E}+02$ & $y$ & Tb-158 & $1.80 \mathrm{E}+02$ & $y$ & & & & ICRP-38 & $1.50 \mathrm{E}+02$ & $y$ \\
\hline Tb-160 & Tb-160 & $7.23 \mathrm{E}+01$ & d & Tb-160 & $7.23 \mathrm{E}+01$ & d & Tb-160 & $7.23 \mathrm{E}+01$ & d & Tb-160 & $7.23 \mathrm{E}+01$ & d & & & & ICRP-38 & $7.23 \mathrm{E}+01$ & d \\
\hline Tb-161 & Tb-161 & $6.91 \mathrm{E}+00$ & $d$ & Tb-161 & $6.91 \mathrm{E}+00$ & $\mathrm{~d}$ & Tb-161 & $6.91 \mathrm{E}+00$ & $d$ & Tb-161 & $6.91 \mathrm{E}+00$ & $\mathrm{~d}$ & & & & ICRP-38 & $6.91 \mathrm{E}+00$ & $d$ \\
\hline Tb-162 & Tb-162 & $7.60 \mathrm{E}+00$ & $\mathrm{~m}$ & & & & & & & Tb-162 & $7.60 \mathrm{E}+00$ & $\mathrm{~m}$ & Tb-162 & $7.60 \mathrm{E}+00$ & $\mathrm{~m}$ & ICRP-107 & $7.60 \mathrm{E}+00$ & $m$ \\
\hline Tb-163 & Tb-163 & $1.95 \mathrm{E}+01$ & $\mathrm{~m}$ & & & & & & & Tb-163 & $1.95 \mathrm{E}+01$ & $\mathrm{~m}$ & Tb-163 & $1.95 \mathrm{E}+01$ & $\mathrm{~m}$ & ICRP-107 & $1.95 \mathrm{E}+01$ & $\mathrm{~m}$ \\
\hline Tb-164 & Tb-164 & $3.00 \mathrm{E}+00$ & $\mathrm{~m}$ & & & & & & & Tb-164 & $3.00 \mathrm{E}+00$ & $\mathrm{~m}$ & Tb-164 & $3.00 \mathrm{E}+00$ & $\mathrm{~m}$ & ICRP-107 & $3.00 \mathrm{E}+00$ & $\mathrm{~m}$ \\
\hline Tb-165 & Tb-165 & $2.11 \mathrm{E}+00$ & $\mathrm{~m}$ & & & & & & & Tb-165 & $2.11 \mathrm{E}+00$ & $m$ & Tb-165 & $2.11 \mathrm{E}+00$ & $m$ & ICRP-107 & $2.11 \mathrm{E}+00$ & $\mathrm{~m}$ \\
\hline Tc-101 & Tc-101 & $1.42 \mathrm{E}+01$ & $\mathrm{~m}$ & Tc-101 & $1.42 \mathrm{E}+01$ & $m$ & Tc-101 & $1.42 \mathrm{E}+01$ & $\mathrm{~m}$ & Tc-101 & $1.42 \mathrm{E}+01$ & $m$ & & & & ICRP-38 & $1.42 \mathrm{E}+01$ & $\mathrm{~m}$ \\
\hline Tc-102 & Tc-102 & $5.28 \mathrm{E}+00$ & $\mathrm{~s}$ & & & & & & & Tc-102 & $5.28 \mathrm{E}+00$ & $\mathrm{~s}$ & & & & ICRP-107 & $5.28 \mathrm{E}+00$ & $\mathrm{~s}$ \\
\hline Tc-102m & Tc-102m & $4.35 \mathrm{E}+00$ & $m$ & & & & & & & Tc-102m & $4.35 \mathrm{E}+00$ & $m$ & Tc-102m & $4.35 \mathrm{E}+00$ & $m$ & ICRP-107 & $4.35 \mathrm{E}+00$ & $\mathrm{~m}$ \\
\hline Tc-104 & Tc-104 & $1.83 \mathrm{E}+01$ & $\mathrm{~m}$ & Tc-104 & $1.82 \mathrm{E}+01$ & $m$ & Tc-104 & $1.82 \mathrm{E}+01$ & $\mathrm{~m}$ & Tc-104 & $1.83 \mathrm{E}+01$ & $m$ & & & & ICRP-38 & $1.82 \mathrm{E}+01$ & $m$ \\
\hline Tc-105 & Tc-105 & $7.60 \mathrm{E}+00$ & $\mathrm{~m}$ & & & & & & & Tc-105 & $7.60 \mathrm{E}+00$ & $\mathrm{~m}$ & & & & ICRP-107 & $7.60 \mathrm{E}+00$ & $\mathrm{~m}$ \\
\hline Tc-91 & Tc-91 & $3.14 \mathrm{E}+00$ & $\mathrm{~m}$ & & & & & & & Tc-91 & $3.14 \mathrm{E}+00$ & $\mathrm{~m}$ & & & & ICRP-107 & $3.14 \mathrm{E}+00$ & $\mathrm{~m}$ \\
\hline Tc-91m & Tc-91m & $3.30 \mathrm{E}+00$ & $m$ & & & & & & & Tc-91m & $3.30 \mathrm{E}+00$ & $\mathrm{~m}$ & & & & ICRP-107 & $3.30 \mathrm{E}+00$ & $m$ \\
\hline Tc-92 & Tc-92 & $4.25 \mathrm{E}+00$ & $\mathrm{~m}$ & & & & & & & Tc-92 & $4.25 \mathrm{E}+00$ & $\mathrm{~m}$ & & & & ICRP-107 & $4.25 \mathrm{E}+00$ & $\mathrm{~m}$ \\
\hline Tc-93 & Tc-93 & $2.75 \mathrm{E}+00$ & $\mathrm{~h}$ & Tc-93 & $2.75 \mathrm{E}+00$ & $\mathrm{~h}$ & Tc-93 & $2.75 \mathrm{E}+00$ & $\mathrm{~h}$ & Tc-93 & $2.75 \mathrm{E}+00$ & $\mathrm{~h}$ & & & & ICRP-38 & $2.75 \mathrm{E}+00$ & $\mathrm{~h}$ \\
\hline Tc-93m & Tc-93m & $4.35 \mathrm{E}+01$ & $\mathrm{~m}$ & Tc-93m & $4.35 \mathrm{E}+01$ & $\mathrm{~m}$ & Tc-93m & $4.35 \mathrm{E}+01$ & $m$ & Tc-93m & $4.35 \mathrm{E}+01$ & $m$ & & & & ICRP-38 & $4.35 \mathrm{E}+01$ & $\mathrm{~m}$ \\
\hline Tc-94 & Tc-94 & $2.93 \mathrm{E}+02$ & $\mathrm{~m}$ & Tc-94 & $2.93 \mathrm{E}+02$ & $\mathrm{~m}$ & Tc-94 & $2.93 \mathrm{E}+02$ & $\mathrm{~m}$ & Tc-94 & $2.93 \mathrm{E}+02$ & $\mathrm{~m}$ & & & & ICRP-38 & $2.93 \mathrm{E}+02$ & $\mathrm{~m}$ \\
\hline Tc-94m & Tc-94m & $5.20 \mathrm{E}+01$ & $m$ & Tc-94m & $5.20 \mathrm{E}+01$ & $\mathrm{~m}$ & Tc-94m & $5.20 \mathrm{E}+01$ & $m$ & Tc-94m & $5.20 \mathrm{E}+01$ & $m$ & & & & ICRP-38 & $5.20 \mathrm{E}+01$ & $\mathrm{~m}$ \\
\hline Tc-95 & Tc-95 & $2.00 \mathrm{E}+01$ & $\mathrm{~h}$ & Tc-95 & $2.00 \mathrm{E}+01$ & $\mathrm{~h}$ & Tc-95 & $2.00 \mathrm{E}+01$ & $\mathrm{~h}$ & Tc-95 & $2.00 \mathrm{E}+01$ & $\mathrm{~h}$ & & & & ICRP-38 & $2.00 \mathrm{E}+01$ & $\mathrm{~h}$ \\
\hline Tc-95m & Tc-95m & $6.10 \mathrm{E}+01$ & $d$ & Tc-95m & $6.10 \mathrm{E}+01$ & $d$ & Tc-95m & $6.10 \mathrm{E}+01$ & $d$ & Tc-95m & $6.10 \mathrm{E}+01$ & $d$ & & & & ICRP-38 & $6.10 \mathrm{E}+01$ & $d$ \\
\hline Tc-96 & Tc-96 & $4.28 \mathrm{E}+00$ & $d$ & Tc-96 & $4.28 \mathrm{E}+00$ & $\mathrm{~d}$ & Tc-96 & $4.28 \mathrm{E}+00$ & $\mathrm{~d}$ & Tc-96 & $4.28 \mathrm{E}+00$ & $\mathrm{~d}$ & & & & ICRP-38 & $4.28 \mathrm{E}+00$ & $d$ \\
\hline Tc-96m & Tc-96m & $5.15 \mathrm{E}+01$ & $\mathrm{~m}$ & Tc-96m & $5.15 \mathrm{E}+01$ & $\mathrm{~m}$ & Tc-96m & $5.15 \mathrm{E}+01$ & $\mathrm{~m}$ & Tc-96m & $5.15 \mathrm{E}+01$ & $\mathrm{~m}$ & & & & ICRP-38 & $5.15 \mathrm{E}+01$ & $\mathrm{~m}$ \\
\hline Tc-97 & Tc-97 & $2.60 \mathrm{E}+06$ & $y$ & Tc-97 & $2.60 \mathrm{E}+06$ & $y$ & Tc-97 & $2.60 \mathrm{E}+06$ & $y$ & Tc-97 & $2.60 \mathrm{E}+06$ & $y$ & & & & ICRP-38 & $2.60 \mathrm{E}+06$ & $y$ \\
\hline Tc-97m & Tc-97m & $9.01 \mathrm{E}+01$ & $d$ & Tc-97m & $8.70 \mathrm{E}+01$ & $\mathrm{~d}$ & Tc-97m & $8.70 \mathrm{E}+01$ & $d$ & Tc-97m & $9.01 \mathrm{E}+01$ & $d$ & & & & ICRP-38 & $8.70 \mathrm{E}+01$ & $d$ \\
\hline Tc-98 & Tc-98 & $4.20 \mathrm{E}+06$ & $y$ & Tc-98 & $4.20 \mathrm{E}+06$ & $y$ & Tc-98 & $4.20 \mathrm{E}+06$ & $y$ & Tc-98 & $4.20 \mathrm{E}+06$ & $y$ & & & & ICRP-38 & $4.20 \mathrm{E}+06$ & $y$ \\
\hline Tc-99 & Tc-99 & $2.11 \mathrm{E}+05$ & $y$ & Tc-99 & $2.13 \mathrm{E}+05$ & $y$ & Tc-99 & $2.13 \mathrm{E}+05$ & $y$ & Tc-99 & $2.11 \mathrm{E}+05$ & $y$ & & & & ICRP-38 & $2.13 \mathrm{E}+05$ & $y$ \\
\hline Tc-99m & Tc-99m & $6.02 \mathrm{E}+00$ & $\mathrm{~h}$ & Tc-99m & $6.02 \mathrm{E}+00$ & $\mathrm{~h}$ & Tc-99m & $6.02 \mathrm{E}+00$ & $\mathrm{~h}$ & Tc-99m & $6.02 \mathrm{E}+00$ & $\mathrm{~h}$ & & & & ICRP-38 & $6.02 \mathrm{E}+00$ & $\mathrm{~h}$ \\
\hline Te-113 & Te-113 & $1.70 \mathrm{E}+00$ & $\mathrm{~m}$ & & & & & & & Te-113 & $1.70 \mathrm{E}+00$ & $\mathrm{~m}$ & & & & ICRP-107 & $1.70 \mathrm{E}+00$ & $\mathrm{~m}$ \\
\hline Te-114 & Te-114 & $1.52 \mathrm{E}+01$ & $m$ & & & & & & & Te-114 & $1.52 \mathrm{E}+01$ & $m$ & & & & ICRP-107 & $1.52 \mathrm{E}+01$ & $\mathrm{~m}$ \\
\hline Te-115 & Te-115 & $5.80 \mathrm{E}+00$ & $\mathrm{~m}$ & & & & & & & Te-115 & $5.80 \mathrm{E}+00$ & $m$ & & & & ICRP-107 & $5.80 \mathrm{E}+00$ & $\mathrm{~m}$ \\
\hline Te-115m & Te-115m & $6.70 \mathrm{E}+00$ & $\mathrm{~m}$ & & & & & & & Te-115m & $6.70 \mathrm{E}+00$ & $m$ & & & & ICRP-107 & $6.70 \mathrm{E}+00$ & $m$ \\
\hline Te-116 & Te-116 & $2.49 \mathrm{E}+00$ & $\mathrm{~h}$ & Te-116 & $2.49 \mathrm{E}+00$ & $\mathrm{~h}$ & Te-116 & $2.49 \mathrm{E}+00$ & $\mathrm{~h}$ & Te-116 & $2.49 \mathrm{E}+00$ & $\mathrm{~h}$ & & & & ICRP-38 & $2.49 \mathrm{E}+00$ & $\mathrm{~h}$ \\
\hline Te-117 & Te-117 & $6.20 \mathrm{E}+01$ & $\mathrm{~m}$ & & & & & & & Te-117 & $6.20 \mathrm{E}+01$ & $\mathrm{~m}$ & Te-117 & $6.20 \mathrm{E}+01$ & $\mathrm{~m}$ & ICRP-107 & $6.20 \mathrm{E}+01$ & $\mathrm{~m}$ \\
\hline Te-118 & Te-118 & $6.00 \mathrm{E}+00$ & $d$ & & & & & & & Te-118 & $6.00 \mathrm{E}+00$ & $d$ & Te-118 & $6.00 \mathrm{E}+00$ & $d$ & ICRP-107 & $6.00 \mathrm{E}+00$ & $d$ \\
\hline Te-119 & Te-119 & $1.61 \mathrm{E}+01$ & $\mathrm{~h}$ & & & & & & & Te-119 & $1.61 \mathrm{E}+01$ & $\mathrm{~h}$ & Te-119 & $1.60 \mathrm{E}+01$ & $\mathrm{~h}$ & ICRP-107 & $1.61 \mathrm{E}+01$ & $\mathrm{~h}$ \\
\hline
\end{tabular}

D-31 


\begin{tabular}{|c|c|c|c|c|c|c|c|c|c|c|c|c|c|c|c|c|c|c|}
\hline \multicolumn{19}{|c|}{ Table D.1 Selection of Radionuclides For Calculating HC-2 and HC-3 TQs (continued) } \\
\hline \multirow{3}{*}{$\begin{array}{l}\text { MASTER } \\
\text { LIST } \\
\text { Te-119m }\end{array}$} & \multicolumn{3}{|c|}{ DOE-STD-1196 } & \multicolumn{3}{|c|}{ FGR-12 } & \multicolumn{3}{|c|}{ ICRP-38 } & \multicolumn{3}{|c|}{ ICRP-107 } & \multicolumn{3}{|c|}{ JAERI } & \multirow{3}{*}{$\begin{array}{c}\begin{array}{c}\text { Selected } \\
\text { Half-Life } \\
\text { Reference }\end{array} \\
\text { ICRP-107 }\end{array}$} & & \\
\hline & \multirow{2}{*}{$\begin{array}{l}\text { Nuclide } \\
\text { Te-119m }\end{array}$} & \multicolumn{2}{|c|}{$\begin{array}{c}\text { Half-Life } \\
\text { (original units) }\end{array}$} & \multirow[t]{2}{*}{ Nuclide } & \multicolumn{2}{|c|}{$\begin{array}{c}\text { Half-Life } \\
\text { (original units) }\end{array}$} & \multirow[t]{2}{*}{ Nuclide } & \multicolumn{2}{|c|}{$\begin{array}{c}\text { Half-Life } \\
\text { (original units) }\end{array}$} & \multirow{2}{*}{$\begin{array}{l}\text { Nuclide } \\
\text { Te-119m }\end{array}$} & \multicolumn{2}{|c|}{$\begin{array}{c}\text { Half-Life } \\
\text { (original units) } \\
\end{array}$} & \multirow{2}{*}{$\begin{array}{l}\text { Nuclide } \\
\text { Te-119m }\end{array}$} & $\begin{array}{r}\text { Half-Lif } \\
\text { (original u }\end{array}$ & its) & & \multicolumn{2}{|c|}{$\begin{array}{c}\text { Selected } \\
\text { Half-Life } \\
\text { (original units) }\end{array}$} \\
\hline & & $4.70 E+00$ & $\mathrm{~d}$ & & & & & & & & $4.70 \mathrm{E}+00$ & $d$ & & $4.70 \mathrm{E}+00$ & $\mathrm{~d}$ & & $4.70 E+00$ & $d$ \\
\hline Te-121 & Te-121 & $1.92 \mathrm{E}+01$ & $d$ & Te-121 & $1.70 \mathrm{E}+01$ & $d$ & Te-121 & $1.70 \mathrm{E}+01$ & d & Te-121 & $1.92 \mathrm{E}+01$ & d & & & & ICRP-38 & $1.70 \mathrm{E}+01$ & d \\
\hline Te-121m & Te-121m & $1.54 \mathrm{E}+02$ & $d$ & Te-121m & $1.54 \mathrm{E}+02$ & d & Te-121m & $1.54 \mathrm{E}+02$ & $d$ & Te-121m & $1.54 \mathrm{E}+02$ & $d$ & & & & ICRP-38 & $1.54 \mathrm{E}+02$ & $d$ \\
\hline Te-123 & Te-123 & $6.00 \mathrm{E}+14$ & $y$ & Te-123 & $1.00 \mathrm{E}+13$ & $y$ & Te-123 & $1.00 \mathrm{E}+13$ & $y$ & Te-123 & $6.00 \mathrm{E}+14$ & $y$ & & & & ICRP-38 & $1.00 \mathrm{E}+13$ & $y$ \\
\hline Te-123m & Te-123m & $1.19 \mathrm{E}+02$ & $d$ & Te-123m & $1.20 \mathrm{E}+02$ & $d$ & Te-123m & $1.20 \mathrm{E}+02$ & $d$ & Te-123m & $1.19 \mathrm{E}+02$ & $d$ & & & & ICRP-38 & $1.20 \mathrm{E}+02$ & $d$ \\
\hline Te-125m & Te-125m & $5.74 \mathrm{E}+01$ & d & Te-125m & $5.80 \mathrm{E}+01$ & $\mathrm{~d}$ & Te-125m & $5.80 \mathrm{E}+01$ & d & Te-125m & $5.74 \mathrm{E}+01$ & d & & & & ICRP-38 & $5.80 \mathrm{E}+01$ & d \\
\hline Te-127 & Te-127 & $9.35 \mathrm{E}+00$ & $\mathrm{~h}$ & Te-127 & $9.35 \mathrm{E}+00$ & $\mathrm{~h}$ & Te-127 & $9.35 \mathrm{E}+00$ & $\mathrm{~h}$ & Te-127 & $9.35 \mathrm{E}+00$ & $\mathrm{~h}$ & & & & ICRP-38 & $9.35 \mathrm{E}+00$ & $\mathrm{~h}$ \\
\hline Te-127m & Te-127m & $1.09 \mathrm{E}+02$ & $d$ & Te-127m & $1.09 \mathrm{E}+02$ & $\mathrm{~d}$ & Te-127m & $1.09 \mathrm{E}+02$ & $d$ & Te-127m & $1.09 \mathrm{E}+02$ & $d$ & & & & ICRP-38 & $1.09 \mathrm{E}+02$ & $d$ \\
\hline Te-129 & Te-129 & $6.96 \mathrm{E}+01$ & $\mathrm{~m}$ & Te-129 & $6.96 \mathrm{E}+01$ & $m$ & Te-129 & $6.96 \mathrm{E}+01$ & $\mathrm{~m}$ & Te-129 & $6.96 \mathrm{E}+01$ & $m$ & & & & ICRP-38 & $6.96 \mathrm{E}+01$ & $\mathrm{~m}$ \\
\hline Te-129m & Te-129m & $3.36 \mathrm{E}+01$ & $d$ & Te-129m & $3.36 \mathrm{E}+01$ & $d$ & Te-129m & $3.36 \mathrm{E}+01$ & $d$ & Te-129m & $3.36 \mathrm{E}+01$ & $d$ & & & & ICRP-38 & $3.36 \mathrm{E}+01$ & $d$ \\
\hline Te-131 & Te-131 & $2.50 \mathrm{E}+01$ & $\mathrm{~m}$ & Te-131 & $2.50 \mathrm{E}+01$ & $\mathrm{~m}$ & Te-131 & $2.50 \mathrm{E}+01$ & $\mathrm{~m}$ & Te-131 & $2.50 \mathrm{E}+01$ & $\mathrm{~m}$ & & & & ICRP-38 & $2.50 \mathrm{E}+01$ & $\mathrm{~m}$ \\
\hline Te-131m & Te-131m & $3.00 \mathrm{E}+01$ & $\mathrm{~h}$ & Te-131m & $3.00 \mathrm{E}+01$ & $\mathrm{~h}$ & Te-131m & $3.00 \mathrm{E}+01$ & $\mathrm{~h}$ & Te-131m & $3.00 \mathrm{E}+01$ & $\mathrm{~h}$ & & & & ICRP-38 & $3.00 \mathrm{E}+01$ & $\mathrm{~h}$ \\
\hline Te-132 & Te-132 & $3.20 \mathrm{E}+00$ & $d$ & Te-132 & $7.82 \mathrm{E}+01$ & $\mathrm{~h}$ & Te-132 & $7.82 \mathrm{E}+01$ & $\mathrm{~h}$ & Te-132 & $3.20 \mathrm{E}+00$ & $d$ & & & & ICRP-38 & $7.82 E+01$ & $\mathrm{~h}$ \\
\hline Te-133 & Te-133 & $1.25 \mathrm{E}+01$ & $m$ & Te-133 & $1.25 \mathrm{E}+01$ & $m$ & Te-133 & $1.25 \mathrm{E}+01$ & $m$ & Te-133 & $1.25 \mathrm{E}+01$ & $m$ & & & & ICRP-38 & $1.25 \mathrm{E}+01$ & $\mathrm{~m}$ \\
\hline Te-133m & Te-133m & $5.54 \mathrm{E}+01$ & $\mathrm{~m}$ & Te-133m & $5.54 \mathrm{E}+01$ & $m$ & Te-133m & $5.54 \mathrm{E}+01$ & $m$ & Te-133m & $5.54 \mathrm{E}+01$ & $m$ & & & & ICRP-38 & $5.54 \mathrm{E}+01$ & $m$ \\
\hline Te-134 & Te-134 & $4.18 \mathrm{E}+01$ & $\mathrm{~m}$ & Te-134 & $4.18 \mathrm{E}+01$ & $\mathrm{~m}$ & Te-134 & $4.18 \mathrm{E}+01$ & $m$ & Te-134 & $4.18 \mathrm{E}+01$ & $\mathrm{~m}$ & & & & ICRP-38 & $4.18 \mathrm{E}+01$ & $\mathrm{~m}$ \\
\hline Th-223 & Th-223 & $6.00 \mathrm{E}-01$ & $\mathrm{~s}$ & & & & & & & Th-223 & $6.00 \mathrm{E}-01$ & $\mathrm{~s}$ & & & & ICRP-107 & $6.00 \mathrm{E}-01$ & $\mathrm{~s}$ \\
\hline Th-224 & Th-224 & $1.05 \mathrm{E}+00$ & $\mathrm{~s}$ & & & & & & & Th-224 & $1.05 \mathrm{E}+00$ & $\mathrm{~s}$ & & & & ICRP-107 & $1.05 \mathrm{E}+00$ & $\mathrm{~s}$ \\
\hline Th-226 & Th-226 & $3.06 \mathrm{E}+01$ & $\mathrm{~m}$ & Th-226 & $3.09 \mathrm{E}+01$ & $\mathrm{~m}$ & Th-226 & $3.09 \mathrm{E}+01$ & $\mathrm{~m}$ & Th-226 & $3.06 \mathrm{E}+01$ & $\mathrm{~m}$ & & & & ICRP-38 & $3.09 \mathrm{E}+01$ & $\mathrm{~m}$ \\
\hline Th-227 & Th-227 & $1.87 \mathrm{E}+01$ & $\mathrm{~d}$ & Th-227 & $1.87 \mathrm{E}+01$ & $\mathrm{~d}$ & Th-227 & $1.87 \mathrm{E}+01$ & $d$ & Th-227 & $1.87 \mathrm{E}+01$ & $\mathrm{~d}$ & & & & ICRP-38 & $1.87 \mathrm{E}+01$ & d \\
\hline Th-228 & Th-228 & $1.91 \mathrm{E}+00$ & $y$ & Th-228 & $1.91 \mathrm{E}+00$ & $y$ & Th-228 & $1.91 \mathrm{E}+00$ & $y$ & Th-228 & $1.91 \mathrm{E}+00$ & $y$ & & & & ICRP-38 & $1.91 \mathrm{E}+00$ & $y$ \\
\hline Th-229 & Th-229 & $7.34 \mathrm{E}+03$ & $\mathrm{y}$ & Th-229 & $7.34 \mathrm{E}+03$ & $y$ & Th-229 & $7.34 \mathrm{E}+03$ & $y$ & Th-229 & $7.34 \mathrm{E}+03$ & $y$ & & & & ICRP-38 & $7.34 \mathrm{E}+03$ & $y$ \\
\hline Th-230 & Th-230 & $7.54 \mathrm{E}+04$ & $y$ & Th-230 & $7.70 \mathrm{E}+04$ & $y$ & Th-230 & $7.70 \mathrm{E}+04$ & $y$ & Th-230 & $7.54 \mathrm{E}+04$ & $y$ & & & & ICRP-38 & $7.70 \mathrm{E}+04$ & $y$ \\
\hline Th-231 & Th-231 & $2.55 \mathrm{E}+01$ & $\mathrm{~h}$ & Th-231 & $2.55 \mathrm{E}+01$ & $\mathrm{~h}$ & Th-231 & $2.55 \mathrm{E}+01$ & $\mathrm{~h}$ & Th-231 & $2.55 \mathrm{E}+01$ & $\mathrm{~h}$ & & & & ICRP-38 & $2.55 \mathrm{E}+01$ & $\mathrm{~h}$ \\
\hline Th-232 & Th-232 & $1.41 \mathrm{E}+10$ & $y$ & Th-232 & $1.41 \mathrm{E}+10$ & $y$ & Th-232 & $1.41 \mathrm{E}+10$ & $y$ & Th-232 & $1.41 \mathrm{E}+10$ & $y$ & & & & ICRP-38 & $1.41 \mathrm{E}+10$ & $y$ \\
\hline Th-233 & Th-233 & $2.23 \mathrm{E}+01$ & $m$ & & & & & & & Th-233 & $2.23 \mathrm{E}+01$ & $\mathrm{~m}$ & Th-233 & $2.23 \mathrm{E}+01$ & $\mathrm{~m}$ & ICRP-107 & $2.23 \mathrm{E}+01$ & $\mathrm{~m}$ \\
\hline Th-234 & Th-234 & $2.41 \mathrm{E}+01$ & $d$ & Th-234 & $2.41 \mathrm{E}+01$ & $d$ & Th-234 & $2.41 \mathrm{E}+01$ & d & Th-234 & $2.41 \mathrm{E}+01$ & $d$ & & & & ICRP-38 & $2.41 \mathrm{E}+01$ & $d$ \\
\hline Th-235 & Th-235 & $7.10 \mathrm{E}+00$ & $\mathrm{~m}$ & & & & & & & Th-235 & $7.10 \mathrm{E}+00$ & $\mathrm{~m}$ & & & & ICRP-107 & $7.10 \mathrm{E}+00$ & $\mathrm{~m}$ \\
\hline Th-236 & Th-236 & $3.75 \mathrm{E}+01$ & $\mathrm{~m}$ & & & & & & & Th-236 & $3.75 \mathrm{E}+01$ & $\mathrm{~m}$ & & & & ICRP-107 & $3.75 E+01$ & $\mathrm{~m}$ \\
\hline Ti-44 & Ti-44 & $6.00 \mathrm{E}+01$ & $y$ & Ti-44 & $4.73 E+01$ & $y$ & Ti-44 & $4.73 \mathrm{E}+01$ & $y$ & Ti-44 & $6.00 \mathrm{E}+01$ & $y$ & & & & ICRP-38 & $4.73 E+01$ & $y$ \\
\hline Ti-45 & $\mathrm{Ti}-45$ & $1.85 \mathrm{E}+02$ & $\mathrm{~m}$ & Ti-45 & $3.08 \mathrm{E}+00$ & $\mathrm{~h}$ & Ti-45 & $3.08 \mathrm{E}+00$ & $\mathrm{~h}$ & $\mathrm{Ti}-45$ & $1.85 \mathrm{E}+02$ & $m$ & & & & ICRP-38 & $3.08 \mathrm{E}+00$ & $\mathrm{~h}$ \\
\hline Ti-51 & Ti-51 & $5.76 \mathrm{E}+00$ & $\mathrm{~m}$ & & & & & & & Ti-51 & $5.76 \mathrm{E}+00$ & $m$ & Ti-51 & $5.76 \mathrm{E}+00$ & $\mathrm{~m}$ & ICRP-107 & $5.76 \mathrm{E}+00$ & $\mathrm{~m}$ \\
\hline Ti-52 & Ti-52 & $1.70 \mathrm{E}+00$ & $\mathrm{~m}$ & & & & & & & Ti-52 & $1.70 \mathrm{E}+00$ & $\mathrm{~m}$ & & & & ICRP-107 & $1.70 \mathrm{E}+00$ & $\mathrm{~m}$ \\
\hline TI-190 & TI-190 & $2.60 \mathrm{E}+00$ & $\mathrm{~m}$ & & & & & & & TI-190 & $2.60 \mathrm{E}+00$ & $\mathrm{~m}$ & TI-190 & $2.60 \mathrm{E}+00$ & $m$ & ICRP-107 & $2.60 \mathrm{E}+00$ & $\mathrm{~m}$ \\
\hline TI-190m & TI-190m & $3.70 \mathrm{E}+00$ & $\mathrm{~m}$ & & & & & & & TI-190m & $3.70 \mathrm{E}+00$ & $m$ & TI-190m & $3.70 \mathrm{E}+00$ & $m$ & ICRP-107 & $3.70 \mathrm{E}+00$ & $\mathrm{~m}$ \\
\hline TI-194 & Tl-194 & $3.30 \mathrm{E}+01$ & $\mathrm{~m}$ & TI-194 & $3.30 \mathrm{E}+01$ & $m$ & TI-194 & $3.30 \mathrm{E}+01$ & $m$ & TI-194 & $3.30 \mathrm{E}+01$ & $m$ & & & & ICRP-38 & $3.30 \mathrm{E}+01$ & $m$ \\
\hline TI-194m & TI-194m & $3.28 \mathrm{E}+01$ & $\mathrm{~m}$ & TI-194m & $3.28 \mathrm{E}+01$ & $\mathrm{~m}$ & TI-194m & $3.28 \mathrm{E}+01$ & $\mathrm{~m}$ & TI-194m & $3.28 \mathrm{E}+01$ & $\mathrm{~m}$ & & & & ICRP-38 & $3.28 \mathrm{E}+01$ & $\mathrm{~m}$ \\
\hline TI-195 & Tl-195 & $1.16 \mathrm{E}+00$ & $\mathrm{~h}$ & Tl-195 & $1.16 \mathrm{E}+00$ & $\mathrm{~h}$ & Tl-195 & $1.16 \mathrm{E}+00$ & $\mathrm{~h}$ & TI-195 & $1.16 \mathrm{E}+00$ & $\mathrm{~h}$ & & & & ICRP-38 & $1.16 \mathrm{E}+00$ & $\mathrm{~h}$ \\
\hline Tl-196 & TI-196 & $1.84 \mathrm{E}+00$ & $\mathrm{~h}$ & & & & & & & TI-196 & $1.84 \mathrm{E}+00$ & $\mathrm{~h}$ & TI-196 & $1.84 \mathrm{E}+00$ & $\mathrm{~h}$ & ICRP-107 & $1.84 \mathrm{E}+00$ & $\mathrm{~h}$ \\
\hline TI-197 & TI-197 & $2.84 \mathrm{E}+00$ & $\mathrm{~h}$ & TI-197 & $2.84 E+00$ & $\mathrm{~h}$ & TI-197 & $2.84 \mathrm{E}+00$ & $\mathrm{~h}$ & TI-197 & $2.84 \mathrm{E}+00$ & $\mathrm{~h}$ & & & & ICRP-38 & $2.84 \mathrm{E}+00$ & $\mathrm{~h}$ \\
\hline
\end{tabular}

D-32 


\begin{tabular}{|c|c|c|c|c|c|c|c|c|c|c|c|c|c|c|c|c|c|c|}
\hline \multicolumn{19}{|c|}{ Table D.1 Selection of Radionuclides For Calculating HC-2 and HC-3 TQs (continued) } \\
\hline \multirow{3}{*}{$\begin{array}{l}\text { MASTER } \\
\text { LIST } \\
\text { Tl-198 }\end{array}$} & \multicolumn{3}{|c|}{ DOE-STD-1196 } & \multicolumn{3}{|c|}{ FGR-12 } & \multicolumn{3}{|c|}{ ICRP-38 } & \multicolumn{3}{|c|}{ ICRP-107 } & \multicolumn{3}{|c|}{ JAERI } & \multirow{3}{*}{$\begin{array}{c}\begin{array}{c}\text { Selected } \\
\text { Half-Life } \\
\text { Reference }\end{array} \\
\text { ICRP-38 }\end{array}$} & \multirow{2}{*}{\multicolumn{2}{|c|}{$\begin{array}{c}\text { Selected } \\
\text { Half-Life } \\
\text { (original units) }\end{array}$}} \\
\hline & \multirow{2}{*}{$\begin{array}{l}\text { Nuclide } \\
\text { TI-198 }\end{array}$} & \multicolumn{2}{|c|}{$\begin{array}{c}\text { Half-Life } \\
\text { (original units) }\end{array}$} & \multirow{2}{*}{$\begin{array}{l}\text { Nuclide } \\
\text { TI-198 }\end{array}$} & \multicolumn{2}{|c|}{$\begin{array}{c}\text { Half-Life } \\
\text { (original units) }\end{array}$} & \multirow{2}{*}{$\begin{array}{l}\text { Nuclide } \\
\text { TI-198 }\end{array}$} & \multicolumn{2}{|c|}{$\begin{array}{c}\text { Half-Life } \\
\text { (original units) }\end{array}$} & \multirow{2}{*}{$\begin{array}{l}\text { Nuclide } \\
\text { Tl-198 }\end{array}$} & \multicolumn{2}{|c|}{$\begin{array}{c}\text { Half-Life } \\
\text { (original units) }\end{array}$} & \multirow[t]{2}{*}{ Nuclide } & $\begin{array}{r}\text { Half-Li } \\
\text { (original }\end{array}$ & its) & & & \\
\hline & & $5.30 \mathrm{E}+00$ & $\mathrm{~h}$ & & $5.30 \mathrm{E}+00$ & $\mathrm{~h}$ & & $5.30 \mathrm{E}+00$ & $\mathrm{~h}$ & & $5.30 \mathrm{E}+00$ & $\mathrm{~h}$ & & & & & $5.30 \mathrm{E}+00$ & $\mathrm{~h}$ \\
\hline TI-198m & Tl-198m & $1.87 \mathrm{E}+00$ & $\mathrm{~h}$ & Tl-198m & $1.87 \mathrm{E}+00$ & $\mathrm{~h}$ & TI-198m & $1.87 \mathrm{E}+00$ & $\mathrm{~h}$ & Tl-198m & $1.87 \mathrm{E}+00$ & $\mathrm{~h}$ & & & & ICRP-38 & $1.87 \mathrm{E}+00$ & $\mathrm{~h}$ \\
\hline TI-199 & TI-199 & $7.42 \mathrm{E}+00$ & $\mathrm{~h}$ & Tl-199 & $7.42 \mathrm{E}+00$ & $\mathrm{~h}$ & Tl-199 & $7.42 \mathrm{E}+00$ & $\mathrm{~h}$ & Tl-199 & $7.42 \mathrm{E}+00$ & $\mathrm{~h}$ & & & & ICRP-38 & $7.42 \mathrm{E}+00$ & $\mathrm{~h}$ \\
\hline TI-200 & TI-200 & $2.61 \mathrm{E}+01$ & $\mathrm{~h}$ & TI-200 & $2.61 \mathrm{E}+01$ & $\mathrm{~h}$ & TI-200 & $2.61 \mathrm{E}+01$ & $\mathrm{~h}$ & TI-200 & $2.61 \mathrm{E}+01$ & $\mathrm{~h}$ & & & & ICRP-38 & $2.61 \mathrm{E}+01$ & $\mathrm{~h}$ \\
\hline TI-201 & TI-201 & $7.29 \mathrm{E}+01$ & $\mathrm{~h}$ & TI-201 & $3.04 \mathrm{E}+00$ & d & TI-201 & $3.04 \mathrm{E}+00$ & $d$ & TI-201 & $7.29 \mathrm{E}+01$ & $\mathrm{~h}$ & & & & ICRP-38 & $3.04 \mathrm{E}+00$ & $d$ \\
\hline TI-202 & TI-202 & $1.22 \mathrm{E}+01$ & $d$ & TI-202 & $1.22 \mathrm{E}+01$ & $d$ & TI-202 & $1.22 \mathrm{E}+01$ & $d$ & TI-202 & $1.22 \mathrm{E}+01$ & $d$ & & & & ICRP-38 & $1.22 \mathrm{E}+01$ & $d$ \\
\hline TI-204 & TI-204 & $3.78 \mathrm{E}+00$ & $y$ & TI-204 & $3.78 \mathrm{E}+00$ & $y$ & TI-204 & $3.78 \mathrm{E}+00$ & $y$ & TI-204 & $3.78 \mathrm{E}+00$ & $y$ & & & & ICRP-38 & $3.78 \mathrm{E}+00$ & $y$ \\
\hline TI-206 & Tl-206 & $4.20 \mathrm{E}+00$ & $\mathrm{~m}$ & TI-206 & $4.20 \mathrm{E}+00$ & $\mathrm{~m}$ & TI-206 & $4.20 \mathrm{E}+00$ & $\mathrm{~m}$ & TI-206 & $4.20 \mathrm{E}+00$ & $\mathrm{~m}$ & TI-206 & $4.20 \mathrm{E}+00$ & $\mathrm{~m}$ & ICRP-38 & $4.20 \mathrm{E}+00$ & $\mathrm{~m}$ \\
\hline TI-206m & TI-206m & $3.74 \mathrm{E}+00$ & $\mathrm{~m}$ & & & & & & & TI-206m & $3.74 \mathrm{E}+00$ & $\mathrm{~m}$ & TI-206m & $3.74 \mathrm{E}+00$ & $\mathrm{~m}$ & ICRP-107 & $3.74 \mathrm{E}+00$ & $\mathrm{~m}$ \\
\hline TI-207 & TI-207 & $4.77 E+00$ & $\mathrm{~m}$ & TI-207 & $4.77 \mathrm{E}+00$ & $\mathrm{~m}$ & TI-207 & $4.77 \mathrm{E}+00$ & $\mathrm{~m}$ & TI-207 & $4.77 \mathrm{E}+00$ & $\mathrm{~m}$ & TI-207 & $4.77 \mathrm{E}+00$ & $\mathrm{~m}$ & ICRP-38 & $4.77 \mathrm{E}+00$ & $\mathrm{~m}$ \\
\hline TI-208 & TI-208 & $3.05 \mathrm{E}+00$ & $\mathrm{~m}$ & TI-208 & $3.07 \mathrm{E}+00$ & $\mathrm{~m}$ & TI-208 & $3.07 \mathrm{E}+00$ & $\mathrm{~m}$ & TI-208 & $3.05 \mathrm{E}+00$ & $m$ & TI-208 & $3.05 \mathrm{E}+00$ & $\mathrm{~m}$ & ICRP-38 & $3.07 E+00$ & $\mathrm{~m}$ \\
\hline TI-209 & Tl-209 & $2.16 \mathrm{E}+00$ & $\mathrm{~m}$ & Tl-209 & $2.20 \mathrm{E}+00$ & $\mathrm{~m}$ & TI-209 & $2.20 \mathrm{E}+00$ & $m$ & Tl-209 & $2.16 \mathrm{E}+00$ & $m$ & Tl-209 & $2.16 \mathrm{E}+00$ & $\mathrm{~m}$ & ICRP-38 & $2.20 \mathrm{E}+00$ & $\mathrm{~m}$ \\
\hline TI-210 & TI-210 & $1.30 \mathrm{E}+00$ & $\mathrm{~m}$ & & & & TI-210 & $1.30 \mathrm{E}+00$ & $\mathrm{~m}$ & TI-210 & $1.30 \mathrm{E}+00$ & $m$ & $\mathrm{Tl}-210$ & $1.30 \mathrm{E}+00$ & $\mathrm{~m}$ & ICRP-38 & $1.30 \mathrm{E}+00$ & $\mathrm{~m}$ \\
\hline Tm-159 & & & & & & & & & & & & & Tm-159 & $9.13 \mathrm{E}+00$ & $\mathrm{~m}$ & JAERI & $9.13 \mathrm{E}+00$ & $\mathrm{~m}$ \\
\hline Tm-161 & Tm-161 & $3.02 E+01$ & $\mathrm{~m}$ & & & & & & & Tm-161 & $3.02 \mathrm{E}+01$ & $\mathrm{~m}$ & & & & ICRP-107 & $3.02 \mathrm{E}+01$ & $m$ \\
\hline Tm-162 & Tm-162 & $2.17 \mathrm{E}+01$ & $\mathrm{~m}$ & Tm-162 & $2.17 \mathrm{E}+01$ & $\mathrm{~m}$ & Tm-162 & $2.17 E+01$ & $\mathrm{~m}$ & Tm-162 & $2.17 \mathrm{E}+01$ & $\mathrm{~m}$ & & & & ICRP-38 & $2.17 \mathrm{E}+01$ & $\mathrm{~m}$ \\
\hline Tm-163 & $\mathrm{Tm}-163$ & $1.81 \mathrm{E}+00$ & $\mathrm{~h}$ & & & & & & & Tm-163 & $1.81 \mathrm{E}+00$ & $\mathrm{~h}$ & Tm-163 & $1.81 \mathrm{E}+00$ & $\mathrm{~h}$ & ICRP-107 & $1.81 \mathrm{E}+00$ & $\mathrm{~h}$ \\
\hline Tm-164 & Tm-164 & $2.00 \mathrm{E}+00$ & $m$ & & & & & & & Tm-164 & $2.00 \mathrm{E}+00$ & $\mathrm{~m}$ & Tm-164 & $2.00 \mathrm{E}+00$ & $\mathrm{~m}$ & ICRP-107 & $2.00 \mathrm{E}+00$ & $\mathrm{~m}$ \\
\hline Tm-165 & Tm-165 & $3.01 E+01$ & $\mathrm{~h}$ & & & & & & & Tm-165 & $3.01 \mathrm{E}+01$ & $\mathrm{~h}$ & Tm-165 & $3.01 \mathrm{E}+01$ & $\mathrm{~h}$ & ICRP-107 & $3.01 \mathrm{E}+01$ & $\mathrm{~h}$ \\
\hline Tm-166 & Tm-166 & $7.70 \mathrm{E}+00$ & $\mathrm{~h}$ & Tm-166 & $7.70 \mathrm{E}+00$ & $\mathrm{~h}$ & Tm-166 & $7.70 \mathrm{E}+00$ & $\mathrm{~h}$ & Tm-166 & $7.70 \mathrm{E}+00$ & $\mathrm{~h}$ & & & & ICRP-38 & $7.70 \mathrm{E}+00$ & $\mathrm{~h}$ \\
\hline Tm-167 & Tm-167 & $9.25 \mathrm{E}+00$ & $d$ & Tm-167 & $9.24 \mathrm{E}+00$ & $d$ & Tm-167 & $9.24 \mathrm{E}+00$ & $d$ & Tm-167 & $9.25 \mathrm{E}+00$ & $d$ & & & & ICRP-38 & $9.24 \mathrm{E}+00$ & $d$ \\
\hline $\mathrm{Tm}-168$ & Tm-168 & $9.31 \mathrm{E}+01$ & d & & & & & & & Tm-168 & $9.31 \mathrm{E}+01$ & $d$ & $\mathrm{Tm}-168$ & $9.31 \mathrm{E}+01$ & $d$ & ICRP-107 & $9.31 \mathrm{E}+01$ & $d$ \\
\hline Tm-170 & Tm-170 & $1.29 \mathrm{E}+02$ & $d$ & Tm-170 & $1.29 \mathrm{E}+02$ & $d$ & Tm-170 & $1.29 \mathrm{E}+02$ & $d$ & Tm-170 & $1.29 \mathrm{E}+02$ & $d$ & & & & ICRP-38 & $1.29 \mathrm{E}+02$ & $d$ \\
\hline Tm-171 & Tm-171 & $1.92 \mathrm{E}+00$ & $y$ & Tm-171 & $1.92 \mathrm{E}+00$ & $y$ & Tm-171 & $1.92 \mathrm{E}+00$ & $y$ & Tm-171 & $1.92 \mathrm{E}+00$ & $y$ & & & & ICRP-38 & $1.92 \mathrm{E}+00$ & $y$ \\
\hline Tm-172 & Tm-172 & $6.36 \mathrm{E}+01$ & $\mathrm{~h}$ & Tm-172 & $6.36 \mathrm{E}+01$ & $\mathrm{~h}$ & Tm-172 & $6.36 \mathrm{E}+01$ & $\mathrm{~h}$ & Tm-172 & $6.36 \mathrm{E}+01$ & $\mathrm{~h}$ & & & & ICRP-38 & $6.36 \mathrm{E}+01$ & $\mathrm{~h}$ \\
\hline Tm-173 & Tm-173 & $8.24 \mathrm{E}+00$ & $\mathrm{~h}$ & Tm-173 & $8.24 \mathrm{E}+00$ & $\mathrm{~h}$ & Tm-173 & $8.24 \mathrm{E}+00$ & $\mathrm{~h}$ & Tm-173 & $8.24 \mathrm{E}+00$ & $\mathrm{~h}$ & & & & ICRP-38 & $8.24 \mathrm{E}+00$ & $\mathrm{~h}$ \\
\hline Tm-174 & Tm-174 & $5.40 \mathrm{E}+00$ & $\mathrm{~m}$ & & & & & & & Tm-174 & $5.40 \mathrm{E}+00$ & $\mathrm{~m}$ & & & & ICRP-107 & $5.40 \mathrm{E}+00$ & $\mathrm{~m}$ \\
\hline Tm-175 & Tm-175 & $1.52 \mathrm{E}+01$ & $\mathrm{~m}$ & Tm-175 & $1.52 \mathrm{E}+01$ & $\mathrm{~m}$ & Tm-175 & $1.52 \mathrm{E}+01$ & $\mathrm{~m}$ & Tm-175 & $1.52 \mathrm{E}+01$ & $\mathrm{~m}$ & & & & ICRP-38 & $1.52 \mathrm{E}+01$ & $\mathrm{~m}$ \\
\hline Tm-176 & Tm-176 & $1.85 \mathrm{E}+00$ & $\mathrm{~m}$ & & & & & & & Tm-176 & $1.85 \mathrm{E}+00$ & $\mathrm{~m}$ & & & & ICRP-107 & $1.85 \mathrm{E}+00$ & $\mathrm{~m}$ \\
\hline U-227 & $\mathrm{U}-227$ & $1.10 \mathrm{E}+00$ & $\mathrm{~m}$ & & & & & & & U-227 & $1.10 \mathrm{E}+00$ & $\mathrm{~m}$ & & & & ICRP-107 & $1.10 \mathrm{E}+00$ & $\mathrm{~m}$ \\
\hline U-228 & U-228 & $9.10 \mathrm{E}+00$ & $\mathrm{~m}$ & & & & & & & U-228 & $9.10 \mathrm{E}+00$ & $m$ & U-228 & $9.10 \mathrm{E}+00$ & $\mathrm{~m}$ & ICRP-107 & $9.10 \mathrm{E}+00$ & $\mathrm{~m}$ \\
\hline$U-230$ & U-230 & $2.08 \mathrm{E}+01$ & $\mathrm{~d}$ & U-230 & $2.08 \mathrm{E}+01$ & $d$ & $\mathrm{U}-230$ & $2.08 \mathrm{E}+01$ & $d$ & $U-230$ & $2.08 \mathrm{E}+01$ & $d$ & & & & ICRP-38 & $2.08 \mathrm{E}+01$ & $d$ \\
\hline U-231 & U-231 & $4.20 \mathrm{E}+00$ & $d$ & U-231 & $4.20 \mathrm{E}+00$ & $d$ & $U-231$ & $4.20 \mathrm{E}+00$ & $d$ & $U-231$ & $4.20 \mathrm{E}+00$ & $d$ & & & & ICRP-38 & $4.20 \mathrm{E}+00$ & $d$ \\
\hline U-232 & $\mathrm{U}-232$ & $6.89 \mathrm{E}+01$ & $y$ & $\mathrm{U}-232$ & $7.20 \mathrm{E}+01$ & $y$ & $\mathrm{U}-232$ & $7.20 \mathrm{E}+01$ & $y$ & $\mathrm{U}-232$ & $6.89 \mathrm{E}+01$ & $y$ & & & & ICRP-38 & $7.20 \mathrm{E}+01$ & $y$ \\
\hline U-233 & U-233 & $1.59 \mathrm{E}+05$ & $y$ & $\mathrm{U}-233$ & $1.59 \mathrm{E}+05$ & $y$ & $\mathrm{U}-233$ & $1.59 \mathrm{E}+05$ & $y$ & $\mathrm{U}-233$ & $1.59 \mathrm{E}+05$ & $y$ & & & & ICRP-38 & $1.59 \mathrm{E}+05$ & $y$ \\
\hline U-234 & U-234 & $2.46 \mathrm{E}+05$ & $y$ & U-234 & $2.45 \mathrm{E}+05$ & $y$ & U-234 & $2.45 \mathrm{E}+05$ & $y$ & U-234 & $2.46 \mathrm{E}+05$ & $y$ & & & & ICRP-38 & $2.45 \mathrm{E}+05$ & $y$ \\
\hline U-235 & U-235 & $7.04 \mathrm{E}+08$ & $y$ & U-235 & $7.04 \mathrm{E}+08$ & $y$ & U-235 & $7.04 \mathrm{E}+08$ & $y$ & U-235 & $7.04 \mathrm{E}+08$ & $y$ & & & & ICRP-38 & $7.04 \mathrm{E}+08$ & $y$ \\
\hline U-235m & $\mathrm{U}-235 \mathrm{~m}$ & $2.60 \mathrm{E}+01$ & $\mathrm{~m}$ & & & & & & & $\mathrm{U}-235 \mathrm{~m}$ & $2.60 \mathrm{E}+01$ & $\mathrm{~m}$ & & & & ICRP-107 & $2.60 \mathrm{E}+01$ & $\mathrm{~m}$ \\
\hline U-236 & U-236 & $2.34 \mathrm{E}+07$ & $y$ & U-236 & $2.34 \mathrm{E}+07$ & $y$ & U-236 & $2.34 \mathrm{E}+07$ & $y$ & U-236 & $2.34 \mathrm{E}+07$ & $y$ & & & & ICRP-38 & $2.34 \mathrm{E}+07$ & $y$ \\
\hline U-237 & U-237 & $6.75 \mathrm{E}+00$ & $d$ & U-237 & $6.75 \mathrm{E}+00$ & $d$ & U-237 & $6.75 \mathrm{E}+00$ & $d$ & U-237 & $6.75 \mathrm{E}+00$ & $d$ & & & & ICRP-38 & $6.75 \mathrm{E}+00$ & $d$ \\
\hline
\end{tabular}

D-33 


\begin{tabular}{|c|c|c|c|c|c|c|c|c|c|c|c|c|c|c|c|c|c|c|}
\hline \multirow{3}{*}{$\begin{array}{l}\text { MASTER } \\
\text { LIST } \\
\text { U-238 }\end{array}$} & \multicolumn{3}{|c|}{ DOE-STD-1196 } & \multicolumn{3}{|c|}{ FGR-12 } & \multicolumn{3}{|c|}{ ICRP-38 } & \multicolumn{3}{|c|}{ ICRP-107 } & \multicolumn{3}{|c|}{ JAERI } & \multirow{3}{*}{$\begin{array}{c}\begin{array}{c}\text { Selected } \\
\text { Half-Life } \\
\text { Reference }\end{array} \\
\text { ICRP-38 }\end{array}$} & \multirow{2}{*}{\multicolumn{2}{|c|}{$\begin{array}{c}\text { Selected } \\
\text { Half-Life } \\
\text { (original units) }\end{array}$}} \\
\hline & \multirow{2}{*}{$\begin{array}{l}\text { Nuclide } \\
\text { U-238 }\end{array}$} & \multicolumn{2}{|c|}{$\begin{array}{c}\text { Half-Life } \\
\text { (original units) }\end{array}$} & \multirow{2}{*}{$\begin{array}{l}\text { Nuclide } \\
\text { U-238 }\end{array}$} & \multicolumn{2}{|c|}{$\begin{array}{c}\text { Half-Life } \\
\text { (original units) }\end{array}$} & \multirow{2}{*}{$\begin{array}{l}\text { Nuclide } \\
\text { U-238 }\end{array}$} & \multicolumn{2}{|c|}{$\begin{array}{c}\text { Half-Life } \\
\text { (original units) }\end{array}$} & \multirow{2}{*}{$\frac{\text { Nuclide }}{\text { U-238 }}$} & \multicolumn{2}{|c|}{$\begin{array}{c}\text { Half-Life } \\
\text { (original units) }\end{array}$} & \multirow[t]{2}{*}{ Nuclide } & \multicolumn{2}{|c|}{$\begin{array}{c}\text { Half-Life } \\
\text { (original units) }\end{array}$} & & & \\
\hline & & $4.47 \mathrm{E}+09$ & $y$ & & $4.47 \mathrm{E}+09$ & $y$ & & $4.47 \mathrm{E}+09$ & $y$ & & $4.47 \mathrm{E}+09$ & $y$ & & & & & $4.47 \mathrm{E}+09$ & $\mathrm{y}$ \\
\hline U-239 & U-239 & $2.35 \mathrm{E}+01$ & $\mathrm{~m}$ & U-239 & $2.35 \mathrm{E}+01$ & $\mathrm{~m}$ & U-239 & $2.35 \mathrm{E}+01$ & $\mathrm{~m}$ & U-239 & $2.35 \mathrm{E}+01$ & $\mathrm{~m}$ & & & & ICRP-38 & $2.35 \mathrm{E}+01$ & $\mathrm{~m}$ \\
\hline$U-240$ & $U-240$ & $1.41 \mathrm{E}+01$ & $\mathrm{~h}$ & $U-240$ & $1.41 \mathrm{E}+01$ & $\mathrm{~h}$ & $U-240$ & $1.41 \mathrm{E}+01$ & $\mathrm{~h}$ & $U-240$ & $1.41 \mathrm{E}+01$ & $\mathrm{~h}$ & & & & ICRP-38 & $1.41 \mathrm{E}+01$ & $\mathrm{~h}$ \\
\hline$U-242$ & U-242 & $1.68 \mathrm{E}+01$ & $\mathrm{~m}$ & & & & & & & $U-242$ & $1.68 \mathrm{E}+01$ & $\mathrm{~m}$ & & & & ICRP-107 & $1.68 \mathrm{E}+01$ & $\mathrm{~m}$ \\
\hline V-47 & V-47 & $3.26 \mathrm{E}+01$ & $\mathrm{~m}$ & V-47 & $3.26 \mathrm{E}+01$ & $m$ & V-47 & $3.26 \mathrm{E}+01$ & $\mathrm{~m}$ & V-47 & $3.26 \mathrm{E}+01$ & $\mathrm{~m}$ & & & & ICRP-38 & $3.26 \mathrm{E}+01$ & $\mathrm{~m}$ \\
\hline V-48 & V-48 & $1.60 \mathrm{E}+01$ & $d$ & V-48 & $1.62 \mathrm{E}+01$ & $\mathrm{~d}$ & V-48 & $1.62 \mathrm{E}+01$ & d & V-48 & $1.60 \mathrm{E}+01$ & $d$ & & & & ICRP-38 & $1.62 \mathrm{E}+01$ & $\mathrm{~d}$ \\
\hline V-49 & V-49 & $3.30 \mathrm{E}+02$ & $d$ & V-49 & $3.30 \mathrm{E}+02$ & $d$ & V-49 & $3.30 \mathrm{E}+02$ & $d$ & V-49 & $3.30 \mathrm{E}+02$ & $d$ & & & & ICRP-38 & $3.30 \mathrm{E}+02$ & $\mathrm{~d}$ \\
\hline V-50 & V-50 & $1.50 \mathrm{E}+17$ & $y$ & & & & & & & V-50 & $1.50 \mathrm{E}+17$ & $y$ & V-50 & $1.50 \mathrm{E}+17$ & $y$ & ICRP-107 & $1.50 \mathrm{E}+17$ & $y$ \\
\hline V-52 & $V-52$ & $3.74 \mathrm{E}+00$ & $\mathrm{~m}$ & & & & & & & V-52 & $3.74 \mathrm{E}+00$ & $\mathrm{~m}$ & $V-52$ & $3.74 \mathrm{E}+00$ & $\mathrm{~m}$ & ICRP-107 & $3.74 \mathrm{E}+00$ & $\mathrm{~m}$ \\
\hline V-53 & V-53 & $1.61 \mathrm{E}+00$ & $\mathrm{~m}$ & & & & & & & V-53 & $1.61 \mathrm{E}+00$ & $\mathrm{~m}$ & $V-53$ & $1.61 \mathrm{E}+00$ & $\mathrm{~m}$ & ICRP-107 & $1.61 \mathrm{E}+00$ & $\mathrm{~m}$ \\
\hline W-176 & & & & W-176 & $2.30 \mathrm{E}+00$ & $\mathrm{~h}$ & W-176 & $2.30 \mathrm{E}+00$ & $\mathrm{~h}$ & & & & & & & ICRP-38 & $2.30 \mathrm{E}+00$ & $\mathrm{~h}$ \\
\hline W-177 & W-177 & $1.32 \mathrm{E}+02$ & $\mathrm{~m}$ & W-177 & $1.35 \mathrm{E}+02$ & $m$ & W-177 & $1.35 \mathrm{E}+02$ & $\mathrm{~m}$ & W-177 & $1.32 \mathrm{E}+02$ & $\mathrm{~m}$ & & & & ICRP-38 & $1.35 \mathrm{E}+02$ & $\mathrm{~m}$ \\
\hline W-178 & W-178 & $2.16 \mathrm{E}+01$ & $d$ & W-178 & $2.17 \mathrm{E}+01$ & $\mathrm{~d}$ & W-178 & $2.17 \mathrm{E}+01$ & $d$ & W-178 & $2.16 \mathrm{E}+01$ & $d$ & & & & ICRP-38 & $2.17 \mathrm{E}+01$ & $\mathrm{~d}$ \\
\hline W-179 & W-179 & $3.71 E+01$ & $m$ & W-179 & $3.75 \mathrm{E}+01$ & $m$ & W-179 & $3.75 \mathrm{E}+01$ & $m$ & W-179 & $3.71 \mathrm{E}+01$ & $m$ & & & & ICRP-38 & $3.75 \mathrm{E}+01$ & $\mathrm{~m}$ \\
\hline W-179m & W-179m & $6.40 \mathrm{E}+00$ & $\mathrm{~m}$ & & & & & & & W-179m & $6.40 \mathrm{E}+00$ & $m$ & W-179m & $6.40 \mathrm{E}+00$ & $\mathrm{~m}$ & ICRP-107 & $6.40 \mathrm{E}+00$ & $\mathrm{~m}$ \\
\hline W-181 & W-181 & $1.21 \mathrm{E}+02$ & $d$ & W-181 & $1.21 \mathrm{E}+02$ & d & W-181 & $1.21 \mathrm{E}+02$ & $d$ & W-181 & $1.21 \mathrm{E}+02$ & $d$ & & & & ICRP-38 & $1.21 \mathrm{E}+02$ & $\mathrm{~d}$ \\
\hline W-185 & W-185 & $7.51 \mathrm{E}+01$ & $d$ & W-185 & $7.51 \mathrm{E}+01$ & $\mathrm{~d}$ & W-185 & $7.51 \mathrm{E}+01$ & $d$ & W-185 & $7.51 \mathrm{E}+01$ & $d$ & & & & ICRP-38 & $7.51 \mathrm{E}+01$ & $\mathrm{~d}$ \\
\hline W-185m & W-185m & $1.60 \mathrm{E}+00$ & $m$ & & & & & & & W-185m & $1.60 \mathrm{E}+00$ & $\mathrm{~m}$ & W-185m & $1.60 \mathrm{E}+00$ & $\mathrm{~m}$ & ICRP-107 & $1.60 \mathrm{E}+00$ & $\mathrm{~m}$ \\
\hline W-187 & W-187 & $2.37 E+01$ & $\mathrm{~h}$ & W-187 & $2.39 \mathrm{E}+01$ & $\mathrm{~h}$ & W-187 & $2.39 \mathrm{E}+01$ & $\mathrm{~h}$ & W-187 & $2.37 \mathrm{E}+01$ & $\mathrm{~h}$ & & & & ICRP-38 & $2.39 \mathrm{E}+01$ & $\mathrm{~h}$ \\
\hline W-188 & W-188 & $6.98 \mathrm{E}+01$ & $\mathrm{~d}$ & W-188 & $6.94 \mathrm{E}+01$ & $d$ & W-188 & $6.94 \mathrm{E}+01$ & $d$ & W-188 & $6.98 \mathrm{E}+01$ & $d$ & & & & ICRP-38 & $6.94 \mathrm{E}+01$ & $\mathrm{~d}$ \\
\hline W-190 & W-190 & $3.00 \mathrm{E}+01$ & $\mathrm{~m}$ & & & & & & & W-190 & $3.00 \mathrm{E}+01$ & $\mathrm{~m}$ & W-190 & $3.00 \mathrm{E}+01$ & $m$ & ICRP-107 & $3.00 \mathrm{E}+01$ & $\mathrm{~m}$ \\
\hline Xe-120 & Xe-120 & $4.00 \mathrm{E}+01$ & $\mathrm{~m}$ & Xe-120 & $4.00 \mathrm{E}+01$ & $\mathrm{~m}$ & Xe-120 & $4.00 \mathrm{E}+01$ & $\mathrm{~m}$ & Xe-120 & $4.00 \mathrm{E}+01$ & $\mathrm{~m}$ & & & & ICRP-38 & $4.00 \mathrm{E}+01$ & $\mathrm{~m}$ \\
\hline Xe-121 & Xe-121 & $4.01 \mathrm{E}+01$ & $m$ & Xe-121 & $4.01 \mathrm{E}+01$ & $\mathrm{~m}$ & Xe-121 & $4.01 \mathrm{E}+01$ & $m$ & Xe-121 & $4.01 \mathrm{E}+01$ & $m$ & & & & ICRP-38 & $4.01 \mathrm{E}+01$ & $\mathrm{~m}$ \\
\hline Xe-122 & Xe-122 & $2.01 \mathrm{E}+01$ & $\mathrm{~h}$ & Xe-122 & $2.01 \mathrm{E}+01$ & $\mathrm{~h}$ & Xe-122 & $2.01 \mathrm{E}+01$ & $\mathrm{~h}$ & Xe-122 & $2.01 \mathrm{E}+01$ & $\mathrm{~h}$ & & & & ICRP-38 & $2.01 \mathrm{E}+01$ & $\mathrm{~h}$ \\
\hline Xe-123 & Xe-123 & $2.08 \mathrm{E}+00$ & $\mathrm{~h}$ & Xe-123 & $2.08 \mathrm{E}+00$ & $\mathrm{~h}$ & Xe-123 & $2.08 \mathrm{E}+00$ & $\mathrm{~h}$ & Xe-123 & $2.08 \mathrm{E}+00$ & $\mathrm{~h}$ & & & & ICRP-38 & $2.08 \mathrm{E}+00$ & $\mathrm{~h}$ \\
\hline Xe-125 & Xe-125 & $1.69 \mathrm{E}+01$ & $h$ & Xe-125 & $1.70 \mathrm{E}+01$ & $\mathrm{~h}$ & Xe-125 & $1.70 \mathrm{E}+01$ & $\mathrm{~h}$ & Xe-125 & $1.69 \mathrm{E}+01$ & $\mathrm{~h}$ & & & & ICRP-38 & $1.70 \mathrm{E}+01$ & $\mathrm{~h}$ \\
\hline Xe-127 & Xe-127 & $3.64 \mathrm{E}+01$ & $d$ & Xe-127 & $3.64 \mathrm{E}+01$ & $d$ & Xe-127 & $3.64 \mathrm{E}+01$ & d & Xe-127 & $3.64 \mathrm{E}+01$ & d & & & & ICRP-38 & $3.64 \mathrm{E}+01$ & d \\
\hline Xe-127m & $\mathrm{Xe}-127 \mathrm{~m}$ & $6.92 \mathrm{E}+01$ & $\mathrm{~s}$ & & & & & & & $\mathrm{Xe}-127 \mathrm{~m}$ & $6.92 \mathrm{E}+01$ & $\mathrm{~s}$ & $X e-127 m$ & $1.15 \mathrm{E}+00$ & $\mathrm{~m}$ & ICRP-107 & $6.92 \mathrm{E}+01$ & $s$ \\
\hline Xe-129m & Xe-129m & $8.88 \mathrm{E}+00$ & $d$ & Xe-129m & $8.00 \mathrm{E}+00$ & $d$ & Xe-129m & $8.00 \mathrm{E}+00$ & $d$ & Xe-129m & $8.88 \mathrm{E}+00$ & $d$ & & & & ICRP-38 & $8.00 \mathrm{E}+00$ & $\mathrm{~d}$ \\
\hline Xe-131m & Xe-131m & $1.18 \mathrm{E}+01$ & $d$ & Xe-131m & $1.19 \mathrm{E}+01$ & d & Xe-131m & $1.19 \mathrm{E}+01$ & $d$ & Xe-131m & $1.18 \mathrm{E}+01$ & $d$ & & & & ICRP-38 & $1.19 \mathrm{E}+01$ & d \\
\hline Xe-133 & Xe-133 & $5.24 \mathrm{E}+00$ & $d$ & Xe-133 & $5.25 \mathrm{E}+00$ & $d$ & Xe-133 & $5.25 \mathrm{E}+00$ & $d$ & Xe-133 & $5.24 \mathrm{E}+00$ & $d$ & & & & ICRP-38 & $5.25 \mathrm{E}+00$ & d \\
\hline Xe-133m & Xe-133m & $2.19 \mathrm{E}+00$ & $d$ & Xe-133m & $2.19 \mathrm{E}+00$ & $d$ & Xe-133m & $2.19 \mathrm{E}+00$ & $d$ & Xe-133m & $2.19 \mathrm{E}+00$ & $d$ & & & & ICRP-38 & $2.19 \mathrm{E}+00$ & d \\
\hline Xe-135 & Xe-135 & $9.14 \mathrm{E}+00$ & $\mathrm{~h}$ & Xe-135 & $9.09 \mathrm{E}+00$ & $\mathrm{~h}$ & Xe-135 & $9.09 \mathrm{E}+00$ & $\mathrm{~h}$ & Xe-135 & $9.14 \mathrm{E}+00$ & $\mathrm{~h}$ & & & & ICRP-38 & $9.09 \mathrm{E}+00$ & $\mathrm{~h}$ \\
\hline Xe-135m & Xe-135m & $1.53 \mathrm{E}+01$ & $m$ & Xe-135m & $1.53 \mathrm{E}+01$ & $\mathrm{~m}$ & $\mathrm{Xe}-135 \mathrm{~m}$ & $1.53 \mathrm{E}+01$ & $m$ & $\mathrm{Xe}-135 \mathrm{~m}$ & $1.53 \mathrm{E}+01$ & $m$ & & & & ICRP-38 & $1.53 \mathrm{E}+01$ & $\mathrm{~m}$ \\
\hline Xe-137 & Xe-137 & $3.82 \mathrm{E}+00$ & $\mathrm{~m}$ & & & & & & & Xe-137 & $3.82 \mathrm{E}+00$ & $m$ & Xe-137 & $3.82 \mathrm{E}+00$ & $\mathrm{~m}$ & ICRP-107 & $3.82 \mathrm{E}+00$ & $\mathrm{~m}$ \\
\hline Xe-138 & Xe-138 & $1.41 \mathrm{E}+01$ & $\mathrm{~m}$ & Xe-138 & $1.42 \mathrm{E}+01$ & $\mathrm{~m}$ & Xe-138 & $1.42 \mathrm{E}+01$ & $\mathrm{~m}$ & Xe-138 & $1.41 \mathrm{E}+01$ & $m$ & & & & ICRP-38 & $1.42 \mathrm{E}+01$ & $\mathrm{~m}$ \\
\hline Y-81 & Y-81 & $7.04 \mathrm{E}+01$ & $\mathrm{~s}$ & & & & & & & Y-81 & $7.04 \mathrm{E}+01$ & $\mathrm{~s}$ & & & & ICRP-107 & $7.04 \mathrm{E}+01$ & $\mathrm{~s}$ \\
\hline Y-83 & Y-83 & $7.08 \mathrm{E}+00$ & $\mathrm{~m}$ & & & & & & & $Y-83$ & $7.08 \mathrm{E}+00$ & $\mathrm{~m}$ & $Y-83$ & $7.08 \mathrm{E}+00$ & $\mathrm{~m}$ & ICRP-107 & $7.08 \mathrm{E}+00$ & $\mathrm{~m}$ \\
\hline Y-83m & $Y-83 m$ & $2.85 \mathrm{E}+00$ & $\mathrm{~m}$ & & & & & & & $Y-83 m$ & $2.85 \mathrm{E}+00$ & $\mathrm{~m}$ & $Y-83 m$ & $2.85 \mathrm{E}+00$ & $\mathrm{~m}$ & ICRP-107 & $2.85 \mathrm{E}+00$ & $\mathrm{~m}$ \\
\hline Y-84m & $\mathrm{Y}-84 \mathrm{~m}$ & $3.95 \mathrm{E}+01$ & $m$ & & & & & & & $Y-84 m$ & $3.95 \mathrm{E}+01$ & $m$ & $Y-84 m$ & $4.00 \mathrm{E}+01$ & $m$ & ICRP-107 & $3.95 \mathrm{E}+01$ & $\mathrm{~m}$ \\
\hline
\end{tabular}

D-34 


\begin{tabular}{|c|c|c|c|c|c|c|c|c|c|c|c|c|c|c|c|c|c|c|}
\hline \multicolumn{19}{|c|}{ Table D.1 Selection of Radionuclides For Calculating HC-2 and HC-3 TQs (continued) } \\
\hline \multirow{3}{*}{$\begin{array}{l}\text { MASTER } \\
\text { LIST } \\
\text { Y-85 }\end{array}$} & \multicolumn{3}{|c|}{ DOE-STD-1196 } & \multicolumn{3}{|c|}{ FGR-12 } & \multicolumn{3}{|c|}{ ICRP-38 } & \multicolumn{3}{|c|}{ ICRP-107 } & \multicolumn{3}{|c|}{ JAERI } & \multirow{3}{*}{$\begin{array}{c}\begin{array}{c}\text { Selected } \\
\text { Half-Life } \\
\text { Reference }\end{array} \\
\text { ICRP-107 }\end{array}$} & & \\
\hline & \multirow{2}{*}{$\begin{array}{l}\text { Nuclide } \\
\text { Y-85 }\end{array}$} & \multicolumn{2}{|c|}{$\begin{array}{c}\text { Half-Life } \\
\text { (original units) }\end{array}$} & \multirow[t]{2}{*}{ Nuclide } & \multicolumn{2}{|c|}{$\begin{array}{c}\text { Half-Life } \\
\text { (original units) }\end{array}$} & \multirow[t]{2}{*}{ Nuclide } & \multicolumn{2}{|c|}{$\begin{array}{c}\text { Half-Life } \\
\text { (original units) }\end{array}$} & \multirow{2}{*}{$\begin{array}{l}\text { Nuclide } \\
\text { Y-85 }\end{array}$} & \multicolumn{2}{|c|}{$\begin{array}{c}\text { Half-Life } \\
\text { (original units) }\end{array}$} & \multirow{2}{*}{$\frac{\text { Nuclide }}{\text { Y-85 }}$} & $\begin{array}{r}\text { Half-Li } \\
\text { (original }\end{array}$ & its) & & \multicolumn{2}{|c|}{$\begin{array}{c}\text { Selected } \\
\text { Half-Life } \\
\text { (original units) }\end{array}$} \\
\hline & & $2.68 \mathrm{E}+00$ & $\mathrm{~h}$ & & & & & & & & $2.68 \mathrm{E}+00$ & $\mathrm{~h}$ & & $2.68 \mathrm{E}+00$ & $\mathrm{~h}$ & & $2.68 \mathrm{E}+00$ & $\mathrm{~h}$ \\
\hline$Y-85 m$ & $Y-85 m$ & $4.86 \mathrm{E}+00$ & $\mathrm{~h}$ & & & & & & & Y-85m & $4.86 \mathrm{E}+00$ & $\mathrm{~h}$ & Y-85m & $4.86 \mathrm{E}+00$ & $\mathrm{~h}$ & ICRP-107 & $4.86 \mathrm{E}+00$ & $\mathrm{~h}$ \\
\hline $\mathrm{Y}-86$ & $\mathrm{Y}-86$ & $1.47 \mathrm{E}+01$ & $h$ & $\mathrm{Y}-86$ & $1.47 \mathrm{E}+01$ & $\mathrm{~h}$ & $\mathrm{Y}-86$ & $1.47 \mathrm{E}+01$ & $\mathrm{~h}$ & $\mathrm{Y}-86$ & $1.47 \mathrm{E}+01$ & $\mathrm{~h}$ & & & & ICRP-38 & $1.47 \mathrm{E}+01$ & $\mathrm{~h}$ \\
\hline$Y-86 m$ & $\mathrm{Y}-86 \mathrm{~m}$ & $4.80 \mathrm{E}+01$ & $\mathrm{~m}$ & $\mathrm{Y}-86 \mathrm{~m}$ & $4.80 \mathrm{E}+01$ & $\mathrm{~m}$ & $\mathrm{Y}-86 \mathrm{~m}$ & $4.80 \mathrm{E}+01$ & $\mathrm{~m}$ & $\mathrm{Y}-86 \mathrm{~m}$ & $4.80 \mathrm{E}+01$ & $\mathrm{~m}$ & & & & ICRP-38 & $4.80 \mathrm{E}+01$ & $\mathrm{~m}$ \\
\hline Y-87 & $\mathrm{Y}-87$ & $7.98 \mathrm{E}+01$ & $\mathrm{~h}$ & Y-87 & $8.03 \mathrm{E}+01$ & $\mathrm{~h}$ & Y-87 & $8.03 E+01$ & $\mathrm{~h}$ & $\mathrm{Y}-87$ & $7.98 \mathrm{E}+01$ & $\mathrm{~h}$ & & & & ICRP-38 & $8.03 \mathrm{E}+01$ & $\mathrm{~h}$ \\
\hline Y-87m & Y-87m & $1.34 \mathrm{E}+01$ & $\mathrm{~h}$ & & & & & & & Y-87m & $1.34 \mathrm{E}+01$ & $\mathrm{~h}$ & $Y-87 m$ & $1.34 \mathrm{E}+01$ & $\mathrm{~h}$ & ICRP-107 & $1.34 \mathrm{E}+01$ & $\mathrm{~h}$ \\
\hline Y-88 & Y-88 & $1.07 \mathrm{E}+02$ & $d$ & $Y-88$ & $1.07 \mathrm{E}+02$ & $d$ & $Y-88$ & $1.07 \mathrm{E}+02$ & $d$ & $Y-88$ & $1.07 \mathrm{E}+02$ & $d$ & & & & ICRP-38 & $1.07 \mathrm{E}+02$ & $\mathrm{~d}$ \\
\hline Y-89m & Y-89m & $1.57 \mathrm{E}+01$ & $s$ & & & & & & & Y-89m & $1.57 \mathrm{E}+01$ & $\mathrm{~s}$ & & & & ICRP-107 & $1.57 \mathrm{E}+01$ & $\mathrm{~s}$ \\
\hline Y-90 & $Y-90$ & $6.41 \mathrm{E}+01$ & $\mathrm{~h}$ & Y-90 & $6.40 \mathrm{E}+01$ & $\mathrm{~h}$ & Y-90 & $6.40 \mathrm{E}+01$ & $\mathrm{~h}$ & $Y-90$ & $6.41 \mathrm{E}+01$ & $\mathrm{~h}$ & & & & ICRP-38 & $6.40 \mathrm{E}+01$ & $\mathrm{~h}$ \\
\hline Y-90m & $\mathrm{Y}-90 \mathrm{~m}$ & $3.19 \mathrm{E}+00$ & $\mathrm{~h}$ & $\mathrm{Y}-90 \mathrm{~m}$ & $3.19 E+00$ & $\mathrm{~h}$ & $\mathrm{Y}-90 \mathrm{~m}$ & $3.19 E+00$ & $\mathrm{~h}$ & $\mathrm{Y}-90 \mathrm{~m}$ & $3.19 \mathrm{E}+00$ & $\mathrm{~h}$ & & & & ICRP-38 & $3.19 \mathrm{E}+00$ & $\mathrm{~h}$ \\
\hline Y-91 & $Y-91$ & $5.85 \mathrm{E}+01$ & $d$ & Y-91 & $5.85 \mathrm{E}+01$ & $d$ & Y-91 & $5.85 \mathrm{E}+01$ & $d$ & $Y-91$ & $5.85 \mathrm{E}+01$ & $d$ & & & & ICRP-38 & $5.85 \mathrm{E}+01$ & $d$ \\
\hline Y-91m & $\mathrm{Y}-91 \mathrm{~m}$ & $4.97 \mathrm{E}+01$ & $\mathrm{~m}$ & Y-91m & $4.97 \mathrm{E}+01$ & $\mathrm{~m}$ & Y-91m & $4.97 \mathrm{E}+01$ & $m$ & $\mathrm{Y}-91 \mathrm{~m}$ & $4.97 \mathrm{E}+01$ & $m$ & & & & ICRP-38 & $4.97 \mathrm{E}+01$ & $\mathrm{~m}$ \\
\hline Y-92 & $\mathrm{Y}-92$ & $3.54 \mathrm{E}+00$ & $\mathrm{~h}$ & $\mathrm{Y}-92$ & $3.54 \mathrm{E}+00$ & $\mathrm{~h}$ & $\mathrm{Y}-92$ & $3.54 \mathrm{E}+00$ & $\mathrm{~h}$ & $\mathrm{Y}-92$ & $3.54 \mathrm{E}+00$ & $\mathrm{~h}$ & & & & ICRP-38 & $3.54 \mathrm{E}+00$ & $\mathrm{~h}$ \\
\hline Y-93 & Y-93 & $1.02 \mathrm{E}+01$ & $\mathrm{~h}$ & $Y-93$ & $1.01 \mathrm{E}+01$ & $\mathrm{~h}$ & $Y-93$ & $1.01 \mathrm{E}+01$ & $\mathrm{~h}$ & $Y-93$ & $1.02 \mathrm{E}+01$ & $\mathrm{~h}$ & & & & ICRP-38 & $1.01 \mathrm{E}+01$ & $\mathrm{~h}$ \\
\hline Y-94 & $Y-94$ & $1.87 \mathrm{E}+01$ & $\mathrm{~m}$ & Y-94 & $1.91 \mathrm{E}+01$ & $m$ & Y-94 & $1.91 \mathrm{E}+01$ & $m$ & $Y-94$ & $1.87 \mathrm{E}+01$ & $m$ & & & & ICRP-38 & $1.91 \mathrm{E}+01$ & $m$ \\
\hline Y-95 & Y-95 & $1.03 \mathrm{E}+01$ & $\mathrm{~m}$ & Y-95 & $1.07 \mathrm{E}+01$ & $\mathrm{~m}$ & Y-95 & $1.07 \mathrm{E}+01$ & $m$ & $Y-95$ & $1.03 \mathrm{E}+01$ & $\mathrm{~m}$ & & & & ICRP-38 & $1.07 \mathrm{E}+01$ & $\mathrm{~m}$ \\
\hline Yb-162 & Yb-162 & $1.89 \mathrm{E}+01$ & $\mathrm{~m}$ & Yb-162 & $1.89 \mathrm{E}+01$ & $\mathrm{~m}$ & Yb-162 & $1.89 \mathrm{E}+01$ & $\mathrm{~m}$ & $Y b-162$ & $1.89 \mathrm{E}+01$ & $\mathrm{~m}$ & & & & ICRP-38 & $1.89 \mathrm{E}+01$ & $\mathrm{~m}$ \\
\hline Yb-163 & Yb-163 & $1.11 \mathrm{E}+01$ & $m$ & & & & & & & Yb-163 & $1.11 \mathrm{E}+01$ & $\mathrm{~m}$ & Yb-163 & $1.11 \mathrm{E}+01$ & $\mathrm{~m}$ & ICRP-107 & $1.11 \mathrm{E}+01$ & $m$ \\
\hline Yb-164 & Yb-164 & $7.58 \mathrm{E}+01$ & $\mathrm{~m}$ & & & & & & & Yb-164 & $7.58 \mathrm{E}+01$ & $\mathrm{~m}$ & Yb-164 & $7.58 \mathrm{E}+01$ & $\mathrm{~m}$ & ICRP-107 & $7.58 \mathrm{E}+01$ & $\mathrm{~m}$ \\
\hline Yb-165 & Yb-165 & $9.90 \mathrm{E}+00$ & $\mathrm{~m}$ & & & & & & & Yb-165 & $9.90 \mathrm{E}+00$ & $m$ & Yb-165 & $9.90 \mathrm{E}+00$ & $m$ & ICRP-107 & $9.90 \mathrm{E}+00$ & $\mathrm{~m}$ \\
\hline Yb-166 & Yb-166 & $5.67 \mathrm{E}+01$ & $\mathrm{~h}$ & Yb-166 & $5.67 \mathrm{E}+01$ & $\mathrm{~h}$ & Yb-166 & $5.67 \mathrm{E}+01$ & $\mathrm{~h}$ & Yb-166 & $5.67 \mathrm{E}+01$ & $\mathrm{~h}$ & & & & ICRP-38 & $5.67 \mathrm{E}+01$ & $\mathrm{~h}$ \\
\hline Yb-167 & Yb-167 & $1.75 \mathrm{E}+01$ & $\mathrm{~m}$ & Yb-167 & $1.75 \mathrm{E}+01$ & $\mathrm{~m}$ & Yb-167 & $1.75 \mathrm{E}+01$ & $\mathrm{~m}$ & Yb-167 & $1.75 \mathrm{E}+01$ & $\mathrm{~m}$ & & & & ICRP-38 & $1.75 \mathrm{E}+01$ & $\mathrm{~m}$ \\
\hline Yb-169 & Yb-169 & $3.20 \mathrm{E}+01$ & $d$ & Yb-169 & $3.20 \mathrm{E}+01$ & $d$ & Yb-169 & $3.20 \mathrm{E}+01$ & $d$ & Yb-169 & $3.20 \mathrm{E}+01$ & $d$ & & & & ICRP-38 & $3.20 \mathrm{E}+01$ & d \\
\hline Yb-175 & Yb-175 & $4.19 \mathrm{E}+00$ & $d$ & Yb-175 & $4.19 \mathrm{E}+00$ & $d$ & Yb-175 & $4.19 E+00$ & $d$ & Yb-175 & $4.19 \mathrm{E}+00$ & $d$ & & & & ICRP-38 & $4.19 \mathrm{E}+00$ & $d$ \\
\hline Yb-177 & Yb-177 & $1.91 \mathrm{E}+00$ & $\mathrm{~h}$ & Yb-177 & $1.90 \mathrm{E}+00$ & $\mathrm{~h}$ & Yb-177 & $1.90 \mathrm{E}+00$ & $\mathrm{~h}$ & Yb-177 & $1.91 \mathrm{E}+00$ & $\mathrm{~h}$ & & & & ICRP-38 & $1.90 \mathrm{E}+00$ & $\mathrm{~h}$ \\
\hline Yb-178 & Yb-178 & $7.40 \mathrm{E}+01$ & $\mathrm{~m}$ & Yb-178 & $7.40 \mathrm{E}+01$ & $m$ & $\mathrm{Yb}-178$ & $7.40 \mathrm{E}+01$ & $m$ & Yb-178 & $7.40 \mathrm{E}+01$ & $m$ & & & & ICRP-38 & $7.40 \mathrm{E}+01$ & $m$ \\
\hline Yb-179 & Yb-179 & $8.00 \mathrm{E}+00$ & $\mathrm{~m}$ & & & & & & & Yb-179 & $8.00 \mathrm{E}+00$ & $\mathrm{~m}$ & & & & ICRP-107 & $8.00 \mathrm{E}+00$ & $\mathrm{~m}$ \\
\hline$Z n-60$ & $Z n-60$ & $2.38 \mathrm{E}+00$ & $\mathrm{~m}$ & & & & & & & $Z n-60$ & $2.38 \mathrm{E}+00$ & $\mathrm{~m}$ & & & & ICRP-107 & $2.38 \mathrm{E}+00$ & $\mathrm{~m}$ \\
\hline $\mathrm{Zn}-61$ & $Z n-61$ & $8.91 \mathrm{E}+01$ & $\mathrm{~s}$ & & & & & & & $Z n-61$ & $8.91 \mathrm{E}+01$ & $\mathrm{~s}$ & & & & ICRP-107 & $8.91 \mathrm{E}+01$ & $\mathrm{~s}$ \\
\hline Zn-62 & Zn-62 & $9.19 \mathrm{E}+00$ & $\mathrm{~h}$ & Zn-62 & $9.26 \mathrm{E}+00$ & $\mathrm{~h}$ & $Z n-62$ & $9.26 \mathrm{E}+00$ & $\mathrm{~h}$ & Zn-62 & $9.19 \mathrm{E}+00$ & $\mathrm{~h}$ & & & & ICRP-38 & $9.26 \mathrm{E}+00$ & $\mathrm{~h}$ \\
\hline Zn-63 & Zn-63 & $3.85 \mathrm{E}+01$ & $\mathrm{~m}$ & Zn-63 & $3.81 \mathrm{E}+01$ & $\mathrm{~m}$ & Zn-63 & $3.81 \mathrm{E}+01$ & $\mathrm{~m}$ & Zn-63 & $3.85 \mathrm{E}+01$ & $m$ & & & & ICRP-38 & $3.81 \mathrm{E}+01$ & $\mathrm{~m}$ \\
\hline $\mathrm{Zn}-65$ & $Z n-65$ & $2.44 \mathrm{E}+02$ & $d$ & $\mathrm{Zn}-65$ & $2.44 \mathrm{E}+02$ & $d$ & Zn-65 & $2.44 \mathrm{E}+02$ & $d$ & $Z n-65$ & $2.44 \mathrm{E}+02$ & $d$ & & & & ICRP-38 & $2.44 \mathrm{E}+02$ & $d$ \\
\hline $\mathrm{Zn}-69$ & $\mathrm{Zn}-69$ & $5.64 \mathrm{E}+01$ & $\mathrm{~m}$ & Zn-69 & $5.70 \mathrm{E}+01$ & $\mathrm{~m}$ & $Z n-69$ & $5.70 \mathrm{E}+01$ & $\mathrm{~m}$ & $Z n-69$ & $5.64 \mathrm{E}+01$ & $\mathrm{~m}$ & & & & ICRP-38 & $5.70 \mathrm{E}+01$ & $\mathrm{~m}$ \\
\hline Zn-69m & Zn-69m & $1.38 \mathrm{E}+01$ & $\mathrm{~h}$ & Zn-69m & $1.38 \mathrm{E}+01$ & $\mathrm{~h}$ & Zn-69m & $1.38 \mathrm{E}+01$ & $\mathrm{~h}$ & Zn-69m & $1.38 \mathrm{E}+01$ & $\mathrm{~h}$ & & & & ICRP-38 & $1.38 \mathrm{E}+01$ & $\mathrm{~h}$ \\
\hline $\mathrm{Zn-71}$ & Zn-71 & $2.45 \mathrm{E}+00$ & $\mathrm{~m}$ & & & & & & & Zn-71 & $2.45 \mathrm{E}+00$ & $m$ & & & & ICRP-107 & $2.45 \mathrm{E}+00$ & $\mathrm{~m}$ \\
\hline Zn-71m & Zn-71m & $3.96 \mathrm{E}+00$ & $\mathrm{~h}$ & Zn-71m & $3.92 \mathrm{E}+00$ & $\mathrm{~h}$ & $\mathrm{Zn}-71 \mathrm{~m}$ & $3.92 \mathrm{E}+00$ & $\mathrm{~h}$ & $\mathrm{Zn}-71 \mathrm{~m}$ & $3.96 \mathrm{E}+00$ & $\mathrm{~h}$ & & & & ICRP-38 & $3.92 \mathrm{E}+00$ & $\mathrm{~h}$ \\
\hline$Z n-72$ & $\mathrm{Zn}-72$ & $4.65 \mathrm{E}+01$ & $\mathrm{~h}$ & $\mathrm{Zn}-72$ & $4.65 \mathrm{E}+01$ & $\mathrm{~h}$ & $\mathrm{Zn}-72$ & $4.65 \mathrm{E}+01$ & $\mathrm{~h}$ & $\mathrm{Zn}-72$ & $4.65 \mathrm{E}+01$ & $\mathrm{~h}$ & & & & ICRP-38 & $4.65 \mathrm{E}+01$ & $\mathrm{~h}$ \\
\hline Zr-85 & $\mathrm{Zr}-85$ & $7.86 \mathrm{E}+00$ & $\mathrm{~m}$ & & & & & & & $\mathrm{Zr}-85$ & $7.86 \mathrm{E}+00$ & $\mathrm{~m}$ & Zr-85 & $7.86 \mathrm{E}+00$ & $\mathrm{~m}$ & ICRP-107 & $7.86 \mathrm{E}+00$ & $\mathrm{~m}$ \\
\hline Zr-86 & Zr-86 & $1.65 \mathrm{E}+01$ & $\mathrm{~h}$ & Zr-86 & $1.65 \mathrm{E}+01$ & $\mathrm{~h}$ & Zr-86 & $1.65 \mathrm{E}+01$ & $\mathrm{~h}$ & Zr-86 & $1.65 \mathrm{E}+01$ & $\mathrm{~h}$ & & & & ICRP-38 & $1.65 \mathrm{E}+01$ & $\mathrm{~h}$ \\
\hline Zr-87 & Zr-87 & $1.68 \mathrm{E}+00$ & $\mathrm{~h}$ & & & & & & & Zr-87 & $1.68 \mathrm{E}+00$ & $\mathrm{~h}$ & $\mathrm{Zr}-87$ & $1.68 \mathrm{E}+00$ & $\mathrm{~h}$ & ICRP-107 & $1.68 \mathrm{E}+00$ & $\mathrm{~h}$ \\
\hline
\end{tabular}

D-35 


\begin{tabular}{|c|c|c|c|c|c|c|c|c|c|c|c|c|c|c|c|c|c|c|}
\hline \multicolumn{19}{|c|}{ Table D.1 Selection of Radionuclides For Calculating HC-2 and HC-3 TQs (continued) } \\
\hline & \multicolumn{3}{|c|}{ DOE-STD-1196 } & \multicolumn{3}{|c|}{ FGR-12 } & \multicolumn{3}{|c|}{ ICRP-38 } & \multicolumn{3}{|c|}{ ICRP-107 } & \multicolumn{3}{|c|}{ JAERI } & \multirow{3}{*}{$\begin{array}{c}\begin{array}{c}\text { Selected } \\
\text { Half-Life } \\
\text { Reference }\end{array} \\
\text { ICRP-38 }\end{array}$} & & \\
\hline $\begin{array}{l}\text { MASTER } \\
\text { LIST }\end{array}$ & \multirow{2}{*}{$\begin{array}{l}\text { Nuclide } \\
\text { Zr-88 } \\
\end{array}$} & \multicolumn{2}{|c|}{$\begin{array}{c}\text { Half-Life } \\
\text { (original units) }\end{array}$} & \multirow{2}{*}{$\begin{array}{l}\text { Nuclide } \\
\text { Zr-88 }\end{array}$} & \multicolumn{2}{|c|}{$\begin{array}{c}\text { Half-Life } \\
\text { (original units) }\end{array}$} & \multirow{2}{*}{$\begin{array}{l}\text { Nuclide } \\
\text { Zr-88 }\end{array}$} & \multicolumn{2}{|c|}{$\begin{array}{c}\text { Half-Life } \\
\text { (original units) }\end{array}$} & \multirow{2}{*}{$\begin{array}{l}\text { Nuclide } \\
\text { Zr-88 }\end{array}$} & \multicolumn{2}{|c|}{$\begin{array}{c}\text { Half-Life } \\
\text { (original units) }\end{array}$} & \multirow[t]{2}{*}{ Nuclide } & \multicolumn{2}{|c|}{$\begin{array}{c}\text { Half-Life } \\
\text { (original units) }\end{array}$} & & \multicolumn{2}{|c|}{$\begin{array}{c}\text { Selected } \\
\text { Half-Life } \\
\text { (original units) }\end{array}$} \\
\hline Zr-88 & & $8.34 \mathrm{E}+01$ & $\mathrm{~d}$ & & $8.34 \mathrm{E}+01$ & $d$ & & $8.34 \mathrm{E}+01$ & $\mathrm{~d}$ & & $8.34 \mathrm{E}+01$ & d & & & & & $8.34 \mathrm{E}+01$ & $\mathrm{~d}$ \\
\hline Zr-89 & Zr-89 & $7.84 \mathrm{E}+01$ & $\mathrm{~h}$ & Zr-89 & $7.84 \mathrm{E}+01$ & $\mathrm{~h}$ & Zr-89 & $7.84 \mathrm{E}+01$ & $\mathrm{~h}$ & Zr-89 & $7.84 \mathrm{E}+01$ & $\mathrm{~h}$ & & & & ICRP-38 & $7.84 \mathrm{E}+01$ & $\mathrm{~h}$ \\
\hline Zr-89m & $\mathrm{Zr}-89 \mathrm{~m}$ & $4.16 \mathrm{E}+00$ & $\mathrm{~m}$ & & & & & & & $\mathrm{Zr}-89 \mathrm{~m}$ & $4.16 \mathrm{E}+00$ & $\mathrm{~m}$ & $\mathrm{Zr}-89 \mathrm{~m}$ & $4.18 \mathrm{E}+00$ & $\mathrm{~m}$ & ICRP-107 & $4.16 \mathrm{E}+00$ & $\mathrm{~m}$ \\
\hline Zr-93 & Zr-93 & $1.53 \mathrm{E}+06$ & $\mathrm{y}$ & Zr-93 & $1.53 \mathrm{E}+06$ & $\mathrm{y}$ & Zr-93 & $1.53 \mathrm{E}+06$ & $y$ & Zr-93 & $1.53 \mathrm{E}+06$ & $\mathrm{y}$ & & & & ICRP-38 & $1.53 \mathrm{E}+06$ & $\mathrm{y}$ \\
\hline Zr-95 & Zr-95 & $6.40 \mathrm{E}+01$ & d & Zr-95 & $6.40 \mathrm{E}+01$ & d & Zr-95 & $6.40 \mathrm{E}+01$ & d & Zr-95 & $6.40 \mathrm{E}+01$ & d & & & & ICRP-38 & $6.40 \mathrm{E}+01$ & d \\
\hline Zr-97 & Zr-97 & $1.67 \mathrm{E}+01$ & $\mathrm{~h}$ & Zr-97 & $1.69 \mathrm{E}+01$ & $\mathrm{~h}$ & Zr-97 & $1.69 \mathrm{E}+01$ & $\mathrm{~h}$ & Zr-97 & $1.67 \mathrm{E}+01$ & $\mathrm{~h}$ & & & & ICRP-38 & $1.69 \mathrm{E}+01$ & $\mathrm{~h}$ \\
\hline
\end{tabular}




\section{APPENDIX E. PRECISION LIMITATIONS FOR THE CALCULATION OF THE EFFECTIVE EXPOSURE DURATION}


(This page is intentionally blank)

E-2 


\section{APPENDIX E. PRECISION LIMITATIONS FOR THE CALCULATION OF THE EFFECTIVE EXPOSURE DURATION}

\section{E.1 Background}

The calculation of updated Hazard Category (HC)- 2 and HC-3 Thresholds Quantities (TQs) was accomplished through the use of Microsoft (MS) Excel 2010 ®, Version 14.0.7184.5000 (32-bit). MS Excel was selected based on its calculational capabilities and its widespread availability, thus allowing for effective sharing of the calculation between developers, reviewers and the end-users.

MS Excel nominally works with 8-byte numbers. Although Excel can display numbers up to 30 decimal places, its precision for a specific number is confined to 15 significant figures. Accordingly, some calculations conducted in MS Excel experience limited accuracy due to the limitation associated with the significant figures retained to describe a number.

\section{E.2 Precision Limitations Associated with the Calculation of the Effective Exposure Duration}

During the calculation of HC-3 pathway specific TQs, these numerical precision limitations of MS Excel were noted to occur. Specifically, the calculation of Contact Times for the Water Ingestion pathway and the Direct Exposure pathway were observed to return inaccurate results for radionuclides with very long half-lives.

For the Water Ingestion pathway and the Direct Exposure pathway, the Contact Time (a.k.a., effective exposure duration) is calculated via the following equation:

$$
\mathrm{CT}=\frac{1-\exp (-\lambda * \mathrm{t})}{\lambda}
$$

Where;

$$
\begin{array}{ll}
\text { CT } & =\text { Contact Time (a.k.a, Effective Exposure Duration) [days] } \\
\lambda & =\text { Radionuclide decay constant }\left(\text { day }^{-1}\right)=\ln (2) / t_{1 / 2} \\
\mathrm{t} & =\text { Actual Exposure Duration [9 days for Water Ingestion pathway and } 1 \text { day for } \\
& \text { Direct Exposure pathway] }
\end{array}
$$

As noted above, the radionuclide decay constant, $\lambda$, is inversely proportional to the radionuclide half-life. Therefore, the radionuclide decay constant becomes smaller for larger evaluated half-life values. A solution to Equation E-1 can be determined by evaluating the limit of $\lambda \rightarrow 0$ by using L'Hôpital's rule (35) per Equation E-2:

$$
\lim _{\lambda \rightarrow 0} \frac{f(\lambda)}{g(\lambda)}=\lim _{\lambda \rightarrow 0} \frac{f^{\prime}(\lambda)}{g^{\prime}(\lambda)}
$$

拉拉㧊 Numerical precision in Microsoft Excel;

https://en.wikipedia.org/wiki/Numeric_precision_in_Microsoft_Excel 
Where;

$\begin{array}{ll}f(\lambda) & =1-\exp (-\lambda t) \\ f^{\prime}(\lambda) & =t^{*} \exp (-\lambda t) \\ g(\lambda) & =\lambda \\ g^{\prime}(\lambda) & =1\end{array}$

Substitution into Equation E-2 yields:

$$
\lim _{\lambda \rightarrow 0} \frac{f^{\prime}(\lambda)}{g^{\prime}(\lambda)}=\lim _{\lambda \rightarrow 0} \frac{t * \exp (-\lambda t)}{1}=t
$$

Based on the results of Equation E-3, it is concluded that as the radionuclide decay constant approaches a value of zero, the calculated value for the $\mathrm{CT}$ is equal to the actual exposure duration, $\mathrm{t}$. With respect to the HC-3 exposure pathways, the consequences of Equation E-3 are as follows:

- For the Water Ingestion pathway: $\mathrm{CT}=9$ days as $\lambda \rightarrow 0$

- For the Direct Exposure pathway: CT $=1$ days as $\lambda \rightarrow 0$

During the calculation of the CT for the Water Ingestion pathway and the Direct Exposure pathway, anomalous values were noted to occur. Specifically, for increasingly large half-life values, the CT did not converge to the expected value as predicted by Equation E-3. The observed behavior of the MS Excel calculation of the CT using Equation E-1 is illustrated in Figure E.1.

- Water Ingestion Pathway: The calculated CT asymptotically approaches an effective exposure duration of 9 days. At a radionuclide half-life equal to $3 \mathrm{E}+14$ days $(\sim 8.22 \mathrm{E}+11$ yrs), the CT value reported by MS Excel drifts from the expected CT value of 9 days. The deviation from the actual CT gradually grows worse as the evaluated radionuclide half-life increases. For radionuclide halflife values greater than $2 \mathrm{E}+17$ days, MS Excel reports a calculated CT value of zero instead of the expected value of 9 days.

- $\quad$ Direct Exposure Pathway: The calculated CT asymptotically approaches an effective exposure duration of 1 day. At a radionuclide half-life equal to $8 \mathrm{E}+12$ days $(\sim 2.19 \mathrm{E}+10 \mathrm{yrs})$, the CT value reported by MS Excel displays small perturbations. The deviation from the actual CT gradually grows worse as the evaluated radionuclide half-life increases. For radionuclide half-life values greater than 2E+16 days, MS Excel reports a calculated CT value of zero instead of the expected value of 1 day.

The radionuclide data set used for the calculation of HC-2 and HC-3 TQs was reviewed to determine the extent of the CT calculation issue for the Water Ingestion pathway and Direct Exposure pathway. Table E.1 lists all the evaluated radionuclides with a reported half-life equal to or greater than $1 \mathrm{E}+12$ days. 


\begin{tabular}{|l|c|}
\hline \multicolumn{2}{|c|}{$\begin{array}{c}\text { Table E.1 Radionuclides with } \\
\text { Half-Life } \geq \\
\text { 1E+12 Days }\end{array}$} \\
\hline Radionuclide & Half-Life (d) \\
\hline Cd-113 & $3.40 \mathrm{E}+18$ \\
\hline Gd-152 & $3.94 \mathrm{E}+16$ \\
\hline Hf-174 & $7.31 \mathrm{E}+17$ \\
\hline In-115 & $1.86 \mathrm{E}+18$ \\
\hline La-138 & $4.93 \mathrm{E}+13$ \\
\hline Lu-176 & $1.31 \mathrm{E}+13$ \\
\hline Nd-144 & $8.36 \mathrm{E}+17$ \\
\hline Os-186 & $7.31 \mathrm{E}+17$ \\
\hline Pt-190 & $2.37 \mathrm{E}+14$ \\
\hline Rb-87 & $1.72 \mathrm{E}+13$ \\
\hline Re-187 & $1.83 \mathrm{E}+13$ \\
\hline Sm-147 & $3.87 \mathrm{E}+13$ \\
\hline Sm-148 & $2.56 \mathrm{E}+18$ \\
\hline Ta-180 & $3.65 \mathrm{E}+15$ \\
\hline Te-123 & $3.65 \mathrm{E}+15$ \\
\hline Th-232 & $5.13 \mathrm{E}+12$ \\
\hline U-238 & $1.63 \mathrm{E}+12$ \\
\hline V-50 & $5.48 \mathrm{E}+19$ \\
\hline
\end{tabular}

In order to work preserve calculational accuracy of the pathway specific TQ values for the radionuclides in Table E.1, a conditional statement was used to calculate the CT for the Water Ingestion pathway and the Direct Exposure pathway.

- If the radionuclide half-life was noted to be less than or equal to $1 \mathrm{E}+12$ days, the CT was calculated using Equation E-1.

- Otherwise, if the radionuclide half-life was noted to be greater than $1 \mathrm{E}+12$ days, the CT was set to be equal to the actual exposure duration (9 days for the Water Ingestion pathway and 1 day for the Direct Exposure pathway).

It was noted that the calculated CT for the Food Ingestion pathway did not display the same precision instability as observed for the Water Ingestion pathway and the Direct Exposure pathway. The equation for the Food Ingestion pathway CT is represented as follows:

$$
\mathrm{CT}=\left(\frac{1-\exp \left(-\left[\left(\lambda_{I}+\lambda_{W}\right) \mathrm{t}_{G}\right]\right)}{\lambda_{I}+\lambda_{W}}\right)
$$


Where;

$$
\begin{array}{ll}
\lambda_{\mathrm{I}} & =\text { Radionuclide decay constant }\left[\mathrm{day}^{-1}\right]=\ln (2) / \mathrm{t}_{1 / 2} \\
\mathrm{t}_{1 / 2} & =\text { Radionuclide half-life }[\text { days }] \\
\lambda_{\mathrm{W}} & =\text { Weathering decay constant }\left[\mathrm{day}^{-1}\right]=\ln (2) / 14 \text { days } \\
\mathrm{t}_{\mathrm{G}} & =\text { Growing season time }[60 \text { days }]
\end{array}
$$

A solution for the Food Ingestion pathway CT can be determined by directly evaluating the limit as $\lambda_{\mathrm{I}}$ approaches zero as follows:

$$
\lim _{\lambda \rightarrow 0} \mathrm{CT}=\left(\frac{1-\exp \left(-\left(\lambda_{W} \mathrm{t}_{G}\right)\right)}{\lambda_{W}}\right)=\frac{1-\exp (-(0.04951 d) *(60 d))}{0.04951 d}=19.2 \mathrm{~d} \quad \text { Eqn E-5 }
$$

As determined per Equation E-5, the calculated CT for the Food Ingestion pathway is equal to 19.2 days as the radionuclide decay constant, $\lambda_{\mathrm{I}}$, goes to zero. As noted in Figure E.1, MS Excel accurately calculates the CT for the Food Ingestion pathway for the entire span of evaluated half-life data (up-to $t_{1 / 2}$ $=1 \mathrm{E}+20$ days). Since the CT for the Food Ingestion pathway did not incur any MS Excel calculation precision errors, no conditional adjustment to the CT calculation was needed. 


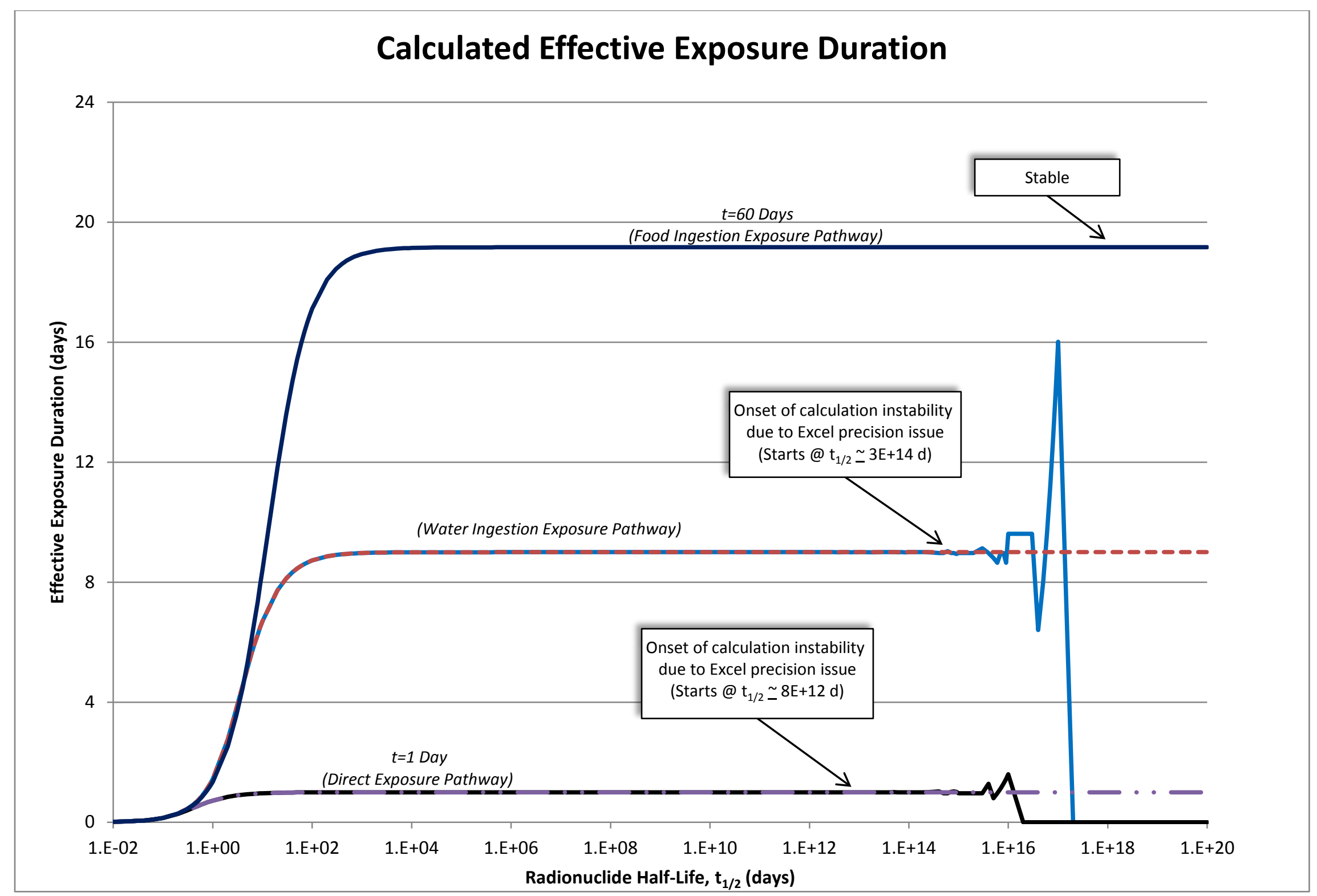

Figure E.1 Accuracy Limitation of MS Excel Regarding the Calculation Exposure Pathway Contact Times 